\title{
FISH COMMUNITY AND FISH HABITAT INVENTORY OF STREAMS AND CONSTRUCTED DRAINS THROUGHOUT AGRICULTURAL AREAS OF MANITOBA (2002 - 2006)
}

D. W. Milani

Fisheries Protection Program

Central and Arctic Region

Fisheries and Oceans Canada

501 University Crescent

Winnipeg, MB

R3T 2N6

2013

Canadian Data Report of

Fisheries and Aquatic Sciences 1247 
Canadian Data Report of

Fisheries and Aquatic Sciences 1247

2013

FISH COMMUNITY AND FISH HABITAT INVENTORY OF STREAMS AND CONSTRUCTED DRAINS THROUGHOUT AGRICULTURAL AREAS OF MANITOBA (2002-2006)

\author{
By \\ D. W. Milani ${ }^{1}$ \\ Fisheries Protection Program \\ Central and Arctic Region \\ Fisheries and Oceans Canada \\ Winnipeg, Manitoba \\ R3T 2N6
}

Aquatic Habitat Management Section

Fisheries Branch

Manitoba Water Stewardship

Winnipeg, Manitoba

R3J 3W3

${ }^{1}$ D. Milani Environmental, Box 34, Boggy Creek, MB R0L0G0 
(c) Her Majesty the Queen in Right of Canada, 2013.

\section{Cat. No. Fs 97-13/1247E-PDF ISSN 1488-5395}

Correct citation for this publication:

Milani, D.W. 2013. Fish community and fish habitat inventory of streams and constructed drains throughout agricultural areas of Manitoba (2002-2006). Can. Data Rep. Fish. Aquat. Sci. 1247: xvi + 6,153 p. 


\section{TABLE OF CONTENTS}

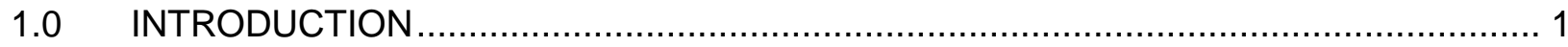

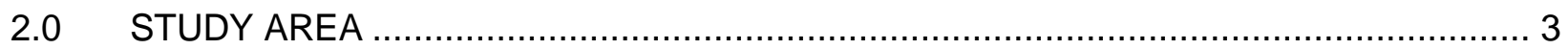

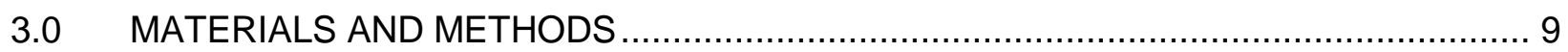

3.1 FISH HABITAT CLASSIFICATION PROTOCOL …......................................... 9

3.2 LOCATION, SELECTION AND NAMING OF SAMPLING REACHES …................14

3.3 BASIC WATER CHEMISTRY MEASUREMENTS .............................................14

3.4 FISH COMMUNITY SAMPLING AND IDENTIFICATION ....................................15

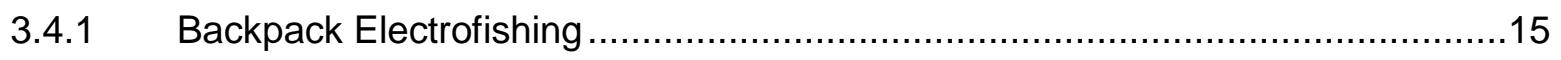

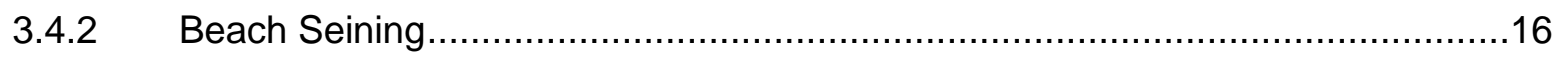

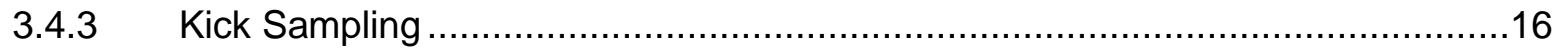

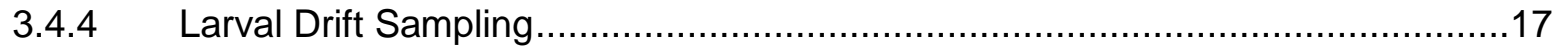

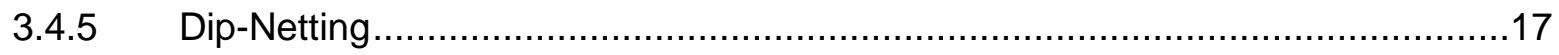

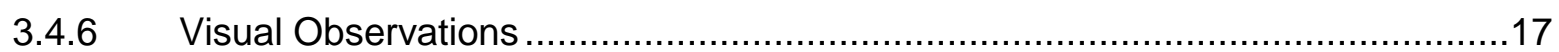

3.4.7 Fish Collection Field Notes ….................................................................

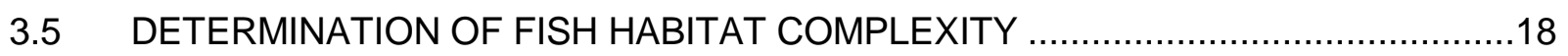

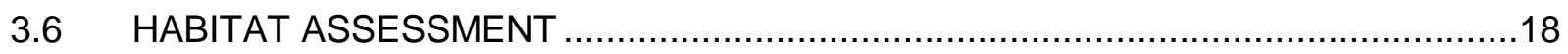

3.6.1 Available Cover/Epifaunal Substrate ......................................................18

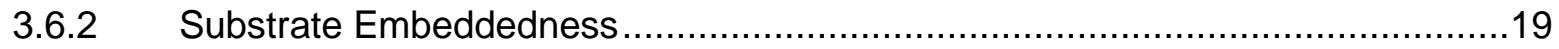

3.6.3 Velocity/Depth Regime........................................................................

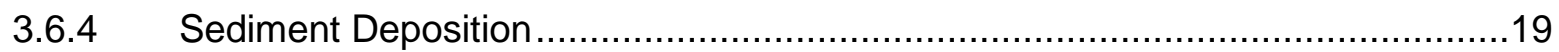

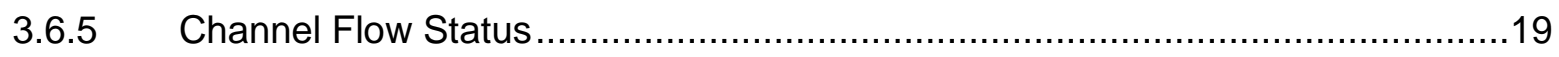

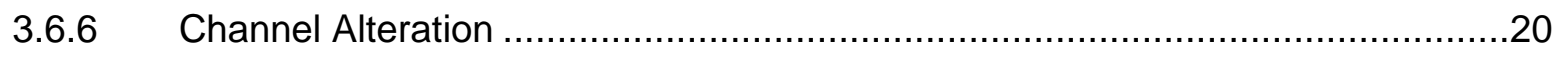

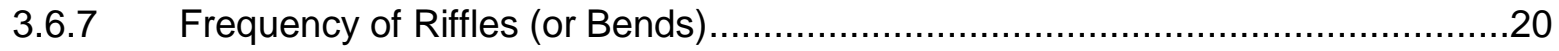

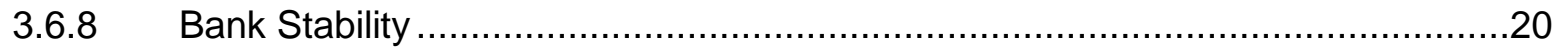

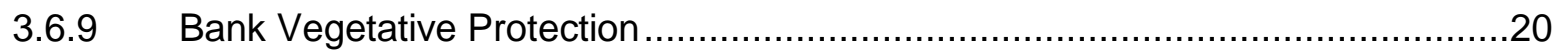

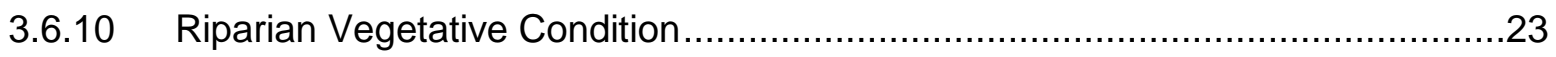

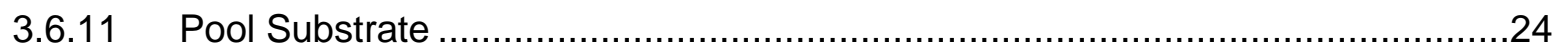

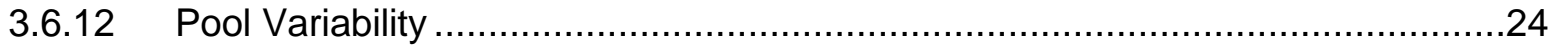

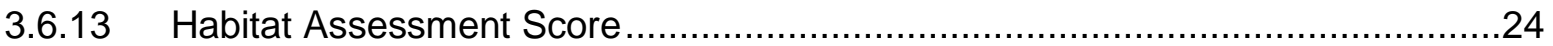

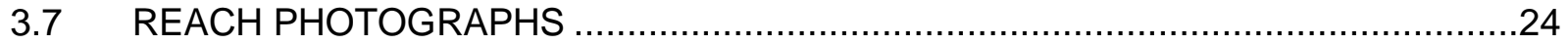

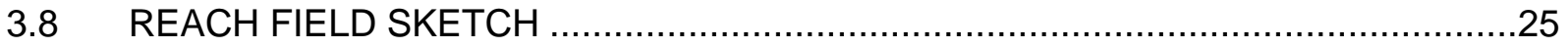

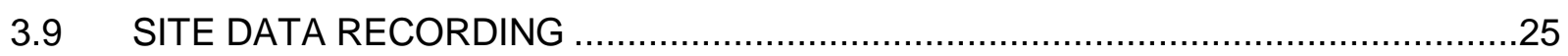




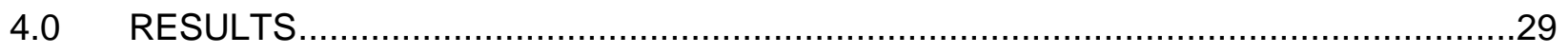

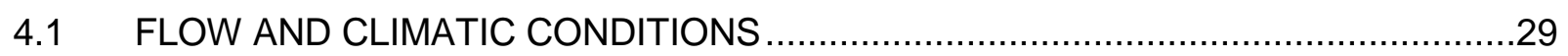

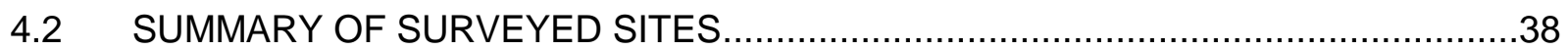

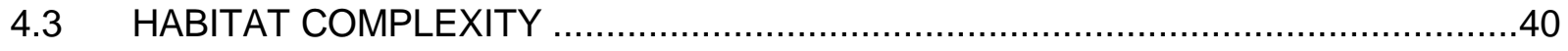

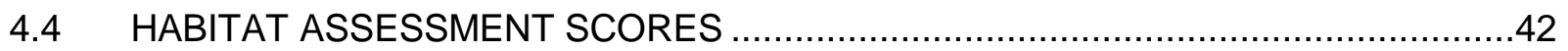

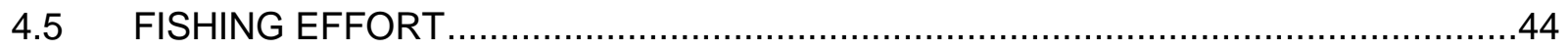

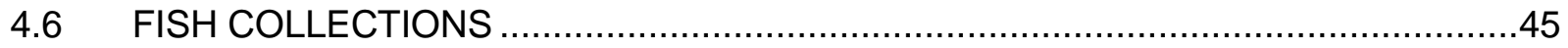

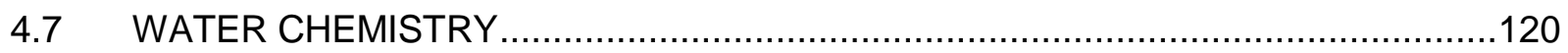

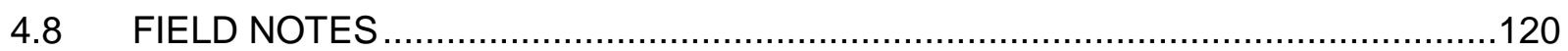

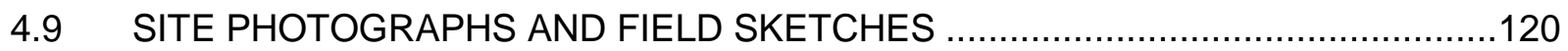

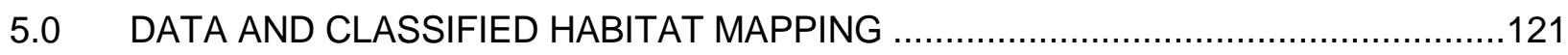

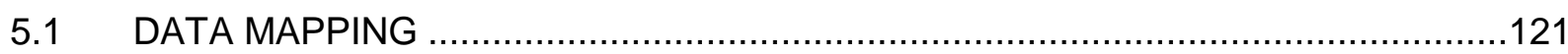

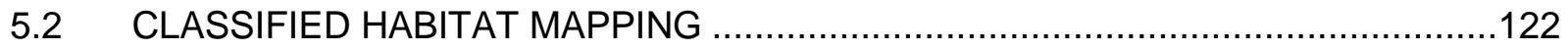

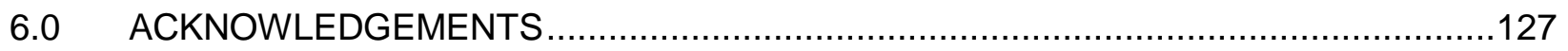

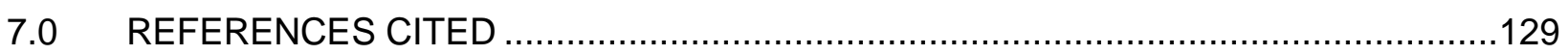

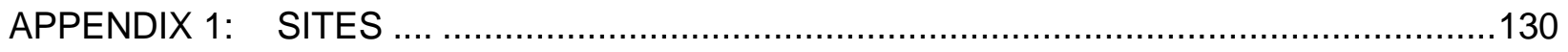

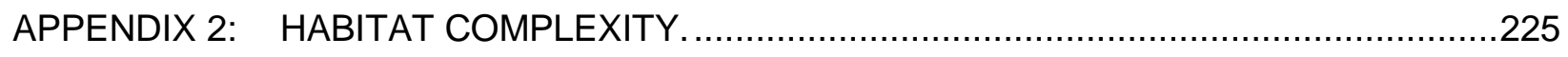

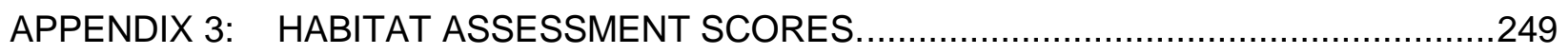

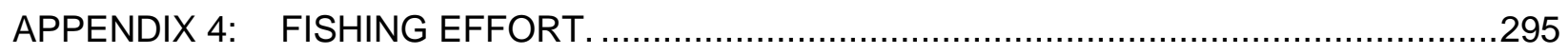

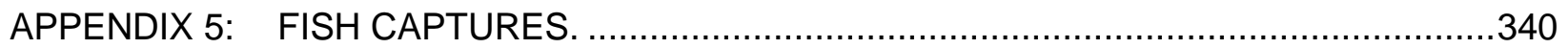

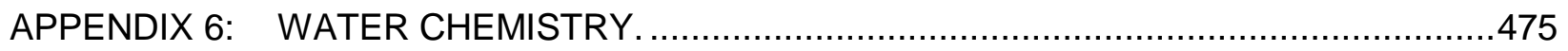

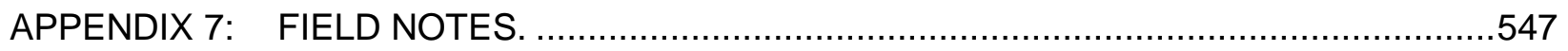

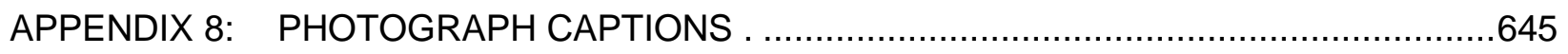

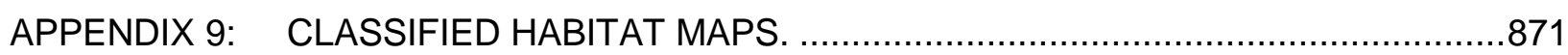

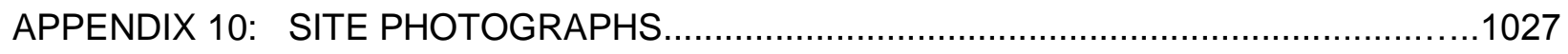

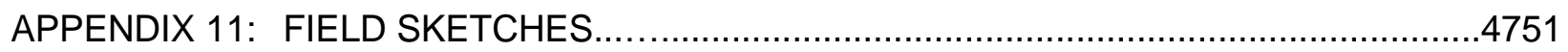

\section{LIST OF FIGURES}

Figure 1: $\quad$ Map showing the location and extent of the study area as defined by the area of coverage provided by all sub-watersheds in the DES map series. The boundary of each DES map in the series is shown.

Figure 2: $\quad$ Fish habitat classification decision flowchart applied to streams and drains within the study area. 10

Figure 3: $\quad$ Three page data sheet adapted from Barbour et al. (1999) and used as a guide to assess and score twelve individual instream 
and riparian habitat parameters at sites where fishing effort was applied between 2002 and 2006 .

Figure 4: $\quad$ Data sheet used to record the survey data gathered at all sites sampled between 2002 and 2006.

Figure 5: A comparison of the precipitation amounts received from September 1, 2001 to August 31, 2002 with historical amounts for the period of record (Agriculture and Agri-Food Canada. Prairie Farm Rehabilitation Administration. Historic Climate Data Map Archive). 29

Figure 6: The intensity and extent of drought conditions across North America in November 2002 (National Oceanic and Atmospheric Administration. National Climate Data Center, Drought Severity Interpretation and Historic Climate Maps).

Figure 7: $\quad$ A comparison of 2002 flows with the historic upper and lower quartile daily discharge for the Assiniboine River at Headingley (05MJ001) and at Devils Creek near Libau (05OJ016). (Environment Canada. Water Survey of Canada. Archived Hydrometric Data).

Figure 8: $\quad$ The intensity and extent of drought conditions across North America on June 30, 2003 (National Oceanic and Atmospheric Administration. National Climate Data Center, Drought Severity Interpretation and Historic Climate Maps)....

Figure 9: $\quad$ A comparison of 2003 flows with the historic upper and lower quartile daily discharge for the Assiniboine River at Headingley (05MJ001). (Environment Canada. Water Survey of Canada. Archived Hydrometric Data)

Figure 10: A comparison of 2003 flows with the historic upper and lower quartile daily discharge for Devils Creek near Libau (05OJ016). (Environment Canada. Water Survey of Canada. Archived Hydrometric Data) 33

Figure 11: A comparison of 2004 flows with the historic upper and lower quartile daily discharge for the Assiniboine River at Headingley (05MJ001) and at Devils Creek near Libau (05OJ016). (Environment Canada. Water Survey of Canada. Archived Hydrometric Data) 35

Figure 12: A comparison of 2005 flows with the historic upper and lower quartile daily discharge for the Assiniboine River at Headingley (05MJ001) and at Devils Creek near Libau (05OJ016). 
(Environment Canada. Water Survey of Canada. Archived Hydrometric Data) 36

Figure 13: A comparison of 2006 flows with the historic upper and lower quartile daily discharge for the Assiniboine River at Headingley (05MJ001) and at Devils Creek near Libau (05OJ016). (Environment Canada. Water Survey of Canada. Archived Hydrometric Data)

Figure 14: The intensity and extent of drought conditions across North America on June 30, 2006. (National Oceanic and Atmospheric Administration. National Climate Data Center, Drought Severity Interpretation and Historic Climate Maps) 38

Figure 15: Map showing the location of 2,371 sites visited from $2002-2006$..... 40

Figure 16: Map showing the distribution of all sites by Habitat Complexity. 41

Figure 17: $\quad$ Percent distribution of Habitat Complexity by DES stream order for all sites surveyed $(n=2,371)$.

Figure 18: Chart showing the distribution of sites across the Habitat Condition scale as determined by the Habitat Assessment Score at each site.

Figure 19: Chart showing the maximum, minimum and median individual habitat parameter scores for each of the twelve parameters assessed to derive the Total Score.

Figure 20: Map displaying the Habitat Condition category derived from the Habitat Assessment Score at all sites assessed.

Figure 21: $\quad$ Map showing the location of 558 sites where Indicator Species were collected.

Figure 22: $\quad$ Map showing the locations of 916 sites where Non-Indicator Species were collected.

Figure 23: $\quad$ Map showing the locations of 271 sites where fishing effort resulted in No Catch.

Figure 24: $\quad$ Showing the composition of Indicator Species collections summarized for DES stream orders 1-3.

Figure 25: $\quad$ Showing the composition of Indicator Species collections summarized for DES stream orders 4 and 5 .

Figure 26: $\quad$ Showing the composition of Indicator Species collections summarized for DES stream orders 6 and 7 . 
Figure 27: $\quad$ Showing the composition of Non-Indicator Species collections summarized for DES stream orders 1 and 2 .

Figure 28: $\quad$ Showing the composition of Non-Indicator Species collections summarized for DES stream order 3.

Figure 29: $\quad$ Showing the composition of Non-Indicator Species collections summarized for DES stream order 4.

Figure 30: $\quad$ Showing the composition of Non-Indicator Species collections summarized for DES stream order 5 .

Figure 31: $\quad$ Showing the composition of Non-Indicator Species collections summarized for DES stream orders 6 and 7 .

Figure 32: $\quad$ Showing the percentage of fishing effort and percentage of each fish capture type (Indicator Species, Non-Indicators Species and No Catch) when distibuted by habitat type (Complex Habitat, Simple Habitat or Transitional Habitat).

Figure 33: $\quad$ Showing the composition (percentage of the entire catch) of each fish capture type (including No Catch as a capture result) when distributed by Habitat Type.

Figure 34: A summary of Chestnut Lamprey collections showing collection sites; maximum, minimum, and median individual parameter and total Habitat Assessment Scores; and the percent of catch by DES stream order.

Figure 35: A summary of Goldeye collections showing collection sites; maximum, minimum and median individual parameter and total Habitat Assessment Scores; and the percent of catch by DES stream order.

Figure 36: A summary of Spotfin Shiner collections showing collection sites; maximum, minimum and median individual parameter and total Habitat Assessment Scores; and the percent of catch by DES stream order.

Figure 37: $\quad$ A summary of Common Carp collections showing collection sites; maximum, minimum and median individual parameter and total Habitat Assessment Scores; and the percent of catch by DES stream order.

Figure 38: A summary of Brassy Minnow collections showing collection sites; maximum, minimum and median individual parameter and total Habitat Assessment Scores; and the percent of catch by DES stream order. 
Figure 39: A summary of Common Shiner collections showing collection sites; maximum, minimum and median individual parameter and total Habitat Assessment Scores; and the percent of catch by DES stream order.

Figure 40: $\quad$ A summary of Pearl Dace collections showing collection sites; maximum, minimum and median individual parameter and total Habitat Assessment Scores; and the percent of catch by DES stream order.

Figure 41: A summary of Hornyhead Chub collections showing collection sites; maximum, minimum and median individual parameter and total Habitat Assessment Scores; and the percent of catch by DES stream order.

Figure 42: $\quad$ A summary of Golden Shiner collections showing collection sites; maximum, minimum and median individual parameter and total Habitat Assessment Scores; and the percent of catch by DES stream order.

Figure 43: $\quad$ A summary of Emerald Shiner collections showing collection sites; maximum, minimum and median individual parameter and total Habitat Assessment Scores; and the percent of catch by DES stream order.

Figure 44: $\quad$ A summary of River Shiner collections showing collection sites; maximum, minimum and median individual parameter and total Habitat Assessment Scores; and the percent of catch by DES stream order.

Figure 45: A summary of Bigmouth Shiner collections showing collection sites; maximum, minimum and median individual parameter and total Habitat Assessment Scores; and the percent of catch by DES stream order.

Figure 46: A summary of Blackchin Shiner collections showing collection sites; maximum, minimum and median individual parameter and total Habitat Assessment Scores; and the percent of catch by DES stream order.

Figure 47: A summary of Blacknose Shiner collections showing collection sites; maximum, minimum and median individual parameter and total Habitat Assessment Scores; and the percent of catch by DES stream order. .73

Figure 48: $\quad$ A summary of Spottail Shiner collections showing collection sites; maximum, minimum and median individual parameter and total 
Habitat Assessment Scores; and the percent of catch by DES stream order.

Figure 49: $\quad$ A summary of Sand Shiner collections showing collection sites; maximum, minimum and median individual parameter and total Habitat Assessment Scores; and the percent of catch by DES stream order.

Figure 50: A summary of Mimic Shiner collections showing collection sites; maximum, minimum and median individual parameter and total Habitat Assessment Scores; and the percent of catch by DES stream order.

Figure 51: A summary of Northern Redbelly Dace collections showing collection sites; maximum, minimum and median individual parameter and total Habitat Assessment Scores; and the percent of catch by DES stream order.

Figure 52: A summary of Finescale Dace collections showing collection sites; maximum, minimum and median individual parameter and total Habitat Assessment Scores; and the percent of catch by DES stream order.

Figure 53: A summary of Fathead Minnow collections showing collection sites; maximum, minimum and median individual parameter and total Habitat Assessment Scores; and the percent of catch by DES stream order.

Figure 54: A summary of Longnose Dace collections showing collection sites; maximum, minimum and median individual parameter and total Habitat Assessment Scores; and the percent of catch by DES stream order.

Figure 55: A summary of Western Blacknose Dace collections showing collection sites; maximum, minimum and median individual parameter and total Habitat Assessment Scores; and the percent of catch by DES stream order.

Figure 56: A summary of Creek Chub collections showing collection sites; maximum, minimum and median individual parameter and total Habitat Assessment Scores; and the percent of catch by DES stream order.

Figure 57: $\quad$ A summary of hybrid minnow (Cyprinidae) collections showing collection sites; maximum, minimum and median individual parameter and total Habitat Assessment Scores; and the percent of catch by DES stream order. 
Figure 58: A summary of unidentified minnow (Cyprinidae) collections showing collection sites; maximum, minimum and median individual parameter and total Habitat Assessment Scores; and the percent of catch by DES stream order.

Figure 59: $\quad$ A summary of Quillback collections showing collection sites; maximum, minimum and median individual parameter and total Habitat Assessment Scores; and the percent of catch by DES stream order.

Figure 60: A summary of White Sucker collections showing collection sites; maximum, minimum and median individual parameter and total Habitat Assessment Scores; and the percent of catch by DES stream order.

Figure 61: A summary of Bigmouth Buffalo collections showing collection sites; maximum, minimum and median individual parameter and total Habitat Assessment Scores; and the percent of catch by DES stream order.

Figure 62: A summary of Silver Redhorse collections showing collection sites; maximum, minimum and median individual parameter and total Habitat Assessment Scores; and the percent of catch by DES stream order.

Figure 63: A summary of Shorthead Redhorse collections showing collection sites; maximum, minimum and median individual parameter and total Habitat Assessment Scores; and the percent of catch by DES stream order.

Figure 64: A summary of unidentified sucker collections showing collection sites; maximum, minimum and median individual parameter and total Habitat Assessment Scores; and the percent of catch by DES stream order. 90

Figure 65: A summary of Black Bullhead collections showing collection sites; maximum, minimum and median individual parameter and total Habitat Assessment Scores; and the percent of catch by DES stream order.

Figure 66: A summary of Channel Catfish collections showing collection sites; maximum, minimum and median individual parameter and total Habitat Assessment Scores; and the percent of catch by DES stream order. 92

Figure 67: A summary of Stonecat collections showing collection sites; maximum, minimum and median individual parameter and total 
Habitat Assessment Scores; and the percent of catch by DES stream order.

Figure 68: A summary of Tadpole Madtom collections showing collection sites; maximum, minimum and median individual parameter and total Habitat Assessment Scores; and the percent of catch by DES stream order.

Figure 69: $\quad$ A summary of Northern Pike collections showing collection sites; maximum, minimum and median individual parameter and total Habitat Assessment Scores; and the percent of catch by DES stream order.

Figure 70: A summary of Central Mudminnow collections showing collection sites; maximum, minimum and median individual parameter and total Habitat Assessment Scores; and the percent of catch by DES stream order. .96

Figure 71: A summary of Rainbow Trout collections showing collection sites; maximum, minimum and median individual parameter and total Habitat Assessment Scores; and the percent of catch by DES stream order.

Figure 72: $\quad$ A summary of Brown Trout collections showing collection sites; maximum, minimum and median individual parameter and total Habitat Assessment Scores; and the percent of catch by DES stream order.

Figure 73: $\quad$ A summary of Brook Trout collections showing collection sites; maximum, minimum and median individual parameter and total Habitat Assessment Scores; and the percent of catch by DES stream order.

Figure 74: A summary of Troutperch collections showing collection sites; maximum, minimum and median individual parameter and total Habitat Assessment Scores; and the percent of catch by DES stream order. 100

Figure 75: A summary of Burbot collections showing collection sites; maximum, minimum and median individual parameter and total Habitat Assessment Scores; and the percent of catch by DES stream order.

Figure 76: A summary of Brook Stickleback collections showing collection sites; maximum, minimum and median individual parameter and total Habitat Assessment Scores; and the percent of catch by DES stream order. 102 
Figure 77: A summary of Mottled Sculpin collections showing collection sites; maximum, minimum and median individual parameter and total Habitat Assessment Scores; and the percent of catch by DES stream order.

Figure 78: $\quad$ A summary of Rock Bass collections showing collection sites; maximum, minimum and median individual parameter and total Habitat Assessment Scores; and the percent of catch by DES stream order.

Figure 79: $\quad$ A summary of Smallmouth Bass collections showing collection sites; maximum, minimum and median individual parameter and total Habitat Assessment Scores; and the percent of catch by DES stream order.

Figure 80: A summary of Black Crappie collections showing collection sites; maximum, minimum and median individual parameter and total Habitat Assessment Scores; and the percent of catch by DES stream order. 106

Figure 81: $\quad$ A summary of lowa Darter collections showing collection sites; maximum, minimum and median individual parameter and total Habitat Assessment Scores; and the percent of catch by DES stream order.

Figure 82: $\quad$ A summary of Johnny Darter collections showing collection sites; maximum, minimum and median individual parameter and total Habitat Assessment Scores; and the percent of catch by DES stream order.

Figure 83: A summary of Yellow Perch collections showing collection sites; maximum, minimum and median individual parameter and total Habitat Assessment Scores; and the percent of catch by DES stream order.

Figure 84: A summary of Logperch collections showing collection sites; maximum, minimum and median individual parameter and total Habitat Assessment Scores; and the percent of catch by DES stream order.

Figure 85: A summary of Blackside Darter collections showing collection sites; maximum, minimum and median individual parameter and total Habitat Assessment Scores; and the percent of catch by DES stream order.

Figure 86: A summary of River Darter collections showing collection sites; maximum, minimum and median individual parameter and total 
Habitat Assessment Scores; and the percent of catch by DES stream order.

Figure 87: A summary of Sauger collections showing collection sites; maximum, minimum and median individual parameter and total Habitat Assessment Scores; and the percent of catch by DES stream order.

Figure 88: A summary of Walleye collections showing collection sites; maximum, minimum and median individual parameter and total Habitat Assessment Scores; and the percent of catch by DES stream order.

Figure 89: A summary of Freshwater Drum collections showing collection sites; maximum, minimum and median individual parameter and total Habitat Assessment Scores; and the percent of catch by DES stream order.

Figure 90: A summary of Egg collections (9-Catostomidae, 1-Percidae) showing collection sites; maximum, minimum and median individual parameter and total Habitat Assessment Scores; and the percent of catch by DES stream order.

Figure 91: A summary of No Catch results showing collection sites; maximum, minimum and median individual parameter and total Habitat Assessment Scores; and the percent of catch by DES stream order.

Figure 92: Composition of the catch summarized by Family and by channel type.

Figure 93: $\quad$ Map showing the area of coverage provided by 154 National Topographic System (NTS) map tiles used to display site locations and classified streams and drains in the study area.

\section{LIST OF TABLES}

Table 1: $\quad$ The list of 114 sub-watersheds in the DES map series that formed the study area showing the DES map number, DES map name, and the area of coverage for each map in the series.

Table 2: $\quad$ List of fish species potentially present within the study area and their status as an Indicator Species (adapted from Stewart and Watkinson. 2004)

Table 3: $\quad$ A summary of all sites visited between 2002 and 2006. The sampling effort has been summarized by year, by sampling crew and by DES stream order. 
Table 4: $\quad$ The number of sites and the number of occasions fishing effort was applied, summarized by year, by sampling crew and by DES stream order.

Table 5: $\quad$ List of the species collected organized by taxonomic family and summarized by DES stream order and by Habitat Complexity.

\section{LIST OF APPENDICES}

Appendix 1: Table listing the site number, date sampled, site name, latitude and longitude (in decimal degrees - DD), DES stream order, DES map number and the corresponding NTS map number for all sites surveyed between 2002 and 2006.

Appendix 2: Habitat complexity $(\mathrm{S}=$ Simple; $\mathrm{C}=\mathrm{Complex}$ ) determination for all sites looking upstream and downstream from each surveyed reach.

Appendix 3: Table showing the twelve individual habitat parameter scores adapted from Barbour et al. (1999) that were assessed at most sites where fishing effort was applied and were totalled to derive the Habitat Assessment Score for each site.

Appendix 4: List of all fishing effort showing the site number, collectors, fishing gear used, amount of fishing effort applied and the fish capture identification (ID) number derived for each occasion where effort was applied.

Appendix 5: List of all fish captures made between 2002 and 2006, showing site number, common name and number (\#) of fish collected, fork (or total ${ }^{\star}$ ) length in millimetres $(\mathrm{mm})$, any comments on the collection and the fish capture identification (ID) number 340

Appendix 6: Results of basic water chemistry and air temperature measurements recorded at most sites where fishing effort was applied between 2002 and 2006.

Appendix 7: List of any additional field notes recorded at sampling sites.

Appendix 8: List of all photographs taken showing the corresponding site number, descriptive caption, direction faced when taken (azimuth in degrees) and the unique file name for each photograph.

Appendix 9: One hundred and fifty-four NTS 1:50,000 map tiles showing sampling sites, fish captures and habitat classification of streams and constructed drains throughout agricultural areas of Manitoba (2002 - 2006). 


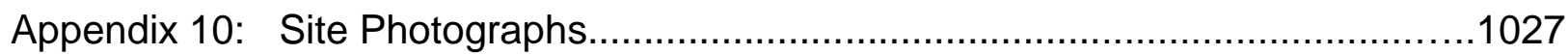

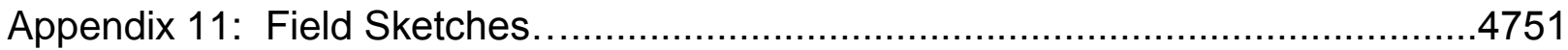

LIST OF SYMBOLS, ABREVIATIONS AND ACRONYMS

\begin{tabular}{|c|c|c|c|}
\hline ITEM & DEFINITION & ITEM & DEFINITION \\
\hline \# & Number & ID & Identification \\
\hline$\%$ & Percent & K & Kick sampler \\
\hline$\%$ SAT & Percent saturation & $\mathrm{km}$ & Kilometre \\
\hline shp & ESRISHAPE file format & $\mathrm{km}^{2}$ & Square kilometre \\
\hline.$t x t$ & Text file format & L & Larval drift trap \\
\hline$\sim$ & Approximately & Length * & Denotes length is Total length, not Fork length \\
\hline$<$ & Less than & $\mathrm{m}$ & Metre \\
\hline$>$ & Greater than & $\mathrm{m} / \mathrm{s}$ & Metres per second \\
\hline$\mu$ & Micrometre & $\mathrm{m}^{2}$ & Square metre \\
\hline$\mu S / \mathrm{cm}$ & Microseimens per centimetre & $\mathrm{m}^{3} / \mathrm{s}$ & Cubic metres per second \\
\hline$A$ & Anecdotal evidence & Max. & Maximum \\
\hline ASL & Above sea level & $\mathrm{mg} / \mathrm{L}$ & Milligrams per litre \\
\hline $\mathrm{B}$ & Beach seine & Min. & Minimum \\
\hline C & Complex Habitat & MLI & Manitoba Lands Initiative (website) \\
\hline cfs & Cubic feet per second & $\mathrm{mm}$ & Millimetre \\
\hline $\mathrm{cm}$ & Centimetre & MWS & Manitoba Water Stewardship \\
\hline $\mathrm{D}$ & Dipnet & $\mathrm{N} / \mathrm{A}$ & Not applicable \\
\hline DC & Direct current & NTS & National Topographic Series \\
\hline DD & Decimal degrees & NTU & Nephalometric Turbidity Units \\
\hline DES & Designation of Drains & $\mathrm{O}$ & Observation \\
\hline DFO & Fisheries and Oceans Canada & ${ }^{\circ} \mathrm{C}$ & Celsius degrees \\
\hline DO & Dissolved oxygen & PR & Provincial Road \\
\hline$E$ & Backpack electrofisher & PTH & Provincial Trunk Highway \\
\hline FIHCS & Fisheries Inventory Habitat Classification System & $\mathrm{S}$ & Simple Habitat \\
\hline GIS & Geographic Information System & SARA & Species at Risk Act \\
\hline GPS & Global Positioning System & YOY & Young-of-year \\
\hline hr. & Hour & Z & Electrofishing boat \\
\hline hrs. & Hours & & \\
\hline
\end{tabular}




\section{ABSTRACT}

Milani, D.W. 2013. Fish community and fish habitat inventory of streams and constructed drains throughout agricultural areas of Manitoba (2002-2006). Can. Data Rep. Fish. Aquat. Sci. 1247: xvi +6,153 p.

Recognizing the importance of well-drained agricultural land and the need to maintain drainage capacity while still protecting valuable fish habitat, the governments of Manitoba and Canada initiated an inventory of the fish community and habitat diversity of streams and constructed drains throughout agricultural areas of southern Manitoba. Over five field seasons between 2002 and 2006, field crews measured basic water chemistry, assessed fish habitat, sampled the fish community and documented conditions at 2,371 sampling sites. These data were used to classify the streams and constructed drains using the habitat classification protocol provided by the Manitoba Drain Maintenance Committee. This report lists and summarizes the fish community and fish habitat data gathered, and provides a series of map tiles displaying the classified habitat throughout the study area.

\section{RÉSUMÉ}

Milani, D.W. 2013. Inventaire des communautés et des habitats de poissons dans les cours d'eau et les canaux de drainage de construction humaine dans les secteurs agricoles du Manitoba (2002-2006). Rapp. stat. can. sci. halieut. aquat. 1247: xvi + $6153 \mathrm{p}$.

Reconnaissant l'importance du drainage adéquat des terres agricoles et la nécessité de maintenir cette capacité tout en protégeant les habitats de poissons importants, les gouvernements du Canada et du Manitoba ont entamé un inventaire de la diversité des communautés et des habitats de poissons dans les cours d'eau et les canaux de drainage de construction humaine des secteurs agricoles du sud du Manitoba. Pendant cinq saisons de travaux sur le terrain, entre 2002 et 2006, les équipes ont mesuré les propriétés chimiques de l'eau, évalué les habitats de poissons, échantillonné les communautés de poissons et consigné les conditions relevées à 2371 sites différents. Les données recueillies ont été utilisées pour classifier les cours d'eau et les canaux de drainage de construction humaine à l'aide du protocole de classification de l'habitat fourni par le Comité d'entretien des drains du Manitoba (Manitoba Drain Maintenance Committee). Le présent rapport présente une liste et un résumé des données recueillies sur les communautés et les habitats de poissons ainsi qu'une série de cartes quadrillées illustrant les habitats recensés dans l'ensemble de la zone visée par l'étude. 


\section{$1.0 \quad$ INTRODUCTION}

In Manitoba, the extent and value of arable farm land, and the pattern of settlement within the province's grid-based agricultural system, has been strongly influenced by the drainage of wet prairie land begun in the mid-1800's and continuing to date (Stunden Bower. 2006). After the creation of the Province in 1870, networks of surface ditches were constructed in several areas of southern Manitoba to drain wet land (Stunden Bower. 2006). These constructed drains helped to facilitate rapid spring runoff. The rapid runoff and increased drainage effectively extended the growing season by allowing early access to fields in the spring, while also helping to reduce flood damage to crops and property. The constructed drains were typically connected to naturally occurring streams. Over the years, sections of many of these streams were channelized and diked to increase flow capacity. Most of the constructed drains and altered stream channels have required periodic maintenance work (e.g. dredging, vegetation removal) to maintain their hydraulic design capacity.

While the Governments of Canada and the Province of Manitoba recognize the important socio-economic benefits derived from well-drained agricultural land, both governments also share the mandate to conserve and protect water resources and fish habitat. In 2001, the Manitoba Drain Maintenance Committee was convened to gain a better understanding of the role of drains as fish habitat and to document the mechanical processes required for effective drain maintenance. The Committee, which consisted of a team of federal and provincial engineers and biologists, was also tasked with streamlining the regulatory review process and preparing a series of documents to guide the drainage practitioner involved in routine drain maintenance work. As part of this process, the Committee developed a fish habitat classification protocol that was to be applied to streams and drainage networks throughout agricultural areas of southern Manitoba, i.e., agro-Manitoba. The fish habitat classification protocol combined existing information on topography, drainage, fish communities and habitat conditions with data obtained from field surveys. The field data required to classify the streams and drainage networks using the Committee's fish habitat classification protocol were collected by Fisheries and Oceans Canada (DFO) over five summer field seasons between 2002 and 2006.

This report documents the results of the field surveys and summarizes the data and methods used to develop the first iteration of classified fish habitat maps for the study area. The classified fish habitat maps were planned to be iterative. The accuracy and function of the future versions will be improved with the inclusion of additional fish community and habitat data and local knowledge over time. 



\subsection{STUDY AREA}

The study area included the network of natural streams and constructed drains throughout agro-Manitoba (Figure 1). These watercourses have been documented by the provincial Water Resources Branch in the Designation of Drains (DES) map series, which is comprised of one hundred and fourteen sub-watershed scale maps that cover an area of more than 110,000 square kilometres $\left(\mathrm{km}^{2}\right)$ and delineate more than $66,500 \mathrm{~km}$ of stream and drainage networks. The DES maps were developed to delineate drains where maintenance was designated as a provincial responsibility and drains where maintenance was designated as a municipal responsibility.

Sampling effort was focused on constructed drains, channelized streams, headwater tributaries and small, wadeable rivers within the study area. Sampling did not include on-farm drainage constructed by landowners to convey water from fields, and did not include roadside ditches unless they were designated as drains by the province or municipality.

A small number of site surveys were conducted on streams located just outside of the area of DES map coverage. Data from these sites have been included in the results.

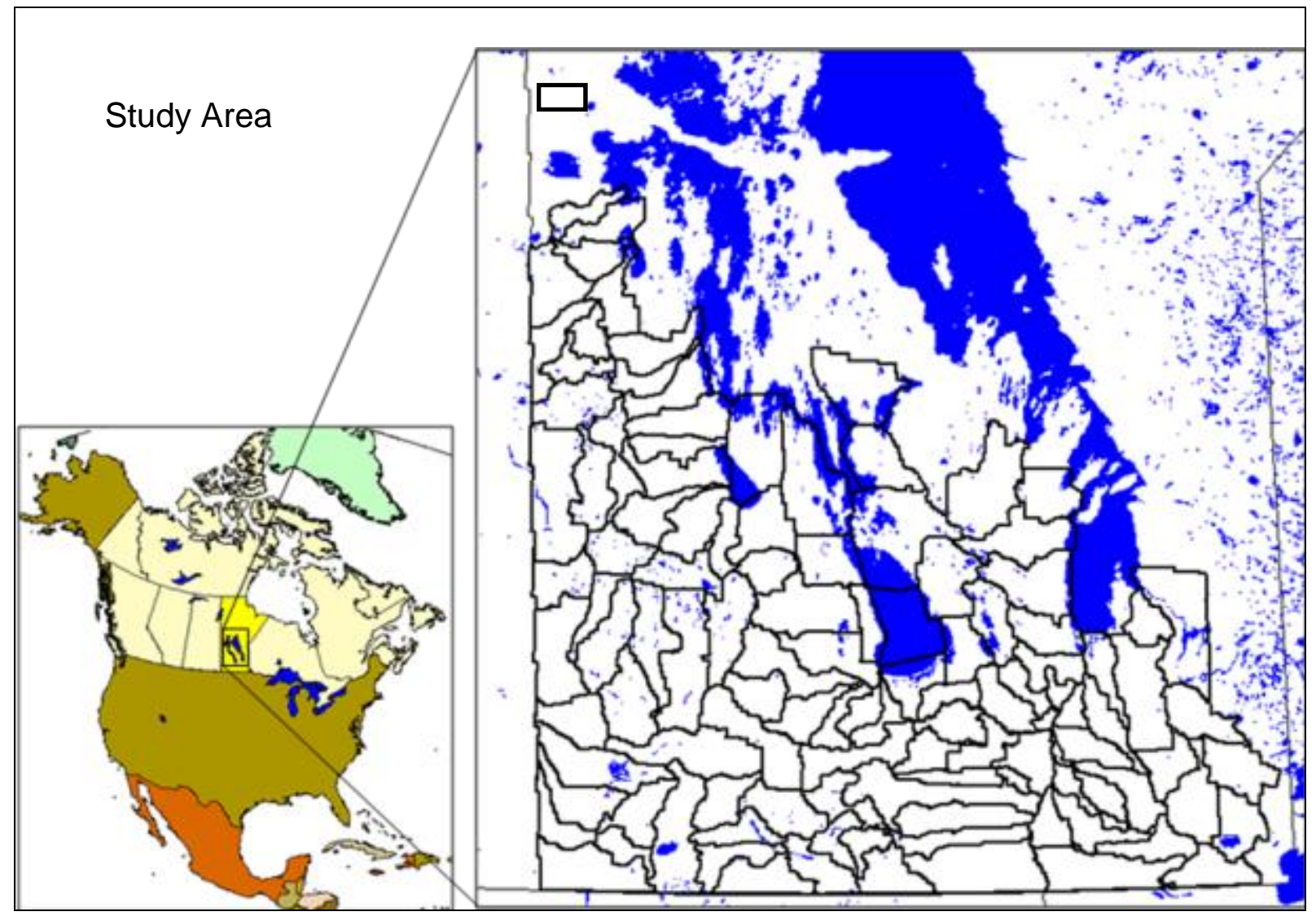

Figure 1: Map showing the location and extent of the study area as defined by the area of coverage provided by all sub-watersheds in the DES map series. The boundary of each DES map in the series is shown. 
The list of the individual DES maps is provided in Table 1. The list provides the DES map number, DES map name and the area of coverage. It should be noted that the series does not have a Map \#1. Nor does it have a Map \#50. As well, there are two maps labelled Map \#31 (\#31W- Lower Interlake Area; and \#31E- East Lower Interlake Area).

Table 1: The list of 114 sub-watersheds in the DES map series that formed the study area showing the DES map number, DES map name, and the area of coverage for each map in the series.

\begin{tabular}{|c|c|c|}
\hline $\begin{array}{l}\text { DES MAP } \\
\text { NUMBER }\end{array}$ & DES MAP NAME & $\begin{array}{c}\text { AREA } \\
\left(\mathrm{km}^{2}\right)\end{array}$ \\
\hline 2 & Lower Roseau River Area & 1707.3 \\
\hline 3 & Upper Rat River Watershed & 787.6 \\
\hline 4 & Lower Rat River Watershed & 828.2 \\
\hline 5 & Marsh River Watershed and Adjacent Areas & 605.7 \\
\hline 6 & Tourond Creek and St. Adolphe Coulee Watershed & 452.5 \\
\hline 7 & Manning Canal Area & 508.1 \\
\hline 8 & Seine River Diversion Watershed & 1007.9 \\
\hline 9 & Lower Seine River Watershed & 723.2 \\
\hline 10 & Brokenhead River Watershed & 1828.1 \\
\hline 11 & Cooks Creek Watershed & 744.2 \\
\hline 12 & Bunns Creek Watershed & 340.1 \\
\hline 13 & Devils Creek Watershed & 706.9 \\
\hline 14 & Catfish Creek Watershed & 618.3 \\
\hline 15 & Riviere aux Marais, Plum River and Adjacent Area & 2095.5 \\
\hline 16 & Shannon Creek Watershed & 834.5 \\
\hline 17 & Tobacco Creek Area & 1199.7 \\
\hline 18 & Norquay Channel Area & 1898.9 \\
\hline 19 & Upper Morris River Area & 508.3 \\
\hline 20 & King Drain Watershed & 168.5 \\
\hline 21 & Elm Creek Watershed & 650.8 \\
\hline 22 & Lower La Salle River Watershed and Adjacent Areas & 848.5 \\
\hline 23 & Central La Salle River Watershed & 672.6 \\
\hline 24 & Upper La Salle River Watershed & 746.5 \\
\hline 25 & Long Lake Area & 520.5 \\
\hline 26 & Sturgeon Creek and Adjacent Watersheds & 850.9 \\
\hline 27 & Grassmere Creek Drain Watershed & 488.9 \\
\hline 28 & Parks Creek and Adjacent Watersheds & 249.4 \\
\hline 29 & Wavey Creek and Adjacent Area & 678.7 \\
\hline
\end{tabular}


Table 1: The list of 114 sub-watersheds in the DES map series that formed the study area showing the DES map number, DES map name, and the area of coverage for each map in the series.

\begin{tabular}{|c|c|c|}
\hline $\begin{array}{l}\text { DES MAP } \\
\text { NUMBER }\end{array}$ & DES MAP NAME & $\begin{array}{c}\text { AREA } \\
\left(\mathrm{km}^{2}\right)\end{array}$ \\
\hline 30 & Netley Creek and Adjacent Area Watersheds & 930.1 \\
\hline $31 \mathrm{E}$ & East Lower Interlake Area & 1360.4 \\
\hline $31 \mathrm{~W}$ & Lower Interlake Area & 1154.5 \\
\hline 32 & Icelandic River Area & 1299.5 \\
\hline 33 & Fisher River Area & 2116.5 \\
\hline 34 & R.M. of Siglunes and R.M. of Eriksdale & 1671.2 \\
\hline 35 & Rat Creek Area & 491.0 \\
\hline 36 & Westbourne Drain Watershed & 443.4 \\
\hline 37 & Squirrel Creek Watershed & 535.0 \\
\hline 38 & Pine Creek Area & 838.2 \\
\hline 39 & Upper Whitemud River Area & 1361.3 \\
\hline 40 & Boggy Creek Watershed & 811.5 \\
\hline 41 & Kinosota Ridge Watershed & 586.2 \\
\hline 42 & Big Grass Marsh Watershed & 1206.4 \\
\hline 43 & Big Grass River Area & 1744.2 \\
\hline 44 & Upper Turtle River Watershed & 703.5 \\
\hline 45 & Lower Turtle River Watershed & 1174.3 \\
\hline 46 & Ochre River Watershed & 576.4 \\
\hline 47 & Vermilion River and Edwards Creek Watershed & 1095.6 \\
\hline 48 & Wilson River Watershed & 970.0 \\
\hline 49 & Fishing River and Mink Creek Watersheds & 614.9 \\
\hline 51 & Upper Roaring River and Ruby River Watersheds & 612.6 \\
\hline 52 & Lower Roaring River and Favel River Watersheds & 551.5 \\
\hline 53 & Lower Swan River and Sinclair River Area & 869.5 \\
\hline 54 & Mary Jane Creek and McCoys Creek Watersheds & 464.7 \\
\hline 55 & Swan Lake and Pilot Creek Watersheds & 553.3 \\
\hline 56 & Crystal Creek, Cypress Creek and Rock Lake Watersheds & 864.8 \\
\hline 57 & Upper Pembina River Watershed & 764.6 \\
\hline 58 & Badger Creek and Long River Watersheds & 1007.8 \\
\hline 59 & Pelican Lake Watershed & 666.9 \\
\hline 60 & Lower Pembina River Watershed & 878.6 \\
\hline 61 & Upper Swan River and Lobstick Creek Watersheds & 877.9 \\
\hline 62 & Plum Creek Area & 1584.9 \\
\hline
\end{tabular}


Table 1: The list of 114 sub-watersheds in the DES map series that formed the study area showing the DES map number, DES map name, and the area of coverage for each map in the series.

\begin{tabular}{|c|c|c|}
\hline $\begin{array}{l}\text { DES MAP } \\
\text { NUMBER }\end{array}$ & DES MAP NAME & $\begin{array}{c}\text { AREA } \\
\left(\mathrm{km}^{2}\right)\end{array}$ \\
\hline 63 & Stony Creek Watershed & 1054.0 \\
\hline 64 & Jackson Creek and Graham Creek Watersheds & 658.3 \\
\hline 65 & Upper Souris River Watershed & 1572.0 \\
\hline 66 & Medora Creek Watershed & 471.4 \\
\hline 67 & Central Souris River Area & 933.3 \\
\hline 68 & Elgin Creek and Whitewater Lake Watersheds & 1804.8 \\
\hline 69 & Lower Souris River Watershed & 820.0 \\
\hline 70 & Oak Creek Watershed & 1086.1 \\
\hline 71 & Cypress River Area & 789.0 \\
\hline 72 & Upper Oak River Watershed & 1254.9 \\
\hline 73 & Lower Oak River and Adjacent Area Watersheds & 1377.7 \\
\hline 74 & Minnewasta Creek and Adjacent Area Watersheds & 1298.3 \\
\hline 75 & Bosshill Creek and Adjacent Area Watersheds & 992.3 \\
\hline 76 & Lower Birdtail Creek Watershed & 882.5 \\
\hline 77 & Upper Birdtail Creek Watershed & 603.3 \\
\hline 78 & Niso Creek, Snake Creek and Adjacent Area Watersheds & 1597.6 \\
\hline 79 & Silver Creek and Adjacent Area Watersheds & 906.8 \\
\hline 80 & Upper Little Saskatchewan River Watershed & 961.4 \\
\hline 81 & Central Little Saskatchewan River Watershed & 1355.6 \\
\hline 82 & Lower Little Saskatchewan River and Adjacent Area & 2065.0 \\
\hline 83 & Lower Whitemouth River Watershed & 1019.6 \\
\hline 84 & Birch River Watershed & 1040.6 \\
\hline 85 & Central Whitemouth River Watershed & 1189.9 \\
\hline 86 & Upper Whitemouth River Watershed & 920.7 \\
\hline 87 & Upper Roseau River Watershed & 945.1 \\
\hline 88 & Upper Brokenhead River Watershed & 801.6 \\
\hline 89 & Upper Woody River Watershed & 1214.3 \\
\hline 90 & Lower Woody River Watershed & 678.6 \\
\hline 91 & Upper Shell River and Adjacent Area Watersheds & 1218.3 \\
\hline 92 & Lower Shell River and Adjacent Area Watersheds & 2301.2 \\
\hline 93 & Upper Valley River and Adjacent Area Watersheds & 912.3 \\
\hline 94 & Central Valley River and Adjacent Area Watersheds & 1112.9 \\
\hline 95 & Lower Valley River Watershed & 1240.1 \\
\hline
\end{tabular}


Table 1: The list of 114 sub-watersheds in the DES map series that formed the study area showing the DES map number, DES map name, and the area of coverage for each map in the series.

\begin{tabular}{|c|l|c|}
\hline $\begin{array}{c}\text { DES MAP } \\
\text { NUMBER }\end{array}$ & \multicolumn{1}{|c|}{ DES MAP NAME } & $\begin{array}{c}\text { AREA } \\
\left(\mathbf{k m}^{2} \mathbf{)}\right.\end{array}$ \\
\hline 96 & Lac du Bonnet Area Watershed & 1995.2 \\
\hline 97 & Bell River and Adjacent Area Watersheds & 606.1 \\
\hline 98 & Little Souris River and Adjacent Area Watersheds & 1800.9 \\
\hline 99 & Epinette Creek, Willow Creek and Adjacent Area Watersheds & 1559.8 \\
\hline 100 & Part of Assiniboine River Watershed & 1176.6 \\
\hline 101 & Portage Delta Watershed & 910.3 \\
\hline 102 & Fork River and Adjacent Area Watersheds & 1043.1 \\
\hline 103 & Garland River and Adjacent Area Watersheds & 902.6 \\
\hline 104 & Pine River Watershed & 588.7 \\
\hline 105 & Sclater River and Duck River Watersheds & 958.3 \\
\hline 106 & Washow Bay and Adjacent Area Watersheds & 1289.7 \\
\hline 107 & Hamlin Drain and Adjacent Area Watersheds & 1211.8 \\
\hline 108 & North Shoal Lake and Adjacent Area Watersheds & 957.0 \\
\hline 109 & West Shoal Lake and Adjacent Area Watershed & 904.2 \\
\hline 110 & Garrioch Creek and Adjacent Area Watersheds & 1069.6 \\
\hline 111 & Birch Creek and Adjacent Area Watersheds & 962.2 \\
\hline 112 & Gypsumville Area Watershed & 1311.8 \\
\hline 113 & Crane River and Adjacent Area Watersheds & 1787.8 \\
\hline 114 & Pasquia Area Watersheds & 800.0 \\
\hline 115 & Steeprock River Watershed & 746.7 \\
\hline
\end{tabular}

Paper copies of the DES maps have been available for decades. These maps were used by the field crews to locate stream and drain survey reaches in the field. Presently, most but not all of the maps in the DES map series are available in an electronic, georeferenced format suitable for use in a Geographic Information System (GIS). 



\subsection{MATERIALS AND METHODS}

Information on the fish communities and habitat conditions within the study area was required to classify the streams and drains in accordance with the fish habitat classification protocol developed by the Manitoba Drain Maintenance Committee (Figure 2). Given the physical size of the study area, the length of stream or drain to be surveyed and the type and amount of data required to classify the watercourse a fairly rapid assessment protocol was needed to gather data in a timely manner. As well, the sampling protocols had to be applied over a wide range of channel types with varied physical characteristics.

The following sections provide a description of the materials and methods used to gather and compile the data required to classify the stream and drainage networks according to the fish habitat classification protocol provided by the Manitoba Drain Maintenance Committee.

\subsection{FISH HABITAT CLASSIFICATION PROTOCOL}

The Manitoba Drain Maintenance Committee developed a fish habitat classification protocol to identify the streams and drains that might be more sensitive to habitat disturbance from routine maintenance work. The protocol involved the following main steps at each sampling reach:

1. Determine if the stream or drain provides direct or indirect fish habitat

2. Determine if fish are present in the stream or drain

3. Determine if indicator fish species are present in the stream or drain

4. Determine if the habitat is simple or complex

5. Classify the type of stream at that sampling reach (Type A, B, C, D or E)

These steps are summarized and illustrated in Figure 2, the Habitat Type Decision Flowchart.

The Habitat Type Decision Flowchart provided the basis for evaluating habitat conditions, habitat diversity and the approach to assessing fish community, and provided a clear and concise result for classifying each stream reach sampled. 


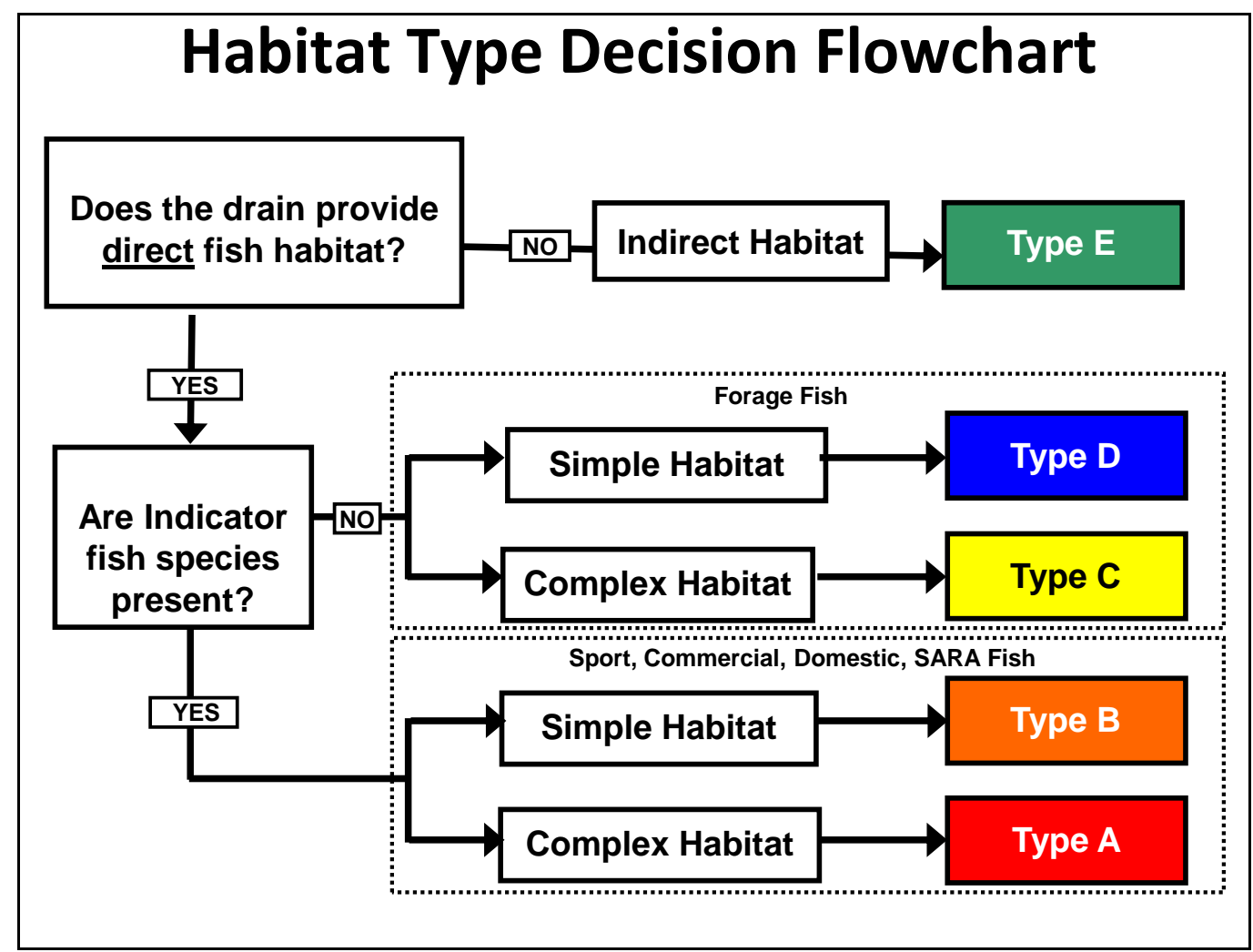

Figure 2: $\quad$ Fish habitat classification decision flowchart applied to streams and drains within the study area.

Direct fish habitat included watercourses where fish can complete any of their life processes, i.e., spawning, rearing, feeding, migration or over-wintering. Indirect fish habitat included ephemeral watercourses that typically have insufficient flow volume or flow duration to allow fish to complete one or more of their life processes. These ephemeral watercourses may not provide habitat for fish to complete one or more of their life processes, but may provide flow and nutrients to downstream areas. These watercourses can also impact downstream areas through the transport of sediment and other deleterious substances.

The fish community decision involved first determining if fish were present in the surveyed reach of the watercourse, and then further defining the fish community based on the presence/absence of "Indicator Species". Indicator Species included largebodied species with commercial, domestic, or sport fishery value such as Northern Pike (Esox lucius), Walleye (Sander vitreus) or White Sucker (Catostomus commersoni). Indicator Species also included any fish listed in Schedule 1 of the Species at Risk Act (SARA). All other fish were designated as "Non-Indicator" Species. A list of the fish species that might be collected within the study area and their status as an Indicator Species or Non-Indicator Species has been prepared based on the distribution maps and species accounts in Stewart and Watkinson (2004) and is provided in Table 2. 
Table 2: List of fish species potentially present within the study area and their status as an Indicator Species (adapted from Stewart and Watkinson. 2004).

\begin{tabular}{|c|c|c|c|c|}
\hline \multirow{2}{*}{$\begin{array}{l}\text { FAMILY } \\
\text { Scientific Name }\end{array}$} & \multirow[b]{2}{*}{ Common Name } & \multicolumn{2}{|c|}{ Indicator Species } & \multirow{2}{*}{$\begin{array}{l}\text { Collected } \\
\text { In Study }\end{array}$} \\
\hline & & Yes & No & \\
\hline \multicolumn{5}{|c|}{ PETROMYZONTIDAE: LAMPREYS } \\
\hline Ichthyomyzon castaneus & Chestnut Lamprey & & $x$ & Yes \\
\hline Ichthyomyzon fossor & Northern Brook Lamprey & & $x$ & No \\
\hline Ichthyomyzon unicuspis & Silver Lamprey & & $x$ & No \\
\hline \multicolumn{5}{|c|}{ ACIPENSERIDAE: STURGEON } \\
\hline Acipenser fulvescens & Lake Sturgeon & $\mathrm{x}$ & & No \\
\hline \multicolumn{5}{|c|}{ HIODONTIDAE: GOLDEYE AND MOONEYE } \\
\hline Hiodon alosoides & Goldeye & $\mathrm{x}$ & & Yes \\
\hline Hiodon tergisus & Mooneye & $\mathrm{X}$ & & No \\
\hline \multicolumn{5}{|c|}{ CYPRINIDAE: MINNOWS AND CARP } \\
\hline Cyprinella spiloptera & Spotfin Shiner & & $x$ & Yes \\
\hline Cyprinus carpio & Common Carp & & $x$ & Yes \\
\hline Hybognathus hankinsoni & Brassy Minnow & & $\mathrm{x}$ & Yes \\
\hline Luxilus cornutus & Common Shiner & & $x$ & Yes \\
\hline Macrhybopsis storeriana & Silver Chub & & $x$ & No \\
\hline Margariscus margarita & Pearl Dace & & $x$ & Yes \\
\hline Nocomis biguttatus & Hornyhead Chub & & $x$ & Yes \\
\hline Notemigonus crysoleucas & Golden Shiner & & $\mathrm{X}$ & Yes \\
\hline Notropis atherinoides & Emerald Shiner & & $\mathrm{X}$ & Yes \\
\hline Notropis blennius & River Shiner & & $x$ & Yes \\
\hline Notropis dorsalis & Bigmouth Shiner & & $x$ & Yes \\
\hline Notropis heterodon & Blackchin Shiner & & $x$ & Yes \\
\hline Notropis heterolepis & Blacknose Shiner & & $x$ & Yes \\
\hline Notropis hudsonius & Spottail Shiner & & $x$ & Yes \\
\hline Notropis percobromus & Carmine Shiner & $x$ & & No \\
\hline Notropis stramineus & Sand Shiner & & $x$ & Yes \\
\hline Notropis texanus & Weed Shiner & & $x$ & No \\
\hline Notropis volucellus & Mimic Shiner & & $x$ & Yes \\
\hline Phoxinus eos & Northern Redbelly Dace & & $x$ & Yes \\
\hline Phoxinus neogaeus & Finescale Dace & & $x$ & Yes \\
\hline Pimephales notatus & Bluntnose Minnow & & $x$ & No \\
\hline Pimephales promelas & Fathead Minnow & & $x$ & Yes \\
\hline Platygobio gracilis & Flathead Chub & & $x$ & No \\
\hline Rhinichthys obtusus & Western Blacknose Dace & & $x$ & Yes \\
\hline
\end{tabular}


Table 2: List of fish species potentially present within the study area and their status as an Indicator Species (adapted from Stewart and Watkinson. 2004).

\begin{tabular}{|c|c|c|c|c|}
\hline \multirow{2}{*}{$\begin{array}{l}\text { FAMILY } \\
\text { Scientific Name }\end{array}$} & \multirow[b]{2}{*}{ Common Name } & \multicolumn{2}{|c|}{ Indicator Species } & \multirow{2}{*}{$\begin{array}{l}\text { Collected } \\
\text { In Study }\end{array}$} \\
\hline & & Yes & No & \\
\hline Rhinichthys cataractae & Longnose Dace & & $\mathrm{X}$ & Yes \\
\hline Semotilus atromaculatus & Creek Chub & & $\mathrm{x}$ & Yes \\
\hline \multicolumn{5}{|l|}{ CATOSTOMIDAE: SUCKERS } \\
\hline Carpiodes cyprinus & Quillback & $\mathrm{X}$ & & Yes \\
\hline Catostomus catostomus & Longnose Sucker & $\mathrm{x}$ & & No \\
\hline Catostomus commersoni & White Sucker & $\mathrm{x}$ & & Yes \\
\hline Ictiobus cyprinellus & Bigmouth Buffalo & $\mathrm{x}$ & & Yes \\
\hline Moxostoma anisurum & Silver Redhorse & $\mathrm{x}$ & & Yes \\
\hline Moxostoma erythrurum & Golden Redhorse & $\mathrm{x}$ & & No \\
\hline Moxostoma macrolepidotum & Shorthead Redhorse & $\mathrm{X}$ & & Yes \\
\hline \multicolumn{5}{|c|}{ ICTALURIDAE: FRESHWATER CATFISHES } \\
\hline Ameiurus melas & Black Bullhead & & $\mathrm{X}$ & Yes \\
\hline Ameiurus natalis & Yellow Bullhead & & $\mathrm{X}$ & No \\
\hline Ameiurus nebulosus & Brown Bullhead & & $\mathrm{X}$ & No \\
\hline Ictalurus punctatus & Channel Catfish & $\mathrm{X}$ & & Yes \\
\hline Noturus flavus & Stonecat & & $\mathrm{X}$ & Yes \\
\hline Noturus gyrinus & Tadpole Madtom & & $\mathrm{X}$ & Yes \\
\hline \multicolumn{5}{|c|}{ ESOCIDAE: PIKE AND MUSKELLUNGE } \\
\hline Esox lucius & Northern Pike & $\mathrm{x}$ & & Yes \\
\hline \multicolumn{5}{|l|}{ UMBRIDAE: MUDMINNOWS } \\
\hline Umbra limi & Central Mudminnow & & $\mathrm{X}$ & Yes \\
\hline \multicolumn{5}{|c|}{ SALMONIDAE: WHITEFISH, TROUT AND SALMON } \\
\hline Coregonus artedi & Cisco & $\mathrm{X}$ & & No \\
\hline Coregonus clupeaformis & Lake Whitefish & $x$ & & No \\
\hline Oncorhynchus mykiss & Rainbow Trout & $x$ & & Yes \\
\hline Salmo trutta & Brown Trout & $x$ & & Yes \\
\hline Salvelinus fontinalis & Brook Trout & $\mathrm{x}$ & & Yes \\
\hline Salvelinus namaycush & Lake Trout & $\mathrm{X}$ & & No \\
\hline \multicolumn{5}{|c|}{ PERCOPSIDAE: TROUTPERCHES } \\
\hline Percopsis omiscomaycus & Troutperch & & $\mathrm{x}$ & Yes \\
\hline \multicolumn{5}{|l|}{ GADIDAE: COD-LIKE FISHES } \\
\hline Lota lota & Burbot & $\mathrm{x}$ & & Yes \\
\hline \multicolumn{5}{|c|}{ GASTEROSTEIDAE: STICKLEBACKS } \\
\hline Culaea inconstans & Brook Stickleback & & $x$ & Yes \\
\hline
\end{tabular}


Table 2: List of fish species potentially present within the study area and their status as an Indicator Species (adapted from Stewart and Watkinson. 2004).

\begin{tabular}{|c|c|c|c|c|}
\hline \multirow{2}{*}{$\begin{array}{l}\text { FAMILY } \\
\text { Scientific Name }\end{array}$} & \multirow[b]{2}{*}{ Common Name } & \multicolumn{2}{|c|}{ Indicator Species } & \multirow{2}{*}{$\begin{array}{l}\text { Collected } \\
\text { In Study }\end{array}$} \\
\hline & & Yes & No & \\
\hline Pungitius pungitius & Ninespine Stickleback & & $x$ & No \\
\hline \multicolumn{5}{|l|}{ COTTIDAE: SCULPINS } \\
\hline Cottus bairdii & Mottled Sculpin & & $\mathrm{x}$ & Yes \\
\hline Cottus cognatus & Slimy Sculpin & & $x$ & No \\
\hline \multicolumn{5}{|c|}{ MORONIDAE: TEMPERATE BASSES } \\
\hline Morone chrysops & White Bass & $\mathrm{X}$ & & No \\
\hline \multicolumn{5}{|c|}{ CENTRARCHIDAE: BASS, CRAPPIES AND SUNFISHES } \\
\hline Ambloplites rupestris & Rock Bass & $\mathrm{x}$ & & Yes \\
\hline Lepomis gibbosus & Pumpkin Seed & $\mathrm{X}$ & & No \\
\hline Lepomis macrochirus & Bluegill & $\mathrm{X}$ & & No \\
\hline Micropterus dolomieu & Smallmouth Bass & $\mathrm{X}$ & & Yes \\
\hline Micrpoterus salmoides & Largemouth Bass & $\mathrm{X}$ & & No \\
\hline Pomoxis annularis & White Crappie & $\mathrm{x}$ & & No \\
\hline Pomoxis nigromaculatus & Black Crappie & $\mathrm{X}$ & & Yes \\
\hline \multicolumn{5}{|c|}{ PERCIDAE: DARTERS, PERCH, SAUGERS AND WALLEYE } \\
\hline Etheostoma exile & lowa Darter & & $x$ & Yes \\
\hline Etheostoma nigrum & Johnny Darter & & $x$ & Yes \\
\hline Perca flavescens & Yellow Perch & $\mathrm{X}$ & & Yes \\
\hline Percina caprodes & Logperch & & $x$ & Yes \\
\hline Percina maculata & Blackside Darter & & $x$ & Yes \\
\hline Percina shumardi & River Darter & & $x$ & Yes \\
\hline Sander canadensis & Sauger & $x$ & & Yes \\
\hline Sander vitreus & Walleye & $x$ & & Yes \\
\hline \multicolumn{5}{|l|}{ SCIANIDAE: DRUMS } \\
\hline Aplodinotus grunniens & Freshwater Drum & $x$ & & Yes \\
\hline
\end{tabular}

Simple Habitat was defined as a linear channel having a trapezoidal cross-section, with a fine, uniform substrate and grassed banks or dikes. All other habitat types were considered to be Complex. 
Classification of the streams and drains was undertaken by selecting representative sections i.e., reaches of each drain or stream for sampling. Sampling reaches were selected with the objective of efficiently gathering data from as many sites as possible over the duration of the study. The focussing of sampling effort on a specific region of the study area was most often determined by local weather and flow conditions. Reaches were selected to allow for the classification of stream segments in the lower, middle and upper portions of individual drainage networks. Where possible, sites with a mix of riffle, run, and pool habitat were selected. Finally, sampling reaches had to be safely wadeable.

All sampling reaches were identified by a unique site number formed by: a single letter that identified the survey crew and their base of operation (Brandon, Dauphin or Winnipeg); the last two digits in the year (02 - 06); and a three-digit number corresponding to each reach in the order they were sampled. For example, the site number D-02-001 identifies the crew from Dauphin; the year 2002; and 001 as the first reach surveyed that year. Crews based in Brandon were assigned the letter " $B$ ", and Winnipeg crews were identified by the letters " $W$ " and " $X$ " in those years when two Winnipeg-based crews were utilized.

Reaches were also identified by name. If the watercourse had an official Geographical Name, that was used to name the reach (e.g. Netley Creek). Where no Geographical Name existed, the reach was identified as an unnamed tributary with the next downstream named feature being included to help describe the reach (e.g. Unnamed Tributary to Netley Creek). The location of each reach was plotted on the corresponding DES map in the field. Hand-held Garmin E-Trex Legend Global Positioning System (GPS) units were used to determine the latitude and longitude in decimal degrees (DD) for each site.

\subsection{BASIC WATER CHEMISTRY MEASUREMENTS}

Prior to commencing any instream work, temperature, dissolved oxygen (DO), $\mathrm{pH}$, conductivity and turbidity of the water were measured. Where possible, these parameters were measured in non-turbulent run habitat. Ambient air temperature was also measured and collected at each site.

Water temperature $\left({ }^{\circ} \mathrm{C}\right)$ was measured at a single location using an alcohol thermometer and the result was used to calibrate the field crews' dissolved oxygen meters.

DO measurements were collected in non-turbulent flow using Hanna Instruments Model HI9143 DO meters. Results were recorded as both milligrams per litre $(\mathrm{mg} / \mathrm{L})$ and the percent saturation (\% SAT). The operation of the DO meter required the probe to be calibrated to the water temperature and altitude (ASL) of the site with each use. The altitude of the reach was determined by the field crews' GPS units. 
The $\mathrm{pH}$ was measured using Hanna Instruments Model HI991300 pH/Conductivity meters. The $\mathrm{pH}$ probe was calibrated at the start of the field season and was recalibrated as required based on the performance against standard $\mathrm{pH}$ solutions and on day-to-day performance in the field.

Conductivity $(\mu \mathrm{S} / \mathrm{cm})$ was measured using Hanna Instruments Model HI991300 $\mathrm{pH} /$ Conductivity meters. The conductivity probe was calibrated at the start of the field season and was recalibrated as required based on the performance against standard conductivity solutions and on day-to-day performance of the instrument in the field.

Turbidity was measured as Nephelometric Turbidity Units (NTUs) using Hanna Instruments HI93703 turbidity meters. Turbidity meters were calibrated at the start of the field season and were recalibrated on a monthly basis or more frequently based on dayto-day performance of the instrument in the field.

\subsection{FISH COMMUNITY SAMPLING AND IDENTIFICATION}

The objectives of the fish community sampling at each reach were to confirm the presence of fish and determine whether Indicator Species were present. The fish community sampling was carried out using at least one of the following fishing methods: backpack electrofisher, beach seine, dip net, larval drift trap or kick sampler. Visual observations of fish were recorded when field crews were confident with their identification of the fish being observed. The fishing effort expended with each fishing method was recorded.

Where possible, all fish collected were identified to species using the keys and species accounts under development and subsequently published by Stewart and Watkinson (2004). In most cases, adult members of those species designated as "Indicator Species" were photographed, measured and released, unless conditions required fish to be released immediately. All length measurements refer to fork-length unless noted with an asterisk, or are stated as total-length. Very small specimens were measured to totallength as noted. Retained fish were first fixed in $10 \%$ formalin and then preserved in $70 \%$ ethanol for later identification in the lab. In some cases, only a representative subsample of non-indicator species was retained. Specimens collected in 2004 and 2005 were provided to the Manitoba Museum.

\subsubsection{Backpack Electrofishing}

Backpack electrofishing was the preferred and most versatile fish sampling method in most situations. All crews were trained and certified for backpack electrofishing using the Ontario Watershed Science Center Electrofishing Manual. As well, the training required a valid CPR Lifesaver (or equivalent) Certificate. Training involved both in class and in the field assessments. Field assessments were continued occasionally throughout the summer. 
Electrofishing was carried out by crews of at least two people, with one person operating the backpack electrofisher and at least one person netting fish and caring for the catch. Smith-Root, Model LR-24 Backpack Electrofishers were used by all field crews. The selection of electrofisher settings (voltage, frequency and duty cycle) was made based on the response of fish collected. The preferred settings produced a strong response in fish of most sizes and yet allowed fish to fully recover within a few minutes after being collected. Typical settings early in the field season were 200 volts (DC), from 15 to 30 hertz frequency, and a $12.5 \%$ duty cycle. As the season progressed and conductivity levels tended to increase, the settings on the electrofisher had to be changed to maintain the preferred response by fish. The preferred response was often regained by adjusting voltage between 200 to 300 volts, incrementally bumping up the frequency to 60 hertz and by increasing the duty cycle up to $25 \%$. Late in the season under low flow and high conductivity conditions, some species, such as Northern Pike, became very difficult to collect due to a strong fright response to physical disturbance and electrical current outside of the effective zone of the electrofisher.

In most situations, electrofishing crews preferred to selectively fish upstream towards likely micro-habitats and available cover using a quiet, randomized approach. In some situations, such as high flow or elevated turbidity when operator visibility was limited, reaches were fished using a more systematic approach. In all cases, care was taken to ensure all habitat types were fished. The effectiveness of electrofishing was often limited in pools with a depth of more than 1.0 metre. Electrofishing effort was recorded as the number of seconds the electrical current was applied times the length of stream in metres $(m)$ that was fished.

\subsubsection{Beach Seining}

Beach seines usually provided the best fish collection method in situations such as deep pools, high conductivity water or very turbid stream conditions where electrofishing was less effective. Beach seining was best in deeper pools with smooth bottoms and few snags.

Field crews had two beach seines $(1 \mathrm{~m} \times 10 \mathrm{~m} \times 3 \mathrm{~mm}$ mesh and $1.8 \mathrm{~m} \times 18 \mathrm{~m} \times 3 \mathrm{~mm}$ mesh) for use, depending on stream conditions. Beach seines were most often drawn upstream through pools toward barriers such as riffles or culverts. Scour pools below culverts were often very productive, especially in late summer and during periods of low flow. Beach seining effort was recorded as the length in metres $(m)$ of stream seined times the average width in metres $(m)$ of stream seined.

\subsubsection{Kick Sampling}

Kick sampling in riffles was an effective way to collect fish eggs and to collect those species of fish that reside at the bottom of higher velocity habitat. Kick sampling was carried out by placing a 500 micrometre $(\mu)$ Nitex mesh, D-framed kick sampling net downstream of the area of the bottom being disturbed by the toe of a boot overturning 
stones and gently stirring up a small areas of the bottom. Fish and any fish eggs were handpicked from the sample and were preserved for later identification.

Additional replicate samples were collected throughout the range of flow velocities and depths present on the riffle until an area of about $1 \mathrm{~m}^{2}$ had been sampled. Kick sampling for eggs allowed the study team the opportunity to collect a life stage of an Indicator Species that may have already left the area after spawning. Kick sampling effort was recorded as the total area in square metres $\left(\mathrm{m}^{2}\right)$ sampled by all replicates combined.

\subsubsection{Larval Drift Sampling}

Larval drift sampling was carried out using $500 \mu$ Nitex mesh drift nets with either a 22 $\mathrm{cm}$ or $30 \mathrm{~cm}$ square opening at the upstream end. Nets were set in flowing waters to collect recently hatched larval fish drifting with the current. Drift nets were often set at a site for the period of time it took a crew to complete a reach survey, i.e., between 0.5 and 3 hours. In other cases drift nets were set overnight. Larval fish, eggs and egg husks were handpicked from the samples and were preserved for later identification. As with kick sampling, larval drift sampling allowed the study team to extend the opportunity to collect a life stage of an Indicator Species.

Larval drift sampling effort was measured in minutes for short sets and in hours and minutes for overnight sets.

\subsubsection{Dip-Netting}

Dip-nets were occasionally used to sample the fish community. Dip-netting with a fine (3 $\mathrm{mm}$ ) mesh, long handled net was effective in standing pools, undercut banks, plunge pools and other situations. Fine mesh dip-nets were effective when used to sweep through dense aquatic vegetation to collect post-larval Northern Pike and other small fishes.

Dip-netting effort was recorded as the number of "arm's length" sweeps made through each habitat type or cover type.

\subsubsection{Visual Observations}

At some sites, it was possible to confirm the presence of Indicator Species such as white sucker when spawning on shallow, riffle habitat, with a simple visual observation. Records of visual observations of fish included the species, approximate number observed, and a description of the observation (e.g., hundreds of White Sucker congregated below culvert).

\subsubsection{Fish Collection Field Notes}

At each site where fishing effort was expended, field crews recorded the effort and noted the catch, including Nil Catch, for each gear type used. Field notes were also 
used to record the number and species of released fish, the length range of released Indicator Species, any observations of fish maturity, spawning activity, spawning colouration, parasites, injuries or other conditions. Crews also noted the presence of obstructions, such as beaver dams or perched culverts that might affect the fish community. Any conditions that might have influenced the effectiveness of the fishing gear used were also noted.

\subsection{DETERMINATION OF FISH HABITAT COMPLEXITY}

The fish habitat classification flowchart defined Simple Habitat as a linear channel having a trapezoidal cross-section, with a fine, uniform substrate and grassed banks or dikes. All other stream conditions were considered to represent Complex Habitat.

At all sites visited, field crews used the definition of Simple Habitat as a guide to classify habitat complexity when looking both upstream and downstream from mid-reach. Linear, constructed drainage networks often had short reaches of more diverse (or more complex) instream and riparian habitat within extensive areas of Simple Habitat. The visual determination of Simple or Complex Habitat was made based on the dominant habitat condition in view of the reach.

\subsection{HABITAT ASSESSMENT}

The rating of twelve instream and riparian zone habitat conditions using the protocols modified and adapted from Barbour et al. (1999) was carried out at sites where fishing effort was expended and where crews were safely able to complete the assessment. The published protocols were modified to include twelve rather than ten habitat parameters for assessment, with the addition of two parameters suitable for assessing pool habitat in low-gradient $(<2 \%)$ stream reaches.

Each parameter was assessed and based on its condition given a numerical score from a low of 0 to a high of 20 . Within that range of scores: the condition of any parameter scoring from 0 to 5 was considered Poor a score from 6 to 10 was considered Marginal; a score from 11 to 15 was described as Sub-Optimal; and a score from 16 to 20 was applied to habitat conditions that were considered to be Optimal for that eco-region.

The following sections provide a brief description of the each parameter assessed. Figure 3 provides the assessment criteria for each habitat parameter and also served as the data collection form used to record the assessment scores at each site.

\subsubsection{Available Cover/Epifaunal Substrate}

Available Cover was ranked based on the relative quantity and variety of natural structures such as fallen trees, logs, woody debris, large rocks and undercut banks that are available to fish as refuge areas, for feeding or for reproduction. A wide variety and/or abundance of submerged structures in the stream provide fish with a large number of niches, potentially increasing the diversity of the fish community. 
Epifaunal Substrate rated the range of microhabitat available to insects and aquatic invertebrates. The most diverse habitat exists when a variety of coarse substrates, such as cobbles and boulders, is present, along with abundant woody debris, logs, and snags. Higher gradient riffle habitat is essential for maintaining some types of aquatic insects. Snags and submerged logs are among the most diverse habitat structures found in lower-gradient streams.

\subsubsection{Substrate Embeddedness}

Substrate Embeddedness rated the extent to which rocks (gravel, cobble, and boulders) were covered or sunken into the silt, sand or mud of the stream bottom. Generally, as rocks become embedded, the surface area available to benthic invertebrates and fish decreases. Embeddedness is a result of sediment movement and deposition and was evaluated in riffle and run habitat. Embeddedness can vary seasonally and from year to year.

\subsubsection{Velocity/Depth Regime}

The stream reaches with the highest quality of fish habitat in the study area were considered to have four Velocity/Depth flow regimes present: (1) Slow/Deep; (2) Slow/Shallow; (3) Fast/Deep; and (4) Fast/Shallow. A water depth greater than $0.5 \mathrm{~m}$ was considered to be deep; and a flow velocity greater than 0.3 metres $/ \mathrm{second}(\mathrm{m} / \mathrm{s})$ was considered to be fast. The occurrence of all four flow regimes helps to ensure that the range of habitat types necessary to maintain a stable aquatic environment are available for use.

\subsubsection{Sediment Deposition}

Sediment Deposition rated the amount of sediment that had accumulated in pools and the changes that have occurred to the stream bottom as a result of deposition. Along with drastic changes to pool substrate, sediment deposition can cause the formation of islands, point bars, mid-channel bars and other features. Heavy sediment deposition can cause erosive flow energy to be diverted into stream banks, which can cause bank instability. In some areas of the study area, the deposition of a uniform substrate type, such as shale, can create an unstable and continually changing environment that limits the growth of aquatic vegetation and reduces the amount of available cover.

\subsubsection{Channel Flow Status}

Channel Flow Status assessed the degree to which the channel was filled with water. When water does not cover much of the stream bed, the amount of viable substrate for aquatic organisms is limited. In high-gradient streams, riffles and cobble substrate are exposed; in low-gradient streams, the decrease in water level exposes logs and snags, thereby reducing the areas of available habitat. 


\subsubsection{Channel Alteration}

Channel Alteration examined the large-scale changes in the shape of the stream channel. Many streams in urban and agricultural areas have been straightened, deepened, or diverted into man-made channels, often for flood control purposes. Channel alteration activities often remove the naturally occurring meanders of the stream, which can shorten the length of the stream, resulting in less habitat area for fish, invertebrates, and plants. Channel alteration is present when artificial embankments, riprap, and other forms of artificial bank stabilization or structures are present; the stream is very straight for significant distances; dams and bridges are present; and/or when other such changes have occurred. Scouring is often associated with channel alteration, particularly in areas downstream of channelized reaches.

\subsubsection{Frequency of Riffles (or Bends)}

Riffles can be a source of high-quality habitat and diverse fauna in streams; therefore, an increased frequency of riffle occurrence usually enhances the diversity of the stream benthic community. For areas where distinct riffles are uncommon, a run/bend ratio can be used as a measure of one characteristic of channel meandering or sinuosity. Higher sinuosity can provide complexity to the stream habitat, which can increase the diversity of available habitats and fauna, and increase the ability of the stream to withstand flow surges when the stream flow fluctuates as a result of storms. The absorption and deflection of this energy by the stream bends also protects the stream from erosion and flooding.

\subsubsection{Bank Stability}

Bank Stability rated the condition of the stream banks and assessed whether the banks are eroded, or have the potential for erosion. Steep banks are more likely to collapse and undergo erosion than gently sloping banks. Signs of erosion included slumping, exposed banks and exposed tree roots.

The assessment of Bank Stability was made for each stream bank. The assessor faced upstream when determining the left or right bank of the stream. It should be noted that this varied from the protocol (Barbour et al. 1999) where the assessor faces downstream. It is much safer and easier to face upstream when working in streams, especially during high water conditions when debris or ice can be running.

\subsubsection{Bank Vegetative Protection}

Bank Vegetative Protection assessed the amount of the stream bank that was covered by vegetation. The root systems of plants growing on stream banks help hold soil in place, thereby reducing the potential for erosion. Banks that have healthy, natural plant growth with a variety of age classes of shrubs and trees are also better at providing shade and woody debris inputs, versus stream banks that are bare or shored up with concrete or riprap. 


\begin{tabular}{|c|c|c|c|c|}
\hline \multirow{2}{*}{$\begin{array}{c}\text { Habitat } \\
\text { Parameter }\end{array}$} & \multicolumn{4}{|c|}{ Condition Category } \\
\hline & Optimal & Sub-Optimal & Marginal & Poor \\
\hline $\begin{array}{l}\text { 1. Available } \\
\text { Cover/ } \\
\text { Epifaunal } \\
\text { Substrate }\end{array}$ & $\begin{array}{l}\text { Greater than } 70 \% \text { of } \\
\text { substrate favourable } \\
\text { for epifaunal } \\
\text { colonization and fish } \\
\text { cover; mix of snags, } \\
\text { submerged logs, } \\
\text { undercut banks, } \\
\text { cobble or other stable } \\
\text { habitat and at stage } \\
\text { to allow full } \\
\text { colonization potential } \\
\text { (i.e., logs/snags that } \\
\text { are not new fall and } \\
\text { not transient). }\end{array}$ & $\begin{array}{l}40-70 \% \text { mix of stable } \\
\text { habitat; well suited for } \\
\text { full colonization } \\
\text { potential; adequate } \\
\text { habitat for } \\
\text { maintenance of } \\
\text { populations; } \\
\text { presence of additional } \\
\text { substrate in the form } \\
\text { of new fall, but not } \\
\text { yet prepared for } \\
\text { colonization (may } \\
\text { rate at high end of } \\
\text { scale). }\end{array}$ & $\begin{array}{l}20-40 \% \text { mix of stable } \\
\text { habitat; habitat } \\
\text { availability less than } \\
\text { desirable; substrate } \\
\text { frequently disturbed } \\
\text { or removed. }\end{array}$ & $\begin{array}{l}\text { Less than } 20 \% \text { stable } \\
\text { habitat; lack of } \\
\text { habitat is obvious; } \\
\text { substrate unstable or } \\
\text { lacking. }\end{array}$ \\
\hline SCORE & $\begin{array}{lllll}20 & 19 & 18 & 17 & 16 \\
\end{array}$ & $\begin{array}{lllll}15 & 14 & 13 & 12 & 11 \\
\end{array}$ & $\begin{array}{lllll}10 & 9 & 8 & 7 & 6 \\
\end{array}$ & \begin{tabular}{llllll|}
5 & 4 & 3 & 2 & 1 & 0 \\
\end{tabular} \\
\hline $\begin{array}{l}\text { 2. Substrate } \\
\text { Embedded- } \\
\text { ness }\end{array}$ & $\begin{array}{l}\text { Gravel, cobble, and } \\
\text { boulder particles are } \\
0-25 \% \text { surrounded by } \\
\text { fine sediment. }\end{array}$ & $\begin{array}{l}\text { Gravel, cobble, and } \\
\text { boulder particles are } \\
25-50 \% \text { surrounded } \\
\text { by fine sediment. }\end{array}$ & $\begin{array}{l}\text { Gravel, cobble, and } \\
\text { boulder particles are } \\
50-75 \% \text { surrounded } \\
\text { by fine sediment. }\end{array}$ & $\begin{array}{l}\text { Gravel, cobble, and } \\
\text { boulder particles are } \\
\text { more than } 75 \% \\
\text { surrounded by fine } \\
\text { sediment. }\end{array}$ \\
\hline SCORE & $\begin{array}{lllll}20 & 19 & 18 & 17 & 16 \\
\end{array}$ & $\begin{array}{lllll}15 & 14 & 13 & 12 & 11 \\
\end{array}$ & $\begin{array}{lllll}10 & 9 & 8 & 7 & 6 \\
\end{array}$ & $\begin{array}{llllll}5 & 4 & 3 & 2 & 1 & 0 \\
\end{array}$ \\
\hline $\begin{array}{l}\text { 3. Velocity / } \\
\text { Depth } \\
\text { Regime }\end{array}$ & $\begin{array}{l}\text { All } 4 \text { velocity/depth } \\
\text { regimes present } \\
\text { (slow-deep, slow- } \\
\text { shallow, fast-deep, } \\
\text { fast-shallow). (slow is } \\
<0.3 \mathrm{~m} / \mathrm{s} \text {, deep is } \\
>0.5 \mathrm{~m} \text { ). }\end{array}$ & $\begin{array}{l}\text { Only } 3 \text { of the } 4 \\
\text { regimes present (if } \\
\text { fast-shallow is } \\
\text { missing, score lower } \\
\text { than if missing other } \\
\text { regimes). }\end{array}$ & $\begin{array}{l}\text { Only } 2 \text { of the } 4 \\
\text { habitat regimes } \\
\text { present (if fast- } \\
\text { shallow or slow- } \\
\text { shallow are missing, } \\
\text { score low). }\end{array}$ & $\begin{array}{l}\text { Dominated by } 1 \\
\text { velocity/depth regime } \\
\text { (usually slow-deep). }\end{array}$ \\
\hline SCORE & $\begin{array}{lllll}20 & 19 & 18 & 17 & 16 \\
\end{array}$ & $\begin{array}{lllll}15 & 14 & 13 & 12 & 11 \\
\end{array}$ & $\begin{array}{lllll}10 & 9 & 8 & 7 & 6 \\
\end{array}$ & $\begin{array}{llllll}5 & 4 & 3 & 2 & 1 & 0 \\
\end{array}$ \\
\hline $\begin{array}{l}\text { 4. Sediment } \\
\text { Deposition }\end{array}$ & $\begin{array}{l}\text { Little or no } \\
\text { enlargement of } \\
\text { islands or point bars } \\
\text { and less than } 5 \% \\
\text { (<20\% for low- } \\
\text { gradient streams) of } \\
\text { the bottom affected } \\
\text { by sediment } \\
\text { deposition. }\end{array}$ & $\begin{array}{l}\text { Some new increase } \\
\text { in bar formation, } \\
\text { mostly from gravel, } \\
\text { sand or fine } \\
\text { sediment; } 5-30 \% \text { (20- } \\
50 \% \text { for low-gradient) } \\
\text { of the bottom } \\
\text { affected; slight } \\
\text { deposition in pools. }\end{array}$ & $\begin{array}{l}\text { Moderate deposition } \\
\text { of new gravel, sand or } \\
\text { fine sediment on old } \\
\text { and new bars; } 30- \\
50 \% \text { ( } 50-80 \% \text { for low- } \\
\text { gradient) of the } \\
\text { bottom affected; } \\
\text { sediment deposits at } \\
\text { obstructions, } \\
\text { constrictions, and } \\
\text { bends; moderate } \\
\text { deposition of pools } \\
\text { prevalent. }\end{array}$ & $\begin{array}{l}\text { Heavy deposits of fine } \\
\text { material, increased } \\
\text { bar development; } \\
\text { more than } 50 \% \text { ( } 80 \% \\
\text { for low-gradient) of } \\
\text { the bottom changing } \\
\text { frequently; pools } \\
\text { almost absent due to } \\
\text { substantial sediment } \\
\text { deposition. }\end{array}$ \\
\hline SCORE & $\begin{array}{lllll}20 & 19 & 18 & 17 & 16 \\
\end{array}$ & $\begin{array}{lllll}15 & 14 & 13 & 12 & 11 \\
\end{array}$ & $\begin{array}{lllll}10 & 9 & 8 & 7 & 6 \\
\end{array}$ & $\begin{array}{llllll}5 & 4 & 3 & 2 & 1 & 0 \\
\end{array}$ \\
\hline
\end{tabular}

Figure 3: $\quad$ Three page data sheet adapted from Barbour et al. (1999) and used as a guide to assess and score twelve individual instream and riparian habitat parameters at sites where fishing effort was applied between 2002 and 2006. 


\begin{tabular}{|c|c|c|c|c|}
\hline \multirow{2}{*}{$\begin{array}{c}\text { Habitat } \\
\text { Parameter }\end{array}$} & \multicolumn{4}{|c|}{ Condition Category } \\
\hline & Optimal & Sub-Optimal & Marginal & Poor \\
\hline $\begin{array}{l}\text { 5. Channel } \\
\text { Flow Status }\end{array}$ & $\begin{array}{l}\text { Water reaches base } \\
\text { of both lower banks, } \\
\text { and minimal amount } \\
\text { of channel substrate } \\
\text { is exposed. }\end{array}$ & $\begin{array}{l}\text { Water fills }>75 \% \text { of } \\
\text { the available channel; } \\
\text { or }<25 \% \text { of channel } \\
\text { substrate is exposed. }\end{array}$ & $\begin{array}{l}\text { Water fills } 25-75 \% \text { of } \\
\text { the available channel, } \\
\text { and/or riffle } \\
\text { substrates are mostly } \\
\text { exposed. }\end{array}$ & $\begin{array}{l}\text { Very little water in } \\
\text { channel and mostly } \\
\text { present as standing } \\
\text { pools. }\end{array}$ \\
\hline SCORE & $\begin{array}{lllll}20 & 19 & 18 & 17 & 16\end{array}$ & $\begin{array}{lllll}15 & 14 & 13 & 12 & 11\end{array}$ & $\begin{array}{lllll}10 & 9 & 8 & 7 & 6 \\
\end{array}$ & $\begin{array}{llllll}5 & 4 & 3 & 2 & 1 & 0\end{array}$ \\
\hline $\begin{array}{l}\text { 6. Channel } \\
\text { Alteration }\end{array}$ & $\begin{array}{l}\text { Recent } \\
\text { channelization or } \\
\text { dredging absent or } \\
\text { minimal; stream with } \\
\text { normal pattern. }\end{array}$ & $\begin{array}{l}\text { Some new } \\
\text { channelization, } \\
\text { usually in areas of } \\
\text { bridge abutments; } \\
\text { evidence of past } \\
\text { channelization, i.e., } \\
\text { dredging, may be } \\
\text { present, but recent } \\
\text { channelization is not } \\
\text { present. }\end{array}$ & $\begin{array}{l}\text { Channelization may } \\
\text { be extensive; } \\
\text { embankments or } \\
\text { shoring structures } \\
\text { present on both } \\
\text { banks; and } 40 \text { to } \\
80 \% \text { of stream reach } \\
\text { channelized and } \\
\text { disrupted. }\end{array}$ & $\begin{array}{l}\text { Banks shored with } \\
\text { gabion or cement; } \\
\text { over } 80 \% \text { of the } \\
\text { stream reach } \\
\text { channelized and } \\
\text { disrupted. In stream } \\
\text { habitat greatly altered } \\
\text { or removed entirely. }\end{array}$ \\
\hline SCORE & $\begin{array}{lllll}20 & 19 & 18 & 17 & 16\end{array}$ & $\begin{array}{lllll}15 & 14 & 13 & 12 & 11\end{array}$ & $\begin{array}{lllll}10 & 9 & 8 & 7 & 6\end{array}$ & $\begin{array}{llllll}5 & 4 & 3 & 2 & 1 & 0\end{array}$ \\
\hline $\begin{array}{l}\text { 7. Frequency } \\
\text { of Riffles (or } \\
\text { Bends) }\end{array}$ & $\begin{array}{l}\text { Occurrence of riffles } \\
\text { relatively frequent; } \\
\text { ratio of distance } \\
\text { between riffles divided } \\
\text { by width of the } \\
\text { stream <7:1 } \\
\text { (generally } 5 \text { to } 7 \text { ); } \\
\text { variety of habitat is } \\
\text { key. In streams } \\
\text { where riffles are } \\
\text { continuous, } \\
\text { placement of } \\
\text { boulders or other } \\
\text { large, natural rest } \\
\text { areas is important. }\end{array}$ & $\begin{array}{l}\text { Occurrence of riffles } \\
\text { infrequent; distance } \\
\text { between riffles divided } \\
\text { by the width of the } \\
\text { stream is between } 7 \\
\text { to } 15 .\end{array}$ & $\begin{array}{l}\text { Occasional riffle or } \\
\text { bend; bottom } \\
\text { contours provide } \\
\text { some habitat; } \\
\text { distance between } \\
\text { riffles divided by the } \\
\text { width of the stream is } \\
\text { between } 15 \text { to } 25 \text {. }\end{array}$ & $\begin{array}{l}\text { Generally all flat } \\
\text { water or shallow } \\
\text { riffles; poor habitat; } \\
\text { distance between } \\
\text { riffles divided by the } \\
\text { width of the stream is } \\
\text { a ratio of }>25 \text {. }\end{array}$ \\
\hline SCORE & $\begin{array}{lllll}20 & 19 & 18 & 17 & 16 \\
\end{array}$ & $\begin{array}{lllll}15 & 14 & 13 & 12 & 11 \\
\end{array}$ & $\begin{array}{lllll}10 & 9 & 8 & 7 & 6 \\
\end{array}$ & $\begin{array}{llllll}5 & 4 & 3 & 2 & 1 & 0\end{array}$ \\
\hline $\begin{array}{l}\text { 8. Bank } \\
\text { Stability } \\
\text { (score each } \\
\text { bank) }\end{array}$ & $\begin{array}{l}\text { Banks stable; } \\
\text { evidence of erosion or } \\
\text { bank failure absent or } \\
\text { minimal; little } \\
\text { potential for future } \\
\text { problems. }<5 \% \text { of } \\
\text { bank affected. }\end{array}$ & $\begin{array}{l}\text { Moderately stable; } \\
\text { infrequent, small } \\
\text { areas of erosion } \\
\text { mostly healed over. } 5- \\
30 \% \text { of bank in reach } \\
\text { has areas of erosion. }\end{array}$ & $\begin{array}{l}\text { Moderately unstable; } \\
30-60 \% \text { of bank in } \\
\text { reach has areas of } \\
\text { erosion; high erosion } \\
\text { potential during } \\
\text { floods. }\end{array}$ & $\begin{array}{l}\text { Unstable; many } \\
\text { eroded areas; "raw" } \\
\text { areas frequent along } \\
\text { straight sections and } \\
\text { bends; obvious bank } \\
\text { sloughing; } 60-100 \% \\
\text { of bank has erosion } \\
\text { scars. }\end{array}$ \\
\hline SCORE & Left Bank 10 & 6 & 5 & 2 \\
\hline SCORE & $\begin{array}{lll}\text { Right Bank } & 10 & 9\end{array}$ & 6 & 4 & 2 \\
\hline
\end{tabular}

Figure 3: Three page data sheet adapted from Barbour et al. (1999) and used as a guide to assess and score twelve individual instream and riparian habitat parameters at sites where fishing effort was applied between 2002 and 2006. 


\begin{tabular}{|c|c|c|c|c|}
\hline \multirow{2}{*}{$\begin{array}{l}\text { Habitat } \\
\text { Parameter }\end{array}$} & \multicolumn{4}{|c|}{ Condition Category } \\
\hline & Optimal & Sub-Optimal & Marginal & Poor \\
\hline $\begin{array}{l}\text { 9. Bank } \\
\text { Vegetative } \\
\text { Protection } \\
\text { (score each } \\
\text { bank) }\end{array}$ & $\begin{array}{l}\text { More than } 90 \% \text { of the } \\
\text { stream bank surfaces } \\
\text { and immediate } \\
\text { riparian zone covered } \\
\text { by native vegetation, } \\
\text { including trees, under } \\
\text { story shrubs, or non- } \\
\text { woody macrophytes; } \\
\text { vegetative disruption } \\
\text { through grazing or } \\
\text { mowing minimal or } \\
\text { not evident; almost all } \\
\text { plants allowed to } \\
\text { grow naturally. }\end{array}$ & $\begin{array}{l}70-90 \% \text { of the stream } \\
\text { bank surfaces } \\
\text { covered by native } \\
\text { vegetation, but one } \\
\text { class of plants is not } \\
\text { well-represented; } \\
\text { disruption evident but } \\
\text { not affecting full plant } \\
\text { growth potential to } \\
\text { any great extent; } \\
\text { more than one-half of } \\
\text { the potential plant } \\
\text { stubble height } \\
\text { remaining. }\end{array}$ & $\begin{array}{l}50-70 \% \text { of the stream } \\
\text { bank surfaces } \\
\text { covered by } \\
\text { vegetation; disruption } \\
\text { obvious; patches of } \\
\text { bare soil or closely } \\
\text { cropped vegetation } \\
\text { common; less than } \\
\text { one-half of the } \\
\text { potential plant } \\
\text { stubble height } \\
\text { remaining. }\end{array}$ & $\begin{array}{l}\text { Less than } 50 \% \text { of the } \\
\text { stream bank surfaces } \\
\text { covered by } \\
\text { vegetation; disruption } \\
\text { of stream bank } \\
\text { vegetation is very } \\
\text { high; vegetation has } \\
\text { been removed to } 5 \\
\text { centimetres or less in } \\
\text { average stubble } \\
\text { height. }\end{array}$ \\
\hline SCORE & $\begin{array}{lll}\text { Left Bank } & 10 & 9\end{array}$ & 7 & 5 & 2 \\
\hline SCORE & Right Bank $10 \quad 9$ & 7 & 4 & 2 \\
\hline $\begin{array}{l}\text { 10. Riparian } \\
\text { Vegetative } \\
\text { Condition } \\
\text { (score each } \\
\text { bank) }\end{array}$ & $\begin{array}{l}\text { Width of riparian zone } \\
>18 \text { meters; human } \\
\text { activities (i.e., parking } \\
\text { lots, roadbeds, clear- } \\
\text { cuts, lawns, or crops) } \\
\text { have not impacted } \\
\text { zone. }\end{array}$ & $\begin{array}{l}\text { Width of riparian zone } \\
12-18 \text { meters; human } \\
\text { activities have } \\
\text { impacted zone only } \\
\text { minimally. }\end{array}$ & $\begin{array}{l}\text { Width of riparian zone } \\
6-12 \text { meters; human } \\
\text { activities have } \\
\text { impacted zone a } \\
\text { great deal. }\end{array}$ & $\begin{array}{l}\text { Width of riparian zone } \\
<6 \text { meters: little or no } \\
\text { riparian vegetation } \\
\text { due to human } \\
\text { activities. }\end{array}$ \\
\hline SCORE & $\begin{array}{lll}\text { Left Bank } & 10 & 9\end{array}$ & 7 & 5 & 2 \\
\hline SCORE & Right Bank $10 \quad 9$ & 7 & 4 & 2 \\
\hline $\begin{array}{l}\text { 11. Pool } \\
\text { Substrate }\end{array}$ & \begin{tabular}{|l|} 
Mixture of substrate \\
materials, with gravel \\
and firm sand \\
prevalent; root mats \\
and submerged \\
vegetation common.
\end{tabular} & $\begin{array}{l}\text { Mixture of soft sand, } \\
\text { mud, or clay; mud } \\
\text { may be dominant; } \\
\text { some root mats and } \\
\text { submerged vegetation } \\
\text { present. }\end{array}$ & $\begin{array}{l}\text { All mud or clay or } \\
\text { sand bottom; little or } \\
\text { no root mat; no } \\
\text { submerged } \\
\text { vegetation. }\end{array}$ & $\begin{array}{l}\text { Hard-pan clay or } \\
\text { bedrock; no root mat } \\
\text { or vegetation. }\end{array}$ \\
\hline SCORE & $\begin{array}{lllll}20 & 19 & 18 & 17 & 16 \\
\end{array}$ & $\begin{array}{lllll}15 & 14 & 13 & 12 & 11 \\
\end{array}$ & $\begin{array}{lllll}10 & 9 & 8 & 7 & 6 \\
\end{array}$ & $\begin{array}{llllll}5 & 4 & 3 & 2 & 1 & 0 \\
\end{array}$ \\
\hline $\begin{array}{l}\text { 12. Pool } \\
\text { Variability }\end{array}$ & \begin{tabular}{|l|} 
Even mix of large- \\
shallow, large-deep, \\
small-shallow, small- \\
deep pools present.
\end{tabular} & $\begin{array}{l}\text { Majority of pools } \\
\text { large-deep; very few } \\
\text { shallow. }\end{array}$ & $\begin{array}{l}\text { Shallow pools much } \\
\text { more prevalent than } \\
\text { deep pools. }\end{array}$ & $\begin{array}{l}\text { Majority of pools } \\
\text { small-shallow or } \\
\text { pools absent. }\end{array}$ \\
\hline SCORE & $\begin{array}{|lllll|}20 & 19 & 18 & 17 & 16 \\
\end{array}$ & \begin{tabular}{lllll|}
15 & 14 & 13 & 12 & 11 \\
\end{tabular} & $\begin{array}{lllll}10 & 9 & 8 & 7 & 6\end{array}$ & \begin{tabular}{llllll|}
5 & 4 & 3 & 2 & 1 & 0
\end{tabular} \\
\hline
\end{tabular}

Figure 3: $\quad$ Three page data sheet adapted from Barbour et al. (1999) and used as a guide to assess and score twelve individual instream and riparian habitat parameters at sites where fishing effort was applied between 2002 and 2006.

\subsubsection{Riparian Vegetative Condition}

Riparian Vegetative Condition assessed the state of vegetation from the wetted-edge of the stream bank out through the riparian zone to upland vegetation. The vegetative 
zone serves as a buffer to pollutants and sediment from runoff, helps build stream banks and control erosion, and provides nutrient inputs to the stream. A relatively undisturbed riparian zone can help support a robust stream system.

\subsubsection{Pool Substrate}

Pool Substrate evaluated the type and condition of substrate found in pools. Substrates such as gravel or sand and rooted aquatic vegetation support a wider variety of organisms than a pool dominated by mud or bedrock and having little or no aquatic vegetation.

\subsubsection{Pool Variability}

Pool Variability rated the mix of pools in a reach according to the size and depth of the pool. The four basic types of pools were: Large/Shallow; Small/Shallow; Large/Deep; and Small/Deep. Any pool dimension greater than one-half the wetted cross-section of the stream or drain was considered large. Any pool greater than $1.0 \mathrm{~m}$ in depth was considered to be deep. A stream with many pool types will typically support a wider variety of aquatic organisms.

\subsubsection{Habitat Assessment Score}

Totalling the habitat condition scores for each of the twelve parameters that were assessed provides a potential total score ranging from 0 to 240 .

Using the same Condition Categories applied to the individual habitat parameters, sites with a Habitat Assessment Score from:

- $0-60$ were considered to be representative of Poor Habitat

- $61-120$ were considered Marginal Habitat

- 121 - 180 were rated as Sub-Optimal Habitat

- $181-240$ were considered to be representative of Optimal Habitat for that ecoregion

\subsection{REACH PHOTOGRAPHS}

A series of digital photographs were taken to document flow, instream and riparian habitat conditions, and channel alterations at each site. Using Olympus Model 4000 or 5000 digital cameras, field crews documented conditions looking both upstream and downstream through the surveyed reach. As well, site photographs were used to document the condition of crossings, culvert inlets and outlets, any apparent effects on the stream from adjacent land use practices, and to provide a photographic record of any Indicator Species or other fish species of interest that were captured and released. 
Along with the photograph number generated by the camera and the direction faced (in degrees) when the photograph was taken, a descriptive caption for each photograph was recorded in the field notes.

\subsection{REACH FIELD SKETCH}

At each site visited a quick field sketch was prepared to document features such as: the wetted channel; minimum and maximum channel widths; locations of riffle, run and pool habitat; maximum and minimum stream depths; cover, bank condition, undercut banks, overhanging vegetation, canopy, emergent and submerged vegetation and boulders; substrate types; woody debris; dams, riparian conditions and any other notable features. As well, each sketch included a North indicator, the location of roads and buildings, and noted adjacent land use activity. Field sketches were scanned and saved as JPEG files.

\subsection{SITE DATA RECORDING}

To ensure that the information gathered at each site visited was collected and recorded in a consistent manner, a Data Sheet was developed to provide field crews with the space to record the following information:

- Site number and name

- Date and time

- Observers names

- Latitude and longitude (DD)

- DES map number

- DES stream order

- Presence/Absence and configuration of dikes

- Indicators of permanent or ephemeral flow

- Weather conditions

- Air temperature and water chemistry

- Fishing effort and statement of catch (or nil catch) for all gear used

- Species, number and length of released fish

- Fish collection sample identification numbers

- Indicator Species collected

- Photograph number, captions and azimuth faced for each photo taken

- Field sketch 
- Local knowledge

- Presence of springs

- Habitat complexity (Simple or Complex) looking upstream and downstream

- Habitat Assessment Scores

- An assessment of visible cattle impacts were recorded in the latter years of the study for a $3^{\text {rd }}$ party but have not been included in the results

Additional space was provided for recording site sketches and other information. Figure 4 provides an example of the data sheet used to record the survey data gathered at all of the sites sampled between 2002 and 2006. 


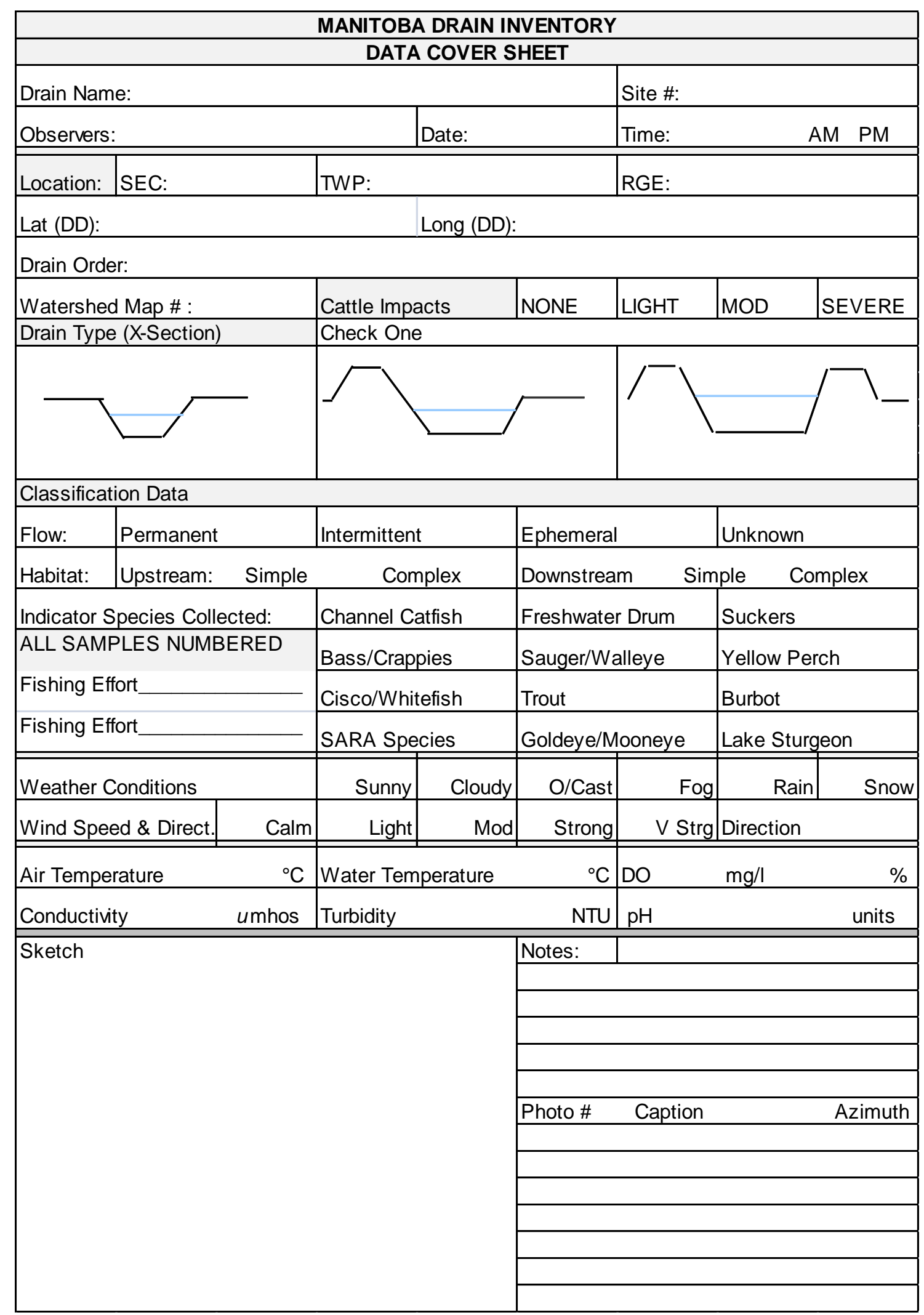

Figure 4: Data sheet used to record the survey data gathered at all sites sampled between 2002 and 2006. 



\subsection{RESULTS}

The data transcribed from each crews' field notes and data sheets have been tabled as a series of data spreadsheets and are included as Appendix 1 through Appendix 8 at the end of this report. Appendix 9 provides a series of NTS map tiles showing the sampling locations and sampling results, and also displays the classified streams and drains in the study area. Site photographs are provided in Appendix 10 and field sketches are included in Appendix 11. These are provided in a searchable PDF binder format.

The following sections provide a summary of the collected data and utilize a series of maps, charts and tables to present the results.

\section{1}

FLOW AND CLIMATIC CONDITIONS

Over the five sampling seasons of the fish community and fish habitat inventory, annual and seasonal flows varied significantly due to climatic conditions. In order to provide some perspective on the range of flow and weather conditions experienced over the course of the inventory, annual precipitation maps, annual drought condition maps and historic hydrometric data are presented in relation to average or normal conditions.

At the start of the inventory in 2002, the Canadian Prairie Provinces were experiencing a third consecutive year of drought conditions (Figure 5).

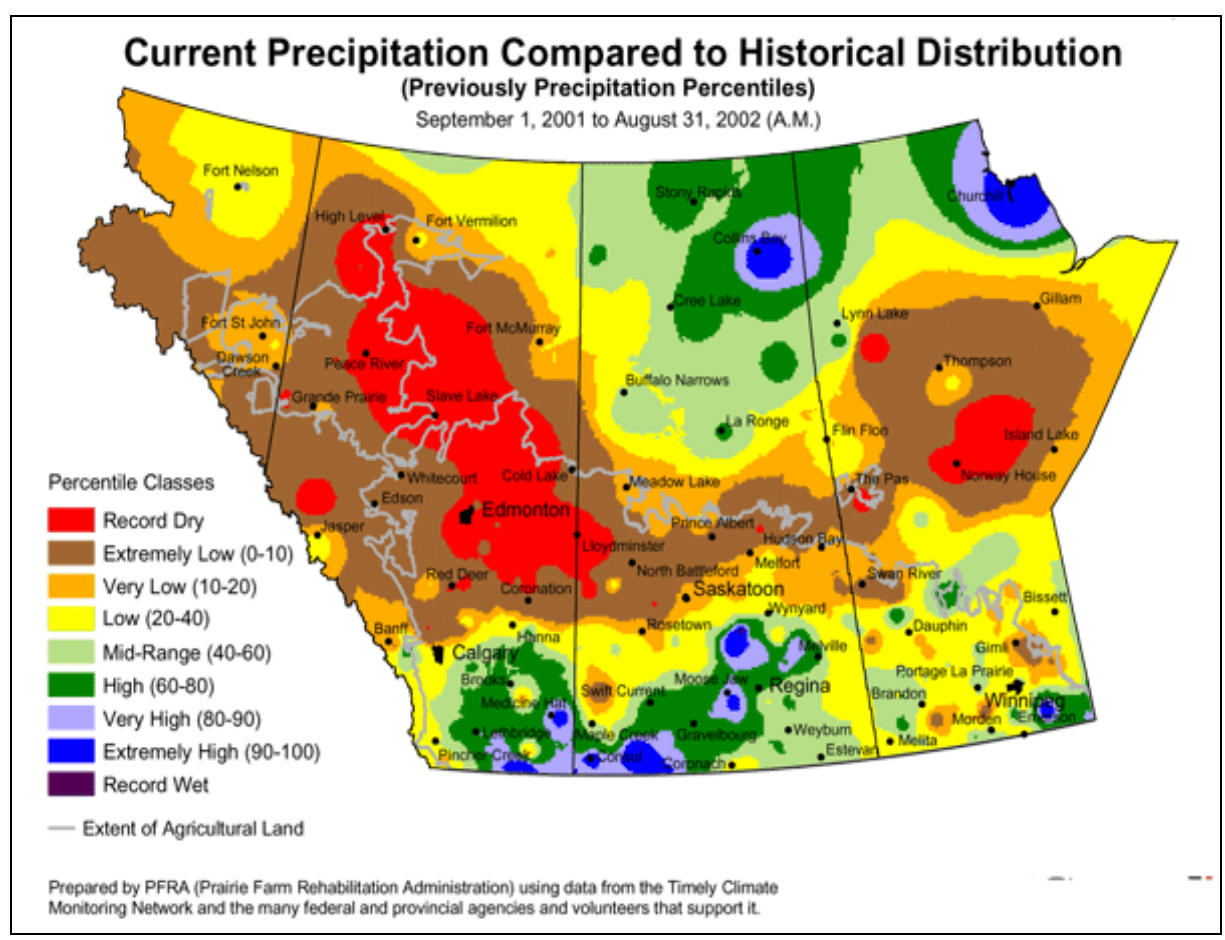

Figure 5: A comparison of the precipitation amounts received from September 1, 2001 to August 31, 2002 with historical amounts for the period of record (Agriculture and Agri-Food Canada. Prairie Farm Rehabilitation Administration. Historic Climate Data Map Archive). 
From the fall of 2001 to the end of the first season of sampling in 2002, precipitation amounts across much of the province were well below normal (Figure 6).

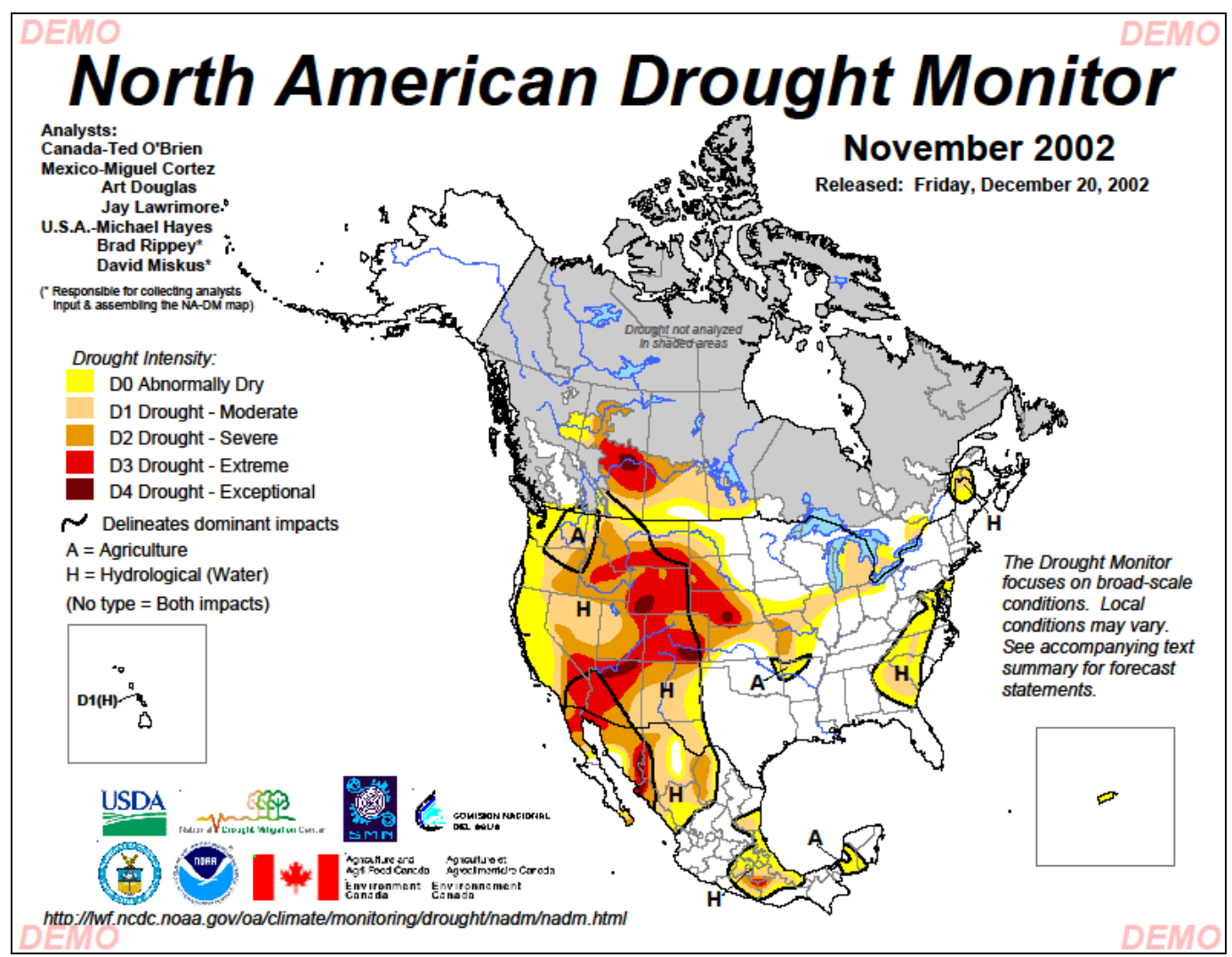

Figure 6: The intensity and extent of drought conditions across North America in November 2002 (National Oceanic and Atmospheric Administration. National Climate Data Center, Drought Severity Interpretation and Historic Climate Maps).

In the first season of sampling, flows in the larger river systems in the study area were well below normal. Many of the smaller constructed drains and channelized streams flowing into the Assiniboine and Red Rivers were dry. However, given the geographic extent of the study area, it was still possible to find and sample streams and drains where instream conditions were near normal for that time of the year. Occasionally, extensive rainfall was experienced and some sampling was carried out under high flow and flood conditions.

The daily discharge hydrographs for the Assiniboine River at Headingley (05MJ001) and Devils Creek near Libau (05OJ016) were selected to provide a comparison of annual flow conditions each year of the inventory (2002-2006) with historic daily flows (Environment Canada, Water Survey of Canada, Archived Hydrometric Data). The hydrometric stations were selected for their long periods of record and the relative size and location of their respective drainage areas. The daily flow hydrographs (Figure 7) compare 2002 conditions with the historic upper and lower quartile flows. 

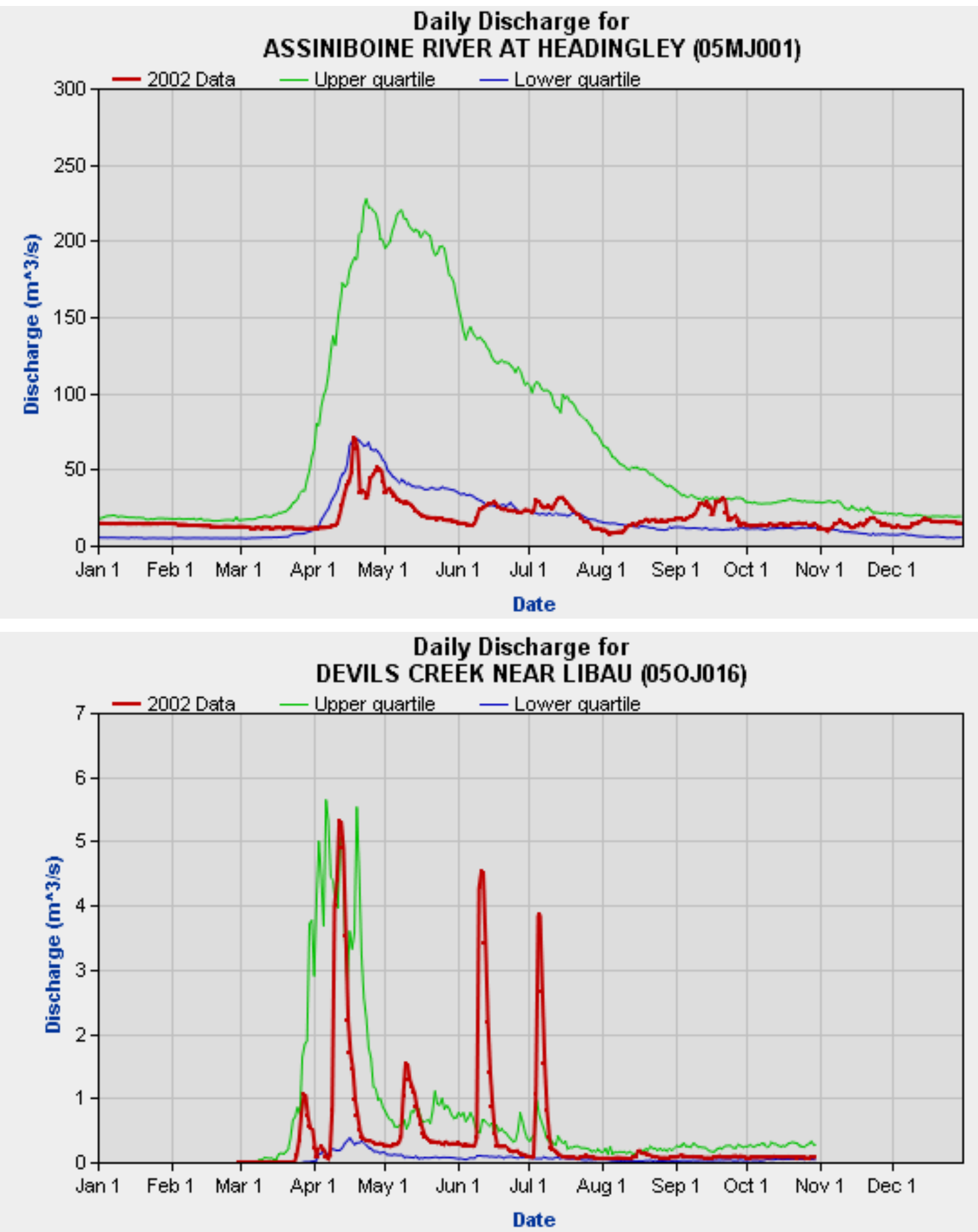

Figure 7: A comparison of 2002 flows with the historic upper and lower quartile daily discharge for the Assiniboine River at Headingley (05MJ001) and at Devils Creek near Libau (050J016). (Environment Canada. Water Survey of Canada. Archived Hydrometric Data). 
Flows in the Assiniboine River were less than the lower quartile historic flow until late summer. The spring peak in Devils Creek was near the upper quartile of historic flows but was relatively short in duration. Several high flow events were experienced after intense summer rainfall events; however, these flows receded very quickly.

In 2003, conditions across the province were even drier than in 2002 and were considered to be near the 1:50 year drought condition (Figure 8).

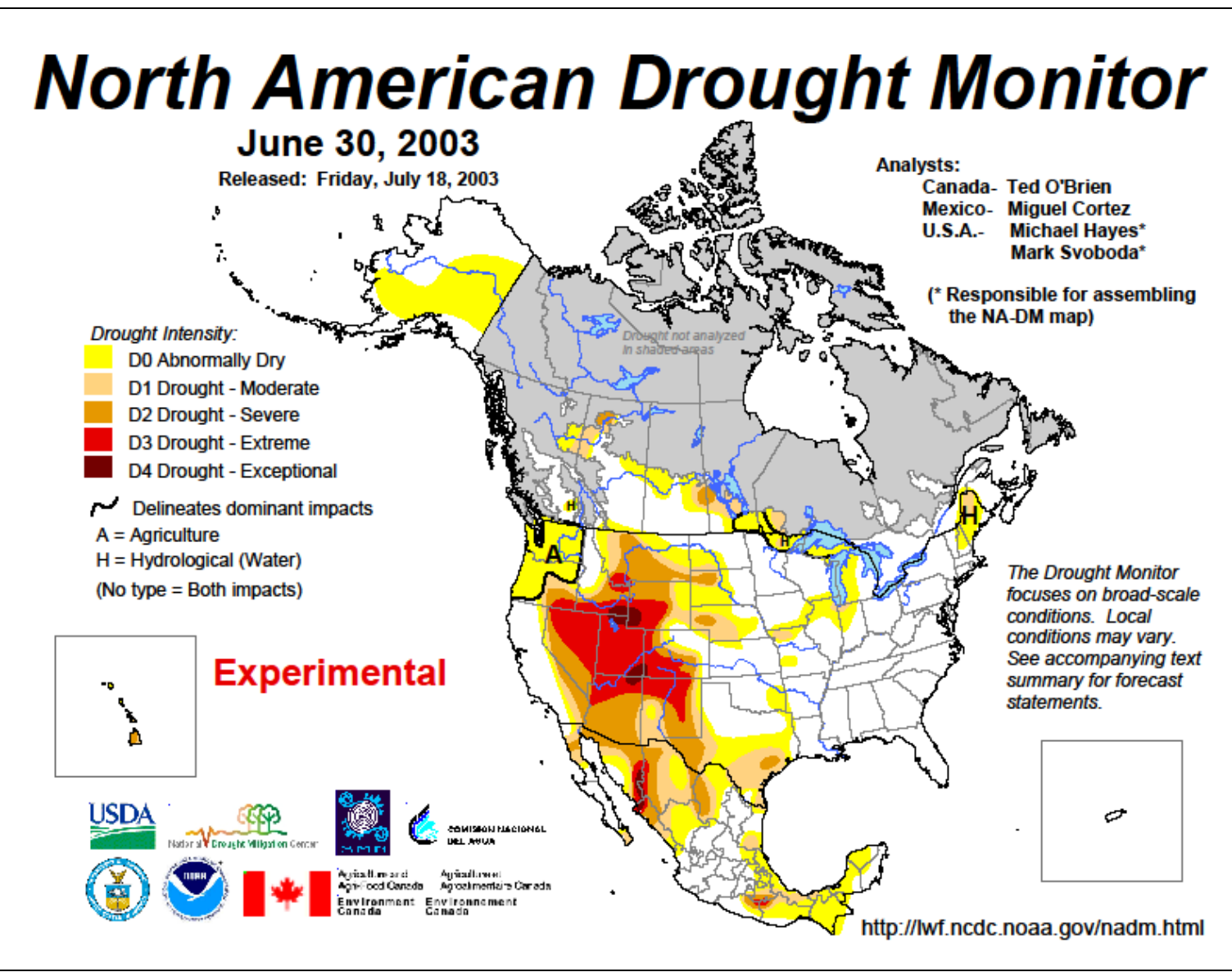

Figure 8: The intensity and extent of drought conditions across North America on June 30, 2003 (National Oceanic and Atmospheric Administration. National Climate Data Center, Drought Severity Interpretation and Historic Climate Maps).

The spring peak in the Assiniboine River at Headingley was near normal but flow quickly receded to a level that was near the lower quartile historic flow amount for the remainder of the year (Figure 9). This was even more dramatically demonstrated in Devils Creek near Libau (Figure 10). 


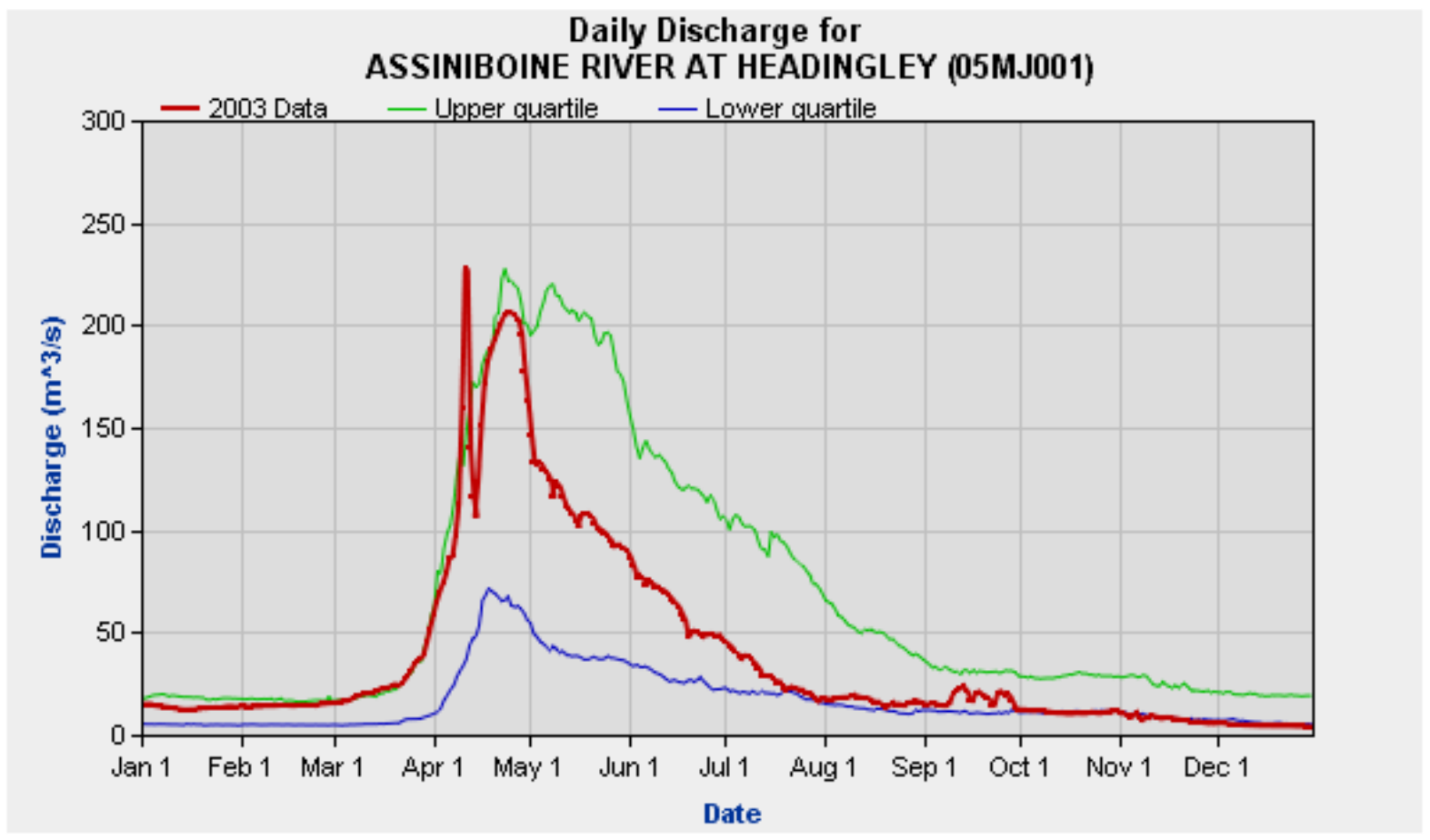

Figure 9: A comparison of 2003 flows with the historic upper and lower quartile daily discharge for the Assiniboine River at Headingley (05MJ001). (Environment Canada. Water Survey of Canada. Archived Hydrometric Data)

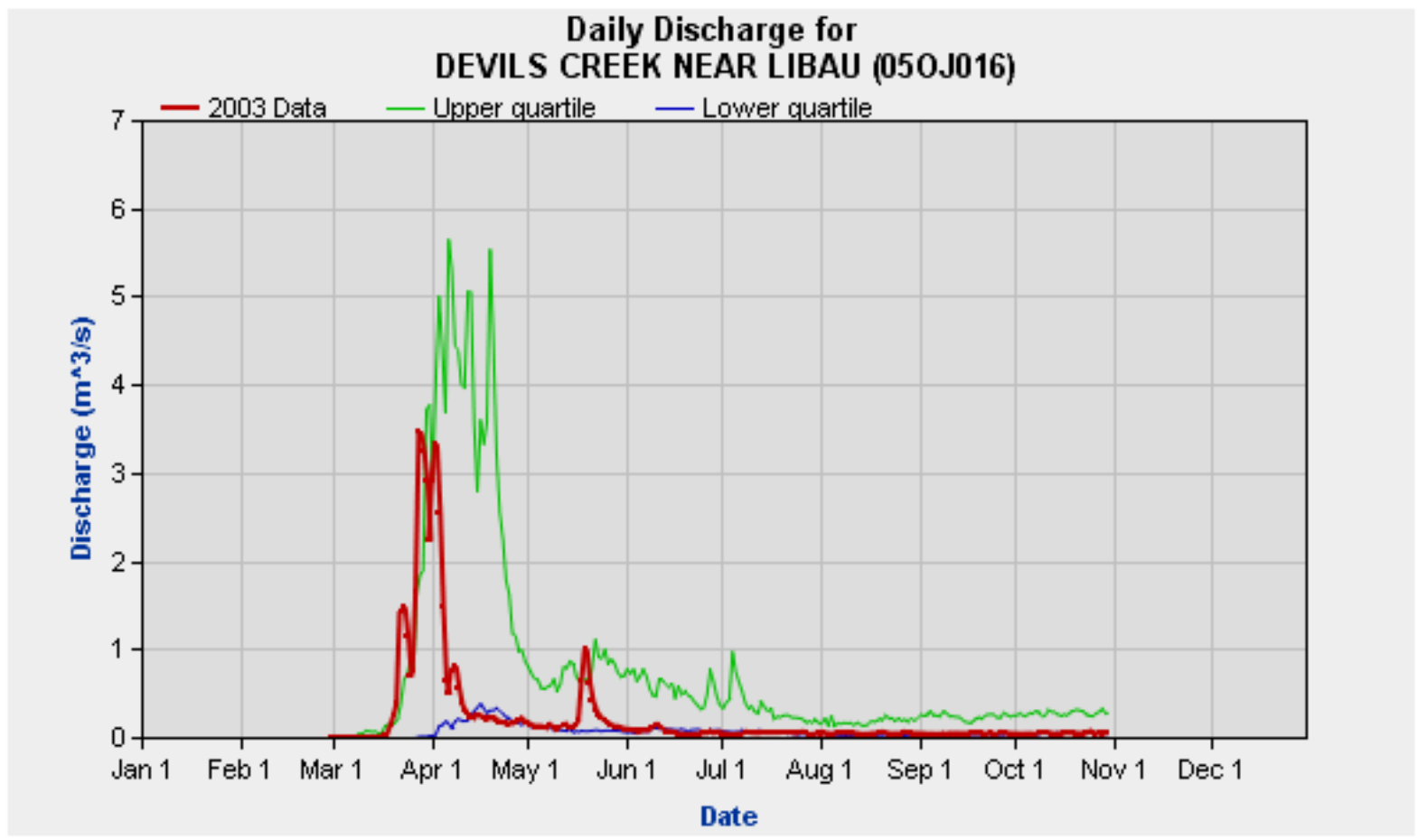

Figure 10: A comparison of 2003 flows with the historic upper and lower quartile daily discharge for Devils Creek near Libau (050J016). (Environment Canada. Water Survey of Canada. Archived Hydrometric Data) 
The spring of 2004 was cold and wet. Across the prairies, the period from May to August was the coldest in 57 years. Summer temperatures in Winnipeg were the coldest on record since 1872 (Environment Canada, Meteorological Services Canada, Archived Meteorological Data).

Spring flows in the Assiniboine River near Headingley were initially near the upper quartile of flows, which receded quickly but were followed by extensive rains beginning in May (Figure 11). Flows then increased and remained seasonally high for the remainder of the year.

The 2004 spring peak flow in Devils Creek was reached prior to April 1 and was well above the upper quartile of historical flows (Figure 11). Flows receded quickly until mid-May, when heavy rains resulted in extremely high seasonal flows in the early summer and the fall of 2004.

In 2005, spring runoff was well above normal and there were extremely wet conditions throughout the summer across the province, with significant overland flooding in some areas (Figure 12).

In 2006, the spring runoff was near normal but conditions over much of the province through the summer were very dry (Figure 13; Figure 14). Conditions ranged from abnormally dry in the southeast areas of the province to moderate drought conditions in west-central Manitoba. 

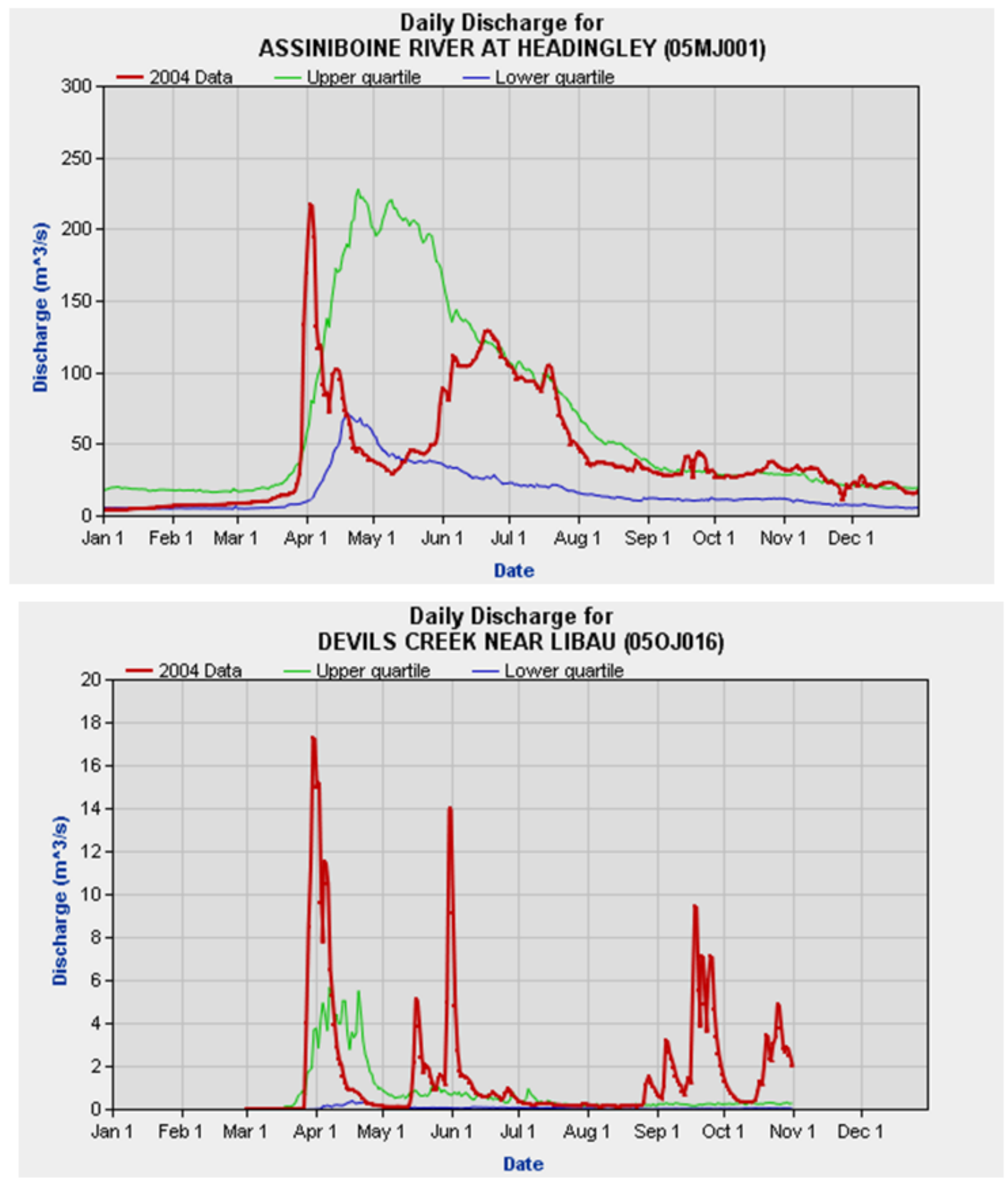

Figure 11: A comparison of 2004 flows with the historic upper and lower quartile daily discharge for the Assiniboine River at Headingley (05MJ001) and at Devils Creek near Libau (05OJ016). (Environment Canada. Water Survey of Canada. Archived Hydrometric Data) 

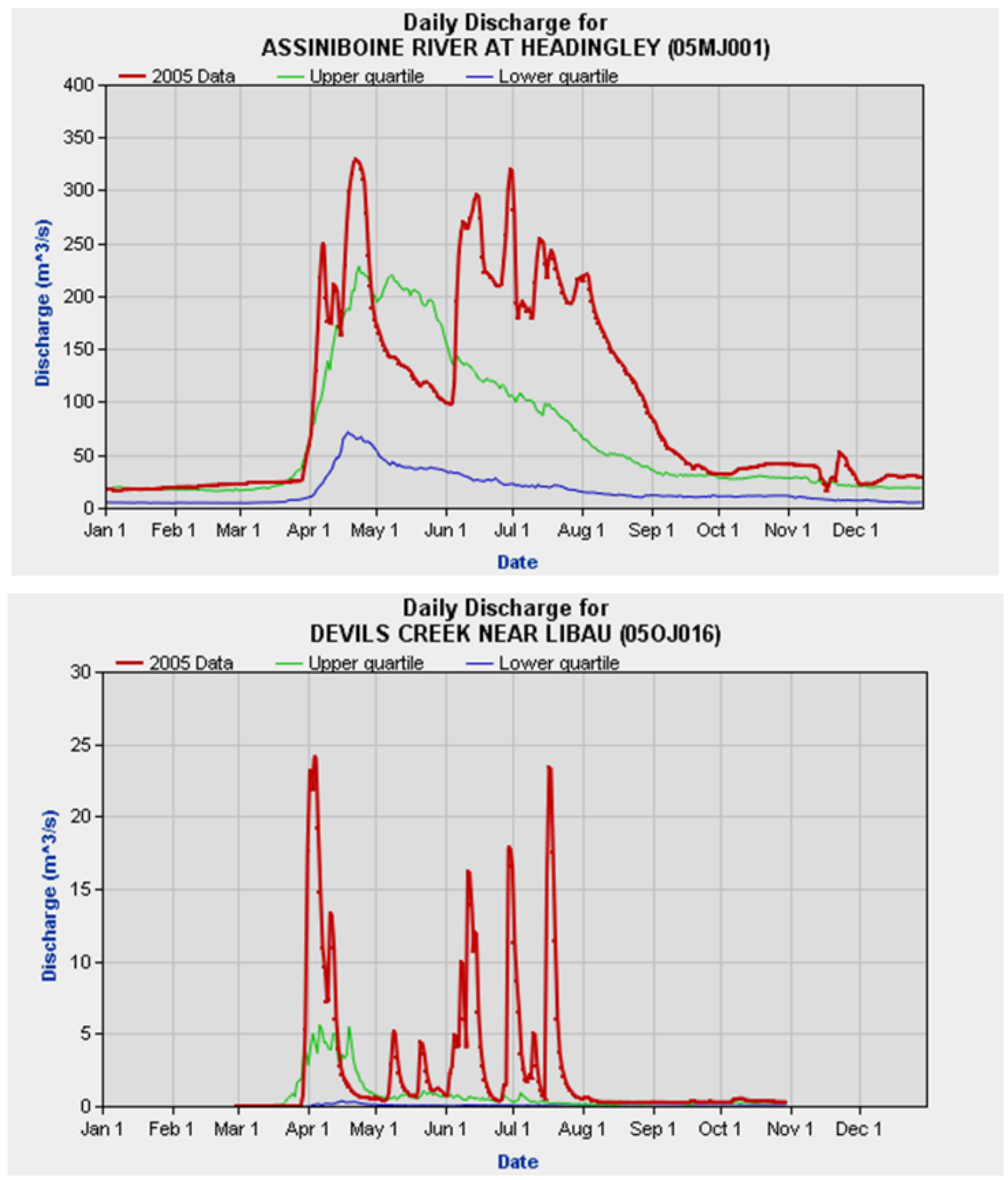

Figure 12: A comparison of 2005 flows with the historic upper and lower quartile daily discharge for the Assiniboine River at Headingley (05MJ001) and at Devils Creek near Libau (050J016). (Environment Canada. Water Survey of Canada. Archived Hydrometric Data) 

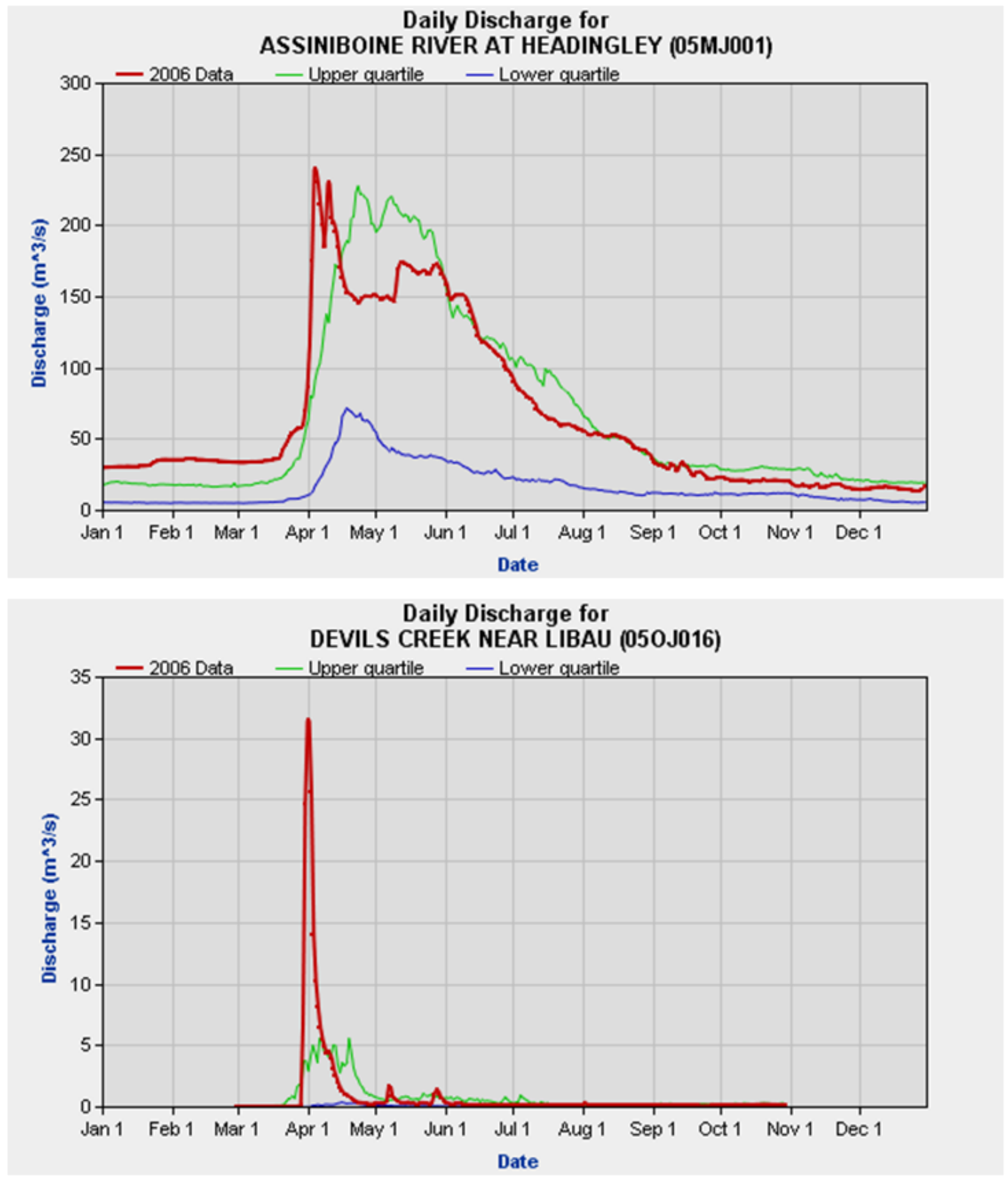

Figure 13: A comparison of 2006 flows with the historic upper and lower quartile daily discharge for the Assiniboine River at Headingley (05MJ001) and at Devils Creek near Libau (050J016). (Environment Canada. Water Survey of Canada. Archived Hydrometric Data) 


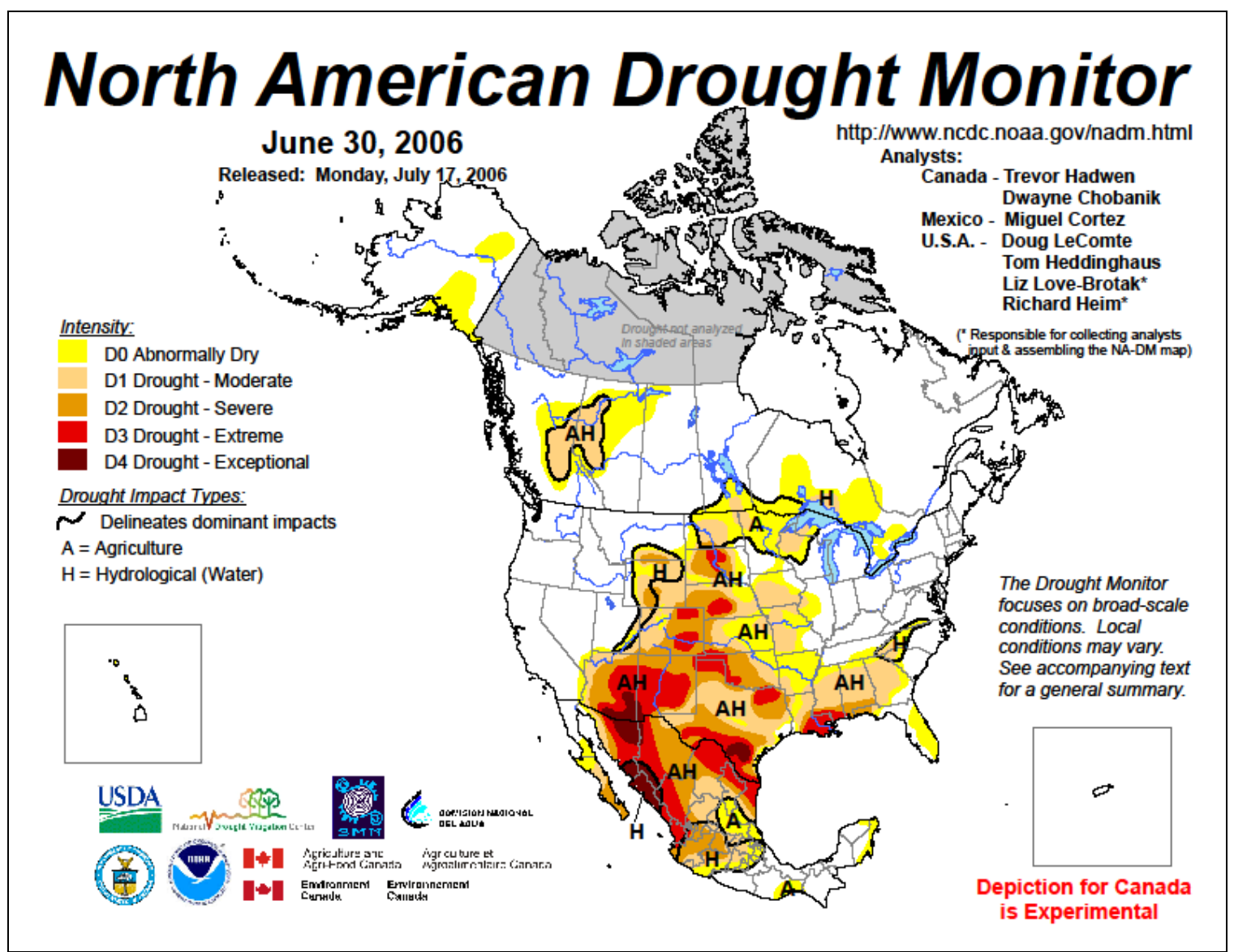

Figure 14: The intensity and extent of drought conditions across North America on June 30, 2006. (National Oceanic and Atmospheric Administration. National Climate Data Center, Drought Severity Interpretation and Historic Climate Maps)

\section{2}

SUMMARY OF SURVEYED SITES

A total of 2,371 sites were visited between the spring of 2002 and the late summer of 2006. A single crew surveyed 201 sites in 2002. In 2003, three crews visited a total of 687 sites. Four crews surveyed 888 sites in 2004. In 2005, three crews sampled 448 sites and in 2006, two crews sampled 147 sites.

A list of all sites showing the site number, the date sampled, the site name, geographic location, corresponding DES map number, DES stream order, and the corresponding NTS map tile number is provided in Appendix 1. 
Table 3: A summary of all sites visited between 2002 and 2006. The sampling effort has been summarized by year, by sampling crew and by DES stream order.

\begin{tabular}{|l|c|c|c|c|c|c|c|c|c|c|}
\hline \multirow{2}{*}{ Year } & \multirow{2}{*}{ Crew } & \multirow{2}{*}{$\begin{array}{c}\text { \# of } \\
\text { Sites }\end{array}$} & \multicolumn{10}{|c|}{ DES Stream Order } \\
\cline { 5 - 12 } & & 1st & 2nd & 3rd & 4th & 5th & 6th & 7th & 8th \\
\hline 2002 & D-02 & 201 & 3 & 31 & 99 & 45 & 22 & 0 & 0 & 1 \\
\hline 2003 & B-03 & 250 & 0 & 45 & 120 & 73 & 11 & 2 & 0 & 0 \\
\hline 2003 & D-03 & 170 & 1 & 45 & 75 & 37 & 11 & 1 & 0 & 0 \\
\hline 2003 & W-03 & 267 & 7 & 101 & 126 & 27 & 6 & 0 & 0 & 0 \\
\hline 2004 & B-04 & 288 & 3 & 67 & 108 & 74 & 32 & 4 & 0 & 0 \\
\hline 2004 & D-04 & 36 & 2 & 3 & 17 & 7 & 7 & 0 & 0 & 0 \\
\hline 2004 & W-04 & 301 & 3 & 161 & 108 & 18 & 7 & 4 & 0 & 0 \\
\hline 2004 & X-04 & 263 & 6 & 43 & 119 & 62 & 27 & 5 & 0 & 0 \\
\hline 2005 & D-05 & 27 & 1 & 8 & 7 & 2 & 9 & 0 & 0 & 0 \\
\hline 2005 & W-05 & 211 & 5 & 58 & 117 & 30 & 1 & 0 & 0 & 0 \\
\hline 2005 & X-05 & 210 & 2 & 30 & 153 & 23 & 2 & 0 & 0 & 0 \\
\hline 2006 & D-06 & 1 & 0 & 0 & 0 & 1 & 0 & 0 & 0 & 0 \\
\hline 2006 & W-06 & 146 & 4 & 36 & 66 & 21 & 11 & 4 & 4 & 0 \\
\hline \multicolumn{2}{|l}{ Total } & 2,371 & 37 & 628 & 1,115 & 420 & 146 & 20 & 4 & 1 \\
\hline
\end{tabular}

The following map shows the location of sampling sites surveyed between 2002 and 2006 (Figure 15). 


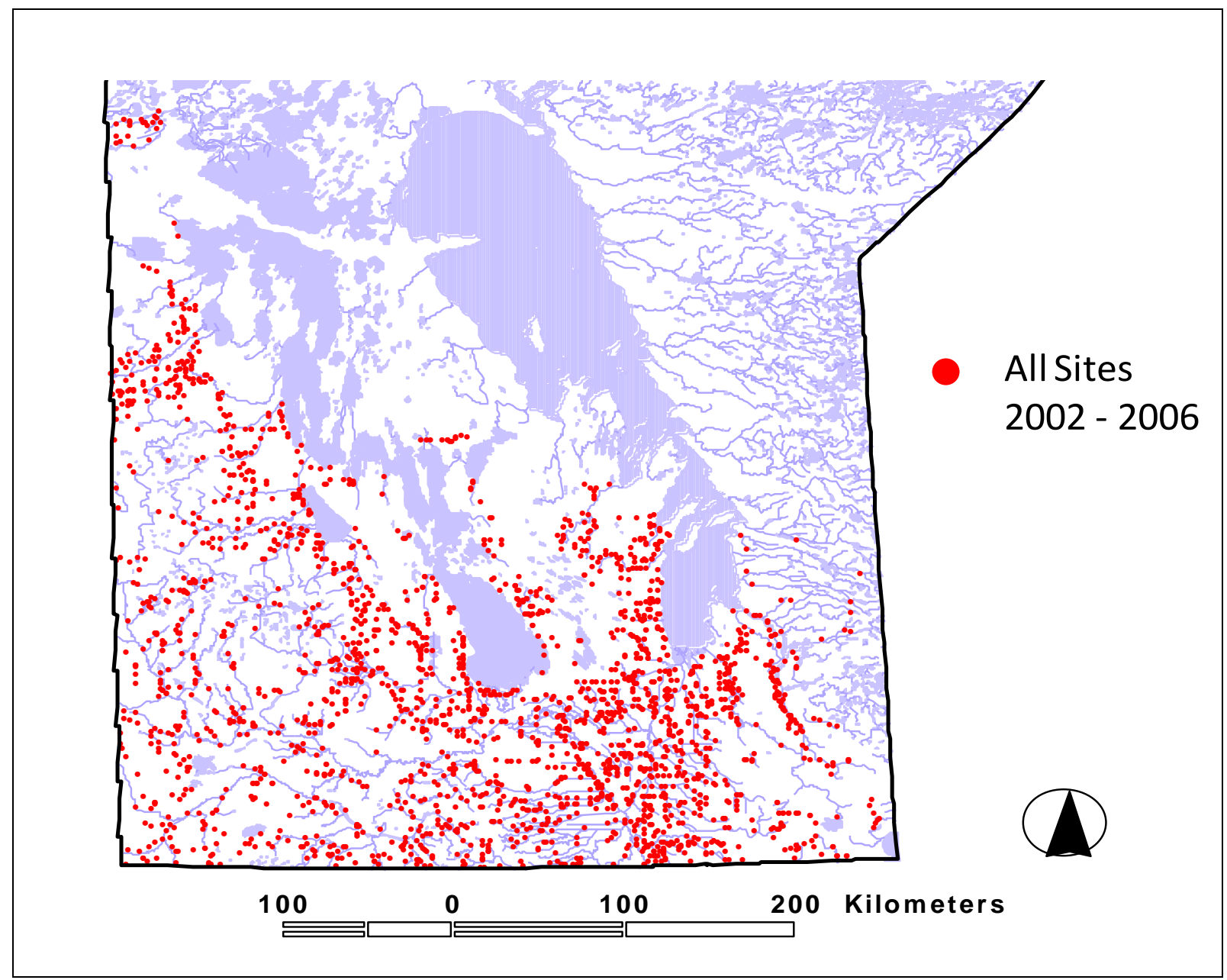

Figure 15: Map showing the location of 2,371 sites visited from $2002-2006$.

\subsection{HABITAT COMPLEXITY}

Field crews used the definition of Simple versus Complex Habitat to classify the upstream and downstream habitat complexity at all 2,371 sites surveyed. The majority $(1,217$ or $51.3 \%)$ of sites surveyed were classified as Complex Habitat. Conditions were classified as Simple Habitat at 645 of 2,371 sites (39.9\%). At 209 sites (8.8\%) the upstream habitat complexity varied from the downstream habitat complexity. These sites were located at distinct changes in channel type and riparian conditions, or were located in transitional zones between complex and simple habitat.

A map of the distribution of sites by habitat complexity is shown in Figure 16.

Figure 17 summarizes the percent distribution of all sites surveyed based on their Habitat Complexity (Complex, Simple or Transitional) and their DES stream order. 


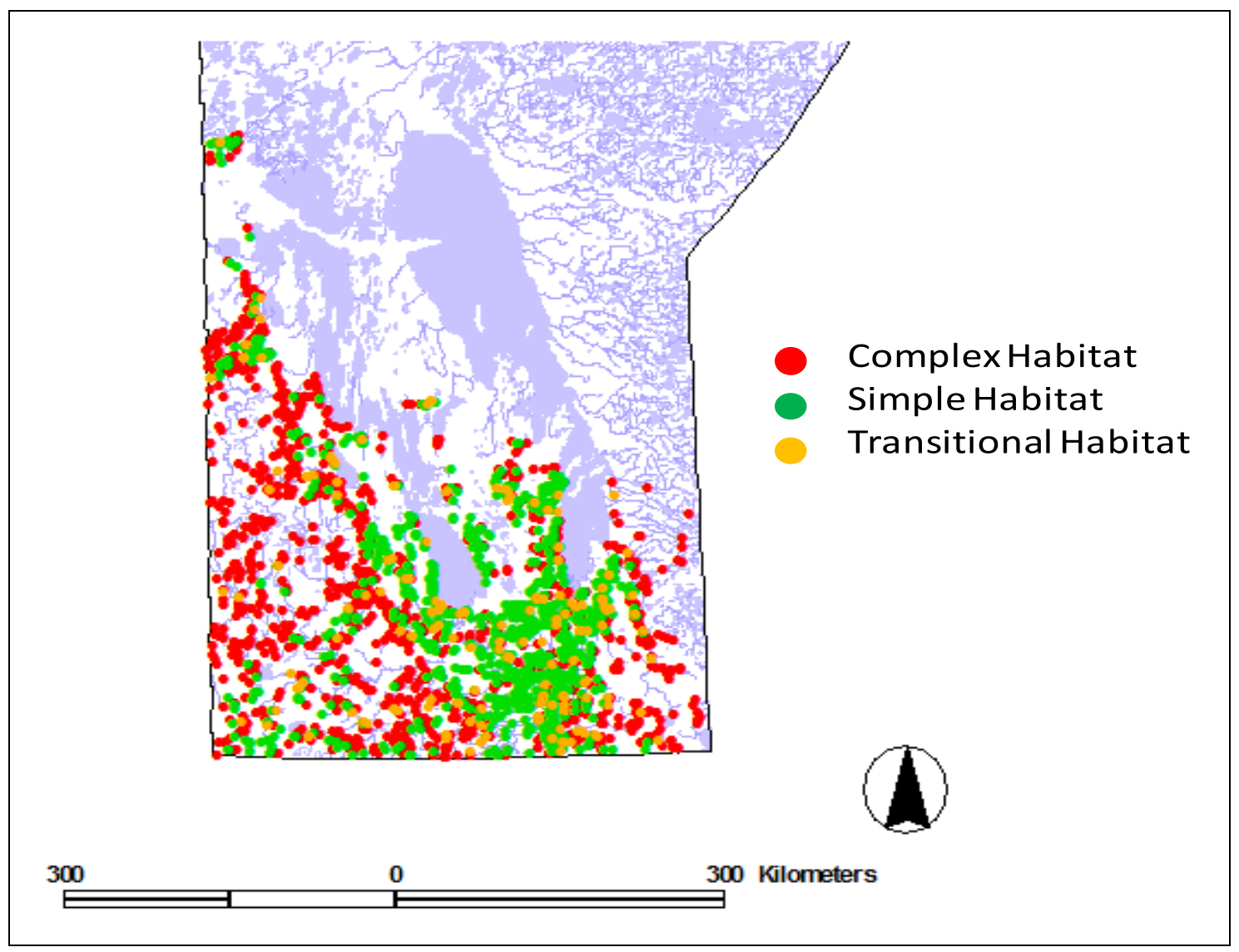

Figure 16: Map showing the distribution of all sites by Habitat Complexity.

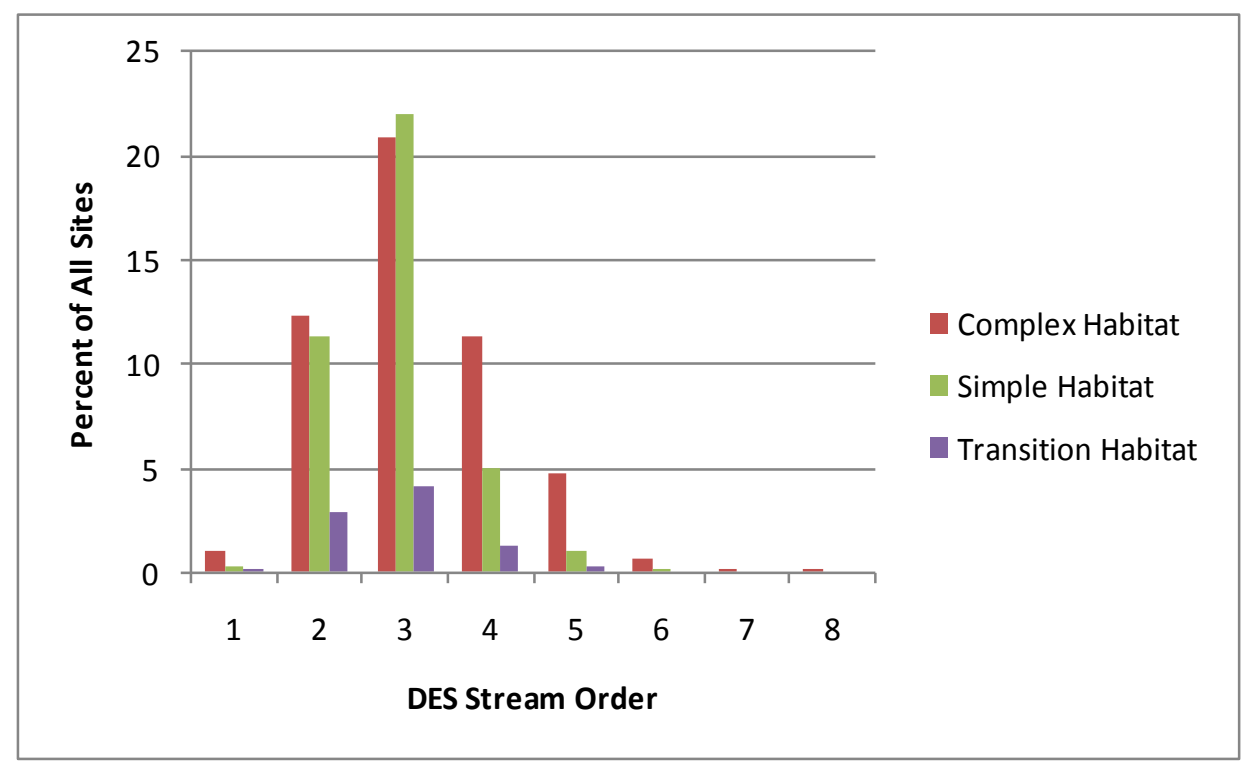

Figure 17: Percent distribution of Habitat Complexity by DES stream order for all sites surveyed ( $n=2,371$ ). 
A list of all sites and their upstream and downstream habitat complexity decision is included in Appendix 2.

\section{4}

\section{HABITAT ASSESSMENT SCORES}

Along with the application of the definition of Simple Habitat to determine Habitat Complexity at all sites surveyed, field crews also assessed and scored twelve instream and riparian habitat parameters at 1,141 of the 1,248 sites where fishing effort was applied. The Habitat Assessment was only completed at those reaches where field crews felt they had spent sufficient time and effort in the sampling of the reach to be familiar with the instream and riparian habitat conditions to be assessed. A list of the sites assessed and the corresponding individual Parameter and Habitat Assessment Scores is included in Appendix 3.

The Habitat Assessment Scores ranged from a high of 225, to a low score of 42, with a median score of 128 . Of all sites where the Habitat Assessment was completed, the majority $(50.3 \%)$ were rated as Sub-Optimal Habitat, followed by Marginal Habitat (41.7\%), Optimal Habitat (7\%) and Poor Habitat (less than 1\%). (Figure18).

At those sites where the Habitat Complexity was determined to be Complex, the Habitat Assessment Scores ranged from a high of 225 , to a low of 60 , with a median score of 146. At sites deemed to be Simple Habitat, the Total Scores ranged from a high of 194 , to a low of 42 , with a median score of 106. At sites where the upstream habitat complexity varied from the downstream habitat complexity, the Total Scores ranged from a high of 191, to a low of 64, with a median score of 116 (Figure 19). Figure 20 provides a map displaying the Habitat Condition category derived from the Total Habitat Assessment Score at all sites assessed.

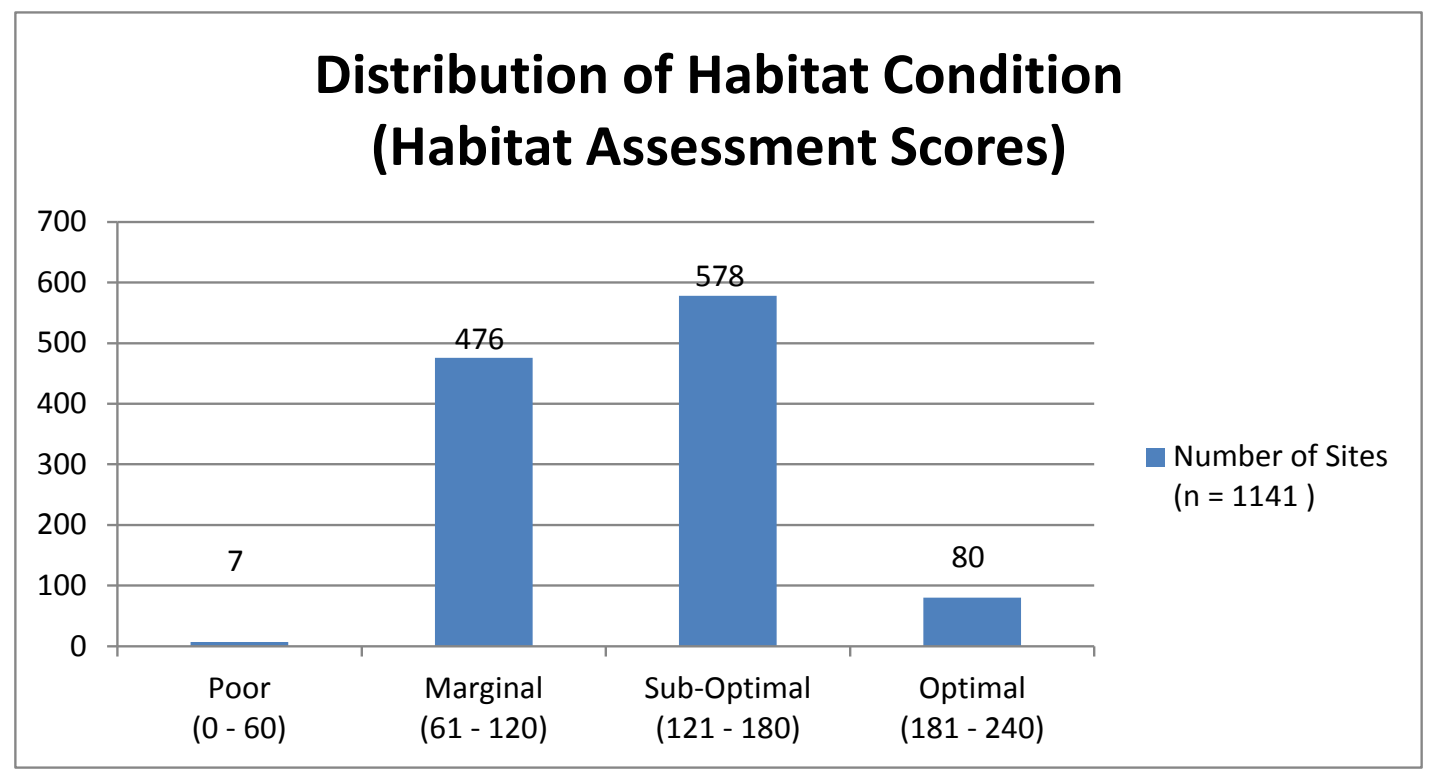

Figure 18: Chart showing the distribution of sites across the Habitat Condition scale as determined by the Habitat Assessment Score at each site. 


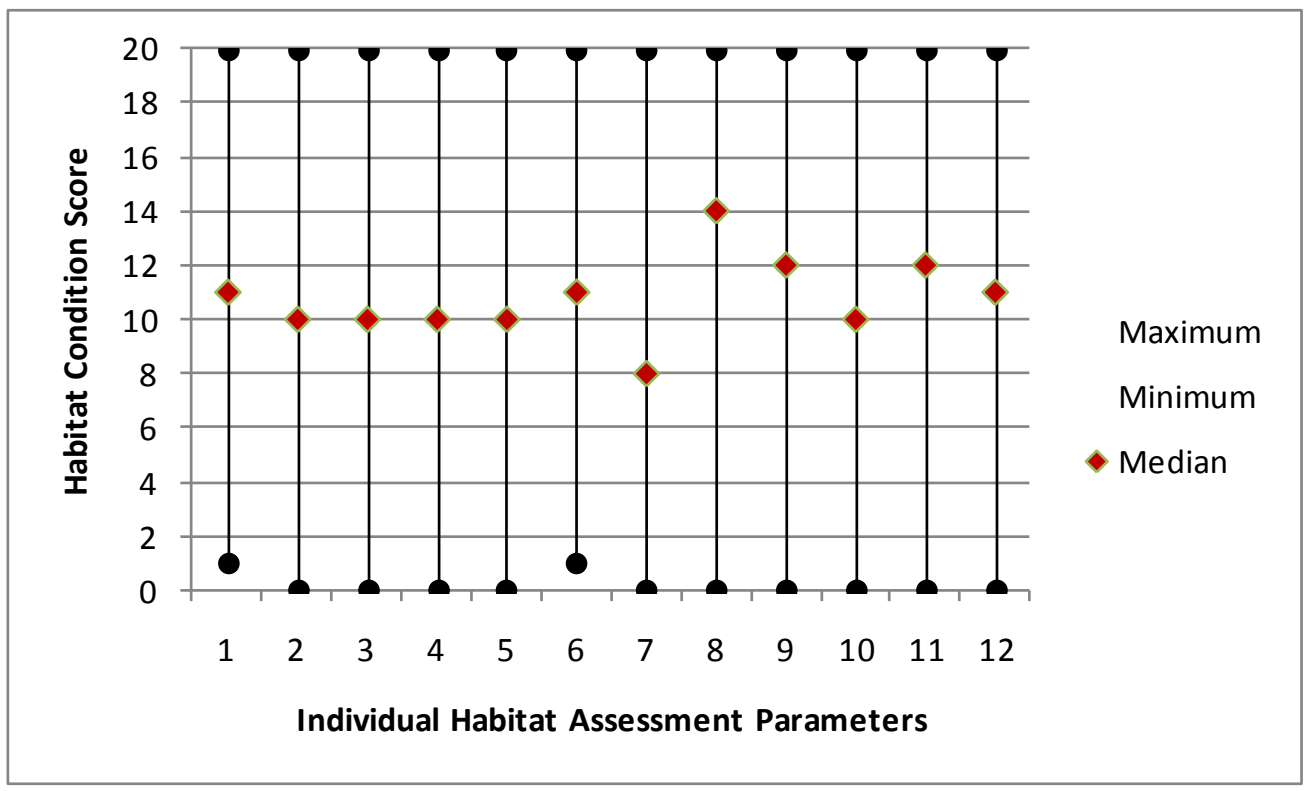

Figure 19: Chart showing the maximum, minimum and median individual habitat parameter scores for each of the twelve parameters assessed to derive the Total Score.

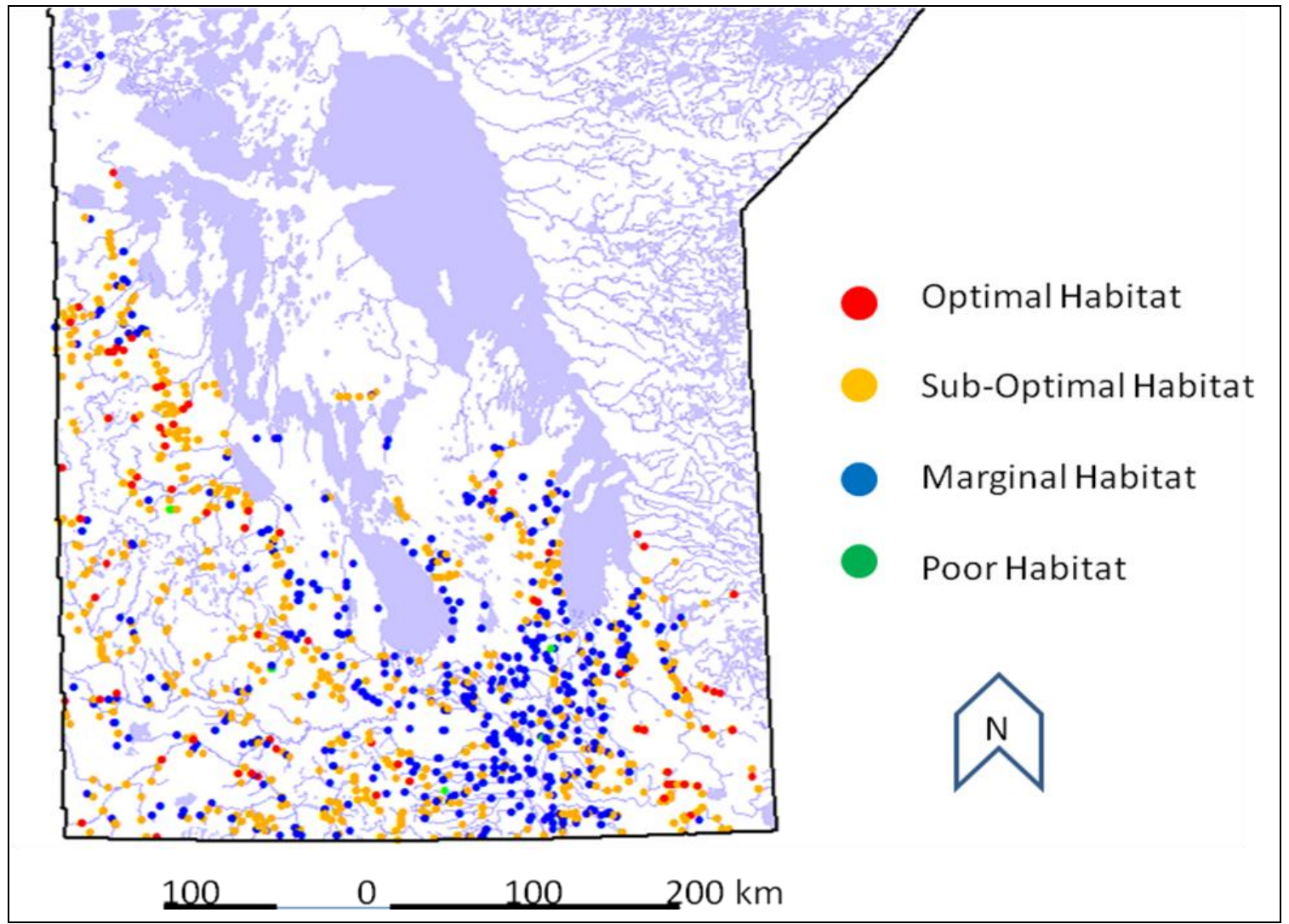

Figure 20: Map displaying the Habitat Condition category derived from the Habitat Assessment Score at all sites assessed. 


\section{$4.5 \quad$ FISHING EFFORT}

Fishing effort was applied on 1,354 occasions with sampling of the fish community being carried out at 1,248 sites. A single gear type was employed at 1,147 of these sites. Sampling effort using two gear types was applied at 96 sites, and three gear types were used to sample the fish community at 5 sites. At 1,123 sites, no fishing effort was applied. These sites were either too dry to fish or were not safe to wade. Some sites were not fished for reasons including nearby aerial crop spraying, mosquito larvaecide application, extreme weather conditions, and property owner denied access. The majority of fishing effort (58\%) was expended in Complex Habitat, with $33 \%$ of fishing effort plied in Simple Habitat and the balance $(9 \%)$ expended in areas of transition between Complex and Simple Habitat.

Backpack electrofishing gear was used on 1,018 occasions. Beach seines were used 155 times. Dip-nets were used on 41 occasions and kick samplers were used on 42 occasions. Larval drift traps were deployed 36 times. Field crew observations were made at 59 sites and anecdotal evidence was utilized at two locations. An electrofishing boat was used at one site. Table 4 provides a summary of the number of sites and the number of occasions fishing effort was applied, summarized by year, by sampling crew and by DES stream order.

Table 4: The number of sites and the number of occasions fishing effort was applied, summarized by year, by sampling crew and by DES stream order.

\begin{tabular}{|c|c|c|c|c|c|c|c|c|c|c|}
\hline \multirow{2}{*}{ Year } & \multirow{2}{*}{ Crew } & \multirow{2}{*}{$\begin{array}{l}\text { \# of } \\
\text { Sites }\end{array}$} & \multicolumn{7}{|c|}{ DES Stream Order } & \multirow{2}{*}{$\begin{array}{c}\text { Total } \\
\text { Occasions }\end{array}$} \\
\hline & & & 1st & 2nd & 3rd & 4th & 5 th & 6th & 7th & \\
\hline 2002 & D-02 & 114 & 0 & 14 & 63 & 31 & 17 & 0 & 0 & 125 \\
\hline 2003 & B-03 & 111 & 0 & 15 & 43 & 45 & 10 & 2 & 0 & 115 \\
\hline 2003 & D-03 & 86 & 0 & 16 & 33 & 30 & 11 & 1 & 0 & 91 \\
\hline 2003 & W-03 & 90 & 2 & 26 & 51 & 12 & 1 & 0 & 0 & 92 \\
\hline 2004 & B-04 & 142 & 0 & 14 & 57 & 54 & 33 & 2 & 0 & 160 \\
\hline 2004 & D-04 & 17 & 0 & 1 & 10 & 6 & 0 & 0 & 0 & 17 \\
\hline 2004 & W-04 & 139 & 3 & 62 & 77 & 10 & 4 & 2 & 0 & 158 \\
\hline 2004 & $X-04$ & 164 & 4 & 27 & 77 & 47 & 21 & 2 & 0 & 178 \\
\hline 2005 & D-05 & 19 & 0 & 4 & 6 & 3 & 8 & 0 & 0 & 21 \\
\hline 2005 & W-05 & 138 & 1 & 40 & 85 & 24 & 0 & 0 & 0 & 150 \\
\hline 2005 & X-05 & 142 & 1 & 19 & 115 & 23 & 3 & 0 & 0 & 161 \\
\hline 2006 & D-06 & 1 & 0 & 0 & 0 & 1 & 0 & 0 & 0 & 1 \\
\hline 2006 & W-06 & 85 & 1 & 17 & 36 & 16 & 7 & 4 & 4 & 85 \\
\hline \multicolumn{2}{|c|}{ Total } & 1248 & 12 & 255 & 653 & 302 & 115 & 13 & 4 & 1354 \\
\hline
\end{tabular}




\subsection{FISH COLLECTIONS}

Over the course of the inventory, fifty-three fish species representing fifteen taxonomic families and comprised of nineteen Indicator Species and thirty-four Non-Indicator Species were collected. As well, a small number of hybrid minnows, unidentified postlarval minnows, unidentified post-larval suckers, and unidentified sucker and Percid family eggs were also collected.

Indicator Species were collected on 741 occasions. Non-Indicator species were collected 2,994 times. The application of fishing effort resulted in No Catch on 281 occasions. In total, 4,016 individual species records and more than 40,000 individual fish were collected from 1,248 sites. The highest number of species collected at one location (X-04-253) was nineteen. This site was located immediately upstream from the Red River.

The following maps provide the locations for all Indicator Species collections (Figure 21), Non-Indicator Species collections (Figure 22) and sites where fishing effort resulted in No Catch (Figure 23).

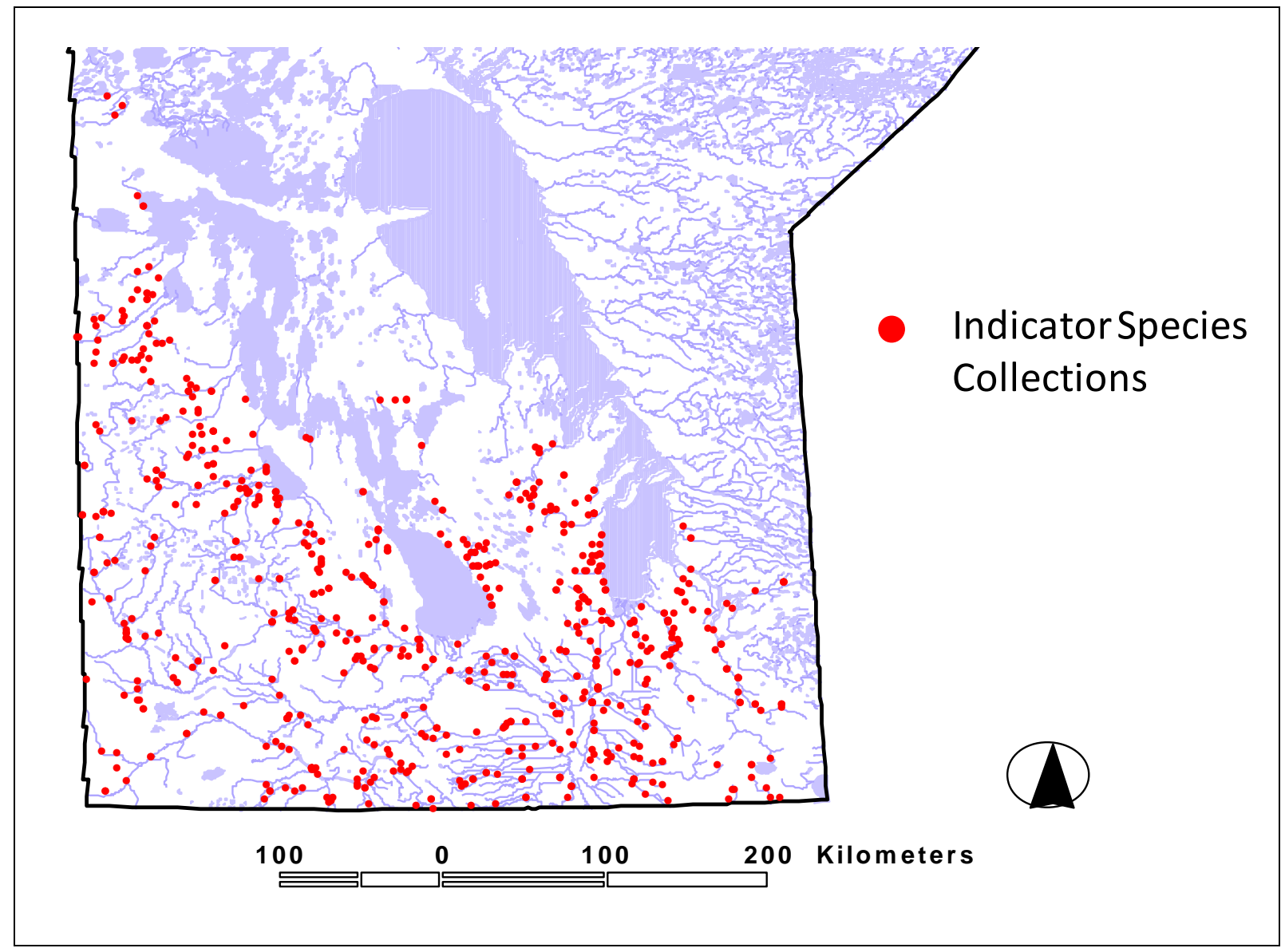

Figure 21: Map showing the location of 558 sites where Indicator Species were collected. 


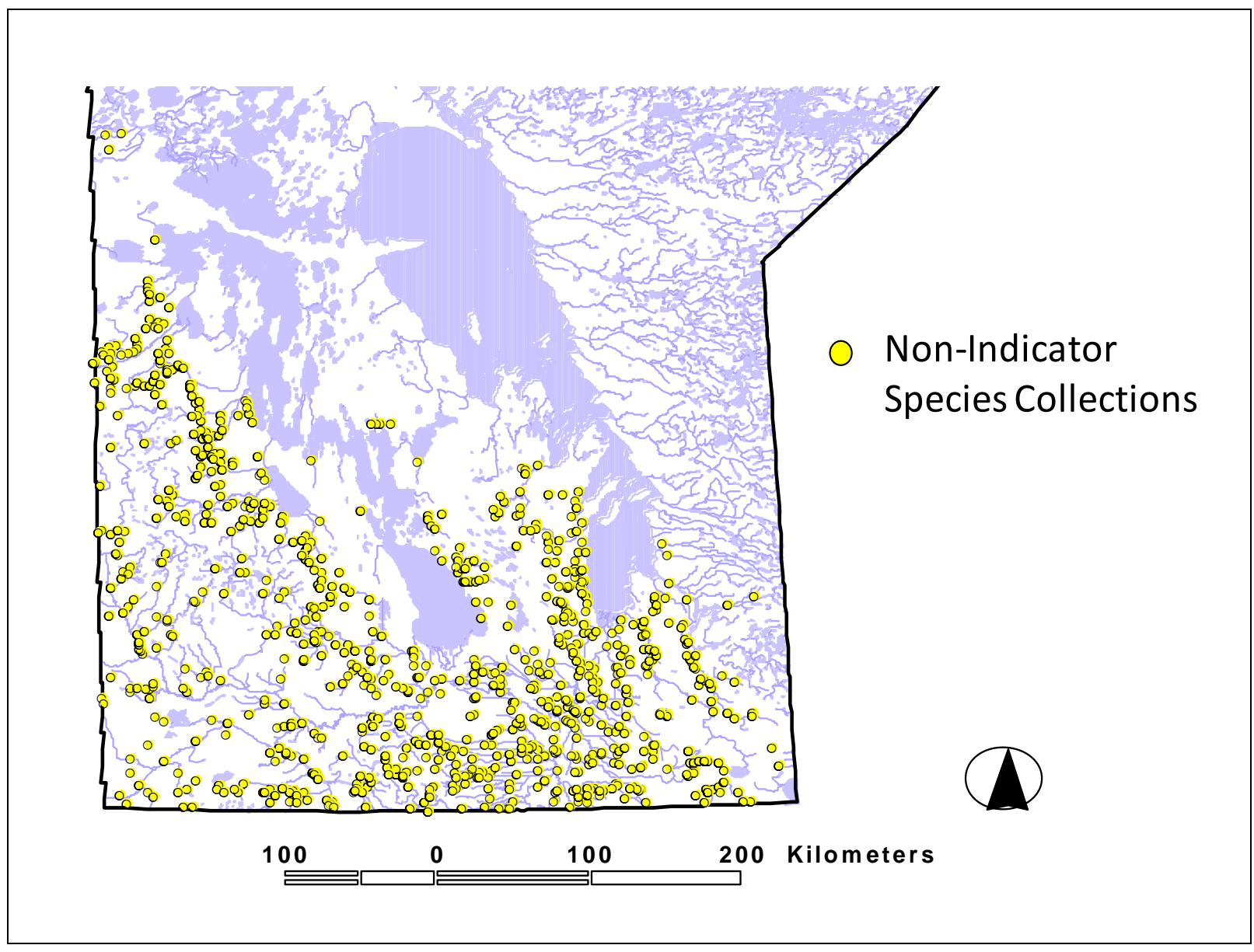

Figure 22: Map showing the locations of 916 sites where Non-Indicator Species were collected. 


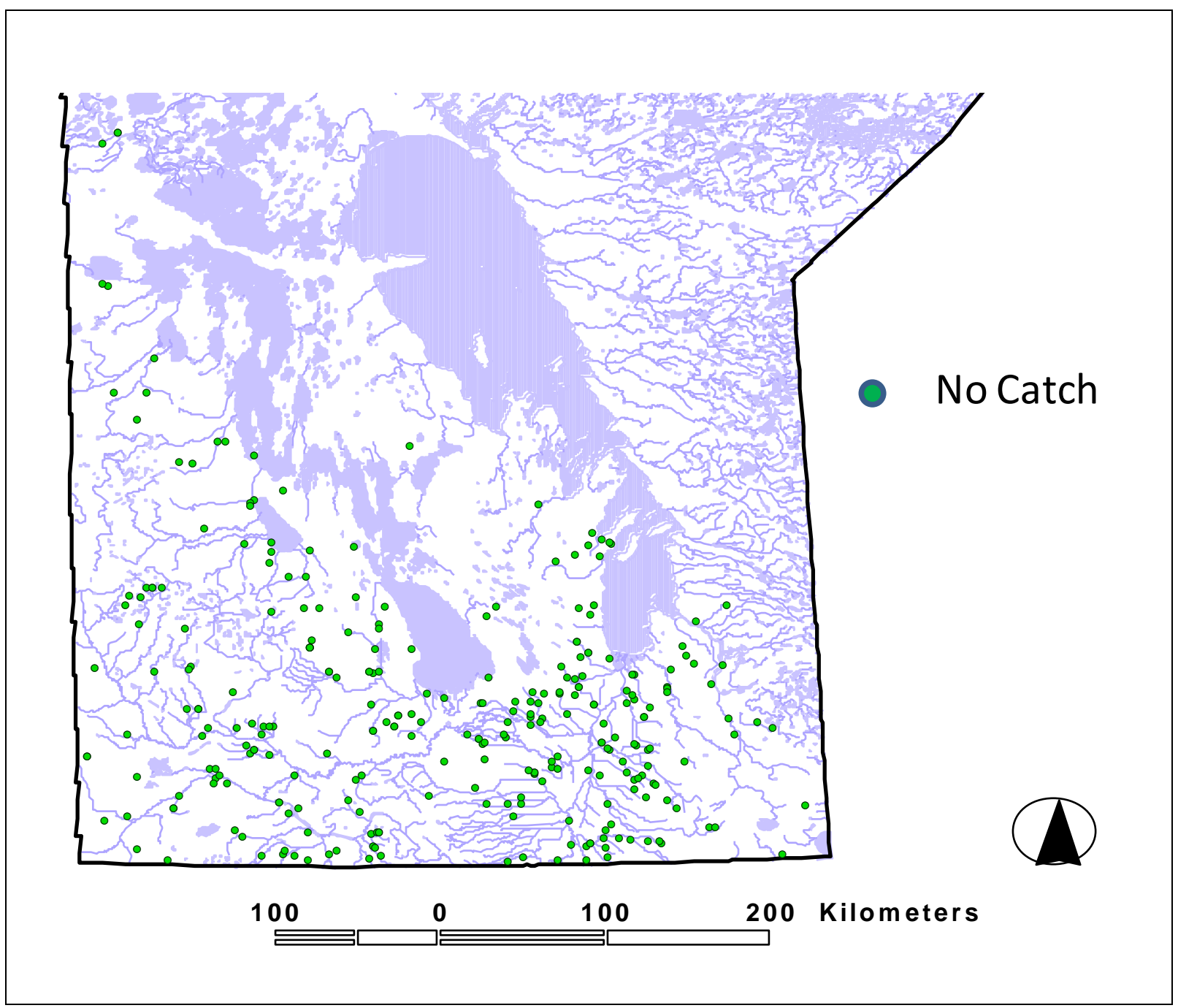

Figure 23: Map showing the locations of 271 sites where fishing effort resulted in No Catch.

The most frequently collected fishes included Fathead Minnow (577 collections), Brook Stickleback (554 collections) and White Sucker (407 collections).

Chestnut Lamprey, Quillback, Blackchin Shiner, Mimic Shiner, Goldeye, and Channel Catfish were the least frequently collected Indicator Species; each of these species was collected at only one location. White Sucker was the most frequently collected Indicator Species from all stream orders sampled.

Table 5 provides the list of all fishes collected, organized by taxonomic family and then summarized by DES stream order and by Habitat Complexity (Complex, Simple or Transition). This table also includes unidentified suckers, unidentified minnows, hybrid minnows, unidentified eggs, and no catch in the summary. 
Table 5: List of the species collected organized by taxonomic family and summarized by DES stream order and by Habitat Complexity.

\begin{tabular}{|c|c|c|c|c|c|c|c|c|c|c|c|}
\hline \multirow{2}{*}{\begin{tabular}{|l} 
FAMILY \\
Common Name \\
\end{tabular}} & \multirow{2}{*}{$\begin{array}{c}\text { \# of } \\
\text { Collections }\end{array}$} & \multicolumn{7}{|c|}{ DES Stream Order } & \multicolumn{3}{|c|}{ Habitat Complexity } \\
\hline & & 1 & 2 & 3 & 4 & 5 & 6 & 7 & Complex & Simple & Transition \\
\hline \multicolumn{12}{|c|}{ PETROMYZONTIDAE - LAMPREYS } \\
\hline Chestnut Lamprey & 1 & & & & 1 & & & & 1 & 0 & 0 \\
\hline \multicolumn{12}{|c|}{ HIODONTIDAE - GOLDEYE and MOONEYE } \\
\hline Goldeye & 1 & & & & 1 & & & & 1 & 0 & 0 \\
\hline \multicolumn{12}{|c|}{ CYPRINIDAE- MINNOWS and CARP } \\
\hline Spotfin Shiner & 4 & & & 1 & & 3 & & & 2 & 2 & 0 \\
\hline Common Carp & 43 & & 9 & 20 & 4 & 9 & 1 & & 17 & 18 & 8 \\
\hline Brassy Minnow & 31 & & 5 & 12 & 9 & 4 & 1 & & 27 & 3 & 1 \\
\hline Common Shiner & 133 & 1 & 10 & 46 & 44 & 27 & 3 & 2 & 115 & 12 & 6 \\
\hline Pearl Dace & 92 & & 23 & 40 & 22 & 7 & & & 70 & 15 & 7 \\
\hline Hornyhead Chub & 5 & & & 5 & & & & & 2 & 0 & 3 \\
\hline Golden Shiner & 3 & & & 1 & 1 & 1 & & & 3 & 0 & 0 \\
\hline Emerald Shiner & 21 & 1 & 4 & 10 & 2 & 3 & 1 & & 15 & 2 & 4 \\
\hline River Shiner & 4 & & & 1 & 1 & 1 & 1 & & 4 & 0 & 0 \\
\hline Bigmouth Shiner & 12 & & & 1 & 6 & 3 & 2 & & 12 & 0 & 0 \\
\hline Blackchin Shiner & 1 & & & & 1 & & & & 0 & 1 & 0 \\
\hline Blacknose Shiner & 28 & & 4 & 12 & 8 & 4 & & & 22 & 5 & 1 \\
\hline Spottail Shiner & 17 & & 5 & 7 & 2 & 2 & 1 & & 6 & 9 & 2 \\
\hline Sand Shiner & 36 & 1 & & 7 & 10 & 9 & 5 & 4 & 27 & 5 & 4 \\
\hline Mimic Shiner & 1 & & 1 & & & & & & 1 & 0 & 0 \\
\hline Northern Redbelly Dace & 77 & & 27 & 32 & 12 & 6 & & & 61 & 8 & 8 \\
\hline Finescale Dace & 148 & 1 & 39 & 69 & 30 & 9 & & & 117 & 21 & 10 \\
\hline Fathead Minnow & 577 & 3 & 93 & 287 & 133 & 52 & 5 & 4 & 346 & 184 & 47 \\
\hline Longnose Dace & 93 & & 7 & 26 & 34 & 18 & 4 & 4 & 85 & 6 & 2 \\
\hline $\begin{array}{l}\text { Western Blacknose } \\
\text { Dace }\end{array}$ & 198 & 2 & 16 & 78 & 69 & 30 & 3 & & 172 & 20 & 6 \\
\hline Creek Chub & 212 & & 24 & 78 & 74 & 30 & 5 & 1 & 178 & 26 & 8 \\
\hline Hybrid minnows & 5 & & 2 & 1 & 2 & & & & 5 & 0 & 0 \\
\hline Unidentified minnows & 16 & & 2 & 6 & 7 & 1 & & & 10 & 4 & 2 \\
\hline \multicolumn{12}{|c|}{ CATOSTOMIDAE - SUCKERS } \\
\hline Quillback & 1 & & & & & & 1 & & 1 & 0 & 0 \\
\hline White Sucker & 407 & 2 & 38 & 178 & 121 & 58 & 6 & 4 & 282 & 96 & 29 \\
\hline Bigmouth Buffalo & 4 & & 1 & 1 & 1 & 1 & & & 1 & 1 & 2 \\
\hline Silver Redhorse & 13 & & & 4 & 3 & 4 & 2 & & 10 & 3 & 0 \\
\hline Shorthead Redhorse & 5 & & & & & 1 & 2 & 2 & 5 & 0 & 0 \\
\hline Eggs (Catostomidae) & 9 & & & 5 & 1 & 3 & & & 6 & 2 & 1 \\
\hline Unidentified Sucker & 8 & & 3 & 2 & & 3 & & & 7 & 1 & 0 \\
\hline
\end{tabular}


Table 5: List of the species collected organized by taxonomic family and summarized by DES stream order and by Habitat Complexity.

\begin{tabular}{|c|c|c|c|c|c|c|c|c|c|c|c|}
\hline \multirow{2}{*}{\begin{tabular}{|l|} 
FAMILY \\
Common Name \\
\end{tabular}} & \multirow{2}{*}{$\begin{array}{c}\text { \# of } \\
\text { Collections }\end{array}$} & \multicolumn{7}{|c|}{ DES Stream Order } & \multicolumn{3}{|c|}{ Habitat Complexity } \\
\hline & & 1 & 2 & 3 & 4 & 5 & 6 & 7 & Complex & Simple & Transition \\
\hline \multicolumn{12}{|c|}{ ICTALURIDAE - FRESHWATER CATFISHES } \\
\hline Black Bullhead & 61 & & 7 & 19 & 19 & 12 & 1 & 3 & 35 & 24 & 2 \\
\hline Channel Catfish & 1 & & & & 1 & & & & 1 & 0 & 0 \\
\hline Stonecat & 2 & & 1 & & & & & 1 & 2 & 0 & 0 \\
\hline Tadpole Madtom & 11 & & 1 & 1 & & 6 & 2 & 1 & 8 & 3 & 0 \\
\hline \multicolumn{12}{|c|}{ ESOCIDAE - PIKE and MUSKELLUNGE } \\
\hline Northern Pike & 182 & & 33 & 78 & 47 & 19 & 3 & 2 & 97 & 65 & 20 \\
\hline \multicolumn{12}{|c|}{ UMBRIDAE - MUDMINNOWS } \\
\hline Central Mudminnow & 259 & & 1 & 58 & 152 & 43 & 5 & & 141 & 87 & 31 \\
\hline \multicolumn{12}{|c|}{ SALMONIDAE - WHITEFISH, CHAR, TROUT and SALMON } \\
\hline Rainbow Trout & 2 & & & 1 & 1 & & & & 2 & 0 & 0 \\
\hline Brown Trout & 2 & & & & 2 & & & & 2 & 0 & 0 \\
\hline Brook Trout & 6 & & & 3 & 2 & 1 & & & 6 & 0 & 0 \\
\hline \multicolumn{12}{|c|}{ PERCOPSIDAE - TROUTPERCHES } \\
\hline Troutperch & 15 & & & 2 & 6 & 3 & 3 & 1 & 14 & 0 & 1 \\
\hline \multicolumn{12}{|c|}{ GADIDAE - CODFISHES } \\
\hline Burbot & 20 & 1 & 1 & 7 & 4 & 5 & 1 & 1 & 16 & 2 & 2 \\
\hline \multicolumn{12}{|c|}{ GASTEROSTEIDAE - STICKLEBACKS } \\
\hline Brook Stickleback & 554 & 7 & 119 & 286 & 116 & 26 & & & 336 & 165 & 53 \\
\hline \multicolumn{12}{|l|}{ COTTIDAE - SCULPINS } \\
\hline Mottled Sculpin & 2 & & & 1 & 1 & & & & 2 & 0 & 0 \\
\hline \multicolumn{12}{|c|}{ CENTRARCHIDAE - BASS, CRAPPIES and SUNFISHES } \\
\hline Rock Bass & 11 & & & 2 & 2 & 5 & 1 & 1 & 9 & 1 & 1 \\
\hline Smallmouth Bass & 2 & & & & 2 & & & & 2 & 0 & 0 \\
\hline Black Crappie & 5 & & & & 3 & 2 & & & 4 & 0 & 1 \\
\hline \multicolumn{12}{|c|}{ PERCIDAE - DARTERS, PERCH, SAUGER and WALLEYE } \\
\hline lowa Darter & 55 & & 15 & 22 & 10 & 8 & & & 43 & 8 & 4 \\
\hline Johnny Darter & 189 & 1 & 21 & 67 & 53 & 37 & 7 & 3 & 165 & 17 & 7 \\
\hline Yellow Perch & 43 & & 9 & 15 & 10 & 7 & 2 & & 25 & 13 & 5 \\
\hline Logperch & 11 & & 1 & 3 & 3 & 3 & 1 & & 8 & 3 & 0 \\
\hline Blackside Darter & 65 & & 8 & 16 & 16 & 18 & 4 & 3 & 60 & 4 & 1 \\
\hline River Darter & 12 & & 1 & 1 & 2 & 7 & 1 & & 12 & 0 & 0 \\
\hline Sauger & 3 & & & & 1 & 2 & & & 3 & 0 & 0 \\
\hline Walleye & 13 & & 3 & 2 & 4 & 3 & & 1 & 8 & 2 & 3 \\
\hline Eggs (Percidae) & 1 & & & & & 1 & & & 1 & 0 & 0 \\
\hline
\end{tabular}


Table 5: List of the species collected organized by taxonomic family and summarized by DES stream order and by Habitat Complexity.

\begin{tabular}{|c|c|c|c|c|c|c|c|c|c|c|c|}
\hline \multirow{2}{*}{$\begin{array}{l}\text { FAMILY } \\
\text { Common Name }\end{array}$} & \multirow{2}{*}{$\begin{array}{c}\text { \# of } \\
\text { Collections }\end{array}$} & \multicolumn{7}{|c|}{ DES Stream Order } & \multicolumn{3}{|c|}{ Habitat Complexity } \\
\hline & & 1 & 2 & 3 & 4 & 5 & 6 & 7 & Complex & Simple & Transition \\
\hline \multicolumn{12}{|c|}{ SCIAENIDAE - DRUMS } \\
\hline Freshwater Drum & 2 & & & 1 & & & 1 & & 0 & 2 & 0 \\
\hline \multicolumn{12}{|l|}{ OTHER } \\
\hline No Catch & 281 & 5 & 60 & 145 & 56 & 13 & 2 & & 136 & 122 & 23 \\
\hline Total & 4016 & 25 & 594 & 1660 & 1112 & 510 & 77 & 38 & 2749 & 962 & 305 \\
\hline
\end{tabular}

Giving consideration to the Habitat Type Decision Flowchart and the status of fish species collected, the most frequent collection and habitat type was Type C - Complex Habitat with Non-Indicator Species (52.9\%), followed by Type D - Simple Habitat with Non-Indicator Species (16.2\%), Type A - Complex Habitat with Indicator Species $(12.2 \%)$ and Type B - Simple Habitat with Indicator Species (4.7\%). Fishing effort in Complex Habitat, and fishing effort in Simple Habitat that resulted in No Catch occurred in $3.4 \%$ and $3.0 \%$ of all collections, respectively. Fishing effort in transitional habitat accounted for the balance of collections $(7.6 \%)$.

Figures 24 to 26 provide the composition of Indicator Species collections summarized by DES stream order.

Figures 27 to 31 provide the composition of Non-Indicator Species collections summarized by DES stream order. Brook Stickleback was the most frequently collected Non-Indicator Species in lower order streams (1-3). No Brook Stickleback were collected from streams with a DES stream order higher than 5 . 

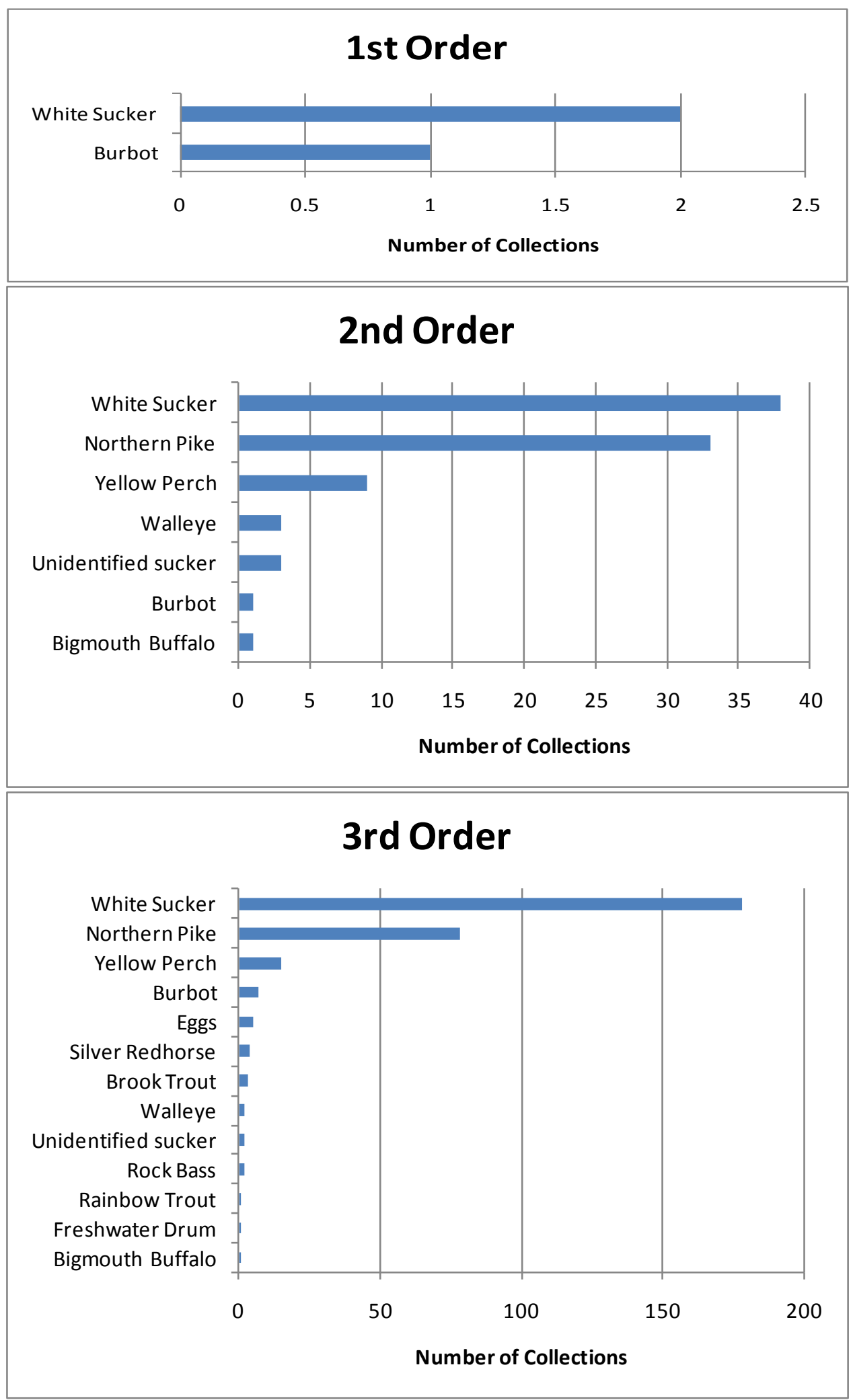

Figure 24: Showing the composition of Indicator Species collections summarized for DES stream orders 1-3. 

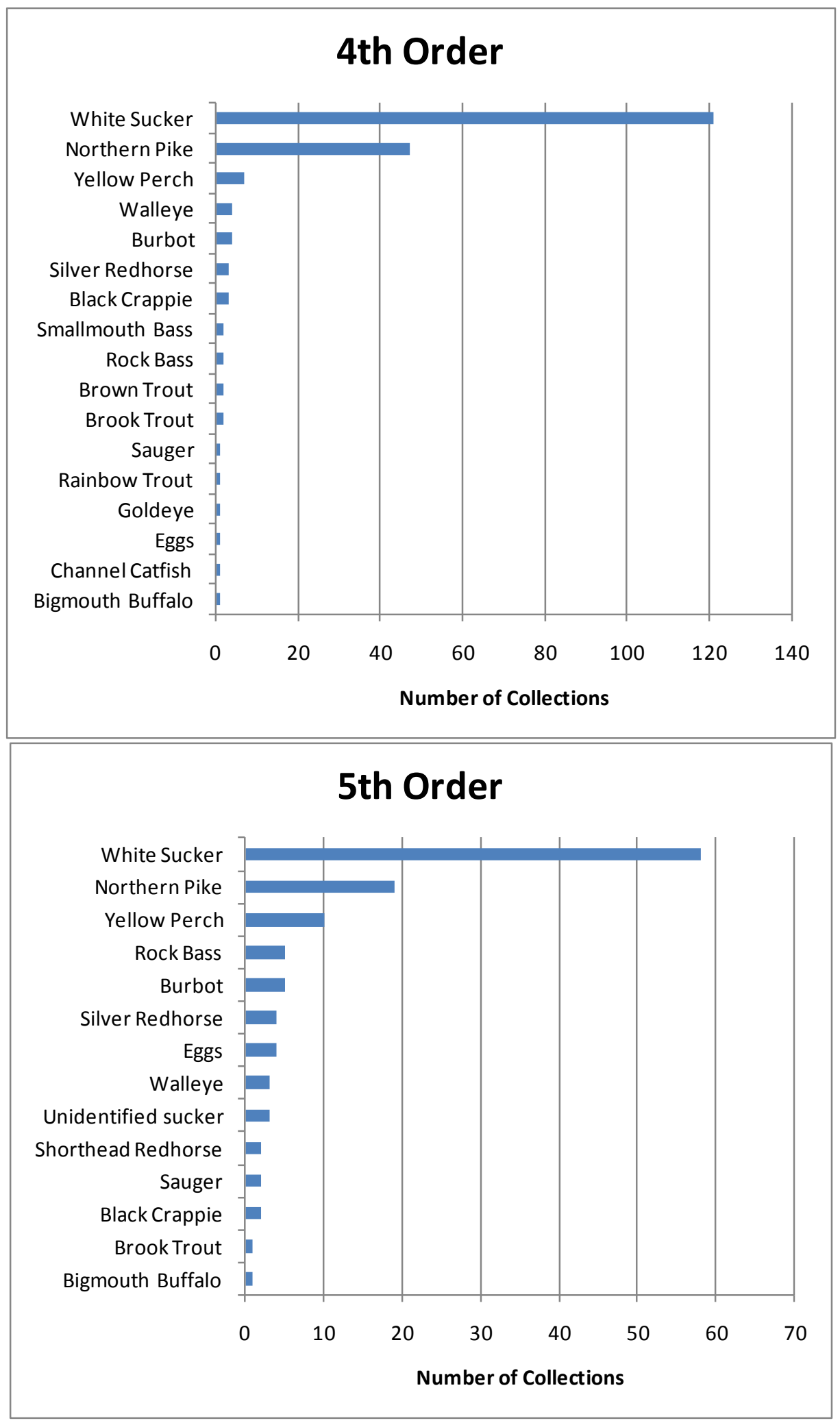

Figure 25: Showing the composition of Indicator Species collections summarized for DES stream orders 4 and 5. 


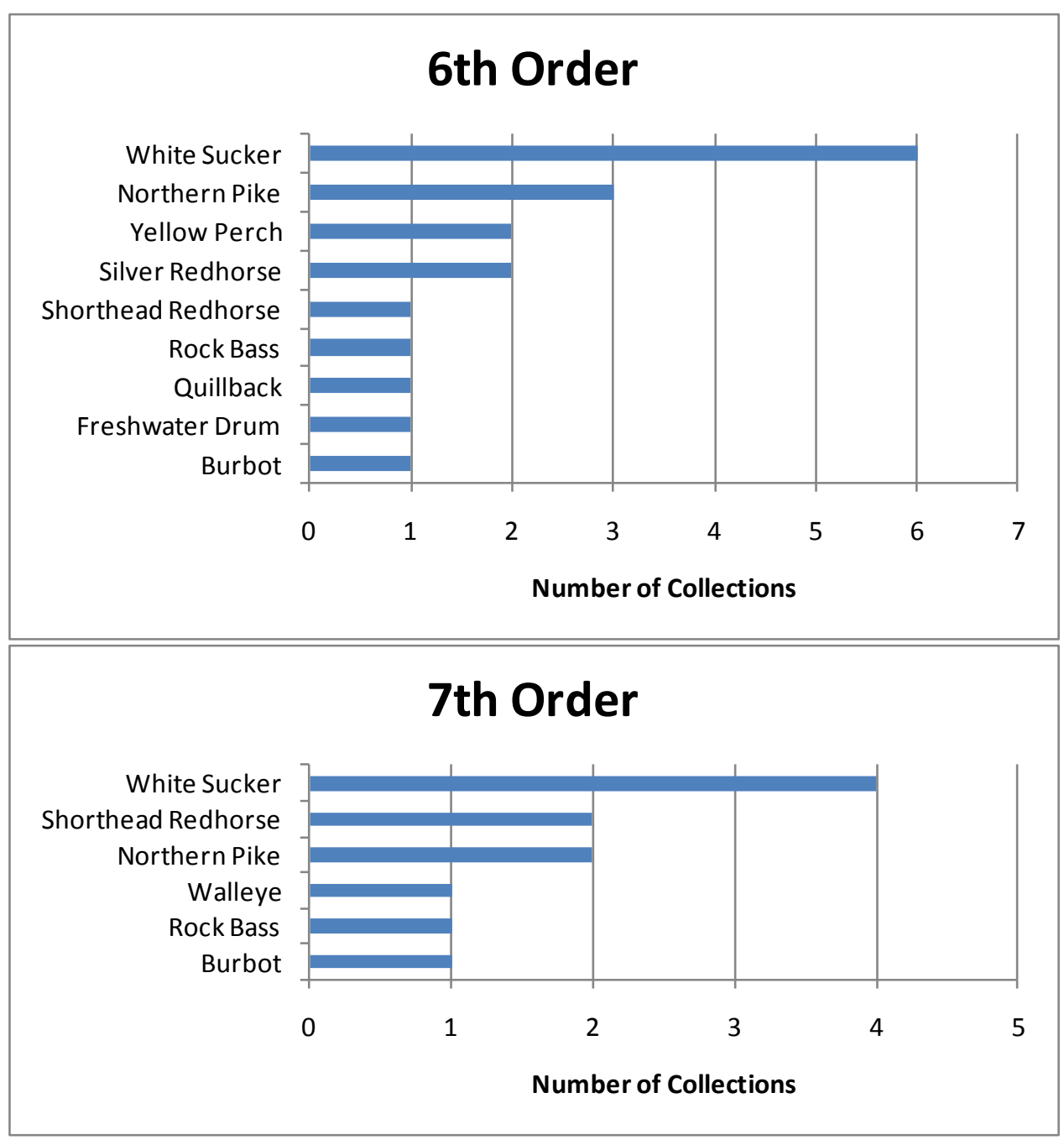

Figure 26: Showing the composition of Indicator Species collections summarized for DES stream orders 6 and 7.

Figure 32 shows the distribution of fishing effort when categorized by habitat type (Complex Habitat, Simple Habitat and Transitional Habitat) and the percentage of each fish capture type (Indicator Species, Non-Indicator Species, and No Catch results) collected from each habitat type.

Figure 33 shows the percentage of Indicator Species collections, Non-Indicator Species collections and No catch results when categorized by Habitat Type.

Figures 34 to 91 provide a summary for each fish species collected with a map showing the number ( $\mathrm{n}$ ) and location of collection sites; maximum, minimum and median scores for individual habitat parameters and the total Habitat Assessment Scores; and the percent of catch by DES stream order. Sites that overlap or are in close proximity may not be displayed as individual locations at the map scale used. 

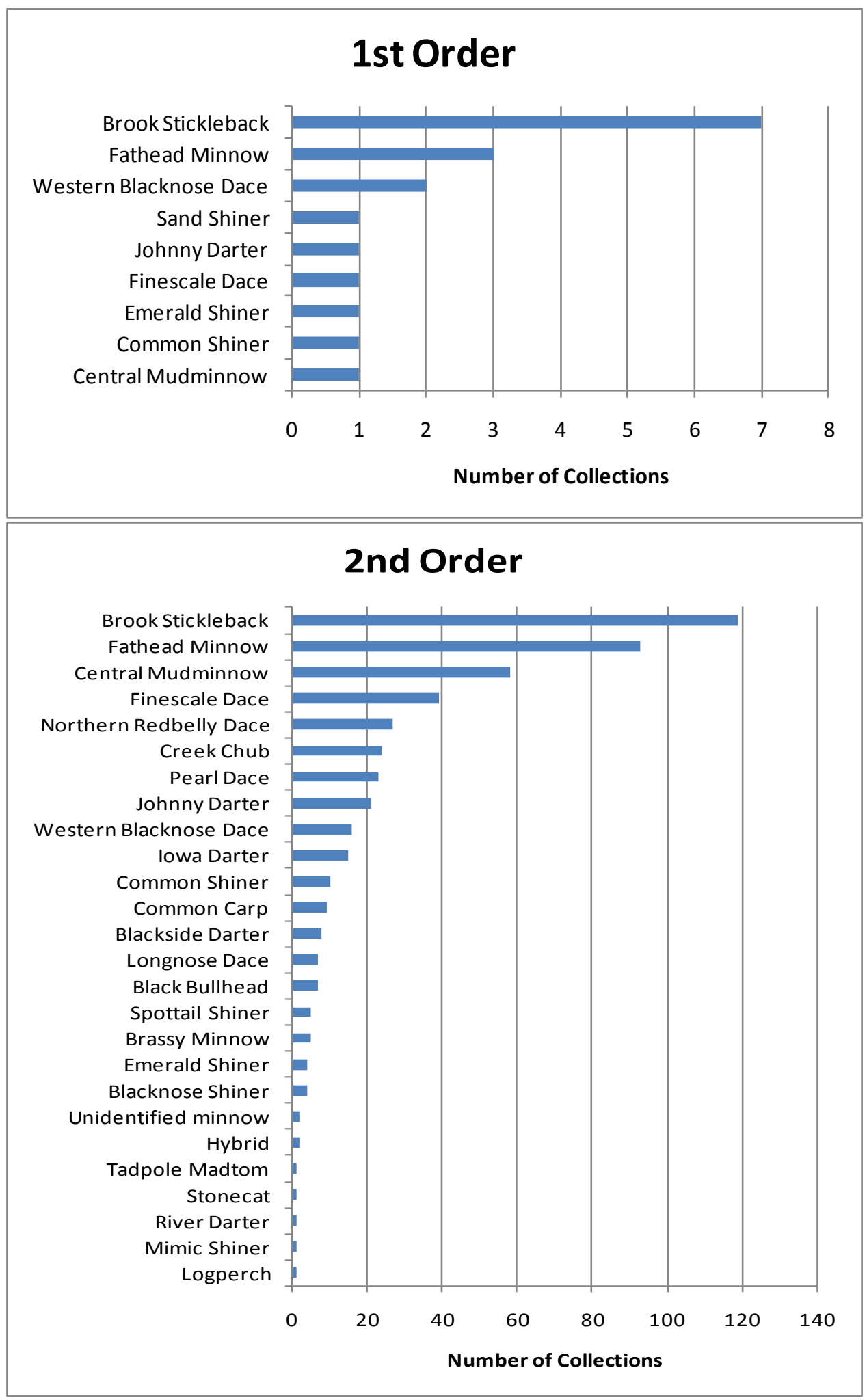

Figure 27: Showing the composition of Non-Indicator Species collections summarized for DES stream orders 1 and 2. 


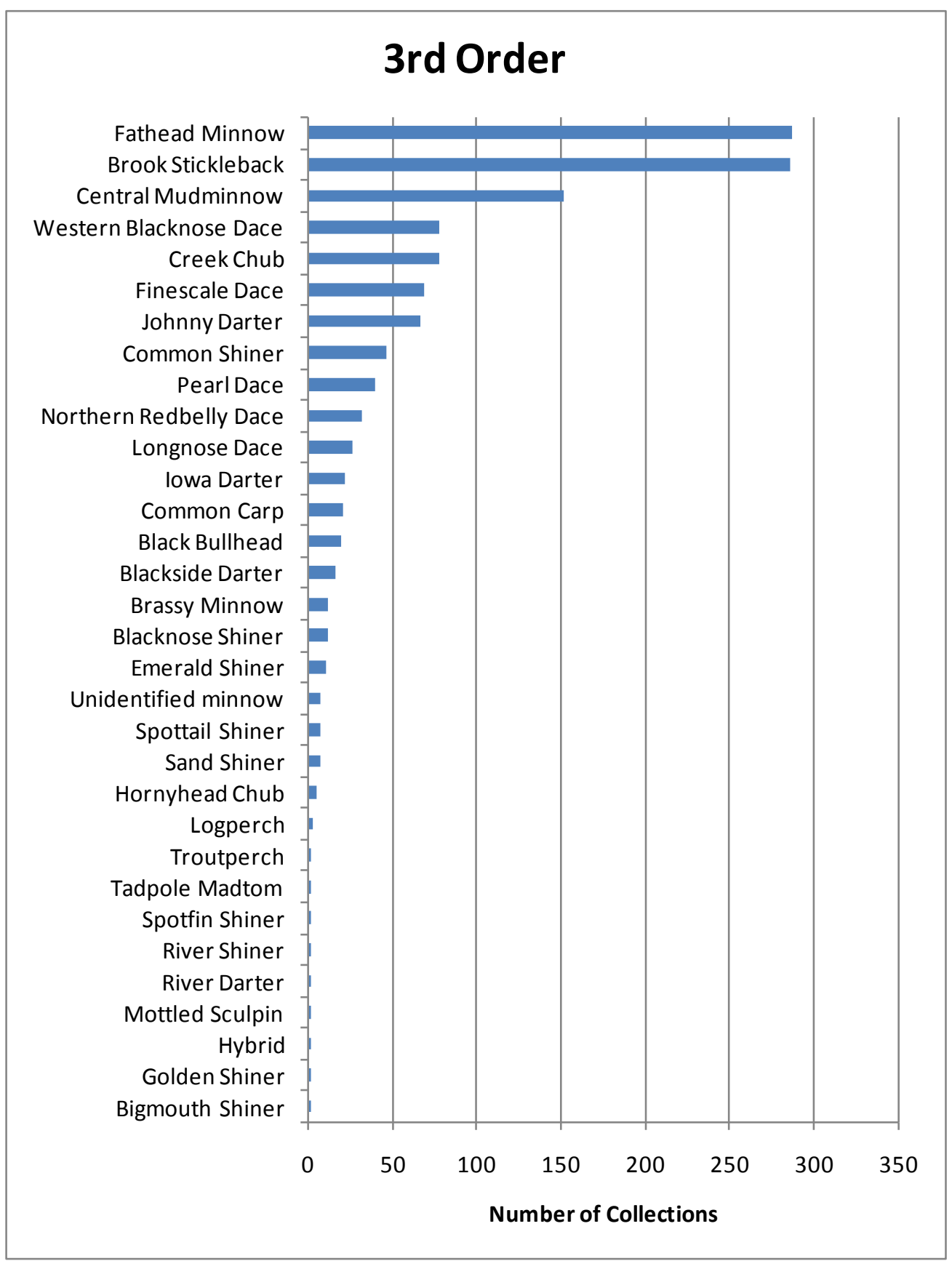

Figure 28: Showing the composition of Non-Indicator Species collections summarized for DES stream order 3. 


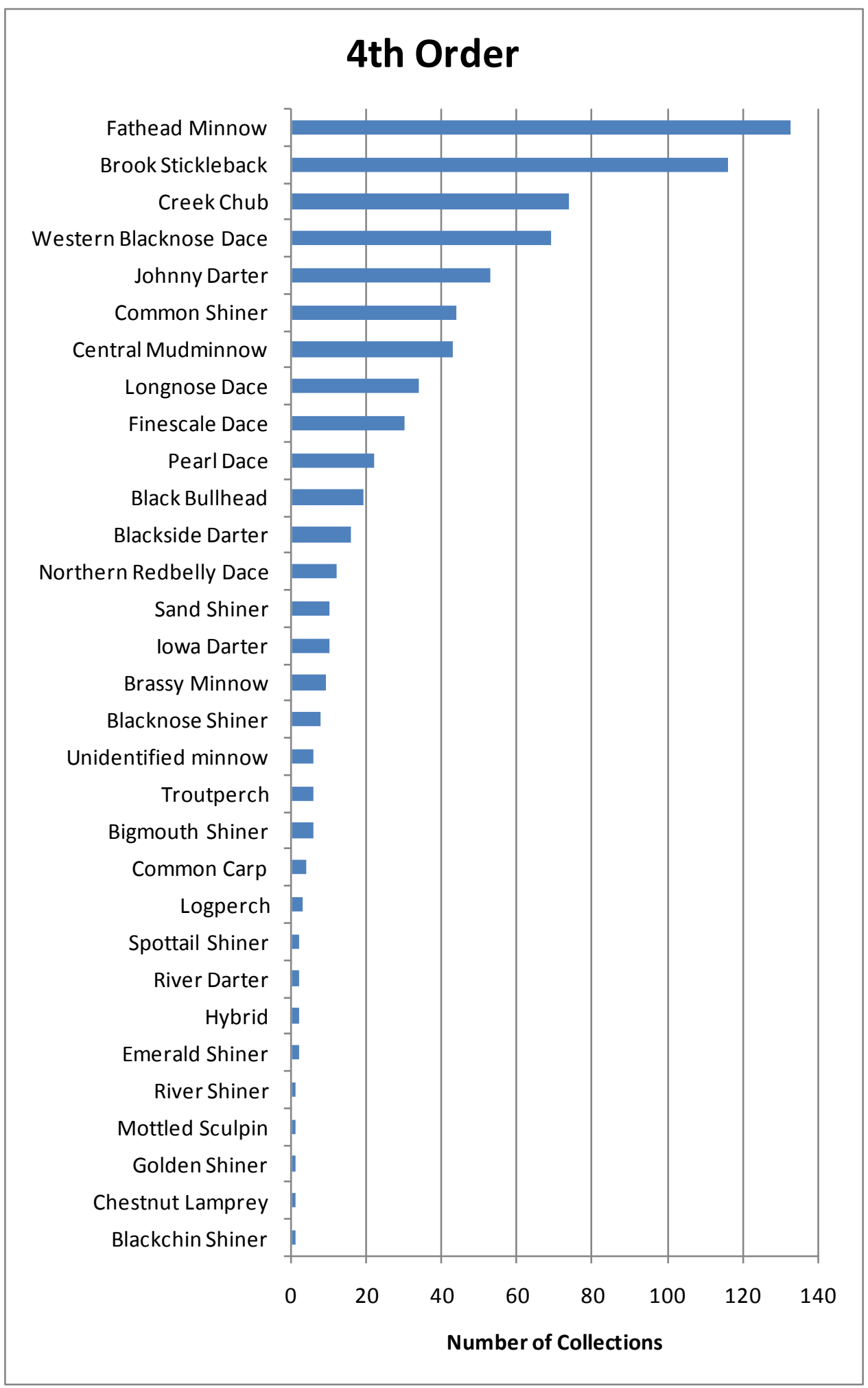

Figure 29: Showing the composition of Non-Indicator Species collections summarized for DES stream order 4. 


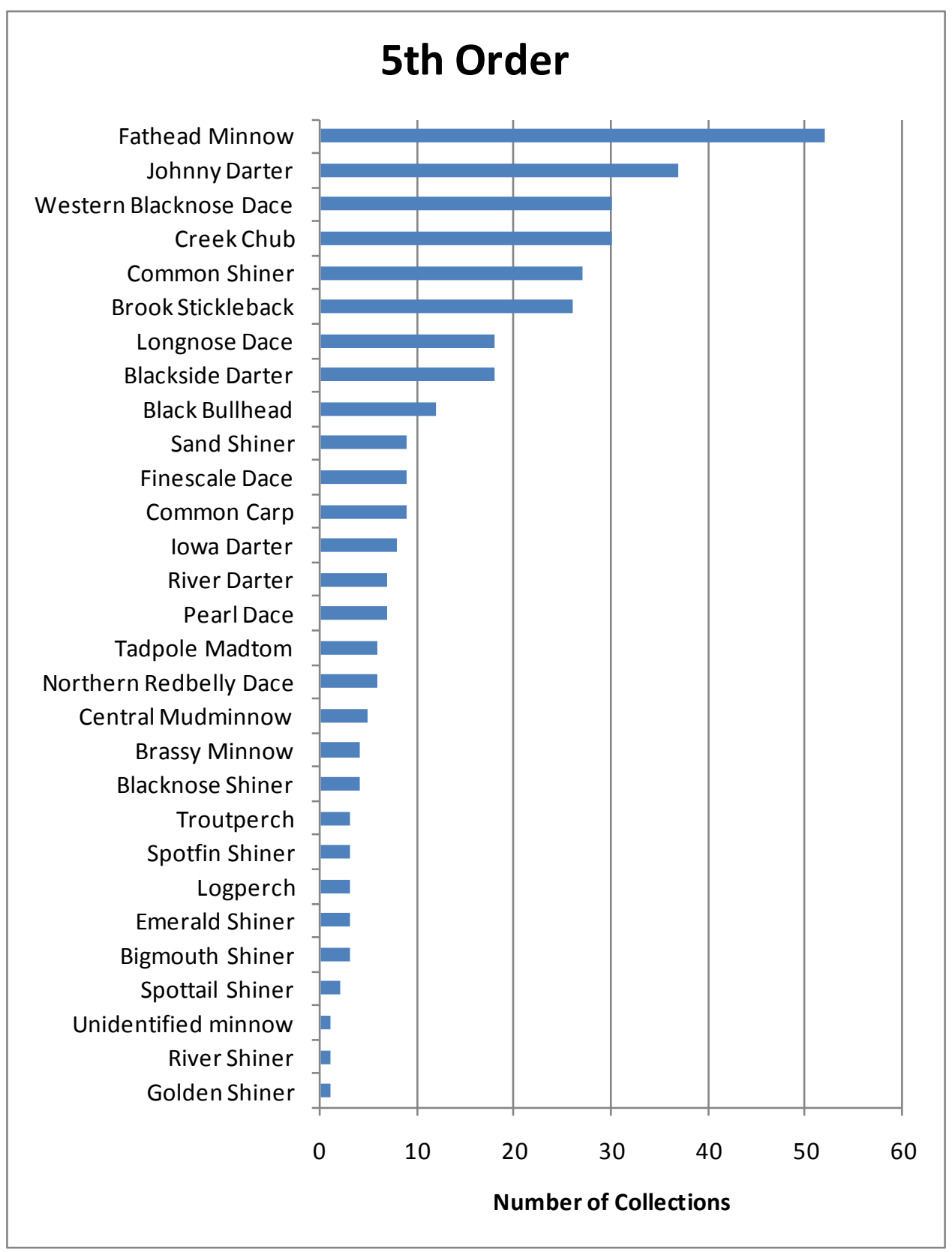

Figure 30: Showing the composition of Non-Indicator Species collections summarized for DES stream order 5 . 

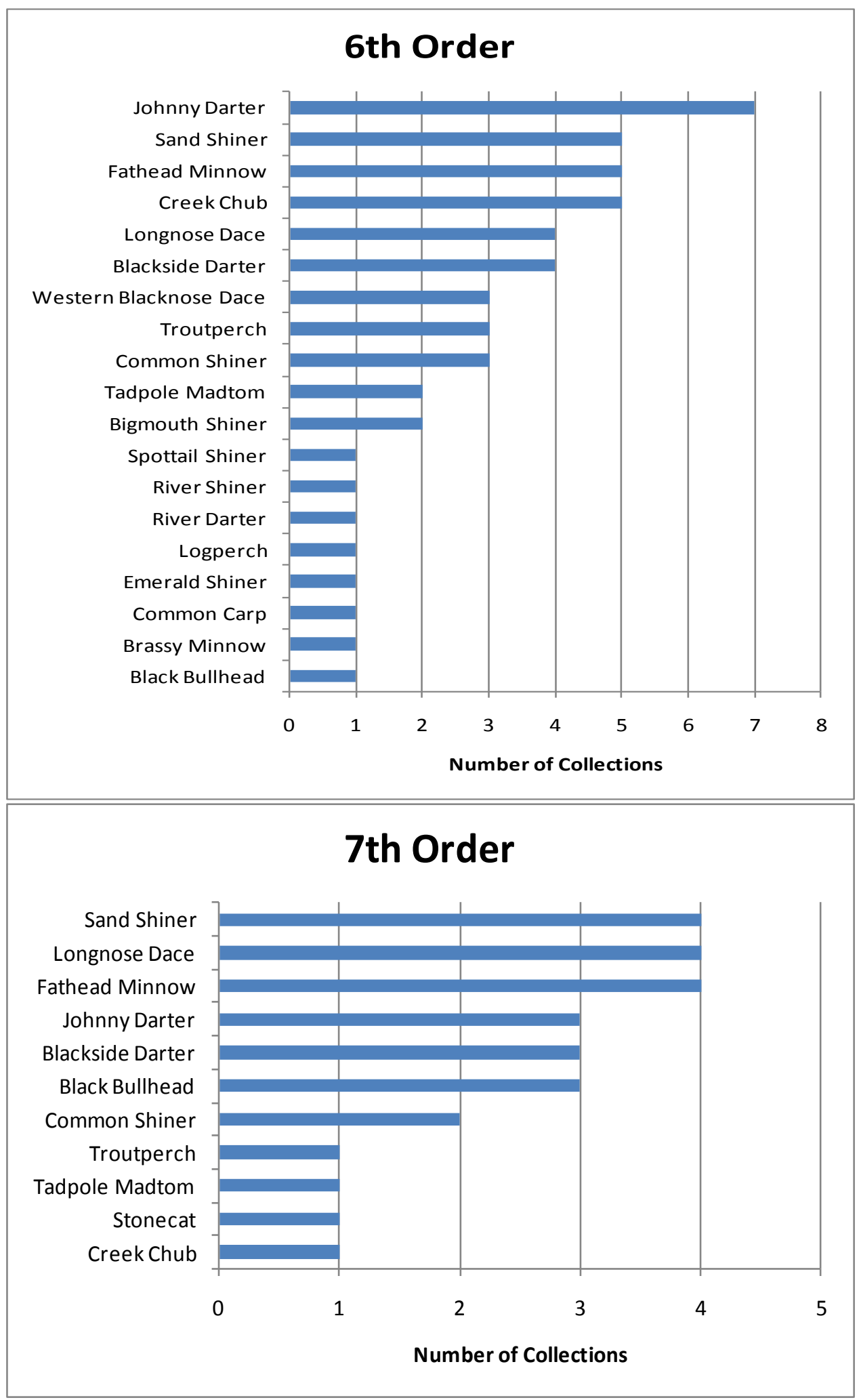

Figure 31: Showing the composition of Non-Indicator Species collections summarized for DES stream orders 6 and 7. 


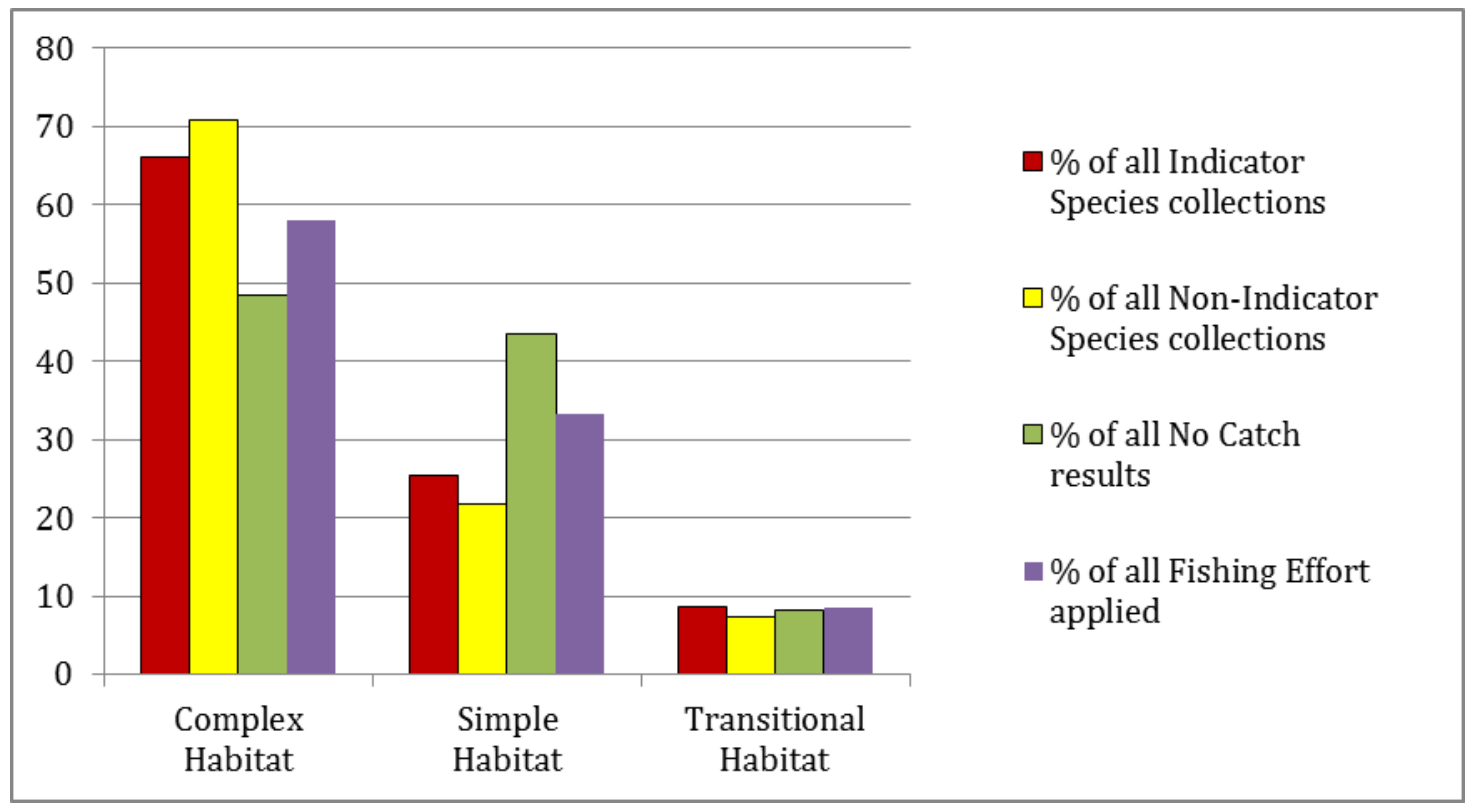

Figure 32: Showing the percentage of fishing effort and percentage of each fish capture type (Indicator Species, Non-Indicators Species and No Catch) when distibuted by habitat type (Complex Habitat, Simple Habitat or Transitional Habitat).

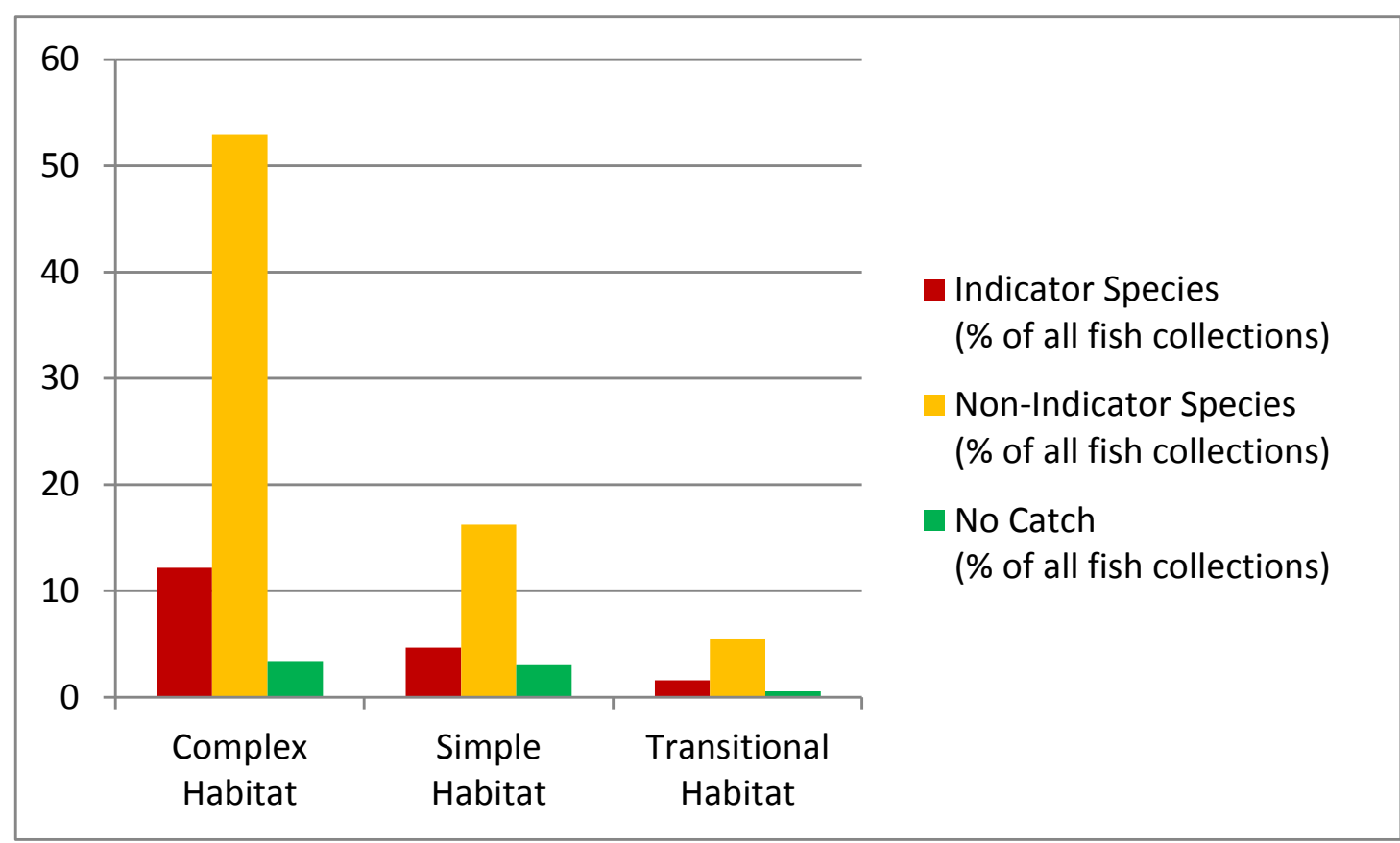

Figure 33: Showing the composition (percentage of the entire catch) of each fish capture type (including No Catch as a capture result) when distributed by Habitat Type.

Figure 92 shows the percent distribution of each species collected by channel type. Channel type was determined by the absence of any dikes; the presence of a dike on one side of the channel only; or by having dykes built on both sides of the channel, isolating it from the floodplain. 

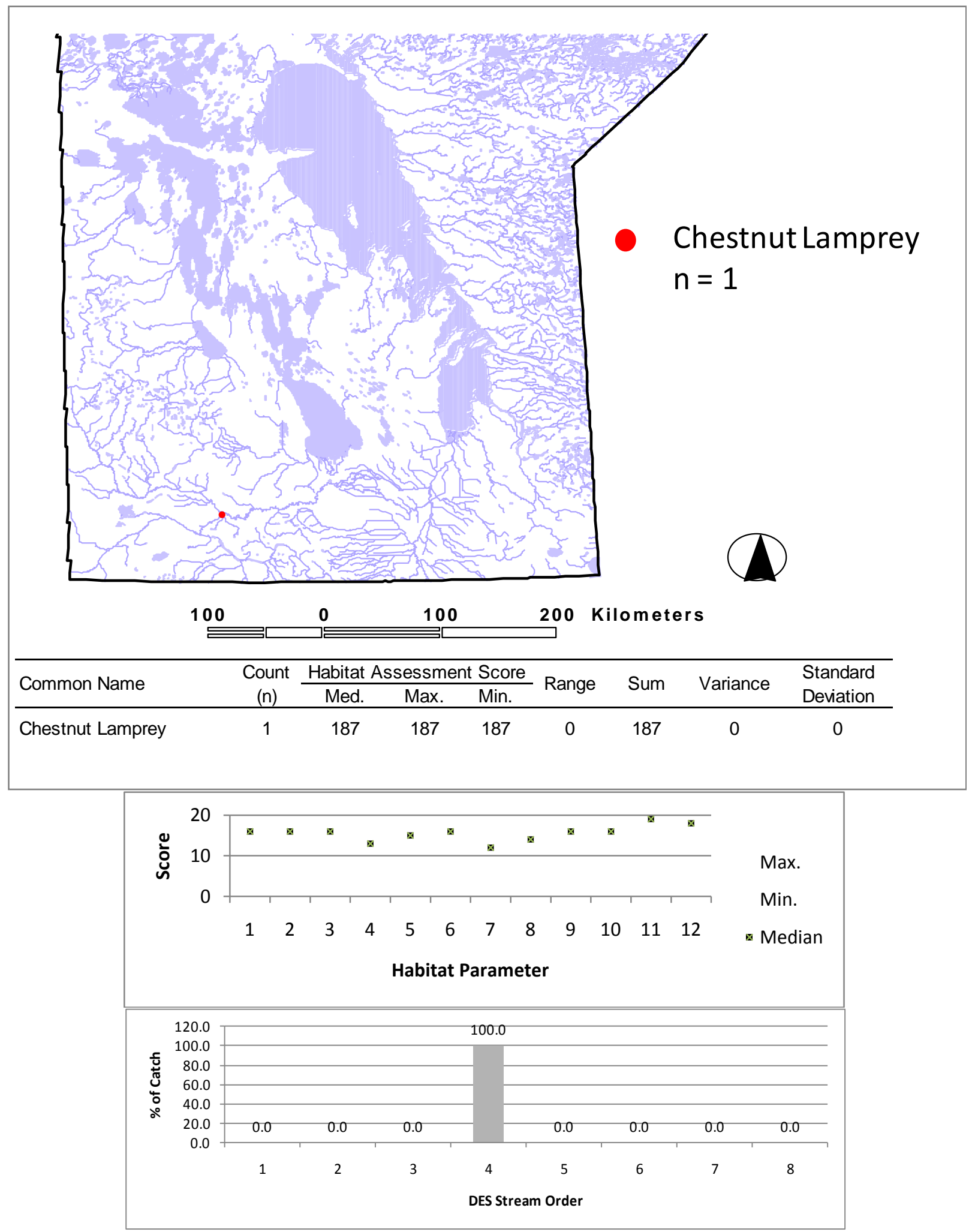

Figure 34: A summary of Chestnut Lamprey collections showing collection sites; maximum, minimum, and median individual parameter and total Habitat Assessment Scores; and the percent of catch by DES stream order. 

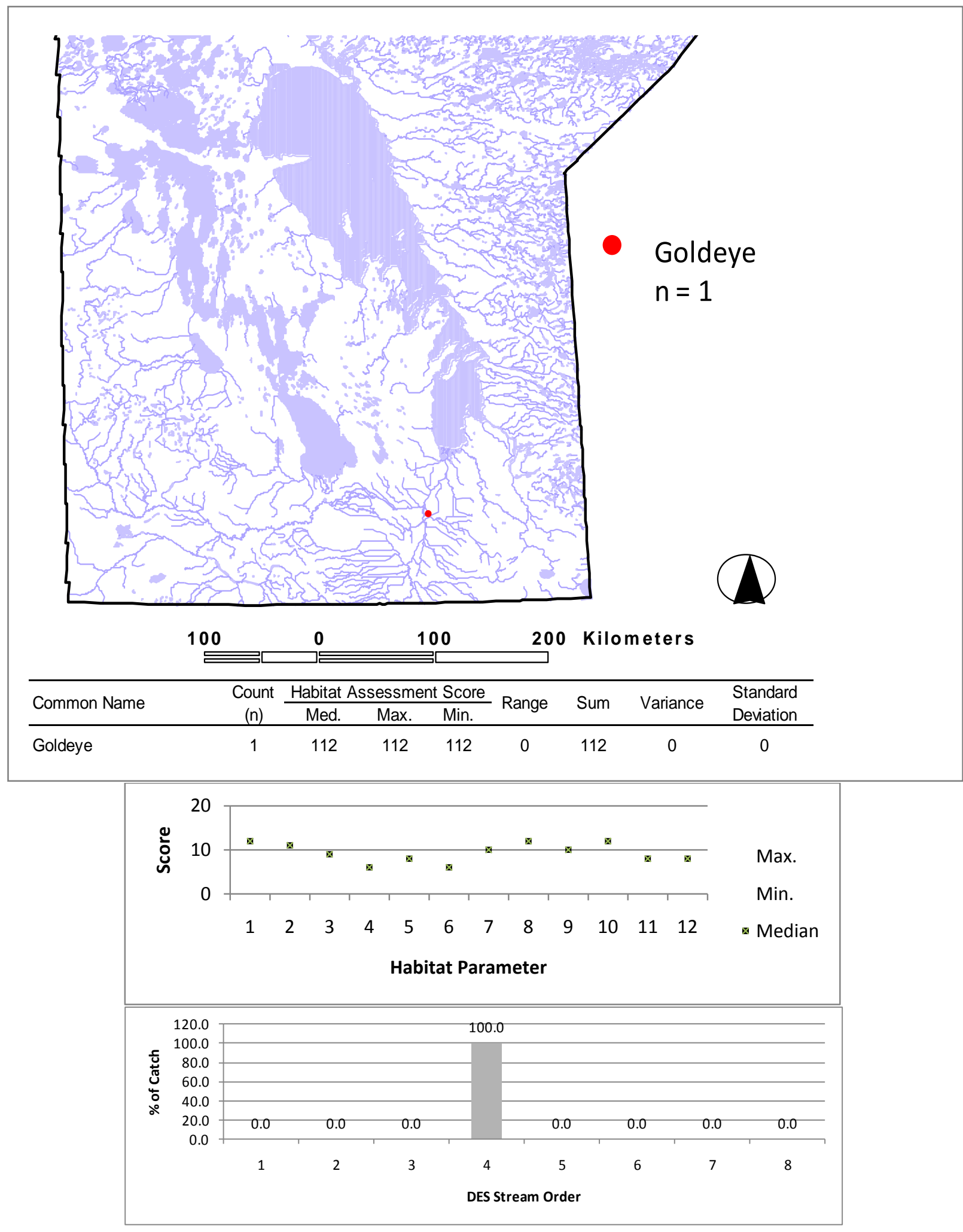

Figure 35: A summary of Goldeye collections showing collection sites; maximum, minimum and median individual parameter and total Habitat Assessment Scores; and the percent of catch by DES stream order. 

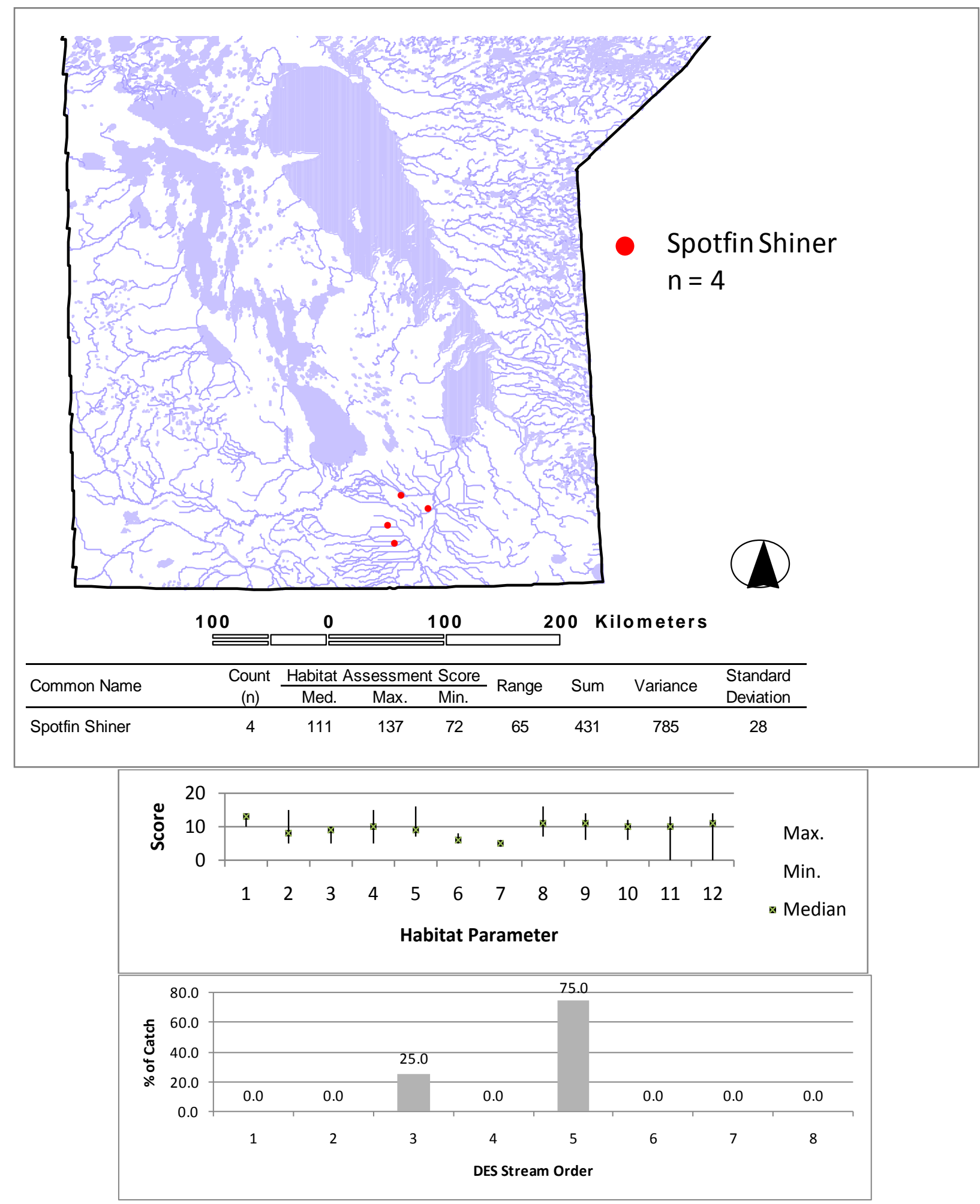

Figure 36: A summary of Spotfin Shiner collections showing collection sites; maximum, minimum and median individual parameter and total Habitat Assessment Scores; and the percent of catch by DES stream order. 

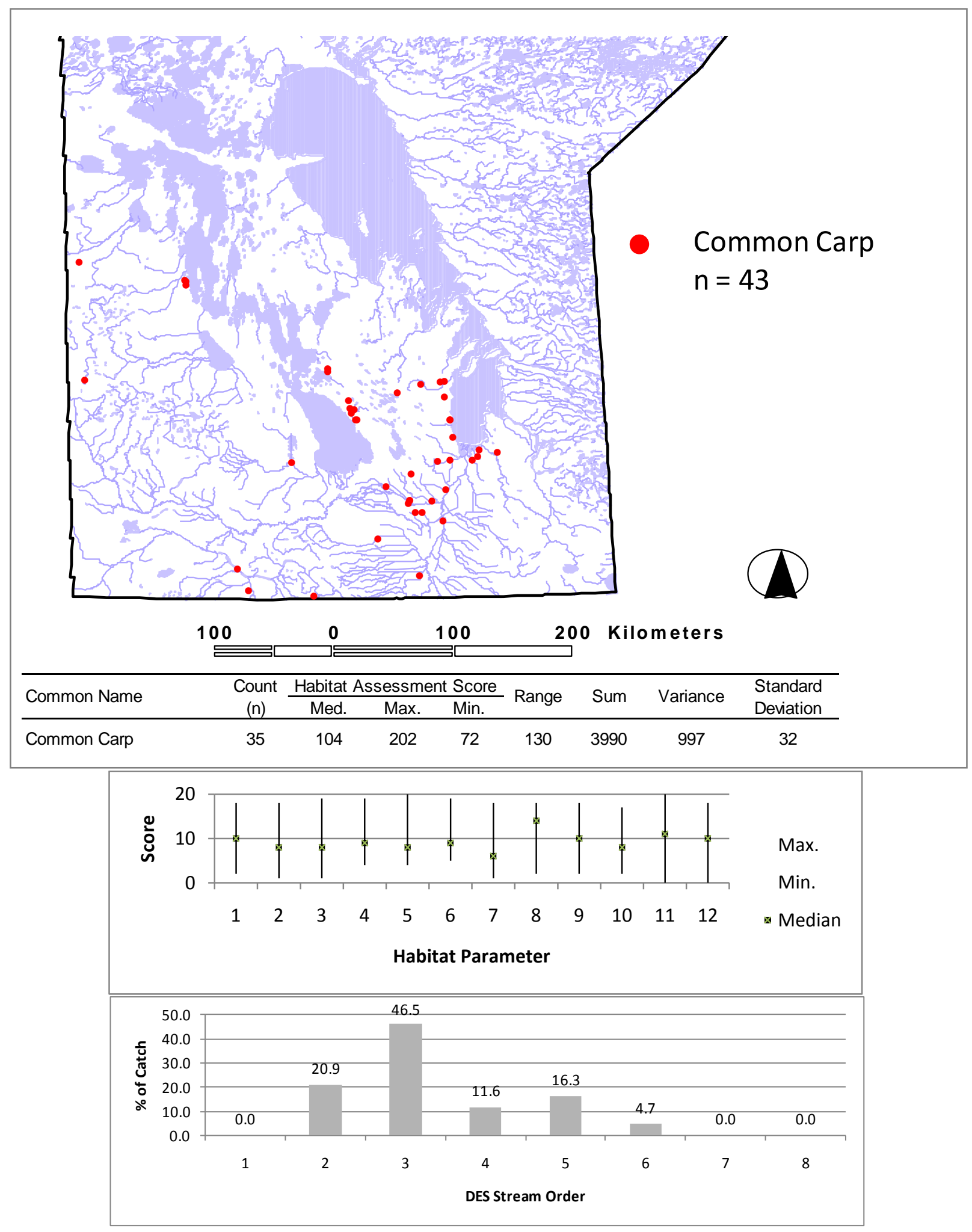

Figure 37: A summary of Common Carp collections showing collection sites; maximum, minimum and median individual parameter and total Habitat Assessment Scores; and the percent of catch by DES stream order. 

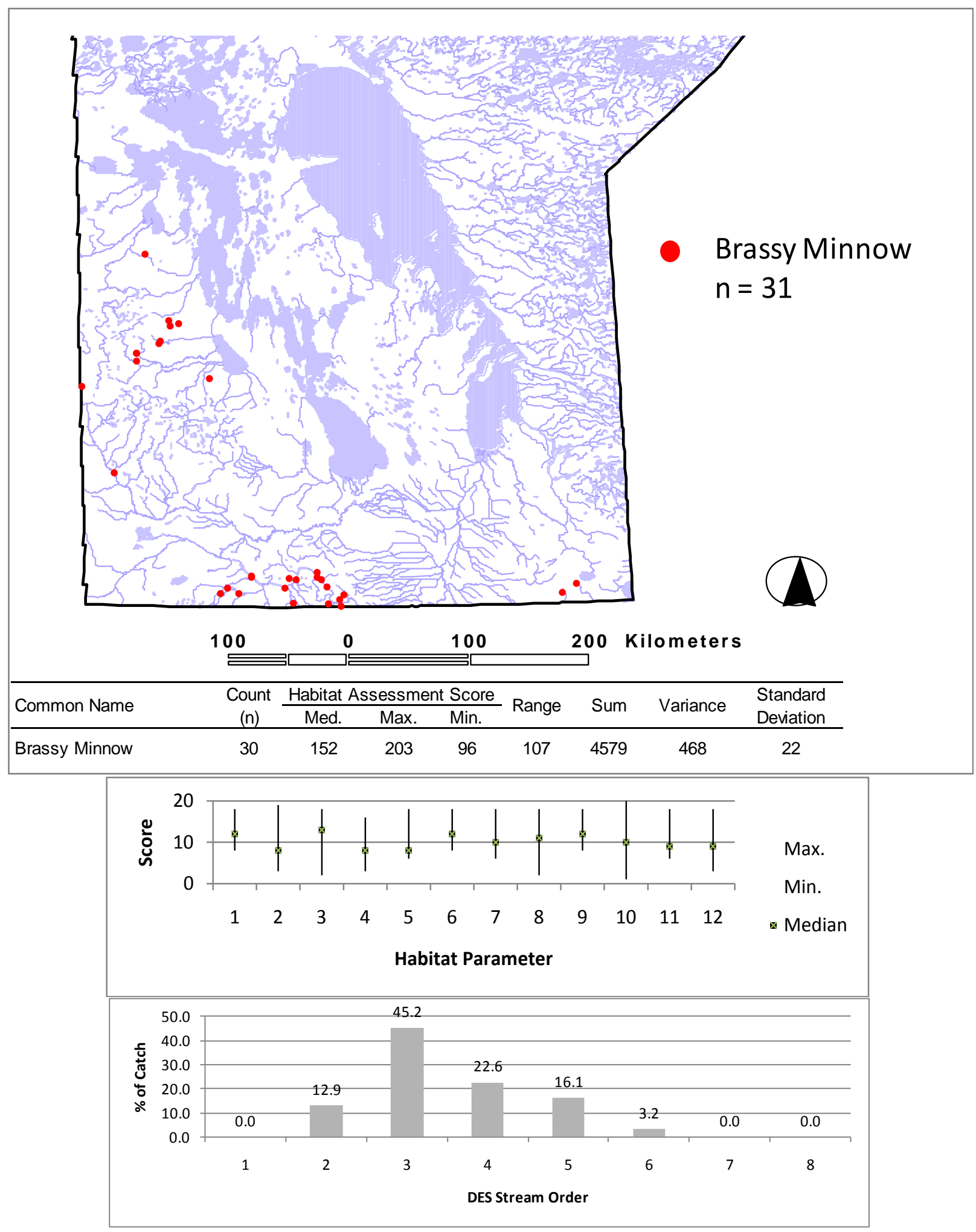

Figure 38: A summary of Brassy Minnow collections showing collection sites; maximum, minimum and median individual parameter and total Habitat Assessment Scores; and the percent of catch by DES stream order. 

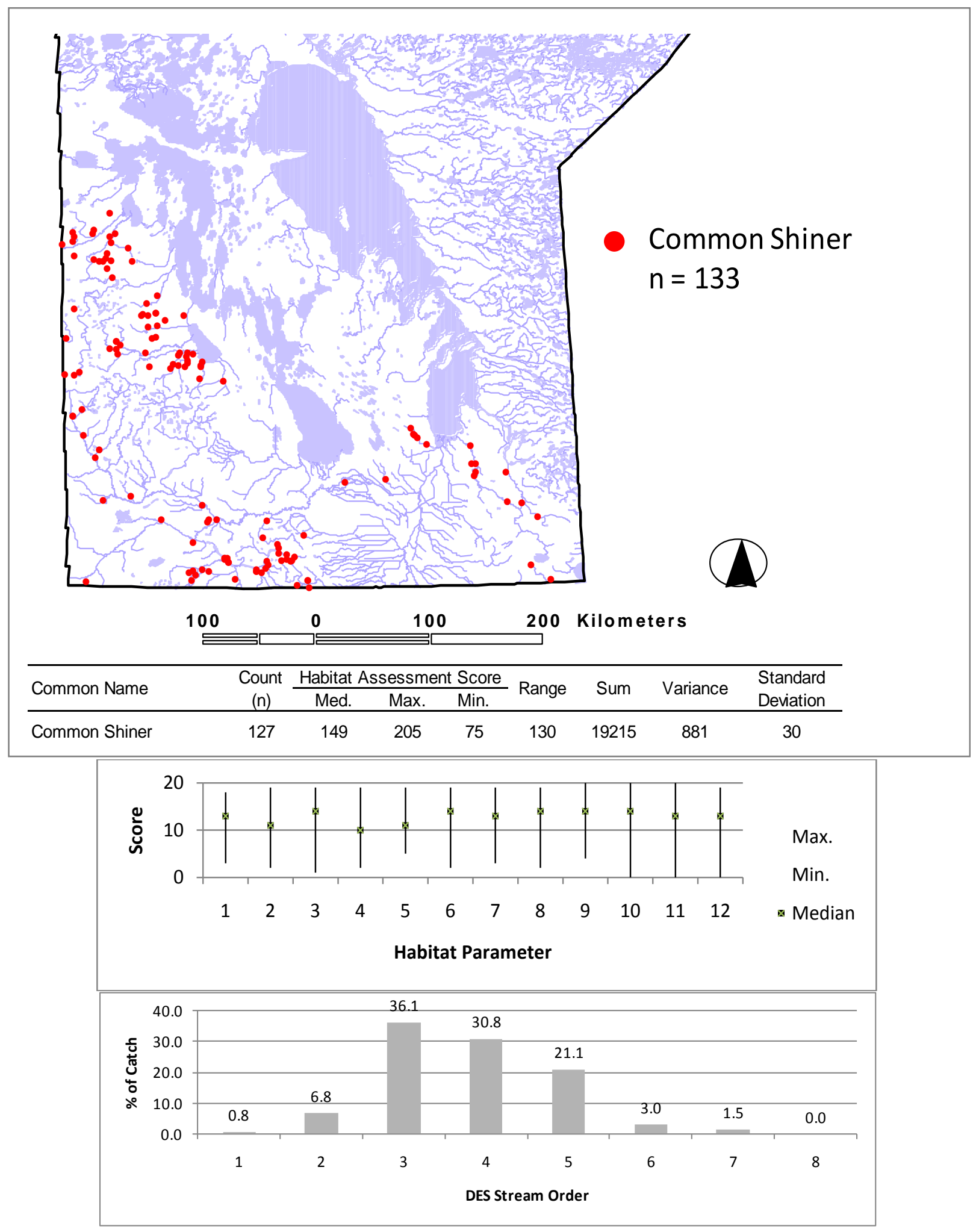

Figure 39: A summary of Common Shiner collections showing collection sites; maximum, minimum and median individual parameter and total Habitat Assessment Scores; and the percent of catch by DES stream order. 


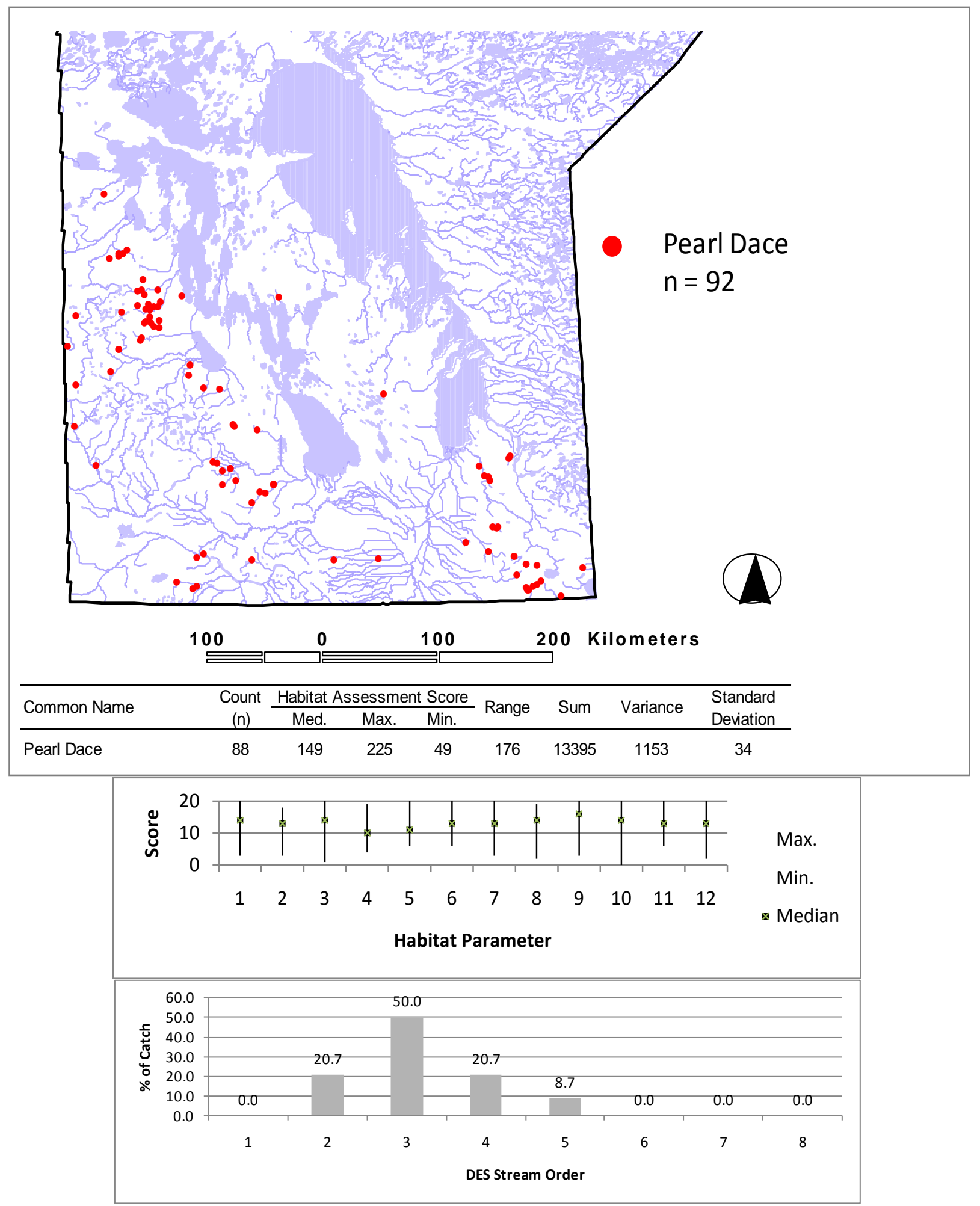

Figure 40: A summary of Pearl Dace collections showing collection sites; maximum, minimum and median individual parameter and total Habitat Assessment Scores; and the percent of catch by DES stream order. 

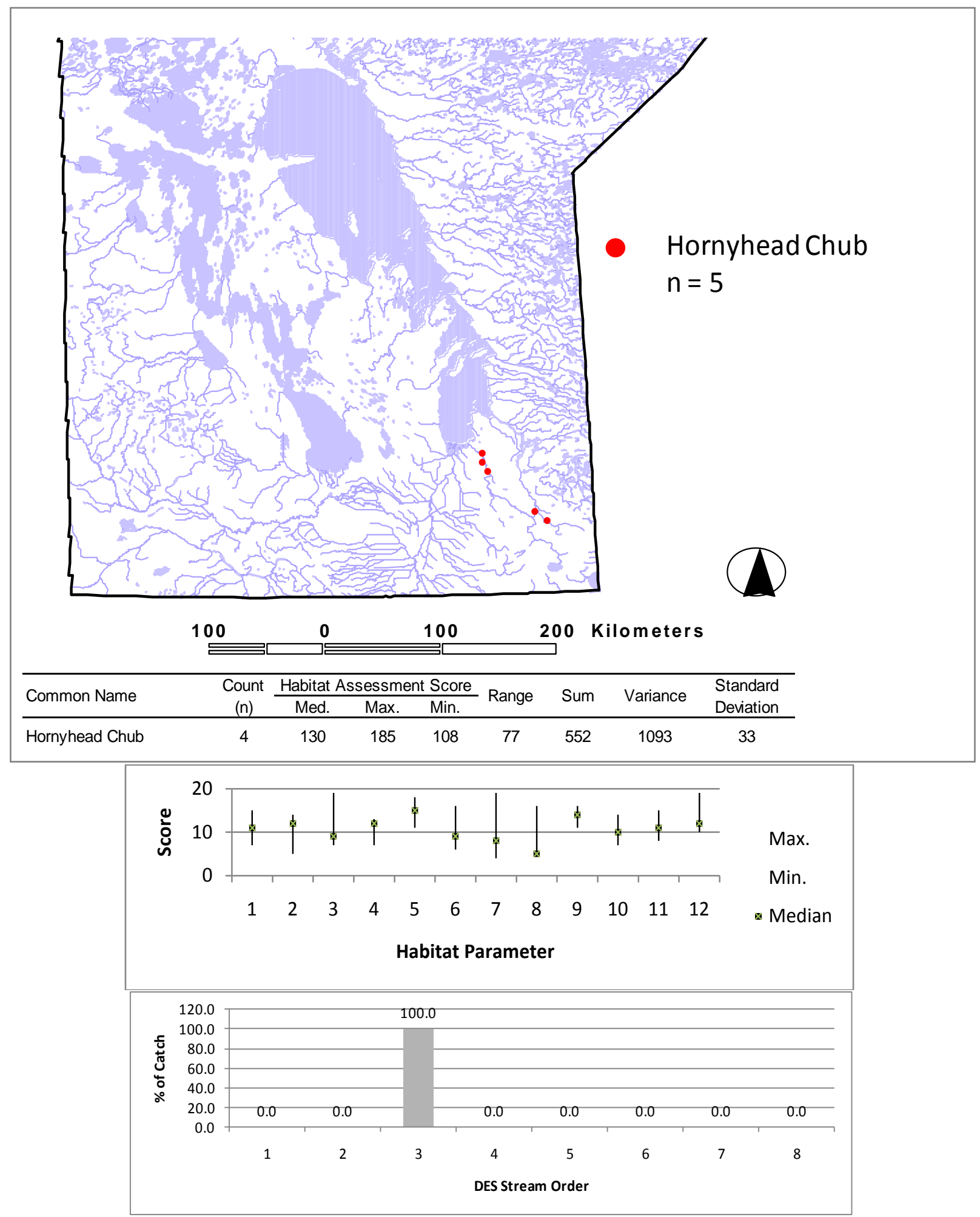

Figure 41: A summary of Hornyhead Chub collections showing collection sites; maximum, minimum and median individual parameter and total Habitat Assessment Scores; and the percent of catch by DES stream order. 


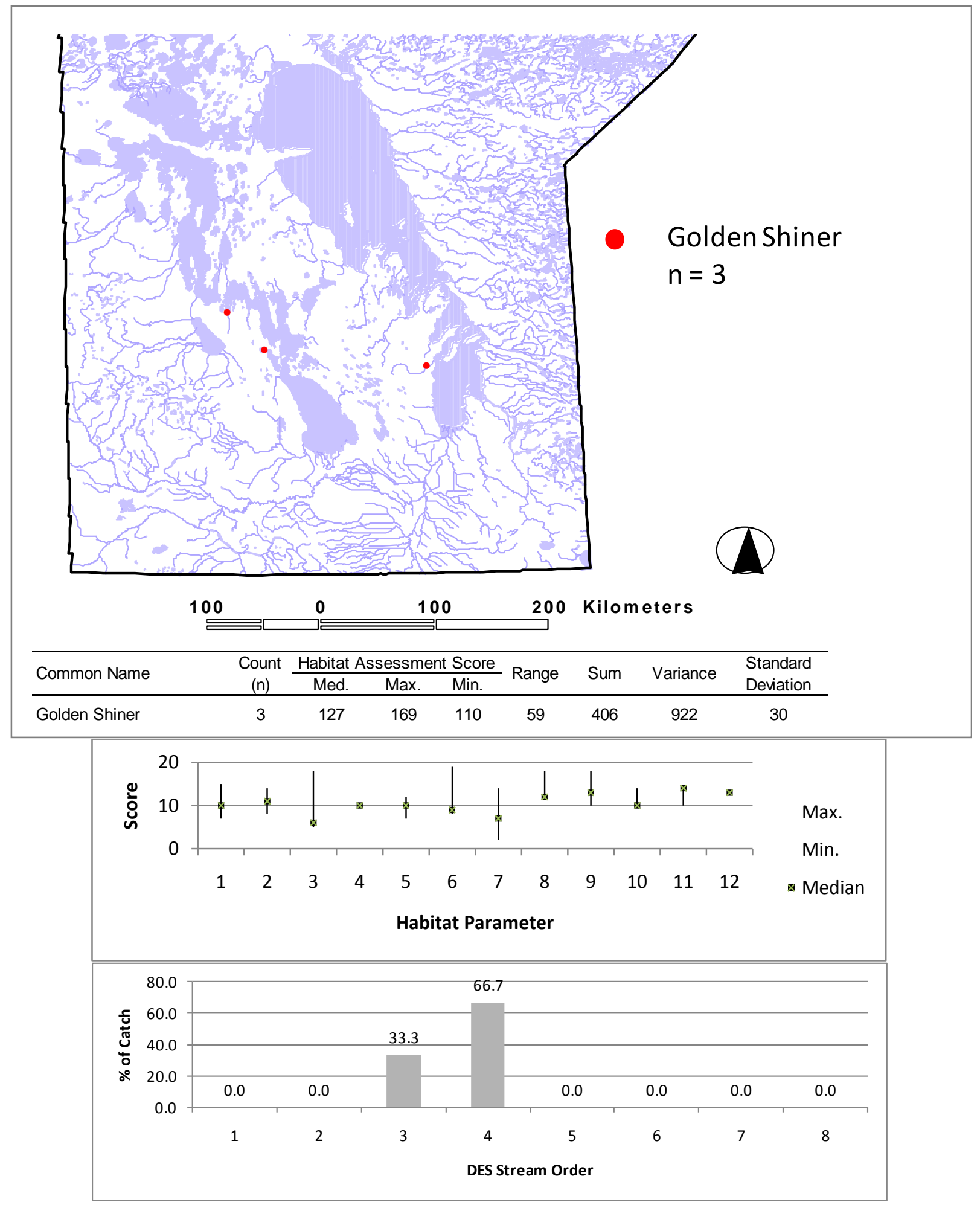

Figure 42: A summary of Golden Shiner collections showing collection sites; maximum, minimum and median individual parameter and total Habitat Assessment Scores; and the percent of catch by DES stream order. 

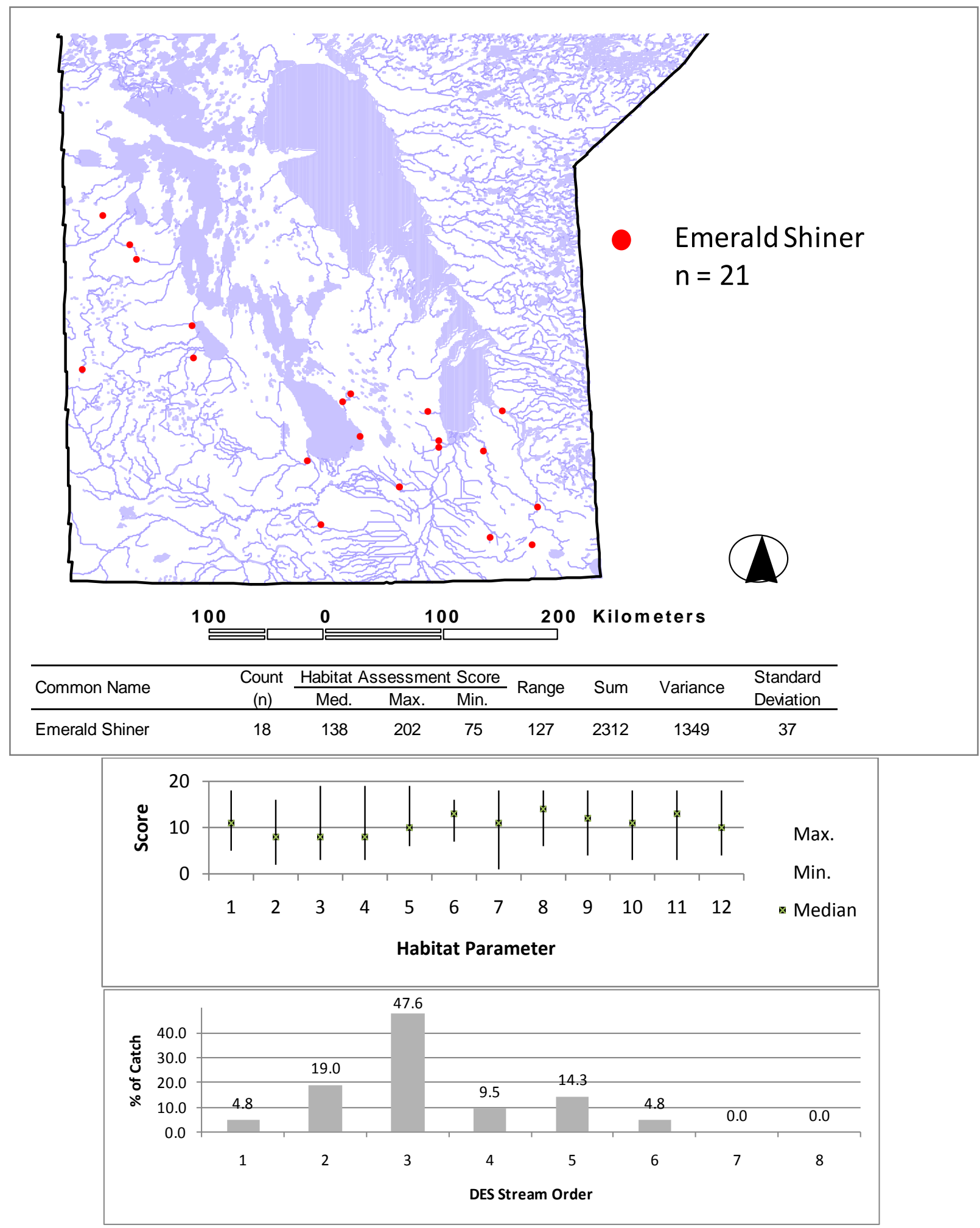

Figure 43: A summary of Emerald Shiner collections showing collection sites; maximum, minimum and median individual parameter and total Habitat Assessment Scores; and the percent of catch by DES stream order. 

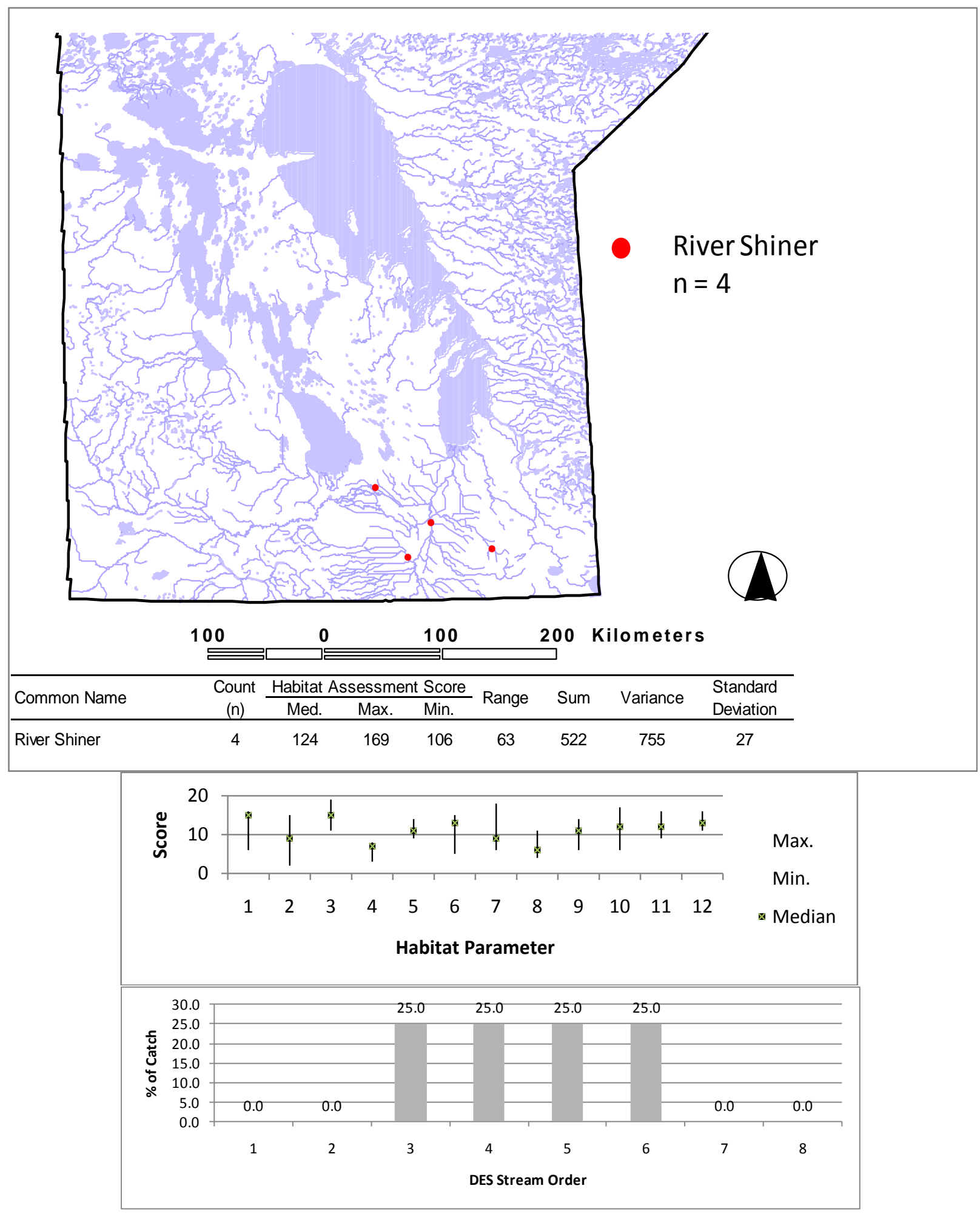

Figure 44: A summary of River Shiner collections showing collection sites; maximum, minimum and median individual parameter and total Habitat Assessment Scores; and the percent of catch by DES stream order. 

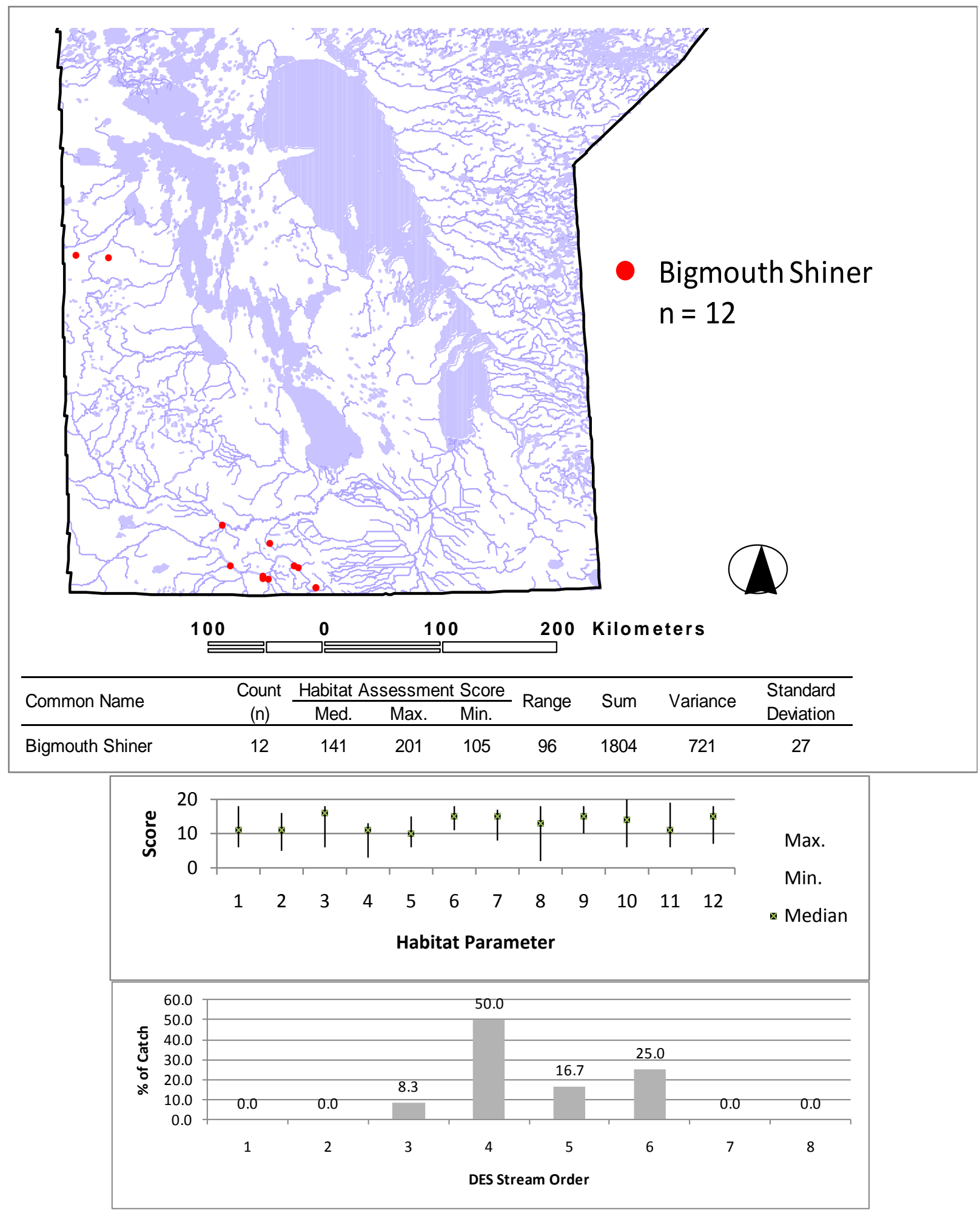

Figure 45: A summary of Bigmouth Shiner collections showing collection sites; maximum, minimum and median individual parameter and total Habitat Assessment Scores; and the percent of catch by DES stream order. 

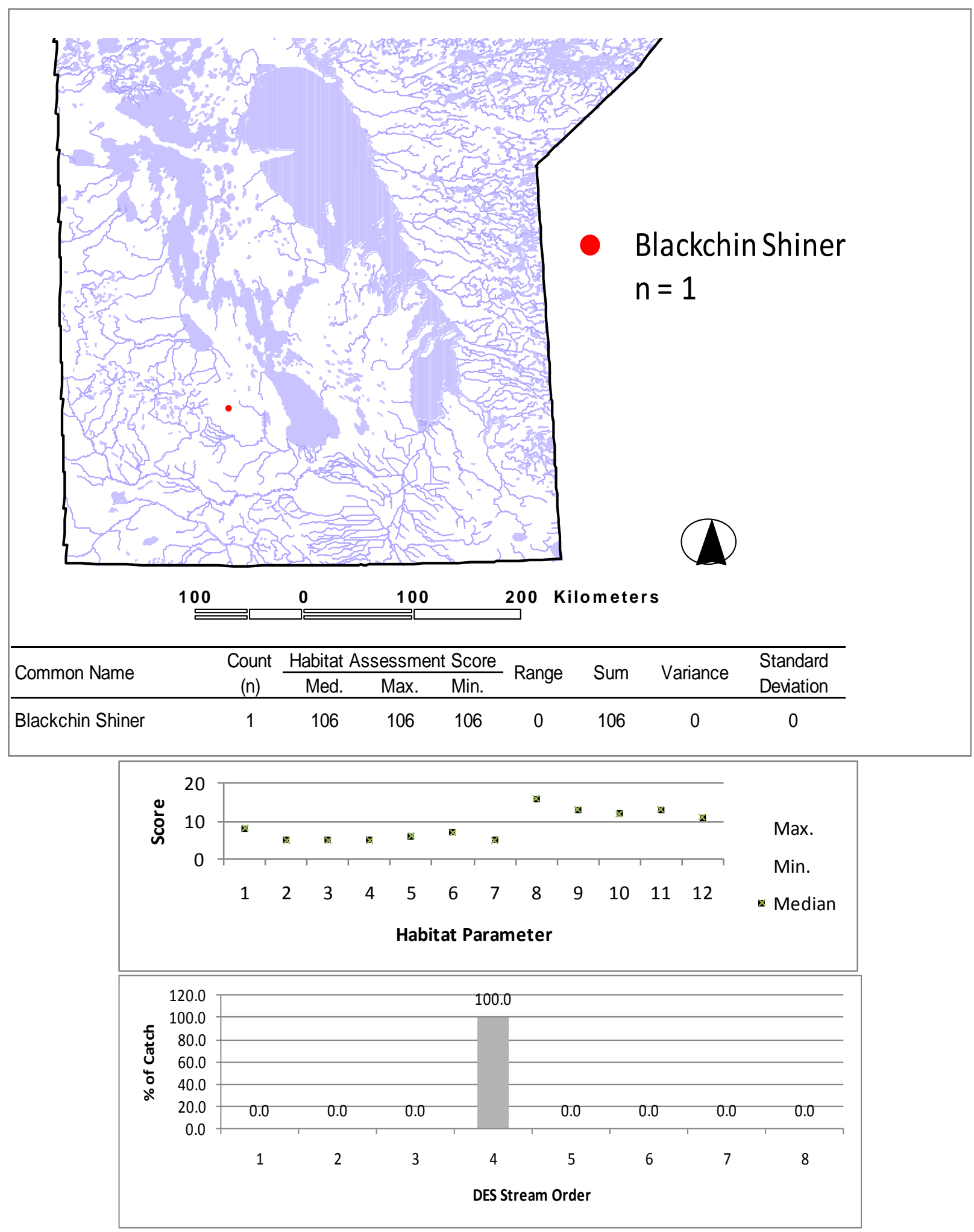

Figure 46: A summary of Blackchin Shiner collections showing collection sites; maximum, minimum and median individual parameter and total Habitat Assessment Scores; and the percent of catch by DES stream order. 

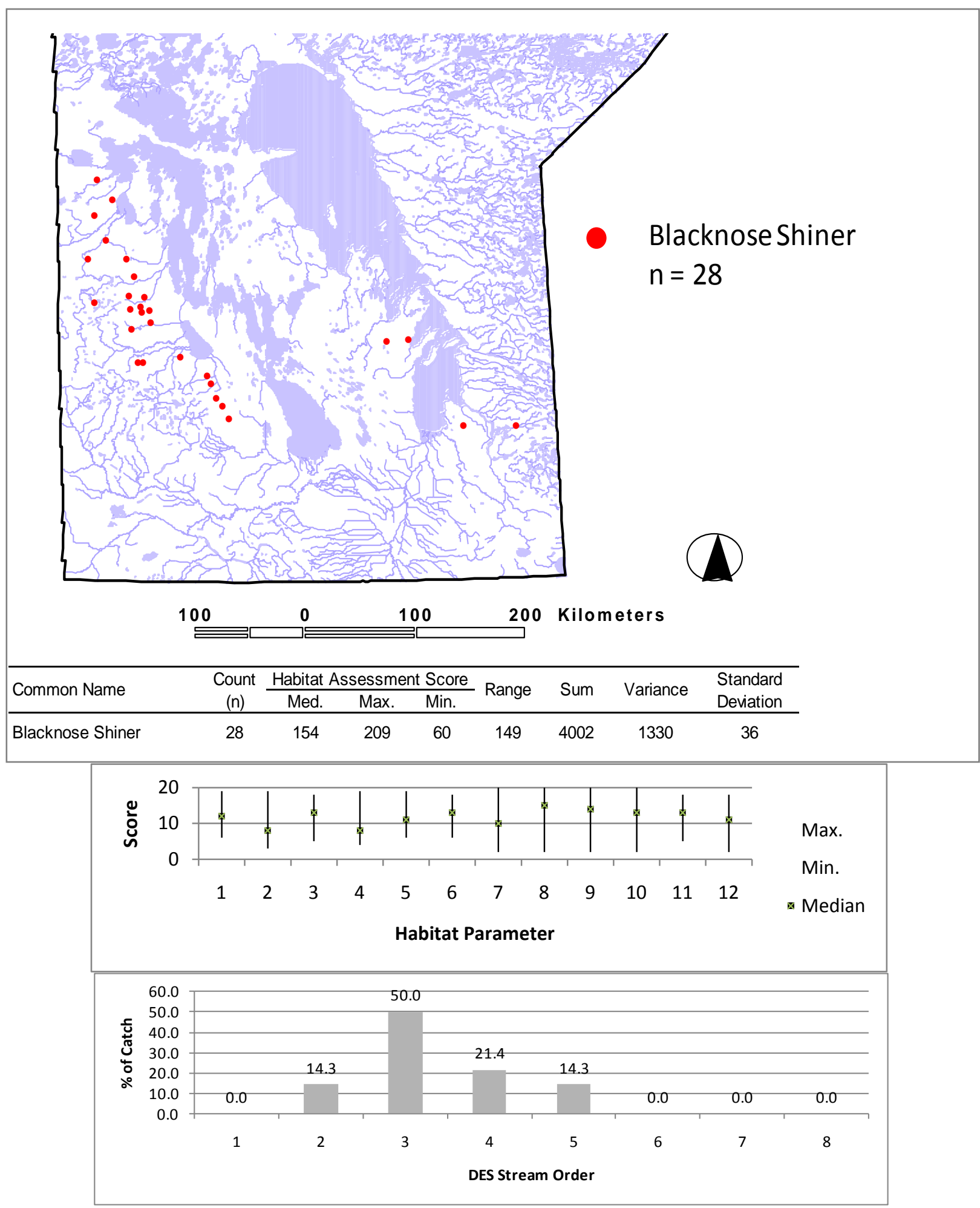

Figure 47: A summary of Blacknose Shiner collections showing collection sites; maximum, minimum and median individual parameter and total Habitat Assessment Scores; and the percent of catch by DES stream order. 


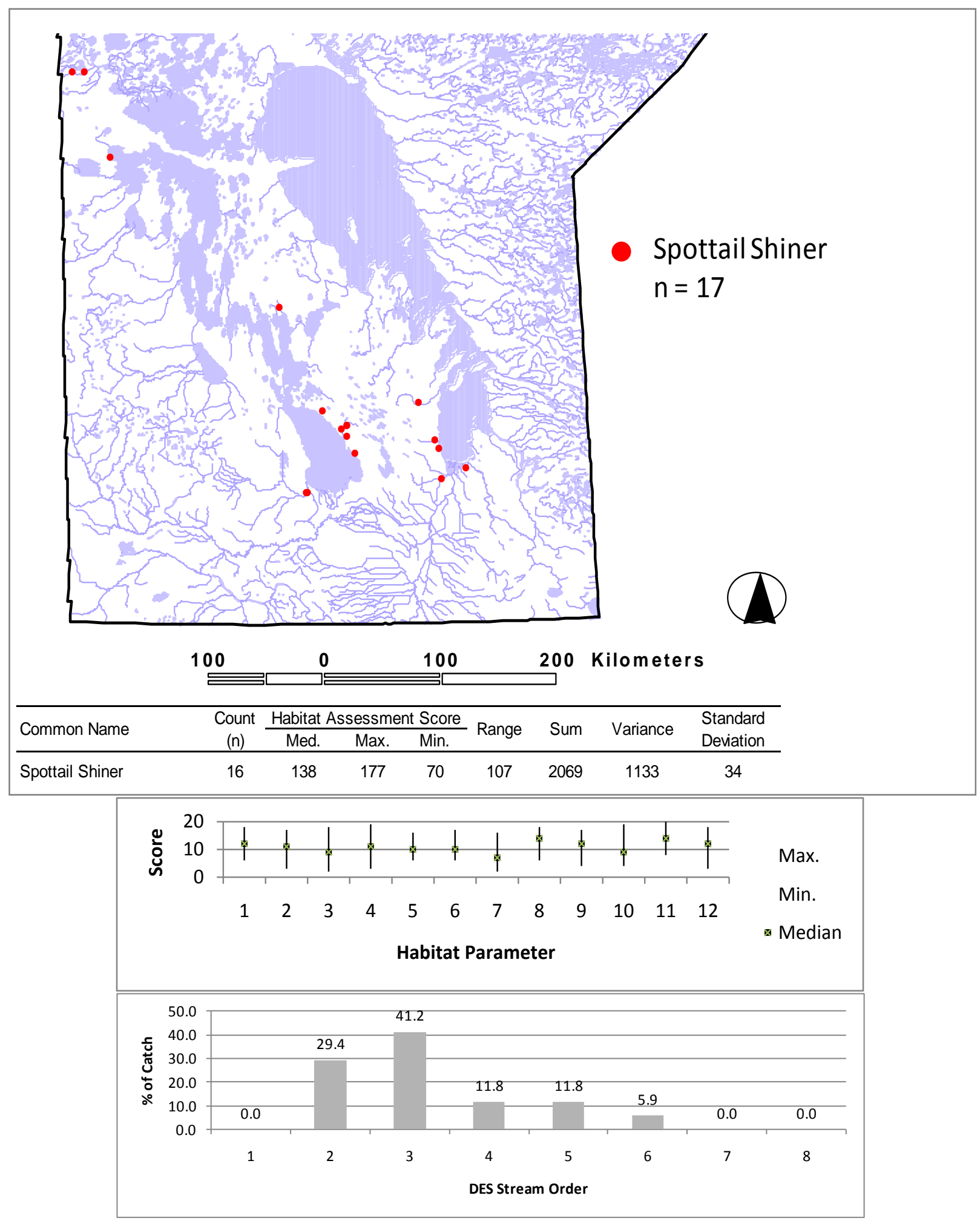

Figure 48: A summary of Spottail Shiner collections showing collection sites; maximum, minimum and median individual parameter and total Habitat Assessment Scores; and the percent of catch by DES stream order. 


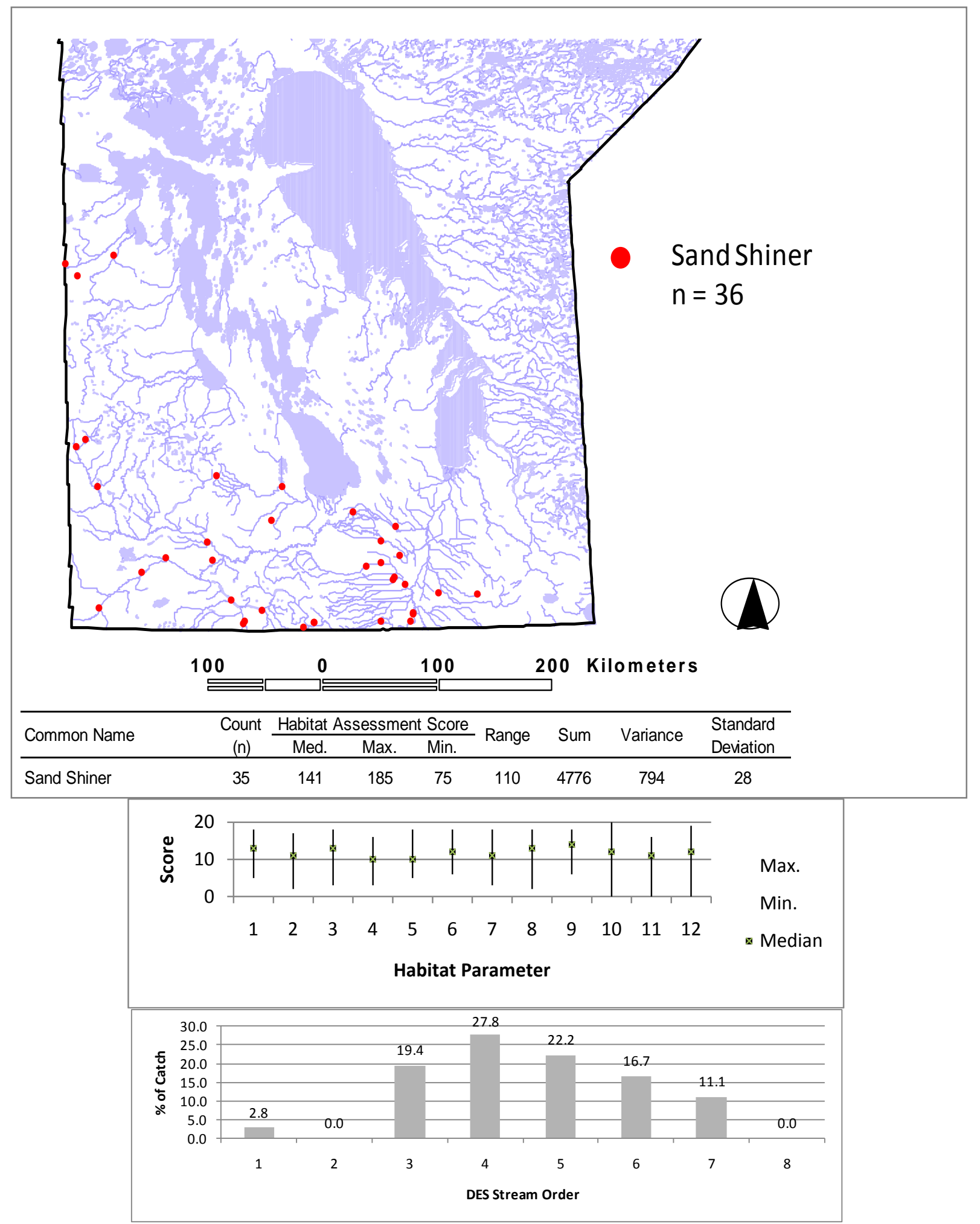

Figure 49: A summary of Sand Shiner collections showing collection sites; maximum, minimum and median individual parameter and total Habitat Assessment Scores; and the percent of catch by DES stream order. 


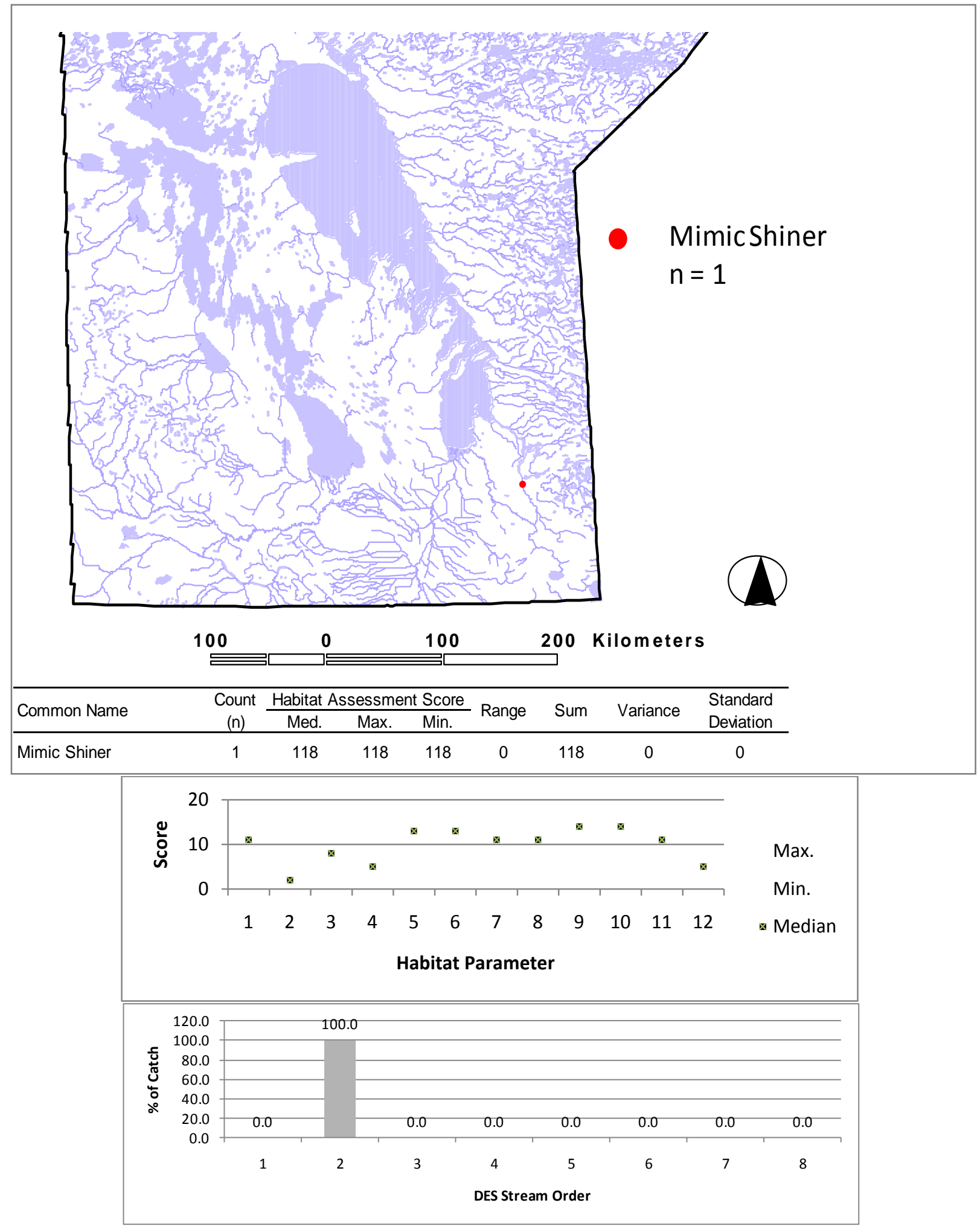

Figure 50: A summary of Mimic Shiner collections showing collection sites; maximum, minimum and median individual parameter and total Habitat Assessment Scores; and the percent of catch by DES stream order. 

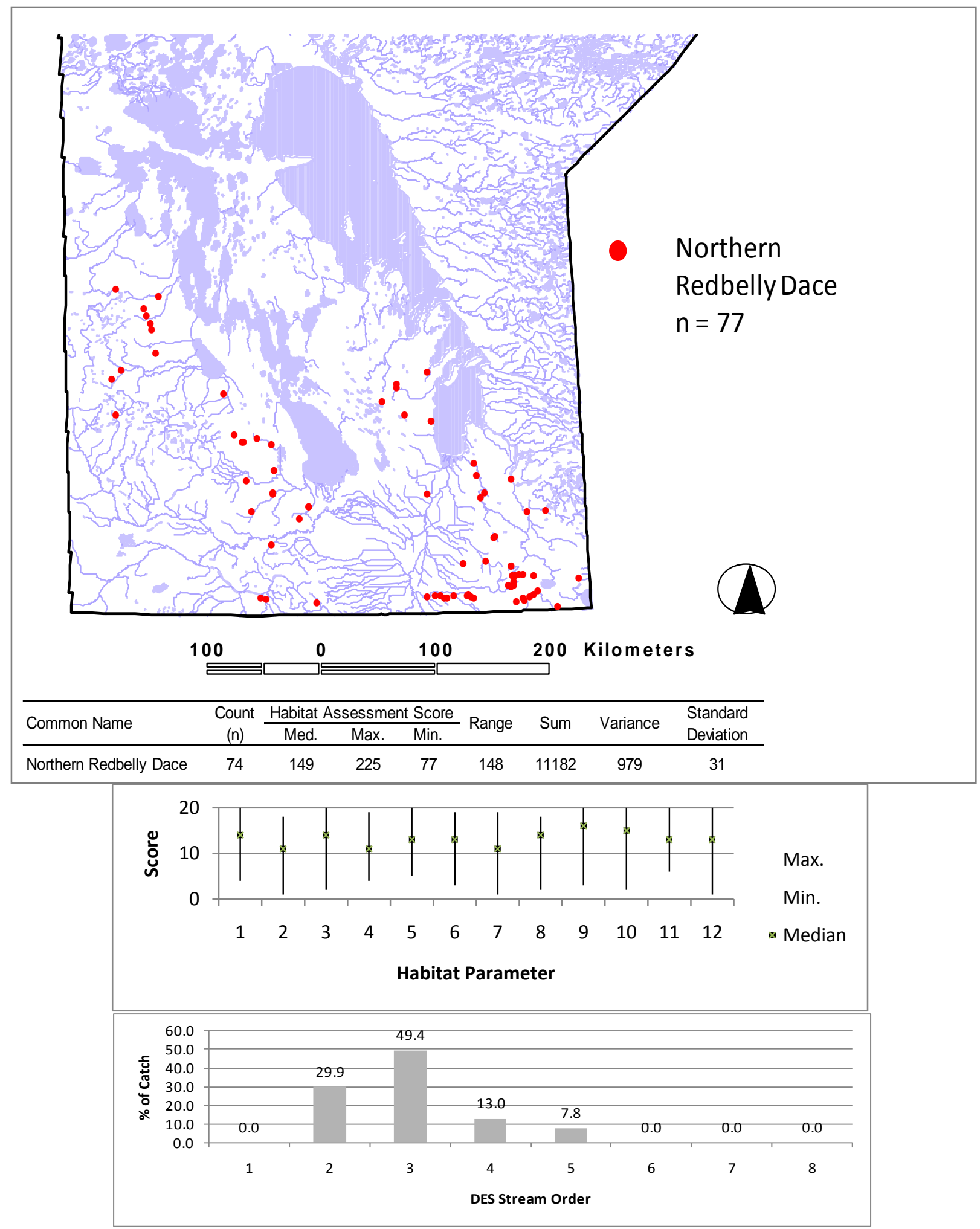

Figure 51: A summary of Northern Redbelly Dace collections showing collection sites; maximum, minimum and median individual parameter and total Habitat Assessment Scores; and the percent of catch by DES stream order. 

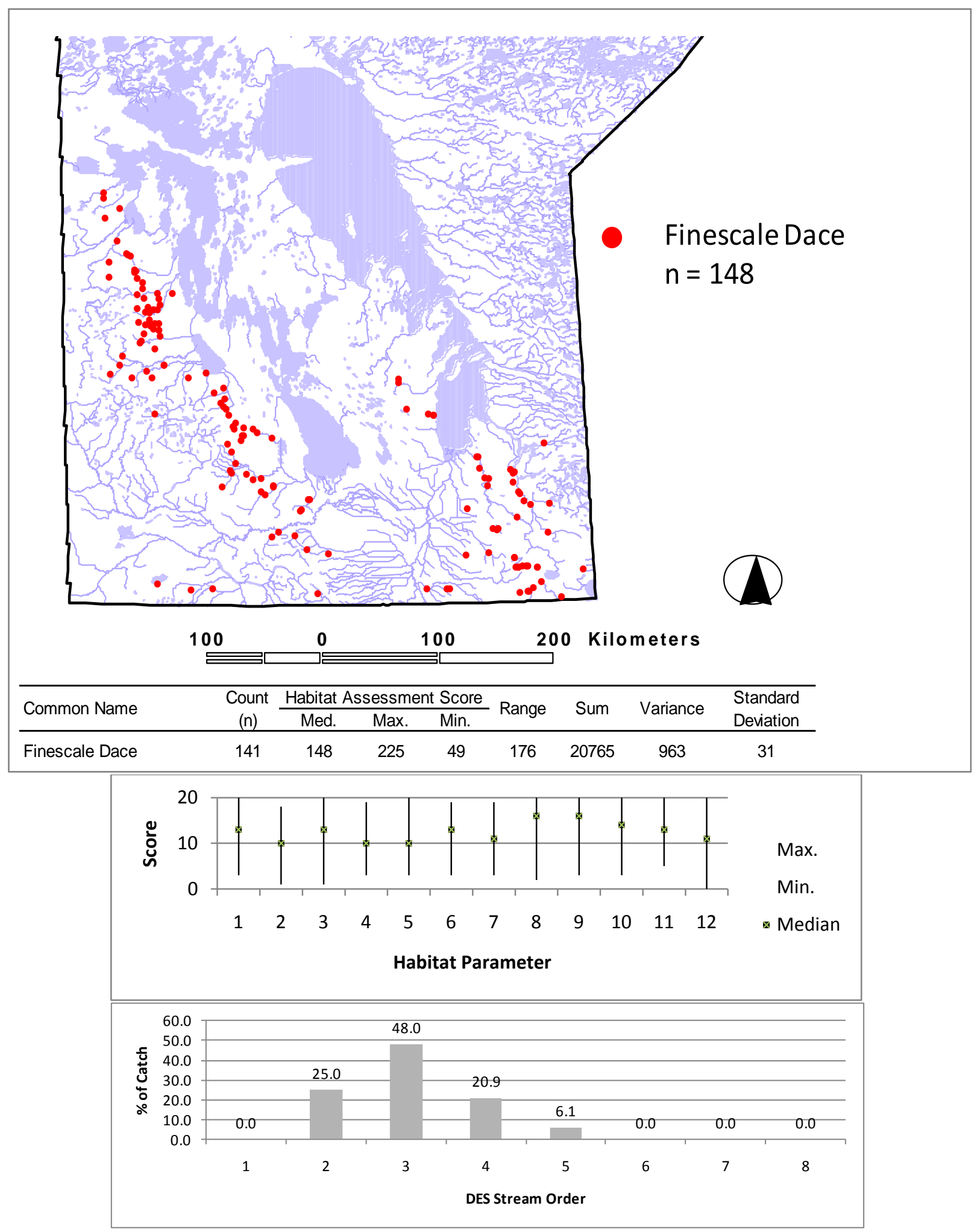

Figure 52: A summary of Finescale Dace collections showing collection sites; maximum, minimum and median individual parameter and total Habitat Assessment Scores; and the percent of catch by DES stream order. 


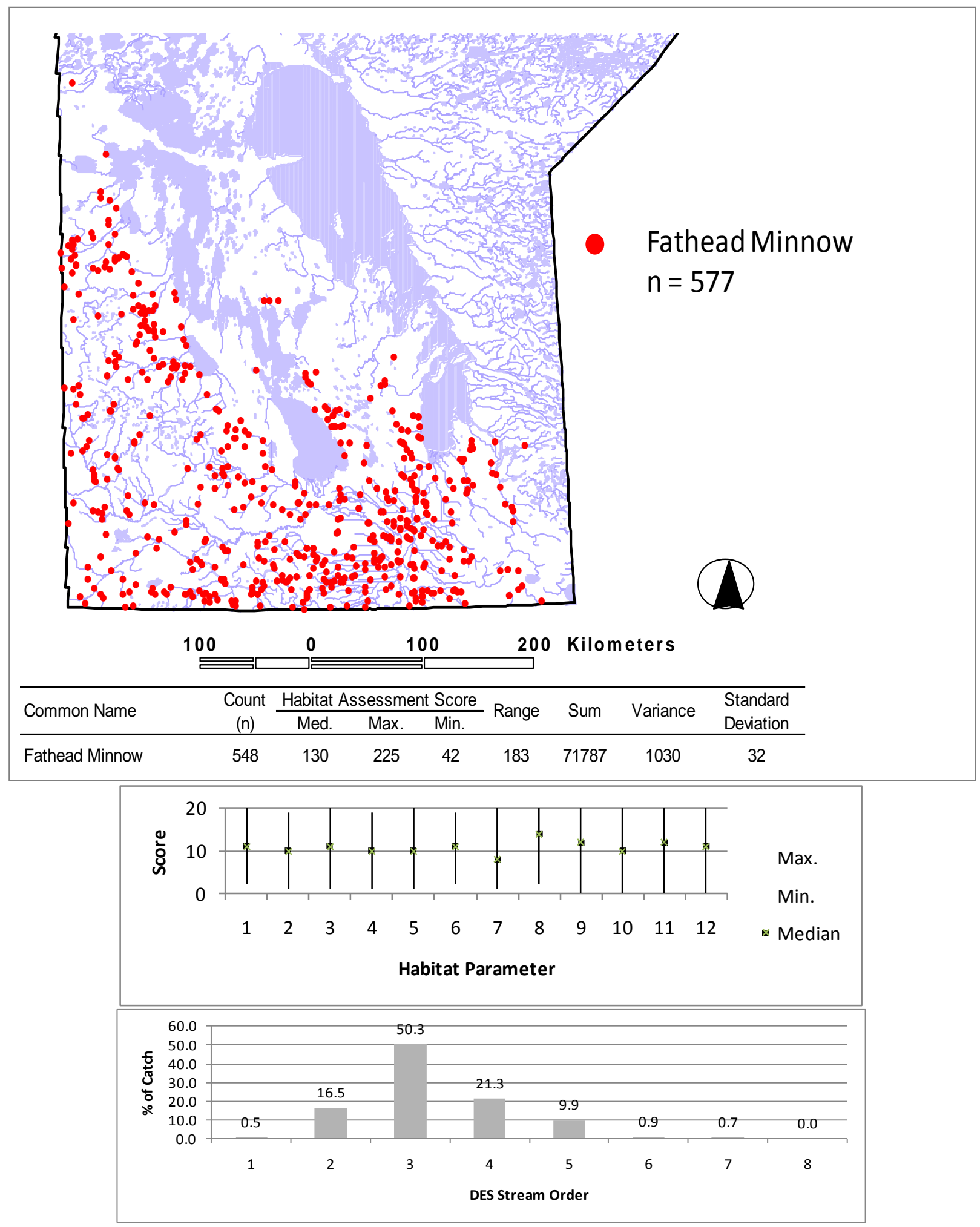

Figure 53: A summary of Fathead Minnow collections showing collection sites; maximum, minimum and median individual parameter and total Habitat Assessment Scores; and the percent of catch by DES stream order. 

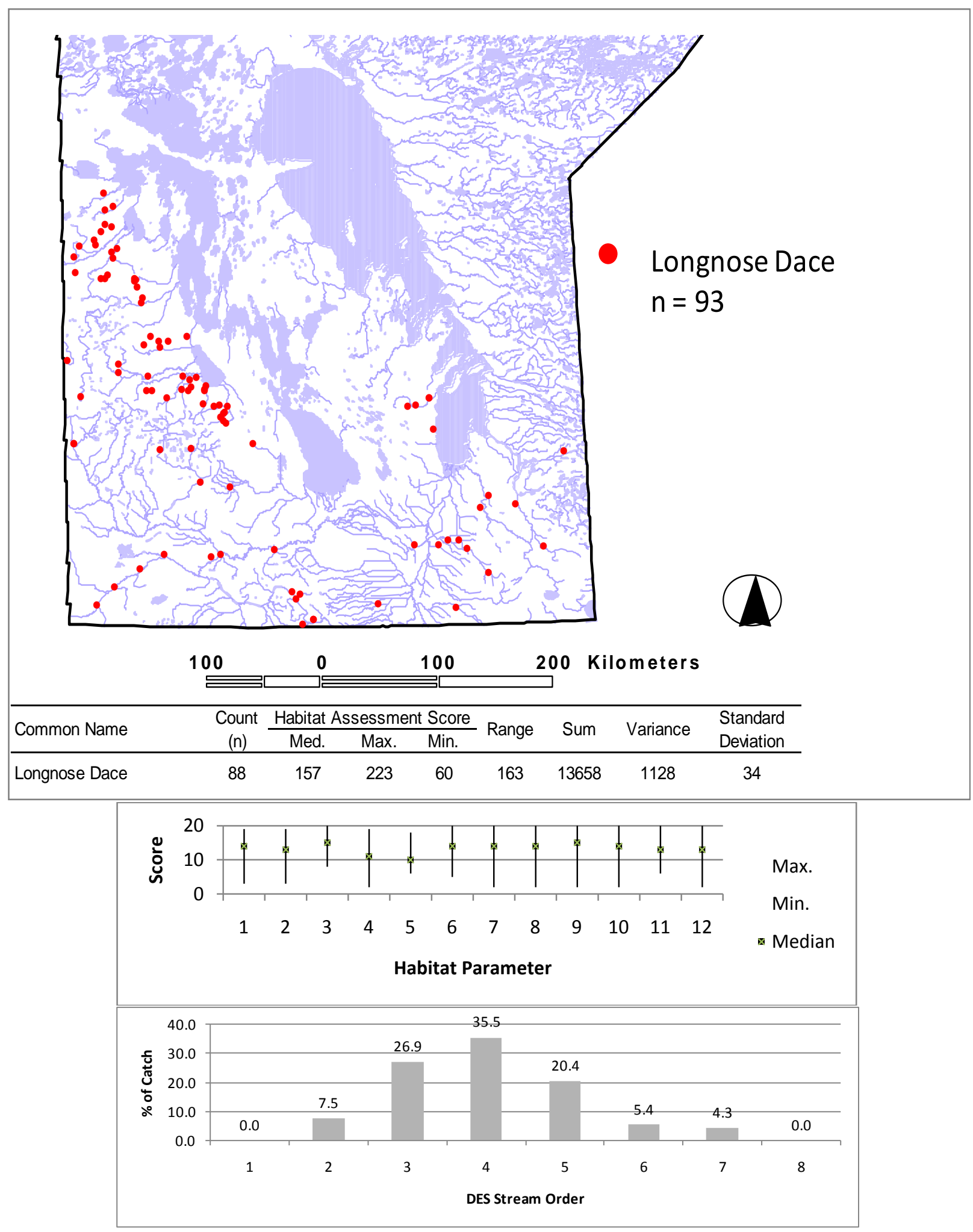

Figure 54: A summary of Longnose Dace collections showing collection sites; maximum, minimum and median individual parameter and total Habitat Assessment Scores; and the percent of catch by DES stream order. 

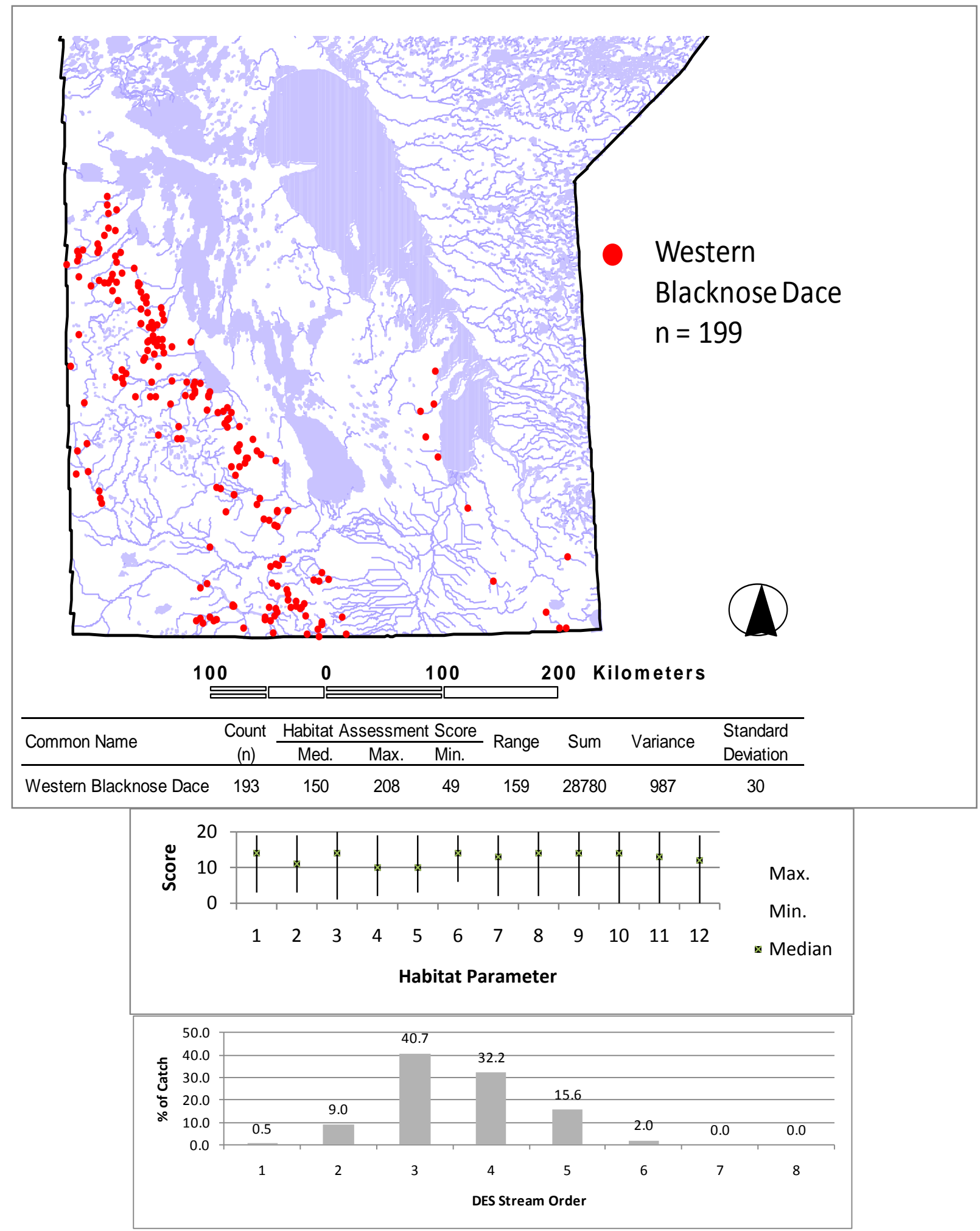

Figure 55: A summary of Western Blacknose Dace collections showing collection sites; maximum, minimum and median individual parameter and total Habitat Assessment Scores; and the percent of catch by DES stream order. 


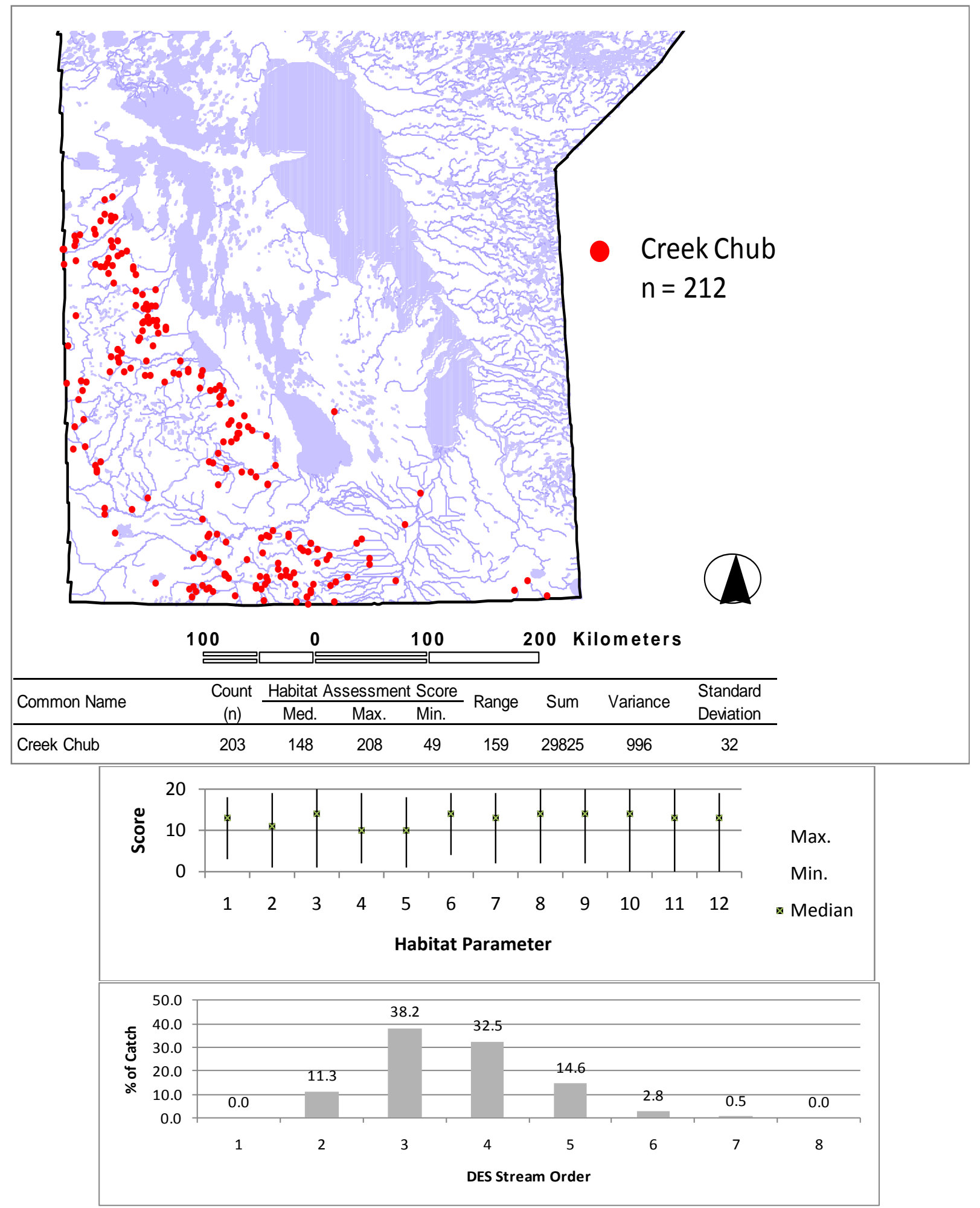

Figure 56: A summary of Creek Chub collections showing collection sites; maximum, minimum and median individual parameter and total Habitat Assessment Scores; and the percent of catch by DES stream order. 

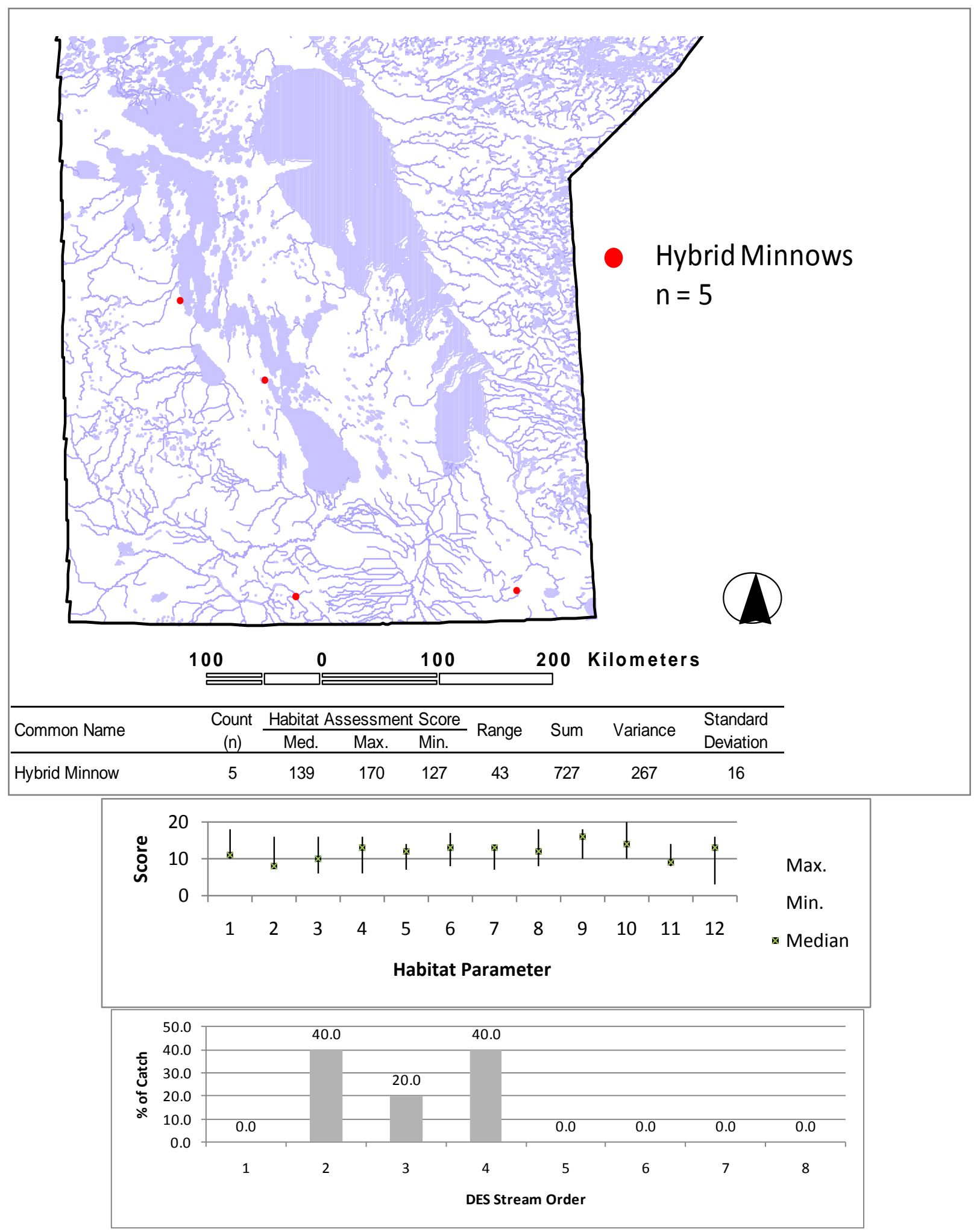

Figure 57: A summary of hybrid minnow (Cyprinidae) collections showing collection sites; maximum, minimum and median individual parameter and total Habitat Assessment Scores; and the percent of catch by DES stream order. 

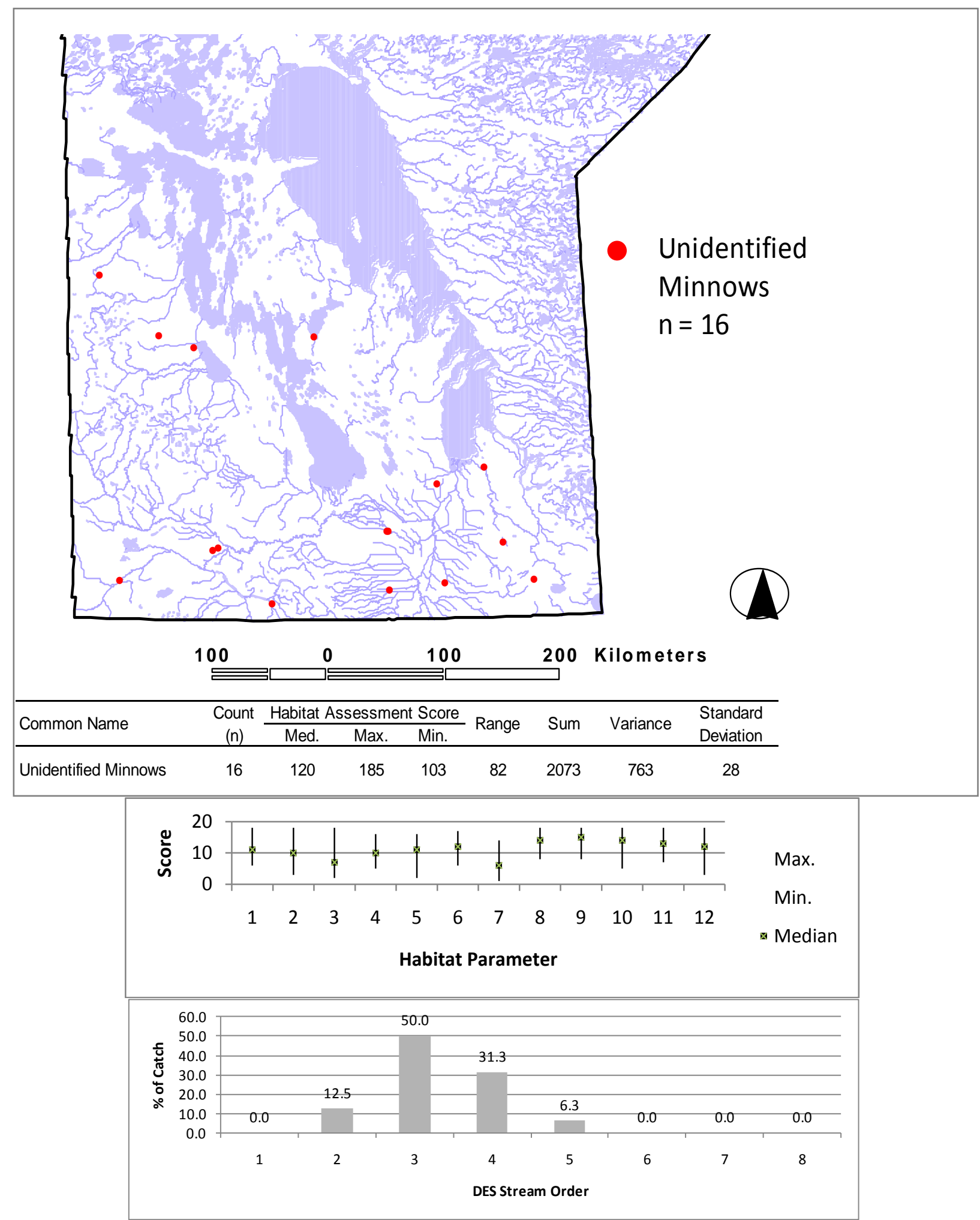

Figure 48: A summary of unidentified minnow (Cyprinidae) collections showing collection sites; maximum, minimum and median individual parameter and total Habitat Assessment Scores; and the percent of catch by DES stream order. 


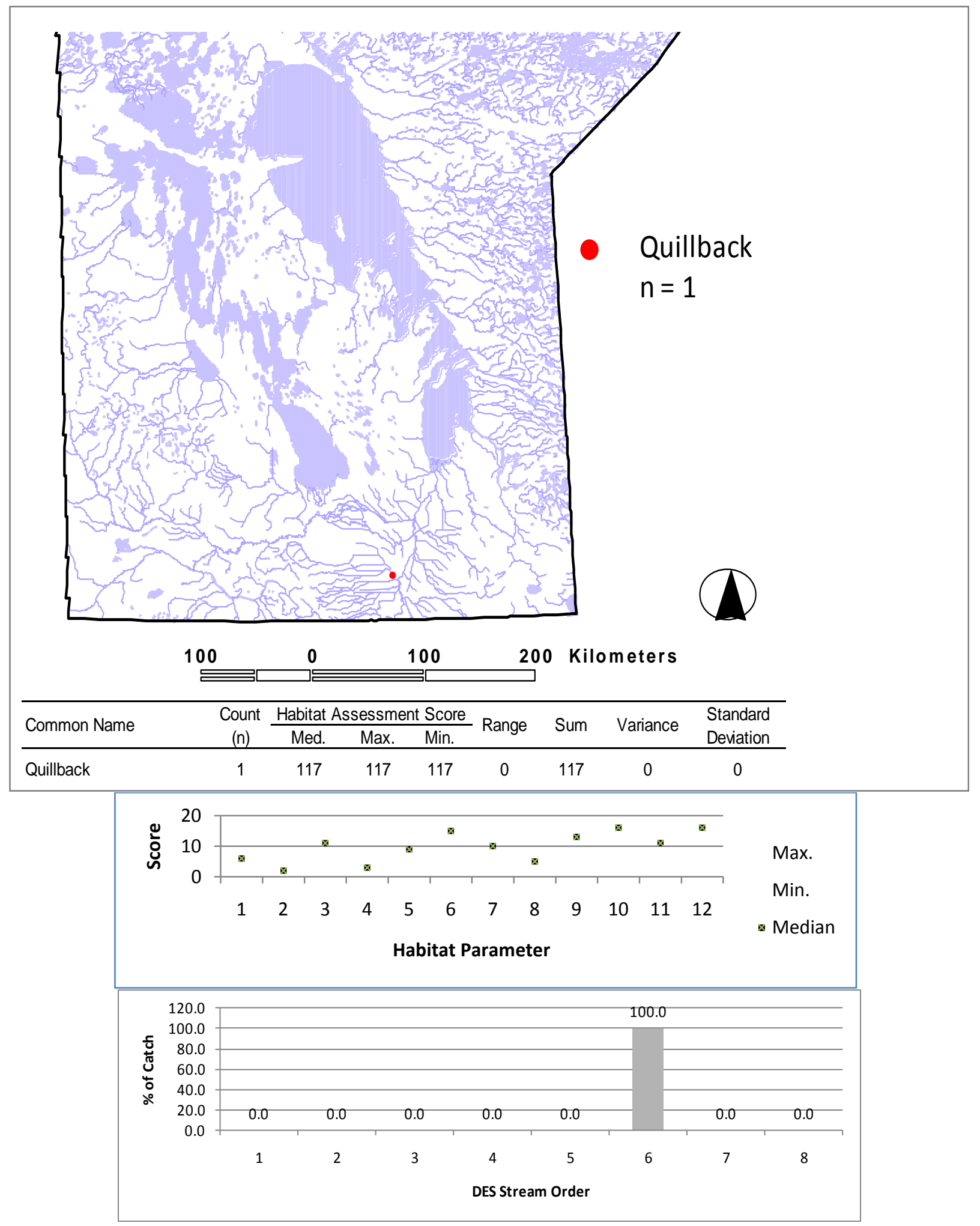

Figure 59: A summary of Quillback collections showing collection sites; maximum, minimum and median individual parameter and total Habitat Assessment Scores; and the percent of catch by DES stream order. 

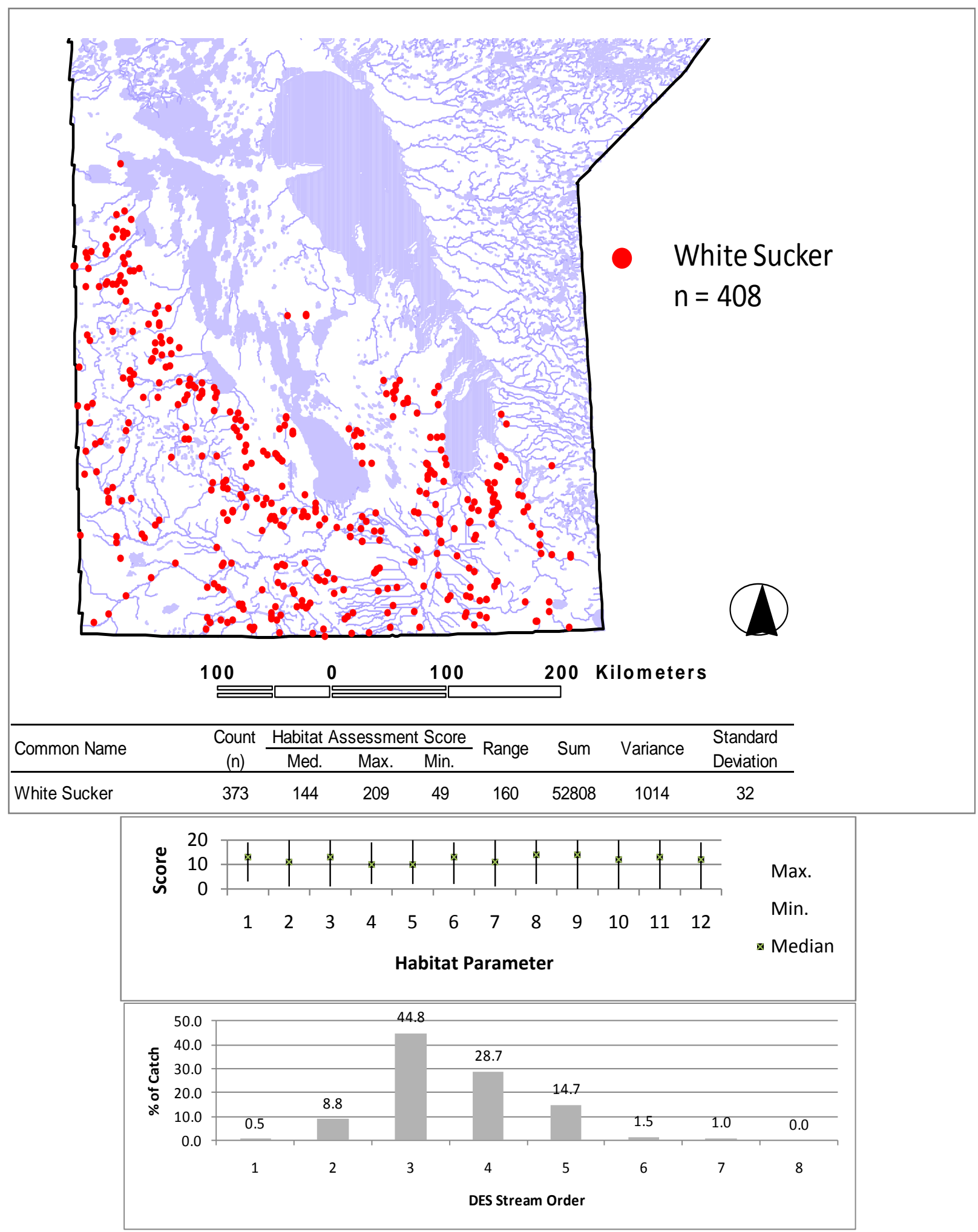

Figure 60: A summary of White Sucker collections showing collection sites; maximum, minimum and median individual parameter and total Habitat Assessment Scores; and the percent of catch by DES stream order. 


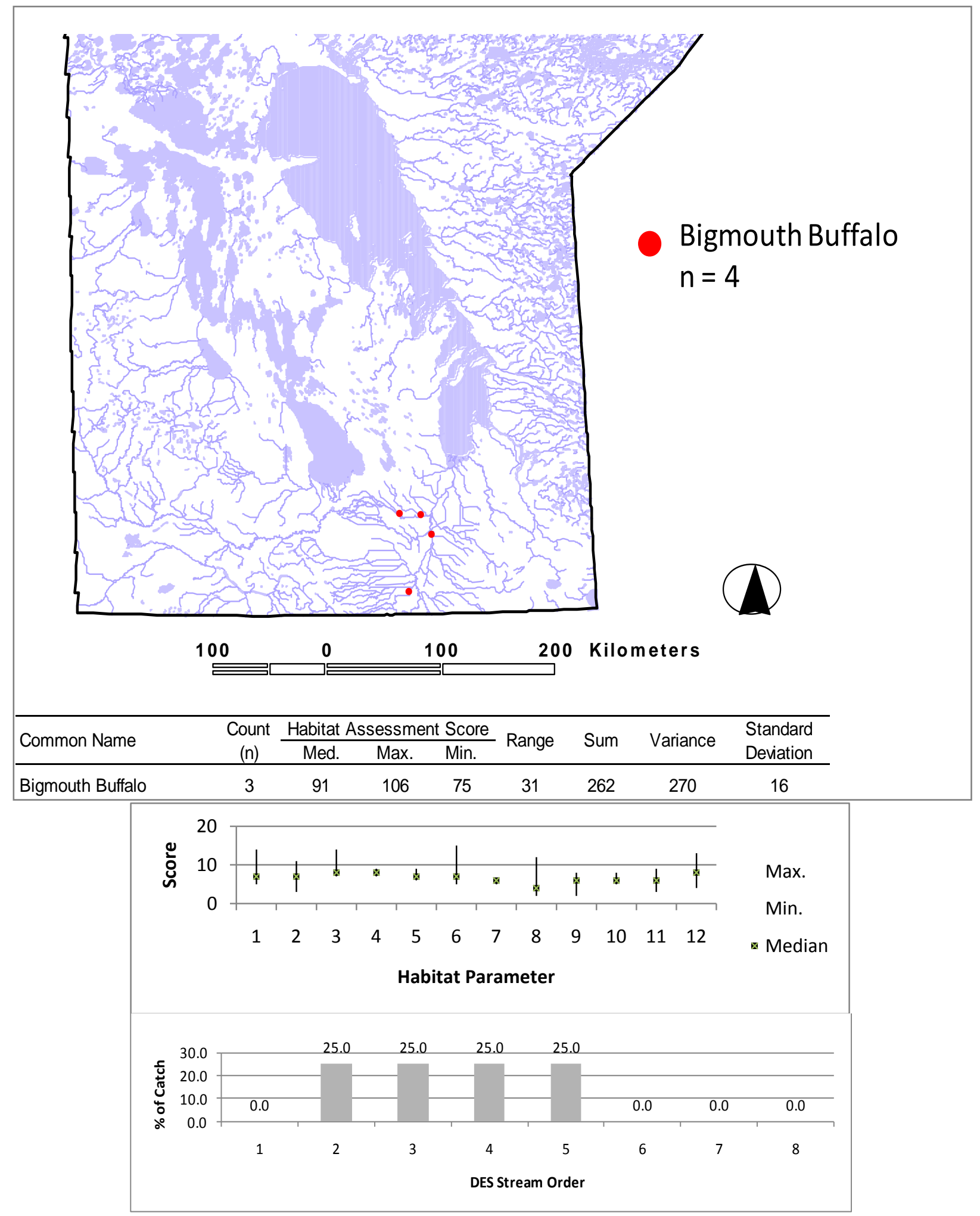

Figure 61: A summary of Bigmouth Buffalo collections showing collection sites; maximum, minimum and median individual parameter and total Habitat Assessment Scores; and the percent of catch by DES stream order. 


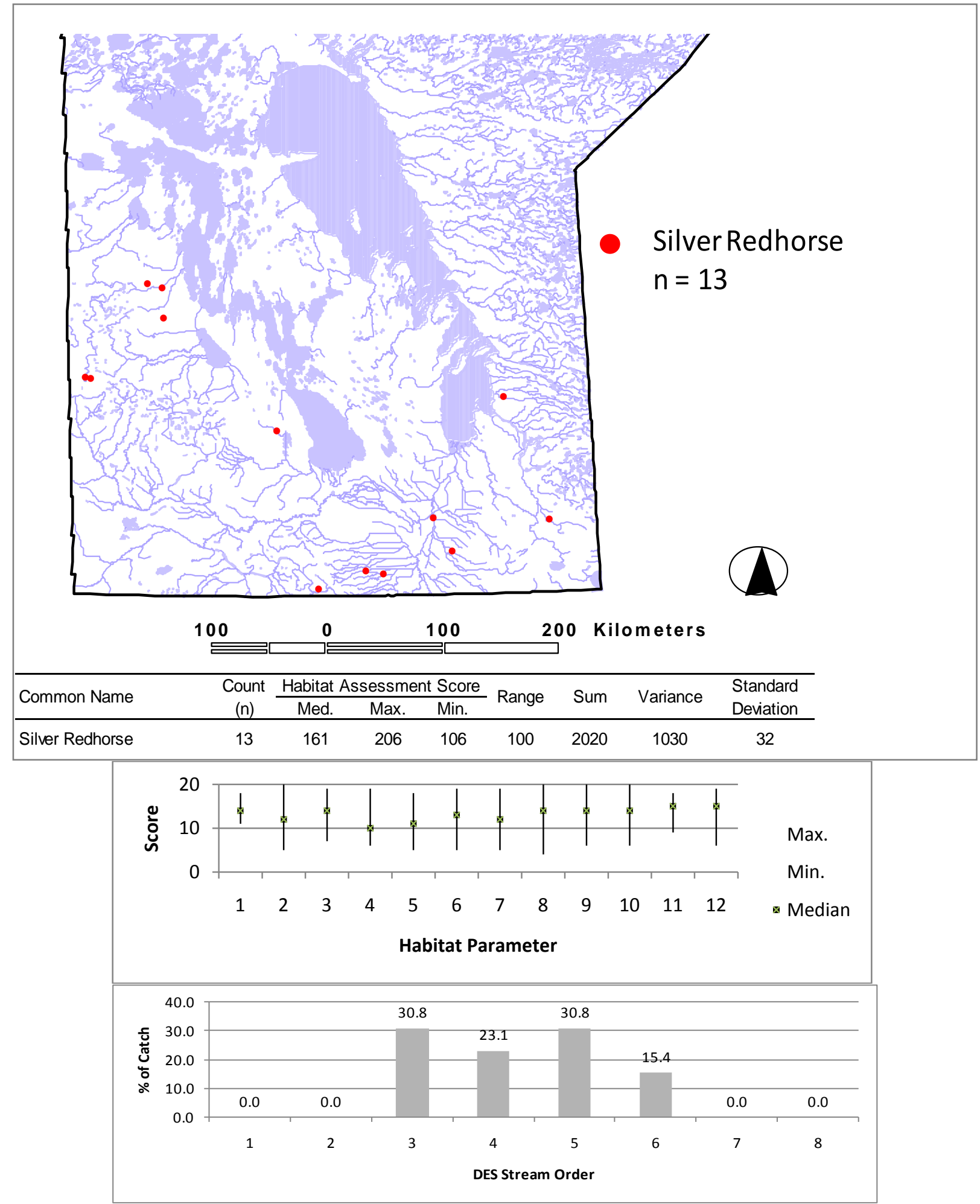

Figure 62: A summary of Silver Redhorse collections showing collection sites; maximum, minimum and median individual parameter and total Habitat Assessment Scores; and the percent of catch by DES stream order. 


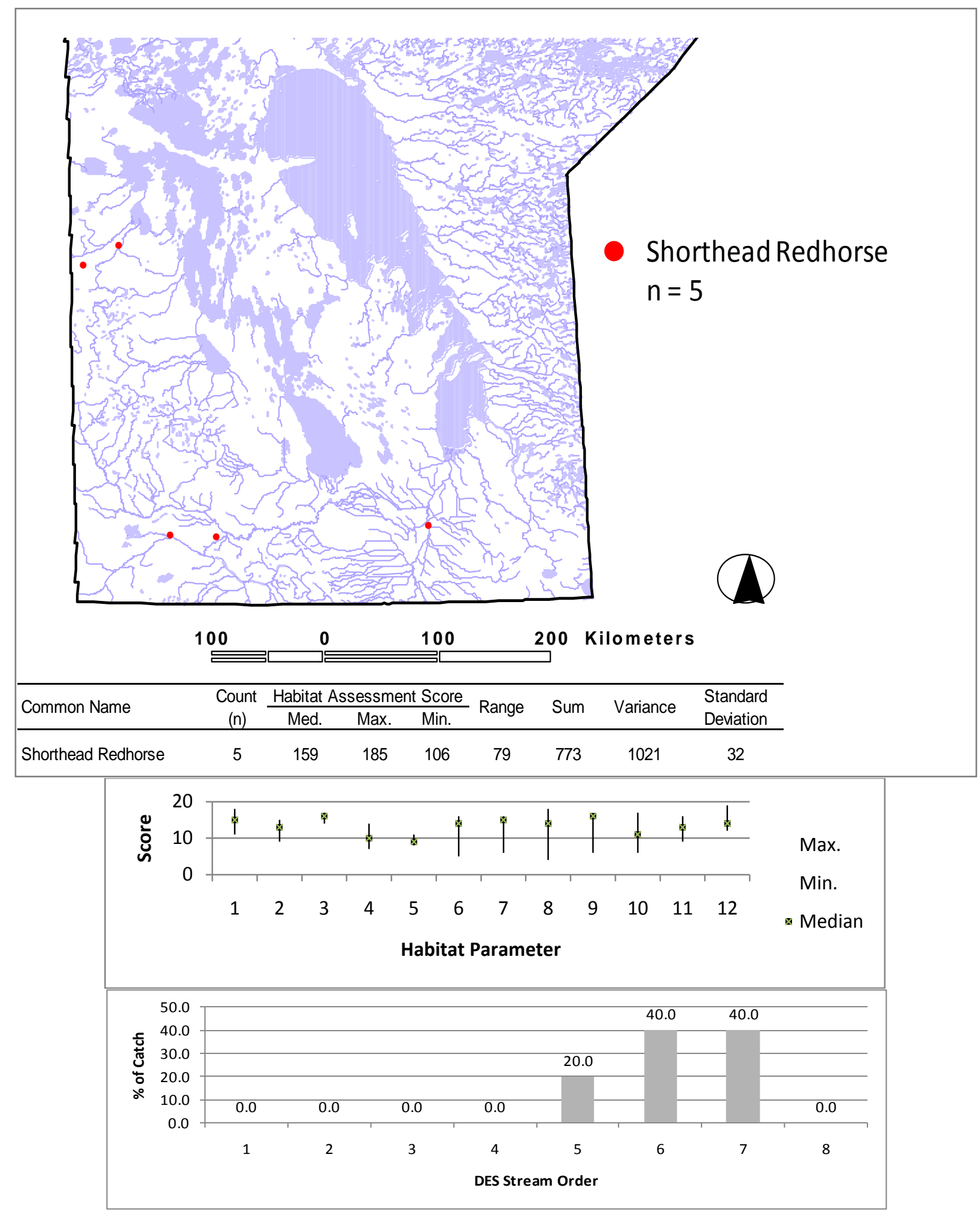

Figure 63: A summary of Shorthead Redhorse collections showing collection sites; maximum, minimum and median individual parameter and total Habitat Assessment Scores; and the percent of catch by DES stream order. 

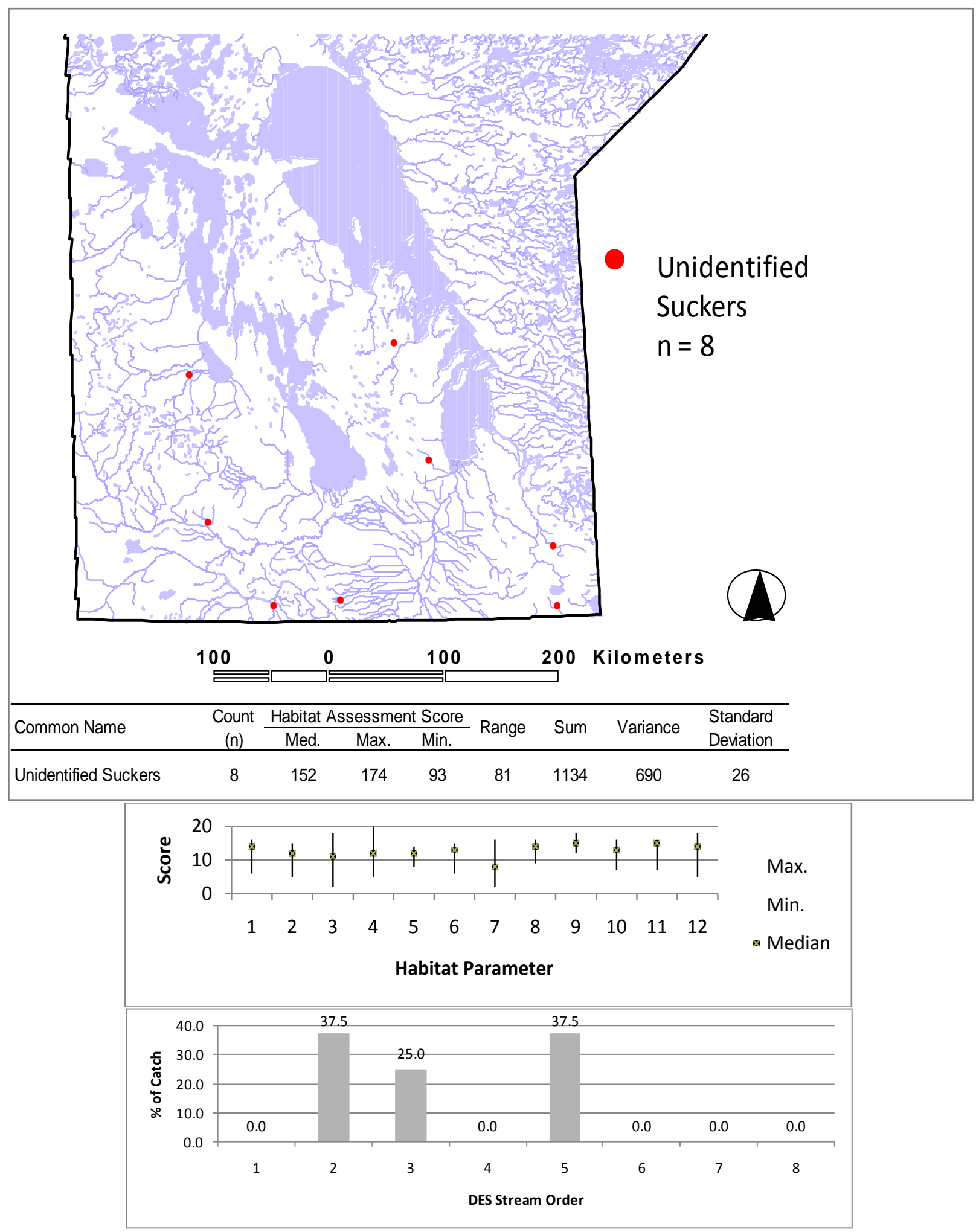

Figure 64: A summary of unidentified sucker collections showing collection sites; maximum, minimum and median individual parameter and total Habitat Assessment Scores; and the percent of catch by DES stream order. 


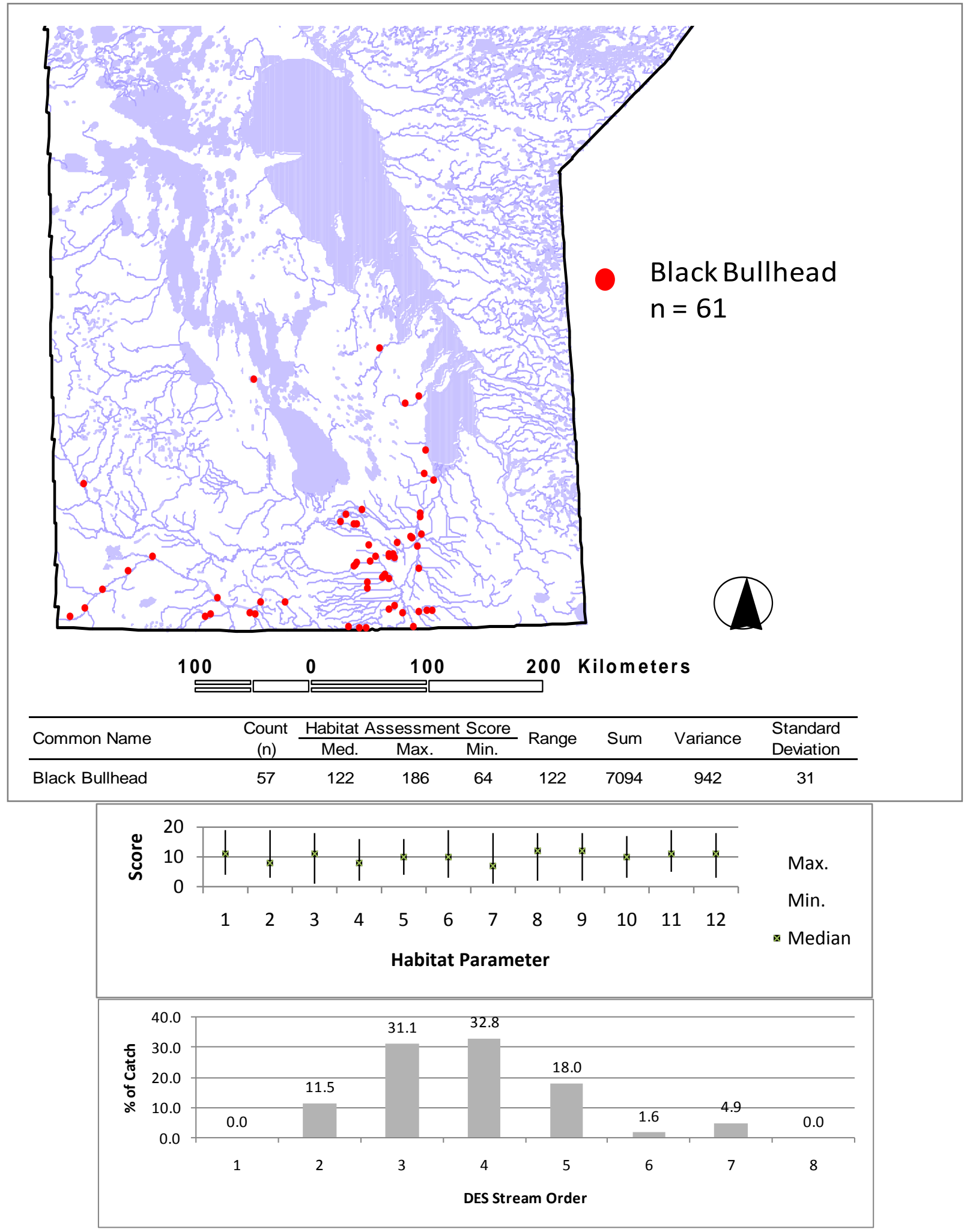

Figure 65: A summary of Black Bullhead collections showing collection sites; maximum, minimum and median individual parameter and total Habitat Assessment Scores; and the percent of catch by DES stream order. 

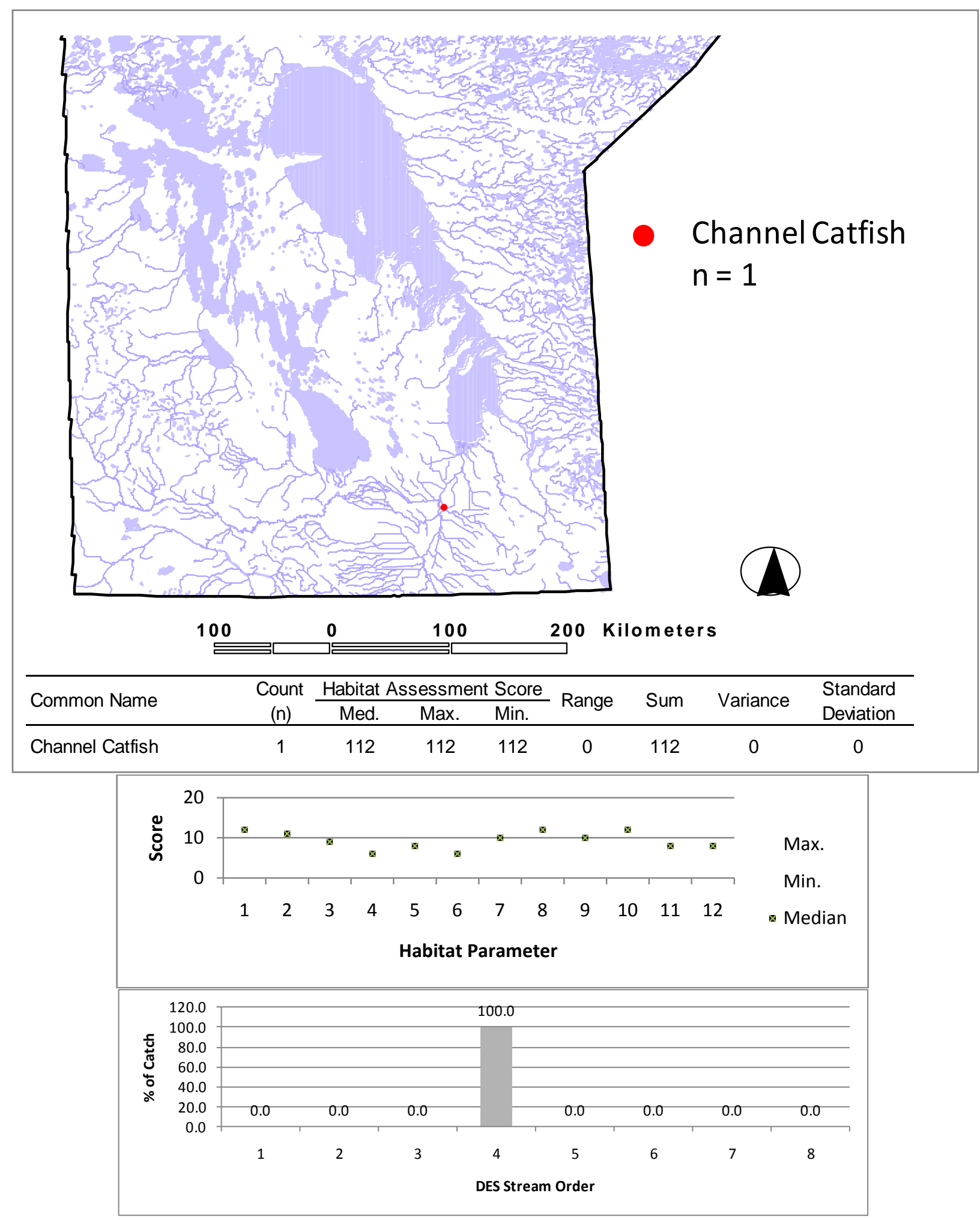

Figure 66: A summary of Channel Catfish collections showing collection sites; maximum, minimum and median individual parameter and total Habitat Assessment Scores; and the percent of catch by DES stream order. 


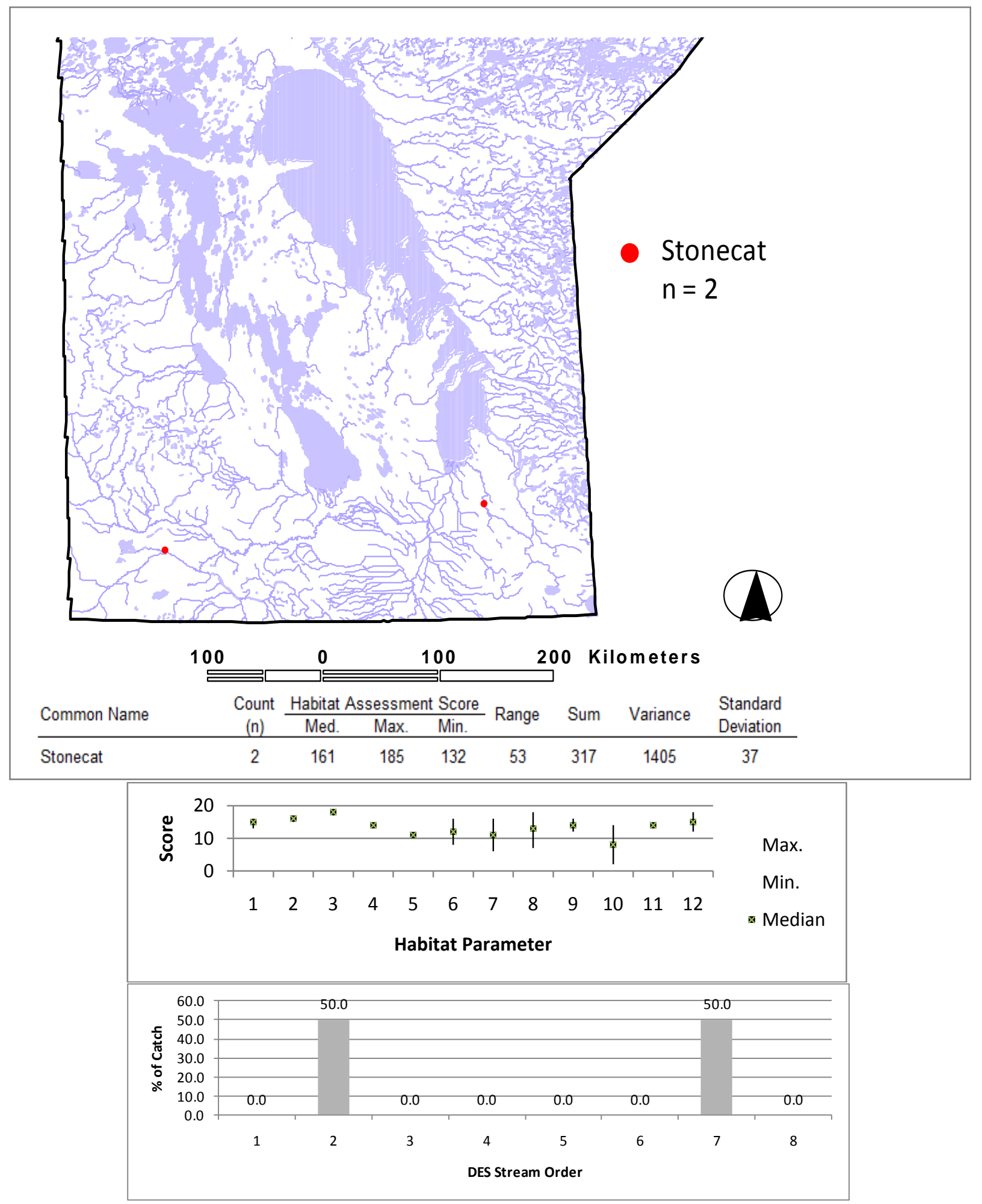

Figure 67: A summary of Stonecat collections showing collection sites; maximum, minimum and median individual parameter and total Habitat Assessment Scores; and the percent of catch by DES stream order. 


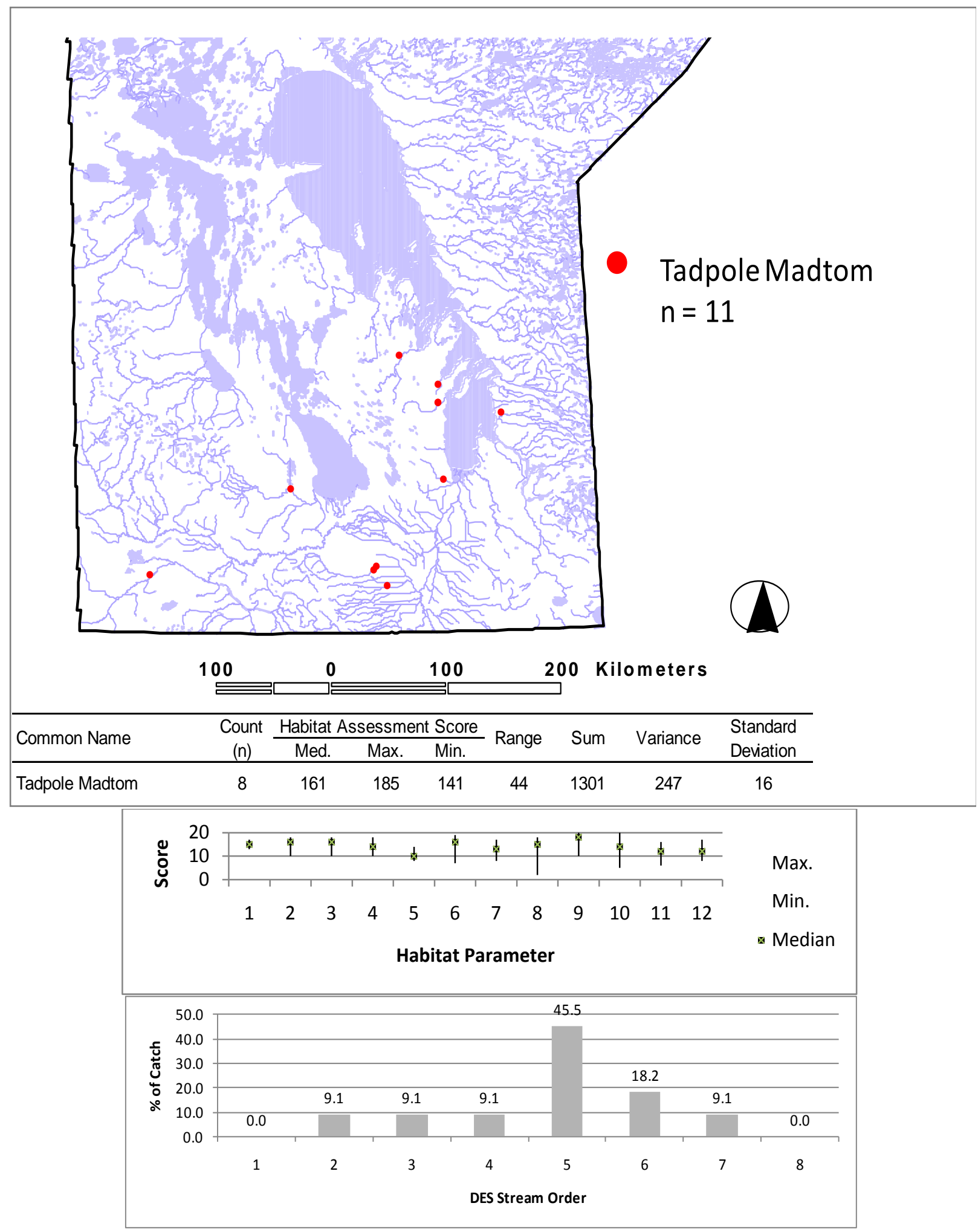

Figure 68: A summary of Tadpole Madtom collections showing collection sites; maximum, minimum and median individual parameter and total Habitat Assessment Scores; and the percent of catch by DES stream order. 

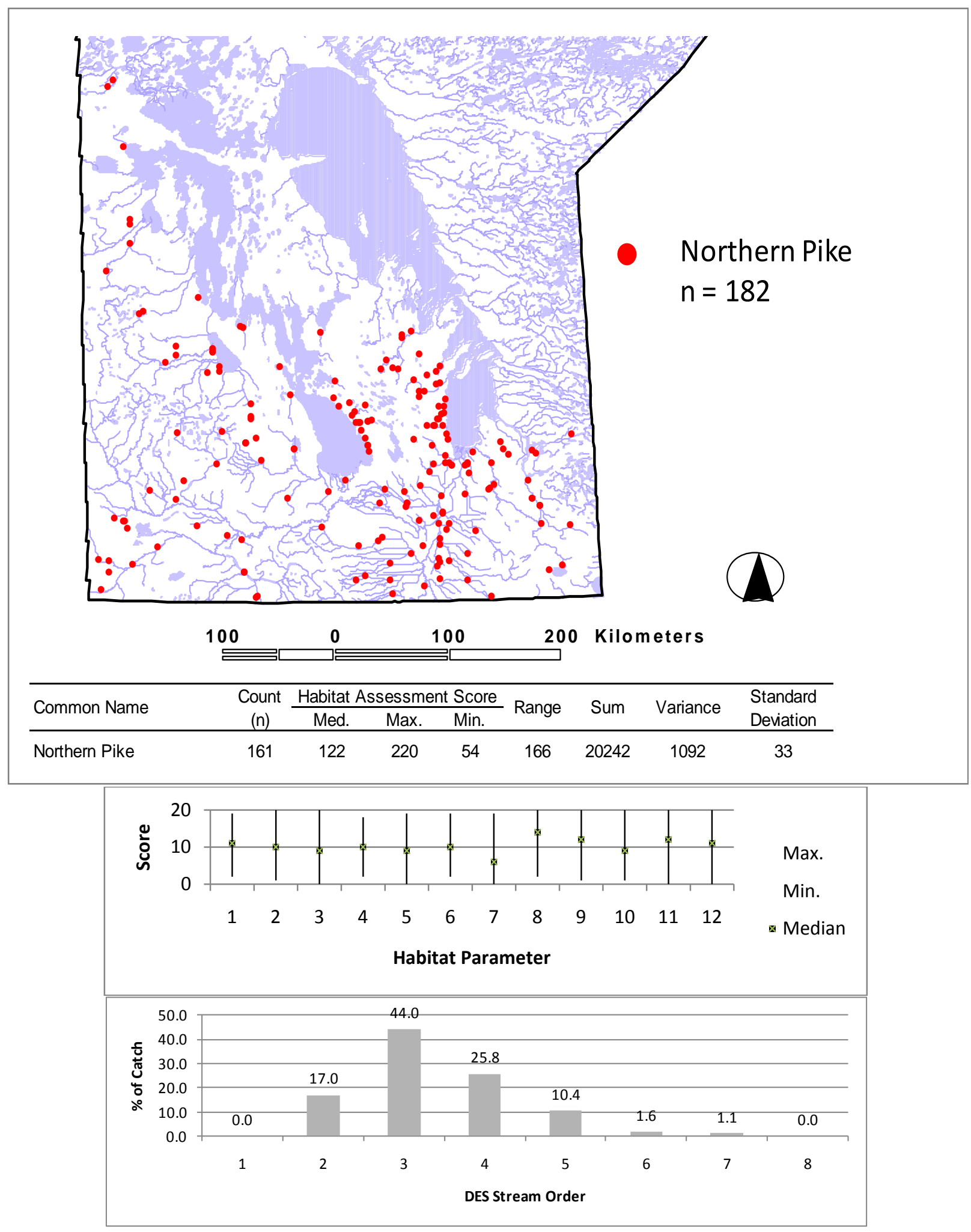

Figure 69: A summary of Northern Pike collections showing collection sites; maximum, minimum and median individual parameter and total Habitat Assessment Scores; and the percent of catch by DES stream order. 


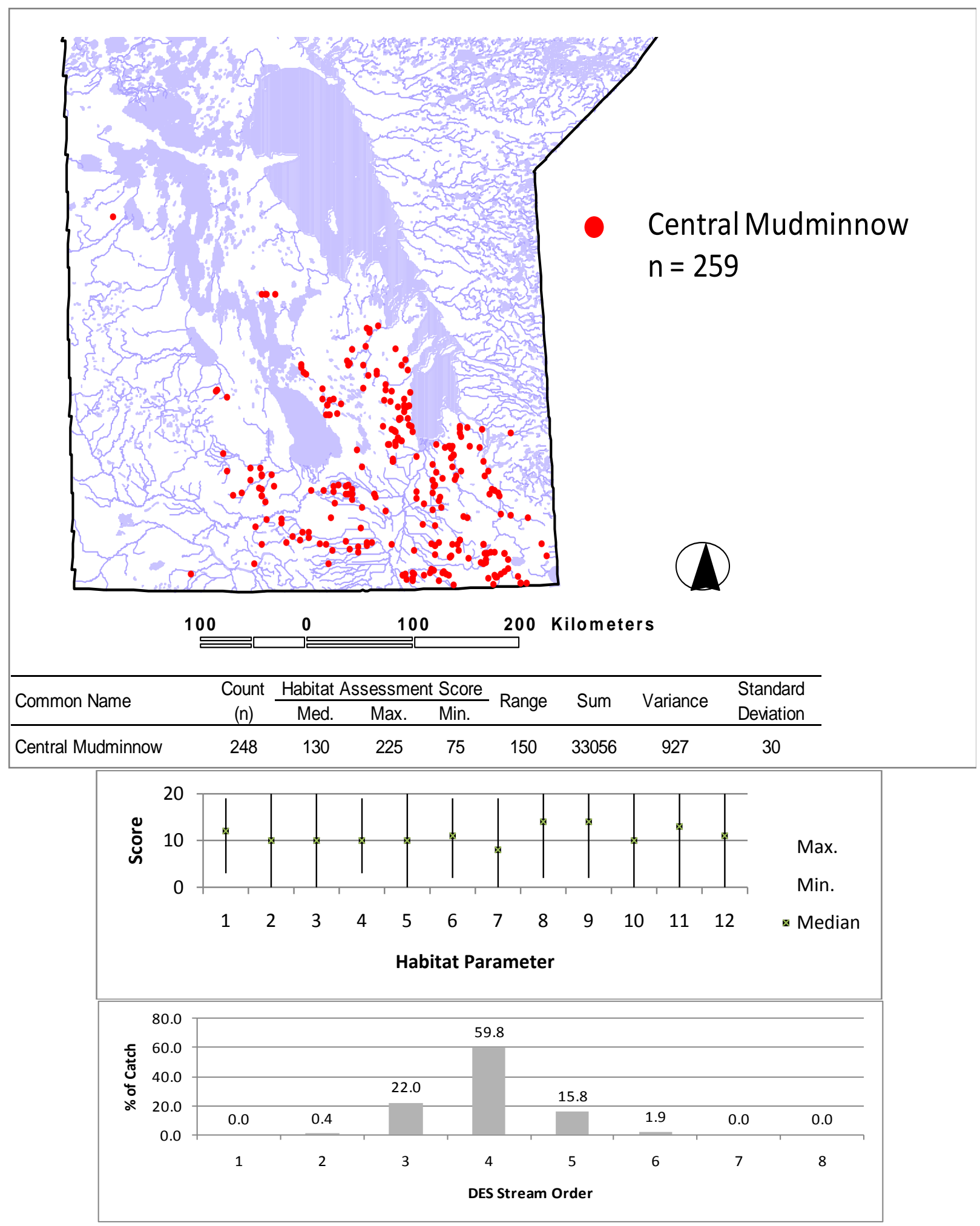

Figure 70: A summary of Central Mudminnow collections showing collection sites; maximum, minimum and median individual parameter and total Habitat Assessment Scores; and the percent of catch by DES stream order. 


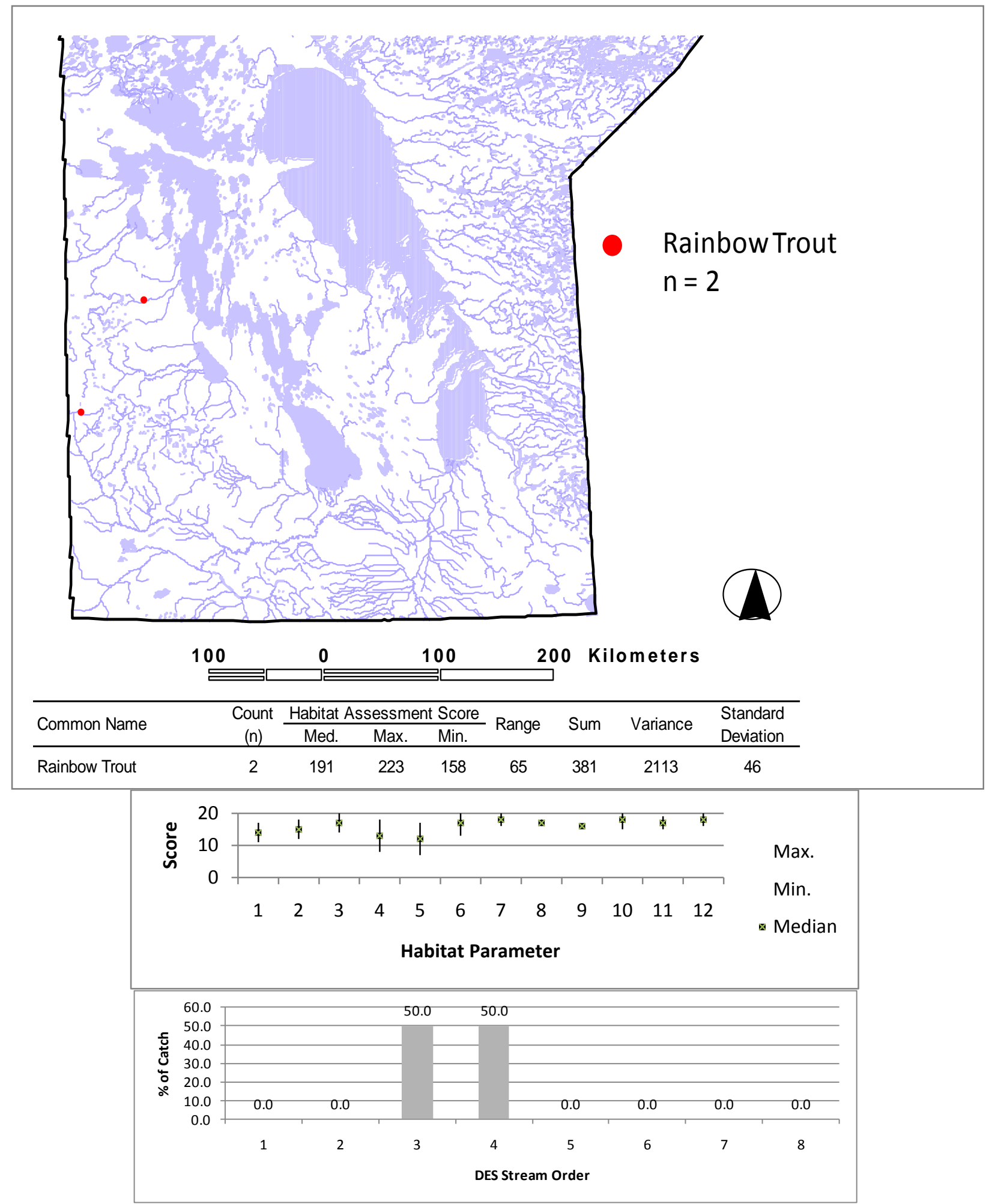

Figure 71: A summary of Rainbow Trout collections showing collection sites; maximum, minimum and median individual parameter and total Habitat Assessment Scores; and the percent of catch by DES stream order. 


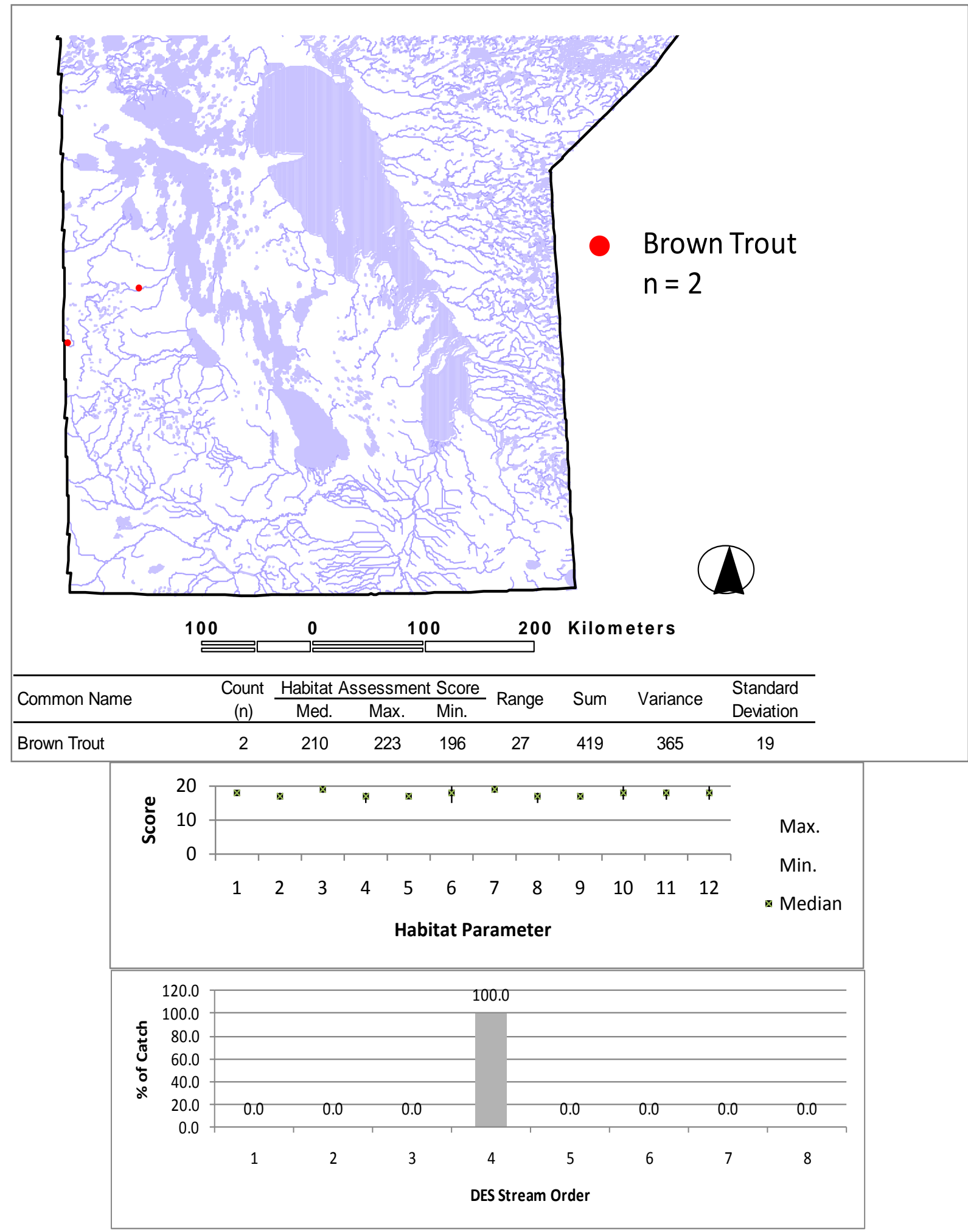

Figure 72: A summary of Brown Trout collections showing collection sites; maximum, minimum and median individual parameter and total Habitat Assessment Scores; and the percent of catch by DES stream order. 

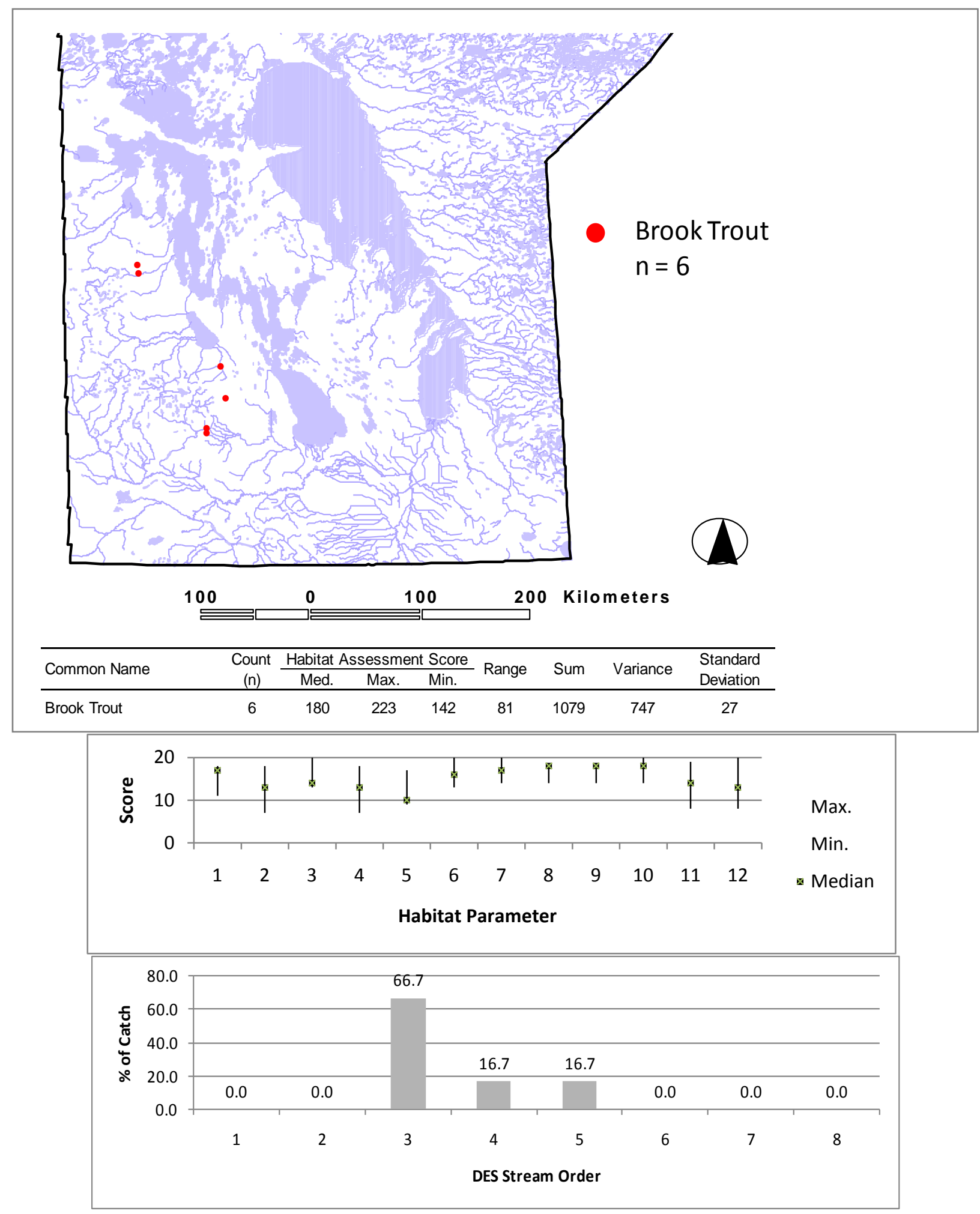

Figure 73: A summary of Brook Trout collections showing collection sites; maximum, minimum and median individual parameter and total Habitat Assessment Scores; and the percent of catch by DES stream order. 

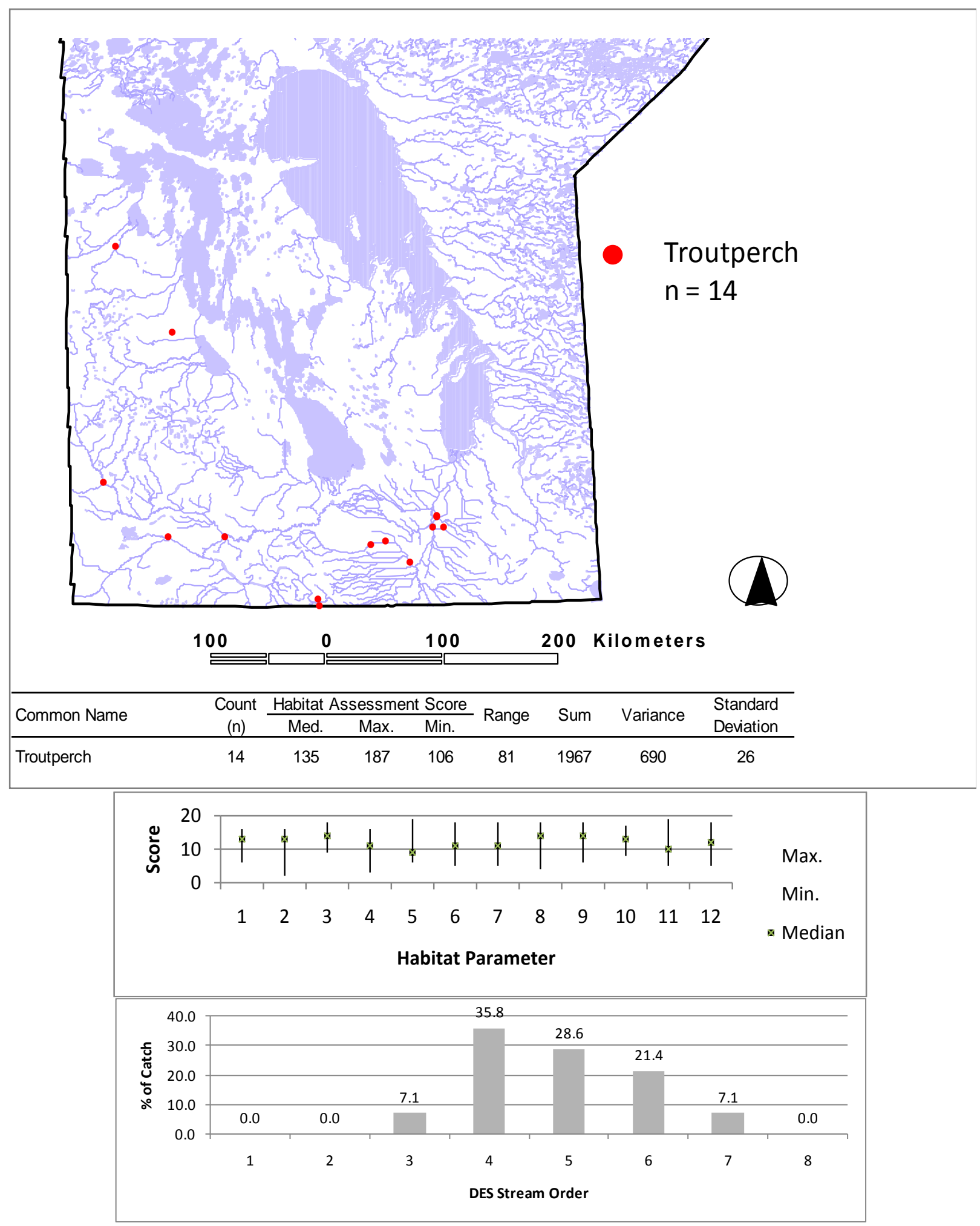

Figure 74: A summary of Troutperch collections showing collection sites; maximum, minimum and median individual parameter and total Habitat Assessment Scores; and the percent of catch by DES stream order. 

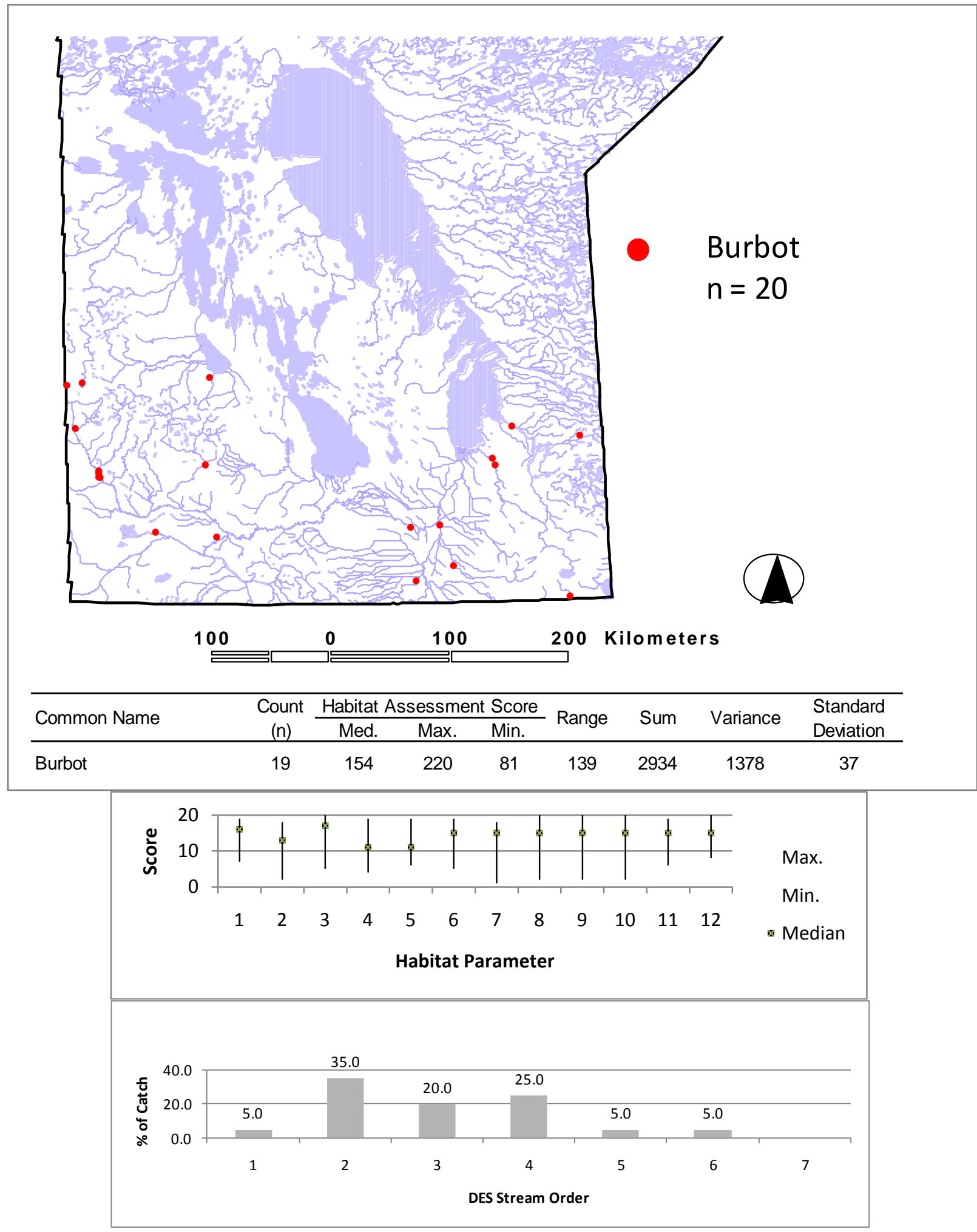

Figure 75: A summary of Burbot collections showing collection sites; maximum, minimum and median individual parameter and total Habitat Assessment Scores; and the percent of catch by DES stream order. 

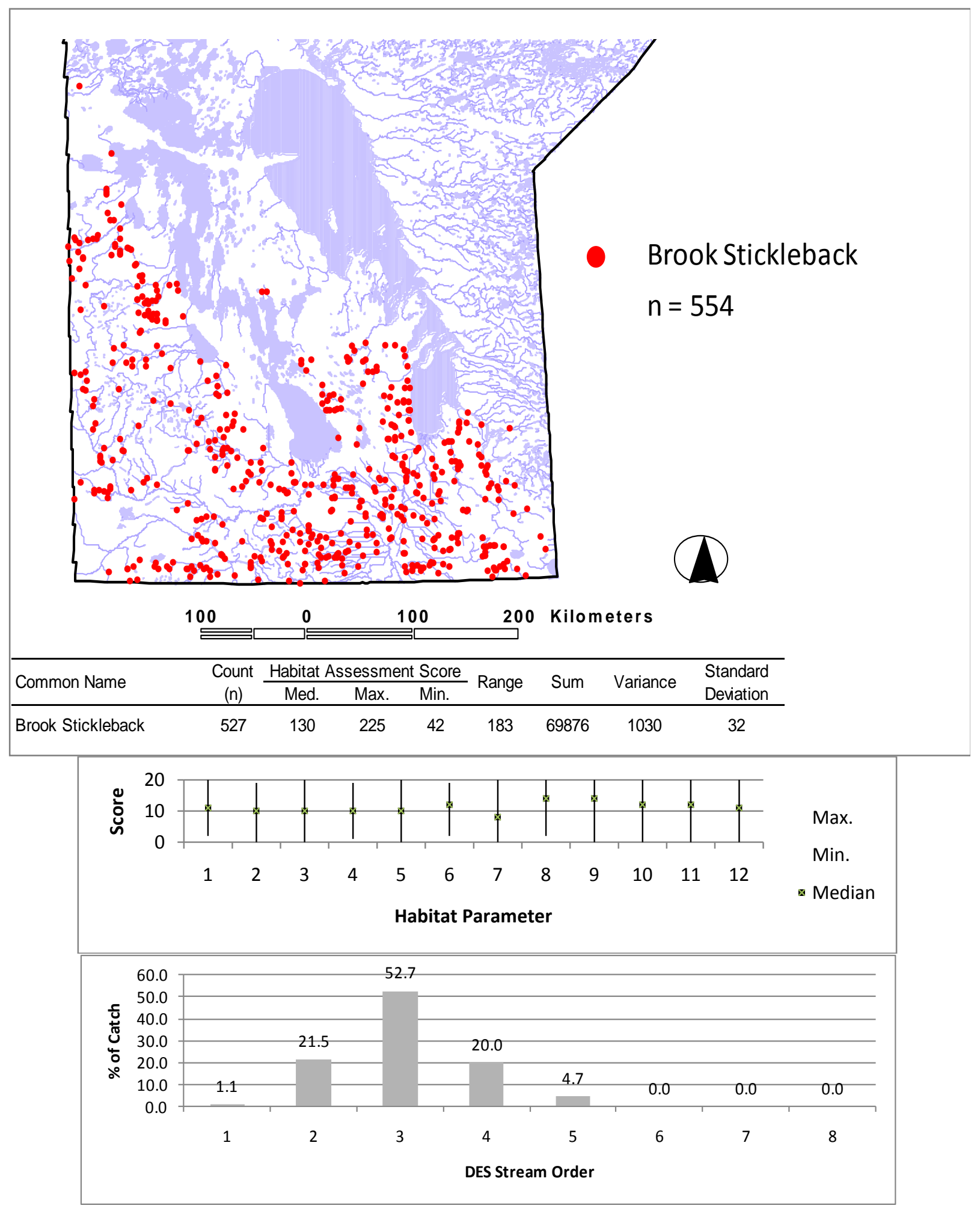

Figure 76: A summary of Brook Stickleback collections showing collection sites; maximum, minimum and median individual parameter and total Habitat Assessment Scores; and the percent of catch by DES stream order. 


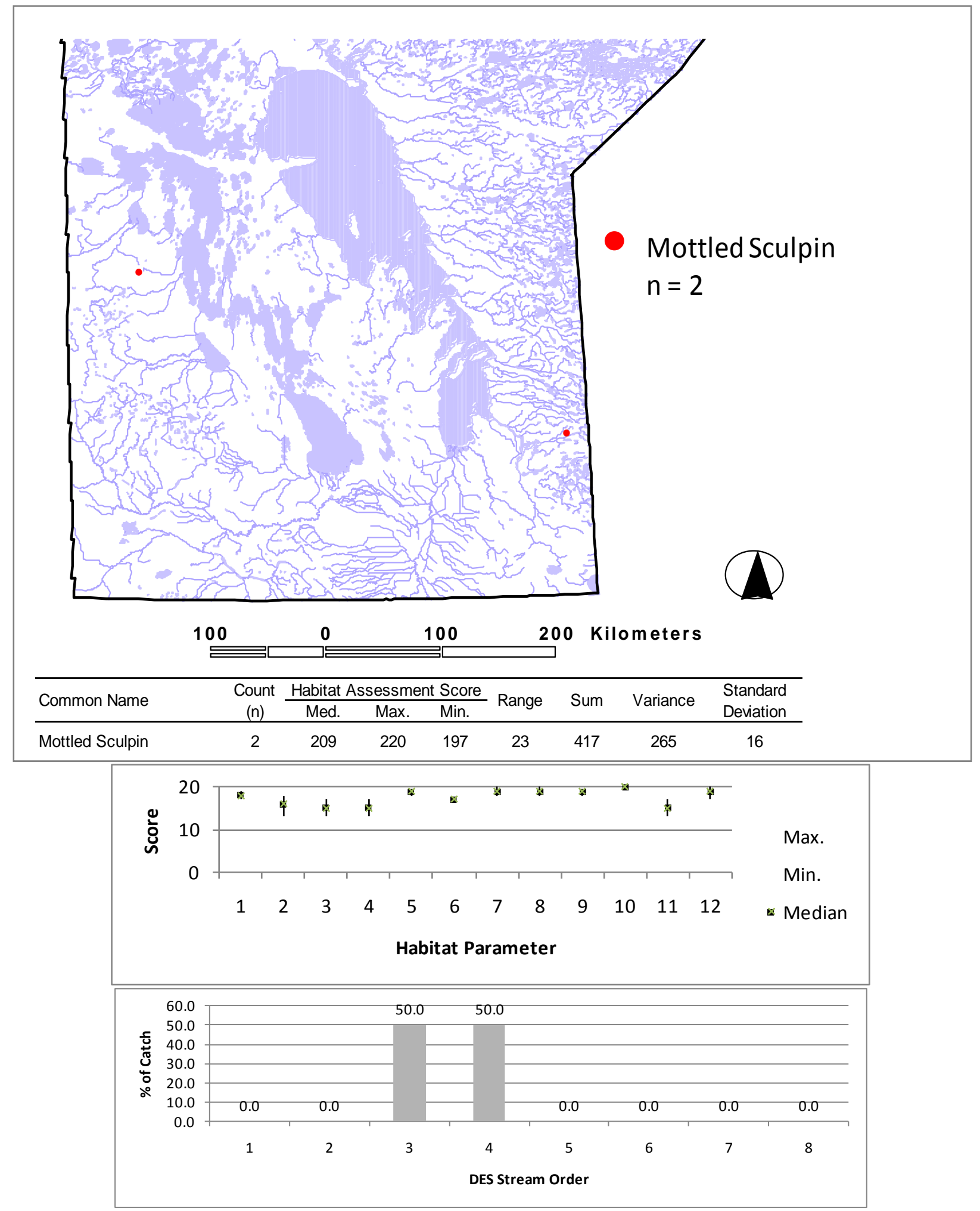

Figure 77: A summary of Mottled Sculpin collections showing collection sites; maximum, minimum and median individual parameter and total Habitat Assessment Scores; and the percent of catch by DES stream order. 


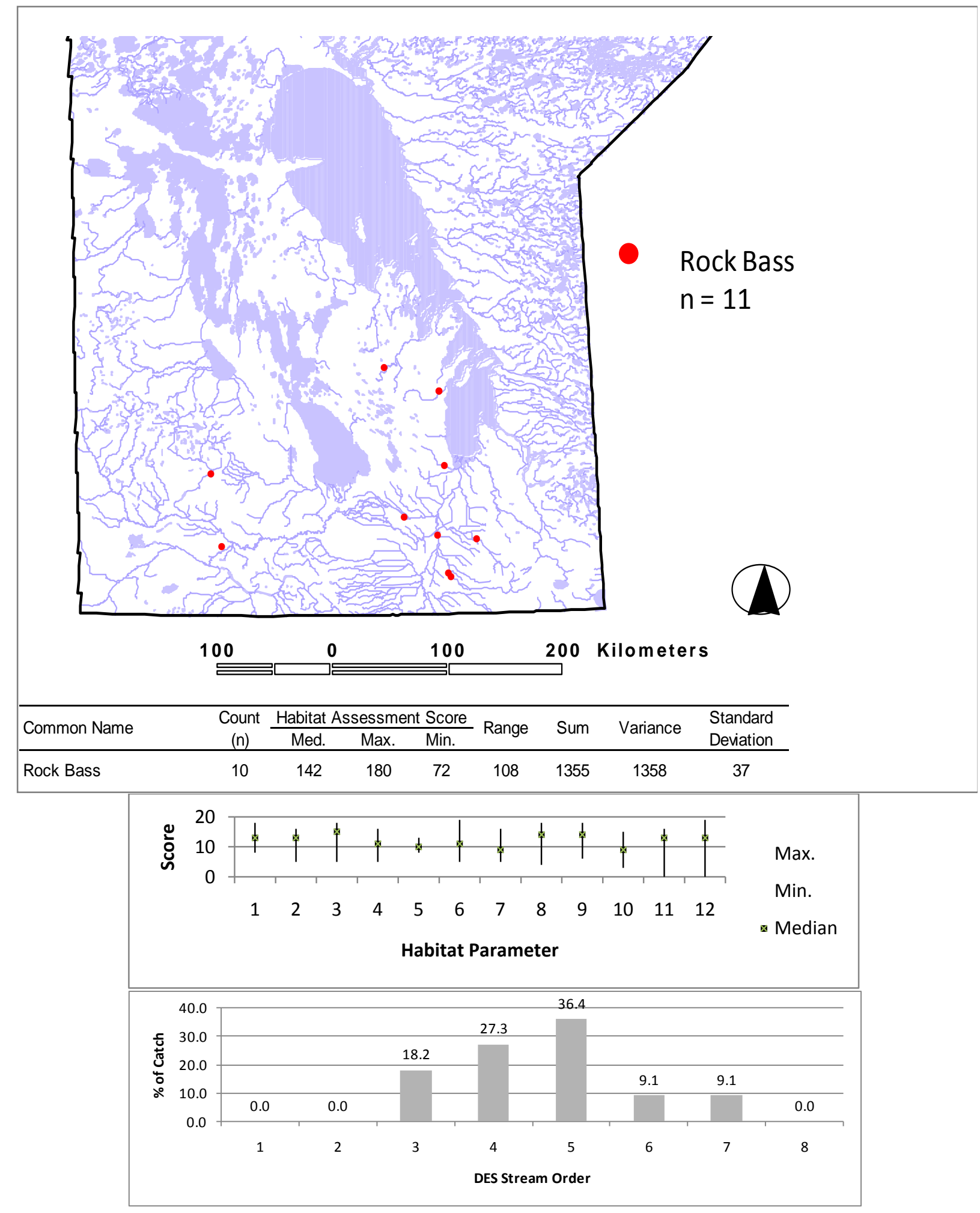

Figure 78: A summary of Rock Bass collections showing collection sites; maximum, minimum and median individual parameter and total Habitat Assessment Scores; and the percent of catch by DES stream order. 


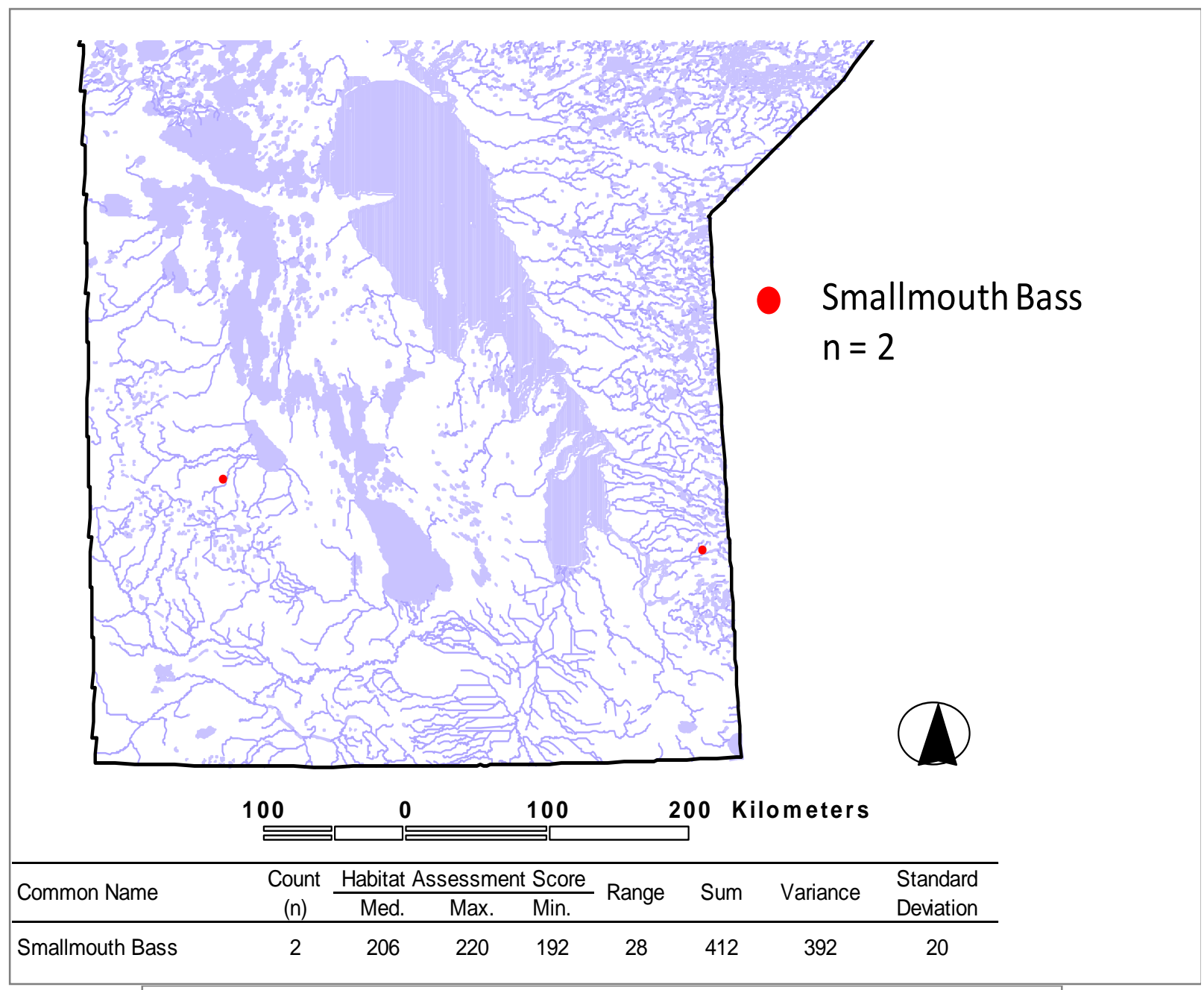

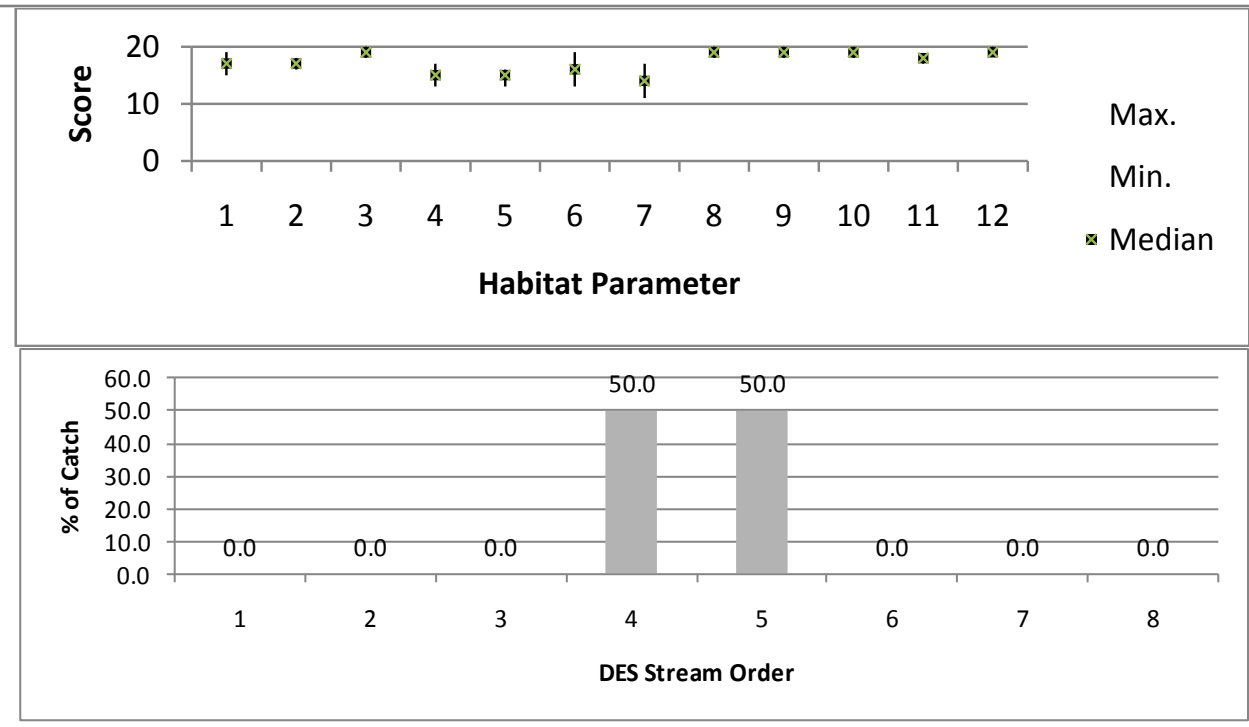

Figure 79: A summary of Smallmouth Bass collections showing collection sites; maximum, minimum and median individual parameter and total Habitat Assessment Scores; and the percent of catch by DES stream order. 

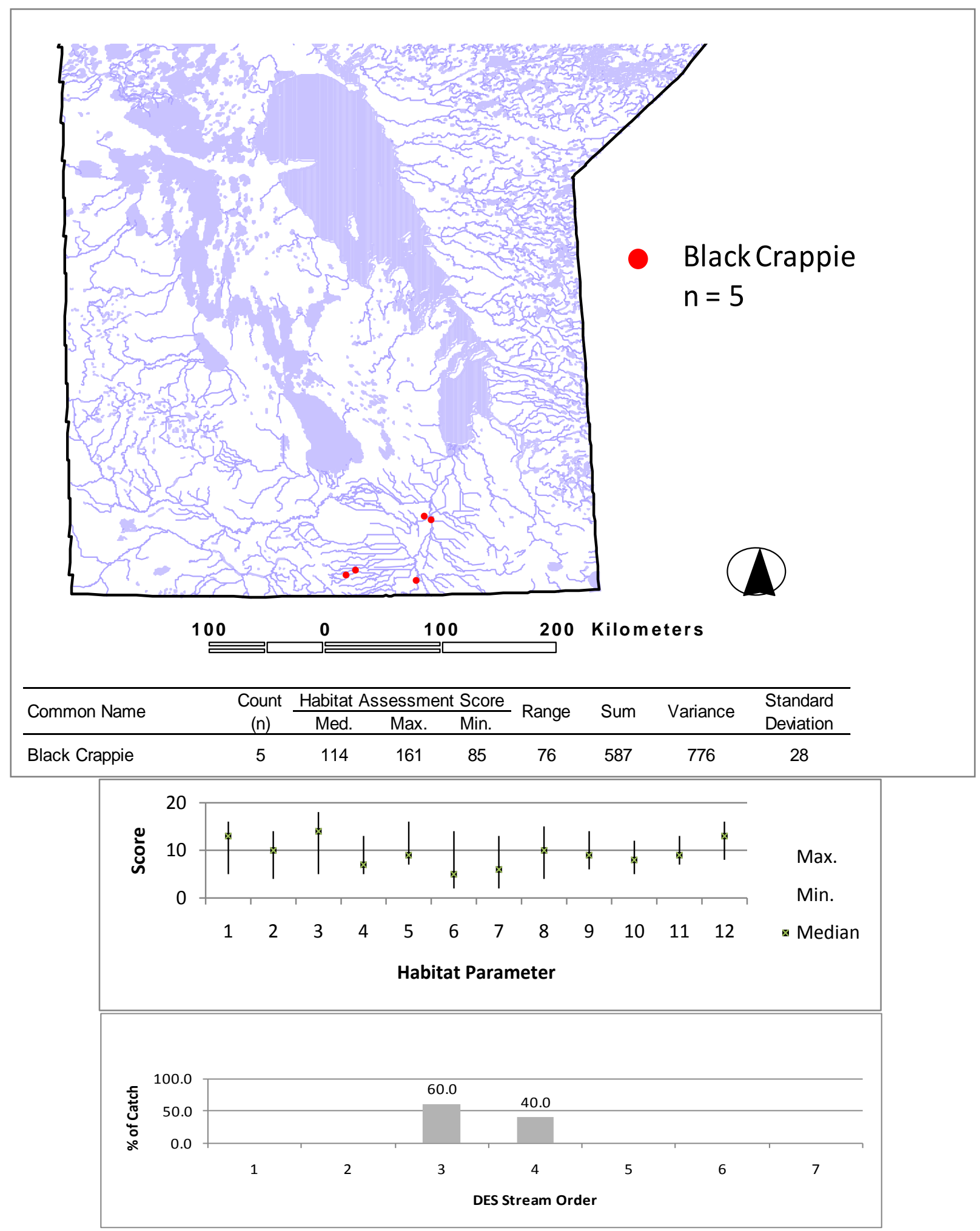

Figure 80: A summary of Black Crappie collections showing collection sites; maximum, minimum and median individual parameter and total Habitat Assessment Scores; and the percent of catch by DES stream order. 

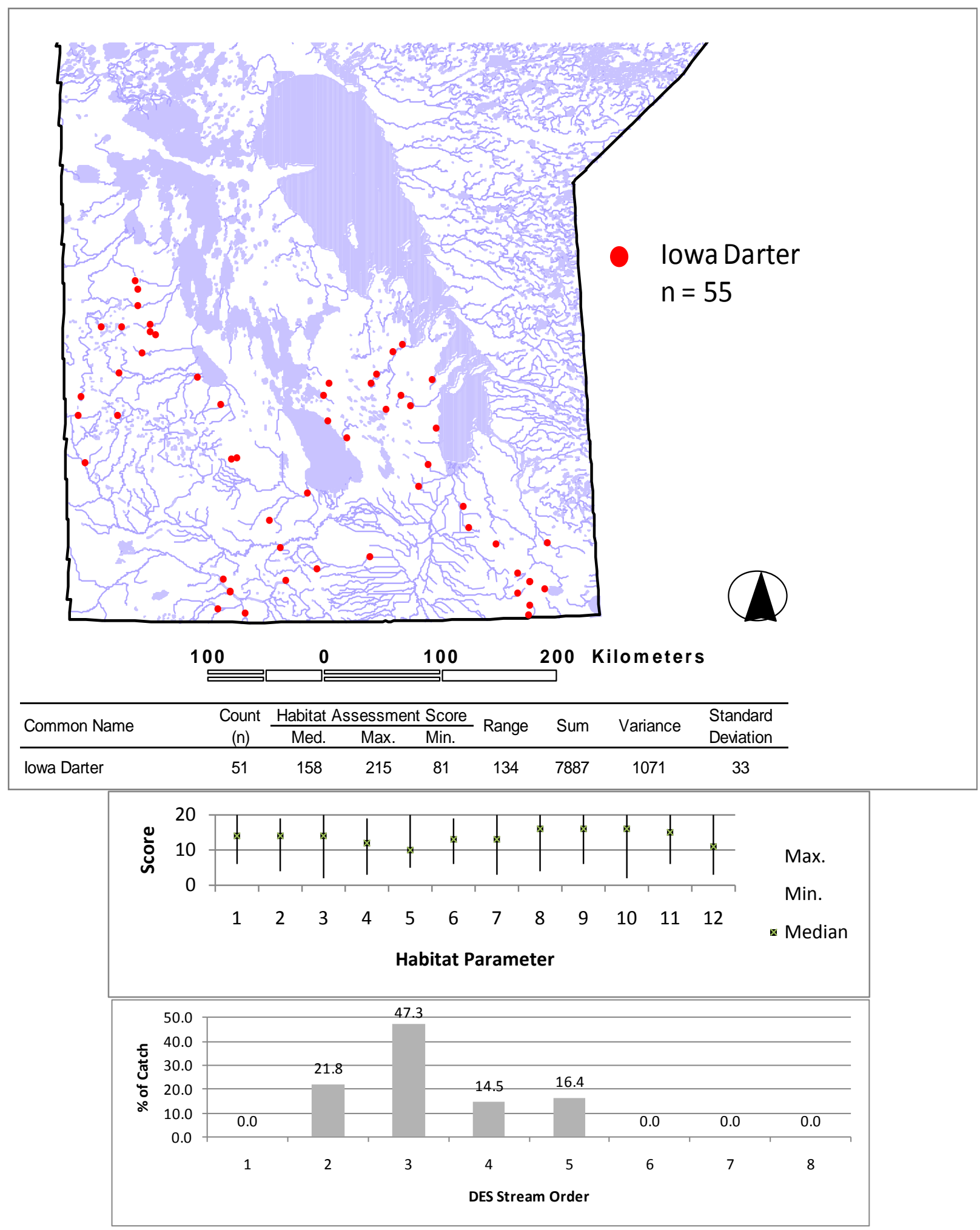

Figure 81: A summary of lowa Darter collections showing collection sites; maximum, minimum and median individual parameter and total Habitat Assessment Scores; and the percent of catch by DES stream order. 


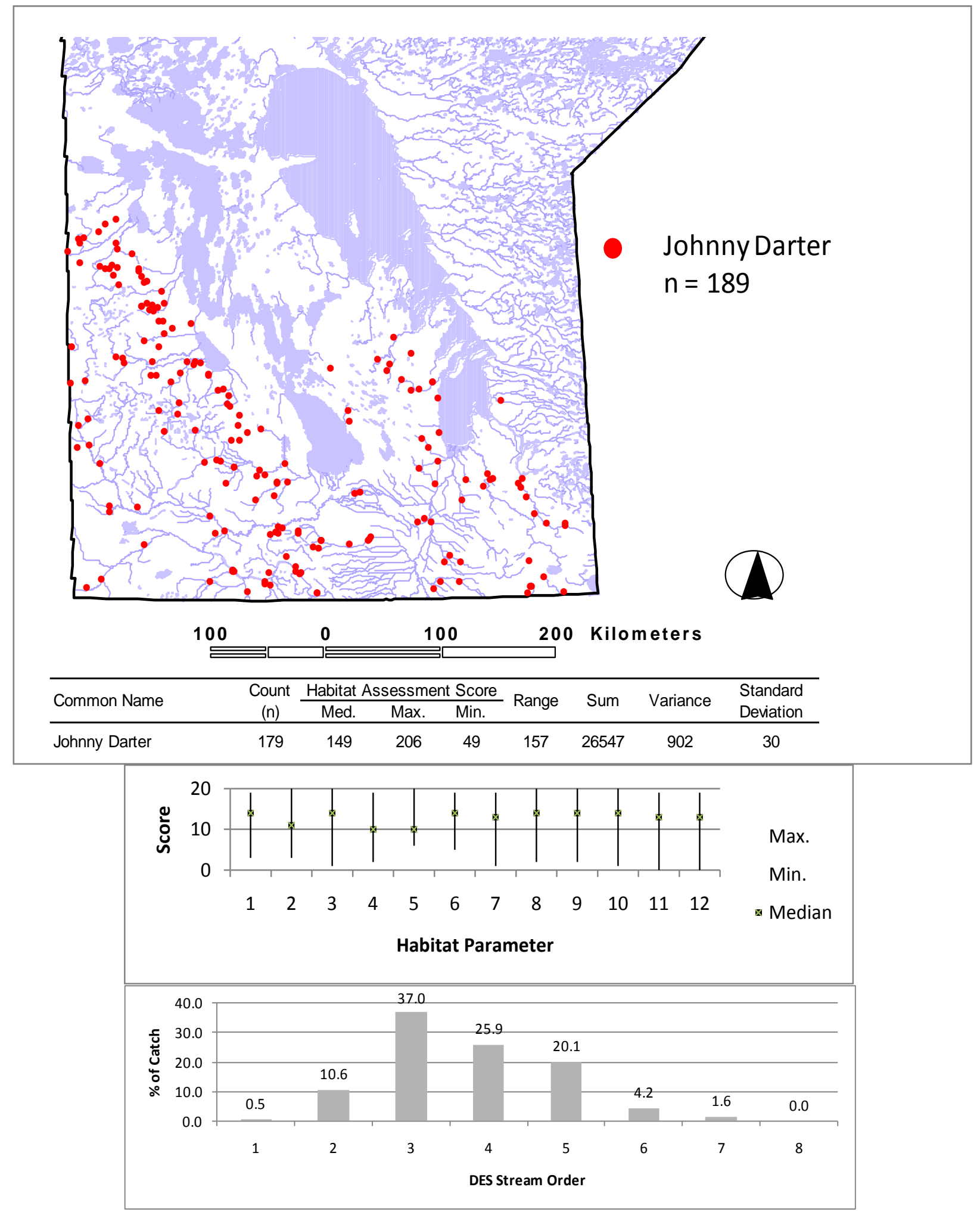

Figure 82: A summary of Johnny Darter collections showing collection sites; maximum, minimum and median individual parameter and total Habitat Assessment Scores; and the percent of catch by DES stream order. 


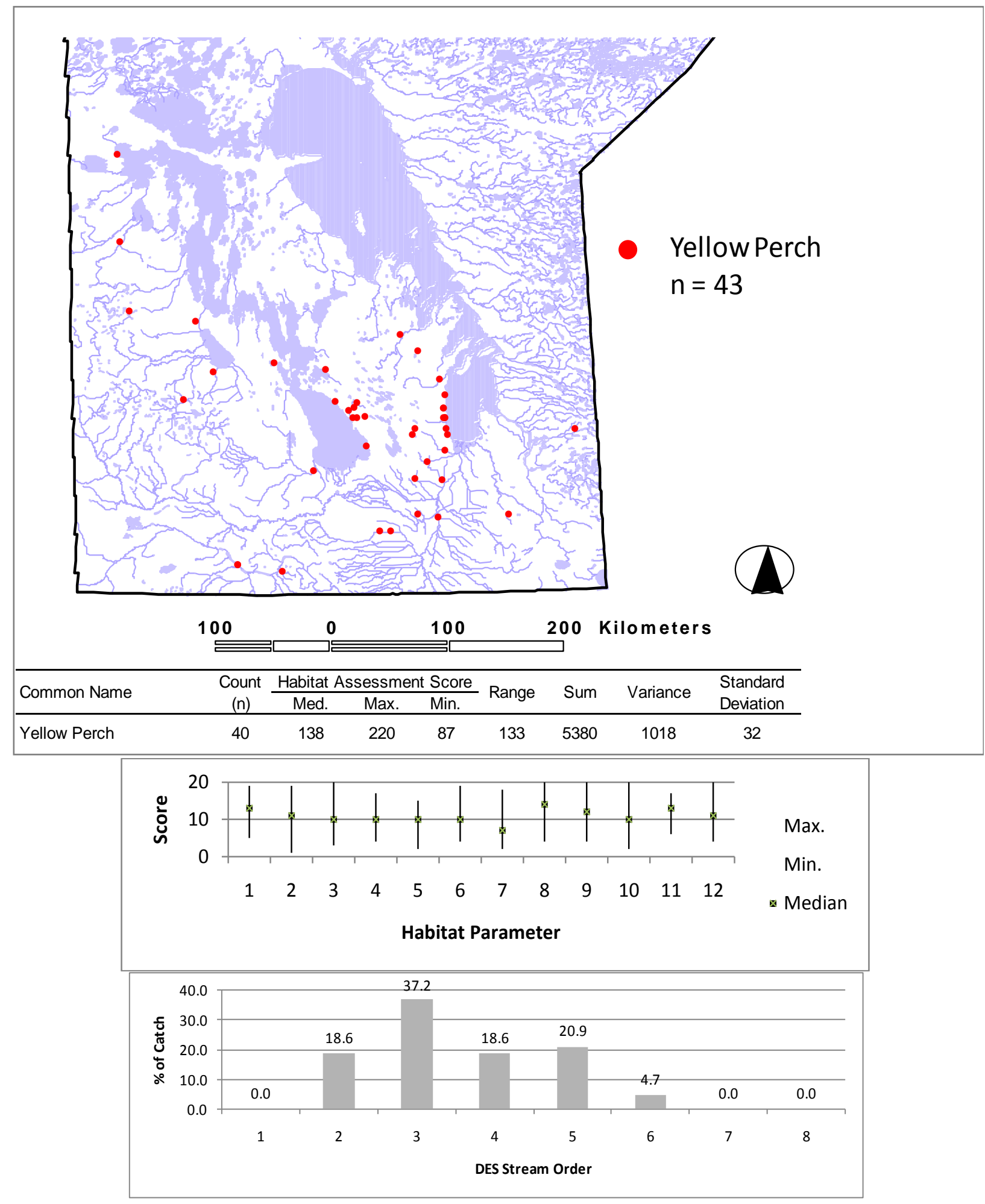

Figure 83: A summary of Yellow Perch collections showing collection sites; maximum, minimum and median individual parameter and total Habitat Assessment Scores; and the percent of catch by DES stream order. 


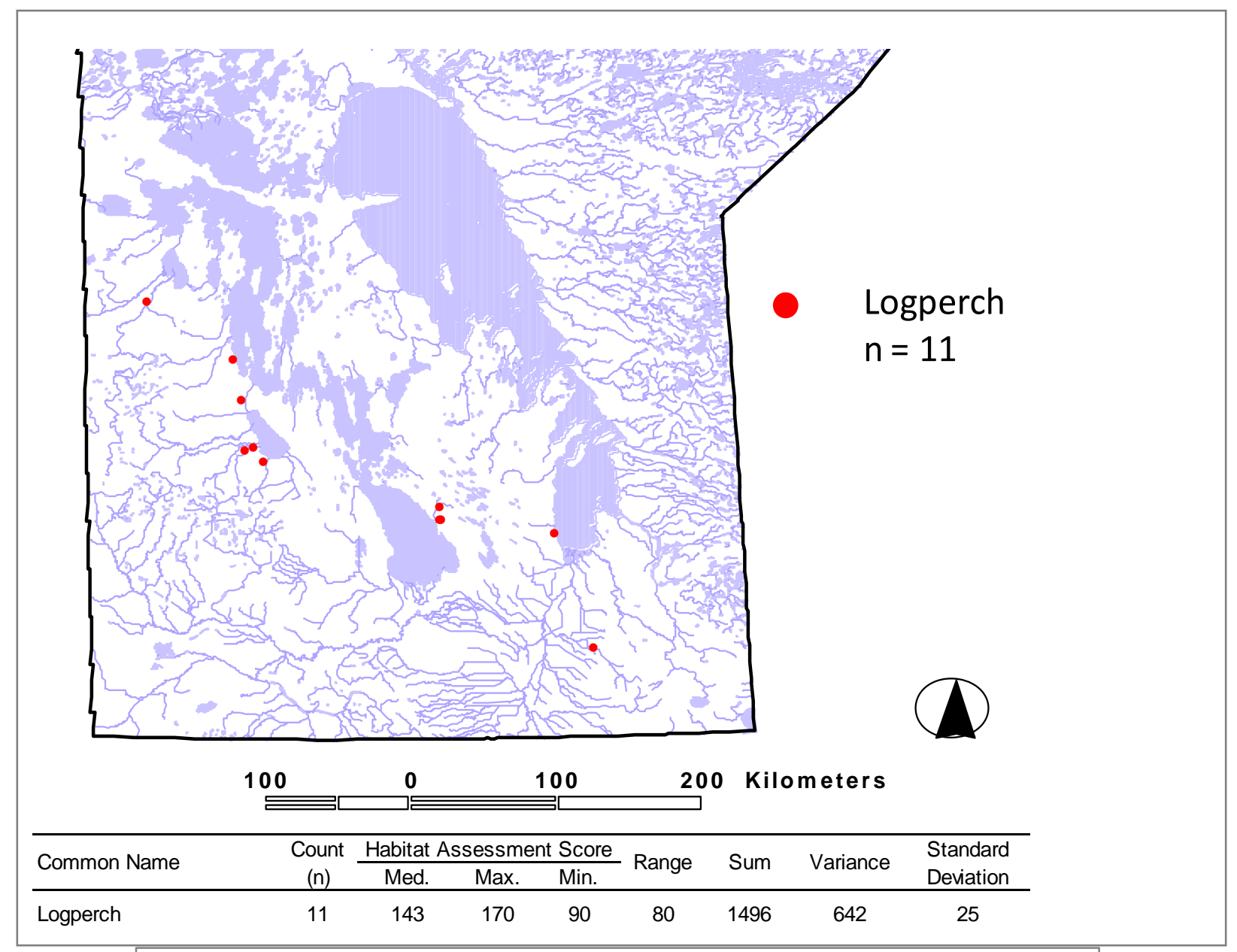

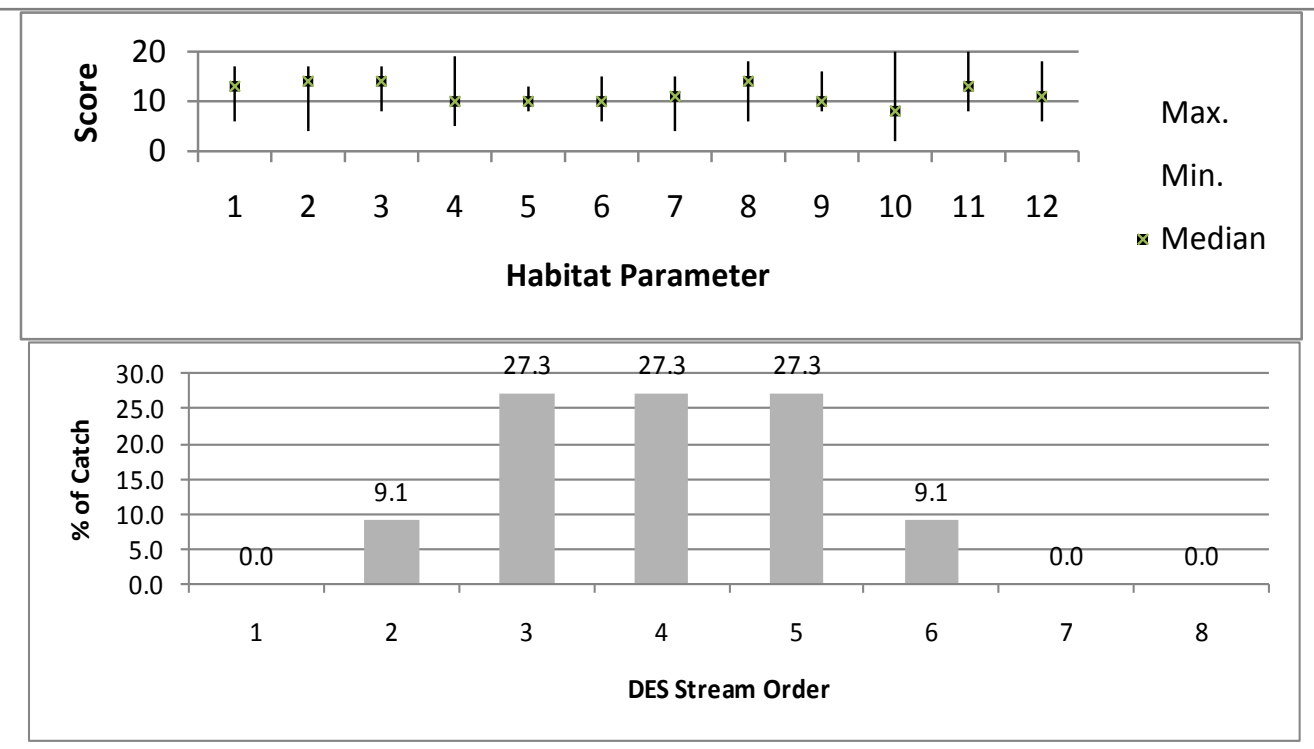

Figure 84: A summary of Logperch collections showing collection sites; maximum, minimum and median individual parameter and total Habitat Assessment Scores; and the percent of catch by DES stream order. 

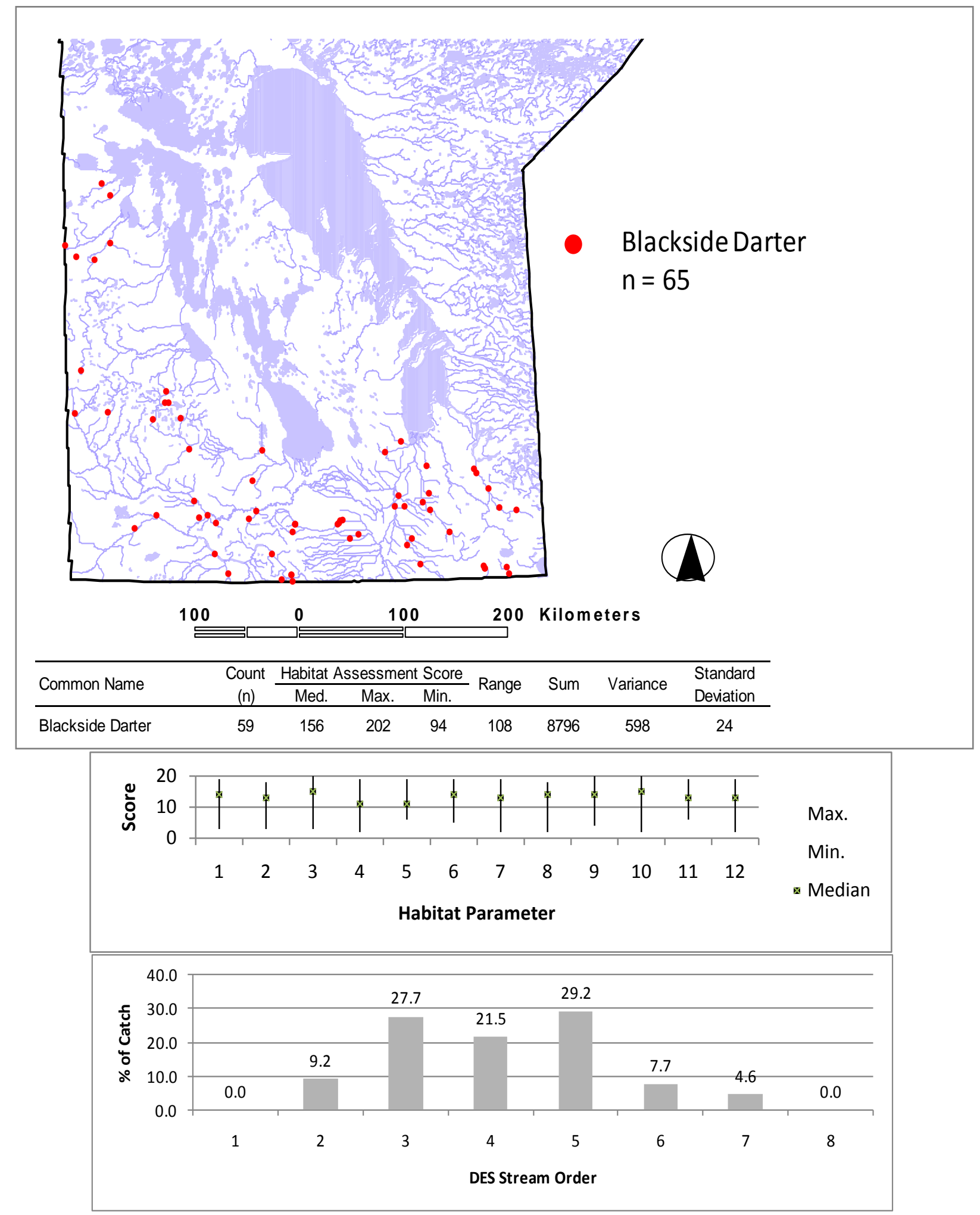

Figure 85: A summary of Blackside Darter collections showing collection sites; maximum, minimum and median individual parameter and total Habitat Assessment Scores; and the percent of catch by DES stream order. 


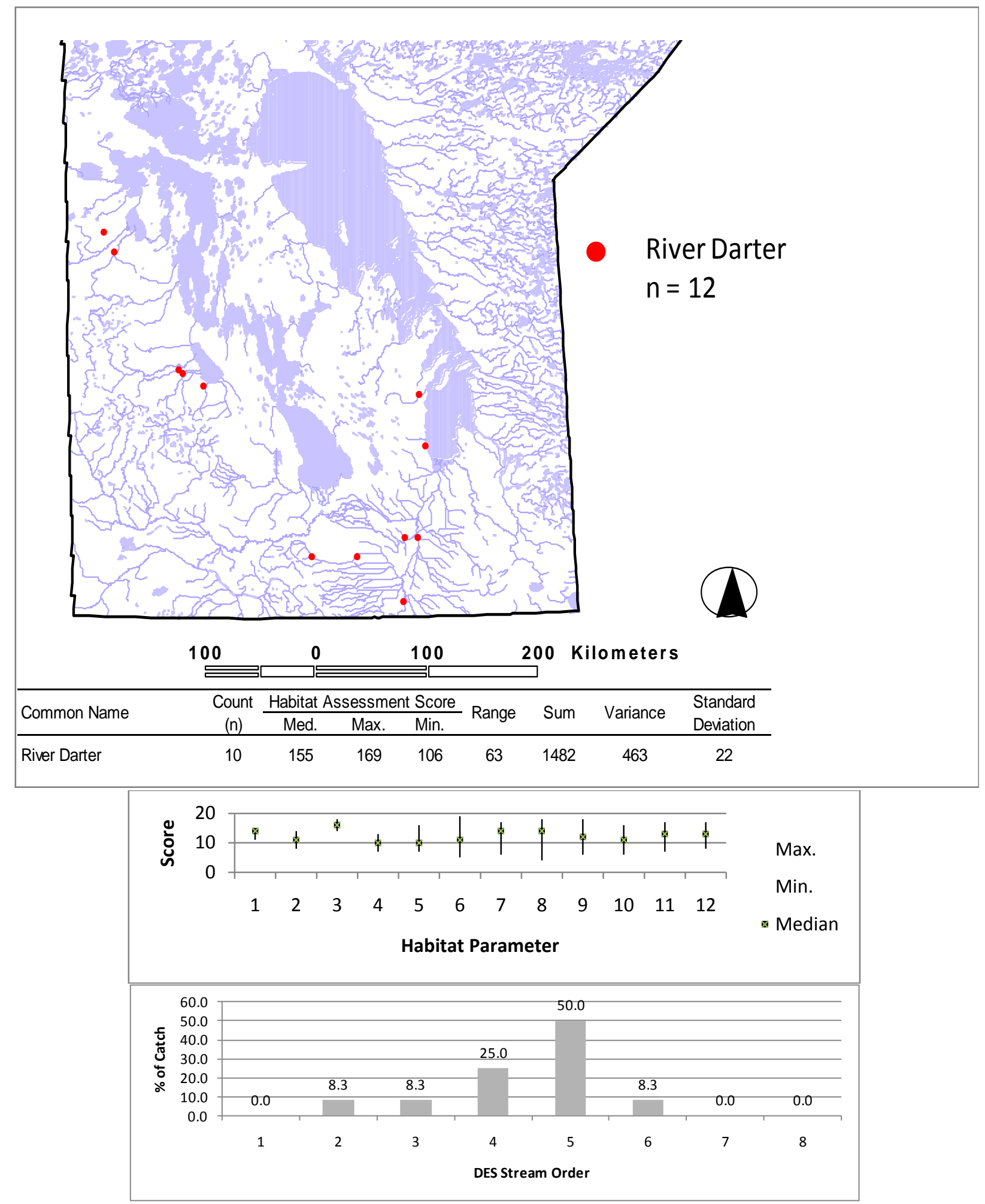

Figure 86: A summary of River Darter collections showing collection sites; maximum, minimum and median individual parameter and total Habitat Assessment Scores; and the percent of catch by DES stream order. 


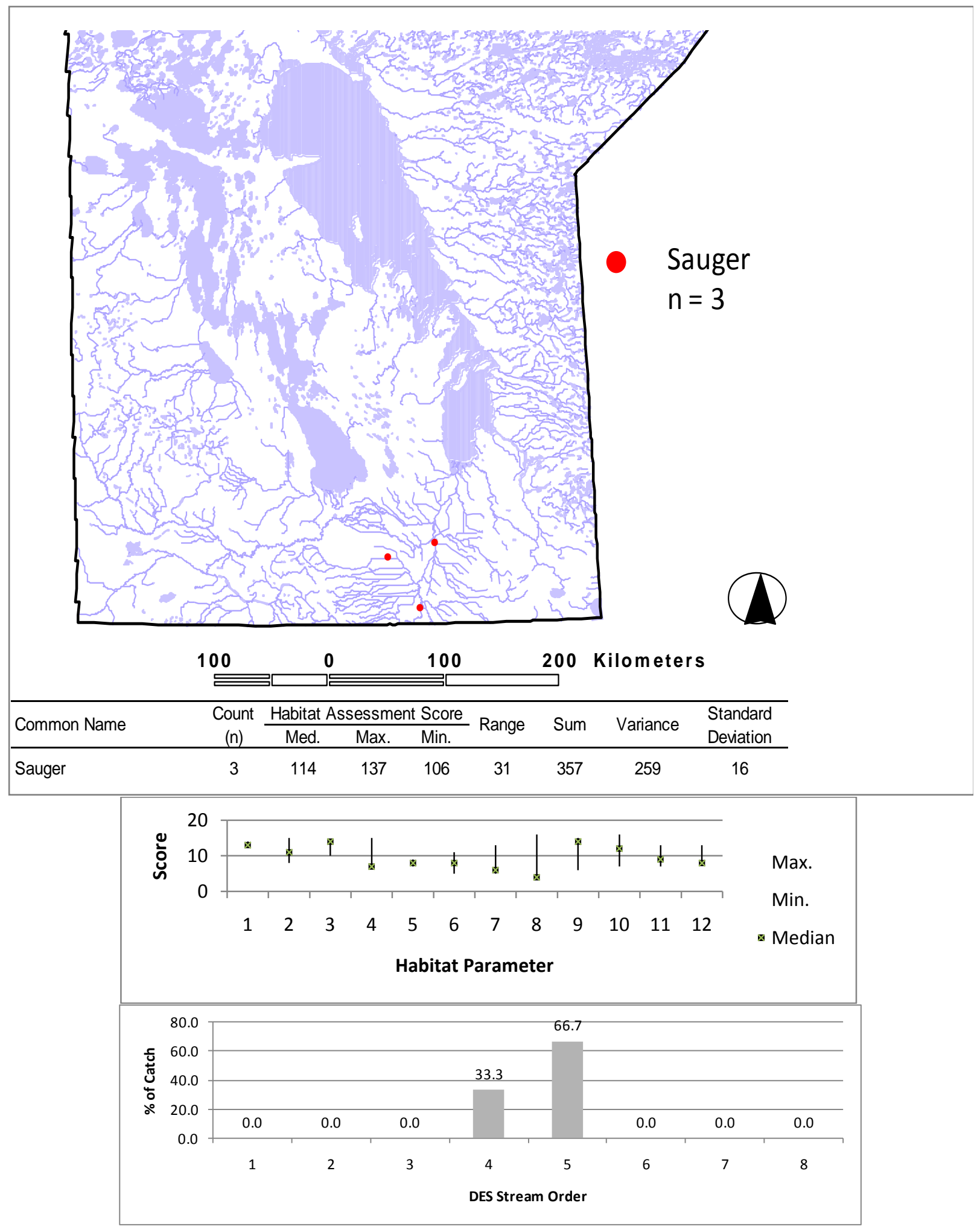

Figure 87: A summary of Sauger collections showing collection sites; maximum, minimum and median individual parameter and total Habitat Assessment Scores; and the percent of catch by DES stream order. 

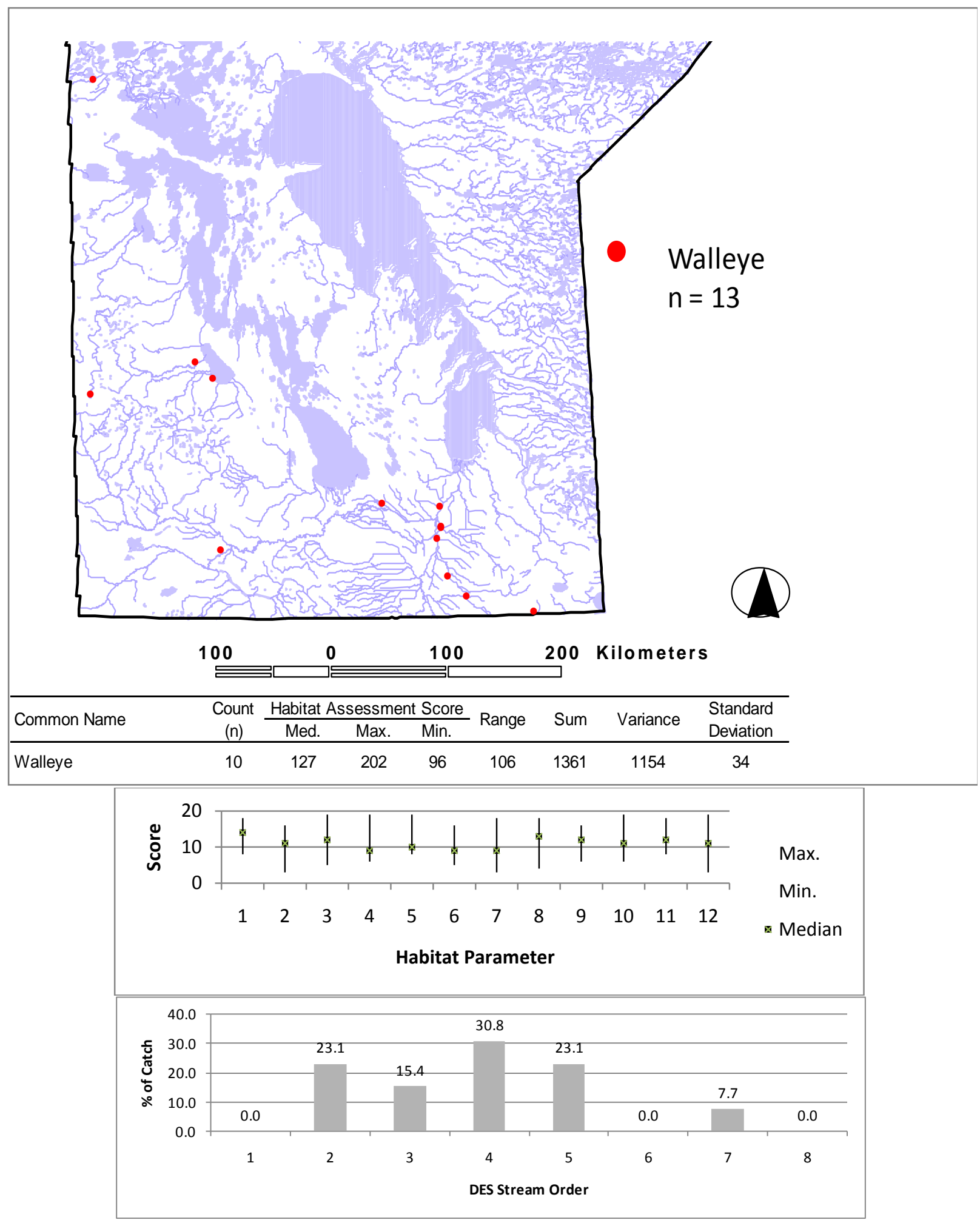

Figure 88: A summary of Walleye collections showing collection sites; maximum, minimum and median individual parameter and total Habitat Assessment Scores; and the percent of catch by DES stream order. 


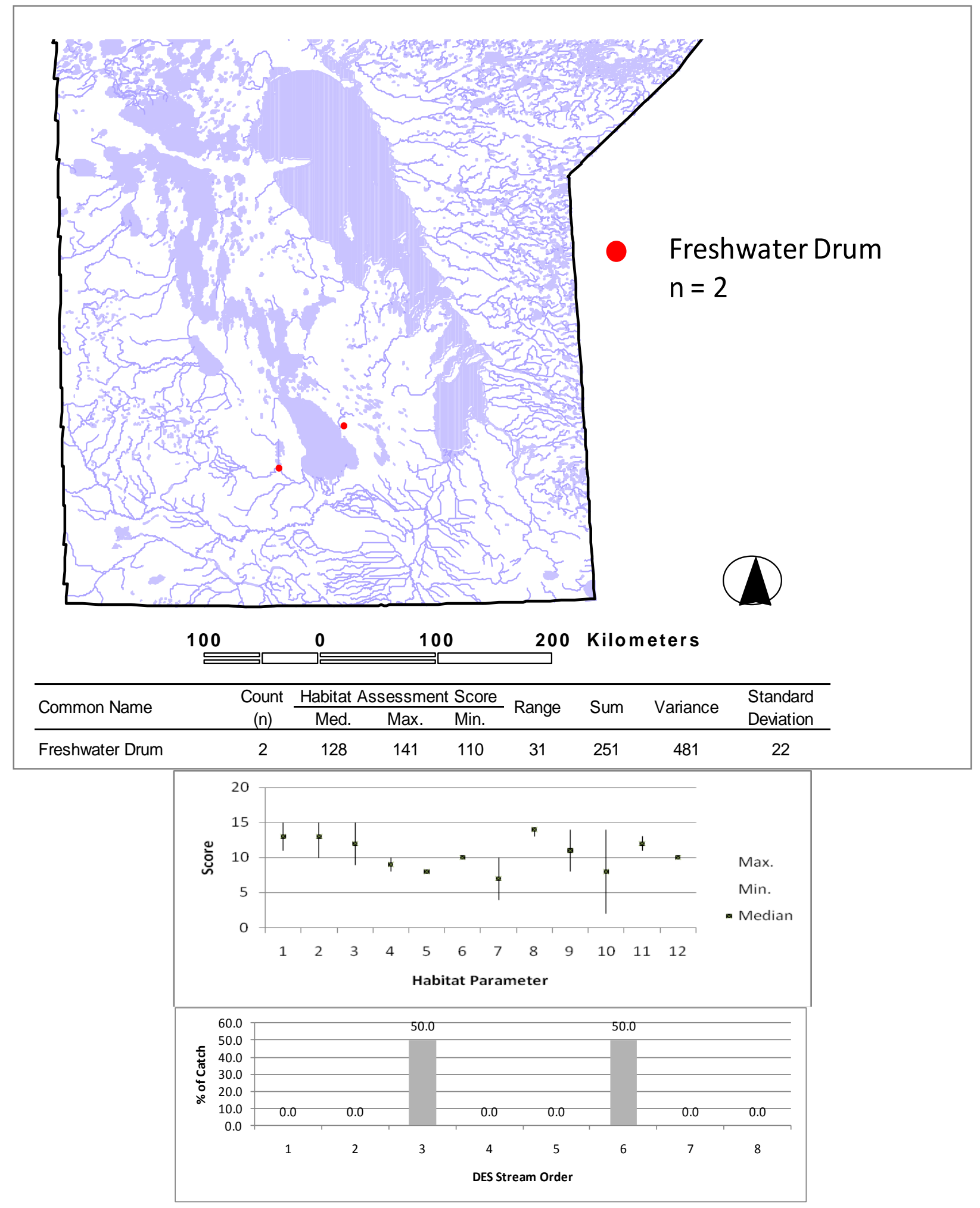

Figure 89: A summary of Freshwater Drum collections showing collection sites; maximum, minimum and median individual parameter and total Habitat Assessment Scores; and the percent of catch by DES stream order. 


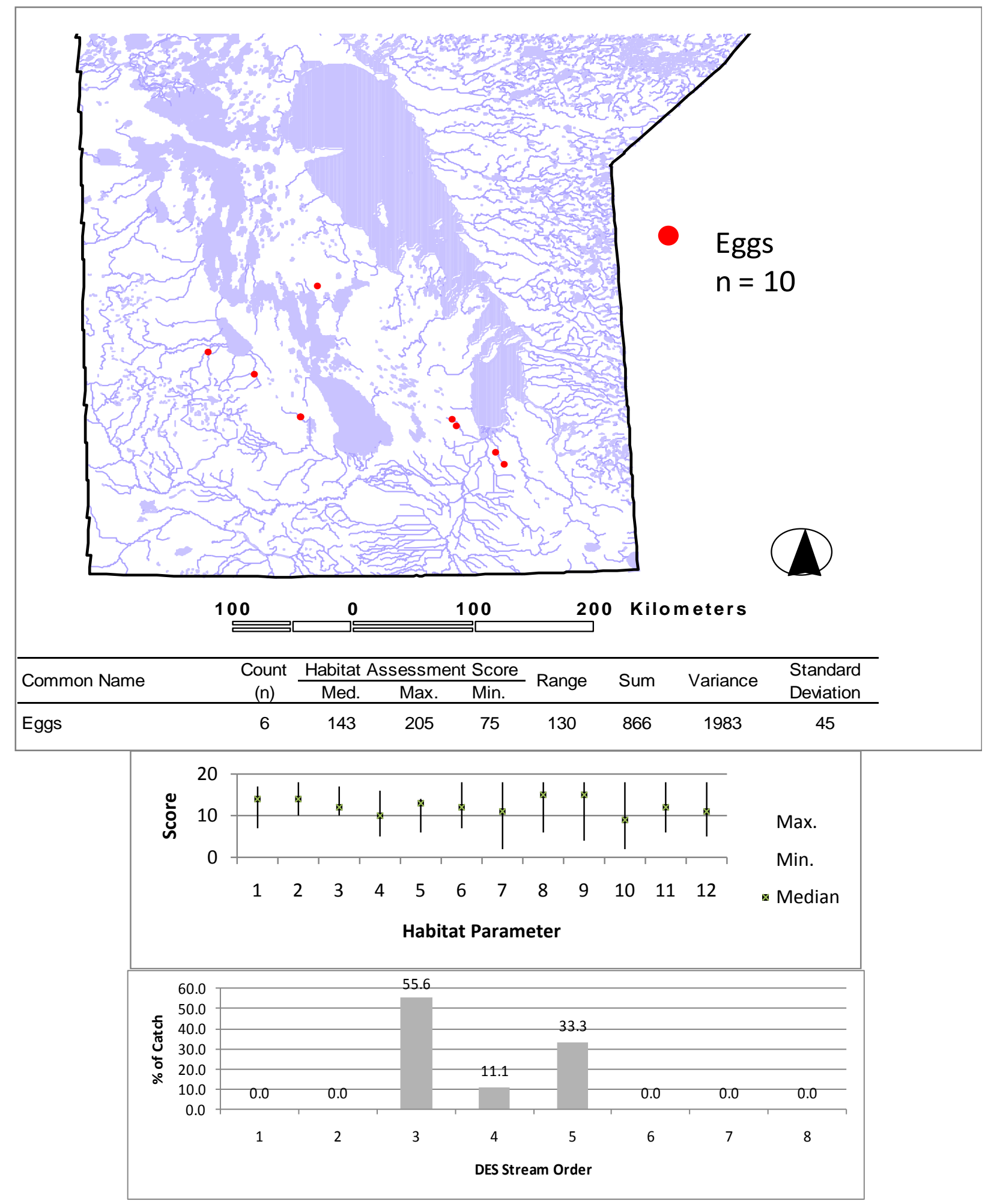

Figure 90: A summary of Egg collections (9-Catostomidae, 1-Percidae) showing collection sites; maximum, minimum and median individual parameter and total Habitat Assessment Scores; and the percent of catch by DES stream order. 

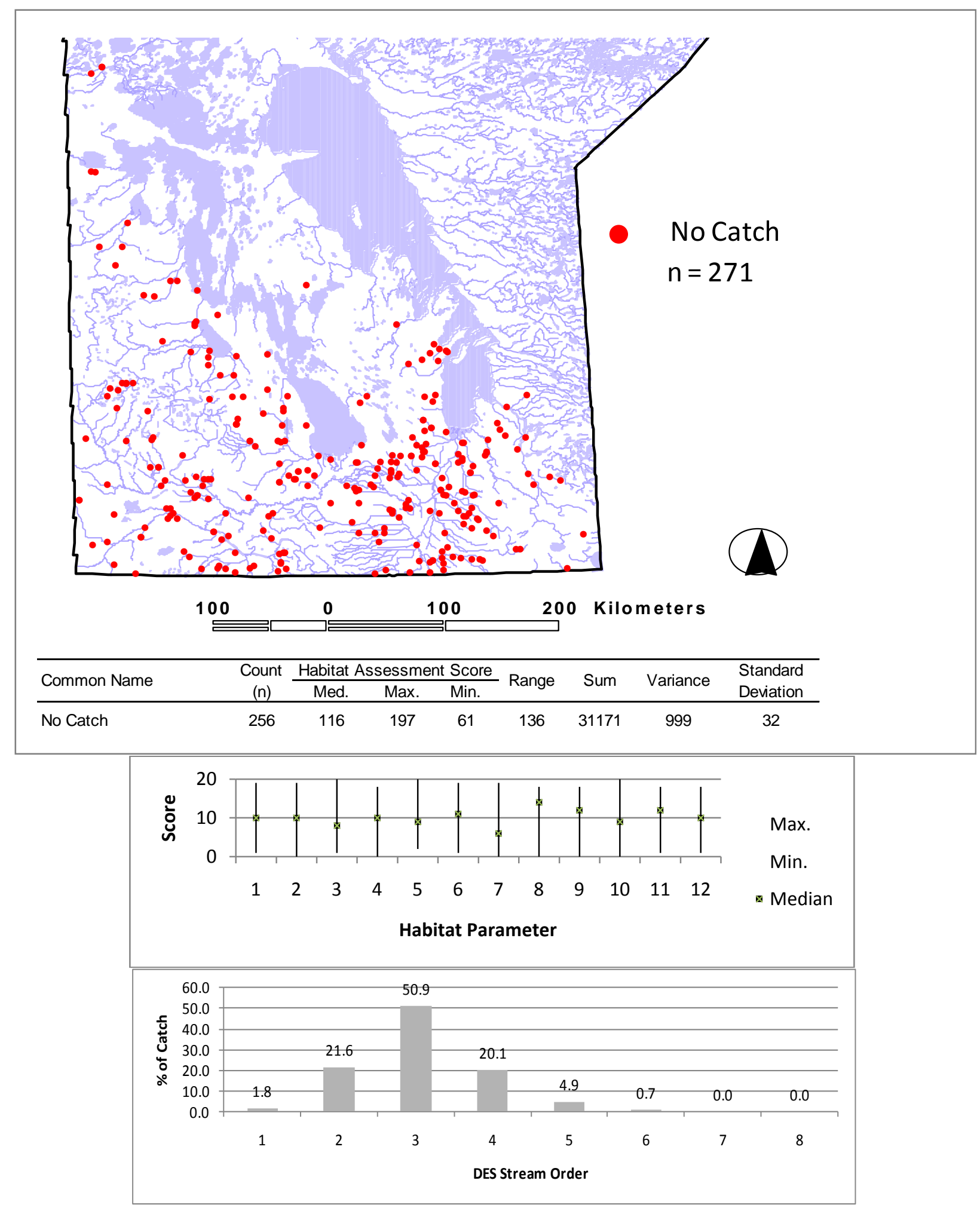

Figure 91: A summary of No Catch results showing collection sites; maximum, minimum and median individual parameter and total Habitat Assessment Scores; and the percent of catch by DES stream order. 


\section{Percent of Catch by Channel Type}

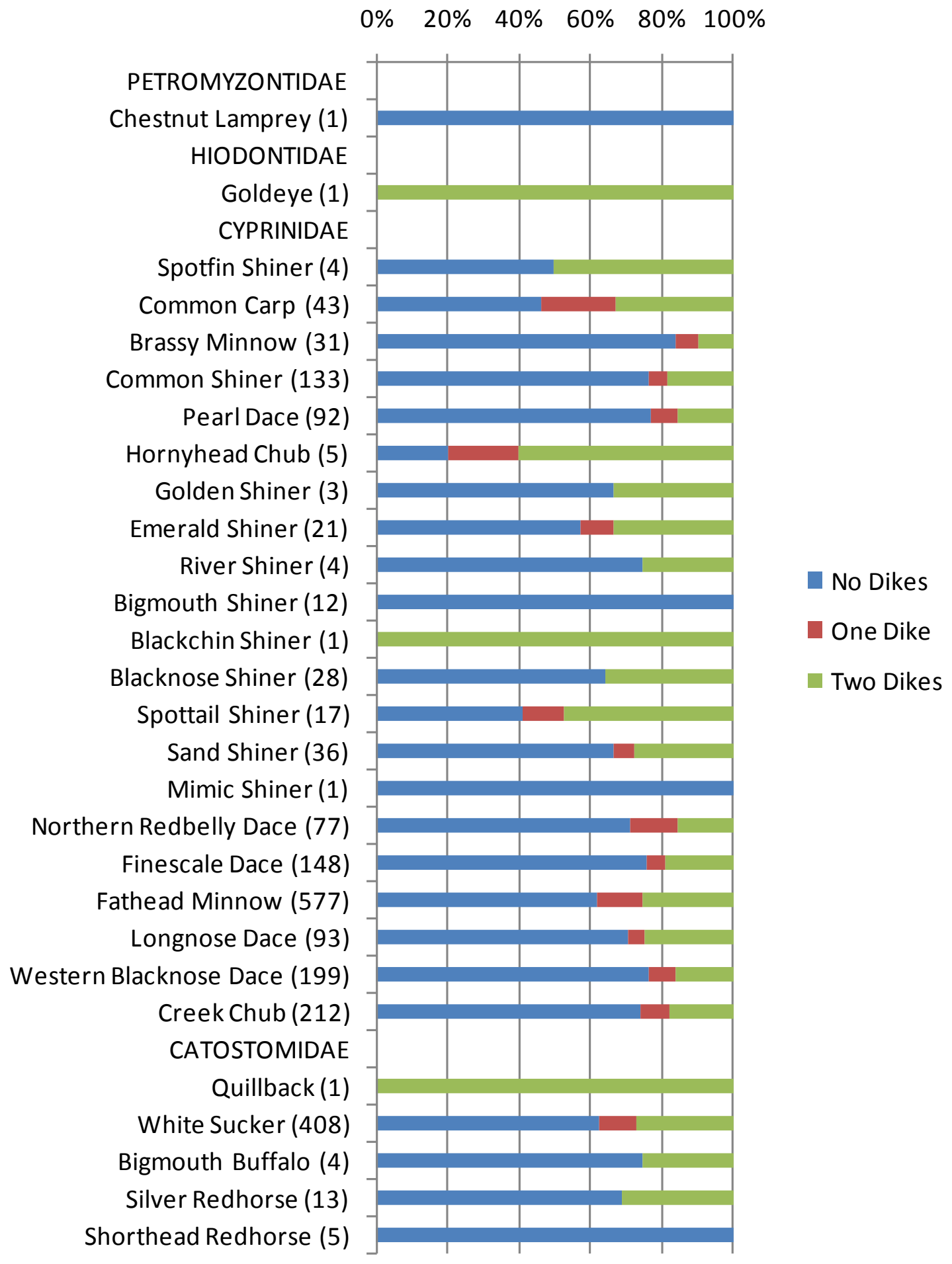

Figure 92: Composition of the catch summarized by Family and by channel type. 


\section{Percent of Catch by Channel Type}

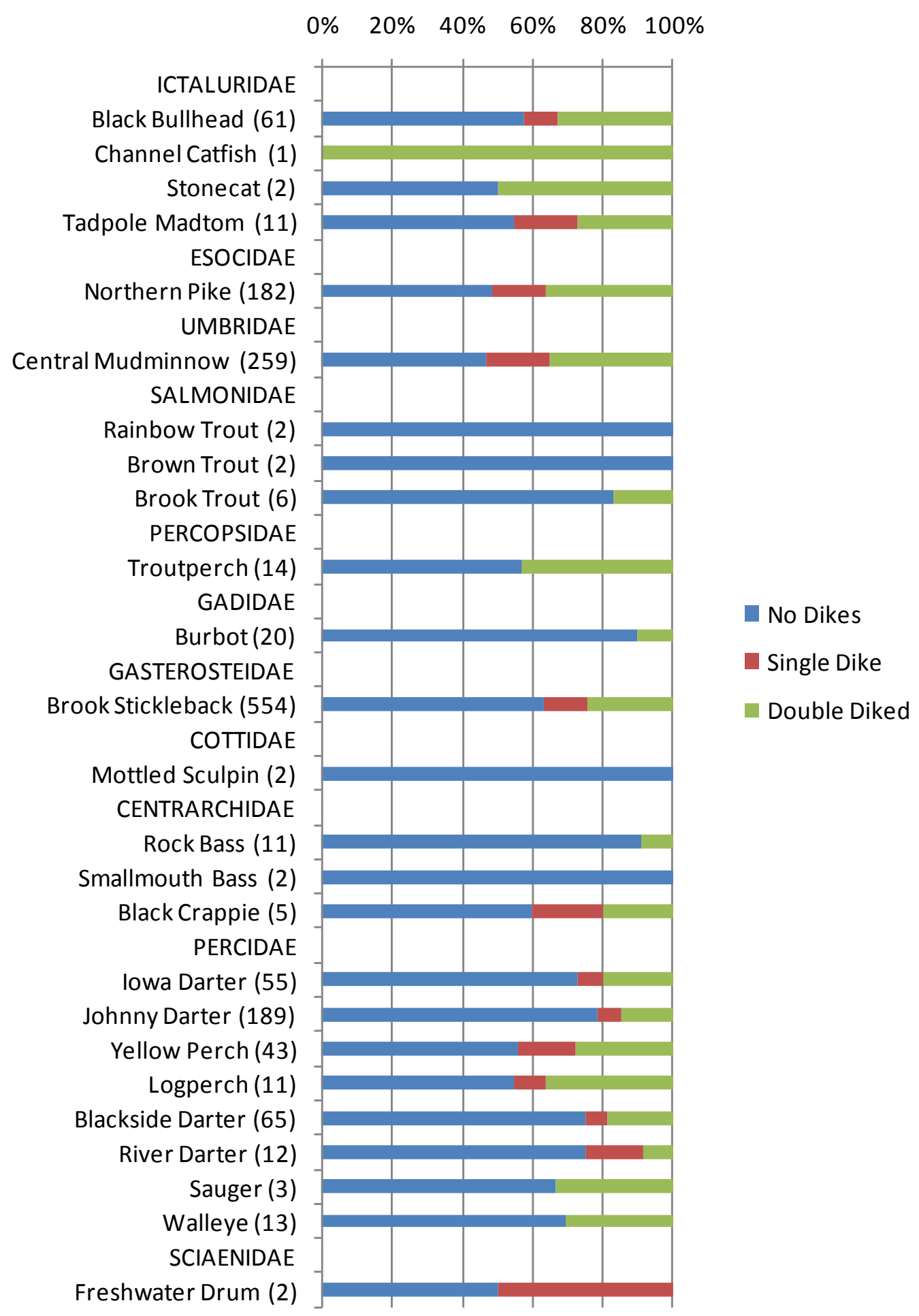

Figure 92: Composition of the catch summarized by Family and by channel type. 


\subsection{WATER CHEMISTRY}

Basic water chemistry measurements were collected at most sites where fishing effort was applied. The author warns that while every effort was made to calibrate and maintain instruments in the field, no effort was made to confirm results from the field with results from certified laboratory analyses.

Water temperature was measured at 1,209 sites and ranged from a low of $0.9^{\circ} \mathrm{C}$ to a high of $29.7^{\circ} \mathrm{C}$.

Dissolved oxygen (DO) was measured at 1,113 sites and ranged from a low of $0.43 \mathrm{mg} / \mathrm{L}$ ( $4 \%$ saturation) to a high of $13.51 \mathrm{mg} / \mathrm{L}$ (135.9\% saturation). The water temperature and altitude at each site was used to calibrate the meter and determine the percent saturation for each DO measurement.

Conductivity was measured at 1,151 sites and ranged from the low of $25 \mu \mathrm{S} / \mathrm{cm}$ to a high of $2000 \mu \mathrm{mhos} / \mathrm{cm}$.

Turbidity was measured at 1,074 sites and ranged from a low of 0 NTU to a high of 300 NTU.

$\mathrm{pH}$ was measured at 1,029 sites and ranged from a low of 6.5 units to a high of $9.24 \mathrm{pH}$ units.

Air temperature was measured at 2,181 sites and results ranged from a low of $-1.0^{\circ} \mathrm{C}$ to a high of $33.5^{\circ} \mathrm{C}$.

The complete list of water chemistry measurements collected from the sampling sites is provided in Appendix 6.

\subsection{FIELD NOTES}

Additional information that field crews deemed to have some relevance to their surveys was recorded as a field note on the data sheets. Crews identified such features as obstructions to fish passage, no flow or low flow conditions, beaver and other wildlife activity, instream and riparian zone features, adjacent land use, and other conditions.

Field notes were recorded at 2,280 sites; a summary of the field notes are provided in Appendix 7.

\subsection{SITE PHOTOGRAPHS AND FIELD SKETCHES}

More than 7,400 site photographs have been included. The list showing site number, descriptive caption, azimuth faced, and the unique number derived for each photograph is included in Appendix 8. A searchable (by site and photo number) PDF binder of all photographs is included in Appendix 10.

Field sketches are also included in a searchable PDF binder in Appendix 11. 


\subsection{DATA AND CLASSIFIED HABITAT MAPPING}

The field data gathered at all sampling sites were mapped and analysed using ESRI Arcview (3.3) Geographic Information System (GIS) software. First, a base map of the study area was constructed using geo-referenced map layers available from the Manitoba Lands Initiative (MLI) website (https://mli2.gov.mb.ca/index.html).

The DES map layers (https://mli2.gov.mb.ca/water resources/des drain index.html) were used to display the lines representing streams and constructed drains that were to be classified based on their habitat type (A to $E$ ). As mentioned previously, most but not all of the 114 sub-watersheds in the series were available in a digital, geo-referenced file format suitable for use in a GIS. Where no geo-referenced DES map was available, the line data representing streams and drains on 1:50,000 or 1:20,000 scale National Topographic Series (NTS) maps were used to display these features. As well, the NTS map layers (https://mli2.gov.mb.ca/topo mapping/index.html) were used to provide the polygon data necessary to display lakes, ponds and other non-linear water bodies

\subsection{DATA MAPPING}

Each of the sampling locations was mapped and displayed as a data point. The colour of each data point was determined by the application of fishing effort and the status of any fish captures (i.e. Indicator Species, Non-Indicator Species, No Catch and No Fishing Effort) and the results were displayed as follows:

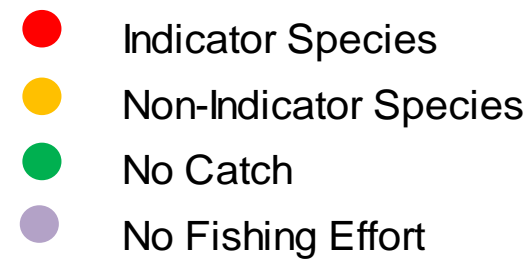

In order for the results to display properly at sites that were visited more than once, or where multiple applications of fishing effort resulted in a different category of fish capture, the effort resulting in the collection of an Indicator Species took precedence over a Non-Indicator Species collection; and a Non-Indicator Species result took precedence over a No Catch result from the same site.

The list of all 2,371 sampling sites was converted from the Excel spreadsheet format shown in Appendix 1, to a text file format (.txt) and was input to the GIS and then converted to an ESRI (.shp) file. The latitude and longitude of each site was used to plot its location. Text files were then created for each of the lists of data provided in Appendices 2-8 (habitat complexity, habitat assessment scores, fishing effort, fish captures, water chemistry, field notes, and captions for site photographs) and these were entered into the GIS where they were joined and linked with their respective data point (sampling site). 
The mapped location of all data points was confirmed visually to ensure they were plotting accurately. Plotting errors were most often a result of field crews transcribing and entering the site latitude and longitude incorrectly. Where numerous drains or streams were located in close proximity, it was often necessary to compare the site photographs with Google Earth ${ }^{\mathrm{TM}}$ or Google Street View ${ }^{\mathrm{TM}}$ images to confirm the plotting accuracy of a site.

The query, charting and analytical tools provided in Arcview were used to sort data, create summary tables, produce maps, charts and other graphics, and to carry out basic statistical analyses of the data.

\subsection{CLASSIFIED HABITAT MAPPING}

The digital DES maps representing the streams and drains that were to be classified were downloaded as geo-referenced ESRI SHAPE (.shp) files. Each map file included the indexed drain lines representing the actual drains in the sub-watershed and the polygon enclosing the map and delineating the drainage boundary of that subwatershed.

The mapping conventions used in the DES drain data sets are fully explained on the MLI website in a document that was prepared by the Geomatics Branch, Land Information Division in response to Water Resource Branch requirements for digital Designation of Drains maps. The document is provided when a user selects an individual DES map for download. The document describes the provincial drainage system, the protocol for drain ordering, the stream segmenting method, and the attributes assigned to each drain segment including drainage basin name, watershed number, DES map number, drain order, topographical features, Geographical Name if any, and the unique numbering code for each drain segment.

Among the topographical attributes assigned to each line (drain) segment by the Geomatics Branch are the following descriptors (feature names):

- Ditch

- Linear Depression

- River/Stream Perennial

- River/Stream Indefinite

- Stream Intermittent

The feature names "Ditch" and "Linear Depression" were assigned to linear, constructed channels and some channelized streams. This attribute correlated well with the field crews' identification of Simple Habitat. Similarly, the line segments assigned the feature name River/Stream Perennial, River/Stream Indefinite, or Stream Intermittent correlated well with the field crews' identification of Complex Habitat. This provided a first step in 
the classification of fish habitat and served to identify and display Simple versus Complex Habitat for those streams and drains within the DES map area of coverage.

In order to display this initial identification of Simple versus Complex Habitat in Arcview GIS, the drain lines with the feature name Linear Depression, or Ditch, were shown as green lines, while the drain lines with any of the River or Stream feature names were displayed in red. Displaying the colour-coded site data points on top of the green, Simple Habitat lines; and the red, Complex Habitat lines, provided the next step in determining the Fish Habitat Classification of the drains and streams in the DES map series. Once the classification of a surveyed reach was determined (e.g. Complex Habitat with Indicator Species) the line segment corresponding to that reach was assigned a habitat type, in this case, Type A.

A new data field (Habitat Type) was then added to the tabular DES line data in Arcview and the classified habitat type for that line segment was entered into the new field. This was completed for all sites and their corresponding line segment. The habitat type at each sampled reach was then displayed and the results were used to guide the application of the habitat type decision flowchart over all of the geo-referenced DES sub-watersheds. The habitat types were displayed as follows:

- Type A

- Type B

- Type C

- Type D

- Type E

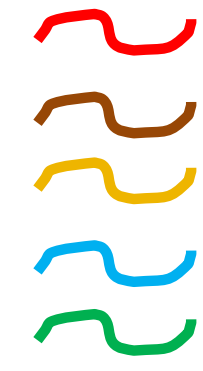

The first iteration of classified habitat maps displayed the classified DES lines (drains) on top of the NTS lines (streams and some drains). The NTS lines were visible in those areas where no DES line data existed. The NTS lines that were visible (i.e. not covered by a DES line) most often represented headwater tributaries that were not mapped as drains on the DES maps. Occasionally, the NTS layer visible beyond the DES line data represented more recent drain construction that was not shown on the older DES maps. The visible NTS line data was identified as Unclassified in the first iteration of the classified habitat maps and was displayed as a purple line. These maps were made available to some drainage practitioners by DFO in 2007.

This unclassified habitat was subsequently classified according to the Fish Habitat Classification Protocol as part of this document preparation. The second iteration of classified habitat maps is provided as a searchable PDF file in Appendix 9. The maps are presented over 155 NTS map tiles at the 1: 50k scale. The NTS map tiles were selected as the 114 sub-watershed maps in the DES series do not have uniform scales. 
Each DES map varies in scale in order to best fit the map to an individual $(38 \mathrm{~cm} \times 90$ $\mathrm{cm})$ paper map sheet.

The 1:50,000 scale National Topographic System (NTS) map tiles used to plot the location of all sampling sites and to show the classification of streams and drains in the study area is shown in Figure 93.

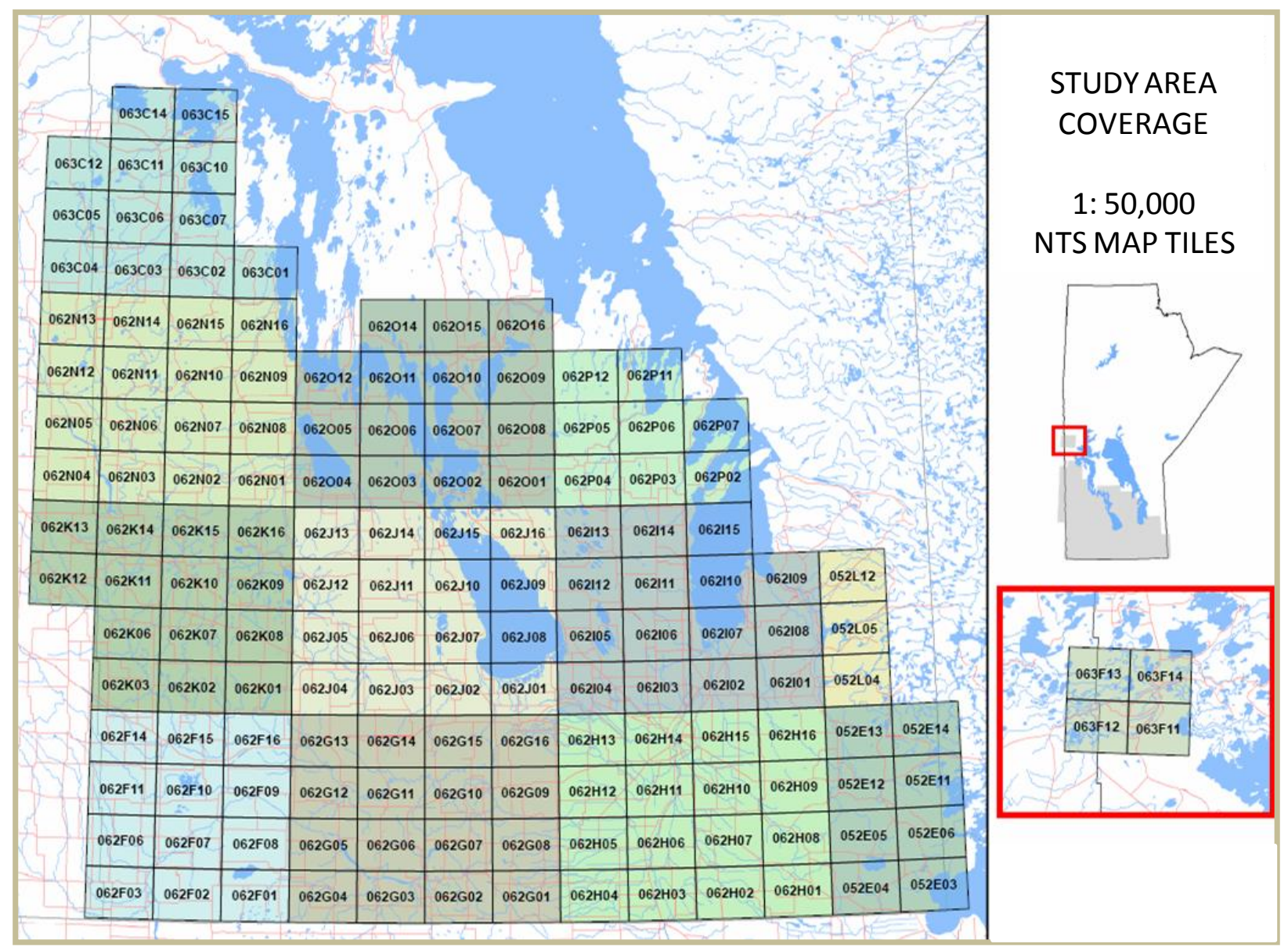

Figure 93: Map showing the area of coverage provided by 154 National Topographic System (NTS) map tiles used to display site locations and classified streams and drains in the study area.

Several principles and methods guided the application of the Fish Habitat Classification Protocol especially in areas that were not extensively sampled.

It was not the intent of the Manitoba Drain Maintenance Committee to create maps displaying a patchwork of habitat types reflecting the timing of routine channel maintenance work. For example, within long segments of linear, constructed drains (Simple Habitat), it is possible to find short drain segments where the channel is attempting to meander within its banks and is starting to assume some of the characteristics of Complex Habitat. If routine drain maintenance was carried out, this would then remove the more diverse channel characteristics that were developing and would bring the drain back to Simple Habitat status. The classification of such drain 
segments as Simple was made to reflect the broad classification of Simple Habitat applied to the rest of the linear drains in that locale.

It was recognized that an individual fish collection at a site can "under" classify habitat. For example, if the presence of an Indicator Species was missed due to poor fishing technique, or difficult fishing conditions, then Type A habitat would be incorrectly classified as Type $C$ habitat. In cases where Indicator Species were known to utilize the system but were not collected at a sampled reach in the lower portions of that system, the higher rated, Type A classification was applied. The decision to apply a higher rating was made based on the information available from a number of sources including discussions with area Fisheries Biologists, Fisheries Branch files including the Fisheries Inventory and Habitat Classification System (FIHCS) data, Manitoba Master Angler records, and the author's field experience.

Field crews typically sampled in the lower, middle and upper reaches of each system in order to efficiently gather the data required to classify habitat. If crews collected an Indicator Species in each of these reaches the resulting classification (Type A or Type B depending on habitat complexity) was extended upstream from the mouth of the system to the uppermost sampling site where Indicator Species were collected.

The locations of breaks between Habitat Types that were dependent on fish community (e.g. between Type A and Type C) were made at obvious locations such as permanent barriers (i.e. dams, perched culverts) or drastic changes in channel morphology (e.g. below the junction of several lower-ordered tributaries) that might modify the composition of the fish community upstream of the feature.

The location of breaks between direct fish habitat (Type A, B, C, and D) and indirect fish habitat (Type E) were made at obvious breaks in habitat connectivity, drastic changes in channel capacity or morphology, the presence of terrestrial vegetation in the channel and the absence of any fish community. Where no breaks in habitat type or channel morphology were evident, the break between direct and indirect fish habitat was made half the distance between features (e.g. half the distance between the uppermost fish collection and the stream origin).

The DES map series describes first-order streams as having less than 1 sq. mile of drainage area. As soon as the drainage area exceeds 1 sq. mile, the DES order changes from first to second order. All DES first-order drains and streams were initially given the Type E classification. Fishing effort was applied at 12 first-order sampling reaches. Fish were collected at eight of these sites. Indicator Species (White Sucker or Burbot) were collected at three sites. Six fish species were collected at one site (X-05229). These sites were appropriately classified as direct fish habitat (Type A, B, C or D). No fishing effort was applied at the other 25 first-order sites surveyed.

More than $80,500 \mathrm{~km}$ of streams and drains have been classified and mapped. The maps are meant to be iterative and will be improved with the addition of new sampling information over time. 



\subsection{ACKNOWLEDGEMENTS}

The author acknowledges the work and support of all members of the Manitoba/Canada Drain Maintenance Committee under the Co-Chairmanship of Beth Hiltz and Joel Hunt. Bruce Webb provided significant support in moving this project forward in its early stages. Todd Schwartz has provided strong guidance, support and good advice through all stages of the project. Jeff Long and Margaret Keast provided advice and reviewed an early draft of the report. Doug Watkinson generously provided laboratory space and his fish identification expertise. Ron Hempel provided invaluable GIS mapping support and technical advice. Maureen Forster and Mark Loudon vastly improved the format and content with their editing and technical review of the draft report. Ernie Watson provided early field assistance and advice on Manitoba's mollusc community.

The author is very pleased to acknowledge the dedication, integrity and hard work of the students involved in the collection of field data. Jaime Clarke, Ashley Wruth, Julie Tingley, Henry Wilson, Ashley Presenger, Vaughn Kachanoski, Jeff Eastman and James Aiken should all take pride in the manner in which they represented DFO over the years of the inventory.

Finally, I must acknowledge the time I was able to spend with Dr. Ken Stewart over the winters of $2004-2006$, when he was contracted to oversee the identification of fish collections. It was an informative, entertaining and exceedingly interesting learning opportunity. He will be sadly missed. 



\subsection{REFERENCES CITED}

Barbour, M.T., Gerritsen, J., Snyder, B.D., and Stribling, J.B. 1999. Rapid bioassessment protocols for use in streams and wadeable rivers: periphyton, benthic macroinvertebrates and fish, Second Edition. Washington, D.C.: U.S. Environmental Protection Agency; Office of Water, Washington, D.C...

Becker, G. C. 1983. Fishes of Wisconsin. University of Wisconsin Press. Madison, WI. $1052 \mathrm{p}$.

Stewart, K. W. and Watkinson, D.A. 2004. The freshwater fishes of Manitoba. University of Manitoba Press, Winnipeg, MB Canada. 278 p.

Stunden Bower, S. 2006. Wet prairie: an environmental history of wetlands, flooding and drainage in agricultural Manitoba, 1810-1980. Thesis (PhD) University of British Columbia. 283 p.

\section{INTERNET DATA SOURCES}

\section{Geographic Information System (GIS) Data}

Province of Manitoba Lands Initiative (MLI). http://web2.gov.mb.ca/mli/mli data/index.html (accessed 28 March 2007)

\section{Hydrometric Data}

Environment Canada. Water Survey of Canada. Archived Hydrometric Data. http://www.wsc.ec.gc.ca/staflo/index e.cfm?cname=main e.cfm (accessed 10 April, 2011).

Drought Severity Interpretation and Historic Climate Maps National Climate Data Center. National Oceanic and Atmospheric Administration. North America Drought Monitor.

http://lwf.ncdc.noaa.gov/oa/climate/monitoring/drought/nadm/nadm.html (accessed 28 April 2012).

Agriculture and Agri-Food Canada. Prairie Farm Rehabilitation Administration. Historic Climate Data Map Archive. http://www4.agr.gc.ca/DW-GS/historicalhistoriques.jspx?lang=eng\&jsEnabled=true (accessed 28 April, 2012).

Environment Canada. Meteorological Services Canada. Archived Meteorological Data. http://www.ec.gc.ca/meteo-weather/default.asp?lang=En\&n=9CA2BD37-1 (accessed 28 April 2012) 
Appendix 1: Table listing the site number, date sampled, site name, latitude and longitude (in decimal degrees DD), DES stream order, DES map number and the corresponding NTS map number for all sites surveyed between 2002 and 2006.

\begin{tabular}{|c|c|c|c|c|c|c|c|}
\hline Site Number & Date & Site Name & $\begin{array}{l}\text { Latitude } \\
\text { (DD) }\end{array}$ & $\begin{array}{l}\text { Longitude } \\
\text { (DD) }\end{array}$ & $\begin{array}{l}\text { DES } \\
\text { Order }\end{array}$ & $\begin{array}{l}\text { DES } \\
\text { Map \# }\end{array}$ & $\begin{array}{l}\text { NTS } \\
\text { Map \# }\end{array}$ \\
\hline D-02-001 & 16-Apr-02 & Sturgeon Creek & 49.87693 & -97.27348 & 4 & 26 & $062 \mathrm{H} 14$ \\
\hline D-02-002 & 17-Apr-02 & La Salle River & 49.69432 & -97.26246 & 5 & 22 & $062 \mathrm{H} 11$ \\
\hline D-02-003 & 19-Apr-02 & Jackfish Creek & 51.13015 & -99.93745 & 4 & 47 & 062004 \\
\hline D-02-004 & 22-Apr-02 & Mink Creek & 51.39807 & -100.45823 & 4 & 49 & 062N08 \\
\hline D-02-005 & 2-May-02 & Turtle River & 50.90373 & -99.50532 & 5 & 45 & $062 J 13$ \\
\hline D-02-006 & 6-May-02 & Unnamed tributary to Assiniboine River & 49.93063 & -98.47507 & 2 & 100 & $062 \mathrm{G} 16$ \\
\hline D-02-007 & 6-May-02 & Rat Creek & 49.84235 & -98.66552 & 3 & 35 & $062 \mathrm{G} 15$ \\
\hline D-02-008 & 7-May-02 & Rat Creek & 50.00472 & -98.45433 & 5 & 35 & 062J01 \\
\hline D-02-009 & 7-May-02 & Norquay Channel & 49.57555 & -97.79270 & 5 & 18 & $062 \mathrm{H} 12$ \\
\hline D-02-010 & 9-May-02 & Manning Canal & 49.59443 & -96.82155 & 4 & 7 & $062 \mathrm{H} 10$ \\
\hline D-02-011 & 9-May-02 & Youville Drain & 49.66448 & -96.85435 & 3 & 8 & $062 \mathrm{H} 10$ \\
\hline D-02-012 & 9-May-02 & Joubert Creek Extension & 49.29627 & -96.50977 & 3 & 4 & $062 \mathrm{H} 07$ \\
\hline D-02-013 & 10-May-02 & Manning Canal & 49.51978 & -96.60098 & 3 & 7 & $062 \mathrm{H} 10$ \\
\hline D-02-014 & 11-May-02 & Main Drain & 49.13332 & -97.14232 & 4 & 2 & $062 \mathrm{H} 03$ \\
\hline D-02-015 & 11-May-02 & Stewart Drain & 49.14073 & -96.98805 & 3 & 2 & $062 \mathrm{H} 02$ \\
\hline D-02-016 & 11-May-02 & Unnamed tributary to Roseau River & 49.20947 & -97.01803 & 3 & 2 & $062 \mathrm{H} 03$ \\
\hline $\mathrm{D}-02-017$ & 11-May-02 & Confluence of unnamed tributary and Roseau River & 49.18372 & -96.82582 & 2 & 2 & 062H02 \\
\hline $\mathrm{D}-02-018$ & 12-May-02 & Selkirk Line Drain & 50.15055 & -96.70206 & 3 & 13 & 062102 \\
\hline D-02-019 & 12-May-02 & Devils Creek & 50.15257 & -96.70889 & 4 & 13 & 062102 \\
\hline D-02-020 & 12-May-02 & Pelletier Drain & 50.23185 & -96.72575 & 3 & 13 & 062102 \\
\hline D-02-021 & 12-May-02 & Township Line Drain & 50.23953 & -96.72597 & 3 & 13 & 062102 \\
\hline D-02-022 & 12-May-02 & Selkirk Line Drain & 50.15055 & -96.70755 & 3 & 13 & 062102 \\
\hline D-02-023 & 12-May-02 & Devils Creek & 50.02843 & -96.61273 & 3 & 13 & 062102 \\
\hline D-02-024 & 12-May-02 & Devils Creek & 49.96167 & -96.55542 & 3 & 13 & $062 \mathrm{H} 15$ \\
\hline D-02-025 & 13-May-02 & Netley Creek & 50.38883 & -97.13925 & 3 & 30 & 062106 \\
\hline
\end{tabular}


Appendix 1: Table listing the site number, date sampled, site name, latitude and longitude (in decimal degrees DD), DES stream order, DES map number and the corresponding NTS map number for all sites surveyed between 2002 and 2006.

\begin{tabular}{|c|c|c|c|c|c|c|c|}
\hline Site Number & Date & Site Name & $\begin{array}{l}\text { Latitude } \\
\text { (DD) }\end{array}$ & $\begin{array}{l}\text { Longitude } \\
\text { (DD) }\end{array}$ & $\begin{array}{l}\text { DES } \\
\text { Order }\end{array}$ & $\begin{array}{l}\text { DES } \\
\text { Map \# }\end{array}$ & $\begin{array}{l}\text { NTS } \\
\text { Map \# }\end{array}$ \\
\hline D-02-026 & 13-May-02 & Netley Creek & 50.40343 & -97.15950 & 3 & 30 & 062106 \\
\hline D-02-027 & 13-May-02 & Netley Creek & 50.47513 & -97.22985 & 3 & 30 & 062106 \\
\hline D-02-028 & 13-May-02 & Netley Creek & 50.46792 & -97.20620 & 3 & 30 & 062106 \\
\hline D-02-029 & 14-May-02 & East Colony Creek & 50.03435 & -97.33232 & 2 & 26 & 062103 \\
\hline D-02-030 & 14-May-02 & Grassmere Creek Drain & 50.01677 & -97.16110 & 5 & 27 & 062103 \\
\hline$D-02-031$ & 15-May-02 & Riding Mountain Drain & 50.52080 & -99.30978 & 5 & 43 & 062J11 \\
\hline$D-02-032$ & 15-May-02 & Big Grass River & 50.46860 & -98.91567 & 5 & 43 & 062J07 \\
\hline D-02-033 & 15-May-02 & Big Grass River & 50.50593 & -98.97175 & 5 & 43 & 062J10 \\
\hline D-02-034 & 16-May-02 & Norgate Drain & 50.68317 & -99.41520 & 4 & 44 & 062J11 \\
\hline D-02-035 & 16-May-02 & Norgate Drain & 50.69803 & -99.39168 & 4 & 44 & 062J11 \\
\hline D-02-036 & 16-May-02 & Turtle River & 50.79348 & -99.40927 & 5 & 44 & 062J14 \\
\hline D-02-037 & 16-May-02 & McKinnon Creek & 50.84567 & -99.47237 & 4 & 44 & 062J14 \\
\hline D-02-038 & 16-May-02 & Harcus Drain & 50.72732 & -98.83317 & 4 & 110 & 062J10 \\
\hline D-02-039 & 16-May-02 & Garrioch Creek & 50.80133 & -98.93403 & 4 & 110 & 062J15 \\
\hline D-02-040 & 16-May-02 & Wilson Creek & 50.75633 & -99.53147 & 3 & 44 & 062J13 \\
\hline D-02-041 & 16-May-02 & Turtle River & 50.90373 & -99.50532 & 5 & 45 & 062J13 \\
\hline D-02-042 & 17-May-02 & Turtle River & 50.90373 & -99.50532 & 5 & 45 & 062J13 \\
\hline D-02-043 & 21-May-02 & Crawford Creek & 51.07362 & -99.81992 & 3 & 46 & 062004 \\
\hline D-02-044 & 21-May-02 & Jackfish Creek & 51.09116 & -99.95771 & 4 & 47 & 062004 \\
\hline D-02-045 & 22-May-02 & McKinnon Creek & 50.78313 & -99.55470 & 3 & 44 & 062J13 \\
\hline D-02-046 & 22-May-02 & Turtle River & 50.90373 & -99.50532 & 5 & 45 & 062J13 \\
\hline D-02-047 & 23-May-02 & South Bay Drain & 51.66407 & -99.99042 & 3 & 102 & 062012 \\
\hline D-02-048 & 23-May-02 & Red Deer Point Drain & 51.70668 & -99.96682 & 3 & 102 & 062012 \\
\hline D-02-049 & 23-May-02 & Bicton Heath Drain & 51.59275 & -99.97164 & 3 & 102 & 062012 \\
\hline D-02-050 & 23-May-02 & Rice Lake Drain & 51.56550 & -99.88557 & 3 & 102 & 062012 \\
\hline
\end{tabular}


Appendix 1: Table listing the site number, date sampled, site name, latitude and longitude (in decimal degrees DD), DES stream order, DES map number and the corresponding NTS map number for all sites surveyed between 2002 and 2006.

\begin{tabular}{|c|c|c|c|c|c|c|c|}
\hline Site Number & Date & Site Name & $\begin{array}{l}\text { Latitude } \\
\text { (DD) }\end{array}$ & $\begin{array}{l}\text { Longitude } \\
\text { (DD) }\end{array}$ & $\begin{array}{l}\text { DES } \\
\text { Order }\end{array}$ & $\begin{array}{l}\text { DES } \\
\text { Map \# }\end{array}$ & $\begin{array}{l}\text { NTS } \\
\text { Map \# }\end{array}$ \\
\hline D-02-051 & 24-May-02 & Unnamed tributary to Ochre River & 50.99687 & -99.82850 & 2 & 46 & $062 J 13$ \\
\hline D-02-052 & 24-May-02 & Ochre River & 50.97818 & -99.77788 & 5 & 46 & 062J13 \\
\hline D-02-053 & 24-May-02 & Kerosene Creek & 50.97812 & -99.74438 & 2 & 45 & 062J13 \\
\hline D-02-054 & 24-May-02 & Henderson Creek & 50.90363 & -99.66882 & 4 & 45 & 062J13 \\
\hline D-02-055 & 24-May-02 & Kergwenan Drain & 50.94843 & -99.55535 & 3 & 45 & 062J13 \\
\hline D-02-056 & 24-May-02 & Henderson Creek & 50.90430 & -99.67117 & 4 & 45 & 062J13 \\
\hline D-02-057 & 24-May-02 & Henderson Creek & 50.92640 & -99.55498 & 4 & 45 & 062J13 \\
\hline D-02-058 & 24-May-02 & Kergwenan Drain & 50.94848 & -99.55466 & 3 & 45 & 062J13 \\
\hline D-02-059 & 27-May-02 & Kergwenan Drain & 50.94863 & -99.53187 & 3 & 45 & 062J13 \\
\hline D-02-060 & 27-May-02 & North Snake Creek & 50.44565 & -99.50560 & 3 & 43 & 062J05 \\
\hline D-02-061 & 28-May-02 & Norquay Channel & 49.56402 & -97.82010 & 5 & 18 & $062 \mathrm{H} 12$ \\
\hline D-02-062 & 28-May-02 & Norquay Channel & 49.53202 & -97.86412 & 5 & 18 & $062 \mathrm{H} 12$ \\
\hline D-02-063 & 28-May-02 & Assiniboine River & 49.93663 & -98.27313 & 8 & 24 & $062 \mathrm{G} 16$ \\
\hline D-02-064 & 29-May-02 & Jordan Creek Drain & 50.38800 & -98.96215 & 4 & 42 & 062J07 \\
\hline D-02-065 & 29-May-02 & Big Grass River & 50.38790 & -98.86935 & 5 & 42 & 062J07 \\
\hline D-02-066 & 29-May-02 & Big Grass River & 50.50593 & -98.97183 & 5 & 43 & 062J10 \\
\hline D-02-067 & 29-May-02 & Big Grass River & 50.55035 & -99.14667 & 5 & 43 & 062J11 \\
\hline D-02-068 & 29-May-02 & Big Grass River Angle Ditch & 50.58859 & -99.19320 & 4 & 43 & 062J11 \\
\hline D-02-069 & 29-May-02 & North Snake Creek & 50.47765 & -99.33518 & 4 & 43 & 062J06 \\
\hline D-02-070 & 30-May-02 & Big Grass River & 50.50593 & -98.97183 & 5 & 43 & 062J10 \\
\hline D-02-071 & 30-May-02 & North Snake Creek & 50.44742 & -99.40038 & 3 & 43 & 062J06 \\
\hline D-02-072 & 3-Jun-02 & Netley Creek & 50.46070 & -97.37798 & 2 & 30 & 062106 \\
\hline D-02-073 & 3-Jun-02 & Netley Creek & 50.38857 & -97.13877 & 3 & 30 & 062106 \\
\hline D-02-074 & 3-Jun-02 & Netley Creek & 50.30882 & -96.99765 & 5 & 30 & 062107 \\
\hline D-02-075 & 4-Jun-02 & Netley Creek & 50.37372 & -97.11280 & 3 & 30 & 062106 \\
\hline
\end{tabular}


Appendix 1: Table listing the site number, date sampled, site name, latitude and longitude (in decimal degrees DD), DES stream order, DES map number and the corresponding NTS map number for all sites surveyed between 2002 and 2006.

\begin{tabular}{|c|c|c|c|c|c|c|c|}
\hline Site Number & Date & Site Name & $\begin{array}{l}\text { Latitude } \\
\text { (DD) }\end{array}$ & $\begin{array}{l}\text { Longitude } \\
\text { (DD) }\end{array}$ & $\begin{array}{l}\text { DES } \\
\text { Order }\end{array}$ & $\begin{array}{l}\text { DES } \\
\text { Map \# }\end{array}$ & $\begin{array}{l}\text { NTS } \\
\text { Map \# }\end{array}$ \\
\hline D-02-076 & 5-Jun-02 & Netley Creek & 50.37372 & -97.11280 & 3 & 30 & 062106 \\
\hline D-02-077 & 5-Jun-02 & Netley Creek & 50.40343 & -97.15950 & 3 & 30 & 062106 \\
\hline D-02-078 & 5-Jun-02 & Netley Creek & 50.45888 & -97.18337 & 3 & 30 & 062106 \\
\hline D-02-079 & 5-Jun-02 & Netley Creek & 50.44797 & -97.16535 & 3 & 30 & 062106 \\
\hline D-02-080 & 5-Jun-02 & Netley Creek & 50.43299 & -97.15985 & 3 & 30 & 062106 \\
\hline D-02-081 & 6-Jun-02 & Willow Creek & 50.57567 & -97.02235 & 4 & $31 \mathrm{E}$ & 062111 \\
\hline D-02-082 & 6-Jun-02 & Willow Creek & 50.58942 & -97.06823 & 4 & $31 \mathrm{E}$ & $062 \mid 11$ \\
\hline D-02-083 & 6-Jun-02 & Bass Drain & 50.57785 & -97.11425 & 4 & $31 \mathrm{E}$ & $062 \mid 11$ \\
\hline D-02-084 & 12-Jun-02 & Burnt Lake Drain & 50.72057 & -98.07592 & 3 & $31 \mathrm{~W}$ & 062J09 \\
\hline D-02-085 & 12-Jun-02 & Swan Creek & 50.77802 & -98.15787 & 3 & $31 \mathrm{~W}$ & 062J16 \\
\hline D-02-086 & 12-Jun-02 & Swan Lake Dam & 50.80042 & -98.16952 & 3 & $31 \mathrm{~W}$ & 062J16 \\
\hline D-02-087 & 12-Jun-02 & Burnt Lake Drain & 50.72977 & -97.90198 & 3 & $31 \mathrm{~W}$ & $062 \mid 12$ \\
\hline D-02-088 & 13-Jun-02 & Wagon Creek & 50.54803 & -98.03767 & 2 & $31 \mathrm{~W}$ & 062J09 \\
\hline D-02-089 & 13-Jun-02 & Hatchery Drain & 50.62405 & -98.09477 & 3 & $31 \mathrm{~W}$ & 062J09 \\
\hline D-02-090 & 13-Jun-02 & Mud Lake Drain & 50.66872 & -98.04380 & 3 & $31 \mathrm{~W}$ & 062J09 \\
\hline D-02-091 & 13-Jun-02 & Mud Lake Drain & 50.69820 & -98.11128 & 3 & $31 \mathrm{~W}$ & 062J09 \\
\hline D-02-092 & 13-Jun-02 & Burnt Lake Drain & 50.76128 & -98.04123 & 3 & $31 \mathrm{~W}$ & 062J16 \\
\hline D-02-093 & 14-Jun-02 & Crawford Creek & 51.08128 & -99.78195 & 3 & 46 & 062004 \\
\hline D-02-094 & 18-Jun-02 & Long Lake Drain & 50.07443 & -98.01000 & 4 & 25 & 062J01 \\
\hline D-02-095 & 18-Jun-02 & Long Lake Drain & 50.01485 & -97.76068 & 4 & 25 & 062104 \\
\hline D-02-096 & 19-Jun-02 & Bachman Drain & 50.12057 & -96.45190 & 3 & 10 & 062101 \\
\hline D-02-097 & 19-Jun-02 & Oneschuk Drain & 50.20783 & -96.46285 & 3 & 10 & 062101 \\
\hline D-02-098 & 19-Jun-02 & $\mathrm{T}$ - Drain & 50.31130 & -96.49982 & 3 & 10 & 062108 \\
\hline D-02-099 & 19-Jun-02 & $\mathrm{T}$ - Drain & 50.31140 & -96.48218 & 3 & 10 & 062108 \\
\hline D-02-100 & 20-Jun-02 & U - Drain & 50.28262 & -96.38058 & 3 & 10 & 062108 \\
\hline
\end{tabular}


Appendix 1: Table listing the site number, date sampled, site name, latitude and longitude (in decimal degrees DD), DES stream order, DES map number and the corresponding NTS map number for all sites surveyed between 2002 and 2006.

\begin{tabular}{|c|c|c|c|c|c|c|c|}
\hline Site Number & Date & Site Name & $\begin{array}{l}\text { Latitude } \\
\text { (DD) }\end{array}$ & $\begin{array}{l}\text { Longitude } \\
\text { (DD) }\end{array}$ & $\begin{array}{l}\text { DES } \\
\text { Order }\end{array}$ & $\begin{array}{l}\text { DES } \\
\text { Map \# }\end{array}$ & $\begin{array}{l}\text { NTS } \\
\text { Map \# }\end{array}$ \\
\hline D-02-101 & 20-Jun-02 & Matychak Drain & 50.17923 & -96.38352 & 3 & 10 & 062101 \\
\hline D-02-102 & 20-Jun-02 & Selkirk Line East & 50.14992 & -96.40622 & 3 & 10 & 062101 \\
\hline D-02-103 & 20-Jun-02 & Bachman East Drain & 50.12028 & -96.36053 & 3 & 10 & 062101 \\
\hline D-02-104 & 20-Jun-02 & Bears Creek & 50.04680 & -96.37635 & 3 & 10 & 062101 \\
\hline D-02-105 & 21-Jun-02 & Jordan Creek Drain & 50.38797 & -99.32143 & 3 & 42 & 062J06 \\
\hline D-02-106 & 21-Jun-02 & Award II Drain & 50.37322 & -99.40058 & 3 & 39 & 062J06 \\
\hline$D-02-107$ & 21-Jun-02 & Unnamed tributary to Award II Drain & 50.40282 & -99.49228 & 2 & 39 & 062J06 \\
\hline D-02-108 & 21-Jun-02 & Conjuring Creek & 50.79505 & -101.29940 & 3 & 92 & $062 \mathrm{~K} 14$ \\
\hline D-02-109 & 26-Jun-02 & Sibbald Creek & 49.92352 & -100.39337 & 3 & 82 & $062 \mathrm{~F} 16$ \\
\hline D-02-110 & 26-Jun-02 & Sibbald Creek & 49.93110 & -100.43728 & 3 & 82 & $062 \mathrm{~F} 16$ \\
\hline D-02-111 & 26-Jun-02 & Sibbald Creek & 49.94523 & -100.43300 & 3 & 82 & $062 \mathrm{~F} 16$ \\
\hline D-02-112 & 3-Jul-02 & Boundary Drain & 51.24383 & -98.37355 & 2 & 34 & 062001 \\
\hline D-02-113 & 3-Jul-02 & Boundary Drain & 51.24385 & -98.36167 & 2 & 34 & 062001 \\
\hline D-02-114 & 3-Jul-02 & Unnamed tributary to Birch Creek Lateral & 51.34210 & -98.46240 & 2 & 111 & 062008 \\
\hline $\mathrm{D}-02-115$ & 3-Jul-02 & Birch Creek & 51.42783 & -98.54363 & 3 & 111 & 062007 \\
\hline$D-02-116$ & 3-Jul-02 & Birch Creek & 51.47097 & -98.52170 & 3 & 111 & 062007 \\
\hline D-02-117 & 3-Jul-02 & Birch Creek & 51.46378 & -98.52258 & 3 & 111 & 062007 \\
\hline D-02-118 & 3-Jul-02 & Birch Creek & 51.43532 & -98.53252 & 3 & 111 & 062007 \\
\hline D-02-119 & 4-Jul-02 & Pioneer Drain & 51.09663 & -98.42030 & 3 & 34 & 062001 \\
\hline D-02-120 & 4-Jul-02 & Confluence of Marne Drain and Pioneer Drain & 51.09672 & -98.43327 & 3 & 34 & 062001 \\
\hline $\mathrm{D}-02-121$ & 4-Jul-02 & Camper Drain & 51.06712 & -98.42022 & 3 & 34 & 062001 \\
\hline D-02-122 & 4-Jul-02 & Little Dog Lake Drain & 51.02313 & -98.39078 & 3 & 34 & 062001 \\
\hline $\mathrm{D}-02-123$ & 4-Jul-02 & Camper Drain & 51.06705 & -98.27943 & 2 & 34 & 062001 \\
\hline$D-02-124$ & 4-Jul-02 & Pioneer Drain & 51.10175 & -98.28295 & 2 & 34 & 062001 \\
\hline D-02-125 & 9-Jul-02 & Broadvalley Drain & 51.08263 & -97.59397 & 4 & 33 & 062P04 \\
\hline
\end{tabular}


Appendix 1: Table listing the site number, date sampled, site name, latitude and longitude (in decimal degrees DD), DES stream order, DES map number and the corresponding NTS map number for all sites surveyed between 2002 and 2006.

\begin{tabular}{|c|c|c|c|c|c|c|c|}
\hline Site Number & Date & Site Name & $\begin{array}{l}\text { Latitude } \\
\text { (DD) }\end{array}$ & $\begin{array}{l}\text { Longitude } \\
\text { (DD) }\end{array}$ & $\begin{array}{l}\text { DES } \\
\text { Order }\end{array}$ & $\begin{array}{l}\text { DES } \\
\text { Map \# }\end{array}$ & $\begin{array}{l}\text { NTS } \\
\text { Map \# }\end{array}$ \\
\hline D-02-126 & 9-Jul-02 & Dumoulin Drain & 51.09735 & -97.56218 & 3 & 33 & 062P04 \\
\hline D-02-127 & 9-Jul-02 & East Letexif Drain & 51.13812 & -97.55582 & 2 & 33 & 062P04 \\
\hline D-02-128 & 9-Jul-02 & Meridian Drain & 51.17092 & -97.49727 & 3 & 33 & 062P03 \\
\hline D-02-129 & 9-Jul-02 & Fisher River & 51.15618 & -97.54577 & 4 & 33 & 062P04 \\
\hline D-02-130 & 9-Jul-02 & Fisher River & 51.10473 & -97.62615 & 3 & 33 & 062P04 \\
\hline$D-02-131$ & 9-Jul-02 & Plishka Drain Lateral & 51.06540 & -97.67282 & 4 & 33 & 062P04 \\
\hline D-02-132 & 10-Jul-02 & Kilkenny Drain & 51.08260 & -97.79030 & 3 & 33 & 062P04 \\
\hline D-02-133 & 10-Jul-02 & Fisher River & 51.12678 & -97.77282 & 4 & 33 & 062P04 \\
\hline D-02-134 & 10-Jul-02 & Leroy Drain & 51.17647 & -97.76693 & 2 & 33 & 062P04 \\
\hline D-02-135 & 10-Jul-02 & Broadvalley Drain & 51.02362 & -97.58398 & 4 & 33 & 062P04 \\
\hline D-02-136 & 10-Jul-02 & Unnamed tributary to Icelandic Drain & 50.86673 & -97.60055 & 3 & 32 & 062113 \\
\hline D-02-137 & 12-Jul-02 & Fishing River & 51.46843 & -100.36797 & 4 & 49 & 062N08 \\
\hline D-02-138 & 12-Jul-02 & Stoney Creek & 51.47278 & -100.36972 & 2 & 49 & 062N08 \\
\hline D-02-139 & 12-Jul-02 & Fishing River & 51.42655 & -100.55722 & 3 & 49 & 062N07 \\
\hline$D-02-140$ & 12-Jul-02 & Mink Creek & 51.41390 & -100.35332 & 4 & 49 & 062N08 \\
\hline$D-02-141$ & 12-Jul-02 & Zoria Drain & 51.39054 & -100.35762 & 1 & 95 & 062N08 \\
\hline D-02-142 & 17-Jul-02 & Shanty Creek & 51.52330 & -100.41665 & 4 & 102 & 062N09 \\
\hline D-02-143 & 17-Jul-02 & North Duck River & 52.03170 & -100.64973 & 4 & 105 & 063C02 \\
\hline D-02-144 & 17-Jul-02 & Old Government Drain & 52.07495 & -101.03702 & 3 & 52 & $063 \mathrm{C03}$ \\
\hline D-02-145 & 17-Jul-02 & East Favel River & 52.11435 & -101.05157 & 4 & 52 & $063 \mathrm{C03}$ \\
\hline D-02-146 & 17-Jul-02 & West Favel River & 52.16528 & -101.04097 & 4 & 52 & $063 C 03$ \\
\hline D-02-147 & 17-Jul-02 & Swinowski Drain & 52.17317 & -100.96590 & 2 & 53 & $063 \mathrm{C02}$ \\
\hline D-02-148 & 17-Jul-02 & Swinowski Drain & 52.17210 & -100.96577 & 4 & 53 & $063 \mathrm{C02}$ \\
\hline D-02-149 & 17-Jul-02 & Sinclair River & 52.17875 & -100.96560 & 5 & 53 & $063 \mathrm{C} 02$ \\
\hline D-02-150 & 18-Jul-02 & Avonlea Drain & 52.17263 & -101.10855 & 3 & 61 & $063 \mathrm{C03}$ \\
\hline
\end{tabular}


Appendix 1: Table listing the site number, date sampled, site name, latitude and longitude (in decimal degrees DD), DES stream order, DES map number and the corresponding NTS map number for all sites surveyed between 2002 and 2006.

\begin{tabular}{|c|c|c|c|c|c|c|c|}
\hline Site Number & Date & Site Name & $\begin{array}{l}\text { Latitude } \\
\text { (DD) }\end{array}$ & $\begin{array}{l}\text { Longitude } \\
\text { (DD) }\end{array}$ & $\begin{array}{l}\text { DES } \\
\text { Order }\end{array}$ & $\begin{array}{l}\text { DES } \\
\text { Map \# }\end{array}$ & $\begin{array}{l}\text { NTS } \\
\text { Map \# }\end{array}$ \\
\hline D-02-151 & 18-Jul-02 & Unnamed tributary to Swan River & 52.18808 & -101.10855 & 2 & 61 & $063 C 03$ \\
\hline D-02-152 & 18-Jul-02 & Unnamed tributary to Swan River & 52.21363 & -101.03717 & 2 & 61 & $063 C 03$ \\
\hline D-02-153 & 18-Jul-02 & Farden Drain & 52.12183 & -100.95838 & 3 & 53 & $063 \mathrm{C} 02$ \\
\hline D-02-154 & 18-Jul-02 & Townline Drain & 52.23073 & -100.94163 & 3 & 53 & 063C02 \\
\hline D-02-155 & 18-Jul-02 & Lalecheur Drain & 52.27095 & -100.94170 & 4 & 53 & $063 C 07$ \\
\hline$D-02-156$ & 18-Jul-02 & Unnamed tributary to Community Pasture Drain & 52.28895 & -100.85368 & 2 & 53 & $063 C 07$ \\
\hline$D-02-157$ & 18-Jul-02 & Renwer Drain & 52.10233 & -100.79868 & 3 & 53 & 063C02 \\
\hline$D-02-158$ & 18-Jul-02 & Jarosz Drain & 52.09987 & -100.83532 & 3 & 53 & $063 \mathrm{C} 02$ \\
\hline D-02-159 & 18-Jul-02 & Jeski Drain & 52.08535 & -101.11763 & 3 & 52 & $063 \mathrm{C} 03$ \\
\hline D-02-160 & 23-Jul-02 & Ochre River & 51.05138 & -99.78653 & 5 & 46 & 062004 \\
\hline D-02-161 & 23-Jul-02 & Scott Creek & 50.84495 & -99.55512 & 4 & 44 & 062J13 \\
\hline D-02-162 & 23-Jul-02 & Tityk Drain & 50.74238 & -99.35180 & 2 & 44 & 062J11 \\
\hline D-02-163 & 24-Jul-02 & Eden Creek & 50.37138 & -99.49287 & 3 & 39 & 062J06 \\
\hline D-02-164 & 24-Jul-02 & Eden Creek & 50.37325 & -99.46195 & 3 & 39 & 062J06 \\
\hline$D-02-165$ & 24-Jul-02 & Eden Creek & 50.34567 & -99.44668 & 3 & 39 & 062J06 \\
\hline$D-02-166$ & 24-Jul-02 & Law Creek & 50.32883 & -99.48478 & 3 & 39 & 062J06 \\
\hline D-02-167 & 24-Jul-02 & Law Creek & 50.34940 & -99.56145 & 3 & 39 & 062J05 \\
\hline D-02-168 & 24-Jul-02 & Unnamed tributary to Spring Creek & 50.33100 & -99.63035 & 1 & 39 & 062J05 \\
\hline D-02-169 & 24-Jul-02 & Unnamed tributary to Spring Creek & 50.30098 & -99.62087 & 2 & 39 & 062J05 \\
\hline D-02-170 & 24-Jul-02 & Stony Creek & 50.26445 & -99.63103 & 4 & 39 & 062J05 \\
\hline D-02-171 & 25-Jul-02 & Bear Cat Creek & 50.18122 & -99.27220 & 2 & 39 & 062J03 \\
\hline $\mathrm{D}-02-172$ & 25-Jul-02 & Unnamed tributary to Bear Cat Creek & 50.16723 & -99.21898 & 2 & 39 & 062J03 \\
\hline $\mathrm{D}-02-173$ & 25-Jul-02 & Helston Drain & 50.12387 & -99.19238 & 3 & 39 & 062J03 \\
\hline $\mathrm{D}-02-174$ & 25-Jul-02 & Unnamed tributary to Bear Creek & 50.07642 & -99.15807 & 2 & 39 & 062J03 \\
\hline D-02-175 & 25-Jul-02 & Bear Creek & 50.11167 & -99.18533 & 3 & 39 & 062J03 \\
\hline
\end{tabular}


Appendix 1: Table listing the site number, date sampled, site name, latitude and longitude (in decimal degrees DD), DES stream order, DES map number and the corresponding NTS map number for all sites surveyed between 2002 and 2006.

\begin{tabular}{|c|c|c|c|c|c|c|c|}
\hline Site Number & Date & Site Name & $\begin{array}{l}\text { Latitude } \\
\text { (DD) }\end{array}$ & $\begin{array}{l}\text { Longitude } \\
\text { (DD) }\end{array}$ & $\begin{array}{l}\text { DES } \\
\text { Order }\end{array}$ & $\begin{array}{l}\text { DES } \\
\text { Map \# }\end{array}$ & $\begin{array}{l}\text { NTS } \\
\text { Map \# }\end{array}$ \\
\hline D-02-176 & 25-Jul-02 & Gillespie Drain & 50.07437 & -99.11040 & 3 & 38 & 062J03 \\
\hline D-02-177 & 25-Jul-02 & Helston Drain & 50.13720 & -99.11050 & 3 & 39 & 062J03 \\
\hline D-02-178 & 25-Jul-02 & Bear Creek & 50.13733 & -99.09235 & 4 & 39 & 062J03 \\
\hline D-02-179 & 30-Jul-02 & Fishtown Creek & 52.46328 & -101.08333 & 3 & 90 & $063 C 06$ \\
\hline D-02-180 & 30-Jul-02 & Fishtown Creek & 52.44917 & -100.99160 & 3 & 90 & $063 C 07$ \\
\hline D-02-181 & 31-Jul-02 & Birch River & 52.39737 & -101.12333 & 3 & 90 & $063 C 06$ \\
\hline D-02-182 & 31-Jul-02 & Indian Birch River & 52.43443 & -100.95175 & 4 & 90 & $063 C 07$ \\
\hline D-02-183 & 31-Jul-02 & Bell River & 52.59112 & -101.09123 & 4 & 97 & $063 C 11$ \\
\hline D-02-184 & 31-Jul-02 & Bell River Drain & 52.62383 & -100.99202 & 4 & 97 & $063 C 10$ \\
\hline D-02-185 & 1-Aug-02 & Martin Creek & 52.40798 & -101.03877 & 2 & 90 & $063 C 06$ \\
\hline D-02-186 & 1-Aug-02 & Robinson Creek & 52.34798 & -101.03883 & 3 & 90 & $063 C 06$ \\
\hline D-02-187 & 1-Aug-02 & Unnamed tributary to Woody River & 52.40201 & -100.89699 & 2 & 90 & $063 C 07$ \\
\hline $\mathrm{D}-02-188$ & 1-Aug-02 & Lalecheur Drain & 52.23075 & -100.89230 & 3 & 53 & $063 \mathrm{C02}$ \\
\hline D-02-189 & 1-Aug-02 & Unnamed tributary to Sinclair River & 52.20128 & -100.91791 & 1 & 53 & $063 \mathrm{C02}$ \\
\hline $\mathrm{D}-02-190$ & 1-Aug-02 & Sinclair River & 52.13920 & -100.77498 & 3 & 53 & $063 \mathrm{C02}$ \\
\hline D-02-191 & 15-Aug-02 & Vermillion River & 50.97573 & -100.25538 & 4 & 47 & $062 \mathrm{~K} 16$ \\
\hline D-02-192 & 16-Aug-02 & Renicker Creek & 51.03708 & -100.50090 & 2 & 48 & 062N02 \\
\hline D-02-193 & 20-Aug-02 & Second Creek & 49.88272 & -97.47458 & 3 & 26 & $062 \mathrm{H} 14$ \\
\hline D-02-194 & 20-Aug-02 & Truro Creek & 49.87796 & -97.22497 & 2 & City & $062 \mathrm{H} 14$ \\
\hline D-02-195 & 21-Aug-02 & Medika Drain & 49.66493 & -95.85633 & 2 & 84 & $052 \mathrm{E} 06$ \\
\hline D-02-196 & 21-Aug-02 & Birch River & 49.64933 & -95.72598 & 3 & 84 & 052E06 \\
\hline D-02-197 & 22-Aug-02 & Simard Creek & 49.44397 & -96.38900 & 3 & 8 & 062H08 \\
\hline D-02-198 & 22-Aug-02 & Marchand West Drain & 49.39978 & -96.41972 & 3 & 8 & 062H08 \\
\hline D-02-199 & 27-Aug-02 & Boyne River & 49.54082 & -98.41438 & 4 & 18 & 062G09 \\
\hline D-02-200 & 27-Aug-02 & Unnamed tributary to Boyne River & 49.54107 & -98.41475 & 2 & 18 & 062G09 \\
\hline
\end{tabular}


Appendix 1: Table listing the site number, date sampled, site name, latitude and longitude (in decimal degrees DD), DES stream order, DES map number and the corresponding NTS map number for all sites surveyed between 2002 and 2006.

\begin{tabular}{|c|c|c|c|c|c|c|c|}
\hline Site Number & Date & Site Name & $\begin{array}{l}\text { Latitude } \\
\text { (DD) }\end{array}$ & $\begin{array}{l}\text { Longitude } \\
\text { (DD) }\end{array}$ & $\begin{array}{l}\text { DES } \\
\text { Order }\end{array}$ & $\begin{array}{l}\text { DES } \\
\text { Map \# }\end{array}$ & $\begin{array}{l}\text { NTS } \\
\text { Map \# }\end{array}$ \\
\hline D-02-201 & 8-Oct-02 & Unnamed tributary to Pipestone Creek & 49.71499 & -100.96235 & 4 & 62 & $062 \mathrm{~F} 10$ \\
\hline B-03-001 & 21-May-03 & Unnamed tributary to Winnipeg River & 50.14912 & -96.06443 & 2 & 96 & 062101 \\
\hline B-03-002 & 23-May-03 & Unnamed tributary to Little Souris River & 49.76832 & -99.96170 & 3 & 98 & $062 \mathrm{G} 13$ \\
\hline B-03-003 & 23-May-03 & Little Souris River & 49.73824 & -99.96701 & 4 & 98 & 062G12 \\
\hline B-03-004 & 23-May-03 & Spring Creek & 49.72432 & -99.80415 & 2 & 98 & $062 \mathrm{G} 12$ \\
\hline B-03-005 & 23-May-03 & Little Souris River & 49.76210 & -99.74825 & 4 & 98 & $062 \mathrm{G} 13$ \\
\hline B-03-006 & 23-May-03 & Five Mile Creek & 49.66508 & -99.71495 & 3 & 98 & $062 \mathrm{G} 12$ \\
\hline B-03-007 & 26-May-03 & Unnamed tributary to Pipestone Creek & 49.70958 & -100.94779 & 4 & 62 & $062 \mathrm{~F} 10$ \\
\hline B-03-008 & 26-May-03 & Unnamed tributary to Pipestone Creek & 49.72197 & -101.07647 & 2 & 62 & $062 \mathrm{~F} 11$ \\
\hline B-03-009 & 26-May-03 & Unnamed tributary to Pipestone Creek & 49.74793 & -101.07660 & 4 & 62 & $062 \mathrm{~F} 11$ \\
\hline B-03-010 & 26-May-03 & Unnamed tributary to Pipestone Creek & 49.77982 & -101.14478 & 4 & 62 & $062 \mathrm{~F} 14$ \\
\hline B-03-011 & 26-May-03 & Belleview Drain & 49.60670 & -100.84925 & 3 & 62 & $062 \mathrm{~F} 10$ \\
\hline B-03-012 & 26-May-03 & Unnamed tributary to Pipestone Creek & 49.56965 & -100.91753 & 2 & 62 & $062 \mathrm{~F} 10$ \\
\hline B-03-013 & 27-May-03 & Gopher Creek & 49.78230 & -100.96335 & 4 & 75 & $062 \mathrm{~F} 15$ \\
\hline B-03-014 & 27-May-03 & Gopher Creek & 49.78353 & -101.07940 & 4 & 75 & $062 \mathrm{~F} 14$ \\
\hline B-03-015 & 27-May-03 & Unnamed tributary to Gopher Creek & 49.82793 & -101.14505 & 3 & 75 & $062 \mathrm{~F} 14$ \\
\hline B-03-016 & 27-May-03 & Gopher Creek & 49.85510 & -101.14462 & 3 & 75 & $062 \mathrm{~F} 14$ \\
\hline B-03-017 & 28-May-03 & Bosshill Creek & 49.84333 & -100.96983 & 4 & 75 & $062 \mathrm{~F} 15$ \\
\hline B-03-018 & 28-May-03 & Bosshill Creek & 49.87185 & -101.12612 & 4 & 75 & $062 \mathrm{~F} 14$ \\
\hline B-03-019 & 28-May-03 & Bosshill Creek & 49.85263 & -101.00868 & 4 & 75 & $062 \mathrm{~F} 14$ \\
\hline B-03-020 & 29-May-03 & Scallion Creek & 49.86872 & -100.93107 & 4 & 75 & $062 \mathrm{~F} 15$ \\
\hline B-03-021 & 29-May-03 & Scallion Creek & 49.88682 & -100.92352 & 4 & 75 & $062 \mathrm{~F} 15$ \\
\hline B-03-022 & 29-May-03 & Little Scallion Creek & 49.89007 & -100.94283 & 3 & 75 & $062 \mathrm{~F} 15$ \\
\hline $\mathrm{B}-03-023$ & 29-May-03 & Unnamed tributary to Assiniboine River & 49.95430 & -100.88923 & 3 & 75 & $062 \mathrm{~F} 15$ \\
\hline B-03-024 & 29-May-03 & Unnamed tributary to Assiniboine River & 49.98372 & -100.89263 & 3 & 75 & $062 \mathrm{~F} 15$ \\
\hline
\end{tabular}


Appendix 1: Table listing the site number, date sampled, site name, latitude and longitude (in decimal degrees DD), DES stream order, DES map number and the corresponding NTS map number for all sites surveyed between 2002 and 2006.

\begin{tabular}{|c|c|c|c|c|c|c|c|}
\hline Site Number & Date & Site Name & $\begin{array}{l}\text { Latitude } \\
\text { (DD) }\end{array}$ & $\begin{array}{l}\text { Longitude } \\
\text { (DD) }\end{array}$ & $\begin{array}{l}\text { DES } \\
\text { Order }\end{array}$ & $\begin{array}{l}\text { DES } \\
\text { Map \# }\end{array}$ & $\begin{array}{l}\text { NTS } \\
\text { Map \# }\end{array}$ \\
\hline B-03-025 & 29-May-03 & Unnamed tributary to Assiniboine River & 49.84485 & -100.78142 & 3 & 73 & $062 \mathrm{~F} 15$ \\
\hline B-03-026 & 29-May-03 & Baileys Creek & 49.82903 & -100.63473 & 3 & 73 & $062 \mathrm{~F} 15$ \\
\hline B-03-027 & 30-May-03 & Baileys Creek & 49.88674 & -100.69514 & 3 & 73 & $062 \mathrm{~F} 15$ \\
\hline B-03-028 & 30-May-03 & Baileys Creek & 49.86023 & -100.66750 & 3 & 73 & $062 \mathrm{~F} 15$ \\
\hline B-03-029 & 30-May-03 & Brierwood Creek & 49.98723 & -100.62242 & 3 & 73 & $062 \mathrm{~F} 15$ \\
\hline B-03-030 & 30-May-03 & Brierwood Creek & 49.99765 & -100.64545 & 3 & 73 & $062 \mathrm{~F} 15$ \\
\hline B-03-031 & 30-May-03 & Unnamed tributary to Brierwood Creek & 50.01937 & -100.49992 & 3 & 73 & $062 \mathrm{~K} 01$ \\
\hline B-03-032 & 2-Jun-03 & Unnamed tributary to Oak River & 49.98980 & -100.47488 & 3 & 73 & $062 \mathrm{~F} 16$ \\
\hline B-03-033 & 2-Jun-03 & Oak River & 49.98020 & -100.46152 & 5 & 72 & $062 \mathrm{~F} 16$ \\
\hline B-03-034 & 2-Jun-03 & Brierwood Creek & 49.92980 & -100.48041 & 4 & 73 & $062 \mathrm{~F} 16$ \\
\hline B-03-035 & 2-Jun-03 & Brierwood Creek & 49.94137 & -100.50777 & 4 & 73 & $062 \mathrm{~F} 15$ \\
\hline B-03-036 & 2-Jun-03 & Unnamed tributary to Oak River & 50.13612 & -100.53102 & 3 & 73 & $062 \mathrm{~K} 02$ \\
\hline B-03-037 & 2-Jun-03 & Brierwood Creek & 50.13753 & -100.68822 & 2 & 73 & $062 \mathrm{~K} 02$ \\
\hline B-03-038 & 2-Jun-03 & Unnamed tributary to Assiniboine River & 50.09148 & -100.82953 & 4 & 73 & $062 \mathrm{~K} 02$ \\
\hline B-03-039 & 3-Jun-03 & West Spring Creek & 50.27000 & -99.94780 & 2 & 81 & 062J05 \\
\hline B-03-040 & 3-Jun-03 & West Spring Creek & 50.24475 & -99.91997 & 2 & 81 & 062J04 \\
\hline B-03-041 & 3-Jun-03 & Sandersons Creek & 50.30702 & -99.81542 & 3 & 81 & 062J05 \\
\hline B-03-042 & 3-Jun-03 & Sandersons Creek & 50.31278 & -99.79482 & 3 & 81 & 062J05 \\
\hline B-03-043 & 3-Jun-03 & Otter Creek & 50.49120 & -99.79595 & 3 & 81 & 062J05 \\
\hline B-03-044 & 3-Jun-03 & Rolling River & 50.54137 & -99.76968 & 4 & 81 & 062J12 \\
\hline B-03-045 & 4-Jun-03 & Unnamed tributary to Assiniboine River & 49.85547 & -100.12103 & 3 & 98 & $062 \mathrm{~F} 16$ \\
\hline B-03-046 & 4-Jun-03 & Unnamed tributary to Willow Creek & 49.92022 & -99.91183 & 3 & 99 & $062 \mathrm{G} 13$ \\
\hline B-03-047 & 4-Jun-03 & Unnamed tributary to Willow Creek & 49.94555 & -99.90587 & 3 & 99 & $062 \mathrm{G} 13$ \\
\hline B-03-048 & 4-Jun-03 & Unnamed tributary to Willow Creek & 49.91632 & -99.86187 & 4 & 99 & $062 \mathrm{G} 13$ \\
\hline B-03-049 & 4-Jun-03 & West Branch Willow Creek & 49.91612 & -99.81395 & 3 & 99 & $062 G 13$ \\
\hline
\end{tabular}


Appendix 1: Table listing the site number, date sampled, site name, latitude and longitude (in decimal degrees DD), DES stream order, DES map number and the corresponding NTS map number for all sites surveyed between 2002 and 2006.

\begin{tabular}{|c|c|c|c|c|c|c|c|}
\hline Site Number & Date & Site Name & $\begin{array}{l}\text { Latitude } \\
\text { (DD) }\end{array}$ & $\begin{array}{l}\text { Longitude } \\
\text { (DD) }\end{array}$ & $\begin{array}{l}\text { DES } \\
\text { Order }\end{array}$ & $\begin{array}{l}\text { DES } \\
\text { Map \# }\end{array}$ & $\begin{array}{l}\text { NTS } \\
\text { Map \# }\end{array}$ \\
\hline B-03-050 & 4-Jun-03 & East Branch Willow Creek & 49.91610 & -99.77523 & 3 & 99 & $062 \mathrm{G} 13$ \\
\hline B-03-051 & 4-Jun-03 & South Boggy Creek & 49.90118 & -99.68998 & 3 & 99 & $062 \mathrm{G} 13$ \\
\hline B-03-052 & 4-Jun-03 & Willow Creek & 49.86105 & -99.82573 & 5 & 99 & $062 \mathrm{G} 13$ \\
\hline B-03-053 & 5-Jun-03 & Broughtons Creek & 50.09167 & -100.22952 & 4 & 82 & $062 K 01$ \\
\hline B-03-054 & 5-Jun-03 & Unnamed tributary to Little Saskatchewan River & 50.13717 & -100.15567 & 3 & 82 & $062 \mathrm{~K} 01$ \\
\hline B-03-055 & 5-Jun-03 & Unnamed tributary to Little Saskatchewan River & 50.13700 & -100.12385 & 2 & 82 & $062 \mathrm{~K} 01$ \\
\hline B-03-056 & 5-Jun-03 & Unnamed tributary to Little Saskatchewan River & 50.12267 & -100.10282 & 2 & 82 & $062 \mathrm{~K} 01$ \\
\hline B-03-057 & 5-Jun-03 & Unnamed tributary to Broughtons Creek & 50.13767 & -100.27625 & 3 & 82 & $062 \mathrm{~K} 01$ \\
\hline B-03-058 & 5-Jun-03 & Broughtons Creek & 50.10805 & -100.28308 & 3 & 82 & $062 \mathrm{~K} 01$ \\
\hline B-03-059 & 5-Jun-03 & Sibbald Creek & 49.92347 & -100.39328 & 3 & 82 & $062 \mathrm{~F} 16$ \\
\hline B-03-060 & 5-Jun-03 & Sibbald Creek & 49.91603 & -100.32795 & 3 & 82 & $062 \mathrm{~F} 16$ \\
\hline B-03-061 & 9-Jun-03 & Arrow River & 50.16688 & -100.80675 & 4 & 74 & $062 \mathrm{~K} 02$ \\
\hline B-03-062 & 9-Jun-03 & Golden Creek & 50.13897 & -100.92018 & 4 & 74 & $062 \mathrm{~K} 02$ \\
\hline B-03-063 & 9-Jun-03 & Arrow Marsh Drain & 50.13738 & -101.01370 & 3 & 74 & $062 \mathrm{~K} 03$ \\
\hline B-03-064 & 10-Jun-03 & Minnewasta Creek & 50.22728 & -101.09483 & 4 & 74 & $062 \mathrm{~K} 03$ \\
\hline B-03-065 & 10-Jun-03 & Minnewasta Creek & 50.35840 & -100.92633 & 4 & 74 & $062 \mathrm{~K} 07$ \\
\hline B-03-066 & 10-Jun-03 & Arrow Creek & 50.48153 & -100.80545 & 4 & 74 & $062 \mathrm{~K} 07$ \\
\hline B-03-067 & 10-Jun-03 & Arrow River & 50.27000 & -100.79797 & 4 & 74 & $062 \mathrm{~K} 07$ \\
\hline B-03-068 & 11-Jun-03 & Unnamed tributary to Birdtail Creek & 50.49123 & -100.93810 & 2 & 77 & $062 \mathrm{~K} 07$ \\
\hline B-03-069 & 11-Jun-03 & Ross Creek & 50.75050 & -100.90390 & 3 & 76 & $062 \mathrm{~K} 15$ \\
\hline B-03-070 & 11-Jun-03 & Konyk Creek & 50.81055 & -100.85747 & 3 & 76 & $062 \mathrm{~K} 15$ \\
\hline B-03-071 & 11-Jun-03 & Tilson Creek & 50.81562 & -100.82885 & 3 & 76 & $062 \mathrm{~K} 15$ \\
\hline B-03-072 & 11-Jun-03 & Giles Creek & 50.51045 & -100.96915 & 2 & 77 & 062K10 \\
\hline B-03-073 & 11-Jun-03 & Unnamed tributary to Birdtail Creek & 50.43212 & -101.07488 & 3 & 77 & $062 \mathrm{~K} 06$ \\
\hline B-03-074 & 11-Jun-03 & Unnamed tributary to Birdtail Creek & 50.36820 & -101.08467 & 2 & 77 & $062 \mathrm{~K} 06$ \\
\hline
\end{tabular}


Appendix 1: Table listing the site number, date sampled, site name, latitude and longitude (in decimal degrees DD), DES stream order, DES map number and the corresponding NTS map number for all sites surveyed between 2002 and 2006.

\begin{tabular}{|c|c|c|c|c|c|c|c|}
\hline Site Number & Date & Site Name & $\begin{array}{l}\text { Latitude } \\
\text { (DD) }\end{array}$ & $\begin{array}{l}\text { Longitude } \\
\text { (DD) }\end{array}$ & $\begin{array}{l}\text { DES } \\
\text { Order }\end{array}$ & $\begin{array}{l}\text { DES } \\
\text { Map \# }\end{array}$ & $\begin{array}{l}\text { NTS } \\
\text { Map \# }\end{array}$ \\
\hline B-03-075 & 12-Jun-03 & Snake Creek & 50.43050 & -101.19068 & 5 & 78 & $062 \mathrm{~K} 06$ \\
\hline B-03-076 & 12-Jun-03 & Snake Creek & 50.38210 & -101.23300 & 5 & 78 & $062 \mathrm{~K} 06$ \\
\hline B-03-077 & 12-Jun-03 & Beaver Creek & 50.36163 & -101.37347 & 5 & 78 & $062 \mathrm{~K} 06$ \\
\hline B-03-078 & 12-Jun-03 & Hotonka Creek & 50.26860 & -101.29165 & 3 & 78 & $062 \mathrm{~K} 06$ \\
\hline B-03-079 & 12-Jun-03 & Scissor Creek & 50.27890 & -101.29193 & 4 & 78 & $062 \mathrm{~K} 06$ \\
\hline B-03-080 & 12-Jun-03 & Brennand Creek & 50.21277 & -101.28711 & 3 & 78 & $062 \mathrm{~K} 03$ \\
\hline B-03-081 & 12-Jun-03 & Wythes Creek & 50.11583 & -101.06575 & 3 & 78 & $062 \mathrm{~K} 03$ \\
\hline B-03-082 & 12-Jun-03 & Niso Creek & 50.10175 & -101.03867 & 4 & 78 & $062 \mathrm{~K} 03$ \\
\hline B-03-083 & 16-Jun-03 & Shannon Creek & 49.28610 & -97.86943 & 4 & 16 & $062 \mathrm{H} 05$ \\
\hline B-03-084 & 16-Jun-03 & Shannon Creek & 49.30098 & -97.83903 & 4 & 16 & $062 \mathrm{H} 05$ \\
\hline B-03-085 & 17-Jun-03 & Shannon Creek & 49.34587 & -97.69873 & 4 & 16 & $062 \mathrm{H} 05$ \\
\hline B-03-086 & 17-Jun-03 & Shannon Creek & 49.32058 & -97.76603 & 4 & 16 & 062H05 \\
\hline B-03-087 & 17-Jun-03 & Buffalo Creek & 49.06048 & -97.68412 & 4 & 15 & $062 \mathrm{H} 04$ \\
\hline B-03-088 & 18-Jun-03 & Riviere Aux Marais & 49.06245 & -97.32473 & 3 & 15 & $062 \mathrm{H} 03$ \\
\hline B-03-089 & 18-Jun-03 & Riviere Aux Marais & 49.11837 & -97.30248 & 4 & 15 & $062 \mathrm{HO3}$ \\
\hline B-03-090 & 18-Jun-03 & Thiessen Drain & 49.19080 & -97.43713 & 2 & 15 & $062 \mathrm{HO3}$ \\
\hline B-03-091 & 18-Jun-03 & Rempel Drain & 49.16230 & -97.46007 & 3 & 15 & $062 \mathrm{H} 03$ \\
\hline B-03-092 & 18-Jun-03 & Knopf Drain & 49.22100 & -97.41400 & 3 & 15 & $062 \mathrm{HO3}$ \\
\hline B-03-093 & 18-Jun-03 & Deadhorse Creek & 49.25097 & -97.64008 & 4 & 15 & $062 \mathrm{H} 05$ \\
\hline B-03-094 & 18-Jun-03 & Hespeler Creek & 49.19303 & -97.70938 & 4 & 15 & $062 \mathrm{H} 04$ \\
\hline B-03-095 & 19-Jun-03 & Deadhorse Creek & 49.22920 & -97.90987 & 4 & 15 & $062 \mathrm{H} 04$ \\
\hline B-03-096 & 19-Jun-03 & Walkof Coulee & 49.16260 & -97.94907 & 4 & 15 & $062 \mathrm{HO4}$ \\
\hline B-03-097 & 19-Jun-03 & Hespeler Creek & 49.16257 & -97.91123 & 3 & 15 & $062 \mathrm{H} 04$ \\
\hline B-03-098 & 19-Jun-03 & Deadhorse Creek & 49.16298 & -98.18523 & 3 & 15 & $062 \mathrm{G} 01$ \\
\hline B-03-099 & 20-Jun-03 & Niso Creek & 50.10175 & -101.03867 & 4 & 78 & $062 \mathrm{~K} 03$ \\
\hline
\end{tabular}


Appendix 1: Table listing the site number, date sampled, site name, latitude and longitude (in decimal degrees DD), DES stream order, DES map number and the corresponding NTS map number for all sites surveyed between 2002 and 2006.

\begin{tabular}{|c|c|c|c|c|c|c|c|}
\hline Site Number & Date & Site Name & $\begin{array}{l}\text { Latitude } \\
\text { (DD) }\end{array}$ & $\begin{array}{l}\text { Longitude } \\
\text { (DD) }\end{array}$ & $\begin{array}{l}\text { DES } \\
\text { Order }\end{array}$ & $\begin{array}{l}\text { DES } \\
\text { Map \# }\end{array}$ & $\begin{array}{l}\text { NTS } \\
\text { Map \# }\end{array}$ \\
\hline B-03-100 & 20-Jun-03 & Niso Creek & 50.07685 & -101.10332 & 4 & 78 & $062 \mathrm{~K} 03$ \\
\hline B-03-101 & 20-Jun-03 & Plum Creek & 49.64145 & -100.39460 & 5 & 62 & 062F09 \\
\hline B-03-102 & 23-Jun-03 & Rolling River & 50.54137 & -99.76968 & 4 & 81 & 062J12 \\
\hline B-03-103 & 23-Jun-03 & Clear Creek & 50.68302 & -100.11407 & 3 & 80 & $062 \mathrm{~K} 09$ \\
\hline B-03-104 & 23-Jun-03 & Heron Creek & 50.71255 & -100.40020 & 2 & 80 & $062 K 09$ \\
\hline B-03-105 & 24-Jun-03 & Oak River & 50.26993 & -100.49682 & 5 & 72 & $062 \mathrm{~K} 08$ \\
\hline B-03-106 & 24-Jun-03 & Unnamed tributary to Oak River & 50.35850 & -100.53780 & 4 & 72 & $062 \mathrm{~K} 07$ \\
\hline B-03-107 & 24-Jun-03 & Wolfe Creek & 50.46837 & -100.57682 & 2 & 72 & 062K07 \\
\hline B-03-108 & 24-Jun-03 & Oak River & 50.47642 & -100.58750 & 3 & 72 & $062 \mathrm{~K} 07$ \\
\hline B-03-109 & 24-Jun-03 & Hales Creek & 49.91252 & -100.50812 & 4 & 73 & $062 \mathrm{~F} 15$ \\
\hline B-03-110 & 26-Jun-03 & Pierce Drain & 50.09297 & -98.99598 & 2 & 38 & 062J02 \\
\hline B-03-111 & 26-Jun-03 & Campbell Drain & 50.03045 & -98.99607 & 3 & 38 & 062J02 \\
\hline B-03-112 & 26-Jun-03 & Pine Creek & 50.02147 & -99.06473 & 3 & 38 & 062J03 \\
\hline B-03-113 & 26-Jun-03 & Boggy Creek & 50.04882 & -99.43942 & 4 & 40 & 062J03 \\
\hline B-03-114 & 26-Jun-03 & Boggy Creek & 50.00503 & -99.56815 & 4 & 40 & 062J04 \\
\hline B-03-115 & 27-Jun-03 & Lepington Drain & 49.98957 & -99.04175 & 3 & 38 & 062G14 \\
\hline B-03-116 & 27-Jun-03 & Pine Creek & 50.00463 & -99.11522 & 3 & 38 & 062J03 \\
\hline B-03-117 & 27-Jun-03 & McCullough Drain & 50.04183 & -99.12720 & 2 & 38 & 062J03 \\
\hline B-03-118 & 27-Jun-03 & Unnamed tributary to Pine Creek & 50.01937 & -99.09002 & 2 & 38 & 062J03 \\
\hline B-03-119 & 2-Jul-03 & Unnamed tributary to Squirrel Creek & 49.87163 & -98.94632 & 3 & 37 & $062 G 15$ \\
\hline B-03-120 & 2-Jul-03 & East Branch Squirrel Creek & 49.93845 & -98.94965 & 4 & 37 & 062G15 \\
\hline B-03-121 & 3-Jul-03 & Unnamed tributary to West Branch Squirrel Creek & 49.89822 & -99.01798 & 2 & 37 & $062 \mathrm{G} 14$ \\
\hline B-03-122 & 3-Jul-03 & West Branch Squirrel Creek & 49.94818 & -98.98330 & 3 & 37 & 062G15 \\
\hline B-03-123 & 3-Jul-03 & Jensen Drain & 50.03410 & -98.83572 & 3 & 37 & 062J02 \\
\hline B-03-124 & 3-Jul-03 & Squirrel Creek & 50.07830 & -98.82420 & 4 & 37 & 062J02 \\
\hline
\end{tabular}


Appendix 1: Table listing the site number, date sampled, site name, latitude and longitude (in decimal degrees DD), DES stream order, DES map number and the corresponding NTS map number for all sites surveyed between 2002 and 2006.

\begin{tabular}{|c|c|c|c|c|c|c|c|}
\hline Site Number & Date & Site Name & $\begin{array}{l}\text { Latitude } \\
\text { (DD) }\end{array}$ & $\begin{array}{l}\text { Longitude } \\
\text { (DD) }\end{array}$ & $\begin{array}{l}\text { DES } \\
\text { Order }\end{array}$ & $\begin{array}{l}\text { DES } \\
\text { Map \# }\end{array}$ & $\begin{array}{l}\text { NTS } \\
\text { Map \# }\end{array}$ \\
\hline B-03-125 & 3-Jul-03 & Old Squirrel Creek & 50.12340 & -98.72118 & 3 & 37 & 062J02 \\
\hline B-03-126 & 4-Jul-03 & Bell Creek & 49.62425 & -100.82662 & 2 & 62 & $062 \mathrm{~F} 10$ \\
\hline B-03-127 & 4-Jul-03 & Stony Creek & 49.39818 & -101.09898 & 3 & 63 & 062F06 \\
\hline B-03-128 & 4-Jul-03 & Stony Creek & 49.45547 & -100.96337 & 3 & 63 & 062F07 \\
\hline B-03-129 & 4-Jul-03 & Stony Creek & 49.45437 & -100.87280 & 4 & 63 & 062F07 \\
\hline B-03-130 & 4-Jul-03 & Maple Lake Drain & 49.48797 & -100.80522 & 4 & 63 & 062F07 \\
\hline B-03-131 & 4-Jul-03 & Bell Creek & 49.63045 & -100.84913 & 2 & 62 & 062F10 \\
\hline B-03-132 & 7-Jul-03 & Stony Creek & 50.27203 & -99.68053 & 4 & 39 & 062J05 \\
\hline B-03-133 & 7-Jul-03 & Logan's Creek & 50.31415 & -99.70532 & 3 & 39 & 062J05 \\
\hline B-03-134 & 7-Jul-03 & Stony Creek & 50.31467 & -99.67837 & 3 & 39 & 062J05 \\
\hline B-03-135 & 7-Jul-03 & Stony Creek & 50.32777 & -99.66738 & 3 & 39 & 062J05 \\
\hline B-03-136 & 7-Jul-03 & Stony Creek & 50.33440 & -99.65313 & 3 & 39 & 062J05 \\
\hline B-03-137 & 7-Jul-03 & Jordan Creek Drain & 50.38812 & -99.32843 & 3 & 42 & 062J06 \\
\hline B-03-138 & 7-Jul-03 & Lach Drain & 50.41770 & -98.98425 & 3 & 42 & 062J07 \\
\hline B-03-139 & 7-Jul-03 & Jordan Creek Drain & 50.38815 & -98.90985 & 4 & 42 & 062J07 \\
\hline B-03-140 & 8-Jul-03 & Black Creek & 49.60505 & -99.80273 & 3 & 69 & $062 \mathrm{G} 12$ \\
\hline B-03-141 & 8-Jul-03 & Unnamed tributary to Black Creek & 49.59530 & -99.80277 & 2 & 69 & $062 \mathrm{G} 12$ \\
\hline B-03-142 & 8-Jul-03 & Black Creek & 49.60432 & -99.73425 & 4 & 69 & $062 \mathrm{G} 12$ \\
\hline B-03-143 & 8-Jul-03 & Black Creek & 49.61945 & -99.67737 & 4 & 69 & $062 \mathrm{G} 12$ \\
\hline B-03-144 & 9-Jul-03 & Unnamed tributary to Souris River & 49.53290 & -100.16113 & 3 & 68 & 062F09 \\
\hline B-03-145 & 9-Jul-03 & Elgin Creek & 49.56193 & -100.25575 & 4 & 68 & 062F09 \\
\hline B-03-146 & 9-Jul-03 & Elgin Creek & 49.53292 & -100.27318 & 4 & 68 & 062F09 \\
\hline B-03-147 & 9-Jul-03 & Unnamed tributary to Elgin Creek & 49.53247 & -100.31080 & 3 & 68 & 062F09 \\
\hline B-03-148 & 9-Jul-03 & Cherry Creek & 49.22800 & -100.08203 & 4 & 68 & 062F01 \\
\hline B-03-149 & 9-Jul-03 & Unnamed tributary to Whitewater Lake & 49.20792 & -100.24327 & 4 & 68 & 062F01 \\
\hline
\end{tabular}


Appendix 1: Table listing the site number, date sampled, site name, latitude and longitude (in decimal degrees DD), DES stream order, DES map number and the corresponding NTS map number for all sites surveyed between 2002 and 2006.

\begin{tabular}{|c|c|c|c|c|c|c|c|}
\hline Site Number & Date & Site Name & $\begin{array}{l}\text { Latitude } \\
\text { (DD) }\end{array}$ & $\begin{array}{l}\text { Longitude } \\
\text { (DD) }\end{array}$ & $\begin{array}{l}\text { DES } \\
\text { Order }\end{array}$ & $\begin{array}{l}\text { DES } \\
\text { Map \# }\end{array}$ & $\begin{array}{l}\text { NTS } \\
\text { Map \# }\end{array}$ \\
\hline B-03-150 & 9-Jul-03 & Unnamed tributary to Whitewater Lake & 49.17758 & -100.32225 & 3 & 68 & 062F01 \\
\hline B-03-151 & 10-Jul-03 & Silver Creek & 50.55635 & -101.36533 & 4 & 79 & $062 \mathrm{~K} 11$ \\
\hline B-03-152 & 10-Jul-03 & Silver Creek & 50.62338 & -101.25680 & 4 & 79 & $062 \mathrm{~K} 11$ \\
\hline B-03-153 & 10-Jul-03 & East Silver Creek & 50.64403 & -101.18287 & 3 & 79 & $062 \mathrm{~K} 11$ \\
\hline B-03-154 & 10-Jul-03 & East Silver Creek & 50.68288 & -101.04655 & 3 & 79 & $062 \mathrm{~K} 11$ \\
\hline B-03-155 & 11-Jul-03 & Silver Creek & 50.68278 & -101.20237 & 3 & 79 & $062 \mathrm{~K} 11$ \\
\hline B-03-156 & 11-Jul-03 & Unnamed tributary to Silver Creek & 50.68278 & -101.22393 & 2 & 79 & $062 \mathrm{~K} 11$ \\
\hline B-03-157 & 11-Jul-03 & Unnamed tributary to Silver Creek & 50.67475 & -101.24587 & 2 & 79 & $062 \mathrm{~K} 11$ \\
\hline B-03-158 & 11-Jul-03 & Thunder Creek & 50.93373 & -101.38012 & 4 & 92 & $062 \mathrm{~K} 14$ \\
\hline B-03-159 & 11-Jul-03 & Bear Creek & 50.95640 & -101.24617 & 3 & 92 & $062 \mathrm{~K} 14$ \\
\hline B-03-160 & 11-Jul-03 & Thunder Creek & 50.88928 & -101.29570 & 4 & 92 & $062 \mathrm{~K} 14$ \\
\hline B-03-161 & 14-Jul-03 & Unnamed tributary to Stony Creek & 50.17818 & -99.52258 & 3 & 39 & 062J04 \\
\hline B-03-162 & 14-Jul-03 & Franklin Creek & 50.21329 & -99.52256 & 2 & 39 & 062J04 \\
\hline B-03-163 & 14-Jul-03 & Whitemud River & 50.13473 & -98.58355 & 6 & 42 & 062J02 \\
\hline B-03-164 & 14-Jul-03 & Unnamed tributary to Whitemud River & 50.18151 & -98.58338 & 3 & 42 & 062J02 \\
\hline B-03-165 & 14-Jul-03 & Unnamed tributary to Whitemud River & 50.21132 & -98.58357 & 3 & 42 & 062J02 \\
\hline B-03-166 & 14-Jul-03 & Unnamed tributary to Whitemud River & 50.22590 & -98.60670 & 2 & 42 & 062J02 \\
\hline B-03-167 & 14-Jul-03 & Unnamed tributary to Whitemud River & 50.24073 & -98.61492 & 2 & 42 & 062J02 \\
\hline B-03-168 & 14-Jul-03 & Unnamed tributary to Lake Manitoba & 50.28452 & -98.61468 & 2 & 41 & 062J07 \\
\hline B-03-169 & 14-Jul-03 & Unnamed tributary to Lake Manitoba & 50.29935 & -98.61482 & 2 & 41 & 062J07 \\
\hline B-03-170 & 14-Jul-03 & Unnamed tributary to Lake Manitoba & 50.31405 & -98.61477 & 2 & 41 & 062J07 \\
\hline B-03-171 & 14-Jul-03 & Unnamed tributary to Lake Manitoba & 50.32870 & -98.61467 & 2 & 41 & 062J07 \\
\hline B-03-172 & 14-Jul-03 & Unnamed tributary to Lake Manitoba & 50.34358 & -98.61465 & 2 & 41 & 062J07 \\
\hline B-03-173 & 14-Jul-03 & Unnamed tributary to Lake Manitoba & 50.35837 & -98.61475 & 2 & 41 & 062J07 \\
\hline B-03-174 & 14-Jul-03 & Unnamed tributary to Lake Manitoba & 50.41748 & -98.61480 & 2 & 41 & 062J07 \\
\hline
\end{tabular}


Appendix 1: Table listing the site number, date sampled, site name, latitude and longitude (in decimal degrees DD), DES stream order, DES map number and the corresponding NTS map number for all sites surveyed between 2002 and 2006.

\begin{tabular}{|c|c|c|c|c|c|c|c|}
\hline Site Number & Date & Site Name & $\begin{array}{l}\text { Latitude } \\
\text { (DD) }\end{array}$ & $\begin{array}{l}\text { Longitude } \\
\text { (DD) }\end{array}$ & $\begin{array}{l}\text { DES } \\
\text { Order }\end{array}$ & $\begin{array}{l}\text { DES } \\
\text { Map \# }\end{array}$ & $\begin{array}{l}\text { NTS } \\
\text { Map \# }\end{array}$ \\
\hline B-03-175 & 14-Jul-03 & Unnamed tributary to Lake Manitoba & 50.43263 & -98.61485 & 2 & 41 & 062J07 \\
\hline B-03-176 & 14-Jul-03 & Unnamed tributary to Lake Manitoba & 50.44737 & -98.61482 & 2 & 41 & 062J07 \\
\hline B-03-177 & 14-Jul-03 & Unnamed tributary to Lake Manitoba & 50.46192 & -98.61480 & 2 & 41 & 062J07 \\
\hline B-03-178 & 14-Jul-03 & Unnamed tributary to Lake Manitoba & 50.47662 & -98.61480 & 2 & 41 & 062J07 \\
\hline B-03-179 & 14-Jul-03 & Carriere Drain & 50.63897 & -98.71737 & 2 & 41 & 062J10 \\
\hline B-03-180 & 14-Jul-03 & Smalley School Drain & 50.66845 & -98.69398 & 3 & 41 & 062J10 \\
\hline B-03-181 & 15-Jul-03 & Whitemud River & 50.17417 & -99.15660 & 6 & 39 & 062J03 \\
\hline B-03-182 & 16-Jul-03 & St. Malo Canal & 49.31078 & -96.98503 & 3 & 4 & 062H07 \\
\hline B-03-183 & 16-Jul-03 & St. Malo Canal & 49.35447 & -96.98485 & 3 & 4 & $062 \mathrm{H} 07$ \\
\hline B-03-184 & 16-Jul-03 & Coulee des Nault & 49.38398 & -96.96207 & 3 & 4 & 062H07 \\
\hline B-03-185 & 16-Jul-03 & Joubert Creek & 49.38455 & -96.88180 & 3 & 4 & 062H07 \\
\hline B-03-186 & 16-Jul-03 & Sarto Creek & 49.40190 & -96.87193 & 3 & 4 & 062H07 \\
\hline B-03-187 & 16-Jul-03 & St. Pierre Creek & 49.46852 & -97.00622 & 3 & 4 & $062 \mathrm{H} 06$ \\
\hline B-03-188 & 16-Jul-03 & Otterburne East Drain & 49.50242 & -97.01925 & 3 & 4 & $062 \mathrm{H} 11$ \\
\hline B-03-189 & 16-Jul-03 & Carey Drain & 49.50227 & -97.08927 & 4 & 4 & $062 \mathrm{H} 11$ \\
\hline B-03-190 & 16-Jul-03 & Joubert Creek & 49.32550 & -96.76338 & 3 & 4 & $062 \mathrm{H} 07$ \\
\hline B-03-191 & 17-Jul-03 & Joubert Creek Extension & 49.28988 & -96.52282 & 3 & 4 & $062 \mathrm{H} 07$ \\
\hline B-03-192 & 17-Jul-03 & Joubert Creek Extension & 49.28152 & -96.60808 & 3 & 4 & $062 \mathrm{H} 07$ \\
\hline B-03-193 & 17-Jul-03 & Rat River & 49.21035 & -96.14815 & 4 & 3 & $062 \mathrm{H} 01$ \\
\hline B-03-194 & 22-Jul-03 & Joe River & 49.01567 & -97.17793 & 3 & 2 & 062H03 \\
\hline B-03-195 & 23-Jul-03 & Main Drain & 49.05962 & -97.06970 & 3 & 2 & 062H03 \\
\hline B-03-196 & 23-Jul-03 & Fredensthal Drain & 49.05958 & -97.07740 & 3 & 2 & 062H03 \\
\hline B-03-197 & 23-Jul-03 & Ridgeville Drain & 49.08818 & -97.05416 & 3 & 2 & 062H03 \\
\hline B-03-198 & 23-Jul-03 & Harlow Drain & 49.12092 & -97.05515 & 3 & 2 & 062H03 \\
\hline B-03-199 & 23-Jul-03 & Stewart Drain & 49.15470 & -97.03335 & 3 & 2 & 062H03 \\
\hline
\end{tabular}


Appendix 1: Table listing the site number, date sampled, site name, latitude and longitude (in decimal degrees DD), DES stream order, DES map number and the corresponding NTS map number for all sites surveyed between 2002 and 2006.

\begin{tabular}{|c|c|c|c|c|c|c|c|}
\hline Site Number & Date & Site Name & $\begin{array}{l}\text { Latitude } \\
\text { (DD) }\end{array}$ & $\begin{array}{l}\text { Longitude } \\
\text { (DD) }\end{array}$ & $\begin{array}{l}\text { DES } \\
\text { Order }\end{array}$ & $\begin{array}{l}\text { DES } \\
\text { Map \# }\end{array}$ & $\begin{array}{l}\text { NTS } \\
\text { Map \# }\end{array}$ \\
\hline B-03-200 & 23-Jul-03 & Riviere Aux Marais & 49.13340 & -97.29205 & 4 & 15 & $062 \mathrm{H} 03$ \\
\hline B-03-201 & 23-Jul-03 & Roseau River & 49.14862 & -97.17067 & 5 & 2 & 062H03 \\
\hline B-03-202 & 23-Jul-03 & Jordan River & 49.14821 & -96.94769 & 3 & 2 & 062H02 \\
\hline B-03-203 & 23-Jul-03 & Gardenton Drain & 49.05935 & -96.68487 & 3 & 2 & $062 \mathrm{H} 02$ \\
\hline B-03-204 & 23-Jul-03 & Gardenton Drain & 49.07408 & -96.68408 & 3 & 2 & 062H02 \\
\hline B-03-205 & 23-Jul-03 & Gardenton Floodway & 49.07564 & -96.62845 & 4 & 2 & $062 \mathrm{H} 02$ \\
\hline B-03-206 & 24-Jul-03 & Vita Drain & 49.17632 & -96.76380 & 4 & 2 & $062 \mathrm{H} 02$ \\
\hline B-03-207 & 24-Jul-03 & Vita Drain & 49.14832 & -96.60662 & 3 & 2 & 062H02 \\
\hline B-03-208 & 24-Jul-03 & Conroy Creek & 49.13778 & -96.58417 & 2 & 2 & 062H02 \\
\hline B-03-209 & 24-Jul-03 & Vita Drain & 49.13327 & -96.52985 & 3 & 2 & 062H02 \\
\hline B-03-210 & 24-Jul-03 & Vita Drain & 49.11175 & -96.41177 & 2 & 2 & $062 \mathrm{H} 01$ \\
\hline B-03-211 & 24-Jul-03 & Roseau River & 49.02895 & -96.52735 & 3 & 2 & 062H02 \\
\hline B-03-212 & 24-Jul-03 & Arbakka Drain & 49.02972 & -96.49340 & 3 & 2 & $062 \mathrm{H} 01$ \\
\hline B-03-213 & 24-Jul-03 & Arbakka Drain & 49.03046 & -96.38191 & 3 & 2 & $062 \mathrm{H} 01$ \\
\hline B-03-214 & 25-Jul-03 & Marsh River & 49.29572 & -97.21220 & 3 & 5 & 062H06 \\
\hline B-03-215 & 25-Jul-03 & Arnaud Drain & 49.29568 & -97.18065 & 3 & 5 & 062H06 \\
\hline B-03-216 & 25-Jul-03 & Ste. Elizabeth Drain & 49.32515 & -97.16527 & 3 & 5 & 062H06 \\
\hline B-03-217 & 25-Jul-03 & Angle Drain & 49.36520 & -97.12025 & 3 & 5 & 062H06 \\
\hline B-03-218 & 25-Jul-03 & Dufrost North Drain & 49.38415 & -97.12040 & 3 & 5 & $062 \mathrm{H} 06$ \\
\hline B-03-219 & 25-Jul-03 & Unnamed tributary to Marsh River & 49.38398 & -97.14470 & 4 & 5 & $062 \mathrm{H} 06$ \\
\hline B-03-220 & 25-Jul-03 & Unnamed tributary to Marsh River & 49.39835 & -97.16553 & 4 & 5 & 062H06 \\
\hline B-03-221 & 25-Jul-03 & Marsh River & 49.44277 & -97.17288 & 5 & 5 & $062 \mathrm{H} 06$ \\
\hline B-03-222 & 25-Jul-03 & Aubigny Drain & 49.41895 & -97.26865 & 3 & 5 & 062H06 \\
\hline B-03-223 & 5-Aug-03 & Meleb Drain & 50.68437 & -97.00048 & 3 & $31 \mathrm{E}$ & 062111 \\
\hline B-03-224 & 5-Aug-03 & Fish Lake Drain & 50.69717 & -96.99617 & 3 & $31 \mathrm{E}$ & 062110 \\
\hline
\end{tabular}


Appendix 1: Table listing the site number, date sampled, site name, latitude and longitude (in decimal degrees DD), DES stream order, DES map number and the corresponding NTS map number for all sites surveyed between 2002 and 2006.

\begin{tabular}{|c|c|c|c|c|c|c|c|}
\hline Site Number & Date & Site Name & $\begin{array}{l}\text { Latitude } \\
\text { (DD) }\end{array}$ & $\begin{array}{l}\text { Longitude } \\
\text { (DD) }\end{array}$ & $\begin{array}{l}\text { DES } \\
\text { Order }\end{array}$ & $\begin{array}{l}\text { DES } \\
\text { Map \# }\end{array}$ & $\begin{array}{l}\text { NTS } \\
\text { Map \# }\end{array}$ \\
\hline B-03-225 & 5-Aug-03 & Drunken River Drain & 50.82783 & -96.99515 & 3 & $31 \mathrm{E}$ & $062 \mid 15$ \\
\hline B-03-226 & 6-Aug-03 & Unnamed tributary to Lake Winnipeg & 50.61078 & -96.98990 & 2 & $31 \mathrm{E}$ & 062110 \\
\hline B-03-227 & 6-Aug-03 & Boundary Creek & 50.50882 & -96.97563 & 4 & $31 \mathrm{E}$ & 062110 \\
\hline B-03-228 & 6-Aug-03 & Boundary Creek & 50.51762 & -96.99892 & 4 & $31 \mathrm{E}$ & $062 \mid 10$ \\
\hline B-03-229 & 6-Aug-03 & Boundary Creek Drain & 50.52147 & -97.00853 & 4 & $31 \mathrm{E}$ & 062111 \\
\hline B-03-230 & 6-Aug-03 & Boundary Creek Drain & 50.50703 & -97.09737 & 4 & $31 \mathrm{E}$ & 062111 \\
\hline B-03-231 & 6-Aug-03 & Bass Drain & 50.57792 & -97.11422 & 4 & $31 \mathrm{E}$ & $062 \mid 11$ \\
\hline B-03-232 & 6-Aug-03 & Peiluck Drain & 50.59580 & -97.16718 & 3 & $31 \mathrm{E}$ & 062111 \\
\hline $\mathrm{B}-03-233$ & 6-Aug-03 & Bass Drain & 50.58107 & -97.13785 & 3 & $31 \mathrm{E}$ & 062111 \\
\hline B-03-234 & 6-Aug-03 & Unnamed tributary to Lake Winnipeg & 50.64015 & -97.06493 & 3 & $31 \mathrm{E}$ & 062111 \\
\hline B-03-235 & 6-Aug-03 & Fish Lake Drain & 50.70648 & -97.06488 & 3 & $31 \mathrm{E}$ & 062111 \\
\hline B-03-236 & 6-Aug-03 & Wheathill Drain & 50.80803 & -97.53253 & 3 & $31 \mathrm{E}$ & $062 \mid 13$ \\
\hline B-03-237 & 7-Aug-03 & Unnamed tributary to Hatchery Drain & 50.61235 & -98.05362 & 2 & $31 \mathrm{~W}$ & 062J09 \\
\hline B-03-238 & 7-Aug-03 & Hatchery Drain & 50.62223 & -98.05957 & 3 & $31 \mathrm{~W}$ & 062J09 \\
\hline B-03-239 & 7-Aug-03 & Swan Creek & 50.68348 & -98.14300 & 5 & $31 \mathrm{~W}$ & 062J09 \\
\hline B-03-240 & 7-Aug-03 & Mud Lake Drain & 50.69818 & -98.11130 & 3 & $31 \mathrm{~W}$ & 062J09 \\
\hline B-03-241 & 7-Aug-03 & Swan Creek & 50.71453 & -98.11113 & 5 & $31 \mathrm{~W}$ & 062J09 \\
\hline B-03-242 & 7-Aug-03 & Burnt Lake Drain & 50.72067 & -98.07552 & 4 & $31 \mathrm{~W}$ & 062J09 \\
\hline B-03-243 & 7-Aug-03 & Hayward Drain & 50.72773 & -98.16783 & 3 & $31 \mathrm{~W}$ & 062J09 \\
\hline B-03-244 & 7-Aug-03 & Swan Lake Dam & 50.80040 & -98.16943 & 3 & $31 \mathrm{~W}$ & 062J16 \\
\hline B-03-245 & 7-Aug-03 & Wagon Creek & 50.55005 & -98.03320 & 2 & $31 \mathrm{~W}$ & 062J09 \\
\hline B-03-246 & 8-Aug-03 & Tugela Creek & 50.45720 & -96.96268 & 3 & 30 & 062107 \\
\hline B-03-247 & 8-Aug-03 & Cochrane Drain & 50.39555 & -96.96815 & 3 & 30 & 062107 \\
\hline B-03-248 & 8-Aug-03 & Fisher Drain & 50.34375 & -96.92898 & 3 & 30 & 062107 \\
\hline B-03-249 & 8-Aug-03 & Ross Creek & 50.32662 & -97.09052 & 4 & 30 & 062106 \\
\hline
\end{tabular}


Appendix 1: Table listing the site number, date sampled, site name, latitude and longitude (in decimal degrees DD), DES stream order, DES map number and the corresponding NTS map number for all sites surveyed between 2002 and 2006.

\begin{tabular}{|c|c|c|c|c|c|c|c|}
\hline Site Number & Date & Site Name & $\begin{array}{l}\text { Latitude } \\
\text { (DD) }\end{array}$ & $\begin{array}{l}\text { Longitude } \\
\text { (DD) }\end{array}$ & $\begin{array}{l}\text { DES } \\
\text { Order }\end{array}$ & $\begin{array}{l}\text { DES } \\
\text { Map \# }\end{array}$ & $\begin{array}{l}\text { NTS } \\
\text { Map \# }\end{array}$ \\
\hline B-03-250 & 8-Aug-03 & Unnamed tributary to Netley Creek & 50.35955 & -97.09643 & 3 & 30 & 062106 \\
\hline D-03-001 & 8-May-03 & Big Grass River & 50.50593 & -98.97183 & 5 & 43 & 062J10 \\
\hline D-03-002 & 10-May-03 & Garland River & 51.65862 & -100.63463 & 3 & 103 & 062N10 \\
\hline D-03-003 & 10-May-03 & Fishing River & 51.46812 & -100.36753 & 4 & 49 & 062N08 \\
\hline D-03-004 & 11-May-03 & Garland River & 51.64702 & -100.43913 & 5 & 103 & 062N09 \\
\hline D-03-005 & 11-May-03 & Mink Creek & 51.41390 & -100.35332 & 4 & 49 & 062N08 \\
\hline D-03-006 & 12-May-03 & Harper Creek & 51.09350 & -100.69538 & 3 & 48 & 062N02 \\
\hline D-03-007 & 12-May-03 & Harper Creek & 51.06652 & -100.72873 & 2 & 48 & 062N02 \\
\hline D-03-008 & 13-May-03 & Turtle River & 51.05060 & -99.53197 & 5 & 45 & 062004 \\
\hline D-03-009 & 13-May-03 & Hanson Creek & 51.05195 & -99.42447 & 3 & 45 & 062003 \\
\hline D-03-010 & 13-May-03 & Hanson Creek & 50.99295 & -99.38610 & 3 & 45 & 062J14 \\
\hline D-03-011 & 13-May-03 & Turtle River & 50.90443 & -99.50482 & 5 & 45 & 062J13 \\
\hline D-03-012 & 13-May-03 & Henderson Creek & 50.91914 & -99.60153 & 4 & 45 & 062J13 \\
\hline D-03-013 & 13-May-03 & Laurier Drain & 50.91914 & -99.60153 & 3 & 45 & 062J13 \\
\hline D-03-014 & 13-May-03 & Kergwenan Drain & 50.94855 & -99.67177 & 3 & 45 & 062J13 \\
\hline D-03-015 & 14-May-03 & Scott Creek & 50.86032 & -99.54002 & 4 & 44 & 062J13 \\
\hline D-03-016 & 14-May-03 & Scott Creek & 50.81627 & -99.59420 & 3 & 44 & 062J13 \\
\hline D-03-017 & 14-May-03 & Dead Ox Creek & 50.70065 & -99.53162 & 2 & 44 & 062J12 \\
\hline D-03-018 & 14-May-03 & Unnamed tributary to Reeve Drain & 50.68327 & -99.52493 & 2 & 44 & 062J12 \\
\hline D-03-019 & 14-May-03 & Reeve Drain & 50.71283 & -99.48490 & 4 & 44 & 062J11 \\
\hline D-03-020 & 14-May-03 & Norgate Drain & 50.65354 & -99.48504 & 3 & 44 & 062J11 \\
\hline $\mathrm{D}-03-021$ & 3-Jun-03 & Unnamed tributary to North Shannon Creek & 49.31062 & -97.93373 & 3 & 16 & 062H05 \\
\hline D-03-022 & 3-Jun-03 & North Shannon Creek & 49.29415 & -97.93373 & 3 & 16 & $062 \mathrm{H} 05$ \\
\hline $\mathrm{D}-03-023$ & 3-Jun-03 & Shannon Creek & 49.27322 & -97.95625 & 4 & 16 & $062 \mathrm{H} 05$ \\
\hline D-03-024 & 3-Jun-03 & Shannon Creek & 49.27134 & -97.97879 & 4 & 16 & 062H05 \\
\hline
\end{tabular}


Appendix 1: Table listing the site number, date sampled, site name, latitude and longitude (in decimal degrees DD), DES stream order, DES map number and the corresponding NTS map number for all sites surveyed between 2002 and 2006.

\begin{tabular}{|c|c|c|c|c|c|c|c|}
\hline Site Number & Date & Site Name & $\begin{array}{l}\text { Latitude } \\
\text { (DD) }\end{array}$ & $\begin{array}{l}\text { Longitude } \\
\text { (DD) }\end{array}$ & $\begin{array}{l}\text { DES } \\
\text { Order }\end{array}$ & $\begin{array}{l}\text { DES } \\
\text { Map \# }\end{array}$ & $\begin{array}{l}\text { NTS } \\
\text { Map \# }\end{array}$ \\
\hline D-03-025 & 3-Jun-03 & Thornhill Coulee & 49.25143 & -98.08298 & 3 & 16 & 062G08 \\
\hline D-03-026 & 3-Jun-03 & Shannon Creek & 49.27365 & -98.13723 & 3 & 16 & 062G08 \\
\hline D-03-027 & 4-Jun-03 & Shannon Creek at Lizard Lake & 49.29650 & -98.38620 & 3 & 16 & 062G08 \\
\hline D-03-028 & 4-Jun-03 & Shannon Creek & 49.29627 & -98.32208 & 3 & 16 & 062G08 \\
\hline D-03-029 & 4-Jun-03 & Shannon Creek & 49.26633 & -98.20980 & 3 & 16 & $062 \mathrm{G} 08$ \\
\hline D-03-030 & 4-Jun-03 & Unnamed tributary to Pembina River & 49.08313 & -98.46998 & 2 & 60 & $062 \mathrm{G} 01$ \\
\hline D-03-031 & 4-Jun-03 & Unnamed tributary to Pembina River & 49.06282 & -98.46978 & 1 & 60 & $062 \mathrm{G} 01$ \\
\hline D-03-032 & 4-Jun-03 & Unnamed tributary to Little Pembina River & 49.10185 & -98.42488 & 2 & 60 & $062 \mathrm{G} 01$ \\
\hline D-03-033 & 4-Jun-03 & Unnamed tributary to Pembina River & 49.01522 & -98.32603 & 2 & 60 & $062 G 01$ \\
\hline D-03-034 & 4-Jun-03 & Unnamed tributary to Pembina River & 49.04385 & -98.15540 & 2 & 60 & $062 G 01$ \\
\hline D-03-035 & 5-Jun-03 & Little Pembina River & 49.17773 & -98.39963 & 2 & 60 & $062 G 01$ \\
\hline D-03-036 & 5-Jun-03 & Pembina River & 49.05787 & -98.46980 & 6 & 60 & 062G01 \\
\hline D-03-037 & 5-Jun-03 & Unnamed tributary to Pembina River & 49.12270 & -98.42507 & 3 & 60 & $062 \mathrm{G} 01$ \\
\hline D-03-038 & 6-Jun-03 & Shell River & 50.96448 & -101.31578 & 5 & 92 & $062 \mathrm{~K} 14$ \\
\hline D-03-039 & 10-Jun-03 & Buffalo Creek & 49.04478 & -97.86363 & 3 & 15 & $062 \mathrm{H} 04$ \\
\hline D-03-040 & 10-Jun-03 & Unnamed tributary to Buffalo Creek & 49.01253 & -97.72898 & 4 & 15 & $062 \mathrm{H} 04$ \\
\hline D-03-041 & 10-Jun-03 & Unnamed tributary to South Buffalo Creek & 49.01555 & -97.71647 & 4 & 15 & $062 \mathrm{H} 04$ \\
\hline D-03-042 & 10-Jun-03 & Unnamed tributary to Buffalo Creek & 49.03018 & -97.68430 & 4 & 15 & $062 \mathrm{H} 04$ \\
\hline D-03-043 & 10-Jun-03 & Buffalo Creek & 49.06043 & -97.70657 & 3 & 15 & $062 \mathrm{H} 04$ \\
\hline D-03-044 & 11-Jun-03 & Reinland Drain & 49.03005 & -97.93112 & 2 & 15 & $062 \mathrm{H} 04$ \\
\hline D-03-045 & 11-Jun-03 & Unnamed tributary to Buffalo Creek & 49.01540 & -97.82960 & 3 & 15 & $062 \mathrm{H} 04$ \\
\hline D-03-046 & 11-Jun-03 & Unnamed tributary to Buffalo Creek & 49.01525 & -97.81882 & 3 & 15 & $062 \mathrm{H} 04$ \\
\hline D-03-047 & 11-Jun-03 & Unnamed tributary to South Buffalo Creek & 49.01528 & -97.81000 & 3 & 15 & $062 \mathrm{H} 04$ \\
\hline D-03-048 & 11-Jun-03 & Unnamed tributary to Buffalo Creek & 49.01542 & -97.74723 & 4 & 15 & $062 \mathrm{H} 04$ \\
\hline D-03-049 & 11-Jun-03 & Unnamed tributary to Buffalo Creek & 49.04492 & -97.69533 & 4 & 15 & $062 \mathrm{H} 04$ \\
\hline
\end{tabular}


Appendix 1: Table listing the site number, date sampled, site name, latitude and longitude (in decimal degrees DD), DES stream order, DES map number and the corresponding NTS map number for all sites surveyed between 2002 and 2006.

\begin{tabular}{|c|c|c|c|c|c|c|c|}
\hline Site Number & Date & Site Name & $\begin{array}{l}\text { Latitude } \\
\text { (DD) }\end{array}$ & $\begin{array}{l}\text { Longitude } \\
\text { (DD) }\end{array}$ & $\begin{array}{l}\text { DES } \\
\text { Order }\end{array}$ & $\begin{array}{l}\text { DES } \\
\text { Map \# }\end{array}$ & $\begin{array}{l}\text { NTS } \\
\text { Map \# }\end{array}$ \\
\hline D-03-050 & 13-Jun-03 & Kaspic Creek & 50.15025 & -99.68313 & 2 & 40 & 062J04 \\
\hline D-03-051 & 13-Jun-03 & Brookdale Drain & 50.12260 & -99.68033 & 2 & 40 & 062J04 \\
\hline D-03-052 & 13-Jun-03 & Kaspic Creek & 50.12265 & -99.63665 & 2 & 40 & 062J04 \\
\hline D-03-053 & 13-Jun-03 & Kaspic Creek & 50.07688 & -99.56842 & 3 & 40 & 062J04 \\
\hline D-03-054 & 13-Jun-03 & Brookdale Drain & 50.05963 & -99.56840 & 3 & 40 & 062J04 \\
\hline D-03-055 & 13-Jun-03 & Brookdale Drain & 50.04885 & -99.57398 & 3 & 40 & 062J04 \\
\hline D-03-056 & 13-Jun-03 & Boggy Creek & 50.00507 & -99.56818 & 4 & 40 & 062J04 \\
\hline D-03-057 & 13-Jun-03 & Brookdale Drain & 50.19263 & -99.45432 & 4 & 40 & 062J03 \\
\hline D-03-058 & 16-Jun-03 & Edwards Creek Drain & 51.15445 & -99.89917 & 5 & 47 & 062004 \\
\hline D-03-059 & 16-Jun-03 & Edwards Creek Drain & 51.13330 & -99.96952 & 4 & 47 & 062004 \\
\hline D-03-060 & 16-Jun-03 & Old Edwards Creek & 51.16988 & -99.95360 & 3 & 47 & 062004 \\
\hline D-03-061 & 17-Jun-03 & Fork River Drain & 51.52227 & -100.36995 & 5 & 102 & 062N09 \\
\hline D-03-062 & 17-Jun-03 & Shanty Creek & 51.50002 & -100.55668 & 3 & 102 & 062N10 \\
\hline D-03-063 & 17-Jun-03 & Shanty Creek & 51.50173 & -100.53325 & 3 & 102 & 062N10 \\
\hline D-03-064 & 17-Jun-03 & Unnamed tributary to Shanty Creek & 51.50895 & -100.53309 & 2 & 102 & $062 \mathrm{~N} 10$ \\
\hline D-03-065 & 17-Jun-03 & Shanty Creek & 51.50721 & -100.46324 & 3 & 102 & 062N09 \\
\hline D-03-066 & 18-Jun-03 & Fork River & 51.53875 & -100.61257 & 3 & 102 & $062 \mathrm{~N} 10$ \\
\hline D-03-067 & 18-Jun-03 & Fork River & 51.56118 & -100.48697 & 4 & 102 & 062N09 \\
\hline D-03-068 & 18-Jun-03 & Fork River & 51.57460 & -100.44858 & 4 & 102 & 062N09 \\
\hline D-03-069 & 18-Jun-03 & Fork River & 51.53658 & -100.40317 & 4 & 102 & 062N09 \\
\hline D-03-070 & 23-Jun-03 & Zoria Drain & 51.32210 & -100.36245 & 3 & 95 & 062N08 \\
\hline D-03-071 & 23-Jun-03 & Drifting River & 51.30253 & -100.35953 & 4 & 95 & 062N08 \\
\hline D-03-072 & 23-Jun-03 & Sulphurspring Creek & 51.33280 & -100.67922 & 2 & 95 & 062N07 \\
\hline D-03-073 & 23-Jun-03 & Unnamed tributary to Mink Creek & 51.34395 & -100.60393 & 2 & 49 & 062N07 \\
\hline D-03-074 & 24-Jun-03 & Sulphurspring Creek & 51.22507 & -100.48492 & 3 & 95 & 062N01 \\
\hline
\end{tabular}


Appendix 1: Table listing the site number, date sampled, site name, latitude and longitude (in decimal degrees DD), DES stream order, DES map number and the corresponding NTS map number for all sites surveyed between 2002 and 2006.

\begin{tabular}{|c|c|c|c|c|c|c|c|}
\hline Site Number & Date & Site Name & $\begin{array}{l}\text { Latitude } \\
\text { (DD) }\end{array}$ & $\begin{array}{l}\text { Longitude } \\
\text { (DD) }\end{array}$ & $\begin{array}{l}\text { DES } \\
\text { Order }\end{array}$ & $\begin{array}{l}\text { DES } \\
\text { Map \# }\end{array}$ & $\begin{array}{l}\text { NTS } \\
\text { Map \# }\end{array}$ \\
\hline D-03-075 & 24-Jun-03 & Sulphurspring Creek & 51.22328 & -100.39150 & 3 & 95 & 062N01 \\
\hline D-03-076 & 24-Jun-03 & Sulphurspring Creek & 51.22713 & -100.36790 & 3 & 95 & 062N01 \\
\hline D-03-077 & 24-Jun-03 & Unnamed tributary to Valley River & 51.20407 & -100.39130 & 3 & 95 & 062N01 \\
\hline D-03-078 & 24-Jun-03 & Browns Creek & 51.09597 & -100.50000 & 4 & 48 & 062N02 \\
\hline D-03-079 & 24-Jun-03 & Wilson River & 51.09765 & -100.62512 & 4 & 48 & 062N02 \\
\hline D-03-080 & 24-Jun-03 & Wilson River & 51.02637 & -100.62508 & 3 & 48 & 062N02 \\
\hline D-03-081 & 24-Jun-03 & Ranch Creek & 51.03698 & -100.43998 & 3 & 48 & 062N01 \\
\hline D-03-082 & 24-Jun-03 & Mineral Creek & 51.06500 & -100.41457 & 3 & 48 & 062N01 \\
\hline D-03-083 & 26-Jun-03 & Ruby Creek & 51.96847 & -101.28397 & 4 & 51 & 062N14 \\
\hline D-03-084 & 26-Jun-03 & Ruby Creek & 51.78025 & -101.35925 & 2 & 51 & $062 N 14$ \\
\hline D-03-085 & 27-Jun-03 & North Pine River & 51.82133 & -100.53383 & 4 & 104 & 062N15 \\
\hline D-03-086 & 27-Jun-03 & South Pine River & 51.79860 & -100.38640 & 4 & 104 & 062N16 \\
\hline D-03-087 & 27-Jun-03 & Pine River Drain & 51.79847 & -100.44093 & 3 & 104 & 062N16 \\
\hline D-03-088 & 2-Jul-03 & Valley River & 51.18470 & -100.85283 & 4 & 94 & 062N02 \\
\hline D-03-089 & 2-Jul-03 & Pleasant Valley Creek & 51.13947 & -100.84117 & 4 & 94 & 062N02 \\
\hline D-03-090 & 2-Jul-03 & Pleasant Valley Dam & 51.05535 & -100.94740 & 4 & 94 & 062N02 \\
\hline D-03-091 & 3-Jul-03 & Munson Creek & 51.19503 & -100.93637 & 3 & 94 & 062N02 \\
\hline D-03-092 & 3-Jul-03 & Short Creek & 51.23353 & -101.03527 & 3 & 94 & 062N03 \\
\hline D-03-093 & 3-Jul-03 & Short Creek & 51.25768 & -101.15483 & 3 & 94 & 062N06 \\
\hline D-03-094 & 8-Jul-03 & Smith Creek & 52.22728 & -101.22763 & 4 & 89 & $063 \mathrm{C03}$ \\
\hline D-03-095 & 8-Jul-03 & Smith Creek & 52.22018 & -101.27542 & 4 & 89 & $063 \mathrm{C03}$ \\
\hline D-03-096 & 8-Jul-03 & Smith Creek & 52.21086 & -101.34689 & 4 & 89 & $063 \mathrm{C03}$ \\
\hline D-03-097 & 9-Jul-03 & Woody River & 52.14353 & -101.59027 & 5 & 89 & $063 C 04$ \\
\hline D-03-098 & 9-Jul-03 & Hart Creek & 52.13817 & -101.60902 & 2 & 89 & $063 C 04$ \\
\hline D-03-099 & 9-Jul-03 & Whitebeech Creek & 52.08535 & -101.58408 & 3 & 89 & $063 C 04$ \\
\hline
\end{tabular}


Appendix 1: Table listing the site number, date sampled, site name, latitude and longitude (in decimal degrees DD), DES stream order, DES map number and the corresponding NTS map number for all sites surveyed between 2002 and 2006.

\begin{tabular}{|c|c|c|c|c|c|c|c|}
\hline Site Number & Date & Site Name & $\begin{array}{l}\text { Latitude } \\
\text { (DD) }\end{array}$ & $\begin{array}{l}\text { Longitude } \\
\text { (DD) }\end{array}$ & $\begin{array}{l}\text { DES } \\
\text { Order }\end{array}$ & $\begin{array}{l}\text { DES } \\
\text { Map \# }\end{array}$ & $\begin{array}{l}\text { NTS } \\
\text { Map \# }\end{array}$ \\
\hline D-03-100 & 9-Jul-03 & Whitebeech Creek & 52.09658 & -101.46588 & 4 & 89 & $063 C 03$ \\
\hline D-03-101 & 9-Jul-03 & Trout Creek & 52.20177 & -101.51448 & 2 & 89 & $063 \mathrm{C} 04$ \\
\hline D-03-102 & 11-Jul-03 & Crawford/Rainbow Creek & 51.13113 & -99.80932 & 2 & 46 & 062004 \\
\hline D-03-103 & 11-Jul-03 & Crawford Creek & 51.08135 & -99.81408 & 3 & 46 & 062004 \\
\hline D-03-104 & 11-Jul-03 & Crooked Creek & 51.12552 & -99.82863 & 3 & 46 & 062004 \\
\hline D-03-105 & 11-Jul-03 & Crawford Creek & 51.12550 & -99.81195 & 2 & 46 & 062004 \\
\hline D-03-106 & 14-Jul-03 & Spring Creek & 50.27143 & -99.58098 & 3 & 39 & 062J05 \\
\hline D-03-107 & 14-Jul-03 & Unnamed tributary to Spring Creek & 50.26977 & -99.51453 & 2 & 39 & 062J05 \\
\hline D-03-108 & 14-Jul-03 & Unnamed tributary to Spring Creek & 50.26972 & -99.48742 & 2 & 39 & 062J06 \\
\hline D-03-109 & 14-Jul-03 & Law Creek & 50.31113 & -99.44677 & 3 & 39 & 062J06 \\
\hline D-03-110 & 14-Jul-03 & Spring Creek & 50.27488 & -99.44682 & 4 & 39 & 062J06 \\
\hline D-03-111 & 14-Jul-03 & Spring Creek & 50.26980 & -99.41103 & 4 & 39 & 062J06 \\
\hline D-03-112 & 15-Jul-03 & Unnamed tributary to Stony Creek & 50.17592 & -99.61320 & 3 & 39 & 062J04 \\
\hline D-03-113 & 15-Jul-03 & Unnamed tributary to Whitemud River & 50.18872 & -99.56810 & 3 & 39 & 062J04 \\
\hline D-03-114 & 15-Jul-03 & Franklin Creek & 50.21309 & -99.56820 & 2 & 39 & 062J04 \\
\hline D-03-115 & 15-Jul-03 & Franklin Creek & 50.21067 & -99.50660 & 2 & 39 & 062J04 \\
\hline D-03-116 & 15-Jul-03 & Unnamed tributary to Stony Creek & 50.19695 & -99.50083 & 3 & 39 & 062J04 \\
\hline D-03-117 & 15-Jul-03 & Stony Creek & 50.21128 & -99.47622 & 5 & 39 & 062J03 \\
\hline D-03-118 & 15-Jul-03 & Eden Creek & 50.43262 & -99.56432 & 3 & 39 & 062J05 \\
\hline D-03-119 & 15-Jul-03 & Unnamed tributary to Eden Creek & 50.35828 & -99.49928 & 3 & 39 & 062J06 \\
\hline D-03-120 & 15-Jul-03 & Snake Creek & 50.28463 & -99.27833 & 5 & 39 & 062J06 \\
\hline $\mathrm{D}-03-121$ & 16-Jul-03 & North Snake Creek & 50.44123 & -99.46982 & 3 & 43 & 062J06 \\
\hline $\mathrm{D}-03-122$ & 16-Jul-03 & Kinch Creek & 50.60957 & -99.44463 & 3 & 43 & 062J11 \\
\hline $\mathrm{D}-03-123$ & 16-Jul-03 & Unnamed tributary to Glencairn Drain & 50.63895 & -99.43853 & 3 & 43 & 062J11 \\
\hline D-03-124 & 16-Jul-03 & Kinch Creek & 50.63898 & -99.41532 & 3 & 43 & 062J11 \\
\hline
\end{tabular}


Appendix 1: Table listing the site number, date sampled, site name, latitude and longitude (in decimal degrees DD), DES stream order, DES map number and the corresponding NTS map number for all sites surveyed between 2002 and 2006.

\begin{tabular}{|c|c|c|c|c|c|c|c|}
\hline Site Number & Date & Site Name & $\begin{array}{l}\text { Latitude } \\
\text { (DD) }\end{array}$ & $\begin{array}{l}\text { Longitude } \\
\text { (DD) }\end{array}$ & $\begin{array}{l}\text { DES } \\
\text { Order }\end{array}$ & $\begin{array}{l}\text { DES } \\
\text { Map \# }\end{array}$ & $\begin{array}{l}\text { NTS } \\
\text { Map \# }\end{array}$ \\
\hline D-03-125 & 16-Jul-03 & Big Grass River Angle Ditch & 50.65390 & -99.25258 & 3 & 43 & 062J11 \\
\hline $\mathrm{D}-03-126$ & 17-Jul-03 & Glencairn Drain & 50.65360 & -99.41518 & 4 & 43 & 062J11 \\
\hline D-03-127 & 17-Jul-03 & Glencairn Drain & 50.65405 & -99.30693 & 4 & 43 & 062J11 \\
\hline D-03-128 & 17-Jul-03 & Potrebka Drain & 50.65390 & -99.22915 & 2 & 43 & 062J11 \\
\hline D-03-129 & 17-Jul-03 & Little Pocket Lake Drain & 50.68343 & -99.25225 & 3 & 43 & 062J11 \\
\hline D-03-130 & 17-Jul-03 & Big Grass River Angle Ditch & 50.68342 & -99.27617 & 3 & 43 & 062J11 \\
\hline D-03-131 & 17-Jul-03 & McLung Creek & 50.57968 & -99.47700 & 2 & 43 & 062J11 \\
\hline D-03-132 & 17-Jul-03 & McLung Creek & 50.58760 & -99.42347 & 3 & 43 & 062J11 \\
\hline D-03-133 & 17-Jul-03 & Big Grass River Angle Ditch & 50.59482 & -99.19725 & 3 & 43 & 062J11 \\
\hline D-03-134 & 17-Jul-03 & Correction Line Drain & 50.59470 & -99.31715 & 3 & 43 & 062J11 \\
\hline D-03-135 & 18-Jul-03 & Kinch Creek & 50.59470 & -99.49342 & 2 & 43 & 062J11 \\
\hline D-03-136 & 18-Jul-03 & Glenella Drain & 50.56497 & -99.45583 & 3 & 43 & 062J11 \\
\hline D-03-137 & 18-Jul-03 & Riding Mountain Drain & 50.53231 & -99.50620 & 2 & 43 & 062J12 \\
\hline D-03-138 & 18-Jul-03 & Pelican Creek & 50.52087 & -99.33138 & 4 & 43 & 062J11 \\
\hline D-03-139 & 18-Jul-03 & North Snake Creek & 50.46893 & -99.58530 & 3 & 43 & 062J05 \\
\hline D-03-140 & 21-Jul-03 & North Duck River & 52.01997 & -100.67997 & 3 & 105 & $063 \mathrm{C02}$ \\
\hline D-03-141 & 22-Jul-03 & Unnamed tributary to Old Government Drain & 52.05612 & -101.00397 & 2 & 52 & $063 \mathrm{C03}$ \\
\hline D-03-142 & 22-Jul-03 & Unnamed tributary to North Duck River & 51.98453 & -100.67992 & 3 & 105 & 062N15 \\
\hline D-03-143 & 22-Jul-03 & Harpiak Drain & 52.00702 & -100.67990 & 2 & 105 & $063 \mathrm{C} 02$ \\
\hline D-03-144 & 22-Jul-03 & Wasyliuk Drain & 51.83262 & -100.58300 & 3 & 105 & 062N15 \\
\hline D-03-145 & 22-Jul-03 & South Duck River & 51.88220 & -100.61292 & 3 & 105 & 062N15 \\
\hline D-03-146 & 22-Jul-03 & Unnamed tributary to South Duck River & 51.88547 & -100.61907 & 2 & 105 & 062N15 \\
\hline D-03-147 & 23-Jul-03 & Mullin Creek & 52.25258 & -101.25148 & 3 & 89 & $063 C 06$ \\
\hline D-03-148 & 23-Jul-03 & Nine Creek & 52.28910 & -101.23303 & 3 & 89 & $063 C 06$ \\
\hline D-03-149 & 23-Jul-03 & Bell Creek & 52.58982 & -101.01525 & 2 & 97 & $063 C 11$ \\
\hline
\end{tabular}


Appendix 1: Table listing the site number, date sampled, site name, latitude and longitude (in decimal degrees DD), DES stream order, DES map number and the corresponding NTS map number for all sites surveyed between 2002 and 2006.

\begin{tabular}{|c|c|c|c|c|c|c|c|}
\hline Site Number & Date & Site Name & $\begin{array}{l}\text { Latitude } \\
\text { (DD) }\end{array}$ & $\begin{array}{l}\text { Longitude } \\
\text { (DD) }\end{array}$ & $\begin{array}{l}\text { DES } \\
\text { Order }\end{array}$ & $\begin{array}{l}\text { DES } \\
\text { Map \# }\end{array}$ & $\begin{array}{l}\text { NTS } \\
\text { Map \# }\end{array}$ \\
\hline D-03-150 & 23-Jul-03 & Glover Creek & 52.56568 & -100.99187 & 2 & 97 & $063 C 10$ \\
\hline D-03-151 & 23-Jul-03 & Bell Creek & 52.56560 & -100.94505 & 2 & 97 & $063 C 10$ \\
\hline D-03-152 & 23-Jul-03 & Bell Creek & 52.55560 & -100.89725 & 3 & 97 & $063 C 10$ \\
\hline D-03-153 & 23-Jul-03 & Stapely Creek & 52.58003 & -100.89705 & 2 & 97 & $063 C 10$ \\
\hline D-03-154 & 23-Jul-03 & Steeprock River & 52.73200 & -101.11510 & 5 & 115 & $063 C 11$ \\
\hline D-03-155 & 23-Jul-03 & Campbells Creek & 52.69747 & -101.11458 & 2 & 115 & $063 C 11$ \\
\hline D-03-156 & 23-Jul-03 & Smith Creek & 52.66252 & -101.11285 & 2 & 115 & $063 C 11$ \\
\hline D-03-157 & 24-Jul-03 & Mafeking Creek & 52.68177 & -101.10958 & 3 & 115 & $063 C 11$ \\
\hline D-03-158 & 24-Jul-03 & Unnamed tributary to Steeprock River & 52.65172 & -101.10378 & 2 & 115 & $063 C 11$ \\
\hline D-03-159 & 24-Jul-03 & Unnamed tributary to Wood Drain & 52.51438 & -101.01538 & 2 & 90 & $063 C 11$ \\
\hline D-03-160 & 24-Jul-03 & Hamel Drain & 52.49287 & -100.99177 & 2 & 90 & $063 \mathrm{C07}$ \\
\hline D-03-161 & 24-Jul-03 & Schlagg Drain & 52.47842 & -100.99180 & 2 & 90 & $063 C 07$ \\
\hline D-03-162 & 24-Jul-03 & Brandt Drain & 52.46373 & -100.99167 & 3 & 90 & $063 C 07$ \\
\hline D-03-163 & 24-Jul-03 & McKinley Drain & 52.43490 & -100.99224 & 3 & 90 & $063 C 07$ \\
\hline D-03-164 & 24-Jul-03 & Indian Birch River & 52.40467 & -100.99170 & 4 & 90 & $063 C 07$ \\
\hline D-03-165 & 24-Jul-03 & Haggarty Creek & 52.36665 & -101.11028 & 3 & 90 & $063 C 06$ \\
\hline D-03-166 & 24-Jul-03 & Robinson Creek & 52.35210 & -101.11052 & 2 & 90 & $063 C 06$ \\
\hline D-03-167 & 25-Jul-03 & Sclater River & 51.92565 & -100.65217 & 3 & 105 & 062N15 \\
\hline D-03-168 & 10-Sep-03 & Unnamed tributary to Boggy River & 49.64085 & -95.49817 & 2 & 84 & 052E10 \\
\hline D-03-169 & 11-Sep-03 & Unnamed tributary to Boggy River & 49.64325 & -95.50053 & 2 & 84 & 052E11 \\
\hline D-03-170 & 11-Sep-03 & Unnamed tributary to Boggy River & 49.62934 & -95.50059 & 2 & 84 & 052E11 \\
\hline W-03-001 & 20-May-03 & Maple Creek & 50.28308 & -96.09032 & 3 & 96 & 062108 \\
\hline W-03-002 & 21-May-03 & Maple Creek & 50.26790 & -96.09853 & 3 & 96 & 062108 \\
\hline W-03-003 & 21-May-03 & Unnamed tributary to Winnipeg River & 50.16363 & -96.07923 & 2 & 96 & 062101 \\
\hline W-03-004 & 21-May-03 & Unnamed tributary to Winnipeg River & 50.17881 & -96.08526 & 2 & 96 & 062101 \\
\hline
\end{tabular}


Appendix 1: Table listing the site number, date sampled, site name, latitude and longitude (in decimal degrees DD), DES stream order, DES map number and the corresponding NTS map number for all sites surveyed between 2002 and 2006.

\begin{tabular}{|c|c|c|c|c|c|c|c|}
\hline Site Number & Date & Site Name & $\begin{array}{l}\text { Latitude } \\
\text { (DD) }\end{array}$ & $\begin{array}{l}\text { Longitude } \\
\text { (DD) }\end{array}$ & $\begin{array}{l}\text { DES } \\
\text { Order }\end{array}$ & $\begin{array}{l}\text { DES } \\
\text { Map \# }\end{array}$ & $\begin{array}{l}\text { NTS } \\
\text { Map \# }\end{array}$ \\
\hline W-03-005 & 21-May-03 & Unnamed tributary to Winnipeg River & 50.48430 & -96.08690 & 2 & 96 & 062108 \\
\hline W-03-006 & 21-May-03 & Maple Creek & 50.44700 & -96.07542 & 4 & 96 & 062108 \\
\hline W-03-007 & 21-May-03 & Unnamed tributary to Winnipeg River & 50.45838 & -96.04228 & 2 & 96 & 062108 \\
\hline W-03-008 & 22-May-03 & Selkirk Line West & 50.14992 & -96.45197 & 3 & 10 & 062101 \\
\hline W-03-009 & 22-May-03 & Beaver Creek & 50.13738 & -96.45190 & 2 & 10 & 062101 \\
\hline W-03-010 & 22-May-03 & Bachman Drain & 50.12057 & -96.46045 & 3 & 10 & 062101 \\
\hline W-03-011 & 22-May-03 & Bachman Drain & 50.12058 & -96.47667 & 3 & 10 & 062101 \\
\hline W-03-012 & 22-May-03 & Unnamed tributary to Brokenhead River & 50.32615 & -96.50957 & 2 & 10 & 062107 \\
\hline W-03-013 & 23-May-03 & Oneschuk Drain & 50.20777 & -96.46305 & 3 & 10 & 062101 \\
\hline W-03-014 & 26-May-03 & Unnamed tributary to Colony Creek & 49.99007 & -97.34428 & 3 & 26 & $062 \mathrm{H} 14$ \\
\hline W-03-015 & 26-May-03 & Colony Creek & 49.99027 & -97.37842 & 3 & 26 & $062 \mathrm{H} 14$ \\
\hline W-03-016 & 26-May-03 & Unnamed tributary to Sturgeon Creek & 49.99027 & -97.40468 & 2 & 26 & $062 \mathrm{H} 14$ \\
\hline W-03-017 & 26-May-03 & Unnamed tributary to Sturgeon Creek & 49.98995 & -97.42855 & 2 & 26 & $062 \mathrm{H} 14$ \\
\hline W-03-018 & 26-May-03 & Old Sturgeon Creek & 49.99022 & -97.48455 & 2 & 26 & $062 \mathrm{H} 14$ \\
\hline W-03-019 & 26-May-03 & Sturgeon Creek & 49.99045 & -97.50505 & 4 & 26 & $062 \mathrm{H} 13$ \\
\hline W-03-020 & 26-May-03 & Fourth Creek & 49.95917 & -97.51802 & 2 & 26 & $062 \mathrm{H} 13$ \\
\hline W-03-021 & 27-May-03 & Third Creek & 49.94900 & -97.52238 & 1 & 26 & $062 \mathrm{H} 13$ \\
\hline W-03-022 & 27-May-03 & $\begin{array}{l}\text { Confluence of Old Sturgeon Creek and Sturgeon } \\
\text { Creek }\end{array}$ & 49.97618 & -97.48253 & 2 & 26 & $062 \mathrm{H} 14$ \\
\hline W-03-023 & 27-May-03 & Unnamed tributary to Sturgeon Creek & 49.96797 & -97.48207 & 2 & 26 & $062 \mathrm{H} 14$ \\
\hline W-03-024 & 27-May-03 & Confluence of Sturgeon Creek and Meridian Drain & 49.92637 & -97.43190 & 3 & 26 & $062 \mathrm{H} 14$ \\
\hline W-03-025 & 27-May-03 & Meridian Drain & 49.94593 & -97.45923 & 3 & 26 & $062 \mathrm{H} 14$ \\
\hline W-03-026 & 27-May-03 & Unnamed tributary to Sturgeon Creek & 49.96072 & -97.39005 & 2 & 26 & $062 \mathrm{H} 14$ \\
\hline W-03-027 & 27-May-03 & Gordon Drain & 50.00480 & -97.32138 & 2 & 26 & 062103 \\
\hline W-03-028 & 28-May-03 & Unnamed tributary to Colony Creek & 50.06382 & -97.46227 & 3 & 26 & 062103 \\
\hline
\end{tabular}


Appendix 1: Table listing the site number, date sampled, site name, latitude and longitude (in decimal degrees DD), DES stream order, DES map number and the corresponding NTS map number for all sites surveyed between 2002 and 2006.

\begin{tabular}{|c|c|c|c|c|c|c|c|}
\hline Site Number & Date & Site Name & $\begin{array}{l}\text { Latitude } \\
\text { (DD) }\end{array}$ & $\begin{array}{l}\text { Longitude } \\
\text { (DD) }\end{array}$ & $\begin{array}{l}\text { DES } \\
\text { Order }\end{array}$ & $\begin{array}{l}\text { DES } \\
\text { Map \# }\end{array}$ & $\begin{array}{l}\text { NTS } \\
\text { Map \# }\end{array}$ \\
\hline W-03-029 & 28-May-03 & Unnamed tributary to Colony Creek & 50.06403 & -97.47753 & 2 & 26 & 062103 \\
\hline W-03-030 & 28-May-03 & East Branch Sturgeon Creek & 50.06382 & -97.54338 & 3 & 26 & 062104 \\
\hline W-03-031 & 28-May-03 & West Branch Sturgeon Creek & 50.06377 & -97.57495 & 4 & 26 & 062104 \\
\hline W-03-032 & 28-May-03 & West Branch Sturgeon Lateral Drain & 50.12300 & -97.65255 & 3 & 26 & 062104 \\
\hline W-03-033 & 28-May-03 & West Branch Sturgeon Creek & 50.18200 & -97.68262 & 3 & 26 & 062104 \\
\hline W-03-034 & 28-May-03 & East Branch Sturgeon Creek & 50.17875 & -97.61833 & 3 & 26 & 062104 \\
\hline W-03-035 & 29-May-03 & Kosc Coulee & 49.67258 & -97.44642 & 3 & 22 & $062 \mathrm{H} 11$ \\
\hline W-03-036 & 29-May-03 & King Drain & 49.62827 & -97.39045 & 4 & 20 & $062 \mathrm{H} 11$ \\
\hline W-03-037 & 29-May-03 & Domain Drain & 49.62078 & -97.29222 & 3 & 22 & $062 \mathrm{H} 11$ \\
\hline W-03-038 & 29-May-03 & Unnamed tributary to Domain Drain & 49.62082 & -97.27158 & 2 & 22 & $062 \mathrm{H} 11$ \\
\hline W-03-039 & 29-May-03 & "B" Drain & 49.62070 & -97.18543 & 3 & 22 & $062 \mathrm{H} 11$ \\
\hline W-03-040 & 29-May-03 & Glenlea Drain & 49.61505 & -97.14280 & 4 & 22 & $062 \mathrm{H} 11$ \\
\hline W-03-041 & 29-May-03 & Atchison Drain & 49.72395 & -97.27128 & 2 & 22 & $062 \mathrm{H} 11$ \\
\hline W-03-042 & 29-May-03 & Unnamed tributary to Atchison Drain & 49.72383 & -97.27648 & 2 & 22 & $062 \mathrm{H} 11$ \\
\hline W-03-043 & 29-May-03 & Oak Bluff Drain & 49.72395 & -97.30212 & 3 & 22 & $062 \mathrm{H} 11$ \\
\hline W-03-044 & 3-Jun-03 & Roblin Drain & 49.50240 & -98.08427 & 3 & 18 & 062G09 \\
\hline W-03-045 & 3-Jun-03 & Unnamed tributary to Boyne River & 49.53158 & -98.30046 & 2 & 18 & 062G0s \\
\hline W-03-046 & 3-Jun-03 & Unnamed tributary to Boyne River & 49.53898 & -98.34342 & 3 & 18 & 062G0S \\
\hline W-03-047 & 3-Jun-03 & Unnamed tributary to Boyne River & 49.54745 & -98.34598 & 2 & 18 & 062G09 \\
\hline W-03-048 & 3-Jun-03 & Unnamed tributary to Boyne River & 49.54742 & -98.39132 & 2 & 18 & 062G09 \\
\hline W-03-049 & 3-Jun-03 & Roseisle Drain & 49.50167 & -98.34128 & 3 & 18 & 062G09 \\
\hline W-03-050 & 4-Jun-03 & Boyne River & 49.54503 & -98.41453 & 4 & 18 & 062G09 \\
\hline W-03-051 & 4-Jun-03 & Confluence of unnamed tributary and Boyne River & 49.54100 & -98.41468 & 2 & 18 & 062G09 \\
\hline W-03-052 & 4-Jun-03 & Unnamed tributary to $7-7 \mathrm{~W}$ Drain & 49.59124 & -98.34601 & 2 & 18 & 062G09 \\
\hline W-03-053 & 4-Jun-03 & Unnamed tributary to Boyne River & 49.65047 & -98.52837 & 2 & 18 & 062G10 \\
\hline
\end{tabular}


Appendix 1: Table listing the site number, date sampled, site name, latitude and longitude (in decimal degrees DD), DES stream order, DES map number and the corresponding NTS map number for all sites surveyed between 2002 and 2006.

\begin{tabular}{|c|c|c|c|c|c|c|c|}
\hline Site Number & Date & Site Name & $\begin{array}{l}\text { Latitude } \\
\text { (DD) }\end{array}$ & $\begin{array}{l}\text { Longitude } \\
\text { (DD) }\end{array}$ & $\begin{array}{l}\text { DES } \\
\text { Order }\end{array}$ & $\begin{array}{l}\text { DES } \\
\text { Map \# }\end{array}$ & $\begin{array}{c}\text { NTS } \\
\text { Map \# }\end{array}$ \\
\hline W-03-054 & 4-Jun-03 & Unnamed tributary to Boyne River & 49.65968 & -98.52833 & 3 & 18 & $062 \mathrm{G} 10$ \\
\hline W-03-055 & 4-Jun-03 & Boyne River & 49.67823 & -98.52853 & 4 & 18 & 062G10 \\
\hline W-03-056 & 4-Jun-03 & Unnamed tributary to Boyne River & 49.64583 & -98.52830 & 2 & 18 & 062G10 \\
\hline W-03-057 & 4-Jun-03 & Unnamed tributary to Boyne River & 49.63575 & -98.52820 & 2 & 18 & 062G10 \\
\hline W-03-058 & 4-Jun-03 & Unnamed tributary to Boyne River & 49.62110 & -98.50843 & 2 & 18 & 062G10 \\
\hline W-03-059 & 4-Jun-03 & Unnamed tributary to Boyne River & 49.62103 & -98.48962 & 3 & 18 & 062G09 \\
\hline W-03-060 & 5-Jun-03 & Unnamed tributary to Boyne River & 49.65068 & -98.59017 & 3 & 18 & 062G10 \\
\hline W-03-061 & 5-Jun-03 & Unnamed tributary to Boyne River & 49.63582 & -98.68477 & 2 & 18 & $062 \mathrm{G} 10$ \\
\hline W-03-062 & 5-Jun-03 & Boyne River & 49.63577 & -98.69358 & 3 & 18 & $062 \mathrm{G} 10$ \\
\hline W-03-063 & 5-Jun-03 & Unnamed tributary to Boyne River & 49.63202 & -98.70882 & 1 & 18 & 062G10 \\
\hline W-03-064 & 5-Jun-03 & Unnamed tributary to Boyne River & 49.63567 & -98.71878 & 3 & 18 & 062G10 \\
\hline W-03-065 & 5-Jun-03 & Boyne River & 49.66523 & -98.68842 & 4 & 18 & 062G10 \\
\hline W-03-066 & 5-Jun-03 & Confluence of unnamed tributary and Boyne River & 49.60243 & -98.68733 & 3 & 18 & $062 \mathrm{G} 10$ \\
\hline W-03-067 & 6-Jun-03 & Lyles Creek & 49.38468 & -98.45752 & 2 & 18 & $062 \mathrm{G} 08$ \\
\hline W-03-068 & 6-Jun-03 & Unnamed tributary to Lyles Creek & 49.38463 & -98.47107 & 2 & 18 & 062G08 \\
\hline W-03-069 & 6-Jun-03 & Unnamed tributary to Lyles Creek & 49.49662 & -98.54500 & 3 & 18 & $062 \mathrm{G} 07$ \\
\hline W-03-070 & 6-Jun-03 & Lyles Creek & 49.47370 & -98.51955 & 3 & 18 & $062 \mathrm{G} 07$ \\
\hline W-03-071 & 9-Jun-03 & Ste. Anne Drain & 49.69302 & -96.65352 & 3 & 9 & $062 \mathrm{H} 10$ \\
\hline W-03-072 & 9-Jun-03 & Confluence of unnamed tributary and Fish Creek & 49.73720 & -96.63925 & 3 & 9 & $062 \mathrm{H} 10$ \\
\hline W-03-073 & 9-Jun-03 & Seine River & 49.66643 & -96.65697 & 3 & 9 & $062 \mathrm{H} 10$ \\
\hline W-03-074 & 9-Jun-03 & Unnamed tributary to Seine River & 49.71545 & -96.82963 & 2 & 9 & $062 \mathrm{H} 10$ \\
\hline W-03-075 & 9-Jun-03 & Unnamed tributary to Seine River & 49.73450 & -96.88756 & 2 & 9 & $062 \mathrm{H} 10$ \\
\hline W-03-076 & 10-Jun-03 & St. Pierre Drain & 49.45025 & -96.98523 & 3 & 4 & $062 \mathrm{H} 07$ \\
\hline W-03-077 & 10-Jun-03 & Coulee Des Nault & 49.38448 & -96.98478 & 3 & 4 & $062 \mathrm{H} 07$ \\
\hline W-03-078 & 10-Jun-03 & Joubert Creek & 49.43750 & -96.98532 & 4 & 4 & $062 \mathrm{H} 07$ \\
\hline
\end{tabular}


Appendix 1: Table listing the site number, date sampled, site name, latitude and longitude (in decimal degrees DD), DES stream order, DES map number and the corresponding NTS map number for all sites surveyed between 2002 and 2006.

\begin{tabular}{|c|c|c|c|c|c|c|c|}
\hline Site Number & Date & Site Name & $\begin{array}{l}\text { Latitude } \\
\text { (DD) }\end{array}$ & $\begin{array}{l}\text { Longitude } \\
\text { (DD) }\end{array}$ & $\begin{array}{l}\text { DES } \\
\text { Order }\end{array}$ & $\begin{array}{l}\text { DES } \\
\text { Map \# }\end{array}$ & $\begin{array}{l}\text { NTS } \\
\text { Map \# }\end{array}$ \\
\hline W-03-079 & 10-Jun-03 & Simard Creek & 49.44385 & -96.38885 & 3 & 8 & $062 \mathrm{H} 08$ \\
\hline W-03-080 & 10-Jun-03 & Marchand East Drain & 49.44377 & -96.40932 & 3 & 8 & $062 \mathrm{H} 08$ \\
\hline W-03-081 & 11-Jun-03 & Unnamed tributary to St. Adolphe Coulee & 49.62057 & -97.06003 & 2 & 6 & $062 \mathrm{H} 11$ \\
\hline W-03-082 & 11-Jun-03 & Prefontaine Drain & 49.64083 & -97.06025 & 3 & 6 & $062 \mathrm{H} 11$ \\
\hline W-03-083 & 11-Jun-03 & Unnamed tributary to St. Adolphe Coulee & 49.65008 & -97.06020 & 2 & 6 & $062 \mathrm{H} 11$ \\
\hline W-03-084 & 11-Jun-03 & Prefontaine Drain & 49.61687 & -96.98053 & 3 & 6 & $062 \mathrm{H} 10$ \\
\hline W-03-085 & 11-Jun-03 & Confluence unnamed tributary and D - 20 Drain & 49.57615 & -97.00297 & 3 & 6 & $062 \mathrm{H} 11$ \\
\hline W-03-086 & 11-Jun-03 & Confluence unnamed tributary and D - 20 Drain & 49.57632 & -96.95752 & 3 & 6 & $062 \mathrm{H} 10$ \\
\hline W-03-087 & 11-Jun-03 & Pansy Drain & 49.38493 & -96.73123 & 3 & 6 & $062 \mathrm{H} 07$ \\
\hline W-03-088 & 11-Jun-03 & Tourond Creek & 49.39945 & -96.71372 & 3 & 6 & $062 \mathrm{H} 07$ \\
\hline W-03-089 & 13-Jun-03 & Carey Drain & 49.44315 & -97.07518 & 3 & 4 & $062 \mathrm{H} 06$ \\
\hline W-03-090 & 13-Jun-03 & Otterburne East Drain & 49.50242 & -96.98490 & 3 & 4 & $062 \mathrm{H} 10$ \\
\hline W-03-091 & 13-Jun-03 & Sarto Creek & 49.40180 & -96.87180 & 3 & 4 & $062 \mathrm{H} 07$ \\
\hline W-03-092 & 13-Jun-03 & Joubert Creek & 49.31282 & -96.71370 & 3 & 4 & $062 \mathrm{H} 07$ \\
\hline W-03-093 & 13-Jun-03 & Joubert Creek Extension & 49.28973 & -96.52280 & 3 & 4 & $062 \mathrm{H} 07$ \\
\hline W-03-094 & 16-Jun-03 & Unnamed tributary to Bog River & 49.86043 & -95.58132 & 1 & 83 & 052E12 \\
\hline W-03-095 & 16-Jun-03 & Bog River & 49.86789 & -95.63941 & 2 & 83 & $052 \mathrm{E} 12$ \\
\hline W-03-096 & 16-Jun-03 & Unnamed tributary to Monk Creek & 49.82672 & -95.90730 & 2 & 83 & 052E12 \\
\hline W-03-097 & 17-Jun-03 & Bog River & 49.89697 & -95.71132 & 3 & 83 & 052E12 \\
\hline W-03-098 & 17-Jun-03 & Unnamed tributary to Bog River & 49.90912 & -95.75447 & 2 & 83 & 052E12 \\
\hline W-03-099 & 17-Jun-03 & Kellner Creek & 49.87572 & -95.96813 & 2 & 83 & 052E12 \\
\hline W-03-100 & 17-Jun-03 & Kellner Creek & 49.85587 & -95.97517 & 2 & 83 & 052E12 \\
\hline W-03-101 & 18-Jun-03 & Unnamed tributary to Monk Creek & 49.88660 & -95.91070 & 2 & 83 & 052E12 \\
\hline W-03-102 & 18-Jun-03 & Unnamed tributary to Monk Creek & 49.90160 & -95.91272 & 1 & 83 & 052E12 \\
\hline W-03-103 & 18-Jun-03 & Unnamed tributary to Whitemouth River & 49.93919 & -95.95471 & 2 & 83 & $052 \mathrm{E} 12$ \\
\hline
\end{tabular}


Appendix 1: Table listing the site number, date sampled, site name, latitude and longitude (in decimal degrees DD), DES stream order, DES map number and the corresponding NTS map number for all sites surveyed between 2002 and 2006.

\begin{tabular}{|c|c|c|c|c|c|c|c|}
\hline Site Number & Date & Site Name & $\begin{array}{l}\text { Latitude } \\
\text { (DD) }\end{array}$ & $\begin{array}{l}\text { Longitude } \\
\text { (DD) }\end{array}$ & $\begin{array}{l}\text { DES } \\
\text { Order }\end{array}$ & $\begin{array}{l}\text { DES } \\
\text { Map \# }\end{array}$ & $\begin{array}{l}\text { NTS } \\
\text { Map \# }\end{array}$ \\
\hline W-03-104 & 18-Jun-03 & Unnamed tributary to Whitemouth River & 49.93357 & -95.95472 & 2 & 83 & $052 \mathrm{E} 12$ \\
\hline W-03-105 & 18-Jun-03 & Kellner Creek & 49.91443 & -95.95153 & 2 & 83 & $052 \mathrm{E} 12$ \\
\hline W-03-106 & 18-Jun-03 & Unnamed tributary to Whitemouth River & 49.92328 & -95.94705 & 2 & 83 & $052 \mathrm{E} 12$ \\
\hline W-03-107 & 18-Jun-03 & Unnamed tributary to Whitemouth River & 49.96665 & -95.98687 & 3 & 83 & 052E12 \\
\hline W-03-108 & 19-Jun-03 & Unnamed tributary to Whitemouth River & 50.07955 & -96.02645 & 2 & 83 & 062101 \\
\hline W-03-109 & 19-Jun-03 & Unnamed tributary to Whitemouth River & 50.06583 & -96.02617 & 1 & 83 & 062101 \\
\hline W-03-110 & 19-Jun-03 & Smith Creek & 50.04162 & -96.00355 & 2 & 83 & 062101 \\
\hline W-03-111 & 19-Jun-03 & Oldenberg Creek & 50.01930 & -96.01522 & 2 & 83 & 062101 \\
\hline W-03-112 & 19-Jun-03 & Unnamed tributary to Whitemouth River & 49.98053 & -96.00300 & 2 & 83 & $062 \mathrm{H} 16$ \\
\hline W-03-113 & 24-Jun-03 & St. Malo Canal & 49.29580 & -96.98470 & 3 & 4 & $062 \mathrm{H} 07$ \\
\hline W-03-114 & 24-Jun-03 & Mosquito Creek & 49.25332 & -96.96297 & 3 & 4 & $062 \mathrm{H} 07$ \\
\hline W-03-115 & 24-Jun-03 & Unnamed tributary to Rat River & 49.26637 & -96.88493 & 2 & 4 & $062 \mathrm{H} 07$ \\
\hline W-03-116 & 24-Jun-03 & Rat River & 49.27293 & -96.84920 & 5 & 4 & $062 \mathrm{H} 07$ \\
\hline W-03-117 & 24-Jun-03 & Unnamed tributary to Coulee des Nault & 49.33240 & -96.84907 & 2 & 4 & $062 \mathrm{H} 07$ \\
\hline W-03-118 & 24-Jun-03 & West Drain & 49.39952 & -96.82648 & 3 & 4 & $062 \mathrm{H} 07$ \\
\hline W-03-119 & 24-Jun-03 & Unnamed tributary to Tourond Creek & 49.50247 & -96.91695 & 2 & 4 & $062 \mathrm{H} 10$ \\
\hline W-03-120 & 25-Jun-03 & Grassmere Creek Drain East Branch & 50.26295 & -97.38963 & 2 & 27 & 062106 \\
\hline W-03-121 & 25-Jun-03 & Grassmere Creek Drain East Branch & 50.18200 & -97.34513 & 2 & 27 & 062103 \\
\hline W-03-122 & 25-Jun-03 & $\begin{array}{l}\text { Unnamed tributary to Grassmere Creek Drain East } \\
\text { Branch }\end{array}$ & 50.18190 & -97.39108 & 3 & 27 & 062103 \\
\hline W-03-123 & 25-Jun-03 & Gramiak Drain & 50.18197 & -97.45967 & 3 & 27 & 062103 \\
\hline W-03-124 & 25-Jun-03 & $\begin{array}{l}\text { Unnamed tributary to Grassmere Creek Drain West } \\
\text { Branch }\end{array}$ & 50.18208 & -97.52868 & 2 & 27 & 062104 \\
\hline W-03-125 & 25-Jun-03 & Grassmere Creek Drain West Branch & 50.18213 & -97.54750 & 2 & 27 & 062104 \\
\hline W-03-126 & 25-Jun-03 & Grassmere Creek Drain West Branch & 50.12278 & -97.48537 & 3 & 27 & 062103 \\
\hline W-03-127 & 25-Jun-03 & Ekhart Drain & 50.12278 & -97.45938 & 3 & 27 & 062103 \\
\hline
\end{tabular}


Appendix 1: Table listing the site number, date sampled, site name, latitude and longitude (in decimal degrees DD), DES stream order, DES map number and the corresponding NTS map number for all sites surveyed between 2002 and 2006.

\begin{tabular}{|c|c|c|c|c|c|c|c|}
\hline Site Number & Date & Site Name & $\begin{array}{l}\text { Latitude } \\
\text { (DD) }\end{array}$ & $\begin{array}{l}\text { Longitude } \\
\text { (DD) }\end{array}$ & $\begin{array}{l}\text { DES } \\
\text { Order }\end{array}$ & $\begin{array}{l}\text { DES } \\
\text { Map \# }\end{array}$ & $\begin{array}{l}\text { NTS } \\
\text { Map \# }\end{array}$ \\
\hline W-03-128 & 25-Jun-03 & Grassmere Creek Drain Center Branch & 50.12285 & -97.39792 & 3 & 27 & 062103 \\
\hline W-03-129 & 25-Jun-03 & Grassmere Creek Drain East Branch & 50.12263 & -97.36648 & 4 & 27 & 062103 \\
\hline W-03-130 & 25-Jun-03 & Grassmere Creek Drain & 50.04478 & -97.32140 & 5 & 27 & 062103 \\
\hline W-03-131 & 25-Jun-03 & Unnamed tributary to Grassmere Creek Drain & 50.06387 & -97.18408 & 2 & 27 & 062103 \\
\hline W-03-132 & 26-Jun-03 & Gamby Drain & 50.01942 & -97.22947 & 2 & 27 & 062103 \\
\hline W-03-133 & 26-Jun-03 & Penitentiary Drain & 50.06400 & -97.20700 & 2 & 27 & 062103 \\
\hline W-03-134 & 26-Jun-03 & $\begin{array}{l}\text { Confluence of unnamed tributary and Grassmere } \\
\text { Creek Drain }\end{array}$ & 50.02533 & -97.18418 & 5 & 27 & 062103 \\
\hline W-03-135 & 26-Jun-03 & City Protection Drain & 50.00497 & -97.15323 & 3 & 27 & 062103 \\
\hline W-03-136 & 26-Jun-03 & Northumberland Drain & 50.01745 & -97.08368 & 3 & 28 & 062103 \\
\hline W-03-137 & 26-Jun-03 & Unnamed tributary to Red River & 50.12470 & -96.95993 & 2 & 28 & 062102 \\
\hline W-03-138 & 26-Jun-03 & Unnamed tributary to Red River & 50.13173 & -96.94842 & 2 & 28 & 062102 \\
\hline W-03-139 & 26-Jun-03 & Mirey Creek & 50.08578 & -96.94880 & 2 & 28 & 062102 \\
\hline W-03-140 & 26-Jun-03 & Bruneau Drain & 50.07313 & -97.04688 & 3 & 28 & 062103 \\
\hline W-03-141 & 26-Jun-03 & Dewar Drain & 50.17443 & -97.04802 & 3 & 29 & 062103 \\
\hline W-03-142 & 26-Jun-03 & Parks Creek & 50.04783 & -97.06845 & 4 & 28 & 062103 \\
\hline W-03-143 & 2-Jul-03 & Dubas Creek & 50.07688 & -96.73875 & 3 & 11 & 062102 \\
\hline W-03-144 & 2-Jul-03 & Devils Creek & 50.07655 & -96.66442 & 3 & 13 & 062102 \\
\hline W-03-145 & 2-Jul-03 & Unnamed tributary to Devils Creek & 50.07662 & -96.62913 & 1 & 13 & 062102 \\
\hline W-03-146 & 2-Jul-03 & Bears Creek & 50.06143 & -96.38153 & 3 & 10 & 062101 \\
\hline W-03-147 & 3-Jul-03 & Unnamed tributary to Brokenhead River & 49.99283 & -96.49843 & 3 & 10 & $062 \mathrm{H} 16$ \\
\hline W-03-148 & 3-Jul-03 & Unnamed tributary to Brokenhead River & 50.00268 & -96.47497 & 3 & 10 & 062101 \\
\hline W-03-149 & 3-Jul-03 & Unnamed tributary to Brokenhead River & 49.95810 & -96.42943 & 2 & 10 & $062 \mathrm{H} 16$ \\
\hline W-03-150 & 3-Jul-03 & Unnamed tributary to Brokenhead River & 49.93580 & -96.42928 & 3 & 10 & $062 \mathrm{H} 16$ \\
\hline W-03-151 & 3-Jul-03 & Coulee St. Onge & 49.66265 & -96.33307 & 2 & 88 & $062 \mathrm{H} 09$ \\
\hline
\end{tabular}


Appendix 1: Table listing the site number, date sampled, site name, latitude and longitude (in decimal degrees DD), DES stream order, DES map number and the corresponding NTS map number for all sites surveyed between 2002 and 2006.

\begin{tabular}{|c|c|c|c|c|c|c|c|}
\hline Site Number & Date & Site Name & $\begin{array}{l}\text { Latitude } \\
\text { (DD) }\end{array}$ & $\begin{array}{l}\text { Longitude } \\
\text { (DD) }\end{array}$ & $\begin{array}{l}\text { DES } \\
\text { Order }\end{array}$ & $\begin{array}{l}\text { DES } \\
\text { Map \# }\end{array}$ & $\begin{array}{c}\text { NTS } \\
\text { Map \# }\end{array}$ \\
\hline W-03-152 & 3-Jul-03 & Brokenhead River & 49.88471 & -96.36641 & 3 & 10 & $062 \mathrm{H} 16$ \\
\hline W-03-153 & 3-Jul-03 & Hazel Creek & 49.87963 & -96.23435 & 3 & 10 & $062 \mathrm{H} 16$ \\
\hline W-03-154 & 4-Jul-03 & Hazel Creek & 49.75037 & -96.04343 & 2 & 88 & $062 \mathrm{H} 16$ \\
\hline W-03-155 & 4-Jul-03 & Brokenhead River & 49.65702 & -96.27400 & 2 & 88 & 062H09 \\
\hline W-03-156 & 4-Jul-03 & Medika Drain & 49.64913 & -95.81577 & 2 & 84 & 052E11 \\
\hline W-03-157 & 4-Jul-03 & Unnamed tributary to Birch River & 49.71938 & -95.83867 & 2 & 84 & $052 \mathrm{E} 11$ \\
\hline W-03-158 & 4-Jul-03 & Medika Drain & 49.73857 & -95.86142 & 3 & 84 & $052 \mathrm{E} 11$ \\
\hline W-03-159 & 5-Jul-03 & Birch River & 49.64823 & -95.72617 & 3 & 84 & 052E11 \\
\hline W-03-160 & 5-Jul-03 & Boggy River & 49.62493 & -95.62003 & 3 & 84 & 052E11 \\
\hline W-03-161 & 5-Jul-03 & Birch River & 49.55398 & -95.65087 & 3 & 84 & 052E11 \\
\hline W-03-162 & 5-Jul-03 & Senchuk Creek & 49.60608 & -95.66868 & 2 & 84 & 052E11 \\
\hline W-03-163 & 5-Jul-03 & Birch River & 49.60775 & -95.62298 & 3 & 84 & $052 \mathrm{E} 11$ \\
\hline W-03-164 & 5-Jul-03 & Unnamed tributary to Whitemouth River & 49.99482 & -96.01707 & 2 & 83 & $062 \mathrm{H} 16$ \\
\hline W-03-165 & 5-Jul-03 & Unnamed tributary to Whitemouth River & 50.03098 & -96.03793 & 2 & 83 & 062101 \\
\hline W-03-166 & 6-Jul-03 & Unnamed tributary to Whitemouth River & 50.06575 & -96.07145 & 2 & 83 & 062101 \\
\hline W-03-167 & 6-Jul-03 & Unnamed tributary to Whitemouth River & 50.06963 & -96.07183 & 2 & 83 & 062101 \\
\hline W-03-168 & 6-Jul-03 & Unnamed tributary to Whitemouth River & 50.09525 & -96.04250 & 2 & 83 & 062101 \\
\hline W-03-169 & 6-Jul-03 & Unnamed tributary to Whitemouth River & 50.05565 & -96.06010 & 2 & 83 & 062101 \\
\hline W-03-170 & 6-Jul-03 & Unnamed tributary to Winnipeg River & 50.11978 & -96.06347 & 2 & 96 & 062101 \\
\hline W-03-171 & 6-Jul-03 & Unnamed tributary to Winnipeg River & 50.14523 & -96.03320 & 2 & 96 & 062101 \\
\hline W-03-172 & 6-Jul-03 & Unnamed tributary to Winnipeg River & 50.16437 & -96.07950 & 2 & 96 & 062101 \\
\hline W-03-173 & 6-Jul-03 & Unnamed tributary to Winnipeg River & 50.17850 & -96.08550 & 2 & 96 & 062101 \\
\hline W-03-174 & 6-Jul-03 & Unnamed tributary to Winnipeg River & 50.21448 & -96.08528 & 3 & 96 & 062101 \\
\hline W-03-175 & 6-Jul-03 & Unnamed tributary to Winnipeg River & 50.22428 & -96.07637 & 2 & 96 & 062101 \\
\hline W-03-176 & 7-Jul-03 & Sprague Drain & 49.02195 & -95.56083 & 3 & 87 & 052E03 \\
\hline
\end{tabular}


Appendix 1: Table listing the site number, date sampled, site name, latitude and longitude (in decimal degrees DD), DES stream order, DES map number and the corresponding NTS map number for all sites surveyed between 2002 and 2006.

\begin{tabular}{|c|c|c|c|c|c|c|c|}
\hline Site Number & Date & Site Name & $\begin{array}{l}\text { Latitude } \\
\text { (DD) }\end{array}$ & $\begin{array}{l}\text { Longitude } \\
\text { (DD) }\end{array}$ & $\begin{array}{l}\text { DES } \\
\text { Order }\end{array}$ & $\begin{array}{l}\text { DES } \\
\text { Map \# }\end{array}$ & $\begin{array}{c}\text { NTS } \\
\text { Map \# }\end{array}$ \\
\hline W-03-177 & 8-Jul-03 & Unnamed tributary to LaSalle River & 49.78353 & -97.61858 & 2 & 23 & $062 \mathrm{H} 13$ \\
\hline W-03-178 & 8-Jul-03 & Cekanauskas Creek & 49.78345 & -97.63843 & 2 & 23 & $062 \mathrm{H} 13$ \\
\hline W-03-179 & 8-Jul-03 & Starbuck Drain & 49.78335 & -97.66022 & 2 & 23 & $062 \mathrm{H} 13$ \\
\hline W-03-180 & 8-Jul-03 & La Salle River & 49.78335 & -97.66657 & 5 & 23 & $062 \mathrm{H} 13$ \\
\hline W-03-181 & 8-Jul-03 & Meakin Creek & 49.78330 & -97.72117 & 3 & 23 & $062 \mathrm{H} 13$ \\
\hline W-03-182 & 8-Jul-03 & Meakin Creek & 49.76052 & -97.66407 & 4 & 23 & $062 \mathrm{H} 13$ \\
\hline W-03-183 & 8-Jul-03 & Bryson Drain & 49.71978 & -97.89165 & 3 & 23 & $062 \mathrm{H} 12$ \\
\hline W-03-184 & 8-Jul-03 & Menard Drain & 49.72412 & -97.77803 & 3 & 23 & $062 \mathrm{H} 12$ \\
\hline W-03-185 & 8-Jul-03 & Codner Drain & 49.75937 & -97.64822 & 3 & 23 & $062 \mathrm{H} 13$ \\
\hline W-03-186 & 9-Jul-03 & Unnamed tributary to Assiniboine River & 49.85423 & -97.47385 & 3 & 23 & $062 \mathrm{H} 14$ \\
\hline W-03-187 & 9-Jul-03 & Barickman Coulee & 49.93102 & -97.68852 & 3 & 23 & $062 \mathrm{H} 13$ \\
\hline W-03-188 & 9-Jul-03 & La Salle River & 49.87533 & -97.75528 & 5 & 24 & $062 \mathrm{H} 13$ \\
\hline W-03-189 & 9-Jul-03 & Scott Coulee & 49.83488 & -97.77790 & 4 & 24 & $062 \mathrm{H} 13$ \\
\hline W-03-190 & 16-Jul-03 & Piney West Drain & 49.08958 & -96.00192 & 3 & 87 & $062 \mathrm{H} 01$ \\
\hline W-03-191 & 16-Jul-03 & Pine Creek Diversion & 49.02165 & -95.97882 & 3 & 87 & 052E03 \\
\hline W-03-192 & 16-Jul-03 & Unnamed tributary to Pine Creek & 49.07487 & -95.95663 & 2 & 87 & $052 \mathrm{E} 03$ \\
\hline W-03-193 & 16-Jul-03 & Unnamed tributary to West Pine Creek & 49.10433 & -95.95682 & 2 & 87 & 052E03 \\
\hline W-03-194 & 16-Jul-03 & West Pine Creek & 49.08950 & -95.94213 & 3 & 87 & $052 \mathrm{E} 03$ \\
\hline W-03-195 & 16-Jul-03 & East Pine Creek & 49.08955 & -95.93167 & 2 & 87 & $052 \mathrm{E} 03$ \\
\hline W-03-196 & 17-Jul-03 & Unnamed tributary to Sprague Creek & 49.04523 & -95.75497 & 2 & 87 & $052 \mathrm{E} 03$ \\
\hline W-03-197 & 17-Jul-03 & Sprague Creek & 49.02337 & -95.63603 & 4 & 87 & $052 \mathrm{E} 03$ \\
\hline W-03-198 & 17-Jul-03 & Mud Creek & 49.02333 & -95.62797 & 3 & 87 & $052 \mathrm{E} 03$ \\
\hline W-03-199 & 17-Jul-03 & Unnamed tributary to Sprague Creek & 49.08948 & -95.66555 & 2 & 87 & $052 \mathrm{E} 03$ \\
\hline W-03-200 & 17-Jul-03 & Unnamed tributary to St. Labre Bog & 49.31128 & -96.07884 & 2 & 85 & 062H08 \\
\hline W-03-201 & 17-Jul-03 & Unnamed tributary to St. Labre Bog & 49.31135 & -96.04650 & 2 & 85 & $062 \mathrm{H} 08$ \\
\hline
\end{tabular}


Appendix 1: Table listing the site number, date sampled, site name, latitude and longitude (in decimal degrees DD), DES stream order, DES map number and the corresponding NTS map number for all sites surveyed between 2002 and 2006.

\begin{tabular}{|c|c|c|c|c|c|c|c|}
\hline Site Number & Date & Site Name & $\begin{array}{l}\text { Latitude } \\
\text { (DD) }\end{array}$ & $\begin{array}{l}\text { Longitude } \\
\text { (DD) }\end{array}$ & $\begin{array}{l}\text { DES } \\
\text { Order }\end{array}$ & $\begin{array}{l}\text { DES } \\
\text { Map \# }\end{array}$ & $\begin{array}{c}\text { NTS } \\
\text { Map \# }\end{array}$ \\
\hline W-03-202 & 18-Jul-03 & Unnamed tributary to St. Labre Bog & 49.31130 & -95.99955 & 2 & 85 & 052E04 \\
\hline W-03-203 & 18-Jul-03 & Unnamed tributary to St. Labre Bog & 49.31125 & -95.94520 & 2 & 85 & $052 \mathrm{E} 04$ \\
\hline W-03-204 & 18-Jul-03 & Unnamed tributary to St. Labre Bog & 49.31125 & -95.92918 & 3 & 85 & 052E04 \\
\hline W-03-205 & 18-Jul-03 & Unnamed tributary to St. Labre Bog & 49.29871 & -95.82512 & 3 & 85 & 052E04 \\
\hline W-03-206 & 31-Jul-03 & Cooks Creek & 49.80893 & -96.63828 & 3 & 11 & $062 \mathrm{H} 15$ \\
\hline W-03-207 & 31-Jul-03 & Edie Creek & 49.84817 & -96.63785 & 3 & 11 & $062 \mathrm{H} 15$ \\
\hline W-03-208 & 31-Jul-03 & Hazelridge Drain & 49.94370 & -96.63645 & 3 & 11 & $062 \mathrm{H} 15$ \\
\hline W-03-209 & 31-Jul-03 & Hazelridge Drain & 49.95120 & -96.70496 & 3 & 11 & $062 \mathrm{H} 15$ \\
\hline W-03-210 & 31-Jul-03 & Edie Creek & 49.88548 & -96.71787 & 3 & 11 & $062 \mathrm{H} 15$ \\
\hline W-03-211 & 31-Jul-03 & Swede Drain & 49.95925 & -96.76455 & 3 & 11 & $062 \mathrm{H} 15$ \\
\hline W-03-212 & 1-Aug-03 & Melrose Drain & 50.04788 & -96.79610 & 3 & 11 & 062102 \\
\hline W-03-213 & 1-Aug-03 & Sapton Drain & 50.01790 & -96.70510 & 3 & 11 & 062102 \\
\hline W-03-214 & 1-Aug-03 & Satans Creek & 50.01810 & -96.73940 & 3 & 11 & 062102 \\
\hline W-03-215 & 1-Aug-03 & Cooks Creek & 50.01820 & -96.77187 & 4 & 11 & 062102 \\
\hline W-03-216 & 1-Aug-03 & Cooks Creek Diversion & 49.90003 & -96.77417 & 4 & 11 & $062 \mathrm{H} 15$ \\
\hline W-03-217 & 1-Aug-03 & South Bibeau Drain & 49.85623 & -96.84383 & 2 & 9 & $062 \mathrm{H} 15$ \\
\hline W-03-218 & 1-Aug-03 & Prairie Centre Line Drain & 49.84137 & -96.86688 & 3 & 9 & $062 \mathrm{H} 15$ \\
\hline W-03-219 & 1-Aug-03 & Prairie Grove Drain & 49.79690 & -96.84412 & 3 & 9 & $062 \mathrm{H} 15$ \\
\hline W-03-220 & 5-Aug-03 & Unnamed tributary to Icelandic River & 50.90545 & -97.08787 & 2 & 32 & 062114 \\
\hline W-03-221 & 5-Aug-03 & Unnamed tributary to Icelandic River & 50.90505 & -97.15793 & 2 & 32 & 062114 \\
\hline W-03-222 & 5-Aug-03 & Bluegoose Drain & 50.90213 & -97.13458 & 3 & 32 & 062114 \\
\hline W-03-223 & 5-Aug-03 & Silver Drain & 50.89027 & -97.22798 & 3 & 32 & 062114 \\
\hline W-03-224 & 5-Aug-03 & Unnamed tributary to Icelandic River & 50.90252 & -97.20465 & 2 & 32 & 062114 \\
\hline W-03-225 & 5-Aug-03 & Framnes Drain & 50.92038 & -97.29783 & 3 & 32 & $062 \mid 14$ \\
\hline W-03-226 & 5-Aug-03 & Vidir Road Drain & 50.97922 & -97.29643 & 3 & 32 & $062 \mid 14$ \\
\hline
\end{tabular}


Appendix 1: Table listing the site number, date sampled, site name, latitude and longitude (in decimal degrees DD), DES stream order, DES map number and the corresponding NTS map number for all sites surveyed between 2002 and 2006.

\begin{tabular}{|c|c|c|c|c|c|c|c|}
\hline Site Number & Date & Site Name & $\begin{array}{l}\text { Latitude } \\
\text { (DD) }\end{array}$ & $\begin{array}{l}\text { Longitude } \\
\text { (DD) }\end{array}$ & $\begin{array}{l}\text { DES } \\
\text { Order }\end{array}$ & $\begin{array}{l}\text { DES } \\
\text { Map \# }\end{array}$ & $\begin{array}{c}\text { NTS } \\
\text { Map \# }\end{array}$ \\
\hline W-03-227 & 5-Aug-03 & Icelandic River & 50.97950 & -97.31817 & 4 & 32 & 062114 \\
\hline W-03-228 & 6-Aug-03 & Unnamed tributary to Icelandic River & 50.99377 & -97.01482 & 2 & 32 & 062114 \\
\hline W-03-229 & 6-Aug-03 & Unnamed tributary to Icelandic River & 51.02325 & -96.99122 & 3 & 32 & 062P02 \\
\hline W-03-230 & 6-Aug-03 & Unnamed tributary to North Crooked Lake Drain & 50.99360 & -97.20338 & 2 & 32 & 062114 \\
\hline W-03-231 & 6-Aug-03 & North Crooked Lake Drain & 50.97890 & -97.17972 & 3 & 32 & 062114 \\
\hline W-03-232 & 6-Aug-03 & South Crooked Lake Drain & 50.94928 & -97.17953 & 3 & 32 & 062114 \\
\hline W-03-233 & 6-Aug-03 & North Crooked Lake Drain & 50.96437 & -97.09773 & 3 & 32 & 062114 \\
\hline W-03-234 & 6-Aug-03 & South Crooked Lake Drain & 50.95537 & -97.10913 & 4 & 32 & 062114 \\
\hline W-03-235 & 6-Aug-03 & Icelandic River & 50.89007 & -97.46315 & 3 & 32 & 062114 \\
\hline W-03-236 & 6-Aug-03 & Icelandic River & 50.94952 & -97.48172 & 4 & 32 & 062114 \\
\hline W-03-237 & 6-Aug-03 & Icelandic River & 50.99407 & -97.41122 & 4 & 32 & 062114 \\
\hline W-03-238 & 7-Aug-03 & Icelandic River & 50.96452 & -97.03750 & 5 & 32 & 062114 \\
\hline W-03-239 & 7-Aug-03 & Rembrandt Drain & 50.89383 & -97.29762 & 4 & 32 & 062114 \\
\hline W-03-240 & 7-Aug-03 & Icelandic River & 50.97785 & -97.46280 & 4 & 32 & 062114 \\
\hline W-03-241 & 7-Aug-03 & Hodgson Road Drain & 51.23405 & -97.03838 & 3 & 106 & 062P03 \\
\hline W-03-242 & 7-Aug-03 & Unnamed tributary to Washow Bay Creek & 51.16348 & -97.03835 & 3 & 106 & 062P03 \\
\hline W-03-243 & 7-Aug-03 & Washow Bay Creek & 51.17887 & -97.01270 & 4 & 106 & 062P03 \\
\hline W-03-244 & 7-Aug-03 & Progress Drain & 51.05295 & -96.99153 & 3 & 106 & 062P02 \\
\hline W-03-245 & 7-Aug-03 & Shorncliff Drain & 51.08250 & -97.13195 & 2 & 106 & 062P03 \\
\hline W-03-246 & 8-Aug-03 & Petrachek Drain & 51.09723 & -97.13207 & 3 & 106 & 062P03 \\
\hline W-03-247 & 8-Aug-03 & Sugar Creek & 51.24375 & -97.01052 & 3 & 106 & 062P03 \\
\hline W-03-248 & 8-Aug-03 & Angle Drain & 51.18622 & -97.03858 & 3 & 106 & 062P03 \\
\hline W-03-249 & 11-Aug-03 & Main Drain No. 2 & 50.47717 & -96.23478 & 3 & 14 & 062108 \\
\hline W-03-250 & 11-Aug-03 & Gusta Drain & 50.45945 & -96.28337 & 3 & 14 & 062108 \\
\hline W-03-251 & 11-Aug-03 & Main Drain No. 1 & 50.42990 & -96.32980 & 4 & 14 & 062108 \\
\hline
\end{tabular}


Appendix 1: Table listing the site number, date sampled, site name, latitude and longitude (in decimal degrees DD), DES stream order, DES map number and the corresponding NTS map number for all sites surveyed between 2002 and 2006.

\begin{tabular}{|c|c|c|c|c|c|c|c|}
\hline Site Number & Date & Site Name & $\begin{array}{l}\text { Latitude } \\
\text { (DD) }\end{array}$ & $\begin{array}{l}\text { Longitude } \\
\text { (DD) }\end{array}$ & $\begin{array}{l}\text { DES } \\
\text { Order }\end{array}$ & $\begin{array}{l}\text { DES } \\
\text { Map \# }\end{array}$ & $\begin{array}{l}\text { NTS } \\
\text { Map \# }\end{array}$ \\
\hline W-03-252 & 11-Aug-03 & Hiebert Drain & 50.41512 & -96.28357 & 3 & 14 & 062108 \\
\hline W-03-253 & 11-Aug-03 & Stead Drain & 50.40292 & -96.44565 & 3 & 14 & 062108 \\
\hline W-03-254 & 11-Aug-03 & Goertz Drain & 50.35584 & -96.37584 & 2 & 14 & 062108 \\
\hline W-03-255 & 12-Aug-03 & Catfish Creek & 50.61822 & -96.37417 & 4 & 14 & 062109 \\
\hline W-03-256 & 12-Aug-03 & Unnamed tributary to Main Drain No. 1 & 50.35615 & -96.28303 & 3 & 14 & 062108 \\
\hline W-03-257 & 12-Aug-03 & Main Drain No. 1 & 50.37103 & -96.35348 & 4 & 14 & 062108 \\
\hline W-03-258 & 12-Aug-03 & Cloverdale Road Drain & 50.18078 & -96.90955 & 3 & 29 & 062102 \\
\hline W-03-259 & 12-Aug-03 & Muckle Creek & 50.24047 & -96.92042 & 2 & 29 & 062102 \\
\hline W-03-260 & 12-Aug-03 & Medicine Creek & 50.24047 & -96.95412 & 2 & 29 & 062102 \\
\hline W-03-261 & 12-Aug-03 & Long Lake Drain & 50.19593 & -97.00739 & 3 & 29 & 062103 \\
\hline W-03-262 & 12-Aug-03 & Dewar Drain & 50.17442 & -97.04823 & 3 & 29 & 062103 \\
\hline W-03-263 & 12-Aug-03 & Wavey Creek & 50.13780 & -97.23012 & 4 & 29 & 062103 \\
\hline W-03-264 & 12-Aug-03 & Jackfish Creek & 50.21010 & -97.25310 & 3 & 29 & 062103 \\
\hline W-03-265 & 12-Aug-03 & Janet Creek & 50.18815 & -97.25308 & 3 & 29 & 062103 \\
\hline W-03-266 & 13-Aug-03 & Unnamed tributary to Steele Drain & 50.16115 & -97.20035 & 1 & 29 & 062103 \\
\hline W-03-267 & 13-Aug-03 & Steele Drain & 50.16515 & -97.20253 & 3 & 29 & 062103 \\
\hline B-04-001 & 1-May-04 & Gordon Drain & 50.01959 & -97.34406 & 2 & 26 & 062103 \\
\hline B-04-002 & 1-May-04 & East Colony Creek & 49.99025 & -97.29995 & 3 & 26 & $062 \mathrm{H} 14$ \\
\hline B-04-003 & 1-May-04 & Grassmere Creek Drain & 50.04464 & -97.32130 & 5 & 27 & 062103 \\
\hline B-04-004 & 1-May-04 & Grassmere Creek Drain East Branch & 50.12028 & -97.36718 & 4 & 27 & 062103 \\
\hline B-04-005 & 1-May-04 & Grassmere Creek Drain & 50.07885 & -97.39043 & 4 & 27 & 062103 \\
\hline B-04-006 & 1-May-04 & Grassmere Creek Drain & 50.04930 & -97.36032 & 4 & 27 & 062103 \\
\hline B-04-007 & 1-May-04 & City Protection Drain & 50.00484 & -97.15317 & 3 & 27 & 062103 \\
\hline B-04-008 & 1-May-04 & Rat Creek & 49.95231 & -98.51443 & 5 & 35 & $062 \mathrm{G} 15$ \\
\hline B-04-009 & 2-May-04 & Unnamed tributary to Gopher Creek & 50.26973 & -98.96138 & 2 & 42 & 062J07 \\
\hline
\end{tabular}


Appendix 1: Table listing the site number, date sampled, site name, latitude and longitude (in decimal degrees DD), DES stream order, DES map number and the corresponding NTS map number for all sites surveyed between 2002 and 2006.

\begin{tabular}{|c|c|c|c|c|c|c|c|}
\hline Site Number & Date & Site Name & $\begin{array}{l}\text { Latitude } \\
\text { (DD) }\end{array}$ & $\begin{array}{l}\text { Longitude } \\
\text { (DD) }\end{array}$ & $\begin{array}{l}\text { DES } \\
\text { Order }\end{array}$ & $\begin{array}{l}\text { DES } \\
\text { Map \# }\end{array}$ & $\begin{array}{l}\text { NTS } \\
\text { Map \# }\end{array}$ \\
\hline B-04-010 & 2-May-04 & Gopher Creek & 50.26950 & -98.93833 & 4 & 42 & 062J07 \\
\hline B-04-011 & 2-May-04 & Pembroke Drain & 50.28488 & -98.88030 & 4 & 42 & 062J07 \\
\hline B-04-012 & 2-May-04 & Big Grass River & 50.50595 & -98.97163 & 5 & 43 & 062J10 \\
\hline B-04-013 & 2-May-04 & Big Grass River & 50.52065 & -98.99210 & 5 & 43 & 062J10 \\
\hline B-04-014 & 2-May-04 & Big Grass River & 50.53693 & -99.01457 & 5 & 43 & 062J11 \\
\hline B-04-015 & 2-May-04 & Big Grass River & 50.55338 & -99.02868 & 5 & 43 & 062J11 \\
\hline B-04-016 & 2-May-04 & Big Grass River & 50.56025 & -99.04787 & 5 & 43 & 062J11 \\
\hline B-04-017 & 2-May-04 & Rat Creek & 49.94523 & -98.52475 & 5 & 35 & $062 \mathrm{G} 15$ \\
\hline B-04-018 & 3-May-04 & King Drain & 49.63542 & -97.38850 & 4 & 20 & $062 \mathrm{H} 11$ \\
\hline B-04-019 & 3-May-04 & King Drain & 49.62836 & -97.41303 & 4 & 20 & $062 \mathrm{H} 11$ \\
\hline B-04-020 & 3-May-04 & Barnland Drain & 49.64019 & -97.43578 & 3 & 20 & $062 \mathrm{H} 11$ \\
\hline B-04-021 & 3-May-04 & Grill Drain & 49.62039 & -97.43536 & 3 & 20 & $062 \mathrm{H} 11$ \\
\hline B-04-022 & 3-May-04 & Manness Drain & 49.62139 & -97.37622 & 3 & 20 & $062 \mathrm{H} 11$ \\
\hline B-04-023 & 4-May-04 & Anderson Creek & 49.36964 & -97.41400 & 3 & 17 & $062 \mathrm{H} 06$ \\
\hline B-04-024 & 4-May-04 & Bell Drain & 49.38442 & -97.41397 & 2 & 17 & 062H06 \\
\hline B-04-025 & 4-May-04 & Russell Drain & 49.42767 & -97.43667 & 2 & 17 & 062H06 \\
\hline B-04-026 & 4-May-04 & Little Morris River & 49.45825 & -97.49894 & 5 & 17 & 062H06 \\
\hline B-04-027 & 4-May-04 & Morris River & 49.38431 & -97.39056 & 6 & 17 & 062H06 \\
\hline B-04-028 & 4-May-04 & Recently constructed overflow channel & 49.42858 & -97.44503 & 6 & 17 & 062H06 \\
\hline B-04-029 & 4-May-04 & Recently constructed overflow channel & 49.43689 & -97.44614 & 6 & 17 & $062 \mathrm{H} 06$ \\
\hline B-04-030 & 4-May-04 & Tobacco Creek & 49.42639 & -97.52575 & 5 & 17 & 062H05 \\
\hline B-04-031 & 6-May-04 & Little Souris River & 49.76141 & -99.93884 & 4 & 98 & $062 \mathrm{G} 13$ \\
\hline B-04-032 & 6-May-04 & Black Creek & 49.60428 & -99.73433 & 4 & 69 & $062 \mathrm{G} 12$ \\
\hline B-04-033 & 6-May-04 & Unnamed tributary to Souris River & 49.44781 & -99.81103 & 3 & 69 & $062 \mathrm{G} 05$ \\
\hline B-04-034 & 6-May-04 & Unnamed tributary to Souris River & 49.39978 & -99.90131 & 3 & 69 & $062 \mathrm{G} 05$ \\
\hline
\end{tabular}


Appendix 1: Table listing the site number, date sampled, site name, latitude and longitude (in decimal degrees DD), DES stream order, DES map number and the corresponding NTS map number for all sites surveyed between 2002 and 2006.

\begin{tabular}{|c|c|c|c|c|c|c|c|}
\hline Site Number & Date & Site Name & $\begin{array}{l}\text { Latitude } \\
\text { (DD) }\end{array}$ & $\begin{array}{l}\text { Longitude } \\
\text { (DD) }\end{array}$ & $\begin{array}{l}\text { DES } \\
\text { Order }\end{array}$ & $\begin{array}{l}\text { DES } \\
\text { Map \# }\end{array}$ & $\begin{array}{c}\text { NTS } \\
\text { Map \# }\end{array}$ \\
\hline B-04-035 & 6-May-04 & Unnamed tributary to Souris River & 49.45785 & -99.95003 & 2 & 69 & $062 \mathrm{G} 05$ \\
\hline B-04-036 & 6-May-04 & Orthez Drain & 49.33333 & -100.01433 & 3 & 59 & 062F08 \\
\hline B-04-037 & 6-May-04 & Orthez Drain & 49.41464 & -99.72844 & 3 & 59 & $062 G 05$ \\
\hline B-04-038 & 6-May-04 & Unnamed tributary to Overend Lake & 49.38475 & -99.67869 & 3 & 59 & $062 \mathrm{G} 05$ \\
\hline B-04-039 & 6-May-04 & Pembina River Diversion & 49.26681 & -99.47092 & 5 & 59 & $062 \mathrm{G} 06$ \\
\hline B-04-040 & 7-May-04 & Unnamed tributary to Cypress River & 49.55072 & -99.12075 & 2 & 71 & $062 \mathrm{G} 11$ \\
\hline B-04-041 & 7-May-04 & Unnamed tributary to Cypress River & 49.56208 & -99.08589 & 2 & 71 & $062 \mathrm{G} 11$ \\
\hline B-04-042 & 7-May-04 & Oxtail Creek & 49.54669 & -99.04222 & 2 & 71 & $062 \mathrm{G} 11$ \\
\hline B-04-043 & 7-May-04 & Tiger Creek Drain & 49.56083 & -99.02922 & 3 & 71 & $062 \mathrm{G} 11$ \\
\hline B-04-044 & 7-May-04 & Rex Creek & 49.62039 & -98.96044 & 4 & 71 & 062G10 \\
\hline B-04-045 & 7-May-04 & Cypress River & 49.66719 & -98.93761 & 5 & 71 & 062G10 \\
\hline B-04-046 & 10-May-04 & Unnamed tributary to Cypress River & 49.65669 & -98.89281 & 3 & 71 & 062G10 \\
\hline B-04-047 & 10-May-04 & Cypress River & 49.59131 & -99.03333 & 4 & 71 & $062 \mathrm{G} 11$ \\
\hline B-04-048 & 10-May-04 & Cypress River & 49.45797 & -99.02133 & 4 & 71 & $062 \mathrm{G} 06$ \\
\hline B-04-049 & 13-May-04 & Unnamed tributary to Birdtail Creek & 50.36822 & -101.08458 & 2 & 77 & $062 \mathrm{~K} 06$ \\
\hline B-04-050 & 13-May-04 & Unnamed tributary to Birdtail Creek & 50.41850 & -101.08397 & 3 & 77 & $062 \mathrm{~K} 06$ \\
\hline B-04-051 & 13-May-04 & Unnamed tributary to Birdtail Creek & 50.42206 & -101.06769 & 2 & 77 & $062 \mathrm{~K} 06$ \\
\hline B-04-052 & 13-May-04 & Unnamed tributary to Birdtail Creek & 50.49128 & -100.93792 & 2 & 77 & $062 K 07$ \\
\hline B-04-053 & 13-May-04 & Birdtail Creek & 50.48450 & -100.96356 & 4 & 77 & $062 K 07$ \\
\hline B-04-054 & 13-May-04 & Unnamed tributary to Birdtail Creek & 50.53549 & -100.95647 & 2 & 77 & $062 \mathrm{~K} 10$ \\
\hline B-04-055 & 13-May-04 & Birdtail Creek & 50.57903 & -100.92614 & 4 & 77 & $062 \mathrm{~K} 10$ \\
\hline B-04-056 & 13-May-04 & Unnamed tributary to Birdtail Creek & 50.63061 & -100.85714 & 2 & 77 & $062 \mathrm{~K} 10$ \\
\hline B-04-057 & 14-May-04 & Konyk Creek & 50.84506 & -100.91611 & 3 & 76 & $062 \mathrm{~K} 15$ \\
\hline B-04-058 & 14-May-04 & Konyk Creek & 50.81608 & -100.88068 & 3 & 76 & $062 \mathrm{~K} 15$ \\
\hline B-04-059 & 14-May-04 & Tilson Creek & 50.83147 & -100.84547 & 3 & 76 & $062 \mathrm{~K} 15$ \\
\hline
\end{tabular}


Appendix 1: Table listing the site number, date sampled, site name, latitude and longitude (in decimal degrees DD), DES stream order, DES map number and the corresponding NTS map number for all sites surveyed between 2002 and 2006.

\begin{tabular}{|c|c|c|c|c|c|c|c|}
\hline Site Number & Date & Site Name & $\begin{array}{l}\text { Latitude } \\
\text { (DD) }\end{array}$ & $\begin{array}{l}\text { Longitude } \\
\text { (DD) }\end{array}$ & $\begin{array}{l}\text { DES } \\
\text { Order }\end{array}$ & $\begin{array}{l}\text { DES } \\
\text { Map \# }\end{array}$ & $\begin{array}{l}\text { NTS } \\
\text { Map \# }\end{array}$ \\
\hline B-04-060 & 14-May-04 & Tilson Creek & 50.82130 & -100.83412 & 3 & 76 & $062 \mathrm{~K} 15$ \\
\hline B-04-061 & 14-May-04 & Unnamed tributary to Birdtail Creek & 50.78614 & -100.76336 & 2 & 76 & $062 \mathrm{~K} 15$ \\
\hline B-04-062 & 14-May-04 & Unnamed tributary to Birdtail Creek & 50.81995 & -100.74431 & 2 & 76 & $062 \mathrm{~K} 15$ \\
\hline B-04-063 & 14-May-04 & Unnamed tributary to Birdtail Creek & 50.83314 & -100.69486 & 2 & 76 & $062 \mathrm{~K} 15$ \\
\hline B-04-064 & 14-May-04 & Birdtail Creek & 50.82543 & -100.74126 & 4 & 76 & $062 \mathrm{~K} 15$ \\
\hline B-04-065 & 17-May-04 & Potts Drain & 50.47658 & -98.93872 & 2 & 43 & 062J07 \\
\hline B-04-066 & 17-May-04 & Jumping Deer Creek & 50.43236 & -98.91561 & 3 & 43 & 062J07 \\
\hline B-04-067 & 17-May-04 & Lach Drain & 50.41761 & -98.93867 & 3 & 42 & 062J07 \\
\hline B-04-068 & 17-May-04 & Whitemud River Cross Ditch & 50.24064 & -98.90497 & 6 & 42 & 062J02 \\
\hline B-04-069 & 18-May-04 & North Snake Creek & 50.44125 & -99.46964 & 3 & 43 & 062J06 \\
\hline B-04-070 & 18-May-04 & Pelican Creek & 50.49157 & -99.45405 & 3 & 43 & 062J06 \\
\hline B-04-071 & 18-May-04 & Badnel Drain & 51.17328 & -99.50061 & 3 & 45 & 062004 \\
\hline B-04-072 & 18-May-04 & Big Grass River & 50.54272 & -99.14681 & 5 & 43 & 062J11 \\
\hline B-04-073 & 19-May-04 & Edwards Creek & 51.12417 & -100.03931 & 4 & 47 & 062N01 \\
\hline B-04-074 & 19-May-04 & Edwards Creek & 51.11133 & -100.06217 & 4 & 47 & 062N01 \\
\hline B-04-075 & 20-May-04 & Valley River & 51.26978 & -100.02300 & 5 & 95 & 062N08 \\
\hline B-04-076 & 20-May-04 & Unnamed tributary to Dauphin Lake & 51.41964 & -99.97544 & 2 & 95 & 062005 \\
\hline B-04-077 & 20-May-04 & Unnamed tributary to Dauphin Lake & 51.39355 & -99.99433 & 2 & 95 & 062005 \\
\hline B-04-078 & 20-May-04 & Mowatt Creek & 51.36878 & -99.99514 & 3 & 95 & 062005 \\
\hline B-04-079 & 20-May-04 & Unnamed tributary to Dauphin Lake & 51.37428 & -99.97167 & 2 & 95 & 062005 \\
\hline B-04-080 & 20-May-04 & Unnamed tributary to Dauphin Lake & 51.34694 & -99.94836 & 2 & 95 & 062005 \\
\hline B-04-081 & 20-May-04 & Unnamed tributary to Dauphin Lake & 51.33236 & -99.94450 & 2 & 95 & 062005 \\
\hline B-04-082 & 20-May-04 & Unnamed tributary to Dauphin Lake & 51.31769 & -99.94853 & 2 & 95 & 062005 \\
\hline B-04-083 & 20-May-04 & Unnamed tributary to Dauphin Lake & 51.28789 & -99.89969 & 2 & 95 & 062005 \\
\hline B-04-084 & 20-May-04 & Unnamed tributary to Dauphin Lake & 51.27311 & -99.89958 & 2 & 95 & 062005 \\
\hline
\end{tabular}


Appendix 1: Table listing the site number, date sampled, site name, latitude and longitude (in decimal degrees DD), DES stream order, DES map number and the corresponding NTS map number for all sites surveyed between 2002 and 2006.

\begin{tabular}{|c|c|c|c|c|c|c|c|}
\hline Site Number & Date & Site Name & $\begin{array}{l}\text { Latitude } \\
\text { (DD) }\end{array}$ & $\begin{array}{l}\text { Longitude } \\
\text { (DD) }\end{array}$ & $\begin{array}{l}\text { DES } \\
\text { Order }\end{array}$ & $\begin{array}{l}\text { DES } \\
\text { Map \# }\end{array}$ & $\begin{array}{l}\text { NTS } \\
\text { Map \# }\end{array}$ \\
\hline B-04-085 & 20-May-04 & Unnamed tributary to Dauphin Lake & 51.25833 & -99.89958 & 2 & 95 & 062005 \\
\hline B-04-086 & 21-May-04 & Salt Creek & 51.14769 & -100.13353 & 3 & 47 & 062N01 \\
\hline B-04-087 & 22-May-04 & North Snake Creek & 50.44125 & -99.46964 & 3 & 43 & 062J06 \\
\hline B-04-088 & 25-May-04 & Unnamed tributary to Assiniboine River & 49.85728 & -99.86858 & 2 & 98 & $062 \mathrm{G} 13$ \\
\hline B-04-089 & 25-May-04 & Unnamed tributary to Assiniboine River & 49.89497 & -100.30139 & 3 & 98 & $062 \mathrm{~F} 16$ \\
\hline B-04-090 & 25-May-04 & Unnamed tributary to Assiniboine River & 49.85133 & -100.37125 & 3 & 98 & $062 \mathrm{~F} 16$ \\
\hline B-04-091 & 25-May-04 & Unnamed tributary to Assiniboine River & 49.84242 & -100.42264 & 3 & 98 & $062 \mathrm{~F} 16$ \\
\hline B-04-092 & 25-May-04 & Oak River & 50.01939 & -100.40935 & 5 & 72 & $062 \mathrm{~K} 01$ \\
\hline B-04-093 & 31-May-04 & Unnamed tributary to Little Saskatchewan River & 49.90142 & -100.08114 & 3 & 82 & $062 \mathrm{~F} 16$ \\
\hline B-04-094 & 31-May-04 & Sibbald Creek & 49.90128 & -100.32069 & 3 & 82 & $062 \mathrm{~F} 16$ \\
\hline B-04-095 & 31-May-04 & Oak River & 50.01955 & -100.40945 & 5 & 72 & $062 \mathrm{~K} 01$ \\
\hline B-04-096 & 1-Jun-04 & Stony Creek & 49.57702 & -101.25330 & 3 & 63 & $062 \mathrm{~F} 11$ \\
\hline B-04-097 & 1-Jun-04 & Stony Creek & 49.44408 & -101.18907 & 3 & 63 & $062 \mathrm{~F} 06$ \\
\hline B-04-098 & 1-Jun-04 & Unnamed tributary to Stony Creek & 49.42897 & -100.90143 & 3 & 63 & 062F07 \\
\hline B-04-099 & 1-Jun-04 & Stony Creek & 49.48790 & -100.85328 & 4 & 63 & 062F07 \\
\hline B-04-100 & 1-Jun-04 & Dooley Coulee & 49.34052 & -100.63583 & 2 & 67 & 062F07 \\
\hline B-04-101 & 1-Jun-04 & Chain Lakes Creek & 49.35897 & -100.60160 & 3 & 67 & 062F07 \\
\hline B-04-102 & 1-Jun-04 & Jackson Creek & 49.30989 & -100.98614 & 3 & 64 & 062F07 \\
\hline B-04-103 & 1-Jun-04 & Graham Creek & 49.25908 & -101.03089 & 3 & 64 & $062 \mathrm{~F} 06$ \\
\hline B-04-104 & 1-Jun-04 & Jackson Creek & 49.36597 & -101.25683 & 2 & 64 & $062 \mathrm{~F} 06$ \\
\hline B-04-105 & 1-Jun-04 & Graham Creek & 49.26667 & -101.16964 & 3 & 64 & $062 \mathrm{~F} 06$ \\
\hline B-04-106 & 2-Jun-04 & Unnamed tributary to Souris River & 49.06566 & -100.64541 & 4 & 65 & 062F02 \\
\hline B-04-107 & 2-Jun-04 & Unnamed tributary to Souris River & 49.02386 & -100.64528 & 3 & 65 & 062F02 \\
\hline B-04-108 & 2-Jun-04 & Unnamed tributary to Souris River & 49.01431 & -100.64050 & 3 & 65 & 062F02 \\
\hline B-04-109 & 2-Jun-04 & Waskada Creek & 49.11769 & -100.95044 & 3 & 65 & 062F02 \\
\hline
\end{tabular}


Appendix 1: Table listing the site number, date sampled, site name, latitude and longitude (in decimal degrees DD), DES stream order, DES map number and the corresponding NTS map number for all sites surveyed between 2002 and 2006.

\begin{tabular}{|c|c|c|c|c|c|c|c|}
\hline Site Number & Date & Site Name & $\begin{array}{l}\text { Latitude } \\
\text { (DD) }\end{array}$ & $\begin{array}{l}\text { Longitude } \\
\text { (DD) }\end{array}$ & $\begin{array}{l}\text { DES } \\
\text { Order }\end{array}$ & $\begin{array}{l}\text { DES } \\
\text { Map \# }\end{array}$ & $\begin{array}{l}\text { NTS } \\
\text { Map \# }\end{array}$ \\
\hline B-04-110 & 2-Jun-04 & Waskada Creek & 49.09069 & -100.91425 & 3 & 65 & $062 \mathrm{~F} 02$ \\
\hline B-04-111 & 2-Jun-04 & Blind River & 49.22203 & -100.99499 & 3 & 65 & 062F02 \\
\hline B-04-112 & 2-Jun-04 & Blind River & 49.26656 & -100.97294 & 4 & 65 & 062F07 \\
\hline B-04-113 & 2-Jun-04 & Antler River & 49.02914 & -101.14039 & 5 & 65 & $062 \mathrm{F03}$ \\
\hline B-04-114 & 3-Jun-04 & North Pembina River & 49.17761 & -100.02306 & 3 & 57 & $062 \mathrm{~F} 01$ \\
\hline B-04-115 & 3-Jun-04 & Pembina River & 49.20250 & -99.78858 & 5 & 57 & $062 \mathrm{G} 04$ \\
\hline B-04-116 & 3-Jun-04 & Starke Creek & 49.13284 & -99.88392 & 3 & 57 & $062 \mathrm{G} 04$ \\
\hline B-04-117 & 4-Jun-04 & Cypress River & 49.47411 & -98.79583 & 4 & 71 & $062 \mathrm{G} 07$ \\
\hline B-04-118 & 4-Jun-04 & Cypress River & 49.49986 & -98.68139 & 3 & 71 & 062G07 \\
\hline B-04-119 & 4-Jun-04 & Somerset Creek & 49.45869 & -98.74119 & 3 & 71 & 062G07 \\
\hline B-04-120 & 4-Jun-04 & Cypress River & 49.44336 & -98.93125 & 4 & 71 & 062G07 \\
\hline B-04-121 & 4-Jun-04 & Cypress River & 49.43286 & -98.95306 & 4 & 71 & 062G07 \\
\hline B-04-122 & 4-Jun-04 & Oak Creek & 49.54978 & -99.46225 & 4 & 70 & $062 \mathrm{G} 11$ \\
\hline B-04-123 & 7-Jun-04 & Oak Creek & 49.62094 & -99.57575 & 4 & 69 & $062 \mathrm{G} 12$ \\
\hline B-04-124 & 7-Jun-04 & Spring Brook & 49.59219 & -99.59825 & 4 & 70 & $062 \mathrm{G} 12$ \\
\hline B-04-125 & 7-Jun-04 & Oak Creek & 49.56197 & -99.51256 & 4 & 70 & $062 \mathrm{G} 12$ \\
\hline B-04-126 & 8-Jun-04 & Unnamed tributary to Oak Creek & 49.52356 & -99.42769 & 3 & 70 & $062 \mathrm{G} 11$ \\
\hline B-04-127 & 8-Jun-04 & Unnamed tributary to Oak Creek & 49.51781 & -99.39553 & 3 & 70 & 062G11 \\
\hline B-04-128 & 8-Jun-04 & Oak Creek & 49.47356 & -99.15081 & 4 & 70 & $062 \mathrm{G} 06$ \\
\hline B-04-129 & 8-Jun-04 & Oak Creek & 49.42917 & -99.13919 & 4 & 70 & 062G06 \\
\hline B-04-130 & 9-Jun-04 & Unnamed tributary to Souris River & 49.53289 & -100.16117 & 3 & 68 & 062F09 \\
\hline B-04-131 & 9-Jun-04 & Unnamed tributary to Souris River & 49.59153 & -100.22000 & 2 & 68 & 062F09 \\
\hline B-04-132 & 9-Jun-04 & Unnamed tributary to Souris River & 49.59144 & -100.22558 & 2 & 68 & 062F09 \\
\hline B-04-133 & 9-Jun-04 & Elgin Creek & 49.53289 & -100.27322 & 4 & 68 & $062 \mathrm{~F} 09$ \\
\hline B-04-134 & 9-Jun-04 & Unnamed tributary to Elgin Creek & 49.53281 & -100.31153 & 3 & 68 & $062 \mathrm{~F} 09$ \\
\hline
\end{tabular}


Appendix 1: Table listing the site number, date sampled, site name, latitude and longitude (in decimal degrees DD), DES stream order, DES map number and the corresponding NTS map number for all sites surveyed between 2002 and 2006.

\begin{tabular}{|c|c|c|c|c|c|c|c|}
\hline Site Number & Date & Site Name & $\begin{array}{l}\text { Latitude } \\
\text { (DD) }\end{array}$ & $\begin{array}{l}\text { Longitude } \\
\text { (DD) }\end{array}$ & $\begin{array}{l}\text { DES } \\
\text { Order }\end{array}$ & $\begin{array}{l}\text { DES } \\
\text { Map \# }\end{array}$ & $\begin{array}{l}\text { NTS } \\
\text { Map \# }\end{array}$ \\
\hline B-04-135 & 9-Jun-04 & Elgin Creek & 49.39964 & -100.20364 & 3 & 68 & 062F08 \\
\hline B-04-136 & 10-Jun-04 & Pipestone Creek & 49.83964 & -101.39572 & 5 & 62 & $062 \mathrm{~F} 14$ \\
\hline B-04-137 & 10-Jun-04 & Unnamed tributary to Assiniboine River & 49.79833 & -100.75761 & 3 & 98 & $062 \mathrm{~F} 15$ \\
\hline B-04-138 & 10-Jun-04 & Flat Creek & 49.76961 & -100.58717 & 2 & 98 & $062 \mathrm{~F} 15$ \\
\hline B-04-139 & 10-Jun-04 & Unnamed tributary to Assiniboine River & 49.85736 & -100.18289 & 2 & 98 & $062 \mathrm{~F} 16$ \\
\hline B-04-140 & 14-Jun-04 & Unnamed tributary to Deadhorse Creek & 49.18411 & -98.22750 & 3 & 15 & $062 \mathrm{G} 01$ \\
\hline B-04-141 & 14-Jun-04 & Unnamed tributary to Deadhorse Creek & 49.13908 & -98.22311 & 2 & 15 & $062 \mathrm{G} 01$ \\
\hline B-04-142 & 14-Jun-04 & Hespeler Drain & 49.12108 & -98.02078 & 2 & 15 & $062 \mathrm{G} 01$ \\
\hline $\mathrm{B}-04-143$ & 14-Jun-04 & Unnamed tributary to Hespeler Drain & 49.11217 & -98.02067 & 3 & 15 & $062 G 01$ \\
\hline B-04-144 & 15-Jun-04 & Crystal Creek & 49.17231 & -98.98747 & 4 & 56 & 062G02 \\
\hline B-04-145 & 15-Jun-04 & Unnamed tributary to Cypress Creek & 49.14792 & -99.09025 & 4 & 56 & $062 G 03$ \\
\hline B-04-146 & 15-Jun-04 & Unnamed tributary to Cypress Creek & 49.11825 & -99.09492 & 4 & 56 & 062G03 \\
\hline B-04-147 & 15-Jun-04 & Unnamed tributary to Cypress Creek & 49.13313 & -99.11740 & 3 & 56 & 062G03 \\
\hline B-04-148 & 15-Jun-04 & Unnamed tributary to Cypress Creek & 49.08853 & -99.03778 & 3 & 56 & 062G03 \\
\hline B-04-149 & 15-Jun-04 & Cypress Creek & 49.13172 & -99.03250 & 5 & 56 & $062 \mathrm{G} 03$ \\
\hline B-04-150 & 16-Jun-04 & Cypress Creek & 49.05917 & -98.97247 & 4 & 56 & $062 \mathrm{G} 02$ \\
\hline B-04-151 & 16-Jun-04 & Unnamed tributary to Cypress Creek & 49.05912 & -98.99333 & 4 & 56 & $062 G 02$ \\
\hline B-04-152 & 16-Jun-04 & Cypress Creek & 49.04403 & -98.96386 & 4 & 56 & 062G02 \\
\hline B-04-153 & 16-Jun-04 & Cypress Creek & 49.19958 & -99.08619 & 5 & 56 & $062 \mathrm{G} 03$ \\
\hline B-04-154 & 16-Jun-04 & Crystal Creek & 49.10331 & -98.90700 & 3 & 56 & 062G02 \\
\hline B-04-155 & 16-Jun-04 & Crystal Creek & 49.12486 & -98.94239 & 4 & 56 & 062G02 \\
\hline B-04-156 & 17-Jun-04 & Crystal Creek & 49.14325 & -98.95742 & 4 & 56 & 062G02 \\
\hline B-04-157 & 17-Jun-04 & Unnamed tributary to Rock Lake & 49.16269 & -99.18836 & 3 & 56 & $062 \mathrm{G} 03$ \\
\hline B-04-158 & 17-Jun-04 & Unnamed tributary to Pembina River & 49.25189 & -99.28253 & 3 & 56 & 062G06 \\
\hline B-04-159 & 18-Jun-04 & Unnamed tributary to Pipestone Creek & 49.70303 & -100.82672 & 4 & 62 & $062 \mathrm{~F} 10$ \\
\hline
\end{tabular}


Appendix 1: Table listing the site number, date sampled, site name, latitude and longitude (in decimal degrees DD), DES stream order, DES map number and the corresponding NTS map number for all sites surveyed between 2002 and 2006.

\begin{tabular}{|c|c|c|c|c|c|c|c|}
\hline Site Number & Date & Site Name & $\begin{array}{l}\text { Latitude } \\
\text { (DD) }\end{array}$ & $\begin{array}{l}\text { Longitude } \\
\text { (DD) }\end{array}$ & $\begin{array}{l}\text { DES } \\
\text { Order }\end{array}$ & $\begin{array}{l}\text { DES } \\
\text { Map \# }\end{array}$ & $\begin{array}{l}\text { NTS } \\
\text { Map \# }\end{array}$ \\
\hline B-04-160 & 18-Jun-04 & Pipestone Creek Diversion & 49.68078 & -100.87214 & 5 & 62 & $062 \mathrm{~F} 10$ \\
\hline B-04-161 & 18-Jun-04 & Pipestone Creek & 49.65081 & -100.91444 & 5 & 62 & $062 \mathrm{~F} 10$ \\
\hline B-04-162 & 18-Jun-04 & Unnamed tributary to Pipestone Creek & 49.60875 & -101.00856 & 5 & 62 & $062 \mathrm{~F} 11$ \\
\hline B-04-163 & 21-Jun-04 & Plum Creek & 49.62578 & -100.30342 & 5 & 62 & 062F09 \\
\hline B-04-164 & 21-Jun-04 & Chain Lakes Creek & 49.44392 & -100.55811 & 4 & 67 & 062F07 \\
\hline B-04-165 & 21-Jun-04 & Chain Lakes Creek & 49.35911 & -100.60161 & 3 & 67 & 062F07 \\
\hline B-04-166 & 21-Jun-04 & Medora Creek & 49.26161 & -100.69153 & 4 & 66 & 062F07 \\
\hline B-04-167 & 21-Jun-04 & Medora Creek & 49.33446 & -100.82761 & 4 & 66 & 062F07 \\
\hline B-04-168 & 22-Jun-04 & Medora Creek & 49.34050 & -100.74631 & 4 & 66 & 062F07 \\
\hline B-04-169 & 22-Jun-04 & Medora Creek & 49.28794 & -100.71458 & 4 & 66 & 062F07 \\
\hline B-04-170 & 22-Jun-04 & Unnamed tributary to Medora Creek & 49.23725 & -100.70172 & 3 & 66 & 062F02 \\
\hline B-04-171 & 22-Jun-04 & Medora Creek & 49.23736 & -100.60933 & 4 & 66 & $062 \mathrm{~F} 02$ \\
\hline B-04-172 & 22-Jun-04 & Unnamed tributary to Gainsborough Creek & 49.14808 & -101.09311 & 2 & 65 & $062 \mathrm{~F} 03$ \\
\hline B-04-173 & 22-Jun-04 & Unnamed tributary to Gainsborough Creek & 49.12025 & -101.13817 & 3 & 65 & $062 \mathrm{~F} 03$ \\
\hline B-04-174 & 22-Jun-04 & Gainsborough Creek & 49.08878 & -101.20569 & 4 & 65 & $062 \mathrm{~F} 03$ \\
\hline B-04-175 & 22-Jun-04 & Gainsborough Creek & 49.08892 & -101.29514 & 4 & 65 & $062 \mathrm{~F} 03$ \\
\hline B-04-176 & 23-Jun-04 & Antler River & 49.04664 & -101.07128 & 5 & 65 & $062 \mathrm{~F} 03$ \\
\hline B-04-177 & 23-Jun-04 & Unnamed tributary to Antler River & 49.04439 & -101.18300 & 3 & 65 & $062 \mathrm{~F} 03$ \\
\hline B-04-178 & 23-Jun-04 & Antler River & 49.00114 & -101.31706 & 5 & 65 & $062 \mathrm{~F} 03$ \\
\hline B-04-179 & 23-Jun-04 & Blind River & 49.17772 & -100.96431 & 3 & 65 & 062F02 \\
\hline B-04-180 & 23-Jun-04 & Unnamed tributary to Blind River & 49.24226 & -100.94073 & 2 & 65 & 062F02 \\
\hline B-04-181 & 28-Jun-04 & Edwards Creek & 51.05178 & -100.07683 & 4 & 47 & 062N01 \\
\hline B-04-182 & 28-Jun-04 & Spruce Creek & 51.09589 & -100.18019 & 4 & 47 & 062N01 \\
\hline B-04-183 & 28-Jun-04 & Unnamed tributary to Vermillion River & 51.08458 & -100.18022 & 1 & 47 & 062N01 \\
\hline B-04-184 & 28-Jun-04 & Keld Drain & 51.03692 & -100.20361 & 3 & 47 & 062N01 \\
\hline
\end{tabular}


Appendix 1: Table listing the site number, date sampled, site name, latitude and longitude (in decimal degrees DD), DES stream order, DES map number and the corresponding NTS map number for all sites surveyed between 2002 and 2006.

\begin{tabular}{|c|c|c|c|c|c|c|c|}
\hline Site Number & Date & Site Name & $\begin{array}{l}\text { Latitude } \\
\text { (DD) }\end{array}$ & $\begin{array}{l}\text { Longitude } \\
\text { (DD) }\end{array}$ & $\begin{array}{l}\text { DES } \\
\text { Order }\end{array}$ & $\begin{array}{l}\text { DES } \\
\text { Map \# }\end{array}$ & $\begin{array}{l}\text { NTS } \\
\text { Map \# }\end{array}$ \\
\hline B-04-185 & 28-Jun-04 & Buck Creek & 50.97667 & -100.27403 & 3 & 47 & $062 \mathrm{~K} 16$ \\
\hline B-04-186 & 29-Jun-04 & Vermillion River & 51.01850 & -100.16978 & 4 & 47 & 062N01 \\
\hline B-04-187 & 29-Jun-04 & Vermillion River & 51.14025 & -100.07489 & 5 & 47 & 062N01 \\
\hline B-04-188 & 29-Jun-04 & Vermillion River & 51.06189 & -100.13847 & 5 & 47 & 062N01 \\
\hline B-04-189 & 30-Jun-04 & Wilson River & 51.19956 & -100.10461 & 5 & 48 & 062N01 \\
\hline B-04-190 & 30-Jun-04 & Mineral Creek & 51.15350 & -100.29794 & 4 & 48 & 062N01 \\
\hline B-04-191 & 5-Jul-04 & Wilson River & 51.16903 & -100.24864 & 5 & 48 & 062N01 \\
\hline B-04-192 & 5-Jul-04 & Unnamed tributary to Wilson River & 51.16544 & -100.25078 & 2 & 48 & 062N01 \\
\hline B-04-193 & 5-Jul-04 & Unnamed tributary to Wilson River & 51.19644 & -100.18053 & 2 & 48 & 062N01 \\
\hline B-04-194 & 6-Jul-04 & Unnamed tributary to Roaring River & 52.04491 & -101.20383 & 1 & 52 & $063 \mathrm{C03}$ \\
\hline B-04-195 & 6-Jul-04 & Unnamed tributary to Minitonas Creek & 51.99772 & -101.19172 & 3 & 52 & 062N14 \\
\hline B-04-196 & 6-Jul-04 & Minitonas Creek & 52.01869 & -101.18006 & 4 & 52 & $063 \mathrm{C03}$ \\
\hline B-04-197 & 6-Jul-04 & Minitonas Creek & 51.99495 & -101.10864 & 3 & 52 & 062N14 \\
\hline B-04-198 & 6-Jul-04 & West Favel River & 51.99749 & -101.05861 & 3 & 52 & 062N14 \\
\hline B-04-199 & 6-Jul-04 & East Favel River & 52.02703 & -101.02784 & 3 & 52 & $063 \mathrm{C03}$ \\
\hline B-04-200 & 6-Jul-04 & Godin Drain & 52.00844 & -100.96553 & 3 & 52 & $063 \mathrm{C} 02$ \\
\hline B-04-201 & 6-Jul-04 & Unnamed tributary to Old Government Drain & 52.04317 & -101.01325 & 2 & 52 & $063 \mathrm{C03}$ \\
\hline B-04-202 & 6-Jul-04 & Unnamed tributary to West Favel River & 52.10511 & -101.08489 & 2 & 52 & $063 \mathrm{C03}$ \\
\hline B-04-203 & 7-Jul-04 & Unnamed tributary to East Favel River & 52.12361 & -101.03714 & 2 & 52 & $063 \mathrm{C03}$ \\
\hline B-04-204 & 7-Jul-04 & Unnamed tributary to East Favel River & 52.17269 & -101.00875 & 3 & 52 & $063 \mathrm{C03}$ \\
\hline B-04-205 & 7-Jul-04 & Old Government Drain & 52.06978 & -101.01336 & 3 & 52 & $063 C 03$ \\
\hline B-04-206 & 7-Jul-04 & Tamarack Creek & 52.09878 & -101.22778 & 2 & 61 & $063 C 03$ \\
\hline B-04-207 & 7-Jul-04 & Silent Creek & 52.02703 & -101.30961 & 2 & 61 & $063 \mathrm{C03}$ \\
\hline B-04-208 & 7-Jul-04 & Unnamed tributary to Hay Creek & 51.96856 & -101.39678 & 2 & 61 & 062N14 \\
\hline B-04-209 & 7-Jul-04 & Hay Creek & 51.96858 & -101.42317 & 2 & 61 & 062N14 \\
\hline
\end{tabular}


Appendix 1: Table listing the site number, date sampled, site name, latitude and longitude (in decimal degrees DD), DES stream order, DES map number and the corresponding NTS map number for all sites surveyed between 2002 and 2006.

\begin{tabular}{|c|c|c|c|c|c|c|c|}
\hline Site Number & Date & Site Name & $\begin{array}{l}\text { Latitude } \\
\text { (DD) }\end{array}$ & $\begin{array}{l}\text { Longitude } \\
\text { (DD) }\end{array}$ & $\begin{array}{l}\text { DES } \\
\text { Order }\end{array}$ & $\begin{array}{l}\text { DES } \\
\text { Map \# }\end{array}$ & $\begin{array}{l}\text { NTS } \\
\text { Map \# }\end{array}$ \\
\hline B-04-210 & 7-Jul-04 & Unnamed tributary to Lobstick Creek & 52.02586 & -101.44215 & 2 & 61 & $063 C 03$ \\
\hline B-04-211 & 7-Jul-04 & Hay Creek & 52.03739 & -101.39447 & 3 & 61 & $063 \mathrm{C03}$ \\
\hline B-04-212 & 7-Jul-04 & Thunderhill Creek & 52.02036 & -101.51369 & 3 & 61 & $063 \mathrm{C} 04$ \\
\hline B-04-213 & 7-Jul-04 & Unnamed tributary to Thunderhill Creek & 52.02347 & -101.53764 & 2 & 61 & $063 \mathrm{C04}$ \\
\hline B-04-214 & 7-Jul-04 & Prairie Creek & 51.83717 & -101.53422 & 4 & 61 & $062 \mathrm{~N} 13$ \\
\hline B-04-215 & 7-Jul-04 & Lobstick Creek & 51.96798 & -101.43967 & 4 & 61 & 062N14 \\
\hline B-04-216 & 7-Jul-04 & Thomas Creek & 51.94547 & -101.41194 & 2 & 61 & 062N14 \\
\hline B-04-217 & 8-Jul-04 & Bowsman River & 52.27122 & -101.39483 & 4 & 89 & $063 C 06$ \\
\hline $\mathrm{B}-04-218$ & 8-Jul-04 & Hubbell Creek & 52.25994 & -101.46400 & 3 & 89 & $063 \mathrm{C} 06$ \\
\hline B-04-219 & 8-Jul-04 & Hubbell Creek & 52.21544 & -101.44208 & 3 & 89 & $063 \mathrm{C03}$ \\
\hline B-04-220 & 8-Jul-04 & Little Kemulch Creek & 52.28898 & -101.19812 & 3 & 89 & $063 \mathrm{C} 06$ \\
\hline B-04-221 & 8-Jul-04 & Unnamed tributary to Little Kemulch Creek & 52.28917 & -101.20694 & 3 & 89 & $063 C 06$ \\
\hline B-04-222 & 8-Jul-04 & Little Kemulch Creek & 52.29083 & -101.19819 & 3 & 89 & $063 C 06$ \\
\hline B-04-223 & 9-Jul-04 & Mineral Creek & 51.15353 & -100.29739 & 4 & 48 & 062N01 \\
\hline B-04-224 & 13-Jul-04 & Kemulch Creek & 52.32444 & -101.21064 & 3 & 89 & $063 C 06$ \\
\hline B-04-225 & 13-Jul-04 & Camp Nine Drain & 52.25467 & -101.20008 & 4 & 89 & $063 C 06$ \\
\hline B-04-226 & 13-Jul-04 & Whitebeech Creek & 52.12278 & -101.41819 & 4 & 89 & $063 C 03$ \\
\hline B-04-227 & 13-Jul-04 & McVey Creek & 52.15936 & -101.56128 & 3 & 89 & $063 \mathrm{C} 04$ \\
\hline B-04-228 & 13-Jul-04 & Trout Creek & 52.23089 & -101.53297 & 2 & 89 & $063 C 04$ \\
\hline B-04-229 & 14-Jul-04 & Swinowski Drain & 52.15217 & -100.96556 & 4 & 53 & $063 \mathrm{C} 02$ \\
\hline B-04-230 & 14-Jul-04 & Cox Drain & 52.11452 & -100.89386 & 3 & 53 & $063 C 02$ \\
\hline B-04-231 & 14-Jul-04 & Cox Drain & 52.08519 & -100.89439 & 3 & 53 & $063 \mathrm{C02}$ \\
\hline B-04-232 & 14-Jul-04 & Jarosz Drain & 52.11444 & -100.84633 & 4 & 53 & $063 \mathrm{C02}$ \\
\hline B-04-233 & 14-Jul-04 & Unnamed tributary to Jarosz Drain & 52.10924 & -100.84645 & 3 & 53 & $063 \mathrm{C} 02$ \\
\hline B-04-234 & 14-Jul-04 & Jarosz Drain & 52.09974 & -100.83506 & 3 & 53 & $063 \mathrm{C02}$ \\
\hline
\end{tabular}


Appendix 1: Table listing the site number, date sampled, site name, latitude and longitude (in decimal degrees DD), DES stream order, DES map number and the corresponding NTS map number for all sites surveyed between 2002 and 2006.

\begin{tabular}{|c|c|c|c|c|c|c|c|}
\hline Site Number & Date & Site Name & $\begin{array}{l}\text { Latitude } \\
\text { (DD) }\end{array}$ & $\begin{array}{l}\text { Longitude } \\
\text { (DD) }\end{array}$ & $\begin{array}{l}\text { DES } \\
\text { Order }\end{array}$ & $\begin{array}{l}\text { DES } \\
\text { Map \# }\end{array}$ & $\begin{array}{l}\text { NTS } \\
\text { Map \# }\end{array}$ \\
\hline B-04-235 & 14-Jul-04 & Renwer Drain & 52.10233 & -100.79867 & 3 & 53 & $063 C 02$ \\
\hline B-04-236 & 14-Jul-04 & Unnamed tributary to Sinclair River & 52.17253 & -100.91789 & 2 & 53 & $063 \mathrm{C} 02$ \\
\hline B-04-237 & 14-Jul-04 & Unnamed tributary to Sinclair River & 52.20192 & -100.97487 & 3 & 53 & $063 \mathrm{C} 02$ \\
\hline B-04-238 & 14-Jul-04 & Lalecheur Drain & 52.25989 & -100.92244 & 4 & 53 & $063 \mathrm{C07}$ \\
\hline B-04-239 & 15-Jul-04 & West Ruby Creek & 51.85978 & -101.43569 & 3 & 51 & 062N14 \\
\hline B-04-240 & 15-Jul-04 & Silver Creek & 51.23142 & -100.81292 & 3 & 93 & 062N02 \\
\hline B-04-241 & 19-Jul-04 & Silver Creek & 51.25811 & -100.85411 & 3 & 93 & 062N07 \\
\hline B-04-242 & 19-Jul-04 & Dry Creek & 51.21467 & -100.62542 & 4 & 93 & 062N02 \\
\hline $\mathrm{B}-04-243$ & 19-Jul-04 & Unnamed tributary to Valley River & 51.15511 & -100.73631 & 3 & 93 & 062N02 \\
\hline B-04-244 & 20-Jul-04 & Vermillion River & 51.16975 & -100.05375 & 5 & 47 & 062N01 \\
\hline B-04-245 & 20-Jul-04 & Hamlin Creek & 51.44911 & -99.51717 & 4 & 107 & 062005 \\
\hline B-04-246 & 20-Jul-04 & Rorketon Drain & 51.44911 & -99.55103 & 3 & 107 & 062005 \\
\hline B-04-247 & 20-Jul-04 & Bretecher Drain & 51.44914 & -99.52744 & 2 & 107 & 062005 \\
\hline B-04-248 & 20-Jul-04 & Defaults Creek & 51.47819 & -99.51722 & 4 & 107 & 062005 \\
\hline B-04-249 & 20-Jul-04 & Rorketon Drain & 51.48672 & -99.55100 & 3 & 107 & 062005 \\
\hline B-04-250 & 20-Jul-04 & Szewczuks Creek & 51.47828 & -99.72250 & 4 & 107 & 062005 \\
\hline B-04-251 & 21-Jul-04 & Bretecher Drain & 51.46378 & -99.52933 & 2 & 107 & 062005 \\
\hline B-04-252 & 21-Jul-04 & Unnamed tributary to Rorketon Drain & 51.47825 & -99.60947 & 3 & 107 & 062005 \\
\hline B-04-253 & 21-Jul-04 & Unnamed tributary to Szewczuks Creek & 51.44914 & -99.75025 & 2 & 107 & 062005 \\
\hline B-04-254 & 21-Jul-04 & Unnamed tributary to Lake Winnipegosis & 51.44919 & -99.77336 & 2 & 107 & 062005 \\
\hline B-04-255 & 21-Jul-04 & German Creek & 51.55278 & -99.80847 & 3 & 107 & 062012 \\
\hline B-04-256 & 21-Jul-04 & Szewczuks Creek & 51.56550 & -99.71881 & 4 & 107 & 062012 \\
\hline B-04-257 & 21-Jul-04 & Lonely Lake Drain & 51.12569 & -99.05358 & 3 & 113 & 062003 \\
\hline B-04-258 & 21-Jul-04 & Unnamed tributary to Lonely Lake & 51.11109 & -99.07079 & 2 & 113 & 062003 \\
\hline B-04-259 & 21-Jul-04 & Unnamed tributary to Lonely Lake & 51.11111 & -99.09664 & 2 & 113 & 062003 \\
\hline
\end{tabular}


Appendix 1: Table listing the site number, date sampled, site name, latitude and longitude (in decimal degrees DD), DES stream order, DES map number and the corresponding NTS map number for all sites surveyed between 2002 and 2006.

\begin{tabular}{|c|c|c|c|c|c|c|c|}
\hline Site Number & Date & Site Name & $\begin{array}{l}\text { Latitude } \\
\text { (DD) }\end{array}$ & $\begin{array}{l}\text { Longitude } \\
\text { (DD) }\end{array}$ & $\begin{array}{l}\text { DES } \\
\text { Order }\end{array}$ & $\begin{array}{l}\text { DES } \\
\text { Map \# }\end{array}$ & $\begin{array}{l}\text { NTS } \\
\text { Map \# }\end{array}$ \\
\hline B-04-260 & 21-Jul-04 & Unnamed tributary to Lonely Lake & 51.16369 & -99.14939 & 3 & 113 & 062003 \\
\hline B-04-261 & 21-Jul-04 & Crane River & 51.39089 & -99.27936 & 2 & 113 & 062006 \\
\hline B-04-262 & 21-Jul-04 & Crane River & 51.50286 & -99.26383 & 3 & 113 & 062011 \\
\hline B-04-263 & 22-Jul-04 & Arrow River & 50.24061 & -100.78728 & 4 & 74 & $062 \mathrm{~K} 02$ \\
\hline B-04-264 & 22-Jul-04 & Arrow River & 50.22583 & -100.77814 & 4 & 74 & $062 \mathrm{~K} 02$ \\
\hline B-04-265 & 22-Jul-04 & Arrow River & 50.18158 & -100.80558 & 4 & 74 & $062 \mathrm{~K} 02$ \\
\hline B-04-266 & 26-Jul-04 & Minnewasta Creek & 50.25517 & -101.02850 & 4 & 74 & $062 \mathrm{~K} 06$ \\
\hline B-04-267 & 4-Aug-04 & Pasquia River Diversion Dam & 53.60809 & -101.63661 & 1 & 114 & $063 \mathrm{~F} 12$ \\
\hline B-04-268 & 4-Aug-04 & Unnamed tributary to Pasquia River & 53.61828 & -101.61606 & 2 & 114 & $063 \mathrm{~F} 12$ \\
\hline B-04-269 & 4-Aug-04 & Drain T & 53.65383 & -101.63703 & 2 & 114 & $063 \mathrm{~F} 12$ \\
\hline B-04-270 & 4-Aug-04 & Z Drain & 53.82206 & -101.27581 & 4 & 114 & 063F14 \\
\hline B-04-271 & 4-Aug-04 & Q Drain/Drain R & 53.79283 & -101.30378 & 3 & 114 & $063 \mathrm{~F} 14$ \\
\hline B-04-272 & 4-Aug-04 & B - 1 Drain & 53.76653 & -101.43375 & 2 & 114 & $063 F 14$ \\
\hline B-04-273 & 5-Aug-04 & Road 8B Drain NW & 53.74844 & -101.40347 & 2 & 114 & $063 F 11$ \\
\hline B-04-274 & 5-Aug-04 & Road 8B Drain SW & 53.74867 & -101.40381 & 2 & 114 & $063 \mathrm{~F} 11$ \\
\hline B-04-275 & 5-Aug-04 & B - 1 Drain & 53.74872 & -101.40331 & 3 & 114 & $063 \mathrm{~F} 11$ \\
\hline B-04-276 & 5-Aug-04 & Drain $\mathrm{P}$ & 53.75836 & -101.58886 & 3 & 114 & $063 \mathrm{~F} 13$ \\
\hline B-04-277 & 5-Aug-04 & Confluence of Drain $\mathrm{O}$ and Drain $\mathrm{L}$ & 53.73992 & -101.52372 & 4 & 114 & $063 F 12$ \\
\hline B-04-278 & 5-Aug-04 & Drain N & 53.72236 & -101.52378 & 2 & 114 & $063 \mathrm{~F} 12$ \\
\hline B-04-279 & 5-Aug-04 & Confluence of Drain 01 and Bon Drain & 53.72933 & -101.64722 & 2 & 114 & $063 \mathrm{~F} 12$ \\
\hline B-04-280 & 5-Aug-04 & Pasquia Lake Drain & 53.65325 & -101.53925 & 3 & 114 & $063 \mathrm{~F} 12$ \\
\hline B-04-281 & 6-Aug-04 & Unnamed tributary to Carrot River & 53.75375 & -101.52378 & 4 & 114 & $063 \mathrm{~F} 13$ \\
\hline B-04-282 & 6-Aug-04 & Road 8 Drain & 53.77572 & -101.28781 & 2 & 114 & $063 \mathrm{~F} 14$ \\
\hline B-04-283 & 6-Aug-04 & Drain III & 53.74872 & -101.33319 & 2 & 114 & $063 \mathrm{~F} 11$ \\
\hline B-04-284 & 6-Aug-04 & Drain II & 53.72294 & -101.37650 & 3 & 114 & $063 \mathrm{~F} 11$ \\
\hline
\end{tabular}


Appendix 1: Table listing the site number, date sampled, site name, latitude and longitude (in decimal degrees DD), DES stream order, DES map number and the corresponding NTS map number for all sites surveyed between 2002 and 2006.

\begin{tabular}{|c|c|c|c|c|c|c|c|}
\hline Site Number & Date & Site Name & $\begin{array}{l}\text { Latitude } \\
\text { (DD) }\end{array}$ & $\begin{array}{l}\text { Longitude } \\
\text { (DD) }\end{array}$ & $\begin{array}{l}\text { DES } \\
\text { Order }\end{array}$ & $\begin{array}{l}\text { DES } \\
\text { Map \# }\end{array}$ & $\begin{array}{l}\text { NTS } \\
\text { Map \# }\end{array}$ \\
\hline B-04-285 & 6-Aug-04 & Pasquia River & 53.75472 & -101.25319 & 4 & 114 & $063 \mathrm{~F} 14$ \\
\hline B-04-286 & 6-Aug-04 & Pasquia Lake Drain & 53.70508 & -101.28900 & 3 & 114 & $063 F 11$ \\
\hline B-04-287 & 6-Aug-04 & Pasquia River & 53.63911 & -101.35728 & 3 & 114 & $063 F 11$ \\
\hline B-04-288 & 6-Aug-04 & Pasquia River & 53.59653 & -101.48961 & 3 & 114 & $063 \mathrm{~F} 11$ \\
\hline D-04-001 & 6-Apr-04 & Turtle River & 51.05778 & -99.52568 & 5 & 45 & 062004 \\
\hline D-04-002 & 6-Apr-04 & Turtle River & 50.90373 & -99.50532 & 5 & 45 & 062J13 \\
\hline D-04-003 & 15-Apr-04 & Unnamed tributary to Glenella Drain & 50.56492 & -99.45598 & 3 & 43 & 062J11 \\
\hline D-04-004 & 15-Apr-04 & Big Grass River & 50.50607 & -98.97130 & 5 & 43 & 062J10 \\
\hline D-04-005 & 15-Apr-04 & Gork Drain & 50.50597 & -99.16970 & 3 & 43 & 062J11 \\
\hline D-04-006 & 16-Apr-04 & North Snake Creek & 50.44160 & -99.47058 & 3 & 43 & 062J06 \\
\hline D-04-007 & 16-Apr-04 & Riding Mountain Drain & 50.52088 & -99.31588 & 5 & 43 & 062J11 \\
\hline D-04-008 & 16-Apr-04 & Big Grass River & 50.52807 & -99.19278 & 5 & 43 & 062J11 \\
\hline D-04-009 & 16-Apr-04 & Big Grass River Angle Ditch & 50.56480 & -99.15730 & 4 & 43 & 062J11 \\
\hline D-04-010 & 2-Jun-04 & Unnamed tributary to Mary Jane Creek & 49.26653 & -98.59518 & 2 & 54 & 062G07 \\
\hline D-04-011 & 2-Jun-04 & Unnamed tributary to Roseisle Creek & 49.45448 & -98.47617 & 2 & 18 & 062G08 \\
\hline D-04-012 & 2-Jun-04 & Mary Jane Creek & 49.28152 & -98.63632 & 4 & 54 & $062 \mathrm{G} 07$ \\
\hline D-04-013 & 3-Jun-04 & North Snake Creek Pond Outlet & 50.44160 & -99.47058 & 3 & 43 & 062J06 \\
\hline D-04-014 & 3-Jun-04 & Turtle River & 50.90373 & -99.50532 & 5 & 45 & 062J13 \\
\hline D-04-015 & 7-Jun-04 & Hanson Creek & 51.08895 & -99.47720 & 3 & 45 & 062003 \\
\hline D-04-016 & 8-Jun-04 & North Snake Creek Pond Outlet & 50.44160 & -99.47058 & 3 & 43 & 062J06 \\
\hline D-04-017 & 9-Jun-04 & Image Creek & 49.92052 & -98.75798 & 3 & 36 & $062 \mathrm{G} 15$ \\
\hline D-04-018 & 9-Jun-04 & Mink Creek & 50.22498 & -99.36050 & 1 & 39 & 062J03 \\
\hline D-04-019 & 9-Jun-04 & Whitemud River & 50.25915 & -99.35390 & 5 & 39 & 062J06 \\
\hline D-04-020 & 10-Jun-04 & North Snake Creek Pond Outlet & 50.44160 & -99.47058 & 3 & 43 & 062J06 \\
\hline D-04-021 & 14-Jun-04 & North Snake Creek Pond Outlet & 50.44160 & -99.47058 & 3 & 43 & 062J06 \\
\hline
\end{tabular}


Appendix 1: Table listing the site number, date sampled, site name, latitude and longitude (in decimal degrees DD), DES stream order, DES map number and the corresponding NTS map number for all sites surveyed between 2002 and 2006.

\begin{tabular}{|c|c|c|c|c|c|c|c|}
\hline Site Number & Date & Site Name & $\begin{array}{l}\text { Latitude } \\
\text { (DD) }\end{array}$ & $\begin{array}{l}\text { Longitude } \\
\text { (DD) }\end{array}$ & $\begin{array}{l}\text { DES } \\
\text { Order }\end{array}$ & $\begin{array}{l}\text { DES } \\
\text { Map \# }\end{array}$ & $\begin{array}{l}\text { NTS } \\
\text { Map \# }\end{array}$ \\
\hline D-04-022 & 15-Jun-04 & Mary Jane Creek & 49.29135 & -98.54482 & 3 & 54 & 062G07 \\
\hline D-04-023 & 15-Jun-04 & Mary Jane Creek & 49.23637 & -98.67435 & 4 & 54 & 062G02 \\
\hline D-04-024 & 15-Jun-04 & Hammond's Creek & 49.28065 & -98.81600 & 2 & 54 & 062G07 \\
\hline D-04-025 & 15-Jun-04 & Unnamed tributary to Hammond's Creek & 49.28068 & -98.75465 & 1 & 54 & $062 \mathrm{G} 07$ \\
\hline D-04-026 & 15-Jun-04 & Hammond's Creek & 49.27210 & -98.74967 & 3 & 54 & $062 \mathrm{G} 07$ \\
\hline D-04-027 & 15-Jun-04 & McCoys Creek & 49.25808 & -98.72620 & 4 & 54 & $062 \mathrm{G} 07$ \\
\hline D-04-028 & 16-Jun-04 & Deadhorse Creek & 49.19037 & -98.11723 & 4 & 15 & $062 \mathrm{G} 01$ \\
\hline D-04-029 & 16-Jun-04 & Deadhorse Creek & 49.23673 & -98.00300 & 4 & 15 & $062 \mathrm{G} 01$ \\
\hline D-04-030 & 22-Jun-04 & Ross Creek & 50.76347 & -100.95047 & 3 & 76 & $062 \mathrm{~K} 15$ \\
\hline D-04-031 & 22-Jun-04 & Ross Creek & 50.75153 & -100.91820 & 3 & 76 & $062 \mathrm{~K} 15$ \\
\hline D-04-032 & 22-Jun-04 & Ross Creek & 50.76020 & -100.92718 & 3 & 76 & $062 \mathrm{~K} 15$ \\
\hline D-04-033 & 23-Jun-04 & Ross Creek & 50.75058 & -100.90397 & 3 & 76 & $062 \mathrm{~K} 15$ \\
\hline D-04-034 & 23-Jun-04 & Ross Creek & 50.75155 & -100.91792 & 3 & 76 & $062 \mathrm{~K} 15$ \\
\hline D-04-035 & 23-Jun-04 & Ross Creek & 50.74240 & -100.89307 & 3 & 76 & $062 \mathrm{~K} 10$ \\
\hline D-04-036 & 7-Jul-04 & Big Boggy Creek & 51.27820 & -101.49030 & 4 & 92 & 062N06 \\
\hline W-04-001 & 3-May-04 & Garrioch Creek & 50.87222 & -98.92098 & 4 & 110 & 062J15 \\
\hline W-04-002 & 3-May-04 & Manitoba House Drain & 50.86018 & -98.85680 & 3 & 110 & 062J15 \\
\hline W-04-003 & 3-May-04 & Bluff Creek & 50.81602 & -98.82763 & 2 & 110 & 062J15 \\
\hline W-04-004 & 3-May-04 & Garrioch Creek at Rochland Drain & 50.80132 & -98.93403 & 4 & 110 & 062J15 \\
\hline W-04-005 & 3-May-04 & Harcus Drain & 50.72738 & -98.83360 & 3 & 110 & 062J10 \\
\hline W-04-006 & 3-May-04 & Unnamed tributary to Harcus Drain & 50.71282 & -98.84508 & 3 & 110 & 062J10 \\
\hline W-04-007 & 3-May-04 & Garrioch Creek & 50.77173 & -98.99650 & 3 & 110 & 062J15 \\
\hline W-04-008 & 3-May-04 & Garrioch Creek & 50.71280 & -99.06638 & 3 & 110 & 062J11 \\
\hline W-04-009 & 4-May-04 & Upper Lake Mary Drain & 50.76795 & -99.08965 & 3 & 110 & 062J14 \\
\hline W-04-010 & 4-May-04 & Smalley School Drain & 50.66840 & -98.71747 & 3 & 41 & 062J10 \\
\hline
\end{tabular}


Appendix 1: Table listing the site number, date sampled, site name, latitude and longitude (in decimal degrees DD), DES stream order, DES map number and the corresponding NTS map number for all sites surveyed between 2002 and 2006.

\begin{tabular}{|c|c|c|c|c|c|c|c|}
\hline Site Number & Date & Site Name & $\begin{array}{l}\text { Latitude } \\
\text { (DD) }\end{array}$ & $\begin{array}{l}\text { Longitude } \\
\text { (DD) }\end{array}$ & $\begin{array}{l}\text { DES } \\
\text { Order }\end{array}$ & $\begin{array}{l}\text { DES } \\
\text { Map \# }\end{array}$ & $\begin{array}{c}\text { NTS } \\
\text { Map \# }\end{array}$ \\
\hline W-04-011 & 4-May-04 & Carriere Drain & 50.63897 & -98.71730 & 2 & 41 & 062J10 \\
\hline W-04-012 & 4-May-04 & Unnamed tributary to Lake Manitoba & 50.41745 & -98.60330 & 2 & 41 & 062J07 \\
\hline W-04-013 & 4-May-04 & Unnamed tributary to Lake Manitoba & 50.43248 & -98.61483 & 2 & 41 & 062J07 \\
\hline W-04-014 & 4-May-04 & Unnamed tributary to Lake Manitoba & 50.47660 & -98.61490 & 2 & 41 & 062J07 \\
\hline W-04-015 & 4-May-04 & Unnamed tributary to Lake Manitoba & 50.50608 & -98.63790 & 3 & 41 & 062J10 \\
\hline W-04-016 & 4-May-04 & Unnamed tributary to Lake Manitoba & 50.31403 & -98.63775 & 2 & 41 & 062J07 \\
\hline W-04-017 & 4-May-04 & Unnamed tributary to Lake Manitoba & 50.34352 & -98.63778 & 2 & 41 & 062J07 \\
\hline W-04-018 & 4-May-04 & Unnamed tributary to Lake Manitoba & 50.28452 & -98.63807 & 2 & 41 & 062J07 \\
\hline W-04-019 & 4-May-04 & House Creek & 50.18173 & -98.49185 & 2 & 101 & 062J01 \\
\hline W-04-020 & 5-May-04 & House Creek & 50.13745 & -98.47415 & 2 & 101 & 062J01 \\
\hline W-04-021 & 5-May-04 & Long Creek & 50.10788 & -98.43333 & 2 & 101 & 062J01 \\
\hline W-04-022 & 5-May-04 & Unnamed tributary to Short Creek & 50.10798 & -98.39068 & 2 & 101 & 062J01 \\
\hline W-04-023 & 5-May-04 & Long Creek & 50.12273 & -98.43840 & 2 & 101 & 062J01 \\
\hline W-04-024 & 5-May-04 & Long Creek & 50.13972 & -98.42405 & 2 & 101 & 062J01 \\
\hline W-04-025 & 5-May-04 & Short Creek & 50.13733 & -98.41893 & 2 & 101 & 062J01 \\
\hline W-04-026 & 5-May-04 & Unnamed tributary to Big Lake & 50.13732 & -98.40683 & 3 & 101 & 062J01 \\
\hline W-04-027 & 5-May-04 & Unnamed tributary to Blind Channel & 50.10760 & -98.33917 & 3 & 101 & 062J01 \\
\hline W-04-028 & 5-May-04 & Blind Channel & 50.10975 & -98.30810 & 2 & 101 & 062J01 \\
\hline W-04-029 & 6-May-04 & Unnamed tributary to Lake Manitoba & 50.10783 & -98.37650 & 3 & 101 & 062J01 \\
\hline W-04-030 & 6-May-04 & Cram Creek & 50.12255 & -98.36398 & 3 & 101 & 062J01 \\
\hline W-04-031 & 6-May-04 & Unnamed tributary to Portage Creek & 50.10982 & -98.26242 & 2 & 101 & 062J01 \\
\hline W-04-032 & 6-May-04 & Portage Creek & 50.10807 & -98.24412 & 2 & 101 & 062J01 \\
\hline W-04-033 & 6-May-04 & Unnamed tributary to Little Bay & 50.13740 & -98.20002 & 2 & 101 & 062J01 \\
\hline W-04-034 & 6-May-04 & Unnamed tributary to Bluebill Bay & 50.11338 & -98.21660 & 3 & 101 & 062J01 \\
\hline W-04-035 & 6-May-04 & Unnamed tributary to Bluebill Bay & 50.13508 & -98.17063 & 3 & 101 & 062J01 \\
\hline
\end{tabular}


Appendix 1: Table listing the site number, date sampled, site name, latitude and longitude (in decimal degrees DD), DES stream order, DES map number and the corresponding NTS map number for all sites surveyed between 2002 and 2006.

\begin{tabular}{|c|c|c|c|c|c|c|c|}
\hline Site Number & Date & Site Name & $\begin{array}{l}\text { Latitude } \\
\text { (DD) }\end{array}$ & $\begin{array}{l}\text { Longitude } \\
\text { (DD) }\end{array}$ & $\begin{array}{l}\text { DES } \\
\text { Order }\end{array}$ & $\begin{array}{l}\text { DES } \\
\text { Map \# }\end{array}$ & $\begin{array}{l}\text { NTS } \\
\text { Map \# }\end{array}$ \\
\hline W-04-036 & 6-May-04 & Unnamed tributary to Lake Frances & 50.24086 & -97.96294 & 2 & 109 & 062104 \\
\hline W-04-037 & 6-May-04 & Wagon Creek & 50.47730 & -97.98753 & 3 & 109 & 062105 \\
\hline W-04-038 & 6-May-04 & Wagon Creek & 50.41833 & -97.83255 & 3 & 109 & 062105 \\
\hline W-04-039 & 10-May-04 & Wagon Creek & 50.47628 & -97.98947 & 3 & 109 & 062105 \\
\hline W-04-040 & 10-May-04 & Wagon Creek & 50.47053 & -97.87562 & 3 & 109 & 062105 \\
\hline W-04-041 & 11-May-04 & Unnamed tributary to Lake St. Martin & 51.76758 & -98.61240 & 2 & 112 & 062015 \\
\hline W-04-042 & 11-May-04 & Byng Drain & 51.75605 & -98.56973 & 2 & 112 & 062015 \\
\hline W-04-043 & 11-May-04 & Partridge Creek East Branch & 51.75317 & -98.66447 & 3 & 112 & 062015 \\
\hline W-04-044 & 11-May-04 & Partridge Creek West Branch & 51.74513 & -98.67057 & 3 & 112 & 062010 \\
\hline W-04-045 & 11-May-04 & Karpaty School Drain & 51.72332 & -98.71190 & 2 & 112 & 062010 \\
\hline W-04-046 & 11-May-04 & Unnamed tributary to Lake Pineimuta & 51.73190 & -98.68837 & 2 & 112 & 062010 \\
\hline W-04-047 & 11-May-04 & Unnamed tributary to Homebrook Drain & 51.74037 & -98.73520 & 2 & 112 & 062010 \\
\hline W-04-048 & 11-May-04 & Unnamed tributary to Homebrook Drain & 51.74038 & -98.73588 & 2 & 112 & 062010 \\
\hline W-04-049 & 11-May-04 & Unnamed tributary to Homebrook Drain & 51.74027 & -98.77108 & 3 & 112 & 062010 \\
\hline W-04-050 & 11-May-04 & Unnamed tributary to Lake Manitoba & 51.74028 & -98.87308 & 3 & 112 & 062010 \\
\hline W-04-051 & 12-May-04 & Powderhorn Creek & 51.74035 & -98.90143 & 2 & 112 & 062010 \\
\hline W-04-052 & 12-May-04 & Basket River & 51.74044 & -98.95285 & 2 & 112 & 062010 \\
\hline W-04-053 & 18-May-04 & North Arm Upper Manning Canal & 49.57397 & -96.68628 & 3 & 7 & $062 \mathrm{H} 10$ \\
\hline W-04-054 & 18-May-04 & Manning Canal & 49.56008 & -96.71538 & 3 & 7 & $062 \mathrm{H} 10$ \\
\hline W-04-055 & 18-May-04 & Chortiz Drain & 49.54575 & -96.80828 & 3 & 7 & $062 \mathrm{H} 10$ \\
\hline W-04-056 & 18-May-04 & $\begin{array}{l}\text { Unnamed tributary to South Arm Upper Manning } \\
\text { Canal }\end{array}$ & 49.54550 & -96.75617 & 2 & 7 & $062 \mathrm{H} 10$ \\
\hline W-04-057 & 18-May-04 & South Arm Upper Manning Canal & 49.54540 & -96.74667 & 3 & 7 & $062 \mathrm{H} 10$ \\
\hline W-04-058 & 18-May-04 & Unnamed tributary to Manning Canal & 49.54545 & -96.72313 & 2 & 7 & $062 \mathrm{H} 10$ \\
\hline W-04-059 & 20-May-04 & Unnamed tributary to Manning Canal & 49.50272 & -96.57833 & 3 & 7 & $062 \mathrm{H} 10$ \\
\hline
\end{tabular}


Appendix 1: Table listing the site number, date sampled, site name, latitude and longitude (in decimal degrees DD), DES stream order, DES map number and the corresponding NTS map number for all sites surveyed between 2002 and 2006.

\begin{tabular}{|c|c|c|c|c|c|c|c|}
\hline Site Number & Date & Site Name & $\begin{array}{l}\text { Latitude } \\
\text { (DD) }\end{array}$ & $\begin{array}{l}\text { Longitude } \\
\text { (DD) }\end{array}$ & $\begin{array}{l}\text { DES } \\
\text { Order }\end{array}$ & $\begin{array}{l}\text { DES } \\
\text { Map \# }\end{array}$ & $\begin{array}{l}\text { NTS } \\
\text { Map \# }\end{array}$ \\
\hline W-04-060 & 20-May-04 & Manning Canal & 49.53220 & -96.65503 & 3 & 7 & $062 \mathrm{H} 10$ \\
\hline W-04-061 & 20-May-04 & South Lateral & 49.42348 & -96.66812 & 3 & 7 & $062 \mathrm{H} 07$ \\
\hline W-04-062 & 20-May-04 & South Lateral & 49.44363 & -96.71378 & 3 & 7 & $062 \mathrm{H} 07$ \\
\hline W-04-063 & 20-May-04 & Unnamed tributary to South Lateral & 49.47297 & -96.75905 & 3 & 7 & $062 \mathrm{H} 07$ \\
\hline W-04-064 & 20-May-04 & South Lateral & 49.50272 & -96.81888 & 4 & 7 & $062 \mathrm{H} 10$ \\
\hline W-04-065 & 20-May-04 & Old South Lateral & 49.57602 & -96.88897 & 2 & 7 & $062 \mathrm{H} 10$ \\
\hline W-04-066 & 20-May-04 & Unnamed tributary to Manning Canal & 49.57562 & -96.84352 & 2 & 7 & $062 \mathrm{H} 10$ \\
\hline W-04-067 & 20-May-04 & North Lateral & 49.62008 & -96.86689 & 3 & 7 & $062 \mathrm{H} 10$ \\
\hline W-04-068 & 20-May-04 & Manning Canal & 49.62412 & -96.88930 & 5 & 7 & $062 \mathrm{H} 10$ \\
\hline W-04-069 & 21-May-04 & Unnamed tributary to Red River & 49.76717 & -96.73003 & 2 & 9 & $062 \mathrm{H} 15$ \\
\hline W-04-070 & 21-May-04 & Station Road Drain & 49.78192 & -96.75282 & 2 & 9 & $062 \mathrm{H} 15$ \\
\hline W-04-071 & 21-May-04 & Lower Youville Drain & 49.73832 & -96.95837 & 3 & 9 & $062 \mathrm{H} 10$ \\
\hline W-04-072 & 25-May-04 & Unnamed tributary to Seine River & 49.73433 & -96.85497 & 2 & 9 & $062 \mathrm{H} 10$ \\
\hline W-04-073 & 25-May-04 & Unnamed tributary to Seine River & 49.75187 & -96.89383 & 2 & 9 & $062 \mathrm{H} 15$ \\
\hline W-04-074 & 25-May-04 & Grove Drain & 49.82693 & -96.91295 & 3 & 9 & $062 \mathrm{H} 15$ \\
\hline W-04-075 & 25-May-04 & South Bibeau Drain & 49.85642 & -96.93553 & 2 & 9 & $062 \mathrm{H} 15$ \\
\hline W-04-076 & 25-May-04 & Prairie Centre Line Drain & 49.84165 & -96.93553 & 3 & 9 & $062 \mathrm{H} 15$ \\
\hline W-04-077 & 26-May-04 & Crescent Lake Overflow & 50.35929 & -97.18275 & 3 & 30 & 062106 \\
\hline W-04-078 & 26-May-04 & Ross Creek & 50.34018 & -97.27545 & 3 & 30 & 062106 \\
\hline W-04-079 & 26-May-04 & Ross Creek & 50.32982 & -97.15962 & 3 & 30 & 062106 \\
\hline W-04-080 & 26-May-04 & Ross Creek & 50.32985 & -97.14817 & 3 & 30 & 062106 \\
\hline W-04-081 & 26-May-04 & Ross Creek & 50.32905 & -97.08793 & 4 & 30 & 062106 \\
\hline W-04-082 & 26-May-04 & Boundary Drain & 50.38882 & -97.11322 & 3 & 30 & 062106 \\
\hline W-04-083 & 27-May-04 & Netley Creek & 50.46780 & -97.20627 & 3 & 30 & 062106 \\
\hline W-04-084 & 27-May-04 & Netley Creek & 50.38883 & -97.13907 & 3 & 30 & 062106 \\
\hline
\end{tabular}


Appendix 1: Table listing the site number, date sampled, site name, latitude and longitude (in decimal degrees DD), DES stream order, DES map number and the corresponding NTS map number for all sites surveyed between 2002 and 2006.

\begin{tabular}{|c|c|c|c|c|c|c|c|}
\hline Site Number & Date & Site Name & $\begin{array}{l}\text { Latitude } \\
\text { (DD) }\end{array}$ & $\begin{array}{l}\text { Longitude } \\
\text { (DD) }\end{array}$ & $\begin{array}{l}\text { DES } \\
\text { Order }\end{array}$ & $\begin{array}{l}\text { DES } \\
\text { Map \# }\end{array}$ & $\begin{array}{c}\text { NTS } \\
\text { Map \# }\end{array}$ \\
\hline W-04-085 & 28-May-04 & Semple Drain & 50.21820 & -96.74898 & 2 & 13 & 062102 \\
\hline W-04-086 & 28-May-04 & Devils Creek & 50.22310 & -96.72545 & 5 & 13 & 062102 \\
\hline W-04-087 & 28-May-04 & Pelletier Drain & 50.23183 & -96.72578 & 3 & 13 & 062102 \\
\hline W-04-088 & 28-May-04 & Township Line Drain & 50.23948 & -96.72607 & 3 & 13 & 062102 \\
\hline W-04-089 & 1-Jun-04 & Unnamed tributary to Seine River & 49.39740 & -96.47009 & 2 & 8 & $062 \mathrm{H} 08$ \\
\hline W-04-090 & 1-Jun-04 & Unnamed tributary to Seine River & 49.39963 & -96.48778 & 2 & 8 & $062 \mathrm{H} 08$ \\
\hline W-04-091 & 1-Jun-04 & Marchand West Drain & 49.37023 & -96.41972 & 3 & 8 & $062 \mathrm{H} 08$ \\
\hline W-04-092 & 1-Jun-04 & Unnamed tributary to Marchand West Drain & 49.34208 & -96.41975 & 3 & 8 & $062 \mathrm{H} 08$ \\
\hline W-04-093 & 1-Jun-04 & Conroy Creek & 49.11848 & -96.55045 & 2 & 2 & $062 \mathrm{H} 02$ \\
\hline W-04-094 & 2-Jun-04 & Unnamed tributary to Rat River & 49.20915 & -96.10252 & 2 & 3 & $062 \mathrm{H} 01$ \\
\hline W-04-095 & 2-Jun-04 & Rat River & 49.25280 & -96.05943 & 2 & 3 & 062H08 \\
\hline W-04-096 & 2-Jun-04 & Unnamed tributary to Rat River & 49.21234 & -96.08126 & 2 & 3 & $062 \mathrm{H} 01$ \\
\hline W-04-097 & 2-Jun-04 & Unnamed tributary to Rat River & 49.22853 & -96.06191 & 3 & 3 & $062 \mathrm{H} 01$ \\
\hline W-04-098 & 2-Jun-04 & Unnamed tributary to Rat River & 49.23567 & -96.05615 & 2 & 3 & $062 \mathrm{H} 01$ \\
\hline W-04-099 & 2-Jun-04 & Rat River & 49.20955 & -96.28653 & 4 & 3 & $062 \mathrm{H} 01$ \\
\hline W-04-100 & 2-Jun-04 & Rat River & 49.22961 & -96.50989 & 4 & 3 & $062 \mathrm{H} 02$ \\
\hline W-04-101 & 3-Jun-04 & Unnamed tributary to Roseau River & 49.16588 & -96.13573 & 2 & 2 & $062 \mathrm{H} 01$ \\
\hline W-04-102 & 3-Jun-04 & Conroy Creek & 49.13343 & -96.57506 & 2 & 2 & 062H02 \\
\hline W-04-103 & 3-Jun-04 & Unnamed tributary to Roseau River & 49.11913 & -96.67323 & 2 & 2 & 062H02 \\
\hline W-04-104 & 3-Jun-04 & Vita South Drain & 49.08125 & -96.51670 & 2 & 2 & 062H02 \\
\hline W-04-105 & 3-Jun-04 & Unnamed tributary to Vita Drain & 49.13940 & -96.62895 & 2 & 2 & $062 \mathrm{H} 02$ \\
\hline W-04-106 & 3-Jun-04 & Unnamed tributary to Vita Drain & 49.16330 & -96.74112 & 2 & 2 & 062H02 \\
\hline W-04-107 & 3-Jun-04 & Roseau River & 49.13395 & -96.76592 & 4 & 2 & $062 \mathrm{H} 02$ \\
\hline W-04-108 & 3-Jun-04 & Jordan River & 49.07413 & -96.84183 & 2 & 2 & 062H02 \\
\hline W-04-109 & 4-Jun-04 & Harlow Drain & 49.09462 & -97.01020 & 3 & 2 & $062 \mathrm{H} 03$ \\
\hline
\end{tabular}


Appendix 1: Table listing the site number, date sampled, site name, latitude and longitude (in decimal degrees DD), DES stream order, DES map number and the corresponding NTS map number for all sites surveyed between 2002 and 2006.

\begin{tabular}{|c|c|c|c|c|c|c|c|}
\hline Site Number & Date & Site Name & $\begin{array}{l}\text { Latitude } \\
\text { (DD) }\end{array}$ & $\begin{array}{l}\text { Longitude } \\
\text { (DD) }\end{array}$ & $\begin{array}{l}\text { DES } \\
\text { Order }\end{array}$ & $\begin{array}{l}\text { DES } \\
\text { Map \# }\end{array}$ & $\begin{array}{c}\text { NTS } \\
\text { Map \# }\end{array}$ \\
\hline W-04-110 & 4-Jun-04 & Ridgeville Drain & 49.07515 & -97.01042 & 2 & 2 & $062 \mathrm{H} 03$ \\
\hline W-04-111 & 4-Jun-04 & Unnamed tributary to Main Drain & 49.04907 & -97.01025 & 2 & 2 & $062 \mathrm{H} 03$ \\
\hline W-04-112 & 4-Jun-04 & Unnamed tributary to Roseau River & 49.22183 & -97.24818 & 3 & 2 & $062 \mathrm{H} 03$ \\
\hline W-04-113 & 5-Jun-04 & Unnamed tributary to Devils Creek & 50.04712 & -96.58980 & 2 & 13 & 062102 \\
\hline W-04-114 & 5-Jun-04 & Unnamed tributary to Devils Creek & 50.02147 & -96.58987 & 2 & 13 & 062102 \\
\hline W-04-115 & 5-Jun-04 & Devils Creek & 50.01765 & -96.61265 & 3 & 13 & 062102 \\
\hline W-04-116 & 5-Jun-04 & Selkirk Line Drain & 50.12033 & -96.63468 & 3 & 13 & 062102 \\
\hline W-04-117 & 7-Jun-04 & Unnamed tributary to Lake Winnipeg & 50.26904 & -96.71061 & 2 & 13 & 062107 \\
\hline W-04-118 & 7-Jun-04 & Unnamed tributary to Libau Drain & 50.26917 & -96.69307 & 2 & 13 & 062107 \\
\hline W-04-119 & 8-Jun-04 & Unnamed tributary to Roseau River & 49.13380 & -96.67138 & 2 & 2 & 062H02 \\
\hline W-04-120 & 8-Jun-04 & Unnamed tributary to Roseau River & 49.14852 & -96.78588 & 2 & 2 & $062 \mathrm{H} 02$ \\
\hline W-04-121 & 8-Jun-04 & Unnamed tributary to Roseau River & 49.14840 & -96.80823 & 2 & 2 & $062 \mathrm{H} 02$ \\
\hline W-04-122 & 8-Jun-04 & Unnamed tributary to Roseau River & 49.11872 & -97.14507 & 2 & 2 & $062 \mathrm{H} 03$ \\
\hline W-04-123 & 8-Jun-04 & Unnamed tributary to Roseau River & 49.11868 & -97.15615 & 2 & 2 & $062 \mathrm{H} 03$ \\
\hline W-04-124 & 8-Jun-04 & Unnamed tributary to Roseau River & 49.11245 & -97.16770 & 2 & 2 & $062 \mathrm{H03}$ \\
\hline W-04-125 & 9-Jun-04 & North Branch Jordan River & 49.12657 & -96.80855 & 2 & 2 & $062 \mathrm{H} 02$ \\
\hline W-04-126 & 9-Jun-04 & Unnamed tributary to Jordon River & 49.12278 & -96.87595 & 2 & 2 & $062 \mathrm{H} 02$ \\
\hline W-04-127 & 9-Jun-04 & Jordan River & 49.11323 & -96.87585 & 3 & 2 & 062H02 \\
\hline W-04-128 & 9-Jun-04 & Unnamed tributary to Stewart Drain & 49.02967 & -96.80806 & 2 & 2 & 062H02 \\
\hline W-04-129 & 9-Jun-04 & Casson Drain & 49.09330 & -96.89842 & 2 & 2 & $062 \mathrm{H} 02$ \\
\hline W-04-130 & 9-Jun-04 & Unnamed tributary to Jordan River & 49.13355 & -96.94365 & 2 & 2 & $062 \mathrm{H} 02$ \\
\hline W-04-131 & 9-Jun-04 & Unnamed tributary to Stewart Drain & 49.13348 & -96.97555 & 2 & 2 & 062H02 \\
\hline W-04-132 & 9-Jun-04 & Unnamed tributary to Ridgeville Drain & 49.10850 & -97.01063 & 2 & 2 & $062 \mathrm{H} 03$ \\
\hline W-04-133 & 9-Jun-04 & 2 Mile Drain & 49.02995 & -97.14483 & 3 & 2 & $062 \mathrm{H} 03$ \\
\hline W-04-134 & 9-Jun-04 & Unnamed tributary to Main Drain & 49.05958 & -97.12238 & 2 & 2 & $062 \mathrm{H} 03$ \\
\hline
\end{tabular}


Appendix 1: Table listing the site number, date sampled, site name, latitude and longitude (in decimal degrees DD), DES stream order, DES map number and the corresponding NTS map number for all sites surveyed between 2002 and 2006.

\begin{tabular}{|c|c|c|c|c|c|c|c|}
\hline Site Number & Date & Site Name & $\begin{array}{l}\text { Latitude } \\
\text { (DD) }\end{array}$ & $\begin{array}{l}\text { Longitude } \\
\text { (DD) }\end{array}$ & $\begin{array}{l}\text { DES } \\
\text { Order }\end{array}$ & $\begin{array}{l}\text { DES } \\
\text { Map \# }\end{array}$ & $\begin{array}{c}\text { NTS } \\
\text { Map \# }\end{array}$ \\
\hline W-04-135 & 9-Jun-04 & Unnamed tributary to Main Drain & 49.05952 & -97.09978 & 2 & 2 & $062 \mathrm{H} 03$ \\
\hline W-04-136 & 10-Jun-04 & Unnamed tributary to Main Drain & 49.05940 & -97.01006 & 2 & 2 & $062 \mathrm{H} 03$ \\
\hline W-04-137 & 10-Jun-04 & Unnamed tributary to Main Drain & 49.03749 & -97.00987 & 2 & 2 & $062 \mathrm{H} 03$ \\
\hline W-04-138 & 10-Jun-04 & Fredensthal Drain & 49.02998 & -97.07740 & 3 & 2 & $062 \mathrm{H} 03$ \\
\hline W-04-139 & 10-Jun-04 & Main Drain & 49.03002 & -97.03255 & 2 & 2 & $062 \mathrm{H03}$ \\
\hline W-04-140 & 10-Jun-04 & Unnamed tributary to Red River & 49.08922 & -97.18963 & 2 & 2 & $062 \mathrm{H} 03$ \\
\hline W-04-141 & 10-Jun-04 & Main Drain & 49.08904 & -97.09997 & 4 & 2 & $062 \mathrm{H} 03$ \\
\hline W-04-142 & 14-Jun-04 & Unnamed tributary to Roseau River & 49.17010 & -97.23482 & 2 & 2 & $062 \mathrm{HO}$ \\
\hline W-04-143 & 14-Jun-04 & Unnamed tributary to Roseau River & 49.14788 & -97.23477 & 2 & 2 & $062 \mathrm{HO3}$ \\
\hline W-04-144 & 14-Jun-04 & Unnamed tributary to Roseau River & 49.13307 & -97.23480 & 2 & 2 & $062 \mathrm{H} 03$ \\
\hline W-04-145 & 14-Jun-04 & Unnamed tributary to Roseau River & 49.16397 & -97.16783 & 2 & 2 & $062 \mathrm{H} 03$ \\
\hline W-04-146 & 14-Jun-04 & Confluence of unnamed tributary and Kyle Drain & 49.20727 & -97.16488 & 2 & 2 & $062 \mathrm{H} 03$ \\
\hline W-04-147 & 14-Jun-04 & Unnamed tributary to Roseau River & 49.20718 & -97.07472 & 2 & 2 & $062 \mathrm{H} 03$ \\
\hline W-04-148 & 14-Jun-04 & Langside Drain & 49.22188 & -97.05220 & 3 & 2 & $062 \mathrm{H} 03$ \\
\hline W-04-149 & 14-Jun-04 & Unnamed tributary to Roseau River & 49.19243 & -97.09728 & 2 & 2 & $062 \mathrm{H03}$ \\
\hline W-04-150 & 14-Jun-04 & Unnamed tributary to Roseau River & 49.23663 & -97.16472 & 2 & 2 & $062 \mathrm{H} 03$ \\
\hline W-04-151 & 14-Jun-04 & Angle Drain & 49.35460 & -97.10588 & 3 & 5 & $062 \mathrm{H} 06$ \\
\hline W-04-152 & 15-Jun-04 & Marsh River & 49.25126 & -97.24764 & 3 & 5 & $062 \mathrm{H} 06$ \\
\hline W-04-153 & 15-Jun-04 & Arnaud Drain & 49.26605 & -97.09638 & 3 & 5 & $062 \mathrm{H} 06$ \\
\hline W-04-154 & 15-Jun-04 & Arnaud Drain & 49.28198 & -97.16517 & 3 & 5 & $062 \mathrm{H} 06$ \\
\hline W-04-155 & 15-Jun-04 & Marsh River & 49.29555 & -97.21288 & 3 & 5 & $062 \mathrm{H} 06$ \\
\hline W-04-156 & 16-Jun-04 & Unnamed tributary to Red River & 49.51712 & -97.21473 & 2 & 5 & $062 \mathrm{H} 11$ \\
\hline W-04-157 & 16-Jun-04 & Lafond Drain & 49.28073 & -97.31868 & 3 & 5 & $062 \mathrm{H} 06$ \\
\hline W-04-158 & 16-Jun-04 & Unnamed tributary to Red River & 49.29550 & -97.31542 & 2 & 5 & $062 \mathrm{H} 06$ \\
\hline W-04-159 & 16-Jun-04 & Aubigny Drain & 49.41369 & -97.26845 & 3 & 5 & $062 \mathrm{H} 06$ \\
\hline
\end{tabular}


Appendix 1: Table listing the site number, date sampled, site name, latitude and longitude (in decimal degrees DD), DES stream order, DES map number and the corresponding NTS map number for all sites surveyed between 2002 and 2006.

\begin{tabular}{|c|c|c|c|c|c|c|c|}
\hline Site Number & Date & Site Name & $\begin{array}{l}\text { Latitude } \\
\text { (DD) }\end{array}$ & $\begin{array}{l}\text { Longitude } \\
\text { (DD) }\end{array}$ & $\begin{array}{l}\text { DES } \\
\text { Order }\end{array}$ & $\begin{array}{l}\text { DES } \\
\text { Map \# }\end{array}$ & $\begin{array}{c}\text { NTS } \\
\text { Map \# }\end{array}$ \\
\hline W-04-160 & 16-Jun-04 & Unnamed tributary to Marsh River & 49.44327 & -97.11060 & 2 & 5 & $062 \mathrm{H} 06$ \\
\hline W-04-161 & 16-Jun-04 & Unnamed tributary to Marsh River & 49.44332 & -97.12847 & 2 & 5 & $062 \mathrm{H} 06$ \\
\hline W-04-162 & 16-Jun-04 & Unnamed tributary to Marsh River & 49.44323 & -97.15303 & 2 & 5 & $062 \mathrm{H} 06$ \\
\hline W-04-163 & 16-Jun-04 & Marsh River & 49.50238 & -97.15582 & 5 & 5 & $062 \mathrm{H} 11$ \\
\hline W-04-164 & 17-Jun-04 & Unnamed tributary to Marsh River & 49.35468 & -97.16530 & 2 & 5 & $062 \mathrm{H} 06$ \\
\hline W-04-165 & 17-Jun-04 & Unnamed tributary to Ste. Elizabeth Drain & 49.32507 & -97.12017 & 2 & 5 & $062 \mathrm{H} 06$ \\
\hline W-04-166 & 17-Jun-04 & Ste. Elizabeth Drain & 49.31043 & -97.13185 & 3 & 5 & $062 \mathrm{H} 06$ \\
\hline W-04-167 & 17-Jun-04 & Dufrost North Drain & 49.38417 & -97.12040 & 3 & 5 & $062 \mathrm{H} 06$ \\
\hline W-04-168 & 17-Jun-04 & Unnamed tributary to Angle Drain & 49.36932 & -97.12027 & 2 & 5 & $062 \mathrm{HOG}$ \\
\hline W-04-169 & 17-Jun-04 & Unnamed tributary to Red River & 49.46242 & -97.24400 & 2 & 5 & $062 \mathrm{H} 06$ \\
\hline W-04-170 & 17-Jun-04 & Unnamed tributary to Red River & 49.44590 & -97.25695 & 2 & 5 & $062 \mathrm{H} 06$ \\
\hline W-04-171 & 17-Jun-04 & Unnamed tributary to Red River & 49.40008 & -97.28423 & 2 & 5 & $062 \mathrm{H} 06$ \\
\hline W-04-172 & 17-Jun-04 & Dufrost North Drain & 49.38410 & -97.14287 & 4 & 5 & $062 \mathrm{H} 06$ \\
\hline W-04-173 & 18-Jun-04 & Prefontaine Drain & 49.63525 & -97.00330 & 3 & 6 & $062 \mathrm{H} 11$ \\
\hline W-04-174 & 18-Jun-04 & D - 20 Drain & 49.57653 & -97.10230 & 4 & 6 & $062 \mathrm{H} 11$ \\
\hline W-04-175 & 21-Jun-04 & Indian Reserve Drain & 50.32857 & -96.65403 & 3 & 13 & 062107 \\
\hline W-04-176 & 21-Jun-04 & $T$ - Drain & 50.31133 & -96.47598 & 3 & 10 & 062108 \\
\hline W-04-177 & 21-Jun-04 & $\mathrm{T}$ - Drain & 50.31135 & -96.48228 & 3 & 10 & 062108 \\
\hline W-04-178 & 21-Jun-04 & Greenwald Drain & 50.29642 & -96.53543 & 3 & 10 & 062107 \\
\hline W-04-179 & 22-Jun-04 & Gusta Drain & 50.47417 & -96.32952 & 3 & 14 & 062108 \\
\hline W-04-180 & 22-Jun-04 & Hiebert Drain & 50.41513 & -96.30658 & 3 & 14 & 062108 \\
\hline W-04-181 & 22-Jun-04 & Unnamed tributary to Lac du Bonnet & 50.33800 & -95.91050 & 2 & 96 & 052L05 \\
\hline W-04-182 & 22-Jun-04 & Unnamed tributary to Lee River & 50.30154 & -95.87763 & 3 & 96 & 052L05 \\
\hline W-04-183 & 22-Jun-04 & Boggy Creek & 50.25090 & -95.88733 & 4 & 96 & 052L05 \\
\hline W-04-184 & 22-Jun-04 & Unnamed tributary to Lac du Bonnet & 50.27956 & -95.96923 & 2 & 96 & 052L05 \\
\hline
\end{tabular}


Appendix 1: Table listing the site number, date sampled, site name, latitude and longitude (in decimal degrees DD), DES stream order, DES map number and the corresponding NTS map number for all sites surveyed between 2002 and 2006.

\begin{tabular}{|c|c|c|c|c|c|c|c|}
\hline Site Number & Date & Site Name & $\begin{array}{l}\text { Latitude } \\
\text { (DD) }\end{array}$ & $\begin{array}{l}\text { Longitude } \\
\text { (DD) }\end{array}$ & $\begin{array}{l}\text { DES } \\
\text { Order }\end{array}$ & $\begin{array}{l}\text { DES } \\
\text { Map \# }\end{array}$ & $\begin{array}{c}\text { NTS } \\
\text { Map \# }\end{array}$ \\
\hline W-04-185 & 23-Jun-04 & Unnamed tributary to Main Drain No. 1 & 50.35618 & -96.32870 & 3 & 14 & 062108 \\
\hline W-04-186 & 23-Jun-04 & Stead Drain & 50.40068 & -96.32997 & 3 & 14 & 062108 \\
\hline W-04-187 & 23-Jun-04 & Unnamed tributary to Main Drain No. 1 & 50.38562 & -96.35370 & 2 & 14 & 062108 \\
\hline W-04-188 & 23-Jun-04 & Unnamed tributary to Maple Creek & 50.26717 & -96.12677 & 2 & 96 & 062108 \\
\hline W-04-189 & 24-Jun-04 & Unnamed tributary to Maskwa River & 50.54777 & -96.09843 & 2 & 96 & 062109 \\
\hline W-04-190 & 24-Jun-04 & Sandy Creek & 50.53553 & -96.09805 & 2 & 96 & 062109 \\
\hline W-04-191 & 24-Jun-04 & Spence Creek & 50.61687 & -96.27338 & 2 & 96 & 062109 \\
\hline W-04-192 & 24-Jun-04 & Unnamed tributary to Winnipeg River & 50.57720 & -96.20660 & 1 & 96 & 062109 \\
\hline W-04-193 & 24-Jun-04 & Unnamed tributary to Winnipeg River & 50.57717 & -96.18497 & 1 & 96 & 062109 \\
\hline W-04-194 & 24-Jun-04 & Unnamed tributary to Lac du Bonnet & 50.39717 & -96.00515 & 2 & 96 & 062108 \\
\hline W-04-195 & 25-Jun-04 & Unnamed tributary to Winnipeg River & 50.45860 & -96.04228 & 2 & 96 & 062108 \\
\hline W-04-196 & 25-Jun-04 & Unnamed tributary near Maple Creek & 50.37199 & -96.02096 & 1 & 96 & 062108 \\
\hline W-04-197 & 25-Jun-04 & Unnamed tributary to Winnipeg River & 50.16585 & -96.04915 & 2 & 96 & 062101 \\
\hline W-04-198 & 29-Jun-04 & Unnamed tributary to Red River & 50.11817 & -96.88590 & 2 & 12 & 062102 \\
\hline W-04-199 & 29-Jun-04 & Unnamed tributary to Red River & 50.08390 & -96.91542 & 2 & 12 & 062102 \\
\hline W-04-200 & 29-Jun-04 & Shkolny Creek & 49.99445 & -96.91095 & 2 & 12 & $062 \mathrm{H} 15$ \\
\hline W-04-201 & 29-Jun-04 & Unnamed tributary to Cook's Creek Diversion & 49.88758 & -96.88860 & 2 & 12 & $062 \mathrm{H} 15$ \\
\hline W-04-202 & 29-Jun-04 & Cook's Creek Diversion & 49.90025 & -96.88823 & 4 & 12 & $062 \mathrm{H} 15$ \\
\hline W-04-203 & 29-Jun-04 & Road Drain & 49.92891 & -96.93368 & 3 & 12 & $062 \mathrm{H} 15$ \\
\hline W-04-204 & 29-Jun-04 & Carr's Creek & 49.94520 & -96.95617 & 3 & 12 & $062 \mathrm{H} 15$ \\
\hline W-04-205 & 29-Jun-04 & Gunn Creek & 50.06470 & -96.93757 & 3 & 12 & 062102 \\
\hline W-04-206 & 29-Jun-04 & Bottomly Creek & 49.98765 & -97.04428 & 2 & 12 & $062 \mathrm{H} 14$ \\
\hline W-04-207 & 5-Jul-04 & Brokenhead River & 49.65023 & -96.27640 & 2 & 88 & 062H09 \\
\hline W-04-208 & 5-Jul-04 & Coulee St. Onge & 49.65780 & -96.32663 & 2 & 88 & $062 \mathrm{H} 09$ \\
\hline W-04-209 & 5-Jul-04 & Brokenhead River & 49.90798 & -96.38345 & 3 & 10 & $062 \mathrm{H} 16$ \\
\hline
\end{tabular}


Appendix 1: Table listing the site number, date sampled, site name, latitude and longitude (in decimal degrees DD), DES stream order, DES map number and the corresponding NTS map number for all sites surveyed between 2002 and 2006.

\begin{tabular}{|c|c|c|c|c|c|c|c|}
\hline Site Number & Date & Site Name & $\begin{array}{l}\text { Latitude } \\
\text { (DD) }\end{array}$ & $\begin{array}{l}\text { Longitude } \\
\text { (DD) }\end{array}$ & $\begin{array}{l}\text { DES } \\
\text { Order }\end{array}$ & $\begin{array}{l}\text { DES } \\
\text { Map \# }\end{array}$ & $\begin{array}{c}\text { NTS } \\
\text { Map \# }\end{array}$ \\
\hline W-04-210 & 5-Jul-04 & Hazel Creek & 49.92720 & -96.38207 & 3 & 10 & $062 \mathrm{H} 16$ \\
\hline W-04-211 & 5-Jul-04 & Unnamed tributary to Brokenhead River & 50.00268 & -96.42370 & 2 & 10 & 062101 \\
\hline$W-04-212$ & 6-Jul-04 & Unnamed tributary to Hazel Creek & 49.99543 & -96.47488 & 2 & 10 & $062 \mathrm{H} 16$ \\
\hline W-04-213 & 6-Jul-04 & Hazel Creek & 50.00293 & -96.45138 & 4 & 10 & 062101 \\
\hline W-04-214 & 6-Jul-04 & Unnamed tributary to Brokenhead River & 50.05487 & -96.49792 & 2 & 10 & 062101 \\
\hline W-04-215 & 6-Jul-04 & Unnamed tributary to Brokenhead River & 50.04697 & -96.47500 & 2 & 10 & 062101 \\
\hline W-04-216 & 6-Jul-04 & Unnamed tributary to Brokenhead River & 50.03205 & -96.40633 & 2 & 10 & 062101 \\
\hline W-04-217 & 6-Jul-04 & Bachman East Drain & 50.07625 & -96.33762 & 3 & 10 & 062101 \\
\hline W-04-218 & 6-Jul-04 & U - Drain & 50.28188 & -96.46685 & 3 & 10 & 062108 \\
\hline W-04-219 & 7-Jul-04 & Unnamed tributary to Brokenhead River & 50.32617 & -96.51290 & 2 & 10 & 062107 \\
\hline W-04-220 & 7-Jul-04 & Unnamed tributary to $\mathrm{T}$ - Drain & 50.32640 & -96.44550 & 2 & 10 & 062108 \\
\hline W-04-221 & 7-Jul-04 & Unnamed tributary to Brokenhead River & 50.25263 & -96.44460 & 2 & 10 & 062108 \\
\hline W-04-222 & 7-Jul-04 & C - Drain & 50.23762 & -96.42967 & 2 & 10 & 062101 \\
\hline W-04-223 & 7-Jul-04 & W - Drain & 50.22278 & -96.45140 & 3 & 10 & 062101 \\
\hline W-04-224 & 7-Jul-04 & Unnamed tributary to Brokenhead River & 50.16463 & -96.42923 & 2 & 10 & 062101 \\
\hline W-04-225 & 7-Jul-04 & Bachman East Drain & 50.12028 & -96.40613 & 3 & 10 & 062101 \\
\hline W-04-226 & 7-Jul-04 & Unnamed tributary to Brokenhead River & 50.13525 & -96.42908 & 2 & 10 & 062101 \\
\hline W-04-227 & 7-Jul-04 & Unnamed tributary to Brokenhead River & 50.10567 & -96.42917 & 2 & 10 & 062101 \\
\hline W-04-228 & 7-Jul-04 & Unnamed tributary to Brokenhead River & 50.16465 & -96.47458 & 2 & 10 & 062101 \\
\hline W-04-229 & 7-Jul-04 & Unnamed tributary to Oneschuk Drain & 50.19423 & -96.52057 & 2 & 10 & 062102 \\
\hline W-04-230 & 7-Jul-04 & Brokenhead Drain & 50.22345 & -96.51960 & 3 & 10 & 062102 \\
\hline W-04-231 & 8-Jul-04 & U - Drain & 50.30468 & -96.21378 & 3 & 10 & 062108 \\
\hline W-04-232 & 8-Jul-04 & Tourond Creek & 49.42138 & -96.75777 & 4 & 6 & $062 \mathrm{H} 07$ \\
\hline W-04-233 & 13-Jul-04 & Garland River & 51.79863 & -100.30252 & 5 & 103 & 062N16 \\
\hline W-04-234 & 13-Jul-04 & Unnamed tributary to Garland River & 51.74997 & -100.37705 & 2 & 103 & 062N09 \\
\hline
\end{tabular}


Appendix 1: Table listing the site number, date sampled, site name, latitude and longitude (in decimal degrees DD), DES stream order, DES map number and the corresponding NTS map number for all sites surveyed between 2002 and 2006.

\begin{tabular}{|c|c|c|c|c|c|c|c|}
\hline Site Number & Date & Site Name & $\begin{array}{l}\text { Latitude } \\
\text { (DD) }\end{array}$ & $\begin{array}{l}\text { Longitude } \\
\text { (DD) }\end{array}$ & $\begin{array}{l}\text { DES } \\
\text { Order }\end{array}$ & $\begin{array}{l}\text { DES } \\
\text { Map \# }\end{array}$ & $\begin{array}{l}\text { NTS } \\
\text { Map \# }\end{array}$ \\
\hline W-04-235 & 13-Jul-04 & Unnamed tributary to Wellburns Creek & 51.79834 & -100.23440 & 2 & 103 & 062N16 \\
\hline W-04-236 & 13-Jul-04 & Unnamed tributary to Wellburns Creek & 51.79860 & -100.22119 & 2 & 103 & 062N16 \\
\hline W-04-237 & 13-Jul-04 & Wellburns Creek & 51.79858 & -100.20442 & 3 & 104 & 062N16 \\
\hline W-04-238 & 13-Jul-04 & Bickels Creek & 51.81133 & -100.10788 & 2 & 103 & 062N16 \\
\hline W-04-239 & 13-Jul-04 & Bonys Creek & 51.75054 & -100.08379 & 2 & 103 & 062N16 \\
\hline W-04-240 & 14-Jul-04 & Unnamed tributary to Lake Winnipegosis & 51.96124 & -100.14326 & 2 & 103 & 062N16 \\
\hline W-04-241 & 14-Jul-04 & Unnamed tributary to Garland River & 51.90586 & -100.24935 & 2 & 103 & 062N16 \\
\hline W-04-242 & 14-Jul-04 & Unnamed tributary to Garland River & 51.88354 & -100.24927 & 3 & 103 & 062N16 \\
\hline W-04-243 & 14-Jul-04 & Wellburns Creek & 51.91243 & -100.15361 & 4 & 103 & 062N16 \\
\hline W-04-244 & 14-Jul-04 & Big Stone Creek & 51.89907 & -100.14469 & 2 & 103 & 062N16 \\
\hline W-04-245 & 14-Jul-04 & Unnamed tributary to Lake Winnipegosis & 51.88085 & -100.13261 & 2 & 103 & 062N16 \\
\hline W-04-246 & 14-Jul-04 & Unnamed tributary to Lake Winnipegosis & 51.85594 & -100.13178 & 3 & 103 & 062N16 \\
\hline W-04-247 & 14-Jul-04 & Flemings Creek & 51.77592 & -100.08390 & 2 & 103 & 062N16 \\
\hline W-04-248 & 14-Jul-04 & Unnamed tributary to Garland River & 51.74044 & -100.31257 & 2 & 103 & 062N09 \\
\hline W-04-249 & 14-Jul-04 & South Garland Creek & 51.62410 & -100.54514 & 4 & 103 & 062N10 \\
\hline W-04-250 & 14-Jul-04 & Unnamed tributary to Garland River & 51.64938 & -100.50958 & 2 & 103 & 062N10 \\
\hline W-04-251 & 15-Jul-04 & Unnamed tributary to South Garland River & 51.63874 & -100.46281 & 2 & 103 & 062N09 \\
\hline W-04-252 & 15-Jul-04 & Garland River & 51.67132 & -100.51053 & 3 & 103 & 062N10 \\
\hline W-04-253 & 15-Jul-04 & North Garland Creek & 51.68706 & -100.56861 & 3 & 103 & 062N10 \\
\hline W-04-254 & 15-Jul-04 & West Favel River & 51.93667 & -101.00797 & 3 & 52 & 062N14 \\
\hline W-04-255 & 15-Jul-04 & Roaring River & 51.85678 & -100.94103 & 3 & 51 & 062N15 \\
\hline W-04-256 & 20-Jul-04 & Tourond Creek & 49.56170 & -97.09455 & 4 & 6 & $062 \mathrm{H} 11$ \\
\hline W-04-257 & 21-Jul-04 & Unnamed tributary to Broadvalley Drain & 50.99417 & -97.62620 & 2 & 33 & $062 I 13$ \\
\hline W-04-258 & 21-Jul-04 & Unnamed tributary to Broadvalley Drain & 51.02358 & -97.60283 & 3 & 33 & 062P04 \\
\hline W-04-259 & 21-Jul-04 & Plishka Drain Lateral & 51.03833 & -97.67302 & 3 & 33 & 062P04 \\
\hline
\end{tabular}


Appendix 1: Table listing the site number, date sampled, site name, latitude and longitude (in decimal degrees DD), DES stream order, DES map number and the corresponding NTS map number for all sites surveyed between 2002 and 2006.

\begin{tabular}{|c|c|c|c|c|c|c|c|}
\hline Site Number & Date & Site Name & $\begin{array}{l}\text { Latitude } \\
\text { (DD) }\end{array}$ & $\begin{array}{l}\text { Longitude } \\
\text { (DD) }\end{array}$ & $\begin{array}{l}\text { DES } \\
\text { Order }\end{array}$ & $\begin{array}{l}\text { DES } \\
\text { Map \# }\end{array}$ & $\begin{array}{l}\text { NTS } \\
\text { Map \# }\end{array}$ \\
\hline$W-04-260$ & 21-Jul-04 & Blind Creek & 51.10487 & -97.76675 & 3 & 33 & 062P04 \\
\hline W-04-261 & 21-Jul-04 & Leroy Drain & 51.17642 & -97.76695 & 2 & 33 & 062P04 \\
\hline W-04-262 & 22-Jul-04 & Goldeye Creek & 51.44112 & -97.37617 & 2 & 33 & 062P06 \\
\hline W-04-263 & 22-Jul-04 & Red Rose Drain & 51.42095 & -97.48582 & 2 & 33 & 062P06 \\
\hline W-04-264 & 22-Jul-04 & Marcynuiks Drain & 51.42102 & -97.53262 & 2 & 33 & 062P05 \\
\hline W-04-265 & 22-Jul-04 & Sunny Valley School Drain & 51.40645 & -97.49728 & 3 & 33 & 062P06 \\
\hline W-04-266 & 22-Jul-04 & Snake Creek & 51.37638 & -97.49468 & 2 & 33 & 062P06 \\
\hline W-04-267 & 22-Jul-04 & Eaglenest Creek & 51.35320 & -97.50470 & 2 & 33 & 062P05 \\
\hline W-04-268 & 22-Jul-04 & Wonder Lake Creek & 51.26485 & -97.59875 & 2 & 33 & 062P05 \\
\hline W-04-269 & 22-Jul-04 & Unnamed tributary to Fisher River & 51.25245 & -97.55322 & 2 & 33 & 062P05 \\
\hline W-04-270 & 22-Jul-04 & Bottle Creek & 51.21523 & -97.50572 & 3 & 33 & 062P04 \\
\hline W-04-271 & 23-Jul-04 & Meridian Drain & 51.18562 & -97.50282 & 3 & 33 & 062P04 \\
\hline W-04-272 & 23-Jul-04 & Hodgson Drain & 51.20043 & -97.58318 & 3 & 33 & 062P04 \\
\hline W-04-273 & 23-Jul-04 & Unnamed tributary to Fisher River & 51.21506 & -97.71415 & 3 & 33 & 062P04 \\
\hline W-04-274 & 23-Jul-04 & Fisher River & 51.18583 & -97.70113 & 4 & 33 & 062P04 \\
\hline W-04-275 & 3-Aug-04 & McTavish Drain & 49.42953 & -97.30061 & 3 & 18 & $062 \mathrm{H} 06$ \\
\hline W-04-276 & 3-Aug-04 & Unnamed tributary to Morris River & 49.45835 & -97.41402 & 2 & 18 & $062 \mathrm{H} 06$ \\
\hline W-04-277 & 3-Aug-04 & Unnamed tributary to Taylor Drain & 49.49517 & -97.30118 & 3 & 18 & 062H06 \\
\hline W-04-278 & 3-Aug-04 & Taylor Drain & 49.50252 & -97.32587 & 3 & 18 & $062 \mathrm{H} 11$ \\
\hline W-04-279 & 3-Aug-04 & Garber Drain & 49.54697 & -97.45207 & 2 & 18 & $062 \mathrm{H} 11$ \\
\hline$W-04-280$ & 3-Aug-04 & Strauss Drain Coulee & 49.56935 & -97.55005 & 3 & 18 & $062 \mathrm{H} 12$ \\
\hline W-04-281 & 3-Aug-04 & Boyne Channel & 49.53210 & -97.54975 & 2 & 18 & $062 \mathrm{H} 12$ \\
\hline W-04-282 & 3-Aug-04 & Unnamed tributary to Morris River & 49.51742 & -97.52717 & 2 & 18 & $062 \mathrm{H} 12$ \\
\hline W-04-283 & 3-Aug-04 & Forrester Drain & 49.50272 & -97.52712 & 3 & 18 & $062 \mathrm{H} 12$ \\
\hline W-04-284 & 3-Aug-04 & Lewis Drain & 49.49737 & -97.50447 & 2 & 18 & $062 \mathrm{H} 05$ \\
\hline
\end{tabular}


Appendix 1: Table listing the site number, date sampled, site name, latitude and longitude (in decimal degrees DD), DES stream order, DES map number and the corresponding NTS map number for all sites surveyed between 2002 and 2006.

\begin{tabular}{|c|c|c|c|c|c|c|c|}
\hline Site Number & Date & Site Name & $\begin{array}{l}\text { Latitude } \\
\text { (DD) }\end{array}$ & $\begin{array}{l}\text { Longitude } \\
\text { (DD) }\end{array}$ & $\begin{array}{l}\text { DES } \\
\text { Order }\end{array}$ & $\begin{array}{l}\text { DES } \\
\text { Map \# }\end{array}$ & $\begin{array}{l}\text { NTS } \\
\text { Map \# }\end{array}$ \\
\hline W-04-285 & 3-Aug-04 & Blackhurst Drain & 49.51675 & -97.50443 & 3 & 18 & $062 \mathrm{H} 12$ \\
\hline W-04-286 & 3-Aug-04 & Morris River & 49.53958 & -97.52727 & 6 & 18 & $062 \mathrm{H} 12$ \\
\hline W-04-287 & 3-Aug-04 & Norquay Channel & 49.57640 & -97.66385 & 5 & 18 & $062 \mathrm{H} 12$ \\
\hline W-04-288 & 4-Aug-04 & Carrothers Drain & 49.57590 & -97.82343 & 2 & 18 & $062 \mathrm{H} 12$ \\
\hline W-04-289 & 4-Aug-04 & Norquay Channel & 49.54562 & -97.84787 & 5 & 18 & $062 \mathrm{H} 12$ \\
\hline W-04-290 & 4-Aug-04 & Unnamed tributary to Pembina River & 49.18080 & -98.46992 & 2 & 60 & $062 \mathrm{G} 01$ \\
\hline W-04-291 & 4-Aug-04 & Unnamed tributary to Pembina River & 49.14356 & -98.46983 & 2 & 60 & $062 \mathrm{G} 01$ \\
\hline W-04-292 & 4-Aug-04 & Mowbray Drain & 49.00107 & -98.48560 & 3 & 60 & $062 \mathrm{G} 01$ \\
\hline W-04-293 & 4-Aug-04 & Unnamed tributary to Snowflake Creek & 49.04457 & -98.62712 & 2 & 60 & 062G02 \\
\hline W-04-294 & 4-Aug-04 & Snowflake Creek & 49.01113 & -98.62690 & 2 & 60 & 062G02 \\
\hline W-04-295 & 4-Aug-04 & Stony Creek & 49.08518 & -98.67189 & 2 & 60 & 062G02 \\
\hline W-04-296 & 4-Aug-04 & Unnamed tributary to Pembina River & 49.22453 & -98.67103 & 2 & 60 & 062G02 \\
\hline W-04-297 & 5-Aug-04 & Pembina River & 49.05790 & -98.46980 & 6 & 60 & $062 G 01$ \\
\hline W-04-298 & 5-Aug-04 & Unnamed tributary to Mowbray Creek & 49.00038 & -98.45417 & 3 & 60 & $062 G 01$ \\
\hline W-04-299 & 5-Aug-04 & Snowflake Creek & 49.02108 & -98.60357 & 5 & 60 & 062G02 \\
\hline W-04-300 & 5-Aug-04 & Pembina River & 49.07525 & -98.58982 & 6 & 60 & 062G02 \\
\hline W-04-301 & 5-Aug-04 & Pembina River & 49.14823 & -98.56415 & 6 & 60 & 062G02 \\
\hline$X-04-001$ & 5-May-04 & Little Morris River & 49.45032 & -97.50435 & 5 & 17 & 062H05 \\
\hline X-04-002 & 5-May-04 & Tobacco Creek & 49.39894 & -97.71056 & 5 & 17 & $062 \mathrm{H} 05$ \\
\hline$X-04-003$ & 5-May-04 & Tobacco Creek & 49.39886 & -97.77350 & 5 & 17 & $062 \mathrm{H} 05$ \\
\hline X-04-004 & 5-May-04 & Graham Creek & 49.38186 & -97.82094 & 4 & 17 & $062 \mathrm{H} 05$ \\
\hline X-04-005 & 5-May-04 & Tobacco Creek & 49.39922 & -97.82094 & 5 & 17 & 062H05 \\
\hline X-04-006 & 6-May-04 & Little Morris River & 49.45364 & -97.45906 & 5 & 17 & $062 \mathrm{H} 06$ \\
\hline$X-04-007$ & 6-May-04 & Unnamed tributary to Little Morris River & 49.45844 & -97.50444 & 2 & 17 & $062 \mathrm{H} 05$ \\
\hline$X-04-008$ & 6-May-04 & Brown Drain & 49.45814 & -97.50453 & 2 & 17 & $062 \mathrm{H} 05$ \\
\hline
\end{tabular}


Appendix 1: Table listing the site number, date sampled, site name, latitude and longitude (in decimal degrees DD), DES stream order, DES map number and the corresponding NTS map number for all sites surveyed between 2002 and 2006.

\begin{tabular}{|c|c|c|c|c|c|c|c|}
\hline Site Number & Date & Site Name & $\begin{array}{l}\text { Latitude } \\
\text { (DD) }\end{array}$ & $\begin{array}{l}\text { Longitude } \\
\text { (DD) }\end{array}$ & $\begin{array}{l}\text { DES } \\
\text { Order }\end{array}$ & $\begin{array}{l}\text { DES } \\
\text { Map \# }\end{array}$ & $\begin{array}{l}\text { NTS } \\
\text { Map \# }\end{array}$ \\
\hline$X-04-009$ & 6-May-04 & Burnett Drain & 49.46853 & -97.50444 & 2 & 17 & 062 H05 \\
\hline$X-04-010$ & 6-May-04 & 4N Drain & 49.43897 & -97.52722 & 3 & 17 & $062 \mathrm{H} 05$ \\
\hline$X-04-011$ & 6-May-04 & 111 Drain & 49.42897 & -97.54989 & 2 & 17 & $062 \mathrm{H} 05$ \\
\hline X-04-012 & 6-May-04 & 110 Drain & 49.41386 & -97.59494 & 2 & 17 & $062 \mathrm{H} 05$ \\
\hline$X-04-013$ & 6-May-04 & Graham Creek & 49.37350 & -98.00145 & 4 & 17 & $062 \mathrm{G} 08$ \\
\hline$X-04-014$ & 6-May-04 & Tobacco Creek & 49.40336 & -98.00158 & 4 & 17 & 062G08 \\
\hline$X-04-015$ & 6-May-04 & Graham Creek & 49.36611 & -98.02442 & 4 & 17 & $062 \mathrm{G} 08$ \\
\hline$X-04-016$ & 6-May-04 & Graham Creek & 49.36856 & -98.09386 & 3 & 17 & 062G08 \\
\hline$X-04-017$ & 6-May-04 & Graham Creek & 49.36536 & -98.09881 & 3 & 17 & 062G08 \\
\hline$X-04-018$ & 6-May-04 & Tobacco Creek & 49.42383 & -98.11492 & 4 & 17 & 062G08 \\
\hline$X-04-019$ & 6-May-04 & Tobacco Creek & 49.42714 & -98.15117 & 3 & 17 & 062G08 \\
\hline$X-04-020$ & 6-May-04 & Tobacco Creek & 49.43594 & -98.20519 & 3 & 17 & 062G08 \\
\hline$X-04-021$ & 6-May-04 & South Tobacco Creek & 49.41381 & -98.21817 & 3 & 17 & 062G08 \\
\hline$X-04-022$ & 6-May-04 & 4N Drain & 49.44417 & -97.77589 & 3 & 17 & $062 \mathrm{H} 05$ \\
\hline$X-04-023$ & 10-May-04 & Morris River & 49.59131 & -97.58239 & 5 & 19 & $062 \mathrm{H} 12$ \\
\hline$X-04-024$ & 10-May-04 & Unnamed tributary to Morris River & 49.59092 & -97.59581 & 2 & 19 & $062 \mathrm{H} 12$ \\
\hline$X-04-025$ & 10-May-04 & Unnamed tributary to Morris River & 49.60620 & -97.58985 & 2 & 19 & $062 \mathrm{H} 12$ \\
\hline$X-04-026$ & 10-May-04 & 11 - A Drain & 49.62086 & -97.59758 & 4 & 19 & $062 \mathrm{H} 12$ \\
\hline$X-04-027$ & 10-May-04 & Unnamed tributary to Morris River & 49.65047 & -97.59586 & 2 & 19 & $062 \mathrm{H} 12$ \\
\hline$X-04-028$ & 10-May-04 & Allison Drain & 49.64139 & -97.61842 & 3 & 19 & $062 \mathrm{H} 12$ \\
\hline$X-04-029$ & 10-May-04 & Castor Drain & 49.63569 & -97.61836 & 2 & 19 & $062 \mathrm{H} 12$ \\
\hline $\mathrm{X}-04-030$ & 10-May-04 & 11 - A Drain & 49.62078 & -97.61836 & 4 & 19 & $062 \mathrm{H} 12$ \\
\hline $\mathrm{X}-04-031$ & 10-May-04 & 11 - A Drain & 49.62067 & -97.64125 & 4 & 19 & $062 \mathrm{H} 12$ \\
\hline $\mathrm{X}-04-032$ & 10-May-04 & 11- A Drain & 49.62089 & -97.70917 & 4 & 19 & $062 \mathrm{H} 12$ \\
\hline X-04-033 & 10-May-04 & 11 - A Drain & 49.63542 & -97.80003 & 4 & 19 & $062 \mathrm{H} 12$ \\
\hline
\end{tabular}


Appendix 1: Table listing the site number, date sampled, site name, latitude and longitude (in decimal degrees DD), DES stream order, DES map number and the corresponding NTS map number for all sites surveyed between 2002 and 2006.

\begin{tabular}{|c|c|c|c|c|c|c|c|}
\hline Site Number & Date & Site Name & $\begin{array}{l}\text { Latitude } \\
\text { (DD) }\end{array}$ & $\begin{array}{l}\text { Longitude } \\
\text { (DD) }\end{array}$ & $\begin{array}{l}\text { DES } \\
\text { Order }\end{array}$ & $\begin{array}{l}\text { DES } \\
\text { Map \# }\end{array}$ & $\begin{array}{l}\text { NTS } \\
\text { Map \# }\end{array}$ \\
\hline$X-04-034$ & 10-May-04 & 11 - A South Lateral Drain & 49.63567 & -97.86858 & 3 & 19 & $062 \mathrm{H} 12$ \\
\hline $\mathrm{X}-04-035$ & 10-May-04 & 11 - A South Lateral Drain & 49.63578 & -97.93731 & 3 & 19 & $062 \mathrm{H} 12$ \\
\hline $\mathrm{X}-04-036$ & 14-May-04 & Northumberland Drain & 50.00731 & -97.05306 & 2 & 28 & 062103 \\
\hline$X-04-037$ & 14-May-04 & Parks Creek & 50.03503 & -97.03986 & 4 & 28 & 062103 \\
\hline$X-04-038$ & 14-May-04 & Mirey Creek & 50.08450 & -96.96453 & 2 & 28 & 062102 \\
\hline$X-04-039$ & 14-May-04 & Unnamed tributary to Red River & 50.11658 & -96.92872 & 2 & 28 & 062102 \\
\hline$X-04-040$ & 14-May-04 & Unnamed tributary to Parks Creek & 50.04736 & -97.06944 & 3 & 28 & 062103 \\
\hline$X-04-041$ & 14-May-04 & Parks Creek & 50.04756 & -97.06756 & 4 & 28 & 062103 \\
\hline$X-04-042$ & 14-May-04 & Dewar Drain & 50.17406 & -97.07156 & 3 & 29 & 062103 \\
\hline$x-04-043$ & 17-May-04 & Big Grass River & 50.50606 & -98.97181 & 5 & 43 & 062J10 \\
\hline$X-04-044$ & 17-May-04 & Alonsa Drain & 50.59472 & -98.89433 & 3 & 43 & 062J10 \\
\hline$X-04-045$ & 17-May-04 & Alonsa Drain & 50.62400 & -98.90339 & 3 & 43 & 062J10 \\
\hline$X-04-046$ & 17-May-04 & Alonsa Drain & 50.56492 & -98.89311 & 3 & 43 & 062J10 \\
\hline$X-04-047$ & 17-May-04 & Jumping Deer Creek & 50.44128 & -99.00803 & 3 & 43 & 062J06 \\
\hline$X-04-048$ & 18-May-04 & Snake Creek & 50.28458 & -99.30431 & 5 & 39 & 062J06 \\
\hline$X-04-049$ & 18-May-04 & Mink Creek & 50.24058 & -99.34892 & 2 & 39 & 062J03 \\
\hline$X-04-050$ & 18-May-04 & Dead Lake Drain & 50.18733 & -98.95072 & 2 & 39 & 062J02 \\
\hline$X-04-051$ & 18-May-04 & Golden Stream & 50.13725 & -98.96419 & 3 & 39 & 062J02 \\
\hline X-04-052 & 19-May-04 & Westbourne Drain & 50.11925 & -98.56033 & 5 & 36 & 062J02 \\
\hline$X-04-053$ & 20-May-04 & Westbourne Drain & 50.13786 & -98.56058 & 5 & 36 & 062J02 \\
\hline X-04-054 & 20-May-04 & Westbourne Drain & 50.09317 & -98.56056 & 5 & 36 & 062J02 \\
\hline X-04-055 & 20-May-04 & Westbourne Drain & 50.06326 & -98.56049 & 5 & 36 & 062J02 \\
\hline X-04-056 & 20-May-04 & Beaver Creek & 50.06347 & -98.70692 & 4 & 36 & 062J02 \\
\hline$X-04-057$ & 20-May-04 & Beaver Creek & 50.01939 & -98.72211 & 4 & 36 & 062J02 \\
\hline X-04-058 & 20-May-04 & Image Creek & 50.02244 & -98.65203 & 3 & 36 & 062J02 \\
\hline
\end{tabular}


Appendix 1: Table listing the site number, date sampled, site name, latitude and longitude (in decimal degrees DD), DES stream order, DES map number and the corresponding NTS map number for all sites surveyed between 2002 and 2006.

\begin{tabular}{|c|c|c|c|c|c|c|c|}
\hline Site Number & Date & Site Name & $\begin{array}{l}\text { Latitude } \\
\text { (DD) }\end{array}$ & $\begin{array}{l}\text { Longitude } \\
\text { (DD) }\end{array}$ & $\begin{array}{l}\text { DES } \\
\text { Order }\end{array}$ & $\begin{array}{l}\text { DES } \\
\text { Map \# }\end{array}$ & $\begin{array}{l}\text { NTS } \\
\text { Map \# }\end{array}$ \\
\hline$X-04-059$ & 20-May-04 & Image Creek & 49.98622 & -98.72103 & 3 & 36 & $062 G 15$ \\
\hline X-04-060 & 20-May-04 & Beaver Creek & 49.97658 & -98.76653 & 4 & 36 & 062G15 \\
\hline$X-04-061$ & 20-May-04 & Anderson Drain & 49.94575 & -98.83131 & 3 & 36 & 062G15 \\
\hline$X-04-062$ & 20-May-04 & Beaver Creek & 49.94567 & -98.87367 & 3 & 36 & $062 \mathrm{G} 15$ \\
\hline$X-04-063$ & 20-May-04 & Westbourne Drain & 50.02625 & -98.56044 & 4 & 36 & 062J02 \\
\hline $\mathrm{X}-04-064$ & 20-May-04 & Westbourne Drain & 50.03408 & -98.56019 & 4 & 36 & 062J02 \\
\hline $\mathrm{X}-04-065$ & 21-May-04 & Long Lake Drain & 50.01478 & -97.76000 & 4 & 25 & 062104 \\
\hline$X-04-066$ & 21-May-04 & Long Lake Drain & 50.04869 & -97.82614 & 4 & 25 & 062104 \\
\hline$X-04-067$ & 25-May-04 & La Salle River & 49.88706 & -97.76722 & 4 & 24 & $062 \mathrm{H} 13$ \\
\hline$X-04-068$ & 25-May-04 & Elm Creek Channel & 49.81264 & -97.78239 & 5 & 21 & $062 \mathrm{H} 13$ \\
\hline$X-04-069$ & 25-May-04 & Elm Creek Channel & 49.68917 & -98.00536 & 3 & 21 & $062 G 09$ \\
\hline X-04-070 & 25-May-04 & Elm Creek Channel & 49.78339 & -98.00567 & 3 & 21 & 062G16 \\
\hline$X-04-071$ & 25-May-04 & Boundary Drain & 49.79819 & -98.00639 & 3 & 21 & $062 \mathrm{G} 16$ \\
\hline$X-04-072$ & 25-May-04 & Crooked Lake Channel & 49.81292 & -98.00272 & 4 & 21 & 062G16 \\
\hline$X-04-073$ & 25-May-04 & Crooked Lake Channel & 49.87194 & -98.14200 & 3 & 21 & 062G16 \\
\hline X-04-074 & 26-May-04 & Long Lake Drain & 50.07411 & -98.01025 & 4 & 25 & 062J01 \\
\hline$X-04-075$ & 26-May-04 & Scotts Drain & 50.01706 & -97.74778 & 3 & 25 & 062104 \\
\hline$X-04-076$ & 26-May-04 & La Salle River & 49.97508 & -97.80231 & 4 & 24 & $062 \mathrm{H} 13$ \\
\hline$X-04-077$ & 26-May-04 & La Salle River & 49.97467 & -97.81311 & 4 & 24 & $062 \mathrm{H} 13$ \\
\hline X-04-078 & 26-May-04 & Elm River Channel & 49.88642 & -97.77781 & 4 & 24 & $062 \mathrm{H} 13$ \\
\hline X-04-079 & 26-May-04 & Meakin Creek & 49.76042 & -97.66406 & 4 & 23 & $062 \mathrm{H} 13$ \\
\hline$X-04-080$ & 26-May-04 & La Salle River & 49.72256 & -97.17261 & 5 & 22 & $062 \mathrm{H} 11$ \\
\hline X-04-081 & 27-May-04 & Cooks Creek Diversion & 49.89472 & -96.93014 & 2 & 12 & $062 \mathrm{H} 15$ \\
\hline$X-04-082$ & 27-May-04 & Springfield Road Drain & 49.94528 & -96.96864 & 3 & 12 & $062 \mathrm{H} 15$ \\
\hline$X-04-083$ & 27-May-04 & Gunn Creek & 50.08339 & -96.93258 & 3 & 12 & 062102 \\
\hline
\end{tabular}


Appendix 1: Table listing the site number, date sampled, site name, latitude and longitude (in decimal degrees DD), DES stream order, DES map number and the corresponding NTS map number for all sites surveyed between 2002 and 2006.

\begin{tabular}{|c|c|c|c|c|c|c|c|}
\hline Site Number & Date & Site Name & $\begin{array}{l}\text { Latitude } \\
\text { (DD) }\end{array}$ & $\begin{array}{l}\text { Longitude } \\
\text { (DD) }\end{array}$ & $\begin{array}{l}\text { DES } \\
\text { Order }\end{array}$ & $\begin{array}{l}\text { DES } \\
\text { Map \# }\end{array}$ & $\begin{array}{l}\text { NTS } \\
\text { Map \# }\end{array}$ \\
\hline$X-04-084$ & 27-May-04 & Bunns Creek & 49.91573 & -97.00213 & 3 & 12 & $062 \mathrm{H} 14$ \\
\hline$X-04-085$ & 27-May-04 & Seine River Diversion & 49.65675 & -96.63917 & 4 & 8 & $062 \mathrm{H} 10$ \\
\hline$X-04-086$ & 27-May-04 & Giroux Drain & 49.57672 & -96.57036 & 3 & 8 & $062 \mathrm{H} 10$ \\
\hline$X-04-087$ & 27-May-04 & Seine River & 49.53228 & -96.50736 & 4 & 8 & $062 \mathrm{H} 10$ \\
\hline$X-04-088$ & 27-May-04 & La Broquerie Drain & 49.50708 & -96.48808 & 3 & 8 & 062H09 \\
\hline X-04-089 & 27-May-04 & Marchand East Drain & 49.44394 & -96.40942 & 3 & 8 & 062H08 \\
\hline X-04-090 & 27-May-04 & Unnamed tributary to Simard Creek & 49.44389 & -96.38883 & 2 & 8 & 062H08 \\
\hline X-04-091 & 28-May-04 & Johnson Drain & 49.62758 & -96.64861 & 3 & 8 & $062 \mathrm{H} 10$ \\
\hline$X-04-092$ & 28-May-04 & Youville Drain & 49.66469 & -96.85431 & 3 & 8 & $062 \mathrm{H} 10$ \\
\hline X-04-093 & 28-May-04 & Tourond Creek & 49.62075 & -97.10789 & 5 & 6 & $062 \mathrm{H} 11$ \\
\hline$X-04-094$ & 28-May-04 & Saint Adolphe Coulee & 49.67975 & -97.09328 & 4 & 6 & $062 \mathrm{H} 11$ \\
\hline$X-04-095$ & 1-Jun-04 & Unnamed tributary to Red River & 49.76892 & -97.15833 & 2 & 22 & $062 \mathrm{H} 14$ \\
\hline $\mathrm{X}-04-096$ & 1-Jun-04 & Unnamed tributary to Red River & 49.71783 & -97.13617 & 2 & 22 & $062 \mathrm{H} 11$ \\
\hline$X-04-097$ & 1-Jun-04 & Unnamed tributary to Red River & 49.70447 & -97.12658 & 2 & 22 & $062 \mathrm{H} 11$ \\
\hline X-04-098 & 1-Jun-04 & Glenlea Drain & 49.61456 & -97.14086 & 4 & 22 & $062 \mathrm{H} 11$ \\
\hline X-04-099 & 1-Jun-04 & Schwartz Drain & 49.60567 & -97.14547 & 3 & 22 & $062 \mathrm{H} 11$ \\
\hline$X-04-100$ & 1-Jun-04 & La Pointe Coulee & 49.54236 & -97.20825 & 3 & 22 & $062 \mathrm{H} 11$ \\
\hline $\mathrm{X}-04-101$ & 1-Jun-04 & Unnamed tributary to Domain Drain & 49.59181 & -97.28739 & 2 & 22 & $062 \mathrm{H} 11$ \\
\hline $\mathrm{X}-04-102$ & 2-Jun-04 & Dubas Drain & 50.14286 & -96.79961 & 3 & 11 & 062102 \\
\hline $\mathrm{X}-04-103$ & 2-Jun-04 & Township Line Drain & 50.23953 & -96.73492 & 3 & 13 & 062102 \\
\hline$X-04-104$ & 2-Jun-04 & Indian Reserve Drain & 50.32856 & -96.65422 & 3 & 13 & 062107 \\
\hline$X-04-105$ & 2-Jun-04 & Libau Drain & 50.26781 & -96.67414 & 3 & 13 & 062107 \\
\hline$X-04-106$ & 3-Jun-04 & Pembina River & 49.26358 & -99.46428 & 5 & 57 & 062G06 \\
\hline $\mathrm{X}-04-107$ & 3-Jun-04 & Unnamed tributary to Pembina River & 49.22406 & -99.42733 & 3 & 57 & 062G03 \\
\hline$X-04-108$ & 3-Jun-04 & Unnamed tributary to Pembina River & 49.20769 & -99.46106 & 3 & 57 & 062G03 \\
\hline
\end{tabular}


Appendix 1: Table listing the site number, date sampled, site name, latitude and longitude (in decimal degrees DD), DES stream order, DES map number and the corresponding NTS map number for all sites surveyed between 2002 and 2006.

\begin{tabular}{|c|c|c|c|c|c|c|c|}
\hline Site Number & Date & Site Name & $\begin{array}{l}\text { Latitude } \\
\text { (DD) }\end{array}$ & $\begin{array}{l}\text { Longitude } \\
\text { (DD) }\end{array}$ & $\begin{array}{l}\text { DES } \\
\text { Order }\end{array}$ & $\begin{array}{l}\text { DES } \\
\text { Map \# }\end{array}$ & $\begin{array}{l}\text { NTS } \\
\text { Map \# }\end{array}$ \\
\hline$X-04-109$ & 3-Jun-04 & Unnamed tributary to Pembina River & 49.21747 & -99.47258 & 2 & 57 & $062 G 03$ \\
\hline$X-04-110$ & 4-Jun-04 & Little Pembina River & 49.15047 & -99.86256 & 4 & 57 & $062 G 04$ \\
\hline$X-04-111$ & 4-Jun-04 & Long River & 49.13689 & -99.57133 & 5 & 58 & $062 G 04$ \\
\hline$X-04-112$ & 4-Jun-04 & Stony Creek & 49.13239 & -99.55444 & 3 & 58 & 062G04 \\
\hline$X-04-113$ & 7-Jun-04 & Unnamed tributary to Badger Creek & 49.08900 & -99.24619 & 3 & 58 & $062 \mathrm{G} 03$ \\
\hline$X-04-114$ & 7-Jun-04 & Badger Creek & 49.07358 & -99.30419 & 5 & 58 & $062 \mathrm{G} 03$ \\
\hline$X-04-115$ & 7-Jun-04 & Jackfish Creek & 49.14172 & -99.66033 & 4 & 58 & $062 \mathrm{G} 04$ \\
\hline$X-04-116$ & 8-Jun-04 & Stony Creek & 49.07308 & -99.68064 & 3 & 58 & $062 \mathrm{G} 04$ \\
\hline$X-04-117$ & 8-Jun-04 & Jackfish Creek & 49.07306 & -99.80794 & 3 & 58 & $062 G 04$ \\
\hline$X-04-118$ & 8-Jun-04 & Long River & 49.05836 & -99.86647 & 3 & 58 & $062 G 04$ \\
\hline$X-04-119$ & 8-Jun-04 & Jackfish Creek & 49.13208 & -99.69117 & 4 & 58 & $062 G 04$ \\
\hline$X-04-120$ & 8-Jun-04 & Gimby Creek & 49.06247 & -99.59261 & 3 & 58 & 062G04 \\
\hline$X-04-121$ & 8-Jun-04 & Gimby Creek & 49.07700 & -99.34664 & 4 & 58 & $062 G 03$ \\
\hline$X-04-122$ & 9-Jun-04 & Unnamed tributary to Badger Creek & 49.04867 & -99.32339 & 4 & 58 & 062G03 \\
\hline$X-04-123$ & 9-Jun-04 & Unnamed tributary to Badger Creek & 49.04389 & -99.33583 & 2 & 58 & 062G03 \\
\hline$X-04-124$ & 9-Jun-04 & Unnamed tributary to Badger Creek & 49.02922 & -99.29225 & 4 & 58 & $062 \mathrm{G} 03$ \\
\hline$X-04-125$ & 10-Jun-04 & Barickman Coulee & 49.94039 & -97.62208 & 4 & 23 & $062 \mathrm{H} 13$ \\
\hline$X-04-126$ & 10-Jun-04 & West Branch La Salle River & 49.93075 & -97.77514 & 3 & 24 & $062 \mathrm{H} 13$ \\
\hline$X-04-127$ & 10-Jun-04 & No. 2 Drain & 49.86100 & -97.49853 & 3 & 23 & $062 \mathrm{H} 14$ \\
\hline$X-04-128$ & 14-Jun-04 & Elm River & 49.91233 & -98.00994 & 3 & 24 & $062 \mathrm{G} 16$ \\
\hline$X-04-129$ & 14-Jun-04 & Overhill Drain & 49.93106 & -98.30517 & 3 & 100 & $062 \mathrm{G} 16$ \\
\hline$X-04-130$ & 14-Jun-04 & Unnamed tributary to Assiniboine River & 49.83942 & -98.43797 & 2 & 100 & $062 \mathrm{G} 16$ \\
\hline$X-04-131$ & 14-Jun-04 & Rossendale Drain & 49.81261 & -98.57419 & 3 & 100 & $062 \mathrm{G} 15$ \\
\hline $\mathrm{X}-04-132$ & 14-Jun-04 & Edwin Drain & 49.93053 & -98.37683 & 3 & 100 & $062 \mathrm{G} 16$ \\
\hline$X-04-133$ & 15-Jun-04 & Deep Creek & 50.03417 & -98.44661 & 3 & 35 & 062J01 \\
\hline
\end{tabular}


Appendix 1: Table listing the site number, date sampled, site name, latitude and longitude (in decimal degrees DD), DES stream order, DES map number and the corresponding NTS map number for all sites surveyed between 2002 and 2006.

\begin{tabular}{|c|c|c|c|c|c|c|c|}
\hline Site Number & Date & Site Name & $\begin{array}{l}\text { Latitude } \\
\text { (DD) }\end{array}$ & $\begin{array}{l}\text { Longitude } \\
\text { (DD) }\end{array}$ & $\begin{array}{l}\text { DES } \\
\text { Order }\end{array}$ & $\begin{array}{l}\text { DES } \\
\text { Map \# }\end{array}$ & $\begin{array}{l}\text { NTS } \\
\text { Map \# }\end{array}$ \\
\hline$X-04-134$ & 15-Jun-04 & Mount Pleasant Drain & 50.01919 & -98.42281 & 3 & 35 & 062J01 \\
\hline$X-04-135$ & 15-Jun-04 & Perch Creek & 50.14461 & -98.55167 & 3 & 35 & 062J02 \\
\hline$X-04-136$ & 15-Jun-04 & New Beaudin Drain & 50.12294 & -98.64964 & 3 & 37 & 062J02 \\
\hline$X-04-137$ & 16-Jun-04 & Pilot Creek & 49.20850 & -98.95067 & 4 & 55 & 062G02 \\
\hline$X-04-138$ & 16-Jun-04 & Pilot Creek & 49.23683 & -98.97469 & 4 & 55 & $062 \mathrm{G} 02$ \\
\hline$X-04-139$ & 16-Jun-04 & Pilot Creek & 49.22167 & -98.90458 & 4 & 55 & $062 \mathrm{G} 02$ \\
\hline$X-04-140$ & 16-Jun-04 & Unnamed tributary to Pembina River & 49.26594 & -98.95253 & 2 & 55 & $062 \mathrm{G} 07$ \\
\hline$X-04-141$ & 17-Jun-04 & McCoys Creek & 49.25494 & -98.79406 & 3 & 54 & $062 \mathrm{G} 07$ \\
\hline$X-04-142$ & 17-Jun-04 & Unnamed tributary to Pembina River & 49.36306 & -98.82119 & 4 & 55 & $062 \mathrm{G} 07$ \\
\hline$X-04-143$ & 17-Jun-04 & Unnamed tributary to Pembina River & 49.39936 & -98.83256 & 3 & 55 & 062G07 \\
\hline$X-04-144$ & 17-Jun-04 & Dry River & 49.33275 & -99.02042 & 3 & 55 & 062G06 \\
\hline$X-04-145$ & 17-Jun-04 & Marrinohurst Creek & 49.24619 & -99.04333 & 2 & 55 & 062G03 \\
\hline$X-04-146$ & 17-Jun-04 & Syndicate Creek & 49.30536 & -98.72886 & 3 & 54 & 062G07 \\
\hline$X-04-147$ & 18-Jun-04 & Unnamed tributary to Pembina River & 49.01547 & -98.13769 & 2 & 60 & $062 G 01$ \\
\hline$X-04-148$ & 18-Jun-04 & Kronsgart Drain & 49.29700 & -97.39139 & 3 & 16 & $062 \mathrm{H} 06$ \\
\hline$X-04-149$ & 21-Jun-04 & Pine Creek & 50.06244 & -98.94994 & 4 & 38 & 062J02 \\
\hline$X-04-150$ & 21-Jun-04 & Gillespie Drain & 50.07822 & -98.95019 & 4 & 38 & 062J02 \\
\hline$X-04-151$ & 21-Jun-04 & Pine Creek & 49.90219 & -99.21439 & 3 & 38 & 062G14 \\
\hline$X-04-152$ & 22-Jun-04 & Pine Creek & 49.95758 & -99.16553 & 3 & 38 & $062 \mathrm{G} 14$ \\
\hline$X-04-153$ & 22-Jun-04 & Pine Creek & 49.88683 & -99.32294 & 1 & 38 & 062G14 \\
\hline$X-04-154$ & 22-Jun-04 & Epinette Creek & 49.74139 & -99.32517 & 2 & 99 & $062 \mathrm{G} 11$ \\
\hline$X-04-155$ & 23-Jun-04 & Boundary Creek & 50.50869 & -96.97562 & 4 & $31 \mathrm{E}$ & 062110 \\
\hline$X-04-156$ & 23-Jun-04 & Boundary Creek Drain & 50.52142 & -97.00469 & 4 & $31 \mathrm{E}$ & $062 \mid 11$ \\
\hline$X-04-157$ & 23-Jun-04 & Bass Drain & 50.57783 & -97.11428 & 4 & $31 \mathrm{E}$ & 062111 \\
\hline$X-04-158$ & 23-Jun-04 & Willow Creek & 50.59575 & -97.11625 & 3 & $31 \mathrm{E}$ & $062 \mid 11$ \\
\hline
\end{tabular}


Appendix 1: Table listing the site number, date sampled, site name, latitude and longitude (in decimal degrees DD), DES stream order, DES map number and the corresponding NTS map number for all sites surveyed between 2002 and 2006.

\begin{tabular}{|c|c|c|c|c|c|c|c|}
\hline Site Number & Date & Site Name & $\begin{array}{l}\text { Latitude } \\
\text { (DD) }\end{array}$ & $\begin{array}{l}\text { Longitude } \\
\text { (DD) }\end{array}$ & $\begin{array}{l}\text { DES } \\
\text { Order }\end{array}$ & $\begin{array}{l}\text { DES } \\
\text { Map \# }\end{array}$ & $\begin{array}{l}\text { NTS } \\
\text { Map \# }\end{array}$ \\
\hline$X-04-159$ & 23-Jun-04 & Bass Drain & 50.58100 & -97.13733 & 3 & $31 \mathrm{E}$ & $062 \mid 11$ \\
\hline$X-04-160$ & 24-Jun-04 & Meleb Drain & 50.68433 & -97.02317 & 3 & $31 \mathrm{E}$ & 062111 \\
\hline$X-04-161$ & 24-Jun-04 & Fish Lake Drain & 50.70653 & -97.04169 & 3 & $31 \mathrm{E}$ & $062 \mid 11$ \\
\hline$X-04-162$ & 24-Jun-04 & Fish Lake Drain & 50.69717 & -96.99592 & 3 & $31 \mathrm{E}$ & 062110 \\
\hline$X-04-163$ & 25-Jun-04 & Bass Drain & 50.58083 & -97.21508 & 3 & $31 \mathrm{E}$ & $062 \mid 11$ \\
\hline$X-04-164$ & 25-Jun-04 & Drunken River & 50.81339 & -96.96725 & 4 & $31 \mathrm{E}$ & $062 \mid 15$ \\
\hline$X-04-165$ & 28-Jun-04 & Unnamed tributary to Lake Winnipeg & 50.88875 & -96.99542 & 2 & 32 & 062115 \\
\hline$X-04-166$ & 28-Jun-04 & Bluegoose Drain & 50.90211 & -97.13447 & 3 & 32 & $062 \mid 14$ \\
\hline$X-04-167$ & 28-Jun-04 & Crooked Lake Drain & 50.95283 & -97.08567 & 4 & 32 & 062114 \\
\hline$X-04-168$ & 28-Jun-04 & Unnamed tributary to Lake Winnipeg & 51.02325 & -96.99119 & 3 & 32 & 062P02 \\
\hline$X-04-169$ & 28-Jun-04 & Unnamed tributary to Lake Winnipeg & 50.75622 & -96.99478 & 2 & $31 \mathrm{E}$ & 062115 \\
\hline$X-04-170$ & 29-Jun-04 & Meleb Drain & 50.68431 & -97.17578 & 3 & $31 \mathrm{E}$ & $062 \mid 11$ \\
\hline $\mathrm{X}-04-171$ & 29-Jun-04 & Fish Lake Drain & 50.74647 & -97.31864 & 3 & $31 \mathrm{E}$ & 062111 \\
\hline$X-04-172$ & 29-Jun-04 & Rembrandt Drain & 50.84669 & -97.29764 & 3 & 32 & $062 \mid 14$ \\
\hline $\mathrm{X}-04-173$ & 29-Jun-04 & Vidir Road Drain & 50.96450 & -97.30358 & 3 & 32 & 062114 \\
\hline$X-04-174$ & 29-Jun-04 & Bifrost Drain & 50.99419 & -97.34522 & 2 & 32 & $062 \mid 14$ \\
\hline $\mathrm{X}-04-175$ & 29-Jun-04 & Sylvan Drain & 50.99386 & -97.41519 & 3 & 32 & 062114 \\
\hline$X-04-176$ & 30-Jun-04 & Icelandic River & 50.97767 & -97.46297 & 4 & 32 & 062114 \\
\hline $\mathrm{X}-04-177$ & 30-Jun-04 & Unnamed tributary to Icelandic River & 50.86640 & -97.60041 & 3 & 32 & 062113 \\
\hline $\mathrm{X}-04-178$ & 1-Jul-04 & Long Lake Drain & 50.24069 & -96.99408 & 3 & 29 & 062102 \\
\hline $\mathrm{X}-04-179$ & 1-Jul-04 & Medicine Creek & 50.24050 & -96.95394 & 2 & 29 & 062102 \\
\hline $\mathrm{X}-04-180$ & 2-Jul-04 & Unnamed tributary to Lake Winnipeg & 51.22981 & -97.15153 & 2 & 106 & 062P03 \\
\hline $\mathrm{X}-04-181$ & 2-Jul-04 & Hodgson Road Drain & 51.22969 & -97.29436 & 3 & 106 & 062P03 \\
\hline $\mathrm{X}-04-182$ & 5-Jul-04 & Crockatt Drain & 50.44806 & -97.66558 & 3 & 108 & 062105 \\
\hline$X-04-183$ & 5-Jul-04 & Crockatt Drain & 50.44061 & -97.64797 & 3 & 108 & 062105 \\
\hline
\end{tabular}


Appendix 1: Table listing the site number, date sampled, site name, latitude and longitude (in decimal degrees DD), DES stream order, DES map number and the corresponding NTS map number for all sites surveyed between 2002 and 2006.

\begin{tabular}{|c|c|c|c|c|c|c|c|}
\hline Site Number & Date & Site Name & $\begin{array}{l}\text { Latitude } \\
\text { (DD) }\end{array}$ & $\begin{array}{l}\text { Longitude } \\
\text { (DD) }\end{array}$ & $\begin{array}{l}\text { DES } \\
\text { Order }\end{array}$ & $\begin{array}{l}\text { DES } \\
\text { Map \# }\end{array}$ & $\begin{array}{l}\text { NTS } \\
\text { Map \# }\end{array}$ \\
\hline$X-04-184$ & 5-Jul-04 & Pine Lake Drain & 50.84783 & -98.36717 & 3 & 34 & 062J16 \\
\hline$X-04-185$ & 5-Jul-04 & Chippewa Creek Drain & 50.77169 & -98.30111 & 3 & 34 & 062J16 \\
\hline$X-04-186$ & 5-Jul-04 & Swan Lake Drain & 50.85986 & -98.13442 & 3 & $31 \mathrm{~W}$ & 062J16 \\
\hline$X-04-187$ & 6-Jul-04 & Swan Creek at Swan Lake Dam & 50.80050 & -98.16958 & 3 & $31 \mathrm{~W}$ & 062J16 \\
\hline$X-04-188$ & 6-Jul-04 & Island Lake Drain & 50.77769 & -97.97169 & 4 & $31 \mathrm{~W}$ & 062113 \\
\hline$X-04-189$ & 6-Jul-04 & Hayward Drain & 50.71333 & -98.14998 & 3 & $31 \mathrm{~W}$ & 062J09 \\
\hline$X-04-190$ & 6-Jul-04 & Burnt Lake Drain & 50.73817 & -97.99503 & 4 & $31 \mathrm{~W}$ & 062112 \\
\hline$X-04-191$ & 6-Jul-04 & Mud Lake Drain & 50.66850 & -98.04153 & 3 & $31 \mathrm{~W}$ & 062J09 \\
\hline$X-04-192$ & 6-Jul-04 & Hatchery Drain & 50.62219 & -98.05953 & 3 & $31 \mathrm{~W}$ & 062J09 \\
\hline$X-04-193$ & 7-Jul-04 & Cloverdale Road Drain & 50.17656 & -96.85775 & 3 & 29 & 062102 \\
\hline$X-04-194$ & 7-Jul-04 & Muckle Creek & 50.22569 & -96.92531 & 2 & 29 & 062102 \\
\hline$X-04-195$ & 7-Jul-04 & Whiskey Ditch & 50.25503 & -96.87928 & 3 & 29 & 062107 \\
\hline$X-04-196$ & 7-Jul-04 & Unnamed tributary to Wavey Creek & 50.24103 & -97.17042 & 2 & 29 & 062103 \\
\hline$X-04-197$ & 7-Jul-04 & Stolars Drain & 50.24106 & -97.20786 & 3 & 29 & 062103 \\
\hline$X-04-198$ & 7-Jul-04 & Jackfish Creek & 50.21161 & -97.21025 & 3 & 29 & 062103 \\
\hline$X-04-199$ & 8-Jul-04 & Unnamed tributary to Lake Winnipeg & 50.61075 & -96.99561 & 2 & $31 \mathrm{E}$ & 062110 \\
\hline$X-04-200$ & 8-Jul-04 & Unnamed tributary to Wavey Creek & 50.23606 & -97.14514 & 2 & 29 & 062103 \\
\hline$X-04-201$ & 8-Jul-04 & Janet Creek & 50.18936 & -97.22992 & 3 & 29 & 062103 \\
\hline$X-04-202$ & 8-Jul-04 & Steele Drain & 50.16614 & -97.20678 & 3 & 29 & 062103 \\
\hline$X-04-203$ & 13-Jul-04 & Piney West Drain & 49.07478 & -96.04603 & 3 & 87 & $062 \mathrm{H} 01$ \\
\hline$X-04-204$ & 13-Jul-04 & Vassar Drain & 49.08852 & -95.82160 & 1 & 87 & 052E03 \\
\hline$X-04-205$ & 13-Jul-04 & Sprague Creek & 49.16308 & -95.79013 & 3 & 87 & 052E03 \\
\hline$X-04-206$ & 13-Jul-04 & Unnamed tributary to Whitemouth Lake & 49.24697 & -95.78717 & 3 & 86 & 052E03 \\
\hline$X-04-207$ & 13-Jul-04 & Unnamed tributary to Sprague Creek & 49.08950 & -95.66553 & 2 & 87 & 052E03 \\
\hline$X-04-208$ & 14-Jul-04 & Sprague Drain & 49.02217 & -95.56126 & 3 & 87 & $052 \mathrm{E} 03$ \\
\hline
\end{tabular}


Appendix 1: Table listing the site number, date sampled, site name, latitude and longitude (in decimal degrees DD), DES stream order, DES map number and the corresponding NTS map number for all sites surveyed between 2002 and 2006.

\begin{tabular}{|c|c|c|c|c|c|c|c|}
\hline Site Number & Date & Site Name & $\begin{array}{l}\text { Latitude } \\
\text { (DD) }\end{array}$ & $\begin{array}{l}\text { Longitude } \\
\text { (DD) }\end{array}$ & $\begin{array}{l}\text { DES } \\
\text { Order }\end{array}$ & $\begin{array}{l}\text { DES } \\
\text { Map \# }\end{array}$ & $\begin{array}{l}\text { NTS } \\
\text { Map \# }\end{array}$ \\
\hline$X-04-209$ & 14-Jul-04 & Mud Creek & 49.02341 & -95.62791 & 3 & 87 & 052Е03 \\
\hline$X-04-210$ & 14-Jul-04 & West Pine Creek & 49.08947 & -95.94203 & 2 & 87 & $052 \mathrm{E} 03$ \\
\hline$X-04-211$ & 14-Jul-04 & Whitemouth River & 49.28156 & -95.61253 & 3 & 86 & $052 \mathrm{E} 03$ \\
\hline$x-04-212$ & 15-Jul-04 & Angle Drain & 51.18628 & -97.03872 & 3 & 106 & 062P03 \\
\hline$X-04-213$ & 15-Jul-04 & Petrachek Drain & 51.09714 & -97.08564 & 3 & 106 & 062P03 \\
\hline$X-04-214$ & 15-Jul-04 & Shorncliff Drain & 51.08256 & -97.18064 & 2 & 106 & $062 \mathrm{P} 03$ \\
\hline$X-04-215$ & 15-Jul-04 & Washow Bay Creek & 51.03801 & -97.20364 & 3 & 106 & 062P03 \\
\hline$X-04-216$ & 16-Jul-04 & Blind Bay Drain & 51.14139 & -96.96833 & 3 & 106 & 062P02 \\
\hline$X-04-217$ & 16-Jul-04 & Unnamed tributary to Kris Johnson Drain & 51.11167 & -96.88558 & 1 & 106 & 062P02 \\
\hline$X-04-218$ & 16-Jul-04 & Unnamed tributary to Ben Johnson Drain & 51.07506 & -96.96806 & 1 & 106 & 062P02 \\
\hline$X-04-219$ & 16-Jul-04 & Progress Drain & 51.05283 & -96.99106 & 3 & 106 & 062P02 \\
\hline$X-04-220$ & 16-Jul-04 & Okno Drain & 51.05281 & -97.08519 & 3 & 106 & 062P03 \\
\hline$X-04-221$ & 16-Jul-04 & Icelandic River & 50.96478 & -97.03881 & 5 & 32 & $062 \mid 14$ \\
\hline$X-04-222$ & 19-Jul-04 & Jackfish Creek & 50.78533 & -100.14486 & 4 & 80 & $062 \mathrm{~K} 16$ \\
\hline$X-04-223$ & 20-Jul-04 & Clear Creek & 50.68628 & -100.17017 & 3 & 80 & $062 \mathrm{~K} 09$ \\
\hline$X-04-224$ & 20-Jul-04 & Unnamed tributary to Little Saskatchewan River & 50.66894 & -100.29931 & 2 & 80 & $062 \mathrm{~K} 09$ \\
\hline$X-04-225$ & 20-Jul-04 & Little Saskatchewan River & 50.53019 & -100.31717 & 5 & 82 & $062 K 09$ \\
\hline$X-04-226$ & 20-Jul-04 & Unnamed tributary to Rolling River & 50.53550 & -99.95367 & 3 & 81 & 062J12 \\
\hline$X-04-227$ & 20-Jul-04 & Whirlpool River & 50.65992 & -99.85317 & 5 & 81 & 062J12 \\
\hline$X-04-228$ & 21-Jul-04 & Swanson Creek & 50.67778 & -99.80328 & 4 & 81 & 062J12 \\
\hline$X-04-229$ & 21-Jul-04 & Unnamed tributary to Rolling River & 50.53586 & -99.83067 & 4 & 81 & 062J12 \\
\hline$X-04-230$ & 21-Jul-04 & Unnamed tributary to Rolling River & 50.55447 & -99.75286 & 2 & 81 & 062J12 \\
\hline$X-04-231$ & 21-Jul-04 & Muskrat Creek & 50.55372 & -99.72328 & 3 & 81 & 062J12 \\
\hline$X-04-232$ & 22-Jul-04 & Unnamed tributary to Octopus Lake & 50.62819 & -99.91883 & 2 & 80 & 062J12 \\
\hline$X-04-233$ & 22-Jul-04 & Rolling River & 50.56431 & -99.88708 & 5 & 81 & 062J12 \\
\hline
\end{tabular}


Appendix 1: Table listing the site number, date sampled, site name, latitude and longitude (in decimal degrees DD), DES stream order, DES map number and the corresponding NTS map number for all sites surveyed between 2002 and 2006.

\begin{tabular}{|c|c|c|c|c|c|c|c|}
\hline Site Number & Date & Site Name & $\begin{array}{l}\text { Latitude } \\
\text { (DD) }\end{array}$ & $\begin{array}{l}\text { Longitude } \\
\text { (DD) }\end{array}$ & $\begin{array}{l}\text { DES } \\
\text { Order }\end{array}$ & $\begin{array}{l}\text { DES } \\
\text { Map \# }\end{array}$ & $\begin{array}{l}\text { NTS } \\
\text { Map \# }\end{array}$ \\
\hline$X-04-234$ & 22-Jul-04 & Whirlpool River & 50.59055 & -99.88620 & 5 & 81 & 062J12 \\
\hline$X-04-235$ & 22-Jul-04 & Unnamed tributary to Otter Lake & 50.51333 & -99.80461 & 3 & 81 & 062J12 \\
\hline$x-04-236$ & 22-Jul-04 & Otter Creek & 50.49147 & -99.81039 & 3 & 81 & 062J05 \\
\hline$X-04-237$ & 22-Jul-04 & Little Saskatchewan River & 50.35897 & -99.90768 & 6 & 81 & 062J05 \\
\hline$X-04-238$ & 22-Jul-04 & West Spring Creek & 50.24475 & -99.91997 & 2 & 81 & 062J04 \\
\hline$X-04-239$ & 22-Jul-04 & Little Saskatchewan River & 50.25297 & -99.82483 & 6 & 81 & 062J05 \\
\hline$X-04-240$ & 26-Jul-04 & Atchison Drain & 49.70928 & -97.26300 & 3 & 22 & $062 \mathrm{H} 11$ \\
\hline$X-04-241$ & 26-Jul-04 & Oak Bluff Drain & 49.70336 & -97.29964 & 4 & 22 & $062 \mathrm{H} 11$ \\
\hline$X-04-242$ & 26-Jul-04 & Kirk Drain & 49.68581 & -97.36825 & 4 & 22 & $062 \mathrm{H} 11$ \\
\hline X-04-243 & 26-Jul-04 & Kosc Coulee & 49.66578 & -97.43617 & 3 & 22 & $062 \mathrm{H} 11$ \\
\hline X-04-244 & 26-Jul-04 & La Salle River & 49.72436 & -97.52569 & 4 & 23 & $062 \mathrm{H} 12$ \\
\hline$X-04-245$ & 26-Jul-04 & La Salle River & 49.67989 & -97.43258 & 4 & 22 & $062 \mathrm{H} 11$ \\
\hline$X-04-246$ & 26-Jul-04 & Baldry Creek & 49.77789 & -97.15297 & 3 & 22 & $062 \mathrm{H} 14$ \\
\hline$X-04-247$ & 28-Jul-04 & Elm Creek Channel & 49.68031 & -98.16461 & 3 & 21 & $062 G 09$ \\
\hline$X-04-248$ & 28-Jul-04 & Elm Creek Channel & 49.67958 & -98.02808 & 3 & 21 & $062 \mathrm{G} 09$ \\
\hline X-04-249 & 28-Jul-04 & La Salle River & 49.96181 & -98.01017 & 2 & 24 & $062 \mathrm{G} 16$ \\
\hline$X-04-250$ & 28-Jul-04 & Unnamed tributary to Mill Creek & 50.01690 & -98.00975 & 3 & 24 & 062J01 \\
\hline$X-04-251$ & 28-Jul-04 & Unnamed tributary to Mill Creek & 50.02264 & -97.99894 & 1 & 24 & 062104 \\
\hline$X-04-252$ & 28-Jul-04 & Unnamed tributary to La Salle River & 49.95289 & -98.13914 & 1 & 24 & 062G16 \\
\hline $\mathrm{X}-04-253$ & 30-Jul-04 & Seine River Diversion & 49.69733 & -97.09975 & 5 & 6 & $062 \mathrm{H} 11$ \\
\hline $\mathrm{X}-04-254$ & 30-Jul-04 & Seine River Diversion & 49.69444 & -96.98753 & 5 & 8 & $062 \mathrm{H} 10$ \\
\hline$X-04-255$ & 30-Jul-04 & Seine River Diversion & 49.69494 & -96.97639 & 4 & 8 & $062 \mathrm{H} 10$ \\
\hline$X-04-256$ & 3-Aug-04 & Seine River & 49.79206 & -97.04700 & 4 & 9 & $062 \mathrm{H} 14$ \\
\hline $\mathrm{X}-04-257$ & 3-Aug-04 & Seine River Diversion & 49.65703 & -96.63856 & 4 & 8 & $062 \mathrm{H} 10$ \\
\hline$X-04-258$ & 4-Aug-04 & Fishing River & 51.46861 & -100.25256 & 4 & 49 & 062N08 \\
\hline
\end{tabular}


Appendix 1: Table listing the site number, date sampled, site name, latitude and longitude (in decimal degrees DD), DES stream order, DES map number and the corresponding NTS map number for all sites surveyed between 2002 and 2006.

\begin{tabular}{|c|c|c|c|c|c|c|c|}
\hline Site Number & Date & Site Name & $\begin{array}{l}\text { Latitude } \\
\text { (DD) }\end{array}$ & $\begin{array}{l}\text { Longitude } \\
\text { (DD) }\end{array}$ & $\begin{array}{l}\text { DES } \\
\text { Order }\end{array}$ & $\begin{array}{l}\text { DES } \\
\text { Map \# }\end{array}$ & $\begin{array}{l}\text { NTS } \\
\text { Map \# }\end{array}$ \\
\hline$X-04-259$ & 5-Aug-04 & Swan River & 52.22781 & -100.99253 & 6 & 53 & $063 \mathrm{C02}$ \\
\hline$X-04-260$ & 5-Aug-04 & Swan River & 52.04094 & -101.44189 & 6 & 61 & $063 \mathrm{C03}$ \\
\hline$X-04-261$ & 6-Aug-04 & Swan River & 52.08581 & -101.32294 & 5 & 61 & $063 \mathrm{C03}$ \\
\hline$X-04-262$ & 6-Aug-04 & Fork River & 51.50759 & -100.01748 & 5 & 102 & 062N09 \\
\hline$X-04-263$ & 6-Aug-04 & Mossy River & 51.44003 & -99.95106 & 6 & 49 & 062005 \\
\hline D-05-001 & 5-Apr-05 & Unnamed tributary to Dauphin Lake & 51.39106 & -100.01853 & 2 & 95 & 062N08 \\
\hline D-05-002 & 5-Apr-05 & Unnamed tributary to Dauphin Lake & 51.35439 & -100.01844 & 2 & 95 & 062N08 \\
\hline D-05-003 & 5-Apr-05 & Unnamed tributary to Dauphin Lake & 51.27311 & -99.88003 & 2 & 95 & 062005 \\
\hline D-05-004 & 14-Apr-05 & Unnamed tributary to Lake Manitoba & 50.21206 & -98.69178 & 2 & 42 & 062J02 \\
\hline D-05-005 & 14-Apr-05 & Unnamed tributary to Lake Manitoba & 50.38783 & -98.67133 & 2 & 41 & 062J07 \\
\hline D-05-006 & 14-Apr-05 & Unnamed tributary to Lake Manitoba & 50.44719 & -98.69319 & 1 & 41 & 062J07 \\
\hline D-05-007 & 14-Apr-05 & Harcus Drain & 50.72706 & -98.83336 & 3 & 110 & 062J10 \\
\hline D-05-008 & 14-Apr-05 & North Leifer Drain & 50.75711 & -98.83531 & 3 & 110 & $062 J 15$ \\
\hline D-05-009 & 14-Apr-05 & Garrioch Creek & 50.87239 & -98.92111 & 4 & 110 & 062J15 \\
\hline D-05-010 & 1-May-05 & North Snake Creek Pond Outlet & 50.44160 & -99.47058 & 3 & 43 & 062J06 \\
\hline D-05-011 & 1-Jun-05 & Seine River & 49.78269 & -97.04454 & 4 & 9 & $062 \mathrm{H} 14$ \\
\hline D-05-012 & 9-Jun-05 & Unnamed tributary to Red River & 49.76958 & -97.16504 & 2 & 22 & $062 \mathrm{H} 14$ \\
\hline D-05-013 & 20-Jul-05 & Pembina River & 49.26419 & -99.47105 & 5 & 57 & 062G06 \\
\hline D-05-014 & 20-Jul-05 & Pelican Lake Enhancement Channel & 49.26678 & -99.47097 & 5 & 59 & 062G06 \\
\hline D-05-015 & 20-Jul-05 & Pelican Lake Enhancement Channel & 49.27395 & -99.47241 & 5 & 59 & 062G06 \\
\hline D-05-016 & 20-Jul-05 & Pembina River & 49.26365 & -99.46445 & 5 & 57 & $062 \mathrm{G} 06$ \\
\hline D-05-017 & 21-Jul-05 & Pelican Lake Diversion Channel & 49.26677 & -99.45235 & 5 & 59 & $062 \mathrm{G} 06$ \\
\hline D-05-018 & 3-Aug-05 & Hatchery Drain & 50.63402 & -97.94788 & 3 & $31 \mathrm{~W}$ & $062 \mid 12$ \\
\hline D-05-019 & 3-Aug-05 & Hatchery Drain & 50.63878 & -97.90388 & 3 & $31 \mathrm{~W}$ & $062 \mid 12$ \\
\hline D-05-020 & 4-Aug-05 & Unnamed tributary to Willow Creek & 50.56619 & -97.12833 & 3 & $31 \mathrm{E}$ & $062 \mid 11$ \\
\hline
\end{tabular}


Appendix 1: Table listing the site number, date sampled, site name, latitude and longitude (in decimal degrees DD), DES stream order, DES map number and the corresponding NTS map number for all sites surveyed between 2002 and 2006.

\begin{tabular}{|c|c|c|c|c|c|c|c|}
\hline Site Number & Date & Site Name & $\begin{array}{l}\text { Latitude } \\
\text { (DD) }\end{array}$ & $\begin{array}{l}\text { Longitude } \\
\text { (DD) }\end{array}$ & $\begin{array}{l}\text { DES } \\
\text { Order }\end{array}$ & $\begin{array}{l}\text { DES } \\
\text { Map \# }\end{array}$ & $\begin{array}{l}\text { NTS } \\
\text { Map \# }\end{array}$ \\
\hline$D-05-021$ & 4-Aug-05 & Unnamed tributary to Bass Drain & 50.63086 & -97.27371 & 2 & $31 \mathrm{E}$ & 062111 \\
\hline D-05-022 & 4-Aug-05 & Unnamed tributary to Willow Creek & 50.63540 & -97.18092 & 3 & $31 \mathrm{E}$ & 062111 \\
\hline $\mathrm{D}-05-023$ & 16-Aug-05 & Pelican Lake Diversion Channel & 49.27403 & -99.47236 & 5 & 59 & $062 G 06$ \\
\hline D-05-024 & 16-Aug-05 & Pembina River & 49.26367 & -99.47133 & 5 & 57 & 062G06 \\
\hline $\mathrm{D}-05-025$ & 17-Aug-05 & Unnamed tributary to Red River & 49.97908 & -97.05069 & 2 & 12 & $062 \mathrm{H} 14$ \\
\hline D-05-026 & 30-Aug-05 & Rat Creek & 49.95207 & -98.51450 & 5 & 35 & $062 \mathrm{G} 15$ \\
\hline D-05-027 & 30-Aug-05 & Rat Creek & 49.95111 & -98.51716 & 5 & 35 & $062 \mathrm{G} 15$ \\
\hline W-05-001 & 2-May-05 & Pembroke Drain & 50.28447 & -98.96647 & 4 & 42 & 062J07 \\
\hline W-05-002 & 2-May-05 & Jordan Creek Drain & 50.38792 & -98.97375 & 4 & 42 & 062J07 \\
\hline W-05-003 & 2-May-05 & Unnamed tributary to Big Grass River & 50.40291 & -98.95155 & 2 & 42 & 062J07 \\
\hline W-05-004 & 2-May-05 & Unnamed tributary to Big Grass River & 50.37311 & -98.96242 & 2 & 42 & 062J07 \\
\hline W-05-005 & 2-May-05 & Pratt Drain & 50.35850 & -98.96231 & 2 & 42 & 062J07 \\
\hline W-05-006 & 2-May-05 & Watson Drain & 50.34367 & -98.96219 & 2 & 42 & 062J07 \\
\hline W-05-007 & 2-May-05 & Crane Drain & 50.32903 & -98.96206 & 3 & 42 & 062J07 \\
\hline W-05-008 & 2-May-05 & Jumping Deer Creek & 50.44292 & -99.05414 & 3 & 43 & 062J06 \\
\hline W-05-009 & 2-May-05 & Jumping Deer Creek & 50.44722 & -99.19606 & 3 & 43 & 062J06 \\
\hline W-05-010 & 2-May-05 & Big Grass River & 50.52775 & -99.19281 & 5 & 43 & 062J11 \\
\hline W-05-011 & 2-May-05 & Jumping Deer Creek & 50.44114 & -99.00800 & 3 & 43 & 062J06 \\
\hline W-05-012 & 3-May-05 & Boggy Creek & 50.10172 & -99.40806 & 4 & 40 & 062J03 \\
\hline W-05-013 & 3-May-05 & Unnamed tributary to Whitemud River & 50.18872 & -99.56792 & 3 & 39 & 062J04 \\
\hline W-05-014 & 3-May-05 & Unnamed tributary to Brookdale Drain & 50.07686 & -99.56847 & 3 & 40 & 062J04 \\
\hline W-05-015 & 3-May-05 & Boggy Creek & 50.00497 & -99.56814 & 4 & 40 & 062J04 \\
\hline W-05-016 & 3-May-05 & Boggy Creek & 49.98911 & -99.63706 & 4 & 40 & $062 \mathrm{G} 13$ \\
\hline W-05-017 & 4-May-05 & Boggy Creek & 50.04881 & -99.67794 & 4 & 40 & 062J04 \\
\hline W-05-018 & 4-May-05 & Boggy Creek & 50.06356 & -99.72294 & 3 & 40 & 062J04 \\
\hline
\end{tabular}


Appendix 1: Table listing the site number, date sampled, site name, latitude and longitude (in decimal degrees DD), DES stream order, DES map number and the corresponding NTS map number for all sites surveyed between 2002 and 2006.

\begin{tabular}{|c|c|c|c|c|c|c|c|}
\hline Site Number & Date & Site Name & $\begin{array}{l}\text { Latitude } \\
\text { (DD) }\end{array}$ & $\begin{array}{l}\text { Longitude } \\
\text { (DD) }\end{array}$ & $\begin{array}{l}\text { DES } \\
\text { Order }\end{array}$ & $\begin{array}{l}\text { DES } \\
\text { Map \# }\end{array}$ & $\begin{array}{c}\text { NTS } \\
\text { Map \# }\end{array}$ \\
\hline W-05-019 & 4-May-05 & Boggy Creek & 50.07833 & -99.75033 & 3 & 40 & 062J04 \\
\hline W-05-020 & 4-May-05 & Spring Creek & 50.24731 & -99.47675 & 3 & 39 & 062J03 \\
\hline W-05-021 & 4-May-05 & Unnamed tributary to Spring Creek & 50.26061 & -99.47686 & 2 & 39 & 062J06 \\
\hline W-05-022 & 4-May-05 & Unnamed tributary to Spring Creek & 50.27533 & -99.47811 & 3 & 39 & 062J06 \\
\hline W-05-023 & 5-May-05 & Scott Drain & 49.83478 & -97.82350 & 3 & 24 & $062 \mathrm{H} 13$ \\
\hline W-05-024 & 5-May-05 & Elm River Channel & 49.88681 & -97.82358 & 4 & 24 & $062 \mathrm{H} 13$ \\
\hline W-05-025 & 5-May-05 & Elm River Relief Channel & 49.89369 & -97.82653 & 3 & 24 & $062 \mathrm{H} 13$ \\
\hline W-05-026 & 5-May-05 & Unnamed tributary to Elm River Relief Channel & 49.90114 & -97.82664 & 2 & 24 & $062 \mathrm{H} 13$ \\
\hline W-05-027 & 5-May-05 & West Branch La Salle River & 49.94058 & -97.80381 & 3 & 24 & $062 \mathrm{H} 13$ \\
\hline W-05-028 & 5-May-05 & La Salle River & 49.96589 & -97.84981 & 3 & 24 & $062 \mathrm{H} 13$ \\
\hline W-05-029 & 5-May-05 & La Salle River & 49.97106 & -97.82678 & 3 & 24 & $062 \mathrm{H} 13$ \\
\hline W-05-030 & 5-May-05 & Unnamed tributary to Barickman Coulee & 49.92294 & -97.61831 & 3 & 23 & $062 \mathrm{H} 13$ \\
\hline W-05-031 & 6-May-05 & Barickman Coulee & 49.94033 & -97.62194 & 4 & 23 & $062 \mathrm{H} 13$ \\
\hline W-05-032 & 6-May-05 & Barickman Coulee & 49.93475 & -97.64881 & 3 & 23 & $062 \mathrm{H} 13$ \\
\hline W-05-033 & 6-May-05 & Meakin Creek & 49.76849 & -97.71004 & 4 & 23 & $062 \mathrm{H} 13$ \\
\hline W-05-034 & 6-May-05 & Franzman Drain & 49.70944 & -97.68669 & 3 & 23 & $062 \mathrm{H} 12$ \\
\hline W-05-035 & 9-May-05 & Unnamed tributary to Codner Drain & 49.68736 & -97.68656 & 3 & 23 & $062 \mathrm{H} 12$ \\
\hline W-05-036 & 9-May-05 & Unnamed tributary to Morris River & 49.59100 & -97.59578 & 2 & 19 & $062 \mathrm{H} 12$ \\
\hline W-05-037 & 9-May-05 & Karlenzie Drain & 49.60608 & -97.59569 & 2 & 19 & $062 \mathrm{H} 12$ \\
\hline W-05-038 & 9-May-05 & Unnamed tributary to Morris River & 49.60608 & -97.59569 & 2 & 19 & $062 \mathrm{H} 12$ \\
\hline W-05-039 & 9-May-05 & Confluence of unnamed tributaries and Morris River & 49.65042 & -97.59567 & 3 & 19 & $062 \mathrm{H} 12$ \\
\hline W-05-040 & 10-May-05 & Allison Drain & 49.65914 & -97.68653 & 3 & 19 & $062 \mathrm{H} 12$ \\
\hline W-05-041 & 10-May-05 & Atchison Drain & 49.70925 & -97.26319 & 3 & 22 & $062 \mathrm{H} 11$ \\
\hline W-05-042 & 10-May-05 & Unnamed tributary to La Salle River & 49.70907 & -97.30420 & 3 & 22 & $062 \mathrm{H} 11$ \\
\hline W-05-043 & 10-May-05 & Kirk Drain & 49.70809 & -97.40036 & 3 & 22 & $062 \mathrm{H} 11$ \\
\hline
\end{tabular}


Appendix 1: Table listing the site number, date sampled, site name, latitude and longitude (in decimal degrees DD), DES stream order, DES map number and the corresponding NTS map number for all sites surveyed between 2002 and 2006.

\begin{tabular}{|c|c|c|c|c|c|c|c|}
\hline Site Number & Date & Site Name & $\begin{array}{l}\text { Latitude } \\
\text { (DD) }\end{array}$ & $\begin{array}{l}\text { Longitude } \\
\text { (DD) }\end{array}$ & $\begin{array}{l}\text { DES } \\
\text { Order }\end{array}$ & $\begin{array}{l}\text { DES } \\
\text { Map \# }\end{array}$ & $\begin{array}{c}\text { NTS } \\
\text { Map \# }\end{array}$ \\
\hline W-05-044 & 10-May-05 & Domain Drain & 49.60597 & -97.28808 & 3 & 22 & $062 \mathrm{H} 11$ \\
\hline W-05-045 & 10-May-05 & Glenlea Drain & 49.63158 & -97.18114 & 2 & 22 & $062 \mathrm{H} 11$ \\
\hline W-05-046 & 11-May-05 & Sturgeon Creek & 49.93083 & -97.41239 & 4 & 26 & $062 \mathrm{H} 14$ \\
\hline W-05-047 & 11-May-05 & Unnamed tributary to Sturgeon Creek & 49.97547 & -97.41258 & 2 & 26 & $062 \mathrm{H} 14$ \\
\hline W-05-048 & 11-May-05 & Colony Creek & 50.03425 & -97.43425 & 3 & 26 & 062103 \\
\hline W-05-049 & 11-May-05 & East Branch Sturgeon Creek & 50.13761 & -97.58572 & 3 & 26 & 062104 \\
\hline W-05-050 & 11-May-05 & West Branch Sturgeon Creek & 50.13764 & -97.63889 & 3 & 26 & 062104 \\
\hline W-05-051 & 12-May-05 & West Branch Sturgeon Creek & 50.07844 & -97.60947 & 4 & 26 & 062104 \\
\hline W-05-052 & 12-May-05 & Grassmere Creek Drain West Branch & 50.13039 & -97.48911 & 3 & 27 & 062103 \\
\hline W-05-053 & 12-May-05 & Grassmere Creek Drain East Branch & 50.13028 & -97.36561 & 4 & 27 & 062103 \\
\hline W-05-054 & 12-May-05 & Confluence of Glenlea Drain and B - Drain & 49.62094 & -97.17116 & 4 & 22 & $062 \mathrm{H} 11$ \\
\hline W-05-055 & 16-May-05 & Unnamed tributary to Pelican Lake & 49.37300 & -99.56348 & 2 & 59 & $062 \mathrm{G} 05$ \\
\hline W-05-056 & 16-May-05 & Unnamed tributary to Overend Lake & 49.39508 & -99.67064 & 3 & 59 & $062 \mathrm{G} 05$ \\
\hline W-05-057 & 17-May-05 & Orthez Drain & 49.39934 & -99.72188 & 3 & 59 & $062 \mathrm{G} 05$ \\
\hline W-05-058 & 17-May-05 & Orthez Drain & 49.41447 & -99.72858 & 3 & 59 & $062 \mathrm{G} 05$ \\
\hline W-05-059 & 17-May-05 & Orthez Drain & 49.35547 & -99.82350 & 3 & 59 & $062 \mathrm{G} 05$ \\
\hline W-05-060 & 17-May-05 & Unnamed tributary to Pelican Lake & 49.34404 & -99.63248 & 2 & 59 & $062 G 05$ \\
\hline W-05-061 & 18-May-05 & Unnamed tributary to Overend Lake & 49.38481 & -99.67861 & 3 & 59 & $062 \mathrm{G} 05$ \\
\hline W-05-062 & 18-May-05 & Waskada Creek & 49.14839 & -100.60064 & 3 & 65 & 062F02 \\
\hline W-05-063 & 18-May-05 & Waskada Creek & 49.11828 & -100.68997 & 3 & 65 & 062F02 \\
\hline W-05-064 & 18-May-05 & Unnamed tributary flowing into North Dakota & 49.01431 & -100.65394 & 3 & 65 & 062F02 \\
\hline W-05-065 & 18-May-05 & Unnamed tributary flowing into North Dakota & 49.01428 & -100.64061 & 3 & 65 & 062F02 \\
\hline W-05-066 & 18-May-05 & Boundary Creek & 49.01603 & -100.55561 & 2 & 65 & 062F02 \\
\hline W-05-067 & 19-May-05 & Unnamed tributary to Waskada Creek & 49.07341 & -100.61581 & 4 & 65 & 062F02 \\
\hline W-05-068 & 19-May-05 & Waskada Creek & 49.10142 & -100.77986 & 3 & 65 & 062F02 \\
\hline
\end{tabular}


Appendix 1: Table listing the site number, date sampled, site name, latitude and longitude (in decimal degrees DD), DES stream order, DES map number and the corresponding NTS map number for all sites surveyed between 2002 and 2006.

\begin{tabular}{|c|c|c|c|c|c|c|c|}
\hline Site Number & Date & Site Name & $\begin{array}{l}\text { Latitude } \\
\text { (DD) }\end{array}$ & $\begin{array}{l}\text { Longitude } \\
\text { (DD) }\end{array}$ & $\begin{array}{l}\text { DES } \\
\text { Order }\end{array}$ & $\begin{array}{l}\text { DES } \\
\text { Map \# }\end{array}$ & $\begin{array}{c}\text { NTS } \\
\text { Map \# }\end{array}$ \\
\hline W-05-069 & 19-May-05 & Waskada Creek & 49.08819 & -100.89836 & 3 & 65 & 062F02 \\
\hline W-05-070 & 19-May-05 & Unnamed tributary to Souris River & 49.02126 & -100.91432 & 2 & 65 & 062F02 \\
\hline W-05-071 & 20-May-05 & South Lateral & 49.59064 & -96.86681 & 4 & 7 & $062 \mathrm{H} 10$ \\
\hline W-05-072 & 20-May-05 & North Arm Upper Manning Canal & 49.58103 & -96.71017 & 3 & 7 & $062 \mathrm{H} 10$ \\
\hline W-05-073 & 24-May-05 & D - 20 Drain & 49.57639 & -97.03756 & 4 & 6 & $062 \mathrm{H} 11$ \\
\hline W-05-074 & 24-May-05 & Tourond Creek & 49.38599 & -96.66807 & 3 & 6 & $062 \mathrm{H} 07$ \\
\hline W-05-075 & 24-May-05 & Pansy Drain & 49.35900 & -96.71342 & 3 & 6 & $062 \mathrm{H} 07$ \\
\hline W-05-076 & 24-May-05 & Tourond Creek & 49.39953 & -96.71353 & 3 & 6 & $062 \mathrm{H} 07$ \\
\hline W-05-077 & 24-May-05 & South Lateral & 49.42353 & -96.66825 & 3 & 7 & $062 \mathrm{H} 07$ \\
\hline W-05-078 & 25-May-05 & Aubigny Drain & 49.41369 & -97.26844 & 3 & 5 & 062H06 \\
\hline W-05-079 & 25-May-05 & Lafond Drain & 49.28081 & -97.27814 & 3 & 5 & 062H06 \\
\hline W-05-080 & 25-May-05 & Marsh River & 49.26272 & -97.23347 & 3 & 5 & $062 \mathrm{H} 06$ \\
\hline W-05-081 & 25-May-05 & Arnaud Drain & 49.26622 & -97.12450 & 3 & 5 & $062 \mathrm{H} 06$ \\
\hline W-05-082 & 25-May-05 & International Boundary Drain & 49.01562 & -97.41369 & 3 & 15 & $062 \mathrm{H03}$ \\
\hline W-05-083 & 26-May-05 & Deadhorse Creek & 49.23353 & -97.97867 & 4 & 15 & $062 \mathrm{H} 04$ \\
\hline W-05-084 & 26-May-05 & Walkof Coulee & 49.15969 & -97.97631 & 3 & 15 & $062 \mathrm{H} 04$ \\
\hline W-05-085 & 26-May-05 & Unnamed tributary to Rosenheim Drain & 49.09125 & -97.88606 & 3 & 15 & $062 \mathrm{H} 04$ \\
\hline W-05-086 & 26-May-05 & Rosenheim Drain & 49.14881 & -97.70681 & 4 & 15 & $062 \mathrm{H} 04$ \\
\hline W-05-087 & 26-May-05 & Marquette Road Drain & 50.07858 & -97.73536 & 2 & 25 & 062104 \\
\hline W-05-088 & 27-May-05 & Scotts Drain & 50.07856 & -97.74989 & 2 & 25 & 062104 \\
\hline W-05-089 & 27-May-05 & Long Lake Drain & 50.04861 & -97.82661 & 4 & 25 & 062104 \\
\hline W-05-090 & 27-May-05 & Deep Creek & 50.06781 & -97.88456 & 3 & 25 & 062104 \\
\hline W-05-091 & 27-May-05 & Unnamed tributary to Long Lake Drain & 50.09331 & -97.87508 & 3 & 25 & 062104 \\
\hline W-05-092 & 27-May-05 & Long Lake Drain & 50.10806 & -97.92947 & 4 & 25 & 062104 \\
\hline W-05-093 & 29-May-05 & Long Lake Drain & 50.07394 & -98.03308 & 4 & 25 & 062J01 \\
\hline
\end{tabular}


Appendix 1: Table listing the site number, date sampled, site name, latitude and longitude (in decimal degrees DD), DES stream order, DES map number and the corresponding NTS map number for all sites surveyed between 2002 and 2006.

\begin{tabular}{|c|c|c|c|c|c|c|c|}
\hline Site Number & Date & Site Name & $\begin{array}{l}\text { Latitude } \\
\text { (DD) }\end{array}$ & $\begin{array}{l}\text { Longitude } \\
\text { (DD) }\end{array}$ & $\begin{array}{l}\text { DES } \\
\text { Order }\end{array}$ & $\begin{array}{l}\text { DES } \\
\text { Map \# }\end{array}$ & $\begin{array}{l}\text { NTS } \\
\text { Map \# }\end{array}$ \\
\hline W-05-094 & 31-May-05 & Tamarack Creek & 52.10678 & -101.21861 & 2 & 61 & $063 \mathrm{C03}$ \\
\hline W-05-095 & 31-May-05 & Avonlea Drain & 52.17261 & -101.21861 & 3 & 61 & $063 \mathrm{C03}$ \\
\hline W-05-096 & 31-May-05 & Hay Creek & 52.02761 & -101.39456 & 3 & 61 & $063 \mathrm{C03}$ \\
\hline W-05-097 & 31-May-05 & Unnamed tributary to Thunderhill Creek & 51.99800 & -101.51864 & 1 & 61 & $062 \mathrm{~N} 13$ \\
\hline W-05-098 & 31-May-05 & Lobstick Creek & 51.93900 & -101.49808 & 4 & 61 & 062N14 \\
\hline W-05-099 & 31-May-05 & Keillor Creek & 51.93908 & -101.43719 & 3 & 61 & 062N14 \\
\hline W-05-100 & 31-May-05 & Unnamed tributary to Lobstick Creek & 51.90994 & -101.53575 & 2 & 61 & $062 \mathrm{~N} 13$ \\
\hline W-05-101 & 1-Jun-05 & Farden Drain & 52.11444 & -100.92703 & 3 & 53 & $063 \mathrm{C02}$ \\
\hline W-05-102 & 1-Jun-05 & Cox Drain & 52.09036 & -100.89388 & 3 & 53 & $063 \mathrm{C02}$ \\
\hline W-05-103 & 1-Jun-05 & Renwer Drain & 52.14356 & -100.79881 & 3 & 53 & $063 \mathrm{C02}$ \\
\hline W-05-104 & 1-Jun-05 & Unnamed tributary to Sinclair River & 52.12181 & -100.75117 & 2 & 53 & $063 \mathrm{C02}$ \\
\hline W-05-105 & 1-Jun-05 & Community Pasture Drain & 52.31814 & -100.90556 & 3 & 53 & $063 \mathrm{CO} 07$ \\
\hline W-05-106 & 2-Jun-05 & Kitzul Drain & 52.34725 & -100.87311 & 3 & 53 & $063 \mathrm{CO} 07$ \\
\hline W-05-107 & 3-Jun-05 & Jeski Drain & 52.08517 & -101.11730 & 3 & 52 & $063 \mathrm{C03}$ \\
\hline W-05-108 & 3-Jun-05 & Unnamed tributary to East Favel River & 52.12367 & -101.03708 & 3 & 52 & $063 \mathrm{C03}$ \\
\hline W-05-109 & 3-Jun-05 & Unnamed tributary to East Favel River & 52.18031 & -101.01340 & 3 & 52 & $063 \mathrm{C03}$ \\
\hline W-05-110 & 4-Jun-05 & Outside Drain & 50.01003 & -96.95503 & 3 & 12 & 062102 \\
\hline W-05-111 & 4-Jun-05 & Gunns Creek & 50.06468 & -96.93758 & 3 & 12 & 062102 \\
\hline W-05-112 & 4-Jun-05 & Bunns Creek & 49.94678 & -97.05650 & 3 & 12 & $062 \mathrm{H} 14$ \\
\hline W-05-113 & 6-Jun-05 & Janet Creek & 50.18942 & -97.23006 & 3 & 29 & 062103 \\
\hline W-05-114 & 6-Jun-05 & Jackfish Creek & 50.22686 & -97.29914 & 3 & 29 & 062103 \\
\hline W-05-115 & 7-Jun-05 & Unnamed tributary to Pembina River & 49.18036 & -98.61261 & 2 & 60 & 062G02 \\
\hline W-05-116 & 7-Jun-05 & Unnamed tributary to Pembina River & 49.14903 & -98.42494 & 2 & 60 & $062 \mathrm{G} 01$ \\
\hline W-05-117 & 7-Jun-05 & Unnamed tributary to Pembina River & 49.10192 & -98.42494 & 2 & 60 & $062 \mathrm{G} 01$ \\
\hline W-05-118 & 8-Jun-05 & Syndicate Creek & 49.30542 & -98.72897 & 3 & 54 & $062 \mathrm{G} 07$ \\
\hline
\end{tabular}


Appendix 1: Table listing the site number, date sampled, site name, latitude and longitude (in decimal degrees DD), DES stream order, DES map number and the corresponding NTS map number for all sites surveyed between 2002 and 2006.

\begin{tabular}{|c|c|c|c|c|c|c|c|}
\hline Site Number & Date & Site Name & $\begin{array}{l}\text { Latitude } \\
\text { (DD) }\end{array}$ & $\begin{array}{l}\text { Longitude } \\
\text { (DD) }\end{array}$ & $\begin{array}{l}\text { DES } \\
\text { Order }\end{array}$ & $\begin{array}{l}\text { DES } \\
\text { Map \# }\end{array}$ & $\begin{array}{c}\text { NTS } \\
\text { Map \# }\end{array}$ \\
\hline W-05-119 & 8-Jun-05 & Unnamed tributary to Pembina River & 49.31039 & -98.81786 & 3 & 54 & $062 \mathrm{G} 07$ \\
\hline W-05-120 & 9-Jun-05 & First Creek & 49.96778 & -97.61342 & 2 & 26 & $062 \mathrm{H} 13$ \\
\hline W-05-121 & 9-Jun-05 & Second Creek & 49.99142 & -97.60275 & 3 & 26 & $062 \mathrm{H} 13$ \\
\hline W-05-122 & 9-Jun-05 & Colony Creek & 49.95969 & -97.33136 & 4 & 26 & $062 \mathrm{H} 14$ \\
\hline W-05-123 & 14-Jun-05 & Unnamed tributary to Bass Drain & 50.59553 & -97.26622 & 1 & $31 \mathrm{E}$ & $062 \mid 11$ \\
\hline W-05-124 & 14-Jun-05 & Unnamed tributary to Bass Drain & 50.61433 & -97.29694 & 2 & $31 \mathrm{E}$ & $062 \mid 11$ \\
\hline W-05-125 & 14-Jun-05 & Unnamed tributary to Bass Drain & 50.62558 & -97.30267 & 1 & $31 \mathrm{E}$ & $062 \mid 11$ \\
\hline W-05-126 & 14-Jun-05 & Unnamed tributary to Hatchery Drain & 50.63931 & -97.96447 & 2 & $31 \mathrm{~W}$ & 062112 \\
\hline W-05-127 & 14-Jun-05 & Hatchery Drain & 50.63428 & -97.94778 & 3 & $31 \mathrm{~W}$ & 062112 \\
\hline W-05-128 & 14-Jun-05 & Unnamed tributary to Burnt Lake Drain & 50.70914 & -97.87856 & 2 & $31 \mathrm{~W}$ & 062112 \\
\hline W-05-129 & 15-Jun-05 & Unnamed tributary to Icelandic River & 50.93531 & -97.32311 & 2 & 32 & 062114 \\
\hline W-05-130 & 15-Jun-05 & Unnamed tributary to Icelandic River & 51.00133 & -97.36833 & 3 & 32 & 062P03 \\
\hline W-05-131 & 15-Jun-05 & Sylvan Drain & 51.02350 & -97.41508 & 3 & 32 & 062P03 \\
\hline W-05-132 & 15-Jun-05 & Unnamed tributary to Shurkas Drain & 50.97933 & -97.52675 & 3 & 32 & 062113 \\
\hline W-05-133 & 15-Jun-05 & Unnamed tributary to Icelandic River & 50.91950 & -97.53719 & 3 & 32 & 062113 \\
\hline W-05-134 & 15-Jun-05 & Silver Drain & 50.83267 & -97.22781 & 3 & 32 & 062114 \\
\hline W-05-135 & 16-Jun-05 & Unnamed tributary to Brokenhead Swamp & 49.70411 & -96.26550 & 2 & 88 & $062 \mathrm{H} 09$ \\
\hline W-05-136 & 16-Jun-05 & Unnamed tributary to St. Labre Bog & 49.31018 & -96.07958 & 2 & 85 & 062H08 \\
\hline W-05-137 & 16-Jun-05 & Unnamed tributary to St. Labre Bog & 49.31010 & -96.04616 & 2 & 85 & 062H08 \\
\hline W-05-138 & 16-Jun-05 & Unnamed tributary to St. Labre Bog & 49.31111 & -95.99988 & 2 & 85 & 0.0052 \\
\hline W-05-139 & 17-Jun-05 & Unnamed tributary to St. Labre Bog & 49.31102 & -95.94611 & 2 & 85 & 0.0052 \\
\hline W-05-140 & 17-Jun-05 & Unnamed tributary to St. Clare Bog & 49.29873 & -95.82503 & 2 & 85 & 0.0052 \\
\hline W-05-141 & 20-Jun-05 & Second Creek & 49.93400 & -97.52911 & 3 & 26 & $062 \mathrm{H} 13$ \\
\hline W-05-142 & 20-Jun-05 & Overhill Drain & 49.82779 & -98.36941 & 3 & 100 & $062 \mathrm{G} 16$ \\
\hline W-05-143 & 20-Jun-05 & Unnamed tributary to Assiniboine River & 49.70964 & -98.74192 & 2 & 100 & 062G10 \\
\hline
\end{tabular}


Appendix 1: Table listing the site number, date sampled, site name, latitude and longitude (in decimal degrees DD), DES stream order, DES map number and the corresponding NTS map number for all sites surveyed between 2002 and 2006.

\begin{tabular}{|c|c|c|c|c|c|c|c|}
\hline Site Number & Date & Site Name & $\begin{array}{l}\text { Latitude } \\
\text { (DD) }\end{array}$ & $\begin{array}{l}\text { Longitude } \\
\text { (DD) }\end{array}$ & $\begin{array}{l}\text { DES } \\
\text { Order }\end{array}$ & $\begin{array}{l}\text { DES } \\
\text { Map \# }\end{array}$ & $\begin{array}{c}\text { NTS } \\
\text { Map \# }\end{array}$ \\
\hline W-05-144 & 20-Jun-05 & Unnamed tributary to Assiniboine River & 49.74797 & -98.78511 & 2 & 100 & $062 \mathrm{G} 10$ \\
\hline W-05-145 & 20-Jun-05 & Rossendale Drain & 49.80581 & -98.50586 & 3 & 100 & $062 \mathrm{G} 15$ \\
\hline W-05-146 & 21-Jun-05 & Bosshill Creek & 49.85806 & -101.07692 & 4 & 75 & 062F14 \\
\hline W-05-147 & 21-Jun-05 & Gopher Creek & 49.90867 & -101.26356 & 3 & 75 & $062 \mathrm{~F} 14$ \\
\hline W-05-148 & 21-Jun-05 & Little Scallion Creek & 49.89017 & -100.94283 & 3 & 75 & $062 \mathrm{~F} 15$ \\
\hline W-05-149 & 22-Jun-05 & Wythes Creek & 50.11614 & -101.06592 & 3 & 78 & $062 K 03$ \\
\hline W-05-150 & 22-Jun-05 & Unnamed tributary to Assiniboine River & 50.13142 & -101.07644 & 2 & 78 & $062 \mathrm{~K} 03$ \\
\hline W-05-151 & 22-Jun-05 & Unnamed tributary to Assiniboine River & 50.15711 & -101.08061 & 3 & 78 & $062 \mathrm{~K} 03$ \\
\hline W-05-152 & 22-Jun-05 & Unnamed tributary to Assiniboine River & 50.17717 & -101.08289 & 3 & 78 & $062 \mathrm{~K} 03$ \\
\hline W-05-153 & 22-Jun-05 & Unnamed tributary to Assiniboine River & 50.15575 & -101.28700 & 2 & 78 & $062 \mathrm{~K} 03$ \\
\hline W-05-154 & 22-Jun-05 & Scissor Creek & 50.27886 & -101.29194 & 4 & 78 & $062 \mathrm{~K} 06$ \\
\hline W-05-155 & 23-Jun-05 & Unnamed tributary to Snake Creek & 50.48519 & -101.13108 & 3 & 78 & $062 K 06$ \\
\hline W-05-156 & 23-Jun-05 & Unnamed tributary to Snake Creek & 50.47800 & -101.13097 & 3 & 78 & $062 K 06$ \\
\hline W-05-157 & 23-Jun-05 & Confluence of unnamed tributary and Snake Creek & 50.48170 & -101.15066 & 4 & 78 & $062 K 06$ \\
\hline W-05-158 & 23-Jun-05 & Unnamed tributary to Silver Creek & 50.70418 & -101.15978 & 2 & 79 & $062 \mathrm{~K} 11$ \\
\hline W-05-159 & 23-Jun-05 & East Silver Creek & 50.76281 & -101.02072 & 3 & 79 & $062 \mathrm{~K} 14$ \\
\hline W-05-160 & 23-Jun-05 & East Silver Creek & 50.69767 & -101.05572 & 3 & 79 & $062 \mathrm{~K} 11$ \\
\hline W-05-161 & 23-Jun-05 & Silver Creek & 50.71236 & -101.18161 & 3 & 79 & $062 \mathrm{~K} 11$ \\
\hline W-05-162 & 23-Jun-05 & Silver Creek & 50.65331 & -101.20086 & 3 & 79 & $062 \mathrm{~K} 11$ \\
\hline W-05-163 & 24-Jun-05 & Unnamed tributary to Overhill Drain & 49.83411 & -98.33002 & 1 & 100 & $062 \mathrm{G} 16$ \\
\hline W-05-164 & 27-Jun-05 & West Pine Creek & 49.11036 & -95.95664 & 2 & 87 & 0.052 \\
\hline W-05-165 & 27-Jun-05 & East Pine Creek & 49.11717 & -95.88944 & 2 & 87 & 0.052 \\
\hline W-05-166 & 27-Jun-05 & Unnamed tributary to Sprague Creek & 49.13303 & -95.83203 & 2 & 87 & 0.052 \\
\hline W-05-167 & 28-Jun-05 & Unnamed tributary to Rat River & 49.22267 & -96.12611 & 2 & 3 & $062 \mathrm{H} 01$ \\
\hline W-05-168 & 11-Jul-05 & Unnamed tributary to Oak River & 50.30550 & -100.48436 & 4 & 72 & 062K08 \\
\hline
\end{tabular}


Appendix 1: Table listing the site number, date sampled, site name, latitude and longitude (in decimal degrees DD), DES stream order, DES map number and the corresponding NTS map number for all sites surveyed between 2002 and 2006.

\begin{tabular}{|c|c|c|c|c|c|c|c|}
\hline Site Number & Date & Site Name & $\begin{array}{l}\text { Latitude } \\
\text { (DD) }\end{array}$ & $\begin{array}{l}\text { Longitude } \\
\text { (DD) }\end{array}$ & $\begin{array}{l}\text { DES } \\
\text { Order }\end{array}$ & $\begin{array}{l}\text { DES } \\
\text { Map \# }\end{array}$ & $\begin{array}{c}\text { NTS } \\
\text { Map \# }\end{array}$ \\
\hline W-05-169 & 12-Jul-05 & Conjuring Creek & 50.80092 & -101.32933 & 3 & 92 & $062 \mathrm{~K} 14$ \\
\hline W-05-170 & 12-Jul-05 & Bell Creek & 50.96033 & -101.45703 & 2 & 92 & $062 \mathrm{~K} 14$ \\
\hline W-05-171 & 12-Jul-05 & Blackbird Creek & 50.94511 & -101.49530 & 3 & 92 & $062 \mathrm{~K} 14$ \\
\hline W-05-172 & 12-Jul-05 & Big Boggy Creek & 51.40622 & -101.51728 & 4 & 92 & 062N05 \\
\hline W-05-173 & 12-Jul-05 & Unnamed tributary to Shell River & 51.55119 & -101.40475 & 3 & 91 & 062N11 \\
\hline W-05-174 & 18-Jul-05 & Boundary Creek Drain & 50.50700 & -97.09517 & 4 & $31 \mathrm{E}$ & $062 \mid 11$ \\
\hline W-05-175 & 18-Jul-05 & Boundary Drain & 50.44794 & -97.11364 & 3 & 30 & 062106 \\
\hline W-05-176 & 18-Jul-05 & Unnamed tributary to Lake Winnipeg & 50.64011 & -97.07947 & 3 & $31 \mathrm{E}$ & 062111 \\
\hline W-05-177 & 19-Jul-05 & Unnamed tributary to Lake Winnipeg & 50.63986 & -97.06167 & 3 & $31 \mathrm{E}$ & 062111 \\
\hline W-05-178 & 19-Jul-05 & Meleb Drain & 50.68431 & -97.06486 & 3 & $31 \mathrm{E}$ & $062 \mid 11$ \\
\hline W-05-179 & 19-Jul-05 & Meleb Drain & 50.69164 & -97.08806 & 3 & $31 \mathrm{E}$ & 062111 \\
\hline W-05-180 & 19-Jul-05 & Meleb Drain & 50.68436 & -97.14219 & 3 & $31 \mathrm{E}$ & $062 \mid 11$ \\
\hline W-05-181 & 19-Jul-05 & Fish Lake Drain & 50.74342 & -97.24019 & 3 & $31 \mathrm{E}$ & 062111 \\
\hline W-05-182 & 19-Jul-05 & Unnamed tributary to Lake Winnipeg & 50.75794 & -97.05736 & 2 & $31 \mathrm{E}$ & 062114 \\
\hline W-05-183 & 19-Jul-05 & Unnamed tributary to Lake Winnipeg & 50.81689 & -97.03317 & 2 & $31 \mathrm{E}$ & 062114 \\
\hline W-05-184 & 20-Jul-05 & Marcynuiks Drain & 51.44994 & -97.58106 & 2 & 33 & 062P05 \\
\hline W-05-185 & 20-Jul-05 & Unnamed tributary to Fisher River & 51.22919 & -97.74333 & 3 & 33 & 062P04 \\
\hline W-05-186 & 25-Jul-05 & Pine Creek & 50.67685 & -95.97487 & 1 & 96 & 052L12 \\
\hline W-05-187 & 25-Jul-05 & Pine Creek & 50.67778 & -95.92272 & 2 & 96 & 052L12 \\
\hline W-05-188 & 25-Jul-05 & Main Drain No. 2 & 50.51231 & -96.28369 & 3 & 14 & 062109 \\
\hline W-05-189 & 26-Jul-05 & Hiebert Drain & 50.41514 & -96.30661 & 3 & 14 & 062108 \\
\hline W-05-190 & 26-Jul-05 & Unnamed tributary to Main Drain No. 1 & 50.35614 & -96.28297 & 3 & 14 & 062108 \\
\hline W-05-191 & 26-Jul-05 & $\mathrm{T}$ - Drain & 50.31192 & -96.44517 & 3 & 10 & 062108 \\
\hline W-05-192 & 26-Jul-05 & Unnamed tributary to Brokenhead River & 50.26761 & -96.42183 & 2 & 10 & 062108 \\
\hline W-05-193 & 26-Jul-05 & C - Drain & 50.23761 & -96.42964 & 2 & 10 & 062101 \\
\hline
\end{tabular}


Appendix 1: Table listing the site number, date sampled, site name, latitude and longitude (in decimal degrees DD), DES stream order, DES map number and the corresponding NTS map number for all sites surveyed between 2002 and 2006.

\begin{tabular}{|c|c|c|c|c|c|c|c|}
\hline Site Number & Date & Site Name & $\begin{array}{l}\text { Latitude } \\
\text { (DD) }\end{array}$ & $\begin{array}{l}\text { Longitude } \\
\text { (DD) }\end{array}$ & $\begin{array}{l}\text { DES } \\
\text { Order }\end{array}$ & $\begin{array}{l}\text { DES } \\
\text { Map \# }\end{array}$ & $\begin{array}{c}\text { NTS } \\
\text { Map \# }\end{array}$ \\
\hline W-05-194 & 27-Jul-05 & W - Drain & 50.22289 & -96.42875 & 3 & 10 & 062101 \\
\hline W-05-195 & 27-Jul-05 & Unnamed tributary to Brokenhead River & 50.09100 & -96.40619 & 2 & 10 & 062101 \\
\hline W-05-196 & 27-Jul-05 & Unnamed tributary to Bachman East Drain & 50.10561 & -96.36014 & 2 & 10 & 062101 \\
\hline W-05-197 & 27-Jul-05 & Boggy Creek & 50.23500 & -95.88561 & 3 & 96 & 052L04 \\
\hline W-05-198 & 28-Jul-05 & Unnamed tributary to Winnipeg River & 50.24477 & -96.06278 & 2 & 96 & 062101 \\
\hline W-05-199 & 2-Aug-05 & Atchison Drain & 49.77669 & -97.31494 & 2 & 22 & $062 \mathrm{H} 14$ \\
\hline W-05-200 & 2-Aug-05 & Lot 16 Drain & 49.79767 & -97.26433 & 3 & 22 & $062 \mathrm{H} 14$ \\
\hline W-05-201 & 2-Aug-05 & Oak Bluff Drain & 49.76808 & -97.34533 & 3 & 22 & $062 \mathrm{H} 14$ \\
\hline W-05-202 & 2-Aug-05 & Kirk Drain & 49.76886 & -97.41347 & 3 & 22 & $062 \mathrm{H} 14$ \\
\hline W-05-203 & 3-Aug-05 & Kellner Drain & 49.90061 & -95.94822 & 2 & 83 & 052E12 \\
\hline W-05-204 & 3-Aug-05 & Unnamed tributary to Whitemouth River & 49.98886 & -95.99892 & 3 & 83 & 052E12 \\
\hline W-05-205 & 3-Aug-05 & Bog River & 50.06544 & -95.98078 & 3 & 83 & 052L04 \\
\hline W-05-206 & 3-Aug-05 & Unnamed tributary to Lac Du Bonnet & 50.23619 & -96.03856 & 2 & 96 & 062101 \\
\hline W-05-207 & 8-Aug-05 & Buffalo Creek & 49.20250 & -97.39175 & 4 & 15 & $062 \mathrm{H} 03$ \\
\hline W-05-208 & 10-Aug-05 & Unnamed tributary to St. Labre Bog & 49.38581 & -96.08506 & 2 & 85 & $062 \mathrm{H} 08$ \\
\hline W-05-209 & 10-Aug-05 & Unnamed tributary to St. Labre Bog & 49.38348 & -96.05265 & 2 & 85 & $062 \mathrm{H} 08$ \\
\hline W-05-210 & 19-May-05 & Unnamed tributary to Antler River & 49.04433 & -101.18306 & 3 & 65 & 062F03 \\
\hline W-05-211 & 31-May-05 & Silent Creek & 52.06844 & -101.32286 & 3 & 61 & $063 \mathrm{C03}$ \\
\hline $\mathrm{X}-05-001$ & 27-Apr-05 & Main Drain & 49.13325 & -97.14244 & 4 & 2 & $062 \mathrm{H} 03$ \\
\hline $\mathrm{X}-05-002$ & 27-Apr-05 & Jordan River & 49.12181 & -96.89823 & 3 & 2 & $062 \mathrm{H} 02$ \\
\hline$X-05-003$ & 29-Apr-05 & Manness Drain & 49.60594 & -97.37429 & 3 & 20 & $062 \mathrm{H} 11$ \\
\hline X-05-004 & 29-Apr-05 & Manness Drain & 49.57653 & -97.36786 & 3 & 20 & $062 \mathrm{H} 11$ \\
\hline X-05-005 & 29-Apr-05 & Barnland Drain & 49.65048 & -97.47648 & 3 & 20 & $062 \mathrm{H} 11$ \\
\hline X-05-006 & 29-Apr-05 & Grill Drain & 49.62058 & -97.45906 & 3 & 20 & $062 \mathrm{H} 11$ \\
\hline X-05-007 & 29-Apr-05 & King Drain & 49.62844 & -97.41300 & 4 & 20 & $062 \mathrm{H} 11$ \\
\hline
\end{tabular}


Appendix 1: Table listing the site number, date sampled, site name, latitude and longitude (in decimal degrees DD), DES stream order, DES map number and the corresponding NTS map number for all sites surveyed between 2002 and 2006.

\begin{tabular}{|c|c|c|c|c|c|c|c|}
\hline Site Number & Date & Site Name & $\begin{array}{l}\text { Latitude } \\
\text { (DD) }\end{array}$ & $\begin{array}{l}\text { Longitude } \\
\text { (DD) }\end{array}$ & $\begin{array}{l}\text { DES } \\
\text { Order }\end{array}$ & $\begin{array}{l}\text { DES } \\
\text { Map \# }\end{array}$ & $\begin{array}{l}\text { NTS } \\
\text { Map \# }\end{array}$ \\
\hline$X-05-008$ & 29-Apr-05 & Bolen Drain & 49.59100 & -97.43586 & 3 & 20 & $062 \mathrm{H} 11$ \\
\hline $\mathrm{X}-05-009$ & 29-Apr-05 & Parker Drain & 49.59869 & -97.45908 & 3 & 20 & $062 \mathrm{H} 11$ \\
\hline$X-05-010$ & 3-May-05 & Unnamed tributary to Morris River & 49.59143 & -97.59757 & 2 & 19 & $062 \mathrm{H} 12$ \\
\hline $\mathrm{X}-05-011$ & 3-May-05 & 11 - A Drain & 49.62064 & -97.64114 & 4 & 19 & $062 \mathrm{H} 12$ \\
\hline$X-05-012$ & 3-May-05 & Unnamed tributary to Morris River & 49.65061 & -97.59586 & 2 & 19 & $062 \mathrm{H} 12$ \\
\hline$X-05-013$ & 3-May-05 & Castor Drain & 49.63569 & -97.61836 & 2 & 19 & $062 \mathrm{H} 12$ \\
\hline$X-05-014$ & 3-May-05 & Allison Drain & 49.65032 & -97.64108 & 3 & 19 & $062 \mathrm{H} 12$ \\
\hline$X-05-015$ & 3-May-05 & Elm Creek Channel & 49.68720 & -98.34610 & 2 & 22 & $062 G 09$ \\
\hline$X-05-016$ & 3-May-05 & Elm Creek Channel & 49.67998 & -98.25515 & 2 & 22 & 062G09 \\
\hline$X-05-017$ & 3-May-05 & Elm Creek Channel & 49.68003 & -98.22343 & 2 & 22 & 062G09 \\
\hline $\mathrm{X}-05-018$ & 4-May-05 & Elm River & 49.92011 & -98.14742 & 3 & 24 & $062 \mathrm{G} 16$ \\
\hline$X-05-019$ & 4-May-05 & Crooked Lake Channel & 49.87172 & -98.14178 & 3 & 21 & $062 \mathrm{G} 16$ \\
\hline $\mathrm{X}-05-020$ & 4-May-05 & Unnamed tributary to Crooked Lake Channel & 49.85717 & -98.14181 & 2 & 21 & $062 \mathrm{G} 16$ \\
\hline$X-05-021$ & 4-May-05 & Kelvin Drain & 49.84706 & -98.14181 & 3 & 21 & $062 \mathrm{G} 16$ \\
\hline $\mathrm{X}-05-022$ & 4-May-05 & Crooked Lake Channel & 49.83303 & -98.05108 & 4 & 21 & $062 \mathrm{G} 16$ \\
\hline$X-05-023$ & 4-May-05 & Boundary Drain & 49.79808 & -98.02394 & 3 & 21 & $062 \mathrm{G} 16$ \\
\hline $\mathrm{X}-05-024$ & 5-May-05 & Elm Creek Channel & 49.81247 & -97.99847 & 5 & 21 & $062 \mathrm{H} 13$ \\
\hline $\mathrm{X}-05-025$ & 5-May-05 & Unnamed tributary to Elm Creek Channel & 49.76862 & -97.89147 & 3 & 23 & $062 \mathrm{H} 13$ \\
\hline $\mathrm{X}-05-026$ & 5-May-05 & Elm Creek Channel & 49.67619 & -98.05094 & 3 & 21 & 062G09 \\
\hline $\mathrm{X}-05-027$ & 6-May-05 & Main Drain & 49.08931 & -97.10008 & 4 & 2 & 062H03 \\
\hline$X-05-028$ & 6-May-05 & Harlow Drain & 49.14072 & -97.10722 & 3 & 2 & $062 \mathrm{H} 03$ \\
\hline$X-05-029$ & 6-May-05 & Stewart Drain & 49.15064 & -97.01036 & 3 & 2 & 062H03 \\
\hline$X-05-030$ & 10-May-05 & North Branch Jordan River & 49.16177 & -96.89996 & 2 & 2 & 062H02 \\
\hline $\mathrm{X}-05-031$ & 10-May-05 & Harlow Drain & 49.09462 & -97.01020 & 3 & 2 & $062 \mathrm{H} 03$ \\
\hline$X-05-032$ & 11-May-05 & Dubas Creek & 50.13681 & -96.79544 & 3 & 11 & 062102 \\
\hline
\end{tabular}


Appendix 1: Table listing the site number, date sampled, site name, latitude and longitude (in decimal degrees DD), DES stream order, DES map number and the corresponding NTS map number for all sites surveyed between 2002 and 2006.

\begin{tabular}{|c|c|c|c|c|c|c|c|}
\hline Site Number & Date & Site Name & $\begin{array}{l}\text { Latitude } \\
\text { (DD) }\end{array}$ & $\begin{array}{l}\text { Longitude } \\
\text { (DD) }\end{array}$ & $\begin{array}{l}\text { DES } \\
\text { Order }\end{array}$ & $\begin{array}{l}\text { DES } \\
\text { Map \# }\end{array}$ & $\begin{array}{c}\text { NTS } \\
\text { Map \# }\end{array}$ \\
\hline$X-05-033$ & 11-May-05 & Melrose Drain & 50.05315 & -96.79610 & 3 & 11 & 062102 \\
\hline X-05-034 & 11-May-05 & Satans Creek & 50.00319 & -96.71794 & 3 & 11 & 062102 \\
\hline$X-05-035$ & 11-May-05 & Satans Creek & 49.99658 & -96.70531 & 3 & 11 & $062 \mathrm{H} 15$ \\
\hline $\mathrm{X}-05-036$ & 11-May-05 & Hazelridge Drain & 49.95150 & -96.70544 & 3 & 11 & $062 \mathrm{H} 15$ \\
\hline$X-05-037$ & 12-May-05 & Hazelridge Drain & 49.95200 & -96.65983 & 3 & 11 & $062 \mathrm{H} 15$ \\
\hline$X-05-038$ & 12-May-05 & Hazelridge Drain & 49.95881 & -96.67997 & 3 & 11 & $062 \mathrm{H} 15$ \\
\hline X-05-039 & 12-May-05 & Sapton Drain & 50.01789 & -96.70497 & 3 & 11 & 062102 \\
\hline$X-05-040$ & 12-May-05 & Dubas Creek & 50.09859 & -96.75004 & 3 & 11 & 062102 \\
\hline$X-05-041$ & 12-May-05 & Swede Drain & 49.91506 & -96.77392 & 3 & 11 & $062 \mathrm{H} 15$ \\
\hline$X-05-042$ & 12-May-05 & Edie Creek & 49.84053 & -96.62567 & 3 & 11 & $062 \mathrm{H} 15$ \\
\hline$X-05-043$ & 12-May-05 & Cooks Creek & 49.79114 & -96.57016 & 3 & 11 & $062 \mathrm{H} 15$ \\
\hline$X-05-044$ & 13-May-05 & Lower Youville Drain & 49.75353 & -96.97953 & 3 & 9 & $062 \mathrm{H} 15$ \\
\hline X-05-045 & 13-May-05 & Fish Creek & 49.73151 & -96.72903 & 3 & 9 & $062 \mathrm{H} 10$ \\
\hline$X-05-046$ & 13-May-05 & Fish Creek & 49.74268 & -96.61623 & 2 & 9 & $062 \mathrm{H} 10$ \\
\hline X-05-047 & 13-May-05 & Inter - Municipal Drain & 49.70792 & -96.69142 & 3 & 9 & $062 \mathrm{H} 10$ \\
\hline$X-05-048$ & 14-May-05 & Prairie Centre Line Drain & 49.84166 & -96.93541 & 3 & 9 & $062 \mathrm{H} 15$ \\
\hline$X-05-049$ & 14-May-05 & South Bibeau Drain & 49.85640 & -96.93536 & 2 & 9 & $062 \mathrm{H} 15$ \\
\hline$X-05-050$ & 14-May-05 & North Bibeau Drain & 49.87113 & -96.93529 & 2 & 9 & $062 \mathrm{H} 15$ \\
\hline$X-05-051$ & 14-May-05 & Old Prairie Grove Drain & 49.79701 & -97.01288 & 3 & 9 & $062 \mathrm{H} 14$ \\
\hline $\mathrm{X}-05-052$ & 14-May-05 & Prairie Station Road Drain & 49.78250 & -96.84386 & 2 & 9 & $062 \mathrm{H} 15$ \\
\hline$X-05-053$ & 15-May-05 & Bagot Drain & 50.01931 & -98.56258 & 3 & 36 & 062J02 \\
\hline$X-05-054$ & 15-May-05 & Westbourne Drain & 50.03403 & -98.56044 & 4 & 36 & 062J02 \\
\hline X-05-055 & 15-May-05 & Bagot Drain & 50.00206 & -98.60625 & 3 & 36 & 062J02 \\
\hline X-05-056 & 15-May-05 & Image Creek & 49.91597 & -98.77031 & 3 & 36 & $062 \mathrm{G} 15$ \\
\hline X-05-057 & 15-May-05 & Image Creek & 49.92058 & -98.76625 & 3 & 36 & $062 \mathrm{G} 15$ \\
\hline
\end{tabular}


Appendix 1: Table listing the site number, date sampled, site name, latitude and longitude (in decimal degrees DD), DES stream order, DES map number and the corresponding NTS map number for all sites surveyed between 2002 and 2006.

\begin{tabular}{|c|c|c|c|c|c|c|c|}
\hline Site Number & Date & Site Name & $\begin{array}{l}\text { Latitude } \\
\text { (DD) }\end{array}$ & $\begin{array}{l}\text { Longitude } \\
\text { (DD) }\end{array}$ & $\begin{array}{l}\text { DES } \\
\text { Order }\end{array}$ & $\begin{array}{l}\text { DES } \\
\text { Map \# }\end{array}$ & $\begin{array}{l}\text { NTS } \\
\text { Map \# }\end{array}$ \\
\hline$X-05-058$ & 16-May-05 & Squirrel Creek East Branch & 49.89706 & -98.93993 & 3 & 37 & $062 \mathrm{G} 15$ \\
\hline$X-05-059$ & 16-May-05 & Squirrel Creek East Branch & 49.88324 & -98.91620 & 3 & 37 & $062 \mathrm{G} 15$ \\
\hline $\mathrm{X}-05-060$ & 16-May-05 & Squirrel Creek East Branch & 49.82750 & -98.90539 & 2 & 37 & $062 \mathrm{G} 15$ \\
\hline $\mathrm{X}-05-061$ & 16-May-05 & Unnamed tributary to Squirrel Creek East Branch & 49.87159 & -98.94632 & 3 & 37 & $062 \mathrm{G} 15$ \\
\hline $\mathrm{X}-05-062$ & 16-May-05 & Unnamed tributary to Squirrel Creek East Branch & 49.88617 & -98.94451 & 3 & 37 & $062 \mathrm{G} 15$ \\
\hline $\mathrm{X}-05-063$ & 16-May-05 & Squirrel Creek West Branch & 49.93228 & -99.10286 & 2 & 38 & $062 \mathrm{G} 14$ \\
\hline$X-05-064$ & 16-May-05 & Beaver Creek & 49.97372 & -98.78950 & 4 & 36 & $062 \mathrm{G} 15$ \\
\hline$X-05-065$ & 16-May-05 & Beaver Creek & 49.93908 & -98.88119 & 3 & 36 & $062 \mathrm{G} 15$ \\
\hline $\mathrm{X}-05-066$ & 17-May-05 & Anderson Drain & 49.93111 & -98.83386 & 3 & 36 & $062 \mathrm{G} 15$ \\
\hline X-05-067 & 17-May-05 & Image Creek & 49.98617 & -98.72092 & 3 & 36 & $062 \mathrm{G} 15$ \\
\hline $\mathrm{X}-05-068$ & 17-May-05 & Bueckert Drain & 49.88158 & -98.61978 & 3 & 35 & $062 \mathrm{G} 15$ \\
\hline $\mathrm{X}-05-069$ & 17-May-05 & Rat Creek & 49.84841 & -98.61985 & 3 & 35 & $062 \mathrm{G} 15$ \\
\hline$X-05-070$ & 18-May-05 & Rat Creek & 49.85700 & -98.61414 & 3 & 35 & $062 \mathrm{G} 15$ \\
\hline $\mathrm{X}-05-071$ & 18-May-05 & Bueckert Drain & 49.87342 & -98.66572 & 3 & 35 & $062 \mathrm{G} 15$ \\
\hline $\mathrm{X}-05-072$ & 18-May-05 & Bagot Creek & 49.88683 & -98.72225 & 4 & 35 & $062 \mathrm{G} 15$ \\
\hline $\mathrm{X}-05-073$ & 18-May-05 & Unnamed tributary to Rat Creek & 49.98975 & -98.45183 & 3 & 35 & $062 \mathrm{G} 16$ \\
\hline $\mathrm{X}-05-074$ & 18-May-05 & Unnamed tributary to Rat Creek & 49.98989 & -98.44025 & 3 & 35 & $062 \mathrm{G} 16$ \\
\hline $\mathrm{X}-05-075$ & 18-May-05 & Mount Pleasant Drain & 50.01921 & -98.42265 & 3 & 35 & 062J01 \\
\hline $\mathrm{X}-05-076$ & 18-May-05 & Rignold Drain & 50.01956 & -98.49171 & 3 & 35 & 062J01 \\
\hline $\mathrm{X}-05-077$ & 18-May-05 & Rat Creek & 49.93071 & -98.55756 & 5 & 35 & $062 \mathrm{G} 15$ \\
\hline $\mathrm{X}-05-078$ & 19-May-05 & Bruneau Drain & 50.07292 & -97.04814 & 3 & 28 & 062103 \\
\hline $\mathrm{X}-05-079$ & 19-May-05 & Parks Creek & 50.09314 & -97.09790 & 3 & 28 & 062103 \\
\hline X-05-080 & 19-May-05 & Parks Creek & 50.04770 & -97.06803 & 4 & 28 & 062103 \\
\hline X-05-081 & 19-May-05 & Wavey Creek & 50.11178 & -97.23006 & 4 & 29 & 062103 \\
\hline X-05-082 & 19-May-05 & Janet Creek & 50.19664 & -97.29425 & 3 & 29 & 062103 \\
\hline
\end{tabular}


Appendix 1: Table listing the site number, date sampled, site name, latitude and longitude (in decimal degrees DD), DES stream order, DES map number and the corresponding NTS map number for all sites surveyed between 2002 and 2006.

\begin{tabular}{|c|c|c|c|c|c|c|c|}
\hline Site Number & Date & Site Name & $\begin{array}{l}\text { Latitude } \\
\text { (DD) }\end{array}$ & $\begin{array}{l}\text { Longitude } \\
\text { (DD) }\end{array}$ & $\begin{array}{l}\text { DES } \\
\text { Order }\end{array}$ & $\begin{array}{l}\text { DES } \\
\text { Map \# }\end{array}$ & $\begin{array}{l}\text { NTS } \\
\text { Map \# }\end{array}$ \\
\hline$X-05-083$ & 19-May-05 & Jackfish Creek & 50.22711 & -97.29917 & 3 & 29 & 062103 \\
\hline$X-05-084$ & 20-May-05 & Janet Creek & 50.18933 & -97.22986 & 3 & 29 & 062103 \\
\hline X-05-085 & 20-May-05 & Jackfish Creek & 50.21167 & -97.23019 & 3 & 29 & 062103 \\
\hline$X-05-086$ & 20-May-05 & Jackfish Creek & 50.30053 & -97.34044 & 3 & 29 & 062106 \\
\hline X-05-087 & 20-May-05 & Janet Creek & 50.25625 & -97.35561 & 3 & 29 & 062106 \\
\hline X-05-088 & 24-May-05 & Main Drain No. 1 & 50.42966 & -96.32975 & 4 & 14 & 062108 \\
\hline X-05-089 & 25-May-05 & Main Drain No. 1 & 50.48850 & -96.33567 & 4 & 14 & 062108 \\
\hline$X-05-090$ & 25-May-05 & Main Drain No. 2 & 50.39917 & -96.09847 & 2 & 14 & 062108 \\
\hline $\mathrm{X}-05-091$ & 25-May-05 & Sand River & 49.27208 & -96.28995 & 3 & 3 & $062 \mathrm{H} 08$ \\
\hline X-05-092 & 25-May-05 & Rat River & 49.22872 & -96.48673 & 4 & 3 & $062 \mathrm{H} 01$ \\
\hline X-05-093 & 27-May-05 & Anderson Creek & 49.36949 & -97.54971 & 3 & 17 & $062 \mathrm{H} 05$ \\
\hline X-05-094 & 27-May-05 & Anderson Creek & 49.36922 & -97.45903 & 3 & 17 & $062 \mathrm{H} 06$ \\
\hline X-05-095 & 30-May-05 & 4N Drain & 49.43883 & -97.52719 & 3 & 17 & $062 \mathrm{H} 05$ \\
\hline X-05-096 & 30-May-05 & 4N Drain & 49.44417 & -97.70811 & 3 & 17 & $062 \mathrm{H} 05$ \\
\hline X-05-097 & 30-May-05 & 4N Drain & 49.44415 & -97.84347 & 3 & 17 & $062 \mathrm{H} 05$ \\
\hline $\mathrm{X}-05-098$ & 30-May-05 & 4N Drain & 49.45814 & -97.86603 & 3 & 17 & $062 \mathrm{H} 05$ \\
\hline $\mathrm{X}-05-099$ & 31-May-05 & 4N Drain & 49.46840 & -97.93363 & 3 & 17 & $062 \mathrm{H} 05$ \\
\hline$X-05-100$ & 31-May-05 & Graham Creek & 49.38451 & -97.81437 & 4 & 17 & $062 \mathrm{H} 05$ \\
\hline$X-05-101$ & 31-May-05 & Graham Creek & 49.35061 & -97.95656 & 4 & 17 & $062 \mathrm{H} 05$ \\
\hline $\mathrm{X}-05-102$ & 31-May-05 & Unnamed tributary to Graham Creek & 49.36862 & -98.04773 & 3 & 17 & 062G08 \\
\hline$X-05-103$ & 31-May-05 & Tobacco Creek & 49.42382 & -98.11493 & 4 & 17 & $062 \mathrm{G} 08$ \\
\hline$X-05-104$ & 31-May-05 & Tobacco Creek & 49.43603 & -98.20525 & 3 & 17 & $062 \mathrm{G} 08$ \\
\hline$X-05-105$ & 1-Jun-05 & Thornhill Coulee & 49.24169 & -98.11436 & 3 & 16 & $062 \mathrm{G} 01$ \\
\hline $\mathrm{X}-05-106$ & 1-Jun-05 & Thornhill Coulee & 49.23257 & -98.18254 & 3 & 16 & $062 \mathrm{G} 01$ \\
\hline$X-05-107$ & 1-Jun-05 & South Tobacco Creek & 49.39525 & -98.22802 & 3 & 17 & $062 \mathrm{G} 08$ \\
\hline
\end{tabular}


Appendix 1: Table listing the site number, date sampled, site name, latitude and longitude (in decimal degrees DD), DES stream order, DES map number and the corresponding NTS map number for all sites surveyed between 2002 and 2006.

\begin{tabular}{|c|c|c|c|c|c|c|c|}
\hline Site Number & Date & Site Name & $\begin{array}{l}\text { Latitude } \\
\text { (DD) }\end{array}$ & $\begin{array}{l}\text { Longitude } \\
\text { (DD) }\end{array}$ & $\begin{array}{l}\text { DES } \\
\text { Order }\end{array}$ & $\begin{array}{l}\text { DES } \\
\text { Map \# }\end{array}$ & $\begin{array}{l}\text { NTS } \\
\text { Map \# }\end{array}$ \\
\hline $\mathrm{X}-05-108$ & 1-Jun-05 & Unnamed tributary to Tobacco Creek & 49.41181 & -98.31839 & 1 & 17 & 062G08 \\
\hline $\mathrm{X}-05-109$ & 1-Jun-05 & Tobacco Creek & 49.42647 & -98.31831 & 2 & 17 & 062G08 \\
\hline $\mathrm{X}-05-110$ & 2-Jun-05 & Shannon Creek & 49.29964 & -98.36381 & 3 & 16 & 062G08 \\
\hline$X-05-111$ & 2-Jun-05 & South Tobacco Creek & 49.36361 & -98.33984 & 3 & 17 & 062G08 \\
\hline$X-05-112$ & 2-Jun-05 & Unnamed tributary to Tobacco Creek & 49.45800 & -98.28667 & 3 & 17 & 062G08 \\
\hline$X-05-113$ & 2-Jun-05 & Graham Creek & 49.37683 & -98.13733 & 3 & 17 & 062G08 \\
\hline $\mathrm{X}-05-114$ & 2-Jun-05 & Unnamed tributary to Graham Creek & 49.33336 & -98.18258 & 3 & 17 & 062G08 \\
\hline$X-05-115$ & 2-Jun-05 & North Shannon Creek & 49.30272 & -98.04692 & 3 & 16 & 062G08 \\
\hline $\mathrm{X}-05-116$ & 2-Jun-05 & Unnamed tributary to North Shannon Creek & 49.31065 & -97.93363 & 3 & 16 & $062 \mathrm{H} 05$ \\
\hline$X-05-117$ & 2-Jun-05 & Kronsgart Drain & 49.28089 & -97.46181 & 3 & 16 & $062 \mathrm{H} 06$ \\
\hline $\mathrm{X}-05-118$ & 6-Jun-05 & Lewis Drain & 49.48786 & -97.59467 & 2 & 18 & $062 \mathrm{H} 05$ \\
\hline$X-05-119$ & 6-Jun-05 & Roblin Drain & 49.51728 & -98.10175 & 3 & 18 & 062G09 \\
\hline$X-05-120$ & 6-Jun-05 & Roblin Drain & 49.50803 & -98.09206 & 3 & 18 & $062 \mathrm{G} 09$ \\
\hline $\mathrm{X}-05-121$ & 6-Jun-05 & Roseisle Drain & 49.48756 & -98.34164 & 3 & 18 & 062G08 \\
\hline $\mathrm{X}-05-122$ & 6-Jun-05 & Roseisle Creek & 49.47114 & -98.45390 & 4 & 18 & 062G08 \\
\hline $\mathrm{X}-05-123$ & 7-Jun-05 & 7 - 7W Drain & 49.53873 & -98.34349 & 3 & 18 & $062 \mathrm{G} 09$ \\
\hline $\mathrm{X}-05-124$ & 7-Jun-05 & Unnamed tributary to Boyne River & 49.66261 & -98.55154 & 3 & 18 & $062 \mathrm{G} 10$ \\
\hline$X-05-125$ & 7-Jun-05 & Boyne River & 49.62100 & -98.69283 & 3 & 18 & $062 \mathrm{G} 10$ \\
\hline$X-05-126$ & 7-Jun-05 & Unnamed tributary to Boyne River & 49.53222 & -98.82569 & 3 & 18 & $062 \mathrm{G} 10$ \\
\hline $\mathrm{X}-05-127$ & 9-Jun-05 & Crystal Creek & 49.11853 & -98.92981 & 3 & 56 & 062G02 \\
\hline$X-05-128$ & 9-Jun-05 & Unnamed tributary to Cypress Creek & 49.16256 & -99.08767 & 4 & 56 & 062G03 \\
\hline$X-05-129$ & 10-Jun-05 & McTavish Drain & 49.42889 & -97.30178 & 3 & 18 & $062 \mathrm{H} 06$ \\
\hline$X-05-130$ & 10-Jun-05 & Unnamed tributary to Taylor Drain & 49.49519 & -97.28966 & 3 & 18 & $062 \mathrm{H} 06$ \\
\hline $\mathrm{X}-05-131$ & 13-Jun-05 & South Boggy Creek & 49.91596 & -99.68198 & 3 & 99 & $062 \mathrm{G} 13$ \\
\hline$X-05-132$ & 13-Jun-05 & West Branch Willow Creek & 49.91606 & -99.81381 & 3 & 99 & $062 \mathrm{G} 13$ \\
\hline
\end{tabular}


Appendix 1: Table listing the site number, date sampled, site name, latitude and longitude (in decimal degrees DD), DES stream order, DES map number and the corresponding NTS map number for all sites surveyed between 2002 and 2006.

\begin{tabular}{|c|c|c|c|c|c|c|c|}
\hline Site Number & Date & Site Name & $\begin{array}{l}\text { Latitude } \\
\text { (DD) }\end{array}$ & $\begin{array}{l}\text { Longitude } \\
\text { (DD) }\end{array}$ & $\begin{array}{l}\text { DES } \\
\text { Order }\end{array}$ & $\begin{array}{l}\text { DES } \\
\text { Map \# }\end{array}$ & $\begin{array}{c}\text { NTS } \\
\text { Map \# }\end{array}$ \\
\hline$X-05-133$ & 13-Jun-05 & East Branch Willow Creek & 49.93461 & -99.77431 & 3 & 99 & $062 \mathrm{G} 13$ \\
\hline$X-05-134$ & 13-Jun-05 & West Branch Willow Creek & 50.04894 & -99.85769 & 3 & 99 & 062J04 \\
\hline$X-05-135$ & 13-Jun-05 & Unnamed tributary to West Branch Willow Creek & 49.96028 & -99.90503 & 3 & 99 & $062 \mathrm{G} 13$ \\
\hline$X-05-136$ & 13-Jun-05 & Unnamed tributary to West Branch Willow Creek & 49.93804 & -99.95778 & 3 & 99 & $062 \mathrm{G} 13$ \\
\hline$X-05-137$ & 14-Jun-05 & Little Souris River & 49.75183 & -99.91536 & 4 & 98 & $062 \mathrm{G} 13$ \\
\hline$X-05-138$ & 14-Jun-05 & Unnamed tributary to Little Souris River & 49.78764 & -99.91625 & 3 & 98 & $062 \mathrm{G} 13$ \\
\hline $\mathrm{X}-05-139$ & 14-Jun-05 & Unnamed tributary to Little Souris River & 49.78350 & -100.00764 & 3 & 98 & $062 \mathrm{~F} 16$ \\
\hline$X-05-140$ & 14-Jun-05 & Little Souris River & 49.68017 & -100.06210 & 3 & 98 & 062F09 \\
\hline$X-05-141$ & 14-Jun-05 & Five Mile Creek & 49.66506 & -99.71475 & 3 & 98 & $062 \mathrm{G} 12$ \\
\hline$X-05-142$ & 15-Jun-05 & Black Creek & 49.61711 & -99.87100 & 3 & 69 & $062 \mathrm{G} 12$ \\
\hline $\mathrm{X}-05-143$ & 15-Jun-05 & Unnamed tributary to Black Creek & 49.59447 & -99.73436 & 3 & 69 & 062G12 \\
\hline$X-05-144$ & 15-Jun-05 & Unnamed tributary to Souris River & 49.44703 & -99.78968 & 3 & 69 & $062 \mathrm{G} 05$ \\
\hline$X-05-145$ & 15-Jun-05 & Unnamed tributary to Souris River & 49.41296 & -99.85779 & 3 & 69 & $062 \mathrm{G} 05$ \\
\hline $\mathrm{X}-05-146$ & 16-Jun-05 & Unnamed tributary to Pembina River & 49.39919 & -98.83250 & 3 & 55 & $062 \mathrm{G} 07$ \\
\hline $\mathrm{X}-05-147$ & 17-Jun-05 & Dry River & 49.33256 & -99.02059 & 3 & 55 & $062 \mathrm{G} 06$ \\
\hline $\mathrm{X}-05-148$ & 17-Jun-05 & Pilot Creek & 49.21954 & -98.88434 & 3 & 55 & 062G02 \\
\hline $\mathrm{X}-05-149$ & 21-Jun-05 & Jackfish Creek & 49.10235 & -99.80224 & 3 & 58 & $062 \mathrm{G} 04$ \\
\hline$X-05-150$ & 21-Jun-05 & Long River & 49.11439 & -99.81772 & 3 & 58 & 062G04 \\
\hline$X-05-151$ & 21-Jun-05 & Long River & 49.16617 & -99.75006 & 4 & 58 & 062G04 \\
\hline $\mathrm{X}-05-152$ & 21-Jun-05 & Unnamed tributary to Jackfish Creek & 49.08774 & -99.69738 & 3 & 58 & $062 \mathrm{G} 04$ \\
\hline$X-05-153$ & 21-Jun-05 & Stony Creek & 49.08786 & -99.66978 & 3 & 58 & 062G04 \\
\hline $\mathrm{X}-05-154$ & 21-Jun-05 & Stony Creek & 49.10953 & -99.61592 & 3 & 58 & $062 \mathrm{G} 04$ \\
\hline$X-05-155$ & 22-Jun-05 & Gimby Creek & 49.06189 & -99.61653 & 3 & 58 & 062G04 \\
\hline $\mathrm{X}-05-156$ & 22-Jun-05 & Gimby Creek & 49.01419 & -99.63494 & 3 & 58 & $062 \mathrm{G} 04$ \\
\hline $\mathrm{X}-05-157$ & 22-Jun-05 & Unnamed tributary to Badger Creek & 49.03019 & -99.48142 & 3 & 58 & 062G03 \\
\hline
\end{tabular}


Appendix 1: Table listing the site number, date sampled, site name, latitude and longitude (in decimal degrees DD), DES stream order, DES map number and the corresponding NTS map number for all sites surveyed between 2002 and 2006.

\begin{tabular}{|c|c|c|c|c|c|c|c|}
\hline Site Number & Date & Site Name & $\begin{array}{l}\text { Latitude } \\
\text { (DD) }\end{array}$ & $\begin{array}{l}\text { Longitude } \\
\text { (DD) }\end{array}$ & $\begin{array}{l}\text { DES } \\
\text { Order }\end{array}$ & $\begin{array}{l}\text { DES } \\
\text { Map \# }\end{array}$ & $\begin{array}{c}\text { NTS } \\
\text { Map \# }\end{array}$ \\
\hline$X-05-158$ & 22-Jun-05 & Unnamed tributary to Badger Creek & 49.06231 & -99.21161 & 3 & 58 & $062 \mathrm{G} 03$ \\
\hline$X-05-159$ & 23-Jun-05 & Unnamed tributary to Elgin Creek & 49.43883 & -100.28564 & 3 & 68 & 062F08 \\
\hline$X-05-160$ & 23-Jun-05 & Unnamed tributary to Elgin Creek & 49.43494 & -100.30797 & 3 & 68 & 062F08 \\
\hline$X-05-161$ & 23-Jun-05 & Unnamed tributary to Elgin Creek & 49.45273 & -100.33055 & 3 & 68 & 062F08 \\
\hline$X-05-162$ & 23-Jun-05 & Elgin Creek & 49.53272 & -100.27288 & 4 & 68 & 062F09 \\
\hline$X-05-163$ & 23-Jun-05 & Unnamed tributary to Souris River & 49.53281 & -100.16100 & 3 & 68 & 062F09 \\
\hline$X-05-164$ & 23-Jun-05 & Unnamed tributary to Souris River & 49.63230 & -100.25747 & 3 & 68 & 062F09 \\
\hline$X-05-165$ & 27-Jun-05 & Unnamed tributary to Whitewater Lake & 49.14680 & -100.33566 & 3 & 68 & $062 \mathrm{~F} 01$ \\
\hline$X-05-166$ & 27-Jun-05 & Glen Creek & 49.14646 & -100.36899 & 2 & 68 & 062F01 \\
\hline$X-05-167$ & 28-Jun-05 & Unnamed tributary to Cherry Creek & 49.18906 & -100.10444 & 3 & 68 & $062 \mathrm{~F} 01$ \\
\hline$X-05-168$ & 28-Jun-05 & Unnamed tributary to Cherry Creek & 49.13150 & -100.14515 & 3 & 68 & 062F01 \\
\hline$X-05-169$ & 28-Jun-05 & Unnamed tributary to Zetterstrom Creek & 49.14667 & -100.17328 & 2 & 68 & $062 \mathrm{~F} 01$ \\
\hline$X-05-170$ & 28-Jun-05 & Unnamed tributary to Turtlehead Creek & 49.08783 & -100.40503 & 3 & 68 & 062F01 \\
\hline$X-05-171$ & 28-Jun-05 & Unnamed tributary to Whitewater Lake & 49.13164 & -100.27933 & 3 & 68 & $062 \mathrm{~F} 01$ \\
\hline $\mathrm{X}-05-172$ & 29-Jun-05 & Graham Creek & 49.25031 & -101.12141 & 3 & 64 & 062F06 \\
\hline$X-05-173$ & 29-Jun-05 & Jackson Creek & 49.35903 & -101.12144 & 2 & 64 & 062F06 \\
\hline$X-05-174$ & 30-Jun-05 & Stony Creek & 49.39814 & -101.09917 & 3 & 63 & 062F06 \\
\hline$X-05-175$ & 30-Jun-05 & Unnamed tributary to Stony Creek & 49.38855 & -101.09919 & 3 & 63 & 062F06 \\
\hline$X-05-176$ & 4-Jul-05 & Stony Creek & 49.69450 & -101.34064 & 3 & 63 & $062 \mathrm{~F} 11$ \\
\hline $\mathrm{X}-05-177$ & 4-Jul-05 & Stony Creek & 49.77258 & -101.39519 & 3 & 63 & $062 \mathrm{~F} 14$ \\
\hline$X-05-178$ & 5-Jul-05 & Unnamed tributary to Oak Creek & 49.38414 & -99.12028 & 3 & 70 & $062 \mathrm{G} 06$ \\
\hline$X-05-179$ & 5-Jul-05 & Unnamed tributary to Oak Creek & 49.35791 & -99.04293 & 2 & 70 & $062 \mathrm{G} 06$ \\
\hline$X-05-180$ & 5-Jul-05 & Unnamed tributary to Oak Creek & 49.41886 & -99.07803 & 2 & 70 & $062 \mathrm{G} 06$ \\
\hline X-05-181 & 6-Jul-05 & Ross Creek & 50.32981 & -97.18265 & 3 & 30 & 062106 \\
\hline$X-05-182$ & 6-Jul-05 & Crescent Lake Overflow & 50.35929 & -97.18277 & 3 & 30 & 062106 \\
\hline
\end{tabular}


Appendix 1: Table listing the site number, date sampled, site name, latitude and longitude (in decimal degrees DD), DES stream order, DES map number and the corresponding NTS map number for all sites surveyed between 2002 and 2006.

\begin{tabular}{|c|c|c|c|c|c|c|c|}
\hline Site Number & Date & Site Name & $\begin{array}{l}\text { Latitude } \\
\text { (DD) }\end{array}$ & $\begin{array}{l}\text { Longitude } \\
\text { (DD) }\end{array}$ & $\begin{array}{l}\text { DES } \\
\text { Order }\end{array}$ & $\begin{array}{l}\text { DES } \\
\text { Map \# }\end{array}$ & $\begin{array}{l}\text { NTS } \\
\text { Map \# }\end{array}$ \\
\hline$X-05-183$ & 6-Jul-05 & Ross Creek & 50.34467 & -97.28536 & 3 & 30 & 062106 \\
\hline$X-05-184$ & 6-Jul-05 & Boundary Drain & 50.44814 & -97.11367 & 3 & 30 & 062106 \\
\hline$X-05-185$ & 7-Jul-05 & Tugela Creek & 50.44929 & -96.95193 & 3 & 30 & 062107 \\
\hline$X-05-186$ & 7-Jul-05 & Fisher Drain & 50.34392 & -96.92894 & 3 & 30 & 062107 \\
\hline$X-05-187$ & 7-Jul-05 & Kilkenny Drain & 51.03822 & -97.79044 & 3 & 33 & 062P04 \\
\hline$X-05-188$ & 7-Jul-05 & Blind Creek & 51.12675 & -97.80772 & 3 & 33 & 062P04 \\
\hline $\mathrm{X}-05-189$ & 8-Jul-05 & Unnamed tributary to Lake Manitoba & 50.41828 & -97.94642 & 3 & 109 & 062105 \\
\hline$X-05-190$ & 11-Jul-05 & Wolfe Creek & 50.46836 & -100.57689 & 2 & 72 & $062 K 07$ \\
\hline $\mathrm{X}-05-191$ & 12-Jul-05 & Unnamed tributary to Shell River & 51.50781 & -101.37669 & 3 & 91 & 062N11 \\
\hline$X-05-192$ & 13-Jul-05 & Valley River & 51.59204 & -100.83935 & 2 & 93 & 062N10 \\
\hline$X-05-193$ & 13-Jul-05 & Garland River & 51.65898 & -100.63445 & 3 & 103 & 062N10 \\
\hline$X-05-194$ & 13-Jul-05 & South Garland Creek & 51.62405 & -100.48668 & 4 & 103 & 062N09 \\
\hline$X-05-195$ & 13-Jul-05 & Stoney Creek & 51.47778 & -100.43888 & 2 & 49 & 062N08 \\
\hline $\mathrm{X}-05-196$ & 14-Jul-05 & Unnamed tributary to Mink Creek & 51.44608 & -100.25256 & 3 & 49 & 062N08 \\
\hline $\mathrm{X}-05-197$ & 14-Jul-05 & Unnamed tributary to Mink Creek & 51.36151 & -100.20517 & 3 & 49 & 062N08 \\
\hline $\mathrm{X}-05-198$ & 14-Jul-05 & Mink Creek & 51.37067 & -100.58016 & 3 & 49 & 062N07 \\
\hline $\mathrm{X}-05-199$ & 14-Jul-05 & West Wilson River & 51.03700 & -100.68750 & 3 & 48 & 062N02 \\
\hline$X-05-200$ & 15-Jul-05 & Mitchell Creek & 51.06656 & -100.76623 & 2 & 48 & 062N02 \\
\hline$X-05-201$ & 3-Aug-05 & Little Pembina River & 49.13200 & -99.91714 & 3 & 57 & $062 \mathrm{G} 04$ \\
\hline $\mathrm{X}-05-202$ & 3-Aug-05 & Unnamed tributary to Little Pembina River & 49.13202 & -99.90420 & 2 & 57 & $062 \mathrm{G} 04$ \\
\hline$X-05-203$ & 3-Aug-05 & North Pembina River & 49.19723 & -99.87854 & 4 & 57 & $062 \mathrm{G} 04$ \\
\hline$X-05-204$ & 4-Aug-05 & Unnamed tributary to Netley Creek & 50.50693 & -97.34049 & 2 & 30 & $062 I 11$ \\
\hline$X-05-205$ & 4-Aug-05 & Unnamed tributary to Netley Creek & 50.49224 & -97.39059 & 2 & 30 & 062106 \\
\hline $\mathrm{X}-05-206$ & 4-Aug-05 & Unnamed tributary to Netley Creek & 50.50689 & -97.29284 & 2 & 30 & $062 \mid 11$ \\
\hline$X-05-207$ & 4-Aug-05 & Cochrane Creek & 50.39789 & -96.97014 & 3 & 30 & 062107 \\
\hline
\end{tabular}


Appendix 1: Table listing the site number, date sampled, site name, latitude and longitude (in decimal degrees DD), DES stream order, DES map number and the corresponding NTS map number for all sites surveyed between 2002 and 2006.

\begin{tabular}{|c|c|c|c|c|c|c|c|}
\hline Site Number & Date & Site Name & $\begin{array}{l}\text { Latitude } \\
\text { (DD) }\end{array}$ & $\begin{array}{l}\text { Longitude } \\
\text { (DD) }\end{array}$ & $\begin{array}{l}\text { DES } \\
\text { Order }\end{array}$ & $\begin{array}{l}\text { DES } \\
\text { Map \# }\end{array}$ & $\begin{array}{c}\text { NTS } \\
\text { Map \# }\end{array}$ \\
\hline$X-05-208$ & 4-Aug-05 & Ekhart Drain & 50.12315 & -97.45950 & 3 & 27 & 062103 \\
\hline$X-05-209$ & 16-Jun-05 & Unnamed Tributary to Mary Jane Creek & 49.25000 & -98.67000 & 1 & 54 & $062 \mathrm{G} 07$ \\
\hline$X-05-210$ & 22-Jun-05 & Gimby Creek & 49.08047 & -99.52628 & 3 & 58 & $062 \mathrm{G} 04$ \\
\hline D-06-001 & 6-Jul-06 & Jackfish Creek & 51.07388 & -99.96062 & 4 & 47 & 062004 \\
\hline W-06-001 & 9-May-06 & Unnamed tributary to Dauphin Lake & 51.39243 & -99.98009 & 2 & 95 & 062005 \\
\hline W-06-002 & 9-May-06 & Unnamed tributary to Dauphin Lake & 51.39122 & -100.01802 & 2 & 95 & 062N08 \\
\hline W-06-003 & 9-May-06 & Wolfe Creek & 50.54119 & -100.51362 & 2 & 72 & $062 \mathrm{~K} 10$ \\
\hline W-06-004 & 9-May-06 & Oak River & 50.55568 & -100.54662 & 3 & 72 & $062 \mathrm{~K} 10$ \\
\hline W-06-005 & 9-May-06 & Unnamed tributary to Minnewasta Creek & 50.43219 & -100.93307 & 2 & 74 & $062 K 07$ \\
\hline W-06-006 & 10-May-06 & Oak River & 50.50592 & -100.57022 & 3 & 72 & $062 \mathrm{~K} 10$ \\
\hline W-06-007 & 10-May-06 & Oak River & 50.47646 & -100.58727 & 3 & 72 & $062 K 07$ \\
\hline W-06-008 & 10-May-06 & Unnamed tributary to Oak River & 50.28474 & -100.49911 & 4 & 72 & $062 K 08$ \\
\hline W-06-009 & 10-May-06 & Arrow River & 50.32896 & -100.81869 & 4 & 74 & $062 K 07$ \\
\hline W-06-010 & 10-May-06 & Arrow River & 50.34209 & -100.83066 & 3 & 74 & $062 K 07$ \\
\hline W-06-011 & 11-May-06 & Bosshill Creek & 49.97514 & -101.27491 & 3 & 75 & 062F14 \\
\hline W-06-012 & 11-May-06 & Gopher Creek & 49.92355 & -101.32630 & 3 & 75 & 062F14 \\
\hline W-06-013 & 11-May-06 & Bosshill Creek & 49.98716 & -101.37812 & 3 & 75 & 062F14 \\
\hline W-06-014 & 11-May-06 & Gopher Creek & 49.93187 & -101.37842 & 3 & 75 & 062F14 \\
\hline W-06-015 & 11-May-06 & Stony Creek & 49.73935 & -101.37824 & 3 & 63 & $062 \mathrm{~F} 11$ \\
\hline W-06-016 & 11-May-06 & Medora Creek & 49.26153 & -100.69221 & 4 & 66 & 062F07 \\
\hline W-06-017 & 12-May-06 & Medora Creek & 49.20846 & -100.53323 & 4 & 66 & 062F02 \\
\hline W-06-018 & 12-May-06 & Waskada Creek & 49.11823 & -100.69012 & 3 & 65 & 062F02 \\
\hline W-06-019 & 15-May-06 & Confluence of unnamed tributary to Lake Manitoba & 50.35737 & -97.94472 & 3 & 109 & 062105 \\
\hline W-06-020 & 15-May-06 & Unnamed tributary to Shoal Lake & 50.30147 & -97.69770 & 3 & 109 & 062105 \\
\hline W-06-021 & 16-May-06 & Unnamed tributary to Scott Coulee & 49.85700 & -97.84700 & 2 & 24 & $062 \mathrm{H} 13$ \\
\hline
\end{tabular}


Appendix 1: Table listing the site number, date sampled, site name, latitude and longitude (in decimal degrees DD), DES stream order, DES map number and the corresponding NTS map number for all sites surveyed between 2002 and 2006.

\begin{tabular}{|c|c|c|c|c|c|c|c|}
\hline Site Number & Date & Site Name & $\begin{array}{l}\text { Latitude } \\
\text { (DD) }\end{array}$ & $\begin{array}{l}\text { Longitude } \\
\text { (DD) }\end{array}$ & $\begin{array}{l}\text { DES } \\
\text { Order }\end{array}$ & $\begin{array}{l}\text { DES } \\
\text { Map \# }\end{array}$ & $\begin{array}{c}\text { NTS } \\
\text { Map \# }\end{array}$ \\
\hline W-06-022 & 16-May-06 & Elm River Channel & 49.88640 & -97.84699 & 4 & 24 & $062 \mathrm{H} 13$ \\
\hline W-06-023 & 16-May-06 & La Salle River & 49.98000 & -97.94100 & 3 & 24 & $062 \mathrm{H} 13$ \\
\hline W-06-024 & 16-May-06 & Long Lake Drain & 50.07863 & -98.12499 & 3 & 25 & 062J01 \\
\hline W-06-025 & 17-May-06 & Hatchery Drain & 50.62218 & -98.04165 & 3 & $31 \mathrm{~W}$ & 062J09 \\
\hline W-06-026 & 17-May-06 & Hatchery Drain & 50.62312 & -98.08789 & 3 & $31 \mathrm{~W}$ & 062J09 \\
\hline W-06-027 & 17-May-06 & Hatchery Drain & 50.62400 & -97.98800 & 3 & $31 \mathrm{~W}$ & $062 \mid 12$ \\
\hline W-06-028 & 17-May-06 & Hatchery Drain & 50.63400 & -97.94800 & 3 & $31 \mathrm{~W}$ & $062 \mid 12$ \\
\hline W-06-029 & 23-May-06 & Tobacco Creek & 49.40518 & -97.59474 & 5 & 17 & 062H05 \\
\hline W-06-030 & 23-May-06 & 4N Drain & 49.44400 & -97.59500 & 3 & 17 & 062H05 \\
\hline W-06-031 & 23-May-06 & Tobacco Creek & 49.39894 & -97.70776 & 5 & 17 & $062 \mathrm{H} 05$ \\
\hline W-06-032 & 24-May-06 & Rex Creek & 49.60600 & -98.93200 & 4 & 71 & 062G10 \\
\hline W-06-033 & 24-May-06 & Pellys Lake & 49.59300 & -98.77900 & 3 & 18 & 062G10 \\
\hline W-06-034 & 24-May-06 & Pellys Lake & 49.55000 & -98.84700 & 3 & 18 & $062 \mathrm{G} 10$ \\
\hline W-06-035 & 24-May-06 & Cypress River & 49.43300 & -98.59100 & 3 & 71 & $062 \mathrm{G} 07$ \\
\hline W-06-036 & 25-May-06 & Cypress River & 49.45031 & -98.63631 & 3 & 71 & $062 \mathrm{G} 07$ \\
\hline W-06-037 & 25-May-06 & Somerset Creek & 49.38500 & -98.67300 & 2 & 71 & $062 \mathrm{G} 07$ \\
\hline W-06-038 & 26-May-06 & Roseisle Creek & 49.48000 & -98.42406 & 4 & 18 & 062G08 \\
\hline W-06-039 & 26-May-06 & Unnamed tributary to Crystal Creek & 49.07966 & -98.87388 & 3 & 56 & 062G02 \\
\hline W-06-040 & 26-May-06 & Crystal Creek & 49.06371 & -98.87382 & 3 & 56 & 062G02 \\
\hline W-06-041 & 26-May-06 & Unnamed tributary to Cypress Creek & 49.01500 & -98.88300 & 2 & 56 & 062G02 \\
\hline W-06-042 & 26-May-06 & Cypress Creek & 49.01500 & -98.92600 & 4 & 56 & 062G02 \\
\hline W-06-043 & 26-May-06 & Unnamed tributary to Cypress Creek & 49.02975 & -98.99400 & 4 & 56 & 062G02 \\
\hline W-06-044 & 26-May-06 & Unnamed tributary to Cypress Creek & 49.04832 & -99.07549 & 3 & 56 & $062 \mathrm{G} 03$ \\
\hline W-06-045 & 26-May-06 & Unnamed tributary to Cypress Creek & 49.14800 & -99.09000 & 4 & 56 & $062 \mathrm{G} 03$ \\
\hline W-06-046 & 27-May-06 & Pilot Creek & 49.22198 & -98.88771 & 4 & 55 & 062G02 \\
\hline
\end{tabular}


Appendix 1: Table listing the site number, date sampled, site name, latitude and longitude (in decimal degrees DD), DES stream order, DES map number and the corresponding NTS map number for all sites surveyed between 2002 and 2006.

\begin{tabular}{|c|c|c|c|c|c|c|c|}
\hline Site Number & Date & Site Name & $\begin{array}{l}\text { Latitude } \\
\text { (DD) }\end{array}$ & $\begin{array}{l}\text { Longitude } \\
\text { (DD) }\end{array}$ & $\begin{array}{l}\text { DES } \\
\text { Order }\end{array}$ & $\begin{array}{l}\text { DES } \\
\text { Map \# }\end{array}$ & $\begin{array}{c}\text { NTS } \\
\text { Map \# }\end{array}$ \\
\hline W-06-047 & 27-May-06 & McCoys Creek & 49.22144 & -98.78123 & 3 & 54 & $062 \mathrm{G} 02$ \\
\hline W-06-048 & 27-May-06 & Unnamed tributary to Mary Jane Creek & 49.25997 & -98.59052 & 2 & 54 & $062 \mathrm{G} 07$ \\
\hline W-06-049 & 27-May-06 & Oak Creek & 49.39854 & -99.20145 & 3 & 70 & 062G06 \\
\hline W-06-050 & 30-May-06 & Unnamed tributary to Red River & 49.97921 & -97.05080 & 2 & 12 & $062 \mathrm{H} 14$ \\
\hline W-06-051 & 30-May-06 & Mirey Creek & 50.08582 & -96.94875 & 2 & 28 & 062102 \\
\hline W-06-052 & 30-May-06 & Mirey Creek & 50.09222 & -96.94435 & 2 & 28 & 062102 \\
\hline W-06-053 & 30-May-06 & Mirey Creek & 50.08458 & -96.96423 & 2 & 28 & 062102 \\
\hline W-06-054 & 30-May-06 & Parks Creek & 50.13735 & -97.11497 & 2 & 28 & 062103 \\
\hline W-06-055 & 30-May-06 & Parks Creek & 50.10798 & -97.10354 & 3 & 28 & 062103 \\
\hline W-06-056 & 31-May-06 & Unnamed tributary to Red River & 49.97992 & -97.05125 & 2 & 12 & $062 \mathrm{H} 14$ \\
\hline W-06-057 & 31-May-06 & Unnamed tributary to Red River & 49.76900 & -97.20800 & 2 & 22 & $062 \mathrm{H} 14$ \\
\hline W-06-058 & 1-Jun-06 & Unnamed tributary to Red River & 49.77341 & -97.17390 & 2 & 22 & $062 \mathrm{H} 14$ \\
\hline W-06-059 & 1-Jun-06 & Unnamed tributary to La Salle River & 49.72404 & -97.31680 & 2 & 22 & $062 \mathrm{H} 11$ \\
\hline W-06-060 & 1-Jun-06 & Unnamed tributary to La Salle River & 49.72400 & -97.34100 & 3 & 22 & $062 \mathrm{H} 11$ \\
\hline W-06-061 & 6-Jun-06 & Unnamed tributary to Overflow Bay & 53.10185 & -101.11202 & 2 & & 063F03 \\
\hline W-06-062 & 6-Jun-06 & Unnamed tributary to Overflow Bay & 53.02513 & -101.06173 & 2 & & 063F03 \\
\hline W-06-063 & 7-Jun-06 & Rice Creek & 52.79695 & -101.24176 & 2 & & $063 \mathrm{C} 14$ \\
\hline W-06-064 & 7-Jun-06 & Rice River & 52.81638 & -101.30812 & 3 & & $063 \mathrm{C} 14$ \\
\hline W-06-065 & 7-Jun-06 & Homestead Creek & 52.82509 & -101.36466 & 4 & & $063 \mathrm{C} 14$ \\
\hline W-06-066 & 7-Jun-06 & Unnamed tributary to Steeprock River & 52.64091 & -101.09954 & 2 & 115 & $063 C 11$ \\
\hline W-06-067 & 8-Jun-06 & Trout Creek & 52.17680 & -101.46600 & 4 & 89 & $063 \mathrm{C03}$ \\
\hline W-06-068 & 8-Jun-06 & Unnamed tributary to Wellburns Creek & 51.65300 & -100.39200 & 2 & 103 & 062N09 \\
\hline W-06-069 & 12-Jun-06 & Rat River & 49.44336 & -97.01239 & 5 & 4 & $062 \mathrm{H} 06$ \\
\hline W-06-070 & 12-Jun-06 & Rat River & 49.32296 & -96.95208 & 5 & 4 & $062 \mathrm{H} 07$ \\
\hline W-06-071 & 13-Jun-06 & Unnamed tributary from Moose Lake & 49.19590 & -95.33530 & 2 & & 052E02 \\
\hline
\end{tabular}


Appendix 1: Table listing the site number, date sampled, site name, latitude and longitude (in decimal degrees DD), DES stream order, DES map number and the corresponding NTS map number for all sites surveyed between 2002 and 2006.

\begin{tabular}{|c|c|c|c|c|c|c|c|}
\hline Site Number & Date & Site Name & $\begin{array}{l}\text { Latitude } \\
\text { (DD) }\end{array}$ & $\begin{array}{l}\text { Longitude } \\
\text { (DD) }\end{array}$ & $\begin{array}{l}\text { DES } \\
\text { Order }\end{array}$ & $\begin{array}{l}\text { DES } \\
\text { Map \# }\end{array}$ & $\begin{array}{c}\text { NTS } \\
\text { Map \# }\end{array}$ \\
\hline W-06-072 & 13-Jun-06 & Unnamed tributary to Moose Lake & 49.22870 & -95.31424 & 1 & & 052E02 \\
\hline W-06-073 & 13-Jun-06 & Unnamed tributary to Moose Lake & 49.24580 & -95.28858 & 2 & & 052E02 \\
\hline W-06-074 & 13-Jun-06 & Unnamed tributary to Sprague Lake & 49.26344 & -95.28133 & 3 & & 052E05 \\
\hline W-06-075 & 13-Jun-06 & Unnamed tributary to Sprague Lake & 49.28949 & -95.32422 & 1 & & $052 E 05$ \\
\hline W-06-076 & 13-Jun-06 & Unnamed tributary to Harrison Creek & 49.34208 & -95.34502 & 1 & & $052 E 05$ \\
\hline W-06-077 & 13-Jun-06 & Harrison Creek & 49.38499 & -95.33482 & 2 & & $052 E 05$ \\
\hline W-06-078 & 14-Jun-06 & Unnamed tributary to Monk Creek & 49.83054 & -95.86144 & 3 & 83 & $052 \mathrm{E} 12$ \\
\hline W-06-079 & 14-Jun-06 & Unnamed tributary to Monk Creek & 49.86023 & -95.87291 & 3 & 83 & $052 \mathrm{E} 12$ \\
\hline W-06-080 & 14-Jun-06 & Monk Creek & 49.84116 & -95.87436 & 3 & 83 & 052E12 \\
\hline W-06-081 & 15-Jun-06 & Bachman Drain & 50.12100 & -96.52100 & 3 & 10 & 062102 \\
\hline W-06-082 & 15-Jun-06 & Selkirk Line West & 50.15030 & -96.52053 & 3 & 10 & 062102 \\
\hline W-06-083 & 20-Jun-06 & Ochre River & 50.92968 & -99.81157 & 5 & 46 & 062J13 \\
\hline W-06-084 & 21-Jun-06 & Unnamed tributary to Thunderhill Creek & 52.01249 & -101.50562 & 3 & 61 & $063 \mathrm{C04}$ \\
\hline W-06-085 & 21-Jun-06 & Thunderhill Creek & 52.00731 & -101.58527 & 2 & 61 & $063 \mathrm{C04}$ \\
\hline W-06-086 & 21-Jun-06 & Watts Creek & 51.70883 & -101.55376 & 3 & 61 & 062N12 \\
\hline W-06-087 & 22-Jun-06 & Hickey Creek & 51.58764 & -101.08872 & 2 & 91 & 062N11 \\
\hline W-06-088 & 22-Jun-06 & Unnamed tributary to Singush Lake & 51.61290 & -100.78924 & 2 & 93 & 062N10 \\
\hline W-06-089 & 22-Jun-06 & Brelinski Creek & 51.75018 & -100.55737 & 3 & 104 & 062N15 \\
\hline W-06-090 & 22-Jun-06 & North Pine River & 51.79865 & -100.60350 & 4 & 104 & 062N15 \\
\hline W-06-091 & 23-Jun-06 & Unnamed tributary to North Pine River & 51.78432 & -100.64757 & 2 & 104 & 062N15 \\
\hline W-06-092 & 23-Jun-06 & South Duck River & 51.89137 & -100.58102 & 3 & 105 & 062N15 \\
\hline W-06-093 & 23-Jun-06 & Unnamed tributary to North Duck River & 51.99058 & -100.66777 & 3 & 105 & 062N15 \\
\hline W-06-094 & 27-Jun-06 & Kris Johnson Drain & 51.11948 & -96.89769 & 3 & 106 & 062P02 \\
\hline W-06-095 & 27-Jun-06 & Blind Bay Drain & 51.15601 & -96.96815 & 2 & 106 & 062P02 \\
\hline W-06-096 & 27-Jun-06 & Washow Bay Creek & 51.06761 & -97.07367 & 4 & 106 & 062P03 \\
\hline
\end{tabular}


Appendix 1: Table listing the site number, date sampled, site name, latitude and longitude (in decimal degrees DD), DES stream order, DES map number and the corresponding NTS map number for all sites surveyed between 2002 and 2006.

\begin{tabular}{|c|c|c|c|c|c|c|c|}
\hline Site Number & Date & Site Name & $\begin{array}{l}\text { Latitude } \\
\text { (DD) }\end{array}$ & $\begin{array}{l}\text { Longitude } \\
\text { (DD) }\end{array}$ & $\begin{array}{l}\text { DES } \\
\text { Order }\end{array}$ & $\begin{array}{l}\text { DES } \\
\text { Map \# }\end{array}$ & $\begin{array}{c}\text { NTS } \\
\text { Map \# }\end{array}$ \\
\hline W-06-097 & 27-Jun-06 & Bump Drain & 51.12646 & -97.02126 & 3 & 106 & $062 \mathrm{P} 03$ \\
\hline W-06-098 & 28-Jun-06 & Swan Creek & 50.77204 & -98.15099 & 3 & $31 \mathrm{~W}$ & 062J16 \\
\hline W-06-099 & 28-Jun-06 & Hayward Drain & 50.72775 & -98.16776 & 3 & $31 \mathrm{~W}$ & 062J09 \\
\hline W-06-100 & 28-Jun-06 & Burnt Lake Drain & 50.72060 & -98.07553 & 3 & $31 \mathrm{~W}$ & 062J09 \\
\hline W-06-101 & 28-Jun-06 & Mud Lake Drain & 50.66878 & -98.04193 & 3 & $31 \mathrm{~W}$ & 062J09 \\
\hline W-06-102 & 28-Jun-06 & Island Lake Drain & 50.76539 & -97.99498 & 3 & $31 \mathrm{~W}$ & 062113 \\
\hline W-06-103 & 28-Jun-06 & Burnt Lake Drain & 50.72985 & -97.90194 & 3 & $31 \mathrm{~W}$ & $062 \mid 12$ \\
\hline W-06-104 & 29-Jun-06 & Ben Johnson Drain & 51.06762 & -96.95456 & 3 & 106 & 062P02 \\
\hline W-06-105 & 29-Jun-06 & Progress Drain & 51.05292 & -96.98521 & 3 & 106 & 062P02 \\
\hline W-06-106 & 29-Jun-06 & Little Dog Lake Drain & 51.00022 & -98.34990 & 3 & 34 & 062001 \\
\hline W-06-107 & 4-Jul-06 & Chippewa Creek Drain & 50.81554 & -98.25094 & 3 & 34 & 062J16 \\
\hline W-06-108 & 4-Jul-06 & Pine Lake Drain & 50.85995 & -98.35152 & 2 & 34 & 062J16 \\
\hline W-06-109 & 4-Jul-06 & Pioneer Drain & 51.09666 & -98.37330 & 3 & 34 & 062001 \\
\hline W-06-110 & 5-Jul-06 & Valley River & 51.16391 & -100.48483 & 5 & 95 & 062N01 \\
\hline W-06-111 & 5-Jul-06 & Drifting River & 51.33844 & -100.48645 & 3 & 95 & 062N08 \\
\hline W-06-112 & 5-Jul-06 & Drifting River & 51.29716 & -100.41457 & 3 & 95 & 062N08 \\
\hline W-06-113 & 6-Jul-06 & Garland River & 51.68959 & -100.36001 & 5 & 103 & 062N09 \\
\hline W-06-114 & 10-Jul-06 & Stony Creek & 50.21115 & -99.47622 & 4 & 39 & 062J03 \\
\hline W-06-115 & 10-Jul-06 & Law Creek & 50.34257 & -99.53876 & 3 & 39 & 062J05 \\
\hline W-06-116 & 11-Jul-06 & Jackfish Creek & 51.03700 & -99.99055 & 4 & 47 & 062004 \\
\hline W-06-117 & 10-Jul-06 & Eden Creek & 50.38138 & -99.51599 & 3 & 39 & 062J05 \\
\hline W-06-118 & 11-Jul-06 & Crawford Creek & 51.03712 & -99.83497 & 2 & 46 & 062004 \\
\hline W-06-119 & 11-Jul-06 & Ochre River & 51.03711 & -99.78922 & 5 & 46 & 062004 \\
\hline W-06-120 & 12-Jul-06 & Unnamed tributary to Henderson Creek & 50.88339 & -99.67184 & 3 & 45 & 062J13 \\
\hline W-06-121 & 12-Jul-06 & Wilson Creek & 50.75718 & -99.53179 & 3 & 44 & 062J13 \\
\hline
\end{tabular}


Appendix 1: Table listing the site number, date sampled, site name, latitude and longitude (in decimal degrees DD), DES stream order, DES map number and the corresponding NTS map number for all sites surveyed between 2002 and 2006.

\begin{tabular}{|c|c|c|c|c|c|c|c|}
\hline Site Number & Date & Site Name & $\begin{array}{l}\text { Latitude } \\
\text { (DD) }\end{array}$ & $\begin{array}{l}\text { Longitude } \\
\text { (DD) }\end{array}$ & $\begin{array}{l}\text { DES } \\
\text { Order }\end{array}$ & $\begin{array}{l}\text { DES } \\
\text { Map \# }\end{array}$ & $\begin{array}{l}\text { NTS } \\
\text { Map \# }\end{array}$ \\
\hline W-06-122 & 12-Jul-06 & Tityk Drain & 50.74221 & -99.43871 & 3 & 44 & 062J11 \\
\hline W-06-123 & 12-Jul-06 & Unnamed tributary to Turtle River & 50.77172 & -99.37975 & 3 & 44 & 062J14 \\
\hline W-06-124 & 18-Jul-06 & Unnamed tributary to Bird River & 50.41358 & -95.66888 & 3 & 96 & 052L05 \\
\hline W-06-125 & 18-Jul-06 & Unnamed tributary to Shellenberg Creek & 50.46681 & -95.46902 & 2 & 96 & 052L06 \\
\hline W-06-126 & 18-Jul-06 & Bird River & 50.46248 & -95.41457 & 4 & 96 & 052L06 \\
\hline W-06-127 & 18-Jul-06 & Unnamed tributary to Sausage Lake & 50.57898 & -95.41947 & 2 & 96 & $052 \mathrm{~L} 11$ \\
\hline W-06-128 & 18-Jul-06 & Unnamed tributary to Rabbit River & 50.65239 & -95.41026 & 4 & 96 & $052 \mathrm{~L} 11$ \\
\hline W-06-129 & 18-Jul-06 & Manigotagan River & 50.83387 & -95.31328 & 5 & & 052L14 \\
\hline W-06-130 & 18-Jul-06 & Cold Creek & 51.05817 & -95.81934 & 3 & & 052M04 \\
\hline W-06-131 & 18-Jul-06 & Manigotagan River & 51.10104 & -96.28401 & 5 & & $062 \mathrm{P} 01$ \\
\hline W-06-132 & 19-Jul-06 & Unnamed tributary to Lake Winnipeg & 51.01153 & -96.23979 & 1 & 96 & $062 \mathrm{P} 01$ \\
\hline W-06-133 & 19-Jul-06 & Black River & 50.86185 & -96.25488 & 6 & 96 & $062 \mid 16$ \\
\hline W-06-134 & 20-Jul-06 & O'Hanley River & 50.78501 & -96.20869 & 6 & 96 & $062 / 16$ \\
\hline W-06-135 & 20-Jul-06 & Pine Creek & 50.57882 & -96.15445 & 2 & 96 & 062109 \\
\hline W-06-136 & 20-Jul-06 & North Coca Cola Creek & 50.47355 & -95.99960 & 2 & 96 & 052L05 \\
\hline W-06-137 & 20-Jul-06 & Maple Creek & 50.44679 & -96.07558 & 4 & 96 & 062108 \\
\hline W-06-138 & 1-Aug-06 & Souris River & 49.17266 & -101.03077 & 7 & 65 & $062 \mathrm{~F} 03$ \\
\hline W-06-139 & 1-Aug-06 & Souris River & 49.49342 & -100.53217 & 7 & 67 & $062 \mathrm{~F} 07$ \\
\hline W-06-140 & 1-Aug-06 & Souris River & 49.61950 & -100.24709 & 7 & 67 & 062F09 \\
\hline W-06-141 & 2-Aug-06 & Souris River & 49.59943 & -99.68279 & 7 & 69 & $062 \mathrm{G} 12$ \\
\hline W-06-142 & 2-Aug-06 & Black Creek & 49.61942 & -99.67744 & 4 & 69 & $062 \mathrm{G} 12$ \\
\hline W-06-143 & 8-Aug-06 & Whitemud River Cross Ditch & 50.24071 & -98.85887 & 6 & 42 & 062J02 \\
\hline W-06-144 & 8-Aug-06 & Whitemud River & 50.23487 & -99.24768 & 6 & 39 & 062J03 \\
\hline W-06-145 & 8-Aug-06 & Whitemud River & 50.25912 & -99.35407 & 5 & 39 & 062J06 \\
\hline W-06-146 & 9-Aug-06 & Joe River & 49.01569 & -97.17751 & 3 & 2 & $062 \mathrm{H} 03$ \\
\hline
\end{tabular}


Appendix 2: Habitat complexity $(S=$ Simple; $C=$ Complex $)$ determination for all sites looking upstream and downstream from each surveyed reach.

\begin{tabular}{|c|c|c|}
\hline \multirow{2}{*}{$\begin{array}{c}\text { Site } \\
\text { Number }\end{array}$} & \multicolumn{2}{|c|}{ Habitat Complexity } \\
\hline & Upstream & Downstream \\
\hline D-02-001 & $\mathrm{C}$ & $\mathrm{C}$ \\
\hline D-02-002 & $\mathrm{C}$ & $\mathrm{C}$ \\
\hline D-02-003 & $\mathrm{C}$ & $\mathrm{C}$ \\
\hline D-02-004 & $\mathrm{C}$ & C \\
\hline D-02-005 & $\mathrm{C}$ & $\mathrm{C}$ \\
\hline D-02-006 & $\mathrm{S}$ & $\mathrm{S}$ \\
\hline D-02-007 & S & $\mathrm{S}$ \\
\hline D-02-008 & C & $\mathrm{S}$ \\
\hline D-02-009 & $\mathrm{C}$ & C \\
\hline D-02-010 & S & S \\
\hline D-02-011 & S & $\mathrm{S}$ \\
\hline D-02-012 & C & $\mathrm{C}$ \\
\hline D-02-013 & C & $\mathrm{S}$ \\
\hline D-02-014 & $\mathrm{S}$ & $\mathrm{C}$ \\
\hline D-02-015 & C & $\mathrm{C}$ \\
\hline D-02-016 & $\mathrm{S}$ & C \\
\hline D-02-017 & $S$ & $\mathrm{C}$ \\
\hline D-02-018 & $\mathrm{S}$ & $\mathrm{S}$ \\
\hline D-02-019 & $\mathrm{C}$ & $\mathrm{S}$ \\
\hline D-02-020 & S & $\mathrm{S}$ \\
\hline D-02-021 & S & $\mathrm{S}$ \\
\hline D-02-022 & $\mathrm{S}$ & $\mathrm{C}$ \\
\hline D-02-023 & $\mathrm{S}$ & $\mathrm{S}$ \\
\hline D-02-024 & $\mathrm{S}$ & $\mathrm{S}$ \\
\hline D-02-025 & $\mathrm{S}$ & $\mathrm{S}$ \\
\hline D-02-026 & S & $\mathrm{S}$ \\
\hline D-02-027 & C & C \\
\hline D-02-028 & C & C \\
\hline D-02-029 & $\mathrm{S}$ & $\mathrm{S}$ \\
\hline D-02-030 & $\mathrm{S}$ & $\mathrm{S}$ \\
\hline D-02-031 & $\mathrm{S}$ & $S$ \\
\hline D-02-032 & C & C \\
\hline D-02-033 & C & C \\
\hline
\end{tabular}

\begin{tabular}{|c|c|c|}
\hline \multirow{2}{*}{$\begin{array}{c}\text { Site } \\
\text { Number }\end{array}$} & \multicolumn{2}{|c|}{ Habitat Complexity } \\
\hline & Upstream & Downstream \\
\hline D-02-034 & $S$ & $\mathrm{~S}$ \\
\hline D-02-035 & C & $\mathrm{C}$ \\
\hline D-02-036 & S & S \\
\hline D-02-037 & C & C \\
\hline D-02-038 & S & S \\
\hline D-02-039 & S & S \\
\hline D-02-040 & C & S \\
\hline D-02-041 & C & C \\
\hline D-02-042 & C & $\mathrm{C}$ \\
\hline D-02-043 & $\mathrm{C}$ & $\mathrm{C}$ \\
\hline D-02-044 & C & C \\
\hline D-02-045 & C & $\mathrm{C}$ \\
\hline D-02-046 & C & $\mathrm{C}$ \\
\hline D-02-047 & C & C \\
\hline D-02-048 & $\mathrm{C}$ & $\mathrm{C}$ \\
\hline D-02-049 & C & $\mathrm{C}$ \\
\hline D-02-050 & $\mathrm{C}$ & $\mathrm{C}$ \\
\hline D-02-051 & S & S \\
\hline D-02-052 & $\mathrm{C}$ & $\mathrm{C}$ \\
\hline D-02-053 & C & C \\
\hline D-02-054 & C & C \\
\hline D-02-055 & $S$ & $S$ \\
\hline D-02-056 & C & $\mathrm{C}$ \\
\hline D-02-057 & C & C \\
\hline D-02-058 & $\mathrm{C}$ & C \\
\hline D-02-059 & $\mathrm{C}$ & C \\
\hline D-02-060 & C & C \\
\hline D-02-061 & C & C \\
\hline D-02-062 & $\mathrm{C}$ & C \\
\hline D-02-063 & C & C \\
\hline D-02-064 & $S$ & C \\
\hline D-02-065 & C & C \\
\hline D-02-066 & C & C \\
\hline
\end{tabular}

\begin{tabular}{|c|c|c|}
\hline \multirow{2}{*}{$\begin{array}{c}\text { Site } \\
\text { Number }\end{array}$} & \multicolumn{2}{|c|}{ Habitat Complexity } \\
\hline & Upstream & Downstream \\
\hline D-02-067 & $\mathrm{S}$ & $\mathrm{C}$ \\
\hline D-02-068 & $\mathrm{S}$ & $\mathrm{S}$ \\
\hline D-02-069 & S & $\mathrm{S}$ \\
\hline D-02-070 & C & C \\
\hline D-02-071 & C & C \\
\hline D-02-072 & C & C \\
\hline D-02-073 & $\mathrm{C}$ & C \\
\hline D-02-074 & C & C \\
\hline D-02-075 & $\mathrm{S}$ & $\mathrm{S}$ \\
\hline D-02-076 & S & S \\
\hline D-02-077 & S & $\mathrm{S}$ \\
\hline D-02-078 & C & C \\
\hline D-02-079 & $\mathrm{C}$ & $\mathrm{C}$ \\
\hline D-02-080 & $\mathrm{S}$ & S \\
\hline D-02-081 & C & $\mathrm{C}$ \\
\hline D-02-082 & $\mathrm{C}$ & C \\
\hline D-02-083 & C & $\mathrm{C}$ \\
\hline D-02-084 & S & $\mathrm{S}$ \\
\hline D-02-085 & S & $\mathrm{S}$ \\
\hline D-02-086 & $\mathrm{C}$ & $\mathrm{S}$ \\
\hline D-02-087 & C & $\mathrm{S}$ \\
\hline D-02-088 & S & $\mathrm{S}$ \\
\hline D-02-089 & S & $\mathrm{S}$ \\
\hline D-02-090 & S & $\mathrm{S}$ \\
\hline D-02-091 & S & $\mathrm{S}$ \\
\hline D-02-092 & $\mathrm{S}$ & $\mathrm{S}$ \\
\hline D-02-093 & C & $\mathrm{S}$ \\
\hline D-02-094 & $\mathrm{S}$ & $\mathrm{S}$ \\
\hline D-02-095 & $\mathrm{S}$ & C \\
\hline D-02-096 & $\mathrm{S}$ & $\mathrm{S}$ \\
\hline D-02-097 & $\mathrm{S}$ & C \\
\hline D-02-098 & $\mathrm{S}$ & S \\
\hline D-02-099 & $S$ & $\mathrm{~S}$ \\
\hline
\end{tabular}


Appendix 2. Habitat complexity $(S=$ Simple; $C=$ Complex $)$ determination for all sites looking upstream and downstream from each surveyed reach.

\begin{tabular}{|c|c|c|}
\hline \multirow{2}{*}{$\begin{array}{c}\text { Site } \\
\text { Number }\end{array}$} & \multicolumn{2}{|c|}{ Habitat Complexity } \\
\hline & Upstream & Downstream \\
\hline D-02-100 & $S$ & $\mathrm{~S}$ \\
\hline D-02-101 & $\mathrm{S}$ & $\mathrm{S}$ \\
\hline D-02-102 & $\mathrm{S}$ & $\mathrm{S}$ \\
\hline D-02-103 & $\mathrm{S}$ & $\mathrm{C}$ \\
\hline D-02-104 & $\mathrm{C}$ & $\mathrm{C}$ \\
\hline D-02-105 & $\mathrm{C}$ & $\mathrm{S}$ \\
\hline D-02-106 & S & $\mathrm{S}$ \\
\hline D-02-107 & $\mathrm{C}$ & $\mathrm{C}$ \\
\hline D-02-108 & $\mathrm{C}$ & $\mathrm{C}$ \\
\hline D-02-109 & $\mathrm{S}$ & $\mathrm{S}$ \\
\hline D-02-110 & $\mathrm{C}$ & $\mathrm{C}$ \\
\hline D-02-111 & $\mathrm{C}$ & $\mathrm{C}$ \\
\hline D-02-112 & $\mathrm{S}$ & $\mathrm{S}$ \\
\hline D-02-113 & $\mathrm{C}$ & $\mathrm{S}$ \\
\hline D-02-114 & $\mathrm{C}$ & $\mathrm{S}$ \\
\hline D-02-115 & $\mathrm{C}$ & $\mathrm{C}$ \\
\hline D-02-116 & $\mathrm{C}$ & $\mathrm{C}$ \\
\hline D-02-117 & $\mathrm{C}$ & $\mathrm{C}$ \\
\hline D-02-118 & $\mathrm{C}$ & $\mathrm{C}$ \\
\hline D-02-119 & $\mathrm{C}$ & $\mathrm{C}$ \\
\hline D-02-120 & $\mathrm{C}$ & $\mathrm{C}$ \\
\hline D-02-121 & $\mathrm{S}$ & $\mathrm{C}$ \\
\hline D-02-122 & $\mathrm{C}$ & $\mathrm{C}$ \\
\hline D-02-123 & $\mathrm{S}$ & $\mathrm{S}$ \\
\hline D-02-124 & $\mathrm{C}$ & $\mathrm{C}$ \\
\hline D-02-125 & $\mathrm{C}$ & $\mathrm{C}$ \\
\hline D-02-126 & $\mathrm{S}$ & $\mathrm{S}$ \\
\hline D-02-127 & $\mathrm{C}$ & $\mathrm{C}$ \\
\hline D-02-128 & C & $\mathrm{C}$ \\
\hline D-02-129 & $\mathrm{C}$ & $\mathrm{C}$ \\
\hline D-02-130 & $\mathrm{S}$ & $\mathrm{S}$ \\
\hline D-02-131 & $S$ & $\mathrm{C}$ \\
\hline D-02-132 & $\mathrm{S}$ & $\mathrm{C}$ \\
\hline D-02-133 & $\mathrm{S}$ & $\mathrm{S}$ \\
\hline
\end{tabular}

\begin{tabular}{|c|c|c|}
\hline \multirow{2}{*}{$\begin{array}{c}\text { Site } \\
\text { Number }\end{array}$} & \multicolumn{2}{|c|}{ Habitat Complexity } \\
\hline & Upstream & Downstream \\
\hline D-02-134 & $\mathrm{S}$ & $\mathrm{S}$ \\
\hline D-02-135 & $\mathrm{C}$ & $\mathrm{C}$ \\
\hline D-02-136 & $\mathrm{C}$ & C \\
\hline D-02-137 & $\mathrm{C}$ & C \\
\hline D-02-138 & $\mathrm{C}$ & $\mathrm{S}$ \\
\hline D-02-139 & $\mathrm{C}$ & $\mathrm{C}$ \\
\hline D-02-140 & C & C \\
\hline D-02-141 & $\mathrm{C}$ & $\mathrm{S}$ \\
\hline D-02-142 & $\mathrm{C}$ & $\mathrm{C}$ \\
\hline D-02-143 & $\mathrm{C}$ & $\mathrm{C}$ \\
\hline D-02-144 & $\mathrm{S}$ & $\mathrm{S}$ \\
\hline D-02-145 & $\mathrm{C}$ & $\mathrm{C}$ \\
\hline D-02-146 & $\mathrm{C}$ & $\mathrm{C}$ \\
\hline D-02-147 & C & C \\
\hline D-02-148 & $\mathrm{C}$ & $\mathrm{C}$ \\
\hline D-02-149 & $\mathrm{C}$ & $\mathrm{C}$ \\
\hline D-02-150 & $\mathrm{S}$ & $\mathrm{S}$ \\
\hline D-02-151 & $\mathrm{S}$ & $\mathrm{C}$ \\
\hline D-02-152 & $\mathrm{C}$ & $\mathrm{C}$ \\
\hline D-02-153 & $\mathrm{S}$ & $\mathrm{S}$ \\
\hline D-02-154 & $\mathrm{S}$ & S \\
\hline D-02-155 & $\mathrm{C}$ & $\mathrm{C}$ \\
\hline D-02-156 & C & C \\
\hline D-02-157 & $\mathrm{S}$ & $\mathrm{S}$ \\
\hline D-02-158 & $\mathrm{S}$ & $\mathrm{S}$ \\
\hline D-02-159 & $\mathrm{S}$ & C \\
\hline D-02-160 & C & C \\
\hline D-02-161 & $\mathrm{C}$ & $\mathrm{C}$ \\
\hline D-02-162 & $\mathrm{S}$ & $S$ \\
\hline D-02-163 & $\mathrm{C}$ & $\mathrm{C}$ \\
\hline D-02-164 & $\mathrm{C}$ & C \\
\hline D-02-165 & C & C \\
\hline D-02-166 & C & C \\
\hline D-02-167 & $\mathrm{C}$ & C \\
\hline
\end{tabular}

\begin{tabular}{|c|c|c|}
\hline \multirow{2}{*}{$\begin{array}{c}\text { Site } \\
\text { Number }\end{array}$} & \multicolumn{2}{|c|}{ Habitat Complexity } \\
\hline & Upstream & Downstream \\
\hline D-02-168 & $\mathrm{C}$ & $\mathrm{C}$ \\
\hline D-02-169 & $\mathrm{C}$ & $\mathrm{C}$ \\
\hline D-02-170 & $\mathrm{C}$ & $\mathrm{C}$ \\
\hline D-02-171 & C & C \\
\hline D-02-172 & $\mathrm{C}$ & $\mathrm{C}$ \\
\hline D-02-173 & $\mathrm{C}$ & $\mathrm{C}$ \\
\hline D-02-174 & $\mathrm{C}$ & C \\
\hline D-02-175 & C & $\mathrm{C}$ \\
\hline D-02-176 & $\mathrm{S}$ & $\mathrm{S}$ \\
\hline D-02-177 & $\mathrm{S}$ & $\mathrm{S}$ \\
\hline D-02-178 & C & C \\
\hline D-02-179 & $\mathrm{C}$ & $\mathrm{C}$ \\
\hline D-02-180 & $\mathrm{C}$ & $\mathrm{C}$ \\
\hline D-02-181 & $\mathrm{C}$ & C \\
\hline D-02-182 & C & $\mathrm{S}$ \\
\hline D-02-183 & $\mathrm{C}$ & $\mathrm{C}$ \\
\hline D-02-184 & C & C \\
\hline D-02-185 & $\mathrm{C}$ & $\mathrm{C}$ \\
\hline D-02-186 & $\mathrm{C}$ & $\mathrm{C}$ \\
\hline D-02-187 & S & $\mathrm{C}$ \\
\hline D-02-188 & $S$ & $\mathrm{~S}$ \\
\hline D-02-189 & $S$ & $\mathrm{~S}$ \\
\hline D-02-190 & $\mathrm{C}$ & $\mathrm{C}$ \\
\hline D-02-191 & $\mathrm{C}$ & $\mathrm{C}$ \\
\hline D-02-192 & $\mathrm{C}$ & $\mathrm{C}$ \\
\hline D-02-193 & $S$ & C \\
\hline D-02-194 & $S$ & $\mathrm{C}$ \\
\hline D-02-195 & $\mathrm{C}$ & $\mathrm{C}$ \\
\hline D-02-196 & $\mathrm{C}$ & C \\
\hline D-02-197 & $\mathrm{C}$ & $\mathrm{C}$ \\
\hline D-02-198 & C & $\mathrm{C}$ \\
\hline D-02-199 & C & C \\
\hline D-02-200 & C & C \\
\hline D-02-201 & $\mathrm{C}$ & $\mathrm{C}$ \\
\hline
\end{tabular}


Appendix 2. Habitat complexity $(S=$ Simple; $C=$ Complex $)$ determination for all sites looking upstream and downstream from each surveyed reach.

\begin{tabular}{|c|c|c|}
\hline \multirow{2}{*}{$\begin{array}{c}\text { Site } \\
\text { Number }\end{array}$} & \multicolumn{2}{|c|}{ Habitat Complexity } \\
\hline & Upstream & Downstream \\
\hline B-03-001 & C & C \\
\hline B-03-002 & $\mathrm{C}$ & $\mathrm{C}$ \\
\hline B-03-003 & $\mathrm{C}$ & $\mathrm{C}$ \\
\hline B-03-004 & $\mathrm{C}$ & $\mathrm{C}$ \\
\hline B-03-005 & $\mathrm{C}$ & $\mathrm{C}$ \\
\hline B-03-006 & $\mathrm{S}$ & $\mathrm{S}$ \\
\hline B-03-007 & $\mathrm{S}$ & $\mathrm{S}$ \\
\hline B-03-008 & C & $\mathrm{C}$ \\
\hline B-03-009 & $\mathrm{C}$ & $\mathrm{C}$ \\
\hline B-03-010 & $\mathrm{C}$ & $\mathrm{C}$ \\
\hline B-03-011 & $\mathrm{S}$ & $\mathrm{S}$ \\
\hline B-03-012 & $\mathrm{C}$ & $\mathrm{C}$ \\
\hline B-03-013 & $\mathrm{C}$ & $\mathrm{S}$ \\
\hline B-03-014 & $\mathrm{C}$ & $\mathrm{C}$ \\
\hline B-03-015 & $\mathrm{C}$ & $\mathrm{C}$ \\
\hline B-03-016 & $\mathrm{C}$ & $\mathrm{C}$ \\
\hline B-03-017 & $\mathrm{C}$ & $\mathrm{C}$ \\
\hline B-03-018 & $\mathrm{C}$ & $\mathrm{C}$ \\
\hline B-03-019 & $\mathrm{C}$ & $\mathrm{C}$ \\
\hline B-03-020 & $\mathrm{C}$ & $\mathrm{C}$ \\
\hline B-03-021 & $\mathrm{C}$ & $\mathrm{C}$ \\
\hline B-03-022 & $\mathrm{C}$ & $\mathrm{C}$ \\
\hline B-03-023 & $\mathrm{C}$ & C \\
\hline B-03-024 & $\mathrm{C}$ & $\mathrm{C}$ \\
\hline B-03-025 & $\mathrm{C}$ & $\mathrm{C}$ \\
\hline B-03-026 & $\mathrm{C}$ & $\mathrm{C}$ \\
\hline B-03-027 & $\mathrm{C}$ & $\mathrm{C}$ \\
\hline B-03-028 & $\mathrm{C}$ & $\mathrm{C}$ \\
\hline B-03-029 & $\mathrm{C}$ & $\mathrm{C}$ \\
\hline B-03-030 & $\mathrm{C}$ & $\mathrm{C}$ \\
\hline B-03-031 & $\mathrm{C}$ & $C$ \\
\hline B-03-032 & $\mathrm{C}$ & $\mathrm{C}$ \\
\hline B-03-033 & $\mathrm{C}$ & $\mathrm{C}$ \\
\hline B-03-034 & $\mathrm{C}$ & $\mathrm{C}$ \\
\hline
\end{tabular}

\begin{tabular}{|c|c|c|}
\hline \multirow{2}{*}{$\begin{array}{c}\text { Site } \\
\text { Number }\end{array}$} & \multicolumn{2}{|c|}{ Habitat Complexity } \\
\hline & Upstream & Downstream \\
\hline B-03-035 & $\mathrm{C}$ & $\mathrm{C}$ \\
\hline B-03-036 & C & $\mathrm{C}$ \\
\hline B-03-037 & $\mathrm{C}$ & $\mathrm{C}$ \\
\hline B-03-038 & C & C \\
\hline B-03-039 & C & $\mathrm{C}$ \\
\hline B-03-040 & $\mathrm{C}$ & $\mathrm{C}$ \\
\hline B-03-041 & C & C \\
\hline B-03-042 & C & C \\
\hline B-03-043 & $\mathrm{C}$ & $\mathrm{C}$ \\
\hline B-03-044 & $\mathrm{C}$ & $\mathrm{C}$ \\
\hline B-03-045 & C & C \\
\hline B-03-046 & $\mathrm{C}$ & $\mathrm{C}$ \\
\hline B-03-047 & C & $\mathrm{C}$ \\
\hline B-03-048 & $\mathrm{C}$ & C \\
\hline B-03-049 & C & C \\
\hline B-03-050 & C & $\mathrm{C}$ \\
\hline B-03-051 & $\mathrm{C}$ & $\mathrm{C}$ \\
\hline B-03-052 & C & $\mathrm{C}$ \\
\hline B-03-053 & $\mathrm{C}$ & $\mathrm{C}$ \\
\hline B-03-054 & C & C \\
\hline B-03-055 & $\mathrm{C}$ & $\mathrm{C}$ \\
\hline B-03-056 & $\mathrm{C}$ & $S$ \\
\hline B-03-057 & C & C \\
\hline B-03-058 & $\mathrm{C}$ & $\mathrm{C}$ \\
\hline B-03-059 & S & S \\
\hline B-03-060 & $\mathrm{C}$ & $\mathrm{C}$ \\
\hline B-03-061 & C & C \\
\hline B-03-062 & C & C \\
\hline B-03-063 & C & C \\
\hline B-03-064 & $S$ & C \\
\hline B-03-065 & $\mathrm{C}$ & C \\
\hline B-03-066 & C & C \\
\hline B-03-067 & C & C \\
\hline B-03-068 & C & C \\
\hline
\end{tabular}

\begin{tabular}{|c|c|c|}
\hline \multirow{2}{*}{$\begin{array}{c}\text { Site } \\
\text { Number }\end{array}$} & \multicolumn{2}{|c|}{ Habitat Complexity } \\
\hline & Upstream & Downstream \\
\hline B-03-069 & $\mathrm{C}$ & $\mathrm{C}$ \\
\hline B-03-070 & $\mathrm{C}$ & $\mathrm{C}$ \\
\hline B-03-071 & $\mathrm{C}$ & $\mathrm{C}$ \\
\hline B-03-072 & $\mathrm{C}$ & $\mathrm{C}$ \\
\hline B-03-073 & $\mathrm{C}$ & $\mathrm{C}$ \\
\hline B-03-074 & $\mathrm{C}$ & $\mathrm{C}$ \\
\hline B-03-075 & $\mathrm{C}$ & $\mathrm{C}$ \\
\hline B-03-076 & $\mathrm{C}$ & C \\
\hline B-03-077 & $\mathrm{C}$ & $\mathrm{C}$ \\
\hline B-03-078 & $\mathrm{S}$ & $\mathrm{C}$ \\
\hline B-03-079 & $\mathrm{C}$ & C \\
\hline B-03-080 & $\mathrm{C}$ & $\mathrm{C}$ \\
\hline B-03-081 & $\mathrm{C}$ & $\mathrm{C}$ \\
\hline B-03-082 & $\mathrm{C}$ & C \\
\hline B-03-083 & $\mathrm{S}$ & $\mathrm{S}$ \\
\hline B-03-084 & $\mathrm{S}$ & $\mathrm{S}$ \\
\hline B-03-085 & $\mathrm{S}$ & $\mathrm{S}$ \\
\hline B-03-086 & $\mathrm{S}$ & $\mathrm{S}$ \\
\hline B-03-087 & $\mathrm{C}$ & $\mathrm{C}$ \\
\hline B-03-088 & $\mathrm{C}$ & C \\
\hline B-03-089 & $\mathrm{C}$ & $\mathrm{C}$ \\
\hline B-03-090 & $\mathrm{S}$ & $\mathrm{S}$ \\
\hline B-03-091 & S & $\mathrm{S}$ \\
\hline B-03-092 & $\mathrm{S}$ & $\mathrm{S}$ \\
\hline B-03-093 & $\mathrm{S}$ & $\mathrm{S}$ \\
\hline B-03-094 & S & $\mathrm{S}$ \\
\hline B-03-095 & $\mathrm{S}$ & $\mathrm{S}$ \\
\hline B-03-096 & $\mathrm{S}$ & $\mathrm{S}$ \\
\hline B-03-097 & C & C \\
\hline B-03-098 & C & C \\
\hline B-03-099 & $\mathrm{C}$ & C \\
\hline B-03-100 & $\mathrm{C}$ & $\mathrm{C}$ \\
\hline B-03-101 & $\mathrm{S}$ & C \\
\hline B-03-102 & C & C \\
\hline
\end{tabular}


Appendix 2. Habitat complexity $(S=$ Simple; $C=$ Complex $)$ determination for all sites looking upstream and downstream from each surveyed reach.

\begin{tabular}{|c|c|c|}
\hline \multirow{2}{*}{$\begin{array}{c}\text { Site } \\
\text { Number }\end{array}$} & \multicolumn{2}{|c|}{ Habitat Complexity } \\
\hline & Upstream & Downstream \\
\hline B-03-103 & C & C \\
\hline B-03-104 & $\mathrm{C}$ & $\mathrm{C}$ \\
\hline B-03-105 & $\mathrm{C}$ & $\mathrm{C}$ \\
\hline B-03-106 & $\mathrm{C}$ & $\mathrm{C}$ \\
\hline B-03-107 & $\mathrm{S}$ & $\mathrm{C}$ \\
\hline B-03-108 & $\mathrm{C}$ & $\mathrm{C}$ \\
\hline B-03-109 & C & C \\
\hline B-03-110 & $\mathrm{S}$ & $\mathrm{S}$ \\
\hline B-03-111 & $\mathrm{S}$ & $\mathrm{S}$ \\
\hline B-03-112 & $\mathrm{C}$ & $\mathrm{C}$ \\
\hline B-03-113 & C & $\mathrm{C}$ \\
\hline B-03-114 & $\mathrm{C}$ & $\mathrm{C}$ \\
\hline B-03-115 & $\mathrm{S}$ & $\mathrm{C}$ \\
\hline B-03-116 & C & $\mathrm{C}$ \\
\hline B-03-117 & $\mathrm{C}$ & $\mathrm{C}$ \\
\hline B-03-118 & $\mathrm{C}$ & $\mathrm{C}$ \\
\hline B-03-119 & $\mathrm{C}$ & $\mathrm{C}$ \\
\hline B-03-120 & $\mathrm{C}$ & $\mathrm{S}$ \\
\hline B-03-121 & $\mathrm{C}$ & $\mathrm{C}$ \\
\hline B-03-122 & $\mathrm{C}$ & $\mathrm{C}$ \\
\hline B-03-123 & $\mathrm{S}$ & $\mathrm{S}$ \\
\hline B-03-124 & $\mathrm{C}$ & $\mathrm{C}$ \\
\hline B-03-125 & $\mathrm{S}$ & $\mathrm{S}$ \\
\hline B-03-126 & $\mathrm{C}$ & $\mathrm{C}$ \\
\hline B-03-127 & $\mathrm{C}$ & $\mathrm{C}$ \\
\hline B-03-128 & $\mathrm{C}$ & $\mathrm{C}$ \\
\hline B-03-129 & $\mathrm{C}$ & $\mathrm{C}$ \\
\hline B-03-130 & $\mathrm{S}$ & $\mathrm{S}$ \\
\hline B-03-131 & $\mathrm{C}$ & $\mathrm{C}$ \\
\hline B-03-132 & $\mathrm{C}$ & $\mathrm{C}$ \\
\hline B-03-133 & $\mathrm{C}$ & $C$ \\
\hline B-03-134 & $\mathrm{C}$ & $\mathrm{C}$ \\
\hline B-03-135 & $\mathrm{C}$ & $\mathrm{C}$ \\
\hline B-03-136 & $\mathrm{C}$ & $\mathrm{C}$ \\
\hline
\end{tabular}

\begin{tabular}{|c|c|c|}
\hline \multirow{2}{*}{$\begin{array}{c}\text { Site } \\
\text { Number }\end{array}$} & \multicolumn{2}{|c|}{ Habitat Complexity } \\
\hline & Upstream & Downstream \\
\hline B-03-137 & $\mathrm{C}$ & $\mathrm{C}$ \\
\hline B-03-138 & $\mathrm{S}$ & $\mathrm{S}$ \\
\hline B-03-139 & $\mathrm{S}$ & $\mathrm{C}$ \\
\hline B-03-140 & $\mathrm{C}$ & $\mathrm{C}$ \\
\hline B-03-141 & $\mathrm{C}$ & $\mathrm{C}$ \\
\hline B-03-142 & $\mathrm{C}$ & $\mathrm{C}$ \\
\hline B-03-143 & C & C \\
\hline B-03-144 & $\mathrm{C}$ & $\mathrm{C}$ \\
\hline B-03-145 & S & $\mathrm{C}$ \\
\hline B-03-146 & $\mathrm{C}$ & $\mathrm{C}$ \\
\hline B-03-147 & S & C \\
\hline B-03-148 & $\mathrm{C}$ & $\mathrm{C}$ \\
\hline B-03-149 & $\mathrm{C}$ & $\mathrm{C}$ \\
\hline B-03-150 & C & C \\
\hline B-03-151 & $\mathrm{C}$ & C \\
\hline B-03-152 & $\mathrm{C}$ & $\mathrm{C}$ \\
\hline B-03-153 & C & C \\
\hline B-03-154 & $\mathrm{C}$ & $\mathrm{C}$ \\
\hline B-03-155 & $\mathrm{C}$ & $\mathrm{C}$ \\
\hline B-03-156 & C & $\mathrm{C}$ \\
\hline B-03-157 & $\mathrm{C}$ & $\mathrm{C}$ \\
\hline B-03-158 & $\mathrm{C}$ & $\mathrm{C}$ \\
\hline B-03-159 & $\mathrm{C}$ & C \\
\hline B-03-160 & $\mathrm{C}$ & $\mathrm{C}$ \\
\hline B-03-161 & $\mathrm{C}$ & $\mathrm{C}$ \\
\hline B-03-162 & C & C \\
\hline B-03-163 & C & C \\
\hline B-03-164 & $S$ & $\mathrm{~S}$ \\
\hline B-03-165 & $S$ & C \\
\hline B-03-166 & $\mathrm{S}$ & $\mathrm{S}$ \\
\hline B-03-167 & $\mathrm{S}$ & $\mathrm{S}$ \\
\hline B-03-168 & $\mathrm{S}$ & $S$ \\
\hline B-03-169 & $\mathrm{S}$ & $\mathrm{S}$ \\
\hline B-03-170 & $\mathrm{S}$ & $\mathrm{S}$ \\
\hline
\end{tabular}

\begin{tabular}{|c|c|c|}
\hline \multirow{2}{*}{$\begin{array}{c}\text { Site } \\
\text { Number }\end{array}$} & \multicolumn{2}{|c|}{ Habitat Complexity } \\
\hline & Upstream & Downstream \\
\hline B-03-171 & $\mathrm{S}$ & $S$ \\
\hline B-03-172 & $\mathrm{S}$ & $\mathrm{S}$ \\
\hline B-03-173 & $\mathrm{S}$ & $\mathrm{S}$ \\
\hline B-03-174 & $\mathrm{S}$ & $\mathrm{S}$ \\
\hline B-03-175 & $\mathrm{S}$ & $\mathrm{S}$ \\
\hline B-03-176 & $\mathrm{S}$ & $\mathrm{S}$ \\
\hline B-03-177 & S & $\mathrm{S}$ \\
\hline B-03-178 & $\mathrm{S}$ & $S$ \\
\hline B-03-179 & $\mathrm{S}$ & $\mathrm{S}$ \\
\hline B-03-180 & $\mathrm{S}$ & $\mathrm{C}$ \\
\hline B-03-181 & $\mathrm{C}$ & C \\
\hline B-03-182 & $\mathrm{S}$ & $\mathrm{S}$ \\
\hline B-03-183 & $\mathrm{S}$ & $\mathrm{C}$ \\
\hline B-03-184 & $\mathrm{S}$ & C \\
\hline B-03-185 & $\mathrm{C}$ & $\mathrm{C}$ \\
\hline B-03-186 & $\mathrm{C}$ & $\mathrm{C}$ \\
\hline B-03-187 & $\mathrm{S}$ & $\mathrm{C}$ \\
\hline B-03-188 & $\mathrm{S}$ & $\mathrm{S}$ \\
\hline B-03-189 & S & $\mathrm{S}$ \\
\hline B-03-190 & $\mathrm{C}$ & C \\
\hline B-03-191 & $\mathrm{C}$ & $\mathrm{S}$ \\
\hline B-03-192 & $\mathrm{S}$ & $\mathrm{S}$ \\
\hline B-03-193 & $\mathrm{C}$ & $\mathrm{C}$ \\
\hline B-03-194 & $\mathrm{C}$ & $\mathrm{C}$ \\
\hline B-03-195 & $\mathrm{S}$ & $\mathrm{S}$ \\
\hline B-03-196 & S & $\mathrm{S}$ \\
\hline B-03-197 & $\mathrm{S}$ & $\mathrm{S}$ \\
\hline B-03-198 & $\mathrm{S}$ & $\mathrm{S}$ \\
\hline B-03-199 & $\mathrm{S}$ & $S$ \\
\hline B-03-200 & C & C \\
\hline B-03-201 & $\mathrm{C}$ & C \\
\hline B-03-202 & $\mathrm{C}$ & C \\
\hline B-03-203 & $\mathrm{S}$ & $S$ \\
\hline B-03-204 & $\mathrm{S}$ & $\mathrm{S}$ \\
\hline
\end{tabular}


Appendix 2. Habitat complexity $(S=$ Simple; $C=$ Complex $)$ determination for all sites looking upstream and downstream from each surveyed reach.

\begin{tabular}{|c|c|c|}
\hline \multirow{2}{*}{$\begin{array}{c}\text { Site } \\
\text { Number }\end{array}$} & \multicolumn{2}{|c|}{ Habitat Complexity } \\
\hline & Upstream & Downstream \\
\hline B-03-205 & $\mathrm{C}$ & C \\
\hline B-03-206 & $S$ & $\mathrm{C}$ \\
\hline B-03-207 & S & $\mathrm{C}$ \\
\hline B-03-208 & $\mathrm{S}$ & $\mathrm{C}$ \\
\hline B-03-209 & $\mathrm{C}$ & $\mathrm{C}$ \\
\hline B-03-210 & $\mathrm{C}$ & $\mathrm{C}$ \\
\hline B-03-211 & C & C \\
\hline B-03-212 & $\mathrm{S}$ & $\mathrm{S}$ \\
\hline B-03-213 & $\mathrm{S}$ & $\mathrm{S}$ \\
\hline B-03-214 & $\mathrm{C}$ & $\mathrm{C}$ \\
\hline B-03-215 & C & $\mathrm{C}$ \\
\hline B-03-216 & $\mathrm{S}$ & $\mathrm{S}$ \\
\hline B-03-217 & S & $\mathrm{S}$ \\
\hline B-03-218 & $\mathrm{S}$ & $\mathrm{S}$ \\
\hline B-03-219 & $S$ & $\mathrm{C}$ \\
\hline B-03-220 & $\mathrm{C}$ & $\mathrm{C}$ \\
\hline B-03-221 & $\mathrm{C}$ & $\mathrm{C}$ \\
\hline B-03-222 & $\mathrm{C}$ & $\mathrm{C}$ \\
\hline $\mathrm{B}-03-223$ & $\mathrm{C}$ & $\mathrm{C}$ \\
\hline B-03-224 & C & $\mathrm{C}$ \\
\hline B-03-225 & $\mathrm{S}$ & $\mathrm{S}$ \\
\hline B-03-226 & $\mathrm{S}$ & $\mathrm{S}$ \\
\hline B-03-227 & $\mathrm{C}$ & C \\
\hline B-03-228 & $\mathrm{C}$ & $\mathrm{C}$ \\
\hline B-03-229 & S & $\mathrm{S}$ \\
\hline B-03-230 & $\mathrm{S}$ & $\mathrm{S}$ \\
\hline B-03-231 & C & $\mathrm{C}$ \\
\hline B-03-232 & $\mathrm{C}$ & $\mathrm{C}$ \\
\hline B-03-233 & $\mathrm{C}$ & $\mathrm{C}$ \\
\hline B-03-234 & $\mathrm{S}$ & $\mathrm{S}$ \\
\hline B-03-235 & $\mathrm{S}$ & $\mathrm{S}$ \\
\hline B-03-236 & $S$ & $S$ \\
\hline B-03-237 & $\mathrm{S}$ & $\mathrm{S}$ \\
\hline B-03-238 & $\mathrm{S}$ & $\mathrm{S}$ \\
\hline
\end{tabular}

\begin{tabular}{|c|c|c|}
\hline \multirow{2}{*}{$\begin{array}{c}\text { Site } \\
\text { Number }\end{array}$} & \multicolumn{2}{|c|}{ Habitat Complexity } \\
\hline & Upstream & Downstream \\
\hline B-03-239 & $\mathrm{C}$ & $\mathrm{C}$ \\
\hline B-03-240 & $\mathrm{S}$ & $\mathrm{S}$ \\
\hline B-03-241 & $\mathrm{S}$ & $\mathrm{S}$ \\
\hline B-03-242 & $\mathrm{S}$ & $\mathrm{S}$ \\
\hline B-03-243 & $\mathrm{S}$ & $\mathrm{S}$ \\
\hline B-03-244 & $\mathrm{C}$ & $\mathrm{S}$ \\
\hline B-03-245 & $\mathrm{S}$ & $\mathrm{S}$ \\
\hline B-03-246 & $\mathrm{S}$ & $\mathrm{S}$ \\
\hline B-03-247 & $\mathrm{S}$ & $\mathrm{S}$ \\
\hline B-03-248 & $\mathrm{S}$ & $\mathrm{S}$ \\
\hline B-03-249 & $\mathrm{C}$ & C \\
\hline B-03-250 & $\mathrm{S}$ & $\mathrm{S}$ \\
\hline D-03-001 & C & C \\
\hline D-03-002 & C & C \\
\hline D-03-003 & C & C \\
\hline D-03-004 & C & C \\
\hline D-03-005 & C & C \\
\hline D-03-006 & $\mathrm{C}$ & C \\
\hline D-03-007 & $\mathrm{S}$ & $\mathrm{C}$ \\
\hline D-03-008 & C & C \\
\hline D-03-009 & $\mathrm{C}$ & $\mathrm{C}$ \\
\hline D-03-010 & $\mathrm{C}$ & $\mathrm{C}$ \\
\hline D-03-011 & C & C \\
\hline D-03-012 & C & C \\
\hline D-03-013 & C & C \\
\hline D-03-014 & C & C \\
\hline D-03-015 & C & C \\
\hline D-03-016 & C & C \\
\hline D-03-017 & C & C \\
\hline D-03-018 & C & C \\
\hline D-03-019 & C & C \\
\hline D-03-020 & $\mathrm{S}$ & $\mathrm{S}$ \\
\hline D-03-021 & C & C \\
\hline D-03-022 & C & C \\
\hline
\end{tabular}

\begin{tabular}{|c|c|c|}
\hline \multirow{2}{*}{$\begin{array}{c}\text { Site } \\
\text { Number }\end{array}$} & \multicolumn{2}{|c|}{ Habitat Complexity } \\
\hline & Upstream & Downstream \\
\hline D-03-023 & $\mathrm{C}$ & $S$ \\
\hline D-03-024 & $\mathrm{C}$ & $\mathrm{C}$ \\
\hline D-03-025 & $\mathrm{C}$ & $\mathrm{C}$ \\
\hline D-03-026 & $\mathrm{S}$ & $\mathrm{C}$ \\
\hline D-03-027 & $\mathrm{C}$ & $\mathrm{C}$ \\
\hline D-03-028 & $\mathrm{C}$ & $\mathrm{C}$ \\
\hline D-03-029 & $\mathrm{C}$ & $\mathrm{C}$ \\
\hline D-03-030 & $\mathrm{C}$ & $\mathrm{C}$ \\
\hline D-03-031 & $\mathrm{C}$ & $\mathrm{C}$ \\
\hline D-03-032 & $\mathrm{C}$ & $\mathrm{C}$ \\
\hline D-03-033 & $\mathrm{C}$ & $\mathrm{C}$ \\
\hline D-03-034 & $\mathrm{S}$ & $\mathrm{C}$ \\
\hline D-03-035 & $\mathrm{C}$ & $\mathrm{C}$ \\
\hline D-03-036 & $\mathrm{C}$ & C \\
\hline D-03-037 & $\mathrm{C}$ & $\mathrm{C}$ \\
\hline D-03-038 & $\mathrm{C}$ & $\mathrm{C}$ \\
\hline D-03-039 & $\mathrm{S}$ & $\mathrm{S}$ \\
\hline D-03-040 & $\mathrm{C}$ & $\mathrm{C}$ \\
\hline D-03-041 & $\mathrm{C}$ & $\mathrm{C}$ \\
\hline D-03-042 & $\mathrm{S}$ & $\mathrm{S}$ \\
\hline D-03-043 & $\mathrm{C}$ & $\mathrm{S}$ \\
\hline D-03-044 & $\mathrm{S}$ & $\mathrm{S}$ \\
\hline D-03-045 & $\mathrm{C}$ & $\mathrm{S}$ \\
\hline D-03-046 & $\mathrm{S}$ & $\mathrm{S}$ \\
\hline D-03-047 & $\mathrm{S}$ & $\mathrm{S}$ \\
\hline D-03-048 & $\mathrm{C}$ & C \\
\hline D-03-049 & $\mathrm{S}$ & $\mathrm{S}$ \\
\hline D-03-050 & $\mathrm{S}$ & $\mathrm{C}$ \\
\hline D-03-051 & $\mathrm{S}$ & $S$ \\
\hline D-03-052 & C & C \\
\hline D-03-053 & $\mathrm{S}$ & $S$ \\
\hline D-03-054 & $S$ & $S$ \\
\hline D-03-055 & $\mathrm{C}$ & C \\
\hline D-03-056 & $\mathrm{C}$ & C \\
\hline
\end{tabular}


Appendix 2. Habitat complexity $(S=$ Simple; $C=$ Complex $)$ determination for all sites looking upstream and downstream from each surveyed reach.

\begin{tabular}{|c|c|c|}
\hline \multirow{2}{*}{$\begin{array}{c}\text { Site } \\
\text { Number }\end{array}$} & \multicolumn{2}{|c|}{ Habitat Complexity } \\
\hline & Upstream & Downstream \\
\hline D-03-057 & $\mathrm{C}$ & $\mathrm{C}$ \\
\hline D-03-058 & C & $\mathrm{C}$ \\
\hline D-03-059 & $\mathrm{C}$ & $\mathrm{C}$ \\
\hline D-03-060 & C & C \\
\hline D-03-061 & $S$ & $\mathrm{~S}$ \\
\hline D-03-062 & $\mathrm{C}$ & $\mathrm{C}$ \\
\hline D-03-063 & C & C \\
\hline D-03-064 & C & C \\
\hline D-03-065 & $\mathrm{S}$ & $\mathrm{S}$ \\
\hline D-03-066 & $\mathrm{C}$ & $\mathrm{C}$ \\
\hline D-03-067 & C & C \\
\hline D-03-068 & C & $\mathrm{C}$ \\
\hline D-03-069 & $\mathrm{C}$ & $\mathrm{C}$ \\
\hline D-03-070 & S & $\mathrm{S}$ \\
\hline D-03-071 & C & C \\
\hline D-03-072 & C & $\mathrm{C}$ \\
\hline D-03-073 & $\mathrm{C}$ & $\mathrm{C}$ \\
\hline D-03-074 & $\mathrm{C}$ & $\mathrm{C}$ \\
\hline D-03-075 & $\mathrm{C}$ & $\mathrm{C}$ \\
\hline D-03-076 & $\mathrm{C}$ & $\mathrm{C}$ \\
\hline D-03-077 & $\mathrm{C}$ & $\mathrm{C}$ \\
\hline D-03-078 & $\mathrm{C}$ & $\mathrm{C}$ \\
\hline D-03-079 & C & C \\
\hline D-03-080 & $\mathrm{C}$ & $\mathrm{C}$ \\
\hline D-03-081 & $\mathrm{C}$ & $\mathrm{C}$ \\
\hline D-03-082 & C & C \\
\hline D-03-083 & C & C \\
\hline D-03-084 & $\mathrm{C}$ & $\mathrm{C}$ \\
\hline D-03-085 & C & C \\
\hline D-03-086 & C & C \\
\hline D-03-087 & $\mathrm{S}$ & $\mathrm{S}$ \\
\hline D-03-088 & $\mathrm{C}$ & C \\
\hline D-03-089 & C & C \\
\hline D-03-090 & C & C \\
\hline
\end{tabular}

\begin{tabular}{|c|c|c|}
\hline \multirow{2}{*}{$\begin{array}{c}\text { Site } \\
\text { Number }\end{array}$} & \multicolumn{2}{|c|}{ Habitat Complexity } \\
\hline & Upstream & Downstream \\
\hline D-03-091 & C & C \\
\hline D-03-092 & $\mathrm{C}$ & $\mathrm{C}$ \\
\hline D-03-093 & $\mathrm{C}$ & C \\
\hline D-03-094 & $\mathrm{C}$ & C \\
\hline D-03-095 & $\mathrm{C}$ & $\mathrm{C}$ \\
\hline D-03-096 & $\mathrm{C}$ & $\mathrm{C}$ \\
\hline D-03-097 & C & C \\
\hline D-03-098 & $\mathrm{C}$ & $\mathrm{C}$ \\
\hline D-03-099 & $\mathrm{C}$ & $\mathrm{C}$ \\
\hline D-03-100 & $\mathrm{C}$ & $\mathrm{C}$ \\
\hline D-03-101 & $\mathrm{C}$ & C \\
\hline D-03-102 & $\mathrm{C}$ & $\mathrm{C}$ \\
\hline D-03-103 & $\mathrm{C}$ & $\mathrm{C}$ \\
\hline D-03-104 & $\mathrm{S}$ & C \\
\hline D-03-105 & $\mathrm{C}$ & $\mathrm{C}$ \\
\hline D-03-106 & $\mathrm{S}$ & $\mathrm{S}$ \\
\hline D-03-107 & $\mathrm{S}$ & $\mathrm{S}$ \\
\hline D-03-108 & $\mathrm{C}$ & $\mathrm{C}$ \\
\hline D-03-109 & $\mathrm{C}$ & $\mathrm{C}$ \\
\hline D-03-110 & C & C \\
\hline D-03-111 & $\mathrm{C}$ & $\mathrm{C}$ \\
\hline D-03-112 & $\mathrm{C}$ & $\mathrm{C}$ \\
\hline D-03-113 & $\mathrm{C}$ & C \\
\hline D-03-114 & $\mathrm{C}$ & $\mathrm{C}$ \\
\hline D-03-115 & $\mathrm{C}$ & $\mathrm{C}$ \\
\hline D-03-116 & C & C \\
\hline D-03-117 & C & C \\
\hline D-03-118 & $\mathrm{C}$ & $\mathrm{C}$ \\
\hline D-03-119 & $\mathrm{S}$ & $S$ \\
\hline D-03-120 & $\mathrm{S}$ & $\mathrm{C}$ \\
\hline D-03-121 & $\mathrm{C}$ & C \\
\hline D-03-122 & C & C \\
\hline D-03-123 & $\mathrm{S}$ & $\mathrm{S}$ \\
\hline D-03-124 & $\mathrm{S}$ & $\mathrm{S}$ \\
\hline
\end{tabular}

\begin{tabular}{|c|c|c|}
\hline \multirow{2}{*}{$\begin{array}{c}\text { Site } \\
\text { Number }\end{array}$} & \multicolumn{2}{|c|}{ Habitat Complexity } \\
\hline & Upstream & Downstream \\
\hline D-03-125 & $\mathrm{S}$ & $S$ \\
\hline D-03-126 & $\mathrm{S}$ & $\mathrm{S}$ \\
\hline D-03-127 & $\mathrm{S}$ & $\mathrm{S}$ \\
\hline D-03-128 & $\mathrm{S}$ & $\mathrm{S}$ \\
\hline D-03-129 & $S$ & $\mathrm{~S}$ \\
\hline D-03-130 & $\mathrm{S}$ & $\mathrm{S}$ \\
\hline D-03-131 & $\mathrm{C}$ & $\mathrm{C}$ \\
\hline D-03-132 & $\mathrm{C}$ & $\mathrm{C}$ \\
\hline D-03-133 & $\mathrm{S}$ & $\mathrm{S}$ \\
\hline D-03-134 & S & $\mathrm{S}$ \\
\hline D-03-135 & $\mathrm{C}$ & C \\
\hline D-03-136 & $\mathrm{S}$ & $\mathrm{S}$ \\
\hline D-03-137 & $\mathrm{C}$ & $\mathrm{C}$ \\
\hline D-03-138 & $\mathrm{S}$ & $\mathrm{S}$ \\
\hline D-03-139 & $\mathrm{C}$ & $\mathrm{C}$ \\
\hline D-03-140 & $\mathrm{C}$ & $\mathrm{C}$ \\
\hline D-03-141 & $\mathrm{S}$ & $\mathrm{S}$ \\
\hline D-03-142 & $\mathrm{C}$ & $\mathrm{C}$ \\
\hline D-03-143 & $\mathrm{C}$ & $\mathrm{C}$ \\
\hline D-03-144 & $\mathrm{C}$ & $\mathrm{C}$ \\
\hline D-03-145 & $\mathrm{C}$ & $\mathrm{C}$ \\
\hline D-03-146 & $\mathrm{C}$ & $\mathrm{C}$ \\
\hline D-03-147 & $\mathrm{C}$ & $\mathrm{C}$ \\
\hline D-03-148 & $\mathrm{C}$ & $\mathrm{C}$ \\
\hline D-03-149 & $\mathrm{C}$ & $\mathrm{C}$ \\
\hline D-03-150 & $\mathrm{C}$ & $\mathrm{S}$ \\
\hline D-03-151 & $\mathrm{S}$ & $\mathrm{S}$ \\
\hline D-03-152 & $\mathrm{S}$ & $\mathrm{C}$ \\
\hline D-03-153 & C & C \\
\hline D-03-154 & C & C \\
\hline D-03-155 & $\mathrm{C}$ & C \\
\hline D-03-156 & $\mathrm{C}$ & $\mathrm{C}$ \\
\hline D-03-157 & $\mathrm{C}$ & C \\
\hline D-03-158 & $\mathrm{C}$ & C \\
\hline
\end{tabular}


Appendix 2. Habitat complexity $(S=$ Simple; $C=$ Complex $)$ determination for all sites looking upstream and downstream from each surveyed reach.

\begin{tabular}{|c|c|c|}
\hline \multirow{2}{*}{$\begin{array}{c}\text { Site } \\
\text { Number }\end{array}$} & \multicolumn{2}{|c|}{ Habitat Complexity } \\
\hline & Upstream & Downstream \\
\hline D-03-159 & $\mathrm{C}$ & $\mathrm{C}$ \\
\hline D-03-160 & $\mathrm{S}$ & $\mathrm{S}$ \\
\hline D-03-161 & $\mathrm{S}$ & $\mathrm{S}$ \\
\hline D-03-162 & S & C \\
\hline D-03-163 & $S$ & $S$ \\
\hline D-03-164 & C & C \\
\hline D-03-165 & C & C \\
\hline D-03-166 & C & C \\
\hline D-03-167 & C & $\mathrm{C}$ \\
\hline D-03-168 & $\mathrm{C}$ & $\mathrm{C}$ \\
\hline D-03-169 & C & C \\
\hline D-03-170 & $\mathrm{C}$ & $\mathrm{C}$ \\
\hline W-03-001 & S & S \\
\hline W-03-002 & $\mathrm{C}$ & $\mathrm{S}$ \\
\hline W-03-003 & C & C \\
\hline W-03-004 & C & $\mathrm{C}$ \\
\hline W-03-005 & $\mathrm{C}$ & $\mathrm{C}$ \\
\hline W-03-006 & C & $\mathrm{C}$ \\
\hline W-03-007 & $\mathrm{C}$ & $\mathrm{C}$ \\
\hline W-03-008 & S & $\mathrm{S}$ \\
\hline W-03-009 & $\mathrm{C}$ & $\mathrm{C}$ \\
\hline W-03-010 & S & $\mathrm{S}$ \\
\hline W-03-011 & S & $\mathrm{S}$ \\
\hline W-03-012 & $S$ & $\mathrm{~S}$ \\
\hline W-03-013 & $\mathrm{S}$ & $\mathrm{C}$ \\
\hline W-03-014 & S & $\mathrm{S}$ \\
\hline W-03-015 & $\mathrm{S}$ & $\mathrm{S}$ \\
\hline W-03-016 & $\mathrm{S}$ & $\mathrm{S}$ \\
\hline W-03-017 & S & $\mathrm{S}$ \\
\hline W-03-018 & $\mathrm{S}$ & $\mathrm{S}$ \\
\hline W-03-019 & $\mathrm{S}$ & $\mathrm{S}$ \\
\hline W-03-020 & $\mathrm{S}$ & $\mathrm{S}$ \\
\hline W-03-021 & $\mathrm{S}$ & $\mathrm{S}$ \\
\hline W-03-022 & $\mathrm{S}$ & $\mathrm{S}$ \\
\hline
\end{tabular}

\begin{tabular}{|c|c|c|}
\hline \multirow{2}{*}{$\begin{array}{c}\text { Site } \\
\text { Number }\end{array}$} & \multicolumn{2}{|c|}{ Habitat Complexity } \\
\hline & Upstream & Downstream \\
\hline W-03-023 & $\mathrm{S}$ & $S$ \\
\hline W-03-024 & $\mathrm{S}$ & S \\
\hline W-03-025 & S & S \\
\hline W-03-026 & $\mathrm{S}$ & $\mathrm{S}$ \\
\hline W-03-027 & $S$ & $S$ \\
\hline W-03-028 & $\mathrm{S}$ & S \\
\hline W-03-029 & S & S \\
\hline W-03-030 & $\mathrm{S}$ & $S$ \\
\hline W-03-031 & $\mathrm{S}$ & S \\
\hline W-03-032 & S & S \\
\hline W-03-033 & $\mathrm{S}$ & $S$ \\
\hline W-03-034 & $\mathrm{S}$ & $S$ \\
\hline W-03-035 & $\mathrm{S}$ & S \\
\hline W-03-036 & $\mathrm{S}$ & S \\
\hline W-03-037 & $\mathrm{S}$ & $\mathrm{S}$ \\
\hline W-03-038 & $\mathrm{S}$ & S \\
\hline W-03-039 & $\mathrm{S}$ & $S$ \\
\hline W-03-040 & $\mathrm{S}$ & $S$ \\
\hline W-03-041 & $\mathrm{S}$ & S \\
\hline W-03-042 & $\mathrm{S}$ & $S$ \\
\hline W-03-043 & $\mathrm{S}$ & $S$ \\
\hline W-03-044 & $\mathrm{C}$ & $\mathrm{C}$ \\
\hline W-03-045 & $\mathrm{S}$ & S \\
\hline W-03-046 & $\mathrm{C}$ & $\mathrm{C}$ \\
\hline W-03-047 & $\mathrm{S}$ & S \\
\hline W-03-048 & $\mathrm{S}$ & S \\
\hline W-03-049 & C & $\mathrm{C}$ \\
\hline W-03-050 & $\mathrm{C}$ & $\mathrm{C}$ \\
\hline W-03-051 & C & $\mathrm{C}$ \\
\hline W-03-052 & $\mathrm{S}$ & $\mathrm{S}$ \\
\hline W-03-053 & $\mathrm{S}$ & $S$ \\
\hline W-03-054 & $\mathrm{C}$ & C \\
\hline W-03-055 & $\mathrm{S}$ & $\mathrm{S}$ \\
\hline W-03-056 & C & C \\
\hline
\end{tabular}

\begin{tabular}{|c|c|c|}
\hline \multirow{2}{*}{$\begin{array}{c}\text { Site } \\
\text { Number }\end{array}$} & \multicolumn{2}{|c|}{ Habitat Complexity } \\
\hline & Upstream & Downstream \\
\hline W-03-057 & $S$ & $\mathrm{~S}$ \\
\hline W-03-058 & $\mathrm{S}$ & $\mathrm{S}$ \\
\hline W-03-059 & $\mathrm{C}$ & $\mathrm{C}$ \\
\hline W-03-060 & $\mathrm{S}$ & $\mathrm{S}$ \\
\hline W-03-061 & $\mathrm{C}$ & $\mathrm{C}$ \\
\hline W-03-062 & $\mathrm{C}$ & $\mathrm{C}$ \\
\hline W-03-063 & $S$ & S \\
\hline W-03-064 & $\mathrm{C}$ & $\mathrm{C}$ \\
\hline W-03-065 & $\mathrm{C}$ & $\mathrm{C}$ \\
\hline W-03-066 & $\mathrm{C}$ & $\mathrm{C}$ \\
\hline W-03-067 & $\mathrm{C}$ & $\mathrm{C}$ \\
\hline W-03-068 & $\mathrm{S}$ & $\mathrm{S}$ \\
\hline W-03-069 & $\mathrm{C}$ & $\mathrm{C}$ \\
\hline W-03-070 & $\mathrm{C}$ & $\mathrm{C}$ \\
\hline W-03-071 & $S$ & $S$ \\
\hline W-03-072 & $\mathrm{S}$ & $\mathrm{S}$ \\
\hline W-03-073 & $\mathrm{C}$ & $\mathrm{C}$ \\
\hline W-03-074 & $\mathrm{C}$ & $\mathrm{C}$ \\
\hline W-03-075 & $\mathrm{C}$ & $\mathrm{C}$ \\
\hline W-03-076 & $S$ & S \\
\hline W-03-077 & $\mathrm{C}$ & $\mathrm{C}$ \\
\hline W-03-078 & S & $\mathrm{C}$ \\
\hline W-03-079 & C & $\mathrm{C}$ \\
\hline W-03-080 & $\mathrm{S}$ & $\mathrm{C}$ \\
\hline W-03-081 & $\mathrm{S}$ & S \\
\hline W-03-082 & S & S \\
\hline W-03-083 & $S$ & $S$ \\
\hline W-03-084 & $S$ & $\mathrm{~S}$ \\
\hline W-03-085 & $S$ & $S$ \\
\hline W-03-086 & $S$ & $S$ \\
\hline W-03-087 & $\mathrm{C}$ & $\mathrm{C}$ \\
\hline W-03-088 & $S$ & $S$ \\
\hline W-03-089 & $\mathrm{S}$ & $S$ \\
\hline W-03-090 & $\mathrm{S}$ & $\mathrm{S}$ \\
\hline
\end{tabular}


Appendix 2. Habitat complexity $(S=$ Simple; $C=$ Complex $)$ determination for all sites looking upstream and downstream from each surveyed reach.

\begin{tabular}{|c|c|c|}
\hline \multirow{2}{*}{$\begin{array}{c}\text { Site } \\
\text { Number }\end{array}$} & \multicolumn{2}{|c|}{ Habitat Complexity } \\
\hline & Upstream & Downstream \\
\hline W-03-091 & $\mathrm{C}$ & $\mathrm{C}$ \\
\hline W-03-092 & C & $\mathrm{C}$ \\
\hline W-03-093 & $\mathrm{C}$ & $\mathrm{C}$ \\
\hline W-03-094 & C & C \\
\hline W-03-095 & $\mathrm{C}$ & $\mathrm{C}$ \\
\hline W-03-096 & $\mathrm{C}$ & $\mathrm{C}$ \\
\hline W-03-097 & C & C \\
\hline W-03-098 & C & C \\
\hline W-03-099 & $\mathrm{C}$ & $\mathrm{C}$ \\
\hline W-03-100 & $\mathrm{C}$ & $\mathrm{C}$ \\
\hline W-03-101 & C & C \\
\hline W-03-102 & $\mathrm{C}$ & $\mathrm{C}$ \\
\hline W-03-103 & $\mathrm{S}$ & $\mathrm{C}$ \\
\hline W-03-104 & C & C \\
\hline W-03-105 & C & C \\
\hline W-03-106 & S & $\mathrm{S}$ \\
\hline W-03-107 & $\mathrm{C}$ & $\mathrm{C}$ \\
\hline W-03-108 & $\mathrm{C}$ & $\mathrm{C}$ \\
\hline W-03-109 & S & $\mathrm{S}$ \\
\hline W-03-110 & $\mathrm{C}$ & $\mathrm{C}$ \\
\hline W-03-111 & $\mathrm{C}$ & $\mathrm{C}$ \\
\hline W-03-112 & $\mathrm{C}$ & $\mathrm{C}$ \\
\hline W-03-113 & $\mathrm{S}$ & $\mathrm{S}$ \\
\hline W-03-114 & $\mathrm{C}$ & $\mathrm{C}$ \\
\hline W-03-115 & C & $\mathrm{C}$ \\
\hline W-03-116 & C & C \\
\hline W-03-117 & $\mathrm{S}$ & $\mathrm{S}$ \\
\hline W-03-118 & $\mathrm{S}$ & $\mathrm{S}$ \\
\hline W-03-119 & S & $\mathrm{S}$ \\
\hline W-03-120 & $\mathrm{S}$ & $\mathrm{S}$ \\
\hline W-03-121 & $\mathrm{S}$ & $\mathrm{S}$ \\
\hline W-03-122 & $\mathrm{S}$ & $\mathrm{S}$ \\
\hline W-03-123 & $\mathrm{S}$ & $\mathrm{S}$ \\
\hline W-03-124 & $\mathrm{S}$ & $\mathrm{S}$ \\
\hline
\end{tabular}

\begin{tabular}{|c|c|c|}
\hline \multirow{2}{*}{$\begin{array}{c}\text { Site } \\
\text { Number }\end{array}$} & \multicolumn{2}{|c|}{ Habitat Complexity } \\
\hline & Upstream & Downstream \\
\hline W-03-125 & $\mathrm{S}$ & $\mathrm{S}$ \\
\hline W-03-126 & S & S \\
\hline W-03-127 & S & S \\
\hline W-03-128 & S & S \\
\hline W-03-129 & $S$ & $S$ \\
\hline W-03-130 & S & S \\
\hline W-03-131 & S & $S$ \\
\hline W-03-132 & S & S \\
\hline W-03-133 & S & $S$ \\
\hline W-03-134 & S & S \\
\hline W-03-135 & S & S \\
\hline W-03-136 & $\mathrm{S}$ & $S$ \\
\hline W-03-137 & S & S \\
\hline W-03-138 & $\mathrm{C}$ & $S$ \\
\hline W-03-139 & C & C \\
\hline W-03-140 & $\mathrm{S}$ & $\mathrm{S}$ \\
\hline W-03-141 & S & S \\
\hline W-03-142 & S & C \\
\hline W-03-143 & S & $S$ \\
\hline W-03-144 & C & $\mathrm{C}$ \\
\hline W-03-145 & S & S \\
\hline W-03-146 & $\mathrm{C}$ & $\mathrm{C}$ \\
\hline W-03-147 & S & $S$ \\
\hline W-03-148 & S & $S$ \\
\hline W-03-149 & S & S \\
\hline W-03-150 & $\mathrm{C}$ & $\mathrm{C}$ \\
\hline W-03-151 & $C$ & $\mathrm{C}$ \\
\hline W-03-152 & C & $\mathrm{C}$ \\
\hline W-03-153 & C & C \\
\hline W-03-154 & C & C \\
\hline W-03-155 & C & $\mathrm{C}$ \\
\hline W-03-156 & C & C \\
\hline W-03-157 & $S$ & C \\
\hline W-03-158 & $\mathrm{C}$ & C \\
\hline
\end{tabular}

\begin{tabular}{|c|c|c|}
\hline \multirow{2}{*}{$\begin{array}{c}\text { Site } \\
\text { Number }\end{array}$} & \multicolumn{2}{|c|}{ Habitat Complexity } \\
\hline & Upstream & Downstream \\
\hline W-03-159 & $\mathrm{C}$ & $\mathrm{C}$ \\
\hline W-03-160 & $\mathrm{C}$ & $\mathrm{C}$ \\
\hline W-03-161 & $\mathrm{C}$ & $\mathrm{C}$ \\
\hline W-03-162 & $\mathrm{C}$ & $\mathrm{C}$ \\
\hline W-03-163 & $\mathrm{C}$ & $\mathrm{C}$ \\
\hline W-03-164 & $\mathrm{C}$ & $\mathrm{C}$ \\
\hline W-03-165 & C & C \\
\hline W-03-166 & S & $\mathrm{S}$ \\
\hline W-03-167 & $S$ & $\mathrm{C}$ \\
\hline W-03-168 & $\mathrm{C}$ & $\mathrm{C}$ \\
\hline W-03-169 & $\mathrm{C}$ & C \\
\hline W-03-170 & $S$ & $\mathrm{~S}$ \\
\hline W-03-171 & $\mathrm{C}$ & $\mathrm{C}$ \\
\hline W-03-172 & C & C \\
\hline W-03-173 & $\mathrm{C}$ & $\mathrm{C}$ \\
\hline W-03-174 & $\mathrm{S}$ & $\mathrm{S}$ \\
\hline W-03-175 & $S$ & $\mathrm{C}$ \\
\hline W-03-176 & $\mathrm{C}$ & $\mathrm{C}$ \\
\hline W-03-177 & S & $\mathrm{S}$ \\
\hline W-03-178 & S & $\mathrm{S}$ \\
\hline W-03-179 & S & $\mathrm{S}$ \\
\hline W-03-180 & $\mathrm{C}$ & $\mathrm{C}$ \\
\hline W-03-181 & C & C \\
\hline W-03-182 & $S$ & $\mathrm{C}$ \\
\hline W-03-183 & S & $\mathrm{S}$ \\
\hline W-03-184 & S & $\mathrm{S}$ \\
\hline W-03-185 & $\mathrm{S}$ & $\mathrm{C}$ \\
\hline W-03-186 & $\mathrm{C}$ & $\mathrm{C}$ \\
\hline W-03-187 & $\mathrm{S}$ & $\mathrm{S}$ \\
\hline W-03-188 & C & $\mathrm{C}$ \\
\hline W-03-189 & $\mathrm{S}$ & $\mathrm{C}$ \\
\hline W-03-190 & $\mathrm{C}$ & $\mathrm{S}$ \\
\hline W-03-191 & $\mathrm{S}$ & $\mathrm{S}$ \\
\hline W-03-192 & $\mathrm{S}$ & $\mathrm{S}$ \\
\hline
\end{tabular}


Appendix 2. Habitat complexity $(S=$ Simple; $C=$ Complex $)$ determination for all sites looking upstream and downstream from each surveyed reach.

\begin{tabular}{|c|c|c|}
\hline \multirow{2}{*}{$\begin{array}{c}\text { Site } \\
\text { Number }\end{array}$} & \multicolumn{2}{|c|}{ Habitat Complexity } \\
\hline & Upstream & Downstream \\
\hline W-03-193 & $\mathrm{C}$ & $\mathrm{C}$ \\
\hline W-03-194 & C & $\mathrm{C}$ \\
\hline W-03-195 & $\mathrm{C}$ & $\mathrm{C}$ \\
\hline W-03-196 & C & C \\
\hline W-03-197 & $\mathrm{C}$ & $\mathrm{C}$ \\
\hline W-03-198 & $\mathrm{C}$ & $\mathrm{C}$ \\
\hline W-03-199 & C & C \\
\hline W-03-200 & C & C \\
\hline W-03-201 & $\mathrm{C}$ & $\mathrm{C}$ \\
\hline W-03-202 & $\mathrm{C}$ & $\mathrm{C}$ \\
\hline W-03-203 & C & C \\
\hline W-03-204 & $\mathrm{C}$ & $\mathrm{C}$ \\
\hline W-03-205 & $\mathrm{C}$ & $\mathrm{C}$ \\
\hline W-03-206 & S & $\mathrm{S}$ \\
\hline W-03-207 & C & S \\
\hline W-03-208 & S & $\mathrm{S}$ \\
\hline W-03-209 & S & $\mathrm{S}$ \\
\hline W-03-210 & S & $\mathrm{S}$ \\
\hline W-03-211 & S & $\mathrm{C}$ \\
\hline W-03-212 & S & $\mathrm{S}$ \\
\hline W-03-213 & S & $\mathrm{S}$ \\
\hline W-03-214 & $\mathrm{C}$ & S \\
\hline W-03-215 & C & C \\
\hline W-03-216 & $S$ & $\mathrm{~S}$ \\
\hline W-03-217 & $\mathrm{S}$ & $\mathrm{S}$ \\
\hline W-03-218 & $\mathrm{S}$ & $\mathrm{S}$ \\
\hline W-03-219 & $\mathrm{S}$ & $\mathrm{S}$ \\
\hline W-03-220 & $\mathrm{S}$ & $\mathrm{S}$ \\
\hline W-03-221 & S & $\mathrm{S}$ \\
\hline W-03-222 & $\mathrm{S}$ & $\mathrm{S}$ \\
\hline W-03-223 & $\mathrm{S}$ & $\mathrm{S}$ \\
\hline W-03-224 & $\mathrm{S}$ & $\mathrm{S}$ \\
\hline W-03-225 & $\mathrm{S}$ & $\mathrm{S}$ \\
\hline W-03-226 & $\mathrm{S}$ & $\mathrm{S}$ \\
\hline
\end{tabular}

\begin{tabular}{|c|c|c|}
\hline \multirow{2}{*}{$\begin{array}{c}\text { Site } \\
\text { Number }\end{array}$} & \multicolumn{2}{|c|}{ Habitat Complexity } \\
\hline & Upstream & Downstream \\
\hline W-03-227 & $\mathrm{S}$ & $\mathrm{S}$ \\
\hline W-03-228 & $\mathrm{S}$ & $\mathrm{S}$ \\
\hline W-03-229 & $\mathrm{S}$ & $\mathrm{C}$ \\
\hline W-03-230 & $\mathrm{S}$ & $\mathrm{S}$ \\
\hline W-03-231 & $S$ & $S$ \\
\hline W-03-232 & $\mathrm{S}$ & S \\
\hline W-03-233 & $\mathrm{S}$ & S \\
\hline W-03-234 & $\mathrm{S}$ & $S$ \\
\hline W-03-235 & $\mathrm{S}$ & $\mathrm{S}$ \\
\hline W-03-236 & $\mathrm{C}$ & $\mathrm{C}$ \\
\hline W-03-237 & $\mathrm{C}$ & $\mathrm{C}$ \\
\hline W-03-238 & $\mathrm{C}$ & $\mathrm{C}$ \\
\hline W-03-239 & $\mathrm{C}$ & $\mathrm{C}$ \\
\hline W-03-240 & $\mathrm{S}$ & $S$ \\
\hline W-03-241 & $\mathrm{C}$ & $\mathrm{C}$ \\
\hline W-03-242 & $\mathrm{C}$ & $\mathrm{C}$ \\
\hline W-03-243 & $\mathrm{C}$ & $\mathrm{C}$ \\
\hline W-03-244 & $\mathrm{S}$ & $S$ \\
\hline W-03-245 & $\mathrm{S}$ & S \\
\hline W-03-246 & $\mathrm{S}$ & $S$ \\
\hline W-03-247 & $\mathrm{C}$ & $\mathrm{C}$ \\
\hline W-03-248 & $\mathrm{S}$ & $\mathrm{S}$ \\
\hline W-03-249 & $\mathrm{C}$ & $\mathrm{C}$ \\
\hline W-03-250 & $\mathrm{S}$ & $\mathrm{S}$ \\
\hline W-03-251 & $\mathrm{S}$ & S \\
\hline W-03-252 & $\mathrm{C}$ & C \\
\hline W-03-253 & $\mathrm{S}$ & $\mathrm{S}$ \\
\hline W-03-254 & $\mathrm{S}$ & $\mathrm{S}$ \\
\hline W-03-255 & C & $\mathrm{C}$ \\
\hline W-03-256 & $\mathrm{S}$ & $\mathrm{S}$ \\
\hline W-03-257 & $\mathrm{S}$ & $S$ \\
\hline W-03-258 & $\mathrm{S}$ & $S$ \\
\hline W-03-259 & $\mathrm{C}$ & C \\
\hline W-03-260 & C & C \\
\hline
\end{tabular}

\begin{tabular}{|c|c|c|}
\hline \multirow{2}{*}{$\begin{array}{c}\text { Site } \\
\text { Number }\end{array}$} & \multicolumn{2}{|c|}{ Habitat Complexity } \\
\hline & Upstream & Downstream \\
\hline W-03-261 & $\mathrm{S}$ & $S$ \\
\hline W-03-262 & $\mathrm{S}$ & $\mathrm{S}$ \\
\hline W-03-263 & $\mathrm{S}$ & $\mathrm{S}$ \\
\hline W-03-264 & $\mathrm{C}$ & $\mathrm{C}$ \\
\hline W-03-265 & $\mathrm{C}$ & $\mathrm{S}$ \\
\hline W-03-266 & $\mathrm{C}$ & $\mathrm{C}$ \\
\hline W-03-267 & S & $\mathrm{S}$ \\
\hline B-04-001 & $\mathrm{S}$ & $S$ \\
\hline B-04-002 & $\mathrm{S}$ & $\mathrm{S}$ \\
\hline B-04-003 & S & $\mathrm{S}$ \\
\hline B-04-004 & $S$ & $\mathrm{~S}$ \\
\hline B-04-005 & $\mathrm{S}$ & $\mathrm{S}$ \\
\hline B-04-006 & $\mathrm{S}$ & $\mathrm{S}$ \\
\hline B-04-007 & $\mathrm{S}$ & $\mathrm{S}$ \\
\hline B-04-008 & $\mathrm{C}$ & $\mathrm{C}$ \\
\hline B-04-009 & $\mathrm{S}$ & $\mathrm{S}$ \\
\hline B-04-010 & $\mathrm{S}$ & $\mathrm{S}$ \\
\hline B-04-011 & $\mathrm{S}$ & $\mathrm{S}$ \\
\hline B-04-012 & $\mathrm{C}$ & $\mathrm{C}$ \\
\hline B-04-013 & $\mathrm{C}$ & C \\
\hline B-04-014 & $\mathrm{C}$ & $\mathrm{C}$ \\
\hline B-04-015 & $\mathrm{C}$ & $\mathrm{C}$ \\
\hline B-04-016 & $\mathrm{C}$ & C \\
\hline B-04-017 & $\mathrm{C}$ & $\mathrm{C}$ \\
\hline B-04-018 & $\mathrm{C}$ & $\mathrm{C}$ \\
\hline B-04-019 & $\mathrm{S}$ & $\mathrm{S}$ \\
\hline B-04-020 & $\mathrm{S}$ & $\mathrm{S}$ \\
\hline B-04-021 & $\mathrm{S}$ & $\mathrm{S}$ \\
\hline B-04-022 & $\mathrm{S}$ & $S$ \\
\hline B-04-023 & $\mathrm{S}$ & $\mathrm{S}$ \\
\hline B-04-024 & $\mathrm{S}$ & $S$ \\
\hline B-04-025 & $S$ & $S$ \\
\hline B-04-026 & $\mathrm{C}$ & C \\
\hline B-04-027 & C & C \\
\hline
\end{tabular}


Appendix 2. Habitat complexity $(S=$ Simple; $C=$ Complex $)$ determination for all sites looking upstream and downstream from each surveyed reach.

\begin{tabular}{|c|c|c|}
\hline \multirow{2}{*}{$\begin{array}{c}\text { Site } \\
\text { Number }\end{array}$} & \multicolumn{2}{|c|}{ Habitat Complexity } \\
\hline & Upstream & Downstream \\
\hline B-04-028 & $\mathrm{S}$ & $\mathrm{S}$ \\
\hline B-04-029 & $S$ & $S$ \\
\hline B-04-030 & S & S \\
\hline B-04-031 & $\mathrm{C}$ & $\mathrm{C}$ \\
\hline B-04-032 & $\mathrm{C}$ & $\mathrm{C}$ \\
\hline B-04-033 & $\mathrm{C}$ & $\mathrm{C}$ \\
\hline B-04-034 & S & $\mathrm{S}$ \\
\hline B-04-035 & C & $\mathrm{C}$ \\
\hline B-04-036 & $\mathrm{S}$ & S \\
\hline B-04-037 & $\mathrm{C}$ & $\mathrm{C}$ \\
\hline B-04-038 & S & $\mathrm{S}$ \\
\hline B-04-039 & $\mathrm{C}$ & $\mathrm{C}$ \\
\hline B-04-040 & $S$ & $\mathrm{~S}$ \\
\hline B-04-041 & $\mathrm{C}$ & C \\
\hline B-04-042 & $\mathrm{C}$ & $\mathrm{C}$ \\
\hline B-04-043 & C & $\mathrm{C}$ \\
\hline B-04-044 & $\mathrm{C}$ & $\mathrm{C}$ \\
\hline B-04-045 & $\mathrm{C}$ & $\mathrm{C}$ \\
\hline B-04-046 & $\mathrm{C}$ & $\mathrm{C}$ \\
\hline B-04-047 & $\mathrm{C}$ & $\mathrm{C}$ \\
\hline B-04-048 & $\mathrm{C}$ & $\mathrm{C}$ \\
\hline B-04-049 & $\mathrm{C}$ & $\mathrm{C}$ \\
\hline B-04-050 & C & C \\
\hline B-04-051 & $\mathrm{C}$ & $\mathrm{C}$ \\
\hline B-04-052 & $\mathrm{C}$ & $\mathrm{C}$ \\
\hline B-04-053 & $\mathrm{C}$ & $\mathrm{C}$ \\
\hline B-04-054 & $\mathrm{C}$ & $\mathrm{C}$ \\
\hline B-04-055 & $\mathrm{C}$ & $\mathrm{C}$ \\
\hline B-04-056 & $\mathrm{C}$ & $\mathrm{C}$ \\
\hline B-04-057 & C & C \\
\hline B-04-058 & $\mathrm{C}$ & $\mathrm{C}$ \\
\hline B-04-059 & C & C \\
\hline B-04-060 & $\mathrm{C}$ & $\mathrm{C}$ \\
\hline B-04-061 & $\mathrm{C}$ & $\mathrm{C}$ \\
\hline
\end{tabular}

\begin{tabular}{|c|c|c|}
\hline \multirow{2}{*}{$\begin{array}{c}\text { Site } \\
\text { Number }\end{array}$} & \multicolumn{2}{|c|}{ Habitat Complexity } \\
\hline & Upstream & Downstream \\
\hline B-04-062 & $\mathrm{C}$ & $\mathrm{C}$ \\
\hline B-04-063 & C & C \\
\hline B-04-064 & C & $\mathrm{C}$ \\
\hline B-04-065 & S & S \\
\hline B-04-066 & S & $\mathrm{S}$ \\
\hline B-04-067 & $\mathrm{S}$ & $\mathrm{S}$ \\
\hline B-04-068 & C & C \\
\hline B-04-069 & C & C \\
\hline B-04-070 & $\mathrm{C}$ & S \\
\hline B-04-071 & S & S \\
\hline B-04-072 & S & S \\
\hline B-04-073 & $\mathrm{C}$ & C \\
\hline B-04-074 & C & $\mathrm{C}$ \\
\hline B-04-075 & $\mathrm{C}$ & $\mathrm{C}$ \\
\hline B-04-076 & $S$ & $S$ \\
\hline B-04-077 & C & C \\
\hline B-04-078 & C & C \\
\hline B-04-079 & C & $\mathrm{C}$ \\
\hline B-04-080 & S & $\mathrm{C}$ \\
\hline B-04-081 & S & $S$ \\
\hline B-04-082 & S & $\mathrm{C}$ \\
\hline B-04-083 & S & $\mathrm{C}$ \\
\hline B-04-084 & S & $S$ \\
\hline B-04-085 & S & $\mathrm{S}$ \\
\hline B-04-086 & S & S \\
\hline B-04-087 & $\mathrm{C}$ & $\mathrm{C}$ \\
\hline B-04-088 & C & C \\
\hline B-04-089 & $S$ & $S$ \\
\hline B-04-090 & C & C \\
\hline B-04-091 & C & C \\
\hline B-04-092 & C & C \\
\hline B-04-093 & C & C \\
\hline B-04-094 & C & C \\
\hline B-04-095 & C & C \\
\hline
\end{tabular}

\begin{tabular}{|c|c|c|}
\hline \multirow{2}{*}{$\begin{array}{c}\text { Site } \\
\text { Number }\end{array}$} & \multicolumn{2}{|c|}{ Habitat Complexity } \\
\hline & Upstream & Downstream \\
\hline B-04-096 & $\mathrm{C}$ & $\mathrm{C}$ \\
\hline B-04-097 & $\mathrm{S}$ & $\mathrm{S}$ \\
\hline B-04-098 & S & $\mathrm{S}$ \\
\hline B-04-099 & $\mathrm{S}$ & $\mathrm{S}$ \\
\hline B-04-100 & $\mathrm{S}$ & $\mathrm{S}$ \\
\hline B-04-101 & $\mathrm{S}$ & $\mathrm{C}$ \\
\hline B-04-102 & C & C \\
\hline B-04-103 & $\mathrm{S}$ & $\mathrm{C}$ \\
\hline B-04-104 & $\mathrm{C}$ & $\mathrm{C}$ \\
\hline B-04-105 & $\mathrm{C}$ & $\mathrm{C}$ \\
\hline B-04-106 & S & $\mathrm{S}$ \\
\hline B-04-107 & $\mathrm{C}$ & $\mathrm{C}$ \\
\hline B-04-108 & $\mathrm{C}$ & $\mathrm{C}$ \\
\hline B-04-109 & $\mathrm{S}$ & $\mathrm{S}$ \\
\hline B-04-110 & $S$ & $\mathrm{~S}$ \\
\hline B-04-111 & $\mathrm{C}$ & C \\
\hline B-04-112 & $\mathrm{C}$ & $\mathrm{C}$ \\
\hline B-04-113 & $\mathrm{C}$ & $\mathrm{C}$ \\
\hline B-04-114 & $\mathrm{C}$ & $\mathrm{C}$ \\
\hline B-04-115 & $\mathrm{C}$ & $\mathrm{C}$ \\
\hline B-04-116 & $\mathrm{C}$ & $\mathrm{C}$ \\
\hline B-04-117 & $\mathrm{C}$ & $\mathrm{C}$ \\
\hline B-04-118 & C & C \\
\hline B-04-119 & $\mathrm{C}$ & $\mathrm{S}$ \\
\hline B-04-120 & $\mathrm{C}$ & $\mathrm{C}$ \\
\hline $\mathrm{B}-04-121$ & $\mathrm{C}$ & C \\
\hline B-04-122 & $\mathrm{C}$ & $\mathrm{C}$ \\
\hline B-04-123 & $\mathrm{C}$ & $\mathrm{C}$ \\
\hline B-04-124 & $\mathrm{C}$ & C \\
\hline B-04-125 & C & C \\
\hline B-04-126 & $\mathrm{C}$ & $\mathrm{C}$ \\
\hline B-04-127 & C & C \\
\hline B-04-128 & $\mathrm{C}$ & C \\
\hline B-04-129 & $\mathrm{C}$ & C \\
\hline
\end{tabular}


Appendix 2. Habitat complexity $(S=$ Simple; $C=$ Complex $)$ determination for all sites looking upstream and downstream from each surveyed reach.

\begin{tabular}{|c|c|c|}
\hline \multirow{2}{*}{$\begin{array}{c}\text { Site } \\
\text { Number }\end{array}$} & \multicolumn{2}{|c|}{ Habitat Complexity } \\
\hline & Upstream & Downstream \\
\hline B-04-130 & $\mathrm{C}$ & $\mathrm{S}$ \\
\hline B-04-131 & $S$ & $\mathrm{~S}$ \\
\hline B-04-132 & S & S \\
\hline B-04-133 & $\mathrm{C}$ & C \\
\hline B-04-134 & $\mathrm{C}$ & $\mathrm{C}$ \\
\hline B-04-135 & $\mathrm{C}$ & $\mathrm{C}$ \\
\hline B-04-136 & C & C \\
\hline B-04-137 & C & $\mathrm{C}$ \\
\hline B-04-138 & $\mathrm{C}$ & $\mathrm{C}$ \\
\hline B-04-139 & $\mathrm{C}$ & $\mathrm{C}$ \\
\hline B-04-140 & $\mathrm{C}$ & $\mathrm{C}$ \\
\hline B-04-141 & $\mathrm{C}$ & $\mathrm{C}$ \\
\hline B-04-142 & $S$ & $\mathrm{C}$ \\
\hline B-04-143 & S & C \\
\hline B-04-144 & $\mathrm{C}$ & $\mathrm{C}$ \\
\hline B-04-145 & C & $\mathrm{C}$ \\
\hline B-04-146 & $\mathrm{C}$ & $\mathrm{C}$ \\
\hline B-04-147 & $\mathrm{C}$ & $\mathrm{C}$ \\
\hline B-04-148 & S & $\mathrm{S}$ \\
\hline B-04-149 & $\mathrm{C}$ & $\mathrm{C}$ \\
\hline B-04-150 & $\mathrm{C}$ & $\mathrm{C}$ \\
\hline B-04-151 & $\mathrm{C}$ & $\mathrm{C}$ \\
\hline B-04-152 & C & C \\
\hline B-04-153 & $\mathrm{C}$ & $\mathrm{C}$ \\
\hline B-04-154 & $\mathrm{C}$ & $\mathrm{C}$ \\
\hline B-04-155 & $\mathrm{C}$ & $\mathrm{C}$ \\
\hline B-04-156 & $\mathrm{C}$ & $\mathrm{C}$ \\
\hline B-04-157 & $\mathrm{C}$ & $\mathrm{C}$ \\
\hline B-04-158 & $\mathrm{C}$ & $\mathrm{C}$ \\
\hline B-04-159 & C & C \\
\hline B-04-160 & $\mathrm{C}$ & $\mathrm{C}$ \\
\hline B-04-161 & C & C \\
\hline B-04-162 & $\mathrm{C}$ & $\mathrm{C}$ \\
\hline B-04-163 & $\mathrm{C}$ & $\mathrm{C}$ \\
\hline
\end{tabular}

\begin{tabular}{|c|c|c|}
\hline \multirow{2}{*}{$\begin{array}{c}\text { Site } \\
\text { Number }\end{array}$} & \multicolumn{2}{|c|}{ Habitat Complexity } \\
\hline & Upstream & Downstream \\
\hline B-04-164 & $\mathrm{C}$ & $\mathrm{C}$ \\
\hline B-04-165 & $\mathrm{C}$ & $\mathrm{C}$ \\
\hline B-04-166 & $\mathrm{C}$ & $\mathrm{C}$ \\
\hline B-04-167 & C & C \\
\hline B-04-168 & $\mathrm{C}$ & $\mathrm{C}$ \\
\hline B-04-169 & $\mathrm{C}$ & $\mathrm{C}$ \\
\hline B-04-170 & $\mathrm{S}$ & C \\
\hline B-04-171 & C & C \\
\hline B-04-172 & $\mathrm{S}$ & $\mathrm{S}$ \\
\hline B-04-173 & $\mathrm{C}$ & $\mathrm{C}$ \\
\hline B-04-174 & C & C \\
\hline B-04-175 & $\mathrm{C}$ & $\mathrm{C}$ \\
\hline B-04-176 & $\mathrm{C}$ & $\mathrm{C}$ \\
\hline B-04-177 & $\mathrm{S}$ & $\mathrm{S}$ \\
\hline B-04-178 & C & C \\
\hline B-04-179 & $\mathrm{C}$ & $\mathrm{C}$ \\
\hline B-04-180 & $\mathrm{C}$ & C \\
\hline B-04-181 & $\mathrm{C}$ & $\mathrm{C}$ \\
\hline B-04-182 & $\mathrm{S}$ & $\mathrm{S}$ \\
\hline B-04-183 & $\mathrm{S}$ & $\mathrm{S}$ \\
\hline B-04-184 & $\mathrm{S}$ & $\mathrm{S}$ \\
\hline B-04-185 & $\mathrm{C}$ & $\mathrm{C}$ \\
\hline B-04-186 & C & C \\
\hline B-04-187 & $\mathrm{C}$ & $\mathrm{C}$ \\
\hline B-04-188 & $\mathrm{C}$ & $\mathrm{C}$ \\
\hline B-04-189 & C & C \\
\hline B-04-190 & C & C \\
\hline B-04-191 & C & C \\
\hline B-04-192 & $S$ & C \\
\hline B-04-193 & $S$ & C \\
\hline B-04-194 & $\mathrm{C}$ & C \\
\hline B-04-195 & C & C \\
\hline B-04-196 & C & C \\
\hline B-04-197 & C & C \\
\hline
\end{tabular}

\begin{tabular}{|c|c|c|}
\hline \multirow{2}{*}{$\begin{array}{c}\text { Site } \\
\text { Number }\end{array}$} & \multicolumn{2}{|c|}{ Habitat Complexity } \\
\hline & Upstream & Downstream \\
\hline B-04-198 & $\mathrm{C}$ & $\mathrm{C}$ \\
\hline B-04-199 & $\mathrm{C}$ & $\mathrm{C}$ \\
\hline B-04-200 & $\mathrm{C}$ & $\mathrm{C}$ \\
\hline B-04-201 & $\mathrm{C}$ & $\mathrm{C}$ \\
\hline B-04-202 & $\mathrm{C}$ & $\mathrm{C}$ \\
\hline B-04-203 & $\mathrm{S}$ & $\mathrm{S}$ \\
\hline B-04-204 & C & C \\
\hline B-04-205 & $\mathrm{S}$ & $\mathrm{S}$ \\
\hline B-04-206 & $\mathrm{C}$ & $\mathrm{C}$ \\
\hline B-04-207 & S & $\mathrm{S}$ \\
\hline B-04-208 & $\mathrm{C}$ & C \\
\hline B-04-209 & $\mathrm{C}$ & $\mathrm{C}$ \\
\hline B-04-210 & $S$ & $\mathrm{~S}$ \\
\hline B-04-211 & $\mathrm{S}$ & $\mathrm{S}$ \\
\hline B-04-212 & $\mathrm{C}$ & $\mathrm{C}$ \\
\hline B-04-213 & $\mathrm{C}$ & C \\
\hline B-04-214 & $\mathrm{C}$ & $\mathrm{C}$ \\
\hline $\mathrm{B}-04-215$ & $\mathrm{C}$ & $\mathrm{C}$ \\
\hline B-04-216 & S & $\mathrm{S}$ \\
\hline B-04-217 & $\mathrm{C}$ & $\mathrm{C}$ \\
\hline B-04-218 & $\mathrm{C}$ & $\mathrm{C}$ \\
\hline B-04-219 & $\mathrm{C}$ & $\mathrm{C}$ \\
\hline B-04-220 & C & C \\
\hline B-04-221 & $\mathrm{C}$ & $\mathrm{C}$ \\
\hline $\mathrm{B}-04-222$ & $\mathrm{C}$ & $\mathrm{C}$ \\
\hline $\mathrm{B}-04-223$ & $\mathrm{C}$ & C \\
\hline B-04-224 & $\mathrm{C}$ & $\mathrm{C}$ \\
\hline B-04-225 & $\mathrm{C}$ & $\mathrm{C}$ \\
\hline B-04-226 & $\mathrm{C}$ & C \\
\hline B-04-227 & C & C \\
\hline B-04-228 & $\mathrm{C}$ & $\mathrm{C}$ \\
\hline B-04-229 & $S$ & $S$ \\
\hline $\mathrm{B}-04-230$ & $\mathrm{~S}$ & $S$ \\
\hline B-04-231 & $\mathrm{S}$ & $\mathrm{S}$ \\
\hline
\end{tabular}


Appendix 2. Habitat complexity $(S=$ Simple; $C=$ Complex $)$ determination for all sites looking upstream and downstream from each surveyed reach.

\begin{tabular}{|c|c|c|}
\hline \multirow{2}{*}{$\begin{array}{c}\text { Site } \\
\text { Number }\end{array}$} & \multicolumn{2}{|c|}{ Habitat Complexity } \\
\hline & Upstream & Downstream \\
\hline B-04-232 & $S$ & $\mathrm{~S}$ \\
\hline B-04-233 & $S$ & $\mathrm{~S}$ \\
\hline B-04-234 & S & $\mathrm{S}$ \\
\hline $\mathrm{B}-04-235$ & $S$ & $\mathrm{~S}$ \\
\hline B-04-236 & $S$ & $\mathrm{~S}$ \\
\hline B-04-237 & S & S \\
\hline B-04-238 & C & C \\
\hline B-04-239 & C & $\mathrm{C}$ \\
\hline B-04-240 & $\mathrm{C}$ & $\mathrm{C}$ \\
\hline B-04-241 & $\mathrm{C}$ & $\mathrm{C}$ \\
\hline B-04-242 & $\mathrm{S}$ & $\mathrm{C}$ \\
\hline B-04-243 & $\mathrm{C}$ & $\mathrm{C}$ \\
\hline B-04-244 & $\mathrm{C}$ & $\mathrm{C}$ \\
\hline B-04-245 & $\mathrm{S}$ & S \\
\hline B-04-246 & $S$ & $S$ \\
\hline B-04-247 & S & $\mathrm{S}$ \\
\hline B-04-248 & $\mathrm{C}$ & $\mathrm{C}$ \\
\hline B-04-249 & $S$ & $\mathrm{~S}$ \\
\hline B-04-250 & S & $\mathrm{S}$ \\
\hline B-04-251 & $S$ & $\mathrm{C}$ \\
\hline B-04-252 & $\mathrm{S}$ & $\mathrm{S}$ \\
\hline B-04-253 & $\mathrm{C}$ & $\mathrm{S}$ \\
\hline B-04-254 & $\mathrm{S}$ & $\mathrm{S}$ \\
\hline B-04-255 & $\mathrm{C}$ & $\mathrm{C}$ \\
\hline B-04-256 & $\mathrm{C}$ & $\mathrm{C}$ \\
\hline B-04-257 & $\mathrm{C}$ & $\mathrm{C}$ \\
\hline B-04-258 & $\mathrm{S}$ & $\mathrm{S}$ \\
\hline B-04-259 & $\mathrm{S}$ & $\mathrm{C}$ \\
\hline B-04-260 & $\mathrm{C}$ & $\mathrm{C}$ \\
\hline B-04-261 & $\mathrm{C}$ & $\mathrm{C}$ \\
\hline B-04-262 & $\mathrm{C}$ & $C$ \\
\hline B-04-263 & $\mathrm{C}$ & $\mathrm{C}$ \\
\hline B-04-264 & $\mathrm{C}$ & $\mathrm{C}$ \\
\hline B-04-265 & $\mathrm{C}$ & $\mathrm{C}$ \\
\hline
\end{tabular}

\begin{tabular}{|c|c|c|}
\hline \multirow{2}{*}{$\begin{array}{c}\text { Site } \\
\text { Number }\end{array}$} & \multicolumn{2}{|c|}{ Habitat Complexity } \\
\hline & Upstream & Downstream \\
\hline B-04-266 & C & C \\
\hline B-04-267 & $\mathrm{C}$ & $\mathrm{C}$ \\
\hline B-04-268 & $\mathrm{C}$ & $\mathrm{C}$ \\
\hline B-04-269 & $\mathrm{C}$ & C \\
\hline B-04-270 & $\mathrm{C}$ & $\mathrm{C}$ \\
\hline B-04-271 & $\mathrm{C}$ & $\mathrm{C}$ \\
\hline B-04-272 & $\mathrm{S}$ & $\mathrm{S}$ \\
\hline B-04-273 & $\mathrm{S}$ & $\mathrm{S}$ \\
\hline B-04-274 & $\mathrm{S}$ & $\mathrm{S}$ \\
\hline B-04-275 & $\mathrm{S}$ & $\mathrm{S}$ \\
\hline B-04-276 & $\mathrm{S}$ & $\mathrm{S}$ \\
\hline B-04-277 & $\mathrm{S}$ & $\mathrm{S}$ \\
\hline B-04-278 & $\mathrm{S}$ & S \\
\hline B-04-279 & $\mathrm{S}$ & $\mathrm{S}$ \\
\hline B-04-280 & $\mathrm{S}$ & $\mathrm{S}$ \\
\hline B-04-281 & $\mathrm{S}$ & $\mathrm{C}$ \\
\hline B-04-282 & $\mathrm{S}$ & $\mathrm{S}$ \\
\hline B-04-283 & $\mathrm{S}$ & $\mathrm{S}$ \\
\hline B-04-284 & $\mathrm{S}$ & $\mathrm{S}$ \\
\hline B-04-285 & C & C \\
\hline B-04-286 & $\mathrm{C}$ & $\mathrm{C}$ \\
\hline B-04-287 & $\mathrm{C}$ & $\mathrm{C}$ \\
\hline B-04-288 & $\mathrm{S}$ & $\mathrm{S}$ \\
\hline D-04-001 & $\mathrm{C}$ & $\mathrm{C}$ \\
\hline D-04-002 & $\mathrm{C}$ & $\mathrm{C}$ \\
\hline D-04-003 & $\mathrm{S}$ & $\mathrm{S}$ \\
\hline D-04-004 & C & C \\
\hline D-04-005 & $\mathrm{S}$ & $\mathrm{S}$ \\
\hline D-04-006 & C & C \\
\hline D-04-007 & $\mathrm{C}$ & $\mathrm{C}$ \\
\hline D-04-008 & $\mathrm{C}$ & C \\
\hline D-04-009 & $S$ & $S$ \\
\hline D-04-010 & C & C \\
\hline D-04-011 & $\mathrm{C}$ & C \\
\hline
\end{tabular}

\begin{tabular}{|c|c|c|}
\hline \multirow{2}{*}{$\begin{array}{c}\text { Site } \\
\text { Number }\end{array}$} & \multicolumn{2}{|c|}{ Habitat Complexity } \\
\hline & Upstream & Downstream \\
\hline D-04-012 & $\mathrm{C}$ & $\mathrm{C}$ \\
\hline D-04-013 & $\mathrm{C}$ & $\mathrm{C}$ \\
\hline D-04-014 & $\mathrm{C}$ & $\mathrm{C}$ \\
\hline D-04-015 & $\mathrm{C}$ & $\mathrm{C}$ \\
\hline D-04-016 & $\mathrm{C}$ & $\mathrm{C}$ \\
\hline D-04-017 & $\mathrm{C}$ & $\mathrm{S}$ \\
\hline D-04-018 & C & C \\
\hline D-04-019 & $\mathrm{C}$ & C \\
\hline D-04-020 & $\mathrm{C}$ & C \\
\hline D-04-021 & $\mathrm{C}$ & $\mathrm{C}$ \\
\hline D-04-022 & S & S \\
\hline D-04-023 & $\mathrm{C}$ & $\mathrm{C}$ \\
\hline D-04-024 & S & $\mathrm{S}$ \\
\hline D-04-025 & $\mathrm{C}$ & C \\
\hline D-04-026 & $\mathrm{C}$ & $\mathrm{C}$ \\
\hline D-04-027 & $\mathrm{C}$ & C \\
\hline D-04-028 & $\mathrm{C}$ & $\mathrm{C}$ \\
\hline D-04-029 & S & $\mathrm{C}$ \\
\hline D-04-030 & $\mathrm{C}$ & $\mathrm{C}$ \\
\hline D-04-031 & $\mathrm{C}$ & $\mathrm{C}$ \\
\hline D-04-032 & $\mathrm{C}$ & $\mathrm{C}$ \\
\hline D-04-033 & $\mathrm{C}$ & $\mathrm{C}$ \\
\hline D-04-034 & C & C \\
\hline D-04-035 & $\mathrm{C}$ & $\mathrm{C}$ \\
\hline D-04-036 & $\mathrm{C}$ & $\mathrm{C}$ \\
\hline W-04-001 & S & $\mathrm{S}$ \\
\hline W-04-002 & $\mathrm{S}$ & $\mathrm{S}$ \\
\hline W-04-003 & $\mathrm{C}$ & $\mathrm{C}$ \\
\hline W-04-004 & $\mathrm{S}$ & $\mathrm{S}$ \\
\hline W-04-005 & $\mathrm{S}$ & $\mathrm{S}$ \\
\hline W-04-006 & $\mathrm{S}$ & $\mathrm{S}$ \\
\hline W-04-007 & $S$ & $\mathrm{~S}$ \\
\hline W-04-008 & $\mathrm{S}$ & $\mathrm{S}$ \\
\hline W-04-009 & $\mathrm{S}$ & $\mathrm{S}$ \\
\hline
\end{tabular}


Appendix 2. Habitat complexity $(S=$ Simple; $C=$ Complex $)$ determination for all sites looking upstream and downstream from each surveyed reach.

\begin{tabular}{|c|c|c|}
\hline \multirow{2}{*}{$\begin{array}{c}\text { Site } \\
\text { Number }\end{array}$} & \multicolumn{2}{|c|}{ Habitat Complexity } \\
\hline & Upstream & Downstream \\
\hline W-04-010 & $S$ & $\mathrm{~S}$ \\
\hline W-04-011 & $S$ & $\mathrm{~S}$ \\
\hline W-04-012 & S & $\mathrm{S}$ \\
\hline W-04-013 & $S$ & $\mathrm{~S}$ \\
\hline W-04-014 & $S$ & $\mathrm{~S}$ \\
\hline W-04-015 & S & S \\
\hline W-04-016 & S & S \\
\hline W-04-017 & $S$ & $S$ \\
\hline W-04-018 & S & $\mathrm{S}$ \\
\hline W-04-019 & S & $\mathrm{C}$ \\
\hline W-04-020 & S & S \\
\hline W-04-021 & $S$ & $S$ \\
\hline W-04-022 & S & S \\
\hline W-04-023 & C & $\mathrm{C}$ \\
\hline W-04-024 & $\mathrm{C}$ & $\mathrm{C}$ \\
\hline W-04-025 & $\mathrm{C}$ & $\mathrm{C}$ \\
\hline W-04-026 & $\mathrm{C}$ & $\mathrm{C}$ \\
\hline W-04-027 & $S$ & $\mathrm{~S}$ \\
\hline W-04-028 & $\mathrm{C}$ & $\mathrm{C}$ \\
\hline W-04-029 & $S$ & $\mathrm{~S}$ \\
\hline W-04-030 & $S$ & $\mathrm{~S}$ \\
\hline W-04-031 & $\mathrm{S}$ & C \\
\hline W-04-032 & $\mathrm{C}$ & C \\
\hline W-04-033 & $S$ & $\mathrm{~S}$ \\
\hline W-04-034 & S & $\mathrm{C}$ \\
\hline W-04-035 & S & S \\
\hline W-04-036 & $\mathrm{C}$ & $\mathrm{S}$ \\
\hline W-04-037 & $\mathrm{S}$ & $\mathrm{S}$ \\
\hline W-04-038 & C & C \\
\hline W-04-039 & $\mathrm{S}$ & $\mathrm{S}$ \\
\hline W-04-040 & $S$ & $\mathrm{~S}$ \\
\hline W-04-041 & $S$ & $\mathrm{C}$ \\
\hline W-04-042 & $\mathrm{S}$ & $\mathrm{S}$ \\
\hline W-04-043 & $\mathrm{S}$ & $\mathrm{S}$ \\
\hline
\end{tabular}

\begin{tabular}{|c|c|c|}
\hline \multirow{2}{*}{$\begin{array}{c}\text { Site } \\
\text { Number }\end{array}$} & \multicolumn{2}{|c|}{ Habitat Complexity } \\
\hline & Upstream & Downstream \\
\hline W-04-044 & $\mathrm{S}$ & $\mathrm{C}$ \\
\hline W-04-045 & S & $\mathrm{S}$ \\
\hline W-04-046 & S & $\mathrm{S}$ \\
\hline W-04-047 & $S$ & $S$ \\
\hline W-04-048 & $S$ & $S$ \\
\hline W-04-049 & $\mathrm{C}$ & $\mathrm{C}$ \\
\hline W-04-050 & C & $\mathrm{C}$ \\
\hline W-04-051 & C & C \\
\hline W-04-052 & $\mathrm{C}$ & $\mathrm{C}$ \\
\hline W-04-053 & S & $\mathrm{C}$ \\
\hline W-04-054 & $\mathrm{C}$ & $S$ \\
\hline W-04-055 & $S$ & $S$ \\
\hline W-04-056 & $\mathrm{S}$ & S \\
\hline W-04-057 & $S$ & S \\
\hline W-04-058 & $\mathrm{S}$ & $\mathrm{C}$ \\
\hline W-04-059 & $\mathrm{S}$ & S \\
\hline W-04-060 & $\mathrm{C}$ & $\mathrm{C}$ \\
\hline W-04-061 & $\mathrm{C}$ & $\mathrm{C}$ \\
\hline W-04-062 & S & $\mathrm{C}$ \\
\hline W-04-063 & $\mathrm{S}$ & $S$ \\
\hline W-04-064 & $\mathrm{C}$ & $\mathrm{C}$ \\
\hline W-04-065 & S & S \\
\hline W-04-066 & $\mathrm{S}$ & $\mathrm{S}$ \\
\hline W-04-067 & $\mathrm{S}$ & $\mathrm{S}$ \\
\hline W-04-068 & $\mathrm{S}$ & $\mathrm{S}$ \\
\hline W-04-069 & $\mathrm{S}$ & S \\
\hline W-04-070 & $\mathrm{S}$ & $\mathrm{S}$ \\
\hline W-04-071 & $S$ & $S$ \\
\hline W-04-072 & $S$ & $\mathrm{C}$ \\
\hline W-04-073 & $S$ & $\mathrm{~S}$ \\
\hline W-04-074 & $\mathrm{S}$ & $\mathrm{S}$ \\
\hline W-04-075 & $\mathrm{S}$ & $\mathrm{S}$ \\
\hline W-04-076 & $\mathrm{S}$ & $\mathrm{S}$ \\
\hline W-04-077 & $\mathrm{S}$ & $\mathrm{S}$ \\
\hline
\end{tabular}

\begin{tabular}{|c|c|c|}
\hline \multirow{2}{*}{$\begin{array}{c}\text { Site } \\
\text { Number }\end{array}$} & \multicolumn{2}{|c|}{ Habitat Complexity } \\
\hline & Upstream & Downstream \\
\hline W-04-078 & $S$ & $\mathrm{~S}$ \\
\hline W-04-079 & $\mathrm{S}$ & $\mathrm{S}$ \\
\hline W-04-080 & S & S \\
\hline W-04-081 & $\mathrm{C}$ & $\mathrm{C}$ \\
\hline W-04-082 & $\mathrm{S}$ & $\mathrm{S}$ \\
\hline W-04-083 & $\mathrm{C}$ & $\mathrm{C}$ \\
\hline W-04-084 & C & C \\
\hline W-04-085 & $\mathrm{C}$ & $\mathrm{C}$ \\
\hline W-04-086 & $\mathrm{C}$ & $\mathrm{C}$ \\
\hline W-04-087 & S & $\mathrm{S}$ \\
\hline W-04-088 & $S$ & $\mathrm{~S}$ \\
\hline W-04-089 & $\mathrm{S}$ & $\mathrm{S}$ \\
\hline W-04-090 & $\mathrm{C}$ & $\mathrm{C}$ \\
\hline W-04-091 & S & C \\
\hline W-04-092 & $\mathrm{C}$ & $\mathrm{C}$ \\
\hline W-04-093 & $\mathrm{C}$ & $\mathrm{C}$ \\
\hline W-04-094 & $\mathrm{C}$ & $\mathrm{C}$ \\
\hline W-04-095 & $\mathrm{C}$ & $\mathrm{C}$ \\
\hline W-04-096 & $\mathrm{C}$ & $\mathrm{C}$ \\
\hline W-04-097 & $\mathrm{C}$ & $\mathrm{C}$ \\
\hline W-04-098 & $\mathrm{C}$ & $\mathrm{C}$ \\
\hline W-04-099 & $\mathrm{C}$ & $\mathrm{C}$ \\
\hline W-04-100 & C & $\mathrm{C}$ \\
\hline W-04-101 & $\mathrm{C}$ & $\mathrm{C}$ \\
\hline W-04-102 & $\mathrm{C}$ & $\mathrm{C}$ \\
\hline W-04-103 & S & S \\
\hline W-04-104 & $\mathrm{C}$ & $\mathrm{C}$ \\
\hline W-04-105 & $\mathrm{C}$ & $\mathrm{S}$ \\
\hline W-04-106 & $\mathrm{S}$ & C \\
\hline W-04-107 & $\mathrm{C}$ & C \\
\hline W-04-108 & $\mathrm{C}$ & $\mathrm{C}$ \\
\hline W-04-109 & $\mathrm{S}$ & C \\
\hline W-04-110 & $\mathrm{S}$ & $\mathrm{S}$ \\
\hline W-04-111 & $\mathrm{S}$ & $\mathrm{S}$ \\
\hline
\end{tabular}


Appendix 2. Habitat complexity $(S=$ Simple; $C=$ Complex $)$ determination for all sites looking upstream and downstream from each surveyed reach.

\begin{tabular}{|c|c|c|}
\hline \multirow{2}{*}{$\begin{array}{c}\text { Site } \\
\text { Number }\end{array}$} & \multicolumn{2}{|c|}{ Habitat Complexity } \\
\hline & Upstream & Downstream \\
\hline W-04-112 & $S$ & $\mathrm{~S}$ \\
\hline W-04-113 & S & $\mathrm{S}$ \\
\hline W-04-114 & S & $\mathrm{S}$ \\
\hline W-04-115 & S & S \\
\hline W-04-116 & $S$ & $S$ \\
\hline W-04-117 & S & S \\
\hline W-04-118 & S & S \\
\hline W-04-119 & S & S \\
\hline W-04-120 & $\mathrm{C}$ & $\mathrm{C}$ \\
\hline W-04-121 & $\mathrm{C}$ & $\mathrm{C}$ \\
\hline W-04-122 & S & S \\
\hline W-04-123 & $S$ & $S$ \\
\hline W-04-124 & S & S \\
\hline W-04-125 & $\mathrm{C}$ & C \\
\hline W-04-126 & $\mathrm{C}$ & C \\
\hline W-04-127 & $\mathrm{C}$ & $\mathrm{C}$ \\
\hline W-04-128 & S & $\mathrm{S}$ \\
\hline W-04-129 & S & $\mathrm{C}$ \\
\hline W-04-130 & $\mathrm{C}$ & $\mathrm{C}$ \\
\hline W-04-131 & S & S \\
\hline W-04-132 & S & $\mathrm{C}$ \\
\hline W-04-133 & S & S \\
\hline W-04-134 & S & $\mathrm{S}$ \\
\hline W-04-135 & $S$ & $\mathrm{~S}$ \\
\hline W-04-136 & S & $\mathrm{S}$ \\
\hline W-04-137 & $\mathrm{C}$ & C \\
\hline W-04-138 & $\mathrm{S}$ & $\mathrm{S}$ \\
\hline W-04-139 & $S$ & $\mathrm{C}$ \\
\hline W-04-140 & S & $\mathrm{S}$ \\
\hline W-04-141 & $\mathrm{S}$ & $\mathrm{S}$ \\
\hline W-04-142 & $S$ & $\mathrm{~S}$ \\
\hline W-04-143 & $\mathrm{S}$ & $\mathrm{S}$ \\
\hline W-04-144 & $\mathrm{S}$ & $\mathrm{S}$ \\
\hline W-04-145 & $\mathrm{C}$ & $\mathrm{C}$ \\
\hline
\end{tabular}

\begin{tabular}{|c|c|c|}
\hline \multirow{2}{*}{$\begin{array}{c}\text { Site } \\
\text { Number }\end{array}$} & \multicolumn{2}{|c|}{ Habitat Complexity } \\
\hline & Upstream & Downstream \\
\hline W-04-146 & $\mathrm{S}$ & $\mathrm{S}$ \\
\hline W-04-147 & S & S \\
\hline W-04-148 & S & S \\
\hline W-04-149 & S & S \\
\hline W-04-150 & $S$ & $S$ \\
\hline W-04-151 & S & S \\
\hline W-04-152 & C & $S$ \\
\hline W-04-153 & S & S \\
\hline W-04-154 & $\mathrm{C}$ & $\mathrm{C}$ \\
\hline W-04-155 & $\mathrm{C}$ & $\mathrm{C}$ \\
\hline W-04-156 & S & S \\
\hline W-04-157 & $\mathrm{S}$ & $\mathrm{C}$ \\
\hline W-04-158 & S & $\mathrm{C}$ \\
\hline W-04-159 & $\mathrm{C}$ & $\mathrm{C}$ \\
\hline W-04-160 & $S$ & $S$ \\
\hline W-04-161 & $\mathrm{S}$ & $\mathrm{S}$ \\
\hline W-04-162 & S & S \\
\hline W-04-163 & C & C \\
\hline W-04-164 & S & $S$ \\
\hline W-04-165 & S & S \\
\hline W-04-166 & S & S \\
\hline W-04-167 & S & $S$ \\
\hline W-04-168 & S & $S$ \\
\hline W-04-169 & S & $\mathrm{C}$ \\
\hline W-04-170 & S & C \\
\hline W-04-171 & S & $\mathrm{C}$ \\
\hline W-04-172 & $S$ & $S$ \\
\hline W-04-173 & $S$ & $S$ \\
\hline W-04-174 & $S$ & $S$ \\
\hline W-04-175 & C & $S$ \\
\hline W-04-176 & C & C \\
\hline W-04-177 & C & S \\
\hline W-04-178 & $\mathrm{C}$ & $S$ \\
\hline W-04-179 & S & S \\
\hline
\end{tabular}

\begin{tabular}{|c|c|c|}
\hline \multirow{2}{*}{$\begin{array}{c}\text { Site } \\
\text { Number }\end{array}$} & \multicolumn{2}{|c|}{ Habitat Complexity } \\
\hline & Upstream & Downstream \\
\hline W-04-180 & $\mathrm{S}$ & $\mathrm{S}$ \\
\hline W-04-181 & $\mathrm{C}$ & $\mathrm{C}$ \\
\hline W-04-182 & $\mathrm{C}$ & $\mathrm{C}$ \\
\hline W-04-183 & $\mathrm{C}$ & $\mathrm{C}$ \\
\hline W-04-184 & $\mathrm{C}$ & $\mathrm{C}$ \\
\hline W-04-185 & S & $\mathrm{S}$ \\
\hline W-04-186 & S & $\mathrm{S}$ \\
\hline W-04-187 & S & $\mathrm{C}$ \\
\hline W-04-188 & $S$ & $\mathrm{~S}$ \\
\hline W-04-189 & S & $\mathrm{C}$ \\
\hline W-04-190 & $\mathrm{C}$ & C \\
\hline W-04-191 & $\mathrm{C}$ & $\mathrm{C}$ \\
\hline W-04-192 & $\mathrm{C}$ & $\mathrm{C}$ \\
\hline W-04-193 & C & C \\
\hline W-04-194 & $\mathrm{C}$ & $\mathrm{C}$ \\
\hline W-04-195 & $\mathrm{C}$ & $\mathrm{C}$ \\
\hline W-04-196 & $\mathrm{C}$ & $\mathrm{C}$ \\
\hline W-04-197 & $\mathrm{C}$ & $\mathrm{C}$ \\
\hline W-04-198 & $\mathrm{C}$ & $\mathrm{C}$ \\
\hline W-04-199 & $\mathrm{C}$ & $\mathrm{S}$ \\
\hline W-04-200 & $\mathrm{C}$ & $\mathrm{C}$ \\
\hline W-04-201 & $\mathrm{S}$ & $\mathrm{S}$ \\
\hline W-04-202 & S & $\mathrm{S}$ \\
\hline W-04-203 & $\mathrm{S}$ & $\mathrm{S}$ \\
\hline W-04-204 & S & $\mathrm{S}$ \\
\hline W-04-205 & S & $\mathrm{S}$ \\
\hline W-04-206 & $\mathrm{C}$ & C \\
\hline W-04-207 & $\mathrm{C}$ & $\mathrm{C}$ \\
\hline W-04-208 & $\mathrm{C}$ & $\mathrm{C}$ \\
\hline W-04-209 & C & $\mathrm{C}$ \\
\hline W-04-210 & $\mathrm{C}$ & $\mathrm{C}$ \\
\hline W-04-211 & $\mathrm{C}$ & $\mathrm{C}$ \\
\hline W-04-212 & $\mathrm{S}$ & $\mathrm{C}$ \\
\hline W-04-213 & $\mathrm{C}$ & $\mathrm{C}$ \\
\hline
\end{tabular}


Appendix 2. Habitat complexity $(S=$ Simple; $C=$ Complex $)$ determination for all sites looking upstream and downstream from each surveyed reach.

\begin{tabular}{|c|c|c|}
\hline \multirow{2}{*}{$\begin{array}{c}\text { Site } \\
\text { Number }\end{array}$} & \multicolumn{2}{|c|}{ Habitat Complexity } \\
\hline & Upstream & Downstream \\
\hline W-04-214 & $S$ & $\mathrm{~S}$ \\
\hline W-04-215 & S & $\mathrm{S}$ \\
\hline W-04-216 & $\mathrm{C}$ & $\mathrm{C}$ \\
\hline W-04-217 & S & S \\
\hline W-04-218 & $S$ & $\mathrm{C}$ \\
\hline W-04-219 & S & S \\
\hline W-04-220 & S & S \\
\hline W-04-221 & S & S \\
\hline W-04-222 & S & $\mathrm{S}$ \\
\hline W-04-223 & S & S \\
\hline W-04-224 & S & S \\
\hline W-04-225 & $S$ & $\mathrm{C}$ \\
\hline W-04-226 & $S$ & $\mathrm{C}$ \\
\hline W-04-227 & S & $\mathrm{S}$ \\
\hline W-04-228 & S & S \\
\hline W-04-229 & S & $\mathrm{S}$ \\
\hline W-04-230 & S & $\mathrm{S}$ \\
\hline W-04-231 & S & $\mathrm{S}$ \\
\hline W-04-232 & $\mathrm{C}$ & $\mathrm{C}$ \\
\hline W-04-233 & C & $\mathrm{C}$ \\
\hline W-04-234 & $\mathrm{C}$ & $\mathrm{C}$ \\
\hline W-04-235 & $\mathrm{C}$ & $\mathrm{C}$ \\
\hline W-04-236 & $\mathrm{C}$ & C \\
\hline W-04-237 & $\mathrm{C}$ & $\mathrm{C}$ \\
\hline W-04-238 & $\mathrm{C}$ & $\mathrm{C}$ \\
\hline W-04-239 & C & C \\
\hline W-04-240 & $\mathrm{C}$ & C \\
\hline W-04-241 & $\mathrm{C}$ & $\mathrm{C}$ \\
\hline W-04-242 & $\mathrm{C}$ & C \\
\hline W-04-243 & $\mathrm{C}$ & $\mathrm{C}$ \\
\hline W-04-244 & $\mathrm{C}$ & $\mathrm{C}$ \\
\hline W-04-245 & $\mathrm{C}$ & C \\
\hline W-04-246 & $\mathrm{C}$ & C \\
\hline W-04-247 & $\mathrm{S}$ & $\mathrm{S}$ \\
\hline
\end{tabular}

\begin{tabular}{|c|c|c|}
\hline \multirow{2}{*}{$\begin{array}{c}\text { Site } \\
\text { Number }\end{array}$} & \multicolumn{2}{|c|}{ Habitat Complexity } \\
\hline & Upstream & Downstream \\
\hline W-04-248 & $\mathrm{C}$ & $\mathrm{C}$ \\
\hline W-04-249 & C & $\mathrm{C}$ \\
\hline W-04-250 & $\mathrm{C}$ & $\mathrm{C}$ \\
\hline W-04-251 & C & C \\
\hline W-04-252 & $\mathrm{C}$ & $\mathrm{C}$ \\
\hline W-04-253 & $\mathrm{C}$ & $\mathrm{C}$ \\
\hline W-04-254 & C & C \\
\hline W-04-255 & C & C \\
\hline W-04-256 & $\mathrm{C}$ & $\mathrm{C}$ \\
\hline W-04-257 & S & $\mathrm{C}$ \\
\hline W-04-258 & S & C \\
\hline W-04-259 & $S$ & $S$ \\
\hline W-04-260 & C & S \\
\hline W-04-261 & S & $S$ \\
\hline W-04-262 & $\mathrm{C}$ & C \\
\hline W-04-263 & $\mathrm{S}$ & $\mathrm{S}$ \\
\hline W-04-264 & S & S \\
\hline W-04-265 & C & C \\
\hline W-04-266 & $\mathrm{C}$ & $\mathrm{C}$ \\
\hline W-04-267 & C & C \\
\hline W-04-268 & $\mathrm{C}$ & $\mathrm{C}$ \\
\hline W-04-269 & $\mathrm{C}$ & $\mathrm{C}$ \\
\hline W-04-270 & S & $S$ \\
\hline W-04-271 & $\mathrm{C}$ & $\mathrm{C}$ \\
\hline W-04-272 & C & $\mathrm{C}$ \\
\hline W-04-273 & S & $S$ \\
\hline W-04-274 & C & C \\
\hline W-04-275 & $S$ & $S$ \\
\hline W-04-276 & $S$ & $S$ \\
\hline W-04-277 & $S$ & $S$ \\
\hline W-04-278 & $S$ & $S$ \\
\hline W-04-279 & S & S \\
\hline W-04-280 & $S$ & $\mathrm{~S}$ \\
\hline W-04-281 & $S$ & $\mathrm{~S}$ \\
\hline
\end{tabular}

\begin{tabular}{|c|c|c|}
\hline \multirow{2}{*}{$\begin{array}{c}\text { Site } \\
\text { Number }\end{array}$} & \multicolumn{2}{|c|}{ Habitat Complexity } \\
\hline & Upstream & Downstream \\
\hline W-04-282 & $\mathrm{S}$ & $\mathrm{S}$ \\
\hline W-04-283 & $S$ & S \\
\hline W-04-284 & S & S \\
\hline W-04-285 & $S$ & S \\
\hline W-04-286 & $\mathrm{C}$ & $\mathrm{C}$ \\
\hline W-04-287 & $\mathrm{C}$ & $\mathrm{C}$ \\
\hline W-04-288 & S & $\mathrm{S}$ \\
\hline W-04-289 & $\mathrm{C}$ & $\mathrm{C}$ \\
\hline W-04-290 & $S$ & $\mathrm{C}$ \\
\hline W-04-291 & S & $\mathrm{C}$ \\
\hline W-04-292 & $\mathrm{C}$ & C \\
\hline W-04-293 & $\mathrm{S}$ & $\mathrm{S}$ \\
\hline W-04-294 & $\mathrm{C}$ & $\mathrm{C}$ \\
\hline W-04-295 & C & C \\
\hline W-04-296 & $\mathrm{C}$ & $\mathrm{C}$ \\
\hline W-04-297 & $\mathrm{C}$ & C \\
\hline W-04-298 & $\mathrm{C}$ & $\mathrm{C}$ \\
\hline W-04-299 & $\mathrm{C}$ & $\mathrm{C}$ \\
\hline W-04-300 & C & $\mathrm{C}$ \\
\hline W-04-301 & $\mathrm{C}$ & $\mathrm{C}$ \\
\hline X-04-001 & S & $\mathrm{S}$ \\
\hline X-04-002 & S & $\mathrm{S}$ \\
\hline$X-04-003$ & S & $\mathrm{S}$ \\
\hline X-04-004 & S & $\mathrm{S}$ \\
\hline X-04-005 & S & S \\
\hline X-04-006 & $\mathrm{C}$ & $\mathrm{C}$ \\
\hline X-04-007 & $S$ & $\mathrm{~S}$ \\
\hline X-04-008 & $S$ & $\mathrm{~S}$ \\
\hline X-04-009 & $S$ & $\mathrm{~S}$ \\
\hline X-04-010 & $S$ & $\mathrm{~S}$ \\
\hline X-04-011 & $S$ & $\mathrm{~S}$ \\
\hline X-04-012 & $S$ & $S$ \\
\hline X-04-013 & $S$ & C \\
\hline X-04-014 & $\mathrm{C}$ & C \\
\hline
\end{tabular}


Appendix 2. Habitat complexity $(S=$ Simple; $C=$ Complex $)$ determination for all sites looking upstream and downstream from each surveyed reach.

\begin{tabular}{|c|c|c|}
\hline \multirow{2}{*}{$\begin{array}{c}\text { Site } \\
\text { Number }\end{array}$} & \multicolumn{2}{|c|}{ Habitat Complexity } \\
\hline & Upstream & Downstream \\
\hline $\mathrm{X}-04-015$ & $S$ & $\mathrm{~S}$ \\
\hline X-04-016 & $\mathrm{C}$ & $\mathrm{C}$ \\
\hline $\mathrm{X}-04-017$ & $\mathrm{C}$ & $\mathrm{C}$ \\
\hline$X-04-018$ & C & $\mathrm{C}$ \\
\hline $\mathrm{X}-04-019$ & $\mathrm{C}$ & $\mathrm{C}$ \\
\hline X-04-020 & $\mathrm{C}$ & $\mathrm{C}$ \\
\hline $\mathrm{X}-04-021$ & $\mathrm{~S}$ & S \\
\hline $\mathrm{X}-04-022$ & $S$ & $S$ \\
\hline $\mathrm{X}-04-023$ & $\mathrm{C}$ & $\mathrm{C}$ \\
\hline X-04-024 & S & S \\
\hline$X-04-025$ & S & S \\
\hline $\mathrm{X}-04-026$ & $S$ & $S$ \\
\hline $\mathrm{X}-04-027$ & S & S \\
\hline $\mathrm{X}-04-028$ & S & S \\
\hline X-04-029 & $\mathrm{S}$ & $\mathrm{S}$ \\
\hline X-04-030 & S & $\mathrm{S}$ \\
\hline $\mathrm{X}-04-031$ & $S$ & $S$ \\
\hline X-04-032 & $S$ & $\mathrm{~S}$ \\
\hline $\mathrm{X}-04-033$ & S & $\mathrm{S}$ \\
\hline $\mathrm{X}-04-034$ & $S$ & $\mathrm{~S}$ \\
\hline X-04-035 & $S$ & $\mathrm{~S}$ \\
\hline X-04-036 & S & $\mathrm{C}$ \\
\hline $\mathrm{X}-04-037$ & $\mathrm{C}$ & C \\
\hline X-04-038 & $S$ & $\mathrm{~S}$ \\
\hline X-04-039 & S & S \\
\hline $\mathrm{X}-04-040$ & S & S \\
\hline X-04-041 & $S$ & $S$ \\
\hline X-04-042 & $\mathrm{S}$ & $\mathrm{S}$ \\
\hline X-04-043 & $\mathrm{C}$ & $\mathrm{C}$ \\
\hline X-04-044 & $\mathrm{S}$ & $\mathrm{S}$ \\
\hline X-04-045 & $S$ & $S$ \\
\hline X-04-046 & $S$ & $S$ \\
\hline X-04-047 & C & $\mathrm{C}$ \\
\hline X-04-048 & $\mathrm{C}$ & $\mathrm{C}$ \\
\hline
\end{tabular}

\begin{tabular}{|c|c|c|}
\hline \multirow{2}{*}{$\begin{array}{c}\text { Site } \\
\text { Number }\end{array}$} & \multicolumn{2}{|c|}{ Habitat Complexity } \\
\hline & Upstream & Downstream \\
\hline X-04-049 & $\mathrm{C}$ & $\mathrm{C}$ \\
\hline $\mathrm{X}-04-050$ & $\mathrm{C}$ & C \\
\hline $\mathrm{X}-04-051$ & $\mathrm{C}$ & $\mathrm{C}$ \\
\hline $\mathrm{X}-04-052$ & $\mathrm{~S}$ & $\mathrm{~S}$ \\
\hline $\mathrm{X}-04-053$ & $\mathrm{~S}$ & $\mathrm{C}$ \\
\hline$X-04-054$ & $\mathrm{~S}$ & $\mathrm{~S}$ \\
\hline$X-04-055$ & $S$ & $\mathrm{~S}$ \\
\hline$X-04-056$ & $\mathrm{~S}$ & $\mathrm{~S}$ \\
\hline $\mathrm{X}-04-057$ & C & C \\
\hline $\mathrm{X}-04-058$ & $\mathrm{~S}$ & $\mathrm{~S}$ \\
\hline$X-04-059$ & $\mathrm{C}$ & C \\
\hline $\mathrm{X}-04-060$ & $\mathrm{~S}$ & $\mathrm{~S}$ \\
\hline $\mathrm{X}-04-061$ & $\mathrm{~S}$ & $\mathrm{~S}$ \\
\hline$X-04-062$ & $\mathrm{~S}$ & C \\
\hline $\mathrm{X}-04-063$ & S & C \\
\hline X-04-064 & $\mathrm{S}$ & $\mathrm{S}$ \\
\hline$X-04-065$ & C & C \\
\hline $\mathrm{X}-04-066$ & $\mathrm{~S}$ & $\mathrm{~S}$ \\
\hline $\mathrm{X}-04-067$ & $\mathrm{C}$ & $\mathrm{C}$ \\
\hline$X-04-068$ & $\mathrm{~S}$ & $\mathrm{~S}$ \\
\hline X-04-069 & $\mathrm{S}$ & $\mathrm{S}$ \\
\hline $\mathrm{X}-04-070$ & S & $\mathrm{S}$ \\
\hline$X-04-071$ & $\mathrm{~S}$ & $\mathrm{~S}$ \\
\hline $\mathrm{X}-04-072$ & $\mathrm{~S}$ & $\mathrm{~S}$ \\
\hline $\mathrm{X}-04-073$ & $\mathrm{~S}$ & $\mathrm{~S}$ \\
\hline$X-04-074$ & $\mathrm{~S}$ & $\mathrm{~S}$ \\
\hline X-04-075 & $\mathrm{S}$ & $\mathrm{S}$ \\
\hline X-04-076 & C & C \\
\hline X-04-077 & C & C \\
\hline X-04-078 & $\mathrm{S}$ & C \\
\hline X-04-079 & $\mathrm{S}$ & C \\
\hline X-04-080 & C & C \\
\hline X-04-081 & $\mathrm{S}$ & $\mathrm{S}$ \\
\hline X-04-082 & $\mathrm{S}$ & $\mathrm{S}$ \\
\hline
\end{tabular}

\begin{tabular}{|c|c|c|}
\hline \multirow{2}{*}{$\begin{array}{c}\text { Site } \\
\text { Number }\end{array}$} & \multicolumn{2}{|c|}{ Habitat Complexity } \\
\hline & Upstream & Downstream \\
\hline X-04-083 & $\mathrm{C}$ & $\mathrm{C}$ \\
\hline X-04-084 & $S$ & $\mathrm{~S}$ \\
\hline $\mathrm{X}-04-085$ & $\mathrm{C}$ & $S$ \\
\hline$X-04-086$ & $S$ & S \\
\hline $\mathrm{X}-04-087$ & $\mathrm{C}$ & C \\
\hline X-04-088 & S & $\mathrm{S}$ \\
\hline$X-04-089$ & C & $\mathrm{C}$ \\
\hline X-04-090 & C & $\mathrm{C}$ \\
\hline X-04-091 & $\mathrm{C}$ & $\mathrm{C}$ \\
\hline X-04-092 & $\mathrm{C}$ & $\mathrm{C}$ \\
\hline X-04-093 & $\mathrm{C}$ & C \\
\hline X-04-094 & $\mathrm{C}$ & C \\
\hline X-04-095 & $\mathrm{C}$ & $\mathrm{C}$ \\
\hline$X-04-096$ & S & $\mathrm{S}$ \\
\hline X-04-097 & $\mathrm{S}$ & $\mathrm{C}$ \\
\hline X-04-098 & S & $\mathrm{S}$ \\
\hline X-04-099 & S & $\mathrm{S}$ \\
\hline $\mathrm{X}-04-100$ & $S$ & $\mathrm{C}$ \\
\hline X-04-101 & S & $\mathrm{S}$ \\
\hline X-04-102 & S & $\mathrm{C}$ \\
\hline $\mathrm{X}-04-103$ & S & $\mathrm{S}$ \\
\hline X-04-104 & $\mathrm{C}$ & $\mathrm{S}$ \\
\hline$X-04-105$ & C & C \\
\hline $\mathrm{X}-04-106$ & $\mathrm{C}$ & $\mathrm{C}$ \\
\hline X-04-107 & $\mathrm{C}$ & $\mathrm{C}$ \\
\hline$X-04-108$ & $\mathrm{C}$ & C \\
\hline X-04-109 & $\mathrm{C}$ & $\mathrm{C}$ \\
\hline X-04-110 & $\mathrm{C}$ & C \\
\hline$X-04-111$ & C & C \\
\hline $\mathrm{X}-04-112$ & $\mathrm{C}$ & C \\
\hline X-04-113 & $S$ & $\mathrm{~S}$ \\
\hline X-04-114 & C & C \\
\hline X-04-115 & $\mathrm{C}$ & $\mathrm{C}$ \\
\hline X-04-116 & $\mathrm{C}$ & $\mathrm{C}$ \\
\hline
\end{tabular}


Appendix 2. Habitat complexity $(S=$ Simple; $C=$ Complex $)$ determination for all sites looking upstream and downstream from each surveyed reach.

\begin{tabular}{|c|c|c|}
\hline \multirow{2}{*}{$\begin{array}{c}\text { Site } \\
\text { Number }\end{array}$} & \multicolumn{2}{|c|}{ Habitat Complexity } \\
\hline & Upstream & Downstream \\
\hline $\mathrm{X}-04-117$ & $S$ & $\mathrm{~S}$ \\
\hline X-04-118 & $\mathrm{C}$ & $\mathrm{C}$ \\
\hline X-04-119 & $\mathrm{C}$ & $\mathrm{C}$ \\
\hline$X-04-120$ & $\mathrm{C}$ & $\mathrm{C}$ \\
\hline $\mathrm{X}-04-121$ & $\mathrm{C}$ & $\mathrm{C}$ \\
\hline $\mathrm{X}-04-122$ & $\mathrm{C}$ & $\mathrm{C}$ \\
\hline$X-04-123$ & $\mathrm{C}$ & $\mathrm{C}$ \\
\hline$X-04-124$ & $\mathrm{C}$ & $\mathrm{C}$ \\
\hline $\mathrm{X}-04-125$ & $\mathrm{~S}$ & $\mathrm{~S}$ \\
\hline $\mathrm{X}-04-126$ & S & $\mathrm{S}$ \\
\hline$X-04-127$ & S & $\mathrm{S}$ \\
\hline $\mathrm{X}-04-128$ & $\mathrm{C}$ & $\mathrm{C}$ \\
\hline X-04-129 & $\mathrm{C}$ & $\mathrm{C}$ \\
\hline$X-04-130$ & $\mathrm{C}$ & $\mathrm{C}$ \\
\hline $\mathrm{X}-04-131$ & $\mathrm{~S}$ & $\mathrm{~S}$ \\
\hline $\mathrm{X}-04-132$ & S & $\mathrm{C}$ \\
\hline X-04-133 & $\mathrm{C}$ & $\mathrm{C}$ \\
\hline X-04-134 & $\mathrm{S}$ & $\mathrm{S}$ \\
\hline $\mathrm{X}-04-135$ & $\mathrm{~S}$ & $\mathrm{C}$ \\
\hline$X-04-136$ & $\mathrm{~S}$ & $\mathrm{C}$ \\
\hline $\mathrm{X}-04-137$ & $\mathrm{C}$ & $\mathrm{C}$ \\
\hline X-04-138 & $\mathrm{C}$ & $\mathrm{C}$ \\
\hline X-04-139 & C & C \\
\hline X-04-140 & $\mathrm{C}$ & $\mathrm{C}$ \\
\hline X-04-141 & $\mathrm{C}$ & $\mathrm{C}$ \\
\hline $\mathrm{X}-04-142$ & $\mathrm{C}$ & $\mathrm{C}$ \\
\hline X-04-143 & $\mathrm{C}$ & $\mathrm{C}$ \\
\hline X-04-144 & $\mathrm{C}$ & $\mathrm{C}$ \\
\hline X-04-145 & C & $\mathrm{C}$ \\
\hline$X-04-146$ & C & C \\
\hline X-04-147 & $\mathrm{C}$ & $\mathrm{C}$ \\
\hline X-04-148 & $\mathrm{C}$ & $S$ \\
\hline X-04-149 & $\mathrm{C}$ & $\mathrm{C}$ \\
\hline X-04-150 & $\mathrm{C}$ & $\mathrm{C}$ \\
\hline
\end{tabular}

\begin{tabular}{|c|c|c|}
\hline \multirow{2}{*}{$\begin{array}{c}\text { Site } \\
\text { Number }\end{array}$} & \multicolumn{2}{|c|}{ Habitat Complexity } \\
\hline & Upstream & Downstream \\
\hline $\mathrm{X}-04-151$ & $\mathrm{C}$ & $\mathrm{C}$ \\
\hline$X-04-152$ & $\mathrm{C}$ & C \\
\hline $\mathrm{X}-04-153$ & $\mathrm{C}$ & $\mathrm{C}$ \\
\hline$X-04-154$ & $\mathrm{C}$ & C \\
\hline $\mathrm{X}-04-155$ & $\mathrm{C}$ & $\mathrm{C}$ \\
\hline$X-04-156$ & $\mathrm{~S}$ & C \\
\hline$X-04-157$ & C & C \\
\hline$X-04-158$ & C & C \\
\hline $\mathrm{X}-04-159$ & C & C \\
\hline$X-04-160$ & $\mathrm{C}$ & $\mathrm{C}$ \\
\hline$X-04-161$ & $\mathrm{C}$ & C \\
\hline $\mathrm{X}-04-162$ & C & C \\
\hline $\mathrm{X}-04-163$ & C & C \\
\hline$X-04-164$ & C & C \\
\hline $\mathrm{X}-04-165$ & $\mathrm{~S}$ & C \\
\hline $\mathrm{X}-04-166$ & $\mathrm{~S}$ & C \\
\hline$X-04-167$ & $\mathrm{~S}$ & $\mathrm{~S}$ \\
\hline$X-04-168$ & $\mathrm{~S}$ & $\mathrm{~S}$ \\
\hline X-04-169 & $\mathrm{C}$ & $\mathrm{C}$ \\
\hline$X-04-170$ & C & C \\
\hline $\mathrm{X}-04-171$ & $\mathrm{C}$ & $\mathrm{C}$ \\
\hline $\mathrm{X}-04-172$ & $\mathrm{C}$ & $\mathrm{C}$ \\
\hline$X-04-173$ & $\mathrm{~S}$ & C \\
\hline $\mathrm{X}-04-174$ & C & C \\
\hline $\mathrm{X}-04-175$ & C & C \\
\hline$X-04-176$ & C & C \\
\hline X-04-177 & C & C \\
\hline X-04-178 & $\mathrm{S}$ & C \\
\hline$X-04-179$ & C & C \\
\hline X-04-180 & C & C \\
\hline X-04-181 & C & C \\
\hline X-04-182 & C & C \\
\hline X-04-183 & C & C \\
\hline X-04-184 & $\mathrm{S}$ & $\mathrm{S}$ \\
\hline
\end{tabular}

\begin{tabular}{|c|c|c|}
\hline \multirow{2}{*}{$\begin{array}{c}\text { Site } \\
\text { Number }\end{array}$} & \multicolumn{2}{|c|}{ Habitat Complexity } \\
\hline & Upstream & Downstream \\
\hline X-04-185 & $\mathrm{C}$ & $\mathrm{S}$ \\
\hline X-04-186 & $\mathrm{S}$ & S \\
\hline $\mathrm{X}-04-187$ & C & $\mathrm{S}$ \\
\hline$X-04-188$ & $\mathrm{C}$ & C \\
\hline X-04-189 & $\mathrm{C}$ & $\mathrm{C}$ \\
\hline X-04-190 & $\mathrm{C}$ & $\mathrm{S}$ \\
\hline X-04-191 & S & S \\
\hline X-04-192 & S & S \\
\hline $\mathrm{X}-04-193$ & $\mathrm{~S}$ & $\mathrm{C}$ \\
\hline X-04-194 & $\mathrm{C}$ & $\mathrm{C}$ \\
\hline X-04-195 & $\mathrm{C}$ & C \\
\hline X-04-196 & $\mathrm{S}$ & $\mathrm{S}$ \\
\hline X-04-197 & $S$ & $S$ \\
\hline X-04-198 & S & C \\
\hline X-04-199 & $S$ & $\mathrm{C}$ \\
\hline X-04-200 & $\mathrm{C}$ & $\mathrm{C}$ \\
\hline$X-04-201$ & S & $\mathrm{S}$ \\
\hline X-04-202 & $S$ & $\mathrm{~S}$ \\
\hline X-04-203 & $\mathrm{C}$ & $\mathrm{C}$ \\
\hline$X-04-204$ & S & $\mathrm{C}$ \\
\hline X-04-205 & $\mathrm{C}$ & $\mathrm{C}$ \\
\hline X-04-206 & $\mathrm{C}$ & $\mathrm{C}$ \\
\hline X-04-207 & $\mathrm{C}$ & $\mathrm{C}$ \\
\hline X-04-208 & $\mathrm{C}$ & C \\
\hline X-04-209 & $\mathrm{C}$ & $\mathrm{C}$ \\
\hline$X-04-210$ & $\mathrm{C}$ & C \\
\hline X-04-211 & $\mathrm{C}$ & $\mathrm{C}$ \\
\hline X-04-212 & $S$ & $\mathrm{~S}$ \\
\hline$X-04-213$ & $S$ & $S$ \\
\hline X-04-214 & $\mathrm{S}$ & $\mathrm{S}$ \\
\hline X-04-215 & $\mathrm{S}$ & $\mathrm{S}$ \\
\hline X-04-216 & $\mathrm{S}$ & $\mathrm{S}$ \\
\hline X-04-217 & $\mathrm{C}$ & $\mathrm{C}$ \\
\hline X-04-218 & $\mathrm{S}$ & $\mathrm{S}$ \\
\hline
\end{tabular}


Appendix 2. Habitat complexity $(S=$ Simple; $C=$ Complex $)$ determination for all sites looking upstream and downstream from each surveyed reach.

\begin{tabular}{|c|c|c|}
\hline \multirow{2}{*}{$\begin{array}{c}\text { Site } \\
\text { Number }\end{array}$} & \multicolumn{2}{|c|}{ Habitat Complexity } \\
\hline & Upstream & Downstream \\
\hline $\mathrm{X}-04-219$ & $S$ & $\mathrm{~S}$ \\
\hline$X-04-220$ & S & $\mathrm{S}$ \\
\hline $\mathrm{X}-04-221$ & $\mathrm{C}$ & $\mathrm{C}$ \\
\hline$X-04-222$ & C & C \\
\hline $\mathrm{X}-04-223$ & $\mathrm{C}$ & $\mathrm{C}$ \\
\hline$X-04-224$ & C & $\mathrm{C}$ \\
\hline$X-04-225$ & C & C \\
\hline$X-04-226$ & C & C \\
\hline $\mathrm{X}-04-227$ & C & $\mathrm{C}$ \\
\hline $\mathrm{X}-04-228$ & $\mathrm{C}$ & $\mathrm{C}$ \\
\hline$X-04-229$ & C & C \\
\hline $\mathrm{X}-04-230$ & $\mathrm{C}$ & $\mathrm{C}$ \\
\hline $\mathrm{X}-04-231$ & $\mathrm{C}$ & $\mathrm{C}$ \\
\hline$X-04-232$ & $\mathrm{C}$ & C \\
\hline $\mathrm{X}-04-233$ & C & C \\
\hline $\mathrm{X}-04-234$ & C & $\mathrm{C}$ \\
\hline$X-04-235$ & $\mathrm{C}$ & $\mathrm{C}$ \\
\hline $\mathrm{X}-04-236$ & $\mathrm{C}$ & $\mathrm{C}$ \\
\hline $\mathrm{X}-04-237$ & $\mathrm{C}$ & $\mathrm{C}$ \\
\hline$X-04-238$ & C & $\mathrm{C}$ \\
\hline$X-04-239$ & $\mathrm{C}$ & $\mathrm{C}$ \\
\hline $\mathrm{X}-04-240$ & S & $\mathrm{S}$ \\
\hline$X-04-241$ & S & $\mathrm{S}$ \\
\hline $\mathrm{X}-04-242$ & $\mathrm{C}$ & $\mathrm{C}$ \\
\hline $\mathrm{X}-04-243$ & $\mathrm{~S}$ & $\mathrm{~S}$ \\
\hline$X-04-244$ & C & C \\
\hline X-04-245 & C & C \\
\hline $\mathrm{X}-04-246$ & $\mathrm{C}$ & $\mathrm{C}$ \\
\hline$X-04-247$ & $S$ & $S$ \\
\hline X-04-248 & C & C \\
\hline X-04-249 & $\mathrm{C}$ & $\mathrm{C}$ \\
\hline$X-04-250$ & $\mathrm{~S}$ & $\mathrm{~S}$ \\
\hline $\mathrm{X}-04-251$ & C & C \\
\hline $\mathrm{X}-04-252$ & $\mathrm{C}$ & C \\
\hline
\end{tabular}

\begin{tabular}{|c|c|c|}
\hline \multirow{2}{*}{$\begin{array}{c}\text { Site } \\
\text { Number }\end{array}$} & \multicolumn{2}{|c|}{ Habitat Complexity } \\
\hline & Upstream & Downstream \\
\hline$X-04-253$ & C & C \\
\hline$X-04-254$ & $\mathrm{C}$ & $\mathrm{C}$ \\
\hline X-04-255 & $\mathrm{C}$ & $\mathrm{C}$ \\
\hline $\mathrm{X}-04-256$ & $\mathrm{C}$ & C \\
\hline $\mathrm{X}-04-257$ & $\mathrm{C}$ & $\mathrm{C}$ \\
\hline $\mathrm{X}-04-258$ & $\mathrm{C}$ & $\mathrm{C}$ \\
\hline$X-04-259$ & C & C \\
\hline$X-04-260$ & $\mathrm{C}$ & $\mathrm{C}$ \\
\hline $\mathrm{X}-04-261$ & $\mathrm{C}$ & $\mathrm{C}$ \\
\hline $\mathrm{X}-04-262$ & $\mathrm{C}$ & $\mathrm{C}$ \\
\hline$X-04-263$ & $\mathrm{C}$ & C \\
\hline D-05-001 & $\mathrm{C}$ & $\mathrm{C}$ \\
\hline D-05-002 & $\mathrm{S}$ & $\mathrm{S}$ \\
\hline D-05-003 & $\mathrm{S}$ & C \\
\hline D-05-004 & $\mathrm{S}$ & $\mathrm{S}$ \\
\hline D-05-005 & $\mathrm{S}$ & $\mathrm{S}$ \\
\hline D-05-006 & $\mathrm{C}$ & $\mathrm{S}$ \\
\hline D-05-007 & $\mathrm{S}$ & $\mathrm{S}$ \\
\hline D-05-008 & $\mathrm{C}$ & $\mathrm{S}$ \\
\hline D-05-009 & $\mathrm{S}$ & $\mathrm{S}$ \\
\hline D-05-010 & $\mathrm{C}$ & $\mathrm{C}$ \\
\hline D-05-011 & $\mathrm{C}$ & $\mathrm{S}$ \\
\hline D-05-012 & $\mathrm{S}$ & $\mathrm{S}$ \\
\hline D-05-013 & $\mathrm{C}$ & $\mathrm{C}$ \\
\hline D-05-014 & $\mathrm{S}$ & $\mathrm{S}$ \\
\hline D-05-015 & $\mathrm{S}$ & $\mathrm{S}$ \\
\hline D-05-016 & C & C \\
\hline D-05-017 & $\mathrm{S}$ & $\mathrm{C}$ \\
\hline D-05-018 & $\mathrm{S}$ & $S$ \\
\hline D-05-019 & $\mathrm{C}$ & $\mathrm{S}$ \\
\hline D-05-020 & $\mathrm{S}$ & $S$ \\
\hline D-05-021 & $S$ & $S$ \\
\hline D-05-022 & $\mathrm{S}$ & $\mathrm{S}$ \\
\hline D-05-023 & $\mathrm{S}$ & C \\
\hline
\end{tabular}

\begin{tabular}{|c|c|c|}
\hline \multirow{2}{*}{$\begin{array}{c}\text { Site } \\
\text { Number }\end{array}$} & \multicolumn{2}{|c|}{ Habitat Complexity } \\
\hline & Upstream & Downstream \\
\hline D-05-024 & $\mathrm{C}$ & $\mathrm{C}$ \\
\hline D-05-025 & $\mathrm{C}$ & $\mathrm{C}$ \\
\hline D-05-026 & $\mathrm{C}$ & $\mathrm{C}$ \\
\hline D-05-027 & $\mathrm{C}$ & $\mathrm{C}$ \\
\hline W-05-001 & S & $\mathrm{S}$ \\
\hline W-05-002 & S & S \\
\hline W-05-003 & S & $S$ \\
\hline W-05-004 & S & S \\
\hline W-05-005 & S & $S$ \\
\hline W-05-006 & S & $\mathrm{S}$ \\
\hline W-05-007 & S & S \\
\hline W-05-008 & $\mathrm{C}$ & $\mathrm{C}$ \\
\hline W-05-009 & S & $\mathrm{S}$ \\
\hline W-05-010 & S & C \\
\hline W-05-011 & $S$ & $\mathrm{~S}$ \\
\hline W-05-012 & $\mathrm{C}$ & C \\
\hline W-05-013 & $\mathrm{C}$ & $\mathrm{C}$ \\
\hline W-05-014 & S & S \\
\hline W-05-015 & $\mathrm{C}$ & $\mathrm{C}$ \\
\hline W-05-016 & S & $\mathrm{S}$ \\
\hline W-05-017 & $\mathrm{C}$ & $\mathrm{C}$ \\
\hline W-05-018 & S & $\mathrm{S}$ \\
\hline W-05-019 & $S$ & $\mathrm{~S}$ \\
\hline W-05-020 & $\mathrm{C}$ & $\mathrm{C}$ \\
\hline W-05-021 & S & $\mathrm{C}$ \\
\hline W-05-022 & $\mathrm{C}$ & $\mathrm{C}$ \\
\hline W-05-023 & $\mathrm{S}$ & $\mathrm{S}$ \\
\hline W-05-024 & $S$ & $\mathrm{~S}$ \\
\hline W-05-025 & $\mathrm{S}$ & $\mathrm{S}$ \\
\hline W-05-026 & $S$ & $\mathrm{~S}$ \\
\hline W-05-027 & $\mathrm{S}$ & $\mathrm{S}$ \\
\hline W-05-028 & $\mathrm{C}$ & C \\
\hline W-05-029 & $\mathrm{S}$ & $\mathrm{S}$ \\
\hline W-05-030 & $\mathrm{S}$ & $\mathrm{S}$ \\
\hline
\end{tabular}


Appendix 2. Habitat complexity $(S=$ Simple; $C=$ Complex $)$ determination for all sites looking upstream and downstream from each surveyed reach.

\begin{tabular}{|c|c|c|}
\hline \multirow{2}{*}{$\begin{array}{c}\text { Site } \\
\text { Number }\end{array}$} & \multicolumn{2}{|c|}{ Habitat Complexity } \\
\hline & Upstream & Downstream \\
\hline W-05-031 & $S$ & $\mathrm{~S}$ \\
\hline W-05-032 & S & $\mathrm{S}$ \\
\hline W-05-033 & S & $\mathrm{S}$ \\
\hline W-05-034 & S & S \\
\hline W-05-035 & $S$ & $S$ \\
\hline W-05-036 & $S$ & $S$ \\
\hline W-05-037 & S & S \\
\hline W-05-038 & S & S \\
\hline W-05-039 & S & $\mathrm{S}$ \\
\hline W-05-040 & S & S \\
\hline W-05-041 & S & S \\
\hline W-05-042 & $S$ & $S$ \\
\hline W-05-043 & $S$ & $S$ \\
\hline W-05-044 & S & S \\
\hline W-05-045 & S & S \\
\hline W-05-046 & S & $\mathrm{S}$ \\
\hline W-05-047 & S & $\mathrm{S}$ \\
\hline W-05-048 & S & $S$ \\
\hline W-05-049 & S & $\mathrm{S}$ \\
\hline W-05-050 & S & S \\
\hline W-05-051 & S & S \\
\hline W-05-052 & S & S \\
\hline W-05-053 & S & C \\
\hline W-05-054 & $S$ & $\mathrm{~S}$ \\
\hline W-05-055 & $\mathrm{C}$ & $\mathrm{C}$ \\
\hline W-05-056 & $\mathrm{C}$ & C \\
\hline W-05-057 & $\mathrm{S}$ & $\mathrm{S}$ \\
\hline W-05-058 & $\mathrm{C}$ & $\mathrm{C}$ \\
\hline W-05-059 & C & C \\
\hline W-05-060 & $\mathrm{S}$ & $\mathrm{C}$ \\
\hline W-05-061 & $\mathrm{C}$ & $\mathrm{C}$ \\
\hline W-05-062 & $\mathrm{S}$ & $\mathrm{S}$ \\
\hline W-05-063 & $\mathrm{S}$ & $\mathrm{S}$ \\
\hline W-05-064 & $\mathrm{S}$ & $\mathrm{S}$ \\
\hline
\end{tabular}

\begin{tabular}{|c|c|c|}
\hline \multirow{2}{*}{$\begin{array}{c}\text { Site } \\
\text { Number }\end{array}$} & \multicolumn{2}{|c|}{ Habitat Complexity } \\
\hline & Upstream & Downstream \\
\hline W-05-065 & $\bar{C}$ & $\mathrm{C}$ \\
\hline W-05-066 & $\mathrm{C}$ & C \\
\hline W-05-067 & $\mathrm{S}$ & S \\
\hline W-05-068 & S & S \\
\hline W-05-069 & $S$ & $\mathrm{~S}$ \\
\hline W-05-070 & S & $S$ \\
\hline W-05-071 & S & $\mathrm{S}$ \\
\hline W-05-072 & $\mathrm{S}$ & S \\
\hline W-05-073 & $\mathrm{S}$ & S \\
\hline W-05-074 & $\mathrm{C}$ & $\mathrm{C}$ \\
\hline W-05-075 & $\mathrm{S}$ & S \\
\hline W-05-076 & $S$ & $S$ \\
\hline W-05-077 & $\mathrm{C}$ & $\mathrm{C}$ \\
\hline W-05-078 & $S$ & S \\
\hline W-05-079 & $\mathrm{S}$ & S \\
\hline W-05-080 & C & $\mathrm{C}$ \\
\hline W-05-081 & $S$ & S \\
\hline W-05-082 & S & S \\
\hline W-05-083 & $\mathrm{C}$ & $\mathrm{C}$ \\
\hline W-05-084 & $\mathrm{S}$ & $\mathrm{C}$ \\
\hline W-05-085 & $\mathrm{C}$ & $\mathrm{S}$ \\
\hline W-05-086 & $S$ & S \\
\hline W-05-087 & S & S \\
\hline W-05-088 & $\mathrm{S}$ & $\mathrm{S}$ \\
\hline W-05-089 & $\mathrm{C}$ & C \\
\hline W-05-090 & C & C \\
\hline W-05-091 & $\mathrm{S}$ & $\mathrm{S}$ \\
\hline W-05-092 & $\mathrm{C}$ & $\mathrm{C}$ \\
\hline W-05-093 & $\mathrm{S}$ & $\mathrm{S}$ \\
\hline W-05-094 & $\mathrm{C}$ & C \\
\hline W-05-095 & $\mathrm{S}$ & $\mathrm{S}$ \\
\hline W-05-096 & $\mathrm{S}$ & $\mathrm{S}$ \\
\hline W-05-097 & $\mathrm{C}$ & $\mathrm{C}$ \\
\hline W-05-098 & $\mathrm{C}$ & C \\
\hline
\end{tabular}

\begin{tabular}{|c|c|c|}
\hline \multirow{2}{*}{$\begin{array}{c}\text { Site } \\
\text { Number }\end{array}$} & \multicolumn{2}{|c|}{ Habitat Complexity } \\
\hline & Upstream & Downstream \\
\hline W-05-099 & $S$ & $S$ \\
\hline W-05-100 & $\mathrm{S}$ & $\mathrm{C}$ \\
\hline W-05-101 & S & S \\
\hline W-05-102 & $\mathrm{S}$ & $\mathrm{C}$ \\
\hline W-05-103 & $S$ & $\mathrm{~S}$ \\
\hline W-05-104 & $\mathrm{S}$ & $\mathrm{S}$ \\
\hline W-05-105 & $\mathrm{C}$ & $\mathrm{C}$ \\
\hline W-05-106 & $\mathrm{C}$ & $\mathrm{C}$ \\
\hline W-05-107 & $\mathrm{C}$ & $\mathrm{C}$ \\
\hline W-05-108 & $\mathrm{C}$ & $\mathrm{C}$ \\
\hline W-05-109 & C & $\mathrm{C}$ \\
\hline W-05-110 & $\mathrm{S}$ & $\mathrm{S}$ \\
\hline W-05-111 & $S$ & $S$ \\
\hline W-05-112 & $\mathrm{C}$ & $\mathrm{C}$ \\
\hline W-05-113 & $S$ & $S$ \\
\hline W-05-114 & $\mathrm{S}$ & C \\
\hline W-05-115 & $\mathrm{C}$ & $\mathrm{C}$ \\
\hline W-05-116 & $\mathrm{C}$ & $\mathrm{C}$ \\
\hline W-05-117 & $\mathrm{C}$ & $\mathrm{C}$ \\
\hline W-05-118 & $\mathrm{C}$ & $\mathrm{C}$ \\
\hline W-05-119 & $\mathrm{C}$ & $\mathrm{C}$ \\
\hline W-05-120 & S & S \\
\hline W-05-121 & S & S \\
\hline W-05-122 & $\mathrm{S}$ & $\mathrm{S}$ \\
\hline W-05-123 & $\mathrm{C}$ & $\mathrm{C}$ \\
\hline W-05-124 & $\mathrm{C}$ & $\mathrm{C}$ \\
\hline W-05-125 & $\mathrm{C}$ & $\mathrm{C}$ \\
\hline W-05-126 & $\mathrm{C}$ & $\mathrm{C}$ \\
\hline W-05-127 & $\mathrm{C}$ & C \\
\hline W-05-128 & $\mathrm{S}$ & $\mathrm{S}$ \\
\hline W-05-129 & $\mathrm{S}$ & $\mathrm{S}$ \\
\hline W-05-130 & $\mathrm{S}$ & $\mathrm{S}$ \\
\hline W-05-131 & $\mathrm{S}$ & $\mathrm{S}$ \\
\hline W-05-132 & $\mathrm{S}$ & $\mathrm{S}$ \\
\hline
\end{tabular}


Appendix 2. Habitat complexity $(S=$ Simple; $C=$ Complex $)$ determination for all sites looking upstream and downstream from each surveyed reach.

\begin{tabular}{|c|c|c|}
\hline \multirow{2}{*}{$\begin{array}{c}\text { Site } \\
\text { Number }\end{array}$} & \multicolumn{2}{|c|}{ Habitat Complexity } \\
\hline & Upstream & Downstream \\
\hline W-05-133 & $S$ & $\mathrm{~S}$ \\
\hline W-05-134 & C & C \\
\hline W-05-135 & $\mathrm{C}$ & $\mathrm{C}$ \\
\hline W-05-136 & C & C \\
\hline W-05-137 & $\mathrm{C}$ & $\mathrm{C}$ \\
\hline W-05-138 & $S$ & $\mathrm{C}$ \\
\hline W-05-139 & C & C \\
\hline W-05-140 & C & C \\
\hline W-05-141 & S & $\mathrm{S}$ \\
\hline W-05-142 & S & $\mathrm{S}$ \\
\hline W-05-143 & C & C \\
\hline W-05-144 & $\mathrm{C}$ & $\mathrm{C}$ \\
\hline W-05-145 & $\mathrm{C}$ & $\mathrm{C}$ \\
\hline W-05-146 & C & C \\
\hline W-05-147 & C & C \\
\hline W-05-148 & C & $\mathrm{C}$ \\
\hline W-05-149 & $\mathrm{C}$ & $\mathrm{C}$ \\
\hline W-05-150 & $\mathrm{C}$ & $\mathrm{C}$ \\
\hline W-05-151 & $\mathrm{C}$ & $\mathrm{C}$ \\
\hline W-05-152 & $\mathrm{C}$ & $\mathrm{C}$ \\
\hline W-05-153 & $\mathrm{C}$ & $\mathrm{C}$ \\
\hline W-05-154 & $\mathrm{C}$ & $\mathrm{C}$ \\
\hline W-05-155 & C & C \\
\hline W-05-156 & $\mathrm{C}$ & $\mathrm{C}$ \\
\hline W-05-157 & $\mathrm{C}$ & $\mathrm{C}$ \\
\hline W-05-158 & C & C \\
\hline W-05-159 & $\mathrm{C}$ & C \\
\hline W-05-160 & $\mathrm{C}$ & $\mathrm{C}$ \\
\hline W-05-161 & $\mathrm{C}$ & C \\
\hline W-05-162 & $\mathrm{C}$ & $\mathrm{C}$ \\
\hline W-05-163 & $\mathrm{S}$ & $\mathrm{S}$ \\
\hline W-05-164 & $\mathrm{C}$ & C \\
\hline W-05-165 & $\mathrm{C}$ & C \\
\hline W-05-166 & $\mathrm{C}$ & $\mathrm{C}$ \\
\hline
\end{tabular}

\begin{tabular}{|c|c|c|}
\hline \multirow{2}{*}{$\begin{array}{c}\text { Site } \\
\text { Number }\end{array}$} & \multicolumn{2}{|c|}{ Habitat Complexity } \\
\hline & Upstream & Downstream \\
\hline W-05-167 & $\bar{C}$ & $\mathrm{C}$ \\
\hline W-05-168 & $\mathrm{C}$ & $\mathrm{C}$ \\
\hline W-05-169 & $\mathrm{C}$ & $\mathrm{C}$ \\
\hline W-05-170 & $\mathrm{C}$ & C \\
\hline W-05-171 & $\mathrm{C}$ & $\mathrm{C}$ \\
\hline W-05-172 & $\mathrm{C}$ & $\mathrm{C}$ \\
\hline W-05-173 & C & C \\
\hline W-05-174 & $\mathrm{S}$ & $\mathrm{S}$ \\
\hline W-05-175 & $\mathrm{C}$ & $\mathrm{C}$ \\
\hline W-05-176 & $\mathrm{S}$ & S \\
\hline W-05-177 & $\mathrm{S}$ & $\mathrm{S}$ \\
\hline W-05-178 & $S$ & S \\
\hline W-05-179 & S & S \\
\hline W-05-180 & S & $S$ \\
\hline W-05-181 & S & $S$ \\
\hline W-05-182 & $\mathrm{S}$ & $\mathrm{S}$ \\
\hline W-05-183 & $\mathrm{S}$ & $\mathrm{S}$ \\
\hline W-05-184 & $\mathrm{C}$ & $\mathrm{C}$ \\
\hline W-05-185 & $\mathrm{C}$ & $\mathrm{C}$ \\
\hline W-05-186 & $\mathrm{C}$ & C \\
\hline W-05-187 & $\mathrm{C}$ & $\mathrm{C}$ \\
\hline W-05-188 & $S$ & $S$ \\
\hline W-05-189 & S & $\mathrm{S}$ \\
\hline W-05-190 & $\mathrm{S}$ & $S$ \\
\hline W-05-191 & $\mathrm{S}$ & S \\
\hline W-05-192 & $\mathrm{S}$ & $S$ \\
\hline W-05-193 & $\mathrm{S}$ & $S$ \\
\hline W-05-194 & $\mathrm{S}$ & $\mathrm{S}$ \\
\hline W-05-195 & $S$ & $S$ \\
\hline W-05-196 & $S$ & $S$ \\
\hline W-05-197 & $\mathrm{C}$ & $\mathrm{C}$ \\
\hline W-05-198 & $S$ & $\mathrm{~S}$ \\
\hline W-05-199 & $\mathrm{S}$ & $S$ \\
\hline W-05-200 & $\mathrm{S}$ & $\mathrm{S}$ \\
\hline
\end{tabular}

\begin{tabular}{|c|c|c|}
\hline \multirow{2}{*}{$\begin{array}{c}\text { Site } \\
\text { Number }\end{array}$} & \multicolumn{2}{|c|}{ Habitat Complexity } \\
\hline & Upstream & Downstream \\
\hline W-05-201 & $\mathrm{S}$ & $\mathrm{S}$ \\
\hline W-05-202 & $\mathrm{S}$ & $\mathrm{S}$ \\
\hline W-05-203 & $\mathrm{C}$ & $\mathrm{C}$ \\
\hline W-05-204 & $\mathrm{C}$ & $\mathrm{C}$ \\
\hline W-05-205 & $\mathrm{C}$ & $\mathrm{C}$ \\
\hline W-05-206 & $\mathrm{S}$ & $\mathrm{S}$ \\
\hline W-05-207 & $S$ & S \\
\hline W-05-208 & $\mathrm{C}$ & $\mathrm{C}$ \\
\hline W-05-209 & $\mathrm{C}$ & C \\
\hline W-05-210 & S & $\mathrm{S}$ \\
\hline W-05-211 & $S$ & $\mathrm{~S}$ \\
\hline $\mathrm{X}-05-001$ & $\mathrm{C}$ & $\mathrm{C}$ \\
\hline $\mathrm{X}-05-002$ & $\mathrm{C}$ & $\mathrm{C}$ \\
\hline $\mathrm{X}-05-003$ & S & S \\
\hline $\mathrm{X}-05-004$ & $S$ & $\mathrm{~S}$ \\
\hline $\mathrm{X}-05-005$ & $\mathrm{~S}$ & $\mathrm{~S}$ \\
\hline $\mathrm{X}-05-006$ & $\mathrm{~S}$ & $\mathrm{~S}$ \\
\hline $\mathrm{X}-05-007$ & S & $\mathrm{S}$ \\
\hline $\mathrm{X}-05-008$ & S & $\mathrm{S}$ \\
\hline $\mathrm{X}-05-009$ & $S$ & $\mathrm{~S}$ \\
\hline $\mathrm{X}-05-010$ & $S$ & $\mathrm{~S}$ \\
\hline $\mathrm{X}-05-011$ & S & $\mathrm{S}$ \\
\hline $\mathrm{X}-05-012$ & S & $\mathrm{S}$ \\
\hline $\mathrm{X}-05-013$ & $S$ & $\mathrm{~S}$ \\
\hline $\mathrm{X}-05-014$ & $\mathrm{~S}$ & $\mathrm{~S}$ \\
\hline $\mathrm{X}-05-015$ & S & $\mathrm{S}$ \\
\hline $\mathrm{X}-05-016$ & $S$ & $S$ \\
\hline $\mathrm{X}-05-017$ & $S$ & $\mathrm{~S}$ \\
\hline $\mathrm{X}-05-018$ & $\mathrm{C}$ & C \\
\hline $\mathrm{X}-05-019$ & $S$ & $S$ \\
\hline $\mathrm{X}-05-020$ & $\mathrm{~S}$ & $\mathrm{~S}$ \\
\hline $\mathrm{X}-05-021$ & $S$ & C \\
\hline $\mathrm{X}-05-022$ & $\mathrm{~S}$ & $S$ \\
\hline $\mathrm{X}-05-023$ & $\mathrm{~S}$ & $\mathrm{~S}$ \\
\hline
\end{tabular}


Appendix 2. Habitat complexity $(S=$ Simple; $C=$ Complex $)$ determination for all sites looking upstream and downstream from each surveyed reach.

\begin{tabular}{|c|c|c|}
\hline \multirow{2}{*}{$\begin{array}{c}\text { Site } \\
\text { Number }\end{array}$} & \multicolumn{2}{|c|}{ Habitat Complexity } \\
\hline & Upstream & Downstream \\
\hline $\mathrm{X}-05-024$ & $S$ & $\mathrm{~S}$ \\
\hline $\mathrm{X}-05-025$ & $\mathrm{~S}$ & $\mathrm{~S}$ \\
\hline $\mathrm{X}-05-026$ & $\mathrm{C}$ & $\mathrm{C}$ \\
\hline$X-05-027$ & $\mathrm{~S}$ & $\mathrm{~S}$ \\
\hline $\mathrm{X}-05-028$ & $\mathrm{~S}$ & $\mathrm{~S}$ \\
\hline X-05-029 & $\mathrm{C}$ & $\mathrm{S}$ \\
\hline $\mathrm{X}-05-030$ & $\mathrm{C}$ & $\mathrm{C}$ \\
\hline $\mathrm{X}-05-031$ & $S$ & $\mathrm{~S}$ \\
\hline $\mathrm{X}-05-032$ & $S$ & $\mathrm{~S}$ \\
\hline X-05-033 & S & $\mathrm{S}$ \\
\hline$X-05-034$ & S & $\mathrm{S}$ \\
\hline $\mathrm{X}-05-035$ & $S$ & $\mathrm{~S}$ \\
\hline X-05-036 & S & $\mathrm{S}$ \\
\hline $\mathrm{X}-05-037$ & S & $\mathrm{S}$ \\
\hline $\mathrm{X}-05-038$ & $\mathrm{~S}$ & $\mathrm{C}$ \\
\hline X-05-039 & S & $\mathrm{S}$ \\
\hline X-05-040 & $S$ & $\mathrm{~S}$ \\
\hline X-05-041 & $S$ & $\mathrm{~S}$ \\
\hline $\mathrm{X}-05-042$ & $\mathrm{~S}$ & $\mathrm{~S}$ \\
\hline$X-05-043$ & $\mathrm{C}$ & $\mathrm{C}$ \\
\hline X-05-044 & $S$ & $\mathrm{~S}$ \\
\hline X-05-045 & $\mathrm{C}$ & $\mathrm{C}$ \\
\hline$X-05-046$ & C & C \\
\hline $\mathrm{X}-05-047$ & $\mathrm{~S}$ & $\mathrm{~S}$ \\
\hline X-05-048 & S & $\mathrm{S}$ \\
\hline X-05-049 & S & $\mathrm{S}$ \\
\hline X-05-050 & $\mathrm{S}$ & $\mathrm{S}$ \\
\hline $\mathrm{X}-05-051$ & $S$ & $\mathrm{~S}$ \\
\hline X-05-052 & $\mathrm{S}$ & $\mathrm{S}$ \\
\hline X-05-053 & $\mathrm{S}$ & $\mathrm{S}$ \\
\hline $\mathrm{X}-05-054$ & $\mathrm{~S}$ & $S$ \\
\hline X-05-055 & $S$ & C \\
\hline X-05-056 & $\mathrm{S}$ & $\mathrm{S}$ \\
\hline X-05-057 & $\mathrm{S}$ & $\mathrm{S}$ \\
\hline
\end{tabular}

\begin{tabular}{|c|c|c|}
\hline \multirow{2}{*}{$\begin{array}{c}\text { Site } \\
\text { Number }\end{array}$} & \multicolumn{2}{|c|}{ Habitat Complexity } \\
\hline & Upstream & Downstream \\
\hline X-05-058 & $\mathrm{C}$ & $\mathrm{C}$ \\
\hline $\mathrm{X}-05-059$ & $\mathrm{C}$ & C \\
\hline $\mathrm{X}-05-060$ & $\mathrm{C}$ & $\mathrm{C}$ \\
\hline $\mathrm{X}-05-061$ & $\mathrm{C}$ & C \\
\hline $\mathrm{X}-05-062$ & $\mathrm{C}$ & $\mathrm{C}$ \\
\hline $\mathrm{X}-05-063$ & $\mathrm{C}$ & $\mathrm{C}$ \\
\hline$X-05-064$ & $\mathrm{~S}$ & $\mathrm{~S}$ \\
\hline$X-05-065$ & $\mathrm{~S}$ & $\mathrm{~S}$ \\
\hline $\mathrm{X}-05-066$ & $\mathrm{~S}$ & $\mathrm{~S}$ \\
\hline $\mathrm{X}-05-067$ & $\mathrm{C}$ & $\mathrm{C}$ \\
\hline$X-05-068$ & $\mathrm{~S}$ & $\mathrm{~S}$ \\
\hline $\mathrm{X}-05-069$ & $S$ & $\mathrm{~S}$ \\
\hline $\mathrm{X}-05-070$ & $\mathrm{~S}$ & $\mathrm{~S}$ \\
\hline $\mathrm{X}-05-071$ & $\mathrm{~S}$ & $\mathrm{~S}$ \\
\hline $\mathrm{X}-05-072$ & S & $\mathrm{S}$ \\
\hline $\mathrm{X}-05-073$ & $\mathrm{~S}$ & $\mathrm{~S}$ \\
\hline$X-05-074$ & $\mathrm{~S}$ & $\mathrm{~S}$ \\
\hline $\mathrm{X}-05-075$ & $\mathrm{~S}$ & S \\
\hline $\mathrm{X}-05-076$ & S & $\mathrm{S}$ \\
\hline$X-05-077$ & C & C \\
\hline $\mathrm{X}-05-078$ & $\mathrm{~S}$ & $\mathrm{~S}$ \\
\hline $\mathrm{X}-05-079$ & S & $\mathrm{S}$ \\
\hline$X-05-080$ & $\mathrm{~S}$ & $\mathrm{~S}$ \\
\hline $\mathrm{X}-05-081$ & $\mathrm{~S}$ & $\mathrm{~S}$ \\
\hline $\mathrm{X}-05-082$ & $\mathrm{~S}$ & $\mathrm{~S}$ \\
\hline$X-05-083$ & $\mathrm{~S}$ & C \\
\hline X-05-084 & $\mathrm{S}$ & $\mathrm{S}$ \\
\hline X-05-085 & $\mathrm{S}$ & $\mathrm{S}$ \\
\hline X-05-086 & $S$ & $S$ \\
\hline X-05-087 & $\mathrm{S}$ & $S$ \\
\hline X-05-088 & $\mathrm{S}$ & $\mathrm{S}$ \\
\hline X-05-089 & $\mathrm{S}$ & $\mathrm{S}$ \\
\hline X-05-090 & C & $\mathrm{S}$ \\
\hline X-05-091 & C & C \\
\hline
\end{tabular}

\begin{tabular}{|c|c|c|}
\hline \multirow{2}{*}{$\begin{array}{c}\text { Site } \\
\text { Number }\end{array}$} & \multicolumn{2}{|c|}{ Habitat Complexity } \\
\hline & Upstream & Downstream \\
\hline X-05-092 & $\mathrm{C}$ & $\mathrm{C}$ \\
\hline X-05-093 & $S$ & $\mathrm{~S}$ \\
\hline X-05-094 & $S$ & $\mathrm{~S}$ \\
\hline X-05-095 & S & S \\
\hline X-05-096 & $S$ & S \\
\hline X-05-097 & S & $S$ \\
\hline$X-05-098$ & S & S \\
\hline X-05-099 & S & S \\
\hline $\mathrm{X}-05-100$ & $\mathrm{~S}$ & S \\
\hline $\mathrm{X}-05-101$ & $\mathrm{C}$ & S \\
\hline$X-05-102$ & S & S \\
\hline $\mathrm{X}-05-103$ & $\mathrm{C}$ & $\mathrm{C}$ \\
\hline X-05-104 & $\mathrm{C}$ & $\mathrm{C}$ \\
\hline$X-05-105$ & C & C \\
\hline X-05-106 & $\mathrm{C}$ & $\mathrm{C}$ \\
\hline X-05-107 & $S$ & $\mathrm{~S}$ \\
\hline$X-05-108$ & $\mathrm{C}$ & C \\
\hline X-05-109 & $S$ & $\mathrm{C}$ \\
\hline $\mathrm{X}-05-110$ & $\mathrm{C}$ & $\mathrm{C}$ \\
\hline$X-05-111$ & $\mathrm{C}$ & $\mathrm{C}$ \\
\hline $\mathrm{X}-05-112$ & $\mathrm{~S}$ & $\mathrm{~S}$ \\
\hline $\mathrm{X}-05-113$ & $S$ & $\mathrm{~S}$ \\
\hline$X-05-114$ & $\mathrm{C}$ & $\mathrm{C}$ \\
\hline X-05-115 & $S$ & $\mathrm{~S}$ \\
\hline $\mathrm{X}-05-116$ & S & $\mathrm{S}$ \\
\hline$X-05-117$ & $S$ & $\mathrm{~S}$ \\
\hline X-05-118 & $\mathrm{S}$ & $\mathrm{S}$ \\
\hline X-05-119 & $S$ & $\mathrm{~S}$ \\
\hline$X-05-120$ & $S$ & C \\
\hline $\mathrm{X}-05-121$ & $S$ & $\mathrm{~S}$ \\
\hline X-05-122 & $\mathrm{C}$ & $\mathrm{C}$ \\
\hline$X-05-123$ & C & C \\
\hline$X-05-124$ & $\mathrm{~S}$ & $\mathrm{~S}$ \\
\hline X-05-125 & $\mathrm{C}$ & $\mathrm{C}$ \\
\hline
\end{tabular}


Appendix 2. Habitat complexity $(S=$ Simple; $C=$ Complex $)$ determination for all sites looking upstream and downstream from each surveyed reach.

\begin{tabular}{|c|c|c|}
\hline \multirow{2}{*}{$\begin{array}{c}\text { Site } \\
\text { Number }\end{array}$} & \multicolumn{2}{|c|}{ Habitat Complexity } \\
\hline & Upstream & Downstream \\
\hline $\mathrm{X}-05-126$ & $\mathrm{C}$ & $\mathrm{C}$ \\
\hline $\mathrm{X}-05-127$ & C & $\mathrm{C}$ \\
\hline $\mathrm{X}-05-128$ & $\mathrm{C}$ & $\mathrm{C}$ \\
\hline$X-05-129$ & S & $\mathrm{S}$ \\
\hline $\mathrm{X}-05-130$ & $S$ & $S$ \\
\hline $\mathrm{X}-05-131$ & $\mathrm{C}$ & $\mathrm{C}$ \\
\hline$X-05-132$ & S & C \\
\hline$X-05-133$ & C & C \\
\hline $\mathrm{X}-05-134$ & S & $\mathrm{S}$ \\
\hline $\mathrm{X}-05-135$ & S & S \\
\hline$X-05-136$ & S & S \\
\hline $\mathrm{X}-05-137$ & $\mathrm{C}$ & $\mathrm{C}$ \\
\hline $\mathrm{X}-05-138$ & S & S \\
\hline$X-05-139$ & S & $\mathrm{S}$ \\
\hline $\mathrm{X}-05-140$ & $\mathrm{C}$ & C \\
\hline $\mathrm{X}-05-141$ & S & $\mathrm{S}$ \\
\hline$X-05-142$ & S & $\mathrm{S}$ \\
\hline $\mathrm{X}-05-143$ & S & $\mathrm{S}$ \\
\hline $\mathrm{X}-05-144$ & $\mathrm{C}$ & $\mathrm{C}$ \\
\hline$X-05-145$ & C & $\mathrm{C}$ \\
\hline $\mathrm{X}-05-146$ & $\mathrm{C}$ & $\mathrm{C}$ \\
\hline $\mathrm{X}-05-147$ & $\mathrm{C}$ & $\mathrm{C}$ \\
\hline$X-05-148$ & S & $\mathrm{S}$ \\
\hline $\mathrm{X}-05-149$ & $S$ & $\mathrm{~S}$ \\
\hline $\mathrm{X}-05-150$ & C & $\mathrm{C}$ \\
\hline$X-05-151$ & C & C \\
\hline $\mathrm{X}-05-152$ & $\mathrm{~S}$ & $\mathrm{~S}$ \\
\hline $\mathrm{X}-05-153$ & $\mathrm{C}$ & $\mathrm{C}$ \\
\hline$X-05-154$ & C & C \\
\hline X-05-155 & C & C \\
\hline X-05-156 & $\mathrm{S}$ & $\mathrm{S}$ \\
\hline X-05-157 & $\mathrm{S}$ & $\mathrm{S}$ \\
\hline $\mathrm{X}-05-158$ & $\mathrm{~S}$ & $\mathrm{~S}$ \\
\hline $\mathrm{X}-05-159$ & $\mathrm{~S}$ & $\mathrm{~S}$ \\
\hline
\end{tabular}

\begin{tabular}{|c|c|c|}
\hline \multirow{2}{*}{$\begin{array}{c}\text { Site } \\
\text { Number }\end{array}$} & \multicolumn{2}{|c|}{ Habitat Complexity } \\
\hline & Upstream & Downstream \\
\hline$X-05-160$ & $\mathrm{~S}$ & $\mathrm{~S}$ \\
\hline $\mathrm{X}-05-161$ & $\mathrm{~S}$ & $\mathrm{~S}$ \\
\hline $\mathrm{X}-05-162$ & S & $\mathrm{S}$ \\
\hline$X-05-163$ & C & C \\
\hline $\mathrm{X}-05-164$ & $\mathrm{C}$ & $\mathrm{C}$ \\
\hline $\mathrm{X}-05-165$ & $\mathrm{~S}$ & S \\
\hline$X-05-166$ & $\mathrm{~S}$ & $\mathrm{~S}$ \\
\hline$X-05-167$ & S & S \\
\hline$X-05-168$ & C & C \\
\hline X-05-169 & $\mathrm{S}$ & $\mathrm{C}$ \\
\hline $\mathrm{X}-05-170$ & C & C \\
\hline $\mathrm{X}-05-171$ & $\mathrm{C}$ & $\mathrm{C}$ \\
\hline $\mathrm{X}-05-172$ & S & S \\
\hline $\mathrm{X}-05-173$ & $\mathrm{~S}$ & $\mathrm{~S}$ \\
\hline$X-05-174$ & $\mathrm{C}$ & $\mathrm{C}$ \\
\hline $\mathrm{X}-05-175$ & S & $\mathrm{S}$ \\
\hline$X-05-176$ & C & C \\
\hline $\mathrm{X}-05-177$ & C & $\mathrm{C}$ \\
\hline $\mathrm{X}-05-178$ & $\mathrm{~S}$ & $\mathrm{C}$ \\
\hline$X-05-179$ & $\mathrm{~S}$ & C \\
\hline $\mathrm{X}-05-180$ & C & C \\
\hline $\mathrm{X}-05-181$ & $\mathrm{~S}$ & $\mathrm{~S}$ \\
\hline$X-05-182$ & $\mathrm{~S}$ & $\mathrm{~S}$ \\
\hline $\mathrm{X}-05-183$ & $\mathrm{~S}$ & $\mathrm{~S}$ \\
\hline $\mathrm{X}-05-184$ & S & $\mathrm{S}$ \\
\hline $\mathrm{X}-05-185$ & $\mathrm{~S}$ & $\mathrm{~S}$ \\
\hline$X-05-186$ & $\mathrm{~S}$ & $S$ \\
\hline $\mathrm{X}-05-187$ & $\mathrm{~S}$ & $\mathrm{~S}$ \\
\hline$X-05-188$ & $S$ & $\mathrm{~S}$ \\
\hline X-05-189 & $\mathrm{S}$ & $\mathrm{S}$ \\
\hline X-05-190 & $\mathrm{S}$ & $\mathrm{S}$ \\
\hline X-05-191 & C & C \\
\hline X-05-192 & C & C \\
\hline X-05-193 & C & C \\
\hline
\end{tabular}

\begin{tabular}{|c|c|c|}
\hline \multirow{2}{*}{$\begin{array}{c}\text { Site } \\
\text { Number }\end{array}$} & \multicolumn{2}{|c|}{ Habitat Complexity } \\
\hline & Upstream & Downstream \\
\hline X-05-194 & $\mathrm{C}$ & $\mathrm{C}$ \\
\hline $\mathrm{X}-05-195$ & $\mathrm{~S}$ & $\mathrm{~S}$ \\
\hline $\mathrm{X}-05-196$ & S & $\mathrm{S}$ \\
\hline $\mathrm{X}-05-197$ & $\mathrm{~S}$ & $\mathrm{~S}$ \\
\hline $\mathrm{X}-05-198$ & $\mathrm{C}$ & $\mathrm{C}$ \\
\hline $\mathrm{X}-05-199$ & $\mathrm{C}$ & $\mathrm{C}$ \\
\hline$X-05-200$ & C & $\mathrm{C}$ \\
\hline$X-05-201$ & $\mathrm{~S}$ & $\mathrm{~S}$ \\
\hline $\mathrm{X}-05-202$ & $\mathrm{~S}$ & $\mathrm{~S}$ \\
\hline $\mathrm{X}-05-203$ & $\mathrm{C}$ & $\mathrm{C}$ \\
\hline$X-05-204$ & $S$ & $\mathrm{~S}$ \\
\hline$X-05-205$ & $\mathrm{C}$ & $\mathrm{S}$ \\
\hline$X-05-206$ & $S$ & $S$ \\
\hline$X-05-207$ & S & S \\
\hline$X-05-208$ & $S$ & $\mathrm{~S}$ \\
\hline $\mathrm{X}-05-209$ & $\mathrm{C}$ & C \\
\hline$X-05-210$ & $\mathrm{C}$ & $\mathrm{C}$ \\
\hline D-06-001 & S & $\mathrm{S}$ \\
\hline W-06-001 & $\mathrm{C}$ & $\mathrm{C}$ \\
\hline W-06-002 & $\mathrm{C}$ & $\mathrm{C}$ \\
\hline W-06-003 & $\mathrm{C}$ & $\mathrm{C}$ \\
\hline W-06-004 & $\mathrm{C}$ & $\mathrm{C}$ \\
\hline W-06-005 & C & C \\
\hline W-06-006 & $\mathrm{C}$ & $\mathrm{C}$ \\
\hline W-06-007 & $\mathrm{C}$ & $\mathrm{C}$ \\
\hline W-06-008 & S & S \\
\hline W-06-009 & $S$ & $\mathrm{~S}$ \\
\hline W-06-010 & $S$ & $\mathrm{~S}$ \\
\hline W-06-011 & $\mathrm{C}$ & C \\
\hline W-06-012 & $\mathrm{C}$ & C \\
\hline W-06-013 & $\mathrm{C}$ & $\mathrm{C}$ \\
\hline W-06-014 & $\mathrm{C}$ & C \\
\hline W-06-015 & $\mathrm{C}$ & C \\
\hline W-06-016 & $\mathrm{S}$ & $\mathrm{S}$ \\
\hline
\end{tabular}


Appendix 2. Habitat complexity $(S=$ Simple; $C=$ Complex $)$ determination for all sites looking upstream and downstream from each surveyed reach.

\begin{tabular}{|c|c|c|}
\hline \multirow{2}{*}{$\begin{array}{c}\text { Site } \\
\text { Number }\end{array}$} & \multicolumn{2}{|c|}{ Habitat Complexity } \\
\hline & Upstream & Downstream \\
\hline W-06-017 & $S$ & $\mathrm{~S}$ \\
\hline W-06-018 & $S$ & $\mathrm{~S}$ \\
\hline W-06-019 & S & $\mathrm{S}$ \\
\hline W-06-020 & C & $\mathrm{S}$ \\
\hline W-06-021 & $S$ & $\mathrm{~S}$ \\
\hline W-06-022 & S & S \\
\hline W-06-023 & C & C \\
\hline W-06-024 & $S$ & $S$ \\
\hline W-06-025 & $\mathrm{S}$ & $S$ \\
\hline W-06-026 & S & S \\
\hline W-06-027 & $\mathrm{S}$ & $\mathrm{S}$ \\
\hline W-06-028 & $\mathrm{C}$ & $\mathrm{C}$ \\
\hline W-06-029 & S & S \\
\hline W-06-030 & $\mathrm{S}$ & S \\
\hline W-06-031 & $\mathrm{S}$ & $\mathrm{S}$ \\
\hline W-06-032 & $\mathrm{C}$ & $\mathrm{C}$ \\
\hline W-06-033 & $\mathrm{C}$ & $\mathrm{C}$ \\
\hline W-06-034 & $\mathrm{C}$ & $\mathrm{C}$ \\
\hline W-06-035 & $\mathrm{C}$ & $\mathrm{C}$ \\
\hline W-06-036 & $\mathrm{S}$ & $\mathrm{C}$ \\
\hline W-06-037 & $\mathrm{S}$ & $\mathrm{C}$ \\
\hline W-06-038 & $\mathrm{C}$ & $\mathrm{C}$ \\
\hline W-06-039 & $\mathrm{C}$ & C \\
\hline W-06-040 & $\mathrm{C}$ & $\mathrm{S}$ \\
\hline W-06-041 & S & $\mathrm{S}$ \\
\hline W-06-042 & $\mathrm{S}$ & $\mathrm{S}$ \\
\hline W-06-043 & $\mathrm{C}$ & $\mathrm{C}$ \\
\hline W-06-044 & $\mathrm{S}$ & $\mathrm{S}$ \\
\hline W-06-045 & C & C \\
\hline W-06-046 & $\mathrm{S}$ & $\mathrm{S}$ \\
\hline W-06-047 & $\mathrm{S}$ & $\mathrm{S}$ \\
\hline W-06-048 & $S$ & $\mathrm{~S}$ \\
\hline W-06-049 & $\mathrm{C}$ & $\mathrm{C}$ \\
\hline W-06-050 & $\mathrm{C}$ & $\mathrm{C}$ \\
\hline
\end{tabular}

\begin{tabular}{|c|c|c|}
\hline \multirow{2}{*}{$\begin{array}{c}\text { Site } \\
\text { Number }\end{array}$} & \multicolumn{2}{|c|}{ Habitat Complexity } \\
\hline & Upstream & Downstream \\
\hline W-06-051 & $\mathrm{C}$ & $\mathrm{C}$ \\
\hline W-06-052 & S & C \\
\hline W-06-053 & S & $\mathrm{S}$ \\
\hline W-06-054 & $S$ & $S$ \\
\hline W-06-055 & $S$ & $S$ \\
\hline W-06-056 & $\mathrm{C}$ & $\mathrm{C}$ \\
\hline W-06-057 & S & S \\
\hline W-06-058 & $\mathrm{S}$ & $S$ \\
\hline W-06-059 & $\mathrm{S}$ & $S$ \\
\hline W-06-060 & S & S \\
\hline W-06-061 & $\mathrm{C}$ & $\mathrm{C}$ \\
\hline W-06-062 & $S$ & $S$ \\
\hline W-06-063 & $\mathrm{S}$ & S \\
\hline W-06-064 & $\mathrm{S}$ & S \\
\hline W-06-065 & $\mathrm{C}$ & $\mathrm{C}$ \\
\hline W-06-066 & $\mathrm{C}$ & $\mathrm{C}$ \\
\hline W-06-067 & $\mathrm{C}$ & $\mathrm{C}$ \\
\hline W-06-068 & $\mathrm{C}$ & $\mathrm{C}$ \\
\hline W-06-069 & $\mathrm{C}$ & $\mathrm{C}$ \\
\hline W-06-070 & $\mathrm{C}$ & $\mathrm{C}$ \\
\hline W-06-071 & $\mathrm{C}$ & $\mathrm{C}$ \\
\hline W-06-072 & $\mathrm{C}$ & $\mathrm{C}$ \\
\hline W-06-073 & C & C \\
\hline W-06-074 & $\mathrm{C}$ & $\mathrm{C}$ \\
\hline W-06-075 & C & C \\
\hline W-06-076 & $\mathrm{C}$ & $\mathrm{C}$ \\
\hline W-06-077 & $\mathrm{C}$ & $\mathrm{C}$ \\
\hline W-06-078 & $\mathrm{C}$ & $\mathrm{C}$ \\
\hline W-06-079 & $\mathrm{C}$ & C \\
\hline W-06-080 & $\mathrm{C}$ & C \\
\hline W-06-081 & $\mathrm{S}$ & $\mathrm{S}$ \\
\hline W-06-082 & $\mathrm{S}$ & $\mathrm{S}$ \\
\hline W-06-083 & $\mathrm{C}$ & $\mathrm{C}$ \\
\hline W-06-084 & $\mathrm{C}$ & $\mathrm{C}$ \\
\hline
\end{tabular}

\begin{tabular}{|c|c|c|}
\hline \multirow{2}{*}{$\begin{array}{c}\text { Site } \\
\text { Number }\end{array}$} & \multicolumn{2}{|c|}{ Habitat Complexity } \\
\hline & Upstream & Downstream \\
\hline W-06-085 & $\mathrm{C}$ & $\mathrm{C}$ \\
\hline W-06-086 & $\mathrm{C}$ & $\mathrm{C}$ \\
\hline W-06-087 & $\mathrm{C}$ & $\mathrm{C}$ \\
\hline W-06-088 & $\mathrm{C}$ & $\mathrm{C}$ \\
\hline W-06-089 & $\mathrm{C}$ & $\mathrm{C}$ \\
\hline W-06-090 & $\mathrm{C}$ & $\mathrm{C}$ \\
\hline W-06-091 & C & C \\
\hline W-06-092 & $\mathrm{C}$ & $\mathrm{C}$ \\
\hline W-06-093 & $\mathrm{C}$ & $\mathrm{C}$ \\
\hline W-06-094 & S & $\mathrm{S}$ \\
\hline W-06-095 & $S$ & $\mathrm{~S}$ \\
\hline W-06-096 & $S$ & $\mathrm{~S}$ \\
\hline W-06-097 & $S$ & $S$ \\
\hline W-06-098 & S & S \\
\hline W-06-099 & $S$ & $\mathrm{~S}$ \\
\hline W-06-100 & $\mathrm{S}$ & $\mathrm{S}$ \\
\hline W-06-101 & $\mathrm{S}$ & $\mathrm{S}$ \\
\hline W-06-102 & S & $\mathrm{S}$ \\
\hline W-06-103 & S & $\mathrm{S}$ \\
\hline W-06-104 & $S$ & S \\
\hline W-06-105 & $S$ & $\mathrm{~S}$ \\
\hline W-06-106 & S & $\mathrm{S}$ \\
\hline W-06-107 & C & $\mathrm{S}$ \\
\hline W-06-108 & $\mathrm{S}$ & $\mathrm{S}$ \\
\hline W-06-109 & $\mathrm{S}$ & $\mathrm{S}$ \\
\hline W-06-110 & $\mathrm{C}$ & C \\
\hline W-06-111 & $\mathrm{C}$ & $\mathrm{C}$ \\
\hline W-06-112 & $\mathrm{C}$ & $\mathrm{C}$ \\
\hline W-06-113 & $\mathrm{C}$ & C \\
\hline W-06-114 & $\mathrm{C}$ & C \\
\hline W-06-115 & $\mathrm{C}$ & $\mathrm{C}$ \\
\hline W-06-116 & $\mathrm{C}$ & C \\
\hline W-06-117 & $\mathrm{C}$ & $\mathrm{C}$ \\
\hline W-06-118 & $\mathrm{S}$ & $\mathrm{C}$ \\
\hline
\end{tabular}


Appendix 2. Habitat complexity $(S=$ Simple; $C=$ Complex $)$ determination for all sites looking upstream and downstream from each surveyed reach.

\begin{tabular}{|c|c|c|}
\hline \multirow{2}{*}{$\begin{array}{c}\text { Site } \\
\text { Number }\end{array}$} & \multicolumn{2}{|c|}{ Habitat Complexity } \\
\cline { 2 - 3 } & Upstream & Downstream \\
\hline W-06-119 & C & C \\
\hline W-06-120 & S & S \\
\hline W-06-121 & C & C \\
\hline W-06-122 & S & S \\
\hline W-06-123 & S & S \\
\hline W-06-124 & C & C \\
\hline W-06-125 & C & C \\
\hline W-06-126 & C & C \\
\hline W-06-127 & C & C \\
\hline W-06-128 & C & C \\
\hline W-06-129 & C & C \\
\hline W-06-130 & C & C \\
\hline W-06-131 & C & C \\
\hline W-06-132 & S & C \\
\hline W-06-133 & C & C \\
\hline W-06-134 & C & C \\
\hline W-06-135 & C & C \\
\hline W-06-136 & C & C \\
\hline W-06-137 & S & S \\
\hline W-06-138 & C & C \\
\hline W-06-139 & C & C \\
\hline W-06-140 & C & C \\
\hline W-06-141 & C & C \\
\hline W-06-142 & C & C \\
\hline W-06-143 & S & S \\
\hline W-06-144 & C & C \\
\hline W-06-145 & C & C \\
\hline W-06-146 & C & C \\
\hline
\end{tabular}


Appendix 3: Table showing the twelve individual habitat parameter scores adapted from Barbour et al. (1999) that were assessed at most sites where fishing effort was applied and were totalled to derive the Habitat Assessment Score for each site.

\begin{tabular}{|c|c|c|c|c|c|c|c|c|c|c|c|c|c|c|c|c|}
\hline \multirow{2}{*}{ Site Number } & \multirow{2}{*}{ 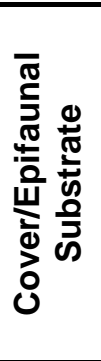 } & \multirow{2}{*}{ 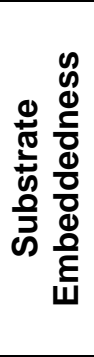 } & \multirow{2}{*}{ 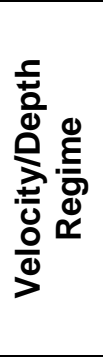 } & \multirow{2}{*}{ 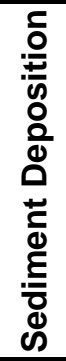 } & \multirow{2}{*}{ 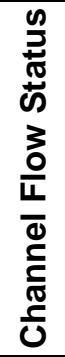 } & \multirow{2}{*}{ 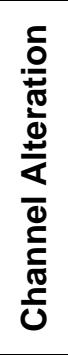 } & \multirow{2}{*}{ 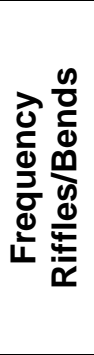 } & \multicolumn{2}{|c|}{ 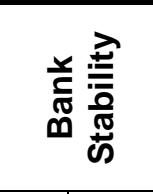 } & \multicolumn{2}{|c|}{ 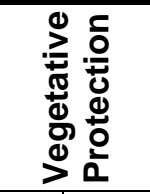 } & \multicolumn{2}{|c|}{ 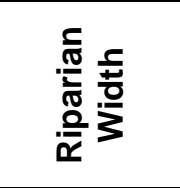 } & \multirow{2}{*}{ 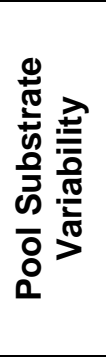 } & \multirow{2}{*}{ 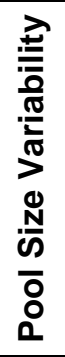 } & \multirow{2}{*}{ 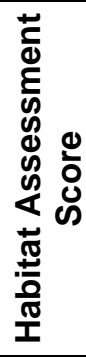 } \\
\hline & & & & & & & & Фృ & $\frac{\text { 돔 }}{\frac{0}{x}}$ & $\Phi$ & $\frac{\text { 돔 }}{\frac{0}{x}}$ & Ф্త & $\frac{\text { 픔 }}{\frac{0}{x}}$ & & & \\
\hline D-02-005 & 12 & 10 & 11 & 5 & 8 & 17 & 14 & 8 & 8 & 9 & 9 & 5 & 4 & 11 & 10 & 141 \\
\hline D-02-008 & 11 & 6 & 13 & 8 & 6 & 8 & 6 & 9 & 9 & 5 & 5 & 5 & 5 & 13 & 13 & 122 \\
\hline D-02-009 & 10 & 13 & 18 & 13 & 8 & 7 & 8 & 9 & 7 & 5 & 8 & 7 & 7 & 10 & 16 & 146 \\
\hline D-02-013 & 3 & 3 & 5 & 3 & 8 & 6 & 6 & 1 & 6 & 1 & 5 & 1 & 6 & 7 & 11 & 72 \\
\hline D-02-014 & 8 & 8 & 5 & 5 & 8 & 3 & 3 & 9 & 9 & 9 & 9 & 4 & 4 & 13 & 0 & 97 \\
\hline D-02-015 & 8 & 0 & 9 & 8 & 10 & 13 & 16 & 9 & 9 & 9 & 9 & 6 & 6 & 15 & 11 & 138 \\
\hline D-02-018 & 9 & 5 & 11 & 8 & 7 & 7 & 7 & 9 & 9 & 4 & 4 & 4 & 5 & 13 & 8 & 110 \\
\hline D-02-026 & 7 & 13 & 12 & 5 & 6 & 7 & 2 & 4 & 2 & 1 & 3 & 1 & 1 & 6 & 5 & 75 \\
\hline D-02-027 & 8 & 7 & 12 & 8 & 8 & 16 & 9 & 9 & 9 & 5 & 4 & 2 & 2 & 13 & 13 & 125 \\
\hline D-02-028 & 17 & 15 & 17 & 16 & 14 & 18 & 18 & 9 & 9 & 9 & 9 & 9 & 9 & 18 & 18 & 205 \\
\hline D-02-031 & 10 & 9 & 8 & 11 & 6 & 8 & 13 & 2 & 7 & 5 & 4 & 3 & 3 & 16 & 7 & 112 \\
\hline D-02-033 & 12 & 12 & 10 & 11 & 11 & 8 & 5 & 7 & 7 & 6 & 7 & 3 & 3 & 11 & 11 & 124 \\
\hline D-02-036 & 7 & 3 & 6 & 11 & 16 & 8 & 4 & 9 & 9 & 9 & 9 & 4 & 4 & 13 & 11 & 123 \\
\hline D-02-043 & 11 & 10 & 13 & 13 & 6 & 12 & 12 & 6 & 7 & 7 & 7 & 3 & 3 & 15 & 13 & 138 \\
\hline D-02-044 & 11 & 12 & 16 & 12 & 7 & 11 & 12 & 9 & 9 & 5 & 5 & 2 & 2 & 14 & 16 & 143 \\
\hline D-02-045 & 6 & 3 & 12 & 5 & 8 & 8 & 10 & 4 & 4 & 5 & 5 & 6 & 6 & 8 & 7 & 97 \\
\hline D-02-056 & 3 & 3 & 13 & 5 & 8 & 10 & 8 & 7 & 7 & 5 & 5 & 6 & 6 & 7 & 8 & 101 \\
\hline D-02-067 & 4 & 6 & 8 & 8 & 6 & 6 & 3 & 8 & 8 & 3 & 3 & 4 & 4 & 8 & 4 & 83 \\
\hline D-02-068 & 8 & 8 & 8 & 10 & 8 & 8 & 3 & 9 & 9 & 5 & 5 & 2 & 2 & 11 & 6 & 102 \\
\hline D-02-069 & 8 & 5 & 11 & 8 & 8 & 8 & 5 & 8 & 8 & 5 & 5 & 4 & 4 & 11 & 8 & 106 \\
\hline D-02-071 & 12 & 12 & 12 & 13 & 9 & 16 & 16 & 4 & 4 & 8 & 8 & 6 & 6 & 16 & 5 & 147 \\
\hline D-02-075 & 3 & 3 & 10 & 6 & 7 & 2 & 4 & 6 & 6 & 4 & 4 & 4 & 4 & 6 & 6 & 75 \\
\hline D-02-078 & 17 & 15 & 16 & 12 & 10 & 15 & 17 & 7 & 7 & 9 & 9 & 7 & 7 & 17 & 16 & 181 \\
\hline D-02-081 & 16 & 13 & 18 & 16 & 11 & 16 & 16 & 7 & 7 & 8 & 5 & 6 & 4 & 18 & 16 & 177 \\
\hline D-02-084 & 6 & 4 & 8 & 6 & 8 & 6 & 4 & 7 & 6 & 4 & 4 & 5 & 5 & 11 & 6 & 90 \\
\hline
\end{tabular}


Appendix 3: Table showing the twelve individual habitat parameter scores adapted from Barbour et al. (1999) that were assessed at most sites where fishing effort was applied and were totalled to derive the Habitat Assessment Score for each site.

\begin{tabular}{|c|c|c|c|c|c|c|c|c|c|c|c|c|c|c|c|c|}
\hline \multirow{2}{*}{ Site Number } & \multirow{2}{*}{ 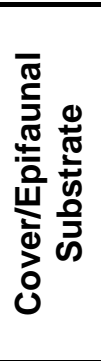 } & \multirow{2}{*}{ 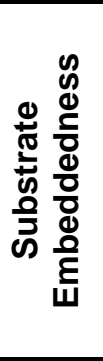 } & \multirow{2}{*}{ 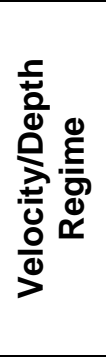 } & \multirow{2}{*}{ 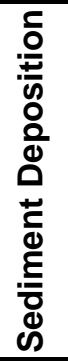 } & \multirow{2}{*}{ 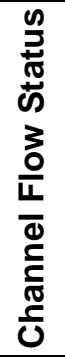 } & \multirow{2}{*}{ 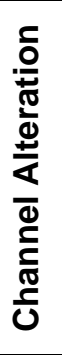 } & \multirow{2}{*}{ 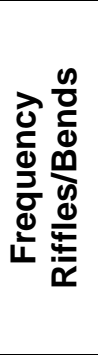 } & \multicolumn{2}{|c|}{ 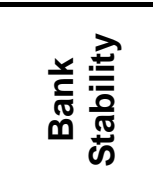 } & \multicolumn{2}{|c|}{ 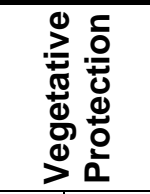 } & \multicolumn{2}{|c|}{ 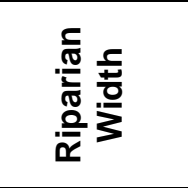 } & \multirow{2}{*}{ 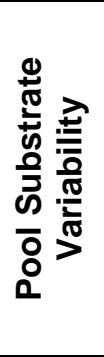 } & \multirow{2}{*}{ 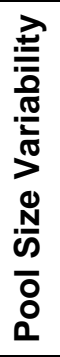 } & \multirow{2}{*}{ 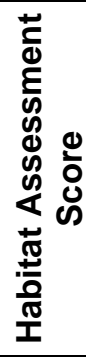 } \\
\hline & & & & & & & & ప্త & $\frac{\text { 동 }}{\frac{0}{x}}$ & ప্త & $\frac{\text { 픙 }}{\frac{9}{x}}$ & Ф્త & $\frac{\tilde{E}}{\frac{\pi}{x}}$ & & & \\
\hline D-02-088 & 4 & 5 & 5 & 11 & 5 & 8 & 4 & 7 & 7 & 7 & 7 & 4 & 5 & 11 & 4 & 94 \\
\hline D-02-089 & 5 & 1 & 3 & 5 & 8 & 8 & 3 & 9 & 9 & 4 & 4 & 6 & 6 & 11 & 5 & 87 \\
\hline D-02-092 & 8 & 8 & 7 & 6 & 8 & 9 & 5 & 8 & 8 & 6 & 6 & 5 & 5 & 11 & 6 & 106 \\
\hline D-02-093 & 18 & 18 & 18 & 13 & 8 & 13 & 16 & 6 & 6 & 8 & 8 & 4 & 7 & 17 & 17 & 177 \\
\hline D-02-094 & 6 & 4 & 6 & 8 & 8 & 8 & 4 & 9 & 9 & 5 & 5 & 5 & 3 & 13 & 6 & 99 \\
\hline D-02-095 & 8 & 3 & 6 & 6 & 9 & 8 & 5 & 6 & 6 & 2 & 2 & 5 & 5 & 13 & 8 & 92 \\
\hline D-02-096 & 5 & 3 & 7 & 6 & 6 & 7 & 3 & 9 & 9 & 4 & 4 & 3 & 3 & 8 & 5 & 82 \\
\hline D-02-100 & 5 & 1 & 6 & 8 & 8 & 8 & 5 & 5 & 5 & 5 & 5 & 5 & 5 & 3 & 11 & 85 \\
\hline D-02-101 & 5 & 2 & 7 & 9 & 8 & 8 & 6 & 5 & 5 & 4 & 4 & 4 & 4 & 6 & 11 & 88 \\
\hline D-02-102 & 5 & 3 & 7 & 10 & 8 & 8 & 5 & 7 & 7 & 5 & 5 & 5 & 5 & 5 & 11 & 96 \\
\hline D-02-104 & 16 & 15 & 16 & 15 & 18 & 16 & 16 & 9 & 9 & 9 & 9 & 7 & 7 & 18 & 13 & 193 \\
\hline D-02-108 & 13 & 8 & 17 & 13 & 17 & 16 & 12 & 7 & 7 & 8 & 8 & 5 & 6 & 14 & 12 & 163 \\
\hline D-02-111 & 11 & 7 & 16 & 9 & 10 & 15 & 10 & 4 & 5 & 8 & 8 & 8 & 8 & 7 & 9 & 135 \\
\hline D-02-116 & 6 & 6 & 5 & 8 & 7 & 13 & 5 & 9 & 9 & 6 & 6 & 6 & 7 & 7 & 3 & 103 \\
\hline D-02-118 & 8 & 6 & 6 & 6 & 3 & 13 & 5 & 9 & 9 & 6 & 6 & 4 & 4 & 8 & 3 & 96 \\
\hline D-02-119 & 10 & 15 & 8 & 16 & 6 & 13 & 6 & 9 & 9 & 7 & 7 & 6 & 6 & 5 & 4 & 127 \\
\hline D-02-121 & 8 & 11 & 8 & 14 & 8 & 10 & 8 & 9 & 9 & 7 & 7 & 6 & 6 & 12 & 4 & 127 \\
\hline D-02-122 & 15 & 10 & 16 & 12 & 7 & 13 & 9 & 9 & 9 & 3 & 3 & 2 & 2 & 16 & 16 & 142 \\
\hline D-02-125 & 14 & 10 & 17 & 13 & 8 & 11 & 7 & 9 & 9 & 8 & 7 & 9 & 8 & 14 & 13 & 157 \\
\hline D-02-126 & 4 & 5 & 7 & 8 & 6 & 6 & 3 & 1 & 2 & 1 & 2 & 1 & 1 & 7 & 33 & 87 \\
\hline D-02-127 & 16 & 13 & 17 & 11 & 15 & 15 & 15 & 9 & 10 & 9 & 9 & 9 & 9 & 16 & 16 & 189 \\
\hline D-02-130 & 6 & 3 & 11 & 5 & 8 & 8 & 4 & 8 & 8 & 7 & 7 & 6 & 6 & 12 & 7 & 106 \\
\hline D-02-131 & 6 & 3 & 5 & 8 & 8 & 9 & 4 & 9 & 9 & 7 & 7 & 1 & 1 & 8 & 11 & 96 \\
\hline D-02-132 & 5 & 5 & 4 & 5 & 5 & 8 & 5 & 9 & 9 & 9 & 8 & 5 & 5 & 10 & 6 & 98 \\
\hline D-02-135 & 11 & 8 & 11 & 7 & 8 & 10 & 6 & 9 & 9 & 8 & 8 & 6 & 6 & 12 & 8 & 127 \\
\hline
\end{tabular}


Appendix 3: Table showing the twelve individual habitat parameter scores adapted from Barbour et al. (1999) that were assessed at most sites where fishing effort was applied and were totalled to derive the Habitat Assessment Score for each site.

\begin{tabular}{|c|c|c|c|c|c|c|c|c|c|c|c|c|c|c|c|c|}
\hline \multirow{2}{*}{ Site Number } & \multirow{2}{*}{ 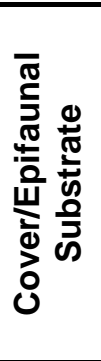 } & \multirow{2}{*}{ 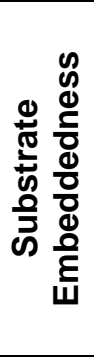 } & \multirow{2}{*}{ 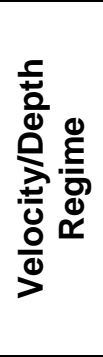 } & \multirow{2}{*}{ 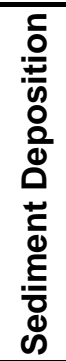 } & \multirow{2}{*}{ 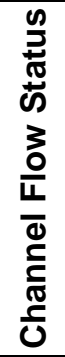 } & \multirow{2}{*}{ 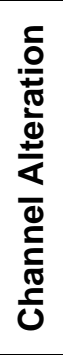 } & \multirow{2}{*}{ 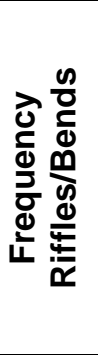 } & \multicolumn{2}{|c|}{ 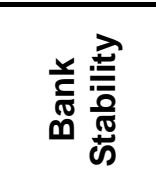 } & \multicolumn{2}{|c|}{ 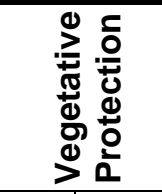 } & \multicolumn{2}{|c|}{ 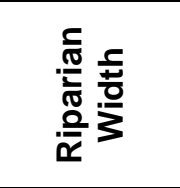 } & \multirow{2}{*}{ 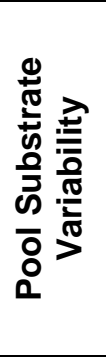 } & \multirow{2}{*}{ 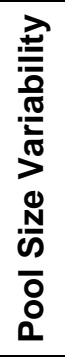 } & \multirow{2}{*}{ 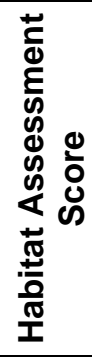 } \\
\hline & & & & & & & & 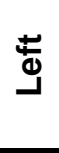 & $\frac{\text { 돔 }}{\frac{0}{x}}$ & Ф્త & $\frac{\text { 돔 }}{\frac{0}{x}}$ & \pm & $\frac{\text { 픔 }}{\frac{0}{x}}$ & & & \\
\hline D-02-137 & 10 & 10 & 13 & 10 & 8 & 8 & 8 & 8 & 8 & 5 & 5 & 6 & 6 & 16 & 7 & 128 \\
\hline D-02-139 & 18 & 18 & 18 & 18 & 13 & 18 & 18 & 10 & 10 & 10 & 10 & 10 & 10 & 18 & 8 & 207 \\
\hline D-02-142 & 16 & 15 & 17 & 15 & 7 & 15 & 13 & 8 & 8 & 8 & 8 & 7 & 7 & 16 & 17 & 177 \\
\hline D-02-147 & 5 & 5 & 6 & 6 & 3 & 6 & 2 & 5 & 5 & 6 & 6 & 5 & 3 & 13 & 11 & 87 \\
\hline D-02-149 & 15 & 12 & 13 & 13 & 7 & 15 & 8 & 8 & 8 & 7 & 7 & 8 & 8 & 16 & 11 & 156 \\
\hline D-02-160 & 13 & 13 & 16 & 8 & 10 & 11 & 15 & 2 & 6 & 4 & 7 & 3 & 6 & 16 & 16 & 146 \\
\hline D-02-161 & 6 & 5 & 10 & 5 & 8 & 8 & 8 & 6 & 3 & 1 & 3 & 2 & 3 & 8 & 8 & 84 \\
\hline D-02-164 & 9 & 8 & 8 & 8 & 6 & 16 & 16 & 9 & 9 & 8 & 9 & 6 & 8 & 8 & 8 & 136 \\
\hline D-02-167 & 10 & 8 & 8 & 8 & 5 & 15 & 16 & 7 & 5 & 7 & 7 & 8 & 7 & 6 & 4 & 121 \\
\hline D-02-170 & 11 & 10 & 13 & 10 & 8 & 14 & 9 & 8 & 8 & 9 & 7 & 8 & 5 & 8 & 8 & 136 \\
\hline D-02-171 & 12 & 8 & 13 & 11 & 10 & 17 & 11 & 9 & 9 & 9 & 9 & 8 & 8 & 8 & 8 & 150 \\
\hline D-02-173 & 14 & 9 & 16 & 8 & 11 & 15 & 13 & 8 & 8 & 8 & 8 & 8 & 8 & 8 & 8 & 150 \\
\hline D-02-178 & 10 & 3 & 7 & 8 & 6 & 10 & 5 & 5 & 7 & 6 & 8 & 5 & 7 & 8 & 8 & 103 \\
\hline D-02-179 & 12 & 8 & 16 & 8 & 7 & 13 & 14 & 8 & 8 & 5 & 5 & 5 & 5 & 13 & 9 & 136 \\
\hline D-02-180 & 8 & 8 & 13 & 8 & 9 & 8 & 11 & 8 & 8 & 5 & 5 & 4 & 4 & 7 & 6 & 112 \\
\hline D-02-181 & 15 & 8 & 18 & 8 & 10 & 16 & 16 & 7 & 7 & 9 & 6 & 9 & 6 & 13 & 8 & 156 \\
\hline D-02-182 & 3 & 1 & 3 & 3 & 6 & 6 & 3 & 6 & 6 & 5 & 6 & 5 & 4 & 13 & 11 & 81 \\
\hline D-02-183 & 13 & 15 & 13 & 11 & 8 & 15 & 18 & 5 & 5 & 9 & 8 & 8 & 8 & 16 & 8 & 160 \\
\hline D-02-184 & 3 & 3 & 8 & 2 & 9 & 8 & 12 & 6 & 7 & 6 & 6 & 8 & 8 & 6 & 8 & 100 \\
\hline D-02-185 & 8 & 3 & 2 & 3 & 3 & 13 & 2 & 5 & 7 & 6 & 8 & 2 & 5 & 8 & 11 & 86 \\
\hline D-02-188 & 7 & 3 & 1 & 8 & 1 & 8 & 3 & 7 & 7 & 6 & 6 & 6 & 6 & 10 & 10 & 89 \\
\hline D-02-190 & 6 & 3 & 6 & 3 & 6 & 13 & 3 & 7 & 5 & 5 & 7 & 4 & 5 & 12 & 8 & 93 \\
\hline D-02-191 & 13 & 13 & 15 & 8 & 7 & 13 & 13 & 5 & 8 & 8 & 6 & 8 & 7 & 10 & 15 & 149 \\
\hline D-02-192 & 7 & 5 & 8 & 6 & 6 & 11 & 2 & 1 & 1 & 1 & 1 & 1 & 1 & 7 & 2 & 60 \\
\hline D-02-193 & 5 & 3 & 7 & 8 & 7 & 7 & 5 & 6 & 6 & 4 & 4 & 3 & 3 & 3 & 4 & 75 \\
\hline
\end{tabular}


Appendix 3: Table showing the twelve individual habitat parameter scores adapted from Barbour et al. (1999) that were assessed at most sites where fishing effort was applied and were totalled to derive the Habitat Assessment Score for each site.

\begin{tabular}{|c|c|c|c|c|c|c|c|c|c|c|c|c|c|c|c|c|}
\hline \multirow{2}{*}{ Site Number } & \multirow{2}{*}{ 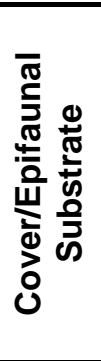 } & \multirow{2}{*}{ 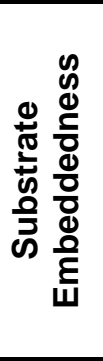 } & \multirow{2}{*}{ 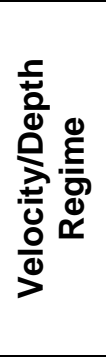 } & \multirow{2}{*}{ 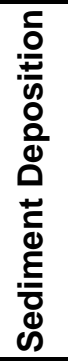 } & \multirow{2}{*}{ 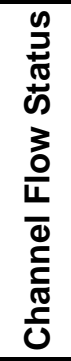 } & \multirow{2}{*}{ 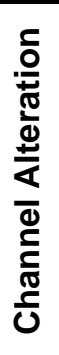 } & \multirow{2}{*}{ 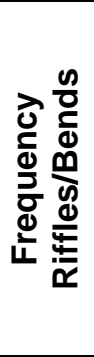 } & \multicolumn{2}{|c|}{ 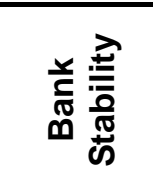 } & \multicolumn{2}{|c|}{ 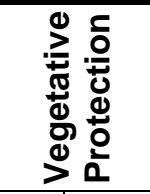 } & \multicolumn{2}{|c|}{ 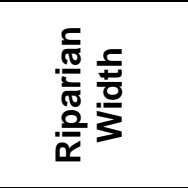 } & \multirow{2}{*}{ 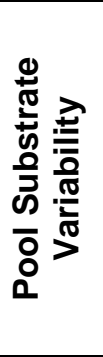 } & \multirow{2}{*}{ 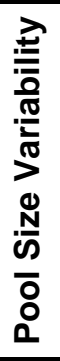 } & \multirow{2}{*}{ 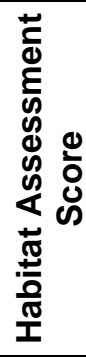 } \\
\hline & & & & & & & & ప্త & $\frac{\text { 동 }}{\frac{0}{x}}$ & ప্త & $\frac{\text { 픙 }}{\frac{9}{x}}$ & Ф્త & $\frac{\tilde{E}}{\frac{\pi}{x}}$ & & & \\
\hline D-02-195 & 8 & 2 & 12 & 8 & 14 & 15 & 15 & 7 & 7 & 9 & 9 & 9 & 9 & 8 & 7 & 139 \\
\hline D-02-196 & 15 & 14 & 19 & 13 & 18 & 16 & 19 & 8 & 8 & 6 & 5 & 5 & 5 & 15 & 19 & 185 \\
\hline D-02-197 & 14 & 14 & 18 & 12 & 7 & 14 & 13 & 2 & 6 & 3 & 6 & 3 & 6 & 11 & 13 & 142 \\
\hline D-02-198 & 10 & 10 & 11 & 10 & 8 & 8 & 8 & 3 & 3 & 6 & 4 & 2 & 3 & 8 & 13 & 107 \\
\hline D-02-199 & 12 & 8 & 16 & 10 & 11 & 17 & 17 & 7 & 5 & 6 & 6 & 5 & 5 & 8 & 16 & 149 \\
\hline D-02-200 & 11 & 13 & 16 & 10 & 16 & 15 & 16 & 6 & 6 & 6 & 6 & 6 & 6 & 16 & 16 & 165 \\
\hline D-02-201 & 8 & 6 & 6 & 5 & 5 & 13 & 5 & 7 & 7 & 5 & 5 & 4 & 4 & 11 & 11 & 102 \\
\hline B-03-001 & 10 & 7 & 12 & 7 & 17 & 15 & 13 & 9 & 9 & 9 & 9 & 8 & 8 & 10 & 13 & 156 \\
\hline B-03-003 & 6 & 5 & 6 & 5 & 5 & 12 & 5 & 5 & 6 & 3 & 3 & 3 & 3 & 9 & 6 & 82 \\
\hline B-03-004 & 10 & 9 & 11 & 11 & 18 & 15 & 8 & 8 & 8 & 8 & 8 & 8 & 8 & 16 & 10 & 156 \\
\hline B-03-005 & 16 & 12 & 18 & 9 & 8 & 14 & 13 & 8 & 8 & 7 & 8 & 9 & 9 & 14 & 16 & 169 \\
\hline B-03-007 & 8 & 6 & 7 & 3 & 13 & 5 & 3 & 5 & 1 & 3 & 0 & 3 & 1 & 13 & 11 & 82 \\
\hline B-03-009 & 8 & 10 & 8 & 9 & 10 & 12 & 3 & 8 & 8 & 7 & 7 & 7 & 4 & 13 & 14 & 128 \\
\hline B-03-012 & 6 & 3 & 9 & 6 & 10 & 11 & 5 & 5 & 7 & 5 & 6 & 3 & 3 & 10 & 5 & 94 \\
\hline B-03-013 & 11 & 10 & 14 & 12 & 11 & 13 & 8 & 6 & 4 & 6 & 3 & 6 & 4 & 16 & 16 & 140 \\
\hline B-03-015 & 8 & 3 & 3 & 8 & 13 & 13 & 1 & 7 & 7 & 5 & 3 & 4 & 3 & 8 & 14 & 100 \\
\hline B-03-016 & 10 & 8 & 11 & 7 & 13 & 15 & 7 & 6 & 8 & 3 & 6 & 6 & 6 & 14 & 12 & 132 \\
\hline B-03-017 & 15 & 11 & 16 & 9 & 7 & 11 & 13 & 4 & 6 & 7 & 7 & 4 & 7 & 19 & 18 & 154 \\
\hline B-03-019 & 9 & 8 & 8 & 8 & 5 & 13 & 4 & 5 & 4 & 4 & 4 & 7 & 7 & 8 & 14 & 108 \\
\hline B-03-020 & 8 & 4 & 6 & 11 & 8 & 18 & 1 & 7 & 7 & 5 & 4 & 4 & 5 & 14 & 6 & 108 \\
\hline B-03-026 & 18 & 10 & 13 & 11 & 10 & 18 & 16 & 8 & 9 & 9 & 9 & 9 & 9 & 13 & 16 & 178 \\
\hline B-03-028 & 10 & 8 & 13 & 6 & 7 & 16 & 14 & 4 & 3 & 3 & 3 & 3 & 3 & 9 & 8 & 110 \\
\hline B-03-030 & 10 & 9 & 7 & 9 & 5 & 11 & 6 & 3 & 4 & 3 & 3 & 4 & 4 & 14 & 11 & 103 \\
\hline B-03-031 & 11 & 11 & 15 & 9 & 15 & 16 & 13 & 7 & 6 & 6 & 6 & 4 & 6 & 16 & 16 & 157 \\
\hline B-03-033 & 14 & 14 & 14 & 17 & 15 & 17 & 15 & 6 & 6 & 6 & 4 & 6 & 6 & 13 & 18 & 171 \\
\hline
\end{tabular}


Appendix 3: Table showing the twelve individual habitat parameter scores adapted from Barbour et al. (1999) that were assessed at most sites where fishing effort was applied and were totalled to derive the Habitat Assessment Score for each site.

\begin{tabular}{|c|c|c|c|c|c|c|c|c|c|c|c|c|c|c|c|c|}
\hline \multirow{2}{*}{ Site Number } & \multirow{2}{*}{ 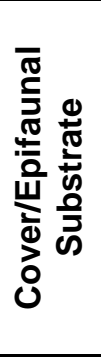 } & \multirow{2}{*}{ 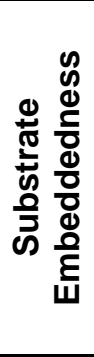 } & \multirow{2}{*}{ 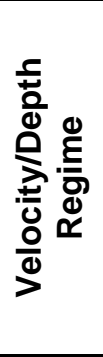 } & \multirow{2}{*}{ 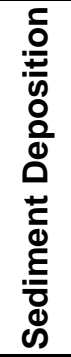 } & \multirow{2}{*}{ 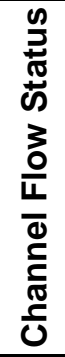 } & \multirow{2}{*}{ 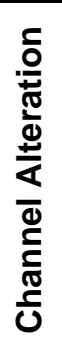 } & \multirow{2}{*}{ 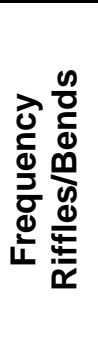 } & \multicolumn{2}{|c|}{ 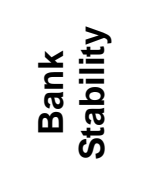 } & \multicolumn{2}{|c|}{ 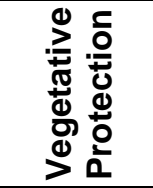 } & \multicolumn{2}{|c|}{$\begin{array}{l}\frac{c}{\sqrt{0}} \\
\frac{2}{\frac{0}{\pi}} \\
\frac{0}{x}\end{array}$} & \multirow{2}{*}{ 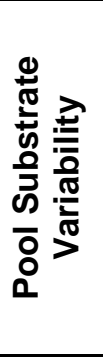 } & \multirow{2}{*}{ 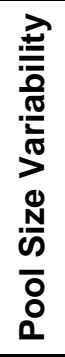 } & \multirow{2}{*}{ 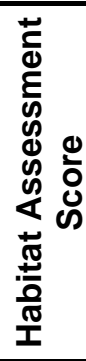 } \\
\hline & & & & & & & & Ф্త & $\begin{array}{l}\frac{}{\frac{1}{\sigma}} \\
\frac{0}{x}\end{array}$ & Ф্త & $\frac{\text { 픔 }}{\frac{0}{\tilde{x}}}$ & Ф્త & $\frac{\tilde{n}}{\frac{\tilde{\sigma}}{\boldsymbol{x}}}$ & & & \\
\hline B-03-035 & 12 & 7 & 4 & 10 & 10 & 12 & 3 & 4 & 3 & 3 & 3 & 6 & 3 & 16 & 13 & 109 \\
\hline B-03-040 & 13 & 15 & 10 & 9 & 8 & 14 & 19 & 4 & 4 & 5 & 8 & 6 & 7 & 16 & 10 & 148 \\
\hline B-03-041 & 14 & 11 & 13 & 11 & 13 & 11 & 11 & 8 & 7 & 6 & 5 & 8 & 5 & 18 & 16 & 157 \\
\hline B-03-048 & 6 & 8 & 7 & 6 & 8 & 12 & 3 & 5 & 7 & 5 & 5 & 5 & 5 & 11 & 12 & 105 \\
\hline B-03-050 & 11 & 11 & 5 & 11 & 11 & 13 & 5 & 8 & 7 & 8 & 7 & 8 & 6 & 18 & 12 & 141 \\
\hline B-03-052 & 16 & 14 & 13 & 20 & 10 & 13 & 16 & 7 & 3 & 6 & 9 & 8 & 5 & 16 & 18 & 174 \\
\hline B-03-053 & 10 & 16 & 9 & 16 & 8 & 16 & 9 & 8 & 8 & 7 & 7 & 7 & 7 & 16 & 6 & 150 \\
\hline B-03-055 & 13 & 10 & 8 & 7 & 7 & 17 & 15 & 2 & 2 & 9 & 8 & 9 & 8 & 10 & 8 & 133 \\
\hline B-03-060 & 14 & 10 & 18 & 9 & 17 & 14 & 13 & 8 & 8 & 9 & 7 & 9 & 7 & 16 & 18 & 177 \\
\hline B-03-061 & 11 & 8 & 13 & 10 & 11 & 13 & 10 & 5 & 7 & 6 & 7 & 6 & 7 & 12 & 12 & 138 \\
\hline B-03-062 & 16 & 13 & 16 & 13 & 14 & 12 & 10 & 8 & 8 & 8 & 8 & 4 & 5 & 13 & 16 & 164 \\
\hline B-03-064 & 15 & 13 & 13 & 8 & 6 & 16 & 18 & 2 & 2 & 5 & 5 & 6 & 6 & 13 & 16 & 144 \\
\hline B-03-065 & 8 & 7 & 12 & 6 & 6 & 7 & 8 & 6 & 6 & 5 & 5 & 5 & 5 & 16 & 16 & 118 \\
\hline B-03-067 & 10 & 11 & 3 & 7 & 13 & 9 & 3 & 6 & 6 & 4 & 4 & 2 & 5 & 11 & 11 & 105 \\
\hline B-03-070 & 16 & 16 & 18 & 10 & 13 & 13 & 18 & 7 & 7 & 9 & 9 & 9 & 9 & 18 & 18 & 190 \\
\hline B-03-071 & 3 & 3 & 9 & 7 & 7 & 3 & 11 & 7 & 7 & 3 & 3 & 1 & 1 & 8 & 3 & 76 \\
\hline B-03-075 & 13 & 11 & 8 & 8 & 7 & 16 & 5 & 9 & 9 & 6 & 6 & 9 & 8 & 10 & 5 & 130 \\
\hline B-03-076 & 16 & 12 & 11 & 13 & 10 & 14 & 7 & 7 & 7 & 9 & 9 & 6 & 6 & 15 & 18 & 160 \\
\hline B-03-077 & 15 & 15 & 16 & 10 & 7 & 13 & 16 & 8 & 8 & 7 & 7 & 8 & 8 & 19 & 19 & 176 \\
\hline B-03-083 & 8 & 3 & 6 & 8 & 14 & 4 & 4 & 7 & 7 & 7 & 7 & 7 & 7 & 16 & 11 & 116 \\
\hline B-03-084 & 8 & 3 & 6 & 15 & 10 & 4 & 4 & 7 & 7 & 7 & 7 & 7 & 7 & 18 & 11 & 121 \\
\hline B-03-085 & 8 & 3 & 6 & 6 & 14 & 4 & 3 & 7 & 7 & 7 & 7 & 7 & 7 & 12 & 11 & 109 \\
\hline B-03-086 & 8 & 3 & 6 & 10 & 15 & 4 & 3 & 7 & 7 & 7 & 7 & 7 & 7 & 12 & 11 & 114 \\
\hline B-03-087 & 10 & 6 & 3 & 8 & 18 & 8 & 3 & 5 & 5 & 4 & 4 & 4 & 4 & 13 & 14 & 109 \\
\hline B-03-088 & 11 & 6 & 10 & 11 & 11 & 13 & 3 & 7 & 7 & 8 & 8 & 7 & 6 & 6 & 13 & 127 \\
\hline
\end{tabular}


Appendix 3: Table showing the twelve individual habitat parameter scores adapted from Barbour et al. (1999) that were assessed at most sites where fishing effort was applied and were totalled to derive the Habitat Assessment Score for each site.

\begin{tabular}{|c|c|c|c|c|c|c|c|c|c|c|c|c|c|c|c|c|}
\hline \multirow{2}{*}{ Site Number } & \multirow{2}{*}{ 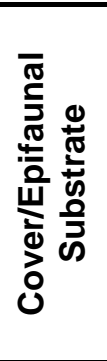 } & \multirow{2}{*}{ 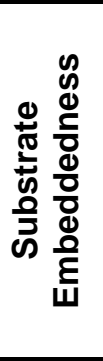 } & \multirow{2}{*}{ 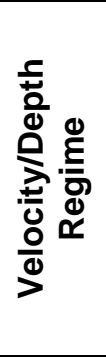 } & \multirow{2}{*}{ 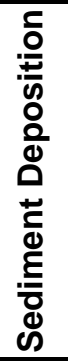 } & \multirow{2}{*}{ 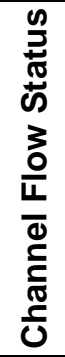 } & \multirow{2}{*}{ 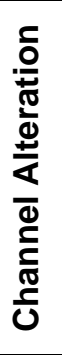 } & \multirow{2}{*}{ 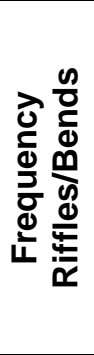 } & \multicolumn{2}{|c|}{ 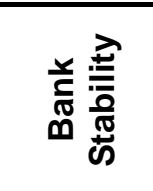 } & \multicolumn{2}{|c|}{ 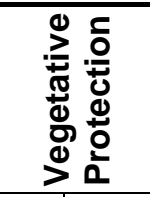 } & \multicolumn{2}{|c|}{ 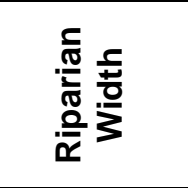 } & \multirow{2}{*}{ 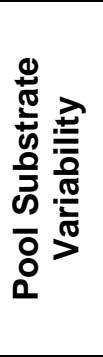 } & \multirow{2}{*}{ 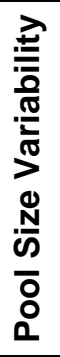 } & \multirow{2}{*}{ 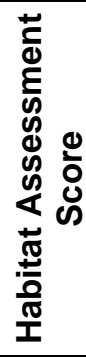 } \\
\hline & & & & & & & & ప্త & $\frac{\text { 픔 }}{\frac{0}{x}}$ & 屯্త & $\frac{\text { 픙 }}{\frac{9}{x}}$ & Ф્త & $\frac{\tilde{E}}{\frac{\pi}{x}}$ & & & \\
\hline B-03-089 & 11 & 8 & 7 & 8 & 11 & 13 & 3 & 9 & 9 & 3 & 5 & 2 & 5 & 11 & 11 & 116 \\
\hline B-03-091 & 6 & 7 & 1 & 10 & 16 & 7 & 3 & 8 & 8 & 7 & 7 & 5 & 5 & 11 & 11 & 112 \\
\hline B-03-093 & 10 & 6 & 3 & 8 & 15 & 7 & 3 & 6 & 8 & 7 & 7 & 7 & 7 & 11 & 11 & 116 \\
\hline B-03-094 & 14 & 7 & 13 & 10 & 11 & 8 & 8 & 7 & 7 & 6 & 6 & 7 & 7 & 15 & 13 & 139 \\
\hline B-03-095 & 12 & 5 & 7 & 6 & 15 & 8 & 6 & 7 & 7 & 7 & 7 & 7 & 7 & 18 & 14 & 133 \\
\hline B-03-097 & 11 & 6 & 6 & 6 & 14 & 7 & 6 & 5 & 7 & 6 & 6 & 6 & 6 & 11 & 11 & 114 \\
\hline B-03-098 & 14 & 5 & 13 & 6 & 8 & 15 & 14 & 2 & 2 & 8 & 8 & 9 & 9 & 13 & 16 & 142 \\
\hline B-03-099 & 12 & 10 & 11 & 6 & 6 & 13 & 11 & 8 & 8 & 9 & 9 & 6 & 6 & 5 & 5 & 125 \\
\hline B-03-101 & 8 & 6 & 5 & 8 & 13 & 11 & 6 & 3 & 2 & 6 & 3 & 6 & 6 & 11 & 11 & 105 \\
\hline B-03-102 & 13 & 14 & 5 & 8 & 10 & 15 & 3 & 7 & 7 & 4 & 8 & 4 & 7 & 13 & 13 & 131 \\
\hline B-03-103 & 11 & 11 & 10 & 8 & 7 & 14 & 18 & 9 & 9 & 9 & 5 & 9 & 5 & 13 & 7 & 145 \\
\hline B-03-104 & 16 & 10 & 16 & 11 & 16 & 13 & 15 & 9 & 9 & 8 & 8 & 5 & 6 & 18 & 18 & 178 \\
\hline B-03-112 & 18 & 6 & 16 & 8 & 15 & 16 & 11 & 9 & 9 & 9 & 8 & 9 & 9 & 15 & 16 & 174 \\
\hline B-03-115 & 11 & 10 & 14 & 11 & 11 & 10 & 11 & 3 & 2 & 6 & 5 & 4 & 4 & 16 & 10 & 128 \\
\hline B-03-116 & 11 & 3 & 10 & 8 & 13 & 13 & 10 & 1 & 8 & 3 & 8 & 4 & 7 & 11 & 14 & 124 \\
\hline B-03-117 & 13 & 11 & 7 & 10 & 8 & 14 & 12 & 8 & 8 & 8 & 8 & 6 & 6 & 7 & 5 & 131 \\
\hline B-03-118 & 16 & 5 & 13 & 7 & 10 & 14 & 11 & 8 & 8 & 7 & 9 & 6 & 7 & 13 & 11 & 145 \\
\hline B-03-119 & 7 & 3 & 6 & 4 & 11 & 13 & 3 & 9 & 9 & 8 & 8 & 8 & 8 & 11 & 6 & 114 \\
\hline B-03-120 & 17 & 8 & 14 & 10 & 9 & 12 & 14 & 3 & 3 & 3 & 6 & 3 & 5 & 18 & 15 & 140 \\
\hline B-03-121 & 11 & 7 & 12 & 6 & 9 & 14 & 10 & 9 & 9 & 8 & 8 & 9 & 9 & 15 & 15 & 151 \\
\hline B-03-122 & 12 & 7 & 11 & 9 & 10 & 12 & 7 & 7 & 6 & 8 & 8 & 6 & 7 & 14 & 18 & 142 \\
\hline B-03-124 & 11 & 8 & 14 & 10 & 6 & 11 & 15 & 4 & 2 & 5 & 4 & 9 & 9 & 9 & 16 & 133 \\
\hline B-03-126 & 10 & 5 & 4 & 7 & 13 & 12 & 6 & 3 & 7 & 4 & 7 & 8 & 8 & 13 & 13 & 120 \\
\hline B-03-132 & 18 & 18 & 15 & 13 & 10 & 18 & 18 & 9 & 9 & 9 & 9 & 8 & 8 & 13 & 16 & 191 \\
\hline B-03-134 & 16 & 13 & 13 & 12 & 9 & 17 & 16 & 9 & 9 & 9 & 9 & 9 & 9 & 16 & 10 & 176 \\
\hline
\end{tabular}


Appendix 3: Table showing the twelve individual habitat parameter scores adapted from Barbour et al. (1999) that were assessed at most sites where fishing effort was applied and were totalled to derive the Habitat Assessment Score for each site.

\begin{tabular}{|c|c|c|c|c|c|c|c|c|c|c|c|c|c|c|c|c|}
\hline \multirow{2}{*}{ Site Number } & \multirow{2}{*}{ 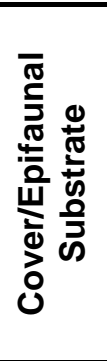 } & \multirow{2}{*}{ 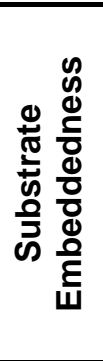 } & \multirow{2}{*}{ 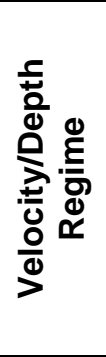 } & \multirow{2}{*}{ 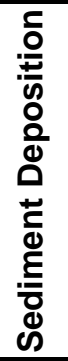 } & \multirow{2}{*}{ 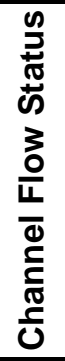 } & \multirow{2}{*}{ 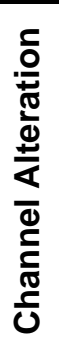 } & \multirow{2}{*}{ 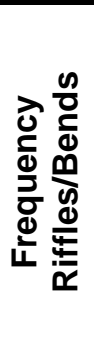 } & \multicolumn{2}{|c|}{ 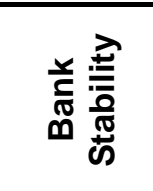 } & \multicolumn{2}{|c|}{ 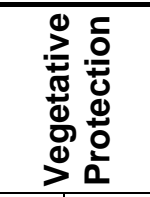 } & \multicolumn{2}{|c|}{ 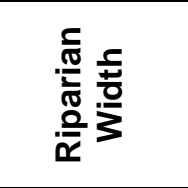 } & \multirow{2}{*}{ 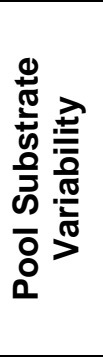 } & \multirow{2}{*}{ 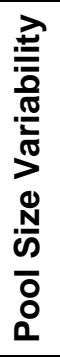 } & \multirow{2}{*}{ 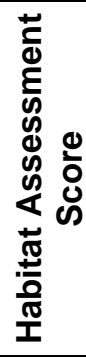 } \\
\hline & & & & & & & & ప্త & $\frac{\text { 픔 }}{\frac{0}{x}}$ & ప্త & $\frac{\overline{\frac{\pi}{0}}}{\frac{0}{x}}$ & 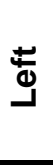 & $\frac{\tilde{E}}{\frac{\pi}{x}}$ & & & \\
\hline B-03-141 & 7 & 11 & 2 & 7 & 2 & 12 & 5 & 4 & 4 & 4 & 4 & 4 & 4 & 17 & 13 & 100 \\
\hline B-03-142 & 9 & 8 & 2 & 7 & 2 & 11 & 6 & 4 & 4 & 4 & 4 & 6 & 6 & 18 & 13 & 104 \\
\hline B-03-143 & 13 & 8 & 3 & 6 & 5 & 12 & 5 & 2 & 2 & 5 & 5 & 7 & 7 & 17 & 15 & 112 \\
\hline B-03-145 & 11 & 11 & 1 & 11 & 6 & 13 & 3 & 7 & 6 & 6 & 5 & 6 & 2 & 14 & 11 & 113 \\
\hline B-03-148 & 6 & 7 & 6 & 7 & 6 & 11 & 5 & 3 & 3 & 3 & 3 & 5 & 5 & 11 & 6 & 87 \\
\hline B-03-150 & 8 & 8 & 3 & 6 & 3 & 12 & 3 & 2 & 7 & 3 & 5 & 6 & 6 & 13 & 13 & 98 \\
\hline B-03-151 & 13 & 13 & 11 & 10 & 10 & 15 & 18 & 2 & 2 & 8 & 8 & 8 & 8 & 9 & 9 & 144 \\
\hline B-03-152 & 14 & 17 & 16 & 6 & 7 & 12 & 18 & 2 & 2 & 6 & 6 & 7 & 7 & 14 & 10 & 144 \\
\hline B-03-158 & 11 & 11 & 2 & 10 & 10 & 13 & 6 & 6 & 8 & 8 & 8 & 6 & 6 & 11 & 12 & 128 \\
\hline B-03-159 & 11 & 10 & 8 & 8 & 5 & 14 & 11 & 2 & 2 & 3 & 3 & 5 & 5 & 13 & 13 & 113 \\
\hline B-03-160 & 7 & 7 & 8 & 7 & 2 & 11 & 5 & 5 & 3 & 6 & 4 & 6 & 6 & 12 & 10 & 99 \\
\hline B-03-163 & 14 & 11 & 13 & 16 & 10 & 13 & 11 & 7 & 7 & 9 & 6 & 9 & 4 & 13 & 13 & 156 \\
\hline B-03-181 & 14 & 12 & 13 & 9 & 9 & 14 & 13 & 6 & 5 & 9 & 8 & 9 & 6 & 11 & 14 & 152 \\
\hline B-03-182 & 10 & 10 & 11 & 10 & 10 & 8 & 6 & 7 & 8 & 7 & 8 & 4 & 4 & 16 & 16 & 135 \\
\hline B-03-183 & 8 & 6 & 5 & 7 & 8 & 8 & 5 & 5 & 5 & 6 & 6 & 4 & 4 & 8 & 11 & 96 \\
\hline B-03-184 & 12 & 7 & 6 & 7 & 10 & 11 & 5 & 7 & 7 & 5 & 5 & 5 & 5 & 10 & 8 & 110 \\
\hline B-03-185 & 16 & 13 & 18 & 8 & 10 & 13 & 14 & 6 & 8 & 7 & 8 & 7 & 8 & 18 & 18 & 172 \\
\hline B-03-189 & 13 & 4 & 4 & 8 & 6 & 8 & 5 & 7 & 7 & 4 & 4 & 4 & 4 & 11 & 6 & 95 \\
\hline B-03-190 & 9 & 10 & 4 & 7 & 8 & 14 & 5 & 9 & 9 & 4 & 7 & 3 & 7 & 11 & 12 & 119 \\
\hline B-03-191 & 10 & 7 & 11 & 7 & 7 & 6 & 6 & 8 & 8 & 6 & 6 & 6 & 6 & 14 & 8 & 116 \\
\hline B-03-192 & 11 & 11 & 16 & 15 & 11 & 7 & 5 & 8 & 8 & 5 & 7 & 4 & 7 & 16 & 13 & 144 \\
\hline B-03-193 & 14 & 11 & 8 & 8 & 16 & 13 & 9 & 7 & 7 & 8 & 8 & 8 & 6 & 13 & 14 & 150 \\
\hline B-03-194 & 12 & 8 & 7 & 8 & 6 & 13 & 6 & 7 & 7 & 6 & 6 & 6 & 6 & 12 & 12 & 122 \\
\hline B-03-199 & 8 & 3 & 3 & 11 & 3 & 8 & 3 & 6 & 6 & 4 & 4 & 4 & 4 & 8 & 3 & 78 \\
\hline B-03-200 & 13 & 8 & 14 & 7 & 7 & 11 & 13 & 2 & 2 & 8 & 3 & 8 & 3 & 7 & 8 & 114 \\
\hline
\end{tabular}


Appendix 3: Table showing the twelve individual habitat parameter scores adapted from Barbour et al. (1999) that were assessed at most sites where fishing effort was applied and were totalled to derive the Habitat Assessment Score for each site.

\begin{tabular}{|c|c|c|c|c|c|c|c|c|c|c|c|c|c|c|c|c|}
\hline \multirow{2}{*}{ Site Number } & \multirow{2}{*}{ 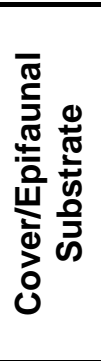 } & \multirow{2}{*}{ 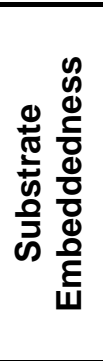 } & \multirow{2}{*}{ 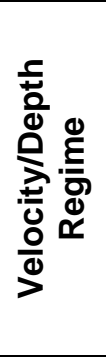 } & \multirow{2}{*}{ 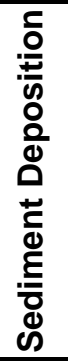 } & \multirow{2}{*}{ 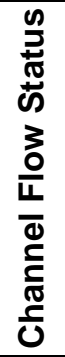 } & \multirow{2}{*}{ 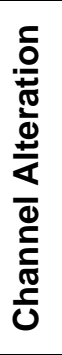 } & \multirow{2}{*}{ 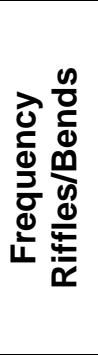 } & \multicolumn{2}{|c|}{ 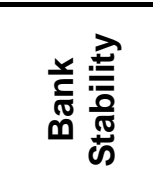 } & \multicolumn{2}{|c|}{ 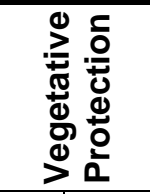 } & \multicolumn{2}{|c|}{ 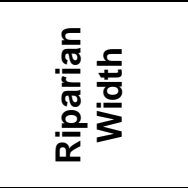 } & \multirow{2}{*}{ 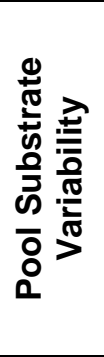 } & \multirow{2}{*}{ 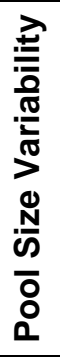 } & \multirow{2}{*}{ 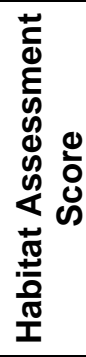 } \\
\hline & & & & & & & & ప্త & $\frac{\text { 동 }}{\frac{0}{x}}$ & ప্త & $\frac{\text { 픙 }}{\frac{9}{x}}$ & Ф્త & $\frac{\tilde{E}}{\frac{\pi}{x}}$ & & & \\
\hline B-03-202 & 13 & 8 & 9 & 8 & 14 & 13 & 6 & 8 & 8 & 7 & 7 & 7 & 7 & 15 & 13 & 143 \\
\hline B-03-204 & 10 & 9 & 3 & 9 & 10 & 6 & 6 & 6 & 6 & 6 & 6 & 6 & 6 & 12 & 6 & 107 \\
\hline B-03-206 & 13 & 8 & 13 & 8 & 10 & 7 & 4 & 5 & 5 & 4 & 4 & 4 & 4 & 14 & 11 & 114 \\
\hline B-03-207 & 11 & 7 & 8 & 8 & 6 & 8 & 12 & 7 & 7 & 4 & 4 & 4 & 4 & 11 & 7 & 108 \\
\hline B-03-209 & 13 & 14 & 15 & 12 & 8 & 13 & 16 & 1 & 1 & 1 & 1 & 2 & 2 & 13 & 14 & 126 \\
\hline B-03-212 & 14 & 12 & 13 & 13 & 8 & 10 & 15 & 4 & 4 & 5 & 5 & 5 & 5 & 15 & 15 & 143 \\
\hline B-03-224 & 17 & 15 & 12 & 10 & 6 & 15 & 13 & 8 & 8 & 9 & 9 & 8 & 8 & 14 & 12 & 164 \\
\hline B-03-226 & 10 & 8 & 9 & 9 & 8 & 7 & 5 & 2 & 2 & 3 & 6 & 6 & 4 & 13 & 10 & 102 \\
\hline B-03-229 & 10 & 10 & 6 & 10 & 8 & 8 & 5 & 6 & 6 & 6 & 6 & 6 & 6 & 11 & 8 & 112 \\
\hline B-03-235 & 13 & 12 & 3 & 8 & 4 & 8 & 5 & 6 & 6 & 5 & 5 & 6 & 6 & 11 & 3 & 101 \\
\hline B-03-239 & 10 & 6 & 3 & 7 & 11 & 7 & 5 & 5 & 5 & 2 & 2 & 3 & 3 & 14 & 12 & 95 \\
\hline B-03-241 & 9 & 7 & 6 & 7 & 4 & 6 & 4 & 5 & 5 & 4 & 4 & 3 & 3 & 11 & 7 & 85 \\
\hline D-03-001 & 11 & 16 & 16 & 13 & 16 & 13 & 11 & 7 & 6 & 6 & 8 & 5 & 6 & 11 & 13 & 158 \\
\hline D-03-002 & 18 & 17 & 18 & 12 & 14 & 14 & 19 & 8 & 6 & 9 & 8 & 9 & 8 & 18 & 18 & 196 \\
\hline D-03-003 & 12 & 16 & 11 & 13 & 18 & 8 & 8 & 7 & 7 & 3 & 3 & 2 & 2 & 16 & 7 & 133 \\
\hline D-03-004 & 8 & 5 & 11 & 8 & 13 & 14 & 8 & 7 & 8 & 6 & 7 & 7 & 8 & 12 & 12 & 134 \\
\hline D-03-005 & 11 & 7 & 13 & 6 & 11 & 7 & 13 & 9 & 9 & 7 & 6 & 5 & 5 & 7 & 8 & 124 \\
\hline D-03-006 & 8 & 8 & 14 & 8 & 7 & 14 & 11 & 3 & 3 & 2 & 2 & 5 & 5 & 7 & 12 & 109 \\
\hline D-03-009 & 4 & 14 & 8 & 10 & 5 & 8 & 3 & 7 & 7 & 3 & 3 & 3 & 3 & 8 & 13 & 99 \\
\hline D-03-010 & 10 & 8 & 8 & 8 & 6 & 10 & 10 & 6 & 6 & 4 & 5 & 4 & 4 & 8 & 6 & 103 \\
\hline D-03-011 & 15 & 13 & 18 & 13 & 14 & 13 & 17 & 9 & 9 & 7 & 7 & 9 & 8 & 14 & 18 & 184 \\
\hline D-03-012 & 10 & 6 & 11 & 8 & 13 & 13 & 15 & 6 & 5 & 3 & 3 & 3 & 4 & 13 & 6 & 119 \\
\hline D-03-015 & 12 & 8 & 13 & 8 & 15 & 8 & 8 & 8 & 8 & 7 & 7 & 7 & 7 & 9 & 10 & 135 \\
\hline D-03-016 & 14 & 14 & 16 & 9 & 13 & 10 & 16 & 9 & 9 & 9 & 9 & 7 & 7 & 8 & 8 & 158 \\
\hline D-03-017 & 8 & 7 & 9 & 3 & 8 & 16 & 16 & 9 & 9 & 9 & 9 & 8 & 7 & 7 & 6 & 131 \\
\hline
\end{tabular}


Appendix 3: Table showing the twelve individual habitat parameter scores adapted from Barbour et al. (1999) that were assessed at most sites where fishing effort was applied and were totalled to derive the Habitat Assessment Score for each site.

\begin{tabular}{|c|c|c|c|c|c|c|c|c|c|c|c|c|c|c|c|c|}
\hline \multirow{2}{*}{ Site Number } & \multirow{2}{*}{ 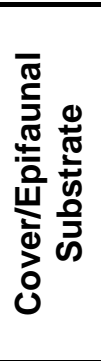 } & \multirow{2}{*}{ 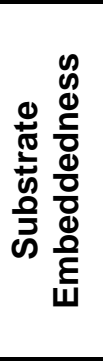 } & \multirow{2}{*}{ 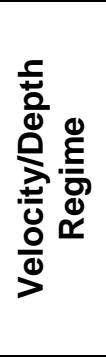 } & \multirow{2}{*}{ 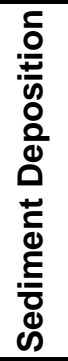 } & \multirow{2}{*}{ 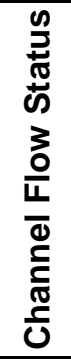 } & \multirow{2}{*}{ 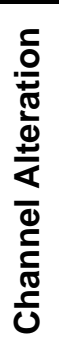 } & \multirow{2}{*}{ 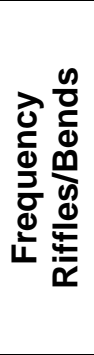 } & \multicolumn{2}{|c|}{ 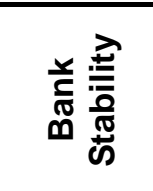 } & \multicolumn{2}{|c|}{ 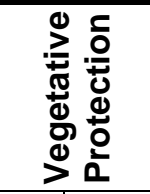 } & \multicolumn{2}{|c|}{ 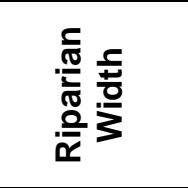 } & \multirow{2}{*}{ 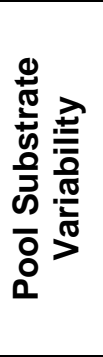 } & \multirow{2}{*}{ 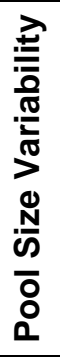 } & \multirow{2}{*}{ 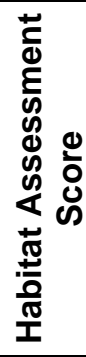 } \\
\hline & & & & & & & & ప্త & $\frac{\text { 동 }}{\frac{0}{x}}$ & ప্త & $\frac{\text { 픙 }}{\frac{9}{x}}$ & Ф્త & $\frac{\tilde{E}}{\frac{\pi}{x}}$ & & & \\
\hline D-03-019 & 15 & 5 & 8 & 8 & 19 & 8 & 3 & 7 & 7 & 7 & 7 & 3 & 5 & 13 & 15 & 130 \\
\hline D-03-023 & 10 & 3 & 7 & 8 & 8 & 6 & 8 & 9 & 9 & 6 & 6 & 5 & 5 & 8 & 6 & 104 \\
\hline D-03-024 & 8 & 6 & 11 & 8 & 8 & 8 & 6 & 3 & 3 & 4 & 4 & 4 & 4 & 10 & 13 & 100 \\
\hline D-03-025 & 14 & 8 & 13 & 5 & 8 & 14 & 13 & 7 & 7 & 7 & 5 & 5 & 4 & 8 & 11 & 129 \\
\hline D-03-026 & 11 & 10 & 8 & 8 & 10 & 15 & 13 & 6 & 6 & 3 & 4 & 5 & 5 & 7 & 11 & 122 \\
\hline D-03-027 & 7 & 3 & 3 & 8 & 5 & 11 & 3 & 9 & 9 & 8 & 8 & 5 & 4 & 6 & 13 & 102 \\
\hline D-03-029 & 10 & 5 & 8 & 6 & 7 & 14 & 11 & 3 & 7 & 6 & 6 & 5 & 5 & 5 & 8 & 106 \\
\hline D-03-035 & 10 & 6 & 8 & 8 & 6 & 14 & 10 & 9 & 8 & 5 & 5 & 6 & 6 & 11 & 13 & 125 \\
\hline D-03-036 & 16 & 11 & 18 & 9 & 11 & 14 & 16 & 8 & 5 & 4 & 9 & 6 & 8 & 11 & 18 & 164 \\
\hline D-03-037 & 11 & 8 & 12 & 8 & 13 & 18 & 16 & 8 & 8 & 8 & 8 & 7 & 6 & 16 & 8 & 155 \\
\hline D-03-038 & 18 & 16 & 19 & 19 & 16 & 13 & 18 & 8 & 9 & 7 & 8 & 9 & 6 & 18 & 18 & 202 \\
\hline D-03-040 & 10 & 5 & 9 & 10 & 13 & 13 & 6 & 5 & 8 & 4 & 4 & 3 & 3 & 6 & 11 & 110 \\
\hline D-03-041 & 5 & 3 & 11 & 5 & 13 & 13 & 6 & 3 & 1 & 3 & 1 & 4 & 4 & 3 & 8 & 83 \\
\hline D-03-044 & 11 & 8 & 14 & 10 & 14 & 8 & 8 & 6 & 8 & 7 & 6 & 5 & 5 & 11 & 12 & 133 \\
\hline D-03-045 & 8 & 3 & 11 & 11 & 16 & 8 & 5 & 8 & 8 & 6 & 4 & 5 & 3 & 13 & 11 & 120 \\
\hline D-03-047 & 12 & 5 & 13 & 8 & 16 & 8 & 5 & 8 & 8 & 5 & 5 & 5 & 5 & 13 & 13 & 129 \\
\hline D-03-049 & 6 & 6 & 9 & 7 & 13 & 6 & 8 & 8 & 8 & 6 & 6 & 5 & 5 & 10 & 11 & 114 \\
\hline D-03-054 & 3 & 3 & 1 & 8 & 6 & 6 & 3 & 1 & 2 & 1 & 2 & 2 & 2 & 6 & 3 & 49 \\
\hline D-03-057 & 10 & 8 & 12 & 13 & 7 & 16 & 8 & 9 & 8 & 9 & 7 & 8 & 5 & 16 & 13 & 149 \\
\hline D-03-058 & 11 & 11 & 16 & 8 & 10 & 8 & 11 & 4 & 4 & 4 & 4 & 4 & 4 & 11 & 11 & 121 \\
\hline D-03-059 & 10 & 10 & 14 & 5 & 10 & 8 & 11 & 7 & 8 & 4 & 5 & 5 & 5 & 11 & 11 & 124 \\
\hline D-03-060 & 13 & 6 & 9 & 8 & 8 & 15 & 13 & 9 & 9 & 8 & 8 & 5 & 5 & 14 & 8 & 138 \\
\hline D-03-061 & 13 & 13 & 16 & 10 & 17 & 13 & 16 & 7 & 8 & 7 & 7 & 6 & 6 & 16 & 16 & 171 \\
\hline D-03-062 & 18 & 16 & 16 & 15 & 16 & 17 & 16 & 9 & 9 & 9 & 9 & 7 & 7 & 13 & 16 & 193 \\
\hline D-03-064 & 15 & 13 & 13 & 13 & 13 & 13 & 15 & 8 & 8 & 6 & 6 & 5 & 5 & 16 & 13 & 162 \\
\hline
\end{tabular}


Appendix 3: Table showing the twelve individual habitat parameter scores adapted from Barbour et al. (1999) that were assessed at most sites where fishing effort was applied and were totalled to derive the Habitat Assessment Score for each site.

\begin{tabular}{|c|c|c|c|c|c|c|c|c|c|c|c|c|c|c|c|c|}
\hline \multirow{2}{*}{ Site Number } & \multirow{2}{*}{ 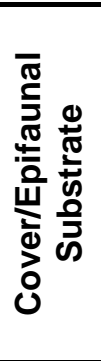 } & \multirow{2}{*}{ 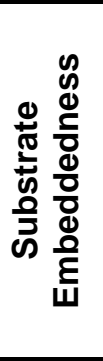 } & \multirow{2}{*}{ 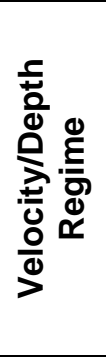 } & \multirow{2}{*}{ 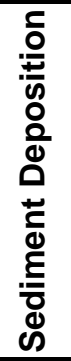 } & \multirow{2}{*}{ 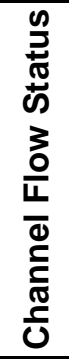 } & \multirow{2}{*}{ 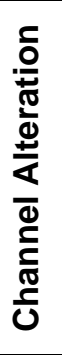 } & \multirow{2}{*}{ 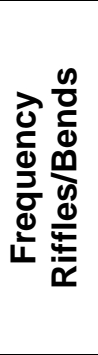 } & \multicolumn{2}{|c|}{ 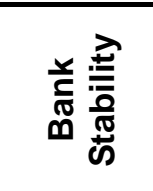 } & \multicolumn{2}{|c|}{ 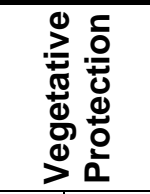 } & \multicolumn{2}{|c|}{ 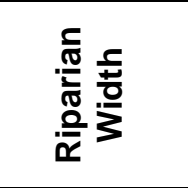 } & \multirow{2}{*}{ 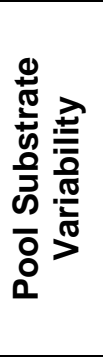 } & \multirow{2}{*}{ 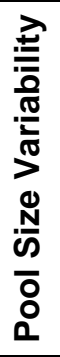 } & \multirow{2}{*}{ 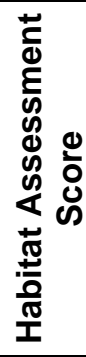 } \\
\hline & & & & & & & & ప্త & $\frac{\text { 동 }}{\frac{0}{x}}$ & ప্త & $\frac{\text { 픙 }}{\frac{9}{x}}$ & 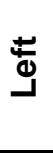 & $\frac{\tilde{E}}{\frac{\pi}{x}}$ & & & \\
\hline D-03-065 & 10 & 8 & 14 & 8 & 11 & 8 & 8 & 8 & 8 & 5 & 5 & 4 & 5 & 13 & 13 & 128 \\
\hline D-03-066 & 18 & 13 & 16 & 13 & 13 & 18 & 14 & 9 & 9 & 9 & 9 & 9 & 9 & 15 & 16 & 190 \\
\hline D-03-067 & 16 & 14 & 17 & 8 & 15 & 16 & 16 & 9 & 9 & 9 & 9 & 9 & 7 & 16 & 17 & 187 \\
\hline D-03-071 & 16 & 11 & 6 & 12 & 8 & 15 & 15 & 9 & 9 & 9 & 9 & 8 & 8 & 16 & 13 & 164 \\
\hline D-03-073 & 13 & 8 & 12 & 11 & 11 & 11 & 11 & 9 & 9 & 9 & 9 & 8 & 8 & 15 & 13 & 157 \\
\hline D-03-075 & 12 & 7 & 8 & 8 & 5 & 16 & 8 & 8 & 8 & 6 & 7 & 7 & 8 & 8 & 13 & 129 \\
\hline D-03-076 & 11 & 8 & 8 & 8 & 5 & 16 & 8 & 8 & 8 & 6 & 6 & 6 & 6 & 8 & 13 & 125 \\
\hline D-03-078 & 11 & 5 & 5 & 5 & 5 & 15 & 8 & 8 & 8 & 7 & 7 & 7 & 7 & 14 & 13 & 125 \\
\hline D-03-081 & 15 & 11 & 16 & 13 & 9 & 15 & 10 & 5 & 7 & 6 & 6 & 6 & 6 & 11 & 16 & 152 \\
\hline D-03-083 & 15 & 6 & 6 & 10 & 3 & 13 & 6 & 7 & 7 & 8 & 8 & 6 & 6 & 14 & 14 & 129 \\
\hline D-03-084 & 3 & 3 & 11 & 6 & 5 & 16 & 15 & 9 & 9 & 6 & 6 & 8 & 8 & 11 & 16 & 132 \\
\hline D-03-085 & 13 & 13 & 16 & 13 & 11 & 15 & 15 & 8 & 8 & 8 & 8 & 7 & 7 & 11 & 16 & 169 \\
\hline D-03-086 & 11 & 8 & 12 & 8 & 6 & 15 & 11 & 6 & 6 & 9 & 9 & 7 & 7 & 13 & 16 & 144 \\
\hline D-03-088 & 18 & 18 & 18 & 16 & 18 & 15 & 16 & 9 & 8 & 9 & 8 & 8 & 6 & 18 & 18 & 203 \\
\hline D-03-089 & 16 & 13 & 17 & 12 & 15 & 16 & 14 & 8 & 7 & 7 & 6 & 7 & 7 & 16 & 17 & 178 \\
\hline D-03-091 & 16 & 8 & 16 & 11 & 10 & 13 & 11 & 9 & 9 & 8 & 8 & 7 & 7 & 16 & 16 & 165 \\
\hline D-03-094 & 11 & 3 & 2 & 8 & 3 & 13 & 8 & 9 & 9 & 9 & 9 & 7 & 6 & 11 & 12 & 120 \\
\hline D-03-095 & 16 & 3 & 3 & 9 & 3 & 13 & 6 & 9 & 9 & 9 & 9 & 7 & 6 & 13 & 14 & 129 \\
\hline D-03-096 & 11 & 3 & 11 & 7 & 8 & 11 & 7 & 6 & 6 & 7 & 7 & 6 & 6 & 14 & 16 & 126 \\
\hline D-03-097 & 10 & 10 & 17 & 10 & 13 & 12 & 15 & 8 & 6 & 9 & 9 & 7 & 7 & 16 & 17 & 166 \\
\hline D-03-098 & 10 & 5 & 8 & 6 & 5 & 13 & 7 & 6 & 6 & 5 & 5 & 5 & 5 & 11 & 13 & 110 \\
\hline D-03-100 & 11 & 5 & 6 & 8 & 5 & 13 & 6 & 8 & 8 & 8 & 8 & 8 & 8 & 11 & 11 & 124 \\
\hline D-03-101 & 13 & 8 & 15 & 14 & 8 & 13 & 13 & 8 & 9 & 9 & 9 & 9 & 9 & 16 & 11 & 164 \\
\hline D-03-102 & 11 & 5 & 11 & 8 & 5 & 13 & 10 & 9 & 9 & 7 & 6 & 6 & 3 & 13 & 13 & 129 \\
\hline D-03-103 & 10 & 11 & 11 & 10 & 6 & 10 & 10 & 9 & 9 & 7 & 5 & 6 & 3 & 10 & 10 & 127 \\
\hline
\end{tabular}


Appendix 3: Table showing the twelve individual habitat parameter scores adapted from Barbour et al. (1999) that were assessed at most sites where fishing effort was applied and were totalled to derive the Habitat Assessment Score for each site.

\begin{tabular}{|c|c|c|c|c|c|c|c|c|c|c|c|c|c|c|c|c|}
\hline \multirow{2}{*}{ Site Number } & \multirow{2}{*}{ 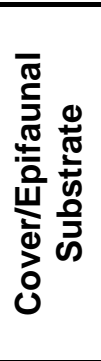 } & \multirow{2}{*}{ 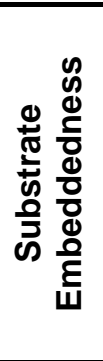 } & \multirow{2}{*}{ 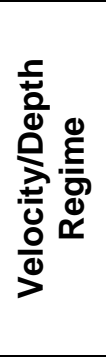 } & \multirow{2}{*}{ 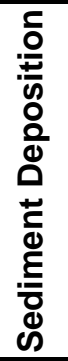 } & \multirow{2}{*}{ 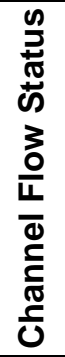 } & \multirow{2}{*}{ 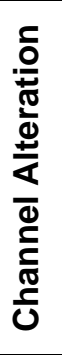 } & \multirow{2}{*}{ 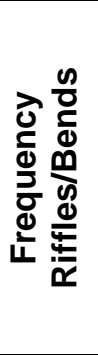 } & \multicolumn{2}{|c|}{ 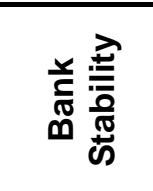 } & \multicolumn{2}{|c|}{ 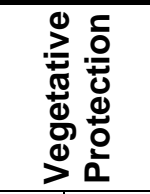 } & \multicolumn{2}{|c|}{ 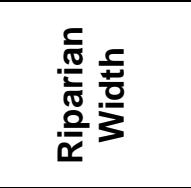 } & \multirow{2}{*}{ 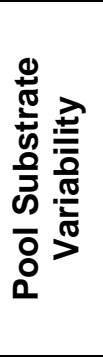 } & \multirow{2}{*}{ 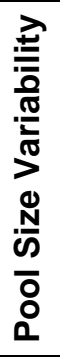 } & \multirow{2}{*}{ 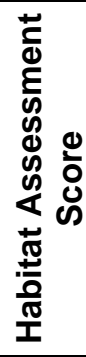 } \\
\hline & & & & & & & & ప্త & $\frac{\text { 동 }}{\frac{0}{x}}$ & ప্త & $\frac{\text { 픔 }}{\frac{0}{x}}$ & 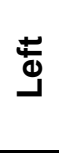 & $\frac{\tilde{E}}{\frac{\pi}{x}}$ & & & \\
\hline D-03-110 & 11 & 2 & 6 & 6 & 5 & 14 & 5 & 6 & 6 & 8 & 8 & 8 & 8 & 13 & 13 & 119 \\
\hline D-03-111 & 11 & 3 & 6 & 6 & 6 & 13 & 7 & 6 & 5 & 9 & 9 & 9 & 9 & 8 & 9 & 116 \\
\hline D-03-117 & 12 & 7 & 16 & 10 & 6 & 15 & 13 & 7 & 8 & 7 & 8 & 7 & 7 & 13 & 15 & 151 \\
\hline D-03-121 & 16 & 11 & 17 & 8 & 12 & 11 & 16 & 9 & 9 & 9 & 9 & 7 & 7 & 15 & 10 & 166 \\
\hline D-03-122 & 11 & 8 & 13 & 8 & 10 & 15 & 14 & 9 & 6 & 9 & 8 & 9 & 6 & 8 & 8 & 142 \\
\hline D-03-124 & 10 & 5 & 11 & 6 & 7 & 10 & 5 & 5 & 5 & 5 & 5 & 5 & 5 & 5 & 8 & 97 \\
\hline D-03-126 & 8 & 5 & 6 & 5 & 5 & 8 & 5 & 8 & 8 & 5 & 5 & 5 & 5 & 7 & 8 & 93 \\
\hline D-03-132 & 10 & 6 & 11 & 8 & 10 & 13 & 11 & 8 & 6 & 8 & 8 & 8 & 8 & 15 & 8 & 138 \\
\hline D-03-134 & 10 & 5 & 7 & 5 & 6 & 10 & 5 & 6 & 7 & 6 & 6 & 5 & 5 & 8 & 10 & 101 \\
\hline D-03-138 & 8 & 5 & 5 & 5 & 6 & 7 & 5 & 8 & 8 & 5 & 8 & 7 & 5 & 11 & 13 & 106 \\
\hline D-03-142 & 17 & 13 & 16 & 13 & 17 & 18 & 17 & 9 & 9 & 9 & 9 & 10 & 10 & 13 & 17 & 197 \\
\hline D-03-143 & 17 & 6 & 16 & 8 & 15 & 14 & 14 & 7 & 8 & 9 & 9 & 8 & 8 & 13 & 9 & 161 \\
\hline D-03-144 & 11 & 14 & 13 & 13 & 7 & 15 & 12 & 9 & 9 & 9 & 9 & 9 & 9 & 16 & 6 & 161 \\
\hline D-03-145 & 18 & 7 & 13 & 7 & 9 & 15 & 17 & 8 & 9 & 9 & 9 & 10 & 10 & 13 & 9 & 163 \\
\hline D-03-152 & 17 & 13 & 12 & 12 & 14 & 13 & 10 & 9 & 9 & 9 & 7 & 9 & 5 & 17 & 13 & 169 \\
\hline D-03-154 & 12 & 16 & 14 & 13 & 13 & 14 & 16 & 5 & 8 & 9 & 9 & 9 & 9 & 7 & 4 & 158 \\
\hline D-03-155 & 11 & 7 & 6 & 7 & 14 & 12 & 5 & 9 & 9 & 7 & 7 & 6 & 6 & 13 & 8 & 127 \\
\hline D-03-156 & 11 & 14 & 10 & 14 & 6 & 13 & 11 & 9 & 9 & 7 & 7 & 6 & 6 & 16 & 3 & 142 \\
\hline D-03-164 & 13 & 10 & 14 & 9 & 7 & 10 & 11 & 7 & 7 & 7 & 7 & 8 & 8 & 11 & 10 & 139 \\
\hline D-03-167 & 11 & 11 & 12 & 8 & 8 & 15 & 15 & 8 & 8 & 9 & 9 & 9 & 9 & 16 & 9 & 157 \\
\hline D-03-168 & 11 & 5 & 18 & 2 & 15 & 10 & 11 & 8 & 8 & 9 & 9 & 7 & 7 & 11 & 13 & 144 \\
\hline D-03-169 & 16 & 5 & 6 & 16 & 16 & 16 & 13 & 9 & 9 & 9 & 9 & 9 & 9 & 13 & 13 & 168 \\
\hline D-03-170 & 18 & 8 & 18 & 13 & 18 & 13 & 15 & 9 & 9 & 8 & 8 & 8 & 8 & 16 & 16 & 185 \\
\hline W-03-001 & 7 & 3 & 4 & 6 & 15 & 8 & 7 & 7 & 8 & 4 & 4 & 2 & 2 & 8 & 3 & 88 \\
\hline W-03-002 & 7 & 6 & 8 & 6 & 15 & 6 & 6 & 6 & 5 & 4 & 3 & 2 & 1 & 8 & 3 & 86 \\
\hline
\end{tabular}


Appendix 3: Table showing the twelve individual habitat parameter scores adapted from Barbour et al. (1999) that were assessed at most sites where fishing effort was applied and were totalled to derive the Habitat Assessment Score for each site.

\begin{tabular}{|c|c|c|c|c|c|c|c|c|c|c|c|c|c|c|c|c|}
\hline \multirow{2}{*}{ Site Number } & \multirow{2}{*}{ 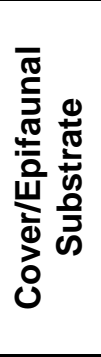 } & \multirow{2}{*}{ 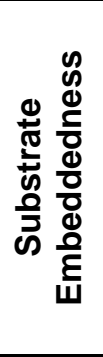 } & \multirow{2}{*}{ 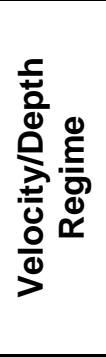 } & \multirow{2}{*}{ 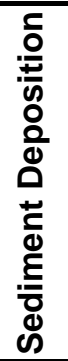 } & \multirow{2}{*}{ 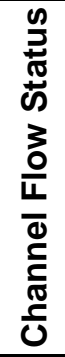 } & \multirow{2}{*}{ 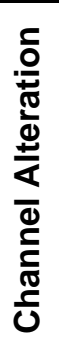 } & \multirow{2}{*}{ 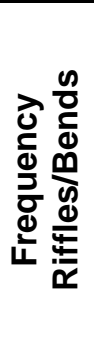 } & \multicolumn{2}{|c|}{ 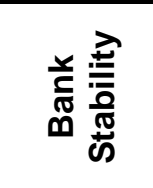 } & \multicolumn{2}{|c|}{ 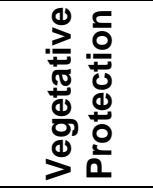 } & \multicolumn{2}{|c|}{$\begin{array}{l}\frac{c}{\sqrt{0}} \\
\frac{2}{\frac{0}{\pi}} \\
\frac{0}{x}\end{array}$} & \multirow{2}{*}{ 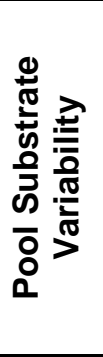 } & \multirow{2}{*}{ 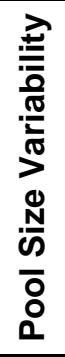 } & \multirow{2}{*}{ 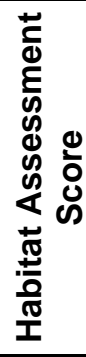 } \\
\hline & & & & & & & & 屯্త & $\begin{array}{l}\frac{}{\frac{1}{\sigma}} \\
\frac{0}{x}\end{array}$ & Ф્త & $\frac{\text { 픔 }}{\frac{0}{\tilde{x}}}$ & 苂 & $\frac{\tilde{n}}{\frac{\tilde{\sigma}}{\boldsymbol{x}}}$ & & & \\
\hline W-03-003 & 8 & 3 & 7 & 5 & 8 & 13 & 8 & 9 & 9 & 9 & 7 & 7 & 3 & 7 & 13 & 116 \\
\hline W-03-004 & 6 & 5 & 8 & 8 & 16 & 11 & 8 & 8 & 8 & 7 & 7 & 6 & 6 & 13 & 13 & 130 \\
\hline W-03-008 & 5 & 8 & 2 & 5 & 5 & 8 & 3 & 5 & 9 & 6 & 6 & 4 & 4 & 13 & 3 & 86 \\
\hline W-03-009 & 5 & 3 & 11 & 7 & 11 & 15 & 11 & 5 & 4 & 4 & 4 & 8 & 8 & 6 & 6 & 108 \\
\hline W-03-010 & 12 & 6 & 12 & 6 & 10 & 8 & 2 & 5 & 6 & 3 & 4 & 1 & 1 & 13 & 9 & 98 \\
\hline W-03-013 & 10 & 10 & 13 & 10 & 11 & 7 & 10 & 4 & 4 & 8 & 6 & 6 & 5 & 11 & 8 & 123 \\
\hline W-03-019 & 9 & 2 & 8 & 2 & 6 & 8 & 3 & 9 & 9 & 5 & 5 & 6 & 6 & 13 & 6 & 97 \\
\hline W-03-020 & 8 & 5 & 3 & 12 & 5 & 1 & 2 & 6 & 5 & 5 & 3 & 3 & 3 & 11 & 2 & 74 \\
\hline W-03-026 & 2 & 2 & 2 & 4 & 2 & 5 & 6 & 7 & 7 & 5 & 5 & 2 & 5 & 9 & 2 & 65 \\
\hline W-03-030 & 11 & 8 & 4 & 8 & 5 & 6 & 2 & 9 & 7 & 5 & 4 & 6 & 6 & 12 & 3 & 96 \\
\hline W-03-035 & 7 & 6 & 3 & 7 & 6 & 8 & 8 & 7 & 7 & 5 & 5 & 3 & 3 & 12 & 4 & 91 \\
\hline W-03-036 & 5 & 5 & 3 & 3 & 6 & 5 & 4 & 6 & 5 & 4 & 4 & 6 & 6 & 6 & 4 & 72 \\
\hline W-03-044 & 15 & 5 & 3 & 8 & 5 & 12 & 8 & 4 & 3 & 7 & 7 & 5 & 4 & 6 & 2 & 94 \\
\hline W-03-046 & 18 & 11 & 5 & 11 & 16 & 13 & 10 & 9 & 9 & 9 & 9 & 9 & 9 & 15 & 8 & 161 \\
\hline W-03-049 & 11 & 11 & 5 & 11 & 16 & 11 & 4 & 9 & 9 & 5 & 7 & 5 & 8 & 14 & 14 & 140 \\
\hline W-03-050 & 19 & 14 & 15 & 14 & 15 & 15 & 13 & 4 & 4 & 8 & 8 & 8 & 8 & 15 & 14 & 174 \\
\hline W-03-055 & 10 & 2 & 5 & 8 & 13 & 6 & 1 & 9 & 9 & 6 & 6 & 3 & 3 & 13 & 13 & 107 \\
\hline W-03-064 & 19 & 3 & 5 & 4 & 10 & 15 & 10 & 8 & 8 & 8 & 8 & 8 & 8 & 12 & 15 & 141 \\
\hline W-03-065 & 13 & 6 & 5 & 5 & 15 & 14 & 8 & 8 & 8 & 7 & 7 & 5 & 5 & 13 & 13 & 132 \\
\hline W-03-066 & 17 & 16 & 13 & 16 & 15 & 15 & 12 & 9 & 9 & 9 & 9 & 9 & 9 & 16 & 10 & 184 \\
\hline W-03-067 & 8 & 10 & 3 & 16 & 16 & 12 & 10 & 9 & 9 & 6 & 6 & 6 & 6 & 13 & 1 & 131 \\
\hline W-03-069 & 15 & 9 & 5 & 9 & 15 & 14 & 10 & 8 & 7 & 8 & 8 & 8 & 8 & 15 & 15 & 154 \\
\hline W-03-070 & 11 & 11 & 15 & 5 & 6 & 11 & 10 & 4 & 4 & 5 & 5 & 7 & 7 & 13 & 11 & 125 \\
\hline W-03-072 & 13 & 13 & 13 & 10 & 6 & 6 & 3 & 6 & 6 & 6 & 6 & 4 & 4 & 13 & 9 & 118 \\
\hline W-03-077 & 15 & 14 & 10 & 13 & 11 & 11 & 8 & 6 & 7 & 8 & 8 & 8 & 8 & 13 & 13 & 153 \\
\hline
\end{tabular}


Appendix 3: Table showing the twelve individual habitat parameter scores adapted from Barbour et al. (1999) that were assessed at most sites where fishing effort was applied and were totalled to derive the Habitat Assessment Score for each site.

\begin{tabular}{|c|c|c|c|c|c|c|c|c|c|c|c|c|c|c|c|c|}
\hline \multirow{2}{*}{ Site Number } & \multirow{2}{*}{ 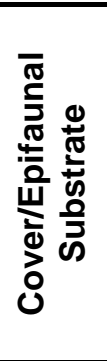 } & \multirow{2}{*}{ 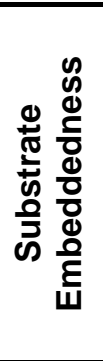 } & \multirow{2}{*}{ 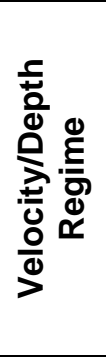 } & \multirow{2}{*}{ 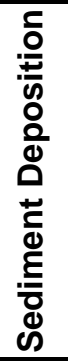 } & \multirow{2}{*}{ 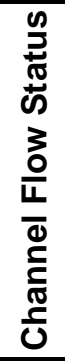 } & \multirow{2}{*}{ 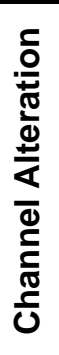 } & \multirow{2}{*}{ 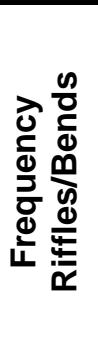 } & \multicolumn{2}{|c|}{ 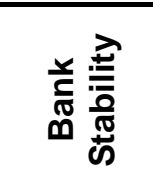 } & \multicolumn{2}{|c|}{ 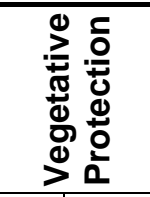 } & \multicolumn{2}{|c|}{ 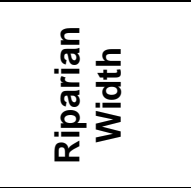 } & \multirow{2}{*}{ 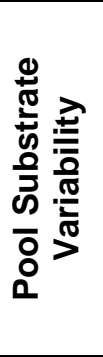 } & \multirow{2}{*}{ 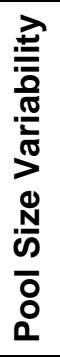 } & \multirow{2}{*}{ 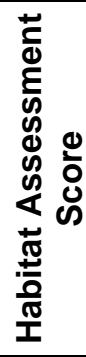 } \\
\hline & & & & & & & & ప্త & $\frac{\text { 픔 }}{\frac{0}{x}}$ & 屯্త & 총 & 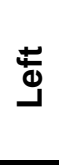 & $\frac{\tilde{E}}{\frac{\pi}{x}}$ & & & \\
\hline W-03-079 & 16 & 7 & 19 & 8 & 14 & 14 & 18 & 4 & 7 & 8 & 8 & 8 & 9 & 16 & 13 & 169 \\
\hline W-03-080 & 11 & 10 & 14 & 5 & 13 & 11 & 8 & 8 & 8 & 7 & 7 & 7 & 5 & 11 & 10 & 135 \\
\hline W-03-084 & 8 & 16 & 2 & 16 & 16 & 10 & 8 & 9 & 9 & 6 & 6 & 2 & 2 & 11 & 3 & 124 \\
\hline W-03-087 & 14 & 16 & 5 & 10 & 15 & 14 & 8 & 8 & 8 & 7 & 7 & 9 & 9 & 17 & 10 & 157 \\
\hline W-03-088 & 13 & 5 & 7 & 8 & 13 & 11 & 8 & 8 & 8 & 7 & 7 & 4 & 4 & 9 & 8 & 120 \\
\hline W-03-091 & 8 & 5 & 8 & 7 & 4 & 12 & 10 & 6 & 6 & 3 & 3 & 6 & 6 & 11 & 5 & 100 \\
\hline W-03-092 & 17 & 10 & 16 & 9 & 11 & 14 & 14 & 8 & 6 & 6 & 6 & 10 & 10 & 16 & 11 & 164 \\
\hline W-03-093 & 15 & 15 & 15 & 16 & 16 & 10 & 5 & 9 & 9 & 8 & 8 & 9 & 9 & 16 & 10 & 170 \\
\hline W-03-094 & 18 & 18 & 8 & 18 & 19 & 15 & 8 & 9 & 9 & 9 & 9 & 10 & 10 & 18 & 15 & 193 \\
\hline W-03-095 & 18 & 18 & 14 & 18 & 16 & 15 & 13 & 9 & 9 & 9 & 9 & 10 & 10 & 18 & 13 & 199 \\
\hline W-03-096 & 13 & 10 & 1 & 5 & 12 & 10 & 5 & 7 & 7 & 8 & 8 & 9 & 8 & 13 & 15 & 131 \\
\hline W-03-097 & 19 & 16 & 12 & 13 & 13 & 15 & 15 & 9 & 9 & 9 & 9 & 10 & 10 & 16 & 14 & 189 \\
\hline W-03-098 & 15 & 13 & 8 & 15 & 15 & 15 & 7 & 8 & 8 & 9 & 9 & 10 & 10 & 15 & 15 & 172 \\
\hline W-03-100 & 13 & 5 & 5 & 5 & 11 & 15 & 7 & 6 & 6 & 3 & 9 & 2 & 8 & 13 & 12 & 120 \\
\hline W-03-101 & 16 & 15 & 15 & 15 & 16 & 15 & 15 & 9 & 9 & 9 & 9 & 8 & 8 & 16 & 10 & 185 \\
\hline W-03-104 & 15 & 16 & 8 & 13 & 18 & 6 & 12 & 9 & 9 & 6 & 6 & 4 & 4 & 13 & 5 & 144 \\
\hline W-03-107 & 18 & 9 & 18 & 10 & 11 & 14 & 13 & 6 & 6 & 8 & 9 & 8 & 8 & 13 & 11 & 162 \\
\hline W-03-110 & 11 & 2 & 8 & 5 & 13 & 13 & 11 & 6 & 5 & 8 & 6 & 8 & 6 & 11 & 5 & 118 \\
\hline W-03-111 & 12 & 1 & 1 & 2 & 13 & 17 & 6 & 8 & 8 & 7 & 7 & 9 & 9 & 7 & 1 & 108 \\
\hline W-03-112 & 17 & 16 & 8 & 9 & 13 & 15 & 15 & 1 & 1 & 8 & 8 & 6 & 6 & 8 & 2 & 133 \\
\hline W-03-113 & 11 & 5 & 5 & 5 & 16 & 10 & 5 & 8 & 8 & 7 & 7 & 8 & 8 & 15 & 5 & 123 \\
\hline W-03-114 & 16 & 13 & 6 & 7 & 16 & 13 & 10 & 9 & 9 & 9 & 9 & 7 & 9 & 13 & 5 & 151 \\
\hline W-03-141 & 9 & 5 & 3 & 10 & 11 & 10 & 1 & 8 & 8 & 8 & 8 & 7 & 7 & 10 & 3 & 108 \\
\hline W-03-142 & 11 & 16 & 2 & 15 & 15 & 11 & 1 & 7 & 6 & 6 & 6 & 5 & 5 & 18 & 1 & 125 \\
\hline W-03-143 & 10 & 5 & 1 & 8 & 11 & 8 & 2 & 7 & 7 & 6 & 6 & 4 & 4 & 11 & 3 & 93 \\
\hline
\end{tabular}


Appendix 3: Table showing the twelve individual habitat parameter scores adapted from Barbour et al. (1999) that were assessed at most sites where fishing effort was applied and were totalled to derive the Habitat Assessment Score for each site.

\begin{tabular}{|c|c|c|c|c|c|c|c|c|c|c|c|c|c|c|c|c|}
\hline \multirow{2}{*}{ Site Number } & \multirow{2}{*}{ 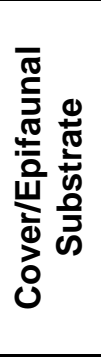 } & \multirow{2}{*}{ 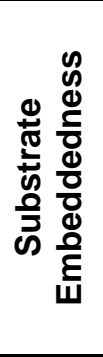 } & \multirow{2}{*}{ 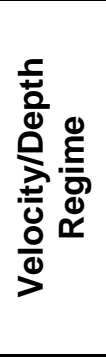 } & \multirow{2}{*}{ 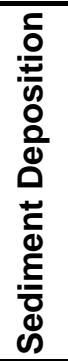 } & \multirow{2}{*}{ 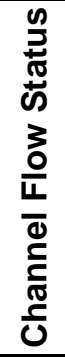 } & \multirow{2}{*}{ 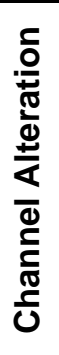 } & \multirow{2}{*}{ 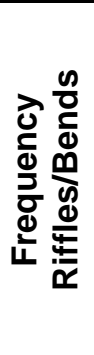 } & \multicolumn{2}{|c|}{ 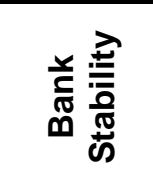 } & \multicolumn{2}{|c|}{ 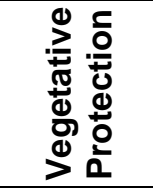 } & \multicolumn{2}{|c|}{$\begin{array}{l}\frac{c}{\sqrt{0}} \\
\frac{2}{\frac{0}{\pi}} \\
\frac{0}{x}\end{array}$} & \multirow{2}{*}{ 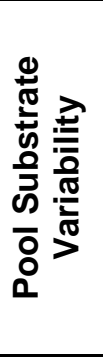 } & \multirow{2}{*}{ 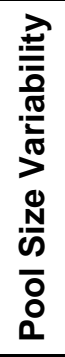 } & \multirow{2}{*}{ 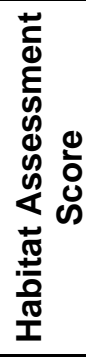 } \\
\hline & & & & & & & & 屯্త & $\begin{array}{l}\frac{}{\frac{1}{\sigma}} \\
\frac{0}{x}\end{array}$ & Ф્త & 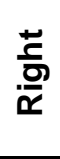 & 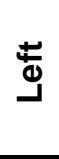 & $\frac{\tilde{n}}{\frac{\tilde{\sigma}}{\boldsymbol{x}}}$ & & & \\
\hline W-03-144 & 13 & 15 & 10 & 15 & 15 & 10 & 13 & 5 & 8 & 7 & 7 & 8 & 8 & 13 & 10 & 157 \\
\hline W-03-146 & 16 & 15 & 15 & 15 & 11 & 15 & 10 & 8 & 8 & 8 & 8 & 8 & 8 & 15 & 15 & 175 \\
\hline W-03-150 & 13 & 13 & 10 & 11 & 15 & 11 & 7 & 8 & 8 & 8 & 8 & 6 & 8 & 15 & 5 & 146 \\
\hline W-03-151 & 15 & 16 & 18 & 13 & 18 & 15 & 15 & 9 & 9 & 9 & 9 & 10 & 10 & 13 & 13 & 192 \\
\hline W-03-154 & 17 & 10 & 5 & 10 & 18 & 15 & 15 & 9 & 9 & 8 & 10 & 6 & 10 & 15 & 15 & 172 \\
\hline W-03-155 & 16 & 15 & 15 & 10 & 16 & 13 & 11 & 8 & 8 & 9 & 9 & 10 & 10 & 15 & 13 & 178 \\
\hline W-03-158 & 13 & 10 & 10 & 10 & 16 & 8 & 5 & 8 & 8 & 8 & 8 & 4 & 4 & 11 & 10 & 133 \\
\hline W-03-162 & 16 & 15 & 10 & 13 & 13 & 15 & 11 & 8 & 8 & 9 & 9 & 8 & 8 & 15 & 5 & 163 \\
\hline W-03-163 & 16 & 16 & 15 & 15 & 13 & 15 & 13 & 8 & 8 & 8 & 9 & 6 & 9 & 13 & 14 & 178 \\
\hline W-03-165 & 17 & 16 & 14 & 13 & 13 & 13 & 15 & 8 & 5 & 8 & 8 & 9 & 9 & 16 & 8 & 172 \\
\hline W-03-167 & 11 & 3 & 3 & 3 & 13 & 13 & 8 & 8 & 8 & 7 & 7 & 5 & 5 & 13 & 13 & 120 \\
\hline W-03-172 & 16 & 15 & 1 & 18 & 18 & 15 & 14 & 9 & 9 & 9 & 9 & 10 & 10 & 14 & 15 & 182 \\
\hline W-03-173 & 13 & 8 & 6 & 16 & 13 & 13 & 8 & 8 & 8 & 8 & 8 & 7 & 8 & 11 & 9 & 144 \\
\hline W-03-176 & 13 & 7 & 3 & 0 & 13 & 8 & 3 & 1 & 1 & 7 & 7 & 10 & 10 & 6 & 3 & 92 \\
\hline W-03-182 & 12 & 3 & 5 & 16 & 13 & 9 & 8 & 8 & 8 & 7 & 7 & 2 & 3 & 13 & 13 & 127 \\
\hline W-03-185 & 13 & 13 & 4 & 10 & 13 & 6 & 5 & 6 & 6 & 6 & 6 & 5 & 5 & 13 & 3 & 114 \\
\hline W-03-186 & 18 & 18 & 1 & 11 & 1 & 14 & 11 & 5 & 5 & 7 & 7 & 9 & 9 & 11 & 1 & 128 \\
\hline W-03-189 & 12 & 18 & 5 & 16 & 8 & 8 & 6 & 8 & 8 & 8 & 8 & 5 & 5 & 16 & 8 & 139 \\
\hline W-03-191 & 10 & 13 & 9 & 14 & 13 & 8 & 3 & 7 & 7 & 6 & 6 & 6 & 6 & 13 & 3 & 124 \\
\hline W-03-194 & 12 & 13 & 3 & 8 & 13 & 13 & 6 & 6 & 6 & 7 & 7 & 9 & 9 & 13 & 5 & 130 \\
\hline W-03-195 & 16 & 9 & 1 & 8 & 20 & 13 & 12 & 8 & 8 & 9 & 9 & 9 & 9 & 13 & 14 & 158 \\
\hline W-03-198 & 19 & 13 & 13 & 8 & 18 & 15 & 16 & 9 & 9 & 9 & 9 & 9 & 9 & 16 & 13 & 185 \\
\hline W-03-201 & 13 & 8 & 6 & 8 & 13 & 15 & 8 & 9 & 9 & 9 & 9 & 10 & 10 & 13 & 8 & 148 \\
\hline W-03-203 & 12 & 13 & 8 & 6 & 7 & 11 & 13 & 2 & 2 & 4 & 4 & 9 & 9 & 13 & 3 & 116 \\
\hline W-03-204 & 13 & 5 & 8 & 5 & 12 & 14 & 8 & 8 & 8 & 9 & 9 & 9 & 8 & 13 & 8 & 137 \\
\hline
\end{tabular}


Appendix 3: Table showing the twelve individual habitat parameter scores adapted from Barbour et al. (1999) that were assessed at most sites where fishing effort was applied and were totalled to derive the Habitat Assessment Score for each site.

\begin{tabular}{|c|c|c|c|c|c|c|c|c|c|c|c|c|c|c|c|c|}
\hline \multirow{2}{*}{ Site Number } & \multirow{2}{*}{ 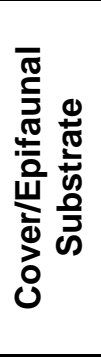 } & \multirow{2}{*}{ 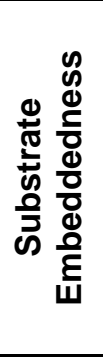 } & \multirow{2}{*}{ 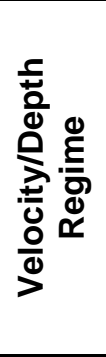 } & \multirow{2}{*}{ 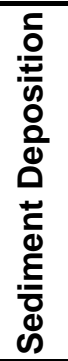 } & \multirow{2}{*}{ 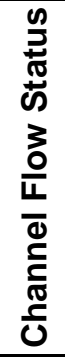 } & \multirow{2}{*}{ 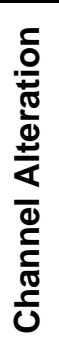 } & \multirow{2}{*}{ 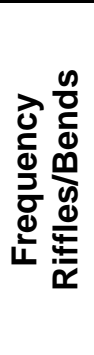 } & \multicolumn{2}{|c|}{ 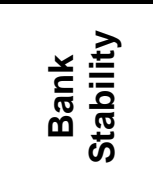 } & \multicolumn{2}{|c|}{ 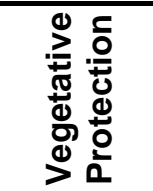 } & \multicolumn{2}{|c|}{ 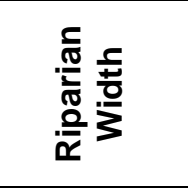 } & \multirow{2}{*}{ 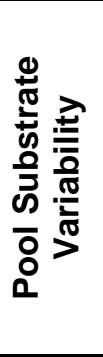 } & \multirow{2}{*}{ 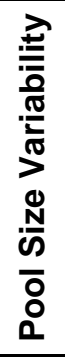 } & \multirow{2}{*}{ 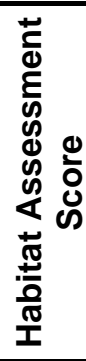 } \\
\hline & & & & & & & & 屯্త & $\begin{array}{l}\frac{}{\frac{1}{\sigma}} \\
\frac{0}{x}\end{array}$ & Ф્త & $\frac{\text { 픔 }}{\frac{0}{\tilde{x}}}$ & 苂 & $\frac{\tilde{n}}{\frac{\tilde{\sigma}}{\boldsymbol{x}}}$ & & & \\
\hline W-03-205 & 11 & 8 & 8 & 8 & 12 & 11 & 11 & 5 & 5 & 7 & 7 & 9 & 9 & 13 & 8 & 132 \\
\hline W-03-206 & 9 & 4 & 3 & 3 & 8 & 11 & 4 & 7 & 7 & 6 & 6 & 5 & 5 & 13 & 3 & 94 \\
\hline W-03-210 & 8 & 8 & 8 & 5 & 8 & 10 & 4 & 5 & 5 & 5 & 5 & 4 & 4 & 11 & 5 & 95 \\
\hline W-03-211 & 9 & 8 & 8 & 3 & 11 & 10 & 4 & 6 & 6 & 6 & 6 & 6 & 6 & 11 & 6 & 106 \\
\hline W-03-223 & 11 & 5 & 2 & 3 & 2 & 9 & 2 & 6 & 6 & 6 & 6 & 2 & 2 & 11 & 3 & 76 \\
\hline W-03-224 & 8 & 3 & 8 & 3 & 7 & 7 & 6 & 4 & 2 & 3 & 2 & 3 & 3 & 8 & 3 & 70 \\
\hline W-03-229 & 13 & 2 & 13 & 3 & 13 & 11 & 7 & 7 & 7 & 7 & 7 & 2 & 2 & 11 & 6 & 111 \\
\hline W-03-237 & 13 & 14 & 13 & 13 & 8 & 11 & 7 & 6 & 5 & 7 & 7 & 2 & 6 & 13 & 8 & 133 \\
\hline W-03-238 & 14 & 16 & 15 & 13 & 13 & 15 & 11 & 8 & 8 & 6 & 9 & 2 & 8 & 13 & 8 & 159 \\
\hline W-03-239 & 14 & 18 & 8 & 15 & 13 & 13 & 13 & 8 & 8 & 8 & 8 & 8 & 8 & 13 & 7 & 162 \\
\hline W-03-247 & 16 & 11 & 5 & 8 & 6 & 14 & 7 & 4 & 4 & 7 & 7 & 9 & 9 & 13 & 3 & 123 \\
\hline W-03-248 & 8 & 5 & 3 & 6 & 10 & 11 & 1 & 8 & 8 & 6 & 6 & 1 & 1 & 11 & 2 & 87 \\
\hline W-03-249 & 11 & 11 & 3 & 10 & 6 & 9 & 4 & 8 & 8 & 8 & 8 & 9 & 9 & 11 & 6 & 121 \\
\hline W-03-251 & 7 & 3 & 8 & 5 & 6 & 6 & 3 & 7 & 7 & 6 & 6 & 2 & 3 & 13 & 5 & 87 \\
\hline W-03-266 & 13 & 8 & 3 & 8 & 13 & 10 & 4 & 8 & 8 & 8 & 8 & 2 & 2 & 13 & 2 & 110 \\
\hline W-03-267 & 10 & 13 & 4 & 10 & 17 & 8 & 3 & 8 & 8 & 7 & 7 & 3 & 3 & 13 & 5 & 119 \\
\hline B-03-249 & 12 & 12 & 4 & 10 & 15 & 11 & 7 & 8 & 8 & 5 & 5 & 4 & 4 & 15 & 13 & 133 \\
\hline B-04-002 & 5 & 2 & 2 & 7 & 3 & 5 & 2 & 7 & 6 & 5 & 5 & 2 & 2 & 7 & 4 & 64 \\
\hline B-04-003 & 8 & 7 & 8 & 8 & 6 & 6 & 5 & 4 & 6 & 5 & 5 & 3 & 3 & 13 & 15 & 102 \\
\hline B-04-004 & 8 & 6 & 7 & 8 & 6 & 7 & 3 & 7 & 7 & 4 & 4 & 7 & 7 & 11 & 8 & 100 \\
\hline B-04-006 & 10 & 10 & 6 & 6 & 2 & 8 & 5 & 7 & 7 & 6 & 6 & 3 & 3 & 15 & 8 & 102 \\
\hline B-04-008 & 11 & 4 & 16 & 7 & 14 & 15 & 9 & 6 & 6 & 8 & 8 & 8 & 6 & 11 & 16 & 145 \\
\hline B-04-010 & 5 & 6 & 9 & 5 & 7 & 3 & 4 & 4 & 7 & 2 & 4 & 2 & 4 & 7 & 8 & 77 \\
\hline B-04-011 & 9 & 7 & 6 & 6 & 5 & 9 & 4 & 3 & 3 & 3 & 3 & 5 & 3 & 13 & 6 & 85 \\
\hline B-04-017 & 15 & 12 & 10 & 9 & 9 & 13 & 8 & 4 & 4 & 8 & 8 & 9 & 9 & 9 & 13 & 140 \\
\hline
\end{tabular}


Appendix 3: Table showing the twelve individual habitat parameter scores adapted from Barbour et al. (1999) that were assessed at most sites where fishing effort was applied and were totalled to derive the Habitat Assessment Score for each site.

\begin{tabular}{|c|c|c|c|c|c|c|c|c|c|c|c|c|c|c|c|c|}
\hline \multirow{2}{*}{ Site Number } & \multirow{2}{*}{ 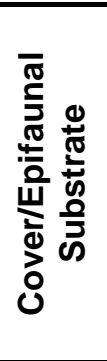 } & \multirow{2}{*}{ 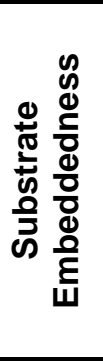 } & \multirow{2}{*}{ 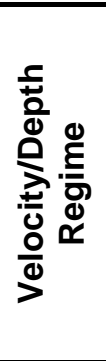 } & \multirow{2}{*}{ 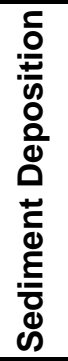 } & \multirow{2}{*}{ 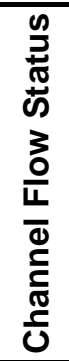 } & \multirow{2}{*}{ 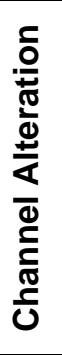 } & \multirow{2}{*}{ 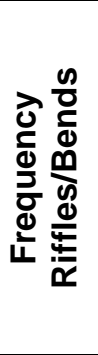 } & \multicolumn{2}{|c|}{ 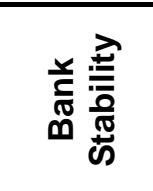 } & \multicolumn{2}{|c|}{ 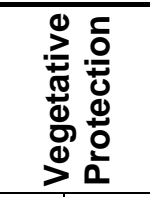 } & \multicolumn{2}{|c|}{ 迹 } & \multirow{2}{*}{ 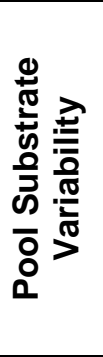 } & \multirow{2}{*}{ 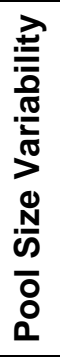 } & \multirow{2}{*}{ 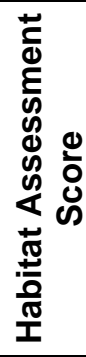 } \\
\hline & & & & & & & & ప্త & $\frac{\text { 픔 }}{\frac{0}{x}}$ & ప্త & 총 & Ф્త & $\frac{\tilde{E}}{\frac{\pi}{x}}$ & & & \\
\hline B-04-018 & 10 & 6 & 2 & 6 & 11 & 16 & 2 & 8 & 7 & 8 & 8 & 6 & 6 & 12 & 12 & 120 \\
\hline B-04-019 & 8 & 6 & 12 & 7 & 12 & 5 & 2 & 3 & 5 & 5 & 5 & 2 & 2 & 6 & 16 & 96 \\
\hline B-04-020 & 5 & 5 & 6 & 8 & 5 & 8 & 5 & 4 & 6 & 5 & 5 & 2 & 2 & 5 & 5 & 76 \\
\hline B-04-021 & 7 & 6 & 4 & 4 & 5 & 5 & 2 & 4 & 4 & 2 & 2 & 2 & 2 & 11 & 4 & 64 \\
\hline $\mathrm{B}-04-022$ & 9 & 11 & 4 & 6 & 8 & 10 & 9 & 7 & 7 & 6 & 6 & 4 & 4 & 13 & 10 & 114 \\
\hline B-04-026 & 7 & 6 & 11 & 8 & 9 & 13 & 9 & 4 & 5 & 6 & 7 & 6 & 6 & 14 & 14 & 125 \\
\hline B-04-027 & 6 & 2 & 11 & 3 & 9 & 15 & 10 & 2 & 3 & 5 & 8 & 8 & 8 & 11 & 16 & 117 \\
\hline B-04-028 & 4 & 5 & 13 & 14 & 12 & 5 & 6 & 5 & 5 & 2 & 2 & 4 & 4 & 6 & 16 & 103 \\
\hline B-04-030 & 13 & 3 & 13 & 6 & 14 & 10 & 6 & 6 & 8 & 8 & 8 & 3 & 3 & 12 & 10 & 123 \\
\hline B-04-031 & 10 & 10 & 6 & 11 & 8 & 11 & 6 & 2 & 2 & 6 & 6 & 7 & 6 & 11 & 13 & 115 \\
\hline B-04-037 & 15 & 11 & 14 & 13 & 8 & 16 & 11 & 7 & 7 & 7 & 7 & 6 & 6 & 13 & 16 & 157 \\
\hline B-04-041 & 8 & 11 & 6 & 11 & 12 & 11 & 4 & 7 & 7 & 5 & 6 & 3 & 5 & 8 & 13 & 117 \\
\hline B-04-043 & 11 & 11 & 2 & 8 & 13 & 10 & 5 & 5 & 5 & 5 & 5 & 4 & 5 & 16 & 11 & 116 \\
\hline B-04-044 & 13 & 6 & 11 & 7 & 13 & 16 & 8 & 6 & 6 & 8 & 7 & 8 & 7 & 13 & 16 & 145 \\
\hline B-04-046 & 10 & 10 & 9 & 11 & 6 & 13 & 7 & 4 & 3 & 4 & 3 & 9 & 9 & 8 & 8 & 114 \\
\hline B-04-047 & 16 & 16 & 20 & 11 & 11 & 19 & 19 & 5 & 5 & 3 & 3 & 5 & 5 & 16 & 18 & 172 \\
\hline B-04-048 & 6 & 5 & 6 & 5 & 8 & 15 & 11 & 5 & 6 & 6 & 6 & 5 & 6 & 7 & 8 & 105 \\
\hline B-04-055 & 11 & 11 & 16 & 11 & 11 & 13 & 16 & 8 & 8 & 6 & 7 & 6 & 9 & 16 & 16 & 165 \\
\hline B-04-058 & 16 & 13 & 13 & 13 & 8 & 15 & 9 & 8 & 8 & 9 & 9 & 6 & 7 & 16 & 16 & 166 \\
\hline B-04-060 & 11 & 6 & 9 & 7 & 6 & 10 & 4 & 8 & 5 & 7 & 5 & 5 & 5 & 9 & 8 & 105 \\
\hline B-04-064 & 17 & 12 & 16 & 11 & 11 & 16 & 6 & 3 & 3 & 9 & 7 & 9 & 7 & 15 & 11 & 153 \\
\hline B-04-066 & 7 & 8 & 3 & 10 & 6 & 7 & 2 & 7 & 7 & 6 & 4 & 6 & 4 & 12 & 11 & 100 \\
\hline B-04-070 & 7 & 8 & 11 & 15 & 8 & 11 & 8 & 8 & 7 & 8 & 5 & 2 & 5 & 13 & 10 & 126 \\
\hline B-04-078 & 11 & 10 & 8 & 8 & 8 & 13 & 3 & 5 & 5 & 6 & 5 & 6 & 4 & 11 & 6 & 109 \\
\hline B-04-086 & 8 & 11 & 6 & 8 & 3 & 8 & 3 & 6 & 4 & 8 & 3 & 5 & 2 & 11 & 13 & 99 \\
\hline
\end{tabular}


Appendix 3: Table showing the twelve individual habitat parameter scores adapted from Barbour et al. (1999) that were assessed at most sites where fishing effort was applied and were totalled to derive the Habitat Assessment Score for each site.

\begin{tabular}{|c|c|c|c|c|c|c|c|c|c|c|c|c|c|c|c|c|}
\hline \multirow{2}{*}{ Site Number } & \multirow{2}{*}{ 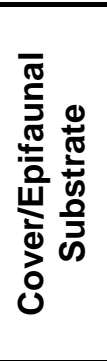 } & \multirow{2}{*}{ 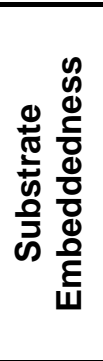 } & \multirow{2}{*}{ 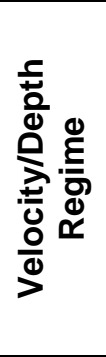 } & \multirow{2}{*}{ 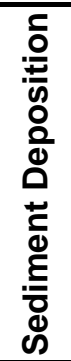 } & \multirow{2}{*}{ 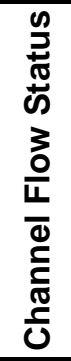 } & \multirow{2}{*}{ 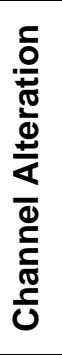 } & \multirow{2}{*}{ 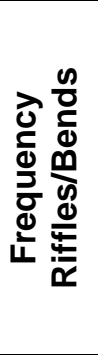 } & \multicolumn{2}{|c|}{ 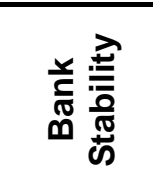 } & \multicolumn{2}{|c|}{ 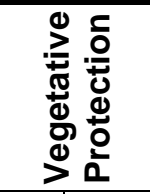 } & \multicolumn{2}{|c|}{ 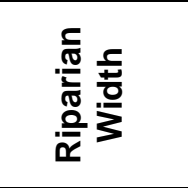 } & \multirow{2}{*}{ 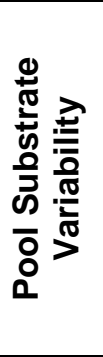 } & \multirow{2}{*}{ 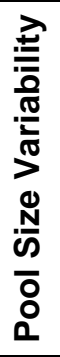 } & \multirow{2}{*}{ 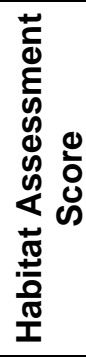 } \\
\hline & & & & & & & & ప্త & $\frac{\text { 동 }}{\frac{0}{x}}$ & 屯্త & $\frac{\text { 픔 }}{\frac{0}{x}}$ & 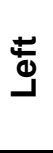 & $\frac{\tilde{E}}{\frac{\pi}{x}}$ & & & \\
\hline B-04-093 & 8 & 12 & 11 & 11 & 13 & 16 & 10 & 3 & 4 & 2 & 2 & 2 & 2 & 13 & 16 & 125 \\
\hline B-04-094 & 10 & 9 & 16 & 9 & 16 & 13 & 8 & 8 & 8 & 7 & 6 & 7 & 6 & 13 & 16 & 152 \\
\hline B-04-095 & 16 & 11 & 16 & 11 & 16 & 16 & 16 & 6 & 8 & 7 & 7 & 7 & 7 & 16 & 16 & 176 \\
\hline B-04-101 & 11 & 11 & 5 & 15 & 19 & 10 & 6 & 8 & 8 & 5 & 5 & 4 & 4 & 13 & 13 & 137 \\
\hline B-04-102 & 11 & 7 & 12 & 9 & 11 & 16 & 12 & 6 & 8 & 4 & 4 & 5 & 5 & 12 & 16 & 138 \\
\hline B-04-105 & 9 & 8 & 11 & 8 & 8 & 8 & 7 & 5 & 5 & 5 & 5 & 2 & 2 & 11 & 8 & 102 \\
\hline B-04-108 & 12 & 10 & 13 & 10 & 16 & 15 & 7 & 8 & 8 & 6 & 6 & 5 & 6 & 11 & 13 & 146 \\
\hline B-04-109 & 4 & 11 & 8 & 10 & 6 & 12 & 4 & 6 & 6 & 2 & 2 & 4 & 4 & 13 & 10 & 102 \\
\hline B-04-110 & 10 & 11 & 11 & 11 & 13 & 8 & 8 & 4 & 4 & 3 & 3 & 2 & 2 & 16 & 16 & 122 \\
\hline B-04-113 & 17 & 13 & 13 & 12 & 13 & 16 & 10 & 7 & 7 & 7 & 7 & 7 & 7 & 17 & 17 & 170 \\
\hline B-04-114 & 15 & 11 & 11 & 8 & 8 & 10 & 11 & 3 & 3 & 8 & 6 & 5 & 5 & 13 & 11 & 128 \\
\hline B-04-116 & 8 & 7 & 11 & 8 & 14 & 9 & 6 & 6 & 6 & 4 & 4 & 2 & 2 & 15 & 14 & 116 \\
\hline B-04-121 & 17 & 16 & 19 & 11 & 15 & 13 & 19 & 3 & 6 & 6 & 7 & 6 & 6 & 18 & 18 & 180 \\
\hline B-04-122 & 11 & 16 & 6 & 11 & 16 & 15 & 5 & 9 & 9 & 8 & 8 & 6 & 9 & 16 & 11 & 156 \\
\hline B-04-123 & 16 & 16 & 16 & 13 & 15 & 16 & 12 & 7 & 7 & 7 & 9 & 7 & 9 & 19 & 18 & 187 \\
\hline B-04-124 & 10 & 11 & 6 & 13 & 11 & 13 & 3 & 7 & 7 & 6 & 6 & 4 & 4 & 11 & 6 & 118 \\
\hline B-04-125 & 18 & 16 & 12 & 14 & 16 & 18 & 8 & 8 & 8 & 8 & 8 & 8 & 8 & 18 & 17 & 185 \\
\hline B-04-126 & 7 & 7 & 6 & 7 & 8 & 11 & 7 & 6 & 6 & 4 & 4 & 5 & 5 & 13 & 16 & 112 \\
\hline B-04-127 & 11 & 11 & 11 & 12 & 13 & 12 & 10 & 7 & 7 & 5 & 5 & 5 & 5 & 13 & 16 & 143 \\
\hline B-04-129 & 18 & 13 & 11 & 14 & 16 & 16 & 9 & 4 & 6 & 8 & 8 & 7 & 7 & 16 & 16 & 169 \\
\hline B-04-131 & 8 & 11 & 6 & 8 & 8 & 7 & 4 & 6 & 6 & 4 & 4 & 4 & 4 & 13 & 8 & 101 \\
\hline B-04-133 & 11 & 13 & 9 & 14 & 14 & 13 & 6 & 6 & 6 & 6 & 6 & 5 & 5 & 13 & 13 & 140 \\
\hline B-04-136 & 14 & 17 & 18 & 14 & 14 & 15 & 17 & 8 & 8 & 7 & 7 & 7 & 7 & 15 & 14 & 182 \\
\hline B-04-140 & 14 & 12 & 13 & 11 & 11 & 13 & 13 & 7 & 6 & 6 & 7 & 6 & 6 & 16 & 16 & 157 \\
\hline B-04-141 & 7 & 8 & 8 & 11 & 10 & 15 & 14 & 3 & 5 & 4 & 4 & 5 & 5 & 13 & 6 & 118 \\
\hline
\end{tabular}


Appendix 3: Table showing the twelve individual habitat parameter scores adapted from Barbour et al. (1999) that were assessed at most sites where fishing effort was applied and were totalled to derive the Habitat Assessment Score for each site.

\begin{tabular}{|c|c|c|c|c|c|c|c|c|c|c|c|c|c|c|c|c|}
\hline \multirow{2}{*}{ Site Number } & \multirow{2}{*}{ 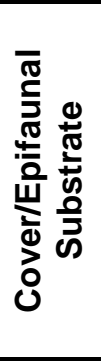 } & \multirow{2}{*}{ 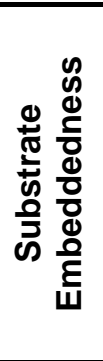 } & \multirow{2}{*}{ 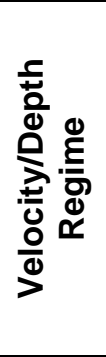 } & \multirow{2}{*}{ 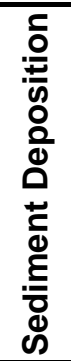 } & \multirow{2}{*}{ 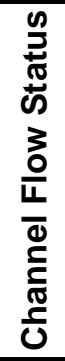 } & \multirow{2}{*}{ 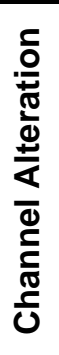 } & \multirow{2}{*}{ 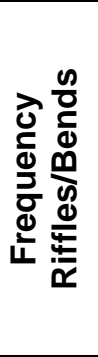 } & \multicolumn{2}{|c|}{ 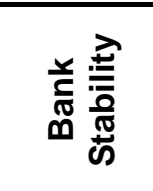 } & \multicolumn{2}{|c|}{ 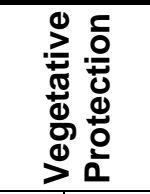 } & \multicolumn{2}{|c|}{ 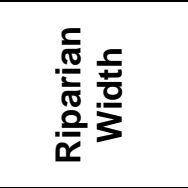 } & \multirow{2}{*}{ 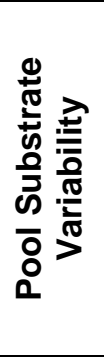 } & \multirow{2}{*}{ 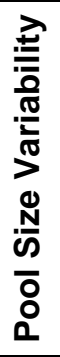 } & \multirow{2}{*}{ 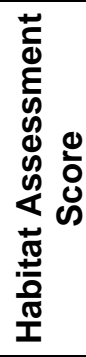 } \\
\hline & & & & & & & & ప্త & $\frac{\text { 동 }}{\frac{0}{x}}$ & ప্త & $\frac{\text { 픙 }}{\frac{9}{x}}$ & Ф્త & $\frac{\tilde{E}}{\frac{\pi}{x}}$ & & & \\
\hline B-04-144 & 17 & 11 & 16 & 10 & 11 & 11 & 16 & 6 & 8 & 7 & 8 & 7 & 7 & 17 & 17 & 169 \\
\hline B-04-145 & 11 & 11 & 11 & 11 & 10 & 11 & 16 & 6 & 6 & 6 & 6 & 6 & 6 & 11 & 7 & 135 \\
\hline B-04-149 & 8 & 9 & 11 & 8 & 8 & 13 & 8 & 7 & 8 & 6 & 8 & 8 & 6 & 7 & 16 & 131 \\
\hline B-04-152 & 8 & 8 & 11 & 7 & 14 & 13 & 7 & 7 & 5 & 6 & 6 & 7 & 7 & 8 & 13 & 127 \\
\hline B-04-153 & 6 & 5 & 11 & 6 & 8 & 13 & 6 & 3 & 4 & 7 & 5 & 5 & 7 & 11 & 10 & 107 \\
\hline B-04-155 & 10 & 10 & 13 & 11 & 14 & 10 & 8 & 5 & 6 & 4 & 5 & 4 & 5 & 11 & 16 & 132 \\
\hline B-04-161 & 9 & 15 & 11 & 14 & 13 & 11 & 6 & 3 & 3 & 6 & 6 & 7 & 7 & 11 & 13 & 135 \\
\hline B-04-163 & 12 & 13 & 3 & 15 & 10 & 13 & 3 & 6 & 6 & 5 & 5 & 4 & 6 & 14 & 15 & 130 \\
\hline B-04-164 & 14 & 10 & 11 & 10 & 11 & 12 & 9 & 7 & 7 & 5 & 5 & 5 & 4 & 13 & 16 & 139 \\
\hline B-04-165 & 13 & 9 & 11 & 8 & 11 & 10 & 10 & 6 & 6 & 6 & 6 & 4 & 4 & 13 & 11 & 128 \\
\hline B-04-167 & 14 & 18 & 15 & 16 & 16 & 16 & 13 & 7 & 5 & 7 & 5 & 5 & 5 & 18 & 18 & 178 \\
\hline B-04-174 & 18 & 16 & 17 & 15 & 16 & 15 & 15 & 8 & 8 & 6 & 6 & 4 & 5 & 19 & 18 & 186 \\
\hline B-04-181 & 11 & 8 & 10 & 8 & 7 & 15 & 14 & 3 & 4 & 9 & 9 & 8 & 8 & 6 & 7 & 127 \\
\hline B-04-186 & 15 & 16 & 18 & 13 & 16 & 13 & 11 & 9 & 9 & 9 & 9 & 9 & 9 & 18 & 18 & 192 \\
\hline B-04-187 & 14 & 8 & 18 & 10 & 13 & 13 & 6 & 6 & 4 & 7 & 7 & 6 & 8 & 11 & 18 & 149 \\
\hline B-04-188 & 10 & 9 & 16 & 10 & 13 & 15 & 8 & 5 & 4 & 6 & 6 & 8 & 6 & 12 & 16 & 144 \\
\hline B-04-189 & 13 & 11 & 18 & 11 & 10 & 11 & 17 & 7 & 7 & 7 & 6 & 7 & 6 & 17 & 17 & 165 \\
\hline B-04-191 & 16 & 14 & 16 & 13 & 14 & 14 & 10 & 5 & 7 & 8 & 8 & 7 & 7 & 13 & 14 & 166 \\
\hline B-04-195 & 15 & 15 & 13 & 15 & 14 & 16 & 10 & 9 & 9 & 8 & 9 & 8 & 8 & 16 & 15 & 180 \\
\hline B-04-196 & 18 & 11 & 15 & 11 & 11 & 16 & 14 & 7 & 7 & 8 & 9 & 8 & 8 & 17 & 16 & 176 \\
\hline B-04-197 & 14 & 14 & 13 & 10 & 14 & 16 & 16 & 9 & 9 & 9 & 9 & 9 & 9 & 16 & 16 & 183 \\
\hline B-04-198 & 18 & 19 & 18 & 13 & 16 & 18 & 18 & 9 & 9 & 9 & 9 & 9 & 9 & 19 & 10 & 203 \\
\hline B-04-199 & 18 & 13 & 18 & 12 & 15 & 18 & 17 & 9 & 9 & 9 & 9 & 9 & 9 & 18 & 18 & 201 \\
\hline B-04-200 & 14 & 14 & 15 & 12 & 13 & 16 & 16 & 8 & 8 & 9 & 9 & 9 & 9 & 18 & 16 & 186 \\
\hline B-04-205 & 10 & 8 & 9 & 13 & 7 & 8 & 8 & 6 & 5 & 6 & 5 & 5 & 5 & 16 & 9 & 120 \\
\hline
\end{tabular}


Appendix 3: Table showing the twelve individual habitat parameter scores adapted from Barbour et al. (1999) that were assessed at most sites where fishing effort was applied and were totalled to derive the Habitat Assessment Score for each site.

\begin{tabular}{|c|c|c|c|c|c|c|c|c|c|c|c|c|c|c|c|c|}
\hline \multirow{2}{*}{ Site Number } & \multirow{2}{*}{ 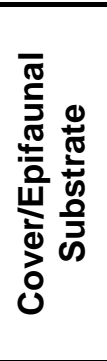 } & \multirow{2}{*}{ 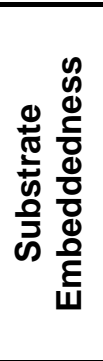 } & \multirow{2}{*}{ 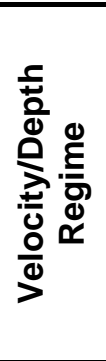 } & \multirow{2}{*}{ 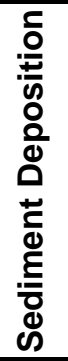 } & \multirow{2}{*}{ 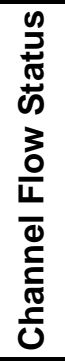 } & \multirow{2}{*}{ 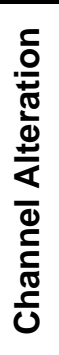 } & \multirow{2}{*}{ 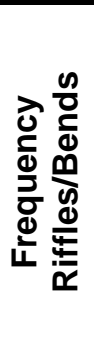 } & \multicolumn{2}{|c|}{ 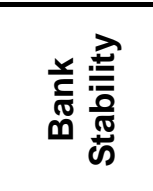 } & \multicolumn{2}{|c|}{ 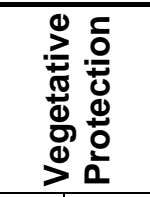 } & \multicolumn{2}{|c|}{ 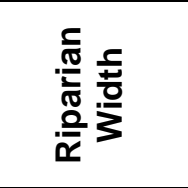 } & \multirow{2}{*}{ 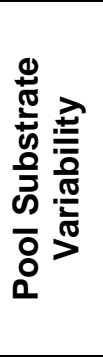 } & \multirow{2}{*}{ 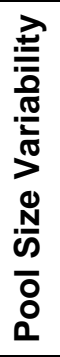 } & \multirow{2}{*}{ 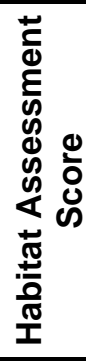 } \\
\hline & & & & & & & & ప্త & $\frac{\text { 픔 }}{\frac{0}{x}}$ & 屯্త & $\frac{\text { 픙 }}{\frac{9}{x}}$ & 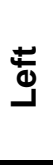 & $\frac{\tilde{E}}{\frac{\pi}{x}}$ & & & \\
\hline B-04-211 & 7 & 8 & 8 & 8 & 5 & 7 & 3 & 5 & 5 & 5 & 5 & 5 & 5 & 14 & 5 & 95 \\
\hline B-04-214 & 9 & 8 & 8 & 6 & 10 & 16 & 5 & 3 & 3 & 8 & 8 & 8 & 8 & 13 & 18 & 131 \\
\hline B-04-215 & 13 & 10 & 8 & 8 & 9 & 16 & 6 & 5 & 5 & 7 & 4 & 7 & 4 & 13 & 18 & 133 \\
\hline B-04-217 & 18 & 15 & 18 & 14 & 16 & 18 & 18 & 9 & 9 & 9 & 9 & 9 & 9 & 16 & 17 & 204 \\
\hline B-04-218 & 13 & 10 & 10 & 11 & 11 & 16 & 15 & 9 & 9 & 9 & 9 & 9 & 9 & 11 & 16 & 167 \\
\hline B-04-219 & 13 & 8 & 8 & 15 & 10 & 15 & 11 & 8 & 8 & 7 & 7 & 5 & 5 & 13 & 11 & 144 \\
\hline B-04-220 & 10 & 16 & 14 & 14 & 8 & 8 & 11 & 5 & 5 & 5 & 5 & 4 & 4 & 13 & 10 & 132 \\
\hline B-04-223 & 12 & 10 & 13 & 10 & 9 & 13 & 8 & 9 & 9 & 9 & 9 & 8 & 8 & 13 & 16 & 156 \\
\hline B-04-224 & 14 & 10 & 13 & 8 & 8 & 16 & 15 & 6 & 6 & 8 & 8 & 8 & 8 & 15 & 16 & 159 \\
\hline B-04-225 & 5 & 6 & 6 & 6 & 6 & 12 & 7 & 3 & 3 & 2 & 2 & 2 & 2 & 11 & 7 & 80 \\
\hline B-04-226 & 12 & 10 & 6 & 10 & 11 & 13 & 6 & 9 & 9 & 9 & 7 & 8 & 7 & 15 & 13 & 145 \\
\hline B-04-228 & 14 & 7 & 10 & 8 & 8 & 16 & 13 & 6 & 6 & 9 & 9 & 9 & 9 & 13 & 7 & 144 \\
\hline B-04-230 & 10 & 11 & 9 & 11 & 8 & 7 & 11 & 7 & 7 & 8 & 5 & 8 & 3 & 13 & 7 & 125 \\
\hline B-04-231 & 10 & 11 & 8 & 7 & 10 & 8 & 6 & 6 & 6 & 6 & 6 & 3 & 3 & 12 & 6 & 108 \\
\hline B-04-232 & 10 & 7 & 11 & 7 & 10 & 6 & 6 & 6 & 6 & 6 & 6 & 4 & 4 & 14 & 16 & 119 \\
\hline B-04-238 & 10 & 14 & 13 & 13 & 10 & 14 & 8 & 6 & 6 & 5 & 5 & 5 & 5 & 18 & 16 & 148 \\
\hline B-04-240 & 17 & 14 & 16 & 14 & 13 & 17 & 14 & 9 & 9 & 9 & 9 & 9 & 8 & 17 & 16 & 191 \\
\hline B-04-241 & 15 & 8 & 15 & 8 & 10 & 18 & 18 & 7 & 5 & 8 & 8 & 5 & 8 & 15 & 16 & 164 \\
\hline B-04-244 & 12 & 10 & 15 & 13 & 8 & 13 & 14 & 7 & 7 & 6 & 5 & 8 & 4 & 16 & 16 & 154 \\
\hline B-04-248 & 7 & 8 & 5 & 10 & 7 & 9 & 2 & 6 & 6 & 7 & 6 & 5 & 5 & 14 & 13 & 110 \\
\hline B-04-249 & 7 & 8 & 5 & 9 & 5 & 6 & 2 & 6 & 6 & 4 & 4 & 5 & 5 & 13 & 8 & 93 \\
\hline B-04-250 & 7 & 7 & 5 & 8 & 7 & 8 & 6 & 8 & 8 & 7 & 7 & 7 & 7 & 13 & 14 & 119 \\
\hline B-04-251 & 9 & 13 & 6 & 11 & 7 & 9 & 1 & 6 & 6 & 6 & 6 & 6 & 6 & 11 & 11 & 114 \\
\hline B-04-256 & 8 & 11 & 7 & 12 & 8 & 8 & 3 & 6 & 6 & 5 & 5 & 8 & 8 & 11 & 11 & 117 \\
\hline B-04-257 & 10 & 11 & 6 & 10 & 12 & 8 & 7 & 6 & 6 & 5 & 5 & 7 & 7 & 14 & 13 & 127 \\
\hline
\end{tabular}


Appendix 3: Table showing the twelve individual habitat parameter scores adapted from Barbour et al. (1999) that were assessed at most sites where fishing effort was applied and were totalled to derive the Habitat Assessment Score for each site.

\begin{tabular}{|c|c|c|c|c|c|c|c|c|c|c|c|c|c|c|c|c|}
\hline \multirow{2}{*}{ Site Number } & \multirow{2}{*}{ 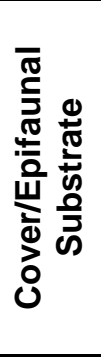 } & \multirow{2}{*}{ 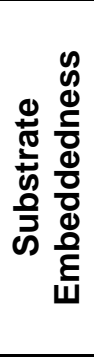 } & \multirow{2}{*}{ 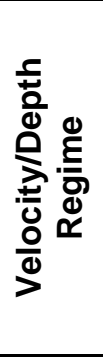 } & \multirow{2}{*}{ 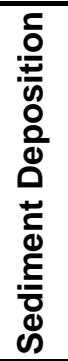 } & \multirow{2}{*}{ 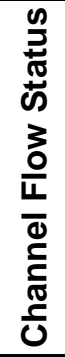 } & \multirow{2}{*}{ 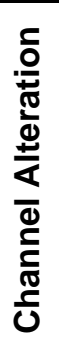 } & \multirow{2}{*}{ 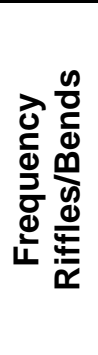 } & \multicolumn{2}{|c|}{ 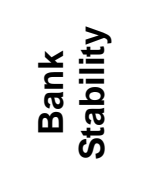 } & \multicolumn{2}{|c|}{ 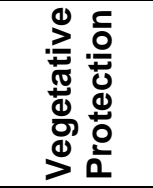 } & \multicolumn{2}{|c|}{$\begin{array}{l}\frac{c}{\sqrt{0}} \\
\frac{2}{\frac{0}{\pi}} \\
\frac{0}{x}\end{array}$} & \multirow{2}{*}{ 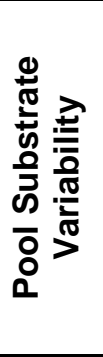 } & \multirow{2}{*}{ 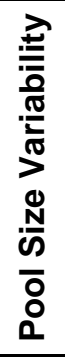 } & \multirow{2}{*}{ 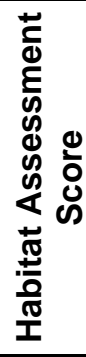 } \\
\hline & & & & & & & & Ф্త & $\begin{array}{l}\frac{}{\frac{1}{\sigma}} \\
\frac{0}{x}\end{array}$ & Ф্త & $\frac{\text { 픔 }}{\frac{0}{\tilde{x}}}$ & 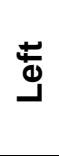 & $\frac{\tilde{n}}{\frac{\tilde{\sigma}}{\boldsymbol{x}}}$ & & & \\
\hline B-04-259 & 10 & 8 & 6 & 10 & 8 & 7 & 7 & 6 & 6 & 5 & 5 & 5 & 5 & 11 & 8 & 107 \\
\hline B-04-263 & 9 & 11 & 6 & 11 & 11 & 11 & 7 & 6 & 6 & 5 & 5 & 5 & 6 & 16 & 16 & 131 \\
\hline B-04-264 & 11 & 10 & 6 & 9 & 13 & 14 & 10 & 6 & 6 & 6 & 6 & 6 & 6 & 12 & 16 & 137 \\
\hline B-04-266 & 11 & 10 & 10 & 9 & 9 & 16 & 10 & 8 & 8 & 8 & 8 & 8 & 8 & 15 & 8 & 146 \\
\hline B-04-270 & 7 & 3 & 3 & 7 & 14 & 8 & 6 & 4 & 4 & 4 & 4 & 4 & 4 & 13 & 13 & 98 \\
\hline B-04-275 & 10 & 10 & 1 & 10 & 13 & 7 & 0 & 5 & 5 & 6 & 6 & 5 & 5 & 14 & 11 & 108 \\
\hline B-04-276 & 10 & 10 & 3 & 10 & 13 & 7 & 2 & 5 & 5 & 2 & 2 & 2 & 2 & 13 & 13 & 99 \\
\hline D-04-011 & 18 & 11 & 16 & 13 & 15 & 16 & 8 & 9 & 9 & 9 & 9 & 5 & 3 & 12 & 17 & 170 \\
\hline D-04-012 & 16 & 18 & 18 & 17 & 17 & 15 & 18 & 5 & 7 & 9 & 9 & 10 & 10 & 20 & 16 & 205 \\
\hline D-04-017 & 5 & 8 & 8 & 13 & 7 & 8 & 3 & 7 & 7 & 7 & 5 & 5 & 3 & 12 & 6 & 104 \\
\hline D-04-022 & 5 & 7 & 13 & 6 & 7 & 5 & 5 & 2 & 6 & 2 & 2 & 2 & 2 & 11 & 8 & 83 \\
\hline D-04-023 & 11 & 8 & 16 & 13 & 7 & 13 & 13 & 4 & 4 & 8 & 8 & 5 & 5 & 8 & 16 & 139 \\
\hline D-04-027 & 15 & 11 & 17 & 9 & 10 & 16 & 14 & 8 & 8 & 8 & 8 & 8 & 8 & 13 & 16 & 169 \\
\hline D-04-028 & 16 & 14 & 18 & 13 & 13 & 14 & 13 & 5 & 8 & 8 & 4 & 4 & 2 & 13 & 16 & 161 \\
\hline D-04-029 & 5 & 4 & 6 & 5 & 8 & 2 & 2 & 6 & 9 & 5 & 5 & 4 & 4 & 8 & 12 & 85 \\
\hline D-04-033 & 16 & 13 & 18 & 13 & 13 & 13 & 13 & 7 & 7 & 7 & 7 & 8 & 8 & 18 & 18 & 179 \\
\hline D-04-034 & 16 & 11 & 16 & 10 & 11 & 13 & 13 & 8 & 8 & 9 & 9 & 8 & 8 & 13 & 16 & 169 \\
\hline D-04-035 & 16 & 13 & 16 & 13 & 13 & 13 & 13 & 9 & 9 & 9 & 9 & 9 & 9 & 11 & 16 & 178 \\
\hline D-04-036 & 18 & 16 & 18 & 15 & 17 & 15 & 18 & 8 & 7 & 7 & 9 & 8 & 8 & 16 & 16 & 196 \\
\hline W-04-006 & 8 & 6 & 1 & 7 & 8 & 6 & 3 & 7 & 7 & 7 & 7 & 8 & 8 & 9 & 2 & 94 \\
\hline W-04-007 & 13 & 16 & 5 & 13 & 13 & 8 & 6 & 7 & 7 & 7 & 7 & 2 & 2 & 17 & 7 & 130 \\
\hline W-04-009 & 10 & 7 & 3 & 7 & 14 & 7 & 1 & 8 & 8 & 8 & 8 & 6 & 6 & 8 & 3 & 104 \\
\hline W-04-013 & 8 & 5 & 4 & 8 & 8 & 7 & 5 & 7 & 7 & 7 & 7 & 1 & 1 & 11 & 12 & 98 \\
\hline W-04-020 & 10 & 7 & 1 & 8 & 8 & 12 & 12 & 9 & 9 & 7 & 7 & 1 & 1 & 11 & 2 & 105 \\
\hline W-04-027 & 3 & 13 & 5 & 13 & 3 & 4 & 6 & 3 & 3 & 1 & 1 & 1 & 1 & 1 & 8 & 66 \\
\hline
\end{tabular}


Appendix 3: Table showing the twelve individual habitat parameter scores adapted from Barbour et al. (1999) that were assessed at most sites where fishing effort was applied and were totalled to derive the Habitat Assessment Score for each site.

\begin{tabular}{|c|c|c|c|c|c|c|c|c|c|c|c|c|c|c|c|c|}
\hline \multirow{2}{*}{ Site Number } & \multirow{2}{*}{ 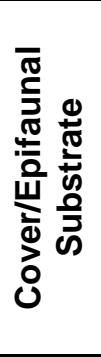 } & \multirow{2}{*}{ 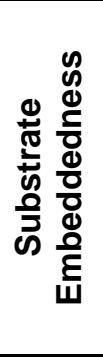 } & \multirow{2}{*}{ 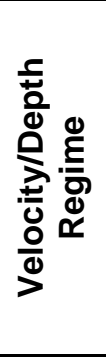 } & \multirow{2}{*}{ 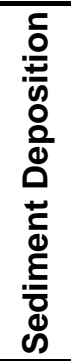 } & \multirow{2}{*}{ 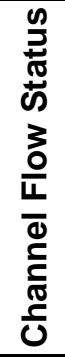 } & \multirow{2}{*}{ 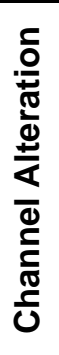 } & \multirow{2}{*}{ 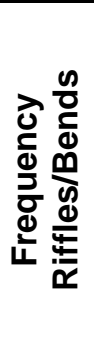 } & \multicolumn{2}{|c|}{ 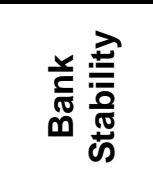 } & \multicolumn{2}{|c|}{ 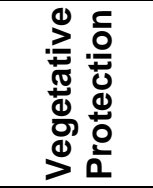 } & \multicolumn{2}{|c|}{$\begin{array}{l}\frac{c}{\sqrt{0}} \\
\frac{2}{\frac{0}{\pi}} \\
\frac{0}{x}\end{array}$} & \multirow{2}{*}{ 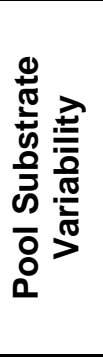 } & \multirow{2}{*}{ 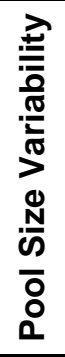 } & \multirow{2}{*}{ 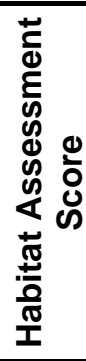 } \\
\hline & & & & & & & & 屯্త & $\begin{array}{l}\frac{}{\frac{1}{\sigma}} \\
\frac{0}{x}\end{array}$ & Ф્త & $\frac{\text { 픔 }}{\frac{0}{\tilde{x}}}$ & 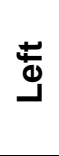 & $\frac{\tilde{n}}{\frac{\tilde{\sigma}}{\boldsymbol{x}}}$ & & & \\
\hline W-04-029 & 3 & 14 & 2 & 3 & 3 & 6 & 2 & 6 & 6 & 5 & 5 & 1 & 1 & 3 & 3 & 63 \\
\hline W-04-032 & 11 & 8 & 8 & 5 & 10 & 13 & 13 & 8 & 8 & 8 & 8 & 10 & 10 & 13 & 9 & 142 \\
\hline W-04-036 & 15 & 15 & 2 & 13 & 3 & 15 & 2 & 8 & 8 & 8 & 8 & 2 & 2 & 5 & 3 & 109 \\
\hline W-04-039 & 8 & 15 & 2 & 16 & 6 & 11 & 2 & 7 & 7 & 7 & 7 & 2 & 2 & 16 & 3 & 111 \\
\hline W-04-040 & 4 & 12 & 2 & 12 & 13 & 11 & 3 & 8 & 8 & 7 & 7 & 2 & 2 & 11 & 3 & 105 \\
\hline W-04-041 & 13 & 13 & 3 & 11 & 14 & 13 & 3 & 8 & 8 & 8 & 4 & 9 & 1 & 12 & 3 & 123 \\
\hline W-04-043 & 11 & 14 & 2 & 11 & 13 & 11 & 4 & 5 & 3 & 7 & 4 & 1 & 1 & 13 & 2 & 102 \\
\hline W-04-044 & 16 & 15 & 3 & 14 & 16 & 13 & 8 & 7 & 7 & 7 & 7 & 2 & 2 & 16 & 4 & 137 \\
\hline W-04-049 & 16 & 18 & 10 & 16 & 14 & 15 & 16 & 8 & 8 & 9 & 7 & 9 & 2 & 18 & 10 & 176 \\
\hline W-04-050 & 15 & 11 & 10 & 8 & 9 & 12 & 13 & 6 & 6 & 6 & 6 & 3 & 3 & 13 & 10 & 131 \\
\hline W-04-051 & 18 & 16 & 10 & 12 & 16 & 17 & 13 & 9 & 9 & 9 & 7 & 9 & 6 & 16 & 10 & 177 \\
\hline W-04-052 & 18 & 16 & 13 & 13 & 18 & 15 & 15 & 8 & 8 & 7 & 7 & 8 & 2 & 16 & 8 & 172 \\
\hline W-04-053 & 9 & 6 & 17 & 4 & 18 & 7 & 10 & 5 & 5 & 6 & 6 & 2 & 2 & 11 & 13 & 121 \\
\hline W-04-054 & 16 & 13 & 16 & 8 & 15 & 12 & 13 & 8 & 6 & 8 & 6 & 2 & 2 & 11 & 11 & 147 \\
\hline W-04-055 & 8 & 16 & 8 & 13 & 15 & 8 & 2 & 7 & 7 & 6 & 6 & 1 & 1 & 17 & 8 & 123 \\
\hline W-04-056 & 8 & 13 & 3 & 10 & 9 & 8 & 2 & 6 & 6 & 4 & 7 & 0 & 0 & 12 & 2 & 90 \\
\hline W-04-057 & 14 & 16 & 13 & 13 & 17 & 10 & 8 & 7 & 7 & 7 & 7 & 0 & 0 & 13 & 12 & 144 \\
\hline W-04-058 & 4 & 1 & 8 & 1 & 10 & 9 & 8 & 7 & 7 & 7 & 7 & 1 & 1 & 9 & 3 & 83 \\
\hline W-04-059 & 14 & 17 & 10 & 16 & 17 & 12 & 11 & 8 & 8 & 6 & 6 & 1 & 1 & 6 & 7 & 140 \\
\hline W-04-060 & 17 & 15 & 14 & 13 & 19 & 16 & 16 & 8 & 8 & 8 & 8 & 2 & 2 & 13 & 12 & 171 \\
\hline W-04-063 & 11 & 16 & 6 & 16 & 11 & 8 & 3 & 8 & 8 & 7 & 7 & 2 & 2 & 16 & 2 & 123 \\
\hline W-04-069 & 1 & 12 & 6 & 16 & 3 & 6 & 6 & 6 & 6 & 6 & 5 & 1 & 1 & 6 & 1 & 82 \\
\hline W-04-070 & 10 & 11 & 5 & 13 & 6 & 7 & 2 & 5 & 6 & 6 & 6 & 1 & 1 & 12 & 6 & 97 \\
\hline W-04-071 & 13 & 13 & 6 & 13 & 8 & 8 & 10 & 6 & 3 & 7 & 3 & 1 & 7 & 12 & 5 & 115 \\
\hline W-04-072 & 14 & 13 & 10 & 13 & 14 & 13 & 13 & 8 & 3 & 7 & 4 & 2 & 2 & 13 & 3 & 132 \\
\hline
\end{tabular}


Appendix 3: Table showing the twelve individual habitat parameter scores adapted from Barbour et al. (1999) that were assessed at most sites where fishing effort was applied and were totalled to derive the Habitat Assessment Score for each site.

\begin{tabular}{|c|c|c|c|c|c|c|c|c|c|c|c|c|c|c|c|c|}
\hline \multirow{2}{*}{ Site Number } & \multirow{2}{*}{ 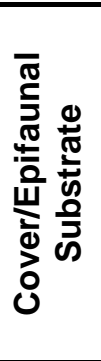 } & \multirow{2}{*}{ 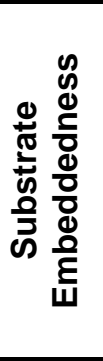 } & \multirow{2}{*}{ 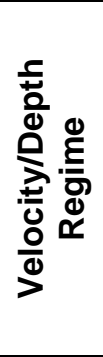 } & \multirow{2}{*}{ 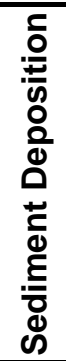 } & \multirow{2}{*}{ 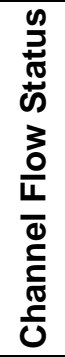 } & \multirow{2}{*}{ 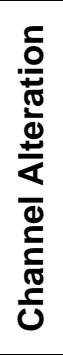 } & \multirow{2}{*}{ 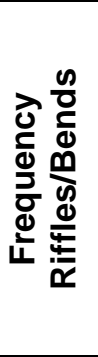 } & \multicolumn{2}{|c|}{ 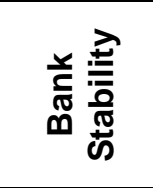 } & \multicolumn{2}{|c|}{ 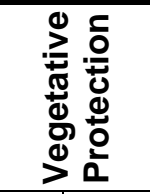 } & \multicolumn{2}{|c|}{ 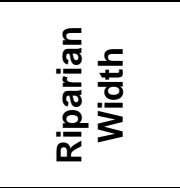 } & \multirow{2}{*}{ 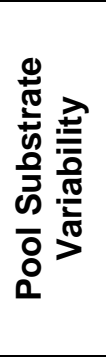 } & \multirow{2}{*}{ 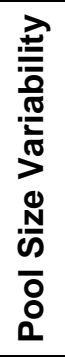 } & \multirow{2}{*}{ 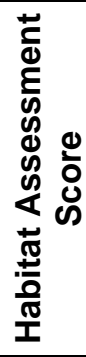 } \\
\hline & & & & & & & & Фృ & $\frac{\text { 돔 }}{\frac{0}{x}}$ & 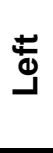 & $\frac{\text { 돔 }}{\frac{0}{x}}$ & 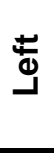 & $\frac{\text { 픔 }}{\frac{0}{x}}$ & & & \\
\hline W-04-074 & 13 & 6 & 13 & 3 & 9 & 8 & 8 & 6 & 6 & 7 & 7 & 2 & 2 & 12 & 8 & 110 \\
\hline W-04-076 & 8 & 17 & 15 & 15 & 15 & 8 & 13 & 7 & 6 & 7 & 7 & 3 & 3 & 9 & 8 & 141 \\
\hline W-04-077 & 8 & 11 & 13 & 8 & 13 & 8 & 3 & 7 & 7 & 6 & 6 & 2 & 2 & 13 & 11 & 118 \\
\hline W-04-078 & 7 & 13 & 8 & 11 & 8 & 3 & 4 & 6 & 3 & 6 & 5 & 1 & 1 & 11 & 2 & 89 \\
\hline W-04-082 & 7 & 8 & 8 & 6 & 7 & 8 & 2 & 6 & 7 & 6 & 7 & 1 & 1 & 13 & 2 & 89 \\
\hline W-04-083 & 15 & 14 & 17 & 15 & 18 & 16 & 13 & 9 & 9 & 9 & 9 & 9 & 8 & 15 & 17 & 193 \\
\hline W-04-084 & 6 & 11 & 4 & 13 & 13 & 6 & 3 & 7 & 6 & 7 & 6 & 4 & 4 & 14 & 8 & 112 \\
\hline W-04-085 & 12 & 5 & 3 & 16 & 18 & 8 & 5 & 9 & 9 & 8 & 8 & 2 & 2 & 13 & 3 & 121 \\
\hline W-04-087 & 8 & 11 & 4 & 8 & 6 & 8 & 3 & 8 & 8 & 7 & 7 & 2 & 2 & 11 & 3 & 96 \\
\hline W-04-088 & 6 & 7 & 8 & 5 & 10 & 7 & 2 & 6 & 7 & 6 & 6 & 1 & 1 & 8 & 2 & 82 \\
\hline W-04-090 & 15 & 16 & 18 & 15 & 18 & 9 & 7 & 8 & 3 & 9 & 5 & 8 & 1 & 12 & 13 & 157 \\
\hline W-04-092 & 12 & 10 & 17 & 10 & 19 & 11 & 8 & 8 & 8 & 9 & 9 & 9 & 9 & 12 & 13 & 164 \\
\hline W-04-093 & 7 & 11 & 8 & 11 & 18 & 7 & 3 & 7 & 7 & 7 & 7 & 1 & 1 & 12 & 8 & 115 \\
\hline W-04-094 & 16 & 10 & 14 & 10 & 20 & 13 & 15 & 8 & 8 & 9 & 9 & 9 & 9 & 13 & 11 & 174 \\
\hline W-04-095 & 18 & 8 & 10 & 6 & 14 & 17 & 13 & 9 & 9 & 9 & 9 & 9 & 9 & 9 & 3 & 152 \\
\hline W-04-097 & 13 & 13 & 12 & 11 & 19 & 13 & 10 & 9 & 9 & 9 & 9 & 9 & 9 & 13 & 8 & 166 \\
\hline W-04-102 & 10 & 15 & 10 & 15 & 14 & 12 & 11 & 9 & 9 & 8 & 8 & 8 & 8 & 12 & 3 & 152 \\
\hline W-04-105 & 8 & 15 & 3 & 15 & 15 & 9 & 3 & 8 & 8 & 8 & 6 & 1 & 3 & 13 & 2 & 117 \\
\hline W-04-106 & 6 & 6 & 9 & 6 & 13 & 6 & 3 & 8 & 8 & 6 & 6 & 7 & 1 & 13 & 8 & 106 \\
\hline W-04-109 & 14 & 15 & 10 & 13 & 13 & 11 & 11 & 8 & 7 & 8 & 6 & 5 & 2 & 16 & 2 & 141 \\
\hline W-04-110 & 10 & 14 & 14 & 11 & 13 & 8 & 10 & 8 & 8 & 7 & 7 & 7 & 7 & 14 & 3 & 141 \\
\hline W-04-111 & 8 & 6 & 8 & 5 & 11 & 8 & 10 & 6 & 6 & 6 & 6 & 0 & 0 & 11 & 4 & 95 \\
\hline W-04-113 & 4 & 2 & 3 & 2 & 12 & 6 & 3 & 4 & 4 & 5 & 5 & 1 & 1 & 8 & 3 & 63 \\
\hline W-04-114 & 11 & 5 & 5 & 3 & 10 & 8 & 3 & 9 & 9 & 9 & 9 & 5 & 3 & 13 & 3 & 105 \\
\hline W-04-115 & 8 & 11 & 6 & 11 & 13 & 8 & 2 & 7 & 7 & 7 & 7 & 4 & 4 & 13 & 8 & 116 \\
\hline
\end{tabular}


Appendix 3: Table showing the twelve individual habitat parameter scores adapted from Barbour et al. (1999) that were assessed at most sites where fishing effort was applied and were totalled to derive the Habitat Assessment Score for each site.

\begin{tabular}{|c|c|c|c|c|c|c|c|c|c|c|c|c|c|c|c|c|}
\hline \multirow{2}{*}{ Site Number } & \multirow{2}{*}{ 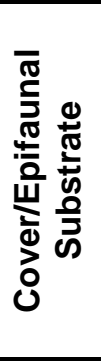 } & \multirow{2}{*}{ 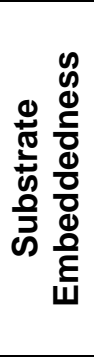 } & \multirow{2}{*}{ 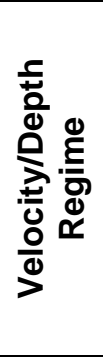 } & \multirow{2}{*}{ 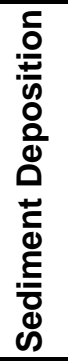 } & \multirow{2}{*}{ 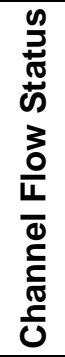 } & \multirow{2}{*}{ 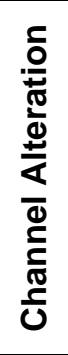 } & \multirow{2}{*}{ 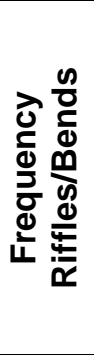 } & \multicolumn{2}{|c|}{ 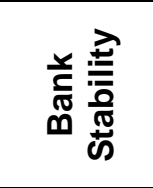 } & \multicolumn{2}{|c|}{ 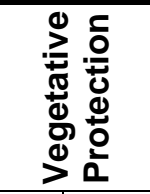 } & \multicolumn{2}{|c|}{ 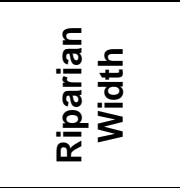 } & \multirow{2}{*}{ 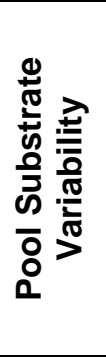 } & \multirow{2}{*}{ 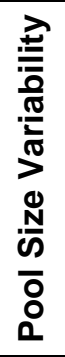 } & \multirow{2}{*}{ 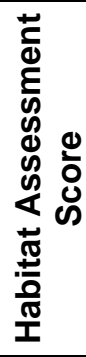 } \\
\hline & & & & & & & & Фృ & $\frac{\text { 돔 }}{\frac{0}{x}}$ & 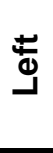 & $\frac{\text { 돔 }}{\frac{0}{x}}$ & Ф্త & $\frac{\text { 픔 }}{\frac{0}{x}}$ & & & \\
\hline W-04-116 & 10 & 15 & 8 & 14 & 7 & 8 & 6 & 4 & 6 & 4 & 7 & 2 & 4 & 10 & 2 & 107 \\
\hline W-04-119 & 8 & 8 & 3 & 9 & 15 & 8 & 2 & 7 & 8 & 7 & 8 & 1 & 9 & 13 & 5 & 111 \\
\hline W-04-120 & 15 & 8 & 18 & 10 & 13 & 9 & 11 & 5 & 1 & 7 & 1 & 9 & 1 & 14 & 14 & 136 \\
\hline W-04-121 & 8 & 9 & 7 & 6 & 12 & 8 & 3 & 8 & 8 & 7 & 7 & 2 & 1 & 11 & 2 & 99 \\
\hline W-04-124 & 5 & 4 & 3 & 10 & 13 & 6 & 3 & 7 & 7 & 7 & 7 & 0 & 2 & 8 & 3 & 85 \\
\hline W-04-126 & 11 & 16 & 15 & 15 & 13 & 11 & 11 & 8 & 8 & 7 & 7 & 6 & 6 & 11 & 9 & 154 \\
\hline W-04-129 & 5 & 10 & 3 & 12 & 20 & 11 & 6 & 8 & 8 & 8 & 8 & 0 & 0 & 11 & 2 & 112 \\
\hline W-04-132 & 7 & 13 & 8 & 13 & 8 & 8 & 5 & 8 & 8 & 8 & 8 & 3 & 3 & 11 & 5 & 116 \\
\hline W-04-135 & 5 & 12 & 2 & 13 & 6 & 6 & 2 & 7 & 7 & 7 & 7 & 0 & 1 & 11 & 3 & 89 \\
\hline W-04-137 & 16 & 14 & 13 & 13 & 10 & 13 & 8 & 8 & 8 & 8 & 8 & 6 & 6 & 13 & 8 & 152 \\
\hline W-04-149 & 2 & 2 & 8 & 2 & 10 & 10 & 1 & 8 & 8 & 6 & 7 & 1 & 1 & 8 & 1 & 75 \\
\hline W-04-151 & 7 & 10 & 8 & 10 & 9 & 9 & 5 & 8 & 6 & 7 & 6 & 1 & 1 & 11 & 7 & 105 \\
\hline W-04-153 & 6 & 11 & 2 & 11 & 10 & 6 & 3 & 7 & 7 & 6 & 6 & 3 & 3 & 10 & 4 & 95 \\
\hline W-04-157 & 6 & 4 & 9 & 4 & 9 & 6 & 4 & 5 & 5 & 5 & 6 & 2 & 2 & 7 & 3 & 77 \\
\hline W-04-159 & 7 & 5 & 1 & 4 & 6 & 8 & 4 & 7 & 7 & 6 & 6 & 0 & 1 & 5 & 8 & 75 \\
\hline W-04-166 & 3 & 6 & 2 & 6 & 6 & 6 & 4 & 7 & 3 & 7 & 4 & 3 & 0 & 6 & 2 & 65 \\
\hline W-04-167 & 5 & 6 & 3 & 5 & 7 & 7 & 2 & 7 & 7 & 7 & 7 & 2 & 4 & 7 & 4 & 80 \\
\hline W-04-171 & 8 & 1 & 3 & 1 & 10 & 10 & 6 & 8 & 8 & 7 & 7 & 2 & 2 & 6 & 5 & 84 \\
\hline W-04-172 & 7 & 2 & 2 & 2 & 7 & 6 & 4 & 7 & 7 & 7 & 7 & 2 & 2 & 6 & 6 & 74 \\
\hline W-04-173 & 10 & 10 & 8 & 10 & 6 & 10 & 5 & 7 & 7 & 7 & 7 & 3 & 2 & 10 & 5 & 107 \\
\hline W-04-174 & 10 & 3 & 2 & 3 & 5 & 8 & 2 & 7 & 7 & 7 & 7 & 3 & 3 & 12 & 4 & 83 \\
\hline W-04-175 & 10 & 5 & 3 & 5 & 10 & 10 & 3 & 8 & 8 & 7 & 7 & 5 & 5 & 12 & 3 & 101 \\
\hline W-04-176 & 8 & 14 & 2 & 13 & 9 & 7 & 5 & 7 & 7 & 7 & 7 & 6 & 4 & 11 & 3 & 110 \\
\hline W-04-177 & 9 & 15 & 18 & 15 & 10 & 6 & 8 & 7 & 7 & 7 & 7 & 4 & 4 & 13 & 9 & 139 \\
\hline W-04-178 & 11 & 5 & 8 & 5 & 10 & 8 & 5 & 7 & 7 & 7 & 7 & 3 & 5 & 12 & 5 & 105 \\
\hline
\end{tabular}


Appendix 3: Table showing the twelve individual habitat parameter scores adapted from Barbour et al. (1999) that were assessed at most sites where fishing effort was applied and were totalled to derive the Habitat Assessment Score for each site.

\begin{tabular}{|c|c|c|c|c|c|c|c|c|c|c|c|c|c|c|c|c|}
\hline \multirow{2}{*}{ Site Number } & \multirow{2}{*}{ 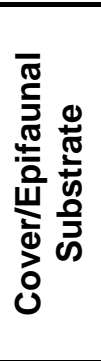 } & \multirow{2}{*}{ 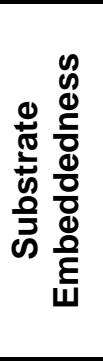 } & \multirow{2}{*}{ 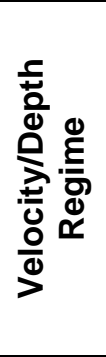 } & \multirow{2}{*}{ 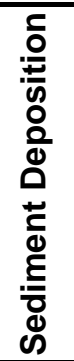 } & \multirow{2}{*}{ 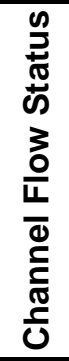 } & \multirow{2}{*}{ 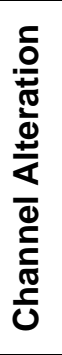 } & \multirow{2}{*}{ 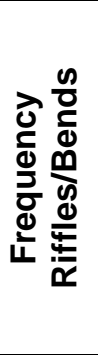 } & \multicolumn{2}{|c|}{ 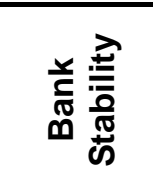 } & \multicolumn{2}{|c|}{ 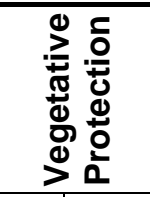 } & \multicolumn{2}{|c|}{ 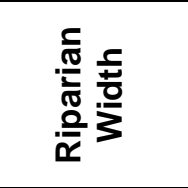 } & \multirow{2}{*}{ 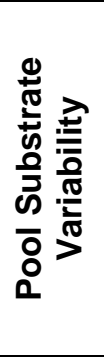 } & \multirow{2}{*}{ 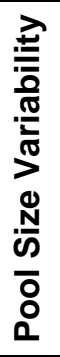 } & \multirow{2}{*}{ 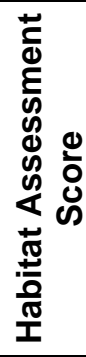 } \\
\hline & & & & & & & & ప্త & $\frac{\text { 픔 }}{\frac{0}{x}}$ & ప্త & $\frac{F}{\frac{\hbar}{x}}$ & 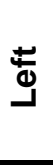 & $\frac{\tilde{E}}{\frac{\pi}{x}}$ & & & \\
\hline W-04-179 & 8 & 10 & 10 & 10 & 8 & 8 & 2 & 5 & 5 & 6 & 6 & 1 & 2 & 11 & 3 & 95 \\
\hline W-04-180 & 8 & 12 & 14 & 10 & 6 & 8 & 3 & 6 & 8 & 7 & 7 & 8 & 1 & 11 & 5 & 114 \\
\hline W-04-181 & 7 & 3 & 2 & 5 & 8 & 13 & 5 & 7 & 7 & 7 & 7 & 5 & 5 & 6 & 3 & 90 \\
\hline W-04-182 & 13 & 13 & 14 & 13 & 12 & 13 & 11 & 8 & 8 & 9 & 9 & 9 & 9 & 14 & 3 & 158 \\
\hline W-04-184 & 11 & 4 & 6 & 3 & 11 & 11 & 5 & 9 & 6 & 8 & 3 & 9 & 1 & 9 & 5 & 101 \\
\hline W-04-186 & 8 & 11 & 8 & 11 & 8 & 7 & 2 & 7 & 7 & 7 & 7 & 3 & 3 & 11 & 4 & 104 \\
\hline W-04-187 & 6 & 10 & 1 & 8 & 8 & 7 & 2 & 8 & 8 & 7 & 7 & 5 & 5 & 11 & 3 & 96 \\
\hline W-04-192 & 15 & 2 & 7 & 6 & 19 & 15 & 14 & 8 & 6 & 8 & 8 & 9 & 9 & 15 & 13 & 154 \\
\hline W-04-193 & 16 & 13 & 9 & 13 & 13 & 15 & 6 & 9 & 9 & 9 & 9 & 9 & 9 & 15 & 11 & 165 \\
\hline W-04-195 & 16 & 13 & 10 & 13 & 13 & 16 & 13 & 7 & 3 & 8 & 4 & 9 & 9 & 13 & 3 & 150 \\
\hline W-04-197 & 16 & 4 & 15 & 4 & 15 & 15 & 13 & 7 & 5 & 7 & 5 & 9 & 9 & 16 & 3 & 143 \\
\hline W-04-200 & 13 & 3 & 3 & 3 & 7 & 13 & 3 & 8 & 8 & 8 & 6 & 8 & 1 & 11 & 3 & 98 \\
\hline W-04-202 & 8 & 6 & 8 & 7 & 10 & 6 & 3 & 8 & 8 & 7 & 7 & 8 & 8 & 11 & 4 & 109 \\
\hline W-04-203 & 10 & 10 & 6 & 6 & 5 & 9 & 1 & 5 & 6 & 3 & 7 & 2 & 2 & 11 & 11 & 94 \\
\hline W-04-207 & 18 & 15 & 18 & 13 & 13 & 17 & 14 & 6 & 6 & 9 & 9 & 9 & 9 & 12 & 17 & 185 \\
\hline W-04-208 & 16 & 18 & 16 & 15 & 14 & 16 & 16 & 9 & 9 & 9 & 9 & 9 & 9 & 16 & 16 & 197 \\
\hline W-04-211 & 13 & 16 & 19 & 13 & 11 & 8 & 6 & 4 & 3 & 6 & 6 & 1 & 1 & 13 & 12 & 132 \\
\hline W-04-212 & 5 & 8 & 1 & 7 & 6 & 6 & 4 & 6 & 6 & 7 & 7 & 1 & 1 & 8 & 5 & 78 \\
\hline W-04-213 & 18 & 16 & 17 & 15 & 13 & 18 & 15 & 3 & 6 & 7 & 9 & 9 & 9 & 15 & 18 & 188 \\
\hline W-04-216 & 10 & 4 & 6 & 4 & 7 & 8 & 3 & 6 & 6 & 7 & 7 & 2 & 2 & 11 & 10 & 93 \\
\hline W-04-217 & 8 & 11 & 10 & 11 & 12 & 10 & 8 & 7 & 7 & 7 & 7 & 4 & 4 & 12 & 8 & 126 \\
\hline W-04-218 & 8 & 13 & 7 & 13 & 11 & 6 & 4 & 8 & 6 & 7 & 7 & 7 & 7 & 11 & 11 & 126 \\
\hline W-04-225 & 7 & 5 & 8 & 7 & 13 & 7 & 5 & 7 & 7 & 7 & 7 & 3 & 5 & 8 & 12 & 108 \\
\hline W-04-231 & 12 & 15 & 10 & 15 & 10 & 10 & 10 & 8 & 6 & 7 & 7 & 6 & 6 & 11 & 8 & 141 \\
\hline W-04-232 & 8 & 14 & 2 & 11 & 11 & 8 & 5 & 5 & 5 & 6 & 6 & 3 & 3 & 14 & 4 & 105 \\
\hline
\end{tabular}


Appendix 3: Table showing the twelve individual habitat parameter scores adapted from Barbour et al. (1999) that were assessed at most sites where fishing effort was applied and were totalled to derive the Habitat Assessment Score for each site.

\begin{tabular}{|c|c|c|c|c|c|c|c|c|c|c|c|c|c|c|c|c|}
\hline \multirow{2}{*}{ Site Number } & \multirow{2}{*}{ 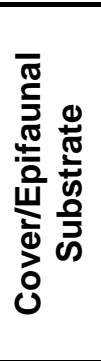 } & \multirow{2}{*}{ 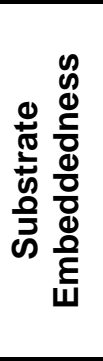 } & \multirow{2}{*}{ 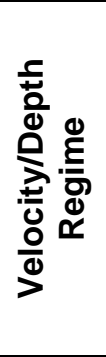 } & \multirow{2}{*}{ 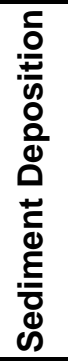 } & \multirow{2}{*}{ 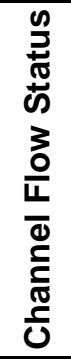 } & \multirow{2}{*}{ 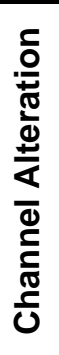 } & \multirow{2}{*}{ 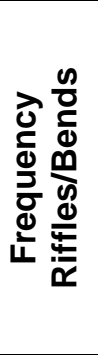 } & \multicolumn{2}{|c|}{ 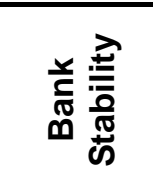 } & \multicolumn{2}{|c|}{ 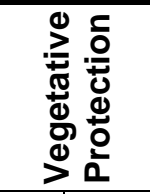 } & \multicolumn{2}{|c|}{ 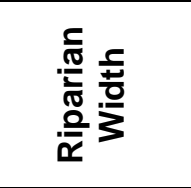 } & \multirow{2}{*}{ 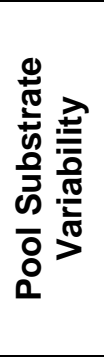 } & \multirow{2}{*}{ 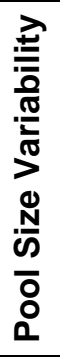 } & \multirow{2}{*}{ 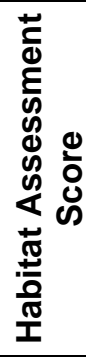 } \\
\hline & & & & & & & & ప্త & $\frac{\text { 동 }}{\frac{0}{x}}$ & ๘্త & $\frac{\text { 듬 }}{\frac{0}{x}}$ & 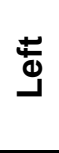 & $\frac{\tilde{E}}{\frac{\pi}{x}}$ & & & \\
\hline W-04-234 & 14 & 17 & 10 & 13 & 10 & 16 & 16 & 6 & 8 & 9 & 9 & 9 & 9 & 13 & 3 & 162 \\
\hline W-04-237 & 16 & 11 & 8 & 11 & 13 & 17 & 13 & 8 & 8 & 9 & 9 & 9 & 9 & 13 & 15 & 169 \\
\hline W-04-238 & 13 & 16 & 8 & 16 & 13 & 15 & 13 & 9 & 9 & 8 & 8 & 10 & 10 & 14 & 8 & 170 \\
\hline W-04-239 & 13 & 16 & 13 & 16 & 13 & 13 & 13 & 9 & 9 & 9 & 9 & 9 & 9 & 13 & 5 & 169 \\
\hline W-04-249 & 15 & 16 & 10 & 16 & 8 & 18 & 11 & 6 & 5 & 8 & 8 & 10 & 10 & 6 & 2 & 149 \\
\hline W-04-250 & 18 & 13 & 10 & 11 & 8 & 17 & 13 & 6 & 7 & 9 & 9 & 10 & 9 & 6 & 3 & 149 \\
\hline W-04-252 & 18 & 16 & 15 & 15 & 12 & 18 & 11 & 9 & 9 & 10 & 10 & 8 & 2 & 10 & 8 & 171 \\
\hline W-04-253 & 16 & 8 & 6 & 8 & 11 & 13 & 8 & 5 & 5 & 7 & 7 & 8 & 8 & 8 & 10 & 128 \\
\hline W-04-254 & 16 & 18 & 10 & 16 & 10 & 17 & 16 & 8 & 8 & 8 & 8 & 10 & 10 & 16 & 5 & 176 \\
\hline W-04-255 & 11 & 5 & 10 & 5 & 13 & 17 & 13 & 6 & 8 & 8 & 8 & 10 & 10 & 11 & 10 & 145 \\
\hline W-04-256 & 10 & 15 & 8 & 15 & 11 & 13 & 5 & 8 & 8 & 8 & 8 & 8 & 7 & 11 & 10 & 145 \\
\hline W-04-260 & 10 & 15 & 3 & 15 & 8 & 11 & 5 & 8 & 8 & 7 & 7 & 4 & 4 & 11 & 3 & 119 \\
\hline W-04-262 & 11 & 11 & 2 & 11 & 7 & 11 & 7 & 8 & 8 & 8 & 8 & 8 & 8 & 11 & 3 & 122 \\
\hline W-04-264 & 8 & 5 & 2 & 5 & 8 & 8 & 2 & 7 & 7 & 7 & 7 & 3 & 5 & 11 & 8 & 93 \\
\hline W-04-265 & 10 & 9 & 4 & 9 & 11 & 12 & 6 & 7 & 7 & 7 & 7 & 3 & 5 & 12 & 6 & 115 \\
\hline W-04-266 & 13 & 16 & 10 & 16 & 10 & 16 & 13 & 8 & 8 & 8 & 8 & 9 & 6 & 13 & 8 & 162 \\
\hline W-04-269 & 9 & 3 & 3 & 3 & 11 & 11 & 6 & 7 & 7 & 8 & 8 & 9 & 9 & 13 & 5 & 112 \\
\hline W-04-271 & 8 & 16 & 6 & 15 & 11 & 15 & 7 & 9 & 9 & 9 & 9 & 7 & 10 & 11 & 15 & 157 \\
\hline W-04-274 & 10 & 16 & 8 & 16 & 8 & 10 & 6 & 7 & 7 & 7 & 7 & 1 & 8 & 6 & 10 & 127 \\
\hline W-04-277 & 2 & 6 & 0 & 7 & 1 & 6 & 2 & 4 & 6 & 5 & 6 & 1 & 3 & 3 & 2 & 54 \\
\hline W-04-286 & 4 & 2 & 1 & 2 & 5 & 11 & 10 & 6 & 5 & 7 & 7 & 8 & 6 & 6 & 3 & 83 \\
\hline W-04-287 & 13 & 15 & 10 & 15 & 8 & 8 & 5 & 8 & 8 & 7 & 7 & 6 & 6 & 13 & 8 & 137 \\
\hline W-04-289 & 10 & 13 & 15 & 11 & 13 & 8 & 8 & 7 & 4 & 7 & 4 & 4 & 4 & 6 & 8 & 122 \\
\hline W-04-297 & 13 & 13 & 18 & 13 & 13 & 18 & 17 & 7 & 5 & 5 & 5 & 7 & 8 & 8 & 11 & 161 \\
\hline W-04-298 & 15 & 16 & 15 & 16 & 11 & 13 & 10 & 8 & 6 & 7 & 7 & 8 & 5 & 11 & 5 & 153 \\
\hline
\end{tabular}


Appendix 3: Table showing the twelve individual habitat parameter scores adapted from Barbour et al. (1999) that were assessed at most sites where fishing effort was applied and were totalled to derive the Habitat Assessment Score for each site.

\begin{tabular}{|c|c|c|c|c|c|c|c|c|c|c|c|c|c|c|c|c|}
\hline \multirow{2}{*}{ Site Number } & \multirow{2}{*}{ 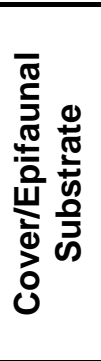 } & \multirow{2}{*}{ 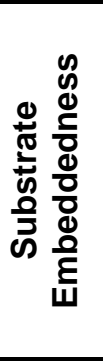 } & \multirow{2}{*}{ 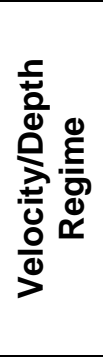 } & \multirow{2}{*}{ 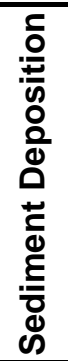 } & \multirow{2}{*}{ 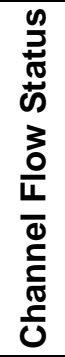 } & \multirow{2}{*}{ 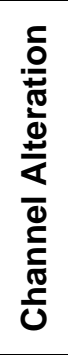 } & \multirow{2}{*}{ 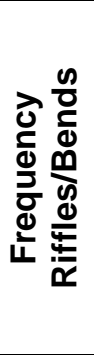 } & \multicolumn{2}{|c|}{ 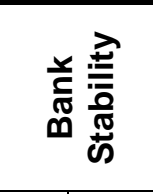 } & \multicolumn{2}{|c|}{ 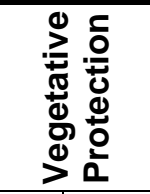 } & \multicolumn{2}{|c|}{ 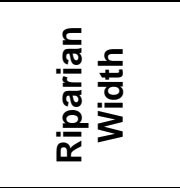 } & \multirow{2}{*}{ 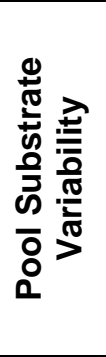 } & \multirow{2}{*}{ 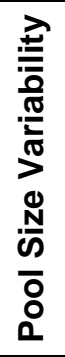 } & \multirow{2}{*}{ 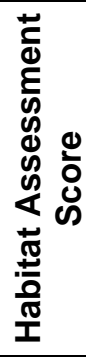 } \\
\hline & & & & & & & & Фృ & $\frac{\text { 돔 }}{\frac{0}{x}}$ & 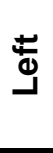 & $\frac{\text { 돔 }}{\frac{0}{x}}$ & Ф্త & $\frac{\text { 픔 }}{\frac{0}{x}}$ & & & \\
\hline W-04-299 & 13 & 16 & 15 & 16 & 13 & 16 & 13 & 8 & 8 & 7 & 7 & 5 & 1 & 6 & 4 & 148 \\
\hline $\mathrm{X}-04-001$ & 11 & 6 & 11 & 5 & 13 & 10 & 6 & 7 & 7 & 6 & 8 & 5 & 7 & 11 & 7 & 120 \\
\hline $\mathrm{X}-04-002$ & 11 & 13 & 17 & 17 & 18 & 11 & 7 & 8 & 8 & 5 & 5 & 7 & 7 & 10 & 16 & 160 \\
\hline X-04-005 & 12 & 12 & 13 & 12 & 6 & 5 & 7 & 2 & 3 & 2 & 3 & 2 & 2 & 13 & 9 & 103 \\
\hline $\mathrm{X}-04-014$ & 11 & 7 & 8 & 10 & 14 & 14 & 4 & 7 & 6 & 7 & 7 & 7 & 6 & 12 & 12 & 132 \\
\hline $\mathrm{X}-04-023$ & 12 & 10 & 6 & 11 & 10 & 10 & 3 & 6 & 6 & 3 & 4 & 3 & 3 & 14 & 7 & 108 \\
\hline $\mathrm{X}-04-026$ & 8 & 6 & 6 & 6 & 9 & 3 & 2 & 2 & 2 & 3 & 3 & 2 & 2 & 6 & 4 & 64 \\
\hline$X-04-031$ & 12 & 7 & 8 & 8 & 9 & 7 & 3 & 5 & 5 & 1 & 1 & 2 & 2 & 14 & 7 & 91 \\
\hline $\mathrm{X}-04-037$ & 14 & 10 & 13 & 8 & 12 & 14 & 13 & 3 & 5 & 7 & 7 & 5 & 5 & 11 & 15 & 142 \\
\hline $\mathrm{X}-04-041$ & 1 & 2 & 7 & 6 & 9 & 6 & 4 & 4 & 4 & 3 & 3 & 1 & 1 & 10 & 9 & 70 \\
\hline X-04-042 & 2 & 1 & 1 & 1 & 14 & 6 & 1 & 4 & 4 & 3 & 3 & 1 & 1 & 0 & 0 & 42 \\
\hline $\mathrm{X}-04-043$ & 16 & 16 & 15 & 9 & 14 & 9 & 8 & 6 & 6 & 6 & 6 & 5 & 5 & 12 & 12 & 145 \\
\hline $\mathrm{X}-04-044$ & 9 & 5 & 8 & 5 & 5 & 3 & 6 & 2 & 3 & 3 & 2 & 2 & 2 & 11 & 6 & 72 \\
\hline X-04-046 & 11 & 8 & 8 & 7 & 6 & 3 & 3 & 3 & 3 & 2 & 3 & 1 & 1 & 11 & 6 & 76 \\
\hline X-04-048 & 10 & 11 & 7 & 13 & 11 & 7 & 2 & 6 & 6 & 4 & 4 & 1 & 2 & 13 & 13 & 110 \\
\hline X-04-049 & 7 & 6 & 6 & 3 & 15 & 10 & 6 & 4 & 4 & 6 & 6 & 6 & 6 & 9 & 9 & 103 \\
\hline $\mathrm{X}-04-051$ & 12 & 8 & 6 & 10 & 16 & 13 & 5 & 4 & 4 & 8 & 8 & 5 & 5 & 13 & 12 & 129 \\
\hline $\mathrm{X}-04-052$ & 10 & 8 & 8 & 7 & 15 & 6 & 3 & 4 & 4 & 3 & 3 & 2 & 2 & 11 & 8 & 94 \\
\hline X-04-053 & 14 & 14 & 15 & 12 & 10 & 8 & 10 & 7 & 6 & 4 & 4 & 3 & 3 & 15 & 15 & 140 \\
\hline $\mathrm{X}-04-054$ & 12 & 14 & 11 & 8 & 14 & 6 & 3 & 6 & 6 & 4 & 4 & 4 & 4 & 11 & 13 & 120 \\
\hline $\mathrm{X}-04-061$ & 5 & 7 & 5 & 11 & 13 & 6 & 0 & 6 & 6 & 3 & 3 & 1 & 1 & 11 & 5 & 83 \\
\hline $\mathrm{X}-04-065$ & 16 & 15 & 16 & 7 & 13 & 11 & 7 & 2 & 4 & 5 & 5 & 3 & 3 & 12 & 11 & 130 \\
\hline X-04-066 & 11 & 11 & 8 & 8 & 12 & 7 & 6 & 6 & 6 & 4 & 4 & 2 & 2 & 14 & 11 & 112 \\
\hline X-04-067 & 12 & 13 & 9 & 13 & 14 & 10 & 7 & 6 & 6 & 5 & 5 & 3 & 4 & 14 & 9 & 130 \\
\hline X-04-068 & 11 & 15 & 8 & 8 & 12 & 7 & 6 & 3 & 4 & 4 & 4 & 2 & 2 & 0 & 0 & 86 \\
\hline
\end{tabular}


Appendix 3: Table showing the twelve individual habitat parameter scores adapted from Barbour et al. (1999) that were assessed at most sites where fishing effort was applied and were totalled to derive the Habitat Assessment Score for each site.

\begin{tabular}{|c|c|c|c|c|c|c|c|c|c|c|c|c|c|c|c|c|}
\hline \multirow{2}{*}{ Site Number } & \multirow{2}{*}{ 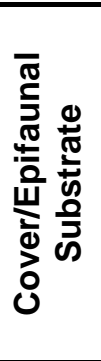 } & \multirow{2}{*}{ 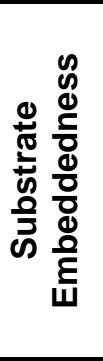 } & \multirow{2}{*}{ 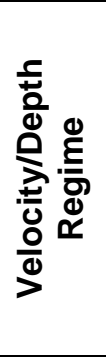 } & \multirow{2}{*}{ 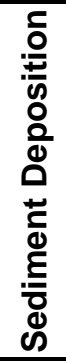 } & \multirow{2}{*}{ 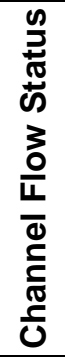 } & \multirow{2}{*}{ 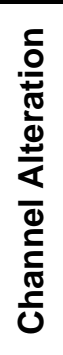 } & \multirow{2}{*}{ 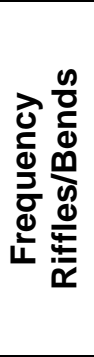 } & \multicolumn{2}{|c|}{ 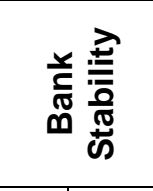 } & \multicolumn{2}{|c|}{ 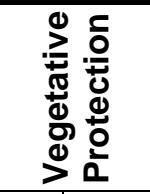 } & \multicolumn{2}{|c|}{ 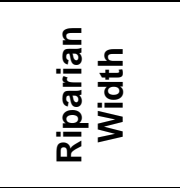 } & \multirow{2}{*}{ 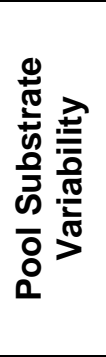 } & \multirow{2}{*}{ 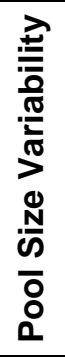 } & \multirow{2}{*}{ 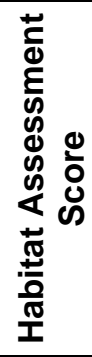 } \\
\hline & & & & & & & & Фృ & $\frac{\text { 돔 }}{\frac{0}{x}}$ & 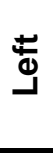 & $\frac{\text { 돔 }}{\frac{0}{x}}$ & Ф্త & $\frac{\text { 픔 }}{\frac{0}{x}}$ & & & \\
\hline $\mathrm{X}-04-071$ & 11 & 14 & 9 & 11 & 11 & 7 & 6 & 6 & 6 & 4 & 4 & 2 & 2 & 12 & 9 & 114 \\
\hline $\mathrm{X}-04-074$ & 7 & 13 & 2 & 10 & 10 & 5 & 4 & 5 & 5 & 2 & 2 & 1 & 2 & 7 & 7 & 82 \\
\hline X-04-077 & 13 & 11 & 9 & 8 & 12 & 14 & 9 & 5 & 4 & 7 & 7 & 5 & 4 & 7 & 12 & 127 \\
\hline X-04-078 & 10 & 9 & 9 & 8 & 10 & 7 & 5 & 6 & 6 & 4 & 4 & 3 & 3 & 11 & 9 & 104 \\
\hline $\mathrm{X}-04-080$ & 13 & 10 & 5 & 10 & 16 & 5 & 5 & 6 & 5 & 5 & 6 & 4 & 4 & 13 & 14 & 121 \\
\hline X-04-081 & 10 & 14 & 9 & 13 & 15 & 5 & 6 & 5 & 5 & 3 & 3 & 4 & 4 & 0 & 0 & 96 \\
\hline $\mathrm{X}-04-082$ & 8 & 10 & 9 & 8 & 9 & 6 & 7 & 6 & 6 & 4 & 4 & 3 & 3 & 9 & 8 & 100 \\
\hline X-04-084 & 10 & 8 & 11 & 7 & 7 & 8 & 8 & 4 & 3 & 4 & 4 & 2 & 2 & 7 & 10 & 95 \\
\hline X-04-088 & 10 & 10 & 9 & 9 & 11 & 7 & 7 & 5 & 5 & 4 & 4 & 1 & 2 & 8 & 9 & 101 \\
\hline X-04-090 & 15 & 12 & 8 & 13 & 16 & 11 & 11 & 4 & 4 & 6 & 6 & 3 & 3 & 16 & 16 & 144 \\
\hline X-04-091 & 15 & 16 & 13 & 13 & 16 & 13 & 14 & 7 & 7 & 8 & 8 & 7 & 8 & 16 & 16 & 177 \\
\hline X-04-092 & 11 & 10 & 8 & 8 & 10 & 7 & 9 & 3 & 3 & 4 & 4 & 2 & 2 & 10 & 9 & 100 \\
\hline X-04-094 & 13 & 13 & 9 & 9 & 13 & 11 & 6 & 3 & 3 & 5 & 5 & 5 & 5 & 12 & 11 & 123 \\
\hline X-04-095 & 9 & 7 & 13 & 7 & 14 & 7 & 7 & 3 & 3 & 4 & 3 & 2 & 2 & 7 & 11 & 99 \\
\hline X-04-097 & 5 & 6 & 6 & 7 & 12 & 8 & 4 & 6 & 6 & 5 & 5 & 4 & 4 & 10 & 9 & 97 \\
\hline X-04-098 & 7 & 5 & 4 & 3 & 14 & 4 & 6 & 4 & 4 & 4 & 4 & 0 & 2 & 7 & 4 & 72 \\
\hline X-04-099 & 7 & 7 & 8 & 7 & 11 & 7 & 3 & 5 & 5 & 4 & 4 & 2 & 2 & 9 & 7 & 88 \\
\hline $\mathrm{X}-04-101$ & 7 & 8 & 10 & 7 & 14 & 6 & 7 & 6 & 6 & 2 & 3 & 1 & 2 & 7 & 11 & 97 \\
\hline X-04-102 & 14 & 11 & 10 & 11 & 11 & 10 & 1 & 5 & 4 & 5 & 4 & 2 & 2 & 14 & 14 & 118 \\
\hline$X-04-103$ & 10 & 8 & 11 & 9 & 12 & 7 & 11 & 4 & 3 & 3 & 3 & 2 & 2 & 12 & 13 & 110 \\
\hline$X-04-106$ & 13 & 7 & 12 & 6 & 14 & 14 & 10 & 3 & 3 & 5 & 5 & 5 & 5 & 0 & 0 & 102 \\
\hline X-04-107 & 11 & 10 & 12 & 9 & 15 & 11 & 14 & 5 & 5 & 7 & 7 & 8 & 8 & 13 & 11 & 146 \\
\hline X-04-109 & 14 & 6 & 12 & 2 & 14 & 11 & 14 & 5 & 5 & 5 & 6 & 6 & 6 & 11 & 11 & 128 \\
\hline $\mathrm{X}-04-110$ & 15 & 15 & 15 & 11 & 15 & 11 & 14 & 4 & 4 & 3 & 3 & 0 & 0 & 16 & 16 & 142 \\
\hline $\mathrm{X}-04-112$ & 11 & 11 & 13 & 11 & 14 & 11 & 11 & 7 & 7 & 7 & 7 & 3 & 3 & 9 & 13 & 138 \\
\hline
\end{tabular}


Appendix 3: Table showing the twelve individual habitat parameter scores adapted from Barbour et al. (1999) that were assessed at most sites where fishing effort was applied and were totalled to derive the Habitat Assessment Score for each site.

\begin{tabular}{|c|c|c|c|c|c|c|c|c|c|c|c|c|c|c|c|c|}
\hline \multirow{2}{*}{ Site Number } & \multirow{2}{*}{ 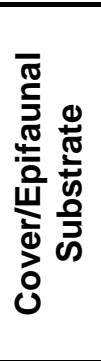 } & \multirow{2}{*}{ 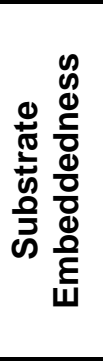 } & \multirow{2}{*}{ 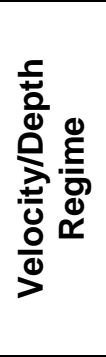 } & \multirow{2}{*}{ 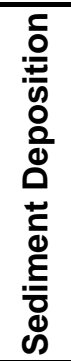 } & \multirow{2}{*}{ 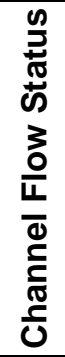 } & \multirow{2}{*}{ 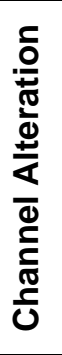 } & \multirow{2}{*}{ 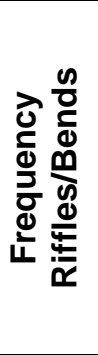 } & \multicolumn{2}{|c|}{ 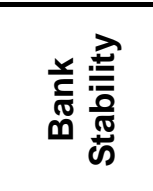 } & \multicolumn{2}{|c|}{ 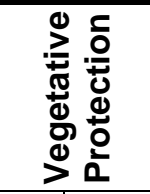 } & \multicolumn{2}{|c|}{ 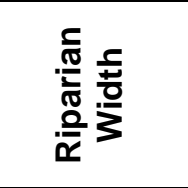 } & \multirow{2}{*}{ 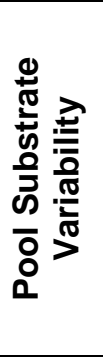 } & \multirow{2}{*}{ 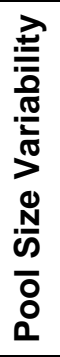 } & \multirow{2}{*}{ 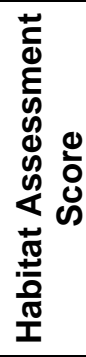 } \\
\hline & & & & & & & & ప্త & $\frac{\text { 동 }}{\frac{0}{x}}$ & 屯্త & $\frac{\text { 픔 }}{\frac{0}{x}}$ & Ф્త & $\frac{\text { 홍 }}{\frac{0}{x}}$ & & & \\
\hline $\mathrm{X}-04-114$ & 15 & 11 & 16 & 10 & 16 & 11 & 11 & 4 & 2 & 5 & 5 & 5 & 5 & 11 & 16 & 143 \\
\hline$X-04-115$ & 14 & 8 & 10 & 7 & 11 & 14 & 11 & 4 & 4 & 7 & 7 & 8 & 8 & 12 & 11 & 136 \\
\hline $\mathrm{X}-04-116$ & 9 & 10 & 8 & 7 & 11 & 11 & 9 & 6 & 6 & 4 & 4 & 5 & 5 & 11 & 7 & 113 \\
\hline $\mathrm{X}-04-118$ & 13 & 10 & 10 & 11 & 13 & 10 & 12 & 5 & 5 & 4 & 6 & 3 & 5 & 11 & 11 & 129 \\
\hline $\mathrm{X}-04-119$ & 14 & 11 & 8 & 9 & 11 & 11 & 10 & 4 & 4 & 6 & 6 & 5 & 5 & 10 & 15 & 129 \\
\hline $\mathrm{X}-04-120$ & 9 & 7 & 8 & 10 & 11 & 12 & 9 & 6 & 6 & 6 & 6 & 4 & 4 & 8 & 13 & 119 \\
\hline $\mathrm{X}-04-121$ & 14 & 8 & 9 & 9 & 13 & 11 & 9 & 5 & 5 & 6 & 5 & 5 & 5 & 0 & 0 & 104 \\
\hline$X-04-122$ & 16 & 14 & 16 & 13 & 14 & 12 & 11 & 5 & 5 & 6 & 6 & 5 & 5 & 16 & 16 & 160 \\
\hline $\mathrm{X}-04-123$ & 11 & 10 & 7 & 10 & 11 & 9 & 7 & 6 & 6 & 5 & 5 & 2 & 2 & 12 & 12 & 115 \\
\hline$X-04-124$ & 10 & 9 & 8 & 13 & 14 & 6 & 5 & 7 & 7 & 4 & 4 & 1 & 1 & 11 & 7 & 107 \\
\hline$X-04-126$ & 7 & 6 & 1 & 8 & 9 & 8 & 0 & 4 & 5 & 4 & 3 & 3 & 3 & 9 & 11 & 81 \\
\hline $\mathrm{X}-04-127$ & 10 & 5 & 7 & 5 & 10 & 6 & 6 & 3 & 4 & 5 & 5 & 3 & 3 & 0 & 0 & 72 \\
\hline $\mathrm{X}-04-128$ & 10 & 6 & 6 & 7 & 11 & 11 & 3 & 6 & 6 & 7 & 7 & 4 & 4 & 7 & 11 & 106 \\
\hline$X-04-129$ & 9 & 7 & 10 & 5 & 10 & 11 & 10 & 5 & 5 & 6 & 6 & 5 & 7 & 7 & 9 & 112 \\
\hline$X-04-130$ & 3 & 2 & 6 & 2 & 10 & 11 & 9 & 4 & 4 & 5 & 5 & 4 & 4 & 6 & 7 & 82 \\
\hline X-04-132 & 13 & 11 & 16 & 10 & 13 & 10 & 11 & 3 & 3 & 4 & 4 & 2 & 1 & 14 & 15 & 130 \\
\hline X-04-133 & 5 & 2 & 6 & 6 & 9 & 8 & 3 & 4 & 4 & 3 & 3 & 3 & 3 & 7 & 11 & 77 \\
\hline $\mathrm{X}-04-137$ & 15 & 15 & 14 & 13 & 10 & 11 & 13 & 4 & 5 & 5 & 5 & 3 & 3 & 10 & 14 & 140 \\
\hline X-04-138 & 14 & 11 & 16 & 8 & 10 & 11 & 14 & 6 & 3 & 6 & 5 & 5 & 5 & 16 & 15 & 145 \\
\hline $\mathrm{X}-04-141$ & 15 & 10 & 14 & 9 & 13 & 14 & 13 & 8 & 8 & 9 & 9 & 7 & 9 & 12 & 12 & 162 \\
\hline$X-04-142$ & 16 & 10 & 13 & 10 & 11 & 13 & 13 & 6 & 8 & 8 & 9 & 6 & 9 & 15 & 10 & 157 \\
\hline X-04-145 & 12 & 11 & 10 & 11 & 10 & 11 & 13 & 6 & 6 & 7 & 7 & 8 & 8 & 12 & 15 & 147 \\
\hline X-04-147 & 12 & 12 & 13 & 11 & 8 & 13 & 12 & 9 & 9 & 6 & 9 & 6 & 8 & 11 & 11 & 150 \\
\hline X-04-149 & 11 & 8 & 11 & 5 & 11 & 12 & 7 & 6 & 6 & 7 & 7 & 8 & 8 & 10 & 11 & 128 \\
\hline X-04-150 & 15 & 8 & 13 & 10 & 10 & 7 & 7 & 4 & 4 & 7 & 5 & 5 & 3 & 11 & 10 & 119 \\
\hline
\end{tabular}


Appendix 3: Table showing the twelve individual habitat parameter scores adapted from Barbour et al. (1999) that were assessed at most sites where fishing effort was applied and were totalled to derive the Habitat Assessment Score for each site.

\begin{tabular}{|c|c|c|c|c|c|c|c|c|c|c|c|c|c|c|c|c|}
\hline \multirow{2}{*}{ Site Number } & \multirow{2}{*}{ 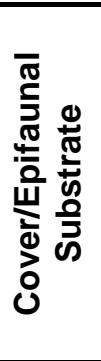 } & \multirow{2}{*}{ 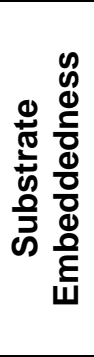 } & \multirow{2}{*}{ 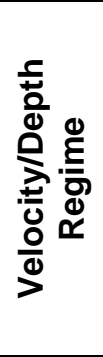 } & \multirow{2}{*}{ 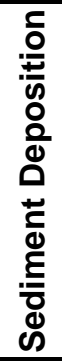 } & \multirow{2}{*}{ 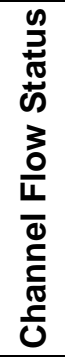 } & \multirow{2}{*}{ 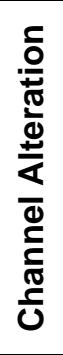 } & \multirow{2}{*}{ 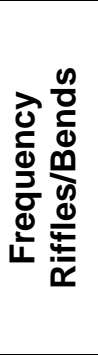 } & \multicolumn{2}{|c|}{ 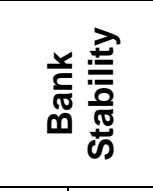 } & \multicolumn{2}{|c|}{ 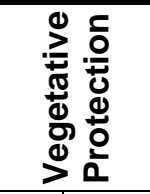 } & \multicolumn{2}{|c|}{ 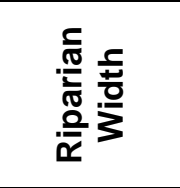 } & \multirow{2}{*}{ 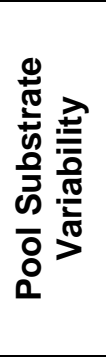 } & \multirow{2}{*}{ 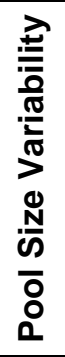 } & \multirow{2}{*}{ 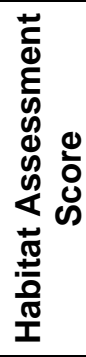 } \\
\hline & & & & & & & & Фృ & $\frac{\text { 돔 }}{\frac{0}{x}}$ & $\Phi$ & $\frac{\text { 돔 }}{\frac{0}{x}}$ & Ф্త & $\frac{\text { 픔 }}{\frac{0}{x}}$ & & & \\
\hline $\mathrm{X}-04-151$ & 10 & 7 & 12 & 5 & 10 & 12 & 12 & 6 & 6 & 7 & 5 & 6 & 3 & 7 & 10 & 118 \\
\hline$X-04-152$ & 7 & 6 & 11 & 7 & 11 & 12 & 10 & 7 & 7 & 7 & 7 & 7 & 7 & 6 & 13 & 125 \\
\hline $\mathrm{X}-04-153$ & 8 & 6 & 5 & 8 & 11 & 16 & 5 & 7 & 7 & 5 & 5 & 7 & 7 & 10 & 15 & 122 \\
\hline X-04-154 & 14 & 13 & 13 & 14 & 10 & 14 & 14 & 8 & 8 & 6 & 6 & 8 & 8 & 18 & 15 & 169 \\
\hline $\mathrm{X}-04-155$ & 15 & 11 & 14 & 11 & 10 & 14 & 14 & 7 & 7 & 6 & 6 & 3 & 3 & 16 & 11 & 148 \\
\hline $\mathrm{X}-04-156$ & 11 & 9 & 13 & 11 & 8 & 7 & 6 & 6 & 6 & 6 & 5 & 6 & 4 & 11 & 11 & 120 \\
\hline $\mathrm{X}-04-157$ & 14 & 16 & 14 & 12 & 13 & 14 & 13 & 9 & 9 & 9 & 9 & 8 & 8 & 16 & 13 & 177 \\
\hline$X-04-159$ & 14 & 15 & 11 & 16 & 11 & 9 & 10 & 8 & 8 & 5 & 7 & 3 & 3 & 16 & 10 & 146 \\
\hline $\mathrm{X}-04-160$ & 14 & 16 & 10 & 15 & 11 & 9 & 6 & 9 & 9 & 6 & 9 & 3 & 6 & 16 & 14 & 153 \\
\hline $\mathrm{X}-04-161$ & 12 & 16 & 10 & 14 & 14 & 7 & 6 & 9 & 9 & 6 & 6 & 5 & 5 & 16 & 14 & 149 \\
\hline$X-04-162$ & 14 & 18 & 14 & 13 & 13 & 13 & 11 & 9 & 9 & 8 & 8 & 9 & 9 & 16 & 15 & 179 \\
\hline $\mathrm{X}-04-163$ & 11 & 12 & 9 & 11 & 13 & 8 & 5 & 6 & 4 & 3 & 5 & 2 & 2 & 16 & 10 & 117 \\
\hline $\mathrm{X}-04-164$ & 15 & 10 & 11 & 13 & 11 & 11 & 9 & 8 & 8 & 7 & 7 & 6 & 6 & 16 & 13 & 151 \\
\hline X-04-167 & 7 & 5 & 1 & 6 & 5 & 8 & 3 & 6 & 6 & 5 & 5 & 5 & 5 & 8 & 14 & 89 \\
\hline X-04-168 & 7 & 3 & 8 & 5 & 8 & 8 & 0 & 6 & 6 & 5 & 5 & 5 & 5 & 11 & 12 & 94 \\
\hline X-04-169 & 13 & 9 & 10 & 13 & 8 & 11 & 5 & 8 & 8 & 6 & 7 & 3 & 6 & 12 & 10 & 129 \\
\hline $\mathrm{X}-04-170$ & 12 & 11 & 7 & 11 & 13 & 10 & 6 & 8 & 8 & 7 & 8 & 5 & 8 & 11 & 9 & 134 \\
\hline$X-04-171$ & 13 & 13 & 11 & 14 & 14 & 10 & 6 & 7 & 7 & 5 & 5 & 3 & 8 & 16 & 11 & 143 \\
\hline $\mathrm{X}-04-172$ & 13 & 18 & 8 & 16 & 7 & 11 & 8 & 8 & 8 & 5 & 8 & 3 & 5 & 16 & 10 & 144 \\
\hline$X-04-175$ & 12 & 6 & 8 & 10 & 6 & 11 & 10 & 7 & 7 & 5 & 5 & 5 & 4 & 11 & 7 & 114 \\
\hline$X-04-176$ & 11 & 12 & 5 & 11 & 5 & 11 & 6 & 8 & 8 & 6 & 6 & 6 & 6 & 11 & 7 & 119 \\
\hline $\mathrm{X}-04-177$ & 12 & 15 & 11 & 13 & 9 & 11 & 11 & 7 & 8 & 6 & 7 & 7 & 6 & 15 & 11 & 149 \\
\hline X-04-178 & 8 & 7 & 5 & 4 & 7 & 8 & 1 & 4 & 4 & 3 & 3 & 4 & 4 & 6 & 9 & 77 \\
\hline $\mathrm{X}-04-179$ & 13 & 6 & 6 & 11 & 10 & 16 & 5 & 8 & 8 & 8 & 8 & 8 & 4 & 12 & 13 & 136 \\
\hline $\mathrm{X}-04-181$ & 11 & 5 & 9 & 4 & 11 & 6 & 6 & 5 & 5 & 5 & 5 & 3 & 8 & 14 & 10 & 107 \\
\hline
\end{tabular}


Appendix 3: Table showing the twelve individual habitat parameter scores adapted from Barbour et al. (1999) that were assessed at most sites where fishing effort was applied and were totalled to derive the Habitat Assessment Score for each site.

\begin{tabular}{|c|c|c|c|c|c|c|c|c|c|c|c|c|c|c|c|c|}
\hline \multirow{2}{*}{ Site Number } & \multirow{2}{*}{ 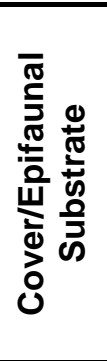 } & \multirow{2}{*}{ 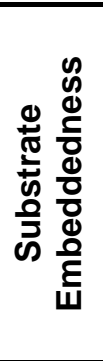 } & \multirow{2}{*}{ 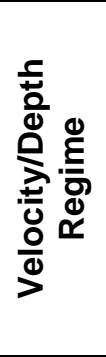 } & \multirow{2}{*}{ 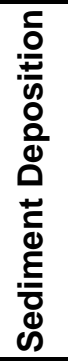 } & \multirow{2}{*}{ 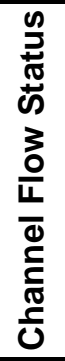 } & \multirow{2}{*}{ 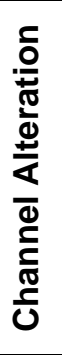 } & \multirow{2}{*}{ 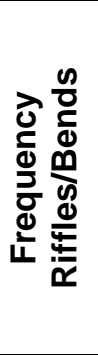 } & \multicolumn{2}{|c|}{ 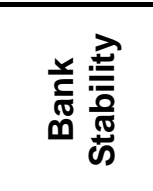 } & \multicolumn{2}{|c|}{ 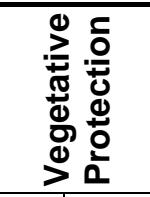 } & \multicolumn{2}{|c|}{ 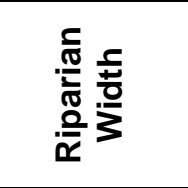 } & \multirow{2}{*}{ 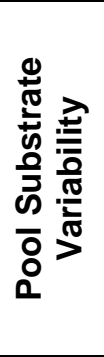 } & \multirow{2}{*}{ 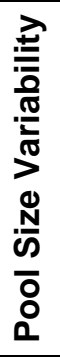 } & \multirow{2}{*}{ 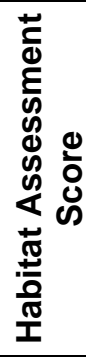 } \\
\hline & & & & & & & & ప্త & $\frac{\text { 픔 }}{\frac{0}{x}}$ & ప্త & $\frac{F}{\frac{\hbar}{x}}$ & 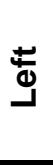 & $\frac{\tilde{E}}{\frac{\pi}{x}}$ & & & \\
\hline $\mathrm{X}-04-182$ & 8 & 8 & 7 & 5 & 9 & 8 & 3 & 6 & 6 & 5 & 5 & 1 & 1 & 7 & 12 & 91 \\
\hline $\mathrm{X}-04-184$ & 9 & 8 & 5 & 10 & 7 & 9 & 2 & 6 & 6 & 6 & 6 & 6 & 6 & 12 & 7 & 105 \\
\hline X-04-185 & 11 & 10 & 9 & 9 & 7 & 10 & 7 & 5 & 5 & 4 & 4 & 1 & 1 & 12 & 8 & 103 \\
\hline X-04-186 & 9 & 7 & 4 & 8 & 6 & 9 & 0 & 6 & 6 & 4 & 4 & 2 & 4 & 13 & 10 & 92 \\
\hline X-04-187 & 12 & 11 & 11 & 13 & 9 & 11 & 5 & 9 & 9 & 6 & 6 & 5 & 5 & 16 & 10 & 138 \\
\hline X-04-190 & 5 & 4 & 7 & 3 & 8 & 8 & 3 & 1 & 1 & 2 & 2 & 1 & 2 & 6 & 11 & 64 \\
\hline X-04-192 & 11 & 15 & 9 & 8 & 8 & 10 & 4 & 6 & 6 & 4 & 4 & 1 & 1 & 13 & 10 & 110 \\
\hline X-04-194 & 11 & 10 & 0 & 7 & 0 & 8 & 0 & 6 & 6 & 5 & 5 & 3 & 3 & 10 & 11 & 85 \\
\hline X-04-195 & 13 & 7 & 7 & 9 & 12 & 10 & 1 & 6 & 6 & 6 & 7 & 4 & 8 & 12 & 14 & 122 \\
\hline X-04-196 & 5 & 5 & 9 & 1 & 12 & 8 & 4 & 4 & 4 & 4 & 4 & 2 & 2 & 6 & 15 & 85 \\
\hline X-04-198 & 12 & 15 & 4 & 10 & 8 & 10 & 2 & 6 & 6 & 4 & 4 & 2 & 2 & 14 & 9 & 108 \\
\hline X-04-199 & 12 & 8 & 6 & 7 & 10 & 10 & 3 & 6 & 6 & 5 & 5 & 5 & 8 & 14 & 13 & 118 \\
\hline X-04-200 & 7 & 11 & 6 & 5 & 8 & 6 & 1 & 6 & 6 & 5 & 5 & 3 & 3 & 8 & 11 & 91 \\
\hline$X-04-201$ & 11 & 12 & 11 & 8 & 6 & 9 & 8 & 7 & 7 & 6 & 6 & 2 & 2 & 8 & 11 & 114 \\
\hline X-04-202 & 8 & 6 & 11 & 5 & 9 & 7 & 3 & 6 & 6 & 4 & 4 & 2 & 2 & 6 & 11 & 90 \\
\hline X-04-203 & 13 & 8 & 3 & 8 & 16 & 11 & 8 & 6 & 6 & 5 & 8 & 3 & 7 & 12 & 13 & 127 \\
\hline X-04-204 & 8 & 6 & 3 & 8 & 9 & 7 & 1 & 6 & 6 & 4 & 6 & 1 & 5 & 8 & 7 & 85 \\
\hline X-04-205 & 14 & 17 & 14 & 15 & 14 & 15 & 13 & 7 & 7 & 7 & 8 & 7 & 7 & 16 & 15 & 176 \\
\hline X-04-206 & 12 & 12 & 9 & 11 & 9 & 10 & 7 & 7 & 7 & 5 & 8 & 5 & 8 & 11 & 12 & 133 \\
\hline X-04-207 & 12 & 14 & 7 & 12 & 14 & 14 & 8 & 7 & 7 & 9 & 9 & 8 & 8 & 15 & 11 & 155 \\
\hline X-04-208 & 12 & 11 & 14 & 5 & 8 & 11 & 8 & 5 & 5 & 5 & 5 & 9 & 9 & 11 & 16 & 134 \\
\hline X-04-209 & 16 & 18 & 18 & 11 & 12 & 15 & 13 & 8 & 8 & 9 & 9 & 8 & 9 & 10 & 15 & 179 \\
\hline $\mathrm{X}-04-210$ & 16 & 14 & 6 & 9 & 12 & 14 & 10 & 5 & 5 & 6 & 6 & 9 & 6 & 13 & 12 & 143 \\
\hline $\mathrm{X}-04-212$ & 7 & 12 & 1 & 6 & 9 & 8 & 6 & 6 & 6 & 4 & 4 & 2 & 2 & 10 & 12 & 95 \\
\hline X-04-213 & 8 & 5 & 2 & 6 & 9 & 8 & 1 & 6 & 6 & 4 & 4 & 2 & 2 & 8 & 12 & 83 \\
\hline
\end{tabular}


Appendix 3: Table showing the twelve individual habitat parameter scores adapted from Barbour et al. (1999) that were assessed at most sites where fishing effort was applied and were totalled to derive the Habitat Assessment Score for each site.

\begin{tabular}{|c|c|c|c|c|c|c|c|c|c|c|c|c|c|c|c|c|}
\hline \multirow{2}{*}{ Site Number } & \multirow{2}{*}{ 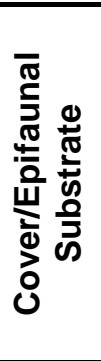 } & \multirow{2}{*}{ 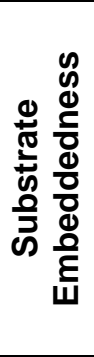 } & \multirow{2}{*}{ 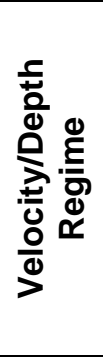 } & \multirow{2}{*}{ 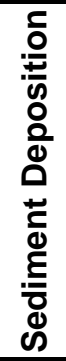 } & \multirow{2}{*}{ 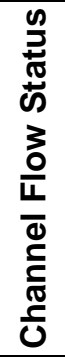 } & \multirow{2}{*}{ 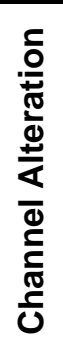 } & \multirow{2}{*}{ 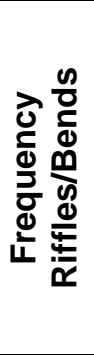 } & \multicolumn{2}{|c|}{ 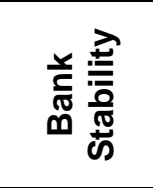 } & \multicolumn{2}{|c|}{ 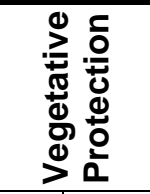 } & \multicolumn{2}{|c|}{ 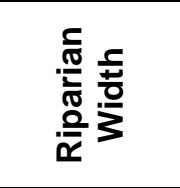 } & \multirow{2}{*}{ 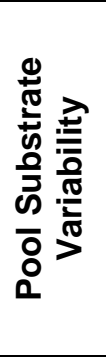 } & \multirow{2}{*}{ 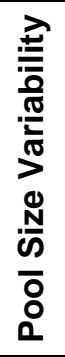 } & \multirow{2}{*}{ 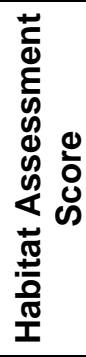 } \\
\hline & & & & & & & & Фృ & $\frac{\text { 돔 }}{\frac{0}{x}}$ & $\Phi$ & $\frac{\text { 돔 }}{\frac{0}{x}}$ & \pm & $\frac{\text { 픔 }}{\frac{0}{x}}$ & & & \\
\hline $\mathrm{X}-04-215$ & 7 & 6 & 1 & 6 & 14 & 8 & 2 & 5 & 6 & 5 & 5 & 2 & 2 & 10 & 11 & 90 \\
\hline$X-04-216$ & 7 & 2 & 1 & 6 & 9 & 8 & 2 & 6 & 6 & 4 & 4 & 3 & 3 & 10 & 11 & 82 \\
\hline$x-04-221$ & 15 & 14 & 18 & 10 & 10 & 19 & 14 & 9 & 9 & 9 & 9 & 5 & 5 & 10 & 13 & 169 \\
\hline $\mathrm{X}-04-222$ & 14 & 16 & 13 & 13 & 9 & 16 & 13 & 8 & 8 & 7 & 7 & 10 & 10 & 14 & 11 & 169 \\
\hline$X-04-223$ & 14 & 14 & 14 & 10 & 8 & 16 & 15 & 9 & 9 & 7 & 7 & 6 & 6 & 14 & 12 & 161 \\
\hline $\mathrm{X}-04-225$ & 11 & 11 & 12 & 11 & 9 & 11 & 7 & 7 & 7 & 7 & 7 & 4 & 4 & 13 & 11 & 132 \\
\hline $\mathrm{X}-04-227$ & 11 & 12 & 13 & 13 & 7 & 15 & 14 & 5 & 5 & 7 & 7 & 9 & 9 & 12 & 11 & 150 \\
\hline$X-04-228$ & 8 & 7 & 7 & 5 & 7 & 15 & 7 & 4 & 4 & 4 & 4 & 9 & 9 & 6 & 11 & 107 \\
\hline $\mathrm{X}-04-232$ & 9 & 9 & 4 & 11 & 10 & 11 & 6 & 7 & 7 & 7 & 7 & 9 & 9 & 11 & 8 & 125 \\
\hline $\mathrm{X}-04-238$ & 11 & 14 & 9 & 12 & 7 & 13 & 18 & 7 & 7 & 6 & 6 & 9 & 9 & 13 & 7 & 148 \\
\hline$X-04-239$ & 14 & 13 & 17 & 10 & 11 & 11 & 11 & 8 & 8 & 7 & 7 & 5 & 5 & 15 & 15 & 157 \\
\hline $\mathrm{X}-04-248$ & 9 & 6 & 6 & 3 & 8 & 10 & 2 & 3 & 3 & 7 & 7 & 5 & 5 & 7 & 11 & 92 \\
\hline $\mathrm{X}-04-249$ & 8 & 10 & 1 & 7 & 10 & 11 & 5 & 6 & 6 & 5 & 5 & 3 & 3 & 12 & 13 & 105 \\
\hline$X-04-251$ & 9 & 13 & 7 & 5 & 13 & 6 & 8 & 7 & 7 & 3 & 3 & 0 & 0 & 0 & 0 & 81 \\
\hline $\mathrm{X}-04-253$ & 14 & 11 & 14 & 7 & 9 & 5 & 6 & 2 & 2 & 3 & 3 & 4 & 4 & 9 & 13 & 106 \\
\hline X-04-255 & 11 & 13 & 13 & 10 & 8 & 5 & 9 & 4 & 4 & 4 & 4 & 7 & 4 & 8 & 13 & 117 \\
\hline $\mathrm{X}-04-256$ & 12 & 11 & 9 & 6 & 8 & 6 & 10 & 6 & 6 & 5 & 5 & 6 & 6 & 8 & 8 & 112 \\
\hline $\mathrm{X}-04-257$ & 12 & 15 & 11 & 11 & 9 & 6 & 7 & 6 & 6 & 5 & 5 & 2 & 2 & 8 & 13 & 118 \\
\hline $\mathrm{X}-04-258$ & 10 & 14 & 13 & 9 & 8 & 8 & 15 & 7 & 7 & 4 & 4 & 5 & 5 & 15 & 9 & 133 \\
\hline$X-04-259$ & 15 & 14 & 17 & 10 & 8 & 11 & 15 & 7 & 7 & 8 & 8 & 7 & 7 & 13 & 12 & 159 \\
\hline$X-04-260$ & 11 & 9 & 16 & 10 & 9 & 14 & 14 & 7 & 7 & 7 & 7 & 3 & 3 & 12 & 14 & 143 \\
\hline $\mathrm{X}-04-262$ & 13 & 16 & 11 & 10 & 8 & 13 & 12 & 7 & 7 & 7 & 7 & 3 & 6 & 12 & 11 & 143 \\
\hline D-05-011 & 16 & 3 & 10 & 11 & 19 & 10 & 18 & 8 & 8 & 6 & 7 & 8 & 8 & 8 & 13 & 153 \\
\hline D-05-013 & 8 & 5 & 16 & 3 & 13 & 13 & 17 & 4 & 6 & 9 & 6 & 9 & 6 & 6 & 10 & 131 \\
\hline D-05-014 & 10 & 3 & 6 & 5 & 6 & 6 & 3 & 9 & 9 & 5 & 5 & 8 & 5 & 6 & 11 & 97 \\
\hline
\end{tabular}


Appendix 3: Table showing the twelve individual habitat parameter scores adapted from Barbour et al. (1999) that were assessed at most sites where fishing effort was applied and were totalled to derive the Habitat Assessment Score for each site.

\begin{tabular}{|c|c|c|c|c|c|c|c|c|c|c|c|c|c|c|c|c|}
\hline \multirow{2}{*}{ Site Number } & \multirow{2}{*}{ 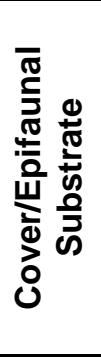 } & \multirow{2}{*}{ 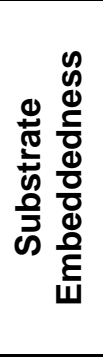 } & \multirow{2}{*}{ 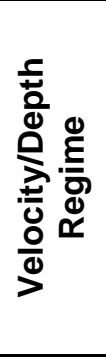 } & \multirow{2}{*}{ 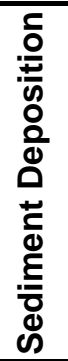 } & \multirow{2}{*}{ 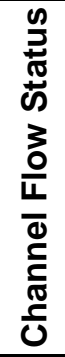 } & \multirow{2}{*}{ 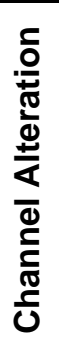 } & \multirow{2}{*}{ 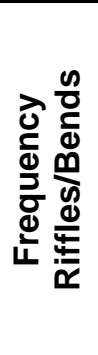 } & \multicolumn{2}{|c|}{ 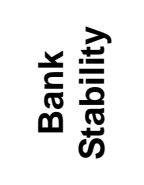 } & \multicolumn{2}{|c|}{ 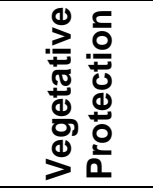 } & \multicolumn{2}{|c|}{$\begin{array}{l}\frac{c}{\sqrt{0}} \\
\frac{2}{\frac{0}{\pi}} \\
\frac{0}{x}\end{array}$} & \multirow{2}{*}{ 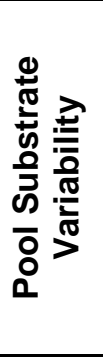 } & \multirow{2}{*}{ 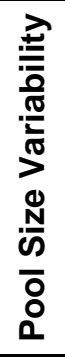 } & \multirow{2}{*}{ 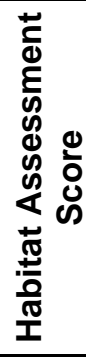 } \\
\hline & & & & & & & & 屯্త & $\begin{array}{l}\frac{}{\frac{1}{\sigma}} \\
\frac{0}{x}\end{array}$ & Ф্త & $\frac{\text { 픔 }}{\frac{0}{\tilde{x}}}$ & 屯্ & $\frac{\tilde{n}}{\frac{\tilde{\sigma}}{\boldsymbol{x}}}$ & & & \\
\hline D-05-015 & 10 & 5 & 6 & 5 & 6 & 6 & 5 & 8 & 8 & 8 & 5 & 8 & 5 & 6 & 13 & 104 \\
\hline D-05-018 & 11 & 8 & 11 & 10 & 13 & 8 & 8 & 8 & 8 & 8 & 8 & 6 & 7 & 15 & 11 & 140 \\
\hline D-05-019 & 12 & 10 & 11 & 11 & 13 & 13 & 6 & 7 & 7 & 8 & 8 & 8 & 8 & 10 & 11 & 143 \\
\hline D-05-020 & 11 & 11 & 10 & 10 & 16 & 10 & 8 & 8 & 8 & 7 & 7 & 5 & 7 & 13 & 11 & 142 \\
\hline D-05-023 & 11 & 10 & 18 & 11 & 12 & 9 & 6 & 7 & 7 & 6 & 6 & 4 & 4 & 8 & 11 & 130 \\
\hline D-05-025 & 13 & 10 & 6 & 10 & 8 & 15 & 15 & 9 & 9 & 8 & 8 & 6 & 8 & 11 & 8 & 144 \\
\hline D-05-026 & 14 & 8 & 7 & 10 & 8 & 15 & 16 & 7 & 7 & 8 & 8 & 8 & 8 & 8 & 11 & 143 \\
\hline W-05-001 & 10 & 6 & 14 & 8 & 7 & 8 & 6 & 7 & 8 & 5 & 5 & 7 & 7 & 7 & 10 & 115 \\
\hline W-05-002 & 9 & 10 & 7 & 8 & 7 & 8 & 3 & 8 & 8 & 5 & 5 & 2 & 1 & 10 & 8 & 99 \\
\hline W-05-009 & 12 & 12 & 14 & 13 & 10 & 13 & 10 & 7 & 7 & 5 & 8 & 4 & 8 & 13 & 10 & 146 \\
\hline W-05-012 & 10 & 6 & 6 & 6 & 10 & 15 & 8 & 8 & 8 & 8 & 6 & 9 & 6 & 13 & 11 & 130 \\
\hline W-05-013 & 11 & 12 & 14 & 9 & 10 & 11 & 7 & 8 & 6 & 5 & 5 & 5 & 6 & 15 & 9 & 133 \\
\hline W-05-014 & 3 & 15 & 12 & 11 & 7 & 5 & 3 & 4 & 5 & 1 & 2 & 3 & 4 & 9 & 8 & 92 \\
\hline W-05-016 & 12 & 13 & 13 & 12 & 14 & 11 & 10 & 7 & 7 & 4 & 4 & 2 & 2 & 11 & 8 & 130 \\
\hline W-05-017 & 14 & 16 & 14 & 5 & 15 & 10 & 16 & 8 & 8 & 7 & 7 & 6 & 6 & 17 & 13 & 162 \\
\hline W-05-019 & 11 & 11 & 13 & 11 & 9 & 11 & 8 & 7 & 7 & 5 & 5 & 1 & 1 & 12 & 11 & 123 \\
\hline W-05-020 & 10 & 10 & 6 & 8 & 6 & 14 & 5 & 9 & 9 & 8 & 8 & 6 & 6 & 11 & 11 & 127 \\
\hline W-05-023 & 6 & 8 & 5 & 5 & 6 & 8 & 2 & 7 & 7 & 5 & 5 & 4 & 4 & 11 & 6 & 89 \\
\hline W-05-024 & 9 & 8 & 10 & 7 & 9 & 8 & 3 & 7 & 7 & 6 & 6 & 7 & 7 & 10 & 9 & 113 \\
\hline W-05-027 & 11 & 9 & 4 & 5 & 6 & 7 & 5 & 6 & 6 & 5 & 5 & 3 & 3 & 13 & 5 & 93 \\
\hline W-05-028 & 13 & 5 & 6 & 5 & 6 & 11 & 10 & 7 & 7 & 5 & 5 & 6 & 4 & 16 & 12 & 118 \\
\hline W-05-030 & 8 & 7 & 3 & 7 & 3 & 11 & 3 & 8 & 8 & 5 & 5 & 5 & 5 & 14 & 8 & 100 \\
\hline W-05-034 & 10 & 7 & 3 & 8 & 5 & 10 & 2 & 7 & 7 & 5 & 5 & 4 & 3 & 11 & 6 & 93 \\
\hline W-05-036 & 8 & 11 & 5 & 10 & 6 & 10 & 5 & 7 & 7 & 4 & 4 & 2 & 2 & 11 & 5 & 97 \\
\hline W-05-037 & 5 & 10 & 5 & 6 & 6 & 6 & 2 & 7 & 8 & 7 & 8 & 3 & 5 & 9 & 5 & 92 \\
\hline
\end{tabular}


Appendix 3: Table showing the twelve individual habitat parameter scores adapted from Barbour et al. (1999) that were assessed at most sites where fishing effort was applied and were totalled to derive the Habitat Assessment Score for each site.

\begin{tabular}{|c|c|c|c|c|c|c|c|c|c|c|c|c|c|c|c|c|}
\hline \multirow{2}{*}{ Site Number } & \multirow{2}{*}{ 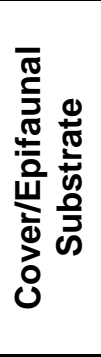 } & \multirow{2}{*}{ 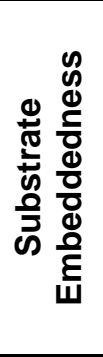 } & \multirow{2}{*}{ 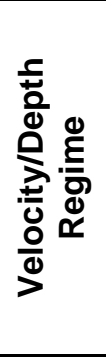 } & \multirow{2}{*}{ 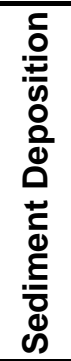 } & \multirow{2}{*}{ 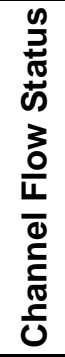 } & \multirow{2}{*}{ 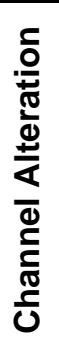 } & \multirow{2}{*}{ 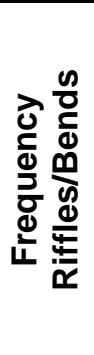 } & \multicolumn{2}{|c|}{ 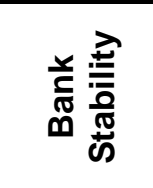 } & \multicolumn{2}{|c|}{ 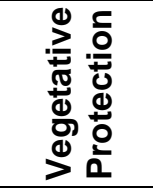 } & \multicolumn{2}{|c|}{$\begin{array}{l}\frac{c}{\sqrt{0}} \\
\frac{2}{\frac{0}{\pi}} \\
\frac{0}{x}\end{array}$} & \multirow{2}{*}{ 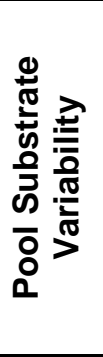 } & \multirow{2}{*}{ 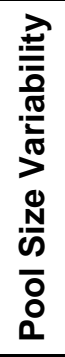 } & \multirow{2}{*}{ 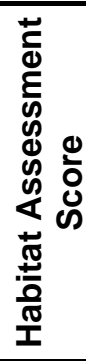 } \\
\hline & & & & & & & & 屯্త & $\begin{array}{l}\frac{}{\frac{1}{\sigma}} \\
\frac{0}{x}\end{array}$ & Ф્త & $\frac{\text { 픔 }}{\frac{0}{\tilde{x}}}$ & 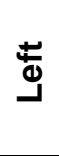 & $\frac{\tilde{n}}{\frac{\tilde{\sigma}}{\boldsymbol{x}}}$ & & & \\
\hline W-05-038 & 9 & 7 & 4 & 8 & 10 & 8 & 2 & 5 & 5 & 5 & 5 & 4 & 3 & 12 & 8 & 95 \\
\hline W-05-041 & 12 & 8 & 8 & 8 & 7 & 11 & 6 & 8 & 8 & 5 & 5 & 2 & 3 & 12 & 11 & 114 \\
\hline W-05-043 & 10 & 11 & 8 & 10 & 7 & 12 & 8 & 6 & 6 & 7 & 7 & 5 & 5 & 13 & 10 & 125 \\
\hline W-05-045 & 10 & 7 & 5 & 3 & 6 & 11 & 4 & 5 & 5 & 2 & 2 & 1 & 1 & 9 & 3 & 74 \\
\hline W-05-046 & 10 & 5 & 13 & 5 & 6 & 11 & 7 & 3 & 3 & 5 & 5 & 3 & 3 & 7 & 13 & 99 \\
\hline W-05-047 & 13 & 10 & 9 & 7 & 7 & 11 & 5 & 7 & 7 & 5 & 5 & 1 & 1 & 14 & 11 & 113 \\
\hline W-05-048 & 7 & 6 & 4 & 6 & 5 & 11 & 5 & 6 & 6 & 5 & 5 & 5 & 5 & 6 & 3 & 85 \\
\hline W-05-049 & 14 & 15 & 8 & 14 & 7 & 14 & 5 & 8 & 8 & 7 & 7 & 8 & 8 & 15 & 6 & 144 \\
\hline W-05-050 & 14 & 15 & 6 & 11 & 13 & 10 & 3 & 8 & 8 & 5 & 5 & 5 & 5 & 13 & 12 & 133 \\
\hline W-05-051 & 16 & 16 & 6 & 14 & 17 & 13 & 11 & 7 & 7 & 5 & 5 & 7 & 7 & 15 & 14 & 160 \\
\hline W-05-052 & 10 & 15 & 5 & 15 & 7 & 11 & 5 & 7 & 7 & 5 & 5 & 3 & 3 & 12 & 9 & 119 \\
\hline W-05-053 & 11 & 11 & 3 & 11 & 7 & 8 & 2 & 7 & 7 & 5 & 5 & 4 & 4 & 13 & 8 & 106 \\
\hline W-05-054 & 6 & 3 & 3 & 1 & 6 & 8 & 3 & 4 & 3 & 3 & 3 & 4 & 4 & 7 & 2 & 60 \\
\hline W-05-055 & 16 & 15 & 9 & 5 & 5 & 16 & 16 & 9 & 8 & 9 & 9 & 9 & 9 & 16 & 8 & 159 \\
\hline W-05-056 & 19 & 18 & 18 & 15 & 10 & 12 & 16 & 8 & 8 & 9 & 9 & 10 & 10 & 18 & 17 & 197 \\
\hline W-05-057 & 8 & 13 & 13 & 11 & 6 & 16 & 10 & 2 & 2 & 2 & 2 & 2 & 2 & 10 & 10 & 109 \\
\hline W-05-058 & 17 & 16 & 14 & 13 & 9 & 18 & 18 & 8 & 8 & 9 & 9 & 9 & 9 & 13 & 17 & 187 \\
\hline W-05-059 & 15 & 16 & 15 & 16 & 13 & 16 & 16 & 9 & 9 & 8 & 8 & 2 & 2 & 17 & 12 & 174 \\
\hline W-05-060 & 8 & 5 & 6 & 4 & 6 & 15 & 11 & 0 & 0 & 4 & 4 & 1 & 1 & 8 & 3 & 76 \\
\hline W-05-062 & 5 & 13 & 2 & 12 & 2 & 18 & 3 & 6 & 6 & 3 & 3 & 1 & 1 & 7 & 13 & 95 \\
\hline W-05-063 & 13 & 13 & 6 & 12 & 3 & 15 & 7 & 9 & 9 & 5 & 5 & 1 & 1 & 11 & 11 & 121 \\
\hline W-05-065 & 16 & 15 & 16 & 13 & 11 & 16 & 16 & 9 & 9 & 5 & 5 & 5 & 5 & 18 & 18 & 177 \\
\hline W-05-066 & 16 & 11 & 16 & 6 & 15 & 16 & 16 & 9 & 9 & 9 & 9 & 9 & 9 & 17 & 18 & 185 \\
\hline W-05-069 & 15 & 15 & 6 & 13 & 9 & 8 & 6 & 8 & 8 & 8 & 8 & 3 & 3 & 13 & 6 & 129 \\
\hline W-05-071 & 11 & 10 & 6 & 10 & 7 & 10 & 5 & 7 & 7 & 5 & 5 & 9 & 7 & 13 & 6 & 118 \\
\hline
\end{tabular}


Appendix 3: Table showing the twelve individual habitat parameter scores adapted from Barbour et al. (1999) that were assessed at most sites where fishing effort was applied and were totalled to derive the Habitat Assessment Score for each site.

\begin{tabular}{|c|c|c|c|c|c|c|c|c|c|c|c|c|c|c|c|c|}
\hline \multirow{2}{*}{ Site Number } & \multirow{2}{*}{ 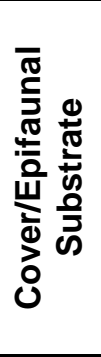 } & \multirow{2}{*}{ 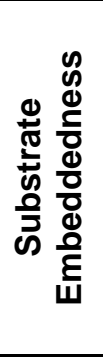 } & \multirow{2}{*}{ 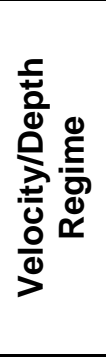 } & \multirow{2}{*}{ 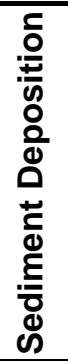 } & \multirow{2}{*}{ 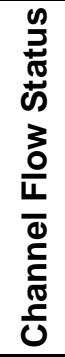 } & \multirow{2}{*}{ 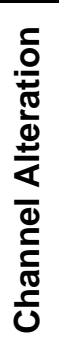 } & \multirow{2}{*}{ 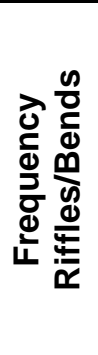 } & \multicolumn{2}{|c|}{ 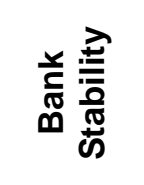 } & \multicolumn{2}{|c|}{ 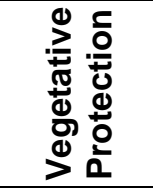 } & \multicolumn{2}{|c|}{$\begin{array}{l}\frac{c}{\sqrt{0}} \\
\frac{2}{\frac{0}{\pi}} \\
\frac{0}{x}\end{array}$} & \multirow{2}{*}{ 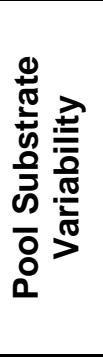 } & \multirow{2}{*}{ 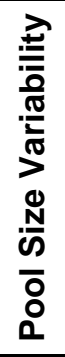 } & \multirow{2}{*}{ 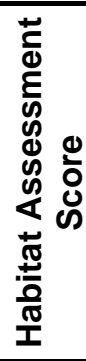 } \\
\hline & & & & & & & & Ф্త & $\begin{array}{l}\frac{}{\frac{1}{\sigma}} \\
\frac{0}{x}\end{array}$ & ప্త & $\frac{\text { 픔 }}{\frac{0}{\tilde{x}}}$ & Ф્త & $\frac{\tilde{n}}{\frac{\tilde{\sigma}}{\boldsymbol{x}}}$ & & & \\
\hline W-05-072 & 10 & 10 & 10 & 10 & 6 & 13 & 10 & 7 & 6 & 5 & 5 & 1 & 1 & 15 & 6 & 115 \\
\hline W-05-073 & 11 & 14 & 6 & 13 & 7 & 10 & 2 & 7 & 7 & 5 & 5 & 3 & 3 & 15 & 13 & 121 \\
\hline W-05-076 & 11 & 11 & 15 & 8 & 14 & 10 & 7 & 8 & 8 & 5 & 5 & 2 & 5 & 13 & 8 & 130 \\
\hline W-05-077 & 11 & 11 & 16 & 10 & 11 & 11 & 10 & 7 & 7 & 6 & 6 & 9 & 1 & 16 & 16 & 148 \\
\hline W-05-078 & 10 & 9 & 6 & 6 & 6 & 11 & 11 & 4 & 4 & 5 & 5 & 2 & 1 & 8 & 6 & 94 \\
\hline W-05-079 & 9 & 5 & 6 & 5 & 6 & 10 & 5 & 5 & 5 & 5 & 5 & 3 & 2 & 10 & 5 & 86 \\
\hline W-05-081 & 10 & 13 & 9 & 11 & 9 & 11 & 7 & 6 & 6 & 6 & 6 & 3 & 5 & 16 & 11 & 129 \\
\hline W-05-082 & 11 & 11 & 6 & 11 & 10 & 12 & 11 & 7 & 7 & 6 & 6 & 3 & 3 & 15 & 11 & 130 \\
\hline W-05-083 & 15 & 12 & 16 & 11 & 9 & 12 & 11 & 5 & 5 & 8 & 8 & 8 & 8 & 14 & 14 & 156 \\
\hline W-05-085 & 10 & 11 & 11 & 11 & 8 & 11 & 10 & 6 & 6 & 5 & 5 & 8 & 8 & 12 & 10 & 132 \\
\hline W-05-086 & 12 & 13 & 10 & 12 & 7 & 8 & 6 & 7 & 7 & 8 & 8 & 8 & 8 & 11 & 10 & 135 \\
\hline W-05-087 & 9 & 10 & 8 & 10 & 8 & 10 & 5 & 7 & 7 & 5 & 5 & 2 & 1 & 11 & 8 & 106 \\
\hline W-05-093 & 10 & 10 & 8 & 10 & 8 & 13 & 11 & 8 & 8 & 6 & 5 & 8 & 7 & 14 & 8 & 134 \\
\hline W-05-094 & 17 & 17 & 12 & 16 & 8 & 12 & 8 & 7 & 7 & 5 & 5 & 5 & 5 & 18 & 17 & 159 \\
\hline W-05-099 & 11 & 12 & 14 & 13 & 11 & 11 & 13 & 2 & 2 & 3 & 3 & 5 & 5 & 11 & 14 & 130 \\
\hline W-05-101 & 7 & 15 & 3 & 15 & 2 & 10 & 2 & 8 & 8 & 5 & 5 & 4 & 2 & 10 & 2 & 98 \\
\hline W-05-102 & 18 & 17 & 19 & 18 & 11 & 14 & 18 & 7 & 8 & 9 & 9 & 5 & 3 & 18 & 17 & 191 \\
\hline W-05-103 & 12 & 11 & 8 & 12 & 10 & 10 & 7 & 7 & 5 & 3 & 5 & 2 & 2 & 16 & 10 & 120 \\
\hline W-05-104 & 16 & 13 & 14 & 12 & 7 & 13 & 16 & 4 & 3 & 3 & 2 & 2 & 2 & 16 & 14 & 137 \\
\hline W-05-105 & 11 & 9 & 12 & 10 & 13 & 11 & 10 & 9 & 9 & 9 & 9 & 8 & 8 & 11 & 11 & 150 \\
\hline W-05-106 & 17 & 11 & 15 & 15 & 15 & 14 & 16 & 7 & 6 & 8 & 8 & 4 & 3 & 17 & 15 & 171 \\
\hline W-05-110 & 10 & 10 & 6 & 10 & 9 & 11 & 3 & 7 & 7 & 5 & 5 & 8 & 6 & 11 & 6 & 114 \\
\hline W-05-112 & 13 & 14 & 14 & 14 & 8 & 9 & 14 & 6 & 6 & 8 & 8 & 7 & 7 & 15 & 14 & 157 \\
\hline W-05-113 & 11 & 12 & 8 & 11 & 7 & 10 & 5 & 7 & 7 & 5 & 5 & 3 & 3 & 12 & 8 & 114 \\
\hline W-05-114 & 10 & 11 & 5 & 12 & 7 & 13 & 7 & 8 & 8 & 5 & 5 & 4 & 4 & 13 & 8 & 120 \\
\hline
\end{tabular}


Appendix 3: Table showing the twelve individual habitat parameter scores adapted from Barbour et al. (1999) that were assessed at most sites where fishing effort was applied and were totalled to derive the Habitat Assessment Score for each site.

\begin{tabular}{|c|c|c|c|c|c|c|c|c|c|c|c|c|c|c|c|c|}
\hline \multirow{2}{*}{ Site Number } & \multirow{2}{*}{ 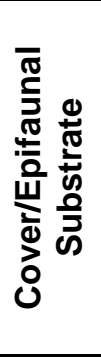 } & \multirow{2}{*}{ 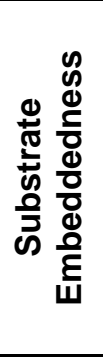 } & \multirow{2}{*}{ 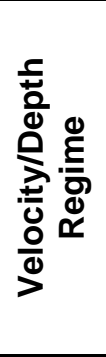 } & \multirow{2}{*}{ 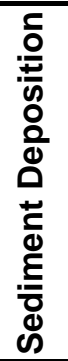 } & \multirow{2}{*}{ 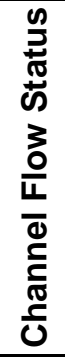 } & \multirow{2}{*}{ 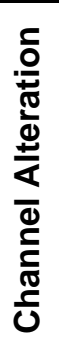 } & \multirow{2}{*}{ 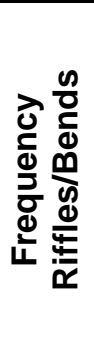 } & \multicolumn{2}{|c|}{ 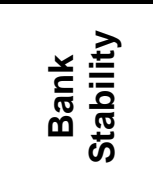 } & \multicolumn{2}{|c|}{ 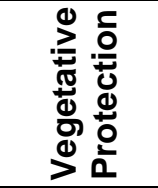 } & \multicolumn{2}{|c|}{$\begin{array}{l}\frac{c}{\sqrt{0}} \\
\frac{2}{\frac{0}{\pi}} \\
\frac{0}{x}\end{array}$} & \multirow{2}{*}{ 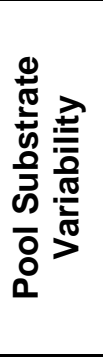 } & \multirow{2}{*}{ 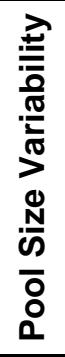 } & \multirow{2}{*}{ 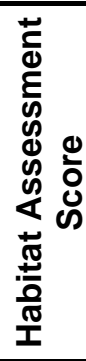 } \\
\hline & & & & & & & & 屯্త & $\begin{array}{l}\frac{}{\frac{1}{\sigma}} \\
\frac{0}{x}\end{array}$ & 屯 & 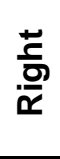 & 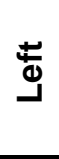 & $\frac{\tilde{n}}{\frac{\tilde{\sigma}}{\boldsymbol{x}}}$ & & & \\
\hline W-05-115 & 9 & 16 & 15 & 14 & 8 & 15 & 14 & 3 & 3 & 5 & 5 & 6 & 8 & 10 & 10 & 141 \\
\hline W-05-116 & 10 & 10 & 11 & 10 & 10 & 11 & 10 & 7 & 7 & 8 & 8 & 9 & 9 & 10 & 10 & 140 \\
\hline W-05-117 & 15 & 13 & 15 & 13 & 6 & 11 & 16 & 5 & 5 & 9 & 9 & 8 & 8 & 9 & 8 & 150 \\
\hline W-05-118 & 16 & 16 & 13 & 14 & 6 & 16 & 16 & 3 & 3 & 8 & 8 & 8 & 8 & 10 & 16 & 161 \\
\hline W-05-119 & 16 & 11 & 15 & 15 & 10 & 16 & 16 & 7 & 6 & 8 & 8 & 8 & 8 & 16 & 17 & 177 \\
\hline W-05-120 & 9 & 8 & 7 & 8 & 18 & 11 & 2 & 8 & 8 & 3 & 3 & 0 & 0 & 11 & 13 & 109 \\
\hline W-05-121 & 8 & 10 & 7 & 9 & 13 & 8 & 4 & 8 & 8 & 8 & 8 & 2 & 2 & 7 & 6 & 108 \\
\hline W-05-122 & 8 & 11 & 8 & 11 & 7 & 10 & 6 & 7 & 7 & 5 & 5 & 5 & 5 & 16 & 6 & 117 \\
\hline W-05-126 & 11 & 15 & 16 & 15 & 20 & 15 & 7 & 9 & 9 & 6 & 6 & 8 & 8 & 16 & 16 & 177 \\
\hline W-05-128 & 8 & 15 & 10 & 15 & 17 & 8 & 3 & 7 & 7 & 8 & 8 & 8 & 8 & 7 & 7 & 136 \\
\hline W-05-129 & 11 & 15 & 15 & 13 & 20 & 11 & 6 & 8 & 8 & 6 & 6 & 3 & 0 & 13 & 9 & 144 \\
\hline W-05-130 & 13 & 15 & 10 & 14 & 15 & 12 & 6 & 8 & 8 & 8 & 8 & 5 & 3 & 14 & 10 & 149 \\
\hline W-05-131 & 13 & 15 & 15 & 15 & 15 & 10 & 6 & 8 & 8 & 5 & 5 & 3 & 3 & 16 & 16 & 153 \\
\hline W-05-132 & 11 & 15 & 6 & 15 & 20 & 11 & 2 & 8 & 8 & 5 & 5 & 1 & 2 & 16 & 8 & 133 \\
\hline W-05-134 & 13 & 15 & 15 & 13 & 16 & 15 & 8 & 9 & 9 & 7 & 9 & 5 & 9 & 17 & 16 & 176 \\
\hline W-05-136 & 19 & 16 & 15 & 18 & 13 & 19 & 19 & 9 & 9 & 10 & 10 & 10 & 10 & 19 & 15 & 211 \\
\hline W-05-137 & 18 & 10 & 15 & 11 & 15 & 17 & 16 & 6 & 6 & 9 & 9 & 9 & 9 & 15 & 16 & 181 \\
\hline W-05-138 & 17 & 14 & 15 & 15 & 8 & 15 & 15 & 8 & 8 & 9 & 9 & 6 & 7 & 15 & 14 & 175 \\
\hline W-05-139 & 20 & 16 & 20 & 15 & 16 & 15 & 18 & 9 & 9 & 10 & 10 & 10 & 10 & 17 & 20 & 215 \\
\hline W-05-140 & 19 & 17 & 19 & 19 & 16 & 19 & 19 & 9 & 9 & 10 & 10 & 10 & 10 & 20 & 19 & 225 \\
\hline W-05-141 & 9 & 16 & 6 & 12 & 7 & 13 & 2 & 7 & 7 & 6 & 6 & 4 & 4 & 14 & 10 & 123 \\
\hline W-05-145 & 15 & 6 & 15 & 5 & 8 & 15 & 11 & 3 & 5 & 9 & 9 & 9 & 9 & 8 & 6 & 133 \\
\hline W-05-146 & 20 & 16 & 20 & 16 & 16 & 17 & 17 & 8 & 8 & 9 & 9 & 9 & 9 & 17 & 18 & 209 \\
\hline W-05-148 & 18 & 16 & 16 & 18 & 12 & 19 & 18 & 8 & 8 & 8 & 8 & 3 & 4 & 19 & 18 & 193 \\
\hline W-05-149 & 18 & 14 & 18 & 13 & 6 & 18 & 17 & 3 & 3 & 6 & 6 & 5 & 5 & 19 & 18 & 169 \\
\hline
\end{tabular}


Appendix 3: Table showing the twelve individual habitat parameter scores adapted from Barbour et al. (1999) that were assessed at most sites where fishing effort was applied and were totalled to derive the Habitat Assessment Score for each site.

\begin{tabular}{|c|c|c|c|c|c|c|c|c|c|c|c|c|c|c|c|c|}
\hline \multirow{2}{*}{ Site Number } & \multirow{2}{*}{ 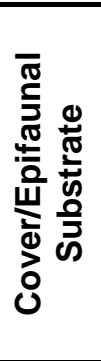 } & \multirow{2}{*}{ 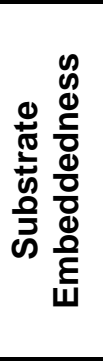 } & \multirow{2}{*}{ 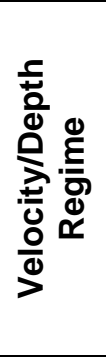 } & \multirow{2}{*}{ 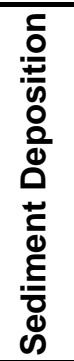 } & \multirow{2}{*}{ 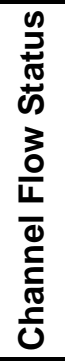 } & \multirow{2}{*}{ 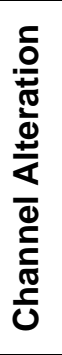 } & \multirow{2}{*}{ 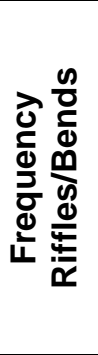 } & \multicolumn{2}{|c|}{ 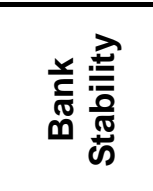 } & \multicolumn{2}{|c|}{ 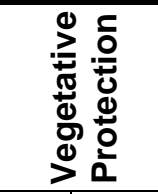 } & \multicolumn{2}{|c|}{ 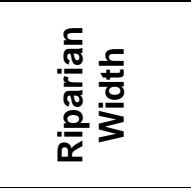 } & \multirow{2}{*}{ 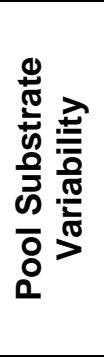 } & \multirow{2}{*}{ 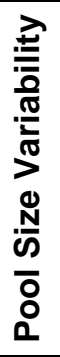 } & \multirow{2}{*}{ 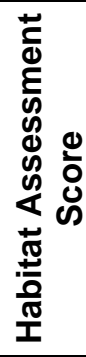 } \\
\hline & & & & & & & & ప্త & $\frac{\text { 듬 }}{\frac{0}{x}}$ & ๘ & $\frac{\text { 픔 }}{\frac{0}{x}}$ & 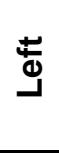 & 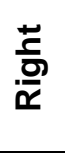 & & & \\
\hline W-05-150 & 16 & 15 & 18 & 11 & 6 & 18 & 16 & 6 & 6 & 9 & 9 & 9 & 9 & 16 & 16 & 180 \\
\hline W-05-151 & 16 & 12 & 17 & 8 & 7 & 17 & 18 & 7 & 7 & 6 & 6 & 5 & 5 & 13 & 10 & 154 \\
\hline W-05-152 & 17 & 16 & 18 & 12 & 18 & 18 & 6 & 9 & 9 & 7 & 7 & 7 & 8 & 15 & 13 & 180 \\
\hline W-05-154 & 15 & 13 & 11 & 16 & 10 & 18 & 9 & 5 & 5 & 6 & 6 & 3 & 3 & 12 & 11 & 143 \\
\hline W-05-157 & 18 & 15 & 16 & 11 & 10 & 18 & 16 & 9 & 9 & 9 & 9 & 9 & 9 & 18 & 18 & 194 \\
\hline W-05-159 & 15 & 13 & 15 & 13 & 8 & 16 & 16 & 8 & 8 & 8 & 8 & 8 & 8 & 15 & 14 & 173 \\
\hline W-05-160 & 16 & 15 & 15 & 16 & 17 & 17 & 17 & 9 & 9 & 7 & 7 & 9 & 9 & 14 & 15 & 192 \\
\hline W-05-161 & 13 & 13 & 15 & 12 & 20 & 16 & 11 & 8 & 8 & 8 & 8 & 7 & 7 & 14 & 16 & 176 \\
\hline W-05-162 & 13 & 11 & 13 & 13 & 20 & 15 & 11 & 8 & 8 & 6 & 6 & 8 & 8 & 14 & 10 & 164 \\
\hline W-05-163 & 6 & 5 & 5 & 6 & 5 & 4 & 5 & 7 & 7 & 4 & 4 & 2 & 2 & 3 & 5 & 70 \\
\hline W-05-164 & 15 & 15 & 16 & 15 & 19 & 16 & 12 & 8 & 7 & 5 & 7 & 1 & 5 & 17 & 16 & 174 \\
\hline W-05-165 & 17 & 11 & 16 & 10 & 17 & 16 & 16 & 8 & 8 & 9 & 9 & 10 & 10 & 15 & 16 & 188 \\
\hline W-05-166 & 17 & 6 & 6 & 10 & 16 & 17 & 13 & 4 & 4 & 10 & 10 & 9 & 10 & 13 & 16 & 161 \\
\hline W-05-167 & 16 & 15 & 18 & 11 & 20 & 15 & 16 & 9 & 9 & 9 & 9 & 10 & 2 & 16 & 18 & 193 \\
\hline W-05-168 & 11 & 13 & 16 & 16 & 18 & 16 & 16 & 7 & 8 & 6 & 8 & 3 & 5 & 12 & 16 & 171 \\
\hline W-05-169 & 11 & 12 & 14 & 8 & 7 & 13 & 16 & 8 & 7 & 8 & 8 & 7 & 8 & 15 & 16 & 158 \\
\hline W-05-170 & 12 & 18 & 8 & 18 & 2 & 15 & 6 & 7 & 7 & 8 & 8 & 8 & 8 & 11 & 8 & 144 \\
\hline W-05-171 & 15 & 13 & 13 & 8 & 13 & 15 & 13 & 9 & 6 & 5 & 9 & 0 & 4 & 9 & 11 & 143 \\
\hline W-05-173 & 12 & 8 & 8 & 15 & 15 & 13 & 15 & 9 & 9 & 9 & 9 & 10 & 10 & 15 & 18 & 175 \\
\hline W-05-176 & 14 & 17 & 10 & 18 & 13 & 11 & 5 & 9 & 9 & 8 & 8 & 2 & 2 & 15 & 11 & 152 \\
\hline W-05-177 & 15 & 17 & 11 & 18 & 15 & 14 & 1 & 8 & 8 & 7 & 7 & 2 & 5 & 16 & 13 & 157 \\
\hline W-05-178 & 16 & 16 & 13 & 15 & 13 & 13 & 6 & 8 & 8 & 7 & 7 & 2 & 2 & 16 & 14 & 156 \\
\hline W-05-180 & 16 & 15 & 16 & 16 & 16 & 10 & 6 & 7 & 7 & 7 & 7 & 1 & 1 & 18 & 15 & 158 \\
\hline W-05-181 & 10 & 15 & 6 & 15 & 15 & 13 & 1 & 8 & 8 & 6 & 9 & 1 & 10 & 15 & 13 & 145 \\
\hline W-05-182 & 19 & 20 & 19 & 14 & 17 & 12 & 16 & 8 & 9 & 8 & 8 & 3 & 2 & 20 & 19 & 194 \\
\hline
\end{tabular}


Appendix 3: Table showing the twelve individual habitat parameter scores adapted from Barbour et al. (1999) that were assessed at most sites where fishing effort was applied and were totalled to derive the Habitat Assessment Score for each site.

\begin{tabular}{|c|c|c|c|c|c|c|c|c|c|c|c|c|c|c|c|c|}
\hline \multirow{2}{*}{ Site Number } & \multirow{2}{*}{ 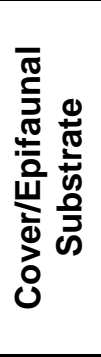 } & \multirow{2}{*}{ 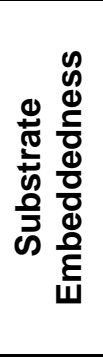 } & \multirow{2}{*}{ 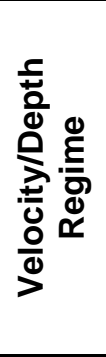 } & \multirow{2}{*}{ 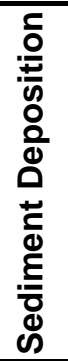 } & \multirow{2}{*}{ 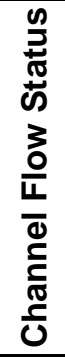 } & \multirow{2}{*}{ 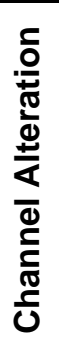 } & \multirow{2}{*}{ 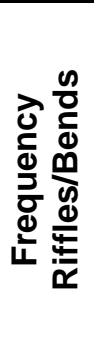 } & \multicolumn{2}{|c|}{ 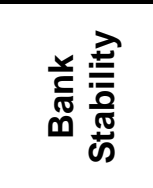 } & \multicolumn{2}{|c|}{ 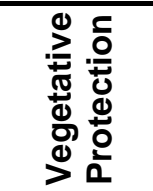 } & \multicolumn{2}{|c|}{$\begin{array}{l}\frac{c}{\sqrt{0}} \\
\frac{2}{\frac{0}{\pi}} \\
\frac{0}{x}\end{array}$} & \multirow{2}{*}{ 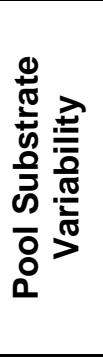 } & \multirow{2}{*}{ 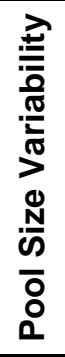 } & \multirow{2}{*}{ 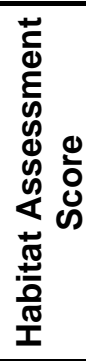 } \\
\hline & & & & & & & & 屯্త & $\begin{array}{l}\frac{}{\frac{1}{\sigma}} \\
\frac{0}{x}\end{array}$ & Ф્త & $\frac{\text { 픔 }}{\frac{0}{\tilde{x}}}$ & 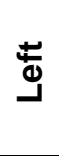 & $\frac{\tilde{n}}{\frac{\tilde{\sigma}}{\boldsymbol{x}}}$ & & & \\
\hline W-05-183 & 2 & 18 & 2 & 18 & 7 & 8 & 8 & 9 & 9 & 8 & 8 & 3 & 2 & 1 & 1 & 104 \\
\hline W-05-185 & 12 & 13 & 6 & 15 & 10 & 16 & 16 & 6 & 6 & 8 & 8 & 10 & 10 & 15 & 15 & 166 \\
\hline W-05-187 & 16 & 11 & 16 & 11 & 20 & 15 & 16 & 6 & 6 & 9 & 9 & 10 & 10 & 10 & 13 & 178 \\
\hline W-05-188 & 3 & 2 & 11 & 4 & 9 & 8 & 1 & 1 & 1 & 4 & 4 & 4 & 4 & 7 & 11 & 74 \\
\hline W-05-189 & 6 & 11 & 7 & 13 & 7 & 8 & 1 & 9 & 9 & 5 & 5 & 3 & 3 & 9 & 9 & 105 \\
\hline W-05-190 & 11 & 10 & 6 & 6 & 6 & 6 & 1 & 8 & 8 & 5 & 5 & 3 & 3 & 13 & 8 & 99 \\
\hline W-05-191 & 7 & 5 & 6 & 5 & 7 & 8 & 1 & 6 & 7 & 4 & 4 & 2 & 3 & 12 & 13 & 90 \\
\hline W-05-193 & 10 & 5 & 7 & 7 & 6 & 8 & 1 & 8 & 8 & 5 & 5 & 2 & 4 & 13 & 9 & 98 \\
\hline W-05-194 & 11 & 7 & 7 & 4 & 6 & 6 & 1 & 9 & 9 & 5 & 5 & 4 & 4 & 11 & 10 & 99 \\
\hline W-05-196 & 8 & 16 & 15 & 16 & 6 & 13 & 7 & 7 & 7 & 8 & 7 & 10 & 3 & 7 & 10 & 140 \\
\hline W-05-200 & 6 & 2 & 17 & 2 & 7 & 5 & 6 & 2 & 2 & 2 & 2 & 3 & 2 & 7 & 8 & 73 \\
\hline W-05-201 & 7 & 5 & 6 & 5 & 6 & 10 & 2 & 7 & 7 & 6 & 3 & 2 & 1 & 10 & 6 & 83 \\
\hline W-05-202 & 6 & 5 & 10 & 7 & 7 & 10 & 5 & 6 & 6 & 6 & 6 & 2 & 2 & 5 & 7 & 90 \\
\hline W-05-203 & 10 & 6 & 16 & 8 & 6 & 15 & 14 & 7 & 7 & 7 & 7 & 7 & 7 & 7 & 16 & 140 \\
\hline W-05-205 & 16 & 11 & 18 & 11 & 11 & 15 & 11 & 8 & 8 & 9 & 9 & 1 & 9 & 13 & 18 & 168 \\
\hline W-05-207 & 7 & 7 & 8 & 9 & 6 & 15 & 6 & 1 & 1 & 1 & 1 & 3 & 2 & 6 & 8 & 81 \\
\hline W-05-208 & 18 & 16 & 15 & 15 & 10 & 16 & 15 & 8 & 8 & 9 & 9 & 10 & 10 & 13 & 20 & 192 \\
\hline W-05-209 & 19 & 17 & 18 & 17 & 16 & 19 & 16 & 8 & 6 & 8 & 8 & 10 & 10 & 20 & 18 & 210 \\
\hline $\mathrm{X}-05-001$ & 8 & 8 & 14 & 7 & 8 & 13 & 10 & 2 & 4 & 3 & 6 & 1 & 4 & 13 & 10 & 111 \\
\hline$X-05-002$ & 10 & 10 & 7 & 12 & 14 & 17 & 12 & 7 & 7 & 8 & 8 & 6 & 9 & 13 & 11 & 151 \\
\hline $\mathrm{X}-05-003$ & 8 & 8 & 2 & 2 & 5 & 8 & 5 & 6 & 6 & 4 & 4 & 2 & 1 & 13 & 7 & 81 \\
\hline X-05-005 & 3 & 2 & 2 & 3 & 3 & 8 & 2 & 7 & 7 & 4 & 4 & 3 & 4 & 7 & 4 & 63 \\
\hline $\mathrm{X}-05-007$ & 9 & 7 & 8 & 7 & 6 & 8 & 6 & 2 & 2 & 3 & 4 & 6 & 6 & 8 & 5 & 87 \\
\hline$X-05-011$ & 9 & 8 & 7 & 7 & 8 & 7 & 2 & 6 & 6 & 4 & 4 & 6 & 4 & 11 & 7 & 96 \\
\hline $\mathrm{X}-05-012$ & 7 & 7 & 6 & 6 & 6 & 7 & 4 & 6 & 6 & 4 & 4 & 2 & 2 & 7 & 8 & 82 \\
\hline
\end{tabular}


Appendix 3: Table showing the twelve individual habitat parameter scores adapted from Barbour et al. (1999) that were assessed at most sites where fishing effort was applied and were totalled to derive the Habitat Assessment Score for each site.

\begin{tabular}{|c|c|c|c|c|c|c|c|c|c|c|c|c|c|c|c|c|}
\hline \multirow{2}{*}{ Site Number } & \multirow{2}{*}{ 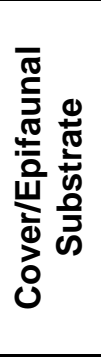 } & \multirow{2}{*}{ 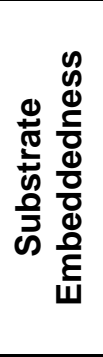 } & \multirow{2}{*}{ 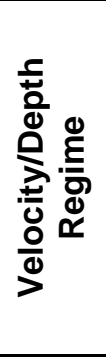 } & \multirow{2}{*}{ 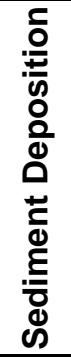 } & \multirow{2}{*}{ 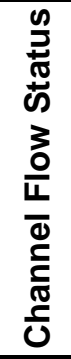 } & \multirow{2}{*}{ 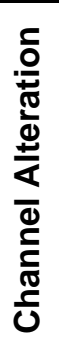 } & \multirow{2}{*}{ 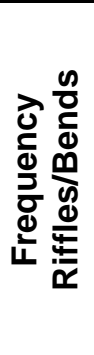 } & \multicolumn{2}{|c|}{ 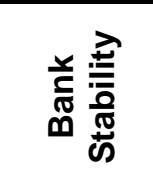 } & \multicolumn{2}{|c|}{ 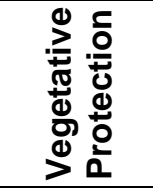 } & \multicolumn{2}{|c|}{$\begin{array}{l}\frac{c}{\sqrt{0}} \\
\frac{2}{\frac{0}{\pi}} \\
\frac{0}{x}\end{array}$} & \multirow{2}{*}{ 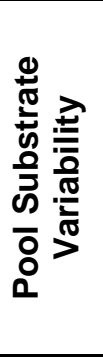 } & \multirow{2}{*}{ 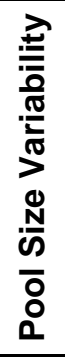 } & \multirow{2}{*}{ 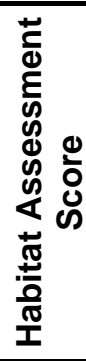 } \\
\hline & & & & & & & & 屯্త & $\begin{array}{l}\frac{}{\frac{1}{\sigma}} \\
\frac{0}{x}\end{array}$ & Ф્త & $\frac{\text { 픔 }}{\frac{0}{\tilde{x}}}$ & 苂 & $\frac{\tilde{n}}{\frac{\tilde{\sigma}}{\boldsymbol{x}}}$ & & & \\
\hline $\mathrm{X}-05-015$ & 9 & 9 & 8 & 10 & 5 & 9 & 4 & 6 & 6 & 5 & 5 & 2 & 2 & 11 & 5 & 96 \\
\hline X-05-018 & 17 & 4 & 15 & 8 & 8 & 16 & 10 & 8 & 9 & 9 & 9 & 6 & 6 & 11 & 16 & 152 \\
\hline $\mathrm{X}-05-020$ & 7 & 11 & 5 & 11 & 7 & 7 & 2 & 6 & 6 & 4 & 4 & 1 & 2 & 11 & 8 & 92 \\
\hline $\mathrm{X}-05-021$ & 18 & 15 & 17 & 14 & 14 & 13 & 10 & 9 & 8 & 9 & 5 & 8 & 3 & 17 & 17 & 177 \\
\hline$X-05-022$ & 10 & 13 & 7 & 9 & 9 & 8 & 3 & 7 & 7 & 4 & 4 & 6 & 6 & 16 & 16 & 125 \\
\hline $\mathrm{X}-05-023$ & 7 & 10 & 14 & 9 & 7 & 7 & 4 & 7 & 8 & 4 & 4 & 3 & 4 & 8 & 6 & 102 \\
\hline $\mathrm{X}-05-024$ & 10 & 14 & 16 & 13 & 13 & 7 & 7 & 3 & 3 & 3 & 3 & 6 & 6 & 8 & 11 & 123 \\
\hline $\mathrm{X}-05-026$ & 8 & 8 & 11 & 7 & 7 & 16 & 10 & 1 & 1 & 4 & 4 & 3 & 3 & 11 & 16 & 110 \\
\hline $\mathrm{X}-05-027$ & 5 & 4 & 4 & 4 & 6 & 7 & 2 & 8 & 8 & 4 & 4 & 5 & 5 & 6 & 4 & 76 \\
\hline X-05-028 & 6 & 7 & 6 & 7 & 5 & 9 & 4 & 6 & 7 & 4 & 4 & 5 & 5 & 8 & 5 & 88 \\
\hline X-05-029 & 12 & 13 & 14 & 12 & 12 & 13 & 7 & 9 & 9 & 8 & 6 & 8 & 3 & 12 & 11 & 149 \\
\hline $\mathrm{X}-05-030$ & 13 & 15 & 14 & 13 & 15 & 14 & 12 & 9 & 9 & 7 & 7 & 5 & 3 & 16 & 10 & 162 \\
\hline $\mathrm{X}-05-031$ & 7 & 10 & 9 & 10 & 8 & 10 & 7 & 8 & 8 & 1 & 1 & 1 & 1 & 10 & 5 & 96 \\
\hline$X-05-032$ & 9 & 13 & 11 & 13 & 14 & 8 & 4 & 9 & 9 & 4 & 4 & 5 & 5 & 12 & 13 & 133 \\
\hline X-05-033 & 7 & 13 & 13 & 12 & 7 & 7 & 6 & 9 & 9 & 1 & 3 & 0 & 3 & 10 & 8 & 108 \\
\hline X-05-035 & 6 & 7 & 8 & 9 & 6 & 6 & 3 & 4 & 3 & 4 & 3 & 3 & 4 & 8 & 7 & 81 \\
\hline$X-05-036$ & 8 & 8 & 7 & 7 & 7 & 8 & 3 & 6 & 6 & 4 & 4 & 6 & 6 & 12 & 8 & 100 \\
\hline X-05-037 & 10 & 11 & 13 & 8 & 8 & 8 & 8 & 8 & 8 & 4 & 4 & 6 & 6 & 15 & 11 & 128 \\
\hline X-05-038 & 14 & 13 & 11 & 13 & 14 & 11 & 10 & 7 & 7 & 8 & 9 & 3 & 8 & 13 & 10 & 151 \\
\hline$X-05-040$ & 8 & 7 & 6 & 9 & 7 & 8 & 3 & 7 & 7 & 5 & 4 & 6 & 4 & 10 & 10 & 101 \\
\hline$X-05-042$ & 12 & 7 & 12 & 9 & 17 & 13 & 5 & 9 & 9 & 8 & 4 & 3 & 3 & 13 & 15 & 139 \\
\hline X-05-043 & 6 & 6 & 7 & 6 & 6 & 7 & 3 & 8 & 4 & 4 & 3 & 4 & 4 & 8 & 8 & 84 \\
\hline $\mathrm{X}-05-044$ & 12 & 11 & 8 & 12 & 16 & 14 & 8 & 7 & 7 & 6 & 5 & 8 & 8 & 13 & 14 & 149 \\
\hline$X-05-046$ & 11 & 8 & 11 & 8 & 8 & 14 & 4 & 7 & 3 & 7 & 2 & 3 & 0 & 8 & 10 & 104 \\
\hline X-05-048 & 11 & 14 & 14 & 14 & 13 & 5 & 11 & 7 & 8 & 5 & 5 & 5 & 5 & 5 & 13 & 135 \\
\hline
\end{tabular}


Appendix 3: Table showing the twelve individual habitat parameter scores adapted from Barbour et al. (1999) that were assessed at most sites where fishing effort was applied and were totalled to derive the Habitat Assessment Score for each site.

\begin{tabular}{|c|c|c|c|c|c|c|c|c|c|c|c|c|c|c|c|c|}
\hline \multirow{2}{*}{ Site Number } & \multirow{2}{*}{ 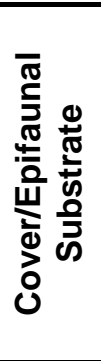 } & \multirow{2}{*}{ 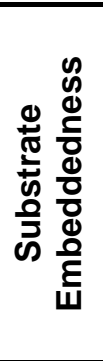 } & \multirow{2}{*}{ 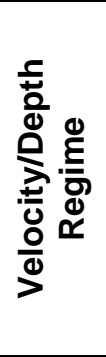 } & \multirow{2}{*}{ 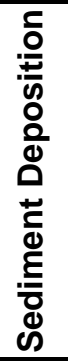 } & \multirow{2}{*}{ 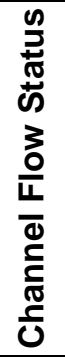 } & \multirow{2}{*}{ 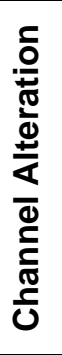 } & \multirow{2}{*}{ 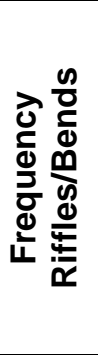 } & \multicolumn{2}{|c|}{ 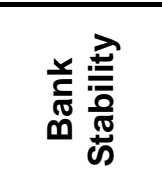 } & \multicolumn{2}{|c|}{ 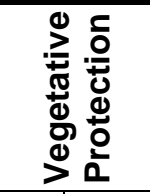 } & \multicolumn{2}{|c|}{ 迹 } & \multirow{2}{*}{ 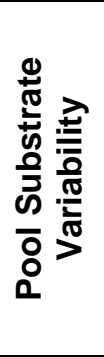 } & \multirow{2}{*}{ 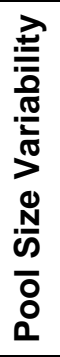 } & \multirow{2}{*}{ 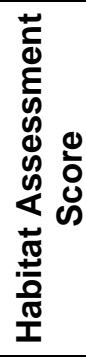 } \\
\hline & & & & & & & & ప্త & $\frac{\text { 동 }}{\frac{0}{x}}$ & Ф્త & $\frac{\text { 픔 }}{\frac{0}{x}}$ & 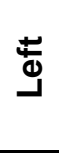 & 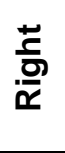 & & & \\
\hline $\mathrm{X}-05-051$ & 9 & 6 & 11 & 6 & 6 & 8 & 6 & 6 & 6 & 5 & 5 & 4 & 3 & 9 & 9 & 99 \\
\hline $\mathrm{X}-05-052$ & 9 & 7 & 16 & 7 & 9 & 8 & 6 & 8 & 8 & 5 & 5 & 4 & 3 & 11 & 10 & 116 \\
\hline $\mathrm{X}-05-054$ & 10 & 8 & 13 & 9 & 7 & 8 & 6 & 8 & 8 & 4 & 4 & 6 & 4 & 9 & 16 & 120 \\
\hline X-05-055 & 10 & 8 & 8 & 8 & 6 & 11 & 6 & 7 & 8 & 7 & 7 & 5 & 4 & 2 & 2 & 99 \\
\hline $\mathrm{X}-05-057$ & 5 & 7 & 5 & 10 & 6 & 8 & 4 & 7 & 7 & 4 & 4 & 3 & 3 & 12 & 11 & 96 \\
\hline $\mathrm{X}-05-058$ & 4 & 1 & 11 & 2 & 9 & 16 & 5 & 6 & 6 & 7 & 7 & 9 & 9 & 7 & 10 & 109 \\
\hline X-05-061 & 8 & 7 & 1 & 7 & 16 & 18 & 4 & 10 & 10 & 9 & 9 & 9 & 9 & 8 & 14 & 139 \\
\hline$X-05-062$ & 16 & 7 & 14 & 6 & 8 & 15 & 12 & 7 & 7 & 9 & 9 & 7 & 7 & 8 & 16 & 148 \\
\hline X-05-064 & 7 & 7 & 6 & 9 & 13 & 8 & 6 & 7 & 7 & 4 & 4 & 6 & 6 & 8 & 14 & 112 \\
\hline X-05-066 & 8 & 8 & 8 & 8 & 7 & 8 & 4 & 7 & 6 & 4 & 4 & 4 & 4 & 14 & 9 & 103 \\
\hline$X-05-067$ & 18 & 11 & 17 & 13 & 9 & 16 & 10 & 8 & 8 & 9 & 9 & 7 & 6 & 11 & 11 & 163 \\
\hline X-05-069 & 6 & 1 & 16 & 5 & 7 & 12 & 7 & 1 & 1 & 3 & 3 & 2 & 2 & 6 & 13 & 85 \\
\hline X-05-070 & 7 & 9 & 7 & 8 & 13 & 11 & 4 & 6 & 5 & 4 & 4 & 3 & 3 & 7 & 13 & 104 \\
\hline X-05-071 & 8 & 9 & 6 & 10 & 6 & 10 & 2 & 7 & 7 & 5 & 5 & 8 & 8 & 10 & 11 & 112 \\
\hline X-05-072 & 8 & 11 & 6 & 12 & 9 & 11 & 4 & 6 & 6 & 5 & 5 & 8 & 5 & 11 & 10 & 117 \\
\hline X-05-079 & 2 & 5 & 1 & 6 & 10 & 8 & 3 & 6 & 6 & 5 & 5 & 5 & 5 & 7 & 13 & 87 \\
\hline X-05-080 & 3 & 5 & 1 & 5 & 8 & 8 & 4 & 7 & 7 & 5 & 5 & 4 & 4 & 7 & 11 & 84 \\
\hline X-05-081 & 2 & 2 & 4 & 2 & 5 & 8 & 2 & 7 & 7 & 4 & 4 & 2 & 2 & 7 & 3 & 61 \\
\hline X-05-083 & 10 & 11 & 3 & 11 & 6 & 11 & 5 & 7 & 7 & 6 & 6 & 2 & 2 & 10 & 8 & 105 \\
\hline X-05-084 & 8 & 9 & 11 & 9 & 6 & 8 & 5 & 7 & 7 & 5 & 5 & 3 & 3 & 8 & 8 & 102 \\
\hline X-05-085 & 7 & 7 & 11 & 11 & 6 & 8 & 4 & 5 & 5 & 4 & 4 & 3 & 2 & 10 & 9 & 96 \\
\hline $\mathrm{X}-05-086$ & 7 & 13 & 6 & 13 & 7 & 9 & 3 & 5 & 5 & 4 & 4 & 1 & 1 & 10 & 8 & 96 \\
\hline $\mathrm{X}-05-088$ & 11 & 17 & 7 & 16 & 14 & 7 & 5 & 7 & 7 & 4 & 4 & 5 & 4 & 10 & 11 & 129 \\
\hline X-05-091 & 16 & 8 & 11 & 8 & 17 & 18 & 4 & 9 & 9 & 10 & 10 & 10 & 10 & 9 & 13 & 162 \\
\hline X-05-093 & 7 & 8 & 7 & 7 & 5 & 8 & 3 & 5 & 6 & 4 & 4 & 4 & 4 & 7 & 7 & 86 \\
\hline
\end{tabular}


Appendix 3: Table showing the twelve individual habitat parameter scores adapted from Barbour et al. (1999) that were assessed at most sites where fishing effort was applied and were totalled to derive the Habitat Assessment Score for each site.

\begin{tabular}{|c|c|c|c|c|c|c|c|c|c|c|c|c|c|c|c|c|}
\hline \multirow{2}{*}{ Site Number } & \multirow{2}{*}{ 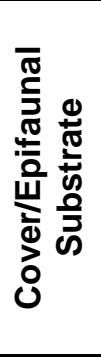 } & \multirow{2}{*}{ 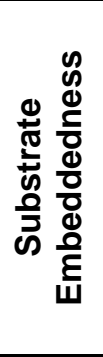 } & \multirow{2}{*}{ 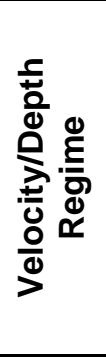 } & \multirow{2}{*}{ 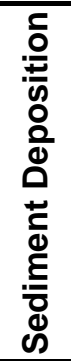 } & \multirow{2}{*}{ 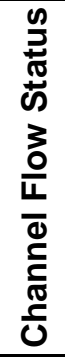 } & \multirow{2}{*}{ 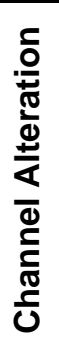 } & \multirow{2}{*}{ 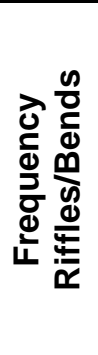 } & \multicolumn{2}{|c|}{ 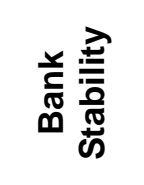 } & \multicolumn{2}{|c|}{ 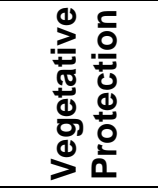 } & \multicolumn{2}{|c|}{$\begin{array}{l}\frac{c}{\sqrt{0}} \\
\frac{2}{\frac{0}{\pi}} \\
\frac{0}{x}\end{array}$} & \multirow{2}{*}{ 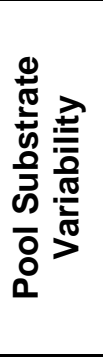 } & \multirow{2}{*}{ 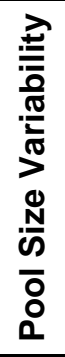 } & \multirow{2}{*}{ 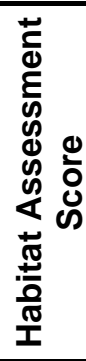 } \\
\hline & & & & & & & & Ф্త & $\begin{array}{l}\frac{}{\frac{1}{\sigma}} \\
\frac{0}{x}\end{array}$ & 屯 & 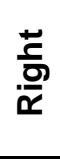 & 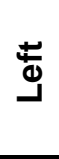 & $\frac{\tilde{n}}{\frac{\tilde{\sigma}}{\boldsymbol{x}}}$ & & & \\
\hline X-05-094 & 4 & 3 & 11 & 3 & 5 & 8 & 6 & 4 & 4 & 4 & 4 & 4 & 4 & 6 & 7 & 77 \\
\hline X-05-095 & 10 & 7 & 12 & 7 & 6 & 8 & 6 & 8 & 7 & 4 & 4 & 5 & 4 & 14 & 16 & 118 \\
\hline X-05-096 & 9 & 11 & 7 & 11 & 6 & 8 & 3 & 6 & 7 & 4 & 4 & 8 & 8 & 9 & 10 & 111 \\
\hline X-05-098 & 8 & 11 & 7 & 11 & 6 & 8 & 3 & 7 & 7 & 4 & 4 & 3 & 3 & 8 & 11 & 101 \\
\hline X-05-099 & 7 & 9 & 11 & 8 & 6 & 9 & 5 & 7 & 7 & 4 & 4 & 2 & 2 & 13 & 10 & 104 \\
\hline$X-05-100$ & 7 & 10 & 6 & 10 & 6 & 8 & 3 & 8 & 8 & 4 & 4 & 7 & 7 & 11 & 9 & 108 \\
\hline $\mathrm{X}-05-102$ & 8 & 7 & 7 & 6 & 5 & 8 & 4 & 7 & 6 & 5 & 5 & 5 & 5 & 9 & 11 & 98 \\
\hline$X-05-103$ & 6 & 3 & 6 & 4 & 15 & 14 & 8 & 5 & 5 & 7 & 7 & 7 & 7 & 9 & 13 & 116 \\
\hline$X-05-104$ & 17 & 13 & 17 & 13 & 8 & 16 & 11 & 5 & 7 & 9 & 9 & 8 & 8 & 17 & 18 & 176 \\
\hline X-05-105 & 10 & 7 & 13 & 6 & 7 & 12 & 10 & 4 & 4 & 6 & 6 & 4 & 4 & 13 & 12 & 118 \\
\hline$X-05-106$ & 11 & 6 & 13 & 5 & 6 & 16 & 10 & 5 & 5 & 7 & 7 & 9 & 9 & 13 & 9 & 131 \\
\hline X-05-107 & 9 & 12 & 11 & 14 & 8 & 8 & 8 & 7 & 7 & 4 & 5 & 4 & 4 & 11 & 14 & 126 \\
\hline X-05-109 & 4 & 6 & 12 & 4 & 6 & 11 & 9 & 6 & 6 & 5 & 5 & 3 & 3 & 6 & 7 & 93 \\
\hline$X-05-111$ & 17 & 14 & 17 & 9 & 8 & 18 & 16 & 9 & 9 & 10 & 10 & 10 & 10 & 11 & 16 & 184 \\
\hline $\mathrm{X}-05-112$ & 6 & 9 & 7 & 6 & 7 & 8 & 4 & 6 & 6 & 5 & 4 & 5 & 5 & 8 & 6 & 92 \\
\hline $\mathrm{X}-05-114$ & 16 & 16 & 11 & 12 & 6 & 16 & 14 & 8 & 8 & 9 & 9 & 8 & 7 & 12 & 10 & 162 \\
\hline$X-05-115$ & 2 & 3 & 3 & 5 & 6 & 7 & 4 & 2 & 1 & 3 & 3 & 3 & 3 & 7 & 6 & 58 \\
\hline $\mathrm{X}-05-117$ & 7 & 7 & 7 & 6 & 6 & 8 & 5 & 8 & 8 & 4 & 4 & 2 & 4 & 11 & 6 & 93 \\
\hline $\mathrm{X}-05-118$ & 6 & 7 & 7 & 7 & 6 & 8 & 3 & 8 & 8 & 4 & 4 & 4 & 4 & 9 & 8 & 93 \\
\hline$X-05-120$ & 8 & 10 & 8 & 11 & 6 & 12 & 7 & 7 & 7 & 6 & 6 & 4 & 4 & 11 & 8 & 115 \\
\hline$X-05-121$ & 8 & 9 & 12 & 15 & 6 & 9 & 6 & 8 & 8 & 4 & 4 & 4 & 4 & 8 & 11 & 116 \\
\hline $\mathrm{X}-05-122$ & 17 & 14 & 15 & 11 & 8 & 16 & 16 & 9 & 9 & 10 & 10 & 8 & 8 & 16 & 16 & 183 \\
\hline $\mathrm{X}-05-123$ & 11 & 5 & 6 & 8 & 16 & 13 & 6 & 9 & 9 & 9 & 9 & 8 & 8 & 10 & 11 & 138 \\
\hline$X-05-125$ & 14 & 3 & 16 & 6 & 13 & 16 & 8 & 3 & 3 & 8 & 8 & 8 & 8 & 13 & 16 & 143 \\
\hline$X-05-126$ & 13 & 9 & 7 & 11 & 6 & 16 & 12 & 4 & 4 & 8 & 8 & 8 & 8 & 11 & 11 & 136 \\
\hline
\end{tabular}


Appendix 3: Table showing the twelve individual habitat parameter scores adapted from Barbour et al. (1999) that were assessed at most sites where fishing effort was applied and were totalled to derive the Habitat Assessment Score for each site.

\begin{tabular}{|c|c|c|c|c|c|c|c|c|c|c|c|c|c|c|c|c|}
\hline \multirow{2}{*}{ Site Number } & \multirow{2}{*}{ 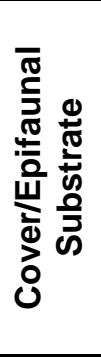 } & \multirow{2}{*}{ 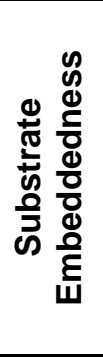 } & \multirow{2}{*}{ 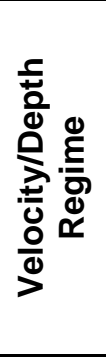 } & \multirow{2}{*}{ 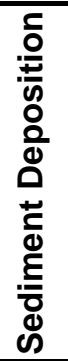 } & \multirow{2}{*}{ 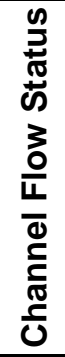 } & \multirow{2}{*}{ 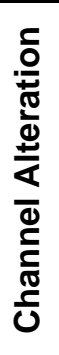 } & \multirow{2}{*}{ 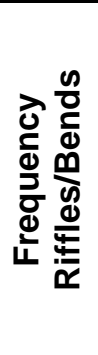 } & \multicolumn{2}{|c|}{ 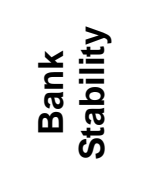 } & \multicolumn{2}{|c|}{ 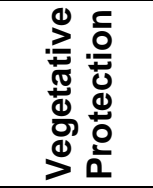 } & \multicolumn{2}{|c|}{$\begin{array}{l}\frac{c}{\sqrt{0}} \\
\frac{2}{\frac{0}{\pi}} \\
\frac{0}{x}\end{array}$} & \multirow{2}{*}{ 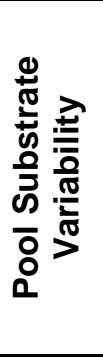 } & \multirow{2}{*}{ 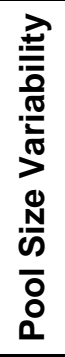 } & \multirow{2}{*}{ 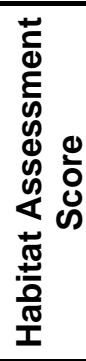 } \\
\hline & & & & & & & & Ф্త & $\begin{array}{l}\frac{}{\frac{1}{\sigma}} \\
\frac{0}{x}\end{array}$ & 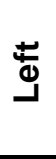 & $\frac{\text { 픔 }}{\frac{0}{\tilde{x}}}$ & 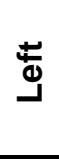 & $\frac{\tilde{n}}{\frac{\tilde{\sigma}}{\boldsymbol{x}}}$ & & & \\
\hline $\mathrm{X}-05-127$ & 9 & 17 & 13 & 14 & 6 & 14 & 10 & 6 & 7 & 5 & 5 & 7 & 7 & 12 & 11 & 143 \\
\hline$X-05-128$ & 10 & 16 & 11 & 13 & 6 & 16 & 16 & 1 & 1 & 5 & 5 & 10 & 10 & 11 & 7 & 138 \\
\hline $\mathrm{X}-05-129$ & 8 & 9 & 6 & 9 & 6 & 8 & 3 & 8 & 8 & 5 & 5 & 4 & 4 & 10 & 11 & 104 \\
\hline $\mathrm{X}-05-130$ & 8 & 7 & 6 & 7 & 6 & 7 & 3 & 8 & 8 & 4 & 4 & 3 & 3 & 8 & 8 & 90 \\
\hline$X-05-132$ & 12 & 13 & 13 & 13 & 8 & 18 & 7 & 9 & 9 & 5 & 5 & 8 & 8 & 13 & 12 & 153 \\
\hline $\mathrm{X}-05-133$ & 10 & 10 & 13 & 9 & 6 & 13 & 8 & 3 & 3 & 4 & 4 & 6 & 6 & 12 & 12 & 119 \\
\hline $\mathrm{X}-05-136$ & 7 & 10 & 12 & 13 & 16 & 12 & 5 & 9 & 9 & 4 & 4 & 5 & 5 & 11 & 13 & 135 \\
\hline$X-05-137$ & 13 & 15 & 16 & 13 & 13 & 16 & 8 & 2 & 2 & 6 & 5 & 8 & 8 & 13 & 13 & 151 \\
\hline $\mathrm{X}-05-138$ & 8 & 10 & 7 & 14 & 12 & 10 & 6 & 8 & 8 & 4 & 4 & 7 & 7 & 12 & 9 & 126 \\
\hline X-05-139 & 8 & 15 & 7 & 16 & 16 & 9 & 2 & 9 & 9 & 6 & 6 & 8 & 3 & 13 & 13 & 140 \\
\hline$X-05-140$ & 9 & 9 & 16 & 6 & 6 & 13 & 14 & 2 & 2 & 3 & 3 & 7 & 7 & 9 & 9 & 115 \\
\hline$X-05-144$ & 14 & 13 & 18 & 13 & 8 & 14 & 9 & 8 & 8 & 7 & 7 & 8 & 8 & 16 & 11 & 162 \\
\hline X-05-145 & 17 & 16 & 18 & 15 & 10 & 17 & 16 & 9 & 9 & 9 & 9 & 10 & 10 & 10 & 10 & 185 \\
\hline$X-05-146$ & 11 & 8 & 12 & 9 & 19 & 15 & 7 & 7 & 9 & 7 & 7 & 7 & 7 & 7 & 13 & 145 \\
\hline X-05-148 & 7 & 8 & 1 & 8 & 10 & 8 & 1 & 8 & 8 & 4 & 4 & 3 & 3 & 6 & 12 & 91 \\
\hline X-05-150 & 17 & 13 & 16 & 9 & 14 & 17 & 10 & 9 & 9 & 9 & 9 & 7 & 7 & 15 & 17 & 178 \\
\hline$X-05-151$ & 12 & 7 & 17 & 6 & 8 & 16 & 7 & 9 & 9 & 8 & 8 & 9 & 9 & 9 & 17 & 151 \\
\hline $\mathrm{X}-05-153$ & 14 & 19 & 14 & 18 & 7 & 18 & 16 & 8 & 9 & 7 & 7 & 7 & 7 & 16 & 8 & 175 \\
\hline X-05-154 & 14 & 19 & 14 & 16 & 8 & 15 & 18 & 6 & 8 & 8 & 8 & 4 & 4 & 12 & 8 & 162 \\
\hline X-05-155 & 16 & 18 & 15 & 15 & 7 & 17 & 11 & 7 & 7 & 6 & 6 & 8 & 8 & 16 & 16 & 173 \\
\hline$X-05-157$ & 7 & 2 & 6 & 5 & 6 & 11 & 5 & 5 & 5 & 6 & 6 & 4 & 4 & 11 & 10 & 93 \\
\hline X-05-162 & 9 & 12 & 13 & 14 & 8 & 14 & 9 & 8 & 8 & 5 & 5 & 5 & 4 & 10 & 12 & 136 \\
\hline $\mathrm{X}-05-163$ & 11 & 9 & 6 & 13 & 7 & 16 & 3 & 9 & 9 & 6 & 6 & 7 & 6 & 13 & 8 & 129 \\
\hline$X-05-164$ & 7 & 9 & 11 & 14 & 7 & 13 & 6 & 9 & 9 & 7 & 7 & 4 & 4 & 11 & 9 & 127 \\
\hline X-05-165 & 7 & 13 & 12 & 13 & 11 & 13 & 3 & 9 & 9 & 5 & 5 & 3 & 3 & 7 & 6 & 119 \\
\hline
\end{tabular}


Appendix 3: Table showing the twelve individual habitat parameter scores adapted from Barbour et al. (1999) that were assessed at most sites where fishing effort was applied and were totalled to derive the Habitat Assessment Score for each site.

\begin{tabular}{|c|c|c|c|c|c|c|c|c|c|c|c|c|c|c|c|c|}
\hline \multirow{2}{*}{ Site Number } & \multirow{2}{*}{ 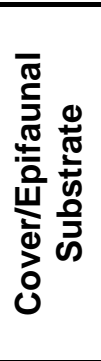 } & \multirow{2}{*}{ 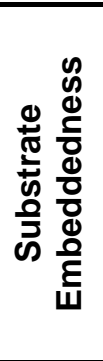 } & \multirow{2}{*}{ 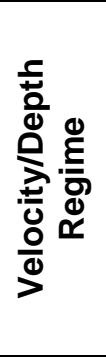 } & \multirow{2}{*}{ 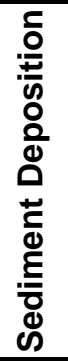 } & \multirow{2}{*}{ 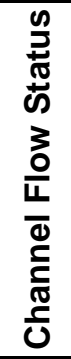 } & \multirow{2}{*}{ 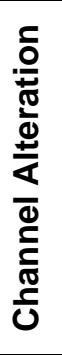 } & \multirow{2}{*}{ 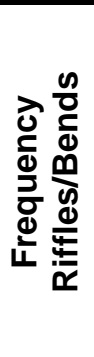 } & \multicolumn{2}{|c|}{ 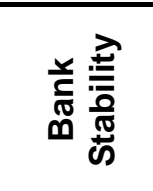 } & \multicolumn{2}{|c|}{ 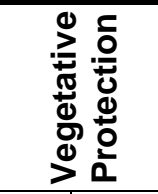 } & \multicolumn{2}{|c|}{ 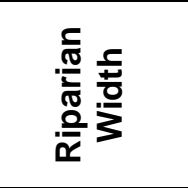 } & \multirow{2}{*}{ 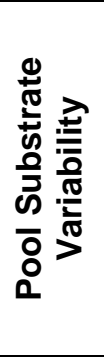 } & \multirow{2}{*}{ 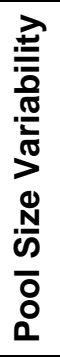 } & \multirow{2}{*}{ 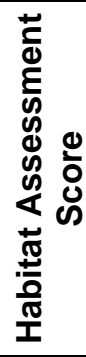 } \\
\hline & & & & & & & & ప্త & $\frac{\text { 듬 }}{\frac{0}{x}}$ & ๘ & $\frac{\text { 픔 }}{\frac{0}{x}}$ & ప্త & $\frac{\tilde{E}}{\frac{\pi}{x}}$ & & & \\
\hline $\mathrm{X}-05-167$ & 7 & 9 & 12 & 11 & 17 & 13 & 7 & 9 & 9 & 6 & 6 & 4 & 5 & 11 & 11 & 137 \\
\hline$X-05-168$ & 11 & 10 & 13 & 10 & 16 & 15 & 12 & 9 & 9 & 8 & 8 & 6 & 8 & 11 & 11 & 157 \\
\hline $\mathrm{X}-05-169$ & 9 & 6 & 8 & 6 & 7 & 11 & 12 & 4 & 5 & 4 & 4 & 4 & 4 & 8 & 8 & 100 \\
\hline $\mathrm{X}-05-171$ & 8 & 9 & 13 & 3 & 14 & 15 & 3 & 5 & 5 & 8 & 8 & 6 & 7 & 8 & 13 & 125 \\
\hline $\mathrm{X}-05-172$ & 9 & 8 & 7 & 11 & 17 & 14 & 7 & 7 & 7 & 4 & 4 & 3 & 4 & 11 & 13 & 126 \\
\hline $\mathrm{X}-05-173$ & 9 & 10 & 11 & 12 & 17 & 14 & 9 & 7 & 7 & 4 & 4 & 4 & 4 & 12 & 12 & 136 \\
\hline $\mathrm{X}-05-176$ & 10 & 8 & 16 & 15 & 18 & 16 & 9 & 6 & 6 & 6 & 6 & 8 & 8 & 12 & 16 & 160 \\
\hline$X-05-177$ & 11 & 18 & 13 & 15 & 18 & 17 & 10 & 9 & 9 & 7 & 7 & 7 & 7 & 14 & 14 & 176 \\
\hline X-05-179 & 6 & 7 & 14 & 4 & 7 & 14 & 12 & 0 & 0 & 1 & 1 & 3 & 2 & 7 & 5 & 83 \\
\hline X-05-181 & 7 & 5 & 11 & 7 & 8 & 6 & 5 & 9 & 9 & 5 & 5 & 3 & 3 & 11 & 12 & 106 \\
\hline$X-05-182$ & 11 & 8 & 11 & 10 & 10 & 7 & 5 & 9 & 9 & 5 & 5 & 3 & 2 & 15 & 15 & 125 \\
\hline $\mathrm{X}-05-183$ & 9 & 9 & 7 & 8 & 7 & 11 & 6 & 9 & 9 & 7 & 7 & 7 & 7 & 11 & 12 & 126 \\
\hline X-05-185 & 8 & 5 & 8 & 7 & 10 & 10 & 4 & 9 & 9 & 4 & 5 & 2 & 2 & 13 & 13 & 109 \\
\hline X-05-186 & 6 & 4 & 7 & 6 & 5 & 8 & 0 & 8 & 8 & 4 & 4 & 4 & 3 & 11 & 9 & 87 \\
\hline X-05-188 & 7 & 4 & 7 & 6 & 6 & 11 & 3 & 4 & 4 & 5 & 5 & 5 & 5 & 13 & 7 & 92 \\
\hline X-05-189 & 7 & 4 & 7 & 6 & 6 & 9 & 2 & 4 & 7 & 3 & 5 & 8 & 1 & 13 & 7 & 89 \\
\hline X-05-190 & 15 & 10 & 6 & 12 & 15 & 11 & 9 & 9 & 9 & 5 & 5 & 8 & 8 & 15 & 14 & 151 \\
\hline X-05-191 & 11 & 7 & 13 & 6 & 7 & 15 & 11 & 8 & 8 & 10 & 10 & 8 & 7 & 12 & 9 & 142 \\
\hline X-05-192 & 15 & 17 & 17 & 11 & 15 & 15 & 18 & 9 & 9 & 9 & 9 & 9 & 9 & 16 & 17 & 195 \\
\hline X-05-193 & 16 & 11 & 14 & 9 & 8 & 15 & 16 & 9 & 7 & 9 & 9 & 9 & 6 & 11 & 8 & 157 \\
\hline X-05-194 & 11 & 4 & 13 & 4 & 6 & 14 & 11 & 9 & 9 & 9 & 9 & 6 & 8 & 8 & 8 & 129 \\
\hline $\mathrm{X}-05-195$ & 7 & 14 & 8 & 14 & 7 & 10 & 6 & 6 & 8 & 9 & 6 & 7 & 4 & 13 & 5 & 124 \\
\hline X-05-196 & 7 & 9 & 9 & 15 & 6 & 8 & 5 & 9 & 9 & 8 & 8 & 3 & 2 & 11 & 7 & 116 \\
\hline X-05-198 & 18 & 6 & 16 & 6 & 8 & 16 & 15 & 9 & 9 & 9 & 9 & 9 & 9 & 16 & 8 & 163 \\
\hline X-05-199 & 17 & 12 & 16 & 9 & 11 & 14 & 11 & 7 & 7 & 8 & 8 & 7 & 6 & 16 & 16 & 165 \\
\hline
\end{tabular}


Appendix 3: Table showing the twelve individual habitat parameter scores adapted from Barbour et al. (1999) that were assessed at most sites where fishing effort was applied and were totalled to derive the Habitat Assessment Score for each site.

\begin{tabular}{|c|c|c|c|c|c|c|c|c|c|c|c|c|c|c|c|c|}
\hline \multirow{2}{*}{ Site Number } & \multirow{2}{*}{ 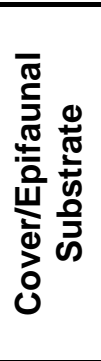 } & \multirow{2}{*}{ 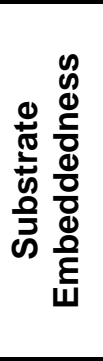 } & \multirow{2}{*}{ 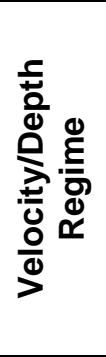 } & \multirow{2}{*}{ 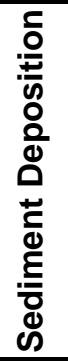 } & \multirow{2}{*}{ 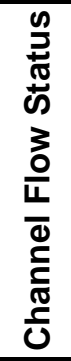 } & \multirow{2}{*}{ 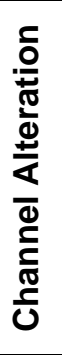 } & \multirow{2}{*}{ 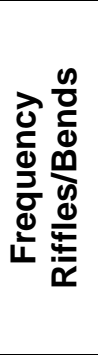 } & \multicolumn{2}{|c|}{ 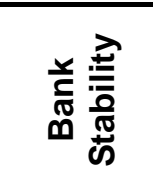 } & \multicolumn{2}{|c|}{ 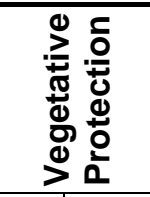 } & \multicolumn{2}{|c|}{ 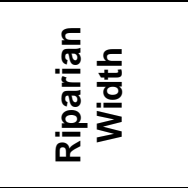 } & \multirow{2}{*}{ 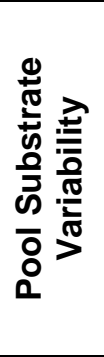 } & \multirow{2}{*}{ 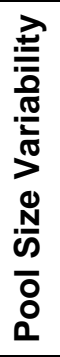 } & \multirow{2}{*}{ 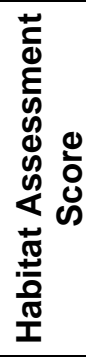 } \\
\hline & & & & & & & & ప্త & $\frac{\text { 픔 }}{\frac{0}{x}}$ & ప্త & $\frac{F}{\frac{\hbar}{x}}$ & 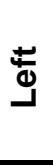 & $\frac{\tilde{E}}{\frac{\pi}{x}}$ & & & \\
\hline $\mathrm{X}-05-200$ & 14 & 12 & 18 & 9 & 11 & 13 & 13 & 8 & 8 & 7 & 7 & 5 & 5 & 13 & 17 & 160 \\
\hline$X-05-202$ & 5 & 5 & 11 & 5 & 6 & 6 & 3 & 5 & 6 & 4 & 4 & 2 & 2 & 9 & 8 & 81 \\
\hline$X-05-204$ & 12 & 7 & 5 & 13 & 14 & 9 & 2 & 9 & 9 & 6 & 6 & 4 & 3 & 13 & 13 & 125 \\
\hline$X-05-207$ & 7 & 4 & 3 & 6 & 6 & 6 & 3 & 8 & 8 & 4 & 4 & 5 & 5 & 12 & 7 & 88 \\
\hline$X-05-208$ & 8 & 4 & 4 & 8 & 12 & 7 & 3 & 9 & 9 & 4 & 4 & 5 & 5 & 13 & 9 & 104 \\
\hline $\mathrm{X}-05-210$ & 9 & 10 & 6 & 8 & 8 & 15 & 7 & 6 & 6 & 7 & 7 & 6 & 6 & 11 & 16 & 128 \\
\hline D-06-001 & 9 & 5 & 9 & 9 & 9 & 9 & 8 & 6 & 6 & 5 & 5 & 4 & 4 & 7 & 6 & 101 \\
\hline W-06-001 & 18 & 10 & 5 & 8 & 11 & 15 & 14 & 9 & 8 & 7 & 6 & 8 & 6 & 15 & 15 & 155 \\
\hline W-06-003 & 15 & 9 & 15 & 16 & 17 & 14 & 13 & 8 & 8 & 6 & 6 & 8 & 8 & 18 & 15 & 176 \\
\hline W-06-004 & 15 & 10 & 13 & 10 & 8 & 13 & 15 & 8 & 8 & 8 & 8 & 9 & 9 & 16 & 16 & 166 \\
\hline W-06-007 & 17 & 16 & 16 & 13 & 10 & 15 & 11 & 8 & 8 & 6 & 6 & 6 & 7 & 17 & 16 & 172 \\
\hline W-06-008 & 14 & 13 & 18 & 12 & 15 & 7 & 13 & 8 & 8 & 3 & 3 & 0 & 0 & 15 & 16 & 145 \\
\hline W-06-009 & 11 & 11 & 5 & 11 & 10 & 16 & 16 & 8 & 8 & 6 & 6 & 5 & 6 & 15 & 11 & 145 \\
\hline W-06-010 & 5 & 7 & 10 & 6 & 9 & 6 & 7 & 3 & 3 & 1 & 1 & 0 & 0 & 7 & 7 & 72 \\
\hline W-06-012 & 11 & 10 & 10 & 6 & 8 & 9 & 11 & 6 & 6 & 4 & 4 & 3 & 3 & 12 & 7 & 110 \\
\hline W-06-015 & 11 & 11 & 15 & 11 & 10 & 15 & 11 & 9 & 9 & 6 & 6 & 0 & 0 & 13 & 10 & 137 \\
\hline W-06-016 & 11 & 10 & 10 & 10 & 10 & 15 & 11 & 7 & 8 & 6 & 6 & 5 & 7 & 14 & 10 & 140 \\
\hline W-06-017 & 11 & 6 & 7 & 5 & 4 & 11 & 11 & 6 & 6 & 2 & 2 & 1 & 1 & 8 & 8 & 89 \\
\hline W-06-018 & 14 & 16 & 10 & 14 & 10 & 11 & 14 & 7 & 7 & 5 & 5 & 3 & 3 & 11 & 12 & 142 \\
\hline W-06-019 & 11 & 15 & 6 & 12 & 10 & 9 & 6 & 7 & 7 & 6 & 6 & 2 & 1 & 8 & 10 & 116 \\
\hline W-06-020 & 10 & 8 & 9 & 6 & 8 & 8 & 7 & 3 & 3 & 5 & 5 & 3 & 3 & 8 & 8 & 94 \\
\hline W-06-021 & 3 & 6 & 5 & 9 & 5 & 10 & 0 & 5 & 7 & 4 & 6 & 1 & 2 & 6 & 3 & 72 \\
\hline W-06-022 & 7 & 6 & 15 & 11 & 7 & 6 & 12 & 5 & 5 & 3 & 3 & 5 & 5 & 7 & 9 & 106 \\
\hline W-06-023 & 19 & 13 & 15 & 13 & 10 & 14 & 13 & 7 & 7 & 9 & 9 & 8 & 8 & 13 & 16 & 174 \\
\hline W-06-025 & 17 & 15 & 10 & 15 & 8 & 10 & 14 & 8 & 8 & 5 & 5 & 5 & 5 & 16 & 10 & 151 \\
\hline
\end{tabular}


Appendix 3: Table showing the twelve individual habitat parameter scores adapted from Barbour et al. (1999) that were assessed at most sites where fishing effort was applied and were totalled to derive the Habitat Assessment Score for each site.

\begin{tabular}{|c|c|c|c|c|c|c|c|c|c|c|c|c|c|c|c|c|}
\hline \multirow{2}{*}{ Site Number } & \multirow{2}{*}{ 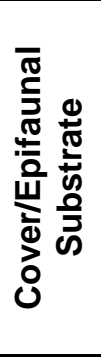 } & \multirow{2}{*}{ 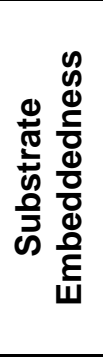 } & \multirow{2}{*}{ 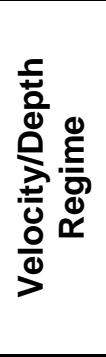 } & \multirow{2}{*}{ 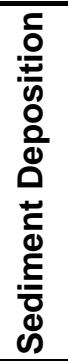 } & \multirow{2}{*}{ 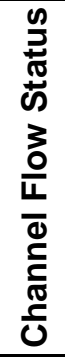 } & \multirow{2}{*}{ 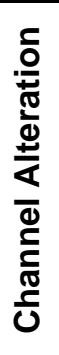 } & \multirow{2}{*}{ 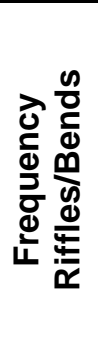 } & \multicolumn{2}{|c|}{ 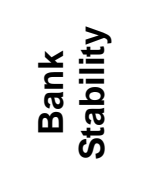 } & \multicolumn{2}{|c|}{ 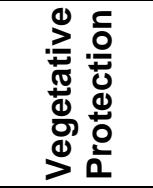 } & \multicolumn{2}{|c|}{$\begin{array}{l}\frac{c}{\sqrt{0}} \\
\frac{2}{\frac{0}{\pi}} \\
\frac{0}{x}\end{array}$} & \multirow{2}{*}{ 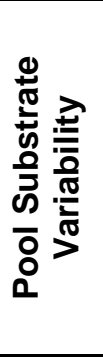 } & \multirow{2}{*}{ 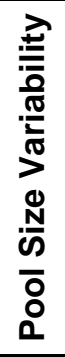 } & \multirow{2}{*}{ 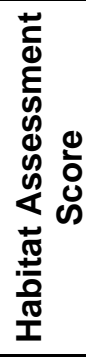 } \\
\hline & & & & & & & & Ф্త & $\begin{array}{l}\frac{}{\frac{1}{\sigma}} \\
\frac{0}{x}\end{array}$ & 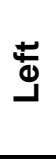 & $\frac{\text { 픔 }}{\frac{0}{\tilde{x}}}$ & 苂 & $\frac{\tilde{n}}{\frac{\tilde{\sigma}}{\boldsymbol{x}}}$ & & & \\
\hline W-06-026 & 17 & 17 & 15 & 19 & 12 & 8 & 7 & 8 & 8 & 5 & 5 & 4 & 4 & 20 & 18 & 167 \\
\hline W-06-027 & 14 & 15 & 13 & 13 & 10 & 9 & 10 & 5 & 5 & 5 & 5 & 0 & 0 & 17 & 18 & 139 \\
\hline W-06-028 & 18 & 16 & 16 & 16 & 13 & 10 & 10 & 6 & 6 & 8 & 8 & 2 & 4 & 17 & 18 & 168 \\
\hline W-06-029 & 12 & 6 & 10 & 10 & 7 & 6 & 4 & 5 & 5 & 3 & 3 & 5 & 5 & 7 & 13 & 101 \\
\hline W-06-030 & 8 & 7 & 6 & 5 & 6 & 8 & 10 & 6 & 6 & 6 & 6 & 8 & 8 & 11 & 10 & 111 \\
\hline W-06-031 & 15 & 14 & 18 & 14 & 11 & 7 & 12 & 1 & 1 & 7 & 7 & 6 & 6 & 11 & 15 & 145 \\
\hline W-06-032 & 15 & 10 & 15 & 10 & 9 & 15 & 11 & 2 & 5 & 6 & 6 & 5 & 5 & 15 & 18 & 147 \\
\hline W-06-036 & 16 & 13 & 6 & 11 & 8 & 11 & 3 & 4 & 4 & 7 & 7 & 5 & 1 & 10 & 6 & 112 \\
\hline W-06-037 & 11 & 10 & 10 & 6 & 7 & 10 & 13 & 5 & 5 & 5 & 5 & 4 & 4 & 6 & 9 & 110 \\
\hline W-06-040 & 10 & 11 & 6 & 11 & 9 & 11 & 5 & 4 & 4 & 6 & 6 & 0 & 0 & 10 & 6 & 99 \\
\hline W-06-043 & 8 & 3 & 2 & 3 & 6 & 15 & 8 & 7 & 7 & 7 & 7 & 3 & 5 & 12 & 3 & 96 \\
\hline W-06-045 & 17 & 15 & 18 & 11 & 11 & 16 & 16 & 7 & 7 & 7 & 7 & 3 & 3 & 16 & 16 & 170 \\
\hline W-06-046 & 10 & 9 & 13 & 6 & 9 & 13 & 13 & 5 & 5 & 4 & 4 & 3 & 2 & 7 & 16 & 119 \\
\hline W-06-050 & 14 & 13 & 10 & 13 & 2 & 13 & 16 & 3 & 3 & 8 & 8 & 8 & 8 & 13 & 0 & 132 \\
\hline W-06-054 & 5 & 5 & 6 & 5 & 8 & 7 & 1 & 7 & 7 & 7 & 7 & 3 & 3 & 8 & 5 & 84 \\
\hline W-06-055 & 7 & 10 & 15 & 10 & 10 & 4 & 2 & 5 & 5 & 0 & 0 & 0 & 0 & 4 & 7 & 79 \\
\hline W-06-056 & 16 & 10 & 10 & 10 & 6 & 15 & 13 & 2 & 2 & 9 & 9 & 6 & 6 & 7 & 5 & 126 \\
\hline W-06-058 & 10 & 5 & 4 & 5 & 4 & 13 & 10 & 8 & 8 & 7 & 7 & 8 & 4 & 12 & 6 & 111 \\
\hline W-06-060 & 14 & 10 & 10 & 10 & 5 & 4 & 5 & 5 & 5 & 5 & 5 & 3 & 3 & 7 & 10 & 101 \\
\hline W-06-061 & 17 & 17 & 18 & 15 & 18 & 19 & 19 & 9 & 9 & 8 & 8 & 9 & 9 & 18 & 19 & 212 \\
\hline W-06-062 & 10 & 10 & 15 & 9 & 11 & 11 & 7 & 9 & 9 & 6 & 6 & 9 & 9 & 12 & 6 & 139 \\
\hline W-06-063 & 8 & 2 & 8 & 1 & 7 & 2 & 14 & 1 & 1 & 1 & 1 & 0 & 0 & 6 & 11 & 63 \\
\hline W-06-064 & 5 & 10 & 15 & 8 & 6 & 7 & 11 & 1 & 1 & 0 & 0 & 0 & 0 & 4 & 6 & 74 \\
\hline W-06-065 & 6 & 6 & 10 & 5 & 8 & 11 & 18 & 6 & 6 & 5 & 5 & 9 & 9 & 12 & 12 & 128 \\
\hline W-06-066 & 16 & 13 & 10 & 11 & 9 & 13 & 16 & 7 & 7 & 7 & 7 & 6 & 6 & 10 & 7 & 145 \\
\hline
\end{tabular}


Appendix 3: Table showing the twelve individual habitat parameter scores adapted from Barbour et al. (1999) that were assessed at most sites where fishing effort was applied and were totalled to derive the Habitat Assessment Score for each site.

\begin{tabular}{|c|c|c|c|c|c|c|c|c|c|c|c|c|c|c|c|c|}
\hline \multirow{2}{*}{ Site Number } & \multirow{2}{*}{ 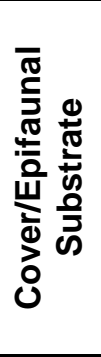 } & \multirow{2}{*}{ 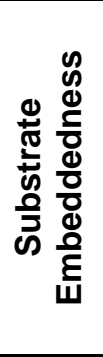 } & \multirow{2}{*}{ 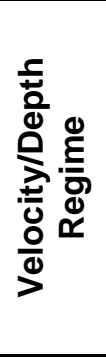 } & \multirow{2}{*}{ 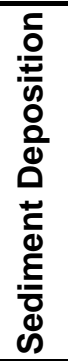 } & \multirow{2}{*}{ 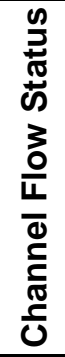 } & \multirow{2}{*}{ 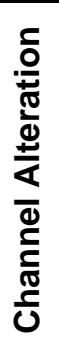 } & \multirow{2}{*}{ 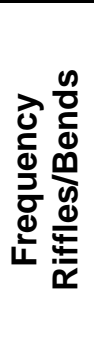 } & \multicolumn{2}{|c|}{ 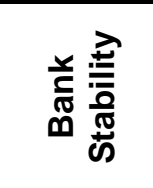 } & \multicolumn{2}{|c|}{ 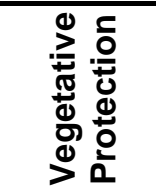 } & \multicolumn{2}{|c|}{ 紊 } & \multirow{2}{*}{ 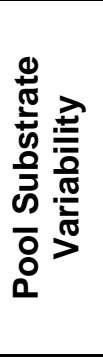 } & \multirow{2}{*}{ 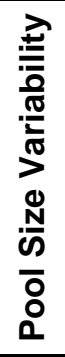 } & \multirow{2}{*}{ 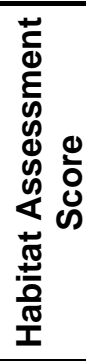 } \\
\hline & & & & & & & & 屯্త & $\begin{array}{l}\frac{}{\frac{1}{\sigma}} \\
\frac{0}{x}\end{array}$ & Ф્త & 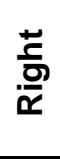 & 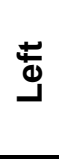 & $\frac{\tilde{n}}{\frac{\tilde{\sigma}}{\boldsymbol{x}}}$ & & & \\
\hline W-06-067 & 16 & 11 & 16 & 11 & 15 & 15 & 16 & 9 & 9 & 9 & 9 & 9 & 9 & 16 & 16 & 186 \\
\hline W-06-068 & 16 & 16 & 19 & 14 & 14 & 12 & 15 & 8 & 8 & 8 & 8 & 9 & 9 & 15 & 16 & 187 \\
\hline W-06-070 & 12 & 10 & 18 & 12 & 10 & 18 & 15 & 7 & 7 & 8 & 8 & 5 & 8 & 16 & 17 & 171 \\
\hline W-06-074 & 16 & 13 & 16 & 7 & 10 & 17 & 14 & 5 & 5 & 9 & 9 & 9 & 9 & 18 & 15 & 172 \\
\hline W-06-076 & 16 & 15 & 13 & 11 & 18 & 15 & 15 & 9 & 9 & 9 & 9 & 10 & 10 & 15 & 18 & 192 \\
\hline W-06-077 & 16 & 14 & 15 & 13 & 7 & 15 & 15 & 3 & 3 & 7 & 7 & 9 & 9 & 14 & 14 & 161 \\
\hline W-06-078 & 15 & 14 & 13 & 10 & 11 & 14 & 14 & 6 & 6 & 7 & 7 & 5 & 5 & 15 & 13 & 155 \\
\hline W-06-079 & 16 & 11 & 15 & 11 & 11 & 16 & 11 & 8 & 8 & 8 & 8 & 8 & 8 & 11 & 16 & 166 \\
\hline W-06-083 & 16 & 18 & 16 & 18 & 16 & 16 & 18 & 10 & 9 & 9 & 9 & 9 & 9 & 16 & 16 & 205 \\
\hline W-06-085 & 14 & 12 & 15 & 11 & 10 & 16 & 17 & 7 & 7 & 8 & 8 & 9 & 9 & 14 & 13 & 170 \\
\hline W-06-087 & 19 & 19 & 10 & 18 & 9 & 18 & 20 & 10 & 10 & 10 & 10 & 10 & 10 & 18 & 18 & 209 \\
\hline W-06-088 & 10 & 6 & 6 & 8 & 13 & 17 & 11 & 9 & 9 & 8 & 8 & 9 & 9 & 13 & 11 & 147 \\
\hline W-06-089 & 17 & 13 & 15 & 16 & 12 & 10 & 16 & 8 & 8 & 5 & 5 & 3 & 3 & 16 & 16 & 163 \\
\hline W-06-090 & 17 & 18 & 20 & 18 & 17 & 20 & 20 & 9 & 9 & 8 & 8 & 10 & 10 & 19 & 20 & 223 \\
\hline W-06-091 & 18 & 16 & 19 & 12 & 15 & 18 & 18 & 9 & 9 & 9 & 9 & 10 & 10 & 18 & 18 & 208 \\
\hline W-06-092 & 11 & 5 & 16 & 5 & 10 & 16 & 14 & 8 & 8 & 8 & 8 & 9 & 5 & 10 & 16 & 149 \\
\hline W-06-093 & 15 & 14 & 14 & 11 & 8 & 16 & 15 & 9 & 9 & 9 & 9 & 9 & 9 & 13 & 7 & 167 \\
\hline W-06-094 & 10 & 8 & 6 & 8 & 9 & 7 & 3 & 8 & 8 & 6 & 6 & 4 & 2 & 12 & 3 & 100 \\
\hline W-06-096 & 14 & 8 & 2 & 6 & 6 & 7 & 5 & 7 & 7 & 6 & 6 & 3 & 2 & 11 & 5 & 95 \\
\hline W-06-097 & 17 & 17 & 17 & 14 & 14 & 9 & 8 & 7 & 7 & 5 & 5 & 4 & 3 & 16 & 15 & 158 \\
\hline W-06-098 & 12 & 9 & 12 & 16 & 6 & 9 & 7 & 8 & 8 & 2 & 2 & 6 & 6 & 13 & 13 & 129 \\
\hline W-06-100 & 17 & 16 & 18 & 11 & 11 & 10 & 9 & 6 & 6 & 5 & 5 & 6 & 6 & 16 & 16 & 158 \\
\hline W-06-102 & 15 & 12 & 10 & 11 & 10 & 12 & 7 & 6 & 6 & 6 & 6 & 4 & 4 & 13 & 8 & 130 \\
\hline W-06-106 & 10 & 15 & 10 & 15 & 6 & 10 & 11 & 8 & 8 & 6 & 6 & 5 & 5 & 15 & 3 & 133 \\
\hline W-06-110 & 18 & 16 & 18 & 15 & 15 & 16 & 16 & 9 & 9 & 9 & 9 & 10 & 9 & 16 & 17 & 202 \\
\hline
\end{tabular}


Appendix 3: Table showing the twelve individual habitat parameter scores adapted from Barbour et al. (1999) that were assessed at most sites where fishing effort was applied and were totalled to derive the Habitat Assessment Score for each site.

\begin{tabular}{|c|c|c|c|c|c|c|c|c|c|c|c|c|c|c|c|c|}
\hline \multirow{2}{*}{ Site Number } & \multirow{2}{*}{ 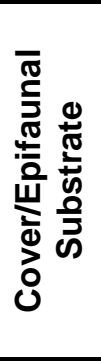 } & \multirow{2}{*}{ 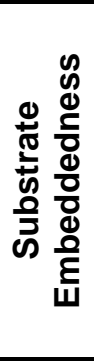 } & \multirow{2}{*}{ 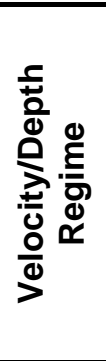 } & \multirow{2}{*}{ 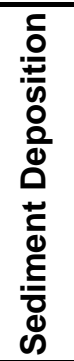 } & \multirow{2}{*}{ 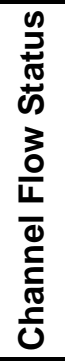 } & \multirow{2}{*}{ 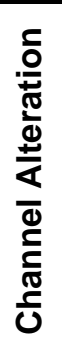 } & \multirow{2}{*}{ 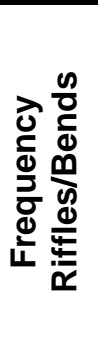 } & \multicolumn{2}{|c|}{ 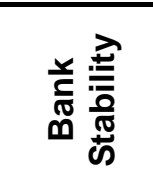 } & \multicolumn{2}{|c|}{ 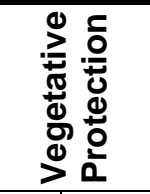 } & \multicolumn{2}{|c|}{ 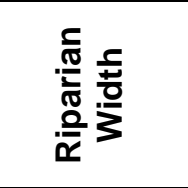 } & \multirow{2}{*}{ 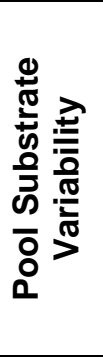 } & \multirow{2}{*}{ 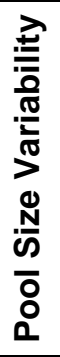 } & \multirow{2}{*}{ 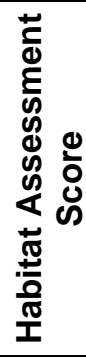 } \\
\hline & & & & & & & & ప্త & $\frac{\text { 동 }}{\frac{0}{x}}$ & ప্త & $\frac{\text { 듬 }}{\frac{0}{x}}$ & 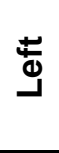 & $\frac{\tilde{E}}{\frac{\pi}{x}}$ & & & \\
\hline W-06-112 & 15 & 11 & 10 & 11 & 6 & 16 & 11 & 9 & 9 & 9 & 9 & 10 & 10 & 15 & 3 & 154 \\
\hline W-06-113 & 18 & 16 & 19 & 13 & 11 & 19 & 19 & 7 & 7 & 10 & 10 & 8 & 8 & 18 & 18 & 201 \\
\hline W-06-114 & 18 & 15 & 19 & 9 & 13 & 17 & 17 & 6 & 6 & 9 & 9 & 5 & 6 & 15 & 15 & 179 \\
\hline W-06-119 & 16 & 16 & 15 & 13 & 6 & 16 & 16 & 7 & 7 & 9 & 9 & 10 & 10 & 16 & 18 & 184 \\
\hline W-06-121 & 11 & 17 & 13 & 9 & 9 & 8 & 12 & 8 & 8 & 8 & 8 & 8 & 8 & 9 & 10 & 146 \\
\hline W-06-124 & 10 & 10 & 15 & 9 & 10 & 18 & 11 & 8 & 8 & 9 & 9 & 10 & 10 & 10 & 10 & 157 \\
\hline W-06-126 & 19 & 18 & 20 & 17 & 13 & 19 & 17 & 10 & 10 & 10 & 10 & 10 & 10 & 17 & 20 & 220 \\
\hline W-06-133 & 15 & 18 & 15 & 18 & 10 & 18 & 17 & 9 & 9 & 10 & 10 & 10 & 10 & 6 & 10 & 185 \\
\hline W-06-134 & 16 & 20 & 19 & 19 & 6 & 19 & 19 & 10 & 10 & 10 & 10 & 10 & 10 & 13 & 15 & 206 \\
\hline W-06-138 & 16 & 13 & 13 & 11 & 10 & 16 & 16 & 5 & 5 & 9 & 9 & 9 & 6 & 10 & 18 & 166 \\
\hline W-06-139 & 16 & 16 & 16 & 14 & 10 & 17 & 16 & 6 & 6 & 9 & 9 & 9 & 7 & 14 & 17 & 182 \\
\hline W-06-140 & 16 & 15 & 17 & 14 & 11 & 16 & 16 & 9 & 9 & 8 & 8 & 6 & 8 & 14 & 18 & 185 \\
\hline W-06-141 & 18 & 13 & 16 & 13 & 8 & 16 & 16 & 8 & 8 & 8 & 8 & 3 & 10 & 16 & 19 & 180 \\
\hline W-06-143 & 15 & 10 & 15 & 10 & 8 & 10 & 10 & 7 & 7 & 7 & 7 & 7 & 7 & 11 & 10 & 141 \\
\hline W-06-144 & 15 & 16 & 15 & 13 & 10 & 18 & 16 & 6 & 6 & 9 & 9 & 9 & 9 & 15 & 15 & 181 \\
\hline W-06-146 & 11 & 13 & 6 & 11 & 5 & 16 & 13 & 9 & 9 & 7 & 7 & 9 & 9 & 15 & 6 & 146 \\
\hline
\end{tabular}


Appendix 4: List of all fishing effort showing the site number, collectors, fishing gear used, amount of fishing effort applied and the fish capture identification (ID) number derived for each occasion where effort was applied.

\begin{tabular}{|c|c|c|c|c|}
\hline Site Number & Collectors & Gear ID & Effort & $\begin{array}{c}\text { Fish Capture ID } \\
\text { (Crew_Date_Gear) }\end{array}$ \\
\hline D-02-002 & $\mathrm{DM}, \mathrm{TS}, \mathrm{NV}, \mathrm{IH}$ & $E$ & 472 seconds $\times 120 \mathrm{~m}$ & D-02-002_170402_E1 \\
\hline D-02-004 & DM, JM, IH & $E$ & 241 seconds $\times 75 \mathrm{~m}$ & D-02-004_220402_E1 \\
\hline D-02-005 & DM, AW & $\mathrm{K}$ & $3 \mathrm{~m}^{2}$ & D-02-005_020502_K1 \\
\hline D-02-008 & $\mathrm{DM}, \mathrm{AW}$ & $E$ & 206 seconds $\times 75 \mathrm{~m}$ & D-02-008_070502_E1 \\
\hline D-02-009 & DM, AW & $E$ & 472 seconds $\times 150 \mathrm{~m}$ & D-02-009_070502_E1 \\
\hline D-02-010 & DM, AW & $E$ & 192 seconds $\times 100 \mathrm{~m}$ & D-02-010_090502_E1 \\
\hline D-02-011 & $\mathrm{DM}, \mathrm{AW}$ & $E$ & 125 seconds $\times 75 \mathrm{~m}$ & D-02-011_090502_E1 \\
\hline D-02-012 & DM, AW & $E$ & 247 seconds $\times 100 \mathrm{~m}$ & D-02-012_090502_E1 \\
\hline D-02-013 & DM, AW & $E$ & 250 seconds $\times 200 \mathrm{~m}$ & D-02-013_100502_E1 \\
\hline D-02-014 & DM, AW & $E$ & 228 seconds $\times 85 \mathrm{~m}$ & D-02-014_110502_E1 \\
\hline D-02-014 & DM, AW & $\mathrm{K}$ & $3 \mathrm{~m}^{2}$ & D-02-014_110502_K1 \\
\hline D-02-015 & DM, AW & $E$ & 435 seconds $\times 75 \mathrm{~m}$ & D-02-015_110502_E1 \\
\hline D-02-016 & DM, AW & $E$ & 140 seconds $\times 50 \mathrm{~m}$ & D-02-016_110502_E1 \\
\hline D-02-018 & DM, AW & $\mathrm{A}$ & Conversation with landowner & D-02-018_120502_A1 \\
\hline D-02-018 & DM, AW & $E$ & 655 seconds $\times 175 \mathrm{~m}$ & D-02-018_120502_E1 \\
\hline D-02-022 & DM, AW & $\mathrm{K}$ & $2 \mathrm{~m}^{2}$ riffle & D-02-022_120502_K1 \\
\hline D-02-023 & DM, AW & $\mathrm{K}$ & $3 \mathrm{~m}^{2}$ riffle & D-02-023_120502_K1 \\
\hline D-02-026 & DM, AW & $E$ & 20 seconds $\times 10 \mathrm{~m}$ & D-02-026_130502_E1 \\
\hline D-02-026 & $\mathrm{DM}, \mathrm{AW}$ & $\mathrm{K}$ & $3 \mathrm{~m}^{2}$ riffle & D-02-026_130502_K1 \\
\hline D-02-027 & DM, AW & $E$ & 595 seconds $\times 100 \mathrm{~m}$ & D-02-027_130502_E1 \\
\hline D-02-028 & DM, AW & $E$ & 330 seconds $\times 100 \mathrm{~m}$ & D-02-028_130502_E1 \\
\hline D-02-028 & DM, AW & $\mathrm{K}$ & $3 \mathrm{~m}^{2}$ riffle & D-02-028_130502_K1 \\
\hline D-02-031 & DM, AW & $E$ & 249 seconds $\times 75 \mathrm{~m}$ & D-02-031_150502_E1 \\
\hline D-02-033 & $\mathrm{DM}, \mathrm{AW}$ & $E$ & 406 seconds $\times 100 \mathrm{~m}$ & D-02-033_150502_E1 \\
\hline D-02-033 & DM, AW & $\mathrm{K}$ & $3 \mathrm{~m}^{2}$ riffle & D-02-033_150502_K1 \\
\hline D-02-034 & DM, AW & A & Conversation with TRWCD Technician & D-02-034_160502_A1 \\
\hline D-02-034 & DM, AW & $E$ & 240 seconds $\times 100 \mathrm{~m}$ & D-02-034_160502_E1 \\
\hline D-02-035 & DM, AW & $E$ & 100 seconds $\times 75 \mathrm{~m}$ & D-02-035_160502_E1 \\
\hline D-02-036 & DM, AW & $\mathrm{E}$ & 499 seconds $\times 150 \mathrm{~m}$ & D-02-036_160502_E1 \\
\hline D-02-037 & DM, AW & $\mathrm{O}$ & Observed dead on rocks & D-02-037_160502_O1 \\
\hline
\end{tabular}


Appendix 4: List of all fishing effort showing the site number, collectors, fishing gear used, amount of fishing effort applied and the fish capture identification (ID) number derived for each occasion where effort was applied.

\begin{tabular}{|c|c|c|c|c|}
\hline Site Number & Collectors & Gear ID & Effort & $\begin{array}{c}\text { Fish Capture ID } \\
\text { (Crew_Date_Gear) }\end{array}$ \\
\hline D-02-043 & $\mathrm{DM}, \mathrm{AW}$ & $E$ & 239 seconds $\times 100 \mathrm{~m}$ & D-02-043_210502_E1 \\
\hline D-02-044 & DM, AW & $E$ & 283 seconds $\times 100 \mathrm{~m}$ & D-02-044_210502_E1 \\
\hline D-02-045 & $\mathrm{DM}, \mathrm{AW}$ & $E$ & 249 seconds $\times 75 \mathrm{~m}$ & D-02-045_220502_E1 \\
\hline D-02-048 & $\mathrm{DM}, \mathrm{AW}$ & $E$ & 39 seconds $\times 30 \mathrm{~m}$ & D-02-048_230502_E1 \\
\hline D-02-051 & DM, AW & $E$ & 183 seconds $\times 75 \mathrm{~m}$ & D-02-051_240502_E1 \\
\hline D-02-054 & DM, AW & $E$ & 138 seconds $\times 75 \mathrm{~m}$ & D-02-054_240502_E1 \\
\hline D-02-056 & DM, AW & $E$ & 510 seconds $\times 125 \mathrm{~m}$ & D-02-056_240502_E1 \\
\hline D-02-058 & DM, AW & $\mathrm{E}$ & 447 seconds $\times 100 \mathrm{~m}$ & D-02-058_240502_E1 \\
\hline D-02-060 & DM, AW & $E$ & 397 seconds $\times 150 \mathrm{~m}$ & D-02-060_270502_E1 \\
\hline D-02-060 & DM, AW & $\mathrm{K}$ & $2 \mathrm{~m}^{2}$ rocks & D-02-060_270502_K1 \\
\hline $\mathrm{D}-02-061$ & DM, AW & $E$ & 697 seconds $\times 100 \mathrm{~m}$ & D-02-061_280502_E1 \\
\hline D-02-062 & DM, AW & $E$ & 398 seconds $\times 50 \mathrm{~m}$ & D-02-062_280502_E1 \\
\hline D-02-065 & DM & $\mathrm{O}$ & Angling spot, 2 dead on shore & D-02-065_290502_01 \\
\hline D-02-065 & DM & $\mathrm{D}$ & Sweeps & D-02-065_290502_D1 \\
\hline D-02-067 & DM, AW & $E$ & 241 seconds $\times 100 \mathrm{~m}$ & D-02-067_290502_E1 \\
\hline $\mathrm{D}-02-068$ & $\mathrm{DM}, \mathrm{AW}$ & $\mathrm{E}$ & 424 seconds $\times 150 \mathrm{~m}$ & D-02-068_290502_E1 \\
\hline D-02-069 & DM, AW & $E$ & 212 seconds $\times 100 \mathrm{~m}$ & D-02-069_290502_E1 \\
\hline D-02-070 & DM, AW & $\mathrm{L}$ & $30 \mathrm{~min}$. & D-02-070_300502_L1 \\
\hline D-02-071 & DM, AW & $E$ & 370 seconds $\times 125 \mathrm{~m}$ & D-02-071_300502_E1 \\
\hline D-02-072 & DM & $\mathrm{O}$ & Observed dead on shore & D-02-072_030602_01 \\
\hline D-02-073 & DM, AW & $\mathrm{L}$ & 18 hour over-night set & D-02-073_030602_L1 \\
\hline D-02-074 & DM, AW & $E$ & 563 seconds $\times 75 \mathrm{~m}$ & D-02-074 $030602 \mathrm{E} 1$ \\
\hline D-02-075 & DM, AW & $\mathrm{E}$ & 560 seconds $\times 75 \mathrm{~m}$ & D-02-075_040602_E1 \\
\hline D-02-076 & $\mathrm{DM}, \mathrm{AW}$ & $E$ & 306 seconds $\times 100 \mathrm{~m}$ & D-02-076_050602_E1 \\
\hline D-02-077 & DM, AW & $E$ & 547 seconds $\times 200 \mathrm{~m}$ & D-02-077_050602_E1 \\
\hline D-02-078 & DM, AW & $E$ & 331 seconds $\times 150 \mathrm{~m}$ & D-02-078_050602_E1 \\
\hline D-02-081 & DM, AW & $E$ & 391 seconds $\times 150 \mathrm{~m}$ & D-02-081_060602_E1 \\
\hline D-02-083 & DM, AW & $E$ & 310 seconds $\times 100 \mathrm{~m}$ & D-02-083_060602_E1 \\
\hline D-02-084 & DM, AW & $E$ & 564 seconds $\times 200 \mathrm{~m}$ & D-02-084_120602_E1 \\
\hline D-02-087 & DM, AW & $E$ & 252 seconds $\times 75 \mathrm{~m}$ & D-02-087_120602_E1 \\
\hline D-02-088 & DM, AW & $\mathrm{E}$ & 211 seconds $\times 100 \mathrm{~m}$ & D-02-088_130602_E1 \\
\hline
\end{tabular}


Appendix 4: List of all fishing effort showing the site number, collectors, fishing gear used, amount of fishing effort applied and the fish capture identification (ID) number derived for each occasion where effort was applied.

\begin{tabular}{|c|c|c|c|c|}
\hline Site Number & Collectors & Gear ID & Effort & $\begin{array}{c}\text { Fish Capture ID } \\
\text { (Crew_Date_Gear) }\end{array}$ \\
\hline D-02-089 & $\mathrm{DM}, \mathrm{AW}$ & $E$ & 451 seconds $\times 150 \mathrm{~m}$ & D-02-089_130602_E1 \\
\hline D-02-092 & $\mathrm{DM}, \mathrm{AW}$ & $E$ & 342 seconds $\times 150 \mathrm{~m}$ & D-02-092_130602_E1 \\
\hline D-02-093 & DM, AW & $E$ & 365 seconds $\times 100 \mathrm{~m}$ & D-02-093_140602_E1 \\
\hline D-02-094 & DM, AW & $E$ & 357 seconds $\times 100 \mathrm{~m}$ & D-02-094_180602_E1 \\
\hline D-02-095 & $\mathrm{DM}, \mathrm{AW}$ & $E$ & 270 seconds $\times 100 \mathrm{~m}$ & D-02-095_180602_E1 \\
\hline D-02-096 & DM, AW, EW & $E$ & 896 seconds $\times 150 \mathrm{~m}$ & D-02-096_190602_E1 \\
\hline D-02-097 & DM, AW, EW & $E$ & 459 seconds $\times 75 \mathrm{~m}$ & D-02-097_190602 E1 \\
\hline D-02-100 & $\mathrm{DM}, \mathrm{AW}$ & $E$ & 275 seconds $\times 100 \mathrm{~m}$ & D-02-100_200602_E1 \\
\hline D-02-101 & $\mathrm{DM}, \mathrm{AW}$ & $E$ & 474 seconds $\times 100 \mathrm{~m}$ & D-02-101_200602_E1 \\
\hline D-02-102 & DM, AW & $E$ & 429 seconds $\times 100 \mathrm{~m}$ & D-02-102_200602_E1 \\
\hline D-02-104 & DM, AW & $E$ & 324 seconds $\times 75 \mathrm{~m}$ & D-02-104_200602_E1 \\
\hline D-02-107 & DM, AW & $\mathrm{E}$ & 100 seconds $\times 50 \mathrm{~m}$ & D-02-107_210602_E1 \\
\hline D-02-108 & $\mathrm{DM}, \mathrm{AW}, \mathrm{KK}$ & $\mathrm{E}$ & 453 seconds $\times 150 \mathrm{~m}$ & D-02-108_210602_E1 \\
\hline D-02-111 & DM, AW & $E$ & 314 seconds $\times 75 \mathrm{~m}$ & D-02-111_260602_E1 \\
\hline D-02-116 & DM, AW & $E$ & 159 seconds $\times 75 \mathrm{~m}$ & D-02-116_030702_E1 \\
\hline D-02-118 & DM, AW & $E$ & 316 seconds $\times 100 \mathrm{~m}$ & D-02-118_030702_E1 \\
\hline D-02-119 & DM, AW & $\mathrm{E}$ & 450 seconds $\times 150 \mathrm{~m}$ & D-02-119_040702_E1 \\
\hline $\mathrm{D}-02-121$ & DM, AW & $\mathrm{E}$ & 227 seconds $\times 100 \mathrm{~m}$ & D-02-121_040702_E1 \\
\hline D-02-122 & $\mathrm{DM}, \mathrm{AW}$ & $E$ & 279 seconds $\times 100 \mathrm{~m}$ & D-02-122_040702_E1 \\
\hline D-02-124 & DM, AW & $\mathrm{B}$ & $5 \mathrm{~m} \times 15 \mathrm{~m}$ & $\mathrm{D}-02-124 \quad 040702 \quad \mathrm{~B} 1$ \\
\hline D-02-125 & DM, AW & $\mathrm{E}$ & 512 seconds $\times 75 \mathrm{~m}$ & D-02-125_090702_E1 \\
\hline$D-02-126$ & $\mathrm{DM}, \mathrm{AW}$ & $\mathrm{B}$ & $5 \mathrm{~m} \times 10 \mathrm{~m}$ & D-02-126_090702_B1 \\
\hline D-02-127 & DM, AW & $\mathrm{E}$ & 446 seconds $\times 150 \mathrm{~m}$ & D-02-127_090702_E1 \\
\hline D-02-130 & DM, AW & $\mathrm{B}$ & $20 \mathrm{~m} \times 8 \mathrm{~m}$ & D-02-130_090702_B1 \\
\hline $\mathrm{D}-02-131$ & DM, AW & $\mathrm{B}$ & $5 \mathrm{~m} \times 20 \mathrm{~m}$ & D-02-131_090702_B1 \\
\hline $\mathrm{D}-02-132$ & DM, AW & $E$ & 195 seconds $\times 75 \mathrm{~m}$ & $\mathrm{D}-02-132 \_100702 \_\mathrm{E} 1$ \\
\hline D-02-135 & DM, AW & $E$ & 206 seconds $\times 75 \mathrm{~m}$ & D-02-135_100702_E1 \\
\hline D-02-137 & DM, AW & $E$ & 365 seconds $\times 150 \mathrm{~m}$ & D-02-137_120702 E1 \\
\hline D-02-138 & DM, AW & $\mathrm{B}$ & $5 \mathrm{~m} \times 15 \mathrm{~m}$ & D-02-138_120702_B1 \\
\hline D-02-139 & DM, AW & $\mathrm{E}$ & 248 seconds $\times 100 \mathrm{~m}$ & D-02-139_120702_E1 \\
\hline$D-02-142$ & DM, AW & $\mathrm{E}$ & 362 seconds $\times 100 \mathrm{~m}$ & D-02-142_170702_E1 \\
\hline
\end{tabular}


Appendix 4: List of all fishing effort showing the site number, collectors, fishing gear used, amount of fishing effort applied and the fish capture identification (ID) number derived for each occasion where effort was applied.

\begin{tabular}{|c|c|c|c|c|}
\hline Site Number & Collectors & Gear ID & Effort & $\begin{array}{c}\text { Fish Capture ID } \\
\text { (Crew_Date_Gear) }\end{array}$ \\
\hline D-02-147 & $\mathrm{DM}, \mathrm{AW}$ & $E$ & 93 seconds $\times 50 \mathrm{~m}$ & D-02-147_170702_E1 \\
\hline D-02-149 & DM, AW & $E$ & 132 seconds $\times 150 \mathrm{~m}$ & D-02-149_170702_E1 \\
\hline D-02-160 & $\mathrm{DM}, \mathrm{AW}$ & $E$ & 343 seconds $\times 100 \mathrm{~m}$ & D-02-160_230702_E1 \\
\hline $\mathrm{D}-02-161$ & $\mathrm{DM}, \mathrm{AW}$ & $E$ & 241 seconds $125 \mathrm{~m}$ & D-02-161_230702_E1 \\
\hline D-02-164 & DM, AW & $E$ & 136 seconds $\times 75 \mathrm{~m}$ & D-02-164_240702_E1 \\
\hline D-02-167 & DM, AW & $\mathrm{B}$ & $5 \mathrm{~m} \times 8 \mathrm{~m}$ & D-02-167_240702_B1 \\
\hline D-02-170 & DM, AW & $E$ & 201 seconds $\times 75 \mathrm{~m}$ & D-02-170_240702_E1 \\
\hline D-02-171 & DM, AW & $\mathrm{E}$ & 148 seconds $\times 30 \mathrm{~m}$ & D-02-171_250702_E1 \\
\hline D-02-173 & DM, AW & $E$ & 100 seconds $\times 50 \mathrm{~m}$ & D-02-173_250702_E1 \\
\hline D-02-178 & DM, AW & $\mathrm{B}$ & $10 \mathrm{~m} \times 8 \mathrm{~m}$ & D-02-178_250702_B1 \\
\hline D-02-179 & DM, AW & $\mathrm{E}$ & 318 seconds $\times 100 \mathrm{~m}$ & D-02-179_300702_E1 \\
\hline D-02-180 & DM, AW & $E$ & 295 seconds $\times 100 \mathrm{~m}$ & D-02-180_300702_E1 \\
\hline D-02-181 & $\mathrm{DM}, \mathrm{AW}$ & $\mathrm{E}$ & 593 seconds $\times 150 \mathrm{~m}$ & D-02-181_310702_E1 \\
\hline D-02-182 & DM, AW & $E$ & 406 seconds $\times 75 \mathrm{~m}$ & D-02-182_310702_E1 \\
\hline $\mathrm{D}-02-183$ & DM, AW & $\mathrm{E}$ & 215 seconds $\times 100 \mathrm{~m}$ & D-02-183_310702_E1 \\
\hline D-02-184 & $\mathrm{DM}, \mathrm{AW}$ & $\mathrm{E}$ & 394 seconds $\times 150 \mathrm{~m}$ & D-02-184_310702_E1 \\
\hline D-02-185 & DM, AW & $E$ & 69 seconds $\times 30 \mathrm{~m}$ & D-02-185_010802_E1 \\
\hline D-02-188 & DM, AW & $E$ & 69 seconds $\times 30 \mathrm{~m}$ & D-02-188_010802_E1 \\
\hline D-02-190 & DM, AW & $E$ & 89 seconds $\times 30 \mathrm{~m}$ & D-02-190_010802_E1 \\
\hline D-02-191 & $\mathrm{DM}, \mathrm{RO}, \mathrm{NV}$ & $\mathrm{E}$ & 19 seconds $\times 100 \mathrm{~m}$ & D-02-191_150802_E1 \\
\hline D-02-192 & $\mathrm{DM}, \mathrm{RO}, \mathrm{NV}$ & $E$ & 319 seconds $\times 50 \mathrm{~m}$ & D-02-192_160802_E1 \\
\hline D-02-193 & DM, BM & $\mathrm{B}$ & $5 \mathrm{~m} \times 15 \mathrm{~m}$ & D-02-193 200802 B1 \\
\hline D-02-193 & $\mathrm{DM}, \mathrm{BM}$ & $E$ & 717 seconds $\times 150 \mathrm{~m}$ & D-02-193_200802_E1 \\
\hline D-02-194 & $\mathrm{DM}, \mathrm{BM}$ & $E$ & 327 seconds $\times 75 \mathrm{~m}$ & D-02-194_200802_E1 \\
\hline D-02-195 & $\mathrm{DM}, \mathrm{BR}$ & $E$ & 429 seconds $\times 125 \mathrm{~m}$ & D-02-195_210802_E1 \\
\hline D-02-196 & $\mathrm{DM}, \mathrm{BR}$ & $E$ & 839 seconds $\times 150 \mathrm{~m}$ & D-02-196_210802_E1 \\
\hline D-02-197 & DM, BT & $\mathrm{B}$ & $5 \mathrm{~m} \times 20 \mathrm{~m}$ & D-02-197_220802_B1 \\
\hline D-02-197 & DM, BT & $\mathrm{B}$ & $5 \mathrm{~m} \times 5 \mathrm{~m}$ & D-02-197_220802_B2 \\
\hline D-02-197 & DM, BT & $E$ & 759 seconds $\times 100 \mathrm{~m}$ & $\mathrm{D}-02-197 \quad 220802 \mathrm{E} 1$ \\
\hline D-02-198 & DM, BT & $E$ & 593 seconds $\times 75 \mathrm{~m}$ & D-02-198_220802_E1 \\
\hline D-02-199 & DM, BL & $\mathrm{E}$ & 494 seconds $\times 100 \mathrm{~m}$ & D-02-199_270802_E1 \\
\hline
\end{tabular}


Appendix 4: List of all fishing effort showing the site number, collectors, fishing gear used, amount of fishing effort applied and the fish capture identification (ID) number derived for each occasion where effort was applied.

\begin{tabular}{|c|c|c|c|c|}
\hline Site Number & Collectors & Gear ID & Effort & $\begin{array}{c}\text { Fish Capture ID } \\
\text { (Crew_Date_Gear) }\end{array}$ \\
\hline D-02-200 & $\mathrm{DM}, \mathrm{BL}$ & $E$ & 340 seconds $\times 75 \mathrm{~m}$ & D-02-200_270802_E1 \\
\hline D-02-201 & DM, JM & $E$ & 376 seconds $\times 100 \mathrm{~m}$ & D-02-201_081002_E1 \\
\hline B-03-001 & JA, HW, AW & $E$ & 433 seconds $\times 75 \mathrm{~m}$ & B-03-001_210503_E1 \\
\hline B-03-003 & $\mathrm{JA}, \mathrm{HW}$ & $\mathrm{E}$ & 400 seconds $\times 100 \mathrm{~m}$ & B-03-003_230503_E1 \\
\hline B-03-004 & JA, HW & $\mathrm{E}$ & 1305 seconds $\times 50 \mathrm{~m}$ & B-03-004_230503_E1 \\
\hline B-03-005 & JA, HW & $E$ & 580 seconds $\times 130 \mathrm{~m}$ & B-03-005_230503_E1 \\
\hline B-03-007 & JA, HW & $E$ & 146 seconds $\times 50 \mathrm{~m}$ & B-03-007_260503_E1 \\
\hline B-03-009 & JA, HW & $E$ & 283 seconds $\times 75 \mathrm{~m}$ & B-03-009_260503_E1 \\
\hline B-03-012 & JA, HW & $E$ & 158 seconds $\times 50 \mathrm{~m}$ & B-03-012_260503_E1 \\
\hline B-03-013 & JA, HW & $E$ & 545 seconds $\times 175 \mathrm{~m}$ & B-03-013_270503_E1 \\
\hline B-03-015 & $\mathrm{JA}, \mathrm{HW}$ & $\mathrm{E}$ & 135 seconds $\times 20 \mathrm{~m}$ & B-03-015_270503_E1 \\
\hline B-03-016 & JA, HW & $E$ & 264 seconds $\times 50 \mathrm{~m}$ & B-03-016_270503_E1 \\
\hline B-03-017 & JA, HW & $\mathrm{E}$ & 393 seconds $\times 75 \mathrm{~m}$ & B-03-017_280503_E1 \\
\hline B-03-019 & JA, HW & $\mathrm{B}$ & $16 \mathrm{~m} \times 40 \mathrm{~m}$ & B-03-019_280503_B1 \\
\hline B-03-020 & JA, HW & $\mathrm{E}$ & 223 seconds $\times 60 \mathrm{~m}$ & B-03-020_290503_E1 \\
\hline B-03-026 & JA, HW & $E$ & 146 seconds $\times 75 \mathrm{~m}$ & B-03-026_290503_E1 \\
\hline B-03-028 & JA, HW & $E$ & 224 seconds $\times 75 \mathrm{~m}$ & B-03-028_300503_E1 \\
\hline B-03-030 & JA, HW & $\mathrm{B}$ & $10 \mathrm{~m} \times 25 \mathrm{~m}$ & B-03-030_300503_B1 \\
\hline B-03-031 & JA, HW & $E$ & 1284 seconds $\times 100 \mathrm{~m}$ & B-03-031_300503_E1 \\
\hline B-03-033 & $\mathrm{JA}, \mathrm{HW}$ & $\mathrm{E}$ & 448 seconds $\times 100 \mathrm{~m}$ & B-03-033_020603_E1 \\
\hline B-03-033 & JA, HW & $\mathrm{L}$ & $5 \mathrm{~min}$. & B-03-033_020603_L1 \\
\hline B-03-035 & JA, HW & $\mathrm{B}$ & $10 \mathrm{~m} \times 45 \mathrm{~m}$ & B-03-035 020603 B1 \\
\hline B-03-040 & JA, HW & $E$ & 223 seconds $\times 75 \mathrm{~m}$ & B-03-040_030603_E1 \\
\hline B-03-041 & JA, HW & $E$ & 409 seconds $\times 125 \mathrm{~m}$ & B-03-041_030603_E1 \\
\hline B-03-048 & JA, HW & $\mathrm{B}$ & $15 \mathrm{~m} \times 60 \mathrm{~m}$ & B-03-048_040603_B1 \\
\hline B-03-050 & JA, HW & $E$ & 344 seconds $\times 120 \mathrm{~m}$ & B-03-050_040603_E1 \\
\hline B-03-052 & $\mathrm{JA}, \mathrm{HW}$ & $E$ & 724 seconds $\times 125 \mathrm{~m}$ & B-03-052_040603_E1 \\
\hline B-03-053 & JA, HW & $E$ & 200 seconds $\times 50 \mathrm{~m}$ & B-03-053_050603_E1 \\
\hline B-03-055 & JA, HW & $E$ & 212 seconds $\times 50 \mathrm{~m}$ & B-03-055_050603_E1 \\
\hline B-03-060 & JA, HW & $E$ & 278 seconds $\times 50 \mathrm{~m}$ & B-03-060_050603_E1 \\
\hline B-03-061 & JA, HW & $\mathrm{E}$ & $322 \mathrm{~m} \times 80 \mathrm{~m}$ & B-03-061_090603_E1 \\
\hline
\end{tabular}


Appendix 4: List of all fishing effort showing the site number, collectors, fishing gear used, amount of fishing effort applied and the fish capture identification (ID) number derived for each occasion where effort was applied.

\begin{tabular}{|c|c|c|c|c|}
\hline Site Number & Collectors & Gear ID & Effort & $\begin{array}{c}\text { Fish Capture ID } \\
\text { (Crew_Date_Gear) }\end{array}$ \\
\hline B-03-062 & JA, HW & $E$ & 270 seconds $\times 50 \mathrm{~m}$ & B-03-062_090603_E1 \\
\hline B-03-064 & JA, HW & $E$ & 162 seconds $\times 100 \mathrm{~m}$ & B-03-064_100603_E1 \\
\hline B-03-065 & $\mathrm{JA}, \mathrm{HW}$ & $E$ & 330 seconds $\times 120 \mathrm{~m}$ & B-03-065_100603_E1 \\
\hline B-03-067 & JA, HW & $E$ & 525 seconds $\times 125 \mathrm{~m}$ & B-03-067_100603_E1 \\
\hline B-03-070 & JA, HW & $\mathrm{E}$ & 386 seconds $\times 150 \mathrm{~m}$ & B-03-070_110603_E1 \\
\hline B-03-071 & JA, HW & $E$ & 332 seconds $\times 100 \mathrm{~m}$ & B-03-071_110603_E1 \\
\hline B-03-075 & JA, HW & $E$ & 375 seconds $\times 75 \mathrm{~m}$ & B-03-075_120603_E1 \\
\hline B-03-076 & JA, HW & $E$ & 233 seconds $\times 75 \mathrm{~m}$ & B-03-076_120603_E1 \\
\hline B-03-077 & JA, HW & $E$ & 549 seconds $\times 75 \mathrm{~m}$ & B-03-077_120603_E1 \\
\hline B-03-083 & JA, HW & $E$ & 336 seconds $\times 30 \mathrm{~m}$ & B-03-083_160603_E1 \\
\hline B-03-084 & $\mathrm{JA}, \mathrm{HW}$ & $\mathrm{E}$ & 321 seconds $\times 40 \mathrm{~m}$ & B-03-084_160603_E1 \\
\hline B-03-085 & JA, HW & $E$ & 423 seconds $\times 100 \mathrm{~m}$ & B-03-085_170603_E1 \\
\hline B-03-086 & JA, HW & $\mathrm{B}$ & $12 \mathrm{~m} \times 30 \mathrm{~m}$ & B-03-086_170603_B1 \\
\hline B-03-087 & JA, HW & $\mathrm{B}$ & $14 \mathrm{~m} \times 20 \mathrm{~m}$ & B-03-087_170603_B1 \\
\hline B-03-088 & JA, HW & $E$ & 382 seconds $\times 100 \mathrm{~m}$ & B-03-088_180603_E1 \\
\hline B-03-089 & JA, HW & $\mathrm{B}$ & $18 \mathrm{~m} \times 30 \mathrm{~m}$ & B-03-089_180603_B1 \\
\hline B-03-091 & JA, HW & $\mathrm{B}$ & $10 \mathrm{~m} \times 20 \mathrm{~m}$ & B-03-091_180603_B1 \\
\hline B-03-093 & JA, HW & $\mathrm{B}$ & $8 \mathrm{~m} \times 25 \mathrm{~m}$ & B-03-093_180603_B1 \\
\hline B-03-094 & JA, HW & $\mathrm{B}$ & $15 \mathrm{~m} \times 30 \mathrm{~m}$ & B-03-094_180603_B1 \\
\hline B-03-094 & $\mathrm{JA}, \mathrm{HW}$ & $\mathrm{E}$ & 325 seconds $\times 75 \mathrm{~m}$ & B-03-094_180603_E1 \\
\hline B-03-095 & JA, HW & $E$ & 533 seconds $\times 100 \mathrm{~m}$ & B-03-095_190603_E1 \\
\hline B-03-097 & JA, HW & $\mathrm{B}$ & $15 \mathrm{~m} \times 30 \mathrm{~m}$ & B-03-097 190603 B1 \\
\hline B-03-098 & JA, HW & $E$ & 325 seconds $\times 75 \mathrm{~m}$ & B-03-098_190603_E1 \\
\hline B-03-099 & JA, HW & $E$ & 301 seconds $\times 75 \mathrm{~m}$ & B-03-099_200603_E1 \\
\hline B-03-101 & JA, HW & $\mathrm{B}$ & $15 \mathrm{~m} \times 30 \mathrm{~m}$ & B-03-101_200603_B1 \\
\hline B-03-102 & JA, HW & $\mathrm{B}$ & $15 \mathrm{~m} \times 25 \mathrm{~m}$ & B-03-102_230603_B1 \\
\hline B-03-103 & $\mathrm{JA}, \mathrm{HW}$ & $E$ & 321 seconds $\times 60 \mathrm{~m}$ & B-03-103_230603_E1 \\
\hline B-03-104 & $\mathrm{JA}, \mathrm{HW}$ & $E$ & 294 seconds $\times 40 \mathrm{~m}$ & B-03-104_230603_E1 \\
\hline B-03-112 & JA, HW & $E$ & 497 seconds $\times 125 \mathrm{~m}$ & B-03-112 260603 E1 \\
\hline B-03-115 & JA, HW & $E$ & 343 seconds $\times 125 \mathrm{~m}$ & B-03-115_270603_E1 \\
\hline B-03-116 & JA, HW & $\mathrm{E}$ & 366 seconds $\times 50 \mathrm{~m}$ & B-03-116_270603_E1 \\
\hline
\end{tabular}


Appendix 4: List of all fishing effort showing the site number, collectors, fishing gear used, amount of fishing effort applied and the fish capture identification (ID) number derived for each occasion where effort was applied.

\begin{tabular}{|c|c|c|c|c|}
\hline Site Number & Collectors & Gear ID & Effort & $\begin{array}{c}\text { Fish Capture ID } \\
\text { (Crew_Date_Gear) }\end{array}$ \\
\hline B-03-117 & JA, HW & $E$ & 126 seconds $\times 30 \mathrm{~m}$ & B-03-117_270603_E1 \\
\hline B-03-118 & JA, HW & $E$ & 265 seconds $\times 60 \mathrm{~m}$ & B-03-118_270603_E1 \\
\hline B-03-119 & $\mathrm{JA}, \mathrm{HW}$ & $E$ & 183 seconds $\times 50 \mathrm{~m}$ & B-03-119_020703_E1 \\
\hline B-03-120 & JA, HW & $\mathrm{E}$ & 223 seconds $\times 50 \mathrm{~m}$ & B-03-120_020703_E1 \\
\hline B-03-121 & JA, HW & $\mathrm{E}$ & 183 seconds $\times 40 \mathrm{~m}$ & B-03-121_030703_E1 \\
\hline B-03-122 & JA, HW & $E$ & 516 seconds $\times 75 \mathrm{~m}$ & B-03-122 030703 E1 \\
\hline B-03-124 & JA, HW & $E$ & 381 seconds $\times 125 \mathrm{~m}$ & B-03-124_030703_E1 \\
\hline B-03-126 & JA, HW & $\mathrm{B}$ & $20 \mathrm{~m} \times 20 \mathrm{~m}$ & B-03-126_040703_B1 \\
\hline B-03-128 & JA, HW & $\mathrm{D}$ & 1 sweep & B-03-128_040703_D1 \\
\hline B-03-132 & JA, HW & $E$ & 89 seconds $\times 30 \mathrm{~m}$ & B-03-132_070703_E1 \\
\hline B-03-134 & $\mathrm{JA}, \mathrm{HW}$ & $\mathrm{E}$ & 111 seconds $\times 30 \mathrm{~m}$ & B-03-134_070703_E1 \\
\hline B-03-136 & JA, HW & $E$ & 280 seconds $\times 40 \mathrm{~m}$ & B-03-136_070703_E1 \\
\hline B-03-141 & JA, HW & $\mathrm{E}$ & 121 seconds $\times 10 \mathrm{~m}$ & B-03-141_080703_E1 \\
\hline B-03-142 & JA, HW & $E$ & 179 seconds $\times 20 \mathrm{~m}$ & B-03-142_080703_E1 \\
\hline B-03-143 & JA, HW & $\mathrm{B}$ & $13 \mathrm{~m} \times 25 \mathrm{~m}$ & B-03-143_080703_B1 \\
\hline B-03-145 & JA, HW & $\mathrm{B}$ & $15 \mathrm{~m} \times 40 \mathrm{~m}$ & B-03-145_090703_B1 \\
\hline B-03-148 & JA, HW & $E$ & 299 seconds $\times 50 \mathrm{~m}$ & B-03-148_090703_E1 \\
\hline B-03-150 & JA, HW & $\mathrm{B}$ & $30 \mathrm{~m} \times 30 \mathrm{~m}$ & B-03-150_090703_B1 \\
\hline B-03-151 & JA, HW & $E$ & 398 seconds $\times 175 \mathrm{~m}$ & B-03-151_100703_E1 \\
\hline B-03-151 & $\mathrm{JA}, \mathrm{HW}$ & $\mathrm{K}$ & $2 \mathrm{~m}^{2}$ & B-03-151_100703_K1 \\
\hline B-03-152 & JA, HW & $E$ & 489 seconds $\times 100 \mathrm{~m}$ & B-03-152_100703_E1 \\
\hline B-03-156 & JA, HW & $\mathrm{O}$ & Observed in pools & B-03-156 $110703 \quad 01$ \\
\hline B-03-157 & JA, HW & $\mathrm{O}$ & Observed in pools & B-03-157_110703_O1 \\
\hline B-03-158 & JA, HW & $\mathrm{B}$ & $15 \mathrm{~m} \times 30 \mathrm{~m}$ & B-03-158_110703_B1 \\
\hline B-03-159 & JA, HW & $E$ & 235 seconds $\times 50 \mathrm{~m}$ & B-03-159_110703_E1 \\
\hline B-03-160 & JA, HW & $\mathrm{B}$ & $10 \mathrm{~m} \times 20 \mathrm{~m}$ & B-03-160_110703_B1 \\
\hline B-03-163 & $\mathrm{JA}, \mathrm{HW}$ & $\mathrm{B}$ & $20 \mathrm{~m} \times 20 \mathrm{~m}$ & B-03-163_140703_B1 \\
\hline B-03-181 & $\mathrm{JA}, \mathrm{HW}$ & $E$ & 436 seconds $\times 75 \mathrm{~m}$ & B-03-181_150703_E1 \\
\hline B-03-182 & JA, HW & $E$ & 254 seconds $\times 30 \mathrm{~m}$ & B-03-182 160703 E1 \\
\hline B-03-183 & JA, HW & $\mathrm{B}$ & $10 \mathrm{~m} \times 30 \mathrm{~m}$ & B-03-183_160703_B1 \\
\hline B-03-184 & JA, HW & $\mathrm{E}$ & 218 seconds $\times 20 \mathrm{~m}$ & B-03-184_160703_E1 \\
\hline
\end{tabular}


Appendix 4: List of all fishing effort showing the site number, collectors, fishing gear used, amount of fishing effort applied and the fish capture identification (ID) number derived for each occasion where effort was applied.

\begin{tabular}{|c|c|c|c|c|}
\hline Site Number & Collectors & Gear ID & Effort & $\begin{array}{c}\text { Fish Capture ID } \\
\text { (Crew_Date_Gear) }\end{array}$ \\
\hline B-03-185 & JA, HW & $E$ & 583 seconds $\times 75 \mathrm{~m}$ & B-03-185_160703_E1 \\
\hline B-03-189 & JA, HW & $\mathrm{B}$ & $10 \mathrm{~m} \times 25 \mathrm{~m}$ & B-03-189_160703_B1 \\
\hline B-03-190 & JA, HW & $\mathrm{B}$ & $15 \mathrm{~m} \times 35 \mathrm{~m}$ & B-03-190_160703_B1 \\
\hline B-03-191 & JA, HW & $E$ & 469 seconds $\times 75 \mathrm{~m}$ & B-03-191_170703_E1 \\
\hline B-03-192 & JA, HW & $\mathrm{B}$ & $10 \mathrm{~m} \times 25 \mathrm{~m}$ & B-03-192_170703_B1 \\
\hline B-03-193 & JA, HW & $\mathrm{B}$ & $10 \mathrm{~m} \times 25 \mathrm{~m}$ & B-03-193_170703_B1 \\
\hline B-03-194 & JA, HW & $\mathrm{B}$ & $6 \mathrm{~m} \times 20 \mathrm{~m}$ & B-03-194_220703_B1 \\
\hline B-03-199 & JA, HW & $E$ & 277 seconds $\times 30 \mathrm{~m}$ & B-03-199_230703_E1 \\
\hline B-03-200 & JA, HW & $\mathrm{B}$ & $10 \mathrm{~m} \times 25 \mathrm{~m}$ & B-03-200_230703_B1 \\
\hline B-03-202 & JA, HW & $E$ & 127 seconds $\times 15 \mathrm{~m}$ & B-03-202_230703_E1 \\
\hline B-03-204 & JA, HW & $\mathrm{B}$ & $10 \mathrm{~m} \times 15 \mathrm{~m}$ & B-03-204_230703_B1 \\
\hline B-03-206 & JA, HW & $E$ & 736 seconds $\times 100 \mathrm{~m}$ & B-03-206_240703_E1 \\
\hline B-03-207 & JA, HW & $E$ & 660 seconds $\times 180 \mathrm{~m}$ & B-03-207_240703_E1 \\
\hline B-03-209 & JA, HW & $\mathrm{B}$ & $8 \mathrm{~m} \times 20 \mathrm{~m}$ & B-03-209_240703_B1 \\
\hline B-03-212 & JA, HW & $E$ & 726 seconds $\times 200 \mathrm{~m}$ & B-03-212_240703_E1 \\
\hline B-03-218 & JA, HW & $\mathrm{D}$ & 1 sweep & B-03-218_250703_D1 \\
\hline B-03-224 & $\mathrm{JA}, \mathrm{HW}, \mathrm{AW}$ & $E$ & 301 seconds $\times 50 \mathrm{~m}$ & B-03-224_050803_E1 \\
\hline B-03-226 & $\mathrm{JA}, \mathrm{HW}, \mathrm{AW}$ & $\mathrm{B}$ & $10 \mathrm{~m} \times 10 \mathrm{~m}$ & B-03-226_060803_B1 \\
\hline B-03-226 & $\mathrm{JA}, \mathrm{HW}, \mathrm{AW}$ & $E$ & 193 seconds $\times 30 \mathrm{~m}$ & B-03-226_060803_E1 \\
\hline B-03-229 & $\mathrm{JA}, \mathrm{HW}, \mathrm{AW}$ & $\mathrm{D}$ & Sweeps & B-03-229_060803_D1 \\
\hline B-03-235 & $\mathrm{JA}, \mathrm{HW}, \mathrm{AW}$ & $E$ & 222 seconds $\times 50 \mathrm{~m}$ & B-03-235_060803_E1 \\
\hline B-03-239 & JA, HW, AW & $\mathrm{B}$ & $15 \mathrm{~m} \times 30 \mathrm{~m}$ & B-03-239_070803_B1 \\
\hline B-03-241 & $\mathrm{JA}, \mathrm{HW}, \mathrm{AW}$ & $E$ & 355 seconds $\times 30 \mathrm{~m}$ & B-03-241_070803_E1 \\
\hline B-03-249 & JA, HW, AW & $\mathrm{B}$ & $12 \mathrm{~m} \times 20 \mathrm{~m}$ & B-03-249_080803_B1 \\
\hline D-03-002 & $\begin{array}{c}\mathrm{DM}, \mathrm{AW}, \mathrm{JT}, \mathrm{JC}, \mathrm{JA}, \\
\mathrm{HW}\end{array}$ & $E$ & 1262 seconds $\times 200 \mathrm{~m}$ & D-03-002_100503_E1 \\
\hline D-03-002 & $\begin{array}{c}\mathrm{DM}, \mathrm{AW}, \mathrm{JT}, \mathrm{JC}, \mathrm{JA}, \\
\mathrm{HW}\end{array}$ & $\mathrm{K}$ & $3 \mathrm{~m}^{2}$ riffle & D-03-002_100503_K1 \\
\hline D-03-003 & $\begin{array}{c}\text { DM, AW, JT, JC, JA, } \\
\text { HW }\end{array}$ & $E$ & 809 seconds $\times 100 \mathrm{~m}$ & D-03-003_100503_E1 \\
\hline
\end{tabular}


Appendix 4: List of all fishing effort showing the site number, collectors, fishing gear used, amount of fishing effort applied and the fish capture identification (ID) number derived for each occasion where effort was applied.

\begin{tabular}{|c|c|c|c|c|}
\hline Site Number & Collectors & Gear ID & Effort & $\begin{array}{c}\text { Fish Capture ID } \\
\text { (Crew_Date_Gear) }\end{array}$ \\
\hline D-03-004 & $\begin{array}{c}\mathrm{DM}, \mathrm{AW}, \mathrm{JT}, \mathrm{JC}, \mathrm{JA} \\
\mathrm{HW}\end{array}$ & $\mathrm{E}$ & 552 seconds $\times 150 \mathrm{~m}$ & D-03-004_110503_E1 \\
\hline D-03-005 & $\begin{array}{c}\mathrm{DM}, \mathrm{AW}, \mathrm{JT}, \mathrm{JC}, \mathrm{JA}, \\
\mathrm{HW}\end{array}$ & $E$ & 402 seconds $\times 80 \mathrm{~m}$ & D-03-005_110503_E1 \\
\hline D-03-006 & $\begin{array}{c}\mathrm{DM}, \mathrm{AW}, \mathrm{JT}, \mathrm{JC}, \mathrm{JA} \\
\mathrm{HW}\end{array}$ & $\mathrm{E}$ & 649 seconds $\times 125 \mathrm{~m}$ & D-03-006_120503_E1 \\
\hline D-03-009 & $\begin{array}{c}\mathrm{DM}, \mathrm{AW}, \mathrm{JT}, \mathrm{JC}, \mathrm{JA} \\
\mathrm{HW}\end{array}$ & $\mathrm{E}$ & 400 seconds $\times 40 \mathrm{~m}$ & D-03-009_130503_E1 \\
\hline D-03-011 & $\begin{array}{c}\mathrm{DM}, \mathrm{AW}, \mathrm{JT}, \mathrm{JC}, \mathrm{JA} \\
\mathrm{HW}\end{array}$ & $\mathrm{E}$ & 575 seconds $\times 75 \mathrm{~m}$ & D-03-011_130503_E1 \\
\hline D-03-011 & $\begin{array}{c}\mathrm{DM}, \mathrm{AW}, \mathrm{JT}, \mathrm{JC}, \mathrm{JA} \\
\mathrm{HW}\end{array}$ & $\mathrm{K}$ & $2 \mathrm{~m}^{2}$ & D-03-011_130503_K1 \\
\hline D-03-012 & $\begin{array}{c}\mathrm{DM}, \mathrm{AW}, \mathrm{JT}, \mathrm{JC}, \mathrm{JA} \\
\mathrm{HW}\end{array}$ & $\mathrm{E}$ & 492 seconds $\times 100 \mathrm{~m}$ & D-03-012_130503_E1 \\
\hline D-03-015 & $\begin{array}{c}\mathrm{DM}, \mathrm{AW}, \mathrm{JT}, \mathrm{JC}, \mathrm{JA} \\
\mathrm{HW}\end{array}$ & $\mathrm{E}$ & 714 seconds $x 125 \mathrm{~m}$ & D-03-015_140503_E1 \\
\hline D-03-016 & $\begin{array}{c}\mathrm{DM}, \mathrm{AW}, \mathrm{JT}, \mathrm{JC}, \mathrm{JA} \\
\mathrm{HW}\end{array}$ & $\mathrm{E}$ & 418 seconds $\times 100 \mathrm{~m}$ & D-03-016_140503 E1 \\
\hline D-03-017 & $\begin{array}{c}\mathrm{DM}, \mathrm{AW}, \mathrm{JT}, \mathrm{JC}, \mathrm{JA} \\
\mathrm{HW}\end{array}$ & $E$ & 200 seconds $\times 60 \mathrm{~m}$ & D-03-017_140503_E1 \\
\hline D-03-019 & $\begin{array}{c}\mathrm{DM}, \mathrm{AW}, \mathrm{JT}, \mathrm{JC}, \mathrm{JA} \\
\mathrm{HW}\end{array}$ & $\mathrm{E}$ & 566 seconds $\times 125 \mathrm{~m}$ & D-03-019_140503_E1 \\
\hline D-03-023 & DM, AW & $E$ & 552 seconds $\times 200 \mathrm{~m}$ & D-03-023_030603_E1 \\
\hline D-03-024 & DM, AW & $\mathrm{B}$ & $10 \mathrm{~m} \times 30 \mathrm{~m}$ & $\mathrm{D}-03-024 \_030603 \_\mathrm{B} 1$ \\
\hline D-03-025 & $\mathrm{DM}, \mathrm{AW}$ & $\mathrm{E}$ & 317 seconds $\times 75 \mathrm{~m}$ & D-03-025_030603_E1 \\
\hline D-03-027 & DM, AW & $\mathrm{B}$ & $10 \mathrm{~m} \times 10 \mathrm{~m}$ & D-03-027_040603_B1 \\
\hline D-03-029 & DM, AW & $E$ & 340 seconds $\times 100 \mathrm{~m}$ & D-03-029_040603_E1 \\
\hline D-03-035 & $\mathrm{DM}, \mathrm{AW}, \mathrm{MK}, \mathrm{SP}, \mathrm{DS}$ & B & $15 \mathrm{~m} \times 50 \mathrm{~m}$ & D-03-035_050603 B1 \\
\hline $\mathrm{D}-03-035$ & $\mathrm{DM}, \mathrm{AW}, \mathrm{MK}, \mathrm{SP}, \mathrm{DS}$ & $E$ & 401 seconds $\times 125 \mathrm{~m}$ & D-03-035_050603_E1 \\
\hline D-03-036 & DM, AW, MK, SP, DS & $E$ & 647 seconds $\times 125 \mathrm{~m}$ & D-03-036_050603_E1 \\
\hline D-03-037 & $\mathrm{DM}, \mathrm{AW}, \mathrm{MK}, \mathrm{SP}, \mathrm{DS}$ & $\mathrm{E}$ & 416 seconds $\times 75 \mathrm{~m}$ & $\mathrm{D}-03-037050603 \mathrm{E} 1$ \\
\hline D-03-038 & $\mathrm{DM}, \mathrm{AW}$ & $E$ & 1089 seconds $\times 150 \mathrm{~m}$ & D-03-038_060603_E1 \\
\hline
\end{tabular}


Appendix 4: List of all fishing effort showing the site number, collectors, fishing gear used, amount of fishing effort applied and the fish capture identification (ID) number derived for each occasion where effort was applied.

\begin{tabular}{|c|c|c|c|c|}
\hline Site Number & Collectors & Gear ID & Effort & $\begin{array}{c}\text { Fish Capture ID } \\
\text { (Crew_Date_Gear) }\end{array}$ \\
\hline D-03-040 & $\mathrm{DM}, \mathrm{AW}$ & $E$ & 314 seconds $\times 100 \mathrm{~m}$ & D-03-040_100603_E1 \\
\hline D-03-041 & DM, AW & $E$ & 269 seconds $\times 100 \mathrm{~m}$ & D-03-041_100603_E1 \\
\hline D-03-044 & $\mathrm{DM}, \mathrm{AW}$ & $E$ & 301 seconds $\times 80 \mathrm{~m}$ & D-03-044_110603_E1 \\
\hline D-03-045 & $\mathrm{DM}, \mathrm{AW}$ & $E$ & 240 seconds $\times 100 \mathrm{~m}$ & D-03-045_110603_E1 \\
\hline D-03-047 & DM, AW & $E$ & 111 seconds $\times 35 \mathrm{~m}$ & D-03-047_110603_E1 \\
\hline D-03-049 & DM, AW & $E$ & 211 seconds $\times 100 \mathrm{~m}$ & D-03-049_110603_E1 \\
\hline D-03-054 & DM, AW & $E$ & 122 seconds $\times 40 \mathrm{~m}$ & D-03-054_130603_E2 \\
\hline D-03-054 & DM, AW & $E$ & 540 seconds $\times 150 \mathrm{~m}$ & D-03-054_130603_E1 \\
\hline D-03-057 & DM, AW & $E$ & 427 seconds $\times 125 \mathrm{~m}$ & D-03-057_130603_E1 \\
\hline D-03-058 & DM, AW & $E$ & 501 seconds $\times 150 \mathrm{~m}$ & D-03-058_160603_E1 \\
\hline D-03-059 & DM, AW & $\mathrm{E}$ & 421 seconds $\times 100 \mathrm{~m}$ & D-03-059_160603_E1 \\
\hline D-03-060 & DM, AW & $E$ & 99 seconds $\times 50 \mathrm{~m}$ & D-03-060_160603_E1 \\
\hline D-03-061 & $\mathrm{DM}, \mathrm{AW}$ & $E$ & 222 seconds $\times 80 \mathrm{~m}$ & D-03-061_170603 E2 \\
\hline D-03-061 & DM, AW & $E$ & 718 seconds $\times 150 \mathrm{~m}$ & D-03-061_170603_E1 \\
\hline D-03-062 & DM, AW & $E$ & 346 seconds $\times 75 \mathrm{~m}$ & D-03-062_170603_E1 \\
\hline D-03-064 & $\mathrm{DM}, \mathrm{AW}$ & $\mathrm{B}$ & $5 \mathrm{~m} \times 10 \mathrm{~m}$ & D-03-064_170603_B1 \\
\hline D-03-065 & DM, AW & $\mathrm{B}$ & $4 \mathrm{~m} \times 50 \mathrm{~m}$ & D-03-065_170603_B1 \\
\hline D-03-066 & DM, AW & $E$ & 452 seconds $\times 75 \mathrm{~m}$ & D-03-066_180603_E1 \\
\hline D-03-067 & DM, AW & $E$ & 262 seconds $\times 50 \mathrm{~m}$ & D-03-067_180603_E1 \\
\hline D-03-071 & $\mathrm{DM}, \mathrm{AW}$ & $\mathrm{B}$ & $8 \mathrm{~m} \times 20 \mathrm{~m}$ & D-03-071_230603_B1 \\
\hline D-03-073 & DM, AW & $E$ & 187 seconds $\times 30 \mathrm{~m}$ & D-03-073_230603_E1 \\
\hline D-03-075 & DM, AW & $E$ & 110 seconds $\times 30 \mathrm{~m}$ & D-03-075 230603 E1 \\
\hline D-03-076 & DM, AW & $E$ & 200 seconds $\times 40 \mathrm{~m}$ & D-03-076_230603_E1 \\
\hline D-03-078 & $\mathrm{DM}, \mathrm{AW}$ & $\mathrm{B}$ & $8 \mathrm{~m} \times 15 \mathrm{~m}$ & D-03-078_240603_B1 \\
\hline D-03-081 & DM, AW & $E$ & 403 seconds $\times 100 \mathrm{~m}$ & D-03-081_240603_E1 \\
\hline D-03-083 & DM, AW & $E$ & 191 seconds $\times 25 \mathrm{~m}$ & D-03-083_260603_E1 \\
\hline D-03-084 & DM, AW & $E$ & 618 seconds $\times 75 \mathrm{~m}$ & D-03-084_260603_E1 \\
\hline D-03-085 & DM, AW & $E$ & 520 seconds $\times 150 \mathrm{~m}$ & D-03-085_270603_E1 \\
\hline D-03-086 & DM, AW & $E$ & 311 seconds $\times 75 \mathrm{~m}$ & D-03-086_270603_E1 \\
\hline D-03-088 & DM, AW & $E$ & 368 seconds $\times 75 \mathrm{~m}$ & D-03-088_020703_E1 \\
\hline D-03-089 & DM, AW & $\mathrm{E}$ & 304 seconds $\times 75 \mathrm{~m}$ & D-03-089_020703_E1 \\
\hline
\end{tabular}


Appendix 4: List of all fishing effort showing the site number, collectors, fishing gear used, amount of fishing effort applied and the fish capture identification (ID) number derived for each occasion where effort was applied.

\begin{tabular}{|c|c|c|c|c|}
\hline Site Number & Collectors & Gear ID & Effort & $\begin{array}{c}\text { Fish Capture ID } \\
\text { (Crew_Date_Gear) }\end{array}$ \\
\hline D-03-090 & $\mathrm{DM}, \mathrm{AW}$ & $\mathrm{D}$ & 10 sweeps below dam spillway & D-03-090_020703_D1 \\
\hline D-03-091 & DM, AW & $E$ & 274 seconds $\times 75 \mathrm{~m}$ & D-03-091_030703_E1 \\
\hline D-03-094 & $\mathrm{DM}, \mathrm{AW}$ & $E$ & 177 seconds $\times 25 \mathrm{~m}$ & D-03-094_080703_E1 \\
\hline D-03-095 & $\mathrm{DM}, \mathrm{AW}$ & $E$ & 195 seconds $\times 25 \mathrm{~m}$ & D-03-095_080703_E1 \\
\hline D-03-096 & DM, AW & $E$ & 374 seconds $\times 35 \mathrm{~m}$ & D-03-096_080703_E1 \\
\hline D-03-097 & DM, AW & $E$ & 579 seconds $\times 150 \mathrm{~m}$ & D-03-097_090703_E1 \\
\hline D-03-098 & DM, AW & $\mathrm{B}$ & $10 \mathrm{~m} \times 20 \mathrm{~m}$ & D-03-098_090703_B1 \\
\hline D-03-100 & DM, AW & $\mathrm{E}$ & 188 seconds $\times 30 \mathrm{~m}$ & D-03-100_090703_E1 \\
\hline D-03-101 & DM, AW & $\mathrm{E}$ & 245 seconds $\times 50 \mathrm{~m}$ & D-03-101_090703_E1 \\
\hline D-03-102 & DM, AW & $E$ & 304 seconds $\times 50 \mathrm{~m}$ & D-03-102_110703_E1 \\
\hline D-03-103 & DM, AW & $\mathrm{E}$ & 326 seconds $\times 100 \mathrm{~m}$ & D-03-103_110703_E1 \\
\hline D-03-105 & DM, AW & $\mathrm{D}$ & 6 sweeps & D-03-105_110703_D1 \\
\hline D-03-110 & $\mathrm{DM}, \mathrm{AW}$ & $\mathrm{E}$ & 133 seconds $\times 30 \mathrm{~m}$ & D-03-110_140703_E1 \\
\hline D-03-111 & DM, AW & $\mathrm{B}$ & $10 \mathrm{~m} \times 20 \mathrm{~m}$ & D-03-111_140703_B1 \\
\hline D-03-117 & DM, AW & $E$ & 107 seconds $\times 75 \mathrm{~m}$ & D-03-117_150703_E1 \\
\hline $\mathrm{D}-03-120$ & $\mathrm{DM}, \mathrm{AW}$ & $\mathrm{O}$ & Observed in pool under bridge & D-03-120_160703_O1 \\
\hline D-03-121 & DM, AW & $E$ & 216 seconds $\times 60 \mathrm{~m}$ & D-03-121_160703_E1 \\
\hline D-03-122 & DM, AW & $E$ & 256 seconds $\times 75 \mathrm{~m}$ & D-03-122_160703_E1 \\
\hline D-03-124 & DM, AW & $E$ & 313 seconds $\times 100 \mathrm{~m}$ & D-03-124_160703_E1 \\
\hline D-03-126 & $\mathrm{DM}, \mathrm{AW}$ & $\mathrm{B}$ & $5 \mathrm{~m} \times 15 \mathrm{~m}$ & D-03-126_170703_B1 \\
\hline D-03-129 & DM, AW & $\mathrm{D}$ & 2 sweeps & D-03-129_170703_D1 \\
\hline D-03-132 & DM, AW & $E$ & 107 seconds $\times 30 \mathrm{~m}$ & D-03-132 170703 E1 \\
\hline D-03-134 & DM, AW & $\mathrm{B}$ & $20 \mathrm{~m} \times 30 \mathrm{~m}$ & D-03-134_170703_B1 \\
\hline D-03-138 & $\mathrm{DM}, \mathrm{AW}$ & $E$ & 144 seconds $\times 30 \mathrm{~m}$ & D-03-138_180703_E1 \\
\hline D-03-142 & AW, JT & $E$ & 540 seconds $\times 200 \mathrm{~m}$ & D-03-142_220703_E1 \\
\hline D-03-143 & AW, JT & $E$ & 816 seconds $\times 150 \mathrm{~m}$ & D-03-143_220703_E1 \\
\hline D-03-144 & AW, JT & $E$ & 662 seconds $\times 150 \mathrm{~m}$ & D-03-144_220703_E1 \\
\hline D-03-145 & AW, JT & $E$ & 402 seconds $\times 150 \mathrm{~m}$ & D-03-145_220703_E1 \\
\hline D-03-152 & AW, JT & $E$ & 432 seconds $\times 150 \mathrm{~m}$ & D-03-152_230703_E1 \\
\hline D-03-154 & AW, JT & $E$ & 756 seconds $\times 200 \mathrm{~m}$ & D-03-154_230703_E1 \\
\hline D-03-155 & AW, JT & $\mathrm{E}$ & 288 seconds $\times 75 \mathrm{~m}$ & D-03-155_230703_E1 \\
\hline
\end{tabular}


Appendix 4: List of all fishing effort showing the site number, collectors, fishing gear used, amount of fishing effort applied and the fish capture identification (ID) number derived for each occasion where effort was applied.

\begin{tabular}{|c|c|c|c|c|}
\hline Site Number & Collectors & Gear ID & Effort & $\begin{array}{c}\text { Fish Capture ID } \\
\text { (Crew_Date_Gear) }\end{array}$ \\
\hline D-03-156 & $\mathrm{AW}, \mathrm{JT}$ & $E$ & 141 seconds $\times 50 \mathrm{~m}$ & D-03-156_230703_E1 \\
\hline D-03-164 & AW, JT & $E$ & 264 seconds $\times 100 \mathrm{~m}$ & D-03-164_240703_E1 \\
\hline D-03-167 & AW, JT & $E$ & 664 seconds $\times 200 \mathrm{~m}$ & D-03-167_250703_E1 \\
\hline D-03-168 & $\mathrm{DM}, \mathrm{NM}, \mathrm{BM}$ & $E$ & 1375 seconds $\times 300 \mathrm{~m}$ & D-03-168_100903_E1 \\
\hline D-03-170 & $\mathrm{DM}, \mathrm{NM}, \mathrm{BM}$ & $\mathrm{E}$ & 1540 seconds $\times 300 \mathrm{~m}$ & D-03-170_110903_E1 \\
\hline W-03-001 & $\begin{array}{c}\mathrm{DM}, \mathrm{AW}, \mathrm{JT}, \mathrm{JC}, \mathrm{JA}, \\
\mathrm{HW}\end{array}$ & $\mathrm{B}$ & $8 \mathrm{~m} \times 30 \mathrm{~m}$ & W-03-001_200503_B1 \\
\hline W-03-002 & $\mathrm{JT}, \mathrm{JC}$ & $\mathrm{B}$ & $8 \mathrm{~m} \times 30 \mathrm{~m}$ & W-03-002_210503_B1 \\
\hline W-03-003 & $\mathrm{DM}, \mathrm{JT}, \mathrm{JC}$ & $E$ & 165 seconds $\times 25 \mathrm{~m}$ & W-03-003_210503_E1 \\
\hline W-03-004 & DM, JT, JC & $E$ & 333 seconds $\times 70 \mathrm{~m}$ & W-03-004_210503_E1 \\
\hline W-03-008 & DM, JT, JC & $E$ & 149 seconds $\times 10 \mathrm{~m}$ & W-03-008_220503_E1 \\
\hline W-03-009 & $\begin{array}{c}\text { DM, AW, JT, JC, JA, } \\
\text { HW }\end{array}$ & $E$ & 361 seconds $\times 75 \mathrm{~m}$ & W-03-009 220503 E1 \\
\hline W-03-010 & DM, JT, JC & $\mathrm{E}$ & 195 seconds $\times 75 \mathrm{~m}$ & W-03-010_220503_E1 \\
\hline W-03-013 & $\begin{array}{c}\text { DM, AW, JT, JC, JA, } \\
\text { HW }\end{array}$ & $E$ & 380 seconds $\times 100 \mathrm{~m}$ & W-03-013_230503_E1 \\
\hline W-03-019 & AW, JT, JC & $E$ & 280 seconds $\times 60 \mathrm{~m}$ & W-03-019_260503_E1 \\
\hline W-03-020 & AW, JT, JC & $E$ & 202 seconds $\times 75 \mathrm{~m}$ & W-03-020_260503_E1 \\
\hline W-03-026 & AW, JT, JC & $\mathrm{B}$ & $10 \mathrm{~m} \times 15 \mathrm{~m}$ & W-03-026_270503_B1 \\
\hline W-03-030 & AW, JT, JC & $\mathrm{B}$ & $8 \mathrm{~m} \times 10 \mathrm{~m}$ & W-03-030_280503_B1 \\
\hline W-03-030 & AW, JT, JC & $E$ & 198 seconds $\times 50 \mathrm{~m}$ & W-03-030_280503_E1 \\
\hline W-03-035 & AW, JT, JC & $E$ & 161 seconds $\times 50 \mathrm{~m}$ & W-03-035_290503_E1 \\
\hline W-03-036 & AW, JT, JC & $E$ & 227 seconds $\times 30 \mathrm{~m}$ & W-03-036_290503_E1 \\
\hline W-03-044 & JT, JC & $\mathrm{B}$ & $6 \mathrm{~m} \times 15 \mathrm{~m}$ & W-03-044_030603_B1 \\
\hline W-03-046 & JT, JC & $E$ & 369 seconds $\times 50 \mathrm{~m}$ & W-03-046_030603_E1 \\
\hline W-03-049 & JT, JC & $E$ & 214 seconds $\times 50 \mathrm{~m}$ & W-03-049_030603_E1 \\
\hline W-03-050 & JT, JC & $E$ & 100 seconds $\times 50 \mathrm{~m}$ & W-03-050_040603_E1 \\
\hline W-03-055 & JT, JC & $\mathrm{B}$ & $5 \mathrm{~m} \times 50 \mathrm{~m}$ & W-03-055_040603_B1 \\
\hline W-03-065 & JT, JC & $\mathrm{D}$ & 25 sweeps & W-03-065_050603_D1 \\
\hline W-03-066 & JT, JC & $\mathrm{D}$ & 2 sweeps & W-03-066_050603_D1 \\
\hline W-03-067 & JT, JC & $E$ & 123 seconds $\times 75 \mathrm{~m}$ & W-03-067_060603_E1 \\
\hline
\end{tabular}


Appendix 4: List of all fishing effort showing the site number, collectors, fishing gear used, amount of fishing effort applied and the fish capture identification (ID) number derived for each occasion where effort was applied.

\begin{tabular}{|c|c|c|c|c|}
\hline Site Number & Collectors & Gear ID & Effort & $\begin{array}{c}\text { Fish Capture ID } \\
\text { (Crew_Date_Gear) }\end{array}$ \\
\hline W-03-069 & JT, JC & $\mathrm{B}$ & $20 \mathrm{~m} \times 40 \mathrm{~m}$ & W-03-069_060603_B1 \\
\hline W-03-070 & JT, JC & $\mathrm{D}$ & One sweep through culvert & W-03-070_060603_D1 \\
\hline W-03-070 & JT, JC & $E$ & 100 seconds $\times 30 \mathrm{~m}$ & W-03-070_060603_E1 \\
\hline W-03-072 & JT, JC & $E$ & 200 seconds $\times 100 \mathrm{~m}$ & W-03-072_090603_E1 \\
\hline W-03-077 & JT, JC & $\mathrm{E}$ & 403 seconds $\times 200 \mathrm{~m}$ & W-03-077 100603 E1 \\
\hline W-03-079 & JT, JC & $E$ & 100 seconds $\times 50 \mathrm{~m}$ & W-03-079_100603_E1 \\
\hline W-03-080 & JT, JC & $\mathrm{D}$ & 1 sweep & W-03-080_100603_D1 \\
\hline W-03-084 & JT, JC & $\mathrm{D}$ & 5 sweeps & W-03-084_110603_D1 \\
\hline W-03-087 & JT, JC & $\mathrm{D}$ & 1 sweep & W-03-087_110603_D1 \\
\hline W-03-088 & JT, JC & $E$ & 75 seconds $\times 10 \mathrm{~m}$ & W-03-088_110603_E1 \\
\hline W-03-091 & JT, JC & $\mathrm{E}$ & 142 seconds $\times 100 \mathrm{~m}$ & W-03-091_130603_E1 \\
\hline W-03-092 & JT, JC & $E$ & 269 seconds $\times 100 \mathrm{~m}$ & W-03-092_130603 E1 \\
\hline W-03-093 & JT, JC & $E$ & 649 seconds $\times 225 \mathrm{~m}$ & W-03-093_130603 E1 \\
\hline W-03-094 & JT, JC & $\mathrm{B}$ & $8 \mathrm{~m} \times 100 \mathrm{~m}$ & W-03-094_160603_B1 \\
\hline W-03-095 & JT, JC & $E$ & 183 seconds $\times 50 \mathrm{~m}$ & W-03-095_160603_E1 \\
\hline W-03-096 & JT, JC & $E$ & 153 seconds $\times 20 \mathrm{~m}$ & W-03-096_160603_E1 \\
\hline W-03-097 & JT, JC & $E$ & 142 seconds $\times 100 \mathrm{~m}$ & W-03-097_170603_E1 \\
\hline W-03-098 & JT, JC & $\mathrm{B}$ & $10 \mathrm{~m} \times 50 \mathrm{~m}$ & W-03-098_170603_B1 \\
\hline W-03-100 & JT, JC & $E$ & 50 seconds $\times 10 \mathrm{~m}$ & W-03-100_170603 E1 \\
\hline W-03-101 & JT, JC & $\mathrm{E}$ & 309 seconds $\times 100 \mathrm{~m}$ & W-03-101_180603_E1 \\
\hline W-03-104 & JT, JC & $E$ & 180 seconds $\times 50 \mathrm{~m}$ & W-03-104_180603_E1 \\
\hline W-03-107 & JT, JC & $E$ & 228 seconds $\times 150 \mathrm{~m}$ & W-03-107_180603_E1 \\
\hline W-03-110 & JT, JC & $E$ & 188 seconds $\times 100 \mathrm{~m}$ & W-03-110_190603_E1 \\
\hline W-03-111 & JT, JC & $E$ & 32 seconds $\times 20 \mathrm{~m}$ & W-03-111_190603_E1 \\
\hline W-03-112 & JT, JC & $E$ & 387 seconds $\times 150 \mathrm{~m}$ & W-03-112_190603_E1 \\
\hline W-03-113 & JT, JC & $E$ & 313 seconds $\times 100 \mathrm{~m}$ & W-03-113_240603_E1 \\
\hline W-03-114 & JT, JC & $E$ & 108 seconds $\times 50 \mathrm{~m}$ & W-03-114_240603_E1 \\
\hline W-03-141 & JT, JC & $\mathrm{D}$ & 1 sweep & W-03-141_260603_D1 \\
\hline W-03-142 & JT, JC & $\mathrm{D}$ & 10 sweeps & W-03-142_260603_D1 \\
\hline W-03-143 & JT, JC & $E$ & 33 seconds $\times 15 \mathrm{~m}$ & W-03-143_020703_E1 \\
\hline W-03-144 & JT, JC & $\mathrm{E}$ & 360 seconds $\times 150 \mathrm{~m}$ & W-03-144_020703_E1 \\
\hline
\end{tabular}


Appendix 4: List of all fishing effort showing the site number, collectors, fishing gear used, amount of fishing effort applied and the fish capture identification (ID) number derived for each occasion where effort was applied.

\begin{tabular}{|c|c|c|c|c|}
\hline Site Number & Collectors & Gear ID & Effort & $\begin{array}{c}\text { Fish Capture ID } \\
\text { (Crew_Date_Gear) }\end{array}$ \\
\hline W-03-146 & JT, JC & $E$ & 280 seconds $\times 100 \mathrm{~m}$ & W-03-146_020703_E1 \\
\hline W-03-150 & JT, JC & $E$ & 243 seconds $\times 75 \mathrm{~m}$ & W-03-150_030703_E1 \\
\hline W-03-151 & JT, JC & $E$ & 66 seconds $\times 50 \mathrm{~m}$ & W-03-151_030703_E1 \\
\hline W-03-154 & JT, JC & $\mathrm{D}$ & 3 sweeps in minnow trap & W-03-154_040703_D1 \\
\hline W-03-155 & JT, JC & $\mathrm{E}$ & 440 seconds $\times 100 \mathrm{~m}$ & W-03-155_040703_E1 \\
\hline W-03-158 & JT, JC & $\mathrm{B}$ & $5 \mathrm{~m} \times 30 \mathrm{~m}$ & W-03-158_040703_B1 \\
\hline W-03-162 & JT, JC & $E$ & 113 seconds $\times 50 \mathrm{~m}$ & W-03-162_050703_E1 \\
\hline W-03-165 & JT, JC & $E$ & 486 seconds $\times 150 \mathrm{~m}$ & W-03-165_050703_E1 \\
\hline W-03-167 & JT, JC & $\mathrm{B}$ & $10 \mathrm{~m} \times 15 \mathrm{~m}$ & W-03-167_060703_B1 \\
\hline W-03-173 & JT, JC & $\mathrm{B}$ & $4 \mathrm{~m} \times 10 \mathrm{~m}$ & W-03-173_060703_B1 \\
\hline W-03-176 & JT, JC & $\mathrm{E}$ & 147 seconds $\times 100 \mathrm{~m}$ & W-03-176_070703_E1 \\
\hline W-03-182 & JT, JC & $\mathrm{B}$ & $5 \mathrm{~m} \times 30 \mathrm{~m}$ & W-03-182_080703_B1 \\
\hline W-03-185 & JT, JC & $\mathrm{B}$ & $10 \mathrm{~m} \times 30 \mathrm{~m}$ & W-03-185_080703_B1 \\
\hline W-03-186 & JT, JC & $\mathrm{D}$ & 6 sweeps & W-03-186_090703_D1 \\
\hline W-03-189 & JT, JC & $\mathrm{B}$ & $7 \mathrm{~m} \times 20 \mathrm{~m}$ & W-03-189_090703_B1 \\
\hline W-03-191 & JT, JC & $E$ & 417 seconds $\times 150 \mathrm{~m}$ & W-03-191_160703_E1 \\
\hline W-03-194 & JT, JC & $E$ & 316 seconds $\times 50 \mathrm{~m}$ & W-03-194_160703_E1 \\
\hline W-03-195 & JT, JC & $\mathrm{B}$ & $6 \mathrm{~m} \times 25 \mathrm{~m}$ & W-03-195_160703_B1 \\
\hline W-03-198 & JT, JC & $E$ & 308 seconds $\times 100 \mathrm{~m}$ & W-03-198_170703_E1 \\
\hline W-03-201 & JT, JC & $\mathrm{B}$ & $8 \mathrm{~m} \times 12 \mathrm{~m}$ & W-03-201_170703_B1 \\
\hline W-03-203 & JT, JC & $E$ & 175 seconds $\times 100 \mathrm{~m}$ & W-03-203_180703_E1 \\
\hline W-03-204 & JT, JC & $E$ & 51 seconds $\times 10 \mathrm{~m}$ & W-03-204_180703_E1 \\
\hline W-03-205 & JT, JC & $E$ & 264 seconds $\times 150 \mathrm{~m}$ & W-03-205_180703_E1 \\
\hline W-03-206 & JT, JC & $E$ & 375 seconds $\times 100 \mathrm{~m}$ & W-03-206_310703_E1 \\
\hline W-03-210 & JT, JC & $E$ & 432 seconds $\times 150 \mathrm{~m}$ & W-03-210_310703_E1 \\
\hline W-03-211 & JT, JC & $\mathrm{B}$ & $12 \mathrm{~m} \times 20 \mathrm{~m}$ & W-03-211_310703_B1 \\
\hline W-03-223 & JT, JC & $E$ & 270 seconds $\times 50 \mathrm{~m}$ & W-03-223_050803_E1 \\
\hline W-03-224 & JT, JC & $E$ & 180 seconds $\times 100 \mathrm{~m}$ & W-03-224_050803_E1 \\
\hline W-03-229 & JT, JC & $\mathrm{B}$ & $8 \mathrm{~m} \times 15 \mathrm{~m}$ & W-03-229_060803_B1 \\
\hline W-03-237 & JT, JC & $E$ & 628 seconds $\times 100 \mathrm{~m}$ & W-03-237_060803_E1 \\
\hline W-03-238 & JT, JC & $\mathrm{E}$ & 619 seconds $\times 150 \mathrm{~m}$ & W-03-238_070803_E1 \\
\hline
\end{tabular}


Appendix 4: List of all fishing effort showing the site number, collectors, fishing gear used, amount of fishing effort applied and the fish capture identification (ID) number derived for each occasion where effort was applied.

\begin{tabular}{|c|c|c|c|c|}
\hline Site Number & Collectors & Gear ID & Effort & $\begin{array}{c}\text { Fish Capture ID } \\
\text { (Crew_Date_Gear) }\end{array}$ \\
\hline W-03-239 & JT, JC & $E$ & 387 seconds $\times 100 \mathrm{~m}$ & W-03-239_070803_E1 \\
\hline W-03-247 & JT, JC & $E$ & 290 seconds $\times 75 \mathrm{~m}$ & W-03-247_080803_E1 \\
\hline W-03-248 & JT, JC & $E$ & 147 seconds $\times 50 \mathrm{~m}$ & W-03-248_080803_E1 \\
\hline W-03-249 & JT, JC & $E$ & 300 seconds $\times 75 \mathrm{~m}$ & W-03-249_110803_E1 \\
\hline W-03-251 & JT, JC & $\mathrm{E}$ & 218 seconds $\times 75 \mathrm{~m}$ & W-03-251_110803_E1 \\
\hline W-03-266 & JT, JC & $E$ & 169 seconds $\times 60 \mathrm{~m}$ & W-03-266 130803 E1 \\
\hline W-03-267 & JT, JC & $\mathrm{B}$ & $3 \mathrm{~m} \times 20 \mathrm{~m}$ & W-03-267_130803_B1 \\
\hline B-04-002 & AW, HW, JE & $E$ & 199 seconds $\times 100 \mathrm{~m}$ & B-04-002_010504_E1 \\
\hline B-04-003 & $\mathrm{AW}, \mathrm{HW}, \mathrm{JE}$ & $\mathrm{B}$ & $11 \mathrm{~m} \times 25 \mathrm{~m}$ & B-04-003_010504_B1 \\
\hline B-04-003 & $\mathrm{AW}, \mathrm{HW}, \mathrm{JE}$ & $E$ & 249 seconds $\times 15 \mathrm{~m}$ & B-04-003_010504_E1 \\
\hline B-04-004 & $\mathrm{AW}, \mathrm{HW}, \mathrm{JE}$ & $\mathrm{E}$ & 564 seconds $\times 150 \mathrm{~m}$ & B-04-004_010504_E1 \\
\hline B-04-006 & $\mathrm{AW}, \mathrm{HW}, \mathrm{JE}$ & $E$ & 300 seconds $\times 80 \mathrm{~m}$ & B-04-006_010504_E1 \\
\hline B-04-008 & $\mathrm{AW}, \mathrm{HW}, \mathrm{JE}$ & $E$ & 615 seconds $\times 100 \mathrm{~m}$ & B-04-008_010504_E1 \\
\hline B-04-010 & AW, HW, JC, JE & $E$ & 482 seconds $\times 109 \mathrm{~m}$ & B-04-010_020504_E1 \\
\hline B-04-010 & $\mathrm{AW}, \mathrm{HW}, \mathrm{JC}, \mathrm{JE}$ & $\mathrm{K}$ & $1 \mathrm{~m}^{2}$ & B-04-010_020504_K1 \\
\hline B-04-011 & AW, HW, JC, JE & $E$ & 224 seconds $\times 90 \mathrm{~m}$ & B-04-011_020504_E1 \\
\hline B-04-012 & AW, HW, JC, JE & $\mathrm{O}$ & Observed spawning & B-04-012_020504_01 \\
\hline B-04-013 & AW, HW, JC, JE & $\mathrm{O}$ & Observed spawning & B-04-013_020504_01 \\
\hline B-04-014 & AW, HW, JC, JE & $\mathrm{O}$ & Observed spawning & B-04-014_020504_01 \\
\hline B-04-015 & $\mathrm{AW}, \mathrm{HW}, \mathrm{JC}, \mathrm{JE}$ & $\mathrm{O}$ & Observed spawning & B-04-015_020504_01 \\
\hline B-04-016 & AW, HW, JC, JE & $\mathrm{O}$ & Observed spawning & B-04-016_020504_01 \\
\hline B-04-017 & $\mathrm{AW}, \mathrm{HW}, \mathrm{JE}, \mathrm{AP}$ & $E$ & 399 seconds $\times 10 \mathrm{~m}$ & B-04-017 020504 E1 \\
\hline B-04-017 & $\mathrm{AW}, \mathrm{HW}, \mathrm{JE}, \mathrm{AP}$ & $\mathrm{K}$ & $1 \mathrm{~m}^{2}$ & B-04-017_020504_K1 \\
\hline B-04-018 & $\mathrm{HW}, \mathrm{JE}, \mathrm{AP}$ & $\mathrm{B}$ & $10 \mathrm{~m} \times 20 \mathrm{~m}$ & B-04-018_030504_B1 \\
\hline B-04-019 & HW, JE, AP & $E$ & 349 seconds $\times 100 \mathrm{~m}$ & B-04-019_030504_E1 \\
\hline B-04-020 & $\mathrm{HW}, \mathrm{JE}, \mathrm{AP}$ & $\mathrm{B}$ & $10 \mathrm{~m} \times 25 \mathrm{~m}$ & B-04-020_030504_B1 \\
\hline B-04-020 & $\mathrm{HW}, \mathrm{JE}, \mathrm{AP}$ & $\mathrm{B}$ & $10 \mathrm{~m} \times 25 \mathrm{~m}$ & B-04-020_030504_B2 \\
\hline B-04-021 & $\mathrm{HW}, \mathrm{JE}, \mathrm{AP}$ & $E$ & 252 seconds $\times 15 \mathrm{~m}$ & B-04-021_030504_E1 \\
\hline B-04-022 & $\mathrm{HW}, \mathrm{JE}, \mathrm{AP}$ & $\mathrm{B}$ & $10 \mathrm{~m} \times 30 \mathrm{~m}$ & B-04-022_030504_B1 \\
\hline B-04-026 & $\mathrm{HW}, \mathrm{JE}, \mathrm{AP}$ & $E$ & 460 seconds $\times 120 \mathrm{~m}$ & B-04-026_040504_E1 \\
\hline B-04-027 & $\mathrm{HW}, \mathrm{JE}, \mathrm{AP}$ & $\mathrm{E}$ & 295 seconds $\times 70 \mathrm{~m}$ & B-04-027_040504_E1 \\
\hline
\end{tabular}


Appendix 4: List of all fishing effort showing the site number, collectors, fishing gear used, amount of fishing effort applied and the fish capture identification (ID) number derived for each occasion where effort was applied.

\begin{tabular}{|c|c|c|c|c|}
\hline Site Number & Collectors & Gear ID & Effort & $\begin{array}{c}\text { Fish Capture ID } \\
\text { (Crew_Date_Gear) }\end{array}$ \\
\hline B-04-028 & $\mathrm{HW}, \mathrm{JE}, \mathrm{AP}$ & $\mathrm{B}$ & $20 \mathrm{~m} \times 20 \mathrm{~m}$ & B-04-028_040504_B1 \\
\hline B-04-030 & $\mathrm{HW}, \mathrm{JE}, \mathrm{AP}$ & $E$ & 451 seconds $\times 71 \mathrm{~m}$ & B-04-030_040504_E1 \\
\hline B-04-031 & $\mathrm{AW}, \mathrm{HW}$ & $\mathrm{B}$ & $18 \mathrm{~m} \times 10 \mathrm{~m}$ & B-04-031_060504_B1 \\
\hline B-04-031 & $\mathrm{AW}, \mathrm{HW}$ & $E$ & 126 seconds $\times 75 \mathrm{~m}$ & B-04-031_060504_E1 \\
\hline B-04-037 & $\mathrm{AW}, \mathrm{HW}$ & $E$ & 257 seconds $\times 75 \mathrm{~m}$ & B-04-037_060504_E1 \\
\hline B-04-039 & $\mathrm{AW}, \mathrm{HW}$ & $\mathrm{O}$ & Observed & B-04-039_060504_01 \\
\hline B-04-041 & $\mathrm{AW}, \mathrm{HW}$ & $\mathrm{B}$ & $20 \mathrm{~m} \times 5 \mathrm{~m}$ & B-04-041_070504_B1 \\
\hline B-04-043 & $\mathrm{AW}, \mathrm{HW}$ & $\mathrm{B}$ & $10 \mathrm{~m} \times 8 \mathrm{~m}$ & B-04-043_070504_B1 \\
\hline B-04-044 & $\mathrm{AW}, \mathrm{HW}$ & $E$ & 310 seconds $\times 150 \mathrm{~m}$ & B-04-044_070504_E1 \\
\hline B-04-045 & $\mathrm{AW}, \mathrm{HW}$ & $\mathrm{K}$ & $2 \mathrm{~m}^{2}$ & B-04-045_070504_K1 \\
\hline B-04-046 & $\mathrm{AW}, \mathrm{HW}$ & $E$ & 233 seconds $\times 75 \mathrm{~m}$ & B-04-046_100504_E1 \\
\hline B-04-047 & $\mathrm{AW}, \mathrm{HW}$ & $E$ & 375 seconds $\times 100 \mathrm{~m}$ & B-04-047_100504_E1 \\
\hline B-04-047 & $\mathrm{AW}, \mathrm{HW}$ & $\mathrm{K}$ & $2 \mathrm{~m}^{2}$ & $\mathrm{~B}-04-047 \quad 100504 \mathrm{~K} 1$ \\
\hline B-04-048 & $\mathrm{AW}, \mathrm{HW}$ & $\mathrm{B}$ & $5 \mathrm{~m} \times 5 \mathrm{~m}$ & B-04-048_100504_B1 \\
\hline B-04-055 & $\mathrm{AW}, \mathrm{HW}$ & $\mathrm{B}$ & $10 \mathrm{~m} \times 15 \mathrm{~m}$ & B-04-055_130504_B1 \\
\hline B-04-055 & $\mathrm{AW}, \mathrm{HW}$ & $\mathrm{E}$ & 370 seconds $\times 65 \mathrm{~m}$ & B-04-055_130504_E1 \\
\hline B-04-058 & $\mathrm{AW}, \mathrm{HW}$ & $E$ & 199 seconds $\times 75 \mathrm{~m}$ & B-04-058_140504_E1 \\
\hline B-04-060 & $\mathrm{AW}, \mathrm{HW}$ & $E$ & 151 seconds $\times 75 \mathrm{~m}$ & B-04-060_140504_E1 \\
\hline B-04-064 & $\mathrm{AW}, \mathrm{HW}$ & $\mathrm{B}$ & $22 \mathrm{~m} \times 25 \mathrm{~m}$ & B-04-064_140504_B1 \\
\hline B-04-066 & $\mathrm{HW}, \mathrm{DM}$ & $\mathrm{E}$ & 148 seconds $\times 30 \mathrm{~m}$ & B-04-066_170504_E1 \\
\hline B-04-069 & HW, DM & $\mathrm{L}$ & $20 \min . \times 3$ & B-04-069_180504_L1 \\
\hline B-04-070 & $\mathrm{HW}, \mathrm{DM}$ & $E$ & 127 seconds $\times 17 \mathrm{~m}$ & B-04-070_180504_E1 \\
\hline B-04-072 & $\mathrm{HW}$ & $\mathrm{K}$ & $4 \mathrm{~m}^{2}$ & B-04-072_180504_K1 \\
\hline B-04-073 & $\mathrm{HW}, \mathrm{DM}$ & $\mathrm{K}$ & $3 \mathrm{~m}^{2}$ & B-04-073_190504_K1 \\
\hline B-04-073 & HW, DM & $\mathrm{O}$ & Observed below structure & B-04-073_190504_O1 \\
\hline B-04-074 & HW, DM & $\mathrm{K}$ & $1 \mathrm{~m}^{2}$ & B-04-074_190504_K1 \\
\hline B-04-075 & $\mathrm{HW}, \mathrm{DM}, \mathrm{IH}$ & $\mathrm{O}$ & Observed walleye larval drift collection & B-04-075_200504_01 \\
\hline B-04-076 & HW, DM & $\mathrm{L}$ & $10 \mathrm{~min}$. & B-04-076_200504_L1 \\
\hline B-04-077 & HW, DM & $\mathrm{L}$ & $10 \mathrm{~min}$. & B-04-077_200504_L1 \\
\hline B-04-078 & $\mathrm{HW}, \mathrm{DM}$ & $\mathrm{E}$ & 190 seconds $\times 15 \mathrm{~m}$ & B-04-078_200504_E1 \\
\hline B-04-078 & HW, DM & $\mathrm{L}$ & $20 \mathrm{~min}$. & B-04-078_200504_L1 \\
\hline
\end{tabular}


Appendix 4: List of all fishing effort showing the site number, collectors, fishing gear used, amount of fishing effort applied and the fish capture identification (ID) number derived for each occasion where effort was applied.

\begin{tabular}{|c|c|c|c|c|}
\hline Site Number & Collectors & Gear ID & Effort & $\begin{array}{c}\text { Fish Capture ID } \\
\text { (Crew_Date_Gear) }\end{array}$ \\
\hline B-04-080 & $\mathrm{HW}, \mathrm{DM}$ & $\mathrm{O}$ & Observed in pool & B-04-080_200504_O1 \\
\hline B-04-083 & HW, DM & $\mathrm{D}$ & $6 \mathrm{~m}^{2}$ & B-04-083_200504_D1 \\
\hline B-04-084 & HW, DM & $\mathrm{O}$ & Observed trapped in pool & B-04-084_200504_O1 \\
\hline B-04-085 & HW, DM & $\mathrm{O}$ & Observed trapped in pool & B-04-085_200504_O1 \\
\hline B-04-086 & $\mathrm{HW}, \mathrm{DM}, \mathrm{AM}$ & $\mathrm{B}$ & $30 \mathrm{~m} \times 8 \mathrm{~m}$ & B-04-086_210504_B1 \\
\hline B-04-086 & $\mathrm{HW}, \mathrm{DM}, \mathrm{AM}$ & $E$ & 143 seconds $\times 15 \mathrm{~m}$ & B-04-086_210504_E1 \\
\hline B-04-087 & $\mathrm{HW}$ & $\mathrm{L}$ & $10 \mathrm{~min}$. & B-04-087_220504_L1 \\
\hline B-04-088 & $\mathrm{HW}$ & $\mathrm{L}$ & $10 \mathrm{~min}$. & B-04-088_250504_L1 \\
\hline B-04-090 & $\mathrm{HW}$ & $\mathrm{L}$ & $10 \mathrm{~min}$. & B-04-090_250504_L1 \\
\hline B-04-092 & HW & $\mathrm{K}$ & $4 \mathrm{~m}^{2}$ & B-04-092_250504_K1 \\
\hline B-04-093 & $\mathrm{AW}, \mathrm{HW}$ & $\mathrm{B}$ & $10 \mathrm{~m} \times 15 \mathrm{~m}$ & B-04-093_310504_B1 \\
\hline B-04-094 & $\mathrm{AW}, \mathrm{HW}$ & $\mathrm{B}$ & $3 \mathrm{~m} \times 15 \mathrm{~m}$ & B-04-094_310504_B1 \\
\hline B-04-095 & $\mathrm{AW}, \mathrm{HW}$ & $\mathrm{B}$ & $15 \mathrm{~m} \times 20 \mathrm{~m}$ & B-04-095_310504_B1 \\
\hline B-04-101 & $\mathrm{AW}, \mathrm{HW}$ & $\mathrm{B}$ & $6 \mathrm{~m} \times 10 \mathrm{~m}$ & B-04-101_010604_B1 \\
\hline B-04-102 & $\mathrm{AW}, \mathrm{HW}$ & $E$ & 267 seconds $\times 75 \mathrm{~m}$ & B-04-102_010604_E1 \\
\hline B-04-104 & $\mathrm{AW}, \mathrm{HW}$ & $\mathrm{L}$ & $30 \mathrm{~min}$. & B-04-104_010604_L1 \\
\hline B-04-105 & $\mathrm{AW}, \mathrm{HW}$ & $\mathrm{E}$ & 116 seconds $\times 20 \mathrm{~m}$ & B-04-105_010604_E1 \\
\hline B-04-108 & $\mathrm{AW}, \mathrm{HW}$ & $\mathrm{B}$ & $20 \mathrm{~m} \times 10 \mathrm{~m}$ & B-04-108_020604_B1 \\
\hline B-04-109 & $\mathrm{AW}, \mathrm{HW}$ & $E$ & 144 seconds $\times 15 \mathrm{~m}$ & B-04-109_020604_E1 \\
\hline B-04-110 & $\mathrm{AW}, \mathrm{HW}$ & $E$ & 168 seconds $\times 75 \mathrm{~m}$ & $\mathrm{~B}-04-110 \quad 020604 \mathrm{E} 1$ \\
\hline B-04-113 & $\mathrm{AW}, \mathrm{HW}$ & $\mathrm{B}$ & $20 \mathrm{~m} \times 12 \mathrm{~m}$ & B-04-113_020604_B1 \\
\hline B-04-113 & $\mathrm{AW}, \mathrm{HW}$ & $\mathrm{E}$ & 105 seconds $\times 15 \mathrm{~m}$ & B-04-113_020604_E1 \\
\hline B-04-114 & $\mathrm{AW}, \mathrm{HW}$ & $E$ & 167 seconds $\times 75 \mathrm{~m}$ & B-04-114_030604_E1 \\
\hline B-04-121 & $\mathrm{AW}, \mathrm{HW}$ & $E$ & 421 seconds $\times 100 \mathrm{~m}$ & B-04-121_040604_E1 \\
\hline B-04-122 & $\mathrm{AW}, \mathrm{HW}$ & $\mathrm{E}$ & 141 seconds $\times 40 \mathrm{~m}$ & B-04-122_040604_E1 \\
\hline B-04-123 & $\mathrm{AW}, \mathrm{HW}$ & $E$ & 306 seconds $\times 100 \mathrm{~m}$ & B-04-123_070604_E1 \\
\hline B-04-124 & $\mathrm{AW}, \mathrm{HW}$ & $E$ & 141 seconds $\times 40 \mathrm{~m}$ & B-04-124_070604_E1 \\
\hline B-04-125 & $\mathrm{AW}, \mathrm{HW}$ & $\mathrm{B}$ & $7 \mathrm{~m} \times 20 \mathrm{~m}$ & B-04-125_070604_B1 \\
\hline B-04-126 & $\mathrm{AW}, \mathrm{HW}$ & $\mathrm{B}$ & $20 \mathrm{~m} \times 20 \mathrm{~m}$ & B-04-126_080604_B1 \\
\hline B-04-127 & $\mathrm{AW}, \mathrm{HW}$ & $\mathrm{E}$ & 302 seconds $\times 80 \mathrm{~m}$ & B-04-127_080604_E1 \\
\hline B-04-129 & $\mathrm{AW}, \mathrm{HW}$ & $\mathrm{E}$ & 261 seconds $\times 100 \mathrm{~m}$ & B-04-129_080604_E1 \\
\hline
\end{tabular}


Appendix 4: List of all fishing effort showing the site number, collectors, fishing gear used, amount of fishing effort applied and the fish capture identification (ID) number derived for each occasion where effort was applied.

\begin{tabular}{|c|c|c|c|c|}
\hline Site Number & Collectors & Gear ID & Effort & $\begin{array}{c}\text { Fish Capture ID } \\
\text { (Crew_Date_Gear) }\end{array}$ \\
\hline B-04-131 & $\mathrm{AW}, \mathrm{HW}$ & $E$ & 144 seconds $\times 15 \mathrm{~m}$ & B-04-131_090604_E1 \\
\hline B-04-133 & $\mathrm{AW}, \mathrm{HW}$ & $E$ & 143 seconds $\times 60 \mathrm{~m}$ & B-04-133_090604_E1 \\
\hline B-04-136 & $\mathrm{AW}, \mathrm{HW}$ & $E$ & 655 seconds $\times 150 \mathrm{~m}$ & B-04-136_100604_E1 \\
\hline B-04-140 & $\mathrm{AW}, \mathrm{HW}$ & $E$ & 246 seconds $\times 100 \mathrm{~m}$ & B-04-140 140604 E1 \\
\hline B-04-141 & $\mathrm{AW}, \mathrm{HW}$ & $\mathrm{D}$ & 2 sweeps & B-04-141_140604_D1 \\
\hline B-04-144 & $\mathrm{AW}, \mathrm{HW}$ & $E$ & 277 seconds $\times 150 \mathrm{~m}$ & B-04-144_150604_E1 \\
\hline B-04-145 & $\mathrm{AW}, \mathrm{HW}$ & $E$ & 339 seconds $\times 110 \mathrm{~m}$ & B-04-145_150604_E1 \\
\hline B-04-147 & $\mathrm{AW}, \mathrm{HW}$ & $\mathrm{O}$ & Observed at impassable culvert & B-04-147_150604_O1 \\
\hline B-04-149 & $\mathrm{AW}, \mathrm{HW}$ & $\mathrm{B}$ & $10 \mathrm{~m} \times 15 \mathrm{~m}$ & B-04-149_150604_B1 \\
\hline B-04-149 & $\mathrm{AW}, \mathrm{HW}$ & $E$ & 318 seconds $\times 125 \mathrm{~m}$ & B-04-149_150604_E1 \\
\hline B-04-152 & $\mathrm{AW}, \mathrm{HW}$ & $\mathrm{B}$ & $5 \mathrm{~m} \times 8 \mathrm{~m}$ & B-04-152_160604_B1 \\
\hline B-04-153 & $\mathrm{AW}, \mathrm{HW}$ & $E$ & 312 seconds $\times 100 \mathrm{~m}$ & B-04-153_160604_E1 \\
\hline B-04-155 & $\mathrm{AW}, \mathrm{HW}$ & $\mathrm{E}$ & 139 seconds $\times 50 \mathrm{~m}$ & B-04-155_160604_E1 \\
\hline B-04-161 & $\mathrm{AW}, \mathrm{HW}$ & $\mathrm{B}$ & $10 \mathrm{~m} \times 5 \mathrm{~m}$ & B-04-161_180604_B1 \\
\hline B-04-161 & $\mathrm{AW}, \mathrm{HW}$ & $E$ & 220 seconds $\times 15 \mathrm{~m}$ & B-04-161_180604_E1 \\
\hline B-04-163 & $\mathrm{AW}, \mathrm{HW}$ & $E$ & $50 \mathrm{~m} \times 131$ seconds & B-04-163_210604_E1 \\
\hline B-04-164 & $\mathrm{AW}, \mathrm{HW}$ & $\mathrm{B}$ & $30 \mathrm{~m} \times 20 \mathrm{~m}$ & B-04-164_210604_B1 \\
\hline B-04-165 & $\mathrm{AW}, \mathrm{HW}$ & $E$ & 139 seconds $\times 100 \mathrm{~m}$ & B-04-165_210604_E1 \\
\hline B-04-167 & $\mathrm{AW}, \mathrm{HW}$ & $\mathrm{B}$ & $30 \mathrm{~m} \times 18 \mathrm{~m}$ & B-04-167_210604_B1 \\
\hline B-04-167 & $\mathrm{AW}, \mathrm{HW}$ & $\mathrm{E}$ & 281 seconds $\times 150 \mathrm{~m}$ & B-04-167 210604 E1 \\
\hline B-04-174 & $\mathrm{AW}, \mathrm{HW}$ & $\mathrm{E}$ & 432 seconds $\times 150 \mathrm{~m}$ & B-04-174_220604_E1 \\
\hline B-04-181 & $\mathrm{AW}, \mathrm{HW}$ & $E$ & 239 seconds $\times 100 \mathrm{~m}$ & B-04-181_280604_E1 \\
\hline B-04-186 & $\mathrm{AW}, \mathrm{HW}$ & $E$ & 674 seconds $\times 150 \mathrm{~m}$ & B-04-186_290604_E1 \\
\hline B-04-187 & $\mathrm{AW}, \mathrm{HW}$ & $\mathrm{D}$ & 1 sweep & B-04-187_290604_D1 \\
\hline B-04-187 & $\mathrm{AW}, \mathrm{HW}$ & $E$ & 761 seconds $\times 150 \mathrm{~m}$ & B-04-187_290604_E1 \\
\hline B-04-188 & $\mathrm{AW}, \mathrm{HW}$ & $E$ & 650 seconds $\times 150 \mathrm{~m}$ & B-04-188_290604_E1 \\
\hline B-04-189 & $\mathrm{AW}, \mathrm{HW}$ & $E$ & 830 seconds $\times 175 \mathrm{~m}$ & B-04-189_300604_E1 \\
\hline B-04-190 & $\mathrm{AW}, \mathrm{HW}$ & $\mathrm{D}$ & 4 sweeps & B-04-190_300604_D1 \\
\hline B-04-191 & $\mathrm{AW}, \mathrm{HW}$ & $E$ & 351 seconds $\times 90 \mathrm{~m}$ & B-04-191_050704_E1 \\
\hline B-04-195 & $\mathrm{AW}, \mathrm{HW}$ & $\mathrm{B}$ & $10 \mathrm{~m} \times 15 \mathrm{~m}$ & B-04-195_060704_B1 \\
\hline B-04-195 & $\mathrm{AW}, \mathrm{HW}$ & $\mathrm{D}$ & 2 sweeps & B-04-195_060704_D1 \\
\hline
\end{tabular}


Appendix 4: List of all fishing effort showing the site number, collectors, fishing gear used, amount of fishing effort applied and the fish capture identification (ID) number derived for each occasion where effort was applied.

\begin{tabular}{|c|c|c|c|c|}
\hline Site Number & Collectors & Gear ID & Effort & $\begin{array}{c}\text { Fish Capture ID } \\
\text { (Crew_Date_Gear) }\end{array}$ \\
\hline B-04-196 & $\mathrm{AW}, \mathrm{HW}$ & $E$ & 530 seconds $\times 50 \mathrm{~m}$ & B-04-196_060704_E1 \\
\hline B-04-197 & $\mathrm{AW}, \mathrm{HW}$ & $E$ & 258 seconds $\times 75 \mathrm{~m}$ & B-04-197_060704_E1 \\
\hline B-04-198 & $\mathrm{AW}, \mathrm{HW}$ & $E$ & 593 seconds $\times 150 \mathrm{~m}$ & B-04-198_060704_E1 \\
\hline B-04-199 & $\mathrm{AW}, \mathrm{HW}$ & $E$ & 475 seconds $\times 150 \mathrm{~m}$ & B-04-199_060704_E1 \\
\hline B-04-200 & $\mathrm{AW}, \mathrm{HW}$ & $E$ & 234 seconds $\times 100 \mathrm{~m}$ & B-04-200_060704_E1 \\
\hline B-04-205 & $\mathrm{AW}, \mathrm{HW}$ & $E$ & 248 seconds $\times 100 \mathrm{~m}$ & B-04-205_070704_E1 \\
\hline B-04-211 & $\mathrm{AW}, \mathrm{HW}$ & $E$ & 119 seconds $\times 35 \mathrm{~m}$ & B-04-211_070704_E1 \\
\hline B-04-214 & $\mathrm{AW}, \mathrm{HW}$ & $E$ & 205 seconds $\times 80 \mathrm{~m}$ & B-04-214_070704_E1 \\
\hline B-04-215 & $\mathrm{AW}, \mathrm{HW}$ & $E$ & 202 seconds $\times 75 \mathrm{~m}$ & B-04-215_070704_E1 \\
\hline B-04-217 & $\mathrm{AW}, \mathrm{HW}$ & $E$ & 531 seconds $\times 100 \mathrm{~m}$ & B-04-217_080704_E1 \\
\hline B-04-218 & $\mathrm{AW}, \mathrm{HW}$ & $\mathrm{E}$ & 153 seconds $\times 70 \mathrm{~m}$ & B-04-218_080704_E1 \\
\hline B-04-219 & $\mathrm{AW}, \mathrm{HW}$ & $E$ & 250 seconds $\times 80 \mathrm{~m}$ & B-04-219_080704_E1 \\
\hline B-04-220 & $\mathrm{AW}, \mathrm{HW}$ & $E$ & 229 seconds $\times 125 \mathrm{~m}$ & B-04-220_080704 E1 \\
\hline B-04-223 & $\mathrm{AW}, \mathrm{HW}$ & $E$ & 436 seconds $\times 80 \mathrm{~m}$ & B-04-223_090704_E1 \\
\hline B-04-224 & $\mathrm{AW}, \mathrm{HW}$ & $\mathrm{E}$ & 264 seconds $\times 70 \mathrm{~m}$ & B-04-224_130704_E1 \\
\hline B-04-225 & $\mathrm{AW}, \mathrm{HW}$ & $\mathrm{E}$ & 175 seconds $\times 70 \mathrm{~m}$ & B-04-225_130704_E1 \\
\hline B-04-226 & $\mathrm{AW}, \mathrm{HW}$ & $\mathrm{B}$ & $12 \mathrm{~m} \times 10 \mathrm{~m}$ & B-04-226_130704_B1 \\
\hline B-04-228 & $\mathrm{AW}, \mathrm{HW}$ & $E$ & 314 seconds $\times 80 \mathrm{~m}$ & B-04-228_130704_E1 \\
\hline B-04-229 & $\mathrm{AW}, \mathrm{HW}$ & $\mathrm{D}$ & 2 sweeps & B-04-229_140704_D1 \\
\hline B-04-230 & $\mathrm{AW}, \mathrm{HW}$ & $\mathrm{E}$ & 290 seconds $\times 70 \mathrm{~m}$ & B-04-230_140704_E1 \\
\hline B-04-231 & $\mathrm{AW}, \mathrm{HW}$ & $E$ & 313 seconds $\times 60 \mathrm{~m}$ & B-04-231_140704_E1 \\
\hline B-04-232 & $\mathrm{AW}, \mathrm{HW}$ & $\mathrm{E}$ & 201 seconds $\times 75 \mathrm{~m}$ & B-04-232 140704 E1 \\
\hline B-04-238 & $\mathrm{AW}, \mathrm{HW}$ & $E$ & 539 seconds $\times 100 \mathrm{~m}$ & B-04-238_140704_E1 \\
\hline B-04-240 & $\mathrm{AW}, \mathrm{HW}$ & $E$ & 248 seconds $\times 85 \mathrm{~m}$ & B-04-240_150704_E1 \\
\hline B-04-241 & $\mathrm{AW}, \mathrm{HW}$ & $\mathrm{B}$ & $10 \mathrm{~m} \times 10 \mathrm{~m}$ & B-04-241_190704_B1 \\
\hline B-04-241 & $\mathrm{AW}, \mathrm{HW}$ & $E$ & 299 seconds $\times 80 \mathrm{~m}$ & B-04-241_190704_E1 \\
\hline B-04-244 & $\mathrm{AW}, \mathrm{HW}$ & $E$ & 703 seconds $\times 115 \mathrm{~m}$ & B-04-244_200704_E1 \\
\hline B-04-248 & $\mathrm{AW}, \mathrm{HW}$ & $\mathrm{B}$ & $8 \mathrm{~m} \times 12 \mathrm{~m}$ & B-04-248_200704_B1 \\
\hline B-04-249 & $\mathrm{AW}, \mathrm{HW}$ & $\mathrm{B}$ & $8 \mathrm{~m} \times 10 \mathrm{~m}$ & B-04-249_200704_B1 \\
\hline B-04-250 & $\mathrm{AW}, \mathrm{HW}$ & $\mathrm{B}$ & $6 \mathrm{~m} \times 8 \mathrm{~m}$ & B-04-250_200704_B1 \\
\hline B-04-257 & $\mathrm{AW}, \mathrm{HW}$ & $\mathrm{B}$ & $10 \mathrm{~m} \times 30 \mathrm{~m}$ & B-04-257_210704_B1 \\
\hline
\end{tabular}


Appendix 4: List of all fishing effort showing the site number, collectors, fishing gear used, amount of fishing effort applied and the fish capture identification (ID) number derived for each occasion where effort was applied.

\begin{tabular}{|c|c|c|c|c|}
\hline Site Number & Collectors & Gear ID & Effort & $\begin{array}{c}\text { Fish Capture ID } \\
\text { (Crew_Date_Gear) }\end{array}$ \\
\hline B-04-259 & $\mathrm{AW}, \mathrm{HW}$ & $\mathrm{B}$ & $7 \mathrm{~m} \times 6 \mathrm{~m}$ & B-04-259_210704_B1 \\
\hline B-04-263 & $\mathrm{AW}, \mathrm{HW}$ & $\mathrm{B}$ & $7 \times 15 \mathrm{~m}$ & B-04-263_220704_B1 \\
\hline B-04-264 & $\mathrm{AW}, \mathrm{HW}$ & $\mathrm{B}$ & $10 \mathrm{~m} \times 15 \mathrm{~m}$ & B-04-264_220704_B1 \\
\hline B-04-266 & $\mathrm{AW}, \mathrm{HW}$ & $E$ & 214 seconds $\times 50 \mathrm{~m}$ & B-04-266_260704_E1 \\
\hline B-04-270 & $\mathrm{AW}, \mathrm{HW}$ & $\mathrm{B}$ & $4 \mathrm{~m} \times 10 \mathrm{~m}$ & B-04-270_040804_B1 \\
\hline B-04-270 & $\mathrm{AW}, \mathrm{HW}$ & $E$ & 100 seconds $\times 10 \mathrm{~m}$ & B-04-270_040804_E1 \\
\hline B-04-272 & $\mathrm{AW}, \mathrm{HW}$ & $\mathrm{O}$ & Observed & B-04-272_040804_01 \\
\hline B-04-275 & $\mathrm{AW}, \mathrm{HW}$ & $\mathrm{B}$ & $10 \mathrm{~m} \times 5 \mathrm{~m}$ & B-04-275_050804_B1 \\
\hline B-04-276 & $\mathrm{AW}, \mathrm{HW}$ & $E$ & 219 seconds $\times 25 \mathrm{~m}$ & B-04-276_050804_E1 \\
\hline B-04-280 & $\mathrm{AW}, \mathrm{HW}$ & $\mathrm{B}$ & $5 \mathrm{~m} \times 10 \mathrm{~m}$ & B-04-280_050804_B1 \\
\hline B-04-286 & $\mathrm{AW}, \mathrm{HW}$ & $\mathrm{O}$ & Observed & B-04-286_060804_01 \\
\hline B-04-287 & $\mathrm{AW}, \mathrm{HW}$ & $\mathrm{O}$ & Observed under bridge & B-04-287_060804_01 \\
\hline D-04-011 & $\mathrm{DM}, \mathrm{EW}, \mathrm{NM}$ & $\mathrm{E}$ & 494 seconds $\times 75 \mathrm{~m}$ & D-04-011_020604_E1 \\
\hline D-04-012 & DM, EW, NM & $E$ & 947 seconds $\times 75 \mathrm{~m}$ & D-04-012_020604_E1 \\
\hline D-04-013 & $\mathrm{DM}$ & $\mathrm{L}$ & $0.5 \mathrm{hr}$. & D-04-013_030604_L1 \\
\hline D-04-015 & DM & $\mathrm{L}$ & $20 \mathrm{hrs}$. & D-04-015_070604_L1 \\
\hline D-04-016 & DM & $\mathrm{L}$ & $0.5 \mathrm{hr}$. & D-04-016_080604_L1 \\
\hline D-04-017 & $\mathrm{DM}, \mathrm{DC}$ & $\mathrm{E}$ & 174 seconds $\times 40 \mathrm{~m}$ & D-04-017_090604_E1 \\
\hline D-04-020 & DM & $\mathrm{L}$ & $0.5 \mathrm{hr}$. & D-04-020_100604_L1 \\
\hline D-04-021 & DM & $\mathrm{L}$ & $0.5 \mathrm{hr}$. & D-04-021 140604 L1 \\
\hline D-04-022 & DM, MK & $\mathrm{E}$ & 388 seconds $\times 75 \mathrm{~m}$ & D-04-022_150604_E1 \\
\hline D-04-023 & DM, MK & $E$ & 566 seconds $\times 100 \mathrm{~m}$ & D-04-023_150604_E1 \\
\hline D-04-027 & $\mathrm{DM}, \mathrm{MK}$ & $\mathrm{E}$ & 988 seconds $\times 100 \mathrm{~m}$ & D-04-027_150604_E1 \\
\hline D-04-028 & DM, BT & $E$ & 679 seconds $\times 75 \mathrm{~m}$ & D-04-028_160604_E1 \\
\hline D-04-029 & DM, BT & $\mathrm{E}$ & 400 seconds $\times 50 \mathrm{~m}$ & D-04-029_160604_E1 \\
\hline D-04-033 & $\mathrm{DM}, \mathrm{DC}$ & $E$ & 686 seconds $\times 125 \mathrm{~m}$ & D-04-033_230604_E1 \\
\hline D-04-034 & $\mathrm{DM}, \mathrm{DC}$ & $E$ & 396 seconds $\times 50 \mathrm{~m}$ & D-04-034_230604_E1 \\
\hline D-04-035 & DM, DC & $E$ & 612 seconds $\times 100 \mathrm{~m}$ & D-04-035_230604_E1 \\
\hline D-04-036 & $\mathrm{DM}, \mathrm{JM}, \mathrm{KK}$ & $E$ & 620 seconds $\times 50 \mathrm{~m}$ & D-04-036_070704_E1 \\
\hline W-04-001 & JT, JC & $\mathrm{O}$ & Observed spawning & W-04-001_030504_01 \\
\hline W-04-004 & JT, JC & $\mathrm{O}$ & Observed spawning & W-04-004_030504_01 \\
\hline
\end{tabular}


Appendix 4: List of all fishing effort showing the site number, collectors, fishing gear used, amount of fishing effort applied and the fish capture identification (ID) number derived for each occasion where effort was applied.

\begin{tabular}{|c|c|c|c|c|}
\hline Site Number & Collectors & Gear ID & Effort & $\begin{array}{c}\text { Fish Capture ID } \\
\text { (Crew_Date_Gear) }\end{array}$ \\
\hline W-04-005 & JT, JC & $\mathrm{O}$ & Observed stranded in pools & W-04-005_030504_01 \\
\hline W-04-006 & JT, JC & $\mathrm{E}$ & 161 seconds $\times 50 \mathrm{~m}$ & W-04-006_030504_E1 \\
\hline W-04-007 & JT, JC & $\mathrm{O}$ & Observed spawning & W-04-007_030504_01 \\
\hline W-04-009 & JT, JC & $E$ & 150 seconds $\times 30 \mathrm{~m}$ & W-04-009_040504_E1 \\
\hline W-04-013 & JT, JC & $\mathrm{B}$ & $6 \mathrm{~m} \times 15 \mathrm{~m}$ & W-04-013_040504_B1 \\
\hline W-04-020 & JT, JC & $\mathrm{B}$ & $6 \mathrm{~m} \times 10 \mathrm{~m}$ & W-04-020_050504_B1 \\
\hline W-04-027 & JT, JC & $E$ & 108 seconds $\times 20 \mathrm{~m}$ & W-04-027_050504_E1 \\
\hline W-04-032 & JT, JC & $E$ & 170 seconds $\times 50 \mathrm{~m}$ & W-04-032_060504_E1 \\
\hline W-04-036 & JT, JC & $\mathrm{E}$ & 100 seconds $\times 45 \mathrm{~m}$ & W-04-036_060504_E1 \\
\hline W-04-039 & JT, JC & $E$ & 172 seconds $\times 50 \mathrm{~m}$ & W-04-039_100504_E1 \\
\hline W-04-040 & JT, JC & $E$ & 117 seconds $\times 75 \mathrm{~m}$ & W-04-040_100504_E1 \\
\hline W-04-041 & JT, JC & $E$ & 126 seconds $\times 30 \mathrm{~m}$ & W-04-041_110504_E1 \\
\hline W-04-043 & JT, JC & $E$ & 537 seconds $\times 50 \mathrm{~m}$ & W-04-043_110504_E1 \\
\hline W-04-044 & JT, JC & $E$ & 569 seconds $\times 150 \mathrm{~m}$ & W-04-044_110504_E1 \\
\hline W-04-049 & JT, JC & $E$ & 516 seconds $\times 150 \mathrm{~m}$ & W-04-049_110504_E1 \\
\hline W-04-049 & JT, JC & $\mathrm{K}$ & $1 \mathrm{~m}^{2}$ & W-04-049_110504_K1 \\
\hline W-04-050 & JT, JC & $E$ & 264 seconds $\times 100 \mathrm{~m}$ & W-04-050_110504_E1 \\
\hline W-04-051 & JT, JC & $E$ & 306 seconds $\times 150 \mathrm{~m}$ & W-04-051_120504_E1 \\
\hline W-04-052 & JT, JC & $E$ & 366 seconds $\times 100 \mathrm{~m}$ & W-04-052_120504_E1 \\
\hline W-04-053 & $\mathrm{JT}, \mathrm{JC}$ & $E$ & 263 seconds $\times 100 \mathrm{~m}$ & W-04-053 180504 E1 \\
\hline W-04-054 & JT, JC & $\mathrm{E}$ & 303 seconds $\times 100 \mathrm{~m}$ & W-04-054_180504_E1 \\
\hline W-04-055 & JT, JC & $E$ & 375 seconds $\times 150 \mathrm{~m}$ & W-04-055_180504_E1 \\
\hline W-04-056 & JT, JC & $E$ & 151 seconds $\times 50 \mathrm{~m}$ & W-04-056_180504_E1 \\
\hline W-04-057 & JT, JC & $E$ & 247 seconds $\times 75 \mathrm{~m}$ & W-04-057_180504_E1 \\
\hline W-04-058 & JT, JC & $E$ & 86 seconds $\times 50 \mathrm{~m}$ & W-04-058_180504_E1 \\
\hline W-04-059 & JT, JC & $E$ & 131 seconds $\times 75 \mathrm{~m}$ & W-04-059_200504_E1 \\
\hline W-04-060 & JT, JC & $E$ & 345 seconds $\times 150 \mathrm{~m}$ & W-04-060_200504_E1 \\
\hline W-04-063 & JT, JC & $E$ & 149 seconds $\times 50 \mathrm{~m}$ & W-04-063_200504_E1 \\
\hline W-04-069 & JT, JC & $E$ & 147 seconds $\times 100 \mathrm{~m}$ & W-04-069_210504_E1 \\
\hline W-04-070 & JT, JC & $E$ & 191 seconds $\times 100 \mathrm{~m}$ & W-04-070_210504_E1 \\
\hline W-04-071 & JT, JC & $E$ & 102 seconds $\times 150 \mathrm{~m}$ & W-04-071_210504_E1 \\
\hline
\end{tabular}


Appendix 4: List of all fishing effort showing the site number, collectors, fishing gear used, amount of fishing effort applied and the fish capture identification (ID) number derived for each occasion where effort was applied.

\begin{tabular}{|c|c|c|c|c|}
\hline Site Number & Collectors & Gear ID & Effort & $\begin{array}{c}\text { Fish Capture ID } \\
\text { (Crew_Date_Gear) }\end{array}$ \\
\hline W-04-072 & JT, JC & $E$ & 256 seconds $\times 150 \mathrm{~m}$ & W-04-072_250504_E1 \\
\hline W-04-074 & JT, JC & $E$ & 401 seconds $\times 150 \mathrm{~m}$ & W-04-074_250504_E1 \\
\hline W-04-074 & JT, JC & $\mathrm{K}$ & $1 \mathrm{~m}^{2}$ & W-04-074_250504_K1 \\
\hline W-04-076 & JT, JC & $E$ & 510 seconds $\times 150 \mathrm{~m}$ & W-04-076_250504_E1 \\
\hline W-04-077 & JT, DM & $\mathrm{E}$ & 283 seconds $\times 100 \mathrm{~m}$ & W-04-077_260504_E1 \\
\hline W-04-077 & JT, DM & $\mathrm{L}$ & $30 \mathrm{~min}$. & W-04-077_260504_L1 \\
\hline W-04-078 & JT, DM & $E$ & 426 seconds $\times 100 \mathrm{~m}$ & W-04-078_260504_E1 \\
\hline W-04-082 & JT, DM & $E$ & 243 seconds $\times 100 \mathrm{~m}$ & W-04-082_260504_E1 \\
\hline W-04-082 & JT, DM & $\mathrm{L}$ & $30 \mathrm{~min}$. & W-04-082_260504_L1 \\
\hline W-04-083 & JT, DM & $E$ & 662 seconds $\times 150 \mathrm{~m}$ & W-04-083_270504_E1 \\
\hline W-04-083 & JT, DM & $\mathrm{K}$ & $1 \mathrm{~m}^{2}$ & W-04-083_270504_K1 \\
\hline W-04-083 & JT, DM & $\mathrm{L}$ & $60 \mathrm{~min}$. & W-04-083_270504_L1 \\
\hline W-04-084 & JT, DM & $\mathrm{D}$ & 10 sweeps over $10 \mathrm{~m}$ & W-04-084_270504_D1 \\
\hline W-04-084 & JT, DM & $E$ & 506 seconds $\times 100 \mathrm{~m}$ & W-04-084_270504_E1 \\
\hline W-04-085 & JT, JC & $\mathrm{B}$ & $50 \mathrm{~m} \times 5 \mathrm{~m}$ & W-04-085_280504_B1 \\
\hline W-04-087 & JT, JC & $\mathrm{L}$ & $15 \mathrm{~min}$. & W-04-087_280504_L1 \\
\hline W-04-088 & JT, JC & $\mathrm{E}$ & 243 seconds $\times 100 \mathrm{~m}$ & W-04-088_280504_E1 \\
\hline W-04-088 & JT, JC & $\mathrm{L}$ & $30 \mathrm{~min}$ & W-04-088_280504_L1 \\
\hline W-04-090 & JT, JC, MK & $E$ & 364 seconds $\times 100 \mathrm{~m}$ & W-04-090_010604_E1 \\
\hline W-04-090 & $\mathrm{JT}, \mathrm{JC}, \mathrm{MK}$ & $\mathrm{L}$ & $30 \mathrm{~min}$. & W-04-090_010604_L1 \\
\hline W-04-092 & JT, JC, MK & $\mathrm{E}$ & 421 seconds $\times 100 \mathrm{~m}$ & W-04-092_010604_E1 \\
\hline W-04-092 & JT, JC, MK & $\mathrm{L}$ & $30 \mathrm{~min}$. & W-04-092_010604_L1 \\
\hline W-04-093 & JT, JC & $E$ & 443 seconds $\times 100 \mathrm{~m}$ & W-04-093_010604_E1 \\
\hline W-04-093 & JT, JC & $\mathrm{L}$ & $30 \mathrm{~min}$. & W-04-093_010604_L1 \\
\hline W-04-094 & JT, JC & $\mathrm{E}$ & 245 seconds $\times 100 \mathrm{~m}$ & W-04-094_020604_E1 \\
\hline W-04-094 & JT, JC & $\mathrm{L}$ & $30 \mathrm{~min}$. & W-04-094_020604_L1 \\
\hline W-04-095 & JT, JC & $E$ & 467 seconds $\times 200 \mathrm{~m}$ & W-04-095_020604_E1 \\
\hline W-04-097 & JT, JC & $E$ & 213 seconds $\times 50 \mathrm{~m}$ & W-04-097_020604_E1 \\
\hline W-04-102 & JT, JC & $E$ & 465 seconds $\times 150 \mathrm{~m}$ & W-04-102_030604_E1 \\
\hline W-04-102 & JT, JC & $\mathrm{K}$ & $1 \mathrm{~m}^{2}$ & W-04-102_030604_K1 \\
\hline W-04-105 & JT, JC & $E$ & 444 seconds $\times 100 \mathrm{~m}$ & W-04-105_030604_E1 \\
\hline
\end{tabular}


Appendix 4: List of all fishing effort showing the site number, collectors, fishing gear used, amount of fishing effort applied and the fish capture identification (ID) number derived for each occasion where effort was applied.

\begin{tabular}{|c|c|c|c|c|}
\hline Site Number & Collectors & Gear ID & Effort & $\begin{array}{c}\text { Fish Capture ID } \\
\text { (Crew_Date_Gear) }\end{array}$ \\
\hline W-04-106 & JT, JC & $E$ & 294 seconds $\times 100 \mathrm{~m}$ & W-04-106_030604_E1 \\
\hline W-04-109 & JT, JC & $E$ & 365 seconds $\times 100 \mathrm{~m}$ & W-04-109_040604_E1 \\
\hline W-04-109 & JT, JC & $\mathrm{K}$ & $1 \mathrm{~m}^{2}$ & W-04-109_040604_K1 \\
\hline W-04-110 & JT, JC & $E$ & 315 seconds $\times 100 \mathrm{~m}$ & W-04-110_040604_E1 \\
\hline W-04-111 & JT, JC & $E$ & 306 seconds $\times 100 \mathrm{~m}$ & W-04-111_040604_E1 \\
\hline W-04-113 & JT, JC & $E$ & 178 seconds $\times 40 \mathrm{~m}$ & W-04-113_050604_E1 \\
\hline W-04-114 & JT, JC & $E$ & 247 seconds $\times 75 \mathrm{~m}$ & W-04-114_050604_E1 \\
\hline W-04-115 & JT, JC & $E$ & 310 seconds $\times 100 \mathrm{~m}$ & W-04-115_050604_E1 \\
\hline W-04-116 & JT, JC & $E$ & 228 seconds $\times 150 \mathrm{~m}$ & W-04-116_050604_E1 \\
\hline W-04-119 & JT, JC & $E$ & 288 seconds $\times 100 \mathrm{~m}$ & W-04-119_080604_E1 \\
\hline W-04-120 & JT, JC & $E$ & 410 seconds $\times 150 \mathrm{~m}$ & W-04-120_080604_E1 \\
\hline W-04-121 & JT, JC & $\mathrm{E}$ & 244 seconds $\times 75 \mathrm{~m}$ & W-04-121_080604_E1 \\
\hline W-04-124 & JT, JC & $\mathrm{E}$ & 154 seconds $\times 75 \mathrm{~m}$ & W-04-124_080604_E1 \\
\hline W-04-126 & JT, JC & $E$ & 388 seconds $\times 100 \mathrm{~m}$ & W-04-126_090604_E1 \\
\hline W-04-129 & JT, JC & $E$ & 293 seconds $\times 100 \mathrm{~m}$ & W-04-129_090604_E1 \\
\hline W-04-132 & JT, JC & $E$ & 386 seconds $\times 100 \mathrm{~m}$ & W-04-132_090604_E1 \\
\hline W-04-135 & JT, JC & $\mathrm{E}$ & 376 seconds $\times 100 \mathrm{~m}$ & W-04-135_090604_E1 \\
\hline W-04-137 & JT, JC & $E$ & 186 seconds $\times 50 \mathrm{~m}$ & W-04-137_100604_E1 \\
\hline W-04-149 & JT, JC & $\mathrm{B}$ & $5 \mathrm{~m} \times 20 \mathrm{~m}$ & W-04-149_140604_B1 \\
\hline W-04-151 & $\mathrm{JT}, \mathrm{JC}$ & $E$ & 629 seconds $\times 150 \mathrm{~m}$ & W-04-151 140604 E1 \\
\hline W-04-153 & JT, JC & $\mathrm{E}$ & 527 seconds $\times 75 \mathrm{~m}$ & W-04-153_150604_E1 \\
\hline W-04-157 & JT, JC & $E$ & 267 seconds $\times 50 \mathrm{~m}$ & W-04-157_160604_E1 \\
\hline W-04-159 & JT, JC & $E$ & 252 seconds $\times 150 \mathrm{~m}$ & W-04-159_160604_E1 \\
\hline W-04-166 & JT, JC & $E$ & 244 seconds $\times 100 \mathrm{~m}$ & W-04-166_170604_E1 \\
\hline W-04-167 & JT, JC & $\mathrm{E}$ & 143 seconds $\times 50 \mathrm{~m}$ & W-04-167_170604_E1 \\
\hline W-04-171 & JT, JC & $E$ & 274 seconds $\times 100 \mathrm{~m}$ & W-04-171_170604_E1 \\
\hline W-04-172 & JT, JC & $E$ & 616 seconds $\times 150 \mathrm{~m}$ & W-04-172_170604_E1 \\
\hline W-04-173 & JT, JC & $E$ & 308 seconds $\times 100 \mathrm{~m}$ & W-04-173_180604_E1 \\
\hline W-04-174 & JT, JC & $E$ & 419 seconds $\times 75 \mathrm{~m}$ & W-04-174_180604_E1 \\
\hline W-04-175 & JT, JC & $E$ & 447 seconds $\times 100 \mathrm{~m}$ & W-04-175_210604_E1 \\
\hline W-04-176 & JT, JC & $E$ & 228 seconds $\times 100 \mathrm{~m}$ & W-04-176_210604_E1 \\
\hline
\end{tabular}


Appendix 4: List of all fishing effort showing the site number, collectors, fishing gear used, amount of fishing effort applied and the fish capture identification (ID) number derived for each occasion where effort was applied.

\begin{tabular}{|c|c|c|c|c|}
\hline Site Number & Collectors & Gear ID & Effort & $\begin{array}{c}\text { Fish Capture ID } \\
\text { (Crew_Date_Gear) }\end{array}$ \\
\hline W-04-177 & JT, JC & $E$ & 423 seconds $\times 75 \mathrm{~m}$ & W-04-177_210604_E1 \\
\hline W-04-178 & JT, JC & $E$ & 96 seconds $\times 50 \mathrm{~m}$ & W-04-178_210604_E1 \\
\hline W-04-179 & JT, JC & $E$ & 148 seconds $\times 50 \mathrm{~m}$ & W-04-179_220604_E1 \\
\hline W-04-180 & JT, JC & $\mathrm{B}$ & $6 \mathrm{~m} \times 20 \mathrm{~m}$ & W-04-180_220604_B1 \\
\hline W-04-181 & JT, JC & $E$ & 308 seconds $\times 75 \mathrm{~m}$ & W-04-181_220604_E1 \\
\hline W-04-182 & JT, JC & $E$ & 242 seconds $\times 120 \mathrm{~m}$ & W-04-182_220604_E1 \\
\hline W-04-184 & JT, JC & $E$ & 232 seconds $\times 75$ m & W-04-184_220604_E1 \\
\hline W-04-186 & JT, JC & $E$ & 312 seconds $\times 75 \mathrm{~m}$ & W-04-186_230604_E1 \\
\hline W-04-187 & JT, JC & $E$ & 175 seconds $\times 100 \mathrm{~m}$ & W-04-187_230604_E1 \\
\hline W-04-192 & JT, JC & $E$ & 361 seconds $\times 100 \mathrm{~m}$ & W-04-192_240604_E1 \\
\hline W-04-193 & JT, JC & $E$ & 242 seconds $\times 100 \mathrm{~m}$ & W-04-193_240604_E1 \\
\hline W-04-195 & JT, JC & $\mathrm{E}$ & 482 seconds $\times 150 \mathrm{~m}$ & W-04-195_250604_E1 \\
\hline W-04-197 & JT, JC & $\mathrm{E}$ & 407 seconds $\times 200 \mathrm{~m}$ & W-04-197_250604_E1 \\
\hline W-04-200 & JT, JC & $E$ & 275 seconds $\times 100 \mathrm{~m}$ & W-04-200_290604_E1 \\
\hline W-04-202 & JT, JC & $\mathrm{B}$ & $30 \mathrm{~m} \times 20 \mathrm{~m}$ & W-04-202_290604_B1 \\
\hline W-04-203 & JT, JC & $E$ & 243 seconds $\times 50 \mathrm{~m}$ & W-04-203_290604_E1 \\
\hline W-04-207 & JT, JC & $E$ & 370 seconds $\times 100 \mathrm{~m}$ & W-04-207_050704_E1 \\
\hline W-04-208 & JT, JC & $E$ & 463 seconds $\times 100 \mathrm{~m}$ & W-04-208_050704_E1 \\
\hline W-04-208 & JT, JC & $\mathrm{K}$ & $1 \mathrm{~m}^{2}$ & W-04-208_050704_K1 \\
\hline W-04-211 & $\mathrm{JT}, \mathrm{JC}$ & $\mathrm{E}$ & 488 seconds $\times 150 \mathrm{~m}$ & W-04-211 $050704 \mathrm{E} 1$ \\
\hline W-04-212 & JT, JC & $\mathrm{E}$ & 334 seconds $\times 150 \mathrm{~m}$ & W-04-212_060704_E1 \\
\hline W-04-213 & JT, JC & $E$ & 249 seconds $\times 100 \mathrm{~m}$ & W-04-213_060704_E1 \\
\hline W-04-216 & JT, JC & $E$ & 247 seconds $\times 100 \mathrm{~m}$ & W-04-216_060704_E1 \\
\hline W-04-217 & JT, JC & $E$ & 517 seconds $\times 150 \mathrm{~m}$ & W-04-217_060704_E1 \\
\hline W-04-218 & JT, JC & $\mathrm{E}$ & 535 seconds $\times 150 \mathrm{~m}$ & W-04-218_060704_E1 \\
\hline W-04-225 & JT, JC & $E$ & 467 seconds $\times 100 \mathrm{~m}$ & W-04-225_070704_E1 \\
\hline W-04-231 & JT, JC & $E$ & 575 seconds $\times 150 \mathrm{~m}$ & W-04-231_080704_E1 \\
\hline W-04-231 & JT, JC & $\mathrm{K}$ & $1 \mathrm{~m}^{2}$ & W-04-231_080704_K1 \\
\hline W-04-232 & JT, JC & $E$ & 327 seconds $\times 100 \mathrm{~m}$ & W-04-232_080704_E1 \\
\hline W-04-233 & JT, JC & $E$ & 327 seconds $\times 100 \mathrm{~m}$ & W-04-233_130704_E1 \\
\hline W-04-234 & JT, JC & $E$ & 468 seconds $\times 150 \mathrm{~m}$ & W-04-234_130704_E1 \\
\hline
\end{tabular}


Appendix 4: List of all fishing effort showing the site number, collectors, fishing gear used, amount of fishing effort applied and the fish capture identification (ID) number derived for each occasion where effort was applied.

\begin{tabular}{|c|c|c|c|c|}
\hline Site Number & Collectors & Gear ID & Effort & $\begin{array}{c}\text { Fish Capture ID } \\
\text { (Crew_Date_Gear) }\end{array}$ \\
\hline W-04-235 & JT, JC & $\mathrm{K}$ & $1 \mathrm{~m}^{2}$ & W-04-235_130704_K1 \\
\hline W-04-237 & JT, JC & $\mathrm{E}$ & 202 seconds $\times 75 \mathrm{~m}$ & W-04-237 130704_E1 \\
\hline W-04-238 & JT, JC & $E$ & 232 seconds $\times 100 \mathrm{~m}$ & W-04-238_130704_E1 \\
\hline W-04-239 & JT, JC & $E$ & 288 seconds $\times 75 \mathrm{~m}$ & W-04-239_130704_E1 \\
\hline W-04-244 & $\mathrm{JT}, \mathrm{JC}$ & $\mathrm{O}$ & Observed & W-04-244_140704_01 \\
\hline W-04-245 & JT, JC & $\mathrm{O}$ & Observed & W-04-245_140704_O1 \\
\hline W-04-246 & JT, JC & $\mathrm{O}$ & Observed & W-04-246_140704_O1 \\
\hline W-04-249 & JT, JC & $E$ & 199 seconds $\times 150 \mathrm{~m}$ & W-04-249_140704_E1 \\
\hline W-04-250 & JT, JC & $E$ & 237 seconds $\times 100 \mathrm{~m}$ & W-04-250_140704_E1 \\
\hline W-04-250 & JT, JC & $\mathrm{K}$ & $1 \mathrm{~m}^{2}$ & W-04-250_140704_K1 \\
\hline W-04-252 & JT, JC & $E$ & 246 seconds $\times 75 \mathrm{~m}$ & W-04-252_150704_E1 \\
\hline W-04-253 & JT, JC & $\mathrm{E}$ & 349 seconds $\times 100 \mathrm{~m}$ & W-04-253_150704_E1 \\
\hline W-04-254 & JT, JC & $\mathrm{E}$ & 291 seconds $\times 150 \mathrm{~m}$ & W-04-254_150704_E1 \\
\hline W-04-254 & JT, JC & $\mathrm{K}$ & $1 \mathrm{~m}^{2}$ & W-04-254_150704_K1 \\
\hline W-04-255 & JT, JC & $E$ & 311 seconds $\times 75 \mathrm{~m}$ & W-04-255_150704_E1 \\
\hline W-04-256 & JT, JC & $E$ & 328 seconds $\times 75 \mathrm{~m}$ & W-04-256_200704_E1 \\
\hline W-04-260 & JT, JC & $\mathrm{E}$ & 324 seconds $\times 100 \mathrm{~m}$ & W-04-260_210704_E1 \\
\hline W-04-262 & JT, JC & $E$ & 233 seconds $\times 100 \mathrm{~m}$ & W-04-262_220704_E1 \\
\hline W-04-264 & JT, JC & $E$ & 375 seconds $\times 100 \mathrm{~m}$ & W-04-264_220704_E1 \\
\hline W-04-265 & $\mathrm{JT}, \mathrm{JC}$ & $E$ & 184 seconds $\times 100 \mathrm{~m}$ & W-04-265 220704 E1 \\
\hline W-04-266 & JT, JC & $\mathrm{E}$ & 582 seconds $\times 150 \mathrm{~m}$ & W-04-266_220704_E1 \\
\hline W-04-266 & JT, JC & $\mathrm{K}$ & $1 \mathrm{~m}^{2}$ & W-04-266_220704_K1 \\
\hline W-04-269 & JT, JC & $E$ & 173 seconds $\times 50 \mathrm{~m}$ & W-04-269_220704_E1 \\
\hline W-04-271 & JT, JC & $\mathrm{B}$ & $11 \mathrm{~m} \times 20 \mathrm{~m}$ & W-04-271_230704_B1 \\
\hline W-04-274 & JT, JC & $\mathrm{E}$ & 388 seconds $\times 100 \mathrm{~m}$ & W-04-274_230704_E1 \\
\hline W-04-277 & JT, JC & $E$ & 222 seconds $\times 45 \mathrm{~m}$ & W-04-277_030804_E1 \\
\hline W-04-286 & JT, JC & $E$ & 78 seconds $\times 25 \mathrm{~m}$ & W-04-286_030804_E1 \\
\hline W-04-287 & JT, JC & $E$ & 701 seconds $\times 150 \mathrm{~m}$ & W-04-287_030804_E1 \\
\hline W-04-289 & JT, JC & $E$ & 322 seconds $\times 100 \mathrm{~m}$ & W-04-289_040804_E1 \\
\hline W-04-297 & JT, JC & $E$ & 153 seconds $\times 100 \mathrm{~m}$ & W-04-297_050804_E1 \\
\hline W-04-298 & JT, JC & $E$ & 99 seconds $\times 100 \mathrm{~m}$ & W-04-298_050804_E1 \\
\hline
\end{tabular}


Appendix 4: List of all fishing effort showing the site number, collectors, fishing gear used, amount of fishing effort applied and the fish capture identification (ID) number derived for each occasion where effort was applied.

\begin{tabular}{|c|c|c|c|c|}
\hline Site Number & Collectors & Gear ID & Effort & $\begin{array}{c}\text { Fish Capture ID } \\
\text { (Crew_Date_Gear) }\end{array}$ \\
\hline W-04-299 & JT, JC & $E$ & 198 seconds $\times 100 \mathrm{~m}$ & W-04-299_050804_E1 \\
\hline $\mathrm{X}-04-001$ & $\mathrm{JE}, \mathrm{AP}, \mathrm{HW}$ & $\mathrm{B}$ & $30 \times 15 \mathrm{~m}$ and $25 \times 10 \mathrm{~m}$ & X-04-001_050504_B1 \\
\hline$X-04-002$ & $\mathrm{JE}, \mathrm{AP}, \mathrm{HW}$ & $\mathrm{B}$ & $40 \mathrm{~m} \times 18 \mathrm{~m}$ & $\mathrm{X}-04-002 \_050504$ B1 \\
\hline $\mathrm{X}-04-005$ & $\mathrm{JE}, \mathrm{AP}, \mathrm{HW}$ & $E$ & 711 seconds $\times 25 \mathrm{~m}$ & X-04-005_050504_E1 \\
\hline$X-04-014$ & JE, AP & $\mathrm{B}$ & $20 \times 4 \mathrm{~m}$ & $\mathrm{X}-04-014 \_060504 \_\mathrm{B} 1$ \\
\hline $\mathrm{X}-04-023$ & JE, AP & $\mathrm{B}$ & $25 \times 4 \mathrm{~m}$ & $\mathrm{X}-04-023 \_100504$ B1 \\
\hline$X-04-026$ & JE, AP & $\mathrm{B}$ & $55 \times 5 \mathrm{~m}$ & $\mathrm{X}-04-026 \_100504$ B1 \\
\hline $\mathrm{X}-04-031$ & JE, AP & $\mathrm{B}$ & $35 \times 4.5 \mathrm{~m}$ & X-04-031_100504_B1 \\
\hline $\mathrm{X}-04-031$ & JE, AP & $\mathrm{E}$ & 491 seconds $\times 45 \mathrm{~m}$ & $\mathrm{X}-04-031 \_100504 \mathrm{E} 1$ \\
\hline$X-04-037$ & JE, AP & $E$ & 842 seconds $\times 100 \mathrm{~m}$ & $\mathrm{X}-04-037 \_140504 \mathrm{E} 1$ \\
\hline$X-04-041$ & JE, AP & $\mathrm{B}$ & $15 \times 2.5 \mathrm{~m}$ & $\mathrm{X}-04-041 \_140504$ B1 \\
\hline X-04-041 & JE, AP & $\mathrm{L}$ & $10 \mathrm{~min}$. & X-04-041_140504_L1 \\
\hline$X-04-042$ & JE, AP & $\mathrm{D}$ & $10 \mathrm{~m}^{2}$ & X-04-042_140504_D1 \\
\hline X-04-043 & JE, AP, HW, DM & $\mathrm{K}$ & $7 \mathrm{~m}^{2}$ & $\mathrm{X}-04-043 \_170504 \mathrm{~K} 1$ \\
\hline $\mathrm{X}-04-044$ & JE, $\mathrm{AP}$ & $E$ & 247 seconds $\times 30 \mathrm{~m}$ & $\mathrm{X}-04-044 \_170504 \_\mathrm{E} 1$ \\
\hline$X-04-046$ & JE, AP & $\mathrm{E}$ & 186 seconds $\times 15 \mathrm{~m}$ & X-04-046_170504_E1 \\
\hline X-04-048 & JE, AP, HW, DM & $\mathrm{B}$ & $25 \times 15 \mathrm{~m}$ & X-04-048_180504_B1 \\
\hline X-04-048 & $\mathrm{JE}, \mathrm{AP}, \mathrm{HW}, \mathrm{DM}$ & $E$ & 161 seconds $\times 20 \mathrm{~m}$ & X-04-048_180504_E1 \\
\hline X-04-049 & JE, AP & $E$ & 305 seconds $\times 20 \mathrm{~m}$ & X-04-049_180504_E1 \\
\hline$X-04-051$ & JE, AP & $\mathrm{E}$ & 418 seconds $\times 30 \mathrm{~m}$ & $\mathrm{X}-04-051 \_180504 \mathrm{E} 1$ \\
\hline $\mathrm{X}-04-052$ & JE, AP & $\mathrm{B}$ & $20 \times 7 \mathrm{~m}$ & X-04-052_190504_B1 \\
\hline$X-04-053$ & JE, AP & $\mathrm{E}$ & 202 seconds $\times 25 \mathrm{~m}$ & X-04-053 200504 E1 \\
\hline X-04-054 & JE, AP & $E$ & 366 seconds $\times 35 \mathrm{~m}$ & X-04-054_200504_E1 \\
\hline $\mathrm{X}-04-055$ & JE, AP & $\mathrm{O}$ & Observed spawning & X-04-055_200504_01 \\
\hline$X-04-056$ & JE, AP & $\mathrm{O}$ & Observed spawning & X-04-056_200504_01 \\
\hline X-04-057 & JE, AP & $\mathrm{O}$ & Observed spawning & $\mathrm{X}-04-057200504$ O1 \\
\hline$X-04-058$ & JE, AP & $\mathrm{O}$ & Observed spawning & X-04-058_200504_01 \\
\hline$X-04-061$ & JE, AP & $E$ & 160 seconds $\times 15 \mathrm{~m}$ & X-04-061_200504_E1 \\
\hline$X-04-065$ & JE, AP & $E$ & 893 seconds $\times 150 \mathrm{~m}$ & X-04-065_210504_E1 \\
\hline$X-04-066$ & JE, AP & $E$ & 470 seconds $\times 50 \mathrm{~m}$ & X-04-066_210504_E1 \\
\hline$X-04-067$ & JE, AP & $\mathrm{E}$ & 229 seconds $\times 8 \mathrm{~m}$ & X-04-067_250504_E1 \\
\hline
\end{tabular}


Appendix 4: List of all fishing effort showing the site number, collectors, fishing gear used, amount of fishing effort applied and the fish capture identification (ID) number derived for each occasion where effort was applied.

\begin{tabular}{|c|c|c|c|c|}
\hline Site Number & Collectors & Gear ID & Effort & $\begin{array}{c}\text { Fish Capture ID } \\
\text { (Crew_Date_Gear) }\end{array}$ \\
\hline $\mathrm{X}-04-068$ & JE, AP & $E$ & 81 seconds $\times 5 \mathrm{~m}$ & X-04-068_250504_E1 \\
\hline X-04-069 & JE, AP & $\mathrm{K}$ & $3 \mathrm{~m}^{2}$ & $\mathrm{X}-04-069 \_250504 \mathrm{~K} 1$ \\
\hline$X-04-071$ & JE, AP & $E$ & 132 seconds $\times 12 \mathrm{~m}$ & X-04-071_250504_E1 \\
\hline $\mathrm{X}-04-072$ & JE, AP & $\mathrm{O}$ & Observed & $\mathrm{X}-04-072 \_250504 \mathrm{O} 1$ \\
\hline X-04-074 & JE, AP & $E$ & 251 seconds $\times 75 \mathrm{~m}$ & X-04-074_260504_E1 \\
\hline X-04-077 & JE, AP & $E$ & 332 seconds $\times 65 \mathrm{~m}$ & X-04-077_260504_E1 \\
\hline$X-04-078$ & JE, AP & $E$ & 298 seconds $\times 15 \mathrm{~m}$ & X-04-078_260504_E1 \\
\hline $\mathrm{X}-04-080$ & JE, AP & $E$ & 428 seconds $\times 30 \mathrm{~m}$ & X-04-080_260504_E1 \\
\hline X-04-081 & JE, AP & $E$ & 262 seconds $\times 20 \mathrm{~m}$ & X-04-081_270504_E1 \\
\hline$X-04-082$ & JE, AP & $\mathrm{B}$ & $3 \mathrm{~m} \times 18 \mathrm{~m}$ & $\mathrm{X}-04-082 \_270504$ B1 \\
\hline$X-04-084$ & JE, AP & $\mathrm{E}$ & 274 seconds $\times 30 \mathrm{~m}$ & $\mathrm{X}-04-084 \_270504 \mathrm{E} 1$ \\
\hline X-04-088 & JE, AP & $\mathrm{L}$ & $20 \mathrm{~min}$. & X-04-088_270504_L1 \\
\hline$X-04-090$ & JE, AP & $\mathrm{E}$ & 201 seconds $\times 20 \mathrm{~m}$ & X-04-090_270504_E1 \\
\hline X-04-091 & JE, AP & $E$ & 307 seconds $\times 50 \mathrm{~m}$ & X-04-091_280504_E1 \\
\hline $\mathrm{X}-04-091$ & JE, AP & $\mathrm{L}$ & 2 min. & X-04-091_280504_L1 \\
\hline X-04-092 & JE, AP & $E$ & 410 seconds $\times 75 \mathrm{~m}$ & X-04-092_280504_E1 \\
\hline X-04-094 & JE, AP & $E$ & 305 seconds $\times 25 \mathrm{~m}$ & X-04-094_280504_E1 \\
\hline X-04-095 & JE, AP & $E$ & 320 seconds $\times 40 \mathrm{~m}$ & X-04-095_010604_E1 \\
\hline X-04-097 & JE, AP & $E$ & 293 seconds $\times 100 \mathrm{~m}$ & X-04-097_010604_E1 \\
\hline $\mathrm{X}-04-098$ & JE, AP & $\mathrm{B}$ & $4 \mathrm{~m} \times 35 \mathrm{~m}$ & X-04-098_010604_B1 \\
\hline X-04-099 & JE, AP & $E$ & 372 seconds $\times 20 \mathrm{~m}$ & X-04-099_010604_E1 \\
\hline$X-04-101$ & JE, AP & $E$ & 305 seconds $\times 15 \mathrm{~m}$ & X-04-101 010604 E1 \\
\hline$X-04-102$ & JE, AP & $E$ & 447 seconds $\times 100 \mathrm{~m}$ & X-04-102_020604_E1 \\
\hline $\mathrm{X}-04-103$ & JE, AP & $E$ & 66 seconds $\times 7 \mathrm{~m}$ & $\mathrm{X}-04-103 \_020604 \mathrm{E} 1$ \\
\hline$X-04-103$ & JE, AP & $\mathrm{K}$ & $3 \mathrm{~m}^{2}$ & X-04-103_020604_K1 \\
\hline X-04-104 & JE, AP & $\mathrm{O}$ & Observed & X-04-104_020604_01 \\
\hline $\mathrm{X}-04-105$ & JE, AP & $\mathrm{O}$ & Observed upstream of culvert & X-04-105_020604_01 \\
\hline$X-04-106$ & JE, AP & $E$ & 575 seconds $\times 80 \mathrm{~m}$ & X-04-106_030604_E1 \\
\hline $\mathrm{X}-04-107$ & JE, AP & $\mathrm{B}$ & $3 \mathrm{~m} \times 25 \mathrm{~m}$ & X-04-107_030604_B1 \\
\hline $\mathrm{X}-04-107$ & JE, AP & $E$ & 640 seconds $\times 80 \mathrm{~m}$ & X-04-107_030604_E1 \\
\hline$X-04-107$ & JE, AP & $\mathrm{O}$ & Observed crossing ford & X-04-107_030604_01 \\
\hline
\end{tabular}


Appendix 4: List of all fishing effort showing the site number, collectors, fishing gear used, amount of fishing effort applied and the fish capture identification (ID) number derived for each occasion where effort was applied.

\begin{tabular}{|c|c|c|c|c|}
\hline Site Number & Collectors & Gear ID & Effort & $\begin{array}{c}\text { Fish Capture ID } \\
\text { (Crew_Date_Gear) }\end{array}$ \\
\hline$X-04-109$ & JE, AP & $E$ & 177 seconds $\times 20 \mathrm{~m}$ & X-04-109_030604_E1 \\
\hline $\mathrm{X}-04-110$ & JE, AP & $E$ & 872 seconds $\times 80 \mathrm{~m}$ & X-04-110_040604_E1 \\
\hline $\mathrm{X}-04-112$ & JE, AP & $E$ & 618 seconds $\times 30 \mathrm{~m}$ & $\mathrm{X}-04-112 \_040604 \mathrm{E} 1$ \\
\hline $\mathrm{X}-04-113$ & JE, AP & $\mathrm{E}$ & 250 seconds $\times 20 \mathrm{~m}$ & $\mathrm{X}-04-113 \_070604 \mathrm{E} 1$ \\
\hline $\mathrm{X}-04-114$ & JE, AP & $E$ & 250 seconds $\times 20 \mathrm{~m}$ & X-04-114_070604_E1 \\
\hline X-04-114 & JE, AP & $\mathrm{K}$ & $2 \mathrm{~m}^{2}$ & $\mathrm{X}-04-114 \_070604 \mathrm{~K} 1$ \\
\hline$X-04-115$ & JE, AP & $E$ & 692 seconds $\times 80 \mathrm{~m}$ & X-04-115_070604_E1 \\
\hline $\mathrm{X}-04-116$ & JE, AP & $E$ & 428 seconds $\times 20 \mathrm{~m}$ & X-04-116_080604_E1 \\
\hline $\mathrm{X}-04-116$ & JE, AP & $\mathrm{L}$ & $45 \mathrm{~min}$. & X-04-116_080604_L1 \\
\hline$X-04-118$ & JE, AP & $E$ & 773 seconds $\times 130 \mathrm{~m}$ & $\mathrm{X}-04-118 \_080604 \mathrm{E} 1$ \\
\hline$X-04-118$ & JE, AP & $\mathrm{L}$ & $45 \mathrm{~min}$. & X-04-118_080604_L1 \\
\hline $\mathrm{X}-04-119$ & JE, AP & $E$ & 419 seconds $\times 25 \mathrm{~m}$ & X-04-119_080604_E1 \\
\hline$X-04-120$ & JE, AP & $\mathrm{E}$ & 281 seconds $\times 30 \mathrm{~m}$ & X-04-120_080604_E1 \\
\hline$X-04-121$ & JE, AP & $E$ & 727 seconds $\times 60 \mathrm{~m}$ & X-04-121_080604_E1 \\
\hline$X-04-122$ & JE, AP & $\mathrm{E}$ & 701 seconds $\times 100 \mathrm{~m}$ & $\mathrm{X}-04-122 \_090604 \_\mathrm{E} 1$ \\
\hline$X-04-123$ & JE, AP & $E$ & 277 seconds $\times 15 \mathrm{~m}$ & X-04-123_090604_E1 \\
\hline $\mathrm{X}-04-124$ & JE, AP & $E$ & 328 seconds $\times 30 \mathrm{~m}$ & X-04-124_090604_E1 \\
\hline$X-04-126$ & JE, AP & $E$ & 596 seconds $\times 70 \mathrm{~m}$ & X-04-126_100604_E1 \\
\hline$X-04-127$ & JE, AP & $E$ & 757 seconds $\times 40 \mathrm{~m}$ & $\mathrm{X}-04-127 \_100604 \mathrm{E} 1$ \\
\hline$X-04-128$ & JE, AP & $\mathrm{E}$ & 512 seconds $\times 40 \mathrm{~m}$ & $\mathrm{X}-04-128 \_140604 \_\mathrm{E} 1$ \\
\hline$X-04-129$ & JE, AP & $E$ & 1125 seconds $\times 120 \mathrm{~m}$ & X-04-129_140604_E1 \\
\hline$X-04-130$ & JE, AP & $E$ & 253 seconds $\times 40$ m & $\mathrm{X}-04-130 \quad 140604 \mathrm{E} 1$ \\
\hline$X-04-132$ & JE, AP & $E$ & 618 seconds $\times 80 \mathrm{~m}$ & $\mathrm{X}-04-132 \_140604$ E1 \\
\hline $\mathrm{X}-04-133$ & JE, AP & $\mathrm{E}$ & 597 seconds $\times 100 \mathrm{~m}$ & $\mathrm{X}-04-133 \_150604 \mathrm{E} 1$ \\
\hline$X-04-137$ & JE, AP & $E$ & 750 seconds $\times 60 \mathrm{~m}$ & X-04-137_160604_E1 \\
\hline X-04-137 & JE, AP & $\mathrm{L}$ & $60 \mathrm{~min}$. & X-04-137_160604_L1 \\
\hline$X-04-138$ & JE, AP & $E$ & 317 seconds $\times 15 \mathrm{~m}$ & X-04-138_160604_E1 \\
\hline$X-04-139$ & JE, AP & $E$ & 354 seconds $\times 50 \mathrm{~m}$ & X-04-139_160604_E1 \\
\hline$X-04-141$ & $\mathrm{JE}, \mathrm{AP}, \mathrm{DM}$ & $E$ & 676 seconds $\times 120 \mathrm{~m}$ & X-04-141_170604 E1 \\
\hline$X-04-142$ & JE, AP & $E$ & 542 seconds $\times 100 \mathrm{~m}$ & $\mathrm{X}-04-142 \_170604 \_\mathrm{E} 1$ \\
\hline$X-04-145$ & JE, AP & $\mathrm{E}$ & 493 seconds $\times 40 \mathrm{~m}$ & $\mathrm{X}-04-145 \_170604 \mathrm{E} 1$ \\
\hline
\end{tabular}


Appendix 4: List of all fishing effort showing the site number, collectors, fishing gear used, amount of fishing effort applied and the fish capture identification (ID) number derived for each occasion where effort was applied.

\begin{tabular}{|c|c|c|c|c|}
\hline Site Number & Collectors & Gear ID & Effort & $\begin{array}{c}\text { Fish Capture ID } \\
\text { (Crew_Date_Gear) }\end{array}$ \\
\hline$X-04-147$ & JE, AP & $E$ & 436 seconds $\times 90 \mathrm{~m}$ & X-04-147_180604_E1 \\
\hline$X-04-149$ & JE, AP & $E$ & 1132 seconds $\times 110 \mathrm{~m}$ & X-04-149_210604_E1 \\
\hline$X-04-149$ & JE, AP & $\mathrm{L}$ & $60 \mathrm{~min}$. & X-04-149_210604_L1 \\
\hline$X-04-150$ & JE, AP & $\mathrm{E}$ & 672 seconds $\times 85 \mathrm{~m}$ & $\mathrm{X}-04-150 \_210604 \mathrm{E} 1$ \\
\hline X-04-151 & JE, AP & $E$ & 688 seconds $\times 65 \mathrm{~m}$ & X-04-151_210604_E1 \\
\hline$X-04-152$ & JE, AP & $\mathrm{B}$ & $6.5 \mathrm{~m} \times 8 \mathrm{~m}$ & $\mathrm{X}-04-152 \_220604$ B1 \\
\hline$X-04-153$ & JE, AP & $E$ & 571 seconds $\times 25 \mathrm{~m}$ & X-04-153_220604_E1 \\
\hline X-04-154 & JE, AP & $E$ & 581 seconds $\times 70 \mathrm{~m}$ & X-04-154_220604_E1 \\
\hline X-04-155 & JE, AP & $E$ & 771 seconds $\times 70 \mathrm{~m}$ & $\mathrm{X}-04-155 \_230604 \mathrm{E} 1$ \\
\hline$X-04-156$ & JE, AP & $E$ & 396 seconds $\times 50 \mathrm{~m}$ & X-04-156_230604_E1 \\
\hline$X-04-157$ & JE, AP & $\mathrm{E}$ & 559 seconds $\times 70 \mathrm{~m}$ & $\mathrm{X}-04-157 \_230604 \mathrm{E} 1$ \\
\hline X-04-159 & JE, AP & $E$ & 65 seconds $\times 8 \mathrm{~m}$ & X-04-159_230604_E1 \\
\hline$X-04-160$ & JE, AP & $\mathrm{E}$ & 242 seconds $\times 25 \mathrm{~m}$ & X-04-160_240604_E1 \\
\hline X-04-161 & JE, AP & $E$ & 435 seconds $\times 100 \mathrm{~m}$ & $\mathrm{X}-04-161 \_240604 \mathrm{E} 1$ \\
\hline$X-04-162$ & JE, AP & $\mathrm{E}$ & 604 seconds $\times 35 \mathrm{~m}$ & $\mathrm{X}-04-162 \_240604 \_\mathrm{E} 1$ \\
\hline$X-04-163$ & JE, AP & $\mathrm{E}$ & 427 seconds $\times 50$ & X-04-163_250604_E1 \\
\hline X-04-164 & JE, AP & $E$ & 907 seconds $\times 90 \mathrm{~m}$ & X-04-164_250604_E1 \\
\hline X-04-165 & JE, AP & $\mathrm{D}$ & $1 \mathrm{~m}^{2}$ & X-04-165_280604_D1 \\
\hline X-04-167 & JE, AP & $E$ & 232 seconds $\times 40 \mathrm{~m}$ & X-04-167_280604_E1 \\
\hline$X-04-168$ & JE, AP & $\mathrm{E}$ & 452 seconds $\times 70 \mathrm{~m}$ & X-04-168_280604_E1 \\
\hline X-04-169 & JE, AP & $E$ & 459 seconds $\times 75 \mathrm{~m}$ & X-04-169_280604_E1 \\
\hline$X-04-170$ & JE, AP & $E$ & 495 seconds $\times 80 \mathrm{~m}$ & X-04-170 290604 E1 \\
\hline $\mathrm{X}-04-171$ & JE, AP & $E$ & 779 seconds $\times 70 \mathrm{~m}$ & X-04-171_290604_E1 \\
\hline $\mathrm{X}-04-172$ & JE, AP & $E$ & 257 seconds $\times 45 \mathrm{~m}$ & $\mathrm{X}-04-172 \_290604 \mathrm{E} 1$ \\
\hline$X-04-175$ & JE, AP & $E$ & 296 seconds $\times 60 \mathrm{~m}$ & X-04-175_290604_E1 \\
\hline $\mathrm{X}-04-176$ & JE, AP & $E$ & 240 seconds $\times 18 \mathrm{~m}$ & X-04-176_300604_E1 \\
\hline $\mathrm{X}-04-177$ & JE, AP & $E$ & 331 seconds $\times 25 \mathrm{~m}$ & X-04-177_300604_E1 \\
\hline$X-04-178$ & JE, AP & $E$ & 347 seconds $\times 30 \mathrm{~m}$ & X-04-178_010704_E1 \\
\hline $\mathrm{X}-04-179$ & JE, AP & $E$ & 222 seconds $\times 10 \mathrm{~m}$ & X-04-179_010704_E1 \\
\hline $\mathrm{X}-04-180$ & JE, AP & $\mathrm{D}$ & $2 \mathrm{~m}^{2}$ & X-04-180_020704_D1 \\
\hline$X-04-181$ & JE, AP & $E$ & 989 seconds $\times 120 \mathrm{~m}$ & X-04-181_020704_E1 \\
\hline
\end{tabular}


Appendix 4: List of all fishing effort showing the site number, collectors, fishing gear used, amount of fishing effort applied and the fish capture identification (ID) number derived for each occasion where effort was applied.

\begin{tabular}{|c|c|c|c|c|}
\hline Site Number & Collectors & Gear ID & Effort & $\begin{array}{c}\text { Fish Capture ID } \\
\text { (Crew_Date_Gear) }\end{array}$ \\
\hline $\mathrm{X}-04-182$ & JE, AP & $E$ & 482 seconds $\times 30 \mathrm{~m}$ & X-04-182_050704_E1 \\
\hline X-04-184 & JE, AP & $E$ & 234 seconds $\times 20 \mathrm{~m}$ & $\mathrm{X}-04-184 \_050704 \mathrm{E} 1$ \\
\hline$X-04-185$ & JE, AP & $E$ & 401 seconds $\times 35 \mathrm{~m}$ & X-04-185_050704_E1 \\
\hline $\mathrm{X}-04-186$ & JE, AP & $E$ & 407 seconds $\times 30 \mathrm{~m}$ & $\mathrm{X}-04-186 \_050704 \mathrm{E} 1$ \\
\hline $\mathrm{X}-04-187$ & JE, AP & $E$ & 389 seconds $\times 17 \mathrm{~m}$ & X-04-187_060704_E1 \\
\hline X-04-188 & JE, AP & $\mathrm{D}$ & $1 \mathrm{~m}^{2}$ & X-04-188_060704_D1 \\
\hline$X-04-190$ & JE, AP & $\mathrm{B}$ & $7 \mathrm{~m} \times 18 \mathrm{~m}$ & X-04-190_060704_B1 \\
\hline $\mathrm{X}-04-192$ & JE, AP & $E$ & 800 seconds $\times 35 \mathrm{~m}$ & X-04-192_060704_E1 \\
\hline X-04-194 & JE, AP & $\mathrm{B}$ & $12 \mathrm{~m} \times 15 \mathrm{~m}$ & X-04-194_070704_B1 \\
\hline X-04-194 & JE, AP & $E$ & 359 seconds $\times 12 \mathrm{~m}$ & X-04-194_070704_E1 \\
\hline X-04-195 & JE, AP & $\mathrm{E}$ & 458 seconds $\times 50 \mathrm{~m}$ & $\mathrm{X}-04-195 \_070704 \mathrm{E} 1$ \\
\hline X-04-196 & JE, AP & $E$ & 275 seconds $\times 35 \mathrm{~m}$ & X-04-196_070704_E1 \\
\hline$X-04-198$ & JE, AP & $\mathrm{E}$ & 445 seconds $\times 30 \mathrm{~m}$ & X-04-198_070704_E1 \\
\hline X-04-199 & JE, AP & $E$ & 485 seconds $\times 50 \mathrm{~m}$ & X-04-199_080704_E1 \\
\hline$X-04-200$ & JE, AP & $\mathrm{E}$ & 273 seconds $\times 15 \mathrm{~m}$ & X-04-200_080704_E1 \\
\hline$X-04-201$ & JE, AP & $E$ & 341 seconds $\times 60 \mathrm{~m}$ & X-04-201_080704_E1 \\
\hline$X-04-202$ & JE, AP & $E$ & 239 seconds $\times 25 \mathrm{~m}$ & X-04-202_080704_E1 \\
\hline$X-04-203$ & JE, AP & $E$ & 658 seconds $\times 40 \mathrm{~m}$ & X-04-203_130704_E1 \\
\hline$X-04-204$ & JE, AP & $E$ & 435 seconds $\times 80 \mathrm{~m}$ & X-04-204_130704_E1 \\
\hline$X-04-205$ & JE, AP & $\mathrm{E}$ & 425 seconds $\times 80 \mathrm{~m}$ & $\mathrm{X}-04-205 \_130704 \mathrm{E} 1$ \\
\hline$X-04-206$ & JE, AP & $E$ & 886 seconds $\times 100 \mathrm{~m}$ & X-04-206_130704_E1 \\
\hline$X-04-207$ & JE, AP & $E$ & 305 seconds $\times 25 \mathrm{~m}$ & $\mathrm{X}-04-207 \quad 130704 \mathrm{E} 1$ \\
\hline$X-04-208$ & JE, AP & $E$ & 781 seconds $\times 90 \mathrm{~m}$ & X-04-208_140704_E1 \\
\hline$X-04-209$ & JE, AP & $E$ & 491 seconds $\times 35 \mathrm{~m}$ & $\mathrm{X}-04-209 \_140704 \mathrm{E} 1$ \\
\hline$X-04-210$ & JE, AP & $E$ & 663 seconds $\times 70 \mathrm{~m}$ & X-04-210_140704_E1 \\
\hline$X-04-211$ & JE, AP & $\mathrm{D}$ & $1 \mathrm{~m}^{2}$ & X-04-211_140704_D1 \\
\hline$X-04-212$ & JE, AP & $E$ & 324 seconds $\times 40 \mathrm{~m}$ & $\mathrm{X}-04-212 \_150704 \_\mathrm{E} 1$ \\
\hline$X-04-213$ & JE, AP & $E$ & 494 seconds $\times 100 \mathrm{~m}$ & X-04-213_150704_E1 \\
\hline$X-04-215$ & JE, AP & $E$ & 1241 seconds $x 180 \mathrm{~m}$ & $\mathrm{X}-04-215 \_150704 \_\mathrm{E} 1$ \\
\hline$X-04-215$ & JE, AP & $\mathrm{O}$ & Observed & $\mathrm{X}-04-215 \_150704 \_\mathrm{O} 1$ \\
\hline$X-04-216$ & JE, AP & $E$ & 499 seconds $\times 75 \mathrm{~m}$ & X-04-216_160704_E1 \\
\hline
\end{tabular}


Appendix 4: List of all fishing effort showing the site number, collectors, fishing gear used, amount of fishing effort applied and the fish capture identification (ID) number derived for each occasion where effort was applied.

\begin{tabular}{|c|c|c|c|c|}
\hline Site Number & Collectors & Gear ID & Effort & $\begin{array}{c}\text { Fish Capture ID } \\
\text { (Crew_Date_Gear) }\end{array}$ \\
\hline$X-04-217$ & JE, AP & $E$ & 186 seconds $\times 20 \mathrm{~m}$ & $\mathrm{X}-04-217 \_160704$ E1 \\
\hline $\mathrm{X}-04-221$ & JE, AP & $\mathrm{E}$ & 558 seconds $\times 35 \mathrm{~m}$ & $\mathrm{X}-04-221 \_160704$ E1 \\
\hline$X-04-222$ & JE, AP & $E$ & 1360 seconds $\times 160 \mathrm{~m}$ & X-04-222_190704_E1 \\
\hline $\mathrm{X}-04-223$ & JE, AP & $E$ & 457 seconds $\times 90 \mathrm{~m}$ & $\mathrm{X}-04-223 \_200704 \mathrm{E} 1$ \\
\hline $\mathrm{X}-04-225$ & JE, AP & $E$ & 490 seconds $\times 85 \mathrm{~m}$ & $\mathrm{X}-04-225 \_200704 \mathrm{E} 1$ \\
\hline $\mathrm{X}-04-226$ & JE, AP & $\mathrm{E}$ & 485 seconds $\times 75 \mathrm{~m}$ & X-04-226_200704_E1 \\
\hline$X-04-228$ & JE, AP & $E$ & 467 seconds $\times 50 \mathrm{~m}$ & X-04-228_210704_E1 \\
\hline $\mathrm{X}-04-230$ & JE, AP & $\mathrm{D}$ & $3 \mathrm{~m}^{2}$ & X-04-230_210704_D1 \\
\hline $\mathrm{X}-04-232$ & JE, AP & $E$ & 267 seconds $\times 25 \mathrm{~m}$ & X-04-232_220704_E1 \\
\hline $\mathrm{X}-04-235$ & JE, AP & $\mathrm{D}$ & $2 \mathrm{~m}^{2}$ & X-04-235_220704_D1 \\
\hline $\mathrm{X}-04-238$ & JE, AP & $E$ & 406 seconds $\times 100 \mathrm{~m}$ & X-04-238_220704_E1 \\
\hline$X-04-239$ & JE, AP & $E$ & 833 seconds $\times 85 \mathrm{~m}$ & X-04-239_220704_E1 \\
\hline$X-04-245$ & JE, AP & $E$ & 333 seconds $\times 50 \mathrm{~m}$ & X-04-245_260704_E1 \\
\hline$X-04-248$ & JE, AP & $E$ & 268 seconds $\times 15 \mathrm{~m}$ & X-04-248_280704_E1 \\
\hline$X-04-249$ & JE, AP & $E$ & 239 seconds $\times 75 \mathrm{~m}$ & X-04-249_280704_E1 \\
\hline$X-04-251$ & JE, AP & $E$ & 305 seconds $\times 45 \mathrm{~m}$ & $\mathrm{X}-04-251 \_280704 \mathrm{E} 1$ \\
\hline $\mathrm{X}-04-253$ & JE, AP & $E$ & 415 seconds $\times 55 \mathrm{~m}$ & $\mathrm{X}-04-253 \_300704 \mathrm{E} 1$ \\
\hline$X-04-255$ & JE, AP & $E$ & 673 seconds $\times 100 \mathrm{~m}$ & X-04-255_300704_E1 \\
\hline$X-04-256$ & JE, AP & $E$ & 661 seconds $\times 40 \mathrm{~m}$ & X-04-256_030804_E1 \\
\hline $\mathrm{X}-04-257$ & JE, AP & $E$ & 716 seconds $\times 60 \mathrm{~m}$ & X-04-257_030804_E1 \\
\hline $\mathrm{X}-04-258$ & JE, AP, DM & $E$ & 1136 seconds $\times 120 \mathrm{~m}$ & $\mathrm{X}-04-258 \_040804 \mathrm{E} 1$ \\
\hline$X-04-259$ & $\mathrm{JE}, \mathrm{AP}, \mathrm{DM}$ & $E$ & 1407 seconds $\times 200 \mathrm{~m}$ & X-04-259_050804_E1 \\
\hline $\mathrm{X}-04-260$ & JE, AP, DM & $E$ & 783 seconds $\times 125 \mathrm{~m}$ & X-04-260_050804_E1 \\
\hline $\mathrm{X}-04-262$ & JE, AP & $E$ & 827 seconds $\times 80 \mathrm{~m}$ & X-04-262_060804_E1 \\
\hline D-05-004 & DM & $\mathrm{O}$ & Observed, pre-spawn & D-05-004_140405_01 \\
\hline D-05-007 & DM & $\mathrm{O}$ & Observed, pre-spawn & D-05-007_140405_01 \\
\hline D-05-008 & DM & $\mathrm{O}$ & Observed, pre-spawn & D-05-008_140405_01 \\
\hline D-05-009 & DM & $\mathrm{O}$ & Observed, pre-spawn & D-05-009_140405_01 \\
\hline D-05-010 & DM & $\mathrm{L}$ & 0.6 hour (40 min.) & D-05-010_010505_L1 \\
\hline D-05-011 & DM, DW, ML & $\mathrm{O}$ & Observed & D-05-011_010605_01 \\
\hline D-05-011 & $\mathrm{DM}, \mathrm{DW}, \mathrm{ML}$ & Z & 1303 seconds & D-05-011_010605_Z1 \\
\hline
\end{tabular}


Appendix 4: List of all fishing effort showing the site number, collectors, fishing gear used, amount of fishing effort applied and the fish capture identification (ID) number derived for each occasion where effort was applied.

\begin{tabular}{|c|c|c|c|c|}
\hline Site Number & Collectors & Gear ID & Effort & $\begin{array}{c}\text { Fish Capture ID } \\
\text { (Crew_Date_Gear) }\end{array}$ \\
\hline D-05-012 & DM, TS & $\mathrm{B}$ & $15 \mathrm{~m} \times 50 \mathrm{~m}$ & D-05-012_090605_B1 \\
\hline D-05-013 & $\mathrm{DM}, \mathrm{AP}$ & $E$ & 494 seconds $\times 40 \mathrm{~m}$ & D-05-013_200705_E1 \\
\hline D-05-014 & $\mathrm{DM}, \mathrm{AP}$ & $\mathrm{B}$ & $5 \mathrm{~m} \times 50 \mathrm{~m}$ & D-05-014_200705_B1 \\
\hline D-05-015 & $\mathrm{DM}, \mathrm{AP}$ & $\mathrm{B}$ & $5 \mathrm{~m} \times 15 \mathrm{~m}$ & D-05-015_200705_B1 \\
\hline D-05-015 & $\mathrm{DM}, \mathrm{AP}$ & $E$ & 535 seconds $\times 80 \mathrm{~m}$ & D-05-015_200705_E1 \\
\hline D-05-017 & $\mathrm{DM}, \mathrm{AP}$ & $\mathrm{D}$ & 5 sweeps & D-05-017_210705_D1 \\
\hline D-05-018 & $\mathrm{DM}, \mathrm{JM}, \mathrm{KK}, \mathrm{KC}$ & $E$ & 501 seconds $\times 50 \mathrm{~m}$ & D-05-018_030805_E1 \\
\hline D-05-019 & $\mathrm{DM}, \mathrm{JM}, \mathrm{KK}, \mathrm{KC}$ & $E$ & 456 seconds $\times 50 \mathrm{~m}$ & D-05-019_030805_E1 \\
\hline D-05-020 & $\mathrm{DM}, \mathrm{JM}, \mathrm{KK}, \mathrm{KC}$ & $\mathrm{E}$ & 585 seconds $\times 75 \mathrm{~m}$ & D-05-020_040805_E1 \\
\hline D-05-021 & $\mathrm{DM}, \mathrm{JM}, \mathrm{KK}, \mathrm{KC}$ & $\mathrm{D}$ & 1 sweep through scour pool & D-05-021_040805_D1 \\
\hline D-05-023 & DM, JC & $\mathrm{E}$ & 442 seconds $\times 60 \mathrm{~m}$ & D-05-023_160805_E1 \\
\hline D-05-025 & DM, RJ & $E$ & 700 seconds $\times 110 \mathrm{~m}$ & D-05-025_170805_E1 \\
\hline D-05-026 & DM, JM & $\mathrm{E}$ & 508 seconds $\times 40 \mathrm{~m}$ & D-05-026_300805_E1 \\
\hline D-05-027 & DM, JM & $E$ & 292 seconds $\times 20 \mathrm{~m}$ & D-05-027_300805_E1 \\
\hline W-05-001 & $\mathrm{JC}, \mathrm{VK}, \mathrm{AP}, \mathrm{AW}$ & $\mathrm{E}$ & 398 seconds $\times 145 \mathrm{~m}$ & W-05-001_020505_E1 \\
\hline W-05-001 & JC, VK, AP, AW & $\mathrm{K}$ & $1 \mathrm{~m}^{2}$ & W-05-001_020505_K1 \\
\hline W-05-001 & JC, VK, AP, AW & $\mathrm{O}$ & Observation & W-05-001_020505_01 \\
\hline W-05-002 & JC, VK, AP, AW & $E$ & 405 seconds $\times 75 \mathrm{~m}$ & W-05-002_020505_E1 \\
\hline W-05-009 & $\mathrm{JC}, \mathrm{VK}, \mathrm{AP}, \mathrm{AW}$ & $E$ & 564 seconds $\times 100 \mathrm{~m}$ & W-05-009_020505E1 \\
\hline W-05-012 & $\mathrm{JC}, \mathrm{VK}, \mathrm{DM}$ & $\mathrm{E}$ & 375 seconds $\times 30 \mathrm{~m}$ & W-05-012_030505_E1 \\
\hline W-05-013 & JC, VK, DM & $E$ & 822 seconds $\times 125 \mathrm{~m}$ & W-05-013_030505_E1 \\
\hline W-05-014 & $\mathrm{JC}, \mathrm{VK}, \mathrm{DM}$ & $E$ & 412 seconds $\times 125 \mathrm{~m}$ & W-05-014 $030505 \mathrm{E} 1$ \\
\hline W-05-016 & JC, VK & $E$ & 145 seconds $\times 80 \mathrm{~m}$ & W-05-016_030505_E1 \\
\hline W-05-016 & $\mathrm{JC}, \mathrm{VK}$ & $\mathrm{O}$ & Observed in deep pool & W-05-016_030505_01 \\
\hline W-05-017 & JC, VK & $E$ & 403 seconds $\times 65 \mathrm{~m}$ & W-05-017_040505_E1 \\
\hline W-05-019 & JC, VK & $E$ & 320 seconds $\times 100 \mathrm{~m}$ & W-05-019_040505_E1 \\
\hline W-05-020 & JC, VK & $E$ & 422 seconds $\times 60 \mathrm{~m}$ & W-05-020_040505_E1 \\
\hline W-05-023 & JC, VK & $E$ & 293 seconds $\times 60 \mathrm{~m}$ & W-05-023_050505_E1 \\
\hline W-05-024 & JC, VK & $E$ & 398 seconds $\times 60 \mathrm{~m}$ & W-05-024_050505_E1 \\
\hline W-05-024 & JC, VK & $\mathrm{O}$ & Observed & W-05-024_050505_01 \\
\hline W-05-027 & JC, VK & $E$ & 300 seconds $\times 80 \mathrm{~m}$ & W-05-027_050505_E1 \\
\hline
\end{tabular}


Appendix 4: List of all fishing effort showing the site number, collectors, fishing gear used, amount of fishing effort applied and the fish capture identification (ID) number derived for each occasion where effort was applied.

\begin{tabular}{|c|c|c|c|c|}
\hline Site Number & Collectors & Gear ID & Effort & $\begin{array}{c}\text { Fish Capture ID } \\
\text { (Crew_Date_Gear) }\end{array}$ \\
\hline W-05-028 & JC, VK & $E$ & 272 seconds $\times 70 \mathrm{~m}$ & W-05-028_050505_E1 \\
\hline W-05-030 & JC, VK & $E$ & 183 seconds $\times 30 \mathrm{~m}$ & W-05-030_050505_E1 \\
\hline W-05-034 & JC, VK & $E$ & 363 seconds $\times 70 \mathrm{~m}$ & W-05-034_060505_E1 \\
\hline W-05-034 & JC, VK & $\mathrm{O}$ & Observed & W-05-034_060505_01 \\
\hline W-05-036 & JC, VK & $\mathrm{E}$ & 291 seconds $\times 70 \mathrm{~m}$ & W-05-036_090505_E1 \\
\hline W-05-037 & JC, VK & $E$ & 248 seconds $\times 60 \mathrm{~m}$ & W-05-037_090505E1 \\
\hline W-05-038 & JC, VK & $E$ & 187 seconds $\times 70 \mathrm{~m}$ & W-05-038_090505E1 \\
\hline W-05-041 & JC, VK & $\mathrm{E}$ & 512 seconds $\times 90 \mathrm{~m}$ & W-05-041_100505_E1 \\
\hline W-05-043 & JC, VK & $E$ & 302 seconds $\times 100 \mathrm{~m}$ & W-05-043_100505_E1 \\
\hline W-05-045 & JC, VK & $E$ & 198 seconds $\times 60 \mathrm{~m}$ & W-05-045_100505_E1 \\
\hline W-05-047 & JC, VK & $\mathrm{E}$ & 193 seconds $\times 60 \mathrm{~m}$ & W-05-047_110505E1 \\
\hline W-05-048 & JC, VK & $\mathrm{E}$ & 192 seconds $\times 60 \mathrm{~m}$ & W-05-048_110505_E1 \\
\hline W-05-049 & JC, VK & $E$ & 357 seconds $\times 75 \mathrm{~m}$ & W-05-049_110505E1 \\
\hline W-05-050 & JC, VK & $E$ & 395 seconds $\times 70 \mathrm{~m}$ & W-05-050_110505_E1 \\
\hline W-05-051 & JC, VK & $E$ & 346 seconds $\times 60 \mathrm{~m}$ & W-05-051_120505_E1 \\
\hline W-05-052 & JC, VK & $E$ & 397 seconds $\times 70 \mathrm{~m}$ & W-05-052_120505_E1 \\
\hline W-05-053 & JC, VK & $E$ & 438 seconds $\times 80 \mathrm{~m}$ & W-05-053_120505_E1 \\
\hline W-05-054 & JC, VK & $E$ & 222 seconds $\times 60 \mathrm{~m}$ & W-05-054_120505_E1 \\
\hline W-05-055 & JC, VK & $E$ & 720 seconds $\times 200 \mathrm{~m}$ & W-05-055_160505E1 \\
\hline W-05-055 & JC, VK & $\mathrm{L}$ & $1 \mathrm{hr}$ & W-05-055_160505_L1 \\
\hline W-05-056 & JC, VK & $\mathrm{E}$ & 806 seconds $\times 200 \mathrm{~m}$ & W-05-056_160505_E1 \\
\hline W-05-057 & JC, VK & $E$ & 473 seconds $\times 100 \mathrm{~m}$ & W-05-057 $170505 \mathrm{E} 1$ \\
\hline W-05-058 & JC, VK & $E$ & 317 seconds $\times 75 \mathrm{~m}$ & W-05-058_170505_E1 \\
\hline W-05-058 & JC, VK & $\mathrm{K}$ & $1 \mathrm{~m}^{2}$ & W-05-058_170505_K1 \\
\hline W-05-059 & JC, VK & $E$ & 334 seconds $\times 40 \mathrm{~m}$ & W-05-059_170505_E1 \\
\hline W-05-060 & JC, VK & $E$ & 245 seconds $\times 30 \mathrm{~m}$ & W-05-060_170505_E1 \\
\hline W-05-062 & JC, VK & $\mathrm{B}$ & $3.5 \mathrm{~m} \times 6 \mathrm{~m}$ & W-05-062_180505_B1 \\
\hline W-05-063 & JC, VK & $E$ & 273 seconds $\times 20 \mathrm{~m}$ & W-05-063_180505E1 \\
\hline W-05-065 & JC, VK & $E$ & 377 seconds $\times 70 \mathrm{~m}$ & W-05-065 $180505 \mathrm{E} 1$ \\
\hline W-05-066 & JC, VK & $E$ & 247 seconds $\times 40 \mathrm{~m}$ & W-05-066_180505_E1 \\
\hline W-05-069 & $\mathrm{JC}, \mathrm{VK}$ & $\mathrm{E}$ & 150 seconds $\times 30 \mathrm{~m}$ & W-05-069_190505_E1 \\
\hline
\end{tabular}


Appendix 4: List of all fishing effort showing the site number, collectors, fishing gear used, amount of fishing effort applied and the fish capture identification (ID) number derived for each occasion where effort was applied.

\begin{tabular}{|c|c|c|c|c|}
\hline Site Number & Collectors & Gear ID & Effort & $\begin{array}{c}\text { Fish Capture ID } \\
\text { (Crew_Date_Gear) }\end{array}$ \\
\hline W-05-071 & JC, VK & $E$ & 323 seconds $\times 120 \mathrm{~m}$ & W-05-071_200505_E1 \\
\hline W-05-072 & JC, VK & $E$ & 273 seconds $\times 80 \mathrm{~m}$ & W-05-072_200505_E1 \\
\hline W-05-073 & JC, VK & $E$ & 327 seconds $\times 80 \mathrm{~m}$ & W-05-073_240505_E1 \\
\hline W-05-076 & JC, VK & $E$ & 700 seconds $\times 50 \mathrm{~m}$ & W-05-076_240505_E1 \\
\hline W-05-077 & JC, VK & $\mathrm{D}$ & 10 sweeps & W-05-077_240505_D1 \\
\hline W-05-077 & JC, VK & $E$ & 335 seconds $\times 100 \mathrm{~m}$ & W-05-077_240505_E1 \\
\hline W-05-078 & JC, VK & $\mathrm{B}$ & $5 \mathrm{~m} \times 7 \mathrm{~m}$ & W-05-078_250505_B1 \\
\hline W-05-079 & JC, VK & $E$ & 442 seconds $\times 100 \mathrm{~m}$ & W-05-079_250505_E1 \\
\hline W-05-081 & JC, VK & $E$ & 490 seconds $\times 100 \mathrm{~m}$ & W-05-081_250505_E1 \\
\hline W-05-082 & JC, VK & $E$ & 245 seconds $\times 80 \mathrm{~m}$ & W-05-082_250505_E1 \\
\hline W-05-083 & JC, VK & $\mathrm{E}$ & 526 seconds $\times 100 \mathrm{~m}$ & W-05-083_260505_E1 \\
\hline W-05-085 & JC, VK & $\mathrm{E}$ & 482 seconds $\times 130 \mathrm{~m}$ & W-05-085_260505_E1 \\
\hline W-05-086 & JC, VK & $\mathrm{E}$ & 187 seconds $\times 50 \mathrm{~m}$ & W-05-086_260505_E1 \\
\hline W-05-087 & JC, VK & $E$ & 230 seconds $\times 70 \mathrm{~m}$ & W-05-087_260505_E1 \\
\hline W-05-093 & JC, VK & $\mathrm{E}$ & 289 seconds $\times 80 \mathrm{~m}$ & W-05-093_290505_E1 \\
\hline W-05-094 & JC, VK & $E$ & 394 seconds $\times 80 \mathrm{~m}$ & W-05-094_310505_E1 \\
\hline W-05-096 & JC, VK & $E$ & 174 seconds $\times 45 \mathrm{~m}$ & W-05-096_310505_E1 \\
\hline W-05-099 & JC, VK & $E$ & 243 seconds $\times 80 \mathrm{~m}$ & W-05-099_310505_E1 \\
\hline W-05-101 & JC, VK & $E$ & 150 seconds $\times 30 \mathrm{~m}$ & W-05-101_010605_E1 \\
\hline W-05-102 & JC, VK & $\mathrm{E}$ & 1053 seconds $\times 275 \mathrm{~m}$ & W-05-102_010605_E1 \\
\hline W-05-103 & JC, VK & $E$ & 447 seconds $\times 125 \mathrm{~m}$ & W-05-103_010605_E1 \\
\hline W-05-104 & JC, VK & $E$ & 953 seconds $\times 230 \mathrm{~m}$ & W-05-104 $010605 \mathrm{E} 1$ \\
\hline W-05-105 & JC, VK & $E$ & 603 seconds $\times 100 \mathrm{~m}$ & W-05-105_010605_E1 \\
\hline W-05-106 & JC, VK & $E$ & 284 seconds $\times 80 \mathrm{~m}$ & W-05-106_020605_E1 \\
\hline W-05-110 & JC, VK & $E$ & 414 seconds $\times 80 \mathrm{~m}$ & W-05-110_040605_E1 \\
\hline W-05-112 & JC, VK & $E$ & 565 seconds $\times 100 \mathrm{~m}$ & W-05-112_040605_E1 \\
\hline W-05-113 & JC, VK & $E$ & 539 seconds $\times 110 \mathrm{~m}$ & W-05-113_060605_E1 \\
\hline W-05-114 & JC, VK & $E$ & 189 seconds $\times 50 \mathrm{~m}$ & W-05-114_060605_E1 \\
\hline W-05-115 & JC, VK & $E$ & 384 seconds $\times 100 \mathrm{~m}$ & W-05-115 $070605 \mathrm{E} 1$ \\
\hline W-05-116 & JC, VK & $E$ & 424 seconds $\times 100 \mathrm{~m}$ & W-05-116_070605_E1 \\
\hline W-05-117 & $\mathrm{JC}, \mathrm{VK}$ & $\mathrm{E}$ & 494 seconds $\times 120 \mathrm{~m}$ & W-05-117_070605_E1 \\
\hline
\end{tabular}


Appendix 4: List of all fishing effort showing the site number, collectors, fishing gear used, amount of fishing effort applied and the fish capture identification (ID) number derived for each occasion where effort was applied.

\begin{tabular}{|c|c|c|c|c|}
\hline Site Number & Collectors & Gear ID & Effort & $\begin{array}{c}\text { Fish Capture ID } \\
\text { (Crew_Date_Gear) }\end{array}$ \\
\hline W-05-118 & JC, VK & $E$ & 393 seconds $\times 100 \mathrm{~m}$ & W-05-118_080605_E1 \\
\hline W-05-119 & JC, VK & $E$ & 384 seconds $\times 100 \mathrm{~m}$ & W-05-119_080605_E1 \\
\hline W-05-120 & JC, VK & $E$ & 174 seconds $\times 80 \mathrm{~m}$ & W-05-120_090605_E1 \\
\hline W-05-121 & JC, VK & $\mathrm{E}$ & 150 seconds $\times 30 \mathrm{~m}$ & W-05-121_090605_E1 \\
\hline W-05-122 & JC, VK & $\mathrm{E}$ & 384 seconds $\times 130 \mathrm{~m}$ & W-05-122_090605_E1 \\
\hline W-05-126 & JC, VK & $E$ & 228 seconds $\times 50 \mathrm{~m}$ & W-05-126_140605_E1 \\
\hline W-05-128 & JC, VK & $E$ & 150 seconds $\times 30 \mathrm{~m}$ & W-05-128_140605_E1 \\
\hline W-05-129 & JC, VK & $\mathrm{E}$ & 150 seconds $\times 30 \mathrm{~m}$ & W-05-129_150605_E1 \\
\hline W-05-130 & JC, VK & $E$ & 150 seconds $\times 30 \mathrm{~m}$ & W-05-130_150605_E1 \\
\hline W-05-130 & JC, VK & $\mathrm{O}$ & Observed & W-05-130_150605_01 \\
\hline W-05-131 & JC, VK & $E$ & 494 seconds $\times 120 \mathrm{~m}$ & W-05-131_150605_E1 \\
\hline W-05-132 & JC, VK & $E$ & 369 seconds $\times 100 \mathrm{~m}$ & W-05-132_150605_E1 \\
\hline W-05-134 & JC, VK & $E$ & 271 seconds $\times 100 \mathrm{~m}$ & W-05-134_150605 E1 \\
\hline W-05-135 & JC, VK & $\mathrm{O}$ & Observed & W-05-135_160605_01 \\
\hline W-05-136 & JC, VK & $E$ & 95 seconds $\times 12 \mathrm{~m}$ & W-05-136_160605_E1 \\
\hline W-05-137 & $\mathrm{JC}, \mathrm{VK}$ & $\mathrm{E}$ & 95 seconds $\times 20 \mathrm{~m}$ & W-05-137_160605_E1 \\
\hline W-05-138 & JC, VK & $E$ & 103 seconds $\times 25 \mathrm{~m}$ & W-05-138_160605_E1 \\
\hline W-05-139 & JC, VK & $E$ & 376 seconds $\times 85 \mathrm{~m}$ & W-05-139_170605_E1 \\
\hline W-05-140 & JC, VK & $E$ & 213 seconds $\times 80 \mathrm{~m}$ & W-05-140_170605_E1 \\
\hline W-05-141 & JC, VK & $\mathrm{E}$ & 223 seconds $\times 100 \mathrm{~m}$ & W-05-141_200605_E1 \\
\hline W-05-145 & JC, VK & $\mathrm{E}$ & 159 seconds $\times 40 \mathrm{~m}$ & W-05-145_200605_E1 \\
\hline W-05-146 & JC, VK & $E$ & 257 seconds $\times 100 \mathrm{~m}$ & W-05-146 $210605 \mathrm{E} 1$ \\
\hline W-05-148 & JC, VK & $E$ & 294 seconds $\times 75 \mathrm{~m}$ & W-05-148_210605_E1 \\
\hline W-05-149 & JC, VK & $\mathrm{E}$ & 376 seconds $\times 150 \mathrm{~m}$ & W-05-149_220605_E1 \\
\hline W-05-150 & JC, VK & $E$ & 324 seconds $\times 110 \mathrm{~m}$ & W-05-150_220605_E1 \\
\hline W-05-151 & JC, VK & $E$ & 293 seconds $\times 200 \mathrm{~m}$ & W-05-151_220605_E1 \\
\hline W-05-152 & JC, VK & $E$ & 449 seconds $\times 200 \mathrm{~m}$ & W-05-152_220605_E1 \\
\hline W-05-154 & JC, VK & $E$ & 219 seconds $\times 50 \mathrm{~m}$ & W-05-154_220605_E1 \\
\hline W-05-157 & JC, VK & $E$ & 261 seconds $\times 100 \mathrm{~m}$ & W-05-157 $230605 \mathrm{E} 1$ \\
\hline W-05-159 & JC, VK & $E$ & 254 seconds $\times 45 \mathrm{~m}$ & W-05-159_230605_E1 \\
\hline W-05-160 & $\mathrm{JC}, \mathrm{VK}$ & $\mathrm{E}$ & 258 seconds $\times 50 \mathrm{~m}$ & W-05-160_230605_E1 \\
\hline
\end{tabular}


Appendix 4: List of all fishing effort showing the site number, collectors, fishing gear used, amount of fishing effort applied and the fish capture identification (ID) number derived for each occasion where effort was applied.

\begin{tabular}{|c|c|c|c|c|}
\hline Site Number & Collectors & Gear ID & Effort & $\begin{array}{c}\text { Fish Capture ID } \\
\text { (Crew_Date_Gear) }\end{array}$ \\
\hline W-05-161 & JC, VK & $E$ & 235 seconds $\times 80 \mathrm{~m}$ & W-05-161_230605_E1 \\
\hline W-05-162 & JC, VK & $E$ & 219 seconds $\times 100 \mathrm{~m}$ & W-05-162_230605_E1 \\
\hline W-05-163 & JC, VK & $E$ & 261 seconds $\times 100 \mathrm{~m}$ & W-05-163_240605_E1 \\
\hline W-05-164 & JC, VK & $E$ & 569 seconds $\times 200 \mathrm{~m}$ & W-05-164_270605_E1 \\
\hline W-05-165 & JC, VK & $E$ & 381 seconds $\times 150 \mathrm{~m}$ & W-05-165_270605_E1 \\
\hline W-05-166 & JC, VK & $E$ & 129 seconds $\times 30 \mathrm{~m}$ & W-05-166_270605_E1 \\
\hline W-05-167 & JC, VK & $E$ & 591 seconds $\times 110 \mathrm{~m}$ & W-05-167_280605_E1 \\
\hline W-05-168 & VK, LT, DM & $E$ & 335 seconds $\times 40 \mathrm{~m}$ & W-05-168 110705 E1 \\
\hline W-05-169 & $\mathrm{VK}, \mathrm{LT}, \mathrm{AW}, \mathrm{AP}, \mathrm{DM}$ & $E$ & 667 seconds $\times 100 \mathrm{~m}$ & W-05-169_120705_E1 \\
\hline W-05-170 & $\mathrm{VK}, \mathrm{LT}, \mathrm{AW}, \mathrm{AP}, \mathrm{DM}$ & $\mathrm{D}$ & $50 \times 0.3 \mathrm{~m}$ & W-05-170_120705_D1 \\
\hline W-05-171 & VK, LT, AW, AP, DM & $E$ & 286 seconds $\times 85 \mathrm{~m}$ & W-05-171_120705_E1 \\
\hline W-05-173 & VK, LT, AW, AP, DM & $E$ & 253 seconds $\times 20 \mathrm{~m}$ & W-05-173_120705_E1 \\
\hline W-05-176 & JC, VK & $E$ & 260 seconds $\times 80 \mathrm{~m}$ & W-05-176_180705_E1 \\
\hline W-05-176 & JC, VK & $\mathrm{O}$ & Observed & W-05-176_180705_01 \\
\hline W-05-177 & JC, VK & $E$ & 708 seconds $\times 80 \mathrm{~m}$ & W-05-177_190705_E1 \\
\hline W-05-178 & JC, VK & $E$ & 638 seconds $\times 150 \mathrm{~m}$ & W-05-178_190705_E1 \\
\hline W-05-180 & JC, VK & $E$ & 450 seconds $\times 60 \mathrm{~m}$ & W-05-180_190705_E1 \\
\hline W-05-181 & JC, VK & $E$ & 609 seconds $\times 100 \mathrm{~m}$ & W-05-181_190705_E1 \\
\hline W-05-182 & JC, VK & $E$ & 868 seconds $\times 200 \mathrm{~m}$ & W-05-182_190705_E1 \\
\hline W-05-183 & JC, VK & $E$ & 416 seconds $\times 80 \mathrm{~m}$ & W-05-183_190705_E1 \\
\hline W-05-185 & JC, VK & $E$ & 416 seconds $\times 50 \mathrm{~m}$ & W-05-185_200705_E1 \\
\hline W-05-187 & JC, AP & $E$ & 60 seconds $\times 7 \mathrm{~m}$ & W-05-187_250705_E1 \\
\hline W-05-188 & JC, AP & $E$ & 531 seconds $\times 40 \mathrm{~m}$ & W-05-188_250705_E1 \\
\hline W-05-189 & JC, AP & $\mathrm{B}$ & $6 \mathrm{~m} \times 10 \mathrm{~m}$ & W-05-189 260705 B1 \\
\hline W-05-190 & JC, AP & $E$ & 362 seconds $\times 20 \mathrm{~m}$ & W-05-190_260705_E1 \\
\hline W-05-190 & JC, AP & $\mathrm{O}$ & Observed & W-05-190_260705_01 \\
\hline W-05-191 & JC, AP & $E$ & 849 seconds $\times 50 \mathrm{~m}$ & W-05-191_260705_E1 \\
\hline W-05-192 & JC, AP & $E$ & 757 seconds $\times 60 \mathrm{~m}$ & W-05-192_260705_E1 \\
\hline W-05-193 & JC, AP & $E$ & 553 seconds $\times 30 \mathrm{~m}$ & W-05-193_260705_E1 \\
\hline W-05-194 & JC, AP & $E$ & 290 seconds $\times 40 \mathrm{~m}$ & W-05-194_270705_E1 \\
\hline W-05-196 & JC, AP & $E$ & 200 seconds $\times 40 \mathrm{~m}$ & W-05-196_270705_E1 \\
\hline
\end{tabular}


Appendix 4: List of all fishing effort showing the site number, collectors, fishing gear used, amount of fishing effort applied and the fish capture identification (ID) number derived for each occasion where effort was applied.

\begin{tabular}{|c|c|c|c|c|}
\hline Site Number & Collectors & Gear ID & Effort & $\begin{array}{c}\text { Fish Capture ID } \\
\text { (Crew_Date_Gear) }\end{array}$ \\
\hline W-05-200 & JC, VK & $E$ & 310 seconds $\times 25 \mathrm{~m}$ & W-05-200_020805_E1 \\
\hline W-05-201 & JC, VK & $E$ & 406 seconds $\times 80 \mathrm{~m}$ & W-05-201_020805_E1 \\
\hline W-05-202 & JC, VK & $E$ & 273 seconds $\times 70 \mathrm{~m}$ & W-05-202_020805_E1 \\
\hline W-05-203 & JC, VK & $E$ & 424 seconds $\times 60 \mathrm{~m}$ & W-05-203_030805_E1 \\
\hline W-05-203 & JC, VK & $\mathrm{O}$ & Observed & W-05-203_030805_01 \\
\hline W-05-205 & JC, VK & $E$ & 389 seconds $\times 80 \mathrm{~m}$ & W-05-205_030805E1 \\
\hline W-05-207 & JC, VK & $E$ & 412 seconds $\times 35 \mathrm{~m}$ & W-05-207_080805E1 \\
\hline W-05-208 & JC, VK & $E$ & 354 seconds $\times 20 \mathrm{~m}$ & W-05-208_100805_E1 \\
\hline W-05-209 & JC, VK, MK & $E$ & 290 seconds $\times 30 \mathrm{~m}$ & W-05-209_100805_E1 \\
\hline$X-05-001$ & $A P, A W, J C$ & $\mathrm{~B}$ & $2 \mathrm{~m} \times 10 \mathrm{~m}$ & X-05-001_270405_B1 \\
\hline$X-05-001$ & $\mathrm{AP}, \mathrm{AW}, \mathrm{JC}$ & $\mathrm{K}$ & $1 \mathrm{~m}^{2}$ & $\mathrm{X}-05-001 \_270405 \mathrm{~K} 1$ \\
\hline $\mathrm{X}-05-002$ & $A P, A W, J C$ & $\mathrm{~B}$ & $5 \mathrm{~m} \times 6 \mathrm{~m}$ & $\mathrm{X}-05-002 \_270405$ B1 \\
\hline$X-05-003$ & $\mathrm{AP}, \mathrm{AW}, \mathrm{JC}, \mathrm{VK}$ & $E$ & 633 seconds $\times 100 \mathrm{~m}$ & X-05-003_290405_E1 \\
\hline $\mathrm{X}-05-005$ & $\mathrm{AP}, \mathrm{AW}, \mathrm{JC}, \mathrm{VK}$ & $E$ & 478 seconds $\times 80 \mathrm{~m}$ & X-05-005_290405_E1 \\
\hline $\mathrm{X}-05-007$ & $\mathrm{AP}, \mathrm{AW}, \mathrm{VK}$ & $\mathrm{E}$ & 328 seconds $\times 120 \mathrm{~m}$ & X-05-007_290405_E1 \\
\hline$X-05-007$ & $\mathrm{AP}, \mathrm{AW}, \mathrm{VK}$ & $\mathrm{O}$ & Observed & X-05-007_290405_01 \\
\hline $\mathrm{X}-05-011$ & AW, AP & $\mathrm{B}$ & $14 \mathrm{~m} \times 10 \mathrm{~m}$ & X-05-011_030505_B1 \\
\hline $\mathrm{X}-05-012$ & $\mathrm{AW}, \mathrm{AP}$ & $\mathrm{B}$ & $12 \mathrm{~m} \times 2 \mathrm{~m}$ & X-05-012_030505_B1 \\
\hline$X-05-015$ & $\mathrm{AW}, \mathrm{AP}$ & $E$ & 217 seconds $\times 15 \mathrm{~m}$ & X-05-015_030505_E1 \\
\hline $\mathrm{X}-05-018$ & $\mathrm{AW}, \mathrm{AP}$ & $\mathrm{E}$ & 299 seconds $\times 100 \mathrm{~m}$ & X-05-018_040505_E1 \\
\hline $\mathrm{X}-05-018$ & $\mathrm{AW}, \mathrm{AP}$ & $\mathrm{O}$ & Observed in culvert & X-05-018_040505_01 \\
\hline$X-05-020$ & $\mathrm{AW}, \mathrm{AP}$ & $E$ & 425 seconds $\times 100 \mathrm{~m}$ & $\mathrm{X}-05-020040505 \mathrm{E} 1$ \\
\hline $\mathrm{X}-05-021$ & $\mathrm{AW}, \mathrm{AP}$ & $\mathrm{E}$ & 276 seconds $\times 100 \mathrm{~m}$ & X-05-021_040505_E1 \\
\hline $\mathrm{X}-05-021$ & $\mathrm{AW}, \mathrm{AP}$ & $\mathrm{O}$ & Observation upstream of barrier & X-05-021_040505_01 \\
\hline$X-05-022$ & $A W, A P$ & $\mathrm{~B}$ & $8 \mathrm{~m} \times 30 \mathrm{~m}$ & X-05-022_040505_B1 \\
\hline $\mathrm{X}-05-022$ & $\mathrm{AW}, \mathrm{AP}$ & $E$ & 112 seconds $\times 15 \mathrm{~m}$ & X-05-022_040505_E1 \\
\hline $\mathrm{X}-05-023$ & $\mathrm{AW}, \mathrm{AP}$ & $E$ & 252 seconds $\times 20 \mathrm{~m}$ & X-05-023_040505_E1 \\
\hline$X-05-023$ & $\mathrm{AW}, \mathrm{AP}$ & $\mathrm{K}$ & $1 \mathrm{~m}^{2}$ & $\mathrm{X}-05-023 \_040505 \mathrm{~K} 1$ \\
\hline$X-05-024$ & $\mathrm{AW}, \mathrm{AP}$ & $\mathrm{E}$ & 672 seconds $\times 100 \mathrm{~m}$ & $\mathrm{X}-05-024050505 \mathrm{E} 1$ \\
\hline$X-05-024$ & $\mathrm{AW}, \mathrm{AP}$ & $\mathrm{K}$ & $1 \mathrm{~m}^{2}$ & X-05-024_050505_K1 \\
\hline$X-05-024$ & $\mathrm{AW}, \mathrm{AP}$ & $\mathrm{O}$ & Observed on riffle & X-05-024_050505_01 \\
\hline
\end{tabular}


Appendix 4: List of all fishing effort showing the site number, collectors, fishing gear used, amount of fishing effort applied and the fish capture identification (ID) number derived for each occasion where effort was applied.

\begin{tabular}{|c|c|c|c|c|}
\hline Site Number & Collectors & Gear ID & Effort & $\begin{array}{c}\text { Fish Capture ID } \\
\text { (Crew_Date_Gear) }\end{array}$ \\
\hline $\mathrm{X}-05-026$ & $A W, A P$ & $E$ & 393 seconds $\times 100 \mathrm{~m}$ & X-05-026_050505_E1 \\
\hline $\mathrm{X}-05-027$ & $\mathrm{AW}, \mathrm{AP}$ & $E$ & 393 seconds $\times 100 \mathrm{~m}$ & $\mathrm{X}-05-027060505 \mathrm{E} 1$ \\
\hline$X-05-028$ & $\mathrm{AW}, \mathrm{AP}$ & $E$ & 230 seconds $\times 75$ & X-05-028_060505_E1 \\
\hline$X-05-029$ & $\mathrm{AW}, \mathrm{AP}$ & $\mathrm{E}$ & 663 seconds $\times 120 \mathrm{~m}$ & X-05-029_060505_E1 \\
\hline $\mathrm{X}-05-030$ & $A W, A P$ & $\mathrm{E}$ & 396 seconds $\times 100 \mathrm{~m}$ & X-05-030_100505_E1 \\
\hline $\mathrm{X}-05-031$ & $\mathrm{AW}, \mathrm{AP}$ & $E$ & 420 seconds $\times 70 \mathrm{~m}$ & X-05-031_100505_E1 \\
\hline$X-05-032$ & $\mathrm{AW}, \mathrm{AP}$ & $E$ & 327 seconds $\times 60 \mathrm{~m}$ & X-05-032_110505E1 \\
\hline $\mathrm{X}-05-033$ & $\mathrm{AW}, \mathrm{AP}$ & $E$ & 250 seconds $\times 60 \mathrm{~m}$ & X-05-033_110505_E1 \\
\hline $\mathrm{X}-05-034$ & $\mathrm{AW}, \mathrm{AP}$ & $E$ & 500 seconds $\times 60 \mathrm{~m}$ & $\mathrm{X}-05-034 \_110505 \mathrm{E} 1$ \\
\hline$X-05-035$ & $\mathrm{AW}, \mathrm{AP}$ & $E$ & 349 seconds $\times 100 \mathrm{~m}$ & $\mathrm{X}-05-035 \_110505 \mathrm{E} 1$ \\
\hline $\mathrm{X}-05-036$ & $\mathrm{AW}, \mathrm{AP}$ & $\mathrm{E}$ & 533 seconds $\times 90 \mathrm{~m}$ & $\mathrm{X}-05-036 \_110505 \mathrm{E} 1$ \\
\hline $\mathrm{X}-05-036$ & $\mathrm{AW}, \mathrm{AP}$ & $\mathrm{O}$ & Dead on banks & X-05-036_110505_01 \\
\hline$X-05-037$ & $A W, A P$ & $E$ & 649 seconds $\times 150 \mathrm{~m}$ & X-05-037_120505_E1 \\
\hline $\mathrm{X}-05-037$ & $\mathrm{AW}, \mathrm{AP}$ & $\mathrm{K}$ & $3 \mathrm{~m}^{2}$ & $\mathrm{X}-05-037 \quad 120505 \mathrm{~K} 1$ \\
\hline $\mathrm{X}-05-038$ & $\mathrm{AW}, \mathrm{AP}$ & $E$ & 569 seconds $\times 110 \mathrm{~m}$ & $\mathrm{X}-05-038 \_120505 \_\mathrm{E} 1$ \\
\hline$X-05-040$ & $\mathrm{AW}, \mathrm{AP}$ & $E$ & 218 seconds $\times 75 \mathrm{~m}$ & $\mathrm{X}-05-040 \_120505 \mathrm{E} 1$ \\
\hline $\mathrm{X}-05-042$ & $\mathrm{AW}, \mathrm{AP}$ & $E$ & 423 seconds $\times 80 \mathrm{~m}$ & X-05-042_120505_E1 \\
\hline $\mathrm{X}-05-044$ & $\mathrm{AW}, \mathrm{AP}$ & $E$ & 259 seconds $\times 70 \mathrm{~m}$ & X-05-044_130505_E1 \\
\hline$X-05-045$ & $A W, A P$ & $E$ & 649 seconds $\times 100 \mathrm{~m}$ & X-05-045_130505E1 \\
\hline$X-05-046$ & $\mathrm{AW}, \mathrm{AP}$ & $\mathrm{B}$ & $8 \mathrm{~m} \times 6 \mathrm{~m}$ & $\mathrm{X}-05-046 \_130505 \_\mathrm{B} 1$ \\
\hline $\mathrm{X}-05-046$ & $\mathrm{AW}, \mathrm{AP}$ & $E$ & 449 seconds $\times 80 \mathrm{~m}$ & X-05-046_130505_E1 \\
\hline$X-05-048$ & $\mathrm{AW}, \mathrm{AP}$ & $E$ & 803 seconds $\times 150 \mathrm{~m}$ & X-05-048 140505 E1 \\
\hline $\mathrm{X}-05-051$ & $\mathrm{AW}, \mathrm{AP}$ & $E$ & 301 seconds $\times 80 \mathrm{~m}$ & X-05-051_140505_E1 \\
\hline $\mathrm{X}-05-051$ & $\mathrm{AW}, \mathrm{AP}$ & $\mathrm{K}$ & $2 \mathrm{~m}^{2}$ & $\mathrm{X}-05-051 \_140505 \_\mathrm{K} 1$ \\
\hline$X-05-052$ & $A W, A P$ & $E$ & 343 seconds $\times 75 \mathrm{~m}$ & X-05-052_140505_E1 \\
\hline X-05-054 & $\mathrm{AW}, \mathrm{AP}$ & $E$ & 525 seconds $\times 120 \mathrm{~m}$ & $\mathrm{X}-05-054 \_150505 \mathrm{E} 1$ \\
\hline $\mathrm{X}-05-055$ & $\mathrm{AW}, \mathrm{AP}$ & $E$ & 370 seconds $\times 80 \mathrm{~m}$ & X-05-055_150505_E1 \\
\hline$X-05-057$ & $\mathrm{AW}, \mathrm{AP}$ & $E$ & 335 seconds $\times 80 \mathrm{~m}$ & X-05-057_150505_E1 \\
\hline $\mathrm{X}-05-060$ & $\mathrm{AW}, \mathrm{AP}$ & $E$ & 201 seconds $\times 30 \mathrm{~m}$ & X-05-060_160505_E1 \\
\hline $\mathrm{X}-05-061$ & $\mathrm{AW}, \mathrm{AP}$ & $E$ & 490 seconds $\times 90 \mathrm{~m}$ & X-05-061_160505_E1 \\
\hline $\mathrm{X}-05-062$ & $A W, A P$ & $\mathrm{E}$ & 436 seconds $\times 100 \mathrm{~m}$ & X-05-062_160505_E1 \\
\hline
\end{tabular}


Appendix 4: List of all fishing effort showing the site number, collectors, fishing gear used, amount of fishing effort applied and the fish capture identification (ID) number derived for each occasion where effort was applied.

\begin{tabular}{|c|c|c|c|c|}
\hline Site Number & Collectors & Gear ID & Effort & $\begin{array}{c}\text { Fish Capture ID } \\
\text { (Crew_Date_Gear) }\end{array}$ \\
\hline $\mathrm{X}-05-062$ & $A W, A P$ & $\mathrm{~K}$ & $3 \mathrm{~m}^{2}$ & X-05-062_160505_K1 \\
\hline $\mathrm{X}-05-064$ & $\mathrm{AW}, \mathrm{AP}$ & $E$ & 552 seconds $\times 80 \mathrm{~m}$ & $\mathrm{X}-05-064 \_160505 \mathrm{E} 1$ \\
\hline$X-05-066$ & $\mathrm{AW}, \mathrm{AP}$ & $E$ & 451 seconds $\times 90 \mathrm{~m}$ & X-05-066_170505_E1 \\
\hline$X-05-067$ & $\mathrm{AW}, \mathrm{AP}$ & $E$ & 567 seconds $\times 150 \mathrm{~m}$ & $\mathrm{X}-05-067$ 170505_E1 \\
\hline $\mathrm{X}-05-067$ & $A W, A P$ & $\mathrm{~L}$ & $19 \mathrm{hr}$ & X-05-067_170505_L1 \\
\hline $\mathrm{X}-05-069$ & $\mathrm{AW}, \mathrm{AP}$ & $E$ & 563 seconds $\times 95 \mathrm{~m}$ & X-05-069_170505_E1 \\
\hline$X-05-070$ & $\mathrm{AW}, \mathrm{AP}$ & $E$ & 330 seconds $\times 85 \mathrm{~m}$ & X-05-070_180505_E1 \\
\hline $\mathrm{X}-05-070$ & $\mathrm{AW}, \mathrm{AP}$ & $\mathrm{L}$ & $17 \mathrm{hr}$. & X-05-070_180505_L1 \\
\hline $\mathrm{X}-05-071$ & $\mathrm{AW}, \mathrm{AP}$ & $E$ & 362 seconds $\times 70 \mathrm{~m}$ & X-05-071_180505_E1 \\
\hline$X-05-072$ & $\mathrm{AW}, \mathrm{AP}$ & $E$ & 480 seconds $\times 90 \mathrm{~m}$ & $\mathrm{X}-05-072 \_180505 \mathrm{E} 1$ \\
\hline$X-05-079$ & $\mathrm{AW}, \mathrm{AP}$ & $\mathrm{B}$ & $15 \mathrm{~m} \times 5 \mathrm{~m}$ & X-05-079_190505_B1 \\
\hline$X-05-080$ & $\mathrm{AW}, \mathrm{AP}$ & $\mathrm{E}$ & 410 seconds $\times 90 \mathrm{~m}$ & X-05-080_190505_E1 \\
\hline$X-05-081$ & $A W, A P$ & $E$ & 185 seconds $\times 50 \mathrm{~m}$ & X-05-081_190505_E1 \\
\hline $\mathrm{X}-05-083$ & $\mathrm{AW}, \mathrm{AP}$ & $E$ & 350 seconds $\times 70 \mathrm{~m}$ & X-05-083_190505_E1 \\
\hline $\mathrm{X}-05-084$ & $\mathrm{AW}, \mathrm{AP}$ & $E$ & 252 seconds $\times 100 \mathrm{~m}$ & X-05-084_200505_E1 \\
\hline$X-05-085$ & $\mathrm{AW}, \mathrm{AP}$ & $\mathrm{D}$ & 1 sweep & X-05-085_200505_D1 \\
\hline X-05-085 & $\mathrm{AW}, \mathrm{AP}$ & $E$ & 231 seconds $\times 80 \mathrm{~m}$ & X-05-085_200505_E1 \\
\hline $\mathrm{X}-05-086$ & $\mathrm{AW}, \mathrm{AP}$ & $E$ & 157 seconds $\times 25 \mathrm{~m}$ & X-05-086_200505_E1 \\
\hline$X-05-088$ & $A W, A P$ & $E$ & 425 seconds $\times 105 \mathrm{~m}$ & X-05-088_240505E1 \\
\hline$X-05-089$ & $\mathrm{AW}, \mathrm{AP}$ & $\mathrm{D}$ & 3 sweeps & X-05-089_250505_D1 \\
\hline $\mathrm{X}-05-091$ & $\mathrm{AW}, \mathrm{AP}$ & $\mathrm{E}$ & 341 seconds $\times 30 \mathrm{~m}$ & X-05-091_250505_E1 \\
\hline$X-05-093$ & $\mathrm{AW}, \mathrm{AP}$ & $\mathrm{B}$ & $5 \mathrm{~m} \times 7 \mathrm{~m}$ & $\mathrm{X}-05-093 \_270505$ \\
\hline X-05-094 & $\mathrm{AW}, \mathrm{AP}$ & $E$ & 514 seconds $\times 100 \mathrm{~m}$ & X-05-094_270505_E1 \\
\hline $\mathrm{X}-05-095$ & $\mathrm{AW}, \mathrm{AP}$ & $E$ & 621 seconds $\times 110 \mathrm{~m}$ & X-05-095_300505_E1 \\
\hline$X-05-096$ & $A W, A P$ & $\mathrm{~B}$ & $30 \mathrm{~m} \times 6 \mathrm{~m}$ & X-05-096_300505_B1 \\
\hline X-05-098 & $\mathrm{AW}, \mathrm{AP}$ & $E$ & 392 seconds $\times 65 \mathrm{~m}$ & X-05-098_300505_E1 \\
\hline X-05-099 & $\mathrm{AW}, \mathrm{AP}$ & $E$ & $10 \mathrm{~m} \times 7 \mathrm{~m}$ & X-05-099_310505_E1 \\
\hline$X-05-100$ & $\mathrm{AW}, \mathrm{AP}$ & $E$ & 325 seconds $\times 45 \mathrm{~m}$ & X-05-100_310505_E1 \\
\hline $\mathrm{X}-05-102$ & $\mathrm{AW}, \mathrm{AP}$ & $E$ & 534 seconds $\times 60 \mathrm{~m}$ & X-05-102 310505 E1 \\
\hline$X-05-103$ & $\mathrm{AW}, \mathrm{AP}$ & $E$ & 332 seconds $\times 75 \mathrm{~m}$ & X-05-103_310505_E1 \\
\hline$X-05-104$ & $A W, A P$ & $\mathrm{E}$ & 502 seconds $\times 110 \mathrm{~m}$ & X-05-104_310505_E1 \\
\hline
\end{tabular}


Appendix 4: List of all fishing effort showing the site number, collectors, fishing gear used, amount of fishing effort applied and the fish capture identification (ID) number derived for each occasion where effort was applied.

\begin{tabular}{|c|c|c|c|c|}
\hline Site Number & Collectors & Gear ID & Effort & $\begin{array}{c}\text { Fish Capture ID } \\
\text { (Crew_Date_Gear) }\end{array}$ \\
\hline $\mathrm{X}-05-105$ & $A W, A P$ & $E$ & 443 seconds $\times 115 \mathrm{~m}$ & X-05-105_010605_E1 \\
\hline $\mathrm{X}-05-106$ & $\mathrm{AW}, \mathrm{AP}$ & $E$ & 448 seconds $\times 85 \mathrm{~m}$ & X-05-106_010605_E1 \\
\hline$X-05-107$ & $\mathrm{AW}, \mathrm{AP}$ & $E$ & 251 seconds $\times 25 \mathrm{~m}$ & X-05-107_010605_E1 \\
\hline$X-05-109$ & $\mathrm{AW}, \mathrm{AP}$ & $E$ & 565 seconds $\times 120 \mathrm{~m}$ & X-05-109_010605_E1 \\
\hline $\mathrm{X}-05-111$ & $A W, A P$ & $E$ & 509 seconds $\times 120 \mathrm{~m}$ & X-05-111_020605_E1 \\
\hline $\mathrm{X}-05-112$ & $\mathrm{AW}, \mathrm{AP}$ & $E$ & 407 seconds $\times 80 \mathrm{~m}$ & $\mathrm{X}-05-112 \_020605 \mathrm{E} 1$ \\
\hline$X-05-114$ & $\mathrm{AW}, \mathrm{AP}$ & $E$ & 400 seconds $\times 100 \mathrm{~m}$ & X-05-114_020605_E1 \\
\hline $\mathrm{X}-05-115$ & $\mathrm{AW}, \mathrm{AP}$ & $\mathrm{B}$ & $10 \mathrm{~m} \times 10 \mathrm{~m}$ & X-05-115_020605_B1 \\
\hline $\mathrm{X}-05-117$ & $\mathrm{AW}, \mathrm{AP}$ & $E$ & 361 seconds $\times 60 \mathrm{~m}$ & $\mathrm{X}-05-117020605 \mathrm{E} 1$ \\
\hline$X-05-118$ & $\mathrm{AW}, \mathrm{AP}$ & $E$ & 394 seconds $\times 90 \mathrm{~m}$ & $\mathrm{X}-05-118 \_060605 \mathrm{E} 1$ \\
\hline$X-05-120$ & $\mathrm{AW}, \mathrm{AP}$ & $\mathrm{E}$ & 337 seconds $\times 80 \mathrm{~m}$ & $\mathrm{X}-05-120 \_060605$ E1 \\
\hline$X-05-121$ & $\mathrm{AW}, \mathrm{AP}$ & $E$ & 272 seconds $\times 50 \mathrm{~m}$ & X-05-121_060605_E1 \\
\hline$X-05-122$ & $A W, A P$ & $\mathrm{E}$ & 479 seconds $\times 100 \mathrm{~m}$ & $\mathrm{X}-05-122 \_060605 \mathrm{E} 1$ \\
\hline $\mathrm{X}-05-123$ & $\mathrm{AW}, \mathrm{AP}$ & $E$ & 332 seconds $\times 50 \mathrm{~m}$ & $\mathrm{X}-05-123 \_070605 \mathrm{E} 1$ \\
\hline$X-05-125$ & $\mathrm{AW}, \mathrm{AP}$ & $E$ & 346 seconds $\times 65 \mathrm{~m}$ & $\mathrm{X}-05-125 \_070605 \_\mathrm{E} 1$ \\
\hline$X-05-126$ & $\mathrm{AW}, \mathrm{AP}$ & $E$ & 431 seconds $\times 85 \mathrm{~m}$ & X-05-126_070605_E1 \\
\hline $\mathrm{X}-05-127$ & $\mathrm{AW}, \mathrm{AP}$ & $E$ & 445 seconds $\times 70 \mathrm{~m}$ & X-05-127_090605_E1 \\
\hline $\mathrm{X}-05-128$ & $\mathrm{AW}, \mathrm{AP}$ & $E$ & 121 seconds $\times 45 \mathrm{~m}$ & X-05-128_090605_E1 \\
\hline$X-05-129$ & $A W, A P$ & $E$ & 343 seconds $\times 70 \mathrm{~m}$ & X-05-129_100605_E1 \\
\hline$X-05-130$ & $\mathrm{AW}, \mathrm{AP}$ & $\mathrm{E}$ & 304 seconds $\times 65 \mathrm{~m}$ & $\mathrm{X}-05-130 \_100605[\mathrm{E} 1$ \\
\hline $\mathrm{X}-05-132$ & $\mathrm{AW}, \mathrm{AP}$ & $E$ & 393 seconds $\times 90 \mathrm{~m}$ & $\mathrm{X}-05-132 \_130605 \_\mathrm{E} 1$ \\
\hline$X-05-133$ & $\mathrm{AW}, \mathrm{AP}$ & $E$ & 210 seconds $\times 45 \mathrm{~m}$ & $\mathrm{X}-05-133 \quad 130605 \mathrm{E} 1$ \\
\hline$X-05-136$ & $\mathrm{AW}, \mathrm{AP}$ & $E$ & 211 seconds $\times 45 \mathrm{~m}$ & $\mathrm{X}-05-136 \_130605 \_\mathrm{E} 1$ \\
\hline $\mathrm{X}-05-137$ & $\mathrm{AW}, \mathrm{AP}$ & $E$ & 502 seconds $\times 100 \mathrm{~m}$ & $\mathrm{X}-05-137 \_140605$ E1 \\
\hline$X-05-138$ & $A W, A P$ & $E$ & 591 seconds $\times 115 \mathrm{~m}$ & X-05-138_140605_E1 \\
\hline X-05-139 & $\mathrm{AW}, \mathrm{AP}$ & $E$ & 286 seconds $\times 80 \mathrm{~m}$ & $\mathrm{X}-05-139 \_140605 \mathrm{E} 1$ \\
\hline$X-05-140$ & $\mathrm{AW}, \mathrm{AP}$ & $E$ & 340 seconds $\times 65 \mathrm{~m}$ & X-05-140_140605_E1 \\
\hline$X-05-140$ & $\mathrm{AW}, \mathrm{AP}$ & $\mathrm{O}$ & Observed & X-05-140_140605_01 \\
\hline$X-05-144$ & $\mathrm{AW}, \mathrm{AP}$ & $E$ & 387 seconds $\times 70 \mathrm{~m}$ & X-05-144_150605 E1 \\
\hline$X-05-145$ & $\mathrm{AW}, \mathrm{AP}$ & $E$ & 446 seconds $\times 90 \mathrm{~m}$ & $\mathrm{X}-05-145 \_150605 \_\mathrm{E} 1$ \\
\hline$X-05-146$ & $A W, A P$ & $\mathrm{E}$ & 706 seconds $\times 160 \mathrm{~m}$ & X-05-146_160605_E1 \\
\hline
\end{tabular}


Appendix 4: List of all fishing effort showing the site number, collectors, fishing gear used, amount of fishing effort applied and the fish capture identification (ID) number derived for each occasion where effort was applied.

\begin{tabular}{|c|c|c|c|c|}
\hline Site Number & Collectors & Gear ID & Effort & $\begin{array}{c}\text { Fish Capture ID } \\
\text { (Crew_Date_Gear) }\end{array}$ \\
\hline $\mathrm{X}-05-148$ & $A W, A P$ & $E$ & 205 seconds $\times 50 \mathrm{~m}$ & X-05-148_170605_E1 \\
\hline$X-05-150$ & $\mathrm{AW}, \mathrm{AP}$ & $E$ & 485 seconds $\times 110 \mathrm{~m}$ & $\mathrm{X}-05-150 \_210605 \mathrm{E} 1$ \\
\hline$X-05-151$ & $\mathrm{AW}, \mathrm{AP}$ & $E$ & 868 seconds $\times 170 \mathrm{~m}$ & $\mathrm{X}-05-151 \_210605 \mathrm{E} 1$ \\
\hline$X-05-153$ & $\mathrm{AW}, \mathrm{AP}$ & $\mathrm{E}$ & 286 seconds $\times 60 \mathrm{~m}$ & $\mathrm{X}-05-153 \_210605$ E1 \\
\hline$X-05-154$ & $\mathrm{AW}, \mathrm{AP}$ & $\mathrm{E}$ & 727 seconds $\times 150 \mathrm{~m}$ & X-05-154_210605_E1 \\
\hline$X-05-155$ & $\mathrm{AW}, \mathrm{AP}$ & $E$ & 605 seconds $\times 110 \mathrm{~m}$ & $\mathrm{X}-05-155 \_220605 \mathrm{E} 1$ \\
\hline$X-05-157$ & $\mathrm{AW}, \mathrm{AP}$ & $E$ & 259 seconds $\times 50 \mathrm{~m}$ & X-05-157_220605_E1 \\
\hline $\mathrm{X}-05-162$ & $\mathrm{AW}, \mathrm{AP}$ & $E$ & 331 seconds $\times 20 \mathrm{~m}$ & X-05-162_230605_E1 \\
\hline $\mathrm{X}-05-163$ & $\mathrm{AW}, \mathrm{AP}$ & $E$ & 198 seconds $\times 40 \mathrm{~m}$ & $\mathrm{X}-05-163 \_230605 \mathrm{E} 1$ \\
\hline$X-05-164$ & $\mathrm{AW}, \mathrm{AP}$ & $E$ & 168 seconds $\times 25 \mathrm{~m}$ & X-05-164_230605_E1 \\
\hline$X-05-165$ & $\mathrm{AW}, \mathrm{AP}$ & $\mathrm{E}$ & 362 seconds $\times 50 \mathrm{~m}$ & $\mathrm{X}-05-165 \_270605$ E1 \\
\hline $\mathrm{X}-05-167$ & $\mathrm{AW}, \mathrm{AP}$ & $E$ & 375 seconds $\times 60 \mathrm{~m}$ & X-05-167_280605_E1 \\
\hline$X-05-168$ & $\mathrm{AW}, \mathrm{AP}$ & $\mathrm{E}$ & 214 seconds $\times 30 \mathrm{~m}$ & X-05-168_280605_E1 \\
\hline X-05-169 & $\mathrm{AW}, \mathrm{AP}$ & $E$ & 316 seconds $\times 70 \mathrm{~m}$ & X-05-169_280605_E1 \\
\hline $\mathrm{X}-05-171$ & $\mathrm{AW}, \mathrm{AP}$ & $E$ & 457 seconds $\times 90 \mathrm{~m}$ & X-05-171_280605_E1 \\
\hline$X-05-172$ & $\mathrm{AW}, \mathrm{AP}$ & $E$ & 269 seconds $\times 60 \mathrm{~m}$ & X-05-172_290605_E1 \\
\hline $\mathrm{X}-05-173$ & $\mathrm{AW}, \mathrm{AP}$ & $E$ & 402 seconds $\times 75 \mathrm{~m}$ & X-05-173_290605_E1 \\
\hline $\mathrm{X}-05-176$ & $\mathrm{AW}, \mathrm{AP}, \mathrm{VK}$ & $E$ & 353 seconds $\times 40 \mathrm{~m}$ & X-05-176_040705_E2 \\
\hline$X-05-176$ & $\mathrm{AW}, \mathrm{AP}, \mathrm{VK}$ & $E$ & 356 seconds $\times 65 \mathrm{~m}$ & X-05-176_040705_E1 \\
\hline$X-05-177$ & $\mathrm{AW}, \mathrm{AP}, \mathrm{VK}$ & $\mathrm{D}$ & 1 sweep through pool & X-05-177_040705_D1 \\
\hline $\mathrm{X}-05-179$ & $\mathrm{AW}, \mathrm{AP}, \mathrm{VK}$ & $E$ & 416 seconds $\times 45 \mathrm{~m}$ & X-05-179_050705_E1 \\
\hline$X-05-181$ & $\mathrm{AW}, \mathrm{AP}, \mathrm{VK}$ & $E$ & 357 seconds $\times 80 \mathrm{~m}$ & X-05-181 060705 E1 \\
\hline$X-05-182$ & $\mathrm{AW}, \mathrm{AP}, \mathrm{VK}$ & $E$ & 339 seconds $\times 85 \mathrm{~m}$ & X-05-182_060705_E1 \\
\hline $\mathrm{X}-05-183$ & $\mathrm{AW}, \mathrm{AP}, \mathrm{VK}$ & $E$ & 286 seconds $\times 40 \mathrm{~m}$ & $\mathrm{X}-05-183 \_060705 \_\mathrm{E} 1$ \\
\hline$X-05-185$ & $\mathrm{AW}, \mathrm{AP}, \mathrm{VK}$ & $E$ & 658 seconds $\times 110 \mathrm{~m}$ & X-05-185_070705_E1 \\
\hline$X-05-186$ & $\mathrm{AW}, \mathrm{AP}$ & $E$ & 405 seconds $\times 65 \mathrm{~m}$ & X-05-186_070705_E1 \\
\hline $\mathrm{X}-05-188$ & $\mathrm{AW}, \mathrm{AP}$ & $E$ & 416 seconds $\times 105 \mathrm{~m}$ & X-05-188_070705_E1 \\
\hline$X-05-189$ & $\mathrm{AW}, \mathrm{AP}$ & $E$ & 280 seconds $\times 50 \mathrm{~m}$ & X-05-189_080705_E1 \\
\hline X-05-189 & $\mathrm{AW}, \mathrm{AP}$ & $\mathrm{O}$ & Observed & X-05-189_080705_01 \\
\hline$X-05-190$ & $\mathrm{DM}, \mathrm{AW}, \mathrm{AP}, \mathrm{VK}, \mathrm{LT}$ & $E$ & 727 seconds $\times 40 \mathrm{~m}$ & X-05-190_110705_E1 \\
\hline$X-05-191$ & $\mathrm{AW}, \mathrm{AP}$ & $E$ & 289 seconds $\times 25 \mathrm{~m}$ & X-05-191_120705_E1 \\
\hline
\end{tabular}


Appendix 4: List of all fishing effort showing the site number, collectors, fishing gear used, amount of fishing effort applied and the fish capture identification (ID) number derived for each occasion where effort was applied.

\begin{tabular}{|c|c|c|c|c|}
\hline Site Number & Collectors & Gear ID & Effort & $\begin{array}{c}\text { Fish Capture ID } \\
\text { (Crew_Date_Gear) }\end{array}$ \\
\hline $\mathrm{X}-05-192$ & $\mathrm{DM}, \mathrm{AW}, \mathrm{AP}, \mathrm{VK}, \mathrm{LT}$ & $E$ & 895 seconds $\times 100 \mathrm{~m}$ & X-05-192_130705_E1 \\
\hline $\mathrm{X}-05-193$ & $\mathrm{DM}, \mathrm{AW}, \mathrm{AP}, \mathrm{VK}, \mathrm{LT}$ & $E$ & 858 seconds $\times 90 \mathrm{~m}$ & X-05-193_130705_E1 \\
\hline$X-05-194$ & $\mathrm{AW}, \mathrm{AP}, \mathrm{VK}, \mathrm{LT}$ & $E$ & 445 seconds $\times 50 \mathrm{~m}$ & X-05-194_130705_E1 \\
\hline $\mathrm{X}-05-195$ & $\mathrm{AW}, \mathrm{AP}, \mathrm{VK}, \mathrm{LT}$ & $E$ & 286 seconds $\times 30 \mathrm{~m}$ & X-05-195_130705_E1 \\
\hline $\mathrm{X}-05-196$ & $\mathrm{AW}, \mathrm{AP}, \mathrm{VK}, \mathrm{LT}$ & $E$ & 352 seconds $\times 39 \mathrm{~m}$ & X-05-196_140705_E1 \\
\hline X-05-198 & $\mathrm{AW}, \mathrm{AP}$ & $E$ & 345 seconds $\times 45 \mathrm{~m}$ & X-05-198_140705_E1 \\
\hline X-05-199 & $\mathrm{AW}, \mathrm{AP}$ & $E$ & 205 seconds $\times 25 \mathrm{~m}$ & X-05-199_140705_E1 \\
\hline$X-05-200$ & $\mathrm{AW}, \mathrm{AP}$ & $E$ & 398 seconds $\times 30 \mathrm{~m}$ & X-05-200_150705_E1 \\
\hline$X-05-201$ & $\mathrm{AW}, \mathrm{AP}$ & $\mathrm{D}$ & 4 sweeps under culvert & X-05-201_030805_D1 \\
\hline$X-05-202$ & $\mathrm{AW}, \mathrm{AP}$ & $\mathrm{B}$ & $4 \mathrm{~m} \times 5 \mathrm{~m}$ & $\mathrm{X}-05-202 \_030805 \_\mathrm{B} 1$ \\
\hline$X-05-204$ & $\mathrm{AW}, \mathrm{AP}$ & $\mathrm{E}$ & 376 seconds $\times 45 \mathrm{~m}$ & X-05-204_040805_E1 \\
\hline$X-05-207$ & $\mathrm{AW}, \mathrm{AP}$ & $E$ & 183 seconds $\times 45 \mathrm{~m}$ & X-05-207_040805_E1 \\
\hline$X-05-208$ & $\mathrm{AW}, \mathrm{AP}$ & $\mathrm{E}$ & 575 seconds $\times 60 \mathrm{~m}$ & X-05-208_040805_E1 \\
\hline$X-05-209$ & $\mathrm{AW}, \mathrm{AP}$ & $E$ & 656 seconds $\times 150 \mathrm{~m}$ & X-05-209_160605_E1 \\
\hline$X-05-210$ & $\mathrm{AW}, \mathrm{AP}$ & $\mathrm{E}$ & 302 seconds $\times 75 \mathrm{~m}$ & $\mathrm{X}-05-210 \_220605 \_\mathrm{E} 1$ \\
\hline D-06-001 & DM, CM, SK & $E$ & 437 seconds $\times 90 \mathrm{~m}$ & D-06-001_060706_E1 \\
\hline W-06-001 & JC, VK & $E$ & 379 seconds $\times 40 \mathrm{~m}$ & W-06-001_090506_E1 \\
\hline W-06-003 & JC, VK & $E$ & 415 seconds $\times 50 \mathrm{~m}$ & W-06-003_090506_E1 \\
\hline W-06-004 & JC, VK & $E$ & 284 seconds $\times 50 \mathrm{~m}$ & W-06-004_090506_E1 \\
\hline W-06-007 & JC, VK & $\mathrm{E}$ & 291 seconds $\times 50 \mathrm{~m}$ & W-06-007_100506_E1 \\
\hline W-06-008 & JC, VK & $E$ & 343 seconds $\times 70 \mathrm{~m}$ & W-06-008_100506_E1 \\
\hline W-06-009 & JC, VK & $E$ & 421 seconds $\times 50 \mathrm{~m}$ & W-06-009_100506_E1 \\
\hline W-06-010 & JC, VK & $\mathrm{B}$ & $3 \mathrm{~m} \times 10 \mathrm{~m}$ & W-06-010_100506_B1 \\
\hline W-06-012 & JC, VK & $\mathrm{E}$ & 183 seconds $\times 20 \mathrm{~m}$ & W-06-012_110506_E1 \\
\hline W-06-015 & JC, VK & $E$ & 190 seconds $\times 30 \mathrm{~m}$ & W-06-015_110506_E1 \\
\hline W-06-016 & JC, VK & $E$ & 331 seconds $\times 90 \mathrm{~m}$ & W-06-016_110506_E1 \\
\hline W-06-017 & JC, VK & $E$ & 378 seconds $\times 80 \mathrm{~m}$ & W-06-017_120506_E1 \\
\hline W-06-018 & JC, VK & $E$ & 168 seconds $\times 20 \mathrm{~m}$ & W-06-018_120506_E1 \\
\hline W-06-019 & JC, VK & $E$ & 308 seconds $\times 40 \mathrm{~m}$ & W-06-019_150506_E1 \\
\hline W-06-020 & JC, VK & $E$ & 387 seconds $\times 80 \mathrm{~m}$ & W-06-020_150506_E1 \\
\hline W-06-021 & $\mathrm{JC}, \mathrm{VK}$ & $\mathrm{E}$ & 167 seconds $\times 80 \mathrm{~m}$ & W-06-021_160506_E1 \\
\hline
\end{tabular}


Appendix 4: List of all fishing effort showing the site number, collectors, fishing gear used, amount of fishing effort applied and the fish capture identification (ID) number derived for each occasion where effort was applied.

\begin{tabular}{|c|c|c|c|c|}
\hline Site Number & Collectors & Gear ID & Effort & $\begin{array}{c}\text { Fish Capture ID } \\
\text { (Crew_Date_Gear) }\end{array}$ \\
\hline W-06-022 & JC, VK & $E$ & 367 seconds $\times 100 \mathrm{~m}$ & W-06-022_160506_E1 \\
\hline W-06-023 & JC, VK & $E$ & 487 seconds $\times 100 \mathrm{~m}$ & W-06-023_160506_E1 \\
\hline W-06-025 & JC, VK & $E$ & 460 seconds $\times 100 \mathrm{~m}$ & W-06-025_170506_E1 \\
\hline W-06-026 & JC, VK & $\mathrm{E}$ & 716 seconds $\times 100 \mathrm{~m}$ & W-06-026_170506_E1 \\
\hline W-06-027 & JC, VK & $\mathrm{B}$ & $5 \mathrm{~m} \times 13 \mathrm{~m}$ & W-06-027 170506_B1 \\
\hline W-06-028 & JC, VK & $E$ & 496 seconds $\times 80 \mathrm{~m}$ & W-06-028_170506_E1 \\
\hline W-06-029 & JC, VK & $E$ & 135 seconds $\times 25 \mathrm{~m}$ & W-06-029_230506_E1 \\
\hline W-06-030 & JC, VK & $E$ & 370 seconds $\times 100 \mathrm{~m}$ & W-06-030_230506_E1 \\
\hline W-06-031 & JC, VK & $E$ & 382 seconds $\times 100 \mathrm{~m}$ & W-06-031_230506_E1 \\
\hline W-06-032 & JC, VK & $E$ & 278 seconds $\times 75 \mathrm{~m}$ & W-06-032_240506_E1 \\
\hline W-06-036 & JC, VK & $\mathrm{E}$ & 194 seconds $\times 80 \mathrm{~m}$ & W-06-036_250506_E1 \\
\hline W-06-037 & JC, VK & $\mathrm{E}$ & 199 seconds $\times 30 \mathrm{~m}$ & W-06-037_250506_E1 \\
\hline W-06-040 & JC, VK & $\mathrm{E}$ & 397 seconds $\times 90 \mathrm{~m}$ & W-06-040_260506_E1 \\
\hline W-06-043 & JC, VK & $E$ & 190 seconds $\times 70 \mathrm{~m}$ & W-06-043_260506_E1 \\
\hline W-06-045 & JC, VK & $\mathrm{E}$ & 204 seconds $\times 80 \mathrm{~m}$ & W-06-045_260506_E1 \\
\hline W-06-046 & JC, VK & $E$ & 180 seconds $\times 80 \mathrm{~m}$ & W-06-046_270506_E1 \\
\hline W-06-049 & JC, VK & $E$ & 530 seconds $\times 90 \mathrm{~m}$ & W-06-049_270506_E1 \\
\hline W-06-054 & JC, VK & $E$ & 391 seconds $\times 100 \mathrm{~m}$ & W-06-054_300506_E1 \\
\hline W-06-055 & JC, VK & $E$ & 194 seconds $\times 80 \mathrm{~m}$ & W-06-055_300506_E1 \\
\hline W-06-056 & JC, VK & $\mathrm{E}$ & 109 seconds $\times 80 \mathrm{~m}$ & W-06-056_310506_E1 \\
\hline W-06-058 & JC, VK & $\mathrm{B}$ & $10 \mathrm{~m} \times 20 \mathrm{~m}$ & W-06-058_010606_B1 \\
\hline W-06-060 & JC, VK & $\mathrm{B}$ & $7 \mathrm{~m} \times 30 \mathrm{~m}$ & W-06-060 $010606 \mathrm{~B} 1$ \\
\hline W-06-061 & JC, VK & $E$ & 385 seconds $\times 70 \mathrm{~m}$ & W-06-061_060606_E1 \\
\hline W-06-062 & JC, VK & $E$ & 445 seconds $\times 60 \mathrm{~m}$ & W-06-062_060606_E1 \\
\hline W-06-064 & JC, VK & $E$ & 323 seconds $\times 60 \mathrm{~m}$ & W-06-064_070606_E1 \\
\hline W-06-065 & JC, VK & $E$ & 289 seconds $\times 90 \mathrm{~m}$ & W-06-065_070606_E1 \\
\hline W-06-066 & JC, VK & $E$ & 349 seconds $\times 60 \mathrm{~m}$ & W-06-066_070606_E1 \\
\hline W-06-067 & JC, VK & $E$ & 438 seconds $\times 100 \mathrm{~m}$ & W-06-067_080606_E1 \\
\hline W-06-068 & JC, VK & $E$ & 438 seconds $\times 120 \mathrm{~m}$ & W-06-068 $080606 \mathrm{E} 1$ \\
\hline W-06-070 & JC, VK & $E$ & 868 seconds $\times 120 \mathrm{~m}$ & W-06-070_120606_E1 \\
\hline W-06-074 & $\mathrm{JC}, \mathrm{VK}$ & $\mathrm{E}$ & 294 seconds $\times 85 \mathrm{~m}$ & W-06-074_130606_E1 \\
\hline
\end{tabular}


Appendix 4: List of all fishing effort showing the site number, collectors, fishing gear used, amount of fishing effort applied and the fish capture identification (ID) number derived for each occasion where effort was applied.

\begin{tabular}{|c|c|c|c|c|}
\hline Site Number & Collectors & Gear ID & Effort & $\begin{array}{c}\text { Fish Capture ID } \\
\text { (Crew_Date_Gear) }\end{array}$ \\
\hline W-06-076 & JC, VK & $E$ & 98 seconds $\times 20 \mathrm{~m}$ & W-06-076_130606_E1 \\
\hline W-06-077 & JC, VK & $E$ & 184 seconds $\times 80 \mathrm{~m}$ & W-06-077_130606_E1 \\
\hline W-06-078 & JC, VK & $E$ & 364 seconds $\times 110 \mathrm{~m}$ & W-06-078_140606_E1 \\
\hline W-06-079 & JC, VK & $E$ & 643 seconds $\times 50 \mathrm{~m}$ & W-06-079_140606_E1 \\
\hline W-06-083 & JC, VK & $\mathrm{E}$ & 1308 seconds $\times 125 \mathrm{~m}$ & W-06-083_200606_E1 \\
\hline W-06-085 & JC, VK & $E$ & 218 seconds $\times 80 \mathrm{~m}$ & W-06-085_210606_E1 \\
\hline W-06-087 & JC, VK & $E$ & 496 seconds $\times 100 \mathrm{~m}$ & W-06-087_220606_E1 \\
\hline W-06-088 & JC, VK & $E$ & 151 seconds $\times 30 \mathrm{~m}$ & W-06-088_220606_E1 \\
\hline W-06-089 & JC, VK & $E$ & 129 seconds $\times 50 \mathrm{~m}$ & W-06-089_220606_E1 \\
\hline W-06-090 & JC, VK & $E$ & 816 seconds $\times 100 \mathrm{~m}$ & W-06-090_220606_E1 \\
\hline W-06-091 & JC, VK & $\mathrm{E}$ & 616 seconds $\times 60 \mathrm{~m}$ & W-06-091_230606_E1 \\
\hline W-06-092 & JC, VK & $E$ & 335 seconds $\times 45 \mathrm{~m}$ & W-06-092_230606_E1 \\
\hline W-06-093 & JC, VK & $E$ & 194 seconds $\times 35 \mathrm{~m}$ & W-06-093_230606_E1 \\
\hline W-06-094 & JC, VK & $E$ & 227 seconds $\times 40 \mathrm{~m}$ & W-06-094_270606_E1 \\
\hline W-06-096 & JC, VK & $\mathrm{E}$ & 945 seconds $\times 120 \mathrm{~m}$ & W-06-096_270606_E1 \\
\hline W-06-097 & JC, VK & $E$ & 445 seconds $\times 80 \mathrm{~m}$ & W-06-097_270606_E1 \\
\hline W-06-098 & JC, VK & $E$ & 369 seconds $\times 50 \mathrm{~m}$ & W-06-098_280606_E1 \\
\hline W-06-099 & JC, VK & $\mathrm{O}$ & Observed, stranded and dead & W-06-099_280606_01 \\
\hline W-06-100 & JC, VK & $E$ & 600 seconds $\times 70 \mathrm{~m}$ & W-06-100_280606_E1 \\
\hline W-06-102 & JC, VK & $\mathrm{D}$ & One sweep & W-06-102_280606_D1 \\
\hline W-06-106 & JC, VK & $\mathrm{E}$ & 205 seconds $\times 50 \mathrm{~m}$ & W-06-106_290606_E1 \\
\hline W-06-110 & JC, VK & $E$ & 479 seconds $\times 70 \mathrm{~m}$ & W-06-110 $050706 \mathrm{E} 1$ \\
\hline W-06-112 & JC, VK & $E$ & 197 seconds $\times 40 \mathrm{~m}$ & W-06-112_050706_E1 \\
\hline W-06-113 & JC, VK & $E$ & 887 seconds $\times 110 \mathrm{~m}$ & W-06-113_060706_E1 \\
\hline W-06-114 & JC, VK & $E$ & 287 seconds $\times 80 \mathrm{~m}$ & W-06-114_100706_E1 \\
\hline W-06-116 & JC, VK & $\mathrm{D}$ & 10 sweeps over cobble & W-06-116_110706_D1 \\
\hline W-06-119 & JC, VK & $E$ & 486 seconds $\times 80 \mathrm{~m}$ & W-06-119_110706_E1 \\
\hline W-06-121 & JC, VK & $E$ & 386 seconds $\times 80 \mathrm{~m}$ & W-06-121_120706_E1 \\
\hline W-06-124 & JC, VK & $E$ & 361 seconds $\times 40 \mathrm{~m}$ & W-06-124 180706 E1 \\
\hline W-06-126 & JC, VK & $E$ & 716 seconds $\times 100 \mathrm{~m}$ & W-06-126_180706_E1 \\
\hline W-06-133 & $\mathrm{JC}, \mathrm{VK}$ & $\mathrm{E}$ & 530 seconds $\times 60 \mathrm{~m}$ & W-06-133_190706_E1 \\
\hline
\end{tabular}


Appendix 4: List of all fishing effort showing the site number, collectors, fishing gear used, amount of fishing effort applied and the fish capture identification (ID) number derived for each occasion where effort was applied.

\begin{tabular}{|c|c|c|c|c|}
\hline Site Number & Collectors & Gear ID & Effort & $\begin{array}{l}\text { Fish Capture ID } \\
\text { (Crew_Date_Gear) }\end{array}$ \\
\hline W-06-134 & $\mathrm{JC}, \mathrm{VK}$ & $\bar{E}$ & 621 seconds $\times 80 \mathrm{~m}$ & W-06-134_200706_E1 \\
\hline W-06-138 & $\mathrm{DM}, \mathrm{JC}$ & $E$ & 329 seconds $\times 50 \mathrm{~m}$ & W-06-138 $010806 \mathrm{E} 1$ \\
\hline W-06-139 & $\mathrm{DM}, \mathrm{JC}$ & $\mathrm{E}$ & 269 seconds $\times 40 \mathrm{~m}$ & W-06-139_010806_E1 \\
\hline W-06-140 & $\mathrm{DM}, \mathrm{JC}$ & $\mathrm{E}$ & 296 seconds $\times 40 \mathrm{~m}$ & W-06-140_010806_E1 \\
\hline W-06-141 & $\mathrm{DM}, \mathrm{JC}$ & $\mathrm{E}$ & 324 seconds $\times 50 \mathrm{~m}$ & W-06-141_010806_E1 \\
\hline W-06-143 & $\mathrm{DM}, \mathrm{JC}$ & $E$ & 349 seconds $\times 30 \mathrm{~m}$ & W-06-143 $020806 \mathrm{E} 1$ \\
\hline W-06-144 & $\mathrm{JC}, \mathrm{VK}$ & $\mathrm{E}$ & 369 seconds $\times 80 \mathrm{~m}$ & W-06-144_080806 E1 \\
\hline W-06-146 & $\mathrm{JC}, \mathrm{VK}$ & $E$ & 209 seconds $\times 60 \mathrm{~m}$ & W-06-146_090806_E1 \\
\hline
\end{tabular}


Appendix 5: List of all fish captures made between 2002 and 2006, showing site number, common name and number (\#) of fish collected, fork (or total ${ }^{\star}$ ) length in millimetres ( $\mathrm{mm}$ ), any comments on the collection and the fish capture identification (ID) number.

\begin{tabular}{|c|c|c|c|c|c|}
\hline Site Number & Common Name & \# & $\begin{array}{c}\text { Fork/Total* } \\
\text { Length }(\mathrm{mm})\end{array}$ & Comments & $\begin{array}{l}\text { Fish Capture } \\
\text { ID Number }\end{array}$ \\
\hline D-02-002 & Creek Chub & 6 & & & D-02-002_170402_E1 \\
\hline D-02-002 & Fathead Minnow & 1 & & & D-02-002_170402_E1 \\
\hline D-02-002 & Johnny Darter & 2 & & & D-02-002_170402_E1 \\
\hline D-02-002 & River Darter & 1 & & & D-02-002_170402_E1 \\
\hline D-02-002 & Longnose Dace & 1 & & & D-02-002_170402_E1 \\
\hline D-02-004 & Common Shiner & 3 & & & D-02-004_220402_E1 \\
\hline D-02-004 & Western Blacknose Dace & 2 & & & D-02-004_220402_E1 \\
\hline D-02-004 & White Sucker & 1 & 200 & & D-02-004_220402_E1 \\
\hline D-02-005 & Eggs & 4 & & $\begin{array}{l}4 \text { Percidae eggs, } 2 \text { viable, }+1.25 \mathrm{~mm} \text {, } \\
\text { golden nucleus, assumed to be Walleye }\end{array}$ & D-02-005_020502_K1 \\
\hline D-02-008 & Northern Pike & 1 & 181 & & D-02-008_070502_E1 \\
\hline D-02-008 & White Sucker & 2 & 390,450 & & D-02-008_070502_E1 \\
\hline D-02-009 & Blackside Darter & 3 & & & D-02-009_070502_E1 \\
\hline D-02-009 & Brook Stickleback & 1 & & & D-02-009_070502_E1 \\
\hline D-02-009 & Creek Chub & 1 & & & D-02-009_070502_E1 \\
\hline D-02-009 & Northern Pike & 1 & 278 & & D-02-009_070502_E1 \\
\hline D-02-009 & White Sucker & 6 & $76-450$ & & D-02-009_070502_E1 \\
\hline D-02-009 & Yellow Perch & 1 & 76 & & D-02-009_070502_E1 \\
\hline D-02-010 & No Catch & & & & D-02-010_090502_E1 \\
\hline D-02-011 & No Catch & & & & D-02-011_090502_E1 \\
\hline D-02-012 & Brook Stickleback & 1 & & & D-02-012_090502_E1 \\
\hline D-02-012 & Central Mudminnow & 1 & & & D-02-012_090502_E1 \\
\hline D-02-013 & No Catch & & & & D-02-013_100502_E1 \\
\hline D-02-014 & Central Mudminnow & 1 & & & D-02-014_110502_E1 \\
\hline D-02-014 & Central Mudminnow & 2 & & & D-02-014_110502_K1 \\
\hline D-02-014 & Fathead Minnow & 1 & & & D-02-014_110502_K1 \\
\hline D-02-014 & Finescale Dace & 1 & & & D-02-014_110502_E1 \\
\hline D-02-015 & Brook Stickleback & 1 & & & D-02-015_110502_E1 \\
\hline D-02-015 & Central Mudminnow & 10 & & & D-02-015_110502_E1 \\
\hline D-02-016 & No Catch & & & & D-02-016_110502_E1 \\
\hline D-02-018 & Brook Stickleback & 5 & & & D-02-018_120502_E1 \\
\hline
\end{tabular}


Appendix 5: List of all fish captures made between 2002 and 2006, showing site number, common name and number (\#) of fish collected, fork (or total ${ }^{\star}$ ) length in millimetres ( $\mathrm{mm}$ ), any comments on the collection and the fish capture identification (ID) number.

\begin{tabular}{|c|c|c|c|c|c|}
\hline Site Number & Common Name & $\#$ & $\begin{array}{l}\text { Fork/Total }{ }^{*} \\
\text { Length }(\mathrm{mm})\end{array}$ & Comments & $\begin{array}{l}\text { Fish Capture } \\
\text { ID Number }\end{array}$ \\
\hline D-02-018 & Central Mudminnow & 5 & & & $\begin{array}{lll}\mathrm{D}-02-018 & 120502 \mathrm{E}\end{array}$ \\
\hline $\mathrm{D}-02-018$ & Northern Pike & & & & D-02-018_120502_A1 \\
\hline D-02-018 & White Sucker & 6 & $60-176$ & & $\mathrm{D}-02-018 \_120502 \mathrm{E} 1$ \\
\hline D-02-022 & Eggs & 8 & & 8 Sucker eggs, 6 viable & $\mathrm{D}-02-022 \quad 120502 \mathrm{~K} 1$ \\
\hline D-02-023 & Eggs & 22 & & 22 Sucker eggs, 14 viable & $\mathrm{D}-02-023 \quad 120502 \mathrm{~K} 1$ \\
\hline D-02-026 & Northern Pike & 2 & 457,495 & & $\mathrm{D}-02-026 \quad 130502 \mathrm{E} 1$ \\
\hline D-02-026 & White Sucker & 2 & 285,291 & & $\mathrm{D}-02-026 \_130502 \mathrm{E} 1$ \\
\hline D-02-026 & Eggs & 42 & & 42 Sucker eggs, 31 viable & $\mathrm{D}-02-026 \quad 130502 \mathrm{~K} 1$ \\
\hline D-02-027 & Brook Stickleback & 7 & & & $\mathrm{D}-02-027 \quad 130502 \mathrm{E} 1$ \\
\hline D-02-027 & Central Mudminnow & 16 & & & $\mathrm{D}-02-027 \quad 130502 \mathrm{E} 1$ \\
\hline D-02-027 & Fathead Minnow & 44 & & & $\mathrm{D}-02-027 \_130502 \mathrm{E} 1$ \\
\hline D-02-028 & Brook Stickleback & 8 & & & $\mathrm{D}-02-028 \quad 130502 \mathrm{E} 1$ \\
\hline D-02-028 & Central Mudminnow & 9 & & & $\mathrm{D}-02-028 \quad 130502 \mathrm{E} 1$ \\
\hline D-02-028 & Fathead Minnow & 40 & & & $\mathrm{D}-02-028 \quad 130502 \mathrm{E} 1$ \\
\hline D-02-028 & White Sucker & 8 & $59-126$ & & $\mathrm{D}-02-028 \_130502 \_\mathrm{E} 1$ \\
\hline D-02-028 & Eggs & 10 & & $\begin{array}{l}\text { Numerous Sucker eggs in kick sample, } \\
\text { mostly viable }\end{array}$ & D-02-028_130502_K1 \\
\hline D-02-031 & Creek Chub & 17 & & & D-02-031_150502_E1 \\
\hline D-02-031 & Fathead Minnow & 7 & & & $\mathrm{D}-02-031 \quad 150502 \mathrm{E} 1$ \\
\hline D-02-031 & Finescale Dace & 16 & & & $\mathrm{D}-02-031 \quad 150502 \mathrm{E} 1$ \\
\hline D-02-031 & Johnny Darter & 3 & & & $\mathrm{D}-02-031 \_150502 \_\mathrm{E} 1$ \\
\hline D-02-031 & Northern Redbelly Dace & 21 & & & D-02-031_150502_E1 \\
\hline D-02-031 & Western Blacknose Dace & 7 & & & $\mathrm{D}-02-031 \quad 150502 \mathrm{E} 1$ \\
\hline D-02-033 & Creek Chub & 6 & & & $\mathrm{D}-02-033 \quad 150502 \mathrm{E} 1$ \\
\hline $\mathrm{D}-02-033$ & Fathead Minnow & 40 & & & D-02-033_150502_E1 \\
\hline D-02-033 & Finescale Dace & 23 & & & D-02-033_150502_E1 \\
\hline D-02-033 & Northern Redbelly Dace & 1 & & & $\mathrm{D}-02-033 \quad 150502 \mathrm{E} 1$ \\
\hline D-02-033 & Silver Redhorse & 1 & 122 & & $\mathrm{D}-02-033 \quad 150502 \mathrm{E} 1$ \\
\hline D-02-033 & Eggs & 19 & & $\begin{array}{l}19 \text { Percidae eggs, } 14 \text { viable, assumed to } \\
\text { be Walleye }+1 \mathrm{~mm} \text {, golden nucleus }\end{array}$ & D-02-033_150502_K1 \\
\hline D-02-033 & Western Blacknose Dace & 9 & & & D-02-033 $150502 \mathrm{E} 1$ \\
\hline
\end{tabular}


Appendix 5: List of all fish captures made between 2002 and 2006, showing site number, common name and number (\#) of fish collected, fork (or total ${ }^{\star}$ ) length in millimetres ( $\mathrm{mm}$ ), any comments on the collection and the fish capture identification (ID) number.

\begin{tabular}{|c|c|c|c|c|c|}
\hline Site Number & Common Name & \# & $\begin{array}{l}\text { Fork/Total* } \\
\text { Length }(\mathrm{mm})\end{array}$ & Comments & $\begin{array}{l}\text { Fish Capture } \\
\text { ID Number }\end{array}$ \\
\hline D-02-033 & White Sucker & 4 & $73-88$ & & D-02-033_150502_E1 \\
\hline D-02-034 & Johnny Darter & 2 & & & D-02-034_160502_E1 \\
\hline D-02-034 & Northern Pike & 1 & & One mature Northern Pike observed & D-02-034_160502_A1 \\
\hline D-02-035 & No Catch & & & & D-02-035_160502_E1 \\
\hline D-02-036 & Brook Stickleback & 8 & & & D-02-036_160502_E1 \\
\hline D-02-036 & Central Mudminnow & 3 & & & D-02-036_160502_E1 \\
\hline D-02-036 & Creek Chub & 1 & & & D-02-036_160502_E1 \\
\hline D-02-036 & Northern Pike & 4 & $140-237$ & & D-02-036_160502_E1 \\
\hline D-02-036 & Western Blacknose Dace & 1 & & & D-02-036_160502_E1 \\
\hline D-02-036 & White Sucker & 3 & $99,108,300$ & & D-02-036_160502_E1 \\
\hline D-02-037 & White Sucker & 1 & & $\begin{array}{l}\text { Mature White Sucker found dead on } \\
\text { cobble under bridge }\end{array}$ & D-02-037_160502_O1 \\
\hline D-02-043 & No Catch & & & & D-02-043_210502_E1 \\
\hline D-02-044 & Blacknose Shiner & 10 & & & D-02-044_210502_E1 \\
\hline D-02-044 & Common Shiner & 10 & & & D-02-044_210502_E1 \\
\hline D-02-044 & Creek Chub & 12 & & & D-02-044_210502_E1 \\
\hline D-02-044 & Emerald Shiner & 8 & & & D-02-044_210502_E1 \\
\hline D-02-044 & Fathead Minnow & 1 & & & D-02-044_210502_E1 \\
\hline D-02-044 & Western Blacknose Dace & 2 & & & D-02-044_210502_E1 \\
\hline D-02-044 & White Sucker & 29 & & & D-02-044_210502_E1 \\
\hline D-02-045 & Brook Stickleback & 1 & & & D-02-045_220502_E1 \\
\hline D-02-045 & Creek Chub & 5 & & & D-02-045_220502_E1 \\
\hline D-02-045 & Fathead Minnow & 8 & & & D-02-045_220502_E1 \\
\hline D-02-045 & Finescale Dace & 1 & & & D-02-045_220502_E1 \\
\hline D-02-045 & Johnny Darter & 2 & & & D-02-045_220502_E1 \\
\hline D-02-045 & Longnose Dace & 2 & & & D-02-045_220502_E1 \\
\hline D-02-045 & Western Blacknose Dace & 1 & & & D-02-045_220502_E1 \\
\hline D-02-045 & White Sucker & 3 & $49-61$ & & D-02-045_220502_E1 \\
\hline D-02-048 & No Catch & & & & D-02-048_230502_E1 \\
\hline D-02-051 & No Catch & & & & D-02-051_240502_E1 \\
\hline D-02-054 & No Catch & & & & D-02-054_240502_E1 \\
\hline
\end{tabular}


Appendix 5: List of all fish captures made between 2002 and 2006, showing site number, common name and number (\#) of fish collected, fork (or total ${ }^{\star}$ ) length in millimetres ( $\mathrm{mm}$ ), any comments on the collection and the fish capture identification (ID) number.

\begin{tabular}{|c|c|c|c|c|c|}
\hline Site Number & Common Name & $\#$ & $\begin{array}{c}\text { Fork/Total* } \\
\text { Length }(\mathrm{mm})\end{array}$ & Comments & $\begin{array}{l}\text { Fish Capture } \\
\text { ID Number }\end{array}$ \\
\hline D-02-056 & Brook Stickleback & 6 & & & D-02-056_240502_E1 \\
\hline D-02-056 & Creek Chub & 1 & & & D-02-056_240502_E1 \\
\hline D-02-056 & Fathead Minnow & 2 & & & D-02-056_240502_E1 \\
\hline D-02-056 & Finescale Dace & 5 & & & D-02-056_240502_E1 \\
\hline D-02-056 & Johnny Darter & 1 & & & D-02-056_240502_E1 \\
\hline D-02-056 & Longnose Dace & 1 & & & D-02-056_240502_E1 \\
\hline D-02-056 & Western Blacknose Dace & 4 & & & D-02-056_240502_E1 \\
\hline D-02-058 & Brook Stickleback & 10 & & & D-02-058_240502_E1 \\
\hline D-02-058 & Creek Chub & 3 & & & D-02-058_240502_E1 \\
\hline D-02-058 & Finescale Dace & 6 & & & D-02-058_240502_E1 \\
\hline D-02-058 & Northern Redbelly Dace & 7 & & & D-02-058_240502_E1 \\
\hline D-02-058 & Western Blacknose Dace & 1 & & & D-02-058_240502_E1 \\
\hline D-02-060 & Brook Stickleback & 1 & & & D-02-060_270502_E1 \\
\hline D-02-060 & Creek Chub & 1 & & & D-02-060_270502_E1 \\
\hline D-02-060 & Johnny Darter & 1 & & & D-02-060_270502_E1 \\
\hline D-02-060 & Western Blacknose Dace & 5 & & & D-02-060_270502_E1 \\
\hline D-02-060 & Finescale Dace & 2 & & & D-02-060_270502_K1 \\
\hline D-02-061 & Black Bullhead & 1 & & & D-02-061_280502_E1 \\
\hline D-02-061 & Blackside Darter & 3 & & & D-02-061_280502_E1 \\
\hline D-02-061 & Iowa Darter & 1 & & & D-02-061 280502 E1 \\
\hline D-02-061 & Johnny Darter & 5 & & & D-02-061_280502_E1 \\
\hline D-02-061 & Tadpole Madtom & 16 & & & D-02-061_280502_E1 \\
\hline D-02-061 & White Sucker & 1 & 96 & & D-02-061_280502_E1 \\
\hline D-02-062 & Black Bullhead & 3 & & & D-02-062_280502_E1 \\
\hline D-02-062 & Blackside Darter & 16 & & & D-02-062_280502_E1 \\
\hline D-02-062 & Common Carp & 3 & & & D-02-062_280502_E1 \\
\hline D-02-062 & Creek Chub & 1 & & & D-02-062_280502_E1 \\
\hline D-02-062 & Johnny Darter & 6 & & & D-02-062_280502_E1 \\
\hline D-02-062 & River Darter & 4 & & & D-02-062_280502_E1 \\
\hline D-02-062 & Tadpole Madtom & 2 & & & D-02-062_280502_E1 \\
\hline D-02-062 & White Sucker & 8 & $226-389$ & & D-02-062_280502_E1 \\
\hline
\end{tabular}


Appendix 5: List of all fish captures made between 2002 and 2006, showing site number, common name and number (\#) of fish collected, fork (or total ${ }^{\star}$ ) length in millimetres ( $\mathrm{mm}$ ), any comments on the collection and the fish capture identification (ID) number.

\begin{tabular}{|c|c|c|c|c|c|}
\hline Site Number & Common Name & \# & $\begin{array}{c}\text { Fork/Total* } \\
\text { Length }(\mathrm{mm})\end{array}$ & Comments & $\begin{array}{l}\text { Fish Capture } \\
\text { ID Number }\end{array}$ \\
\hline D-02-065 & Northern Pike & 2 & & Dead, found on shore & D-02-065_290502_01 \\
\hline D-02-065 & Northern Pike & 2 & 390,451 & & D-02-065_290502_D1 \\
\hline D-02-067 & Creek Chub & 4 & & & D-02-067_290502_E1 \\
\hline D-02-067 & Fathead Minnow & 25 & & & D-02-067_290502_E1 \\
\hline D-02-067 & Finescale Dace & 37 & & & D-02-067_290502_E1 \\
\hline D-02-067 & Johnny Darter & 2 & & & D-02-067_290502_E1 \\
\hline D-02-067 & Northern Redbelly Dace & 18 & & & D-02-067_290502_E1 \\
\hline D-02-067 & Pearl Dace & 7 & & & D-02-067_290502_E1 \\
\hline D-02-067 & Western Blacknose Dace & 17 & & & D-02-067_290502_E1 \\
\hline D-02-067 & White Sucker & 5 & $45-168$ & & D-02-067_290502_E1 \\
\hline D-02-068 & Creek Chub & 25 & & & D-02-068_290502_E1 \\
\hline D-02-068 & Fathead Minnow & 38 & & & D-02-068_290502_E1 \\
\hline D-02-068 & Finescale Dace & 7 & & & D-02-068_290502_E1 \\
\hline D-02-068 & Longnose Dace & 2 & & & D-02-068_290502_E1 \\
\hline D-02-068 & Western Blacknose Dace & 5 & & & D-02-068_290502_E1 \\
\hline D-02-068 & White Sucker & 8 & $59-301$ & & D-02-068_290502_E1 \\
\hline D-02-069 & Brook Stickleback & 18 & & & D-02-069_290502_E1 \\
\hline D-02-069 & Creek Chub & 11 & & & D-02-069_290502_E1 \\
\hline D-02-069 & Finescale Dace & 11 & & & D-02-069_290502_E1 \\
\hline D-02-069 & Northern Pike & 2 & 160,180 & & D-02-069_290502_E1 \\
\hline D-02-069 & Western Blacknose Dace & 2 & & & D-02-069_290502_E1 \\
\hline D-02-069 & White Sucker & 17 & 53-189 & & D-02-069_290502_E1 \\
\hline D-02-070 & Eggs & 10 & & Sucker eggs & D-02-070_300502_L1 \\
\hline D-02-071 & Brook Stickleback & 9 & & & D-02-071_300502_E1 \\
\hline D-02-071 & Creek Chub & 4 & & & D-02-071_300502_E1 \\
\hline D-02-071 & Fathead Minnow & 10 & & & D-02-071_300502_E1 \\
\hline D-02-071 & lowa Darter & 1 & & & D-02-071_300502_E1 \\
\hline D-02-071 & Johnny Darter & 3 & & & D-02-071_300502_E1 \\
\hline D-02-071 & Western Blacknose Dace & 14 & & & D-02-071_300502_E1 \\
\hline D-02-071 & White Sucker & 11 & $80-109$ & & D-02-071_300502_E1 \\
\hline D-02-072 & Northern Pike & & & Observed dead on shore & D-02-072_030602_01 \\
\hline
\end{tabular}


Appendix 5: List of all fish captures made between 2002 and 2006, showing site number, common name and number (\#) of fish collected, fork (or total ${ }^{\star}$ ) length in millimetres ( $\mathrm{mm}$ ), any comments on the collection and the fish capture identification (ID) number.

\begin{tabular}{|c|c|c|c|c|c|}
\hline Site Number & Common Name & \# & $\begin{array}{c}\text { Fork/Total* } \\
\text { Length }(\mathrm{mm})\end{array}$ & Comments & $\begin{array}{l}\text { Fish Capture } \\
\text { ID Number }\end{array}$ \\
\hline D-02-072 & Yellow Perch & & & Observed dead on shore & D-02-072_030602_01 \\
\hline D-02-073 & White Sucker & 4 & $12^{*}$ & Larvae, $12 \mathrm{~mm}$ total length & D-02-073_030602_L1 \\
\hline D-02-074 & Black Bullhead & 1 & & & D-02-074_030602_E1 \\
\hline D-02-074 & Blackside Darter & 3 & & & D-02-074_030602_E1 \\
\hline D-02-074 & Common Shiner & 3 & & & D-02-074_030602_E1 \\
\hline D-02-074 & Emerald Shiner & 1 & & & D-02-074_030602_E1 \\
\hline D-02-074 & Northern Pike & 1 & 181 & & D-02-074_030602_E1 \\
\hline D-02-074 & Rock Bass & 4 & $70-120$ & & $\mathrm{D}-02-074 \quad 030602 \mathrm{E} 1$ \\
\hline D-02-074 & Tadpole Madtom & 1 & & & D-02-074_030602_E1 \\
\hline D-02-074 & White Sucker & 1 & 109 & & D-02-074_030602_E1 \\
\hline D-02-074 & Yellow Perch & 1 & 98 & & D-02-074_030602_E1 \\
\hline D-02-075 & Central Mudminnow & 8 & & & D-02-075_040602_E1 \\
\hline D-02-075 & Common Shiner & 20 & & & D-02-075_040602_E1 \\
\hline D-02-075 & Fathead Minnow & 5 & & & D-02-075_040602_E1 \\
\hline D-02-075 & White Sucker & 1 & 279 & & D-02-075_040602_E1 \\
\hline D-02-076 & Brook Stickleback & 4 & & & D-02-076_050602_E1 \\
\hline D-02-076 & Central Mudminnow & 17 & & & D-02-076_050602_E1 \\
\hline D-02-076 & Fathead Minnow & 4 & & & D-02-076_050602_E1 \\
\hline D-02-076 & Iowa Darter & 6 & & & D-02-076_050602_E1 \\
\hline D-02-076 & Johnny Darter & 1 & & & D-02-076_050602_E1 \\
\hline D-02-077 & Central Mudminnow & 1 & & & D-02-077_050602_E1 \\
\hline D-02-077 & Common Shiner & 7 & & & D-02-077_050602_E1 \\
\hline D-02-077 & Fathead Minnow & 3 & & & D-02-077_050602_E1 \\
\hline D-02-077 & White Sucker & 1 & 111 & & D-02-077_050602_E1 \\
\hline D-02-078 & Brook Stickleback & 2 & & & D-02-078_050602_E1 \\
\hline D-02-078 & Central Mudminnow & 8 & & & D-02-078_050602_E1 \\
\hline D-02-078 & Common Shiner & 30 & $122-160$ & & D-02-078_050602_E1 \\
\hline D-02-078 & Fathead Minnow & 17 & & & D-02-078_050602_E1 \\
\hline D-02-078 & Johnny Darter & 1 & & & $\mathrm{D}-02-078 \_050602 \_\mathrm{E} 1$ \\
\hline D-02-078 & White Sucker & 6 & $80-161$ & & D-02-078_050602_E1 \\
\hline D-02-081 & Central Mudminnow & 5 & & & D-02-081_060602_E1 \\
\hline
\end{tabular}


Appendix 5: List of all fish captures made between 2002 and 2006, showing site number, common name and number (\#) of fish collected, fork (or total ${ }^{\star}$ ) length in millimetres $(\mathrm{mm})$, any comments on the collection and the fish capture identification (ID) number.

\begin{tabular}{|c|c|c|c|c|c|}
\hline Site Number & Common Name & \# & $\begin{array}{c}\text { Fork/Total* } \\
\text { Length }(\mathrm{mm})\end{array}$ & Comments & $\begin{array}{l}\text { Fish Capture } \\
\text { ID Number }\end{array}$ \\
\hline D-02-081 & Northern Pike & 1 & 190 & & D-02-081_060602_E1 \\
\hline D-02-081 & Spottail Shiner & 25 & & & D-02-081_060602_E1 \\
\hline D-02-081 & White Sucker & 5 & $83-133$ & & D-02-081_060602_E1 \\
\hline D-02-083 & Central Mudminnow & 11 & & & D-02-083_060602_E1 \\
\hline D-02-083 & Emerald Shiner & 1 & & & D-02-083_060602_E1 \\
\hline D-02-084 & Brook Stickleback & 4 & & & D-02-084_120602_E1 \\
\hline D-02-084 & Central Mudminnow & 14 & & & D-02-084_120602_E1 \\
\hline D-02-084 & Fathead Minnow & 6 & & & D-02-084_120602_E1 \\
\hline D-02-084 & Logperch & 9 & & & D-02-084_120602_E1 \\
\hline D-02-084 & Spottail Shiner & 59 & & & D-02-084_120602_E1 \\
\hline D-02-084 & Yellow Perch & 13 & $70-75$ & & D-02-084_120602_E1 \\
\hline D-02-087 & Brook Stickleback & 2 & & & D-02-087_120602_E1 \\
\hline D-02-087 & Central Mudminnow & 1 & & & D-02-087_120602_E1 \\
\hline D-02-087 & Fathead Minnow & 3 & & & D-02-087_120602_E1 \\
\hline D-02-088 & Northern Pike & 5 & $202-366$ & & D-02-088_130602_E1 \\
\hline D-02-089 & Brook Stickleback & 3 & & & D-02-089_130602_E1 \\
\hline D-02-089 & Central Mudminnow & 3 & & & D-02-089_130602_E1 \\
\hline D-02-089 & Common Carp & 1 & 405 & & D-02-089_130602_E1 \\
\hline D-02-089 & Fathead Minnow & 1 & & & D-02-089_130602_E1 \\
\hline D-02-089 & Northern Pike & 1 & 261 & & D-02-089_130602_E1 \\
\hline D-02-089 & Yellow Perch & 5 & $66-106$ & & D-02-089_130602_E1 \\
\hline D-02-092 & Brook Stickleback & 5 & & & D-02-092_130602_E1 \\
\hline D-02-092 & Central Mudminnow & 6 & & & D-02-092_130602_E1 \\
\hline D-02-092 & Emerald Shiner & 1 & & & D-02-092_130602_E1 \\
\hline D-02-092 & Fathead Minnow & 39 & & & D-02-092_130602_E1 \\
\hline D-02-092 & White Sucker & 1 & 59 & & D-02-092_130602_E1 \\
\hline D-02-092 & Yellow Perch & 15 & $50-66$ & & D-02-092_130602_E1 \\
\hline D-02-093 & Brook Stickleback & 2 & & & D-02-093_140602_E1 \\
\hline D-02-093 & Common Shiner & 3 & & & D-02-093_140602_E1 \\
\hline D-02-093 & Creek Chub & 12 & & & D-02-093_140602_E1 \\
\hline D-02-093 & Fathead Minnow & 2 & & & D-02-093_140602_E1 \\
\hline
\end{tabular}


Appendix 5: List of all fish captures made between 2002 and 2006, showing site number, common name and number (\#) of fish collected, fork (or total ${ }^{\star}$ ) length in millimetres ( $\mathrm{mm}$ ), any comments on the collection and the fish capture identification (ID) number.

\begin{tabular}{|c|c|c|c|c|c|}
\hline Site Number & Common Name & \# & $\begin{array}{c}\text { Fork/Total* } \\
\text { Length }(\mathrm{mm})\end{array}$ & Comments & $\begin{array}{l}\text { Fish Capture } \\
\text { ID Number }\end{array}$ \\
\hline D-02-093 & Finescale Dace & 7 & & & D-02-093_140602_E1 \\
\hline D-02-093 & Longnose Dace & 1 & & & D-02-093_140602_E1 \\
\hline D-02-093 & Western Blacknose Dace & 3 & & & D-02-093_140602_E1 \\
\hline D-02-093 & White Sucker & 3 & & & D-02-093_140602_E1 \\
\hline D-02-094 & Fathead Minnow & 7 & & & D-02-094_180602_E1 \\
\hline D-02-095 & No Catch & & & & D-02-095_180602_E1 \\
\hline D-02-096 & Brook Stickleback & 3 & & & D-02-096_190602_E1 \\
\hline D-02-096 & Central Mudminnow & 1 & & & D-02-096_190602_E1 \\
\hline D-02-096 & Common Shiner & 2 & & & D-02-096_190602_E1 \\
\hline D-02-096 & Fathead Minnow & 8 & & & D-02-096_190602_E1 \\
\hline D-02-097 & Brook Stickleback & 13 & & Spawning & D-02-097_190602_E1 \\
\hline D-02-097 & Central Mudminnow & 6 & & Spawning & D-02-097_190602_E1 \\
\hline D-02-097 & Emerald Shiner & 17 & & & D-02-097_190602_E1 \\
\hline D-02-097 & Finescale Dace & 1 & & & D-02-097_190602_E1 \\
\hline D-02-097 & Hornyhead Chub & 2 & & & D-02-097_190602_E1 \\
\hline D-02-097 & Northern Redbelly Dace & 1 & & & D-02-097_190602_E1 \\
\hline D-02-097 & Pearl Dace & 2 & & & D-02-097_190602_E1 \\
\hline D-02-100 & White Sucker & 1 & 119 & & D-02-100_200602_E1 \\
\hline D-02-101 & White Sucker & 2 & $113-120$ & & D-02-101_200602_E1 \\
\hline D-02-102 & White Sucker & 1 & 281 & & D-02-102_200602_E1 \\
\hline D-02-104 & Brook Stickleback & 4 & & & D-02-104_200602_E1 \\
\hline D-02-104 & Finescale Dace & 2 & & & D-02-104_200602_E1 \\
\hline D-02-104 & Northern Redbelly Dace & 2 & & & D-02-104_200602_E1 \\
\hline D-02-104 & White Sucker & 4 & 109-135 & & D-02-104_200602_E1 \\
\hline D-02-107 & Fathead Minnow & 2 & & & D-02-107_210602_E1 \\
\hline D-02-108 & Brook Stickleback & 9 & & & D-02-108_210602_E1 \\
\hline D-02-111 & Creek Chub & 10 & & Young-of-the-Year (YOY) & D-02-111_260602_E1 \\
\hline D-02-111 & Fathead Minnow & 5 & & & D-02-111_260602_E1 \\
\hline D-02-116 & Unidentified minnow & 1 & $10.2^{*}$ & Post-larval stage & D-02-116_030702_E1 \\
\hline D-02-118 & Northern Pike & 1 & 80 & YOY-Several observed & D-02-118_030702_E1 \\
\hline D-02-119 & Brook Stickleback & 7 & & & D-02-119_040702_E1 \\
\hline
\end{tabular}


Appendix 5: List of all fish captures made between 2002 and 2006, showing site number, common name and number (\#) of fish collected, fork (or total ${ }^{\star}$ ) length in millimetres ( $\mathrm{mm}$ ), any comments on the collection and the fish capture identification (ID) number.

\begin{tabular}{|c|c|c|c|c|c|}
\hline Site Number & Common Name & \# & $\begin{array}{c}\text { Fork/Total* } \\
\text { Length }(\mathrm{mm})\end{array}$ & Comments & $\begin{array}{l}\text { Fish Capture } \\
\text { ID Number }\end{array}$ \\
\hline D-02-119 & Central Mudminnow & 2 & & & D-02-119_040702_E1 \\
\hline D-02-119 & Common Carp & 1 & & YOY & D-02-119_040702_E1 \\
\hline D-02-119 & Fathead Minnow & 19 & & Spawning & D-02-119_040702_E1 \\
\hline D-02-121 & Brook Stickleback & 5 & & & $\mathrm{D}-02-121$ 040702_E1 \\
\hline D-02-121 & Central Mudminnow & 3 & & & D-02-121_040702_E1 \\
\hline D-02-121 & Common Carp & 1 & & & D-02-121_040702_E1 \\
\hline D-02-121 & Fathead Minnow & 46 & & & D-02-121_040702_E1 \\
\hline D-02-121 & Yellow Perch & 1 & 123 & & D-02-121_040702_E1 \\
\hline D-02-122 & Central Mudminnow & 3 & & & D-02-122_040702_E1 \\
\hline D-02-122 & Fathead Minnow & 7 & & Spawning & D-02-122_040702_E1 \\
\hline D-02-124 & Brook Stickleback & 51 & & & D-02-124_040702_B1 \\
\hline D-02-124 & Fathead Minnow & 230 & & & D-02-124_040702_B1 \\
\hline D-02-124 & Iowa Darter & 2 & & & D-02-124_040702_B1 \\
\hline D-02-124 & Johnny Darter & 7 & & & D-02-124_040702_B1 \\
\hline D-02-125 & Brook Stickleback & 2 & & & D-02-125_090702_E1 \\
\hline D-02-125 & Central Mudminnow & 8 & & & D-02-125_090702_E1 \\
\hline D-02-125 & Johnny Darter & 2 & & & D-02-125_090702_E1 \\
\hline D-02-125 & White Sucker & 5 & $71-100$ & & D-02-125_090702_E1 \\
\hline D-02-126 & Brook Stickleback & 5 & & & D-02-126_090702_B1 \\
\hline D-02-126 & Northern Pike & 1 & 451 & & D-02-126_090702_B1 \\
\hline D-02-126 & White Sucker & 4 & $189-301$ & & D-02-126_090702_B1 \\
\hline D-02-127 & Johnny Darter & 6 & & & D-02-127_090702_E1 \\
\hline D-02-127 & White Sucker & 4 & $140-160$ & & D-02-127_090702_E1 \\
\hline D-02-130 & Northern Pike & 2 & 195,200 & & D-02-130_090702_B1 \\
\hline D-02-131 & White Sucker & 4 & $90-100$ & Juvenile & D-02-131_090702_B1 \\
\hline D-02-132 & Brook Stickleback & 3 & & & D-02-132_100702_E1 \\
\hline D-02-132 & Central Mudminnow & 18 & & & D-02-132_100702_E1 \\
\hline D-02-135 & White Sucker & 1 & 348 & & D-02-135_100702_E1 \\
\hline D-02-137 & Brook Stickleback & 38 & & & D-02-137_120702_E1 \\
\hline D-02-137 & Fathead Minnow & 41 & & & D-02-137_120702_E1 \\
\hline D-02-137 & Finescale Dace & 3 & & & D-02-137_120702_E1 \\
\hline
\end{tabular}


Appendix 5: List of all fish captures made between 2002 and 2006, showing site number, common name and number (\#) of fish collected, fork (or total ${ }^{\star}$ ) length in millimetres ( $\mathrm{mm}$ ), any comments on the collection and the fish capture identification (ID) number.

\begin{tabular}{|c|c|c|c|c|c|}
\hline Site Number & Common Name & \# & $\begin{array}{c}\text { Fork/Total* } \\
\text { Length }(\mathrm{mm})\end{array}$ & Comments & $\begin{array}{l}\text { Fish Capture } \\
\text { ID Number }\end{array}$ \\
\hline D-02-137 & Longnose Dace & 1 & & & D-02-137_120702_E1 \\
\hline D-02-137 & Western Blacknose Dace & 13 & & $10 \mathrm{YOY}$ & D-02-137_120702_E1 \\
\hline D-02-138 & Brook Stickleback & 28 & & & D-02-138_120702_B1 \\
\hline D-02-138 & Creek Chub & 22 & & & D-02-138_120702_B1 \\
\hline D-02-138 & Fathead Minnow & 25 & & & D-02-138_120702_B1 \\
\hline D-02-139 & Creek Chub & 7 & & & D-02-139_120702_E1 \\
\hline D-02-139 & Fathead Minnow & 13 & & & D-02-139_120702_E1 \\
\hline D-02-139 & Finescale Dace & 11 & & 3 YOY & D-02-139_120702_E1 \\
\hline D-02-139 & Longnose Dace & 3 & & $1 \mathrm{YOY}$ & D-02-139_120702_E1 \\
\hline D-02-139 & Western Blacknose Dace & 3 & & & D-02-139_120702_E1 \\
\hline D-02-139 & White Sucker & 3 & & YOY & D-02-139_120702_E1 \\
\hline D-02-142 & Brook Stickleback & 4 & & & D-02-142_170702_E1 \\
\hline D-02-142 & Creek Chub & 11 & & & D-02-142_170702_E1 \\
\hline D-02-142 & Finescale Dace & 3 & & & D-02-142_170702_E1 \\
\hline D-02-142 & Iowa Darter & 1 & & & D-02-142_170702_E1 \\
\hline D-02-142 & Johnny Darter & 3 & & & D-02-142_170702_E1 \\
\hline D-02-142 & Western Blacknose Dace & 4 & & & D-02-142_170702_E1 \\
\hline D-02-147 & Brook Stickleback & 36 & & & D-02-147_170702_E1 \\
\hline D-02-147 & Creek Chub & 21 & & YOY & D-02-147_170702_E1 \\
\hline D-02-149 & Blacknose Shiner & 4 & & & D-02-149_170702_E1 \\
\hline D-02-149 & Blackside Darter & 7 & & & D-02-149_170702_E1 \\
\hline D-02-149 & Common Shiner & 2 & & & D-02-149_170702_E1 \\
\hline D-02-149 & Creek Chub & 6 & & & D-02-149_170702_E1 \\
\hline D-02-149 & Johnny Darter & 13 & & & D-02-149_170702_E1 \\
\hline D-02-149 & Longnose Dace & 3 & & & D-02-149_170702_E1 \\
\hline D-02-149 & Western Blacknose Dace & 13 & & & D-02-149_170702_E1 \\
\hline D-02-149 & White Sucker & 7 & $31-34^{*}$ & YOY & D-02-149_170702_E1 \\
\hline D-02-160 & Common Shiner & 1 & & & D-02-160_230702_E1 \\
\hline D-02-160 & Johnny Darter & 5 & & & D-02-160_230702_E1 \\
\hline D-02-160 & Logperch & 2 & & & D-02-160_230702_E1 \\
\hline D-02-160 & Longnose Dace & 10 & & & D-02-160_230702_E1 \\
\hline
\end{tabular}


Appendix 5: List of all fish captures made between 2002 and 2006, showing site number, common name and number (\#) of fish collected, fork (or total ${ }^{\star}$ ) length in millimetres ( $\mathrm{mm}$ ), any comments on the collection and the fish capture identification (ID) number.

\begin{tabular}{|c|c|c|c|c|c|}
\hline Site Number & Common Name & \# & $\begin{array}{l}\text { Fork/Total* } \\
\text { Length (mm) }\end{array}$ & Comments & $\begin{array}{l}\text { Fish Capture } \\
\text { ID Number }\end{array}$ \\
\hline D-02-160 & River Darter & 4 & & & D-02-160_230702_E1 \\
\hline D-02-160 & Western Blacknose Dace & 7 & & & D-02-160_230702_E1 \\
\hline D-02-161 & Blacknose Shiner & 7 & & & D-02-161_230702_E1 \\
\hline D-02-161 & Central Mudminnow & 1 & & & D-02-161_230702_E1 \\
\hline D-02-161 & Creek Chub & 7 & & & D-02-161_230702_E1 \\
\hline D-02-161 & Longnose Dace & 5 & & & D-02-161_230702_E1 \\
\hline D-02-161 & Western Blacknose Dace & 5 & & & D-02-161_230702_E1 \\
\hline D-02-164 & Brook Stickleback & 2 & & & D-02-164_240702_E1 \\
\hline D-02-164 & Finescale Dace & 4 & & & D-02-164_240702_E1 \\
\hline D-02-164 & Western Blacknose Dace & 16 & & & D-02-164_240702_E1 \\
\hline D-02-167 & Brook Stickleback & 16 & & & D-02-167_240702_B1 \\
\hline D-02-167 & Creek Chub & 19 & $14^{*}$ & YOY & D-02-167_240702_B1 \\
\hline D-02-170 & Brook Stickleback & 1 & & & D-02-170_240702_E1 \\
\hline D-02-170 & Creek Chub & 17 & & & D-02-170_240702_E1 \\
\hline D-02-170 & Fathead Minnow & 10 & & & D-02-170_240702_E1 \\
\hline D-02-170 & Johnny Darter & 1 & & & D-02-170_240702_E1 \\
\hline D-02-170 & Pearl Dace & 2 & & & D-02-170_240702_E1 \\
\hline D-02-170 & Western Blacknose Dace & 7 & & & D-02-170_240702_E1 \\
\hline D-02-170 & White Sucker & 15 & $66-116$ & & D-02-170_240702_E1 \\
\hline D-02-171 & Brook Stickleback & 11 & & & D-02-171_250702_E1 \\
\hline D-02-171 & Creek Chub & 5 & & & D-02-171_250702_E1 \\
\hline D-02-171 & Fathead Minnow & 2 & & & D-02-171_250702_E1 \\
\hline D-02-171 & Finescale Dace & 10 & & & D-02-171_250702_E1 \\
\hline D-02-171 & Northern Redbelly Dace & 3 & & & D-02-171_250702_E1 \\
\hline D-02-171 & White Sucker & 2 & 110 & Immature & D-02-171_250702_E1 \\
\hline D-02-173 & Fathead Minnow & 12 & & & D-02-173_250702_E1 \\
\hline D-02-173 & Finescale Dace & 29 & & & D-02-173_250702_E1 \\
\hline D-02-173 & Johnny Darter & 5 & & & D-02-173_250702_E1 \\
\hline D-02-173 & Western Blacknose Dace & 2 & & & D-02-173_250702_E1 \\
\hline D-02-173 & White Sucker & 5 & $73-155$ & & D-02-173_250702_E1 \\
\hline D-02-178 & Brook Stickleback & 6 & & & D-02-178_250702_B1 \\
\hline
\end{tabular}


Appendix 5: List of all fish captures made between 2002 and 2006, showing site number, common name and number (\#) of fish collected, fork (or total ${ }^{\star}$ ) length in millimetres ( $\mathrm{mm}$ ), any comments on the collection and the fish capture identification (ID) number.

\begin{tabular}{|c|c|c|c|c|c|}
\hline Site Number & Common Name & \# & $\begin{array}{c}\text { Fork/Total* } \\
\text { Length }(\mathrm{mm})\end{array}$ & Comments & $\begin{array}{l}\text { Fish Capture } \\
\text { ID Number }\end{array}$ \\
\hline D-02-178 & Central Mudminnow & 3 & & & D-02-178_250702_B1 \\
\hline D-02-178 & Creek Chub & 15 & & & D-02-178_250702_B1 \\
\hline D-02-178 & Fathead Minnow & 77 & & & D-02-178_250702_B1 \\
\hline D-02-178 & Finescale Dace & 82 & & & D-02-178_250702_B1 \\
\hline D-02-178 & Johnny Darter & 5 & & & D-02-178_250702_B1 \\
\hline D-02-178 & White Sucker & 68 & $33^{*}-111$ & & D-02-178_250702_B1 \\
\hline D-02-179 & Brook Stickleback & 4 & & & D-02-179_300702_E1 \\
\hline D-02-179 & Creek Chub & 4 & & & D-02-179_300702_E1 \\
\hline D-02-179 & Finescale Dace & 25 & & & D-02-179_300702_E1 \\
\hline D-02-179 & Longnose Dace & 3 & & & D-02-179_300702_E1 \\
\hline D-02-179 & Western Blacknose Dace & 3 & & & D-02-179_300702_E1 \\
\hline D-02-179 & White Sucker & 9 & $76-170$ & & D-02-179_300702_E1 \\
\hline D-02-180 & Central Mudminnow & 1 & & & D-02-180_300702_E1 \\
\hline D-02-180 & Common Shiner & 1 & & & D-02-180_300702_E1 \\
\hline D-02-180 & Creek Chub & 24 & & & D-02-180_300702_E1 \\
\hline D-02-180 & Fathead Minnow & 3 & & & D-02-180_300702_E1 \\
\hline D-02-180 & Johnny Darter & 4 & & & D-02-180_300702_E1 \\
\hline D-02-180 & Longnose Dace & 3 & & & D-02-180_300702_E1 \\
\hline D-02-180 & Northern Pike & 4 & $122-160$ & & D-02-180_300702_E1 \\
\hline D-02-180 & Western Blacknose Dace & 1 & & & D-02-180_300702_E1 \\
\hline D-02-180 & White Sucker & 5 & $88-100$ & & D-02-180_300702_E1 \\
\hline D-02-181 & Blacknose Shiner & 6 & & & D-02-181_310702_E1 \\
\hline D-02-181 & Creek Chub & 1 & & & D-02-181_310702_E1 \\
\hline D-02-181 & Emerald Shiner & 1 & & & D-02-181_310702_E1 \\
\hline D-02-181 & Johnny Darter & 2 & & & D-02-181_310702_E1 \\
\hline D-02-181 & Longnose Dace & 10 & & & D-02-181_310702_E1 \\
\hline D-02-181 & River Darter & 1 & & & D-02-181_310702_E1 \\
\hline D-02-181 & Western Blacknose Dace & 3 & & & D-02-181_310702_E1 \\
\hline D-02-181 & White Sucker & 4 & $100-103$ & & D-02-181_310702_E1 \\
\hline D-02-182 & Creek Chub & 21 & & & D-02-182_310702_E1 \\
\hline D-02-182 & White Sucker & 1 & 251 & & D-02-182_310702_E1 \\
\hline
\end{tabular}


Appendix 5: List of all fish captures made between 2002 and 2006, showing site number, common name and number (\#) of fish collected, fork (or total ${ }^{\star}$ ) length in millimetres ( $\mathrm{mm}$ ), any comments on the collection and the fish capture identification (ID) number.

\begin{tabular}{|c|c|c|c|c|c|}
\hline Site Number & Common Name & \# & $\begin{array}{c}\text { Fork/Total* } \\
\text { Length }(\mathrm{mm})\end{array}$ & Comments & $\begin{array}{l}\text { Fish Capture } \\
\text { ID Number }\end{array}$ \\
\hline D-02-183 & Creek Chub & 1 & & & D-02-183_310702_E1 \\
\hline D-02-183 & Longnose Dace & 8 & & & D-02-183_310702_E1 \\
\hline D-02-183 & Western Blacknose Dace & 3 & & & D-02-183_310702_E1 \\
\hline D-02-183 & White Sucker & 4 & $38-41^{*}$ & YOY & $\mathrm{D}-02-183 \_310702 \mathrm{E} 1$ \\
\hline D-02-184 & Blackside Darter & 4 & & & D-02-184_310702_E1 \\
\hline D-02-184 & Creek Chub & 4 & & & D-02-184_310702_E1 \\
\hline D-02-184 & Fathead Minnow & 7 & & & $\mathrm{D}-02-184$ 310702_E1 \\
\hline D-02-184 & Longnose Dace & 12 & & & D-02-184_310702_E1 \\
\hline D-02-184 & Western Blacknose Dace & 15 & & & D-02-184_310702_E1 \\
\hline D-02-184 & White Sucker & 5 & $72-83$ & & D-02-184_310702_E1 \\
\hline D-02-185 & Brook Stickleback & 49 & & & $\mathrm{D}-02-185010802 \mathrm{E} 1$ \\
\hline D-02-188 & Brook Stickleback & 41 & & & D-02-188_010802_E1 \\
\hline D-02-188 & Creek Chub & 111 & & & D-02-188_010802 E1 \\
\hline D-02-190 & Brook Stickleback & 1 & & & $\mathrm{D}-02-190 \_010802 \mathrm{E} 1$ \\
\hline D-02-190 & Creek Chub & 2 & & & D-02-190_010802_E1 \\
\hline D-02-190 & Emerald Shiner & 4 & & & D-02-190_010802_E1 \\
\hline D-02-190 & Fathead Minnow & 1 & & & D-02-190_010802_E1 \\
\hline D-02-190 & Finescale Dace & 5 & & & D-02-190_010802_E1 \\
\hline D-02-190 & Johnny Darter & 4 & & & $\mathrm{D}-02-190 \_010802 \mathrm{E} 1$ \\
\hline D-02-190 & White Sucker & 4 & $38-40^{*}$ & YOY & $\mathrm{D}-02-190 \_010802 \_\mathrm{E} 1$ \\
\hline D-02-191 & Creek Chub & 29 & & $9 \mathrm{YOY}$ & D-02-191_150802_E1 \\
\hline D-02-191 & Johnny Darter & 9 & & $3 \mathrm{YOY}$ & D-02-191_150802_E1 \\
\hline D-02-191 & Longnose Dace & 1 & & & D-02-191_150802_E1 \\
\hline D-02-191 & Western Blacknose Dace & 31 & & & D-02-191_150802_E1 \\
\hline D-02-191 & White Sucker & 2 & 90,253 & & D-02-191_150802_E1 \\
\hline D-02-192 & Blacknose Shiner & 6 & & & $\mathrm{D}-02-192 \_160802$ E1 \\
\hline D-02-192 & Brook Stickleback & 4 & & & D-02-192_160802_E1 \\
\hline D-02-192 & Creek Chub & 55 & 42 & YOY & $\mathrm{D}-02-192 \_160802 \_\mathrm{E} 1$ \\
\hline D-02-192 & Johnny Darter & 4 & & & D-02-192_160802_E1 \\
\hline D-02-192 & Longnose Dace & 10 & & & D-02-192_160802_E1 \\
\hline D-02-192 & Western Blacknose Dace & 30 & & & D-02-192_160802_E1 \\
\hline
\end{tabular}


Appendix 5: List of all fish captures made between 2002 and 2006, showing site number, common name and number (\#) of fish collected, fork (or total ${ }^{\star}$ ) length in millimetres ( $\mathrm{mm}$ ), any comments on the collection and the fish capture identification (ID) number.

\begin{tabular}{|c|c|c|c|c|c|}
\hline Site Number & Common Name & \# & $\begin{array}{l}\text { Fork/Total* } \\
\text { Length (mm) }\end{array}$ & Comments & $\begin{array}{l}\text { Fish Capture } \\
\text { ID Number }\end{array}$ \\
\hline D-02-192 & White Sucker & 7 & $47-140$ & & D-02-192_160802_E1 \\
\hline D-02-193 & Bigmouth Buffalo & 2 & & YOY & D-02-193_200802_E1 \\
\hline D-02-193 & Brook Stickleback & 2 & & & D-02-193_200802_B1 \\
\hline D-02-193 & Brook Stickleback & 2 & & & D-02-193_200802_E1 \\
\hline D-02-193 & Central Mudminnow & 4 & & & D-02-193_200802_B1 \\
\hline D-02-193 & Central Mudminnow & 4 & & & D-02-193_200802_E1 \\
\hline D-02-193 & Common Carp & 1 & & YOY & D-02-193_200802_B1 \\
\hline D-02-193 & Common Carp & 23 & & YOY & D-02-193_200802_E1 \\
\hline D-02-193 & Emerald Shiner & 2 & & & D-02-193_200802_B1 \\
\hline D-02-193 & Emerald Shiner & 6 & & & D-02-193_200802_E1 \\
\hline D-02-193 & Northern Pike & 1 & 204 & & D-02-193_200802_E1 \\
\hline D-02-193 & Northern Pike & 4 & $200-214$ & & D-02-193_200802_B1 \\
\hline D-02-193 & Sand Shiner & 4 & & & D-02-193_200802_E1 \\
\hline D-02-194 & Bigmouth Buffalo & 2 & & YOY & D-02-194_200802_E1 \\
\hline D-02-194 & Brook Stickleback & 1 & & & D-02-194_200802_E1 \\
\hline D-02-194 & Common Carp & 66 & & & D-02-194_200802_E1 \\
\hline D-02-194 & Fathead Minnow & 7 & & & D-02-194_200802_E1 \\
\hline D-02-194 & White Sucker & 2 & 50,56 & YOY & D-02-194_200802_E1 \\
\hline D-02-195 & Central Mudminnow & 3 & & & D-02-195_210802_E1 \\
\hline D-02-195 & Emerald Shiner & 1 & & & D-02-195_210802_E1 \\
\hline D-02-195 & Northern Pike & 2 & $138-139$ & Immature & D-02-195_210802_E1 \\
\hline D-02-195 & White Sucker & 2 & $142-193$ & Immature & D-02-195_210802_E1 \\
\hline D-02-196 & Blackside Darter & 13 & & & D-02-196_210802_E1 \\
\hline D-02-196 & Central Mudminnow & 7 & & & D-02-196_210802_E1 \\
\hline D-02-196 & Hornyhead Chub & 6 & $5-9^{*}$ & YOY & D-02-196_210802_E1 \\
\hline D-02-196 & Iowa Darter & 2 & & & D-02-196_210802_E1 \\
\hline D-02-196 & Johnny Darter & 3 & & & D-02-196_210802_E1 \\
\hline D-02-196 & Longnose Dace & 13 & & YOY & D-02-196_210802_E1 \\
\hline D-02-196 & Silver Redhorse & 1 & 40 & & D-02-196_210802_E1 \\
\hline D-02-196 & White Sucker & 2 & 38,88 & & D-02-196_210802_E1 \\
\hline D-02-197 & Blackside Darter & 7 & & & D-02-197_220802_E1 \\
\hline
\end{tabular}


Appendix 5: List of all fish captures made between 2002 and 2006, showing site number, common name and number (\#) of fish collected, fork (or total ${ }^{\star}$ ) length in millimetres ( $\mathrm{mm}$ ), any comments on the collection and the fish capture identification (ID) number.

\begin{tabular}{|c|c|c|c|c|c|}
\hline Site Number & Common Name & \# & $\begin{array}{c}\text { Fork/Total* } \\
\text { Length }(\mathrm{mm})\end{array}$ & Comments & $\begin{array}{l}\text { Fish Capture } \\
\text { ID Number }\end{array}$ \\
\hline D-02-197 & Brook Stickleback & 5 & & & D-02-197_220802_E1 \\
\hline D-02-197 & Central Mudminnow & 3 & & & D-02-197_220802_E1 \\
\hline D-02-197 & Longnose Dace & 10 & & & D-02-197_220802_E1 \\
\hline D-02-197 & Western Blacknose Dace & 6 & & & D-02-197_220802_E1 \\
\hline D-02-197 & White Sucker & 2 & $28-31^{*}$ & YOY & D-02-197_220802_B1 \\
\hline D-02-197 & White Sucker & 2 & 220,225 & & D-02-197_220802_B2 \\
\hline D-02-197 & White Sucker & 12 & $28^{*}-195$ & 4 YOY,28 mm, immature @ 195 mm & D-02-197_220802_E1 \\
\hline D-02-198 & Fathead Minnow & 5 & & & D-02-198_220802_E1 \\
\hline D-02-198 & Brook Stickleback & 10 & & & D-02-198_220802_E1 \\
\hline D-02-198 & Central Mudminnow & 4 & & & D-02-198_220802_E1 \\
\hline D-02-198 & Emerald Shiner & 5 & & & D-02-198_220802_E1 \\
\hline D-02-198 & White Sucker & 2 & $40-42$ & YOY & D-02-198_220802_E1 \\
\hline D-02-199 & Blackside Darter & 5 & & & D-02-199_270802_E1 \\
\hline D-02-199 & Central Mudminnow & 5 & & & D-02-199_270802_E1 \\
\hline D-02-199 & Creek Chub & 36 & $20^{*}$ & YOY & D-02-199_270802_E1 \\
\hline D-02-199 & Fathead Minnow & 7 & & & D-02-199_270802_E1 \\
\hline D-02-199 & Johnny Darter & 16 & $5^{*}$ & YOY & D-02-199_270802_E1 \\
\hline D-02-199 & Western Blacknose Dace & 4 & & & D-02-199_270802_E1 \\
\hline D-02-199 & White Sucker & 29 & $44-215$ & $28 \mathrm{YOY}$ & D-02-199_270802_E1 \\
\hline D-02-200 & Blackside Darter & 1 & & & D-02-200_270802_E1 \\
\hline D-02-200 & Creek Chub & 22 & & & D-02-200_270802_E1 \\
\hline D-02-200 & Emerald Shiner & 6 & & & D-02-200_270802_E1 \\
\hline D-02-200 & Fathead Minnow & 7 & & & D-02-200_270802_E1 \\
\hline D-02-200 & Johnny Darter & 8 & & & D-02-200_270802_E1 \\
\hline D-02-200 & River Darter & 1 & & & D-02-200_270802_E1 \\
\hline D-02-200 & White Sucker & 12 & $51-60$ & YOY & D-02-200_270802_E1 \\
\hline D-02-201 & Northern Pike & 1 & 170 & Immature & D-02-201_081002_E1 \\
\hline B-03-001 & Brook Stickleback & 5 & & & B-03-001_210503_E1 \\
\hline B-03-001 & Finescale Dace & 9 & & & B-03-001_210503_E1 \\
\hline B-03-003 & No Catch & & & & B-03-003_230503_E1 \\
\hline B-03-004 & No Catch & & & & B-03-004_230503_E1 \\
\hline
\end{tabular}


Appendix 5: List of all fish captures made between 2002 and 2006, showing site number, common name and number (\#) of fish collected, fork (or total ${ }^{\star}$ ) length in millimetres ( $\mathrm{mm}$ ), any comments on the collection and the fish capture identification (ID) number.

\begin{tabular}{|c|c|c|c|c|c|}
\hline Site Number & Common Name & \# & $\begin{array}{l}\text { Fork/Total }{ }^{*} \\
\text { Length }(\mathrm{mm})\end{array}$ & Comments & $\begin{array}{l}\text { Fish Capture } \\
\text { ID Number }\end{array}$ \\
\hline B-03-005 & Blackside Darter & 2 & & & B-03-005_230503_E1 \\
\hline B-03-005 & Common Shiner & 3 & & & B-03-005_230503_E1 \\
\hline B-03-005 & Creek Chub & 2 & & & B-03-005_230503_E1 \\
\hline B-03-005 & Johnny Darter & 8 & & & B-03-005_230503_E1 \\
\hline B-03-005 & Sand Shiner & 2 & & & B-03-005_230503_E1 \\
\hline B-03-005 & Western Blacknose Dace & 6 & & & B-03-005_230503_E1 \\
\hline B-03-005 & White Sucker & 9 & $51^{*}-116$ & & B-03-005_230503_E1 \\
\hline B-03-007 & Northern Pike & 11 & $30-42^{*}$ & YOY & B-03-007_260503_E1 \\
\hline B-03-009 & Northern Pike & 1 & $35^{\star}$ & YOY & B-03-009_260503_E1 \\
\hline B-03-012 & No Catch & & & & B-03-012_260503_E1 \\
\hline B-03-013 & Brook Stickleback & 24 & & & B-03-013_270503_E1 \\
\hline B-03-013 & Common Shiner & 5 & & & B-03-013_270503_E1 \\
\hline B-03-013 & Creek Chub & 3 & & & B-03-013_270503_E1 \\
\hline B-03-013 & Fathead Minnow & 16 & & & B-03-013_270503_E1 \\
\hline B-03-013 & Johnny Darter & 7 & & & B-03-013_270503_E1 \\
\hline B-03-013 & White Sucker & 2 & 114,116 & & B-03-013_270503_E1 \\
\hline B-03-015 & Brook Stickleback & 5 & & & B-03-015_270503_E1 \\
\hline B-03-016 & Brook Stickleback & 4 & & Released & B-03-016_270503_E1 \\
\hline B-03-017 & Brook Stickleback & 50 & & & B-03-017_280503_E1 \\
\hline B-03-017 & Creek Chub & 2 & & & B-03-017_280503_E1 \\
\hline B-03-017 & Fathead Minnow & 63 & & & B-03-017_280503_E1 \\
\hline B-03-017 & Johnny Darter & 1 & & & B-03-017_280503_E1 \\
\hline B-03-017 & White Sucker & 4 & $94,135,136,200$ & Released1@200 mm & B-03-017_280503_E1 \\
\hline B-03-019 & Brook Stickleback & 33 & & & B-03-019_280503_B1 \\
\hline B-03-019 & Fathead Minnow & 4 & & & B-03-019_280503_B1 \\
\hline B-03-020 & Brook Stickleback & 2 & & Released & B-03-020_290503_E1 \\
\hline B-03-026 & Common Shiner & 45 & & Released1@120 mm & B-03-026_290503_E1 \\
\hline B-03-026 & Creek Chub & 55 & & & B-03-026_290503_E1 \\
\hline B-03-026 & Fathead Minnow & 9 & & & B-03-026_290503_E1 \\
\hline B-03-026 & Johnny Darter & 5 & & & B-03-026_290503_E1 \\
\hline B-03-026 & White Sucker & 3 & $116-122$ & Released $1 @ 120$ mm & B-03-026_290503_E1 \\
\hline
\end{tabular}


Appendix 5: List of all fish captures made between 2002 and 2006, showing site number, common name and number (\#) of fish collected, fork (or total ${ }^{*}$ ) length in millimetres $(\mathrm{mm})$, any comments on the collection and the fish capture identification (ID) number.

\begin{tabular}{|c|c|c|c|c|c|}
\hline Site Number & Common Name & \# & $\begin{array}{l}\text { Fork/Total }{ }^{*} \\
\text { Length }(\mathrm{mm})\end{array}$ & Comments & $\begin{array}{l}\text { Fish Capture } \\
\text { ID Number }\end{array}$ \\
\hline B-03-028 & Brook Stickleback & 13 & & & B-03-028_300503_E1 \\
\hline B-03-028 & Fathead Minnow & 6 & & & B-03-028_300503_E1 \\
\hline $\mathrm{B}-03-028$ & White Sucker & 3 & $115,118,300$ & Released mature female @ 300 mm & B-03-028_300503_E1 \\
\hline B-03-030 & Fathead Minnow & 173 & & Released an additional 150 & B-03-030_300503_B1 \\
\hline B-03-030 & Northern Pike & 19 & $29-34^{*}$ & All YOY, 6 released & B-03-030_300503_B1 \\
\hline B-03-031 & No Catch & & & & B-03-031_300503_E1 \\
\hline B-03-033 & Brook Stickleback & 1 & & Released & B-03-033_020603_E1 \\
\hline B-03-033 & White Sucker & 10 & $9-13^{*}$ & Post-larval stage & B-03-033_020603_L1 \\
\hline B-03-035 & Brook Stickleback & 1 & & & B-03-035_020603_B1 \\
\hline B-03-035 & White Sucker & 5 & $67-81$ & & B-03-035_020603_B1 \\
\hline B-03-040 & Brook Stickleback & 1 & & Released & B-03-040_030603_E1 \\
\hline B-03-041 & White Sucker & 1 & 500 & Released & B-03-041_030603_E1 \\
\hline B-03-041 & Brook Stickleback & 1 & & Released & B-03-041_030603_E1 \\
\hline B-03-048 & No Catch & & & & B-03-048_040603_B1 \\
\hline B-03-050 & No Catch & & & & B-03-050_040603_E1 \\
\hline B-03-052 & Unidentified sucker & 10 & $12-19^{*}$ & Post-larval stage, recent hatch & B-03-052_040603_E1 \\
\hline B-03-053 & Northern Pike & 6 & $500-670$ & Released & B-03-053_050603_E1 \\
\hline B-03-055 & No Catch & & & & B-03-055_050603_E1 \\
\hline B-03-060 & Brook Stickleback & 1 & & & B-03-060_050603_E1 \\
\hline B-03-060 & Fathead Minnow & 3 & & & B-03-060_050603_E1 \\
\hline B-03-060 & Northern Pike & 1 & 326 & $\begin{array}{l}\text { Released, other Northern Pike observed, } \\
\text { unable to collect }\end{array}$ & B-03-060_050603_E1 \\
\hline B-03-061 & White Sucker & 2 & 180,200 & Released & B-03-061_090603_E1 \\
\hline $\mathrm{B}-03-062$ & Brook Stickleback & 2 & & Released & B-03-062_090603_E1 \\
\hline B-03-062 & Fathead Minnow & 22 & & Released 4 male and 14 female spawners & B-03-062_090603_E1 \\
\hline B-03-062 & White Sucker & 2 & 170,200 & Released & B-03-062_090603_E1 \\
\hline B-03-064 & Black Bullhead & 1 & $190^{*}$ & Released & B-03-064_100603_E1 \\
\hline B-03-064 & Creek Chub & 27 & & Released1@140 mm & B-03-064_100603_E1 \\
\hline B-03-064 & Fathead Minnow & 11 & & & B-03-064_100603_E1 \\
\hline B-03-064 & Pearl Dace & 1 & & & B-03-064_100603_E1 \\
\hline B-03-064 & Johnny Darter & 1 & & & B-03-064_100603_E1 \\
\hline
\end{tabular}


Appendix 5: List of all fish captures made between 2002 and 2006, showing site number, common name and number (\#) of fish collected, fork (or total ${ }^{\star}$ ) length in millimetres ( $\mathrm{mm}$ ), any comments on the collection and the fish capture identification (ID) number.

\begin{tabular}{|c|c|c|c|c|c|}
\hline Site Number & Common Name & \# & $\begin{array}{c}\text { Fork/Total* } \\
\text { Length }(\mathrm{mm})\end{array}$ & Comments & $\begin{array}{l}\text { Fish Capture } \\
\text { ID Number }\end{array}$ \\
\hline B-03-064 & Sand Shiner & 3 & & & B-03-064_100603_E1 \\
\hline B-03-064 & Western Blacknose Dace & 24 & & & B-03-064_100603_E1 \\
\hline B-03-064 & White Sucker & 1 & 65 & & B-03-064_100603_E1 \\
\hline B-03-065 & Fathead Minnow & 2 & & Released & B-03-065_100603_E1 \\
\hline B-03-067 & No Catch & & & & B-03-067_100603_E1 \\
\hline B-03-070 & Brook Stickleback & 34 & & Released 12 & B-03-070_110603_E1 \\
\hline B-03-070 & Fathead Minnow & 2 & & Released & B-03-070_110603_E1 \\
\hline B-03-070 & Iowa Darter & 4 & & & B-03-070_110603_E1 \\
\hline B-03-070 & White Sucker & 4 & 300,330 & Released $2 @$ 400, 600 mm & B-03-070_110603_E1 \\
\hline B-03-071 & No Catch & & & & B-03-071_110603_E1 \\
\hline B-03-075 & Brook Stickleback & 6 & & Released & B-03-075_120603_E1 \\
\hline B-03-075 & Fathead Minnow & 2 & & Released & B-03-075_120603_E1 \\
\hline B-03-076 & Common Shiner & 11 & & & B-03-076_120603 E1 \\
\hline B-03-076 & Creek Chub & 6 & & Released 1 & B-03-076_120603_E1 \\
\hline B-03-076 & Fathead Minnow & 3 & & & B-03-076_120603_E1 \\
\hline B-03-076 & lowa Darter & 1 & & & B-03-076_120603_E1 \\
\hline B-03-076 & Johnny Darter & 4 & & & B-03-076_120603_E1 \\
\hline B-03-076 & Western Blacknose Dace & 6 & & & B-03-076_120603_E1 \\
\hline B-03-076 & White Sucker & 9 & $23^{*}-345$ & 8 YOY, released $1 @ 345$ mm & B-03-076_120603_E1 \\
\hline B-03-077 & Creek Chub & 17 & & Released 7 & B-03-077_120603_E1 \\
\hline B-03-077 & Fathead Minnow & 2 & & & B-03-077_120603_E1 \\
\hline B-03-077 & Johnny Darter & 3 & & & B-03-077_120603_E1 \\
\hline B-03-077 & Western Blacknose Dace & 55 & & & B-03-077_120603_E1 \\
\hline B-03-077 & White Sucker & 13 & $31^{*}-120$ & Released 3 @ 90, 95, 120 mm, 3 are YOY & B-03-077_120603_E1 \\
\hline B-03-083 & Brook Stickleback & 155 & & Released an additional 100 & B-03-083_160603_E1 \\
\hline B-03-083 & Fathead Minnow & 104 & & & B-03-083_160603_E1 \\
\hline B-03-084 & Brook Stickleback & 10 & & & B-03-084_160603_E1 \\
\hline B-03-084 & Fathead Minnow & 10 & & & B-03-084_160603_E1 \\
\hline B-03-085 & Black Bullhead & 12 & & Released 10 & B-03-085_170603_E1 \\
\hline B-03-085 & Northern Pike & 1 & 130 & Released & B-03-085_170603_E1 \\
\hline B-03-085 & Brook Stickleback & 16 & & Released 10 & B-03-085_170603_E1 \\
\hline
\end{tabular}


Appendix 5: List of all fish captures made between 2002 and 2006, showing site number, common name and number (\#) of fish collected, fork (or total ${ }^{\star}$ ) length in millimetres ( $\mathrm{mm}$ ), any comments on the collection and the fish capture identification (ID) number.

\begin{tabular}{|c|c|c|c|c|c|}
\hline Site Number & Common Name & \# & $\begin{array}{c}\text { Fork/Total* } \\
\text { Length }(\mathrm{mm})\end{array}$ & Comments & $\begin{array}{l}\text { Fish Capture } \\
\text { ID Number }\end{array}$ \\
\hline B-03-085 & Central Mudminnow & 2 & & & B-03-085_170603_E1 \\
\hline B-03-085 & Creek Chub & 1 & & & B-03-085_170603_E1 \\
\hline B-03-085 & Fathead Minnow & 33 & & & B-03-085_170603_E1 \\
\hline B-03-086 & No Catch & & & & B-03-086_170603_B1 \\
\hline B-03-087 & Fathead Minnow & 35 & & & B-03-087_170603_B1 \\
\hline B-03-087 & Northern Pike & 2 & 460,640 & Released & B-03-087_170603_B1 \\
\hline B-03-087 & Sand Shiner & 27 & & & B-03-087_170603_B1 \\
\hline B-03-087 & White Sucker & 123 & $29-34^{*}$ & All YOY & B-03-087_170603_B1 \\
\hline B-03-088 & Sand Shiner & 6 & & & B-03-088_180603_E1 \\
\hline B-03-088 & White Sucker & 1 & $25^{\star}$ & YOY & B-03-088_180603_E1 \\
\hline B-03-089 & Fathead Minnow & 18 & & & B-03-089_180603_B1 \\
\hline B-03-089 & Sand Shiner & 3 & & & B-03-089_180603_B1 \\
\hline B-03-091 & Black Bullhead & 1 & $160^{*}$ & Released & B-03-091_180603_B1 \\
\hline B-03-091 & Fathead Minnow & 2 & & Released & B-03-091_180603_B1 \\
\hline B-03-093 & Brook Stickleback & 1 & & & B-03-093_180603_B1 \\
\hline B-03-093 & Fathead Minnow & 10 & & Released 8 & B-03-093_180603_B1 \\
\hline B-03-093 & Unidentified minnow & 8 & $15-18^{*}$ & Post-larval stage & B-03-093_180603_B1 \\
\hline B-03-093 & White Sucker & 1 & $20^{*}$ & YOY & B-03-093_180603_B1 \\
\hline B-03-094 & Fathead Minnow & 1 & & & B-03-094_180603_B1 \\
\hline B-03-094 & Northern Pike & 3 & $110,116,130$ & Released & B-03-094_180603_B1 \\
\hline B-03-094 & Silver Redhorse & 25 & $21-30^{*}$ & YOY & B-03-094_180603_B1 \\
\hline B-03-094 & Longnose Dace & 6 & & Released & B-03-094_180603_E1 \\
\hline B-03-094 & White Sucker & 1 & 330 & Released & B-03-094_180603_E1 \\
\hline B-03-095 & Brook Stickleback & 20 & & Released 6 & B-03-095_190603_E1 \\
\hline B-03-095 & Silver Redhorse & 1 & $27^{*}$ & YOY & B-03-095_190603_E1 \\
\hline B-03-097 & Brook Stickleback & 13 & & & B-03-097_190603_B1 \\
\hline B-03-097 & Fathead Minnow & 37 & & & B-03-097_190603_B1 \\
\hline B-03-098 & Creek Chub & 1 & & & B-03-098_190603_E1 \\
\hline B-03-098 & Troutperch & 2 & & & B-03-098_190603_E1 \\
\hline B-03-098 & Western Blacknose Dace & 6 & & Released & B-03-098_190603_E1 \\
\hline B-03-098 & White Sucker & 1 & 111 & & B-03-098_190603_E1 \\
\hline
\end{tabular}


Appendix 5: List of all fish captures made between 2002 and 2006, showing site number, common name and number (\#) of fish collected, fork (or total ${ }^{\star}$ ) length in millimetres ( $\mathrm{mm}$ ), any comments on the collection and the fish capture identification (ID) number.

\begin{tabular}{|c|c|c|c|c|c|}
\hline Site Number & Common Name & \# & $\begin{array}{c}\text { Fork/Total* } \\
\text { Length }(\mathrm{mm})\end{array}$ & Comments & $\begin{array}{l}\text { Fish Capture } \\
\text { ID Number }\end{array}$ \\
\hline B-03-099 & Troutperch & 2 & & & B-03-099_200603_E1 \\
\hline B-03-101 & Burbot & 1 & $222^{*}$ & & B-03-101_200603_B1 \\
\hline B-03-101 & Fathead Minnow & 6 & & Released & B-03-101_200603_B1 \\
\hline B-03-102 & Northern Pike & 1 & 220 & Released & B-03-102_230603_B1 \\
\hline B-03-102 & White Sucker & 1 & 420 & Released & B-03-102_230603_B1 \\
\hline B-03-103 & Blackside Darter & 2 & & & B-03-103_230603_E1 \\
\hline B-03-103 & Western Blacknose Dace & 2 & & & B-03-103_230603_E1 \\
\hline B-03-103 & White Sucker & 4 & $80-92$ & Released & B-03-103_230603_E1 \\
\hline B-03-104 & Brook Stickleback & 2 & & & B-03-104_230603_E1 \\
\hline B-03-104 & Fathead Minnow & 5 & & & B-03-104_230603_E1 \\
\hline B-03-104 & Finescale Dace & 2 & & & B-03-104_230603_E1 \\
\hline B-03-104 & Johnny Darter & 1 & & & B-03-104_230603_E1 \\
\hline B-03-104 & Western Blacknose Dace & 5 & & & B-03-104_230603_E1 \\
\hline B-03-112 & White Sucker & 1 & 110 & Released & B-03-112_260603_E1 \\
\hline B-03-115 & Finescale Dace & 1 & & & B-03-115_270603_E1 \\
\hline B-03-115 & Pearl Dace & 18 & & & B-03-115_270603_E1 \\
\hline B-03-115 & Western Blacknose Dace & 6 & & & B-03-115_270603_E1 \\
\hline B-03-115 & White Sucker & 1 & 160 & Released & B-03-115_270603_E1 \\
\hline B-03-116 & Fathead Minnow & 4 & $70-75$ & Released 2 & B-03-116_270603_E1 \\
\hline B-03-116 & Pearl Dace & 2 & & & B-03-116_270603_E1 \\
\hline B-03-116 & Western Blacknose Dace & 8 & & & B-03-116_270603_E1 \\
\hline B-03-116 & White Sucker & 2 & 110,130 & Released & B-03-116_270603_E1 \\
\hline B-03-117 & Brook Stickleback & 1 & & Released & B-03-117_270603_E1 \\
\hline B-03-118 & Brook Stickleback & 6 & & & B-03-118_270603_E1 \\
\hline B-03-118 & Central Mudminnow & 6 & & & B-03-118_270603_E1 \\
\hline B-03-118 & Finescale Dace & 8 & & & B-03-118_270603_E1 \\
\hline B-03-118 & White Sucker & 2 & $21,26^{*}$ & YOY & B-03-118_270603_E1 \\
\hline B-03-119 & Central Mudminnow & 2 & & Released & B-03-119_020703_E1 \\
\hline B-03-120 & Central Mudminnow & 9 & & & B-03-120_020703_E1 \\
\hline B-03-120 & Northern Pike & 3 & 88 & YOY, released 1 immature Northern Pike & B-03-120_020703_E1 \\
\hline B-03-120 & Western Blacknose Dace & 1 & & & B-03-120_020703_E1 \\
\hline
\end{tabular}


Appendix 5: List of all fish captures made between 2002 and 2006, showing site number, common name and number (\#) of fish collected, fork (or total ${ }^{\star}$ ) length in millimetres ( $\mathrm{mm}$ ), any comments on the collection and the fish capture identification (ID) number.

\begin{tabular}{|c|c|c|c|c|c|}
\hline Site Number & Common Name & \# & $\begin{array}{c}\text { Fork/Total* } \\
\text { Length }(\mathrm{mm})\end{array}$ & Comments & $\begin{array}{l}\text { Fish Capture } \\
\text { ID Number }\end{array}$ \\
\hline B-03-120 & White Sucker & 2 & 105 & Released 1 & B-03-120_020703_E1 \\
\hline B-03-121 & Iowa Darter & 1 & & & B-03-121_030703_E1 \\
\hline B-03-122 & Blackside Darter & 2 & & & B-03-122_030703_E1 \\
\hline B-03-122 & Brook Stickleback & 6 & & & B-03-122_030703_E1 \\
\hline B-03-122 & Central Mudminnow & 5 & & Released & B-03-122_030703_E1 \\
\hline B-03-122 & Fathead Minnow & 3 & & & B-03-122_030703_E1 \\
\hline B-03-122 & Johnny Darter & 4 & & & B-03-122_030703_E1 \\
\hline B-03-122 & Sand Shiner & 42 & & & B-03-122_030703_E1 \\
\hline B-03-122 & Western Blacknose Dace & 1 & & & B-03-122_030703_E1 \\
\hline B-03-122 & White Sucker & 16 & $29 *-175$ & 14 YOY, 2 juvenile & B-03-122_030703_E1 \\
\hline B-03-124 & Central Mudminnow & 1 & & & B-03-124_030703_E1 \\
\hline B-03-124 & Johnny Darter & 4 & & & B-03-124_030703_E1 \\
\hline B-03-124 & Western Blacknose Dace & 8 & & & B-03-124_030703_E1 \\
\hline B-03-124 & White Sucker & 2 & $24,26^{*}$ & YOY & B-03-124_030703_E1 \\
\hline B-03-126 & Creek Chub & 27 & & YOY & B-03-126_040703_B1 \\
\hline B-03-128 & Fathead Minnow & 7 & & Released & B-03-128_040703_D1 \\
\hline B-03-132 & Brook Trout & 1 & 170 & Released & B-03-132_070703_E1 \\
\hline B-03-132 & Creek Chub & 7 & & & B-03-132_070703_E1 \\
\hline B-03-132 & Johnny Darter & 3 & & & B-03-132_070703_E1 \\
\hline B-03-132 & Pearl Dace & 3 & & & B-03-132_070703_E1 \\
\hline B-03-132 & White Sucker & & 95,120 & Released & B-03-132_070703_E1 \\
\hline B-03-132 & Western Blacknose Dace & 31 & & & B-03-132_070703_E1 \\
\hline B-03-134 & Brook Trout & 3 & $130-145$ & Released & B-03-134_070703_E1 \\
\hline B-03-134 & White Sucker & 2 & 150,210 & Released 1 & B-03-134_070703_E1 \\
\hline B-03-136 & Brook Stickleback & 6 & & & B-03-136_070703_E1 \\
\hline B-03-136 & Sand Shiner & 3 & & & B-03-136_070703_E1 \\
\hline B-03-136 & White Sucker & 150 & $19-27^{*}$ & YOY & B-03-136_070703_E1 \\
\hline B-03-141 & Brook Stickleback & 17 & & Released & B-03-141_080703_E1 \\
\hline B-03-142 & Brook Stickleback & 5 & & & B-03-142_080703_E1 \\
\hline B-03-142 & Unidentified minnow & 9 & & Post-larval shiners & B-03-142_080703_E1 \\
\hline B-03-143 & Brook Stickleback & 2 & & & B-03-143_080703_B1 \\
\hline
\end{tabular}


Appendix 5: List of all fish captures made between 2002 and 2006, showing site number, common name and number (\#) of fish collected, fork (or total ${ }^{\star}$ ) length in millimetres ( $\mathrm{mm}$ ), any comments on the collection and the fish capture identification (ID) number.

\begin{tabular}{|c|c|c|c|c|c|}
\hline Site Number & Common Name & \# & $\begin{array}{c}\text { Fork/Total* } \\
\text { Length }(\mathrm{mm})\end{array}$ & Comments & $\begin{array}{l}\text { Fish Capture } \\
\text { ID Number }\end{array}$ \\
\hline B-03-143 & Common Shiner & 124 & & Released 80 & B-03-143_080703_B1 \\
\hline B-03-143 & Creek Chub & 40 & & Released & B-03-143_080703_B1 \\
\hline B-03-143 & Fathead Minnow & 24 & & Released 20 & B-03-143_080703_B1 \\
\hline B-03-143 & Unidentified minnow & 65 & & Post-larval dace & B-03-143_080703_B1 \\
\hline B-03-143 & White Sucker & 23 & $140-210$ & Released 20 & B-03-143_080703_B1 \\
\hline B-03-145 & No Catch & & & & B-03-145_090703_B1 \\
\hline B-03-148 & No Catch & & & & B-03-148_090703_E1 \\
\hline B-03-150 & Creek Chub & 2 & & & B-03-150_090703_B1 \\
\hline B-03-150 & Brook Stickleback & 2 & & & B-03-150_090703_B1 \\
\hline B-03-150 & Fathead Minnow & 25 & & & B-03-150_090703_B1 \\
\hline B-03-150 & Finescale Dace & 2 & & & B-03-150_090703_B1 \\
\hline B-03-151 & Blackside Darter & 1 & & & B-03-151_100703_E1 \\
\hline B-03-151 & Burbot & 1 & & & B-03-151_100703_E1 \\
\hline B-03-151 & Common Shiner & 1 & & Released & B-03-151_100703_K1 \\
\hline B-03-151 & Common Shiner & 6 & & & B-03-151_100703_E1 \\
\hline B-03-151 & Creek Chub & 7 & & Released 4 male and 14 female spawners & B-03-151_100703_E1 \\
\hline B-03-151 & Johnny Darter & 1 & & & B-03-151_100703_E1 \\
\hline B-03-151 & Longnose Dace & 3 & & Released & B-03-151_100703_K1 \\
\hline B-03-151 & Longnose Dace & 6 & & Released $3 @ 90,95,120$ mm, 3 are YOY & B-03-151_100703_E1 \\
\hline B-03-151 & Pearl Dace & 3 & & & B-03-151_100703_E1 \\
\hline B-03-151 & Sand Shiner & 7 & & & B-03-151_100703_E1 \\
\hline B-03-151 & Western Blacknose Dace & 23 & & & B-03-151_100703_E1 \\
\hline B-03-151 & White Sucker & 1 & 70 & & B-03-151_100703_E1 \\
\hline B-03-152 & Common Shiner & 1 & & & B-03-152_100703_E1 \\
\hline B-03-152 & Creek Chub & 16 & & Released 2 & B-03-152_100703_E1 \\
\hline B-03-152 & Johnny Darter & 4 & & & B-03-152_100703_E1 \\
\hline B-03-152 & Sand Shiner & 3 & & & B-03-152_100703_E1 \\
\hline B-03-152 & Western Blacknose Dace & 12 & & & B-03-152_100703_E1 \\
\hline $\mathrm{B}-03-152$ & White Sucker & 3 & $25^{\star}, 145,210$ & 1 YOY, also released 2 others & B-03-152 100703 E1 \\
\hline B-03-156 & Fathead Minnow & & & Observed in standing pools & B-03-156_110703_O1 \\
\hline B-03-157 & Fathead Minnow & & & Observed in standing pools & B-03-157_110703_O1 \\
\hline
\end{tabular}


Appendix 5: List of all fish captures made between 2002 and 2006, showing site number, common name and number (\#) of fish collected, fork (or total ${ }^{\star}$ ) length in millimetres ( $\mathrm{mm}$ ), any comments on the collection and the fish capture identification (ID) number.

\begin{tabular}{|c|c|c|c|c|c|}
\hline Site Number & Common Name & \# & $\begin{array}{c}\text { Fork/Total* } \\
\text { Length }(\mathrm{mm})\end{array}$ & Comments & $\begin{array}{l}\text { Fish Capture } \\
\text { ID Number }\end{array}$ \\
\hline B-03-158 & Brook Stickleback & 11 & & & B-03-158_110703_B1 \\
\hline B-03-158 & Common Shiner & 1 & & Released & B-03-158_110703_B1 \\
\hline B-03-158 & Fathead Minnow & 49 & & Released 40 & B-03-158_110703_B1 \\
\hline B-03-158 & Pearl Dace & 29 & & & B-03-158_110703_B1 \\
\hline B-03-158 & White Sucker & 10 & $111-200$ & Released 9 & B-03-158_110703_B1 \\
\hline B-03-159 & Creek Chub & 4 & & & B-03-159_110703_E1 \\
\hline B-03-159 & Silver Redhorse & 24 & $21-27^{*}$ & YOY & B-03-159_110703_E1 \\
\hline B-03-160 & Brook Stickleback & 15 & & & B-03-160_110703_B1 \\
\hline B-03-160 & Creek Chub & 1 & & Released & B-03-160_110703_B1 \\
\hline B-03-160 & Fathead Minnow & 50 & & Released & B-03-160_110703_B1 \\
\hline B-03-163 & Emerald Shiner & 6 & & & B-03-163_140703_B1 \\
\hline B-03-163 & Spottail Shiner & 6 & & & B-03-163_140703_B1 \\
\hline B-03-163 & Yellow Perch & 1 & 115 & Released & B-03-163_140703_B1 \\
\hline B-03-181 & Creek Chub & 25 & & $23 \mathrm{YOY}$ & B-03-181_150703_E1 \\
\hline B-03-181 & Johnny Darter & 5 & & & B-03-181_150703_E1 \\
\hline B-03-181 & Western Blacknose Dace & 4 & & & B-03-181_150703_E1 \\
\hline B-03-181 & White Sucker & 1 & $28^{*}$ & YOY & B-03-181_150703_E1 \\
\hline B-03-182 & Sand Shiner & 1 & & & B-03-182_160703_E1 \\
\hline B-03-182 & Unidentified minnow & 4 & & Post-larval dace & B-03-182_160703_E1 \\
\hline B-03-183 & Central Mudminnow & 1 & & & B-03-183_160703_B1 \\
\hline B-03-183 & Fathead Minnow & 22 & & & B-03-183_160703_B1 \\
\hline B-03-183 & Northern Pike & 1 & 160 & Released & B-03-183_160703_B1 \\
\hline B-03-183 & Rock Bass & 1 & $22^{*}$ & YOY & B-03-183_160703_B1 \\
\hline B-03-183 & Walleye & 2 & 67,325 & Released 1, 1 YOY & B-03-183_160703_B1 \\
\hline B-03-184 & Fathead Minnow & 8 & & & B-03-184_160703_E1 \\
\hline B-03-185 & Blackside Darter & 3 & & & B-03-185_160703_E1 \\
\hline B-03-185 & Johnny Darter & 1 & & & B-03-185_160703_E1 \\
\hline B-03-185 & Silver Redhorse & 4 & $200-220$ & Released & B-03-185_160703_E1 \\
\hline B-03-189 & Black Bullhead & 1 & 170 & Released & B-03-189_160703_B1 \\
\hline B-03-189 & Fathead Minnow & 19 & & & B-03-189_160703_B1 \\
\hline B-03-189 & Northern Pike & 3 & $90,270,285$ & Released & B-03-189_160703_B1 \\
\hline
\end{tabular}


Appendix 5: List of all fish captures made between 2002 and 2006, showing site number, common name and number (\#) of fish collected, fork (or total ${ }^{\star}$ ) length in millimetres ( $\mathrm{mm}$ ), any comments on the collection and the fish capture identification (ID) number.

\begin{tabular}{|c|c|c|c|c|c|}
\hline Site Number & Common Name & \# & $\begin{array}{c}\text { Fork/Total* } \\
\text { Length }(\mathrm{mm})\end{array}$ & Comments & $\begin{array}{l}\text { Fish Capture } \\
\text { ID Number }\end{array}$ \\
\hline B-03-189 & White Sucker & 18 & $34-44^{*}$ & YOY & B-03-189_160703_B1 \\
\hline B-03-190 & Fathead Minnow & 13 & & & B-03-190_160703_B1 \\
\hline B-03-190 & Johnny Darter & 1 & & & B-03-190_160703_B1 \\
\hline B-03-190 & White Sucker & 65 & $40-51^{*}$ & YOY & B-03-190_160703_B1 \\
\hline B-03-191 & Brook Stickleback & 7 & & & B-03-191_170703_E1 \\
\hline B-03-191 & Central Mudminnow & 20 & & Released 17 & B-03-191_170703_E1 \\
\hline B-03-191 & Sand Shiner & 7 & & & B-03-191_170703_E1 \\
\hline B-03-191 & White Sucker & 2 & 108,110 & YOY released & B-03-191_170703_E1 \\
\hline B-03-192 & Brook Stickleback & 1 & & Released & B-03-192_170703_B1 \\
\hline B-03-192 & Fathead Minnow & 9 & & Released & B-03-192_170703_B1 \\
\hline B-03-192 & White Sucker & 1 & 190 & Released & B-03-192_170703_B1 \\
\hline B-03-193 & No Catch & & & & B-03-193_170703_B1 \\
\hline B-03-194 & Black Bullhead & 57 & & Released 50 YOY & B-03-194_220703_B1 \\
\hline B-03-194 & Fathead Minnow & 307 & & Released 200 & B-03-194_220703_B1 \\
\hline B-03-199 & No Catch & & & & B-03-199_230703_E1 \\
\hline B-03-200 & Black Bullhead & 13 & $145-200$ & Released 12 & B-03-200_230703_B1 \\
\hline $\mathrm{B}-03-200$ & Black Crappie & 3 & $133,165,180$ & Released & B-03-200_230703_B1 \\
\hline B-03-200 & Sand Shiner & 4 & & & B-03-200_230703_B1 \\
\hline B-03-200 & Northern Pike & 5 & $350-405$ & Released & B-03-200_230703_B1 \\
\hline B-03-200 & River Darter & 1 & & & B-03-200_230703_B1 \\
\hline B-03-200 & Sauger & 1 & 310 & Released mature Sauger @ 310 mm & B-03-200_230703_B1 \\
\hline B-03-202 & Black Bullhead & 1 & & Released & B-03-202_230703_E1 \\
\hline B-03-202 & Brook Stickleback & 9 & & & B-03-202_230703_E1 \\
\hline B-03-202 & Fathead Minnow & 21 & & & B-03-202_230703_E1 \\
\hline $\mathrm{B}-03-202$ & Northern Redbelly Dace & 30 & & & B-03-202_230703_E1 \\
\hline B-03-204 & Brook Stickleback & 265 & & Released & B-03-204_230703_B1 \\
\hline B-03-204 & Central Mudminnow & 2 & & Released & B-03-204_230703_B1 \\
\hline B-03-204 & Fathead Minnow & 2 & & Released & B-03-204_230703_B1 \\
\hline B-03-204 & White Sucker & 1 & $46^{*}$ & YOY & B-03-204_230703_B1 \\
\hline B-03-206 & Brook Stickleback & 1 & & Released & B-03-206_240703_E1 \\
\hline B-03-206 & Central Mudminnow & 23 & & & B-03-206_240703_E1 \\
\hline
\end{tabular}


Appendix 5: List of all fish captures made between 2002 and 2006, showing site number, common name and number (\#) of fish collected, fork (or total ${ }^{*}$ ) length in millimetres $(\mathrm{mm})$, any comments on the collection and the fish capture identification (ID) number.

\begin{tabular}{|c|c|c|c|c|c|}
\hline Site Number & Common Name & \# & $\begin{array}{c}\text { Fork/Total* } \\
\text { Length }(\mathrm{mm})\end{array}$ & Comments & $\begin{array}{l}\text { Fish Capture } \\
\text { ID Number }\end{array}$ \\
\hline B-03-206 & Northern Pike & 2 & 140,155 & Released & B-03-206_240703_E1 \\
\hline B-03-206 & Walleye & 2 & 108,114 & YOY & B-03-206_240703_E1 \\
\hline B-03-206 & White Sucker & 11 & $42-49^{*}$ & Released 9 YOY & B-03-206_240703_E1 \\
\hline B-03-207 & Brook Stickleback & 12 & & Released 8 & B-03-207_240703_E1 \\
\hline B-03-207 & Central Mudminnow & 4 & & Released 3 & B-03-207_240703_E1 \\
\hline B-03-207 & Fathead Minnow & 54 & & Released 43 & B-03-207_240703_E1 \\
\hline B-03-207 & Northern Redbelly Dace & 40 & & & B-03-207_240703_E1 \\
\hline B-03-207 & White Sucker & 1 & 135 & Released & B-03-207_240703_E1 \\
\hline B-03-209 & Brook Stickleback & 25 & & Released & B-03-209_240703_B1 \\
\hline B-03-209 & Fathead Minnow & 11 & & Released & B-03-209_240703_B1 \\
\hline B-03-209 & White Sucker & 24 & & YOY released & B-03-209_240703_B1 \\
\hline $\mathrm{B}-03-212$ & Central Mudminnow & 4 & & Released & B-03-212_240703_E1 \\
\hline $\mathrm{B}-03-212$ & Fathead Minnow & 3 & & Released & B-03-212_240703_E1 \\
\hline B-03-212 & Northern Pike & 6 & $150-350$ & Released & B-03-212_240703_E1 \\
\hline B-03-218 & Northern Pike & 1 & 210 & Released & B-03-218_250703_D1 \\
\hline B-03-218 & Fathead Minnow & 7 & & Released & B-03-218_250703_D1 \\
\hline $\mathrm{B}-03-224$ & Brook Stickleback & 39 & & & B-03-224_050803_E1 \\
\hline B-03-224 & Central Mudminnow & 7 & & & B-03-224_050803_E1 \\
\hline $\mathrm{B}-03-224$ & Fathead Minnow & 36 & & & B-03-224_050803_E1 \\
\hline $\mathrm{B}-03-224$ & Finescale Dace & 8 & & & B-03-224_050803_E1 \\
\hline B-03-224 & Northern Redbelly Dace & 8 & & & B-03-224_050803_E1 \\
\hline B-03-224 & Yellow Perch & 1 & 120 & & B-03-224_050803_E1 \\
\hline $\mathrm{B}-03-226$ & Brook Stickleback & 1 & & & B-03-226_060803_E1 \\
\hline $\mathrm{B}-03-226$ & Brook Stickleback & 6 & & & B-03-226_060803_B1 \\
\hline B-03-226 & Common Carp & 1 & & & B-03-226_060803_B1 \\
\hline B-03-226 & Common Carp & 1 & & & B-03-226_060803_E1 \\
\hline B-03-226 & Fathead Minnow & 2 & & & B-03-226_060803_E1 \\
\hline $\mathrm{B}-03-226$ & Yellow Perch & 3 & $42,52,58$ & & B-03-226_060803_B1 \\
\hline B-03-226 & Yellow Perch & 3 & & Released & B-03-226_060803_E1 \\
\hline B-03-229 & Brook Stickleback & 100 & & & B-03-229_060803_D1 \\
\hline B-03-229 & Fathead Minnow & 100 & & & B-03-229_060803_D1 \\
\hline
\end{tabular}


Appendix 5: List of all fish captures made between 2002 and 2006, showing site number, common name and number (\#) of fish collected, fork (or total ${ }^{\star}$ ) length in millimetres ( $\mathrm{mm}$ ), any comments on the collection and the fish capture identification (ID) number.

\begin{tabular}{|c|c|c|c|c|c|}
\hline Site Number & Common Name & \# & $\begin{array}{c}\text { Fork/Total* } \\
\text { Length }(\mathrm{mm})\end{array}$ & Comments & $\begin{array}{l}\text { Fish Capture } \\
\text { ID Number }\end{array}$ \\
\hline B-03-229 & Western Blacknose Dace & 10 & & & B-03-229_060803_D1 \\
\hline B-03-235 & Brook Stickleback & 25 & & & B-03-235_060803_E1 \\
\hline B-03-235 & Central Mudminnow & 9 & & & B-03-235_060803_E1 \\
\hline B-03-235 & Fathead Minnow & 7 & & & B-03-235_060803_E1 \\
\hline B-03-235 & Finescale Dace & 5 & & & B-03-235_060803_E1 \\
\hline B-03-239 & Brook Stickleback & 9 & & Many released & B-03-239_070803_B1 \\
\hline B-03-239 & Common Carp & 6 & & & B-03-239_070803_B1 \\
\hline B-03-239 & Emerald Shiner & 4 & & Many released & B-03-239_070803_B1 \\
\hline B-03-239 & Fathead Minnow & 114 & & Many released & B-03-239_070803_B1 \\
\hline B-03-239 & Northern Pike & 1 & 141 & & B-03-239_070803_B1 \\
\hline B-03-239 & Spottail Shiner & 4 & & & B-03-239_070803_B1 \\
\hline B-03-239 & Yellow Perch & 2 & 85,122 & & B-03-239_070803_B1 \\
\hline B-03-241 & Common Carp & 8 & & & B-03-241_070803_E1 \\
\hline B-03-241 & Creek Chub & 3 & & & B-03-241_070803_E1 \\
\hline B-03-241 & Northern Pike & 2 & & YOY, several others observed & B-03-241_070803_E1 \\
\hline B-03-249 & Fathead Minnow & 64 & & & B-03-249_080803_B1 \\
\hline D-03-002 & No Catch & & & Invertebrates only & D-03-002_100503_K1 \\
\hline D-03-002 & Blacknose Shiner & 5 & & & D-03-002_100503_E1 \\
\hline D-03-002 & Brook Stickleback & 5 & & & D-03-002_100503_E1 \\
\hline D-03-002 & Johnny Darter & 11 & & & D-03-002_100503_E1 \\
\hline D-03-002 & Western Blacknose Dace & 6 & & & D-03-002_100503_E1 \\
\hline D-03-003 & Brook Stickleback & 4 & & Spawning & D-03-003_100503_E1 \\
\hline D-03-003 & Finescale Dace & 4 & & & D-03-003_100503_E1 \\
\hline D-03-003 & Pearl Dace & 23 & & & D-03-003_100503_E1 \\
\hline D-03-004 & Blacknose Shiner & 6 & & & D-03-004_110503_E1 \\
\hline D-03-004 & Brook Stickleback & 2 & & & D-03-004_110503_E1 \\
\hline D-03-004 & Creek Chub & 1 & & & D-03-004_110503_E1 \\
\hline D-03-004 & Fathead Minnow & 3 & & & D-03-004_110503_E1 \\
\hline D-03-004 & Finescale Dace & 3 & & & D-03-004_110503_E1 \\
\hline D-03-004 & Johnny Darter & 3 & & & D-03-004_110503_E1 \\
\hline D-03-004 & Pearl Dace & 6 & & & D-03-004_110503_E1 \\
\hline
\end{tabular}


Appendix 5: List of all fish captures made between 2002 and 2006, showing site number, common name and number (\#) of fish collected, fork (or total ${ }^{\star}$ ) length in millimetres ( $\mathrm{mm}$ ), any comments on the collection and the fish capture identification (ID) number.

\begin{tabular}{|c|c|c|c|c|c|}
\hline Site Number & Common Name & \# & $\begin{array}{c}\text { Fork/Total* } \\
\text { Length }(\mathrm{mm})\end{array}$ & Comments & $\begin{array}{l}\text { Fish Capture } \\
\text { ID Number }\end{array}$ \\
\hline D-03-004 & Western Blacknose Dace & 10 & & & D-03-004_110503_E1 \\
\hline D-03-005 & Blacknose Shiner & 5 & & & D-03-005_110503_E1 \\
\hline D-03-005 & Common Shiner & 17 & & & D-03-005_110503_E1 \\
\hline D-03-005 & Creek Chub & 9 & & Released 5 & D-03-005_110503_E1 \\
\hline D-03-005 & Fathead Minnow & 1 & & & D-03-005_110503_E1 \\
\hline D-03-005 & Finescale Dace & 3 & & & D-03-005_110503_E1 \\
\hline D-03-005 & Johnny Darter & 7 & & & D-03-005_110503_E1 \\
\hline D-03-005 & Longnose Dace & 4 & & & D-03-005_110503_E1 \\
\hline D-03-005 & Western Blacknose Dace & 20 & & & D-03-005_110503_E1 \\
\hline D-03-005 & White Sucker & 2 & 91,112 & & D-03-005_110503_E1 \\
\hline D-03-006 & Brook Stickleback & 7 & & & D-03-006_120503_E1 \\
\hline D-03-006 & Creek Chub & 1 & & & D-03-006_120503_E1 \\
\hline D-03-009 & Brook Stickleback & 20 & & Released & D-03-009_130503_E1 \\
\hline D-03-011 & Brook Trout & 1 & 132 & & D-03-011_130503_E1 \\
\hline D-03-011 & Common Shiner & 11 & & & D-03-011_130503_E1 \\
\hline D-03-011 & Creek Chub & 1 & & & D-03-011_130503_E1 \\
\hline D-03-011 & Longnose Dace & 3 & & & D-03-011_130503_E1 \\
\hline D-03-011 & Western Blacknose Dace & 1 & & & D-03-011_130503_E1 \\
\hline D-03-011 & White Sucker & 10 & $51-161$ & & D-03-011_130503_E1 \\
\hline D-03-011 & No Catch & & & Invertebrates only & D-03-011_130503_K1 \\
\hline D-03-012 & Blacknose Shiner & 3 & & & D-03-012_130503_E1 \\
\hline D-03-012 & Creek Chub & 1 & & & D-03-012_130503_E1 \\
\hline D-03-012 & Iowa Darter & 1 & & & D-03-012_130503_E1 \\
\hline D-03-012 & Johnny Darter & 9 & & & D-03-012_130503_E1 \\
\hline D-03-012 & Longnose Dace & 15 & & & D-03-012_130503_E1 \\
\hline D-03-012 & Pearl Dace & 11 & & & D-03-012_130503_E1 \\
\hline D-03-012 & Western Blacknose Dace & 19 & & & D-03-012_130503_E1 \\
\hline D-03-012 & White Sucker & 1 & 76 & & D-03-012_130503_E1 \\
\hline D-03-015 & Brook Stickleback & 6 & & & D-03-015_140503_E1 \\
\hline D-03-015 & Central Mudminnow & 6 & & & D-03-015_140503_E1 \\
\hline D-03-015 & Creek Chub & 3 & & & D-03-015_140503_E1 \\
\hline
\end{tabular}


Appendix 5: List of all fish captures made between 2002 and 2006, showing site number, common name and number (\#) of fish collected, fork (or total ${ }^{\star}$ ) length in millimetres ( $\mathrm{mm}$ ), any comments on the collection and the fish capture identification (ID) number.

\begin{tabular}{|c|c|c|c|c|c|}
\hline Site Number & Common Name & \# & $\begin{array}{l}\text { Fork/Total* } \\
\text { Length }(\mathrm{mm})\end{array}$ & Comments & $\begin{array}{l}\text { Fish Capture } \\
\text { ID Number }\end{array}$ \\
\hline D-03-015 & Finescale Dace & 5 & & & D-03-015_140503_E1 \\
\hline D-03-015 & Johnny Darter & 6 & & & D-03-015_140503_E1 \\
\hline D-03-015 & Longnose Dace & 6 & & & D-03-015_140503_E1 \\
\hline D-03-015 & Western Blacknose Dace & 9 & & & D-03-015_140503_E1 \\
\hline D-03-015 & White Sucker & 10 & $45-320$ & & D-03-015_140503_E1 \\
\hline D-03-016 & Finescale Dace & 4 & & & D-03-016_140503_E1 \\
\hline D-03-016 & Longnose Dace & 11 & & & D-03-016_140503_E1 \\
\hline D-03-016 & Western Blacknose Dace & 28 & & & D-03-016_140503_E1 \\
\hline D-03-017 & No Catch & & & & D-03-017_140503_E1 \\
\hline D-03-019 & Blacknose Shiner & 3 & & & D-03-019_140503_E1 \\
\hline D-03-019 & Finescale Dace & 1 & & & D-03-019_140503_E1 \\
\hline D-03-019 & White Sucker & 9 & $30 *-96$ & & D-03-019_140503_E1 \\
\hline D-03-023 & Brook Stickleback & 1 & & & D-03-023 030603 E1 \\
\hline D-03-023 & Fathead Minnow & 10 & & Spawning & D-03-023_030603_E1 \\
\hline D-03-024 & Brook Stickleback & 24 & & & D-03-024_030603_B1 \\
\hline D-03-024 & Fathead Minnow & 309 & & & D-03-024_030603_B1 \\
\hline D-03-025 & Brook Stickleback & 12 & & & D-03-025_030603_E1 \\
\hline D-03-025 & Central Mudminnow & 1 & & & D-03-025_030603_E1 \\
\hline D-03-025 & Fathead Minnow & 2 & & & D-03-025_030603_E1 \\
\hline D-03-027 & Brook Stickleback & 105 & & Released & D-03-027_040603_B1 \\
\hline D-03-027 & Fathead Minnow & 200 & & Released & D-03-027_040603_B1 \\
\hline D-03-029 & Brook Stickleback & 6 & & & D-03-029 040603 E1 \\
\hline D-03-029 & Fathead Minnow & 26 & & Spawning & D-03-029_040603_E1 \\
\hline D-03-035 & Brook Stickleback & 7 & & & D-03-035_050603_E1 \\
\hline D-03-035 & Brook Stickleback & 24 & & Released & D-03-035_050603_B1 \\
\hline D-03-035 & Creek Chub & 3 & & Released & D-03-035_050603_B1 \\
\hline D-03-035 & Fathead Minnow & 26 & & Spawning & D-03-035_050603_E1 \\
\hline D-03-035 & Fathead Minnow & 100 & & Released & D-03-035_050603_B1 \\
\hline D-03-036 & Bigmouth Shiner & 3 & & & D-03-036_050603_E1 \\
\hline D-03-036 & Blackside Darter & 1 & & & D-03-036_050603_E1 \\
\hline D-03-036 & Common Shiner & 11 & & & D-03-036_050603_E1 \\
\hline
\end{tabular}


Appendix 5: List of all fish captures made between 2002 and 2006, showing site number, common name and number (\#) of fish collected, fork (or total ${ }^{\star}$ ) length in millimetres ( $\mathrm{mm}$ ), any comments on the collection and the fish capture identification (ID) number.

\begin{tabular}{|c|c|c|c|c|c|}
\hline Site Number & Common Name & \# & $\begin{array}{c}\text { Fork/Total* } \\
\text { Length }(\mathrm{mm})\end{array}$ & Comments & $\begin{array}{l}\text { Fish Capture } \\
\text { ID Number }\end{array}$ \\
\hline D-03-036 & Creek Chub & 2 & & & D-03-036_050603_E1 \\
\hline D-03-036 & Fathead Minnow & 29 & & & D-03-036_050603_E1 \\
\hline D-03-036 & Johnny Darter & 1 & & & D-03-036_050603_E1 \\
\hline D-03-036 & Longnose Dace & 2 & & & D-03-036_050603_E1 \\
\hline D-03-036 & Sand Shiner & 75 & & & D-03-036_050603_E1 \\
\hline D-03-036 & Silver Redhorse & 8 & $61-264$ & $7 \mathrm{YOY}, 61-69 \mathrm{~mm}$ & D-03-036_050603_E1 \\
\hline D-03-036 & Western Blacknose Dace & 5 & & & D-03-036_050603_E1 \\
\hline D-03-037 & Brook Stickleback & 8 & & & D-03-037_050603_E1 \\
\hline D-03-037 & Creek Chub & 3 & & & D-03-037_050603_E1 \\
\hline D-03-037 & Fathead Minnow & 4 & & & D-03-037_050603_E1 \\
\hline D-03-037 & Western Blacknose Dace & 3 & & & D-03-037_050603_E1 \\
\hline D-03-038 & Blackside Darter & 20 & & & D-03-038_060603_E1 \\
\hline D-03-038 & Brook Stickleback & 1 & & & D-03-038_060603_E1 \\
\hline D-03-038 & Burbot & 1 & & & D-03-038_060603_E1 \\
\hline D-03-038 & Common Carp & 10 & & Released & D-03-038_060603_E1 \\
\hline D-03-038 & Common Shiner & 10 & & & D-03-038_060603_E1 \\
\hline D-03-038 & Creek Chub & 1 & & & D-03-038_060603_E1 \\
\hline D-03-038 & Emerald Shiner & 22 & & & D-03-038_060603_E1 \\
\hline D-03-038 & Fathead Minnow & 6 & & & D-03-038_060603_E1 \\
\hline D-03-038 & Iowa Darter & 1 & & & D-03-038_060603_E1 \\
\hline D-03-038 & Johnny Darter & 6 & & & D-03-038_060603_E1 \\
\hline D-03-038 & Longnose Dace & 6 & & & D-03-038_060603_E1 \\
\hline D-03-038 & Silver Redhorse & 3 & $111-136$ & & D-03-038_060603_E1 \\
\hline D-03-038 & Walleye & 1 & 280 & Mature, released & D-03-038_060603_E1 \\
\hline D-03-038 & Western Blacknose Dace & 2 & & & D-03-038_060603_E1 \\
\hline D-03-038 & White Sucker & 1 & 176 & & D-03-038_060603_E1 \\
\hline D-03-040 & Black Bullhead & 1 & $250^{*}$ & & D-03-040_100603_E1 \\
\hline D-03-041 & Fathead Minnow & 23 & & & D-03-041_100603_E1 \\
\hline D-03-044 & Black Bullhead & 1 & & & D-03-044_110603_E1 \\
\hline D-03-044 & Fathead Minnow & 25 & & Spawning & D-03-044_110603_E1 \\
\hline D-03-044 & White Sucker & 3 & $68-108$ & & D-03-044_110603_E1 \\
\hline
\end{tabular}


Appendix 5: List of all fish captures made between 2002 and 2006, showing site number, common name and number (\#) of fish collected, fork (or total ${ }^{\star}$ ) length in millimetres ( $\mathrm{mm}$ ), any comments on the collection and the fish capture identification (ID) number.

\begin{tabular}{|c|c|c|c|c|c|}
\hline Site Number & Common Name & \# & $\begin{array}{c}\text { Fork/Total* } \\
\text { Length }(\mathrm{mm})\end{array}$ & Comments & $\begin{array}{l}\text { Fish Capture } \\
\text { ID Number }\end{array}$ \\
\hline D-03-045 & No Catch & & & & D-03-045_110603_E1 \\
\hline D-03-047 & Black Bullhead & 3 & $110-120$ & & D-03-047_110603_E1 \\
\hline D-03-049 & No Catch & & & & D-03-049_110603_E1 \\
\hline D-03-054 & Brook Stickleback & 49 & & Released & D-03-054_130603_E2 \\
\hline D-03-054 & Creek Chub & 16 & & & D-03-054_130603_E1 \\
\hline D-03-054 & Finescale Dace & 16 & & & D-03-054_130603_E1 \\
\hline D-03-054 & Johnny Darter & 7 & & & D-03-054_130603_E1 \\
\hline D-03-054 & Pearl Dace & 3 & & & D-03-054_130603_E1 \\
\hline D-03-054 & Western Blacknose Dace & 20 & & & D-03-054_130603_E1 \\
\hline D-03-054 & White Sucker & 1 & 44 & YOY & D-03-054_130603_E2 \\
\hline D-03-054 & White Sucker & 2 & 102,172 & & D-03-054_130603_E1 \\
\hline D-03-057 & Fathead Minnow & 20 & & & D-03-057_130603_E1 \\
\hline D-03-057 & Finescale Dace & 1 & & & D-03-057_130603_E1 \\
\hline D-03-057 & White Sucker & 2 & $21^{*}, 220$ & $1 \mathrm{YOY}$ & D-03-057_130603_E1 \\
\hline D-03-058 & Common Shiner & 15 & & & D-03-058_160603_E1 \\
\hline D-03-058 & Fathead Minnow & 2 & & & D-03-058_160603_E1 \\
\hline D-03-058 & Iowa Darter & 6 & & & D-03-058_160603_E1 \\
\hline D-03-058 & Johnny Darter & 4 & & & D-03-058_160603_E1 \\
\hline D-03-058 & Logperch & 5 & & & D-03-058_160603_E1 \\
\hline D-03-058 & Longnose Dace & 6 & & & D-03-058_160603_E1 \\
\hline D-03-058 & Western Blacknose Dace & 3 & & & D-03-058_160603_E1 \\
\hline D-03-059 & Common Shiner & 14 & & & D-03-059_160603_E1 \\
\hline D-03-059 & Johnny Darter & 2 & & & D-03-059_160603_E1 \\
\hline D-03-059 & Logperch & 4 & & & D-03-059_160603_E1 \\
\hline D-03-059 & Longnose Dace & 6 & & & D-03-059_160603_E1 \\
\hline D-03-059 & Pearl Dace & 2 & & & D-03-059_160603_E1 \\
\hline D-03-059 & Western Blacknose Dace & 11 & & & D-03-059_160603_E1 \\
\hline D-03-060 & Common Shiner & 32 & & & D-03-060_160603_E1 \\
\hline D-03-060 & Fathead Minnow & 3 & & & D-03-060_160603_E1 \\
\hline D-03-060 & Johnny Darter & 4 & & & D-03-060_160603_E1 \\
\hline D-03-060 & Western Blacknose Dace & 3 & & & D-03-060_160603_E1 \\
\hline
\end{tabular}


Appendix 5: List of all fish captures made between 2002 and 2006, showing site number, common name and number (\#) of fish collected, fork (or total ${ }^{*}$ ) length in millimetres $(\mathrm{mm})$, any comments on the collection and the fish capture identification (ID) number.

\begin{tabular}{|c|c|c|c|c|c|}
\hline Site Number & Common Name & \# & $\begin{array}{l}\text { Fork/Total* } \\
\text { Length (mm) }\end{array}$ & Comments & $\begin{array}{l}\text { Fish Capture } \\
\text { ID Number }\end{array}$ \\
\hline D-03-060 & White Sucker & 6 & $175-199$ & Released 2 & D-03-060_160603_E1 \\
\hline D-03-061 & Blacknose Shiner & 3 & & & D-03-061_170603_E1 \\
\hline D-03-061 & Brassy Minnow & 2 & & & D-03-061_170603_E2 \\
\hline D-03-061 & Brook Stickleback & 3 & & & D-03-061_170603_E2 \\
\hline D-03-061 & Brook Stickleback & 8 & & & D-03-061_170603_E1 \\
\hline D-03-061 & Common Shiner & 4 & & & D-03-061_170603_E2 \\
\hline D-03-061 & Common Shiner & 11 & & & D-03-061_170603_E1 \\
\hline D-03-061 & Creek Chub & 6 & & & D-03-061_170603_E2 \\
\hline D-03-061 & Creek Chub & 10 & & & D-03-061_170603_E1 \\
\hline D-03-061 & Fathead Minnow & 4 & & & D-03-061_170603_E1 \\
\hline D-03-061 & Finescale Dace & 6 & & & D-03-061_170603_E1 \\
\hline D-03-061 & Johnny Darter & 2 & & & D-03-061_170603_E2 \\
\hline D-03-061 & Pearl Dace & 1 & & & D-03-061_170603_E1 \\
\hline D-03-061 & Silver Redhorse & 3 & $24-26^{*}$ & YOY & D-03-061_170603_E1 \\
\hline D-03-061 & Western Blacknose Dace & 7 & & & D-03-061_170603_E1 \\
\hline D-03-061 & White Sucker & 3 & $58,83,160$ & & D-03-061_170603_E1 \\
\hline D-03-061 & White Sucker & 4 & $80-120$ & & D-03-061_170603_E2 \\
\hline D-03-062 & Common Shiner & 6 & & & D-03-062_170603_E1 \\
\hline D-03-062 & Creek Chub & 2 & & & D-03-062_170603_E1 \\
\hline D-03-062 & Pearl Dace & 2 & & & D-03-062_170603_E1 \\
\hline D-03-062 & Western Blacknose Dace & 19 & & & D-03-062_170603_E1 \\
\hline D-03-062 & White Sucker & 1 & $22^{*}$ & YOY & D-03-062_170603_E1 \\
\hline D-03-064 & Brook Stickleback & 15 & & & D-03-064_170603_B1 \\
\hline D-03-064 & Common Shiner & 14 & & & D-03-064_170603_B1 \\
\hline D-03-064 & Creek Chub & 2 & & & D-03-064_170603_B1 \\
\hline D-03-064 & Fathead Minnow & 3 & & & D-03-064_170603_B1 \\
\hline D-03-064 & Finescale Dace & 9 & & & D-03-064_170603_B1 \\
\hline D-03-064 & Pearl Dace & 1 & & & D-03-064_170603_B1 \\
\hline D-03-064 & Western Blacknose Dace & 3 & & & D-03-064_170603_B1 \\
\hline D-03-065 & Blacknose Shiner & 12 & & & D-03-065_170603_B1 \\
\hline D-03-065 & Brassy Minnow & 5 & & & D-03-065_170603_B1 \\
\hline
\end{tabular}


Appendix 5: List of all fish captures made between 2002 and 2006, showing site number, common name and number (\#) of fish collected, fork (or total ${ }^{\star}$ ) length in millimetres $(\mathrm{mm})$, any comments on the collection and the fish capture identification (ID) number.

\begin{tabular}{|c|c|c|c|c|c|}
\hline Site Number & Common Name & \# & $\begin{array}{c}\text { Fork/Total* } \\
\text { Length }(\mathrm{mm})\end{array}$ & Comments & $\begin{array}{l}\text { Fish Capture } \\
\text { ID Number }\end{array}$ \\
\hline D-03-065 & Brook Stickleback & 2 & & & D-03-065_170603_B1 \\
\hline D-03-065 & Common Shiner & 83 & & & D-03-065_170603_B1 \\
\hline D-03-065 & Creek Chub & 7 & & & D-03-065_170603_B1 \\
\hline D-03-065 & Fathead Minnow & 4 & & & D-03-065_170603_B1 \\
\hline D-03-065 & Finescale Dace & 1 & & & D-03-065_170603_B1 \\
\hline D-03-065 & Longnose Dace & 2 & & & D-03-065_170603_B1 \\
\hline D-03-065 & Northern Redbelly Dace & 6 & & & D-03-065_170603_B1 \\
\hline D-03-065 & Pearl Dace & 1 & & & D-03-065_170603_B1 \\
\hline D-03-065 & Western Blacknose Dace & 3 & & & D-03-065_170603_B1 \\
\hline D-03-065 & White Sucker & 29 & $25^{*}-200$ & $25 \mathrm{YOY}$ & D-03-065_170603_B1 \\
\hline D-03-066 & Blacknose Shiner & 11 & & & D-03-066_180603_E1 \\
\hline D-03-066 & Brook Stickleback & 5 & & & D-03-066_180603_E1 \\
\hline D-03-066 & Finescale Dace & 2 & & & D-03-066_180603_E1 \\
\hline D-03-067 & Blacknose Shiner & 2 & & & D-03-067_180603_E1 \\
\hline D-03-067 & Brassy Minnow & 60 & & & D-03-067_180603_E1 \\
\hline D-03-067 & Brook Stickleback & 1 & & & D-03-067_180603_E1 \\
\hline D-03-067 & Creek Chub & 9 & & & D-03-067_180603_E1 \\
\hline D-03-067 & Fathead Minnow & 48 & & & D-03-067_180603_E1 \\
\hline D-03-067 & Finescale Dace & 18 & & & D-03-067_180603_E1 \\
\hline D-03-067 & Iowa Darter & 1 & & & D-03-067_180603_E1 \\
\hline D-03-067 & Northern Redbelly Dace & 15 & & & D-03-067_180603_E1 \\
\hline D-03-067 & Pearl Dace & 1 & & & D-03-067_180603_E1 \\
\hline D-03-067 & Western Blacknose Dace & 36 & & & D-03-067_180603_E1 \\
\hline D-03-067 & White Sucker & 1 & 112 & & D-03-067_180603_E1 \\
\hline D-03-071 & Common Shiner & 45 & & & D-03-071_230603_B1 \\
\hline D-03-071 & Northern Pike & 1 & 116 & YOY & D-03-071_230603_B1 \\
\hline D-03-071 & White Sucker & 1 & $32^{*}$ & YOY & D-03-071_230603_B1 \\
\hline D-03-073 & Blacknose Shiner & 2 & & & D-03-073_230603_E1 \\
\hline D-03-073 & Brassy Minnow & 2 & & & D-03-073_230603_E1 \\
\hline D-03-073 & Brook Stickleback & 1 & & & D-03-073_230603_E1 \\
\hline D-03-073 & Creek Chub & 1 & & & D-03-073_230603_E1 \\
\hline
\end{tabular}


Appendix 5: List of all fish captures made between 2002 and 2006, showing site number, common name and number (\#) of fish collected, fork (or total ${ }^{\star}$ ) length in millimetres $(\mathrm{mm})$, any comments on the collection and the fish capture identification (ID) number.

\begin{tabular}{|c|c|c|c|c|c|}
\hline Site Number & Common Name & \# & $\begin{array}{c}\text { Fork/Total* } \\
\text { Length }(\mathrm{mm})\end{array}$ & Comments & $\begin{array}{l}\text { Fish Capture } \\
\text { ID Number }\end{array}$ \\
\hline D-03-073 & Fathead Minnow & 9 & & & D-03-073_230603_E1 \\
\hline D-03-073 & Finescale Dace & 36 & & & D-03-073_230603_E1 \\
\hline D-03-073 & Johnny Darter & 1 & & & D-03-073_230603_E1 \\
\hline D-03-073 & Pearl Dace & 1 & & & D-03-073_230603_E1 \\
\hline D-03-073 & Western Blacknose Dace & 2 & & & D-03-073_230603_E1 \\
\hline D-03-073 & White Sucker & 13 & $76-102$ & & D-03-073_230603_E1 \\
\hline D-03-075 & No Catch & & & & D-03-075_230603_E1 \\
\hline D-03-076 & Fathead Minnow & 4 & & Released & D-03-076_230603_E1 \\
\hline D-03-076 & Northern Pike & 2 & 166,172 & Released & D-03-076_230603_E1 \\
\hline D-03-076 & Brook Stickleback & 3 & & Released & D-03-076_230603_E1 \\
\hline D-03-078 & Brook Stickleback & 100 & & Released & D-03-078_240603_B1 \\
\hline D-03-078 & Fathead Minnow & 100 & & Released & D-03-078_240603_B1 \\
\hline D-03-078 & Finescale Dace & 1 & & Released & D-03-078_240603_B1 \\
\hline D-03-081 & Blacknose Shiner & 1 & & & D-03-081_240603_E1 \\
\hline D-03-081 & Common Shiner & 10 & & & D-03-081_240603_E1 \\
\hline D-03-081 & Creek Chub & 1 & & & D-03-081_240603_E1 \\
\hline D-03-081 & Fathead Minnow & 4 & & & D-03-081_240603_E1 \\
\hline D-03-081 & Finescale Dace & 5 & & & D-03-081_240603_E1 \\
\hline D-03-081 & Johnny Darter & 1 & & & D-03-081_240603_E1 \\
\hline D-03-081 & Longnose Dace & 3 & & & D-03-081 240603 E1 \\
\hline D-03-081 & Western Blacknose Dace & 7 & & & D-03-081_240603_E1 \\
\hline D-03-083 & Northern Pike & 1 & 300 & Released & D-03-083_260603_E1 \\
\hline D-03-083 & Western Blacknose Dace & 2 & & Released & D-03-083_260603_E1 \\
\hline D-03-083 & White Sucker & 1 & 220 & & D-03-083_260603_E1 \\
\hline D-03-084 & Brook Stickleback & 27 & & & D-03-084_260603_E1 \\
\hline D-03-084 & Fathead Minnow & 127 & & & D-03-084_260603_E1 \\
\hline D-03-085 & White Sucker & 10 & & & D-03-085_270603_E1 \\
\hline D-03-086 & Brook Stickleback & 32 & & & D-03-086_270603_E1 \\
\hline D-03-086 & Creek Chub & 5 & & & D-03-086_270603_E1 \\
\hline D-03-086 & Fathead Minnow & 53 & & & D-03-086_270603_E1 \\
\hline D-03-086 & Finescale Dace & 21 & & & D-03-086_270603_E1 \\
\hline
\end{tabular}


Appendix 5: List of all fish captures made between 2002 and 2006, showing site number, common name and number (\#) of fish collected, fork (or total ${ }^{\star}$ ) length in millimetres $(\mathrm{mm})$, any comments on the collection and the fish capture identification (ID) number.

\begin{tabular}{|c|c|c|c|c|c|}
\hline Site Number & Common Name & \# & $\begin{array}{c}\text { Fork/Total* } \\
\text { Length }(\mathrm{mm})\end{array}$ & Comments & $\begin{array}{l}\text { Fish Capture } \\
\text { ID Number }\end{array}$ \\
\hline D-03-086 & Johnny Darter & 3 & & & D-03-086_270603_E1 \\
\hline D-03-086 & Northern Redbelly Dace & 2 & & & D-03-086_270603_E1 \\
\hline D-03-086 & Pearl Dace & 1 & & & D-03-086_270603_E1 \\
\hline D-03-086 & Silver Redhorse & 1 & $26^{*}$ & YOY & D-03-086_270603_E1 \\
\hline D-03-086 & Western Blacknose Dace & 15 & & & D-03-086_270603_E1 \\
\hline D-03-086 & White Sucker & 1 & 171 & & D-03-086_270603_E1 \\
\hline D-03-088 & Brassy Minnow & 1 & & & D-03-088_020703_E1 \\
\hline D-03-088 & Common Shiner & 33 & & & D-03-088_020703_E1 \\
\hline D-03-088 & Creek Chub & 5 & & & D-03-088_020703_E1 \\
\hline D-03-088 & Fathead Minnow & 3 & & & D-03-088_020703_E1 \\
\hline D-03-088 & Iowa Darter & 1 & & & D-03-088_020703_E1 \\
\hline D-03-088 & Johnny Darter & 16 & & & D-03-088_020703_E1 \\
\hline D-03-088 & Longnose Dace & 9 & & & D-03-088_020703_E1 \\
\hline D-03-088 & Western Blacknose Dace & 18 & & & D-03-088_020703_E1 \\
\hline D-03-088 & White Sucker & 2 & 107,114 & & D-03-088_020703_E1 \\
\hline D-03-089 & Common Shiner & 12 & & & D-03-089_020703_E1 \\
\hline D-03-089 & Creek Chub & 10 & & & D-03-089_020703_E1 \\
\hline D-03-089 & Fathead Minnow & 24 & & & D-03-089_020703_E1 \\
\hline D-03-089 & Finescale Dace & 3 & & & D-03-089_020703_E1 \\
\hline D-03-089 & Johnny Darter & 18 & & & D-03-089_020703_E1 \\
\hline D-03-089 & Northern Redbelly Dace & 4 & & & D-03-089_020703_E1 \\
\hline D-03-089 & Western Blacknose Dace & 24 & & & D-03-089_020703_E1 \\
\hline D-03-089 & White Sucker & 8 & $83-216$ & 216 is mature male & D-03-089_020703_E1 \\
\hline D-03-090 & Brook Stickleback & 100 & & Released & D-03-090_020703_D1 \\
\hline D-03-090 & Creek Chub & 100 & & Released & D-03-090_020703_D1 \\
\hline D-03-090 & Fathead Minnow & 100 & & Released & D-03-090_020703_D1 \\
\hline D-03-090 & Northern Redbelly Dace & 50 & & Released & D-03-090_020703_D1 \\
\hline D-03-090 & Finescale Dace & 50 & & Released & D-03-090_020703_D1 \\
\hline D-03-090 & Pearl Dace & 10 & & Released & D-03-090_020703_D1 \\
\hline D-03-091 & Brook Stickleback & 1 & & & D-03-091_030703_E1 \\
\hline D-03-091 & Common Shiner & 3 & & & D-03-091_030703_E1 \\
\hline
\end{tabular}


Appendix 5: List of all fish captures made between 2002 and 2006, showing site number, common name and number (\#) of fish collected, fork (or total ${ }^{\star}$ ) length in millimetres ( $\mathrm{mm}$ ), any comments on the collection and the fish capture identification (ID) number.

\begin{tabular}{|c|c|c|c|c|c|}
\hline Site Number & Common Name & \# & $\begin{array}{c}\text { Fork/Total* } \\
\text { Length }(\mathrm{mm})\end{array}$ & Comments & $\begin{array}{l}\text { Fish Capture } \\
\text { ID Number }\end{array}$ \\
\hline D-03-091 & Creek Chub & 4 & & & D-03-091_030703_E1 \\
\hline D-03-091 & Fathead Minnow & 11 & & & D-03-091_030703_E1 \\
\hline D-03-091 & Johnny Darter & 7 & & & D-03-091_030703_E1 \\
\hline D-03-091 & Western Blacknose Dace & 6 & & & D-03-091_030703_E1 \\
\hline D-03-091 & White Sucker & 20 & $46-350$ & $15 \mathrm{YOY}$ & D-03-091_030703_E1 \\
\hline D-03-094 & Brook Stickleback & 1 & & Released & D-03-094_080703_E1 \\
\hline D-03-095 & Brook Stickleback & 4 & & & D-03-095_080703_E1 \\
\hline D-03-096 & Brook Stickleback & 6 & & & D-03-096_080703_E1 \\
\hline D-03-097 & Blackside Darter & 12 & & & D-03-097_090703_E1 \\
\hline D-03-097 & Common Shiner & 17 & & & D-03-097_090703_E1 \\
\hline D-03-097 & Creek Chub & 12 & & & D-03-097_090703_E1 \\
\hline D-03-097 & Johnny Darter & 7 & & & D-03-097_090703_E1 \\
\hline D-03-097 & Sand Shiner & 1 & & & D-03-097_090703_E1 \\
\hline D-03-097 & Western Blacknose Dace & 1 & & & D-03-097_090703_E1 \\
\hline D-03-097 & White Sucker & 3 & $71-116$ & & D-03-097_090703_E1 \\
\hline D-03-098 & Brook Stickleback & 50 & & & D-03-098_090703_B1 \\
\hline D-03-098 & Creek Chub & 10 & & & D-03-098_090703_B1 \\
\hline D-03-098 & Fathead Minnow & 13 & & & D-03-098_090703_B1 \\
\hline D-03-098 & White Sucker & 220 & $51-57$ & YOY & D-03-098_090703_B1 \\
\hline D-03-100 & Brook Stickleback & 13 & & & D-03-100_090703_E1 \\
\hline D-03-100 & Fathead Minnow & 1 & & & D-03-100_090703_E1 \\
\hline D-03-101 & Brook Stickleback & 10 & & & D-03-101_090703_E1 \\
\hline D-03-101 & Fathead Minnow & 7 & & & D-03-101_090703_E1 \\
\hline D-03-102 & No Catch & & & & D-03-102_110703_E1 \\
\hline D-03-103 & Northern Pike & 2 & 86,96 & YOY-7 others observed & D-03-103_110703_E1 \\
\hline D-03-105 & Northern Pike & 1 & & YOY & D-03-105_110703_D1 \\
\hline D-03-105 & Walleye & 1 & & YOY & D-03-105_110703_D1 \\
\hline D-03-105 & White Sucker & 1 & & YOY & D-03-105_110703_D1 \\
\hline D-03-110 & Brook Stickleback & 24 & & & D-03-110_140703_E1 \\
\hline D-03-110 & Central Mudminnow & 4 & & & D-03-110_140703_E1 \\
\hline D-03-111 & Brook Stickleback & 55 & & & D-03-111_140703_B1 \\
\hline
\end{tabular}


Appendix 5: List of all fish captures made between 2002 and 2006, showing site number, common name and number (\#) of fish collected, fork (or total ${ }^{\star}$ ) length in millimetres ( $\mathrm{mm}$ ), any comments on the collection and the fish capture identification (ID) number.

\begin{tabular}{|c|c|c|c|c|c|}
\hline Site Number & Common Name & \# & $\begin{array}{l}\text { Fork/Total* } \\
\text { Length }(\mathrm{mm})\end{array}$ & Comments & $\begin{array}{l}\text { Fish Capture } \\
\text { ID Number }\end{array}$ \\
\hline D-03-111 & Fathead Minnow & 87 & & & D-03-111_140703_B1 \\
\hline D-03-111 & Finescale Dace & 2 & & & D-03-111_140703_B1 \\
\hline D-03-111 & White Sucker & 5 & $40-170$ & 4 YOY & D-03-111_140703_B1 \\
\hline D-03-117 & Brook Stickleback & 57 & & & D-03-117_150703_E1 \\
\hline D-03-117 & Fathead Minnow & 34 & & & D-03-117_150703_E1 \\
\hline D-03-117 & Finescale Dace & 2 & & & D-03-117_150703_E1 \\
\hline D-03-117 & Johnny Darter & 2 & & & D-03-117_150703_E1 \\
\hline D-03-117 & Pearl Dace & 1 & & & D-03-117_150703_E1 \\
\hline D-03-117 & Western Blacknose Dace & 1 & & & D-03-117_150703_E1 \\
\hline D-03-117 & White Sucker & 56 & $28-38^{*}$ & YOY & D-03-117_150703_E1 \\
\hline D-03-120 & Northern Pike & 10 & $150-250$ & $\begin{array}{l}\text { Observed under bridge, } 2 \text { year/size } \\
\text { classes }\end{array}$ & D-03-120_160703_01 \\
\hline D-03-121 & Northern Pike & 3 & $85-100$ & YOY & D-03-121_160703_E1 \\
\hline D-03-122 & Brook Trout & 1 & 138 & & D-03-122_160703_E1 \\
\hline D-03-122 & Creek Chub & 1 & & & D-03-122_160703_E1 \\
\hline D-03-122 & Finescale Dace & 13 & & & D-03-122_160703_E1 \\
\hline D-03-122 & Pearl Dace & 1 & & & D-03-122_160703_E1 \\
\hline D-03-122 & Western Blacknose Dace & 16 & & & D-03-122_160703_E1 \\
\hline D-03-124 & Blacknose Shiner & 11 & & & D-03-124_160703_E1 \\
\hline D-03-124 & Creek Chub & 2 & & & D-03-124_160703_E1 \\
\hline D-03-124 & Fathead Minnow & 9 & & & D-03-124_160703_E1 \\
\hline D-03-124 & Finescale Dace & 14 & & & D-03-124_160703_E1 \\
\hline D-03-124 & Western Blacknose Dace & 22 & & & D-03-124_160703_E1 \\
\hline D-03-124 & White Sucker & 1 & $34^{*}$ & YOY & D-03-124_160703_E1 \\
\hline D-03-126 & Northern Pike & 10 & $200-250$ & Released & D-03-126_170703_B1 \\
\hline D-03-129 & Creek Chub & 10 & & & D-03-129_170703_D1 \\
\hline D-03-129 & Fathead Minnow & 10 & & & D-03-129_170703_D1 \\
\hline D-03-129 & Western Blacknose Dace & 10 & & & D-03-129_170703_D1 \\
\hline D-03-132 & Brook Stickleback & 5 & & & D-03-132_170703_E1 \\
\hline D-03-132 & Fathead Minnow & 1 & & & D-03-132_170703_E1 \\
\hline D-03-132 & Finescale Dace & 32 & & & D-03-132_170703_E1 \\
\hline
\end{tabular}


Appendix 5: List of all fish captures made between 2002 and 2006, showing site number, common name and number (\#) of fish collected, fork (or total ${ }^{\star}$ ) length in millimetres $(\mathrm{mm})$, any comments on the collection and the fish capture identification (ID) number.

\begin{tabular}{|c|c|c|c|c|c|}
\hline Site Number & Common Name & \# & $\begin{array}{c}\text { Fork/Total* } \\
\text { Length }(\mathrm{mm})\end{array}$ & Comments & $\begin{array}{l}\text { Fish Capture } \\
\text { ID Number }\end{array}$ \\
\hline D-03-132 & Johnny Darter & $\overline{1}$ & & & D-03-132_170703_E1 \\
\hline D-03-132 & Northern Redbelly Dace & 15 & & & D-03-132_170703_E1 \\
\hline D-03-132 & Pearl Dace & 1 & & Released & D-03-132_170703_E1 \\
\hline D-03-132 & Western Blacknose Dace & 8 & & & D-03-132_170703_E1 \\
\hline D-03-132 & White Sucker & 1 & $29^{*}$ & YOY & D-03-132_170703_E1 \\
\hline D-03-134 & Brook Stickleback & 12 & & Released 10 & D-03-134_170703_B1 \\
\hline D-03-134 & Creek Chub & 139 & & Released 100 & D-03-134_170703_B1 \\
\hline D-03-134 & Fathead Minnow & 143 & & Released 100 & D-03-134_170703_B1 \\
\hline D-03-134 & Finescale Dace & 13 & & & D-03-134_170703_B1 \\
\hline D-03-138 & Brook Stickleback & 3 & & Released & D-03-138_180703_E1 \\
\hline D-03-138 & Blackchin Shiner & 19 & & & D-03-138_180703_E1 \\
\hline D-03-138 & Blacknose Shiner & 3 & & & D-03-138_180703_E1 \\
\hline D-03-138 & Creek Chub & 4 & & & D-03-138_180703_E1 \\
\hline D-03-138 & Fathead Minnow & 1 & & & D-03-138_180703_E1 \\
\hline D-03-138 & Finescale Dace & 24 & & & D-03-138_180703_E1 \\
\hline D-03-138 & Northern Redbelly Dace & 5 & & & D-03-138_180703_E1 \\
\hline D-03-138 & Western Blacknose Dace & 2 & & & D-03-138_180703_E1 \\
\hline D-03-142 & Brook Stickleback & 3 & & & D-03-142_220703_E1 \\
\hline D-03-142 & Creek Chub & 6 & & & D-03-142_220703_E1 \\
\hline D-03-142 & Finescale Dace & 4 & & & D-03-142_220703_E1 \\
\hline D-03-142 & Johnny Darter & 15 & & & D-03-142_220703_E1 \\
\hline D-03-142 & Longnose Dace & 3 & & & D-03-142_220703_E1 \\
\hline D-03-142 & Mottled Sculpin & 2 & & & D-03-142_220703_E1 \\
\hline D-03-142 & Western Blacknose Dace & 17 & & & D-03-142_220703_E1 \\
\hline D-03-143 & Blacknose Shiner & 5 & & & D-03-143_220703_E1 \\
\hline D-03-143 & Common Shiner & 1 & & & D-03-143_220703_E1 \\
\hline D-03-143 & Creek Chub & 10 & & & D-03-143_220703_E1 \\
\hline D-03-143 & Emerald Shiner & 4 & & & D-03-143_220703_E1 \\
\hline D-03-143 & Finescale Dace & 6 & & & D-03-143_220703_E1 \\
\hline D-03-143 & Iowa Darter & 2 & & & D-03-143_220703_E1 \\
\hline D-03-143 & Johnny Darter & 6 & & & D-03-143_220703_E1 \\
\hline
\end{tabular}


Appendix 5: List of all fish captures made between 2002 and 2006, showing site number, common name and number (\#) of fish collected, fork (or total ${ }^{\star}$ ) length in millimetres $(\mathrm{mm})$, any comments on the collection and the fish capture identification (ID) number.

\begin{tabular}{|c|c|c|c|c|c|}
\hline Site Number & Common Name & \# & $\begin{array}{l}\text { Fork/Total* } \\
\text { Length }(\mathrm{mm})\end{array}$ & Comments & $\begin{array}{l}\text { Fish Capture } \\
\text { ID Number }\end{array}$ \\
\hline D-03-143 & Longnose Dace & 4 & & & D-03-143_220703_E1 \\
\hline D-03-143 & Western Blacknose Dace & 35 & & & D-03-143_220703_E1 \\
\hline D-03-144 & Blacknose Shiner & 4 & & & D-03-144_220703_E1 \\
\hline D-03-144 & Brook Stickleback & 13 & & & D-03-144_220703_E1 \\
\hline D-03-144 & Finescale Dace & 8 & & & D-03-144_220703_E1 \\
\hline D-03-144 & Longnose Dace & 20 & & & D-03-144_220703_E1 \\
\hline D-03-144 & Silver Redhorse & 8 & $20-31^{*}$ & YOY & D-03-144_220703_E1 \\
\hline D-03-144 & Western Blacknose Dace & 77 & & & D-03-144_220703_E1 \\
\hline D-03-145 & Brook Stickleback & 4 & & & D-03-145_220703_E1 \\
\hline D-03-145 & Brook Trout & 5 & $77-190$ & 2 larger observed & D-03-145_220703_E1 \\
\hline D-03-145 & Johnny Darter & 6 & & & D-03-145_220703_E1 \\
\hline D-03-145 & Western Blacknose Dace & 27 & & & D-03-145_220703_E1 \\
\hline D-03-152 & Blacknose Shiner & 8 & & & D-03-152_230703_E1 \\
\hline D-03-152 & Brook Stickleback & 14 & & & D-03-152_230703_E1 \\
\hline D-03-152 & Fathead Minnow & 1 & & & D-03-152_230703_E1 \\
\hline D-03-152 & Finescale Dace & 2 & & & D-03-152_230703_E1 \\
\hline D-03-152 & White Sucker & 4 & $170-300$ & & D-03-152_230703_E1 \\
\hline D-03-154 & Blacknose Shiner & 3 & & & D-03-154_230703_E1 \\
\hline D-03-154 & Blackside Darter & 3 & & & D-03-154_230703_E1 \\
\hline D-03-154 & Longnose Dace & 10 & & & D-03-154_230703_E1 \\
\hline D-03-154 & Western Blacknose Dace & 3 & & & D-03-154_230703_E1 \\
\hline D-03-155 & Brook Stickleback & 14 & & & D-03-155_230703_E1 \\
\hline D-03-155 & Fathead Minnow & 6 & & & D-03-155_230703_E1 \\
\hline D-03-155 & Finescale Dace & 3 & & & D-03-155_230703_E1 \\
\hline D-03-156 & Brook Stickleback & 60 & & & D-03-156_230703_E1 \\
\hline D-03-156 & Western Blacknose Dace & 1 & & & D-03-156_230703_E1 \\
\hline D-03-164 & Brook Stickleback & 3 & & & D-03-164_240703_E1 \\
\hline D-03-164 & Creek Chub & 9 & & & D-03-164_240703_E1 \\
\hline D-03-164 & Fathead Minnow & 2 & & & D-03-164_240703_E1 \\
\hline D-03-164 & Northern Pike & 3 & $122-200$ & $1 \mathrm{YOY}$ & D-03-164_240703_E1 \\
\hline D-03-164 & White Sucker & 3 & $122-210$ & Released 1 & D-03-164_240703_E1 \\
\hline
\end{tabular}


Appendix 5: List of all fish captures made between 2002 and 2006, showing site number, common name and number (\#) of fish collected, fork (or total ${ }^{\star}$ ) length in millimetres ( $\mathrm{mm}$ ), any comments on the collection and the fish capture identification (ID) number.

\begin{tabular}{|c|c|c|c|c|c|}
\hline Site Number & Common Name & \# & $\begin{array}{c}\text { Fork/Total* } \\
\text { Length }(\mathrm{mm})\end{array}$ & Comments & $\begin{array}{l}\text { Fish Capture } \\
\text { ID Number }\end{array}$ \\
\hline D-03-167 & Creek Chub & 1 & & & D-03-167_250703_E1 \\
\hline D-03-167 & Finescale Dace & 2 & & & D-03-167_250703_E1 \\
\hline D-03-167 & lowa Darter & 1 & & & D-03-167_250703_E1 \\
\hline D-03-167 & Johnny Darter & 4 & & & D-03-167_250703_E1 \\
\hline D-03-167 & Longnose Dace & 6 & & & D-03-167_250703_E1 \\
\hline D-03-167 & Western Blacknose Dace & 11 & & & D-03-167_250703_E1 \\
\hline D-03-168 & Brook Stickleback & 2 & & $\begin{array}{l}\text { Observed while electrofishing, unable to } \\
\text { collect. }\end{array}$ & D-03-168_100903_E1 \\
\hline D-03-168 & Johnny Darter & 1 & & $\begin{array}{l}\text { Observed while electrofishing, unable to } \\
\text { collect. }\end{array}$ & D-03-168_100903_E1 \\
\hline D-03-168 & Northern Pike & 2 & & $\begin{array}{l}\text { Observed while electrofishing, unable to } \\
\text { collect. One estimated @ } 200 \text { mm, one @ } \\
350 \text { mm. }\end{array}$ & D-03-168_100903_E1 \\
\hline D-03-168 & White Sucker & 6 & $91-202$ & & D-03-168_100903_E1 \\
\hline D-03-170 & Blackside Darter & 1 & & & D-03-170_110903_E1 \\
\hline D-03-170 & Central Mudminnow & 1 & & & D-03-170_110903_E1 \\
\hline D-03-170 & Johnny Darter & 2 & & & D-03-170_110903_E1 \\
\hline D-03-170 & Western Blacknose Dace & 2 & & & D-03-170_110903_E1 \\
\hline D-03-170 & White Sucker & 5 & $124-266$ & & D-03-170_110903_E1 \\
\hline W-03-001 & Central Mudminnow & 1 & & & W-03-001_200503_B1 \\
\hline W-03-001 & Pearl Dace & 3 & & & W-03-001_200503_B1 \\
\hline W-03-001 & White Sucker & 16 & $360-470$ & Post-spawn, released & W-03-001_200503_B1 \\
\hline W-03-002 & Brook Stickleback & 8 & & Released & W-03-002_210503_B1 \\
\hline W-03-002 & Pearl Dace & 2 & & Released & W-03-002_210503_B1 \\
\hline W-03-002 & Fathead Minnow & 1 & & Released & W-03-002_210503_B1 \\
\hline W-03-003 & No Catch & & & & W-03-003_210503_E1 \\
\hline W-03-004 & Finescale Dace & 6 & & & W-03-004_210503_E1 \\
\hline W-03-008 & No Catch & & & & W-03-008_220503_E1 \\
\hline W-03-009 & No Catch & & & & W-03-009_220503_E1 \\
\hline W-03-010 & No Catch & & & & W-03-010_220503_E1 \\
\hline W-03-013 & Brook Stickleback & 9 & & & W-03-013_230503_E1 \\
\hline
\end{tabular}


Appendix 5: List of all fish captures made between 2002 and 2006, showing site number, common name and number (\#) of fish collected, fork (or total ${ }^{\star}$ ) length in millimetres ( $\mathrm{mm}$ ), any comments on the collection and the fish capture identification (ID) number.

\begin{tabular}{|c|c|c|c|c|c|}
\hline Site Number & Common Name & $\#$ & $\begin{array}{c}\text { Fork/Total* } \\
\text { Length }(\mathrm{mm})\end{array}$ & Comments & $\begin{array}{l}\text { Fish Capture } \\
\text { ID Number }\end{array}$ \\
\hline W-03-013 & Central Mudminnow & 3 & & & W-03-013_230503_E1 \\
\hline W-03-019 & Common Shiner & 1 & & & W-03-019_260503_E1 \\
\hline W-03-019 & Northern Pike & 3 & $21-26^{*}$ & YOY & W-03-019_260503_E1 \\
\hline W-03-020 & No Catch & & & & W-03-020_260503_E1 \\
\hline W-03-026 & Brook Stickleback & 3 & & & W-03-026_270503_B1 \\
\hline W-03-026 & Fathead Minnow & 79 & & & W-03-026_270503_B1 \\
\hline W-03-030 & Brook Stickleback & 3 & & & W-03-030_280503_B1 \\
\hline W-03-030 & Fathead Minnow & 1 & & & W-03-030_280503_B1 \\
\hline W-03-030 & No Catch & & & & W-03-030_280503_E1 \\
\hline W-03-035 & No Catch & & & & W-03-035_290503_E1 \\
\hline W-03-036 & No Catch & & & & W-03-036_290503_E1 \\
\hline W-03-044 & No Catch & & & & W-03-044_030603_B1 \\
\hline W-03-046 & Brook Stickleback & 6 & & & W-03-046 030603 E1 \\
\hline W-03-046 & Fathead Minnow & 4 & & & W-03-046_030603_E1 \\
\hline W-03-049 & Brook Stickleback & 5 & & Released 3 & W-03-049_030603_E1 \\
\hline W-03-050 & Blackside Darter & 6 & & & W-03-050_040603_E1 \\
\hline W-03-050 & Fathead Minnow & 1 & & & W-03-050_040603_E1 \\
\hline W-03-050 & Johnny Darter & 2 & & & W-03-050_040603_E1 \\
\hline W-03-050 & White Sucker & 1 & 85 & & W-03-050_040603_E1 \\
\hline W-03-055 & Brook Stickleback & 1 & & & W-03-055_040603_B1 \\
\hline W-03-055 & Northern Pike & 5 & $30^{\star}-41$ & YOY & W-03-055_040603_B1 \\
\hline W-03-065 & Brook Stickleback & 25 & & & W-03-065_050603_D1 \\
\hline W-03-065 & Central Mudminnow & 4 & & & W-03-065_050603_D1 \\
\hline W-03-066 & Brook Stickleback & 1 & & & W-03-066_050603_D1 \\
\hline W-03-066 & Creek Chub & 1 & & & W-03-066_050603_D1 \\
\hline W-03-066 & Fathead Minnow & 1 & & & W-03-066_050603_D1 \\
\hline W-03-066 & Johnny Darter & 1 & & & W-03-066_050603_D1 \\
\hline W-03-067 & Brook Stickleback & 6 & & & W-03-067_060603_E1 \\
\hline W-03-069 & Brook Stickleback & 6 & & Spawning & W-03-069_060603_B1 \\
\hline W-03-069 & Central Mudminnow & 5 & & & W-03-069_060603_B1 \\
\hline W-03-069 & Creek Chub & 3 & & & W-03-069_060603_B1 \\
\hline
\end{tabular}


Appendix 5: List of all fish captures made between 2002 and 2006, showing site number, common name and number (\#) of fish collected, fork (or total ${ }^{\star}$ ) length in millimetres ( $\mathrm{mm}$ ), any comments on the collection and the fish capture identification (ID) number.

\begin{tabular}{|c|c|c|c|c|c|}
\hline Site Number & Common Name & \# & $\begin{array}{c}\text { Fork/Total* } \\
\text { Length }(\mathrm{mm})\end{array}$ & Comments & $\begin{array}{l}\text { Fish Capture } \\
\text { ID Number }\end{array}$ \\
\hline W-03-069 & Fathead Minnow & 11 & & Spawning & W-03-069_060603_B1 \\
\hline W-03-069 & Finescale Dace & 8 & & & W-03-069_060603_B1 \\
\hline W-03-069 & White Sucker & 7 & $118-137$ & & W-03-069_060603_B1 \\
\hline W-03-070 & Common Shiner & 16 & & & W-03-070_060603_E1 \\
\hline W-03-070 & Creek Chub & 81 & & 63 are YOY & W-03-070_060603_E1 \\
\hline W-03-070 & Fathead Minnow & 22 & & & W-03-070_060603_D1 \\
\hline W-03-070 & Fathead Minnow & 30 & & & W-03-070_060603_E1 \\
\hline W-03-070 & Johnny Darter & 5 & & & W-03-070_060603_E1 \\
\hline W-03-070 & Western Blacknose Dace & 6 & & & W-03-070_060603_E1 \\
\hline W-03-070 & White Sucker & 2 & 55,70 & & W-03-070_060603_E1 \\
\hline W-03-072 & No Catch & & & & W-03-072_090603_E1 \\
\hline W-03-077 & No Catch & & & & W-03-077_100603_E1 \\
\hline W-03-079 & Brook Stickleback & 5 & & & W-03-079_100603_E1 \\
\hline W-03-079 & Fathead Minnow & 4 & & & W-03-079_100603_E1 \\
\hline W-03-079 & Finescale Dace & 6 & & & W-03-079_100603_E1 \\
\hline W-03-079 & River Shiner & 1 & & & W-03-079_100603_E1 \\
\hline W-03-079 & Northern Redbelly Dace & 5 & & & W-03-079_100603_E1 \\
\hline W-03-079 & Pearl Dace & 1 & & & W-03-079_100603_E1 \\
\hline W-03-080 & Brook Stickleback & 6 & & & W-03-080_100603_D1 \\
\hline W-03-084 & Brook Stickleback & 17 & & & W-03-084_110603_D1 \\
\hline W-03-087 & Brook Stickleback & 13 & & & W-03-087_110603_D1 \\
\hline W-03-088 & Brook Stickleback & 32 & & & W-03-088_110603_E1 \\
\hline W-03-088 & Central Mudminnow & 10 & & & W-03-088_110603_E1 \\
\hline W-03-091 & Brook Stickleback & 2 & & & W-03-091_130603_E1 \\
\hline W-03-092 & Brook Stickleback & 3 & & & W-03-092_130603_E1 \\
\hline W-03-092 & Central Mudminnow & 6 & & & W-03-092_130603_E1 \\
\hline W-03-092 & White Sucker & 42 & $21-30 *$ & & W-03-092_130603_E1 \\
\hline W-03-093 & Brook Stickleback & 1 & & & W-03-093_130603_E1 \\
\hline W-03-093 & Central Mudminnow & 4 & & & W-03-093_130603_E1 \\
\hline W-03-093 & White Sucker & 1 & 87 & & W-03-093_130603_E1 \\
\hline W-03-094 & No Catch & & & & W-03-094_160603_B1 \\
\hline
\end{tabular}


Appendix 5: List of all fish captures made between 2002 and 2006, showing site number, common name and number (\#) of fish collected, fork (or total ${ }^{\star}$ ) length in millimetres ( $\mathrm{mm}$ ), any comments on the collection and the fish capture identification (ID) number.

\begin{tabular}{|c|c|c|c|c|c|}
\hline Site Number & Common Name & \# & $\begin{array}{c}\text { Fork/Total* } \\
\text { Length }(\mathrm{mm})\end{array}$ & Comments & $\begin{array}{l}\text { Fish Capture } \\
\text { ID Number }\end{array}$ \\
\hline W-03-095 & Brook Stickleback & 4 & & & W-03-095_160603_E1 \\
\hline W-03-095 & Finescale Dace & 3 & & & W-03-095_160603_E1 \\
\hline W-03-095 & Northern Redbelly Dace & 2 & & & W-03-095_160603_E1 \\
\hline W-03-096 & No Catch & & & & W-03-096_160603_E1 \\
\hline W-03-097 & No Catch & & & & W-03-097_170603_E1 \\
\hline W-03-098 & Brook Stickleback & 3 & & & W-03-098_170603_B1 \\
\hline W-03-100 & Brook Stickleback & 2 & & & W-03-100_170603_E1 \\
\hline W-03-100 & Central Mudminnow & 2 & & & W-03-100_170603_E1 \\
\hline W-03-101 & Central Mudminnow & 2 & & & W-03-101_180603_E1 \\
\hline W-03-104 & No Catch & & & & W-03-104_180603_E1 \\
\hline W-03-107 & Finescale Dace & 1 & & & W-03-107_180603_E1 \\
\hline W-03-110 & Brook Stickleback & 9 & & & W-03-110_190603_E1 \\
\hline W-03-110 & Fathead Minnow & 3 & & & W-03-110_190603_E1 \\
\hline W-03-110 & Mimic Shiner & 1 & & & W-03-110_190603_E1 \\
\hline W-03-111 & Brook Stickleback & 1 & & & W-03-111_190603_E1 \\
\hline W-03-112 & Blackside Darter & 9 & & & W-03-112_190603_E1 \\
\hline W-03-112 & Brook Stickleback & 2 & & & W-03-112_190603_E1 \\
\hline W-03-112 & Finescale Dace & 2 & & & W-03-112_190603_E1 \\
\hline W-03-112 & Johnny Darter & 3 & & & W-03-112_190603_E1 \\
\hline W-03-113 & White Sucker & 1 & 141 & & W-03-113_240603_E1 \\
\hline W-03-114 & No Catch & & & & W-03-114_240603_E1 \\
\hline W-03-141 & Unidentified minnow & 16 & & Post-larval stage & W-03-141_260603_D1 \\
\hline W-03-142 & Brook Stickleback & 116 & & & W-03-142_260603_D1 \\
\hline W-03-142 & Northern Redbelly Dace & 4 & & & W-03-142_260603_D1 \\
\hline W-03-143 & No Catch & & & & W-03-143_020703_E1 \\
\hline W-03-144 & Blackside Darter & 1 & & & W-03-144_020703_E1 \\
\hline W-03-144 & Brook Stickleback & 3 & & & W-03-144_020703_E1 \\
\hline W-03-144 & Central Mudminnow & 1 & & & W-03-144_020703_E1 \\
\hline W-03-144 & Johnny Darter & 8 & & & W-03-144_020703_E1 \\
\hline W-03-144 & Western Blacknose Dace & 8 & & & W-03-144_020703_E1 \\
\hline W-03-144 & White Sucker & 1 & 44 & YOY & W-03-144_020703_E1 \\
\hline
\end{tabular}


Appendix 5: List of all fish captures made between 2002 and 2006, showing site number, common name and number (\#) of fish collected, fork (or total ${ }^{\star}$ ) length in millimetres ( $\mathrm{mm}$ ), any comments on the collection and the fish capture identification (ID) number.

\begin{tabular}{|c|c|c|c|c|c|}
\hline Site Number & Common Name & \# & $\begin{array}{c}\text { Fork/Total* } \\
\text { Length }(\mathrm{mm})\end{array}$ & Comments & $\begin{array}{l}\text { Fish Capture } \\
\text { ID Number }\end{array}$ \\
\hline W-03-146 & Brook Stickleback & 14 & & & W-03-146_020703_E1 \\
\hline W-03-146 & Johnny Darter & 4 & & & W-03-146_020703_E1 \\
\hline W-03-150 & Brook Stickleback & 8 & & & W-03-150_030703_E1 \\
\hline W-03-150 & White Sucker & 8 & $33-47$ & YOY & W-03-150_030703_E1 \\
\hline W-03-151 & Finescale Dace & 47 & & & W-03-151_030703_E1 \\
\hline W-03-154 & Brook Stickleback & 2 & & & W-03-154_040703_D1 \\
\hline W-03-154 & Common Shiner & 2 & & & W-03-154_040703_D1 \\
\hline W-03-154 & Finescale Dace & 2 & & & W-03-154_040703_D1 \\
\hline W-03-155 & Brook Stickleback & 7 & & & W-03-155_040703_E1 \\
\hline W-03-155 & Finescale Dace & 7 & & & W-03-155_040703_E1 \\
\hline W-03-155 & Northern Redbelly Dace & 1 & & & W-03-155_040703_E1 \\
\hline W-03-155 & Pearl Dace & 1 & & & W-03-155_040703_E1 \\
\hline W-03-158 & Fathead Minnow & 1 & & & W-03-158_040703_B1 \\
\hline W-03-158 & Brook Stickleback & 25 & & & W-03-158_040703_B1 \\
\hline W-03-158 & Hornyhead Chub & 8 & & SARA species & W-03-158_040703_B1 \\
\hline W-03-158 & Johnny Darter & 1 & & & W-03-158_040703_B1 \\
\hline W-03-158 & Common Shiner & 48 & & & W-03-158_040703_B1 \\
\hline W-03-158 & White Sucker & 6 & $27-23^{*}$ & YOY & W-03-158_040703_B1 \\
\hline W-03-162 & Brook Stickleback & 12 & & & W-03-162_050703_E1 \\
\hline W-03-162 & Common Shiner & 12 & & & W-03-162_050703_E1 \\
\hline W-03-162 & Finescale Dace & 1 & & & W-03-162_050703_E1 \\
\hline W-03-162 & Unidentified sucker & 3 & $12-14^{*}$ & Post-larval stage, recent hatch & W-03-162_050703_E1 \\
\hline W-03-165 & Blackside Darter & 7 & & & W-03-165_050703_E1 \\
\hline W-03-165 & Central Mudminnow & 1 & & & W-03-165_050703_E1 \\
\hline W-03-165 & Johnny Darter & 12 & & & W-03-165_050703_E1 \\
\hline W-03-165 & Longnose Dace & 1 & & & W-03-165_050703_E1 \\
\hline W-03-165 & Common Shiner & 6 & & & W-03-165_050703_E1 \\
\hline W-03-165 & White Sucker & 1 & $32^{*}$ & YOY & W-03-165_050703_E1 \\
\hline W-03-167 & Brook Stickleback & 10 & & & W-03-167_060703_B1 \\
\hline W-03-167 & Finescale Dace & 1 & & & W-03-167_060703_B1 \\
\hline W-03-173 & Brook Stickleback & 10 & & & W-03-173_060703_B1 \\
\hline
\end{tabular}


Appendix 5: List of all fish captures made between 2002 and 2006, showing site number, common name and number (\#) of fish collected, fork (or total ${ }^{\star}$ ) length in millimetres ( $\mathrm{mm}$ ), any comments on the collection and the fish capture identification (ID) number.

\begin{tabular}{|c|c|c|c|c|c|}
\hline Site Number & Common Name & \# & $\begin{array}{c}\text { Fork/Total* } \\
\text { Length }(\mathrm{mm})\end{array}$ & Comments & $\begin{array}{l}\text { Fish Capture } \\
\text { ID Number }\end{array}$ \\
\hline W-03-173 & Fathead Minnow & 3 & & & W-03-173_060703_B1 \\
\hline W-03-173 & White Sucker & 4 & $24-29 *$ & YOY & W-03-173_060703_B1 \\
\hline W-03-176 & No Catch & & & & W-03-176_070703_E1 \\
\hline W-03-182 & Sand Shiner & 3 & & & W-03-182_080703_B1 \\
\hline W-03-182 & Brook Stickleback & 5 & & & W-03-182_080703_B1 \\
\hline W-03-182 & Fathead Minnow & 3 & & & W-03-182_080703_B1 \\
\hline W-03-182 & Unidentified minnow & $100 \mathrm{~s}$ & & Post-larval stage & W-03-182_080703_B1 \\
\hline W-03-185 & Brook Stickleback & 12 & & & W-03-185_080703_B1 \\
\hline W-03-185 & Central Mudminnow & 2 & & & W-03-185_080703_B1 \\
\hline W-03-185 & Fathead Minnow & 21 & & & W-03-185_080703_B1 \\
\hline W-03-185 & Unidentified minnow & 105 & & Post-larval stage & W-03-185_080703_B1 \\
\hline W-03-186 & Brook Stickleback & 5 & & & W-03-186_090703_D1 \\
\hline W-03-186 & Central Mudminnow & 1 & & & W-03-186_090703_D1 \\
\hline W-03-186 & Fathead Minnow & 21 & & & W-03-186_090703_D1 \\
\hline W-03-189 & Brook Stickleback & 1 & & & W-03-189_090703_B1 \\
\hline W-03-189 & Central Mudminnow & 12 & & & W-03-189_090703_B1 \\
\hline W-03-191 & Brook Stickleback & 7 & & & W-03-191_160703_E1 \\
\hline W-03-191 & Central Mudminnow & 3 & & & W-03-191_160703_E1 \\
\hline W-03-191 & Iowa Darter & 2 & & & W-03-191_160703_E1 \\
\hline W-03-191 & Johnny Darter & 3 & & & W-03-191_160703_E1 \\
\hline W-03-191 & Walleye & 1 & 85 & Escaped dip-net & W-03-191_160703_E1 \\
\hline W-03-194 & Blackside Darter & 3 & & & W-03-194_160703_E1 \\
\hline W-03-194 & Central Mudminnow & 1 & & & W-03-194_160703_E1 \\
\hline W-03-194 & Fathead Minnow & 3 & & & W-03-194_160703_E1 \\
\hline W-03-194 & Finescale Dace & 13 & & & W-03-194_160703_E1 \\
\hline W-03-194 & Johnny Darter & 1 & & & W-03-194_160703_E1 \\
\hline W-03-194 & Pearl Dace & 8 & & & W-03-194_160703_E1 \\
\hline W-03-195 & Brook Stickleback & 18 & & & W-03-195_160703_B1 \\
\hline W-03-195 & Central Mudminnow & 2 & & & W-03-195_160703_B1 \\
\hline W-03-195 & Finescale Dace & 12 & & & W-03-195_160703_B1 \\
\hline W-03-195 & Johnny Darter & 1 & & & W-03-195_160703_B1 \\
\hline
\end{tabular}


Appendix 5: List of all fish captures made between 2002 and 2006, showing site number, common name and number (\#) of fish collected, fork (or total ${ }^{\star}$ ) length in millimetres ( $\mathrm{mm}$ ), any comments on the collection and the fish capture identification (ID) number.

\begin{tabular}{|c|c|c|c|c|c|}
\hline Site Number & Common Name & \# & $\begin{array}{c}\text { Fork/Total* } \\
\text { Length }(\mathrm{mm})\end{array}$ & Comments & $\begin{array}{l}\text { Fish Capture } \\
\text { ID Number }\end{array}$ \\
\hline W-03-195 & Pearl Dace & 26 & & & W-03-195_160703_B1 \\
\hline W-03-195 & White Sucker & 2 & 132,133 & & W-03-195_160703_B1 \\
\hline W-03-198 & Central Mudminnow & 2 & & & W-03-198_170703_E1 \\
\hline W-03-198 & Western Blacknose Dace & 2 & & & W-03-198_170703_E1 \\
\hline W-03-201 & Brook Stickleback & 25 & & & W-03-201_170703_B1 \\
\hline W-03-201 & Central Mudminnow & 12 & & & W-03-201_170703_B1 \\
\hline W-03-201 & Finescale Dace & 36 & & & W-03-201_170703_B1 \\
\hline W-03-201 & Northern Redbelly Dace & 17 & & & W-03-201_170703_B1 \\
\hline W-03-203 & Brook Stickleback & 72 & & & W-03-203_180703_E1 \\
\hline W-03-203 & Central Mudminnow & 1 & & & W-03-203_180703_E1 \\
\hline W-03-203 & Pearl Dace & 1 & & & W-03-203_180703_E1 \\
\hline W-03-203 & Fathead Minnow & 2 & & & W-03-203_180703_E1 \\
\hline W-03-203 & Finescale Dace & 48 & & & W-03-203_180703_E1 \\
\hline W-03-203 & lowa Darter & 3 & & & W-03-203_180703_E1 \\
\hline W-03-203 & Johnny Darter & 50 & & & W-03-203_180703_E1 \\
\hline W-03-204 & Brook Stickleback & 9 & & & W-03-204_180703_E1 \\
\hline W-03-204 & Emerald Shiner & 10 & & & W-03-204_180703_E1 \\
\hline W-03-204 & Finescale Dace & 7 & & & W-03-204_180703_E1 \\
\hline W-03-204 & Unidentified minnow & 100 & & Post-larval stage & W-03-204_180703_E1 \\
\hline W-03-205 & Brook Stickleback & 10 & & & W-03-205_180703_E1 \\
\hline W-03-205 & Central Mudminnow & 6 & & & W-03-205_180703_E1 \\
\hline W-03-205 & Finescale Dace & 103 & & & W-03-205_180703_E1 \\
\hline W-03-206 & Blackside Darter & 1 & & & W-03-206_310703_E1 \\
\hline W-03-206 & Brook Stickleback & 11 & & & W-03-206_310703_E1 \\
\hline W-03-206 & Central Mudminnow & 4 & & & W-03-206_310703_E1 \\
\hline W-03-206 & Iowa Darter & 2 & & & W-03-206_310703_E1 \\
\hline W-03-206 & White Sucker & 6 & $30^{*}-44$ & YOY & W-03-206_310703_E1 \\
\hline W-03-210 & Central Mudminnow & 3 & & & W-03-210_310703_E1 \\
\hline W-03-210 & Johnny Darter & 5 & & & W-03-210_310703_E1 \\
\hline W-03-211 & Northern Pike & 24 & $120-151$ & YOY, 22 released & W-03-211_310703_B1 \\
\hline W-03-223 & Northern Pike & 5 & $101-140$ & YOY & W-03-223_050803_E1 \\
\hline
\end{tabular}


Appendix 5: List of all fish captures made between 2002 and 2006, showing site number, common name and number (\#) of fish collected, fork (or total ${ }^{\star}$ ) length in millimetres ( $\mathrm{mm}$ ), any comments on the collection and the fish capture identification (ID) number.

\begin{tabular}{|c|c|c|c|c|c|}
\hline Site Number & Common Name & \# & $\begin{array}{c}\text { Fork/Total* } \\
\text { Length }(\mathrm{mm})\end{array}$ & Comments & $\begin{array}{l}\text { Fish Capture } \\
\text { ID Number }\end{array}$ \\
\hline W-03-224 & Black Bullhead & 150 & & YOY, 99 released & W-03-224_050803_E1 \\
\hline W-03-224 & Johnny Darter & 2 & & & W-03-224_050803_E1 \\
\hline W-03-224 & Longnose Dace & 1 & & & W-03-224_050803_E1 \\
\hline W-03-224 & Spottail Shiner & 17 & & & W-03-224_050803_E1 \\
\hline W-03-224 & Western Blacknose Dace & 6 & & & W-03-224_050803_E1 \\
\hline W-03-229 & No Catch & & & & W-03-229_060803_B1 \\
\hline W-03-237 & Brook Stickleback & 41 & & & W-03-237_060803_E1 \\
\hline W-03-237 & Fathead Minnow & 13 & & Spawning & W-03-237_060803_E1 \\
\hline W-03-237 & Finescale Dace & 1 & & & W-03-237_060803_E1 \\
\hline W-03-237 & Iowa Darter & 2 & & & W-03-237_060803_E1 \\
\hline W-03-237 & Johnny Darter & 20 & & & W-03-237_060803_E1 \\
\hline W-03-237 & White Sucker & 10 & $65-200$ & Released 6 & W-03-237_060803_E1 \\
\hline W-03-238 & Johnny Darter & 12 & & & W-03-238_070803_E1 \\
\hline W-03-238 & Longnose Dace & 16 & & & W-03-238_070803_E1 \\
\hline W-03-238 & Rock Bass & 16 & $31-121$ & 5 YOY & W-03-238_070803_E1 \\
\hline W-03-238 & Tadpole Madtom & 1 & & & W-03-238_070803_E1 \\
\hline W-03-238 & Western Blacknose Dace & 30 & & & W-03-238_070803_E1 \\
\hline W-03-238 & White Sucker & 3 & $50,60,63$ & YOY & W-03-238_070803_E1 \\
\hline W-03-239 & Central Mudminnow & 7 & & & W-03-239_070803_E1 \\
\hline W-03-239 & Iowa Darter & 6 & & & W-03-239_070803_E1 \\
\hline W-03-239 & Johnny Darter & 56 & & & W-03-239_070803_E1 \\
\hline W-03-239 & Longnose Dace & 10 & & & W-03-239_070803_E1 \\
\hline W-03-239 & Northern Pike & 5 & $140-200$ & YOY, 4 released & W-03-239_070803_E1 \\
\hline W-03-239 & White Sucker & 4 & $97-125$ & & W-03-239_070803_E1 \\
\hline W-03-247 & Blacknose Shiner & 15 & & & W-03-247_080803_E1 \\
\hline W-03-247 & Western Blacknose Dace & 25 & & & W-03-247_080803_E1 \\
\hline W-03-248 & Brook Stickleback & 3 & & & W-03-248_080803_E1 \\
\hline W-03-249 & Brook Stickleback & 25 & & & W-03-249_110803_E1 \\
\hline W-03-249 & Central Mudminnow & 4 & & & W-03-249_110803_E1 \\
\hline W-03-249 & White Sucker & 13 & $27-42^{*}$ & YOY & W-03-249_110803_E1 \\
\hline W-03-251 & Blacknose Shiner & 10 & & & W-03-251_110803_E1 \\
\hline
\end{tabular}


Appendix 5: List of all fish captures made between 2002 and 2006, showing site number, common name and number (\#) of fish collected, fork (or total ${ }^{\star}$ ) length in millimetres ( $\mathrm{mm}$ ), any comments on the collection and the fish capture identification (ID) number.

\begin{tabular}{|c|c|c|c|c|c|}
\hline Site Number & Common Name & \# & $\begin{array}{l}\text { Fork/Total* } \\
\text { Length }(\mathrm{mm})\end{array}$ & Comments & $\begin{array}{l}\text { Fish Capture } \\
\text { ID Number }\end{array}$ \\
\hline W-03-251 & Brook Stickleback & 3 & & & W-03-251_110803_E1 \\
\hline W-03-251 & Fathead Minnow & 3 & & & W-03-251_110803_E1 \\
\hline W-03-266 & No Catch & & & & W-03-266_130803_E1 \\
\hline W-03-267 & Brook Stickleback & 5 & & & W-03-267_130803_B1 \\
\hline W-03-267 & Fathead Minnow & 52 & & & W-03-267_130803_B1 \\
\hline W-03-267 & Northern Pike & 2 & 220,230 & Released & W-03-267_130803_B1 \\
\hline B-04-002 & No Catch & & & & B-04-002_010504_E1 \\
\hline B-04-003 & Fathead Minnow & 1 & & & B-04-003_010504_B1 \\
\hline B-04-003 & Northern Pike & 1 & 520 & Ripe male, released & B-04-003_010504_E1 \\
\hline B-04-003 & White Sucker & 1 & 490 & Spent female, released & B-04-003_010504_E1 \\
\hline B-04-004 & No Catch & & & & B-04-004_010504_E1 \\
\hline B-04-006 & White Sucker & 100's & & Dead, trapped in pools & B-04-006_010504_E1 \\
\hline B-04-006 & Yellow Perch & 100's & & Dead, trapped in pools & B-04-006_010504_E1 \\
\hline B-04-008 & Brook Stickleback & 12 & & & B-04-008_010504_E1 \\
\hline B-04-008 & Fathead Minnow & 7 & & & B-04-008_010504_E1 \\
\hline B-04-008 & Finescale Dace & 1 & & & B-04-008_010504_E1 \\
\hline B-04-008 & Northern Redbelly Dace & 1 & & & B-04-008_010504_E1 \\
\hline B-04-010 & Brook Stickleback & 15 & & & B-04-010_020504_E1 \\
\hline B-04-010 & Fathead Minnow & 20 & & & B-04-010_020504_E1 \\
\hline B-04-010 & No Catch & & & Invertebrates only & B-04-010_020504_K1 \\
\hline B-04-010 & Northern Redbelly Dace & 1 & & $\begin{array}{l}\text { A large operculum (unidentified) is } \\
\text { included in the sample }\end{array}$ & B-04-010_020504_E1 \\
\hline B-04-011 & No Catch & & & & B-04-011_020504_E1 \\
\hline B-04-012 & White Sucker & 100's & & Congregated on spawning riffle & B-04-012_020504_O1 \\
\hline B-04-013 & White Sucker & 100's & & Congregated on spawning riffle & B-04-013_020504_01 \\
\hline B-04-014 & White Sucker & 100 's & & Congregated on spawning riffle & B-04-014_020504_O1 \\
\hline B-04-015 & White Sucker & 100's & & Congregated on spawning riffle & B-04-015_020504_01 \\
\hline B-04-016 & White Sucker & 100's & & Congregated on spawning riffle & B-04-016_020504_01 \\
\hline B-04-017 & Brook Stickleback & 1 & & & B-04-017_020504_E1 \\
\hline B-04-017 & Fathead Minnow & 6 & & & B-04-017_020504_E1 \\
\hline B-04-017 & Finescale Dace & 1 & & & B-04-017_020504_E1 \\
\hline
\end{tabular}


Appendix 5: List of all fish captures made between 2002 and 2006, showing site number, common name and number (\#) of fish collected, fork (or total ${ }^{\star}$ ) length in millimetres ( $\mathrm{mm}$ ), any comments on the collection and the fish capture identification (ID) number.

\begin{tabular}{|c|c|c|c|c|c|}
\hline Site Number & Common Name & \# & $\begin{array}{c}\text { Fork/Total* } \\
\text { Length }(\mathrm{mm})\end{array}$ & Comments & $\begin{array}{l}\text { Fish Capture } \\
\text { ID Number }\end{array}$ \\
\hline B-04-017 & No Catch & & & Invertebrates only & B-04-017_020504_K1 \\
\hline B-04-018 & Black Bullhead & 3 & & & B-04-018_030504_B1 \\
\hline B-04-018 & Brook Stickleback & 2 & & & B-04-018_030504_B1 \\
\hline B-04-018 & Fathead Minnow & 3 & & & B-04-018_030504_B1 \\
\hline B-04-019 & Black Bullhead & 4 & & & B-04-019_030504_E1 \\
\hline B-04-019 & Brook Stickleback & 6 & & & B-04-019_030504_E1 \\
\hline B-04-019 & Fathead Minnow & 7 & & & B-04-019_030504_E1 \\
\hline B-04-020 & Black Bullhead & 8 & & & B-04-020_030504_B1 \\
\hline B-04-020 & Fathead Minnow & 6 & & & B-04-020_030504_B1 \\
\hline B-04-020 & Fathead Minnow & 8 & & & B-04-020_030504_B1 \\
\hline B-04-020 & No Catch & & & Beach seine failed & B-04-020_030504_B2 \\
\hline B-04-020 & Sand Shiner & 17 & & & B-04-020_030504_B1 \\
\hline B-04-021 & Black Bullhead & 1 & $170^{*}$ & Released & B-04-021_030504_E1 \\
\hline B-04-022 & Brook Stickleback & 3 & & & B-04-022_030504_B1 \\
\hline B-04-022 & Fathead Minnow & 16 & & Plus one Orconectes virilis & B-04-022_030504_B1 \\
\hline B-04-022 & White Sucker & 1 & $14^{*}$ & YOY & B-04-022_030504_B1 \\
\hline B-04-026 & Black Bullhead & 9 & & Released & B-04-026_040504_E1 \\
\hline B-04-026 & Fathead Minnow & 16 & & Plus one Anodonta spp. mussel & B-04-026_040504_E1 \\
\hline B-04-027 & Fathead Minnow & 9 & & & B-04-027_040504_E1 \\
\hline B-04-027 & Quillback & 2 & 403,424 & Released & B-04-027_040504_E1 \\
\hline B-04-027 & River Shiner & 3 & & & B-04-027_040504_E1 \\
\hline B-04-027 & Sand Shiner & 15 & & & B-04-027_040504_E1 \\
\hline B-04-027 & Troutperch & 3 & & & B-04-027_040504_E1 \\
\hline B-04-028 & Black Bullhead & 250 & $60-100^{*}$ & Released & B-04-028_040504_B1 \\
\hline B-04-028 & Northern Pike & 1 & 520 & Released & B-04-028_040504_B1 \\
\hline B-04-030 & Fathead Minnow & 8 & & & B-04-030_040504_E1 \\
\hline B-04-030 & Sand Shiner & 1 & & & B-04-030_040504_E1 \\
\hline B-04-031 & No Catch & & & & B-04-031_060504_E1 \\
\hline B-04-031 & No Catch & & & & B-04-031_060504_B1 \\
\hline B-04-037 & No Catch & & & & B-04-037_060504_E1 \\
\hline
\end{tabular}


Appendix 5: List of all fish captures made between 2002 and 2006, showing site number, common name and number (\#) of fish collected, fork (or total ${ }^{\star}$ ) length in millimetres ( $\mathrm{mm}$ ), any comments on the collection and the fish capture identification (ID) number.

\begin{tabular}{|c|c|c|c|c|c|}
\hline Site Number & Common Name & \# & $\begin{array}{l}\text { Fork/Total* } \\
\text { Length }(\mathrm{mm})\end{array}$ & Comments & $\begin{array}{l}\text { Fish Capture } \\
\text { ID Number }\end{array}$ \\
\hline B-04-039 & White Sucker & 100's & & $\begin{array}{l}\text { Congregated below structure, no } \\
\text { upstream passage }\end{array}$ & B-04-039_060504_01 \\
\hline B-04-041 & No Catch & & & & B-04-041_070504_B1 \\
\hline B-04-043 & Fathead Minnow & 3 & & & B-04-043_070504_B1 \\
\hline B-04-044 & Brook Stickleback & 3 & & & B-04-044_070504_E1 \\
\hline B-04-044 & Common Shiner & 1 & & & B-04-044_070504_E1 \\
\hline B-04-044 & Creek Chub & 2 & & & B-04-044_070504_E1 \\
\hline B-04-044 & Finescale Dace & 3 & & & B-04-044_070504_E1 \\
\hline B-04-044 & Johnny Darter & 2 & & & B-04-044_070504_E1 \\
\hline B-04-044 & Northern Redbelly Dace & 2 & & & B-04-044_070504_E1 \\
\hline B-04-044 & Western Blacknose Dace & 2 & & & B-04-044_070504_E1 \\
\hline B-04-044 & White Sucker & 1 & 157 & & B-04-044_070504_E1 \\
\hline B-04-045 & Blackside Darter & 1 & & $\begin{array}{l}\text { Kick sample contains stone fly nymphs } \\
\text { and pea clams, suggesting riffle habitat } \\
\text { and stony substrate }\end{array}$ & B-04-045_070504_K1 \\
\hline B-04-045 & Johnny Darter & 1 & & & B-04-045_070504_K1 \\
\hline B-04-045 & Longnose Dace & 8 & & & B-04-045_070504_K1 \\
\hline B-04-046 & Brook Stickleback & 13 & & & B-04-046_100504_E1 \\
\hline B-04-046 & Central Mudminnow & 1 & & & B-04-046_100504_E1 \\
\hline B-04-046 & Creek Chub & 32 & & Released $2(170,135 \mathrm{~mm})$ & B-04-046_100504_E1 \\
\hline B-04-046 & Fathead Minnow & 13 & & & B-04-046_100504_E1 \\
\hline B-04-046 & Finescale Dace & 2 & & & B-04-046_100504_E1 \\
\hline B-04-046 & Iowa Darter & 3 & & & B-04-046_100504_E1 \\
\hline B-04-046 & Johnny Darter & 5 & & & B-04-046_100504_E1 \\
\hline B-04-046 & Western Blacknose Dace & 2 & & & B-04-046_100504_E1 \\
\hline B-04-047 & Blackside Darter & 2 & & & B-04-047_100504_E1 \\
\hline B-04-047 & Central Mudminnow & 1 & & & B-04-047_100504_E1 \\
\hline B-04-047 & Creek Chub & 4 & & & B-04-047_100504_E1 \\
\hline B-04-047 & Fathead Minnow & 2 & & & B-04-047_100504_E1 \\
\hline B-04-047 & Johnny Darter & 1 & & & B-04-047_100504_E1 \\
\hline B-04-047 & No Catch & & & Invertebrates only & B-04-047_100504_K1 \\
\hline
\end{tabular}


Appendix 5: List of all fish captures made between 2002 and 2006, showing site number, common name and number (\#) of fish collected, fork (or total ${ }^{\star}$ ) length in millimetres ( $\mathrm{mm}$ ), any comments on the collection and the fish capture identification (ID) number.

\begin{tabular}{|c|c|c|c|c|c|}
\hline Site Number & Common Name & \# & $\begin{array}{l}\text { Fork/Total* } \\
\text { Length }(\mathrm{mm})\end{array}$ & Comments & $\begin{array}{l}\text { Fish Capture } \\
\text { ID Number }\end{array}$ \\
\hline B-04-047 & Western Blacknose Dace & 2 & & & B-04-047_100504_E1 \\
\hline B-04-047 & White Sucker & 14 & 53-169 & $\begin{array}{l}3 \text { specimens, } 140 \mathrm{~mm}(2 \mathrm{male})-169 \mathrm{~mm} \\
\text { (female), all mature }\end{array}$ & B-04-047_100504_E1 \\
\hline B-04-048 & Bigmouth Shiner & 10 & & & B-04-048_100504_B1 \\
\hline B-04-048 & Common Shiner & 138 & & & B-04-048_100504_B1 \\
\hline B-04-048 & Creek Chub & 10 & & & B-04-048_100504_B1 \\
\hline B-04-048 & Fathead Minnow & 16 & & & B-04-048_100504_B1 \\
\hline B-04-048 & Western Blacknose Dace & 20 & & & B-04-048_100504_B1 \\
\hline B-04-048 & White Sucker & 20 & $48-105$ & $\begin{array}{l}\text { Largest was female with developed eggs - } \\
\text { probably dwarf }\end{array}$ & B-04-048_100504_B1 \\
\hline B-04-055 & Blackside Darter & 2 & & & B-04-055_130504_E1 \\
\hline B-04-055 & No Catch & & & & B-04-055_130504_B1 \\
\hline B-04-055 & White Sucker & 1 & 67 & & B-04-055_130504_E1 \\
\hline B-04-058 & No Catch & & & & B-04-058_140504_E1 \\
\hline B-04-060 & No Catch & & & & B-04-060_140504_E1 \\
\hline B-04-064 & No Catch & & & & B-04-064_140504_B1 \\
\hline B-04-066 & No Catch & & & & B-04-066_170504_E1 \\
\hline B-04-069 & Northern Pike & 75 & $12-14^{*}$ & $\begin{array}{l}\text { YOY, } 18 \text { unidentifiable but likely Northern } \\
\text { Pike }\end{array}$ & B-04-069_180504_L1 \\
\hline B-04-070 & No Catch & & & & B-04-070_180504_E1 \\
\hline B-04-072 & No Catch & & & & B-04-072_180504_K1 \\
\hline B-04-073 & No Catch & & & Invertebrates only & B-04-073_190504_K1 \\
\hline B-04-073 & White Sucker & 100's & & Many observed below control structure & B-04-073_190504_01 \\
\hline B-04-074 & Eggs & 4 & & Sucker eggs, most likely White Sucker & B-04-074_190504_K1 \\
\hline B-04-075 & Walleye & & & $\begin{array}{l}\text { Annual drift samples by Fisheries Branch, } \\
\text { Dauphin }\end{array}$ & B-04-075_200504_01 \\
\hline B-04-076 & No Catch & & & & B-04-076_200504_L1 \\
\hline B-04-077 & No Catch & & & & B-04-077_200504_L1 \\
\hline B-04-078 & No Catch & & & & B-04-078_200504_E1 \\
\hline B-04-078 & Unidentified minnow & 2 & & Post-larval stage & B-04-078_200504_L1 \\
\hline B-04-080 & Fathead Minnow & & & Many observed in pool & B-04-080_200504_01 \\
\hline
\end{tabular}


Appendix 5: List of all fish captures made between 2002 and 2006, showing site number, common name and number (\#) of fish collected, fork (or total ${ }^{\star}$ ) length in millimetres ( $\mathrm{mm}$ ), any comments on the collection and the fish capture identification (ID) number.

\begin{tabular}{|c|c|c|c|c|c|}
\hline Site Number & Common Name & \# & $\begin{array}{l}\text { Fork/Total* } \\
\text { Length }(\mathrm{mm})\end{array}$ & Comments & $\begin{array}{l}\text { Fish Capture } \\
\text { ID Number }\end{array}$ \\
\hline B-04-083 & Northern Pike & 15 & $350-480$ & $\begin{array}{l}\text { Adult Northern Pike and many larval } \\
\text { Northern Pike trapped in pools }\end{array}$ & B-04-083_200504_D1 \\
\hline B-04-084 & Northern Pike & 7 & & Adult Northern Pike trapped in pools & B-04-084_200504_01 \\
\hline B-04-085 & Northern Pike & & & $\begin{array}{l}\text { Adults and larval Northern Pike trapped in } \\
\text { pools }\end{array}$ & B-04-085_200504_01 \\
\hline B-04-086 & Fathead Minnow & 5 & & & B-04-086_210504_E1 \\
\hline B-04-086 & White Sucker & 1 & & Adult released & B-04-086_210504_B1 \\
\hline B-04-087 & No Catch & & & & B-04-087_220504_L1 \\
\hline B-04-088 & No Catch & & & & B-04-088_250504_L1 \\
\hline B-04-090 & No Catch & & & & B-04-090_250504_L1 \\
\hline B-04-092 & No Catch & & & Invertebrates only & B-04-092_250504_K1 \\
\hline B-04-093 & No Catch & & & & B-04-093_310504_B1 \\
\hline B-04-094 & No Catch & & & & B-04-094_310504_B1 \\
\hline B-04-095 & No Catch & & & & B-04-095_310504_B1 \\
\hline B-04-101 & No Catch & & & & B-04-101_010604_B1 \\
\hline B-04-102 & No Catch & & & & B-04-102_010604_E1 \\
\hline B-04-104 & Northern Pike & 1 & 62 & $\begin{array}{l}\text { Plus } 2 \text { tadpole shrimp (Lepidurus spp.) } \\
\text { indicator of intermittent water }\end{array}$ & B-04-104_010604_L1 \\
\hline B-04-105 & No Catch & & & & B-04-105_010604_E1 \\
\hline B-04-108 & No Catch & & & & B-04-108_020604_B1 \\
\hline B-04-109 & Fathead Minnow & 2 & & Released males with spawning tubercles & B-04-109_020604_E1 \\
\hline B-04-110 & Brook Stickleback & 13 & & & B-04-110_020604_E1 \\
\hline B-04-110 & Fathead Minnow & 4 & & & B-04-110_020604_E1 \\
\hline B-04-113 & Common Shiner & 18 & & & B-04-113_020604_E1 \\
\hline B-04-113 & Fathead Minnow & 22 & & & B-04-113_020604_B1 \\
\hline B-04-114 & No Catch & & & & B-04-114_030604_E1 \\
\hline B-04-121 & Central Mudminnow & 1 & & & B-04-121_040604_E1 \\
\hline B-04-121 & Fathead Minnow & 2 & & & B-04-121_040604_E1 \\
\hline B-04-121 & Western Blacknose Dace & 4 & & & B-04-121_040604_E1 \\
\hline B-04-121 & White Sucker & 3 & $58-72$ & & B-04-121_040604_E1 \\
\hline B-04-122 & Blackside Darter & 2 & & & B-04-122_040604_E1 \\
\hline
\end{tabular}


Appendix 5: List of all fish captures made between 2002 and 2006, showing site number, common name and number (\#) of fish collected, fork (or total ${ }^{\star}$ ) length in millimetres ( $\mathrm{mm}$ ), any comments on the collection and the fish capture identification (ID) number.

\begin{tabular}{|c|c|c|c|c|c|}
\hline Site Number & Common Name & \# & $\begin{array}{c}\text { Fork/Total* }^{*} \\
\text { Length }(\mathrm{mm})\end{array}$ & Comments & $\begin{array}{l}\text { Fish Capture } \\
\text { ID Number }\end{array}$ \\
\hline B-04-122 & Brook Stickleback & 2 & & & B-04-122_040604_E1 \\
\hline B-04-122 & Creek Chub & 3 & & 1 male & B-04-122_040604_E1 \\
\hline B-04-122 & Fathead Minnow & 4 & & 1 male with spawning color & B-04-122_040604_E1 \\
\hline B-04-123 & Bigmouth Shiner & 1 & & & B-04-123_070604_E1 \\
\hline B-04-123 & Blackside Darter & 1 & & & B-04-123_070604_E1 \\
\hline B-04-123 & Brook Stickleback & 1 & & & B-04-123_070604_E1 \\
\hline B-04-123 & Chestnut Lamprey & 1 & $239 *$ & $\begin{array}{l}\text { Free eggs in abdomen - probably } \\
\text { spawning, bicuspid inner teeth, pigmented } \\
\text { lateral line }\end{array}$ & B-04-123_070604_E1 \\
\hline B-04-123 & Common Shiner & 2 & & & B-04-123_070604_E1 \\
\hline B-04-123 & Creek Chub & 1 & & & B-04-123_070604_E1 \\
\hline B-04-123 & Johnny Darter & 1 & & & B-04-123 $070604 \mathrm{E} 1$ \\
\hline B-04-123 & Longnose Dace & 1 & & & B-04-123_070604_E1 \\
\hline B-04-123 & Troutperch & 1 & & & B-04-123_070604_E1 \\
\hline B-04-123 & White Sucker & 4 & $58-86$ & & B-04-123_070604_E1 \\
\hline B-04-124 & No Catch & & & & B-04-124_070604_E1 \\
\hline B-04-125 & Northern Pike & 21 & $37-54^{*}$ & All YOY & B-04-125_070604_B1 \\
\hline B-04-126 & Fathead Minnow & 3 & & Released 2 male, 1 female spawners & B-04-126_080604_B1 \\
\hline B-04-127 & Fathead Minnow & 3 & & & B-04-127_080604_E1 \\
\hline B-04-129 & No Catch & & & & B-04-129_080604_E1 \\
\hline B-04-131 & No Catch & & & & B-04-131_090604_E1 \\
\hline B-04-133 & No Catch & & & & B-04-133_090604_E1 \\
\hline B-04-136 & White Sucker & 1 & 158 & Post-spawn male & B-04-136_100604_E1 \\
\hline B-04-140 & Brook Stickleback & 1 & & & B-04-140_140604_E1 \\
\hline B-04-140 & Fathead Minnow & 1 & & & B-04-140_140604_E1 \\
\hline B-04-140 & Unidentified sucker & 1 & $10^{*}$ & Post-larval stage & B-04-140_140604_E1 \\
\hline B-04-141 & Brook Stickleback & 1 & & & B-04-141_140604_D1 \\
\hline B-04-141 & Fathead Minnow & 12 & & & B-04-141_140604_D1 \\
\hline B-04-141 & White Sucker & 1 & 63 & & B-04-141_140604_D1 \\
\hline B-04-144 & Common Shiner & 2 & & & B-04-144_150604_E1 \\
\hline B-04-144 & Creek Chub & 3 & & Plus 1 crayfish - Orconectes virilis & B-04-144_150604_E1 \\
\hline
\end{tabular}


Appendix 5: List of all fish captures made between 2002 and 2006, showing site number, common name and number (\#) of fish collected, fork (or total ${ }^{*}$ ) length in millimetres $(\mathrm{mm})$, any comments on the collection and the fish capture identification (ID) number.

\begin{tabular}{|c|c|c|c|c|c|}
\hline Site Number & Common Name & \# & $\begin{array}{l}\text { Fork/Total* } \\
\text { Length }(\mathrm{mm})\end{array}$ & Comments & $\begin{array}{l}\text { Fish Capture } \\
\text { ID Number }\end{array}$ \\
\hline B-04-144 & Fathead Minnow & 6 & & 2 spawning males & B-04-144_150604_E1 \\
\hline B-04-144 & Western Blacknose Dace & 7 & & & B-04-144_150604_E1 \\
\hline B-04-144 & White Sucker & 3 & $79-140$ & $140 \mathrm{~mm}$ is immature male & B-04-144_150604_E1 \\
\hline B-04-145 & Bigmouth Shiner & 1 & & & B-04-145_150604_E1 \\
\hline B-04-145 & Black Bullhead & 2 & & Released $1 @ 191 \mathrm{~mm}$ & B-04-145_150604_E1 \\
\hline B-04-145 & Brook Stickleback & 3 & & & B-04-145_150604_E1 \\
\hline B-04-145 & Common Shiner & 2 & & 1 male with spawning color & B-04-145_150604_E1 \\
\hline B-04-145 & Creek Chub & 2 & 158 & 1 spawning male & B-04-145_150604_E1 \\
\hline B-04-145 & Fathead Minnow & 34 & & & B-04-145_150604_E1 \\
\hline B-04-145 & Western Blacknose Dace & 7 & & & B-04-145_150604_E1 \\
\hline B-04-147 & Fathead Minnow & $300+$ & & Congregated at impassable culvert & B-04-147_150604_O1 \\
\hline B-04-149 & Bigmouth Shiner & 1 & & & B-04-149_150604_E1 \\
\hline B-04-149 & Black Bullhead & 31 & $70-190^{*}$ & Released 26 & B-04-149_150604_B1 \\
\hline B-04-149 & Common Shiner & 2 & & & B-04-149_150604_E1 \\
\hline B-04-149 & Creek Chub & 1 & & & B-04-149_150604_E1 \\
\hline B-04-149 & Fathead Minnow & 1 & & & B-04-149_150604_E1 \\
\hline B-04-149 & Fathead Minnow & 33 & & & B-04-149_150604_B1 \\
\hline B-04-149 & Johnny Darter & 2 & & Spawning colors & B-04-149_150604_B1 \\
\hline B-04-149 & Northern Redbelly Dace & 6 & & & B-04-149_150604_E1 \\
\hline B-04-149 & Unidentified minnow & 1 & & Post-larval stage & B-04-149_150604_B1 \\
\hline B-04-149 & Unidentified sucker & 3 & $18-19^{*}$ & $\begin{array}{l}\text { Post larval stage. Plus } 2 \text { Orconectes virilis } \\
\text { (crayfish) }\end{array}$ & B-04-149_150604_B1 \\
\hline B-04-149 & Western Blacknose Dace & 1 & & & B-04-149_150604_B1 \\
\hline B-04-149 & White Sucker & 2 & $18^{*}$ & One larvae, one post larvae & B-04-149_150604_B1 \\
\hline B-04-152 & No Catch & & & & B-04-152_160604_B1 \\
\hline B-04-153 & Brook Stickleback & 1 & & & B-04-153_160604_E1 \\
\hline B-04-153 & Fathead Minnow & 28 & & & B-04-153_160604_E1 \\
\hline B-04-153 & White Sucker & 28 & $10-14^{*}$ & $\begin{array}{l}\text { Largest is consistent with White Sucker - } \\
\text { there is less certainty with the smallest } \\
\text { larvae but they appear to grade into the } \\
\text { larger ones with increasing size }\end{array}$ & B-04-153_160604_E1 \\
\hline
\end{tabular}


Appendix 5: List of all fish captures made between 2002 and 2006, showing site number, common name and number (\#) of fish collected, fork (or total ${ }^{\star}$ ) length in millimetres ( $\mathrm{mm}$ ), any comments on the collection and the fish capture identification (ID) number.

\begin{tabular}{|c|c|c|c|c|c|}
\hline Site Number & Common Name & \# & $\begin{array}{l}\text { Fork/Total* } \\
\text { Length }(\mathrm{mm})\end{array}$ & Comments & $\begin{array}{l}\text { Fish Capture } \\
\text { ID Number }\end{array}$ \\
\hline B-04-155 & No Catch & & & & B-04-155_160604_E1 \\
\hline B-04-161 & Fathead Minnow & 11 & & & B-04-161_180604_B1 \\
\hline B-04-161 & Northern Pike & 2 & 50,55 & 2 Neuroptera nymphs, 1 fingernail clam & B-04-161_180604_B1 \\
\hline B-04-161 & White Sucker & 1 & 95 & & B-04-161_180604_E1 \\
\hline B-04-163 & No Catch & & & & B-04-163_210604_E1 \\
\hline B-04-164 & No Catch & & & & B-04-164_210604_B1 \\
\hline B-04-165 & No Catch & & & & B-04-165_210604_E1 \\
\hline B-04-167 & Black Bullhead & 6 & & & B-04-167_210604_E1 \\
\hline B-04-167 & Fathead Minnow & 7 & & & B-04-167_210604_E1 \\
\hline B-04-167 & Longnose Dace & 1 & & & B-04-167_210604_E1 \\
\hline B-04-167 & Northern Pike & 2 & 66,400 & $\begin{array}{l}\text { One adult 400mm escaped seine, } 1 \text { post } \\
\text { larval Northern Pike @ } 66 \text { mm collected }\end{array}$ & B-04-167_210604_E1 \\
\hline B-04-167 & Unidentified minnow & 1 & & $\begin{array}{l}\text { Post larval stage. Plus } 2 \text { Orconectes virilis } \\
\text { (crayfish) }\end{array}$ & B-04-167_210604_B1 \\
\hline B-04-167 & White Sucker & 469 & $18-24^{*}$ & All YOY & B-04-167_210604_E1 \\
\hline B-04-174 & Black Bullhead & 3 & & & B-04-174_220604_E1 \\
\hline B-04-174 & Fathead Minnow & 28 & & 21 are YOY & B-04-174_220604_E1 \\
\hline B-04-174 & Johnny Darter & 2 & & & B-04-174_220604_E1 \\
\hline B-04-174 & Northern Pike & 4 & $150-650$ & Released & B-04-174_220604_E1 \\
\hline B-04-174 & White Sucker & 8 & $76-163$ & $163 \mathrm{~mm}$ is an immature male & B-04-174_220604_E1 \\
\hline B-04-181 & Common Shiner & 3 & & & B-04-181_280604_E1 \\
\hline $\mathrm{B}-04-181$ & Creek Chub & 5 & & & B-04-181_280604_E1 \\
\hline B-04-181 & Longnose Dace & 1 & & & B-04-181_280604_E1 \\
\hline B-04-181 & Western Blacknose Dace & 6 & & & B-04-181_280604_E1 \\
\hline B-04-186 & Brook Stickleback & 1 & & & B-04-186_290604_E1 \\
\hline B-04-186 & Common Shiner & 1 & & & B-04-186_290604_E1 \\
\hline B-04-186 & Fathead Minnow & 4 & & & B-04-186_290604_E1 \\
\hline B-04-186 & Smallmouth Bass & 4 & $\begin{array}{l}270,310,390 \\
420\end{array}$ & Released & B-04-186_290604_E1 \\
\hline B-04-186 & White Sucker & 13 & $86-131,210,400$ & $\begin{array}{l}\text { Released } 1 @ 400 \mathrm{~mm}, 210 \mathrm{~mm} \text { is } \\
\text { immature male }\end{array}$ & B-04-186_290604_E1 \\
\hline
\end{tabular}


Appendix 5: List of all fish captures made between 2002 and 2006, showing site number, common name and number (\#) of fish collected, fork (or total ${ }^{\star}$ ) length in millimetres ( $\mathrm{mm}$ ), any comments on the collection and the fish capture identification (ID) number.

\begin{tabular}{|c|c|c|c|c|c|}
\hline Site Number & Common Name & $\#$ & $\begin{array}{l}\text { Fork/Total* } \\
\text { Length }(\mathrm{mm})\end{array}$ & Comments & $\begin{array}{l}\text { Fish Capture } \\
\text { ID Number }\end{array}$ \\
\hline B-04-187 & Common Shiner & 5 & & & B-04-187_290604_D1 \\
\hline B-04-187 & Fathead Minnow & 8 & & & B-04-187_290604_D1 \\
\hline B-04-187 & Unidentified sucker & 17 & $12-15^{*}$ & $\begin{array}{l}\text { May be } C \text {. commersoni but not certain due } \\
\text { to poor condition of specimens. Definitely } \\
\text { Catostomid }\end{array}$ & B-04-187_290604_D1 \\
\hline B-04-187 & White Sucker & 1 & $31^{*}$ & $\begin{array}{l}\text { Plus } 2 \text { unidentified Catostomus post- } \\
\text { larvae }\end{array}$ & B-04-187_290604_E1 \\
\hline B-04-188 & Common Shiner & 23 & & 20 are $Y O Y$ & B-04-188_290604_E1 \\
\hline B-04-188 & Creek Chub & & & & B-04-188_290604_E1 \\
\hline B-04-188 & Fathead Minnow & & & & B-04-188_290604_E1 \\
\hline B-04-188 & Johnny Darter & & & & B-04-188_290604_E1 \\
\hline B-04-188 & White Sucker & 28 & $10-15^{*}$ & & B-04-188_290604_E1 \\
\hline B-04-189 & Fathead Minnow & 1 & & & B-04-189_300604_E1 \\
\hline B-04-189 & River Darter & 1 & & & B-04-189_300604_E1 \\
\hline B-04-189 & White Sucker & 73 & $10-19^{*}$ & All YOY & B-04-189_300604_E1 \\
\hline B-04-190 & Fathead Minnow & 4 & & & B-04-190_300604_D1 \\
\hline B-04-190 & Finescale Dace & 6 & & & B-04-190_300604_D1 \\
\hline B-04-191 & Fathead Minnow & 2 & & & B-04-191_050704_E1 \\
\hline B-04-191 & Western Blacknose Dace & 1 & & & B-04-191_050704_E1 \\
\hline B-04-191 & White Sucker & 10 & $10-18^{*}$ & Post-larvae & B-04-191_050704_E1 \\
\hline B-04-195 & Blacknose Shiner & 13 & & All YOY & B-04-195_060704_B1 \\
\hline B-04-195 & Unidentified minnow & 7 & & $\begin{array}{l}\text { Post larval stage. Plus } 2 \text { Orconectes virilis } \\
\text { (crayfish) }\end{array}$ & B-04-195_060704_D1 \\
\hline B-04-195 & White Sucker & 23 & $21-23^{*}$ & All YOY & B-04-195_060704_B1 \\
\hline B-04-196 & Blackside Darter & 1 & & & B-04-196_060704_E1 \\
\hline B-04-196 & Common Shiner & 7 & 130 & Released 1 & B-04-196_060704_E1 \\
\hline B-04-196 & Creek Chub & 4 & & & B-04-196_060704_E1 \\
\hline B-04-196 & Fathead Minnow & 2 & & & B-04-196_060704_E1 \\
\hline B-04-196 & Johnny Darter & 4 & & & B-04-196_060704_E1 \\
\hline B-04-196 & Western Blacknose Dace & 4 & & & B-04-196_060704_E1 \\
\hline B-04-196 & White Sucker & 3 & $63,180,210$ & $\begin{array}{l}\text { Released } 2 \text { adults }(210,180 \mathrm{~mm}) 63 \mathrm{~mm} \\
\text { kept }\end{array}$ & B-04-196_060704_E1 \\
\hline
\end{tabular}


Appendix 5: List of all fish captures made between 2002 and 2006, showing site number, common name and number (\#) of fish collected, fork (or total ${ }^{\star}$ ) length in millimetres ( $\mathrm{mm}$ ), any comments on the collection and the fish capture identification (ID) number.

\begin{tabular}{|c|c|c|c|c|c|}
\hline Site Number & Common Name & \# & $\begin{array}{l}\text { Fork/Total* } \\
\text { Length (mm) }\end{array}$ & Comments & $\begin{array}{l}\text { Fish Capture } \\
\text { ID Number }\end{array}$ \\
\hline B-04-197 & Common Shiner & 1 & & & B-04-197_060704_E1 \\
\hline B-04-197 & Creek Chub & 7 & 120 & Released 1 (120 mm) & B-04-197_060704_E1 \\
\hline B-04-197 & Fathead Minnow & 7 & & & B-04-197_060704_E1 \\
\hline B-04-197 & Johnny Darter & 7 & & & B-04-197_060704_E1 \\
\hline B-04-197 & Longnose Dace & 3 & & & B-04-197_060704_E1 \\
\hline B-04-197 & Western Blacknose Dace & 16 & & & B-04-197_060704_E1 \\
\hline B-04-197 & White Sucker & 2 & 180,200 & Released & B-04-197_060704_E1 \\
\hline B-04-198 & Common Shiner & 5 & & & B-04-198_060704_E1 \\
\hline B-04-198 & Creek Chub & 5 & & & B-04-198_060704_E1 \\
\hline B-04-198 & Johnny Darter & 1 & & & B-04-198_060704_E1 \\
\hline B-04-198 & Longnose Dace & 4 & & & B-04-198_060704_E1 \\
\hline B-04-198 & Western Blacknose Dace & 3 & & & B-04-198_060704_E1 \\
\hline B-04-198 & White Sucker & 1 & 74 & & B-04-198_060704_E1 \\
\hline B-04-199 & Bigmouth Shiner & 7 & & & B-04-199_060704_E1 \\
\hline B-04-199 & Common Shiner & 4 & & & B-04-199_060704_E1 \\
\hline B-04-199 & Creek Chub & 10 & & & B-04-199_060704_E1 \\
\hline B-04-199 & Fathead Minnow & 3 & & & B-04-199_060704_E1 \\
\hline B-04-199 & Johnny Darter & 4 & & Plus one Orconectes virilis & B-04-199_060704_E1 \\
\hline B-04-199 & Longnose Dace & 3 & & & B-04-199_060704_E1 \\
\hline B-04-199 & Western Blacknose Dace & 9 & & & B-04-199_060704_E1 \\
\hline B-04-199 & White Sucker & 3 & $43-59$ & & B-04-199_060704_E1 \\
\hline B-04-200 & Common Shiner & 6 & & & B-04-200_060704_E1 \\
\hline B-04-200 & Creek Chub & 11 & & & B-04-200_060704_E1 \\
\hline B-04-200 & Fathead Minnow & 5 & & & B-04-200_060704_E1 \\
\hline B-04-200 & Johnny Darter & 3 & & & B-04-200_060704_E1 \\
\hline B-04-200 & Western Blacknose Dace & 7 & & & B-04-200_060704_E1 \\
\hline B-04-200 & White Sucker & 2 & $121-127$ & 1 immature male, 1 mature female & B-04-200_060704_E1 \\
\hline B-04-205 & Brook Stickleback & 1 & & & B-04-205_070704_E1 \\
\hline B-04-205 & Common Shiner & 1 & & & B-04-205_070704_E1 \\
\hline B-04-205 & Creek Chub & 4 & & & B-04-205_070704_E1 \\
\hline B-04-205 & Fathead Minnow & 21 & & & B-04-205_070704_E1 \\
\hline
\end{tabular}


Appendix 5: List of all fish captures made between 2002 and 2006, showing site number, common name and number (\#) of fish collected, fork (or total ${ }^{\star}$ ) length in millimetres ( $\mathrm{mm}$ ), any comments on the collection and the fish capture identification (ID) number.

\begin{tabular}{|c|c|c|c|c|c|}
\hline Site Number & Common Name & \# & $\begin{array}{c}\text { Fork/Total* } \\
\text { Length }(\mathrm{mm})\end{array}$ & Comments & $\begin{array}{l}\text { Fish Capture } \\
\text { ID Number }\end{array}$ \\
\hline B-04-205 & Finescale Dace & 2 & & & B-04-205_070704_E1 \\
\hline B-04-205 & Pearl Dace & 2 & & & B-04-205_070704_E1 \\
\hline B-04-205 & Western Blacknose Dace & 8 & & & B-04-205_070704_E1 \\
\hline B-04-205 & White Sucker & 5 & $90-117$ & 3 female, 2 male & B-04-205_070704_E1 \\
\hline B-04-211 & Brook Stickleback & 3 & & Released & B-04-211_070704_E1 \\
\hline B-04-211 & Fathead Minnow & 2 & & Released & B-04-211_070704_E1 \\
\hline B-04-214 & Brook Stickleback & 1 & & & B-04-214_070704_E1 \\
\hline B-04-214 & Fathead Minnow & 7 & & & B-04-214_070704_E1 \\
\hline B-04-215 & Fathead Minnow & 1 & & & B-04-215_070704_E1 \\
\hline B-04-215 & White Sucker & 23 & $16-17^{*}$ & All YOY & B-04-215_070704_E1 \\
\hline B-04-217 & Creek Chub & 1 & & & B-04-217_080704_E1 \\
\hline B-04-217 & Fathead Minnow & 1 & & & B-04-217_080704_E1 \\
\hline B-04-217 & Johnny Darter & 2 & & & B-04-217_080704_E1 \\
\hline B-04-217 & Longnose Dace & 5 & & & B-04-217_080704_E1 \\
\hline B-04-217 & Western Blacknose Dace & 6 & & & B-04-217_080704_E1 \\
\hline B-04-217 & White Sucker & 2 & $106-124$ & & B-04-217_080704_E1 \\
\hline B-04-218 & Common Shiner & 3 & & & B-04-218_080704_E1 \\
\hline B-04-218 & Creek Chub & 12 & & Released 2 (190, $150 \mathrm{~mm})$ & B-04-218_080704_E1 \\
\hline B-04-218 & Fathead Minnow & 3 & & & B-04-218_080704_E1 \\
\hline B-04-218 & Johnny Darter & 12 & & & B-04-218_080704_E1 \\
\hline B-04-218 & Western Blacknose Dace & 21 & & & B-04-218_080704_E1 \\
\hline B-04-218 & White Sucker & 3 & $72-114$ & $114 \mathrm{~mm}$ is immature male & B-04-218_080704_E1 \\
\hline B-04-219 & Common Shiner & 10 & & & B-04-219_080704_E1 \\
\hline B-04-219 & Creek Chub & 8 & & & B-04-219_080704_E1 \\
\hline B-04-219 & Fathead Minnow & 5 & & & B-04-219_080704_E1 \\
\hline B-04-219 & Johnny Darter & 1 & & & B-04-219_080704_E1 \\
\hline B-04-219 & Western Blacknose Dace & 11 & & & B-04-219_080704_E1 \\
\hline B-04-219 & White Sucker & 2 & 105,126 & $126 \mathrm{~mm}$ is an immature male & B-04-219_080704_E1 \\
\hline B-04-220 & Common Shiner & 1 & & & B-04-220_080704_E1 \\
\hline B-04-220 & Creek Chub & 24 & & & B-04-220_080704_E1 \\
\hline B-04-220 & Fathead Minnow & 1 & & & B-04-220_080704_E1 \\
\hline
\end{tabular}


Appendix 5: List of all fish captures made between 2002 and 2006, showing site number, common name and number (\#) of fish collected, fork (or total ${ }^{\star}$ ) length in millimetres ( $\mathrm{mm}$ ), any comments on the collection and the fish capture identification (ID) number.

\begin{tabular}{|c|c|c|c|c|c|}
\hline Site Number & Common Name & \# & $\begin{array}{c}\text { Fork/Total* } \\
\text { Length }(\mathrm{mm})\end{array}$ & Comments & $\begin{array}{l}\text { Fish Capture } \\
\text { ID Number }\end{array}$ \\
\hline B-04-220 & Longnose Dace & 9 & & & B-04-220_080704_E1 \\
\hline B-04-220 & Western Blacknose Dace & 11 & & & B-04-220_080704_E1 \\
\hline B-04-220 & White Sucker & 3 & $91-102$ & & B-04-220_080704_E1 \\
\hline B-04-223 & Brook Stickleback & 1 & & & B-04-223_090704_E1 \\
\hline B-04-223 & Fathead Minnow & 5 & & & B-04-223_090704_E1 \\
\hline B-04-223 & Finescale Dace & 15 & & & B-04-223_090704_E1 \\
\hline B-04-224 & Creek Chub & 9 & & Released 1 (210 mm) & B-04-224_130704_E1 \\
\hline B-04-224 & Fathead Minnow & 7 & & & B-04-224_130704_E1 \\
\hline B-04-224 & Johnny Darter & 2 & & & B-04-224_130704_E1 \\
\hline B-04-224 & Longnose Dace & 8 & & & B-04-224_130704_E1 \\
\hline B-04-224 & Western Blacknose Dace & 14 & & & B-04-224_130704_E1 \\
\hline B-04-224 & White Sucker & 4 & $99-156$ & 1 female, 3 male & B-04-224_130704_E1 \\
\hline B-04-225 & Brook Stickleback & 12 & & & B-04-225_130704_E1 \\
\hline B-04-225 & Common Shiner & 3 & & Released 1 (140 mm) & B-04-225_130704_E1 \\
\hline B-04-225 & Western Blacknose Dace & 2 & & & B-04-225_130704_E1 \\
\hline B-04-225 & White Sucker & 8 & $15-18^{*}$ & All YOY & B-04-225_130704_E1 \\
\hline B-04-226 & Fathead Minnow & 1 & & & B-04-226_130704_B1 \\
\hline B-04-226 & White Sucker & 9 & $28-33^{*}$ & All YOY & B-04-226_130704_B1 \\
\hline B-04-228 & Brook Stickleback & 3 & & Released & B-04-228_130704_E1 \\
\hline B-04-229 & Brook Stickleback & 4 & & Released & B-04-229_140704_D1 \\
\hline B-04-230 & Brook Stickleback & 6 & & & B-04-230_140704_E1 \\
\hline B-04-230 & Fathead Minnow & 2 & & & B-04-230_140704_E1 \\
\hline B-04-230 & Pearl Dace & 7 & & 4 are $\mathrm{YOY}$ & B-04-230_140704_E1 \\
\hline B-04-230 & White Sucker & 1 & 90 & & B-04-230_140704_E1 \\
\hline B-04-231 & Brook Stickleback & 2 & & & B-04-231_140704_E1 \\
\hline B-04-231 & Fathead Minnow & 7 & & Male with spawning color & B-04-231_140704_E1 \\
\hline B-04-231 & Western Blacknose Dace & 2 & & & B-04-231_140704_E1 \\
\hline B-04-232 & Creek Chub & 2 & & & B-04-232_140704_E1 \\
\hline B-04-232 & Fathead Minnow & 1 & & & B-04-232_140704_E1 \\
\hline B-04-232 & Pearl Dace & 1 & & & B-04-232_140704_E1 \\
\hline B-04-232 & White Sucker & 1 & 113 & Immature male & B-04-232_140704_E1 \\
\hline
\end{tabular}


Appendix 5: List of all fish captures made between 2002 and 2006, showing site number, common name and number (\#) of fish collected, fork (or total ${ }^{\star}$ ) length in millimetres ( $\mathrm{mm}$ ), any comments on the collection and the fish capture identification (ID) number.

\begin{tabular}{|c|c|c|c|c|c|}
\hline Site Number & Common Name & \# & $\begin{array}{l}\text { Fork/Total* } \\
\text { Length (mm) }\end{array}$ & Comments & $\begin{array}{l}\text { Fish Capture } \\
\text { ID Number }\end{array}$ \\
\hline $\mathrm{B}-04-238$ & Common Shiner & 5 & & & B-04-238_140704_E1 \\
\hline B-04-238 & Finescale Dace & 1 & & & B-04-238_140704_E1 \\
\hline B-04-238 & Longnose Dace & 2 & & & B-04-238_140704_E1 \\
\hline $\mathrm{B}-04-238$ & Western Blacknose Dace & 5 & & & B-04-238_140704_E1 \\
\hline $\mathrm{B}-04-238$ & White Sucker & 5 & $17-23^{*}$ & All YOY & B-04-238_140704_E1 \\
\hline B-04-240 & Brook Stickleback & 1 & & & B-04-240_150704_E1 \\
\hline $\mathrm{B}-04-240$ & Common Shiner & 5 & & 4 juveniles & B-04-240_150704_E1 \\
\hline B-04-240 & Creek Chub & 1 & & Juvenile & B-04-240_150704_E1 \\
\hline B-04-240 & Fathead Minnow & 11 & & & B-04-240_150704_E1 \\
\hline B-04-240 & Finescale Dace & 29 & & & B-04-240_150704_E1 \\
\hline $\mathrm{B}-04-240$ & Western Blacknose Dace & 4 & & & B-04-240_150704_E1 \\
\hline B-04-240 & White Sucker & 6 & $51-87$ & Juveniles & B-04-240_150704_E1 \\
\hline $\mathrm{B}-04-241$ & Brassy Minnow & 5 & & & B-04-241_190704_B1 \\
\hline B-04-241 & Brassy Minnow & 13 & & & B-04-241_190704_E1 \\
\hline B-04-241 & Common Shiner & 32 & & & B-04-241_190704_B1 \\
\hline $\mathrm{B}-04-241$ & Common Shiner & 34 & & & B-04-241_190704_E1 \\
\hline B-04-241 & Creek Chub & 5 & & Plus one Orconectes virilis & B-04-241_190704_B1 \\
\hline B-04-241 & Creek Chub & 7 & & & B-04-241_190704_E1 \\
\hline $\mathrm{B}-04-241$ & Fathead Minnow & 2 & & & B-04-241_190704_B1 \\
\hline B-04-241 & Longnose Dace & 1 & & & B-04-241_190704_E1 \\
\hline B-04-241 & Pearl Dace & 1 & & & B-04-241_190704_E1 \\
\hline B-04-241 & Pearl Dace & 1 & & & B-04-241_190704_B1 \\
\hline B-04-241 & Western Blacknose Dace & 2 & & & B-04-241_190704_B1 \\
\hline B-04-241 & Western Blacknose Dace & 5 & & & B-04-241_190704_E1 \\
\hline B-04-241 & White Sucker & 4 & $45-144$ & $144 \mathrm{~mm}$ is an immature male & B-04-241_190704_B1 \\
\hline B-04-241 & White Sucker & 5 & $36^{*}-123$ & 3 are YOY & B-04-241_190704_E1 \\
\hline B-04-244 & Common Shiner & 7 & & & B-04-244_200704_E1 \\
\hline B-04-244 & Creek Chub & 2 & & & B-04-244_200704_E1 \\
\hline B-04-244 & Fathead Minnow & 3 & & & B-04-244_200704_E1 \\
\hline B-04-244 & Johnny Darter & 2 & & & B-04-244_200704_E1 \\
\hline B-04-244 & Longnose Dace & 3 & & & B-04-244_200704_E1 \\
\hline
\end{tabular}


Appendix 5: List of all fish captures made between 2002 and 2006, showing site number, common name and number (\#) of fish collected, fork (or total ${ }^{\star}$ ) length in millimetres ( $\mathrm{mm}$ ), any comments on the collection and the fish capture identification (ID) number.

\begin{tabular}{|c|c|c|c|c|c|}
\hline Site Number & Common Name & \# & $\begin{array}{l}\text { Fork/Total* } \\
\text { Length }(\mathrm{mm})\end{array}$ & Comments & $\begin{array}{l}\text { Fish Capture } \\
\text { ID Number }\end{array}$ \\
\hline B-04-244 & River Darter & 3 & & & B-04-244_200704_E1 \\
\hline B-04-244 & Western Blacknose Dace & 1 & & & B-04-244_200704_E1 \\
\hline B-04-248 & Golden Shiner & 8 & & & B-04-248_200704_B1 \\
\hline B-04-248 & Northern Pike & 1 & 148 & & B-04-248_200704_B1 \\
\hline B-04-249 & Northern Pike & 4 & $110-144$ & & B-04-249_200704_B1 \\
\hline B-04-250 & No Catch & & & & B-04-250_200704_B1 \\
\hline B-04-257 & Black Bullhead & 1 & & Released & B-04-257_210704_B1 \\
\hline B-04-257 & Fathead Minnow & 4 & & & B-04-257_210704_B1 \\
\hline B-04-257 & Golden Shiner & 121 & & & B-04-257_210704_B1 \\
\hline B-04-257 & Hybrid & 3 & & $\begin{array}{l}\text { Possible Fathead Minnow X Common } \\
\text { Shiner hybrid, dorsal fin ray problem }\end{array}$ & B-04-257_210704_B1 \\
\hline B-04-257 & Northern Pike & 14 & $97-130$ & All YOY & B-04-257_210704_B1 \\
\hline B-04-257 & Yellow Perch & 8 & $38^{*}-103$ & $1 \mathrm{YOY}$ & B-04-257_210704_B1 \\
\hline B-04-259 & No Catch & & & & B-04-259_210704_B1 \\
\hline B-04-263 & Fathead Minnow & & & $\begin{array}{l}\text { Beach seine failed observed several } \\
\text { Fathead Minnows escape }\end{array}$ & B-04-263_220704_B1 \\
\hline B-04-263 & Brook Stickleback & & & $\begin{array}{l}\text { Beach seine failed observed several } \\
\text { Brook Sticklebacks escape }\end{array}$ & B-04-263_220704_B1 \\
\hline B-04-264 & Brook Stickleback & 8 & & Released & B-04-264_220704_B1 \\
\hline B-04-264 & Fathead Minnow & 30 & & Released & B-04-264_220704_B1 \\
\hline B-04-266 & Brook Stickleback & 19 & & $17 \mathrm{YOY}$ & B-04-266_260704_E1 \\
\hline B-04-266 & Common Shiner & 27 & & Plus one Orconectes virilis YOY & B-04-266_260704_E1 \\
\hline B-04-266 & Creek Chub & 8 & & All YOY & B-04-266_260704_E1 \\
\hline B-04-266 & White Sucker & 5 & $20-29^{*}$ & All YOY & B-04-266_260704_E1 \\
\hline B-04-270 & No Catch & & & & B-04-270_040804_B1 \\
\hline B-04-270 & No Catch & & & & B-04-270_040804_E1 \\
\hline B-04-272 & Spottail Shiner & 100's & & Many below gated box culvert & B-04-272_040804_01 \\
\hline B-04-272 & Walleye & 1 & 200 & Observed & B-04-272_040804_01 \\
\hline B-04-275 & No Catch & & & & B-04-275_050804_B1 \\
\hline B-04-276 & Spottail Shiner & 4 & & Released & B-04-276_050804_E1 \\
\hline B-04-280 & Brook Stickleback & 12 & & & B-04-280_050804_B1 \\
\hline
\end{tabular}


Appendix 5: List of all fish captures made between 2002 and 2006, showing site number, common name and number (\#) of fish collected, fork (or total ${ }^{*}$ ) length in millimetres $(\mathrm{mm})$, any comments on the collection and the fish capture identification (ID) number.

\begin{tabular}{|c|c|c|c|c|c|}
\hline Site Number & Common Name & \# & $\begin{array}{l}\text { Fork/Total* } \\
\text { Length }(\mathrm{mm})\end{array}$ & Comments & $\begin{array}{l}\text { Fish Capture } \\
\text { ID Number }\end{array}$ \\
\hline B-04-280 & Fathead Minnow & 5 & & & B-04-280_050804_B1 \\
\hline B-04-286 & Northern Pike & & & Observed & B-04-286_060804_01 \\
\hline B-04-287 & Northern Pike & 2 & & $\begin{array}{l}\text { Small Northern Pike observed under } \\
\text { bridge }\end{array}$ & B-04-287_060804_O1 \\
\hline D-04-011 & No Catch & & & & D-04-011_020604_E1 \\
\hline D-04-012 & Brook Stickleback & 32 & & & D-04-012_020604_E1 \\
\hline D-04-012 & Common Shiner & 16 & & & D-04-012_020604_E1 \\
\hline D-04-012 & Creek Chub & 8 & & & D-04-012_020604_E1 \\
\hline D-04-012 & Fathead Minnow & 63 & & & D-04-012_020604_E1 \\
\hline D-04-012 & Longnose Dace & 3 & & & D-04-012_020604_E1 \\
\hline D-04-012 & Western Blacknose Dace & 19 & & & D-04-012_020604_E1 \\
\hline D-04-012 & White Sucker & 5 & $55-107$ & & D-04-012_020604_E1 \\
\hline D-04-013 & Northern Pike & 78 & $13-29^{*}$ & & D-04-013_030604_L1 \\
\hline D-04-013 & lowa Darter & 1 & & & D-04-013_030604_L1 \\
\hline D-04-015 & No Catch & & & & D-04-015_070604_L1 \\
\hline D-04-016 & Northern Pike & 9 & $17-28^{*}$ & $\begin{array}{l}\text { YOY, measured to tip of notocord } \\
\text { extension }\end{array}$ & D-04-016_080604_L1 \\
\hline D-04-017 & No Catch & & & & D-04-017_090604_E1 \\
\hline D-04-020 & No Catch & & & & D-04-020_100604_L1 \\
\hline D-04-021 & No Catch & & & & D-04-021_140604_L1 \\
\hline D-04-022 & Fathead Minnow & 7 & & Released & D-04-022_150604_E1 \\
\hline D-04-023 & Bigmouth Shiner & 1 & & & D-04-023_150604_E1 \\
\hline D-04-023 & Black Bullhead & 1 & $125^{*}$ & & D-04-023_150604_E1 \\
\hline D-04-023 & Brassy Minnow & 2 & & & D-04-023_150604_E1 \\
\hline D-04-023 & Brook Stickleback & 1 & & & D-04-023_150604_E1 \\
\hline D-04-023 & Common Shiner & 15 & & & D-04-023_150604_E1 \\
\hline D-04-023 & Creek Chub & 6 & & & D-04-023_150604_E1 \\
\hline D-04-023 & Fathead Minnow & 17 & & & D-04-023_150604_E1 \\
\hline D-04-023 & Hybrid & 1 & & $\begin{array}{l}42 \text { lateral line scales, dusky peritoneum, } \\
\text { plus no barbel, Creek Chub x Brassy } \\
\text { Minnow hybrids }\end{array}$ & D-04-023_150604_E1 \\
\hline
\end{tabular}


Appendix 5: List of all fish captures made between 2002 and 2006, showing site number, common name and number (\#) of fish collected, fork (or total ${ }^{\star}$ ) length in millimetres $(\mathrm{mm})$, any comments on the collection and the fish capture identification (ID) number.

\begin{tabular}{|c|c|c|c|c|c|}
\hline Site Number & Common Name & \# & $\begin{array}{c}\text { Fork/Total* } \\
\text { Length }(\mathrm{mm})\end{array}$ & Comments & $\begin{array}{l}\text { Fish Capture } \\
\text { ID Number }\end{array}$ \\
\hline D-04-023 & Hybrid & $\overline{1}$ & & $\begin{array}{l}44 \text { lateral line scales, also no barbel, pale } \\
\text { peritoneum, Creek Chub x Common } \\
\text { Shiner hybrids }\end{array}$ & D-04-023_150604_E1 \\
\hline D-04-023 & Johnny Darter & 4 & & 1 spawning male & D-04-023_150604_E1 \\
\hline D-04-023 & Longnose Dace & 1 & & & D-04-023_150604_E1 \\
\hline D-04-023 & Western Blacknose Dace & 35 & & & D-04-023_150604_E1 \\
\hline D-04-023 & White Sucker & 14 & $71-127$ & 127 is a mature female, rest are males & D-04-023_150604_E1 \\
\hline D-04-027 & Bigmouth Shiner & 4 & & & D-04-027_150604_E1 \\
\hline D-04-027 & Blackside Darter & 3 & & & D-04-027_150604_E1 \\
\hline D-04-027 & Brassy Minnow & 1 & & & D-04-027_150604_E1 \\
\hline D-04-027 & Common Shiner & 23 & & & D-04-027_150604_E1 \\
\hline D-04-027 & Creek Chub & 10 & & & D-04-027_150604_E1 \\
\hline D-04-027 & Fathead Minnow & 7 & & & D-04-027_150604_E1 \\
\hline D-04-027 & Johnny Darter & 2 & & & D-04-027_150604_E1 \\
\hline D-04-027 & Western Blacknose Dace & 3 & & & D-04-027_150604_E1 \\
\hline D-04-027 & White Sucker & 2 & 57,62 & & D-04-027_150604_E1 \\
\hline D-04-028 & Black Crappie & 5 & $70-110$ & Released & D-04-028_160604_E1 \\
\hline D-04-028 & Creek Chub & 5 & up to 169 & Mature, ripe adults present & D-04-028_160604_E1 \\
\hline D-04-028 & Fathead Minnow & 8 & & & D-04-028_160604_E1 \\
\hline D-04-028 & Northern Pike & 1 & 300 & Released & D-04-028_160604_E1 \\
\hline D-04-028 & White Sucker & 12 & $48-89$ & & D-04-028_160604_E1 \\
\hline D-04-029 & Black Crappie & 4 & $69-120$ & Released 1 (120 mm) & D-04-029_160604_E1 \\
\hline D-04-029 & Brook Stickleback & 21 & & & D-04-029_160604_E1 \\
\hline D-04-029 & Fathead Minnow & 94 & & & D-04-029_160604_E1 \\
\hline D-04-029 & Northern Pike & 1 & 650 & Released & D-04-029_160604_E1 \\
\hline D-04-033 & Fathead Minnow & 2 & & Released & D-04-033_230604_E1 \\
\hline D-04-034 & No Catch & & & Invertebrates only & D-04-034_230604_E1 \\
\hline D-04-035 & Fathead Minnow & 1 & & & D-04-035_230604_E1 \\
\hline D-04-035 & Northern Redbelly Dace & 1 & & & D-04-035_230604_E1 \\
\hline D-04-035 & White Sucker & 4 & $51-98$ & & D-04-035_230604_E1 \\
\hline D-04-036 & Brown Trout & 3 & 203-269 & Released & D-04-036_070704_E1 \\
\hline
\end{tabular}


Appendix 5: List of all fish captures made between 2002 and 2006, showing site number, common name and number (\#) of fish collected, fork (or total ${ }^{*}$ ) length in millimetres $(\mathrm{mm})$, any comments on the collection and the fish capture identification (ID) number.

\begin{tabular}{|c|c|c|c|c|c|}
\hline Site Number & Common Name & \# & $\begin{array}{l}\text { Fork/Total* } \\
\text { Length }(\mathrm{mm})\end{array}$ & Comments & $\begin{array}{l}\text { Fish Capture } \\
\text { ID Number }\end{array}$ \\
\hline D-04-036 & Common Shiner & 1 & & & D-04-036_070704_E1 \\
\hline D-04-036 & Creek Chub & 12 & & Released 10 & D-04-036_070704_E1 \\
\hline D-04-036 & Johnny Darter & 11 & & Released 10 & D-04-036_070704_E1 \\
\hline D-04-036 & Longnose Dace & 14 & & Released 10 & D-04-036_070704_E1 \\
\hline D-04-036 & Pearl Dace & 1 & & & D-04-036_070704_E1 \\
\hline D-04-036 & Western Blacknose Dace & 23 & & Released 20 & D-04-036_070704_E1 \\
\hline D-04-036 & White Sucker & 4 & 60,96 & Released 2, $96 \mathrm{~mm}$ is immature female & D-04-036_070704_E1 \\
\hline W-04-001 & White Sucker & 100's & & $\begin{array}{l}\text { Congregated on spawning riffle upstream } \\
\text { and downstream from crossing }\end{array}$ & W-04-001_030504_01 \\
\hline W-04-004 & White Sucker & 100's & & Congregated on spawning riffle & W-04-004 03050401 \\
\hline W-04-005 & White Sucker & 4 & & Stranded in pool, 2 dead and 2 alive & W-04-005_030504_01 \\
\hline W-04-006 & No Catch & & & & W-04-006_030504_E1 \\
\hline W-04-007 & White Sucker & 100's & & Congregated on spawning riffle & W-04-007_030504_01 \\
\hline W-04-009 & No Catch & & & & W-04-009_040504_E1 \\
\hline W-04-013 & No Catch & & & & W-04-013_040504_B1 \\
\hline W-04-020 & No Catch & & & & W-04-020_050504_B1 \\
\hline W-04-027 & No Catch & & & & W-04-027_050504_E1 \\
\hline W-04-032 & Northern Pike & 3 & & 3 other Northern Pike observed & W-04-032_060504_E1 \\
\hline W-04-036 & No Catch & & & & W-04-036_060504_E1 \\
\hline W-04-039 & Fathead Minnow & 12 & & & W-04-039_100504_E1 \\
\hline W-04-039 & Northern Pike & 1 & 400 & Released & W-04-039_100504_E1 \\
\hline W-04-039 & Spottail Shiner & 16 & & 3 mature, 12 in pre-spawn condition & W-04-039_100504_E1 \\
\hline W-04-039 & White Sucker & 2 & 390,430 & Released & W-04-039_100504_E1 \\
\hline W-04-040 & Fathead Minnow & 22 & & & W-04-040_100504_E1 \\
\hline W-04-040 & White Sucker & 7 & $286-407$ & Released & W-04-040_100504_E1 \\
\hline W-04-041 & No Catch & & & & W-04-041_110504_E1 \\
\hline W-04-043 & White Sucker & 7 & $355-395$ & Released & W-04-043_110504_E1 \\
\hline W-04-044 & White Sucker & 3 & & 3 White Sucker observed & W-04-044_110504_E1 \\
\hline W-04-049 & Central Mudminnow & 2 & & & W-04-049_110504_E1 \\
\hline W-04-049 & Fathead Minnow & 14 & & & W-04-049_110504_E1 \\
\hline
\end{tabular}


Appendix 5: List of all fish captures made between 2002 and 2006, showing site number, common name and number (\#) of fish collected, fork (or total ${ }^{\star}$ ) length in millimetres ( $\mathrm{mm}$ ), any comments on the collection and the fish capture identification (ID) number.

\begin{tabular}{|c|c|c|c|c|c|}
\hline Site Number & Common Name & \# & $\begin{array}{c}\text { Fork/Total* } \\
\text { Length }(\mathrm{mm})\end{array}$ & Comments & $\begin{array}{l}\text { Fish Capture } \\
\text { ID Number }\end{array}$ \\
\hline W-04-049 & Eggs & & & $\begin{array}{l}\text { Sucker eggs, most likely White Sucker. } \\
\text { Invertebrates in sample }\end{array}$ & W-04-049_110504_K1 \\
\hline W-04-050 & Brook Stickleback & 2 & & & W-04-050_110504_E1 \\
\hline W-04-050 & Central Mudminnow & 8 & & & W-04-050_110504_E1 \\
\hline W-04-050 & Fathead Minnow & 8 & & & W-04-050_110504_E1 \\
\hline W-04-050 & Pearl Dace & 1 & & & W-04-050_110504_E1 \\
\hline W-04-051 & Central Mudminnow & 2 & & & W-04-051_120504_E1 \\
\hline W-04-051 & Spottail Shiner & 11 & & & W-04-051_120504_E1 \\
\hline W-04-051 & White Sucker & 1 & & Adult released & W-04-051_120504_E1 \\
\hline W-04-052 & Brook Stickleback & 1 & & & W-04-052_120504_E1 \\
\hline W-04-052 & Central Mudminnow & 17 & & & W-04-052_120504_E1 \\
\hline W-04-052 & Fathead Minnow & 1 & & & W-04-052_120504_E1 \\
\hline W-04-053 & No Catch & & & & W-04-053_180504_E1 \\
\hline W-04-054 & White Sucker & 3 & $190-400$ & Released & W-04-054_180504_E1 \\
\hline W-04-055 & Brook Stickleback & 1 & & & W-04-055_180504_E1 \\
\hline W-04-055 & Fathead Minnow & 6 & & & W-04-055_180504_E1 \\
\hline W-04-056 & No Catch & & & & W-04-056_180504_E1 \\
\hline W-04-057 & Fathead Minnow & 8 & & & W-04-057_180504_E1 \\
\hline W-04-058 & No Catch & & & & W-04-058_180504_E1 \\
\hline W-04-059 & No Catch & & & & W-04-059_200504_E1 \\
\hline W-04-060 & Fathead Minnow & 4 & & & W-04-060_200504_E1 \\
\hline W-04-060 & Pearl Dace & 1 & & & W-04-060_200504_E1 \\
\hline W-04-060 & White Sucker & 1 & 116 & & W-04-060_200504_E1 \\
\hline W-04-063 & No Catch & & & & W-04-063_200504_E1 \\
\hline W-04-069 & No Catch & & & & W-04-069_210504_E1 \\
\hline W-04-070 & No Catch & & & & W-04-070_210504_E1 \\
\hline W-04-071 & No Catch & & & & W-04-071_210504_E1 \\
\hline W-04-072 & Longnose Dace & 1 & & Plus Orconectes virilis & W-04-072_250504_E1 \\
\hline W-04-074 & Brook Stickleback & 5 & & & W-04-074_250504_E1 \\
\hline W-04-074 & Fathead Minnow & 3 & & & W-04-074_250504_E1 \\
\hline W-04-074 & No Catch & & & Invertebrates: Annelids, Dipteran larvae & W-04-074_250504_K1 \\
\hline
\end{tabular}


Appendix 5: List of all fish captures made between 2002 and 2006, showing site number, common name and number (\#) of fish collected, fork (or total ${ }^{*}$ ) length in millimetres $(\mathrm{mm})$, any comments on the collection and the fish capture identification (ID) number.

\begin{tabular}{|c|c|c|c|c|c|}
\hline Site Number & Common Name & \# & $\begin{array}{c}\text { Fork/Total* } \\
\text { Length }(\mathrm{mm})\end{array}$ & Comments & $\begin{array}{l}\text { Fish Capture } \\
\text { ID Number }\end{array}$ \\
\hline W-04-076 & Fathead Minnow & 1 & & & W-04-076_250504_E1 \\
\hline W-04-077 & Central Mudminnow & 1 & & Released & W-04-077_260504_E1 \\
\hline W-04-077 & No Catch & & & & W-04-077_260504_L1 \\
\hline W-04-078 & Brook Stickleback & 3 & & & W-04-078_260504_E1 \\
\hline W-04-078 & Central Mudminnow & 8 & & & W-04-078_260504_E1 \\
\hline W-04-082 & No Catch & & & & W-04-082_260504_E1 \\
\hline W-04-082 & No Catch & & & & W-04-082_260504_L1 \\
\hline W-04-083 & Central Mudminnow & 9 & & & W-04-083_270504_E1 \\
\hline W-04-083 & Fathead Minnow & 8 & & & W-04-083_270504_E1 \\
\hline W-04-083 & No Catch & & & & W-04-083_270504_K1 \\
\hline W-04-083 & No Catch & & & & W-04-083_270504_L1 \\
\hline W-04-084 & Common Shiner & 3 & & & W-04-084_270504_E1 \\
\hline W-04-084 & Unidentified sucker & 1 & $12^{*}$ & Unidentified larval suckers & W-04-084_270504_D1 \\
\hline W-04-085 & Northern Pike & 2 & $22,24^{*}$ & $\begin{array}{l}\text { Also unidentified amphibian eggs, } \\
\text { Gastropod eggs, large Dipteran larvae, } \\
\text { and small Coleoptera larvae }\end{array}$ & W-04-085_280504_B1 \\
\hline W-04-087 & Northern Pike & 8 & $17-25^{*}$ & & W-04-087_280504_L1 \\
\hline W-04-088 & No Catch & & & & W-04-088_280504_E1 \\
\hline W-04-088 & Northern Pike & 4 & $16-19^{*}$ & & W-04-088_280504_L1 \\
\hline W-04-090 & Brook Stickleback & 38 & & & W-04-090_010604_E1 \\
\hline W-04-090 & Central Mudminnow & 30 & & & W-04-090_010604_E1 \\
\hline W-04-090 & Fathead Minnow & 8 & & & W-04-090_010604_E1 \\
\hline W-04-090 & No Catch & & & Invertebrates only & W-04-090_010604_L1 \\
\hline W-04-092 & Brook Stickleback & 1 & & & W-04-092_010604_L1 \\
\hline W-04-092 & Central Mudminnow & 1 & & & W-04-092_010604_E1 \\
\hline W-04-092 & No Catch & & & Possibly one unidentified fish egg & W-04-092_010604_L1 \\
\hline W-04-093 & Central Mudminnow & 7 & & & W-04-093_010604_E1 \\
\hline W-04-093 & No Catch & & & Invertebrates only & W-04-093_010604_L1 \\
\hline W-04-093 & Northern Redbelly Dace & 2 & & & W-04-093_010604_E1 \\
\hline W-04-094 & Brook Stickleback & 11 & & & W-04-094_020604_E1 \\
\hline W-04-094 & Central Mudminnow & 9 & & & W-04-094_020604_E1 \\
\hline
\end{tabular}


Appendix 5: List of all fish captures made between 2002 and 2006, showing site number, common name and number (\#) of fish collected, fork (or total ${ }^{\star}$ ) length in millimetres ( $\mathrm{mm}$ ), any comments on the collection and the fish capture identification (ID) number.

\begin{tabular}{|c|c|c|c|c|c|}
\hline Site Number & Common Name & \# & $\begin{array}{l}\text { Fork/Total* } \\
\text { Length }(\mathrm{mm})\end{array}$ & Comments & $\begin{array}{l}\text { Fish Capture } \\
\text { ID Number }\end{array}$ \\
\hline W-04-094 & lowa Darter & 2 & & & W-04-094_020604_E1 \\
\hline W-04-094 & No Catch & & & Invertebrates only & W-04-094_020604_L1 \\
\hline W-04-094 & Northern Redbelly Dace & 6 & & & W-04-094_020604_E1 \\
\hline W-04-095 & Brook Stickleback & 36 & & & W-04-095_020604_E1 \\
\hline W-04-095 & Central Mudminnow & 7 & & & W-04-095_020604_E1 \\
\hline W-04-095 & Hybrid & 1 & & $\begin{array}{l}\text { Finescale Dace and Northern Redbelly } \\
\text { Dace hybrid }\end{array}$ & W-04-095_020604_E1 \\
\hline W-04-095 & Northern Redbelly Dace & 9 & & & W-04-095_020604_E1 \\
\hline W-04-095 & White Sucker & 4 & $53-59$ & & W-04-095_020604_E1 \\
\hline W-04-097 & Brook Stickleback & 4 & & & W-04-097_020604_E1 \\
\hline W-04-097 & Central Mudminnow & 2 & & & W-04-097_020604_E1 \\
\hline W-04-097 & Northern Redbelly Dace & 5 & & & W-04-097_020604_E1 \\
\hline W-04-097 & Pearl Dace & 7 & & & W-04-097_020604_E1 \\
\hline W-04-102 & Central Mudminnow & 1 & & & W-04-102_030604_E1 \\
\hline W-04-102 & Fathead Minnow & 1 & & & W-04-102_030604_E1 \\
\hline W-04-102 & No Catch & & & Invertebrates plus 2 tadpoles & W-04-102_030604_K1 \\
\hline W-04-102 & Northern Redbelly Dace & 1 & & & W-04-102_030604_E1 \\
\hline W-04-105 & Central Mudminnow & 2 & & & W-04-105_030604_E1 \\
\hline W-04-105 & Fathead Minnow & 2 & & & W-04-105_030604_E1 \\
\hline W-04-105 & Northern Redbelly Dace & 3 & & & W-04-105_030604_E1 \\
\hline W-04-106 & Brook Stickleback & 1 & & & W-04-106_030604_E1 \\
\hline W-04-106 & Central Mudminnow & 1 & & & W-04-106_030604_E1 \\
\hline W-04-109 & Central Mudminnow & 12 & & & W-04-109_040604_E1 \\
\hline W-04-109 & Fathead Minnow & 5 & & & W-04-109_040604_E1 \\
\hline W-04-109 & No Catch & & & & W-04-109_040604_K1 \\
\hline W-04-110 & Central Mudminnow & 1 & & & W-04-110_040604_E1 \\
\hline W-04-111 & Fathead Minnow & 1 & & & W-04-111_040604_E1 \\
\hline W-04-113 & Fathead Minnow & 25 & & & W-04-113_050604_E1 \\
\hline W-04-113 & White Sucker & 1 & 67 & & W-04-113_050604_E1 \\
\hline W-04-114 & Brook Stickleback & 2 & & & W-04-114_050604_E1 \\
\hline W-04-114 & Central Mudminnow & 3 & & & W-04-114_050604_E1 \\
\hline
\end{tabular}


Appendix 5: List of all fish captures made between 2002 and 2006, showing site number, common name and number (\#) of fish collected, fork (or total ${ }^{\star}$ ) length in millimetres ( $\mathrm{mm}$ ), any comments on the collection and the fish capture identification (ID) number.

\begin{tabular}{|c|c|c|c|c|c|}
\hline Site Number & Common Name & \# & $\begin{array}{c}\text { Fork/Total* } \\
\text { Length }(\mathrm{mm})\end{array}$ & Comments & $\begin{array}{l}\text { Fish Capture } \\
\text { ID Number }\end{array}$ \\
\hline W-04-115 & No Catch & & & & W-04-115_050604_E1 \\
\hline W-04-116 & Fathead Minnow & 30 & & 3 spawning males & W-04-116_050604_E1 \\
\hline W-04-116 & White Sucker & 4 & $57-91$ & $57 \mathrm{~mm}$ is an immature male & W-04-116_050604_E1 \\
\hline W-04-119 & No Catch & & & & W-04-119_080604_E1 \\
\hline W-04-120 & Blackside Darter & 1 & & & W-04-120_080604_E1 \\
\hline W-04-120 & Brook Stickleback & 3 & & & W-04-120_080604_E1 \\
\hline W-04-120 & Central Mudminnow & 2 & & & W-04-120_080604_E1 \\
\hline W-04-120 & Fathead Minnow & 24 & & & W-04-120_080604_E1 \\
\hline W-04-120 & Johnny Darter & 2 & & & W-04-120_080604_E1 \\
\hline W-04-120 & Longnose Dace & 3 & & & W-04-120_080604_E1 \\
\hline W-04-120 & Northern Redbelly Dace & 5 & & & W-04-120_080604_E1 \\
\hline W-04-120 & White Sucker & 2 & 83,128 & & W-04-120_080604_E1 \\
\hline W-04-121 & No Catch & & & & W-04-121_080604_E1 \\
\hline W-04-124 & No Catch & & & & W-04-124_080604_E1 \\
\hline W-04-126 & Brook Stickleback & 19 & & & W-04-126_090604_E1 \\
\hline W-04-126 & Central Mudminnow & 12 & & & W-04-126_090604_E1 \\
\hline W-04-126 & Fathead Minnow & 4 & & & W-04-126_090604_E1 \\
\hline W-04-126 & Finescale Dace & 1 & & & W-04-126_090604_E1 \\
\hline W-04-126 & Northern Redbelly Dace & 55 & & & W-04-126_090604_E1 \\
\hline W-04-129 & Brook Stickleback & 1 & & & W-04-129_090604_E1 \\
\hline W-04-132 & Brook Stickleback & 1 & & & W-04-132_090604_E1 \\
\hline W-04-132 & Fathead Minnow & 1 & & & W-04-132_090604_E1 \\
\hline W-04-135 & Fathead Minnow & 5 & & & W-04-135_090604_E1 \\
\hline W-04-137 & No Catch & & & & W-04-137_100604_E1 \\
\hline W-04-149 & Northern Pike & 1 & 305 & Released & W-04-149_140604_B1 \\
\hline W-04-151 & Fathead Minnow & 16 & & Male with spawning color & W-04-151_140604_E1 \\
\hline W-04-151 & Northern Pike & 1 & 68 & & W-04-151_140604_E1 \\
\hline W-04-153 & Brook Stickleback & 11 & & & W-04-153_150604_E1 \\
\hline W-04-153 & Fathead Minnow & 17 & & Male with spawning color & W-04-153_150604_E1 \\
\hline W-04-157 & No Catch & & & & W-04-157_160604_E1 \\
\hline W-04-159 & Brook Stickleback & 1 & & & W-04-159_160604_E1 \\
\hline
\end{tabular}


Appendix 5: List of all fish captures made between 2002 and 2006, showing site number, common name and number (\#) of fish collected, fork (or total ${ }^{*}$ ) length in millimetres $(\mathrm{mm})$, any comments on the collection and the fish capture identification (ID) number.

\begin{tabular}{|c|c|c|c|c|c|}
\hline Site Number & Common Name & \# & $\begin{array}{c}\text { Fork/Total* } \\
\text { Length }(\mathrm{mm})\end{array}$ & Comments & $\begin{array}{l}\text { Fish Capture } \\
\text { ID Number }\end{array}$ \\
\hline W-04-159 & Fathead Minnow & 3 & & & W-04-159_160604_E1 \\
\hline W-04-166 & Northern Pike & 1 & 68 & & W-04-166_170604_E1 \\
\hline W-04-167 & Brook Stickleback & 1 & & & W-04-167_170604_E1 \\
\hline W-04-167 & Fathead Minnow & 16 & & & W-04-167_170604_E1 \\
\hline W-04-171 & Brook Stickleback & 4 & & & W-04-171_170604_E1 \\
\hline W-04-171 & Fathead Minnow & 111 & & & W-04-171_170604_E1 \\
\hline W-04-172 & Fathead Minnow & 9 & & & W-04-172_170604_E1 \\
\hline W-04-173 & Fathead Minnow & 12 & & Male with spawning colors & W-04-173_180604_E1 \\
\hline W-04-173 & Northern Pike & 3 & 87 & Released 2 YOY & W-04-173_180604_E1 \\
\hline W-04-174 & Brook Stickleback & 1 & & & W-04-174_180604_E1 \\
\hline W-04-175 & Central Mudminnow & 1 & & & W-04-175_210604_E1 \\
\hline W-04-175 & Northern Pike & 4 & $56-74$ & Released 1 & W-04-175_210604_E1 \\
\hline W-04-175 & Spottail Shiner & 2 & & & W-04-175_210604_E1 \\
\hline W-04-176 & Brook Stickleback & 39 & & & W-04-176_210604_E1 \\
\hline W-04-176 & Central Mudminnow & 2 & & & W-04-176_210604_E1 \\
\hline W-04-176 & Fathead Minnow & 1 & & Spawning male & W-04-176_210604_E1 \\
\hline W-04-176 & Finescale Dace & 7 & & & W-04-176_210604_E1 \\
\hline W-04-176 & Unidentified minnow & 3 & & 2 post-larva, 1 YOY & W-04-176_210604_E1 \\
\hline W-04-177 & Brook Stickleback & 12 & & & W-04-177_210604_E1 \\
\hline W-04-177 & Central Mudminnow & 2 & & & W-04-177_210604_E1 \\
\hline W-04-177 & Fathead Minnow & 2 & & & W-04-177_210604_E1 \\
\hline W-04-177 & Finescale Dace & 4 & & & W-04-177_210604_E1 \\
\hline W-04-177 & Northern Redbelly Dace & 4 & & & W-04-177_210604_E1 \\
\hline W-04-178 & Brook Stickleback & 2 & & & W-04-178_210604_E1 \\
\hline W-04-178 & Central Mudminnow & 1 & & & W-04-178_210604_E1 \\
\hline W-04-179 & Brook Stickleback & 3 & & & W-04-179_220604_E1 \\
\hline W-04-179 & Central Mudminnow & 1 & & & W-04-179_220604_E1 \\
\hline W-04-179 & Fathead Minnow & 3 & & & W-04-179_220604_E1 \\
\hline W-04-180 & No Catch & & & & W-04-180_220604_B1 \\
\hline W-04-181 & Northern Pike & 4 & $30-34^{*}$ & & W-04-181_220604_E1 \\
\hline W-04-182 & Northern Pike & 3 & $28-30^{*}$ & & W-04-182_220604_E1 \\
\hline
\end{tabular}


Appendix 5: List of all fish captures made between 2002 and 2006, showing site number, common name and number (\#) of fish collected, fork (or total ${ }^{*}$ ) length in millimetres $(\mathrm{mm})$, any comments on the collection and the fish capture identification (ID) number.

\begin{tabular}{|c|c|c|c|c|c|}
\hline Site Number & Common Name & \# & $\begin{array}{c}\text { Fork/Total* } \\
\text { Length }(\mathrm{mm})\end{array}$ & Comments & $\begin{array}{l}\text { Fish Capture } \\
\text { ID Number }\end{array}$ \\
\hline W-04-184 & No Catch & & & & W-04-184_220604_E1 \\
\hline W-04-186 & Central Mudminnow & 2 & & & W-04-186_230604_E1 \\
\hline W-04-186 & Fathead Minnow & 1 & & & W-04-186_230604_E1 \\
\hline W-04-187 & Brook Stickleback & 2 & & & W-04-187_230604_E1 \\
\hline W-04-187 & Fathead Minnow & 2 & & & W-04-187_230604_E1 \\
\hline W-04-192 & Brook Stickleback & 1 & & & W-04-192_240604_E1 \\
\hline W-04-192 & Burbot & 1 & 186 & & W-04-192_240604_E1 \\
\hline W-04-192 & Emerald Shiner & 1 & & & W-04-192_240604_E1 \\
\hline W-04-193 & No Catch & & & & W-04-193_240604_E1 \\
\hline W-04-195 & Brook Stickleback & 16 & & & W-04-195_250604_E1 \\
\hline W-04-195 & Central Mudminnow & 5 & & & W-04-195_250604_E1 \\
\hline W-04-195 & Fathead Minnow & 13 & & & W-04-195_250604_E1 \\
\hline W-04-197 & Central Mudminnow & 2 & & & W-04-197_250604_E1 \\
\hline W-04-197 & Fathead Minnow & 1 & & & W-04-197_250604_E1 \\
\hline W-04-197 & Finescale Dace & 7 & & & W-04-197_250604_E1 \\
\hline W-04-197 & Northern Redbelly Dace & 4 & & & W-04-197_250604_E1 \\
\hline W-04-197 & White Sucker & 1 & 252 & Released & W-04-197_250604_E1 \\
\hline W-04-200 & Brook Stickleback & 9 & & & W-04-200_290604_E1 \\
\hline W-04-202 & Brook Stickleback & 2 & & & W-04-202_290604_B1 \\
\hline W-04-202 & White Sucker & 24 & $18-217$ & 23 are $\mathrm{YOY}, 217 \mathrm{~mm}$ is an immature male & W-04-202_290604_B1 \\
\hline W-04-203 & Fathead Minnow & 6 & & & W-04-203_290604_E1 \\
\hline W-04-207 & Brook Stickleback & 14 & & & W-04-207_050704_E1 \\
\hline W-04-207 & Central Mudminnow & 2 & & & W-04-207_050704_E1 \\
\hline W-04-207 & Finescale Dace & 2 & & & W-04-207_050704_E1 \\
\hline W-04-207 & Northern Redbelly Dace & 1 & & & W-04-207_050704_E1 \\
\hline W-04-207 & Pearl Dace & 15 & & & W-04-207_050704_E1 \\
\hline W-04-207 & Unidentified minnow & 27 & & Post-larvae (may be Pearl Dace) & W-04-207_050704_E1 \\
\hline W-04-208 & Brook Stickleback & 30 & & & W-04-208_050704_E1 \\
\hline W-04-208 & Central Mudminnow & 31 & & & W-04-208_050704_E1 \\
\hline W-04-208 & Iowa Darter & 1 & & & W-04-208_050704_E1 \\
\hline W-04-208 & No Catch & & & Invertebrates only & W-04-208_050704_K1 \\
\hline
\end{tabular}


Appendix 5: List of all fish captures made between 2002 and 2006, showing site number, common name and number (\#) of fish collected, fork (or total ${ }^{*}$ ) length in millimetres $(\mathrm{mm})$, any comments on the collection and the fish capture identification (ID) number.

\begin{tabular}{|c|c|c|c|c|c|}
\hline Site Number & Common Name & \# & $\begin{array}{c}\text { Fork/Total* } \\
\text { Length }(\mathrm{mm})\end{array}$ & Comments & $\begin{array}{l}\text { Fish Capture } \\
\text { ID Number }\end{array}$ \\
\hline W-04-208 & Pearl Dace & 14 & & & W-04-208_050704_E1 \\
\hline W-04-211 & Central Mudminnow & 3 & & & W-04-211_050704_E1 \\
\hline W-04-211 & Common Shiner & 16 & & & W-04-211_050704_E1 \\
\hline W-04-211 & Fathead Minnow & 3 & & & W-04-211_050704_E1 \\
\hline W-04-211 & Northern Redbelly Dace & 7 & & & W-04-211_050704_E1 \\
\hline W-04-211 & Stonecat & 1 & & & W-04-211_050704_E1 \\
\hline W-04-211 & White Sucker & 1 & 70 & & W-04-211_050704_E1 \\
\hline W-04-212 & Northern Pike & 2 & 88 & Released $1(80 \mathrm{~mm})$ & W-04-212_060704_E1 \\
\hline W-04-213 & Johnny Darter & 3 & & & W-04-213_060704_E1 \\
\hline W-04-213 & Longnose Dace & 1 & & & W-04-213_060704_E1 \\
\hline W-04-213 & Northern Pike & 1 & 90 & & W-04-213_060704_E1 \\
\hline W-04-216 & Central Mudminnow & 2 & & & W-04-216_060704_E1 \\
\hline W-04-216 & Common Shiner & 2 & & & W-04-216_060704_E1 \\
\hline W-04-216 & Fathead Minnow & 1 & & & W-04-216_060704_E1 \\
\hline W-04-216 & Northern Pike & 1 & 97 & & W-04-216_060704_E1 \\
\hline W-04-216 & White Sucker & 1 & 23 & YOY & W-04-216_060704_E1 \\
\hline W-04-217 & Brook Stickleback & 15 & & & W-04-217_060704_E1 \\
\hline W-04-217 & Central Mudminnow & 8 & & & W-04-217_060704_E1 \\
\hline W-04-217 & Fathead Minnow & 1 & & & W-04-217_060704_E1 \\
\hline W-04-217 & Johnny Darter & 8 & & & W-04-217_060704_E1 \\
\hline W-04-217 & Pearl Dace & 12 & & & W-04-217_060704_E1 \\
\hline W-04-217 & White Sucker & 5 & $57-143$ & & W-04-217_060704_E1 \\
\hline W-04-218 & Burbot & 1 & 31 & YOY & W-04-218_060704_E1 \\
\hline W-04-218 & Central Mudminnow & 7 & & & W-04-218_060704_E1 \\
\hline W-04-218 & Common Shiner & 3 & & & W-04-218_060704_E1 \\
\hline W-04-218 & Fathead Minnow & 1 & & & W-04-218_060704_E1 \\
\hline W-04-218 & Hornyhead Chub & 1 & & & W-04-218_060704_E1 \\
\hline W-04-218 & White Sucker & 2 & 72,86 & & W-04-218_060704_E1 \\
\hline W-04-225 & Brook Stickleback & 1 & & & W-04-225_070704_E1 \\
\hline W-04-225 & Common Shiner & 5 & & & W-04-225_070704_E1 \\
\hline W-04-225 & Fathead Minnow & 3 & & & W-04-225_070704_E1 \\
\hline
\end{tabular}


Appendix 5: List of all fish captures made between 2002 and 2006, showing site number, common name and number (\#) of fish collected, fork (or total ${ }^{*}$ ) length in millimetres $(\mathrm{mm})$, any comments on the collection and the fish capture identification (ID) number.

\begin{tabular}{|c|c|c|c|c|c|}
\hline Site Number & Common Name & $\#$ & $\begin{array}{c}\text { Fork/Total* } \\
\text { Length }(\mathrm{mm})\end{array}$ & Comments & $\begin{array}{l}\text { Fish Capture } \\
\text { ID Number }\end{array}$ \\
\hline W-04-225 & Finescale Dace & 6 & & & W-04-225 070704 E1 \\
\hline W-04-225 & Hornyhead Chub & 1 & & & W-04-225_070704_E1 \\
\hline W-04-225 & Johnny Darter & 4 & & & W-04-225_070704_E1 \\
\hline W-04-225 & Pearl Dace & 3 & & & W-04-225_070704_E1 \\
\hline W-04-225 & White Sucker & 9 & $19-255$ & Released 3 (150-255 mm) & W-04-225_070704_E1 \\
\hline W-04-231 & Brook Stickleback & 1 & & & W-04-231_080704_E1 \\
\hline W-04-231 & Central Mudminnow & 26 & & & W-04-231_080704_E1 \\
\hline W-04-231 & No Catch & & & Invertebrates only & W-04-231_080704_K1 \\
\hline W-04-231 & Northern Pike & 3 & $76-87$ & Released 1 (76 mm) & W-04-231_080704_E1 \\
\hline W-04-232 & Central Mudminnow & 8 & & & W-04-232_080704_E1 \\
\hline W-04-232 & Northern Pike & 4 & $93-250$ & Released $2(102,250 \mathrm{~mm})$ & W-04-232_080704_E1 \\
\hline W-04-233 & No Catch & & & & W-04-233_130704_E1 \\
\hline W-04-234 & Brook Stickleback & 30 & & & W-04-234_130704_E1 \\
\hline W-04-234 & Finescale Dace & 54 & & 45 are $Y O Y$ & W-04-234_130704_E1 \\
\hline W-04-234 & Western Blacknose Dace & 3 & & & W-04-234_130704_E1 \\
\hline W-04-235 & No Catch & & & Invertebrates only & W-04-235_130704_K1 \\
\hline W-04-237 & Brook Stickleback & 8 & & & W-04-237_130704_E1 \\
\hline W-04-237 & Finescale Dace & 47 & & 43 are YOY & W-04-237_130704_E1 \\
\hline W-04-238 & Brook Stickleback & 27 & & & W-04-238_130704_E1 \\
\hline W-04-238 & Fathead Minnow & 2 & & & W-04-238_130704_E1 \\
\hline W-04-238 & Hybrid & 22 & & $\begin{array}{l}\text { Hybrid swarm of Finescale Dace, Northern } \\
\text { Redbelly Dace, and Pearl Dace; none } \\
\text { could be identified to species }\end{array}$ & W-04-238_130704_E1 \\
\hline W-04-238 & Logperch & 1 & & & W-04-238_130704_E1 \\
\hline W-04-239 & Brook Stickleback & 89 & & Many are YOY & W-04-239_130704_E1 \\
\hline W-04-239 & Fathead Minnow & 2 & & & W-04-239_130704_E1 \\
\hline W-04-239 & Northern Pike & 1 & 110 & & W-04-239_130704_E1 \\
\hline W-04-239 & Pearl Dace & 2 & & & W-04-239_130704_E1 \\
\hline W-04-244 & Common Carp & 100's & & Observed in pool & W-04-244_140704_O1 \\
\hline W-04-245 & Common Carp & 100's & & Observed in pool & W-04-245_140704_01 \\
\hline
\end{tabular}


Appendix 5: List of all fish captures made between 2002 and 2006, showing site number, common name and number (\#) of fish collected, fork (or total ${ }^{\star}$ ) length in millimetres ( $\mathrm{mm}$ ), any comments on the collection and the fish capture identification (ID) number.

\begin{tabular}{|c|c|c|c|c|c|}
\hline Site Number & Common Name & \# & $\begin{array}{c}\text { Fork/Total* } \\
\text { Length }(\mathrm{mm})\end{array}$ & Comments & $\begin{array}{l}\text { Fish Capture } \\
\text { ID Number }\end{array}$ \\
\hline W-04-246 & Common Carp & 100's & & $\begin{array}{l}\text { Observed upstream and downstream from } \\
\text { culverts }\end{array}$ & W-04-246_140704_01 \\
\hline W-04-249 & Brook Stickleback & 1 & & & W-04-249_140704_E1 \\
\hline W-04-249 & Creek Chub & 19 & & & W-04-249_140704_E1 \\
\hline W-04-249 & Fathead Minnow & 7 & & & W-04-249_140704_E1 \\
\hline W-04-249 & Finescale Dace & 2 & & & W-04-249_140704_E1 \\
\hline W-04-249 & Johnny Darter & 3 & & & W-04-249_140704_E1 \\
\hline W-04-249 & Northern Redbelly Dace & 15 & & & W-04-249_140704_E1 \\
\hline W-04-249 & Pearl Dace & 2 & & & W-04-249_140704_E1 \\
\hline W-04-249 & Western Blacknose Dace & 9 & & & W-04-249_140704_E1 \\
\hline W-04-250 & Brook Stickleback & 124 & & & W-04-250_140704_E1 \\
\hline W-04-250 & Creek Chub & 39 & & & W-04-250_140704_E1 \\
\hline W-04-250 & Fathead Minnow & 6 & & & W-04-250_140704_E1 \\
\hline W-04-250 & Finescale Dace & 2 & & & W-04-250_140704_E1 \\
\hline W-04-250 & Johnny Darter & 2 & & & W-04-250_140704_E1 \\
\hline W-04-250 & No Catch & & & Invertebrates only & W-04-250_140704_K1 \\
\hline W-04-250 & White Sucker & 2 & 49,91 & & W-04-250_140704_E1 \\
\hline W-04-252 & Creek Chub & 14 & & & W-04-252_150704_E1 \\
\hline W-04-252 & Fathead Minnow & 1 & & & W-04-252_150704_E1 \\
\hline W-04-252 & Finescale Dace & 4 & & & W-04-252_150704_E1 \\
\hline W-04-252 & Johnny Darter & 3 & & & W-04-252_150704_E1 \\
\hline W-04-252 & Pearl Dace & 5 & & & W-04-252_150704_E1 \\
\hline W-04-252 & Western Blacknose Dace & 12 & & & W-04-252_150704_E1 \\
\hline W-04-252 & White Sucker & 1 & 174 & Mature male & W-04-252_150704_E1 \\
\hline W-04-253 & Brook Stickleback & 8 & & & W-04-253_150704_E1 \\
\hline W-04-253 & Fathead Minnow & 3 & & & W-04-253_150704_E1 \\
\hline W-04-253 & Johnny Darter & 1 & & & W-04-253_150704_E1 \\
\hline W-04-253 & Northern Redbelly Dace & 1 & & & W-04-253_150704_E1 \\
\hline W-04-254 & Common Shiner & 8 & & & W-04-254_150704_E1 \\
\hline W-04-254 & Creek Chub & 5 & & & W-04-254_150704_E1 \\
\hline W-04-254 & Finescale Dace & 1 & & & W-04-254_150704_E1 \\
\hline
\end{tabular}


Appendix 5: List of all fish captures made between 2002 and 2006, showing site number, common name and number (\#) of fish collected, fork (or total ${ }^{\star}$ ) length in millimetres $(\mathrm{mm})$, any comments on the collection and the fish capture identification (ID) number.

\begin{tabular}{|c|c|c|c|c|c|}
\hline Site Number & Common Name & \# & $\begin{array}{c}\text { Fork/Total* } \\
\text { Length }(\mathrm{mm})\end{array}$ & Comments & $\begin{array}{l}\text { Fish Capture } \\
\text { ID Number }\end{array}$ \\
\hline W-04-254 & Johnny Darter & 3 & & & W-04-254_150704_E1 \\
\hline W-04-254 & No Catch & & & Invertebrates only & W-04-254_150704_K1 \\
\hline W-04-254 & Western Blacknose Dace & 20 & & & W-04-254_150704_E1 \\
\hline W-04-254 & White Sucker & 2 & 77,80 & & W-04-254_150704_E1 \\
\hline W-04-255 & Common Shiner & 2 & & & W-04-255_150704_E1 \\
\hline W-04-255 & Creek Chub & 2 & & & W-04-255_150704_E1 \\
\hline W-04-255 & Johnny Darter & 4 & & & W-04-255_150704_E1 \\
\hline W-04-255 & Northern Redbelly Dace & 1 & & & W-04-255_150704_E1 \\
\hline W-04-255 & Western Blacknose Dace & 16 & & & W-04-255_150704_E1 \\
\hline W-04-255 & White Sucker & 5 & $57-103$ & Released 1 & W-04-255_150704_E1 \\
\hline W-04-256 & Northern Pike & 1 & 145 & & W-04-256_200704_E1 \\
\hline W-04-260 & Brook Stickleback & 7 & & & W-04-260_210704_E1 \\
\hline W-04-260 & Central Mudminnow & 8 & & & W-04-260_210704_E1 \\
\hline W-04-260 & Iowa Darter & 4 & & & W-04-260_210704_E1 \\
\hline W-04-260 & Northern Pike & 2 & $76-146$ & Released 1 (76 mm) & W-04-260_210704_E1 \\
\hline W-04-262 & Central Mudminnow & 3 & & & W-04-262_220704_E1 \\
\hline W-04-262 & Iowa Darter & 3 & & & W-04-262_220704_E1 \\
\hline W-04-262 & Northern Pike & 2 & 117,118 & & W-04-262_220704_E1 \\
\hline W-04-264 & Central Mudminnow & 2 & & & W-04-264_220704_E1 \\
\hline W-04-264 & Unidentified sucker & 1 & & Unidentified post-larval stage & W-04-264_220704_E1 \\
\hline W-04-265 & Central Mudminnow & 1 & & & W-04-265_220704_E1 \\
\hline W-04-265 & Northern Pike & 1 & 66 & YOY & W-04-265_220704_E1 \\
\hline W-04-266 & Black Bullhead & 1 & & & W-04-266_220704_E1 \\
\hline W-04-266 & Central Mudminnow & 7 & & & W-04-266_220704_E1 \\
\hline W-04-266 & Iowa Darter & 4 & & & W-04-266_220704_E1 \\
\hline W-04-266 & Johnny Darter & 1 & & & W-04-266_220704_E1 \\
\hline W-04-266 & No Catch & & & Invertebrates only & W-04-266_220704_K1 \\
\hline W-04-266 & Northern Pike & 3 & $98-203$ & Released 1 (203 mm) & W-04-266_220704_E1 \\
\hline W-04-266 & Tadpole Madtom & 1 & & & W-04-266_220704_E1 \\
\hline W-04-266 & Yellow Perch & 1 & 90 & & W-04-266_220704_E1 \\
\hline W-04-269 & Brook Stickleback & 2 & & & W-04-269_220704_E1 \\
\hline
\end{tabular}


Appendix 5: List of all fish captures made between 2002 and 2006, showing site number, common name and number (\#) of fish collected, fork (or total ${ }^{*}$ ) length in millimetres $(\mathrm{mm})$, any comments on the collection and the fish capture identification (ID) number.

\begin{tabular}{|c|c|c|c|c|c|}
\hline Site Number & Common Name & \# & $\begin{array}{c}\text { Fork/Total* } \\
\text { Length }(\mathrm{mm})\end{array}$ & Comments & $\begin{array}{l}\text { Fish Capture } \\
\text { ID Number }\end{array}$ \\
\hline W-04-269 & Central Mudminnow & 4 & & & W-04-269_220704_E1 \\
\hline W-04-271 & White Sucker & 3 & $32-33$ & YOY & W-04-271_230704_B1 \\
\hline W-04-274 & Brook Stickleback & 1 & & YOY & W-04-274_230704_E1 \\
\hline W-04-274 & Iowa Darter & 3 & & & W-04-274_230704_E1 \\
\hline W-04-274 & Johnny Darter & 13 & & & W-04-274_230704_E1 \\
\hline W-04-274 & Northern Pike & 4 & 105,106 & Released 2 & W-04-274_230704_E1 \\
\hline W-04-274 & Rock Bass & 2 & 127 & Released 1 & W-04-274_230704_E1 \\
\hline W-04-274 & White Sucker & 3 & 73,81 & Released 1 & W-04-274_230704_E1 \\
\hline W-04-277 & Northern Pike & 1 & 130 & Observed 5 others & W-04-277_030804_E1 \\
\hline W-04-286 & No Catch & & & & W-04-286_030804_E1 \\
\hline W-04-287 & Black Bullhead & 30 & & Released 23 & W-04-287_030804_E1 \\
\hline W-04-287 & Central Mudminnow & 1 & & & W-04-287_030804_E1 \\
\hline W-04-287 & Sand Shiner & 9 & & & W-04-287_030804_E1 \\
\hline W-04-287 & Sauger & 2 & 240,280 & Released & W-04-287_030804_E1 \\
\hline W-04-287 & Spotfin Shiner & 1 & & & W-04-287_030804_E1 \\
\hline W-04-287 & Troutperch & 2 & & & W-04-287_030804_E1 \\
\hline W-04-287 & Yellow Perch & 2 & 113,117 & Both look like maturing males & W-04-287_030804_E1 \\
\hline W-04-289 & Black Bullhead & 66 & & 12 YOY, 40 juveniles released & W-04-289_040804_E1 \\
\hline W-04-289 & Blackside Darter & 7 & & & W-04-289_040804_E1 \\
\hline W-04-289 & Fathead Minnow & 1 & & & W-04-289_040804_E1 \\
\hline W-04-289 & Johnny Darter & 3 & & Juveniles & W-04-289_040804_E1 \\
\hline W-04-289 & Northern Pike & 1 & 90 & Released & W-04-289_040804_E1 \\
\hline W-04-289 & Sand Shiner & 5 & & Adults & W-04-289_040804_E1 \\
\hline W-04-289 & Troutperch & 5 & & Released & W-04-289_040804_E1 \\
\hline W-04-289 & White Sucker & 3 & $157,305,457$ & Released 2 mature White Sucker & W-04-289_040804_E1 \\
\hline W-04-297 & Bigmouth Shiner & 1 & & & W-04-297_050804_E1 \\
\hline W-04-297 & Blackside Darter & 5 & & Juveniles & W-04-297_050804_E1 \\
\hline W-04-297 & Brassy Minnow & 77 & & & W-04-297_050804_E1 \\
\hline W-04-297 & Common Shiner & 32 & & 7 YOY, 20 others released & W-04-297_050804_E1 \\
\hline W-04-297 & Creek Chub & 1 & & & W-04-297_050804_E1 \\
\hline W-04-297 & Fathead Minnow & 5 & & & W-04-297_050804_E1 \\
\hline
\end{tabular}


Appendix 5: List of all fish captures made between 2002 and 2006, showing site number, common name and number (\#) of fish collected, fork (or total ${ }^{\star}$ ) length in millimetres $(\mathrm{mm})$, any comments on the collection and the fish capture identification (ID) number.

\begin{tabular}{|c|c|c|c|c|c|}
\hline Site Number & Common Name & \# & $\begin{array}{c}\text { Fork/Total* } \\
\text { Length }(\mathrm{mm})\end{array}$ & Comments & $\begin{array}{l}\text { Fish Capture } \\
\text { ID Number }\end{array}$ \\
\hline W-04-297 & Johnny Darter & 12 & & 3 YOY & W-04-297_050804_E1 \\
\hline W-04-297 & Longnose Dace & 10 & & & W-04-297_050804_E1 \\
\hline W-04-297 & Sand Shiner & 14 & & 7 YOY & W-04-297_050804_E1 \\
\hline W-04-297 & Troutperch & 6 & & Juveniles & W-04-297_050804_E1 \\
\hline W-04-297 & White Sucker & 5 & & All YOY & W-04-297_050804_E1 \\
\hline W-04-298 & Blackside Darter & 1 & & & W-04-298_050804_E1 \\
\hline W-04-298 & Brassy Minnow & 22 & & All YOY & W-04-298_050804_E1 \\
\hline W-04-298 & Brook Stickleback & 15 & & & W-04-298_050804_E1 \\
\hline W-04-298 & Common Shiner & 10 & & & W-04-298_050804_E1 \\
\hline W-04-298 & Creek Chub & 3 & & & W-04-298_050804_E1 \\
\hline W-04-298 & Fathead Minnow & 192 & & All YOY & W-04-298_050804_E1 \\
\hline W-04-298 & Troutperch & 1 & & & W-04-298_050804_E1 \\
\hline W-04-298 & Western Blacknose Dace & 11 & & & W-04-298_050804_E1 \\
\hline W-04-298 & White Sucker & 17 & $44-55$ & Released 10 & W-04-298_050804_E1 \\
\hline W-04-299 & Blackside Darter & 2 & & & W-04-299_050804_E1 \\
\hline W-04-299 & Brassy Minnow & 14 & & & W-04-299_050804_E1 \\
\hline W-04-299 & Brook Stickleback & 7 & & & W-04-299_050804_E1 \\
\hline W-04-299 & Common Carp & 1 & & YOY & W-04-299_050804_E1 \\
\hline W-04-299 & Common Shiner & 25 & & & W-04-299_050804_E1 \\
\hline W-04-299 & Creek Chub & 2 & & $1 \mathrm{YOY}$ & W-04-299_050804_E1 \\
\hline W-04-299 & Fathead Minnow & 3 & & 2 YOY & W-04-299_050804_E1 \\
\hline W-04-299 & Longnose Dace & 13 & & 3 YOY & W-04-299_050804_E1 \\
\hline W-04-299 & Sand Shiner & 9 & & & W-04-299_050804_E1 \\
\hline W-04-299 & Western Blacknose Dace & 10 & & 1 YOY, 1 mature & W-04-299_050804_E1 \\
\hline W-04-299 & White Sucker & 4 & $40-57$ & & W-04-299_050804_E1 \\
\hline $\mathrm{X}-04-001$ & Brook Stickleback & 1 & & & X-04-001_050504_B1 \\
\hline X-04-001 & Fathead Minnow & 689 & & & X-04-001_050504_B1 \\
\hline$X-04-001$ & Sand Shiner & 80 & & & X-04-001_050504_B1 \\
\hline $\mathrm{X}-04-002$ & No Catch & & & & X-04-002_050504_B1 \\
\hline X-04-005 & No Catch & & & & X-04-005_050504_E1 \\
\hline X-04-014 & No Catch & & & & X-04-014_060504_B1 \\
\hline
\end{tabular}


Appendix 5: List of all fish captures made between 2002 and 2006, showing site number, common name and number (\#) of fish collected, fork (or total ${ }^{*}$ ) length in millimetres $(\mathrm{mm})$, any comments on the collection and the fish capture identification (ID) number.

\begin{tabular}{|c|c|c|c|c|c|}
\hline Site Number & Common Name & \# & $\begin{array}{l}\text { Fork/Total* } \\
\text { Length (mm) }\end{array}$ & Comments & $\begin{array}{l}\text { Fish Capture } \\
\text { ID Number }\end{array}$ \\
\hline $\mathrm{X}-04-023$ & Fathead Minnow & 28 & & & X-04-023_100504_B1 \\
\hline $\mathrm{X}-04-026$ & Black Bullhead & 2 & & & X-04-026_100504_B1 \\
\hline $\mathrm{X}-04-026$ & Brook Stickleback & 1 & & & X-04-026_100504_B1 \\
\hline $\mathrm{X}-04-031$ & Fathead Minnow & 3 & & & X-04-031_100504_E1 \\
\hline $\mathrm{X}-04-031$ & No Catch & & & & X-04-031_100504_B1 \\
\hline $\mathrm{X}-04-037$ & Brook Stickleback & 1 & & & X-04-037_140504_E1 \\
\hline $\mathrm{X}-04-037$ & Fathead Minnow & 15 & & & $\mathrm{X}-04-037 \_140504 \_\mathrm{E} 1$ \\
\hline $\mathrm{X}-04-037$ & Johnny Darter & 1 & & & X-04-037_140504_E1 \\
\hline $\mathrm{X}-04-037$ & Yellow Perch & 1 & 73 & & X-04-037_140504_E1 \\
\hline$X-04-041$ & No Catch & & & & X-04-041_140504_B1 \\
\hline $\mathrm{X}-04-041$ & No Catch & & & & X-04-041_140504_L1 \\
\hline $\mathrm{X}-04-042$ & Brook Stickleback & 1 & & & X-04-042_140504_D1 \\
\hline $\mathrm{X}-04-042$ & Fathead Minnow & 1 & & & X-04-042_140504_D1 \\
\hline $\mathrm{X}-04-043$ & Eggs & & & $\begin{array}{l}\text { Sucker eggs in one vial, invertebrates in a } \\
\text { second vial }\end{array}$ & X-04-043_170504_K1 \\
\hline $\mathrm{X}-04-044$ & No Catch & & & & X-04-044_170504_E1 \\
\hline $\mathrm{X}-04-046$ & No Catch & & & & X-04-046_170504_E1 \\
\hline $\mathrm{X}-04-048$ & No Catch & & & & X-04-048_180504_B1 \\
\hline $\mathrm{X}-04-048$ & No Catch & & & & X-04-048_180504_E1 \\
\hline $\mathrm{X}-04-049$ & Brook Stickleback & 3 & & & X-04-049_180504_E1 \\
\hline $\mathrm{X}-04-051$ & Brook Stickleback & 2 & & & X-04-051_180504_E1 \\
\hline $\mathrm{X}-04-051$ & Central Mudminnow & 9 & & & X-04-051_180504_E1 \\
\hline$X-04-052$ & Fathead Minnow & 1 & & & X-04-052_190504_B1 \\
\hline $\mathrm{X}-04-053$ & Brook Stickleback & 1 & & & X-04-053_200504_E1 \\
\hline $\mathrm{X}-04-053$ & Fathead Minnow & 90 & & & X-04-053_200504_E1 \\
\hline $\mathrm{X}-04-053$ & Iowa Darter & 1 & & & X-04-053_200504_E1 \\
\hline $\mathrm{X}-04-053$ & Spottail Shiner & 4 & & & X-04-053_200504_E1 \\
\hline $\mathrm{X}-04-053$ & White Sucker & 15 & & Congregation of ripe, mature fish & X-04-053_200504_E1 \\
\hline $\mathrm{X}-04-054$ & Fathead Minnow & 1 & & & X-04-054_200504_E1 \\
\hline $\mathrm{X}-04-054$ & White Sucker & 100's & & Congregation of ripe, mature fish & X-04-054_200504_E1 \\
\hline $\mathrm{X}-04-055$ & White Sucker & 100's & & Congregation of ripe, mature fish & X-04-055_200504_01 \\
\hline
\end{tabular}


Appendix 5: List of all fish captures made between 2002 and 2006, showing site number, common name and number (\#) of fish collected, fork (or total ${ }^{*}$ ) length in millimetres $(\mathrm{mm})$, any comments on the collection and the fish capture identification (ID) number.

\begin{tabular}{|c|c|c|c|c|c|}
\hline Site Number & Common Name & \# & $\begin{array}{l}\text { Fork/Total* } \\
\text { Length }(\mathrm{mm})\end{array}$ & Comments & $\begin{array}{l}\text { Fish Capture } \\
\text { ID Number }\end{array}$ \\
\hline $\mathrm{X}-04-056$ & White Sucker & 100's & & Congregation of ripe, mature fish & $\mathrm{X}-04-056 \_200504 \_01$ \\
\hline $\mathrm{X}-04-057$ & White Sucker & 100's & & Congregation of ripe, mature fish & X-04-057_200504_01 \\
\hline $\mathrm{X}-04-058$ & White Sucker & 100's & & Congregation of ripe, mature fish & X-04-058_200504_01 \\
\hline $\mathrm{X}-04-061$ & No Catch & & & & X-04-061_200504_E1 \\
\hline $\mathrm{X}-04-065$ & Black Bullhead & 8 & & & X-04-065_210504_E1 \\
\hline $\mathrm{X}-04-065$ & Brook Stickleback & 1 & & & X-04-065_210504_E1 \\
\hline $\mathrm{X}-04-065$ & Common Carp & 2 & & & X-04-065_210504_E1 \\
\hline $\mathrm{X}-04-065$ & Fathead Minnow & 10 & & & X-04-065_210504_E1 \\
\hline $\mathrm{X}-04-065$ & Northern Pike & 1 & 803 & Released & X-04-065_210504_E1 \\
\hline$X-04-065$ & River Shiner & 10 & & & $\mathrm{X}-04-065 \_210504 \_\mathrm{E} 1$ \\
\hline $\mathrm{X}-04-065$ & Walleye & 1 & 287 & Released & X-04-065_210504_E1 \\
\hline $\mathrm{X}-04-066$ & White Sucker & 2 & $48-642$ & Adults also observed & X-04-066_210504_E1 \\
\hline $\mathrm{X}-04-067$ & Brook Stickleback & 1 & & & X-04-067_250504_E1 \\
\hline $\mathrm{X}-04-067$ & Central Mudminnow & 1 & & & $\mathrm{X}-04-067250504 \mathrm{E} 1$ \\
\hline $\mathrm{X}-04-067$ & Fathead Minnow & 11 & & & X-04-067_250504_E1 \\
\hline $\mathrm{X}-04-067$ & White Sucker & 1 & & Adult, dead on shore & X-04-067_250504_E1 \\
\hline $\mathrm{X}-04-068$ & White Sucker & 100 's & & $\begin{array}{l}\text { Many spawning at site so did not fish very } \\
\text { much area }\end{array}$ & X-04-068_250504_E1 \\
\hline $\mathrm{X}-04-069$ & No Catch & & & & X-04-069_250504_K1 \\
\hline $\mathrm{X}-04-071$ & Brook Stickleback & 1 & & & X-04-071_250504_E1 \\
\hline $\mathrm{X}-04-072$ & White Sucker & 100's & & Many observed downstream of riffle & $\mathrm{X}-04-072 \_250504 \_01$ \\
\hline $\mathrm{X}-04-074$ & No Catch & & & & X-04-074_260504_E1 \\
\hline $\mathrm{X}-04-077$ & Central Mudminnow & 3 & & & X-04-077_260504_E1 \\
\hline $\mathrm{X}-04-078$ & Brook Stickleback & 2 & & & X-04-078_260504_E1 \\
\hline $\mathrm{X}-04-078$ & Central Mudminnow & 2 & & & X-04-078_260504_E1 \\
\hline $\mathrm{X}-04-078$ & Fathead Minnow & 7 & & & X-04-078_260504_E1 \\
\hline $\mathrm{X}-04-080$ & Black Crappie & 3 & $45-80$ & Released & X-04-080_260504_E1 \\
\hline $\mathrm{X}-04-080$ & Fathead Minnow & 20 & & & X-04-080_260504_E1 \\
\hline $\mathrm{X}-04-080$ & Johnny Darter & 1 & & & X-04-080_260504_E1 \\
\hline $\mathrm{X}-04-080$ & Spotfin Shiner & 1 & & & X-04-080_260504_E1 \\
\hline $\mathrm{X}-04-081$ & Central Mudminnow & 1 & & & X-04-081_270504_E1 \\
\hline
\end{tabular}


Appendix 5: List of all fish captures made between 2002 and 2006, showing site number, common name and number (\#) of fish collected, fork (or total ${ }^{*}$ ) length in millimetres $(\mathrm{mm})$, any comments on the collection and the fish capture identification (ID) number.

\begin{tabular}{|c|c|c|c|c|c|}
\hline Site Number & Common Name & \# & $\begin{array}{c}\text { Fork/Total* } \\
\text { Length }(\mathrm{mm})\end{array}$ & Comments & $\begin{array}{l}\text { Fish Capture } \\
\text { ID Number }\end{array}$ \\
\hline $\mathrm{X}-04-081$ & Fathead Minnow & 8 & & & X-04-081_270504_E1 \\
\hline $\mathrm{X}-04-082$ & Fathead Minnow & 2 & & & X-04-082_270504_B1 \\
\hline $\mathrm{X}-04-084$ & No Catch & & & & X-04-084_270504_E1 \\
\hline X-04-088 & Fathead Minnow & 1 & & & X-04-088_270504_L1 \\
\hline X-04-090 & Central Mudminnow & 1 & & & X-04-090_270504_E1 \\
\hline $\mathrm{X}-04-091$ & No Catch & & & & X-04-091_280504_L1 \\
\hline X-04-091 & Northern Pike & 1 & & Adult, released & X-04-091_280504_E1 \\
\hline X-04-092 & White Sucker & 1 & & Ripe, pre-spawn male, 255 mm dwarf & X-04-092_280504_E1 \\
\hline $\mathrm{X}-04-094$ & Brook Stickleback & 2 & & & X-04-094_280504_E1 \\
\hline X-04-094 & Fathead Minnow & 19 & & & X-04-094_280504_E1 \\
\hline X-04-095 & Black Bullhead & 1 & $205^{*}$ & Released & X-04-095_010604_E1 \\
\hline X-04-095 & Fathead Minnow & 4 & & & X-04-095_010604_E1 \\
\hline X-04-097 & Brook Stickleback & 1 & & & X-04-097_010604_E1 \\
\hline X-04-097 & Fathead Minnow & 2 & & & X-04-097_010604_E1 \\
\hline X-04-098 & Brook Stickleback & 1 & & & X-04-098_010604_B1 \\
\hline X-04-098 & Fathead Minnow & 1 & & & X-04-098_010604_B1 \\
\hline X-04-099 & No Catch & & & & X-04-099_010604_E1 \\
\hline $\mathrm{X}-04-101$ & Fathead Minnow & 1 & & Released & X-04-101_010604_E1 \\
\hline $\mathrm{X}-04-102$ & Fathead Minnow & 1 & & & $\mathrm{X}-04-102 \_020604 \_\mathrm{E} 1$ \\
\hline X-04-103 & Common Carp & 1 & 643 & Released, many observed & X-04-103_020604_E1 \\
\hline $\mathrm{X}-04-103$ & No Catch & & & Invertebrates only & X-04-103_020604_K1 \\
\hline X-04-104 & Common Carp & 100's & & Many observed downstream of riffle & X-04-104_020604_01 \\
\hline X-04-105 & Common Carp & 100's & & Many observed upstream of culvert & X-04-105_020604_01 \\
\hline X-04-106 & Brook Stickleback & 1 & & & X-04-106_030604_E1 \\
\hline $\mathrm{X}-04-106$ & Common Shiner & 6 & & & X-04-106_030604_E1 \\
\hline $\mathrm{X}-04-106$ & Creek Chub & 9 & & & X-04-106_030604_E1 \\
\hline X-04-106 & Fathead Minnow & 6 & & & X-04-106_030604_E1 \\
\hline$X-04-106$ & Johnny Darter & 1 & & Age 1+ & X-04-106_030604_E1 \\
\hline $\mathrm{X}-04-106$ & Sand Shiner & 1 & & & X-04-106_030604_E1 \\
\hline $\mathrm{X}-04-106$ & Western Blacknose Dace & 5 & & & X-04-106_030604_E1 \\
\hline $\mathrm{X}-04-106$ & White Sucker & 1 & 130 & & X-04-106_030604_E1 \\
\hline
\end{tabular}


Appendix 5: List of all fish captures made between 2002 and 2006, showing site number, common name and number (\#) of fish collected, fork (or total ${ }^{*}$ ) length in millimetres $(\mathrm{mm})$, any comments on the collection and the fish capture identification (ID) number.

\begin{tabular}{|c|c|c|c|c|c|}
\hline Site Number & Common Name & \# & $\begin{array}{c}\text { Fork/Total* } \\
\text { Length }(\mathrm{mm})\end{array}$ & Comments & $\begin{array}{l}\text { Fish Capture } \\
\text { ID Number }\end{array}$ \\
\hline $\mathrm{X}-04-107$ & Brook Stickleback & 1 & & & X-04-107_030604_E1 \\
\hline $\mathrm{X}-04-107$ & Common Shiner & 1 & & & X-04-107_030604_E1 \\
\hline$X-04-107$ & Common Shiner & 20 & & & X-04-107_030604_B1 \\
\hline X-04-107 & Creek Chub & 1 & & & X-04-107_030604_E1 \\
\hline $\mathrm{X}-04-107$ & Fathead Minnow & 3 & & & X-04-107_030604_B1 \\
\hline $\mathrm{X}-04-107$ & Fathead Minnow & 4 & & & X-04-107_030604_E1 \\
\hline $\mathrm{X}-04-107$ & White Sucker & 2 & & Observed swimming across ford crossing & X-04-107_030604_01 \\
\hline X-04-109 & No Catch & & & & X-04-109_030604_E1 \\
\hline $\mathrm{X}-04-110$ & Brook Stickleback & 37 & & Plus 1 Anodonta grandis empty shell & X-04-110_040604_E1 \\
\hline$X-04-110$ & Central Mudminnow & 2 & & & X-04-110_040604_E1 \\
\hline X-04-110 & Common Shiner & 2 & & & X-04-110_040604_E1 \\
\hline $\mathrm{X}-04-110$ & Creek Chub & 3 & & & X-04-110_040604_E1 \\
\hline$X-04-110$ & Fathead Minnow & 41 & & & X-04-110_040604_E1 \\
\hline $\mathrm{X}-04-110$ & Pearl Dace & 6 & & & X-04-110_040604_E1 \\
\hline $\mathrm{X}-04-110$ & Western Blacknose Dace & 10 & & & X-04-110_040604_E1 \\
\hline $\mathrm{X}-04-110$ & White Sucker & 2 & 56,58 & & X-04-110_040604_E1 \\
\hline $\mathrm{X}-04-112$ & Black Bullhead & 3 & & & X-04-112_040604_E1 \\
\hline $\mathrm{X}-04-112$ & Fathead Minnow & 1 & & & X-04-112_040604_E1 \\
\hline $\mathrm{X}-04-112$ & White Sucker & 4 & & Released 3 (150-255 mm) & X-04-112_040604_E1 \\
\hline X-04-113 & No Catch & & & & X-04-113_070604_E1 \\
\hline $\mathrm{X}-04-114$ & Blackside Darter & 2 & & & X-04-114_070604_E1 \\
\hline $\mathrm{X}-04-114$ & Brook Stickleback & 4 & & & X-04-114_070604_E1 \\
\hline X-04-114 & Fathead Minnow & 247 & & & X-04-114_070604_E1 \\
\hline X-04-114 & Iowa Darter & 1 & & & X-04-114_070604_E1 \\
\hline $\mathrm{X}-04-114$ & Johnny Darter & 6 & & 2 ripe males & X-04-114_070604_E1 \\
\hline X-04-114 & No Catch & & & & X-04-114_070604_K1 \\
\hline X-04-114 & Sand Shiner & 2 & & & X-04-114_070604_E1 \\
\hline$X-04-114$ & White Sucker & 4 & $59-80$ & & X-04-114_070604_E1 \\
\hline $\mathrm{X}-04-115$ & Brook Stickleback & 1 & & & X-04-115_070604_E1 \\
\hline $\mathrm{X}-04-115$ & Common Shiner & 3 & & & X-04-115_070604_E1 \\
\hline $\mathrm{X}-04-115$ & Creek Chub & 5 & & & X-04-115_070604_E1 \\
\hline
\end{tabular}


Appendix 5: List of all fish captures made between 2002 and 2006, showing site number, common name and number (\#) of fish collected, fork (or total ${ }^{\star}$ ) length in millimetres $(\mathrm{mm})$, any comments on the collection and the fish capture identification (ID) number.

\begin{tabular}{|c|c|c|c|c|c|}
\hline Site Number & Common Name & \# & $\begin{array}{c}\text { Fork/Total* } \\
\text { Length }(\mathrm{mm})\end{array}$ & Comments & $\begin{array}{l}\text { Fish Capture } \\
\text { ID Number }\end{array}$ \\
\hline $\mathrm{X}-04-115$ & Fathead Minnow & 5 & & & $\mathrm{X}-04-115 \_070604 \_\mathrm{E} 1$ \\
\hline $\mathrm{X}-04-115$ & Finescale Dace & 2 & & Possible hybrid with Pearl Dace & X-04-115_070604_E1 \\
\hline$X-04-115$ & Western Blacknose Dace & 8 & & & X-04-115_070604_E1 \\
\hline $\mathrm{X}-04-116$ & Fathead Minnow & 4 & & & $\mathrm{X}-04-116 \_080604 \_\mathrm{E} 1$ \\
\hline $\mathrm{X}-04-116$ & No Catch & & & & X-04-116_080604_L1 \\
\hline $\mathrm{X}-04-118$ & Brook Stickleback & 1 & & & X-04-118_080604_E1 \\
\hline$X-04-118$ & Common Shiner & 1 & & & X-04-118_080604_E1 \\
\hline $\mathrm{X}-04-118$ & Creek Chub & 3 & & & $\mathrm{X}-04-118 \_080604 \mathrm{E} 1$ \\
\hline $\mathrm{X}-04-118$ & No Catch & & & & X-04-118_080604_L1 \\
\hline$X-04-118$ & White Sucker & 1 & 95 & & X-04-118_080604_E1 \\
\hline $\mathrm{X}-04-119$ & Brook Stickleback & 4 & & & $\mathrm{X}-04-119 \_080604 \_\mathrm{E} 1$ \\
\hline $\mathrm{X}-04-119$ & Creek Chub & 1 & & & X-04-119_080604_E1 \\
\hline$X-04-119$ & Fathead Minnow & 24 & & & X-04-119_080604_E1 \\
\hline $\mathrm{X}-04-119$ & Western Blacknose Dace & 2 & & & X-04-119_080604_E1 \\
\hline $\mathrm{X}-04-119$ & White Sucker & 1 & 61 & & $\mathrm{X}-04-119 \_080604 \_\mathrm{E} 1$ \\
\hline$X-04-120$ & No Catch & & & & X-04-120_080604_E1 \\
\hline $\mathrm{X}-04-121$ & Common Carp & 1 & 670 & Released & X-04-121_080604_E1 \\
\hline $\mathrm{X}-04-121$ & Common Shiner & 2 & & & X-04-121_080604_E1 \\
\hline$X-04-121$ & Creek Chub & 2 & & & X-04-121_080604_E1 \\
\hline $\mathrm{X}-04-121$ & Fathead Minnow & 21 & & & X-04-121_080604_E1 \\
\hline $\mathrm{X}-04-121$ & Western Blacknose Dace & 3 & & & X-04-121_080604_E1 \\
\hline$X-04-121$ & White Sucker & 1 & 62 & & X-04-121_080604_E1 \\
\hline $\mathrm{X}-04-122$ & Fathead Minnow & 2 & & & X-04-122_090604_E1 \\
\hline $\mathrm{X}-04-122$ & Northern Pike & 7 & $40-55$ & & X-04-122_090604_E1 \\
\hline$X-04-122$ & Sand Shiner & 2 & & & X-04-122_090604_E1 \\
\hline $\mathrm{X}-04-122$ & White Sucker & 1 & $13^{*}$ & YOY & X-04-122_090604_E1 \\
\hline $\mathrm{X}-04-123$ & Fathead Minnow & 1 & & & X-04-123_090604_E1 \\
\hline$X-04-123$ & Northern Pike & 5 & $48-59$ & YOY & X-04-123_090604_E1 \\
\hline $\mathrm{X}-04-124$ & Fathead Minnow & 5 & & & X-04-124_090604_E1 \\
\hline $\mathrm{X}-04-126$ & Central Mudminnow & 2 & & & X-04-126_100604_E1 \\
\hline$X-04-127$ & Common Carp & 1 & 539 & & $\mathrm{X}-04-127 \_100604 \_\mathrm{E} 1$ \\
\hline
\end{tabular}


Appendix 5: List of all fish captures made between 2002 and 2006, showing site number, common name and number (\#) of fish collected, fork (or total ${ }^{\star}$ ) length in millimetres ( $\mathrm{mm}$ ), any comments on the collection and the fish capture identification (ID) number.

\begin{tabular}{|c|c|c|c|c|c|}
\hline Site Number & Common Name & \# & $\begin{array}{c}\text { Fork/Total* } \\
\text { Length }(\mathrm{mm})\end{array}$ & Comments & $\begin{array}{l}\text { Fish Capture } \\
\text { ID Number }\end{array}$ \\
\hline $\mathrm{X}-04-127$ & Fathead Minnow & 16 & & & X-04-127_100604_E1 \\
\hline $\mathrm{X}-04-127$ & Northern Pike & 1 & 347 & Released & X-04-127_100604_E1 \\
\hline$X-04-127$ & Rock Bass & 3 & $221-262$ & Released & X-04-127_100604_E1 \\
\hline X-04-127 & Spotfin Shiner & 3 & & & X-04-127_100604_E1 \\
\hline $\mathrm{X}-04-128$ & Black Bullhead & 1 & & & X-04-128_140604_E1 \\
\hline $\mathrm{X}-04-128$ & Brook Stickleback & 1 & & & X-04-128_140604_E1 \\
\hline $\mathrm{X}-04-128$ & Central Mudminnow & 2 & & & $\mathrm{X}-04-128 \_140604 \_\mathrm{E} 1$ \\
\hline X-04-128 & White Sucker & 1 & 272 & Released & X-04-128_140604_E1 \\
\hline $\mathrm{X}-04-129$ & Brook Stickleback & 58 & & & X-04-129_140604_E1 \\
\hline$X-04-129$ & Central Mudminnow & 4 & & & X-04-129_140604_E1 \\
\hline X-04-129 & Fathead Minnow & 53 & & & X-04-129_140604_E1 \\
\hline X-04-129 & White Sucker & 2 & 212,249 & Released & X-04-129_140604_E1 \\
\hline$X-04-130$ & Fathead Minnow & 1 & & & $\mathrm{X}-04-130 \_140604$ E1 \\
\hline $\mathrm{X}-04-132$ & Brook Stickleback & 79 & & Plus 2 tadpoles (Rana spp) & $\mathrm{X}-04-132 \_140604 \_\mathrm{E} 1$ \\
\hline $\mathrm{X}-04-132$ & Fathead Minnow & 45 & & & X-04-132_140604_E1 \\
\hline $\mathrm{X}-04-133$ & Fathead Minnow & 9 & & & X-04-133_150604_E1 \\
\hline $\mathrm{X}-04-137$ & Brook Stickleback & 14 & & & X-04-137_160604_E1 \\
\hline $\mathrm{X}-04-137$ & Common Shiner & 4 & & & X-04-137_160604_E1 \\
\hline $\mathrm{X}-04-137$ & Creek Chub & 7 & & & X-04-137_160604_E1 \\
\hline X-04-137 & Fathead Minnow & 38 & & & X-04-137_160604_E1 \\
\hline $\mathrm{X}-04-137$ & No Catch & & & & X-04-137_160604_L1 \\
\hline $\mathrm{X}-04-137$ & Western Blacknose Dace & 7 & & & $\mathrm{X}-04-137 \_160604 \_\mathrm{E} 1$ \\
\hline $\mathrm{X}-04-137$ & Yellow Perch & 1 & & & X-04-137_160604_E1 \\
\hline X-04-138 & Black Bullhead & 3 & & Released 1 (222 mm) & X-04-138_160604_E1 \\
\hline $\mathrm{X}-04-138$ & Brassy Minnow & 33 & & & X-04-138_160604_E1 \\
\hline $\mathrm{X}-04-138$ & Brook Stickleback & 3 & & & X-04-138_160604_E1 \\
\hline X-04-138 & Common Shiner & 1 & & & X-04-138_160604_E1 \\
\hline X-04-138 & Creek Chub & 2 & & & X-04-138_160604_E1 \\
\hline $\mathrm{X}-04-138$ & Fathead Minnow & 368 & & Ripe adults \& juveniles & X-04-138_160604_E1 \\
\hline $\mathrm{X}-04-138$ & Western Blacknose Dace & 6 & & & X-04-138_160604_E1 \\
\hline X-04-139 & No Catch & & & & X-04-139_160604_E1 \\
\hline
\end{tabular}


Appendix 5: List of all fish captures made between 2002 and 2006, showing site number, common name and number (\#) of fish collected, fork (or total ${ }^{\star}$ ) length in millimetres ( $\mathrm{mm}$ ), any comments on the collection and the fish capture identification (ID) number.

\begin{tabular}{|c|c|c|c|c|c|}
\hline Site Number & Common Name & $\#$ & $\begin{array}{l}\text { Fork/Total* } \\
\text { Length }(\mathrm{mm})\end{array}$ & Comments & $\begin{array}{l}\text { Fish Capture } \\
\text { ID Number }\end{array}$ \\
\hline $\mathrm{X}-04-141$ & Common Shiner & 9 & & & $\mathrm{X}-04-141$ 170604_E1 \\
\hline $\mathrm{X}-04-141$ & Creek Chub & 13 & & & X-04-141_170604_E1 \\
\hline$X-04-141$ & Fathead Minnow & 8 & & & X-04-141_170604_E1 \\
\hline $\mathrm{X}-04-141$ & Western Blacknose Dace & 4 & & & $\mathrm{X}-04-141$ 170604_E1 \\
\hline $\mathrm{X}-04-141$ & White Sucker & 10 & $141-208$ & $\begin{array}{l}4 \text { kept }(141-158 \mathrm{~mm}), 6 \text { released }(154- \\
208 \mathrm{~mm})\end{array}$ & X-04-141_170604_E1 \\
\hline $\mathrm{X}-04-142$ & Brook Stickleback & 18 & & & X-04-142_170604_E1 \\
\hline$X-04-142$ & Common Shiner & 5 & & & X-04-142_170604_E1 \\
\hline $\mathrm{X}-04-142$ & Creek Chub & 14 & & & $\mathrm{X}-04-142$ 170604_E1 \\
\hline $\mathrm{X}-04-142$ & Fathead Minnow & 8 & & & X-04-142_170604_E1 \\
\hline$X-04-142$ & lowa Darter & 1 & & & X-04-142_170604_E1 \\
\hline $\mathrm{X}-04-142$ & Western Blacknose Dace & 19 & & & X-04-142_170604_E1 \\
\hline $\mathrm{X}-04-142$ & White Sucker & 1 & 134 & Immature male & X-04-142_170604_E1 \\
\hline$X-04-145$ & Brassy Minnow & 8 & & & X-04-145_170604_E1 \\
\hline $\mathrm{X}-04-145$ & Brook Stickleback & 2 & & & X-04-145_170604_E1 \\
\hline $\mathrm{X}-04-145$ & Central Mudminnow & 4 & & & X-04-145_170604_E1 \\
\hline$X-04-145$ & Creek Chub & 18 & & & X-04-145_170604_E1 \\
\hline $\mathrm{X}-04-145$ & Fathead Minnow & 11 & & & X-04-145_170604_E1 \\
\hline $\mathrm{X}-04-145$ & Johnny Darter & 4 & & & X-04-145_170604_E1 \\
\hline$X-04-145$ & Western Blacknose Dace & 7 & & & X-04-145_170604_E1 \\
\hline $\mathrm{X}-04-145$ & White Sucker & 1 & 78 & & X-04-145_170604_E1 \\
\hline $\mathrm{X}-04-147$ & Brook Stickleback & 3 & & & $\mathrm{X}-04-147 \_180604$ E1 \\
\hline$X-04-147$ & Creek Chub & 9 & & & X-04-147_180604_E1 \\
\hline $\mathrm{X}-04-147$ & Fathead Minnow & 39 & & Mature, ripe males present & X-04-147_180604_E1 \\
\hline $\mathrm{X}-04-147$ & Western Blacknose Dace & 18 & & Ripe adults & X-04-147_180604_E1 \\
\hline$X-04-147$ & White Sucker & 1 & 53 & & $\mathrm{X}-04-147 \quad 180604$ E1 \\
\hline X-04-149 & Brook Stickleback & 1 & & YOY & X-04-149_210604_E1 \\
\hline $\mathrm{X}-04-149$ & Central Mudminnow & 10 & & & X-04-149_210604_E1 \\
\hline$X-04-149$ & Creek Chub & 1 & & & X-04-149_210604_E1 \\
\hline $\mathrm{X}-04-149$ & Finescale Dace & 3 & & & X-04-149_210604_E1 \\
\hline $\mathrm{X}-04-149$ & Johnny Darter & 3 & & & X-04-149_210604_E1 \\
\hline
\end{tabular}


Appendix 5: List of all fish captures made between 2002 and 2006, showing site number, common name and number (\#) of fish collected, fork (or total ${ }^{*}$ ) length in millimetres $(\mathrm{mm})$, any comments on the collection and the fish capture identification (ID) number.

\begin{tabular}{|c|c|c|c|c|c|}
\hline Site Number & Common Name & \# & $\begin{array}{l}\text { Fork/Total }{ }^{*} \\
\text { Length }(\mathrm{mm})\end{array}$ & Comments & $\begin{array}{l}\text { Fish Capture } \\
\text { ID Number }\end{array}$ \\
\hline $\mathrm{X}-04-149$ & No Catch & & & & X-04-149_210604_L1 \\
\hline $\mathrm{X}-04-149$ & Northern Redbelly Dace & 1 & & & X-04-149_210604_E1 \\
\hline$X-04-149$ & Pearl Dace & 12 & & 10 are $\mathrm{YOY}$ & X-04-149_210604_E1 \\
\hline $\mathrm{X}-04-149$ & Western Blacknose Dace & 10 & & & X-04-149_210604_E1 \\
\hline $\mathrm{X}-04-149$ & White Sucker & 1 & 91 & & X-04-149_210604_E1 \\
\hline $\mathrm{X}-04-150$ & Central Mudminnow & 4 & & & X-04-150_210604_E1 \\
\hline$X-04-150$ & Creek Chub & 2 & & & X-04-150_210604_E1 \\
\hline $\mathrm{X}-04-150$ & Fathead Minnow & 4 & & & X-04-150_210604_E1 \\
\hline $\mathrm{X}-04-150$ & Finescale Dace & 1 & & & X-04-150_210604_E1 \\
\hline$X-04-150$ & Johnny Darter & 13 & & & X-04-150_210604_E1 \\
\hline $\mathrm{X}-04-150$ & Northern Redbelly Dace & 1 & & & X-04-150_210604_E1 \\
\hline $\mathrm{X}-04-150$ & Pearl Dace & 5 & & & $\mathrm{X}-04-150 \_210604 \mathrm{E} 1$ \\
\hline$X-04-150$ & Western Blacknose Dace & 25 & & & X-04-150_210604_E1 \\
\hline$X-04-150$ & White Sucker & 5 & $49-172$ & $\begin{array}{l}143 \mathrm{~mm} \text { is a spawning male, } 1 @ 172 \\
\text { released }\end{array}$ & X-04-150_210604_E1 \\
\hline $\mathrm{X}-04-151$ & Brook Stickleback & 26 & & & X-04-151_210604_E1 \\
\hline$X-04-151$ & Central Mudminnow & 7 & & & X-04-151_210604_E1 \\
\hline $\mathrm{X}-04-151$ & Fathead Minnow & 7 & & & X-04-151_210604_E1 \\
\hline $\mathrm{X}-04-151$ & Johnny Darter & 6 & & & X-04-151_210604_E1 \\
\hline$X-04-151$ & Northern Redbelly Dace & 81 & & & X-04-151_210604_E1 \\
\hline $\mathrm{X}-04-151$ & Pearl Dace & 1 & & & X-04-151_210604_E1 \\
\hline $\mathrm{X}-04-151$ & White Sucker & 5 & $50-125$ & & X-04-151_210604_E1 \\
\hline$X-04-152$ & Brook Stickleback & 1 & & & $\mathrm{X}-04-152 \_220604$ B1 \\
\hline $\mathrm{X}-04-153$ & Brook Stickleback & 3 & & & X-04-153_220604_E1 \\
\hline $\mathrm{X}-04-153$ & Central Mudminnow & 5 & & & $\mathrm{X}-04-153 \_220604 \mathrm{E} 1$ \\
\hline$X-04-153$ & Fathead Minnow & 3 & & & X-04-153_220604_E1 \\
\hline $\mathrm{X}-04-154$ & No Catch & & & Stocked with trout in past & X-04-154_220604_E1 \\
\hline $\mathrm{X}-04-155$ & Black Bullhead & 1 & & & X-04-155_230604_E1 \\
\hline$X-04-155$ & Central Mudminnow & 11 & & & X-04-155_230604_E1 \\
\hline $\mathrm{X}-04-155$ & Fathead Minnow & 5 & & & X-04-155_230604_E1 \\
\hline $\mathrm{X}-04-155$ & Johnny Darter & 2 & & & X-04-155_230604_E1 \\
\hline
\end{tabular}


Appendix 5: List of all fish captures made between 2002 and 2006, showing site number, common name and number (\#) of fish collected, fork (or total ${ }^{\star}$ ) length in millimetres ( $\mathrm{mm}$ ), any comments on the collection and the fish capture identification (ID) number.

\begin{tabular}{|c|c|c|c|c|c|}
\hline Site Number & Common Name & \# & $\begin{array}{l}\text { Fork/Total* } \\
\text { Length }(\mathrm{mm})\end{array}$ & Comments & $\begin{array}{l}\text { Fish Capture } \\
\text { ID Number }\end{array}$ \\
\hline $\mathrm{X}-04-155$ & Logperch & 1 & & & $\mathrm{X}-04-155 \_230604 \_\mathrm{E} 1$ \\
\hline $\mathrm{X}-04-155$ & Northern Pike & 1 & 64 & YOY & X-04-155_230604_E1 \\
\hline$X-04-155$ & River Darter & 1 & & Plus 2 Orconectes virilis & X-04-155_230604_E1 \\
\hline $\mathrm{X}-04-155$ & Spottail Shiner & 9 & & & X-04-155_230604_E1 \\
\hline $\mathrm{X}-04-155$ & White Sucker & 2 & 84,99 & & $\mathrm{X}-04-155 \_230604$ E1 \\
\hline $\mathrm{X}-04-155$ & Yellow Perch & 22 & $60-85$ & Released 20 & X-04-155_230604_E1 \\
\hline$X-04-156$ & Brook Stickleback & 5 & & Released 20 & $\mathrm{X}-04-156 \_230604$ E1 \\
\hline $\mathrm{X}-04-156$ & Central Mudminnow & 36 & & Released 20 & X-04-156_230604_E1 \\
\hline $\mathrm{X}-04-157$ & Central Mudminnow & 1 & & & X-04-157_230604_E1 \\
\hline$X-04-157$ & Northern Pike & 3 & $73-76$ & & X-04-157_230604_E1 \\
\hline X-04-159 & Central Mudminnow & 1 & & & X-04-159_230604_E1 \\
\hline $\mathrm{X}-04-159$ & Northern Pike & 3 & $69-92$ & Released & X-04-159_230604_E1 \\
\hline$X-04-160$ & Central Mudminnow & 10 & & & $\mathrm{X}-04-160 \_240604 \_\mathrm{E} 1$ \\
\hline X-04-160 & Northern Pike & 4 & $62-83$ & $\begin{array}{l}\text { YOY Northern Pike released, } 1 \text { Tadpole } \\
\text { shrimp bagged with sample - presence } \\
\text { indicates that water dries up for much of } \\
\text { the year }\end{array}$ & X-04-160_240604_E1 \\
\hline $\mathrm{X}-04-161$ & No Catch & & & Suckers use drain & X-04-161_240604_E1 \\
\hline$X-04-162$ & Brook Stickleback & 4 & & & X-04-162_240604_E1 \\
\hline $\mathrm{X}-04-162$ & Central Mudminnow & 4 & & & X-04-162_240604_E1 \\
\hline $\mathrm{X}-04-162$ & Fathead Minnow & 21 & & & X-04-162_240604_E1 \\
\hline$X-04-162$ & Finescale Dace & 14 & & & $\mathrm{X}-04-162 \_240604$ E1 \\
\hline $\mathrm{X}-04-162$ & lowa Darter & 6 & & & X-04-162_240604_E1 \\
\hline $\mathrm{X}-04-162$ & Longnose Dace & 3 & & & X-04-162_240604_E1 \\
\hline$X-04-162$ & Northern Pike & 1 & 84 & & X-04-162_240604_E1 \\
\hline $\mathrm{X}-04-162$ & Northern Redbelly Dace & 3 & & & X-04-162_240604_E1 \\
\hline $\mathrm{X}-04-162$ & White Sucker & 1 & 58 & & X-04-162_240604_E1 \\
\hline$X-04-162$ & Yellow Perch & 10 & $58-66$ & & $\mathrm{X}-04-162 \_240604 \_\mathrm{E} 1$ \\
\hline $\mathrm{X}-04-163$ & Fathead Minnow & 1 & & & X-04-163_250604_E1 \\
\hline $\mathrm{X}-04-163$ & Northern Pike & 6 & $64-84$ & $\begin{array}{l}5 \text { donated to New Icelandic Heritage } \\
\text { Museum }\end{array}$ & X-04-163_250604_E1 \\
\hline
\end{tabular}


Appendix 5: List of all fish captures made between 2002 and 2006, showing site number, common name and number (\#) of fish collected, fork (or total ${ }^{\star}$ ) length in millimetres ( $\mathrm{mm}$ ), any comments on the collection and the fish capture identification (ID) number.

\begin{tabular}{|c|c|c|c|c|c|}
\hline Site Number & Common Name & \# & $\begin{array}{l}\text { Fork/Total* } \\
\text { Length }(\mathrm{mm})\end{array}$ & Comments & $\begin{array}{l}\text { Fish Capture } \\
\text { ID Number }\end{array}$ \\
\hline $\mathrm{X}-04-164$ & Brook Stickleback & 1 & & & X-04-164_250604_E1 \\
\hline $\mathrm{X}-04-164$ & Central Mudminnow & 1 & & & X-04-164_250604_E1 \\
\hline$X-04-164$ & Johnny Darter & 1 & & & X-04-164_250604_E1 \\
\hline $\mathrm{X}-04-164$ & Northern Pike & 14 & $57-72$ & $\begin{array}{l}8 \text { donated to New Icelandic Heritage } \\
\text { Museum }\end{array}$ & X-04-164_250604_E1 \\
\hline X-04-164 & Yellow Perch & 1 & 71 & & X-04-164_250604_E1 \\
\hline $\mathrm{X}-04-165$ & Brook Stickleback & 7 & & & X-04-165_280604_D1 \\
\hline$X-04-167$ & Common Carp & 100's & & Many observed & X-04-167_280604_E1 \\
\hline $\mathrm{X}-04-167$ & Northern Pike & 1 & 44 & & X-04-167_280604_E1 \\
\hline $\mathrm{X}-04-168$ & Brook Stickleback & 2 & & & X-04-168_280604_E1 \\
\hline$X-04-168$ & Central Mudminnow & 1 & & & X-04-168_280604_E1 \\
\hline $\mathrm{X}-04-169$ & Northern Pike & 2 & $85-95$ & Plus one Lepidurus spp. & X-04-169_280604_E1 \\
\hline $\mathrm{X}-04-170$ & No Catch & & & & X-04-170_290604_E1 \\
\hline$X-04-171$ & Brook Stickleback & 17 & & & X-04-171_290604_E1 \\
\hline $\mathrm{X}-04-171$ & Central Mudminnow & 80 & & & X-04-171_290604_E1 \\
\hline $\mathrm{X}-04-171$ & Fathead Minnow & 6 & & $\begin{array}{l}7 \text { post-larval Cyprinids in sample may also } \\
\text { be Fathead Minnow }\end{array}$ & X-04-171_290604_E1 \\
\hline$X-04-171$ & Finescale Dace & 1 & & & X-04-171_290604_E1 \\
\hline X-04-171 & Northern Redbelly Dace & 1 & & & X-04-171_290604_E1 \\
\hline $\mathrm{X}-04-172$ & Central Mudminnow & 1 & & & X-04-172_290604_E1 \\
\hline$X-04-172$ & Northern Pike & 2 & 81,247 & Adult released & X-04-172_290604_E1 \\
\hline $\mathrm{X}-04-175$ & Brook Stickleback & 15 & & & X-04-175_290604_E1 \\
\hline X-04-175 & Central Mudminnow & 1 & & $\begin{array}{l}\text { Donated to New Icelandic Heritage } \\
\text { Museum }\end{array}$ & X-04-175_290604_E1 \\
\hline$X-04-175$ & Fathead Minnow & 27 & & & X-04-175_290604_E1 \\
\hline $\mathrm{X}-04-175$ & Northern Redbelly Dace & 40 & & & X-04-175_290604_E1 \\
\hline $\mathrm{X}-04-175$ & White Sucker & 38 & $37-60$ & $37 \mathrm{YOY}$ & X-04-175_290604_E1 \\
\hline$X-04-176$ & Brook Stickleback & 7 & & $\begin{array}{l}\text { Plus } 3 \text { Physa spp. snails and } 1 \text { beetle } \\
\text { larvae }\end{array}$ & X-04-176_300604_E1 \\
\hline $\mathrm{X}-04-176$ & Fathead Minnow & 2 & & & X-04-176_300604_E1 \\
\hline $\mathrm{X}-04-176$ & White Sucker & 32 & $18-25^{*}$ & & X-04-176_300604_E1 \\
\hline
\end{tabular}


Appendix 5: List of all fish captures made between 2002 and 2006, showing site number, common name and number (\#) of fish collected, fork (or total ${ }^{\star}$ ) length in millimetres ( $\mathrm{mm}$ ), any comments on the collection and the fish capture identification (ID) number.

\begin{tabular}{|c|c|c|c|c|c|}
\hline Site Number & Common Name & \# & $\begin{array}{l}\text { Fork/Total* } \\
\text { Length (mm) }\end{array}$ & Comments & $\begin{array}{l}\text { Fish Capture } \\
\text { ID Number }\end{array}$ \\
\hline $\mathrm{X}-04-177$ & Brook Stickleback & 35 & & & X-04-177_300604_E1 \\
\hline $\mathrm{X}-04-177$ & Central Mudminnow & 44 & & Mostly YOY & X-04-177_300604_E1 \\
\hline $\mathrm{X}-04-177$ & Common Carp & 4 & $600-650$ & Released & $\mathrm{X}-04-177 \_300604 \mathrm{E} 1$ \\
\hline $\mathrm{X}-04-177$ & Fathead Minnow & 32 & & & X-04-177_300604_E1 \\
\hline $\mathrm{X}-04-177$ & Iowa Darter & 2 & & & X-04-177_300604_E1 \\
\hline $\mathrm{X}-04-177$ & Northern Redbelly Dace & 1 & & & X-04-177_300604_E1 \\
\hline $\mathrm{X}-04-177$ & Pearl Dace & 1 & & & X-04-177_300604_E1 \\
\hline $\mathrm{X}-04-177$ & White Sucker & 12 & $18-26^{*}$ & YOY & X-04-177_300604_E1 \\
\hline $\mathrm{X}-04-178$ & Common Carp & 4 & $600-650$ & Released & X-04-178_010704_E1 \\
\hline$X-04-178$ & Emerald Shiner & 3 & & & X-04-178_010704_E1 \\
\hline $\mathrm{X}-04-178$ & Fathead Minnow & 4 & & 2 males in spawning colors & X-04-178_010704_E1 \\
\hline $\mathrm{X}-04-178$ & Johnny Darter & 1 & & & X-04-178_010704_E1 \\
\hline $\mathrm{X}-04-178$ & Northern Pike & 2 & $83-92$ & & X-04-178_010704_E1 \\
\hline $\mathrm{X}-04-179$ & Northern Pike & 1 & 91 & & X-04-179_010704_E1 \\
\hline $\mathrm{X}-04-179$ & Spottail Shiner & 1 & & & X-04-179_010704_E1 \\
\hline$X-04-180$ & Brook Stickleback & 3 & & Plus one dragon fly larvae & X-04-180_020704_D1 \\
\hline $\mathrm{X}-04-180$ & Central Mudminnow & 1 & & Plus one dragon fly larvae & X-04-180_020704_D1 \\
\hline $\mathrm{X}-04-181$ & Blacknose Shiner & 2 & & & X-04-181_020704_E1 \\
\hline $\mathrm{X}-04-181$ & Brook Stickleback & 1 & & & X-04-181_020704_E1 \\
\hline $\mathrm{X}-04-181$ & Fathead Minnow & 1 & & & X-04-181_020704_E1 \\
\hline $\mathrm{X}-04-181$ & Johnny Darter & 1 & & & X-04-181_020704_E1 \\
\hline $\mathrm{X}-04-181$ & Northern Pike & 3 & $53-72$ & & X-04-181_020704_E1 \\
\hline $\mathrm{X}-04-181$ & Yellow Perch & 1 & 68 & & X-04-181_020704_E1 \\
\hline $\mathrm{X}-04-182$ & Brook Stickleback & 32 & & & X-04-182_050704_E1 \\
\hline$X-04-182$ & Fathead Minnow & 4 & & & X-04-182_050704_E1 \\
\hline $\mathrm{X}-04-184$ & Northern Pike & 1 & 77 & Tail damaged & X-04-184_050704_E1 \\
\hline $\mathrm{X}-04-184$ & Spottail Shiner & 1 & & & X-04-184_050704_E1 \\
\hline $\mathrm{X}-04-185$ & Fathead Minnow & 14 & & & X-04-185_050704_E1 \\
\hline $\mathrm{X}-04-185$ & lowa Darter & 10 & & & X-04-185_050704_E1 \\
\hline $\mathrm{X}-04-185$ & Northern Pike & 8 & $78-191$ & Released 6 & X-04-185_050704_E1 \\
\hline $\mathrm{X}-04-185$ & Yellow Perch & 9 & $63-91$ & Released 7 & X-04-185_050704_E1 \\
\hline
\end{tabular}


Appendix 5: List of all fish captures made between 2002 and 2006, showing site number, common name and number (\#) of fish collected, fork (or total ${ }^{\star}$ ) length in millimetres ( $\mathrm{mm}$ ), any comments on the collection and the fish capture identification (ID) number.

\begin{tabular}{|c|c|c|c|c|c|}
\hline Site Number & Common Name & \# & $\begin{array}{c}\text { Fork/Total* } \\
\text { Length }(\mathrm{mm})\end{array}$ & Comments & $\begin{array}{l}\text { Fish Capture } \\
\text { ID Number }\end{array}$ \\
\hline $\mathrm{X}-04-186$ & Brook Stickleback & 17 & & & X-04-186_050704_E1 \\
\hline X-04-186 & Central Mudminnow & 10 & & & X-04-186_050704_E1 \\
\hline$X-04-187$ & Common Carp & 100's & & Many observed & X-04-187_060704_E1 \\
\hline X-04-187 & Fathead Minnow & 1 & & Male in spawning color & X-04-187_060704_E1 \\
\hline X-04-187 & Northern Pike & 18 & $91-131$ & Released 16 & X-04-187_060704_E1 \\
\hline $\mathrm{X}-04-188$ & Northern Pike & 1 & 76 & & X-04-188_060704_D1 \\
\hline $\mathrm{X}-04-190$ & White Sucker & 55 & $23-33^{*}$ & $\begin{array}{l}\text { All YOY; } 5 \text { Corixidae, } 1 \text { Physid snail, } 1 \\
\text { dragon fly nymph, and a tadpole shrimp. } \\
\text { Tadpole shrimp suggests site goes dry }\end{array}$ & X-04-190_060704_B1 \\
\hline X-04-192 & Central Mudminnow & 1 & & & X-04-192_060704_E1 \\
\hline X-04-192 & Fathead Minnow & 1 & & & X-04-192_060704_E1 \\
\hline X-04-192 & Freshwater Drum & 1 & 682 & Released & X-04-192_060704_E1 \\
\hline X-04-192 & Johnny Darter & 1 & & & X-04-192_060704_E1 \\
\hline X-04-192 & Logperch & 1 & & & X-04-192_060704_E1 \\
\hline X-04-192 & Northern Pike & 3 & $50-117$ & & X-04-192_060704_E1 \\
\hline X-04-194 & Brook Stickleback & 2 & & & X-04-194_070704_E1 \\
\hline X-04-194 & Central Mudminnow & 1 & & & X-04-194_070704_E1 \\
\hline X-04-194 & Northern Pike & 2 & 69,71 & & X-04-194_070704_E1 \\
\hline X-04-194 & Northern Pike & 95 & & YOY released & X-04-194_070704_B1 \\
\hline X-04-195 & Black Bullhead & 1 & & Found dead onsite & X-04-195_070704_E1 \\
\hline X-04-195 & Brook Stickleback & 15 & & & X-04-195_070704_E1 \\
\hline X-04-195 & Fathead Minnow & 6 & & & X-04-195_070704_E1 \\
\hline X-04-196 & No Catch & & & & X-04-196_070704_E1 \\
\hline X-04-198 & Blackside Darter & 2 & & & X-04-198_070704_E1 \\
\hline X-04-198 & White Sucker & 1 & 138 & & X-04-198_070704_E1 \\
\hline X-04-198 & Yellow Perch & 2 & $68-79$ & & X-04-198_070704_E1 \\
\hline X-04-199 & Brook Stickleback & 9 & & & X-04-199_080704_E1 \\
\hline X-04-199 & Yellow Perch & 6 & $92-122$ & Released & X-04-199_080704_E1 \\
\hline $\mathrm{X}-04-200$ & Common Carp & 2 & 497,669 & Released & X-04-200_080704_E1 \\
\hline$X-04-200$ & Northern Pike & 2 & 90,99 & & X-04-200_080704_E1 \\
\hline X-04-201 & Brook Stickleback & 3 & & & X-04-201_080704_E1 \\
\hline
\end{tabular}


Appendix 5: List of all fish captures made between 2002 and 2006, showing site number, common name and number (\#) of fish collected, fork (or total ${ }^{*}$ ) length in millimetres $(\mathrm{mm})$, any comments on the collection and the fish capture identification (ID) number.

\begin{tabular}{|c|c|c|c|c|c|}
\hline Site Number & Common Name & \# & $\begin{array}{l}\text { Fork/Total* } \\
\text { Length }(\mathrm{mm})\end{array}$ & Comments & $\begin{array}{l}\text { Fish Capture } \\
\text { ID Number }\end{array}$ \\
\hline $\mathrm{X}-04-202$ & No Catch & & & & X-04-202_080704_E1 \\
\hline$X-04-203$ & Brook Stickleback & 1 & & & X-04-203_130704_E1 \\
\hline$X-04-203$ & Central Mudminnow & 2 & & & X-04-203_130704_E1 \\
\hline $\mathrm{X}-04-203$ & Finescale Dace & 2 & & & X-04-203_130704_E1 \\
\hline $\mathrm{X}-04-203$ & Northern Redbelly Dace & 1 & & & X-04-203_130704_E1 \\
\hline $\mathrm{X}-04-204$ & Brook Stickleback & 10 & & Plus one Rana spp. tadpole & X-04-204_130704_E1 \\
\hline$X-04-205$ & Brassy Minnow & 3 & & & X-04-205_130704_E1 \\
\hline $\mathrm{X}-04-205$ & Brook Stickleback & 13 & & & X-04-205_130704_E1 \\
\hline$X-04-205$ & Central Mudminnow & 9 & & & X-04-205_130704_E1 \\
\hline$X-04-205$ & Common Shiner & 1 & & & X-04-205_130704_E1 \\
\hline $\mathrm{X}-04-205$ & Creek Chub & 6 & & & X-04-205_130704_E1 \\
\hline $\mathrm{X}-04-205$ & Fathead Minnow & 4 & & $1 \mathrm{YOY}$ & X-04-205_130704_E1 \\
\hline$X-04-205$ & Finescale Dace & 5 & & & X-04-205_130704_E1 \\
\hline$X-04-205$ & Johnny Darter & 7 & & & $\mathrm{X}-04-205 \_130704 \mathrm{E} 1$ \\
\hline $\mathrm{X}-04-205$ & Northern Redbelly Dace & 37 & & & X-04-205_130704_E1 \\
\hline$X-04-205$ & Pearl Dace & 16 & & & X-04-205_130704_E1 \\
\hline $\mathrm{X}-04-205$ & Western Blacknose Dace & 3 & & & X-04-205_130704_E1 \\
\hline $\mathrm{X}-04-205$ & White Sucker & 1 & $19^{*}$ & $\begin{array}{l}\text { Definitely a Catostomid, fairly confident is } \\
\text { White Sucker }\end{array}$ & X-04-205_130704_E1 \\
\hline$X-04-206$ & Central Mudminnow & 14 & & & X-04-206_130704_E1 \\
\hline$X-04-206$ & Iowa Darter & 1 & & & X-04-206_130704_E1 \\
\hline $\mathrm{X}-04-206$ & Northern Pike & 1 & 109 & & X-04-206_130704_E1 \\
\hline$X-04-206$ & White Sucker & 1 & 79 & & X-04-206_130704_E1 \\
\hline $\mathrm{X}-04-207$ & Blackside Darter & 1 & & & $\mathrm{X}-04-207 \_130704 \_\mathrm{E} 1$ \\
\hline $\mathrm{X}-04-207$ & Brook Stickleback & 4 & & & X-04-207_130704_E1 \\
\hline$X-04-207$ & Central Mudminnow & 12 & & & X-04-207_130704_E1 \\
\hline $\mathrm{X}-04-207$ & Unidentified sucker & 1 & 9 & Larval stage & X-04-207_130704_E1 \\
\hline $\mathrm{X}-04-208$ & Brook Stickleback & 14 & & & X-04-208_140704_E1 \\
\hline$X-04-208$ & Central Mudminnow & 19 & & & X-04-208_140704_E1 \\
\hline$X-04-208$ & Common Shiner & 3 & & & X-04-208_140704_E1 \\
\hline $\mathrm{X}-04-208$ & Creek Chub & 6 & & & X-04-208_140704_E1 \\
\hline
\end{tabular}


Appendix 5: List of all fish captures made between 2002 and 2006, showing site number, common name and number (\#) of fish collected, fork (or total ${ }^{\star}$ ) length in millimetres ( $\mathrm{mm}$ ), any comments on the collection and the fish capture identification (ID) number.

\begin{tabular}{|c|c|c|c|c|c|}
\hline Site Number & Common Name & \# & $\begin{array}{l}\text { Fork/Total* } \\
\text { Length (mm) }\end{array}$ & Comments & $\begin{array}{l}\text { Fish Capture } \\
\text { ID Number }\end{array}$ \\
\hline$X-04-208$ & Fathead Minnow & 1 & & & X-04-208_140704_E1 \\
\hline $\mathrm{X}-04-208$ & Finescale Dace & 1 & & & X-04-208_140704_E1 \\
\hline$X-04-208$ & Johnny Darter & 7 & & One spawning male & X-04-208_140704_E1 \\
\hline $\mathrm{X}-04-208$ & Northern Redbelly Dace & 16 & & & X-04-208_140704_E1 \\
\hline$X-04-208$ & Pearl Dace & 2 & & & X-04-208_140704_E1 \\
\hline $\mathrm{X}-04-208$ & Western Blacknose Dace & 3 & & & X-04-208_140704_E1 \\
\hline$X-04-208$ & White Sucker & 2 & 96,130 & & X-04-208_140704_E1 \\
\hline $\mathrm{X}-04-209$ & Blackside Darter & 2 & & & X-04-209_140704_E1 \\
\hline$X-04-209$ & Burbot & 1 & 304 & Released & X-04-209_140704_E1 \\
\hline$X-04-209$ & Central Mudminnow & 6 & & & X-04-209_140704_E1 \\
\hline $\mathrm{X}-04-210$ & Blackside Darter & 1 & & & X-04-210_140704_E1 \\
\hline $\mathrm{X}-04-210$ & Brassy Minnow & 6 & & & X-04-210_140704_E1 \\
\hline$X-04-210$ & Brook Stickleback & 13 & & & X-04-210_140704_E1 \\
\hline $\mathrm{X}-04-210$ & Central Mudminnow & 10 & & 4 YOY & X-04-210_140704_E1 \\
\hline $\mathrm{X}-04-210$ & Creek Chub & 1 & & & X-04-210_140704_E1 \\
\hline $\mathrm{X}-04-210$ & Fathead Minnow & 2 & & & X-04-210_140704_E1 \\
\hline $\mathrm{X}-04-210$ & Johnny Darter & 9 & & & X-04-210_140704_E1 \\
\hline $\mathrm{X}-04-210$ & Northern Redbelly Dace & 69 & & & X-04-210_140704_E1 \\
\hline$X-04-210$ & Pearl Dace & 3 & & & X-04-210_140704_E1 \\
\hline $\mathrm{X}-04-210$ & White Sucker & 1 & $19^{*}$ & YOY & X-04-210_140704_E1 \\
\hline $\mathrm{X}-04-211$ & Northern Pike & 2 & 85,82 & 100's of YOY Northern Pike observed & X-04-211_140704_D1 \\
\hline $\mathrm{X}-04-212$ & No Catch & & & & X-04-212_150704_E1 \\
\hline $\mathrm{X}-04-213$ & No Catch & & & & X-04-213_150704_E1 \\
\hline $\mathrm{X}-04-215$ & No Catch & & & & X-04-215_150704_E1 \\
\hline$X-04-215$ & Northern Pike & 5 & & 5 - YOY observed unable to collect & X-04-215_150704_O1 \\
\hline $\mathrm{X}-04-216$ & No Catch & & & & X-04-216_160704_E1 \\
\hline $\mathrm{X}-04-217$ & No Catch & & & & $\mathrm{X}-04-217 \_160704 \_\mathrm{E} 1$ \\
\hline $\mathrm{X}-04-221$ & Black Bullhead & 3 & & & X-04-221_160704_E1 \\
\hline $\mathrm{X}-04-221$ & Common Carp & 1 & 163 & & X-04-221_160704_E1 \\
\hline $\mathrm{X}-04-221$ & Golden Shiner & 1 & & & X-04-221_160704_E1 \\
\hline $\mathrm{X}-04-221$ & Johnny Darter & 2 & & & X-04-221_160704_E1 \\
\hline
\end{tabular}


Appendix 5: List of all fish captures made between 2002 and 2006, showing site number, common name and number (\#) of fish collected, fork (or total ${ }^{\star}$ ) length in millimetres ( $\mathrm{mm}$ ), any comments on the collection and the fish capture identification (ID) number.

\begin{tabular}{|c|c|c|c|c|c|}
\hline Site Number & Common Name & \# & $\begin{array}{l}\text { Fork/Total* } \\
\text { Length }(\mathrm{mm})\end{array}$ & Comments & $\begin{array}{l}\text { Fish Capture } \\
\text { ID Number }\end{array}$ \\
\hline $\mathrm{X}-04-221$ & Longnose Dace & 39 & & & X-04-221_160704_E1 \\
\hline $\mathrm{X}-04-221$ & Northern Pike & 1 & 364 & Released & $\mathrm{X}-04-221 \_160704 \_\mathrm{E} 1$ \\
\hline$X-04-221$ & River Darter & 1 & & $\begin{array}{l}\text { Plus } 2 \text { crayfish (probably Orconectes } \\
\text { virilis) }\end{array}$ & X-04-221_160704_E1 \\
\hline$X-04-221$ & Rock Bass & 16 & $50-147$ & $\begin{array}{l}\text { Released } 5 \text { Common Shiners, some } \\
\text { mature females and small males with } \\
\text { tubercles }\end{array}$ & X-04-221_160704_E1 \\
\hline $\mathrm{X}-04-221$ & Tadpole Madtom & 3 & & Released 1 & X-04-221_160704_E1 \\
\hline $\mathrm{X}-04-221$ & White Sucker & 2 & $123-141$ & Immature, 1 released & X-04-221_160704_E1 \\
\hline$X-04-221$ & Yellow Perch & 1 & 91 & & X-04-221_160704_E1 \\
\hline $\mathrm{X}-04-222$ & Blackside Darter & 9 & & $1 \mathrm{YOY}$ & $\mathrm{X}-04-222 \_190704 \_\mathrm{E} 1$ \\
\hline $\mathrm{X}-04-222$ & Johnny Darter & 7 & & & X-04-222_190704_E1 \\
\hline$X-04-222$ & Western Blacknose Dace & 6 & & & X-04-222_190704_E1 \\
\hline $\mathrm{X}-04-222$ & White Sucker & 45 & $22 *-134$ & $\begin{array}{l}\text { Released } 12,134 \mathrm{~mm} \text { is an immature } \\
\text { male, } 19 \text { are YOY }\end{array}$ & $\mathrm{X}-04-222 \_190704 \_\mathrm{E} 1$ \\
\hline $\mathrm{X}-04-222$ & Yellow Perch & 1 & 67 & & X-04-222_190704_E1 \\
\hline$X-04-223$ & Blackside Darter & 3 & & & X-04-223_200704_E1 \\
\hline $\mathrm{X}-04-223$ & Brook Stickleback & 3 & & & X-04-223_200704_E1 \\
\hline $\mathrm{X}-04-223$ & Johnny Darter & 5 & & & X-04-223_200704_E1 \\
\hline $\mathrm{X}-04-223$ & Western Blacknose Dace & 93 & & & X-04-223_200704_E1 \\
\hline $\mathrm{X}-04-223$ & White Sucker & 2 & 139,146 & Spent female, 1 released & X-04-223_200704_E1 \\
\hline $\mathrm{X}-04-225$ & Blackside Darter & 2 & & & X-04-225_200704_E1 \\
\hline$X-04-225$ & Johnny Darter & 9 & & & X-04-225_200704_E1 \\
\hline $\mathrm{X}-04-225$ & Longnose Dace & 1 & & & X-04-225_200704_E1 \\
\hline $\mathrm{X}-04-225$ & Northern Pike & 2 & & & X-04-225_200704_E1 \\
\hline$X-04-225$ & White Sucker & 1 & 132 & $\begin{array}{l}\text { Male, length is approximate as the body } \\
\text { was bent }\end{array}$ & X-04-225_200704_E1 \\
\hline $\mathrm{X}-04-226$ & Blackside Darter & 4 & & & X-04-226_200704_E1 \\
\hline$X-04-226$ & Johnny Darter & 1 & & & X-04-226_200704_E1 \\
\hline $\mathrm{X}-04-226$ & Longnose Dace & 1 & & & X-04-226_200704_E1 \\
\hline $\mathrm{X}-04-226$ & White Sucker & 35 & $18^{*}-148$ & $148 \mathrm{~mm}$ is an immature male, 34 are $\mathrm{YOY}$ & X-04-226_200704_E1 \\
\hline$X-04-228$ & No Catch & & & & X-04-228_210704_E1 \\
\hline
\end{tabular}


Appendix 5: List of all fish captures made between 2002 and 2006, showing site number, common name and number (\#) of fish collected, fork (or total ${ }^{\star}$ ) length in millimetres ( $\mathrm{mm}$ ), any comments on the collection and the fish capture identification (ID) number.

\begin{tabular}{|c|c|c|c|c|c|}
\hline Site Number & Common Name & \# & $\begin{array}{l}\text { Fork/Total* } \\
\text { Length (mm) }\end{array}$ & Comments & $\begin{array}{l}\text { Fish Capture } \\
\text { ID Number }\end{array}$ \\
\hline $\mathrm{X}-04-230$ & Brook Stickleback & 1 & & & X-04-230_210704_D1 \\
\hline $\mathrm{X}-04-230$ & Fathead Minnow & 22 & & & X-04-230_210704_D1 \\
\hline$X-04-232$ & Brook Stickleback & 23 & & & X-04-232_220704_E1 \\
\hline $\mathrm{X}-04-235$ & Fathead Minnow & 1 & & & X-04-235_220704_D1 \\
\hline $\mathrm{X}-04-235$ & Brook Stickleback & 1 & & & X-04-235_220704_D1 \\
\hline $\mathrm{X}-04-238$ & Brook Stickleback & 21 & & & X-04-238_220704_E1 \\
\hline$X-04-238$ & Fathead Minnow & 1 & & & X-04-238_220704_E1 \\
\hline $\mathrm{X}-04-239$ & Blackside Darter & 17 & & & X-04-239_220704_E1 \\
\hline $\mathrm{X}-04-239$ & Burbot & 3 & $273-304$ & Released & X-04-239_220704_E1 \\
\hline$X-04-239$ & Johnny Darter & 1 & & & X-04-239_220704_E1 \\
\hline $\mathrm{X}-04-239$ & Longnose Dace & 16 & & & X-04-239_220704_E1 \\
\hline$X-04-239$ & Northern Pike & 2 & 87,100 & YOY & X-04-239_220704_E1 \\
\hline$X-04-239$ & Rock Bass & 1 & 134 & & X-04-239_220704_E1 \\
\hline $\mathrm{X}-04-239$ & White Sucker & 7 & $31 *-386$ & Largest released, 5 YOY in sample & X-04-239_220704_E1 \\
\hline $\mathrm{X}-04-245$ & Burbot & 1 & $304^{*}$ & Released & X-04-245_260704_E1 \\
\hline$X-04-248$ & Brook Stickleback & 17 & & & X-04-248_280704_E1 \\
\hline $\mathrm{X}-04-248$ & Fathead Minnow & 2 & & & X-04-248_280704_E1 \\
\hline $\mathrm{X}-04-249$ & Brook Stickleback & 21 & & & X-04-249_280704_E1 \\
\hline $\mathrm{X}-04-249$ & Central Mudminnow & 4 & & & X-04-249_280704_E1 \\
\hline $\mathrm{X}-04-249$ & Common Shiner & 3 & & & X-04-249_280704_E1 \\
\hline $\mathrm{X}-04-249$ & Fathead Minnow & 15 & & & X-04-249_280704_E1 \\
\hline$X-04-249$ & Johnny Darter & 1 & & & X-04-249_280704_E1 \\
\hline $\mathrm{X}-04-251$ & Brook Stickleback & 1 & & & X-04-251_280704_E1 \\
\hline $\mathrm{X}-04-251$ & Fathead Minnow & 1 & & & X-04-251_280704_E1 \\
\hline$X-04-251$ & Sand Shiner & 1 & & & X-04-251_280704_E1 \\
\hline $\mathrm{X}-04-251$ & White Sucker & 2 & 39,45 & & X-04-251_280704_E1 \\
\hline $\mathrm{X}-04-253$ & Bigmouth Buffalo & 3 & & YOY & X-04-253_300704_E1 \\
\hline $\mathrm{X}-04-253$ & Black Bullhead & 1 & & & X-04-253_300704_E1 \\
\hline$X-04-253$ & Black Crappie & 1 & & YOY & X-04-253_300704_E1 \\
\hline$X-04-253$ & Blackside Darter & 15 & & & X-04-253_300704_E1 \\
\hline$X-04-253$ & Burbot & 2 & $92,100^{*}$ & & X-04-253_300704_E1 \\
\hline
\end{tabular}


Appendix 5: List of all fish captures made between 2002 and 2006, showing site number, common name and number (\#) of fish collected, fork (or total ${ }^{\star}$ ) length in millimetres $(\mathrm{mm})$, any comments on the collection and the fish capture identification (ID) number.

\begin{tabular}{|c|c|c|c|c|c|}
\hline Site Number & Common Name & \# & $\begin{array}{l}\text { Fork/Total* } \\
\text { Length }(\mathrm{mm})\end{array}$ & Comments & $\begin{array}{l}\text { Fish Capture } \\
\text { ID Number }\end{array}$ \\
\hline $\mathrm{X}-04-253$ & Common Carp & 1 & & & X-04-253_300704_E1 \\
\hline $\mathrm{X}-04-253$ & Fathead Minnow & 5 & & $1 \mathrm{YOY}$ & X-04-253_300704_E1 \\
\hline$X-04-253$ & Johnny Darter & 1 & & & X-04-253_300704_E1 \\
\hline X-04-253 & Northern Pike & 1 & 116 & YOY & X-04-253_300704_E1 \\
\hline $\mathrm{X}-04-253$ & River Darter & 18 & & & X-04-253_300704_E1 \\
\hline $\mathrm{X}-04-253$ & River Shiner & 13 & & & X-04-253_300704_E1 \\
\hline $\mathrm{X}-04-253$ & Rock Bass & 6 & 57,78 & & X-04-253_300704_E1 \\
\hline X-04-253 & Sauger & 3 & 203-295 & Released & X-04-253_300704_E1 \\
\hline $\mathrm{X}-04-253$ & Shorthead Redhorse & 3 & $83-360$ & $\begin{array}{l}\text { Juveniles in sample, } 1 \text { released }(360 \mathrm{~mm}) \text {, } \\
\text { kept }(83,126 \mathrm{~mm})\end{array}$ & X-04-253_300704_E1 \\
\hline$X-04-253$ & Silver Redhorse & 1 & 93 & Juvenile & X-04-253_300704_E1 \\
\hline $\mathrm{X}-04-253$ & Troutperch & 10 & & & X-04-253_300704_E1 \\
\hline $\mathrm{X}-04-253$ & Walleye & 2 & 58,59 & YOY & X-04-253_300704_E1 \\
\hline$X-04-253$ & White Sucker & 6 & $12^{*}-88$ & 4 YOY & X-04-253_300704_E1 \\
\hline X-04-253 & Yellow Perch & 1 & & YOY & X-04-253_300704_E1 \\
\hline $\mathrm{X}-04-255$ & Blackside Darter & 3 & & & X-04-255_300704_E1 \\
\hline $\mathrm{X}-04-255$ & Fathead Minnow & 8 & & & X-04-255_300704_E1 \\
\hline $\mathrm{X}-04-255$ & Longnose Dace & 1 & & & X-04-255_300704_E1 \\
\hline $\mathrm{X}-04-255$ & Northern Pike & 1 & 114 & & X-04-255_300704_E1 \\
\hline$X-04-255$ & Troutperch & 1 & & & X-04-255_300704_E1 \\
\hline $\mathrm{X}-04-256$ & Black Bullhead & 1 & & & X-04-256_030804_E1 \\
\hline X-04-256 & Blackside Darter & 3 & & & X-04-256_030804_E1 \\
\hline$X-04-256$ & Channel Catfish & 1 & 333 & Released & X-04-256_030804_E1 \\
\hline $\mathrm{X}-04-256$ & Fathead Minnow & 3 & & & X-04-256_030804_E1 \\
\hline $\mathrm{X}-04-256$ & Goldeye & 2 & $64-69$ & & X-04-256_030804_E1 \\
\hline$X-04-256$ & Northern Pike & 2 & $121-136$ & & X-04-256_030804_E1 \\
\hline $\mathrm{X}-04-256$ & Troutperch & 1 & & & X-04-256_030804_E1 \\
\hline $\mathrm{X}-04-256$ & Walleye & 1 & 77 & & X-04-256_030804_E1 \\
\hline $\mathrm{X}-04-256$ & White Sucker & 1 & 110 & & X-04-256_030804_E1 \\
\hline$X-04-257$ & Logperch & 7 & & & X-04-257_030804_E1 \\
\hline$X-04-257$ & Blackside Darter & 21 & & & X-04-257_030804_E1 \\
\hline
\end{tabular}


Appendix 5: List of all fish captures made between 2002 and 2006, showing site number, common name and number (\#) of fish collected, fork (or total ${ }^{*}$ ) length in millimetres $(\mathrm{mm})$, any comments on the collection and the fish capture identification (ID) number.

\begin{tabular}{|c|c|c|c|c|c|}
\hline Site Number & Common Name & \# & $\begin{array}{l}\text { Fork/Total* } \\
\text { Length (mm) }\end{array}$ & Comments & $\begin{array}{l}\text { Fish Capture } \\
\text { ID Number }\end{array}$ \\
\hline$X-04-257$ & Longnose Dace & 9 & & & X-04-257_030804_E1 \\
\hline $\mathrm{X}-04-257$ & Rock Bass & 1 & 84 & & X-04-257_030804_E1 \\
\hline$X-04-258$ & Brook Stickleback & 8 & & & X-04-258_040804_E1 \\
\hline $\mathrm{X}-04-258$ & Common Shiner & 42 & & All YOY & X-04-258_040804_E1 \\
\hline $\mathrm{X}-04-258$ & Creek Chub & 6 & & 4 YOY or Age 1 & X-04-258_040804_E1 \\
\hline $\mathrm{X}-04-258$ & Fathead Minnow & 6 & & 1 YOY & X-04-258_040804_E1 \\
\hline$X-04-258$ & Johnny Darter & 4 & & & X-04-258_040804_E1 \\
\hline $\mathrm{X}-04-258$ & Longnose Dace & 1 & & & X-04-258_040804_E1 \\
\hline $\mathrm{X}-04-258$ & Troutperch & 1 & 104 & & X-04-258_040804_E1 \\
\hline$X-04-258$ & Western Blacknose Dace & 10 & & $8 \mathrm{YOY}$ & X-04-258_040804_E1 \\
\hline $\mathrm{X}-04-258$ & White Sucker & 10 & $41 *-181$ & $\begin{array}{l}\text { Released } 5 \text { (98-181 mm), } 5 \text { kept (41-91 } \\
\mathrm{mm})\end{array}$ & X-04-258_040804_E1 \\
\hline $\mathrm{X}-04-259$ & Common Shiner & 14 & & & X-04-259_050804_E1 \\
\hline $\mathrm{X}-04-259$ & Creek Chub & 13 & & 12 juveniles & X-04-259_050804_E1 \\
\hline X-04-259 & Fathead Minnow & 5 & & & X-04-259_050804_E1 \\
\hline$X-04-259$ & Johnny Darter & 3 & & & X-04-259_050804_E1 \\
\hline$X-04-259$ & Logperch & 7 & & & X-04-259_050804_E1 \\
\hline$X-04-259$ & Longnose Dace & 19 & & 2 YOY & X-04-259_050804_E1 \\
\hline$X-04-259$ & Northern Pike & 1 & & $\begin{array}{l}\text { Caught by angler on upstream side of } \\
\text { raised ford crossing }\end{array}$ & X-04-259_050804_E1 \\
\hline$X-04-259$ & River Darter & 10 & & 8 YOY or $1+$ & X-04-259_050804_E1 \\
\hline$X-04-259$ & Sand Shiner & 96 & & $30 \%$ YOY and $1+$ & X-04-259_050804_E1 \\
\hline $\mathrm{X}-04-259$ & Shorthead Redhorse & 4 & $86-119$ & & X-04-259_050804_E1 \\
\hline $\mathrm{X}-04-259$ & Troutperch & 3 & & & X-04-259_050804_E1 \\
\hline $\mathrm{X}-04-259$ & Western Blacknose Dace & 1 & & & X-04-259_050804_E1 \\
\hline$X-04-259$ & White Sucker & 1 & 126 & & X-04-259_050804_E1 \\
\hline$X-04-259$ & Yellow Perch & 34 & & 27 are YOY or $1+$ & X-04-259_050804_E1 \\
\hline $\mathrm{X}-04-260$ & Bigmouth Shiner & 23 & & & X-04-260_050804_E1 \\
\hline$X-04-260$ & Blackside Darter & 12 & & & X-04-260_050804_E1 \\
\hline$X-04-260$ & Common Carp & 1 & & YOY & X-04-260_050804_E1 \\
\hline $\mathrm{X}-04-260$ & Common Shiner & 23 & & & X-04-260_050804_E1 \\
\hline
\end{tabular}


Appendix 5: List of all fish captures made between 2002 and 2006, showing site number, common name and number (\#) of fish collected, fork (or total ${ }^{\star}$ ) length in millimetres ( $\mathrm{mm}$ ), any comments on the collection and the fish capture identification (ID) number.

\begin{tabular}{|c|c|c|c|c|c|}
\hline Site Number & Common Name & \# & $\begin{array}{l}\text { Fork/Total* } \\
\text { Length }(\mathrm{mm})\end{array}$ & Comments & $\begin{array}{l}\text { Fish Capture } \\
\text { ID Number }\end{array}$ \\
\hline$X-04-260$ & Creek Chub & 5 & & & X-04-260_050804_E1 \\
\hline $\mathrm{X}-04-260$ & Johnny Darter & 10 & & & X-04-260_050804_E1 \\
\hline$X-04-260$ & Longnose Dace & 8 & & & X-04-260_050804_E1 \\
\hline $\mathrm{X}-04-260$ & Sand Shiner & 369 & & & X-04-260_050804_E1 \\
\hline $\mathrm{X}-04-260$ & Shorthead Redhorse & 3 & $68,119,161$ & & X-04-260_050804_E1 \\
\hline $\mathrm{X}-04-260$ & Western Blacknose Dace & 8 & & & X-04-260_050804_E1 \\
\hline$X-04-262$ & Brook Stickleback & 1 & & & X-04-262_060804_E1 \\
\hline $\mathrm{X}-04-262$ & Common Shiner & 4 & & All YOY & X-04-262_060804_E1 \\
\hline $\mathrm{X}-04-262$ & Fathead Minnow & 1 & & & $\mathrm{X}-04-262 \_060804 \_\mathrm{E} 1$ \\
\hline$X-04-262$ & Johnny Darter & 5 & & 4 YOY & $\mathrm{X}-04-262 \_060804 \_\mathrm{E} 1$ \\
\hline $\mathrm{X}-04-262$ & Logperch & 7 & $101-108^{*}$ & Released 4 & X-04-262_060804_E1 \\
\hline $\mathrm{X}-04-262$ & Longnose Dace & 3 & & & $\mathrm{X}-04-262 \_060804 \_\mathrm{E} 1$ \\
\hline$X-04-262$ & Western Blacknose Dace & 2 & & YOY & $\mathrm{X}-04-262 \_060804$ E1 \\
\hline $\mathrm{X}-04-262$ & Yellow Perch & 2 & & $78-87 \mathrm{~mm}$ & $\mathrm{X}-04-262 \_060804 \_\mathrm{E} 1$ \\
\hline D-05-004 & White Sucker & several & & $\begin{array}{l}\text { Observed congregated on hard substrate } \\
\text { downstream of culvert }\end{array}$ & D-05-004_140405_01 \\
\hline D-05-007 & White Sucker & several & & $\begin{array}{l}\text { Observed congregated on cobble and } \\
\text { boulders under and around bridge. }\end{array}$ & D-05-007_140405_01 \\
\hline D-05-008 & White Sucker & several & & $\begin{array}{l}\text { Observed congregated on cobble and } \\
\text { boulders under and around bridge. }\end{array}$ & D-05-008_140405_01 \\
\hline D-05-009 & White Sucker & several & & $\begin{array}{l}\text { Observed congregated on cobble and } \\
\text { boulders under and around bridge. }\end{array}$ & D-05-009_140405_01 \\
\hline D-05-009 & Northern Pike & 2 & & $\begin{array}{l}\text { Immature, observed in submerged } \\
\text { grasses }\end{array}$ & D-05-009_140405_01 \\
\hline D-05-010 & No Catch & & & & D-05-010_010505_L1 \\
\hline D-05-011 & Fathead Minnow & 1 & & & D-05-011_010605_Z1 \\
\hline D-05-011 & Northern Pike & several & & $\begin{array}{l}\text { Several adults observed but unable to } \\
\text { collect }\end{array}$ & D-05-011_010605_01 \\
\hline D-05-011 & Troutperch & 1 & & & D-05-011_010605_Z1 \\
\hline D-05-011 & Walleye & 2 & 246,586 & Released & D-05-011_010605_Z1 \\
\hline D-05-011 & White Sucker & 5 & $330-421$ & Released, 1 ripe female & D-05-011_010605_Z1 \\
\hline D-05-012 & Black Bullhead & 1 & 189 & & D-05-012_090605_B1 \\
\hline
\end{tabular}


Appendix 5: List of all fish captures made between 2002 and 2006, showing site number, common name and number (\#) of fish collected, fork (or total ${ }^{*}$ ) length in millimetres $(\mathrm{mm})$, any comments on the collection and the fish capture identification (ID) number.

\begin{tabular}{|c|c|c|c|c|c|}
\hline Site Number & Common Name & \# & $\begin{array}{l}\text { Fork/Total* } \\
\text { Length }(\mathrm{mm})\end{array}$ & Comments & $\begin{array}{l}\text { Fish Capture } \\
\text { ID Number }\end{array}$ \\
\hline D-05-012 & Northern Pike & 4 & $204-242$ & Released & D-05-012_090605_B1 \\
\hline D-05-013 & Bigmouth Shiner & 4 & 65 & & D-05-013_200705_E1 \\
\hline D-05-013 & Blackside Darter & 1 & & Juvenile & D-05-013_200705_E1 \\
\hline D-05-013 & Brassy Minnow & 3 & $60-66$ & & D-05-013_200705_E1 \\
\hline D-05-013 & Common Shiner & 1 & & & D-05-013_200705_E1 \\
\hline D-05-013 & Creek Chub & 3 & $98-115$ & & D-05-013_200705_E1 \\
\hline D-05-013 & Fathead Minnow & 133 & & & D-05-013_200705_E1 \\
\hline D-05-013 & lowa Darter & 1 & & Juvenile & D-05-013_200705_E1 \\
\hline D-05-013 & Johnny Darter & 14 & $30-50^{*}$ & Juveniles & D-05-013_200705_E1 \\
\hline D-05-013 & Western Blacknose Dace & 9 & & & D-05-013_200705_E1 \\
\hline D-05-013 & White Sucker & 23 & $38-51$ & YOY & D-05-013_200705_E1 \\
\hline D-05-014 & Black Bullhead & 1 & $38^{*}$ & Plus 4 dragonfly larvae & D-05-014_200705_B1 \\
\hline D-05-014 & Brook Stickleback & 2 & $23-24^{*}$ & & D-05-014_200705_B1 \\
\hline D-05-014 & Fathead Minnow & 333 & 13-18 & YOY & D-05-014_200705_B1 \\
\hline D-05-014 & Johnny Darter & 1 & $48^{*}$ & & D-05-014_200705_B1 \\
\hline D-05-014 & Northern Pike & 2 & $151-152$ & & D-05-014_200705_B1 \\
\hline D-05-014 & Yellow Perch & 3 & $47-50$ & & D-05-014_200705_B1 \\
\hline D-05-015 & Common Shiner & 2 & & & D-05-015_200705_E1 \\
\hline D-05-015 & Creek Chub & 3 & & & D-05-015_200705_B1 \\
\hline D-05-015 & Fathead Minnow & 2 & & & D-05-015_200705_B1 \\
\hline D-05-015 & Johnny Darter & 2 & & & D-05-015_200705_E1 \\
\hline D-05-015 & Johnny Darter & 7 & & & D-05-015_200705_B1 \\
\hline D-05-015 & Northern Pike & 2 & 131,187 & & D-05-015_200705_B1 \\
\hline D-05-015 & White Sucker & 34 & $31^{*}-441$ & $\begin{array}{l}29 \text { YOY \& } 2 \text { immature. Released } 2 \text { White } \\
\text { Sucker }(441,404 \mathrm{~mm})\end{array}$ & D-05-015_200705_B1 \\
\hline D-05-017 & White Sucker & 10 & 51 & Immature & D-05-017_210705_D1 \\
\hline D-05-017 & Fathead Minnow & 10 & & & D-05-017_210705_D1 \\
\hline D-05-017 & Common Shiner & 10 & & & D-05-017_210705_D1 \\
\hline D-05-018 & Northern Pike & 5 & 147,160 & $\begin{array}{l}\text { Released } 3 \text { Northern Pike }(160,158,153 \\
\mathrm{mm})\end{array}$ & D-05-018_030805_E1 \\
\hline D-05-019 & Brook Stickleback & 10 & & Released & D-05-019_030805_E1 \\
\hline
\end{tabular}


Appendix 5: List of all fish captures made between 2002 and 2006, showing site number, common name and number (\#) of fish collected, fork (or total ${ }^{\star}$ ) length in millimetres ( $\mathrm{mm}$ ), any comments on the collection and the fish capture identification (ID) number.

\begin{tabular}{|c|c|c|c|c|c|}
\hline Site Number & Common Name & \# & $\begin{array}{l}\text { Fork/Total* } \\
\text { Length }(\mathrm{mm})\end{array}$ & Comments & $\begin{array}{l}\text { Fish Capture } \\
\text { ID Number }\end{array}$ \\
\hline D-05-019 & Northern Pike & 1 & 201 & Released & D-05-019_030805_E1 \\
\hline D-05-020 & Brook Stickleback & 11 & $25-60^{*}$ & & D-05-020_040805_E1 \\
\hline D-05-020 & Central Mudminnow & 1 & $62^{*}$ & & D-05-020_040805_E1 \\
\hline D-05-020 & Fathead Minnow & 10 & $33-57$ & & D-05-020_040805_E1 \\
\hline D-05-021 & Brook Stickleback & 12 & $26-41^{*}$ & & D-05-021_040805_D1 \\
\hline D-05-021 & Fathead Minnow & 4 & $30-61$ & & D-05-021_040805_D1 \\
\hline D-05-023 & Brassy Minnow & 3 & 68 & & D-05-023_160805_E1 \\
\hline D-05-023 & Brook Stickleback & 11 & $27-57^{*}$ & Plus 20 unidentified Amphipods & D-05-023_160805_E1 \\
\hline D-05-023 & Common Carp & 5 & & 2 YOY, 2 immature & D-05-023_160805_E1 \\
\hline D-05-023 & Common Shiner & 5 & $82-111$ & & D-05-023_160805_E1 \\
\hline D-05-023 & Creek Chub & 6 & $73-101$ & Released 5 additional Creek Chub & D-05-023_160805_E1 \\
\hline D-05-023 & Fathead Minnow & 702 & $15-63$ & & D-05-023_160805_E1 \\
\hline D-05-023 & lowa Darter & 18 & $41-55^{*}$ & & D-05-023_160805_E1 \\
\hline D-05-023 & Northern Pike & 1 & 210 & & D-05-023_160805_E1 \\
\hline D-05-023 & Western Blacknose Dace & 8 & $64-71$ & & D-05-023_160805_E1 \\
\hline D-05-023 & White Sucker & 13 & $63-430$ & $\begin{array}{l}1 \text { YOY \& } 7 \text { immature, released } 3 \text { adults } \\
(430,406,404 \mathrm{~mm})\end{array}$ & D-05-023_160805_E1 \\
\hline D-05-023 & Yellow Perch & 1 & 100 & & D-05-023_160805_E1 \\
\hline D-05-025 & Black Bullhead & 7 & $30-48^{*}$ & & D-05-025_170805_E1 \\
\hline D-05-025 & Brook Stickleback & 3 & $32-42^{*}$ & $\begin{array}{l}\text { Released an additional } 3 \text { Brook } \\
\text { Stickleback }\end{array}$ & D-05-025_170805_E1 \\
\hline D-05-025 & Common Carp & 68 & $56-108$ & Released an additional 30 Common Carp & D-05-025_170805_E1 \\
\hline D-05-025 & Creek Chub & 1 & 87 & Released 2 additional Creek Chubs & D-05-025_170805_E1 \\
\hline D-05-025 & Fathead Minnow & 10 & $25-62$ & $\begin{array}{l}\text { Released an additional } 30 \text { Fathead } \\
\text { Minnows }\end{array}$ & D-05-025_170805_E1 \\
\hline D-05-025 & Walleye & 3 & $121-127$ & & D-05-025_170805_E1 \\
\hline D-05-025 & White Sucker & 7 & $58-76$ & & D-05-025_170805_E1 \\
\hline D-05-026 & Brook Stickleback & 145 & $28-67^{*}$ & & D-05-026_300805_E1 \\
\hline D-05-026 & Fathead Minnow & 44 & $25-72$ & & D-05-026_300805_E1 \\
\hline D-05-026 & Northern Redbelly Dace & 1 & 37 & & D-05-026_300805_E1 \\
\hline D-05-027 & Brook Stickleback & 105 & $25-65^{*}$ & & D-05-027_300805_E1 \\
\hline
\end{tabular}


Appendix 5: List of all fish captures made between 2002 and 2006, showing site number, common name and number (\#) of fish collected, fork (or total ${ }^{\star}$ ) length in millimetres ( $\mathrm{mm}$ ), any comments on the collection and the fish capture identification (ID) number.

\begin{tabular}{|c|c|c|c|c|c|}
\hline Site Number & Common Name & \# & $\begin{array}{c}\text { Fork/Total* } \\
\text { Length }(\mathrm{mm})\end{array}$ & Comments & $\begin{array}{l}\text { Fish Capture } \\
\text { ID Number }\end{array}$ \\
\hline D-05-027 & Fathead Minnow & 15 & $25-70$ & & D-05-027_300805_E1 \\
\hline D-05-027 & White Sucker & 5 & $65-90$ & & D-05-027_300805_E1 \\
\hline W-05-001 & No Catch & & & & W-05-001_020505_E1 \\
\hline W-05-001 & No Catch & & & & W-05-001_020505_K1 \\
\hline W-05-001 & White Sucker & $>20$ & & Dead on riffle rocks & W-05-001_020505_01 \\
\hline W-05-002 & Fathead Minnow & 1 & 25 & & W-05-002_020505_E1 \\
\hline W-05-009 & Brook Stickleback & 1 & $38^{*}$ & & W-05-009_020505_E1 \\
\hline W-05-009 & Fathead Minnow & 33 & $19-64$ & & W-05-009_020505_E1 \\
\hline W-05-012 & Brook Stickleback & 11 & $24-48^{*}$ & & W-05-012_030505_E1 \\
\hline W-05-012 & Central Mudminnow & 1 & $48^{*}$ & & W-05-012_030505_E1 \\
\hline W-05-012 & Fathead Minnow & 1 & 56 & & W-05-012_030505_E1 \\
\hline W-05-012 & Pearl Dace & 3 & $97-113$ & & W-05-012_030505_E1 \\
\hline W-05-012 & White Sucker & 5 & $100-240^{*}$ & Released, already spawned & W-05-012_030505_E1 \\
\hline W-05-013 & Brook Stickleback & 18 & $42-64^{*}$ & & W-05-013_030505_E1 \\
\hline W-05-013 & Fathead Minnow & 18 & $23-47$ & & W-05-013_030505_E1 \\
\hline W-05-013 & Pearl Dace & 2 & 65,69 & & W-05-013_030505_E1 \\
\hline W-05-014 & Brook Stickleback & 2 & $37-43^{*}$ & & W-05-014_030505_E1 \\
\hline W-05-014 & Fathead Minnow & 6 & $31-40$ & & W-05-014_030505_E1 \\
\hline W-05-014 & White Sucker & 1 & $345^{*}$ & Released & W-05-014_030505_E1 \\
\hline W-05-016 & White Sucker & 1 & $175^{*}$ & Released & W-05-016_030505_E1 \\
\hline W-05-016 & White Sucker & $>30$ & & Observed congregated in deep pool & W-05-016_030505_01 \\
\hline W-05-017 & White Sucker & 3 & $192.5-270^{*}$ & Released, saw many others & W-05-017_040505_E1 \\
\hline W-05-019 & Fathead Minnow & 2 & 69,74 & & W-05-019_040505_E1 \\
\hline W-05-020 & Brook Stickleback & 9 & $29-60^{*}$ & & W-05-020_040505_E1 \\
\hline W-05-020 & Fathead Minnow & 2 & $27-34$ & & W-05-020_040505_E1 \\
\hline W-05-023 & No Catch & & & & W-05-023_050505_E1 \\
\hline W-05-024 & Black Bullhead & 1 & $151^{*}$ & & W-05-024_050505_E1 \\
\hline W-05-024 & Brook Stickleback & 3 & $44-49^{*}$ & & W-05-024_050505_E1 \\
\hline W-05-024 & Central Mudminnow & 2 & $84-89^{*}$ & & W-05-024_050505_E1 \\
\hline W-05-024 & Northern Pike & 1 & & Observed & W-05-024_050505_01 \\
\hline W-05-027 & No Catch & & & & W-05-027_050505_E1 \\
\hline
\end{tabular}


Appendix 5: List of all fish captures made between 2002 and 2006, showing site number, common name and number (\#) of fish collected, fork (or total ${ }^{\star}$ ) length in millimetres ( $\mathrm{mm}$ ), any comments on the collection and the fish capture identification (ID) number.

\begin{tabular}{|c|c|c|c|c|c|}
\hline Site Number & Common Name & \# & $\begin{array}{l}\text { Fork/Total* } \\
\text { Length (mm) }\end{array}$ & Comments & $\begin{array}{l}\text { Fish Capture } \\
\text { ID Number }\end{array}$ \\
\hline W-05-028 & Central Mudminnow & 2 & $67,69^{*}$ & & W-05-028_050505_E1 \\
\hline W-05-030 & No Catch & & & & W-05-030_050505_E1 \\
\hline W-05-034 & Black Bullhead & 1 & & Observed & W-05-034_060505_01 \\
\hline W-05-034 & Brook Stickleback & 2 & $46,58^{*}$ & & W-05-034_060505_E1 \\
\hline W-05-036 & No Catch & & & & W-05-036_090505_E1 \\
\hline W-05-037 & No Catch & & & & W-05-037_090505_E1 \\
\hline W-05-038 & No Catch & & & & W-05-038_090505_E1 \\
\hline W-05-041 & Brook Stickleback & 1 & $32^{*}$ & & W-05-041_100505_E1 \\
\hline W-05-041 & Fathead Minnow & 5 & $44-72$ & & W-05-041_100505_E1 \\
\hline W-05-043 & No Catch & & & & W-05-043_100505_E1 \\
\hline W-05-045 & Brook Stickleback & 2 & $50,63^{*}$ & & W-05-045_100505_E1 \\
\hline W-05-045 & Fathead Minnow & 1 & 65 & & W-05-045_100505_E1 \\
\hline W-05-047 & Brook Stickleback & 2 & $36,40^{*}$ & & W-05-047_110505_E1 \\
\hline W-05-047 & Fathead Minnow & 5 & $27-55$ & & W-05-047_110505_E1 \\
\hline W-05-048 & Fathead Minnow & 3 & $33-56$ & & W-05-048_110505_E1 \\
\hline W-05-049 & No Catch & & & & W-05-049_110505_E1 \\
\hline W-05-050 & Central Mudminnow & 1 & $71^{*}$ & & W-05-050_110505_E1 \\
\hline W-05-050 & Fathead Minnow & 1 & 61 & & W-05-050_110505_E1 \\
\hline W-05-051 & No Catch & & & & W-05-051_120505_E1 \\
\hline W-05-052 & No Catch & & & & W-05-052_120505_E1 \\
\hline W-05-053 & No Catch & & & & W-05-053_120505_E1 \\
\hline W-05-054 & Fathead Minnow & 5 & $50-65$ & & W-05-054_120505_E1 \\
\hline W-05-055 & Brook Stickleback & 27 & $28-52^{*}$ & & W-05-055_160505_E1 \\
\hline W-05-055 & Creek Chub & 2 & $107-124$ & & W-05-055_160505_E1 \\
\hline W-05-055 & Fathead Minnow & 4 & $34-64$ & & W-05-055_160505_E1 \\
\hline W-05-055 & Iowa Darter & 1 & $41^{*}$ & & W-05-055_160505_E1 \\
\hline W-05-055 & No Catch & & & & W-05-055_160505_L1 \\
\hline W-05-056 & Brook Stickleback & 25 & $37-63^{*}$ & & W-05-056_160505_E1 \\
\hline W-05-056 & White Sucker & 4 & $51-75$ & & W-05-056_160505_E1 \\
\hline W-05-057 & Brook Stickleback & 57 & $35-59^{*}$ & & W-05-057_170505_E1 \\
\hline W-05-057 & Fathead Minnow & 15 & $29-51$ & & W-05-057_170505_E1 \\
\hline
\end{tabular}


Appendix 5: List of all fish captures made between 2002 and 2006, showing site number, common name and number (\#) of fish collected, fork (or total ${ }^{\star}$ ) length in millimetres ( $\mathrm{mm}$ ), any comments on the collection and the fish capture identification (ID) number.

\begin{tabular}{|c|c|c|c|c|c|}
\hline Site Number & Common Name & \# & $\begin{array}{l}\text { Fork/Total* } \\
\text { Length }(\mathrm{mm})\end{array}$ & Comments & $\begin{array}{l}\text { Fish Capture } \\
\text { ID Number }\end{array}$ \\
\hline W-05-058 & Brook Stickleback & 22 & $30-48^{*}$ & & W-05-058_170505_E1 \\
\hline W-05-058 & Creek Chub & 3 & $39-57$ & & W-05-058_170505_E1 \\
\hline W-05-058 & Fathead Minnow & 3 & $28-39$ & & W-05-058_170505_E1 \\
\hline W-05-058 & No Catch & & & & W-05-058_170505_K1 \\
\hline W-05-058 & White Sucker & 4 & $152.5-175^{*}$ & Released, 3 male, 1 female & W-05-058_170505_E1 \\
\hline W-05-059 & Fathead Minnow & 3 & $48-55$ & & W-05-059_170505_E1 \\
\hline W-05-060 & No Catch & & & & W-05-060_170505_E1 \\
\hline W-05-062 & Brook Stickleback & 28 & $37-69^{*}$ & & W-05-062_180505_B1 \\
\hline W-05-062 & Fathead Minnow & 21 & $35-60$ & & W-05-062_180505_B1 \\
\hline W-05-063 & Brook Stickleback & 3 & & Released & W-05-063_180505_E1 \\
\hline W-05-065 & Brook Stickleback & 62 & $24-64^{*}$ & In fish collection labeled W-05-068 & W-05-065_180505_E1 \\
\hline W-05-065 & Fathead Minnow & 23 & $29-75$ & $\begin{array}{l}10 \text { Gammarus lacustris included in fish } \\
\text { collection }\end{array}$ & W-05-065_180505_E1 \\
\hline W-05-066 & Brook Stickleback & 24 & $23-61^{*}$ & & W-05-066_180505_E1 \\
\hline W-05-066 & Fathead Minnow & 10 & $32-47$ & & W-05-066_180505_E1 \\
\hline W-05-069 & No Catch & & & & W-05-069_190505_E1 \\
\hline W-05-071 & Brook Stickleback & 8 & $36-64^{*}$ & & W-05-071_200505_E1 \\
\hline W-05-071 & Central Mudminnow & 5 & $40-62^{*}$ & & W-05-071_200505_E1 \\
\hline W-05-071 & Fathead Minnow & 10 & $27-43$ & & W-05-071_200505_E1 \\
\hline W-05-072 & Brook Stickleback & 1 & $44^{*}$ & & W-05-072_200505_E1 \\
\hline W-05-072 & Central Mudminnow & 1 & $50^{*}$ & & W-05-072_200505_E1 \\
\hline W-05-072 & Fathead Minnow & 1 & 39 & & W-05-072_200505_E1 \\
\hline W-05-073 & No Catch & & & & W-05-073_240505_E1 \\
\hline W-05-076 & Brook Stickleback & 7 & $35-60 *$ & & W-05-076_240505_E1 \\
\hline W-05-076 & Central Mudminnow & 23 & $30-96^{*}$ & & W-05-076_240505_E1 \\
\hline W-05-076 & White Sucker & 1 & 124 & & W-05-076_240505_E1 \\
\hline W-05-077 & Fathead Minnow & 49 & $33-69$ & & W-05-077_240505_E1 \\
\hline W-05-077 & Brook Stickleback & 19 & $29-49^{*}$ & & W-05-077_240505_E1 \\
\hline W-05-077 & Central Mudminnow & 2 & $34-59^{*}$ & & W-05-077_240505_E1 \\
\hline W-05-077 & Finescale Dace & 90 & $29-44$ & & W-05-077_240505_E1 \\
\hline W-05-077 & No Catch & & & & W-05-077_240505_D1 \\
\hline
\end{tabular}


Appendix 5: List of all fish captures made between 2002 and 2006, showing site number, common name and number (\#) of fish collected, fork (or total ${ }^{\star}$ ) length in millimetres ( $\mathrm{mm}$ ), any comments on the collection and the fish capture identification (ID) number.

\begin{tabular}{|c|c|c|c|c|c|}
\hline Site Number & Common Name & \# & $\begin{array}{c}\text { Fork/Total* } \\
\text { Length }(\mathrm{mm})\end{array}$ & Comments & $\begin{array}{l}\text { Fish Capture } \\
\text { ID Number }\end{array}$ \\
\hline W-05-077 & Northern Redbelly Dace & 4 & $34-35$ & & W-05-077_240505_E1 \\
\hline W-05-078 & Brook Stickleback & 3 & $49-57^{*}$ & & W-05-078_250505_B1 \\
\hline W-05-078 & Central Mudminnow & 1 & $58^{*}$ & & W-05-078_250505_B1 \\
\hline W-05-078 & Fathead Minnow & 267 & $26-74$ & & W-05-078_250505_B1 \\
\hline W-05-078 & White Sucker & 1 & 66 & Specimens badly decomposed & W-05-078_250505_B1 \\
\hline W-05-079 & Fathead Minnow & 11 & $44-72$ & & W-05-079_250505_E1 \\
\hline W-05-081 & Brook Stickleback & 1 & $69^{*}$ & & W-05-081_250505_E1 \\
\hline W-05-081 & Fathead Minnow & 6 & $34-58$ & & W-05-081_250505_E1 \\
\hline W-05-082 & No Catch & & & & W-05-082_250505_E1 \\
\hline W-05-083 & Brook Stickleback & 74 & $28-54^{*}$ & & W-05-083_260505_E1 \\
\hline W-05-083 & Creek Chub & 2 & $43-53$ & & W-05-083_260505_E1 \\
\hline W-05-083 & Fathead Minnow & 39 & $20-70$ & & W-05-083_260505_E1 \\
\hline W-05-085 & Brook Stickleback & 6 & & & W-05-085_260505_E1 \\
\hline W-05-086 & Brook Stickleback & 1 & $71^{*}$ & & W-05-086_260505_E1 \\
\hline W-05-086 & Fathead Minnow & 4 & $35-48$ & & W-05-086_260505_E1 \\
\hline W-05-087 & No Catch & & & & W-05-087_260505_E1 \\
\hline W-05-093 & No Catch & & & & W-05-093_290505_E1 \\
\hline W-05-094 & No Catch & & & & W-05-094_310505_E1 \\
\hline W-05-096 & Brook Stickleback & 3 & $43-59^{*}$ & & W-05-096_310505_E1 \\
\hline W-05-096 & Fathead Minnow & 1 & 77 & Ripe male & W-05-096_310505_E1 \\
\hline W-05-099 & Brook Stickleback & 3 & $42-67^{*}$ & & W-05-099_310505_E1 \\
\hline W-05-101 & No Catch & & & & W-05-101_010605_E1 \\
\hline W-05-102 & Brook Stickleback & 23 & $38-53^{*}$ & & W-05-102_010605_E1 \\
\hline W-05-102 & Creek Chub & 1 & 123 & & W-05-102_010605_E1 \\
\hline W-05-102 & Fathead Minnow & 1 & 67 & & W-05-102_010605_E1 \\
\hline W-05-102 & Pearl Dace & 4 & $65-89$ & & W-05-102_010605_E1 \\
\hline W-05-103 & Brassy Minnow & 2 & 74,85 & & W-05-103_010605_E1 \\
\hline W-05-103 & Brook Stickleback & 57 & $27-54^{*}$ & & W-05-103_010605_E1 \\
\hline W-05-103 & Fathead Minnow & 21 & $39-68$ & & W-05-103_010605_E1 \\
\hline
\end{tabular}


Appendix 5: List of all fish captures made between 2002 and 2006, showing site number, common name and number (\#) of fish collected, fork (or total ${ }^{\star}$ ) length in millimetres ( $\mathrm{mm}$ ), any comments on the collection and the fish capture identification (ID) number.

\begin{tabular}{|c|c|c|c|c|c|}
\hline Site Number & Common Name & \# & $\begin{array}{c}\text { Fork/Total* } \\
\text { Length }(\mathrm{mm})\end{array}$ & Comments & $\begin{array}{l}\text { Fish Capture } \\
\text { ID Number }\end{array}$ \\
\hline W-05-103 & Finescale Dace & 25 & $34-77$ & $\begin{array}{l}\text { NOTE: some of these looked atypical. } \\
\text { Mainly the scales seemed too large for } \\
\text { any Phoxinus. However, lateral line was } \\
\text { incomplete; mouth \& pigmentation were } \\
\text { ok. }\end{array}$ & W-05-103_010605_E1 \\
\hline W-05-103 & Pearl Dace & 135 & $37-109$ & & W-05-103_010605_E1 \\
\hline W-05-104 & Brook Stickleback & 14 & $25-41^{*}$ & & W-05-104_010605_E1 \\
\hline W-05-104 & Common Shiner & 1 & 65 & & W-05-104_010605_E1 \\
\hline W-05-104 & Fathead Minnow & 1 & 42 & & W-05-104_010605_E1 \\
\hline W-05-104 & Finescale Dace & 51 & $33-63$ & & W-05-104_010605_E1 \\
\hline W-05-104 & Western Blacknose Dace & 3 & $42-83$ & & W-05-104_010605_E1 \\
\hline W-05-105 & Brook Stickleback & 8 & $30-57^{*}$ & & W-05-105_010605_E1 \\
\hline W-05-105 & Fathead Minnow & 2 & $29-37$ & & W-05-105_010605_E1 \\
\hline W-05-106 & No Catch & & & & W-05-106_020605_E1 \\
\hline W-05-110 & Brook Stickleback & 9 & $24-45^{*}$ & & W-05-110_040605_E1 \\
\hline W-05-110 & Fathead Minnow & 6 & $27-38$ & & W-05-110_040605_E1 \\
\hline W-05-112 & Black Bullhead & 1 & $190^{*}$ & Released & W-05-112_040605_E1 \\
\hline W-05-112 & Brook Stickleback & 1 & $51^{*}$ & & W-05-112_040605_E1 \\
\hline W-05-112 & Fathead Minnow & 249 & $21-71$ & & W-05-112_040605_E1 \\
\hline W-05-112 & Northern Pike & 1 & $400^{*}$ & Released & W-05-112_040605_E1 \\
\hline W-05-112 & White Sucker & 6 & $67-195^{*}$ & Released, 1 male, 1 female & W-05-112_040605_E1 \\
\hline W-05-113 & Brook Stickleback & 40 & $33-64^{*}$ & & W-05-113_060605_E1 \\
\hline W-05-113 & Central Mudminnow & 1 & $64^{*}$ & & W-05-113_060605_E1 \\
\hline W-05-113 & Iowa Darter & 6 & $33-49^{*}$ & & W-05-113_060605_E1 \\
\hline W-05-113 & Johnny Darter & 4 & $32-42^{*}$ & & W-05-113_060605_E1 \\
\hline
\end{tabular}


Appendix 5: List of all fish captures made between 2002 and 2006, showing site number, common name and number (\#) of fish collected, fork (or total ${ }^{\star}$ ) length in millimetres $(\mathrm{mm})$, any comments on the collection and the fish capture identification (ID) number.

\begin{tabular}{|c|c|c|c|c|c|}
\hline Site Number & Common Name & \# & $\begin{array}{c}\text { Fork/Total* } \\
\text { Length }(\mathrm{mm})\end{array}$ & Comments & $\begin{array}{l}\text { Fish Capture } \\
\text { ID Number }\end{array}$ \\
\hline W-05-114 & White Sucker & 82 & $10-15^{\star}$ & $\begin{array}{l}\text { Post-larvae, estimated length Note: } \\
\text { specimens in poor condition, limp and } \\
\text { fragile; overall slender, elongate body } \\
\text { form at a TL of about } 10-15 \mathrm{~mm} \text { is } \\
\text { consistent with Catostomidae; lower } \\
\text { portion of pharyngeal arch found on a } \\
\text { detached, smashed head- teeth consistent } \\
\text { with Catostomidae; dorsal ray count (2 } \\
\text { specimens) } 11 \text { in range of } C \text {. commersoni; } \\
\text { counted } 42 \text { \& } 44 \text { centra on } 2 \text { specimens - } \\
\text { a bit low for C. commersoni, but centra } \\
\text { may have been missed; terminal, oblique } \\
\text { mouth with protrusible premaxillae and } \\
\text { short lower jaw is consistent with larval } \\
\text { Catostomids. ID- Catostomidae with } \\
\text { confidence, C. commersoni on basis of } \\
\text { vertebral and dorsal ray counts. }\end{array}$ & W-05-114_060605_E1 \\
\hline W-05-115 & Brassy Minnow & 1 & 51 & & W-05-115_070605_E1 \\
\hline W-05-115 & Brook Stickleback & 7 & $34-48^{*}$ & & W-05-115_070605_E1 \\
\hline W-05-115 & Creek Chub & 18 & $30-54$ & & W-05-115_070605_E1 \\
\hline W-05-115 & Fathead Minnow & 3 & $39-53$ & & W-05-115_070605_E1 \\
\hline W-05-115 & Western Blacknose Dace & 2 & 40,74 & & W-05-115_070605_E1 \\
\hline W-05-116 & Fathead Minnow & 2 & 62,69 & & W-05-116_070605_E1 \\
\hline W-05-117 & Brassy Minnow & 3 & $79-93$ & & W-05-117_070605_E1 \\
\hline W-05-117 & Creek Chub & 5 & $59-126$ & Released 28 others & W-05-117_070605_E1 \\
\hline W-05-117 & Fathead Minnow & 3 & $64-78$ & Released 11 & W-05-117_070605_E1 \\
\hline W-05-117 & Finescale Dace & 3 & & Released & W-05-117_070605_E1 \\
\hline W-05-117 & Northern Redbelly Dace & 13 & & Released (red spawning colour) & W-05-117_070605_E1 \\
\hline W-05-117 & Western Blacknose Dace & 21 & $66-80$ & Released 11 & W-05-117_070605_E1 \\
\hline W-05-118 & Brassy Minnow & 1 & 48 & & W-05-118_080605_E1 \\
\hline W-05-118 & Brook Stickleback & 6 & $36-49^{*}$ & & W-05-118_080605_E1 \\
\hline W-05-118 & Common Shiner & 10 & $25-79$ & & W-05-118_080605_E1 \\
\hline
\end{tabular}


Appendix 5: List of all fish captures made between 2002 and 2006, showing site number, common name and number (\#) of fish collected, fork (or total ${ }^{\star}$ ) length in millimetres $(\mathrm{mm})$, any comments on the collection and the fish capture identification (ID) number.

\begin{tabular}{|c|c|c|c|c|c|}
\hline Site Number & Common Name & \# & $\begin{array}{c}\text { Fork/Total* } \\
\text { Length }(\mathrm{mm})\end{array}$ & Comments & $\begin{array}{l}\text { Fish Capture } \\
\text { ID Number }\end{array}$ \\
\hline W-05-118 & Creek Chub & 22 & $33-72$ & & W-05-118_080605_E1 \\
\hline W-05-118 & Fathead Minnow & 3 & $43-44$ & & W-05-118_080605_E1 \\
\hline W-05-118 & Johnny Darter & 2 & $37,39^{*}$ & & W-05-118_080605_E1 \\
\hline W-05-118 & Longnose Dace & 1 & 59 & & W-05-118_080605_E1 \\
\hline W-05-118 & Western Blacknose Dace & 61 & $25-72$ & & W-05-118_080605_E1 \\
\hline W-05-118 & White Sucker & 2 & 46,52 & & W-05-118_080605_E1 \\
\hline W-05-119 & Brook Stickleback & 216 & $25-50^{*}$ & & W-05-119_080605_E1 \\
\hline W-05-119 & Common Shiner & 1 & 34 & & W-05-119_080605_E1 \\
\hline W-05-119 & Creek Chub & 1 & 52 & & W-05-119_080605_E1 \\
\hline W-05-119 & Western Blacknose Dace & 3 & $34-89$ & & W-05-119_080605_E1 \\
\hline W-05-120 & No Catch & & & & W-05-120_090605_E1 \\
\hline W-05-121 & No Catch & & & & W-05-121_090605_E1 \\
\hline W-05-122 & Brook Stickleback & 5 & $36-52^{*}$ & & W-05-122_090605_E1 \\
\hline W-05-122 & Fathead Minnow & 24 & $39-63$ & & W-05-122_090605_E1 \\
\hline W-05-126 & No Catch & & & & W-05-126_140605_E1 \\
\hline W-05-128 & No Catch & & & & W-05-128_140605_E1 \\
\hline W-05-129 & Common Carp & 1 & 700 & Released & W-05-129_150605_E1 \\
\hline W-05-130 & No Catch & & & & W-05-130_150605_E1 \\
\hline W-05-130 & Northern Pike & 1 & & Observed & W-05-130_150605_01 \\
\hline W-05-131 & Brook Stickleback & 23 & $18-53^{*}$ & & W-05-131_150605_E1 \\
\hline W-05-131 & Central Mudminnow & 2 & $81-100^{\star}$ & & W-05-131_150605_E1 \\
\hline W-05-131 & Fathead Minnow & 4 & $30-66$ & & W-05-131_150605_E1 \\
\hline W-05-131 & Finescale Dace & 47 & $32-50$ & & W-05-131_150605_E1 \\
\hline W-05-131 & Northern Redbelly Dace & 334 & $30-45$ & $\begin{array}{l}\text { NOTE: There appears to be a lot of } \\
\text { morphological integration between } P \text {. eos } \\
\& P \text {. neogaeus in this sample. } \\
\text { Assignment to species was more or less } \\
\text { arbitrary in several specimens of each } \\
\text { spec. Is there anything (like obstruction of } \\
\text { fish passage) that might explain } \\
\text { hybridization here? }\end{array}$ & W-05-131_150605_E1 \\
\hline W-05-131 & White Sucker & 3 & $79-111$ & & W-05-131_150605_E1 \\
\hline
\end{tabular}


Appendix 5: List of all fish captures made between 2002 and 2006, showing site number, common name and number (\#) of fish collected, fork (or total ${ }^{\star}$ ) length in millimetres ( $\mathrm{mm}$ ), any comments on the collection and the fish capture identification (ID) number.

\begin{tabular}{|c|c|c|c|c|c|}
\hline Site Number & Common Name & \# & $\begin{array}{c}\text { Fork/Total* } \\
\text { Length }(\mathrm{mm})\end{array}$ & Comments & $\begin{array}{l}\text { Fish Capture } \\
\text { ID Number }\end{array}$ \\
\hline W-05-132 & Brook Stickleback & 4 & $23-53^{*}$ & & W-05-132_150605_E1 \\
\hline W-05-132 & Central Mudminnow & 1 & $57^{*}$ & & W-05-132_150605_E1 \\
\hline W-05-134 & Brook Stickleback & 2 & $22,31^{*}$ & & W-05-134_150605_E1 \\
\hline W-05-134 & Central Mudminnow & 1 & $98^{*}$ & & W-05-134_150605_E1 \\
\hline W-05-135 & Yellow Perch & 1 & & Observed & W-05-135_160605_01 \\
\hline W-05-136 & Brook Stickleback & 204 & $23-68^{*}$ & & W-05-136_160605_E1 \\
\hline W-05-136 & Central Mudminnow & 14 & $38-93^{*}$ & & W-05-136_160605_E1 \\
\hline W-05-136 & Finescale Dace & 3 & $43-50$ & & W-05-136_160605_E1 \\
\hline W-05-136 & Northern Redbelly Dace & 1 & 60 & & W-05-136_160605_E1 \\
\hline W-05-137 & Brook Stickleback & 63 & $36-68^{*}$ & & W-05-137_160605_E1 \\
\hline W-05-137 & Central Mudminnow & 23 & $38-97^{*}$ & & W-05-137_160605_E1 \\
\hline W-05-137 & Fathead Minnow & 2 & 51,66 & & W-05-137_160605_E1 \\
\hline W-05-137 & Finescale Dace & 11 & $30-60$ & & W-05-137_160605_E1 \\
\hline W-05-137 & Northern Redbelly Dace & 23 & $28-45$ & & W-05-137_160605_E1 \\
\hline W-05-138 & Brook Stickleback & 355 & $22-58^{*}$ & Dytiscid larvae, Caddis spp. cases & W-05-138_160605_E1 \\
\hline W-05-138 & Central Mudminnow & 153 & $41-103^{*}$ & & W-05-138_160605_E1 \\
\hline W-05-138 & Finescale Dace & 21 & $37-61$ & & W-05-138_160605_E1 \\
\hline W-05-138 & Northern Redbelly Dace & 2 & 40,49 & & W-05-138_160605_E1 \\
\hline W-05-139 & Brook Stickleback & 7 & $32-55^{*}$ & & W-05-139_170605_E1 \\
\hline W-05-139 & Fathead Minnow & 10 & $25-70$ & & W-05-139_170605_E1 \\
\hline W-05-139 & Finescale Dace & 1 & 42 & & W-05-139_170605_E1 \\
\hline W-05-139 & lowa Darter & 3 & $39-59^{*}$ & & W-05-139_170605_E1 \\
\hline W-05-139 & Northern Redbelly Dace & 42 & $24-56$ & & W-05-139_170605_E1 \\
\hline W-05-139 & Pearl Dace & 2 & 54,88 & & W-05-139_170605_E1 \\
\hline W-05-140 & Brook Stickleback & 54 & $17-60^{*}$ & & W-05-140_170605_E1 \\
\hline W-05-140 & Central Mudminnow & 66 & $36-86^{*}$ & & W-05-140_170605_E1 \\
\hline W-05-140 & Fathead Minnow & 17 & $23-42$ & & W-05-140_170605_E1 \\
\hline W-05-140 & Finescale Dace & 7 & $30-76$ & & W-05-140_170605_E1 \\
\hline W-05-140 & Northern Redbelly Dace & 394 & $25-61$ & & W-05-140_170605_E1 \\
\hline W-05-140 & Pearl Dace & 1 & 46 & & W-05-140_170605_E1 \\
\hline W-05-141 & No Catch & & & & W-05-141_200605_E1 \\
\hline
\end{tabular}


Appendix 5: List of all fish captures made between 2002 and 2006, showing site number, common name and number (\#) of fish collected, fork (or total ${ }^{*}$ ) length in millimetres $(\mathrm{mm})$, any comments on the collection and the fish capture identification (ID) number.

\begin{tabular}{|c|c|c|c|c|c|}
\hline Site Number & Common Name & \# & $\begin{array}{c}\text { Fork/Total* } \\
\text { Length }(\mathrm{mm})\end{array}$ & Comments & $\begin{array}{l}\text { Fish Capture } \\
\text { ID Number }\end{array}$ \\
\hline W-05-145 & Fathead Minnow & 20 & $43-67$ & & W-05-145_200605_E1 \\
\hline W-05-146 & Brook Stickleback & 18 & $20-61^{*}$ & & W-05-146_210605_E1 \\
\hline W-05-146 & Fathead Minnow & 144 & $35-63$ & & W-05-146_210605_E1 \\
\hline W-05-148 & Brook Stickleback & 54 & $21-48^{*}$ & & W-05-148_210605_E1 \\
\hline W-05-148 & Fathead Minnow & 9 & $46-77$ & & W-05-148_210605_E1 \\
\hline W-05-149 & Brook Stickleback & 83 & $30-62^{*}$ & & W-05-149_220605_E1 \\
\hline W-05-149 & Burbot & 43 & $38-67^{*}$ & & W-05-149_220605_E1 \\
\hline W-05-149 & Fathead Minnow & 34 & $32-74$ & & W-05-149_220605_E1 \\
\hline W-05-149 & Western Blacknose Dace & 1 & 55 & & W-05-149_220605_E1 \\
\hline W-05-150 & Brook Stickleback & 20 & $28-57^{*}$ & & W-05-150_220605_E1 \\
\hline W-05-150 & Burbot & 3 & $40-48^{*}$ & & W-05-150_220605_E1 \\
\hline W-05-150 & Fathead Minnow & 6 & $31-47$ & & W-05-150_220605_E1 \\
\hline W-05-150 & White Sucker & 1 & 155 & & W-05-150_220605_E1 \\
\hline W-05-151 & Brook Stickleback & 10 & $32-49^{*}$ & & W-05-151_220605_E1 \\
\hline W-05-151 & Burbot & 3 & $50-57^{*}$ & & W-05-151_220605_E1 \\
\hline W-05-151 & Creek Chub & 3 & $41-75$ & & W-05-151_220605_E1 \\
\hline W-05-151 & Fathead Minnow & 2 & 41,44 & & W-05-151_220605_E1 \\
\hline W-05-151 & Western Blacknose Dace & 7 & $34-44$ & & W-05-151_220605_E1 \\
\hline W-05-151 & White Sucker & 2 & 45,74 & & W-05-151_220605_E1 \\
\hline W-05-152 & Brassy Minnow & 1 & 38 & & W-05-152_220605_E1 \\
\hline W-05-152 & Brook Stickleback & 52 & $22-37^{*}$ & & W-05-152_220605_E1 \\
\hline W-05-152 & Burbot & 12 & $37-57^{*}$ & & W-05-152_220605_E1 \\
\hline W-05-152 & Common Shiner & 1 & 35 & & W-05-152_220605_E1 \\
\hline W-05-152 & Creek Chub & 10 & $41-61$ & & W-05-152_220605_E1 \\
\hline W-05-152 & Fathead Minnow & 3 & $35-38$ & & W-05-152_220605_E1 \\
\hline W-05-154 & No Catch & & & & W-05-154_220605_E1 \\
\hline W-05-157 & Brook Stickleback & 8 & $43-53^{*}$ & & W-05-157_230605_E1 \\
\hline W-05-157 & Fathead Minnow & 22 & $29-42$ & & W-05-157_230605_E1 \\
\hline W-05-159 & No Catch & & & & W-05-159_230605_E1 \\
\hline W-05-160 & No Catch & & & & W-05-160_230605_E1 \\
\hline W-05-161 & Brook Stickleback & 337 & $20-56^{*}$ & & W-05-161_230605_E1 \\
\hline
\end{tabular}


Appendix 5: List of all fish captures made between 2002 and 2006, showing site number, common name and number (\#) of fish collected, fork (or total ${ }^{*}$ ) length in millimetres $(\mathrm{mm})$, any comments on the collection and the fish capture identification (ID) number.

\begin{tabular}{|c|c|c|c|c|c|}
\hline Site Number & Common Name & \# & $\begin{array}{l}\text { Fork/Total* } \\
\text { Length }(\mathrm{mm})\end{array}$ & Comments & $\begin{array}{l}\text { Fish Capture } \\
\text { ID Number }\end{array}$ \\
\hline W-05-161 & Fathead Minnow & 5 & $30-56$ & & W-05-161_230605_E1 \\
\hline W-05-162 & Brook Stickleback & 1 & $19^{*}$ & & W-05-162_230605_E1 \\
\hline W-05-162 & White Sucker & 3 & $88-114$ & & W-05-162_230605_E1 \\
\hline W-05-163 & Brook Stickleback & 110 & $16-34^{*}$ & & W-05-163_240605_E1 \\
\hline W-05-164 & Blackside Darter & 2 & $58,70^{*}$ & & W-05-164_270605_E1 \\
\hline W-05-164 & Brook Stickleback & 4 & $21-40^{*}$ & & W-05-164_270605_E1 \\
\hline W-05-164 & Central Mudminnow & 1 & $122^{*}$ & & W-05-164_270605_E1 \\
\hline W-05-164 & Iowa Darter & 1 & $43^{*}$ & & W-05-164_270605_E1 \\
\hline W-05-164 & Northern Redbelly Dace & 12 & $29-50$ & & W-05-164_270605_E1 \\
\hline W-05-164 & Pearl Dace & 4 & $16-98$ & & W-05-164_270605_E1 \\
\hline W-05-165 & Brook Stickleback & 33 & $21-90^{*}$ & & W-05-165_270605_E1 \\
\hline W-05-165 & Central Mudminnow & 26 & $35-86^{*}$ & & W-05-165_270605_E1 \\
\hline W-05-165 & Finescale Dace & 1 & 42 & & W-05-165_270605_E1 \\
\hline W-05-165 & Northern Redbelly Dace & 4 & $34-52$ & & W-05-165_270605_E1 \\
\hline W-05-165 & Pearl Dace & 7 & $51-106$ & & W-05-165_270605_E1 \\
\hline W-05-166 & Brook Stickleback & 6 & $35-44^{*}$ & & W-05-166_270605_E1 \\
\hline W-05-166 & Central Mudminnow & 1 & $40^{*}$ & & W-05-166_270605_E1 \\
\hline W-05-166 & Fathead Minnow & 2 & 23,61 & & W-05-166_270605_E1 \\
\hline W-05-166 & Northern Redbelly Dace & 6 & $38-59$ & & W-05-166_270605_E1 \\
\hline W-05-166 & Pearl Dace & 2 & 65,67 & & W-05-166_270605_E1 \\
\hline W-05-167 & Central Mudminnow & 8 & $42-83$ & & W-05-167_280605_E1 \\
\hline W-05-167 & Northern Redbelly Dace & 4 & $24-38$ & $\begin{array}{l}\text { Look like } P \text {. eos, but possible hybrid with } \\
P \text {. neogaeus }\end{array}$ & W-05-167_280605_E1 \\
\hline W-05-168 & No Catch & & & & W-05-168_110705_E1 \\
\hline W-05-169 & Brook Stickleback & 111 & $21-67^{*}$ & & W-05-169_120705_E1 \\
\hline W-05-169 & Creek Chub & 5 & $94-139$ & & W-05-169_120705_E1 \\
\hline W-05-169 & Fathead Minnow & 101 & $51-73$ & & W-05-169_120705_E1 \\
\hline W-05-169 & Iowa Darter & 1 & $47^{*}$ & & W-05-169_120705_E1 \\
\hline W-05-169 & Rainbow Trout & 1 & 240 & Released & W-05-169_120705_E1 \\
\hline W-05-169 & White Sucker & 4 & $103-201$ & & W-05-169_120705_E1 \\
\hline W-05-170 & Brook Stickleback & 4 & $37-48^{*}$ & & W-05-170_120705_D1 \\
\hline
\end{tabular}


Appendix 5: List of all fish captures made between 2002 and 2006, showing site number, common name and number (\#) of fish collected, fork (or total ${ }^{\star}$ ) length in millimetres ( $\mathrm{mm}$ ), any comments on the collection and the fish capture identification (ID) number.

\begin{tabular}{|c|c|c|c|c|c|}
\hline Site Number & Common Name & \# & $\begin{array}{c}\text { Fork/Total* } \\
\text { Length }(\mathrm{mm})\end{array}$ & Comments & $\begin{array}{l}\text { Fish Capture } \\
\text { ID Number }\end{array}$ \\
\hline W-05-171 & Brassy Minnow & 1 & 61 & & W-05-171_120705_E1 \\
\hline W-05-171 & Burbot & 4 & $52-54^{*}$ & & W-05-171_120705_E1 \\
\hline W-05-171 & Common Shiner & 10 & $35-69$ & & W-05-171_120705_E1 \\
\hline W-05-171 & Creek Chub & 14 & $77-135$ & & W-05-171_120705_E1 \\
\hline W-05-171 & Fathead Minnow & 21 & $47-75$ & & W-05-171_120705_E1 \\
\hline W-05-171 & Johnny Darter & 6 & $35-60^{*}$ & & W-05-171_120705_E1 \\
\hline W-05-171 & White Sucker & 3 & $66-80$ & & W-05-171_120705_E1 \\
\hline W-05-173 & Brook Stickleback & 3 & $30-35^{*}$ & & W-05-173_120705_E1 \\
\hline W-05-173 & Common Shiner & 7 & $37-80$ & & W-05-173_120705_E1 \\
\hline W-05-173 & Creek Chub & 3 & 54-183 & & W-05-173_120705_E1 \\
\hline W-05-173 & Fathead Minnow & 1 & 54 & & W-05-173_120705_E1 \\
\hline W-05-173 & Pearl Dace & 2 & 64,68 & & W-05-173_120705_E1 \\
\hline W-05-173 & Western Blacknose Dace & 2 & 42 & & W-05-173_120705_E1 \\
\hline W-05-173 & White Sucker & 1 & 57 & & W-05-173_120705_E1 \\
\hline W-05-176 & No Catch & & & & W-05-176_180705_E1 \\
\hline W-05-176 & Northern Pike & 1 & & & W-05-176_180705_O1 \\
\hline W-05-177 & Central Mudminnow & 2 & $70,86^{*}$ & & W-05-177_190705_E1 \\
\hline W-05-177 & Northern Pike & 2 & 154,158 & Observed several others & W-05-177_190705_E1 \\
\hline W-05-178 & Brook Stickleback & 128 & $23-54^{*}$ & & W-05-178_190705_E1 \\
\hline W-05-178 & Central Mudminnow & 19 & $35-94^{*}$ & & W-05-178_190705_E1 \\
\hline W-05-178 & White Sucker & 5 & $52-139$ & & W-05-178_190705_E1 \\
\hline W-05-180 & Brook Stickleback & 17 & $30-40^{*}$ & & W-05-180_190705_E1 \\
\hline W-05-180 & Central Mudminnow & 1 & $89^{*}$ & Invertebrate sample & W-05-180_190705_E1 \\
\hline W-05-180 & Western Blacknose Dace & 1 & 30 & & W-05-180_190705_E1 \\
\hline W-05-180 & White Sucker & 16 & $37^{*}-107$ & & W-05-180_190705_E1 \\
\hline W-05-181 & Brook Stickleback & 42 & $25-51^{*}$ & & W-05-181_190705_E1 \\
\hline W-05-181 & Central Mudminnow & 6 & $29-83^{*}$ & Invertebrate sample & W-05-181_190705_E1 \\
\hline W-05-182 & Central Mudminnow & 4 & $63-80^{*}$ & & W-05-182_190705_E1 \\
\hline W-05-182 & Northern Pike & 5 & $118-137$ & $>20$ others observed but not collected & W-05-182_190705_E1 \\
\hline W-05-183 & Brook Stickleback & 11 & $22-65^{*}$ & & W-05-183_190705_E1 \\
\hline W-05-183 & Common Carp & 30 & $34-57$ & All YOY & W-05-183_190705_E1 \\
\hline
\end{tabular}


Appendix 5: List of all fish captures made between 2002 and 2006, showing site number, common name and number (\#) of fish collected, fork (or total ${ }^{\star}$ ) length in millimetres ( $\mathrm{mm}$ ), any comments on the collection and the fish capture identification (ID) number.

\begin{tabular}{|c|c|c|c|c|c|}
\hline Site Number & Common Name & $\#$ & $\begin{array}{l}\text { Fork/Total }^{*} \\
\text { Length }(\mathrm{mm})\end{array}$ & Comments & $\begin{array}{l}\text { Fish Capture } \\
\text { ID Number }\end{array}$ \\
\hline W-05-185 & Brook Stickleback & 4 & $23-27^{*}$ & & W-05-185_200705 E1 \\
\hline W-05-185 & Central Mudminnow & 8 & $36-81^{*}$ & & W-05-185_200705_E1 \\
\hline W-05-187 & No Catch & & & & W-05-187_250705_E1 \\
\hline W-05-188 & White Sucker & 7 & $41-46$ & YOY, released & W-05-188 250705 E1 \\
\hline W-05-189 & Northern Pike & 2 & $145-340$ & Released 1 & W-05-189_260705 B1 \\
\hline W-05-189 & White Sucker & 2 & $41-46$ & Released 19 & W-05-189_260705_B1 \\
\hline W-05-190 & No Catch & & & & W-05-190_260705_E1 \\
\hline W-05-190 & Northern Pike & 1 & & Observed & W-05-190_260705_01 \\
\hline W-05-191 & Brook Stickleback & 62 & $23-45^{*}$ & & W-05-191_260705E1 \\
\hline W-05-191 & Central Mudminnow & 32 & $38-103^{*}$ & & W-05-191_260705_E1 \\
\hline W-05-191 & Common Carp & 8 & $35-77$ & & W-05-191_260705_E1 \\
\hline W-05-191 & Fathead Minnow & 3 & $27-30$ & & W-05-191_260705_E1 \\
\hline W-05-192 & No Catch & & & & W-05-192_260705E1 \\
\hline W-05-193 & Central Mudminnow & 1 & $47^{*}$ & & W-05-193_260705_E1 \\
\hline W-05-193 & Northern Pike & 1 & 170 & & W-05-193_260705_E1 \\
\hline W-05-193 & White Sucker & 1 & 45 & & W-05-193 $260705 \mathrm{E} 1$ \\
\hline W-05-194 & Burbot & 1 & $107^{*}$ & & W-05-194_270705_E1 \\
\hline W-05-194 & Central Mudminnow & 2 & $45^{\star}$ & & W-05-194_270705_E1 \\
\hline W-05-194 & White Sucker & 1 & 50 & & W-05-194_270705_E1 \\
\hline W-05-196 & Brook Stickleback & 25 & $24-44^{*}$ & & W-05-196_270705_E1 \\
\hline W-05-196 & Finescale Dace & 13 & $34-61$ & & W-05-196_270705_E1 \\
\hline W-05-196 & Longnose Dace & 1 & 70 & & W-05-196_270705_E1 \\
\hline W-05-196 & Pearl Dace & 46 & 40,113 & & W-05-196 $270705 \mathrm{E} 1$ \\
\hline W-05-196 & White Sucker & 2 & 151,221 & & W-05-196_270705_E1 \\
\hline W-05-200 & Brook Stickleback & 1 & $38^{*}$ & & W-05-200_020805_E1 \\
\hline W-05-200 & Fathead Minnow & 3 & $33-55$ & & W-05-200_020805_E1 \\
\hline W-05-201 & Brook Stickleback & 2 & $37-49^{*}$ & & W-05-201_020805_E1 \\
\hline W-05-201 & Common Carp & 16 & $23-75$ & & W-05-201_020805E1 \\
\hline W-05-201 & Fathead Minnow & 3 & $23-43$ & & W-05-201_020805_E1 \\
\hline W-05-202 & Common Carp & 4 & $22-47$ & & W-05-202_020805_E1 \\
\hline W-05-202 & Fathead Minnow & 1 & 24 & & W-05-202_020805_E1 \\
\hline
\end{tabular}


Appendix 5: List of all fish captures made between 2002 and 2006, showing site number, common name and number (\#) of fish collected, fork (or total ${ }^{\star}$ ) length in millimetres ( $\mathrm{mm}$ ), any comments on the collection and the fish capture identification (ID) number.

\begin{tabular}{|c|c|c|c|c|c|}
\hline Site Number & Common Name & \# & $\begin{array}{c}\text { Fork/Total* } \\
\text { Length }(\mathrm{mm})\end{array}$ & Comments & $\begin{array}{l}\text { Fish Capture } \\
\text { ID Number }\end{array}$ \\
\hline W-05-203 & Brook Stickleback & & & & W-05-203_030805_E1 \\
\hline W-05-203 & Central Mudminnow & 4 & $34-61^{*}$ & & W-05-203_030805_E1 \\
\hline W-05-203 & Fathead Minnow & 2 & 26,33 & & W-05-203_030805_E1 \\
\hline W-05-203 & Finescale Dace & 2 & 19,30 & & W-05-203_030805_E1 \\
\hline W-05-203 & Johnny Darter & 11 & $20-31^{*}$ & & W-05-203_030805_E1 \\
\hline W-05-203 & Northern Pike & 1 & 116 & & W-05-203_030805_E1 \\
\hline W-05-203 & Northern Pike & 4 & $300-460$ & Observed & W-05-203_030805_01 \\
\hline W-05-203 & White Sucker & 16 & $31-37$ & & W-05-203_030805_E1 \\
\hline W-05-205 & Brook Stickleback & 16 & $28-45^{*}$ & & W-05-205_030805_E1 \\
\hline W-05-205 & Central Mudminnow & 20 & $54-84^{*}$ & & W-05-205_030805_E1 \\
\hline W-05-205 & Johnny Darter & 6 & $48-55^{*}$ & 5 Caddis fly larvae, 1 snail & W-05-205_030805_E1 \\
\hline W-05-205 & Northern Pike & 1 & 123 & & W-05-205_030805_E1 \\
\hline W-05-205 & White Sucker & 1 & 165 & & W-05-205_030805_E1 \\
\hline W-05-207 & Bigmouth Buffalo & 2 & $56-85^{*}$ & & W-05-207_080805_E1 \\
\hline W-05-207 & Black Bullhead & 23 & $33-48^{*}$ & & W-05-207_080805_E1 \\
\hline W-05-207 & Burbot & 35 & $66-115^{*}$ & & W-05-207_080805_E1 \\
\hline W-05-207 & Common Carp & 95 & $49-153$ & & W-05-207_080805_E1 \\
\hline W-05-207 & Creek Chub & 11 & $72-99$ & & W-05-207_080805_E1 \\
\hline W-05-207 & Fathead Minnow & 55 & $19-64$ & & W-05-207_080805_E1 \\
\hline W-05-207 & White Sucker & 1 & 79 & & W-05-207_080805_E1 \\
\hline W-05-208 & Brook Stickleback & 26 & $27-54^{*}$ & & W-05-208_100805_E1 \\
\hline W-05-208 & Central Mudminnow & 5 & $36-65^{*}$ & & W-05-208_100805_E1 \\
\hline W-05-208 & Finescale Dace & 6 & $29-51$ & & W-05-208_100805_E1 \\
\hline W-05-208 & Northern Redbelly Dace & 3 & $26-58$ & & W-05-208_100805_E1 \\
\hline W-05-208 & Pearl Dace & 35 & $32-109$ & & W-05-208_100805_E1 \\
\hline W-05-209 & Brook Stickleback & 73 & $24-56^{*}$ & & W-05-209_100805_E1 \\
\hline W-05-209 & Central Mudminnow & 48 & $48-95^{*}$ & & W-05-209_100805_E1 \\
\hline W-05-209 & Finescale Dace & 2 & $30-56$ & & W-05-209_100805_E1 \\
\hline W-05-209 & Iowa Darter & 1 & $49^{*}$ & & W-05-209_100805_E1 \\
\hline W-05-209 & Northern Redbelly Dace & 21 & $20-51$ & & W-05-209_100805_E1 \\
\hline W-05-209 & Pearl Dace & 22 & $40-69$ & & W-05-209_100805_E1 \\
\hline
\end{tabular}


Appendix 5: List of all fish captures made between 2002 and 2006, showing site number, common name and number (\#) of fish collected, fork (or total ${ }^{\star}$ ) length in millimetres ( $\mathrm{mm}$ ), any comments on the collection and the fish capture identification (ID) number.

\begin{tabular}{|c|c|c|c|c|c|}
\hline Site Number & Common Name & \# & $\begin{array}{l}\text { Fork/Total* } \\
\text { Length }(\mathrm{mm})\end{array}$ & Comments & $\begin{array}{l}\text { Fish Capture } \\
\text { ID Number }\end{array}$ \\
\hline $\mathrm{X}-04-001$ & Black Bullhead & 8 & & Released 6 & X-04-001_050504_B1 \\
\hline $\mathrm{X}-05-001$ & No Catch & & & 1 Orconectes virilis & X-05-001_270405_B1 \\
\hline $\mathrm{X}-05-001$ & No Catch & & & & X-05-001_270405_K1 \\
\hline X-05-002 & Brook Stickleback & 608 & $26-53^{*}$ & & X-05-002_270405_B1 \\
\hline $\mathrm{X}-05-002$ & Fathead Minnow & 56 & $29-43$ & & X-05-002_270405_B1 \\
\hline $\mathrm{X}-05-002$ & Finescale Dace & 9 & $30-35$ & & X-05-002_270405_B1 \\
\hline $\mathrm{X}-05-002$ & Northern Redbelly Dace & 149 & $23-40$ & & X-05-002_270405_B1 \\
\hline $\mathrm{X}-05-003$ & Black Bullhead & 1 & $127^{*}$ & & X-05-003_290405_E1 \\
\hline $\mathrm{X}-05-003$ & Brook Stickleback & 3 & $39-55^{*}$ & & X-05-003_290405_E1 \\
\hline $\mathrm{X}-05-003$ & Fathead Minnow & 1 & 61 & & X-05-003_290405_E1 \\
\hline X-05-005 & Fathead Minnow & 1 & 38 & & X-05-005_290405_E1 \\
\hline $\mathrm{X}-05-007$ & Fathead Minnow & 19 & $28-62$ & & X-05-007_290405_E1 \\
\hline$X-05-007$ & White Sucker & 3 & $240-480$ & $\begin{array}{l}\text { Released, } 2 \text { females }(240-440 \mathrm{~mm}) ; 1 \\
\text { male }(480 \mathrm{~mm}) \text { post spawn, Sucker eggs } \\
\text { collected }\end{array}$ & X-05-007_290405_E1 \\
\hline $\mathrm{X}-05-007$ & White Sucker & 30 & & Observed in culvert & X-05-007_290405_01 \\
\hline $\mathrm{X}-05-011$ & Fathead Minnow & 1 & 22 & & X-05-011_030505_B1 \\
\hline$X-05-012$ & Brook Stickleback & 1 & $21^{*}$ & & $\mathrm{X}-05-012 \_030505$ B1 \\
\hline $\mathrm{X}-05-012$ & Fathead Minnow & 101 & $25-43$ & & $\mathrm{X}-05-012 \_030505 \_\mathrm{B} 1$ \\
\hline $\mathrm{X}-05-015$ & No Catch & & & & $\mathrm{X}-05-015 \_030505$ E1 \\
\hline$X-05-018$ & Brook Stickleback & 24 & $29-59^{*}$ & & X-05-018_040505_E1 \\
\hline X-05-018 & Central Mudminnow & 4 & $54-92^{*}$ & Released 1 & X-05-018 040505 E1 \\
\hline $\mathrm{X}-05-018$ & Fathead Minnow & 8 & $28-74$ & & $\mathrm{X}-05-018 \_040505$ E1 \\
\hline $\mathrm{X}-05-018$ & White Sucker & 15 & $56-58$ & $\begin{array}{l}\text { Released } 12,134 \mathrm{~mm} \text { is an immature } \\
\text { male, } 19 \text { are YOY }\end{array}$ & X-05-018_040505_E1 \\
\hline X-05-018 & White Sucker & 30 & & Observed in culvert & X-05-018_040505_01 \\
\hline $\mathrm{X}-05-020$ & No Catch & & & & X-05-020_040505_E1 \\
\hline $\mathrm{X}-05-021$ & White Sucker & 1 & 480 & Released & X-05-021_040505_E1 \\
\hline $\mathrm{X}-05-021$ & White Sucker & 2 & & $\begin{array}{l}\text { Congregated on spawning riffle upstream } \\
\text { of barrier }\end{array}$ & X-05-021_040505_01 \\
\hline $\mathrm{X}-05-022$ & No Catch & & & & X-05-022_040505_E1 \\
\hline
\end{tabular}


Appendix 5: List of all fish captures made between 2002 and 2006, showing site number, common name and number (\#) of fish collected, fork (or total ${ }^{*}$ ) length in millimetres $(\mathrm{mm})$, any comments on the collection and the fish capture identification (ID) number.

\begin{tabular}{|c|c|c|c|c|c|}
\hline Site Number & Common Name & \# & $\begin{array}{l}\text { Fork } / \text { Total } \\
\text { Length }(\mathrm{mm})\end{array}$ & Comments & $\begin{array}{l}\text { Fish Capture } \\
\text { ID Number }\end{array}$ \\
\hline $\mathrm{X}-05-022$ & No Catch & & & & X-05-022_040505_B1 \\
\hline $\mathrm{X}-05-023$ & Brook Stickleback & 3 & $40-46^{*}$ & & X-05-023_040505_E1 \\
\hline $\mathrm{X}-05-023$ & Fathead Minnow & 4 & $40-46$ & & X-05-023_040505_E1 \\
\hline $\mathrm{X}-05-023$ & No Catch & & & & X-05-023_040505_K1 \\
\hline $\mathrm{X}-05-024$ & Brook Stickleback & 6 & $38-53^{*}$ & & X-05-024_050505_E1 \\
\hline $\mathrm{X}-05-024$ & Central Mudminnow & 4 & $59-82^{*}$ & & X-05-024_050505_E1 \\
\hline $\mathrm{X}-05-024$ & Fathead Minnow & 24 & $28-66$ & & X-05-024_050505_E1 \\
\hline $\mathrm{X}-05-024$ & No Catch & & & & X-05-024_050505_K1 \\
\hline$X-05-024$ & White Sucker & 30 & & $\begin{array}{l}\text { Adult White Suckers observed } \\
\text { congregated on riffle }\end{array}$ & X-05-024_050505_01 \\
\hline $\mathrm{X}-05-026$ & Brook Stickleback & 8 & $30-47^{*}$ & & X-05-026_050505_E1 \\
\hline $\mathrm{X}-05-026$ & Central Mudminnow & 2 & $44,55^{\star}$ & & X-05-026_050505_E1 \\
\hline $\mathrm{X}-05-026$ & Fathead Minnow & 3 & $34-37$ & & X-05-026_050505_E1 \\
\hline $\mathrm{X}-05-027$ & Brook Stickleback & 1 & $50^{*}$ & & X-05-027_060505_E1 \\
\hline $\mathrm{X}-05-027$ & Central Mudminnow & 7 & $44-66^{*}$ & & X-05-027_060505_E1 \\
\hline $\mathrm{X}-05-027$ & Fathead Minnow & 8 & $26-52$ & & X-05-027_060505_E1 \\
\hline $\mathrm{X}-05-027$ & Johnny Darter & 1 & $42^{*}$ & & X-05-027_060505_E1 \\
\hline $\mathrm{X}-05-028$ & Black Bullhead & 1 & $122^{*}$ & & X-05-028_060505_E1 \\
\hline $\mathrm{X}-05-028$ & Brook Stickleback & 2 & $38,47^{*}$ & & X-05-028_060505_E1 \\
\hline$X-05-028$ & Central Mudminnow & 4 & $44-54^{*}$ & & X-05-028_060505_E1 \\
\hline $\mathrm{X}-05-028$ & Fathead Minnow & 10 & $27-48$ & & X-05-028_060505_E1 \\
\hline $\mathrm{X}-05-028$ & Northern Redbelly Dace & 3 & $25-28$ & & X-05-028_060505_E1 \\
\hline$X-05-029$ & Black Bullhead & 1 & $105^{*}$ & & X-05-029_060505_E1 \\
\hline $\mathrm{X}-05-029$ & Brook Stickleback & 7 & $38-54^{*}$ & & X-05-029_060505_E1 \\
\hline $\mathrm{X}-05-029$ & Central Mudminnow & 5 & $53-89^{*}$ & & X-05-029_060505_E1 \\
\hline $\mathrm{X}-05-029$ & Fathead Minnow & 7 & $26-66$ & & X-05-029_060505_E1 \\
\hline$X-05-029$ & Johnny Darter & 2 & $48,65^{\star}$ & & X-05-029_060505_E1 \\
\hline$X-05-029$ & Northern Redbelly Dace & 9 & $28-40$ & & X-05-029_060505_E1 \\
\hline $\mathrm{X}-05-030$ & No Catch & & & & X-05-030_100505_E1 \\
\hline $\mathrm{X}-05-031$ & Brook Stickleback & 9 & $48-65^{*}$ & & X-05-031_100505_E1 \\
\hline $\mathrm{X}-05-031$ & Central Mudminnow & 5 & $53-65^{*}$ & & X-05-031_100505_E1 \\
\hline
\end{tabular}


Appendix 5: List of all fish captures made between 2002 and 2006, showing site number, common name and number (\#) of fish collected, fork (or total ${ }^{\star}$ ) length in millimetres ( $\mathrm{mm}$ ), any comments on the collection and the fish capture identification (ID) number.

\begin{tabular}{|c|c|c|c|c|c|}
\hline Site Number & Common Name & \# & $\begin{array}{l}\text { Fork/Total* } \\
\text { Length }(\mathrm{mm})\end{array}$ & Comments & $\begin{array}{l}\text { Fish Capture } \\
\text { ID Number }\end{array}$ \\
\hline $\mathrm{X}-05-031$ & Fathead Minnow & 5 & $29-68$ & & X-05-031_100505_E1 \\
\hline $\mathrm{X}-05-032$ & No Catch & & & & X-05-032_110505_E1 \\
\hline$X-05-033$ & No Catch & & & & X-05-033_110505_E1 \\
\hline $\mathrm{X}-05-034$ & Central Mudminnow & 1 & $71^{*}$ & & X-05-034_110505_E1 \\
\hline $\mathrm{X}-05-035$ & Brook Stickleback & 2 & $30^{*}$ & & X-05-035_110505_E1 \\
\hline $\mathrm{X}-05-035$ & Iowa Darter & 1 & $44^{*}$ & & X-05-035_110505_E1 \\
\hline$X-05-036$ & Central Mudminnow & 1 & & Released & X-05-036_110505_E1 \\
\hline X-05-036 & White Sucker & 100's & & $\begin{array}{l}\text { Observed many dead (killed by humans) } \\
\text { along bank }\end{array}$ & X-05-036_110505_01 \\
\hline $\mathrm{X}-05-037$ & No Catch & & & & X-05-037_120505_E1 \\
\hline$X-05-037$ & No Catch & & & & $\mathrm{X}-05-037 \_120505 \_\mathrm{K} 1$ \\
\hline $\mathrm{X}-05-038$ & Brook Stickleback & 3 & $45-58^{*}$ & & X-05-038_120505_E1 \\
\hline $\mathrm{X}-05-038$ & Central Mudminnow & 1 & $96^{*}$ & & X-05-038_120505_E1 \\
\hline$X-05-038$ & Fathead Minnow & 3 & $42-50$ & & X-05-038_120505_E1 \\
\hline X-05-038 & White Sucker & 1 & 220 & Released & X-05-038_120505_E1 \\
\hline $\mathrm{X}-05-040$ & No Catch & & & & X-05-040_120505_E1 \\
\hline$X-05-042$ & Brook Stickleback & 2 & $31,41^{*}$ & & X-05-042_120505_E1 \\
\hline $\mathrm{X}-05-042$ & Central Mudminnow & 2 & $45,50^{*}$ & One additional released & X-05-042_120505_E1 \\
\hline $\mathrm{X}-05-042$ & Fathead Minnow & 2 & 26,29 & & X-05-042 120505 E1 \\
\hline$X-05-042$ & Finescale Dace & 4 & $22-35$ & & X-05-042_120505_E1 \\
\hline $\mathrm{X}-05-042$ & White Sucker & 1 & 220 & Released & X-05-042_120505_E1 \\
\hline $\mathrm{X}-05-044$ & No Catch & & & & X-05-044_130505_E1 \\
\hline$X-05-045$ & Blackside Darter & 2 & $77^{*}$ & & X-05-045_130505_E1 \\
\hline $\mathrm{X}-05-045$ & Brook Stickleback & 1 & $42^{*}$ & & X-05-045_130505_E1 \\
\hline $\mathrm{X}-05-045$ & Central Mudminnow & 1 & $56^{*}$ & & X-05-045_130505_E1 \\
\hline$X-05-045$ & Longnose Dace & 4 & $65-78$ & & X-05-045_130505_E1 \\
\hline $\mathrm{X}-05-046$ & Brook Stickleback & 2 & $37,38^{*}$ & & X-05-046_130505_E1 \\
\hline $\mathrm{X}-05-046$ & Central Mudminnow & 5 & $43-59^{*}$ & & X-05-046_130505_E1 \\
\hline$X-05-046$ & Fathead Minnow & 2 & 25,29 & & X-05-046_130505_E1 \\
\hline $\mathrm{X}-05-046$ & No Catch & & & & X-05-046_130505_B1 \\
\hline X-05-048 & Brook Stickleback & 1 & $58^{*}$ & & X-05-048_140505_E1 \\
\hline
\end{tabular}


Appendix 5: List of all fish captures made between 2002 and 2006, showing site number, common name and number (\#) of fish collected, fork (or total ${ }^{\star}$ ) length in millimetres ( $\mathrm{mm}$ ), any comments on the collection and the fish capture identification (ID) number.

\begin{tabular}{|c|c|c|c|c|c|}
\hline Site Number & Common Name & \# & $\begin{array}{l}\text { Fork/Total* } \\
\text { Length (mm) }\end{array}$ & Comments & $\begin{array}{l}\text { Fish Capture } \\
\text { ID Number }\end{array}$ \\
\hline $\mathrm{X}-05-048$ & Central Mudminnow & 1 & $59^{*}$ & & X-05-048_140505_E1 \\
\hline $\mathrm{X}-05-048$ & Fathead Minnow & 15 & $27-68$ & & X-05-048_140505_E1 \\
\hline $\mathrm{X}-05-051$ & No Catch & & & & $\mathrm{X}-05-051 \_140505 \_\mathrm{K} 1$ \\
\hline $\mathrm{X}-05-051$ & No Catch & & & & X-05-051_140505_E1 \\
\hline $\mathrm{X}-05-052$ & Brook Stickleback & 2 & $43,57^{*}$ & & X-05-052_140505_E1 \\
\hline $\mathrm{X}-05-052$ & Central Mudminnow & 2 & $68,69^{*}$ & & X-05-052_140505_E1 \\
\hline $\mathrm{X}-05-052$ & Fathead Minnow & 6 & $31-60$ & & X-05-052_140505_E1 \\
\hline $\mathrm{X}-05-054$ & Brook Stickleback & 1 & & Released & X-05-054_150505_E1 \\
\hline $\mathrm{X}-05-055$ & No Catch & & & & X-05-055_150505_E1 \\
\hline$X-05-057$ & No Catch & & & & X-05-057_150505_E1 \\
\hline $\mathrm{X}-05-060$ & Central Mudminnow & 1 & & Released & X-05-060_160505_E1 \\
\hline $\mathrm{X}-05-061$ & Central Mudminnow & 1 & $70^{*}$ & & X-05-061_160505_E1 \\
\hline $\mathrm{X}-05-062$ & No Catch & & & & $\mathrm{X}-05-062 \_160505 \_\mathrm{E} 1$ \\
\hline $\mathrm{X}-05-062$ & No Catch & & & & X-05-062_160505_K1 \\
\hline $\mathrm{X}-05-064$ & Central Mudminnow & 2 & $42,43^{*}$ & Released $1(70 \mathrm{~mm})$ & X-05-064_160505_E1 \\
\hline $\mathrm{X}-05-066$ & Brook Stickleback & 4 & $38-43^{*}$ & & X-05-066_170505_E1 \\
\hline $\mathrm{X}-05-066$ & Fathead Minnow & 2 & 48,57 & & X-05-066_170505_E1 \\
\hline $\mathrm{X}-05-067$ & No Catch & & & & X-05-067_170505_E1 \\
\hline$X-05-067$ & No Catch & & & & X-05-067_170505_L1 \\
\hline $\mathrm{X}-05-069$ & Brook Stickleback & 271 & $29-66^{*}$ & & X-05-069_170505_E1 \\
\hline $\mathrm{X}-05-069$ & Fathead Minnow & 24 & $37-68$ & & X-05-069_170505_E1 \\
\hline $\mathrm{X}-05-069$ & Finescale Dace & 29 & $29-53$ & & X-05-069_170505_E1 \\
\hline $\mathrm{X}-05-069$ & Northern Redbelly Dace & 8 & $36-43$ & & X-05-069_170505_E1 \\
\hline $\mathrm{X}-05-070$ & Brook Stickleback & 4 & $21-49^{*}$ & & X-05-070_180505_E1 \\
\hline $\mathrm{X}-05-070$ & Finescale Dace & 1 & 41 & & X-05-070_180505_E1 \\
\hline $\mathrm{X}-05-070$ & No Catch & & & & X-05-070_180505_L1 \\
\hline $\mathrm{X}-05-071$ & Brook Stickleback & 3 & $50-52^{*}$ & & X-05-071_180505_E1 \\
\hline $\mathrm{X}-05-072$ & Brook Stickleback & 14 & $30-47^{*}$ & & X-05-072_180505_E1 \\
\hline $\mathrm{X}-05-072$ & Fathead Minnow & 5 & $22-33$ & & X-05-072_180505_E1 \\
\hline $\mathrm{X}-05-079$ & Brook Stickleback & 4 & $45-60^{*}$ & & X-05-079_190505_B1 \\
\hline $\mathrm{X}-05-079$ & Fathead Minnow & 1 & 42 & & X-05-079_190505_B1 \\
\hline
\end{tabular}


Appendix 5: List of all fish captures made between 2002 and 2006, showing site number, common name and number (\#) of fish collected, fork (or total ${ }^{\star}$ ) length in millimetres ( $\mathrm{mm}$ ), any comments on the collection and the fish capture identification (ID) number.

\begin{tabular}{|c|c|c|c|c|c|}
\hline Site Number & Common Name & \# & $\begin{array}{c}\text { Fork/Total* } \\
\text { Length }(\mathrm{mm})\end{array}$ & Comments & $\begin{array}{l}\text { Fish Capture } \\
\text { ID Number }\end{array}$ \\
\hline $\mathrm{X}-05-080$ & Brook Stickleback & 3 & $49^{*}$ & & X-05-080_190505_E1 \\
\hline $\mathrm{X}-05-080$ & Fathead Minnow & 1 & 40 & & X-05-080_190505_E1 \\
\hline $\mathrm{X}-05-081$ & No Catch & & & & X-05-081_190505_E1 \\
\hline $\mathrm{X}-05-083$ & No Catch & & & & X-05-083_190505_E1 \\
\hline $\mathrm{X}-05-084$ & Brook Stickleback & 63 & $32-55^{*}$ & & X-05-084_200505_E1 \\
\hline $\mathrm{X}-05-084$ & Central Mudminnow & 4 & $54-62^{*}$ & & X-05-084_200505_E1 \\
\hline $\mathrm{X}-05-084$ & Fathead Minnow & 1 & 29 & & X-05-084_200505_E1 \\
\hline $\mathrm{X}-05-085$ & Central Mudminnow & 2 & $59,64^{*}$ & & X-05-085_200505_D1 \\
\hline $\mathrm{X}-05-085$ & No Catch & & & & X-05-085_200505_E1 \\
\hline $\mathrm{X}-05-086$ & No Catch & & & & X-05-086_200505_E1 \\
\hline $\mathrm{X}-05-088$ & Brook Stickleback & 35 & $34-60^{*}$ & Invertebrate sample & X-05-088_240505_E1 \\
\hline $\mathrm{X}-05-088$ & Central Mudminnow & 4 & $55-92^{*}$ & & X-05-088_240505_E1 \\
\hline $\mathrm{X}-05-088$ & Fathead Minnow & 4 & $58-71$ & & X-05-088_240505_E1 \\
\hline $\mathrm{X}-05-088$ & White Sucker & 2 & 55,150 & & X-05-088_240505_E1 \\
\hline $\mathrm{X}-05-089$ & Brook Stickleback & 15 & $29-53^{*}$ & & X-05-089_250505_D1 \\
\hline $\mathrm{X}-05-089$ & Central Mudminnow & 1 & $48^{*}$ & & X-05-089_250505_D1 \\
\hline $\mathrm{X}-05-091$ & Central Mudminnow & 13 & $57-85^{*}$ & & X-05-091_250505_E1 \\
\hline $\mathrm{X}-05-093$ & Fathead Minnow & 3 & $54-70$ & & X-05-093_270505_B1 \\
\hline $\mathrm{X}-05-094$ & Brook Stickleback & 1 & $54^{*}$ & & X-05-094_270505_E1 \\
\hline $\mathrm{X}-05-094$ & Fathead Minnow & 8 & $45-71$ & & X-05-094_270505_E1 \\
\hline $\mathrm{X}-05-095$ & Black Bullhead & 1 & $158^{*}$ & & X-05-095_300505_E1 \\
\hline $\mathrm{X}-05-095$ & Central Mudminnow & 2 & $77-111^{*}$ & & X-05-095_300505_E1 \\
\hline $\mathrm{X}-05-095$ & Fathead Minnow & 4 & $42-57$ & & X-05-095_300505_E1 \\
\hline $\mathrm{X}-05-096$ & No Catch & & & & X-05-096_300505_B1 \\
\hline X-05-098 & Central Mudminnow & 1 & $60^{*}$ & & X-05-098_300505_E1 \\
\hline X-05-098 & Fathead Minnow & 23 & $31-59$ & $\begin{array}{l}8 \text { tadpole shrimp (Hepidurus arcticus) - } \\
\text { Indicate temporary water that goes dry } \\
\text { seasonally. Eggs have dormant stage } \\
\text { and hatch when area is flooded in spring. }\end{array}$ & X-05-098_300505_E1 \\
\hline X-05-099 & Brook Stickleback & 1 & $22^{*}$ & & X-05-099_310505_E1 \\
\hline X-05-099 & Fathead Minnow & 45 & $27-57$ & & X-05-099_310505_E1 \\
\hline
\end{tabular}


Appendix 5: List of all fish captures made between 2002 and 2006, showing site number, common name and number (\#) of fish collected, fork (or total ${ }^{\star}$ ) length in millimetres ( $\mathrm{mm}$ ), any comments on the collection and the fish capture identification (ID) number.

\begin{tabular}{|c|c|c|c|c|c|}
\hline Site Number & Common Name & \# & $\begin{array}{l}\text { Fork/Total* } \\
\text { Length (mm) }\end{array}$ & Comments & $\begin{array}{l}\text { Fish Capture } \\
\text { ID Number }\end{array}$ \\
\hline $\mathrm{X}-05-100$ & Brook Stickleback & 2 & $47^{*}$ & & X-05-100_310505_E1 \\
\hline $\mathrm{X}-05-100$ & Central Mudminnow & 3 & $57-68^{*}$ & & X-05-100_310505_E1 \\
\hline $\mathrm{X}-05-100$ & Fathead Minnow & 116 & $24-79$ & & X-05-100_310505_E1 \\
\hline $\mathrm{X}-05-100$ & White Sucker & 1 & 69 & & X-05-100_310505_E1 \\
\hline $\mathrm{X}-05-102$ & Brook Stickleback & 3 & $39-41^{*}$ & & X-05-102_310505_E1 \\
\hline $\mathrm{X}-05-102$ & Central Mudminnow & 3 & $52-62^{*}$ & & X-05-102_310505_E1 \\
\hline $\mathrm{X}-05-102$ & Fathead Minnow & 18 & $26-78$ & Many in spawning colours observed & X-05-102_310505_E1 \\
\hline $\mathrm{X}-05-103$ & Brook Stickleback & 3 & $44-57^{*}$ & & X-05-103_310505_E1 \\
\hline $\mathrm{X}-05-103$ & Central Mudminnow & 2 & $84,95^{\star}$ & & X-05-103_310505_E1 \\
\hline$X-05-104$ & Brook Stickleback & 9 & $41-59^{*}$ & & X-05-104_310505_E1 \\
\hline $\mathrm{X}-05-104$ & Central Mudminnow & 1 & $78^{*}$ & & X-05-104_310505_E1 \\
\hline $\mathrm{X}-05-104$ & Creek Chub & 7 & $38-147$ & One additional $200 \mathrm{~mm}$ released & X-05-104_310505_E1 \\
\hline$X-05-104$ & Fathead Minnow & 15 & $38-80$ & & X-05-104_310505_E1 \\
\hline $\mathrm{X}-05-105$ & Brook Stickleback & 13 & $35-55^{*}$ & & X-05-105_010605_E1 \\
\hline $\mathrm{X}-05-105$ & Fathead Minnow & 45 & $35-76$ & & X-05-105_010605_E1 \\
\hline $\mathrm{X}-05-106$ & Brook Stickleback & 53 & $18-36^{*}$ & & X-05-106_010605_E1 \\
\hline $\mathrm{X}-05-106$ & Fathead Minnow & 8 & $23-67$ & & X-05-106_010605_E1 \\
\hline $\mathrm{X}-05-107$ & Brook Stickleback & 61 & $45-69^{*}$ & & X-05-107_010605_E1 \\
\hline $\mathrm{X}-05-107$ & Creek Chub & 17 & $60-158$ & & X-05-107_010605_E1 \\
\hline $\mathrm{X}-05-107$ & Fathead Minnow & 90 & $53-72$ & & X-05-107_010605_E1 \\
\hline $\mathrm{X}-05-107$ & Pearl Dace & 3 & $63-67$ & & X-05-107_010605_E1 \\
\hline $\mathrm{X}-05-107$ & White Sucker & 4 & $106-171$ & & X-05-107_010605_E1 \\
\hline $\mathrm{X}-05-109$ & Brook Stickleback & 1 & $52^{*}$ & & X-05-109_010605_E1 \\
\hline $\mathrm{X}-05-109$ & Fathead Minnow & 5 & $28-118$ & & X-05-109_010605_E1 \\
\hline$X-05-111$ & Brook Stickleback & 4 & $33-60^{*}$ & & X-05-111_020605_E1 \\
\hline $\mathrm{X}-05-111$ & Creek Chub & 8 & $62-133$ & & X-05-111_020605_E1 \\
\hline $\mathrm{X}-05-111$ & Fathead Minnow & 1 & 67 & & X-05-111_020605_E1 \\
\hline $\mathrm{X}-05-111$ & White Sucker & 3 & $100-167$ & & X-05-111_020605_E1 \\
\hline $\mathrm{X}-05-112$ & Brook Stickleback & 24 & $42-68^{*}$ & & X-05-112_020605_E1 \\
\hline
\end{tabular}


Appendix 5: List of all fish captures made between 2002 and 2006, showing site number, common name and number (\#) of fish collected, fork (or total ${ }^{\star}$ ) length in millimetres ( $\mathrm{mm}$ ), any comments on the collection and the fish capture identification (ID) number.

\begin{tabular}{|c|c|c|c|c|c|}
\hline Site Number & Common Name & $\#$ & $\begin{array}{l}\text { Fork/Total* } \\
\text { Length }(\mathrm{mm})\end{array}$ & Comments & $\begin{array}{l}\text { Fish Capture } \\
\text { ID Number }\end{array}$ \\
\hline $\mathrm{X}-05-112$ & Fathead Minnow & 30 & $42-70$ & $\begin{array}{l}1 \text { vial with } 7 \text { young of the year Cyprinids - } \\
\text { species unidentified, vial filled with } 70 \% \\
\text { ethanol bottled with } P \text {. promelas }\end{array}$ & X-05-112_020605_E1 \\
\hline $\mathrm{X}-05-112$ & Finescale Dace & 4 & $48-58$ & & X-05-112_020605_E1 \\
\hline $\mathrm{X}-05-114$ & Brook Stickleback & 15 & $28-50 *$ & & X-05-114_020605_E1 \\
\hline$X-05-114$ & Fathead Minnow & 55 & $21-74$ & & X-05-114_020605_E1 \\
\hline $\mathrm{X}-05-115$ & Fathead Minnow & 1 & & Released & X-05-115_020605_B1 \\
\hline $\mathrm{X}-05-117$ & Brook Stickleback & 2 & $51,53^{*}$ & & X-05-117_020605_E1 \\
\hline$X-05-117$ & Fathead Minnow & 9 & $39-67$ & & X-05-117_020605_E1 \\
\hline $\mathrm{X}-05-118$ & Brook Stickleback & 2 & $15,47^{*}$ & & X-05-118_060605_E1 \\
\hline $\mathrm{X}-05-118$ & Fathead Minnow & 7 & $43-71$ & & X-05-118_060605_E1 \\
\hline$X-05-120$ & Fathead Minnow & 2 & 35,49 & & $\mathrm{X}-05-120 \_060605 \mathrm{E} 1$ \\
\hline $\mathrm{X}-05-120$ & Johnny Darter & 1 & $55^{\star}$ & Mature male & X-05-120_060605_E1 \\
\hline $\mathrm{X}-05-120$ & Northern Pike & 2 & 72,75 & & X-05-120_060605_E1 \\
\hline$X-05-121$ & Brook Stickleback & 2 & $42,49^{*}$ & & X-05-121_060605_E1 \\
\hline $\mathrm{X}-05-121$ & Central Mudminnow & 1 & $102^{*}$ & & X-05-121_060605_E1 \\
\hline $\mathrm{X}-05-121$ & Creek Chub & 5 & $73-160$ & & X-05-121_060605_E1 \\
\hline$X-05-121$ & Fathead Minnow & 9 & $21-81$ & & X-05-121_060605_E1 \\
\hline $\mathrm{X}-05-121$ & Western Blacknose Dace & 1 & 67 & & X-05-121_060605_E1 \\
\hline $\mathrm{X}-05-121$ & White Sucker & 3 & $37-101$ & & X-05-121_060605_E1 \\
\hline$X-05-122$ & Blackside Darter & 1 & $46^{*}$ & Juvenile & $\mathrm{X}-05-122 \_060605 \mathrm{E} 1$ \\
\hline $\mathrm{X}-05-122$ & Brook Stickleback & 8 & $40-65^{*}$ & & X-05-122_060605_E1 \\
\hline $\mathrm{X}-05-122$ & Central Mudminnow & 1 & $63^{*}$ & & X-05-122_060605_E1 \\
\hline$X-05-122$ & Creek Chub & 14 & $40-143$ & & $\mathrm{X}-05-122 \_060605 \mathrm{E} 1$ \\
\hline $\mathrm{X}-05-122$ & Fathead Minnow & 7 & $37-77$ & & X-05-122_060605_E1 \\
\hline $\mathrm{X}-05-122$ & Iowa Darter & 1 & $46^{*}$ & & X-05-122_060605_E1 \\
\hline$X-05-122$ & Johnny Darter & 22 & $39-61^{*}$ & 4 spawning males & X-05-122_060605_E1 \\
\hline $\mathrm{X}-05-122$ & Western Blacknose Dace & 51 & $33-86$ & & $\mathrm{X}-05-122 \_060605 \mathrm{E} 1$ \\
\hline $\mathrm{X}-05-122$ & White Sucker & 3 & $67-155$ & & X-05-122_060605_E1 \\
\hline$X-05-123$ & Brook Stickleback & 11 & $33-46^{*}$ & & X-05-123_070605_E1 \\
\hline $\mathrm{X}-05-123$ & Central Mudminnow & 2 & $48,52^{*}$ & & X-05-123_070605_E1 \\
\hline
\end{tabular}


Appendix 5: List of all fish captures made between 2002 and 2006, showing site number, common name and number (\#) of fish collected, fork (or total ${ }^{\star}$ ) length in millimetres ( $\mathrm{mm}$ ), any comments on the collection and the fish capture identification (ID) number.

\begin{tabular}{|c|c|c|c|c|c|}
\hline Site Number & Common Name & \# & $\begin{array}{l}\text { Fork/Total* } \\
\text { Length (mm) }\end{array}$ & Comments & $\begin{array}{l}\text { Fish Capture } \\
\text { ID Number }\end{array}$ \\
\hline $\mathrm{X}-05-123$ & Fathead Minnow & 15 & $37-62$ & & X-05-123_070605_E1 \\
\hline $\mathrm{X}-05-125$ & Brook Stickleback & 12 & $26-47^{*}$ & & $\mathrm{X}-05-125 \_070605 \_\mathrm{E} 1$ \\
\hline$X-05-125$ & Central Mudminnow & 3 & $42-85^{*}$ & & $\mathrm{X}-05-125 \_070605 \mathrm{E} 1$ \\
\hline $\mathrm{X}-05-125$ & Creek Chub & 7 & $31-122$ & & X-05-125_070605_E1 \\
\hline $\mathrm{X}-05-125$ & Finescale Dace & 2 & 44,45 & & X-05-125_070605_E1 \\
\hline $\mathrm{X}-05-125$ & Johnny Darter & 1 & $34^{*}$ & & X-05-125_070605_E1 \\
\hline$X-05-125$ & White Sucker & 1 & 220 & Released & X-05-125_070605_E1 \\
\hline $\mathrm{X}-05-126$ & Brook Stickleback & 24 & $32-67^{*}$ & & X-05-126_070605_E1 \\
\hline $\mathrm{X}-05-126$ & Fathead Minnow & 5 & $34-41$ & & X-05-126_070605_E1 \\
\hline$X-05-127$ & No Catch & & & & X-05-127_090605_E1 \\
\hline $\mathrm{X}-05-128$ & Bigmouth Shiner & 3 & $31-46$ & & X-05-128_090605_E1 \\
\hline $\mathrm{X}-05-128$ & Brassy Minnow & 11 & $45-52$ & & X-05-128_090605_E1 \\
\hline$X-05-128$ & Brook Stickleback & 11 & $34-46^{*}$ & & X-05-128_090605_E1 \\
\hline $\mathrm{X}-05-128$ & Common Shiner & 19 & 33-101 & & X-05-128_090605_E1 \\
\hline $\mathrm{X}-05-128$ & Creek Chub & 24 & $37-166$ & & X-05-128_090605_E1 \\
\hline $\mathrm{X}-05-128$ & Fathead Minnow & 10 & $30-45$ & & X-05-128_090605_E1 \\
\hline $\mathrm{X}-05-128$ & Johnny Darter & 6 & $43-62^{*}$ & & X-05-128_090605_E1 \\
\hline $\mathrm{X}-05-128$ & Sand Shiner & 1 & 40 & & X-05-128_090605_E1 \\
\hline $\mathrm{X}-05-128$ & Western Blacknose Dace & 19 & $28-83$ & & X-05-128_090605_E1 \\
\hline $\mathrm{X}-05-128$ & White Sucker & 16 & $55-77$ & & X-05-128_090605_E1 \\
\hline$X-05-129$ & Fathead Minnow & 4 & $31-51$ & & X-05-129_100605_E1 \\
\hline $\mathrm{X}-05-130$ & Brook Stickleback & 1 & $22^{*}$ & & X-05-130_100605_E1 \\
\hline $\mathrm{X}-05-130$ & Fathead Minnow & 11 & $53-80$ & & X-05-130_100605_E1 \\
\hline $\mathrm{X}-05-132$ & No Catch & & & & X-05-132_130605_E1 \\
\hline$X-05-133$ & Fathead Minnow & 8 & $48-61$ & & X-05-133_130605_E1 \\
\hline $\mathrm{X}-05-136$ & No Catch & & & & X-05-136_130605_E1 \\
\hline $\mathrm{X}-05-137$ & Brook Stickleback & 1 & $61^{*}$ & & $\mathrm{X}-05-137 \_140605 \_\mathrm{E} 1$ \\
\hline $\mathrm{X}-05-137$ & Fathead Minnow & 17 & $40-57$ & & X-05-137_140605_E1 \\
\hline $\mathrm{X}-05-138$ & Fathead Minnow & 2 & 47,48 & & X-05-138_140605_E1 \\
\hline $\mathrm{X}-05-139$ & No Catch & & & & X-05-139_140605_E1 \\
\hline $\mathrm{X}-05-140$ & Brook Stickleback & 43 & $47-68^{*}$ & & X-05-140_140605_E1 \\
\hline
\end{tabular}


Appendix 5: List of all fish captures made between 2002 and 2006, showing site number, common name and number (\#) of fish collected, fork (or total ${ }^{*}$ ) length in millimetres $(\mathrm{mm})$, any comments on the collection and the fish capture identification (ID) number.

\begin{tabular}{|c|c|c|c|c|c|}
\hline Site Number & Common Name & \# & $\begin{array}{c}\text { Fork/Total* } \\
\text { Length }(\mathrm{mm})\end{array}$ & Comments & $\begin{array}{l}\text { Fish Capture } \\
\text { ID Number }\end{array}$ \\
\hline $\mathrm{X}-05-140$ & Fathead Minnow & 139 & $44-62$ & & X-05-140_140605_E1 \\
\hline $\mathrm{X}-05-140$ & Northern Pike & 1 & 500 & Observed but unable to collect & X-05-140_140605_01 \\
\hline$X-05-144$ & Brook Stickleback & 412 & $19-61^{*}$ & & X-05-144_150605_E1 \\
\hline X-05-144 & Creek Chub & 11 & $52-129$ & & X-05-144_150605_E1 \\
\hline $\mathrm{X}-05-144$ & Fathead Minnow & 179 & $41-70$ & & X-05-144_150605_E1 \\
\hline $\mathrm{X}-05-144$ & Pearl Dace & 2 & 61,77 & & X-05-144_150605_E1 \\
\hline $\mathrm{X}-05-144$ & Western Blacknose Dace & 2 & 72,76 & & X-05-144_150605_E1 \\
\hline X-05-144 & White Sucker & 12 & $68-120$ & & X-05-144_150605_E1 \\
\hline $\mathrm{X}-05-145$ & Brook Stickleback & 60 & $18-55^{\star}$ & & X-05-145_150605_E1 \\
\hline$X-05-145$ & Common Shiner & 2 & $57-87$ & & X-05-145_150605_E1 \\
\hline X-05-145 & Creek Chub & 15 & $57-136$ & & X-05-145_150605_E1 \\
\hline $\mathrm{X}-05-145$ & Fathead Minnow & 26 & $40-69$ & & X-05-145_150605_E1 \\
\hline$X-05-145$ & Pearl Dace & 15 & 44-119 & & X-05-145_150605_E1 \\
\hline X-05-145 & Western Blacknose Dace & 6 & $48-77$ & & X-05-145_150605_E1 \\
\hline X-05-145 & White Sucker & 5 & $70-173$ & & X-05-145_150605_E1 \\
\hline$X-05-146$ & Brook Stickleback & 23 & $30-65^{*}$ & sample number X-05-146a & X-05-146_160605_E1 \\
\hline X-05-146 & Common Shiner & 11 & $25-136$ & sample number X-05-146a & X-05-146_160605_E1 \\
\hline X-05-146 & Fathead Minnow & 40 & $20-150$ & sample number X-05-146a & X-05-146_160605_E1 \\
\hline $\mathrm{X}-05-146$ & Johnny Darter & 14 & $32-42^{*}$ & sample number X-05-146a & X-05-146_160605_E1 \\
\hline X-05-146 & Western Blacknose Dace & 143 & $22-90$ & sample number X-05-146a & X-05-146_160605_E1 \\
\hline X-05-146 & White Sucker & 1 & 61 & sample number X-05-146a & X-05-146_160605_E1 \\
\hline $\mathrm{X}-05-148$ & No Catch & & & & X-05-148_170605_E1 \\
\hline $\mathrm{X}-05-150$ & Brassy Minnow & 12 & $42-56$ & & X-05-150_210605_E1 \\
\hline $\mathrm{X}-05-150$ & Brook Stickleback & 15 & $16-48^{*}$ & & X-05-150_210605_E1 \\
\hline $\mathrm{X}-05-150$ & Common Shiner & 15 & $32-114$ & & X-05-150_210605_E1 \\
\hline $\mathrm{X}-05-150$ & Creek Chub & 20 & $35-131$ & & X-05-150_210605_E1 \\
\hline $\mathrm{X}-05-150$ & Fathead Minnow & 4 & $18-53$ & & X-05-150_210605_E1 \\
\hline$X-05-150$ & Western Blacknose Dace & 23 & $33-54$ & & X-05-150_210605_E1 \\
\hline $\mathrm{X}-05-150$ & White Sucker & 88 & $18-19^{*}, 150$ & Young of the year bottled separately & X-05-150_210605_E1 \\
\hline $\mathrm{X}-05-151$ & Brassy Minnow & 12 & $38-54$ & & X-05-151_210605_E1 \\
\hline $\mathrm{X}-05-151$ & Brook Stickleback & 27 & $21-51^{*}$ & & X-05-151_210605_E1 \\
\hline
\end{tabular}


Appendix 5: List of all fish captures made between 2002 and 2006, showing site number, common name and number (\#) of fish collected, fork (or total ${ }^{\star}$ ) length in millimetres ( $\mathrm{mm}$ ), any comments on the collection and the fish capture identification (ID) number.

\begin{tabular}{|c|c|c|c|c|c|}
\hline Site Number & Common Name & \# & $\begin{array}{l}\text { Fork/Total* } \\
\text { Length (mm) }\end{array}$ & Comments & $\begin{array}{l}\text { Fish Capture } \\
\text { ID Number }\end{array}$ \\
\hline$X-05-151$ & Common Shiner & 46 & $27-50$ & & X-05-151_210605_E1 \\
\hline $\mathrm{X}-05-151$ & Creek Chub & 15 & $35-115$ & & X-05-151_210605_E1 \\
\hline$X-05-151$ & Fathead Minnow & 1 & 35 & & X-05-151_210605_E1 \\
\hline $\mathrm{X}-05-151$ & Johnny Darter & 3 & $44-50^{*}$ & & X-05-151_210605_E1 \\
\hline $\mathrm{X}-05-151$ & Western Blacknose Dace & 24 & $30-84$ & & X-05-151_210605_E1 \\
\hline $\mathrm{X}-05-153$ & No Catch & & & & X-05-153_210605_E1 \\
\hline $\mathrm{X}-05-154$ & Black Bullhead & 2 & $115,117^{*}$ & (sample number X-05-154a) & X-05-154_210605_E1 \\
\hline $\mathrm{X}-05-154$ & Brassy Minnow & 2 & 53,69 & (sample number X-05-154a) & X-05-154_210605_E1 \\
\hline $\mathrm{X}-05-154$ & Brook Stickleback & 12 & $47-63^{*}$ & (sample number X-05-154a) & X-05-154_210605_E1 \\
\hline$X-05-154$ & Creek Chub & 1 & 93 & (sample number X-05-154a) & X-05-154_210605_E1 \\
\hline $\mathrm{X}-05-154$ & Fathead Minnow & 117 & $37-68$ & (sample number X-05-154a) & X-05-154_210605_E1 \\
\hline $\mathrm{X}-05-154$ & Iowa Darter & 1 & $50^{*}$ & (sample number X-05-154a) & X-05-154_210605_E1 \\
\hline$X-05-154$ & White Sucker & 1 & 75 & (sample number X-05-154a) & X-05-154_210605_E1 \\
\hline $\mathrm{X}-05-155$ & Brook Stickleback & 10 & $29-80^{*}$ & & X-05-155_220605_E1 \\
\hline $\mathrm{X}-05-155$ & Fathead Minnow & 26 & $36-79$ & & X-05-155_220605_E1 \\
\hline$X-05-157$ & No Catch & & & & X-05-157_220605_E1 \\
\hline $\mathrm{X}-05-162$ & Fathead Minnow & 20 & $41-55$ & & X-05-162_230605_E1 \\
\hline $\mathrm{X}-05-163$ & No Catch & & & & X-05-163_230605_E1 \\
\hline $\mathrm{X}-05-164$ & No Catch & & & & X-05-164_230605_E1 \\
\hline $\mathrm{X}-05-165$ & Brook Stickleback & 3 & $32-44^{*}$ & & X-05-165_270605_E1 \\
\hline $\mathrm{X}-05-165$ & Fathead Minnow & 58 & $28-58$ & & X-05-165_270605_E1 \\
\hline $\mathrm{X}-05-167$ & Brook Stickleback & 22 & $23-63^{*}$ & & X-05-167_280605_E1 \\
\hline $\mathrm{X}-05-167$ & Fathead Minnow & 19 & $45-58$ & & X-05-167_280605_E1 \\
\hline $\mathrm{X}-05-167$ & Pearl Dace & 22 & $51-70$ & & X-05-167_280605_E1 \\
\hline$X-05-168$ & Brook Stickleback & 97 & $23-60 *$ & & X-05-168_280605_E1 \\
\hline $\mathrm{X}-05-169$ & Fathead Minnow & 2 & 53,60 & & X-05-169_280605_E1 \\
\hline $\mathrm{X}-05-168$ & Fathead Minnow & 31 & $28-60$ & & X-05-168_280605_E1 \\
\hline $\mathrm{X}-05-169$ & Brook Stickleback & 122 & $20-60^{*}$ & & X-05-169_280605_E1 \\
\hline $\mathrm{X}-05-171$ & Brook Stickleback & 95 & $46-70^{*}$ & & X-05-171_280605_E1 \\
\hline $\mathrm{X}-05-171$ & Fathead Minnow & 12 & $32-62$ & & X-05-171_280605_E1 \\
\hline $\mathrm{X}-05-172$ & Fathead Minnow & 3 & $42-62$ & & X-05-172_290605_E1 \\
\hline
\end{tabular}


Appendix 5: List of all fish captures made between 2002 and 2006, showing site number, common name and number (\#) of fish collected, fork (or total ${ }^{\star}$ ) length in millimetres ( $\mathrm{mm}$ ), any comments on the collection and the fish capture identification (ID) number.

\begin{tabular}{|c|c|c|c|c|c|}
\hline Site Number & Common Name & \# & $\begin{array}{c}\text { Fork/Total* } \\
\text { Length }(\mathrm{mm})\end{array}$ & Comments & $\begin{array}{l}\text { Fish Capture } \\
\text { ID Number }\end{array}$ \\
\hline $\mathrm{X}-05-172$ & Northern Pike & 2 & 122,151 & & X-05-172_290605_E1 \\
\hline $\mathrm{X}-05-173$ & Fathead Minnow & 11 & $51-64$ & & X-05-173_290605_E1 \\
\hline $\mathrm{X}-05-173$ & Northern Pike & 1 & 146 & & X-05-173_290605_E1 \\
\hline $\mathrm{X}-05-176$ & No Catch & & & & X-05-176_040705_E1 \\
\hline $\mathrm{X}-05-176$ & No Catch & & & & X-05-176_040705_E2 \\
\hline $\mathrm{X}-05-177$ & Brook Stickleback & 37 & $22-61^{*}$ & & X-05-177_040705_D1 \\
\hline $\mathrm{X}-05-179$ & No Catch & & & & $\mathrm{X}-05-179$ 050705_E1 \\
\hline $\mathrm{X}-05-181$ & Brook Stickleback & 133 & $26-52^{*}$ & & X-05-181_060705_E1 \\
\hline $\mathrm{X}-05-181$ & Central Mudminnow & 35 & $55-87^{*}$ & & X-05-181_060705_E1 \\
\hline $\mathrm{X}-05-181$ & Fathead Minnow & 3 & $22-47$ & & X-05-181_060705_E1 \\
\hline $\mathrm{X}-05-181$ & White Sucker & 3 & $29-39^{*}$ & & X-05-181_060705_E1 \\
\hline $\mathrm{X}-05-182$ & Brook Stickleback & 119 & $20-62^{*}$ & Invertebrate sample & X-05-182_060705_E1 \\
\hline $\mathrm{X}-05-182$ & Central Mudminnow & 62 & $28-115^{*}$ & & $\mathrm{X}-05-182 \quad 060705 \mathrm{E} 1$ \\
\hline $\mathrm{X}-05-182$ & White Sucker & 3 & $39-91$ & & $\mathrm{X}-05-182 \_060705$ E1 \\
\hline $\mathrm{X}-05-183$ & Brook Stickleback & 1 & $18^{*}$ & & X-05-183_060705_E1 \\
\hline$X-05-183$ & Central Mudminnow & 2 & $42,43^{*}$ & & X-05-183_060705_E1 \\
\hline $\mathrm{X}-05-185$ & Central Mudminnow & 5 & $34-82^{*}$ & & X-05-185_070705_E1 \\
\hline $\mathrm{X}-05-185$ & Common Carp & 1 & 830 & Released & X-05-185_070705_E1 \\
\hline $\mathrm{X}-05-185$ & Northern Pike & 2 & $93-102$ & & X-05-185_070705_E1 \\
\hline $\mathrm{X}-05-185$ & White Sucker & 1 & 65 & & X-05-185_070705_E1 \\
\hline $\mathrm{X}-05-185$ & Yellow Perch & 1 & 113 & & X-05-185_070705_E1 \\
\hline $\mathrm{X}-05-186$ & No Catch & & & & X-05-186_070705_E1 \\
\hline $\mathrm{X}-05-188$ & Brook Stickleback & 2 & & Released & X-05-188_070705_E1 \\
\hline $\mathrm{X}-05-188$ & Central Mudminnow & 1 & & Released & X-05-188_070705_E1 \\
\hline$X-05-189$ & Northern Pike & 1 & 80 & Observed & X-05-189_080705_01 \\
\hline $\mathrm{X}-05-189$ & Northern Pike & 1 & 121 & & X-05-189_080705_E1 \\
\hline $\mathrm{X}-05-190$ & Brook Stickleback & 48 & $21-49^{*}$ & & X-05-190_110705_E1 \\
\hline $\mathrm{X}-05-190$ & Fathead Minnow & 6 & $19-48$ & & X-05-190_110705_E1 \\
\hline $\mathrm{X}-05-191$ & White Sucker & 1 & & Released & X-05-191_120705_E1 \\
\hline $\mathrm{X}-05-192$ & Iowa Darter & 60 & $35-59^{*}$ & & X-05-192_130705_E1 \\
\hline$X-05-192$ & Northern Pike & 1 & 123 & & X-05-192_130705_E1 \\
\hline
\end{tabular}


Appendix 5: List of all fish captures made between 2002 and 2006, showing site number, common name and number (\#) of fish collected, fork (or total ${ }^{*}$ ) length in millimetres $(\mathrm{mm})$, any comments on the collection and the fish capture identification (ID) number.

\begin{tabular}{|c|c|c|c|c|c|}
\hline Site Number & Common Name & \# & $\begin{array}{l}\text { Fork/Total* } \\
\text { Length (mm) }\end{array}$ & Comments & $\begin{array}{l}\text { Fish Capture } \\
\text { ID Number }\end{array}$ \\
\hline $\mathrm{X}-05-192$ & Pearl Dace & 5 & $29-77$ & & X-05-192_130705_E1 \\
\hline $\mathrm{X}-05-192$ & White Sucker & 13 & $44-106$ & & X-05-192_130705_E1 \\
\hline $\mathrm{X}-05-192$ & Yellow Perch & 4 & $72-80$ & & $\mathrm{X}-05-192 \_130705 \mathrm{E} 1$ \\
\hline $\mathrm{X}-05-193$ & Brook Stickleback & 2 & $35,45^{\star}$ & & X-05-193_130705_E1 \\
\hline $\mathrm{X}-05-193$ & Creek Chub & 4 & $119-126$ & & X-05-193_130705_E1 \\
\hline $\mathrm{X}-05-193$ & Fathead Minnow & 10 & $38-66$ & & X-05-193_130705_E1 \\
\hline $\mathrm{X}-05-193$ & Finescale Dace & 15 & $38-66$ & & X-05-193_130705_E1 \\
\hline $\mathrm{X}-05-193$ & Johnny Darter & 5 & $42-55^{*}$ & & X-05-193_130705_E1 \\
\hline $\mathrm{X}-05-193$ & Pearl Dace & 22 & $51-83$ & & X-05-193_130705_E1 \\
\hline$X-05-193$ & Western Blacknose Dace & 63 & $51-85$ & & X-05-193_130705_E1 \\
\hline $\mathrm{X}-05-193$ & White Sucker & 3 & $82-118$ & & X-05-193_130705_E1 \\
\hline $\mathrm{X}-05-194$ & Brook Stickleback & 1 & $54^{*}$ & & X-05-194_130705_E1 \\
\hline$X-05-194$ & Common Shiner & 16 & $52-98$ & & X-05-194_130705_E1 \\
\hline X-05-194 & Creek Chub & 7 & $36-141$ & & X-05-194_130705_E1 \\
\hline $\mathrm{X}-05-194$ & Fathead Minnow & 2 & 41,50 & & X-05-194_130705_E1 \\
\hline $\mathrm{X}-05-194$ & Finescale Dace & 2 & 40,60 & & X-05-194_130705_E1 \\
\hline $\mathrm{X}-05-194$ & Iowa Darter & 1 & $39^{*}$ & & X-05-194_130705_E1 \\
\hline X-05-194 & Johnny Darter & 4 & $40-49^{*}$ & & X-05-194_130705_E1 \\
\hline$X-05-194$ & Pearl Dace & 42 & $40-87$ & & X-05-194_130705_E1 \\
\hline X-05-194 & Western Blacknose Dace & 9 & $29-77$ & & X-05-194_130705_E1 \\
\hline $\mathrm{X}-05-195$ & Brook Stickleback & 63 & $22-54^{*}$ & & X-05-195_130705_E1 \\
\hline $\mathrm{X}-05-195$ & Fathead Minnow & 17 & $46-61$ & & X-05-195_130705_E1 \\
\hline $\mathrm{X}-05-195$ & Finescale Dace & 83 & $46-61$ & & X-05-195_130705_E1 \\
\hline $\mathrm{X}-05-195$ & Pearl Dace & 16 & $35-67$ & & X-05-195_130705_E1 \\
\hline$X-05-195$ & Unidentified minnow & 2 & $16,17^{*}$ & YOY & X-05-195_130705_E1 \\
\hline $\mathrm{X}-05-195$ & Western Blacknose Dace & 40 & $39-62$ & & X-05-195_130705_E1 \\
\hline $\mathrm{X}-05-196$ & Brook Stickleback & 99 & $22-40^{*}$ & & X-05-196_140705_E1 \\
\hline $\mathrm{X}-05-196$ & Creek Chub & 1 & 55 & & X-05-196_140705_E1 \\
\hline $\mathrm{X}-05-198$ & Brassy Minnow & 1 & 72 & & X-05-198_140705_E1 \\
\hline $\mathrm{X}-05-198$ & Brook Stickleback & 21 & $35-56^{*}$ & & X-05-198_140705_E1 \\
\hline $\mathrm{X}-05-198$ & Creek Chub & 1 & 138 & & X-05-198_140705_E1 \\
\hline
\end{tabular}


Appendix 5: List of all fish captures made between 2002 and 2006, showing site number, common name and number (\#) of fish collected, fork (or total ${ }^{\star}$ ) length in millimetres ( $\mathrm{mm}$ ), any comments on the collection and the fish capture identification (ID) number.

\begin{tabular}{|c|c|c|c|c|c|}
\hline Site Number & Common Name & \# & $\begin{array}{c}\text { Fork/Total* } \\
\text { Length }(\mathrm{mm})\end{array}$ & Comments & $\begin{array}{l}\text { Fish Capture } \\
\text { ID Number }\end{array}$ \\
\hline $\mathrm{X}-05-198$ & Fathead Minnow & 7 & $52-66$ & & X-05-198_140705_E1 \\
\hline $\mathrm{X}-05-198$ & Finescale Dace & 90 & $32-78$ & & X-05-198_140705_E1 \\
\hline $\mathrm{X}-05-198$ & Iowa Darter & 1 & $37^{*}$ & & X-05-198_140705_E1 \\
\hline $\mathrm{X}-05-198$ & Pearl Dace & 4 & $50-110$ & & X-05-198_140705_E1 \\
\hline $\mathrm{X}-05-198$ & Western Blacknose Dace & 15 & $33-66$ & & X-05-198_140705_E1 \\
\hline $\mathrm{X}-05-198$ & White Sucker & 9 & $59-130$ & & X-05-198_140705_E1 \\
\hline $\mathrm{X}-05-199$ & Brook Stickleback & 72 & $33-65^{*}$ & & X-05-199_140705_E1 \\
\hline $\mathrm{X}-05-199$ & Fathead Minnow & 81 & $39-74$ & & X-05-199_140705_E1 \\
\hline $\mathrm{X}-05-199$ & Finescale Dace & 158 & $40-71$ & & X-05-199_140705_E1 \\
\hline $\mathrm{X}-05-199$ & Western Blacknose Dace & 3 & $40-60$ & & X-05-199_140705_E1 \\
\hline $\mathrm{X}-05-199$ & White Sucker & 16 & $64-87$ & & X-05-199_140705_E1 \\
\hline $\mathrm{X}-05-200$ & Brook Stickleback & 9 & $34-59^{*}$ & & X-05-200_150705_E1 \\
\hline$X-05-200$ & Creek Chub & 1 & 47 & & X-05-200_150705_E1 \\
\hline $\mathrm{X}-05-201$ & Brook Stickleback & 15 & $29-40^{*}$ & & X-05-201_030805_D1 \\
\hline $\mathrm{X}-05-201$ & Fathead Minnow & 1 & 35 & & X-05-201_030805_D1 \\
\hline$X-05-201$ & Finescale Dace & 2 & 38,48 & & X-05-201_030805_D1 \\
\hline $\mathrm{X}-05-202$ & Brook Stickleback & 13 & $30-62^{*}$ & & X-05-202_030805_B1 \\
\hline $\mathrm{X}-05-202$ & Common Shiner & 2 & 36,38 & & X-05-202_030805_B1 \\
\hline $\mathrm{X}-05-202$ & Creek Chub & 3 & $35-41$ & & $\mathrm{X}-05-202 \_030805$ B1 \\
\hline $\mathrm{X}-05-202$ & Fathead Minnow & 2 & 61,62 & & $\mathrm{X}-05-202 \_030805 \_\mathrm{B} 1$ \\
\hline $\mathrm{X}-05-202$ & Pearl Dace & 4 & $40-50$ & & X-05-202_030805_B1 \\
\hline$X-05-202$ & Western Blacknose Dace & 8 & $39-43$ & & $\mathrm{X}-05-202 \_030805$ B1 \\
\hline $\mathrm{X}-05-204$ & Brook Stickleback & 6 & $35-45^{*}$ & & X-05-204_040805_E1 \\
\hline$X-05-204$ & Central Mudminnow & 4 & $53-90^{*}$ & & X-05-204_040805_E1 \\
\hline$X-05-204$ & Yellow Perch & 4 & $56-66$ & & X-05-204_040805_E1 \\
\hline $\mathrm{X}-05-207$ & Brook Stickleback & 99 & $23-49^{*}$ & & X-05-207_040805_E1 \\
\hline $\mathrm{X}-05-208$ & Common Carp & 32 & $79-125$ & & X-05-208_040805_E1 \\
\hline$X-05-209$ & Brook Stickleback & 23 & $30-65^{*}$ & (sample number X-05-146b) & X-05-209_160605_E1 \\
\hline $\mathrm{X}-05-209$ & Common Shiner & 11 & $25-136$ & (sample number X-05-146b) & X-05-209_160605_E1 \\
\hline $\mathrm{X}-05-209$ & Fathead Minnow & 40 & $20-150$ & (sample number X-05-146b) & X-05-209_160605_E1 \\
\hline$X-05-209$ & Johnny Darter & 14 & $32-42^{*}$ & (sample number X-05-146b) & X-05-209_160605_E1 \\
\hline
\end{tabular}


Appendix 5: List of all fish captures made between 2002 and 2006, showing site number, common name and number (\#) of fish collected, fork (or total ${ }^{\star}$ ) length in millimetres ( $\mathrm{mm}$ ), any comments on the collection and the fish capture identification (ID) number.

\begin{tabular}{|c|c|c|c|c|c|}
\hline Site Number & Common Name & \# & $\begin{array}{c}\text { Fork/Total* } \\
\text { Length }(\mathrm{mm})\end{array}$ & Comments & $\begin{array}{l}\text { Fish Capture } \\
\text { ID Number }\end{array}$ \\
\hline $\mathrm{X}-05-209$ & Western Blacknose Dace & 143 & $22-90$ & (sample number X-05-146b) & X-05-209_160605_E1 \\
\hline $\mathrm{X}-05-209$ & White Sucker & 1 & 61 & (sample number X-05-146b) & X-05-209_160605_E1 \\
\hline $\mathrm{X}-05-210$ & Brook Stickleback & 6 & $42-50 *$ & (sample number X-05-146b) & X-05-210_220605_E1 \\
\hline $\mathrm{X}-05-210$ & Fathead Minnow & 20 & $38-58$ & (sample number X-05-146b) & X-05-210_220605_E1 \\
\hline D-06-001 & Creek Chub & 2 & 150,160 & Released & D-06-001_060706_E1 \\
\hline D-06-001 & Northern Pike & 1 & & Immature & D-06-001_060706_E1 \\
\hline D-06-001 & Common Shiner & 6 & & & D-06-001_060706_E1 \\
\hline D-06-001 & Western Blacknose Dace & 8 & & & D-06-001_060706_E1 \\
\hline D-06-001 & Longnose Dace & 4 & & & D-06-001_060706_E1 \\
\hline D-06-001 & White Sucker & 1 & 186 & Immature & D-06-001_060706_E1 \\
\hline W-06-001 & Emerald Shiner & 3 & $22-23$ & & W-06-001_090506_E1 \\
\hline W-06-001 & Fathead Minnow & 8 & $20-40$ & & W-06-001_090506_E1 \\
\hline W-06-003 & Fathead Minnow & 11 & $30-46$ & & W-06-003_090506_E1 \\
\hline W-06-004 & No Catch & & & & W-06-004_090506_E1 \\
\hline W-06-007 & Fathead Minnow & 2 & 41,42 & & W-06-007_100506_E1 \\
\hline W-06-008 & No Catch & & & & W-06-008_100506_E1 \\
\hline W-06-009 & Fathead Minnow & 1 & & Released & W-06-009_100506_E1 \\
\hline W-06-010 & Brook Stickleback & 162 & $54-70^{*}$ & Released additional 300 & W-06-010_100506_B1 \\
\hline W-06-010 & Fathead Minnow & 227 & $33-71$ & Released additional 400 & W-06-010_100506_B1 \\
\hline W-06-012 & Brook Stickleback & 1 & $45^{\star}$ & Ripe female & W-06-012 110506 E1 \\
\hline W-06-012 & Fathead Minnow & 13 & $23-36$ & & W-06-012_110506_E1 \\
\hline W-06-015 & Fathead Minnow & 11 & $52-70$ & All in spawning condition & W-06-015_110506_E1 \\
\hline W-06-016 & Fathead Minnow & 201 & $29-52$ & & W-06-016_110506_E1 \\
\hline W-06-017 & Brook Stickleback & 13 & $40-61^{*}$ & & W-06-017_120506_E1 \\
\hline W-06-017 & Fathead Minnow & 27 & $26-69$ & & W-06-017_120506_E1 \\
\hline W-06-018 & Brook Stickleback & 23 & $31-58^{*}$ & & W-06-018_120506_E1 \\
\hline W-06-018 & Fathead Minnow & 60 & $24-69$ & & W-06-018 120506 E1 \\
\hline W-06-019 & Brook Stickleback & 6 & $37-48^{*}$ & & W-06-019_150506_E1 \\
\hline W-06-019 & Emerald Shiner & 66 & $21-40$ & Released an additional 150 & W-06-019_150506_E1 \\
\hline W-06-019 & Fathead Minnow & 18 & $27-55$ & & W-06-019_150506_E1 \\
\hline W-06-019 & Northern Pike & 1 & 227 & & W-06-019_150506_E1 \\
\hline
\end{tabular}


Appendix 5: List of all fish captures made between 2002 and 2006, showing site number, common name and number (\#) of fish collected, fork (or total ${ }^{\star}$ ) length in millimetres ( $\mathrm{mm}$ ), any comments on the collection and the fish capture identification (ID) number.

\begin{tabular}{|c|c|c|c|c|c|}
\hline Site Number & Common Name & \# & $\begin{array}{l}\text { Fork/Total* } \\
\text { Length }(\mathrm{mm})\end{array}$ & Comments & $\begin{array}{l}\text { Fish Capture } \\
\text { ID Number }\end{array}$ \\
\hline W-06-019 & Yellow Perch & 3 & $59-70$ & & W-06-019_150506_E1 \\
\hline W-06-020 & Brook Stickleback & 8 & $35-53^{*}$ & & W-06-020_150506_E1 \\
\hline W-06-020 & Central Mudminnow & 10 & $35-68^{*}$ & $\begin{array}{l}\text { Note: Numerous Amphipods in sample: } 7 \\
\text { Limnaea snails, } 1 \text { Unionia snail }\end{array}$ & W-06-020_150506_E1 \\
\hline W-06-020 & Fathead Minnow & 25 & $38-62$ & & W-06-020_150506_E1 \\
\hline W-06-021 & No Catch & & & & W-06-021_160506_E1 \\
\hline W-06-022 & Black Bullhead & 1 & $208^{*}$ & & W-06-022_160506_E1 \\
\hline W-06-022 & Brook Stickleback & 2 & $40,52^{*}$ & & W-06-022_160506_E1 \\
\hline W-06-022 & Central Mudminnow & 8 & $50-105^{\star}$ & & W-06-022_160506_E1 \\
\hline W-06-022 & White Sucker & 1 & 92 & Immature & W-06-022_160506_E1 \\
\hline W-06-023 & Black Bullhead & 1 & $54^{*}$ & & W-06-023_160506_E1 \\
\hline W-06-023 & Brook Stickleback & 1 & $52^{*}$ & & W-06-023_160506_E1 \\
\hline W-06-023 & Central Mudminnow & 1 & $96^{*}$ & & W-06-023_160506_E1 \\
\hline W-06-023 & Fathead Minnow & 74 & $33-65$ & & W-06-023_160506_E1 \\
\hline W-06-023 & Johnny Darter & 2 & $49,54^{*}$ & & W-06-023_160506_E1 \\
\hline W-06-023 & White Sucker & 3 & $85-147$ & Released one mature White Sucker & W-06-023_160506_E1 \\
\hline W-06-025 & Brook Stickleback & 9 & $35-41^{*}$ & & W-06-025_170506_E1 \\
\hline W-06-025 & Central Mudminnow & 1 & $49^{*}$ & & W-06-025_170506_E1 \\
\hline W-06-025 & Fathead Minnow & 21 & $32-64$ & & W-06-025 170506 E1 \\
\hline W-06-025 & Northern Pike & 1 & 233 & & W-06-025_170506_E1 \\
\hline W-06-025 & White Sucker & 1 & 76 & & W-06-025_170506_E1 \\
\hline W-06-025 & Yellow Perch & 1 & 60 & & W-06-025_170506_E1 \\
\hline W-06-026 & Brook Stickleback & 1 & $34^{*}$ & & W-06-026_170506_E1 \\
\hline W-06-026 & Common Carp & 1 & 600 & Released & W-06-026_170506_E1 \\
\hline W-06-026 & Fathead Minnow & 36 & $31-61$ & $\begin{array}{l}\text { All larger specimens have a rosy lateral } \\
\text { pigmentation }\end{array}$ & W-06-026_170506_E1 \\
\hline W-06-026 & lowa Darter & 1 & $39^{*}$ & & W-06-026_170506_E1 \\
\hline W-06-026 & Logperch & 2 & $74,82^{*}$ & & W-06-026_170506_E1 \\
\hline W-06-026 & Spottail Shiner & 1 & 71 & & W-06-026_170506_E1 \\
\hline W-06-027 & Brook Stickleback & 13 & $30-43^{*}$ & & W-06-027_170506_B1 \\
\hline W-06-027 & Fathead Minnow & 98 & $28-60$ & & W-06-027_170506_B1 \\
\hline
\end{tabular}


Appendix 5: List of all fish captures made between 2002 and 2006, showing site number, common name and number (\#) of fish collected, fork (or total ${ }^{*}$ ) length in millimetres $(\mathrm{mm})$, any comments on the collection and the fish capture identification (ID) number.

\begin{tabular}{|c|c|c|c|c|c|}
\hline Site Number & Common Name & \# & $\begin{array}{l}\text { Fork/Total* } \\
\text { Length }(\mathrm{mm})\end{array}$ & Comments & $\begin{array}{l}\text { Fish Capture } \\
\text { ID Number }\end{array}$ \\
\hline W-06-027 & White Sucker & 1 & & Released 1 mature White Sucker & W-06-027_170506_B1 \\
\hline W-06-028 & Brook Stickleback & 17 & $33-48^{*}$ & & W-06-028_170506_E1 \\
\hline W-06-028 & Central Mudminnow & 4 & $50-71^{*}$ & & W-06-028_170506_E1 \\
\hline W-06-028 & Fathead Minnow & 20 & $34-59$ & & W-06-028_170506_E1 \\
\hline W-06-028 & Northern Pike & 1 & & Released mature Northern Pike & W-06-028_170506_E1 \\
\hline W-06-028 & Yellow Perch & 3 & $59-62$ & & W-06-028_170506_E1 \\
\hline W-06-029 & Central Mudminnow & 2 & $60-73^{*}$ & & W-06-029_230506_E1 \\
\hline W-06-029 & Fathead Minnow & 58 & $32-56$ & & W-06-029_230506_E1 \\
\hline W-06-029 & Spotfin Shiner & 1 & 61 & & W-06-029_230506_E1 \\
\hline W-06-029 & White Sucker & 4 & $95-105$ & & W-06-029_230506_E1 \\
\hline W-06-030 & Blackside Darter & 1 & $57^{*}$ & & W-06-030_230506_E1 \\
\hline W-06-030 & Brook Stickleback & 3 & $48-55^{*}$ & & W-06-030_230506_E1 \\
\hline W-06-030 & Central Mudminnow & 5 & $63-80^{*}$ & & W-06-030_230506_E1 \\
\hline W-06-030 & Fathead Minnow & 14 & $42-56$ & & W-06-030_230506_E1 \\
\hline W-06-031 & Black Bullhead & 2 & $155,173^{*}$ & & W-06-031_230506_E1 \\
\hline W-06-031 & Blackside Darter & 6 & $56-85^{*}$ & & W-06-031_230506_E1 \\
\hline W-06-031 & Central Mudminnow & 1 & $53^{*}$ & Tail broken & W-06-031_230506_E1 \\
\hline W-06-031 & Creek Chub & 3 & 94-131 & & W-06-031_230506_E1 \\
\hline W-06-031 & Fathead Minnow & 20 & $39-58$ & & W-06-031_230506_E1 \\
\hline W-06-031 & Pearl Dace & 1 & 128 & & W-06-031_230506_E1 \\
\hline W-06-031 & Tadpole Madtom & 1 & $75^{\star}$ & & W-06-031_230506_E1 \\
\hline W-06-031 & White Sucker & 5 & $83-282$ & $\begin{array}{l}\text { One is mature male, } 197 \mathrm{~mm} \text { spawning } \\
\text { tubercles }\end{array}$ & W-06-031_230506_E1 \\
\hline W-06-032 & Brook Stickleback & 4 & $44-50^{*}$ & All sexually mature females & W-06-032_240506_E1 \\
\hline W-06-032 & Creek Chub & 22 & $94-167$ & $\begin{array}{l}\text { Mature ripe female }(167 \mathrm{~mm}), \text { released } 15 \\
\text { Creek Chubs }\end{array}$ & W-06-032_240506_E1 \\
\hline W-06-032 & Fathead Minnow & 49 & $36-70$ & Many sexually mature & W-06-032_240506_E1 \\
\hline W-06-032 & Johnny Darter & 2 & $66,67^{*}$ & Sexually mature & W-06-032_240506_E1 \\
\hline W-06-032 & Western Blacknose Dace & 1 & 79 & Sexually mature & W-06-032_240506_E1 \\
\hline W-06-032 & White Sucker & 20 & $93-120$ & Released 14,immature White Suckers & W-06-032_240506_E1 \\
\hline W-06-036 & Brook Stickleback & 39 & $38-53^{*}$ & & W-06-036_250506_E1 \\
\hline
\end{tabular}


Appendix 5: List of all fish captures made between 2002 and 2006, showing site number, common name and number (\#) of fish collected, fork (or total ${ }^{\star}$ ) length in millimetres ( $\mathrm{mm}$ ), any comments on the collection and the fish capture identification (ID) number.

\begin{tabular}{|c|c|c|c|c|c|}
\hline Site Number & Common Name & $\#$ & $\begin{array}{l}\text { Fork/Total }{ }^{\star} \\
\text { Length }(\mathrm{mm})\end{array}$ & Comments & $\begin{array}{l}\text { Fish Capture } \\
\text { ID Number }\end{array}$ \\
\hline W-06-036 & Central Mudminnow & 7 & $50-104^{*}$ & & W-06-036_250506_E1 \\
\hline W-06-036 & Fathead Minnow & 23 & $40-70$ & $\begin{array}{l}\text { Most males \& females in spawning } \\
\text { condition (ripe) }\end{array}$ & W-06-036_250506_E1 \\
\hline W-06-037 & Brook Stickleback & 3 & & Released & W-06-037_250506_E1 \\
\hline W-06-040 & No Catch & & & & W-06-040_260506_E1 \\
\hline W-06-043 & Brassy Minnow & 1 & 80 & & W-06-043_260506_E1 \\
\hline W-06-043 & Brook Stickleback & 5 & $44-62^{*}$ & & W-06-043_260506_E1 \\
\hline W-06-043 & Creek Chub & 1 & 109 & & W-06-043_260506_E1 \\
\hline W-06-043 & Fathead Minnow & 11 & $52-70$ & & W-06-043_260506_E1 \\
\hline W-06-043 & Western Blacknose Dace & 2 & 73,77 & & W-06-043_260506_E1 \\
\hline W-06-043 & White Sucker & 2 & 160,163 & & W-06-043_260506_E1 \\
\hline W-06-045 & Brook Stickleback & 1 & $52^{*}$ & & W-06-045 260506 E1 \\
\hline W-06-045 & Common Shiner & 16 & $29-117$ & $\begin{array}{l}\text { Released } 5 \text { Common Shiners, some } \\
\text { mature females and small males with } \\
\text { tubercles }\end{array}$ & W-06-045_260506_E1 \\
\hline W-06-045 & Creek Chub & 17 & $51-173$ & Released 7 other Creek Chub & W-06-045_260506_E1 \\
\hline W-06-045 & Fathead Minnow & 8 & 49 & Released 7 other Fathead Minnows & W-06-045_260506_E1 \\
\hline W-06-045 & Johnny Darter & 2 & $49-55^{*}$ & $\begin{array}{l}\text { Smallest is mature female, largest is } \\
\text { mature male }\end{array}$ & W-06-045_260506_E1 \\
\hline W-06-045 & Northern Redbelly Dace & 3 & & & W-06-045_260506_E1 \\
\hline W-06-045 & Western Blacknose Dace & 17 & $48-74$ & $\begin{array}{l}\text { Released } 7 \text { other Western Blacknose } \\
\text { Dace, some mature males \& females in } \\
\text { sample }\end{array}$ & W-06-045_260506_E1 \\
\hline W-06-045 & White Sucker & 17 & $53-82$ & $\begin{array}{l}\text { Released } 11 \text { other White Suckers } \\
\text { immature }\end{array}$ & W-06-045_260506_E1 \\
\hline W-06-046 & No Catch & & & & W-06-046_270506_E1 \\
\hline W-06-049 & Brook Stickleback & 4 & $47-61^{*}$ & & W-06-049_270506_E1 \\
\hline W-06-049 & Creek Chub & 2 & 94,142 & Largest is a mature male & W-06-049_270506_E1 \\
\hline W-06-049 & Fathead Minnow & 62 & $39-68$ & & W-06-049_270506_E1 \\
\hline W-06-049 & Pearl Dace & 7 & $73-92$ & & W-06-049_270506_E1 \\
\hline W-06-049 & White Sucker & 1 & 176 & Female, mature & W-06-049_270506_E1 \\
\hline W-06-054 & Fathead Minnow & 49 & $29-62$ & & W-06-054_300506_E1 \\
\hline
\end{tabular}


Appendix 5: List of all fish captures made between 2002 and 2006, showing site number, common name and number (\#) of fish collected, fork (or total ${ }^{\star}$ ) length in millimetres ( $\mathrm{mm}$ ), any comments on the collection and the fish capture identification (ID) number.

\begin{tabular}{|c|c|c|c|c|c|}
\hline Site Number & Common Name & \# & $\begin{array}{c}\text { Fork/Total* } \\
\text { Length }(\mathrm{mm})\end{array}$ & Comments & $\begin{array}{l}\text { Fish Capture } \\
\text { ID Number }\end{array}$ \\
\hline W-06-055 & Brook Stickleback & 1 & $55^{\star}$ & & W-06-055_300506_E1 \\
\hline W-06-055 & Fathead Minnow & 16 & $37-64$ & $\begin{array}{l}\text { Many have a rosy pigment along the } \\
\text { lateral line }\end{array}$ & W-06-055_300506_E1 \\
\hline W-06-055 & White Sucker & 1 & 90 & & W-06-055_300506_E1 \\
\hline W-06-056 & Brook Stickleback & 5 & $38-56^{*}$ & & W-06-056_310506_E1 \\
\hline W-06-056 & Fathead Minnow & 15 & $36-49$ & & W-06-056_310506_E1 \\
\hline W-06-056 & White Sucker & 1 & 102 & Immature & W-06-056_310506_E1 \\
\hline W-06-058 & Black Bullhead & 1 & $193^{*}$ & & W-06-058_010606_B1 \\
\hline W-06-058 & Fathead Minnow & 30 & $43-58$ & & W-06-058_010606_B1 \\
\hline W-06-060 & Black Bullhead & 4 & $89,195^{\star}$ & Released 2 Black Bullheads & W-06-060_010606_B1 \\
\hline W-06-060 & Brook Stickleback & 2 & $47,48^{*}$ & & W-06-060_010606_B1 \\
\hline W-06-060 & Central Mudminnow & 2 & $100,108^{*}$ & & W-06-060_010606_B1 \\
\hline W-06-060 & Fathead Minnow & 254 & $37-63$ & Released 200 Fathead Minnows & W-06-060_010606_B1 \\
\hline W-06-060 & Northern Pike & 3 & $40-44$ & All are YOY & W-06-060_010606_B1 \\
\hline W-06-060 & White Sucker & 2 & 97,115 & & W-06-060_010606_B1 \\
\hline W-06-060 & Yellow Perch & 1 & 98 & & W-06-060_010606_B1 \\
\hline W-06-061 & Northern Pike & 3 & $198-233$ & Mature & W-06-061_060606_E1 \\
\hline W-06-062 & Brook Stickleback & 1 & $45^{\star}$ & & W-06-062_060606_E1 \\
\hline W-06-062 & Fathead Minnow & 42 & $25-68$ & & W-06-062_060606_E1 \\
\hline W-06-062 & Spottail Shiner & 3 & $40-81$ & & W-06-062_060606_E1 \\
\hline W-06-062 & White Sucker & 2 & 69,85 & Immature & W-06-062_060606_E1 \\
\hline W-06-062 & Yellow Perch & 3 & $56-128$ & Immature & W-06-062_060606_E1 \\
\hline W-06-064 & No Catch & & & & W-06-064_070606_E1 \\
\hline W-06-065 & No Catch & & & & W-06-065_070606_E1 \\
\hline W-06-066 & Brook Stickleback & 103 & $36-59^{*}$ & & W-06-066_070606_E1 \\
\hline W-06-066 & Fathead Minnow & 17 & $42-65$ & & W-06-066_070606_E1 \\
\hline W-06-066 & Finescale Dace & 14 & $47-74$ & Many show characteristics of $P$. eos & W-06-066_070606_E1 \\
\hline W-06-066 & Pearl Dace & 2 & 43,72 & & W-06-066_070606_E1 \\
\hline W-06-067 & Brook Stickleback & 2 & $43,49^{*}$ & & W-06-067_080606_E1 \\
\hline W-06-067 & Common Shiner & 17 & $65-142$ & Several are mature males with tubercles & W-06-067_080606_E1 \\
\hline W-06-067 & Creek Chub & 1 & 46 & & W-06-067_080606_E1 \\
\hline
\end{tabular}


Appendix 5: List of all fish captures made between 2002 and 2006, showing site number, common name and number (\#) of fish collected, fork (or total ${ }^{\star}$ ) length in millimetres ( $\mathrm{mm}$ ), any comments on the collection and the fish capture identification (ID) number.

\begin{tabular}{|c|c|c|c|c|c|}
\hline Site Number & Common Name & \# & $\begin{array}{c}\text { Fork/Total* } \\
\text { Length }(\mathrm{mm})\end{array}$ & Comments & $\begin{array}{l}\text { Fish Capture } \\
\text { ID Number }\end{array}$ \\
\hline W-06-067 & Fathead Minnow & 4 & $36-62$ & & W-06-067_080606_E1 \\
\hline W-06-067 & Longnose Dace & 10 & $62-86$ & In spawning colours & W-06-067_080606_E1 \\
\hline W-06-067 & Western Blacknose Dace & 2 & 64,75 & & W-06-067_080606_E1 \\
\hline W-06-068 & Brook Stickleback & 4 & $49-56^{*}$ & & W-06-068_080606_E1 \\
\hline W-06-068 & Creek Chub & 1 & 151 & & W-06-068_080606_E1 \\
\hline W-06-068 & Fathead Minnow & 2 & $52-67$ & & W-06-068_080606_E1 \\
\hline W-06-068 & Finescale Dace & 9 & $57-85$ & & W-06-068_080606_E1 \\
\hline W-06-068 & Pearl Dace & 1 & 83 & & W-06-068_080606_E1 \\
\hline W-06-070 & Blackside Darter & 3 & $56-74^{*}$ & & W-06-070_120606_E1 \\
\hline W-06-070 & Burbot & 4 & 133-162* & & W-06-070_120606_E1 \\
\hline W-06-070 & Johnny Darter & 3 & $40-52^{*}$ & & W-06-070_120606_E1 \\
\hline W-06-070 & Rock Bass & 1 & 95 & & W-06-070_120606_E1 \\
\hline W-06-070 & White Sucker & 2 & $21,24^{*}$ & Immature & W-06-070_120606_E1 \\
\hline W-06-078 & Blackside Darter & 1 & $55^{\star}$ & & W-06-078_140606_E1 \\
\hline W-06-078 & Brook Stickleback & 1 & $39^{*}$ & & W-06-078_140606_E1 \\
\hline W-06-078 & Central Mudminnow & 4 & $42-82^{*}$ & & W-06-078_140606_E1 \\
\hline W-06-078 & Fathead Minnow & 1 & 37 & & W-06-078_140606_E1 \\
\hline W-06-078 & Northern Pike & 1 & 308 & Mature, released & W-06-078_140606_E1 \\
\hline W-06-078 & White Sucker & 2 & 110,300 & $300 \mathrm{~mm}$ released, $110 \mathrm{~mm}$ immature & W-06-078_140606_E1 \\
\hline W-06-074 & Brook Stickleback & 26 & $31-46^{*}$ & & W-06-074_130606_E1 \\
\hline W-06-074 & Central Mudminnow & 11 & $58-91^{*}$ & & W-06-074_130606_E1 \\
\hline W-06-074 & Finescale Dace & 9 & $36-67$ & $\begin{array}{l}P \text {. neogaeus/ } P \text {. eos hybrids in sample, a } \\
\text { few specimens identified as } P \text {. eos show } \\
\text { some characteristics of } P \text {. neogaeus }\end{array}$ & W-06-074_130606_E1 \\
\hline W-06-074 & Northern Redbelly Dace & 44 & $31-74$ & & W-06-074_130606_E1 \\
\hline W-06-074 & Pearl Dace & 16 & $49-62$ & & W-06-074_130606_E1 \\
\hline W-06-076 & No Catch & & & & W-06-076_130606_E1 \\
\hline W-06-077 & Brook Stickleback & 6 & $27-58^{*}$ & & W-06-077_130606_E1 \\
\hline W-06-077 & Central Mudminnow & 37 & $42-81^{*}$ & & W-06-077_130606_E1 \\
\hline W-06-078 & Northern Pike & 1 & 308 & Released 1 mature Northern Pike & W-06-078_140606_E1 \\
\hline W-06-078 & White Sucker & 1 & & Released 1 mature White Sucker & W-06-078_140606_E1 \\
\hline
\end{tabular}


Appendix 5: List of all fish captures made between 2002 and 2006, showing site number, common name and number (\#) of fish collected, fork (or total ${ }^{\star}$ ) length in millimetres ( $\mathrm{mm}$ ), any comments on the collection and the fish capture identification (ID) number.

\begin{tabular}{|c|c|c|c|c|c|}
\hline Site Number & Common Name & \# & $\begin{array}{c}\text { Fork/Total* } \\
\text { Length }(\mathrm{mm})\end{array}$ & Comments & $\begin{array}{l}\text { Fish Capture } \\
\text { ID Number }\end{array}$ \\
\hline W-06-079 & Brook Stickleback & 7 & $44-58^{*}$ & & W-06-079_140606_E1 \\
\hline W-06-079 & Central Mudminnow & 3 & $51-75^{*}$ & & W-06-079_140606_E1 \\
\hline W-06-079 & Fathead Minnow & 1 & 28 & & W-06-079_140606_E1 \\
\hline W-06-079 & Finescale Dace & 2 & 43,66 & & W-06-079_140606_E1 \\
\hline W-06-079 & Northern Redbelly Dace & 4 & $32-45$ & & W-06-079_140606_E1 \\
\hline W-06-083 & Common Shiner & 2 & 69,87 & & W-06-083_200606_E1 \\
\hline W-06-083 & Creek Chub & 2 & 91,99 & & W-06-083_200606_E1 \\
\hline W-06-083 & Longnose Dace & 6 & $34-73$ & & W-06-083_200606_E1 \\
\hline W-06-083 & Pearl Dace & 1 & 45 & & W-06-083_200606_E1 \\
\hline W-06-083 & Western Blacknose Dace & 13 & $52-87$ & & W-06-083_200606_E1 \\
\hline W-06-083 & White Sucker & 1 & 70 & Immature & W-06-083_200606_E1 \\
\hline W-06-085 & Brook Stickleback & 5 & $37-60^{*}$ & & W-06-085_210606_E1 \\
\hline W-06-085 & Creek Chub & 3 & $75-108$ & & W-06-085_210606_E1 \\
\hline W-06-085 & Fathead Minnow & 21 & $41-83$ & & W-06-085_210606_E1 \\
\hline W-06-087 & Blacknose Shiner & 2 & 26,30 & & W-06-087_220606_E1 \\
\hline W-06-087 & Brook Stickleback & 21 & $38-65^{*}$ & & W-06-087_220606_E1 \\
\hline W-06-087 & Fathead Minnow & 142 & $24-32$ & & W-06-087_220606_E1 \\
\hline W-06-087 & Iowa Darter & 24 & $37-51^{*}$ & & W-06-087_220606_E1 \\
\hline W-06-087 & White Sucker & 3 & $69-71$ & Immature & W-06-087_220606_E1 \\
\hline W-06-088 & Fathead Minnow & 10 & & & W-06-088 220606 E1 \\
\hline W-06-088 & Northern Pike & 1 & 210 & Released, immature & W-06-088_220606_E1 \\
\hline W-06-089 & Creek Chub & 5 & $89-144$ & Post spawn male in sample & W-06-089_220606_E1 \\
\hline W-06-089 & Finescale Dace & 1 & 58 & & W-06-089_220606_E1 \\
\hline W-06-089 & Pearl Dace & 10 & $71-116$ & Some in spawning colours & W-06-089_220606_E1 \\
\hline W-06-089 & Western Blacknose Dace & 6 & $47-66$ & & W-06-089_220606_E1 \\
\hline W-06-089 & White Sucker & 5 & $95-110$ & Immature & W-06-089_220606_E1 \\
\hline W-06-090 & Brook Trout & 1 & 60 & Immature & W-06-090_220606_E1 \\
\hline W-06-090 & Brown Trout & 1 & 68 & Immature & W-06-090_220606_E1 \\
\hline W-06-090 & Longnose Dace & 12 & $63-94$ & & W-06-090_220606_E1 \\
\hline W-06-090 & Pearl Dace & 1 & 94 & & W-06-090_220606_E1 \\
\hline W-06-090 & Rainbow Trout & 1 & & Released, immature & W-06-090_220606_E1 \\
\hline
\end{tabular}


Appendix 5: List of all fish captures made between 2002 and 2006, showing site number, common name and number (\#) of fish collected, fork (or total ${ }^{\star}$ ) length in millimetres ( $\mathrm{mm}$ ), any comments on the collection and the fish capture identification (ID) number.

\begin{tabular}{|c|c|c|c|c|c|}
\hline Site Number & Common Name & \# & $\begin{array}{c}\text { Fork/Total* } \\
\text { Length }(\mathrm{mm})\end{array}$ & Comments & $\begin{array}{l}\text { Fish Capture } \\
\text { ID Number }\end{array}$ \\
\hline W-06-091 & Brook Stickleback & 46 & $28-43^{*}$ & & W-06-091_230606_E1 \\
\hline W-06-091 & Creek Chub & 2 & $94-98$ & & W-06-091_230606_E1 \\
\hline W-06-091 & Fathead Minnow & 5 & $34-72$ & & W-06-091_230606_E1 \\
\hline W-06-091 & Finescale Dace & 5 & $50-75$ & & W-06-091_230606_E1 \\
\hline W-06-091 & Iowa Darter & 1 & $50^{*}$ & & W-06-091_230606_E1 \\
\hline W-06-091 & Pearl Dace & 10 & $60-112$ & & W-06-091_230606_E1 \\
\hline W-06-091 & Western Blacknose Dace & 11 & $39-72$ & & W-06-091_230606_E1 \\
\hline W-06-092 & Brook Stickleback & 10 & $39-78^{*}$ & & W-06-092_230606_E1 \\
\hline W-06-092 & Fathead Minnow & 2 & 42,52 & & W-06-092_230606_E1 \\
\hline W-06-092 & Finescale Dace & 1 & 42 & & W-06-092_230606_E1 \\
\hline W-06-092 & Johnny Darter & 4 & $39-54^{*}$ & One spawning male in sample & W-06-092_230606_E1 \\
\hline W-06-092 & Pearl Dace & 3 & $59-111$ & & W-06-092_230606_E1 \\
\hline W-06-092 & Western Blacknose Dace & 10 & $40-85$ & & W-06-092_230606_E1 \\
\hline W-06-093 & Brook Stickleback & 2 & $39,42^{*}$ & & W-06-093_230606_E1 \\
\hline W-06-093 & Fathead Minnow & 2 & 32,62 & & W-06-093_230606_E1 \\
\hline W-06-093 & Finescale Dace & 5 & $45-55$ & & W-06-093_230606_E1 \\
\hline W-06-093 & Longnose Dace & 2 & $38-73$ & & W-06-093_230606_E1 \\
\hline W-06-093 & Western Blacknose Dace & 4 & $60-82$ & & W-06-093_230606_E1 \\
\hline W-06-094 & No Catch & & & & W-06-094_270606_E1 \\
\hline W-06-096 & Central Mudminnow & 3 & $64-88^{*}$ & & W-06-096_270606_E1 \\
\hline W-06-096 & Northern Pike & 2 & 97,97 & & W-06-096_270606_E1 \\
\hline W-06-096 & White Sucker & 1 & 126 & & W-06-096_270606_E1 \\
\hline W-06-097 & Brook Stickleback & 35 & $29-56^{*}$ & & W-06-097_270606_E1 \\
\hline W-06-097 & Central Mudminnow & 30 & $33-82^{*}$ & & W-06-097_270606_E1 \\
\hline W-06-097 & lowa Darter & 2 & $39,51^{*}$ & & W-06-097_270606_E1 \\
\hline W-06-097 & Northern Pike & 2 & 78,85 & Immature & W-06-097_270606_E1 \\
\hline W-06-097 & Northern Redbelly Dace & 2 & 36,47 & $\begin{array}{l}\text { Largest specimen agrees more closely } \\
\text { with } P \text {. erythrugaster than } P \text {. eos in } \\
\text { Becker (1983). Confirmed by D. } \\
\text { Watkinson as } P \text {. eos, NOT } P \text {. } \\
\text { erythrugaster }\end{array}$ & W-06-097_270606_E1 \\
\hline
\end{tabular}


Appendix 5: List of all fish captures made between 2002 and 2006, showing site number, common name and number (\#) of fish collected, fork (or total ${ }^{*}$ ) length in millimetres $(\mathrm{mm})$, any comments on the collection and the fish capture identification (ID) number.

\begin{tabular}{|c|c|c|c|c|c|}
\hline Site Number & Common Name & \# & $\begin{array}{c}\text { Fork/Total* } \\
\text { Length }(\mathrm{mm})\end{array}$ & Comments & $\begin{array}{l}\text { Fish Capture } \\
\text { ID Number }\end{array}$ \\
\hline W-06-097 & Tadpole Madtom & 1 & $86^{*}$ & Mature female & W-06-097_270606_E1 \\
\hline W-06-097 & White Sucker & 6 & $65-87$ & Immature & W-06-097_270606_E1 \\
\hline W-06-098 & Brook Stickleback & 4 & $25-36^{*}$ & & W-06-098_280606_E1 \\
\hline W-06-098 & Central Mudminnow & 6 & $32-84^{*}$ & & W-06-098_280606_E1 \\
\hline W-06-098 & Fathead Minnow & 16 & $22-63$ & & W-06-098_280606_E1 \\
\hline W-06-098 & White Sucker & 1 & 45 & YOY & W-06-098_280606_E1 \\
\hline W-06-099 & Common Carp & 15 & & Stranded, dead on site, various sizes & W-06-099-280606_01 \\
\hline W-06-100 & Brook Stickleback & 2 & $31-36^{*}$ & & W-06-100_280606_E1 \\
\hline W-06-100 & Central Mudminnow & 1 & $115^{*}$ & & W-06-100_280606_E1 \\
\hline W-06-100 & Fathead Minnow & 15 & $45-70$ & & W-06-100_280606_E1 \\
\hline W-06-100 & Johnny Darter & 4 & $73-87^{*}$ & & W-06-100_280606_E1 \\
\hline W-06-100 & Spottail Shiner & 4 & $83-93$ & & W-06-100_280606_E1 \\
\hline W-06-100 & White Sucker & 6 & $43-127$ & All immature & W-06-100_280606_E1 \\
\hline W-06-102 & Brook Stickleback & 7 & & Released & W-06-102_280606_D1 \\
\hline W-06-102 & Central Mudminnow & 6 & & Released & W-06-102_280606_D1 \\
\hline W-06-102 & Fathead Minnow & 5 & & Released & W-06-102_280606_D1 \\
\hline W-06-106 & Brook Stickleback & 1 & $42^{*}$ & & W-06-106_290606_E1 \\
\hline W-06-106 & Central Mudminnow & 3 & $84-102^{*}$ & & W-06-106_290606_E1 \\
\hline W-06-106 & Fathead Minnow & 10 & $46-64$ & Spawning males & W-06-106_290606_E1 \\
\hline W-06-106 & Iowa Darter & 6 & $41-47^{*}$ & & W-06-106_290606_E1 \\
\hline W-06-106 & Northern Pike & 1 & 93 & Immature & W-06-106_290606_E1 \\
\hline W-06-110 & Common Shiner & 7 & $65-81$ & & W-06-110_050706_E1 \\
\hline W-06-110 & Creek Chub & 3 & $67-88$ & & W-06-110_050706_E1 \\
\hline W-06-110 & Johnny Darter & 7 & $42-57^{*}$ & & W-06-110_050706_E1 \\
\hline W-06-110 & Longnose Dace & 10 & $50-68$ & One very full of Ligula spp. (tapeworm) & W-06-110_050706_E1 \\
\hline W-06-110 & Northern Pike & 1 & 110 & & W-06-110_050706_E1 \\
\hline W-06-110 & Western Blacknose Dace & 2 & 52,70 & & W-06-110_050706_E1 \\
\hline W-06-110 & White Sucker & 1 & $26^{*}$ & YOY & W-06-110_050706_E1 \\
\hline W-06-112 & Common Shiner & 30 & $31-78$ & & W-06-112_050706_E1 \\
\hline W-06-112 & Creek Chub & 8 & $44-115$ & & W-06-112_050706_E1 \\
\hline W-06-112 & Fathead Minnow & 4 & $15-53$ & 3 smallest are YOY & W-06-112_050706_E1 \\
\hline
\end{tabular}


Appendix 5: List of all fish captures made between 2002 and 2006, showing site number, common name and number (\#) of fish collected, fork (or total ${ }^{\star}$ ) length in millimetres ( $\mathrm{mm}$ ), any comments on the collection and the fish capture identification (ID) number.

\begin{tabular}{|c|c|c|c|c|c|}
\hline Site Number & Common Name & \# & $\begin{array}{c}\text { Fork/Total* } \\
\text { Length }(\mathrm{mm})\end{array}$ & Comments & $\begin{array}{l}\text { Fish Capture } \\
\text { ID Number }\end{array}$ \\
\hline W-06-112 & Finescale Dace & 11 & $38-53$ & & W-06-112_050706_E1 \\
\hline W-06-112 & Johnny Darter & 15 & $29-44^{*}$ & & W-06-112_050706_E1 \\
\hline W-06-112 & Northern Redbelly Dace & 2 & 42,43 & & W-06-112_050706_E1 \\
\hline W-06-112 & Western Blacknose Dace & 31 & $30-56$ & & W-06-112_050706_E1 \\
\hline W-06-112 & White Sucker & 6 & $27^{*}-116$ & Some YOY & W-06-112_050706_E1 \\
\hline W-06-113 & Brook Stickleback & 9 & $29-41^{*}$ & & W-06-113_060706_E1 \\
\hline W-06-113 & Common Shiner & 2 & 63,90 & & W-06-113_060706_E1 \\
\hline W-06-113 & Fathead Minnow & 11 & $25-58$ & & W-06-113_060706_E1 \\
\hline W-06-113 & Finescale Dace & 8 & $53-69$ & & W-06-113_060706_E1 \\
\hline W-06-113 & Johnny Darter & 8 & $46-60^{*}$ & & W-06-113_060706_E1 \\
\hline W-06-113 & Pearl Dace & 9 & $71-106$ & & W-06-113_060706_E1 \\
\hline W-06-113 & Western Blacknose Dace & 9 & $41-84$ & & W-06-113_060706_E1 \\
\hline W-06-114 & Brook Stickleback & 5 & $32-35^{*}$ & & W-06-114_100706_E1 \\
\hline W-06-114 & Creek Chub & 48 & $26-122$ & & W-06-114_100706_E1 \\
\hline W-06-114 & Fathead Minnow & 65 & $29-76$ & & W-06-114_100706_E1 \\
\hline W-06-114 & Johnny Darter & 7 & $44-61^{*}$ & & W-06-114_100706_E1 \\
\hline W-06-114 & Longnose Dace & 4 & $57-72$ & & W-06-114_100706_E1 \\
\hline W-06-114 & Pearl Dace & 16 & $32-63$ & & W-06-114_100706_E1 \\
\hline W-06-114 & Western Blacknose Dace & 2 & 35,49 & & W-06-114_100706_E1 \\
\hline W-06-114 & White Sucker & 17 & $25-55$ & All immature & W-06-114_100706_E1 \\
\hline W-06-116 & Brassy Minnow & 3 & $61-76$ & & W-06-116_110706_D1 \\
\hline W-06-116 & Common Shiner & 12 & $53-67$ & & W-06-116_110706_D1 \\
\hline W-06-116 & Fathead Minnow & 2 & 49,51 & & W-06-116_110706_D1 \\
\hline W-06-116 & Finescale Dace & 3 & $41-47$ & & W-06-116_110706_D1 \\
\hline W-06-116 & Longnose Dace & 9 & $37-58$ & & W-06-116_110706_D1 \\
\hline W-06-116 & Pearl Dace & 3 & $51-59$ & & W-06-116_110706_D1 \\
\hline W-06-116 & Western Blacknose Dace & 24 & $35-72$ & & W-06-116_110706_D1 \\
\hline W-06-116 & White Sucker & 1 & $34^{*}$ & Immature & W-06-116_110706_D1 \\
\hline W-06-119 & Burbot & 1 & $245^{*}$ & Released & W-06-119_110706_E1 \\
\hline W-06-119 & Common Shiner & 4 & $44-89$ & & W-06-119_110706_E1 \\
\hline W-06-119 & Creek Chub & 3 & $82-156$ & & W-06-119_110706_E1 \\
\hline
\end{tabular}


Appendix 5: List of all fish captures made between 2002 and 2006, showing site number, common name and number (\#) of fish collected, fork (or total ${ }^{\star}$ ) length in millimetres ( $\mathrm{mm}$ ), any comments on the collection and the fish capture identification (ID) number.

\begin{tabular}{|c|c|c|c|c|c|}
\hline Site Number & Common Name & \# & $\begin{array}{c}\text { Fork/Total* } \\
\text { Length }(\mathrm{mm})\end{array}$ & Comments & $\begin{array}{l}\text { Fish Capture } \\
\text { ID Number }\end{array}$ \\
\hline W-06-119 & Johnny Darter & 1 & $45^{\star}$ & & W-06-119_110706_E1 \\
\hline W-06-119 & Longnose Dace & 8 & $45-84$ & & W-06-119_110706_E1 \\
\hline W-06-119 & Western Blacknose Dace & 3 & $43-50$ & & W-06-119_110706_E1 \\
\hline W-06-119 & White Sucker & 11 & $51-72,300$ & $\begin{array}{l}\text { Released } 1 \text { mature White Sucker, kept } 10 \\
\text { immature White Suckers }\end{array}$ & W-06-119_110706_E1 \\
\hline W-06-119 & Yellow Perch & 1 & 100 & Released & W-06-119_110706_E1 \\
\hline W-06-121 & Brook Stickleback & 29 & $17-48^{*}$ & & W-06-121_120706_E1 \\
\hline W-06-121 & Fathead Minnow & 2 & $24-61$ & & W-06-121_120706_E1 \\
\hline W-06-121 & Finescale Dace & 1 & 45 & & W-06-121_120706_E1 \\
\hline W-06-121 & Johnny Darter & 3 & $21-53^{*}$ & & W-06-121_120706_E1 \\
\hline W-06-121 & Longnose Dace & 2 & $55-79$ & & W-06-121_120706_E1 \\
\hline W-06-121 & White Sucker & 1 & 43 & Immature & W-06-121_120706_E1 \\
\hline W-06-124 & Blacknose Shiner & 1 & 51 & & W-06-124_180706_E1 \\
\hline W-06-124 & Brook Stickleback & 4 & $33-34^{*}$ & & W-06-124_180706_E1 \\
\hline W-06-124 & Central Mudminnow & 1 & $73^{*}$ & & W-06-124_180706_E1 \\
\hline W-06-124 & Fathead Minnow & 1 & 23 & & W-06-124_180706_E1 \\
\hline W-06-124 & Finescale Dace & 3 & 33-35 & & W-06-124_180706_E1 \\
\hline W-06-124 & White Sucker & 3 & $29-31^{*}$ & YOY & W-06-124_180706_E1 \\
\hline W-06-126 & Burbot & 2 & $166^{*}$ & Released 1 Burbot & W-06-126_180706_E1 \\
\hline W-06-126 & Smallmouth Bass & 1 & & Released 1 Smallmouth Bass, immature & W-06-126_180706_E1 \\
\hline W-06-126 & Longnose Dace & 1 & 89 & & W-06-126_180706_E1 \\
\hline W-06-126 & Mottled Sculpin & 2 & $86^{*}$ & Released 1 Mottled Sculpin & W-06-126_180706_E1 \\
\hline W-06-126 & Northern Pike & 2 & 104 & Released 1 immature Northern Pike & W-06-126_180706_E1 \\
\hline W-06-126 & Yellow Perch & 1 & 85 & & W-06-126_180706_E1 \\
\hline W-06-133 & Tadpole Madtom & 1 & $58^{*}$ & & W-06-133_190706_E1 \\
\hline W-06-133 & White Sucker & 1 & 93 & & W-06-133_190706_E1 \\
\hline W-06-134 & Johnny Darter & 1 & $38^{*}$ & & W-06-134_200706_E1 \\
\hline W-06-134 & Silver Redhorse & 4 & $67-141$ & & W-06-134_200706_E1 \\
\hline W-06-134 & White Sucker & 4 & $39-41$ & Immature & W-06-134_200706_E1 \\
\hline W-06-138 & Black Bullhead & 2 & $38,106^{*}$ & & W-06-138_010806_E1 \\
\hline W-06-138 & Fathead Minnow & 266 & $25-61$ & & W-06-138_010806_E1 \\
\hline
\end{tabular}


Appendix 5: List of all fish captures made between 2002 and 2006, showing site number, common name and number (\#) of fish collected, fork (or total ${ }^{*}$ ) length in millimetres $(\mathrm{mm})$, any comments on the collection and the fish capture identification (ID) number.

\begin{tabular}{|c|c|c|c|c|c|}
\hline Site Number & Common Name & \# & $\begin{array}{c}\text { Fork/Total* } \\
\text { Length }(\mathrm{mm})\end{array}$ & Comments & $\begin{array}{l}\text { Fish Capture } \\
\text { ID Number }\end{array}$ \\
\hline W-06-138 & Johnny Darter & 5 & $34-40^{*}$ & & W-06-138_010806_E1 \\
\hline W-06-138 & Longnose Dace & 24 & $88-101$ & & W-06-138_010806_E1 \\
\hline W-06-138 & Sand Shiner & 17 & $49-59$ & & W-06-138_010806_E1 \\
\hline W-06-138 & White Sucker & 2 & 54,116 & & W-06-138_010806_E1 \\
\hline W-06-139 & Black Bullhead & 8 & $37-41^{*}$ & All YOY & W-06-139_010806_E1 \\
\hline W-06-139 & Blackside Darter & 1 & $81^{*}$ & & W-06-139_010806_E1 \\
\hline W-06-139 & Fathead Minnow & 30 & $23-37$ & & W-06-139_010806_E1 \\
\hline W-06-139 & Johnny Darter & 1 & $39^{*}$ & & W-06-139_010806_E1 \\
\hline W-06-139 & Longnose Dace & 2 & $37-62$ & & W-06-139_010806_E1 \\
\hline W-06-139 & Northern Pike & 2 & $310-315$ & Released 2 mature Northern Pike & W-06-139_010806_E1 \\
\hline W-06-139 & Sand Shiner & 3 & 23-34 & & W-06-139_010806_E1 \\
\hline W-06-139 & Tadpole Madtom & 1 & $68^{*}$ & & W-06-139_010806_E1 \\
\hline W-06-139 & White Sucker & 1 & 54 & & W-06-139_010806_E1 \\
\hline W-06-140 & Black Bullhead & 6 & $93-112^{*}$ & & W-06-140_010806_E1 \\
\hline W-06-140 & Blackside Darter & 12 & $52-75^{*}$ & & W-06-140_010806_E1 \\
\hline W-06-140 & Common Shiner & 6 & 93-111 & & W-06-140_010806_E1 \\
\hline W-06-140 & Fathead Minnow & 1 & 57 & & W-06-140_010806_E1 \\
\hline W-06-140 & Longnose Dace & 16 & $42-87$ & & W-06-140_010806_E1 \\
\hline W-06-140 & Sand Shiner & 52 & $58-69$ & & W-06-140_010806_E1 \\
\hline W-06-140 & Shorthead Redhorse & 1 & 140 & & W-06-140_010806_E1 \\
\hline W-06-140 & Stonecat & 1 & $127^{*}$ & & W-06-140_010806_E1 \\
\hline W-06-140 & Troutperch & 4 & $48-75$ & & W-06-140_010806_E1 \\
\hline W-06-140 & White Sucker & 2 & 61,73 & & W-06-140_010806_E1 \\
\hline W-06-141 & Blackside Darter & 16 & $41-55^{*}$ & & W-06-141_010806_E1 \\
\hline W-06-141 & Burbot & 1 & $139^{*}$ & & W-06-141_010806_E1 \\
\hline W-06-141 & Common Shiner & 2 & 90,117 & & W-06-141_010806_E1 \\
\hline W-06-141 & Creek Chub & 1 & 121 & & W-06-141_010806_E1 \\
\hline W-06-141 & Fathead Minnow & 3 & $50-58$ & & W-06-141_010806_E1 \\
\hline W-06-141 & Johnny Darter & 4 & $35-49^{*}$ & & W-06-141_010806_E1 \\
\hline W-06-141 & Longnose Dace & 56 & $27-76$ & & W-06-141_010806_E1 \\
\hline W-06-141 & Northern Pike & 1 & 350 & Released, mature & W-06-141_010806_E1 \\
\hline
\end{tabular}


Appendix 5: List of all fish captures made between 2002 and 2006, showing site number, common name and number (\#) of fish collected, fork (or total ${ }^{\star}$ ) length in millimetres $(\mathrm{mm})$, any comments on the collection and the fish capture identification (ID) number.

\begin{tabular}{|c|c|c|c|c|c|}
\hline Site Number & Common Name & \# & $\begin{array}{c}\text { Fork/Total* } \\
\text { Length }(\mathrm{mm})\end{array}$ & Comments & $\begin{array}{l}\text { Fish Capture } \\
\text { ID Number }\end{array}$ \\
\hline W-06-141 & Rock Bass & 1 & & Observed, unable to collect & W-06-141_010806_E1 \\
\hline W-06-141 & Sand Shiner & 1 & 61 & & W-06-141_010806_E1 \\
\hline W-06-141 & Shorthead Redhorse & 5 & $220-290$ & & W-06-141_010806_E1 \\
\hline W-06-141 & Walleye & 2 & 87,125 & & W-06-141_010806_E1 \\
\hline W-06-141 & White Sucker & 2 & $50-130$ & & W-06-141_010806_E1 \\
\hline W-06-143 & Blackside Darter & 3 & $61-70^{*}$ & & W-06-143_020806_E1 \\
\hline W-06-143 & Common Carp & 6 & $66-90$ & & W-06-143_020806_E1 \\
\hline W-06-143 & Creek Chub & 8 & $66-106$ & & W-06-143_020806_E1 \\
\hline W-06-143 & Fathead Minnow & 7 & $20-44$ & Smallest 5 are YOY & W-06-143_020806_E1 \\
\hline W-06-143 & Freshwater Drum & 1 & 400 & Released mature Freshwater Drum & W-06-143_020806_E1 \\
\hline W-06-143 & Johnny Darter & 24 & $28-63^{*}$ & & W-06-143_020806_E1 \\
\hline W-06-143 & Sand Shiner & 3 & $31-54$ & & W-06-143_020806_E1 \\
\hline W-06-143 & Tadpole Madtom & 2 & $78,79^{*}$ & & W-06-143_020806_E1 \\
\hline W-06-144 & No Catch & & & & W-06-144_080806_E1 \\
\hline W-06-146 & No Catch & & & & W-06-146_090806_E1 \\
\hline
\end{tabular}


Appendix 6: Results of basic water chemistry and air temperature measurements recorded at most sites where fishing effort was applied between 2002 and 2006.

\begin{tabular}{|c|c|c|c|c|c|c|c|c|}
\hline \multirow{2}{*}{ Site Number } & \multirow{2}{*}{ Date } & \multirow{2}{*}{$\begin{array}{l}\text { Air Temp. } \\
\left({ }^{\circ} \mathrm{C}\right)\end{array}$} & \multirow{2}{*}{$\begin{array}{c}\text { Water Temp. } \\
\left({ }^{\circ} \mathrm{C}\right)\end{array}$} & \multicolumn{2}{|c|}{ Dissolved Oxygen } & \multirow{2}{*}{$\begin{array}{c}\text { Conductivity } \\
(\mu \mathrm{S} / \mathrm{cm})\end{array}$} & \multirow{2}{*}{$\begin{array}{l}\text { Turbidity } \\
\text { (NTU) }\end{array}$} & \multirow{2}{*}{$\begin{array}{c}\mathrm{pH} \\
\text { (units) }\end{array}$} \\
\hline & & & & $(\mathrm{mg} / \mathrm{L})$ & (\% SAT) & & & \\
\hline D-02-001 & 16-Apr-02 & 4.6 & 1.6 & 10.40 & 100 & 425 & & 7.40 \\
\hline $\mathrm{D}-02-002$ & 17-Apr-02 & 4 & 1.1 & & & 265 & & 7.41 \\
\hline D-02-003 & 19-Apr-02 & -1 & 0.9 & 11.50 & 99 & 415 & & 8.54 \\
\hline D-02-004 & 22-Apr-02 & & 5.0 & 11.10 & 98.8 & & & \\
\hline D-02-005 & 2-May-02 & & 6.5 & 10.69 & 98.6 & 510 & & 8.41 \\
\hline$D-02-006$ & 6-May-02 & & & & & & & \\
\hline D-02-007 & 6-May-02 & & & & & & & \\
\hline D-02-008 & 7-May-02 & 5 & 6.2 & 9.98 & 90.8 & 661 & & 8.78 \\
\hline D-02-009 & 7-May-02 & 13 & 9.5 & 10.78 & 107.5 & 597 & & 8.68 \\
\hline D-02-010 & 9-May-02 & 2 & & & & & & \\
\hline D-02-011 & 9-May-02 & 2 & & & & & & \\
\hline $\mathrm{D}-02-012$ & 9-May-02 & 2 & & & & & & \\
\hline D-02-013 & 10-May-02 & & 14.4 & 8.96 & 101 & 449 & & 8.66 \\
\hline $\mathrm{D}-02-014$ & 11-Мау-02 & 14 & 8.9 & 7.84 & 78.4 & 866 & & 7.32 \\
\hline D-02-015 & 11-May-02 & 20.5 & 12.0 & 8.94 & 96 & 502 & & 7.33 \\
\hline D-02-016 & 11-May-02 & 20 & & & & & & \\
\hline D-02-017 & 11-May-02 & & & & & & & \\
\hline D-02-018 & 12-May-02 & 17 & 11.8 & 9.71 & 103.6 & 558 & & 7.32 \\
\hline D-02-019 & 12-May-02 & & & & & & & \\
\hline D-02-020 & 12-May-02 & & & & & & & \\
\hline $\mathrm{D}-02-021$ & 12-May-02 & & & & & & & \\
\hline D-02-022 & 12-May-02 & & & & & & & \\
\hline$D-02-023$ & 12-May-02 & & & & & & & \\
\hline D-02-024 & 12-May-02 & & & & & & & \\
\hline D-02-025 & 13-May-02 & & & & & & & \\
\hline D-02-026 & 13-May-02 & & 11.0 & 8.81 & 93.2 & 408 & & 8.68 \\
\hline $\mathrm{D}-02-027$ & 13-May-02 & & 12.9 & 9.91 & 99 & 386 & & 7.33 \\
\hline D-02-028 & 13-May-02 & & 11.3 & 6.50 & 69 & 385 & & 8.58 \\
\hline D-02-029 & 14-May-02 & & & & & & & \\
\hline D-02-030 & 14-May-02 & & & & & & & \\
\hline D-02-031 & 15-May-02 & & 11.7 & 5.37 & 56.1 & 605 & & 8.28 \\
\hline D-02-032 & 15-May-02 & & & & & & & \\
\hline D-02-033 & 15-Мау-02 & & 13.5 & 6.74 & 74.6 & 540 & & 8.66 \\
\hline
\end{tabular}


Appendix 6: Results of basic water chemistry and air temperature measurements recorded at most sites where fishing effort was applied between 2002 and 2006.

\begin{tabular}{|c|c|c|c|c|c|c|c|c|}
\hline Site Number & Date & $\begin{array}{l}\text { Air Temp. } \\
\left({ }^{\circ} \mathrm{C}\right)\end{array}$ & $\begin{array}{c}\text { Water Temp. } \\
\left({ }^{\circ} \mathrm{C}\right)\end{array}$ & \multicolumn{2}{|c|}{ Dissolved Oxygen } & $\begin{array}{c}\text { Conductivity } \\
(\mu \mathrm{S} / \mathrm{cm})\end{array}$ & $\begin{array}{l}\text { Turbidity } \\
\text { (NTU) }\end{array}$ & $\begin{array}{c}\mathrm{pH} \\
\text { (units) }\end{array}$ \\
\hline D-02-034 & 16-May-02 & & & & & & & \\
\hline D-02-035 & 16-May-02 & & & & & & & \\
\hline D-02-036 & 16-Мay-02 & 11.5 & 7.0 & 6.87 & 64 & 567 & & 8.76 \\
\hline D-02-037 & 16-May-02 & & & & & & & \\
\hline D-02-038 & 16-May-02 & & & & & & & \\
\hline $\mathrm{D}-02-039$ & 16-Мay-02 & & & & & & & \\
\hline D-02-040 & 16-May-02 & & & & & & & \\
\hline $\mathrm{D}-02-041$ & 16-May-02 & & 14.4 & & & & & \\
\hline D-02-042 & 17-May-02 & & 5.4 & & & & & \\
\hline$D-02-043$ & 21-May-02 & 25.5 & 13.3 & 11.34 & 123.2 & 545 & & 8.17 \\
\hline D-02-044 & 21-May-02 & 26 & 16.1 & 12.17 & 140.4 & 497 & & 8.43 \\
\hline D-02-045 & 22-May-02 & 6 & 10.0 & 13.51 & 135.9 & 486 & & 8.20 \\
\hline D-02-046 & 22-May-02 & & 12.0 & & & & & \\
\hline $\mathrm{D}-02-047$ & 23-Мay-02 & & & & & & & \\
\hline D-02-048 & 23-May-02 & & & & & & & \\
\hline D-02-049 & 23-May-02 & & & & & & & \\
\hline D-02-050 & 23-May-02 & & & & & & & \\
\hline $\mathrm{D}-02-051$ & 24-May-02 & & 5.2 & 11.23 & 100.7 & 642 & & 7.78 \\
\hline D-02-052 & 24-May-02 & & & & & & & \\
\hline D-02-053 & 24-May-02 & & & & & & & \\
\hline D-02-054 & 24-May-02 & & & & & & & \\
\hline D-02-055 & 24-May-02 & & & & & & & \\
\hline D-02-056 & 24-May-02 & & 10.4 & 10.39 & 110.2 & 518 & & 7.98 \\
\hline D-02-057 & 24-May-02 & & & & & & & \\
\hline $\mathrm{D}-02-058$ & 24-May-02 & & & & & & & \\
\hline D-02-059 & 27-May-02 & & 16.1 & 5.32 & 62.7 & 932 & & 7.78 \\
\hline D-02-060 & 27-May-02 & 27 & 11.7 & 5.53 & 57.6 & 453 & & 8.50 \\
\hline D-02-061 & 28-May-02 & 28.5 & 17.5 & 8.17 & 100 & 583 & & 7.32 \\
\hline $\mathrm{D}-02-062$ & 28-May-02 & & & & & & & \\
\hline D-02-063 & 28-May-02 & & & & & & & \\
\hline $\mathrm{D}-02-064$ & 29-May-02 & & & & & & & \\
\hline D-02-065 & 29-May-02 & & 17.2 & 4.88 & 54.6 & 536 & & 7.97 \\
\hline$D-02-066$ & 29-May-02 & & 17.0 & & & & & \\
\hline
\end{tabular}


Appendix 6: Results of basic water chemistry and air temperature measurements recorded at most sites where fishing effort was applied between 2002 and 2006.

\begin{tabular}{|c|c|c|c|c|c|c|c|c|}
\hline \multirow{2}{*}{ Site Number } & \multirow{2}{*}{ Date } & \multirow{2}{*}{$\begin{array}{l}\text { Air Temp. } \\
\left({ }^{\circ} \mathrm{C}\right)\end{array}$} & \multirow{2}{*}{$\begin{array}{c}\text { Water Temp. } \\
\left({ }^{\circ} \mathrm{C}\right)\end{array}$} & \multicolumn{2}{|c|}{ Dissolved Oxygen } & \multirow{2}{*}{$\begin{array}{c}\text { Conductivity } \\
(\mu \mathrm{S} / \mathrm{cm})\end{array}$} & \multirow{2}{*}{$\begin{array}{c}\text { Turbidity } \\
\text { (NTU) }\end{array}$} & \multirow{2}{*}{$\begin{array}{c}\mathrm{pH} \\
\text { (units) }\end{array}$} \\
\hline & & & & $(\mathrm{mg} / \mathrm{L})$ & (\% SAT) & & & \\
\hline D-02-067 & 29-May-02 & 22 & 21.5 & 7.30 & 90 & 598 & & 8.69 \\
\hline D-02-068 & 29-Мау-02 & 25 & 18.5 & 6.35 & 75.7 & 575 & & 7.32 \\
\hline D-02-069 & 29-May-02 & 26 & 21.1 & 6.83 & 76.1 & 496 & & 8.60 \\
\hline D-02-070 & 30-May-02 & & & & & & & \\
\hline D-02-071 & 30-May-02 & 29 & 19.3 & 7.35 & 90.4 & 519 & & 7.11 \\
\hline D-02-072 & 3-Jun-02 & & 18.0 & & & & & \\
\hline D-02-073 & 3-Jun-02 & & 18.0 & & & & & \\
\hline D-02-074 & 3-Jun-02 & 24 & 19.5 & 8.03 & 99 & 652 & & 7.11 \\
\hline D-02-075 & 4-Jun-02 & & 17.9 & 6.55 & 77.6 & 520 & & 7.29 \\
\hline D-02-076 & 5-Jun-02 & & & & & & & \\
\hline D-02-077 & 5-Jun-02 & 26 & 20.6 & 8.23 & 102.6 & 498 & & 7.42 \\
\hline $\mathrm{D}-02-078$ & 5-Jun-02 & 26.5 & 23.5 & 7.74 & 102.9 & 495 & & 7.39 \\
\hline D-02-079 & 5-Jun-02 & & & & & & & \\
\hline D-02-080 & 5-Jun-02 & & & & & & & \\
\hline D-02-081 & 6-Jun-02 & 15 & 17.6 & 6.70 & 78.1 & 565 & & 7.11 \\
\hline D-02-082 & 6-Jun-02 & & & & & & & \\
\hline D-02-083 & 6-Jun-02 & 16.5 & 16.3 & 6.26 & 71.7 & 580 & & 7.23 \\
\hline D-02-084 & 12-Jun-02 & 17.5 & 18.3 & 10.36 & 121.4 & 563 & & 7.11 \\
\hline D-02-085 & 12-Jun-02 & & & 6.20 & & & & \\
\hline D-02-086 & 12-Jun-02 & & & & & & & \\
\hline D-02-087 & 12-Jun-02 & 17.5 & 20.3 & 8.30 & 100.8 & 611 & & 7.12 \\
\hline D-02-088 & 13-Jun-02 & 16 & 15.3 & 4.71 & 52.6 & 960 & & 7.22 \\
\hline D-02-089 & 13-Jun-02 & 16 & 18.5 & 7.15 & 87.4 & 1015 & & 7.26 \\
\hline D-02-090 & 13-Jun-02 & & & & & & & \\
\hline D-02-091 & 13-Jun-02 & 16 & & & & & & \\
\hline D-02-092 & 13-Jun-02 & 17.5 & 21.5 & 6.66 & 85.5 & 549 & & 7.82 \\
\hline D-02-093 & 14-Jun-02 & 16.5 & 18.1 & 5.60 & 67.9 & 604 & & 6.80 \\
\hline D-02-094 & 18-Jun-02 & 20 & 20.8 & 3.20 & 40.5 & 573 & & 6.59 \\
\hline D-02-095 & 18-Jun-02 & 20 & 23.1 & 9.45 & 126.5 & 1382 & & 8.40 \\
\hline D-02-096 & 19-Jun-02 & 22 & 19.1 & 5.86 & 72.7 & 961 & & 7.83 \\
\hline D-02-097 & 19-Jun-02 & 25 & 20.7 & 5.80 & 74.5 & 517 & & 7.35 \\
\hline D-02-098 & 19-Jun-02 & & & & & & & \\
\hline D-02-099 & 19-Jun-02 & & & & & & & \\
\hline
\end{tabular}


Appendix 6: Results of basic water chemistry and air temperature measurements recorded at most sites where fishing effort was applied between 2002 and 2006.

\begin{tabular}{|c|c|c|c|c|c|c|c|c|}
\hline \multirow{2}{*}{ Site Number } & \multirow{2}{*}{ Date } & \multirow{2}{*}{$\begin{array}{l}\text { Air Temp. } \\
\left({ }^{\circ} \mathrm{C}\right)\end{array}$} & \multirow{2}{*}{$\begin{array}{c}\text { Water Temp. } \\
\left({ }^{\circ} \mathrm{C}\right)\end{array}$} & \multicolumn{2}{|c|}{ Dissolved Oxygen } & \multirow{2}{*}{$\begin{array}{c}\text { Conductivity } \\
(\mu \mathrm{S} / \mathrm{cm})\end{array}$} & \multirow{2}{*}{$\begin{array}{c}\text { Turbidity } \\
\text { (NTU) }\end{array}$} & \multirow{2}{*}{$\begin{array}{c}\mathrm{pH} \\
\text { (units) }\end{array}$} \\
\hline & & & & $(\mathrm{mg} / \mathrm{L})$ & (\% SAT) & & & \\
\hline D-02-100 & 20-Jun-02 & & 15.6 & 6.43 & 74 & 444 & & 7.19 \\
\hline $\mathrm{D}-02-101$ & 20-Jun-02 & 18 & 14.9 & 7.83 & 89.3 & 342 & & 7.28 \\
\hline $\mathrm{D}-02-102$ & 20-Jun-02 & 21 & 15.7 & 7.06 & 81.8 & 284 & & 7.28 \\
\hline D-02-103 & 20-Jun-02 & & & & & & & \\
\hline D-02-104 & 20-Jun-02 & 25 & 20.1 & 7.10 & 90.6 & 234 & & 7.51 \\
\hline $\mathrm{D}-02-105$ & 21-Jun-02 & 22 & & & & & & \\
\hline D-02-106 & 21-Jun-02 & 25 & & & & & & \\
\hline D-02-107 & 21-Jun-02 & 26 & 17.3 & 7.28 & 88.1 & 460 & & 7.72 \\
\hline D-02-108 & 21-Jun-02 & & 22.2 & 3.60 & 47.3 & 1379 & & 7.11 \\
\hline D-02-109 & 26-Jun-02 & & & & & & & \\
\hline$D-02-110$ & 26-Jun-02 & & & & & & & \\
\hline $\mathrm{D}-02-111$ & 26-Jun-02 & 28 & 25.4 & 8.41 & 118.2 & 803 & & 7.18 \\
\hline D-02-112 & 3-Jul-02 & & & & & & & \\
\hline $\mathrm{D}-02-113$ & 3-Jul-02 & & & & & & & \\
\hline$D-02-114$ & 3-Jul-02 & & & & & & & \\
\hline D-02-115 & 3-Jul-02 & & 20.8 & 1.00 & 13 & 852 & & \\
\hline D-02-116 & 3-Jul-02 & & 20.5 & 6.19 & 81.8 & 572 & & 6.85 \\
\hline $\mathrm{D}-02-117$ & 3-Jul-02 & & & & & & & \\
\hline$D-02-118$ & 3-Jul-02 & & 19.4 & 5.85 & 68 & 658 & & \\
\hline $\mathrm{D}-02-119$ & 4-Jul-02 & & 16.1 & 7.27 & 84.6 & 635 & & 7.73 \\
\hline D-02-120 & 4-Jul-02 & & & & & & & \\
\hline $\mathrm{D}-02-121$ & 4-Jul-02 & & 16.1 & 8.75 & 91.9 & 592 & & 8.12 \\
\hline$D-02-122$ & 4-Jul-02 & & 16.9 & 8.14 & 96.4 & 710 & & 8.26 \\
\hline D-02-123 & 4-Jul-02 & & & & & & & \\
\hline$D-02-124$ & 4-Jul-02 & & 17.3 & 7.88 & 94.1 & 636 & & 7.76 \\
\hline$D-02-125$ & 9-Jul-02 & & 20.6 & 10.24 & 131.5 & 467 & & 7.13 \\
\hline$D-02-126$ & 9-Jul-02 & & 22.7 & 10.23 & 136.3 & 476 & & 7.58 \\
\hline D-02-127 & 9-Jul-02 & & 21.2 & 9.46 & 123.8 & 478 & & 7.96 \\
\hline $\mathrm{D}-02-128$ & 9-Jul-02 & & & & & & & \\
\hline D-02-129 & 9-Jul-02 & & & & & & & \\
\hline D-02-130 & 9-Jul-02 & & 21.3 & 5.94 & 77.2 & 716 & & 7.69 \\
\hline $\mathrm{D}-02-131$ & 9-Jul-02 & & 21.0 & 6.55 & 84.7 & 501 & & 7.96 \\
\hline D-02-132 & 10-Jul-02 & & 18.0 & 6.95 & 84.8 & 414 & & 8.01 \\
\hline
\end{tabular}


Appendix 6: Results of basic water chemistry and air temperature measurements recorded at most sites where fishing effort was applied between 2002 and 2006.

\begin{tabular}{|c|c|c|c|c|c|c|c|c|}
\hline \multirow{2}{*}{ Site Number } & \multirow{2}{*}{ Date } & \multirow{2}{*}{$\begin{array}{l}\text { Air Temp. } \\
\left({ }^{\circ} \mathrm{C}\right)\end{array}$} & \multirow{2}{*}{$\begin{array}{c}\text { Water Temp. } \\
\left({ }^{\circ} \mathrm{C}\right)\end{array}$} & \multicolumn{2}{|c|}{ Dissolved Oxygen } & \multirow{2}{*}{$\begin{array}{c}\text { Conductivity } \\
(\mu \mathrm{S} / \mathrm{cm})\end{array}$} & \multirow{2}{*}{$\begin{array}{l}\text { Turbidity } \\
\text { (NTU) }\end{array}$} & \multirow{2}{*}{$\begin{array}{c}\mathrm{pH} \\
\text { (units) }\end{array}$} \\
\hline & & & & (mg/L) & (\% SAT) & & & \\
\hline D-02-133 & 10-Jul-02 & & & & & & & \\
\hline D-02-134 & 10-Jul-02 & & & & & & & \\
\hline D-02-135 & 10-Jul-02 & & 17.9 & 8.43 & 102 & 514 & & 6.95 \\
\hline D-02-136 & 10-Jul-02 & & & & & & & \\
\hline D-02-137 & 12-Jul-02 & & 21.5 & 6.23 & 81 & 476 & & 7.96 \\
\hline D-02-138 & 12-Jul-02 & 27.5 & 25.3 & 10.86 & 148 & 836 & & 7.60 \\
\hline D-02-139 & 12-Jul-02 & & 21.8 & 7.14 & 94.2 & 433 & & 6.95 \\
\hline D-02-140 & 12-Jul-02 & & & & & & & \\
\hline D-02-141 & 12-Jul-02 & & & & & & & \\
\hline D-02-142 & 17-Jul-02 & 25 & 22.3 & 6.46 & 85.4 & 423 & & 7.23 \\
\hline D-02-143 & 17-Jul-02 & & & & & & & \\
\hline D-02-144 & 17-Jul-02 & & & & & & & \\
\hline D-02-145 & 17-Jul-02 & & & & & & & \\
\hline D-02-146 & 17-Jul-02 & & & & & & & \\
\hline D-02-147 & 17-Jul-02 & & 23.4 & 8.44 & 114.1 & 606 & & 7.36 \\
\hline D-02-148 & 17-Jul-02 & & & & & & & \\
\hline D-02-149 & 17-Jul-02 & & 26.9 & 6.59 & 94.4 & 405 & & 7.79 \\
\hline D-02-150 & 18-Jul-02 & & & & & & & \\
\hline D-02-151 & 18-Jul-02 & & & & & & & \\
\hline D-02-152 & 18-Jul-02 & & & & & & & \\
\hline D-02-153 & 18-Jul-02 & & & & & & & \\
\hline D-02-154 & 18-Jul-02 & & & & & & & \\
\hline D-02-155 & 18-Jul-02 & & & & & & & \\
\hline D-02-156 & 18-Jul-02 & & & & & & & \\
\hline D-02-157 & 18-Jul-02 & & & & & & & \\
\hline D-02-158 & 18-Jul-02 & & & & & & & \\
\hline D-02-159 & 18-Jul-02 & & & & & & & \\
\hline D-02-160 & 23-Jul-02 & 15 & 20.9 & 7.05 & 90.6 & 441 & & 7.06 \\
\hline D-02-161 & 23-Jul-02 & 15 & 21.0 & 8.42 & 108.7 & 447 & & 7.14 \\
\hline D-02-162 & 23-Jul-02 & & & & & & & \\
\hline D-02-163 & 24-Jul-02 & & & & & & & \\
\hline D-02-164 & 24-Jul-02 & & 16.5 & 7.16 & 84.2 & 617 & & 7.71 \\
\hline D-02-165 & 24-Jul-02 & & & & & & & \\
\hline
\end{tabular}


Appendix 6: Results of basic water chemistry and air temperature measurements recorded at most sites where fishing effort was applied between 2002 and 2006.

\begin{tabular}{|c|c|c|c|c|c|c|c|c|}
\hline \multirow{2}{*}{ Site Number } & \multirow{2}{*}{ Date } & \multirow{2}{*}{$\begin{array}{l}\text { Air Temp. } \\
\left({ }^{\circ} \mathrm{C}\right)\end{array}$} & \multirow{2}{*}{$\begin{array}{c}\text { Water Temp. } \\
\left({ }^{\circ} \mathrm{C}\right)\end{array}$} & \multicolumn{2}{|c|}{ Dissolved Oxygen } & \multirow{2}{*}{$\begin{array}{c}\text { Conductivity } \\
(\mu \mathrm{S} / \mathrm{cm})\end{array}$} & \multirow{2}{*}{$\begin{array}{l}\text { Turbidity } \\
\text { (NTU) }\end{array}$} & \multirow{2}{*}{$\begin{array}{c}\mathrm{pH} \\
\text { (units) }\end{array}$} \\
\hline & & & & (mg/L) & (\% SAT) & & & \\
\hline D-02-166 & 24-Jul-02 & & & & & & & \\
\hline D-02-167 & 24-Jul-02 & & 17.4 & 8.19 & 98.6 & 768 & & 7.79 \\
\hline D-02-168 & 24-Jul-02 & & & & & & & \\
\hline D-02-169 & 24-Jul-02 & & & & & & & \\
\hline D-02-170 & 24-Jul-02 & 25 & 21.6 & 7.83 & 102.4 & 603 & & 8.08 \\
\hline D-02-171 & 25-Jul-02 & & 15.9 & 7.08 & 82.4 & 461 & & 7.82 \\
\hline D-02-172 & 25-Jul-02 & 21 & & & & & & \\
\hline D-02-173 & 25-Jul-02 & 25 & 18.8 & 7.51 & 93 & 483 & & 7.79 \\
\hline D-02-174 & 25-Jul-02 & & & & & & & \\
\hline D-02-175 & 25-Jul-02 & & & & & & & \\
\hline D-02-176 & 25-Jul-02 & & & & & & & \\
\hline D-02-177 & 25-Jul-02 & & & & & & & \\
\hline D-02-178 & 25-Jul-02 & & 24.7 & 8.95 & 124.5 & 493 & & 6.95 \\
\hline D-02-179 & 30-Jul-02 & 25 & 21.4 & 8.20 & 107.3 & 431 & & 7.33 \\
\hline D-02-180 & 30-Jul-02 & 23 & 21.6 & 8.95 & 116 & 781 & & 7.47 \\
\hline D-02-181 & 31-Jul-02 & & 18.1 & 8.82 & 107.3 & 464 & & 7.36 \\
\hline D-02-182 & 31-Jul-02 & 25 & 17.8 & 4.82 & 59.7 & 395 & & 8.68 \\
\hline D-02-183 & 31-Jul-02 & & 20.5 & 7.96 & 102 & 372 & & 8.28 \\
\hline D-02-184 & 31-Jul-02 & & 21.7 & 8.15 & 106.7 & 379 & & 8.24 \\
\hline D-02-185 & 1-Aug-02 & & 15.4 & 0.00 & 0 & 754 & & 7.20 \\
\hline D-02-186 & 1-Aug-02 & & & & & & & \\
\hline D-02-187 & 1-Aug-02 & & & & & & & \\
\hline D-02-188 & 1-Aug-02 & & 19.1 & 12.54 & & 227 & & 8.63 \\
\hline D-02-189 & 1-Aug-02 & & & & & & & \\
\hline D-02-190 & 1-Aug-02 & & 19.5 & 7.61 & 94.7 & 253 & & 7.76 \\
\hline D-02-191 & 15-Aug-02 & 20 & 17.8 & 8.33 & 101.4 & 593 & & 8.93 \\
\hline D-02-192 & 16-Aug-02 & 25 & 21.5 & 7.66 & 83.9 & 640 & & 8.21 \\
\hline D-02-193 & 20-Aug-02 & 24 & 21.2 & 12.42 & & 1225 & & 8.94 \\
\hline D-02-194 & 20-Aug-02 & & & & & & & \\
\hline D-02-195 & 21-Aug-02 & 23 & 17.6 & 8.67 & 104.5 & 269 & & 7.88 \\
\hline D-02-196 & 21-Aug-02 & 23 & 18.8 & 8.55 & 105.9 & 143 & & 7.52 \\
\hline D-02-197 & 22-Aug-02 & 25 & 14.4 & 7.16 & 83.9 & 392 & & 8.16 \\
\hline D-02-198 & 22-Aug-02 & 26 & 21.9 & 10.41 & 139 & 481 & & 8.58 \\
\hline
\end{tabular}


Appendix 6: Results of basic water chemistry and air temperature measurements recorded at most sites where fishing effort was applied between 2002 and 2006.

\begin{tabular}{|c|c|c|c|c|c|c|c|c|}
\hline \multirow{2}{*}{ Site Number } & \multirow{2}{*}{ Date } & \multirow{2}{*}{$\begin{array}{l}\text { Air Temp. } \\
\left({ }^{\circ} \mathrm{C}\right)\end{array}$} & \multirow{2}{*}{$\begin{array}{c}\text { Water Temp. } \\
\left({ }^{\circ} \mathrm{C}\right)\end{array}$} & \multicolumn{2}{|c|}{ Dissolved Oxygen } & \multirow{2}{*}{$\begin{array}{c}\text { Conductivity } \\
(\mu \mathrm{S} / \mathrm{cm})\end{array}$} & \multirow{2}{*}{$\begin{array}{l}\text { Turbidity } \\
\text { (NTU) }\end{array}$} & \multirow{2}{*}{$\begin{array}{c}\mathrm{pH} \\
\text { (units) }\end{array}$} \\
\hline & & & & (mg/L) & (\% SAT) & & & \\
\hline D-02-199 & 27-Aug-02 & 20.5 & 14.4 & 9.55 & 107.4 & 626 & & 8.14 \\
\hline D-02-200 & 27-Aug-02 & 21 & 16.2 & 8.64 & 102 & 520 & & 8.02 \\
\hline D-02-201 & 8-Oct-02 & 5 & & & & & & \\
\hline B-03-001 & 21-May-03 & 19 & 8.2 & 10.48 & 96.6 & 174 & 24.98 & 7.94 \\
\hline B-03-002 & 23-May-03 & 17 & & & & & & \\
\hline B-03-003 & 23-May-03 & 17.5 & 13.2 & 6.88 & 70.8 & 950 & 1.11 & 8.25 \\
\hline B-03-004 & 23-May-03 & 17 & 13.4 & 7.36 & 82 & 700 & 0.00 & 8.05 \\
\hline B-03-005 & 23-May-03 & 17 & 16.0 & 9.84 & 116 & 679 & 2.54 & 8.43 \\
\hline B-03-006 & 23-May-03 & 17 & & & & & & \\
\hline B-03-007 & 26-May-03 & 21 & 16.2 & 9.28 & 113.8 & 749 & 2.87 & 7.90 \\
\hline B-03-008 & 26-May-03 & 21 & & & & & & \\
\hline B-03-009 & 26-May-03 & 26 & 19.4 & 8.01 & 104.4 & 1321 & 0.5 & 7.97 \\
\hline B-03-010 & 26-May-03 & 29 & & & & & & \\
\hline B-03-011 & 26-May-03 & 29 & & & & & & \\
\hline B-03-012 & 26-May-03 & 27 & 25.2 & & & 358 & 1.72 & 8.70 \\
\hline B-03-013 & 27-May-03 & 19 & 17.5 & 4.62 & 57.6 & 903 & 1.89 & 8.23 \\
\hline B-03-014 & 27-May-03 & 21 & & & & & & \\
\hline B-03-015 & 27-May-03 & 22 & 18.3 & 3.75 & 48.9 & 1054 & 0.15 & 7.78 \\
\hline B-03-016 & 27-May-03 & 27.5 & 19.9 & 10.94 & & 1010 & 1.02 & 8.56 \\
\hline B-03-017 & 28-May-03 & 25.5 & 18.5 & 8.83 & 104.7 & 1085 & 10.72 & 8.64 \\
\hline B-03-018 & 28-May-03 & 28 & & & & & & \\
\hline B-03-019 & 28-May-03 & 26 & 24.0 & 13.40 & & 1185 & 4.46 & 8.73 \\
\hline B-03-020 & 29-May-03 & 22.5 & 16.9 & 1.80 & 27.8 & 755 & 0.00 & 7.63 \\
\hline B-03-021 & 29-May-03 & 22 & & & & & & \\
\hline B-03-022 & 29-May-03 & 22 & & & & & & \\
\hline B-03-023 & 29-May-03 & 22 & & & & & & \\
\hline B-03-024 & 29-May-03 & 22 & & & & & & \\
\hline B-03-025 & 29-May-03 & 25 & & & & & & \\
\hline B-03-026 & 29-May-03 & 21 & 19.0 & 6.80 & 89.7 & 751 & 2.94 & 8.04 \\
\hline B-03-027 & 30-May-03 & 10 & & & & & & \\
\hline B-03-028 & 30-May-03 & 10 & 12.6 & 6.95 & 77.8 & 700 & 0.46 & 8.05 \\
\hline B-03-029 & 30-May-03 & 12 & & & & & & \\
\hline B-03-030 & 30-May-03 & 14 & 17.6 & 10.89 & 137.5 & 1296 & 13.01 & 8.92 \\
\hline
\end{tabular}


Appendix 6: Results of basic water chemistry and air temperature measurements recorded at most sites where fishing effort was applied between 2002 and 2006.

\begin{tabular}{|c|c|c|c|c|c|c|c|c|}
\hline \multirow{2}{*}{ Site Number } & \multirow{2}{*}{ Date } & \multirow{2}{*}{$\begin{array}{l}\text { Air Temp. } \\
\left({ }^{\circ} \mathrm{C}\right)\end{array}$} & \multirow{2}{*}{$\begin{array}{c}\text { Water Temp. } \\
\left({ }^{\circ} \mathrm{C}\right)\end{array}$} & \multicolumn{2}{|c|}{ Dissolved Oxygen } & \multirow{2}{*}{$\begin{array}{c}\text { Conductivity } \\
(\mu \mathrm{S} / \mathrm{cm})\end{array}$} & \multirow{2}{*}{$\begin{array}{l}\text { Turbidity } \\
\text { (NTU) }\end{array}$} & \multirow{2}{*}{$\begin{array}{c}\mathrm{pH} \\
\text { (units) }\end{array}$} \\
\hline & & & & (mg/L) & (\% SAT) & & & \\
\hline B-03-031 & 30-May-03 & 15 & 19.4 & 11.70 & 152.8 & 1724 & 43.05 & 8.56 \\
\hline B-03-032 & 2-Jun-03 & 20 & & & & & & \\
\hline B-03-033 & 2-Jun-03 & 16 & 15.8 & 6.94 & 82.6 & 1195 & 2.19 & 8.27 \\
\hline B-03-034 & 2-Jun-03 & 17 & & & & & & \\
\hline B-03-035 & 2-Jun-03 & 22 & 18.7 & 11.54 & 144 & 1688 & 4.69 & 8.28 \\
\hline B-03-036 & 2-Jun-03 & 21 & & & & & & \\
\hline B-03-037 & 2-Jun-03 & 20 & & & & & & \\
\hline B-03-038 & 2-Jun-03 & 20 & & & & & & \\
\hline B-03-039 & 3-Jun-03 & 14 & & & & & & \\
\hline B-03-040 & 3-Jun-03 & 14 & 13.0 & 8.70 & 100 & 900 & 0.00 & 8.20 \\
\hline B-03-041 & 3-Jun-03 & 15 & 13.7 & 7.30 & 87.9 & 624 & 7.87 & 8.10 \\
\hline B-03-042 & 3-Jun-03 & 18 & & & & & & \\
\hline B-03-043 & 3-Jun-03 & 17 & & & & & & \\
\hline B-03-044 & 3-Jun-03 & & & & & & & \\
\hline B-03-045 & 4-Jun-03 & 17 & & & & & & \\
\hline B-03-046 & 4-Jun-03 & 17 & & & & & & \\
\hline B-03-047 & 4-Jun-03 & 14 & & & & & & \\
\hline B-03-048 & 4-Jun-03 & 18 & 16.9 & 7.40 & 90 & 1475 & 0.74 & 8.25 \\
\hline B-03-049 & 4-Jun-03 & 17 & & & & & & \\
\hline B-03-050 & 4-Jun-03 & 17 & 18.2 & 6.25 & 77.7 & 1591 & 2.26 & 7.91 \\
\hline B-03-051 & 4-Jun-03 & 20 & & & & & & \\
\hline B-03-052 & 4-Jun-03 & 20 & 20.1 & 6.04 & 79.6 & 1057 & 1.76 & 8.20 \\
\hline B-03-053 & 5-Jun-03 & 16 & 18.0 & 10.73 & 137.6 & 1508 & 3.12 & 6.85 \\
\hline B-03-054 & 5-Jun-03 & 18 & & & & & & \\
\hline B-03-055 & 5-Jun-03 & 18 & 15.7 & 8.39 & 105 & 1378 & 6.84 & 6.85 \\
\hline B-03-056 & 5-Jun-03 & 19 & & & & & & \\
\hline B-03-057 & 5-Jun-03 & 17 & & & & & & \\
\hline B-03-058 & 5-Jun-03 & 19 & & & & & & \\
\hline B-03-059 & 5-Jun-03 & 19 & & & & & & \\
\hline B-03-060 & 5-Jun-03 & 19 & 18.8 & 8.08 & 101 & 588 & 1.73 & 6.86 \\
\hline B-03-061 & 9-Jun-03 & 10 & 15.1 & 5.95 & 73.9 & 963 & 0.27 & 8.06 \\
\hline B-03-062 & 9-Jun-03 & 12 & 14.9 & 8.53 & 101.6 & $>2000$ & 2.39 & 8.49 \\
\hline B-03-063 & 9-Jun-03 & 12 & & & & & & \\
\hline
\end{tabular}


Appendix 6: Results of basic water chemistry and air temperature measurements recorded at most sites where fishing effort was applied between 2002 and 2006.

\begin{tabular}{|c|c|c|c|c|c|c|c|c|}
\hline \multirow{2}{*}{ Site Number } & \multirow{2}{*}{ Date } & \multirow{2}{*}{$\begin{array}{l}\text { Air Temp. } \\
\left({ }^{\circ} \mathrm{C}\right)\end{array}$} & \multirow{2}{*}{$\begin{array}{c}\text { Water Temp. } \\
\left({ }^{\circ} \mathrm{C}\right)\end{array}$} & \multicolumn{2}{|c|}{ Dissolved Oxygen } & \multirow{2}{*}{$\begin{array}{c}\text { Conductivity } \\
(\mu \mathrm{S} / \mathrm{cm})\end{array}$} & \multirow{2}{*}{$\begin{array}{l}\text { Turbidity } \\
\text { (NTU) }\end{array}$} & \multirow{2}{*}{$\begin{array}{c}\mathrm{pH} \\
\text { (units) }\end{array}$} \\
\hline & & & & (mg/L) & (\% SAT) & & & \\
\hline B-03-064 & 10-Jun-03 & 21.5 & 13.7 & 10.39 & 116.4 & 651 & 2.01 & 8.49 \\
\hline B-03-065 & 10-Jun-03 & 20 & 15.4 & 9.58 & 118.5 & 949 & 5.11 & 6.86 \\
\hline B-03-066 & 10-Jun-03 & 23 & & & & & & \\
\hline B-03-067 & 10-Jun-03 & 23 & 18.4 & 10.77 & 141.5 & 850 & 6.2 & 6.86 \\
\hline B-03-068 & 11-Jun-03 & 17 & & & & & & \\
\hline B-03-069 & 11-Jun-03 & 18 & & & & & & \\
\hline B-03-070 & 11-Jun-03 & 21 & 14.4 & 7.85 & 96.2 & 372 & 6.31 & 8.20 \\
\hline B-03-071 & 11-Jun-03 & 14 & 16.0 & 8.94 & 113.4 & 350 & 7.32 & 6.85 \\
\hline B-03-072 & 11-Jun-03 & 16 & & & & & & \\
\hline B-03-073 & 11-Jun-03 & 15 & & & & & & \\
\hline B-03-074 & 11-Jun-03 & 15 & & & & & & \\
\hline B-03-075 & 12-Jun-03 & 10 & 13.4 & 2.71 & 32.7 & 844 & 2.2 & 7.35 \\
\hline B-03-076 & 12-Jun-03 & 23 & 15.2 & 6.23 & 73 & 523 & 11.77 & 6.85 \\
\hline B-03-077 & 12-Jun-03 & 15 & 8.9 & 12.48 & & 490 & 10.96 & 6.86 \\
\hline B-03-078 & 12-Jun-03 & 17 & & & & & & \\
\hline B-03-079 & 12-Jun-03 & 18 & & & & & & \\
\hline B-03-080 & 12-Jun-03 & 18 & & & & & & \\
\hline B-03-081 & 12-Jun-03 & 18 & & & & & & \\
\hline B-03-082 & 12-Jun-03 & 18 & 18.9 & 7.76 & 98.3 & 1265 & 2.3 & 6.86 \\
\hline B-03-083 & 16-Jun-03 & 24 & 25.7 & 7.75 & 105.9 & 972 & 8.07 & \\
\hline B-03-084 & 16-Jun-03 & 24 & 27.3 & 8.03 & 113.8 & 837 & 4.24 & 8.05 \\
\hline B-03-085 & 17-Jun-03 & 21 & 23.5 & 6.28 & 82.2 & 520 & 12.94 & 7.66 \\
\hline B-03-086 & 17-Jun-03 & 25 & 25.9 & 6.37 & 87.3 & 751 & 16.89 & 7.92 \\
\hline B-03-087 & 17-Jun-03 & 20 & 26.7 & 8.80 & 122 & 785 & 7.23 & 6.86 \\
\hline B-03-088 & 18-Jun-03 & 21 & 22.5 & 7.63 & 98 & 606 & 1.2 & 7.68 \\
\hline B-03-089 & 18-Jun-03 & 24 & 23.1 & 8.64 & 112 & 599 & 3.48 & 7.90 \\
\hline B-03-090 & 18-Jun-03 & 24 & & & & & & \\
\hline B-03-091 & 18-Jun-03 & 23 & 23.5 & 1.45 & 19 & 283 & 177 & 6.86 \\
\hline B-03-092 & 18-Jun-03 & 24 & & & & & & \\
\hline B-03-093 & 18-Jun-03 & 21 & 25.9 & 8.73 & 119.7 & 887 & 11.19 & 6.86 \\
\hline B-03-094 & 18-Jun-03 & 19 & 26.6 & 11.59 & 160.3 & 833 & 0.79 & 6.86 \\
\hline B-03-095 & 19-Jun-03 & 17 & 20.0 & 5.84 & 71.2 & 705 & 0.00 & 7.73 \\
\hline B-03-096 & 19-Jun-03 & 21 & & & & & & \\
\hline
\end{tabular}


Appendix 6: Results of basic water chemistry and air temperature measurements recorded at most sites where fishing effort was applied between 2002 and 2006.

\begin{tabular}{|c|c|c|c|c|c|c|c|c|}
\hline \multirow{2}{*}{ Site Number } & \multirow{2}{*}{ Date } & \multirow{2}{*}{$\begin{array}{c}\text { Air Temp. } \\
\left({ }^{\circ} \mathrm{C}\right)\end{array}$} & \multirow{2}{*}{$\begin{array}{c}\text { Water Temp. } \\
\left({ }^{\circ} \mathrm{C}\right)\end{array}$} & \multicolumn{2}{|c|}{ Dissolved Oxygen } & \multirow{2}{*}{$\begin{array}{c}\text { Conductivity } \\
(\mu \mathrm{S} / \mathrm{cm})\end{array}$} & \multirow{2}{*}{$\begin{array}{c}\text { Turbidity } \\
\text { (NTU) }\end{array}$} & \multirow{2}{*}{$\begin{array}{c}\mathrm{pH} \\
\text { (units) }\end{array}$} \\
\hline & & & & (mg/L) & (\% SAT) & & & \\
\hline B-03-097 & 19-Jun-03 & 23 & 21.5 & 7.41 & 94.6 & 925 & 14.48 & 8.03 \\
\hline B-03-098 & 19-Jun-03 & 23 & 23.5 & 5.99 & 82 & 1407 & 13.7 & 8.09 \\
\hline B-03-099 & 20-Jun-03 & 22 & 17.9 & 6.66 & 83 & 1099 & 1.27 & 7.80 \\
\hline B-03-100 & 20-Jun-03 & & & & & & & \\
\hline B-03-101 & 20-Jun-03 & 24 & 24.9 & 9.89 & 143 & 714 & 3.77 & 8.65 \\
\hline B-03-102 & 23-Jun-03 & 21 & 18.0 & 8.59 & 100.7 & 295 & 2.26 & 8.05 \\
\hline B-03-103 & 23-Jun-03 & 21 & 24.9 & 9.67 & 147.9 & 341 & 2.25 & 7.59 \\
\hline B-03-104 & 23-Jun-03 & 23 & 20.2 & 6.87 & 95.5 & 393 & 3.04 & 7.90 \\
\hline B-03-105 & 24-Jun-03 & 13 & & & & & & \\
\hline B-03-106 & 24-Jun-03 & 14 & & & & & & \\
\hline B-03-107 & 24-Jun-03 & 15 & & & & & & \\
\hline B-03-108 & 24-Jun-03 & 15 & & & & & & \\
\hline B-03-109 & 24-Jun-03 & 17 & & & & & & \\
\hline B-03-110 & 26-Jun-03 & 17 & & & & & & \\
\hline B-03-111 & 26-Jun-03 & 17 & & & & & & \\
\hline B-03-112 & 26-Jun-03 & 17 & 16.0 & 9.94 & 115.7 & 324 & 6.03 & 8.31 \\
\hline B-03-113 & 26-Jun-03 & 18 & & & & & & \\
\hline B-03-114 & 26-Jun-03 & 18 & & & & & & \\
\hline B-03-115 & 27-Jun-03 & 14.5 & 14.0 & 8.37 & 93.7 & 347 & 9.07 & 8.07 \\
\hline B-03-116 & 27-Jun-03 & 17 & 15.5 & 9.62 & 108.7 & 273 & 6.68 & 6.86 \\
\hline B-03-117 & 27-Jun-03 & 18 & 14.7 & 7.13 & 80.1 & 323 & 1.68 & 7.85 \\
\hline B-03-118 & 27-Jun-03 & 18 & 21.1 & 7.06 & 91.2 & 284 & 2.67 & 7.70 \\
\hline B-03-119 & 2-Jul-03 & 22 & 22.6 & 4.62 & 61.4 & 321 & 4.01 & 7.56 \\
\hline B-03-120 & 2-Jul-03 & 24 & 23.4 & 6.99 & 93.8 & 352 & 5.35 & 7.98 \\
\hline B-03-121 & 3-Jul-03 & 18 & 19.9 & 3.53 & 45 & 259 & 6.63 & 7.36 \\
\hline B-03-122 & 3-Jul-03 & 20 & 20.5 & 8.25 & 104.5 & 323 & 2.7 & 8.05 \\
\hline B-03-123 & 3-Jul-03 & 24 & & & & & & \\
\hline B-03-124 & 3-Jul-03 & 24 & 24.8 & 10.49 & 148.1 & 326 & 13.29 & 8.34 \\
\hline B-03-125 & 3-Jul-03 & 26 & & & & & & \\
\hline B-03-126 & 4-Jul-03 & 18 & 21.9 & 9.84 & 133.4 & 528 & 2.52 & \\
\hline B-03-127 & 4-Jul-03 & 23 & & & & & & \\
\hline B-03-128 & 4-Jul-03 & 23 & & & & & & \\
\hline B-03-129 & 4-Jul-03 & 23 & & & & & & \\
\hline
\end{tabular}


Appendix 6: Results of basic water chemistry and air temperature measurements recorded at most sites where fishing effort was applied between 2002 and 2006.

\begin{tabular}{|c|c|c|c|c|c|c|c|c|}
\hline \multirow{2}{*}{ Site Number } & \multirow{2}{*}{ Date } & \multirow{2}{*}{$\begin{array}{l}\text { Air Temp. } \\
\left({ }^{\circ} \mathrm{C}\right)\end{array}$} & \multirow{2}{*}{$\begin{array}{c}\text { Water Temp. } \\
\left({ }^{\circ} \mathrm{C}\right)\end{array}$} & \multicolumn{2}{|c|}{ Dissolved Oxygen } & \multirow{2}{*}{$\begin{array}{c}\text { Conductivity } \\
(\mu \mathrm{S} / \mathrm{cm})\end{array}$} & \multirow{2}{*}{$\begin{array}{l}\text { Turbidity } \\
\text { (NTU) }\end{array}$} & \multirow{2}{*}{$\begin{array}{c}\mathrm{pH} \\
\text { (units) }\end{array}$} \\
\hline & & & & (mg/L) & (\% SAT) & & & \\
\hline B-03-130 & 4-Jul-03 & 24 & & & & & & \\
\hline B-03-131 & 4-Jul-03 & 24 & & & & & & \\
\hline B-03-132 & 7-Jul-03 & 14 & 15.3 & 9.73 & 116.7 & 424 & 2.89 & 8.38 \\
\hline B-03-133 & 7-Jul-03 & 14 & & & & & & \\
\hline B-03-134 & 7-Jul-03 & 15 & 13.9 & 5.17 & 63.3 & 415 & 3.99 & 7.53 \\
\hline B-03-135 & 7-Jul-03 & 16 & & & & & & \\
\hline B-03-136 & 7-Jul-03 & 13 & 18.2 & 3.18 & 43.3 & 501 & 11.9 & 7.76 \\
\hline B-03-137 & 7-Jul-03 & 13 & & & & & & \\
\hline B-03-138 & 7-Jul-03 & 18 & & & & & & \\
\hline B-03-139 & 7-Jul-03 & 18 & & & & & & \\
\hline B-03-140 & 8-Jul-03 & 14 & & & & & & \\
\hline B-03-141 & 8-Jul-03 & 16 & 17.3 & 9.91 & 121.3 & 1045 & 6.4 & 8.08 \\
\hline B-03-142 & 8-Jul-03 & 16 & 17.2 & 8.50 & 104 & 1125 & 5.22 & 7.74 \\
\hline B-03-143 & 8-Jul-03 & 16 & 20.4 & 7.01 & 90.3 & 746 & 6.52 & 8.05 \\
\hline B-03-144 & 9-Jul-03 & 14 & & & & & & \\
\hline B-03-145 & 9-Jul-03 & 14 & 18.6 & 8.58 & 108.7 & 720 & 2.57 & 8.69 \\
\hline B-03-146 & 9-Jul-03 & 14 & & & & & & \\
\hline B-03-147 & 9-Jul-03 & 14 & & & & & & \\
\hline B-03-148 & 9-Jul-03 & 14 & 20.8 & 12.69 & & 985 & 48.14 & 8.31 \\
\hline B-03-149 & 9-Jul-03 & 17 & & & & & & \\
\hline B-03-150 & 9-Jul-03 & 15 & 22.4 & 13.27 & & 1194 & 22.65 & \\
\hline B-03-151 & 10-Jul-03 & 15 & 18.5 & 8.29 & 105.5 & 643 & 6.13 & 8.29 \\
\hline B-03-152 & 10-Jul-03 & 18 & 21.5 & 8.69 & 121.8 & 711 & 20.33 & 8.16 \\
\hline B-03-153 & 10-Jul-03 & 21 & & & & & & \\
\hline B-03-154 & 10-Jul-03 & 21 & & & & & & \\
\hline B-03-155 & 11-Jul-03 & 19 & & & & & & \\
\hline B-03-156 & 11-Jul-03 & 19 & & & & & & \\
\hline B-03-157 & 11-Jul-03 & 19 & & & & & & \\
\hline B-03-158 & 11-Jul-03 & 20.5 & 18.8 & 7.45 & 98.3 & 597 & 7.32 & 8.23 \\
\hline B-03-159 & 11-Jul-03 & 24 & 18.5 & 12.35 & 162.5 & 645 & 6.02 & 8.16 \\
\hline B-03-160 & 11-Jul-03 & 24 & 23.6 & 9.42 & 139.5 & 927 & 13.00 & 8.15 \\
\hline B-03-161 & 14-Jul-03 & 18 & & & & & & \\
\hline B-03-162 & 14-Jul-03 & 19 & & & & & & \\
\hline
\end{tabular}


Appendix 6: Results of basic water chemistry and air temperature measurements recorded at most sites where fishing effort was applied between 2002 and 2006.

\begin{tabular}{|c|c|c|c|c|c|c|c|c|}
\hline \multirow{2}{*}{ Site Number } & \multirow{2}{*}{ Date } & \multirow{2}{*}{$\begin{array}{l}\text { Air Temp. } \\
\left({ }^{\circ} \mathrm{C}\right)\end{array}$} & \multirow{2}{*}{$\begin{array}{c}\text { Water Temp. } \\
\left({ }^{\circ} \mathrm{C}\right)\end{array}$} & \multicolumn{2}{|c|}{ Dissolved Oxygen } & \multirow{2}{*}{$\begin{array}{c}\text { Conductivity } \\
(\mu \mathrm{S} / \mathrm{cm})\end{array}$} & \multirow{2}{*}{$\begin{array}{l}\text { Turbidity } \\
\text { (NTU) }\end{array}$} & \multirow{2}{*}{$\begin{array}{c}\mathrm{pH} \\
\text { (units) }\end{array}$} \\
\hline & & & & (mg/L) & (\% SAT) & & & \\
\hline B-03-163 & 14-Jul-03 & 23 & 23.3 & 3.97 & 52.3 & 361 & 55 & 8.01 \\
\hline B-03-164 & 14-Jul-03 & 23 & & & & & & \\
\hline B-03-165 & 14-Jul-03 & 23 & & & & & & \\
\hline B-03-166 & 14-Jul-03 & 17 & & & & & & \\
\hline B-03-167 & 14-Jul-03 & 19 & & & & & & \\
\hline B-03-168 & 14-Jul-03 & 19 & & & & & & \\
\hline B-03-169 & 14-Jul-03 & 19 & & & & & & \\
\hline B-03-170 & 14-Jul-03 & 19 & & & & & & \\
\hline B-03-171 & 14-Jul-03 & 19 & & & & & & \\
\hline B-03-172 & 14-Jul-03 & 20 & & & & & & \\
\hline B-03-173 & 14-Jul-03 & 20 & & & & & & \\
\hline B-03-174 & 14-Jul-03 & 20 & & & & & & \\
\hline B-03-175 & 14-Jul-03 & 20 & & & & & & \\
\hline B-03-176 & 14-Jul-03 & 20 & & & & & & \\
\hline B-03-177 & 14-Jul-03 & 21 & & & & & & \\
\hline B-03-178 & 14-Jul-03 & 21 & & & & & & \\
\hline B-03-179 & 14-Jul-03 & 21 & & & & & & \\
\hline B-03-180 & 14-Jul-03 & 21 & & & & & & \\
\hline B-03-181 & 15-Jul-03 & 22 & 19.0 & 6.15 & 75.3 & 349 & 49.54 & 8.07 \\
\hline B-03-182 & 16-Jul-03 & 20 & 21.2 & 4.84 & 60.4 & 335 & 5.4 & 7.69 \\
\hline B-03-183 & 16-Jul-03 & 22 & 20.9 & 5.36 & 65.1 & 311 & 27.33 & 8.14 \\
\hline B-03-184 & 16-Jul-03 & 23 & 21.8 & 3.74 & 47.4 & 350 & 3.24 & 7.66 \\
\hline B-03-185 & 16-Jul-03 & 23 & 24.4 & 6.74 & 90.3 & 262 & 9.08 & 7.95 \\
\hline B-03-186 & 16-Jul-03 & 25 & & & & & & \\
\hline B-03-187 & 16-Jul-03 & 25 & & & & & & \\
\hline B-03-188 & 16-Jul-03 & 25 & & & & & & \\
\hline B-03-189 & 16-Jul-03 & 23 & 24.6 & 6.60 & 85 & 296 & 18.29 & 7.51 \\
\hline B-03-190 & 16-Jul-03 & 24 & 26.0 & 7.85 & 105.1 & 255 & 7.82 & 8.25 \\
\hline B-03-191 & 17-Jul-03 & 15 & 18.9 & 5.92 & 72 & 298 & 3.7 & 7.65 \\
\hline B-03-192 & 17-Jul-03 & 18 & 18.9 & 8.67 & 106 & 253 & 2.00 & 7.59 \\
\hline B-03-193 & 17-Jul-03 & 23 & 21.0 & 8.23 & 104.8 & 250 & 2.54 & 7.78 \\
\hline B-03-194 & 22-Jul-03 & 29 & 26.8 & 11.69 & 165.5 & 529 & 6.84 & 9.05 \\
\hline B-03-195 & 23-Jul-03 & 17 & & & & & & \\
\hline
\end{tabular}


Appendix 6: Results of basic water chemistry and air temperature measurements recorded at most sites where fishing effort was applied between 2002 and 2006.

\begin{tabular}{|c|c|c|c|c|c|c|c|c|}
\hline \multirow{2}{*}{ Site Number } & \multirow{2}{*}{ Date } & \multirow{2}{*}{$\begin{array}{l}\text { Air Temp. } \\
\left({ }^{\circ} \mathrm{C}\right)\end{array}$} & \multirow{2}{*}{$\begin{array}{c}\text { Water Temp. } \\
\left({ }^{\circ} \mathrm{C}\right)\end{array}$} & \multicolumn{2}{|c|}{ Dissolved Oxygen } & \multirow{2}{*}{$\begin{array}{c}\text { Conductivity } \\
(\mu \mathrm{S} / \mathrm{cm})\end{array}$} & \multirow{2}{*}{$\begin{array}{l}\text { Turbidity } \\
\text { (NTU) }\end{array}$} & \multirow{2}{*}{$\begin{array}{c}\mathrm{pH} \\
\text { (units) }\end{array}$} \\
\hline & & & & (mg/L) & (\% SAT) & & & \\
\hline B-03-196 & 23-Jul-03 & 17 & & & & & & \\
\hline B-03-197 & 23-Jul-03 & 19 & & & & & & \\
\hline B-03-198 & 23-Jul-03 & 18 & & & & & & \\
\hline B-03-199 & 23-Jul-03 & 20 & 16.3 & 7.14 & 81 & 356 & 22.91 & 7.91 \\
\hline B-03-200 & 23-Jul-03 & 23 & 23.1 & 10.51 & 136 & 482 & 73 & 8.35 \\
\hline B-03-201 & 23-Jul-03 & 27 & & & & & & \\
\hline B-03-202 & 23-Jul-03 & 25 & 20.8 & 4.70 & 59.5 & 332 & 3.56 & 7.52 \\
\hline B-03-203 & 23-Jul-03 & 27 & & & & & & \\
\hline B-03-204 & 23-Jul-03 & 27 & 23.3 & 4.89 & 65.9 & 375 & 1.11 & 7.48 \\
\hline B-03-205 & 23-Jul-03 & 25 & & & & & & \\
\hline B-03-206 & 24-Jul-03 & 18 & 19.5 & 4.32 & 53.4 & 290 & 5.00 & 7.97 \\
\hline B-03-207 & 24-Jul-03 & 18 & 20.7 & 7.77 & 98.4 & 288 & 10.20 & 8.23 \\
\hline B-03-208 & 24-Jul-03 & 23 & & & & & & \\
\hline B-03-209 & 24-Jul-03 & 27 & 23.9 & 8.28 & 110.9 & 294 & 15.23 & 6.89 \\
\hline B-03-210 & 24-Jul-03 & 27 & & & & & & \\
\hline B-03-211 & 24-Jul-03 & 27 & & & & & & \\
\hline B-03-212 & 24-Jul-03 & 30 & 27.5 & 12.05 & & 271 & 1.92 & 8.19 \\
\hline B-03-213 & 24-Jul-03 & 31 & & & & & & \\
\hline B-03-214 & 25-Jul-03 & 21 & & & & & & \\
\hline B-03-215 & 25-Jul-03 & 21 & & & & & & \\
\hline B-03-216 & 25-Jul-03 & 21 & & & & & & \\
\hline B-03-217 & 25-Jul-03 & 23 & & & & & & \\
\hline B-03-218 & 25-Jul-03 & 23 & & & & & & \\
\hline B-03-219 & 25-Jul-03 & 21 & & & & & & \\
\hline B-03-220 & 25-Jul-03 & 23 & & & & & & \\
\hline B-03-221 & 25-Jul-03 & 25 & & & & & & \\
\hline B-03-222 & 25-Jul-03 & 25 & & & & & & \\
\hline B-03-223 & 5-Aug-03 & 23 & & & & & & \\
\hline B-03-224 & 5-Aug-03 & 25 & 23.4 & 7.69 & 103.5 & 580 & 18.56 & 8.15 \\
\hline B-03-225 & 5-Aug-03 & 24 & & & & & & \\
\hline B-03-226 & 6-Aug-03 & 15 & 18.8 & 1.99 & 22.7 & 244 & 4.16 & 7.94 \\
\hline B-03-227 & 6-Aug-03 & 23.5 & & & & & & \\
\hline B-03-228 & 6-Aug-03 & 23.5 & & & & & & \\
\hline
\end{tabular}


Appendix 6: Results of basic water chemistry and air temperature measurements recorded at most sites where fishing effort was applied between 2002 and 2006.

\begin{tabular}{|c|c|c|c|c|c|c|c|c|}
\hline \multirow{2}{*}{ Site Number } & \multirow{2}{*}{ Date } & \multirow{2}{*}{$\begin{array}{l}\text { Air Temp. } \\
\left({ }^{\circ} \mathrm{C}\right)\end{array}$} & \multirow{2}{*}{$\begin{array}{c}\text { Water Temp. } \\
\left({ }^{\circ} \mathrm{C}\right)\end{array}$} & \multicolumn{2}{|c|}{ Dissolved Oxygen } & \multirow{2}{*}{$\begin{array}{c}\text { Conductivity } \\
(\mu \mathrm{S} / \mathrm{cm})\end{array}$} & \multirow{2}{*}{$\begin{array}{l}\text { Turbidity } \\
\text { (NTU) }\end{array}$} & \multirow{2}{*}{$\begin{array}{c}\mathrm{pH} \\
\text { (units) }\end{array}$} \\
\hline & & & & (mg/L) & (\% SAT) & & & \\
\hline B-03-229 & 6-Aug-03 & 23.5 & & & & & & \\
\hline B-03-230 & 6-Aug-03 & 23.5 & & & & & & \\
\hline B-03-231 & 6-Aug-03 & 24 & & & & & & \\
\hline B-03-232 & 6-Aug-03 & 24 & & & & & & \\
\hline B-03-233 & 6-Aug-03 & 24 & & & & & & \\
\hline B-03-234 & 6-Aug-03 & 25 & & & & & & \\
\hline B-03-235 & 6-Aug-03 & 25 & 27.3 & 11.39 & 159.6 & 446 & 4.9 & 8.29 \\
\hline B-03-236 & 6-Aug-03 & 24 & & & & & & \\
\hline B-03-237 & 7-Aug-03 & 21 & & & & & & \\
\hline B-03-238 & 7-Aug-03 & 21 & & & & & & \\
\hline B-03-239 & 7-Aug-03 & 26 & 21.3 & 6.32 & 78.8 & 528 & 21.32 & \\
\hline B-03-240 & 7-Aug-03 & 25 & & & & & & \\
\hline B-03-241 & 7-Aug-03 & 25 & & & & 689 & 26.59 & 8.44 \\
\hline B-03-242 & 7-Aug-03 & 26 & & & & & & \\
\hline B-03-243 & 7-Aug-03 & 27 & & & & & & \\
\hline B-03-244 & 7-Aug-03 & 29 & & & & & & \\
\hline B-03-245 & 7-Aug-03 & 28 & & & & & & \\
\hline B-03-246 & 8-Aug-03 & 18 & & & & & & \\
\hline B-03-247 & 8-Aug-03 & 18 & & & & & & \\
\hline B-03-248 & 8-Aug-03 & 18 & & & & & & \\
\hline B-03-249 & 8-Aug-03 & 23 & 22.7 & & & 442 & 1.74 & 7.86 \\
\hline B-03-250 & 8-Aug-03 & 27 & & & & & & \\
\hline B-03-273 & 5-Aug-04 & 17 & & & & & & \\
\hline D-03-001 & 8-May-03 & & 15.3 & 8.74 & 98 & 487 & 1.42 & $7-7.5^{*}$ \\
\hline D-03-002 & 10-May-03 & 0 & 6.5 & 9.44 & 92.5 & 303 & 1.15 & 8.19 \\
\hline D-03-003 & 10-May-03 & 4.5 & 8.5 & 9.82 & 96.4 & 424 & 6.4 & 8.47 \\
\hline D-03-004 & 11-May-03 & 11 & 6.5 & 9.97 & 92 & 425 & 6.15 & 8.16 \\
\hline D-03-005 & 11-May-03 & 14.5 & 10.6 & 10.40 & 107.6 & 949 & 3.2 & 8.34 \\
\hline D-03-006 & 12-May-03 & 17 & 13.8 & 11.92 & 129 & 588 & 6.26 & $7-7.5^{*}$ \\
\hline D-03-007 & 12-May-03 & & & & & & & \\
\hline D-03-008 & 13-May-03 & 10 & & & & & & \\
\hline D-03-009 & 13-May-03 & 18 & 11.1 & 7.06 & 72.1 & 516 & 1.06 & $7-7.5^{\star}$ \\
\hline D-03-010 & 13-May-03 & & & & & & & \\
\hline
\end{tabular}


Appendix 6: Results of basic water chemistry and air temperature measurements recorded at most sites where fishing effort was applied between 2002 and 2006.

\begin{tabular}{|c|c|c|c|c|c|c|c|c|}
\hline \multirow{2}{*}{ Site Number } & \multirow{2}{*}{ Date } & \multirow{2}{*}{$\begin{array}{l}\text { Air Temp. } \\
\left({ }^{\circ} \mathrm{C}\right)\end{array}$} & \multirow{2}{*}{$\begin{array}{l}\text { Water Temp. } \\
\left({ }^{\circ} \mathrm{C}\right)\end{array}$} & \multicolumn{2}{|c|}{ Dissolved Oxygen } & \multirow{2}{*}{$\begin{array}{c}\text { Conductivity } \\
(\mu \mathrm{S} / \mathrm{cm})\end{array}$} & \multirow{2}{*}{$\begin{array}{l}\text { Turbidity } \\
\text { (NTU) }\end{array}$} & \multirow{2}{*}{$\begin{array}{c}\text { pH } \\
\text { (units) }\end{array}$} \\
\hline & & & & (mg/L) & (\% SAT) & & & \\
\hline D-03-011 & 13-May-03 & 24 & 15.5 & 13.32 & & 442 & 2.48 & $7-7.5^{*}$ \\
\hline D-03-012 & 13-May-03 & 25 & 17.7 & 12.24 & & 435 & 5.35 & 8.65 \\
\hline D-03-013 & 13-May-03 & 25 & & & & & & \\
\hline D-03-014 & 13-May-03 & 20 & & & & & & \\
\hline D-03-015 & 14-May-03 & 14 & 9.0 & 10.17 & 99.9 & 416 & 26.10 & $7-7.5^{\star}$ \\
\hline D-03-016 & 14-May-03 & 25 & 12.7 & 9.45 & 101.2 & 381 & 4.57 & $7-7.5^{\star}$ \\
\hline D-03-017 & 14-May-03 & 25 & 14.0 & 9.27 & 105.6 & 555 & 1.42 & $7-7.5^{*}$ \\
\hline D-03-018 & 14-May-03 & 25 & & & & & & \\
\hline D-03-019 & 14-May-03 & 26 & 17.2 & 9.76 & 117.3 & 239 & 5.11 & $7-7.5^{*}$ \\
\hline D-03-020 & 14-May-03 & 25 & & & & & & \\
\hline D-03-021 & 3-Jun-03 & 18 & & & & & & \\
\hline D-03-022 & 3-Jun-03 & 19 & & & & & & \\
\hline D-03-023 & 3-Jun-03 & 20 & 18.1 & 8.65 & 103.5 & & 20.35 & 8.05 \\
\hline D-03-024 & 3-Jun-03 & 21 & 18.5 & 7.73 & 93.1 & & 10.57 & 8.15 \\
\hline D-03-025 & 3-Jun-03 & 22 & 19.3 & 9.19 & 110.7 & & 4.86 & 8.14 \\
\hline D-03-026 & 3-Jun-03 & 20 & 17.5 & 7.73 & 92.4 & & 13.38 & 8.24 \\
\hline D-03-027 & 4-Jun-03 & & 15.5 & 8.20 & 92.3 & & 3.47 & 6.90 \\
\hline D-03-028 & 4-Jun-03 & 18 & & & & & & \\
\hline D-03-029 & 4-Jun-03 & 19 & 15.5 & 8.98 & 106.3 & & 11.32 & 8.25 \\
\hline D-03-030 & 4-Jun-03 & & & & & & & \\
\hline D-03-031 & 4-Jun-03 & 19 & & & & & & \\
\hline D-03-032 & 4-Jun-03 & 18 & & & & & & \\
\hline D-03-033 & 4-Jun-03 & & & & & & & \\
\hline D-03-034 & 4-Jun-03 & & & & & & & \\
\hline D-03-035 & 5-Jun-03 & & 16.8 & 6.16 & 73 & & 3.91 & 8.13 \\
\hline D-03-036 & 5-Jun-03 & 19 & 20.0 & 7.02 & 89.7 & & 17.69 & 8.39 \\
\hline D-03-037 & 5-Jun-03 & 20 & 20.1 & 11.47 & 140 & & 3.8 & 8.64 \\
\hline D-03-038 & 6-Jun-03 & & 16.2 & 7.35 & 91.4 & 895 & 9.32 & 6.90 \\
\hline D-03-039 & 10-Jun-03 & 15 & & & & & & \\
\hline D-03-040 & 10-Jun-03 & & 18.0 & 8.74 & 110.9 & 1601 & 2.73 & 7.21 \\
\hline D-03-041 & 10-Jun-03 & 18 & 16.6 & 7.94 & 98 & 1887 & 2.62 & 7.37 \\
\hline D-03-042 & 10-Jun-03 & & & & & & & \\
\hline D-03-043 & 10-Jun-03 & 21 & & & & & & \\
\hline
\end{tabular}


Appendix 6: Results of basic water chemistry and air temperature measurements recorded at most sites where fishing effort was applied between 2002 and 2006.

\begin{tabular}{|c|c|c|c|c|c|c|c|c|}
\hline \multirow{2}{*}{ Site Number } & \multirow{2}{*}{ Date } & \multirow{2}{*}{$\begin{array}{l}\text { Air Temp. } \\
\left({ }^{\circ} \mathrm{C}\right)\end{array}$} & \multirow{2}{*}{$\begin{array}{c}\text { Water Temp. } \\
\left({ }^{\circ} \mathrm{C}\right)\end{array}$} & \multicolumn{2}{|c|}{ Dissolved Oxygen } & \multirow{2}{*}{$\begin{array}{c}\text { Conductivity } \\
(\mu \mathrm{S} / \mathrm{cm})\end{array}$} & \multirow{2}{*}{$\begin{array}{l}\text { Turbidity } \\
\text { (NTU) }\end{array}$} & \multirow{2}{*}{$\begin{array}{c}\mathrm{pH} \\
\text { (units) }\end{array}$} \\
\hline & & & & (mg/L) & (\% SAT) & & & \\
\hline D-03-044 & 11-Jun-03 & 16 & 14.3 & 6.98 & 86.8 & 871 & 244 & 7.48 \\
\hline D-03-045 & 11-Jun-03 & 17 & 17.0 & 6.17 & 73 & 1711 & 5.15 & 7.39 \\
\hline D-03-046 & 11-Jun-03 & & & & & & & \\
\hline D-03-047 & 11-Jun-03 & 17 & 17.8 & 6.23 & 75 & 1790 & 3.43 & 7.27 \\
\hline D-03-048 & 11-Jun-03 & & & & & & & \\
\hline D-03-049 & 11-Jun-03 & & 20.0 & 9.15 & 115.7 & 1810 & 2.88 & 7.81 \\
\hline D-03-050 & 13-Jun-03 & & & & & & & \\
\hline D-03-051 & 13-Jun-03 & & & & & & & \\
\hline D-03-052 & 13-Jun-03 & & & & & & & \\
\hline D-03-053 & 13-Jun-03 & & & & & & & \\
\hline D-03-054 & 13-Jun-03 & 20 & 18.8 & 12.08 & 159.4 & 1006 & 1.1 & 8.64 \\
\hline D-03-055 & 13-Jun-03 & 23 & & & & & & \\
\hline D-03-056 & 13-Jun-03 & 23 & & & & & & \\
\hline D-03-057 & 13-Jun-03 & 24 & 18.6 & 7.51 & 94.3 & 823 & 5.54 & 6.91 \\
\hline D-03-058 & 16-Jun-03 & & 22.3 & 7.48 & 97.2 & 589 & 3.89 & 7.70 \\
\hline D-03-059 & 16-Jun-03 & 18 & 23.8 & 8.33 & 113 & 487 & 4.12 & 8.04 \\
\hline D-03-060 & 16-Jun-03 & 27 & 24.4 & 6.90 & 95 & 507 & 3.57 & 7.67 \\
\hline D-03-061 & 17-Jun-03 & 20 & 19.9 & 6.64 & 87.7 & 423 & 2.35 & 7.52 \\
\hline D-03-062 & 17-Jun-03 & 22 & 18.6 & 6.80 & 88.8 & 321 & 5.82 & 7.74 \\
\hline D-03-063 & 17-Jun-03 & 21 & & & & & & \\
\hline D-03-064 & 17-Jun-03 & 22 & 20.6 & 5.84 & 79.4 & 490 & 9.12 & 6.91 \\
\hline D-03-065 & 17-Jun-03 & & 22.2 & 7.17 & 101.4 & 354 & 11.15 & 8.05 \\
\hline D-03-066 & 18-Jun-03 & & 15.4 & 7.28 & 89.5 & 304 & 3.3 & 6.92 \\
\hline D-03-067 & 18-Jun-03 & 23 & 19.4 & 7.29 & 94.3 & 361 & 6.42 & 7.63 \\
\hline D-03-068 & 18-Jun-03 & 25 & & & & & & \\
\hline D-03-069 & 18-Jun-03 & 25 & & & & & & \\
\hline D-03-070 & 23-Jun-03 & 23 & & & & & & \\
\hline D-03-071 & 23-Jun-03 & 21 & 21.5 & 6.74 & 89.8 & 1058 & 2.12 & 8.21 \\
\hline D-03-072 & 23-Jun-03 & & & & & & 2.03 & \\
\hline D-03-073 & 23-Jun-03 & 23 & 21.4 & 6.52 & 88.5 & 601 & 11.64 & 6.93 \\
\hline D-03-074 & 24-Jun-03 & & & & & & & \\
\hline D-03-075 & 24-Jun-03 & 20 & 11.4 & 0.43 & 4.6 & $>2000$ & 13.52 & 8.60 \\
\hline D-03-076 & 24-Jun-03 & 20 & 16.0 & 7.26 & 87.8 & $>2000$ & 5.68 & 8.47 \\
\hline
\end{tabular}


Appendix 6: Results of basic water chemistry and air temperature measurements recorded at most sites where fishing effort was applied between 2002 and 2006.

\begin{tabular}{|c|c|c|c|c|c|c|c|c|}
\hline \multirow{2}{*}{ Site Number } & \multirow{2}{*}{ Date } & \multirow{2}{*}{$\begin{array}{l}\text { Air Temp. } \\
\left({ }^{\circ} \mathrm{C}\right)\end{array}$} & \multirow{2}{*}{$\begin{array}{c}\text { Water Temp. } \\
\left({ }^{\circ} \mathrm{C}\right)\end{array}$} & \multicolumn{2}{|c|}{ Dissolved Oxygen } & \multirow{2}{*}{$\begin{array}{c}\text { Conductivity } \\
(\mu \mathrm{S} / \mathrm{cm})\end{array}$} & \multirow{2}{*}{$\begin{array}{l}\text { Turbidity } \\
\text { (NTU) }\end{array}$} & \multirow{2}{*}{$\begin{array}{c}\mathrm{pH} \\
\text { (units) }\end{array}$} \\
\hline & & & & (mg/L) & (\% SAT) & & & \\
\hline D-03-077 & 24-Jun-03 & 21 & & & & & & \\
\hline D-03-078 & 24-Jun-03 & 17 & 19.8 & 8.04 & 103.4 & 1013 & 1.09 & 8.17 \\
\hline D-03-079 & 24-Jun-03 & 17 & & & & & & \\
\hline D-03-080 & 24-Jun-03 & 16 & & & & & & \\
\hline D-03-081 & 24-Jun-03 & 16 & 18.4 & 7.00 & 89.2 & 627 & 0.92 & 8.53 \\
\hline D-03-082 & 24-Jun-03 & 17 & & & & & & \\
\hline D-03-083 & 26-Jun-03 & 19 & 18.8 & 7.40 & 105.4 & 737 & 0.54 & 8.53 \\
\hline D-03-084 & 26-Jun-03 & 16 & 13.2 & 3.91 & 49 & 381 & 14.57 & 7.98 \\
\hline D-03-085 & 27-Jun-03 & 18 & 15.1 & 7.34 & 89 & 339 & 6.3 & 8.57 \\
\hline D-03-086 & 27-Jun-03 & & 18.0 & 6.89 & 84.5 & 552 & 3.58 & 6.93 \\
\hline D-03-087 & 27-Jun-03 & & & & & & & \\
\hline D-03-088 & 2-Jul-03 & 21 & 21.8 & 7.27 & 92.6 & 337 & 5.02 & 8.82 \\
\hline D-03-089 & 2-Jul-03 & 22 & 22.9 & 7.41 & 100.1 & 484 & 11.61 & 8.60 \\
\hline D-03-090 & 2-Jul-03 & 27 & & & & & & \\
\hline D-03-091 & 3-Jul-03 & 18 & 17.3 & 6.14 & 79.4 & 1083 & 6.42 & 8.28 \\
\hline D-03-092 & 3-Jul-03 & 22 & & & & & & \\
\hline D-03-093 & 3-Jul-03 & 21 & & & & & & \\
\hline D-03-094 & 8-Jul-03 & 18 & 14.8 & 2.08 & 38.2 & 629 & 16.02 & 8.14 \\
\hline D-03-095 & 8-Jul-03 & 19 & 16.2 & 7.01 & 88.5 & 602 & 0.81 & 8.15 \\
\hline D-03-096 & 8-Jul-03 & 21 & 16.7 & 6.44 & 79.4 & 725 & 4.46 & 8.44 \\
\hline D-03-097 & 9-Jul-03 & 15 & 15.6 & 8.13 & 96.1 & 488 & 3.79 & 8.08 \\
\hline D-03-098 & 9-Jul-03 & 16 & 12.5 & 7.09 & 84.3 & 648 & 1.93 & 8.49 \\
\hline D-03-099 & 9-Jul-03 & & & & & & & \\
\hline D-03-100 & 9-Jul-03 & 18 & 20.1 & 8.49 & 103.8 & 995 & 0.41 & 6.93 \\
\hline D-03-101 & 9-Jul-03 & 20 & 17.7 & 6.33 & 80.1 & 516 & 2.45 & 8.50 \\
\hline D-03-102 & 11-Jul-03 & 19 & 16.5 & 4.70 & 57 & 570 & 4.76 & 8.16 \\
\hline D-03-103 & 11-Jul-03 & 19 & 18.3 & 7.32 & 90.7 & 499 & 0.71 & 8.63 \\
\hline D-03-104 & 11-Jul-03 & & & & & & & \\
\hline D-03-105 & 11-Jul-03 & 23 & & & & & & \\
\hline D-03-106 & 14-Jul-03 & 18 & & & & & & \\
\hline D-03-107 & 14-Jul-03 & 19 & & & & & & \\
\hline D-03-108 & 14-Jul-03 & 19 & & & & & & \\
\hline D-03-109 & 14-Jul-03 & 16 & & & & & & \\
\hline
\end{tabular}


Appendix 6: Results of basic water chemistry and air temperature measurements recorded at most sites where fishing effort was applied between 2002 and 2006.

\begin{tabular}{|c|c|c|c|c|c|c|c|c|}
\hline \multirow{2}{*}{ Site Number } & \multirow{2}{*}{ Date } & \multirow{2}{*}{$\begin{array}{l}\text { Air Temp. } \\
\left({ }^{\circ} \mathrm{C}\right)\end{array}$} & \multirow{2}{*}{$\begin{array}{c}\text { Water Temp. } \\
\left({ }^{\circ} \mathrm{C}\right)\end{array}$} & \multicolumn{2}{|c|}{ Dissolved Oxygen } & \multirow{2}{*}{$\begin{array}{c}\text { Conductivity } \\
(\mu \mathrm{S} / \mathrm{cm})\end{array}$} & \multirow{2}{*}{$\begin{array}{l}\text { Turbidity } \\
\text { (NTU) }\end{array}$} & \multirow{2}{*}{$\begin{array}{c}\mathrm{pH} \\
\text { (units) }\end{array}$} \\
\hline & & & & (mg/L) & (\% SAT) & & & \\
\hline D-03-110 & 14-Jul-03 & 18 & 20.6 & 9.04 & 122.8 & 1139 & 42.41 & 8.47 \\
\hline D-03-111 & 14-Jul-03 & 18 & 22.2 & 6.01 & 79 & 604 & 26.52 & 8.59 \\
\hline D-03-112 & 15-Jul-03 & 21 & & & & & & \\
\hline D-03-113 & 15-Jul-03 & 21 & & & & & & \\
\hline D-03-114 & 15-Jul-03 & 21 & & & & & & \\
\hline D-03-115 & 15-Jul-03 & 22 & & & & & & \\
\hline D-03-116 & 15-Jul-03 & 24 & & & & & & \\
\hline D-03-117 & 15-Jul-03 & 23 & 23.6 & 5.47 & 75.5 & 699 & 11.51 & 6.92 \\
\hline D-03-118 & 15-Jul-03 & 24 & & & & & & \\
\hline D-03-119 & 15-Jul-03 & 26 & & & & & & \\
\hline D-03-120 & 15-Jul-03 & 30 & & & & & & \\
\hline D-03-121 & 16-Jul-03 & 22 & 16.3 & 7.15 & 89.8 & 464 & 1.45 & 7.15 \\
\hline D-03-122 & 16-Jul-03 & 23 & 21.6 & 6.78 & 89.6 & 502 & 2.56 & 7.13 \\
\hline D-03-123 & 16-Jul-03 & & & & & & & \\
\hline D-03-124 & 16-Jul-03 & 23 & 26.4 & 7.86 & 111.8 & 532 & 4.15 & 7.00 \\
\hline D-03-125 & 16-Jul-03 & 21 & & & & & & \\
\hline D-03-126 & 17-Jul-03 & 17 & 17.0 & 6.69 & 78.8 & 612 & 9.11 & 6.97 \\
\hline D-03-127 & 17-Jul-03 & 19 & & & & & & \\
\hline D-03-128 & 17-Jul-03 & 20 & & & & & & \\
\hline D-03-129 & 17-Jul-03 & 21 & & & & & & \\
\hline D-03-130 & 17-Jul-03 & 22 & & & & & & \\
\hline D-03-131 & 17-Jul-03 & 22 & & & & & & \\
\hline D-03-132 & 17-Jul-03 & 24 & 18.2 & 8.15 & 99.2 & 614 & 2.35 & 7.20 \\
\hline D-03-133 & 17-Jul-03 & 24 & & & & & & \\
\hline D-03-134 & 17-Jul-03 & 24 & 20.8 & 5.25 & 74.6 & 551 & 3.36 & $7-7.5^{\star}$ \\
\hline D-03-135 & 18-Jul-03 & 19 & & & & & & \\
\hline D-03-136 & 18-Jul-03 & 24 & & & & & & \\
\hline D-03-137 & 18-Jul-03 & 24 & 14.5 & & & 430 & & \\
\hline D-03-138 & 18-Jul-03 & 28 & 24.6 & 8.06 & 124.1 & 582 & 3.44 & 7.27 \\
\hline D-03-139 & 18-Jul-03 & 26 & & & & & & \\
\hline D-03-140 & 21-Jul-03 & 24 & & & & & & \\
\hline D-03-141 & 22-Jul-03 & 18 & & & & & & \\
\hline D-03-142 & 22-Jul-03 & 19 & 17.2 & 6.62 & 83.5 & 425 & 8.14 & 6.91 \\
\hline
\end{tabular}


Appendix 6: Results of basic water chemistry and air temperature measurements recorded at most sites where fishing effort was applied between 2002 and 2006.

\begin{tabular}{|c|c|c|c|c|c|c|c|c|}
\hline \multirow{2}{*}{ Site Number } & \multirow{2}{*}{ Date } & \multirow{2}{*}{$\begin{array}{l}\text { Air Temp. } \\
\left({ }^{\circ} \mathrm{C}\right)\end{array}$} & \multirow{2}{*}{$\begin{array}{c}\text { Water Temp. } \\
\left({ }^{\circ} \mathrm{C}\right)\end{array}$} & \multicolumn{2}{|c|}{ Dissolved Oxygen } & \multirow{2}{*}{$\begin{array}{c}\text { Conductivity } \\
(\mu \mathrm{S} / \mathrm{cm})\end{array}$} & \multirow{2}{*}{$\begin{array}{l}\text { Turbidity } \\
\text { (NTU) }\end{array}$} & \multirow{2}{*}{$\begin{array}{c}\mathrm{pH} \\
\text { (units) }\end{array}$} \\
\hline & & & & (mg/L) & (\% SAT) & & & \\
\hline D-03-143 & 22-Jul-03 & 26 & 19.1 & 6.80 & 92.3 & 436 & 2.8 & 6.91 \\
\hline D-03-144 & 22-Jul-03 & 26 & 21.0 & 5.66 & 76.4 & 423 & 2.21 & 7.84 \\
\hline D-03-145 & 22-Jul-03 & 27 & 20.2 & 4.20 & 75 & 429 & 3.13 & 6.90 \\
\hline D-03-146 & 22-Jul-03 & 28 & & & & & & \\
\hline D-03-147 & 23-Jul-03 & 18 & & & & & & \\
\hline D-03-148 & 23-Jul-03 & 18 & & & & & & \\
\hline D-03-149 & 23-Jul-03 & 20 & & & & & & \\
\hline D-03-150 & 23-Jul-03 & 23 & & & & & & \\
\hline D-03-151 & 23-Jul-03 & 24 & & & & & & \\
\hline D-03-152 & 23-Jul-03 & 24 & 19.2 & 1.99 & 26.5 & 443 & 7.4 & 6.91 \\
\hline D-03-153 & 23-Jul-03 & 26.5 & & & & & & \\
\hline D-03-154 & 23-Jul-03 & 20 & 19.4 & 10.32 & 132.3 & 339 & 2.72 & 6.91 \\
\hline D-03-155 & 23-Jul-03 & 29 & 19.2 & 7.76 & 92.5 & 460 & 0.00 & 6.90 \\
\hline D-03-156 & 23-Jul-03 & 32 & 21.2 & 11.15 & 149.6 & 233 & 0.00 & 6.90 \\
\hline D-03-157 & 24-Jul-03 & 26 & & & & & & \\
\hline D-03-158 & 24-Jul-03 & 26 & & & & & & \\
\hline D-03-159 & 24-Jul-03 & 25 & & & & & & \\
\hline D-03-160 & 24-Jul-03 & 26 & & & & & & \\
\hline D-03-161 & 24-Jul-03 & 26 & & & & & & \\
\hline D-03-162 & 24-Jul-03 & 26 & & & & & & \\
\hline D-03-163 & 24-Jul-03 & 28 & & & & & & \\
\hline D-03-164 & 24-Jul-03 & 28 & 22.6 & 9.14 & 126.1 & 600 & 6.62 & 6.90 \\
\hline D-03-165 & 24-Jul-03 & 27 & & & & & & \\
\hline D-03-166 & 24-Jul-03 & 27 & & & & & & \\
\hline D-03-167 & 25-Jul-03 & 22 & 19.4 & 9.08 & 117.4 & 400 & 5.12 & 6.90 \\
\hline D-03-168 & 10-Sep-03 & 14.5 & 18.6 & 5.23 & 64.2 & 190 & 0.75 & \\
\hline D-03-169 & 11-Sep-03 & 13 & 17.6 & 4.36 & 52.6 & 168 & 0.00 & \\
\hline D-03-170 & 11-Sep-03 & 16.5 & 17.6 & 5.96 & 71.7 & 181 & 2.75 & 6.81 \\
\hline W-03-001 & 20-May-03 & 17.5 & 14.2 & 9.48 & 102.6 & 280 & 30.1 & 7.84 \\
\hline W-03-002 & 21-May-03 & 13 & 0.9 & 5.51 & 49.9 & 251 & 16.42 & 7.74 \\
\hline W-03-003 & 21-May-03 & 18 & 9.1 & 8.43 & 84.7 & 266 & 9.12 & 8.03 \\
\hline W-03-004 & 21-May-03 & 18 & 10.1 & 11.10 & 109.6 & 251 & 5.70 & $7-7.5^{\star}$ \\
\hline W-03-005 & 21-May-03 & 18 & & & & & & \\
\hline
\end{tabular}


Appendix 6: Results of basic water chemistry and air temperature measurements recorded at most sites where fishing effort was applied between 2002 and 2006.

\begin{tabular}{|c|c|c|c|c|c|c|c|c|}
\hline \multirow{2}{*}{ Site Number } & \multirow{2}{*}{ Date } & \multirow{2}{*}{$\begin{array}{l}\text { Air Temp. } \\
\left({ }^{\circ} \mathrm{C}\right)\end{array}$} & \multirow{2}{*}{$\begin{array}{c}\text { Water Temp. } \\
\left({ }^{\circ} \mathrm{C}\right)\end{array}$} & \multicolumn{2}{|c|}{ Dissolved Oxygen } & \multirow{2}{*}{$\begin{array}{c}\text { Conductivity } \\
(\mu \mathrm{S} / \mathrm{cm})\end{array}$} & \multirow{2}{*}{$\begin{array}{l}\text { Turbidity } \\
\text { (NTU) }\end{array}$} & \multirow{2}{*}{$\begin{array}{c}\mathrm{pH} \\
\text { (units) }\end{array}$} \\
\hline & & & & (mg/L) & (\% SAT) & & & \\
\hline W-03-006 & 21-May-03 & 18 & & & & & & \\
\hline W-03-007 & 21-May-03 & 18 & & & & & & \\
\hline W-03-008 & 22-May-03 & 17 & 11.0 & 5.38 & 54.2 & 739 & 0.25 & $7-7.5^{*}$ \\
\hline W-03-009 & 22-May-03 & 20 & 10.3 & 6.49 & 65.5 & 401 & 2.02 & $7-7.5^{*}$ \\
\hline W-03-010 & 22-May-03 & 21 & 15.0 & & & 729 & 1.08 & $7-7.5^{*}$ \\
\hline W-03-011 & 22-May-03 & 21 & & & & & & \\
\hline W-03-012 & 22-May-03 & 25 & & & & & & \\
\hline W-03-013 & 23-May-03 & 25 & 16.0 & 8.20 & 92.7 & 464 & 0.00 & $7-7.5^{*}$ \\
\hline W-03-014 & 26-May-03 & 27.5 & & & & & & \\
\hline W-03-015 & 26-May-03 & 27.5 & & & & & & \\
\hline W-03-016 & 26-May-03 & 28 & & & & & & \\
\hline W-03-017 & 26-May-03 & 28 & & & & & & \\
\hline W-03-018 & 26-May-03 & 28 & & & & & & \\
\hline W-03-019 & 26-May-03 & 28 & 23.1 & 10.84 & 140.4 & 1023 & 1.22 & \\
\hline W-03-020 & 26-May-03 & 27 & 24.7 & 9.56 & 127.5 & 497 & 13.97 & 8.50 \\
\hline W-03-021 & 27-May-03 & 25 & & & & & & \\
\hline W-03-022 & 27-May-03 & 27 & & & & & & \\
\hline W-03-023 & 27-May-03 & 28 & & & & & & \\
\hline W-03-024 & 27-May-03 & 28 & & & & & & \\
\hline W-03-025 & 27-May-03 & 25 & & & & & & \\
\hline W-03-026 & 27-May-03 & 25 & 22.2 & 11.94 & & 1402 & 10.67 & 9.09 \\
\hline W-03-027 & 27-May-03 & 24 & & & & & & \\
\hline W-03-028 & 28-May-03 & 24 & & & & & & \\
\hline W-03-029 & 28-May-03 & 25.5 & & & & & & \\
\hline W-03-030 & 28-May-03 & 24.5 & 20.1 & 9.68 & 118.4 & 820 & 2.32 & \\
\hline W-03-031 & 28-May-03 & 25 & & & & & & \\
\hline W-03-032 & 28-May-03 & 28 & & & & & & \\
\hline W-03-033 & 28-May-03 & 28 & 23.2 & 5.96 & 72.2 & 646 & 2.38 & $7-7.5^{*}$ \\
\hline W-03-034 & 28-May-03 & 28 & & & & & & \\
\hline W-03-035 & 29-May-03 & 22 & 16.4 & 8.48 & 94.6 & 1484 & 0.00 & 8.56 \\
\hline W-03-036 & 29-May-03 & 30 & 18.0 & 4.86 & 61.8 & 1383 & 3.47 & 8.12 \\
\hline W-03-037 & 29-May-03 & 29.5 & & & & & & \\
\hline W-03-038 & 29-May-03 & 29.5 & & & & & & \\
\hline
\end{tabular}


Appendix 6: Results of basic water chemistry and air temperature measurements recorded at most sites where fishing effort was applied between 2002 and 2006.

\begin{tabular}{|c|c|c|c|c|c|c|c|c|}
\hline \multirow{2}{*}{ Site Number } & \multirow{2}{*}{ Date } & \multirow{2}{*}{$\begin{array}{l}\text { Air Temp. } \\
\left({ }^{\circ} \mathrm{C}\right)\end{array}$} & \multirow{2}{*}{$\begin{array}{c}\text { Water Temp. } \\
\left({ }^{\circ} \mathrm{C}\right)\end{array}$} & \multicolumn{2}{|c|}{ Dissolved Oxygen } & \multirow{2}{*}{$\begin{array}{c}\text { Conductivity } \\
(\mu \mathrm{S} / \mathrm{cm})\end{array}$} & \multirow{2}{*}{$\begin{array}{l}\text { Turbidity } \\
\text { (NTU) }\end{array}$} & \multirow{2}{*}{$\begin{array}{c}\mathrm{pH} \\
\text { (units) }\end{array}$} \\
\hline & & & & (mg/L) & (\% SAT) & & & \\
\hline W-03-039 & 29-May-03 & 28.5 & & & & & & \\
\hline W-03-040 & 29-May-03 & 28 & & & & & & \\
\hline W-03-041 & 29-May-03 & 26 & & & & & & \\
\hline W-03-042 & 29-May-03 & 26 & & & & & & \\
\hline W-03-043 & 29-May-03 & 26 & & & & & & \\
\hline W-03-044 & 3-Jun-03 & 18 & 17.2 & 8.48 & 88.1 & 1350 & 37.91 & 7.80 \\
\hline W-03-045 & 3-Jun-03 & 18.5 & & & & & & \\
\hline W-03-046 & 3-Jun-03 & 18 & 14.9 & 6.42 & 66.3 & 555 & 5.14 & 7.50 \\
\hline W-03-047 & 3-Jun-03 & 18 & & & & & & \\
\hline W-03-048 & 3-Jun-03 & 20 & & & & & & \\
\hline W-03-049 & 3-Jun-03 & 20 & 17.0 & 12.80 & & 697 & 0.00 & $7-7.5^{*}$ \\
\hline W-03-050 & 4-Jun-03 & 20 & 17.7 & 9.68 & 115.3 & 753 & 8.00 & $7-7.5^{*}$ \\
\hline W-03-051 & 4-Jun-03 & 20 & & & & & & \\
\hline W-03-052 & 4-Jun-03 & 18.5 & & & & & & \\
\hline W-03-053 & 4-Jun-03 & 20 & & & & & & \\
\hline W-03-054 & 4-Jun-03 & 20 & & & & & & \\
\hline W-03-055 & 4-Jun-03 & 20 & 16.9 & 10.90 & 128 & 913 & 10.00 & $7-7.5^{*}$ \\
\hline W-03-056 & 4-Jun-03 & 18 & & & & & & \\
\hline W-03-057 & 4-Jun-03 & 17.5 & & & & & & \\
\hline W-03-058 & 4-Jun-03 & 17.5 & & & & & & \\
\hline W-03-059 & 4-Jun-03 & 17.5 & & & & & & \\
\hline W-03-060 & 5-Jun-03 & 18.5 & & & & & & \\
\hline W-03-061 & 5-Jun-03 & 20 & & & & & & \\
\hline W-03-062 & 5-Jun-03 & 20 & & & & & & \\
\hline W-03-063 & 5-Jun-03 & 21 & & & & & & \\
\hline W-03-064 & 5-Jun-03 & 22 & 17.3 & 7.86 & 95 & 1335 & 11.70 & $7-7.5^{*}$ \\
\hline W-03-065 & 5-Jun-03 & 24 & 18.4 & 9.52 & 118.4 & 868 & 67 & $7-7.5^{\star}$ \\
\hline W-03-066 & 5-Jun-03 & 24 & 19.8 & 7.01 & 89.2 & 541 & 77 & $7-7.5^{*}$ \\
\hline W-03-067 & 6-Jun-03 & 15 & 15.5 & 6.24 & 73.1 & 861 & 79 & 7.90 \\
\hline W-03-068 & 6-Jun-03 & 15 & & & & & & \\
\hline W-03-069 & 6-Jun-03 & 18 & 17.3 & 5.62 & 68 & 635 & 76 & $7-7.5^{*}$ \\
\hline W-03-070 & 6-Jun-03 & 19 & 17.0 & 3.37 & 40.1 & 468 & 255 & $7-7.5^{*}$ \\
\hline W-03-071 & 9-Jun-03 & 20 & & & & & & \\
\hline
\end{tabular}


Appendix 6: Results of basic water chemistry and air temperature measurements recorded at most sites where fishing effort was applied between 2002 and 2006.

\begin{tabular}{|c|c|c|c|c|c|c|c|c|}
\hline \multirow{2}{*}{ Site Number } & \multirow{2}{*}{ Date } & \multirow{2}{*}{$\begin{array}{l}\text { Air Temp. } \\
\left({ }^{\circ} \mathrm{C}\right)\end{array}$} & \multirow{2}{*}{$\begin{array}{c}\text { Water Temp. } \\
\left({ }^{\circ} \mathrm{C}\right)\end{array}$} & \multicolumn{2}{|c|}{ Dissolved Oxygen } & \multirow{2}{*}{$\begin{array}{c}\text { Conductivity } \\
(\mu \mathrm{S} / \mathrm{cm})\end{array}$} & \multirow{2}{*}{$\begin{array}{l}\text { Turbidity } \\
\text { (NTU) }\end{array}$} & \multirow{2}{*}{$\begin{array}{c}\mathrm{pH} \\
\text { (units) }\end{array}$} \\
\hline & & & & (mg/L) & (\% SAT) & & & \\
\hline W-03-072 & 9-Jun-03 & 20 & 14.4 & 8.16 & 92.8 & 512 & 276 & 8.09 \\
\hline W-03-073 & 9-Jun-03 & 20 & 19.4 & 8.17 & 98.9 & 343 & $>300$ & $7-7.5^{\star}$ \\
\hline W-03-074 & 9-Jun-03 & 17 & & & & & & \\
\hline W-03-075 & 9-Jun-03 & 17 & & & & & & \\
\hline W-03-076 & 10-Jun-03 & 16 & & & & & & \\
\hline W-03-077 & 10-Jun-03 & 17 & 16.0 & 8.10 & 92.2 & 569 & 33.78 & $7-7.5^{\star}$ \\
\hline W-03-078 & 10-Jun-03 & 18 & 17.7 & 7.45 & 87.6 & 399 & $>300$ & $7-7.5^{*}$ \\
\hline W-03-079 & 10-Jun-03 & 21 & 14.2 & 8.36 & 90.5 & 306 & 174 & $7-7.5^{*}$ \\
\hline W-03-080 & 10-Jun-03 & 24 & 22.1 & 7.72 & 97.8 & 481 & 127 & $7-7.5^{*}$ \\
\hline W-03-081 & 11-Jun-03 & 20 & & & & & & \\
\hline W-03-082 & 11-Jun-03 & 21 & & & & & & \\
\hline W-03-083 & 11-Jun-03 & 21 & & & & & & \\
\hline W-03-084 & 11-Jun-03 & 22 & 18.9 & 11.21 & 133 & 1120 & 26.04 & 8.40 \\
\hline W-03-085 & 11-Jun-03 & 23 & & & & & & \\
\hline W-03-086 & 11-Jun-03 & 24 & & & & & & \\
\hline W-03-087 & 11-Jun-03 & 24 & 19.0 & 11.10 & 134.4 & 411 & 47.84 & \\
\hline W-03-088 & 11-Jun-03 & 24 & 25.0 & 9.24 & 126.8 & 426 & 61 & \\
\hline W-03-089 & 13-Jun-03 & 22 & & & & & & \\
\hline W-03-090 & 13-Jun-03 & 23 & & & & & & \\
\hline W-03-091 & 13-Jun-03 & 24 & 20.0 & 10.96 & 137.3 & 905 & 82 & 8.50 \\
\hline W-03-092 & 13-Jun-03 & 24 & 21.2 & 8.70 & 110.5 & 350 & 78 & \\
\hline W-03-093 & 13-Jun-03 & 26 & 23.4 & 9.87 & 129.4 & 459 & 52 & \\
\hline W-03-094 & 16-Jun-03 & 29 & 21.3 & 0.57 & 20 & 49 & 44.97 & \\
\hline W-03-095 & 16-Jun-03 & 27.5 & 23.0 & 6.93 & 91.2 & 25 & 65 & \\
\hline W-03-096 & 16-Jun-03 & 27 & 23.6 & 4.02 & 6.1 & 449 & 0.00 & \\
\hline W-03-097 & 17-Jun-03 & 24 & 19.6 & 4.95 & 62.3 & 55 & 122 & \\
\hline W-03-098 & 17-Jun-03 & 27 & 16.8 & 2.78 & 32.1 & 68 & 9.99 & \\
\hline W-03-099 & 17-Jun-03 & 31 & & & & & & \\
\hline W-03-100 & 17-Jun-03 & 30 & 22.4 & 9.55 & 124.5 & 212 & 0.00 & \\
\hline W-03-101 & 18-Jun-03 & 17 & 13.3 & 7.89 & 85.4 & 125 & 84 & \\
\hline W-03-102 & 18-Jun-03 & 21 & & & & & & \\
\hline W-03-103 & 18-Jun-03 & 21.5 & & & & & & \\
\hline W-03-104 & 18-Jun-03 & 22.5 & 17.3 & 5.01 & 59.1 & 99 & 7.06 & \\
\hline
\end{tabular}


Appendix 6: Results of basic water chemistry and air temperature measurements recorded at most sites where fishing effort was applied between 2002 and 2006.

\begin{tabular}{|c|c|c|c|c|c|c|c|c|}
\hline \multirow{2}{*}{ Site Number } & \multirow{2}{*}{ Date } & \multirow{2}{*}{$\begin{array}{l}\text { Air Temp. } \\
\left({ }^{\circ} \mathrm{C}\right)\end{array}$} & \multirow{2}{*}{$\begin{array}{c}\text { Water Temp. } \\
\left({ }^{\circ} \mathrm{C}\right)\end{array}$} & \multicolumn{2}{|c|}{ Dissolved Oxygen } & \multirow{2}{*}{$\begin{array}{c}\text { Conductivity } \\
(\mu \mathrm{S} / \mathrm{cm})\end{array}$} & \multirow{2}{*}{$\begin{array}{l}\text { Turbidity } \\
\text { (NTU) }\end{array}$} & \multirow{2}{*}{$\begin{array}{c}\mathrm{pH} \\
\text { (units) }\end{array}$} \\
\hline & & & & (mg/L) & (\% SAT) & & & \\
\hline W-03-105 & 18-Jun-03 & 23 & & & & & & \\
\hline W-03-106 & 18-Jun-03 & 22 & & & & & & \\
\hline W-03-107 & 18-Jun-03 & 24 & 17.9 & 6.90 & 83.7 & 112 & 260 & \\
\hline W-03-108 & 19-Jun-03 & 18 & & & & & & \\
\hline W-03-109 & 19-Jun-03 & 18 & & & & & & \\
\hline W-03-110 & 19-Jun-03 & 21 & 13.3 & 7.02 & 76.5 & 99 & $>300$ & \\
\hline W-03-111 & 19-Jun-03 & 26 & 15.2 & 6.97 & 78 & 81 & 20.82 & \\
\hline W-03-112 & 19-Jun-03 & 28 & 18.1 & 7.74 & 92.8 & 109 & $>300$ & \\
\hline W-03-113 & 24-Jun-03 & 17 & 19.6 & 6.52 & 81.2 & 432 & 269 & 8.09 \\
\hline W-03-114 & 24-Jun-03 & 21 & 19.8 & 6.31 & 76.5 & 424 & 87 & $7-7.5^{*}$ \\
\hline W-03-115 & 24-Jun-03 & 19.5 & & & & & & \\
\hline W-03-116 & 24-Jun-03 & 20 & & & & & & \\
\hline W-03-117 & 24-Jun-03 & 23 & & & & & & \\
\hline W-03-118 & 24-Jun-03 & 24 & & & & & & \\
\hline W-03-119 & 24-Jun-03 & 20.5 & & & & & & \\
\hline W-03-120 & 25-Jun-03 & 18.5 & & & & & & \\
\hline W-03-121 & 25-Jun-03 & 15 & & & & & & \\
\hline W-03-122 & 25-Jun-03 & 15 & & & & & & \\
\hline W-03-123 & 25-Jun-03 & 13.5 & & & & & & \\
\hline W-03-124 & 25-Jun-03 & 13.5 & & & & & & \\
\hline W-03-125 & 25-Jun-03 & 13.5 & & & & & & \\
\hline W-03-126 & 25-Jun-03 & 13 & & & & & & \\
\hline W-03-127 & 25-Jun-03 & 15 & & & & & & \\
\hline W-03-128 & 25-Jun-03 & 15 & & & & & & \\
\hline W-03-129 & 25-Jun-03 & 15 & & & & & & \\
\hline W-03-130 & 25-Jun-03 & 14 & & & & & & \\
\hline W-03-131 & 25-Jun-03 & 13 & & & & & & \\
\hline W-03-132 & 26-Jun-03 & 15 & & & & & & \\
\hline W-03-133 & 26-Jun-03 & 15 & & & & & & \\
\hline W-03-134 & 26-Jun-03 & 16 & & & & & & \\
\hline W-03-135 & 26-Jun-03 & 16 & & & & & & \\
\hline W-03-136 & 26-Jun-03 & 16 & & & & & & \\
\hline W-03-137 & 26-Jun-03 & 15 & & & & & & \\
\hline
\end{tabular}


Appendix 6: Results of basic water chemistry and air temperature measurements recorded at most sites where fishing effort was applied between 2002 and 2006.

\begin{tabular}{|c|c|c|c|c|c|c|c|c|}
\hline \multirow{2}{*}{ Site Number } & \multirow{2}{*}{ Date } & \multirow{2}{*}{$\begin{array}{l}\text { Air Temp. } \\
\left({ }^{\circ} \mathrm{C}\right)\end{array}$} & \multirow{2}{*}{$\begin{array}{c}\text { Water Temp. } \\
\left({ }^{\circ} \mathrm{C}\right)\end{array}$} & \multicolumn{2}{|c|}{ Dissolved Oxygen } & \multirow{2}{*}{$\begin{array}{c}\text { Conductivity } \\
(\mu \mathrm{S} / \mathrm{cm})\end{array}$} & \multirow{2}{*}{$\begin{array}{l}\text { Turbidity } \\
\text { (NTU) }\end{array}$} & \multirow{2}{*}{$\begin{array}{c}\mathrm{pH} \\
\text { (units) }\end{array}$} \\
\hline & & & & (mg/L) & (\% SAT) & & & \\
\hline W-03-138 & 26-Jun-03 & 15 & & & & & & \\
\hline W-03-139 & 26-Jun-03 & 15 & & & & & & \\
\hline W-03-140 & 26-Jun-03 & 16.5 & & & & & & \\
\hline W-03-141 & 26-Jun-03 & 16.5 & 17.0 & 10.28 & 115.1 & 1291 & 0.84 & \\
\hline W-03-142 & 26-Jun-03 & 16.5 & 17.8 & 9.90 & 116.6 & 680 & 160 & \\
\hline W-03-143 & 2-Jul-03 & 26 & 23.0 & 2.70 & 44.4 & 822 & 35.81 & \\
\hline W-03-144 & 2-Jul-03 & 27 & 26.2 & 6.57 & 90 & 469 & 243 & \\
\hline W-03-145 & 2-Jul-03 & 26 & & & & & & \\
\hline W-03-146 & 2-Jul-03 & 27.5 & 24.8 & 8.85 & 118.5 & 203 & 52 & \\
\hline W-03-147 & 3-Jul-03 & 20 & & & & & & \\
\hline W-03-148 & 3-Jul-03 & 19 & & & & & & \\
\hline W-03-149 & 3-Jul-03 & 19 & & & & & & \\
\hline W-03-150 & 3-Jul-03 & 21 & 21.4 & 3.93 & 49.9 & 470 & 0.00 & \\
\hline W-03-151 & 3-Jul-03 & 26 & 23.3 & 6.39 & 84 & 292 & 7.57 & \\
\hline W-03-152 & 3-Jul-03 & 26 & & & & & & \\
\hline W-03-153 & 3-Jul-03 & 26 & & & & & & \\
\hline W-03-154 & 4-Jul-03 & 20 & 20.8 & 4.91 & 60.9 & 348 & 0.00 & \\
\hline W-03-155 & 4-Jul-03 & 27 & 20.3 & 7.99 & 97.5 & 290 & 64 & \\
\hline W-03-156 & 4-Jul-03 & 24 & & & & & & \\
\hline W-03-157 & 4-Jul-03 & 24.5 & & & & & & \\
\hline W-03-158 & 4-Jul-03 & 25 & 22.9 & & & 229 & $>300$ & \\
\hline W-03-159 & 5-Jul-03 & 20 & & & & & & \\
\hline W-03-160 & 5-Jul-03 & 20 & & & & & & \\
\hline W-03-161 & 5-Jul-03 & 22 & & & & & & \\
\hline W-03-162 & 5-Jul-03 & 21 & 20.1 & 1.86 & 23.4 & 224 & 0.00 & \\
\hline W-03-163 & 5-Jul-03 & 22 & 21.0 & 6.18 & 76.5 & 95 & 113 & \\
\hline W-03-164 & 5-Jul-03 & 23 & & & & & & \\
\hline W-03-165 & 5-Jul-03 & 25 & 22.9 & 6.00 & 70 & 153 & $>300$ & \\
\hline W-03-166 & 6-Jul-03 & 21.5 & & & & & & \\
\hline W-03-167 & 6-Jul-03 & 22 & 17.2 & 9.98 & 116.2 & 277 & 105 & \\
\hline W-03-168 & 6-Jul-03 & 25 & & & & & & \\
\hline W-03-169 & 6-Jul-03 & 25 & & & & & & \\
\hline W-03-170 & 6-Jul-03 & 25 & & & & & & \\
\hline
\end{tabular}


Appendix 6: Results of basic water chemistry and air temperature measurements recorded at most sites where fishing effort was applied between 2002 and 2006.

\begin{tabular}{|c|c|c|c|c|c|c|c|c|}
\hline \multirow{2}{*}{ Site Number } & \multirow{2}{*}{ Date } & \multirow{2}{*}{$\begin{array}{l}\text { Air Temp. } \\
\left({ }^{\circ} \mathrm{C}\right)\end{array}$} & \multirow{2}{*}{$\begin{array}{c}\text { Water Temp. } \\
\left({ }^{\circ} \mathrm{C}\right)\end{array}$} & \multicolumn{2}{|c|}{ Dissolved Oxygen } & \multirow{2}{*}{$\begin{array}{c}\text { Conductivity } \\
(\mu \mathrm{S} / \mathrm{cm})\end{array}$} & \multirow{2}{*}{$\begin{array}{l}\text { Turbidity } \\
\text { (NTU) }\end{array}$} & \multirow{2}{*}{$\begin{array}{c}\mathrm{pH} \\
\text { (units) }\end{array}$} \\
\hline & & & & (mg/L) & (\% SAT) & & & \\
\hline W-03-171 & 6-Jul-03 & 22.5 & & & & & & \\
\hline W-03-172 & 6-Jul-03 & 23.5 & 19.9 & 5.28 & 63.8 & 240 & $>300$ & \\
\hline W-03-173 & 6-Jul-03 & 24.5 & 21.1 & 6.73 & 83.6 & 280 & 141 & \\
\hline W-03-174 & 6-Jul-03 & 25 & & & & & & \\
\hline W-03-175 & 6-Jul-03 & 25 & & & & & & \\
\hline W-03-176 & 7-Jul-03 & 19 & 20.1 & 3.26 & 41.6 & 120 & 59 & \\
\hline W-03-177 & 8-Jul-03 & 17 & & & & & & \\
\hline W-03-178 & 8-Jul-03 & 17 & & & & & & \\
\hline W-03-179 & 8-Jul-03 & 17 & & & & & & \\
\hline W-03-180 & 8-Jul-03 & 17 & & & & & & \\
\hline W-03-181 & 8-Jul-03 & 17 & & & & & & \\
\hline W-03-182 & 8-Jul-03 & 18 & 19.6 & 2.31 & 27.7 & 961 & 95 & \\
\hline W-03-183 & 8-Jul-03 & 18 & & & & & & \\
\hline W-03-184 & 8-Jul-03 & 18 & & & & & & \\
\hline W-03-185 & 8-Jul-03 & 19 & 18.5 & 6.30 & 78.3 & 884 & 132 & \\
\hline W-03-186 & 9-Jul-03 & 19 & 17.6 & 2.70 & 31.4 & 1190 & 188 & \\
\hline W-03-187 & 9-Jul-03 & 20 & & & & & & \\
\hline W-03-188 & 9-Jul-03 & 20 & & & & & & \\
\hline W-03-189 & 9-Jul-03 & 20 & 19.0 & 12.12 & 146.7 & 366 & 33.42 & \\
\hline W-03-190 & 16-Jul-03 & 27 & & & & & & \\
\hline W-03-191 & 16-Jul-03 & 28 & 23.8 & 6.74 & 87.4 & 309 & 245 & \\
\hline W-03-192 & 16-Jul-03 & 29.5 & & & & & & \\
\hline W-03-193 & 16-Jul-03 & 30 & & & & & & \\
\hline W-03-194 & 16-Jul-03 & 30 & 27.7 & 5.78 & 97.2 & 379 & 96 & \\
\hline W-03-195 & 16-Jul-03 & 30 & & & & 295 & 56 & \\
\hline W-03-196 & 17-Jul-03 & 18 & & & & & & \\
\hline W-03-197 & 17-Jul-03 & 20 & & & & & & \\
\hline W-03-198 & 17-Jul-03 & 23 & 20.1 & 2.88 & 34.6 & 90 & 13.08 & \\
\hline W-03-199 & 17-Jul-03 & 23 & & & & & & \\
\hline W-03-200 & 17-Jul-03 & 24 & & & & & & \\
\hline W-03-201 & 17-Jul-03 & 24 & 19.8 & 3.67 & 44.2 & 379 & 51 & \\
\hline W-03-202 & 18-Jul-03 & 18 & & & & & & \\
\hline W-03-203 & 18-Jul-03 & 18 & 16.6 & 6.46 & 77.2 & 302 & 0.00 & \\
\hline
\end{tabular}


Appendix 6: Results of basic water chemistry and air temperature measurements recorded at most sites where fishing effort was applied between 2002 and 2006.

\begin{tabular}{|c|c|c|c|c|c|c|c|c|}
\hline \multirow{2}{*}{ Site Number } & \multirow{2}{*}{ Date } & \multirow{2}{*}{$\begin{array}{l}\text { Air Temp. } \\
\left({ }^{\circ} \mathrm{C}\right)\end{array}$} & \multirow{2}{*}{$\begin{array}{c}\text { Water Temp. } \\
\left({ }^{\circ} \mathrm{C}\right)\end{array}$} & \multicolumn{2}{|c|}{ Dissolved Oxygen } & \multirow{2}{*}{$\begin{array}{c}\text { Conductivity } \\
(\mu \mathrm{S} / \mathrm{cm})\end{array}$} & \multirow{2}{*}{$\begin{array}{c}\text { Turbidity } \\
\text { (NTU) }\end{array}$} & \multirow{2}{*}{$\begin{array}{c}\mathrm{pH} \\
\text { (units) }\end{array}$} \\
\hline & & & & (mg/L) & (\% SAT) & & & \\
\hline W-03-204 & 18-Jul-03 & 20 & 17.9 & 2.97 & 35.8 & 423 & 0.69 & \\
\hline W-03-205 & 18-Jul-03 & 20 & 19.4 & 10.21 & 129.1 & 329 & 13.09 & \\
\hline W-03-206 & 31-Jul-03 & 16 & 19.4 & & & 400 & 34.74 & 7.99 \\
\hline W-03-207 & 31-Jul-03 & 23.4 & & & & & & \\
\hline W-03-208 & 31-Jul-03 & 23 & & & & & & \\
\hline W-03-209 & 31-Jul-03 & 23.6 & & & & & & \\
\hline W-03-210 & 31-Jul-03 & 23.4 & 20.9 & 10.96 & 136.7 & 711 & 4.94 & \\
\hline W-03-211 & 31-Jul-03 & 25.2 & 23.7 & 6.31 & 86.6 & $>2000$ & 0.00 & \\
\hline W-03-212 & 1-Aug-03 & 24.1 & & & & & & \\
\hline W-03-213 & 1-Aug-03 & 24.1 & & & & & & \\
\hline W-03-214 & 1-Aug-03 & 24.1 & & & & & & \\
\hline W-03-215 & 1-Aug-03 & 25 & & & & & & \\
\hline W-03-216 & 1-Aug-03 & 23.3 & & & & & & \\
\hline W-03-217 & 1-Aug-03 & 24.8 & & & & & & \\
\hline W-03-218 & 1-Aug-03 & 25 & & & & & & \\
\hline W-03-219 & 1-Aug-03 & 25 & & & & & & \\
\hline W-03-220 & 5-Aug-03 & 23.6 & & & & & & \\
\hline W-03-221 & 5-Aug-03 & 23.6 & & & & & & \\
\hline W-03-222 & 5-Aug-03 & 24 & & & & & & \\
\hline W-03-223 & 5-Aug-03 & 28 & 23.7 & 5.36 & 75.1 & 504 & 7.71 & \\
\hline W-03-224 & 5-Aug-03 & 28.8 & 27.0 & & & 1267 & 44.2 & \\
\hline W-03-225 & 5-Aug-03 & 27.3 & & & & & & \\
\hline W-03-226 & 5-Aug-03 & 27.3 & & & & & & \\
\hline W-03-227 & 5-Aug-03 & 27.3 & 26.2 & 6.83 & 89.5 & 424 & 2.81 & \\
\hline W-03-228 & 6-Aug-03 & 17 & & & & & & \\
\hline W-03-229 & 6-Aug-03 & 21 & 10.0 & & & 634 & 3.15 & \\
\hline W-03-230 & 6-Aug-03 & 23 & & & & & & \\
\hline W-03-231 & 6-Aug-03 & 24.9 & & & & & & \\
\hline W-03-232 & 6-Aug-03 & 24.4 & & & & & & \\
\hline W-03-233 & 6-Aug-03 & 28.1 & & & & & & \\
\hline W-03-234 & 6-Aug-03 & 27 & & & & & & \\
\hline W-03-235 & 6-Aug-03 & 25.8 & & & & & & \\
\hline W-03-236 & 6-Aug-03 & 26.8 & & & & & & \\
\hline
\end{tabular}


Appendix 6: Results of basic water chemistry and air temperature measurements recorded at most sites where fishing effort was applied between 2002 and 2006.

\begin{tabular}{|c|c|c|c|c|c|c|c|c|}
\hline \multirow{2}{*}{ Site Number } & \multirow{2}{*}{ Date } & \multirow{2}{*}{$\begin{array}{c}\text { Air Temp. } \\
\left({ }^{\circ} \mathrm{C}\right)\end{array}$} & \multirow{2}{*}{$\begin{array}{c}\text { Water Temp. } \\
\left({ }^{\circ} \mathrm{C}\right)\end{array}$} & \multicolumn{2}{|c|}{ Dissolved Oxygen } & \multirow{2}{*}{$\begin{array}{c}\text { Conductivity } \\
(\mu \mathrm{S} / \mathrm{cm})\end{array}$} & \multirow{2}{*}{$\begin{array}{c}\text { Turbidity } \\
\text { (NTU) }\end{array}$} & \multirow{2}{*}{$\begin{array}{c}\text { pH } \\
\text { (units) }\end{array}$} \\
\hline & & & & $(\mathrm{mg} / \mathrm{L})$ & $(\%$ SAT $)$ & & & \\
\hline W-03-237 & 6-Aug-03 & 28 & 17.3 & & & 532 & 12.59 & \\
\hline W-03-238 & 7-Aug-03 & 22.1 & 22.0 & & & 610 & 9.02 & 7.90 \\
\hline W-03-239 & 7-Aug-03 & 22.3 & 16.0 & & & 502 & $>300$ & \\
\hline W-03-240 & 7-Aug-03 & 27.5 & & & & & & \\
\hline W-03-241 & 7-Aug-03 & 29.5 & & & & & & \\
\hline W-03-242 & 7-Aug-03 & 30 & & & & & & \\
\hline W-03-243 & 7-Aug-03 & 29 & & & & & & \\
\hline W-03-244 & 7-Aug-03 & 30 & & & & & & \\
\hline W-03-245 & 7-Aug-03 & 30 & & & & & & \\
\hline W-03-246 & 8-Aug-03 & 22 & & & & & & \\
\hline W-03-247 & 8-Aug-03 & 25 & 21.1 & & & 303 & 2.79 & 7.21 \\
\hline W-03-248 & 8-Aug-03 & 28 & 20.0 & & & 479 & 5.08 & 7.20 \\
\hline W-03-249 & 11-Aug-03 & 28.9 & 20.6 & & & 257 & 0.24 & 7.03 \\
\hline W-03-250 & 11-Aug-03 & 30 & & & & & & \\
\hline W-03-251 & 11-Aug-03 & 30 & 26.3 & & & 316 & 4.82 & 9.16 \\
\hline W-03-252 & 11-Aug-03 & 32.5 & & & & & & \\
\hline W-03-253 & 11-Aug-03 & 33 & & & & & & \\
\hline W-03-254 & 11-Aug-03 & 33.5 & & & & & & \\
\hline W-03-255 & 12-Aug-03 & 20.3 & & & & & & \\
\hline W-03-256 & 12-Aug-03 & 25.6 & & & & & & \\
\hline W-03-257 & 12-Aug-03 & 25.6 & & & & & & \\
\hline W-03-258 & 12-Aug-03 & 25.3 & & & & & & \\
\hline W-03-259 & 12-Aug-03 & 25.3 & & & & & & \\
\hline W-03-260 & 12-Aug-03 & 27.7 & & & & & & \\
\hline W-03-261 & 12-Aug-03 & 27.7 & & & & & & \\
\hline W-03-262 & 12-Aug-03 & 27.7 & & & & & & \\
\hline W-03-263 & 12-Aug-03 & 28 & & & & & & \\
\hline W-03-264 & 12-Aug-03 & 28 & & & & & & \\
\hline W-03-265 & 12-Aug-03 & 28 & & & & & & \\
\hline W-03-266 & 13-Aug-03 & 24.3 & 21.1 & & & 806 & 13.43 & 7.76 \\
\hline W-03-267 & 13-Aug-03 & 24.7 & 21.5 & & & 795 & 0 & 7.99 \\
\hline B-04-001 & 1-May-04 & 8.0 & & & & & & \\
\hline B-04-002 & 1-May-04 & 8.0 & 4.9 & 8.53 & 77.3 & 547 & 10.02 & 7.98 \\
\hline
\end{tabular}


Appendix 6: Results of basic water chemistry and air temperature measurements recorded at most sites where fishing effort was applied between 2002 and 2006.

\begin{tabular}{|c|c|c|c|c|c|c|c|c|}
\hline \multirow{2}{*}{ Site Number } & \multirow{2}{*}{ Date } & \multirow{2}{*}{$\begin{array}{l}\text { Air Temp. } \\
\left({ }^{\circ} \mathrm{C}\right)\end{array}$} & \multirow{2}{*}{$\begin{array}{c}\text { Water Temp. } \\
\left({ }^{\circ} \mathrm{C}\right)\end{array}$} & \multicolumn{2}{|c|}{ Dissolved Oxygen } & \multirow{2}{*}{$\begin{array}{c}\text { Conductivity } \\
(\mu \mathrm{S} / \mathrm{cm})\end{array}$} & \multirow{2}{*}{$\begin{array}{l}\text { Turbidity } \\
\text { (NTU) }\end{array}$} & \multirow{2}{*}{$\begin{array}{c}\mathrm{pH} \\
\text { (units) }\end{array}$} \\
\hline & & & & (mg/L) & (\% SAT) & & & \\
\hline B-04-003 & 1-May-04 & 8.0 & 6.5 & 8.82 & 85.0 & 455 & 24.79 & 8.11 \\
\hline B-04-004 & 1-May-04 & 8.0 & 6.5 & 8.42 & 82.0 & 358 & 75 & 7.50 \\
\hline B-04-005 & 1-May-04 & 12.0 & & & & & & \\
\hline B-04-006 & 1-May-04 & 12.0 & 6.6 & 8.43 & 79.6 & 536 & 1.13 & 8.17 \\
\hline B-04-007 & 1-May-04 & 9.0 & & & & & & \\
\hline B-04-008 & 1-May-04 & 12.0 & 11.4 & 10.24 & 111.3 & 674 & 1.66 & 6.74 \\
\hline B-04-009 & 2-May-04 & 8.0 & & & & & & \\
\hline B-04-010 & 2-May-04 & 10.0 & 4.3 & 8.32 & 73.1 & 809 & 0.00 & 7.76 \\
\hline B-04-011 & 2-May-04 & 10.0 & 8.1 & 7.01 & 73.0 & 805 & 0.87 & 7.75 \\
\hline B-04-012 & 2-May-04 & 12.0 & 9.4 & 9.80 & 97.5 & 523 & 3.92 & 8.21 \\
\hline B-04-013 & 2-May-04 & 12.0 & & & & & & \\
\hline B-04-014 & 2-May-04 & 14.0 & 10.3 & 9.33 & 90.5 & 579 & 4.53 & 7.34 \\
\hline B-04-015 & 2-May-04 & 12.0 & 9.7 & 9.60 & 98.6 & 588 & 0.52 & 8.04 \\
\hline B-04-016 & 2-May-04 & 17.0 & & & & & & \\
\hline B-04-017 & 2-May-04 & 12.0 & 12.3 & & & 700 & 1.48 & 7.88 \\
\hline B-04-018 & 3-May-04 & 6.0 & 7.2 & 10.20 & 95.8 & 1043 & 34.28 & 7.77 \\
\hline B-04-019 & 3-May-04 & 7.0 & 8.0 & 10.79 & 91.5 & 751 & 35.30 & 7.96 \\
\hline B-04-020 & 3-May-04 & 8.0 & 8.4 & 10.63 & 99.8 & 674 & 20.68 & 7.90 \\
\hline B-04-021 & 3-May-04 & 6.0 & 9.0 & 10.63 & 95.2 & 741 & 6.27 & 7.75 \\
\hline B-04-022 & 3-May-04 & 5.0 & 10.1 & 10.72 & 100.6 & 1394 & 33.23 & 7.85 \\
\hline B-04-023 & 4-May-04 & 6.0 & & & & & & \\
\hline B-04-024 & 4-May-04 & 6.0 & & & & & & \\
\hline B-04-025 & 4-May-04 & 6.0 & & & & & & \\
\hline B-04-026 & 4-May-04 & 5.0 & 6.4 & 10.07 & 90.0 & 888 & 15.88 & 7.85 \\
\hline B-04-027 & 4-May-04 & 8.0 & 9.5 & 13.21 & & 491 & 77 & 8.61 \\
\hline B-04-028 & 4-May-04 & 10.0 & 14.0 & 10.18 & 100.0 & 875 & 55 & 8.04 \\
\hline B-04-029 & 4-May-04 & 12.0 & 14.0 & & & & & \\
\hline B-04-030 & 4-May-04 & 12.0 & 10.1 & 11.30 & 100.1 & 880 & 14.17 & 7.84 \\
\hline B-04-031 & 6-May-04 & 9.0 & 9.4 & 10.66 & 100.1 & 1350 & 6.34 & 8.14 \\
\hline B-04-032 & 6-May-04 & 9.0 & & & & & & \\
\hline B-04-033 & 6-May-04 & 9.0 & & & & & & \\
\hline B-04-034 & 6-May-04 & 9.0 & & & & & & \\
\hline B-04-035 & 6-May-04 & 9.0 & & & & & & \\
\hline
\end{tabular}


Appendix 6: Results of basic water chemistry and air temperature measurements recorded at most sites where fishing effort was applied between 2002 and 2006.

\begin{tabular}{|c|c|c|c|c|c|c|c|c|}
\hline \multirow{2}{*}{ Site Number } & \multirow{2}{*}{ Date } & \multirow{2}{*}{$\begin{array}{l}\text { Air Temp. } \\
\left({ }^{\circ} \mathrm{C}\right)\end{array}$} & \multirow{2}{*}{$\begin{array}{c}\text { Water Temp. } \\
\left({ }^{\circ} \mathrm{C}\right)\end{array}$} & \multicolumn{2}{|c|}{ Dissolved Oxygen } & \multirow{2}{*}{$\begin{array}{c}\text { Conductivity } \\
(\mu \mathrm{S} / \mathrm{cm})\end{array}$} & \multirow{2}{*}{$\begin{array}{l}\text { Turbidity } \\
\text { (NTU) }\end{array}$} & \multirow{2}{*}{$\begin{array}{c}\mathrm{pH} \\
\text { (units) }\end{array}$} \\
\hline & & & & (mg/L) & (\% SAT) & & & \\
\hline B-04-036 & 6-May-04 & 9.0 & & & & & & \\
\hline B-04-037 & 6-May-04 & 9.0 & 11.8 & 6.97 & 75.7 & 782 & 1.46 & 7.20 \\
\hline B-04-038 & 6-May-04 & 10.0 & & & & & & \\
\hline B-04-039 & 6-May-04 & 10.0 & & & & & & \\
\hline B-04-040 & 7-May-04 & 12.0 & & & & & & \\
\hline B-04-041 & 7-May-04 & 12.0 & 8.6 & 4.60 & 44.5 & 691 & 0.97 & \\
\hline B-04-042 & 7-May-04 & 12.0 & & & & & & \\
\hline B-04-043 & 7-May-04 & 12.0 & 8.5 & 6.56 & 65.3 & 1318 & 0.80 & \\
\hline B-04-044 & 7-May-04 & 12.0 & 11.5 & 10.32 & 104.5 & 1268 & 19.59 & \\
\hline B-04-045 & 7-May-04 & 14.0 & & & & & & \\
\hline B-04-046 & 10-May-04 & 10.0 & 10.8 & 10.28 & 107.9 & 926 & 10.85 & \\
\hline B-04-047 & 10-May-04 & 10.0 & 13.6 & 9.27 & 102.0 & 853 & 6.00 & \\
\hline B-04-048 & 10-May-04 & 12.0 & 16.6 & 10.82 & 121.5 & 768 & 2.59 & \\
\hline B-04-049 & 13-May-04 & 3.0 & & & & & & \\
\hline B-04-050 & 13-May-04 & 4.0 & & & & & & \\
\hline B-04-051 & 13-May-04 & 4.0 & & & & & & \\
\hline B-04-052 & 13-May-04 & 3.0 & & & & & & \\
\hline B-04-053 & 13-May-04 & 4.0 & & & & & & \\
\hline B-04-054 & 13-May-04 & 5.0 & & & & & & \\
\hline B-04-055 & 13-May-04 & 5.0 & 8.4 & 10.83 & 111.5 & 839 & 7.80 & \\
\hline B-04-056 & 13-May-04 & 4.0 & & & & & & \\
\hline B-04-057 & 14-May-04 & 3.0 & & & & & & \\
\hline B-04-058 & 14-May-04 & 3.0 & 4.7 & 8.86 & 86.0 & 1112 & 5.21 & \\
\hline B-04-059 & 14-May-04 & 4.0 & & & & & & \\
\hline B-04-060 & 14-May-04 & 4.0 & 5.4 & 9.48 & 93.9 & 534 & 4.30 & \\
\hline B-04-061 & 14-May-04 & 8.0 & & & & & & \\
\hline B-04-062 & 14-May-04 & 5.0 & & & & & & \\
\hline B-04-063 & 14-May-04 & 5.0 & & & & & & \\
\hline B-04-064 & 14-May-04 & 8.0 & 10.5 & 9.45 & 96.8 & 706 & 7.60 & \\
\hline B-04-065 & 17-May-04 & 19.0 & & & & & & \\
\hline B-04-066 & 17-May-04 & 19.0 & 14.4 & 10.07 & 108.8 & 510 & 3.62 & \\
\hline B-04-067 & 17-May-04 & 20.0 & & & & & & \\
\hline B-04-068 & 17-May-04 & 20.0 & & & & & & \\
\hline
\end{tabular}


Appendix 6: Results of basic water chemistry and air temperature measurements recorded at most sites where fishing effort was applied between 2002 and 2006.

\begin{tabular}{|c|c|c|c|c|c|c|c|c|}
\hline \multirow{2}{*}{ Site Number } & \multirow{2}{*}{ Date } & \multirow{2}{*}{$\begin{array}{l}\text { Air Temp. } \\
\left({ }^{\circ} \mathrm{C}\right)\end{array}$} & \multirow{2}{*}{$\begin{array}{c}\text { Water Temp. } \\
\left({ }^{\circ} \mathrm{C}\right)\end{array}$} & \multicolumn{2}{|c|}{ Dissolved Oxygen } & \multirow{2}{*}{$\begin{array}{c}\text { Conductivity } \\
(\mu \mathrm{S} / \mathrm{cm})\end{array}$} & \multirow{2}{*}{$\begin{array}{l}\text { Turbidity } \\
\text { (NTU) }\end{array}$} & \multirow{2}{*}{$\begin{array}{c}\mathrm{pH} \\
\text { (units) }\end{array}$} \\
\hline & & & & (mg/L) & (\% SAT) & & & \\
\hline B-04-069 & 18-May-04 & 20.0 & 11.4 & 10.47 & 111.8 & 534 & 7.64 & \\
\hline B-04-070 & 18-May-04 & 20.0 & 13.3 & 10.26 & 118.5 & 432 & 4.57 & \\
\hline B-04-071 & 18-May-04 & 26.0 & & & & & & \\
\hline B-04-072 & 18-May-04 & 25.0 & 17.6 & 10.31 & 120.9 & 637 & 6.40 & \\
\hline B-04-073 & 19-May-04 & & 12.7 & 9.14 & 97.5 & 320 & 15.55 & 7.45 \\
\hline B-04-074 & 19-May-04 & & & & & & & \\
\hline B-04-075 & 20-May-04 & 9.0 & 11.0 & 8.47 & 85.9 & 693 & 1.15 & 7.45 \\
\hline B-04-076 & 20-May-04 & 9.0 & 10.1 & 8.11 & 81.2 & 590 & 0.00 & 7.45 \\
\hline B-04-077 & 20-May-04 & 9.0 & 10.4 & 4.71 & 47.0 & 714 & 2.64 & 7.45 \\
\hline B-04-078 & 20-May-04 & 9.0 & 10.9 & 4.62 & 46.9 & 718 & 2.70 & 7.47 \\
\hline B-04-079 & 20-May-04 & 9.0 & 10.5 & 5.66 & 57.4 & 963 & 3.35 & 7.48 \\
\hline B-04-080 & 20-May-04 & 10.0 & & & & & & \\
\hline B-04-081 & 20-May-04 & 10.0 & & & & & & \\
\hline B-04-082 & 20-May-04 & 10.0 & & & & & & \\
\hline B-04-083 & 20-May-04 & 10.0 & & & & & & \\
\hline B-04-084 & 20-May-04 & 10.0 & & & & & & \\
\hline B-04-085 & 20-May-04 & 10.0 & & & & & & \\
\hline B-04-086 & 21-May-04 & & 10.1 & 6.37 & 63.5 & 554 & 0.86 & 7.47 \\
\hline B-04-087 & 22-May-04 & -1.0 & & & & & & \\
\hline B-04-088 & 25-May-04 & 5.0 & & & & & & \\
\hline B-04-089 & 25-May-04 & 5.0 & & & & & & \\
\hline B-04-090 & 25-May-04 & 5.0 & & & & & & \\
\hline B-04-091 & 25-May-04 & 5.0 & & & & & & \\
\hline B-04-092 & 25-May-04 & 5.0 & 9.7 & 9.57 & 100.3 & 1479 & 3.94 & 7.46 \\
\hline B-04-093 & 31-May-04 & 11.0 & 16.5 & 6.91 & 76.8 & 1381 & 3.64 & 7.45 \\
\hline B-04-094 & 31-May-04 & 11.0 & 12.5 & 6.24 & 66.8 & 715 & 11.89 & 7.45 \\
\hline B-04-095 & 31-May-04 & 12.0 & 12.8 & 8.08 & 89.6 & 1592 & 3.71 & 7.45 \\
\hline B-04-096 & 1-Jun-04 & 10.0 & & & & & & \\
\hline B-04-097 & 1-Jun-04 & 11.0 & & & & & & \\
\hline B-04-098 & 1-Jun-04 & 11.0 & & & & & & \\
\hline B-04-099 & 1-Jun-04 & 11.0 & & & & & & \\
\hline B-04-100 & 1-Jun-04 & 10.0 & & & & & & \\
\hline B-04-101 & 1-Jun-04 & 10.0 & 12.7 & 7.12 & 79.8 & 551 & 18.36 & 7.45 \\
\hline
\end{tabular}


Appendix 6: Results of basic water chemistry and air temperature measurements recorded at most sites where fishing effort was applied between 2002 and 2006.

\begin{tabular}{|c|c|c|c|c|c|c|c|c|}
\hline \multirow{2}{*}{ Site Number } & \multirow{2}{*}{ Date } & \multirow{2}{*}{$\begin{array}{l}\text { Air Temp. } \\
\left({ }^{\circ} \mathrm{C}\right)\end{array}$} & \multirow{2}{*}{$\begin{array}{l}\text { Water Temp. } \\
\left({ }^{\circ} \mathrm{C}\right)\end{array}$} & \multicolumn{2}{|c|}{ Dissolved Oxygen } & \multirow{2}{*}{$\begin{array}{c}\text { Conductivity } \\
(\mu \mathrm{S} / \mathrm{cm})\end{array}$} & \multirow{2}{*}{$\begin{array}{l}\text { Turbidity } \\
\text { (NTU) }\end{array}$} & \multirow{2}{*}{$\begin{array}{c}\text { pH } \\
\text { (units) }\end{array}$} \\
\hline & & & & (mg/L) & (\% SAT) & & & \\
\hline B-04-102 & 1-Jun-04 & 12.0 & 16.0 & 6.81 & 80.6 & 706 & 1.21 & 7.44 \\
\hline B-04-103 & 1-Jun-04 & 13.0 & & & & & & \\
\hline B-04-104 & 1-Jun-04 & 11.0 & & & & & & \\
\hline B-04-105 & 1-Jun-04 & 12.0 & 12.5 & 7.21 & 80.5 & 587 & 2.29 & 7.45 \\
\hline B-04-106 & 2-Jun-04 & 16.0 & & & & & & \\
\hline B-04-107 & 2-Jun-04 & 18.0 & & & & & & \\
\hline B-04-108 & 2-Jun-04 & 18.0 & 16.3 & 8.53 & 105.6 & 808 & 0.69 & 7.44 \\
\hline B-04-109 & 2-Jun-04 & 17.0 & 22.8 & 9.16 & 126.6 & 273 & 13.59 & 7.43 \\
\hline B-04-110 & 2-Jun-04 & 19.0 & 20.8 & 8.92 & 115.6 & 747 & 2.66 & 7.43 \\
\hline B-04-111 & 2-Jun-04 & 20.0 & & & & & & \\
\hline B-04-112 & 2-Jun-04 & 21.0 & & & & & & \\
\hline B-04-113 & 2-Jun-04 & 18.0 & 17.2 & 9.40 & 115.6 & 780 & 2.25 & 7.44 \\
\hline B-04-114 & 3-Jun-04 & 18.0 & 15.6 & 4.34 & 55.8 & 402 & 6.68 & 7.44 \\
\hline B-04-115 & 3-Jun-04 & 18.0 & & & & & & \\
\hline B-04-116 & 3-Jun-04 & 18.0 & 17.3 & 8.05 & 105.8 & 493 & 5.06 & 7.44 \\
\hline B-04-117 & 4-Jun-04 & 17.0 & & & & & & \\
\hline B-04-118 & 4-Jun-04 & 24.0 & & & & & & \\
\hline B-04-119 & 4-Jun-04 & 24.0 & & & & & & \\
\hline B-04-120 & 4-Jun-04 & 24.0 & & & & & & \\
\hline B-04-121 & 4-Jun-04 & 25.0 & 19.0 & 9.00 & 114.5 & 562 & 5.81 & 7.44 \\
\hline B-04-122 & 4-Jun-04 & 26.0 & 21.7 & 6.30 & 83.9 & 557 & 6.48 & 7.43 \\
\hline B-04-123 & 7-Jun-04 & 20.0 & 17.0 & 7.81 & 92.1 & 643 & 11.92 & 7.44 \\
\hline B-04-124 & 7-Jun-04 & 20.0 & 18.2 & 4.00 & 49.0 & 1354 & 2.45 & 7.44 \\
\hline B-04-125 & 7-Jun-04 & 20.0 & 20.0 & 5.63 & 73.6 & 549 & 6.12 & 7.43 \\
\hline B-04-126 & 8-Jun-04 & 12.0 & 15.2 & 7.80 & 88.6 & 857 & 5.06 & 7.70 \\
\hline B-04-127 & 8-Jun-04 & 13.0 & 15.7 & 6.52 & 74.6 & 952 & 2.80 & \\
\hline B-04-128 & 8-Jun-04 & 15.0 & & & & & & \\
\hline B-04-129 & 8-Jun-04 & 16.0 & 17.3 & 8.51 & 103.8 & 705 & 4.78 & 7.59 \\
\hline B-04-130 & 9-Jun-04 & 13.0 & & & & & & \\
\hline B-04-131 & 9-Jun-04 & 13.0 & 13.1 & 9.24 & 111.5 & $\mathrm{~N} / \mathrm{A}$ & 0.75 & \\
\hline B-04-132 & 9-Jun-04 & 16.0 & & & & & & \\
\hline B-04-133 & 9-Jun-04 & 15.0 & 17.6 & 7.88 & 98.0 & $\mathrm{~N} / \mathrm{A}$ & 1.21 & \\
\hline B-04-134 & 9-Jun-04 & 17.0 & & & & & & \\
\hline
\end{tabular}


Appendix 6: Results of basic water chemistry and air temperature measurements recorded at most sites where fishing effort was applied between 2002 and 2006.

\begin{tabular}{|c|c|c|c|c|c|c|c|c|}
\hline \multirow{2}{*}{ Site Number } & \multirow{2}{*}{ Date } & \multirow{2}{*}{$\begin{array}{l}\text { Air Temp. } \\
\left({ }^{\circ} \mathrm{C}\right)\end{array}$} & \multirow{2}{*}{$\begin{array}{c}\text { Water Temp. } \\
\left({ }^{\circ} \mathrm{C}\right)\end{array}$} & \multicolumn{2}{|c|}{ Dissolved Oxygen } & \multirow{2}{*}{$\begin{array}{c}\text { Conductivity } \\
(\mu \mathrm{S} / \mathrm{cm})\end{array}$} & \multirow{2}{*}{$\begin{array}{l}\text { Turbidity } \\
\text { (NTU) }\end{array}$} & \multirow{2}{*}{$\begin{array}{c}\mathrm{pH} \\
\text { (units) }\end{array}$} \\
\hline & & & & (mg/L) & (\% SAT) & & & \\
\hline B-04-135 & 9-Jun-04 & 18.0 & & & & & & \\
\hline B-04-136 & 10-Jun-04 & 10.0 & 17.2 & 8.62 & 107.6 & $\mathrm{~N} / \mathrm{A}$ & 4.77 & \\
\hline B-04-137 & 10-Jun-04 & 13.0 & & & & & & \\
\hline B-04-138 & 10-Jun-04 & 13.0 & & & & & & \\
\hline B-04-139 & 10-Jun-04 & 13.0 & & & & & & \\
\hline B-04-140 & 14-Jun-04 & 12.0 & 15.3 & 10.53 & 124.6 & $\mathrm{~N} / \mathrm{A}$ & 7.59 & \\
\hline B-04-141 & 14-Jun-04 & 12.0 & 15.5 & 9.70 & 114.6 & $\mathrm{~N} / \mathrm{A}$ & 19.29 & \\
\hline B-04-142 & 14-Jun-04 & 12.0 & & & & & & \\
\hline B-04-143 & 14-Jun-04 & 12.0 & & & & & & \\
\hline B-04-144 & 15-Jun-04 & 15.0 & 15.2 & 6.97 & 85.9 & 600 & 10.67 & 8.38 \\
\hline B-04-145 & 15-Jun-04 & 13.0 & 15.7 & 7.34 & 89.6 & 772 & 0.53 & 8.20 \\
\hline B-04-146 & 15-Jun-04 & 15.0 & & & & & & \\
\hline B-04-147 & 15-Jun-04 & 19.0 & & & & & & \\
\hline B-04-148 & 15-Jun-04 & 18.0 & & & & & & \\
\hline B-04-149 & 15-Jun-04 & 15.0 & 18.5 & 7.38 & 92.3 & 511 & 13.89 & 8.03 \\
\hline B-04-150 & 16-Jun-04 & 16.0 & & & & & & \\
\hline B-04-151 & 16-Jun-04 & 17.0 & & & & & & \\
\hline B-04-152 & 16-Jun-04 & 18.0 & 17.6 & 7.36 & 91.7 & 488 & & 7.65 \\
\hline B-04-153 & 16-Jun-04 & 21.0 & 21.4 & 7.09 & 95.0 & 634 & 8.97 & 8.40 \\
\hline B-04-154 & 16-Jun-04 & 23.0 & & & & & & \\
\hline B-04-155 & 16-Jun-04 & 24.0 & 21.0 & 9.41 & 124.6 & 604 & 2.57 & 8.17 \\
\hline B-04-156 & 17-Jun-04 & 19.0 & & & & & & \\
\hline B-04-157 & 17-Jun-04 & 19.0 & & & & & & \\
\hline B-04-158 & 17-Jun-04 & 19.0 & 19.0 & & & & & \\
\hline B-04-159 & 18-Jun-04 & 15.0 & & & & & & \\
\hline B-04-160 & 18-Jun-04 & 15.0 & & & & & & \\
\hline B-04-161 & 18-Jun-04 & 15.0 & 15.7 & 8.09 & 98.6 & 1022 & 32.99 & 8.53 \\
\hline B-04-162 & 18-Jun-04 & 15.0 & & & & & & \\
\hline B-04-163 & 21-Jun-04 & 13.0 & 16.9 & 10.80 & 127.1 & 1348 & 4.27 & 7.64 \\
\hline B-04-164 & 21-Jun-04 & 15.0 & 15.5 & 6.60 & 77 & 844 & 3.69 & 7.99 \\
\hline B-04-165 & 21-Jun-04 & 15.0 & 16.0 & 7.76 & 94.7 & 842 & 3.52 & 8.11 \\
\hline B-04-166 & 21-Jun-04 & 14.0 & 14.0 & & & & & \\
\hline B-04-167 & 21-Jun-04 & 17.0 & 17.3 & 9.08 & 122 & 985 & 0.75 & 7.64 \\
\hline
\end{tabular}


Appendix 6: Results of basic water chemistry and air temperature measurements recorded at most sites where fishing effort was applied between 2002 and 2006.

\begin{tabular}{|c|c|c|c|c|c|c|c|c|}
\hline \multirow{2}{*}{ Site Number } & \multirow{2}{*}{ Date } & \multirow{2}{*}{$\begin{array}{l}\text { Air Temp. } \\
\left({ }^{\circ} \mathrm{C}\right)\end{array}$} & \multirow{2}{*}{$\begin{array}{c}\text { Water Temp. } \\
\left({ }^{\circ} \mathrm{C}\right)\end{array}$} & \multicolumn{2}{|c|}{ Dissolved Oxygen } & \multirow{2}{*}{$\begin{array}{c}\text { Conductivity } \\
(\mu \mathrm{S} / \mathrm{cm})\end{array}$} & \multirow{2}{*}{$\begin{array}{l}\text { Turbidity } \\
\text { (NTU) }\end{array}$} & \multirow{2}{*}{$\begin{array}{c}\mathrm{pH} \\
\text { (units) }\end{array}$} \\
\hline & & & & (mg/L) & (\% SAT) & & & \\
\hline B-04-168 & 22-Jun-04 & 12.0 & & & & & & \\
\hline B-04-169 & 22-Jun-04 & 13.0 & & & & & & \\
\hline B-04-170 & 22-Jun-04 & 13.0 & & & & & & \\
\hline B-04-171 & 22-Jun-04 & 14.0 & & & & & & \\
\hline B-04-172 & 22-Jun-04 & 15.0 & & & & & & \\
\hline B-04-173 & 22-Jun-04 & 20.0 & 15.5 & 3.43 & 41 & 777 & 0.00 & 7.74 \\
\hline B-04-174 & 22-Jun-04 & 21.0 & 17.7 & 8.13 & 101.7 & 542 & 3.99 & 7.64 \\
\hline B-04-175 & 22-Jun-04 & 20.0 & & & & & & \\
\hline B-04-176 & 23-Jun-04 & 12.0 & & & & & & \\
\hline B-04-177 & 23-Jun-04 & 12.0 & & & & & & \\
\hline B-04-178 & 23-Jun-04 & 13.0 & & & & & & \\
\hline B-04-179 & 23-Jun-04 & 14.0 & & & & & & \\
\hline B-04-180 & 23-Jun-04 & & & & & & & \\
\hline B-04-181 & 28-Jun-04 & 30.0 & 21.2 & 8.42 & 113 & 499 & 5.68 & 7.64 \\
\hline B-04-182 & 28-Jun-04 & 31.0 & & & & & & \\
\hline B-04-183 & 28-Jun-04 & 31.0 & & & & & & \\
\hline B-04-184 & 28-Jun-04 & 31.0 & & & & & & \\
\hline B-04-185 & 28-Jun-04 & 29.0 & & & & & & \\
\hline B-04-186 & 29-Jun-04 & 24.0 & 14.8 & 9.30 & 106.7 & 490 & 50 & 8.06 \\
\hline B-04-187 & 29-Jun-04 & 31.0 & 22.7 & 7.60 & 100 & 551 & 20.00 & 8.02 \\
\hline B-04-188 & 29-Jun-04 & 31.0 & 23.4 & 7.87 & 104 & 519 & 24.76 & 8.03 \\
\hline B-04-189 & 30-Jun-04 & 17.0 & 26.4 & 7.86 & 99 & 766 & 7.18 & 8.02 \\
\hline B-04-190 & 30-Jun-04 & 18.0 & & & & & & \\
\hline B-04-191 & 5-Jul-04 & 18.0 & 17.5 & 8.44 & 99 & 765 & 9.62 & 8.51 \\
\hline B-04-192 & 5-Jul-04 & 18.0 & & & & & & \\
\hline B-04-193 & 5-Jul-04 & 19.0 & & & & & & \\
\hline B-04-194 & 6-Jul-04 & 15.0 & & & & & & \\
\hline B-04-195 & 6-Jul-04 & 17.0 & 16.4 & 6.65 & 77.6 & 601 & 3.04 & 8.48 \\
\hline B-04-196 & 6-Jul-04 & 19.0 & 17.1 & 8.63 & 110.1 & 522 & 10.99 & 8.04 \\
\hline B-04-197 & 6-Jul-04 & 21.0 & 19.4 & 8.25 & 106.3 & 454 & 2.41 & 9.24 \\
\hline B-04-198 & 6-Jul-04 & 21.0 & 19.5 & 8.14 & 106 & 241 & 5.01 & 8.03 \\
\hline B-04-199 & 6-Jul-04 & 22.0 & 18.8 & 8.03 & 102.1 & 365 & 11.54 & 9.15 \\
\hline B-04-200 & 6-Jul-04 & 21.0 & 20.6 & 7.58 & 101.7 & 384 & 7.28 & 8.98 \\
\hline
\end{tabular}


Appendix 6: Results of basic water chemistry and air temperature measurements recorded at most sites where fishing effort was applied between 2002 and 2006.

\begin{tabular}{|c|c|c|c|c|c|c|c|c|}
\hline \multirow{2}{*}{ Site Number } & \multirow{2}{*}{ Date } & \multirow{2}{*}{$\begin{array}{l}\text { Air Temp. } \\
\left({ }^{\circ} \mathrm{C}\right)\end{array}$} & \multirow{2}{*}{$\begin{array}{l}\text { Water Temp. } \\
\left({ }^{\circ} \mathrm{C}\right)\end{array}$} & \multicolumn{2}{|c|}{ Dissolved Oxygen } & \multirow{2}{*}{$\begin{array}{c}\text { Conductivity } \\
(\mu \mathrm{S} / \mathrm{cm})\end{array}$} & \multirow{2}{*}{$\begin{array}{l}\text { Turbidity } \\
\text { (NTU) }\end{array}$} & \multirow{2}{*}{$\begin{array}{c}\text { pH } \\
\text { (units) }\end{array}$} \\
\hline & & & & (mg/L) & (\% SAT) & & & \\
\hline B-04-201 & 6-Jul-04 & 20.0 & & & & & & \\
\hline B-04-202 & 6-Jul-04 & 16.0 & & & & & & \\
\hline B-04-203 & 7-Jul-04 & 16.0 & & & & & & \\
\hline B-04-204 & 7-Jul-04 & 18.0 & & & & & & \\
\hline B-04-205 & 7-Jul-04 & 19.0 & 18.7 & 7.62 & 92.1 & 705 & 3.97 & 8.74 \\
\hline B-04-206 & 7-Jul-04 & 21.0 & & & & & & \\
\hline B-04-207 & 7-Jul-04 & 23.0 & & & & & & \\
\hline B-04-208 & 7-Jul-04 & 21.0 & & & & & & \\
\hline B-04-209 & 7-Jul-04 & 22.0 & & & & & & \\
\hline B-04-210 & 7-Jul-04 & 24.0 & & & & & & \\
\hline B-04-211 & 7-Jul-04 & 23.0 & 22.2 & 9.44 & 123.7 & 1536 & 3.26 & 8.75 \\
\hline B-04-212 & 7-Jul-04 & 23.0 & & & & & & \\
\hline B-04-213 & 7-Jul-04 & 22.0 & & & & & & \\
\hline B-04-214 & 7-Jul-04 & 23.0 & 22.3 & 7.71 & 110.1 & 622 & 16.52 & 9.01 \\
\hline B-04-215 & 7-Jul-04 & 23.0 & 22.8 & 7.06 & 107.6 & 1224 & 6.86 & 8.67 \\
\hline B-04-216 & 7-Jul-04 & 23.0 & & & & & & \\
\hline B-04-217 & 8-Jul-04 & 13.0 & 15.0 & 8.20 & 96.9 & 246 & 5.27 & 9.13 \\
\hline B-04-218 & 8-Jul-04 & 13.0 & 16.3 & 7.71 & 94.8 & 528 & 14.40 & 8.92 \\
\hline B-04-219 & 8-Jul-04 & 13.0 & 18.3 & 7.09 & 86 & 530 & 11.74 & 8.76 \\
\hline B-04-220 & 8-Jul-04 & 12.0 & 17.3 & 8.64 & 104 & 710 & 19.50 & 8.24 \\
\hline B-04-221 & 8-Jul-04 & 18.0 & & & & & & \\
\hline B-04-222 & 8-Jul-04 & 17.0 & & & & & & \\
\hline B-04-223 & 9-Jul-04 & 19.0 & 18.9 & 8.15 & 97.3 & 751 & 4.06 & 9.06 \\
\hline B-04-224 & 13-Jul-04 & 14.0 & 16.9 & 8.73 & 103 & 713 & 66 & 9.15 \\
\hline B-04-225 & 13-Jul-04 & 21.0 & 19.7 & 3.53 & 43.8 & 871 & 3.37 & 8.63 \\
\hline B-04-226 & 13-Jul-04 & 24.0 & 25.6 & 6.82 & 92.3 & 907 & 7.44 & \\
\hline B-04-227 & 13-Jul-04 & 25.0 & & & & & & \\
\hline B-04-228 & 13-Jul-04 & 26.0 & 24.2 & 7.12 & 109.1 & 488 & 9.75 & \\
\hline B-04-229 & 14-Jul-04 & 20.0 & & & & & & \\
\hline B-04-230 & 14-Jul-04 & 22.0 & 19.4 & 7.41 & 89.5 & 586 & 4.70 & 8.48 \\
\hline B-04-231 & 14-Jul-04 & 25.0 & 19.7 & 6.49 & 79.5 & 600 & 3.30 & 9.19 \\
\hline B-04-232 & 14-Jul-04 & 28.0 & 22.0 & 8.07 & 99 & 478 & 5.83 & 8.71 \\
\hline B-04-233 & 14-Jul-04 & 28.0 & & & & & & \\
\hline
\end{tabular}


Appendix 6: Results of basic water chemistry and air temperature measurements recorded at most sites where fishing effort was applied between 2002 and 2006.

\begin{tabular}{|c|c|c|c|c|c|c|c|c|}
\hline \multirow{2}{*}{ Site Number } & \multirow{2}{*}{ Date } & \multirow{2}{*}{$\begin{array}{c}\text { Air Temp. } \\
\left({ }^{\circ} \mathrm{C}\right)\end{array}$} & \multirow{2}{*}{$\begin{array}{l}\text { Water Temp. } \\
\left({ }^{\circ} \mathrm{C}\right)\end{array}$} & \multicolumn{2}{|c|}{ Dissolved Oxygen } & \multirow{2}{*}{$\begin{array}{c}\text { Conductivity } \\
(\mu \mathrm{S} / \mathrm{cm})\end{array}$} & \multirow{2}{*}{$\begin{array}{c}\text { Turbidity } \\
\text { (NTU) }\end{array}$} & \multirow{2}{*}{$\begin{array}{c}\text { pH } \\
\text { (units) }\end{array}$} \\
\hline & & & & (mg/L) & (\% SAT) & & & \\
\hline B-04-234 & 14-Jul-04 & 29.0 & & & & & & \\
\hline B-04-235 & 14-Jul-04 & 28.0 & & & & & & \\
\hline B-04-236 & 14-Jul-04 & 28.0 & & & & & & \\
\hline B-04-237 & 14-Jul-04 & 29.0 & & & & & & \\
\hline B-04-238 & 14-Jul-04 & 30.0 & 27.1 & 8.22 & 112.4 & 217 & 2.98 & 8.66 \\
\hline B-04-239 & 15-Jul-04 & 19.0 & & & & & & \\
\hline B-04-240 & 15-Jul-04 & 25.0 & 25.6 & 6.93 & 92.9 & 630 & 4.16 & 8.95 \\
\hline B-04-241 & 19-Jul-04 & 30.0 & 25.2 & 7.06 & 102.7 & 384 & 11.43 & 8.95 \\
\hline B-04-242 & 19-Jul-04 & 31.0 & & & & & & \\
\hline B-04-243 & 19-Jul-04 & 30.0 & & & & & & \\
\hline B-04-244 & 20-Jul-04 & 22.0 & 25.4 & 5.83 & 79.6 & 622 & 10.07 & 8.75 \\
\hline B-04-245 & 20-Jul-04 & 23.0 & & & & & & \\
\hline B-04-246 & 20-Jul-04 & 24.0 & & & & & & \\
\hline B-04-247 & 20-Jul-04 & 25.0 & & & & & & \\
\hline B-04-248 & 20-Jul-04 & 25.0 & 25.4 & 4.95 & 65.3 & $>2000$ & 5.13 & 8.68 \\
\hline B-04-249 & 20-Jul-04 & 26.0 & 24.9 & 6.22 & 82.5 & 1486 & 2.16 & 8.35 \\
\hline B-04-250 & 20-Jul-04 & 29.0 & 27.3 & 3.76 & 51.6 & 1095 & 1.54 & 8.17 \\
\hline B-04-251 & 21-Jul-04 & 16.0 & 21.1 & 2.70 & 33 & $>2000$ & 3.61 & 7.93 \\
\hline B-04-252 & 21-Jul-04 & 18.0 & & & & & & \\
\hline B-04-253 & 21-Jul-04 & 18.0 & & & & & & \\
\hline B-04-254 & 21-Jul-04 & 19.0 & & & & & & \\
\hline B-04-255 & 21-Jul-04 & 18.0 & & & & & & \\
\hline B-04-256 & 21-Jul-04 & 18.0 & 22.1 & 0.83 & 10.5 & 1083 & 4.81 & 7.98 \\
\hline B-04-257 & 21-Jul-04 & 16.0 & 22.3 & 6.05 & 75 & 1075 & 9.71 & 8.82 \\
\hline B-04-258 & 21-Jul-04 & 18.0 & & & & & & \\
\hline B-04-259 & 21-Jul-04 & 17.0 & 21.5 & 8.54 & 105 & 745 & 2.29 & 8.95 \\
\hline B-04-260 & 21-Jul-04 & 19.0 & & & & & & \\
\hline B-04-261 & 21-Jul-04 & 18.0 & & & & & & \\
\hline B-04-262 & 21-Jul-04 & 18.0 & & & & & & \\
\hline B-04-263 & 22-Jul-04 & 17.0 & 19.1 & 3.47 & 35.7 & 841 & 3.10 & 8.25 \\
\hline B-04-264 & 22-Jul-04 & 18.0 & 20.5 & 3.89 & 51.1 & 832 & 6.81 & 8.23 \\
\hline B-04-265 & 22-Jul-04 & 19.0 & & & & & & \\
\hline B-04-266 & 26-Jul-04 & 23.0 & 19.0 & 5.63 & 72.3 & 1076 & 3.92 & 8.75 \\
\hline
\end{tabular}


Appendix 6: Results of basic water chemistry and air temperature measurements recorded at most sites where fishing effort was applied between 2002 and 2006.

\begin{tabular}{|c|c|c|c|c|c|c|c|c|}
\hline \multirow{2}{*}{ Site Number } & \multirow{2}{*}{ Date } & \multirow{2}{*}{$\begin{array}{l}\text { Air Temp. } \\
\left({ }^{\circ} \mathrm{C}\right)\end{array}$} & \multirow{2}{*}{$\begin{array}{l}\text { Water Temp. } \\
\left({ }^{\circ} \mathrm{C}\right)\end{array}$} & \multicolumn{2}{|c|}{ Dissolved Oxygen } & \multirow{2}{*}{$\begin{array}{c}\text { Conductivity } \\
(\mu \mathrm{S} / \mathrm{cm})\end{array}$} & \multirow{2}{*}{$\begin{array}{l}\text { Turbidity } \\
\text { (NTU) }\end{array}$} & \multirow{2}{*}{$\begin{array}{c}\mathrm{pH} \\
\text { (units) }\end{array}$} \\
\hline & & & & (mg/L) & (\% SAT) & & & \\
\hline B-04-267 & 4-Aug-04 & 21.0 & & & & & & \\
\hline B-04-268 & 4-Aug-04 & 21.0 & & & & & & \\
\hline B-04-269 & 4-Aug-04 & 22.0 & & & & & & \\
\hline B-04-270 & 4-Aug-04 & 23 & 21.8 & 7.76 & 123.30 & 470 & 3.91 & 8.05 \\
\hline B-04-271 & 4-Aug-04 & 23 & & & & & & \\
\hline B-04-272 & 4-Aug-04 & 24 & & & & & & \\
\hline B-04-274 & 5-Aug-04 & 17 & & & & & & \\
\hline B-04-275 & 5-Aug-04 & 17 & 16.8 & 3.82 & 43.80 & 358.0 & 2.11 & 8.04 \\
\hline B-04-276 & 5-Aug-04 & 20 & 19.8 & 4.90 & 58.50 & 452.0 & 0.00 & 8.04 \\
\hline B-04-277 & 5-Aug-04 & 25 & & & & & & \\
\hline B-04-278 & 5-Aug-04 & 26 & & & & & & \\
\hline B-04-279 & 5-Aug-04 & 25 & & & & & & \\
\hline B-04-280 & 5-Aug-04 & 25 & & & & & & \\
\hline B-04-281 & 6-Aug-04 & 21 & & & & & & \\
\hline B-04-282 & 6-Aug-04 & 21 & & & & & & \\
\hline B-04-283 & 6-Aug-04 & 21 & & & & & & \\
\hline B-04-284 & 6-Aug-04 & 21 & & & & & & \\
\hline B-04-285 & 6-Aug-04 & 23 & & & & & & \\
\hline B-04-286 & 6-Aug-04 & 23 & & & & & & \\
\hline B-04-287 & 6-Aug-04 & 23 & & & & & & \\
\hline B-04-288 & 6-Aug-04 & 23 & & & & & & \\
\hline D-04-001 & 6-Apr-04 & & & & & & & \\
\hline D-04-002 & 6-Apr-04 & & & & & & & \\
\hline D-04-003 & 15-Apr-04 & & & & & & & \\
\hline D-04-004 & 15-Apr-04 & & 5.8 & & & & & \\
\hline D-04-005 & 15-Apr-04 & & & & & & & \\
\hline D-04-006 & 16-Apr-04 & & 2.8 & & & & & \\
\hline D-04-007 & 16-Apr-04 & & 2.3 & & & & & \\
\hline D-04-008 & 16-Apr-04 & & 3.8 & & & & & \\
\hline D-04-009 & 16-Apr-04 & & & & & & & \\
\hline D-04-010 & 2-Jun-04 & 14.0 & & & & & & \\
\hline D-04-011 & 2-Jun-04 & 14.0 & 12.8 & & & 358 & 7.11 & 7.74 \\
\hline D-04-012 & 2-Jun-04 & 15.0 & 15.7 & & & 461 & 4.82 & 8.30 \\
\hline
\end{tabular}


Appendix 6: Results of basic water chemistry and air temperature measurements recorded at most sites where fishing effort was applied between 2002 and 2006.

\begin{tabular}{|c|c|c|c|c|c|c|c|c|}
\hline \multirow{2}{*}{ Site Number } & \multirow{2}{*}{ Date } & \multirow{2}{*}{$\begin{array}{l}\text { Air Temp. } \\
\left({ }^{\circ} \mathrm{C}\right)\end{array}$} & \multirow{2}{*}{$\begin{array}{l}\text { Water Temp. } \\
\left({ }^{\circ} \mathrm{C}\right)\end{array}$} & \multicolumn{2}{|c|}{ Dissolved Oxygen } & \multirow{2}{*}{$\begin{array}{c}\text { Conductivity } \\
(\mu \mathrm{S} / \mathrm{cm})\end{array}$} & \multirow{2}{*}{$\begin{array}{l}\text { Turbidity } \\
\text { (NTU) }\end{array}$} & \multirow{2}{*}{$\begin{array}{c}\text { pH } \\
\text { (units) }\end{array}$} \\
\hline & & & & (mg/L) & (\% SAT) & & & \\
\hline D-04-013 & 3-Jun-04 & 14.0 & 15.2 & & & 312 & 18.56 & 7.71 \\
\hline D-04-014 & 3-Jun-04 & 18.0 & & & & & & \\
\hline D-04-015 & 7-Jun-04 & 14.0 & & & & & & \\
\hline D-04-016 & 8-Jun-04 & 10.0 & 13.5 & & & 339 & 7.89 & 7.75 \\
\hline D-04-017 & 9-Jun-04 & 12.0 & 14.6 & & & 437 & 3.06 & 7.86 \\
\hline D-04-018 & 9-Jun-04 & 15.0 & & & & & & \\
\hline D-04-019 & 9-Jun-04 & & & & & & & \\
\hline D-04-020 & 10-Jun-04 & 10.9 & 14.8 & & & 343 & 2.09 & 8.19 \\
\hline D-04-021 & 14-Jun-04 & 12.0 & 14.9 & & & 320 & 1.16 & 8.18 \\
\hline D-04-022 & 15-Jun-04 & 14.0 & 14.2 & 8.79 & 86.2 & 833 & 1.38 & 7.02 \\
\hline D-04-023 & 15-Jun-04 & 16.0 & 12.4 & 8.82 & 95.3 & 656 & 0.96 & 7.02 \\
\hline D-04-024 & 15-Jun-04 & 14.0 & & & & & & \\
\hline D-04-025 & 15-Jun-04 & & & & & & & \\
\hline D-04-026 & 15-Jun-04 & & & & & & & \\
\hline D-04-027 & 15-Jun-04 & 17.0 & 16.7 & 7.74 & 91.5 & 525 & 9.59 & 7.02 \\
\hline D-04-028 & 16-Jun-04 & 19.0 & 15.3 & 7.10 & 85.7 & 937 & 13.74 & 8.02 \\
\hline D-04-029 & 16-Jun-04 & 21.0 & 16.4 & 5.96 & 73.6 & 1277 & 10.68 & 8.20 \\
\hline D-04-030 & 22-Jun-04 & 13.0 & & & & & & \\
\hline D-04-031 & 22-Jun-04 & & & & & & & \\
\hline D-04-032 & 22-Jun-04 & & & & & & & \\
\hline D-04-033 & 23-Jun-04 & 13.0 & 13.4 & 7.20 & 83.4 & 712 & 2.14 & 7.80 \\
\hline D-04-034 & 23-Jun-04 & 11.0 & 13.9 & 7.79 & 91.6 & 669 & 1.36 & 7.88 \\
\hline D-04-035 & 23-Jun-04 & 14.0 & 17.0 & 8.59 & 104.7 & 720 & 0.85 & 8.18 \\
\hline D-04-036 & 7-Jul-04 & 14.0 & 15.0 & 9.40 & 112.7 & 404 & 5.80 & 8.12 \\
\hline W-04-001 & 3-May-04 & 8.3 & 8.3 & 9.36 & 90.7 & 331 & 6.51 & 8.28 \\
\hline W-04-002 & 3-May-04 & 9.6 & & & & & & \\
\hline W-04-003 & 3-May-04 & 10.8 & & & & & & \\
\hline W-04-004 & 3-May-04 & 7.8 & & & & & & \\
\hline W-04-005 & 3-May-04 & 8.1 & & & & & & \\
\hline W-04-006 & 3-May-04 & 15.6 & 9.5 & 8.85 & 86.1 & 442 & 8.87 & 8.27 \\
\hline W-04-007 & 3-May-04 & 11.7 & 10.5 & 8.27 & 83.4 & 279 & 0.00 & 7.83 \\
\hline W-04-008 & 3-May-04 & 9.3 & & & & & & \\
\hline W-04-009 & 4-May-04 & 2.7 & 4.3 & 11.15 & 96.6 & 567 & 0.00 & 8.55 \\
\hline
\end{tabular}


Appendix 6: Results of basic water chemistry and air temperature measurements recorded at most sites where fishing effort was applied between 2002 and 2006.

\begin{tabular}{|c|c|c|c|c|c|c|c|c|}
\hline \multirow{2}{*}{ Site Number } & \multirow{2}{*}{ Date } & \multirow{2}{*}{$\begin{array}{l}\text { Air Temp. } \\
\left({ }^{\circ} \mathrm{C}\right)\end{array}$} & \multirow{2}{*}{$\begin{array}{c}\text { Water Temp. } \\
\left({ }^{\circ} \mathrm{C}\right)\end{array}$} & \multicolumn{2}{|c|}{ Dissolved Oxygen } & \multirow{2}{*}{$\begin{array}{c}\text { Conductivity } \\
(\mu \mathrm{S} / \mathrm{cm})\end{array}$} & \multirow{2}{*}{$\begin{array}{l}\text { Turbidity } \\
\text { (NTU) }\end{array}$} & \multirow{2}{*}{$\begin{array}{c}\mathrm{pH} \\
\text { (units) }\end{array}$} \\
\hline & & & & (mg/L) & (\% SAT) & & & \\
\hline W-04-010 & 4-May-04 & 3.8 & & & & & & \\
\hline W-04-011 & 4-May-04 & 3.8 & & & & & & \\
\hline W-04-012 & 4-May-04 & 5.5 & & & & & & \\
\hline W-04-013 & 4-May-04 & 7.8 & 9.4 & 11.02 & 107.1 & 344 & 6.81 & 8.55 \\
\hline W-04-014 & 4-May-04 & 7.6 & & & & & & \\
\hline W-04-015 & 4-May-04 & 8.9 & & & & & & \\
\hline W-04-016 & 4-May-04 & 8.1 & & & & & & \\
\hline W-04-017 & 4-May-04 & 7.1 & & & & & & \\
\hline W-04-018 & 4-May-04 & 7.5 & & & & & & \\
\hline W-04-019 & 4-May-04 & 7.1 & & & & & & \\
\hline W-04-020 & 5-May-04 & 9.3 & 5.6 & 11.78 & 109.2 & 1018 & 2.66 & 8.54 \\
\hline W-04-021 & 5-May-04 & 15.4 & & & & & & \\
\hline W-04-022 & 5-May-04 & 14.7 & & & & & & \\
\hline W-04-023 & 5-May-04 & 18.6 & & & & & & \\
\hline W-04-024 & 5-May-04 & 18.4 & & & & & & \\
\hline W-04-025 & 5-May-04 & 18.4 & & & & & & \\
\hline W-04-026 & 5-May-04 & 18.4 & & & & & & \\
\hline W-04-027 & 5-May-04 & 22.6 & 9.6 & 11.23 & 109.7 & 812 & 5.76 & 8.70 \\
\hline W-04-028 & 5-May-04 & 12.6 & & & & & & \\
\hline W-04-029 & 6-May-04 & 4.1 & 5.4 & 0.79 & 6.1 & 434 & 7.76 & 7.84 \\
\hline W-04-030 & 6-May-04 & 4 & & & & & & \\
\hline W-04-031 & 6-May-04 & 3 & & & & & & \\
\hline W-04-032 & 6-May-04 & 3 & 6.5 & 6.07 & 54.3 & 795 & 2.24 & 7.84 \\
\hline W-04-033 & 6-May-04 & 7.9 & & & & & & \\
\hline W-04-034 & 6-May-04 & 7.9 & & & & & & \\
\hline W-04-035 & 6-May-04 & 9.6 & & & & & & \\
\hline W-04-036 & 6-May-04 & 7.1 & 7.8 & 8.77 & 81.6 & 441 & 3.27 & 8.22 \\
\hline W-04-037 & 6-May-04 & 9.9 & & & & & & \\
\hline W-04-038 & 6-May-04 & 7.9 & & & & & & \\
\hline W-04-039 & 10-May-04 & 10 & 7.5 & 3.47 & 78.7 & 404 & 6.89 & 8.23 \\
\hline W-04-040 & 10-May-04 & 7.1 & 12.7 & 9.10 & 87.3 & 292 & 40.67 & 8.21 \\
\hline W-04-041 & 11-May-04 & 3.2 & 4.6 & 3.57 & 27.6 & 937 & 3.59 & 7.42 \\
\hline W-04-042 & 11-May-04 & 3.2 & & & & & & \\
\hline
\end{tabular}


Appendix 6: Results of basic water chemistry and air temperature measurements recorded at most sites where fishing effort was applied between 2002 and 2006.

\begin{tabular}{|c|c|c|c|c|c|c|c|c|}
\hline \multirow{2}{*}{ Site Number } & \multirow{2}{*}{ Date } & \multirow{2}{*}{$\begin{array}{l}\text { Air Temp. } \\
\left({ }^{\circ} \mathrm{C}\right)\end{array}$} & \multirow{2}{*}{$\begin{array}{c}\text { Water Temp. } \\
\left({ }^{\circ} \mathrm{C}\right)\end{array}$} & \multicolumn{2}{|c|}{ Dissolved Oxygen } & \multirow{2}{*}{$\begin{array}{c}\text { Conductivity } \\
(\mu \mathrm{S} / \mathrm{cm})\end{array}$} & \multirow{2}{*}{$\begin{array}{l}\text { Turbidity } \\
\text { (NTU) }\end{array}$} & \multirow{2}{*}{$\begin{array}{c}\mathrm{pH} \\
\text { (units) }\end{array}$} \\
\hline & & & & (mg/L) & (\% SAT) & & & \\
\hline W-04-043 & 11-May-04 & 4 & 5.2 & 3.75 & 29.3 & 635 & 0.23 & 7.79 \\
\hline W-04-044 & 11-May-04 & 4 & 6.8 & 6.83 & 57.8 & 323 & 0.37 & 7.89 \\
\hline W-04-045 & 11-May-04 & 8.3 & & & & & & \\
\hline W-04-046 & 11-May-04 & 8.3 & & & & & & \\
\hline W-04-047 & 11-May-04 & 8.5 & & & & & & \\
\hline W-04-048 & 11-May-04 & 8.5 & & & & & & \\
\hline W-04-049 & 11-May-04 & 8.5 & 9.7 & 3.45 & 30.7 & 262 & 3.12 & 8.24 \\
\hline W-04-050 & 11-May-04 & 15 & 15.3 & & & 281 & 6.94 & 8.32 \\
\hline W-04-051 & 12-May-04 & 1.7 & 6.6 & 6.32 & 51.2 & 269 & 1.59 & 7.92 \\
\hline W-04-052 & 12-May-04 & 1.7 & 6.5 & & & 279 & 0.00 & 8.00 \\
\hline W-04-053 & 18-May-04 & 13.5 & 12.6 & $\mathrm{~N} / \mathrm{A}$ & & 320 & 33.03 & 8.11 \\
\hline W-04-054 & 18-May-04 & 23.1 & 13.1 & $\mathrm{~N} / \mathrm{A}$ & & 402 & 8.58 & 8.11 \\
\hline W-04-055 & 18-May-04 & 18.8 & 15.8 & $\mathrm{~N} / \mathrm{A}$ & & 441 & 0.00 & 7.87 \\
\hline W-04-056 & 18-May-04 & 21.2 & 18.5 & $\mathrm{~N} / \mathrm{A}$ & & 578 & 2.85 & 8.77 \\
\hline W-04-057 & 18-May-04 & 25.1 & 18.3 & $\mathrm{~N} / \mathrm{A}$ & & 321 & 2.53 & 8.09 \\
\hline W-04-058 & 18-May-04 & 26.0 & 23.0 & $\mathrm{~N} / \mathrm{A}$ & & 386 & 10.99 & 8.74 \\
\hline W-04-059 & 20-May-04 & 6.5 & 8.5 & $\mathrm{~N} / \mathrm{A}$ & & 306 & 14.03 & 7.95 \\
\hline W-04-060 & 20-May-04 & 7.3 & 8.2 & $\mathrm{~N} / \mathrm{A}$ & & 390 & 9.04 & 7.85 \\
\hline W-04-061 & 20-May-04 & 10.5 & & & & & & \\
\hline W-04-062 & 20-May-04 & 10.5 & & & & & & \\
\hline W-04-063 & 20-May-04 & 12.3 & 14.6 & $\mathrm{~N} / \mathrm{A}$ & & 349 & 1.50 & 7.87 \\
\hline W-04-064 & 20-May-04 & 12.3 & & & & & & \\
\hline W-04-065 & 20-May-04 & 12.3 & & & & & & \\
\hline W-04-066 & 20-May-04 & 12.3 & & & & & & \\
\hline W-04-067 & 20-May-04 & 12.3 & & & & & & \\
\hline W-04-068 & 20-May-04 & 13.0 & & & & & & \\
\hline W-04-069 & 21-May-04 & 8.3 & 8.1 & $\mathrm{~N} / \mathrm{A}$ & & 376 & 41.62 & 7.85 \\
\hline W-04-070 & 21-May-04 & 9.1 & 9.8 & $\mathrm{~N} / \mathrm{A}$ & & 331 & 5.63 & 7.85 \\
\hline W-04-071 & 21-May-04 & 9.4 & 10.1 & $\mathrm{~N} / \mathrm{A}$ & & 394 & 0.46 & 7.85 \\
\hline W-04-072 & 25-May-04 & 6.8 & 9.3 & 10.43 & 91.5 & 451 & 4.18 & 7.85 \\
\hline W-04-073 & 25-May-04 & 6.8 & & & & & & \\
\hline W-04-074 & 25-May-04 & 8.4 & 9.7 & 11.11 & 98.1 & 398 & 3.05 & 7.85 \\
\hline W-04-075 & 25-May-04 & 7.5 & & & & & & \\
\hline
\end{tabular}


Appendix 6: Results of basic water chemistry and air temperature measurements recorded at most sites where fishing effort was applied between 2002 and 2006.

\begin{tabular}{|c|c|c|c|c|c|c|c|c|}
\hline \multirow{2}{*}{ Site Number } & \multirow{2}{*}{ Date } & \multirow{2}{*}{$\begin{array}{l}\text { Air Temp. } \\
\left({ }^{\circ} \mathrm{C}\right)\end{array}$} & \multirow{2}{*}{$\begin{array}{c}\text { Water Temp. } \\
\left({ }^{\circ} \mathrm{C}\right)\end{array}$} & \multicolumn{2}{|c|}{ Dissolved Oxygen } & \multirow{2}{*}{$\begin{array}{c}\text { Conductivity } \\
(\mu \mathrm{S} / \mathrm{cm})\end{array}$} & \multirow{2}{*}{$\begin{array}{l}\text { Turbidity } \\
\text { (NTU) }\end{array}$} & \multirow{2}{*}{$\begin{array}{c}\mathrm{pH} \\
\text { (units) }\end{array}$} \\
\hline & & & & (mg/L) & (\% SAT) & & & \\
\hline W-04-076 & 25-May-04 & 7.5 & 11.1 & 10.80 & 97.4 & 433 & 2.27 & 7.85 \\
\hline W-04-077 & 26-May-04 & 6.9 & 8.4 & & & 376 & 3.43 & 7.85 \\
\hline W-04-078 & 26-May-04 & 9.1 & 9.5 & & & 277 & 5.33 & 7.85 \\
\hline W-04-079 & 26-May-04 & 7.5 & & & & & & \\
\hline W-04-080 & 26-May-04 & 7.5 & & & & & & \\
\hline W-04-081 & 26-May-04 & 7.5 & & & & & & \\
\hline W-04-082 & 26-May-04 & 9.9 & 11.7 & & & 375 & 0.12 & 7.85 \\
\hline W-04-083 & 27-May-04 & 10.7 & 10.1 & 9.22 & 92.7 & 280 & 1.47 & 7.07 \\
\hline W-04-084 & 27-May-04 & 12.7 & 14.6 & 9.10 & 106.7 & 292 & 0.98 & 7.07 \\
\hline W-04-085 & 28-May-04 & 14.3 & 11.1 & 2.85 & 30.5 & 266 & 0.00 & 7.07 \\
\hline W-04-086 & 28-May-04 & 15.0 & & & & & & \\
\hline W-04-087 & 28-May-04 & 15.0 & 13.3 & 8.10 & 91.9 & 377 & 0.29 & 7.98 \\
\hline W-04-088 & 28-May-04 & 17.0 & 14.4 & 11.01 & 122.7 & 324 & 2.01 & 7.07 \\
\hline W-04-089 & 1-Jun-04 & 13.0 & & & & & & \\
\hline W-04-090 & 1-Jun-04 & 13.3 & 12.5 & 5.99 & 63.9 & 427 & 0.64 & 7.95 \\
\hline W-04-091 & 1-Jun-04 & 18.0 & & & & & & \\
\hline W-04-092 & 1-Jun-04 & 23.8 & 13.8 & 6.57 & 75.5 & 443 & 0.58 & 7.95 \\
\hline W-04-093 & 1-Jun-04 & 20.0 & 21.5 & 9.43 & 120.0 & 402 & 0.00 & 7.97 \\
\hline W-04-094 & 2-Jun-04 & 17.7 & 12.7 & 6.77 & 73.3 & 269 & 0.81 & 7.85 \\
\hline W-04-095 & 2-Jun-04 & 18.2 & 12.2 & 8.07 & 87.6 & 184 & 0.43 & 7.95 \\
\hline W-04-096 & 2-Jun-04 & 20.7 & & & & & & \\
\hline W-04-097 & 2-Jun-04 & 21.5 & 14.8 & 5.15 & 59.6 & 323 & 1.54 & 7.97 \\
\hline W-04-098 & 2-Jun-04 & 22.0 & & & & & & \\
\hline W-04-099 & 2-Jun-04 & 24.7 & & & & & & \\
\hline W-04-100 & 2-Jun-04 & 24.7 & & & & & & \\
\hline W-04-101 & 3-Jun-04 & 11.5 & & & & & & \\
\hline W-04-102 & 3-Jun-04 & 22.0 & 18.0 & 6.18 & 74.9 & 431 & 0.16 & 7.95 \\
\hline W-04-103 & 3-Jun-04 & 22.0 & & & & & & \\
\hline W-04-104 & 3-Jun-04 & 22.0 & & & & & & \\
\hline W-04-105 & 3-Jun-04 & 25.0 & 20.0 & 6.18 & 79.5 & 446 & 0.60 & 7.88 \\
\hline W-04-106 & 0-Jan-00 & 25.0 & 22.2 & 7.48 & 99.7 & 455 & 2.89 & 7.97 \\
\hline W-04-107 & 3-Jun-04 & 25.0 & & & & & & \\
\hline W-04-108 & 3-Jun-04 & 25.0 & & & & & & \\
\hline
\end{tabular}


Appendix 6: Results of basic water chemistry and air temperature measurements recorded at most sites where fishing effort was applied between 2002 and 2006.

\begin{tabular}{|c|c|c|c|c|c|c|c|c|}
\hline \multirow{2}{*}{ Site Number } & \multirow{2}{*}{ Date } & \multirow{2}{*}{$\begin{array}{l}\text { Air Temp. } \\
\left({ }^{\circ} \mathrm{C}\right)\end{array}$} & \multirow{2}{*}{$\begin{array}{c}\text { Water Temp. } \\
\left({ }^{\circ} \mathrm{C}\right)\end{array}$} & \multicolumn{2}{|c|}{ Dissolved Oxygen } & \multirow{2}{*}{$\begin{array}{c}\text { Conductivity } \\
(\mu \mathrm{S} / \mathrm{cm})\end{array}$} & \multirow{2}{*}{$\begin{array}{l}\text { Turbidity } \\
\text { (NTU) }\end{array}$} & \multirow{2}{*}{$\begin{array}{c}\mathrm{pH} \\
\text { (units) }\end{array}$} \\
\hline & & & & (mg/L) & (\% SAT) & & & \\
\hline W-04-109 & 4-Jun-04 & 15.0 & 16.2 & 5.07 & 59.4 & 580 & 1.92 & 7.95 \\
\hline W-04-110 & 4-Jun-04 & 18.0 & 18.2 & 4.31 & 53.8 & 530 & 0.75 & 7.97 \\
\hline W-04-111 & 4-Jun-04 & 25.0 & 19.4 & 7.17 & 91.1 & 592 & 6.29 & 7.97 \\
\hline W-04-112 & 4-Jun-04 & 25.0 & & & & & & \\
\hline W-04-113 & 5-Jun-04 & 23.0 & 19.9 & 4.80 & 60.0 & 633 & 19.86 & 7.53 \\
\hline W-04-114 & 5-Jun-04 & 25.0 & 18.5 & 7.08 & 88.3 & 555 & 2.35 & 7.95 \\
\hline W-04-115 & 5-Jun-04 & 25.0 & 22.3 & 6.52 & 86.0 & 467 & 3.52 & 7.97 \\
\hline W-04-116 & 5-Jun-04 & 25.0 & 22.5 & 12.67 & & 430 & 0.64 & 7.97 \\
\hline W-04-117 & 7-Jun-04 & 15.0 & & & & & & \\
\hline W-04-118 & 7-Jun-04 & 15.0 & & & & & & \\
\hline W-04-119 & 8-Jun-04 & 15.0 & 15.2 & 7.83 & 88.6 & 391 & 0.23 & 7.87 \\
\hline W-04-120 & 8-Jun-04 & 15.0 & 16.8 & 7.13 & 83.3 & 394 & 2.73 & 7.95 \\
\hline W-04-121 & 8-Jun-04 & 15.0 & 18.3 & 8.44 & 102.1 & 380 & 0.60 & 7.95 \\
\hline W-04-122 & 8-Jun-04 & 15.0 & & & & & & \\
\hline W-04-123 & 8-Jun-04 & 15.0 & & & & & & \\
\hline W-04-124 & 8-Jun-04 & 15.0 & 18.3 & 9.02 & 107.4 & 751 & 14.14 & 7.95 \\
\hline W-04-125 & 9-Jun-04 & 13.0 & & & & & & \\
\hline W-04-126 & 9-Jun-04 & 13.0 & 13.7 & 5.53 & 59.9 & 416 & 0.00 & 7.95 \\
\hline W-04-127 & 9-Jun-04 & 15.0 & & & & & & \\
\hline W-04-128 & 9-Jun-04 & 15.0 & & & & & & \\
\hline W-04-129 & 9-Jun-04 & 21.0 & 14.3 & 6.40 & 70.9 & 408 & 0.73 & 7.95 \\
\hline W-04-130 & 9-Jun-04 & 21.0 & & & & & & \\
\hline W-04-131 & 9-Jun-04 & 21.0 & & & & & & \\
\hline W-04-132 & 9-Jun-04 & 21.0 & 19.7 & 5.29 & 65.0 & 499 & 1.65 & 7.97 \\
\hline W-04-133 & 9-Jun-04 & 21.0 & & & & & & \\
\hline W-04-134 & 9-Jun-04 & 21.0 & & & & & & \\
\hline W-04-135 & 9-Jun-04 & 21.0 & 20.6 & 7.83 & 99.0 & 443 & 5.36 & 7.97 \\
\hline W-04-136 & 10-Jun-04 & 15.0 & & & & & & \\
\hline W-04-137 & 10-Jun-04 & 15.0 & 15.2 & 4.44 & 50.7 & 609 & 0.28 & 7.95 \\
\hline W-04-138 & 10-Jun-04 & 15.0 & & & & & & \\
\hline W-04-139 & 10-Jun-04 & 18.0 & & & & & & \\
\hline W-04-140 & 10-Jun-04 & 18.0 & & & & & & \\
\hline W-04-141 & 10-Jun-04 & 18.0 & & & & & & \\
\hline
\end{tabular}


Appendix 6: Results of basic water chemistry and air temperature measurements recorded at most sites where fishing effort was applied between 2002 and 2006.

\begin{tabular}{|c|c|c|c|c|c|c|c|c|}
\hline \multirow{2}{*}{ Site Number } & \multirow{2}{*}{ Date } & \multirow{2}{*}{$\begin{array}{l}\text { Air Temp. } \\
\left({ }^{\circ} \mathrm{C}\right)\end{array}$} & \multirow{2}{*}{$\begin{array}{c}\text { Water Temp. } \\
\left({ }^{\circ} \mathrm{C}\right)\end{array}$} & \multicolumn{2}{|c|}{ Dissolved Oxygen } & \multirow{2}{*}{$\begin{array}{c}\text { Conductivity } \\
(\mu \mathrm{S} / \mathrm{cm})\end{array}$} & \multirow{2}{*}{$\begin{array}{l}\text { Turbidity } \\
\text { (NTU) }\end{array}$} & \multirow{2}{*}{$\begin{array}{c}\mathrm{pH} \\
\text { (units) }\end{array}$} \\
\hline & & & & (mg/L) & (\% SAT) & & & \\
\hline W-04-142 & 14-Jun-04 & 15.0 & & & & & & \\
\hline W-04-143 & 14-Jun-04 & 15.0 & & & & & & \\
\hline W-04-144 & 14-Jun-04 & 15.0 & & & & & & \\
\hline W-04-145 & 14-Jun-04 & 15.0 & & & & & & \\
\hline W-04-146 & 14-Jun-04 & 15.0 & & & & & & \\
\hline W-04-147 & 14-Jun-04 & 18.0 & & & & & & \\
\hline W-04-148 & 14-Jun-04 & 18.0 & & & & & & \\
\hline W-04-149 & 14-Jun-04 & 18.0 & 16.5 & 8.25 & 94.9 & 271 & 12.05 & 7.87 \\
\hline W-04-150 & 14-Jun-04 & 18.0 & & & & & & \\
\hline W-04-151 & 14-Jun-04 & 20.0 & 18.1 & 13.32 & & 346 & 8.49 & 7.87 \\
\hline W-04-152 & 15-Jun-04 & 17.0 & & & & & & \\
\hline W-04-153 & 15-Jun-04 & 17.0 & 14.6 & 6.99 & 78.1 & 309 & 2.09 & 7.87 \\
\hline W-04-154 & 15-Jun-04 & 18.0 & & & & & & \\
\hline W-04-155 & 15-Jun-04 & 18.0 & & & & & & \\
\hline W-04-156 & 16-Jun-04 & 13.0 & & & & & & \\
\hline W-04-157 & 16-Jun-04 & 18.0 & 13.5 & 8.90 & 96.2 & 305 & 118 & 7.87 \\
\hline W-04-158 & 16-Jun-04 & 18.0 & & & & & & \\
\hline W-04-159 & 16-Jun-04 & 19.0 & 16.8 & 12.14 & & 372 & 19.07 & 7.87 \\
\hline W-04-160 & 16-Jun-04 & 22.0 & & & & & & \\
\hline W-04-161 & 16-Jun-04 & 22.0 & & & & & & \\
\hline W-04-162 & 16-Jun-04 & 22.0 & & & & & & \\
\hline W-04-163 & 16-Jun-04 & 22.0 & & & & & & \\
\hline W-04-164 & 17-Jun-04 & 19.0 & & & & & & \\
\hline W-04-165 & 17-Jun-04 & 19.0 & & & & & & \\
\hline W-04-166 & 17-Jun-04 & 22.0 & 16.8 & 8.07 & 93.0 & 317 & 6.69 & 7.04 \\
\hline W-04-167 & 17-Jun-04 & 22.0 & 18.2 & 6.52 & 79.4 & 326 & 11.75 & 7.04 \\
\hline W-04-168 & 17-Jun-04 & 25.0 & & & & & & \\
\hline W-04-169 & 17-Jun-04 & 23.0 & & & & & & \\
\hline W-04-170 & 17-Jun-04 & 20.0 & & & & & & \\
\hline W-04-171 & 17-Jun-04 & 24.0 & 20.0 & 10.61 & 130.5 & 269 & 75 & 7.04 \\
\hline W-04-172 & 17-Jun-04 & 24.0 & 21.4 & 8.56 & 110.5 & 344 & 10.69 & \\
\hline W-04-173 & 18-Jun-04 & 13.0 & 13.0 & 9.76 & 102.5 & 625 & 4.43 & \\
\hline W-04-174 & 18-Jun-04 & 15.0 & 13.5 & 5.80 & 62.9 & 554 & 3.44 & \\
\hline
\end{tabular}


Appendix 6: Results of basic water chemistry and air temperature measurements recorded at most sites where fishing effort was applied between 2002 and 2006.

\begin{tabular}{|c|c|c|c|c|c|c|c|c|}
\hline \multirow{2}{*}{ Site Number } & \multirow{2}{*}{ Date } & \multirow{2}{*}{$\begin{array}{l}\text { Air Temp. } \\
\left({ }^{\circ} \mathrm{C}\right)\end{array}$} & \multirow{2}{*}{$\begin{array}{c}\text { Water Temp. } \\
\left({ }^{\circ} \mathrm{C}\right)\end{array}$} & \multicolumn{2}{|c|}{ Dissolved Oxygen } & \multirow{2}{*}{$\begin{array}{c}\text { Conductivity } \\
(\mu \mathrm{S} / \mathrm{cm})\end{array}$} & \multirow{2}{*}{$\begin{array}{l}\text { Turbidity } \\
\text { (NTU) }\end{array}$} & \multirow{2}{*}{$\begin{array}{c}\mathrm{pH} \\
\text { (units) }\end{array}$} \\
\hline & & & & (mg/L) & (\% SAT) & & & \\
\hline W-04-175 & 21-Jun-04 & 15.0 & 15.0 & 9.50 & 104.5 & 266 & 2.14 & \\
\hline W-04-176 & 21-Jun-04 & 16.0 & 16.7 & 10.20 & 116.5 & 371 & 2.72 & \\
\hline W-04-177 & 21-Jun-04 & 18.0 & 16.6 & 9.02 & 107.5 & 348 & 3.58 & \\
\hline W-04-178 & 21-Jun-04 & 19.0 & 18.0 & 9.78 & 114.4 & 246 & 0.00 & \\
\hline W-04-179 & 22-Jun-04 & 11.0 & 13.5 & 4.95 & 53.6 & 296 & 0.00 & \\
\hline W-04-180 & 22-Jun-04 & 11.0 & 12.3 & 6.04 & 63.6 & 252 & 2.38 & \\
\hline W-04-181 & 22-Jun-04 & 14.0 & 14.6 & 7.53 & 83.4 & 117 & 2.08 & \\
\hline W-04-182 & 22-Jun-04 & 15.0 & 16.5 & 6.02 & 68.5 & 215 & 5.91 & \\
\hline W-04-183 & 22-Jun-04 & 18.0 & & & & & & \\
\hline W-04-184 & 22-Jun-04 & 16.0 & 16.7 & 6.37 & 73.4 & 143 & 1.99 & \\
\hline W-04-185 & 23-Jun-04 & 11.0 & & & & & & \\
\hline W-04-186 & 23-Jun-04 & 11.0 & 11.8 & 2.76 & 28.5 & 324 & 0.00 & \\
\hline W-04-187 & 23-Jun-04 & 10.0 & 13.4 & 5.68 & 63.7 & 350 & 1.51 & \\
\hline W-04-188 & 23-Jun-04 & 11.0 & & & & & & \\
\hline W-04-189 & 24-Jun-04 & 15.0 & & & & & & \\
\hline W-04-190 & 24-Jun-04 & 15.0 & & & & & & \\
\hline W-04-191 & 24-Jun-04 & 15.0 & & & & & & \\
\hline W-04-192 & 24-Jun-04 & 15.0 & 10.4 & 7.75 & 79.8 & 130 & 10.56 & \\
\hline W-04-193 & 24-Jun-04 & 15.0 & 11.6 & 7.20 & 77.2 & 104 & 19.40 & \\
\hline W-04-194 & 24-Jun-04 & 14.0 & & & & & & \\
\hline W-04-195 & 25-Jun-04 & 9.0 & 11.7 & 7.24 & 74.5 & 235 & 7.84 & \\
\hline W-04-196 & 25-Jun-04 & 9.0 & & & & & & \\
\hline W-04-197 & 25-Jun-04 & 10.0 & 10.7 & 8.20 & 82.5 & 66 & 11.43 & \\
\hline W-04-198 & 29-Jun-04 & 22.0 & & & & & & \\
\hline W-04-199 & 29-Jun-04 & 22.0 & & & & & & \\
\hline W-04-200 & 29-Jun-04 & 23.0 & 16.9 & 5.19 & 63.2 & 330 & 1.95 & \\
\hline W-04-201 & 29-Jun-04 & 28.0 & & & & & & \\
\hline W-04-202 & 29-Jun-04 & 32.0 & 20.7 & 6.40 & 85.8 & 255 & 1.86 & \\
\hline W-04-203 & 29-Jun-04 & 32.0 & 19.1 & 5.22 & 68.5 & 312 & 0.00 & \\
\hline W-04-204 & 29-Jun-04 & 32.0 & & & & & & \\
\hline W-04-205 & 29-Jun-04 & 32.0 & & & & & & \\
\hline W-04-206 & 29-Jun-04 & 32.0 & & & & & & \\
\hline W-04-207 & 5-Jul-04 & 22.0 & 15.4 & 6.59 & 75.5 & 227 & 2.32 & 7.88 \\
\hline
\end{tabular}


Appendix 6: Results of basic water chemistry and air temperature measurements recorded at most sites where fishing effort was applied between 2002 and 2006.

\begin{tabular}{|c|c|c|c|c|c|c|c|c|}
\hline \multirow{2}{*}{ Site Number } & \multirow{2}{*}{ Date } & \multirow{2}{*}{$\begin{array}{l}\text { Air Temp. } \\
\left({ }^{\circ} \mathrm{C}\right)\end{array}$} & \multirow{2}{*}{$\begin{array}{l}\text { Water Temp. } \\
\left({ }^{\circ} \mathrm{C}\right)\end{array}$} & \multicolumn{2}{|c|}{ Dissolved Oxygen } & \multirow{2}{*}{$\begin{array}{c}\text { Conductivity } \\
(\mu \mathrm{S} / \mathrm{cm})\end{array}$} & \multirow{2}{*}{$\begin{array}{l}\text { Turbidity } \\
\text { (NTU) }\end{array}$} & \multirow{2}{*}{$\begin{array}{c}\text { pH } \\
\text { (units) }\end{array}$} \\
\hline & & & & (mg/L) & (\% SAT) & & & \\
\hline W-04-208 & 5-Jul-04 & 23.0 & 17.4 & 4.81 & 60.0 & 200 & 0.08 & \\
\hline W-04-209 & 5-Jul-04 & 22.0 & & & & & & \\
\hline W-04-210 & 5-Jul-04 & 22.0 & & & & & & \\
\hline W-04-211 & 5-Jul-04 & 22.0 & 19.1 & 8.29 & 102.0 & 187 & 0.00 & \\
\hline W-04-212 & 6-Jul-04 & 22.0 & 15.6 & 8.37 & 94.7 & 200 & 24.88 & \\
\hline W-04-213 & 6-Jul-04 & 22.0 & 17.9 & 7.02 & 83.4 & 164 & 2.68 & \\
\hline W-04-214 & 6-Jul-04 & 22.0 & & & & & & \\
\hline W-04-215 & 6-Jul-04 & 23.0 & & & & & & \\
\hline W-04-216 & 6-Jul-04 & 23.0 & 18.3 & 7.47 & 95.5 & 117 & 0.76 & \\
\hline W-04-217 & 6-Jul-04 & 24.0 & 17.5 & 7.52 & 95.0 & 163 & 216 & \\
\hline W-04-218 & 6-Jul-04 & 24.0 & 21.9 & 8.31 & 109.4 & 207 & 0.98 & \\
\hline W-04-219 & 7-Jul-04 & 19.0 & & & & & & \\
\hline W-04-220 & 7-Jul-04 & 20.0 & & & & & & \\
\hline W-04-221 & 7-Jul-04 & 20.0 & & & & & & \\
\hline W-04-222 & 7-Jul-04 & 20.0 & & & & & & \\
\hline W-04-223 & 7-Jul-04 & 23.0 & & & & & & \\
\hline W-04-224 & 7-Jul-04 & 23.0 & & & & & & \\
\hline W-04-225 & 7-Jul-04 & 24.0 & 16.9 & 7.66 & 91.2 & 181 & 17.95 & \\
\hline W-04-226 & 7-Jul-04 & 24.0 & & & & & & \\
\hline W-04-227 & 7-Jul-04 & 24.0 & & & & & & \\
\hline W-04-228 & 7-Jul-04 & 25.0 & & & & & & \\
\hline W-04-229 & 7-Jul-04 & 25.0 & & & & & & \\
\hline W-04-230 & 7-Jul-04 & 26.0 & & & & & & \\
\hline W-04-231 & 8-Jul-04 & 24.0 & 16.6 & 3.74 & 43.1 & 131 & 0.72 & \\
\hline W-04-232 & 8-Jul-04 & 20.0 & 21.3 & 8.87 & 109.9 & 219 & 1.05 & \\
\hline W-04-233 & 13-Jul-04 & 18.0 & & & & & & \\
\hline W-04-234 & 13-Jul-04 & 20.0 & 18.0 & 6.20 & 73.7 & 654 & 0.00 & 7.99 \\
\hline W-04-235 & 13-Jul-04 & 23.0 & & & & & & \\
\hline W-04-236 & 13-Jul-04 & 23.0 & & & & & & \\
\hline W-04-237 & 13-Jul-04 & 25.0 & 21.4 & 6.46 & 83.9 & 591 & 1.53 & \\
\hline W-04-238 & 13-Jul-04 & 25.0 & 23.0 & 6.25 & 87.2 & 556 & 0.81 & \\
\hline W-04-239 & 13-Jul-04 & 26.0 & 23.0 & 4.46 & 60.9 & 536 & 4.81 & \\
\hline W-04-240 & 14-Jul-04 & 25.0 & & & & & & \\
\hline
\end{tabular}


Appendix 6: Results of basic water chemistry and air temperature measurements recorded at most sites where fishing effort was applied between 2002 and 2006.

\begin{tabular}{|c|c|c|c|c|c|c|c|c|}
\hline \multirow{2}{*}{ Site Number } & \multirow{2}{*}{ Date } & \multirow{2}{*}{$\begin{array}{l}\text { Air Temp. } \\
\left({ }^{\circ} \mathrm{C}\right)\end{array}$} & \multirow{2}{*}{$\begin{array}{c}\text { Water Temp. } \\
\left({ }^{\circ} \mathrm{C}\right)\end{array}$} & \multicolumn{2}{|c|}{ Dissolved Oxygen } & \multirow{2}{*}{$\begin{array}{c}\text { Conductivity } \\
(\mu \mathrm{S} / \mathrm{cm})\end{array}$} & \multirow{2}{*}{$\begin{array}{l}\text { Turbidity } \\
\text { (NTU) }\end{array}$} & \multirow{2}{*}{$\begin{array}{c}\mathrm{pH} \\
\text { (units) }\end{array}$} \\
\hline & & & & (mg/L) & (\% SAT) & & & \\
\hline W-04-241 & 14-Jul-04 & 26.0 & & & & & & \\
\hline W-04-242 & 14-Jul-04 & 26.0 & & & & & & \\
\hline W-04-243 & 14-Jul-04 & 27.0 & & & & & & \\
\hline W-04-244 & 14-Jul-04 & 27.0 & & & & & & \\
\hline W-04-245 & 14-Jul-04 & 27.0 & & & & & & \\
\hline W-04-246 & 14-Jul-04 & 27.0 & & & & & & \\
\hline W-04-247 & 14-Jul-04 & 27.0 & & & & & & \\
\hline W-04-248 & 14-Jul-04 & 29.0 & & & & & & \\
\hline W-04-249 & 14-Jul-04 & 30.0 & 22.3 & 6.35 & 87.3 & 556 & 2.80 & 8.50 \\
\hline W-04-250 & 14-Jul-04 & 30.0 & 21.2 & 6.08 & 83.5 & 617 & 11.35 & 7.80 \\
\hline W-04-251 & 15-Jul-04 & 20.0 & & & & & & \\
\hline W-04-252 & 15-Jul-04 & 20.0 & 19.0 & 6.36 & 84.1 & 511 & 1.63 & 7.77 \\
\hline W-04-253 & 15-Jul-04 & 28.0 & 19.7 & 3.62 & 51.7 & 619 & 1.29 & 7.42 \\
\hline W-04-254 & 15-Jul-04 & 28.0 & 21.8 & 6.25 & 90.4 & 331 & 2.59 & 7.81 \\
\hline W-04-255 & 15-Jul-04 & 28.0 & 24.3 & 6.26 & 92.2 & 222 & 0.85 & 7.74 \\
\hline W-04-256 & 20-Jul-04 & 30.0 & 21.8 & 5.78 & 80.1 & 660 & 4.81 & 7.95 \\
\hline W-04-257 & 21-Jul-04 & 21.0 & & & & & & \\
\hline W-04-258 & 21-Jul-04 & 21.0 & & & & & & \\
\hline W-04-259 & 21-Jul-04 & 21.0 & & & & & & \\
\hline W-04-260 & 21-Jul-04 & 20.0 & 22.8 & 2.48 & 31.7 & & 0.00 & \\
\hline W-04-261 & 21-Jul-04 & 18.0 & & & & & & \\
\hline W-04-262 & 22-Jul-04 & 16.0 & 16.1 & 5.23 & 59.1 & & 0.62 & \\
\hline W-04-263 & 22-Jul-04 & 20.0 & & & & & & \\
\hline W-04-264 & 22-Jul-04 & 20.0 & 19.7 & 6.08 & 73.9 & & 22.12 & \\
\hline W-04-265 & 22-Jul-04 & 20.0 & 20.7 & 6.05 & 75.1 & & 2.89 & \\
\hline W-04-266 & 22-Jul-04 & 20.0 & 19.7 & 7.31 & 90.7 & & 1.78 & \\
\hline W-04-267 & 22-Jul-04 & 18.0 & & & & & & \\
\hline W-04-268 & 22-Jul-04 & 18.0 & & & & & & \\
\hline W-04-269 & 22-Jul-04 & 18.0 & 18.0 & 8.16 & 98.5 & & 0.43 & \\
\hline W-04-270 & 22-Jul-04 & 20.0 & & & & & & \\
\hline W-04-271 & 23-Jul-04 & 11.0 & 13.5 & 3.11 & 32.9 & & 0.01 & \\
\hline W-04-272 & 23-Jul-04 & 12.0 & & & & & & \\
\hline W-04-273 & 23-Jul-04 & 12.0 & & & & & & \\
\hline
\end{tabular}


Appendix 6: Results of basic water chemistry and air temperature measurements recorded at most sites where fishing effort was applied between 2002 and 2006.

\begin{tabular}{|c|c|c|c|c|c|c|c|c|}
\hline \multirow{2}{*}{ Site Number } & \multirow{2}{*}{ Date } & \multirow{2}{*}{$\begin{array}{l}\text { Air Temp. } \\
\left({ }^{\circ} \mathrm{C}\right)\end{array}$} & \multirow{2}{*}{$\begin{array}{c}\text { Water Temp. } \\
\left({ }^{\circ} \mathrm{C}\right)\end{array}$} & \multicolumn{2}{|c|}{ Dissolved Oxygen } & \multirow{2}{*}{$\begin{array}{c}\text { Conductivity } \\
(\mu \mathrm{S} / \mathrm{cm})\end{array}$} & \multirow{2}{*}{$\begin{array}{l}\text { Turbidity } \\
\text { (NTU) }\end{array}$} & \multirow{2}{*}{$\begin{array}{c}\mathrm{pH} \\
\text { (units) }\end{array}$} \\
\hline & & & & (mg/L) & (\% SAT) & & & \\
\hline W-04-274 & 23-Jul-04 & 17.0 & 17.3 & 8.78 & 101.5 & & 2.37 & \\
\hline W-04-275 & 3-Aug-04 & 24.0 & & & & & & \\
\hline W-04-276 & 3-Aug-04 & 24.0 & & & & & & \\
\hline W-04-277 & 3-Aug-04 & 24.0 & 22.1 & 9.21 & 116.8 & & 4.30 & \\
\hline W-04-278 & 3-Aug-04 & 24.0 & & & & & & \\
\hline W-04-279 & 3-Aug-04 & 24.0 & & & & & & \\
\hline W-04-280 & 3-Aug-04 & 25.0 & & & & & & \\
\hline W-04-281 & 3-Aug-04 & 26.0 & & & & & & \\
\hline W-04-282 & 3-Aug-04 & 26.0 & & & & & & \\
\hline W-04-283 & 3-Aug-04 & 26.0 & & & & & & \\
\hline W-04-284 & 3-Aug-04 & 26.0 & & & & & & \\
\hline W-04-285 & 3-Aug-04 & 26.0 & & & & & & \\
\hline W-04-286 & 3-Aug-04 & 26.0 & 25.9 & 9.18 & 124.6 & & 19.64 & \\
\hline W-04-287 & 3-Aug-04 & 26.0 & 25.7 & 7.32 & 99.9 & & 24.24 & \\
\hline W-04-288 & 4-Aug-04 & 19.0 & & & & & & \\
\hline W-04-289 & 4-Aug-04 & 19.0 & 21.3 & 6.75 & 84.6 & & 14.76 & \\
\hline W-04-290 & 4-Aug-04 & 23.0 & & & & & & \\
\hline W-04-291 & 4-Aug-04 & 23.0 & & & & & & \\
\hline W-04-292 & 4-Aug-04 & 23.0 & & & & & & \\
\hline W-04-293 & 4-Aug-04 & 24.0 & & & & & & \\
\hline W-04-294 & 4-Aug-04 & 24.0 & & & & & & \\
\hline W-04-295 & 4-Aug-04 & 24.0 & & & & & & \\
\hline W-04-296 & 4-Aug-04 & 24.0 & & & & & & \\
\hline W-04-297 & 5-Aug-04 & 13.0 & 18.6 & 8.09 & 95.5 & & 10.84 & \\
\hline W-04-298 & 5-Aug-04 & 22.0 & 20.6 & 10.71 & 133.0 & & 5.12 & \\
\hline W-04-299 & 5-Aug-04 & 23.0 & 21.5 & 9.08 & 114.4 & & 3.44 & \\
\hline W-04-300 & 5-Aug-04 & 24.0 & & & & & & \\
\hline W-04-301 & 5-Aug-04 & 25.0 & & & & & & \\
\hline $\mathrm{X}-04-001$ & 5-May-04 & 6 & 7.3 & 10.00 & 97.8 & 882 & 24.02 & 7.98 \\
\hline$X-04-002$ & 5-May-04 & 8 & 8.4 & 9.66 & 97.4 & 907 & 4.99 & 7.99 \\
\hline X-04-003 & 5-May-04 & 10 & & & & & & \\
\hline X-04-004 & 5-May-04 & 10 & & & & & & \\
\hline$X-04-005$ & 5-May-04 & 10 & 10.2 & 9.24 & 96.2 & 908 & 2.38 & $7-7.5^{*}$ \\
\hline
\end{tabular}


Appendix 6: Results of basic water chemistry and air temperature measurements recorded at most sites where fishing effort was applied between 2002 and 2006.

\begin{tabular}{|c|c|c|c|c|c|c|c|c|}
\hline \multirow{2}{*}{ Site Number } & \multirow{2}{*}{ Date } & \multirow{2}{*}{$\begin{array}{l}\text { Air Temp. } \\
\left({ }^{\circ} \mathrm{C}\right)\end{array}$} & \multirow{2}{*}{$\begin{array}{c}\text { Water Temp. } \\
\left({ }^{\circ} \mathrm{C}\right)\end{array}$} & \multicolumn{2}{|c|}{ Dissolved Oxygen } & \multirow{2}{*}{$\begin{array}{c}\text { Conductivity } \\
(\mu \mathrm{S} / \mathrm{cm})\end{array}$} & \multirow{2}{*}{$\begin{array}{l}\text { Turbidity } \\
\text { (NTU) }\end{array}$} & \multirow{2}{*}{$\begin{array}{c}\mathrm{pH} \\
\text { (units) }\end{array}$} \\
\hline & & & & (mg/L) & (\% SAT) & & & \\
\hline $\mathrm{X}-04-006$ & 6-May-04 & 6 & & & & & & \\
\hline X-04-007 & 6-May-04 & 6 & & & & & & \\
\hline X-04-008 & 6-May-04 & 6 & & & & & & \\
\hline X-04-009 & 6-May-04 & 6 & & & & & & \\
\hline $\mathrm{X}-04-010$ & 6-May-04 & 6 & & & & & & \\
\hline X-04-011 & 6-May-04 & 6 & & & & & & \\
\hline$X-04-012$ & 6-May-04 & 7 & & & & & & \\
\hline X-04-013 & 6-May-04 & 10 & & & & & & \\
\hline X-04-014 & 6-May-04 & 10 & 10.1 & 8.99 & 94.2 & 945 & 15.04 & $7-7.5^{*}$ \\
\hline$X-04-015$ & 6-May-04 & 10 & & & & & & \\
\hline X-04-016 & 6-May-04 & 10 & & & & & & \\
\hline $\mathrm{X}-04-017$ & 6-May-04 & 10 & & & & & & \\
\hline X-04-018 & 6-May-04 & 10 & & & & & & \\
\hline X-04-019 & 6-May-04 & 10 & & & & & & \\
\hline $\mathrm{X}-04-020$ & 6-May-04 & 10 & & & & & & \\
\hline X-04-021 & 6-May-04 & 10 & 12.1 & & & 761 & 7.31 & 7.88 \\
\hline X-04-022 & 6-May-04 & 7 & & & & & & \\
\hline X-04-023 & 10-May-04 & 6 & 10.2 & 7.94 & 82.5 & 456 & 21.41 & 7.46 \\
\hline X-04-024 & 10-May-04 & 6 & & & & & & \\
\hline X-04-025 & 10-May-04 & 6 & & & & & & \\
\hline X-04-026 & 10-May-04 & 6 & 10.6 & 8.85 & 91.1 & 460 & 30.29 & 7.50 \\
\hline X-04-027 & 10-May-04 & 6 & & & & & & \\
\hline X-04-028 & 10-May-04 & 6 & & & & & & \\
\hline X-04-029 & 10-May-04 & 6 & & & & & & \\
\hline X-04-030 & 10-May-04 & 8 & & & & & & \\
\hline $\mathrm{X}-04-031$ & 10-May-04 & 8 & 12.0 & 10.19 & 106.1 & 441 & 16.99 & 7.37 \\
\hline X-04-032 & 10-May-04 & 10 & & & & & & \\
\hline $\mathrm{X}-04-033$ & 10-May-04 & 10 & & & & & & \\
\hline X-04-034 & 10-May-04 & 10 & & & & & & \\
\hline X-04-035 & 10-May-04 & 10 & & & & & & \\
\hline X-04-036 & 14-May-04 & 2 & & & & & & \\
\hline $\mathrm{X}-04-037$ & 14-May-04 & 2 & 4.0 & 11.55 & 101.1 & 1562 & 9.61 & \\
\hline$X-04-038$ & 14-May-04 & 3 & & & & & & \\
\hline
\end{tabular}


Appendix 6: Results of basic water chemistry and air temperature measurements recorded at most sites where fishing effort was applied between 2002 and 2006.

\begin{tabular}{|c|c|c|c|c|c|c|c|c|}
\hline \multirow{2}{*}{ Site Number } & \multirow{2}{*}{ Date } & \multirow{2}{*}{$\begin{array}{l}\text { Air Temp. } \\
\left({ }^{\circ} \mathrm{C}\right)\end{array}$} & \multirow{2}{*}{$\begin{array}{c}\text { Water Temp. } \\
\left({ }^{\circ} \mathrm{C}\right)\end{array}$} & \multicolumn{2}{|c|}{ Dissolved Oxygen } & \multirow{2}{*}{$\begin{array}{c}\text { Conductivity } \\
(\mu \mathrm{S} / \mathrm{cm})\end{array}$} & \multirow{2}{*}{$\begin{array}{l}\text { Turbidity } \\
\text { (NTU) }\end{array}$} & \multirow{2}{*}{$\begin{array}{c}\mathrm{pH} \\
\text { (units) }\end{array}$} \\
\hline & & & & (mg/L) & (\% SAT) & & & \\
\hline X-04-039 & 14-May-04 & 3 & & & & & & \\
\hline X-04-040 & 14-May-04 & 3 & & & & & & \\
\hline X-04-041 & 14-May-04 & 3 & 7.4 & 9.40 & 90 & 1786 & 16.91 & 7.06 \\
\hline X-04-042 & 14-May-04 & 2 & 5.5 & 10.10 & 89.9 & 1191 & 231 & 6.60 \\
\hline X-04-043 & 17-May-04 & 10 & 12.3 & 9.46 & 104.2 & 616 & 0.68 & \\
\hline X-04-044 & 17-May-04 & 13 & 14.9 & 10.60 & 122.9 & 411 & 0.00 & 7.26 \\
\hline X-04-045 & 17-May-04 & 16 & & & & & & \\
\hline X-04-046 & 17-May-04 & 13 & 18.6 & 9.89 & 123.3 & 435 & 0.00 & 7.74 \\
\hline X-04-047 & 17-May-04 & 13 & & & & & & \\
\hline X-04-048 & 18-May-04 & 16 & 11.9 & 7.11 & 77.6 & 808 & 0.78 & \\
\hline X-04-049 & 18-May-04 & 21 & 13.6 & 4.04 & 47.2 & 542 & 0.00 & 7.07 \\
\hline $\mathrm{X}-04-050$ & 18-May-04 & 23 & & & & & & \\
\hline X-04-051 & 18-May-04 & 25 & 16.7 & 7.55 & 91.4 & 490 & 6.74 & \\
\hline X-04-052 & 19-May-04 & 17 & 12.6 & & & 656 & 1.40 & 7.42 \\
\hline X-04-053 & 20-May-04 & 5 & 9.9 & 8.75 & 90 & 683 & 0.37 & 6.69 \\
\hline X-04-054 & 20-May-04 & 5 & 10.2 & 8.69 & 90.2 & 685 & 0.00 & 7.56 \\
\hline X-04-055 & 20-May-04 & 6 & & & & & & \\
\hline X-04-056 & 20-May-04 & 7 & 12.0 & 10.07 & 106.6 & 735 & 0.00 & 7.31 \\
\hline X-04-057 & 20-May-04 & 10 & & & & & & \\
\hline X-04-058 & 20-May-04 & 10 & & & & & & \\
\hline X-04-059 & 20-May-04 & 11 & & & & & & \\
\hline X-04-060 & 20-May-04 & 11 & & & & & & \\
\hline X-04-061 & 20-May-04 & 13 & 18.0 & & & 623 & 0.00 & 7.94 \\
\hline X-04-062 & 20-May-04 & 13 & & & & & & \\
\hline X-04-063 & 20-May-04 & 13 & & & & & & \\
\hline X-04-064 & 20-May-04 & 13 & & & & & & \\
\hline X-04-065 & 21-May-04 & 4 & 10.9 & 7.18 & 75.7 & 556 & 6.22 & 7.37 \\
\hline $\mathrm{X}-04-066$ & 21-May-04 & 5 & 10.3 & 6.91 & 70 & 517 & 0.00 & 7.13 \\
\hline$X-04-067$ & 25-May-04 & 6 & 11.5 & 8.95 & 96.2 & 572 & 2.14 & 7.40 \\
\hline X-04-068 & 25-May-04 & 5 & 11.1 & 8.44 & 88.2 & 478 & 17.67 & 7.55 \\
\hline X-04-069 & 25-May-04 & 6 & 11.2 & 9.98 & 104.3 & 561 & 0.00 & 6.50 \\
\hline X-04-070 & 25-May-04 & 6 & & & & & & \\
\hline$X-04-071$ & 25-May-04 & 6 & 10.6 & 9.55 & 98.6 & 444 & 0.00 & 7.53 \\
\hline
\end{tabular}


Appendix 6: Results of basic water chemistry and air temperature measurements recorded at most sites where fishing effort was applied between 2002 and 2006.

\begin{tabular}{|c|c|c|c|c|c|c|c|c|}
\hline \multirow{2}{*}{ Site Number } & \multirow{2}{*}{ Date } & \multirow{2}{*}{$\begin{array}{l}\text { Air Temp. } \\
\left({ }^{\circ} \mathrm{C}\right)\end{array}$} & \multirow{2}{*}{$\begin{array}{c}\text { Water Temp. } \\
\left({ }^{\circ} \mathrm{C}\right)\end{array}$} & \multicolumn{2}{|c|}{ Dissolved Oxygen } & \multirow{2}{*}{$\begin{array}{c}\text { Conductivity } \\
(\mu \mathrm{S} / \mathrm{cm})\end{array}$} & \multirow{2}{*}{$\begin{array}{l}\text { Turbidity } \\
\text { (NTU) }\end{array}$} & \multirow{2}{*}{$\begin{array}{c}\mathrm{pH} \\
\text { (units) }\end{array}$} \\
\hline & & & & (mg/L) & (\% SAT) & & & \\
\hline X-04-072 & 25-May-04 & 6 & 10.7 & 9.96 & 103.3 & 450 & 0.00 & 7.68 \\
\hline X-04-073 & 25-May-04 & 9 & & & & & & \\
\hline X-04-074 & 26-May-04 & 7 & 9.8 & 6.07 & 64.8 & 461 & 0.00 & 7.08 \\
\hline$X-04-075$ & 26-May-04 & 6 & & & & & & \\
\hline X-04-076 & 26-May-04 & 6 & & & & & & \\
\hline X-04-077 & 26-May-04 & 10 & 10.9 & 8.47 & 89.7 & 585 & 3.51 & 7.55 \\
\hline X-04-078 & 26-May-04 & 7 & 9.8 & 10.73 & 113.3 & 569 & 7.06 & 7.77 \\
\hline X-04-079 & 26-May-04 & 7 & & & & & & \\
\hline X-04-080 & 26-May-04 & 8 & 11.6 & 10.70 & 111.4 & 483 & 44.61 & 7.15 \\
\hline X-04-081 & 27-May-04 & 10 & 10.2 & 8.64 & 87.8 & 451 & 32.28 & \\
\hline X-04-082 & 27-May-04 & 10 & 11.8 & 8.98 & 93.4 & 546 & 0.76 & 7.20 \\
\hline X-04-083 & 27-May-04 & 10 & & & & & & \\
\hline X-04-084 & 27-May-04 & 11 & 17.5 & 13.46 & & 1281 & 0.00 & 7.84 \\
\hline X-04-085 & 27-May-04 & 15 & & & & & & \\
\hline X-04-086 & 27-May-04 & 15 & & & & & & \\
\hline X-04-087 & 27-May-04 & 15 & & & & & & \\
\hline X-04-088 & 27-May-04 & 15 & 17.0 & 10.29 & 136.8 & 534 & 0.00 & 7.73 \\
\hline X-04-089 & 27-May-04 & 13 & & & & & & \\
\hline X-04-090 & 27-May-04 & 13 & 11.9 & 7.94 & 90.4 & 320 & 3.32 & 7.03 \\
\hline X-04-091 & 28-May-04 & 11 & 10.0 & 4.77 & 59.8 & 653 & 0.80 & 7.22 \\
\hline X-04-092 & 28-May-04 & 12 & 12.6 & 9.81 & 112.8 & 928 & 3.27 & 7.38 \\
\hline X-04-093 & 28-May-04 & 15 & & & & & & \\
\hline X-04-094 & 28-May-04 & 15 & 16.1 & & & 1022 & 7.06 & 8.05 \\
\hline X-04-095 & 1-Jun-04 & 13 & 12.4 & 4.96 & 54.6 & 426 & 32.11 & \\
\hline X-04-096 & 1-Jun-04 & 13 & & & & & & \\
\hline X-04-097 & 1-Jun-04 & 15 & 14.0 & 5.62 & 70.3 & 267 & 151 & 6.94 \\
\hline X-04-098 & 1-Jun-04 & 16 & 15.3 & 5.82 & 68.2 & 142 & 254 & 6.54 \\
\hline X-04-099 & 1-Jun-04 & 16 & 16.5 & 5.63 & 70.9 & 100 & $>300$ & 6.69 \\
\hline X-04-100 & 1-Jun-04 & 19 & & & & & & \\
\hline X-04-101 & 1-Jun-04 & 19 & 21.0 & 6.88 & 91.6 & 132 & 254 & 7.06 \\
\hline X-04-102 & 2-Jun-04 & 13 & 15.7 & 7.48 & 88.6 & 414 & 3.30 & 7.29 \\
\hline X-04-103 & 2-Jun-04 & 16 & 18.2 & 7.26 & 87.6 & 411 & 9.18 & 7.52 \\
\hline X-04-104 & 2-Jun-04 & 20 & & & & & & \\
\hline
\end{tabular}


Appendix 6: Results of basic water chemistry and air temperature measurements recorded at most sites where fishing effort was applied between 2002 and 2006.

\begin{tabular}{|c|c|c|c|c|c|c|c|c|}
\hline \multirow{2}{*}{ Site Number } & \multirow{2}{*}{ Date } & \multirow{2}{*}{$\begin{array}{l}\text { Air Temp. } \\
\left({ }^{\circ} \mathrm{C}\right)\end{array}$} & \multirow{2}{*}{$\begin{array}{c}\text { Water Temp. } \\
\left({ }^{\circ} \mathrm{C}\right)\end{array}$} & \multicolumn{2}{|c|}{ Dissolved Oxygen } & \multirow{2}{*}{$\begin{array}{c}\text { Conductivity } \\
(\mu \mathrm{S} / \mathrm{cm})\end{array}$} & \multirow{2}{*}{$\begin{array}{l}\text { Turbidity } \\
\text { (NTU) }\end{array}$} & \multirow{2}{*}{$\begin{array}{c}\mathrm{pH} \\
\text { (units) }\end{array}$} \\
\hline & & & & (mg/L) & (\% SAT) & & & \\
\hline X-04-105 & 2-Jun-04 & 20 & & & & & & \\
\hline X-04-106 & 3-Jun-04 & 19 & 15.0 & 6.22 & 81.3 & 674 & 112 & 7.46 \\
\hline X-04-107 & 3-Jun-04 & 20 & 19.8 & 7.11 & 97.6 & 1055 & 12.75 & 6.66 \\
\hline$X-04-108$ & 3-Jun-04 & 20 & & & & & & \\
\hline X-04-109 & 3-Jun-04 & 20 & 23.0 & 4.96 & 72.1 & 993 & 0.00 & 7.42 \\
\hline $\mathrm{X}-04-110$ & 4-Jun-04 & 20 & 17.1 & 6.72 & 91.5 & 613 & 0.00 & 7.55 \\
\hline$X-04-111$ & 4-Jun-04 & 20 & & & & & & \\
\hline X-04-112 & 4-Jun-04 & 16 & 19.6 & 7.04 & 96.6 & 634 & 0.00 & 7.48 \\
\hline X-04-113 & 7-Jun-04 & 15 & 18.1 & 6.86 & 92.7 & 596 & 0.00 & 7.46 \\
\hline$X-04-114$ & 7-Jun-04 & 25 & 18.8 & 5.96 & 80.6 & 789 & 9.93 & 7.63 \\
\hline $\mathrm{X}-04-115$ & 7-Jun-04 & 25 & 21.1 & 7.26 & 102.4 & 888 & 0.00 & 8.03 \\
\hline X-04-116 & 8-Jun-04 & 9 & 13.8 & 4.34 & 56.2 & 618 & 0.00 & 7.43 \\
\hline X-04-117 & 8-Jun-04 & 10 & & & & & & \\
\hline$X-04-118$ & 8-Jun-04 & 11 & 16.1 & 7.33 & 97.3 & 519 & 4.49 & 8.05 \\
\hline X-04-119 & 8-Jun-04 & 13 & 15.2 & 6.90 & 87.9 & 940 & 0.00 & 7.83 \\
\hline X-04-120 & 8-Jun-04 & 15 & 16.0 & 6.85 & 89.4 & 799 & 0.00 & 7.92 \\
\hline X-04-121 & 8-Jun-04 & 17 & 16.9 & 7.67 & 102.8 & 768 & 0.00 & 7.88 \\
\hline$X-04-122$ & 9-Jun-04 & 9 & 14.3 & 6.22 & 79.7 & 742 & 3.63 & 7.38 \\
\hline$X-04-123$ & 9-Jun-04 & 13 & 16.2 & 7.28 & 92.1 & 748 & 3.80 & 7.46 \\
\hline X-04-124 & 9-Jun-04 & 15 & 16.8 & 8.38 & 110.1 & 825 & 1.75 & 7.60 \\
\hline$X-04-125$ & 10-Jun-04 & 13 & & & & & & \\
\hline X-04-126 & 10-Jun-04 & 13 & 16.6 & 5.03 & 60.8 & 708 & 0.00 & 7.00 \\
\hline X-04-127 & 10-Jun-04 & 13 & 17.0 & 6.59 & 78.5 & 577 & 39.44 & 7.25 \\
\hline$X-04-128$ & 14-Jun-04 & 10 & 15.7 & 9.69 & 111.5 & 522 & 0.00 & 8.19 \\
\hline X-04-129 & 14-Jun-04 & 11 & 13.2 & 7.93 & 88.2 & 720 & 33.92 & 7.78 \\
\hline $\mathrm{X}-04-130$ & 14-Jun-04 & 11 & 16.1 & 9.88 & 117.7 & 611 & 0.00 & 8.09 \\
\hline$X-04-131$ & 14-Jun-04 & 12 & & & & & & \\
\hline $\mathrm{X}-04-132$ & 14-Jun-04 & 11 & 15.4 & 5.75 & 67.6 & 651 & 13.48 & 7.35 \\
\hline$X-04-133$ & 15-Jun-04 & 11 & 14.2 & 8.03 & 90.1 & 932 & 0.00 & 7.73 \\
\hline$X-04-134$ & 15-Jun-04 & 11 & & & & & & \\
\hline X-04-135 & 15-Jun-04 & 11 & & & & & & \\
\hline X-04-136 & 15-Jun-04 & 10 & & & & & & \\
\hline$X-04-137$ & 16-Jun-04 & 20 & 16.3 & 8.40 & 106.8 & 686 & 0.28 & 7.70 \\
\hline
\end{tabular}


Appendix 6: Results of basic water chemistry and air temperature measurements recorded at most sites where fishing effort was applied between 2002 and 2006.

\begin{tabular}{|c|c|c|c|c|c|c|c|c|}
\hline \multirow{2}{*}{ Site Number } & \multirow{2}{*}{ Date } & \multirow{2}{*}{$\begin{array}{l}\text { Air Temp. } \\
\left({ }^{\circ} \mathrm{C}\right)\end{array}$} & \multirow{2}{*}{$\begin{array}{c}\text { Water Temp. } \\
\left({ }^{\circ} \mathrm{C}\right)\end{array}$} & \multicolumn{2}{|c|}{ Dissolved Oxygen } & \multirow{2}{*}{$\begin{array}{c}\text { Conductivity } \\
(\mu \mathrm{S} / \mathrm{cm})\end{array}$} & \multirow{2}{*}{$\begin{array}{l}\text { Turbidity } \\
\text { (NTU) }\end{array}$} & \multirow{2}{*}{$\begin{array}{c}\mathrm{pH} \\
\text { (units) }\end{array}$} \\
\hline & & & & (mg/L) & (\% SAT) & & & \\
\hline X-04-138 & 16-Jun-04 & 23 & 19.0 & 6.76 & 90.6 & 778 & 0.13 & 7.59 \\
\hline X-04-139 & 16-Jun-04 & 23 & 20.5 & 5.89 & 83.3 & 507 & 0.00 & 6.97 \\
\hline $\mathrm{X}-04-140$ & 16-Jun-04 & 23 & & & & & & \\
\hline$X-04-141$ & 17-Jun-04 & 20 & 17.8 & 6.69 & 93.9 & 479 & 5.30 & 7.74 \\
\hline $\mathrm{X}-04-142$ & 17-Jun-04 & 20 & 15.3 & 7.99 & 99.7 & 709 & 0.00 & 7.65 \\
\hline $\mathrm{X}-04-143$ & 17-Jun-04 & 20 & & & & & & \\
\hline$X-04-144$ & 17-Jun-04 & 20 & & & & & & \\
\hline X-04-145 & 17-Jun-04 & 23 & 20.3 & 6.63 & 90 & 516 & 0.00 & 7.61 \\
\hline X-04-146 & 17-Jun-04 & 13 & & & & & & \\
\hline$X-04-147$ & 18-Jun-04 & 10 & 11.5 & 7.46 & 84.2 & 596 & 0.00 & 7.36 \\
\hline X-04-148 & 18-Jun-04 & 14 & & & & & & \\
\hline X-04-149 & 21-Jun-04 & 13 & 15.3 & 8.28 & 97.4 & 484 & 3.74 & 7.72 \\
\hline$X-04-150$ & 21-Jun-04 & 13 & 14.9 & 10.07 & 117.9 & 607 & 0.00 & 7.65 \\
\hline X-04-151 & 21-Jun-04 & 17 & 15.5 & 7.29 & 86.7 & 450 & 5.19 & 7.27 \\
\hline X-04-152 & 22-Jun-04 & 12 & 13.7 & 7.27 & 83.9 & 454 & 1.06 & 7.53 \\
\hline X-04-153 & 22-Jun-04 & 14 & 15.3 & 4.43 & 52.8 & 438 & 0.00 & 7.11 \\
\hline X-04-154 & 22-Jun-04 & 12 & 17.5 & 7.63 & 96.2 & 468 & 0.00 & 7.64 \\
\hline X-04-155 & 23-Jun-04 & 10 & 14.5 & 8.77 & 97 & 498 & 0.14 & 6.72 \\
\hline X-04-156 & 23-Jun-04 & 10 & & & & & & \\
\hline X-04-157 & 23-Jun-04 & 11 & 16.7 & 9.05 & 106.9 & 488 & 0.00 & 7.94 \\
\hline X-04-158 & 23-Jun-04 & 11 & & & & & & \\
\hline X-04-159 & 23-Jun-04 & 11 & 15.8 & 10.85 & 126.2 & 562 & 0.00 & 8.01 \\
\hline X-04-160 & 24-Jun-04 & 9 & 12.5 & 9.04 & 99.2 & 435 & 0.00 & 6.60 \\
\hline X-04-161 & 24-Jun-04 & 9 & 13.0 & 9.95 & 108.9 & 469 & 0.00 & 7.85 \\
\hline X-04-162 & 24-Jun-04 & 9 & 13.2 & 8.89 & 95.3 & 466 & 4.05 & 7.85 \\
\hline $\mathrm{X}-04-163$ & 25-Jun-04 & 10 & 11.7 & 5.53 & 58.6 & 594 & 0.00 & 7.11 \\
\hline X-04-164 & 25-Jun-04 & 10 & 12.2 & 7.77 & 83.2 & 466 & 0.00 & \\
\hline X-04-165 & 28-Jun-04 & 26 & & & & & & \\
\hline X-04-166 & 28-Jun-04 & 26 & & & & & & \\
\hline$X-04-167$ & 28-Jun-04 & 26 & 20.0 & 4.41 & 55.2 & 639 & 112 & 7.37 \\
\hline X-04-168 & 28-Jun-04 & 26 & 16.3 & 10.80 & 128 & 738 & 0.00 & 7.18 \\
\hline X-04-169 & 28-Jun-04 & 26 & 24.3 & 9.41 & 126.5 & 440 & 0.00 & 8.01 \\
\hline$X-04-170$ & 29-Jun-04 & 21 & 19.8 & 5.07 & 63.3 & 417 & 0.00 & 7.47 \\
\hline
\end{tabular}


Appendix 6: Results of basic water chemistry and air temperature measurements recorded at most sites where fishing effort was applied between 2002 and 2006.

\begin{tabular}{|c|c|c|c|c|c|c|c|c|}
\hline \multirow{2}{*}{ Site Number } & \multirow{2}{*}{ Date } & \multirow{2}{*}{$\begin{array}{l}\text { Air Temp. } \\
\left({ }^{\circ} \mathrm{C}\right)\end{array}$} & \multirow{2}{*}{$\begin{array}{c}\text { Water Temp. } \\
\left({ }^{\circ} \mathrm{C}\right)\end{array}$} & \multicolumn{2}{|c|}{ Dissolved Oxygen } & \multirow{2}{*}{$\begin{array}{c}\text { Conductivity } \\
(\mu \mathrm{S} / \mathrm{cm})\end{array}$} & \multirow{2}{*}{$\begin{array}{l}\text { Turbidity } \\
\text { (NTU) }\end{array}$} & \multirow{2}{*}{$\begin{array}{c}\mathrm{pH} \\
\text { (units) }\end{array}$} \\
\hline & & & & (mg/L) & (\% SAT) & & & \\
\hline X-04-171 & 29-Jun-04 & 24 & 20.4 & 6.52 & 83.2 & 478 & 0.29 & 7.52 \\
\hline $\mathrm{X}-04-172$ & 29-Jun-04 & 27 & 20.4 & 10.75 & 139.9 & 640 & 0.00 & 7.68 \\
\hline $\mathrm{X}-04-173$ & 29-Jun-04 & 25 & & & & & & \\
\hline X-04-174 & 29-Jun-04 & 26 & & & & & & \\
\hline $\mathrm{X}-04-175$ & 29-Jun-04 & 30 & 25.8 & 9.29 & 131.4 & 540 & 3.19 & 7.13 \\
\hline $\mathrm{X}-04-176$ & 30-Jun-04 & 15 & 17.5 & 3.93 & 47.2 & 634 & 0.00 & 6.83 \\
\hline$X-04-177$ & 30-Jun-04 & 15 & 18.5 & 7.49 & 92.9 & 460 & 20.51 & 7.99 \\
\hline X-04-178 & 1-Jul-04 & 17 & 17.6 & 5.28 & 64.2 & 980 & 29.69 & 8.54 \\
\hline X-04-179 & 1-Jul-04 & 20 & 16.9 & 5.88 & 68.7 & 918 & 8.72 & 6.84 \\
\hline$X-04-180$ & 2-Jul-04 & 17 & & & & & & \\
\hline X-04-181 & 2-Jul-04 & 17 & 17.7 & 5.87 & 69 & 327 & 0.00 & 7.15 \\
\hline X-04-182 & 5-Jul-04 & 15 & 15.3 & 2.63 & 29.9 & 1419 & 4.54 & 6.79 \\
\hline X-04-183 & 5-Jul-04 & 15 & & & & & & \\
\hline X-04-184 & 5-Jul-04 & 20 & 20.2 & 5.97 & 77.6 & 497 & 0.00 & 7.23 \\
\hline X-04-185 & 5-Jul-04 & 20 & 24.6 & 11.51 & 157.5 & 375 & 12.14 & 6.80 \\
\hline X-04-186 & 5-Jul-04 & 17 & 22.6 & 10.36 & 138.9 & 659 & 0.00 & 8.09 \\
\hline X-04-187 & 6-Jul-04 & 15 & 16.6 & 6.48 & 76.5 & 539 & 1.22 & 7.36 \\
\hline X-04-188 & 6-Jul-04 & 20 & & & & & & \\
\hline X-04-189 & 6-Jul-04 & 18 & & & & & & \\
\hline X-04-190 & 6-Jul-04 & 20 & 16.8 & 4.39 & 59 & 731 & $>300$ & 7.35 \\
\hline X-04-191 & 6-Jul-04 & 23 & & & & & & \\
\hline X-04-192 & 6-Jul-04 & 25 & 23.4 & 11.46 & 154.6 & 648 & 3.98 & 8.28 \\
\hline X-04-193 & 7-Jul-04 & 16 & & & & & & \\
\hline X-04-194 & 7-Jul-04 & 20 & 18.3 & 5.55 & 67.4 & 991 & 1.81 & 7.58 \\
\hline X-04-195 & 7-Jul-04 & 25 & 24.5 & 8.34 & 115.8 & 564 & 0.00 & 8.01 \\
\hline X-04-196 & 7-Jul-04 & 27 & 12.6 & 5.95 & 63.5 & 785 & 32.59 & 7.05 \\
\hline X-04-197 & 7-Jul-04 & 27 & & & & & & \\
\hline X-04-198 & 7-Jul-04 & 27 & 21.5 & 11.49 & 154.1 & 703 & 0.00 & 8.40 \\
\hline X-04-199 & 8-Jul-04 & 15 & 19.1 & 5.08 & 61.8 & 633 & 5.00 & 7.50 \\
\hline$X-04-200$ & 8-Jul-04 & 17 & 18.5 & 3.03 & 36.6 & 1698 & 228 & 8.34 \\
\hline X-04-201 & 8-Jul-04 & 17 & 18.9 & 9.49 & 123.4 & 549 & 2.73 & 6.70 \\
\hline X-04-202 & 8-Jul-04 & 16 & 15.7 & 8.60 & 102.7 & 806 & 0.00 & 7.39 \\
\hline$X-04-203$ & 13-Jul-04 & 16 & 20.9 & 3.78 & 51 & 605 & 0.00 & 7.36 \\
\hline
\end{tabular}


Appendix 6: Results of basic water chemistry and air temperature measurements recorded at most sites where fishing effort was applied between 2002 and 2006.

\begin{tabular}{|c|c|c|c|c|c|c|c|c|}
\hline \multirow{2}{*}{ Site Number } & \multirow{2}{*}{ Date } & \multirow{2}{*}{$\begin{array}{l}\text { Air Temp. } \\
\left({ }^{\circ} \mathrm{C}\right)\end{array}$} & \multirow{2}{*}{$\begin{array}{c}\text { Water Temp. } \\
\left({ }^{\circ} \mathrm{C}\right)\end{array}$} & \multicolumn{2}{|c|}{ Dissolved Oxygen } & \multirow{2}{*}{$\begin{array}{c}\text { Conductivity } \\
(\mu \mathrm{S} / \mathrm{cm})\end{array}$} & \multirow{2}{*}{$\begin{array}{l}\text { Turbidity } \\
\text { (NTU) }\end{array}$} & \multirow{2}{*}{$\begin{array}{c}\mathrm{pH} \\
\text { (units) }\end{array}$} \\
\hline & & & & (mg/L) & (\% SAT) & & & \\
\hline X-04-204 & 13-Jul-04 & 15 & 22.2 & 3.03 & 41.8 & 382 & 0.00 & 6.80 \\
\hline X-04-205 & 13-Jul-04 & 17 & 22.2 & 6.42 & 89.6 & 231 & 0.04 & 7.31 \\
\hline X-04-206 & 13-Jul-04 & 22 & 20.8 & 5.83 & 80.8 & 350 & 0.00 & 7.24 \\
\hline$X-04-207$ & 13-Jul-04 & 26 & 23.4 & 3.93 & 56.4 & 170 & 0.00 & 6.68 \\
\hline X-04-208 & 14-Jul-04 & 23 & 17.8 & 4.50 & 59.7 & 157 & 0.00 & 6.58 \\
\hline X-04-209 & 14-Jul-04 & 26 & 19.6 & 6.48 & 84.5 & 120 & 0.00 & 6.79 \\
\hline$X-04-210$ & 14-Jul-04 & 25 & 21.9 & 6.40 & 90.1 & 363 & 0.00 & 7.36 \\
\hline X-04-211 & 14-Jul-04 & 27 & & & & & & \\
\hline $\mathrm{X}-04-212$ & 15-Jul-04 & 26 & 22.8 & 4.69 & 62 & 404 & 0.00 & 7.34 \\
\hline$X-04-213$ & 15-Jul-04 & 27 & 24.6 & 6.61 & 91.3 & 533 & 0.00 & 7.07 \\
\hline X-04-214 & 15-Jul-04 & 20 & & & & & & \\
\hline X-04-215 & 15-Jul-04 & 23 & 24.7 & 6.62 & 91.4 & 555 & 0.00 & 7.51 \\
\hline X-04-216 & 16-Jul-04 & 17 & 12.9 & 2.55 & 28 & 412 & 0.00 & 6.73 \\
\hline $\mathrm{X}-04-217$ & 16-Jul-04 & 21 & 20.5 & 4.67 & 60.3 & 661 & 0.00 & 7.11 \\
\hline X-04-218 & 16-Jul-04 & 21 & & & & & & \\
\hline X-04-219 & 16-Jul-04 & 21 & & & & & & \\
\hline X-04-220 & 16-Jul-04 & 23 & & & & & & \\
\hline$X-04-221$ & 16-Jul-04 & 24 & 24.3 & 7.37 & 101 & 604 & 30.79 & 6.86 \\
\hline $\mathrm{X}-04-222$ & 19-Jul-04 & 30 & 27.5 & 7.48 & 124 & 332 & 0.73 & 7.91 \\
\hline X-04-223 & 20-Jul-04 & 20 & 21.6 & 4.08 & 61.4 & 512 & 0.00 & 7.17 \\
\hline X-04-224 & 20-Jul-04 & 20 & & & & & & \\
\hline X-04-225 & 20-Jul-04 & 20 & 23.8 & 4.58 & 69.5 & 561 & 0.00 & 7.46 \\
\hline X-04-226 & 20-Jul-04 & 23 & & & & & & \\
\hline$X-04-227$ & 20-Jul-04 & 26 & 21.3 & 6.18 & 94 & 392 & 0.00 & 7.53 \\
\hline X-04-228 & 21-Jul-04 & 16 & 19.3 & 2.61 & 38.3 & 458 & 3.63 & 7.06 \\
\hline $\mathrm{X}-04-229$ & 21-Jul-04 & 19 & & & & & & \\
\hline$X-04-230$ & 21-Jul-04 & 16 & & & & & & \\
\hline $\mathrm{X}-04-231$ & 21-Jul-04 & 16 & & & & & & \\
\hline$X-04-232$ & 22-Jul-04 & 15 & 14.9 & 4.40 & 56.8 & 542 & 0.00 & 6.85 \\
\hline$X-04-233$ & 22-Jul-04 & 15 & & & & & & \\
\hline X-04-234 & 22-Jul-04 & 16 & & & & & & \\
\hline X-04-235 & 22-Jul-04 & 16 & & & & & & \\
\hline$X-04-236$ & 22-Jul-04 & 17 & & & & & & \\
\hline
\end{tabular}


Appendix 6: Results of basic water chemistry and air temperature measurements recorded at most sites where fishing effort was applied between 2002 and 2006.

\begin{tabular}{|c|c|c|c|c|c|c|c|c|}
\hline \multirow{2}{*}{ Site Number } & \multirow{2}{*}{ Date } & \multirow{2}{*}{$\begin{array}{l}\text { Air Temp. } \\
\left({ }^{\circ} \mathrm{C}\right)\end{array}$} & \multirow{2}{*}{$\begin{array}{c}\text { Water Temp. } \\
\left({ }^{\circ} \mathrm{C}\right)\end{array}$} & \multicolumn{2}{|c|}{ Dissolved Oxygen } & \multirow{2}{*}{$\begin{array}{c}\text { Conductivity } \\
(\mu \mathrm{S} / \mathrm{cm})\end{array}$} & \multirow{2}{*}{$\begin{array}{l}\text { Turbidity } \\
\text { (NTU) }\end{array}$} & \multirow{2}{*}{$\begin{array}{c}\mathrm{pH} \\
\text { (units) }\end{array}$} \\
\hline & & & & (mg/L) & (\% SAT) & & & \\
\hline $\mathrm{X}-04-237$ & 22-Jul-04 & 16 & & & & & & \\
\hline $\mathrm{X}-04-238$ & 22-Jul-04 & 17 & 20.2 & 7.79 & 109.3 & 923 & 0.00 & 7.67 \\
\hline X-04-239 & 22-Jul-04 & 21 & 22.7 & 6.56 & 95.9 & 659 & 7.81 & 7.86 \\
\hline$X-04-240$ & 26-Jul-04 & 25 & & & & & & \\
\hline X-04-241 & 26-Jul-04 & 25 & & & & & & \\
\hline $\mathrm{X}-04-242$ & 26-Jul-04 & 25 & & & & & & \\
\hline$X-04-243$ & 26-Jul-04 & 25 & & & & & & \\
\hline X-04-244 & 26-Jul-04 & 26 & & & & & & \\
\hline $\mathrm{X}-04-245$ & 26-Jul-04 & 27 & & & & & & \\
\hline$X-04-246$ & 26-Jul-04 & 27 & & & & & & \\
\hline X-04-247 & 28-Jul-04 & 16 & 20.2 & 7.48 & 96.3 & 925 & 0.00 & 7.46 \\
\hline $\mathrm{X}-04-248$ & 28-Jul-04 & 16 & & & & & & \\
\hline$X-04-249$ & 28-Jul-04 & 18 & 23.0 & 4.89 & 64.1 & 867 & 0.00 & 7.31 \\
\hline $\mathrm{X}-04-250$ & 28-Jul-04 & 18 & & & & & & \\
\hline $\mathrm{X}-04-251$ & 28-Jul-04 & 17 & 23.7 & 8.28 & 112.2 & 856 & 58 & 8.02 \\
\hline$X-04-252$ & 28-Jul-04 & 15 & & & & & & \\
\hline X-04-253 & 30-Jul-04 & 14 & 20.3 & 7.24 & 89.3 & 736 & 40.07 & 7.97 \\
\hline X-04-254 & 30-Jul-04 & 14 & & & & & & \\
\hline $\mathrm{X}-04-255$ & 30-Jul-04 & 15 & 20.5 & 7.62 & 96.7 & 521 & 33.98 & 7.73 \\
\hline X-04-256 & 3-Aug-04 & 23 & 19.8 & & & 451 & 37.86 & 6.68 \\
\hline$X-04-257$ & 3-Aug-04 & 22 & 20.8 & & & 428 & 15.69 & 7.54 \\
\hline X-04-258 & 4-Aug-04 & 23 & 24.2 & 8.70 & 118.6 & 415 & 0.00 & 7.86 \\
\hline X-04-259 & 5-Aug-04 & 25 & 19.7 & 6.73 & 84.7 & 425 & 18.71 & 7.55 \\
\hline X-04-260 & 5-Aug-04 & 25 & 21.6 & 7.03 & 96.9 & 370 & 10.94 & 7.75 \\
\hline X-04-261 & 6-Aug-04 & 23 & & & & & & \\
\hline $\mathrm{X}-04-262$ & 6-Aug-04 & 21 & 21.7 & 7.54 & 97.8 & 460 & 3.58 & 7.67 \\
\hline$X-04-263$ & 6-Aug-04 & 22 & & & & & & \\
\hline D-05-001 & 31-Dec-37 & 4 & 2.0 & & & & & \\
\hline D-05-002 & 31-Dec-37 & 4 & 2.0 & & & & & \\
\hline D-05-003 & 31-Dec-37 & 6 & 2.0 & & & & & \\
\hline D-05-004 & 30-May-84 & 8 & 9.0 & & & & & \\
\hline D-05-005 & 30-May-84 & 10 & 9.0 & & & & & \\
\hline D-05-006 & 30-May-84 & 10 & 9.0 & & & & & \\
\hline
\end{tabular}


Appendix 6: Results of basic water chemistry and air temperature measurements recorded at most sites where fishing effort was applied between 2002 and 2006.

\begin{tabular}{|c|c|c|c|c|c|c|c|c|}
\hline \multirow{2}{*}{ Site Number } & \multirow{2}{*}{ Date } & \multirow{2}{*}{$\begin{array}{l}\text { Air Temp. } \\
\left({ }^{\circ} \mathrm{C}\right)\end{array}$} & \multirow{2}{*}{$\begin{array}{c}\text { Water Temp. } \\
\left({ }^{\circ} \mathrm{C}\right)\end{array}$} & \multicolumn{2}{|c|}{ Dissolved Oxygen } & \multirow{2}{*}{$\begin{array}{c}\text { Conductivity } \\
(\mu \mathrm{S} / \mathrm{cm})\end{array}$} & \multirow{2}{*}{$\begin{array}{l}\text { Turbidity } \\
\text { (NTU) }\end{array}$} & \multirow{2}{*}{$\begin{array}{c}\mathrm{pH} \\
\text { (units) }\end{array}$} \\
\hline & & & & (mg/L) & (\% SAT) & & & \\
\hline D-05-007 & 30-May-84 & 10 & 9.0 & & & & & \\
\hline D-05-008 & 30-May-84 & 11 & 9.0 & & & & & \\
\hline D-05-009 & 30-May-84 & 8 & 9.0 & & & & & \\
\hline D-05-010 & 4-Oct-28 & 2 & & 11.16 & 111.8 & 484 & 4.11 & 7.50 \\
\hline D-05-011 & 12-Jan-29 & 18.7 & 18.2 & 6.16 & 75.7 & 616 & 78 & 7.48 \\
\hline D-05-012 & 24-Jan-48 & & & & & & & \\
\hline D-05-013 & 4-Jul-49 & 24 & 24.1 & 6.20 & 87.9 & 467 & 46.51 & 8.20 \\
\hline D-05-014 & 4-Jul-49 & 24 & 24.1 & 8.86 & 130.9 & 631 & 8.84 & 8.66 \\
\hline D-05-015 & 4-Jul-49 & 26 & 23.6 & 8.54 & 123.8 & 757 & 39.29 & 8.54 \\
\hline D-05-016 & 4-Jul-49 & 26 & & & & & & \\
\hline D-05-017 & 19-Nov-76 & 23 & & & & & & \\
\hline D-05-018 & 3-May-84 & 27.4 & 24.0 & 5.31 & 77.5 & 560 & 3.65 & 7.01 \\
\hline D-05-019 & 3-May-84 & 25 & 26.8 & 6.46 & 94.1 & 579 & 2.05 & 7.01 \\
\hline D-05-020 & 19-Sep-11 & 23 & 21.0 & 3.47 & 44.7 & 387 & 4.01 & 7.50 \\
\hline D-05-021 & 19-Sep-11 & & & & & & & \\
\hline D-05-022 & 19-Sep-11 & & & & & & & \\
\hline D-05-023 & 7-Apr-40 & 20 & 18.7 & 8.50 & 99.8 & 699 & 18.53 & 8.30 \\
\hline D-05-024 & 7-Apr-40 & 22 & & & & & & \\
\hline D-05-025 & 24-Aug-67 & 19 & 18.4 & 5.29 & 61.3 & 626 & 12.17 & 8.59 \\
\hline D-05-026 & 29-Jul-23 & 20 & 19.9 & 3.71 & 44.3 & 416 & 17.95 & 7.50 \\
\hline D-05-027 & 29-Jul-23 & 20 & 19.9 & 3.71 & 44.3 & 416 & 17.95 & 7.50 \\
\hline W-05-001 & 2-May-05 & 4 & 4.1 & 12.18 & 110 & 434 & 0.00 & \\
\hline W-05-002 & 2-May-05 & 5 & 7.4 & 13.04 & & 405 & 11.50 & 6.50 \\
\hline W-05-003 & 2-May-05 & 4 & & & & & & \\
\hline W-05-004 & 2-May-05 & 4 & & & & & & \\
\hline W-05-005 & 2-May-05 & 4 & & & & & & \\
\hline W-05-006 & 2-May-05 & 4 & & & & & & \\
\hline W-05-007 & 2-May-05 & 4 & & & & & & \\
\hline W-05-008 & 2-May-05 & 5 & & & & & & \\
\hline W-05-009 & 2-May-05 & 6 & 11.5 & 11.65 & 125 & 624 & 11.04 & 6.50 \\
\hline W-05-010 & 2-May-05 & 5 & & & & & & \\
\hline W-05-011 & 2-May-05 & 6 & & & & & & \\
\hline W-05-012 & 3-May-05 & 5 & 5.7 & 9.39 & 86.6 & 779 & 6.02 & 7.00 \\
\hline
\end{tabular}


Appendix 6: Results of basic water chemistry and air temperature measurements recorded at most sites where fishing effort was applied between 2002 and 2006.

\begin{tabular}{|c|c|c|c|c|c|c|c|c|}
\hline \multirow{2}{*}{ Site Number } & \multirow{2}{*}{ Date } & \multirow{2}{*}{$\begin{array}{l}\text { Air Temp. } \\
\left({ }^{\circ} \mathrm{C}\right)\end{array}$} & \multirow{2}{*}{$\begin{array}{c}\text { Water Temp. } \\
\left({ }^{\circ} \mathrm{C}\right)\end{array}$} & \multicolumn{2}{|c|}{ Dissolved Oxygen } & \multirow{2}{*}{$\begin{array}{c}\text { Conductivity } \\
(\mu \mathrm{S} / \mathrm{cm})\end{array}$} & \multirow{2}{*}{$\begin{array}{l}\text { Turbidity } \\
\text { (NTU) }\end{array}$} & \multirow{2}{*}{$\begin{array}{c}\mathrm{pH} \\
\text { (units) }\end{array}$} \\
\hline & & & & (mg/L) & (\% SAT) & & & \\
\hline W-05-013 & 3-May-05 & 7 & 9.0 & 10.08 & 102.3 & 1514 & 10.44 & 7.00 \\
\hline W-05-014 & 3-May-05 & 7 & 7.2 & 11.97 & 147.5 & 1563 & 1.82 & 7.00 \\
\hline W-05-015 & 3-May-05 & 8 & & & & & & \\
\hline W-05-016 & 3-May-05 & 8 & 12.8 & 11.82 & 132.5 & 1519 & 7.19 & 7.00 \\
\hline W-05-017 & 4-May-05 & 4 & 7.7 & 10.32 & 103 & 1550 & 1.79 & 7.00 \\
\hline W-05-018 & 4-May-05 & 5 & & & & & & \\
\hline W-05-019 & 4-May-05 & 10 & 9.8 & 10.44 & 106 & 1484 & 2.39 & 7.00 \\
\hline W-05-020 & 4-May-05 & 11 & 9.3 & 12.28 & 127.2 & 1425 & 0.00 & 7.25 \\
\hline W-05-021 & 4-May-05 & 12 & & & & & & \\
\hline W-05-022 & 4-May-05 & 10 & & & & & & \\
\hline W-05-023 & 5-May-05 & 10 & 13.0 & 8.22 & 90.1 & 568 & 1.35 & 7.00 \\
\hline W-05-024 & 5-May-05 & 14 & 14.7 & 11.41 & 129.8 & 770 & 2.14 & 7.50 \\
\hline W-05-025 & 5-May-05 & 14 & & & & & & \\
\hline W-05-026 & 5-May-05 & 15 & & & & & & \\
\hline W-05-027 & 5-May-05 & 14 & 15.7 & 4.94 & 54.7 & 630 & 0.00 & 6.50 \\
\hline W-05-028 & 5-May-05 & 15 & 14.7 & 13.46 & & 677 & 4.05 & 7.00 \\
\hline W-05-029 & 5-May-05 & 18 & & & & & & \\
\hline W-05-030 & 5-May-05 & 8 & 11.2 & 2.46 & 25.4 & 798 & 0.00 & 6.50 \\
\hline W-05-031 & 6-May-05 & 9 & & & & & & \\
\hline W-05-032 & 6-May-05 & 10 & & & & & & \\
\hline W-05-033 & 6-May-05 & 13 & & & & & & \\
\hline W-05-034 & 6-May-05 & 18 & 11.5 & 8.15 & 82.8 & 487 & 5.32 & 6.50 \\
\hline W-05-035 & 9-May-05 & 18 & & & & & & \\
\hline W-05-036 & 9-May-05 & 4 & 12.3 & 3.65 & 38.7 & 355 & 68 & \\
\hline W-05-037 & 9-May-05 & 7 & 11.9 & 3.75 & 38.8 & 322 & $>300$ & \\
\hline W-05-038 & 9-May-05 & 8 & 12.6 & 3.41 & 36 & 640 & 15.71 & 6.50 \\
\hline W-05-039 & 9-May-05 & 8 & & & & & & \\
\hline W-05-040 & 10-May-05 & 8 & & & & & & \\
\hline W-05-041 & 10-May-05 & 7 & 9.9 & 8.15 & 80.9 & 486 & 2.75 & 6.50 \\
\hline W-05-042 & 10-May-05 & 5 & & & & & & \\
\hline W-05-043 & 10-May-05 & 5 & 9.9 & 13.08 & & 514 & 3.80 & 6.50 \\
\hline W-05-044 & 10-May-05 & 4 & & & & & & \\
\hline W-05-045 & 10-May-05 & 5 & 10.0 & 10.52 & 105.2 & 613 & 16.06 & \\
\hline
\end{tabular}


Appendix 6: Results of basic water chemistry and air temperature measurements recorded at most sites where fishing effort was applied between 2002 and 2006.

\begin{tabular}{|c|c|c|c|c|c|c|c|c|}
\hline \multirow{2}{*}{ Site Number } & \multirow{2}{*}{ Date } & \multirow{2}{*}{$\begin{array}{l}\text { Air Temp. } \\
\left({ }^{\circ} \mathrm{C}\right)\end{array}$} & \multirow{2}{*}{$\begin{array}{l}\text { Water Temp. } \\
\left({ }^{\circ} \mathrm{C}\right)\end{array}$} & \multicolumn{2}{|c|}{ Dissolved Oxygen } & \multirow{2}{*}{$\begin{array}{c}\text { Conductivity } \\
(\mu \mathrm{S} / \mathrm{cm})\end{array}$} & \multirow{2}{*}{$\begin{array}{l}\text { Turbidity } \\
\text { (NTU) }\end{array}$} & \multirow{2}{*}{$\begin{array}{c}\mathrm{pH} \\
\text { (units) }\end{array}$} \\
\hline & & & & (mg/L) & (\% SAT) & & & \\
\hline W-05-046 & 11-May-05 & -1 & 5.4 & 10.44 & 95.3 & 716 & & 6.50 \\
\hline W-05-047 & 11-May-05 & 0 & 5.4 & & & 1495 & 5.33 & 6.50 \\
\hline W-05-048 & 11-May-05 & 2 & 9.5 & & & 349 & 14.26 & 7.25 \\
\hline W-05-049 & 11-May-05 & 4 & 9.4 & 12.63 & 123.6 & 883 & 0.00 & 6.50 \\
\hline W-05-050 & 11-May-05 & 7 & 10.3 & 12.23 & 118.4 & 682 & 3.73 & 7.00 \\
\hline W-05-051 & 12-May-05 & 7 & 10.4 & 11.88 & 118.8 & 953 & 0.00 & 7.00 \\
\hline W-05-052 & 12-May-05 & 7 & 9.5 & 9.57 & 92.6 & 541 & 0.00 & \\
\hline W-05-053 & 12-May-05 & 5 & 10.4 & 10.43 & 110 & 606 & 2.13 & 6.50 \\
\hline W-05-054 & 12-May-05 & 8 & 13.4 & 12.46 & 138.4 & 512 & 14.55 & 6.50 \\
\hline W-05-055 & 16-May-05 & 12 & 15.4 & 10.21 & 114.4 & 631 & 0.55 & 7.00 \\
\hline W-05-056 & 16-May-05 & 15 & 14.4 & 7.08 & 79.2 & 1026 & 1.69 & 6.50 \\
\hline W-05-057 & 16-May-05 & 8 & 12.4 & 8.04 & 86.6 & 784 & 7.41 & 6.50 \\
\hline W-05-058 & 17-May-05 & 12 & 14.4 & 6.91 & 76.6 & 793 & 0.93 & 7.00 \\
\hline W-05-059 & 17-May-05 & 15 & 15.4 & 10.57 & 110.5 & 891 & 0.00 & 7.25 \\
\hline W-05-060 & 18-May-05 & 10 & 4.7 & 8.41 & 93 & 1184 & 0.00 & 6.50 \\
\hline W-05-061 & 17-May-05 & 8 & & & & & & \\
\hline W-05-062 & 17-May-05 & 15 & 22.5 & 10.53 & 147.4 & $>2000$ & 2.78 & 8.75 \\
\hline W-05-063 & 18-May-05 & 17 & 16.0 & 7.74 & 106.4 & 853 & 0.00 & 7.25 \\
\hline W-05-064 & 18-May-05 & 17 & & & & & & \\
\hline W-05-065 & 18-May-05 & 20 & 23.7 & 7.50 & 106.1 & 1780 & 0.00 & 8.00 \\
\hline W-05-066 & 18-May-05 & 20 & 19.2 & 6.25 & 83.4 & 895 & 1.00 & 7.50 \\
\hline W-05-067 & 19-May-05 & 16 & & & & & & \\
\hline W-05-068 & 19-May-05 & 18 & & & & & & \\
\hline W-05-069 & 19-May-05 & 20 & 19.8 & 3.39 & 45.4 & 1145 & 0.22 & 6.50 \\
\hline W-05-070 & 19-May-05 & 20 & & & & & & \\
\hline W-05-071 & 20-May-05 & 17 & 16.6 & 8.21 & 104 & 488 & 2.67 & 7.00 \\
\hline W-05-072 & 20-May-05 & 20 & 22.9 & 8.80 & 124.5 & 629 & 19.91 & 7.00 \\
\hline W-05-073 & 24-May-05 & 15 & 17.3 & 5.69 & 51.5 & 1119 & 0.53 & 6.50 \\
\hline W-05-074 & 24-May-05 & 18 & & & & & & \\
\hline W-05-075 & 24-May-05 & 18 & & & & & & \\
\hline W-05-076 & 24-May-05 & 18 & 16.8 & 6.14 & 77.9 & 544 & 7.03 & 6.50 \\
\hline W-05-077 & 24-May-05 & 17 & 16.8 & 4.17 & 53.1 & 413 & 0.42 & 7.00 \\
\hline W-05-078 & 25-May-05 & 15 & 16.5 & 7.84 & 89.1 & 638 & 30.31 & 6.50 \\
\hline
\end{tabular}


Appendix 6: Results of basic water chemistry and air temperature measurements recorded at most sites where fishing effort was applied between 2002 and 2006.

\begin{tabular}{|c|c|c|c|c|c|c|c|c|}
\hline \multirow{2}{*}{ Site Number } & \multirow{2}{*}{ Date } & \multirow{2}{*}{$\begin{array}{l}\text { Air Temp. } \\
\left({ }^{\circ} \mathrm{C}\right)\end{array}$} & \multirow{2}{*}{$\begin{array}{c}\text { Water Temp. } \\
\left({ }^{\circ} \mathrm{C}\right)\end{array}$} & \multicolumn{2}{|c|}{ Dissolved Oxygen } & \multirow{2}{*}{$\begin{array}{c}\text { Conductivity } \\
(\mu \mathrm{S} / \mathrm{cm})\end{array}$} & \multirow{2}{*}{$\begin{array}{l}\text { Turbidity } \\
\text { (NTU) }\end{array}$} & \multirow{2}{*}{$\begin{array}{c}\mathrm{pH} \\
\text { (units) }\end{array}$} \\
\hline & & & & (mg/L) & (\% SAT) & & & \\
\hline W-05-079 & 25-May-05 & 16 & 20.5 & 9.64 & 119.2 & 435 & 92 & 6.50 \\
\hline W-05-080 & 25-May-05 & 17 & & & & & & \\
\hline W-05-081 & 25-May-05 & 17 & 19.5 & & & 511 & 1.31 & 7.00 \\
\hline W-05-082 & 25-May-05 & 15 & 17.7 & 5.15 & 60.5 & 1004 & 23.21 & \\
\hline W-05-083 & 26-May-05 & 12 & 14.1 & 6.99 & 75.8 & 1336 & 5.26 & 6.50 \\
\hline W-05-084 & 26-May-05 & 16 & & & & & & \\
\hline W-05-085 & 26-May-05 & 16 & 15.7 & 10.03 & 112.9 & 1667 & 1.30 & 8.25 \\
\hline W-05-086 & 26-May-05 & 15 & 14.2 & 8.16 & 88.9 & $>2000$ & 0.00 & 7.00 \\
\hline W-05-087 & 26-May-05 & 10 & 10.3 & 9.36 & 95.4 & 821 & 0.00 & 6.50 \\
\hline W-05-088 & 27-May-05 & 11 & & & & & & \\
\hline W-05-089 & 27-May-05 & 12 & & & & & & \\
\hline W-05-090 & 27-May-05 & 12 & & & & & & \\
\hline W-05-091 & 27-May-05 & 10 & & & & & & \\
\hline W-05-092 & 27-May-05 & 12 & & & & & & \\
\hline W-05-093 & 29-May-05 & 14 & 17.1 & 6.18 & 71.8 & 596 & 1.99 & 7.00 \\
\hline W-05-094 & 31-May-05 & 18 & 17.3 & 5.31 & 63.8 & 1683 & 7.52 & 7.50 \\
\hline W-05-095 & 31-May-05 & 20 & & & & & & \\
\hline W-05-096 & 31-May-05 & 20 & 18.5 & 6.86 & 82 & 1751 & 15.58 & 7.50 \\
\hline W-05-097 & 31-May-05 & 20 & & & & & & \\
\hline W-05-098 & 31-May-05 & 24 & & & & & & \\
\hline W-05-099 & 31-May-05 & 20 & 18.8 & 9.12 & 114.1 & 1099 & 11.87 & 7.00 \\
\hline W-05-100 & 31-May-05 & 21 & & & & & & \\
\hline W-05-101 & 1-Jun-05 & 14 & 14.4 & 5.12 & 58.5 & 1117 & 2.85 & 7.50 \\
\hline W-05-102 & 1-Jun-05 & 14 & 13.3 & 7.90 & 87.9 & 601 & 3.19 & 6.50 \\
\hline W-05-103 & 1-Jun-05 & 14 & 17.4 & 5.39 & 65.3 & 759 & 3.11 & 7.50 \\
\hline W-05-104 & 1-Jun-05 & 15 & 16.7 & 7.50 & 90.2 & 603 & 3.56 & \\
\hline W-05-105 & 1-Jun-05 & 16 & 16.8 & 7.28 & 85.5 & 401 & 7.18 & 7.00 \\
\hline W-05-106 & 2-Jun-05 & 14 & 15.4 & 5.75 & 67.1 & 525 & 4.65 & 6.50 \\
\hline W-05-107 & 3-Jun-05 & 12 & & & & & & \\
\hline W-05-108 & 3-Jun-05 & 14 & & & & & & \\
\hline W-05-109 & 3-Jun-05 & 14 & & & & & & \\
\hline W-05-110 & 4-Jun-05 & 20 & 19.4 & 5.09 & 62.2 & 1129 & 9.01 & 8.00 \\
\hline W-05-111 & 4-Jun-05 & 20 & & & & & & \\
\hline
\end{tabular}


Appendix 6: Results of basic water chemistry and air temperature measurements recorded at most sites where fishing effort was applied between 2002 and 2006.

\begin{tabular}{|c|c|c|c|c|c|c|c|c|}
\hline \multirow{2}{*}{ Site Number } & \multirow{2}{*}{ Date } & \multirow{2}{*}{$\begin{array}{l}\text { Air Temp. } \\
\left({ }^{\circ} \mathrm{C}\right)\end{array}$} & \multirow{2}{*}{$\begin{array}{c}\text { Water Temp. } \\
\left({ }^{\circ} \mathrm{C}\right)\end{array}$} & \multicolumn{2}{|c|}{ Dissolved Oxygen } & \multirow{2}{*}{$\begin{array}{c}\text { Conductivity } \\
(\mu \mathrm{S} / \mathrm{cm})\end{array}$} & \multirow{2}{*}{$\begin{array}{l}\text { Turbidity } \\
\text { (NTU) }\end{array}$} & \multirow{2}{*}{$\begin{array}{c}\mathrm{pH} \\
\text { (units) }\end{array}$} \\
\hline & & & & (mg/L) & (\% SAT) & & & \\
\hline W-05-112 & 4-Jun-05 & 20 & 19.9 & 10.59 & 131.4 & 1421 & 20.53 & 8.75 \\
\hline W-05-113 & 6-Jun-05 & 15 & 13.7 & & & 546 & 16.43 & 8.00 \\
\hline W-05-114 & 6-Jun-05 & 15 & 14.6 & 10.45 & 115 & 486 & 5.37 & 7.00 \\
\hline W-05-115 & 7-Jun-05 & 16 & 15.0 & 7.04 & 82.4 & 997 & 3.78 & 7.00 \\
\hline W-05-116 & 7-Jun-05 & 15 & 13.4 & 6.66 & 74.8 & 1141 & 30.47 & 7.50 \\
\hline W-05-117 & 7-Jun-05 & 14 & 15.4 & 6.80 & 79.1 & & 9.71 & 7.25 \\
\hline W-05-118 & 8-Jun-05 & 14 & 9.8 & 7.90 & 87.8 & 1023 & 4.30 & 7.50 \\
\hline W-05-119 & 8-Jun-05 & 14 & 13.0 & 7.65 & 85.4 & 406 & 20.22 & 7.00 \\
\hline W-05-120 & 9-Jun-05 & 14 & 14.1 & 6.35 & 72.2 & & 88 & 6.50 \\
\hline W-05-121 & 9-Jun-05 & 15 & $13 . .9$ & 5.62 & 60.4 & & 72 & 6.50 \\
\hline W-05-122 & 9-Jun-05 & 15 & 15.5 & 12.19 & 135.5 & & 53 & 8.50 \\
\hline W-05-123 & 14-Jun-05 & 15 & 17.0 & 3.00 & 34.6 & 38 & 172 & 7.00 \\
\hline W-05-124 & 14-Jun-05 & & & & & & & \\
\hline W-05-125 & 14-Jun-05 & & & & & & & \\
\hline W-05-126 & 14-Jun-05 & 16 & 11.6 & 5.40 & 53.5 & 722 & 0.27 & 7.50 \\
\hline W-05-127 & 14-Jun-05 & & & & & & & \\
\hline W-05-128 & 14-Jun-05 & 14 & 18.3 & 4.92 & 58.2 & 261 & 0.00 & 7.00 \\
\hline W-05-129 & 15-Jun-05 & 15 & 16.2 & 5.73 & 64.5 & & & 7.00 \\
\hline W-05-130 & 15-Jun-05 & 16 & 17.5 & 3.78 & 42.8 & & 0.00 & 7.00 \\
\hline W-05-131 & 15-Jun-05 & 22 & 20.5 & 12.67 & & & 0.00 & 7.50 \\
\hline W-05-132 & 15-Jun-05 & 22 & 20.0 & 7.58 & 83.1 & 764 & 0.00 & 7.00 \\
\hline W-05-133 & 15-Jun-05 & 22 & & & & & & \\
\hline W-05-134 & 15-Jun-05 & 22 & 21.4 & 8.16 & 97.1 & 807 & 0.00 & 7.25 \\
\hline W-05-135 & 16-Jun-05 & 23 & & & & & & \\
\hline W-05-136 & 16-Jun-05 & 25 & 19.7 & 3.94 & 41.1 & & 0.00 & \\
\hline W-05-137 & 16-Jun-05 & 25 & 17.8 & 7.08 & 74.4 & 489 & 0.00 & 7.00 \\
\hline W-05-138 & 16-Jun-05 & 25 & 21.4 & 5.81 & 76.9 & 481 & 0.00 & 6.50 \\
\hline W-05-139 & 17-Jun-05 & 24 & 19.1 & 8.09 & 101.2 & 253 & 0.00 & 7.25 \\
\hline W-05-140 & 17-Jun-05 & 25 & 19.8 & 7.48 & 96.2 & 482 & 0.00 & 6.50 \\
\hline W-05-141 & 20-Jun-05 & 24 & 24.6 & 9.20 & 128.8 & 1831 & 0.00 & 7.50 \\
\hline W-05-142 & 20-Jun-05 & 25 & & & & & & \\
\hline W-05-143 & 20-Jun-05 & 28 & & & & & & \\
\hline W-05-144 & 20-Jun-05 & 27 & & & & & & \\
\hline
\end{tabular}


Appendix 6: Results of basic water chemistry and air temperature measurements recorded at most sites where fishing effort was applied between 2002 and 2006.

\begin{tabular}{|c|c|c|c|c|c|c|c|c|}
\hline \multirow{2}{*}{ Site Number } & \multirow{2}{*}{ Date } & \multirow{2}{*}{$\begin{array}{l}\text { Air Temp. } \\
\left({ }^{\circ} \mathrm{C}\right)\end{array}$} & \multirow{2}{*}{$\begin{array}{c}\text { Water Temp. } \\
\left({ }^{\circ} \mathrm{C}\right)\end{array}$} & \multicolumn{2}{|c|}{ Dissolved Oxygen } & \multirow{2}{*}{$\begin{array}{c}\text { Conductivity } \\
(\mu \mathrm{S} / \mathrm{cm})\end{array}$} & \multirow{2}{*}{$\begin{array}{l}\text { Turbidity } \\
\text { (NTU) }\end{array}$} & \multirow{2}{*}{$\begin{array}{c}\mathrm{pH} \\
\text { (units) }\end{array}$} \\
\hline & & & & (mg/L) & (\% SAT) & & & \\
\hline W-05-145 & 20-Jun-05 & 28 & 18.4 & 7.68 & 109.9 & 1348 & 0.00 & 8.00 \\
\hline W-05-146 & 21-Jun-05 & 21 & 24.0 & 4.83 & 66.8 & $>2000$ & 0.00 & 8.00 \\
\hline W-05-147 & 21-Jun-05 & 24 & & & & & & \\
\hline W-05-148 & 21-Jun-05 & 25 & 24.8 & 3.87 & 53.5 & $>2000$ & 0.00 & 7.00 \\
\hline W-05-149 & 22-Jun-05 & 20 & 18.5 & 8.13 & 101.6 & 431 & & 7.25 \\
\hline W-05-150 & 22-Jun-05 & 22 & 21.2 & 6.20 & 81.7 & $>2000$ & & 8.00 \\
\hline W-05-151 & 22-Jun-05 & 22 & 22.6 & 8.23 & 111 & 1284 & & 7.50 \\
\hline W-05-152 & 22-Jun-05 & 25 & 22.9 & 8.13 & 113.3 & 936 & & 7.75 \\
\hline W-05-153 & 22-Jun-05 & 27 & & & & & & \\
\hline W-05-154 & 22-Jun-05 & 28 & 22.9 & 7.34 & 113.5 & 801 & & 8.00 \\
\hline W-05-155 & 23-Jun-05 & 18 & & & & & & \\
\hline W-05-156 & 23-Jun-05 & 20 & & & & & & \\
\hline W-05-157 & 23-Jun-05 & 20 & 21.9 & 7.05 & 95 & 200 & & 7.50 \\
\hline W-05-158 & 23-Jun-05 & 19 & & & & & & \\
\hline W-05-159 & 23-Jun-05 & 20 & 21.8 & 4.57 & 62.2 & 809 & & 7.00 \\
\hline W-05-160 & 23-Jun-05 & 22 & 20.9 & 1.21 & 16.5 & 993 & & 7.00 \\
\hline W-05-161 & 23-Jun-05 & 22 & 22.4 & 3.66 & 53.3 & 594 & & 7.00 \\
\hline W-05-162 & 23-Jun-05 & 27 & 24.2 & 4.92 & 71.5 & 1074 & & 6.50 \\
\hline W-05-163 & 24-Jun-05 & 25 & 22.7 & 6.88 & 101.2 & 770 & & 7.00 \\
\hline W-05-164 & 27-Jun-05 & 21 & 18.0 & 5.88 & 75.3 & 295 & & 6.50 \\
\hline W-05-165 & 27-Jun-05 & 20 & 18.2 & 6.59 & 84.2 & 306 & & \\
\hline W-05-166 & 27-Jun-05 & 18 & 19.2 & 3.28 & 42.3 & 251 & & 6.50 \\
\hline W-05-167 & 28-Jun-05 & 20 & 13.8 & 4.01 & 50.4 & 271 & & 6.50 \\
\hline W-05-168 & 11-Jul-05 & 24 & 27.7 & 7.70 & 112.4 & 1613 & 7.56 & 7.39 \\
\hline W-05-169 & 12-Jul-05 & 27 & 23.4 & 5.61 & 84 & 838 & 0.00 & 8.21 \\
\hline W-05-170 & 12-Jul-05 & 29 & 20.0 & 8.95 & 119.6 & 1307 & 0.00 & 7.41 \\
\hline W-05-171 & 12-Jul-05 & 30 & 25.3 & 5.97 & 94.8 & 658 & 36.10 & 8.15 \\
\hline W-05-172 & 12-Jul-05 & 30 & & & & & & \\
\hline W-05-173 & 12-Jul-05 & 30 & 27.1 & 5.53 & 87.7 & 444 & 3.94 & 6.86 \\
\hline W-05-174 & 18-Jul-05 & 18 & & & & & & \\
\hline W-05-175 & 18-Jul-05 & 18 & & & & & & \\
\hline W-05-176 & 18-Jul-05 & 21 & 19.8 & 5.88 & 73.3 & 320 & 3.41 & 7.36 \\
\hline W-05-177 & 19-Jul-05 & 22 & 17.9 & 5.19 & 70.4 & 319 & 1.30 & 7.34 \\
\hline
\end{tabular}


Appendix 6: Results of basic water chemistry and air temperature measurements recorded at most sites where fishing effort was applied between 2002 and 2006.

\begin{tabular}{|c|c|c|c|c|c|c|c|c|}
\hline \multirow{2}{*}{ Site Number } & \multirow{2}{*}{ Date } & \multirow{2}{*}{$\begin{array}{l}\text { Air Temp. } \\
\left({ }^{\circ} \mathrm{C}\right)\end{array}$} & \multirow{2}{*}{$\begin{array}{c}\text { Water Temp. } \\
\left({ }^{\circ} \mathrm{C}\right)\end{array}$} & \multicolumn{2}{|c|}{ Dissolved Oxygen } & \multirow{2}{*}{$\begin{array}{c}\text { Conductivity } \\
(\mu \mathrm{S} / \mathrm{cm})\end{array}$} & \multirow{2}{*}{$\begin{array}{l}\text { Turbidity } \\
\text { (NTU) }\end{array}$} & \multirow{2}{*}{$\begin{array}{c}\mathrm{pH} \\
\text { (units) }\end{array}$} \\
\hline & & & & (mg/L) & (\% SAT) & & & \\
\hline W-05-178 & 19-Jul-05 & 22 & 20.0 & 5.26 & 64 & 360 & 3.78 & 7.52 \\
\hline W-05-179 & 19-Jul-05 & 24 & & & & & & \\
\hline W-05-180 & 19-Jul-05 & 23 & 21.4 & 6.08 & 83.1 & 398 & 4.18 & 7.86 \\
\hline W-05-181 & 19-Jul-05 & 25 & 23.5 & 7.07 & 94 & 420 & 2.20 & 7.80 \\
\hline W-05-182 & 19-Jul-05 & 26 & 23.0 & 6.52 & 80.7 & 389 & 0.00 & 8.03 \\
\hline W-05-183 & 19-Jul-05 & 26 & 22.9 & 7.28 & 96.4 & 395 & 0.00 & 7.97 \\
\hline W-05-184 & 20-Jul-05 & 22 & & & & & & \\
\hline W-05-185 & 20-Jul-05 & 24 & 19.7 & 3.19 & 40.2 & 488 & 1.65 & 7.41 \\
\hline W-05-186 & 25-Jul-05 & 22 & & & & & & \\
\hline W-05-187 & 25-Jul-05 & 24 & 18.2 & 1.13 & 13.9 & 46 & 3.90 & \\
\hline W-05-188 & 25-Jul-05 & 21 & 20.3 & 5.19 & 76.8 & 204 & 34.55 & 7.02 \\
\hline W-05-189 & 26-Jul-05 & 10 & 15.4 & 4.52 & 51.3 & 376 & 0.23 & 7.29 \\
\hline W-05-190 & 26-Jul-05 & 15 & 15.9 & 5.71 & 64.9 & 491 & 9.61 & 7.58 \\
\hline W-05-191 & 26-Jul-05 & 21 & 20.8 & 10.94 & 136 & 400 & 3.16 & 8.41 \\
\hline W-05-192 & 26-Jul-05 & 23 & & & & & & \\
\hline W-05-193 & 26-Jul-05 & 22 & 22.2 & 11.31 & 144.5 & 349 & 4.54 & 8.41 \\
\hline W-05-194 & 27-Jul-05 & 21 & 18.3 & 6.92 & 81.1 & 314 & 8.35 & 7.59 \\
\hline W-05-195 & 27-Jul-05 & 20 & & & & & & \\
\hline W-05-196 & 27-Jul-05 & 20 & 16.2 & 9.86 & 111.5 & 364 & 14.59 & 8.11 \\
\hline W-05-197 & 27-Jul-05 & 20 & & & & & & \\
\hline W-05-198 & 28-Jul-05 & 19 & & & & & & \\
\hline W-05-199 & 2-Aug-05 & 24 & & & & & & \\
\hline W-05-200 & 2-Aug-05 & 28 & 27.3 & 5.35 & 75.1 & 167 & 122 & 7.50 \\
\hline W-05-201 & 2-Aug-05 & 30 & 29.6 & 2.31 & 32.8 & 181 & $>300$ & 7.05 \\
\hline W-05-202 & 2-Aug-05 & 33 & 29.7 & 2.21 & 32.2 & 166 & 212 & 6.92 \\
\hline W-05-203 & 3-Aug-05 & 20 & 23.2 & 6.33 & 84 & 266 & 14.23 & 7.36 \\
\hline W-05-204 & 3-Aug-05 & 25 & & & & & & \\
\hline W-05-205 & 3-Aug-05 & 25 & 22.0 & 7.23 & 93.6 & 147 & 8.92 & 7.05 \\
\hline W-05-206 & 3-Aug-05 & 26 & & & & & & \\
\hline W-05-207 & 8-Aug-05 & 30 & 26.0 & 7.57 & 107.1 & 1882 & 87 & 8.13 \\
\hline W-05-208 & 10-Aug-05 & 22 & 14.7 & 6.72 & 79 & 384 & 19.36 & 7.40 \\
\hline W-05-209 & 10-Aug-05 & 20 & 21.8 & & & 391 & 16.69 & 7.35 \\
\hline W-05-210 & 19-May-05 & 21 & & & & & & \\
\hline
\end{tabular}


Appendix 6: Results of basic water chemistry and air temperature measurements recorded at most sites where fishing effort was applied between 2002 and 2006.

\begin{tabular}{|c|c|c|c|c|c|c|c|c|}
\hline \multirow{2}{*}{ Site Number } & \multirow{2}{*}{ Date } & \multirow{2}{*}{$\begin{array}{l}\text { Air Temp. } \\
\left({ }^{\circ} \mathrm{C}\right)\end{array}$} & \multirow{2}{*}{$\begin{array}{c}\text { Water Temp. } \\
\left({ }^{\circ} \mathrm{C}\right)\end{array}$} & \multicolumn{2}{|c|}{ Dissolved Oxygen } & \multirow{2}{*}{$\begin{array}{c}\text { Conductivity } \\
(\mu \mathrm{S} / \mathrm{cm})\end{array}$} & \multirow{2}{*}{$\begin{array}{l}\text { Turbidity } \\
\text { (NTU) }\end{array}$} & \multirow{2}{*}{$\begin{array}{c}\mathrm{pH} \\
\text { (units) }\end{array}$} \\
\hline & & & & (mg/L) & (\% SAT) & & & \\
\hline W-05-211 & 31-May-05 & 19 & & & & & & \\
\hline $\mathrm{X}-05-001$ & $27 / 04 / 2005$ & 1 & 5.5 & 10.76 & 95 & & 11.96 & \\
\hline X-05-002 & $27 / 04 / 2005$ & -1 & 6.8 & 10.50 & 92.6 & & 11.39 & \\
\hline$X-05-003$ & 29/04/2005 & 1 & 4.3 & 10.18 & 91.7 & 974 & 16.07 & \\
\hline X-05-004 & $29 / 04 / 2005$ & 1 & & & & & & \\
\hline X-05-005 & $29 / 04 / 2005$ & 1 & 9.0 & 9.84 & 101.8 & 499 & 67 & 6.50 \\
\hline$X-05-006$ & 29/04/2005 & 1 & & & & & & \\
\hline X-05-007 & $29 / 04 / 2005$ & 1 & 6.5 & 9.47 & 89.2 & 583 & 6.00 & 6.50 \\
\hline X-05-008 & $29 / 04 / 2005$ & 1 & & & & & & \\
\hline X-05-009 & 29/04/2005 & 1 & & & & & & \\
\hline X-05-010 & $03 / 05 / 2005$ & 6 & & & & & & \\
\hline $\mathrm{X}-05-011$ & $03 / 05 / 2005$ & 7 & 6.4 & 10.35 & 98 & 445 & 17.89 & 6.75 \\
\hline X-05-012 & 03/05/2005 & 7 & 5.9 & 9.81 & 95.6 & 352 & 57 & \\
\hline $\mathrm{X}-05-013$ & $03 / 05 / 2005$ & 10 & & & & & & \\
\hline X-05-014 & 03/05/2005 & 10 & & & & & & \\
\hline X-05-015 & $03 / 05 / 2005$ & 13 & 11.6 & 11.82 & 130.1 & 477 & 0.05 & \\
\hline X-05-016 & $03 / 05 / 2005$ & 13 & & & & & & \\
\hline X-05-017 & 03/05/2005 & 13 & & & & & & \\
\hline X-05-018 & $04 / 05 / 2005$ & 9 & 7.4 & 10.46 & 105.2 & 569 & 17.59 & 7.00 \\
\hline X-05-019 & $04 / 05 / 2005$ & 13 & & & & & & \\
\hline X-05-020 & 04/05/2005 & 15 & 8.7 & 10.34 & 105.7 & 347 & 13.37 & 7.50 \\
\hline X-05-021 & $04 / 05 / 2005$ & 13 & 11.5 & 10.79 & 115.6 & 411 & 3.99 & 7.25 \\
\hline X-05-022 & 04/05/2005 & 16 & 12.4 & 9.14 & 102.2 & 381 & 5.78 & 7.50 \\
\hline X-05-023 & 04/05/2005 & 13 & 16.3 & & & 403 & 0.00 & 7.50 \\
\hline X-05-024 & $05 / 05 / 2005$ & 9 & 10.5 & 5.62 & 58.1 & 388 & 3.19 & 6.75 \\
\hline $\mathrm{X}-05-025$ & $05 / 05 / 2005$ & 14 & & & & & & \\
\hline$X-05-026$ & $05 / 05 / 2005$ & 17 & 12.6 & 9.69 & 107.5 & 580 & 15.99 & 6.75 \\
\hline $\mathrm{X}-05-027$ & $06 / 05 / 2005$ & 17 & 15.5 & 9.40 & 108.2 & 479 & 30.21 & 7.25 \\
\hline $\mathrm{X}-05-028$ & 06/05/2005 & 21 & 17.3 & 12.24 & 148 & 524 & 0.51 & 7.25 \\
\hline$X-05-029$ & 06/05/2005 & 21 & 15.7 & 9.83 & 116.4 & 425 & 13.76 & 7.00 \\
\hline X-05-030 & $10 / 05 / 2005$ & 4 & 10.8 & 8.76 & 91.7 & 391 & 4.36 & \\
\hline $\mathrm{X}-05-031$ & $10 / 05 / 2005$ & 4 & 10.4 & 9.85 & 103.1 & 685 & 6.20 & 7.00 \\
\hline $\mathrm{X}-05-032$ & $11 / 05 / 2005$ & 5 & 5.2 & 9.77 & 88.3 & 454 & 21.33 & \\
\hline
\end{tabular}


Appendix 6: Results of basic water chemistry and air temperature measurements recorded at most sites where fishing effort was applied between 2002 and 2006.

\begin{tabular}{|c|c|c|c|c|c|c|c|c|}
\hline \multirow{2}{*}{ Site Number } & \multirow{2}{*}{ Date } & \multirow{2}{*}{$\begin{array}{l}\text { Air Temp. } \\
\left({ }^{\circ} \mathrm{C}\right)\end{array}$} & \multirow{2}{*}{$\begin{array}{c}\text { Water Temp. } \\
\left({ }^{\circ} \mathrm{C}\right)\end{array}$} & \multicolumn{2}{|c|}{ Dissolved Oxygen } & \multirow{2}{*}{$\begin{array}{c}\text { Conductivity } \\
(\mu \mathrm{S} / \mathrm{cm})\end{array}$} & \multirow{2}{*}{$\begin{array}{l}\text { Turbidity } \\
\text { (NTU) }\end{array}$} & \multirow{2}{*}{$\begin{array}{c}\mathrm{pH} \\
\text { (units) }\end{array}$} \\
\hline & & & & (mg/L) & (\% SAT) & & & \\
\hline $\mathrm{X}-05-033$ & $11 / 05 / 2005$ & 1 & 6.2 & 12.55 & 121.2 & 499 & 8.81 & 7.50 \\
\hline X-05-034 & $11 / 05 / 2005$ & 2 & & & & & & \\
\hline X-05-035 & $11 / 05 / 2005$ & 2 & 7.7 & 9.85 & 96.7 & 548 & 33.02 & 6.75 \\
\hline$X-05-036$ & $11 / 05 / 2005$ & 3 & 10.2 & 11.72 & 122.1 & 435 & 1.59 & 7.25 \\
\hline X-05-037 & $12 / 05 / 2005$ & 6 & 8.3 & 11.86 & 116.4 & 438 & 7.70 & 7.00 \\
\hline X-05-038 & $12 / 05 / 2005$ & 7 & 9.5 & 11.91 & 121.6 & 432 & 9.88 & 6.75 \\
\hline X-05-039 & $12 / 05 / 2005$ & 11 & & & & & & \\
\hline X-05-040 & $12 / 05 / 2005$ & 11 & 9.9 & & & 493 & 28.13 & 6.75 \\
\hline X-05-041 & $12 / 05 / 2005$ & 11 & & & & & & \\
\hline$X-05-042$ & $12 / 05 / 2005$ & 11 & 12.9 & & & 344 & 29.71 & 6.75 \\
\hline X-05-043 & $12 / 05 / 2005$ & 8 & & & & & & \\
\hline X-05-044 & $13 / 05 / 2005$ & 5 & 8.7 & & & 627 & 0.00 & \\
\hline X-05-045 & $13 / 05 / 2005$ & 5 & 8.7 & 8.54 & 85 & 409 & 26.43 & 6.50 \\
\hline X-05-046 & $13 / 05 / 2005$ & 5 & 8.5 & 8.41 & 83.3 & 396 & 3.37 & 6.50 \\
\hline X-05-047 & $13 / 05 / 2005$ & 4 & & & & & & \\
\hline X-05-048 & $14 / 05 / 2005$ & 7 & 7.4 & 8.74 & 84.8 & 514 & 7.33 & 6.50 \\
\hline X-05-049 & $14 / 05 / 2005$ & 4 & & & & & & \\
\hline X-05-050 & $14 / 05 / 2005$ & 5 & & & & & & \\
\hline X-05-051 & $14 / 05 / 2005$ & 5 & 7.4 & 11.61 & 114.1 & 837 & 0.00 & 6.75 \\
\hline X-05-052 & $14 / 05 / 2005$ & 5 & 7.5 & 9.38 & 93.8 & 402 & 8.55 & 6.75 \\
\hline X-05-053 & $15 / 05 / 2005$ & 9 & & & & & & \\
\hline X-05-054 & $15 / 05 / 2005$ & 10 & 5.2 & 11.59 & 108.6 & 829 & 13.14 & 7.00 \\
\hline X-05-055 & $15 / 05 / 2005$ & 12 & 10.2 & 12.36 & 129.3 & 815 & 0.93 & 7.00 \\
\hline X-05-056 & $15 / 05 / 2005$ & 10 & & & & & & \\
\hline X-05-057 & $15 / 05 / 2005$ & 10 & 12.2 & 13.24 & & 450 & 2.71 & 7.00 \\
\hline $\mathrm{X}-05-058$ & $16 / 05 / 2005$ & 12 & & & & & & \\
\hline X-05-059 & $16 / 05 / 2005$ & 14 & & & & & & \\
\hline $\mathrm{X}-05-060$ & $16 / 05 / 2005$ & 14 & 10.6 & 9.60 & 103.3 & 278 & 6.80 & 6.50 \\
\hline $\mathrm{X}-05-061$ & $16 / 05 / 2005$ & 16 & 10.7 & 9.24 & 101.5 & 313 & 2.53 & 6.50 \\
\hline X-05-062 & $16 / 05 / 2005$ & 16 & 10.9 & 9.16 & 102.3 & 314 & 2.19 & 7.00 \\
\hline X-05-063 & $16 / 05 / 2005$ & 16 & & & & & & \\
\hline $\mathrm{X}-05-064$ & $16 / 05 / 2005$ & 16 & 15.2 & 9.35 & 110.8 & 600 & 4.84 & 7.00 \\
\hline$X-05-065$ & $16 / 05 / 2005$ & 17 & & & & & & \\
\hline
\end{tabular}


Appendix 6: Results of basic water chemistry and air temperature measurements recorded at most sites where fishing effort was applied between 2002 and 2006.

\begin{tabular}{|c|c|c|c|c|c|c|c|c|}
\hline \multirow{2}{*}{ Site Number } & \multirow{2}{*}{ Date } & \multirow{2}{*}{$\begin{array}{l}\text { Air Temp. } \\
\left({ }^{\circ} \mathrm{C}\right)\end{array}$} & \multirow{2}{*}{$\begin{array}{c}\text { Water Temp. } \\
\left({ }^{\circ} \mathrm{C}\right)\end{array}$} & \multicolumn{2}{|c|}{ Dissolved Oxygen } & \multirow{2}{*}{$\begin{array}{c}\text { Conductivity } \\
(\mu \mathrm{S} / \mathrm{cm})\end{array}$} & \multirow{2}{*}{$\begin{array}{l}\text { Turbidity } \\
\text { (NTU) }\end{array}$} & \multirow{2}{*}{$\begin{array}{c}\mathrm{pH} \\
\text { (units) }\end{array}$} \\
\hline & & & & (mg/L) & (\% SAT) & & & \\
\hline X-05-066 & $17 / 05 / 2005$ & 14 & 13.3 & 10.89 & 124 & 488 & 13.50 & 7.25 \\
\hline X-05-067 & $17 / 05 / 2005$ & 14 & 13.7 & 11.13 & 127.8 & 526 & 0.00 & 7.25 \\
\hline X-05-068 & $17 / 05 / 2005$ & 16 & & & & & & \\
\hline X-05-069 & $17 / 05 / 2005$ & 20 & 17.3 & 8.26 & 101.7 & 474 & 6.40 & 7.00 \\
\hline X-05-070 & $18 / 05 / 2005$ & 13 & 12.7 & 6.81 & 76.4 & 534 & 4.80 & 6.75 \\
\hline X-05-071 & $18 / 05 / 2005$ & 12 & 14.8 & 6.00 & 70.5 & 440 & 6.28 & 6.75 \\
\hline$X-05-072$ & $18 / 05 / 2005$ & 14 & 14.4 & 7.53 & 88.7 & 432 & 2.07 & 7.25 \\
\hline X-05-073 & $18 / 05 / 2005$ & 16 & & & & & & \\
\hline X-05-074 & $18 / 05 / 2005$ & 16 & & & & & & \\
\hline$X-05-075$ & $18 / 05 / 2005$ & 16 & & & & & & \\
\hline X-05-076 & $18 / 05 / 2005$ & 15 & & & & & & \\
\hline X-05-077 & $18 / 05 / 2005$ & 16 & & & & & & \\
\hline X-05-078 & $19 / 05 / 2005$ & 15 & & & & & & \\
\hline X-05-079 & $19 / 05 / 2005$ & 14 & 14.2 & 2.23 & 24.5 & 602 & 15.25 & 6.75 \\
\hline X-05-080 & $19 / 05 / 2005$ & 15 & 14.6 & 4.71 & 54 & 638 & 19.50 & 6.75 \\
\hline X-05-081 & $19 / 05 / 2005$ & 16 & 18.1 & & & 779 & 2.01 & 7.00 \\
\hline X-05-082 & $19 / 05 / 2005$ & 20 & & & & & & \\
\hline X-05-083 & $19 / 05 / 2005$ & 17 & 18.6 & 10.26 & 125 & 339 & 1.78 & 7.25 \\
\hline X-05-084 & $20 / 05 / 05$ & 24 & 20.1 & & & 592 & 25.85 & 8.00 \\
\hline X-05-085 & $20 / 05 / 05$ & 25 & 21.1 & & & 400 & 11.89 & 7.75 \\
\hline X-05-086 & $20 / 05 / 05$ & 25 & 23.3 & 6.57 & 87.8 & 348 & 50 & 7.50 \\
\hline X-05-087 & $20 / 05 / 05$ & 26 & & & & & & \\
\hline X-05-088 & $24 / 05 / 05$ & 17 & 18.2 & 7.32 & 87.8 & 441 & 2.50 & 6.75 \\
\hline X-05-089 & $25 / 05 / 05$ & 17 & & & & & & \\
\hline X-05-090 & $25 / 05 / 05$ & 20 & & & & & & \\
\hline $\mathrm{X}-05-091$ & $25 / 05 / 05$ & 17 & 12.8 & 6.98 & 81.3 & 172 & 3.29 & \\
\hline X-05-092 & $25 / 05 / 05$ & 17 & & & & & & \\
\hline X-05-093 & $27 / 05 / 05$ & 7 & 10.9 & 5.51 & 58.8 & 660 & 33.90 & 7.25 \\
\hline X-05-094 & $27 / 05 / 05$ & 7 & 10.3 & 11.43 & 117.6 & 603 & 28.84 & 6.75 \\
\hline X-05-095 & $30 / 05 / 05$ & 24 & 17.4 & 9.29 & 112.3 & 650 & 7.34 & 8.00 \\
\hline X-05-096 & $30 / 05 / 05$ & 24 & 18.8 & 5.78 & 73.5 & 696 & 6.02 & 7.50 \\
\hline X-05-097 & $30 / 05 / 05$ & 19 & & & & & & \\
\hline X-05-098 & $30 / 05 / 05$ & 24 & 19.0 & 6.10 & 76.1 & 678 & 92 & 7.00 \\
\hline
\end{tabular}


Appendix 6: Results of basic water chemistry and air temperature measurements recorded at most sites where fishing effort was applied between 2002 and 2006.

\begin{tabular}{|c|c|c|c|c|c|c|c|c|}
\hline \multirow{2}{*}{ Site Number } & \multirow{2}{*}{ Date } & \multirow{2}{*}{$\begin{array}{l}\text { Air Temp. } \\
\left({ }^{\circ} \mathrm{C}\right)\end{array}$} & \multirow{2}{*}{$\begin{array}{l}\text { Water Temp. } \\
\left({ }^{\circ} \mathrm{C}\right)\end{array}$} & \multicolumn{2}{|c|}{ Dissolved Oxygen } & \multirow{2}{*}{$\begin{array}{c}\text { Conductivity } \\
(\mu \mathrm{S} / \mathrm{cm})\end{array}$} & \multirow{2}{*}{$\begin{array}{c}\text { Turbidity } \\
\text { (NTU) }\end{array}$} & \multirow{2}{*}{$\begin{array}{c}\text { pH } \\
\text { (units) }\end{array}$} \\
\hline & & & & (mg/L) & (\% SAT) & & & \\
\hline X-05-099 & $30 / 05 / 05$ & 23 & 21.8 & 9.13 & 121.7 & 639 & 1.10 & 7.00 \\
\hline X-05-100 & 31-May-05 & 24 & 18.0 & 4.98 & 61.8 & 944 & 31.74 & 7.00 \\
\hline X-05-101 & 31-May-05 & 25 & & & & & & \\
\hline$X-05-102$ & 31-May-05 & 23 & 18.3 & 9.76 & 123.6 & 995 & 13.88 & 6.75 \\
\hline X-05-103 & 31-May-05 & 22 & 18.4 & 8.68 & 110.2 & 752 & 62 & 7.00 \\
\hline X-05-104 & 31-May-05 & 24 & 18.4 & 9.03 & 114.2 & 788 & 11.58 & 7.00 \\
\hline$X-05-105$ & 1-Jun-05 & 18 & 16.6 & 5.70 & 75.5 & 1091 & 21.25 & 6.75 \\
\hline X-05-106 & 1-Jun-05 & 20 & 18.1 & 7.89 & 102 & 1033 & 26.62 & 7.75 \\
\hline X-05-107 & 1-Jun-05 & 21 & 17.5 & 7.23 & 92.7 & 628 & 259 & 7.00 \\
\hline$X-05-108$ & 1-Jun-05 & 23 & & & & & & \\
\hline X-05-109 & 1-Jun-05 & 22 & 17.1 & 7.34 & 92 & 611 & 145 & 7.00 \\
\hline X-05-110 & 2-Jun-05 & 20 & & & & & & \\
\hline X-05-111 & 2-Jun-05 & 24 & 16.5 & 8.32 & 103 & 500 & 91 & 7.50 \\
\hline $\mathrm{X}-05-112$ & 2-Jun-05 & 20 & 21.7 & 9.08 & 122.7 & 788 & 9.42 & 7.75 \\
\hline X-05-113 & 2-Jun-05 & 20 & & & & & & \\
\hline X-05-114 & 2-Jun-05 & 20 & 20.5 & 9.00 & 123.8 & 1126 & 16.33 & 7.50 \\
\hline X-05-115 & 2-Jun-05 & 20 & 21.4 & 10.06 & 135.7 & 886 & 25.08 & 8.25 \\
\hline X-05-116 & 2-Jun-05 & 20 & & & & & & \\
\hline $\mathrm{X}-05-117$ & 2-Jun-05 & 20 & 20.7 & 11.35 & 146.9 & 370 & 7.62 & 7.00 \\
\hline X-05-118 & 6-Jun-05 & 14 & 14.8 & 4.03 & 46 & 377 & 53 & 7.00 \\
\hline X-05-119 & 6-Jun-05 & 17 & & & & & & \\
\hline$X-05-120$ & 6-Jun-05 & 18 & 19.5 & 9.74 & 123.9 & 575 & 20.80 & 7.00 \\
\hline X-05-121 & 6-Jun-05 & 23 & 17.7 & 7.62 & 94.2 & 545 & 152 & 7.00 \\
\hline$X-05-122$ & 6-Jun-05 & 25 & 20.1 & 7.94 & 107 & 245 & 12.06 & 8.00 \\
\hline X-05-123 & 7-Jun-05 & 10 & 15.4 & 4.57 & 54.4 & 270 & 7.92 & 7.00 \\
\hline$X-05-124$ & 7-Jun-05 & 10 & & & & & & \\
\hline$X-05-125$ & 7-Jun-05 & 10 & 12.5 & 8.44 & 95.3 & 496 & 7.03 & 7.00 \\
\hline $\mathrm{X}-05-126$ & 7-Jun-05 & 14 & 13.9 & 7.25 & 86.5 & 665 & 0.00 & 7.25 \\
\hline$X-05-127$ & 9-Jun-05 & 15 & 13.0 & 4.78 & 57.5 & 1004 & 16.33 & 7.53 \\
\hline$X-05-128$ & 9-Jun-05 & 16 & 17.2 & 8.49 & 111.3 & 851 & 12.55 & 7.93 \\
\hline X-05-129 & 10-Jun-05 & 15 & 14.9 & 4.27 & 49.2 & $>2000$ & 3.64 & 7.46 \\
\hline $\mathrm{X}-05-130$ & 10-Jun-05 & 15 & 15.5 & 7.86 & 92 & 1196 & 17.57 & 7.91 \\
\hline $\mathrm{X}-05-131$ & 13-Jun-05 & 18 & & & & & & \\
\hline
\end{tabular}


Appendix 6: Results of basic water chemistry and air temperature measurements recorded at most sites where fishing effort was applied between 2002 and 2006.

\begin{tabular}{|c|c|c|c|c|c|c|c|c|}
\hline \multirow{2}{*}{ Site Number } & \multirow{2}{*}{ Date } & \multirow{2}{*}{$\begin{array}{l}\text { Air Temp. } \\
\left({ }^{\circ} \mathrm{C}\right)\end{array}$} & \multirow{2}{*}{$\begin{array}{c}\text { Water Temp. } \\
\left({ }^{\circ} \mathrm{C}\right)\end{array}$} & \multicolumn{2}{|c|}{ Dissolved Oxygen } & \multirow{2}{*}{$\begin{array}{c}\text { Conductivity } \\
(\mu \mathrm{S} / \mathrm{cm})\end{array}$} & \multirow{2}{*}{$\begin{array}{l}\text { Turbidity } \\
\text { (NTU) }\end{array}$} & \multirow{2}{*}{$\begin{array}{c}\mathrm{pH} \\
\text { (units) }\end{array}$} \\
\hline & & & & (mg/L) & (\% SAT) & & & \\
\hline X-05-132 & 13-Jun-05 & 19 & 9.5 & 7.02 & 94.9 & $>2000$ & 13.79 & 8.06 \\
\hline X-05-133 & 13-Jun-05 & 19 & 18.4 & 7.67 & 100.9 & $>2000$ & 0.45 & 7.94 \\
\hline $\mathrm{X}-05-134$ & 13-Jun-05 & 19 & & & & & & \\
\hline X-05-135 & 13-Jun-05 & 17 & & & & & & \\
\hline $\mathrm{X}-05-136$ & 13-Jun-05 & 17 & 18.8 & & & 1151 & 0.00 & 7.86 \\
\hline X-05-137 & 14-Jun-05 & 15 & 17.7 & 5.57 & 71.5 & 1271 & 3.83 & 7.82 \\
\hline$X-05-138$ & 14-Jun-05 & 15 & 15.8 & 4.51 & 56 & 1291 & 4.68 & 7.69 \\
\hline X-05-139 & 14-Jun-05 & 15 & 16.3 & 2.89 & 35.8 & 911 & 10.45 & 7.68 \\
\hline $\mathrm{X}-05-140$ & 14-Jun-05 & 15 & 16.1 & 6.23 & 77.6 & 1223 & 0.00 & 8.06 \\
\hline$X-05-141$ & 14-Jun-05 & 15 & & & & & & \\
\hline X-05-142 & 15-Jun-05 & 19 & & & & & & \\
\hline $\mathrm{X}-05-143$ & 15-Jun-05 & 19 & 16.8 & 6.55 & 83.5 & $>2000$ & 0.00 & 7.58 \\
\hline X-05-144 & 15-Jun-05 & 21 & 18.1 & & & 1550 & 0.00 & 7.93 \\
\hline X-05-145 & 15-Jun-05 & 23 & 19.6 & & & 1399 & 0.00 & 7.92 \\
\hline X-05-146 & 16-Jun-05 & 25 & 20.5 & & & 682 & 42.84 & 7.55 \\
\hline X-05-147 & 17-Jun-05 & 23 & & & & & & \\
\hline X-05-148 & 17-Jun-05 & 23 & 19.5 & & & 420 & 4.09 & 6.69 \\
\hline X-05-149 & 21-Jun-05 & 24 & & & & & & \\
\hline X-05-150 & 21-Jun-05 & 25 & 22.0 & & & 547 & 19.39 & 8.26 \\
\hline X-05-151 & 21-Jun-05 & 26 & 22.8 & & & 612 & 36.49 & 8.31 \\
\hline X-05-152 & 21-Jun-05 & 27 & & & & & & \\
\hline X-05-153 & 21-Jun-05 & 30 & 25.5 & & & 953 & 5.57 & 8.27 \\
\hline X-05-154 & 21-Jun-05 & 30 & 27.2 & & & 1015 & 9.66 & 8.20 \\
\hline X-05-155 & 22-Jun-05 & 28 & 23.8 & & & 969 & 0.00 & 8.18 \\
\hline X-05-156 & 22-Jun-05 & 29 & & & & & & \\
\hline $\mathrm{X}-05-157$ & 22-Jun-05 & 29 & 25.0 & & & 1944 & 3.86 & 8.33 \\
\hline$X-05-158$ & 22-Jun-05 & 30 & & & & & & \\
\hline $\mathrm{X}-05-159$ & 23-Jun-05 & 20 & & & & & & \\
\hline X-05-160 & 23-Jun-05 & 20 & & & & & & \\
\hline$X-05-161$ & 23-Jun-05 & 20 & & & & & & \\
\hline X-05-162 & 23-Jun-05 & 25 & 25.1 & & & 1224 & 0.00 & 8.04 \\
\hline $\mathrm{X}-05-163$ & 23-Jun-05 & 25 & 22.9 & & & 1417 & 0.00 & 7.65 \\
\hline$X-05-164$ & 23-Jun-05 & 20 & 24.5 & & & 908 & 6.07 & 7.70 \\
\hline
\end{tabular}


Appendix 6: Results of basic water chemistry and air temperature measurements recorded at most sites where fishing effort was applied between 2002 and 2006.

\begin{tabular}{|c|c|c|c|c|c|c|c|c|}
\hline \multirow{2}{*}{ Site Number } & \multirow{2}{*}{ Date } & \multirow{2}{*}{$\begin{array}{l}\text { Air Temp. } \\
\left({ }^{\circ} \mathrm{C}\right)\end{array}$} & \multirow{2}{*}{$\begin{array}{c}\text { Water Temp. } \\
\left({ }^{\circ} \mathrm{C}\right)\end{array}$} & \multicolumn{2}{|c|}{ Dissolved Oxygen } & \multirow{2}{*}{$\begin{array}{c}\text { Conductivity } \\
(\mu \mathrm{S} / \mathrm{cm})\end{array}$} & \multirow{2}{*}{$\begin{array}{l}\text { Turbidity } \\
\text { (NTU) }\end{array}$} & \multirow{2}{*}{$\begin{array}{c}\mathrm{pH} \\
\text { (units) }\end{array}$} \\
\hline & & & & (mg/L) & (\% SAT) & & & \\
\hline $\mathrm{X}-05-165$ & 27-Jun-05 & 20 & 16.4 & 4.21 & 57.4 & 783 & 54 & 7.80 \\
\hline X-05-166 & 27-Jun-05 & 20 & & & & & & \\
\hline X-05-167 & 28-Jun-05 & 14 & 14.9 & 4.50 & 58.5 & 671 & 4.96 & 7.53 \\
\hline$X-05-168$ & 28-Jun-05 & 16 & 15.2 & 6.30 & 84 & 448 & 123 & 8.07 \\
\hline X-05-169 & 28-Jun-05 & 15 & 16.2 & 4.60 & 61.9 & 616 & 0.00 & 7.59 \\
\hline $\mathrm{X}-05-170$ & 28-Jun-05 & 21 & & & & & & \\
\hline$X-05-171$ & 28-Jun-05 & 24 & 16.6 & 6.35 & 86.6 & 425 & $>300$ & 7.94 \\
\hline X-05-172 & 29-Jun-05 & 16 & 17.8 & 4.26 & 56.7 & 795 & 0.00 & 7.91 \\
\hline $\mathrm{X}-05-173$ & 29-Jun-05 & 16 & 17.2 & 4.85 & 64.7 & 733 & 0.00 & 7.93 \\
\hline$X-05-174$ & 30-Jun-05 & 19 & & & & & & \\
\hline X-05-175 & 30-Jun-05 & 19 & & & & & & \\
\hline $\mathrm{X}-05-176$ & 4-Jul-05 & 22 & 19.9 & 5.52 & 77.6 & 894 & 0.00 & 7.60 \\
\hline X-05-177 & 4-Jul-05 & 24 & 20.8 & 4.68 & 66.8 & 987 & 2.72 & 7.40 \\
\hline X-05-178 & 5-Jul-05 & 25 & 21.5 & 5.78 & 83.4 & 929 & 9.57 & 8.12 \\
\hline X-05-179 & 5-Jul-05 & 25 & & & & & & \\
\hline X-05-180 & 5-Jul-05 & 25 & & & & & & \\
\hline X-05-181 & 6-Jul-05 & 25 & 7.9 & 9.34 & 125.8 & 396 & 113 & 7.00 \\
\hline X-05-182 & 6-Jul-05 & 25 & 21.5 & 7.79 & 113.3 & 382 & 26.21 & 7.83 \\
\hline X-05-183 & 6-Jul-05 & 25 & 23.7 & 7.33 & 114.2 & 376 & 14.40 & 7.68 \\
\hline X-05-184 & 6-Jul-05 & 25 & & & & & & \\
\hline X-05-185 & 7-Jul-05 & 22 & 19.9 & 4.29 & 60.2 & 419 & 5.08 & 7.48 \\
\hline X-05-186 & 7-Jul-05 & 25 & 23.3 & 9.99 & 149.4 & 467 & 8.11 & 7.83 \\
\hline X-05-187 & 7-Jul-05 & 30 & & & & & & \\
\hline X-05-188 & 7-Jul-05 & 31 & 27.5 & 6.27 & 93.2 & 511 & 0.00 & 7.76 \\
\hline X-05-189 & 8-Jul-05 & 31 & 23.3 & 4.19 & 58.1 & 515 & 6.58 & 7.36 \\
\hline X-05-190 & 11-Jul-05 & 27 & 25.8 & 3.39 & 54.5 & 824 & 0.00 & 7.50 \\
\hline X-05-191 & 12-Jul-05 & 29 & 22.7 & 5.60 & 84.7 & 448 & 10.14 & 7.78 \\
\hline X-05-192 & 13-Jul-05 & 28 & 22.6 & 6.05 & 97.7 & 211 & 1.91 & 7.48 \\
\hline $\mathrm{X}-05-193$ & 13-Jul-05 & 29 & 20.8 & 6.04 & 89.9 & 447 & 2.12 & 7.59 \\
\hline X-05-194 & 13-Jul-05 & 30 & 24.5 & 5.08 & 81.4 & 526 & 4.39 & 7.76 \\
\hline X-05-195 & 13-Jul-05 & 32 & 26.0 & 6.88 & 104.2 & 543 & 2.19 & 8.35 \\
\hline X-05-196 & 14-Jul-05 & 25 & 26.4 & 1.47 & 20.6 & 372 & 5.54 & 6.81 \\
\hline$X-05-197$ & 14-Jul-05 & 27 & & & & & & \\
\hline
\end{tabular}


Appendix 6: Results of basic water chemistry and air temperature measurements recorded at most sites where fishing effort was applied between 2002 and 2006.

\begin{tabular}{|c|c|c|c|c|c|c|c|c|}
\hline \multirow{2}{*}{ Site Number } & \multirow{2}{*}{ Date } & \multirow{2}{*}{$\begin{array}{l}\text { Air Temp. } \\
\left({ }^{\circ} \mathrm{C}\right)\end{array}$} & \multirow{2}{*}{$\begin{array}{c}\text { Water Temp. } \\
\left({ }^{\circ} \mathrm{C}\right)\end{array}$} & \multicolumn{2}{|c|}{ Dissolved Oxygen } & \multirow{2}{*}{$\begin{array}{c}\text { Conductivity } \\
(\mu \mathrm{S} / \mathrm{cm})\end{array}$} & \multirow{2}{*}{$\begin{array}{l}\text { Turbidity } \\
\text { (NTU) }\end{array}$} & \multirow{2}{*}{$\begin{array}{c}\mathrm{pH} \\
\text { (units) }\end{array}$} \\
\hline & & & & (mg/L) & (\% SAT) & & & \\
\hline $\mathrm{X}-05-198$ & 14-Jul-05 & 25 & 22.9 & 6.23 & 92.9 & 367 & 12.86 & 8.13 \\
\hline X-05-199 & 14-Jul-05 & 26 & 24.7 & 6.24 & 95.5 & 449 & 23.57 & 8.09 \\
\hline X-05-200 & 15-Jul-05 & 25 & 19.3 & 6.16 & 87.2 & 497 & 14.66 & 8.07 \\
\hline$X-05-201$ & 3-Aug-05 & 20 & & & & & & \\
\hline X-05-202 & 3-Aug-05 & 21 & 22.9 & 6.94 & 104 & 548 & 7.53 & 8.23 \\
\hline X-05-203 & 3-Aug-05 & 19 & 23.4 & 2.34 & 35.1 & 942 & 0.00 & 7.64 \\
\hline$X-05-204$ & 4-Aug-05 & 23 & 21.1 & 1.08 & 14.2 & 449 & 7.69 & 7.27 \\
\hline X-05-205 & 4-Aug-05 & 22 & & & & & & \\
\hline X-05-206 & 4-Aug-05 & 22 & & & & & & \\
\hline$X-05-207$ & 4-Aug-05 & 21 & 19.3 & 9.05 & 118 & 654 & 15.48 & 7.82 \\
\hline X-05-208 & 4-Aug-05 & 24 & 23.9 & 11.13 & 150 & 330 & 18.39 & 8.31 \\
\hline X-05-209 & 16-Jun-05 & 20 & & & & & & \\
\hline$X-05-210$ & 22-Jun-05 & 24 & 23.3 & & & 986 & 5.95 & 8.29 \\
\hline D-06-001 & 6-Jul-06 & 22 & 22.5 & 8.23 & 107.9 & 429 & 8.18 & 8.38 \\
\hline W-06-001 & 9-May-06 & 8 & 13.0 & 2.49 & 27.2 & 873 & 13.37 & 7.34 \\
\hline W-06-002 & 9-May-06 & 12 & & & & & & \\
\hline W-06-003 & 9-May-06 & 10 & 12.8 & 6.33 & 70.5 & 933 & 5.06 & 8.00 \\
\hline W-06-004 & 9-May-06 & 10 & 12.9 & 6.40 & 70.9 & 687 & 0.00 & 7.61 \\
\hline W-06-005 & 9-May-06 & 10 & & & & & & \\
\hline W-06-006 & 10-May-06 & 8 & & & & & & \\
\hline W-06-007 & 10-May-06 & 8 & 9.1 & 6.80 & 73.3 & 852 & 2.76 & 6.92 \\
\hline W-06-008 & 10-May-06 & 8 & 9.3 & 9.43 & 106.2 & $>2000$ & 7.82 & 7.93 \\
\hline W-06-009 & 10-May-06 & 11 & 11.5 & 8.18 & 99.4 & 1056 & 0.00 & 7.39 \\
\hline W-06-010 & 10-May-06 & 14 & 14.3 & 11.79 & 151.8 & 537 & 0.00 & 8.53 \\
\hline W-06-011 & 11-May-06 & 8 & & & & & & \\
\hline W-06-012 & 11-May-06 & 9 & 12.0 & 8.82 & 105 & 1478 & 0.00 & 7.28 \\
\hline W-06-013 & 11-May-06 & 10 & & & & & & \\
\hline W-06-014 & 11-May-06 & 14 & & & & & & \\
\hline W-06-015 & 11-May-06 & 14 & 14.3 & 10.10 & 130.1 & 1323 & 2.05 & 8.48 \\
\hline W-06-016 & 11-May-06 & 16 & 16.2 & 11.07 & 148.8 & 1360 & 0.00 & 6.91 \\
\hline W-06-017 & 11-May-06 & 9 & 10.6 & 8.24 & 96.4 & $>2000$ & 0.00 & 8.33 \\
\hline W-06-018 & 12-May-06 & 9 & 10.2 & 7.68 & 98.7 & $>2000$ & 10.65 & 6.91 \\
\hline W-06-019 & 12-May-06 & 7 & 12.4 & 7.29 & 91 & 837 & 20.59 & 8.01 \\
\hline
\end{tabular}


Appendix 6: Results of basic water chemistry and air temperature measurements recorded at most sites where fishing effort was applied between 2002 and 2006.

\begin{tabular}{|c|c|c|c|c|c|c|c|c|}
\hline \multirow{2}{*}{ Site Number } & \multirow{2}{*}{ Date } & \multirow{2}{*}{$\begin{array}{l}\text { Air Temp. } \\
\left({ }^{\circ} \mathrm{C}\right)\end{array}$} & \multirow{2}{*}{$\begin{array}{c}\text { Water Temp. } \\
\left({ }^{\circ} \mathrm{C}\right)\end{array}$} & \multicolumn{2}{|c|}{ Dissolved Oxygen } & \multirow{2}{*}{$\begin{array}{c}\text { Conductivity } \\
(\mu \mathrm{S} / \mathrm{cm})\end{array}$} & \multirow{2}{*}{$\begin{array}{l}\text { Turbidity } \\
\text { (NTU) }\end{array}$} & \multirow{2}{*}{$\begin{array}{c}\mathrm{pH} \\
\text { (units) }\end{array}$} \\
\hline & & & & (mg/L) & (\% SAT) & & & \\
\hline W-06-020 & 15-May-06 & 10 & 14.5 & 8.50 & 109.6 & 360 & 0.00 & 7.63 \\
\hline W-06-021 & 15-May-06 & 12 & 13.4 & 4.39 & 53.7 & 477 & 44.69 & 7.30 \\
\hline W-06-022 & 16-May-06 & 15 & 15.8 & 8.63 & 116.9 & 949 & 30.07 & 8.48 \\
\hline W-06-023 & 16-May-06 & 16 & 15.2 & 9.93 & 130.8 & 776 & 16.67 & 8.40 \\
\hline W-06-024 & 16-May-06 & 19 & & & & & & \\
\hline W-06-025 & 16-May-06 & 16 & 16.6 & 8.55 & 103 & 736 & 5.44 & 8.39 \\
\hline W-06-026 & 17-May-06 & 17 & 17.0 & 7.08 & 86.5 & 747 & 1.59 & 8.15 \\
\hline W-06-027 & 17-May-06 & 18 & 20.9 & 10.37 & 133.9 & 727 & 4.64 & 8.68 \\
\hline W-06-028 & 17-May-06 & 19 & 20.1 & 9.87 & 125.7 & 725 & 3.38 & 8.54 \\
\hline W-06-029 & 17-May-06 & 12 & 16.7 & 5.52 & 67.3 & 1192 & 41.83 & 7.92 \\
\hline W-06-030 & 23-May-06 & 24 & 16.4 & 6.69 & 82 & 1450 & 11.91 & 8.17 \\
\hline W-06-031 & 23-May-06 & 27 & 17.5 & 8.33 & 101.5 & 1171 & 27.57 & 8.28 \\
\hline W-06-032 & 23-May-06 & 12 & 19.0 & 4.91 & 60.4 & 1422 & 21.85 & 8.05 \\
\hline W-06-033 & 24-May-06 & 16 & & & & & & \\
\hline W-06-034 & 24-May-06 & 17 & & & & & & \\
\hline W-06-035 & 24-May-06 & 17 & & & & & & \\
\hline W-06-036 & 24-May-06 & 11 & 16.3 & 2.55 & 42.1 & 954 & 2.44 & 7.71 \\
\hline W-06-037 & 25-May-06 & 12 & & & & & & \\
\hline W-06-038 & 25-May-06 & 12 & & & & & & \\
\hline W-06-039 & 26-May-06 & 12 & & & & & & \\
\hline W-06-040 & 26-May-06 & 12 & 14.7 & 4.03 & 47.5 & 833 & 0.00 & 6.91 \\
\hline W-06-041 & 26-May-06 & 12 & & & & & & \\
\hline W-06-042 & 26-May-06 & 14 & & & & & & \\
\hline W-06-043 & 26-May-06 & 16 & 18.4 & 8.59 & 105.8 & 1339 & 29.41 & 8.33 \\
\hline W-06-044 & 26-May-06 & 16 & & & & & & \\
\hline W-06-045 & 26-May-06 & 17 & 18.2 & 9.24 & 123.1 & 947 & 0.07 & 8.38 \\
\hline W-06-046 & 26-May-06 & 12 & 16.0 & 2.03 & 25.7 & 1103 & 0.00 & 7.54 \\
\hline W-06-047 & 27-May-06 & 14 & & & & & & \\
\hline W-06-048 & 27-May-06 & 14 & & & & & & \\
\hline W-06-049 & 27-May-06 & 16 & 17.6 & 6.37 & 84 & 905 & 6.54 & 8.40 \\
\hline W-06-050 & 27-May-06 & 17 & & & & & & \\
\hline W-06-051 & 30-May-06 & 17 & & & & & & \\
\hline W-06-052 & 30-May-06 & 17 & & & & & & \\
\hline
\end{tabular}


Appendix 6: Results of basic water chemistry and air temperature measurements recorded at most sites where fishing effort was applied between 2002 and 2006.

\begin{tabular}{|c|c|c|c|c|c|c|c|c|}
\hline \multirow{2}{*}{ Site Number } & \multirow{2}{*}{ Date } & \multirow{2}{*}{$\begin{array}{l}\text { Air Temp. } \\
\left({ }^{\circ} \mathrm{C}\right)\end{array}$} & \multirow{2}{*}{$\begin{array}{c}\text { Water Temp. } \\
\left({ }^{\circ} \mathrm{C}\right)\end{array}$} & \multicolumn{2}{|c|}{ Dissolved Oxygen } & \multirow{2}{*}{$\begin{array}{c}\text { Conductivity } \\
(\mu \mathrm{S} / \mathrm{cm})\end{array}$} & \multirow{2}{*}{$\begin{array}{l}\text { Turbidity } \\
\text { (NTU) }\end{array}$} & \multirow{2}{*}{$\begin{array}{c}\mathrm{pH} \\
\text { (units) }\end{array}$} \\
\hline & & & & (mg/L) & (\% SAT) & & & \\
\hline W-06-053 & 30-May-06 & 18 & & & & & & \\
\hline W-06-054 & 30-May-06 & 21 & 20.5 & 11.34 & 160 & 910 & 65 & 8.80 \\
\hline W-06-055 & 30-May-06 & 22 & 20.5 & & & 876 & 26.52 & 9.01 \\
\hline W-06-056 & 30-May-06 & 18 & 16.9 & 7.31 & 86 & 1366 & 2.33 & 8.91 \\
\hline W-06-057 & 31-May-06 & 17 & & & & & & \\
\hline W-06-058 & 31-May-06 & 17 & 18.0 & 8.99 & 109 & 795 & 16.14 & 8.33 \\
\hline W-06-059 & 1-Jun-06 & 23 & & & & & & \\
\hline W-06-060 & 1-Jun-06 & 24 & 17.7 & 7.99 & 99.7 & 1793 & 41.60 & 8.45 \\
\hline W-06-061 & 1-Jun-06 & 25 & 20.3 & 6.61 & 93.8 & 169 & 0.00 & 7.55 \\
\hline W-06-062 & 6-Jun-06 & 22 & 21.0 & 4.74 & 60.79 & 1028 & 38.17 & 7.44 \\
\hline W-06-063 & 6-Jun-06 & 20 & 12.9 & 9.69 & 105.5 & 501 & $>300$ & 8.59 \\
\hline W-06-064 & 7-Jun-06 & 20 & 14.3 & 9.14 & 106.7 & 298 & $>300$ & 8.53 \\
\hline W-06-065 & 7-Jun-06 & 22 & 14.6 & 9.45 & 110.8 & 486 & $>300$ & 8.64 \\
\hline W-06-066 & 7-Jun-06 & 22 & 14.5 & 7.96 & 95.9 & 562 & 45.62 & 8.66 \\
\hline W-06-067 & 7-Jun-06 & 14 & 15.2 & 7.43 & 85.8 & 691 & 2.47 & 8.11 \\
\hline W-06-068 & 8-Jun-06 & 15 & 16.3 & 7.75 & 94.8 & 560 & 11.40 & 8.09 \\
\hline W-06-069 & 8-Jun-06 & 17 & & & & & & \\
\hline W-06-070 & 12-Jun-06 & 20 & 23.0 & 8.57 & 120.5 & 410 & 5.17 & 8.58 \\
\hline W-06-071 & 12-Jun-06 & 17 & & & & & & \\
\hline W-06-072 & 13-Jun-06 & 17 & & & & & & \\
\hline W-06-073 & 13-Jun-06 & 17 & & & & & & \\
\hline W-06-074 & 13-Jun-06 & 17 & 21.0 & 6.97 & 92.6 & 280 & 1.69 & 7.50 \\
\hline W-06-075 & 13-Jun-06 & 19 & & & & & & \\
\hline W-06-076 & 13-Jun-06 & 22 & 19.3 & 6.86 & 84.8 & 102 & 5.51 & 6.91 \\
\hline W-06-077 & 13-Jun-06 & 22 & 19.6 & 2.44 & 33.5 & 519 & 23.24 & 7.41 \\
\hline W-06-078 & 13-Jun-06 & 20 & 21.8 & 8.32 & 111 & 165 & 15.28 & 8.01 \\
\hline W-06-079 & 14-Jun-06 & 24 & 20.7 & 6.50 & 88.3 & 133 & 6.66 & 7.41 \\
\hline W-06-080 & 14-Jun-06 & 24 & & & & & & \\
\hline W-06-081 & 14-Jun-06 & 22 & & & & & & \\
\hline W-06-082 & 15-Jun-06 & 22 & & & & & & \\
\hline W-06-083 & 15-Jun-06 & 20 & 19.5 & 8.19 & 110.7 & 494 & 0.00 & 8.54 \\
\hline W-06-084 & 20-Jun-06 & 15 & & & & & & \\
\hline W-06-085 & 21-Jun-06 & 15 & 17.4 & 7.09 & 89.1 & 1150 & 15.43 & 7.92 \\
\hline
\end{tabular}


Appendix 6: Results of basic water chemistry and air temperature measurements recorded at most sites where fishing effort was applied between 2002 and 2006.

\begin{tabular}{|c|c|c|c|c|c|c|c|c|}
\hline \multirow{2}{*}{ Site Number } & \multirow{2}{*}{ Date } & \multirow{2}{*}{$\begin{array}{l}\text { Air Temp. } \\
\left({ }^{\circ} \mathrm{C}\right)\end{array}$} & \multirow{2}{*}{$\begin{array}{c}\text { Water Temp. } \\
\left({ }^{\circ} \mathrm{C}\right)\end{array}$} & \multicolumn{2}{|c|}{ Dissolved Oxygen } & \multirow{2}{*}{$\begin{array}{c}\text { Conductivity } \\
(\mu \mathrm{S} / \mathrm{cm})\end{array}$} & \multirow{2}{*}{$\begin{array}{l}\text { Turbidity } \\
\text { (NTU) }\end{array}$} & \multirow{2}{*}{$\begin{array}{c}\mathrm{pH} \\
\text { (units) }\end{array}$} \\
\hline & & & & (mg/L) & (\% SAT) & & & \\
\hline W-06-086 & 21-Jun-06 & 15 & & & & & & \\
\hline W-06-087 & 21-Jun-06 & 13 & 16.9 & 6.40 & 89.4 & 341 & 1.95 & 7.92 \\
\hline W-06-088 & 22-Jun-06 & 16 & 19.3 & 6.94 & 102.1 & 262 & 10.31 & 7.81 \\
\hline W-06-089 & 22-Jun-06 & 17 & 20.8 & 8.08 & 112.8 & 485 & 2.47 & 8.53 \\
\hline W-06-090 & 22-Jun-06 & 20 & 19.4 & 8.42 & 113.6 & 302 & 3.82 & 8.61 \\
\hline W-06-091 & 22-Jun-06 & 19 & 17.1 & 5.49 & 71.3 & 565 & 0.00 & 7.82 \\
\hline W-06-092 & 23-Jun-06 & 20 & 18.3 & 7.92 & 104.6 & 509 & 3.09 & 8.37 \\
\hline W-06-093 & 23-Jun-06 & 20 & 19.6 & 6.52 & 87.7 & 489 & 33.26 & 8.37 \\
\hline W-06-094 & 23-Jun-06 & 20 & 18.6 & 2.55 & 31.2 & 286 & 62 & 6.91 \\
\hline W-06-095 & 27-Jun-06 & 21 & & & & & & \\
\hline W-06-096 & 27-Jun-06 & 26 & 24.2 & 9.79 & 130.3 & 592 & 14.28 & 8.67 \\
\hline W-06-097 & 27-Jun-06 & 28 & 17.7 & 6.67 & 84.6 & 504 & 5.37 & 7.88 \\
\hline W-06-098 & 27-Jun-06 & 20 & 22.9 & 8.68 & 116.7 & 633 & 45.36 & 8.37 \\
\hline W-06-099 & 28-Jun-06 & 22 & & & & & & \\
\hline W-06-100 & 28-Jun-06 & 22 & 24.3 & 8.56 & 125.1 & 608 & 9.94 & 8.38 \\
\hline W-06-101 & 28-Jun-06 & 26 & & & & & & \\
\hline W-06-102 & 28-Jun-06 & 26 & 26.2 & 5.97 & 111.5 & 567 & 2.03 & 8.46 \\
\hline W-06-103 & 28-Jun-06 & 26 & & & & & & \\
\hline W-06-104 & 28-Jun-06 & 18 & & & & & & \\
\hline W-06-105 & 29-Jun-06 & 18 & & & & & & \\
\hline W-06-106 & 29-Jun-06 & 24 & 24.7 & 7.99 & 118.5 & 806 & 36.24 & 8.57 \\
\hline W-06-107 & 29-Jun-06 & 25 & & & & & & \\
\hline W-06-108 & 4-Jul-06 & 25 & & & & & & \\
\hline W-06-109 & 4-Jul-06 & 25 & & & & & & \\
\hline W-06-110 & 4-Jul-06 & 26 & 23.5 & 8.69 & 123 & 455 & 27.80 & 8.46 \\
\hline W-06-111 & 5-Jul-06 & 25 & & & & & & \\
\hline W-06-112 & 5-Jul-06 & 29 & 28.4 & 7.52 & 110.6 & 800 & 15.11 & 8.08 \\
\hline W-06-113 & 5-Jul-06 & 20 & 21.3 & 7.84 & 102.4 & 511 & 15.05 & 8.22 \\
\hline W-06-114 & 6-Jul-06 & 20 & 19.8 & 9.59 & 122.4 & 693 & 29.37 & 8.37 \\
\hline W-06-115 & 10-Jul-06 & 26 & & & & & & \\
\hline W-06-116 & 10-Jul-06 & 20 & & & & & & \\
\hline W-06-117 & 11-Jul-06 & 26 & & & & & & \\
\hline W-06-118 & 10-Jul-06 & 22 & & & & & & \\
\hline
\end{tabular}


Appendix 6: Results of basic water chemistry and air temperature measurements recorded at most sites where fishing effort was applied between 2002 and 2006.

\begin{tabular}{|c|c|c|c|c|c|c|c|c|}
\hline \multirow{2}{*}{ Site Number } & \multirow{2}{*}{ Date } & \multirow{2}{*}{$\begin{array}{l}\text { Air Temp. } \\
\left({ }^{\circ} \mathrm{C}\right)\end{array}$} & \multirow{2}{*}{$\begin{array}{c}\text { Water Temp. } \\
\left({ }^{\circ} \mathrm{C}\right)\end{array}$} & \multicolumn{2}{|c|}{ Dissolved Oxygen } & \multirow{2}{*}{$\begin{array}{c}\text { Conductivity } \\
(\mu \mathrm{S} / \mathrm{cm})\end{array}$} & \multirow{2}{*}{$\begin{array}{l}\text { Turbidity } \\
\text { (NTU) }\end{array}$} & \multirow{2}{*}{$\begin{array}{c}\mathrm{pH} \\
\text { (units) }\end{array}$} \\
\hline & & & & (mg/L) & (\% SAT) & & & \\
\hline W-06-119 & 11-Jul-06 & 22 & 21.1 & 8.93 & 116.1 & 530 & 2.23 & 8.45 \\
\hline W-06-120 & 11-Jul-06 & 23 & & & & & & \\
\hline W-06-121 & 12-Jul-06 & 25 & 24.5 & 8.75 & 121.6 & 655 & 0.00 & 8.27 \\
\hline W-06-122 & 12-Jul-06 & 27 & & & & & & \\
\hline W-06-123 & 12-Jul-06 & 27 & & & & & & \\
\hline W-06-124 & 12-Jul-06 & 20 & 21.4 & 4.38 & 56.9 & 212 & 66 & 7.31 \\
\hline W-06-125 & 18-Jul-06 & 22 & & & & & & \\
\hline W-06-126 & 18-Jul-06 & 22 & 23.9 & 7.19 & 97.1 & 35 & 16.45 & 7.32 \\
\hline W-06-127 & 18-Jul-06 & 24 & & & & & & \\
\hline W-06-128 & 18-Jul-06 & 24 & & & & & & \\
\hline W-06-129 & 18-Jul-06 & 24 & & & & & & \\
\hline W-06-130 & 18-Jul-06 & 22 & & & & & & \\
\hline W-06-131 & 18-Jul-06 & 25 & & & & & & \\
\hline W-06-132 & 18-Jul-06 & 26 & & & & & & \\
\hline W-06-133 & 19-Jul-06 & 26 & 25.4 & 4.20 & 60.7 & 33 & 36.99 & 6.72 \\
\hline W-06-134 & 19-Jul-06 & 31 & 25.5 & 5.71 & 79.6 & 73 & 241 & 6.75 \\
\hline W-06-135 & 20-Jul-06 & 29 & & & & & & \\
\hline W-06-136 & 20-Jul-06 & 29 & & & & & & \\
\hline W-06-137 & 20-Jul-06 & 30 & & & & & & \\
\hline W-06-138 & 1-Aug-06 & 24 & 22.5 & 5.50 & 75.7 & 1362 & 117 & 7.22 \\
\hline W-06-139 & 1-Aug-06 & 27 & 23.9 & 8.40 & 111.6 & 1260 & 143 & 7.18 \\
\hline W-06-140 & 1-Aug-06 & 29 & 24.9 & 9.57 & 1323 & 1248 & 281 & 7.34 \\
\hline W-06-141 & 1-Aug-06 & 20 & 22.9 & 7.19 & 93.9 & 1320 & 18.21 & 7.27 \\
\hline W-06-142 & 2-Aug-06 & 22 & & & & & & \\
\hline W-06-143 & 8-Aug-06 & 30 & 25.0 & 5.92 & 91.4 & 840 & 107 & 7.31 \\
\hline W-06-144 & 8-Aug-06 & 30 & 26.2 & 11.41 & 161.7 & 852 & 26.11 & 6.54 \\
\hline W-06-145 & 8-Aug-06 & 30 & & & & & & \\
\hline W-06-146 & 9-Aug-06 & 30 & 21.3 & 7.34 & 106 & 954 & 8.08 & 7.08 \\
\hline
\end{tabular}




\section{Appendix 7: List of any additional field notes recorded at sampling sites.}

\begin{tabular}{|c|c|}
\hline $\begin{array}{l}\text { Site } \\
\text { Number }\end{array}$ & Field Notes \\
\hline D-02-001 & Test protocols. \\
\hline D-02-002 & Test electrofisher. \\
\hline D-02-003 & Channelized natural stream. Minimum spring flows. \\
\hline D-02-005 & $\begin{array}{l}\text { Walleye parts collected on-site (cleaned, from another location?). Raised ford crossing. Downstream natural; upstream diked } \\
\text { (both sides) and channelized. }\end{array}$ \\
\hline D-02-006 & Recent construction. Some algae growth. No aquatic vegetation or diverse habitat. \\
\hline D-02-007 & Flow estimate $1 \mathrm{cfs}$ (cubic feet per second). Some meanders forming. No cattails or other aquatic vegetation. No hard substrate. \\
\hline $\mathrm{D}-02-008$ & White Suckers still spawning. Fairly diverse habitat. \\
\hline D-02-009 & Bank slumping. South side of channel recently cleaned. \\
\hline D-02-010 & Very turbid. \\
\hline D-02-011 & Running less turbid than Manning Canal. \\
\hline D-02-012 & Local knowledge: local says fish (not sure what kind) are often in the ditch. \\
\hline D-02-013 & $\begin{array}{l}\text { Very recent dredging. Spoils on east bank. Uniform bottom (clay/muck). Local knowledge: local says Northern Pike are present } \\
\text { during high water. }\end{array}$ \\
\hline D-02-014 & $\begin{array}{l}\text { Upstream of sheet pile weir. Upstream has simple habitat, downstream is natural habitat. Heavily silted upstream of weir and } \\
\text { some areas downstream of weir. }\end{array}$ \\
\hline D-02-015 & A large beaver dam 100 m downstream. Local says suckers present during high water. Flooded willow and cattails. \\
\hline D-02-016 & Culvert is steeply pitched - likely obstructs fish passage. Flow estimate $=1 \mathrm{cfs}$. \\
\hline D-02-017 & $\begin{array}{l}\text { Note how drain outlet to river is perched above river level. This effect may prevent upstream movement of fish during periods of } \\
\text { low river flow. }\end{array}$ \\
\hline D-02-018 & $\begin{array}{l}\text { Well defined thalweg. Local knowledge: local says often Northern Pike in drain. There is a steeply graded riffle } 200 \mathrm{~m} \\
\text { downstream. }\end{array}$ \\
\hline D-02-019 & Recent works have been done. Water very turbid. Some areas of bank slumping. \\
\hline D-02-020 & Recent works downstream of crossing. \\
\hline D-02-021 & More recent cleanout than Selkirk Line Drain. Poorly defined thalweg, uniform trapezoid channel, no undercut banks. \\
\hline $\mathrm{D}-02-022$ & Kick sampled $2 \mathrm{~m}^{2} ;$ lots of sucker eggs and benthic invertebrates. \\
\hline D-02-023 & Kick sampled $2 \mathrm{~m}^{2}$; sucker eggs and a few invertebrates. Riffle still has good flow/gradient. \\
\hline D-02-026 & $\begin{array}{l}\text { Kick sampled, sucker eggs and invertebrates. Right bank slumping, left bank recently cleaned; riffle has areas of hydraulic jump } \\
\text { (oxygen input). }\end{array}$ \\
\hline D-02-027 & Confined cattle operation $75 \mathrm{~m}$ from stream. Residence lawn to edge of bank. Remnant beaver dam. \\
\hline D-02-028 & $\begin{array}{l}\text { Kick sampled, sucker eggs and invertebrates. There is substantial beaver activity on floodplain. One corrugated metal pipe; } 40 \% \\
\text { pool, } 30 \% \text { riffle, } 30 \% \text { run. }\end{array}$ \\
\hline D-02-029 & No flow. \\
\hline $\mathrm{D}-02-030$ & All run/pool habitat. Low flow. \\
\hline
\end{tabular}




\section{Appendix 7: List of any additional field notes recorded at sampling sites.}

\begin{tabular}{|c|c|}
\hline $\begin{array}{l}\text { Site } \\
\text { Number }\end{array}$ & Field Notes \\
\hline D-02-031 & Flow estimate $=<1.5 \mathrm{cfs}$. Not recently dredged. Extensive undercut banks. Mean pool depth is $0.3 \mathrm{~m}$. \\
\hline D-02-033 & Spawning suckers and Fathead Minnow observed. Kick sampled Percidae eggs and Trichoptera on constructed riffle. \\
\hline D-02-034 & Local knowledge: Turtle River Watershed employee says Northern Pike are present in high water. \\
\hline $\mathrm{D}-02-035$ & Willow lined wetted perimeter. Very heavy sediment deposition except on the cobble at culvert inlets/outlets. \\
\hline D-02-036 & Some submerged vegetation and undercut banks. Mostly run habitat with an occasional scour pool. \\
\hline $\mathrm{D}-02-037$ & White Sucker congregated on cobble/ boulders under bridge. \\
\hline $\mathrm{D}-02-038$ & Dry. \\
\hline D-02-039 & Very low flow. Standing pools. \\
\hline D-02-040 & One large pool. \\
\hline D-02-041 & Found several egg husks and nematodes in drift sample; reset drift trap at 17:15. \\
\hline D-02-042 & Sample contained egg husks but no larvae. \\
\hline $\mathrm{D}-02-043$ & No recent maintenance work. Grassed banks with few willows. \\
\hline D-02-044 & Lots of submerged cobble/boulder; max depth is $1.4 \mathrm{~m}$. \\
\hline $\mathrm{D}-02-045$ & Undercut banks and overhanging vegetation. Shale trap/control structure. Grassed riparian area with willows. \\
\hline D-02-046 & Pulled larval drift trap, sample appeared empty. \\
\hline D-02-047 & $\begin{array}{l}\text { Standing pools only. Pasture upstream has moderate bank impacts and a dugout constructed midstream. There is very limited } \\
\text { buffer vegetation. }\end{array}$ \\
\hline D-02-048 & Heavy cattle impacts on left bank downstream of crossing in pasture. Standing pools. \\
\hline D-02-049 & Dry. \\
\hline D-02-050 & Dry. \\
\hline D-02-051 & $\begin{array}{l}\text { Woody debris instream. Thalweg is well defined meanders within channel. Thalweg length is about } 2 \mathrm{X} \text { channel length. Pasture on } \\
\text { right bank; right bank well vegetated with poplar. }\end{array}$ \\
\hline $\mathrm{D}-02-052$ & Site of shale cleanout. \\
\hline $\mathrm{D}-02-053$ & No flow. Cattle impacts. \\
\hline D-02-054 & Flow estimate $=1.5 \mathrm{cfs}$. \\
\hline D-02-055 & Heavy algal growth downstream of cultivated fields. Recent maintenance works upstream. \\
\hline D-02-056 & $\begin{array}{l}\text { Shale and sand deposition. Some scour pools. Appears old trees have been removed. Tops of banks grassed, sides of banks are } \\
\text { treed. }\end{array}$ \\
\hline D-02-057 & Cattle impacts. \\
\hline $\mathrm{D}-02-058$ & Perched culverts. \\
\hline D-02-059 & Backwater area of Turtle River with lots of deadfall, undercut banks, overhanging vegetation, canopy, and pools. \\
\hline $\mathrm{D}-02-060$ & Shale substrate. Site of shale removal. Habitat is mostly run with some small shallow riffles. \\
\hline $\mathrm{D}-02-061$ & Only able to fish edges and gradient control structure. Bank vegetation is burnt and some bank slumping present. \\
\hline D-02-062 & section only. \\
\hline
\end{tabular}


Appendix 7: List of any additional field notes recorded at sampling sites.

\begin{tabular}{|c|c|}
\hline $\begin{array}{l}\text { Site } \\
\text { Number }\end{array}$ & Field Notes \\
\hline $\mathrm{D}-02-063$ & Photo of culvert perched above water level. \\
\hline D-02-064 & Dry. \\
\hline D-02-065 & $\begin{array}{l}\text { Two dead Northern Pike on shore, site is a popular angling spot. Incoming drains are perched above water level and culverts are } \\
\text { gated. }\end{array}$ \\
\hline $\mathrm{D}-02-066$ & Set a larval drift trap. \\
\hline D-02-067 & Flows through a cow pasture downstream of reach. \\
\hline $\mathrm{D}-02-068$ & Lots of undercut banks. Some burning of bank vegetation upstream. No other recent works. \\
\hline D-02-069 & Well defined thalweg. No recent work except bank vegetation mowed to edge of wetted channel. \\
\hline $\mathrm{D}-02-070$ & Pulled a larval drift trap at 09:30 - sucker eggs but no larvae. \\
\hline D-02-071 & Cow pasture upstream with heavy bank impacts. Natural stream conditions observed downstream. \\
\hline D-02-072 & $\begin{array}{l}\text { Set a larval drift trap at 13:30. Local knowledge: Water Resources staff seeding the area have seen Yellow Perch and Northern } \\
\text { Pike in past years. }\end{array}$ \\
\hline D-02-073 & Set a larval drift trap at 14:10. \\
\hline $\mathrm{D}-02-074$ & Lots of undercut banks, boulders, submerged vegetation, and woody debris. Site is beside a Golf course. \\
\hline $\mathrm{D}-02-075$ & No recent cleanout; heavy sediment in pools. \\
\hline $\mathrm{D}-02-076$ & Checked larval drift trap at 08:30. \\
\hline D-02-077 & Bank slumping on right bank; left bank has bare spots. \\
\hline D-02-078 & Cattle impacts upstream of bridge. Some bank slumping and exposed root wads. \\
\hline D-02-079 & Running very clear. Lots of good healthy riparian vegetation. \\
\hline $\mathrm{D}-02-080$ & Some meanders starting to form upstream. \\
\hline D-02-081 & Cow pasture on right bank; bank slump on bend. \\
\hline $\mathrm{D}-02-082$ & Four corrugated metal pipes. \\
\hline D-02-083 & Lots of beaver activity (dam upstream). \\
\hline $\mathrm{D}-02-084$ & Cow pasture upstream of bridge. Cobble/boulder placed to stabilize banks downstream of bridge. \\
\hline D-02-085 & Culverts are perched $0.1 \mathrm{~m}$. Low flow (very slight trickle). 4 corrugated metal pipe culverts present. \\
\hline $\mathrm{D}-02-087$ & Two corrugated metal pipes. \\
\hline D-02-088 & Saw four Northern Pike. Channel fairly choked with cattails, heavy filamentous algae growth. Bank scour below culvert. \\
\hline D-02-089 & Riparian area all forage. \\
\hline $\mathrm{D}-02-090$ & Cement box bridge structure. Drain is choked with cattails. \\
\hline D-02-091 & Choked with cattails, No flow. Pasture upstream. \\
\hline D-02-092 & Heavy growth of filamentous algae observed. Pasture upstream. \\
\hline D-02-093 & Lots of instream boulders and vegetation (some bulrush). \\
\hline D-02-094 & Instream cattails. Banks are forage plants only. \\
\hline D-02-095 & Undercut banks above present water level. Lots of algal growth in backwater area \\
\hline
\end{tabular}


Appendix 7: List of any additional field notes recorded at sampling sites.

\begin{tabular}{|c|c|}
\hline $\begin{array}{l}\text { Site } \\
\text { Number }\end{array}$ & Field Notes \\
\hline D-02-096 & Gradient control structure $100 \mathrm{~m}$ upstream. Areas of bank scour. \\
\hline D-02-097 & $\begin{array}{l}\text { Numerous larval and juvenile minnows observed. Two corrugated metal pipes, perched. Bank slumping, right bank recently } \\
\text { disturbed. }\end{array}$ \\
\hline D-02-098 & Choked with cattail. Three corrugated metal pipes present. \\
\hline D-02-099 & Cement V-notch weir. \\
\hline D-02-100 & $\begin{array}{l}\text { Heavy rains overnight. Some undercut banks and channel is starting to meander due to bank slumping. Channel has a U-shaped } \\
\text { cross-section. }\end{array}$ \\
\hline D-02-101 & Two corrugated metal pipes. Channel is beginning to meander; sediment deposition present; similar to U-Drain (clay substrate). \\
\hline D-02-102 & Channel has U-shaped cross-section and has a well-defined thalweg. Clay substrate. Three corrugated metal pipes present. \\
\hline D-02-103 & Water is very turbid from the south; inflow from the north is clear. \\
\hline D-02-104 & Stream is natural with some channelization 1 mile upstream and has cattle impacts downstream. \\
\hline D-02-105 & No flow. There is a concrete dam with lots of silt infilling upstream of the dam. Some bank slump is present. \\
\hline D-02-106 & Raised crossing with $0.7 \mathrm{~m}$ corrugated metal pipes. No flow. Cattails and sedges in channel. \\
\hline $\mathrm{D}-02-107$ & There are cattle trails and bank impacts. A $2.0 \mathrm{~m}$ culvert is perched about $0.4 \mathrm{~m}$. Flow $=0.2 \mathrm{cfs}$. \\
\hline D-02-108 & Local knowledge: Rainbow Trout and White Sucker present in spring from Spear Lake. Beaver activity. \\
\hline D-02-109 & Standing pools only. Lots of willows along channel; banks are stable. \\
\hline D-02-110 & Active beaver pond; cow trails; native vegetation on banks (shrubs, willows, poplar, oak). \\
\hline D-02-111 & Ford crossing; undercut banks. \\
\hline D-02-112 & $\begin{array}{l}\text { Scour pool downstream; some scour upstream; lady slippers on banks; diverse aquatic and riparian vegetation; large spruce } \\
\text { across drain. }\end{array}$ \\
\hline $\mathrm{D}-02-113$ & 1/2 mile upstream: large still across drain, more lady slippers. \\
\hline D-02-114 & Very slight trickle of flow; scour pool (diameter is $0.5 \mathrm{~m}$ ); two corrugated metal pipes present. \\
\hline $\mathrm{D}-02-115$ & Near anoxic conditions, no fish collected. \\
\hline D-02-116 & Water is very turbid. Cobble/boulders present under bridge, the rest is sand/clay. \\
\hline D-02-117 & Looking at cow pasture. \\
\hline D-02-118 & Some flow; no recent works, moderate sediment deposition except bridge area. \\
\hline D-02-119 & Limestone outcrops; max depth is $0.6 \mathrm{~m}$. \\
\hline D-02-120 & Dry. One standing pool and 1 corrugated metal pipe present. \\
\hline D-02-121 & Some recent cleanout upstream (2-3 years). There are three corrugated metal pipes and water depth is $0.4 \mathrm{~m}$. \\
\hline $\mathrm{D}-02-122$ & Inflow from tributary; cattle pasture. \\
\hline $\mathrm{D}-02-123$ & Very slight trickle of flow; dike mowed and baled to $3 \mathrm{~m}$ from water's edge; two corrugated metal pipes. \\
\hline D-02-124 & There is a slight trickle of flow, a perched culvert, and cattle pasture downstream - bank impacts. \\
\hline D-02-125 & $\begin{array}{l}\text { Lots of springs in area. Local knowledge: local says Northern Pike usually present. There are lots of undercut banks, } \\
\text { overhanging vegetation. It's good habitat. }\end{array}$ \\
\hline
\end{tabular}


Appendix 7: List of any additional field notes recorded at sampling sites.

\begin{tabular}{|c|c|}
\hline $\begin{array}{c}\text { Site } \\
\text { Number }\end{array}$ & Field Notes \\
\hline $\mathrm{D}-02-126$ & Beach seined scour pool. There are cattle impacts downstream with crossing and manure pile. \\
\hline D-02-127 & Diverse habitat. Some cattle trails. Four corrugated metal pipes present. \\
\hline D-02-128 & Backwater pools. Four corrugated metal pipes. \\
\hline D-02-129 & Banks are a mix of hay meadow and willows. Submerged vegetation, emergent vegetation, canopy, and undercut banks present. \\
\hline $\mathrm{D}-02-130$ & Two Northern Pike caught, one released. Heavy vegetation in channel except in large scour pool. \\
\hline D-02-131 & Cultivated to within $3 \mathrm{~m}$ of water's edge. \\
\hline D-02-132 & Dense cattail growth. \\
\hline D-02-133 & Emergent vegetation (pond lily, arrowhead) suggests water permanence. Dense cattails. \\
\hline D-02-134 & No flow. Standing pools. \\
\hline D-02-135 & Local knowledge: landowner says usually Northern Pike each spring. Spring fed. Beaver activity. \\
\hline D-02-136 & Flow estimate $=0.1 \mathrm{cfs}$. Culvert perched by $0.3 \mathrm{~m}$. Recently cleaned trapezoidal channel, with a hard bottom. \\
\hline D-02-137 & Lots of young of the year Brook Stickleback, Creek Chub, and Fathead Minnow. Natural channel upstream. Hayed downstream. \\
\hline D-02-138 & No flow. Standing pools. Lots of boulder/cobble associated with bridge. Upstream is forested. \\
\hline D-02-140 & Photo of Water Survey of Canada hydrometric station. \\
\hline D-02-141 & Culvert replacement with sediment/erosion controls in place. \\
\hline D-02-142 & Some shale is being trapped at constrictions. Historic shale cleanouts are evident. Mowed highway right-of-way. \\
\hline D-02-143 & Photo of Water Survey of Canada hydrometric station. \\
\hline D-02-144 & Dry. \\
\hline $\mathrm{D}-02-145$ & No flow. Standing pools. \\
\hline D-02-146 & Low flow, 0.2 cfs estimated. Culverts perched $0.3 \mathrm{~m}$ above water level. Channel is natural. \\
\hline D-02-147 & Lots of young of the year Creek Chub and Brook Stickleback. Beaver activity. No flow. Standing pools. \\
\hline D-02-149 & Cattle trails and trampled banks. \\
\hline D-02-150 & No flow. Standing pools. \\
\hline $\mathrm{D}-02-151$ & Dry. Rill. \\
\hline D-02-152 & Dry except for standing pools. V-shaped, deeply incised channel. \\
\hline D-02-153 & Dry. \\
\hline D-02-154 & Dry. \\
\hline $\mathrm{D}-02-155$ & Dry. Scour pool below culvert. Deeply incised channel. \\
\hline D-02-156 & Dry. \\
\hline D-02-157 & Standing pools. \\
\hline D-02-158 & No flow. \\
\hline $\mathrm{D}-02-159$ & Dry. \\
\hline $\mathrm{D}-02-160$ & Local knowledge: anecdotal evidence of Northern Pike, Walleye, and White Sucker. Constructed riffles? \\
\hline D-02-161 & Permanent flow? Shale deposition. \\
\hline
\end{tabular}


Appendix 7: List of any additional field notes recorded at sampling sites.

\begin{tabular}{|c|c|}
\hline $\begin{array}{c}\text { Site } \\
\text { Number }\end{array}$ & Field Notes \\
\hline D-02-162 & Very slight trickle of flow. Lots of standing water in heavily vegetated channel. \\
\hline $\mathrm{D}-02-163$ & Dry. \\
\hline D-02-164 & Local knowledge: landowner says flow all winter, suckers in high water, occasional Northern Pike, too many beaver dams \\
\hline $\mathrm{D}-02-165$ & Low flow 0.2 cfs estimated. Lots of beaver activity. Extensive beaver flood upstream. \\
\hline D-02-166 & Dry. Broad grassy floodplain. Boulder/cobble downstream of culvert. \\
\hline D-02-167 & 100's Brook Stickleback and Creek Chub. Heavy cattle impacts upstream. Bare ground, widened. \\
\hline D-02-168 & Dry. Small diameter corrugated metal pipe. \\
\hline $\mathrm{D}-02-169$ & Grassed waterway. Dry. \\
\hline D-02-170 & U-shaped channel with lots of undercut banks and overhanging vegetation. \\
\hline D-02-171 & Spring fed. Lots of undercut banks and overhanging vegetation. Grated culvert to reduce beaver activity. \\
\hline $\mathrm{D}-02-172$ & Cattle impacts. Culvert perched $0.4 \mathrm{~m}$ above water level. \\
\hline $\mathrm{D}-02-173$ & Spring fed. Firm sand/gravel bottom. \\
\hline D-02-174 & Lots of beaver activity. Submerged and emergent vegetation present. \\
\hline $\mathrm{D}-02-175$ & Cattle impacts. Banks trampled and channel widened at cattle crossing. \\
\hline D-02-176 & Natural channel upstream, channelized downstream. Spring fed. $100 \%$ of flow from 1st-order tributary from SW. \\
\hline $\mathrm{D}-02-177$ & Channelized downstream from crossing. \\
\hline D-02-178 & Extensive cobble/boulder armouring and infilling associated with bridge. \\
\hline D-02-179 & A small sheet pile dam below culvert (landowner installed) has created a deep pool below culvert. \\
\hline $\mathrm{D}-02-180$ & Emergent vegetation (mostly cattails) and submerged vegetation present. Silt/sand deposition at constrictions and bends. \\
\hline D-02-181 & Pools to $1.2 \mathrm{~m}$ deep. Natural riparian vegetation. \\
\hline D-02-182 & Hayed to near top of right bank. Heavily vegetated instream. Old ford crossing. \\
\hline D-02-183 & Extensive boulder armouring with geotextile on banks. Sediment deposition on bars. \\
\hline $\mathrm{D}-02-184$ & Numerous stoneflies indicate good water quality. Hardened/fenced cattle watering site. \\
\hline D-02-185 & No flow standing pools. No measurable dissolved oxygen. 100's of Brook Stickleback. \\
\hline D-02-186 & No flow. Dry. \\
\hline $\mathrm{D}-02-187$ & Dry. \\
\hline D-02-188 & No flow standing pools only. Well oxygenated at time of site visit. \\
\hline D-02-189 & Dry. \\
\hline D-02-190 & Severe cattle impacts. Raised crossing with small diameter, corrugated metal pipe $200 \mathrm{~m}$ downstream. \\
\hline D-02-192 & Severe bank erosion downstream of culverts. Closely cropped riparian vegetation and mature trees only. \\
\hline D-02-193 & Numerous Northern Pike observed. Many young of the year Common Carp. \\
\hline D-02-195 & Northern Pike $(141,137 \mathrm{~mm})$ and White Sucker $(193,142 \mathrm{~mm})$ caught. \\
\hline D-02-197 & Released two White Sucker @ 280 mm and 300 mm. Recent bank stabilization works. \\
\hline D-02-198 & Local knowledge: landowner says flows all year. Northern Pike every spring. \\
\hline
\end{tabular}


Appendix 7: List of any additional field notes recorded at sampling sites.

\begin{tabular}{|c|c|}
\hline $\begin{array}{l}\text { Site } \\
\text { Number }\end{array}$ & Field Notes \\
\hline D-02-199 & Released White Sucker $215 \mathrm{~mm}$. Observed numerous other White Sucker. \\
\hline D-02-201 & Northern Pike 220 - 255 mm. Spring-fed pools. \\
\hline B-03-001 & Only small immature fish, no mature fish caught. Kick sample effort was $2 \mathrm{~m}^{2}$. Beaver activity observed. \\
\hline B-03-003 & Local knowledge: Farmer mentioned large fish show up after rains and Northern Pike caught 3 miles downstream \\
\hline B-03-004 & Velocity $(\mathrm{m} / \mathrm{s})$ at perched corrugated metal pipe $=0.57$ (upstream) and 0.01 (downstream). \\
\hline B-03-006 & Dry and channelized. \\
\hline B-03-007 & Water flows over road at low level crossing. \\
\hline B-03-008 & Standing water only, three corrugated metal pipes. \\
\hline B-03-009 & One Northern Pike caught. \\
\hline B-03-011 & Channelized to road edge, very dry. \\
\hline B-03-012 & $\begin{array}{l}\text { Water quality poor (very eutrophic, high amounts of algae). Two culverts perched } 1 \mathrm{~m} \text {. Velocity }(\mathrm{m} / \mathrm{sec}) \text { upstream }=0.2 \text {, } \\
\text { downstream }=0.75 \text {, Holding/storage ponds present } 1 \text { mile upstream. }\end{array}$ \\
\hline B-03-013 & $\begin{array}{l}\text { Channelized downstream. Kick sample } 2 \mathrm{~m}^{2} \text {, found nothing perhaps due to geotextile under rocks. Landowner mentioned creek } \\
\text { floods every spring. Released a Creek Chub }(19 \mathrm{~cm}) \text {, missed a lot of fish in pool east of PTH \#83. }\end{array}$ \\
\hline B-03-014 & Horses grazing up to creek on south side of road. \\
\hline B-03-016 & Flows through cattle pasture. Missed four Brook Stickleback. \\
\hline B-03-017 & Released a $200 \mathrm{~mm}$ White Sucker. DU control structure just upstream, lots of fish in pool below structure. \\
\hline B-03-018 & Road crossing flooded. Brook Stickleback observed. Site unsuitable to fish. Lots of beaver activity. \\
\hline B-03-019 & Corrugated metal pipe $2.9 \mathrm{~m}$ diameter. \\
\hline B-03-020 & Released two Brook Stickleback. No flow. \\
\hline B-03-021 & No flow, standing pools. \\
\hline $\mathrm{B}-03-022$ & Cattle pasture to southeast of crossing. \\
\hline B-03-023 & $40 \mathrm{~cm}$ diameter corrugated metal pipe perched $20 \mathrm{~cm}$. Dry. \\
\hline B-03-024 & $40 \mathrm{~cm}$ diameter culvert, dry. \\
\hline B-03-025 & North corrugated metal pipe perched and severe erosion observed. \\
\hline B-03-026 & Released eight Creek Chub $(180,130,125,110,135,135,110,110 \mathrm{~mm}) 1$ Common Shiner $(120 \mathrm{~mm})$ and 1 White Sucker $(120 \mathrm{~mm})$. \\
\hline B-03-027 & Two perched corrugated metal pipes; east corrugated metal pipe perched $10 \mathrm{~cm}$, west corrugated metal pipe perched $3 \mathrm{~cm}$. \\
\hline B-03-029 & Recent roadwork and corrugated metal pipe installed, cement chunks used for rip-rap. \\
\hline B-03-030 & Released seven small Northern Pike and 150 plus Fathead Minnow. \\
\hline B-03-031 & Culvert velocity (corrugated metal pipe) upstream is $0.06 \mathrm{~m} / \mathrm{s}$ downstream is $0.07 \mathrm{~m} / \mathrm{s}$ \\
\hline B-03-032 & Three corrugated metal pipes at $190 \mathrm{~cm}$. Little or no flow. \\
\hline B-03-033 & Released two Brook Stickleback. \\
\hline B-03-034 & Major scouring downstream of corrugated metal pipe observed. \\
\hline B-03-035 & Many crayfish. \\
\hline
\end{tabular}


Appendix 7: List of any additional field notes recorded at sampling sites.

\begin{tabular}{|c|c|}
\hline $\begin{array}{l}\text { Site } \\
\text { Number }\end{array}$ & Field Notes \\
\hline B-03-036 & Dry. \\
\hline B-03-037 & Dry. \\
\hline B-03-038 & Control structure has backed up flow. \\
\hline B-03-039 & Dry. \\
\hline B-03-040 & Released one Brook Stickleback. \\
\hline B-03-041 & Released one Brook Stickleback and one White Sucker $(500 \mathrm{~mm})$. Concrete shoring present around culverts. \\
\hline B-03-042 & Low level crossing creates a bit of riffle, but habitat is mostly marshy. \\
\hline B-03-043 & Reach is within a pasture, but very natural. Low flow with maximum depth of $25 \mathrm{~cm}$. \\
\hline B-03-044 & Not complete. \\
\hline B-03-045 & Two gated corrugated metal pipes. \\
\hline B-03-046 & No flow, wide marsh. Low area. \\
\hline B-03-047 & Creek consists mainly of vegetated marshy areas. \\
\hline B-03-048 & Little flow, mainly a few large standing pools. \\
\hline B-03-049 & Two corrugated metal pipes with $2.45 \mathrm{~m}$ diameters. \\
\hline B-03-051 & Most of creek is deep with a very soft, mucky bottom (not suitable to wade and fish). \\
\hline B-03-052 & Caught a number of immature fish. Appears sewage from farm up the hill is pumped directly into creek. \\
\hline B-03-053 & Released six Northern Pike $(700,700,700,600,600,500 \mathrm{~mm})$. \\
\hline B-03-054 & Very low flow. \\
\hline B-03-055 & Culvert perched $12 \mathrm{~cm}$. Rocks up to base at slope of 1:1.5. \\
\hline B-03-056 & Pasture on both sides. Dry. Beaver dam 1 mile north at crossing on PTH \#24. \\
\hline B-03-057 & Water backed up due to beaver dams. \\
\hline B-03-058 & Extensive beaver dams. \\
\hline B-03-059 & Channelized, very little flow. \\
\hline $\mathrm{B}-03-060$ & Certain we saw another mature Northern Pike. \\
\hline B-03-061 & Corrugated metal pipe upstream velocity $0.47 \mathrm{~m} / \mathrm{s}$, downstream velocity $0.57 \mathrm{~m} / \mathrm{s}$, and is perched $190 \mathrm{~cm}$. \\
\hline B-03-062 & Released two White Sucker $(200 \mathrm{~mm}, 170 \mathrm{~mm})$, four male and 14 female Fathead Minnows, and two Brook Stickleback. \\
\hline B-03-063 & No flow and a lot of algal growth. \\
\hline B-03-064 & Released: 1 Black Bullhead $(190 \mathrm{~mm}), 1$ Creek Chub $(140 \mathrm{~mm})$. \\
\hline B-03-065 & $\begin{array}{l}\text { Electrofishing effort was } 330 \mathrm{sec} \times 120 \mathrm{~m} \text {. Released } 2 \text { Fathead Minnows. } 180 \mathrm{~cm} \text { corrugated metal pipe perched } 10 \mathrm{~cm} \text { with } \\
\text { upstream velocity of } 0.14 \mathrm{~m} / \mathrm{s} \text { and downstream of } 0.31 \text { and } 120 \mathrm{~cm} \text { diameter pipe perched } 10 \mathrm{~cm} \text { with no flow. }\end{array}$ \\
\hline B-03-066 & Very marshy without much water movement. \\
\hline B-03-067 & Electrofishing effort was $525 \mathrm{sec} \times 125 \mathrm{~m}$. No catch. Very little flow. \\
\hline B-03-069 & No flow. Standing pools with lots of algae, very deep ditch along road. \\
\hline B-03-070 & Released: two White Sucker $(400,600 \mathrm{~mm})$, two male Fathead Minnows, and 12 Brook Stickleback. \\
\hline
\end{tabular}


Appendix 7: List of any additional field notes recorded at sampling sites.

\begin{tabular}{|c|c|}
\hline $\begin{array}{l}\text { Site } \\
\text { Number }\end{array}$ & Field Notes \\
\hline B-03-071 & Reach is channelized. \\
\hline B-03-074 & Water in standing pools only. \\
\hline B-03-075 & Released: two Fathead Minnow and 6 Brook Stickleback. \\
\hline B-03-076 & Released one White Sucker (345 mm), 1 Creek Chub $(215 \mathrm{~mm})$. \\
\hline B-03-077 & Released seven Creek Chub $(110,140,120,125,105,135,120 \mathrm{~mm}), 3$ White Sucker $(120,90,95 \mathrm{~mm})$. \\
\hline B-03-078 & Corrugated metal pipe with cement bottom. \\
\hline B-03-079 & No flow. \\
\hline B-03-082 & Both electrofisher batteries dead must return to site later. \\
\hline B-03-083 & Dip netted large number of Brook Stickleback and Fathead Minnows. \\
\hline B-03-084 & Electrofishing effort was $321 \mathrm{sec} \times 40 \mathrm{~m}$. Saw a large number of Brook Stickleback and Fathead Minnow. \\
\hline B-03-085 & Released 11 bullhead @ $120-175 \mathrm{~mm}$ and one immature Northern Pike $(130 \mathrm{~mm})$. \\
\hline B-03-086 & Above lowest gradient control structure. One beach seine haul $30 \times 12 \mathrm{~m}$, with no catch. \\
\hline B-03-087 & Released two Northern Pike $(460,640 \mathrm{~mm})$. \\
\hline B-03-088 & Lots of algae and duckweed. \\
\hline B-03-090 & Three corrugated metal pipes. \\
\hline B-03-091 & Water high and turbid. One problematic beach seine haul, released one Black Bullhead and two Fathead Minnows. \\
\hline B-03-093 & Released eight Fathead Minnows. \\
\hline B-03-094 & Released three Northern Pike $(110,115,130 \mathrm{~mm})$. \\
\hline B-03-095 & Missed a number of fish. Caught three salamanders. Released six Brook Stickleback. \\
\hline B-03-097 & Lots of tadpoles observed. \\
\hline B-03-098 & Released six Western Blacknose Dace. \\
\hline B-03-101 & One beach seine haul $30 \times 15 \mathrm{~m}$ released six Fathead Minnows and one Burbot. \\
\hline B-03-102 & Released one White Sucker (420 mm), saw Northern Pike as well. \\
\hline B-03-103 & See cover sheet about bridge. Released four White Sucker $(80,85,90,92 \mathrm{~mm})$. \\
\hline $\mathrm{B}-03-110$ & Very dry. \\
\hline B-03-111 & Gradient control structures dry. \\
\hline B-03-112 & Released one White Sucker $(110 \mathrm{~mm})$. \\
\hline B-03-115 & Released one White Sucker $(160 \mathrm{~mm})$. Eroded cattle watering site. Channelized. \\
\hline B-03-116 & Released two White Sucker $(130,110 \mathrm{~mm})$, two Fathead Minnows. \\
\hline B-03-117 & Released one Brook Stickleback. Thick bush riparian area on west side. Cattle pasture on east side. \\
\hline B-03-119 & $\begin{array}{l}\text { Released two Mudminnow. Local knowledge- two farmers mentioned catching Northern Pike } 1 / 4 \text { mile downstream in small lake. } \\
\text { One cemented in corrugated metal pipe, } 3.5 \mathrm{~m} \times 2.5 \mathrm{~m} \text {. }\end{array}$ \\
\hline $\mathrm{B}-03-120$ & Released one White Sucker, one Northern Pike. Local knowledge- local again mentioned Northern Pike in stream. \\
\hline B-03-122 & Released three White Sucker $(175,128,30 \mathrm{~mm})$, and five Mudminnow. \\
\hline
\end{tabular}


Appendix 7: List of any additional field notes recorded at sampling sites.

\begin{tabular}{|c|c|}
\hline $\begin{array}{l}\text { Site } \\
\text { Number }\end{array}$ & Field Notes \\
\hline B-03-123 & Dry. \\
\hline B-03-124 & Low flow. Channelized in past with some erosion having occurred since. \\
\hline B-03-125 & No flow with stagnant water. Channelized and diked. \\
\hline B-03-126 & Channel wide and deep with low flow. \\
\hline B-03-127 & Dry. \\
\hline B-03-128 & Dry. Cattle grazing in creek bed. \\
\hline B-03-130 & Dry. Cattle grazing in creek bed. \\
\hline B-03-132 & Released one Brook Trout $(170 \mathrm{~mm})$ and two White Sucker $(120 \mathrm{~mm}, 95 \mathrm{~mm})$. \\
\hline B-03-133 & $\begin{array}{l}\text { Three corrugated metal pipes: west one diameter } 50 \mathrm{~cm} \text { and perched by } 30 \mathrm{~cm} \text {; middle one diameter } 90 \mathrm{~cm} \text { and perched by } 5 \\
\mathrm{~cm} \text {; east one diameter was } 30 \mathrm{~cm} \text { not perched with. A trickle of flow. }\end{array}$ \\
\hline B-03-134 & Released one White Sucker $(150 \mathrm{~mm})$ and three Brook Trout $(135-145 \mathrm{~mm})$. Kick sample was $2 \mathrm{~m}$ squared. \\
\hline B-03-135 & Recent construction on PR \#471 ditch causing bare soil to wash into creek. \\
\hline B-03-136 & Missed habitat assessment. \\
\hline B-03-137 & Dry. \\
\hline B-03-139 & Runs along PR \#265. \\
\hline B-03-140 & No flow. \\
\hline B-03-141 & Released 17 Brook Sticklebacks. \\
\hline B-03-142 & Pasture to west. One $4.5 \mathrm{~m}$ diameter corrugated metal pipe. \\
\hline B-03-143 & Released approximately 80 Common Shiner, 40 White Sucker, 20 Fathead Minnows, 40 Creek Chub, and 20 Brook Stickleback. \\
\hline B-03-145 & Beach seine haul was $40 \mathrm{~m} \times 15 \mathrm{~m}$, no catch. \\
\hline B-03-146 & Dry. Extensively grazed by cattle. \\
\hline B-03-147 & Dry. Major erosion caused by cattle. \\
\hline B-03-148 & Electrofishing effort of $289 \mathrm{sec} \times 40 \mathrm{~m}$ resulted in no catch. Extensive erosion caused by cattle. \\
\hline B-03-149 & Dry. Cattle grazing in creek bed. \\
\hline B-03-150 & Large deep pool, $30 \mathrm{~m} \times 30 \mathrm{~m}$. Pasture. \\
\hline B-03-151 & $\begin{array}{l}\text { Released four Creek Chub }(120,130,110,100 \mathrm{~mm}) \text {. South corrugated metal pipe velocity upstream } 0.34 \mathrm{~m} / \mathrm{s} \text {, and downstream } \\
\text { was } 0.43 \mathrm{~m} / \mathrm{s} \text { Kick sample } 2 \mathrm{~m} \text { squared of riffle habitat and released three Longnose Dace and one Common Shiner from sample. }\end{array}$ \\
\hline B-03-152 & Released two White Sucker $(210,145 \mathrm{~mm})$, and two Creek Chub $(205,100 \mathrm{~mm})$. \\
\hline B-03-153 & Stagnant and marshy. \\
\hline B-03-154 & Dry. \\
\hline B-03-155 & Dry. \\
\hline B-03-156 & Fatheads in standing pool. \\
\hline B-03-157 & Fatheads in standing pool. \\
\hline
\end{tabular}




\section{Appendix 7: List of any additional field notes recorded at sampling sites.}

\begin{tabular}{|c|c|}
\hline $\begin{array}{l}\text { Site } \\
\text { Number }\end{array}$ & Field Notes \\
\hline B-03-158 & $\begin{array}{l}\text { Released nine White Sucker }(160,200,135,120,130,140,125,120,115 \mathrm{~mm}) \text {, One Common Shiner (120 mm), and } 40 \text { Fathead } \\
\text { Minnows. Pasture. }\end{array}$ \\
\hline B-03-159 & Two large corrugated metal pipes perched $10 \mathrm{~cm}$. \\
\hline B-03-160 & Released 15 Brook Sticklebacks, 50 Fathead Minnows, and one Creek Chub. \\
\hline B-03-163 & Large sediment trap and manmade riffle. \\
\hline B-03-181 & Missed a large sucker. \\
\hline B-03-182 & Old crossing is now a riffle. \\
\hline B-03-183 & Released one Walleye $(325 \mathrm{~mm})$ and one Northern Pike $(160 \mathrm{~mm})$. \\
\hline B-03-184 & Electrofishing effort was $218 \mathrm{sec}$ X $20 \mathrm{~m}$. \\
\hline B-03-185 & Missed four larger fish. \\
\hline B-03-186 & Creek flows through pasture. Manure pile $10 \mathrm{~m}$ from drain. \\
\hline B-03-189 & Released three Northern Pike $(285,270,90 \mathrm{~mm})$, and one bullhead $(170 \mathrm{~mm})$. \\
\hline B-03-190 & Water Survey of Canada hydrometric station on North side of PR \#403. Hog barn on left bank. \\
\hline B-03-191 & Released 17 Mudminnow, and one White Sucker $(110 \mathrm{~mm})$. \\
\hline B-03-192 & Released nine Fathead Minnows, one White Sucker $(190 \mathrm{~mm})$, and one Brook Stickleback. \\
\hline B-03-193 & Beach seine attempt failed. \\
\hline B-03-194 & Released approximately 50 young of the year bullheads, 200 Fathead Minnows, and one adult Black Bullhead ( $285 \mathrm{~mm})$. \\
\hline B-03-195 & Channelized Some standing water full of algae. \\
\hline B-03-196 & Channelized. \\
\hline B-03-197 & Hayed dikes. \\
\hline B-03-198 & Channelized. Hayed dikes. Dry. \\
\hline B-03-199 & One standing pool, channelized and diked. \\
\hline B-03-200 & $\begin{array}{l}\text { Released } 5 \text { Northern Pike }(455,385,520,365,350 \mathrm{~mm}), 1 \text { Sauger }(310 \mathrm{~mm}) \text {, } 3 \text { Black Crappies }(130,165,180 \mathrm{~mm}), 12 \text { Black } \\
\text { Bullheads }(200,170,170,170,165,190,180,180,155,185,145,175 \mathrm{~mm}) \text {. Gradient Control Structure present. }\end{array}$ \\
\hline B-03-201 & Dominion City Dam and reservoir. \\
\hline $\mathrm{B}-03-202$ & Abundance of small fish. Spotted one bullhead. Too much vegetation to fish, standing water only. \\
\hline B-03-203 & $\begin{array}{l}\text { Cattle grazing in stream on north bank. Gravel pit } 10 \mathrm{~m} \text { from stream on right bank. Too much vegetation to fish. Standing water } \\
\text { only. }\end{array}$ \\
\hline B-03-204 & Released one small White Sucker, two Fathead Minnows, two central Mudminnow, and 265 Brook Stickleback. \\
\hline B-03-206 & $\begin{array}{l}\text { Released two Northern Pike (155, } 140 \mathrm{~mm}), 23 \text { Mudminnow, nine White Sucker, and one Brook Stickleback. Channelized and } \\
\text { diked. }\end{array}$ \\
\hline B-03-207 & $\begin{array}{l}\text { Missed four large fish, released one White Sucker }(135 \mathrm{~mm}) 43 \text { Fathead Minnows, three Mudminnow, and eight Brook } \\
\text { Stickleback. }\end{array}$ \\
\hline B-03-208 & Cattle grazing on east side of road. \\
\hline
\end{tabular}


Appendix 7: List of any additional field notes recorded at sampling sites.

\begin{tabular}{|c|c|}
\hline $\begin{array}{l}\text { Site } \\
\text { Number }\end{array}$ & Field Notes \\
\hline B-03-209 & $\begin{array}{l}\text { Released } 24 \text { young of the year White Sucker, } 11 \text { Fathead Minnows, and } 25 \text { Brook Stickleback. There was extensive erosion from } \\
\text { cattle on North side of PR \#201. }\end{array}$ \\
\hline B-03-210 & Standing water only. Channelized. \\
\hline B-03-211 & Boulder dam with old mill and channel on left bank. \\
\hline B-03-212 & Released four Mudminnow, and three Fathead Minnows. Stunned, but could not net, six Northern Pike. \\
\hline B-03-213 & No flow. \\
\hline B-03-214 & Marshy. \\
\hline B-03-215 & Dry. \\
\hline B-03-216 & Dry. \\
\hline B-03-217 & Dry. \\
\hline B-03-218 & Dry except one tiny pool in which we netted one Northern Pike $(210 \mathrm{~mm})$ and seven Fathead Minnow. \\
\hline B-03-219 & Low level crossing. Dry. \\
\hline B-03-220 & No flow and marshy. \\
\hline B-03-221 & Deep, and marshy. Flow seemed to be reversed. \\
\hline $\mathrm{B}-03-222$ & Dry with three perched corrugated metal pipes. \\
\hline B-03-223 & Two corrugated metal pipes. Dry. In fairly natural/unaltered condition. \\
\hline B-03-224 & $\begin{array}{l}\text { Local knowledge-Hasn't been running until recent rains according to landowner, water much lower than usual and Yellow Perch } \\
\text { have been caught here other years. }\end{array}$ \\
\hline B-03-225 & Dry. Dense cattail growth. \\
\hline B-03-226 & Very low flow. Channelized. Deep and wide. \\
\hline B-03-227 & Little or no flow evident. One large corrugated metal pipe. Wetted area completely filled with vegetation. \\
\hline $\mathrm{B}-03-228$ & Two corrugated metal pipes. Dry. \\
\hline B-03-229 & $\begin{array}{l}\text { No flow. Channelized. Approach across drain with two corrugated metal pipes. Dip netted 100's of Brook Stickleback, Fathead } \\
\text { Minnow, and ten Western Blacknose Dace. }\end{array}$ \\
\hline $\mathrm{B}-03-230$ & Dry. Reach has one v-notch weir; two more weirs within the next $1 / 2 \mathrm{~km}$ upstream. \\
\hline B-03-231 & Dry. Exposed substrate has a good mix of cobble, boulders and gravel. Would be good riffle habitat during spring flows. \\
\hline B-03-232 & No flow. Very dry but with some wetted areas of marsh vegetation. \\
\hline $\mathrm{B}-03-233$ & Dry. Good mix of hard substrate present. \\
\hline B-03-234 & Channelized. Dry. Bank vegetation mostly grasses. \\
\hline B-03-235 & Channelized and diked. Four corrugated metal pipes at crossing. \\
\hline B-03-236 & Dry. Channelized. Cattle grazing upstream, cattle crossing downstream of crossing. \\
\hline B-03-237 & Heavy cattail growth in stream. Very minor cattle impacts. \\
\hline $\mathrm{B}-03-238$ & attle grazing on both sides of crossing. Lots of hummocks from grazing evident. \\
\hline
\end{tabular}




\section{Appendix 7: List of any additional field notes recorded at sampling sites.}

\begin{tabular}{|c|c|}
\hline $\begin{array}{l}\text { Site } \\
\text { Number }\end{array}$ & Field Notes \\
\hline B-03-239 & $\begin{array}{l}\text { Released quite a few Fathead Minnows, Brook Stickleback, shiners. Local knowledge: landowner says water at lowest since drain } \\
\text { alteration in } 1994 .\end{array}$ \\
\hline B-03-240 & Dry. Dense cattail growth. \\
\hline B-03-241 & Standing pools only. Observed other small Northern Pike that we couldn't catch. Both dikes hayed. \\
\hline B-03-242 & No flow. Narrow riparian zone, affected by cattle grazing. Channelized and diked. \\
\hline B-03-243 & Very dry. Some impacts (erosion) from cattle. \\
\hline B-03-244 & No flow through dam. \\
\hline B-03-245 & Recent clean out downstream of corrugated metal pipes. \\
\hline B-03-246 & Dry. \\
\hline B-03-247 & Tiny trickle of flow through corrugated metal pipe. \\
\hline B-03-248 & Dry. Bank vegetation mowed. \\
\hline B-03-249 & Wide and deep channel. Local knowledge: local farmer mentioned that sucker and Northern Pike have been caught at this site. \\
\hline B-03-250 & Dry. Recent dredging and alteration. \\
\hline B-03-273 & Dry; mowed channel and roadside bank. \\
\hline D-03-001 & At riffle $75 \mathrm{~m}$ upstream of bridge crossing. \\
\hline D-03-002 & $\begin{array}{l}\text { One culvert perched } 0.4 \mathrm{~m} \text {; the other culvert slightly perched. Kick sampled for invertebrates }(\mathrm{E}=3 \mathrm{~m} 2) ; \mathrm{Flow}=2.17 \mathrm{~m} / \mathrm{s} \text { at } \\
\text { downstream end of culvert and } 1.25 \mathrm{~m} / \mathrm{s} \text { at upstream end of culvert. }\end{array}$ \\
\hline D-03-003 & Constructed riffles. Released 13 Pearl Dace and two spawning Brook Sticklebacks. \\
\hline D-03-005 & Water Survey of Canada hydrometric station. Control structure/weir present. \\
\hline $\mathrm{D}-03-006$ & Cattle impacts include bank trampled and slumping. \\
\hline D-03-008 & At concrete dam with five bays. \\
\hline D-03-009 & Twenty Brook Stickleback released. Very few shrubs and no trees in riparian area. \\
\hline D-03-011 & $\begin{array}{l}\text { Caught suckers and a Brook Trout (Fork }=13.2 \mathrm{~cm} \text {, Total length }=14.0 \mathrm{~cm} \text { ). Upstream has heavy sediment deposition. Riffles } \\
\text { are prevalent downstream of crossing. Inflow to culvert has a velocity of } 0.35 \mathrm{~m} / \mathrm{s} \text {; velocity culvert outflow is } 0.48 \mathrm{~m} / \mathrm{s} \text {; all other } \\
\text { culvert velocities are lower; kick sampled } 2 \mathrm{~m} \text { sq. for invertebrates. }\end{array}$ \\
\hline D-03-012 & Cattle have impacted the banks and there is evidence of vehicle crossings. \\
\hline D-03-013 & Dry. Cattle impacts and vehicle/tractor crossings through channel. \\
\hline D-03-015 & $\begin{array}{l}\text { The best habitat is under the bridge and around beaver dam. Heavy shale deposition present, } 5 \text { mature White Sucker } \\
\text { congregated under bridge. }\end{array}$ \\
\hline D-03-016 & $\begin{array}{l}\text { Heavy sediment deposited above shale trap (full); shale is being deposited downstream. General habitat quality is poorer } \\
\text { downstream of shale trap. }\end{array}$ \\
\hline D-03-017 & No catch. Shale deposition in pools. \\
\hline D-03-019 & No riffles. \\
\hline $\mathrm{D}-03-020$ & Very slight trickle of water. \\
\hline
\end{tabular}




\section{Appendix 7: List of any additional field notes recorded at sampling sites.}

\begin{tabular}{|c|c|}
\hline $\begin{array}{l}\text { Site } \\
\text { Number }\end{array}$ & Field Notes \\
\hline D-03-021 & o flow; standing pool under bridge and upstream. \\
\hline $\mathrm{D}-03-022$ & No flow; standing pools only. \\
\hline $\mathrm{D}-03-023$ & Fatheads spawning. Some bank instability in area of culverts and some undercut banks. \\
\hline D-03-024 & Significant bank slumping. The velocity at the downstream end of the culvert was $1.13 \mathrm{~m} / \mathrm{s}$. Some minor cattle impacts present. \\
\hline D-03-025 & Boulders associated with bridge (upstream and downstream). There are some undercut banks and woody debris. \\
\hline D-03-026 & $\begin{array}{l}\text { Minnows attempting to move upstream are limited by the slope and shallow water over cement structure }(0.7 \mathrm{~m} \text { to } 1.0 \mathrm{~m} \mathrm{head}) \text {. } \\
\text { Bank vegetation cropped to within } 5 \mathrm{~m} \text { from water's edge upstream of crossing. }\end{array}$ \\
\hline D-03-027 & $\begin{array}{l}\text { Lizard Lake Ducks Unlimited project has } 2 \text { water level controls (site was below } 2 \text { nd control structure). Culverts are perched } 0.6 \mathrm{~m} \\
\text { (likely intentional to limit fish ingress to the marsh). } 1000 \mathrm{~s} \text { of Fathead Minnow and } 100 \mathrm{~s} \text { of Brook Stickleback were caught. }\end{array}$ \\
\hline D-03-028 & Cement box culvert in disrepair and is marked as a hazard to public safety. \\
\hline D-03-029 & Shale substrate in the pools. Released Brook Sticklebacks and spawning Fathead Minnows. \\
\hline D-03-030 & Culverts are perched. \\
\hline D-03-031 & Culverts are perched. \\
\hline D-03-032 & Culvert is perched $5-7 \mathrm{~m}$ by scour \\
\hline D-03-033 & Culverts are backwatered. There are cattle trails downstream of crossing. \\
\hline D-03-035 & $\begin{array}{l}\text { Bridge to be replaced. Minor cattle impacts. 100s of Fathead Minnow, dozens of Brook Stickleback and } 3 \text { Creek Chub released } \\
\text { from seine haul. }\end{array}$ \\
\hline D-03-036 & Released 1 Silver Redhorse (Fork length $=264 \mathrm{~mm}$ ). Cattle impacts; fences across river. \\
\hline D-03-037 & $\begin{array}{l}\text { There is a dam } 150 \text { m upstream. Collected stonefly larvae (Order Plecoptera) and the presence of bulrushes indicate permanent } \\
\text { flow. }\end{array}$ \\
\hline D-03-038 & $\begin{array}{l}\text { There is a weir upstream of reach not passable at this flow. Released Common Carp, Walleye, White Sucker, Silver Redhorse, } \\
\text { Longnose Dace, Blackside Darter, Johnny Darter, Common Shiner, and emerald shiners. }\end{array}$ \\
\hline D-03-039 & $\begin{array}{l}\text { Very high flow (recent rains). Bankfull stage in un-diked section. Culvert \# } 2 \text { blocked by woody debris at upstream end. Culvert } \\
\text { velocities: } \# 1-\text { upstream }=1.19 \mathrm{~m} / \mathrm{s} \text {, downstream }=1.37 \mathrm{~m} / \mathrm{s} ; \# 2-\text { upstream }=0.19 \mathrm{~m} / \mathrm{s} \text {, downstream }=0.2 \mathrm{~m} / \mathrm{s} ; \# 3-\text { upstream }= \\
1.10 \mathrm{~m} / \mathrm{s}, \text { downstream }=1.39 \mathrm{~m} / \mathrm{s} \text {. }\end{array}$ \\
\hline D-03-040 & $\begin{array}{l}\text { Channel is poorly defined upstream. Heavy recent rains. Catch is one Black Bullhead. There is a sewage/water reservoir } 300 \mathrm{~m} \\
\text { upstream of reach. }\end{array}$ \\
\hline D-03-041 & Severe cattle impacts present downstream of crossing, the banks are trampled bare. Culverts are perched by 0.3 and $0.4 \mathrm{~m}$. \\
\hline D-03-043 & Lagoon is $0.5 \mathrm{~km}$ east of crossing. \\
\hline D-03-044 & $\begin{array}{l}\text { Site is by PTH \#32. Woody debris and willows on bank. Fatheads are spawning and caught one bullhead and three White } \\
\text { Sucker. }\end{array}$ \\
\hline D-03-045 & $\begin{array}{l}\text { Water is running very clear in comparison to local drains. No catch. Substrate is heavily vegetated }<\text { banks have grasses and } \\
\text { willows. Recent heavy rains. }\end{array}$ \\
\hline
\end{tabular}




\section{Appendix 7: List of any additional field notes recorded at sampling sites.}

\begin{tabular}{|c|c|}
\hline $\begin{array}{l}\text { Site } \\
\text { Number }\end{array}$ & Field Notes \\
\hline D-03-047 & $\begin{array}{l}\text { There is a cement ford crossing with a small culvert (diameter }=0.3 \mathrm{~m} \text { ). Released } 3 \text { Black Bullheads (lengths }=120 \mathrm{~mm}, 116 \mathrm{~mm} \text {, } \\
110 \mathrm{~mm} \text { ). The flow is } 1.09 \mathrm{~m} / \mathrm{s} \text { at upstream end and } 3.04 \mathrm{~m} / \mathrm{s} \text { at downstream end of culvert. Some undercut banks and well } \\
\text { defined thalweg. }\end{array}$ \\
\hline D-03-048 & Cattle instream on site. \\
\hline D-03-049 & $\begin{array}{l}\text { Banks are grassed. There are some scour holes present where the gated culverts are located. A ford crossing within the reach } \\
\text { provides some riffle habitat. The culvert diameter is } 2.8 \mathrm{~m} \text {. }\end{array}$ \\
\hline D-03-050 & There is no flow; dry with the odd pool. \\
\hline D-03-051 & Dry. Standing pools only. \\
\hline D-03-052 & Culvert perched $0.4 \mathrm{~m}$. No flow, standing pools only. Cattle impacts present \\
\hline D-03-053 & Dry; scour pool downstream of bridge $(5 \mathrm{~m} \times 3 \mathrm{~m} \times 0.3 \mathrm{~m})$. Reach is a grassed waterway. \\
\hline D-03-054 & $\begin{array}{l}\text { Recent clean out? Already } 15-20 \mathrm{~cm} \text { of silt deposited. Trickle of flow. Cultivated to edge of drain. Only Brook Stickleback present. } \\
\text { Site was very turbid after sampling ( } 34.68 \mathrm{NTU}) \text {. Local knowledge: landowner said Rainbow Trout and White Sucker come up } \\
\text { from Lake Irwin in high water years. No cobble except under bridge. }\end{array}$ \\
\hline D-03-055 & Marsh habitat; bulrushes in small pockets indicate permanent pools of water. \\
\hline D-03-056 & Marsh habitat; lots of bulrushes. \\
\hline D-03-057 & Site is adjacent to PR \#465, $100 \mathrm{~m}$ don \\
\hline D-03-058 & $\begin{array}{l}\text { Local knowledge: anecdotal evidence of many kinds of indicator species. Geoweb ford crossing. Undercut banks. Moderate } \\
\text { deposition of shale, sand and silt. There have been recent rains. }\end{array}$ \\
\hline D-03-059 & $\begin{array}{l}\text { Site is at raised ford crossing; mid-channel culvert velocities }(\mathrm{m} / \mathrm{s}) \text { : \#1 upstream }=0.22 \text {, downstream }=1.05 ; \# 2 \text { upstream }=0.11 \text {, } \\
\text { downstream }=1.08 \text {. Shale and silt deposition. Constructed riffles at gradient control structures/silt traps. }\end{array}$ \\
\hline D-03-060 & $\begin{array}{l}\text { Released } 2 \text { White Sucker. Lots of submerged vegetation and grasses present. No riffle habitat, some diversity at numerous } \\
\text { bends. }\end{array}$ \\
\hline D-03-061 & There is a good mix of hard substrate: sand/ gravel/ cobble/ boulders. \\
\hline D-03-062 & $\begin{array}{l}\text { Local knowledge: landowner ( } 100 \mathrm{~m} \text { south) indicated suckers and Northern Pike present years ago. Dam in Ethelbert removed } \\
\text { now. Upper Fork River stocked with trout in 1996. Good mix of riffle/run/pool habitat. Overhanging vegetation, canopy, undercut } \\
\text { banks, and woody debris present. }\end{array}$ \\
\hline D-03-063 & Culvert slightly perched at this water level. \\
\hline D-03-064 & $\begin{array}{l}\text { Local knowledge: landowner (one mile west) says Northern Pike and suckers present years ago. Cattle impacts upstream of } \\
\text { crossing. Beaver dam removed upstream, last week. Pool immediately downstream of crossing was beach seined. }\end{array}$ \\
\hline D-03-065 & $\begin{array}{l}\text { Released White Sucker, Creek Chub, Brook Stickleback, Common Shiner, Fathead Minnow, and Western Blacknose Dace. } \\
\text { Some submerged vegetation and undercut banks present. }\end{array}$ \\
\hline D-03-066 & \\
\hline
\end{tabular}




\section{Appendix 7: List of any additional field notes recorded at sampling sites.}

\begin{tabular}{|c|c|}
\hline $\begin{array}{l}\text { Site } \\
\text { Number }\end{array}$ & Field Notes \\
\hline D-03-067 & $\begin{array}{l}\text { Culvert velocities } \mathrm{m} / \mathrm{s}: \# 1 \text { (Northern most corrugated metal pipe) upstream }=0.96 \text {, downstream }=1.63 ; \# 2 \text { upstream }=0.45, \\
\text { downstream }=1.90 ; \# 3 \text { upstream }=0.95 \text {, downstream }=1.22 \text {, perched } 0.2 \mathrm{~m} ; \# 4 \text { upstream }=0.24 \text {, downstream }=1.39 \text {, perched } \\
0.3 \mathrm{~m} ; \# 5 \text { (middle culvert) upstream }=\mathrm{n} / \mathrm{a} \text {, downstream }=1.5 \text {, perched } 0.3 \mathrm{~m} ; \text { Riparian area is limited by lawn upstream. }\end{array}$ \\
\hline D-03-068 & There is lots of submerged vegetation and overhanging vegetation present. Some signs of beaver activity. \\
\hline D-03-069 & Loon Lake Wetland upstream of crossing. \\
\hline D-03-070 & Dry with standing pools. No flow. \\
\hline D-03-071 & $\begin{array}{l}\text { Three } 2.8 \mathrm{~m} \text { corrugated metal pipes embedded with beaver screens on upstream end. There is a natural meander but no riffles. } \\
\text { Pool substrate is mostly sand and some silt. }\end{array}$ \\
\hline D-03-072 & There was a recent beaver dam removal Very little flow. \\
\hline D-03-073 & There was a very recent beaver dam removal. \\
\hline D-03-074 & No flow. \\
\hline D-03-075 & Slight trickle of flow; very strong sulphur dioxide odour; very low oxygen. No catch. \\
\hline D-03-076 & $\begin{array}{l}\text { Released two Northern Pike (166, } 172 \mathrm{~mm}) \text {, three Fathead Minnows, four Brook Stickleback. Higher dissolved oxygen at this } \\
\text { reach. }\end{array}$ \\
\hline D-03-077 & Slight trickle of flow through middle culvert. \\
\hline D-03-078 & Hundreds of Brook Stickleback and Fathead Minnow released plus one Finescale Dace. \\
\hline D-03-079 & Minor cattle impacts from cattle access/pasture upstream. No flow, pools only. \\
\hline D-03-080 & Culvert slightly perched. Just a trickle of flow. \\
\hline D-03-081 & Reach is at a natural section of stream. \\
\hline D-03-082 & Dry. No flow. \\
\hline D-03-083 & $\begin{array}{l}\text { There is a beaver dam } 25 \mathrm{~m} \text { upstream of crossing. No flow; standing pools. Released one } 300 \mathrm{~mm} \text { Northern Pike and one } 220 \\
\mathrm{~mm} \text { White Sucker. Also released two Western Blacknose Dace. }\end{array}$ \\
\hline D-03-084 & $\begin{array}{l}\text { Site is at a ford crossing. Beavers have removed all poplar trees within } 20 \mathrm{~m} \text { of banks. There is a very slight trickle of flow } \\
\text { (approx. } 0.2 \mathrm{cfs}) \text {. Low oxygen conditions present, DO in pool was } 3.51 \mathrm{mg} / \mathrm{L}(43.4 \%) \text {. Stickleback and Fathead Minnows } \\
\text { present. }\end{array}$ \\
\hline D-03-085 & $\begin{array}{l}\text { There is moderate sand/silt deposition with very little instream cover. It is a known trout stream (naturalized); caught White } \\
\text { Sucker. }\end{array}$ \\
\hline D-03-086 & $\begin{array}{l}\text { There was a recent removal of beaver dams downstream. Existing beaver dams catching sediment. All age classes of riparian } \\
\text { vegetation present. White Sucker were caught. }\end{array}$ \\
\hline D-03-087 & There is a trickle of flow and heavy cattail growth is present. \\
\hline D-03-088 & $\begin{array}{l}\text { There is a cow pasture with moderate bank trampling } 200 \mathrm{~m} \text { downstream of bridge. Landowner (south of pasture on downstream } \\
\text { side of bridge) says Northern Pike were always in the stream before the Gilbert Dam was built (used to over winter in beaver } \\
\text { ponds). White Sucker were caught. }\end{array}$ \\
\hline
\end{tabular}




\section{Appendix 7: List of any additional field notes recorded at sampling sites.}

\begin{tabular}{|c|c|}
\hline $\begin{array}{l}\text { Site } \\
\text { Number }\end{array}$ & Field Notes \\
\hline D-03-089 & $\begin{array}{l}\text { Landowner immediately East says it flows all year since the dam has been installed on Pleasant Valley Lake; released two (200 } \\
\mathrm{mm}) \text { White Sucker. }\end{array}$ \\
\hline D-03-090 & $\begin{array}{l}\text { Water level gauge present. Extensive algae bloom on lake. 1000s of Brook Stickleback and Fathead Minnow, lesser numbers of } \\
\text { Creek Chub, Finescale Dace, Northern Redbelly Dace and Pearl Dace present at spillway outfall. Dam was constructed in } 1971 .\end{array}$ \\
\hline D-03-091 & Released one $350 \mathrm{~mm}$ White Sucker. \\
\hline D-03-092 & Marsh habitat. \\
\hline D-03-093 & No flow; marsh habitat. \\
\hline D-03-094 & $\begin{array}{l}\text { Released one Brook Stickleback. Reach is immediately downstream of recent riparian vegetation removal. Very low oxygen. No } \\
\text { flow. Beaver dam at upstream end of culvert. }\end{array}$ \\
\hline D-03-095 & Good dissolved oxygen conditions. Brook Stickleback only (young of year). \\
\hline D-03-096 & $\begin{array}{l}\text { Flow estimated to be }>2 \text { cfs - } 3 \text { cfs. Cattle have access and are crossing immediately upstream of road crossing. Only Brook } \\
\text { Stickleback collected. }\end{array}$ \\
\hline D-03-097 & $\begin{array}{l}\text { White Sucker collected. The ford crossing has } 12 \text { small diameter }(0.8 \mathrm{~m}) \text { culverts. There is not a lot of woody debris. Lots of } \\
\text { sediment deposition. There is evidence of sediment cleanouts and there is very little submerged vegetation. }\end{array}$ \\
\hline D-03-098 & $\begin{array}{l}\text { Slight trickle of flow. Young of year suckers present. The reach has lots of instream vegetation. Upstream of culvert crossing has } \\
\text { a smaller riparian vegetation zone width. }\end{array}$ \\
\hline D-03-099 & Very low gradient area. Marsh adjacent to creek. \\
\hline D-03-100 & $\begin{array}{l}\text { A beaver dam is present } 50 \mathrm{~m} \text { downstream of crossing. There is low flow estimated at } 2 \text { cfs. Collected only Brook Sticklebacks } \\
\text { and Fathead Minnows. Local knowledge: landowner said creek was dry until the rains last week. }\end{array}$ \\
\hline D-03-101 & Plunge pool present below culvert (perched $0.2 \mathrm{~m}$ ). Pasture on upstream side of road, minor cattle impacts. \\
\hline D-03-102 & $\begin{array}{l}\text { Zero catch. Local knowledge: campground owner says lots of 'minnows' } 3 \text { weeks ago - no flow for a few weeks, then it started } \\
\text { flowing again with the recent rains. Known fish bearing stream (I. Hagenson). Stream rehabilitation work done to return it to old } \\
\text { channel by TRWCD. }\end{array}$ \\
\hline D-03-103 & $\begin{array}{l}\text { Frequent bends present (natural meander). Rehabilitation site by TRWCD to put creek back into old channel. Low flow. Several } \\
\text { other young of year Northern Pike observed. }\end{array}$ \\
\hline $\mathrm{D}-03-104$ & Channel is dry. I. Hagenson says Northern Pike and Common Carp are present in the spring. \\
\hline D-03-105 & $\begin{array}{l}\text { Local knowledge: landowner caught young of year Northern Pike, suckers, and Walleye in the stream two weeks earlier. There } \\
\text { has been enhancement of riffles upstream of reach. }\end{array}$ \\
\hline D-03-106 & Dry. Standing pool only. \\
\hline $\mathrm{D}-03-107$ & Mostly grassed waterway. Poorly defined channel thalweg. \\
\hline D-03-108 & Dry except for pool under bridge. Channel is grassed waterway upstream of bridge. \\
\hline D-03-109 & $\begin{array}{l}\text { Dry. No flow. Small scour pool present below bridge. Lightly grazed pasture downstream of crossing. Good riparian area } \\
\text { upstream of crossing. }\end{array}$ \\
\hline D-03-110 & rickle of flow. Reinforced cemented in culverts. \\
\hline
\end{tabular}


Appendix 7: List of any additional field notes recorded at sampling sites.

\begin{tabular}{|c|c|}
\hline $\begin{array}{l}\text { Site } \\
\text { Number }\end{array}$ & Field Notes \\
\hline D-03-111 & Cattle impacts present upstream of crossing. \\
\hline D-03-112 & No flow. Scour pool present downstream from bridge. \\
\hline D-03-113 & Dry. \\
\hline D-03-114 & Dry Well armoured culverts (both upstream and downstream ends). \\
\hline D-03-115 & Channel has some undercut banks, boulders, and woody debris. \\
\hline D-03-116 & Dry; scour pools below culverts. \\
\hline D-03-117 & $\begin{array}{l}\text { Collected White Sucker. Large pool present. There is only a trickle of flow. Undercut banks and over-hanging vegetation present. } \\
\text { Lots of shale deposition. }\end{array}$ \\
\hline D-03-118 & Dry. No flow; grassed channel. \\
\hline D-03-119 & Dry. Some down cutting of channel and some areas of bank slump. Field is cultivated almost to edge of the bank. \\
\hline D-03-120 & $\begin{array}{l}\text { Standing pools; no flow. Small Northern Pike (2 year classes) are in pool below bridge. Local knowledge: landowner says } \\
\text { Walleye, Northern Pike, and suckers present in high water years. }\end{array}$ \\
\hline D-03-121 & Collected young of year Northern Pike. Shale/silt in pools. Culvert is perched by $0.7 \mathrm{~m}$. There is a shale trap $200 \mathrm{~m}$ upstream. \\
\hline D-03-122 & Large amount of shale deposition. Caught a $138 \mathrm{~mm}$ long Brook Trout. \\
\hline D-03-123 & Standing pools only. \\
\hline D-03-124 & Heavy shale deposition dominates substrate. Channel straightened and diked on both sides. Grassed banks. \\
\hline D-03-125 & Heavy cattail growth in Big Grass River Ditch. \\
\hline D-03-126 & $\begin{array}{l}\text { Caught ten Northern Pike }(200-250 \mathrm{~mm}) \text {. There are areas with heavy cattail growth in pools and areas with well-defined thalweg } \\
\text { (no cattail growth); the riparian area is grassed. }\end{array}$ \\
\hline D-03-127 & There has been a recent cleanout at the reach and a more recent cleanout one mile upstream; trapezoidal channel. \\
\hline D-03-128 & Dry; grassed riparian area. \\
\hline D-03-129 & $\begin{array}{l}\text { There is good riparian vegetation along road allowance. No flow; standing pools. Channel is choked with cattails downstream of } \\
\text { culvert. Creek Chub, Fathead Minnow, and Western Blacknose Dace were in one pool (10's of each). }\end{array}$ \\
\hline D-03-130 & Trickle of flow; standing pools. Some pools have bulrush growth indicating permanently wetted conditions. \\
\hline D-03-131 & Cattle impacts upstream and downstream. Shale cleanouts. Dugout constructed instream. \\
\hline D-03-132 & $\begin{array}{l}\text { Over } 100 \text { dead Cyprinids (Pearl Dace, Western Blacknose Dace) observed. Local knowledge: landowner said the dead fish } \\
\text { showed up after the Monday rains. Recent crop spraying in the area. }\end{array}$ \\
\hline D-03-133 & A riffle is present under bridge. Flow estimate $3-5$ cfs. \\
\hline D-03-134 & Banks are grassed. Dense growth of cattails. Released hundreds of Creek Chub, Fathead Minnows, and a few Brook Stickleback. \\
\hline D-03-135 & Shale deposits embed boulders. Canopy cover provided by riparian vegetation. Springs present - flow over and under shale. \\
\hline D-03-136 & Heavy cattails/grasses instream. Very long culverts $(50 \mathrm{~m})$ that angle under PR \#261. Small standing pools with a trickle of flow. \\
\hline D-03-137 & No fish observed. Estimate flow at $1.5-2$ cfs (cold spring water). There is no flow two miles downstream - under the shale? \\
\hline D-03-138 & There is heavy cattail growth. Released hundreds of Creek Chub, Fathead Minnows, and a few Brook Stickleback. \\
\hline D-03-139 & pasture exists downstream of crossing - presently lightly grazed Springs are likely in area. \\
\hline
\end{tabular}




\section{Appendix 7: List of any additional field notes recorded at sampling sites.}

\begin{tabular}{|c|c|}
\hline $\begin{array}{l}\text { Site } \\
\text { Number }\end{array}$ & Field Notes \\
\hline D-03-140 & Banks are impacted by cattle; cattle have direct access to water. \\
\hline $\mathrm{D}-03-141$ & Dry. Small scour pool on downstream side. \\
\hline D-03-142 & $\begin{array}{l}\text { Site is at ford crossing. A lot of gravel, cobble, undercut banks, and overhanging vegetation (natural section) with a good } \\
\text { riffle/pool/riffle sequence present. }\end{array}$ \\
\hline D-03-143 & $\begin{array}{l}\text { Dissolved oxygen in pool above riffle is } 56.9 \%(4.4 \mathrm{mg} / \mathrm{L}) \text {. Local knowledge: landowner says there are Brook Trout in the North } \\
\text { and South Duck River. }\end{array}$ \\
\hline D-03-144 & $\begin{array}{l}\text { Western Blacknose Dace, Brook Stickleback, Longnose Dace, and Creek Chub were collected. Minor cattle impacts upstream of } \\
\text { crossing. Wooden box culvert under highway. }\end{array}$ \\
\hline D-03-145 & $\begin{array}{l}\text { One } 190 \mathrm{~mm} \text { long Brook Trout was collected; couldn't catch two others that were approximately } 190-250 \mathrm{~mm} \text {. Lots of undercut } \\
\text { banks, boulders, logs, and overhanging vegetation present. There are cement box culverts and the ditch is mowed by PTH \#10. } \\
\text { Boulders are serving as a sediment trap. }\end{array}$ \\
\hline D-03-146 & The landowner upstream of culvert has mowed lawn to edge of stream. Cattle have impacted the banks downstream of culvert. \\
\hline D-03-147 & There is vegetation throughout the channel. Dry. \\
\hline D-03-148 & Small amount of flow. Lots of overhanging vegetation and canopy. \\
\hline D-03-149 & Vegetation is present throughout channel. Reach is dry. \\
\hline $\mathrm{D}-03-150$ & Dry; flows into Swan Lake. \\
\hline D-03-151 & Dry; pool below culvert. \\
\hline D-03-152 & $\begin{array}{l}\text { Local knowledge: Landowner one mile south says Northern Pike and suckers have problems accessing drains because of beaver } \\
\text { dams. There are lots of cattails, arrowhead, and lily pads present in pools; lots of undercut banks present and one riffle } \\
\text { downstream of bridge. Upstream of culvert has good riparian area. }\end{array}$ \\
\hline D-03-153 & $\begin{array}{l}\text { There is a trickle of flow through culvert, although it is mostly blocked by woody debris placed by beavers. The beaver pond } \\
\text { flooding over road } 10 \mathrm{~m} \text { upstream of crossing. }\end{array}$ \\
\hline D-03-154 & $\begin{array}{l}\text { Mostly riffle/run habitat with two deep pools and cover provided by fallen trees. Small rock/ cobble/ gravel substrate. Little or no } \\
\text { aquatic vegetation. The banks are mowed by PTH \#10 and the right bank is armoured upstream of the bridge. }\end{array}$ \\
\hline D-03-155 & $\begin{array}{l}\text { Dense aquatic vegetation and heavy algae growth. No riffles; few bends; and riparian vegetation up to } 15 \mathrm{~m} \text { upstream and } \\
\text { downstream of bridge is mowed. }\end{array}$ \\
\hline D-03-156 & $\begin{array}{l}\text { There are no pools; it's a shallow stream (approx. } 3 \text { inches deep) with mowed riparian area }(15 \mathrm{~m}) \text { from bridge to forest. } \\
\text { Vegetation from top of bank to the stream has been left untouched. }\end{array}$ \\
\hline D-03-157 & Shallow riffle/run sequence. Water Survey of Canada hydrometric station present. \\
\hline $\mathrm{D}-03-158$ & Dry. Recent rock infill upstream and downstream of culverts. \\
\hline D-03-159 & A recent culvert replacement; old culvert filled by beavers with mud and woody debris. \\
\hline $\mathrm{D}-03-160$ & Dry. \\
\hline D-03-161 & asses and cattails in channel. \\
\hline
\end{tabular}




\section{Appendix 7: List of any additional field notes recorded at sampling sites.}

\begin{tabular}{|c|c|}
\hline $\begin{array}{l}\text { Site } \\
\text { Number }\end{array}$ & Field Notes \\
\hline D-03-162 & $\begin{array}{l}\text { Small shallow pools upstream and downstream of culvert. Meandering channel with well-defined thalweg. Lots of emergent and } \\
\text { submerged vegetation. }\end{array}$ \\
\hline D-03-163 & Dry with standing pools $>$ No flow. \\
\hline D-03-164 & $\begin{array}{l}\text { Heavy sand/silt deposits in pools. Upstream of bridge has good riparian vegetation (all stages). Stream beginning to meander } \\
\text { forming undercut banks. Released one } 210 \mathrm{~mm} \text { White Sucker and one } 200 \mathrm{~mm} \text { Northern Pike. Instream vegetation is providing } \\
\text { some cover and shade. }\end{array}$ \\
\hline D-03-165 & Trickle of flow. Very shallow. \\
\hline D-03-166 & Trickle of flow. Thalweg is approximately 6 inches wide upstream of crossing. The culvert is perched approximately 6 inches. \\
\hline D-03-167 & $\begin{array}{l}\text { Shale deposition present. No aquatic vegetation. Flow through left side of cement box culvert only; middle culvert plugged with } \\
\text { shale. All fine shale/sand substrate in pools and other deposition areas. }\end{array}$ \\
\hline D-03-168 & Fairly heavy rains last night. Observed 2 Northern Pike and 2 Brook Stickleback. \\
\hline D-03-169 & $\begin{array}{l}\text { Natural reach with U-shaped channel cross-section. Good undercut banks, overhanging vegetation. Deposition largely organic } \\
\text { debris. }\end{array}$ \\
\hline D-03-170 & Just upstream of Shoal Lake aqueduct siphon. Recent siphon cleanout? \\
\hline W-03-001 & $\begin{array}{l}\text { Released four Central Mudminnow. Cultivated fields both sides, little to no riparian width. Recent rains Sunday and Monday } \\
\text { (May 18, 19). One large unknown fish observed. Sixteen Suckers caught and released. Stream depth uniform } \sim 1 \mathrm{~m} \text {. East bank } \\
\text { diked. }\end{array}$ \\
\hline W-03-002 & $\begin{array}{l}\text { Six Brook Stickleback and two Creek Chub released from beach seine. Agricultural field goes right to edge of bank downstream } \\
\text { of reach. Right bank eroded by culvert. One natural bend natural and one artificial riffle downstream of culvert. A few boulders } \\
\text { and cobbles. }\end{array}$ \\
\hline W-03-003 & No fish caught. Large beaver dam 100 m downstream. Soft, silt bottom. \\
\hline W-03-004 & $\begin{array}{l}\text { Flow velocity: upstream culvert }=0.68 \mathrm{~m} / \mathrm{s} \text {, downstream }=1.34 \mathrm{~m} / \mathrm{s} \text {. Recent rains. Uniform depth. Site off PTH \#11. Four } \\
\text { culverts west of PTH \#11, two culverts east of PTH \#11. }\end{array}$ \\
\hline W-03-005 & Off PTH \#11; North of Lac Du Bonnet. Round cement culvert. Small cattle herd (15 cow/calf pairs) with access to drain. \\
\hline W-03-006 & Off Maple Creek Road. Three perched culverts and one embedded culvert (with fastest flow). No flow in 2nd culvert from east. \\
\hline W-03-007 & Off PTH \#11. Beaver dam west of culvert. \\
\hline W-03-008 & $\begin{array}{l}\text { Electrofishing effort was } 149 \mathrm{~s} \text { by } 10 \mathrm{~m} . \text { No fish caught. Heavy cattail vegetation upstream of crossing. Light rain May 21/03. } \\
\text { Vegetative bank protection dominated by grasses. }\end{array}$ \\
\hline W-03-009 & Natural section. Not much gravel, cobble or boulders. Not many riffles, more bends. Riparian area good except along road. \\
\hline W-03-010 & $\begin{array}{l}\text { Electrofishing effort was } 196 \mathrm{sec} \times 75 \mathrm{~m} .{ }^{*} \text { No fish caught. Flow velocities: culvert inlet }=0.53 \mathrm{~m} / \mathrm{s} \text {; culvert outlet }=1.04 \mathrm{~m} / \mathrm{s} \text {. } \\
\text { Culvert armoured with boulders and concrete materials, some unstable. Gradient control structure - sheet-pile weir. One riffle }\end{array}$ \\
\hline W-03-011 & $\begin{array}{l}\text { Flow velocity: culvert inlet }=0.58 \mathrm{~m} / \mathrm{s} \text {; culvert outlet }=1.69 \mathrm{~m} / \mathrm{s} \text {. Culvert armoured with boulders and concrete. Raised crossing } \\
\text { working as a control structure. Gradient control structures every mile. }\end{array}$ \\
\hline W-03-012 & Low flow. \\
\hline
\end{tabular}


Appendix 7: List of any additional field notes recorded at sampling sites.

\begin{tabular}{|c|c|}
\hline $\begin{array}{l}\text { Site } \\
\text { Number }\end{array}$ & Field Notes \\
\hline W-03-013 & Two culverts perched $0.3 \mathrm{~m}$ at downstream end. \\
\hline W-03-014 & Adjacent to Icelandic horse farm. Standing pool. Grassed banks, no trees or shrubs. Off PR. \#236. \\
\hline W-03-015 & $\begin{array}{l}\text { Stream is dry except for standing pool. East of PR \#236. No flow, heavy instream cattails. Grassed banks, no trees. Three } \\
\text { culverts, one gated culvert. }\end{array}$ \\
\hline W-03-016 & $\begin{array}{l}\text { Dry except for small standing pool. Stream recently cleaned out. Some grass growing in channel downstream of culverts. } \\
\text { Grassed banks, no trees. Two culverts ( } 1 \mathrm{~m} \text { in diameter) perched above small ( } 0.3 \mathrm{~m} \text { in diameter) culvert. }\end{array}$ \\
\hline W-03-017 & Standing shallow pool downstream of reach. MB Hydro power lines (photos 51-56). Located off PR \#236. \\
\hline W-03-018 & Upstream agricultural field right to diked banks (on east bank). Located off PR \# 221. Two culverts present. \\
\hline W-03-019 & $\begin{array}{l}\text { Three young of the year Northern Pike collected. Electrofishing was very difficult because of high conductivity. A lot of instream } \\
\text { vegetation. Submerged vegetation providing some cover. One riffle under bridge, two bends. Banks covered by grasses west of } \\
\text { PTH \#2. }\end{array}$ \\
\hline W-03-020 & $\begin{array}{l}\text { No fish caught. Recently dredged, piles of mud on banks. Some instream vegetation (Bulrushes, Arrowheads). No trees, } \\
\text { grasses only, bare banks on north side (right bank) and on south side (left bank). Upstream no water at culvert, marshy further } \\
\text { upstream. Hard mud bottom after cleanout. }\end{array}$ \\
\hline W-03-021 & Agricultural land. No trees in riparian area. Drain is dry. Agricultural fields run to edge of drain bank. \\
\hline W-03-022 & $\begin{array}{l}\text { Lots of instream vegetation (Coontails). Boulders and cobble along right bank. } 3 \text { gated culverts (perched) from Old Sturgeon } \\
\text { Creek. Old Sturgeon Creek is dry. }\end{array}$ \\
\hline W-03-023 & $\begin{array}{l}\text { Joins up with Sturgeon Creek (4th order). } 2 \text { nd order drain is dry. Sturgeon Creek uniform depth of } 0.2 \mathrm{~m} \text {. Two gated culverts are } \\
0.1 \mathrm{~m} \text { above creek bed. }\end{array}$ \\
\hline W-03-024 & Farm adjacent to Meridian Drain. Possible hog barn? \\
\hline W-03-025 & $\begin{array}{l}\text { No flow. Standing pools only. Grassed banks. At junction of Meridian drain and unnamed second and first order drains. } \\
\text { Approximately } 40 \mathrm{~m} \text { of Meridian drain upstream of crossing have recently been cleaned out. }\end{array}$ \\
\hline W-03-026 & Some cattails. Field ends at top of bank (where bank slopes to water). Mats of algae present. One pool. \\
\hline W-03-027 & Off PR \# 236. Very recently cleaned out. One standing pool. \\
\hline W-03-028 & Diked downstream of culverts (left bank). Grassed banks upstream. All dry except for one standing pool. \\
\hline W-03-029 & Off Meridian Road. (RM of Woodlands). Junction of 2-2nd order drains. Dry. \\
\hline W-03-030 & $\begin{array}{l}\text { Electrofisher would not work effectively in highly conductive water. Beach seine haul was } 10 \mathrm{~m} \times 8 \mathrm{~m} \text {. Stream uniform depth } 0.3 \\
\mathrm{~m} \text {. Standing pool under bridge. Gravel pile under bridge. Grassy banks. Marsh downstream of bridge. }\end{array}$ \\
\hline W-03-031 & Junction of 2 nd and 3rd order drains. 2nd order drain is dry. \\
\hline W-03-032 & Dry. No trees, only grasses in riparian zone. \\
\hline W-03-033 & Trickle of flow. Lots of instream vegetation (cattails). Early succession shrubs and trees growing in riparian area. \\
\hline W-03-034 & No flow, some standing pools. Young trees growing along banks. \\
\hline W-03-035 & $\begin{array}{l}\text { No fish caught. Heavy filamentous algae. Very bad rotten egg smell (hydrogen sulphide). No riffles in reach, five be } \\
\text { view. One deep scour pool below culvert. Grassed banks. }\end{array}$ \\
\hline
\end{tabular}




\section{Appendix 7: List of any additional field notes recorded at sampling sites.}

\begin{tabular}{|c|c|}
\hline $\begin{array}{l}\text { Site } \\
\text { Number }\end{array}$ & Field Notes \\
\hline W-03-036 & Problems with electrofisher because of high conductivity. No fish caught. Riparian zone greater than $18 \mathrm{~m}$. \\
\hline W-03-037 & Very low flow. 2 very large culverts ( 2 $\mathrm{m}$ in diameter). \\
\hline W-03-038 & Dry. Standing pools upstream and downstream of culverts. \\
\hline W-03-039 & No flow. Standing pools only. Left bank, downstream of culverts, is bare. Some grass starting to establish. \\
\hline W-03-040 & Perched culverts (approx. $0.2 \mathrm{~m}$ ). Standing pools with dense algae growth. \\
\hline W-03-041 & Dry. Left bank bare upstream. Some grass growing on left bank, downstream of culverts. \\
\hline W-03-043 & Extensive algae bloom in pools. \\
\hline W-03-044 & $\begin{array}{l}\text { Beach seine effort } 6 \mathrm{~m} \times 15 \mathrm{~m} \text {. No catch. Long culverts. Significant bank slump and erosion on right bank upstream from culvert. } \\
\text { Flow very slow downstream from culvert. No riffles, one pool, many bends both upstream and downstream of reach. Upstream } \\
\text { left bank has greater than } 18 \mathrm{~m} \text { riparian zone. }\end{array}$ \\
\hline W-03-045 & North of Stephenfield Lake along gravel road. \\
\hline W-03-046 & $\begin{array}{l}\text { Undercut banks. Dense emergent vegetation. Three culverts, no riffles but good run and pool habitat although both pools in reach } \\
\text { are shallow. }\end{array}$ \\
\hline W-03-047 & Mostly surrounded by shrubs and grasses. \\
\hline W-03-048 & $\begin{array}{l}\text { No flow. Dry. Appears to be a lot of vegetation in the active channel. Diked on both sides at stream bends. Road on south and } \\
\text { west side. Evidence of potential new road. Downed trees. }\end{array}$ \\
\hline W-03-049 & $\begin{array}{l}\text { One Brook Stickleback caught; three others observed but could not catch. No riffles, or fast moving water, no bends, deep } \\
\text { thalweg in center of channel. Mostly grasses and a few shrubs in riparian zone. Dry above rocks and weir. }\end{array}$ \\
\hline W-03-050 & $\begin{array}{l}\text { A lot of crayfish and Gammarus. Problems with electrofisher in high conductivity water. Farmer stopped to enquire about beaver } \\
\text { dams. He said there are a lot of dams upstream. Cattle crossing river, trampled banks }\end{array}$ \\
\hline W-03-051 & Fences on either side of the road. \\
\hline W-03-052 & Completely dry. \\
\hline W-03-053 & $\begin{array}{l}\text { Wooden bridge. Marsh like habitat upstream and downstream from bridge. Dry upstream and downstream of the marsh area } \\
\text { about } 100 \mathrm{~m} \text { upstream and downstream of the crossing. }\end{array}$ \\
\hline W-03-054 & Very marshy, a lot of emergent and submerged vegetation. \\
\hline W-03-055 & $\begin{array}{l}\text { Five juvenile Northern Pike caught. Wooden bridge. Tree rows around cultivated fields, none adjacent to stream. Mainly grass } \\
\text { and shrubs on banks. Water extremely turbid, no riffles, slow/ deep and slow/ shallow flow regimes. No bends, deep pools. }\end{array}$ \\
\hline W-03-056 & Marshy. \\
\hline W-03-057 & $\begin{array}{l}\text { Spoil material from cleanout on top of dikes. Culverts upstream perched } 0.2 \mathrm{~m} \text {. Culverts downstream embedded in bottom of } \\
\text { drain. Downstream south side diked. }\end{array}$ \\
\hline W-03-058 & $\begin{array}{l}\text { Mostly grasses and some small trees and shrubs along the banks. Virtually no riparian zone width as field is cultivated to the } \\
\text { bank. Mostly marsh habitat in reach. }\end{array}$ \\
\hline W-03-059 & ecent rain. \\
\hline
\end{tabular}




\section{Appendix 7: List of any additional field notes recorded at sampling sites.}

\begin{tabular}{|c|c|}
\hline $\begin{array}{l}\text { Site } \\
\text { Number }\end{array}$ & Field Notes \\
\hline W-03-060 & $\begin{array}{l}\text { Large sprayer parked near reach. Concrete bridge with two channels. Completely overgrown, especially upstream from bridge. } \\
\text { Fields cultivated to banks both upstream and downstream. }\end{array}$ \\
\hline W-03-061 & Lots of canopy, deadfall, woody debris. Crosses PTH \#2 just east of Treherne and PR \#242. \\
\hline W-03-062 & $\begin{array}{l}\text { Large culverts. Short manicured lawn downstream from culvert. Hay bales placed in pool. Downstream area fenced. Bank } \\
\text { slump and exposed banks upstream from crossing. Manicured lawn upstream from crossing on right bank. }\end{array}$ \\
\hline W-03-063 & Unmapped drain. Not on Designation of Drains map. \\
\hline W-03-064 & $\begin{array}{l}\text { Many dead trees along right bank, downstream from crossing. Uniform slow/deep flow regime. Would have been good fishing, } \\
\text { but impossible because of depth and vertical banks }(\sim 1.8 \mathrm{~m}) \text {. Clay, very slippery bottom. No riffles, many bends. }\end{array}$ \\
\hline W-03-065 & $\begin{array}{l}\text { Problems with electrofisher. Used a dipnet to make fish collection. Two culverts. Some bends in reach. Vegetative bank } \\
\text { protection shrubs and grasses. }\end{array}$ \\
\hline W-03-066 & $\begin{array}{l}\text { Large culverts } 3.5 \mathrm{~m} \text { in diameter. Very hilly country. No cropland in immediate area. Natural meanders and bends. Riffles } \\
\text { associated with culverts. }\end{array}$ \\
\hline W-03-068 & Unmapped ponded area. No flow. Marsh habitat. Manicured lawn along PTH \#23. \\
\hline W-03-069 & $\begin{array}{l}\text { Released four White Sucker. (Lengths: } 144 \mathrm{~mm}, 140 \mathrm{~mm}, 135 \mathrm{~mm}, 130 \mathrm{~mm} \text { ) Clam caught. Bridge has steel piles and treated } \\
\text { wood abutments. No riffles, bends only. Grasses and shrubs dominate riparian zone. Beaver dam. }\end{array}$ \\
\hline W-03-070 & $\begin{array}{l}\text { Blind sweep } 10 \mathrm{~m} \text { inside culvert, } 12 \text { shiners caught. This sample separated from electrofishing sample. Downstream end of } \\
\text { culvert perched } 0.3 \mathrm{~m} \text {. A lot of woody debris jammed upstream from culvert. Culverts } \sim 3 \mathrm{~m} \text { diameter, and are rusted. Loose } \\
\text { geotextile. }\end{array}$ \\
\hline W-03-071 & Concrete box culverts and bridge. West of Perimeter Hwy (PTH \#101). \\
\hline W-03-072 & $\begin{array}{l}\text { Electrofishing effort was } 200 \mathrm{sec} \times 100 \mathrm{~m} \text {. No fish caught, saw crayfish and freshwater shrimp. Low flow. Mean width of channel } \\
1.5 \mathrm{~m} \text {. Small pool downstream from bridge, old posts sticking out of pool. A lot of aquatic vegetation in pool downstream from } \\
\text { bridge. A lot of cattails upstream from bridge. }\end{array}$ \\
\hline W-03-073 & Too deep to fish mid channel. Fished edges of emergent vegetation. Crossing PR \#210 at Town of Ste. Anne. \\
\hline W-03-074 & Runs into Seine River. Located north of PR \#405. Little standing water, low flow, some submerged vegetation. \\
\hline W-03-075 & Dry. Streambed overgrown with grasses. \\
\hline W-03-076 & A lot of standing water. Thick cattail debris. \\
\hline W-03-077 & $\begin{array}{l}\text { Electrofishing effort was } 403 \mathrm{sec} \times 200 \mathrm{~m} \text {. No fish caught. Looked like good habitat. Three deep pools in reach. Lots of } \\
\text { submerged vegetation and woody debris. Remnant beaver dam downstream from bridge. Canopy of trees downstream of bridge. }\end{array}$ \\
\hline W-03-078 & $\begin{array}{l}\text { Upstream gated culvert closest to bridge open } 85 \% \text {, upstream gated culvert farthest from bridge open } 15 \% \text {. Downstream: right } \\
\text { bank undercut, some slumping evident, large boulder field. }\end{array}$ \\
\hline W-03-079 & Two large bodied fish observed, but could not catch. Fence crosses west 2nd order, see diagram. Sand bottom. Two bridges, \\
\hline W-03-080 & $\begin{array}{l}\text { Upstream channel lined with vegetation and undercut banks. Downstream from culvert (perched } 0.2 \mathrm{~m} \text { ), fast moving water, } \\
\text { cobble field continues to fence. Right bank has undercut banks. One metre downstream of culvert a broken wooden sign lies in } \\
\text { stream forming plunge pool. }\end{array}$ \\
\hline
\end{tabular}




\section{Appendix 7: List of any additional field notes recorded at sampling sites.}

\begin{tabular}{|c|c|}
\hline $\begin{array}{l}\text { Site } \\
\text { Number }\end{array}$ & Field Notes \\
\hline W-03-081 & lot of cattails and other vegetation upstream from culverts. Low flow, mud bottom. Appears to have been cleaned recently? \\
\hline W-03-082 & ated culvert southeast corner is larger than rest. \\
\hline W-03-083 & ry except for one standing pool $\sim 0.3 \mathrm{~m}$ deep. Sand bars. Exposed mud banks present. \\
\hline W-03-084 & $\begin{array}{l}\text { Fields cultivated to stream bank. Pool has relatively hard, stable bottom. Streambed is mostly covered by submerged vegetation. } \\
\text { Upstream trees and shrubs line banks, while downstream fields run to edge of bank. Uniform depth. Max } 0.6 \mathrm{~m} \text { in pool. No } \\
\text { riffles, three bends in reach. }\end{array}$ \\
\hline W-03-085 & Dry upstream and downstream from bridge, with the exception of one algae covered pool upstream from bridge. \\
\hline W-03-086 & $\begin{array}{l}\text { Two main culverts of D-20 Drain wrapped in geotextile fabric. Water surface completely covered by algae. Downstream only one } \\
\text { small pool free of algae cover. }\end{array}$ \\
\hline W-03-087 & $\begin{array}{l}\text { Blind sweep effort over } 100 \mathrm{~m} \text {. A lot of emergent vegetation (cattails) and submerged vegetation. Majority of catch include } \\
\text { tadpoles and invertebrates. Depth uniform shallow. No riffles, three bends. Three culverts and one gated culvert. Downstream } \\
\text { from culverts }(10 \mathrm{~m}) \text { has areas of minor bank erosion. }\end{array}$ \\
\hline W-03-088 & $\begin{array}{l}\text { Max depth in pools } 1 \mathrm{~m} \text {. Slow moving. Sand bottom. Downstream from culverts is sand plume (deposition below three culverts. } \\
\text { Slow/shallow, slow/deep flow regimes. No riffles. Sand deposits on both banks. Grasses and shrubs along banks. }\end{array}$ \\
\hline W-03-089 & $\begin{array}{l}\text { Carey Drain ( } 3^{\text {rd }} \text { order) which runs perpendicular to PR \#205; } 2 \text { nd order drain joins from the east which runs parallel to PR \#205. } \\
\text { Located } 1 \text { mile west of town of Carey. }\end{array}$ \\
\hline W-03-090 & $\begin{array}{l}\text { Otterburne East Drain (3rd order, downstream) runs perpendicular to PTH \#59, 2nd order (upstream). Unnamed 2nd order runs } \\
\text { perpendicular to Otterburne East Drain (from north). Very little flow. Small riffle less than } 0.2 \mathrm{~m} \text { deep. }\end{array}$ \\
\hline W-03-091 & Very marshy. A lot of aquatic vegetation. \\
\hline W-03-092 & $\begin{array}{l}\text { Upstream: shallow, fast moving, bottom is rock and gravel in riffles. Downstream: stream bottom is mud with gravel and sand } \\
\text { bars. Thalweg meanders through center of channel. }\end{array}$ \\
\hline W-03-093 & $\begin{array}{l}\text { Upstream: right bank is diked, old growth, almost completely overgrown. Riffles free from instream vegetation. A lot of emergent } \\
\text { (cattails, bulrushes) and submerged vegetation. Mainly channelized but good habitat. Riparian excellent. No deep/ fast flow } \\
\text { regime. Box culvert in concrete bridge. Uniform depth } 0.4-0.6 \mathrm{~m} \text {. }\end{array}$ \\
\hline W-03-094 & $\begin{array}{l}\text { Beach seine haul was } 100 \mathrm{~m} \times 8 \mathrm{~m} \text {, downstream from culvert. No fish caught. Not a mapped drain. Circled approximate area on } \\
\text { map. Runs perpendicular to PTH \#44, } 2 \text { miles west of Rennie. A lot of shrubs and native grasses. Submerged vegetation } \\
\text { throughout reach. }\end{array}$ \\
\hline W-03-095 & Railway ties across 2 culverts. No fast/ deep flow regime. Located 2 miles west of Rennie, crossing PTH \#44. \\
\hline W-03-096 & No fish caught. Heavy aquatic vegetation throughout reach. A lot of organic deposition in pools. Cement culver. \\
\hline W-03-097 & $\begin{array}{l}\text { No fish caught. Saw very tiny minnows, could not catch. A lot of overhanging vegetation. Riffles caused by debris jams and } \\
\text { channel constrictions. A lot of rock substrate. No fast/ shallow flow regime. }\end{array}$ \\
\hline W-03-098 & $\begin{array}{l}\text { Debris and debris screen on upstream end of culvert may limit passage of larger fish. Riparian condition excellent. Flow regime } \\
\text { slow/ deep and slow/ shallow. }\end{array}$ \\
\hline W-03-099 & ailway. No apparent flow. $\subseteq$ \\
\hline
\end{tabular}




\section{Appendix 7: List of any additional field notes recorded at sampling sites.}

\begin{tabular}{|c|c|}
\hline $\begin{array}{l}\text { Site } \\
\text { Number }\end{array}$ & Field Notes \\
\hline W-03-100 & $\begin{array}{l}\text { Fishing effort discontinued because of thunderstorm. Upstream lots of cattails and submerged vegetation. Two culverts. No riffle. } \\
\text { Exposed banks. Left bank to road edge }=1 \mathrm{~m} \text {. Located } 1.5 \text { mile south PTH } \# 15 \text {. }\end{array}$ \\
\hline W-03-101 & $\begin{array}{l}\text { Local said that wells were dry prior to recent rains (June 17th). Riffles caused by constricted channel and instream vegetation. } \\
\text { Max. channel width }=0.5 \mathrm{~m} \text {. No fast/deep flow regime. Two culverts, drain is flowing through one only. One deep narrow pool in } \\
\text { reach. }\end{array}$ \\
\hline W-03-102 & 1st order upstream, 2nd order downstream (according to map). \\
\hline W-03-103 & $\begin{array}{l}\text { Culverts } 2 \mathrm{~m} \text { diameter, embedded in streambed. Cannot fish, as the trickle of flow percolating through boulders and rocks. } \\
\text { Stream width less than } 0.3 \mathrm{~m} \text {. Nice habitat, with a lot of overhanging vegetation downstream of culverts. }\end{array}$ \\
\hline W-03-104 & $\begin{array}{l}\text { No fish caught. Upstream of riffle long grasses providing cover. A lot of embedded cobble and rock in pool upstream of culvert. } \\
\text { One culvert. Cobble and rock common throughout reach. }\end{array}$ \\
\hline W-03-105 & Off PR \#406. \\
\hline W-03-106 & Unmapped field drainage. Completely dry. Vegetation choked. \\
\hline W-03-107 & $\begin{array}{l}\text { Concrete box culvert } 5 \mathrm{~m} \text { high. Good vegetative bank protection, trees shrubs and tall grasses. Overhanging vegetation and } \\
\text { trees. Farms either side of stream on upstream side. Moderate flow. PTH \#44 diked } 25 \mathrm{~m} \text { above. Diverse habitat. Some bank } \\
\text { slump downstream on both banks. }\end{array}$ \\
\hline W-03-108 & No flow. Small pools upstream and downstream from culvert. \\
\hline W-03-109 & One mile north of River Hills. \\
\hline W-03-110 & $\begin{array}{l}\text { Downstream both banks eroded with exposed mud banks. Turbulent flows (riffles) caused of woody debris. Deeply embedded } \\
\text { cobbles. Three culverts. two miles east of PTH \#11. Runs under gravel road. }\end{array}$ \\
\hline W-03-111 & Slow/ shallow flow regime. Many bends, no riffles, no pools. Uniform depth. \\
\hline W-03-112 & $\begin{array}{l}\text { Local knowledge: Stream permanent. Floods can be significant even after one day of rain. Large fish don't usually use this } \\
\text { drain. In } 1997 \text { flood drain was } \sim 50 \mathrm{~m} \text { wide. Fast/ shallow and slow/ shallow flow regimes present. Two culverts perched } 0.2 \mathrm{~m} \text {. }\end{array}$ \\
\hline W-03-113 & $\begin{array}{l}\text { Flooded. Recent rains June } 21 \text { and 22. A lot of instream vegetation. Small dike and road provides barrier. One bridge, three } \\
\text { gated culverts. No riffles, channelized. Grass banks. Uniform depth. }\end{array}$ \\
\hline W-03-114 & $\begin{array}{l}\text { No fish caught. Effort discontinued because of heavy instream vegetation (reeds), could not walk through with electrofisher. Local } \\
\text { knowledge: bullheads, Northern Pike, suckers have been caught here. Uniform depth. }\end{array}$ \\
\hline W-03-115 & No flow. Marsh habitat. \\
\hline W-03-116 & $\begin{array}{l}\text { Barbed wire attached to bridge (upstream side). Cows have direct access to river. Downstream side grass overhangs banks } \\
\text { High water due to recent rains June } 21 \text { and } 22 \text {. Can see lots of minnows from bridge. No access to river because farmer has } \\
\text { completely fenced. }\end{array}$ \\
\hline W-03-117 & Marshy, No flow. Downstream a lot of woody debris instream. \\
\hline W-03-118 & $\begin{array}{l}\text { No flow. Marshy. Downstream side no flow, small pools ( } \sim 10 \mathrm{~m} \text { long and } 3 \mathrm{~m} \text { wide). PR \#205 provides dike on north side. } \\
\text { Stream runs parallel to PR \#205. }\end{array}$ \\
\hline
\end{tabular}




\section{Appendix 7: List of any additional field notes recorded at sampling sites.}

\begin{tabular}{|c|c|}
\hline $\begin{array}{l}\text { Site } \\
\text { Number }\end{array}$ & Field Notes \\
\hline W-03-119 & $\begin{array}{l}\text { Slight trickle of flow downstream of culvert. Upstream side instream vegetation completely fills the channel. 2nd order runs into } \\
\text { Tourond Creek. }\end{array}$ \\
\hline W-03-120 & Choked with vegetation. Dry. A lot of cattails. Culvert completely overgrown. No standing water. \\
\hline W-03-121 & Dry. Diked east side (road side). \\
\hline W-03-122 & Dry. 2nd order runs into 3rd order. \\
\hline W-03-123 & Dry. \\
\hline W-03-124 & $\begin{array}{l}\text { Pool } 0.5 \mathrm{~m} \text { across on upstream side of crossing. Downstream looks very overgrown with shrubs, possibly dry for extended } \\
\text { periods of time. Diked on road (east) side. }\end{array}$ \\
\hline W-03-125 & $\begin{array}{l}\text { Many culverts and roads. Culverts go under grass meridian of PTH \#7, emerges on either side, then crosses PTH \#7 running } \\
\text { perpendicular to the } 2 \text { nd mile road from Perimeter (PTH \#101). Pools shallow, filled with instream vegetation. }\end{array}$ \\
\hline W-03-126 & Dry upstream and downstream of crossing. Major hydro lines in this area. Lightly raining. \\
\hline W-03-127 & Dry. Field goes to edge of bank on downstream side. Reach is located at RM of Woodlands and Roc \\
\hline W-03-128 & Dry. \\
\hline W-03-129 & $\begin{array}{l}\text { Upstream: appears new grass seeded, starting to germinate. Downstream: lots of growing grasses, and well established native } \\
\text { grasses. }\end{array}$ \\
\hline W-03-130 & $\begin{array}{l}\text { A lot dense mats of instream vegetation in pools. Pools upstream and downstream of bridge. Dry patches upstream and } \\
\text { downstream } 20 \text { m either direction from pools. 2nd order runs into 5th order (Grassmere Creek). }\end{array}$ \\
\hline W-03-131 & $\begin{array}{l}\text { Downstream side completely filled with instream vegetation (cattails, bulrushes). Dry } 10 \mathrm{~m} \text { upstream and downstream of culverts. } \\
\text { Marshy area upstream and downstream of culverts up to } 10 \mathrm{~m} \text { either direction. }\end{array}$ \\
\hline W-03-132 & $\begin{array}{l}\text { Many culverts and roads. Culverts go under grass meridian of PTH \#7, emerges on either side. Pools shallow, filled with } \\
\text { instream vegetation. Crosses PTH \#7. }\end{array}$ \\
\hline W-03-133 & $\begin{array}{l}\text { A lot of rainfall on June } 25 \text { th is the only reason for little water in drain. Earlier in day on June } 25 \text { th drain was dry, therefore no } \\
\text { point to fish. Isolated pools upstream } \times 3 \text { and downstream. Mud road east side provides dike. }\end{array}$ \\
\hline W-03-134 & $\begin{array}{l}\text { Water level is down } 0.5 \mathrm{~m} \text {. Max water depth } 0.2 \mathrm{~m} \text {. Dense growth of vegetation in stream. Mud road acts like a dike for } 2 \mathrm{nd} \\
\text { order drain. }\end{array}$ \\
\hline W-03-135 & $\begin{array}{l}\text { Gated culverts open. No flow. Dry. Upstream: instream vegetation (cattails). Downstream: a few unconnected pools, most likely } \\
\text { due to recent rains, June 25th. }\end{array}$ \\
\hline W-03-136 & $\begin{array}{l}\text { All culverts are corrugated metal except the second most northern ones, which are concrete. The concrete culverts are more } \\
\text { embedded than the metal ones. The third east-west culvert is very close to the road (Northumberland Rd), which provides a } \\
\text { natural dike. }\end{array}$ \\
\hline W-03-137 & No flow. Dry. Upstream side: small pool $1 \mathrm{~m}^{2}$. Diked on one side (north side road). \\
\hline W-03-138 & Downstream side: emergent vegetation scattered throughout. Only $0.2 \mathrm{~m}$ deep upstream. \\
\hline W-03-139 & $\begin{array}{l}\text { A lot of instream vegetation (cattails). No flow. Deep standing pool under bridge, dry } 10 \mathrm{~m} \text { upstream and downstream of pool } \\
\text { except for several other unconnected standing pools. Crosses PTH \#44, } 0.5 \text { miles west of Lockport. }\end{array}$ \\
\hline
\end{tabular}




\section{Appendix 7: List of any additional field notes recorded at sampling sites.}

\begin{tabular}{|c|c|}
\hline $\begin{array}{l}\text { Site } \\
\text { Number }\end{array}$ & Field Notes \\
\hline W-03-140 & nallow $(0.1 \mathrm{~m})$ standing wat \\
\hline W-03-141 & $\begin{array}{l}\text { Could not electrofish or beach seine because of very dense instream vegetation and filamentous algae. Dip-net sweeps in open } \\
\text { areas. }\end{array}$ \\
\hline W-03-142 & $\begin{array}{l}\text { Junction of Parks Creek with Bruneau Drain and unnamed 2nd order drain. Bruneau Drain has shallow }(0.1 \mathrm{~m}) \text { pools. Unnamed } \\
\text { drain is dry. Grassed banks with a few shrubs. }\end{array}$ \\
\hline W-03-143 & $\begin{array}{l}\text { Electrofishing effort limited by dense instream vegetation (cattails) in channel. Standing pools only with a trickle of flow. Closely } \\
\text { mowed lawn at boulevards and to stream bank on upstream side of crossing. }\end{array}$ \\
\hline W-03-144 & $\begin{array}{l}\text { Upstream of crossing is diked both sides. Downstream: not diked. Flow regime fast/ deep and slow/ deep. Three bridges in view. } \\
\text { Channelized. Banks moderately unstable. }\end{array}$ \\
\hline W-03-145 & Dry. Channel is densely vegetated with cattails. Not diked. \\
\hline W-03-146 & $\begin{array}{l}\text { Water level recently dropped } 0.5 \mathrm{~m} \text {. Downstream from crossing substrate in riffles are quite embedded. No fast/deep flow regime. } \\
\text { two bridges. Deep pools below riffles. }\end{array}$ \\
\hline W-03-147 & $\begin{array}{l}\text { Only a few inches of standing water downstream. A lot of submerged vegetation throughout. Culvert \#1 is dry, surrounded by } \\
\text { mud and grasses. Cultivated fields surrounding drains. Small shallow pools upstream. Boulders and cobble armouring on } \\
\text { upstream ends of culverts. }\end{array}$ \\
\hline W-03-148 & $\begin{array}{l}\text { Little to no flow, one shallow pool downstream culverts. A lot of submerged vegetation. Few standing pools upstream side. } \\
\text { Exposed mud banks upstream and downstream of crossing. 1st order runs into 3rd order then into Whitemouth River } 1 \text { mile west. } \\
\text { Upstream: diked both sides. Downstream: diked road (north) side. }\end{array}$ \\
\hline W-03-149 & $\begin{array}{l}\text { Downstream: shallow, dense vegetation. Upstream: Dry. Overgrown. On northwest side deposition of sand and gravel. Some } \\
\text { areas exposed soil on northwest bank. Diked roadside. }\end{array}$ \\
\hline W-03-150 & $\begin{array}{l}\text { Local knowledge: talked to local drain on Colony land, local's land adjacent to this. He has seen minnows in this drain before. } \\
\text { Channelized. Diked north side. Two culverts. Two riffles (turbulent flow constrictions), no bends in reach. Long grasses and } \\
\text { wildflowers on banks. }\end{array}$ \\
\hline W-03-151 & $\begin{array}{l}\text { Flooded due to recent rains June } 28 \text { and 29. Firm gravel and silt bottom. A lot of instream vegetation. Moderate sediment } \\
\text { deposition on inside bends, small areas of bank scour on outside bends, center of channel rocky with little sediment deposition. }\end{array}$ \\
\hline W-03-152 & $\begin{array}{l}\text { Nice habitat. Observed lots of minnows. Flooding due to recent rains. Lots of submerged vegetation (entire stream). Crosses } \\
\text { PTH \#15 east of the town of Norse. }\end{array}$ \\
\hline W-03-153 & $\begin{array}{l}\text { Excellent habitat and riparian zone. Many minnows observed as well as larger fish. Very flooded due to recent rains (June } 28 \text {, } \\
\text { 29). Crosses PTH \#15. }\end{array}$ \\
\hline W-03-154 & $\begin{array}{l}\text { Effort for fishing - Local minnow catchers gave us samples because we would not have been able to fish this one due to depth ( } \\
2.0 \mathrm{~m} \text { ) and soft bottom). Fishing effort called dip-net sweeps in "minnow trap" in sample \# W-03-154. }\end{array}$ \\
\hline W-03-155 & $\begin{array}{l}\text { Could see a lot of fish (larger and smaller) but unable to catch. Tried bumping up volts, frequency and cycles with little success. } \\
\text { Metal quad crossing on downstream side (north of westbound PTH \#1). Crosses PTH \#1. }\end{array}$ \\
\hline
\end{tabular}




\section{Appendix 7: List of any additional field notes recorded at sampling sites.}

\begin{tabular}{|c|c|}
\hline $\begin{array}{l}\text { Site } \\
\text { Number }\end{array}$ & Field Notes \\
\hline W-03-156 & $\begin{array}{l}\text { Unmapped small standing pool upstream side of crossing. Marshy habitat downstream with dense algae, pondweed, cattails and } \\
\text { other aquatic vegetation. }\end{array}$ \\
\hline W-03-157 & $\begin{array}{l}\text { Unmapped. Pools are very shallow. Small culvert southwest is perched } 0.3 \mathrm{~m} \text {, Large culvert (north-south) perched } 0.1 \mathrm{~m} \text { above } \\
\text { pool. Water levels dropped } 0.5 \mathrm{~m} \text {. Diked roadside upstream, not diked downstream. }\end{array}$ \\
\hline W-03-158 & $\begin{array}{l}\text { Slow/deep and slow/shallow flow regimes present. Channelized, two culverts. No riffles, no bends in reach. Diked west side. } \\
\text { Crosses PR \#507. }\end{array}$ \\
\hline W-03-159 & Water levels recently dropped $0.5 \mathrm{~m}$. \\
\hline W-03-160 & Excellent habitat. Water levels down $0.3 \mathrm{~m}$. Riffles from instream vegetation/woody debris. \\
\hline W-03-161 & $\begin{array}{l}\text { Nice habitat. Little to no alteration. One bridge. Completely surrounded by marsh and flooded land. Water is down } 0.3 \mathrm{~m} \text { from } \\
\text { earlier levels. }\end{array}$ \\
\hline W-03-162 & $\begin{array}{l}\text { Local knowledge: Numbers of fish have significantly declined in the last } 30 \text { yrs. Suckers were prevalent in the past, now cannot } \\
\text { be found due to salt from highway or Chlorine from Winnipeg. Aqueduct. Neighbour used to net suckers in the spring. }\end{array}$ \\
\hline W-03-163 & $\begin{array}{l}\text { Water levels recently dropped } 0.3 \mathrm{~m} \text {. Shallow around bridge, then steep drop off into scour pool. Crosses River Road west of } \\
\text { Braintree, south of PTH \#1. }\end{array}$ \\
\hline W-03-164 & $\begin{array}{l}\text { Cannot access drain because farmer has completely fenced. Flowing slightly. Crosses PTH \#11/44 } 2.5 \text { miles north of } \\
\text { Whitemouth. }\end{array}$ \\
\hline W-03-165 & $\begin{array}{l}\text { Water levels down } 0.3 \mathrm{~m} \text {. No fast/deep flow regime. One cement culvert. Several riffles and bends in reach. Right bank eroded } \\
\text { upstream side of crossing. Adjacent lands are cultivated fields and pasture. }\end{array}$ \\
\hline W-03-166 & Upstream: standing pool with cattails, dry 5 m upstream. Downstream: Mostly dry with marshy pools. \\
\hline W-03-167 & $\begin{array}{l}\text { Strong chemical smell in the drain. Good habitat downstream side (less human impact). Agricultural field } 6 \mathrm{~m} \text { from drain bank } \\
\text { upstream side. A lot of farm activity upstream side. Max pool depth } 0.7 \mathrm{~m} \text {. Slow/ deep flow regime present. Box culvert, }\end{array}$ \\
\hline W-03-168 & Two miles south of Power House, runs into Whitemouth River. \\
\hline W-03-169 & Southeast (small) culvert perched $0.2 \mathrm{~m}$ trickling into small pool. Two shallow pools upstream (max depth $0.2 \mathrm{~m}$ ). Bank erosion. \\
\hline W-03-170 & $\begin{array}{l}\text { Confluence of two } 1 \text { st order tributaries. Crosses PTH \#11 and becomes } 2 \text { nd order. Dry except for standing pool that is } \\
\text { surrounded by instream vegetation and completely fenced off. }\end{array}$ \\
\hline W-03-171 & $\begin{array}{l}\text { Trickle of flow only. Upstream side, small pool } 0.5 \mathrm{~m} \text { wide. Downstream side small pool } 0.5 \mathrm{~m} \text { wide. Parallel to PR \#211 } \\
\text { (upstream) crosses PR \#211 (downstream). }\end{array}$ \\
\hline W-03-172 & $\begin{array}{l}\text { Could not fish because far too deep, vertical drop from banks. Trees provide canopy over stream. Pool upstream is } 0.8 \mathrm{~m} \text { deep } \\
\text { (inaccessible for fishing because of overhanging vegetation and woody debris). Downstream very deep could not seine because } \\
\text { of straight drop off at water's edge and debris. Dominated by slow/deep flow regime. }\end{array}$ \\
\hline W-03-173 & $\begin{array}{l}\text { Downstream side: north culvert is perched } 0.1 \mathrm{~m} \text { but is flowing; south culvert has no flow but is embedded and wetted. Crosses } \\
\text { PTH \#11, runs into Winnipeg. River. }\end{array}$ \\
\hline W-03-174 & Dry. Grasses and shrubs in channel. \\
\hline W-03-175 & grown with grasses. \\
\hline
\end{tabular}




\section{Appendix 7: List of any additional field notes recorded at sampling sites.}

\begin{tabular}{|c|c|}
\hline $\begin{array}{l}\text { Site } \\
\text { Number }\end{array}$ & Field Notes \\
\hline W-03-176 & $\begin{array}{l}\text { Two box culverts. Severe bank erosion and bank slump present along upstream banks. Bottom is primarily soil (due to banks } \\
\text { falling in?). Crosses PTH \#12, } 5.5 \text { miles west of Middlebro. }\end{array}$ \\
\hline W-03-177 & Culverts are grown over. Parallel to PR \#332, 0.5 miles north of Starbuck. \\
\hline W-03-178 & Completely dry and overgrown. 1 mi northwest of Starbuck. \\
\hline W-03-179 & Two miles northwest of Starbuck \\
\hline W-03-180 & Submerged vegetation throughout. Lots of woody debris both submerged and on surface. No flow apparent. \\
\hline W-03-181 & Complex Habitat at the confluence of two tributary drains (2nd order). \\
\hline W-03-182 & No flow, standing pools only. No riffles, three bends in view. Banks are gr \\
\hline W-03-183 & Diked roadside (19W, Dakota School Rd.). \\
\hline W-03-184 & Standing pool under bridge. Dry upstream and downstream from crossing. \\
\hline W-03-185 & $\begin{array}{l}\text { Mainly mud bottom with extensive root mat and submerged vegetation. Standing water with little visible flow. Channelized, five } \\
\text { culverts under PTH \#2, three culverts and bridge under rail line. No riffles, two bends in reach. Some exposed mud banks } \\
\text { although mostly grass covered. }\end{array}$ \\
\hline W-03-186 & $\begin{array}{l}\text { Water quality was done in standing pool. Water levels appear to have dropped } 1.0 \mathrm{~m} \text {. Bank slump and undercuts present along } \\
\text { each bank. Soils exposed on each bank. Standing pools only; no flow. One bridge. Many bends. }\end{array}$ \\
\hline W-03-187 & Dry. A lot of vegetation in the channel. \\
\hline W-03-188 & Slow water movement Maximum depth $0.6 \mathrm{~m}$. Low flow. \\
\hline W-03-189 & $\begin{array}{l}\text { Max depth } 0.7 \mathrm{~m} \text { at center of channel downstream side (between culverts and bridge). Both sides diked upstream, not diked } \\
\text { downstream. Slow/shallow flow regime. Water levels down } 0.6 \mathrm{~m} \text {. Channelized, one bridge (PR \#248), and two large culverts. } \\
\text { No riffles, one bend in reach. }\end{array}$ \\
\hline W-03-190 & Piney West Drain: 3rd order, upstream side has a standing pool above and below the culvert. Dry beyond, no flow. \\
\hline W-03-191 & $\begin{array}{l}\text { One Walleye (approximately. 5") got out of net. Fished downstream side of bridge. Moderate cattle impacts (trails, crossing, } \\
\text { manure. trampled banks) along both banks. Cows cross on riffle. }\end{array}$ \\
\hline W-03-192 & Diked on roadside of drain only. Dry. No flow. \\
\hline W-03-193 & Significant vegetation growth in channel, mostly grasses. Small pool below culvert. \\
\hline W-03-194 & Two salamanders caught, 1 foot in length, released. Simple habitat. Bridge well armoured with cobble and small boulders. \\
\hline W-03-195 & $\begin{array}{l}\text { Problem with dissolved oxygen meter. Some flooding into riparian area. Cobble armouring around bridge. Many bends, no riffles. } \\
\text { Very soft bottom. }\end{array}$ \\
\hline W-03-196 & $\begin{array}{l}\text { Two smaller tributary drains join on the south side of the gravel road and flow through culvert along north side of road. Pool ( } 0.1 \\
\text { m) and no apparent flow. }\end{array}$ \\
\hline W-03-197 & $\begin{array}{l}\text { Turbulent areas created by organic debris, channel constriction. Nice habitat with cover provided by overhanging vegetation, } \\
\text { woody debris, instream vegetation, undercut banks. Some areas of eroded mud banks exposed on both banks. Nice complex } \\
\text { habitat. Crosses PTH 1\#2, southeast of Sprague ( } 1.5 \text { mile). }\end{array}$ \\
\hline
\end{tabular}




\section{Appendix 7: List of any additional field notes recorded at sampling sites.}

\begin{tabular}{|c|c|}
\hline $\begin{array}{l}\text { Site } \\
\text { Number }\end{array}$ & Field Notes \\
\hline W-03-198 & $\begin{array}{l}\text { Slow/ shallow flow regime missing. Bridge crossing. Downstream turbulent areas created by woody debris and vegetation. Deep } \\
(>1.0 \mathrm{~m}) \text { pools. Deeply undercut banks. Firm gravel substrate with significant organic debris. }\end{array}$ \\
\hline W-03-199 & Large culvert, $70 \%$ submerged. Very deep pools with no apparent flow. Slow/deep flow regime. Natural, complex habitat. \\
\hline W-03-200 & Dense grasses throughout channel. Standing, stagnant pools. Oily sheen on surface. \\
\hline W-03-201 & $\begin{array}{l}\text { Water depth recently dropped } 0.3 \mathrm{~m} \text {. Bog/marsh habitat dominates surrounding area. Nice, complex habitat, low to no visible flow, } \\
\text { mostly standing water, dominated by slow/deep flow regime. Grate over culvert. No riffle habitat. Frequent bends. }\end{array}$ \\
\hline W-03-202 & $\begin{array}{l}\text { Culverts perched } 0.1-0.2 \mathrm{~m} \text {. Little flow. Shallow pools (maximum depth } 0.3 \mathrm{~m} \text { ). Nice habitat, complex, but little water. Located } \\
1.5 \text { miles southeast of St. Labre. }\end{array}$ \\
\hline W-03-203 & $\begin{array}{l}\text { Upstream side of crossing has some areas of exposed mud on banks. Frequent sand and gravel bars. Very little flow. Mostly } \\
\text { shallow pools }(0.2 \mathrm{~m}) \text {. Downstream side, exposed mud on both banks. Rocks piled across channel } 10 \mathrm{~m} \text { downstream from culvert } \\
\text { create a shallow pool }(0.2 \mathrm{~m}) \text {. }\end{array}$ \\
\hline W-03-204 & $\begin{array}{l}\text { Fishing effort discontinued because of extremely soft bottom. Submerged vegetation throughout pool. Slow/shallow and } \\
\text { slow/deep flow regimes. Two culverts in crossing. No riffles in reach. }\end{array}$ \\
\hline W-03-205 & $\begin{array}{l}\text { Upstream side of crossing both banks are bare, exposed mud. Marshy. Two well armoured culverts. No riffles or bends within } \\
\text { reach. }\end{array}$ \\
\hline W-03-206 & $\begin{array}{l}\text { Water depth recently dropped } 0.5 \mathrm{~m} \text {. Thalweg meandering within banks. Slightly undercut banks. No riffles, } 1 \text { bend in reach. } \\
\text { Grassed banks. Diked on both banks. }\end{array}$ \\
\hline W-03-207 & Upstream: Dry. Bare mud. Downstream one pool $(8 \mathrm{~m} \times 2 \mathrm{~m})$ at culverts. Dry beyond pool. Diked on both sides. \\
\hline W-03-208 & $\begin{array}{l}\text { Pool under bridge and downstream of bridge ( } 5 \mathrm{~m} \text { wide). Diked on both sides. Unnamed tributary flows west into northbound } \\
\text { Hazelridge drain as it turns and flows west under PTH \#12. }\end{array}$ \\
\hline W-03-209 & Dry. Terrestrial vegetation throughout inactive channel upstream and downstream of crossing. \\
\hline W-03-210 & $\begin{array}{l}\text { Water level down } 0.6 \mathrm{~m} \text {. Both banks have exposed mud areas. Downstream shallow riffle at box culvert. Upstream: max depth } \\
0.2 \mathrm{~m} \text {. Slow/shallow and fast/shallow flow regimes. Channelized. Cement box culvert. Vegetative bank protection is dominated } \\
\text { by grasses. }\end{array}$ \\
\hline W-03-211 & $\begin{array}{l}\text { Twenty-two Northern Pike }(75-150 \mathrm{~mm}) \text { collected. Released } 20 \text {. Water level recently dropped } .3 \mathrm{~m} \text {. Slow/deep and slow/shallow } \\
\text { flow regimes present. Channelized. Two large culverts. No riffles and no bends in reach }\end{array}$ \\
\hline W-03-212 & Dry. Diked on both sides. \\
\hline W-03-213 & Dry. Cattails throughout drain. Diked on both sides. \\
\hline W-03-214 & No flow, One small pool downstream of crossing. Streambed consists of exposed mud. Diked on both sides. \\
\hline W-03-215 & $\begin{array}{l}\text { Water level recently dropped } 0.7 \mathrm{~m} \text {. Soil exposed on both banks. Local knowledge: Common Carp, Northern Pike, suckers and } \\
\text { perch use this creek. Water levels higher than normal - water from southeast corner of Manitoba. }\end{array}$ \\
\hline W-03-216 & No flow, some standing pools. Exposed mud banks. Diked both sides. \\
\hline W-03-217 & PR \#206. \\
\hline
\end{tabular}


Appendix 7: List of any additional field notes recorded at sampling sites.

\begin{tabular}{|c|c|}
\hline $\begin{array}{l}\text { Site } \\
\text { Number }\end{array}$ & Field Notes \\
\hline W-03-218 & $\begin{array}{l}\text { Vegetation completely fills channel. One small algae covered pool downstream of culverts. Dry upstream and downstream from } \\
\text { the pool. Diked both sides. }\end{array}$ \\
\hline W-03-219 & Upstream (north drain) has on shallow standing pool. \\
\hline W-03-220 & Recent rains this morning and for several days previous. \\
\hline W-03-221 & Dry. Three culverts in crossing, two are corrugated metal pipes and one is concrete. Diked roadside. \\
\hline W-03-222 & $\begin{array}{l}\text { Dry. Channelized. Runs into the Icelandic River. Moderately dense vegetation in the dry channel. Diked both sides downstream } \\
\text { of culverts. Diked on roadside upstream of culverts. }\end{array}$ \\
\hline W-03-223 & $\begin{array}{l}\text { Five Northern Pike caught. Recent rains. No flow, with very little standing water. Pool downstream of culvert maximum water } \\
\text { depth of } 0.5 \mathrm{~m} \text {. Exposed dry mud patches. No riffles or bends in reach. }\end{array}$ \\
\hline W-03-224 & $\begin{array}{l}\text { Released } 99 \text { Bullheads. Kept a few in the fish collection. Slow/ shallow and fast/ shallow flow regimes present. } 2 \text { culverts: } 1 \\
\text { metal and } 1 \text { concrete. Channelized and diked both sides. No bends. A few areas of turbulent flow created by vegetation } \\
\text { constricting channel. }\end{array}$ \\
\hline W-03-225 & $\begin{array}{l}\text { Dry. Recent rains. Completely overgrown with vegetation. Diked roadside. Crosses PR \#223 (PR \#226 on map). Runs parallel } \\
\text { with Framnes Road. }\end{array}$ \\
\hline W-03-226 & Dry. Four north-south culverts are concrete, east-west gated culvert is corrugated metal. \\
\hline W-03-227 & Shallow, max depth of $0.3 \mathrm{~m}$. Dense aquatic vegetation. Minimal flow, slow movement of water. \\
\hline W-03-228 & Dry. 3 large diameter culverts. Diked on roadside. \\
\hline W-03-229 & $\begin{array}{l}\text { No catch. Observed } 2 \text { fish, 150mm in length, unable to collect. Water temperature (10C) very cold, perhaps spring fed. Two } \\
\text { parallel drains created to mitigate flood hazard? (DES Map is not accurate } 2 \text { extra 3rd order channels on either side of unnamed } \\
\text { 3rd order are not mapped). }\end{array}$ \\
\hline W-03-230 & Dry. Diked roadside. Junction of two 2nd orders upstream to make 3rd order downstream at junction of PR \#329 and PR \#326. \\
\hline W-03-231 & Dry. Grasses throughout channel. Diked on both sides. \\
\hline W-03-232 & Dry. Exposed soil in upstream bed and on right bank upstream. Diked on roadside. \\
\hline W-03-233 & Dry. Overgrown with grasses. Channelized and diked on both sides. \\
\hline W-03-234 & Dry. \\
\hline W-03-235 & $\begin{array}{l}\text { Vegetation throughout channel. Marshy, little to no flow. Bank scour downstream of box culverts right bank and left bank. Diked } \\
\text { on both sides. }\end{array}$ \\
\hline W-03-236 & Upstream: 1 shallow standing pool. Downstream is dry. Huge metal arch culvert like a bridge. Dikes hayed.. Diked both sides. \\
\hline W-03-237 & $\begin{array}{l}\text { Released } 6 \text { White Sucker ranging ( } 150 \text { to } 200 \mathrm{~mm}) \text {. DO meter problem. Cover provided by overhanging vegetation and } \\
\text { submerged vegetation. Three of four regimes present, fast/ deep missing. Flooded upstream of beaver dam }\end{array}$ \\
\hline W-03-238 & $\begin{array}{l}\text { Released } 3 \text { Rock Bass }(120 \mathrm{~mm}, 20 \mathrm{~mm}, 110 \mathrm{~mm}) \text {. Three of four velocity regimes present, fast/ deep missing. Flooded } \\
\text { backwater pool upstream from bridge. }\end{array}$ \\
\hline W-03-239 & $\begin{array}{l}\text { Released } 4 \text { Northern Pike }(200 \mathrm{~mm}, 170 \mathrm{~mm}, 150 \mathrm{~mm}, 150 \mathrm{~mm}) .{ }^{* *} \text { Local Knowledge: spring fed. Fast/shallow and slow/ deep } \\
\text { regimes present. Channelized upstream side only. Two culverts in crossing. four riffles and two bends in reach. }\end{array}$ \\
\hline
\end{tabular}




\section{Appendix 7: List of any additional field notes recorded at sampling sites.}

\begin{tabular}{|c|c|}
\hline $\begin{array}{l}\text { Site } \\
\text { Number }\end{array}$ & Field Notes \\
\hline W-03-240 & ry. Terrestrial vegetation / shrubs throughout channel. Shurkas Drain enters river at this point. Diked on both sides. \\
\hline W-03-241 & $\begin{array}{l}\text { Shallow pools upstream and downstream of culverts }(0.2 \mathrm{~m} \text { deep). Cobble and boulders throughout channel. Diked roadside (left } \\
\text { bank). }\end{array}$ \\
\hline W-03-242 & $\begin{array}{l}\text { Standing water upstream and downstream, completely covered with duckweed. Perched gated culvert. Manure pile } 5 \mathrm{~m} \text { from } \\
\text { right bank. Little to no water movement. Stagnant, standing water. Runs into Washow Bay Creek. }\end{array}$ \\
\hline W-03-243 & Too deep to fish, major waterway. Runs into Lake Winnipeg. \\
\hline W-03-244 & $\begin{array}{l}\text { Marshy habitat upstream and downstream of crossing. Many bulrushes. Little to no flow. Gated culvert on inflow is closed. } \\
\text { Diked on both sides. Runs into Lake Winnipeg. }\end{array}$ \\
\hline W-03-245 & Diked on both sides. \\
\hline W-03-246 & Dry upstream. Marshy downstream. Diked roadside (left bank). \\
\hline W-03-247 & $\begin{array}{l}\text { Water level recently dropped } 1.0 \mathrm{~m} \text {. Exposed mud banks. Bank slump upstream, both banks and downstream right bank. } \\
\text { Instream vegetation throughout upstream and downstream of bridge. Very thick instream vegetation downstream side. Water } \\
\text { levels too low otherwise banks would be undercut. Many submerged logs downstream. Slow/shallow and slow/deep flow } \\
\text { regimes present. }\end{array}$ \\
\hline W-03-248 & $\begin{array}{l}\text { Water levels recently dropped } 0.6 \mathrm{~m} \text {. Slow/shallow flow regime only. Channelized. Three culverts in crossing. Grasses line } \\
\text { banks. Garbage in the drain. Diked roadside. }\end{array}$ \\
\hline W-03-249 & $\begin{array}{l}\text { Water levels recently dropped about } 1.3 \mathrm{~m} \text {. Little to no visible flow. Slow/shallow flow regime present. Channelized, diked both } \\
\text { sides. Rocky bottom upstream and under the bridge. Vegetative bank protection consists of grasses, shrubs, and trees. Good } \\
\text { riparian conditions. }\end{array}$ \\
\hline W-03-250 & Dry, vegetation throughout channel. Diked both sides. \\
\hline W-03-251 & $\begin{array}{l}\text { Substrate }>75 \% \text { embedded upstream side. Slow/shallow and fast/shallow flow regimes present. One small riffle downstream of } \\
\text { bridge, one culvert under bridge, three gated culverts on inflows. Diked on both sides. Vegetative bank protection consists of } \\
\text { grasses only. Mud dominant substrate in pool. }\end{array}$ \\
\hline W-03-252 & Dry. Very overgrown. Diked roadside. Poplar forest on both banks. \\
\hline W-03-253 & Dry. Lots of cattails in the channel. \\
\hline W-03-254 & $\begin{array}{l}\text { Downstream: pool filled with vegetation. Diked both sides. Upstream: Marshy. Diked roadside. One culvert, one closed gated } \\
\text { culvert. }\end{array}$ \\
\hline W-03-255 & Too deep to fish. Exposed mud banks. \\
\hline W-03-256 & $\begin{array}{l}\text { Two culverts, one gated culvert, and one wooden bridge. Small culvert running north-south is perched } 2 \text { feet above pool and sits } \\
\text { higher than large culvert (which is embedded). Sixteen metre long pool starting upstream going under bridge and ending } \\
\text { downstream. (Downstream pool } 4 \mathrm{~m} \times 0.5 \mathrm{~m} \text { ) Pool depth about } 0.5 \mathrm{~m} \text {. }\end{array}$ \\
\hline W-03-257 & Dry on upstream side of crossing. Dense instream vegetation. Pool downstream culverts $10 \mathrm{~m} \times 12 \mathrm{~m}$. Diked both sides. \\
\hline W-03-258 & with cattails. Diked both sides. \\
\hline
\end{tabular}




\section{Appendix 7: List of any additional field notes recorded at sampling sites.}

\begin{tabular}{|c|c|}
\hline $\begin{array}{l}\text { Site } \\
\text { Number }\end{array}$ & Field Notes \\
\hline W-03-259 & $\begin{array}{l}\text { Too deep to fish. Upstream side of crossing water level is about } 1.5 \mathrm{~m} \text { higher than downstream. Backed up by perched culvert. } \\
\text { No flow. }\end{array}$ \\
\hline W-03-260 & Very soft bottom - not fishable at this point. Dense submerged vegetation mats. No flow is evident. \\
\hline W-03-261 & $\begin{array}{l}\text { No flow, standing shallow pools. Downstream: } 5 \mathrm{~m} \times 8 \mathrm{~m} \text { standing pool. Diked both sides. Crosses Meadowdale Road, } 4.5 \mathrm{miles} \\
\text { south of PR \#515. }\end{array}$ \\
\hline W-03-262 & $\begin{array}{l}\text { Trickle of flow, standing shallow pools. Six culverts, two running east-west for Dewar Drain, four running north-south for ditches. } \\
\text { two on either side of PTH \#8. }\end{array}$ \\
\hline W-03-263 & Dry. Terrestrial grasses throughout channel. Diked both sides upstream, road (east) side downstream. \\
\hline W-03-264 & Dry. Long grasses throughout channel. \\
\hline W-03-265 & Dry. No defined channel upstream of crossing. Diked both sides downstream of crossing. \\
\hline W-03-266 & $\begin{array}{l}\text { No fish caught. Electrofisher kept shutting off because of high conductivity. Strong mineral smell in drain. Three natural springs in } \\
\text { immediate area. Slow/shallow flow regime dominant. One culvert in crossing. Channelized with one bend in reach. Diked on both } \\
\text { sides. }\end{array}$ \\
\hline W-03-267 & $\begin{array}{l}\text { Two Northern Pike released (both } 230 \mathrm{~mm} \text { total length). Three springs in immediate area. Dominated by slow/deep flow regime. } \\
\text { Channelized, and diked both sides. Two human made bends in channel. Vegetative bank protection consists mainly of long } \\
\text { grasses and wild flowers. }\end{array}$ \\
\hline B-04-001 & No flow. Standing pools only. Grassed banks. \\
\hline B-04-002 & Site just north of access road for Perimeter Highway No flow. Grassed banks. \\
\hline B-04-003 & Released one White Sucker $(490 \mathrm{~mm})$ - in rough shape (spent) and one ripe Northern Pike $(520 \mathrm{~mm})$. \\
\hline B-04-004 & Site south on dike from PTH \#67. Very little flow except from the small corrugated metal pipes draining the field. \\
\hline B-04-005 & Both banks mowed. \\
\hline B-04-006 & $\begin{array}{l}\text { No flow, standing pools only. Starting to meander within dikes. Several dead White Sucker and Yellow Perch washed up under } \\
\text { bridge. }\end{array}$ \\
\hline B-04-007 & Moderate growth of cattails in stream. No flow. \\
\hline B-04-008 & $\begin{array}{l}\text { Lots of woody debris blocking corrugated metal pipes at upstream end of crossing and at many points on channel Numerous } \\
\text { beaver dams are present. }\end{array}$ \\
\hline B-04-009 & Dry except for a couple small pools. \\
\hline B-04-010 & Recent cleanout downstream of bridge, no instream vegetation, no vegetative protection in ditches to the north. \\
\hline B-04-011 & $\begin{array}{l}\text { Marshy area, lots of submerged vegetation. Cultivated fields on both sides of the drain. No catch but looks like good Northern } \\
\text { Pike spawning habitat. }\end{array}$ \\
\hline B-04-012 & Estimated 300 to 500 White Sucker spawning on the riffles created by the gradient control structure. \\
\hline B-04-013 & Reach located one mile upstream from a White Sucker spawning riffle. Some White Sucker on site. Natural section of stream. \\
\hline B-04-014 & Large riffle present and spawning suckers are present on the riffle and upstream. \\
\hline B-04-015 & nt control structure. \\
\hline
\end{tabular}




\section{Appendix 7: List of any additional field notes recorded at sampling sites.}

\begin{tabular}{|c|c|}
\hline $\begin{array}{l}\text { Site } \\
\text { Number }\end{array}$ & Field Notes \\
\hline B-04-016 & Large riffle (approximately $150 \mathrm{~m}$ long) with good flow. Spawning White Sucker present. \\
\hline B-04-017 & $\begin{array}{l}\text { Local knowledge: local says stream flows all year. Riffle was constructed by his neighbour. A dam is present about } 1 / 2 \text { mile } \\
\text { downstream to maintain water for potato crop irrigation. Mainly slow/ deep flow and fast/shallow flow regime. Good riparian area } \\
\text { with all age classes of woody vegetation including decadent trees. }\end{array}$ \\
\hline B-04-018 & $\begin{array}{l}\text { No flow, standing pools only. } 1 / 4 \text { mile upstream from confluence with La Salle River. Evidence of very high water in the past. } \\
\text { Cultivated fields surround drain south of bridge. Much healthier riparian area upstream of crossing. Mainly a soft bottom. }\end{array}$ \\
\hline B-04-019 & Two large perched corrugated metal pipes ( $3 \mathrm{~m}$ diameter). Cultivated fields on both sides. Maximum pool depth $0.5 \mathrm{~m}$. \\
\hline B-04-020 & One $3 \mathrm{~m}$ diameter corrugated metal pipe. Slight trickle of flow from recent rain, mostly standing pools. Heavy silt deposition. \\
\hline B-04-021 & All slow/ shallow flow regime. Released a Black Bullhead $(170 \mathrm{~mm})$. \\
\hline B-04-022 & Organic substrate. \\
\hline B-04-023 & No flow, very shallow standing pools only. 3 corrugated metal pipes $(0.3 \mathrm{~m})$ diameter. \\
\hline B-04-024 & No flow. Three corrugated metal pipes armoured with gabion baskets on upstream end of culverts. \\
\hline B-04-025 & $\begin{array}{l}\text { Dry, no flow. Channel has been mowed. 2-0.9 m diameter corrugated metal pipes and one-lower set of } 0.4 \mathrm{~m} \text { diameter corrugated } \\
\text { metal pipes. }\end{array}$ \\
\hline B-04-026 & Released three bullheads $(170 \mathrm{~mm}, 125 \mathrm{~mm}, 125 \mathrm{~mm})$. Evidence that water level was much higher in early spring. \\
\hline B-04-027 & Released 2 Quillback (403mm, 424mm). Past flooding evident and high cut banks on left. Good meander sequences. \\
\hline B-04-028 & $\begin{array}{l}\text { Released } 250 \text { bullheads }(60 \mathrm{~mm} \text { to } 100 \mathrm{~mm}) \text {, one }(520 \mathrm{~mm}) \text { Northern Pike. Three corrugated metal pipes about } 50 \mathrm{~m} \text { long and } 50 \\
\mathrm{~cm} \text { diameter. Local knowledge: Local mentioned that Walleye have been caught downstream in spring and that this Diversion } \\
\text { usually dries up }\end{array}$ \\
\hline B-04-029 & Newly constructed channel. \\
\hline B-04-030 & Mowed to about $6 \mathrm{~m}$ from stream. Lots of instream vegetation. Good flow $\mathrm{cc}$ \\
\hline B-04-031 & Severe cattle impacts on upstream banks. No catch. \\
\hline B-04-032 & Pasture upstream of crossing. \\
\hline B-04-033 & Moderate cattle impacts to ban \\
\hline B-04-034 & No flow, standing pools only. \\
\hline B-04-035 & Almost no flow. Two corrugated metal pipes perched about $1 \mathrm{~m}$. Moderate cattle impacts. Some bank erosion. Good canopy. \\
\hline B-04-036 & No flow, standing pools only. \\
\hline B-04-037 & $\begin{array}{l}\text { Pasture north of crossing. Natural section of stream south of crossing. Trickle of flow. Washed our low level crossing on closed } \\
\text { road. }\end{array}$ \\
\hline B-04-038 & Low flow through small diameter corrugated metal pipes. \\
\hline B-04-039 & $\begin{array}{l}\text { Hundreds of adult White Sucker congregated in pool below water control structure outlet. No fish passage is possible but fish still } \\
\text { trying to go farther upstream. Weir on Pembina River upstream about } 200 \text { m directs flow back through diversion. }\end{array}$ \\
\hline B-04-040 & $\begin{array}{l}\text { No flow. Standing pools only. Two corrugated metal pipes } 1.5 \mathrm{~m} \text { in diameter. Marsh like conditions. Pasture on both sides of the } \\
\text { drain. }\end{array}$ \\
\hline
\end{tabular}


Appendix 7: List of any additional field notes recorded at sampling sites.

\begin{tabular}{|c|c|}
\hline $\begin{array}{l}\text { Site } \\
\text { Number }\end{array}$ & Field Notes \\
\hline B-04-041 & Marsh habitat. No catch. Low flow. \\
\hline B-04-042 & Trickle of flow. Cattle have access to stream downstream of crossing. \\
\hline B-04-043 & Mostly cobble substrate. Very little flow. \\
\hline B-04-044 & $\begin{array}{l}\text { Local knowledge: local says that suckers and Northern Pike used to come up into stream. Cover provided mainly by undercut } \\
\text { banks. }\end{array}$ \\
\hline B-04-045 & $\begin{array}{l}\text { Four corrugated metal pipes ( } 1 \mathrm{~m} \text { diameter) perched } 0.2 \mathrm{~m} \text {. Good riffle habitat upstream and downstream of crossing. Flow } \\
\text { velocities }(\mathrm{m} / \mathrm{s}) \text { through culverts (inlets, outlets): \#1 }(0.62,1.15), \# 2(0.42,1.53), \# 3(0.36,1.41), \# 4(0.36,1.11) \text {. }\end{array}$ \\
\hline B-04-046 & $\begin{array}{l}\text { Released two Creek Chub }(135 \mathrm{~mm}, 170 \mathrm{~mm}) \text {. Culvert perched } 1 \mathrm{~m} \text {. Cattle impacts upstream of crossing, eroded banks } \\
\text { downstream of crossing. }\end{array}$ \\
\hline B-04-047 & $\begin{array}{l}\text { Five, } 2 \mathrm{~m} \text { diameter corrugated metal pipes in crossing. Good riffle pool sequences. Significant scour holes. Minor bank scour. } \\
\text { Diverse habitat. }\end{array}$ \\
\hline B-04-048 & Low flow. Heavy shale deposits. Beaver dam downstream of crossing. \\
\hline B-04-049 & No flow. Moderate cattle impacts are evident upstream of crossing. \\
\hline B-04-050 & No flow, channel is dry. Good riparian conditions. Corrugated metal pipes perched about $0.75 \mathrm{~m}$. \\
\hline B-04-051 & Dry. Recent snow accumulated in drain. \\
\hline B-04-052 & $\begin{array}{l}\text { No flow. Standing pools only. Very narrow riparian zone. Moderate cattle impacts to south and land cultivated to the edge of the } \\
\text { banks. }\end{array}$ \\
\hline B-04-053 & $\begin{array}{l}\text { Fairly natural reach with cover provided by overhanging vegetation. Some cultivated areas immediately adjacent to the riparian } \\
\text { zone. }\end{array}$ \\
\hline B-04-054 & No flow. Large marsh to north. Water Survey control structure on the Birdtail River east of reach. \\
\hline B-04-055 & Riffle habitat covered in a dense mat of filamentous algae. \\
\hline B-04-056 & Trickle of flow. Situated about 1 mile upstream from Birdtail River. \\
\hline B-04-057 & Trickle of flow only. Good, healthy riparian area. \\
\hline B-04-058 & $\begin{array}{l}\text { Many deep pools present. Lots of log jams and debris dams. Many beaver dams in area. One smooth metal pipe (1.3 m } \\
\text { diameter). }\end{array}$ \\
\hline B-04-059 & Corrugated metal pipes perched $0.7 \mathrm{~m}$. Pond leveler installed. Water backed up by beavers to the north. \\
\hline B-04-060 & Shallow stream with lots of shallow riffles. Moderately heavy gravel and shale deposition. \\
\hline B-04-061 & $\begin{array}{l}\text { Corrugated metal pipes perched } 0.75 \mathrm{~m} \text {. Mostly standing water except for plunge pool at the downstream end of the culverts. Lots } \\
\text { of overhanging vegetation. }\end{array}$ \\
\hline B-04-062 & Trickle of flow from recent snow. \\
\hline B-04-063 & Little flow, Mostly standing pools. \\
\hline B-04-064 & Lots of overhanging vegetation and deep pools. Cement box bridge. \\
\hline B-04-065 & Melt water pools from recent snow and rain. No flow. \\
\hline B-04-066 & Mostly standing water. No flow. Channelized. Dense submerged vegetation. \\
\hline
\end{tabular}


Appendix 7: List of any additional field notes recorded at sampling sites.

\begin{tabular}{|c|c|}
\hline $\begin{array}{c}\text { Site } \\
\text { Number }\end{array}$ & Field Notes \\
\hline B-04-067 & Two corrugated metal pipes (1.3 m diameter). \\
\hline B-04-068 & Diked but meandering within dikes. Quite a bit of woody vegetation along banks. \\
\hline B-04-069 & Outflow from pond $9^{\circ} \mathrm{C}$. Larval Northern Pike drifting out of pond. \\
\hline B-04-070 & Site at the point where natural section of stream has been channelized into a ditch. \\
\hline B-04-071 & Standing pools only. No flow. \\
\hline B-04-072 & Newly constructed riffle in mid reach is flooded. Some embeddedness of the riffle in clay deposits. \\
\hline B-04-073 & Suckers trying to move over gradient control structure at Mountain road (some success). \\
\hline B-04-074 & Riffles just east of PTH \#5, downstream of major shale trap. \\
\hline B-04-075 & Site with a number of riffles where provincial Fisheries Branch monitors annual Walleye drift. \\
\hline B-04-076 & Slight trickle of flow. Channelized. \\
\hline B-04-077 & Two corrugated metal pipes. Slight trickle of flow. No catch. \\
\hline B-04-078 & Rock armoured crossing. No adult fish caught. \\
\hline B-04-079 & Limited submerged vegetation and overhanging vegetation. \\
\hline B-04-080 & Two - $1.3 \mathrm{~m}$ corrugated metal pipes. Broken bales block culvert with a school of Fathead Minnows trapped by the bales. \\
\hline B-04-081 & Three - $1.3 \mathrm{~m}$ corrugated metal pipes. No flow with shallow standing pools. Lots of algae and plant biomass in channel. \\
\hline B-04-082 & Three - $1.3 \mathrm{~m}$ corrugated metal pipes. Little flow. Some bank slumping to the east. \\
\hline B-04-083 & Quite a number of adult Northern Pike trapped in pool at base of corrugated metal pipes. \\
\hline B-04-084 & Adult Northern Pike stranded in pool at base of two $-1.3 \mathrm{~m}$ corrugated metal pipes (one with a crushed inlet). \\
\hline B-04-085 & Stranded adult Northern Pike downstream of corrugated metal pipes and hundreds of larval Northern Pike upstream of crossing \\
\hline B-04-086 & Released one White Sucker. Channelized section of drain. \\
\hline B-04-087 & No catch in short drift net set. \\
\hline B-04-088 & Just mosquito larvae and Gammarus caught in drift trap. Some flow after rains, but mostly standing water. \\
\hline B-04-089 & No flow, channel not well defined, one corrugated metal pipes $-1.3 \mathrm{~m}$. \\
\hline B-04-090 & Lots of overhanging vegetation. Enough flow to set drift trap. \\
\hline B-04-091 & Much of stream valley has been burnt. No flow. Mostly standing pools. \\
\hline B-04-092 & Pasture to the south. Extensive riffle/pool sequences. \\
\hline B-04-093 & Cattle erosion and heavy grazing have resulted in severe impacts to riparian vegetation. \\
\hline B-04-094 & Recent heavy rains have created high flow. Some beaver activity in area. \\
\hline B-04-095 & Same site as B-04-092 but fished this time. \\
\hline B-04-096 & Marshy, standing pools only. \\
\hline B-04-097 & No flow, standing pools only. Dense growth of cattails. \\
\hline B-04-098 & No flow, some standing pools upstream. \\
\hline B-04-099 & No defined channel. Marshy with cattails and grasses instream. \\
\hline B-04-100 & ater flowing over well grown brome grass indicates that the drain has been dry through most of spring until rec \\
\hline
\end{tabular}




\section{Appendix 7: List of any additional field notes recorded at sampling sites.}

\begin{tabular}{|c|c|}
\hline $\begin{array}{l}\text { Site } \\
\text { Number }\end{array}$ & Field Notes \\
\hline B-04-101 & $\begin{array}{l}\text { Major flow from recent rains. Local councilor mentioned that Northern Pike have been known to come upstream from Souris } \\
\text { River, but not sure how far upstream they go. }\end{array}$ \\
\hline B-04-102 & Minor cattle impacts. Marsh vegetation and lots of frogs. \\
\hline B-04-103 & Lots of woody debris at the upstream end of the corrugated metal pipes. Only a trickle of flow. \\
\hline B-04-104 & Mostly standing pools with little flow although water levels are still fairly high. \\
\hline B-04-105 & Flowing from recent rains. Likely it was previously dry. \\
\hline B-04-106 & $\begin{array}{l}\text { Flowing from rains, but terrestrial grasses make up only instream vegetation, } 2 \text { corrugated metal pipes }(0.9 \mathrm{~m} \text { and } 0.4 \mathrm{~m}) \text {. Fields } \\
\text { cultivated to drain edge. }\end{array}$ \\
\hline B-04-107 & One 2 m corrugated metal pipe. Dammed upstream. \\
\hline B-04-108 & Deep pool at downstream end of corrugated metal pipes, Lots of cattails. Flooded riparian zone. No catch. \\
\hline B-04-109 & Cattle instream and moderate impacts evident in riparian zone. Released two male Fathead Minnow with breeding tubercles. \\
\hline B-04-110 & Site of a Turtle Mountain CD - Sustainable Resource Project. Two corrugated metal pipes ( $3 \mathrm{~m}$ and $2 \mathrm{~m}$ diameter) both flattened. \\
\hline B-04-111 & Extremely flooded. \\
\hline B-04-112 & Site at confluence with the Souris River. Very deep pool at PTH \#3 crossing. \\
\hline B-04-113 & Local knowledge: local farmer says he used to catch Northern Pike, suckers, and the odd Walleye in the spring. \\
\hline B-04-114 & $\begin{array}{l}\text { Good flow from recent rains. Sign on site indicates "Another Turtle River Watershed Conservation District Sustainable Resource } \\
\text { Project". }\end{array}$ \\
\hline B-04-115 & Minor cattle impacts upstream, moderate cattle impacts downstream High water levels due to recent rains. \\
\hline B-04-116 & Stream is quite deep. \\
\hline B-04-117 & Water levels high with some flooding of riparian areas from recent rains. \\
\hline B-04-118 & Flooded with no defined channel. \\
\hline B-04-119 & Severe cattle impacts on banks and cattle fed $10 \mathrm{~m}$ from stream. Lots of bank slumping along road. \\
\hline B-04-120 & Very deep pool on downstream side of crossing. Cattle access to stream downstream from crossing. \\
\hline B-04-121 & Severe cattle impacts upstream. Rock armouring placed to stabilize banks seems to be contributing to erosion downstream. \\
\hline B-04-122 & High water. Flooded. No riffles evident. \\
\hline B-04-123 & Sand substrate dominates with some gravel and cobble. \\
\hline B-04-124 & Lots of cattails instream and very marshy upstream of crossing. \\
\hline B-04-125 & Noticed a mink swimming upstream. \\
\hline B-04-126 & $\begin{array}{l}\text { Corrugated metal pipes in sections joined by cement. Most of the flow is moving under cement. Released two male and one } \\
\text { female Fathead Minnow. Light cattle impacts on banks. }\end{array}$ \\
\hline B-04-127 & Good flow. Marshy upstream. \\
\hline B-04-128 & Large beaver dam upstream of crossing. Minor cattle and goat impacts downstream. \\
\hline B-04-129 & Lots of flow. Pool downstream of crossing too deep to fish. No catch. \\
\hline B-04-130 & Trickle of flow. Mostly standing pools. Channel is vegetated with cattails, grasses, sedges. \\
\hline
\end{tabular}




\section{Appendix 7: List of any additional field notes recorded at sampling sites.}

\begin{tabular}{|c|c|}
\hline $\begin{array}{l}\text { Site } \\
\text { Number }\end{array}$ & Field Notes \\
\hline B-04-131 & No flow. Lots of standing water. Dense submerged vegetation. \\
\hline B-04-132 & No flow. Two - $1.3 \mathrm{~m}$ corrugated metal pipes. \\
\hline B-04-133 & Few riffles, lots of pools. \\
\hline B-04-134 & Parts of drain choked with cattails. \\
\hline B-04-135 & Moderate cattle impacts. \\
\hline B-04-136 & Lots of riffles and pools. Quite a wide area to try and electrofish. \\
\hline B-04-137 & Corrugated metal pipes inlet set at control height. No flow. \\
\hline B-04-138 & Marsh habitat. One $1.3 \mathrm{~m}$ corrugated metal pipe. Flooded inlets and outlets- no pictures. \\
\hline B-04-139 & Marsh habitat. No flow. \\
\hline B-04-140 & Moderate cattle impacts. Flooded grasses along banks. \\
\hline B-04-141 & Large corrugated metal pipes - Approximately $4 \mathrm{~m}$ diameter. \\
\hline B-04-142 & Standing pools from recent rain. Moderate cattle impacts upstream of crossing. \\
\hline B-04-143 & $\begin{array}{l}\text { Severe cattle impacts upstream of culvert - stream channel widened and banks bare because of cattle trampling banks and } \\
\text { crossing stream }\end{array}$ \\
\hline B-04-144 & Good riffle/run/pool sequences. Bulrush indicate permanent flow/wetted conditions. \\
\hline B-04-145 & Released one $191 \mathrm{~mm}$ Black Bullhead. \\
\hline B-04-146 & Lots of tadpoles. \\
\hline B-04-147 & $\begin{array}{l}\text { Corrugated metal pipes at steep incline at inlet - barrier to fish passage. Approx. 200-300 adult Fathead Minnow downstream of } \\
\text { corrugated metal pipes. }\end{array}$ \\
\hline B-04-148 & Little flow. Mostly standing pools. \\
\hline B-04-149 & $\begin{array}{l}\text { Had to electro-fish the morning of June } 16,04 \text { because of the heavy rain and hail. Released } 26 \text { Black Bullheads from } 70 \mathrm{~mm} \text { to } \\
190 \mathrm{~mm} \text {. Local knowledge: local says they used to catch Northern Pike here a few years ago, also mentioned Northern Pike were } \\
\text { stocked in Crystal Creek near town. }\end{array}$ \\
\hline B-04-150 & Cormorants fishing downstream of crossing Culvert with cement revetment is perched and presents a barrier to fish passage. \\
\hline B-04-151 & No flow over ford crossing, but some water is flowing under/through the shale crossing. Dip netted many Fathead Minnows. \\
\hline B-04-152 & $\begin{array}{l}\text { At location of old ford crossing, road closed because crossing has deteriorated. Turbulent flow over and through crossing is } \\
\text { aerating the pool immediately downstream. Moderate cattle impacts upstream of crossing. No catch. }\end{array}$ \\
\hline B-04-153 & Shale substrate. \\
\hline B-04-154 & Marshy with good flow. \\
\hline B-04-155 & A water survey station located onsite. Weir downstream of bridge prevents fish passage. No catch. \\
\hline B-04-157 & Marsh like habitat. \\
\hline B-04-158 & Lots of water backed up upstream of culvert, downstream of crossing channel is very dry. No flow. Perc \\
\hline B-04-159 & High water levels. \\
\hline B-04-160 & es. Starting to meander within dikes. \\
\hline
\end{tabular}




\section{Appendix 7: List of any additional field notes recorded at sampling sites.}

\begin{tabular}{|c|c|}
\hline $\begin{array}{l}\text { Site } \\
\text { Number }\end{array}$ & Field Notes \\
\hline B-04-161 & Diked on east side. \\
\hline B-04-162 & Water level too high to fish (at bankfull stage). \\
\hline B-04-163 & Hydrometric station on site. Dense submerged vegetation and floating algae mats. \\
\hline B-04-164 & Lots of submerged vegetation. Deep scour pool. Moderate cattle impacts. \\
\hline B-04-167 & $\begin{array}{l}\text { Lots of areas too deep for electro-fishing. Fished the edges. Observed one adult Northern Pike escape beach seine } \\
\text { (approximately } 400 \mathrm{~mm} \text { ). }\end{array}$ \\
\hline B-04-168 & Very marshy. No defined channel. Dense aquatic vegetation. \\
\hline B-04-169 & Lots of vegetation instream. \\
\hline B-04-170 & Mostly standing water. \\
\hline B-04-171 & Marsh habitat. Little to no flow. One small corrugated metal pipe through low level ford crossing. \\
\hline B-04-172 & Completely dry. Grassed banks. Poor riparian width. Most likely an ephemeral channel. \\
\hline B-04-173 & $\begin{array}{l}\text { Didn't fish because dissolved oxygen was very low. Lots of algae and cattails instream on upstream side of corrugated metal } \\
\text { pipes and lots of grasses instream downstream of corrugated metal pipes. }\end{array}$ \\
\hline B-04-174 & $\begin{array}{l}\text { Large (greater than } 1.5 \mathrm{~m} \text { ) culvert surrounded by a cement armouring. Released } 2 \text { Black Bullheads }(150 \mathrm{~mm}, 185 \mathrm{~mm}), \mathrm{missed} 4 \\
\text { Northern Pike (about } 150 \mathrm{~mm}, 300 \mathrm{~mm}, 350 \mathrm{~mm} \text {, and } 650 \mathrm{~mm} \text { ). Velocity entering corrugated metal pipes }=1.56 \mathrm{~m} / \mathrm{s}\end{array}$ \\
\hline B-04-175 & Moderate cattle impacts upstream of bridge. \\
\hline B-04-176 & Too deep to fish. \\
\hline B-04-177 & Standing pools only. Too deep for culvert inlet/outlet photos. \\
\hline B-04-178 & Too deep to fish. \\
\hline B-04-179 & Marsh like habitat. Low flow. Minor cattle impacts. \\
\hline B-04-180 & Too deep to fish. \\
\hline B-04-181 & Lots of shale, but diverse fish community although no indicator species. \\
\hline B-04-182 & Dry with grasses and cattails in channel. \\
\hline B-04-183 & Dry with grasses and cattails in channel. \\
\hline B-04-184 & Channelized. \\
\hline B-04-185 & Good flow. \\
\hline B-04-186 & Released two White Sucker $(210 \mathrm{~mm}, 400 \mathrm{~mm})$ and 4 Smallmouth Bass $(270 \mathrm{~mm}, 310 \mathrm{~mm}, 390 \mathrm{~mm}$, and $420 \mathrm{~mm})$. \\
\hline B-04-187 & Released one Creek Chub. Observed one adult White Sucker. \\
\hline B-04-188 & Some slumping on right bank and some sediment deposition on banks. \\
\hline B-04-189 & Lots of riffles and a few deep pools over reach. Very few adult fish present. \\
\hline B-04-190 & Some flow. Culverts (corrugated metal pipes) perched $30 \mathrm{~cm}$. Very shallow upstream of crossing. \\
\hline B-04-191 & Diverse habitat but very few fish caught. \\
\hline B-04-192 & Standing pool. No flow. \\
\hline B-04-193 & Channelized, No Flow. \\
\hline
\end{tabular}




\section{Appendix 7: List of any additional field notes recorded at sampling sites.}

\begin{tabular}{|c|c|}
\hline $\begin{array}{l}\text { Site } \\
\text { Number }\end{array}$ & Field Notes \\
\hline B-04-194 & Dry with lots of grasses, shrubs, and trees growing in the drain. \\
\hline B-04-195 & No larger fish caught. Beaver dam at upstream end of corrugated metal pipes is currently acting as barrier to fish passage. \\
\hline B-04-196 & Released two White Sucker $(210 \mathrm{~mm}, 180 \mathrm{~mm})$, and one Common Shiner $(130 \mathrm{~mm})$. \\
\hline B-04-197 & Released two White Sucker (180 mm, $200 \mathrm{~mm})$ and one Creek Chub (120 mm). \\
\hline B-04-199 & Ford crossing. Wide riparian zone. Mostly pools with some riffle habitat. \\
\hline B-04-201 & Stream has some flow. Good shading provided by canopy. \\
\hline B-04-202 & No flow, one standing pool upstream. Channel not well defined. \\
\hline B-04-203 & No flow. Beaver dam upstream of crossing. \\
\hline B-04-204 & No flow. Marsh habitat. \\
\hline B-04-206 & Marsh habitat. \\
\hline B-04-207 & No flow. Standing pools. Dense algae growth. \\
\hline B-04-208 & No flow. Canopy. \\
\hline B-04-209 & No flow. Standing pools. Most of the channel in the reach is dry. Good cover provided by canopy. \\
\hline B-04-210 & No flow. Grassed waterway. \\
\hline B-04-211 & Released three Brook Sticklebacks and two Fathead Minnows. \\
\hline B-04-212 & Very high water levels, backed up and flooding willows. \\
\hline B-04-213 & Marsh habitat. \\
\hline B-04-214 & Lots of beaver activity. \\
\hline B-04-215 & Some flow but mostly standing pools. \\
\hline B-04-218 & Lots of beaver activity. Released two Creek Chub $(190 \mathrm{~mm}, 150 \mathrm{~mm})$. \\
\hline B-04-219 & Flowing, but with many beaver dams. \\
\hline B-04-220 & $\begin{array}{l}\text { Released one White Sucker }(180 \mathrm{~mm}) \text { and } 4 \text { Creek Chub }(180 \mathrm{~mm}, 120 \mathrm{~mm}, 90 \mathrm{~mm} \text {, and } 80 \mathrm{~mm}) \text {. Meanders forming upstream of } \\
\text { crossing. }\end{array}$ \\
\hline B-04-221 & Almost complete canopy cover. \\
\hline B-04-222 & Ditch recently had brush cleared out of it. Upstream of corrugated metal pipes has almost complete canopy. \\
\hline B-04-223 & Fish were not reacting very well to electrofisher settings. Tried a range of settings. High conductivity. \\
\hline B-04-224 & Released one Creek Chub $(210 \mathrm{~mm})$. Corrugated metal pipes perched $20 \mathrm{~cm}$. \\
\hline B-04-225 & Cattle impacts very evident downstream. Released one Common Shiner $(140 \mathrm{~mm})$. Woody debris blocking culvert inlet. \\
\hline B-04-226 & Trickle of flow through corrugated metal pipes. Lots of pond lilies. \\
\hline B-04-227 & $\begin{array}{l}\text { Some flow. Dense submerged vegetation. Large beaver dam } 10 \mathrm{~m} \text { downstream of crossing. Lots of YOY Brook Stickleback. } \\
\text { Many tadpoles. }\end{array}$ \\
\hline B-04-228 & $\begin{array}{l}\text { Mostly shallow riffle habitat. Very few pools. Released three Brook Sticklebacks. Missed two larger minnows, possibly Creek } \\
\text { Chub. }\end{array}$ \\
\hline
\end{tabular}




\section{Appendix 7: List of any additional field notes recorded at sampling sites.}

\begin{tabular}{|c|c|}
\hline $\begin{array}{l}\text { Site } \\
\text { Number }\end{array}$ & Field Notes \\
\hline B-04-229 & $\begin{array}{l}\text { No flow. Standing pool at downstream end of corrugated metal pipes. No defined channel downstream. Choked with cattails. } \\
\text { Released four Brook Stickleback. }\end{array}$ \\
\hline B-04-230 & $\begin{array}{l}\text { Local knowledge: local farmer mentioned that Northern Pike and suckers used to be fished upstream of here using trap nets, but } \\
\text { thinks beaver activity downstream is preventing upstream passage in recent years. Three } 1.3 \mathrm{~m} \text { diameter corrugated metal pipes. }\end{array}$ \\
\hline B-04-231 & Low flow. Dense submerged vegetation. \\
\hline B-04-233 & At junction with Jarosz Drain. No flow. Standing pools. Choked with vegetation. \\
\hline B-04-234 & Some flow but both tributaries are choked with vegetation. Would be useful to fish this in the spring. \\
\hline B-04-235 & Dense cattail growth in channel. Standing pools only. \\
\hline B-04-236 & Dry. No flow. \\
\hline B-04-237 & No flow. Marsh habitat. \\
\hline B-04-238 & Meandering between banks. Substrate gravel and cobble with very little sediment deposition. \\
\hline B-04-239 & Standing water only. Marsh habitat. \\
\hline B-04-240 & $\begin{array}{l}\text { Lots of riffles and overhanging willows downstream of corrugated metal pipes. Upstream is pond-like with lots of submerged } \\
\text { vegetation. Local knowledge: local farmer said they see Northern Pike in the stream in the spring. }\end{array}$ \\
\hline B-04-242 & Dry. Standing pool downstream of corrugated metal pipes. Lots of algae. Poor riparian vegetation upstream of crossing. \\
\hline B-04-243 & Standing pools only. Pools very shallow with submerged vegetation. \\
\hline B-04-244 & Riprap (broken cement on banks). Missed netting a couple of large Common Carp. \\
\hline B-04-245 & Upstream side of crossing is choked with vegetation. \\
\hline B-04-246 & No flow. Standing pool at corrugated metal pipes outlets. Drain is choked with cattails. \\
\hline B-04-247 & No flow. Dense vegetation in channel. Reach is in channelized section. \\
\hline B-04-248 & A posted sign states that these waters are closed to fishing between certain dates (not listed). \\
\hline B-04-249 & $\begin{array}{l}\text { Pool beneath corrugated metal pipes; channel choked with cattails downstream of pool and also upstream of corrugated metal } \\
\text { pipes. }\end{array}$ \\
\hline B-04-250 & Drain choked with cattails upstream of crossing and submerged vegetation downstream of crossing. Trickle of flow only. No catch. \\
\hline B-04-251 & $\begin{array}{l}\text { Did not fish. Very low dissolved oxygen. One standing pool at base of corrugated metal pipes. Remainder drain is choked with } \\
\text { vegetation. }\end{array}$ \\
\hline B-04-252 & Dry. Choked with cattails. \\
\hline B-04-254 & Dry. No defined channel. \\
\hline B-04-255 & Standing pools. No flow. Dense mats of algae. \\
\hline B-04-256 & Extremely low dissolved oxygen in the drain therefore we didn't fish. \\
\hline B-04-257 & Released seven Yellow Perch, 66 young of the year Northern Pike, and one Black Bullhead. \\
\hline B-04-258 & Dry with no defined/active channel. \\
\hline B-04-259 & No catch. Dense growth of cattails on the upstream side of the culverts. \\
\hline B-04-260 & No flow, some standing water. \\
\hline
\end{tabular}




\section{Appendix 7: List of any additional field notes recorded at sampling sites.}

\begin{tabular}{|c|c|}
\hline $\begin{array}{l}\text { Site } \\
\text { Number }\end{array}$ & Field Notes \\
\hline B-04-261 & Slight trickle of flow. Drain is choked with vegetation and algae. \\
\hline B-04-262 & Lots of water backed up into drain from lake. Wide channel (approximately $50 \mathrm{~m}$ ) Sign saying "No fishing from bridge". \\
\hline B-04-263 & $\begin{array}{l}\text { Beach seine attempted, but the pool was too wide and deep; Observed several Brook Stickleback and Fathead Minnows. Low } \\
\text { dissolved oxygen. }\end{array}$ \\
\hline B-04-264 & Released 30 Fathead Minnow and eight Brook Stickleback. \\
\hline B-04-265 & Turbid water. Very little flow. Dense algae growth. \\
\hline B-04-266 & Lots of beaver dams in the area. \\
\hline B-04-267 & No flow over dam. Standing pools only. A boat launch is located on the upstream side of the dam. Lots of debris left by fishers. \\
\hline B-04-268 & $\begin{array}{l}\text { Unnamed drain leading to Pasquia River (approximately } 1 / 4 \text { mile north of the river). Excellent riparian area. Cannot see the } \\
\text { culverts due to dense cattail growth. }\end{array}$ \\
\hline B-04-269 & Marsh habitat with dense aquatic vegetation. Too deep to electrofish or seine. \\
\hline B-04-270 & No Catch. Most of channel too deep to fish. Dense submerged vegetation. No flow. Sta \\
\hline B-04-271 & Standing water. No flow. Choked with vegetation. \\
\hline B-04-272 & $\begin{array}{l}\text { Too deep to fish. Saw a Walleye and dip netted some Spottail Shiner at the outlet of the gated corrugated metal pipes of the } \\
\text { control structure; gate closed so no fish passage is possible. Dense submerged vegetation. }\end{array}$ \\
\hline B-04-274 & Dry; mowed channel and roadside bank. \\
\hline B-04-275 & $\begin{array}{l}\text { Deep pool at base of corrugated metal pipes. Channel choked with grasses and emergent vegetation; no water movement; tons } \\
\text { of zooplankton. }\end{array}$ \\
\hline B-04-276 & $\begin{array}{l}\text { No flow through pump station. Gated at both inlet and outlet. Very deep and } 80 \% \text { full of tall submerged vegetation. Released four } \\
\text { Spottail Shiner. }\end{array}$ \\
\hline B-04-277 & Lots of submerged vegetation on both drains; deep (over $1.3 \mathrm{~m}$ ). \\
\hline B-04-278 & Dense growth of cattails and sedges in the channel. \\
\hline B-04-279 & Dry. No flow. \\
\hline B-04-280 & Upstream backed up and very deep, downstream most of channel filled with submerged vegetation. \\
\hline B-04-281 & Gated control structures/ pump station. No fish passage. Lots of submerged vegetation and too deep to fish. \\
\hline B-04-282 & Dry, ditches to south mowed. \\
\hline B-04-283 & Two corrugated metal pipes hidden in grass so no photos of them. \\
\hline B-04-284 & Standing water only. Drain is choked with vegetation. \\
\hline B-04-285 & Deep water - staff gauge reads $1.5 \mathrm{~m}$, lots of submerged vegetation some emergent vegetation. \\
\hline B-04-286 & $\begin{array}{l}\text { Water being pumped from station into Pasquia River. Local knowledge: local water stewardship employee says they see Northern } \\
\text { Pike in drain, surveying and cleanout scheduled for next two days on drain. }\end{array}$ \\
\hline B-04-287 & $\begin{array}{l}\text { Two small Northern Pike could be seen under bridge. Too deep to fish. Lots of emergent vegetation and submerged vegetation } \\
\text { present. }\end{array}$ \\
\hline B-04-288 & ts of emergent vegetation and submerged vegetation. Too deep to fish. \\
\hline
\end{tabular}


Appendix 7: List of any additional field notes recorded at sampling sites.

\begin{tabular}{|c|c|}
\hline $\begin{array}{l}\text { Site } \\
\text { Number }\end{array}$ & Field Notes \\
\hline D-04-001 & Spring flows over Walleye spawning riffle. No fish observed. \\
\hline D-04-002 & Spring flows through raised crossing. No fish observed. \\
\hline D-04-003 & Dry. No flow. \\
\hline D-04-005 & Low flow conditions. \\
\hline D-04-006 & $1.5 \mathrm{~m}$ deep plunge pool below PTH \#5 crossing. \\
\hline D-04-007 & $1.1 \mathrm{~m}$ deep pool below riffle. \\
\hline D-04-008 & $0.6 \mathrm{~m}$ deep pool $50 \mathrm{~m}$ downstream from bridge. \\
\hline D-04-009 & Old WSC hydrometric station. Sheet pile weir still in place blocking fish passage at this flow. \\
\hline D-04-010 & Headwater storage project. \\
\hline D-04-011 & Future headwater storage planned at this site. \\
\hline D-04-012 & Site visit by Manitou Grade 8 Class. \\
\hline D-04-013 & Very high flows in North Snake Creek. Increased flows out of pond. 100's of larval Northern Pike in 30 minute larval drift trap set. \\
\hline D-04-014 & High flows over raised crossing. \\
\hline D-04-015 & 20-hour larval drift trap set -no catch. \\
\hline D-04-016 & Numerous larval Northern Pike and predacious diving beetles in sample. \\
\hline D-04-017 & Nil catch. Perched culvert 4 miles downstream. Site of future water withdrawal. \\
\hline D-04-018 & Breach through old PTH \#16 \\
\hline D-04-019 & Culvert outlet velocity $2 \mathrm{~m} / \mathrm{s}$ \\
\hline D-04-020 & Nil catch in larval drift trap. \\
\hline D-04-021 & Nil catch in larval drift trap. \\
\hline D-04-022 & Cultivated to water's edge. \\
\hline D-04-023 & Minor cattle impacts downstream from bridge crossing. \\
\hline D-04-024 & No flow. Pooled water from recent heavy rains. \\
\hline D-04-025 & Cattle access to stream. Water storage structure (upright corrugated metal pipe) on upstream side of road. \\
\hline D-04-026 & Headwater storage project. \\
\hline D-04-028 & New condominium development on stream bank. Recent works have not re-vegetated. \\
\hline D-04-029 & Good flows after recent rains. Crappie washed out of reservoir upstream. \\
\hline $\mathrm{D}-04-030$ & Slightly perched corrugated metal pipe culvert. Beaver dam in upstream end of culvert. \\
\hline D-04-031 & Old railway trestle bridge piles in stream. Deck of trestle has been removed. \\
\hline D-04-032 & Culvert plugged with woody debris and creek is flowing across gravel road. \\
\hline D-04-033 & Culvert perched above bed level. Good riffle/run/pool sequences. \\
\hline D-04-034 & Good riffle pool sequences. Good mix of habitat. No fish? Blockages downstream ? \\
\hline D-04-035 & $\begin{array}{l}\text { Old railway trestle piles in place, old bridge decking removed, Old, large remnant beaver dams throughout reach. Debris jam on } \\
\text { upstream side of bridge piles. }\end{array}$ \\
\hline
\end{tabular}




\section{Appendix 7: List of any additional field notes recorded at sampling sites.}

\begin{tabular}{|c|c|}
\hline $\begin{array}{l}\text { Site } \\
\text { Number }\end{array}$ & Field Notes \\
\hline D-04-036 & $\begin{array}{l}\text { Natural section stocked with Brown Trout. Released } 20 \text { Western Blacknose Dace, } 11 \text { Creek Chub, } 10 \text { Longnose Dace, } 13 \text { Johnny } \\
\text { Darter. Perched culvert at low flows. }\end{array}$ \\
\hline W-04-001 & $\begin{array}{l}\text { Unnamed 2nd order runs into drain, and is dry. Quad tracks upstream and downstream of bridge and throughout stream. Suckers } \\
\text { spawning upstream and downstream side of bridge crossing. Barbwire fence across drain. Diked both sides. }\end{array}$ \\
\hline W-04-002 & Stream has slight trickle of flow. Diked right bank. \\
\hline W-04-003 & Grasses throughout drain upstream and downstream of crossing. Downstream side has standing pools. No flow. Not diked. \\
\hline W-04-004 & $\begin{array}{l}\text { Garrioch Creek: upstream is 4th order; downstream is 3rd order, Rockland Drain is 3rd order. Suckers spawning. Local } \\
\text { knowledge: Pike used this creek earlier this year, usually creek is dry in summer except after heavy rains. On downstream right } \\
\text { bank sign indicating "Riparian Stewardship Program, Dan and Jen Sherman and Family, Land Water Partnerships" Diked both } \\
\text { banks. }\end{array}$ \\
\hline W-04-005 & $\begin{array}{l}\text { Stranded White Sucker }(n=4) \text { found upstream of bridge crossing, two alive, two dead. Upstream side has a shallow pool }(0.2 \\
\text { m). Very little flow. One small riffle upstream side of bridge. Rock barrier likely limits fish passage at upstream side of box culvert. } \\
\text { Diked both banks. }\end{array}$ \\
\hline W-04-006 & $\begin{array}{l}\text { No fish caught. Consistent depth 0.3-0.6 m. Dominated by slow/ deep flow regime. Upstream diked both banks, downstream } \\
\text { diked left bank only. }\end{array}$ \\
\hline W-04-007 & $\begin{array}{l}\text { White Sucker spawning on site. Cattle Impacts - cows have free access to drain. Submerged and emergent vegetation } \\
\text { throughout reach. Crossing has culvert armoured with cement filled bags on geotextile. Good flow. Diked both banks. }\end{array}$ \\
\hline W-04-008 & Inflow with gated culvert (open) on upstream side right bank. Dry. Dense growth of vegetation in channel. Diked right bank. \\
\hline W-04-009 & $\begin{array}{l}\text { No fish caught. Water depth } 0.3 \mathrm{~m} \text {, some submerged vegetation on bottom of stream, emergent vegetation along water's edge, } \\
\text { predominantly long grasses. Flow regime slow/ shallow. Channelized, no riffles, no bends. Riparian vegetative protection mainly } \\
\text { grasses. Diked both banks. }\end{array}$ \\
\hline W-04-010 & Trickle of flow. Submerged and emergent vegetation throughout channel Lots of litter in drain. Diked both banks. \\
\hline W-04-011 & Diked roadside. \\
\hline W-04-012 & No flow. Diked south bank. \\
\hline W-04-013 & No fish caught. Channelized, a few deep pools, no riffle habitat. Infrequent bends upstream of crossing. Diked south side. \\
\hline W-04-014 & $\begin{array}{l}\text { Diked on one side (road). Pool downstream } \sim 0.2 \mathrm{~m} \text { deep. Dry upstream. Dense mat of dead vegetation clogging culverts. Grassed } \\
\text { banks. Diked south bank. }\end{array}$ \\
\hline W-04-015 & Diked both banks. \\
\hline W-04-016 & Diked roadside. \\
\hline W-04-017 & Grassed channel. Downstream side of crossing is dry below standing pool extending $10 \mathrm{~m}$ downstream. Diked south bank. \\
\hline W-04-018 & Diked on one side (road). Simple habitat upstream and downstream. \\
\hline W-04-019 & Not diked. Complex habitat upstream and downstream. \\
\hline W-04-020 & Not diked. Complex habitat upstream and downstream? No flo \\
\hline W-04-021 & ts of dry cattails throughout channel. Predominantly marshland with unconnected standing po \\
\hline
\end{tabular}


Appendix 7: List of any additional field notes recorded at sampling sites.

\begin{tabular}{|c|c|}
\hline $\begin{array}{l}\text { Site } \\
\text { Number }\end{array}$ & Field Notes \\
\hline W-04-022 & $\begin{array}{l}\text { Major crossing road work, recently completed, road improvements, new culverts, geotextile fabric with straw and grass fibers } \\
\text { protecting exposed banks and extending into the wetted channel. Diked road side }\end{array}$ \\
\hline W-04-023 & Marsh habitat upstream and downstream of crossing. Not diked. \\
\hline W-04-024 & Not diked. \\
\hline W-04-025 & Gated culvert closed on downstream side. Not diked. \\
\hline W-04-026 & Culverts gated on downstream side, all three culverts closed. Not diked. \\
\hline W-04-027 & $\begin{array}{l}\text { No fish caught. Many patches of exposed soil on banks. Road improvements underway. Substrate mainly cobble and silt. } 1 \text { large } \\
(10 \mathrm{~m} \times 20 \mathrm{~m}) \text { deep pool on downstream side of crossing. }\end{array}$ \\
\hline W-04-028 & Cattle impacts - cows have access to channel upstream and downstream. Not diked. \\
\hline W-04-029 & $\begin{array}{l}\text { Did not fish due to strong sewage smell and low dissolved oxygen. Riprap bottom prevalent, Geotextile upstream and } \\
\text { downstream. Road resurfacing along PR \#227. Dominated by one velocity regime- slow/deep. Diked both banks. }\end{array}$ \\
\hline W-04-030 & Not diked. \\
\hline W-04-031 & Diked south bank. \\
\hline W-04-032 & $\begin{array}{l}\text { No fish caught, but three Northern Pike observed on downstream side of bridge crossing. Slow/ shallow and slow/ deep velocity } \\
\text { regimes present. Cement box culvert. Numerous bends present, Upstream right bank riparian zone closely mowed to water's } \\
\text { edge from bridge to } 200 \text { upstream. Marshy habitat with mostly shallow pools. One deep pool in reach. Not diked. }\end{array}$ \\
\hline W-04-033 & $\begin{array}{l}\text { Dry. Cultivated field to edge of drain and mud road. Channel has been cultivated upstream and downstream of crossing. Not } \\
\text { diked. }\end{array}$ \\
\hline W-04-034 & Not diked. \\
\hline W-04-035 & No flow. Dry upstream. One small shallow pool (depth $=0.15 \mathrm{~m}$ ) downstream of crossing. Not diked. \\
\hline W-04-036 & No fish caught. Slow/ shallow flow regime. No flow. One culvert, no riffles, no real bends. Not diked. \\
\hline W-04-037 & Slight trickle of flow. Not diked. \\
\hline W-04-038 & Not diked. \\
\hline W-04-039 & $\begin{array}{l}\text { Released two White Sucker }(430 \mathrm{~mm}, 390 \mathrm{~mm} \text { FL) and Northern Pike }(400 \mathrm{~mm}) \text {. Dense aquatic vegetation. Standing water, little } \\
\text { to no flow. Channelized with no riffles or bends. Cultivated field at edge of drain. Grassed banks. Slow/ shallow flow regime } \\
\text { present. Lots of submerged vegetation covering bottom of channel except in deeper pools. Two culverts at access road, three at } \\
\text { PTH \#6. Not diked. }\end{array}$ \\
\hline W-04-040 & $\begin{array}{l}\text { Seven White Sucker released ( } 286 \mathrm{~mm}-407 \mathrm{~mm} \text { FL). Very slow flow velocities and very shallow although water fills }>75 \% \text { of } \\
\text { available channel. Two culverts in crossing. Not diked. Very slight bends throughout (meandering). Grasses and a few shrubs line } \\
\text { the banks. Pasture at edge of bank. Channel fenced off on both upstream and downstream sides. }\end{array}$ \\
\hline W-04-041 & No fish caught. Submerged and emergent vegetation throughout. Maximum pool depth $0.6 \mathrm{~m}$. Not diked. \\
\hline W-04-042 & Diked both sides. \\
\hline W-04-043 & $\begin{array}{l}\text { Seven White Sucker released ( } 355 \mathrm{~mm}-395 \mathrm{~mm} \text { FL). Slow/ shallow flow regime present. One culvert, channelized. Field } \\
\text { cultivated to edge of channel. Uniform water depth } \sim 0.6 \mathrm{~m} \text { max. Diked roadside (north). }\end{array}$ \\
\hline
\end{tabular}


Appendix 7: List of any additional field notes recorded at sampling sites.

\begin{tabular}{|c|c|}
\hline $\begin{array}{l}\text { Site } \\
\text { Number }\end{array}$ & Field Notes \\
\hline W-04-044 & $\begin{array}{l}\text { Three suckers observed but unable to collect. Local knowledge: suckers use this drain. Lots of emergent and submerged } \\
\text { vegetation along banks. Not diked. Slow/ shallow flow regime present. Bridge crossing, channelized. Three bends in reach. }\end{array}$ \\
\hline W-04-045 & Diked north bank, roadside. One culvert at PR \#513. Small pool upstream, marshy downstream. \\
\hline W-04-046 & Diked roadside, east. \\
\hline W-04-047 & Small standing pools on either side of culvert. Dry upstream and downstream of crossing. Diked both sides. \\
\hline W-04-048 & Diked both sides downstream, roadside upstream. Submerged and emergent vegetation throughout channel. \\
\hline W-04-049 & Diked both sides. $>25 \%$ of channel substrate is exposed. Four culverts under PR \#328. Riffle-run-pool sequenc \\
\hline W-04-050 & ked roadside, east. Culverts downstream side are perched $0.2 \mathrm{~m}$. Three culverts in crossing. \\
\hline W-04-051 & One White Sucker released, others observed. Bridge crossing with good flow. Not diked. \\
\hline W-04-052 & $\begin{array}{l}\text { Local knowledge: many beaver dams, thought we wouldn't catch anything because fish couldn't get past beaver dam. In past, } \\
\text { when water is high fish passed but got stuck behind dam in fall- "thousands of fish" couldn't get back to lake. Not diked. Five } \\
\text { bends in sight. Pockets of submerged vegetation throughout reach. Substrate dominated by sand/gravel/cobble. }\end{array}$ \\
\hline W-04-053 & $\begin{array}{l}\text { Flooded - due to recent snow (May 11) and rain (May 15). Not diked. No fish caught. Bridge with box culvert. Channelized. One } \\
\text { bend upstream of crossing and } 2 \text { riffles downstream of crossing. Right bank: upstream side mud banks, downstream, some } \\
\text { exposed mud. Vegetative bank protection consists of grasses. }\end{array}$ \\
\hline W-04-054 & $\begin{array}{l}\text { Flooded. Three suckers caught }(190-400 \mathrm{~mm} \text {, Fork length). Not diked. } 1 \text { bridge, channelized in the past. Four bends in sight. } \\
\text { Horse pasture upstream left bank (East). Gravel deposit downstream of bridge. Patchy vegetation on banks. Trickle of inflow from } \\
\text { east and west tributaries downstream of bridge crossing. }\end{array}$ \\
\hline W-04-055 & ach. Channelized. 3 culverts. Grass banks \\
\hline W-04-056 & fish caught. Channelized, two culverts. Roadside cultivated field to edge of water. Diked on I \\
\hline W-04-057 & $\begin{array}{l}\text { Dense vegetation throughout channel (long grasses). Flooded because of recent snows and rains ( } 30 \mathrm{~mm} \text { of rain). Flow regime- } \\
\text { no fast/ deep. Channelized, two culverts. Not diked. No riparian vegetation on upstream side, cultivated field to edge. }\end{array}$ \\
\hline W-04-058 & $\begin{array}{l}\text { No fish caught. Substrate - immediately downstream and upstream of crossing is cobble with submerged and emergent } \\
\text { vegetation. The rest of the channel has a heavy deposit of sediment. Flow regime - slow/ shallow and fast/shallow. Water fills } \\
25 \% \text { of available channel. Not diked. Upstream - channelized, downstream - many bends. Two culvert crossings in sight. No real } \\
\text { riffles. Yard and fields to water's edge. }\end{array}$ \\
\hline W-04-059 & $\begin{array}{l}\text { No fish caught. Recent rains May 19/04, 30 mm. Channelized, one culvert. Diked both sides. Riffles upstream and downstream } \\
\text { of culvert for } 10 \mathrm{~m} \text { each side. Grassed banks. Little aquatic vegetation. }\end{array}$ \\
\hline W-04-060 & $\begin{array}{l}\text { Suckers caught. Flooded due to recent rains } 30 \mathrm{~mm} \text {. Local knowledge: two years ago flooded like present, foot long Northern } \\
\text { Pike were using drain on upstream side; drain dries in summer. Emergent and submerged vegetation throughout, good flow. No } \\
\text { fast/ deep flow regime. Banks flooded. Channelized in past, one bridge, not diked. Six bends in view, riffles at channel } \\
\text { constrictions. Some riprap used to stabilize left bank on downstream side, } 15 \mathrm{~m} \text { downstream of bridge. }\end{array}$ \\
\hline
\end{tabular}




\section{Appendix 7: List of any additional field notes recorded at sampling sites.}

\begin{tabular}{|c|c|}
\hline $\begin{array}{l}\text { Site } \\
\text { Number }\end{array}$ & Field Notes \\
\hline W-04-061 & $\begin{array}{l}\text { Very flooded. Local knowledge: RCMP Doug (dive team with Experimental Lakes Area) - says stream never dries up; upstream } \\
1.6 \mathrm{~km} \text { is a large marsh area, lots of water, beaver dams; water levels always high and flooded at this time of year; } 7 \text { years ago } \\
\text { drain was dredged. Diked one side right bank (north). }\end{array}$ \\
\hline W-04-062 & $\begin{array}{l}\text { Very flooded, deep and fast water. Water levels at top of culverts } \sim 2 \mathrm{~m} \text { deep. Recent rains May 19, } 30 \mathrm{~mm} \text {. Upstream-diked } \\
\text { both sides. Downstream-diked roadside (north). }\end{array}$ \\
\hline W-04-063 & $\begin{array}{l}\text { No fish caught. Channelized, two culverts, diked both sides. One riffle, no bends. Vegetative bank protection - grasses. Riparian - } \\
\text { road on North side, fields on South sides. Gated culverts on inflows are closed. }\end{array}$ \\
\hline W-04-064 & Upstream - not diked. Downstream - diked both banks. \\
\hline W-04-065 & Diked both sides. \\
\hline W-04-066 & Diked road (west) side. \\
\hline W-04-067 & Diked both sides. \\
\hline W-04-068 & Diked both sides. Cattle access to drain. \\
\hline W-04-069 & $\begin{array}{l}\text { No fish caught. Very little water in channel. Channelized, one culvert, diked both sides. One riffle in corrugated metal culvert. } \\
\text { Stream substrate - mud and firm clay. No pools. Mud banks exposed, recently dredged? Probably dry before recent rains. }\end{array}$ \\
\hline W-04-070 & $\begin{array}{l}\text { No fish caught. Submerged vegetation throughout drain. Channel diked both sides. Three culverts. Upstream and downstream of } \\
\text { culverts are shallow pools. }\end{array}$ \\
\hline W-04-071 & $\begin{array}{l}\text { No fish caught. Electrofisher not working properly-kept cutting out and could not fish deep pool downstream of culverts. } \\
\text { Channelized, two culverts. Diked both sides. No riffles, three bends in reach. Very steep banks on roadside (right bank) have } \\
\text { areas of bare sand exposed, with potential for erosion. Left bank vegetation protection consists of grasses. Upstream side of } \\
\text { culverts banks are covered with straw. }\end{array}$ \\
\hline W-04-072 & $\begin{array}{l}\text { Diked both banks and channelized, one bridge. Right bank slump } 50 \mathrm{~m} \text { downstream from bridge. Vegetative bank protection - } \\
\text { short grasses. Rock dam at edge of Seine River in mouth of unnamed 2nd order - appears to block fish passage. Flow trickling } \\
\text { through rocks. }\end{array}$ \\
\hline W-04-073 & Diked road (west) side. Water depth $0.2 \mathrm{~m}$, little to no flow. Water level appears to have receded recently. \\
\hline W-04-074 & $\begin{array}{l}\text { Channelized, diked both sides, one bridge. Submerged vegetation mid channel and edges lined with emergent vegetation. } \\
\text { Gravel/ cobble substrate predominate. Perched, closed gated culverts - still draining water from adjacent area. }\end{array}$ \\
\hline W-04-075 & Diked both sides. \\
\hline W-04-076 & Channelized, box culvert (cement), diked both banks. Vegetative bank protection - grasses. \\
\hline W-04-077 & $\begin{array}{l}\text { No fish caught electrofishing. Larval drift trap set for } 30 \text { minutes- caught one central Mudminnow. Only one riffle downstream of } \\
\text { culverts. Three culverts, Channelized and diked both banks. Vegetative bank protection - short grasses prevalent. }\end{array}$ \\
\hline W-04-078 & $\begin{array}{l}\text { One riffle downstream of culverts. Three culverts. Channelized - extensive on upstream side, less on downstream side of } \\
\text { crossing. Diked both banks upstream side and diked south bank only on downstream side. Bank stability poor on downstream } \\
\text { side. Vegetative bank protection - grasses. Horses have free access to drain upstream side of crossing. }\end{array}$ \\
\hline W-04-079 & Dense instream vegetation throughout reach. Diked both sides. \\
\hline
\end{tabular}




\section{Appendix 7: List of any additional field notes recorded at sampling sites.}

\begin{tabular}{|c|c|}
\hline $\begin{array}{l}\text { Site } \\
\text { Number }\end{array}$ & Field Notes \\
\hline W-04-080 & d order tributary runs north into Ross Creek and 2nd order tributary runs south into Ross Creek. Diked both sides. \\
\hline W-04-081 & eaver dam (height $1 \mathrm{~m}$ ). Diked both sides. \\
\hline W-04-082 & $\begin{array}{l}\text { No fish caught. Larval drift trap set for } 30 \mathrm{~min} \text {. Channelized and diked both banks. Four culverts. Vegetative bank protection } \\
\text { grasses. No pools present in reach. }\end{array}$ \\
\hline W-04-083 & $\begin{array}{l}\text { Local knowledge - some Northern Pike used drain in past. Suckers also use drain, but due to beaver dams upstream fish } \\
\text { passage is blocked. Banks flooded. One culvert, Not diked. Riffles all backwatered by flooding. Vegetative bank protection - } \\
\text { grasses prevalent. Deep pools common. }\end{array}$ \\
\hline W-04-084 & $\begin{array}{l}\text { Lots of submerged vegetation throughout channel. Eight inflows with gated culverts. Bridge crossing. Diked both banks and lots of } \\
\text { riprap used to stabilize banks. Water levels down } 0.3 \mathrm{~m} \text {. Uniform depth } 0.3 \mathrm{~m} \text { maximum in thalweg. Vegetative bank protection - } \\
\text { grasses prevalent. }\end{array}$ \\
\hline W-04-085 & $\begin{array}{l}\text { Larval Northern Pike caught. No apparent flow. Flooded above banks } \sim 0.6 \mathrm{~m} \text { upstream side is channelized and diked both } \\
\text { banks. Flat water, two bends in reach. Grassed banks. Uniform water depth } \sim 1 \mathrm{~m} \text {. }\end{array}$ \\
\hline W-04-086 & Not diked. \\
\hline W-04-087 & $\begin{array}{l}\text { Larval drift trap effort = } 15 \mathrm{~min} \text {. Larval Northern Pike caught. Didn't need to set larval drift trap for longer than } 15 \text { minutes because } \\
\text { lots of larval Northern Pike seen in pool downstream of culvert. } 1 \text { culvert. Channelized and diked both sides. No riffles, no bends. } \\
\text { Vegetative bank protection - grasses. Banks } 5 \mathrm{~m} \text { high. Larval Northern Pike caught in pool - depth } 0.45 \mathrm{~m} \text { upstream side very little } \\
\text { water except for shallow pools. }\end{array}$ \\
\hline W-04-088 & $\begin{array}{l}\text { Larval drift trap effort }=30 \mathrm{~min} \text {. Larval Northern Pike caught. Water depth down } 0.3 \mathrm{~m} \text {. Channelized, two culverts, diked both } \\
\text { sides. Maximum water depth } 0.2 \mathrm{~m} \text {. }\end{array}$ \\
\hline W-04-089 & $\begin{array}{l}\text { Fish passage blocked by flattened culvert and sand deposition. Submerged vegetation throughout; grasses. Recent rains May 29- } \\
31(\sim 50 \mathrm{~mm}) \text {. Not diked. }\end{array}$ \\
\hline W-04-090 & $\begin{array}{l}\text { Larval drift trap effort = } 30 \mathrm{~min} \text {. Fast flow, riffle at downstream end of culvert. Recent rains May } 29-31.50 \mathrm{~mm} \text {. Local knowledge: } \\
\text { drain dredged } 2 \text { years ago and flow is usually higher. Overhanging vegetation, woody debris and some submerged vegetation for } \\
\text { available cover. Flow regime: little slow/shallow at edge of drain, flooded surrounding areas. Channelized, diked west bank, one } \\
\text { culvert. Eroded downstream side of culvert on right bank for } 5 \mathrm{~m} \text {. Flow velocity: culvert inlet: } 1.59 \mathrm{~m} / \mathrm{s} \text {; culvert outlet } 2.25 \mathrm{~m} / \mathrm{s}\end{array}$ \\
\hline W-04-091 & $\begin{array}{l}\text { Local knowledge: Drain flooded three times this year to extent of flowing over road. Severely flooded. } 30 \mathrm{~m} \text { of Balcaen Road is } \\
\text { under water. Could not take inlet or outlet pictures submerged culverts. Too deep to electrofish. Diked both banks. }\end{array}$ \\
\hline W-04-092 & Flooded banks more than $0.6 \mathrm{~m}$. Channelized. Diked left bank (north). No riffles, one bend in reach. \\
\hline W-04-093 & $\begin{array}{l}\text { Submerged vegetation throughout drain. Banks flooded. Channelized, two culverts, diked road (north) side. Vegetative bank } \\
\text { protection mainly grasses. }\end{array}$ \\
\hline W-04-094 & $\begin{array}{l}\text { Submerged vegetation and overhanging vegetation through drain on upstream side of culvert starting } 10 \mathrm{~m} \text { upstream of culvert. } \\
\text { downstream deep and un-wadeable. Drain is flooded } 1 \mathrm{~m} \text { above banks, banks soft. Bottom has very little sediment deposition. } \\
\text { One large culvert. Diked downstream left bank (north), and upstream right bank (south). two riffles and two bends in reach. Deep } \\
\text { centre channel } 1.5 \mathrm{~m} \text {. }\end{array}$ \\
\hline
\end{tabular}




\section{Appendix 7: List of any additional field notes recorded at sampling sites.}

\begin{tabular}{|c|c|}
\hline $\begin{array}{l}\text { Site } \\
\text { Number }\end{array}$ & Field Notes \\
\hline W-04-095 & $\begin{array}{l}\text { Lots of woody debris, little submerged vegetation and emergent vegetation. Two culverts, riprap for stability. Not diked, many } \\
\text { bends. Water depth shallow } \sim 0.3 \mathrm{~m} \text {. }\end{array}$ \\
\hline W-04-096 & Pool downstream side of culvert depth $\sim 0.5 \mathrm{~m}$. Area flooded because of recent rains, not diked. \\
\hline W-04-097 & Banks flooded. Two culverts, not diked. No riffles or bends in view. Centre channel $\sim 1.5 \mathrm{~m}$ deep. \\
\hline W-04-098 & $\begin{array}{l}\text { Submerged vegetation throughout. Very little water on upstream side and standing pool on downstream side. Poorly defined } \\
\text { channel. Pool } \sim 0.3 \mathrm{~m} \text { deep. }\end{array}$ \\
\hline W-04-099 & Deep and very flooded. Surface of water $0.3 \mathrm{~m}$ below bridge. Trees in water, banks flooded. \\
\hline W-04-100 & Water level $1 \mathrm{~m}$ from bottom of bridge. Area severely flooded. \\
\hline W-04-101 & Slight trickle of flow. Not diked. \\
\hline W-04-102 & $\begin{array}{l}\text { Drain bottom - gravel, sand and vegetation. Diked downstream side both banks. Many bends. No riffles. Vegetative bank } \\
\text { protection - downstream grasses, upstream grasses and shrubs. }\end{array}$ \\
\hline W-04-103 & Not diked. \\
\hline W-04-104 & Diked left bank (north). \\
\hline W-04-105 & $\begin{array}{l}\text { One culvert, channelized, diked roadside (west) bank. No riffles, no bends. Vegetative bank protection left bank - grasses, trees } \\
\text { shrubs, right bank - grasses. }\end{array}$ \\
\hline W-04-106 & Channelized, diked west bank (road side). Two culverts. Vegetative bank protection - predominantly grasses. \\
\hline W-04-107 & $\begin{array}{l}\text { Flooded. Cannot tell if diked on right bank on downstream side. Riffles caused by submerged trees and shrubs constricting } \\
\text { channel. }\end{array}$ \\
\hline W-04-108 & Not diked. \\
\hline W-04-109 & $\begin{array}{l}\text { No undercut banks, snags or submerged logs. Primarily cobble and gravel on downstream side of culverts and heavy instream } \\
\text { vegetation upstream side of culverts. Water level has dropped a few inches. Three culverts, diked roadside. } 50 \mathrm{~m} \text { of riffles } \\
\text { downstream of culverts, five bends in sight. Vegetative bank protection - primarily grasses. }\end{array}$ \\
\hline W-04-110 & $\begin{array}{l}\text { Rocks present } 5 \mathrm{~m} \text { upstream and downstream of bridge. Cover provided by a few places where banks are undercut. Some } \\
\text { submerged vegetation and instream vegetation. Water depth in center channel is } 1.5 \mathrm{~m} \text { deep, with gravel bottom downstream of } \\
\text { bridge. Turbulent flow at channel constrictions. No snags, no submerged logs. Flooded. Channelized, diked left bank (north). } \\
\text { Bridge. Grasses prevalent. }\end{array}$ \\
\hline W-04-111 & $\begin{array}{l}\text { One culvert, diked right bank (south) on upstream side and both banks downstream of crossing. Two bends, two riffles in reach. } \\
\text { Grassed banks. Field cultivated to edge of drain on upstream side. Clay bottom dominant. }\end{array}$ \\
\hline W-04-112 & Flooded. Unable to locate flooded culverts. \\
\hline W-04-113 & White Sucker caught. Heavily silted reach. Diked both banks. \\
\hline W-04-114 & Saw two very large fish - probably Common Carp. Channelized, one culvert. Diked both banks, no riffles, no bends. \\
\hline W-04-115 & $\begin{array}{l}\text { No fish caught. Flow regime - slow/ shallow and slow/ deep. Water levels have dropped } 0.3 \mathrm{~m} \text {. Channelized, diked both banks. } \\
\text { Two large culverts, two small culverts. Vegetative bank protection - grasses prevalent. }\end{array}$ \\
\hline
\end{tabular}




\section{Appendix 7: List of any additional field notes recorded at sampling sites.}

\begin{tabular}{|c|c|}
\hline $\begin{array}{l}\text { Site } \\
\text { Number }\end{array}$ & Field Notes \\
\hline W-04-116 & $\begin{array}{l}\text { Two juvenile White Sucker released ( 60 mm long). Channelized, diked left bank (east) one culvert, one bridge. Little submerged } \\
\text { vegetation. }\end{array}$ \\
\hline W-04-117 & Not diked. Instream vegetation throughout. \\
\hline W-04-118 & Not diked. \\
\hline W-04-119 & $\begin{array}{l}\text { No fish caught. Recent rains June 5-6/04. Lots of emergent and submerged vegetation. Grasses prevalent. Area flooded. } \\
\text { Channelized, one culvert. Diked north bank (roadside) }\end{array}$ \\
\hline W-04-120 & $\begin{array}{l}\text { Undercut banks, cobble bottom, instream vegetation. Cobbles } 50-75 \% \text { embedded at bank edges. Channelized, two culverts. } \\
\text { Diked right bank (east, road). Grasses prevalent. }\end{array}$ \\
\hline W-04-121 & $\begin{array}{l}\text { No fish caught. Emergent vegetation and submerged vegetation only, no logs, no snags. Channelized, two culverts, diked right } \\
\text { bank (west, road). No riffles, no bends. Grassed banks. }\end{array}$ \\
\hline W-04-122 & Diked both banks. \\
\hline W-04-123 & Diked rail side (west). Mainly standing water. \\
\hline W-04-124 & $\begin{array}{l}\text { No fish caught. Little flow. No pools, no bends, no riffles. Channelized, diked right bank (west, road). Water depth } 0.5 \mathrm{~m} \text { deep. } \\
\text { Grasses prevalent. }\end{array}$ \\
\hline W-04-125 & Not diked. \\
\hline W-04-126 & One culvert. Not diked. Some bends, no riffles, long grasses prevalent. Vegetation natural with gravel bottom throughout reach. \\
\hline W-04-127 & Not diked. \\
\hline W-04-128 & Diked left bank (north, road). \\
\hline W-04-129 & $\begin{array}{l}\text { No fish caught. Surrounding fields flooded. Channelized in parts, one culvert. Diked right bank (west) upstream, downstream not } \\
\text { diked. No riffles. Cannot tell where banks are with flooding, grasses prevalent. }\end{array}$ \\
\hline W-04-130 & Diked road (North) side. Flooded, $0.5 \mathrm{~m}$ deep at downstream side of culvert. \\
\hline W-04-131 & Not diked. Cows have access to drain. \\
\hline W-04-132 & $\begin{array}{l}\text { Flooded adjacent fields. Channelized, two culverts, diked both banks downstream side, not diked upstream side. Grasses } \\
\text { prevalent }\end{array}$ \\
\hline W-04-133 & Diked both sides downstream, diked left bank (north, road) upstream side. \\
\hline W-04-134 & Diked right bank (road, West), water level down $0.5 \mathrm{~m}$. \\
\hline W-04-135 & $\begin{array}{l}\text { Algae (filamentous) provides a lot of cover. Water level down } 1 \mathrm{~m} \text {, channelized, diked right bank (road, west). Two culverts, } \\
\text { grasses prevalent. }\end{array}$ \\
\hline W-04-136 & $\begin{array}{l}\text { Not diked. Cultivated field to edge of drain on downstream side. No flow upstream side. Not sure where drain comes from on } \\
\text { upstream side - no defined drain channel. }\end{array}$ \\
\hline W-04-137 & $\begin{array}{l}\text { No fish caught, could only fish } 50 \mathrm{~m} \text { because dense vegetation } 25 \mathrm{~m} \text { upstream and downstream of culverts. } 2 \text { culverts, not diked. } \\
\text { Few riffles at channel constrictions. }\end{array}$ \\
\hline W-04-138 & Diked right bank (west, road). \\
\hline W-04-139 & Diked both banks \\
\hline
\end{tabular}




\section{Appendix 7: List of any additional field notes recorded at sampling sites.}

\begin{tabular}{|c|c|}
\hline $\begin{array}{l}\text { Site } \\
\text { Number }\end{array}$ & Field Notes \\
\hline W-04-140 & $\begin{array}{l}\text { Diked right bank (north, road). Vegetation throughout channel. No flow, dry upstream and downstream, with some marshy areas. } \\
\text { Cultivated field to edge of drain. Diked north bank and cultivated field left bank (south). }\end{array}$ \\
\hline W-04-141 & Diked both banks, mud road submerged in water. Drain is flooded. \\
\hline W-04-142 & Field cultivated to edge of drain downstream right bank. Marshy except for standing pool downstream culvert. \\
\hline W-04-143 & Water depth $0.2 \mathrm{~m}$, Little flow. Diked both banks. \\
\hline W-04-144 & $\begin{array}{l}\text { Pool downstream side of culvert } ~ 0.2 \mathrm{~m} \text { deep, } 1 \mathrm{~m} \text { wide, and } 3 \mathrm{~m} \text { long. Diked both banks on downstream side and diked left bank } \\
\text { (PR \# 201) upstream side. }\end{array}$ \\
\hline W-04-145 & Not diked. \\
\hline W-04-146 & $\begin{array}{l}\text { Diked both banks downstream side. Reach at junction of unnamed tributary and Kyle Drain. Diked right bank (south) upstream } \\
\text { side. }\end{array}$ \\
\hline W-04-147 & $\begin{array}{l}\text { Small pool upstream side of culvert is shallow } \sim 0.2 \mathrm{~m} \text { deep. Diked both sides. Downstream side gravel bottom from culvert } \\
\text { extending } 5 \mathrm{~m} \text { south (downstream). Pool downstream } 0.4 \mathrm{~m} \text { deep and is } 8 \mathrm{~m} \times 1 \mathrm{~m} \text {. }\end{array}$ \\
\hline W-04-148 & $\begin{array}{l}\text { Drain is not flowing, standing pool on upstream side of culvert. On downstream side dry beyond standing pools. Diked roadside } \\
\text { (west) left bank. }\end{array}$ \\
\hline W-04-149 & $\begin{array}{l}\text { Northern Pike (305 mm fork length) released. Flow regime - slow/shallow and slow/deep. Water has dropped } 2 \mathrm{~m} \text {. Channelized, } \\
\text { one culvert, diked both banks. Pool substrate mainly clay and mud, } 0.5 \mathrm{~m} \text { deep silt downstream of culvert. }\end{array}$ \\
\hline W-04-150 & Slight trickle of flow with standing pools. Diked left bank (north, road). \\
\hline W-04-151 & Trickle of flow. Channelized, diked right bank (west), one bridge. Vegetative bank protection - grasses. \\
\hline W-04-152 & Water very turbid, all mud channel. Not diked. \\
\hline W-04-153 & $\begin{array}{l}\text { Water depth down } 1 \mathrm{~m} \text {. Channelized, diked both banks, five culverts one cement box culvert, five gated culverts. Vegetative bank } \\
\text { protection - grasses. Pool substrate mud with little submerged vegetation. }\end{array}$ \\
\hline W-04-154 & Not diked. \\
\hline W-04-155 & Not diked. \\
\hline W-04-156 & Perched culvert on field access road, steep drop to downstream side of culvert. Diked left bank (road, north) \\
\hline W-04-157 & $\begin{array}{l}\text { No fish caught. Substrate Embedded: }>75 \% \text { surrounded by fine sediment on upstream side of culverts. Water level down } 1 \mathrm{~m} \text {. } \\
\text { Channelized, diked both banks on upstream side, not diked on downstream side. Cement box culvert downstream side and four } \\
\text { large culverts. Banks unstable, exposed mud shoreline on both banks upstream of crossing. Vegetative bank protection - grasses } \\
0.5 \mathrm{~m} \text { above mud shoreline, water level down } 1 \mathrm{~m} \text {. }\end{array}$ \\
\hline W-04-158 & $\begin{array}{l}\text { Upstream side of culverts looks like a tractor drove in the drain for entire length of drain (grass in drain cut short). Standing pools } \\
\text { on downstream side. Upstream side grasses except for scour pool by culvert. }\end{array}$ \\
\hline W-04-159 & $\begin{array}{l}\text { Primarily clay bottom. No flow. Channelized, diked both banks downstream side, not diked upstream side. Three culverts and } \\
\text { riprap. No riffles, four bends in view. Vegetative bank protection- grasses. }\end{array}$ \\
\hline W-04-160 & Standing pools $\sim 0.2 \mathrm{~m}$ deep throughout downstream side of crossing. Diked right bank (west). \\
\hline W-04-161 & wnstream side of culverts is mainly marshy. No flow. Upstream side has standing pools. Diked left bank (west). \\
\hline
\end{tabular}




\section{Appendix 7: List of any additional field notes recorded at sampling sites.}

\begin{tabular}{|c|c|}
\hline $\begin{array}{l}\text { Site } \\
\text { Number }\end{array}$ & Field Notes \\
\hline W-04-162 & Very shallow, Heavy instream vegetation. Not diked. \\
\hline W-04-163 & Mud shoreline right bank and left bank because water level has dropped $1 \mathrm{~m}$. Water is very turbid. \\
\hline W-04-164 & Diked both banks \\
\hline W-04-165 & Diked left bank (north, road). \\
\hline W-04-166 & $\begin{array}{l}\text { One Northern Pike caught. Dredged material from upstream side on right bank. Exposed soil right bank. Channelized, diked both } \\
\text { banks, four culverts, one gated culvert. Vegetative bank protection - grasses left bank, eroded bare soil patch on upstream side of } \\
\text { right bank for } 3 \mathrm{~m} \text {. Pool substrate, few boulders. Maximum water depth } 0.3 \mathrm{~m} \text {. }\end{array}$ \\
\hline W-04-167 & Channelized, two culverts. Four gated culverts. Diked both banks. No riffles, no bends. Vegetative bank protection - grasses. \\
\hline W-04-168 & Diked left bank (north, road). \\
\hline W-04-169 & Not diked. \\
\hline W-04-170 & Not diked. \\
\hline W-04-171 & $\begin{array}{l}\text { Channelized, upstream side not diked, two culverts. Vegetative bank protection - grasses. Pool substrate is clay. Pools upstream } \\
\text { and downstream of culverts. }\end{array}$ \\
\hline W-04-172 & Channelized, diked both banks, three culverts. Vegetative bank protection - grasses. \\
\hline W-04-173 & $\begin{array}{l}\text { Channelized, diked both banks on downstream side. Upstream side diked left bank (west, road). One bend in reach. Vegetative } \\
\text { bank protection - grasses. }\end{array}$ \\
\hline W-04-174 & Channelized, diked both banks. Vegetative bank protection- grasses. \\
\hline W-04-175 & $\begin{array}{l}\text { Four Northern Pike caught, one juvenile released. Substrate - gravel bed downstream side of culvert. Submerged vegetation, } \\
\text { undercut banks, no snags, logs or cobble. Flow regime - slow/deep, water depth dropped } 1 \mathrm{~m} \text {. Channelized, diked both banks } \\
\text { upstream, and diked right bank downstream. Two culverts. Vegetative bank protection - grasses. }\end{array}$ \\
\hline W-04-176 & $\begin{array}{l}\text { Sediment deposition at edges. Channelized, diked both banks. Bridges upstream and downstream and dam structure } \\
\text { downstream. No riffles, no bends. Vegetative bank protection - grasses. }\end{array}$ \\
\hline W-04-177 & Channelized, diked both banks. Dam structure is barrier to fish passage. Vegetative bank protection - grasses. \\
\hline W-04-178 & $\begin{array}{l}\text { Had to quit electrofishing due to downpour. Flow regime - slow/ shallow and slow/ deep. Channelized, diked both banks, two } \\
\text { gated culverts. No riffles, no bends. Vegetative bank protection - grasses. Deep pool under bridge from centre of channel to right } \\
\text { bank }\end{array}$ \\
\hline W-04-179 & $\begin{array}{l}\text { Channelized, two culverts, diked both sides downstream and diked left bank (north, road) upstream. No riffles, no bends. Bank } \\
\text { stability is poor due to very steep slopes on both banks. Vegetative bank protection - grasses. Riparian vegetation zone: } \\
\text { upstream - field and road to edge of bank. }\end{array}$ \\
\hline W-04-180 & $\begin{array}{l}\text { No fish caught. Channelized, diked both banks, one culvert. Steep right bank is unstable. Vegetative bank protection - grasses. } \\
\text { Riparian vegetation zone - minimal on roadside. One deep pool on downstream side of culvert. }\end{array}$ \\
\hline W-04-181 & $\begin{array}{l}\text { Four Northern Pike caught. Marshy. Water level down } 0.3 \mathrm{~m} \text {. Two culverts. No riffles, three bends in sight. Vegetative bank } \\
\text { protection - grasses. }\end{array}$ \\
\hline
\end{tabular}




\section{Appendix 7: List of any additional field notes recorded at sampling sites.}

\begin{tabular}{|c|c|}
\hline $\begin{array}{l}\text { Site } \\
\text { Number }\end{array}$ & Field Notes \\
\hline W-04-182 & $\begin{array}{l}\text { Five young of the year Northern Pike caught. Channel alteration - two culverts and riprap. Many bends, riffle on upstream side of } \\
\text { culvert. Vegetative bank protection - forest and grasses. Pool variability - deep pools on downstream side of culverts. }\end{array}$ \\
\hline W-04-183 & Not diked, complex habitat. Appears that water has dropped at least a few meters. \\
\hline W-04-184 & $\begin{array}{l}\text { No fish caught. Flow regime - slow/ shallow and slow/deep. Channel flow status - water levels have dropped } 0.3 \mathrm{~m} \text {. Channelized } \\
\text { on downstream side. One culvert. Vegetative bank protection - left bank forest and right bank grasses. }\end{array}$ \\
\hline W-04-185 & $\begin{array}{l}\text { Dry upstream and downstream from bridge crossing. Vegetation throughout active channel. Small shallow pool at bridge crossing, } \\
\text { diked both sides. }\end{array}$ \\
\hline W-04-186 & $\begin{array}{l}\text { Channelized, diked both banks, three culverts. Pool variability - pools only immediately upstream and downstream of culverts - } \\
\text { upstream pool is shallow, downstream deep. }\end{array}$ \\
\hline W-04-187 & $\begin{array}{l}\text { Channelized, two culverts, diked both banks upstream and downstream. No riffles, no bends. Vegetative bank protection - } \\
\text { grasses. }\end{array}$ \\
\hline W-04-188 & Diked both banks. \\
\hline W-04-189 & Not diked. \\
\hline W-04-190 & Not diked \\
\hline W-04-191 & Not diked. \\
\hline W-04-192 & $\begin{array}{l}\text { Flow regime - slow/deep, slow/shallow. Channel flow status - flooded } 2 \mathrm{~m} \text { outside of banks. Two culverts. No riffles, many bends. } \\
\text { Eroded bank on downstream side. Vegetative bank protection - trees and grasses. Not diked. }\end{array}$ \\
\hline W-04-193 & $\begin{array}{l}\text { No fish caught. Epifaunal substrate - undercut banks, woody debris, snags and cobble at culverts. Flow regime - fast/ shallow, } \\
\text { fast/deep. Channel flow status - water level has dropped } 1 \mathrm{~m} \text { (previously flooded). Not channelized. Not diked, two culverts. } \\
\text { Riparian vegetation zone - downstream side left bank } 12 \mathrm{~m} \text { to mowed yard. }\end{array}$ \\
\hline W-04-194 & Dry with some marshy areas. Instream vegetation and shrubs throughout - drain completely overgrown. Not diked. \\
\hline W-04-195 & Water level down $2 \mathrm{~m}$. Lots of woody debris, overhanging vegetation and undercut banks. One culvert. Not diked. \\
\hline W-04-196 & Not diked, complex habitat. \\
\hline W-04-197 & $\begin{array}{l}\text { One White Sucker }(252 \mathrm{~mm}) \text { released. Flow regime - no slow deep. One culvert, not diked. Vegetative bank protection - grasses. } \\
\text { Water level down } 1 \mathrm{~m} .\end{array}$ \\
\hline W-04-198 & Not diked. Dry. \\
\hline W-04-199 & No flow. Not diked. \\
\hline W-04-200 & Vegetative bank protection - shrubs, grasses and trees. Not diked. \\
\hline W-04-201 & Diked both banks. \\
\hline W-04-202 & $\begin{array}{l}\text { Flow regime - slow/deep and slow/shallow. Channelized, diked both banks. Five large culverts and two other small culverts on } \\
\text { upstream side. Vegetative bank protection - grasses. One pool on downstream side - large, deep } 30 \mathrm{~m} \times 20 \mathrm{~m} \times 1.5 \mathrm{~m} \text {. }\end{array}$ \\
\hline W-04-203 & $\begin{array}{l}\text { Epifaunal substrate - gravel/cobble bottom and instream vegetation. Embeddedness - very embedded at edges but not in center } \\
\text { channel. Channel flow status - very little water except for pool on downstream side of culverts. Channelized, two culverts, diked } \\
\text { both banks. Vegetative bank protection - grasses. Trickle of flow only. }\end{array}$ \\
\hline
\end{tabular}




\section{Appendix 7: List of any additional field notes recorded at sampling sites.}

\begin{tabular}{|c|c|}
\hline $\begin{array}{l}\text { Site } \\
\text { Number }\end{array}$ & Field Notes \\
\hline W-04-204 & Diked both banks. \\
\hline W-04-205 & On upstream side of drain grass is mowed close to edge of drain - $1 \mathrm{~m}$ to edge on right bank. Diked both banks. \\
\hline W-04-206 & Small standing pools upstream and downstream of culverts - otherwise drain is dry. Not diked. \\
\hline W-04-207 & $\begin{array}{l}\text { Water level has dropped } 1 \mathrm{~m} \text {. Nice habitat. Undercut banks. Sediment deposition at banks and in pools. Flow regime - no } \\
\text { fast/deep. One bridge, no riffles, many bends. Vegetative bank protection - grasses, shrubs and natural forest (all age classes). } \\
\text { Pool substrate - sand dominant, some cobble and boulders, little submerged vegetation. Not diked. }\end{array}$ \\
\hline W-04-208 & $\begin{array}{l}\text { Water levels have dropped } 0.5 \mathrm{~m} \text {. Three culverts. Not diked. Many bends. Pool on downstream side of crossing is very deep }>1.5 \\
\mathrm{~m} \text {. }\end{array}$ \\
\hline W-04-209 & Not diked. Complex habitat. \\
\hline W-04-210 & $\begin{array}{l}\text { Not diked. Water levels appear to have dropped } \sim 1 \mathrm{~m} \text {. Local knowledge: approximately one week ago aerial spraying was } \\
\text { conducted on a windy day, chemicals are responsible for killing riparian vegetation along Hazel Creek. Dying trees visible along } \\
\text { stream edge. }\end{array}$ \\
\hline W-04-211 & $\begin{array}{l}\text { One Stonecat and one sucker caught. Sand, cobble and gravel bottom. Some instream vegetation. Sediment deposition at edges. } \\
\text { Water down } 0.6 \mathrm{~m} \text {. Channelized, diked both banks, one culvert. Vegetative bank protection - upstream: grasses, downstream: } \\
\text { grasses and shrubs. Pool substrate - mainly soft sand. }\end{array}$ \\
\hline W-04-212 & $\begin{array}{l}\text { One Northern Pike released }(80 \mathrm{~mm}) \text {. Channelized, two gated culverts, diked both banks. (two level dike) No riffles, no bends. } \\
\text { Vegetative bank protection- grasses. Possible depth of } 2 \mathrm{~m} \text { in spring, based on high water marks. }\end{array}$ \\
\hline W-04-213 & $\begin{array}{l}\text { One Northern Pike caught. Note: electrofishing discontinued because water was too deep and fast. One bridge, not diked. } \\
\text { Vegetative bank protection - mature forest and grasses. }\end{array}$ \\
\hline W-04-214 & Diked roadside. \\
\hline W-04-215 & Drain is dry. Lots of cattails on upstream side, long grasses on downstream side. Diked right bank (west, road). \\
\hline W-04-216 & $\begin{array}{l}\text { Flow regime - slow/ shallow and slow/ deep. Channelized, diked both banks, one culvert. No riffles, no bends. Very steep banks } \\
\text { around culverts. Vegetative bank protection - grasses. Local knowledge: both upstream and downstream of culverts was dredged } \\
\text { last summer (2003) }\end{array}$ \\
\hline W-04-217 & Vegetative bank protection - long grasses. Drain is beginning to meander within its channel. Water level down $0.5 \mathrm{~m}$. \\
\hline W-04-218 & $\begin{array}{l}\text { Flow regime - slow/shallow and slow/deep. Riprap, bridge, and gated culverts. Channelized, diked both banks. Drain in beginning } \\
\text { to meander within its channel on downstream side. Vegetative bank protection - grasses. Deep pool on upstream side of } \\
\text { crossing. }\end{array}$ \\
\hline W-04-219 & Drain is dry. Aquatic vegetation throughout. Diked right bank (east, road side) \\
\hline W-04-220 & $\begin{array}{l}\text { Culvert \#2 (East) is flattened. Pool upstream side is } 4 \mathrm{~m} \text { wide and } 2 \mathrm{~m} \text { long. Pool downstream side is } 7 \mathrm{~m} \text { wide and } 5 \mathrm{~m} \text { long. } \\
\text { Drain is dry upstream and downstream of crossing beyond pools. Diked both banks upstream side, diked right bank (east, road) } \\
\text { on downstream side. }\end{array}$ \\
\hline W-04-221 & $\begin{array}{l}\text { Drain is dry except for one pool ( } 1 \mathrm{~m} \times 4 \mathrm{~m} \text { ) on downstream side of culvert. Diked both banks downstream side, diked left bank } \\
\text { (north, road) upstream side of crossing. }\end{array}$ \\
\hline
\end{tabular}




\section{Appendix 7: List of any additional field notes recorded at sampling sites.}

\begin{tabular}{|c|c|}
\hline $\begin{array}{l}\text { Site } \\
\text { Number }\end{array}$ & Field Notes \\
\hline W-04-222 & iked both banks. \\
\hline W-04-223 & Dry upstream and downstream. Little standing water with in channel. Drain completely overgrown. Diked both banks. \\
\hline W-04-224 & Drain is dry. Diked left bank (north, road.) \\
\hline W-04-225 & $\begin{array}{l}\text { Three suckers caught and released }(150-255 \mathrm{~mm}) \text {. Few undercut clay banks. Sedges and cattails (instream vegetation). Flow } \\
\text { regime - slow/deep and slow/shallow. Water level down } 0.5 \mathrm{~m} \text {. Channelized, diked both banks, bridge crossing. No riffles. Drain } \\
\text { starting to meander on downstream side of crossing. Vegetative bank protection - grasses. Riparian - mowed on downstream side } \\
\text { (left bank) for } 200 \mathrm{~m} \text {. Pool substrate - mainly clay bottom. }\end{array}$ \\
\hline W-04-226 & Drain overgrown on downstream side. Not diked downstream side,. Diked left bank (road) on upstream side. \\
\hline W-04-227 & $\begin{array}{l}\text { Drain overgrown. Dry upstream and downstream. Lots of instream vegetation throughout drain. Cattails at upstream side of } \\
\text { culvert. Diked both banks. }\end{array}$ \\
\hline W-04-228 & Drain dry except for one pool on downstream side of culvert (pool size: $2 \mathrm{~m} \times 4 \mathrm{~m}$ ). Diked both banks. \\
\hline W-04-229 & Diked right bank (north, road.) \\
\hline W-04-230 & Diked both banks. \\
\hline W-04-231 & $\begin{array}{l}\text { Three juvenile Northern Pike caught, one released }(76 \mathrm{~mm}) \text {. Kick sampled } 1 \mathrm{~m}^{2} \text {. Flow regime - fast/deep and slow/deep. } \\
\text { Channelized, diked both banks, three culverts, one gated culvert. Drain is starting to meander within its channel on downstream } \\
\text { side of crossing. Vegetative bank protection - mainly grasses, a few shrubs. Riparian vegetation zone on upstream side left bank } \\
\sim 6 \mathrm{~m} \text { wide on diked roadside. }\end{array}$ \\
\hline W-04-232 & $\begin{array}{l}\text { Four juvenile Northern Pike caught, two released }(1-250 \mathrm{~mm}, 1-102 \mathrm{~mm}) \text {. Cobble bottom, lots of submerged and emergent } \\
\text { vegetation. Water level has dropped } 1 \mathrm{~m} \text {. Sediment deposition mainly at bank edges. Channelized, three main culverts. One } \\
\text { gated and one regular culvert running into drain from tributaries. Diked both banks upstream side, diked right bank downstream } \\
\text { side. No riffles, two bends in sight. }\end{array}$ \\
\hline W-04-233 & Not diked. Water level down $\sim 1 \mathrm{~m}$. Local knowledge: suckers and Common Carp used this drain three weeks ago. \\
\hline W-04-234 & $\begin{array}{l}\text { Pool substrate mainly cobble and gravel. Flow regime - fast/ deep and slow/ deep. Not diked. Cattle have access to drain on } \\
\text { downstream side of bridge. }\end{array}$ \\
\hline W-04-235 & Not diked. \\
\hline W-04-236 & Not diked. \\
\hline W-04-237 & $\begin{array}{l}\text { Electrofishing discontinued because water was too deep. Lots of submerged vegetation. Not diked. No riffles, few bends in view } \\
\text { upstream and downstream. Vegetative bank protection - shrubs, grasses and trees. Pool substrate - gravel and silt with dense } \\
\text { submerged vegetation. }\end{array}$ \\
\hline W-04-238 & $\begin{array}{l}\text { Cover provided by submerged vegetation. Channel flow status - flooded adjacent grasses. Water level down } \sim 1 \mathrm{~m} \text { minimum and } \\
\text { dropping quickly. Not diked, one culvert. Riffles caused by channel constrictions. Pool substrate - gravel, boulders and cobble. }\end{array}$ \\
\hline W-04-239 & $\begin{array}{l}\text { One Northern Pike caught, } 30 \text { Brook Stickleback released. Fish passage blocked because upstream side of culvert is blocked } \\
\text { with a metal grate. Flow regime - no fast/deep. Not channelized, not diked, two cement culverts. }\end{array}$ \\
\hline W-04-240 & Not diked. Large beaver dam (8 $\mathrm{m}$ wide). \\
\hline
\end{tabular}




\section{Appendix 7: List of any additional field notes recorded at sampling sites.}

\begin{tabular}{|c|c|}
\hline $\begin{array}{l}\text { Site } \\
\text { Number }\end{array}$ & Field Notes \\
\hline W-04-241 & $\begin{array}{l}\text { Not diked. Two beaver dams upstream side of bridge. Trickle of flow, mainly deep standing pools. Oily sheen on water. Too deep } \\
\text { to fish. }\end{array}$ \\
\hline W-04-242 & Not diked. Too deep to fish. Fenced off on upstream side of bridge crossing. \\
\hline W-04-243 & ot diked. Water level down $2 \mathrm{~m}$. Dense submerged vegetation in pools. \\
\hline W-04-244 & ot diked. Many Common Carp seen in the pool (upstream of culvert). Too deep to take inlet and outlet photos (submerged). \\
\hline W-04-245 & Not diked. Many Common Carp seen. Water level down $1 \mathrm{~m}$. Very deep drain. Water mainly standing, (slow movement) \\
\hline W-04-246 & Many Common Carp seen on upstream side of culvert. Not diked. \\
\hline W-04-247 & $\begin{array}{l}\text { Not diked. Drain is dry upstream and downstream. Some marshy areas. Cement culverts. No outlet pictur } \\
\text { completely overgrown. }\end{array}$ \\
\hline W-04-248 & t diked. Unable to fish because water depth. \\
\hline W-04-249 & $\begin{array}{l}\text { Local knowledge: usually trout are found in this creek. Flow regime - fast/shallow and slow/shallow. Low water level. Lots of } \\
\text { woody debris, forming dam on upstream side. Both banks diked, not channelized. } 3 \text { culverts. No riffles, run and pools only. Pool } \\
\text { substrate - cobble. No pools-uniform depth } \sim 0.3 \mathrm{~m}\end{array}$ \\
\hline W-04-250 & $\begin{array}{l}\text { Two White Sucker caught. Flow regime - fast/shallow and slow/shallow. Water level down } 1 \mathrm{~m} \text {. Not diked, not channelized, one } \\
\text { culvert. One riffle downstream side of culverts, many bends. Pool substrate - sandy bottom. One pool downstream side of culverts } \\
\text { (shallow) }\end{array}$ \\
\hline W-04-251 & Not diked. Drain is flowing. Drain is completely overgrown on both sides and access is difficult on downstream side. \\
\hline W-04-252 & $\begin{array}{l}\text { One sucker caught. Flow regime - no fast/deep. Not diked. One bridge. Riparian zone is less than } 6 \mathrm{~m} \text { on right bank downstream } \\
\text { side for } 30 \mathrm{~m} \text { from bridge. Nice diverse habitat. }\end{array}$ \\
\hline W-04-253 & $\begin{array}{l}\text { Culvert \#1 on downstream side filled with woody debris - little water getting through. Drain has a foul odour. Tractor tracks around } \\
\text { drain - exposed mud areas surrounding drain. Flow regime - slow/deep and slow/shallow. Two culverts, not diked. Pool substrate } \\
\text { - no submerged vegetation. }\end{array}$ \\
\hline W-04-254 & $\begin{array}{l}\text { No undercut banks. Flow regime - fast/shallow and slow/shallow. Water level has dropped } \sim 1 \mathrm{~m} \text {. Two large culverts, not diked. } \\
\text { Many riffles and bends. Pool substrate - algae covered cobble. }\end{array}$ \\
\hline W-04-255 & $\begin{array}{l}\text { Dense instream vegetation. Flow regime - fast/shallow and slow/shallow, water level down } 1 \mathrm{~m} \text {. Two large culverts, not diked. No } \\
\text { riffles (except associated with culverts). Many bends. }\end{array}$ \\
\hline W-04-256 & $\begin{array}{l}\text { One Northern Pike caught. Cover provided by submerged vegetation. Flow regime- slow/deep and slow/shallow. One bridge, not } \\
\text { diked. No riffles, two bends in reach. Vegetative bank protection - long grasses and shrubs. }\end{array}$ \\
\hline W-04-257 & Diked left bank (north, road). \\
\hline W-04-258 & $\begin{array}{l}\text { Diked right bank (north, road). Drain is dry upstream and downstream, except for one pool on downstream side of culverts. Lots c } \\
\text { instream vegetation- dominated by cattails. }\end{array}$ \\
\hline & \\
\hline
\end{tabular}




\section{Appendix 7: List of any additional field notes recorded at sampling sites.}

\begin{tabular}{|c|c|}
\hline $\begin{array}{l}\text { Site } \\
\text { Number }\end{array}$ & Field Notes \\
\hline W-04-260 & $\begin{array}{l}\text { Two Northern Pike caught, one Northern Pike }(76 \mathrm{~mm}) \text { released. Problem with pH meter. Cover provided by lots of instream } \\
\text { vegetation. Flow regime - slow/shallow. Channelized, diked both banks, three culverts. No riffles, drain is starting to meander } \\
\text { within its dikes. Vegetative bank protection -very long grasses and shrubs. }\end{array}$ \\
\hline W-04-261 & Diked both banks. Standing pools present, very overgrown with vegetation. \\
\hline W-04-262 & $\begin{array}{l}\text { Two Northern Pike caught. Cover provided by lots of instream and overhanging vegetation, and undercut banks. Flow regime - } \\
\text { slow/ shallow, some slow/ deep (max depth } 0.75 \mathrm{~m} \text { ). Water level has dropped } 1 \mathrm{~m} \text {. Not diked, not channelized, two culverts. No } \\
\text { riffles, stream is meandering. Vegetative bank protection - upstream and downstream grasses and shrubs, downstream - forest. }\end{array}$ \\
\hline W-04-263 & Diked right bank (south, along road). \\
\hline W-04-264 & $\begin{array}{l}\text { Local knowledge: suckers used the drain this spring. Flow regime - slow/shallow. Channelized, diked both banks, two culverts, } \\
\text { one gated culvert. No riffles, no bends. Steep dikes. Vegetative bank protection - grasses. Pool substrate - submerged } \\
\text { vegetation. }\end{array}$ \\
\hline W-04-265 & $\begin{array}{l}\text { One Northern Pike caught. Flow regime - slow/shallow. Water levels have dropped } \sim 1 \mathrm{~m} \text {. Not diked. Five culverts in drain } \\
\text { crossing, one culvert on tributary crossing. No riffles, two bends in sight. Vegetative bank protection - grasses. Pool substrate - } \\
\text { mainly mud. }\end{array}$ \\
\hline W-04-266 & $\begin{array}{l}\text { One Northern Pike }(203 \mathrm{~mm}) \text { released. One Yellow Perch caught. Kick sampled } 1 \mathrm{~m}^{2} \text {. Flow regime - fast/deep and slow/deep. } \\
\text { Not diked, not channelized, one bridge. Vegetative bank protection - grasses, shrubs, bulrushes. Undercut banks. }\end{array}$ \\
\hline W-04-267 & Not diked. \\
\hline W-04-268 & $\begin{array}{l}\text { Diked right bank (east) by road) No flow. Standing pool upstream of crossing. Dense submerged vegetation throughout drain. No } \\
\text { culvert outlet picture (inaccessible). }\end{array}$ \\
\hline W-04-269 & $\begin{array}{l}\text { Couldn't fish more due to extremely dense submerged vegetation and sediment. Cover provided by submerged vegetation and a } \\
\text { few undercut banks. Flow regime - slow/shallow. Water has dropped } 0.5 \mathrm{~m} \text {. Not diked. Three culverts. No riffles, one bend in } \\
\text { reach. }\end{array}$ \\
\hline W-04-270 & $\begin{array}{l}\text { Not diked upstream side. Diked right bank (North) downstream side. Drain is dry upstream and downstream beyond marshy area } \\
\text { under and around bridge. Drain is heavily overgrown. }\end{array}$ \\
\hline W-04-271 & $\begin{array}{l}\text { Three suckers caught. Flow regime - slow/deep and slow/shallow. Water level has dropped } 2 \mathrm{~m} \text {. Not diked, not channelized, one } \\
\text { large diameter culvert. No riffles. Marshy area. One very deep pool on downstream side of crossing. }\end{array}$ \\
\hline W-04-272 & $\begin{array}{l}\text { Not diked. No flow. Long grasses and sedges throughout drain upstream and downstream of standing pool. One standing pool on } \\
\text { downstream side of culvert }(3 \mathrm{~m} \times 5 \mathrm{~m}) \text {. }\end{array}$ \\
\hline W-04-273 & Not diked. Upstream of the crossing the channel has been altered due to agricultural practices. \\
\hline W-04-274 & $\begin{array}{l}\text { Released two Northern Pike, one White Sucker and one Rock Bass. Epifaunal substrate - lots of riprap, little instream vegetation } \\
\text { or submerged vegetation. Flow regime - slow/deep and slow/shallow. Not diked. Not channelized. Bridge crossing. Lots of riprap } \\
\text { around and under bridge. No riffles. Vegetative bank protection - mainly grasses with a few shrubs. Left bank - field cultivated field } \\
\text { right to top of bank. }\end{array}$ \\
\hline
\end{tabular}




\section{Appendix 7: List of any additional field notes recorded at sampling sites.}

\begin{tabular}{|c|c|}
\hline $\begin{array}{l}\text { Site } \\
\text { Number }\end{array}$ & Field Notes \\
\hline W-04-275 & $\begin{array}{l}\text { Drain is dry. Lots of grasses and cattails throughout the channel. Standing pool under the bridge. Diked left bank (south, road) on } \\
\text { upstream and right bank on downstream side. }\end{array}$ \\
\hline W-04-276 & Drain is dry. Dredged on upstream side. Diked on right bank (south, PR \#205). \\
\hline W-04-277 & $\begin{array}{l}\text { Five Northern Pike seen, one caught. Fished pool on downstream side of culverts. Clay and mud bottom. No water movement, } \\
\text { just one shallow pool on downstream side of culverts. One small pool on upstream side. Diked both banks, channelized, three } \\
\text { main culverts, four small culverts from tributaries. No riffles, no bends. Vegetative bank protection - grasses. No vegetation in } \\
\text { pools. Riparian on upstream side of culverts is } 12 \mathrm{~m} \text { wide. }\end{array}$ \\
\hline W-04-278 & Not diked. Dry. Channel filled with vegetation. \\
\hline W-04-279 & Diked both banks. Several standing pools, with a maximum depth $0.3 \mathrm{~m}$. \\
\hline W-04-280 & Not diked. One standing pool on downstream side of crossing. Very turbid and dense instream vegetation. No flow. \\
\hline W-04-281 & Diked both banks. Small standing pool on downstream side of culverts. No flow. \\
\hline W-04-282 & Diked left bank (road). No flow, standing pool. \\
\hline W-04-283 & Diked left bank (south, road). Dry upstream and downstream from bridge crossing. Sediment deposit around and under bridge. \\
\hline W-04-284 & Not diked. \\
\hline W-04-285 & Diked both banks. Dry, except for standing pools upstream and downstream of culverts. \\
\hline W-04-286 & $\begin{array}{l}\text { No fish caught, had to discontinue electrofishing because sediment was too deep. Water depth } 0.25 \mathrm{~m} \text {. Sediment depth at least } \\
0.5 \mathrm{~m} \text { in some areas. Flow regime - slow/shallow only. Water levels have dropped } \sim 3 \mathrm{~m} \text {. Diked both banks. Wooden bridge, old } \\
\text { piles. No riffles, five bends in sight. Some bank slump. Vegetative bank protection - grasses. No pools. Very little submerged } \\
\text { vegetation (embedded in silt). }\end{array}$ \\
\hline W-04-287 & $\begin{array}{l}\text { Thirty bullheads }(240 \mathrm{~mm}) \text {, three Yellow Perch }(250 \mathrm{~mm}) \text {, two Sauger }(280 \mathrm{~mm}, 300 \mathrm{~mm}) \text { released. Substrate covered with } \\
\text { submerged vegetation. Overhanging vegetation (grasses) along banks. Diked both banks. Channelized. Paved raised crossing } \\
\text { with six culverts. No real bends, one riffle on downstream side of ford crossing. Vegetative bank protection - grasses. }\end{array}$ \\
\hline W-04-288 & Not diked. \\
\hline W-04-289 & $\begin{array}{l}\text { Forty bullheads, one Northern Pike }(90 \mathrm{~mm}) \text {, and two White Sucker }(305,457 \mathrm{~mm}) \text { released. Troutperch - some samples kept } \\
\text { and five released. Flow regime - no fast/deep. Channelized, diked both banks. Riprap dam. No real bends, one riffle through } \\
\text { riprap dam. Eroded banks with slump and scours evident. Vegetative bank protection - grasses. Pool substrate - mainly cobble } \\
\text { and clays, no submerged vegetation. }\end{array}$ \\
\hline W-04-290 & Not diked. Pool on downstream side of culvert is $3 \mathrm{~m}$ (wide) $\times 8 \mathrm{~m}$ (long). Grass throughout pool and appears very shallow. \\
\hline W-04-291 & Not diked. Cattails and tall grasses in dry channel. No flow. \\
\hline W-04-292 & Diked both banks. Cattle have direct access to drain on downstream side of culverts. \\
\hline W-04-293 & Not diked. Drain is completely overgrown with cattails and tall grasses. Dry. \\
\hline W-04-294 & Not diked. Couldn't fish because access blocked by fence. Cattle upstream $15 \mathrm{~m}$ from crossing have direct access to drain. \\
\hline W-04-295 & $\begin{array}{l}\text { Not diked. Cattle have direct access to drain on upstream side of culverts. Slight trickle, heavy vegetation (instream vegetation - } \\
\text { bulrushes), standing pool on downstream side of culverts. }\end{array}$ \\
\hline
\end{tabular}


Appendix 7: List of any additional field notes recorded at sampling sites.

\begin{tabular}{|c|c|}
\hline $\begin{array}{l}\text { Site } \\
\text { Number }\end{array}$ & Field Notes \\
\hline W-04-296 & Not diked. Drain is dry. \\
\hline W-04-297 & $\begin{array}{l}\text { Released one White Sucker and } 20 \text { shiners. Cattle have direct access to river on upstream side of culvert bridge structure. Cattle } \\
\text { impacts on banks and streambed. Water level down } 0.5 \mathrm{~m} \text {. Not diked, not channelized, one culvert bridge structure with three } \\
\text { very large culvert openings. Shale bars present. Vegetative bank protection - grasses, shrubs and forest back from shale } \\
\text { shoreline. Pool substrate - shale bottom, no submerged vegetation, little emergent vegetation. }\end{array}$ \\
\hline W-04-298 & $\begin{array}{l}\text { Suckers caught, } 10 \text { juvenile suckers released. Water has dropped } 2 \mathrm{~m} \text {. Epifaunal substrate - cobble and snags. Flow regime - no } \\
\text { fast/deep. Not diked, not channelized. Weir and bridge. Many bends, no riffles. Vegetation protection - mostly grasses. Shallow } \\
\text { pools or pools absent - except where we fished. }\end{array}$ \\
\hline W-04-299 & $\begin{array}{l}\text { Suckers caught. Moderate cattle impacts - have direct access to creek. Flow regime - no fast/deep. Not diked, not channelized. } \\
\text { Lots of riprap, one culvert structure with four large culvert openings. One riffle, bends in view. Banks protected with riprap. } \\
\text { Vegetative bank protection - grasses and shrubs. Pool substrate - lots of boulders/cobble. }\end{array}$ \\
\hline W-04-300 & Not diked. Water level down $3 \mathrm{~m}$. \\
\hline W-04-301 & Not diked. Water level down $\sim 3 \mathrm{~m}$. Local knowledge: Northern Pike and Walleye use this river. Cattle have direct access to river. \\
\hline $\mathrm{X}-04-001$ & $\begin{array}{l}\text { Low level crossing, with four } 1.25 \text { m corrugated metal pipes, left bank mowed, riffle upstream. Released six bullheads (100-200 } \\
\text { mm fork length).One bank diked. }\end{array}$ \\
\hline $\mathrm{X}-04-002$ & $\begin{array}{l}\text { Cultivated land on both sides. Large cement dam } 6 \mathrm{~m} \text { high, } 8 \mathrm{~m} \text { long, } 3 \text { tiers. Fast flowing water. Both banks diked. Complex } \\
\text { habitat upstream and downstream.. }\end{array}$ \\
\hline $\mathrm{X}-04-003$ & Too deep to fish. Diked on 1 bank. \\
\hline $\mathrm{X}-04-004$ & Both banks diked. Complex habitat upstream and downstream. \\
\hline $\mathrm{X}-04-005$ & Drain is surrounded by cultivated fields. Both banks diked. \\
\hline $\mathrm{X}-04-006$ & No dikes. \\
\hline X-04-007 & No flow. No dikes. \\
\hline $\mathrm{X}-04-008$ & No flow. No dikes. \\
\hline $\mathrm{X}-04-009$ & No flow. Perched corrugated metal pipe. No dikes. \\
\hline $\mathrm{X}-04-010$ & A little flow from yesterday's rain, but was dry previously. No dikes downstream, one bank diked upstream. \\
\hline $\mathrm{X}-04-011$ & No flow. Standing pools at crossings. \\
\hline $\mathrm{X}-04-012$ & No flow, diked on one side. \\
\hline $\mathrm{X}-04-013$ & Too deep to fish. \\
\hline $\mathrm{X}-04-014$ & No catch. Crossing is at PTH \#3. Pool depth under bridge $>2 \mathrm{~m}$. \\
\hline $\mathrm{X}-04-015$ & Diked on both sides. \\
\hline $\mathrm{X}-04-016$ & Too deep to fish. Diked on northwest side. \\
\hline $\mathrm{X}-04-017$ & Local knowledge: local has seen fish here before. \\
\hline $\mathrm{X}-04-018$ & Not diked. Too deep to fish. Livestock have access to creek. \\
\hline $\mathrm{X}-04-019$ & Not fishable due to right bank wire fencing. Not diked. \\
\hline
\end{tabular}


Appendix 7: List of any additional field notes recorded at sampling sites.

\begin{tabular}{|c|c|}
\hline $\begin{array}{l}\text { Site } \\
\text { Number }\end{array}$ & Field Notes \\
\hline $\mathrm{X}-04-020$ & Too deep on downstream side to fish and barbed wire fence upstream. Not diked. \\
\hline $\mathrm{X}-04-021$ & Dissolved oxygen meter not working. Too deep to fish. Not diked. \\
\hline $\mathrm{X}-04-022$ & Diked on both sides. \\
\hline $\mathrm{X}-04-023$ & $\begin{array}{l}\text { Downstream right bank no agricultural land. Left bank cultivated land }>20 \mathrm{~m} \text { away, upstream left bank cultivated land }<5 \mathrm{~m} \text { away. } \\
\text { Right bank mowed grass. }\end{array}$ \\
\hline $\mathrm{X}-04-024$ & Cultivated land around. Very dry. No flow. Lots of vegetation in drain. Rip rap in front of two outer corrugated metal pipes. \\
\hline $\mathrm{X}-04-025$ & Burned cuttings blocking drain. No flow. Lots of vegetation in drain. Cultivated land all around and close to drain. \\
\hline $\mathrm{X}-04-026$ & Cultivated land all around. Mowed grass. Downstream lots of vegetation. Local knowledge: completely dry by July. \\
\hline $\mathrm{X}-04-027$ & Land cultivated very close to drain. Banks mowed to water's edge. \\
\hline $\mathrm{X}-04-028$ & Land cultivated very close to drain. Burned on right bank. Very low water. No flow. \\
\hline $\mathrm{X}-04-029$ & No flow. Cultivated land all around, $25 \mathrm{~m}$ from drain. Mowed grass left bank. \\
\hline $\mathrm{X}-04-030$ & Cultivated land around. No flow. Very shallow. Low cement crossing. \\
\hline $\mathrm{X}-04-031$ & Burned grass along banks. Cultivated land all around. Local knowledge: Drain dries up in summer. \\
\hline $\mathrm{X}-04-032$ & Low level crossing flooded. \\
\hline $\mathrm{X}-04-033$ & No flow. \\
\hline $\mathrm{X}-04-034$ & No flow. Perched corrugated metal pipes. \\
\hline $\mathrm{X}-04-035$ & Corrugated metal pipes blocked heavily vegetation debris, some burnt debris. No flow. \\
\hline $\mathrm{X}-04-036$ & One corrugated metal pipe overgrown. Low water level. \\
\hline $\mathrm{X}-04-037$ & Lots of overhanging vegetation over cut banks. Metal debris in creek. \\
\hline $\mathrm{X}-04-038$ & $\begin{array}{l}\text { Very shallow. Probably dries up in summer. Mowed grass right to water edge. Lots of submerged vegetation. Submerged } \\
\text { vegetation is lawn grass. }\end{array}$ \\
\hline $\mathrm{X}-04-039$ & Perched corrugated metal pipe $(0.7 \mathrm{~m})$. Flow goes into small corrugated metal pipe only. Length of corrugated metal pipes $\sim 30 \mathrm{~m}$. \\
\hline $\mathrm{X}-04-040$ & Culvert near highway is perched $(0.3 \mathrm{~m})$. If flow is less by $5 \mathrm{~cm}$ both corrugated metal pipes would be perched. \\
\hline $\mathrm{X}-04-041$ & Cultivated land around. Lots of flow coming out of ditch culverts. \\
\hline $\mathrm{X}-04-042$ & Cultivated land around. Lots of snow in drain. Small culvert on left bank on downstream side of crossing perched (0.1 m). \\
\hline $\mathrm{X}-04-043$ & Long riffle with high flow velocity. \\
\hline $\mathrm{X}-04-044$ & One crayfish observed. \\
\hline $\mathrm{X}-04-045$ & Adjacent to cattle pasture. \\
\hline $\mathrm{X}-04-046$ & Area where Kitner drain enters Alonsa drain. Melt water flow. \\
\hline $\mathrm{X}-04-047$ & Upstream there is a dugout pool in drain. Light cattle impacts. \\
\hline $\mathrm{X}-04-048$ & Cultivated land right to drain on right bank of downstream side. \\
\hline $\mathrm{X}-04-049$ & Land around drain is flooded. Wagon wheels at culvert inlets hold plant debris. \\
\hline $\mathrm{X}-04-050$ & Too deep to fish. Control structure upstream holding back water. \\
\hline $\mathrm{X}-04-051$ & Bridge crossing is very low. Beaver dam holding back flow. \\
\hline
\end{tabular}




\section{Appendix 7: List of any additional field notes recorded at sampling sites.}

\begin{tabular}{|c|c|}
\hline $\begin{array}{l}\text { Site } \\
\text { Number }\end{array}$ & Field Notes \\
\hline $\mathrm{X}-04-052$ & Cultivated land around. High velocity flow. \\
\hline $\mathrm{X}-04-053$ & $\begin{array}{l}\text { Cultivated land around. High velocity long riffle. Corrugated metal pipes would be perched if water drops } 0.20 \mathrm{~m} \text {. Corrugated } \\
\text { metal pipe inlet is at a } 45 \text { degree angle. } 10-15 \text { visible White Sucker at culvert outlet. }\end{array}$ \\
\hline $\mathrm{X}-04-054$ & Spawning suckers observed. Cultivated land around. High velocity flow. \\
\hline $\mathrm{X}-04-055$ & Observed spawning White Sucker. \\
\hline $\mathrm{X}-04-056$ & $\begin{array}{l}\text { Mowed grass right to creek. Observed spawning White Sucker. Gravel, sand and cobble substrate. No suckers up Binden Drain. } \\
\text { Suckers here are showing more spawning colours than those downstream from this site. }\end{array}$ \\
\hline $\mathrm{X}-04-057$ & $\begin{array}{l}\text { Right bank turbid water from where 2nd order drain enters creek. Spawning White Sucker observed. Lots of overhanging } \\
\text { vegetation. }\end{array}$ \\
\hline $\mathrm{X}-04-058$ & Observed spawning White Sucker \\
\hline$X-04-059$ & No suckers observed. \\
\hline $\mathrm{X}-04-060$ & No suckers observed. \\
\hline $\mathrm{X}-04-061$ & Simple habitat but grass bottom. Possible cattle pasture upstream (evidence from electric fence). No catch. \\
\hline $\mathrm{X}-04-062$ & Wire fence across creek on upstream and downstream sides of dirt road. \\
\hline $\mathrm{X}-04-063$ & Area where 2nd order tributary meets Westbourne Drain. \\
\hline $\mathrm{X}-04-064$ & Low level crossing flooded. No culverts visible under crossing. No fish passage when water levels are low. \\
\hline$X-04-065$ & Released Northern Pike $(803 \mathrm{~mm})$, Walleye $(297 \mathrm{~mm})$, four and bullheads. Local knowledge: catfish and Walleye come up creek. \\
\hline $\mathrm{X}-04-066$ & Observed two adult White Sucker. \\
\hline $\mathrm{X}-04-067$ & $\begin{array}{l}\text { Dead sucker on right bank. Dam is } 1.5 \mathrm{~m} \text { high. High velocity flow. Cobble, gravel, woody debris at foot of dam. Downstream from } \\
\text { dam rotting vegetation, lots of methane production. Local knowledge: farmers block La Salle River further upstream with small } \\
\text { culverts that prevent fish passage. }\end{array}$ \\
\hline $\mathrm{X}-04-068$ & Long man-made riffle. One huge slumped bank (left bank). All suckers released. Other suckers observed. \\
\hline $\mathrm{X}-04-069$ & Gradient control structure. No spawning suckers observed. \\
\hline $\mathrm{X}-04-070$ & $\begin{array}{l}\text { Corrugated metal pipes for 2nd order drain closed. Inlet to Elm Creek channel from } 2 \text { nd order drain has been recently cleaned } \\
\text { out. }\end{array}$ \\
\hline $\mathrm{X}-04-071$ & Constructed riffle. \\
\hline $\mathrm{X}-04-072$ & Observed suckers downstream of riffle. Left bank muddy. \\
\hline $\mathrm{X}-04-073$ & Substrate is pure clay and black soil. Very shallow. \\
\hline $\mathrm{X}-04-074$ & $\begin{array}{l}\text { Oily film on surface of water. Deposition of organic matter. Rocks under bridge. Not much flow. Just grass and reeds on banks. } \\
\text { No catch. }\end{array}$ \\
\hline $\mathrm{X}-04-075$ & Cultivated land around. \\
\hline $\mathrm{X}-04-076$ & Too deep to fish. \\
\hline $\mathrm{X}-04-077$ & Methane bubbles coming out of substrate. Lots of bends, one riffle. Some sticks and logs in water. \\
\hline $\mathrm{X}-04-078$ & Not much submerged vegetation. Grass only along banks. \\
\hline
\end{tabular}




\section{Appendix 7: List of any additional field notes recorded at sampling sites.}

\begin{tabular}{|c|c|}
\hline $\begin{array}{l}\text { Site } \\
\text { Number }\end{array}$ & Field Notes \\
\hline $\mathrm{X}-04-079$ & Top of corrugated metal pipes submerged by at least $0.3 \mathrm{~m}$. Too deep to fish. \\
\hline $\mathrm{X}-04-080$ & Only able to fish along shore, because too deep and high velocity. Three Black Crappies released $(\sim 80 \mathrm{~mm}, \sim 75 \mathrm{~mm}, \sim 45 \mathrm{~mm})$. \\
\hline $\mathrm{X}-04-081$ & Too deep to fish in pools. Mostly large rocks on man-made riffle. One visible riffle. Grass only on banks. High velocity flow. \\
\hline $\mathrm{X}-04-082$ & Long grass along banks. Submerged vegetation present. Area where Carr's Creek flows into Springfield Road Drain. \\
\hline $\mathrm{X}-04-083$ & Too dry to fish. No eggs found on riffles. Very low flow. \\
\hline X-04-084 & No Catch. \\
\hline $\mathrm{X}-04-085$ & Too deep to fish. Very high velocity flow. \\
\hline $\mathrm{X}-04-086$ & Mud blocking culvert inlet. Reeds growing at culvert outlet. \\
\hline $\mathrm{X}-04-087$ & Too deep to fish. Severe cattle impacts. Poor habitat, all muddy clay substrate. \\
\hline $\mathrm{X}-04-088$ & High velocity flow. Cultivated land around. Cattle pasture on right bank directly adjacent to drain. Mostly grass along banks. \\
\hline $\mathrm{X}-04-089$ & Perched corrugated metal pipe $(0.5 \mathrm{~m})$. Slumped left bank. \\
\hline $\mathrm{X}-04-090$ & $\begin{array}{l}\text { Local knowledge: lots of Northern Pike caught in upper Seine River, water depth drops here to } \sim 15 \mathrm{~cm} \text { in summer. Small trees } \\
\text { and grass along banks. }\end{array}$ \\
\hline $\mathrm{X}-04-091$ & Water level very high. Flooded land and over road. Lots of bends, not as many riffles. \\
\hline $\mathrm{X}-04-092$ & $\begin{array}{l}\text { Ripe male White Sucker ( } 255 \mathrm{~mm} \text {, release) mature dwarf? Scale samples taken for aging. Undercut banks, gravel, some rocks. } \\
\text { Grass only along banks. Shallow pools have gravel substrate. }\end{array}$ \\
\hline $\mathrm{X}-04-093$ & Too deep to fish. \\
\hline $\mathrm{X}-04-094$ & Lots of spawning Fathead Minnows. One visible man-made riffle under bridge. \\
\hline X-04-095 & $\begin{array}{l}\text { One Black Bullhead }(205 \mathrm{~mm}) \text { released. Two other bullheads observed. One visible riffle. Long grass and some small trees on } \\
\text { banks. Shopping carts in drain. }\end{array}$ \\
\hline $\mathrm{X}-04-096$ & Simple habitat but with submerged grass substrate. Lots of flooded land. \\
\hline $\mathrm{X}-04-097$ & Lots of flooded land. Submerged grass substrate. Grass and some trees along banks. \\
\hline $\mathrm{X}-04-098$ & No catch using seine. Channel depth is $1.5 \mathrm{~m}$. Only fast/deep flow regime. Only grasses along banks. Pools absent. \\
\hline $\mathrm{X}-04-099$ & $\begin{array}{l}\text { No catch. Deep channel through the middle of the drain under the bridge } \sim 1 \mathrm{~m} \text { deep. Grasses only along banks. Some } \\
\text { submerged vegetation and rocks under bridge, rest of drain is mud/clay bottom. }\end{array}$ \\
\hline $\mathrm{X}-04-100$ & Fish passage is blocked by debris upstream. \\
\hline $\mathrm{X}-04-101$ & $\begin{array}{l}\text { Spawning male Fathead Minnow accidentally released. Flood control structure on Domain Drain. One visible riffle. Drain is just } \\
\text { inside the Red River floodway west dike. }\end{array}$ \\
\hline $\mathrm{X}-04-102$ & Undercut banks. Generally all flat water. Lots of submerged vegetation. \\
\hline $\mathrm{X}-04-103$ & $\begin{array}{l}\text { Lots of Common Carp observed. Common Carp }(643 \mathrm{~mm}) \text { released. Gravel, sand, some submerged vegetation in pools. } \\
\text { Undercut banks. Just grasses along banks. }\end{array}$ \\
\hline X-04-104 & Many Common Carp observed. Too deep to fish. \\
\hline $\mathrm{X}-04-105$ & d upstream of culvert inlet. Very high veloc \\
\hline
\end{tabular}




\section{Appendix 7: List of any additional field notes recorded at sampling sites.}

\begin{tabular}{|c|c|}
\hline $\begin{array}{l}\text { Site } \\
\text { Number }\end{array}$ & Field Notes \\
\hline $\mathrm{X}-04-106$ & $\begin{array}{l}\text { Local knowledge: suckers observed in river. Undercut banks. Submerged vegetation. Overhanging vegetation. Lacking rocks. } \\
\text { Lots of bends, no riffles. Trees, shrubs and grasses along banks. }\end{array}$ \\
\hline $\mathrm{X}-04-107$ & $\begin{array}{l}\text { Road is flooded } \sim 0.3 \mathrm{~m} \text {. Two suckers observed crossing the low level crossing. Lots of bends. One long riffle. Large trees, shrubs } \\
\text { and grasses along banks. Some root mat and some gravel in pools. }\end{array}$ \\
\hline $\mathrm{X}-04-108$ & Cannot fish because of electric fence. Many riffles. One huge slumped bank $\sim \mathrm{m}$ high. \\
\hline $\mathrm{X}-04-109$ & No catch. Many riffles and bends. Some exposed banks. Not much submerged vegetation. Lots of sediment deposition. \\
\hline $\mathrm{X}-04-110$ & $\begin{array}{l}\text { Moderate cattle impact. River runs through pasture. Trampled bank. No woody debris. Many bends. Bare soil and closely cropped } \\
\text { vegetation along banks. }\end{array}$ \\
\hline $\mathrm{X}-04-111$ & Corrugated metal pipe's diameter is $3 \mathrm{~m}$. Not able to fish because of electric fence. \\
\hline $\mathrm{X}-04-112$ & $\begin{array}{l}\text { Four juvenile White Sucker and two bullhead accidentally released. White Sucker caught above and below waterfall. Submerged } \\
\text { grasses, cobble/boulder substrate and woody debris provide cover. Light cattle impact on downstream side of crossing. Shrubs, } \\
\text { trees and grasses along banks. Large deep pools. }\end{array}$ \\
\hline $\mathrm{X}-04-113$ & Not correctly mapped. Unable to seine due to dense submerged vegetation. Simple habitat with submerged vegetation. \\
\hline $\mathrm{X}-04-114$ & $\begin{array}{l}\text { Local knowledge: suckers use drain, Northern Pike come up creek, but not seen in last two years. High velocity flow over flooded } \\
\text { low level crossing. Dead Common Carp on right bank. Grasses and small woody plants along banks. Mostly deep pools. Obvious } \\
\text { bank slumping on right bank. }\end{array}$ \\
\hline $\mathrm{X}-04-115$ & $\begin{array}{l}\text { Right corrugated metal pipe has buildup of debris at outlet. Lots of undercut banks. Trees and grasses along banks. Very little } \\
\text { root mats in pools. }\end{array}$ \\
\hline $\mathrm{X}-04-116$ & Cattle impacts. Many obstructions to fish passage on this creek. Grasses and small bushes along banks. No larval drift catch. \\
\hline $\mathrm{X}-04-117$ & Very little flow, all from recent rainfall. \\
\hline $\mathrm{X}-04-118$ & $\begin{array}{l}\text { Flooded low level crossing. Long riffle/deep pool/run/riffle sequence. Complex habitat at riffle. More channelization upstream. } \\
\text { Undercut banks downstream. Downstream right bank has more vegetative protection. }\end{array}$ \\
\hline $\mathrm{X}-04-119$ & Cattle impacts. One riffle and some bends upstream. All mud/clay bottom with some submerged vegetation in pools. \\
\hline $\mathrm{X}-04-120$ & No Catch. Pool substrate is mud and decomposing organic matter \\
\hline $\mathrm{X}-04-121$ & Cattle impacts. Common Carp $(670 \mathrm{~mm})$ released. One other adult Common Carp observed. \\
\hline $\mathrm{X}-04-122$ & $\begin{array}{l}\text { No flow through two right corrugated metal pipes. Local knowledge: suckers come up drain, Northern Pike caught slightly further } \\
\text { upstream. Undercut banks. }\end{array}$ \\
\hline $\mathrm{X}-04-123$ & $\begin{array}{l}\text { Some bends. Agricultural land very close to drain. No visible riffles. Few shrubs, mostly grasses along banks. Some root mats } \\
\text { and submerged vegetation in pools. }\end{array}$ \\
\hline $\mathrm{X}-04-124$ & $\begin{array}{l}\text { Flooded low level crossing. Submerged vegetation with few rocks in drain. Only long grasses along banks. Pool substrate is } \\
\text { choked with submerged vegetation. Upstream is agricultural land, downstream is grown-in cattle pasture. }\end{array}$ \\
\hline $\mathrm{X}-04-125$ & No flow. Downstream, cultivated fields right up to drain. \\
\hline $\mathrm{X}-04-126$ & $\begin{array}{l}\text { Simple habitat but with submerged vegetation. Methane production in sediments. Only slow/deep velocity/depth regime. Flat } \\
\text { water (no riffles). Little submerged vegetation in pools. }\end{array}$ \\
\hline
\end{tabular}




\section{Appendix 7: List of any additional field notes recorded at sampling sites.}

\begin{tabular}{|c|c|}
\hline $\begin{array}{l}\text { Site } \\
\text { Number }\end{array}$ & Field Notes \\
\hline $\mathrm{X}-04-127$ & $\begin{array}{l}\text { Simple habitat but with submerged vegetation. Large dead Common Carp caught in rocks. Dead bullhead caught in rocks. } \\
\text { Corrugated metal pipe outlet perched } 0.3 \mathrm{~m} \text {. Rock Bass }(234 \mathrm{~mm}, 221 \mathrm{~mm}, 262 \mathrm{~mm}) \text { released. Northern Pike ( } 347 \mathrm{~mm}) \text { released. } \\
\text { Common Carp }(539 \mathrm{~mm}) \text { released. Habitat composed of undercut banks, limestone and flooded bushes. One riffle observed at } \\
\text { culvert outlet. Upstream only grasses along banks. Downstream there are bushes, grasses and small trees along banks. Pools } \\
\text { too deep to fish. }\end{array}$ \\
\hline $\mathrm{X}-04-128$ & White Sucker $(272 \mathrm{~mm})$ released. Dense submerged vegetation throughout. Mud deposits. Some bends. \\
\hline $\mathrm{X}-04-129$ & $\begin{array}{l}\text { White Sucker }(212 \mathrm{~mm}, 249 \mathrm{~mm}) \text { released. Other White Sucker observed. Logs, some submerged vegetation and some } \\
\text { undercut banks provide cover. Lots of bends and small riffles. Mostly grasses along banks. }\end{array}$ \\
\hline $\mathrm{X}-04-130$ & Only run-off into drain. High sediment deposition. Lots of bends. Trees and grasses along banks. \\
\hline $\mathrm{X}-04-131$ & Very little flow. Water likely from recent rainfall. \\
\hline $\mathrm{X}-04-132$ & $\begin{array}{l}\text { Cut banks, rocks, woody debris, but lacking submerged vegetation. Moderate sediment deposition in pools. High velocity outflow } \\
\text { from corrugated metal pipes. Drain channelized upstream. Riffles and bends downstream. Only long grasses and bare mud along } \\
\text { banks. }\end{array}$ \\
\hline $\mathrm{X}-04-133$ & $\begin{array}{l}\text { Unable to seine due to dense submerged vegetation. High methane production. Lots of long bends. Grasses and reeds along } \\
\text { banks. }\end{array}$ \\
\hline $\mathrm{X}-04-134$ & Simple habitat with submerged vegetation. \\
\hline $\mathrm{X}-04-135$ & Cultivated land around. Low flow. Lots of vegetation. \\
\hline $\mathrm{X}-04-136$ & Simple habitat with submerged vegetation. Pool is too deep to fish. \\
\hline $\mathrm{X}-04-137$ & $\begin{array}{l}\text { No larval catch. Large unidentified fish observed. Cattle impacts. Large deep pool upstream. Shale substrate. Stocked Walleye } \\
\text { sign at dam and gate entrance. Undercut banks, rocks, and lots of submerged vegetation provide cover. Grasses only along } \\
\text { banks. }\end{array}$ \\
\hline $\mathrm{X}-04-138$ & $\begin{array}{l}\text { Black bullhead }(222 \mathrm{~mm}) \text { released. Perched culvert } 0.5 \mathrm{~m} \text {. Electric fence along both sides of the road. Sand bars and shale } \\
\text { sediment deposits. Bends in channel. Pasture upstream. One large, deep pool fished. }\end{array}$ \\
\hline $\mathrm{X}-04-139$ & No catch. Some methane production. Deep pool. Cut banks. \\
\hline $\mathrm{X}-04-140$ & Culvert outlet perched outlet $(0.35 \mathrm{~m})$. \\
\hline $\mathrm{X}-04-141$ & $\begin{array}{l}\text { Beaver dam upstream. Bulrush and arrowhead in creek. Six White Sucker }(208 \mathrm{~mm}, 195 \mathrm{~mm}, 149 \mathrm{~mm}, 173 \mathrm{~mm}, 188 \mathrm{~mm}, 154 \\
\mathrm{mm}) \text { released. Grasses, shrubs and trees along banks. }\end{array}$ \\
\hline $\mathrm{X}-04-142$ & $\begin{array}{l}\text { Lots of bends. Lots of tree branches and woody debris upstream. High sediment deposition. Sand and shale sediment deposits. } \\
\text { Cultivated field in close proximity to left bank. }\end{array}$ \\
\hline $\mathrm{X}-04-143$ & Inlet and outlet not accessible due to barbed wire fencing. \\
\hline $\mathrm{X}-04-144$ & Lakes formed by stream at inlet and outlet. Too deep to fish. Cattle impacts downstream. \\
\hline $\mathrm{X}-04-145$ & $\begin{array}{l}\text { Culvert outlet is perched }(0.3 \mathrm{~m}) \text {. One elevated corrugated metal pipe set higher than other culvert is perched } 1.5 \mathrm{~m} \text { at inlet and } 2 \\
\mathrm{~m} \text { at outlet. Lots of bends. }\end{array}$ \\
\hline $\mathrm{X}-04-146$ & \\
\hline
\end{tabular}




\section{Appendix 7: List of any additional field notes recorded at sampling sites.}

\begin{tabular}{|c|c|}
\hline $\begin{array}{l}\text { Site } \\
\text { Number }\end{array}$ & Field Notes \\
\hline $\mathrm{X}-04-147$ & $\begin{array}{l}\text { Lots of spawning Western Blacknose Dace. Shale substrate. Corrugated metal pipe is perched } 0.5 \mathrm{~m} \text {. Shale sediment } \\
\text { deposition. }\end{array}$ \\
\hline $\mathrm{X}-04-148$ & $\begin{array}{l}\text { Old bridge abutments under bridge. Too deep to fish. Heavy sediment deposition downstream is blocking most of the flow. } \\
\text { Cultivated fields right up to drain downstream. }\end{array}$ \\
\hline $\mathrm{X}-04-149$ & Lots of submerged logs. Sand substrate with no rocks. No catch from larval drift trap. Submerged woody debris in pools. \\
\hline $\mathrm{X}-04-150$ & White Sucker $(172 \mathrm{~mm})$ released. Back eddies along banks. One riffle under bridge. No submerged vegetation in pools. \\
\hline $\mathrm{X}-04-151$ & $\begin{array}{l}\text { Beaver dam in drain. Two large mud bars. Heavy deposits of sediment and obstructions at bends. Bends are frequent and riffle is } \\
\text { below beaver dam. Right bank not protected because of mowed lawn/park area. }\end{array}$ \\
\hline $\mathrm{X}-04-152$ & $\begin{array}{l}\text { Drain located in marsh (no agricultural fields). Dike downstream on left bank. Mud substrate only. Channel alteration at crossing. } \\
\text { Bends are long. Riffle habitat associated with outlet only. Mostly long grass along banks. }\end{array}$ \\
\hline $\mathrm{X}-04-153$ & $\begin{array}{l}\text { Only able to fish along banks because creek is too deep. Methane gas production in sediments. Marsh habitat. Lily pads in creek. } \\
\text { Headwaters of Pine Creek. }\end{array}$ \\
\hline $\mathrm{X}-04-154$ & $\begin{array}{l}\text { No catch. Good habitat: Undercut banks, submerged vegetation, cobble and logs in drain. Natural Resource Officer said this drain } \\
\text { was stocked with trout and that people fish this creek. Lots of bends, and riffles created by logs. Channel alteration at bridge only. }\end{array}$ \\
\hline $\mathrm{X}-04-155$ & $\begin{array}{l}\text { Ten Yellow Perch released }(66-85 \mathrm{~mm}) \text {. Riffle in culvert. Lots of bends, one natural riffle in reach. Grass downstream is mowed } \\
\sim 6 \mathrm{~m} \text { away. Cobble substrate throughout. }\end{array}$ \\
\hline $\mathrm{X}-04-156$ & $\begin{array}{l}\text { Bulrush in drain. } 20 \text { Central Mudminnow released. Good habitat downstream. Local knowledge: suckers and Northern Pike come } \\
\text { up drain in spring. Drain is usually dry by this time of the year. Beaver dams downstream. Only grasses upstream along banks, } \\
\text { but forest downstream. Channel is altered mostly upstream. Bends and riffles downstream only. }\end{array}$ \\
\hline $\mathrm{X}-04-157$ & More young of the year Northern Pike observed. Many beaver dams downstream. \\
\hline $\mathrm{X}-04-158$ & No flow. \\
\hline $\mathrm{X}-04-159$ & One young of the year Northern Pike $(94 \mathrm{~mm})$ released. No bends. \\
\hline $\mathrm{X}-04-160$ & $\begin{array}{l}\text { Simple habitat but with submerged vegetation. Observed young of the year Northern Pike. Two Northern Pike }(83 \mathrm{~mm}, 73 \mathrm{~mm}) \\
\text { released. One riffle in reach. Potential for riffles when water is lower. }\end{array}$ \\
\hline $\mathrm{X}-04-161$ & $\begin{array}{l}\text { No catch. Simple habitat but with submerged vegetation. Bulrush and filamentous algae mats in drain. Local knowledge: suckers } \\
\text { come up drain in spring. Drain dries up in summer. Northern Pike may be coming down from Fish Lake. }\end{array}$ \\
\hline $\mathrm{X}-04-162$ & High flow velocity. Two large Common Carp observed in pool at outlets. Outlets perched $0.4 \mathrm{~m}$. \\
\hline $\mathrm{X}-04-163$ & $\begin{array}{l}\text { Simple habitat but with diverse submerged vegetation. Five young of the year Northern Pike donated to the New Iceland Heritage } \\
\text { Museum. }\end{array}$ \\
\hline $\mathrm{X}-04-164$ & A lot of submerged vegetation in drain. Eight Northern Pike donated to the New Iceland Heritage Museum. \\
\hline$X-04-165$ & Trickle of flow. \\
\hline $\mathrm{X}-04-166$ & Dry. Not diked upstream on west bank. \\
\hline $\mathrm{X}-04-167$ & Many Common Carp ob \\
\hline $\mathrm{X}-04-168$ & id and submerged vegetation substrate. Long grasses along banks. \\
\hline
\end{tabular}




\section{Appendix 7: List of any additional field notes recorded at sampling sites.}

\begin{tabular}{|c|c|}
\hline $\begin{array}{l}\text { Site } \\
\text { Number }\end{array}$ & Field Notes \\
\hline $\mathrm{X}-04-169$ & $\begin{array}{l}\text { Both Northern Pike collected below perched culverts. No catch above perched culverts. Bulrush and arrowhead in drain. } \\
\text { Channelization upstream. Slow flow though drain. Grasses all along banks, bushes downstream. Forest upstream on right bank. } \\
\text { One bend downstream. Pools only deep at corrugated metal pipe outlets. }\end{array}$ \\
\hline $\mathrm{X}-04-170$ & $\begin{array}{l}\text { No catch. Arrowhead and bulrush in drain. A lot of submerged vegetation in drain. No visible riffles. Potential for shallow riffles. } \\
\text { One deep pool }(>0.5 \mathrm{~m}) \text {. }\end{array}$ \\
\hline $\mathrm{X}-04-171$ & Bulrush around drain. Culvert inlet submerged and outlet perched $0.3 \mathrm{~m}$. One riffle at outlet. \\
\hline $\mathrm{X}-04-172$ & $\begin{array}{l}\text { Northern Pike }(247 \mathrm{~mm}) \text { released. No bends, with occasional riffle. Large cobble near culvert outlet. Local knowledge: many large } \\
\text { Northern Pike caught in drain earlier this year. }\end{array}$ \\
\hline $\mathrm{X}-04-173$ & Dry. \\
\hline $\mathrm{X}-04-174$ & Very little flow through culverts, more flow through rocks. \\
\hline $\mathrm{X}-04-175$ & One Mudminnow $(74 \mathrm{~mm})$ donated to the New lceland Heritage Museum. Cobble and dense algae mats in pools. \\
\hline $\mathrm{X}-04-176$ & $\begin{array}{l}\text { Sediment deposition mostly from gravel, sand and fine sediment in corrugated metal pipes. Very little water in channel. Long } \\
\text { bends. Mostly long grasses and some shrubs along banks. Cobble sized hard substrate found only near culverts. }\end{array}$ \\
\hline $\mathrm{X}-04-177$ & $\begin{array}{l}\text { One young of the year Brook Stickleback released. Outlet perched } 0.3 \mathrm{~m} \text {. Bulrush around drain. Continuous riffle because of } \\
\text { shallow water. Upstream the right bank is close to a cultivated field. One deep pool. }\end{array}$ \\
\hline $\mathrm{X}-04-178$ & $\begin{array}{l}\text { Four Common Carp released }(634 \mathrm{~mm}, 620 \mathrm{~mm}, 600-650 \mathrm{~mm}) \text {. No flow through right corrugated metal pipe. Dirt along left bank } \\
\text { and right bank upstream. Patches of bare soil along both banks. }\end{array}$ \\
\hline $\mathrm{X}-04-179$ & Corrugated metal pipes blocked by debris. Deposits of organic matter along bottom. No riffles, some bends. \\
\hline $\mathrm{X}-04-180$ & Too deep to fish. Drains Lee and Otter Lakes into Fisher Bay (Lake Winnipeg). Bulrush present in drain. \\
\hline $\mathrm{X}-04-181$ & $\begin{array}{l}\text { Bulrush in drain. Patches of eroded bank. Heavy muddy clay deposits. Less than } 25 \% \text { of channel substrate is exposed. No } \\
\text { bends, some small riffles in reach. }\end{array}$ \\
\hline $\mathrm{X}-04-182$ & $\begin{array}{l}\text { Cattle impact. Bulrush present in drain. Local knowledge: pelicans in drain eating } \sim 10 \mathrm{~cm} \text { long fish. Lake stocked with Northern } \\
\text { Pike } \sim 35 \text { years ago. }\end{array}$ \\
\hline $\mathrm{X}-04-183$ & Drain full of bulrush and cattails. Cattle impact. \\
\hline $\mathrm{X}-04-184$ & $\begin{array}{l}\text { One large Common Carp observed in drain. Arrowhead observed in drain. Diked only on right bank upstream. All flat water. Long } \\
\text { grasses, some bushes and reeds along bank. Long grasses in riparian zone. }\end{array}$ \\
\hline $\mathrm{X}-04-185$ & $\begin{array}{l}\text { Arrowhead and bulrush in drain. Moderate cattle-impacts. A lot of algae in drain. Six Northern Pike (199 mm, } 99 \mathrm{~mm}, 99 \mathrm{~mm}, 81 \\
\mathrm{~mm}, 84 \mathrm{~mm}, 93 \mathrm{~mm}) \text { released. Seven Yellow Perch }(93 \mathrm{~mm}, 71 \mathrm{~mm}, 73 \mathrm{~mm}, 68 \mathrm{~mm}, 69 \mathrm{~mm}, 68 \mathrm{~mm}, 66 \mathrm{~mm}) \text { released. Long } \\
\text { grass outside fence, grazed grass inside fence. }\end{array}$ \\
\hline $\mathrm{X}-04-186$ & Dense growth of cattails growing in drain. No riffles, no bends. Some exposed banks. Pool only present under bridge. \\
\hline $\mathrm{X}-04-187$ & $\begin{array}{l}\text { Sixteen Northern Pike }(107 \mathrm{~mm}, 130 \mathrm{~mm}, 124 \mathrm{~mm}, 131 \mathrm{~mm}, 109 \mathrm{~mm}, 124 \mathrm{~mm}, 120 \mathrm{~mm}, 120 \mathrm{~mm}, 118 \mathrm{~mm}, 91 \mathrm{~mm}, 108 \mathrm{~mm}, 107 \\
\mathrm{mm}, 112 \mathrm{~mm}, 112 \mathrm{~mm}, 116 \mathrm{~mm}, 110 \mathrm{~mm}) \text { released. High velocity flow across dam. Some long bends. Grasses and reeds along } \\
\text { banks. }\end{array}$ \\
\hline
\end{tabular}




\section{Appendix 7: List of any additional field notes recorded at sampling sites.}

\begin{tabular}{|c|c|}
\hline $\begin{array}{l}\text { Site } \\
\text { Number }\end{array}$ & Field Notes \\
\hline $\mathrm{X}-04-188$ & $\begin{array}{l}\text { Many young of the year Northern Pike observed. Two large Common Carp observed. Arrowhead in drain. Island Lake Drain } \\
\text { appears to have been recently cleaned or channelized. }\end{array}$ \\
\hline $\mathrm{X}-04-189$ & No flow. \\
\hline $\mathrm{X}-04-190$ & Moderate cattle impacts. Heavy deposits of mud. All flat water. Many exposed banks. All mud substrate in pools. \\
\hline $\mathrm{X}-04-191$ & No flow. Dense cattail growth in drain. \\
\hline $\mathrm{X}-04-192$ & $\begin{array}{l}\text { Freshwater Drum }(682 \mathrm{~mm}) \text { released. Sand deposit at culvert inlet. Just grasses and reeds along banks. One deep pool } \\
\text { downstream. }\end{array}$ \\
\hline $\mathrm{X}-04-193$ & No flow. \\
\hline X-04-194 & Arrowhead in drain. No flow, standing pool. Organic debris at inlets. Mostly grasses along banks upstream. One deep pool. \\
\hline X-04-195 & $\begin{array}{l}\text { Dead adult bullhead found in drain. Drain is flowing in the opposite direction of its usual flow. No riffles, no bends. Small trees and } \\
\text { long grasses along banks. }\end{array}$ \\
\hline $\mathrm{X}-04-196$ & $\begin{array}{l}\text { Local knowledge: adult Northern Pike and Common Carp caught in drain during spring. Limestone along left bank at outlet. Some } \\
\text { deep riffles due to high-velocity flow over gravel substrate. Mud and clay substrate in pools. }\end{array}$ \\
\hline $\mathrm{X}-04-197$ & No flow. \\
\hline $\mathrm{X}-04-198$ & $\begin{array}{l}\text { Dense mats of filamentous algae throughout most of drain. All flat water. Long grasses only along banks. Mud and submerged } \\
\text { vegetation dominate pool substrate. }\end{array}$ \\
\hline $\mathrm{X}-04-199$ & $\begin{array}{l}\text { Five Yellow Perch ( } 84 \mathrm{~mm}, 88 \mathrm{~mm}, 122 \mathrm{~mm}, 88 \mathrm{~mm}, 81 \mathrm{~mm} \text { ) released. Lots of submerged vegetation. No riffles. Some long } \\
\text { bends. Long grasses and reeds along banks. Mud and dense submerged vegetation dominate pool substrate. }\end{array}$ \\
\hline $\mathrm{X}-04-200$ & $\begin{array}{l}\text { Two Common Carp: (669 mm, } 497 \mathrm{~mm} \text { ) released. Many Common Carp observed. Corrugated metal pipe culvert outlets are gated } \\
\text { and closed. All long grasses along banks. Moderately deep pools present. }\end{array}$ \\
\hline $\mathrm{X}-04-201$ & $\begin{array}{l}\text { Arrowhead in drain. Channel meanders within drain. Turbulent flow, shallow }(\sim 0.03 \mathrm{~m}) \text { gravel. Shrubs and grasses along banks. } \\
\text { Mud, clay and some fine gravel present in pools. One deep pool at corrugated metal pipe outlet. }\end{array}$ \\
\hline $\mathrm{X}-04-202$ & Dead bushes and trees sunken at outlets. Small riffles at outlet. Only long grasses along banks. \\
\hline$X-04-203$ & $\begin{array}{l}\text { Only able to fish along banks because pools are too deep (over } 1.5 \mathrm{~m} \text { ). Flooded grasses along banks. No riffles. Left bank only } \\
\text { grasses, right bank all ages of trees and grasses. Submerged vegetation in pools. }\end{array}$ \\
\hline $\mathrm{X}-04-204$ & $\begin{array}{l}\text { Many tadpoles observed. Flooded long grasses along banks. Rocks embedded in detritus. All water is slow/ shallow flow regime.. } \\
\text { No bends or riffles. Right bank has long grasses and bushes. Left bank only has long grasses and is adjacent to railway tracks. }\end{array}$ \\
\hline $\mathrm{X}-04-205$ & Fine gravel deposits. Only channelized next to road. Undercut banks, overhanging vegetation. Riffle-run-pool sequences. \\
\hline$X-04-206$ & $\begin{array}{l}\text { Inlets blocked by vegetation, debris and logs. Bulrush and arrowheads in drain. Cyprinids or Catostomids }(\sim 100-150 \mathrm{~mm}) \\
\text { observed. Unable to collect. Undercut banks. Channelized along gravel road. Left bank has long grasses. Right bank has all } \\
\text { classes of vegetation. Submerged woody debris in pools. }\end{array}$ \\
\hline X-04-207 & $\begin{array}{l}\text { Local knowledge: adult Northern Pike caught in Sprague Creek, White Sucker runs out of Whitemouth Lake. Occasional riffle. } \\
\text { Gravel and cobble in pool. One deep pool present. }\end{array}$ \\
\hline$X-04-208$ & Heavy deposits of mud and some fine gravel. Many undercut banks. Long grasses, small bushes and reeds along both banks. \\
\hline
\end{tabular}




\section{Appendix 7: List of any additional field notes recorded at sampling sites.}

\begin{tabular}{|c|c|}
\hline $\begin{array}{l}\text { Site } \\
\text { Number }\end{array}$ & Field Notes \\
\hline $\mathrm{X}-04-209$ & $\begin{array}{l}\text { Burbot }(304 \mathrm{~mm}) \text { released. Other Burbot observed. Undercut banks. Lots of cobble. Rocks along bank are very unstable. } \\
\text { Upstream left bank is near grass field. }\end{array}$ \\
\hline $\mathrm{X}-04-210$ & $\begin{array}{l}\text { Local knowledge: "bass" caught in drain (information from Manitoba conservation receptionist). At least one young of the year } \\
\text { White Sucker in seine sample. One White Sucker }(\sim 100-120 \mathrm{~mm}) \text { observed. Cobble, submerged vegetation and undercut banks } \\
\text { present. No riffles. Many bends. Mud with submerged vegetation in pools. Some cobble around bridge. }\end{array}$ \\
\hline $\mathrm{X}-04-211$ & $\begin{array}{l}\text { Too deep to fish. Thousands of young of the year Northern Pike observed. Very high velocity flow through corrugated metal pipe. } \\
\text { River is marshy. }\end{array}$ \\
\hline $\mathrm{X}-04-212$ & $\begin{array}{l}\text { No catch. Piles of cleared dredged plant matter along banks. All flat water. Few long bends. Long grasses only. Small amount of } \\
\text { submerged vegetation. }\end{array}$ \\
\hline $\mathrm{X}-04-213$ & $\begin{array}{l}\text { No catch. Arrowhead in drain. A lot of submerged vegetation in drain. No riffles or bends. Only grasses along banks. Pool } \\
\text { substrate consists of mud and submerged vegetation. }\end{array}$ \\
\hline $\mathrm{X}-04-214$ & Not mapped. Very little flow through drain. \\
\hline $\mathrm{X}-04-215$ & $\begin{array}{l}\text { No catch but five young of the year Northern Pike observed. No riffles, few very long bends. Some patches of loose gravel along } \\
\text { banks. Long grasses and some shrubs along banks. Some submerged vegetation along banks. }\end{array}$ \\
\hline $\mathrm{X}-04-216$ & $\begin{array}{l}\text { No catch. Simple habitat but with submerged vegetation. Dense mats of submerged vegetation. Deposits of organic matter along } \\
\text { bottom of drain. No riffles, few bends. Long grasses along banks. Pool substrate consists of mud, submerged vegetation and } \\
\text { decomposing organic matter. }\end{array}$ \\
\hline $\mathrm{X}-04-217$ & No catch. Simple habitat. Submerged vegetation. Many frogs and tadpoles in drain. \\
\hline$X-04-218$ & No flow. Dry. Ben Johnson drain is also dry. \\
\hline$X-04-219$ & Marginal habitat. Too deep to fish. \\
\hline $\mathrm{X}-04-220$ & Drain is dry. \\
\hline$X-04-221$ & $\begin{array}{l}\text { One Northern Pike }(364 \mathrm{~mm}) \text { released. One White Sucker }(141 \mathrm{~mm}) \text { released. Five Rock Bass }(147 \mathrm{~mm}, 68 \mathrm{~mm}, 70 \mathrm{~mm}, 61 \mathrm{~mm}) \\
\text { released. One Common Carp }(163 \mathrm{~mm}) \text { released. One Tadpole Madtom }(61 \mathrm{~mm}) \text { released. Long riffle } 20 \mathrm{~m} \text {. Three Rock Bass, } \\
\text { one Tadpole Madtom, one Common Carp donated to the Icelandic Heritage Museum. }\end{array}$ \\
\hline $\mathrm{X}-04-222$ & $\begin{array}{l}\text { Two White Sucker }(142 \mathrm{~mm}, 143 \mathrm{~mm}) \text { released. Ten YOY White Sucker }(\sim 50-70 \mathrm{~mm}) \text { released. Most riffle substrates are } \\
\text { exposed. Long grasses and shrubs along banks. Pool substrate is dominated by cobble with some submerged vegetation. }\end{array}$ \\
\hline $\mathrm{X}-04-223$ & $\begin{array}{l}\text { One White Sucker (139 mm released). Arrowhead in drain. Embeddedness } 30 \% \text { in riffle but } ~ 75 \% \text { embeddedness in pools. Most } \\
\text { riffle substrates are exposed. Many bends and riffles. No mature trees in immediate reach but present upstream and downstream. }\end{array}$ \\
\hline $\mathrm{X}-04-224$ & No flow through drain. \\
\hline $\mathrm{X}-04-225$ & $\begin{array}{l}\text { Cement blocks make up } ~ 30 \% \text { of riffle. Slow/shallow velocity/depth regime found only along banks. Most riffle substrates are } \\
\text { exposed. One visible riffle. Many long bends. Shrubs and long grasses along banks. Pool substrate consists of mud and rock with } \\
\text { some submerged vegetation. }\end{array}$ \\
\hline $\mathrm{X}-04-226$ & Too deep to fish. Submerged vegetation throughout drain. \\
\hline $\mathrm{X}-04-227$ & Many riffles and bends. Some eroded areas along banks. Pool substrate consists of mud and roch \\
\hline
\end{tabular}




\section{Appendix 7: List of any additional field notes recorded at sampling sites.}

\begin{tabular}{|c|c|}
\hline $\begin{array}{l}\text { Site } \\
\text { Number }\end{array}$ & Field Notes \\
\hline $\mathrm{X}-04-228$ & $\begin{array}{l}\text { No catch. Left corrugated metal pipe blocked at inlet. Right corrugated metal pipe blocked at outlet. Potential for riffles if water } \\
\text { was not held back by a beaver dam. Long grasses along banks. }\end{array}$ \\
\hline $\mathrm{X}-04-229$ & Too deep to fish. \\
\hline $\mathrm{X}-04-230$ & Too deep in pool to fish. Right bank wire fence prevents access to riffle downstream. \\
\hline $\mathrm{X}-04-231$ & No access to inlet or outlet because of wire fence. \\
\hline $\mathrm{X}-04-232$ & $\begin{array}{l}\text { Grates on inlet and outlet. Inlet extension also covered by grates. Marsh habitat around drain. Flow is altered by grates on } \\
\text { corrugated metal pipe. Drain has some bends but no riffles. Pool substrate dominated by mud with some root mats, submerged } \\
\text { vegetation and some fine gravel. }\end{array}$ \\
\hline $\mathrm{X}-04-233$ & Riffle $\sim 100 \mathrm{~m}$ upstream from culvert crossing. Too deep to fish. Many exposed mud banks. \\
\hline $\mathrm{X}-04-234$ & Too deep to fish. \\
\hline $\mathrm{X}-04-235$ & Many mud bars downstream. \\
\hline $\mathrm{X}-04-236$ & Arrowhead in drain. Too deep to fish. \\
\hline $\mathrm{X}-04-237$ & Too deep to fish. \\
\hline$X-04-238$ & $\begin{array}{l}\text { Almost no flow through right corrugated metal pipe. Most riffle substrates are exposed. The substrate is cement at outlets. The } \\
\text { creek is almost all riffles due to low flow. Long grasses and small shrubs along banks. Pool substrate consists of a mixture of soft } \\
\text { sand and fine gravel. }\end{array}$ \\
\hline $\mathrm{X}-04-239$ & $\begin{array}{l}\text { One White Sucker }(386 \mathrm{~mm}) \text { released. Three Burbot }(273 \mathrm{~mm}, 283 \mathrm{~mm}, 304 \mathrm{~mm}) \text { released. Two other adult White Sucker } \\
\text { observed. Large dam present upstream. Rip rap along banks. }\end{array}$ \\
\hline $\mathrm{X}-04-240$ & $\begin{array}{l}\text { No flow through drain. Cultivated land around. Some standing water present in drain. Drain is choked with algae and submerged } \\
\text { vegetation. }\end{array}$ \\
\hline $\mathrm{X}-04-241$ & Wooden bridge allows dirt to fall into drain underneath. Standing pool. Drain is choked with algae. \\
\hline $\mathrm{X}-04-242$ & Drain is dry with standing pools upstream. Cattle impact present. Pools are choked with algae and submerged vegetation. \\
\hline$X-04-243$ & Standing pool with little/no flow. Drain is choked with vegetation. \\
\hline $\mathrm{X}-04-244$ & Too deep to fish. Many large trees/logs and debris create a jam under the bridge. \\
\hline $\mathrm{X}-04-245$ & $\begin{array}{l}\text { Burbot }(304 \mathrm{~mm}) \text { released. Other Burbot observed. Undercut banks. Lots of cobble. Rocks along bank are very unstable. } \\
\text { Upstream left bank is near grass field. }\end{array}$ \\
\hline$X-04-246$ & Trickle of flow through drain. Mud substrate only. Drain is blocked with dead plant matter and garbage downstream. \\
\hline $\mathrm{X}-04-247$ & Pool too deep to fish. Most of drain is choked with cattails. \\
\hline $\mathrm{X}-04-248$ & Heavy deposits of fine sediment. Upstream, the top of the water is covered in mats of floating vegetation. \\
\hline$X-04-249$ & $\begin{array}{l}\text { A lot of floating vegetation mats on top of water. Methane or hydrogen sulphide production in sediments. All flat water with long } \\
\text { bends. Mostly long grasses along banks. }\end{array}$ \\
\hline $\mathrm{X}-04-250$ & h drain. Dense cattails throughout drain upstream. Diked on left bank downstrean \\
\hline
\end{tabular}




\section{Appendix 7: List of any additional field notes recorded at sampling sites.}

\begin{tabular}{|c|c|}
\hline $\begin{array}{l}\text { Site } \\
\text { Number }\end{array}$ & Field Notes \\
\hline $\mathrm{X}-04-251$ & $\begin{array}{l}\text { Dead White Sucker found at outflow. Outflow is } \sim 150 \mathrm{~m} \text { from Assiniboine River. Very high velocity flow. Other pipe at outlet may } \\
\text { be drawing water from Mill Creek to Assiniboine River. Some of the surrounding areas are flooded. One long riffle at outflow. Only } \\
\text { mowed grasses along banks. }\end{array}$ \\
\hline $\mathrm{X}-04-252$ & Too deep to fish. High velocity flow. Reach is $150 \mathrm{~m}$ from Assiniboine River. \\
\hline $\mathrm{X}-04-253$ & $\begin{array}{l}\text { Very long riffle. Very heavy deposits of fine sediment and silt. Cement substrate on riffle. Lots of layered cobble. Many raw banks. } \\
\text { Mud dominant with large cobble in pools. Shorthead Redhorse }(360 \mathrm{~mm}) \text {, White Sucker }(288 \mathrm{~mm}) \text {, Sauger }(295 \mathrm{~mm}, 242 \mathrm{~mm} \text { and } \\
203 \mathrm{~mm} \text { ) released. }\end{array}$ \\
\hline $\mathrm{X}-04-254$ & Too deep to fish upstream and downstream of riffle. Water Survey of Canada hydrometric station at reach. \\
\hline $\mathrm{X}-04-255$ & $\begin{array}{l}\text { Observed bird flying away with fish in its mouth. Some bare banks. Man-made riffle. Long grasses along banks. Mud with some } \\
\text { cobble in pools. }\end{array}$ \\
\hline $\mathrm{X}-04-256$ & $\begin{array}{l}5.5 \mathrm{~m} \text { fall at siphon inlet prevents fish passage. High velocity flow. Dead Walleye or Sauger (adult) found at siphon overflow } \\
\text { outlet. Unable to calibrate dissolved oxygen meter. Channel catfish }(333 \mathrm{~mm}) \text { released. Layered cobble at overflow outlet. Bushes } \\
\text { and grasses along banks. Mud with occasional rock in pools. }\end{array}$ \\
\hline $\mathrm{X}-04-257$ & Dissolved oxygen meter not calibrating. Man-made riffle. Shrubs and long grasses along banks. Mud and boulders in pools. \\
\hline $\mathrm{X}-04-258$ & Five White Sucker $(181 \mathrm{~mm}, 147 \mathrm{~mm}, 124 \mathrm{~mm}, 140 \mathrm{~mm}$, and $98 \mathrm{~mm})$ released. Few bends. Only long grasses along banks. \\
\hline $\mathrm{X}-04-259$ & $\begin{array}{l}\text { Observed fishermen catching adult Northern Pike. High velocity flow at riffle. Flooded low-level crossing. Denil fish passageway } \\
\text { full of debris. Large slumped bank at bend. All classes of vegetation along banks. Sand, gravel and little vegetation in pools. }\end{array}$ \\
\hline$X-04-260$ & $\begin{array}{l}\text { Local knowledge: Walleye caught at crossing and upstream. Many sucker runs in past. Outlet velocities vary between } 1.33 \text { and } \\
2.1 \mathrm{~m} / \mathrm{s} \text { Channelized only at crossing. Build-up of sticks and logs at culvert inlets. }\end{array}$ \\
\hline $\mathrm{X}-04-261$ & Too deep to fish. Velocity at outlets measured between 0.59 and $1.29 \mathrm{~m} / \mathrm{s}$ Velocity at inlets measured between 0.65 and $1.17 \mathrm{~m} / \mathrm{s}$ \\
\hline $\mathrm{X}-04-262$ & $\begin{array}{l}\text { Arrowhead in river. Four Logperch }(104 \mathrm{~mm}, 103 \mathrm{~mm}, 101 \mathrm{~mm} \text { and } 108 \mathrm{~mm}) \text { released. Boulders and cobble throughout reach. } \\
\text { One slumped bank. Not much submerged vegetation. }\end{array}$ \\
\hline$X-04-263$ & High flow velocity. Head at dam is $0.65 \mathrm{~m}$ above water downstream of dam. Too deep to fish. \\
\hline D-05-001 & At bankfull stage. Scheduled for bridge replacement. \\
\hline D-05-002 & Very low flow. \\
\hline D-05-003 & High flow velocities through culvert $(1.2 \mathrm{~m} / \mathrm{s})$. No signs of fish movement. \\
\hline D-05-004 & White Sucker congregating upstream and downstream of culverts. No passage problems at this flow velocity. \\
\hline D-05-005 & No fish evident. Fairly high velocities through culvert. Some cobble/boulder substrate. \\
\hline D-05-006 & Almost dry. Trickle of flow only. \\
\hline D-05-007 & Lots of large White Sucker congregating in drain. Cement, open bottom, box culvert. \\
\hline D-05-008 & $\begin{array}{l}\text { Lot of large White Sucker congregating. Local knowledge indicates not enough flow duration anymore. Used to have Walleye } \\
\text { spawning in drain. }\end{array}$ \\
\hline D-05-009 & $\begin{array}{l}\text { Lots of White Sucker congregating. Two small Northern Pike visible in grass cover. Local knowledge indicates sucker and } \\
\text { Northern Pike run most years that have good flow. No run last year (2004). }\end{array}$ \\
\hline
\end{tabular}




\section{Appendix 7: List of any additional field notes recorded at sampling sites.}

\begin{tabular}{|c|c|}
\hline $\begin{array}{l}\text { Site } \\
\text { Number }\end{array}$ & Field Notes \\
\hline D-05-010 & Outflow from pond sampled for larval drift. No catch. New ice cover on pond. A lot of Gammarus spp. in sample. \\
\hline D-05-011 & Electrofisher boat operated by M. Lowdon and D. Watkinson. \\
\hline D-05-013 & $\begin{array}{l}\text { Appears the sheet pile weir directs flow toward the inlet of Enhancement Channel. Heavy sediment (shale) deposits on banks, } \\
\text { point bars and the inlet to the Enhancement Channel. }\end{array}$ \\
\hline D-05-014 & $\begin{array}{l}\text { Enhancement channel has no flow. Mouth of the inlet channel at the Pembina River weir is blocked with sediment. Fish in the } \\
\text { inlet are trapped at this time. }\end{array}$ \\
\hline D-05-015 & Observed two Northern Pike when electrofishing - unable to collect. Released two White Sucker $(441,404 \mathrm{~mm})$ \\
\hline D-05-016 & Water over the road three weeks ago. Heavy shale deposits on banks. \\
\hline D-05-017 & $\begin{array}{l}\text { Upstream end of diversion channel is well armoured. Too much flow to wade safely (near bankfull flow). Dip netted White Sucker, } \\
\text { dace, Fathead Minnow, Common Shiner. Local says Common Carp recently moved into the area. Previously there were no } \\
\text { Common Carp in Pelican Lake until screens in control structure were removed? }\end{array}$ \\
\hline D-05-018 & Released three Northern Pike $(160,158,153 \mathrm{~mm})$ \\
\hline D-05-019 & Released one Northern Pike $(201 \mathrm{~mm})$ and ten Brook Stickleback \\
\hline D-05-021 & Dip netted and released dozen of Brook Stickleback and a four Fathead Minnows \\
\hline D-05-022 & Almost dry, no flow \\
\hline D-05-023 & Released three White Sucker $(430,406,404 \mathrm{~mm})$ and five Creek Chub. \\
\hline $\mathrm{D}-05-024$ & Cattle access to river, moderate impacts \\
\hline D-05-025 & $\begin{array}{l}\text { Released }>30 \text { Common Carp D/S of Burr Oak Bay culverts. Released }>30 \text { Fathead Minnows, three Brook Sticklebacks upstream } \\
\text { of culverts (impassable at this flow, perched } 0.5 \mathrm{~m} \text { ). Upstream storage in constructed retention ponds associated with a housing } \\
\text { development. Small amount of purple loosestrife on site. }\end{array}$ \\
\hline D-05-026 & Some erosion damage around culvert. Some areas of heavy sediment deposition upstream of culverts. \\
\hline $\mathrm{D}-05-027$ & Suckers caught only in areas of flow turbulence (channel restriction around rock dam) \\
\hline W-05-001 & $\begin{array}{l}\text { More than } 20 \text { suckers dead on shore. Gated culverts on north side of right bank, downstream of the cobble armouring. A weir and } \\
\text { riffle have been constructed. There is moderate cattail growth at water's edge. }\end{array}$ \\
\hline W-05-002 & There is a mat of terrestrial grasses in channel. \\
\hline W-05-003 & Diked. Surrounded by cultivated fields. \\
\hline W-05-004 & Diked. \\
\hline W-05-005 & Not diked. \\
\hline W-05-006 & Diked around cultivated fields. \\
\hline W-05-007 & Diked. Surrounded by cultivated fields. \\
\hline W-05-008 & $\begin{array}{l}\text { Not diked. Light cattle impacts downstream. Upstream brush is very thick. Deep thalweg channel. Brush and vegetation collecting } \\
\text { on barbed wire fence. }\end{array}$ \\
\hline W-05-009 & $\begin{array}{l}\text { Diked. Local knowledge: no large fish observed in stream. Lots of submerged vegetation. Thalweg beginning to meander within } \\
\text { channel. }\end{array}$ \\
\hline
\end{tabular}




\section{Appendix 7: List of any additional field notes recorded at sampling sites.}

\begin{tabular}{|c|c|}
\hline $\begin{array}{l}\text { Site } \\
\text { Number }\end{array}$ & Field Notes \\
\hline W-05-010 & Diked. \\
\hline W-05-011 & Not diked. \\
\hline W-05-012 & Not diked. White Sucker released. Culverts in poor condition. \\
\hline W-05-013 & Not diked. Fairly natural stream conditions. \\
\hline W-05-014 & Not diked. Farmer drives tractor through drain. Post-spawn White Sucker collected. \\
\hline W-05-015 & Not diked. Marsh habitat. \\
\hline W-05-016 & Diked along railway tracks. $30+$ suckers observed in the deep pool (attempts to collect them resulted in wet waders). \\
\hline W-05-017 & Not diked. Many other suckers observed but not captured. Light cattle impacts. Stream is meandering naturally. \\
\hline W-05-018 & Diked. The two smaller culverts are perched. \\
\hline W-05-019 & $\begin{array}{l}\text { Not diked; Local knowledge: clay soils, short spring runoff, stream usually stops running in May, dries up expect for occasional } \\
\text { rain. Terrestrial grass throughout channel. }\end{array}$ \\
\hline W-05-020 & Not diked. Trees and shrubs in water. \\
\hline W-05-021 & Not diked. \\
\hline W-05-022 & Not diked. Substantial amounts of woody debris in drain and plugging culvert. \\
\hline W-05-023 & Diked. \\
\hline W-05-024 & Diked. Dense growth of cattails. Northern Pike observed but not caught. \\
\hline W-05-025 & Not diked. \\
\hline W-05-026 & Not diked. \\
\hline W-05-027 & Diked. Dense aquatic vegetation. \\
\hline W-05-028 & Diked. Channelized. Dense instream vegetation growth. \\
\hline W-05-029 & Water down $\sim 1 \mathrm{~m}$. Observed ten large fish moving upstream through culvert. Not diked. \\
\hline W-05-030 & Standing pools only. Vegetative protection: grass and cattails. Diked roadside. No fish caught. \\
\hline W-05-031 & Not diked. \\
\hline W-05-032 & Not diked. Perched culvert outlets. \\
\hline W-05-033 & Diked right bank downstream of crossing. \\
\hline W-05-034 & $\begin{array}{l}\text { Observed one bullhead but did not catch. Diked both sides. Farmer came and burned directly up to drain. Samples were } \\
\text { mislabeled as "Menard Drain" but have correct site number. Only slow/shallow flow regime present. Pools on either side of } \\
\text { crossing. Vegetative protection: all grasses. }\end{array}$ \\
\hline W-05-035 & Diked both sides. Farmer came by and burned grass and other riparian vegetation. \\
\hline W-05-036 & $\begin{array}{l}\text { Diked both sides upstream, not diked downstream. Instream vegetation dominated by reeds. Vegetative protection: grasses. No } \\
\text { fish caught. }\end{array}$ \\
\hline W-05-037 & $\begin{array}{l}\text { Diked both sides. Vegetative bank protection: grasses. Only slow/shallow flow regime present. All flat water, no riffles, no bends, } \\
\text { a few pools. No fish caught. }\end{array}$ \\
\hline
\end{tabular}




\section{Appendix 7: List of any additional field notes recorded at sampling sites.}

\begin{tabular}{|c|c|}
\hline $\begin{array}{l}\text { Site } \\
\text { Number }\end{array}$ & Field Notes \\
\hline W-05-038 & $\begin{array}{l}\text { Diked both sides. Vegetative bank protection: grasses. Only slow/shallow flow regime present. All flat water, no riffles, no bends, } \\
\text { a few pools. }\end{array}$ \\
\hline W-05-039 & Diked on right bank. \\
\hline W-05-040 & Diked both sides. Farmer came by and burned grass and other riparian vegetation. \\
\hline W-05-041 & Not diked. Velocity/depth regimes present: slow/ shallow, slow/deep. Vegetative protection: all grasses. \\
\hline W-05-042 & Not diked. \\
\hline W-05-043 & Not diked. No fish caught. \\
\hline W-05-044 & Not diked. Too deep to fish. \\
\hline W-05-045 & Channelized, but not diked. Only slow/ shallow flow regime present. Vegetative protection: all grasses. \\
\hline W-05-046 & $\begin{array}{l}\text { Not diked. No turbidity data, meter and vials frozen. Water filling less than } 25 \% \text { of the channel but still too deep to fish. Instream } \\
\text { vegetation present. Three of four velocity depth regimes present - missing fast/shallow. Vegetative protection: all grasses. }\end{array}$ \\
\hline W-05-047 & $\begin{array}{l}\text { Channelized. Diked roadside. Velocity/depth regimes: slow/shallow and slow/deep. Vegetative protection: all grasses. } 1 \text { large } \\
\text { scour pool at downstream end of culverts. }\end{array}$ \\
\hline W-05-048 & $\begin{array}{l}\text { Velocity depth regime: slow/ shallow; vegetative protection: all grasses. Channelized. Not diked. No riffles, } 1 \text { bend in reach. } \\
\text { Substrate is hardpan clay in some areas. }\end{array}$ \\
\hline W-05-049 & Diked both sides. Slow/ shallow and slow/deep velocity/depth regimes present. No fish caught. \\
\hline W-05-050 & $\begin{array}{l}\text { Channelized and diked both sides. Slow/deep and slow/shallow flow regimes present. No riffles no bends. Bank vegetative } \\
\text { protection: all grasses. }\end{array}$ \\
\hline W-05-051 & $\begin{array}{l}\text { Channelized and diked both sides. Slow/deep and slow/shallow flow regimes present. No riffles, } 3 \text { bends in reach. Vegetative } \\
\text { protection: all grasses. No fish caught. }\end{array}$ \\
\hline W-05-052 & $\begin{array}{l}\text { Not diked. Velocity/depth regime: slow/ shallow; vegetative protection: all grasses; pool substrate is matted submerged } \\
\text { vegetation. }\end{array}$ \\
\hline W-05-053 & Channelized and diked both sides. Velocity/depth regime: slow/shallow; vegetative protection: all grasses; no riffles, no bends. \\
\hline W-05-054 & $\begin{array}{l}\text { Channelized. Diked both sides. Velocity/depth regime: slow/shallow. Heavy sediment deposition, cobble very embedded. No } \\
\text { riffles, no bends. Vegetative protection: all grasses. Very little vegetation in drain, mostly along edges. Had to discontinuing } \\
\text { fishing - heavy sediment was too difficult to wade. }\end{array}$ \\
\hline W-05-055 & Not diked. Moderate cattle impacts downstream. \\
\hline W-05-056 & Not Diked, Inlet of culvert perched $0.2 \mathrm{~m}$. Reach in very deep ravine surrounded by pastures. Caught White Sucker. \\
\hline W-05-057 & $\begin{array}{l}\text { Not diked, Heavy cattle impacts, a section of left bank upstream from bridge has slumped and been trampled by cattle. No } \\
\text { fast/deep flow regime. }\end{array}$ \\
\hline W-05-058 & $\begin{array}{l}\text { Light cattle impacts upstream. Not diked. Suckers caught. Shale dominant substrate type. Moderate growth of aquatic vegetation } \\
\text { in drain. Suckers spawning upstream from washed out road crossing. Four White Sucker caught and released. }\end{array}$ \\
\hline W-05-059 & No cattle impacts. Not diked. No fast/deep flow regime. \\
\hline W-05-060 & t diked. No cattle impacts. Many bends created by bank slum \\
\hline
\end{tabular}




\section{Appendix 7: List of any additional field notes recorded at sampling sites.}

\begin{tabular}{|c|c|}
\hline $\begin{array}{l}\text { Site } \\
\text { Number }\end{array}$ & Field Notes \\
\hline W-05-061 & $\begin{array}{l}\text { Not diked. Light cattle impacts downstream, severe bank erosion. Culverts are perched at inlet and outlet about } 0.2 \mathrm{~m} \text {. Did not } \\
\text { fish because of electric fence upstream and deep pool immediately downstream of culvert. Flow velocity at culvert inlet }=0.7 \mathrm{~m} / \mathrm{s} \text {. }\end{array}$ \\
\hline W-05-062 & Not diked. No cattle impacts. Water level has recently dropped $0.5 \mathrm{~m}$. \\
\hline W-05-063 & Not diked. No cattle impacts. Released three Brook Stickleback. \\
\hline W-05-064 & Not diked. No cattle impacts. \\
\hline W-05-065 & Not diked. No cattle impacts. \\
\hline W-05-066 & Not diked. No cattle impacts. Not channelized. \\
\hline W-05-067 & Not diked. Moderate cattle impacts. \\
\hline W-05-068 & Diked roadside downstream; not diked upstream No cattle impacts. \\
\hline W-05-069 & Diked both sides. No cattle impacts. Slow/shallow and slow/deep flow regimes present. \\
\hline W-05-070 & Not diked. Light cattle impacts. \\
\hline W-05-071 & $\begin{array}{l}\text { Diked both sides. No cattle impacts. Slow/shallow and slow/deep flow regimes present. No riffles, no bends. Channelized, } 2 \text { large } \\
(4 \mathrm{~m}) \text { culverts. }\end{array}$ \\
\hline W-05-072 & Not diked. Light cattle impacts. Slow/ shallow and fast/ shallow regimes present. \\
\hline W-05-073 & $\begin{array}{l}\text { Diked both sides. No cattle impacts. }{ }^{* *} \text { Local knowledge: (from local who resides at farm yard right bank downstream bridge } \\
\text { crossing) main channel usually dries up, pool remains under bridge trapping Northern Pike and "silver- coloured fish that might be } \\
\text { bass" - we thought they might be suckers. Slow/deep and slow/shallow flow regimes present. Channelized with no riffles. No fish } \\
\text { caught. }\end{array}$ \\
\hline W-05-074 & Not diked. No cattle impacts. Water $\sim 0.75 \mathrm{~m}$ deep with dense aquatic vegetation. Substantial rain all weekend. \\
\hline W-05-075 & Diked both sides. No cattle impacts. Too deep to fish. Substantial rain all weekend. \\
\hline W-05-076 & $\begin{array}{l}\text { Diked both sides. No cattle impacts. }{ }^{* *} \text { Local knowledge: local farmer says he has caught Northern Pike here, drain is always } \\
\text { flowing and drain has to be dredged every few years. Caught one sucker, saw two others approximately the same length, but } \\
\text { couldn't catch. Heavy sediment at mouth of culvert outlet. Riffles habitat has very little to no cobble/boulder. Turbulent flow over } \\
\text { mud/clay. }\end{array}$ \\
\hline W-05-077 & Not diked. Moderate cattle impacts upstream of crossing. Culverts. Not channelized. \\
\hline W-05-078 & Not diked. No cattle impacts. Slow/ deep and slow/ shallow flow regimes present. \\
\hline W-05-079 & $\begin{array}{l}\text { Diked roadside. No cattle impacts. Previously dredged. Slow/shallow and fast/shallow flow regimes present. Channelized. Three } \\
\text { culverts, riffles downstream of culverts }\end{array}$ \\
\hline W-05-080 & Diked right bank. No cattle impacts. Ford crossing with Geoweb columns filled with sand and gravel. \\
\hline W-05-081 & Not diked. No cattle impacts. Slow/deep and slow/shallow flow regimes present. \\
\hline W-05-082 & $\begin{array}{l}\text { Not diked. No cattle impacts. Bottom covered in root mat and submerged vegetation, marshy. Slow/ shallow and slow/ deep flow } \\
\text { regimes present. Frequent bends. Bank vegetation consists of grasses with a few shrubs. No fish caught. Water has dropped } 0.5 \\
\mathrm{~m} \text { recently. }\end{array}$ \\
\hline W-05-083 & Not diked. Light cattle impacts. Ford crossing without culvert. Water down $0.6 \mathrm{~m}$ since spring freshet. \\
\hline
\end{tabular}




\section{Appendix 7: List of any additional field notes recorded at sampling sites.}

\begin{tabular}{|c|c|}
\hline $\begin{array}{l}\text { Site } \\
\text { Number }\end{array}$ & Field Notes \\
\hline W-05-084 & ot diked. No cattle impacts. \\
\hline W-05-085 & $\begin{array}{l}\text { Not diked. Light cattle impacts. Hog barn less than } 1 \text { mile north. Only missing fast/deep flow regime. Not channelized, few riffles } \\
\text { and bends. }\end{array}$ \\
\hline W-05-086 & Diked both sides. No cattle impacts. Slow/ shallow and slow/deep flow regimes present. \\
\hline W-05-087 & $\begin{array}{l}\text { Diked roadside. No cattle impacts. No fish caught. Slow/shallow and fast/shallow flow regimes present. Channelized. Vegetative } \\
\text { bank protection consists of mix of grasses and woody vegetation. }\end{array}$ \\
\hline W-05-088 & Not diked. No cattle impacts upstream, severe cattle impacts downstream. \\
\hline W-05-089 & Diked both sides downstream; not diked upstream. No cattle impacts. \\
\hline W-05-090 & Not diked. No cattle impacts. \\
\hline W-05-091 & Not diked. No cattle impacts. \\
\hline W-05-092 & Not diked. Light cattle impacts. \\
\hline W-05-093 & $\begin{array}{l}\text { Diked both sides upstream; not diked downstream. No cattle impacts. Slow/deep and slow/shallow flow regimes present. No } \\
\text { riffles, few bends. }\end{array}$ \\
\hline W-05-094 & Not channelized or diked. Riffle habitat associated with cobble and boulders at culvert inlet and outlet. No cattle impacts. \\
\hline W-05-095 & Culvert inlet drops vertically about $1.6 \mathrm{~m}$, then flows through a horizontal culvert (for water storage?). \\
\hline W-05-096 & $\begin{array}{l}\text { Diked left bank upstream; and diked both sides downstream from culvert crossing, Creek meanders quite a bit upstream from } \\
\text { crossing. }\end{array}$ \\
\hline W-05-097 & Not diked. No cattle impacts. Water is backed up upstream $($ by $>0.6 \mathrm{~m}$ ) by a gated culvert. \\
\hline W-05-098 & Not diked. No cattle impacts. \\
\hline W-05-099 & $\begin{array}{l}\text { Not diked. Moderate to severe cattle impacts, banks are trampled and falling into creek. Slow/shallow, slow/deep and fast/shallow } \\
\text { flow regimes are present in reach. }\end{array}$ \\
\hline W-05-100 & Not diked. Severe cattle impacts. Channel has been severely trampled upstream of crossing. \\
\hline W-05-101 & Diked left bank. No cattle impacts. Slow/shallow flow regime present. Two culverts. Channelized. \\
\hline W-05-102 & Diked roadside. No cattle impacts. \\
\hline W-05-103 & $\begin{array}{l}\text { Diked both sides. No cattle impacts. Slow/ shallow and fast/ shallow flow regimes present. Channelized. } 4 \text { culverts. Dense } \\
\text { aquatic vegetation. }\end{array}$ \\
\hline W-05-104 & $\begin{array}{l}\text { Diked roadside (left bank). No cattle impacts. Drain is adjacent to pastures but cattle do not have access to drain. No slow/deep } \\
\text { flow regime. Recently cleaned out with backhoe. }\end{array}$ \\
\hline W-05-105 & Not diked. No cattle impacts. No fast/ deep flow regime. Channelized upstream, not channelized downstream. \\
\hline W-05-106 & Diked roadside. No cattle impacts. Very heavy rains overnight. Slow/ shallow flow regime not present. \\
\hline W-05-107 & Not diked. Moderate cattle impacts. \\
\hline W-05-108 & Not diked. Deeply incised channel (approximately > $10 \mathrm{~m}$ ) No cattle impacts. \\
\hline W-05-109 & Not diked. No cattle impacts. Crushed culvert and beaver dam upstream of crossing. \\
\hline W-05-110 & o cattle impacts. Slow/ deep and slow/ shallow flow regimes prese \\
\hline
\end{tabular}




\section{Appendix 7: List of any additional field notes recorded at sampling sites.}

\begin{tabular}{|c|c|}
\hline $\begin{array}{l}\text { Site } \\
\text { Number }\end{array}$ & Field Notes \\
\hline W-05-111 & Diked both sides. No cattle impacts. \\
\hline W-05-112 & $\begin{array}{l}\text { Not diked. No cattle impacts. Released: four White Sucker (two males, two females @ } 150 \text { - } 185 \text { mm); Black Bullhead @ } 180 \\
\text { mm; and one Northern Pike @ } 260 \text { mm (All are fork lengths). }\end{array}$ \\
\hline W-05-113 & $\begin{array}{l}\text { Diked both sides. No cattle impacts. Slow/deep and slow/shallow flow regimes present. Channelized, no riffles, thalweg } \\
\text { meandering within banks. }\end{array}$ \\
\hline W-05-114 & $\begin{array}{l}\text { Not diked. No cattle impacts. Slow/shallow only flow regime present. Not channelized. No riffles, three bends in sight from culvert } \\
\text { crossing. Mostly gravel substrate in pools. }\end{array}$ \\
\hline W-05-115 & $\begin{array}{l}\text { Not diked. Light cattle impacts. Fast/ deep flow regime missing. Banks are highly undercut and slumping. Shale substrate in } \\
\text { pools. }\end{array}$ \\
\hline W-05-116 & Not diked. No cattle impacts. Missing fast/deep flow regime. Not channelized, frequent bends. \\
\hline W-05-117 & $\begin{array}{l}\text { Not diked. No cattle impacts. Released: } 28 \text { Creek Chub, } 13 \text { Northern Redbelly Dace (vibrant red spawning colours), three brassy } \\
\text { minnows (vibrant yellow spawning colours), } 11 \text { Western Blacknose Dace, } 11 \text { Fathead Minnows. Lots of cobble. Many riffles } \\
\text { downstream, and a few upstream of reach. Missing fast/ deep flow regime. }\end{array}$ \\
\hline W-05-118 & $\begin{array}{l}\text { Not diked. Moderate cattle impacts. Missing fast/deep flow regime. Juvenile suckers caught. Lots of dead fall but no new tree } \\
\text { growth in riparian area. Cattle have access to drain. Shale substrate in pools. }\end{array}$ \\
\hline W-05-119 & Not diked. No cattle impacts. Missing fast/deep flow regime. Small amounts of aquatic vegetation. \\
\hline W-05-120 & Not diked. Velocity depth regime includes only slow moving water. Fields cultivated to the edge of the water. \\
\hline W-05-121 & Not diked. Surrounded by agricultural land. \\
\hline W-05-122 & Not diked. Surrounded by agricultural land. \\
\hline W-05-123 & Diked by the road. Too deep to fish. Beaver activity and one beaver carcass. \\
\hline W-05-124 & Not diked. No well-defined channel. Culvert overgrown by grass. \\
\hline W-05-125 & Not diked. No cattle impacts. \\
\hline W-05-126 & Not diked. Surrounded by agricultural land. No catch. Marshy conditions. \\
\hline W-05-127 & Not diked. No cattle impacts. \\
\hline W-05-128 & Not diked. Velocity depth regime includes fast/deep and fast/shallow. Riparian area is grassed. Standing pools present. \\
\hline W-05-129 & $\begin{array}{l}\text { Diked on one side, Common Carp observed in drain and } 700 \mathrm{~mm} \text { Common Carp collected. Some flooded areas, water to field } \\
\text { edges. }\end{array}$ \\
\hline W-05-130 & $\begin{array}{l}\text { Diked on one side. No cattle impacts. Northern Pike observed in the pool but not caught. Slow/ deep and slow/ shallow flow } \\
\text { regimes present. }\end{array}$ \\
\hline W-05-131 & Diked both sides. No cattle impacts. \\
\hline W-05-132 & Diked on one side. Fairly dense aquatic vegetation growth. \\
\hline W-05-133 & Dikes present and roads also serves as a dike. Significant flooding in the area. \\
\hline W-05-134 & $\begin{array}{l}\text { Diked on one bank. Perched culverts downstream of road, flowing fast at full capacity, Weir downstream preventing fish } \\
\text { movement upstream. No bends and very few riffles. }\end{array}$ \\
\hline
\end{tabular}




\section{Appendix 7: List of any additional field notes recorded at sampling sites.}

\begin{tabular}{|c|c|}
\hline $\begin{array}{l}\text { Site } \\
\text { Number }\end{array}$ & Field Notes \\
\hline W-05-135 & Not diked. Marsh habitat. Yellow Perch observed but unable to collect. Too deep to fish effectively.. \\
\hline W-05-136 & Not diked. No cattle impacts. Marshy/wet conditions. \\
\hline W-05-137 & Not diked. Fast/ deep flow regime missing. Many bends and riffles. Flow has eroded and disconnected a section of culvert. \\
\hline W-05-138 & Not diked, No cattle impacts. Fast/deep flow regime absent. \\
\hline W-05-139 & Not diked. No cattle impacts. Cobble placed around culverts. Forested riparian area. \\
\hline W-05-140 & Not diked. No cattle impacts. Cobble placed around culverts. Forested riparian area. \\
\hline W-05-141 & Not diked. Surrounded by agricultural fields, $50 \mathrm{~m}$ from a garbage dump. Slow/deep and slow/shallow flow regimes present. \\
\hline W-05-142 & $\begin{array}{l}\text { Diked on one side by road. Cleaned out this spring by a farmer. Dense floating vegetation. Local knowledge: according to a local } \\
\text { farmer, drain flows all summer, has seen suckers and Common Carp. }\end{array}$ \\
\hline W-05-143 & Not diked. No flow. \\
\hline W-05-144 & Not diked. No cattle impacts. Marshy/ wet conditions. \\
\hline W-05-145 & Not diked. No cattle impacts. Soft substrate. There is a vertical culvert with water coming out of it adjacent to reach. \\
\hline W-05-146 & $\begin{array}{l}\text { Not diked. No cattle impacts. Turbidity meter not working - reads everything as } 0.00 \text {. Woody debris, submerged logs, undercut } \\
\text { banks and cobble provide cover. One culvert. Many riffles and bends downstream. Bank slumping around culvert. }\end{array}$ \\
\hline W-05-147 & Not diked. Light cattle impacts (bison). \\
\hline W-05-148 & Not diked. Light cattle impacts. \\
\hline W-05-149 & Not diked. No cattle impacts. Turbidity meter not working. \\
\hline W-05-150 & Not diked. No cattle impacts. Suckers caught. \\
\hline W-05-151 & Not diked. No cattle impacts. Turbidity meter not working. \\
\hline W-05-152 & Not diked. Light cattle impacts. Flooded upstream: woody debris blocking culverts. \\
\hline W-05-153 & Not diked. Light cattle impacts. \\
\hline W-05-154 & Not diked. Moderate cattle impacts. Herd of cattle on right bank downstream of culvert. Deep/fast velocity depth regime missing. \\
\hline W-05-155 & $\begin{array}{l}\text { Not diked. No cattle impacts. Perched culvert outlet. Woody debris and vegetation covering fence creating a backwater pool } \\
\text { upstream. Just a trickle of flow through culvert. }\end{array}$ \\
\hline W-05-156 & Not diked. Moderate cattle impacts. \\
\hline W-05-157 & $\begin{array}{l}\text { Not diked. Fishing stopped due to thunderstorm. }{ }^{* *} \text { Local knowledge: tributary usually dries up in the summer. This is the first } \\
\text { time the 4th order has run in two years at this time of year. Cattle have access to drain upstream. }\end{array}$ \\
\hline W-05-158 & Not diked. Couldn't find culverts - submerged due to beaver activity. \\
\hline W-05-159 & Not diked. Deep/fast flow regime absent. \\
\hline W-05-160 & Not diked. \\
\hline W-05-161 & Not diked. \\
\hline W-05-162 & $\begin{array}{l}\text { Not diked. Suckers caught. Heavy sediment deposition downstream of culverts. Flooded adjacent fields. Banks } \\
\text { culverts. No pictures of site taken -digital camera not working. }\end{array}$ \\
\hline
\end{tabular}




\section{Appendix 7: List of any additional field notes recorded at sampling sites.}

\begin{tabular}{|c|c|}
\hline $\begin{array}{l}\text { Site } \\
\text { Number }\end{array}$ & Field Notes \\
\hline W-05-163 & $\begin{array}{l}\text { Diked roadside (left bank). Only slow/shallow velocity/depth regime present. Dredged earlier this year. }{ }^{* *} \text { No pictures of site taken } \\
\text { - digital camera not working. }\end{array}$ \\
\hline W-05-164 & Not diked. Moderate cattle impacts. Some flooded areas. \\
\hline W-05-165 & Not diked. No direct cattle impacts. Marshy conditions. \\
\hline W-05-166 & Not diked. No direct cattle impacts. Marshy conditions. \\
\hline W-05-167 & Not diked. No direct cattle impacts. Marshy conditions. \\
\hline W-05-168 & Not diked. Severe cattle impacts. No fish caught. \\
\hline W-05-169 & $\begin{array}{l}\text { Not diked. Light cattle impacts. Boulder, cobble, submerged vegetation and undercut banks present. Rainbow Trout caught (and } \\
\text { released) and White Sucker caught. Relatively high gradient outflow with placement of boulders forming pools. High frequency of } \\
\text { riffles. }\end{array}$ \\
\hline W-05-170 & Not diked. Spring fed creek; spring located at the base of hill. Piles of woody debris deposited in riparian area from spring flows. \\
\hline W-05-171 & Not diked. White Sucker and Burbot caught. Culvert outlet perched $\sim 1 \mathrm{~m}$. Right bank is cultivated to edge. \\
\hline W-05-172 & Not diked. No cattle impacts. \\
\hline W-05-173 & Not diked. White Sucker caught. ${ }^{* *}$ Sample mislabeled as W-05-172. \\
\hline W-05-174 & $\begin{array}{l}\text { Diked roadside (left bank, south). Four weirs within a mile of reach; water drops } \sim 1 \mathrm{~m} / \text { weir. Weirs are cement and stone } \\
\text { structures. All fast moving water. Rocky substrate present with mostly grassy bank vegetation. }\end{array}$ \\
\hline W-05-175 & Diked both sides. No cattle impacts. Flooded adjacent fields. \\
\hline W-05-176 & $\begin{array}{l}\text { Diked roadside (left bank, south). No cattle impacts. Larger fish (Northern Pike) observed but unable to collect. Slow/deep and } \\
\text { fast/deep velocity/depth regimes present. }\end{array}$ \\
\hline W-05-177 & $\begin{array}{l}\text { Diked road side (right bank, south) No cattle impacts. Observed } 15 \text { other small Northern Pike that we couldn't catch. Some } \\
\text { cobble and lots of submerged vegetation present. Missing slow/shallow velocity/depth regime. Channelized, no riffles or bends. } \\
\text { Area fished had good riparian vegetative zone width, but all along rest of drain width is }<6 \text { metres. }\end{array}$ \\
\hline W-05-178 & $\begin{array}{l}\text { Diked road side (left bank, south) No cattle impacts. Channelized. Submerged vegetation provides cover and creates riffle father } \\
\text { upstream of culvert crossing. }\end{array}$ \\
\hline W-05-179 & Not diked. Moderate cattle impacts. \\
\hline W-05-180 & $\begin{array}{l}\text { Diked left bank. Severe cattle impacts. Drain substrate downstream of culvert crossing is pummeled by cattle. Bottom changing } \\
\text { frequently. Culverts are submerged. Too deep to fish immediately upstream and downstream of culvert crossing. }\end{array}$ \\
\hline W-05-181 & $\begin{array}{l}\text { Diked road side (right bank, south) No cattle impacts. Lots of submerged vegetation, no undercut banks, snags or woody debris } \\
\text { present. Channelized with no riffles or bends. }\end{array}$ \\
\hline W-05-182 & Diked roadside (left bank, south). No cattle impacts. Drain is full of Northern Pike - we saw >20 in addition to our catch. \\
\hline W-05-183 & Diked both sides. Drain dredged this year or last - minimal/ poor habitat for fish. Fast/ shallow only flow regime present. \\
\hline W-05-184 & Not diked. No cattle impacts. \\
\hline W-05-185 & $\begin{array}{l}\text { Not diked, no cattle impacts. Choked with algae and other aquatic vegetation. Slow/ deep and slow/ shallow regimes pres } \\
\text { Not channelized, no riffles, many bends. }\end{array}$ \\
\hline
\end{tabular}




\section{Appendix 7: List of any additional field notes recorded at sampling sites.}

\begin{tabular}{|c|c|}
\hline $\begin{array}{l}\text { Site } \\
\text { Number }\end{array}$ & Field Notes \\
\hline W-05-186 & $\begin{array}{l}\text { Too deep to fish, abundant algae growth. Many large logs and woody debris in creek. No access through gate. Not diked. No } \\
\text { cattle impacts. }\end{array}$ \\
\hline W-05-187 & $\begin{array}{l}\text { Too deep to fish beyond road overflow and edges. No fish caught. Not diked. No cattle impacts. All four velocity/depth regimes } \\
\text { present. }\end{array}$ \\
\hline W-05-188 & $\begin{array}{l}\text { Young of the year suckers caught and released. Not diked. No cattle impacts. Many eroded areas; bare exposed banks frequent } \\
\text { along straight sections. No submerged vegetation. No cattle impacts. Diked right bank (south) side. Slow/deep, slow/shallow, } \\
\text { and fast/ deep velocity/depth regimes present. }\end{array}$ \\
\hline W-05-189 & $\begin{array}{l}\text { Diked both sides. No cattle impacts. }{ }^{* *} \text { Released } 1 \text { Northern Pike (fork length }=145 \mathrm{~mm} \text { ) }{ }^{* *} \text { Released } 19 \text { young of the year } \\
\text { suckers (same size as those in sample) Note: Samples labeled 3rd order tributary to Goertz drain. Cattails and undercut banks } \\
\text { present. Mud and sand substrate, little root mat, no submerged vegetation. } 1 \text { deep pool at culvert outlet. Slow/ deep and slow/ } \\
\text { shallow flow regimes present. }\end{array}$ \\
\hline W-05-190 & $\begin{array}{l}\text { Upstream: diked left bank (road, north side); downstream: diked both sides. No cattle impacts. }{ }^{* *} \text { Observed Northern Pike (small } \\
\sim 5 \mathrm{~cm} \text { in length) }{ }^{* *} \text { Local knowledge: suckers come up in spring; occasional Northern Pike caught. Channelized. All grasses } \\
\text { upstream. } 25 \% \text { tree covered banks downstream reach. All mud, some rood mats, and submerged vegetation present. } \\
\text { Slow/shallow, slow/deep flow regimes present. }\end{array}$ \\
\hline W-05-191 & $\begin{array}{l}\text { Dense algae and submerged vegetation growth. Diked roadside. No cattle impacts. Lots of filamentous algae in drain. } \\
\text { Slow/shallow and slow/deep regimes present. Bank vegetation consists of grass with some mud/soil exposed. Mud substrate with } \\
\text { heavy instream and submerged vegetation. }\end{array}$ \\
\hline W-05-192 & $\begin{array}{l}\text { Diked both sides. No cattle impacts. Choked with cattails upstream and downstream of culvert crossing. No flow, very little } \\
\text { standing water in drain. }\end{array}$ \\
\hline W-05-193 & $\begin{array}{l}\text { Diked both sides. No cattle impacts. }{ }^{*} \text { Sucker caught. Slow/deep and slow/shallow regimes present. No bends or riffles. Clay } \\
\text { banks. Bank vegetation is mainly grasses with a few shrubs. Submerged vegetation and instream vegetation present. Mud, clay, } \\
\text { sand and gravel substrate in pools. }\end{array}$ \\
\hline W-05-194 & $\begin{array}{l}\text { Diked both sides. No cattle impacts }{ }^{* *} \text { Young of the year White Sucker and Burbot caught. Dense submerged vegetation, } \\
\text { instream vegetation, present. Some rocks at culvert, undercut banks present. Good habitat around culvert, but marginal-poor } \\
\text { habitat further upstream and downstream of culvert. No riffles or bends. }\end{array}$ \\
\hline W-05-195 & Diked both sides. No cattle impacts. No flow. \\
\hline W-05-196 & $\begin{array}{l}\text { Diked both sides. No cattle impacts. }{ }^{*} \text { Suckers caught. Substrate mostly exposed. Channelized in past, starting to meander. } \\
\text { Riffles downstream every } 30 \mathrm{~m} \text {; some meanders. Pool substrate: clay, cobble and small boulders. No aquatic vegetation. Very } \\
\text { few small deep pools. }\end{array}$ \\
\hline W-05-197 & Too deep to fish. Large waterfall is potential barrier to fish passage. Natural habitat and waterfall. Not diked. No cattle impacts. \\
\hline W-05-198 & No cattle impacts. Not diked. Drain is choked w \\
\hline W-05-199 & impacts. Not diked. Crosses PTH \#3. \\
\hline W-05-200 & No cattle impacts. Diked both sides. Clay substrate. \\
\hline
\end{tabular}




\section{Appendix 7: List of any additional field notes recorded at sampling sites.}

\begin{tabular}{|c|c|}
\hline $\begin{array}{l}\text { Site } \\
\text { Number }\end{array}$ & Field Notes \\
\hline W-05-201 & $\begin{array}{l}\text { Diked roadside (left bank). No cattle impacts. Some submerged vegetation and emergent vegetation. Slow/deep and } \\
\text { slow/shallow regimes present. Bottom changing frequently. Channelized, cobble present to stabilize banks downstream of } \\
\text { culvert. Many bends upstream of } 2 \text { nd culvert crossing (man-made) which is upstream from a natural ford crossing. Mud/clay } \\
\text { substrate in pools. }\end{array}$ \\
\hline W-05-202 & $\begin{array}{l}\text { Diked road side (right bank). No cattle impacts. Slow/ deep and slow/ shallow flow regimes present. Clay substrate with a layer } \\
\text { of vegetation. }\end{array}$ \\
\hline W-05-203 & $\begin{array}{l}\text { No cattle impact. Not diked. }{ }^{* *} \text { Caught young of the year White Sucker and Northern Pike. Saw four other Northern Pike - a large } \\
\text { one of } \sim 500 \mathrm{~mm} \text {. All four velocity/depth regimes present. No channel alteration except around bridge. Many bends. Riffles } \\
\text { caused by fast water on bends. Mud and sand substrate in pools. }\end{array}$ \\
\hline W-05-204 & Moderate cattle impacts. Not diked. \\
\hline W-05-205 & $\begin{array}{l}\text { Not diked. No cattle impacts. }{ }^{* *} \text { Sucker and Northern Pike caught. Two culverts, possibly three ( } 1 \text { hidden). Many bends. Banks } \\
\text { are stable except for along road washout. Pool substrate: parts with firm gravel and parts with soft sand and dense vegetation. }\end{array}$ \\
\hline W-05-206 & Diked roadside (left bank). No cattle impacts \\
\hline W-05-207 & $\begin{array}{l}\text { Not diked, no cattle impacts. }{ }^{* *} \text { Caught bullheads, Burbot and lots of young of the year Common Carp. Slow/deep and } \\
\text { slow/shallow regimes present. Occasional bend, no riffles. Mud substrate. One deep pool under bridge, small shallow pools } \\
\text { downstream of bridge. }\end{array}$ \\
\hline W-05-208 & $\begin{array}{l}\text { Not diked, no cattle impacts. Fast/ deep flow regime missing. Natural channel, one small wooden quad/walking bridge. Many } \\
\text { bends, a few riffles. Woody debris, undercut banks and some rocks and gravel provide cover. }\end{array}$ \\
\hline W-05-209 & $\begin{array}{l}\text { Not diked, no cattle impacts. }{ }^{* *} \text { Dissolved oxygen meter not working. Natural, many bends with a few riffles upstream of culvert } \\
\text { crossing. }\end{array}$ \\
\hline W-05-210 & Not diked, light cattle impacts. Too deep to electrofish downstream of culverts. Fenced off on both sides. \\
\hline W-05-211 & Not diked, no cattle impacts. \\
\hline $\mathrm{X}-05-001$ & Some deep pools. No fish caught. Electrofisher and conductivity meter not working. \\
\hline $\mathrm{X}-05-002$ & $\begin{array}{l}\text { Electrofisher and conductivity meter not working. More flow through north culvert. Some boulders and undercut banks. Small } \\
\text { bends. Mostly mud substrate. Slow/ deep, slow/ shallow flow regimes present. }\end{array}$ \\
\hline $\mathrm{X}-05-003$ & Silt substrate at upstream of culvert. Lots of cattails in drain. No flow. Grassed banks. Mud and clay substrate. \\
\hline $\mathrm{X}-05-004$ & Trickle of flow. Cattails in stream. Standing shallow pool. \\
\hline $\mathrm{X}-05-005$ & Moderate cattail growth. Grasses in drain. No flow. Missed three minnows. Slow/shallow flow regime present. Grassed banks. \\
\hline $\mathrm{X}-05-006$ & Mowed grass banks. Cattails in drain. \\
\hline $\mathrm{X}-05-007$ & $\begin{array}{l}\text { Two sucker eggs found. Released three White Sucker } 240-440 \mathrm{~mm} \text { female, } 480 \mathrm{~mm} \text { post spawn male. Suckers in poor condition } \\
\text { Very low flow. No fish passage over riffle. Bank slumping on downstream side. Mowed grass banks. }\end{array}$ \\
\hline $\mathrm{X}-05-008$ & No flow. Small pools of water surrounded by cattails. \\
\hline $\mathrm{X}-05-009$ & No flow. Drain chokec \\
\hline $\mathrm{X}-05-010$ & etation upstream. Drain is dry further upstream. \\
\hline
\end{tabular}




\section{Appendix 7: List of any additional field notes recorded at sampling sites.}

\begin{tabular}{|c|c|}
\hline $\begin{array}{l}\text { Site } \\
\text { Number }\end{array}$ & Field Notes \\
\hline $\mathrm{X}-05-011$ & eep water at culvert crossing only. Shallow water with emergent vegetation upstream and downstream. Grassed banks. \\
\hline $\mathrm{X}-05-012$ & Grassed banks. Mud, clay, silt and some submerged vegetation in pool. Deep pools at crossings. \\
\hline $\mathrm{X}-05-013$ & $\begin{array}{l}\text { Standing scour pools below culverts. Dry upstream and downstream. No flow. Moderate growth of cattails in drain. Heavy growth } \\
\text { of terrestrial grasses in drain. }\end{array}$ \\
\hline $\mathrm{X}-05-014$ & No flow. Very shallow standing water. High silt deposition at inlets. \\
\hline $\mathrm{X}-05-015$ & $\begin{array}{l}\text { Little flow through drain. Terrestrial grasses in drain upstream and downstream of culvert. Algae growing in drain. Cobble, } \\
\text { vegetation and algae provide some cover. Slow/shallow and fast/shallow flow regimes. One shallow riffle in reach. Grassed } \\
\text { banks, with some shrubs downstream. }\end{array}$ \\
\hline $\mathrm{X}-05-016$ & Some flow. Heavy growth of terrestrial grasses in channel. \\
\hline $\mathrm{X}-05-017$ & $\begin{array}{l}\text { Very poor riparian zone with only mud and almost no grass along banks (due to clean-out). A new drain connects with channel at } \\
\text { outflow. }\end{array}$ \\
\hline $\mathrm{X}-05-018$ & $\begin{array}{l}\text { Approximately } 30 \text { suckers in culvert, below woody debris (obstruction?). Released } 12 \text { White Sucker }(120-410 \mathrm{~mm}) \text { and one } \\
\text { Mudminnow }(100 \mathrm{~mm}) \text {. Cobble substrate associated with culvert. Cobbles } 75 \% \text { embedded downstream of culvert. }\end{array}$ \\
\hline $\mathrm{X}-05-019$ & $\begin{array}{l}\text { Heavy sediment deposition, cobble and boulders are completely embedded. Lack of riparian area width. Fields cultivated almost } \\
\text { to edge of Crooked Lake Channel and also upstream on unnamed tributary. }\end{array}$ \\
\hline $\mathrm{X}-05-020$ & $\begin{array}{l}\text { No catch. Dense growth of grasses in stream. Left bank upstream of culvert crossing has no riparian area width as field is } \\
\text { cultivated right to drain. Substantial algae growth in drain. Slow/shallow flow regime only. }\end{array}$ \\
\hline $\mathrm{X}-05-021$ & $\begin{array}{l}\text { Potential barrier to fish passage during low flow at bridge located } 80 \mathrm{~m} \text { downstream. } 2 \text { spawning suckers observed upstream of } \\
\text { barrier. One White Sucker released (fork length }=480 \mathrm{~mm} \text { ) showing spawning tubercles on anal and caudal fin. Right bank is } \\
\text { grassed with a few small trees, left bank has native vegetation. Well defined thalweg with a good mix of run and pool sequences. } \\
\text { Mostly channelized upstream of bridge with moderate cattail growth. All four velocity depth regimes present. }\end{array}$ \\
\hline $\mathrm{X}-05-022$ & $\begin{array}{l}\text { No catch with seine. Electrofisher and conductivity meter not working. More flow through north culvert. Some boulders and } \\
\text { undercut banks. Small bends. Mostly mud substrate. }\end{array}$ \\
\hline $\mathrm{X}-05-023$ & $\begin{array}{l}\text { Constructed riffle at site. Deep pool with a depth greater than } 1.5 \mathrm{~m} \text {. Generally shallow water upstream and downstream with } \\
\text { moderate cattail growth. Grassed banks. }\end{array}$ \\
\hline $\mathrm{X}-05-024$ & $\begin{array}{l}\text { Five adult suckers observed moving from the pool towards the riffle. Banks stable upstream but high erosion potential } \\
\text { downstream of riffle with sediments infilling pool. Clay is dominant in pool. Note: invertebrate sample accidentally labeled as X- } \\
04-024 \text {. }\end{array}$ \\
\hline $\mathrm{X}-05-025$ & No flow. Dense mat of filamentous algae. Dry except for standing pools. Standing pools are too deep to fish. \\
\hline $\mathrm{X}-05-026$ & $\begin{array}{l}\text { Cattle paths leading to drain. Many bare, muddy patches along banks. Many bends, bottom contours, and one riffle provide } \\
\text { habitat diversity. Pools are a mix of large shallow, small shallow, and small deep. }\end{array}$ \\
\hline $\mathrm{X}-05-027$ & $\begin{array}{l}\text { Dense algae growth. Downstream is choked with cattails. No culverts at cement ford crossing. Slow/shallow flow regime only. No } \\
\text { riffles. Mowed/ burned grass in riparian zone. }\end{array}$ \\
\hline $\mathrm{X}-05-028$ & ome flow. Dense algae growth. \\
\hline
\end{tabular}




\section{Appendix 7: List of any additional field notes recorded at sampling sites.}

\begin{tabular}{|c|c|}
\hline $\begin{array}{l}\text { Site } \\
\text { Number }\end{array}$ & Field Notes \\
\hline $\mathrm{X}-05-029$ & $\begin{array}{l}\text { Habitat assessed for reach fished. Immediately downstream of reach, there are severe cattle impacts with high erosion potential } \\
\text { and a lack of stable substrate. Stream natural with no cattle impacts upstream of crossing. }\end{array}$ \\
\hline $\mathrm{X}-05-030$ & $\begin{array}{l}\text { No catch. High flow velocity through culvert. Undercut banks, rocks, cobble, and lots of submerged vegetation. Sediment } \\
\text { deposition, mostly sand and gravel. Many bends. }\end{array}$ \\
\hline $\mathrm{X}-05-031$ & $\begin{array}{l}\text { High velocity outflow. Moderate to heavy growth of terrestrial vegetation in drain. Cobble only present at riffle. Slow/shallow and } \\
\text { fast/ shallow flow regimes only. One riffle visible in reach. Grassed banks are mowed down to edge of drain. }\end{array}$ \\
\hline $\mathrm{X}-05-032$ & $\begin{array}{l}\text { Deep run and pools. No catch. Observed approximately } 15 \mathrm{~cm} \text { long fish darting out of reeds. High flow velocity through culverts. } \\
\text { Inlets angle slightly downward - at low water levels, culverts may be perched. Slow/deep, slow/shallow, and fast/deep flow } \\
\text { regimes. All flat water. Grassed banks have been mowed. }\end{array}$ \\
\hline $\mathrm{X}-05-033$ & $\begin{array}{l}\text { No catch. Likely dry before the recent rains; drain is filled with terrestrial grass. Slow/shallow, slow/deep, and fast/shallow flow } \\
\text { regimes. Grassed banks, with left bank mowed to the water's edge. }\end{array}$ \\
\hline $\mathrm{X}-05-034$ & Recently cleaned out, with raw areas on right bank. Heavy sediment deposition. \\
\hline $\mathrm{X}-05-035$ & $\begin{array}{l}\text { Recent clean-out of cattails downstream. Few fish caught, mostly around culverts. Slow/shallow and fast/shallow flow regimes. } \\
\text { Many exposed areas along banks. Closely cropped vegetation. }\end{array}$ \\
\hline $\mathrm{X}-05-036$ & Dead suckers (obviously killed by people) observed in drain and along banks. One Fathead Minnow released. \\
\hline $\mathrm{X}-05-037$ & Caught one Mudminnow, saw one larger fish that escaped.. Heavy submerged vegetation growth. Mowed grass along banks. \\
\hline $\mathrm{X}-05-038$ & Released one White Sucker (fork length $=220 \mathrm{~mm}$ ). Undercut banks and deadfall provide cover. \\
\hline $\mathrm{X}-05-039$ & Drain is choked with cattails. Max depth is $0.3 \mathrm{~m}$. No flow. \\
\hline $\mathrm{X}-05-040$ & $\begin{array}{l}\text { No catch. Heavy cattail growth. Slow/ deep and slow/ shallow flow regimes. All flat water. One very deep pool located } \\
\text { downstream of culvert crossing. }\end{array}$ \\
\hline $\mathrm{X}-05-041$ & No flow. Dense growth of grasses in stream. Cattails have been mowed upstream of crossing. \\
\hline $\mathrm{X}-05-042$ & Released one White Sucker (fork length=220 mm) and one Central Mudminnow (total length $=190 \mathrm{~mm}$ ). All flat water. \\
\hline $\mathrm{X}-05-043$ & Culverts are almost submerged and the creek is too deep to fish. Area around stream is flooded. Many dead trees. \\
\hline$X-05-044$ & $\begin{array}{l}\text { No catch. Grasses growing in much of the drain. Dense growth of algae. Deep pool downstream from culverts. Slow/deep and } \\
\text { slow/shallow flow regimes. Banks are grassed. Mud and submerged vegetation present in pools. }\end{array}$ \\
\hline $\mathrm{X}-05-045$ & Slow/deep and fast/deep flow regimes. Undercut banks, rocks and submerged vegetation provide some cover. \\
\hline $\mathrm{X}-05-046$ & $\begin{array}{l}\text { Local knowledge: farmer (living approx. } 200 \mathrm{~m} \text { south) says that Northern Pike and suckers come up this creek. Along right bank, } \\
\text { the field is cultivated to the very edge of the creek. Mud substrate in pool. }\end{array}$ \\
\hline $\mathrm{X}-05-047$ & Very high flow velocity. Depths more than $1.5 \mathrm{~m}$ in pools and run habitat. \\
\hline $\mathrm{X}-05-048$ & Cement and cobble present in sections of drain. Grassed banks with a few shrubs. \\
\hline $\mathrm{X}-05-049$ & Drain is choked with cattails upstream and downstream of culvert crossing. No flow. \\
\hline $\mathrm{X}-05-050$ & ittle flow through drain. Choked with cattails upstream and downstream of crossing. \\
\hline $\mathrm{X}-05-051$ & $\begin{array}{l}\text { Heavy sediment deposition downstream of ford crossing. Some dense algae growing in the str } \\
\text { effort. }\end{array}$ \\
\hline
\end{tabular}




\section{Appendix 7: List of any additional field notes recorded at sampling sites.}

\begin{tabular}{|c|c|}
\hline $\begin{array}{l}\text { Site } \\
\text { Number }\end{array}$ & Field Notes \\
\hline $\mathrm{X}-05-052$ & $\begin{array}{l}\text { Very deep drain downstream of bridge with heavy growth of terrestrial grasses. Drain is choked with cattails } 75 \mathrm{~m} \text { upstream of } \\
\text { bridge. Slow/deep and slow/shallow flow regimes. }\end{array}$ \\
\hline $\mathrm{X}-05-053$ & No flow. Terrestrial grasses growing in drain. \\
\hline $\mathrm{X}-05-054$ & One Brook Stickleback caught and released. There are no culverts at ford crossing. Grassed banks. \\
\hline $\mathrm{X}-05-055$ & $\begin{array}{l}\text { At the upstream side of the culvert crossing, the field is cultivated to the edge of the drain on the right bank. There is also an } \\
\text { abundance of grasses and cattails in the drain. Downstream of the crossing, habitat is complex, characterized by complete } \\
\text { canopy cover and an abundance of woody debris and deadfall in the drain. Excessive amounts of straw from the field upstream } \\
\text { have accumulated in the drain, downstream of the crossing. }\end{array}$ \\
\hline $\mathrm{X}-05-056$ & $\begin{array}{l}\text { There is a very deep pool downstream of the culvert, otherwise, the drain is shallow and filled with terrestrial grasses. There is no } \\
\text { defined thalweg. }\end{array}$ \\
\hline $\mathrm{X}-05-057$ & No catch. \\
\hline $\mathrm{X}-05-058$ & Highly flooded marsh area that is too deep and wide to fish. \\
\hline $\mathrm{X}-05-059$ & Marshy, flooded area that is too deep to fish. The pool downstream of the culvert was greater than 2 metres deep. \\
\hline $\mathrm{X}-05-060$ & $\begin{array}{l}\text { One Central Mudminnow was released. Beavers blocked the culvert inlet and flooded the upstream. The road has washed out } \\
\text { beside the culvert and substantial amounts of sediments have been deposited downstream of the crossing. We sank up to } 1 \mathrm{~m} \\
\text { deep in the sediments and could not finish fishing the site. Slow/shallow, slow/deep, and fast/deep water flow regimes present. }\end{array}$ \\
\hline $\mathrm{X}-05-061$ & $\begin{array}{l}\text { Channel is greater than } 1 \mathrm{~m} \text { deep, reaching the base of both banks. Slow/deep flow regime predominant. Wide riparian area, with } \\
\text { native vegetation. }\end{array}$ \\
\hline $\mathrm{X}-05-062$ & $\begin{array}{l}\text { No catch. Two good riffles were in the reach and there was an abundance of snags, woody debris, and undercut banks. } \\
\text { Numerous Gammarus spp. Fish passage upstream is blocked due to a } 3 \text { m vertical drop into the culvert. }\end{array}$ \\
\hline $\mathrm{X}-05-063$ & $\begin{array}{l}\text { The area is flooded, with willows, shrubs, and tall grass flooded downstream of the crossing, and upstream of the crossing is } \\
\text { marshy, with lots of open water and heavy growth of cattails along the edges. There is no defined channel downstream of the } \\
\text { crossing, except for the very deep pool (>2 m) below the culvert. }\end{array}$ \\
\hline $\mathrm{X}-05-064$ & $\begin{array}{l}\text { One } 90 \mathrm{~mm} \text { Central Mudminnow was released. The upstream end of the culvert is almost totally blocked by woody debris. } \\
\text { Slow/deep and slow/shallow flow regimes were present. The banks are grassed. One riffle located in the reach. The majority of } \\
\text { the pools were large and deep. }\end{array}$ \\
\hline $\mathrm{X}-05-065$ & Recent removal of riparian vegetation downstream. \\
\hline $\mathrm{X}-05-066$ & Abundance of submerged vegetation and terrestrial grass in drain. \\
\hline $\mathrm{X}-05-067$ & $\begin{array}{l}\text { No catch with electrofisher and larval drift effort. There is optimal substrate and cover within the reach, with lots of deadfall, } \\
\text { undercut banks, overhanging vegetation, and some areas of complete canopy cover. A large riffle is present upstream. All four } \\
\text { flow regimes present. }\end{array}$ \\
\hline $\mathrm{X}-05-068$ & The drain is choked with cattails, except for a standing pool. \\
\hline $\mathrm{X}-05-069$ & Severe banks slumping downstream, with parts of the banks falling into the creek. Very high erosion potential. \\
\hline $\mathrm{X}-05-070$ & A larval drift trap was set for 17 hours (17:30-10:30). No larval fish present in sample. Banks are grassed. \\
\hline
\end{tabular}




\section{Appendix 7: List of any additional field notes recorded at sampling sites.}

\begin{tabular}{|c|c|}
\hline $\begin{array}{l}\text { Site } \\
\text { Number }\end{array}$ & Field Notes \\
\hline $\mathrm{X}-05-071$ & $\begin{array}{l}\text { Channel is shallow and filled with terrestrial grasses, though deep pools are present at the culvert inlet and outlet. No defined } \\
\text { thalweg. Slow/ deep and slow/ shallow flow regimes. }\end{array}$ \\
\hline $\mathrm{X}-05-072$ & Numerous caddisflies present in sample. Slow/deep and fast/shallow flow regimes predominant. \\
\hline $\mathrm{X}-05-073$ & Culvert inlets and outlets are choked with cattails. Pools are too deep to fish ( $>1.5 \mathrm{~m})$. No flow - standing pools only. \\
\hline $\mathrm{X}-05-074$ & $\begin{array}{l}\text { Channel is filled with cattails, grasses and sedges. Upstream is choked with cattails. Low to no flow. Pool associated with the } \\
\text { cement box culvert was too deep to fish. }\end{array}$ \\
\hline $\mathrm{X}-05-075$ & $\begin{array}{l}\text { No flow. Shallow, standing water except for a deep pool downstream of the culvert. Too deep to seine (>1.75 m). Moderate } \\
\text { growth of cattails in upstream and the drain is choked with cattails downstream of the pool. }\end{array}$ \\
\hline $\mathrm{X}-05-076$ & $\begin{array}{l}\text { Mostly dry, though some standing shallow pools are present. Substantial amounts of stubble have washed downstream from } \\
\text { ditches around cultivated fields. }\end{array}$ \\
\hline $\mathrm{X}-05-077$ & Culvert is perched approximately $0.2 \mathrm{~m}$. There is a drop culvert with a trickle of flow. \\
\hline $\mathrm{X}-05-078$ & Almost dry. No flow. \\
\hline $\mathrm{X}-05-079$ & Very low dissolved oxygen. Banks are covered in long grass, with a few small shrubs. \\
\hline $\mathrm{X}-05-080$ & Poor habitat with heavy deposits of sediments. Grassed banks. \\
\hline $\mathrm{X}-05-081$ & Extremely heavy growth of algae instream. Heavy sedimentation. No catch. Slow/shallow flow regime only. \\
\hline $\mathrm{X}-05-082$ & Dry. \\
\hline $\mathrm{X}-05-083$ & No catch. \\
\hline $\mathrm{X}-05-084$ & Shallow water throughout most of drain. A $1 \mathrm{~m}$ deep pool is associated with the culvert. \\
\hline $\mathrm{X}-05-085$ & $\begin{array}{l}\text { Slow/deep, slow/shallow and fast/shallow flow regimes present. Banks are grassed and moderately unstable. Larval fish caught } \\
\text { with one dipnet sweep. }\end{array}$ \\
\hline $\mathrm{X}-05-086$ & No catch. Slow/deep and slow/shallow flow regimes present. Narrow small riparian area width $(<6 \mathrm{~m})$. Grassed banks. \\
\hline $\mathrm{X}-05-087$ & No flow. Standing pool present. \\
\hline $\mathrm{X}-05-088$ & $\begin{array}{l}\text { Councilor for the area says that suckers and Northern Pike are frequently caught at this site. The last Walleye caught was } 20 \\
\text { years ago. The riffle under the bridge was dry, though there were high flow velocities through two culverts below the riffle. Deep } \\
\text { run with some undercut banks and lots of submerged boulders and rock. Caddisflies were abundant. Velocities for the right } \\
\text { culvert were: inlet }=0.68 \mathrm{~m} / \mathrm{s} \text {, outlet }=1.01 \mathrm{~m} / \mathrm{s} \text {. Velocities for the left culvert were: inlet }=0.4 \mathrm{~m} / \mathrm{s} \text {, outlet }=1.02 \mathrm{~m} / \mathrm{s} \text {. }\end{array}$ \\
\hline $\mathrm{X}-05-089$ & $\begin{array}{l}\text { Too deep to fish (> } 1.25 \mathrm{~m} \text { deep at edges). Bank slumping and erosion scars are evident downstream, but are mostly healed } \\
\text { over. Very turbid water. }\end{array}$ \\
\hline $\mathrm{X}-05-090$ & $\begin{array}{l}\text { Mix of mature trees, shrubs, and young trees in riparian area upstream of crossing. No flow. Standing shallow water with } \\
\text { terrestrial grasses and cattails instream downstream of the culverts. Terrestrial grasses in channel upstream as well. }\end{array}$ \\
\hline $\mathrm{X}-05-091$ & $\begin{array}{l}\text { Water levels were very deep so only the edges of the river were fished. The riparian area was flooded and marshy. The right inlet } \\
\text { was submerged and there was a high velocity outflow from the culverts. } 20-30 \text { fish }(\sim 20 \mathrm{~cm}) \text { were observed upstream of the right } \\
\text { culvert in a backwater pool. }\end{array}$ \\
\hline $\mathrm{X}-05-092$ & Water levels were too high to fish. Riparian area is flooded approximately $80 \mathrm{~m}$ on each side of the ban \\
\hline
\end{tabular}




\section{Appendix 7: List of any additional field notes recorded at sampling sites.}

\begin{tabular}{|c|c|}
\hline $\begin{array}{l}\text { Site } \\
\text { Number }\end{array}$ & Field Notes \\
\hline $\mathrm{X}-05-093$ & anks are slumping around the pool, downstream of the culverts. Slow/ deep and slow/ shallow flow regimes. Banks are grassed. \\
\hline $\mathrm{X}-05-094$ & urush instream. Slow/deep, slow/shallow and fast/shallow flow regimes present. Grassed banks, moderately unstable. \\
\hline $\mathrm{X}-05-095$ & Bulrush instream. Grassed banks. \\
\hline $\mathrm{X}-05-096$ & No catch but a minnow was observed in the pool. Bulrush present in drain. Slow/deep and slow/shallow flow regimes present. \\
\hline $\mathrm{X}-05-097$ & Pool too deep fish $(>1.5 \mathrm{~m})$. Choked with cattails and bulrush. \\
\hline $\mathrm{X}-05-098$ & Deep pool (> $1.25 \mathrm{~m}$ ) associated with bridge. Otherwise, water is shallow with bulrush and cattails instream. Banks are grassed. \\
\hline $\mathrm{X}-05-100$ & Choked with cattails, except for deep pool associated with bridge. \\
\hline $\mathrm{X}-05-101$ & $\begin{array}{l}\text { Natural section with 3-5 m wide riparian upstream of culvert crossing. Willows and mature trees overhanging the water's edge. } \\
\text { Very large pool downstream of culvert crossing, with erosion on left banks and light cattle impacts. Gradient control structure (weir } \\
\text { and constructed riffle) downstream of large pool. Too deep to fish. }\end{array}$ \\
\hline $\mathrm{X}-05-102$ & $\begin{array}{l}\text { Many spawning Fathead Minnow. Choked with cattails throughout most of drain. Rocks with emergent vegetation and some } \\
\text { submerged vegetation as cover. High sediment deposition under bridge. Slow/ shallow and slow/ deep flow regimes. Grassed } \\
\text { riparian area with some shrubs. }\end{array}$ \\
\hline $\mathrm{X}-05-103$ & $\begin{array}{l}\text { Natural section with bulrush present below bridge. Slow/deep and fast/shallow flow regimes. Heavy deposits of fine sediments. } \\
\text { Cattle impacted banks. Mud and clay substrate. }\end{array}$ \\
\hline $\mathrm{X}-05-104$ & $\begin{array}{l}\text { Good habitat. Released a Creek Chub (fork length }=20 \mathrm{~cm} \text { ) with red spawning tubercles. Mix of snags, submerged logs, undercut } \\
\text { banks and cobble for cover. All four velocity regimes present. One large riffle within reach and many bends. Wide riparian area } \\
\text { with native vegetation. }\end{array}$ \\
\hline $\mathrm{X}-05-105$ & $\begin{array}{l}\text { Banks are unstable downstream of bridge crossing, with high erosion potential. Mix of deep and shallow pools upstream with lots } \\
\text { of submerged vegetation and woody debris in stream. Two small riffles and several bends in reach. Some bulrush present near } \\
\text { pools and willows and mature trees as riparian vegetation. Poor habitat downstream, with eroded banks, submerged vegetation } \\
\text { as the only cover, and mainly grass for riparian vegetation. }\end{array}$ \\
\hline$X-05-106$ & $\begin{array}{l}\text { Fished a natural section with native riparian vegetation. Bulrush in stream. Substantial sediment deposition downstream. Slow/ } \\
\text { deep, slow/ shallow and fast/ shallow flow regimes. Banks moderately unstable and slumping. Light to moderate cattle impacts. }\end{array}$ \\
\hline $\mathrm{X}-05-107$ & $\begin{array}{l}\text { Only fished the constructed riffle up to the weir because the water was too deep upstream and downstream. Released hundreds } \\
\text { of Fathead Minnows and Creek Chub. Creek is beginning to meander within its banks. Slow/shallow, fast/shallow, and fast/deep } \\
\text { regimes in reach. Grassed banks with some shrubs growing on the right bank upstream of the constructed riffle. }\end{array}$ \\
\hline $\mathrm{X}-05-108$ & Cement box culvert. Stream flowing through a yard, where grass is mowed to the edges. \\
\hline $\mathrm{X}-05-109$ & $\begin{array}{l}\text { Heavy sediment deposition under bridge, forming bars. Hard clay substrate. Slow/deep, slow/shallow, and fast/shallow flow } \\
\text { regimes. Riparian area approximately } 6 \mathrm{~m} \text { wide downstream of bridge and impacted by cattle upstream of bridge. }\end{array}$ \\
\hline
\end{tabular}




\section{Appendix 7: List of any additional field notes recorded at sampling sites.}

\begin{tabular}{|c|c|}
\hline $\begin{array}{l}\text { Site } \\
\text { Number }\end{array}$ & Field Notes \\
\hline $\mathrm{X}-05-110$ & $\begin{array}{l}\text { At a natural section of the creek with a beaver dam upstream of the culvert crossing. There is an abundance of old and new tree } \\
\text { fall. Abundant overhanging vegetation and also complete canopy in sections along creek. Water was flowing at an extremely high } \\
\text { velocity: } 1.58 \mathrm{~m} / \mathrm{s} \text { at inlet of culvert; } 1.56 \mathrm{~m} / \mathrm{s} \text { in run, approximately } 1.5 \mathrm{~m} \text { in front of the culvert; } 1.41 \mathrm{~m} / \mathrm{s} \text { at outlet; } 1.13 \mathrm{~m} / \mathrm{s} \text { in } \\
\text { rapids, approximately } 2.5 \mathrm{~m} \text { downstream of culvert outlet. Mostly riffle and rapid habitat downstream of culvert, with run and } \\
\text { plunge pool upstream. Banks are severely eroded and down cut downstream of the culvert. A local said that the outflow to } \\
\text { Shannon Creek from Lizard Lake has been opened up due to recent heavy rains. }\end{array}$ \\
\hline $\mathrm{X}-05-111$ & $\begin{array}{l}\text { Natural section of stream. Undercut banks, overhanging vegetation, deadfall, logs, boulders, and submerged vegetation provide } \\
\text { cover. All four flow regimes present. Riffle substrates were mostly exposed. Excellent riparian vegetation and zone width. Even } \\
\text { mix of pool sizes, with root mat and submerged vegetation. }\end{array}$ \\
\hline $\mathrm{X}-05-112$ & $\begin{array}{l}\text { A low-level cement ford crossing is present at the site, with a culvert located immediately West, adjacent to the crossing, through } \\
\text { which the water is flowing. Moderate deposition of sediment. Mainly grassed banks, with a few mature trees. Cattle have widened } \\
\text { the channel and created an abundance of hummocks in and adjacent to the creek. }\end{array}$ \\
\hline $\mathrm{X}-05-113$ & $\begin{array}{l}\text { No flow. Shallow pool and standing water with terrestrial grasses growing in the stream. Sand substrate with high sediment } \\
\text { deposition. No submerged vegetation. Patches of bare soil are visible on the banks, but mostly healed over. }\end{array}$ \\
\hline $\mathrm{X}-05-114$ & $\begin{array}{l}\text { Downstream of bridge is a natural section of stream with abundant overhanging vegetation, deadfall, sections of complete } \\
\text { canopy, and an abundance of boulders and cobble in riffles and pools. Light cattle impacts upstream of bridge, resulting in } \\
\text { hummocks approximately } 1 \mathrm{~m} \text { wide on each side of the stream. Slow/deep, slow/shallow and fast/shallow flow regimes. Riffle } \\
\text { substrate was mostly exposed. Two deep pools within reach, but mostly shallow pools present. }\end{array}$ \\
\hline $\mathrm{X}-05-115$ & $\begin{array}{l}\text { tly disturbed by cattle. Severe cattle impacts on } \\
\text { ed. One riffle present upstream of culvert. }\end{array}$ \\
\hline $\mathrm{X}-05-116$ & Too deep to fish. Trickle of flow. Cattails and gra \\
\hline $\mathrm{X}-05-117$ & $\begin{array}{l}\text { Low flow. Moderate cattail growth in drain. Downstream of bridge, water fills only } 5-10 \% \text { of channel, though slow/deep } \\
\text { slow/shallow flow regimes are present upstream of bridge. Grassed banks. No riffle habitat. }\end{array}$ \\
\hline $\mathrm{X}-05-118$ & Dense algae growth. Slow/deep and slow/shallow flow regimes. Grassed banks. \\
\hline $\mathrm{X}-05-119$ & from \\
\hline$X-05-120$ & $\begin{array}{l}\text { Moderate algae growth in stream. Submerged vegetation, woody debris, and snags as cover. Fast/shallow and slow/shallow flow } \\
\text { regimes. }\end{array}$ \\
\hline $\mathrm{X}-05-121$ & $\begin{array}{l}\text { Well defined thalweg upstream of culvert crossing. Downstream of crossing is choked with terrestrial grasses and cattails. } \\
\text { Emergent vegetation and some boulders as cover. Slow/deep, slow/shallow and fast/shallow flow regimes present. Two shallow } \\
\text { riffles present. Grassed banks. }\end{array}$ \\
\hline-122 & $\begin{array}{l}\text { Natural section of the stream with a good riffle/run/pool sequence and excellent riparian vegetation. Slow/deep, slow/shallow, and } \\
\text { fast/shallow flow regimes. }\end{array}$ \\
\hline $\mathrm{X}-05-123$ & section of stream with good riparian vegetation and area. There are no rif \\
\hline
\end{tabular}




\section{Appendix 7: List of any additional field notes recorded at sampling sites.}

\begin{tabular}{|c|c|}
\hline $\begin{array}{l}\text { Site } \\
\text { Number }\end{array}$ & Field Notes \\
\hline $\mathrm{X}-05-124$ & $\begin{array}{l}\text { o flow. Shallow, standing water only. Terrestrial grasses fill drain. The tributary runs through a pasture downstream of the bridge } \\
\text { ossing. }\end{array}$ \\
\hline $\mathrm{X}-05-125$ & $\begin{array}{l}\text { Outlet is perched approximately } 30 \mathrm{~cm} \text {. Released one White Sucker (FL-220 mm). Bulrush is present along banks upstream of } \\
\text { culvert. Severe cattle impacts with raw areas on banks upstream of the crossing. Abundance of root mats and deadfall in the } \\
\text { stream. All four velocity-depths are present. }\end{array}$ \\
\hline $\mathrm{X}-05-126$ & $\begin{array}{l}\text { Salamander found in the drain. Slow/deep and slow/shallow flow regimes.. Stream has many bends. Banks are moderately } \\
\text { unstable due to cattle impacts and bank slumping. }\end{array}$ \\
\hline $\mathrm{X}-05-127$ & $\begin{array}{l}\text { No catch. Salamander caught. Bulrush in creek. Slow/deep, slow/shallow and fast/shallow flow regimes present. Grassed banks } \\
\text { with moderate cattle impacts. }\end{array}$ \\
\hline $\mathrm{X}-05-128$ & $\begin{array}{l}\text { Some bulrush near the culverts. Severe scouring of banks and cattle impacts. Many shallow riffles. Grasses and small shrubs on } \\
\text { banks. }\end{array}$ \\
\hline $\mathrm{X}-05-129$ & rain is choked with cattails. Some bulrush present. Lots of submerged vegetation in pool under bridge. \\
\hline $\mathrm{X}-05-131$ & o to fish. Marshy $r$ \\
\hline $\mathrm{X}-05-132$ & $\sim 2 \mathrm{~cm}$ ) obser \\
\hline $\mathrm{X}-05-133$ & $\begin{array}{l}\text { Severe cattle impacts downstream of crossing. Bulrush present in stream, as well as a variety of other emergent vegetation. } \\
\text { Marshy upstream of crossing. }\end{array}$ \\
\hline $\mathrm{X}-05-134$ & $\begin{array}{l}\text { No picture of outlets due to excessive growth of the grasses. Heavy growth of terrestrial grasses in drain. Flowing due to recent } \\
\text { rains. }\end{array}$ \\
\hline $\mathrm{X}-05-135$ & am of the culvert crossing is choked with cattails. A large pool is located below the outlets and was $1.5 \mathrm{~m}$ deep at the edge. \\
\hline $\mathrm{X}-05-136$ & No catch. \\
\hline $\mathrm{X}-05-137$ & $\begin{array}{l}\text { Bulrush present. Many cattle trails, closely cropped vegetation, and raw areas along banks upstream of the crossing. Bank } \\
\text { scouring also present. Banks covered by native vegetation downstream of crossing. All four velocity-depth regimes present. }\end{array}$ \\
\hline $\mathrm{X}-05-138$ & Bulrush in drain downstream of culvert crossing. Substrate disturbed by livestock accessing the drain. \\
\hline $\mathrm{X}-05-139$ & Sparse bulrush growing in drain. Fields adjacent to the drain are flooded. Slow/ deep and slow/ shallow flow regimes. \\
\hline $\mathrm{X}-05-140$ & $\begin{array}{l}\text { Local says that stream is dry most of the year. Recent beaver dam removal upstream. The outlet is perched } \sim 30 \mathrm{~cm} \text {. At inlet, } \\
\text { there is a potential barrier to fish passage where soil and cattail growth is forming and creating a drop in front of the inlet. } 5 \mathrm{~cm} \\
\text { Northern Pike observed. }\end{array}$ \\
\hline $\mathrm{X}-05-141$ & Choked with cattails upstream and downstream. Deep pool associated with culvert. \\
\hline $\mathrm{X}-05-142$ & $\begin{array}{l}\text { Choked with cattails downstream of culvert crossing and full of terrestrial grasses upstream of culvert crossing. Likely only flowing } \\
\text { because of recent heavy rains. Low flow. }\end{array}$ \\
\hline $\mathrm{X}-05-143$ & Large deep pool downstream of culvert $(>1.5 \mathrm{~m})$. Too deep to fish. Fish $(\sim$ length $2-3 \mathrm{~cm})$ observed in pool. Abundance of \\
\hline
\end{tabular}




\section{Appendix 7: List of any additional field notes recorded at sampling sites.}

\begin{tabular}{|c|c|}
\hline $\begin{array}{l}\text { Site } \\
\text { Number }\end{array}$ & Field Notes \\
\hline $\mathrm{X}-05-144$ & $\begin{array}{l}\text { Released hundreds of Brook Stickleback and Central Mudminnow. Inlets were submerged and one outlet was perched } \sim 0.5 \mathrm{~m} \text {. } \\
\text { DO meter would not calibrate. Suboptimal cover with trees, root mats, cobble, and some submerged vegetation. All four velocity- } \\
\text { depths present. Many riffles at outlet and flat water upstream and downstream. }\end{array}$ \\
\hline $\mathrm{X}-05-145$ & $\begin{array}{l}\text { Dissolved oxygen meter would not calibrate. Bulrush present. Light cattle impacts upstream of crossing. Optimal substrate and } \\
\text { cover, with undercut banks, cobble, boulders, deadfall, overhanging vegetation and submerged vegetation. All four velocity-depth } \\
\text { regimes present and very little sediment deposition or embeddedness. Relatively frequent occurrence of riffles and good riparian } \\
\text { zone width and vegetation. }\end{array}$ \\
\hline $\mathrm{X}-05-146$ & $\begin{array}{l}\text { Could not cross farmer's fences to get the inlet pictures. Some bulrush present. High turbidity and deep, fast water made fishing } \\
\text { difficult. Missed several minnows and two large White Sucker (fork length= } 25 \mathrm{~cm} \text { ). Water was primarily deep run with some } \\
\text { shallow run and deep pools. }\end{array}$ \\
\hline $\mathrm{X}-05-147$ & Cattle trails leading to the river upstream of the culvert crossing. Very wide channel and too deep to fish. \\
\hline$X-05-148$ & $\begin{array}{l}\text { Dissolved oxygen meter would not calibrate. No catch. Heavy growth of terrestrial grass in drain downstream of bridge. Water } \\
\text { smells like decomposing vegetation. }\end{array}$ \\
\hline $\mathrm{X}-05-149$ & No flow. Standing pools below culverts. Grass too tall to take culvert inlet and outlet pictures. \\
\hline $\mathrm{X}-05-150$ & $\begin{array}{l}\text { Dissolved oxygen meter would not calibrate. Bulrush present at river edges. Thousands of young-of-year fish present. Culvert } \\
\text { outlet was perched approximately } 0.2 \mathrm{~m} \text {. Even mix of large deep, small shallow, large shallow and small deep pools. Stable banks } \\
\text { with good riparian vegetation. }\end{array}$ \\
\hline $\mathrm{X}-05-151$ & $\begin{array}{l}\text { Arrowhead and bulrush present. Sand bars have formed at outlet. Suboptimal substrate and cover, with limited submerged } \\
\text { vegetation, undercut banks, overhanging vegetation, emergent vegetation and some rock. Stable banks with wide riparian zones } \\
\text { consisting of native vegetation. }\end{array}$ \\
\hline $\mathrm{X}-05-152$ & Choked with cattails upstream and downstream of culvert crossing. Little flow. \\
\hline $\mathrm{X}-05-153$ & $\begin{array}{l}\text { Dissolved oxygen meter would not calibrate. No catch. Bulrush in creek. Substrate comprised of boulders, cobble, and gravel, } \\
\text { with abundant submerged vegetation. }\end{array}$ \\
\hline$X-05-154$ & $\begin{array}{l}\text { Dissolved oxygen meter would not calibrate. Outlet was perched } \sim 25 \mathrm{~cm} \text {. Bulrush and sedges in creek. Severe cattle impacts } \\
\text { downstream of reach. Left bank is raw and highly unstable. Hundreds of minnows observed in plunge pool beneath culvert. } \\
\text { Relatively frequent occurrence of riffles. Slow/deep, slow/shallow and fast/shallow flow regimes present. }\end{array}$ \\
\hline $\mathrm{X}-05-155$ & $\begin{array}{l}\text { Abundant of cobble, boulders, sedges and a variety of submerged vegetation in creek. Many small shrubs growing along bank } \\
\text { and overhanging parts of the creek. Heavy growth of algae on cobble in riffles. Dissolved oxygen meter would not calibrate. }\end{array}$ \\
\hline $\mathrm{X}-05-156$ & $\begin{array}{l}\text { Choked with grasses, sedges and cattails upstream of crossing. Downstream is choked with cattails. An abundance of bushes are } \\
\text { present in the riparian zone downstream of the crossing. }\end{array}$ \\
\hline $\mathrm{X}-05-157$ & $\begin{array}{l}\text { No catch. High sediment deposition. Cobble and rock are } 75-100 \% \text { buried with sediments. Lots of submerged vegetation, } \\
\text { arrowhead, and sedges in stream. Cattails present in channel downstream of bridge. Cattle have created hummocks and bare } \\
\text { exposed sections along edge of creek. }\end{array}$ \\
\hline $\mathrm{X}-05-158$ & oked with cattails upstream and downstream of culvert crossing. Trickle of flow through culvert. \\
\hline
\end{tabular}




\section{Appendix 7: List of any additional field notes recorded at sampling sites.}

\begin{tabular}{|c|c|}
\hline $\begin{array}{l}\text { Site } \\
\text { Number }\end{array}$ & Field Notes \\
\hline $\mathrm{X}-05-159$ & $\begin{array}{l}\text { Pools present below inlet and outlet were too deep to seine. Bulrush present. Choked with cattails upstream and downstream of } \\
\text { pools. }\end{array}$ \\
\hline $\mathrm{X}-05-160$ & $\begin{array}{l}\text { Wide, flat channel with shallow water, except for the pool located at the outlet. Choked with cattails upstream and downstream of } \\
\text { crossing. }\end{array}$ \\
\hline $\mathrm{X}-05-161$ & Raw areas along banks upstream of crossing due to cattle impacts. Drain is choked with cattails. Slight flow through culverts. \\
\hline$X-05-162$ & $\begin{array}{l}\text { Most of reach was too deep to fish. We were only able to fish below the bridge. DO meter would not calibrate. Slow/ deep, slow/ } \\
\text { shallow and fast/ shallow flow regimes present. }\end{array}$ \\
\hline $\mathrm{X}-05-163$ & $\begin{array}{l}\text { DO meter would not calibrate. Choked with emergent vegetation upstream of culvert crossing. Abundant growth of bulrush. No } \\
\text { catch. Variety of submerged vegetation and emergent vegetation species in drain. }\end{array}$ \\
\hline $\mathrm{X}-05-164$ & $\begin{array}{l}\text { Dissolved oxygen meter would not calibrate. Heavy growth of cattails and floating macrophytes upstream and downstream of } \\
\text { culvert crossing. Heavy growth of algae. No catch. }\end{array}$ \\
\hline $\mathrm{X}-05-165$ & $\begin{array}{l}\text { Riparian area is flooded. Heavy growth of terrestrial grasses in drain downstream of culvert crossing. A small riffle is associated } \\
\text { with the outlet. Banks are mainly grassed, with a few willows and shrubs. }\end{array}$ \\
\hline$X-05-166$ & $\begin{array}{l}\text { Very high flow velocity through culverts due to recent rains. Area around creek and upstream of crossing is flooded. Combination } \\
\text { of high velocity flow and cattle impacts have resulted in bank erosion. Outlet is perched } \sim 2 \mathrm{~m} \text {, causing a barrier to fish passage. } \\
\text { Unable to fish because of high barbed wire fencing. }\end{array}$ \\
\hline $\mathrm{X}-05-167$ & Lots of flow due to recent rain. Bulrush present in channel. Some cattle impacts. \\
\hline $\mathrm{X}-05-168$ & $\begin{array}{l}\text { High flow due to recent rain. Culvert is submerged. Bulrush present in creek. Three riffles within reach. Stable banks with native } \\
\text { riparian vegetation. }\end{array}$ \\
\hline $\mathrm{X}-05-169$ & $\begin{array}{l}\text { Raw banks downstream of culverts due to cattle crossing the creek. Two riffles located within reach. Culvert outlets perched } \sim 0.2 \\
\text { m. }\end{array}$ \\
\hline $\mathrm{X}-05-170$ & $\begin{array}{l}\text { Inlet is submerged, no photo taken. Plunge pool below outlet with fast, deep run. Flooded riparian area upstream of crossing. } \\
\text { Creek was too fast and deep to fish. }\end{array}$ \\
\hline $\mathrm{X}-05-171$ & $\begin{array}{l}\text { Long run upstream of culvert crossing. Highly turbid water made it difficult to see fish. Approximately } 200 \mathrm{~m} \text { downstream of } \\
\text { crossing, a straw bedding area for cattle is situated on the edge of the right bank, extending into the creek. Large riffle at the } \\
\text { culvert outlet. }\end{array}$ \\
\hline $\mathrm{X}-05-172$ & $\begin{array}{l}\text { Flow is predominantly deep, fast run. The grassed edges of the channel were fished because the water flow was too high to wade } \\
\text { the creek. Some cattle trails are present along the edges of the creek. }\end{array}$ \\
\hline $\mathrm{X}-05-173$ & $\begin{array}{l}\text { Slow/ shallow, slow/ deep, and fast/ deep flow regimes present. Channel is greater than } 1.5 \mathrm{~m} \text { deep except for below bridge. } \\
\text { Arrowhead and a variety of aquatic vegetation present. }\end{array}$ \\
\hline $\mathrm{X}-05-174$ & $\begin{array}{l}\text { High water levels due to recent rains. Shrubs and trees within riparian zone are flooded. Mostly run habitat except for the flooded, } \\
\text { grassy edges. }\end{array}$ \\
\hline $\mathrm{X}-05-175$ & $\begin{array}{l}\text { Fields adjacent to drain are severely flooded due to recent rain. Shallow water and the presence of terrestrial grasses in drain } \\
\text { suggest that it is usually dry. }\end{array}$ \\
\hline
\end{tabular}




\section{Appendix 7: List of any additional field notes recorded at sampling sites.}

\begin{tabular}{|c|c|}
\hline $\begin{array}{l}\text { Site } \\
\text { Number }\end{array}$ & Field Notes \\
\hline $\mathrm{X}-05-176$ & $\begin{array}{l}\text { No catch. Water is at bank full stage. Excessive growth of grasses within the channel. Bulrush present in some sections. } \\
\text { Moderate cattle impacts throughout pasture. Cattle have widened and flattened the channel. }\end{array}$ \\
\hline $\mathrm{X}-05-177$ & $\begin{array}{l}\text { Local farmer says this creek dries up almost every summer. Some overhanging vegetation and root mats present. Abundance of } \\
\text { submerged vegetation. Rock/cobble recently deposited at culvert. }\end{array}$ \\
\hline $\mathrm{X}-05-178$ & Bulrush present in tributary. High water levels. \\
\hline $\mathrm{X}-05-179$ & $\begin{array}{l}\text { Banks are raw and muddy due to severe cattle impacts and bank scouring. Many point bars within drain. Usually dries up with low } \\
\text { water levels. }\end{array}$ \\
\hline $\mathrm{X}-05-180$ & Culvert inlets and left outlet submerged. Part of drain has washed over the road. Bulrush present in drain. \\
\hline $\mathrm{X}-05-181$ & $\begin{array}{l}\text { Submerged vegetation, root mats and cattail growth along edges of drain. Slow/deep, slow/shallow and fast/shallow flow regimes } \\
\text { present. Grassed banks. Two hog farms nearby, located NE and NW of drain. }\end{array}$ \\
\hline $\mathrm{X}-05-182$ & Deep channel (>2 m) downstream of culvert crossing. Slow/shallow, slow/deep and fast/deep flow regimes present. \\
\hline $\mathrm{X}-05-183$ & Stable banks with some trees and shrubs present. Marginal substrate and cover. \\
\hline $\mathrm{X}-05-184$ & $\begin{array}{l}\text { The fields to the north were flooded and very turbid water. Flow into Boundary drain from the } 2 \text { nd order tributary. Local said that } \\
\text { the stream usually dries up in the summer. }\end{array}$ \\
\hline $\mathrm{X}-05-185$ & $\begin{array}{l}\text { Released one Common Carp }(T L=830 \mathrm{~mm}) \text {. Slow/shallow and slow/deep flow regimes. Grassed banks are mowed almost to } \\
\text { edges of streams in some places though mature trees are present on banks. }\end{array}$ \\
\hline$X-05-186$ & $\begin{array}{l}\text { Local has seen fish come up the drain in the spring during previous years but not this year. All flat water with no bends or shallow } \\
\text { riffles. Moderately stable, grassed banks. }\end{array}$ \\
\hline $\mathrm{X}-05-187$ & Water flow at $>75 \%$ bank full status. Too deep to fish, culverts almost submerged. \\
\hline $\mathrm{X}-05-188$ & $\begin{array}{l}\text { No flow through the left culvert. Bulrush present in drain. Dense growth of algae. Released two Brook Sticklebacks and one } \\
\text { Central Mudminnow. Downstream from the crossing, the riparian zone is }<2 \mathrm{~m} \text {. Riparian zone is }>18 \mathrm{~m} \text { upstream of the crossing }\end{array}$ \\
\hline $\mathrm{X}-05-189$ & $\begin{array}{l}\text { Lots of submerged vegetation and filamentous algae. Observed one Northern Pike (total length } \sim 8 \mathrm{~cm} \text { ). Mostly slow } / \text { shallow flow } \\
\text { regime. }\end{array}$ \\
\hline $\mathrm{X}-05-190$ & Substantial cattail growth along both banks. No riffles within reach. A few bends are present. \\
\hline $\mathrm{X}-05-191$ & $\begin{array}{l}\text { Suboptimal substrate and cover, with snags, deadfall, undercut banks, overhanging vegetation, and submerged vegetation. } \\
\text { Slow/deep, slow/shallow, and fast/shallow flow regimes were present. }\end{array}$ \\
\hline $\mathrm{X}-05-192$ & $\begin{array}{l}\text { Mostly a braided stream pattern with native vegetation (primarily sedges, grasses and shrubs) in the wide riparian zone. One } \\
\text { deep pool ( }>2 \mathrm{~m} \text { ) present within reach. Substrate was primarily organic material. Little cover present. Relatively frequent } \\
\text { occurrence of riffles and even mix of pools. The right culvert was perched } 0.5 \mathrm{~m} \text { and the left culvert was perched } 0.75 \mathrm{~m}\end{array}$ \\
\hline $\mathrm{X}-05-193$ & $\begin{array}{l}\text { High frequency of riffles within reach. Both culverts are perched and flow is only occurring through the left culvert. The right } \\
\text { culvert is perched } 0.2 \mathrm{~m} \text { higher than the left culvert. }\end{array}$ \\
\hline $\mathrm{X}-05-194$ & $\begin{array}{l}\text { Heavy deposits of sand and gravel. Lots of snags, deadfall, undercut banks and overhanging vegetation in stream as cover. } \\
\text { Stable banks with native riparian vegetation. }\end{array}$ \\
\hline $\mathrm{X}-05-195$ & rched 0.5 to $0.75 \mathrm{~m}$. Abund \\
\hline
\end{tabular}




\section{Appendix 7: List of any additional field notes recorded at sampling sites.}

\begin{tabular}{|c|c|}
\hline $\begin{array}{l}\text { Site } \\
\text { Number }\end{array}$ & Field Notes \\
\hline $\mathrm{X}-05-196$ & Abundance of terrestrial grasses and shrubs growing in channel. Slow/shallow and fast/shallow flow regimes. \\
\hline $\mathrm{X}-05-197$ & Trickle of flow. Drain is choked with cattail and some willows are growing within the drain, downstream of the culvert crossing. \\
\hline $\mathrm{X}-05-198$ & $\begin{array}{l}\text { Frequent riffles and an abundance of undercut banks and snags as cover. Canopy upstream with a mix of overhanging vegetation } \\
\text { and canopy downstream of crossing. Deposits of silt and sand on bends and at obstructions. Deposits of silt and sand also } \\
\text { present on banks due to high water. }\end{array}$ \\
\hline $\mathrm{X}-05-199$ & $\begin{array}{l}\text { Thousands of minnows present beneath culvert, which is perched } 0.1 \mathrm{~m} \text {. Lots of woody debris, undercut banks, overhanging } \\
\text { vegetation, and cobble in the channel downstream of the crossing. }\end{array}$ \\
\hline $\mathrm{X}-05-200$ & $\begin{array}{l}\text { Suboptimal substrate and cover, with cobble, submerged vegetation, undercut banks, and overhanging vegetation. Slow/deep, } \\
\text { slow/shallow, fast/deep and fast/shallow flow regimes were present. Downstream of bridge, the riparian zone is less than } 6 \mathrm{~m} \text {. } \\
\text { Upstream of the crossing, the riparian zone width is approximately } 15 \mathrm{~m} \text {. }\end{array}$ \\
\hline $\mathrm{X}-05-201$ & Drain is heavily lined with dead algae mats. Dry, with a small pool at the inlets only. Inlets were perched $0.2 \mathrm{~m}$. \\
\hline $\mathrm{X}-05-202$ & Bulrush, sedges, and arrowhead in drain. Recently replaced culvert. Large backwater pool downstream of culvert. \\
\hline $\mathrm{X}-05-203$ & $\begin{array}{l}\text { Unable to fish because the electrofisher was not working. Many raw areas along banks due to cattle crossing the stream. High } \\
\text { erosion potential during floods. }\end{array}$ \\
\hline $\mathrm{X}-05-204$ & Channel was $>1.5 \mathrm{~m}$ deep. Fished the drain from the banks. Tall grass and young shrubs on the banks. \\
\hline $\mathrm{X}-05-205$ & $\begin{array}{l}\text { Stream was dry } 2 \text { miles upstream of crossing. Shallow, standing pools present at inlets and outlets only. Choked with cattails. } \\
\text { Willows growing in channel, upstream of culvert crossing. }\end{array}$ \\
\hline $\mathrm{X}-05-206$ & $\begin{array}{l}\text { Wet meadow dominated by sedges and grasses. No flow. Small shallow pool present at outlet. Local says that Northern Pike can } \\
\text { be found in the drain located at the outlet of Norris Lake. }\end{array}$ \\
\hline $\mathrm{X}-05-207$ & Heavy growth of algae. \\
\hline $\mathrm{X}-05-208$ & Marginal habitat. Stable, grassed banks. \\
\hline $\mathrm{X}-05-210$ & Abundant sedges, arrowhead and bulrush. A few cattails present as well. No riffles but a moderate amount of bends are present. \\
\hline D-06-001 & Light cattle impacts. Shale substrate predominant. Mowed banks. Released two Creek Chub @ 150, 160 mm. \\
\hline W-06-001 & $\begin{array}{l}\text { Drain is back flowing from Lake Dauphin - yesterday strong South winds. Little to no flow. Site is at a ford crossing. Two culverts } \\
\text { present; no riffles, a few bends. Marshy habitat. Diked both sides upstream, not diked downstream. }\end{array}$ \\
\hline W-06-002 & Not diked. \\
\hline W-06-003 & Moderate cattle impacts. Deep/fast flow regime missing. Not diked. \\
\hline W-06-004 & No catch. Light cattle impacts. Deep/fast flow regime missing. Not diked. \\
\hline W-06-005 & Not diked. \\
\hline W-06-006 & Not diked. \\
\hline W-06-007 & $\begin{array}{l}\text { Local knowledge: suckers spawned at site last week. Most years Northern Pike also spawn here. There is a weir } 1 \text { mile upstream } \\
\text { in the neighbour's property (fish blockage). Beaver dams are the main barriers to fish passage in the area. Other notes: Two } \\
\text { culverts, no channelization. Not diked downstream, diked roadside upstream. }\end{array}$ \\
\hline W-06-008 & Severe cattle impacts. No catch. Not diked. \\
\hline
\end{tabular}




\section{Appendix 7: List of any additional field notes recorded at sampling sites.}

\begin{tabular}{|c|c|}
\hline $\begin{array}{l}\text { Site } \\
\text { Number }\end{array}$ & Field Notes \\
\hline W-06-009 & eleased a Fathead Minnow. Slow/shallow and slow/deep regimes present. One large culvert. Many bends present. Not diked. \\
\hline W-06-010 & $\begin{array}{l}\text { Released twice as many Fathead Minnow and Brook Stickleback as kept. Channel ploughed under } 100 \% \text {. Natural bends present. } \\
\text { Not diked. }\end{array}$ \\
\hline W-06-011 & Marshy habitat. Too deep to fish. Not diked. \\
\hline W-06-012 & $\begin{array}{l}\text { Moderate cattle impacts downstream. Slow/ shallow and fast/ shallow regimes present. Large earth dam to hold back water } \\
\text { upstream of site. Cattle have access to drain. Not diked. }\end{array}$ \\
\hline W-06-013 & ( \\
\hline W-06-014 & Not diked. \\
\hline W-06-015 & $\begin{array}{l}\text { Not diked. Moderate cattle impacts. Fast/deep flow regime missing. Evidence of past beaver activity. Few bends and riffles. } \\
\text { Pasture downstream of culverts. Bank vegetation is all grasses. }\end{array}$ \\
\hline W-06-016 & $\begin{array}{l}\text { Not diked. Lots of submerged vegetation with some cobble under bridge. Banks are undercut in some places. A few riffles and } \\
\text { bends present. Bank vegetation is all grass. }\end{array}$ \\
\hline W-06-017 & $\begin{array}{l}\text { Not diked. Slow/deep and slow/shallow regimes present. Some minor cattle impacts. Low flow has exposed many riffles. Very few } \\
\text { bends. Banks upstream of culverts slumping. The only deep pool is under the bridge. }\end{array}$ \\
\hline W-06-018 & Not diked. Low flow, slow velocity. All woody vegetation has been removed. Little submerged and instream vegetation present. \\
\hline W-06-019 & $\begin{array}{l}\text { Channelized. Diked roadside downstream. Diked both sides upstream. Released } \sim 150 \text { shiners. Water level rising due to inflow } \\
\text { from lake. 3rd order tributary blocked by grate to prevent Common Carp from moving into Lake Francis. Gate has created a small } \\
\text { waterfall to pool downstream. Lots of shiners and Common Carp. Some rocks and undercut banks present. Slow/deep and } \\
\text { slow/shallow regimes present. No riffles or bends. Bank vegetation mainly grasses. }\end{array}$ \\
\hline W-06-020 & $\begin{array}{l}\text { Not diked. Severe cattle impacts. Slow/deep and slow/shallow regimes present. Soft sediment and woody debris present. } \\
\text { Channel has been straightened and all vegetation has been removed. Heavy sediment deposition. }\end{array}$ \\
\hline W-06-021 & $\begin{array}{l}\text { Diked road side, right bank. No catch. Slow/shallow regime present. Channelized, no riffles or bends, bank vegetation consists of } \\
\text { grasses. }\end{array}$ \\
\hline W-06-022 & Diked both sides. Deep/fast regime missing. Substrate is mainly mud/silt. \\
\hline W-06-023 & Not diked. Released one White Sucker. Fast/deep flow regime missing. Many bends and a few riffles are present. \\
\hline W-06-024 & Diked both sides. Severe cattle impacts present. \\
\hline W-06-025 & $\begin{array}{l}\text { Diked both sides. Saw } 40+\text { suckers at drain upon first inspection. Drain is channelized, the riffles are caused by channel } \\
\text { constrictions and vegetation, and there are no bends, but thalweg is meandering within banks. Bank vegetation consists of } \\
\text { grasses. Not enough water for deep pools to form. }\end{array}$ \\
\hline W-06-026 & $\begin{array}{l}\text { Diked both sides. Released one Common Carp } \sim 60 \mathrm{~cm} \text { in length. Saw suckers and Northern Pike but couldn't catch them. } \\
\text { Deep/fast regime missing. Bank vegetation is mowed/cleared. }\end{array}$ \\
\hline W-06-027 & $\begin{array}{l}\text { Channelized and left bank diked. Moderate cattle impacts. Released one sucker. Fast/deep regime absent. One set of riffles. } \\
\text { Cattle impacts are affecting bank stability. Bank vegetation is mainly grass. Pool substrate is highly variable. }\end{array}$ \\
\hline W-06-028 & k upstream, not diked downstream. Released one Northern Pike. \\
\hline
\end{tabular}




\section{Appendix 7: List of any additional field notes recorded at sampling sites.}

\begin{tabular}{|c|c|}
\hline $\begin{array}{l}\text { Site } \\
\text { Number }\end{array}$ & Field Notes \\
\hline W-06-029 & $\begin{array}{l}\text { Diked both sides. Slow/deep and slow/shallow flow regimes present. Riparian vegetation cleared and mowed. Pool substrate } \\
\text { consists of soft sediment with some submerged vegetation. The majority of pools are deep. }\end{array}$ \\
\hline W-06-030 & Diked both sides. Slow/deep and slow/ shallow regimes present. No riffles, meandering within bank. Banks are grasses only. \\
\hline W-06-031 & Diked both sides. Suckers and bullheads caught. Banks very unstable and slumping. No woody vegetation on banks, all grasses. \\
\hline W-06-032 & $\begin{array}{l}\text { Diked both sides. Released } 14 \text { suckers, } 15 \text { Creek Chub. Stream is flowing under culvert on downstream side - culvert has } \\
\text { become a barrier to fish passage. Diverse substrate provides lots of habitat. Old cattle pasture - in the past, cattle had access to } \\
\text { drain upstream and downstream of crossing. Vegetative protection consists of grasses. }\end{array}$ \\
\hline W-06-033 & Diked one side downstream from crossing (spoil pile). \\
\hline W-06-034 & Not diked. \\
\hline W-06-035 & Not diked. culvert outlet perched $\sim 1.1 \mathrm{~m}$. \\
\hline W-06-036 & $\begin{array}{l}\text { Not diked downstream, diked roadside upstream. Pool downstream of culverts too deep to fish. Slow/ deep and slow/ shallow } \\
\text { regimes present. Channelized along road upstream, not channelized downstream. No riffles or bends. Vegetative protection } \\
\text { consists of grasses. }\end{array}$ \\
\hline W-06-037 & $\begin{array}{l}\text { Not diked. Released three Brook Stickleback. Slow/deep and slow/shallow flow regimes present. A few bends in reach. Deep } \\
\text { pools much more prevalent than shallow pools. }\end{array}$ \\
\hline W-06-038 & Not diked. Nice habitat. \\
\hline W-06-039 & Not diked. Marshy habitat. \\
\hline W-06-040 & $\begin{array}{l}\text { Not diked upstream, diked roadside downstream. No fish caught. Slow/deep and slow/shallow regimes present. Channelized } \\
\text { downstream of crossing. No riffles, no bends downstream, meandering within banks. Vegetative protection consists of all grasses. } \\
\text { Marshy habitat upstream. }\end{array}$ \\
\hline W-06-041 & Not diked. \\
\hline W-06-042 & Not diked. Moderate cattle impacts. \\
\hline W-06-043 & $\begin{array}{l}\text { Not diked downstream, diked roadside upstream. Caught suckers. Slow/shallow flow regime present. No riffles, three bends in } \\
\text { sight. Deep pools on either side of culverts. }\end{array}$ \\
\hline W-06-044 & Not diked. Local knowledge: farmer told us the drain only flows 2 weeks per year. \\
\hline W-06-045 & $\begin{array}{l}\text { Not diked. Released: } 11 \text { suckers, five shiners, three Redbelly Dace, seven Western Blacknose Dace, seven Fathead Minnows, } \\
\text { and seven Creek Chub. Light cattle impacts; pasture on both sides of crossing. }\end{array}$ \\
\hline W-06-046 & $\begin{array}{l}\text { Not diked. No catch. Heavy thunderstorm the previous night. Substrate is mostly mud with some nature riffles and some } \\
\text { channelization present. No submerged vegetation. }\end{array}$ \\
\hline W-06-047 & Not diked downstream, diked right bank upstream. Water control structure upstream. \\
\hline W-06-048 & ong railway tracks downstream. \\
\hline W-06-049 & t bank eroded at bend downstream of culverts. \\
\hline
\end{tabular}




\section{Appendix 7: List of any additional field notes recorded at sampling sites.}

\begin{tabular}{|c|c|}
\hline $\begin{array}{l}\text { Site } \\
\text { Number }\end{array}$ & Field Notes \\
\hline W-06-050 & $\begin{array}{l}\text { Not diked. Very little water 2-3" deep, trickling through drain. Nice habitat if more water were present. Stream drops } 4-5 \mathrm{ft} \text {. through } \\
\text { culvert. Eroded immediately upstream and downstream of culvert. Almost completely shaded by trees. Large rocks around } \\
\text { culvert, mud substrate. }\end{array}$ \\
\hline W-06-051 & Not diked. Box culvert. \\
\hline W-06-052 & Not diked. Dry. \\
\hline W-06-053 & Not diked. \\
\hline W-06-054 & $\begin{array}{l}\text { Channelized and diked both sides. Slow/deep and slow/shallow regimes present. Vegetative bank protection consists of grasses. } \\
\text { All mud/clay substrate with a bit of sand immediately upstream of culverts. Appears to have migrated from road shoulder. }\end{array}$ \\
\hline W-06-055 & Diked right bank. Fast/deep regime absent. Substrate all clay with some instream vegetation present. \\
\hline W-06-056 & $\begin{array}{l}\text { Not diked. Caught suckers. Slow/shallow and fast/shallow regimes present. Not channelized. Boulders around culverts. Many } \\
\text { bends with a few riffles. Tree roots are holding banks together. }\end{array}$ \\
\hline W-06-057 & Not diked. \\
\hline W-06-058 & $\begin{array}{l}\text { Not diked. Bullheads caught. No inlet pictures taken - sprayed with mosquito larvaecide seconds prior to taking pictures. A few } \\
\text { bends present, and a large pool upstream of crossing. }\end{array}$ \\
\hline W-06-059 & Not diked. \\
\hline W-06-060 & $\begin{array}{l}\text { Not diked. Released numerous Fathead Minnow, two bullheads. Caught bullhead, Common Carp, White Sucker. Saw but unable } \\
\text { to catch one large Northern Pike } \sim 650 \mathrm{~mm} \text {. Slow shallow and slow/deep regimes present. Vegetative bank protection is grass } \\
\text { only. }\end{array}$ \\
\hline W-06-061 & $\begin{array}{l}\text { Not diked. Caught Northern Pike, saw two other Northern Pike but could not catch. Rust line on culvert outlet suggests high water } \\
\text { mark is 2-3" above what is present today. Woody debris throughout channel. }\end{array}$ \\
\hline W-06-062 & $\begin{array}{l}\text { Diked both sides downstream, upstream diked one side. Caught sucker. No snags, undercut banks or cobble. Some cover } \\
\text { provided by submerged vegetation. Fast/deep flow regime absent. Channelized with no riffles. Bank vegetative protection is } \\
\text { grasses. One deep pool downstream of culvert. }\end{array}$ \\
\hline W-06-063 & $\begin{array}{l}\text { Diked both sides downstream, upstream not diked. Recently cleaned and graded. No bends, infrequent riffles observed. Banks } \\
\text { are very unstable and raw. No submerged vegetation. Substrate consists of sand, silt and cobble. The majority of pools are } \\
\text { shallow. }\end{array}$ \\
\hline W-06-064 & $\begin{array}{l}\text { Diked both sides. No catch. Only cobble substrate present. Slow/ deep regime absent. Stream is meandering but banks have } \\
\text { been recently reshaped or cleaned. Many riffles due to low water level, a few bends present. Pool substrate consists of rocks and } \\
\text { fine sediment. }\end{array}$ \\
\hline W-06-065 & Not diked. No catch. Fast/ shallow and fast/ deep present. Pool substrate is mostly gravel/ cobble and boulders. \\
\hline W-06-066 & Not diked. Fast/ shallow and slow/ shallow flow regimes. \\
\hline W-06-067 & Not diked. Many riffles and bends. Riffles substrate rocks/gravel, with submerged vegetation and woody debris. \\
\hline W-06-068 & Not diked. Beaver dam removed recently. Numerous riffles. \\
\hline W-06-069 & Not diked. Water level is down $\sim 0.3 \mathrm{~m}$. \\
\hline
\end{tabular}




\section{Appendix 7: List of any additional field notes recorded at sampling sites.}

\begin{tabular}{|c|c|}
\hline $\begin{array}{l}\text { Site } \\
\text { Number }\end{array}$ & Field Notes \\
\hline W-06-070 & Not diked. Suckers seen but not caught. Caught Burbot, Rock Bass and darter. \\
\hline W-06-071 & $\begin{array}{l}\text { Not diked. Drain crosses gravel road into campground. Dike around Moose Lake and dam (metal control structure) to ensure high } \\
\text { water levels in the lake. Water is } \sim 3 " \text { at deepest point. }\end{array}$ \\
\hline W-06-072 & Not diked. \\
\hline W-06-073 & Not diked. Beaver blocked culverts at inlet, outlet is crushed. There is a trickle of water moving through crossing. \\
\hline W-06-074 & Not diked. A few bends, fewer riffles. \\
\hline W-06-075 & Diked both sides. \\
\hline W-06-076 & $\begin{array}{l}\text { Not diked. Attempted to fish edges. No catch. Too deep to fish main channel. Fast/shallow flow regime only present in culvert. } \\
\text { Flooded upstream. No riffles, many bends. Observed large fish entering culverts - looked like a Common Carp or sucker. }\end{array}$ \\
\hline W-06-077 & Not diked. Fast/ deep regime absent. A little submerged vegetation present. \\
\hline W-06-078 & Not diked. Northern Pike and sucker caught; one Northern Pike released. \\
\hline W-06-079 & Not diked. Fast/deep regime absent. Many bends and a few riffles downstream of culverts. \\
\hline W-06-080 & Not diked. \\
\hline W-06-081 & Diked both sides. \\
\hline W-06-082 & Diked both sides. \\
\hline W-06-083 & Not diked. Sucker caught. Ford crossing. \\
\hline W-06-084 & Not diked. \\
\hline W-06-085 & Not diked. Fast/ deep flow regime absent. \\
\hline W-06-086 & Not diked. \\
\hline W-06-087 & Not diked. Slow/shallow and fast/shallow regimes present. \\
\hline W-06-088 & $\begin{array}{l}\text { Not diked. Released one Northern Pike and five Fathead Minnows. Pike had different colouring - had a black stripe off center } \\
\text { along head and had a blue tinge underneath jaw. Snags and undercut banks present, No cobble. Marshy, vegetated substrate. } \\
\text { Slow/ deep and slow/shallow regimes present. Many bends, no riffles. }\end{array}$ \\
\hline W-06-089 & Not diked. Suckers caught. Fast/deep regime absent. Cattle grazing on both sides of crossing. Moderate cattle impacts. \\
\hline W-06-090 & $\begin{array}{l}\text { Not diked. Released one Rainbow Trout. Kept one immature Brown Trout, one immature Brook Trout. Tags in sample read "Pine } \\
\text { River" instead of North Pine River. Nice habitat. }\end{array}$ \\
\hline W-06-091 & Not diked. Local knowledge: local told us he's never seen drain dried up and he has seen fish (species?) use it. \\
\hline W-06-092 & Not diked. Saw one Northern Pike. Many beaver dams, mix of snags, submerged logs, and undercut banks. Many bends. \\
\hline W-06-093 & Not diked. Fast/ deep regime absent. \\
\hline W-06-094 & $\begin{array}{l}\text { Diked both sides. No fish caught. Slow/shallow and slow/deep regimes present. Channelized. Three gated culverts at crossing. } \\
\text { No riffles or bends. Vegetative protection consists of grass with a few shrubs. Pool substrate is mainly mud. }\end{array}$ \\
\hline W-06-095 & Diked roadside. \\
\hline
\end{tabular}




\section{Appendix 7: List of any additional field notes recorded at sampling sites.}

\begin{tabular}{|c|c|}
\hline $\begin{array}{l}\text { Site } \\
\text { Number }\end{array}$ & Field Notes \\
\hline W-06-096 & $\begin{array}{l}\text { Diked both sides. Saw three Northern Pike } 75-100 \mathrm{~mm} \text { in length and } 1 \text { much larger } \sim 300 \mathrm{~mm} \text { stuck in pool by culverts. DO meter } \\
\text { may not be working (algae decomposing, no riffles, little flow). Caught Northern Pike and White Sucker. Some cobble by culverts. } \\
\text { Algae and submerged vegetation provides only cover. Slow shallow regime present. Channelized, no riffles or bends in sight. } \\
\text { Vegetative bank protection consists of grasses. }\end{array}$ \\
\hline W-06-097 & Diked roadside. White Sucker, Black Bullhead and Northern Pike caught. Culverts are perched $\sim 0.15 \mathrm{~m}$. \\
\hline W-06-098 & Diked both sides. White Sucker caught. No fast/deep flow regime. Very few deep pools present. \\
\hline W-06-099 & Not diked. More than 15 large, dead Common Carp in drain. \\
\hline W-06-100 & $\begin{array}{l}\text { Diked roadside. White Sucker and Yellow Perch caught. Observed Northern Pike but could not catch. Vegetative bank protection } \\
\text { consists of grasses. }\end{array}$ \\
\hline W-06-101 & Diked both sides downstream, right bank upstream. \\
\hline W-06-102 & $\begin{array}{l}\text { Diked both sides. Released several Mudminnow, Brook Stickleback and Fathead Minnows. Fast/shallow and slow/shallow } \\
\text { regimes present. Vegetative protection consists of grasses. }\end{array}$ \\
\hline W-06-103 & Not diked. \\
\hline W-06-104 & Diked both sides. Water level has dropped $0.3 \mathrm{~m}$. Channelized. \\
\hline W-06-105 & Diked both sides. \\
\hline W-06-106 & $\begin{array}{l}\text { Diked both sides downstream, diked right bank upstream. Small cobble and a bit of vegetation are the only cover. Fast/shallow } \\
\text { and slow/shallow regimes present. one riffle and three bends in reach. Vegetative bank protection consists of grasses. Pool } \\
\text { substrate is mainly gravel/cobble. }\end{array}$ \\
\hline W-06-107 & Not diked. \\
\hline W-06-108 & Diked east side. Channelized, no bends. \\
\hline W-06-109 & Diked left bank and on roadside. \\
\hline W-06-110 & Not diked. Observed a mature White Sucker but could not catch. \\
\hline W-06-111 & Not diked. \\
\hline W-06-112 & Not diked. Fast/shallow and slow/shallow regimes present. A few bends in channel. \\
\hline W-06-113 & Not diked. \\
\hline W-06-114 & Not diked. Moderate cattle impacts. Suckers caught. \\
\hline W-06-115 & Not diked. \\
\hline W-06-116 & Not diked. Lots of fish in cobble downstream of culvert. Rocks and banks too difficult to traverse with electrofisher. \\
\hline W-06-117 & Not diked. \\
\hline W-06-118 & Not diked. \\
\hline W-06-119 & $\begin{array}{l}\text { Not diked. Yellow Perch, White Sucker and Burbot caught. Released six suckers. Fast deep regime absent. Ford crossing and } \\
\text { many bends. Very little root mat and instream vegetation. }\end{array}$ \\
\hline W-06-120 & Diked roadside. Overhanging vegetation throughout. Only about $0.1 \mathrm{~m}$. of water in stream. \\
\hline W-06-121 & ed left bank upstream, diked both sides downstream. Sucker caught. All shale substrate. \\
\hline
\end{tabular}




\section{Appendix 7: List of any additional field notes recorded at sampling sites.}

\begin{tabular}{|c|c|}
\hline $\begin{array}{l}\text { Site } \\
\text { Number }\end{array}$ & Field Notes \\
\hline W-06-122 & Diked both sides. \\
\hline W-06-123 & Diked roadside. \\
\hline W-06-124 & Diked downstream. Lots of mud, woody debris and submerged logs. Some cobble downstream of bridge. A few bends present. \\
\hline W-06-125 & Dry. Not diked. \\
\hline W-06-126 & $\begin{array}{l}\text { Not diked. Released one immature Smallmouth Bass, one sculpin (same as in sample), one Northern Pike and one Burbot (same } \\
\text { size as those in samples). Substrate consists of boulders and cobble with vegetation and mud along the water's edge. }\end{array}$ \\
\hline W-06-127 & Not diked. Large beaver using culvert. \\
\hline W-06-128 & Not diked. Water appears to have dropped $0.3 \mathrm{~m}$. \\
\hline W-06-129 & Not diked. \\
\hline W-06-130 & Not diked. \\
\hline W-06-131 & Not diked. \\
\hline W-06-132 & Not diked. \\
\hline W-06-133 & $\begin{array}{l}\text { Not diked. White Sucker were caught. Lots of cobble and rocks present. Fast deep regime absent. Many riffles and bends } \\
\text { present. Pool substrate consists of root mat and mud. }\end{array}$ \\
\hline W-06-134 & $\begin{array}{l}\text { Not diked. Caught White Sucker and Shorthead Redhorse. Saw one Northern Pike and bullhead but could not collect. Substrate } \\
\text { consisted mostly of cobble sized rocks. }\end{array}$ \\
\hline W-06-135 & Not diked \\
\hline W-06-136 & Not diked. Stream was back flowing from the lake - flowing east when it should be flowing west. \\
\hline W-06-137 & Not diked. \\
\hline W-06-138 & $\begin{array}{l}\text { Caught sucker, bullhead. Site at Snyder Dam. No fast/deep regime. Not channelized or diked; one riffle, many bends. Dense } \\
\text { duckweed present. No submerged vegetation or instream vegetation within fished reach. }\end{array}$ \\
\hline W-06-139 & Caught Northern Pike, bullhead, Sauger and Tadpole Madtom. Site at Hartney Dam. Not diked or channelized; many bends. \\
\hline W-06-140 & $\begin{array}{l}\text { Caught bullhead, Silver Redhorse, White Sucker, Troutperch and Stonecat. Lots of algae present. Site at Souris Dam. Not diked } \\
\text { or channelized; three riffles in sight, many bends. }\end{array}$ \\
\hline W-06-141 & $\begin{array}{l}\text { Did initial (front page) assessment on August } 1 \text { st; did water quality, habitat assessment and fishing on August } 2 \text {. For consistency } \\
\text { site assessment and pictures } 15-21 \text { recorded as August } 1 \text {. Not diked or channelized. Many bends, four in sight downstream of } \\
\text { dam. Released one Northern Pike } 350 \mathrm{~mm} \text {, five Shorthead Redhorse } 220-290 \mathrm{~mm} \text {. Saw a Rock Bass. Local knowledge: local has } \\
\text { seen Walleye upstream of dam - he thinks they came from Oak Lake. That was when the water was much higher and there was } \\
\text { less than } 1 \mathrm{~m} \text { drop over the dam. }\end{array}$ \\
\hline W-06-142 & No flow; standing pools only. Not diked. Dry at PR \#344 crossing. \\
\hline W-06-143 & $\begin{array}{l}\text { Diked left bank. Released large Freshwater Drum } \sim 650 \mathrm{~mm} \text { and Black Bullhead. Fast deep regime absent. Channelized, no } \\
\text { bends, two riffles on either side of bridge. Grassed banks. }\end{array}$ \\
\hline W-06-144 & Not diked. Fast/deep flow regime missing. \\
\hline W-06-145 & t diked. culvert outlet perched $0.3 \mathrm{~m}$. \\
\hline
\end{tabular}


Appendix 7: List of any additional field notes recorded at sampling sites.

Site Number

Field Notes

W-06-146

No flow, standing pools upstream of crossing. Not diked. Maximum pool depth (1.3 m) under bridge. Dense vegetation would

have made it impossible to catch fish. No undercut banks, very little cobble and snags. No riffles, many bends. 
Appendix 8: List of all photographs taken showing the corresponding site number, descriptive caption, direction faced when taken (azimuth in degrees) and the unique file name for each photograph.

\begin{tabular}{|c|c|c|c|}
\hline $\begin{array}{c}\text { Site } \\
\text { Number }\end{array}$ & Photo Description & Azimuth & File Name \\
\hline D-02-003 & Looking across ford crossing, looking east & 90 & D-02-003_1-01 \\
\hline D-02-003 & Looking upstream & 180 & D-02-003_1-02 \\
\hline D-02-003 & Looking downstream & 360 & D-02-003_1-03 \\
\hline D-02-005 & Looking upstream & 180 & D-02-005_1-04 \\
\hline D-02-005 & Looking downstream & 360 & D-02-005_1-05 \\
\hline D-02-006 & Looking east & 90 & D-02-006_1-06 \\
\hline D-02-006 & Looking west & 270 & D-02-006_1-07 \\
\hline D-02-007 & Looking east & 90 & D-02-007_1-08 \\
\hline D-02-007 & Looking west & 270 & D-02-007_1-09 \\
\hline D-02-008 & Looking downstream & 0 & D-02-008_1-10 \\
\hline D-02-008 & Looking upstream & 180 & D-02-008_1-11 \\
\hline D-02-009 & Looking upstream from bottom of reach & 270 & D-02-009_1-12 \\
\hline D-02-009 & Looking downstream from bottom of reach & 90 & D-02-009_1-13 \\
\hline D-02-009 & Looking at bank slump on north side of channel & 0 & D-02-009_1-14 \\
\hline D-02-009 & Looking north at gradient control structure & 0 & D-02-009_1-15 \\
\hline D-02-010 & Looking downstream & 110 & D-02-010_1-18 \\
\hline D-02-010 & Looking upstream & 290 & D-02-010_1-19 \\
\hline D-02-011 & Looking downstream & 0 & D-02-011_1-16 \\
\hline D-02-011 & Looking upstream & 135 & D-02-011_1-17 \\
\hline D-02-012 & Looking downstream & 270 & D-02-012_1-20 \\
\hline D-02-012 & Looking upstream & 90 & D-02-012_1-21 \\
\hline D-02-013 & Looking downstream from bottom of reach & 0 & D-02-013_1-22 \\
\hline D-02-013 & Looking upstream through reach & 180 & D-02-013_1-23 \\
\hline D-02-014 & Looking upstream through reach & 135 & D-02-014_1-24 \\
\hline D-02-014 & Looking upstream from below weir & 135 & D-02-014_1-25 \\
\hline D-02-014 & Looking downstream from bridge & 305 & D-02-014_1-26 \\
\hline D-02-015 & Looking upstream & 100 & D-02-015_1-27 \\
\hline D-02-015 & Looking downstream & 300 & D-02-015_1-28 \\
\hline D-02-016 & Looking upstream from crossing & 0 & D-02-016_1-29 \\
\hline D-02-016 & Looking downstream from crossing & 180 & D-02-016_1-30 \\
\hline D-02-017 & Looking at perched drain at confluence of Roseau River at PTH 59 & 0 & D-02-017_1-31 \\
\hline D-02-018 & Looking upstream from bridge & 90 & D-02-018_1-32 \\
\hline D-02-018 & Looking downstream from bridge & 270 & D-02-018_1-33 \\
\hline
\end{tabular}


Appendix 8: List of all photographs taken showing the corresponding site number, descriptive caption, direction faced when taken (azimuth in degrees) and the unique file name for each photograph.

\begin{tabular}{|c|c|c|c|}
\hline $\begin{array}{c}\text { Site } \\
\text { Number }\end{array}$ & Photo Description & Azimuth & File Name \\
\hline D-02-019 & Looking downstream from bridge & 0 & D-02-019_1-34 \\
\hline D-02-019 & Looking upstream from below bridge & 180 & D-02-019_1-35 \\
\hline D-02-019 & Looking downstream from $50 \mathrm{~m}$ below bridge & 0 & D-02-019_1-36 \\
\hline D-02-020 & Looking upstream & 90 & D-02-020_2-01 \\
\hline D-02-020 & Looking downstream & 270 & D-02-020_2-02 \\
\hline D-02-021 & Looking upstream & 90 & D-02-021_2-03 \\
\hline D-02-021 & Looking downstream & 270 & D-02-021_2-04 \\
\hline D-02-022 & Photo of kick sampling the riffle & 0 & D-02-022_2-05 \\
\hline D-02-022 & Photo of kick sampling the riffle & 0 & D-02-022_2-06 \\
\hline D-02-025 & Looking downstream from bridge & 315 & D-02-025_2-07 \\
\hline D-02-025 & Looking upstream from bridge & 135 & D-02-025_2-08 \\
\hline D-02-026 & Photo of kick sampling below culverts & 0 & D-02-026_2-09 \\
\hline D-02-026 & Photo of kick sampling below culverts & 0 & D-02-026_2-10 \\
\hline D-02-026 & Photo of picking through sample & 0 & D-02-026_2-11 \\
\hline D-02-026 & Photo of picking through sample & 0 & D-02-026_2-12 \\
\hline D-02-026 & Looking upstream through reach & 135 & D-02-026_2-13 \\
\hline D-02-026 & Looking downstream from crossing & 0 & D-02-026_2-14 \\
\hline D-02-027 & Looking upstream from crossing & 270 & D-02-027_2-15 \\
\hline D-02-027 & Looking downstream from crossing & 135 & D-02-027_2-16 \\
\hline D-02-028 & Looking upstream from road & 270 & D-02-028_2-17 \\
\hline D-02-028 & Looking downstream from road & 90 & D-02-028_2-18 \\
\hline D-02-029 & Looking downstream & 180 & D-02-029_2-19 \\
\hline D-02-029 & Looking upstream & 10 & D-02-029_2-20 \\
\hline D-02-030 & Looking upstream & 280 & D-02-030_2-21 \\
\hline D-02-030 & Looking downstream & 100 & D-02-030_2-22 \\
\hline D-02-031 & Looking upstream from bottom of reach & 270 & D-02-031_2-23 \\
\hline D-02-031 & Looking downstream from top of reach & 90 & D-02-031_2-24 \\
\hline D-02-032 & Looking upstream & 130 & D-02-032_2-25 \\
\hline D-02-032 & Looking downstream & 320 & D-02-032_2-26 \\
\hline D-02-033 & Looking upstream through riffle at ford crossing & 300 & D-02-033_2-27 \\
\hline D-02-033 & Looking downstream through riffle at ford crossing & 120 & D-02-033_2-28 \\
\hline D-02-033 & Looking downstream through riffle at ford crossing & 120 & D-02-033_2-29 \\
\hline D-02-034 & Looking upstream & 180 & D-02-034_2-30 \\
\hline
\end{tabular}


Appendix 8: List of all photographs taken showing the corresponding site number, descriptive caption, direction faced when taken (azimuth in degrees) and the unique file name for each photograph.

\begin{tabular}{|c|c|c|c|}
\hline $\begin{array}{c}\text { Site } \\
\text { Number }\end{array}$ & Photo Description & Azimuth & File Name \\
\hline D-02-034 & Looking downstream & 90 & D-02-034_2-31 \\
\hline D-02-035 & Looking upstream from crossing & 200 & D-02-035_2-32 \\
\hline D-02-035 & Looking downstream from crossing & 20 & D-02-035_2-33 \\
\hline D-02-036 & Looking upstream from middle of reach & 140 & D-02-036_2-34 \\
\hline D-02-036 & Looking downstream from middle of reach & 315 & D-02-036_2-35 \\
\hline D-02-038 & Looking downstream through reach at minor cattle impacts & 180 & D-02-038_2-36 \\
\hline D-02-038 & Looking downstream through reach at minor cattle impacts & 180 & D-02-038_2-37 \\
\hline D-02-039 & Looking downstream & 90 & D-02-039_3-01 \\
\hline D-02-039 & Looking upstream & 270 & D-02-039_3-02 \\
\hline D-02-039 & Looking downstream & 10 & D-02-039_3-03 \\
\hline D-02-039 & Looking upstream & 190 & D-02-039_3-04 \\
\hline D-02-040 & Looking upstream at bank stabilization & 270 & D-02-040_3-05 \\
\hline D-02-040 & Looking at site of last year's bank stability & 270 & D-02-040_3-06 \\
\hline D-02-040 & Looking at site of last year's bank stability & 270 & D-02-040_3-07 \\
\hline D-02-040 & Looking downstream from bridge at bank stabilization & 90 & D-02-040_3-08 \\
\hline D-02-043 & Looking upstream from middle of reach & 270 & D-02-043_3-09 \\
\hline D-02-043 & Looking downstream from middle of reach & 0 & D-02-043_3-10 \\
\hline D-02-044 & Looking upstream from bottom of reach & 180 & D-02-044_3-11 \\
\hline D-02-044 & Looking upstream from bridge & 180 & D-02-044_3-12 \\
\hline D-02-044 & Looking downstream from bridge & 0 & D-02-044_3-13 \\
\hline D-02-045 & Looking at gradient control structure & 90 & D-02-045_3-14 \\
\hline D-02-045 & Looking upstream from top of reach & 270 & D-02-045_3-15 \\
\hline D-02-045 & Looking upstream from bottom of reach & 270 & D-02-045_3-16 \\
\hline D-02-047 & Looking upstream from ford crossing & 270 & D-02-047_3-17 \\
\hline D-02-047 & Looking downstream from ford crossing & 90 & D-02-047_3-18 \\
\hline D-02-048 & Looking upstream from crossing & 200 & D-02-048_3-19 \\
\hline D-02-048 & Looking downstream from crossing & 20 & D-02-048_3-20 \\
\hline D-02-051 & Looking upstream & 180 & D-02-051_3-21 \\
\hline D-02-051 & Looking downstream & 0 & D-02-051_3-22 \\
\hline D-02-052 & Looking downstream at shale cleanout & 350 & D-02-052_3-23 \\
\hline D-02-052 & Looking upstream at shale cleanout & 90 & D-02-052_3-24 \\
\hline D-02-053 & Looking upstream & 200 & D-02-053_4-01 \\
\hline D-02-053 & Looking downstream & 40 & D-02-053_4-02 \\
\hline
\end{tabular}


Appendix 8: List of all photographs taken showing the corresponding site number, descriptive caption, direction faced when taken (azimuth in degrees) and the unique file name for each photograph.

\begin{tabular}{|c|c|c|c|}
\hline $\begin{array}{c}\text { Site } \\
\text { Number }\end{array}$ & Photo Description & Azimuth & File Name \\
\hline D-02-055 & Looking upstream & 270 & D-02-055_4-03 \\
\hline D-02-055 & Looking downstream & 90 & $D-02-055 \quad 4-04$ \\
\hline D-02-056 & Looking upstream from bridge & 270 & D-02-056_4-05 \\
\hline D-02-056 & Looking downstream from bridge & 90 & D-02-056_4-06 \\
\hline D-02-057 & Looking at cattle impact upstream & 270 & D-02-057_4-07 \\
\hline D-02-057 & Looking downstream & 90 & D-02-057_4-08 \\
\hline D-02-058 & Looking at perched culverts upstream & 270 & D-02-058_4-09 \\
\hline D-02-060 & Looking upstream from crossing & 270 & D-02-060_4-10 \\
\hline D-02-060 & Looking upstream at perched culverts & 100 & D-02-060_4-11 \\
\hline D-02-060 & Looking downstream from crossing & 90 & D-02-060_4-12 \\
\hline D-02-061 & Looking upstream & 270 & D-02-061_4-13 \\
\hline D-02-061 & Looking downstream & 90 & D-02-061_4-14 \\
\hline D-02-062 & Looking upstream & 270 & D-02-062_4-15 \\
\hline D-02-062 & Photo (pan) over riffle upstream & 270 & D-02-062_4-16 \\
\hline D-02-062 & Looking at dam upstream of gradient control structure & 270 & D-02-062_4-17 \\
\hline D-02-063 & Photo of Assiniboine River (perched culverts) & 180 & D-02-063_4-18 \\
\hline D-02-063 & Photo of Assiniboine River (perched culverts) & 180 & D-02-063_4-19 \\
\hline D-02-064 & Looking downstream & 90 & D-02-064_4-20 \\
\hline D-02-064 & Looking upstream & 270 & D-02-064_4-21 \\
\hline D-02-065 & Looking upstream from bridge & 0 & D-02-065_4-22 \\
\hline D-02-065 & Looking downstream from bridge & 180 & D-02-065_4-23 \\
\hline D-02-067 & Looking upstream from bridge & 180 & D-02-067_4-24 \\
\hline D-02-067 & Looking downstream from bridge & 60 & D-02-067_4-25 \\
\hline D-02-068 & Looking upstream from middle of reach & 345 & D-02-068_5-01 \\
\hline D-02-068 & Looking downstream from middle of reach & 135 & D-02-068_5-02 \\
\hline D-02-069 & Looking upstream from bottom of reach & 350 & D-02-069_5-03 \\
\hline D-02-069 & Looking downstream from bottom of reach & 200 & D-02-069_5-04 \\
\hline D-02-070 & Photo of checking larval drift trap & 0 & D-02-070_5-05 \\
\hline D-02-070 & Photo of checking larval drift trap & 0 & D-02-070_5-06 \\
\hline D-02-071 & Looking downstream from bridge & 90 & D-02-071_5-07 \\
\hline D-02-071 & Looking upstream from bridge & 270 & D-02-071_5-08 \\
\hline D-02-074 & Looking upstream from bridge & 290 & D-02-074_5-09 \\
\hline D-02-074 & Looking downstream from bridge & 100 & D-02-074_5-10 \\
\hline
\end{tabular}


Appendix 8: List of all photographs taken showing the corresponding site number, descriptive caption, direction faced when taken (azimuth in degrees) and the unique file name for each photograph.

\begin{tabular}{|c|c|c|c|}
\hline $\begin{array}{c}\text { Site } \\
\text { Number }\end{array}$ & Photo Description & Azimuth & File Name \\
\hline D-02-075 & Looking downstream from ford crossing & 135 & D-02-075_5-11 \\
\hline D-02-075 & Looking upstream at ford crossing & 315 & D-02-075_5-12 \\
\hline D-02-075 & Looking upstream from ford crossing & 315 & D-02-075_5-13 \\
\hline D-02-077 & Looking upstream & 0 & D-02-077_5-14 \\
\hline D-02-077 & Looking downstream & 180 & D-02-077_5-15 \\
\hline D-02-077 & Looking upstream at perched culverts & 0 & D-02-077_5-16 \\
\hline D-02-078 & Looking upstream from bridge & 315 & D-02-078_5-17 \\
\hline D-02-078 & Looking downstream from bridge & 135 & D-02-078_5-18 \\
\hline D-02-079 & Looking upstream from bridge & 300 & D-02-079_5-19 \\
\hline D-02-079 & Looking downstream from bridge & 120 & D-02-079_5-20 \\
\hline D-02-080 & Looking upstream from crossing & 310 & D-02-080_5-21 \\
\hline D-02-080 & Looking downstream from crossing & 135 & D-02-080_5-22 \\
\hline D-02-081 & Looking upstream from PTH 8 & 270 & D-02-081_5-23 \\
\hline D-02-081 & Looking downstream from PTH 8 & 90 & D-02-081_5-24 \\
\hline D-02-082 & Looking upstream & 215 & D-02-082_6-01 \\
\hline D-02-082 & Looking downstream & 50 & D-02-082_6-02 \\
\hline D-02-082 & Looking downstream & 50 & D-02-082_6-03 \\
\hline D-02-083 & Looking upstream from bridge & 270 & D-02-083_6-04 \\
\hline D-02-083 & Looking downstream from bridge & 90 & D-02-083_6-05 \\
\hline D-02-084 & Looking upstream from top of reach & 30 & D-02-084_6-06 \\
\hline D-02-084 & Looking downstream from top of reach & 270 & D-02-084_6-07 \\
\hline D-02-085 & Looking upstream from ford crossing & 300 & D-02-085_6-08 \\
\hline D-02-085 & Looking downstream from ford crossing & 140 & D-02-085_6-09 \\
\hline D-02-085 & Looking upstream at culverts (ford crossing) & 300 & D-02-085_6-10 \\
\hline D-02-086 & Looking at Swan Lake dam & 80 & D-02-086_6-11 \\
\hline D-02-086 & Looking at Swan Lake dam & 80 & D-02-086_6-12 \\
\hline D-02-086 & Looking at Swan Lake dam & 80 & D-02-086_6-13 \\
\hline D-02-087 & Looking upstream & 90 & D-02-087_6-14 \\
\hline D-02-087 & Looking downstream & 300 & D-02-087_6-15 \\
\hline D-02-088 & Looking upstream from crossing & 120 & D-02-088_6-16 \\
\hline D-02-088 & Looking downstream from crossing & 240 & D-02-088_6-17 \\
\hline D-02-089 & Looking upstream from top of reach & 90 & D-02-089_6-18 \\
\hline D-02-089 & Looking downstream from top of reach & 270 & D-02-089_6-19 \\
\hline
\end{tabular}


Appendix 8: List of all photographs taken showing the corresponding site number, descriptive caption, direction faced when taken (azimuth in degrees) and the unique file name for each photograph.

\begin{tabular}{|c|c|c|c|}
\hline $\begin{array}{c}\text { Site } \\
\text { Number }\end{array}$ & Photo Description & Azimuth & File Name \\
\hline D-02-090 & Looking at Mud Lake Drain choked with vegetation & 270 & D-02-090_6-20 \\
\hline D-02-091 & Looking upstream from crossing & 100 & D-02-091_6-21 \\
\hline D-02-091 & Looking downstream from crossing & 290 & D-02-091_6-22 \\
\hline D-02-091 & Looking upstream at cow pasture $75 \mathrm{~m}$ from crossing & 100 & D-02-091_6-23 \\
\hline D-02-092 & Looking upstream from bottom of reach & 120 & D-02-092_6-24 \\
\hline D-02-092 & Looking downstream from bridge & 280 & D-02-092_6-25 \\
\hline D-02-093 & Looking downstream from top of reach & 90 & D-02-093_7-01 \\
\hline D-02-093 & Looking upstream from bridge & 270 & D-02-093_7-02 \\
\hline D-02-094 & Looking upstream from bridge & 280 & D-02-094_7-03 \\
\hline D-02-094 & Looking downstream from bridge & 90 & D-02-094_7-04 \\
\hline D-02-095 & Looking upstream from bridge & 280 & D-02-095_7-05 \\
\hline D-02-095 & Looking downstream from bridge & 100 & D-02-095_7-06 \\
\hline D-02-096 & Looking upstream from crossing & 270 & D-02-096_7-07 \\
\hline D-02-096 & Looking downstream from crossing & 90 & D-02-096_7-08 \\
\hline D-02-096 & Looking downstream from gradient control structure & 90 & D-02-096_7-09 \\
\hline D-02-096 & Looking upstream at gradient control structure & 270 & D-02-096_7-10 \\
\hline D-02-097 & Looking upstream from bottom of reach & 270 & D-02-097_7-11 \\
\hline D-02-097 & Looking downstream from crossing & 90 & D-02-097_7-12 \\
\hline D-02-097 & Looking upstream from crossing & 270 & D-02-097_7-13 \\
\hline D-02-098 & Looking upstream from crossing & 90 & D-02-098_7-14 \\
\hline D-02-098 & Looking downstream from crossing & 270 & D-02-098_7-15 \\
\hline D-02-099 & Looking upstream at cement v-notch weir & 90 & D-02-099_7-16 \\
\hline D-02-099 & Looking upstream from weir & 90 & D-02-099_7-17 \\
\hline D-02-099 & Looking downstream from weir & 270 & D-02-099_7-18 \\
\hline D-02-100 & Looking upstream from top of reach & 90 & D-02-100_7-19 \\
\hline D-02-100 & Looking downstream from top of reach & 270 & D-02-100_7-20 \\
\hline D-02-101 & Looking upstream from crossing & 90 & D-02-101_7-21 \\
\hline D-02-101 & Looking downstream from crossing & 270 & D-02-101_7-22 \\
\hline D-02-102 & Looking upstream from crossing & 90 & D-02-102_7-23 \\
\hline D-02-102 & Looking downstream from crossing & 270 & D-02-102_7-24 \\
\hline D-02-103 & Looking upstream & 180 & D-02-103_8-01 \\
\hline D-02-103 & Looking downstream & 270 & D-02-103_8-02 \\
\hline D-02-103 & Looking at clear inflow from culverts & 90 & D-02-103_8-03 \\
\hline
\end{tabular}


Appendix 8: List of all photographs taken showing the corresponding site number, descriptive caption, direction faced when taken (azimuth in degrees) and the unique file name for each photograph.

\begin{tabular}{|c|c|c|c|}
\hline $\begin{array}{c}\text { Site } \\
\text { Number }\end{array}$ & Photo Description & Azimuth & File Name \\
\hline $\mathrm{D}-02-104$ & Looking upstream from bridge & 180 & D-02-104_8-04 \\
\hline $\mathrm{D}-02-104$ & Looking downstream from bridge & 0 & D-02-104_8-05 \\
\hline $\mathrm{D}-02-105$ & Looking upstream at dam & 250 & D-02-105_8-06 \\
\hline $\mathrm{D}-02-105$ & Looking downstream from dam & 90 & D-02-105_8-07 \\
\hline $\mathrm{D}-02-106$ & Looking upstream from crossing & 0 & D-02-106_8-08 \\
\hline $\mathrm{D}-02-106$ & Looking downstream from crossing & 180 & D-02-106_8-09 \\
\hline $\mathrm{D}-02-106$ & Looking at windblown straw \& topsoil infilling & 0 & D-02-106_8-10 \\
\hline $\mathrm{D}-02-107$ & Looking upstream from crossing & 270 & D-02-107_8-11 \\
\hline $\mathrm{D}-02-107$ & Looking downstream from crossing & 90 & D-02-107_8-12 \\
\hline D-02-107 & Looking at perched culverts & 270 & D-02-107_8-13 \\
\hline D-02-108 & Looking downstream from bridge & 250 & D-02-108_8-14 \\
\hline $\mathrm{D}-02-108$ & Looking upstream from bridge & 100 & D-02-108_8-15 \\
\hline $\mathrm{D}-02-109$ & Looking upstream & 270 & D-02-109_8-21 \\
\hline D-02-109 & Looking downstream & 90 & D-02-109_8-22 \\
\hline $\mathrm{D}-02-110$ & Looking upstream from crossing & 350 & D-02-110_8-23 \\
\hline $\mathrm{D}-02-110$ & Looking downstream from crossing & 170 & D-02-110_8-24 \\
\hline $\mathrm{D}-02-111$ & Looking upstream from ford crossing & 10 & D-02-111_9-01 \\
\hline $\mathrm{D}-02-111$ & Looking downstream from ford crossing & 190 & D-02-111_9-02 \\
\hline $\mathrm{D}-02-111$ & Looking across ford, crossing looking east & 90 & D-02-111_9-03 \\
\hline $\mathrm{D}-02-112$ & Looking upstream from crossing & 90 & D-02-112_9-04 \\
\hline $\mathrm{D}-02-112$ & Looking downstream from crossing & 270 & D-02-112_9-05 \\
\hline $\mathrm{D}-02-113$ & Looking upstream & 90 & D-02-113_9-06 \\
\hline $\mathrm{D}-02-113$ & Looking downstream & 270 & D-02-113_9-07 \\
\hline $\mathrm{D}-02-113$ & Looking at lady slippers growing on banks of Boundary Drain & 90 & D-02-113_9-08 \\
\hline $\mathrm{D}-02-114$ & Looking upstream & 90 & D-02-114_9-09 \\
\hline $\mathrm{D}-02-114$ & Looking downstream & 270 & D-02-114_9-10 \\
\hline $\mathrm{D}-02-115$ & Looking upstream from crossing & 30 & D-02-115_9-11 \\
\hline $\mathrm{D}-02-115$ & Looking downstream from crossing & 150 & D-02-115_9-12 \\
\hline $\mathrm{D}-02-116$ & Looking upstream from bridge & 30 & D-02-116_9-13 \\
\hline $\mathrm{D}-02-116$ & Looking downstream from bridge & 180 & D-02-116_9-14 \\
\hline $\mathrm{D}-02-117$ & Looking upstream at cow pasture & 40 & D-02-117_9-15 \\
\hline $\mathrm{D}-02-117$ & Looking downstream & 220 & D-02-117_9-16 \\
\hline $\mathrm{D}-02-118$ & Looking upstream from bridge & 20 & D-02-118_9-17 \\
\hline
\end{tabular}


Appendix 8: List of all photographs taken showing the corresponding site number, descriptive caption, direction faced when taken (azimuth in degrees) and the unique file name for each photograph.

\begin{tabular}{|c|c|c|c|}
\hline $\begin{array}{c}\text { Site } \\
\text { Number }\end{array}$ & Photo Description & Azimuth & File Name \\
\hline D-02-118 & Looking downstream from bridge & 330 & D-02-118_9-18 \\
\hline D-02-119 & Looking upstream from crossing & 90 & D-02-119_9-19 \\
\hline D-02-119 & Looking upstream from crossing & 90 & D-02-119_9-20 \\
\hline D-02-119 & Looking downstream from crossing & 270 & D-02-119_9-21 \\
\hline D-02-120 & Looking upstream & 345 & D-02-120_9-22 \\
\hline D-02-120 & Looking downstream & 225 & D-02-120_9-23 \\
\hline D-02-121 & Looking upstream from crossing & 90 & D-02-121_10-01 \\
\hline D-02-121 & Looking downstream from crossing & 270 & D-02-121_10-02 \\
\hline D-02-122 & Looking downstream from tributary crossing & 300 & $\mathrm{D}-02-122 \_10-03$ \\
\hline D-02-122 & Looking upstream at culverts & 130 & D-02-122_10-04 \\
\hline D-02-122 & Looking upstream from crossing & 130 & D-02-122_10-05 \\
\hline D-02-123 & Looking upstream from crossing & 90 & $\mathrm{D}-02-123 \_10-06$ \\
\hline D-02-123 & Looking downstream from crossing & 270 & D-02-123_10-07 \\
\hline D-02-124 & Looking downstream at cow pasture & 270 & D-02-124_10-08 \\
\hline D-02-124 & Looking upstream & 90 & D-02-124_10-09 \\
\hline D-02-125 & Looking upstream from bridge & 120 & D-02-125_10-10 \\
\hline D-02-125 & Looking downstream from bridge & 290 & D-02-125_10-11 \\
\hline D-02-126 & Looking upstream from crossing & 150 & $\mathrm{D}-02-126 \_10-12$ \\
\hline D-02-126 & Looking downstream from crossing & 320 & D-02-126_10-13 \\
\hline D-02-127 & Looking upstream from crossing & 110 & D-02-127 $10-14$ \\
\hline D-02-127 & Looking downstream from crossing & 280 & D-02-127_10-15 \\
\hline D-02-127 & Looking downstream from crossing & 280 & D-02-127_10-16 \\
\hline D-02-128 & Looking upstream & 105 & D-02-128_10-17 \\
\hline D-02-128 & Looking downstream & 320 & D-02-128_10-18 \\
\hline D-02-129 & Looking downstream from bridge & 270 & D-02-129_10-19 \\
\hline D-02-129 & Looking upstream from bridge & 45 & D-02-129_10-20 \\
\hline D-02-130 & Looking upstream from crossing & 270 & D-02-130_10-21 \\
\hline D-02-130 & Looking downstream from crossing & 90 & D-02-130_10-22 \\
\hline D-02-131 & Looking downstream from crossing & 90 & D-02-131_10-23 \\
\hline D-02-131 & Looking upstream to the south & 180 & D-02-131_10-24 \\
\hline D-02-132 & Looking upstream from crossing & 180 & D-02-132_11-01 \\
\hline D-02-132 & Looking downstream from crossing & 0 & D-02-132_11-02 \\
\hline D-02-133 & Looking downstream from crossing & 320 & D-02-133_11-03 \\
\hline
\end{tabular}


Appendix 8: List of all photographs taken showing the corresponding site number, descriptive caption, direction faced when taken (azimuth in degrees) and the unique file name for each photograph.

\begin{tabular}{|c|c|c|c|}
\hline $\begin{array}{c}\text { Site } \\
\text { Number }\end{array}$ & Photo Description & Azimuth & File Name \\
\hline D-02-133 & Looking upstream from crossing & 160 & D-02-133_11-04 \\
\hline D-02-134 & Looking upstream from crossing & 330 & D-02-134_11-05 \\
\hline D-02-134 & Looking downstream from crossing & 90 & D-02-134_11-06 \\
\hline D-02-135 & Looking upstream from crossing & 230 & D-02-135_11-07 \\
\hline D-02-135 & Looking downstream from crossing & 50 & D-02-135_11-08 \\
\hline D-02-136 & Looking downstream from PTH 17 & 45 & D-02-136_11-09 \\
\hline D-02-136 & Looking upstream from PTH 17 & 210 & D-02-136_11-10 \\
\hline D-02-137 & Looking upstream from under bridge & 270 & D-02-137_11-11 \\
\hline D-02-137 & Looking downstream from under bridge & 90 & $\mathrm{D}-02-137 \quad 11-12$ \\
\hline D-02-137 & Looking downstream from bridge & 70 & $\mathrm{D}-02-137 \quad 11-13$ \\
\hline D-02-137 & Looking upstream from bridge & 90 & D-02-137_11-14 \\
\hline D-02-138 & Looking downstream from bridge & 90 & $\mathrm{D}-02-138 \_11-15$ \\
\hline D-02-138 & Looking upstream from bridge & 270 & D-02-138_11-16 \\
\hline D-02-139 & Looking upstream from bridge & 270 & D-02-139_11-17 \\
\hline D-02-139 & Looking downstream from bridge & 90 & D-02-139_11-18 \\
\hline D-02-140 & Looking downstream (east) at WSC station and cableway & 100 & D-02-140_11-19 \\
\hline D-02-141 & Looking at culvert replacement on Zoria Drain & 90 & D-02-141_11-20 \\
\hline D-02-141 & Looking at culvert replacement on Zoria Drain & 330 & D-02-141_11-21 \\
\hline D-02-141 & Looking at culvert replacement on Zoria Drain & 330 & D-02-141_11-22 \\
\hline D-02-141 & Looking at culvert replacement on Zoria Drain & 330 & D-02-141_11-23 \\
\hline D-02-141 & Looking at culvert replacement on Zoria Drain & 330 & D-02-141_11-24 \\
\hline $\mathrm{D}-02-142$ & Looking upstream from bridge & 270 & D-02-142_12-01 \\
\hline D-02-142 & Looking downstream from bridge & 90 & D-02-142_12-02 \\
\hline D-02-143 & Looking at WSC station & 210 & D-02-143_12-03 \\
\hline D-02-143 & Looking upstream past WSC station & 270 & D-02-143_12-04 \\
\hline D-02-144 & Looking upstream & 90 & D-02-144_12-05 \\
\hline D-02-144 & Looking downstream & 290 & D-02-144_12-06 \\
\hline D-02-145 & Looking upstream & 180 & D-02-145_12-07 \\
\hline D-02-145 & Looking downstream & 0 & D-02-145_12-08 \\
\hline D-02-146 & Looking upstream from crossing & 180 & D-02-146_12-09 \\
\hline D-02-146 & Looking downstream from crossing & 0 & D-02-146_12-10 \\
\hline D-02-147 & Looking upstream from crossing & 140 & D-02-147_12-11 \\
\hline D-02-147 & Looking downstream from crossing & 320 & $\mathrm{D}-02-147 \quad 12-12$ \\
\hline
\end{tabular}


Appendix 8: List of all photographs taken showing the corresponding site number, descriptive caption, direction faced when taken (azimuth in degrees) and the unique file name for each photograph.

\begin{tabular}{|c|c|c|c|}
\hline $\begin{array}{c}\text { Site } \\
\text { Number }\end{array}$ & Photo Description & Azimuth & File Name \\
\hline D-02-148 & Looking downstream at rock/boulder infilling and bank slumping & 20 & D-02-148_12-13 \\
\hline D-02-149 & Looking upstream from crossing & 120 & D-02-149_12-14 \\
\hline D-02-149 & Looking downstream from crossing & 320 & D-02-149_12-15 \\
\hline D-02-150 & Looking downstream from bridge & 90 & D-02-150_12-19 \\
\hline D-02-150 & Looking upstream from bridge & 270 & D-02-150_12-20 \\
\hline D-02-151 & Looking upstream & 230 & D-02-151_12-21 \\
\hline D-02-151 & Looking downstream & 100 & D-02-151_12-22 \\
\hline D-02-152 & Looking upstream & 270 & D-02-152_12-23 \\
\hline D-02-152 & Looking downstream & 90 & D-02-152_12-24 \\
\hline D-02-152 & Looking upstream & 270 & D-02-152_13-01 \\
\hline D-02-152 & Looking downstream & 90 & D-02-152_13-02 \\
\hline D-02-153 & Looking upstream & 270 & D-02-153_13-03 \\
\hline D-02-153 & Looking downstream & 90 & D-02-153_13-04 \\
\hline D-02-154 & Looking upstream from crossing & 90 & D-02-154_13-05 \\
\hline D-02-154 & Looking downstream from crossing & 270 & D-02-154_13-06 \\
\hline D-02-155 & Looking upstream from crossing & 90 & D-02-155_13-07 \\
\hline D-02-155 & Looking downstream from crossing & 270 & D-02-155_13-08 \\
\hline D-02-156 & Looking upstream & 140 & D-02-156_13-09 \\
\hline D-02-156 & Looking downstream & 270 & D-02-156_13-10 \\
\hline D-02-157 & Looking downstream & 180 & D-02-157_13-11 \\
\hline D-02-157 & Looking upstream & 0 & $\mathrm{D}-02-157 \_13-12$ \\
\hline D-02-157 & Looking east at armour on culverts & 90 & D-02-157_13-13 \\
\hline D-02-158 & Looking downstream & 315 & D-02-158_13-14 \\
\hline D-02-158 & Looking upstream & 120 & D-02-158_13-15 \\
\hline D-02-159 & Looking upstream & 180 & D-02-159_13-16 \\
\hline D-02-159 & Looking downstream & 35 & D-02-159_13-17 \\
\hline D-02-160 & Looking upstream at constructed riffles & 120 & D-02-160_13-18 \\
\hline D-02-160 & Looking downstream at constructed riffles & 230 & D-02-160_13-19 \\
\hline D-02-161 & Looking downstream from crossing & 40 & D-02-161_13-20 \\
\hline D-02-161 & Looking upstream from crossing & 230 & D-02-161_13-21 \\
\hline D-02-162 & Looking upstream & 270 & D-02-162_13-22 \\
\hline D-02-162 & Looking downstream & 90 & D-02-162_13-23 \\
\hline D-02-162 & Looking upstream at culvert & 270 & D-02-162_13-24 \\
\hline
\end{tabular}


Appendix 8: List of all photographs taken showing the corresponding site number, descriptive caption, direction faced when taken (azimuth in degrees) and the unique file name for each photograph.

\begin{tabular}{|c|c|c|c|}
\hline $\begin{array}{c}\text { Site } \\
\text { Number }\end{array}$ & Photo Description & Azimuth & File Name \\
\hline D-02-162 & Looking upstream at culvert & 230 & D-02-162_14-01 \\
\hline D-02-163 & Looking upstream from bridge & 300 & D-02-163_14-02 \\
\hline D-02-163 & Looking downstream from bridge & 80 & D-02-163_14-03 \\
\hline D-02-164 & Looking downstream from crossing & 160 & D-02-164_14-04 \\
\hline D-02-164 & Looking upstream from crossing & 315 & D-02-164_14-05 \\
\hline D-02-165 & Looking upstream & 90 & D-02-165_14-06 \\
\hline D-02-165 & Looking downstream & 240 & D-02-165_14-07 \\
\hline D-02-166 & Looking upstream from crossing & 315 & D-02-166_14-08 \\
\hline D-02-166 & Looking downstream from crossing & 180 & D-02-166_14-09 \\
\hline D-02-167 & Looking downstream from road crossing & 135 & D-02-167_14-10 \\
\hline D-02-167 & Looking at perched culvert & 270 & D-02-167_14-11 \\
\hline D-02-167 & Looking upstream from crossing & 315 & D-02-167_14-12 \\
\hline D-02-168 & Looking upstream & 290 & D-02-168_14-13 \\
\hline D-02-168 & Looking downstream & 120 & D-02-168_14-14 \\
\hline D-02-169 & Looking upstream from road & 335 & D-02-169_14-15 \\
\hline D-02-169 & Looking downstream from road & 170 & D-02-169_14-16 \\
\hline D-02-170 & Looking upstream from crossing & 270 & D-02-170_14-17 \\
\hline D-02-170 & Looking downstream from crossing & 90 & D-02-170_14-18 \\
\hline D-02-171 & Looking upstream & 270 & D-02-171_14-19 \\
\hline D-02-171 & Looking downstream & 90 & D-02-171_14-20 \\
\hline D-02-171 & Looking downstream & 90 & D-02-171_14-21 \\
\hline $\mathrm{D}-02-172$ & Looking upstream from road & 275 & $\mathrm{D}-02-172 \_14-22$ \\
\hline D-02-172 & Looking downstream from road & 100 & D-02-172_14-23 \\
\hline D-02-172 & Photo of perched culvert & 275 & D-02-172_14-24 \\
\hline D-02-173 & Looking upstream & 270 & D-02-173_15-01 \\
\hline D-02-173 & Looking upstream & 270 & D-02-173_15-02 \\
\hline D-02-173 & Looking downstream & 75 & D-02-173_15-03 \\
\hline D-02-174 & Looking upstream from PR 352 & 270 & D-02-174_15-04 \\
\hline D-02-174 & Looking downstream from PR 352 & 90 & D-02-174_15-05 \\
\hline D-02-175 & Looking downstream from PR 352 & 45 & D-02-175_15-07 \\
\hline D-02-175 & Looking downstream at cattle impacts & 90 & D-02-175_15-08 \\
\hline D-02-175 & Looking upstream from PR 352 & 270 & D-02-175_15-09 \\
\hline D-02-176 & Looking upstream (at 1st order tributary) & 180 & $\mathrm{D}-02-176 \_15-10$ \\
\hline
\end{tabular}


Appendix 8: List of all photographs taken showing the corresponding site number, descriptive caption, direction faced when taken (azimuth in degrees) and the unique file name for each photograph.

\begin{tabular}{|c|c|c|c|}
\hline $\begin{array}{c}\text { Site } \\
\text { Number }\end{array}$ & Photo Description & Azimuth & File Name \\
\hline $\mathrm{D}-02-176$ & Looking upstream (mainstream) & 270 & D-02-176_15-11 \\
\hline D-02-176 & Looking downstream from PR 352 & 90 & $\mathrm{D}-02-176 \_15-12$ \\
\hline $\mathrm{D}-02-177$ & Looking upstream from road & 270 & $\mathrm{D}-02-177 \quad 15-13$ \\
\hline D-02-177 & Looking downstream from road & 90 & D-02-177_15-14 \\
\hline D-02-178 & Looking downstream from bridge & 315 & D-02-178_15-15 \\
\hline D-02-178 & Looking upstream from bridge & 250 & D-02-178_15-16 \\
\hline D-02-179 & Looking downstream from mid-reach & 220 & D-02-179_15-17 \\
\hline D-02-179 & Looking upstream from mid-reach & 285 & D-02-179_15-18 \\
\hline D-02-179 & Looking upstream at culverts and low head dam & 350 & D-02-179_15-19 \\
\hline D-02-180 & Looking upstream from crossing & 270 & D-02-180_15-20 \\
\hline D-02-180 & Looking downstream from crossing & 90 & D-02-180_15-21 \\
\hline D-02-181 & Photo of Dave and Ashley fishing & $\mathrm{N} / \mathrm{A}$ & D-02-181_15-22 \\
\hline D-02-181 & Looking upstream from bridge & 270 & D-02-181_15-23 \\
\hline D-02-181 & Looking downstream from bridge & 90 & D-02-181_15-24 \\
\hline D-02-182 & Looking downstream from bridge & 50 & D-02-182_16-01 \\
\hline D-02-182 & Looking upstream from bridge & 225 & D-02-182_16-02 \\
\hline D-02-183 & Looking upstream from PTH 10 crossing & 270 & D-02-183_16-03 \\
\hline D-02-183 & Looking downstream from PTH 10 crossing & 90 & D-02-183_16-04 \\
\hline D-02-184 & Looking upstream from mid reach & 180 & D-02-184_16-05 \\
\hline D-02-184 & Looking downstream from mid reach & 0 & D-02-184_16-06 \\
\hline D-02-185 & Looking upstream from crossing & 310 & D-02-185_16-07 \\
\hline D-02-185 & Looking upstream from crossing & 310 & D-02-185_16-08 \\
\hline D-02-185 & Looking downstream from crossing & 130 & D-02-185_16-09 \\
\hline D-02-186 & Looking downstream from PR 366 & 70 & D-02-186_16-10 \\
\hline D-02-186 & Looking upstream from PR 366 & 230 & D-02-186_16-11 \\
\hline D-02-187 & Looking upstream from crossing & 180 & D-02-187_16-12 \\
\hline D-02-188 & Looking upstream from crossing & 275 & D-02-188_16-13 \\
\hline D-02-188 & Looking downstream from crossing & 90 & D-02-188_16-14 \\
\hline D-02-189 & Looking upstream from crossing & 270 & D-02-189_16-15 \\
\hline D-02-189 & Looking downstream from crossing & 90 & D-02-189_16-16 \\
\hline D-02-190 & Looking upstream from crossing & 300 & D-02-190_16-17 \\
\hline D-02-190 & Looking downstream from crossing & 140 & D-02-190_16-18 \\
\hline D-02-191 & Photo of beach seining pool & 80 & D-02-191_17-01 \\
\hline
\end{tabular}


Appendix 8: List of all photographs taken showing the corresponding site number, descriptive caption, direction faced when taken (azimuth in degrees) and the unique file name for each photograph.

\begin{tabular}{|c|c|c|c|}
\hline $\begin{array}{c}\text { Site } \\
\text { Number }\end{array}$ & Photo Description & Azimuth & File Name \\
\hline D-02-191 & Looking upstream from mid-reach & 280 & D-02-191_17-02 \\
\hline D-02-191 & Looking downstream from mid-reach & 20 & D-02-191_17-03 \\
\hline D-02-192 & Looking upstream from crossing & 180 & D-02-192_17-04 \\
\hline D-02-192 & Looking downstream from crossing & 0 & D-02-192_17-05 \\
\hline D-02-193 & Looking upstream & 315 & D-02-193_17-06 \\
\hline D-02-193 & Looking downstream & 180 & D-02-193_17-07 \\
\hline D-02-195 & Looking upstream from crossing & 180 & D-02-195_17-08 \\
\hline $\mathrm{D}-02-195$ & Looking downstream from crossing & 0 & D-02-195_17-09 \\
\hline D-02-196 & Looking upstream from mid-reach & 225 & D-02-196_17-10 \\
\hline D-02-196 & Looking downstream from mid-reach & 50 & D-02-196_17-11 \\
\hline D-02-197 & Looking upstream from bridge & 170 & D-02-197_17-12 \\
\hline D-02-197 & Looking downstream from bridge & 315 & D-02-197_17-13 \\
\hline D-02-197 & Looking at recent bank stabilization work & $\mathrm{N} / \mathrm{A}$ & D-02-197_17-14 \\
\hline D-02-197 & Looking at recent bank stabilization work & $\mathrm{N} / \mathrm{A}$ & $\mathrm{D}-02-197 \quad 17-15$ \\
\hline D-02-197 & Looking at recent bank stabilization work & $\mathrm{N} / \mathrm{A}$ & D-02-197_17-16 \\
\hline D-02-197 & Looking at recent bank stabilization work & $\mathrm{N} / \mathrm{A}$ & D-02-197_17-17 \\
\hline D-02-198 & Looking upstream from crossing & 180 & D-02-198_17-18 \\
\hline D-02-198 & Looking downstream from crossing & 0 & D-02-198_17-19 \\
\hline D-02-198 & Looking downstream & 0 & D-02-198_17-20 \\
\hline D-02-198 & Looking upstream at culverts & 180 & D-02-198_17-21 \\
\hline D-02-198 & Looking at substrate & $\mathrm{N} / \mathrm{A}$ & D-02-198_17-22 \\
\hline D-02-201 & Looking downstream from bridge & 90 & D-02-201_19-01 \\
\hline D-02-201 & Looking upstream from bridge & 270 & D-02-201_19-02 \\
\hline D-02-201 & Looking south across dry portion of channel & 180 & D-02-201_19-03 \\
\hline B-03-001 & Looking upstream from PTH 11 & 280 & B-03-001_P5210001 \\
\hline B-03-001 & Looking downstream from PTH 11 & 120 & B-03-001_P5210002 \\
\hline B-03-001 & Looking upstream at concrete overpass & 280 & B-03-001_P5210003 \\
\hline B-03-002 & Looking upstream from PTH 10 crossing & 270 & B-03-002_P5230001 \\
\hline B-03-002 & Looking downstream from PTH 10 crossing & 90 & B-03-002_P5230002 \\
\hline B-03-003 & Looking upstream from PR 349 crossing & 180 & B-03-003_P5230003 \\
\hline B-03-003 & Looking downstream from PR 349 crossing & 360 & B-03-003_P5230004 \\
\hline B-03-004 & Looking upstream from road & 190 & B-03-004_P5230005 \\
\hline B-03-004 & Looking downstream from road & 320 & B-03-004_P5230006 \\
\hline
\end{tabular}


Appendix 8: List of all photographs taken showing the corresponding site number, descriptive caption, direction faced when taken (azimuth in degrees) and the unique file name for each photograph.

\begin{tabular}{|c|c|c|c|}
\hline $\begin{array}{c}\text { Site } \\
\text { Number }\end{array}$ & Photo Description & Azimuth & File Name \\
\hline B-03-004 & Looking upstream at culvert & 180 & B-03-004 P5230007 \\
\hline B-03-005 & Looking upstream from road & 310 & B-03-005 P5230008 \\
\hline B-03-005 & Looking downstream from road & 130 & B-03-005_P5230009 \\
\hline B-03-005 & Looking upstream at five large culverts from $20 \mathrm{~m}$ east of road & $\mathrm{N} / \mathrm{A}$ & B-03-005_P5230010 \\
\hline B-03-006 & Looking upstream from PTH 453 Bridge & 260 & B-03-006_P5230011 \\
\hline B-03-006 & Looking downstream from PTH 453 Bridge & 80 & B-03-006 P5230012 \\
\hline B-03-007 & Looking upstream from road & 270 & B-03-007_P5260001 \\
\hline B-03-007 & Looking downstream from road & 110 & B-03-007_P5260002 \\
\hline B-03-007 & Looking upstream at ford crossing from $5 \mathrm{~m}$ downstream from road & 270 & B-03-007_P5260003 \\
\hline B-03-008 & Looking upstream from PTH 252 & 280 & B-03-008 P5260004 \\
\hline B-03-008 & Looking downstream from PTH 252 & 110 & B-03-008_P5260005 \\
\hline B-03-009 & Looking upstream from PTH 252 & 270 & B-03-009_P5260006 \\
\hline B-03-009 & Looking downstream from PTH 252 & 90 & B-03-009 P5260007 \\
\hline B-03-010 & Looking upstream from road & 270 & B-03-010 P5260008 \\
\hline B-03-010 & Looking downstream from road & 90 & B-03-010_P5260009 \\
\hline B-03-011 & Looking upstream from road & 270 & B-03-011_P5260010 \\
\hline B-03-011 & Looking downstream from road & 90 & B-03-011_P5260011 \\
\hline B-03-011 & Canada Goose Refuge sign & $\mathrm{N} / \mathrm{A}$ & B-03-011_P5260012 \\
\hline B-03-012 & Looking upstream from road & 240 & B-03-012_P5260013 \\
\hline B-03-012 & Looking downstream from road & 340 & B-03-012_P5260014 \\
\hline B-03-012 & Looking upstream at 2 perched $0.3 \mathrm{~m}$ diameter culverts & 240 & B-03-012 P5260015 \\
\hline B-03-012 & Looking downstream at 2 perched $0.3 \mathrm{~m}$ diameter culverts & 340 & B-03-012_P5260016 \\
\hline B-03-013 & Looking upstream from PTH 83 crossing & 270 & B-03-013_P5270001 \\
\hline B-03-013 & Looking downstream from PTH 83 crossing & 90 & B-03-013_P5270002 \\
\hline B-03-014 & Looking downstream from road & 150 & B-03-014_P5270003 \\
\hline B-03-014 & Looking upstream from road & 280 & B-03-014_P5270004 \\
\hline B-03-014 & Looking at culvert at upstream end & 270 & B-03-014_P5270005 \\
\hline B-03-015 & Looking upstream from road & 270 & B-03-015_P5270006 \\
\hline B-03-015 & Looking downstream from road & 90 & B-03-015 P5270007 \\
\hline B-03-016 & Looking upstream from $20 \mathrm{~m}$ east of low level crossing & 290 & B-03-016 P5270008 \\
\hline B-03-016 & Looking downstream from $20 \mathrm{~m}$ east of low level crossing & 200 & B-03-016_P5270009 \\
\hline B-03-017 & Looking upstream from dike/control structure & 360 & B-03-017_P5280001 \\
\hline B-03-017 & Looking downstream from dike/control structure & 180 & B-03-017 P5280002 \\
\hline
\end{tabular}


Appendix 8: List of all photographs taken showing the corresponding site number, descriptive caption, direction faced when taken (azimuth in degrees) and the unique file name for each photograph.

\begin{tabular}{|c|c|c|c|}
\hline $\begin{array}{c}\text { Site } \\
\text { Number }\end{array}$ & Photo Description & Azimuth & File Name \\
\hline B-03-017 & Looking upstream at culvert catchments & 300 & B-03-017_P5280003 \\
\hline B-03-017 & Looking upstream at small reservoir from $30 \mathrm{~m}$ east of control structure & 360 & B-03-017_P5280004 \\
\hline $\mathrm{B}-03-017$ & Looking upstream at perched culverts from $20 \mathrm{~m}$ downstream & 340 & B-03-017_P5280005 \\
\hline B-03-018 & Looking upstream from road & 300 & B-03-018_P5280006 \\
\hline B-03-018 & Looking downstream from road & 150 & B-03-018_P5280007 \\
\hline B-03-018 & Looking across flooded crossing from east side looking west & 270 & B-03-018_P5280008 \\
\hline B-03-019 & Looking upstream from road & 270 & B-03-019_P5280009 \\
\hline B-03-019 & Looking downstream from road & 90 & B-03-019_P5280010 \\
\hline B-03-019 & Looking upstream at $290 \mathrm{~cm}$ diameter culvert & 300 & B-03-019_P5280011 \\
\hline B-03-020 & Looking upstream from road & 10 & B-03-020_P5290001 \\
\hline $\mathrm{B}-03-020$ & Looking downstream from road & 160 & B-03-020_P5290002 \\
\hline $\mathrm{B}-03-021$ & Looking upstream from bridge & 180 & B-03-021_P5290003 \\
\hline B-03-021 & Looking downstream from bridge & 360 & B-03-021_P5290004 \\
\hline B-03-022 & Looking upstream from bridge & 320 & B-03-022 P5290005 \\
\hline B-03-022 & Looking downstream from bridge & 100 & B-03-022_P5290006 \\
\hline B-03-023 & Looking upstream from road & 270 & B-03-023_P5290007 \\
\hline B-03-023 & Looking downstream from road & 90 & B-03-023 P5290008 \\
\hline B-03-023 & Looking upstream at culvert perched $0.4 \mathrm{~m}$ from $15 \mathrm{~m}$ east of road & 270 & B-03-023 P5290009 \\
\hline B-03-024 & Looking upstream from road & 270 & B-03-024_P5290010 \\
\hline B-03-024 & Looking downstream from road & 90 & B-03-024_P5290011 \\
\hline B-03-025 & Looking upstream from south culvert in west ditch & 350 & B-03-025_P5290012 \\
\hline B-03-025 & Looking downstream from south culvert & 60 & B-03-025_P5290013 \\
\hline B-03-025 & Looking upstream from north culvert & 290 & B-03-025_P5290014 \\
\hline B-03-025 & Looking downstream from north culvert & 110 & B-03-025_P5290015 \\
\hline B-03-025 & Looking upstream at north culvert perched $1.1 \mathrm{~m}$ & N/A & B-03-025 P5290016 \\
\hline B-03-025 & Looking upstream at south culvert perched $0.6 \mathrm{~m}$ & N/A & B-03-025 P5290017 \\
\hline B-03-026 & Looking upstream from road & 340 & B-03-026_P5290018 \\
\hline B-03-026 & Looking downstream from road & 70 & B-03-026_P5290019 \\
\hline B-03-026 & Looking upstream at low level crossing/riffle & 360 & B-03-026 P5290020 \\
\hline B-03-027 & Looking upstream from road & 350 & B-03-027_P5300001 \\
\hline B-03-027 & Looking downstream from road & 180 & B-03-027_P5300002 \\
\hline B-03-027 & Looking upstream at 2 perched culverts & 300 & B-03-027_P5300003 \\
\hline B-03-028 & Looking upstream from road & 350 & B-03-028 P5300004 \\
\hline
\end{tabular}


Appendix 8: List of all photographs taken showing the corresponding site number, descriptive caption, direction faced when taken (azimuth in degrees) and the unique file name for each photograph.

\begin{tabular}{|c|c|c|c|}
\hline $\begin{array}{c}\text { Site } \\
\text { Number }\end{array}$ & Photo Description & Azimuth & File Name \\
\hline B-03-028 & Looking upstream from road & 110 & B-03-028_P5300005 \\
\hline B-03-028 & Looking upstream at culvert from $10 \mathrm{~m}$ east of road & $\mathrm{N} / \mathrm{A}$ & B-03-028_P5300006 \\
\hline B-03-028 & Looking downstream at culvert from $10 \mathrm{~m}$ west of road & $\mathrm{N} / \mathrm{A}$ & B-03-028 P5300007 \\
\hline B-03-029 & Looking upstream from road & 275 & B-03-029_P5300008 \\
\hline B-03-029 & Looking downstream from road & 120 & B-03-029_P5300009 \\
\hline $\mathrm{B}-03-030$ & Looking upstream from road & 290 & B-03-030_P5300010 \\
\hline B-03-030 & Looking downstream from road & 90 & B-03-030_P5300011 \\
\hline B-03-031 & Looking upstream from road & 360 & B-03-031_P5300012 \\
\hline B-03-031 & Looking downstream from road & 180 & B-03-031_P5300013 \\
\hline B-03-031 & Looking upstream at 1 culvert from $10 \mathrm{~m}$ south of road & 340 & B-03-031_P5300014 \\
\hline B-03-032 & Looking upstream from road & 40 & B-03-032 P6020001 \\
\hline B-03-032 & Looking downstream from road & 180 & B-03-032 P6020002 \\
\hline B-03-032 & Looking upstream at 3 culverts from $20 \mathrm{~m}$ south of road & 40 & B-03-032_P6020003 \\
\hline B-03-033 & Looking upstream from road & 50 & B-03-033_P6020004 \\
\hline B-03-033 & Looking downstream from road & 330 & B-03-033_P6020005 \\
\hline B-03-034 & Looking upstream from road & 30 & B-03-034_P6020006 \\
\hline B-03-034 & Looking downstream from road & 190 & B-03-034_P6020007 \\
\hline B-03-034 & Looking downstream at scouring from west metal pipe culvert & 190 & B-03-034_P6020008 \\
\hline B-03-034 & & $\mathrm{N} / \mathrm{A}$ & B-03-034_P6020009 \\
\hline B-03-034 & Looking upstream at east perched culvert & N/A & B-03-034_P6020010 \\
\hline B-03-035 & Looking upstream from road & 220 & B-03-035_P6020011 \\
\hline B-03-035 & Looking downstream from road & 30 & B-03-035_P6020012 \\
\hline B-03-036 & Looking upstream form road & 120 & B-03-036_P6020013 \\
\hline B-03-036 & Looking downstream from road & 290 & B-03-036_P6020014 \\
\hline B-03-037 & Looking upstream form road & 360 & B-03-037_P6020015 \\
\hline B-03-037 & Looking downstream from road & 90 & B-03-037_P6020016 \\
\hline B-03-038 & Looking upstream form road & 90 & B-03-038 P6020017 \\
\hline B-03-038 & Looking downstream from road & 270 & B-03-038_P6020018 \\
\hline B-03-038 & Looking upstream at control structure from road & 140 & B-03-038_P6020019 \\
\hline B-03-038 & Looking at beaver deterrent & 90 & B-03-038_P6020020 \\
\hline B-03-039 & Looking upstream from road & 360 & B-03-039 P6030001 \\
\hline B-03-039 & Looking downstream from road & 80 & B-03-039_P6030002 \\
\hline B-03-040 & Looking upstream from PR 355 & 360 & B-03-040_P6030003 \\
\hline
\end{tabular}


Appendix 8: List of all photographs taken showing the corresponding site number, descriptive caption, direction faced when taken (azimuth in degrees) and the unique file name for each photograph.

\begin{tabular}{|c|c|c|c|}
\hline $\begin{array}{c}\text { Site } \\
\text { Number }\end{array}$ & Photo Description & Azimuth & File Name \\
\hline B-03-040 & Looking downstream from PR 355 & 195 & B-03-040_P6030004 \\
\hline B-03-040 & Looking upstream at 2- $2.9 \mathrm{~m}$ diameter culverts & 20 & B-03-040 P6030005 \\
\hline B-03-041 & Looking upstream from PR 262 & 130 & B-03-041_P6030006 \\
\hline B-03-041 & Looking upstream from PR 262 & 260 & B-03-041_P6030007 \\
\hline B-03-041 & Looking upstream at cemented culverts from 30m west of PR 262 & $\mathrm{~N} / \mathrm{A}$ & B-03-041_P6030008 \\
\hline B-03-042 & Looking upstream from low grade crossing & 270 & B-03-042_P6030009 \\
\hline B-03-042 & Looking downstream from low grade crossing & 90 & B-03-042_P6030010 \\
\hline B-03-043 & Looking upstream from $30 \mathrm{~m}$ south of trail & 270 & B-03-043_P6030011 \\
\hline B-03-043 & Looking upstream from $30 \mathrm{~m}$ south of trail & 90 & B-03-043_P6030012 \\
\hline B-03-044 & Looking upstream from bridge & 130 & B-03-044_P6030013 \\
\hline B-03-044 & Looking downstream from bridge & 255 & B-03-044_P6030014 \\
\hline B-03-045 & Looking upstream from road & 30 & B-03-045_P6040001 \\
\hline B-03-045 & Looking downstream from road & 250 & B-03-045_P6040002 \\
\hline B-03-045 & Looking downstream at south gated culvert & $\mathrm{N} / \mathrm{A}$ & B-03-045_P6040003 \\
\hline B-03-046 & Looking upstream from road & 270 & B-03-046_P6040004 \\
\hline B-03-046 & Looking downstream from road & 90 & B-03-046_P6040005 \\
\hline B-03-047 & Looking upstream from road & 310 & B-03-047_P6040006 \\
\hline B-03-047 & Looking downstream from road & 180 & B-03-047_P6040007 \\
\hline B-03-048 & Looking upstream from west crossing & 360 & B-03-048_P6040008 \\
\hline B-03-048 & Looking downstream from west crossing & 90 & B-03-048_P6040009 \\
\hline B-03-048 & Looking at upstream end of crossing & 90 & B-03-048_P6040010 \\
\hline B-03-049 & Looking upstream from crossing & 360 & B-03-049_P6040011 \\
\hline B-03-049 & Looking at downstream of crossing & 180 & B-03-049_P6040012 \\
\hline B-03-049 & Looking downstream from end of culverts & $\mathrm{N} / \mathrm{A}$ & B-03-049_P6040013 \\
\hline B-03-050 & Looking upstream from crossing & 270 & B-03-050 P6040014 \\
\hline B-03-050 & Looking at downstream of crossing & 180 & B-03-050 P6040015 \\
\hline B-03-051 & Looking upstream from crossing & 200 & B-03-051_P6040016 \\
\hline B-03-051 & Looking at downstream of crossing & 360 & B-03-051_P6040017 \\
\hline B-03-052 & Looking upstream from crossing & 10 & B-03-052_P6040018 \\
\hline B-03-052 & Looking at downstream of crossing & 270 & B-03-052_P6040019 \\
\hline B-03-052 & Photo of algae bloom & N/A & B-03-052_P6040020 \\
\hline B-03-052 & Photo of pumping into water? & $\mathrm{N} / \mathrm{A}$ & B-03-052_P6040021 \\
\hline B-03-053 & Looking upstream from point east of PTH 250 & 280 & B-03-053_P6050001 \\
\hline
\end{tabular}


Appendix 8: List of all photographs taken showing the corresponding site number, descriptive caption, direction faced when taken (azimuth in degrees) and the unique file name for each photograph.

\begin{tabular}{|c|c|c|c|}
\hline $\begin{array}{c}\text { Site } \\
\text { Number }\end{array}$ & Photo Description & Azimuth & File Name \\
\hline B-03-053 & Downstream from point east of PTH 250 & 120 & B-03-053_P6050002 \\
\hline B-03-054 & Looking downstream from crossing & 100 & B-03-054_P6050003 \\
\hline B-03-054 & Looking upstream from crossing & 300 & B-03-054_P6050004 \\
\hline B-03-055 & Looking upstream from crossing & 280 & B-03-055_P6050005 \\
\hline B-03-055 & Looking downstream from crossing & 80 & B-03-055_P6050006 \\
\hline B-03-055 & Looking upstream at perched culvert & $\mathrm{N} / \mathrm{A}$ & B-03-055 P6050007 \\
\hline B-03-056 & Looking upstream from crossing & 360 & B-03-056_P6050008 \\
\hline B-03-056 & Looking downstream from crossing & 90 & B-03-056_P6050009 \\
\hline B-03-057 & Looking upstream from crossing & 360 & B-03-057_P6050010 \\
\hline B-03-057 & Looking downstream from crossing & 180 & B-03-057_P6050011 \\
\hline B-03-057 & Looking upstream through culvert at beaver dam & $\mathrm{N} / \mathrm{A}$ & B-03-057_P6050012 \\
\hline B-03-058 & Looking upstream from crossing & 320 & B-03-058_P6050013 \\
\hline B-03-058 & Looking downstream from crossing & 140 & B-03-058_P6050014 \\
\hline B-03-059 & Looking upstream from crossing & 270 & B-03-059 P6050015 \\
\hline B-03-059 & Looking downstream from crossing & 90 & B-03-059_P6050016 \\
\hline B-03-060 & Looking upstream from crossing & $?$ & B-03-060_P6050017 \\
\hline B-03-060 & Looking downstream from crossing & $?$ & B-03-060 P6050018 \\
\hline B-03-061 & Looking upstream from road & 50 & B-03-061_P6090001 \\
\hline B-03-061 & Looking downstream from road & 200 & B-03-061_P6090002 \\
\hline B-03-061 & Looking upstream at $2.9 \mathrm{~m}$ diameter culvert perched $0.6 \mathrm{~m}$ & 70 & B-03-061_P6090003 \\
\hline B-03-062 & Looking upstream from $10 \mathrm{~m}$ south of crossing & 10 & B-03-062 P6090004 \\
\hline B-03-062 & Looking downstream from $20 \mathrm{~m}$ north of crossing & 210 & B-03-062_P6090005 \\
\hline B-03-063 & Looking upstream from crossing on PTH 24 & 170 & B-03-063_P6090006 \\
\hline B-03-063 & Looking upstream from crossing on PTH 24 & 350 & B-03-063_P6090007 \\
\hline B-03-064 & Looking upstream from washed out culvert & 95 & B-03-064_P6100001 \\
\hline B-03-064 & Looking downstream from washed out culvert & 260 & B-03-064_P6100002 \\
\hline B-03-064 & Looking at railway crossing & 155 & B-03-064_P6100003 \\
\hline B-03-064 & Photo of Black Bullhead & $\mathrm{N} / \mathrm{A}$ & B-03-064_P6100004 \\
\hline B-03-064 & Photo of Black Bullhead & $\mathrm{N} / \mathrm{A}$ & B-03-064 P6100005 \\
\hline B-03-064 & Photo of Black Bullhead & N/A & B-03-064_P6100006 \\
\hline B-03-064 & Photo of Creek Chub & $\mathrm{N} / \mathrm{A}$ & B-03-064_P6100007 \\
\hline B-03-065 & Looking upstream from crossing & 50 & B-03-065_P6100008 \\
\hline B-03-065 & Looking downstream from crossing & 220 & B-03-065 P6100009 \\
\hline
\end{tabular}


Appendix 8: List of all photographs taken showing the corresponding site number, descriptive caption, direction faced when taken (azimuth in degrees) and the unique file name for each photograph.

\begin{tabular}{|c|c|c|c|}
\hline $\begin{array}{c}\text { Site } \\
\text { Number }\end{array}$ & Photo Description & Azimuth & File Name \\
\hline B-03-065 & Looking upstream at west $1.8 \mathrm{~m}$ diameter culvert perched $0.10 \mathrm{~m}$ & $\mathrm{~N} / \mathrm{A}$ & B-03-065_P6100010 \\
\hline B-03-066 & Looking upstream from PTH 16 crossing & 360 & B-03-066_P6100011 \\
\hline B-03-066 & Looking upstream from PTH 16 crossing & 180 & B-03-066 P6100012 \\
\hline B-03-067 & Looking upstream from PR 355 crossing & 330 & B-03-067_P6100013 \\
\hline B-03-067 & Looking downstream from PR 355 crossing & 150 & B-03-067_P6100014 \\
\hline B-03-068 & Looking upstream from road & 10 & B-03-068_P6110001 \\
\hline B-03-068 & Looking downstream from road & 190 & B-03-068_P6110002 \\
\hline B-03-069 & Looking upstream from road & 300 & B-03-069 P6110003 \\
\hline B-03-069 & Looking downstream from road & 80 & B-03-069_P6110004 \\
\hline B-03-070 & Looking upstream from road & 270 & B-03-070_P6110005 \\
\hline B-03-070 & Looking downstream from road & 90 & B-03-070 P6110006 \\
\hline B-03-070 & Looking upstream at $0.85 \mathrm{~m}$ diameter perched culvert & $\mathrm{N} / \mathrm{A}$ & B-03-070 P6110007 \\
\hline B-03-071 & Looking upstream from PR 264 crossing & 360 & B-03-071_P6110008 \\
\hline B-03-071 & Looking downstream from PR 264 crossing & 180 & B-03-071_P6110009 \\
\hline B-03-072 & Looking upstream from road & 310 & B-03-072_P6110010 \\
\hline B-03-072 & Looking downstream from road & 90 & B-03-072_P6110011 \\
\hline B-03-073 & Looking upstream from PTH 42 crossing & 10 & B-03-073_P6110012 \\
\hline B-03-073 & Looking downstream from PTH 42 crossing & 190 & B-03-073_P6110013 \\
\hline B-03-074 & Looking upstream from road & 70 & B-03-074_P6110014 \\
\hline B-03-074 & Looking downstream from road & 250 & B-03-074_P6110015 \\
\hline B-03-075 & Looking upstream from PTH 42 crossing & 360 & B-03-075_P6120001 \\
\hline B-03-075 & Looking downstream from PTH 42 crossing & 200 & B-03-075_P6120002 \\
\hline B-03-076 & Looking upstream from CN tracks & 10 & B-03-076_P6120003 \\
\hline B-03-076 & Looking downstream from $\mathrm{CN}$ tracks & 195 & B-03-076_P6120004 \\
\hline B-03-076 & Photo of White Sucker & $\mathrm{N} / \mathrm{A}$ & B-03-076_P6120005 \\
\hline B-03-076 & Photo of White Sucker & $\mathrm{N} / \mathrm{A}$ & B-03-076_P6120006 \\
\hline B-03-076 & Photo of Creek Chub & $\mathrm{N} / \mathrm{A}$ & B-03-076 P6120007 \\
\hline B-03-076 & Photo of Creek Chub & $\mathrm{N} / \mathrm{A}$ & B-03-076_P6120008 \\
\hline B-03-077 & Looking upstream from bridge & 330 & B-03-077_P6120009 \\
\hline B-03-077 & Looking downstream from bridge & 100 & B-03-077_P6120010 \\
\hline B-03-078 & Looking upstream from road & 90 & B-03-078 P6120011 \\
\hline B-03-078 & Looking upstream from road & 270 & B-03-078_P6120012 \\
\hline B-03-079 & Looking upstream from road & 10 & B-03-079_P6120013 \\
\hline
\end{tabular}


Appendix 8: List of all photographs taken showing the corresponding site number, descriptive caption, direction faced when taken (azimuth in degrees) and the unique file name for each photograph.

\begin{tabular}{|c|c|c|c|}
\hline $\begin{array}{c}\text { Site } \\
\text { Number }\end{array}$ & Photo Description & Azimuth & File Name \\
\hline B-03-079 & Looking downstream from road & 170 & B-03-079_P6120014 \\
\hline B-03-080 & Looking upstream from PR 256 crossing & 340 & B-03-080 P6120015 \\
\hline B-03-080 & Looking downstream from PR 256 crossing & 110 & B-03-080_P6120016 \\
\hline B-03-080 & Looking upstream at 4 culverts from $20 \mathrm{~m}$ south of road & 340 & B-03-080_P6120017 \\
\hline B-03-081 & Looking upstream from PR 467 crossing & 250 & B-03-081_P6120018 \\
\hline B-03-081 & Looking downstream from PR 467 crossing & 10 & B-03-081_P6120019 \\
\hline B-03-082 & Looking upstream from PTH 83 crossing & 200 & B-03-082_P6120020 \\
\hline B-03-082 & Looking downstream from PTH 83 crossing & 20 & B-03-082_P6120021 \\
\hline B-03-083 & Looking upstream from control structure & 280 & B-03-083_P6160001 \\
\hline B-03-083 & Looking downstream from control structure & 80 & B-03-083 P6160002 \\
\hline B-03-083 & Looking upstream at control structure from $30 \mathrm{~m}$ downstream & 280 & B-03-083_P6160003 \\
\hline B-03-084 & Looking upstream from control structure & 280 & B-03-084_P6160004 \\
\hline B-03-084 & Looking downstream from control structure & 80 & B-03-084_P6160005 \\
\hline B-03-084 & Looking upstream at control structure from $30 \mathrm{~m}$ downstream & 280 & B-03-084_P6160006 \\
\hline B-03-085 & Looking upstream from control structure & 280 & B-03-085_P6170001 \\
\hline B-03-085 & Looking downstream from control structure & 80 & B-03-085_P6170002 \\
\hline B-03-085 & Looking upstream at control structure from $30 \mathrm{~m}$ downstream & 280 & B-03-085_P6170003 \\
\hline B-03-086 & Looking upstream from control structure & 280 & B-03-086 P6170004 \\
\hline B-03-086 & Looking downstream from control structure & 80 & B-03-086_P6170005 \\
\hline B-03-086 & Looking upstream at control structure from 30m downstream & 280 & B-03-086_P6170006 \\
\hline B-03-087 & Looking upstream from crossing & 270 & B-03-087_P6170007 \\
\hline B-03-087 & Looking downstream from crossing & 90 & B-03-087_P6170008 \\
\hline B-03-088 & Looking upstream from crossing & 310 & B-03-088_P6180001 \\
\hline B-03-088 & Looking downstream from crossing & 90 & B-03-088_P6180002 \\
\hline B-03-089 & Looking upstream from crossing & 180 & B-03-089 P6180003 \\
\hline B-03-089 & Looking downstream from crossing & 360 & B-03-089_P6180004 \\
\hline B-03-090 & Looking upstream from crossing & 270 & B-03-090_P6180005 \\
\hline B-03-090 & Looking downstream from crossing & 90 & B-03-090_P6180006 \\
\hline B-03-091 & Looking upstream from crossing & 270 & B-03-091_P6180007 \\
\hline B-03-091 & Looking downstream from crossing & 360 & B-03-091_P6180008 \\
\hline B-03-092 & Looking upstream from crossing & 110 & B-03-092_P6180009 \\
\hline B-03-092 & Looking downstream from crossing & 75 & B-03-092_P6180010 \\
\hline B-03-092 & Looking downstream at 4 culverts from $30 \mathrm{~m}$ upstream & $\mathrm{N} / \mathrm{A}$ & B-03-092_P6180011 \\
\hline
\end{tabular}


Appendix 8: List of all photographs taken showing the corresponding site number, descriptive caption, direction faced when taken (azimuth in degrees) and the unique file name for each photograph.

\begin{tabular}{|c|c|c|c|}
\hline $\begin{array}{c}\text { Site } \\
\text { Number }\end{array}$ & Photo Description & Azimuth & File Name \\
\hline B-03-093 & Looking upstream from crossing & 270 & B-03-093_P6180012 \\
\hline B-03-093 & Looking downstream from crossing & 90 & B-03-093 P6180013 \\
\hline B-03-094 & Looking upstream from base of control structure & 275 & B-03-094_P6180014 \\
\hline B-03-094 & Looking downstream from base of control structure & 85 & B-03-094_P6180015 \\
\hline B-03-094 & Looking upstream at control structure from $10 \mathrm{~m}$ downstream & $\mathrm{N} / \mathrm{A}$ & B-03-094_P6180016 \\
\hline B-03-095 & Looking upstream from control structure & 290 & B-03-095_P6190001 \\
\hline B-03-095 & Looking downstream from control structure & 80 & B-03-095_P6190002 \\
\hline B-03-095 & Looking upstream at control structure from $30 \mathrm{~m}$ downstream & N/A & B-03-095_P6190003 \\
\hline B-03-096 & Looking upstream from southeast quarter of stream junction & 300 & B-03-096_P6190004 \\
\hline B-03-096 & Looking downstream from southeast quarter of stream junction & 80 & B-03-096 P6190005 \\
\hline B-03-097 & Looking upstream from right bank at steam junction & 360 & B-03-097_P6190006 \\
\hline B-03-097 & Looking downstream from right bank at stream junction & 180 & B-03-097_P6190007 \\
\hline B-03-098 & Looking upstream from ford crossing & 200 & B-03-098_P6190008 \\
\hline B-03-098 & Looking downstream from ford crossing & 360 & B-03-098_P6190009 \\
\hline B-03-099 & Looking upstream from crossing & 200 & B-03-099_P6200001 \\
\hline B-03-099 & Looking downstream from crossing & 20 & B-03-099_P6200002 \\
\hline B-03-100 & Looking upstream from crossing & 280 & B-03-100_P6200003 \\
\hline B-03-100 & Looking downstream from crossing & 90 & B-03-100 P6200004 \\
\hline B-03-101 & Looking upstream from crossing & 270 & B-03-101_P6200005 \\
\hline B-03-101 & Looking downstream from crossing & 90 & B-03-101_P6200006 \\
\hline B-03-102 & Looking upstream from bridge & 130 & B-03-102_P6230001 \\
\hline B-03-102 & Looking downstream from bridge & 255 & B-03-102_P6230002 \\
\hline B-03-103 & Looking upstream from crossing & 170 & B-03-103_P6230003 \\
\hline B-03-103 & Looking downstream from crossing & 340 & B-03-103_P6230004 \\
\hline B-03-104 & Looking upstream from crossing & 320 & B-03-104_P6230005 \\
\hline B-03-104 & Looking downstream from crossing & 280 & B-03-104_P6230006 \\
\hline B-03-104 & Photo of turbulent flow created by deadfall & 20 & B-03-104_P6230007 \\
\hline B-03-105 & Looking upstream from crossing & 340 & B-03-105_P6240001 \\
\hline B-03-105 & Looking downstream from crossing & 160 & B-03-105_P6240002 \\
\hline B-03-106 & Looking upstream from crossing & 360 & B-03-106_P6240003 \\
\hline B-03-106 & Looking downstream from crossing & 180 & B-03-106_P6240004 \\
\hline B-03-107 & Looking upstream from crossing & 270 & B-03-107_P6240005 \\
\hline B-03-107 & Looking downstream from crossing & 80 & B-03-107_P6240006 \\
\hline
\end{tabular}


Appendix 8: List of all photographs taken showing the corresponding site number, descriptive caption, direction faced when taken (azimuth in degrees) and the unique file name for each photograph.

\begin{tabular}{|c|c|c|c|}
\hline $\begin{array}{c}\text { Site } \\
\text { Number }\end{array}$ & Photo Description & Azimuth & File Name \\
\hline B-03-108 & Looking upstream from crossing & 360 & B-03-108_P6240007 \\
\hline B-03-108 & Looking downstream from crossing & 90 & B-03-108_P6240008 \\
\hline B-03-109 & Looking upstream from crossing & 250 & B-03-109_P6240009 \\
\hline B-03-109 & Looking downstream from crossing & 110 & B-03-109_P6240010 \\
\hline B-03-110 & Looking upstream from crossing & 270 & B-03-110_P6260001 \\
\hline B-03-110 & Looking downstream from crossing & 90 & B-03-110_P6260002 \\
\hline B-03-110 & Looking upstream from control structure & 190 & B-03-110_P6260003 \\
\hline B-03-111 & Looking downstream from control structure & 350 & B-03-111_P6260004 \\
\hline B-03-111 & Looking upstream at control structure & $\mathrm{N} / \mathrm{A}$ & B-03-111_P6260005 \\
\hline B-03-112 & Looking upstream from bridge & 270 & B-03-112_P6260006 \\
\hline B-03-112 & Looking downstream from bridge & 95 & B-03-112_P6260007 \\
\hline B-03-113 & Looking upstream from bridge & 180 & B-03-113_P6260008 \\
\hline B-03-113 & Looking downstream from bridge & 360 & B-03-113_P6260009 \\
\hline B-03-114 & Looking upstream from bridge & 270 & B-03-114_P6260010 \\
\hline B-03-114 & Looking downstream from bridge & 90 & B-03-114_P6260011 \\
\hline B-03-115 & Looking upstream from close to old bridge structure & 180 & B-03-115_P6270001 \\
\hline B-03-115 & Looking downstream from close to old bridge structure & 350 & B-03-115_P6270002 \\
\hline B-03-116 & Looking upstream from crossing & 170 & B-03-116_P6270003 \\
\hline B-03-116 & Looking downstream from crossing & 360 & B-03-116_P6270004 \\
\hline B-03-117 & Looking upstream from crossing & 190 & B-03-117_P6270005 \\
\hline B-03-117 & Looking downstream from crossing & 135 & B-03-117_P6270006 \\
\hline B-03-118 & Looking upstream from crossing & 280 & B-03-118_P6270007 \\
\hline B-03-118 & Looking downstream from crossing & 180 & B-03-118_P6270008 \\
\hline B-03-119 & Looking upstream from crossing & 200 & B-03-119_P7020001 \\
\hline B-03-119 & Looking downstream from crossing & 20 & B-03-119_P7020002 \\
\hline B-03-120 & Looking upstream from crossing & 260 & B-03-120_P7020003 \\
\hline B-03-120 & Looking downstream from crossing & 80 & B-03-120_P7020004 \\
\hline B-03-121 & Looking upstream from crossing & 180 & B-03-121_P7030001 \\
\hline B-03-121 & Looking downstream from crossing & 360 & B-03-121_P7030002 \\
\hline B-03-122 & Looking upstream from crossing & 230 & B-03-122_P7030003 \\
\hline B-03-122 & Looking downstream from crossing & 10 & B-03-122_P7030004 \\
\hline B-03-122 & Looking upstream from intersection & 180 & B-03-122_P7030005 \\
\hline B-03-122 & Looking downstream from intersection & 360 & B-03-122_P7030006 \\
\hline
\end{tabular}


Appendix 8: List of all photographs taken showing the corresponding site number, descriptive caption, direction faced when taken (azimuth in degrees) and the unique file name for each photograph.

\begin{tabular}{|c|c|c|c|}
\hline $\begin{array}{c}\text { Site } \\
\text { Number }\end{array}$ & Photo Description & Azimuth & File Name \\
\hline B-03-123 & Looking upstream from $10 \mathrm{~m}$ west of road & 180 & B-03-123 P7030007 \\
\hline B-03-123 & Looking downstream from $10 \mathrm{~m}$ west of road & 10 & B-03-123_P7030008 \\
\hline B-03-124 & Looking upstream from crossing & 360 & B-03-124_P7030009 \\
\hline B-03-124 & Looking downstream from crossing & 180 & B-03-124_P7030010 \\
\hline B-03-125 & Looking upstream from crossing & 250 & B-03-125 P7030011 \\
\hline B-03-125 & Looking downstream from crossing & 20 & B-03-125_P7030012 \\
\hline B-03-126 & Looking upstream from bridge & 250 & B-03-126_P7040001 \\
\hline B-03-126 & Looking downstream from bridge & 140 & B-03-126 P7040002 \\
\hline B-03-127 & Looking upstream from crossing & 250 & B-03-127 P7040003 \\
\hline B-03-127 & Looking downstream from crossing & 40 & B-03-127_P7040004 \\
\hline B-03-128 & Looking upstream from crossing & 270 & B-03-128_P7040005 \\
\hline B-03-128 & Looking downstream from crossing & 90 & B-03-128 P7040006 \\
\hline B-03-129 & Looking upstream from crossing & 260 & B-03-129 P7040007 \\
\hline B-03-129 & Looking downstream from crossing & 10 & B-03-129 P7040008 \\
\hline B-03-130 & Looking upstream from crossing & 320 & B-03-130_P7040009 \\
\hline B-03-130 & Looking downstream from crossing & 170 & B-03-130 P7040010 \\
\hline B-03-130 & Photo of cattle grazing in drain & N/A & B-03-130 P7040011 \\
\hline B-03-131 & Looking upstream from crossing & 190 & B-03-131_P7040012 \\
\hline B-03-131 & Looking downstream from crossing & 80 & B-03-131_P7040013 \\
\hline B-03-132 & Looking upstream from under former railway crossing & 10 & B-03-132 P7070001 \\
\hline B-03-132 & Looking downstream from under former railway crossing & 195 & B-03-132 P7070002 \\
\hline B-03-132 & Photo of Brook Trout & $\mathrm{N} / \mathrm{A}$ & B-03-132_P7070003 \\
\hline B-03-133 & Looking upstream from crossing & 320 & B-03-133_P7070004 \\
\hline B-03-133 & Looking downstream from crossing & 150 & B-03-133 P7070005 \\
\hline B-03-133 & Looking upstream at culvert & $\mathrm{N} / \mathrm{A}$ & B-03-133_P7070006 \\
\hline B-03-134 & Looking upstream from crossing & 100 & B-03-134_P7070007 \\
\hline B-03-134 & Looking downstream from crossing & 250 & B-03-134_P7070008 \\
\hline B-03-135 & Looking upstream from crossing & 15 & B-03-135 P7070009 \\
\hline B-03-135 & Looking downstream from crossing & 200 & B-03-135 P7070010 \\
\hline B-03-135 & Photo of north ditch of PR 471 to east & 90 & B-03-135_P7070011 \\
\hline B-03-135 & Photo of north ditch of PR 471 to west & 270 & B-03-135 P7070012 \\
\hline B-03-136 & Looking upstream from crossing & 15 & B-03-136 P7070013 \\
\hline B-03-136 & Looking downstream from crossing & 270 & B-03-136 P7070014 \\
\hline
\end{tabular}


Appendix 8: List of all photographs taken showing the corresponding site number, descriptive caption, direction faced when taken (azimuth in degrees) and the unique file name for each photograph.

\begin{tabular}{|c|c|c|c|}
\hline $\begin{array}{c}\text { Site } \\
\text { Number }\end{array}$ & Photo Description & Azimuth & File Name \\
\hline B-03-137 & Looking upstream from crossing & 270 & B-03-137_P7070015 \\
\hline B-03-137 & Looking downstream from crossing & 140 & B-03-137_P7070016 \\
\hline B-03-138 & Looking upstream from crossing & 270 & B-03-138_P7070017 \\
\hline B-03-138 & Looking downstream from crossing & 90 & B-03-138_P7070018 \\
\hline B-03-139 & Looking upstream from crossing & 270 & B-03-139_P7070019 \\
\hline B-03-139 & Looking downstream from crossing & 90 & B-03-139_P7070020 \\
\hline B-03-140 & Looking upstream from crossing & 45 & B-03-140_P7080001 \\
\hline B-03-140 & Looking downstream from crossing & 220 & B-03-140_P7080002 \\
\hline B-03-140 & Photo of culvert & $\mathrm{N} / \mathrm{A}$ & B-03-140_P7080003 \\
\hline B-03-141 & Looking upstream from crossing & 280 & B-03-141_P7080004 \\
\hline B-03-141 & Looking downstream from crossing & 90 & B-03-141_P7080005 \\
\hline B-03-141 & Looking upstream at perched culvert & $\mathrm{N} / \mathrm{A}$ & B-03-141_P7080006 \\
\hline B-03-142 & Looking upstream from crossing & 270 & B-03-142_P7080007 \\
\hline B-03-142 & Looking downstream from crossing & 90 & B-03-142_P7080008 \\
\hline B-03-142 & Looking upstream at culvert & $\mathrm{N} / \mathrm{A}$ & B-03-142_P7080009 \\
\hline B-03-143 & Looking upstream from crossing of gravel road & 340 & B-03-143_P7080010 \\
\hline B-03-143 & Looking downstream from crossing of gravel road & 100 & B-03-143_P7080011 \\
\hline B-03-143 & Looking downstream from crossing of PR 344 & 90 & B-03-143_P7080013 \\
\hline B-03-143 & Looking upstream at cement culverts from 30m west of PR 344 & 100 & B-03-143_P7080014 \\
\hline B-03-144 & Looking upstream from crossing & 140 & B-03-144_P7090001 \\
\hline B-03-144 & Looking downstream from crossing & 50 & B-03-144_P7090002 \\
\hline B-03-145 & Looking upstream from crossing & 210 & B-03-145_P7090003 \\
\hline B-03-145 & Looking downstream from crossing & 50 & B-03-145_P7090004 \\
\hline B-03-146 & Looking upstream from crossing & 180 & B-03-146_P7090005 \\
\hline B-03-146 & Looking downstream from crossing & 340 & B-03-146_P7090006 \\
\hline B-03-147 & Looking upstream from crossing & 170 & B-03-147_P7090007 \\
\hline B-03-147 & Looking downstream from crossing & 40 & B-03-147_P7090008 \\
\hline B-03-148 & Looking upstream from crossing & 100 & B-03-148_P7090009 \\
\hline B-03-148 & Looking downstream from crossing & 250 & B-03-148_P7090010 \\
\hline B-03-148 & Looking upstream at culverts & $\mathrm{N} / \mathrm{A}$ & B-03-148_P7090011 \\
\hline B-03-149 & Looking upstream from crossing & 200 & B-03-149_P7090012 \\
\hline B-03-149 & Looking downstream from crossing & 360 & B-03-149_P7090013 \\
\hline B-03-150 & Looking upstream from crossing & 220 & B-03-150_P7090014 \\
\hline
\end{tabular}


Appendix 8: List of all photographs taken showing the corresponding site number, descriptive caption, direction faced when taken (azimuth in degrees) and the unique file name for each photograph.

\begin{tabular}{|c|c|c|c|}
\hline $\begin{array}{c}\text { Site } \\
\text { Number }\end{array}$ & Photo Description & Azimuth & File Name \\
\hline B-03-150 & Looking downstream from crossing & 65 & B-03-150_P7090015 \\
\hline B-03-151 & Looking upstream from crossing & 60 & B-03-151_P7100001 \\
\hline B-03-151 & Looking downstream from crossing & 300 & B-03-151_P7100002 \\
\hline B-03-151 & Looking upstream at culverts & $\mathrm{N} / \mathrm{A}$ & B-03-151_P7100003 \\
\hline B-03-151 & Looking upstream at riffles & $\mathrm{N} / \mathrm{A}$ & B-03-151_P7100004 \\
\hline B-03-152 & Looking upstream from crossing & 300 & B-03-152_P7100005 \\
\hline B-03-152 & Looking downstream from crossing & 210 & B-03-152_P7100006 \\
\hline B-03-153 & Looking upstream from crossing & 90 & B-03-153_P7100007 \\
\hline B-03-153 & Looking downstream from crossing & 280 & B-03-153_P7100008 \\
\hline B-03-154 & Looking upstream from crossing & 10 & B-03-154_P7100009 \\
\hline B-03-154 & Looking downstream from crossing & 180 & B-03-154_P7100010 \\
\hline B-03-155 & Looking upstream from crossing & 10 & B-03-155_P7110001 \\
\hline B-03-155 & Looking downstream from crossing & 180 & B-03-155_P7110002 \\
\hline B-03-156 & Looking upstream from crossing & 20 & B-03-156_P7110003 \\
\hline B-03-156 & Looking downstream from crossing & 200 & B-03-156_P7110004 \\
\hline B-03-157 & Looking upstream from crossing & 10 & B-03-157_P7110005 \\
\hline B-03-157 & Looking downstream from crossing & 160 & B-03-157_P7110006 \\
\hline B-03-157 & Photo of two perched most easterly culverts & $\mathrm{N} / \mathrm{A}$ & B-03-157_P7110007 \\
\hline B-03-157 & Looking downstream at outflow of west culvert & 220 & B-03-157_P7110008 \\
\hline B-03-157 & Photo of west culvert & N/A & B-03-157_P7110009 \\
\hline B-03-158 & Looking upstream from crossing & 180 & B-03-158_P7110010 \\
\hline B-03-158 & Looking downstream from crossing & 360 & B-03-158_P7110011 \\
\hline B-03-159 & Looking upstream from crossing & 110 & B-03-159_P7110012 \\
\hline B-03-159 & Looking downstream from crossing & 290 & B-03-159_P7110013 \\
\hline B-03-160 & Looking upstream from crossing & 160 & B-03-160_P7110014 \\
\hline B-03-160 & Looking downstream from crossing & 300 & B-03-160 P7110015 \\
\hline B-03-161 & Looking upstream from crossing & 310 & B-03-161_P7140001 \\
\hline B-03-161 & Looking downstream from crossing & 140 & B-03-161_P7140002 \\
\hline B-03-162 & Looking upstream from crossing & 200 & B-03-162_P7140003 \\
\hline B-03-162 & Looking downstream from crossing & 80 & B-03-162_P7140004 \\
\hline B-03-163 & Looking upstream from middle of control structure & 10 & B-03-163_P7140005 \\
\hline B-03-163 & Looking downstream from middle of control structure & 210 & B-03-163_P7140006 \\
\hline B-03-163 & Looking across control structure from right bank & $\mathrm{N} / \mathrm{A}$ & B-03-163_P7140007 \\
\hline
\end{tabular}


Appendix 8: List of all photographs taken showing the corresponding site number, descriptive caption, direction faced when taken (azimuth in degrees) and the unique file name for each photograph.

\begin{tabular}{|c|c|c|c|}
\hline $\begin{array}{c}\text { Site } \\
\text { Number }\end{array}$ & Photo Description & Azimuth & File Name \\
\hline B-03-164 & Looking upstream from crossing & 270 & 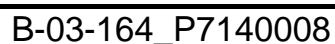 \\
\hline B-03-164 & Looking downstream from crossing & 90 & B-03-164_P7140009 \\
\hline B-03-165 & Looking upstream from crossing & 180 & B-03-165_P7140010 \\
\hline B-03-165 & Looking upstream from crossing at second order drains & 270 & B-03-165_P7140011 \\
\hline B-03-165 & Looking downstream from crossing & 360 & B-03-165 P7140012 \\
\hline B-03-166 & Looking upstream from crossing & 270 & B-03-166_P7140013 \\
\hline B-03-166 & Looking downstream from crossing & 90 & B-03-166_P7140014 \\
\hline B-03-167 & Looking upstream from crossing & 270 & B-03-167_P7140015 \\
\hline B-03-167 & Looking downstream from crossing & 90 & B-03-167 P7140016 \\
\hline B-03-168 & Looking upstream from crossing & 270 & B-03-168 P7140017 \\
\hline B-03-168 & Looking downstream from crossing & 90 & B-03-168_P7140018 \\
\hline B-03-169 & Looking upstream from crossing & 270 & B-03-169 P7140019 \\
\hline B-03-169 & Looking downstream from crossing & 90 & B-03-169 P7140020 \\
\hline B-03-170 & Looking upstream from crossing & 270 & B-03-170 P7140021 \\
\hline B-03-170 & Looking downstream from crossing & 90 & B-03-170_P7140022 \\
\hline B-03-171 & Looking upstream from crossing & 270 & B-03-171 P7140023 \\
\hline B-03-171 & Looking downstream from crossing & 90 & B-03-171 P7140024 \\
\hline B-03-172 & Looking upstream from crossing & 270 & B-03-172_P7140025 \\
\hline B-03-172 & Looking downstream from crossing & 90 & B-03-172_P7140026 \\
\hline B-03-173 & Looking upstream from crossing & 270 & B-03-173 P7140027 \\
\hline B-03-173 & Looking downstream from crossing & 90 & B-03-173 P7140028 \\
\hline B-03-174 & Looking upstream from crossing & 270 & B-03-174_P7140029 \\
\hline B-03-174 & Looking downstream from crossing & 90 & B-03-174_P7140030 \\
\hline B-03-175 & Looking upstream from crossing & 270 & B-03-175_P7140031 \\
\hline B-03-175 & Looking downstream from crossing & 90 & B-03-175 P7140032 \\
\hline B-03-176 & Looking upstream from crossing & 270 & B-03-176_P7140033 \\
\hline B-03-176 & Looking downstream from crossing & 90 & B-03-176_P7140034 \\
\hline B-03-177 & Looking upstream from crossing & 270 & B-03-177 P7140035 \\
\hline B-03-177 & Looking downstream from crossing & 90 & B-03-177 P7140036 \\
\hline B-03-178 & Looking upstream from crossing & 270 & B-03-178_P7140037 \\
\hline B-03-178 & Looking downstream from crossing & 90 & B-03-178_P7140038 \\
\hline B-03-179 & Looking upstream from crossing & 270 & B-03-179 P7140039 \\
\hline B-03-179 & Looking downstream from crossing & 90 & B-03-179 P7140040 \\
\hline
\end{tabular}


Appendix 8: List of all photographs taken showing the corresponding site number, descriptive caption, direction faced when taken (azimuth in degrees) and the unique file name for each photograph.

\begin{tabular}{|c|c|c|c|}
\hline $\begin{array}{c}\text { Site } \\
\text { Number }\end{array}$ & Photo Description & Azimuth & File Name \\
\hline B-03-180 & Looking upstream from crossing & 270 & B-03-180_P7140041 \\
\hline B-03-180 & Looking downstream from crossing & 90 & B-03-180 P7140042 \\
\hline B-03-181 & Looking upstream from crossing & 260 & B-03-181_P7150001 \\
\hline B-03-181 & Looking downstream from crossing & 150 & B-03-181_P7150002 \\
\hline B-03-182 & Looking upstream from crossing & 180 & B-03-182_P7160001 \\
\hline B-03-182 & Looking downstream from crossing & 360 & B-03-182_P7160002 \\
\hline B-03-182 & Looking at former crossing & 90 & B-03-182_P7160003 \\
\hline B-03-183 & Looking upstream from crossing & 180 & B-03-183_P7160004 \\
\hline B-03-183 & Looking downstream from crossing & 360 & B-03-183_P7160005 \\
\hline B-03-183 & Photo of Walleye & N/A & B-03-183 P7160006 \\
\hline B-03-183 & Photo of Northern Pike & $\mathrm{N} / \mathrm{A}$ & B-03-183_P7160007 \\
\hline B-03-184 & Looking upstream from crossing & 90 & B-03-184_P7160008 \\
\hline B-03-184 & Looking downstream from crossing & 260 & B-03-184_P7160009 \\
\hline B-03-185 & Looking upstream from crossing & 200 & B-03-185 P7160010 \\
\hline B-03-185 & Looking downstream from crossing & 350 & B-03-185_P7160011 \\
\hline B-03-186 & Looking upstream from crossing & 130 & B-03-186_P7160012 \\
\hline B-03-186 & Looking downstream from crossing & 310 & B-03-186_P7160021 \\
\hline B-03-187 & Looking upstream from crossing & 50 & B-03-187_P7160013 \\
\hline B-03-187 & Looking downstream from crossing & 310 & B-03-187_P7160014 \\
\hline B-03-188 & Looking upstream from crossing & 90 & B-03-188_P7160015 \\
\hline B-03-188 & Looking downstream from crossing & 180 & B-03-188_P7160016 \\
\hline B-03-189 & Looking upstream from crossing & 130 & B-03-189_P7160017 \\
\hline B-03-189 & Looking downstream from crossing & 10 & B-03-189_P7160018 \\
\hline B-03-189 & Photo of Northern Pike & $\mathrm{N} / \mathrm{A}$ & B-03-189_P7160019 \\
\hline B-03-189 & Photo of Black Bullhead & $\mathrm{N} / \mathrm{A}$ & B-03-189 P7160020 \\
\hline B-03-190 & Looking upstream from crossing & 300 & B-03-190 P7160022 \\
\hline B-03-190 & Looking downstream from crossing & 160 & B-03-190_P7160023 \\
\hline B-03-191 & Looking upstream from crossing & 90 & B-03-191_P7170001 \\
\hline B-03-191 & Looking downstream from crossing & 260 & B-03-191_P7170002 \\
\hline B-03-192 & Looking upstream from riffle & 95 & B-03-192_P7170003 \\
\hline B-03-192 & Looking downstream from riffle & 265 & B-03-192_P7170004 \\
\hline B-03-192 & Looking upstream at riffles & $\mathrm{N} / \mathrm{A}$ & B-03-192_P7170005 \\
\hline B-03-193 & Looking upstream from crossing & 250 & B-03-193_P7170006 \\
\hline
\end{tabular}


Appendix 8: List of all photographs taken showing the corresponding site number, descriptive caption, direction faced when taken (azimuth in degrees) and the unique file name for each photograph.

\begin{tabular}{|c|c|c|c|}
\hline $\begin{array}{c}\text { Site } \\
\text { Number }\end{array}$ & Photo Description & Azimuth & File Name \\
\hline B-03-193 & Looking downstream from crossing & 120 & B-03-193_P7170007 \\
\hline B-03-194 & Looking upstream from crossing & 100 & B-03-194_P7200001 \\
\hline B-03-194 & Looking downstream from crossing & 20 & B-03-194_P7220002 \\
\hline B-03-195 & Looking upstream from crossing & 170 & B-03-195_P7230001 \\
\hline B-03-195 & Looking downstream from crossing & 350 & B-03-195_P7230002 \\
\hline B-03-196 & Looking upstream from crossing & 180 & B-03-196_P7230003 \\
\hline B-03-196 & Looking downstream from crossing & 350 & B-03-196_P7230004 \\
\hline B-03-197 & Looking upstream from crossing & 90 & B-03-197_P7230005 \\
\hline B-03-197 & Looking downstream from crossing & 270 & B-03-197_P7230006 \\
\hline B-03-198 & Looking upstream from crossing & 130 & B-03-198_P7230007 \\
\hline B-03-198 & Looking downstream from crossing & 300 & B-03-198_P7230008 \\
\hline B-03-199 & Looking upstream from crossing & 110 & B-03-199_P7230009 \\
\hline B-03-199 & Looking downstream from crossing & 200 & B-03-199_P7230010 \\
\hline B-03-200 & Looking at aerated pond from gravel road & 270 & B-03-200 P7230011 \\
\hline B-03-200 & Looking at outlet of culvert from left bank & $\mathrm{N} / \mathrm{A}$ & B-03-200_P7230012 \\
\hline $\mathrm{B}-03-200$ & Looking downstream from outlet of culvert & 10 & B-03-200_P7230013 \\
\hline B-03-200 & Looking upstream from outlet of culvert & 160 & B-03-200_P7230014 \\
\hline B-03-200 & Looking across control structure from right bank & 90 & B-03-200 P7230015 \\
\hline B-03-200 & Looking upstream at control structure from $10 \mathrm{~m}$ downstream & $\mathrm{N} / \mathrm{A}$ & B-03-200_P7230016 \\
\hline B-03-200 & Looking downstream at pool from base of control structure & 360 & B-03-200_P7230017 \\
\hline $\mathrm{B}-03-200$ & Photo of Sauger & $\mathrm{N} / \mathrm{A}$ & B-03-200_P7230018 \\
\hline B-03-200 & Photo of Sauger & N/A & B-03-200_P7230019 \\
\hline B-03-200 & Photo of Sauger & $\mathrm{N} / \mathrm{A}$ & B-03-200_P7230020 \\
\hline B-03-200 & Photo of Black Crappie & $\mathrm{N} / \mathrm{A}$ & B-03-200_P7230021 \\
\hline B-03-200 & Photo of Black Crappie & N/A & B-03-200 P7230022 \\
\hline B-03-200 & Bad photo of Black Crappie & N/A & B-03-200 P7230023 \\
\hline B-03-200 & Photo of Black Bullhead & $\mathrm{N} / \mathrm{A}$ & B-03-200_P7230024 \\
\hline B-03-201 & Looking upstream from control structure & 100 & B-03-201_P7230025 \\
\hline B-03-201 & Looking downstream from control structure & 280 & B-03-201_P7230026 \\
\hline B-03-201 & Looking across structure from left bank & N/A & B-03-201_P7230027 \\
\hline B-03-201 & Looking upstream at control structure & N/A & B-03-201_P7230028 \\
\hline B-03-202 & Looking upstream from crossing & 180 & B-03-202_P7230029 \\
\hline B-03-202 & Looking downstream from crossing & 360 & B-03-202_P7230030 \\
\hline
\end{tabular}


Appendix 8: List of all photographs taken showing the corresponding site number, descriptive caption, direction faced when taken (azimuth in degrees) and the unique file name for each photograph.

\begin{tabular}{|c|c|c|c|}
\hline $\begin{array}{c}\text { Site } \\
\text { Number }\end{array}$ & Photo Description & Azimuth & File Name \\
\hline B-03-203 & Looking upstream from crossing & 180 & B-03-203_P7230031 \\
\hline B-03-203 & Looking downstream from crossing & 360 & B-03-203 P7230032 \\
\hline B-03-204 & Looking upstream from crossing & 180 & B-03-204_P7230033 \\
\hline B-03-204 & Looking downstream from crossing & 360 & B-03-204_P7230034 \\
\hline B-03-205 & Looking upstream from crossing & 150 & B-03-205_P7230035 \\
\hline B-03-205 & Looking downstream from crossing & 360 & B-03-205_P7230036 \\
\hline B-03-206 & Looking upstream from bridge & 90 & B-03-206_P7240001 \\
\hline B-03-206 & Looking downstream from bridge & 270 & B-03-206_P7240002 \\
\hline B-03-206 & Looking upstream at riffles & $\mathrm{N} / \mathrm{A}$ & B-03-206_P7240003 \\
\hline B-03-206 & Photo of Walleye & N/A & B-03-206 P7240004 \\
\hline B-03-207 & Looking upstream from bridge & 90 & B-03-207_P7240005 \\
\hline B-03-207 & Looking downstream from bridge & 360 & B-03-207_P7240006 \\
\hline B-03-207 & Photo of White Sucker & $\mathrm{N} / \mathrm{A}$ & B-03-207_P7240007 \\
\hline B-03-207 & Photo of Fathead Minnow & $\mathrm{N} / \mathrm{A}$ & B-03-207_P7240008 \\
\hline B-03-208 & Looking upstream from crossing & 110 & B-03-208_P7240009 \\
\hline B-03-208 & Looking downstream from crossing & 300 & B-03-208_P7240010 \\
\hline B-03-209 & Looking upstream from crossing & 120 & B-03-209_P7240011 \\
\hline B-03-209 & Looking downstream from crossing & 360 & B-03-209 P7240012 \\
\hline B-03-210 & Looking upstream from crossing & 70 & B-03-210_P7240013 \\
\hline B-03-210 & Looking downstream from crossing & 280 & B-03-210_P7240014 \\
\hline $\mathrm{B}-03-211$ & Looking upstream from dam at left bank & 160 & B-03-211_P7240015 \\
\hline B-03-211 & Looking downstream from dam at left bank & 270 & B-03-211_P7240016 \\
\hline B-03-211 & Photo of old mill? & $\mathrm{N} / \mathrm{A}$ & B-03-211_P7240017 \\
\hline B-03-211 & Looking at dam from left bank & $\mathrm{N} / \mathrm{A}$ & B-03-211_P7240018 \\
\hline B-03-212 & Looking upstream from point in stream & 90 & B-03-212 P7240019 \\
\hline B-03-212 & Looking downstream from point in stream & 270 & B-03-212 P7240020 \\
\hline B-03-212 & Photo of boulder crossing located $400 \mathrm{~m}$ upstream from sample reach & $\mathrm{N} / \mathrm{A}$ & B-03-212_P7240021 \\
\hline B-03-213 & Looking upstream from crossing & 90 & B-03-213_P7240022 \\
\hline B-03-213 & Looking downstream from crossing & 270 & B-03-213_P7240023 \\
\hline B-03-214 & Looking upstream from crossing & 190 & B-03-214_P7250001 \\
\hline B-03-214 & Looking downstream from crossing & 40 & B-03-214_P7250002 \\
\hline B-03-215 & Looking upstream from bridge & 220 & B-03-215_P7250003 \\
\hline B-03-215 & Looking downstream from bridge & 360 & B-03-215_P7250004 \\
\hline
\end{tabular}


Appendix 8: List of all photographs taken showing the corresponding site number, descriptive caption, direction faced when taken (azimuth in degrees) and the unique file name for each photograph.

\begin{tabular}{|c|c|c|c|}
\hline $\begin{array}{c}\text { Site } \\
\text { Number }\end{array}$ & Photo Description & Azimuth & File Name \\
\hline B-03-216 & Looking upstream from crossing & 90 & $\begin{array}{lll}\text { B-03-216 } & \text { P7250005 }\end{array}$ \\
\hline B-03-216 & Looking downstream from crossing & 280 & B-03-216_P7250006 \\
\hline B-03-217 & Looking upstream from crossing & 140 & B-03-217_P7250007 \\
\hline B-03-217 & Looking downstream from crossing & 320 & B-03-217_P7250008 \\
\hline B-03-218 & Looking upstream from crossing & 90 & B-03-218 P7250009 \\
\hline B-03-218 & Looking downstream from crossing & 270 & B-03-218 P7250010 \\
\hline B-03-218 & Photo of small pool & $\mathrm{N} / \mathrm{A}$ & B-03-218_P7250011 \\
\hline B-03-219 & Looking upstream from crossing & 90 & B-03-219 P7250012 \\
\hline B-03-219 & Looking downstream from crossing & 360 & B-03-219 P7250013 \\
\hline B-03-219 & Looking upstream of crossing & $\mathrm{N} / \mathrm{A}$ & B-03-219_P7250014 \\
\hline B-03-220 & Looking upstream from crossing & 95 & B-03-220_P7250015 \\
\hline B-03-220 & Looking downstream from crossing & 210 & B-03-220 P7250016 \\
\hline B-03-221 & Looking upstream from crossing & 220 & B-03-221_P7250017 \\
\hline B-03-221 & Looking downstream from crossing & 20 & B-03-221_P7250018 \\
\hline B-03-222 & Looking upstream from crossing & 120 & B-03-222_P7250019 \\
\hline B-03-222 & Looking downstream from crossing & 280 & B-03-222 P7250020 \\
\hline B-03-222 & Looking upstream of culverts & N/A & B-03-222 P7250021 \\
\hline B-03-223 & Looking upstream from crossing & 280 & B-03-223_P8050001 \\
\hline B-03-223 & Looking downstream from crossing & 222 & B-03-223_P8050002 \\
\hline B-03-224 & Looking upstream from crossing & 245 & B-03-224 P8050003 \\
\hline B-03-224 & Looking downstream from crossing & 110 & B-03-224 P8050004 \\
\hline B-03-224 & Looking upstream at perched culverts & $\mathrm{N} / \mathrm{A}$ & B-03-224_P8050005 \\
\hline B-03-225 & Looking upstream from crossing & 360 & B-03-225_P8050006 \\
\hline B-03-225 & Looking downstream from crossing & 90 & B-03-225_P8050007 \\
\hline B-03-226 & Looking upstream from PTH 9 bridge crossing & 270 & B-03-226_P8060001 \\
\hline B-03-226 & Looking downstream from PTH 9 bridge crossing & 90 & B-03-226_P8060002 \\
\hline B-03-226 & Photo of "danger rocks" sign & $\mathrm{N} / \mathrm{A}$ & B-03-226_P8060003 \\
\hline B-03-226 & Looking upstream from rocks & 270 & B-03-226 P8060004 \\
\hline B-03-226 & Looking downstream from rocks & 90 & B-03-226 P8060005 \\
\hline B-03-227 & Looking upstream from crossing & 200 & B-03-227_P8060006 \\
\hline $\mathrm{B}-03-227$ & Looking downstream from crossing & 50 & B-03-227_P8060007 \\
\hline B-03-228 & Looking upstream from crossing & 270 & B-03-228 P8060008 \\
\hline B-03-228 & Looking downstream from crossing & 90 & B-03-228 P8060009 \\
\hline
\end{tabular}


Appendix 8: List of all photographs taken showing the corresponding site number, descriptive caption, direction faced when taken (azimuth in degrees) and the unique file name for each photograph.

\begin{tabular}{|c|c|c|c|}
\hline $\begin{array}{c}\text { Site } \\
\text { Number }\end{array}$ & Photo Description & Azimuth & File Name \\
\hline B-03-229 & Looking upstream from crossing & 270 & B-03-229 P8060010 \\
\hline B-03-229 & Looking downstream from crossing & 90 & B-03-229_P8060011 \\
\hline B-03-230 & Looking upstream from v-notch weir & 270 & B-03-230_P8060012 \\
\hline B-03-230 & Looking downstream from v-notch weir & 90 & B-03-230 P8060013 \\
\hline B-03-230 & Looking upstream at weir from $20 \mathrm{~m}$ downstream & 270 & B-03-230 P8060014 \\
\hline B-03-230 & Looking upstream at weir from $10 \mathrm{~m}$ downstream & 270 & B-03-230 P8060015 \\
\hline B-03-230 & Photo of toad & $\mathrm{N} / \mathrm{A}$ & B-03-230_P8060016 \\
\hline B-03-230 & Photo of toad & N/A & B-03-230 P8060017 \\
\hline B-03-231 & Looking upstream from bridge & 230 & B-03-231 P8060018 \\
\hline B-03-231 & Looking downstream from bridge & 65 & B-03-231_P8060019 \\
\hline B-03-232 & Looking upstream from crossing & 310 & B-03-232_P8060020 \\
\hline B-03-232 & Looking downstream from crossing & 75 & B-03-232 P8060021 \\
\hline B-03-233 & Looking upstream from crossing & 270 & B-03-233 P8060022 \\
\hline B-03-233 & Looking downstream from crossing & 90 & B-03-233 P8060023 \\
\hline B-03-234 & Looking upstream from crossing & 270 & B-03-234_P8060024 \\
\hline B-03-234 & Looking downstream from crossing & 90 & B-03-234 P8060025 \\
\hline B-03-235 & Looking upstream from crossing & 270 & B-03-235 P8060026 \\
\hline B-03-235 & Looking downstream from crossing & 90 & B-03-235_P8060027 \\
\hline B-03-236 & Looking upstream from crossing & 290 & B-03-236_P8060028 \\
\hline B-03-236 & Looking downstream from crossing & 120 & B-03-236 P8060029 \\
\hline B-03-237 & Looking upstream from crossing & 150 & B-03-237 P8070001 \\
\hline B-03-237 & Looking downstream from crossing & 330 & B-03-237_P8070002 \\
\hline B-03-238 & Looking upstream from crossing & 270 & B-03-238_P8070003 \\
\hline B-03-238 & Looking downstream from crossing & 90 & B-03-238_P8070004 \\
\hline B-03-239 & Photo of ford crossing & $\mathrm{N} / \mathrm{A}$ & B-03-239 P8070005 \\
\hline B-03-239 & Looking upstream from crossing & 180 & B-03-239_P8070006 \\
\hline B-03-239 & Looking downstream from crossing & 10 & B-03-239_P8070007 \\
\hline B-03-240 & Looking upstream from crossing & 270 & B-03-240 P8070008 \\
\hline B-03-240 & Looking downstream from crossing & 90 & B-03-240 P8070009 \\
\hline B-03-241 & Looking upstream from bridge & 75 & B-03-241_P8070010 \\
\hline B-03-241 & Looking downstream from bridge & 215 & B-03-241_P8070011 \\
\hline B-03-242 & Looking upstream from bridge & 340 & B-03-242 P8070012 \\
\hline B-03-242 & Looking downstream from bridge & 240 & B-03-242 P8070013 \\
\hline
\end{tabular}


Appendix 8: List of all photographs taken showing the corresponding site number, descriptive caption, direction faced when taken (azimuth in degrees) and the unique file name for each photograph.

\begin{tabular}{|c|c|c|c|}
\hline $\begin{array}{c}\text { Site } \\
\text { Number }\end{array}$ & Photo Description & Azimuth & File Name \\
\hline B-03-243 & Looking upstream from crossing & 110 & B-03-243_P8070014 \\
\hline B-03-243 & Looking downstream from crossing & 270 & B-03-243_P8070015 \\
\hline B-03-244 & Looking upstream from dam & 270 & B-03-244_P8070016 \\
\hline $\mathrm{B}-03-244$ & Looking downstream from dam & 80 & B-03-244_P8070017 \\
\hline B-03-244 & Looking upstream at dam & $\mathrm{N} / \mathrm{A}$ & B-03-244_P8070018 \\
\hline B-03-244 & Looking at staff gauge & $\mathrm{N} / \mathrm{A}$ & B-03-244_P8070019 \\
\hline B-03-245 & Looking upstream from crossing & 160 & B-03-245_P8070020 \\
\hline B-03-245 & Looking downstream from crossing & 230 & B-03-245_P8070021 \\
\hline B-03-246 & Looking upstream from crossing & 340 & B-03-246_P8080001 \\
\hline B-03-246 & Looking downstream from crossing & 140 & B-03-246_P8080002 \\
\hline $\mathrm{B}-03-247$ & Looking upstream from crossing & 330 & B-03-247_P8080003 \\
\hline B-03-247 & Looking downstream from crossing & 135 & B-03-247_P8080004 \\
\hline B-03-248 & Looking upstream from crossing & 270 & B-03-248_P8080005 \\
\hline B-03-248 & Looking downstream from crossing & 90 & B-03-248_P8080006 \\
\hline B-03-249 & Looking upstream from bridge & 270 & B-03-249_P8080009 \\
\hline B-03-249 & Looking downstream from bridge & 30 & B-03-249_P8080010 \\
\hline B-03-250 & Looking upstream from dike & 270 & B-03-250_P8080011 \\
\hline B-03-250 & Looking downstream from dike & 90 & B-03-250_P8080012 \\
\hline B-03-250 & Photo of culverts and Geotextile cloth & $\mathrm{N} / \mathrm{A}$ & B-03-250_P8080013 \\
\hline D-03-001 & Looking upstream from mid-reach & 270 & D-03-001_P5080001 \\
\hline D-03-001 & Looking downstream from mid-reach & 90 & D-03-001_P5080002 \\
\hline D-03-001 & Looking across low head dam & 0 & D-03-001_P5080003 \\
\hline D-03-002 & Looking upstream across road & 340 & D-03-002_P5100001 \\
\hline D-03-002 & Looking upstream from road & 340 & D-03-002_P5100002 \\
\hline D-03-002 & Looking downstream from road & 110 & D-03-002_P5100003 \\
\hline D-03-002 & Looking upstream from below beaver dam & 270 & D-03-002_P5100004 \\
\hline D-03-002 & Looking upstream at perched culverts & 360 & D-03-002_P5100005 \\
\hline D-03-003 & Looking upstream from bottom of reach & 270 & D-03-003_P5100006 \\
\hline D-03-003 & Looking downstream from top of reach & 90 & D-03-003_P5100007 \\
\hline D-03-004 & Looking upstream from bridge crossing & 260 & D-03-004_P5110008 \\
\hline D-03-004 & Looking downstream from bridge crossing & 80 & D-03-004_P5110009 \\
\hline D-03-005 & Looking upstream from bottom of reach & 270 & D-03-005_P5110010 \\
\hline D-03-005 & Looking upstream at gradient control structure & 270 & D-03-005_P5110011 \\
\hline
\end{tabular}


Appendix 8: List of all photographs taken showing the corresponding site number, descriptive caption, direction faced when taken (azimuth in degrees) and the unique file name for each photograph.

\begin{tabular}{|c|c|c|c|}
\hline $\begin{array}{c}\text { Site } \\
\text { Number }\end{array}$ & Photo Description & Azimuth & File Name \\
\hline D-03-005 & Looking downstream from gradient control structure & 90 & D-03-005_P5110012 \\
\hline D-03-006 & Looking upstream from bridge crossing & 270 & D-03-006_P5120001 \\
\hline D-03-006 & Looking downstream from bridge crossing & 90 & D-03-006 P5120002 \\
\hline D-03-007 & Looking downstream from bridge crossing & 0 & D-03-007_P5120003 \\
\hline D-03-007 & Looking upstream from bridge crossing & 180 & D-03-007_P5120004 \\
\hline $\mathrm{D}-03-007$ & Looking upstream from bridge crossing & 180 & D-03-007_P5120005 \\
\hline D-03-008 & Looking $125 \mathrm{~m}$ upstream from dam & $\mathrm{N} / \mathrm{A}$ & D-03-008_P5130001 \\
\hline D-03-008 & Looking downstream 125 m upstream from dam & N/A & D-03-008_P5130002 \\
\hline D-03-008 & Looking upstream from $30 \mathrm{~m}$ downstream of dam & $\mathrm{N} / \mathrm{A}$ & D-03-008_P5130003 \\
\hline D-03-009 & Looking upstream from crossing & 180 & D-03-009_P5130004 \\
\hline D-03-009 & Looking upstream from $30 \mathrm{~m}$ back from crossing & 180 & D-03-009 P5130005 \\
\hline D-03-010 & Looking upstream from bridge crossing & 210 & D-03-010 P5130006 \\
\hline D-03-010 & Looking downstream from bridge crossing & 30 & D-03-010_P5130007 \\
\hline D-03-010 & Looking east at Verhaeghe Drain & 90 & D-03-010_P5130008 \\
\hline D-03-011 & Looking upstream from concrete raised crossing & 180 & D-03-011_P5130009 \\
\hline $\mathrm{D}-03-011$ & Looking downstream from concrete raised crossing & 0 & D-03-011_P5130010 \\
\hline D-03-011 & Looking upstream from $20 \mathrm{~m}$ downstream of raised crossing & 180 & D-03-011_P5130011 \\
\hline D-03-012 & Looking upstream from top of reach at junction of Laurier Drain & 270 & D-03-012_P5130012 \\
\hline D-03-012 & Looking downstream from top of reach & 80 & D-03-012 P5130013 \\
\hline D-03-012 & Looking upstream from bottom of reach & 270 & D-03-012 P5130014 \\
\hline D-03-013 & Looking upstream from junction of Laurier Drain and Henderson Creek & 180 & D-03-013_P5130015 \\
\hline D-03-014 & Looking upstream from bridge crossing & $\mathrm{N} / \mathrm{A}$ & D-03-014_P5130016 \\
\hline D-03-014 & Looking downstream from bridge crossing & $\mathrm{N} / \mathrm{A}$ & D-03-014_P5130017 \\
\hline D-03-015 & Looking upstream from crossing & 215 & D-03-015 P5140001 \\
\hline $\mathrm{D}-03-015$ & Looking downstream from crossing & 45 & D-03-015_P5140002 \\
\hline D-03-015 & Looking west at incoming drain & 270 & D-03-015 P5140003 \\
\hline D-03-016 & Looking upstream from top of control structure (sediment trap) & 190 & D-03-016 P5140004 \\
\hline $\mathrm{D}-03-016$ & Looking downstream from top of control structure & 10 & D-03-016_P5140005 \\
\hline $\mathrm{D}-03-016$ & Looking upstream at sediment trap & 190 & D-03-016_P5140006 \\
\hline $\mathrm{D}-03-017$ & Looking upstream from crossing & 270 & D-03-017_P5140007 \\
\hline D-03-017 & Looking downstream from crossing & 90 & D-03-017_P5140008 \\
\hline D-03-017 & Looking upstream at culvert & 270 & D-03-017_P5140009 \\
\hline D-03-017 & Photo of completing habitat assessment at Dead Ox Creek & N/A & D-03-017_P5140010 \\
\hline
\end{tabular}


Appendix 8: List of all photographs taken showing the corresponding site number, descriptive caption, direction faced when taken (azimuth in degrees) and the unique file name for each photograph.

\begin{tabular}{|c|c|c|c|}
\hline $\begin{array}{c}\text { Site } \\
\text { Number }\end{array}$ & Photo Description & Azimuth & File Name \\
\hline D-03-018 & Looking upstream from bridge on PTH 19 & 0 & D-03-018_P5140011 \\
\hline D-03-018 & Looking downstream from bridge on PTH 19 & 180 & $\mathrm{D}-03-018 \mathrm{P} 5140012$ \\
\hline D-03-019 & Looking upstream from bridge crossing & 270 & D-03-019_P5140013 \\
\hline D-03-019 & Looking downstream from bridge crossing & 90 & D-03-019_P5140014 \\
\hline D-03-020 & Looking upstream from bridge crossing & 270 & D-03-020_P5140015 \\
\hline D-03-020 & Looking downstream from bridge crossing & 90 & D-03-020_P5140016 \\
\hline D-03-020 & Looking upstream at concrete bridge from $10 \mathrm{~m}$ below crossing & 310 & D-03-020_P5140017 \\
\hline $\mathrm{D}-03-021$ & Looking upstream from crossing & 290 & D-03-021_P6030001 \\
\hline D-03-021 & Looking downstream from crossing & 100 & D-03-021_P6030002 \\
\hline D-03-022 & Looking upstream from crossing & 270 & $\mathrm{D}-03-022 \_\mathrm{P} 6030003$ \\
\hline D-03-022 & Looking downstream from crossing & 95 & D-03-022_P6030004 \\
\hline D-03-023 & Looking upstream from crossing & 270 & D-03-023_P6030005 \\
\hline D-03-023 & Looking downstream from crossing & 90 & D-03-023_P6030006 \\
\hline D-03-023 & Looking north at culverts & 0 & $\mathrm{D}-03-023 \mathrm{P} 6030007$ \\
\hline D-03-023 & Looking east from $100 \mathrm{~m}$ upstream of culverts & 90 & D-03-023_P6030008 \\
\hline D-03-024 & Looking upstream from crossing & 270 & D-03-024_P6030009 \\
\hline $\mathrm{D}-03-024$ & Looking downstream from crossing & 120 & D-03-024_P6030010 \\
\hline D-03-024 & Looking downstream from $50 \mathrm{~m}$ above crossing & $\mathrm{N} / \mathrm{A}$ & $\mathrm{D}-03-024 \_\mathrm{P} 6030011$ \\
\hline D-03-024 & Looking downstream from $30 \mathrm{~m}$ south of crossing & 90 & D-03-024_P6030012 \\
\hline D-03-025 & Looking upstream from crossing & 180 & D-03-025_P6030013 \\
\hline D-03-025 & Looking downstream from crossing & 0 & D-03-025_P6030014 \\
\hline D-03-025 & Looking upstream under bridge & 0 & $\mathrm{D}-03-025+\mathrm{P} 6030015$ \\
\hline D-03-026 & Looking upstream from concrete bridge crossing & 305 & D-03-026_P6030016 \\
\hline D-03-026 & Looking downstream from crossing & 130 & D-03-026_P6030017 \\
\hline D-03-026 & Looking upstream under bridge - note limits to fish passage & 250 & $\mathrm{D}-03-026 \_\mathrm{P} 6030018$ \\
\hline D-03-026 & Looking at critical habitat sign $50 \mathrm{~m}$ SE of crossing & 90 & $\mathrm{D}-03-026 \mathrm{P} 6030019$ \\
\hline D-03-027 & Looking upstream from crossing at Lizard Lake & 270 & D-03-027_P6040001 \\
\hline D-03-027 & Looking downstream from crossing & 90 & D-03-027_P6040002 \\
\hline D-03-027 & Looking upstream at perched culverts & 270 & D-03-027_P6040003 \\
\hline D-03-028 & Looking upstream from crossing & 250 & $\mathrm{D}-03-028 \mathrm{P} 6040004$ \\
\hline $\mathrm{D}-03-028$ & Looking downstream from crossing & 155 & D-03-028_P6040005 \\
\hline D-03-028 & Looking east at structure & 90 & D-03-028_P6040006 \\
\hline D-03-028 & Looking west at structure & 270 & D-03-028_P6040007 \\
\hline
\end{tabular}


Appendix 8: List of all photographs taken showing the corresponding site number, descriptive caption, direction faced when taken (azimuth in degrees) and the unique file name for each photograph.

\begin{tabular}{|c|c|c|c|}
\hline $\begin{array}{c}\text { Site } \\
\text { Number }\end{array}$ & Photo Description & Azimuth & File Name \\
\hline D-03-029 & Looking upstream from crossing & 220 & $\begin{array}{ll}D-03-029 & P 6040008\end{array}$ \\
\hline D-03-029 & Looking downstream from crossing & 30 & D-03-029_P6040009 \\
\hline $\mathrm{D}-03-030$ & Looking upstream from crossing & 285 & D-03-030_P6040010 \\
\hline D-03-030 & Looking downstream from crossing & 140 & D-03-030 P6040011 \\
\hline D-03-030 & Looking upstream at perched culverts & 270 & D-03-030 P6040012 \\
\hline D-03-031 & Looking upstream from crossing & 270 & D-03-031_P6040013 \\
\hline $\mathrm{D}-03-031$ & Looking downstream from crossing & 120 & D-03-031_P6040014 \\
\hline D-03-031 & Looking west at perched culverts & 270 & D-03-031_P6040015 \\
\hline D-03-032 & Looking upstream from crossing & 270 & D-03-032 P6040016 \\
\hline $\mathrm{D}-03-032$ & Looking downstream from crossing & 90 & D-03-032 P6040017 \\
\hline D-03-032 & Looking west at downstream end of culverts & 270 & $\mathrm{D}-03-032 \mathrm{P} 6040018$ \\
\hline $\mathrm{D}-03-033$ & Looking upstream from crossing & 0 & $\mathrm{D}-03-033 \quad \mathrm{P} 6040019$ \\
\hline D-03-033 & Looking downstream from crossing & 180 & D-03-033 P6040020 \\
\hline D-03-034 & Looking upstream from crossing & 300 & D-03-034_P6040021 \\
\hline D-03-034 & Looking downstream from crossing & 45 & D-03-034_P6040022 \\
\hline $\mathrm{D}-03-034$ & Looking downstream from crossing & 180 & $\mathrm{D}-03-034 \mathrm{P} 6040023$ \\
\hline D-03-035 & Looking upstream from crossing & 0 & D-03-035 P6050001 \\
\hline D-03-035 & Looking downstream from crossing & 180 & D-03-035_P6050002 \\
\hline D-03-036 & Looking upstream from crossing & 245 & D-03-036_P6050003 \\
\hline D-03-036 & Looking downstream from crossing & 60 & $\mathrm{D}-03-036 \mathrm{P} 6050004$ \\
\hline D-03-036 & Looking downstream at Dave, Shawna, and Daniel fishing & 90 & D-03-036 P6050005 \\
\hline D-03-037 & Looking upstream from crossing & 330 & D-03-037_P6050006 \\
\hline D-03-037 & Looking downstream from crossing & 150 & D-03-037_P6050007 \\
\hline D-03-038 & Looking upstream at dam & 90 & $\mathrm{D}-03-038 \mathrm{P} 6060001$ \\
\hline D-03-038 & Looking upstream at dam middle across to north & 90 & $\mathrm{D}-03-038 \mathrm{P} 6060002$ \\
\hline D-03-038 & Looking upstream at dam middle across to south & 90 & D-03-038_P6060003 \\
\hline D-03-038 & Looking downstream from just above weir & 200 & D-03-038_P6060004 \\
\hline D-03-038 & Looking upstream at Inglis Beach Park & 110 & D-03-038 P6060005 \\
\hline $\mathrm{D}-03-038$ & Looking across base of dam (north) & 0 & $\mathrm{D}-03-038 \quad \mathrm{P} 6060006$ \\
\hline D-03-038 & Looking across base of dam (north) & 0 & $\mathrm{D}-03-038 \mathrm{P} 6060007$ \\
\hline D-03-038 & Looking across base of dam (north) & 0 & $\mathrm{D}-03-038 \mathrm{P} 6060008$ \\
\hline D-03-038 & Looking upstream at Bear Creek & $\mathrm{N} / \mathrm{A}$ & D-03-038 P6060009 \\
\hline D-03-039 & Looking upstream from crossing & 270 & D-03-039 P6100001 \\
\hline
\end{tabular}


Appendix 8: List of all photographs taken showing the corresponding site number, descriptive caption, direction faced when taken (azimuth in degrees) and the unique file name for each photograph.

\begin{tabular}{|c|c|c|c|}
\hline $\begin{array}{c}\text { Site } \\
\text { Number }\end{array}$ & Photo Description & Azimuth & File Name \\
\hline D-03-039 & Looking downstream from crossing & 90 & D-03-039_P6100002 \\
\hline D-03-039 & Looking downstream through culverts from upstream of culverts & 140 & D-03-039-P6100003 \\
\hline D-03-039 & Looking at downstream end of culverts from just below culverts & 210 & D-03-039_P6100004 \\
\hline D-03-040 & Looking upstream from crossing & 290 & D-03-040_P6100005 \\
\hline D-03-040 & Looking downstream from crossing & 110 & D-03-040_P6100006 \\
\hline D-03-041 & Looking upstream from crossing & 180 & D-03-041_P6100007 \\
\hline D-03-041 & Looking upstream from crossing & 180 & D-03-041_P6100008 \\
\hline D-03-041 & Looking downstream from crossing & 0 & D-03-041_P6100009 \\
\hline D-03-041 & Looking upstream from $50 \mathrm{~m}$ below crossing & 200 & D-03-041_P6100010 \\
\hline D-03-042 & Looking upstream from bridge crossing & 180 & D-03-042_P6100011 \\
\hline D-03-042 & Looking downstream from bridge crossing & 0 & D-03-042_P6100012 \\
\hline D-03-043 & Looking upstream from crossing & 270 & D-03-043_P6100013 \\
\hline D-03-043 & Looking downstream from crossing & 90 & D-03-043_P6100014 \\
\hline D-03-044 & Looking upstream from bridge crossing & 270 & D-03-044_P6110001 \\
\hline D-03-044 & Looking downstream from bridge crossing & 90 & D-03-044_P6110002 \\
\hline D-03-045 & Looking upstream from bottom of reach & 270 & D-03-045_P6110003 \\
\hline D-03-045 & Looking upstream from mid-reach & 180 & D-03-045_P6110004 \\
\hline D-03-045 & Looking downstream from mid-reach & 90 & D-03-045 P6110005 \\
\hline D-03-046 & Looking upstream from bridge crossing & 270 & D-03-046_P6110006 \\
\hline D-03-046 & Looking downstream from bridge crossing & 90 & D-03-046_P6110007 \\
\hline D-03-046 & Looking at downstream end of bridge & $\mathrm{N} / \mathrm{A}$ & D-03-046_P6110008 \\
\hline D-03-047 & Looking upstream from ford crossing & 270 & D-03-047_P6110009 \\
\hline D-03-047 & Looking downstream from ford crossing & 90 & D-03-047_P6110010 \\
\hline D-03-047 & Looking southwest across downstream end of ford crossing & 210 & D-03-047_P6110011 \\
\hline $\mathrm{D}-03-047$ & Looking southwest across downstream end of ford crossing & 210 & D-03-047_P6110012 \\
\hline D-03-048 & Looking upstream from crossing & 0 & D-03-048_P6110013 \\
\hline D-03-048 & Looking downstream from crossing & 120 & D-03-048_P6110014 \\
\hline D-03-048 & Looking west up incoming 3rd order drain & 270 & D-03-048_P6110015 \\
\hline D-03-049 & Looking upstream from crossing & 180 & D-03-049_P6110016 \\
\hline D-03-049 & Looking downstream from crossing & 0 & D-03-049_P6110017 \\
\hline $\mathrm{D}-03-050$ & Looking upstream from crossing & 290 & D-03-050_P6130001 \\
\hline D-03-050 & Looking downstream from crossing & 90 & D-03-050_P6130002 \\
\hline D-03-051 & Looking upstream from bridge crossing & 10 & D-03-051_P6130003 \\
\hline
\end{tabular}


Appendix 8: List of all photographs taken showing the corresponding site number, descriptive caption, direction faced when taken (azimuth in degrees) and the unique file name for each photograph.

\begin{tabular}{|c|c|c|c|}
\hline $\begin{array}{c}\text { Site } \\
\text { Number }\end{array}$ & Photo Description & Azimuth & File Name \\
\hline D-03-051 & Looking downstream from bridge crossing & 190 & D-03-051_P6130004 \\
\hline D-03-052 & Looking upstream from culvert crossing & 0 & D-03-052 P6130005 \\
\hline $\mathrm{D}-03-052$ & Looking downstream from culvert crossing & 180 & D-03-052_P6130006 \\
\hline D-03-052 & Looking upstream at downstream end of perched culverts & 0 & D-03-052_P6130007 \\
\hline $\mathrm{D}-03-053$ & Looking upstream from bridge crossing & 290 & D-03-053_P6130008 \\
\hline D-03-053 & Looking downstream from bridge crossing & 90 & D-03-053_P6130009 \\
\hline D-03-054 & Looking upstream from bridge crossing & 220 & D-03-054_P6130010 \\
\hline D-03-054 & Looking downstream from bridge crossing & 45 & D-03-054_P6130011 \\
\hline $\mathrm{D}-03-055$ & Looking upstream from bridge crossing & 360 & D-03-055_P6130012 \\
\hline D-03-055 & Looking downstream from bridge crossing & 180 & D-03-055_P6130013 \\
\hline D-03-055 & Looking at lady slippers on site & $\mathrm{N} / \mathrm{A}$ & D-03-055_P6130014 \\
\hline D-03-056 & Looking upstream from bridge crossing & 270 & D-03-056_P6130015 \\
\hline D-03-056 & Looking downstream from bridge crossing & 90 & D-03-056_P6130016 \\
\hline D-03-057 & Looking upstream from mid-reach & 180 & D-03-057_P6130017 \\
\hline D-03-057 & Looking downstream from mid-reach & 0 & D-03-057_P6130018 \\
\hline $\mathrm{D}-03-058$ & Looking upstream from ford crossing & 270 & D-03-058_P6160001 \\
\hline D-03-058 & Looking upstream from $50 \mathrm{~m}$ below crossing & 270 & D-03-058_P6160002 \\
\hline D-03-058 & Looking downstream from ford crossing & 90 & D-03-058 P6160003 \\
\hline D-03-059 & Looking upstream from raised crossing & 270 & D-03-059_P6160004 \\
\hline D-03-059 & Looking downstream from raised crossing & 90 & D-03-059_P6160005 \\
\hline D-03-059 & Looking upstream at raised crossing from $20 \mathrm{~m}$ downstream & 280 & D-03-059_P6160006 \\
\hline D-03-060 & Looking downstream from culvert crossing & 10 & D-03-060_P6160007 \\
\hline D-03-060 & Looking upstream from culvert crossing & 180 & D-03-060_P6160008 \\
\hline $\mathrm{D}-03-061$ & Looking upstream from bridge crossing & 270 & D-03-061_P6170001 \\
\hline D-03-061 & Looking downstream from bridge crossing & 90 & D-03-061_P6170002 \\
\hline D-03-062 & Looking upstream from bridge crossing & 270 & D-03-062_P6170003 \\
\hline $\mathrm{D}-03-062$ & Looking downstream from bridge crossing & 90 & D-03-062_P6170004 \\
\hline $\mathrm{D}-03-063$ & Looking upstream from culvert crossing & 210 & D-03-063_P6170005 \\
\hline $\mathrm{D}-03-063$ & Looking downstream from culvert crossing & 65 & D-03-063_P6170006 \\
\hline D-03-063 & Looking upstream at downstream end of culverts & 310 & D-03-063_P6170007 \\
\hline D-03-064 & Looking upstream from culvert crossing & 270 & D-03-064_P6170008 \\
\hline D-03-064 & Looking downstream from culvert crossing & 90 & D-03-064_P6170009 \\
\hline D-03-065 & Looking upstream from bridge crossing & 180 & D-03-065_P6170010 \\
\hline
\end{tabular}


Appendix 8: List of all photographs taken showing the corresponding site number, descriptive caption, direction faced when taken (azimuth in degrees) and the unique file name for each photograph.

\begin{tabular}{|c|c|c|c|}
\hline $\begin{array}{c}\text { Site } \\
\text { Number }\end{array}$ & Photo Description & Azimuth & File Name \\
\hline D-03-065 & Looking downstream from bridge crossing & 0 & D-03-065_P6170011 \\
\hline D-03-066 & Looking upstream from bridge crossing & 250 & D-03-066 P6180001 \\
\hline $\mathrm{D}-03-066$ & Looking downstream from bridge crossing & 80 & D-03-066_P6180002 \\
\hline D-03-067 & Looking upstream from culvert crossing & 225 & D-03-067_P6180003 \\
\hline D-03-067 & Looking downstream from culvert crossing & 100 & D-03-067_P6180004 \\
\hline D-03-067 & Looking at downstream end of culverts \#1 and 2 (northern most) & 270 & D-03-067_P6180005 \\
\hline D-03-067 & Looking at downstream end of perched culvert \#5 (middle culvert) & 270 & D-03-067_P6180006 \\
\hline D-03-067 & Looking at downstream end of perched culverts \# 3 and 4 & 270 & D-03-067_P6180007 \\
\hline $\mathrm{D}-03-068$ & Looking upstream from bridge crossing & 200 & D-03-068_P6180008 \\
\hline D-03-068 & Looking downstream from bridge crossing & 90 & D-03-068_P6180009 \\
\hline D-03-068 & Looking upstream at downstream end of bridge & 200 & D-03-068_P6180010 \\
\hline D-03-069 & Looking upstream from bridge crossing & 300 & D-03-069_P6180011 \\
\hline D-03-069 & Looking downstream from bridge crossing & 140 & D-03-069_P6180012 \\
\hline D-03-070 & Looking upstream from culvert crossing & 0 & D-03-070_P6230001 \\
\hline D-03-070 & Looking downstream from culvert crossing & 180 & D-03-070_P6230002 \\
\hline $\mathrm{D}-03-070$ & Looking upstream at downstream end of culverts & 30 & D-03-070_P6230003 \\
\hline D-03-071 & Looking upstream from culvert crossing & 350 & D-03-071_P6230004 \\
\hline D-03-071 & Looking downstream from culvert crossing & 180 & D-03-071_P6230005 \\
\hline D-03-071 & Looking southeast at upstream end of culverts & 100 & D-03-071_P6230006 \\
\hline D-03-071 & Looking northeast at downstream end of culverts & 40 & D-03-071_P6230007 \\
\hline D-03-072 & Looking upstream from bridge crossing & 0 & D-03-072_P6230008 \\
\hline $\mathrm{D}-03-072$ & Looking downstream from bridge crossing & 180 & D-03-072_P6230009 \\
\hline D-03-072 & Looking southeast at upstream end of bridge & 135 & D-03-072_P6230010 \\
\hline $\mathrm{D}-03-073$ & Looking upstream from culvert crossing & 270 & D-03-073_P6230011 \\
\hline $\mathrm{D}-03-073$ & Looking downstream from culvert crossing & 90 & D-03-073_P6230012 \\
\hline D-03-073 & Looking west at downstream end of perched culverts & 270 & D-03-073_P6230013 \\
\hline D-03-074 & Looking upstream from bridge crossing & 300 & D-03-074_P6240001 \\
\hline D-03-074 & Looking downstream from bridge crossing & 180 & D-03-074_P6240002 \\
\hline D-03-075 & Looking upstream from bridge crossing & 250 & D-03-075_P6240003 \\
\hline D-03-075 & Looking downstream from bridge crossing & 90 & D-03-075_P6240004 \\
\hline D-03-076 & Looking upstream from culvert crossing & 270 & D-03-076_P6240005 \\
\hline D-03-076 & Looking downstream from culvert crossing & 90 & D-03-076_P6240006 \\
\hline D-03-077 & Looking upstream from culvert crossing & 300 & D-03-077_P6240007 \\
\hline
\end{tabular}


Appendix 8: List of all photographs taken showing the corresponding site number, descriptive caption, direction faced when taken (azimuth in degrees) and the unique file name for each photograph.

\begin{tabular}{|c|c|c|c|}
\hline $\begin{array}{c}\text { Site } \\
\text { Number }\end{array}$ & Photo Description & Azimuth & File Name \\
\hline D-03-077 & Looking downstream from culvert crossing & 120 & D-03-077_P6240008 \\
\hline D-03-077 & Looking west at downstream end of culverts & 270 & D-03-077_P6240009 \\
\hline D-03-078 & Looking upstream from culvert crossing & 170 & D-03-078_P6240010 \\
\hline D-03-078 & Looking downstream from culvert crossing & 0 & D-03-078_P6240011 \\
\hline D-03-079 & Looking upstream from culvert crossing & 270 & D-03-079_P6240012 \\
\hline D-03-079 & Looking downstream from culvert crossing & 90 & D-03-079_P6240013 \\
\hline D-03-080 & Looking upstream from culvert crossing & 100 & D-03-080_P6240014 \\
\hline $\mathrm{D}-03-080$ & Looking downstream from culvert crossing & 270 & D-03-080_P6240015 \\
\hline D-03-081 & Looking upstream from bridge crossing & 210 & D-03-081_P6240016 \\
\hline D-03-081 & Looking downstream from bridge crossing & 30 & D-03-081_P6240017 \\
\hline D-03-082 & Looking upstream from bridge crossing & 140 & D-03-082_P6240018 \\
\hline D-03-082 & Looking downstream from bridge crossing & 215 & D-03-082_P6240019 \\
\hline D-03-083 & Looking upstream from bridge crossing & 180 & D-03-083_P6260005 \\
\hline D-03-083 & Looking downstream from bridge crossing & 45 & D-03-083_P6260006 \\
\hline D-03-084 & Looking upstream from $10 \mathrm{~m}$ from top of reach & 200 & D-03-084_P6260001 \\
\hline D-03-084 & Looking downstream from silt fences & 25 & D-03-084_P6260002 \\
\hline D-03-084 & Looking west at silt fences & 270 & D-03-084_P6260003 \\
\hline D-03-084 & Looking downstream from mid-reach & 360 & D-03-084_P6260004 \\
\hline D-03-085 & Looking upstream from bridge crossing & 300 & D-03-085_P6270001 \\
\hline D-03-085 & Looking downstream from bridge crossing & 90 & D-03-085_P6270002 \\
\hline D-03-086 & Looking upstream from bridge crossing & 190 & D-03-086_P6270003 \\
\hline D-03-086 & Looking downstream from bridge crossing & 10 & D-03-086_P6270004 \\
\hline D-03-086 & Looking north at cows instream & 0 & D-03-086_P6270005 \\
\hline D-03-087 & Looking upstream from bridge crossing & 270 & D-03-087_P6270006 \\
\hline D-03-087 & Looking downstream from bridge crossing & 90 & D-03-087_P6270007 \\
\hline D-03-088 & Looking upstream from bridge crossing & 0 & D-03-088_P7020001 \\
\hline D-03-088 & Looking downstream from bridge crossing & 180 & D-03-088_P7020002 \\
\hline D-03-089 & Looking upstream from bridge crossing & 180 & D-03-089_P7020003 \\
\hline D-03-089 & Looking downstream from bridge crossing & 0 & D-03-089_P7020004 \\
\hline D-03-090 & Looking south at Pleasant Valley Lake water control structure & 180 & D-03-090_P7020005 \\
\hline $\mathrm{D}-03-090$ & Looking downstream from dam & 50 & D-03-090_P7020006 \\
\hline D-03-091 & Looking upstream from bridge crossing & 180 & D-03-091_P7030001 \\
\hline D-03-091 & Looking downstream from bridge crossing & 0 & D-03-091_P7030002 \\
\hline
\end{tabular}


Appendix 8: List of all photographs taken showing the corresponding site number, descriptive caption, direction faced when taken (azimuth in degrees) and the unique file name for each photograph.

\begin{tabular}{|c|c|c|c|}
\hline $\begin{array}{c}\text { Site } \\
\text { Number }\end{array}$ & Photo Description & Azimuth & File Name \\
\hline D-03-092 & Looking upstream from culvert crossing & 270 & D-03-092_P7030003 \\
\hline D-03-092 & Looking downstream from culvert crossing & 30 & D-03-092 P7030004 \\
\hline $\mathrm{D}-03-093$ & Looking upstream from culvert crossing & 0 & D-03-093_P7030005 \\
\hline D-03-093 & Looking downstream from culvert crossing & 135 & D-03-093_P7030006 \\
\hline D-03-094 & Looking upstream from culvert crossing & 260 & D-03-094_P7080001 \\
\hline D-03-094 & Looking downstream from culvert crossing & 80 & D-03-094_P7080002 \\
\hline D-03-094 & Looking north at beaver dam at upstream end of culvert & 0 & D-03-094_P7080003 \\
\hline D-03-095 & Looking upstream from culvert crossing & 255 & D-03-095_P7080004 \\
\hline D-03-095 & Looking downstream from culvert crossing & 100 & D-03-095_P7080005 \\
\hline D-03-096 & Looking upstream from culvert crossing & 280 & D-03-096_P7080006 \\
\hline D-03-096 & Looking downstream from culvert crossing & 125 & D-03-096_P7080007 \\
\hline D-03-097 & Looking upstream from ford crossing & 0 & D-03-097_P7090001 \\
\hline D-03-097 & Looking downstream from ford crossing & 200 & D-03-097_P7090002 \\
\hline D-03-097 & Looking southeast at upstream end of culverts & 150 & D-03-097_P7090003 \\
\hline D-03-097 & Looking northeast at downstream end of culverts & 45 & D-03-097_P7090004 \\
\hline $\mathrm{D}-03-098$ & Looking upstream from culvert crossing & 280 & D-03-098_P7090005 \\
\hline D-03-098 & Looking downstream from culvert crossing & 120 & D-03-098_P7090006 \\
\hline D-03-099 & Looking upstream from culvert crossing & 190 & D-03-099_P7090007 \\
\hline D-03-099 & Looking downstream from culvert crossing & 10 & D-03-099_P7090008 \\
\hline $\mathrm{D}-03-100$ & Looking upstream from culvert crossing & 240 & D-03-100_P7090009 \\
\hline D-03-100 & Looking downstream from culvert crossing & 45 & D-03-100_P7090010 \\
\hline D-03-101 & Looking upstream from culvert crossing & 50 & D-03-101_P7090011 \\
\hline D-03-101 & Looking downstream from culvert crossing & 180 & D-03-101_P7090012 \\
\hline $\mathrm{D}-03-101$ & Looking northeast at culvert perched $0.2 \mathrm{~m}$ & 45 & D-03-101_P7090013 \\
\hline $\mathrm{D}-03-102$ & Looking upstream from culvert crossing & 220 & D-03-102_P7110001 \\
\hline D-03-102 & Looking downstream from culvert crossing & 0 & D-03-102_P7110002 \\
\hline $\mathrm{D}-03-103$ & Looking upstream from culvert crossing & 260 & D-03-103_P7110003 \\
\hline $\mathrm{D}-03-103$ & Looking downstream from weir & 90 & D-03-103_P7110004 \\
\hline D-03-103 & Looking downstream from culvert crossing & 0 & D-03-103_P7110005 \\
\hline D-03-103 & Looking west of sheet pile weir and upstream & 270 & D-03-103_P7110006 \\
\hline $\mathrm{D}-03-103$ & Looking downstream (east) at upstream end of culvert and weir & 90 & D-03-103_P7110007 \\
\hline D-03-104 & Looking upstream from culvert crossing & 180 & D-03-104_P7110008 \\
\hline D-03-104 & Looking downstream from culvert crossing & 0 & D-03-104_P7110009 \\
\hline
\end{tabular}


Appendix 8: List of all photographs taken showing the corresponding site number, descriptive caption, direction faced when taken (azimuth in degrees) and the unique file name for each photograph.

\begin{tabular}{|c|c|c|c|}
\hline $\begin{array}{c}\text { Site } \\
\text { Number }\end{array}$ & Photo Description & Azimuth & File Name \\
\hline D-03-104 & Looking south at downstream end of culvert & 180 & D-03-104_P7110010 \\
\hline D-03-105 & Looking upstream from culvert crossing & 245 & D-03-105 P7110011 \\
\hline D-03-105 & Looking downstream from culvert crossing & 0 & D-03-105_P7110012 \\
\hline $\mathrm{D}-03-106$ & Looking upstream from bridge crossing & 345 & D-03-106_P7140001 \\
\hline D-03-106 & Looking downstream from bridge crossing & 150 & D-03-106_P7140002 \\
\hline D-03-107 & Looking upstream from bridge crossing & 180 & D-03-107_P7140003 \\
\hline D-03-107 & Looking downstream from bridge crossing & 310 & D-03-107_P7140004 \\
\hline D-03-108 & Looking upstream from bridge crossing & 0 & D-03-108_P7140005 \\
\hline D-03-108 & Looking downstream from bridge crossing & 160 & D-03-108_P7140006 \\
\hline D-03-109 & Looking upstream from culvert crossing & 270 & D-03-109_P7140007 \\
\hline D-03-109 & Looking downstream from culvert crossing & 90 & D-03-109_P7140008 \\
\hline D-03-110 & Looking upstream from culvert crossing & 270 & D-03-110_P7140009 \\
\hline D-03-110 & Looking downstream from culvert crossing & 60 & D-03-110_P7140010 \\
\hline D-03-110 & Looking upstream at downstream end of culverts & 300 & D-03-110_P7140011 \\
\hline D-03-111 & Looking upstream from culvert crossing & 0 & D-03-111_P7140012 \\
\hline D-03-111 & Looking downstream from culvert crossing & 180 & D-03-111_P7140013 \\
\hline $\mathrm{D}-03-112$ & Looking upstream from culvert crossing & 270 & D-03-112_P7150001 \\
\hline D-03-112 & Looking downstream from culvert crossing & 90 & D-03-112 P7150002 \\
\hline D-03-113 & Looking upstream from culvert crossing & 270 & D-03-113_P7150003 \\
\hline D-03-113 & Looking downstream from culvert crossing & 90 & D-03-113_P7150004 \\
\hline D-03-114 & Looking upstream from culvert crossing & 300 & D-03-114_P7150005 \\
\hline D-03-114 & Looking downstream from culvert crossing & 130 & D-03-114_P7150006 \\
\hline D-03-115 & Looking upstream from culvert crossing & 0 & D-03-115_P7150007 \\
\hline D-03-115 & Looking downstream from culvert crossing & 185 & D-03-115_P7150008 \\
\hline D-03-116 & Looking upstream from culvert crossing & 300 & D-03-116_P7150009 \\
\hline D-03-116 & Looking upstream from culvert crossing & 260 & D-03-116 P7150010 \\
\hline D-03-116 & Looking downstream from culvert crossing & 140 & D-03-116_P7150011 \\
\hline D-03-117 & Looking upstream from culvert crossing & 290 & D-03-117_P7150012 \\
\hline D-03-117 & Looking downstream from culvert crossing & 90 & D-03-117_P7150013 \\
\hline D-03-118 & Looking upstream from culvert crossing & 0 & D-03-118 P7150014 \\
\hline $\mathrm{D}-03-118$ & Looking downstream from culvert crossing & 260 & D-03-118_P7150015 \\
\hline D-03-119 & Looking upstream from culvert crossing & 270 & D-03-119_P7150016 \\
\hline D-03-119 & Looking downstream from culvert crossing & 90 & D-03-119_P7150017 \\
\hline
\end{tabular}


Appendix 8: List of all photographs taken showing the corresponding site number, descriptive caption, direction faced when taken (azimuth in degrees) and the unique file name for each photograph.

\begin{tabular}{|c|c|c|c|}
\hline $\begin{array}{c}\text { Site } \\
\text { Number }\end{array}$ & Photo Description & Azimuth & File Name \\
\hline D-03-120 & Looking upstream from bridge crossing & 270 & D-03-120_P7150018 \\
\hline $\mathrm{D}-03-120$ & Looking downstream from bridge crossing & 150 & D-03-120_P7150019 \\
\hline D-03-121 & Looking upstream from PTH 5 culvert crossing & 270 & D-03-121 P7160001 \\
\hline D-03-121 & Looking downstream from PTH 5 culvert crossing & 90 & D-03-121_P7160002 \\
\hline D-03-121 & Looking north at downstream end of perched culvert & 350 & D-03-121_P7160003 \\
\hline $\mathrm{D}-03-121$ & Looking west at sediment trap $200 \mathrm{~m}$ upstream from crossing & 270 & D-03-121_P7160004 \\
\hline D-03-121 & Looking downstream at outside bend of creek $200 \mathrm{~m}$ above crossing & 110 & D-03-121_P7160005 \\
\hline D-03-122 & Looking upstream from bridge crossing & 190 & D-03-122 P7160006 \\
\hline $\mathrm{D}-03-122$ & Looking downstream from bridge crossing & 20 & D-03-122_P7160007 \\
\hline D-03-123 & Looking upstream from culvert crossing & 180 & D-03-123_P7160008 \\
\hline D-03-123 & Looking downstream from culvert crossing & 0 & D-03-123_P7160009 \\
\hline D-03-124 & Looking upstream from culvert crossing & 180 & D-03-124_P7160010 \\
\hline D-03-124 & Looking downstream from culvert crossing & 0 & D-03-124_P7160011 \\
\hline D-03-125 & Looking upstream from bridge crossing & 350 & D-03-125_P7160012 \\
\hline $\mathrm{D}-03-125$ & Looking downstream from bridge crossing & 170 & D-03-125_P7160013 \\
\hline D-03-125 & Looking west up Glencairn Drain & 270 & D-03-125_P7160014 \\
\hline D-03-126 & Looking upstream from culvert crossing & 270 & D-03-126_P7170001 \\
\hline D-03-126 & Looking downstream from culvert crossing & 90 & D-03-126_P7170002 \\
\hline D-03-126 & Looking upstream at Kinch Creek & 180 & D-03-126_P7170003 \\
\hline D-03-127 & Looking upstream from bridge crossing & 270 & D-03-127_P7170004 \\
\hline D-03-127 & Looking downstream from bridge crossing & 90 & D-03-127_P7170005 \\
\hline D-03-128 & Looking upstream from culvert crossing & 0 & D-03-128_P7170006 \\
\hline D-03-128 & Looking downstream from culvert crossing & 180 & D-03-128 P7170007 \\
\hline $\mathrm{D}-03-129$ & Looking upstream from culvert crossing & 0 & D-03-129_P7170008 \\
\hline D-03-129 & Looking downstream from culvert crossing & 270 & D-03-129_P7170009 \\
\hline D-03-130 & Looking upstream from culvert crossing & 330 & D-03-130_P7170010 \\
\hline D-03-130 & Looking downstream from culvert crossing & 150 & D-03-130 P7170011 \\
\hline D-03-131 & Looking upstream from bridge crossing & 190 & D-03-131_P7170012 \\
\hline D-03-131 & Looking downstream from bridge crossing & 45 & D-03-131_P7170013 \\
\hline $\mathrm{D}-03-132$ & Looking upstream from culvert crossing & 270 & D-03-132_P7170014 \\
\hline $\mathrm{D}-03-132$ & Looking downstream from culvert crossing & 45 & D-03-132_P7170015 \\
\hline D-03-132 & Looking west at downstream end of culverts & 270 & D-03-132_P7170016 \\
\hline D-03-132 & Close up of dead fish accumulated at downstream end of culvert & $\mathrm{N} / \mathrm{A}$ & D-03-132_P7170017 \\
\hline
\end{tabular}


Appendix 8: List of all photographs taken showing the corresponding site number, descriptive caption, direction faced when taken (azimuth in degrees) and the unique file name for each photograph.

\begin{tabular}{|c|c|c|c|}
\hline $\begin{array}{c}\text { Site } \\
\text { Number }\end{array}$ & Photo Description & Azimuth & File Name \\
\hline D-03-133 & Looking upstream from bridge crossing & 0 & D-03-133_P7170018 \\
\hline D-03-133 & Looking downstream from bridge crossing & 180 & D-03-133 P7170019 \\
\hline D-03-133 & Looking west up Correction Line Drain & 270 & D-03-133_P7170020 \\
\hline $\mathrm{D}-03-134$ & Looking upstream from culvert crossing & 270 & D-03-134_P7170021 \\
\hline D-03-134 & Looking downstream from culvert crossing & 90 & D-03-134_P7170022 \\
\hline D-03-135 & Looking upstream from culvert crossing & 180 & D-03-135 P7180001 \\
\hline D-03-135 & Looking downstream from culvert crossing & 0 & D-03-135_P7180002 \\
\hline D-03-135 & Looking south at downstream end of culvert & 180 & D-03-135_P7180003 \\
\hline D-03-136 & Looking upstream from culvert crossing & 210 & D-03-136 P7180004 \\
\hline $\mathrm{D}-03-136$ & Looking downstream from culvert crossing & 90 & $\mathrm{D}-03-136 \mathrm{P} 7180005$ \\
\hline D-03-137 & Looking upstream from ford crossing & 270 & D-03-137_P7180006 \\
\hline $\mathrm{D}-03-137$ & Looking downstream from ford crossing & 125 & D-03-137_P7180007 \\
\hline D-03-138 & Looking upstream on Pelican Creek from culvert crossing & 180 & D-03-138 P7180008 \\
\hline D-03-138 & Looking downstream on Pelican Creek from culvert crossing & 90 & D-03-138 P7180009 \\
\hline D-03-138 & Looking upstream from above confluence with Pelican Ck. & 220 & D-03-138_P7180010 \\
\hline D-03-139 & Looking upstream from culvert crossing & 270 & D-03-139_P7180011 \\
\hline D-03-139 & Looking downstream from culvert crossing & 90 & D-03-139 P7180012 \\
\hline $\mathrm{D}-03-140$ & Looking upstream from bridge crossing & 270 & D-03-140 P7210001 \\
\hline D-03-140 & Looking downstream from bridge crossing & 90 & D-03-140_P7210002 \\
\hline $\mathrm{D}-03-141$ & Looking upstream from culvert crossing & 180 & D-03-141_P7220003 \\
\hline D-03-141 & Looking downstream from culvert crossing & 0 & D-03-141_P7220004 \\
\hline $\mathrm{D}-03-142$ & Looking upstream from gravel ford crossing & 290 & D-03-142_P7220005 \\
\hline D-03-142 & Looking downstream from gravel ford crossing & 90 & D-03-142_P7220006 \\
\hline D-03-143 & Looking upstream from bridge crossing & 270 & D-03-143 P7220007 \\
\hline D-03-143 & Looking downstream from bridge crossing & 90 & D-03-143 P7220008 \\
\hline D-03-144 & Looking upstream at wooden box culverts from $10 \mathrm{~m}$ downstream & 240 & D-03-144_P7220009 \\
\hline D-03-144 & Looking upstream from PTH 10 & 250 & D-03-144_P7220010 \\
\hline D-03-144 & Looking downstream from PTH 10 & 70 & D-03-144_P7220011 \\
\hline D-03-145 & Looking upstream from PTH 10 & 270 & $\mathrm{D}-03-145 \mathrm{P} 7220012$ \\
\hline D-03-145 & Looking downstream from PTH 10 & 100 & D-03-145 P7220013 \\
\hline $\mathrm{D}-03-146$ & Looking upstream from culvert crossing & 0 & D-03-146_P7220014 \\
\hline D-03-146 & Looking downstream from culvert crossing & 180 & D-03-146 P7220015 \\
\hline D-03-147 & Looking upstream from culvert crossing & 270 & D-03-147 P7230001 \\
\hline
\end{tabular}


Appendix 8: List of all photographs taken showing the corresponding site number, descriptive caption, direction faced when taken (azimuth in degrees) and the unique file name for each photograph.

\begin{tabular}{|c|c|c|c|}
\hline $\begin{array}{c}\text { Site } \\
\text { Number }\end{array}$ & Photo Description & Azimuth & File Name \\
\hline D-03-147 & Looking downstream from culvert crossing & 90 & D-03-147_P7230002 \\
\hline $\mathrm{D}-03-148$ & Looking upstream from culvert crossing & 0 & D-03-148_P7230003 \\
\hline D-03-148 & Looking downstream from culvert crossing & 180 & D-03-148 P7230004 \\
\hline D-03-149 & Looking upstream from culvert crossing & 310 & D-03-149_P7230005 \\
\hline D-03-149 & Looking downstream from culvert crossing & 120 & D-03-149_P7230006 \\
\hline $\mathrm{D}-03-150$ & Looking upstream from culvert crossing & 260 & D-03-150_P7230007 \\
\hline D-03-150 & Looking downstream from culvert crossing & 80 & D-03-150_P7230008 \\
\hline D-03-151 & Looking upstream from culvert crossing & 0 & D-03-151_P7230009 \\
\hline $\mathrm{D}-03-151$ & Looking downstream from culvert crossing & 180 & D-03-151_P7230010 \\
\hline $\mathrm{D}-03-152$ & Looking upstream from bridge crossing & 270 & D-03-152_P7230011 \\
\hline D-03-152 & Looking downstream from bridge crossing & 90 & D-03-152_P7230012 \\
\hline D-03-153 & Looking upstream from culvert crossing & 140 & D-03-153_P7230013 \\
\hline D-03-153 & Looking downstream from culvert crossing & 90 & D-03-153_P7230014 \\
\hline D-03-154 & Looking upstream from PTH 10 & 220 & D-03-154_P7230015 \\
\hline $\mathrm{D}-03-154$ & Looking downstream from PTH 10 & 30 & D-03-154_P7230016 \\
\hline D-03-155 & Looking upstream from bridge crossing & 250 & D-03-155_P7230017 \\
\hline D-03-155 & Looking downstream from bridge crossing & 70 & D-03-155_P7230018 \\
\hline D-03-156 & Looking upstream from PTH 10 & 280 & D-03-156_P7230019 \\
\hline D-03-156 & Looking downstream from PTH 10 & 90 & D-03-156_P7230020 \\
\hline D-03-157 & Looking upstream from bridge crossing & 260 & D-03-157_P7240001 \\
\hline D-03-157 & Looking downstream from bridge crossing & 80 & D-03-157_P7240002 \\
\hline D-03-158 & Looking upstream from PTH 10 & 250 & D-03-158_P7240003 \\
\hline D-03-158 & Looking downstream from PTH 10 & 30 & D-03-158 P7240004 \\
\hline D-03-159 & Looking upstream from culvert crossing & 0 & D-03-159_P7240005 \\
\hline D-03-159 & Looking west at flow from beaver pond & 300 & D-03-159_P7240006 \\
\hline D-03-159 & Looking downstream from culvert crossing & 180 & D-03-159_P7240007 \\
\hline D-03-160 & Looking upstream from culvert crossing & 270 & D-03-160_P7240008 \\
\hline D-03-160 & Looking downstream from culvert crossing & 90 & D-03-160_P7240009 \\
\hline D-03-161 & Looking upstream from culvert crossing & 270 & D-03-161_P7240010 \\
\hline D-03-161 & Looking downstream from culvert crossing & 90 & D-03-161_P7240011 \\
\hline $\mathrm{D}-03-162$ & Looking upstream from culvert crossing & 270 & D-03-162_P7240012 \\
\hline D-03-162 & Looking downstream from culvert crossing & 90 & D-03-162_P7240013 \\
\hline D-03-163 & Looking upstream from culvert crossing & 270 & D-03-163_P7240014 \\
\hline
\end{tabular}


Appendix 8: List of all photographs taken showing the corresponding site number, descriptive caption, direction faced when taken (azimuth in degrees) and the unique file name for each photograph.

\begin{tabular}{|c|c|c|c|}
\hline $\begin{array}{c}\text { Site } \\
\text { Number }\end{array}$ & Photo Description & Azimuth & File Name \\
\hline D-03-163 & Looking downstream from culvert crossing & 90 & D-03-163_P7240015 \\
\hline D-03-164 & Looking upstream from bridge crossing & 270 & D-03-164_P7240016 \\
\hline $\mathrm{D}-03-164$ & Looking downstream from bridge crossing & 90 & D-03-164_P7240017 \\
\hline D-03-165 & Looking upstream from PR 266 culvert crossing & 270 & D-03-165_P7240018 \\
\hline D-03-165 & Looking downstream from PR 266 culvert crossing & 90 & D-03-165_P7240019 \\
\hline D-03-166 & Looking upstream from culvert crossing & 270 & D-03-166_P7240020 \\
\hline D-03-166 & Looking downstream from culvert crossing & 90 & D-03-166_P7240021 \\
\hline D-03-166 & Looking west at perched culvert & $\mathrm{N} / \mathrm{A}$ & D-03-166_P7240022 \\
\hline D-03-167 & Looking upstream from PTH 10 & 240 & D-03-167_P7250001 \\
\hline D-03-167 & Looking downstream from PTH 10 & 100 & D-03-167_P7250002 \\
\hline W-03-001 & Electrofishing crew & 270 & W-03-001_D1_001 \\
\hline W-03-001 & Looking upstream 60 m north (downstream) from PR 317 & 90 & W-03-001_D1_002 \\
\hline W-03-001 & Looking downstream 60 m north from PR 317 & 270 & W-03-001_D1_003 \\
\hline W-03-002 & Looking upstream 20 m downstream from culvert & 270 & W-03-002_D1_021 \\
\hline W-03-002 & Looking upstream from culvert & 270 & W-03-002_D1_022 \\
\hline W-03-002 & Looking downstream from culvert & 90 & W-03-002_D1_023 \\
\hline W-03-002 & Looking downstream after bend & 360 & W-03-002_D1_024 \\
\hline W-03-003 & Looking upstream from gravel road at top of reach & 180 & W-03-003_D1_004 \\
\hline W-03-003 & Looking upstream (small tributary from west) & 270 & W-03-003_D1_005 \\
\hline W-03-003 & Looking upstream at culvert from mid-reach & 180 & W-03-003_D1_006 \\
\hline W-03-003 & Looking downstream from mid-reach at PTH 11 culverts & 90 & W-03-003_D1_007 \\
\hline W-03-003 & Looking downstream from PTH 11 & 90 & W-03-003_D1_008 \\
\hline W-03-003 & Looking downstream from PTH 11 & 90 & W-03-003_D1_009 \\
\hline W-03-003 & Looking downstream through reach & 360 & W-03-003_D1_010 \\
\hline W-03-004 & Looking upstream from culvert going west PTH 11 & 270 & W-03-004_D1_011 \\
\hline W-03-004 & Looking upstream towards other culvert & 270 & W-03-004_D1_012 \\
\hline W-03-004 & Looking downstream from culvert going east PTH 11 & 180 & W-03-004_D1_013 \\
\hline W-03-005 & Looking upstream from culvert & 210 & W-03-005_D1_014 \\
\hline W-03-005 & Looking downstream from culvert & 0 & W-03-005_D1_015 \\
\hline W-03-006 & Looking upstream from crossing & 150 & W-03-006_D1_016 \\
\hline W-03-006 & Looking downstream from crossing & 300 & W-03-006_D1_017 \\
\hline W-03-006 & Looking downstream at crossing & 270 & W-03-006_D1_018 \\
\hline W-03-007 & Looking upstream from PTH 11 & 220 & W-03-007_D1_019 \\
\hline
\end{tabular}


Appendix 8: List of all photographs taken showing the corresponding site number, descriptive caption, direction faced when taken (azimuth in degrees) and the unique file name for each photograph.

\begin{tabular}{|c|c|c|c|}
\hline $\begin{array}{c}\text { Site } \\
\text { Number }\end{array}$ & Photo Description & Azimuth & File Name \\
\hline W-03-007 & Looking downstream from PTH 11 & 330 & W-03-007_D1_020 \\
\hline W-03-008 & Looking upstream from culverts (west) & 270 & W-03-008_D1_025 \\
\hline W-03-008 & Looking downstream from culverts (east) & 90 & W-03-008_D1_026 \\
\hline W-03-009 & Looking upstream from crossing & 270 & W-03-009_D1_027 \\
\hline W-03-009 & Looking downstream from crossing & 150 & W-03-009_D1_028 \\
\hline W-03-009 & Looking upstream at culvert & 270 & W-03-009_D1_029 \\
\hline W-03-010 & Looking upstream from culvert & 270 & W-03-010_D1_030 \\
\hline W-03-010 & Looking downstream from culvert & 90 & W-03-010_D1_031 \\
\hline W-03-010 & Looking downstream from culvert & 90 & W-03-010_D1_032 \\
\hline W-03-011 & Looking upstream west of culvert & 270 & W-03-011_D1_033 \\
\hline W-03-011 & Looking downstream east of culvert & 90 & W-03-011_D1_034 \\
\hline W-03-012 & Looking upstream from gravel road & 360 & W-03-012_D1_035 \\
\hline W-03-013 & Looking upstream from culverts (east) & 90 & W-03-013_D1_036 \\
\hline W-03-013 & Looking downstream from culverts (west) & 270 & W-03-013_D1_037 \\
\hline W-03-013 & Looking downstream from culverts to bridge & 270 & W-03-013_D1_038 \\
\hline W-03-013 & Looking upstream at east side of perched culverts & 90 & W-03-013_D1_039 \\
\hline W-03-014 & Looking upstream from crossing & 360 & W-03-014_D1_040 \\
\hline W-03-014 & Looking downstream from crossing & 180 & W-03-014_D1_041 \\
\hline W-03-015 & Looking upstream from crossing & 320 & W-03-015_D1_042 \\
\hline W-03-015 & Looking downstream from crossing & 95 & W-03-015_D1_043 \\
\hline W-03-015 & Looking downstream from crossing & 95 & W-03-015_D1_044 \\
\hline W-03-016 & Looking upstream from crossing & 315 & W-03-016_D1_045 \\
\hline W-03-016 & Looking downstream from crossing & 100 & W-03-016_D1_046 \\
\hline W-03-016 & Looking downstream at culverts & 160 & W-03-016_D1_047 \\
\hline W-03-017 & Looking upstream from reach & 270 & W-03-017_D1_048 \\
\hline W-03-017 & Looking downstream from reach & 90 & W-03-017_D1_049 \\
\hline W-03-017 & Looking south at bend downstream & 180 & W-03-017_D1_050 \\
\hline W-03-017 & Looking south of road at Manitoba Hydro plant & 180 & W-03-017_D1_051 \\
\hline W-03-017 & Looking south of road at Manitoba Hydro plant & 180 & W-03-017_D1_052 \\
\hline W-03-017 & Looking south of road at Manitoba Hydro plant & 180 & W-03-017_D1_053 \\
\hline W-03-017 & Looking north of road at Manitoba Hydro plant & 360 & W-03-017_D1_054 \\
\hline W-03-017 & Looking north of road at Manitoba Hydro plant & 360 & W-03-017_D1_055 \\
\hline W-03-017 & Looking north of road at Manitoba Hydro plant & 360 & W-03-017_D1_056 \\
\hline
\end{tabular}


Appendix 8: List of all photographs taken showing the corresponding site number, descriptive caption, direction faced when taken (azimuth in degrees) and the unique file name for each photograph.

\begin{tabular}{|c|c|c|c|}
\hline $\begin{array}{c}\text { Site } \\
\text { Number }\end{array}$ & Photo Description & Azimuth & File Name \\
\hline W-03-018 & Looking upstream from culverts at reach & 360 & W-03-018_D1_057 \\
\hline W-03-018 & Looking downstream from culverts & 155 & W-03-018_D1_058 \\
\hline W-03-019 & Looking upstream from $20 \mathrm{~m}$ downstream of crossing & 0 & W-03-019_D1_059 \\
\hline W-03-019 & Looking upstream from crossing & 0 & W-03-019_D1_060 \\
\hline W-03-019 & Looking downstream from crossing & 180 & W-03-019_D1_061 \\
\hline $\mathrm{W}-03-020$ & Looking upstream from crossing & 315 & W-03-020_D1_062 \\
\hline W-03-020 & Looking downstream from crossing & 105 & W-03-020_D1_063 \\
\hline W-03-021 & Looking upstream at culverts & 270 & W-03-021_D1_064 \\
\hline W-03-021 & Looking downstream at culverts & 90 & W-03-021_D1_065 \\
\hline W-03-022 & Looking upstream at Sturgeon Creek & 315 & W-03-022_D1_066 \\
\hline W-03-022 & Looking downstream at Sturgeon Creek & 180 & W-03-022_D1_067 \\
\hline W-03-022 & Looking upstream at culverts from Old Sturgeon Creek & 30 & W-03-022 D1_068 \\
\hline W-03-022 & Looking upstream at Sturgeon Creek & 30 & W-03-022_D1_069 \\
\hline W-03-023 & Looking upstream at confluence of Sturgeon Creek and 2nd order drain & 270 & W-03-023_D1_070 \\
\hline W-03-023 & Looking upstream at gated culverts & 270 & W-03-023_D1_071 \\
\hline W-03-024 & Looking upstream at Meridian Drain & 0 & W-03-024_D1_072 \\
\hline W-03-025 & Looking upstream at 2nd order drain from crossing & 320 & W-03-025_D1_073 \\
\hline W-03-025 & Looking upstream at Meridian Drain & 360 & W-03-025_D1_074 \\
\hline W-03-025 & Looking downstream at Meridian Drain & 135 & W-03-025_D1_075 \\
\hline W-03-026 & Looking upstream from crossing & 315 & W-03-026_D1_076 \\
\hline W-03-026 & Looking downstream from crossing & 155 & W-03-026_D1_077 \\
\hline W-03-026 & Photo of Ashley \& Julie stuck in the gumbo & $\mathrm{N} / \mathrm{A}$ & W-03-026_D1_078 \\
\hline W-03-026 & Looking downstream $5 \mathrm{~m}$ east of crossing & 180 & W-03-026_D1_079 \\
\hline W-03-027 & Looking upstream from crossing & 270 & W-03-027_D1_080 \\
\hline W-03-027 & Looking downstream from crossing & 90 & W-03-027_D1_081 \\
\hline W-03-027 & Looking upstream at culverts $20 \mathrm{~m}$ back & 270 & W-03-027_D1_082 \\
\hline W-03-028 & Looking upstream from crossing & 315 & W-03-028 D1_083 \\
\hline $\mathrm{W}-03-028$ & Looking downstream from crossing & 140 & W-03-028_D1_084 \\
\hline W-03-028 & Looking at incoming drain & 270 & W-03-028_D1_085 \\
\hline W-03-029 & Looking upstream at junction of streams & 345 & W-03-029_D1_086 \\
\hline W-03-029 & Looking downstream at junction of 2nd order streams & 90 & W-03-029_D1_087 \\
\hline W-03-030 & Looking upstream from crossing & 0 & W-03-030_D1_088 \\
\hline W-03-030 & Looking downstream from crossing & 180 & W-03-030_D1_089 \\
\hline
\end{tabular}


Appendix 8: List of all photographs taken showing the corresponding site number, descriptive caption, direction faced when taken (azimuth in degrees) and the unique file name for each photograph.

\begin{tabular}{|c|c|c|c|}
\hline $\begin{array}{c}\text { Site } \\
\text { Number }\end{array}$ & Photo Description & Azimuth & File Name \\
\hline W-03-031 & Looking downstream from crossing & 140 & W-03-031_D1_090 \\
\hline W-03-031 & Looking upstream from crossing & 340 & W-03-031_D1_091 \\
\hline W-03-031 & Looking at incoming drain upstream from crossing & 360 & W-03-031_D1_092 \\
\hline W-03-031 & Looking downstream at junction of 2nd order and 3rd order drains & 180 & W-03-031_D1_093 \\
\hline W-03-032 & Looking upstream from crossing & 0 & W-03-032_D1_094 \\
\hline W-03-032 & Looking downstream from crossing & 180 & W-03-032_D1_095 \\
\hline W-03-033 & Looking upstream from crossing & 360 & W-03-033_D1_096 \\
\hline W-03-033 & Looking upstream from crossing & 360 & W-03-033 D1_097 \\
\hline W-03-033 & Looking downstream from crossing & 180 & W-03-033_D1_098 \\
\hline W-03-034 & Looking upstream from crossing & 90 & W-03-034_D1_099 \\
\hline W-03-034 & Looking downstream from crossing & 270 & W-03-034_D1_100 \\
\hline W-03-034 & Looking upstream at cement culverts & 90 & W-03-034_D1_101 \\
\hline W-03-034 & Looking downstream from PTH 6 & 270 & W-03-034_D1_102 \\
\hline W-03-035 & Looking upstream at culverts from crossing & 0 & W-03-035_D1_103 \\
\hline W-03-035 & Looking downstream from crossing & 180 & W-03-035_D1_104 \\
\hline W-03-036 & Looking upstream from crossing & 270 & W-03-036_D1_105 \\
\hline W-03-036 & Looking downstream from crossing & 90 & W-03-036_D1_106 \\
\hline W-03-037 & Looking upstream from crossing & 180 & W-03-037_D1_107 \\
\hline W-03-037 & Looking downstream from crossing & 30 & W-03-037_D1_108 \\
\hline W-03-038 & Looking upstream from crossing & 170 & W-03-038D1_109 \\
\hline W-03-038 & Looking downstream from crossing & 350 & W-03-038_D1_110 \\
\hline W-03-039 & Looking upstream from crossing & 270 & W-03-039_D1_111 \\
\hline W-03-039 & Looking downstream from crossing & 90 & W-03-039_D1_112 \\
\hline W-03-040 & Looking upstream from crossing & 90 & W-03-040_D1_113 \\
\hline W-03-040 & Looking downstream from crossing & 270 & W-03-040_D1_114 \\
\hline W-03-041 & Looking upstream from crossing & 360 & W-03-041_D1_115 \\
\hline W-03-041 & Looking downstream from crossing & 180 & W-03-041 D1 116 \\
\hline W-03-042 & Looking upstream from crossing & 330 & W-03-042_D1_117 \\
\hline W-03-042 & Looking downstream from crossing & 165 & W-03-042_D1_118 \\
\hline W-03-043 & Looking upstream from crossing & 360 & W-03-043_D1_119 \\
\hline W-03-043 & Looking downstream from crossing & 180 & W-03-043 D1_120 \\
\hline W-03-044 & Looking upstream from PR 245 & 120 & W-03-044_D1_121 \\
\hline W-03-044 & Looking downstream from top of culvert & 350 & W-03-044 D1_122 \\
\hline
\end{tabular}


Appendix 8: List of all photographs taken showing the corresponding site number, descriptive caption, direction faced when taken (azimuth in degrees) and the unique file name for each photograph.

\begin{tabular}{|c|c|c|c|}
\hline $\begin{array}{c}\text { Site } \\
\text { Number }\end{array}$ & Photo Description & Azimuth & File Name \\
\hline W-03-044 & Looking further downstream from top of culvert & 350 & W-03-044_D1_123 \\
\hline W-03-044 & Looking upstream $10 \mathrm{~m}$ south of downstream culvert & 120 & W-03-044_D1_124 \\
\hline W-03-045 & Looking upstream from crossing & 0 & W-03-045 D1 125 \\
\hline W-03-045 & Looking upstream $10 \mathrm{~m}$ back from culvert & 270 & W-03-045_D1_126 \\
\hline W-03-045 & Looking downstream at culvert & 90 & W-03-045_D1_127 \\
\hline W-03-046 & Looking upstream from PR 240 & 45 & W-03-046_D1_128 \\
\hline W-03-046 & Looking downstream from PR 240 & 180 & W-03-046_D1_129 \\
\hline W-03-046 & Looking further downstream from PR 240 & 180 & W-03-046 D1 130 \\
\hline W-03-047 & Looking upstream from crossing & 0 & W-03-047_D1_131 \\
\hline W-03-047 & Looking downstream from culverts & 120 & W-03-047_D1_132 \\
\hline W-03-048 & Looking upstream from crossing & 0 & W-03-048_D1_133 \\
\hline W-03-048 & Looking downstream from crossing & 90 & W-03-048 D1_134 \\
\hline W-03-049 & Looking upstream at Roseisle Drain $50 \mathrm{~m}$ south of Roseisle Creek & 180 & W-03-049_D1_135 \\
\hline W-03-049 & Looking downstream at Roseisle Drain $50 \mathrm{~m}$ south of Roseisle Creek & 0 & W-03-049_D1_136 \\
\hline W-03-049 & Looking upstream $100 \mathrm{~m}$ from creek at weir & 180 & W-03-049_D1_137 \\
\hline W-03-049 & Looking downstream at Roseisle Creek & 50 & W-03-049_D1_138 \\
\hline W-03-049 & Looking upstream at Roseisle Creek & 300 & W-03-049_D1_139 \\
\hline W-03-050 & Looking upstream at bridge & 270 & W-03-050_D1_140 \\
\hline W-03-050 & Looking downstream at bridge & 90 & W-03-050_D1_141 \\
\hline W-03-050 & Looking upstream 300m downstream from bridge & 270 & W-03-050_D1_142 \\
\hline W-03-051 & Looking upstream of 2nd order drain & 220 & W-03-051_D1_143 \\
\hline W-03-051 & Looking downstream at confluence of 2 nd order with Boyne River & 80 & W-03-051_D1_144 \\
\hline W-03-051 & Looking upstream Boyne River $20 \mathrm{~m}$ downstream of junction & 320 & W-03-051_D1_145 \\
\hline W-03-051 & Looking downstream Boyne River upstream from culvert at junction & 100 & W-03-051_D1_146 \\
\hline W-03-052 & Looking upstream from PR 240 & 270 & W-03-052_D1_147 \\
\hline W-03-052 & Looking downstream from PR 240 & 90 & W-03-052_D1_148 \\
\hline W-03-053 & Looking upstream from crossing & 270 & W-03-053 D1 149 \\
\hline W-03-053 & Looking downstream from crossing & 90 & W-03-053_D1_150 \\
\hline W-03-054 & Looking upstream from crossing & 270 & W-03-054_D1_151 \\
\hline W-03-054 & Looking upstream from crossing & 270 & W-03-054_D1_152 \\
\hline W-03-054 & Looking upstream from crossing & 270 & W-03-054_D1_153 \\
\hline W-03-054 & Looking downstream from crossing & 90 & W-03-054_D1_154 \\
\hline W-03-054 & Looking downstream from crossing & 90 & W-03-054_D1_155 \\
\hline
\end{tabular}


Appendix 8: List of all photographs taken showing the corresponding site number, descriptive caption, direction faced when taken (azimuth in degrees) and the unique file name for each photograph.

\begin{tabular}{|c|c|c|c|}
\hline $\begin{array}{c}\text { Site } \\
\text { Number }\end{array}$ & Photo Description & Azimuth & File Name \\
\hline W-03-054 & Looking downstream from crossing & 105 & W-03-054_D1_156 \\
\hline W-03-055 & Looking upstream from crossing & 270 & W-03-055_D1_157 \\
\hline W-03-055 & Looking downstream from crossing & 90 & W-03-055 D1 158 \\
\hline W-03-056 & Looking upstream $2 \mathrm{~m}$ south of culvert from crossing & 345 & W-03-056_D1_159 \\
\hline W-03-056 & Looking downstream $2 \mathrm{~m}$ north of culvert from crossing & 155 & W-03-056_D1_160 \\
\hline W-03-057 & Looking upstream from crossing & 270 & W-03-057_D1_161 \\
\hline W-03-057 & Looking upstream $20 \mathrm{~m}$ south of culverts & 270 & W-03-057_D1_162 \\
\hline W-03-057 & Looking downstream from crossing & 90 & W-03-057D1_163 \\
\hline W-03-057 & Looking at dredged matter & 120 & W-03-057_D1_164 \\
\hline W-03-058 & Looking upstream from crossing & 180 & W-03-058_D1_165 \\
\hline W-03-058 & Looking downstream from crossing & 0 & W-03-058_D1_166 \\
\hline W-03-059 & Looking upstream from crossing & 210 & W-03-059 D1_167 \\
\hline W-03-059 & Looking downstream from crossing & 0 & W-03-059_D1_168 \\
\hline W-03-059 & Looking at dead wood & 180 & W-03-059_D1_169 \\
\hline W-03-060 & Looking upstream from crossing & 190 & W-03-060_D1_170 \\
\hline W-03-060 & Looking downstream from crossing & 45 & W-03-060_D1_171 \\
\hline W-03-061 & Looking downstream from culvert (backwards from regular order) & 0 & W-03-061_D1_172 \\
\hline W-03-061 & Looking downstream from PTH 2 & 0 & W-03-061_D1_173 \\
\hline W-03-061 & Looking upstream from culvert & 100 & W-03-061_D1_174 \\
\hline W-03-061 & Looking upstream from PTH 2 & 180 & W-03-061_D1_175 \\
\hline W-03-062 & Looking downstream from PTH 2 & 0 & W-03-062_D1_176 \\
\hline W-03-062 & Looking upstream from PTH 2, $20 \mathrm{~m}$ back from culvert & 180 & W-03-062_D1_177 \\
\hline W-03-062 & Looking downstream from culvert & 0 & W-03-062 D1 178 \\
\hline W-03-063 & Looking upstream from culvert & 180 & W-03-063_D1_179 \\
\hline W-03-063 & Looking downstream from culvert & 0 & W-03-063_D1_180 \\
\hline W-03-064 & Looking upstream from crossing & 180 & W-03-064_D1_181 \\
\hline W-03-064 & Looking downstream from crossing & 0 & W-03-064 D1 182 \\
\hline W-03-064 & Looking downstream from crossing at woody debris & 43 & W-03-064_D1_183 \\
\hline W-03-065 & Looking upstream from middle of culvert & 230 & W-03-065_D1_184 \\
\hline W-03-065 & Looking upstream between culverts & 190 & W-03-065_D1_185 \\
\hline W-03-065 & Looking upstream from $5 \mathrm{~m}$ back of east culvert & 230 & W-03-065 D1_186 \\
\hline W-03-065 & Looking downstream from east culvert & 20 & W-03-065_D1_187 \\
\hline W-03-065 & Looking downstream from west culvert & 70 & W-03-065 D1_188 \\
\hline
\end{tabular}


Appendix 8: List of all photographs taken showing the corresponding site number, descriptive caption, direction faced when taken (azimuth in degrees) and the unique file name for each photograph.

\begin{tabular}{|c|c|c|c|}
\hline $\begin{array}{c}\text { Site } \\
\text { Number }\end{array}$ & Photo Description & Azimuth & File Name \\
\hline W-03-065 & Photo of scenery along PR 242 & 270 & W-03-065_D1_189 \\
\hline W-03-066 & Looking upstream $5 \mathrm{~m}$ west of upstream culvert & 110 & W-03-066_D1_190 \\
\hline W-03-066 & Looking upstream from top of culvert at culvert & 110 & W-03-066_D1_191 \\
\hline W-03-066 & Looking at culvert 10m upstream & 300 & W-03-066_D1_192 \\
\hline W-03-066 & Looking downstream from culverts & 110 & W-03-066_D1_193 \\
\hline W-03-066 & Photo of scenic landscape & $\mathrm{N} / \mathrm{A}$ & W-03-066_D1_194 \\
\hline W-03-066 & Photo of scenic landscape & $\mathrm{N} / \mathrm{A}$ & W-03-066_D1_195 \\
\hline W-03-066 & Photo of scenic landscape & $\mathrm{N} / \mathrm{A}$ & W-03-066_D1_196 \\
\hline W-03-066 & Photo of scenic landscape & $\mathrm{N} / \mathrm{A}$ & W-03-066_D1_197 \\
\hline W-03-066 & Photo of scenic landscape & $\mathrm{N} / \mathrm{A}$ & W-03-066_D1_198 \\
\hline W-03-066 & Photo of scenic landscape & $\mathrm{N} / \mathrm{A}$ & W-03-066_D1_199 \\
\hline W-03-067 & Looking upstream from crossing & 180 & W-03-067_D1_200 \\
\hline W-03-067 & Looking downstream from crossing at fisheries officers & 345 & W-03-067_D1_201 \\
\hline W-03-067 & Looking downstream from cement crossing & 0 & W-03-067_D1_202 \\
\hline W-03-068 & Looking upstream from PTH 23 & 180 & W-03-068_D1_203 \\
\hline W-03-068 & Looking downstream from PTH 23 & 0 & W-03-068_D1_204 \\
\hline W-03-071 & Looking upstream from crossing at culverts \& PTH 12 & 145 & W-03-071_D1_205 \\
\hline W-03-071 & Looking downstream from crossing & 250 & W-03-071_D1_206 \\
\hline W-03-072 & Looking upstream at 2nd order from crossing & 0 & W-03-072_D1_207 \\
\hline W-03-072 & Looking upstream Fish Creek from bridge & 90 & W-03-072_D1_208 \\
\hline W-03-072 & Looking downstream Fish Creek from bridge & 270 & W-03-072_D1_209 \\
\hline W-03-073 & Looking upstream Seine River from crossing & 110 & W-03-073_D1_210 \\
\hline W-03-073 & Looking downstream Seine River from crossing & 270 & W-03-073_D1_211 \\
\hline W-03-074 & Looking upstream from crossing & 135 & W-03-074_D1_212 \\
\hline W-03-074 & Looking downstream from crossing & 300 & W-03-074_D1_213 \\
\hline W-03-075 & Looking upstream from culvert & 140 & W-03-075_D1_214 \\
\hline W-03-075 & Looking downstream from culvert & 0 & W-03-075_D1_215 \\
\hline W-03-076 & Looking upstream from crossing & 80 & W-03-076_D1_216 \\
\hline W-03-076 & Looking downstream from crossing & 270 & W-03-076_D1_217 \\
\hline W-03-076 & Looking downstream 10m upstream from culverts & 270 & W-03-076_D1_218 \\
\hline W-03-077 & Looking upstream from bridge & 90 & W-03-077_D1_219 \\
\hline W-03-077 & Looking downstream from bridge & 270 & W-03-077_D1_220 \\
\hline W-03-077 & Looking downstream from bridge & 270 & W-03-077_D1_221 \\
\hline
\end{tabular}


Appendix 8: List of all photographs taken showing the corresponding site number, descriptive caption, direction faced when taken (azimuth in degrees) and the unique file name for each photograph.

\begin{tabular}{|c|c|c|c|}
\hline $\begin{array}{c}\text { Site } \\
\text { Number }\end{array}$ & Photo Description & Azimuth & File Name \\
\hline W-03-078 & Looking upstream from bridge & 90 & W-03-078_D1_222 \\
\hline W-03-078 & Looking downstream from bridge & 270 & W-03-078_D1_223 \\
\hline W-03-079 & Looking upstream from bridge & 140 & W-03-079 D1_224 \\
\hline W-03-079 & Looking downstream from bridge & 360 & W-03-079_D1_225 \\
\hline W-03-080 & Looking upstream from Pine Drive crossing & 180 & W-03-080_D1_226 \\
\hline W-03-080 & Looking downstream from culvert from PR 210 & 90 & W-03-080_D1_227 \\
\hline W-03-081 & Looking upstream from crossing & 90 & W-03-081_D1_228 \\
\hline W-03-081 & Looking downstream from crossing & 260 & W-03-081_D1_229 \\
\hline W-03-082 & Looking upstream from bridge & 90 & W-03-082_D1_230 \\
\hline W-03-082 & Looking south at gated culvert from bridge & 180 & W-03-082_D1_231 \\
\hline W-03-082 & Looking downstream from bridge & 290 & W-03-082 D1_232 \\
\hline W-03-083 & Looking upstream from crossing & 90 & W-03-083 D1_233 \\
\hline W-03-083 & Looking downstream from crossing & 270 & W-03-083_D1_234 \\
\hline W-03-084 & Looking upstream from culverts & 160 & W-03-084_D1_235 \\
\hline W-03-084 & Looking downstream from culvers & 270 & W-03-084 D1_236 \\
\hline W-03-085 & Looking upstream from dike of unnamed 3rd order & 180 & W-03-085_D1_237 \\
\hline W-03-085 & Looking upstream from bridge of D-20 Drain & 90 & W-03-085_D1_238 \\
\hline W-03-085 & Looking downstream from bridge of D-20 Drain & 270 & W-03-085_D1_239 \\
\hline W-03-086 & Looking upstream from road of unnamed 3rd order drain & 180 & W-03-086 D1_240 \\
\hline W-03-086 & Looking upstream of D-20 Drain & 90 & W-03-086_D1_241 \\
\hline W-03-086 & Looking downstream of D-20 Drain & 270 & W-03-086_D1_242 \\
\hline W-03-087 & Looking upstream from crossing & 180 & W-03-087_D1_243 \\
\hline W-03-087 & Looking downstream from crossing & 360 & W-03-087 D1_244 \\
\hline W-03-088 & Looking upstream from PR 205 & 125 & W-03-088_D1_245 \\
\hline W-03-088 & Looking at pool & 310 & W-03-088_D1_246 \\
\hline W-03-088 & Looking downstream from PR 205 & 360 & W-03-088 D1 247 \\
\hline W-03-089 & Looking upstream Carey Drain from crossing & 120 & W-03-089 D1_248 \\
\hline W-03-089 & Looking upstream unnamed 2nd order drain from crossing & 90 & W-03-089_D1_249 \\
\hline W-03-089 & Looking downstream Carey Drain from crossing & 0 & W-03-089_D1_250 \\
\hline W-03-090 & Looking upstream Otterburne East Drain from PTH 59 & 95 & W-03-090 D1 251 \\
\hline W-03-090 & Looking upstream unnamed 2nd order drain from Gagnon Road & 0 & W-03-090 D1_252 \\
\hline W-03-090 & Looking downstream Otterburne East Drain from PTH 59 & 270 & W-03-090_D1_253 \\
\hline W-03-091 & Looking upstream from crossing & 155 & W-03-091_D1_254 \\
\hline
\end{tabular}


Appendix 8: List of all photographs taken showing the corresponding site number, descriptive caption, direction faced when taken (azimuth in degrees) and the unique file name for each photograph.

\begin{tabular}{|c|c|c|c|}
\hline $\begin{array}{c}\text { Site } \\
\text { Number }\end{array}$ & Photo Description & Azimuth & File Name \\
\hline W-03-091 & Looking downstream from crossing & 300 & W-03-091_D1_255 \\
\hline W-03-092 & Looking upstream from crossing & 75 & W-03-092 D1_256 \\
\hline W-03-092 & Looking downstream from crossing & 300 & W-03-092_D1_257 \\
\hline W-03-092 & Looking downstream from culverts $10 \mathrm{~m}$ upstream & 300 & W-03-092_D1_258 \\
\hline W-03-093 & Looking upstream from PTH 12 & 90 & W-03-093_D1_259 \\
\hline W-03-093 & Looking downstream from PTH 12 & 270 & W-03-093_D1_260 \\
\hline W-03-094 & Looking upstream from crossing & 270 & W-03-094_D1_261 \\
\hline W-03-094 & Looking downstream from crossing & 340 & W-03-094_D1_262 \\
\hline W-03-095 & Looking upstream from PTH 44 & 180 & W-03-095_D1_263 \\
\hline W-03-095 & Looking downstream from PTH 44 & 360 & W-03-095 D1_264 \\
\hline W-03-096 & Looking upstream from crossing & 270 & W-03-096_D1_265 \\
\hline W-03-096 & Looking downstream from crossing & 90 & W-03-096_D1_266 \\
\hline W-03-097 & Looking upstream from PTH 44 & 10 & W-03-097_D1_267 \\
\hline W-03-097 & Looking downstream from PTH 44 & 200 & W-03-097_D1_268 \\
\hline W-03-098 & Looking upstream from PTH 44 & 360 & W-03-098_D1_269 \\
\hline W-03-098 & Looking downstream from PTH 44 & 180 & W-03-098_D1_270 \\
\hline W-03-099 & Looking upstream from PTH 15 & 180 & W-03-099_D1_271 \\
\hline W-03-099 & Looking downstream from PTH 15 & 45 & W-03-099 D1_272 \\
\hline W-03-100 & Looking upstream from crossing & 110 & W-03-100_D1_273 \\
\hline W-03-100 & Looking downstream from crossing & 300 & W-03-100_D1_274 \\
\hline W-03-101 & Looking upstream from PTH 11 & 130 & W-03-101_D2_001 \\
\hline W-03-101 & Looking downstream from PTH 11 & 250 & W-03-101_D2_002 \\
\hline W-03-102 & Looking upstream from PTH 11 & 90 & W-03-102_D2_003 \\
\hline W-03-102 & Looking downstream from PTH 11 & 270 & W-03-102_D2_004 \\
\hline W-03-103 & Looking upstream from PR 406 & 330 & W-03-103_D2_005 \\
\hline W-03-103 & Looking downstream from PR 406 & 140 & W-03-103 D2 006 \\
\hline W-03-103 & Looking downstream at lip of culvert (photo sideways) & 140 & W-03-103_D2_007 \\
\hline W-03-104 & Looking upstream from crossing & 225 & W-03-104_D2_008 \\
\hline W-03-104 & Looking downstream from crossing & 35 & W-03-104_D2_009 \\
\hline W-03-105 & Looking upstream from Kellner Creek & 280 & W-03-105 D2 010 \\
\hline W-03-105 & Looking downstream from Kellner Creek & 40 & W-03-105_D2_011 \\
\hline W-03-106 & Looking upstream from PR 406 & 270 & W-03-106_D2_012 \\
\hline W-03-106 & Looking downstream from PR 406 & 90 & W-03-106_D2_013 \\
\hline
\end{tabular}


Appendix 8: List of all photographs taken showing the corresponding site number, descriptive caption, direction faced when taken (azimuth in degrees) and the unique file name for each photograph.

\begin{tabular}{|c|c|c|c|}
\hline $\begin{array}{c}\text { Site } \\
\text { Number }\end{array}$ & Photo Description & Azimuth & File Name \\
\hline W-03-107 & Looking upstream from box culvert & 190 & W-03-107_D2_014 \\
\hline W-03-107 & Looking downstream from box culvert & 20 & W-03-107_D2_015 \\
\hline W-03-107 & Looking downstream from box culvert at bank scour & 350 & W-03-107 D2 016 \\
\hline W-03-108 & Looking upstream from PR 408 & 90 & W-03-108_D2_017 \\
\hline W-03-108 & Looking downstream from PR 408 & 270 & W-03-108_D2_018 \\
\hline W-03-109 & Looking upstream from PR 408 & 90 & W-03-109_D2_019 \\
\hline W-03-109 & Looking downstream from PR 408 & 270 & W-03-109_D2_020 \\
\hline W-03-110 & Looking upstream Smith Creek & 100 & $\mathrm{~W}-03-110 \mathrm{D} 2021$ \\
\hline W-03-110 & Looking downstream Smith Creek & 340 & W-03-110_D2_022 \\
\hline W-03-111 & Looking upstream from $100 \mathrm{~m}$ northeast of PR 408 & 180 & W-03-111_D2_023 \\
\hline W-03-111 & Looking downstream from $100 \mathrm{~m}$ northeast of PR 408 & 0 & W-03-111 D2 024 \\
\hline W-03-112 & Looking upstream from PTH 11 & 230 & W-03-112 D2 025 \\
\hline W-03-112 & Looking downstream from PTH 11 & 30 & W-03-112_D2_026 \\
\hline W-03-112 & Looking downstream from PTH 11, 20 m upstream at culverts & 245 & W-03-112_D2_027 \\
\hline W-03-113 & Looking upstream from crossing & 180 & W-03-113_D2_028 \\
\hline W-03-113 & Looking downstream from crossing & 0 & W-03-113_D2_029 \\
\hline W-03-114 & Looking upstream from PR 218 & 150 & W-03-114_D2_030 \\
\hline W-03-114 & Looking downstream from PR 218 & 300 & W-03-114_D2_031 \\
\hline W-03-114 & Looking at dog kennels left bank upstream from PR 218 & 120 & W-03-114 D2 032 \\
\hline W-03-115 & Looking upstream from PTH 59 & 120 & W-03-115 D2_033 \\
\hline W-03-115 & Looking downstream from PTH 59 & 350 & W-03-115_D2_034 \\
\hline W-03-116 & Looking upstream from bridge & 90 & W-03-116_D2_035 \\
\hline W-03-116 & Looking downstream from bridge & 270 & W-03-116 D2 036 \\
\hline W-03-117 & Looking upstream from PR 216 & 160 & W-03-117_D2_037 \\
\hline W-03-117 & Looking downstream from PR 216 & 270 & W-03-117_D2_038 \\
\hline W-03-118 & Looking upstream from crossing & 90 & W-03-118_D2_041 \\
\hline W-03-118 & Looking downstream from crossing & 270 & W-03-118 D2 042 \\
\hline W-03-118 & Looking downstream from crossing & 270 & W-03-118_D2_043 \\
\hline W-03-119 & Looking upstream from PR 303 & 180 & W-03-119_D2_044 \\
\hline W-03-119 & Looking downstream from PR 303 & 0 & W-03-119_D2_045 \\
\hline W-03-120 & Looking at culvert $1 \mathrm{~m}$ upstream from culvert & 180 & W-03-120 D2 046 \\
\hline W-03-120 & Looking upstream from PR 517 & 0 & W-03-120_D2_047 \\
\hline W-03-120 & Looking downstream from PR 517 & 180 & W-03-120_D2_048 \\
\hline
\end{tabular}


Appendix 8: List of all photographs taken showing the corresponding site number, descriptive caption, direction faced when taken (azimuth in degrees) and the unique file name for each photograph.

\begin{tabular}{|c|c|c|c|}
\hline $\begin{array}{c}\text { Site } \\
\text { Number }\end{array}$ & Photo Description & Azimuth & File Name \\
\hline W-03-121 & Looking upstream from PR 323 & 0 & W-03-121_D2_049 \\
\hline W-03-121 & Looking downstream from PR 323 & 180 & W-03-121_D2_050 \\
\hline W-03-122 & Looking upstream 2nd order from road 3E & 270 & W-03-122_D2_051 \\
\hline W-03-122 & Looking upstream 3rd order from PR 323 & 0 & W-03-122_D2_052 \\
\hline W-03-122 & Looking downstream 3rd order from PR 323 & 180 & W-03-122_D2_053 \\
\hline W-03-123 & Looking upstream from PR 322 & 0 & W-03-123_D2_054 \\
\hline W-03-123 & Looking downstream from PR 322 & 180 & W-03-123_D2_055 \\
\hline W-03-124 & Looking upstream from PR 323 & 0 & W-03-124_D2_056 \\
\hline W-03-124 & Looking downstream from PR 323 & 180 & W-03-124_D2_057 \\
\hline W-03-125 & Looking upstream from PR 323 & 0 & W-03-125_D2_058 \\
\hline W-03-125 & Looking downstream from PR 323 & 80 & W-03-125_D2_059 \\
\hline W-03-126 & Looking upstream from PTH 67 & 0 & W-03-126_D2_060 \\
\hline W-03-126 & Looking downstream from PTH 67 & 180 & W-03-126_D2_061 \\
\hline W-03-127 & Looking upstream from PTH 67 & 320 & W-03-127_D2_062 \\
\hline W-03-127 & Looking downstream from PTH 67 & 120 & W-03-127_D2_063 \\
\hline W-03-128 & Looking upstream from PTH 67 & 0 & W-03-128_D2_064 \\
\hline W-03-128 & Looking downstream from PTH 67 & 180 & W-03-128_D2_065 \\
\hline W-03-129 & Looking upstream from PTH 67 & 0 & W-03-129_D2_066 \\
\hline W-03-129 & Looking downstream from PTH 67 & 180 & W-03-129_D2_067 \\
\hline W-03-129 & Looking upstream at gated culvert from Grassmere Drain & 270 & W-03-129_D2_068 \\
\hline W-03-130 & Looking upstream from PR 236 & 270 & W-03-130_D2_069 \\
\hline W-03-130 & Looking downstream from PR 236 & 90 & W-03-130_D2_070 \\
\hline W-03-131 & Looking upstream from PR 321 & 0 & W-03-131_D2_071 \\
\hline W-03-131 & Looking downstream from PR 321 & 180 & W-03-131_D2_072 \\
\hline W-03-132 & Looking upstream from PTH 7 & 270 & W-03-132_D2_073 \\
\hline W-03-132 & Looking downstream from PTH 7 & 90 & W-03-132_D2_074 \\
\hline W-03-133 & Looking upstream from crossing & 270 & W-03-133_D2_075 \\
\hline W-03-133 & Looking downstream from crossing & 180 & W-03-133_D2_076 \\
\hline W-03-134 & Looking upstream from junction & 310 & W-03-134_D2_077 \\
\hline W-03-134 & Looking downstream from junction & 110 & W-03-134_D2_078 \\
\hline W-03-134 & Looking directly at Grassmere Creek from junction & 180 & W-03-134_D2_079 \\
\hline W-03-135 & Looking upstream from Toshack Road & 200 & W-03-135_D2_080 \\
\hline W-03-135 & Looking downstream from Toshack Road & 20 & W-03-135_D2_081 \\
\hline
\end{tabular}


Appendix 8: List of all photographs taken showing the corresponding site number, descriptive caption, direction faced when taken (azimuth in degrees) and the unique file name for each photograph.

\begin{tabular}{|c|c|c|c|}
\hline $\begin{array}{c}\text { Site } \\
\text { Number }\end{array}$ & Photo Description & Azimuth & File Name \\
\hline W-03-136 & Looking upstream from crossing & 270 & W-03-136_D2_082 \\
\hline W-03-136 & Looking downstream from crossing & 90 & W-03-136 D2 083 \\
\hline W-03-137 & Looking upstream from PR 230 & 270 & W-03-137_D2_084 \\
\hline W-03-137 & Looking downstream from PR 230 & 90 & W-03-137_D2_085 \\
\hline W-03-138 & Looking upstream from PR 230 & 270 & W-03-138_D2_086 \\
\hline W-03-138 & Looking downstream from PR 230 & 90 & W-03-138 D2 087 \\
\hline W-03-139 & Looking upstream from PTH 44 & 205 & W-03-139_D2_088 \\
\hline W-03-139 & Looking downstream from PTH 44 & 30 & W-03-139_D2_089 \\
\hline W-03-139 & Looking at wooden bridge $10 \mathrm{~m}$ downstream & 170 & W-03-139 D2 090 \\
\hline W-03-140 & Looking upstream from PTH 8 & 90 & W-03-140 D2 091 \\
\hline W-03-140 & Looking downstream from PTH 8 & 270 & W-03-140_D2_092 \\
\hline W-03-141 & Looking upstream from PTH 8 & 270 & W-03-141_D2_093 \\
\hline W-03-141 & Looking downstream from PTH 8 & 90 & W-03-141 D2 094 \\
\hline W-03-142 & Looking upstream from PTH 8 & 270 & W-03-142 D2 095 \\
\hline W-03-142 & Looking downstream from PTH 8 & 90 & W-03-142_D2_096 \\
\hline W-03-143 & Looking upstream from PTH 44 & 180 & W-03-143_D2_097 \\
\hline W-03-143 & Looking downstream from PTH 44 & 0 & W-03-143 D2 098 \\
\hline W-03-143 & Looking downstream from $\mathrm{N}$ most gravel road & 0 & W-03-143 D2 099 \\
\hline W-03-144 & Looking upstream from PTH 44 & 160 & W-03-144_D2_100 \\
\hline W-03-144 & Looking downstream from PTH 44 & 355 & W-03-144_D2_101 \\
\hline W-03-144 & Looking downstream from north most crossing (paved) & 345 & W-03-144 D2 102 \\
\hline W-03-145 & Looking upstream from PTH 44 & 30 & W-03-145_D2_103 \\
\hline W-03-145 & Looking downstream from Middle Boulevard & 0 & W-03-145_D2_104 \\
\hline W-03-145 & Looking downstream from PTH 44 & 180 & W-03-145 D2 105 \\
\hline W-03-146 & Looking upstream from crossing & 180 & W-03-146 D2 106 \\
\hline W-03-146 & Looking downstream from crossing & 0 & W-03-146_D2_107 \\
\hline W-03-147 & Looking upstream 2nd order parallel to PR 302 & 180 & W-03-147_D2_108 \\
\hline W-03-147 & Looking upstream 2nd order perpendicular to PR 302 & 270 & W-03-147_D2_109 \\
\hline W-03-147 & Looking downstream 3rd order from PR 302 & 90 & W-03-147 D2 110 \\
\hline W-03-148 & Looking upstream from crossing & 180 & W-03-148 D2 111 \\
\hline W-03-148 & Looking downstream from crossing & 90 & W-03-148_D2_112 \\
\hline W-03-148 & Looking upstream 1st order & 0 & W-03-148 D2 113 \\
\hline W-03-148 & Looking upstream 3rd order from 15m back & 180 & W-03-148 D2 114 \\
\hline
\end{tabular}


Appendix 8: List of all photographs taken showing the corresponding site number, descriptive caption, direction faced when taken (azimuth in degrees) and the unique file name for each photograph.

\begin{tabular}{|c|c|c|c|}
\hline $\begin{array}{c}\text { Site } \\
\text { Number }\end{array}$ & Photo Description & Azimuth & File Name \\
\hline W-03-149 & Looking upstream from crossing & 270 & W-03-149 D2 115 \\
\hline W-03-149 & Looking downstream from crossing & 90 & W-03-149_D2 116 \\
\hline W-03-150 & Looking upstream from crossing & 270 & W-03-150_D2_117 \\
\hline W-03-150 & Looking downstream from crossing & 90 & W-03-150 D2 118 \\
\hline W-03-151 & Looking upstream from east side of PTH 1 & 270 & W-03-151 D2 119 \\
\hline W-03-151 & Looking downstream from east side of PTH 1 median & 360 & W-03-151_D2_120 \\
\hline W-03-151 & Looking upstream from west side of PTH 1 & 360 & W-03-151_D2_121 \\
\hline W-03-152 & Looking upstream from PTH 15 & 185 & W-03-152 D2 122 \\
\hline W-03-152 & Looking downstream from PTH 15 & 0 & W-03-152 D2 123 \\
\hline W-03-153 & Looking upstream from PTH 15 & 180 & W-03-153_D2_124 \\
\hline W-03-153 & Looking upstream from PTH 15 & 180 & W-03-153_D2_125 \\
\hline W-03-153 & Looking upstream from PTH 15 & 140 & W-03-153 D2 126 \\
\hline W-03-153 & Looking downstream from PTH 15 & 0 & W-03-153 D2 127 \\
\hline W-03-153 & Looking downstream from PTH 15 & 310 & W-03-153 D2 128 \\
\hline W-03-153 & Looking downstream from PTH 15 & 290 & W-03-153_D2_129 \\
\hline W-03-154 & Looking upstream from PR 506 & 90 & W-03-154 D2 130 \\
\hline W-03-154 & Looking downstream from PR 506 & 270 & W-03-154 D2 131 \\
\hline W-03-155 & Looking upstream from PTH 1 eastbound & 180 & W-03-155 D2 132 \\
\hline W-03-155 & Looking downstream from PTH 1 eastbound & 0 & W-03-155_D2_133 \\
\hline W-03-155 & Looking downstream from PTH 1 westbound & 0 & W-03-155 D2 134 \\
\hline W-03-156 & Looking upstream from PTH 1 & 180 & W-03-156 D2 135 \\
\hline W-03-156 & Looking downstream from PTH 1 & 0 & W-03-156_D2_136 \\
\hline W-03-157 & Looking upstream from PR 506 & 180 & W-03-157 D2 137 \\
\hline W-03-157 & Looking downstream from PR 506 & 0 & W-03-157 D2 138 \\
\hline W-03-158 & Looking upstream from PR 507 & 180 & W-03-158_D2_139 \\
\hline W-03-158 & Looking downstream from PR 507 & 0 & W-03-158 D2 140 \\
\hline W-03-159 & Looking upstream from PTH 1 eastbound & 180 & W-03-159_D2_141 \\
\hline W-03-159 & Looking downstream from PTH 1 eastbound & 300 & W-03-159 D2 142 \\
\hline W-03-159 & Looking downstream from PTH 1 westbound & 360 & W-03-159 D2 143 \\
\hline W-03-160 & Looking upstream from PR 308 & 90 & W-03-160 D2 144 \\
\hline W-03-160 & Looking downstream from PR 308 & 270 & W-03-160 D2 145 \\
\hline W-03-161 & Looking upstream from PR 503 & 60 & W-03-161 D2 146 \\
\hline W-03-161 & Looking downstream from PR 503 & 200 & W-03-161_D2_147 \\
\hline
\end{tabular}


Appendix 8: List of all photographs taken showing the corresponding site number, descriptive caption, direction faced when taken (azimuth in degrees) and the unique file name for each photograph.

\begin{tabular}{|c|c|c|c|}
\hline $\begin{array}{c}\text { Site } \\
\text { Number }\end{array}$ & Photo Description & Azimuth & File Name \\
\hline W-03-162 & Looking upstream from River Road & 270 & W-03-162_D2_148 \\
\hline W-03-162 & Looking downstream from River Road & 360 & W-03-162 D2 149 \\
\hline W-03-163 & Looking upstream River Road & 90 & W-03-163_D2_150 \\
\hline W-03-163 & Looking downstream River Road & 270 & W-03-163_D2_151 \\
\hline W-03-164 & Looking upstream PTH 11 & 220 & W-03-164_D2_152 \\
\hline W-03-164 & Looking downstream PTH 11 & 75 & W-03-164_D2_153 \\
\hline W-03-164 & Looking downstream PTH 11 (zoomed in) & 75 & W-03-164_D2_154 \\
\hline W-03-165 & Looking upstream from box culvert & 200 & W-03-165_D2_155 \\
\hline W-03-165 & Looking downstream from box culvert & 90 & W-03-165_D2_156 \\
\hline W-03-166 & Looking upstream from crossing & 270 & W-03-166 D2 157 \\
\hline W-03-166 & Looking downstream from crossing & 90 & W-03-166_D2_158 \\
\hline W-03-167 & Looking upstream from PTH 11 & 315 & W-03-167_D2_159 \\
\hline W-03-167 & Looking downstream from PTH 11 & 135 & W-03-167_D2_160 \\
\hline W-03-168 & Looking upstream from crossing & 270 & W-03-168_D2_161 \\
\hline W-03-168 & Looking downstream from crossing & 90 & W-03-168_D2_162 \\
\hline W-03-169 & Looking upstream from culverts & 200 & W-03-169_D2_163 \\
\hline W-03-169 & Looking downstream from culverts & 100 & W-03-169_D2_164 \\
\hline W-03-169 & Looking at upstream eroded bank & 90 & W-03-169 D2 165 \\
\hline W-03-170 & Looking upstream north 1st order from grass crossing & 0 & W-03-170_D2_166 \\
\hline W-03-170 & Looking upstream south 1st order from grass crossing & 180 & W-03-170_D2_167 \\
\hline W-03-170 & Looking downstream 2nd order from PTH 11 & 270 & W-03-170_D2_168 \\
\hline W-03-171 & Looking upstream from PR 211 & 0 & W-03-171_D2_169 \\
\hline W-03-171 & Looking downstream from PR 211 & 180 & W-03-171_D2_170 \\
\hline W-03-172 & Looking upstream from culvert & 270 & W-03-172_D2_172 \\
\hline W-03-172 & Looking downstream from PTH 11 & 90 & W-03-172 D2 173 \\
\hline W-03-173 & Looking upstream from north culvert & 300 & W-03-173 D2 174 \\
\hline W-03-173 & Looking downstream from north culvert & 90 & W-03-173_D2_175 \\
\hline W-03-174 & Looking upstream 2nd order from culvert & 180 & W-03-174_D2_176 \\
\hline W-03-174 & Looking downstream 3rd order from PTH 11 & 90 & W-03-174_D2_177 \\
\hline W-03-175 & Looking upstream from crossing & 300 & W-03-175 D2 178 \\
\hline W-03-175 & Looking downstream from crossing & 120 & W-03-175_D2_179 \\
\hline W-03-176 & Looking upstream from PTH 11 & 100 & W-03-176_D2_180 \\
\hline W-03-176 & Looking downstream from PTH 11 & 240 & W-03-176_D2_181 \\
\hline
\end{tabular}


Appendix 8: List of all photographs taken showing the corresponding site number, descriptive caption, direction faced when taken (azimuth in degrees) and the unique file name for each photograph.

\begin{tabular}{|c|c|c|c|}
\hline $\begin{array}{c}\text { Site } \\
\text { Number }\end{array}$ & Photo Description & Azimuth & File Name \\
\hline W-03-177 & Looking upstream from crossing & 0 & W-03-177_D2_182 \\
\hline W-03-177 & Looking downstream from crossing & 180 & W-03-177 D2 183 \\
\hline W-03-178 & Looking upstream from crossing & 315 & W-03-178_D2_184 \\
\hline W-03-178 & Looking downstream from crossing & 135 & W-03-178_D2_185 \\
\hline W-03-179 & Looking upstream from crossing & 310 & W-03-179_D2_186 \\
\hline W-03-179 & Looking downstream from crossing & 170 & W-03-179_D2_187 \\
\hline W-03-180 & Looking upstream from crossing & 340 & W-03-180_D2_188 \\
\hline W-03-180 & Looking downstream from crossing & 165 & W-03-180_D2_189 \\
\hline W-03-181 & Looking upstream from crossing & 330 & W-03-181_D2_190 \\
\hline W-03-181 & Looking downstream from crossing & 170 & W-03-181_D2 191 \\
\hline W-03-182 & Looking upstream from mud road & 250 & W-03-182_D2_192 \\
\hline W-03-182 & Looking downstream from mud road & 70 & W-03-182_D2_193 \\
\hline W-03-183 & Looking upstream PTH 2 & 0 & W-03-183_D2_194 \\
\hline W-03-183 & Looking downstream PTH 2 & 180 & W-03-183_D2_195 \\
\hline W-03-183 & Looking upstream from railway crossing of 3 culverts & 0 & W-03-183_D2_196 \\
\hline W-03-184 & Looking upstream from PR 248 & 270 & W-03-184_D2_197 \\
\hline W-03-184 & Looking downstream from PR 248 & 90 & W-03-184_D2_198 \\
\hline W-03-185 & Looking upstream from PTH 2 & 180 & W-03-185 D2 199 \\
\hline W-03-185 & Looking downstream from PTH 2 & 0 & W-03-185_D2_200 \\
\hline W-03-185 & Looking downstream from railway track & 0 & W-03-185_D2_201 \\
\hline W-03-186 & Looking downstream from bridge (backwards from regular order) & 315 & W-03-186_D2_202 \\
\hline W-03-186 & Looking upstream from bridge & 180 & W-03-186_D2_203 \\
\hline W-03-187 & Looking upstream from crossing & 300 & W-03-187_D2_204 \\
\hline W-03-187 & Looking downstream from crossing & 90 & W-03-187_D2_205 \\
\hline W-03-188 & Looking upstream from PR 248 & 290 & W-03-188 D2 206 \\
\hline W-03-188 & Looking downstream from PR 248 & 110 & W-03-188D2 207 \\
\hline W-03-189 & Looking upstream from PR 248 & 270 & W-03-189_D2_208 \\
\hline W-03-189 & Looking downstream from PR 248 & 90 & W-03-189_D2_209 \\
\hline W-03-190 & Looking upstream from PR 201, Piney West Drain & 0 & W-03-190_D2_210 \\
\hline W-03-190 & Looking upstream unnamed 2nd order drain from PR 201 & 270 & W-03-190 D2 211 \\
\hline W-03-190 & Looking downstream Piney West Drain from PR 201 & 180 & W-03-190_D2_212 \\
\hline W-03-191 & Looking upstream PTH 89 & 90 & W-03-191_D2_213 \\
\hline W-03-191 & Looking downstream PTH 89 & 270 & W-03-191_D2_214 \\
\hline
\end{tabular}


Appendix 8: List of all photographs taken showing the corresponding site number, descriptive caption, direction faced when taken (azimuth in degrees) and the unique file name for each photograph.

\begin{tabular}{|c|c|c|c|}
\hline $\begin{array}{c}\text { Site } \\
\text { Number }\end{array}$ & Photo Description & Azimuth & File Name \\
\hline W-03-192 & Looking upstream from crossing & 270 & W-03-192_D2_215 \\
\hline W-03-192 & Looking downstream from crossing & 90 & W-03-192_D2_216 \\
\hline W-03-193 & Looking upstream from crossing & 270 & W-03-193_D2_217 \\
\hline W-03-193 & Looking downstream from crossing & 90 & W-03-193_D2_218 \\
\hline W-03-194 & Looking upstream from PTH 12 & 0 & W-03-194_D2_219 \\
\hline W-03-194 & Looking downstream from PTH 12 & 180 & W-03-194_D2_220 \\
\hline W-03-195 & Looking upstream from PTH 12 & 0 & W-03-195_D2_221 \\
\hline W-03-195 & Looking downstream from PTH 12 & 180 & W-03-195_D2_222 \\
\hline W-03-196 & Looking upstream 2nd order from crossing & 90 & W-03-196_D3_001 \\
\hline W-03-196 & Looking upstream 2nd order from crossing & 270 & W-03-196_D3_002 \\
\hline W-03-196 & Looking downstream 3rd order from crossing & 0 & W-03-196_D3_003 \\
\hline W-03-197 & Looking upstream PTH 12 & 0 & W-03-197_D3_004 \\
\hline W-03-197 & Looking downstream PTH 12 & 180 & W-03-197_D3_005 \\
\hline W-03-197 & Looking at bank slump on downstream side of bridge on left bank & 270 & W-03-197_D3_006 \\
\hline W-03-198 & Looking upstream from PTH 12 & 30 & W-03-198_D3_007 \\
\hline W-03-198 & Looking downstream from PTH 12 & 200 & W-03-198_D3_008 \\
\hline W-03-199 & Looking upstream from crossing & 0 & W-03-199_D3_009 \\
\hline W-03-199 & Looking downstream from crossing & 180 & W-03-199_D3_010 \\
\hline W-03-200 & Looking upstream from crossing & 210 & W-03-200_D3_011 \\
\hline W-03-200 & Looking downstream from crossing & 280 & W-03-200_D3_012 \\
\hline W-03-201 & Looking upstream from crossing & 130 & W-03-201_D3_013 \\
\hline W-03-201 & Looking downstream from crossing & 350 & W-03-201_D3_014 \\
\hline W-03-202 & Looking upstream from crossing & 180 & W-03-202_D3_015 \\
\hline W-03-202 & Looking downstream from crossing & 0 & W-03-202_D3_016 \\
\hline W-03-203 & Looking upstream from crossing & 180 & W-03-203_D3_017 \\
\hline W-03-203 & Looking downstream from crossing & 0 & W-03-203_D3_018 \\
\hline W-03-204 & Looking upstream from crossing & 100 & W-03-204_D3_019 \\
\hline W-03-204 & Looking downstream from crossing & 340 & W-03-204_D3_020 \\
\hline W-03-205 & Looking upstream from crossing & 180 & W-03-205_D3_021 \\
\hline W-03-205 & Looking downstream from crossing & 0 & W-03-205_D3_022 \\
\hline W-03-206 & Looking upstream from PTH 12 & 120 & W-03-206_D3_023 \\
\hline W-03-206 & Looking downstream from PTH 12 & 260 & W-03-206_D3_024 \\
\hline W-03-207 & Looking upstream from PTH 12 & 130 & W-03-207_D3_025 \\
\hline
\end{tabular}


Appendix 8: List of all photographs taken showing the corresponding site number, descriptive caption, direction faced when taken (azimuth in degrees) and the unique file name for each photograph.

\begin{tabular}{|c|c|c|c|}
\hline $\begin{array}{c}\text { Site } \\
\text { Number }\end{array}$ & Photo Description & Azimuth & File Name \\
\hline W-03-207 & Looking downstream from PTH 12 & 280 & W-03-207_D3_026 \\
\hline W-03-208 & Looking upstream unnamed 2nd order from PTH 12 & 90 & W-03-208 D3 027 \\
\hline W-03-208 & Looking upstream Hazelridge Drain from gravel crossing & 180 & W-03-208_D3_028 \\
\hline W-03-208 & Looking downstream Hazelridge Drain from PTH 12 & 270 & W-03-208_D3_029 \\
\hline W-03-209 & Looking upstream from PR 306 & 70 & W-03-209_D3_030 \\
\hline W-03-209 & Looking downstream from PR 306 & 280 & W-03-209_D3_031 \\
\hline W-03-210 & Looking upstream from PTH 15 & 270 & W-03-210_D3_032 \\
\hline W-03-210 & Looking downstream from PTH 15 & 0 & W-03-210_D3_033 \\
\hline W-03-211 & Looking upstream 2nd order from grass crossing & 270 & W-03-211_D3_034 \\
\hline W-03-211 & Looking upstream Swede Drain from Hazelridge Road & 180 & W-03-211_D3 035 \\
\hline W-03-211 & Looking downstream Swede Drain from Hazelridge Road & 0 & W-03-211_D3_036 \\
\hline W-03-212 & Looking upstream from crossing & 180 & W-03-212_D3_037 \\
\hline W-03-212 & Looking downstream from crossing & 0 & W-03-212_D3_038 \\
\hline W-03-213 & Looking upstream from PR 306 & 100 & W-03-213_D3_039 \\
\hline W-03-213 & Looking downstream from PR 306 & 280 & W-03-213_D3_040 \\
\hline W-03-214 & Looking upstream from Sapton Road & 140 & W-03-214_D3_041 \\
\hline W-03-214 & Looking downstream from Sapton Road. & 60 & W-03-214_D3_042 \\
\hline W-03-215 & Looking upstream from Sapton Road & 180 & W-03-215 D3 043 \\
\hline W-03-215 & Looking downstream from Sapton Road. & 0 & W-03-215_D3_044 \\
\hline W-03-216 & Looking upstream Swede Drain from Corbet Road & 180 & W-03-216_D3_045 \\
\hline W-03-216 & Looking upstream Cooks Creek Diversion from Cooks Creek Road & 90 & W-03-216_D3_046 \\
\hline W-03-216 & Looking downstream Cooks Creek Diversion from Cooks Creek Road & 270 & W-03-216_D3_047 \\
\hline W-03-217 & Looking upstream from PR 206 & 90 & W-03-217_D3_048 \\
\hline W-03-217 & Looking downstream from PR 206 & 270 & W-03-217_D3_049 \\
\hline W-03-218 & Looking upstream from gravel crossing & 90 & W-03-218_D3_050 \\
\hline W-03-218 & Looking downstream from gravel crossing & 270 & W-03-218D3 051 \\
\hline W-03-219 & Looking upstream from PR 206 & 90 & W-03-219_D3_052 \\
\hline W-03-219 & Looking downstream from PR 206 & 270 & W-03-219_D3_053 \\
\hline W-03-220 & Looking upstream from PTH 68 & 180 & W-03-220_D3_054 \\
\hline W-03-220 & Looking downstream from PTH 68 & 0 & W-03-220_D3 055 \\
\hline W-03-221 & Looking upstream from PTH 68 & 90 & W-03-221_D3_056 \\
\hline W-03-221 & Looking downstream from PTH 68 & 270 & W-03-221_D3_057 \\
\hline W-03-222 & Looking upstream from PTH 68 & 220 & W-03-222_D3_058 \\
\hline
\end{tabular}


Appendix 8: List of all photographs taken showing the corresponding site number, descriptive caption, direction faced when taken (azimuth in degrees) and the unique file name for each photograph.

\begin{tabular}{|c|c|c|c|}
\hline $\begin{array}{c}\text { Site } \\
\text { Number }\end{array}$ & Photo Description & Azimuth & File Name \\
\hline W-03-222 & Looking downstream from PTH 68 & 40 & W-03-222 D3 059 \\
\hline W-03-223 & Looking upstream from gravel culvert crossing & 210 & W-03-223_D3_060 \\
\hline W-03-223 & Looking downstream from gravel culvert crossing & 310 & W-03-223_D3_061 \\
\hline W-03-224 & Looking upstream from PTH 68 & 160 & W-03-224_D3_062 \\
\hline W-03-224 & Looking downstream from PTH 68 & 345 & W-03-224_D3_063 \\
\hline W-03-225 & Looking upstream from PR 233 & 280 & W-03-225_D3_064 \\
\hline W-03-225 & Looking downstream from PR 233 & 90 & W-03-225_D3_065 \\
\hline W-03-226 & Looking upstream from farmer's road & 0 & W-03-226 D3 066 \\
\hline W-03-226 & Looking downstream from farmer's road & 180 & W-03-226_D3_067 \\
\hline W-03-227 & Looking upstream Icelandic River & 290 & W-03-227_D3_068 \\
\hline W-03-227 & Looking downstream Icelandic River & 80 & W-03-227 D3 069 \\
\hline W-03-228 & Looking upstream from PTH 8 & 270 & W-03-228_D3_070 \\
\hline W-03-228 & Looking downstream from PTH 8 & 90 & W-03-228_D3_071 \\
\hline W-03-229 & Looking upstream 2nd order from Road $137 \mathrm{~N}$ & 0 & W-03-229_D3_072 \\
\hline W-03-229 & Looking upstream 2nd order from PTH 8 & 260 & W-03-229 D3 073 \\
\hline W-03-229 & Looking downstream 3rd order from PTH 8 & 70 & W-03-229_D3_074 \\
\hline W-03-229 & Looking downstream at un-mapped drain (north) & 70 & W-03-229_D3_075 \\
\hline W-03-229 & Looking downstream at un-mapped drain (south) & 70 & W-03-229 D3 076 \\
\hline W-03-230 & Looking upstream from PR 326 & 0 & W-03-230_D3_077 \\
\hline W-03-230 & Looking downstream from PR 326 & 180 & W-03-230_D3_078 \\
\hline W-03-231 & Looking upstream from Road 12E & 260 & W-03-231_D3_079 \\
\hline W-03-231 & Looking downstream from Road 12E & 80 & W-03-231_D3_080 \\
\hline W-03-232 & Looking upstream from crossing & 290 & W-03-232_D3_081 \\
\hline W-03-232 & Looking downstream from crossing & 130 & W-03-232_D3_082 \\
\hline W-03-233 & Looking upstream from gravel culvert crossing & 40 & W-03-233_D3_083 \\
\hline W-03-233 & Looking downstream from gravel culvert crossing & 150 & W-03-233_D3_084 \\
\hline W-03-233 & Photo of canola field & $\mathrm{N} / \mathrm{A}$ & W-03-233_D3_085 \\
\hline W-03-233 & Photo of canola field & $\mathrm{N} / \mathrm{A}$ & W-03-233_D3_086 \\
\hline W-03-234 & Looking upstream from crossing & 260 & W-03-234_D3_087 \\
\hline W-03-234 & Looking downstream from crossing & 80 & W-03-234_D3_088 \\
\hline W-03-235 & Looking upstream from PTH 68 & 160 & W-03-235 D3 089 \\
\hline W-03-235 & Looking downstream from PTH 68 & 300 & W-03-235_D3_090 \\
\hline W-03-236 & Looking upstream from crossing & 230 & W-03-236_D3_091 \\
\hline
\end{tabular}


Appendix 8: List of all photographs taken showing the corresponding site number, descriptive caption, direction faced when taken (azimuth in degrees) and the unique file name for each photograph.

\begin{tabular}{|c|c|c|c|}
\hline $\begin{array}{c}\text { Site } \\
\text { Number }\end{array}$ & Photo Description & Azimuth & File Name \\
\hline W-03-236 & Looking downstream from crossing & 60 & W-03-236_D3_092 \\
\hline W-03-236 & Looking upstream at large culvert & 230 & W-03-236_D3_093 \\
\hline W-03-237 & Looking upstream from PR 329 & 160 & W-03-237_D3_094 \\
\hline W-03-237 & Looking downstream from PR 329 & 40 & W-03-237_D3_095 \\
\hline W-03-238 & Looking upstream from bridge crossing & 270 & W-03-238_D3_096 \\
\hline W-03-238 & Looking downstream from bridge crossing & 340 & W-03-238_D3_097 \\
\hline W-03-239 & Looking upstream from crossing & 205 & W-03-239_D3_098 \\
\hline W-03-239 & Looking downstream from crossing & 40 & W-03-239_D3_099 \\
\hline W-03-240 & Looking upstream from gravel culvert crossing & 270 & W-03-240_D3_100 \\
\hline W-03-240 & Looking downstream from gravel culvert crossing & 85 & W-03-240_D3_101 \\
\hline W-03-241 & Looking upstream from gravel crossing & 180 & W-03-241_D3_102 \\
\hline W-03-241 & Looking downstream from gravel crossing & 320 & W-03-241_D3_103 \\
\hline W-03-242 & Looking upstream from gravel crossing & 260 & W-03-242_D3_104 \\
\hline W-03-242 & Looking downstream from gravel crossing & 80 & W-03-242_D3_105 \\
\hline W-03-242 & Looking at dried manure & 80 & W-03-242_D3_106 \\
\hline W-03-242 & Looking at manure field \& banks & 0 & W-03-242_D3_107 \\
\hline W-03-243 & Looking upstream from PR 234 from bridge & 240 & W-03-243_D3_108 \\
\hline W-03-243 & Looking downstream from PR 243 from bridge & 100 & W-03-243_D3_109 \\
\hline W-03-244 & Looking upstream from PTH 8 & 250 & W-03-244_D3_110 \\
\hline W-03-244 & Looking downstream from PTH 8 & 80 & W-03-244_D3_111 \\
\hline W-03-245 & Looking upstream from Road 14E & 270 & W-03-245_D3_112 \\
\hline W-03-245 & Looking downstream from Road 14E & 90 & W-03-245_D3_113 \\
\hline W-03-246 & Looking upstream from PR 233 & 270 & W-03-246_D3_114 \\
\hline W-03-246 & Looking downstream from PR 233 & 90 & W-03-246_D3_115 \\
\hline W-03-247 & Looking upstream from bridge at PR 234 & 310 & W-03-247_D3_116 \\
\hline W-03-247 & Looking downstream from bridge at PR 234 & 120 & W-03-247_D3_117 \\
\hline W-03-248 & Looking upstream from crossing & 320 & W-03-248_D3_118 \\
\hline W-03-248 & Looking downstream from crossing & 180 & W-03-248_D3_119 \\
\hline W-03-249 & Looking upstream from bridge crossing & 130 & W-03-249_D3_120 \\
\hline W-03-249 & Looking downstream from bridge crossing & 325 & W-03-249_D3_121 \\
\hline W-03-250 & Looking upstream from PR 304 & 180 & W-03-250_D3_122 \\
\hline W-03-250 & Looking downstream from PR 304 & 0 & W-03-250_D3_123 \\
\hline W-03-251 & Looking upstream from Gusta Road at bridge crossing & 180 & W-03-251_D3_124 \\
\hline
\end{tabular}


Appendix 8: List of all photographs taken showing the corresponding site number, descriptive caption, direction faced when taken (azimuth in degrees) and the unique file name for each photograph.

\begin{tabular}{|c|c|c|c|}
\hline $\begin{array}{c}\text { Site } \\
\text { Number }\end{array}$ & Photo Description & Azimuth & File Name \\
\hline W-03-251 & Looking downstream from Gusta Road at bridge crossing & 0 & W-03-251_D3_125 \\
\hline W-03-252 & Looking upstream from crossing & 90 & W-03-252 D3 126 \\
\hline W-03-252 & Looking downstream from crossing & 270 & W-03-252_D3_127 \\
\hline W-03-253 & Looking upstream from culvert crossing (paved road) & 270 & W-03-253_D3_128 \\
\hline W-03-253 & Looking downstream from culvert crossing (paved road) & 90 & W-03-253_D3_129 \\
\hline W-03-254 & Looking upstream from crossing & 260 & W-03-254_D3_130 \\
\hline W-03-254 & Looking downstream from crossing & 140 & W-03-254_D3_131 \\
\hline W-03-255 & Looking upstream from PTH 11 at bridge crossing & 120 & W-03-255_D3_132 \\
\hline W-03-255 & Looking downstream from PTH 11 at bridge crossing & 300 & W-03-255_D3_133 \\
\hline W-03-255 & Looking at Winnipeg River from PTH 11, at Fort Alexander Reserve & 160 & W-03-255 D3 134 \\
\hline W-03-255 & Looking at Winnipeg River from PTH 11, at Fort Alexander Reserve & $\mathrm{N} / \mathrm{A}$ & W-03-255_D3_135 \\
\hline W-03-256 & Looking upstream 2nd order from gravel crossing at wood bridge & 60 & W-03-256_D3_136 \\
\hline W-03-256 & Looking upstream 3rd order from gravel crossing at wood bridge & 180 & W-03-256_D3_137 \\
\hline W-03-256 & Looking downstream 3rd order from gravel crossing & 180 & W-03-256_D3_138 \\
\hline W-03-257 & Looking upstream from gravel crossing & 180 & W-03-257_D3_139 \\
\hline W-03-257 & Looking downstream from gravel crossing & 0 & W-03-257_D3_140 \\
\hline W-03-258 & Looking upstream from PTH 9 & 205 & W-03-258_D3_141 \\
\hline W-03-258 & Looking downstream from PTH 9 & 80 & W-03-258 D3 142 \\
\hline W-03-259 & Looking upstream from PR 515 & 180 & W-03-259_D3_143 \\
\hline W-03-259 & Looking downstream from PR 515 & 0 & W-03-259_D3_144 \\
\hline W-03-260 & Looking upstream at culvert (from rocks north of pool) & 180 & W-03-260_D3_145 \\
\hline $\mathrm{W}-03-260$ & Looking upstream from PR 515 & 180 & W-03-260_D3_146 \\
\hline W-03-260 & Looking downstream from PR 515 & 0 & W-03-260_D3_147 \\
\hline W-03-261 & Looking upstream from gravel road crossing & 195 & W-03-261_D3_148 \\
\hline W-03-261 & Looking downstream from gravel road crossing & 335 & W-03-261_D3_149 \\
\hline W-03-262 & Looking upstream from PTH 8 & 270 & W-03-262 D3 150 \\
\hline W-03-262 & Looking downstream from PTH 8 & 90 & W-03-262_D3_151 \\
\hline W-03-263 & Looking upstream from PTH 67 & 180 & W-03-263_D3_152 \\
\hline W-03-263 & Looking downstream from PTH 67 & 0 & W-03-263_D3_153 \\
\hline W-03-264 & Looking upstream from PTH 7 & 305 & W-03-264_D3 154 \\
\hline W-03-264 & Looking downstream from PTH 7 & 105 & W-03-264_D3_155 \\
\hline W-03-265 & Looking upstream from PTH 7 & 270 & W-03-265_D3_156 \\
\hline W-03-265 & Looking downstream from PTH 7 & 170 & W-03-265_D3_157 \\
\hline
\end{tabular}


Appendix 8: List of all photographs taken showing the corresponding site number, descriptive caption, direction faced when taken (azimuth in degrees) and the unique file name for each photograph.

\begin{tabular}{|c|c|c|c|}
\hline $\begin{array}{c}\text { Site } \\
\text { Number }\end{array}$ & Photo Description & Azimuth & File Name \\
\hline W-03-266 & Looking upstream from dike crossing & 105 & W-03-266 D3 158 \\
\hline W-03-267 & Looking upstream from left bank dike & 280 & W-03-267_D3_159 \\
\hline W-03-267 & Looking downstream from left bank dike & 160 & W-03-267_D3_160 \\
\hline B-04-001 & Looking upstream from culvert crossing & 310 & B-04-001 P5010001 \\
\hline B-04-001 & Looking downstream from culvert crossing & 135 & B-04-001 P5010002 \\
\hline B-04-001 & Looking downstream towards culvert inlet & $\mathrm{N} / \mathrm{A}$ & B-04-001_P5010003 \\
\hline B-04-001 & Looking upstream towards culvert inlet & $\mathrm{N} / \mathrm{A}$ & B-04-001_P5010004 \\
\hline B-04-002 & Looking upstream from crossing of access road & 315 & B-04-002 P5010005 \\
\hline B-04-002 & Looking downstream from crossing of access road & 130 & B-04-002 P5010006 \\
\hline B-04-002 & Looking upstream at culverts & 125 & B-04-002 P5010007 \\
\hline B-04-002 & Looking downstream at culverts & 315 & B-04-002_P5010008 \\
\hline B-04-002 & Looking upstream at culvert crossing on the westbound Perimeter & 125 & B-04-002 P5010009 \\
\hline B-04-003 & Looking downstream from bridge & 90 & B-04-003 P5010010 \\
\hline B-04-003 & Looking upstream from bridge & 270 & B-04-003 P5010011 \\
\hline B-04-003 & Looking upstream at rocks and pool under bridge & $\mathrm{N} / \mathrm{A}$ & B-04-003_P5010012 \\
\hline B-04-003 & Photo of White Sucker caught in seine haul & N/A & B-04-003 P5010013 \\
\hline B-04-003 & Photo of Northern Pike caught in seine haul & N/A & B-04-003 P5010014 \\
\hline B-04-004 & Looking upstream from low-level crossing & 360 & B-04-004_P5010015 \\
\hline B-04-004 & Looking downstream from low-level crossing & 180 & B-04-004_P5010016 \\
\hline B-04-004 & Looking at culvert bringing flow in from ditch outside of dike & 90 & B-04-004 P5010017 \\
\hline B-04-005 & Looking upstream from bridge & 270 & B-04-005 P5010018 \\
\hline B-04-005 & Looking downstream from bridge & 90 & B-04-005_P5010019 \\
\hline B-04-006 & Looking upstream from under bridge & 110 & B-04-006_P5010020 \\
\hline B-04-006 & Looking downstream from under bridge & 230 & B-04-006_P5010021 \\
\hline B-04-006 & Photo of suckers stranded after higher waters (spawning?) & $\mathrm{N} / \mathrm{A}$ & B-04-006 P5010022 \\
\hline B-04-006 & Photo of garbage and dead Yellow Perch washed onto rocks & $\mathrm{N} / \mathrm{A}$ & B-04-006_P5010023 \\
\hline B-04-007 & Looking upstream from gravel road crossing & 45 & B-04-007_P5010024 \\
\hline B-04-007 & Looking downstream from gravel road crossing & 225 & B-04-007 P5010025 \\
\hline B-04-007 & Looking upstream at culverts & 45 & B-04-007 P5010026 \\
\hline B-04-008 & Looking upstream from culvert crossing & 270 & B-04-008_P5010027 \\
\hline B-04-008 & Looking downstream from culvert crossing & 90 & B-04-008_P5010028 \\
\hline B-04-008 & Looking downstream at culvert inlet & 45 & B-04-008 P5010029 \\
\hline B-04-008 & Looking upstream at culvert outlet & 270 & B-04-008_P5010030 \\
\hline
\end{tabular}


Appendix 8: List of all photographs taken showing the corresponding site number, descriptive caption, direction faced when taken (azimuth in degrees) and the unique file name for each photograph.

\begin{tabular}{|c|c|c|c|}
\hline $\begin{array}{c}\text { Site } \\
\text { Number }\end{array}$ & Photo Description & Azimuth & File Name \\
\hline B-04-009 & Looking upstream from crossing of PR 480 & 270 & B-04-009 P5020001 \\
\hline B-04-009 & Looking downstream from crossing of PR 480 & 90 & B-04-009_P5020002 \\
\hline B-04-009 & Looking up east ditch downstream from PR 480 crossing & 360 & B-04-009_P5020003 \\
\hline B-04-009 & Looking upstream at outlet of $1.5 \mathrm{~m}$ culverts & $\mathrm{N} / \mathrm{A}$ & B-04-009_P5020004 \\
\hline B-04-010 & Looking upstream from bridge crossing & 270 & B-04-010 P5020005 \\
\hline B-04-010 & Looking downstream from bridge crossing & 90 & B-04-010 P5020006 \\
\hline B-04-010 & Looking south at gated culvert running into drain & 180 & B-04-010_P5020007 \\
\hline B-04-010 & Looking west at recently dredged portion of the drain & 90 & B-04-010 P5020008 \\
\hline B-04-010 & Looking at south at recently dredged ditches that flow into drain & 180 & B-04-010 P5020009 \\
\hline B-04-011 & Looking upstream from end of reach & 270 & B-04-011_P5020010 \\
\hline B-04-011 & Looking downstream from end of reach & 90 & B-04-011_P5020011 \\
\hline B-04-012 & Looking downstream from riffles & 170 & B-04-012 P5020012 \\
\hline B-04-012 & Looking upstream from riffles & 340 & B-04-012 P5020013 \\
\hline B-04-012 & Looking at spawning suckers & N/A & B-04-012 P5020014 \\
\hline B-04-012 & Looking at spawning suckers & $\mathrm{N} / \mathrm{A}$ & B-04-012_P5020015 \\
\hline B-04-012 & Looking at spawning suckers & N/A & B-04-012 P5020016 \\
\hline B-04-012 & Looking at suckers moving upstream of gradient control structure & N/A & B-04-012 P5020017 \\
\hline B-04-012 & Looking at suckers moving upstream of gradient control structure & $\mathrm{N} / \mathrm{A}$ & B-04-012_P5020018 \\
\hline B-04-013 & Looking upstream from bridge crossing & 300 & B-04-013_P5020019 \\
\hline B-04-013 & Looking downstream from bridge crossing & 100 & B-04-013 P5020020 \\
\hline B-04-014 & Looking upstream from riffles & 350 & B-04-014 P5020021 \\
\hline B-04-014 & Looking downstream from riffles & 170 & B-04-014_P5020022 \\
\hline B-04-014 & Looking at washed up suckers (note spawning colors on them) & $\mathrm{N} / \mathrm{A}$ & B-04-014_P5020023 \\
\hline B-04-015 & Looking upstream from control structure & $\mathrm{N} / \mathrm{A}$ & B-04-015_P5020024 \\
\hline B-04-015 & Looking downstream from control structure & $\mathrm{N} / \mathrm{A}$ & B-04-015 P5020025 \\
\hline B-04-015 & Photo of spawning suckers at the riffle & $\mathrm{N} / \mathrm{A}$ & B-04-015_P5020026 \\
\hline B-04-016 & Looking upstream from bottom of riffle & 280 & B-04-016_P5020027 \\
\hline B-04-016 & Looking downstream from bottom of riffle & 45 & B-04-016 P5020028 \\
\hline B-04-017 & Looking upstream from riffle & 180 & B-04-017 P5020029 \\
\hline B-04-017 & Looking downstream from riffle & 360 & B-04-017_P5020030 \\
\hline B-04-018 & Looking upstream from bridge crossing & 340 & B-04-018_P5030001 \\
\hline B-04-018 & Looking downstream from bridge crossing & 160 & B-04-018 P5030002 \\
\hline B-04-019 & Looking upstream from gravel road crossing & 270 & B-04-019_P5030003 \\
\hline
\end{tabular}


Appendix 8: List of all photographs taken showing the corresponding site number, descriptive caption, direction faced when taken (azimuth in degrees) and the unique file name for each photograph.

\begin{tabular}{|c|c|c|c|}
\hline $\begin{array}{c}\text { Site } \\
\text { Number }\end{array}$ & Photo Description & Azimuth & File Name \\
\hline B-04-019 & Looking downstream from gravel road crossing & 90 & B-04-019 P5030004 \\
\hline B-04-019 & Looking upstream at culvert outlet & $\mathrm{N} / \mathrm{A}$ & B-04-019_P5030005 \\
\hline B-04-020 & Looking upstream from dirt road crossing & 270 & B-04-020_P5030006 \\
\hline B-04-020 & Looking downstream from dirt road crossing & 90 & B-04-020 P5030007 \\
\hline B-04-020 & Looking upstream at culvert outlet & N/A & B-04-020 P5030008 \\
\hline B-04-021 & Looking downstream from gravel road crossing & 90 & B-04-021_P5030009 \\
\hline B-04-021 & Looking upstream at culvert outlet & 300 & B-04-021_P5030010 \\
\hline B-04-021 & Looking at released Black Bullhead (170mm) & $\mathrm{N} / \mathrm{A}$ & B-04-021_P5030011 \\
\hline B-04-022 & Looking downstream from reach & 360 & B-04-022 P5030012 \\
\hline B-04-022 & Looking upstream from reach & 180 & B-04-022 P5030013 \\
\hline B-04-023 & Looking upstream from crossing & 270 & B-04-023_P5040001 \\
\hline B-04-023 & Looking downstream from crossing & 90 & B-04-023 P5040002 \\
\hline B-04-024 & Looking upstream from crossing & 270 & B-04-024 P5040003 \\
\hline B-04-024 & Looking downstream from crossing & 90 & B-04-024_P5040004 \\
\hline B-04-024 & Looking upstream at culvert outlet & $\mathrm{N} / \mathrm{A}$ & B-04-024_P5040005 \\
\hline B-04-025 & Looking upstream from road crossing & N/A & B-04-025 P5040006 \\
\hline B-04-025 & Looking downstream from road crossing & N/A & B-04-025 P5040007 \\
\hline B-04-026 & Looking upstream from crossing & 195 & B-04-026_P5040008 \\
\hline B-04-026 & Looking downstream from crossing & 10 & B-04-026_P5040009 \\
\hline B-04-026 & Looking at released Black Bullhead $(170 \mathrm{~mm})$ & N/A & B-04-026 P5040010 \\
\hline B-04-026 & Looking at released Black Bullhead (125mm) & $\mathrm{N} / \mathrm{A}$ & B-04-026 P5040011 \\
\hline B-04-027 & Looking upstream & 360 & B-04-027_P5040012 \\
\hline B-04-027 & Looking downstream & 160 & B-04-027_P5040013 \\
\hline B-04-027 & Looking at released quillback $(403 \mathrm{~mm})$ & $\mathrm{N} / \mathrm{A}$ & B-04-027 P5040014 \\
\hline B-04-027 & Looking at released quillback (424mm) & $\mathrm{N} / \mathrm{A}$ & B-04-027_P5040015 \\
\hline B-04-027 & Head shot of the $424 \mathrm{~mm}$ Quillback & $\mathrm{N} / \mathrm{A}$ & B-04-027_P5040016 \\
\hline B-04-028 & Looking upstream from road crossing & 340 & B-04-028_P5040017 \\
\hline B-04-028 & Looking downstream from road crossing & 150 & B-04-028 P5040018 \\
\hline B-04-028 & Looking across downstream end of road crossing & $\mathrm{N} / \mathrm{A}$ & B-04-028 P5040019 \\
\hline B-04-029 & Looking upstream from riffle & 360 & B-04-029_P5040020 \\
\hline B-04-029 & Looking downstream from riffle & 180 & B-04-029 P5040021 \\
\hline B-04-029 & Looking at riffle & 315 & B-04-029 P5040022 \\
\hline B-04-030 & Looking upstream from middle of reach & 360 & B-04-030 P5040023 \\
\hline
\end{tabular}


Appendix 8: List of all photographs taken showing the corresponding site number, descriptive caption, direction faced when taken (azimuth in degrees) and the unique file name for each photograph.

\begin{tabular}{|c|c|c|c|}
\hline $\begin{array}{c}\text { Site } \\
\text { Number }\end{array}$ & Photo Description & Azimuth & File Name \\
\hline B-04-030 & Looking downstream from middle of reach & 180 & B-04-030_P5040024 \\
\hline B-04-031 & Looking upstream from culvert crossing & 270 & B-04-031_P5060001 \\
\hline B-04-031 & Looking downstream from culvert crossing & 90 & B-04-031_P5060002 \\
\hline B-04-031 & Looking upstream at culvert outlets & 250 & B-04-031_P5060003 \\
\hline B-04-031 & Looking downstream at culvert inlets & 90 & B-04-031_P5060004 \\
\hline B-04-032 & Looking upstream from culvert crossing & 270 & B-04-032 P5060005 \\
\hline B-04-032 & Looking downstream from culvert crossing & 90 & B-04-032_P5060006 \\
\hline B-04-033 & Looking upstream from culvert crossing & 90 & B-04-033_P5060009 \\
\hline B-04-033 & Looking downstream from culvert crossing & 270 & B-04-033 P5060010 \\
\hline B-04-033 & Looking south at inflow from 3rd order tributary (note perched culvert) & 200 & B-04-033_P5060011 \\
\hline B-04-034 & Looking upstream from culvert crossing & 270 & B-04-034_P5060012 \\
\hline B-04-034 & Looking downstream from culvert crossing & 90 & B-04-034_P5060013 \\
\hline B-04-035 & Looking upstream from culvert crossing & 180 & B-04-035 P5060014 \\
\hline B-04-035 & Looking downstream from culvert crossing & 360 & B-04-035 P5060015 \\
\hline B-04-035 & Looking at culvert outlets & 300 & B-04-035_P5060016 \\
\hline B-04-036 & Looking upstream from culvert crossing & 270 & B-04-036_P5060017 \\
\hline B-04-036 & Looking downstream from culvert crossing & 90 & B-04-036 P5060018 \\
\hline B-04-037 & Looking upstream from low-level crossing & 350 & B-04-037_P5060019 \\
\hline B-04-037 & Looking downstream from low-level crossing & 170 & B-04-037_P5060020 \\
\hline B-04-038 & Looking upstream from culvert crossing & 150 & B-04-038_P5060021 \\
\hline B-04-038 & Looking downstream from culvert crossing & 90 & B-04-038 P5060022 \\
\hline B-04-038 & Looking downstream at large culvert inlet & 360 & B-04-038_P5060023 \\
\hline B-04-038 & Looking downstream at small culvert inlet & 360 & B-04-038_P5060024 \\
\hline B-04-039 & $\begin{array}{l}\text { Looking at } 100 \text { s of White Suckers in a pool downstream of a water control structure - } \\
\text { passage to upstream spawning riffles is blocked }\end{array}$ & $\mathrm{N} / \mathrm{A}$ & B-04-039_P5060025 \\
\hline B-04-039 & $\begin{array}{l}\text { Looking at } 100 \text { s of White Suckers in a pool downstream of a water control structure - } \\
\text { passage to upstream spawning riffles is blocked }\end{array}$ & $\mathrm{N} / \mathrm{A}$ & B-04-039_P5060026 \\
\hline B-04-039 & $\begin{array}{l}\text { Looking at } 100 \text { s of White Suckers in a pool downstream of a water control structure - } \\
\text { passage to upstream spawning riffles is blocked }\end{array}$ & $\mathrm{N} / \mathrm{A}$ & B-04-039_P5060027 \\
\hline B-04-039 & $\begin{array}{l}\text { Looking at } 100 \text { s of White Suckers in a pool downstream of a water control structure - } \\
\text { passage to upstream spawning riffles is blocked }\end{array}$ & $\mathrm{N} / \mathrm{A}$ & B-04-039_P5060028 \\
\hline B-04-039 & Looking upstream from crossing & 180 & B-04-039 P5060029 \\
\hline B-04-039 & Looking upstream from crossing & 200 & B-04-039 P5060030 \\
\hline
\end{tabular}


Appendix 8: List of all photographs taken showing the corresponding site number, descriptive caption, direction faced when taken (azimuth in degrees) and the unique file name for each photograph.

\begin{tabular}{|c|c|c|c|}
\hline $\begin{array}{c}\text { Site } \\
\text { Number }\end{array}$ & Photo Description & Azimuth & File Name \\
\hline B-04-039 & Looking downstream from crossing & 360 & B-04-039_P5060031 \\
\hline B-04-039 & Looking upstream at culvert outlets & $\mathrm{N} / \mathrm{A}$ & B-04-039-P5060032 \\
\hline B-04-039 & Looking downstream at water control structure & 335 & B-04-039_P5060033 \\
\hline B-04-039 & Looking upstream at Pembina River weir & 210 & B-04-039_P5060034 \\
\hline B-04-039 & Looking downstream from above weir & 125 & B-04-039_P5060035 \\
\hline B-04-040 & Looking upstream from gravel road crossing & 125 & B-04-040_P5070001 \\
\hline B-04-040 & Looking downstream from gravel road crossing & 70 & B-04-040_P5070002 \\
\hline B-04-041 & Looking upstream from culvert crossing & 360 & B-04-041_P5070003 \\
\hline B-04-041 & Looking downstream from culvert crossing & 180 & B-04-041_P5070004 \\
\hline B-04-042 & Looking upstream from gravel road crossing & 150 & B-04-042 P5070005 \\
\hline B-04-042 & Looking downstream from gravel road crossing & 330 & B-04-042_P5070006 \\
\hline B-04-043 & Looking upstream from culvert crossing & 90 & B-04-043_P5070007 \\
\hline B-04-043 & Looking downstream from culvert crossing & 250 & B-04-043_P5070008 \\
\hline B-04-043 & Looking downstream at culvert inlet & 340 & B-04-043_P5070009 \\
\hline B-04-043 & Looking upstream at culvert outlet & 160 & B-04-043_P5070010 \\
\hline B-04-044 & Looking upstream from culvert crossing & 90 & B-04-044_P5070011 \\
\hline B-04-044 & Looking downstream from culvert crossing & 180 & B-04-044_P5070012 \\
\hline B-04-044 & Looking upstream at culvert outlet & 140 & B-04-044_P5070013 \\
\hline B-04-044 & Looking at downstream at culvert inlet & 250 & B-04-044_P5070014 \\
\hline B-04-045 & Looking upstream from culvert crossing & 230 & B-04-045_P5070015 \\
\hline B-04-045 & Looking downstream from culvert crossing & 115 & B-04-045_P5070016 \\
\hline B-04-045 & Looking upstream at perched culverts & $\mathrm{N} / \mathrm{A}$ & B-04-045_P5070017 \\
\hline B-04-046 & Looking upstream from culvert crossing & 90 & B-04-046_P5100001 \\
\hline B-04-046 & Looking downstream from culvert crossing & 270 & B-04-046_P5100002 \\
\hline B-04-046 & Looking downstream at culvert inlet & 320 & B-04-046 P5100003 \\
\hline B-04-046 & Looking upstream at culvert outlet & 70 & B-04-046_P5100004 \\
\hline B-04-046 & Looking at released Creek Chub $(170 \mathrm{~mm})$ & $\mathrm{N} / \mathrm{A}$ & B-04-046_P5100005 \\
\hline B-04-046 & Looking at released Creek Chub $(170 \mathrm{~mm})$ & $\mathrm{N} / \mathrm{A}$ & B-04-046_P5100006 \\
\hline B-04-046 & Looking at Northern Redbelly Dace & N/A & B-04-046_P5100007 \\
\hline B-04-047 & Looking upstream from culvert crossing & 200 & B-04-047_P5100008 \\
\hline B-04-047 & Looking downstream from culvert crossing & 30 & B-04-047_P5100009 \\
\hline B-04-047 & Looking downstream at culvert inlets & 35 & B-04-047_P5100010 \\
\hline B-04-047 & Looking upstream at culvert outlets & 160 & B-04-047_P5100011 \\
\hline
\end{tabular}


Appendix 8: List of all photographs taken showing the corresponding site number, descriptive caption, direction faced when taken (azimuth in degrees) and the unique file name for each photograph.

\begin{tabular}{|c|c|c|c|}
\hline $\begin{array}{c}\text { Site } \\
\text { Number }\end{array}$ & Photo Description & Azimuth & File Name \\
\hline B-04-048 & Looking upstream from bridge crossing & 135 & B-04-048 P5100012 \\
\hline B-04-048 & Looking downstream from bridge crossing & 330 & B-04-048_P5100013 \\
\hline B-04-049 & Looking upstream from crossing & 65 & B-04-049_P5130001 \\
\hline B-04-049 & Looking downstream from crossing & 250 & B-04-049 P5130002 \\
\hline B-04-049 & Looking downstream at inlet of cement box culvert & 240 & B-04-049 P5130003 \\
\hline B-04-050 & Looking upstream from road crossing & 350 & B-04-050 P5130004 \\
\hline B-04-050 & Looking downstream from road crossing & 170 & B-04-050_P5130005 \\
\hline B-04-050 & Looking at perched culvert & $\mathrm{N} / \mathrm{A}$ & B-04-050 P5130006 \\
\hline B-04-051 & Looking upstream from culvert crossing & 350 & B-04-051 P5130007 \\
\hline B-04-051 & Looking downstream from culvert crossing & 170 & B-04-051_P5130008 \\
\hline B-04-052 & Looking upstream from road crossing & 20 & B-04-052_P5130009 \\
\hline B-04-052 & Looking downstream from road crossing & 200 & B-04-052 P5130010 \\
\hline B-04-053 & Looking upstream from bridge crossing & 20 & B-04-053 P5130011 \\
\hline B-04-053 & Looking downstream from bridge crossing & 190 & B-04-053 P5130012 \\
\hline B-04-054 & Looking upstream from road crossing & 360 & B-04-054_P5130013 \\
\hline B-04-054 & Looking downstream from road crossing & 190 & B-04-054 P5130014 \\
\hline B-04-055 & Looking upstream from bridge crossing & 10 & B-04-055 P5130015 \\
\hline B-04-055 & Looking downstream from bridge crossing & 80 & B-04-055 P5130016 \\
\hline B-04-056 & Looking upstream from culvert crossing & 45 & B-04-056_P5130017 \\
\hline B-04-056 & Looking downstream from culvert crossing & 270 & B-04-056 P5130018 \\
\hline B-04-057 & Looking upstream from bridge crossing & 25 & B-04-057 P5140001 \\
\hline B-04-057 & Looking downstream from bridge crossing & 220 & B-04-057_P5140002 \\
\hline B-04-058 & Looking upstream from road crossing & 260 & B-04-058_P5140003 \\
\hline B-04-058 & Looking downstream from road crossing & 110 & B-04-058_P5140004 \\
\hline B-04-058 & Looking upstream at metal pipe outlet & $\mathrm{N} / \mathrm{A}$ & B-04-058 P5140005 \\
\hline B-04-058 & Photo of side view of metal pipe inlet & $\mathrm{N} / \mathrm{A}$ & B-04-058_P5140006 \\
\hline B-04-059 & Looking upstream from culvert crossing & 350 & B-04-059_P5140007 \\
\hline B-04-059 & Looking downstream from culvert crossing & 180 & B-04-059 P5140008 \\
\hline B-04-059 & Looking upstream at perched culvert outlet & $\mathrm{N} / \mathrm{A}$ & B-04-059 P5140009 \\
\hline B-04-059 & Photo of side view of inlet & N/A & B-04-059_P5140010 \\
\hline B-04-060 & Looking upstream from bridge crossing & 260 & B-04-060_P5140011 \\
\hline B-04-060 & Looking downstream from bridge crossing & 100 & B-04-060 P5140012 \\
\hline B-04-061 & Looking upstream from culvert crossing & 180 & B-04-061_P5140013 \\
\hline
\end{tabular}


Appendix 8: List of all photographs taken showing the corresponding site number, descriptive caption, direction faced when taken (azimuth in degrees) and the unique file name for each photograph.

\begin{tabular}{|c|c|c|c|}
\hline $\begin{array}{c}\text { Site } \\
\text { Number }\end{array}$ & Photo Description & Azimuth & File Name \\
\hline B-04-061 & Looking downstream from culvert crossing & 0 & B-04-061_P5140014 \\
\hline B-04-061 & Looking upstream at perched culvert & N/A & B-04-061_P5140015 \\
\hline B-04-062 & Looking upstream from road crossing & 160 & B-04-062_P5140016 \\
\hline B-04-062 & Looking upstream from road crossing & 130 & B-04-062_P5140017 \\
\hline B-04-063 & Looking upstream from road crossing & 80 & B-04-063_P5140018 \\
\hline B-04-063 & Looking downstream from road crossing & 290 & B-04-063_P5140019 \\
\hline B-04-064 & Looking upstream from bridge crossing & 95 & B-04-064_P5140020 \\
\hline B-04-064 & Looking downstream from bridge crossing & 270 & B-04-064_P5140021 \\
\hline B-04-065 & Looking upstream from bend & 270 & B-04-065_P5170001 \\
\hline B-04-065 & Looking downstream from bend & 0 & B-04-065 P5170002 \\
\hline B-04-065 & Looking east along roadside to Big Grass Marsh & N/A & B-04-065_P5170003 \\
\hline B-04-066 & Looking upstream from middle of reach & 270 & B-04-066_P5170004 \\
\hline B-04-066 & Looking downstream from middle of reach & 90 & B-04-066_P5170005 \\
\hline B-04-067 & Looking upstream from culvert crossing & 270 & B-04-067_P5170006 \\
\hline B-04-067 & Looking downstream from culvert crossing & 90 & B-04-067_P5170007 \\
\hline B-04-067 & Looking upstream at culvert outlets & $\mathrm{N} / \mathrm{A}$ & B-04-067_P5170008 \\
\hline B-04-068 & Looking upstream from bridge crossing & 270 & B-04-068_P5170009 \\
\hline B-04-068 & Looking downstream from bridge crossing & 90 & B-04-068 P5170010 \\
\hline B-04-069 & Looking upstream from culvert crossing & 290 & B-04-069_P5180001 \\
\hline B-04-069 & Looking downstream from culvert crossing & 95 & B-04-069_P5180002 \\
\hline B-04-069 & Looking upstream at culvert outlet & N/A & B-04-069_P5180003 \\
\hline B-04-069 & Looking at pond filled by backflow & 5 & B-04-069_P5180004 \\
\hline B-04-069 & Looking at pond filled by backflow & 310 & B-04-069_P5180005 \\
\hline B-04-069 & Looking at pond filled by backflow & 295 & B-04-069_P5180006 \\
\hline B-04-069 & Looking at pond outlet & $\mathrm{N} / \mathrm{A}$ & B-04-069 P5180007 \\
\hline B-04-071 & Looking upstream from bridge crossing & 50 & B-04-071_P5180008 \\
\hline B-04-071 & Looking downstream from bridge crossing & 210 & B-04-071_P5180009 \\
\hline B-04-072 & Looking upstream from constructed riffle & 180 & B-04-072_P5180010 \\
\hline B-04-072 & Looking downstream from constructed riffle & 360 & B-04-072_P5180011 \\
\hline B-04-072 & Looking across area & 250 & B-04-072_P5180012 \\
\hline B-04-073 & Looking south across drop structure & 180 & B-04-073_P5190001 \\
\hline B-04-073 & Looking upstream from drop structure & 270 & B-04-073_P5190002 \\
\hline B-04-073 & Looking downstream from drop structure & 90 & B-04-073_P5190003 \\
\hline
\end{tabular}


Appendix 8: List of all photographs taken showing the corresponding site number, descriptive caption, direction faced when taken (azimuth in degrees) and the unique file name for each photograph.

\begin{tabular}{|c|c|c|c|}
\hline $\begin{array}{c}\text { Site } \\
\text { Number }\end{array}$ & Photo Description & Azimuth & File Name \\
\hline B-04-073 & Looking upstream from drop structure & 270 & B-04-073 P5190004 \\
\hline B-04-074 & Looking upstream from middle of reach & 270 & B-04-074_P5190005 \\
\hline B-04-074 & Looking downstream from middle of reach & 90 & B-04-074_P5190006 \\
\hline B-04-075 & Looking upstream from middle of reach & 200 & B-04-075 P5200001 \\
\hline B-04-075 & Looking downstream from middle of reach & 340 & B-04-075 P5200002 \\
\hline B-04-075 & Looking downstream over riffle & 330 & B-04-075_P5200003 \\
\hline B-04-076 & Looking upstream from middle of reach & 270 & B-04-076_P5200004 \\
\hline B-04-076 & Looking downstream from middle of reach & 90 & B-04-076_P5200005 \\
\hline B-04-077 & Looking upstream from culvert crossing & 270 & B-04-077 P5200006 \\
\hline B-04-077 & Looking downstream from culvert crossing & 90 & B-04-077_P5200007 \\
\hline B-04-078 & Looking upstream from culvert crossing & 200 & B-04-078_P5200008 \\
\hline B-04-078 & Looking downstream from culvert crossing & 80 & B-04-078 P5200009 \\
\hline B-04-079 & Looking upstream from culvert crossing & 300 & B-04-079 P5200010 \\
\hline B-04-079 & Looking downstream from culvert crossing & 20 & B-04-079 P5200011 \\
\hline B-04-080 & Looking upstream from culvert crossing & 270 & B-04-080_P5200012 \\
\hline B-04-080 & Looking upstream from culvert crossing & 45 & B-04-080 P5200013 \\
\hline B-04-081 & Looking upstream from culvert crossing & 270 & B-04-081_P5200014 \\
\hline B-04-081 & Looking downstream from culvert crossing & 0 & B-04-081_P5200015 \\
\hline B-04-081 & Looking downstream at culvert inlets & 90 & B-04-081_P5200016 \\
\hline B-04-081 & Looking upstream at culvert outlets & 270 & B-04-081 P5200017 \\
\hline B-04-082 & Looking upstream from culvert crossing & 270 & B-04-082 P5200018 \\
\hline B-04-082 & Looking downstream from culvert crossing & 90 & B-04-082_P5200019 \\
\hline B-04-082 & Looking downstream from culvert inlets & 95 & B-04-082_P5200020 \\
\hline B-04-082 & Looking upstream at culvert outlets & 275 & B-04-082_P5200021 \\
\hline B-04-083 & Looking upstream from culvert crossing & 270 & B-04-083_P5200022 \\
\hline B-04-083 & Looking downstream from culvert crossing & 90 & B-04-083_P5200023 \\
\hline B-04-083 & Looking downstream at culvert inlets & 95 & B-04-083_P5200024 \\
\hline B-04-083 & Looking upstream at culvert outlets & 260 & B-04-083 P5200025 \\
\hline B-04-084 & Photo of stranded Northern Pike & $\mathrm{N} / \mathrm{A}$ & B-04-084 P5200026 \\
\hline B-04-084 & Photo of stranded Northern Pike & N/A & B-04-084_P5200027 \\
\hline B-04-084 & Photo of stranded Northern Pike & $\mathrm{N} / \mathrm{A}$ & B-04-084_P5200028 \\
\hline B-04-084 & Looking upstream from culvert crossing & 270 & B-04-084 P5200029 \\
\hline B-04-084 & Looking downstream from culvert crossing & 90 & B-04-084 P5200030 \\
\hline
\end{tabular}


Appendix 8: List of all photographs taken showing the corresponding site number, descriptive caption, direction faced when taken (azimuth in degrees) and the unique file name for each photograph.

\begin{tabular}{|c|c|c|c|}
\hline $\begin{array}{c}\text { Site } \\
\text { Number }\end{array}$ & Photo Description & Azimuth & File Name \\
\hline B-04-084 & Looking downstream at culvert inlets & 135 & B-04-084 P5200031 \\
\hline B-04-084 & Looking upstream at culvert outlets & 270 & B-04-084_P5200032 \\
\hline B-04-085 & Looking upstream from culvert crossing & 270 & B-04-085_P5200033 \\
\hline B-04-085 & Looking downstream from culvert crossing & 90 & B-04-085 P5200034 \\
\hline B-04-085 & Photo of injured Northern Pike with infection & N/A & B-04-085 P5200035 \\
\hline B-04-085 & Photo of injured Northern Pike with infection & $\mathrm{N} / \mathrm{A}$ & B-04-085_P5200036 \\
\hline B-04-085 & Photo of injured Northern Pike with infection & $\mathrm{N} / \mathrm{A}$ & B-04-085_P5200037 \\
\hline B-04-085 & Looking at culvert inlets & $\mathrm{N} / \mathrm{A}$ & B-04-085 P5200038 \\
\hline B-04-086 & Looking upstream from bridge & 270 & B-04-086 P5210001 \\
\hline B-04-086 & Looking downstream from bridge & 90 & B-04-086 P5210002 \\
\hline B-04-087 & Looking at drift trap & $\mathrm{N} / \mathrm{A}$ & B-04-087_P5210003 \\
\hline B-04-087 & Looking at drift trap & N/A & B-04-087 P5210004 \\
\hline B-04-088 & Looking upstream from road crossing & 10 & B-04-088 P5250001 \\
\hline B-04-088 & Looking downstream from road crossing & 190 & B-04-088 P5250002 \\
\hline B-04-088 & Looking at drift trap & $\mathrm{N} / \mathrm{A}$ & B-04-088_P5250003 \\
\hline B-04-089 & Looking upstream from road crossing & 270 & B-04-089_P5250004 \\
\hline B-04-089 & Looking downstream from road crossing & 90 & B-04-089 P5250005 \\
\hline B-04-090 & Looking upstream from crossing of first tributary & 80 & B-04-090 P5250006 \\
\hline B-04-090 & Looking upstream from crossing of second tributary & 100 & B-04-090_P5250007 \\
\hline B-04-090 & Looking downstream from crossing & 270 & B-04-090 P5250008 \\
\hline B-04-091 & Looking upstream from crossing of 455 & 180 & B-04-091_P5250009 \\
\hline B-04-091 & Looking downstream from crossing of 455 & 360 & B-04-091_P5250010 \\
\hline B-04-092 & Looking upstream from bridge & 345 & B-04-092_P5250011 \\
\hline B-04-092 & Looking downstream from bridge & 200 & B-04-092_P5250012 \\
\hline B-04-093 & Looking upstream from bridge crossing & 360 & B-04-093_P5310001 \\
\hline B-04-093 & Looking downstream from bridge crossing & 200 & B-04-093_P5310002 \\
\hline B-04-094 & Looking upstream from bridge crossing & 90 & B-04-094_P5310003 \\
\hline B-04-094 & Looking downstream from bridge crossing & 270 & B-04-094 P5310004 \\
\hline B-04-095 & Looking upstream from bridge crossing & 360 & B-04-095 P5310005 \\
\hline B-04-095 & Looking downstream from bridge crossing & 225 & B-04-095 P5310006 \\
\hline B-04-095 & Looking downstream from bridge crossing & N/A & B-04-095_P5310007 \\
\hline B-04-096 & Looking upstream from bridge crossing & 360 & B-04-096 P6010001 \\
\hline B-04-096 & Looking downstream from bridge crossing & 180 & B-04-096_P6010002 \\
\hline
\end{tabular}


Appendix 8: List of all photographs taken showing the corresponding site number, descriptive caption, direction faced when taken (azimuth in degrees) and the unique file name for each photograph.

\begin{tabular}{|c|c|c|c|}
\hline $\begin{array}{c}\text { Site } \\
\text { Number }\end{array}$ & Photo Description & Azimuth & File Name \\
\hline B-04-097 & Looking upstream from culvert crossing & 310 & B-04-097 P6010003 \\
\hline B-04-097 & Looking downstream from culvert crossing & 90 & B-04-097_P6010004 \\
\hline B-04-097 & Looking at metal pipe outlets & 195 & B-04-097_P6010005 \\
\hline B-04-098 & Looking upstream from culvert crossing & 180 & B-04-098_P6010006 \\
\hline B-04-098 & Looking downstream from culvert crossing & 280 & B-04-098 P6010007 \\
\hline B-04-098 & Looking at culvert outlets & $\mathrm{N} / \mathrm{A}$ & B-04-098_P6010008 \\
\hline B-04-099 & Looking upstream from culvert crossing & 180 & B-04-099_P6010009 \\
\hline B-04-099 & Looking downstream from culvert crossing & 310 & B-04-099-P6010010 \\
\hline B-04-099 & Looking at culvert outlets & $\mathrm{N} / \mathrm{A}$ & B-04-099 P6010011 \\
\hline B-04-100 & Looking upstream from culvert crossing & 210 & B-04-100 P6010012 \\
\hline B-04-100 & Looking downstream from culvert crossing & 10 & B-04-100_P6010013 \\
\hline B-04-101 & Looking upstream from bridge crossing & 110 & B-04-101_P6010014 \\
\hline B-04-101 & Looking downstream from bridge crossing & 270 & B-04-101_P6010015 \\
\hline B-04-102 & Looking upstream from bridge crossing & 290 & B-04-102 P6010016 \\
\hline B-04-102 & Looking downstream from bridge crossing & 155 & B-04-102_P6010017 \\
\hline B-04-103 & Looking upstream from culvert crossing & 290 & B-04-103 P6010018 \\
\hline B-04-103 & Looking downstream from culvert crossing & 110 & B-04-103 P6010019 \\
\hline B-04-104 & Looking upstream from bridge crossing & 250 & B-04-104_P6010020 \\
\hline B-04-104 & Looking downstream from bridge crossing & 100 & B-04-104_P6010021 \\
\hline B-04-105 & Looking upstream from bridge crossing & 350 & B-04-105 P6010022 \\
\hline B-04-105 & Looking downstream from bridge crossing & 180 & B-04-105 P6010023 \\
\hline B-04-106 & Looking upstream from culvert crossing & 190 & B-04-106_P6020001 \\
\hline B-04-106 & Looking downstream from culvert crossing & 270 & B-04-106_P6020002 \\
\hline B-04-107 & Looking upstream from culvert crossing & 120 & B-04-107_P6020003 \\
\hline B-04-107 & Looking downstream from culvert crossing & 225 & B-04-107_P6020004 \\
\hline B-04-108 & Looking upstream from culvert crossing & 50 & B-04-108_P6020005 \\
\hline B-04-108 & Looking downstream from culvert crossing & 190 & B-04-108_P6020006 \\
\hline B-04-108 & Looking at culvert inlets & $\mathrm{N} / \mathrm{A}$ & B-04-108 P6020007 \\
\hline B-04-108 & Looking at culvert outlets & $\mathrm{N} / \mathrm{A}$ & B-04-108 P6020008 \\
\hline B-04-109 & Looking upstream from culvert crossing & 350 & B-04-109_P6020009 \\
\hline B-04-109 & Looking downstream from culvert crossing & 180 & B-04-109 P6020010 \\
\hline B-04-109 & Looking at culvert outlets & $\mathrm{N} / \mathrm{A}$ & B-04-109 P6020011 \\
\hline B-04-110 & Looking upstream from culvert crossing & 110 & B-04-110 P6020012 \\
\hline
\end{tabular}


Appendix 8: List of all photographs taken showing the corresponding site number, descriptive caption, direction faced when taken (azimuth in degrees) and the unique file name for each photograph.

\begin{tabular}{|c|c|c|c|}
\hline $\begin{array}{c}\text { Site } \\
\text { Number }\end{array}$ & Photo Description & Azimuth & File Name \\
\hline B-04-110 & Looking downstream from culvert crossing & 300 & B-04-110_P6020013 \\
\hline B-04-110 & Looking across riffle and culvert outlets & $\mathrm{N} / \mathrm{A}$ & B-04-110 P6020014 \\
\hline B-04-111 & Looking upstream from road & 180 & B-04-111_P6020015 \\
\hline B-04-111 & Looking downstream from road & 360 & B-04-111_P6020016 \\
\hline B-04-111 & Looking at flooded river valley & $\mathrm{N} / \mathrm{A}$ & B-04-111_P6020017 \\
\hline B-04-111 & Looking at flooded river valley & $\mathrm{N} / \mathrm{A}$ & B-04-111_P6020018 \\
\hline B-04-111 & Looking at flooded river valley & $\mathrm{N} / \mathrm{A}$ & B-04-111_P6020019 \\
\hline B-04-111 & Looking at flooded river valley & N/A & B-04-111_P6020020 \\
\hline B-04-112 & Looking upstream from cement box culvert & 180 & B-04-112_P6020021 \\
\hline B-04-112 & Looking downstream from cement box culvert & 360 & B-04-112 P6020022 \\
\hline B-04-112 & Looking at inlet of cement box culvert & N/A & B-04-112_P6020023 \\
\hline B-04-113 & Looking upstream from bridge crossing & 180 & B-04-113_P6020024 \\
\hline B-04-113 & Looking downstream from bridge crossing & 45 & B-04-113_P6020025 \\
\hline B-04-114 & Looking upstream from cement box culvert & 200 & B-04-114_P6030001 \\
\hline B-04-114 & Looking downstream from cement box culvert & 20 & B-04-114_P6030002 \\
\hline B-04-114 & Looking at outflow from dam & $\mathrm{N} / \mathrm{A}$ & B-04-114_P6030003 \\
\hline B-04-115 & Looking upstream from bridge crossing & 270 & B-04-115_P6030004 \\
\hline B-04-115 & Looking downstream from bridge crossing & 115 & B-04-115 P6030005 \\
\hline B-04-116 & Looking upstream from cement supported metal pipe & 360 & B-04-116_P6030006 \\
\hline B-04-116 & Looking downstream from cement supported metal pipe & 180 & B-04-116_P6030007 \\
\hline B-04-116 & Looking at culvert outlet & N/A & B-04-116_P6030008 \\
\hline B-04-117 & Looking upstream from bridge crossing & 90 & B-04-117_P6040001 \\
\hline B-04-117 & Looking downstream from bridge crossing & 270 & B-04-117_P6040002 \\
\hline B-04-118 & Looking upstream from bridge crossing & 140 & B-04-118_P6040003 \\
\hline B-04-118 & Looking downstream from bridge crossing & 50 & B-04-118_P6040004 \\
\hline B-04-118 & Looking downstream from bridge crossing & 260 & B-04-118_P6040005 \\
\hline B-04-119 & Looking upstream from culvert crossing & 170 & B-04-119_P6040006 \\
\hline B-04-119 & Looking downstream from culvert crossing & 360 & B-04-119_P6040007 \\
\hline B-04-119 & Looking west across culvert outlet & N/A & B-04-119_P6040008 \\
\hline B-04-120 & Looking downstream from bridge crossing & 130 & B-04-120_P6040009 \\
\hline B-04-120 & Looking upstream from bridge crossing & 300 & B-04-120_P6040010 \\
\hline B-04-120 & Looking upstream from bridge crossing & 335 & B-04-120_P6040011 \\
\hline B-04-121 & Looking upstream from bridge crossing & 90 & B-04-121_P6040012 \\
\hline
\end{tabular}


Appendix 8: List of all photographs taken showing the corresponding site number, descriptive caption, direction faced when taken (azimuth in degrees) and the unique file name for each photograph.

\begin{tabular}{|c|c|c|c|}
\hline $\begin{array}{c}\text { Site } \\
\text { Number }\end{array}$ & Photo Description & Azimuth & File Name \\
\hline B-04-121 & Looking downstream from bridge crossing & 270 & B-04-121_P6040013 \\
\hline B-04-122 & Looking upstream from bridge crossing & 90 & B-04-122 P6040014 \\
\hline B-04-122 & Looking downstream from bridge crossing & 270 & B-04-122_P6040015 \\
\hline B-04-123 & Looking upstream from bridge crossing & 100 & B-04-123_P6070001 \\
\hline B-04-123 & Looking downstream from bridge crossing & 250 & B-04-123_P6070002 \\
\hline B-04-124 & Looking upstream from bridge crossing & 250 & B-04-124_P6070003 \\
\hline B-04-124 & Looking downstream from bridge crossing & 120 & B-04-124_P6070004 \\
\hline B-04-125 & Looking upstream from bridge crossing & 180 & B-04-125_P6070005 \\
\hline B-04-125 & Looking downstream from bridge crossing & 360 & B-04-125_P6070006 \\
\hline B-04-126 & Looking upstream from culvert crossing & 270 & B-04-126_P6080001 \\
\hline B-04-126 & Looking downstream from culvert crossing & 90 & B-04-126_P6080002 \\
\hline B-04-126 & Looking at culvert outlets & $\mathrm{N} / \mathrm{A}$ & B-04-126_P6080003 \\
\hline B-04-126 & Looking at culvert inlets & $\mathrm{N} / \mathrm{A}$ & B-04-126_P6080004 \\
\hline B-04-127 & Looking upstream from culvert crossing & 160 & B-04-127_P6080005 \\
\hline B-04-127 & Looking downstream from culvert crossing & 340 & B-04-127_P6080006 \\
\hline B-04-127 & Looking at culvert outlets & $\mathrm{N} / \mathrm{A}$ & B-04-127_P6080007 \\
\hline B-04-127 & Looking at culvert inlets & $\mathrm{N} / \mathrm{A}$ & B-04-127_P6080008 \\
\hline B-04-128 & Looking upstream from culvert crossing & 310 & B-04-128_P6080009 \\
\hline B-04-128 & Looking downstream from culvert crossing & 170 & B-04-128_P6080010 \\
\hline B-04-128 & Looking at culvert inlets & N/A & B-04-128_P6080011 \\
\hline$B-04-128$ & Looking at culvert outlets & N/A & B-04-128_P6080012 \\
\hline B-04-129 & Looking upstream from culvert crossing & 170 & B-04-129_P6080013 \\
\hline B-04-129 & Looking downstream from culvert crossing & 350 & B-04-129_P6080014 \\
\hline B-04-129 & Looking at culvert inlets & $\mathrm{N} / \mathrm{A}$ & B-04-129_P6080015 \\
\hline B-04-129 & Looking at culvert outlets & $\mathrm{N} / \mathrm{A}$ & B-04-129 P6080016 \\
\hline B-04-130 & Looking upstream from culvert crossing & 170 & B-04-130 P6090001 \\
\hline B-04-130 & Looking downstream from culvert crossing & 45 & B-04-130_P6090002 \\
\hline B-04-130 & Looking at culvert inlets & $\mathrm{N} / \mathrm{A}$ & B-04-130_P6090003 \\
\hline B-04-130 & Looking at culvert outlets & N/A & B-04-130_P6090004 \\
\hline B-04-131 & Looking upstream from cement box culvert & 180 & B-04-131_P6090005 \\
\hline B-04-131 & Looking downstream from cement box culvert & 360 & B-04-131_P6090006 \\
\hline B-04-132 & Looking upstream from culvert crossing & 230 & B-04-132_P6090007 \\
\hline B-04-132 & Looking downstream from culvert crossing & 200 & B-04-132_P6090008 \\
\hline
\end{tabular}


Appendix 8: List of all photographs taken showing the corresponding site number, descriptive caption, direction faced when taken (azimuth in degrees) and the unique file name for each photograph.

\begin{tabular}{|c|c|c|c|}
\hline $\begin{array}{c}\text { Site } \\
\text { Number }\end{array}$ & Photo Description & Azimuth & File Name \\
\hline B-04-133 & Looking upstream from bridge crossing & 180 & B-04-133 P6090009 \\
\hline B-04-133 & Looking downstream from bridge crossing & 360 & B-04-133_P6090010 \\
\hline B-04-134 & Looking upstream from bridge crossing & 180 & B-04-134_P6090011 \\
\hline B-04-134 & Looking downstream from bridge crossing & 30 & B-04-134 P6090012 \\
\hline B-04-135 & Looking upstream from culvert crossing & 180 & B-04-135 P6090013 \\
\hline B-04-135 & Looking downstream from culvert crossing & 360 & B-04-135_P6090014 \\
\hline B-04-136 & Looking upstream from middle of reach & 10 & B-04-136_P6100001 \\
\hline B-04-136 & Looking downstream from middle of reach & 170 & B-04-136_P6100002 \\
\hline B-04-137 & Looking upstream from culvert crossing & 110 & B-04-137 P6100003 \\
\hline B-04-137 & Looking downstream from culvert crossing & 290 & B-04-137_P6100004 \\
\hline B-04-137 & Looking at culvert inlets & $\mathrm{N} / \mathrm{A}$ & B-04-137_P6100005 \\
\hline B-04-138 & Looking upstream from highway & 160 & B-04-138 P6100006 \\
\hline B-04-138 & Looking downstream from highway & 360 & B-04-138 P6100007 \\
\hline B-04-139 & Looking upstream from culvert crossing & 180 & B-04-139 P6100008 \\
\hline B-04-139 & Looking downstream from culvert crossing & 360 & B-04-139_P6100009 \\
\hline B-04-140 & Looking upstream from box-style bridge & 210 & B-04-140 P6140001 \\
\hline B-04-140 & Looking downstream from box-style bridge & 75 & B-04-140 P6140002 \\
\hline B-04-140 & Looking at culvert outlet & 250 & B-04-140_P6140003 \\
\hline B-04-141 & Looking upstream from culvert crossing & 70 & B-04-141_P6140004 \\
\hline B-04-141 & Looking downstream from culvert crossing & N/A & B-04-141 P6140005 \\
\hline B-04-141 & Looking at culvert outlets & $\mathrm{N} / \mathrm{A}$ & B-04-141 P6140006 \\
\hline B-04-142 & Looking upstream from culvert crossing & 225 & B-04-142_P6140007 \\
\hline B-04-142 & Looking downstream from culvert crossing & 100 & B-04-142_P6140008 \\
\hline B-04-142 & Looking at culvert outlets & $\mathrm{N} / \mathrm{A}$ & B-04-142 P6140009 \\
\hline B-04-142 & Looking at culvert inlets & $\mathrm{N} / \mathrm{A}$ & B-04-142 P6140010 \\
\hline B-04-143 & Looking upstream from culvert crossing & 270 & B-04-143_P6140011 \\
\hline B-04-143 & Looking downstream from culvert crossing & 90 & B-04-143_P6140012 \\
\hline B-04-143 & Looking at culvert inlets & $\mathrm{N} / \mathrm{A}$ & B-04-143 P6140013 \\
\hline B-04-143 & Looking at culvert outlets & $\mathrm{N} / \mathrm{A}$ & B-04-143 P6140014 \\
\hline B-04-144 & Looking upstream from bridge crossing & 110 & B-04-144_P6150001 \\
\hline B-04-144 & Looking downstream from bridge crossing & 270 & B-04-144_P6150002 \\
\hline B-04-145 & Looking upstream from culvert crossing & 175 & $\mathrm{~B}-04-145$ P6150003 \\
\hline B-04-145 & Looking downstream from culvert crossing & 360 & B-04-145 P6150004 \\
\hline
\end{tabular}


Appendix 8: List of all photographs taken showing the corresponding site number, descriptive caption, direction faced when taken (azimuth in degrees) and the unique file name for each photograph.

\begin{tabular}{|c|c|c|c|}
\hline $\begin{array}{c}\text { Site } \\
\text { Number }\end{array}$ & Photo Description & Azimuth & File Name \\
\hline B-04-145 & Looking at culvert inlets & $\mathrm{N} / \mathrm{A}$ & B-04-145_P6150005 \\
\hline B-04-145 & Looking at culvert outlets & N/A & B-04-145 P6150006 \\
\hline B-04-146 & Looking upstream from culvert crossing & 200 & B-04-146 P6150007 \\
\hline B-04-146 & Looking downstream from culvert crossing & 360 & B-04-146_P6150008 \\
\hline B-04-146 & Looking at culvert inlets & $\mathrm{N} / \mathrm{A}$ & B-04-146_P6150009 \\
\hline B-04-146 & Looking at culvert outlets & $\mathrm{N} / \mathrm{A}$ & B-04-146_P6150010 \\
\hline B-04-147 & Looking upstream from culvert crossing & 230 & B-04-147_P6150011 \\
\hline B-04-147 & Looking downstream from culvert crossing & 360 & B-04-147_P6150012 \\
\hline B-04-147 & Looking at culvert outlets & N/A & B-04-147_P6150013 \\
\hline B-04-147 & Looking at culvert inlets & $\mathrm{N} / \mathrm{A}$ & B-04-147_P6150014 \\
\hline B-04-148 & Looking upstream from culvert crossing & 180 & B-04-148 P6150015 \\
\hline B-04-148 & Looking downstream from culvert crossing & 360 & B-04-148_P6150016 \\
\hline B-04-149 & Looking upstream from middle of reach & 360 & B-04-149_P6160001 \\
\hline B-04-149 & Looking downstream from middle of reach & 200 & B-04-149_P6160002 \\
\hline B-04-149 & Looking upstream at weir from highway & $\mathrm{N} / \mathrm{A}$ & B-04-149_P6160003 \\
\hline B-04-149 & Looking upstream from weir & 180 & B-04-149_P6160004 \\
\hline B-04-150 & Looking upstream from culvert crossing & 160 & B-04-150_P6160005 \\
\hline B-04-150 & Looking downstream from culvert crossing & 360 & B-04-150_P6160006 \\
\hline B-04-150 & Looking at culvert outlets & $\mathrm{N} / \mathrm{A}$ & B-04-150 P6160007 \\
\hline B-04-150 & Looking at lip of cement infill & N/A & B-04-150 P6160008 \\
\hline B-04-150 & Looking upstream through culvert & N/A & B-04-150_P6160009 \\
\hline B-04-151 & Looking upstream from ford crossing & 180 & B-04-151_P6160010 \\
\hline B-04-151 & Looking downstream from ford crossing & 360 & B-04-151_P6160011 \\
\hline B-04-152 & Looking upstream from old ford crossing & 90 & B-04-152 P6160012 \\
\hline B-04-152 & Looking downstream from old ford crossing & 270 & B-04-152_P6160013 \\
\hline B-04-153 & Looking upstream from bridge crossing & 180 & B-04-153 P6160014 \\
\hline B-04-153 & Looking downstream from bridge crossing & 360 & B-04-153_P6160015 \\
\hline B-04-153 & Looking west at inflow from 3rd order tributary & 290 & B-04-153_P6160016 \\
\hline B-04-153 & Looking at beaver dam from $125 \mathrm{~m}$ upstream of bridge & 230 & B-04-153_P6160017 \\
\hline B-04-154 & Looking upstream from culvert crossing & 150 & B-04-154_P6160018 \\
\hline B-04-154 & Looking downstream from culvert crossing & 310 & B-04-154_P6160019 \\
\hline B-04-155 & Looking upstream from bridge crossing on highway & 130 & B-04-155_P6160020 \\
\hline B-04-155 & Looking downstream from bridge crossing on highway & 270 & B-04-155_P6160021 \\
\hline
\end{tabular}


Appendix 8: List of all photographs taken showing the corresponding site number, descriptive caption, direction faced when taken (azimuth in degrees) and the unique file name for each photograph.

\begin{tabular}{|c|c|c|c|}
\hline $\begin{array}{c}\text { Site } \\
\text { Number }\end{array}$ & Photo Description & Azimuth & File Name \\
\hline $\mathrm{B}-04-156$ & Looking upstream at control structure & $\mathrm{N} / \mathrm{A}$ & B-04-156 P6170001 \\
\hline B-04-156 & Looking upstream from control structure & 360 & B-04-156_P6170002 \\
\hline B-04-156 & Looking across control structure & $\mathrm{N} / \mathrm{A}$ & B-04-156_P6170003 \\
\hline B-04-156 & Looking downstream from control structure & 180 & B-04-156 P6170004 \\
\hline B-04-157 & Looking upstream from culvert crossing & 180 & B-04-157_P6170005 \\
\hline B-04-157 & Looking downstream from culvert crossing & 360 & B-04-157_P6170006 \\
\hline B-04-158 & Looking upstream from culvert crossing & 45 & B-04-158_P6170007 \\
\hline B-04-158 & Looking downstream from culvert crossing & 180 & B-04-158 P6170008 \\
\hline B-04-158 & Looking at culvert outlets & $\mathrm{N} / \mathrm{A}$ & B-04-158 P6170009 \\
\hline B-04-158 & Looking at culvert inlets & $\mathrm{N} / \mathrm{A}$ & B-04-158_P6170010 \\
\hline B-04-159 & Looking upstream from bridge crossing & 270 & B-04-159 P6180001 \\
\hline B-04-159 & Looking downstream from bridge crossing & 90 & B-04-159_P6180002 \\
\hline B-04-160 & Looking upstream from bridge crossing & 270 & B-04-160_P6180003 \\
\hline B-04-160 & Looking downstream from bridge crossing & 90 & B-04-160_P6180004 \\
\hline B-04-161 & Looking upstream from bridge crossing & 180 & B-04-161 P6180005 \\
\hline B-04-161 & Looking downstream from bridge crossing & 360 & B-04-161_P6180006 \\
\hline B-04-162 & Looking upstream from bridge crossing & 285 & B-04-162_P6180007 \\
\hline B-04-162 & Looking downstream from bridge crossing & 110 & B-04-162 P6180008 \\
\hline B-04-163 & Looking upstream from bridge crossing & 230 & B-04-163_P6210001 \\
\hline B-04-163 & Looking downstream from bridge crossing & 45 & B-04-163_P6210002 \\
\hline B-04-164 & Looking upstream from culvert crossing & 180 & B-04-164_P6210003 \\
\hline B-04-164 & Looking downstream from culvert crossing & 340 & B-04-164_P6210004 \\
\hline B-04-164 & Looking at main culvert inlets & $\mathrm{N} / \mathrm{A}$ & B-04-164_P6210005 \\
\hline B-04-164 & Looking at older culvert inlets & $\mathrm{N} / \mathrm{A}$ & B-04-164_P6210006 \\
\hline B-04-164 & Looking at main culvert outlets & $\mathrm{N} / \mathrm{A}$ & B-04-164_P6210007 \\
\hline B-04-164 & Looking at older culvert outlets & $\mathrm{N} / \mathrm{A}$ & B-04-164_P6210008 \\
\hline B-04-165 & Looking upstream from bridge crossing & 105 & B-04-165_P6210009 \\
\hline B-04-165 & Looking downstream from bridge crossing & 260 & B-04-165_P6210010 \\
\hline B-04-166 & Looking upstream from bridge crossing & 80 & B-04-166_P6210011 \\
\hline B-04-166 & Looking downstream from bridge crossing & 260 & B-04-166_P6210012 \\
\hline B-04-167 & Looking upstream from bridge crossing & 90 & B-04-167 P6210013 \\
\hline B-04-167 & Looking downstream from bridge crossing & 270 & B-04-167_P6210014 \\
\hline B-04-168 & Looking upstream from culvert crossing & 65 & B-04-168_P6220001 \\
\hline
\end{tabular}


Appendix 8: List of all photographs taken showing the corresponding site number, descriptive caption, direction faced when taken (azimuth in degrees) and the unique file name for each photograph.

\begin{tabular}{|c|c|c|c|}
\hline $\begin{array}{c}\text { Site } \\
\text { Number }\end{array}$ & Photo Description & Azimuth & File Name \\
\hline B-04-168 & Looking downstream from culvert crossing & 180 & B-04-168_P6220002 \\
\hline B-04-168 & Looking downstream from culvert crossing & 140 & B-04-168_P6220003 \\
\hline B-04-168 & Looking downstream from culvert crossing & 230 & B-04-168_P6220004 \\
\hline B-04-168 & Looking at culvert outlets & $\mathrm{N} / \mathrm{A}$ & B-04-168_P6220005 \\
\hline B-04-169 & Looking upstream from culvert crossing & 130 & B-04-169_P6220006 \\
\hline B-04-169 & Looking downstream from culvert crossing & 330 & B-04-169_P6220007 \\
\hline B-04-169 & Looking at culvert inlets & $\mathrm{N} / \mathrm{A}$ & B-04-169_P6220008 \\
\hline B-04-169 & Looking at culvert outlets & N/A & B-04-169_P6220009 \\
\hline B-04-170 & Looking upstream from culvert crossing & 190 & B-04-170_P6220010 \\
\hline B-04-170 & Looking downstream from culvert crossing & 10 & B-04-170 P6220011 \\
\hline B-04-171 & Looking upstream from ford crossing & 155 & B-04-171_P6220012 \\
\hline B-04-171 & Looking downstream from ford crossing & 300 & B-04-171_P6220013 \\
\hline B-04-172 & Looking upstream from bridge crossing & 310 & B-04-172_P6220014 \\
\hline B-04-172 & Looking downstream from bridge crossing & 110 & B-04-172_P6220015 \\
\hline B-04-173 & Looking upstream from culvert crossing & 280 & B-04-173_P6220016 \\
\hline B-04-173 & Looking downstream from culvert crossing & 90 & B-04-173_P6220017 \\
\hline B-04-173 & Looking at culvert inlets & $\mathrm{N} / \mathrm{A}$ & B-04-173_P6220018 \\
\hline B-04-173 & Looking at culvert outlets & N/A & B-04-173 P6220019 \\
\hline B-04-174 & Looking upstream from culvert crossing & 270 & B-04-174_P6220020 \\
\hline B-04-174 & Looking downstream from culvert crossing & 135 & B-04-174_P6220021 \\
\hline B-04-174 & Looking at culvert outlets & N/A & B-04-174_P6220022 \\
\hline B-04-175 & Looking upstream from bridge crossing & 270 & B-04-175_P6220023 \\
\hline B-04-175 & Looking downstream from bridge crossing & 105 & B-04-175_P6220024 \\
\hline B-04-176 & Looking upstream from bridge crossing & 260 & B-04-176_P6230001 \\
\hline B-04-176 & Looking downstream from bridge crossing & 300 & B-04-176 P6230002 \\
\hline B-04-177 & Looking upstream from culvert crossing & 270 & B-04-177_P6230003 \\
\hline B-04-177 & Looking downstream from culvert crossing & 30 & B-04-177_P6230004 \\
\hline B-04-178 & Looking upstream from bridge crossing & 300 & B-04-178_P6230005 \\
\hline B-04-178 & Looking downstream from bridge crossing & 105 & B-04-178_P6230006 \\
\hline B-04-179 & Looking upstream from bridge crossing & 180 & B-04-179_P6230007 \\
\hline B-04-179 & Looking downstream from bridge crossing & 360 & B-04-179_P6230008 \\
\hline B-04-180 & Looking upstream from culvert crossing & 160 & B-04-180_P6230009 \\
\hline B-04-180 & Looking downstream from culvert crossing & 330 & B-04-180_P6230010 \\
\hline
\end{tabular}


Appendix 8: List of all photographs taken showing the corresponding site number, descriptive caption, direction faced when taken (azimuth in degrees) and the unique file name for each photograph.

\begin{tabular}{|c|c|c|c|}
\hline $\begin{array}{c}\text { Site } \\
\text { Number }\end{array}$ & Photo Description & Azimuth & File Name \\
\hline $\mathrm{B}-04-180$ & Looking upstream at culvert outlets & $\mathrm{N} / \mathrm{A}$ & B-04-180 P6230011 \\
\hline B-04-180 & Looking at culvert outlets & $\mathrm{N} / \mathrm{A}$ & B-04-180 P6230012 \\
\hline B-04-181 & Looking upstream from bridge crossing & 200 & B-04-181_P6280001 \\
\hline B-04-181 & Looking downstream from bridge crossing & 360 & B-04-181_P6280002 \\
\hline B-04-182 & Looking upstream from culvert crossing & 270 & B-04-182_P6280003 \\
\hline B-04-182 & Looking downstream from culvert crossing & 90 & B-04-182 P6280004 \\
\hline B-04-182 & Looking at culvert inlets & $\mathrm{N} / \mathrm{A}$ & B-04-182_P6280005 \\
\hline B-04-182 & Looking at culvert outlets & $\mathrm{N} / \mathrm{A}$ & B-04-182_P6280006 \\
\hline B-04-183 & Looking upstream from road & 175 & B-04-183_P6280007 \\
\hline B-04-183 & Looking downstream from road & 105 & B-04-183_P6280008 \\
\hline B-04-183 & Looking at 1st order tributary & 300 & B-04-183 P6280009 \\
\hline B-04-184 & Looking upstream from bridge crossing & 270 & B-04-184_P6280010 \\
\hline B-04-184 & Looking downstream from bridge crossing & 90 & B-04-184_P6280011 \\
\hline B-04-185 & Looking upstream from culvert crossing & 290 & B-04-185 P6280012 \\
\hline B-04-185 & Looking downstream from culvert crossing & 135 & B-04-185 P6280013 \\
\hline B-04-185 & Looking at culvert inlets & $\mathrm{N} / \mathrm{A}$ & B-04-185_P6280014 \\
\hline B-04-185 & Looking at culvert outlets & $\mathrm{N} / \mathrm{A}$ & B-04-185_P6280015 \\
\hline B-04-186 & Looking upstream from spillway & 120 & B-04-186 P6290016 \\
\hline B-04-186 & Looking across spillway & $\mathrm{N} / \mathrm{A}$ & B-04-186_P6290017 \\
\hline B-04-186 & Looking downstream from spillway & 310 & B-04-186_P6290018 \\
\hline B-04-186 & Looking upstream at control structure outlet & 180 & B-04-186_P6290019 \\
\hline B-04-186 & Looking downstream from base of control outlet pool & 310 & B-04-186_P6290020 \\
\hline B-04-186 & Looking at the Smallmouth Bass (released) & $\mathrm{N} / \mathrm{A}$ & B-04-186_P6290021 \\
\hline B-04-186 & Looking at the Smallmouth Bass (released) & $\mathrm{N} / \mathrm{A}$ & B-04-186_P6290022 \\
\hline B-04-186 & Looking at the Smallmouth Bass (released) & $\mathrm{N} / \mathrm{A}$ & B-04-186_P6290023 \\
\hline B-04-186 & Looking at the Smallmouth Bass (released) & $\mathrm{N} / \mathrm{A}$ & B-04-186_P6290024 \\
\hline B-04-186 & Looking at the Smallmouth Bass (released) & $\mathrm{N} / \mathrm{A}$ & B-04-186_P6290025 \\
\hline B-04-186 & Looking at the White Sucker released & $\mathrm{N} / \mathrm{A}$ & B-04-186_P6290026 \\
\hline B-04-186 & Looking over reach at base of dam & 360 & B-04-186_P6290027 \\
\hline B-04-186 & Looking upstream from control structure on dam & 180 & B-04-186_P6290028 \\
\hline B-04-187 & Looking upstream from bottom of reach & 190 & B-04-187 P6290029 \\
\hline B-04-187 & Looking downstream from bottom of reach & 10 & B-04-187_P6290030 \\
\hline B-04-188 & Photo of $200 \mathrm{~mm}$ Creek Chub & 290 & B-04-188_P6290031 \\
\hline
\end{tabular}


Appendix 8: List of all photographs taken showing the corresponding site number, descriptive caption, direction faced when taken (azimuth in degrees) and the unique file name for each photograph.

\begin{tabular}{|c|c|c|c|}
\hline $\begin{array}{c}\text { Site } \\
\text { Number }\end{array}$ & Photo Description & Azimuth & File Name \\
\hline B-04-188 & Looking upstream from middle of reach & $\mathrm{N} / \mathrm{A}$ & B-04-188_P6290032 \\
\hline B-04-188 & Looking downstream from middle of reach & 45 & B-04-188_P6290033 \\
\hline B-04-189 & Looking upstream from middle of reach & 340 & B-04-189_P6300001 \\
\hline B-04-189 & Looking downstream from middle of reach & 150 & B-04-189_P6300002 \\
\hline B-04-190 & Looking upstream from culvert crossing & 270 & B-04-190_P6300003 \\
\hline B-04-190 & Looking downstream from culvert crossing & 80 & B-04-190_P6300004 \\
\hline B-04-191 & Looking upstream from low level crossing & 200 & B-04-191_P7050001 \\
\hline B-04-191 & Looking downstream from low level crossing & 20 & B-04-191_P7050002 \\
\hline B-04-191 & Looking at low level crossing outlets & $\mathrm{N} / \mathrm{A}$ & B-04-191_P7050003 \\
\hline B-04-192 & Looking upstream from beaver dam & 180 & B-04-192_P7050004 \\
\hline B-04-192 & Looking downstream from beaver dam & 360 & B-04-192_P7050005 \\
\hline B-04-192 & Looking across beaver dam & $\mathrm{N} / \mathrm{A}$ & B-04-192_P7050006 \\
\hline B-04-193 & Looking upstream from lane & 180 & B-04-193_P7050007 \\
\hline B-04-193 & Looking downstream from lane & 360 & B-04-193_P7050008 \\
\hline B-04-194 & Looking upstream from culvert crossing & 180 & B-04-194_P7060001 \\
\hline B-04-194 & Looking downstream from culvert crossing & 360 & B-04-194_P7060002 \\
\hline B-04-194 & Looking at culvert inlets & $\mathrm{N} / \mathrm{A}$ & B-04-194_P7060003 \\
\hline B-04-195 & Looking upstream from culvert crossing & 180 & B-04-195_P7060004 \\
\hline B-04-195 & Looking downstream from culvert crossing & 360 & B-04-195_P7060005 \\
\hline B-04-195 & Looking at culvert inlets & $\mathrm{N} / \mathrm{A}$ & B-04-195_P7060006 \\
\hline B-04-195 & Looking at culvert outlets & $\mathrm{N} / \mathrm{A}$ & B-04-195_P7060007 \\
\hline B-04-196 & Looking upstream from bridge crossing & 180 & B-04-196_P7060008 \\
\hline B-04-196 & Looking downstream from bridge crossing & 260 & B-04-196_P7060009 \\
\hline B-04-196 & Looking at 210mm White Sucker (released) & $\mathrm{N} / \mathrm{A}$ & B-04-196_P7060010 \\
\hline B-04-196 & Looking at 180mm White Sucker (released) & $\mathrm{N} / \mathrm{A}$ & B-04-196_P7060011 \\
\hline B-04-196 & Looking upstream from culvert crossing & $\mathrm{N} / \mathrm{A}$ & B-04-196_P7060012 \\
\hline B-04-196 & Looking at $120 \mathrm{~mm}$ Common Shiner (released) & 90 & B-04-196_P7060013 \\
\hline B-04-197 & Looking downstream from culvert crossing & 270 & B-04-197_P7060014 \\
\hline B-04-197 & Looking at culvert outlets & $\mathrm{N} / \mathrm{A}$ & B-04-197_P7060015 \\
\hline B-04-197 & Looking at culvert inlets & $\mathrm{N} / \mathrm{A}$ & B-04-197_P7060016 \\
\hline B-04-197 & Looking at 180mm White Sucker (released) & $\mathrm{N} / \mathrm{A}$ & B-04-197_P7060017 \\
\hline B-04-197 & Looking upstream from ford crossing & $\mathrm{N} / \mathrm{A}$ & B-04-197_P7060018 \\
\hline
\end{tabular}


Appendix 8: List of all photographs taken showing the corresponding site number, descriptive caption, direction faced when taken (azimuth in degrees) and the unique file name for each photograph.

\begin{tabular}{|c|c|c|c|}
\hline $\begin{array}{c}\text { Site } \\
\text { Number }\end{array}$ & Photo Description & Azimuth & File Name \\
\hline B-04-197 & Looking at 120mm White Sucker (released) & $\mathrm{N} / \mathrm{A}$ & B-04-197_P7060019 \\
\hline B-04-197 & Looking at $120 \mathrm{~mm}$ Creek Chub released & $\mathrm{N} / \mathrm{A}$ & B-04-197_P7060020 \\
\hline B-04-198 & Looking at $120 \mathrm{~mm}$ Creek Chub released & 160 & B-04-198_P7060021 \\
\hline B-04-198 & Looking downstream from gravel ford crossing & 10 & B-04-198_P7060022 \\
\hline B-04-199 & Looking upstream from ford crossing & 190 & B-04-199_P7060023 \\
\hline B-04-199 & Looking downstream from ford crossing & 360 & B-04-199_P7060024 \\
\hline B-04-200 & Looking upstream from ford crossing & 100 & B-04-200_P7060025 \\
\hline B-04-200 & Looking downstream from ford crossing & 270 & B-04-200_P7060026 \\
\hline B-04-201 & Looking upstream from culvert crossing & 135 & B-04-201_P7060027 \\
\hline B-04-201 & Looking downstream from culvert crossing & 305 & B-04-201_P7060028 \\
\hline B-04-201 & Looking at culvert inlets & $\mathrm{N} / \mathrm{A}$ & B-04-201_P7060029 \\
\hline B-04-201 & Looking at culvert outlets & $\mathrm{N} / \mathrm{A}$ & B-04-201_P7060030 \\
\hline B-04-202 & Looking upstream from culvert crossing & 270 & B-04-202_P7070001 \\
\hline B-04-202 & Looking downstream from culvert crossing & 90 & B-04-202_P7070002 \\
\hline B-04-203 & Looking upstream from culvert crossing & 160 & B-04-203_P7070003 \\
\hline B-04-203 & Looking downstream from culvert crossing & 340 & B-04-203_P7070004 \\
\hline B-04-204 & Looking upstream from culvert crossing & 180 & B-04-204_P7070005 \\
\hline B-04-204 & Looking downstream from culvert crossing & 360 & B-04-204_P7070006 \\
\hline B-04-205 & Looking upstream from culvert crossing & 90 & B-04-205_P7070007 \\
\hline B-04-205 & Looking downstream from culvert crossing & 270 & B-04-205_P7070008 \\
\hline B-04-205 & Looking at culvert inlets & $\mathrm{N} / \mathrm{A}$ & B-04-205_P7070009 \\
\hline B-04-205 & Looking at culvert outlets & $\mathrm{N} / \mathrm{A}$ & B-04-205_P7070010 \\
\hline B-04-206 & Looking upstream from culvert crossing & 270 & B-04-206_P7070011 \\
\hline B-04-206 & Looking downstream from culvert crossing & 50 & B-04-206_P7070012 \\
\hline B-04-206 & Looking at beaver deceiver & $\mathrm{N} / \mathrm{A}$ & B-04-206_P7070013 \\
\hline B-04-207 & Looking upstream from cement box culvert & 150 & B-04-207_P7070014 \\
\hline B-04-207 & Looking downstream from cement box culvert & 330 & B-04-207_P7070015 \\
\hline B-04-208 & Looking upstream from culvert crossing & 180 & B-04-208_P7070016 \\
\hline B-04-208 & Looking downstream from culvert crossing & 360 & B-04-208_P7070017 \\
\hline B-04-209 & Looking upstream from culvert crossing & 180 & B-04-209_P7070018 \\
\hline B-04-209 & Looking downstream from culvert crossing & 360 & B-04-209_P7070019 \\
\hline B-04-210 & Looking upstream from culvert crossing & 150 & B-04-210_P7070020 \\
\hline B-04-210 & Looking downstream from culvert crossing & 25 & B-04-210_P7070021 \\
\hline
\end{tabular}


Appendix 8: List of all photographs taken showing the corresponding site number, descriptive caption, direction faced when taken (azimuth in degrees) and the unique file name for each photograph.

\begin{tabular}{|c|c|c|c|}
\hline $\begin{array}{c}\text { Site } \\
\text { Number }\end{array}$ & Photo Description & Azimuth & File Name \\
\hline B-04-211 & Looking upstream from culvert crossing & 100 & B-04-211 P7070022 \\
\hline B-04-211 & Looking downstream from culvert crossing & 311 & B-04-211_P7070023 \\
\hline B-04-213 & Looking upstream from bridge crossing & 270 & B-04-213_P7070024 \\
\hline B-04-213 & Looking downstream from bridge crossing & 100 & B-04-213 P7070025 \\
\hline B-04-214 & Looking upstream from culvert crossing & 180 & B-04-214 P7070026 \\
\hline B-04-214 & Looking downstream from culvert crossing & 360 & B-04-214_P7070027 \\
\hline B-04-214 & Looking at culvert inlets & $\mathrm{N} / \mathrm{A}$ & B-04-214_P7070028 \\
\hline B-04-215 & Looking upstream from bridge crossing & 180 & B-04-215 P7070029 \\
\hline B-04-215 & Looking downstream from bridge crossing & 360 & B-04-215 P7070030 \\
\hline B-04-216 & Looking upstream from culvert crossing & 100 & B-04-216_P7070031 \\
\hline B-04-216 & Looking downstream from culvert crossing & 270 & B-04-216_P7070032 \\
\hline B-04-217 & Looking upstream from bridge crossing & 270 & B-04-217 P7080001 \\
\hline B-04-217 & Looking downstream from bridge crossing & 90 & B-04-217 P7080002 \\
\hline B-04-218 & Looking upstream from culvert crossing & 300 & B-04-218 P7080003 \\
\hline B-04-218 & Looking downstream from culvert crossing & 160 & B-04-218_P7080004 \\
\hline B-04-219 & Looking upstream from bridge crossing & 300 & B-04-219_P7080005 \\
\hline B-04-219 & Looking downstream from bridge crossing & 100 & B-04-219 P7080006 \\
\hline B-04-220 & Looking upstream from bridge crossing & 300 & B-04-220 P7080007 \\
\hline B-04-220 & Looking downstream from bridge crossing & 110 & B-04-220_P7080008 \\
\hline B-04-220 & Looking at $180 \mathrm{~mm}$ Creek Chub (released) & N/A & B-04-220 P7080009 \\
\hline B-04-220 & Looking upstream from culvert crossing & $\mathrm{N} / \mathrm{A}$ & B-04-220 P7080010 \\
\hline B-04-220 & Looking at $180 \mathrm{~mm}$ White Sucker (released) & $\mathrm{N} / \mathrm{A}$ & B-04-220_P7080011 \\
\hline B-04-221 & Looking downstream from culvert crossing & 360 & B-04-221_P7080012 \\
\hline B-04-221 & Longnose Dace in spawning condition & 135 & B-04-221_P7080013 \\
\hline B-04-222 & Looking upstream from culvert crossing & 120 & B-04-222 P7080014 \\
\hline B-04-222 & Looking downstream from culvert crossing & 360 & B-04-222_P7080015 \\
\hline B-04-223 & Looking upstream from culvert crossing & 270 & B-04-223_P7090001 \\
\hline B-04-223 & Looking downstream from culvert crossing & 90 & B-04-223 P7090002 \\
\hline B-04-223 & Looking at culvert inlets & $\mathrm{N} / \mathrm{A}$ & B-04-223 P7090003 \\
\hline B-04-223 & Looking at culvert outlets & N/A & B-04-223_P7090004 \\
\hline B-04-224 & Looking upstream from culvert crossing on PTH 10 & 280 & B-04-224_P7130001 \\
\hline B-04-224 & Looking downstream from culvert crossing on PTH 10 & 90 & B-04-224 P7130002 \\
\hline B-04-224 & Looking at culvert outlets & N/A & B-04-224 P7130003 \\
\hline
\end{tabular}


Appendix 8: List of all photographs taken showing the corresponding site number, descriptive caption, direction faced when taken (azimuth in degrees) and the unique file name for each photograph.

\begin{tabular}{|c|c|c|c|}
\hline $\begin{array}{c}\text { Site } \\
\text { Number }\end{array}$ & Photo Description & Azimuth & File Name \\
\hline $\mathrm{B}-04-224$ & Looking upstream from crossing & $\mathrm{N} / \mathrm{A}$ & B-04-224 P7130004 \\
\hline B-04-225 & Looking at $210 \mathrm{~mm}$ Creek Chub (released) & 270 & B-04-225 P7130005 \\
\hline B-04-225 & Looking downstream from culvert crossing & 90 & B-04-225_P7130006 \\
\hline $\mathrm{B}-04-225$ & Looking at culvert inlets & $\mathrm{N} / \mathrm{A}$ & B-04-225 P7130007 \\
\hline B-04-225 & Looking at culvert outlets & $\mathrm{N} / \mathrm{A}$ & B-04-225_P7130008 \\
\hline B-04-225 & Looking at Common Shiner (released) & $\mathrm{N} / \mathrm{A}$ & B-04-225 P7130009 \\
\hline B-04-226 & Looking upstream from culvert crossing & 270 & B-04-226_P7130010 \\
\hline B-04-226 & Looking downstream from culvert crossing & 90 & B-04-226_P7130011 \\
\hline B-04-226 & Looking at culvert inlets & $\mathrm{N} / \mathrm{A}$ & B-04-226 P7130012 \\
\hline B-04-226 & Looking at culvert outlets & $\mathrm{N} / \mathrm{A}$ & B-04-226_P7130013 \\
\hline B-04-227 & Looking upstream from culvert crossing & 270 & B-04-227 P7130014 \\
\hline B-04-227 & Looking downstream from culvert crossing & 90 & B-04-227_P7130015 \\
\hline B-04-228 & Looking upstream from culvert crossing & 360 & B-04-228_P7130016 \\
\hline B-04-228 & Looking downstream from culvert crossing & 200 & B-04-228 P7130017 \\
\hline B-04-229 & Looking upstream from culvert crossing & 180 & B-04-229 P7140001 \\
\hline B-04-229 & Looking downstream from culvert crossing & 360 & B-04-229_P7140002 \\
\hline B-04-229 & Looking at culvert inlets & $\mathrm{N} / \mathrm{A}$ & B-04-229_P7140003 \\
\hline B-04-229 & Looking at culvert outlets & $\mathrm{N} / \mathrm{A}$ & B-04-229 P7140004 \\
\hline B-04-230 & Looking upstream from culvert crossing & 180 & B-04-230_P7140005 \\
\hline B-04-230 & Looking downstream from culvert crossing & 360 & B-04-230_P7140006 \\
\hline B-04-230 & Looking at culvert outlets & $\mathrm{N} / \mathrm{A}$ & B-04-230 P7140007 \\
\hline B-04-230 & Looking at culvert inlets & $\mathrm{N} / \mathrm{A}$ & B-04-230_P7140008 \\
\hline B-04-231 & Looking upstream from crossing at inflow from west 3rd order tributary & 220 & B-04-231_P7140009 \\
\hline B-04-231 & Looking upstream from crossing at inflow from east 2nd order tributary & 175 & B-04-231_P7140010 \\
\hline B-04-231 & Looking downstream from culvert crossing & 35 & B-04-231_P7140011 \\
\hline B-04-231 & Looking at inlet of west branch & $\mathrm{N} / \mathrm{A}$ & B-04-231_P7140012 \\
\hline B-04-231 & Looking at inlet of east branch & $\mathrm{N} / \mathrm{A}$ & B-04-231_P7140013 \\
\hline B-04-231 & Looking at outlet of east branch & $\mathrm{N} / \mathrm{A}$ & B-04-231_P7140014 \\
\hline B-04-231 & Looking at outlet of west branch & $\mathrm{N} / \mathrm{A}$ & B-04-231_P7140015 \\
\hline B-04-232 & Looking upstream from bridge crossing & 180 & B-04-232_P7140016 \\
\hline B-04-232 & Looking downstream from bridge crossing & 360 & B-04-232 P7140017 \\
\hline B-04-233 & Looking upstream from bridge crossing & 330 & B-04-233_P7140018 \\
\hline $\mathrm{B}-04-233$ & Looking downstream from bridge crossing & 10 & B-04-233_P7140019 \\
\hline
\end{tabular}


Appendix 8: List of all photographs taken showing the corresponding site number, descriptive caption, direction faced when taken (azimuth in degrees) and the unique file name for each photograph.

\begin{tabular}{|c|c|c|c|}
\hline $\begin{array}{c}\text { Site } \\
\text { Number }\end{array}$ & Photo Description & Azimuth & File Name \\
\hline $\mathrm{B}-04-234$ & Looking upstream from culvert crossing on PTH 10 & 135 & B-04-234 P7140020 \\
\hline B-04-234 & Looking downstream from culvert crossing on PTH 10 & 300 & B-04-234_P7140021 \\
\hline B-04-235 & Looking upstream from culvert crossing & 180 & B-04-235_P7140022 \\
\hline B-04-235 & Looking downstream from culvert crossing & 360 & B-04-235_P7140023 \\
\hline B-04-236 & Looking upstream from culvert crossing & 90 & B-04-236_P7140024 \\
\hline B-04-236 & Looking downstream from culvert crossing & 270 & B-04-236 P7140025 \\
\hline B-04-237 & Looking upstream from culvert crossing & 10 & B-04-237_P7140026 \\
\hline B-04-237 & Looking downstream from culvert crossing & 215 & B-04-237_P7140027 \\
\hline B-04-238 & Looking upstream from bridge crossing & 180 & B-04-238 P7140028 \\
\hline B-04-238 & Looking downstream from bridge crossing & 360 & B-04-238_P7140029 \\
\hline B-04-239 & Looking upstream from culvert crossing & 270 & B-04-239 P7150001 \\
\hline B-04-239 & Looking downstream from culvert crossing & 90 & B-04-239_P7150002 \\
\hline B-04-240 & Looking upstream from culvert crossing & 210 & B-04-240_P7150003 \\
\hline B-04-240 & Looking upstream from culvert crossing & 270 & B-04-240 P7150004 \\
\hline B-04-240 & Looking upstream from culvert crossing & 230 & B-04-240 P7150005 \\
\hline B-04-240 & Looking downstream from culvert crossing & 95 & B-04-240_P7150006 \\
\hline B-04-240 & Looking at culvert inlets & $\mathrm{N} / \mathrm{A}$ & B-04-240_P7150007 \\
\hline B-04-240 & Looking at culvert outlets & $\mathrm{N} / \mathrm{A}$ & B-04-240 P7150008 \\
\hline B-04-241 & Looking upstream from culvert crossing & 245 & B-04-241_P7190009 \\
\hline B-04-241 & Looking downstream from culvert crossing & 80 & B-04-241_P7190010 \\
\hline B-04-241 & Looking at culvert inlets & $\mathrm{N} / \mathrm{A}$ & B-04-241_P7190011 \\
\hline B-04-242 & Looking upstream from culvert crossing & 280 & B-04-242_P7190012 \\
\hline B-04-242 & Looking at culvert inlet & 90 & B-04-242_P7190013 \\
\hline B-04-242 & Looking downstream from culvert crossing & $\mathrm{N} / \mathrm{A}$ & B-04-242_P7190014 \\
\hline B-04-243 & Looking upstream from culvert crossing & 175 & B-04-243_P7190015 \\
\hline B-04-243 & Looking downstream from culvert crossing & 360 & B-04-243_P7190016 \\
\hline B-04-244 & Looking upstream from bridge crossing & 190 & B-04-244_P7200001 \\
\hline B-04-244 & Looking downstream from bridge crossing & 15 & B-04-244_P7200002 \\
\hline B-04-245 & Looking upstream from culvert crossing & 150 & B-04-245_P7200003 \\
\hline B-04-245 & Looking downstream from culvert crossing & 340 & B-04-245_P7200004 \\
\hline B-04-245 & Looking at culvert outlets & $\mathrm{N} / \mathrm{A}$ & $\mathrm{B}-04-245 \mathrm{P} 7200005$ \\
\hline B-04-245 & Looking at culvert inlets & $\mathrm{N} / \mathrm{A}$ & B-04-245_P7200006 \\
\hline B-04-246 & Looking upstream from culvert crossing & 180 & B-04-246_P7200007 \\
\hline
\end{tabular}


Appendix 8: List of all photographs taken showing the corresponding site number, descriptive caption, direction faced when taken (azimuth in degrees) and the unique file name for each photograph.

\begin{tabular}{|c|c|c|c|}
\hline $\begin{array}{c}\text { Site } \\
\text { Number }\end{array}$ & Photo Description & Azimuth & File Name \\
\hline B-04-246 & Looking downstream from culvert crossing & 360 & B-04-246_P7200008 \\
\hline B-04-246 & Looking at culvert inlets & $\mathrm{N} / \mathrm{A}$ & B-04-246_P7200009 \\
\hline B-04-246 & Looking at culvert outlets & $\mathrm{N} / \mathrm{A}$ & B-04-246_P7200010 \\
\hline B-04-247 & Looking upstream from bridge crossing & 180 & B-04-247_P7200011 \\
\hline B-04-247 & Looking downstream from bridge crossing & 360 & B-04-247_P7200012 \\
\hline B-04-248 & Looking upstream from bridge crossing & 180 & B-04-248_P7200013 \\
\hline B-04-248 & Looking downstream from bridge crossing & 325 & B-04-248_P7200014 \\
\hline B-04-249 & Looking upstream from culvert crossing & 180 & B-04-249_P7200015 \\
\hline B-04-249 & Looking downstream from culvert crossing & 360 & B-04-249_P7200016 \\
\hline B-04-249 & Looking at culvert outlets & N/A & B-04-249_P7200017 \\
\hline B-04-250 & Photo of 5 Northern Pike (released) & 180 & B-04-250_P7200018 \\
\hline B-04-250 & Looking upstream from culvert crossing & 10 & B-04-250_P7200019 \\
\hline B-04-250 & Looking downstream from culvert crossing & $\mathrm{N} / \mathrm{A}$ & B-04-250_P7200020 \\
\hline B-04-251 & Looking upstream from culvert crossing & 180 & B-04-251_P7210001 \\
\hline B-04-251 & Looking downstream from culvert crossing & 360 & B-04-251_P7210002 \\
\hline B-04-252 & Looking upstream from culvert crossing & 180 & B-04-252_P7210003 \\
\hline B-04-252 & Looking downstream from culvert crossing & 360 & B-04-252_P7210004 \\
\hline B-04-253 & Looking upstream from culvert crossing & N/A & B-04-253_P7210005 \\
\hline B-04-253 & Looking downstream from culvert crossing & $\mathrm{N} / \mathrm{A}$ & B-04-253_P7210006 \\
\hline B-04-254 & Looking upstream from culvert crossing & 180 & B-04-254_P7210007 \\
\hline B-04-254 & Looking downstream from culvert crossing & 360 & B-04-254_P7210008 \\
\hline B-04-255 & Looking upstream from culvert crossing & 210 & B-04-255_P7210009 \\
\hline B-04-255 & Looking downstream from culvert crossing & 45 & B-04-255_P7210010 \\
\hline B-04-256 & Looking upstream from bridge crossing & 180 & B-04-256_P7210011 \\
\hline B-04-256 & Looking downstream from bridge crossing & 360 & B-04-256 P7210012 \\
\hline B-04-257 & Looking upstream from bridge crossing & 290 & B-04-257_P7210013 \\
\hline B-04-257 & Looking downstream from bridge crossing & 60 & B-04-257_P7210014 \\
\hline$B-04-258$ & Looking upstream from culvert crossing & 180 & B-04-258_P7210015 \\
\hline$B-04-258$ & Looking downstream from culvert crossing & 360 & B-04-258_P7210016 \\
\hline B-04-259 & Looking upstream from culvert crossing & 180 & B-04-259_P7210017 \\
\hline B-04-259 & Looking downstream from culvert crossing & 360 & B-04-259_P7210018 \\
\hline B-04-260 & Looking upstream from bridge crossing & 325 & B-04-260_P7210019 \\
\hline B-04-260 & Looking downstream from bridge crossing & 130 & B-04-260_P7210020 \\
\hline
\end{tabular}


Appendix 8: List of all photographs taken showing the corresponding site number, descriptive caption, direction faced when taken (azimuth in degrees) and the unique file name for each photograph.

\begin{tabular}{|c|c|c|c|}
\hline $\begin{array}{c}\text { Site } \\
\text { Number }\end{array}$ & Photo Description & Azimuth & File Name \\
\hline B-04-261 & Looking upstream from bridge crossing & 170 & B-04-261 P7210021 \\
\hline B-04-261 & Looking downstream from bridge crossing & 360 & B-04-261_P7210022 \\
\hline B-04-262 & Looking upstream from bridge crossing & 180 & B-04-262_P7210023 \\
\hline B-04-262 & Looking downstream from bridge crossing & 360 & B-04-262_P7210024 \\
\hline B-04-263 & Looking upstream from culvert crossing & 340 & B-04-263_P7220001 \\
\hline B-04-263 & Looking downstream from culvert crossing & 150 & B-04-263_P7220002 \\
\hline B-04-263 & Looking at culvert outlets & N/A & B-04-263_P7220003 \\
\hline B-04-264 & Looking upstream from metal pipe crossing & 360 & B-04-264_P7220004 \\
\hline B-04-264 & Looking downstream from metal pipe crossing & 180 & B-04-264_P7220005 \\
\hline B-04-264 & Looking at culvert outlets & N/A & B-04-264_P7220006 \\
\hline B-04-264 & Looking at culvert inlets & N/A & B-04-264_P7220007 \\
\hline B-04-265 & Looking upstream from culvert crossing & 360 & B-04-265_P7220008 \\
\hline B-04-265 & Looking downstream from culvert crossing & 180 & $\mathrm{~B}-04-265 \mathrm{P} 7220009$ \\
\hline B-04-265 & Looking at culvert outlets & N/A & B-04-265 P7220010 \\
\hline B-04-266 & Looking upstream from culvert crossing & 350 & B-04-266_P7260001 \\
\hline B-04-266 & Looking downstream from culvert crossing & 195 & B-04-266_P7260002 \\
\hline B-04-267 & Looking upstream from dam & 190 & B-04-267 P8040001 \\
\hline B-04-267 & Looking upstream from dam & 125 & B-04-267_P8040002 \\
\hline B-04-267 & Looking downstream from dam & 45 & B-04-267_P8040003 \\
\hline B-04-267 & Looking across dam & 135 & B-04-267_P8040004 \\
\hline B-04-268 & Looking upstream from road crossing & 360 & B-04-268 P8040005 \\
\hline B-04-268 & Looking downstream & 180 & B-04-268_P8040006 \\
\hline B-04-269 & Looking upstream from dike & 270 & B-04-269_P8040007 \\
\hline B-04-269 & Looking downstream from dike & 90 & B-04-269_P8040008 \\
\hline B-04-270 & Looking upstream from bridge & 270 & B-04-270 P8040009 \\
\hline B-04-270 & Looking downstream from bridge & 90 & B-04-270 P8040010 \\
\hline B-04-271 & Looking upstream on Q Drain from control structure & 135 & B-04-271_P8040011 \\
\hline B-04-271 & Looking upstream on Drain $\mathrm{R}$ from approach & 180 & B-04-271_P8040012 \\
\hline B-04-271 & Looking downstream on Drain $\mathrm{R}$ from approach & 360 & B-04-271 P8040013 \\
\hline B-04-271 & Looking downstream on Drain $\mathrm{R} / \mathrm{Z}$ & 10 & B-04-271_P8040014 \\
\hline B-04-271 & Looking at old channel to Pasquia from control structure & 45 & B-04-271_P8040015 \\
\hline B-04-272 & Looking upstream from water control structure & 180 & B-04-272_P8040016 \\
\hline B-04-272 & Looking downstream at water control structure & N/A & B-04-272 P8040017 \\
\hline
\end{tabular}


Appendix 8: List of all photographs taken showing the corresponding site number, descriptive caption, direction faced when taken (azimuth in degrees) and the unique file name for each photograph.

\begin{tabular}{|c|c|c|c|}
\hline $\begin{array}{c}\text { Site } \\
\text { Number }\end{array}$ & Photo Description & Azimuth & File Name \\
\hline B-04-272 & Looking downstream from dike behind control structure & 360 & B-04-272 P8040018 \\
\hline B-04-273 & Looking upstream from culvert crossing & 225 & B-04-273_P8050001 \\
\hline B-04-273 & Looking downstream from crossing at confluence with B-1 Drain & 45 & B-04-273_P8050002 \\
\hline B-04-273 & Looking at inlet & $\mathrm{N} / \mathrm{A}$ & B-04-273_P8050003 \\
\hline B-04-273 & Looking at outlet & $\mathrm{N} / \mathrm{A}$ & B-04-273_P8050004 \\
\hline B-04-274 & Looking upstream from culvert crossing & 225 & B-04-274_P8050005 \\
\hline B-04-274 & Looking downstream from culvert crossing & 45 & B-04-274_P8050006 \\
\hline B-04-274 & Looking at culvert inlets & $\mathrm{N} / \mathrm{A}$ & B-04-274_P8050007 \\
\hline B-04-274 & Looking at culvert outlets & $\mathrm{N} / \mathrm{A}$ & B-04-274_P8050008 \\
\hline B-04-275 & Looking upstream from culvert crossing & 135 & B-04-275_P8050009 \\
\hline B-04-275 & Looking downstream from culvert crossing & 315 & $\mathrm{~B}-04-275 \mathrm{P} 8050010$ \\
\hline B-04-275 & Looking at culvert inlets & $\mathrm{N} / \mathrm{A}$ & B-04-275_P8050011 \\
\hline B-04-275 & Looking at culvert outlets & $\mathrm{N} / \mathrm{A}$ & B-04-275_P8050012 \\
\hline B-04-276 & Looking upstream from pump station & 180 & B-04-276_P8050013 \\
\hline B-04-276 & Looking at culvert inlets & $\mathrm{N} / \mathrm{A}$ & $\mathrm{B}-04-276 \mathrm{P} 8050014$ \\
\hline B-04-276 & Looking upstream from outlet & 260 & B-04-276_P8050015 \\
\hline B-04-276 & Looking at culvert outlets & $\mathrm{N} / \mathrm{A}$ & B-04-276_P8050016 \\
\hline B-04-277 & Looking upstream at Drain $\mathrm{O}$ from $10 \mathrm{~m}$ north of the drain junction & 140 & B-04-277 P8050017 \\
\hline B-04-277 & Looking upstream at Drain L from culvert crossing & 235 & B-04-277_P8050018 \\
\hline B-04-277 & Looking downstream from culvert crossing & 350 & B-04-277_P8050019 \\
\hline B-04-278 & Looking upstream from culvert crossing & 80 & B-04-278_P8050020 \\
\hline B-04-278 & Looking downstream from culvert crossing & 270 & B-04-278_P8050021 \\
\hline B-04-279 & Looking upstream from crossing & 350 & B-04-279_P8050022 \\
\hline B-04-279 & Looking upstream at 1st order & 190 & B-04-279_P8050023 \\
\hline B-04-279 & Looking upstream at Bon Drain & 10 & B-04-279_P8050024 \\
\hline B-04-279 & Looking upstream at Bon Drain & 180 & B-04-279_P8050025 \\
\hline B-04-279 & Looking downstream on Drain 01 & 270 & B-04-279_P8050026 \\
\hline B-04-280 & Looking upstream from control structure & 270 & B-04-280_P8050027 \\
\hline B-04-280 & Looking downstream from control structure & 90 & B-04-280_P8050028 \\
\hline B-04-280 & Looking at control structure & $\mathrm{N} / \mathrm{A}$ & B-04-280_P8050029 \\
\hline B-04-280 & Looking upstream at downstream end of culverts & $\mathrm{N} / \mathrm{A}$ & $\mathrm{B}-04-280 \mathrm{P} 8050030$ \\
\hline B-04-281 & Looking upstream from control structure & 180 & B-04-281_P8060001 \\
\hline B-04-281 & Looking downstream from control structure & 360 & B-04-281_P8060002 \\
\hline
\end{tabular}


Appendix 8: List of all photographs taken showing the corresponding site number, descriptive caption, direction faced when taken (azimuth in degrees) and the unique file name for each photograph.

\begin{tabular}{|c|c|c|c|}
\hline $\begin{array}{c}\text { Site } \\
\text { Number }\end{array}$ & Photo Description & Azimuth & File Name \\
\hline B-04-281 & Looking at culverts outlets & $\mathrm{N} / \mathrm{A}$ & B-04-281 P8060003 \\
\hline B-04-281 & Looking at culvert inlets & $\mathrm{N} / \mathrm{A}$ & B-04-281_P8060004 \\
\hline B-04-282 & Looking upstream from culvert crossing & 125 & B-04-282_P8060005 \\
\hline B-04-282 & Looking downstream from culvert crossing & 295 & B-04-282_P8060006 \\
\hline B-04-282 & Looking upstream at Road 8C Drain S/E (1st order) & 225 & B-04-282_P8060007 \\
\hline B-04-282 & Looking upstream at the other 1st order drain & 225 & B-04-282 P8060008 \\
\hline B-04-282 & Looking at culvert inlets & $\mathrm{N} / \mathrm{A}$ & B-04-282_P8060009 \\
\hline B-04-282 & Looking at culvert outlets & $\mathrm{N} / \mathrm{A}$ & B-04-282_P8060010 \\
\hline B-04-283 & Looking upstream from culvert crossing & $\mathrm{N} / \mathrm{A}$ & B-04-283 P8060011 \\
\hline B-04-283 & Looking downstream from culvert crossing & $\mathrm{N} / \mathrm{A}$ & B-04-283_P8060012 \\
\hline B-04-284 & Looking upstream from culvert crossing & $\mathrm{N} / \mathrm{A}$ & B-04-284 P8060013 \\
\hline B-04-284 & Looking downstream from culvert crossing & $\mathrm{N} / \mathrm{A}$ & B-04-284_P8060014 \\
\hline B-04-285 & Looking upstream from culvert crossing & 80 & B-04-285_P8060015 \\
\hline B-04-285 & Looking downstream from culvert crossing & 300 & B-04-285 P8060016 \\
\hline B-04-286 & Looking upstream from behind pump station & 100 & B-04-286 P8060017 \\
\hline B-04-286 & Looking downstream from pump station & 260 & B-04-286_P8060018 \\
\hline B-04-286 & Looking at culvert outlets & $\mathrm{N} / \mathrm{A}$ & B-04-286_P8060019 \\
\hline B-04-286 & Looking upstream from pump station & 270 & B-04-286 P8060020 \\
\hline B-04-286 & Looking downstream at inlet of pump station & $\mathrm{N} / \mathrm{A}$ & B-04-286_P8060021 \\
\hline B-04-287 & Looking upstream from bridge crossing & 180 & B-04-287_P8060022 \\
\hline B-04-287 & Looking downstream from bridge crossing & 360 & B-04-287_P8060023 \\
\hline B-04-288 & Looking upstream from culvert crossing & 270 & B-04-288_P8060024 \\
\hline B-04-288 & Looking downstream from culvert crossing & 90 & B-04-288_P8060025 \\
\hline D-04-001 & Looking upstream through riffle & 340 & D-04-001_P4060001 \\
\hline $\mathrm{D}-04-001$ & Looking upstream through riffle & 340 & D-04-001_P4060002 \\
\hline D-04-001 & Looking downstream through riffle & 200 & D-04-001_P4060003 \\
\hline D-04-002 & Looking east across raised crossing & 110 & D-04-002_P4060004 \\
\hline $\mathrm{D}-04-002$ & Looking east across raised crossing & 110 & D-04-002_P4060005 \\
\hline $\mathrm{D}-04-003$ & Looking upstream from culvert crossing & 200 & D-04-003_P4150006 \\
\hline D-04-003 & Looking downstream from culvert crossing & 90 & D-04-003_P4150007 \\
\hline D-04-003 & Looking at culvert outlet & 270 & $\mathrm{D}-04-003 \quad \mathrm{P} 4150008$ \\
\hline D-04-003 & Looking at culvert inlet & 180 & D-04-003_P4150009 \\
\hline D-04-004 & Looking upstream from below gradient control structure (riffle) & 270 & D-04-004_P4150010 \\
\hline
\end{tabular}


Appendix 8: List of all photographs taken showing the corresponding site number, descriptive caption, direction faced when taken (azimuth in degrees) and the unique file name for each photograph.

\begin{tabular}{|c|c|c|c|}
\hline $\begin{array}{c}\text { Site } \\
\text { Number }\end{array}$ & Photo Description & Azimuth & File Name \\
\hline D-04-004 & Looking downstream from below gradient control structure (riffle) & 90 & D-04-004_P4150011 \\
\hline D-04-005 & Looking upstream from culvert crossing at small riffle & 270 & $\mathrm{D}-04-005 \mathrm{P} 4150012$ \\
\hline D-04-005 & Looking downstream from culvert crossing at small riffle & 90 & D-04-005_P4150013 \\
\hline D-04-005 & Looking at culvert outlet & 270 & D-04-005_P4150014 \\
\hline D-04-005 & Looking at culvert inlet & 90 & D-04-005_P4150015 \\
\hline D-04-005 & Close-up photo of channel constriction & 270 & $\mathrm{D}-04-005 \mathrm{P} 4150016$ \\
\hline D-04-006 & Looking southwest at perched culvert outlet & 220 & D-04-006_P4150017 \\
\hline D-04-008 & Looking upstream from bridge crossing & 290 & D-04-008_P4160018 \\
\hline D-04-008 & Looking downstream from bridge crossing & 90 & $\mathrm{D}-04-008 \quad \mathrm{P} 4160019$ \\
\hline D-04-009 & Looking upstream from below WSC weir & 300 & D-04-009 P4160020 \\
\hline D-04-009 & Looking downstream from WSC weir & 90 & D-04-009_P4160021 \\
\hline $\mathrm{D}-04-010$ & Looking upstream from culvert crossing & 110 & D-04-010_P6020001 \\
\hline D-04-010 & Looking downstream from culvert crossing & 300 & D-04-010_P6020002 \\
\hline D-04-010 & Looking at culvert outlet & 270 & $\mathrm{D}-04-010 \mathrm{P} 6020003$ \\
\hline $\mathrm{D}-04-010$ & Looking at culvert inlet & 270 & $\mathrm{D}-04-010 \mathrm{P} 6020004$ \\
\hline $\mathrm{D}-04-011$ & Looking upstream from culvert crossing & 270 & D-04-011_P6020005 \\
\hline D-04-011 & Looking downstream from culvert crossing & 45 & D-04-011 P6020006 \\
\hline D-04-011 & Looking at culvert outlet & 0 & D-04-011_P6020007 \\
\hline D-04-011 & Looking at culvert inlet & 0 & D-04-011_P6020008 \\
\hline $\mathrm{D}-04-012$ & Looking upstream from large diameter culvert crossing & 90 & D-04-012_P6020009 \\
\hline D-04-012 & Looking downstream from large diameter culver crossing & 260 & D-04-012_P6020010 \\
\hline $\mathrm{D}-04-012$ & Looking at culvert inlet & 0 & D-04-012_P6020011 \\
\hline D-04-012 & Photo of Manitou grade 8 class visit & $\mathrm{n} / \mathrm{a}$ & D-04-012_P6020012 \\
\hline $\mathrm{D}-04-012$ & Photo of Manitou grade 8 class visit & $\mathrm{n} / \mathrm{a}$ & D-04-012_P6020013 \\
\hline D-04-012 & Photo of Manitou grade 8 class visit & $\mathrm{n} / \mathrm{a}$ & D-04-012 P6020014 \\
\hline D-04-012 & Photo of Manitou grade 8 class visit & $\mathrm{n} / \mathrm{a}$ & D-04-012 P6020015 \\
\hline D-04-013 & Looking upstream from PTH 5 culvert crossing & 270 & D-04-013_P6030001 \\
\hline $\mathrm{D}-04-013$ & Looking downstream from PTH 5 culvert crossing & 90 & D-04-013_P6030002 \\
\hline D-04-013 & Looking at culvert outlet & 340 & $\mathrm{D}-04-013 \quad \mathrm{P} 6030003$ \\
\hline D-04-013 & Looking at culvert inlet & 160 & D-04-013 P6030004 \\
\hline D-04-013 & Looking north west at pond outlet and larval drift trap & 340 & D-04-013_P6030005 \\
\hline D-04-014 & Looking east at flow over raised crossing & 90 & D-04-014_P6030006 \\
\hline D-04-015 & Looking upstream from raised crossing & 270 & D-04-015_P6070001 \\
\hline
\end{tabular}


Appendix 8: List of all photographs taken showing the corresponding site number, descriptive caption, direction faced when taken (azimuth in degrees) and the unique file name for each photograph.

\begin{tabular}{|c|c|c|c|}
\hline $\begin{array}{c}\text { Site } \\
\text { Number }\end{array}$ & Photo Description & Azimuth & File Name \\
\hline D-04-015 & Looking downstream from raised crossing & 90 & D-04-015_P6070002 \\
\hline D-04-015 & Looking at culvert outlet & 180 & D-04-015_P6070003 \\
\hline D-04-015 & Looking at culvert inlet & 180 & D-04-015_P6070004 \\
\hline D-04-016 & Looking upstream from pond outlet & 0 & D-04-016_P6080001 \\
\hline D-04-016 & Looking downstream from pond outlet & 180 & D-04-016_P6080002 \\
\hline D-04-017 & Looking upstream from culvert crossing & 270 & D-04-017_P6090003 \\
\hline D-04-017 & Looking downstream from culvert crossing & 90 & D-04-017_P6090004 \\
\hline D-04-017 & Looking at culvert outlet & 0 & D-04-017_P6090005 \\
\hline D-04-017 & Looking at culvert inlet & 340 & D-04-017_P6090006 \\
\hline D-04-018 & Looking upstream from old road bed & 180 & D-04-018_P6090007 \\
\hline D-04-018 & Looking east across breach in old road bed & 90 & D-04-018_P6090008 \\
\hline D-04-019 & Looking upstream from culvert crossing & 270 & D-04-019_P6090009 \\
\hline D-04-019 & Looking downstream from culvert crossing & 90 & D-04-019_P6090010 \\
\hline D-04-019 & Looking at culvert outlet & 0 & D-04-019_P6090011 \\
\hline D-04-019 & Looking at culvert inlet & 0 & D-04-019_P6090012 \\
\hline D-04-020 & Looking upstream at pond outflow & 0 & D-04-020_P6100001 \\
\hline D-04-020 & Looking downstream from pond outflow & 180 & D-04-020_P6100002 \\
\hline D-04-020 & Looking upstream at north Snake Creek & 270 & D-04-020_P6100003 \\
\hline D-04-022 & Looking upstream from bridge crossing & 120 & D-04-022_P6150001 \\
\hline D-04-022 & Looking downstream from bridge crossing & 270 & D-04-022_P6150002 \\
\hline D-04-023 & Looking upstream from bridge crossing & 270 & D-04-023_P6150003 \\
\hline D-04-023 & Looking downstream from bridge crossing & 90 & D-04-023_P6150004 \\
\hline D-04-024 & Looking upstream from culvert crossing & 200 & D-04-024_P6150005 \\
\hline D-04-024 & Looking downstream from culvert crossing & 60 & D-04-024_P6150006 \\
\hline D-04-025 & Looking upstream from culvert crossing & 0 & D-04-025_P6150007 \\
\hline D-04-025 & Looking downstream from culvert crossing & 180 & D-04-025_P6150008 \\
\hline D-04-026 & Looking upstream from culvert crossing & 90 & D-04-026_P6150009 \\
\hline D-04-026 & Looking downstream from culvert crossing & 270 & D-04-026_P6150010 \\
\hline D-04-026 & Looking at culvert outlet perched $2 \mathrm{~m}$ & 0 & D-04-026_P6150011 \\
\hline D-04-026 & Looking at culvert inlet & 180 & D-04-026_P6150012 \\
\hline D-04-027 & Looking upstream from bridge crossing & 300 & D-04-027_P6150013 \\
\hline D-04-027 & Looking downstream from bridge crossing & 90 & D-04-027_P6150014 \\
\hline D-04-028 & Looking upstream from walking bridge & 180 & D-04-028_P6160001 \\
\hline
\end{tabular}


Appendix 8: List of all photographs taken showing the corresponding site number, descriptive caption, direction faced when taken (azimuth in degrees) and the unique file name for each photograph.

\begin{tabular}{|c|c|c|c|}
\hline $\begin{array}{c}\text { Site } \\
\text { Number }\end{array}$ & Photo Description & Azimuth & File Name \\
\hline D-04-028 & Looking downstream from walking bridge & 0 & D-04-028_P6160002 \\
\hline D-04-028 & Looking downstream at west bank condo development & $\mathrm{n} / \mathrm{a}$ & D-04-028_P6160003 \\
\hline D-04-028 & Looking downstream at west bank condo development & $\mathrm{n} / \mathrm{a}$ & D-04-028_P6160004 \\
\hline $\mathrm{D}-04-028$ & Looking downstream at roof downspouts from condo development & $\mathrm{n} / \mathrm{a}$ & D-04-028_P6160005 \\
\hline D-04-028 & Looking at concrete riprap on east bank & $\mathrm{n} / \mathrm{a}$ & D-04-028_P6160006 \\
\hline D-04-028 & Looking downstream at concrete riprap & $\mathrm{n} / \mathrm{a}$ & D-04-028_P6160007 \\
\hline D-04-028 & Looking downstream from railway bridge & $\mathrm{n} / \mathrm{a}$ & D-04-028_P6160008 \\
\hline D-04-028 & Looking upstream from railway bridge & $\mathrm{n} / \mathrm{a}$ & D-04-028_P6160009 \\
\hline $\mathrm{D}-04-029$ & Looking upstream from gradient control structure (riffle) & 270 & D-04-029_P6160010 \\
\hline $\mathrm{D}-04-029$ & Looking downstream from gradient control structure & 90 & D-04-029_P6160011 \\
\hline D-04-029 & Looking upstream at gradient control structure & 270 & D-04-029_P6160012 \\
\hline D-04-029 & Looking north at gradient control structure & 0 & D-04-029 P6160013 \\
\hline $\mathrm{D}-04-030$ & Looking upstream from culvert crossing & 270 & D-04-030_P6220001 \\
\hline D-04-030 & Looking downstream from culvert crossing & 90 & D-04-030_P6220002 \\
\hline $\mathrm{D}-04-030$ & Looking at culvert outlet & $\mathrm{n} / \mathrm{a}$ & D-04-030_P6220003 \\
\hline D-04-030 & Looking at culvert inlet & $\mathrm{n} / \mathrm{a}$ & $\mathrm{D}-04-030 \mathrm{P} 6220004$ \\
\hline D-04-030 & Looking downstream at beaver dam in culvert & $\mathrm{n} / \mathrm{a}$ & D-04-030_P6220005 \\
\hline $\mathrm{D}-04-030$ & Looking upstream at beaver dam in culvert & $\mathrm{n} / \mathrm{a}$ & D-04-030_P6220006 \\
\hline $\mathrm{D}-04-030$ & Looking upstream at beaver dam in culvert & $\mathrm{n} / \mathrm{a}$ & D-04-030_P6220007 \\
\hline D-04-032 & Looking upstream from culvert crossing & 0 & $\mathrm{D}-04-032 \mathrm{P} 6220008$ \\
\hline $\mathrm{D}-04-032$ & Looking downstream from culvert crossing & 180 & D-04-032_P6220009 \\
\hline $\mathrm{D}-04-032$ & Looking south across flow over gravel road & 180 & D-04-032_P6220010 \\
\hline D-04-032 & Looking at plugged culvert inlet & $\mathrm{n} / \mathrm{a}$ & D-04-032_P6220011 \\
\hline $\mathrm{D}-04-032$ & Looking at culvert outlet & $\mathrm{n} / \mathrm{a}$ & D-04-032_P6220012 \\
\hline $\mathrm{D}-04-033$ & Looking upstream from culver crossing & 300 & D-04-033_P6230001 \\
\hline $\mathrm{D}-04-033$ & Looking downstream from culvert crossing & 90 & D-04-033_P6230002 \\
\hline $\mathrm{D}-04-033$ & Looking at culvert outlet & $\mathrm{n} / \mathrm{a}$ & $\mathrm{D}-04-033 \quad \mathrm{P} 6230003$ \\
\hline D-04-033 & Looking at culvert inlet & $\mathrm{n} / \mathrm{a}$ & D-04-033_P6230004 \\
\hline D-04-034 & Looking upstream from old railway trestle & 0 & D-04-034_P6230005 \\
\hline $\mathrm{D}-04-034$ & Looking downstream from old railway trestle & 180 & D-04-034_P6230006 \\
\hline $\mathrm{D}-04-035$ & Looking upstream from old rail bed & 220 & $\mathrm{D}-04-035 \mathrm{P} 6230007$ \\
\hline D-04-035 & Looking east at old railway trestle bridge piles & 90 & $\mathrm{D}-04-035+\mathrm{P} 6230008$ \\
\hline D-04-035 & Looking downstream from old rail bed & 40 & D-04-035_P6230009 \\
\hline
\end{tabular}


Appendix 8: List of all photographs taken showing the corresponding site number, descriptive caption, direction faced when taken (azimuth in degrees) and the unique file name for each photograph.

\begin{tabular}{|c|c|c|c|}
\hline $\begin{array}{c}\text { Site } \\
\text { Number }\end{array}$ & Photo Description & Azimuth & File Name \\
\hline W-04-001 & Looking upstream from bridge crossing & 200 & W-04-001 P5030001 \\
\hline W-04-001 & Looking downstream from bridge crossing & 20 & W-04-001_P5030002 \\
\hline W-04-001 & Looking at spawning suckers, upstream side of bridge crossing & $\mathrm{N} / \mathrm{A}$ & W-04-001_P5030003 \\
\hline W-04-001 & Looking at spawning suckers, upstream side of bridge crossing & $\mathrm{N} / \mathrm{A}$ & W-04-001_P5030004 \\
\hline W-04-001 & Looking at spawning bed $15 \mathrm{~m}$ upstream from bridge crossing & 120 & W-04-001 P5030005 \\
\hline W-04-002 & Looking upstream Manitoba House Drain from culvert crossing & 240 & W-04-002 P5030006 \\
\hline W-04-002 & Looking downstream Manitoba House Drain from culvert crossing & 70 & W-04-002_P5030007 \\
\hline W-04-002 & Looking upstream at downstream side of culvert crossing & 300 & W-04-002 P5030008 \\
\hline W-04-002 & Looking at culverts $10 \mathrm{~m}$ upstream & 110 & W-04-002 P5030009 \\
\hline W-04-002 & Looking at dead sucker $6 \mathrm{~m}$ downstream from culvert crossing & $\mathrm{N} / \mathrm{A}$ & W-04-002_P5030010 \\
\hline W-04-003 & Looking upstream from culvert crossing (Bluff Creek Road) & 200 & W-04-003 P5030011 \\
\hline W-04-003 & Looking downstream from culvert crossing (Bluff Creek Road) & 350 & W-04-003_P5030012 \\
\hline W-04-004 & Looking upstream Rochland Drain at bridge crossing (PTH 50) & 180 & W-04-004 P5030013 \\
\hline W-04-004 & Looking upstream Garrioch Creek at bridge crossing (PTH 50) & 200 & W-04-004_P5030014 \\
\hline W-04-004 & Looking downstream from bridge at 4th order Garrioch Creek & 20 & W-04-004_P5030015 \\
\hline W-04-004 & Looking down from bridge at spawning suckers & $\mathrm{N} / \mathrm{A}$ & W-04-004_P5030016 \\
\hline W-04-005 & Photo of Julie with sucker & 0 & W-04-005 P5030017 \\
\hline W-04-005 & Looking downstream at box culvert/bridge crossing (PTH 50) & 90 & W-04-005_P5030018 \\
\hline W-04-005 & Looking upstream from box culvert crossing & 270 & W-04-005_P5030019 \\
\hline W-04-005 & Looking downstream from box culvert crossing & 90 & W-04-005_P5030020 \\
\hline W-04-006 & Looking upstream from culvert crossing & 0 & W-04-006 P5030021 \\
\hline W-04-006 & Looking downstream from culvert crossing & 180 & W-04-006_P5030022 \\
\hline W-04-007 & Looking upstream from culvert crossing (PR 260) & 200 & W-04-007_P5030023 \\
\hline W-04-007 & Looking downstream from culvert crossing (PR 260) & 80 & W-04-007 P5030024 \\
\hline W-04-007 & Looking downstream from culvert crossing (PR 260) at cattle & 80 & W-04-007_P5030025 \\
\hline W-04-007 & Looking at spawning suckers & $\mathrm{N} / \mathrm{A}$ & W-04-007_P5030026 \\
\hline W-04-007 & Looking at spawning suckers & $\mathrm{N} / \mathrm{A}$ & W-04-007_P5030027 \\
\hline W-04-007 & Photo of culvert $10 \mathrm{~m}$ upstream & 80 & W-04-007 P5030028 \\
\hline W-04-008 & Looking upstream from culvert crossing & 240 & W-04-008 P5030029 \\
\hline W-04-008 & Looking downstream from culvert crossing & 110 & W-04-008 P5030030 \\
\hline W-04-009 & Looking upstream from right bank & 270 & W-04-009_P5040001 \\
\hline W-04-009 & Looking downstream from right bank & 80 & W-04-009 P5040002 \\
\hline W-04-010 & Looking upstream from culvert crossing & 270 & W-04-010 P5040003 \\
\hline
\end{tabular}


Appendix 8: List of all photographs taken showing the corresponding site number, descriptive caption, direction faced when taken (azimuth in degrees) and the unique file name for each photograph.

\begin{tabular}{|c|c|c|c|}
\hline $\begin{array}{c}\text { Site } \\
\text { Number }\end{array}$ & Photo Description & Azimuth & File Name \\
\hline W-04-010 & Looking downstream from culvert crossing & 90 & W-04-010 P5040004 \\
\hline W-04-011 & Looking upstream from culvert crossing & 270 & W-04-011_P5040005 \\
\hline W-04-011 & Looking downstream from culvert crossing & 90 & W-04-011_P5040006 \\
\hline W-04-012 & Looking upstream from culvert crossing & 270 & W-04-012_P5040007 \\
\hline W-04-012 & Looking downstream from culvert crossing & 90 & W-04-012 P5040008 \\
\hline W-04-012 & Looking downstream from $10 \mathrm{~m}$ upstream of culvert crossing & 60 & W-04-012 P5040009 \\
\hline W-04-013 & Looking upstream from culvert crossing & 0 & W-04-013_P5040010 \\
\hline W-04-013 & Looking downstream from culvert crossing & 90 & W-04-013 P5040011 \\
\hline W-04-014 & Looking upstream from culvert crossing & 270 & W-04-014 P5040012 \\
\hline W-04-014 & Looking downstream from culvert crossing & 90 & W-04-014_P5040013 \\
\hline W-04-015 & Looking upstream from $5 \mathrm{~m}$ downstream of culvert crossing & 260 & W-04-015 P5040014 \\
\hline W-04-015 & Looking downstream from $5 \mathrm{~m}$ downstream of culvert crossing & 80 & W-04-015_P5040015 \\
\hline W-04-015 & Looking at burnt banks \& old culvert & 210 & W-04-015 P5040016 \\
\hline W-04-015 & Looking from culvert crossing (gravel road) looking at pool & 350 & W-04-015 P5040017 \\
\hline W-04-016 & Looking upstream from culvert crossing & 270 & W-04-016_P5040018 \\
\hline W-04-016 & Looking downstream from culvert crossing & 90 & W-04-016_P5040019 \\
\hline W-04-017 & Looking upstream from culvert crossing & 260 & W-04-017 P5040020 \\
\hline W-04-017 & Looking downstream from culvert crossing & 85 & W-04-017_P5040021 \\
\hline W-04-018 & Looking upstream from culvert crossing & 230 & W-04-018_P5040022 \\
\hline W-04-018 & Looking downstream from culvert crossing & 60 & W-04-018_P5040023 \\
\hline W-04-019 & Looking upstream from culvert crossing & 170 & W-04-019 P5040024 \\
\hline W-04-019 & Looking downstream from culvert crossing & 350 & W-04-019_P5040025 \\
\hline W-04-020 & Looking upstream from box culvert crossing & 270 & W-04-020_P5050001 \\
\hline W-04-020 & Looking downstream from box culvert crossing & 325 & W-04-020 P5050002 \\
\hline W-04-021 & Looking upstream from culvert crossing (PR 227) & 170 & W-04-021_P5050003 \\
\hline W-04-021 & Looking downstream from culvert crossing (PR 227) & 350 & W-04-021_P5050004 \\
\hline W-04-021 & Looking further downstream from culvert crossing & 270 & W-04-021_P5050005 \\
\hline W-04-021 & Looking further upstream from culvert crossing & 155 & W-04-021_P5050006 \\
\hline W-04-022 & Looking upstream from culvert crossing & 180 & W-04-022 P5050007 \\
\hline W-04-022 & Looking downstream from culvert crossing & 0 & W-04-022_P5050008 \\
\hline $\mathrm{W}-04-022$ & Looking at east side view of culverts at riprap (downstream) & 240 & W-04-022_P5050009 \\
\hline $\mathrm{W}-04-022$ & Looking at east side view of culverts at riprap (upstream) & 270 & W-04-022_P5050010 \\
\hline W-04-023 & Looking upstream from culvert crossing & 180 & W-04-023_P5050011 \\
\hline
\end{tabular}


Appendix 8: List of all photographs taken showing the corresponding site number, descriptive caption, direction faced when taken (azimuth in degrees) and the unique file name for each photograph.

\begin{tabular}{|c|c|c|c|}
\hline $\begin{array}{c}\text { Site } \\
\text { Number }\end{array}$ & Photo Description & Azimuth & File Name \\
\hline W-04-023 & Looking downstream from culvert crossing & 0 & W-04-023_P5050012 \\
\hline W-04-024 & Looking upstream from north bank shore & 180 & W-04-024_P5050013 \\
\hline W-04-024 & Looking downstream from north bank shore & 340 & W-04-024 P5050014 \\
\hline W-04-025 & Looking upstream from culvert crossing & 180 & W-04-025_P5050015 \\
\hline W-04-025 & Looking downstream from culvert crossing & 0 & W-04-025_P5050016 \\
\hline W-04-025 & Looking at closed gated culverts on downstream end of culvert & $\mathrm{N} / \mathrm{A}$ & W-04-025_P5050017 \\
\hline W-04-026 & Looking upstream from culvert crossing & 180 & W-04-026_P5050018 \\
\hline W-04-026 & Looking downstream from culvert crossing & 0 & W-04-026 P5050019 \\
\hline W-04-026 & Looking down at gated culvert on downstream side & N/A & W-04-026_P5050020 \\
\hline W-04-027 & Looking upstream from culvert crossing (PR 227) & 140 & W-04-027_P5050021 \\
\hline W-04-027 & Looking downstream from culvert crossing (PR 227) & 0 & W-04-027 P5050022 \\
\hline W-04-028 & Looking upstream from culvert crossing & 120 & W-04-028_P5050023 \\
\hline W-04-028 & Looking downstream from culvert crossing & 260 & W-04-028_P5050024 \\
\hline W-04-028 & Looking at cows with access to channel downstream side & 270 & W-04-028_P5050025 \\
\hline W-04-029 & Looking upstream from culvert crossing & 180 & W-04-029_P5060001 \\
\hline $\mathrm{W}-04-029$ & Looking downstream from culvert crossing & 0 & W-04-029_P5060002 \\
\hline W-04-030 & Looking upstream from culvert crossing & 200 & W-04-030_P5060003 \\
\hline W-04-030 & Looking downstream from culvert crossing & 0 & W-04-030_P5060004 \\
\hline W-04-031 & Looking upstream from culvert crossing & 200 & W-04-031_P5060005 \\
\hline W-04-031 & Looking downstream form culvert crossing & 95 & W-04-031_P5060006 \\
\hline W-04-032 & Looking upstream from box culvert crossing & 180 & W-04-032_P5060007 \\
\hline W-04-032 & Looking downstream from box culvert crossing & 0 & W-04-032_P5060008 \\
\hline W-04-033 & Looking upstream from culvert crossing & 170 & W-04-033_P5060009 \\
\hline W-04-033 & Looking downstream from culvert crossing & 330 & W-04-033_P5060010 \\
\hline W-04-034 & Looking upstream from culvert crossing & 250 & W-04-034_P5060011 \\
\hline W-04-034 & Looking downstream from culvert crossing & 70 & W-04-034_P5060012 \\
\hline W-04-035 & Looking upstream from culvert crossing & 235 & W-04-035 P5060013 \\
\hline W-04-035 & Looking downstream from culvert crossing & 150 & W-04-035_P5060014 \\
\hline W-04-035 & Looking downstream 5m upstream looking at riprap \& box culvert & 80 & W-04-035_P5060015 \\
\hline W-04-036 & Looking upstream from culvert crossing (PR 411) & 200 & W-04-036_P5060016 \\
\hline W-04-036 & Looking downstream from culvert crossing (PR 411) & 0 & W-04-036_P5060017 \\
\hline W-04-037 & Looking upstream from culvert crossing & 160 & W-04-037_P5060018 \\
\hline W-04-037 & Looking downstream from culvert crossing & 230 & W-04-037_P5060019 \\
\hline
\end{tabular}


Appendix 8: List of all photographs taken showing the corresponding site number, descriptive caption, direction faced when taken (azimuth in degrees) and the unique file name for each photograph.

\begin{tabular}{|c|c|c|c|}
\hline $\begin{array}{c}\text { Site } \\
\text { Number }\end{array}$ & Photo Description & Azimuth & File Name \\
\hline W-04-037 & Looking downstream from culvert crossing from PTH 6 & 270 & W-04-037_P5060020 \\
\hline W-04-038 & Looking upstream from culvert crossing (PR 415) & 330 & W-04-038_P5060021 \\
\hline W-04-038 & Looking downstream from culvert crossing (PR 415) & 180 & W-04-038 P5060022 \\
\hline W-04-039 & Looking upstream from culvert crossing & 90 & W-04-039_P5100001 \\
\hline W-04-039 & Looking downstream from culvert crossing & 250 & W-04-039_P5100002 \\
\hline W-04-039 & Looking at White Sucker (released) & $\mathrm{N} / \mathrm{A}$ & W-04-039_P5100003 \\
\hline W-04-039 & Looking at White Sucker (released) & $\mathrm{N} / \mathrm{A}$ & W-04-039_P5100004 \\
\hline W-04-039 & Looking at Northern Pike (released) & N/A & W-04-039 P5100005 \\
\hline W-04-039 & Looking at Northern Pike with very light colouration & $\mathrm{N} / \mathrm{A}$ & W-04-039_P5100006 \\
\hline W-04-040 & Looking upstream from culvert crossing & 140 & W-04-040_P5100007 \\
\hline W-04-040 & Looking downstream from culvert crossing & 300 & W-04-040 P5100008 \\
\hline W-04-040 & Looking at White Sucker (released) & N/A & W-04-040 P5100009 \\
\hline W-04-040 & Looking at White Sucker (released) & $\mathrm{N} / \mathrm{A}$ & W-04-040_P5100010 \\
\hline W-04-040 & Looking at White Sucker (released) & $\mathrm{N} / \mathrm{A}$ & W-04-040_P5100011 \\
\hline W-04-040 & Looking at White Sucker (released) & $\mathrm{N} / \mathrm{A}$ & W-04-040_P5100012 \\
\hline W-04-041 & Looking upstream from east bank & 320 & W-04-041_P5110001 \\
\hline W-04-041 & Looking downstream from east bank & 140 & W-04-041_P5110002 \\
\hline W-04-042 & Looking upstream from culvert crossing & 100 & W-04-042_P5110003 \\
\hline W-04-042 & Looking downstream from culvert crossing & 290 & W-04-042 P5110004 \\
\hline W-04-043 & Looking upstream from culvert crossing & 360 & W-04-043_P5110005 \\
\hline W-04-043 & Looking downstream from culvert crossing & 180 & W-04-043_P5110006 \\
\hline W-04-043 & Looking at White Sucker (released) & $\mathrm{N} / \mathrm{A}$ & W-04-043_P5110007 \\
\hline W-04-044 & Looking upstream from bridge crossing & 310 & W-04-044_P5110008 \\
\hline W-04-044 & Looking downstream from bridge crossing & 140 & W-04-044_P5110009 \\
\hline W-04-045 & Looking upstream from culvert crossing & 340 & W-04-045_P5110010 \\
\hline W-04-045 & Looking downstream from culvert crossing & 170 & W-04-045_P5110011 \\
\hline W-04-046 & Looking upstream from culvert crossing & 0 & W-04-046 P5110012 \\
\hline W-04-046 & Looking downstream from culvert crossing & 180 & W-04-046_P5110013 \\
\hline W-04-047 & Looking upstream from culvert crossing & 345 & W-04-047_P5110014 \\
\hline W-04-047 & Looking downstream from culvert crossing & 210 & W-04-047_P5110015 \\
\hline W-04-048 & Looking upstream from culvert crossing & 0 & W-04-048_P5110016 \\
\hline W-04-048 & Looking downstream from culvert crossing & 180 & W-04-048_P5110017 \\
\hline W-04-049 & Looking upstream from culvert crossing & 0 & W-04-049_P5110018 \\
\hline
\end{tabular}


Appendix 8: List of all photographs taken showing the corresponding site number, descriptive caption, direction faced when taken (azimuth in degrees) and the unique file name for each photograph.

\begin{tabular}{|c|c|c|c|}
\hline $\begin{array}{c}\text { Site } \\
\text { Number }\end{array}$ & Photo Description & Azimuth & File Name \\
\hline W-04-049 & Looking downstream from culvert crossing & 180 & W-04-049_P5110019 \\
\hline W-04-050 & Looking upstream 2nd order (east) from mid channel & 90 & W-04-050_P5110020 \\
\hline W-04-050 & Looking upstream 2nd order (west) from mid channel & 270 & W-04-050 P5110021 \\
\hline W-04-050 & Looking downstream 3rd order from culvert crossing & 180 & W-04-050_P5110022 \\
\hline W-04-050 & Looking upstream at perched culverts & 20 & W-04-050_P5110023 \\
\hline W-04-051 & Looking upstream from bridge crossing & 300 & W-04-051_P5120001 \\
\hline W-04-051 & Looking downstream from bridge crossing & 180 & W-04-051_P5120002 \\
\hline W-04-051 & looking at White Sucker (released) & N/A & W-04-051_P5120003 \\
\hline W-04-052 & Looking at cattle & 30 & W-04-052_P5120004 \\
\hline W-04-052 & Looking downstream from bridge crossing & 180 & W-04-052_P5120005 \\
\hline W-04-053 & Looking upstream from bridge crossing & 130 & W-04-053 P5180001 \\
\hline W-04-053 & Looking downstream from bridge crossing & 270 & W-04-053 P5180002 \\
\hline W-04-054 & Looking upstream from bridge crossing & 160 & W-04-054_P5180003 \\
\hline W-04-054 & Looking downstream from bridge crossing & 330 & W-04-054_P5180004 \\
\hline W-04-054 & Looking at White Sucker (released) & $\mathrm{N} / \mathrm{A}$ & W-04-054_P5180005 \\
\hline W-04-054 & Looking at White Sucker (released) & $\mathrm{N} / \mathrm{A}$ & W-04-054_P5180006 \\
\hline W-04-054 & Photo of 2nd sucker (moving) & N/A & W-04-054_P5180007 \\
\hline W-04-054 & Photo of 2nd sucker (moving) & $\mathrm{N} / \mathrm{A}$ & W-04-054_P5180008 \\
\hline W-04-054 & Looking at White Sucker (released) & $\mathrm{N} / \mathrm{A}$ & W-04-054_P5180009 \\
\hline W-04-055 & Looking upstream from culvert crossing & 180 & W-04-055 P5180010 \\
\hline W-04-055 & Looking downstream from culvert crossing & 0 & W-04-055_P5180011 \\
\hline W-04-056 & Looking upstream from culvert crossing & 180 & W-04-056_P5180012 \\
\hline W-04-056 & Looking downstream from culvert crossing & 0 & W-04-056 P5180013 \\
\hline W-04-057 & Looking upstream from culvert crossing & 160 & W-04-057_P5180014 \\
\hline W-04-057 & Looking downstream from culvert crossing & 0 & W-04-057_P5180015 \\
\hline W-04-058 & Looking upstream from culvert crossing & 180 & W-04-058 P5180016 \\
\hline W-04-058 & Looking downstream from culvert crossing & 25 & W-04-058 P5180017 \\
\hline W-04-059 & Looking upstream from culvert crossing & 180 & W-04-059_P5200001 \\
\hline W-04-059 & Looking downstream from culvert crossing & 0 & W-04-059_P5200002 \\
\hline W-04-060 & Looking upstream from bridge crossing & 50 & W-04-060_P5200003 \\
\hline W-04-060 & Looking upstream from bridge crossing & 180 & W-04-060_P5200004 \\
\hline W-04-060 & Looking downstream from bridge crossing & 0 & W-04-060_P5200005 \\
\hline W-04-060 & Looking downstream from bridge crossing & 250 & W-04-060_P5200006 \\
\hline
\end{tabular}


Appendix 8: List of all photographs taken showing the corresponding site number, descriptive caption, direction faced when taken (azimuth in degrees) and the unique file name for each photograph.

\begin{tabular}{|c|c|c|c|}
\hline $\begin{array}{c}\text { Site } \\
\text { Number }\end{array}$ & Photo Description & Azimuth & File Name \\
\hline W-04-061 & Looking upstream from culvert crossing & 90 & W-04-061_P5200007 \\
\hline W-04-061 & Looking downstream from culvert crossing & 180 & W-04-061_P5200008 \\
\hline W-04-062 & Looking upstream from culvert crossing & 110 & W-04-062 P5200009 \\
\hline W-04-062 & Looking downstream from culvert crossing & 285 & W-04-062_P5200010 \\
\hline W-04-063 & Looking upstream from culvert crossing & 100 & W-04-063_P5200011 \\
\hline W-04-063 & Looking downstream from culvert crossing & 270 & W-04-063_P5200012 \\
\hline W-04-064 & Looking upstream from culvert crossing & 180 & W-04-064_P5200013 \\
\hline W-04-064 & Looking downstream from culvert crossing & 325 & W-04-064_P5200014 \\
\hline W-04-065 & Looking upstream from culvert crossing & 180 & W-04-065_P5200015 \\
\hline W-04-065 & Looking downstream from culvert crossing & 0 & W-04-065_P5200016 \\
\hline W-04-066 & Looking upstream from culvert crossing & 180 & W-04-066 P5200017 \\
\hline W-04-066 & Looking downstream from culvert crossing & 0 & W-04-066 P5200018 \\
\hline W-04-067 & Looking upstream from culvert crossing & 90 & W-04-067_P5200019 \\
\hline W-04-067 & Looking downstream from culvert crossing & 270 & W-04-067_P5200020 \\
\hline W-04-068 & Looking upstream from right grass bank east side & 140 & W-04-068_P5200021 \\
\hline W-04-068 & Looking downstream from right grass bank east side & 0 & W-04-068_P5200022 \\
\hline W-04-068 & Looking at cattle & 120 & W-04-068_P5200023 \\
\hline W-04-068 & Looking at cattle & 70 & W-04-068_P5200024 \\
\hline W-04-069 & Looking upstream from culvert crossing & 90 & W-04-069 P5210001 \\
\hline W-04-069 & Looking downstream from culvert crossing & 270 & W-04-069_P5210002 \\
\hline W-04-070 & Looking upstream from culvert crossing & 90 & W-04-070_P5210003 \\
\hline W-04-070 & Looking downstream from culvert crossing & 270 & W-04-070_P5210004 \\
\hline W-04-071 & Looking upstream from culvert crossing & 110 & W-04-071_P5210005 \\
\hline W-04-071 & Looking downstream from culvert crossing & 270 & W-04-071_P5210006 \\
\hline W-04-072 & Looking upstream from bridge crossing (PR 207) & 0 & W-04-072_P5250001 \\
\hline W-04-072 & Looking directly down at riffle & $\mathrm{N} / \mathrm{A}$ & W-04-072_P5250002 \\
\hline W-04-072 & Looking downstream from bridge crossing (PR 207) & 180 & W-04-072 P5250003 \\
\hline W-04-072 & Looking downstream at bank slump $50 \mathrm{~m}$ downstream from bridge & 180 & W-04-072_P5250004 \\
\hline W-04-073 & Looking upstream from culvert crossing (PR 207) & 0 & W-04-073_P5250005 \\
\hline W-04-073 & Looking downstream from culvert crossing (PR 207) & 180 & W-04-073_P5250006 \\
\hline W-04-074 & Looking upstream from bridge crossing & 180 & W-04-074_P5250007 \\
\hline W-04-074 & Looking downstream from bridge crossing & 0 & W-04-074_P5250008 \\
\hline W-04-074 & Looking at perched gated culverts from upstream side of bridge & 110 & W-04-074_P5250009 \\
\hline
\end{tabular}


Appendix 8: List of all photographs taken showing the corresponding site number, descriptive caption, direction faced when taken (azimuth in degrees) and the unique file name for each photograph.

\begin{tabular}{|c|c|c|c|}
\hline $\begin{array}{c}\text { Site } \\
\text { Number }\end{array}$ & Photo Description & Azimuth & File Name \\
\hline W-04-074 & Looking at bank slump / erosion downstream from bridge & 290 & W-04-074_P5250010 \\
\hline W-04-075 & Looking upstream from culvert crossing (PR 207) & 180 & W-04-075_P5250011 \\
\hline W-04-075 & Looking downstream from culvert crossing (PR 207) & 0 & W-04-075_P5250012 \\
\hline W-04-076 & Looking upstream from box culvert crossing (PR 207) & 180 & W-04-076_P5250013 \\
\hline W-04-076 & Looking downstream from box culvert crossing (PR 207) & 0 & W-04-076_P5250014 \\
\hline W-04-077 & Looking upstream from culvert crossing & 270 & W-04-077_P5260001 \\
\hline W-04-077 & Looking downstream from culvert crossing & 90 & W-04-077_P5260002 \\
\hline W-04-077 & Looking at culvert inlet & 120 & W-04-077_P5260003 \\
\hline W-04-077 & Looking at culvert outlet & 220 & W-04-077_P5260004 \\
\hline W-04-078 & Looking upstream from culvert crossing & 300 & W-04-078_P5260005 \\
\hline W-04-078 & Looking downstream from culvert crossing & 130 & W-04-078_P5260006 \\
\hline W-04-078 & Looking at culvert inlet & 270 & W-04-078_P5260007 \\
\hline W-04-078 & Looking at culvert outlet & 100 & W-04-078_P5260008 \\
\hline W-04-078 & Looking at horse in stream - upstream side of culvert & 300 & W-04-078_P5260009 \\
\hline W-04-079 & Looking upstream from culvert crossing & 270 & W-04-079_P5260010 \\
\hline W-04-079 & Looking downstream from culvert crossing & 90 & W-04-079_P5260011 \\
\hline W-04-079 & Looking at culvert inlet & 160 & W-04-079_P5260012 \\
\hline W-04-079 & Looking at culvert outlet & 310 & W-04-079_P5260013 \\
\hline W-04-080 & Looking upstream Ross Creek & 270 & W-04-080_P5260014 \\
\hline W-04-080 & Looking downstream Ross Creek & 90 & W-04-080_P5260015 \\
\hline W-04-080 & Looking upstream 2nd order drain & 0 & W-04-080_P5260016 \\
\hline W-04-080 & Looking upstream 2nd order drain & 0 & W-04-080_P5260017 \\
\hline W-04-080 & Looking upstream at 3rd order drain across Ross Creek & 180 & W-04-080_P5260018 \\
\hline W-04-081 & Looking upstream $10 \mathrm{~m}$ below beaver dam & 270 & W-04-081_P5260019 \\
\hline W-04-081 & Looking downstream 10m above beaver dam & 90 & W-04-081_P5260020 \\
\hline W-04-082 & Looking upstream from culvert crossing (PTH 17) & 0 & W-04-082_P5260021 \\
\hline W-04-082 & Looking downstream from culvert crossing (PTH 17) & 180 & W-04-082_P5260022 \\
\hline W-04-082 & Looking at culvert inlet & 210 & W-04-082_P5260023 \\
\hline W-04-082 & Looking at culvert outlet & 300 & W-04-082_P5260024 \\
\hline W-04-083 & Looking upstream from culvert crossing & 240 & W-04-083_P5270001 \\
\hline W-04-083 & Looking downstream from culvert crossing & 130 & W-04-083_P5270002 \\
\hline W-04-083 & Looking at culvert inlet & 40 & W-04-083_P5270003 \\
\hline W-04-083 & Looking at culvert outlet & 280 & W-04-083_P5270004 \\
\hline
\end{tabular}


Appendix 8: List of all photographs taken showing the corresponding site number, descriptive caption, direction faced when taken (azimuth in degrees) and the unique file name for each photograph.

\begin{tabular}{|c|c|c|c|}
\hline $\begin{array}{c}\text { Site } \\
\text { Number }\end{array}$ & Photo Description & Azimuth & File Name \\
\hline W-04-084 & Looking upstream from bridge crossing (PTH 17) & 300 & W-04-084_P5270005 \\
\hline W-04-084 & Looking downstream from bridge crossing (PTH 17) & 160 & W-04-084 P5270006 \\
\hline W-04-085 & Looking upstream from right bank (north side) & 200 & W-04-085_P5280001 \\
\hline W-04-085 & Looking downstream from right bank (north side) & 90 & W-04-085_P5280002 \\
\hline W-04-086 & Looking upstream from bridge crossing & 140 & W-04-086_P5280003 \\
\hline W-04-086 & Looking downstream from bridge crossing & 300 & W-04-086_P5280004 \\
\hline W-04-086 & Looking further upstream from bridge crossing & 160 & W-04-086_P5280005 \\
\hline W-04-087 & Looking upstream from culvert crossing & 90 & W-04-087_P5280006 \\
\hline W-04-087 & Looking downstream from culvert crossing & 270 & W-04-087_P5280007 \\
\hline W-04-087 & Looking at culvert inlet & 270 & W-04-087 P5280008 \\
\hline W-04-087 & Looking at culvert outlet & 90 & W-04-087_P5280009 \\
\hline W-04-088 & Looking upstream from culvert crossing & 90 & W-04-088_P5280010 \\
\hline W-04-088 & Looking downstream from culvert crossing & 270 & W-04-088_P5280011 \\
\hline W-04-088 & Looking at culvert inlet & 220 & W-04-088_P5280012 \\
\hline W-04-088 & Looking at culvert outlet & 120 & W-04-088_P5280013 \\
\hline W-04-089 & Looking upstream from culvert crossing & 180 & W-04-089_P6010001 \\
\hline W-04-089 & Looking down at whirlpool & $\mathrm{N} / \mathrm{A}$ & W-04-089_P6010002 \\
\hline W-04-089 & Looking downstream from culvert crossing & 0 & W-04-089 P6010003 \\
\hline W-04-089 & Looking down at culvert & $\mathrm{N} / \mathrm{A}$ & W-04-089_P6010004 \\
\hline W-04-090 & Looking upstream from culvert crossing & 180 & W-04-090_P6010005 \\
\hline W-04-090 & Looking downstream from culvert crossing & 0 & W-04-090_P6010006 \\
\hline W-04-090 & Looking at culvert inlet & 0 & W-04-090_P6010007 \\
\hline W-04-090 & Looking at culvert outlet & 180 & W-04-090_P6010008 \\
\hline W-04-090 & Looking at eroded right bank on downstream side of culvert for $10 \mathrm{~m}$ & 200 & W-04-090_P6010009 \\
\hline W-04-091 & Looking upstream from culvert crossing & 180 & W-04-091_P6010010 \\
\hline W-04-091 & Looking downstream from culvert crossing & 0 & W-04-091_P6010011 \\
\hline W-04-091 & Looking at flooded road (Balcaen Road) & 90 & W-04-091_P6010012 \\
\hline W-04-092 & Looking upstream 3rd order from road at junction & 120 & W-04-092_P6010013 \\
\hline W-04-092 & Looking upstream Marchand West Drain from road at junction & 180 & W-04-092_P6010014 \\
\hline W-04-092 & Looking downstream Marchand West Drain from rd. at junction. & 0 & W-04-092_P6010015 \\
\hline W-04-093 & Looking upstream from culvert crossing & 90 & W-04-093_P6010016 \\
\hline W-04-093 & Looking downstream from culvert crossing & 270 & W-04-093_P6010017 \\
\hline W-04-093 & Looking at culvert inlet & 240 & W-04-093_P6010018 \\
\hline
\end{tabular}


Appendix 8: List of all photographs taken showing the corresponding site number, descriptive caption, direction faced when taken (azimuth in degrees) and the unique file name for each photograph.

\begin{tabular}{|c|c|c|c|}
\hline $\begin{array}{c}\text { Site } \\
\text { Number }\end{array}$ & Photo Description & Azimuth & File Name \\
\hline W-04-093 & Looking at culvert outlet & 130 & W-04-093 P6010019 \\
\hline W-04-094 & Looking upstream from culvert crossing & 100 & W-04-094_P6020001 \\
\hline W-04-094 & Looking upstream from culvert crossing at dam & 100 & W-04-094_P6020002 \\
\hline W-04-094 & Looking downstream from culvert crossing & 280 & W-04-094_P6020003 \\
\hline W-04-094 & Looking at culvert inlet & 0 & W-04-094_P6020007 \\
\hline W-04-094 & Looking at culvert outlet & 100 & W-04-094_P6020008 \\
\hline W-04-095 & Looking upstream from culvert crossing & 0 & W-04-095_P6020009 \\
\hline W-04-095 & Looking downstream from culvert crossing & 180 & W-04-095_P6020010 \\
\hline W-04-095 & Looking at culvert inlet & 240 & W-04-095_P6020011 \\
\hline W-04-095 & Looking at culvert outlet & 300 & W-04-095_P6020012 \\
\hline W-04-096 & Looking upstream from culvert \#1 crossing & 90 & W-04-096 P6020013 \\
\hline W-04-096 & Looking downstream from culvert \#1 crossing & 270 & W-04-096_P6020014 \\
\hline W-04-096 & Looking upstream from culvert \#2 crossing & 90 & W-04-096_P6020015 \\
\hline W-04-096 & Looking downstream from culvert \#2 crossing & 270 & W-04-096 P6020016 \\
\hline W-04-097 & Looking upstream from culvert crossing & 80 & W-04-097_P6020017 \\
\hline W-04-097 & Looking downstream from culvert crossing & 250 & W-04-097_P6020018 \\
\hline W-04-097 & Looking at culvert inlet & 300 & W-04-097_P6020019 \\
\hline W-04-097 & Looking at culvert outlet & 140 & W-04-097 P6020020 \\
\hline W-04-098 & Looking upstream from culvert crossing & 120 & W-04-098_P6020021 \\
\hline W-04-098 & Looking downstream from culvert crossing & 290 & W-04-098_P6020022 \\
\hline W-04-098 & Looking at culvert inlet & 200 & W-04-098_P6020023 \\
\hline W-04-098 & Looking at culvert outlet & 90 & W-04-098_P6020024 \\
\hline W-04-099 & Looking upstream from bridge crossing & 130 & W-04-099_P6020025 \\
\hline W-04-099 & Looking downstream from bridge crossing & 250 & W-04-099_P6020026 \\
\hline W-04-099 & Looking at flooded area on upstream side (south) of bridge & 180 & W-04-099_P6020027 \\
\hline W-04-099 & Looking at flooded area on upstream side (north) of bridge & 80 & W-04-099_P6020028 \\
\hline W-04-099 & Looking upstream from bridge $10 \mathrm{~m}$ downstream & 50 & W-04-099_P6020029 \\
\hline W-04-099 & Looking at flooding further downstream & 250 & W-04-099_P6020030 \\
\hline W-04-100 & Looking upstream from bridge crossing & 0 & W-04-100_P6020031 \\
\hline W-04-100 & Looking upstream from bridge crossing & 110 & W-04-100_P6020032 \\
\hline W-04-100 & Looking downstream from bridge crossing & 200 & W-04-100_P6020033 \\
\hline W-04-100 & Looking downstream from bridge crossing & 310 & W-04-100_P6020034 \\
\hline W-04-101 & Looking upstream from culvert crossing & 130 & W-04-101_P6030001 \\
\hline
\end{tabular}


Appendix 8: List of all photographs taken showing the corresponding site number, descriptive caption, direction faced when taken (azimuth in degrees) and the unique file name for each photograph.

\begin{tabular}{|c|c|c|c|}
\hline $\begin{array}{c}\text { Site } \\
\text { Number }\end{array}$ & Photo Description & Azimuth & File Name \\
\hline W-04-101 & Looking downstream from culvert crossing & 280 & W-04-101_P6030002 \\
\hline W-04-101 & Looking at culvert inlet & 295 & W-04-101_P6030003 \\
\hline W-04-101 & Looking at culvert outlet & 95 & W-04-101_P6030004 \\
\hline W-04-102 & Looking upstream from bridge crossing (PR 201) & 0 & W-04-102_P6030005 \\
\hline W-04-102 & Looking downstream from bridge crossing (PR 201) & 180 & W-04-102_P6030006 \\
\hline W-04-103 & Looking upstream from culvert crossing & 180 & W-04-103_P6030007 \\
\hline W-04-103 & Looking downstream from culvert crossing & 0 & W-04-103_P6030008 \\
\hline W-04-103 & Looking at culvert outlet & 220 & W-04-103 P6030009 \\
\hline W-04-103 & Looking at culvert inlet & 300 & W-04-103_P6030010 \\
\hline W-04-104 & Looking upstream from culvert crossing & 280 & W-04-104_P6030011 \\
\hline W-04-104 & Looking downstream from culvert crossing & 80 & W-04-104 P6030012 \\
\hline W-04-104 & Looking at whirlpool at culvert inlet & 80 & W-04-104_P6030013 \\
\hline W-04-104 & Looking at culvert outlet & 70 & W-04-104_P6030014 \\
\hline W-04-104 & Looking at previously dredged drain downstream side & 305 & W-04-104_P6030015 \\
\hline W-04-104 & Looking at current dredged drain upstream side & 110 & W-04-104_P6030016 \\
\hline W-04-104 & Looking down at whirlpool at culvert inlet & $\mathrm{N} / \mathrm{A}$ & W-04-104_P6030017 \\
\hline W-04-104 & Looking down at whirlpool at culvert inlet & N/A & W-04-104_P6030018 \\
\hline W-04-105 & Looking upstream from culvert crossing & 170 & W-04-105_P6030019 \\
\hline W-04-105 & Looking downstream from culvert crossing & 330 & W-04-105 P6030020 \\
\hline W-04-105 & Looking at culvert inlet & 20 & W-04-105 P6030021 \\
\hline W-04-105 & Looking at culvert outlet & 130 & W-04-105_P6030022 \\
\hline W-04-106 & Looking upstream from culvert crossing & 180 & W-04-106_P6030023 \\
\hline W-04-106 & Looking downstream from culvert crossing & 0 & W-04-106 P6030024 \\
\hline W-04-106 & Looking at culvert inlet & 45 & W-04-106_P6030025 \\
\hline W-04-106 & Looking at culvert outlet & 120 & W-04-106_P6030026 \\
\hline W-04-107 & Looking upstream from bridge crossing & 170 & W-04-107 P6030027 \\
\hline W-04-107 & Looking upstream at flooded right bank & 230 & W-04-107_P6030028 \\
\hline W-04-107 & Looking downstream from bridge crossing & 300 & W-04-107_P6030029 \\
\hline W-04-107 & Looking downstream at flooded left bank & 360 & W-04-107_P6030030 \\
\hline W-04-108 & Looking upstream from culvert crossing & 180 & W-04-108_P6030031 \\
\hline W-04-108 & Looking downstream from culvert crossing & 0 & W-04-108_P6030032 \\
\hline W-04-109 & Looking upstream from culvert crossing & 125 & W-04-109_P6040001 \\
\hline W-04-109 & Looking downstream from culvert crossing & 300 & W-04-109_P6040002 \\
\hline
\end{tabular}


Appendix 8: List of all photographs taken showing the corresponding site number, descriptive caption, direction faced when taken (azimuth in degrees) and the unique file name for each photograph.

\begin{tabular}{|c|c|c|c|}
\hline $\begin{array}{c}\text { Site } \\
\text { Number }\end{array}$ & Photo Description & Azimuth & File Name \\
\hline W-04-109 & Looking further downstream from south downstream culvert & 330 & W-04-109_P6040003 \\
\hline W-04-109 & Looking at culvert inlet & 250 & W-04-109_P6040004 \\
\hline W-04-109 & Looking at culvert outlet & 90 & W-04-109 P6040005 \\
\hline W-04-110 & Looking upstream bridge crossing (PR 218) & 30 & W-04-110_P6040006 \\
\hline W-04-110 & Looking downstream bridge crossing (PR 218) & 230 & W-04-110_P6040007 \\
\hline W-04-111 & Looking upstream from culvert crossing & 280 & W-04-111_P6040008 \\
\hline W-04-111 & Looking downstream from culvert crossing & 100 & W-04-111_P6040009 \\
\hline W-04-111 & Looking at culvert inlet & 350 & W-04-111_P6040010 \\
\hline W-04-111 & Looking at culvert outlet & 60 & W-04-111_P6040011 \\
\hline W-04-112 & Looking upstream from west bank & 0 & W-04-112_P6040012 \\
\hline W-04-112 & Looking upstream from centre ( $40 \mathrm{~m}$ from either side) & 0 & W-04-112 P6040013 \\
\hline W-04-112 & Looking upstream from east bank & 0 & W-04-112_P6040014 \\
\hline W-04-112 & Looking downstream from east bank & 180 & W-04-112_P6040015 \\
\hline W-04-112 & Looking downstream from centre - east branch & 135 & W-04-112_P6040016 \\
\hline W-04-112 & Looking downstream from centre - west branch & 205 & W-04-112_P6040017 \\
\hline W-04-112 & Looking downstream from west bank & 190 & W-04-112_P6040018 \\
\hline W-04-112 & Looking downstream from west bank - looking at east branch & 120 & W-04-112_P6040019 \\
\hline W-04-113 & Looking upstream from culvert crossing & 180 & W-04-113_P6050001 \\
\hline W-04-113 & Looking downstream from culvert crossing & 270 & W-04-113 P6050002 \\
\hline W-04-113 & Looking at culvert inlet & 270 & W-04-113_P6050003 \\
\hline W-04-113 & Looking at culvert outlet & 90 & W-04-113_P6050004 \\
\hline W-04-113 & Looking at waterfall running into 2 nd order drain & 90 & W-04-113_P6050005 \\
\hline W-04-114 & Looking downstream from culvert crossing & 300 & W-04-114_P6050006 \\
\hline W-04-114 & Looking upstream from culvert crossing & 150 & W-04-114_P6050007 \\
\hline W-04-114 & Looking at culvert outlet & 110 & W-04-114_P6050008 \\
\hline W-04-114 & Looking at culvert inlet & 280 & W-04-114_P6050009 \\
\hline W-04-115 & Looking upstream from culvert crossing & 180 & W-04-115 P6050010 \\
\hline W-04-115 & Looking downstream from culvert crossing & 0 & W-04-115_P6050011 \\
\hline W-04-115 & Looking at culvert inlet & 0 & W-04-115_P6050012 \\
\hline W-04-115 & Looking at culvert outlet & 180 & W-04-115_P6050013 \\
\hline W-04-116 & Looking upstream from bridge crossing & 180 & W-04-116_P6050014 \\
\hline W-04-116 & Looking downstream from bridge crossing & 0 & W-04-116_P6050015 \\
\hline W-04-117 & Looking upstream from culvert crossing & 180 & W-04-117_P6070001 \\
\hline
\end{tabular}


Appendix 8: List of all photographs taken showing the corresponding site number, descriptive caption, direction faced when taken (azimuth in degrees) and the unique file name for each photograph.

\begin{tabular}{|c|c|c|c|}
\hline $\begin{array}{c}\text { Site } \\
\text { Number }\end{array}$ & Photo Description & Azimuth & File Name \\
\hline W-04-117 & Looking downstream from culvert crossing & 340 & $\begin{array}{ll}\mathrm{W}-04-117 & \mathrm{P} 6070002\end{array}$ \\
\hline W-04-117 & Looking at culvert inlet & 340 & W-04-117_P6070003 \\
\hline W-04-117 & Looking at culvert outlet & 180 & W-04-117_P6070004 \\
\hline W-04-118 & Looking upstream from culvert crossing & 175 & W-04-118_P6070005 \\
\hline W-04-118 & Looking downstream from culvert crossing & 20 & W-04-118 P6070006 \\
\hline W-04-118 & Looking at culvert inlet & 5 & W-04-118_P6070007 \\
\hline W-04-118 & Looking at culvert outlet & 185 & W-04-118_P6070008 \\
\hline W-04-119 & Looking upstream from culvert crossing & 90 & W-04-119 P6080001 \\
\hline W-04-119 & Looking downstream from culvert crossing & 270 & W-04-119 P6080002 \\
\hline W-04-119 & Looking at culvert inlet & 300 & W-04-119 P6080003 \\
\hline W-04-119 & Looking at culvert outlet & 60 & W-04-119_P6080004 \\
\hline W-04-120 & Looking upstream from culvert crossing & 180 & W-04-120_P6080005 \\
\hline W-04-120 & Looking downstream from culvert crossing & 0 & W-04-120 P6080006 \\
\hline W-04-120 & Looking at culvert inlet & 330 & W-04-120_P6080007 \\
\hline W-04-120 & Looking at culvert outlet & 160 & W-04-120_P6080008 \\
\hline W-04-120 & Looking downstream from right bank at eroded banks & 0 & W-04-120 P6080009 \\
\hline W-04-120 & Looking downstream from right bank at eroded banks & 0 & W-04-120 P6080010 \\
\hline W-04-121 & Looking upstream from culvert crossing & 180 & W-04-121_P6080011 \\
\hline W-04-121 & Looking downstream from culvert crossing & 0 & W-04-121_P6080012 \\
\hline W-04-121 & Looking at culvert inlet & 35 & W-04-121 P6080013 \\
\hline W-04-121 & Looking at culvert outlet & 160 & W-04-121 P6080014 \\
\hline W-04-122 & Looking upstream from culvert crossing & 180 & W-04-122_P6080015 \\
\hline W-04-122 & Looking downstream from culvert crossing & 0 & W-04-122_P6080016 \\
\hline W-04-122 & Looking at culvert inlet & 10 & W-04-122 P6080017 \\
\hline W-04-122 & Looking at culvert outlet & 130 & W-04-122 P6080018 \\
\hline W-04-123 & Looking upstream from culvert crossing & 0 & W-04-123_P6080019 \\
\hline W-04-123 & Looking downstream from culvert crossing & 180 & W-04-123_P6080020 \\
\hline W-04-123 & Looking at culvert inlet & 180 & W-04-123 P6080021 \\
\hline W-04-123 & Looking at culvert outlet & 0 & W-04-123 P6080022 \\
\hline W-04-124 & Looking upstream from right bank & 180 & W-04-124_P6080023 \\
\hline W-04-124 & Looking downstream from right bank & 0 & W-04-124_P6080024 \\
\hline W-04-125 & Looking upstream from culvert crossing & 90 & W-04-125 P6090001 \\
\hline W-04-125 & Looking downstream from culvert crossing & 270 & W-04-125 P6090002 \\
\hline
\end{tabular}


Appendix 8: List of all photographs taken showing the corresponding site number, descriptive caption, direction faced when taken (azimuth in degrees) and the unique file name for each photograph.

\begin{tabular}{|c|c|c|c|}
\hline $\begin{array}{c}\text { Site } \\
\text { Number }\end{array}$ & Photo Description & Azimuth & File Name \\
\hline W-04-125 & Looking at culvert inlet - Photo taken looking straight down at culvert & $\mathrm{N} / \mathrm{A}$ & W-04-125_P6090003 \\
\hline W-04-125 & Looking at culvert outlet - Photo taken looking straight down at culvert & $\mathrm{N} / \mathrm{A}$ & W-04-125_P6090004 \\
\hline W-04-126 & Looking upstream from culvert crossing & 80 & W-04-126_P6090005 \\
\hline W-04-126 & Looking downstream from culvert crossing & 290 & W-04-126_P6090006 \\
\hline W-04-126 & Looking at culvert inlet & 0 & W-04-126_P6090007 \\
\hline W-04-126 & Looking at culvert outlet & 60 & W-04-126_P6090008 \\
\hline W-04-127 & Looking upstream from culvert crossing & 120 & W-04-127_P6090009 \\
\hline W-04-127 & Looking downstream from culvert crossing & 300 & W-04-127_P6090010 \\
\hline W-04-128 & Looking upstream from culvert crossing & 90 & W-04-128_P6090011 \\
\hline W-04-128 & Looking downstream from culvert crossing & 270 & W-04-128_P6090012 \\
\hline W-04-128 & Looking at culvert inlet & 230 & W-04-128_P6090013 \\
\hline W-04-128 & Looking at culvert outlet & 100 & W-04-128_P6090014 \\
\hline W-04-129 & Looking upstream from culvert crossing & 180 & W-04-129_P6090015 \\
\hline W-04-129 & Looking downstream from culvert crossing & 270 & W-04-129_P6090016 \\
\hline W-04-129 & Looking at culvert inlet & 240 & W-04-129_P6090017 \\
\hline W-04-129 & Looking at culvert outlet & 60 & W-04-129_P6090018 \\
\hline W-04-130 & Looking upstream from culvert crossing & 120 & W-04-130_P6090019 \\
\hline W-04-130 & Looking downstream from culvert crossing & 340 & W-04-130_P6090020 \\
\hline W-04-130 & Looking at culvert inlet & 0 & W-04-130_P6090021 \\
\hline W-04-130 & Looking at culvert outlet & 180 & W-04-130_P6090022 \\
\hline W-04-131 & Looking upstream from culvert crossing & 180 & W-04-131_P6090023 \\
\hline W-04-131 & Looking downstream from culvert crossing & 0 & W-04-131_P6090024 \\
\hline W-04-131 & Looking at culvert inlet & 320 & W-04-131_P6090025 \\
\hline W-04-131 & Looking at culvert outlet & 160 & W-04-131_P6090026 \\
\hline W-04-132 & Looking upstream from culvert crossing & 90 & W-04-132_P6090027 \\
\hline W-04-132 & Looking downstream from culvert crossing & 300 & W-04-132_P6090028 \\
\hline W-04-132 & Looking at culvert inlet & 270 & W-04-132_P6090029 \\
\hline W-04-132 & Looking at culvert outlet & 90 & W-04-132_P6090030 \\
\hline W-04-133 & Looking upstream from culvert crossing & 140 & W-04-133_P6090031 \\
\hline W-04-133 & Looking downstream from culvert crossing & 290 & W-04-133_P6090032 \\
\hline W-04-133 & Looking at culvert inlet & 240 & W-04-133_P6090033 \\
\hline W-04-133 & Looking at culvert outlet & 140 & W-04-133_P6090034 \\
\hline W-04-134 & Looking upstream from culvert crossing & 180 & W-04-134_P6090035 \\
\hline
\end{tabular}


Appendix 8: List of all photographs taken showing the corresponding site number, descriptive caption, direction faced when taken (azimuth in degrees) and the unique file name for each photograph.

\begin{tabular}{|c|c|c|c|}
\hline $\begin{array}{c}\text { Site } \\
\text { Number }\end{array}$ & Photo Description & Azimuth & File Name \\
\hline W-04-134 & Looking downstream from culvert crossing & 0 & W-04-134 P6090036 \\
\hline W-04-134 & Looking at culvert inlet & 25 & W-04-134_P6090037 \\
\hline W-04-134 & Looking at culvert outlet & 150 & W-04-134_P6090038 \\
\hline W-04-135 & Looking upstream from culvert crossing & 180 & W-04-135 P6090039 \\
\hline W-04-135 & Looking downstream from culvert crossing & 0 & W-04-135_P6090040 \\
\hline W-04-135 & Looking at culvert outlet & 180 & W-04-135 P6090041 \\
\hline W-04-135 & Looking at culvert inlet & 0 & W-04-135_P6090042 \\
\hline W-04-136 & Looking upstream from culvert crossing & 140 & W-04-136_P6100001 \\
\hline W-04-136 & Looking downstream from culvert crossing & 240 & W-04-136_P6100002 \\
\hline W-04-136 & Looking at culvert inlet & 45 & W-04-136_P6100003 \\
\hline W-04-136 & Looking at culvert outlet & 270 & W-04-136 P6100004 \\
\hline W-04-137 & Looking upstream from culvert crossing & 90 & W-04-137_P6100005 \\
\hline W-04-137 & Looking downstream from culvert crossing & 270 & W-04-137_P6100006 \\
\hline W-04-137 & Looking further downstream from culvert crossing & 270 & W-04-137 P6100007 \\
\hline W-04-137 & Looking at culvert inlet & 230 & W-04-137 P6100008 \\
\hline W-04-137 & Looking at culvert outlet & 90 & W-04-137_P6100009 \\
\hline W-04-138 & Looking upstream from culvert crossing & 180 & W-04-138_P6100010 \\
\hline W-04-138 & Looking downstream from culvert crossing & 0 & W-04-138 P6100011 \\
\hline W-04-138 & Looking at culvert inlet & 0 & W-04-138_P6100012 \\
\hline W-04-138 & Looking at culvert outlet & 150 & W-04-138_P6100013 \\
\hline W-04-139 & Looking upstream from culvert crossing & 180 & W-04-139 P6100014 \\
\hline W-04-139 & Looking downstream from culvert crossing & 0 & W-04-139_P6100015 \\
\hline W-04-139 & Looking at culvert inlet & 50 & W-04-139_P6100016 \\
\hline W-04-139 & Looking at culvert outlet & 120 & W-04-139_P6100017 \\
\hline W-04-140 & Looking upstream from mud road right bank (north bank) & 115 & W-04-140_P6100018 \\
\hline W-04-140 & Looking downstream from mud road right bank (north bank) & 260 & W-04-140_P6100019 \\
\hline W-04-141 & Looking upstream from ford crossing (flooded road) & 130 & W-04-141_P6100020 \\
\hline W-04-141 & Looking downstream from ford crossing (flooded road) & 0 & W-04-141_P6100021 \\
\hline W-04-141 & Looking at submerged road & 120 & W-04-141_P6100022 \\
\hline W-04-142 & Looking upstream from culvert crossing & 150 & W-04-142_P6140001 \\
\hline W-04-142 & Looking downstream from culvert crossing & 280 & W-04-142_P6140002 \\
\hline W-04-142 & Looking at culvert inlet & 230 & W-04-142_P6140003 \\
\hline W-04-142 & Looking at culvert outlet & 40 & W-04-142_P6140004 \\
\hline
\end{tabular}


Appendix 8: List of all photographs taken showing the corresponding site number, descriptive caption, direction faced when taken (azimuth in degrees) and the unique file name for each photograph.

\begin{tabular}{|c|c|c|c|}
\hline $\begin{array}{c}\text { Site } \\
\text { Number }\end{array}$ & Photo Description & Azimuth & File Name \\
\hline W-04-143 & Looking upstream from culvert crossing & 90 & W-04-143_P6140005 \\
\hline W-04-143 & Looking downstream from culvert crossing & 270 & W-04-143 P6140006 \\
\hline W-04-143 & Looking at culvert inlet & 115 & W-04-143_P6140007 \\
\hline W-04-143 & Looking at culvert outlet & 240 & W-04-143_P6140008 \\
\hline W-04-144 & Looking upstream from culvert crossing & 140 & W-04-144_P6140009 \\
\hline W-04-144 & Looking downstream from culvert crossing & 320 & W-04-144_P6140010 \\
\hline W-04-144 & Looking at culvert inlet & 300 & W-04-144_P6140011 \\
\hline W-04-144 & Looking at culvert outlet & 100 & W-04-144_P6140012 \\
\hline W-04-145 & Looking upstream from culvert crossing & 90 & W-04-145 P6140013 \\
\hline W-04-145 & Looking downstream from culvert crossing & 240 & W-04-145 P6140014 \\
\hline W-04-145 & Looking at culvert inlet & 230 & W-04-145_P6140015 \\
\hline W-04-145 & Looking at culvert outlet & 100 & W-04-145_P6140016 \\
\hline W-04-146 & Looking upstream Kyle Drain at culvert crossing & 90 & W-04-146_P6140017 \\
\hline W-04-146 & Looking downstream Kyle Drain at culvert crossing & 270 & W-04-146 P6140018 \\
\hline W-04-146 & Looking at Kyle Drain culvert inlet & 260 & W-04-146_P6140019 \\
\hline W-04-146 & Looking at Kyle Drain culvert outlet & 95 & W-04-146_P6140020 \\
\hline W-04-146 & Looking upstream 2nd order drain at culvert crossing & 90 & W-04-146 P6140021 \\
\hline W-04-146 & Looking downstream 2nd order drain at culvert crossing & 270 & W-04-146 P6140022 \\
\hline W-04-146 & Looking at 2nd order culvert inlet & 270 & W-04-146_P6140023 \\
\hline W-04-146 & Looking at 2nd order culvert outlet & 40 & W-04-146_P6140024 \\
\hline W-04-147 & Looking upstream from culvert crossing & 0 & W-04-147 P6140025 \\
\hline W-04-147 & Looking downstream from culvert crossing & 180 & W-04-147_P6140026 \\
\hline W-04-147 & Looking at culvert inlet & 140 & W-04-147_P6140027 \\
\hline W-04-147 & Looking at culvert outlet & 0 & W-04-147_P6140028 \\
\hline W-04-148 & Looking upstream from culvert crossing & 0 & W-04-148 P6140029 \\
\hline W-04-148 & Looking downstream from culvert crossing & 180 & W-04-148_P6140030 \\
\hline W-04-148 & Looking at culvert inlet & 180 & W-04-148_P6140031 \\
\hline W-04-148 & Looking at culvert outlet & 350 & W-04-148_P6140032 \\
\hline W-04-149 & Looking upstream 2nd order \#1 at culvert crossing & 0 & W-04-149 P6140033 \\
\hline W-04-149 & Looking downstream 2nd order \#1 at culvert crossing & 180 & W-04-149P6140034 \\
\hline W-04-149 & Looking downstream 2nd order \#2 from gravel road & 90 & W-04-149_P6140035 \\
\hline W-04-149 & Looking at culvert inlet & 180 & W-04-149_P6140036 \\
\hline W-04-149 & Looking at culvert outlet Photo taken looking straight down at culvert & 0 & W-04-149 P6140037 \\
\hline
\end{tabular}


Appendix 8: List of all photographs taken showing the corresponding site number, descriptive caption, direction faced when taken (azimuth in degrees) and the unique file name for each photograph.

\begin{tabular}{|c|c|c|c|}
\hline $\begin{array}{c}\text { Site } \\
\text { Number }\end{array}$ & Photo Description & Azimuth & File Name \\
\hline W-04-149 & Photo of Northern Pike & $\mathrm{N} / \mathrm{A}$ & W-04-149_P6140038 \\
\hline W-04-149 & Photo of Northern Pike & $\mathrm{N} / \mathrm{A}$ & W-04-149 P6140039 \\
\hline W-04-150 & Looking upstream from culvert crossing & 90 & W-04-150_P6140040 \\
\hline W-04-150 & Looking downstream from culvert crossing & 270 & W-04-150_P6140041 \\
\hline W-04-150 & Looking at culvert inlet & 270 & W-04-150_P6140042 \\
\hline W-04-150 & Looking at culvert outlet & 90 & W-04-150 P6140043 \\
\hline W-04-151 & Looking upstream from bridge crossing (PTH 23) & 160 & W-04-151_P6140044 \\
\hline W-04-151 & Looking downstream from bridge crossing (PTH 23) & 0 & W-04-151_P6140045 \\
\hline W-04-152 & Looking upstream from culvert crossing & 210 & W-04-152 P6150001 \\
\hline W-04-152 & Looking downstream from culvert crossing & 0 & W-04-152 P6150002 \\
\hline W-04-152 & Looking at culvert inlet & 30 & W-04-152_P6150003 \\
\hline W-04-152 & Looking at culvert outlet & 170 & W-04-152_P6150004 \\
\hline W-04-152 & Photo of barren soil bank on downstream side & 90 & W-04-152 P6150005 \\
\hline W-04-152 & Photo of barren soil bank on upstream side & 140 & W-04-152 P6150006 \\
\hline W-04-153 & Looking upstream from culvert crossing & 90 & W-04-153_P6150007 \\
\hline W-04-153 & Looking downstream from culvert crossing & 270 & W-04-153_P6150008 \\
\hline W-04-153 & Looking at culvert inlet & 270 & W-04-153 P6150009 \\
\hline W-04-153 & Looking at culvert outlet & 90 & W-04-153 P6150010 \\
\hline W-04-154 & Looking upstream from bridge crossing & 130 & W-04-154_P6150011 \\
\hline W-04-154 & Looking downstream from bridge crossing & 290 & W-04-154_P6150012 \\
\hline W-04-155 & Looking upstream from culvert crossing & 200 & W-04-155 P6150013 \\
\hline W-04-155 & Looking downstream from culvert crossing & 40 & W-04-155_P6150014 \\
\hline W-04-156 & Looking upstream from culvert crossing & 110 & W-04-156_P6160001 \\
\hline W-04-156 & Looking downstream from culvert crossing & 290 & W-04-156 P6160002 \\
\hline W-04-156 & Looking at culvert inlet & 315 & W-04-156 P6160003 \\
\hline W-04-156 & Looking at culvert outlet & 120 & W-04-156_P6160004 \\
\hline W-04-157 & Looking upstream from culvert crossing (PR 246) & 80 & W-04-157_P6160005 \\
\hline W-04-157 & Looking downstream from culvert crossing (PR 246) & 260 & W-04-157_P6160006 \\
\hline W-04-157 & Looking at culvert inlet & 210 & W-04-157 P6160007 \\
\hline W-04-157 & Looking at culvert outlet & 140 & W-04-157_P6160008 \\
\hline W-04-158 & Looking upstream from culvert crossing & 130 & W-04-158_P6160009 \\
\hline W-04-158 & Looking downstream from culvert crossing & 270 & W-04-158 P6160010 \\
\hline W-04-158 & Looking at culvert inlet & 290 & W-04-158 P6160011 \\
\hline
\end{tabular}


Appendix 8: List of all photographs taken showing the corresponding site number, descriptive caption, direction faced when taken (azimuth in degrees) and the unique file name for each photograph.

\begin{tabular}{|c|c|c|c|}
\hline $\begin{array}{c}\text { Site } \\
\text { Number }\end{array}$ & Photo Description & Azimuth & File Name \\
\hline W-04-158 & Looking at culvert outlet & 140 & $\begin{array}{ll}W-04-158 & P 6160012\end{array}$ \\
\hline W-04-159 & Looking upstream from culvert crossing & 220 & W-04-159_P6160013 \\
\hline W-04-159 & Looking downstream from culvert crossing & 0 & W-04-159_P6160014 \\
\hline W-04-159 & Looking at culvert inlet & 220 & W-04-159_P6160015 \\
\hline W-04-159 & Looking at culvert outlet & 0 & W-04-159 P6160016 \\
\hline W-04-160 & Looking upstream from culvert crossing & 140 & W-04-160 P6160017 \\
\hline W-04-160 & Looking downstream from culvert crossing & 0 & W-04-160_P6160018 \\
\hline W-04-160 & Looking at culvert inlet & 0 & W-04-160 P6160019 \\
\hline W-04-160 & Looking at culvert outlet & 100 & W-04-160 P6160020 \\
\hline W-04-161 & Looking upstream from culvert crossing & 120 & W-04-161_P6160021 \\
\hline W-04-161 & Looking downstream from culvert crossing & 335 & W-04-161_P6160022 \\
\hline W-04-161 & Looking at culvert inlet from dike & 355 & W-04-161 P6160023 \\
\hline W-04-161 & Looking at culvert outlet & 180 & W-04-161 P6160024 \\
\hline W-04-162 & Looking upstream from culvert crossing & 140 & W-04-162 P6160025 \\
\hline W-04-162 & Looking downstream from culvert crossing & 315 & W-04-162_P6160026 \\
\hline W-04-162 & Looking at culvert inlet & 350 & W-04-162 P6160027 \\
\hline W-04-162 & Looking at culvert outlet & 180 & W-04-162 P6160028 \\
\hline W-04-163 & Looking upstream from bridge crossing (PR 303) & 200 & W-04-163_P6160029 \\
\hline W-04-163 & Looking downstream from bridge crossing (PR 303) & 315 & W-04-163_P6160030 \\
\hline W-04-164 & Looking upstream from culvert crossing (PR 200) & 90 & W-04-164 P6170001 \\
\hline W-04-164 & Looking downstream from culvert crossing (PR 200) & 270 & W-04-164 P6170002 \\
\hline W-04-164 & Looking at culvert outlet & 90 & W-04-164_P6170003 \\
\hline W-04-164 & Looking at culvert inlet & 270 & W-04-164_P6170004 \\
\hline W-04-165 & Looking upstream from culvert crossing & 90 & W-04-165 P6170005 \\
\hline W-04-165 & Looking downstream from culvert crossing & 270 & W-04-165 P6170006 \\
\hline W-04-165 & Looking at culvert inlet & 270 & W-04-165 P6170007 \\
\hline W-04-165 & Looking at culvert outlet & 90 & W-04-165_P6170008 \\
\hline W-04-166 & Looking upstream from culvert crossing & 180 & W-04-166 P6170009 \\
\hline W-04-166 & Looking at dredged matter on right bank on upstream side & 210 & W-04-166 P6170010 \\
\hline W-04-166 & Looking downstream from culvert crossing & 300 & W-04-166_P6170011 \\
\hline W-04-166 & Looking at culvert inlet & 300 & W-04-166 P6170012 \\
\hline W-04-166 & Looking at culvert outlet & 150 & W-04-166 P6170013 \\
\hline W-04-167 & Looking upstream from culvert crossing & 90 & W-04-167 P6170014 \\
\hline
\end{tabular}


Appendix 8: List of all photographs taken showing the corresponding site number, descriptive caption, direction faced when taken (azimuth in degrees) and the unique file name for each photograph.

\begin{tabular}{|c|c|c|c|}
\hline $\begin{array}{c}\text { Site } \\
\text { Number }\end{array}$ & Photo Description & Azimuth & File Name \\
\hline W-04-167 & Looking downstream from culvert crossing & 270 & W-04-167_P6170015 \\
\hline W-04-167 & Looking at culvert inlet & 250 & W-04-167_P6170016 \\
\hline W-04-167 & Looking at culvert outlet & 115 & W-04-167_P6170017 \\
\hline W-04-167 & Looking at perched gated culvert \#1 on upstream side left bank & 0 & W-04-167_P6170018 \\
\hline W-04-167 & Looking at perched gated culvert \#2 on upstream side right bank & 180 & W-04-167_P6170019 \\
\hline W-04-168 & Looking upstream from culvert crossing & 90 & W-04-168 P6170020 \\
\hline W-04-168 & Looking downstream from culvert crossing & 270 & W-04-168_P6170021 \\
\hline W-04-168 & Looking at culvert inlet & 270 & W-04-168_P6170022 \\
\hline W-04-168 & Looking at culvert outlet & 110 & W-04-168 P6170023 \\
\hline W-04-168 & Looking at eroded bank (right bank) on downstream side & 180 & W-04-168 P6170024 \\
\hline W-04-169 & Looking upstream from culvert crossing & 200 & W-04-169_P6170025 \\
\hline W-04-169 & Looking downstream from culvert crossing & 310 & W-04-169_P6170026 \\
\hline W-04-169 & Looking at culvert inlet & 240 & W-04-169_P6170027 \\
\hline W-04-169 & Looking at culvert outlet & 120 & W-04-169 P6170028 \\
\hline W-04-170 & Looking upstream from culvert crossing & 110 & W-04-170_P6170029 \\
\hline W-04-170 & Looking downstream from culvert crossing & 330 & W-04-170_P6170030 \\
\hline W-04-170 & Looking at culvert inlet & 280 & W-04-170 P6170031 \\
\hline W-04-170 & Looking at culvert outlet & 130 & W-04-170 P6170032 \\
\hline W-04-171 & Looking upstream from culvert crossing (PR 246) & 90 & W-04-171_P6170033 \\
\hline W-04-171 & Looking downstream from culvert crossing (PR 246) & 270 & W-04-171_P6170034 \\
\hline W-04-171 & Looking at culvert inlet & 270 & W-04-171_P6170035 \\
\hline W-04-171 & Looking at culvert outlet & 90 & W-04-171_P6170036 \\
\hline W-04-172 & Looking upstream from culvert crossing & 90 & W-04-172_P6170037 \\
\hline W-04-172 & Looking downstream from culvert crossing & 270 & W-04-172_P6170038 \\
\hline W-04-172 & Looking at culvert inlet & 270 & W-04-172 P6170039 \\
\hline W-04-172 & Looking at culvert outlet & 90 & W-04-172 P6170040 \\
\hline W-04-173 & Looking upstream from bridge crossing & 80 & W-04-173_P6180001 \\
\hline W-04-173 & Looking downstream from bridge crossing & 265 & W-04-173_P6180002 \\
\hline W-04-174 & Looking upstream from left bank & 80 & W-04-174 P6180003 \\
\hline W-04-174 & Looking downstream from left bank & 270 & W-04-174_P6180004 \\
\hline W-04-175 & Looking upstream from culvert crossing & 160 & W-04-175_P6210001 \\
\hline W-04-175 & Looking downstream from culvert crossing & 330 & W-04-175 P6210002 \\
\hline W-04-175 & Looking at culvert inlet & 290 & W-04-175 P6210003 \\
\hline
\end{tabular}


Appendix 8: List of all photographs taken showing the corresponding site number, descriptive caption, direction faced when taken (azimuth in degrees) and the unique file name for each photograph.

\begin{tabular}{|c|c|c|c|}
\hline $\begin{array}{c}\text { Site } \\
\text { Number }\end{array}$ & Photo Description & Azimuth & File Name \\
\hline W-04-175 & Looking at culvert outlet & 180 & W-04-175_P6210004 \\
\hline W-04-176 & Looking upstream from edge of right bank & 65 & W-04-176 P6210005 \\
\hline W-04-176 & Looking downstream from edge of right bank & 290 & W-04-176_P6210006 \\
\hline W-04-177 & Looking upstream from V-notch weir (5m back) & 270 & W-04-177_P6210007 \\
\hline W-04-177 & Looking upstream from V-notch weir & 270 & W-04-177_P6210008 \\
\hline W-04-177 & Looking downstream from V-notch weir & 90 & W-04-177_P6210009 \\
\hline W-04-178 & Looking upstream from bridge crossing & 180 & W-04-178_P6210010 \\
\hline W-04-178 & Looking downstream from bridge crossing & 0 & W-04-178_P6210011 \\
\hline W-04-179 & Looking upstream Gusta Drain from culvert crossing & 90 & W-04-179_P6220001 \\
\hline W-04-179 & Looking downstream Main Drain \#1 from culvert crossing & 300 & W-04-179_P6220002 \\
\hline W-04-179 & Looking at culvert inlet & 270 & W-04-179_P6220003 \\
\hline W-04-179 & Looking at culvert outlet & 40 & W-04-179_P6220004 \\
\hline W-04-180 & Looking upstream from culvert crossing & 90 & W-04-180_P6220005 \\
\hline W-04-180 & Looking downstream from culvert crossing & 270 & W-04-180_P6220006 \\
\hline W-04-180 & Looking at culvert inlet & 270 & W-04-180_P6220007 \\
\hline W-04-180 & Looking at culvert outlet & 110 & W-04-180_P6220008 \\
\hline W-04-181 & Looking upstream from culvert crossing & 170 & W-04-181_P6220009 \\
\hline W-04-181 & Looking downstream from culvert crossing & 350 & W-04-181_P6220010 \\
\hline W-04-181 & Looking at culvert inlet & 330 & W-04-181_P6220011 \\
\hline W-04-181 & Looking at culvert outlet & 150 & W-04-181_P6220012 \\
\hline W-04-182 & Looking upstream from culvert crossing & 260 & W-04-182_P6220013 \\
\hline W-04-182 & Looking downstream from culvert crossing & 150 & W-04-182_P6220014 \\
\hline W-04-182 & Looking at culvert outlet & 220 & W-04-182_P6220015 \\
\hline W-04-182 & Looking at culvert inlet & 80 & W-04-182_P6220016 \\
\hline W-04-183 & Looking upstream from culvert crossing & 240 & W-04-183_P6220017 \\
\hline W-04-183 & Looking downstream from culvert crossing & 30 & W-04-183_P6220018 \\
\hline W-04-183 & Looking at culvert inlet & 50 & W-04-183_P6220019 \\
\hline W-04-183 & Looking at culvert outlet & 240 & W-04-183_P6220020 \\
\hline W-04-184 & Looking upstream from edge of right bank & 180 & W-04-184_P6220021 \\
\hline W-04-184 & Looking downstream from edge of right bank & 40 & W-04-184_P6220022 \\
\hline W-04-185 & Looking upstream from bridge crossing & 90 & W-04-185_P6230001 \\
\hline W-04-185 & Looking downstream from bridge crossing & 270 & W-04-185_P6230002 \\
\hline W-04-186 & Looking upstream Stead Drain & 280 & W-04-186_P6230003 \\
\hline
\end{tabular}


Appendix 8: List of all photographs taken showing the corresponding site number, descriptive caption, direction faced when taken (azimuth in degrees) and the unique file name for each photograph.

\begin{tabular}{|c|c|c|c|}
\hline $\begin{array}{c}\text { Site } \\
\text { Number }\end{array}$ & Photo Description & Azimuth & File Name \\
\hline W-04-186 & Looking downstream at confluence of Stead Drain and Main Drain \#1 & 100 & W-04-186_P6230004 \\
\hline W-04-186 & Looking at culvert inlet & 90 & W-04-186 P6230005 \\
\hline W-04-186 & Looking at culvert outlet & 280 & W-04-186_P6230006 \\
\hline W-04-187 & Looking upstream from culvert crossing (on dike) & 260 & W-04-187_P6230007 \\
\hline W-04-187 & Looking downstream from $10 \mathrm{~m}$ S of culvert crossing (on dike) & 25 & W-04-187_P6230008 \\
\hline W-04-187 & Mistake Photo & $\mathrm{N} / \mathrm{A}$ & W-04-187_P6230009 \\
\hline W-04-187 & Looking at culvert inlet & 50 & W-04-187_P6230010 \\
\hline W-04-187 & Looking at culvert outlet & 220 & W-04-187_P6230011 \\
\hline W-04-188 & Looking upstream from culvert crossing & 270 & W-04-188 P6230012 \\
\hline W-04-188 & Looking downstream from culvert crossing & 90 & W-04-188 P6230013 \\
\hline W-04-188 & Looking at culvert outlet & 310 & W-04-188_P6230014 \\
\hline W-04-188 & Looking at culvert inlet & 30 & W-04-188_P6230015 \\
\hline W-04-189 & Looking upstream from culvert crossing & 180 & W-04-189_P6240001 \\
\hline W-04-189 & Looking downstream from culvert crossing & 0 & W-04-189 P6240002 \\
\hline W-04-189 & Looking at culvert inlet & 0 & W-04-189_P6240003 \\
\hline W-04-189 & Looking at culvert outlet & 180 & W-04-189_P6240004 \\
\hline W-04-190 & Looking upstream from culvert crossing & 90 & W-04-190 P6240005 \\
\hline W-04-190 & Looking downstream from culvert crossing & 290 & W-04-190 P6240006 \\
\hline W-04-190 & Looking at culvert outlet & 130 & W-04-190_P6240007 \\
\hline W-04-191 & Looking upstream from culvert crossing & 90 & W-04-191_P6240008 \\
\hline W-04-191 & Looking downstream from culvert crossing & 270 & W-04-191_P6240009 \\
\hline W-04-191 & Looking at culvert inlet & 200 & W-04-191_P6240010 \\
\hline W-04-191 & Looking at culvert outlet & 100 & W-04-191_P6240011 \\
\hline W-04-192 & Looking upstream from culvert crossing & 0 & W-04-192_P6240012 \\
\hline W-04-192 & Looking downstream from culvert crossing & 200 & W-04-192 P6240013 \\
\hline W-04-192 & Looking at culvert inlet & 215 & W-04-192_P6240014 \\
\hline W-04-192 & Looking at culvert outlet & 0 & W-04-192_P6240015 \\
\hline W-04-193 & Looking upstream from culvert crossing & 0 & W-04-193_P6240016 \\
\hline W-04-193 & Looking downstream from culvert crossing & 180 & W-04-193 P6240017 \\
\hline W-04-193 & Looking at culvert inlet & 180 & W-04-193 P6240018 \\
\hline W-04-193 & Looking at culvert outlet & 0 & W-04-193_P6240019 \\
\hline W-04-194 & Looking upstream from culvert crossing & 220 & W-04-194_P6240020 \\
\hline W-04-194 & Looking downstream from culvert crossing & 40 & W-04-194 P6240021 \\
\hline
\end{tabular}


Appendix 8: List of all photographs taken showing the corresponding site number, descriptive caption, direction faced when taken (azimuth in degrees) and the unique file name for each photograph.

\begin{tabular}{|c|c|c|c|}
\hline $\begin{array}{c}\text { Site } \\
\text { Number }\end{array}$ & Photo Description & Azimuth & File Name \\
\hline W-04-194 & Looking at culvert inlet & 40 & W-04-194 P6240022 \\
\hline W-04-194 & Looking at culvert outlet & 220 & W-04-194_P6240023 \\
\hline W-04-195 & Looking upstream from culvert crossing & 160 & W-04-195_P6250001 \\
\hline W-04-195 & Looking downstream from culvert crossing & 0 & W-04-195_P6250002 \\
\hline W-04-195 & Looking at culvert inlet & 0 & W-04-195_P6250003 \\
\hline W-04-195 & Looking at culvert outlet & 180 & W-04-195 P6250004 \\
\hline W-04-196 & Looking downstream from PTH 11 & 250 & W-04-196_P6250005 \\
\hline W-04-196 & Looking downstream from PTH 11 (zoomed in) & 250 & W-04-196_P6250006 \\
\hline W-04-197 & Looking upstream from culvert crossing & 70 & W-04-197 P6250007 \\
\hline W-04-197 & Looking downstream from culvert crossing & 310 & W-04-197_P6250008 \\
\hline W-04-197 & Looking at culvert inlet & 220 & W-04-197_P6250009 \\
\hline W-04-197 & Looking at culvert outlet & 115 & W-04-197_P6250010 \\
\hline W-04-197 & Photo of White Sucker & $\mathrm{N} / \mathrm{A}$ & W-04-197 P6250011 \\
\hline W-04-197 & Photo of bear off PTH 11 & N/A & W-04-197_P6250012 \\
\hline W-04-197 & Photo of bear off PTH 11 & $\mathrm{~N} / \mathrm{A}$ & W-04-197_P6250013 \\
\hline W-04-198 & Looking upstream from culvert crossing & 200 & W-04-198_P6290001 \\
\hline W-04-198 & Looking downstream from culvert crossing & 350 & W-04-198 P6290002 \\
\hline W-04-198 & Looking at culvert inlet & 330 & W-04-198 P6290003 \\
\hline W-04-198 & Looking at culvert outlet & 210 & W-04-198_P6290004 \\
\hline W-04-199 & Looking downstream from culvert crossing & 0 & W-04-199_P6290005 \\
\hline W-04-199 & Looking upstream from culvert crossing & 180 & W-04-199_P6290006 \\
\hline W-04-199 & Looking at culvert outlet & 180 & W-04-199_P6290007 \\
\hline W-04-199 & Looking at culvert inlet & 0 & W-04-199_P6290008 \\
\hline W-04-200 & Looking upstream from culvert crossing & 40 & W-04-200_P6290009 \\
\hline W-04-200 & Looking downstream from culvert crossing & 280 & W-04-200 P6290010 \\
\hline W-04-200 & Looking at culvert inlet & 280 & W-04-200 P6290011 \\
\hline W-04-200 & Looking at culvert outlet & 40 & W-04-200_P6290012 \\
\hline W-04-201 & Looking upstream from culvert crossing & 180 & W-04-201_P6290013 \\
\hline W-04-201 & Looking downstream from culvert crossing & 280 & W-04-201_P6290014 \\
\hline W-04-201 & Looking at culvert inlet & 280 & W-04-201_P6290015 \\
\hline W-04-201 & Looking at culvert outlet & 90 & W-04-201_P6290016 \\
\hline W-04-202 & Looking upstream from culvert crossing & 80 & W-04-202 P6290017 \\
\hline W-04-202 & Looking downstream from culvert crossing & 260 & W-04-202 P6290018 \\
\hline
\end{tabular}


Appendix 8: List of all photographs taken showing the corresponding site number, descriptive caption, direction faced when taken (azimuth in degrees) and the unique file name for each photograph.

\begin{tabular}{|c|c|c|c|}
\hline $\begin{array}{c}\text { Site } \\
\text { Number }\end{array}$ & Photo Description & Azimuth & File Name \\
\hline W-04-202 & Looking at culvert inlet & 230 & W-04-202_P6290019 \\
\hline W-04-202 & Looking at culvert outlet & 130 & W-04-202 P6290020 \\
\hline W-04-203 & Looking upstream from culvert crossing (PR 207) & 90 & W-04-203_P6290021 \\
\hline W-04-203 & Looking downstream from culvert crossing (PR 207) & 270 & W-04-203_P6290022 \\
\hline W-04-203 & Looking at culvert inlet & 270 & W-04-203_P6290023 \\
\hline W-04-203 & Looking at culvert outlet & 90 & W-04-203 P6290024 \\
\hline W-04-204 & Looking upstream from culvert crossing & 70 & W-04-204_P6290025 \\
\hline W-04-204 & Looking downstream from culvert crossing & 260 & W-04-204_P6290026 \\
\hline W-04-204 & Looking at culvert inlet & 290 & W-04-204 P6290027 \\
\hline W-04-204 & Looking at culvert outlet & 60 & W-04-204_P6290028 \\
\hline W-04-205 & Looking upstream from culvert crossing & 240 & W-04-205_P6290029 \\
\hline W-04-205 & Looking downstream from culvert crossing & 0 & W-04-205_P6290030 \\
\hline W-04-205 & Looking at culvert inlet & 0 & W-04-205_P6290031 \\
\hline W-04-205 & Looking at culvert outlet & 240 & W-04-205 P6290032 \\
\hline W-04-206 & Looking upstream from culvert crossing (PR 208) & 180 & W-04-206_P6290033 \\
\hline W-04-206 & Looking downstream from culvert crossing (PR 208) & 250 & W-04-206_P6290034 \\
\hline W-04-206 & Looking at culvert inlet & 280 & W-04-206 P6290035 \\
\hline W-04-206 & Looking at culvert outlet & 120 & W-04-206 P6290036 \\
\hline W-04-207 & Looking upstream from bridge crossing & 180 & W-04-207_P7050001 \\
\hline W-04-207 & Looking downstream from bridge crossing & 300 & W-04-207_P7050002 \\
\hline W-04-208 & Looking upstream from culvert crossing & 140 & W-04-208 P7050003 \\
\hline W-04-208 & Looking downstream from culvert crossing & 50 & W-04-208_P7050004 \\
\hline W-04-208 & Looking at culvert inlet & 330 & W-04-208_P7050005 \\
\hline W-04-208 & Looking at culvert outlet & 160 & W-04-208 P7050006 \\
\hline W-04-209 & Looking upstream from bridge crossing & 130 & W-04-209 P7050007 \\
\hline W-04-209 & Looking downstream from bridge crossing & 290 & W-04-209 P7050008 \\
\hline W-04-210 & Looking upstream from bridge crossing & 180 & W-04-210_P7050009 \\
\hline W-04-210 & Looking downstream from bridge crossing & 270 & W-04-210_P7050010 \\
\hline W-04-211 & Looking upstream from culvert crossing & 140 & W-04-211_P7050011 \\
\hline W-04-211 & Looking downstream from culvert crossing & 320 & W-04-211_P7050012 \\
\hline W-04-211 & Looking at culvert inlet & 40 & W-04-211_P7050013 \\
\hline W-04-211 & Looking at culvert outlet & 140 & W-04-211 P7050014 \\
\hline W-04-211 & Looking at bank slump on left bank downstream side & 25 & W-04-211 P7050015 \\
\hline
\end{tabular}


Appendix 8: List of all photographs taken showing the corresponding site number, descriptive caption, direction faced when taken (azimuth in degrees) and the unique file name for each photograph.

\begin{tabular}{|c|c|c|c|}
\hline $\begin{array}{c}\text { Site } \\
\text { Number }\end{array}$ & Photo Description & Azimuth & File Name \\
\hline W-04-211 & Looking across drain at bank slump on right bank & 290 & W-04-211_P7050016 \\
\hline W-04-212 & Looking upstream 2nd order & 160 & W-04-212 P7060001 \\
\hline W-04-212 & Looking downstream 3rd order & 320 & W-04-212_P7060002 \\
\hline W-04-212 & Looking at culvert inlet & 300 & W-04-212_P7060003 \\
\hline W-04-212 & Looking at culvert outlet & 250 & W-04-212_P7060004 \\
\hline W-04-212 & Looking upstream 3rd order \#2 & 280 & W-04-212 P7060005 \\
\hline W-04-213 & Looking upstream from bridge crossing & 135 & W-04-213_P7060006 \\
\hline W-04-213 & Looking downstream from bridge crossing & 265 & W-04-213_P7060007 \\
\hline W-04-213 & Looking at woody debris from left bank upstream of bridge & 265 & W-04-213 P7060008 \\
\hline W-04-213 & Photo of bank scour on downstream side (left bank) & 300 & W-04-213 P7060009 \\
\hline W-04-213 & Photo of bank scour further downstream (left bank) & 330 & W-04-213_P7060010 \\
\hline W-04-214 & Looking upstream from culvert crossing & 180 & W-04-214_P7060011 \\
\hline W-04-214 & Looking downstream from culvert crossing & 0 & W-04-214_P7060012 \\
\hline W-04-214 & Looking at culvert inlet & 0 & W-04-214 P7060013 \\
\hline W-04-214 & Looking at culvert outlet & 180 & W-04-214_P7060014 \\
\hline W-04-215 & Looking upstream from culvert crossing & 180 & W-04-215_P7060015 \\
\hline W-04-215 & Looking downstream from culvert crossing & 0 & W-04-215 P7060016 \\
\hline W-04-215 & Looking at culvert inlet & 0 & W-04-215 P7060017 \\
\hline W-04-215 & Looking at culvert outlet & 180 & W-04-215_P7060018 \\
\hline W-04-216 & Looking upstream from culvert crossing & 110 & W-04-216_P7060019 \\
\hline W-04-216 & Looking downstream from culvert crossing & 250 & W-04-216_P7060020 \\
\hline W-04-216 & Looking at culvert inlet & 260 & W-04-216_P7060021 \\
\hline W-04-216 & Looking at culvert outlet & 120 & W-04-216_P7060022 \\
\hline W-04-217 & Looking upstream from culvert crossing & 75 & W-04-217_P7060023 \\
\hline W-04-217 & Looking downstream from culvert crossing & 270 & W-04-217_P7060024 \\
\hline W-04-217 & Looking at culvert inlet & 305 & W-04-217_P7060025 \\
\hline W-04-217 & Looking at culvert outlet & 60 & W-04-217_P7060026 \\
\hline W-04-218 & Looking upstream bridge crossing & 90 & W-04-218_P7060027 \\
\hline W-04-218 & Looking downstream bridge crossing & 270 & W-04-218 P7060028 \\
\hline W-04-219 & Looking upstream from culvert crossing & 90 & W-04-219 P7070001 \\
\hline W-04-219 & Looking downstream from culvert crossing & 180 & W-04-219_P7070002 \\
\hline W-04-219 & Looking at culvert inlet & 290 & W-04-219 P7070003 \\
\hline W-04-219 & Looking at culvert outlet & 0 & W-04-219 P7070004 \\
\hline
\end{tabular}


Appendix 8: List of all photographs taken showing the corresponding site number, descriptive caption, direction faced when taken (azimuth in degrees) and the unique file name for each photograph.

\begin{tabular}{|c|c|c|c|}
\hline $\begin{array}{c}\text { Site } \\
\text { Number }\end{array}$ & Photo Description & Azimuth & File Name \\
\hline W-04-220 & Looking upstream from culvert crossing & 0 & W-04-220_P7070005 \\
\hline W-04-220 & Looking downstream from culvert crossing & 180 & W-04-220_P7070006 \\
\hline W-04-220 & Looking at culvert inlet & 180 & W-04-220_P7070007 \\
\hline W-04-220 & Looking at culvert outlet & 320 & W-04-220_P7070008 \\
\hline W-04-221 & Looking upstream from culvert crossing & 40 & W-04-221_P7070009 \\
\hline W-04-221 & Looking downstream from culvert crossing & 270 & W-04-221_P7070010 \\
\hline W-04-221 & Looking at culvert inlet & 210 & W-04-221_P7070011 \\
\hline W-04-221 & Looking at culvert outlet & 140 & W-04-221_P7070012 \\
\hline W-04-222 & Looking upstream from culvert crossing & 90 & W-04-222_P7070013 \\
\hline W-04-222 & Looking downstream from culvert crossing & 270 & W-04-222_P7070014 \\
\hline W-04-222 & Looking at culvert inlet & 230 & W-04-222_P7070015 \\
\hline W-04-222 & Looking at culvert outlet & 100 & W-04-222_P7070016 \\
\hline W-04-223 & Looking upstream from culvert crossing & 90 & W-04-223_P7070017 \\
\hline W-04-223 & Looking downstream from culvert crossing & 270 & W-04-223_P7070018 \\
\hline W-04-223 & Looking at culvert inlet & 220 & W-04-223_P7070019 \\
\hline W-04-223 & Looking at culvert outlet & 110 & W-04-223_P7070020 \\
\hline W-04-224 & Looking upstream from culvert crossing & 90 & W-04-224_P7070021 \\
\hline W-04-224 & Looking downstream from culvert crossing & 270 & W-04-224_P7070022 \\
\hline W-04-224 & Looking at culvert inlet & 220 & W-04-224_P7070023 \\
\hline W-04-224 & Looking at culvert outlet & 120 & W-04-224_P7070024 \\
\hline W-04-225 & Looking upstream bridge crossing & 90 & W-04-225_P7070025 \\
\hline W-04-225 & Looking downstream bridge crossing & 270 & W-04-225_P7070026 \\
\hline W-04-226 & Looking upstream from culvert crossing & 70 & W-04-226_P7070027 \\
\hline W-04-226 & Looking downstream from culvert crossing & 300 & W-04-226_P7070028 \\
\hline W-04-226 & Looking at culvert inlet & 230 & W-04-226_P7070029 \\
\hline W-04-226 & Looking at culvert outlet & 90 & W-04-226_P7070030 \\
\hline W-04-227 & Looking upstream from culvert crossing & 270 & W-04-227_P7070031 \\
\hline W-04-227 & Looking downstream from culvert crossing & 90 & W-04-227_P7070032 \\
\hline W-04-227 & Looking at culvert inlet & 140 & W-04-227_P7070033 \\
\hline W-04-227 & Looking at culvert outlet & 320 & W-04-227_P7070034 \\
\hline W-04-228 & Looking upstream from culvert crossing & 265 & W-04-228_P7070035 \\
\hline W-04-228 & Looking downstream from culvert crossing & 90 & W-04-228_P7070036 \\
\hline W-04-228 & Looking at culvert inlet & 120 & W-04-228_P7070037 \\
\hline
\end{tabular}


Appendix 8: List of all photographs taken showing the corresponding site number, descriptive caption, direction faced when taken (azimuth in degrees) and the unique file name for each photograph.

\begin{tabular}{|c|c|c|c|}
\hline $\begin{array}{c}\text { Site } \\
\text { Number }\end{array}$ & Photo Description & Azimuth & File Name \\
\hline W-04-228 & Looking at culvert outlet & 220 & W-04-228_P7070038 \\
\hline W-04-229 & Looking upstream from culvert crossing & 270 & W-04-229 P7070039 \\
\hline W-04-229 & Looking downstream from culvert crossing & 180 & W-04-229_P7070040 \\
\hline W-04-229 & Looking at culvert inlet & 120 & W-04-229_P7070041 \\
\hline W-04-229 & Looking at culvert outlet & 250 & W-04-229_P7070042 \\
\hline W-04-230 & Looking upstream from culvert crossing & 270 & W-04-230 P7070043 \\
\hline W-04-230 & Looking downstream from culvert crossing & 90 & W-04-230_P7070044 \\
\hline W-04-230 & Looking at culvert inlet & 100 & W-04-230_P7070045 \\
\hline W-04-230 & Looking at culvert outlet & 220 & W-04-230 P7070046 \\
\hline W-04-231 & Looking upstream from culvert crossing & 60 & W-04-231_P7080001 \\
\hline W-04-231 & Looking downstream from culvert crossing & 270 & W-04-231_P7080002 \\
\hline W-04-231 & Looking at culvert inlet & 270 & W-04-231_P7080003 \\
\hline W-04-231 & Looking at culvert outlet & 115 & W-04-231_P7080004 \\
\hline W-04-232 & Looking upstream from culvert crossing & 90 & W-04-232 P7080005 \\
\hline W-04-232 & Looking downstream from culvert crossing & 270 & W-04-232_P7080006 \\
\hline W-04-232 & Looking at culvert inlet & 270 & W-04-232_P7080007 \\
\hline W-04-232 & Looking at culvert outlet & 160 & W-04-232 P7080008 \\
\hline W-04-233 & Looking upstream from bridge crossing & 140 & W-04-233 P7130001 \\
\hline W-04-233 & Looking downstream from bridge crossing & 340 & W-04-233_P7130002 \\
\hline W-04-234 & Looking upstream from bridge crossing & 170 & W-04-234_P7130003 \\
\hline W-04-234 & Looking downstream from bridge crossing & 10 & W-04-234 P7130004 \\
\hline W-04-235 & Looking upstream from culvert crossing & 180 & W-04-235_P7130005 \\
\hline W-04-235 & Looking downstream from culvert crossing & 0 & W-04-235_P7130006 \\
\hline W-04-235 & Looking at culvert inlet & 0 & W-04-235 P7130007 \\
\hline W-04-235 & Looking at culvert outlet & 180 & W-04-235 P7130008 \\
\hline W-04-236 & Looking upstream from culvert crossing & 260 & W-04-236_P7130009 \\
\hline W-04-236 & Looking downstream from culvert crossing & 340 & W-04-236_P7130010 \\
\hline W-04-236 & Looking at culvert inlet & 340 & W-04-236_P7130011 \\
\hline W-04-236 & Looking at culvert outlet & 190 & W-04-236 P7130012 \\
\hline W-04-237 & Looking upstream from bridge crossing & 190 & W-04-237_P7130013 \\
\hline W-04-237 & Looking downstream from bridge crossing & 310 & W-04-237_P7130014 \\
\hline W-04-238 & Looking upstream from culvert crossing & 290 & W-04-238 P7130015 \\
\hline W-04-238 & Looking downstream from culvert crossing & 130 & W-04-238 P7130016 \\
\hline
\end{tabular}


Appendix 8: List of all photographs taken showing the corresponding site number, descriptive caption, direction faced when taken (azimuth in degrees) and the unique file name for each photograph.

\begin{tabular}{|c|c|c|c|}
\hline $\begin{array}{c}\text { Site } \\
\text { Number }\end{array}$ & Photo Description & Azimuth & File Name \\
\hline W-04-238 & Looking at culvert inlet & 50 & W-04-238_P7130017 \\
\hline W-04-238 & Looking at culvert outlet & 270 & W-04-238_P7130018 \\
\hline W-04-239 & Looking downstream from culvert crossing & 90 & W-04-239_P7130019 \\
\hline W-04-239 & Looking upstream from culvert crossing & 270 & W-04-239_P7130020 \\
\hline W-04-239 & Looking at culvert inlet & 80 & W-04-239_P7130021 \\
\hline W-04-239 & Looking at culvert outlet & 320 & W-04-239_P7130022 \\
\hline W-04-240 & Looking upstream from culvert crossing & 270 & W-04-240_P7140001 \\
\hline W-04-240 & Looking upstream at beaver dam & 270 & W-04-240_P7140002 \\
\hline W-04-240 & Looking downstream from culvert crossing & 90 & W-04-240_P7140003 \\
\hline W-04-240 & Looking at culvert inlet & 150 & W-04-240_P7140004 \\
\hline W-04-240 & Looking at culvert outlet & 220 & W-04-240_P7140005 \\
\hline W-04-241 & Looking upstream from bridge crossing & 270 & W-04-241_P7140006 \\
\hline W-04-241 & Looking downstream from bridge crossing & 90 & W-04-241_P7140007 \\
\hline W-04-241 & Looking straight down at beaver dam \#2 from bridge & $\mathrm{N} / \mathrm{A}$ & W-04-241_P7140008 \\
\hline W-04-242 & Looking upstream from bridge crossing & 230 & W-04-242_P7140009 \\
\hline W-04-242 & Looking downstream from bridge crossing & 110 & W-04-242_P7140010 \\
\hline W-04-243 & Looking upstream from bridge crossing & 240 & W-04-243_P7140011 \\
\hline W-04-243 & Looking downstream from bridge crossing & 30 & W-04-243_P7140012 \\
\hline W-04-244 & Looking upstream from culvert crossing & 250 & W-04-244_P7140013 \\
\hline W-04-244 & Looking downstream from culvert crossing & 120 & W-04-244_P7140014 \\
\hline W-04-245 & Looking upstream from culvert crossing & 250 & W-04-245_P7140015 \\
\hline W-04-245 & Looking downstream from culvert crossing & 20 & W-04-245_P7140016 \\
\hline W-04-245 & Looking at culvert outlet & 270 & W-04-245_P7140017 \\
\hline W-04-246 & Looking upstream from culvert crossing & 230 & W-04-246_P7140018 \\
\hline W-04-246 & Looking downstream from culvert crossing & 90 & W-04-246_P7140019 \\
\hline W-04-246 & Looking at culvert inlet & 90 & W-04-246_P7140020 \\
\hline W-04-246 & Looking at culvert outlet & 180 & W-04-246_P7140021 \\
\hline W-04-247 & Looking upstream from culvert crossing & 220 & W-04-247_P7140022 \\
\hline W-04-247 & Looking downstream from culvert crossing & 30 & W-04-247_P7140023 \\
\hline W-04-247 & Looking at culvert inlet & 180 & W-04-247_P7140024 \\
\hline W-04-248 & Looking upstream from culvert crossing & 230 & W-04-248_P7140025 \\
\hline W-04-248 & Looking downstream from culvert crossing & 80 & W-04-248_P7140026 \\
\hline W-04-248 & Looking at culvert inlet & 150 & W-04-248_P7140027 \\
\hline
\end{tabular}


Appendix 8: List of all photographs taken showing the corresponding site number, descriptive caption, direction faced when taken (azimuth in degrees) and the unique file name for each photograph.

\begin{tabular}{|c|c|c|c|}
\hline $\begin{array}{c}\text { Site } \\
\text { Number }\end{array}$ & Photo Description & Azimuth & File Name \\
\hline W-04-248 & Looking at culvert outlet & 150 & $\begin{array}{ll}W-04-248 & \text { P7140028 }\end{array}$ \\
\hline W-04-249 & Looking upstream from culvert crossing & 110 & W-04-249_P7140029 \\
\hline W-04-249 & Looking downstream from culvert crossing & 305 & W-04-249_P7140030 \\
\hline W-04-249 & Looking at culvert inlet & 300 & W-04-249 P7140031 \\
\hline W-04-249 & Looking at culvert outlet & 135 & W-04-249 P7140032 \\
\hline W-04-250 & Looking upstream from culvert crossing & 255 & W-04-250 P7140033 \\
\hline W-04-250 & Looking downstream from culvert crossing & 80 & W-04-250_P7140034 \\
\hline$W-04-250$ & Looking at culvert inlet & 130 & W-04-250 P7140035 \\
\hline W-04-250 & Looking at culvert outlet & 280 & W-04-250 P7140036 \\
\hline W-04-251 & Looking upstream from culvert crossing & 250 & W-04-251_P7150001 \\
\hline W-04-251 & Looking downstream from culvert crossing & 60 & W-04-251_P7150002 \\
\hline W-04-251 & Looking at culvert inlet & 130 & W-04-251 P7150003 \\
\hline W-04-251 & Looking at culvert outlet & 305 & W-04-251 P7150004 \\
\hline W-04-252 & Looking upstream from bridge crossing & 230 & W-04-252 P7150005 \\
\hline W-04-252 & Looking downstream from bridge crossing & 60 & W-04-252_P7150006 \\
\hline W-04-253 & Looking upstream from culvert crossing & 250 & W-04-253 P7150007 \\
\hline W-04-253 & Looking upstream from culvert crossing & 225 & W-04-253 P7150008 \\
\hline W-04-253 & Looking downstream from culvert crossing & 60 & W-04-253_P7150009 \\
\hline W-04-253 & Looking at culvert inlet & 100 & W-04-253_P7150010 \\
\hline W-04-253 & Looking at culvert outlet & 280 & W-04-253 P7150011 \\
\hline W-04-254 & Looking upstream from culvert crossing & 180 & W-04-254 P7150012 \\
\hline W-04-254 & Looking downstream from culvert crossing & 0 & W-04-254_P7150013 \\
\hline W-04-254 & Looking at culvert inlet & 0 & W-04-254_P7150014 \\
\hline W-04-254 & Looking at culvert outlet & 180 & W-04-254 P7150015 \\
\hline W-04-255 & Looking upstream from culvert crossing & 95 & W-04-255 P7150016 \\
\hline W-04-255 & Looking downstream from culvert crossing & 260 & W-04-255 P7150017 \\
\hline W-04-255 & Looking at culvert inlet & 260 & W-04-255_P7150018 \\
\hline W-04-255 & Looking at south culvert inlet at woody debris & 230 & W-04-255 P7150019 \\
\hline W-04-255 & Looking at culvert outlet & 45 & W-04-255 P7150020 \\
\hline W-04-256 & Looking upstream from bridge crossing (PR 305) & 180 & W-04-256_P7200001 \\
\hline W-04-256 & Looking downstream from bridge crossing (PR 305) & 0 & W-04-256 P7200002 \\
\hline W-04-257 & Looking upstream from culvert crossing & 270 & W-04-257 P7210001 \\
\hline W-04-257 & Looking downstream from culvert crossing & 90 & W-04-257 P7210002 \\
\hline
\end{tabular}


Appendix 8: List of all photographs taken showing the corresponding site number, descriptive caption, direction faced when taken (azimuth in degrees) and the unique file name for each photograph.

\begin{tabular}{|c|c|c|c|}
\hline $\begin{array}{c}\text { Site } \\
\text { Number }\end{array}$ & Photo Description & Azimuth & File Name \\
\hline W-04-257 & Looking at culvert inlet & 90 & W-04-257 P7210003 \\
\hline W-04-257 & Looking at culvert outlet & 270 & W-04-257_P7210004 \\
\hline W-04-258 & Looking upstream from culvert crossing & 250 & W-04-258_P7210005 \\
\hline W-04-258 & Looking downstream from culvert crossing & 100 & W-04-258 P7210006 \\
\hline W-04-258 & Looking at culvert inlet & 90 & W-04-258_P7210007 \\
\hline W-04-258 & Looking at culvert outlet & 250 & W-04-258_P7210008 \\
\hline W-04-259 & Looking upstream from culvert crossing & 180 & W-04-259_P7210009 \\
\hline W-04-259 & Looking downstream from culvert crossing & 0 & W-04-259_P7210010 \\
\hline W-04-259 & Looking at culvert inlet & 0 & W-04-259 P7210011 \\
\hline W-04-259 & Looking at culvert outlet & 180 & W-04-259_P7210012 \\
\hline W-04-260 & Looking upstream from culvert crossing & 230 & W-04-260 P7210013 \\
\hline W-04-260 & Looking downstream from culvert crossing & 75 & W-04-260_P7210014 \\
\hline W-04-260 & Looking at culvert inlet & 80 & W-04-260_P7210015 \\
\hline W-04-260 & Looking at culvert outlet & 335 & W-04-260_P7210016 \\
\hline W-04-261 & Looking upstream from culvert crossing & 270 & W-04-261 P7210017 \\
\hline W-04-261 & Looking downstream from culvert crossing & 90 & W-04-261_P7210018 \\
\hline W-04-261 & Looking at culvert inlet & 180 & W-04-261_P7210019 \\
\hline W-04-261 & Looking at culvert outlet & 220 & W-04-261_P7210020 \\
\hline W-04-262 & Looking upstream from culvert crossing & 295 & W-04-262_P7220001 \\
\hline W-04-262 & Looking downstream from culvert crossing & 130 & W-04-262_P7220002 \\
\hline W-04-262 & Looking at culvert inlet & 190 & W-04-262 P7220003 \\
\hline W-04-262 & Looking at culvert outlet & 320 & W-04-262_P7220004 \\
\hline W-04-263 & Looking upstream from intersection & 0 & W-04-263_P7220005 \\
\hline W-04-263 & Looking downstream from intersection & 90 & W-04-263_P7220006 \\
\hline W-04-264 & Looking upstream from culvert crossing & 270 & W-04-264_P7220007 \\
\hline W-04-264 & Looking downstream from culvert crossing & 90 & W-04-264_P7220008 \\
\hline W-04-264 & Looking at culvert inlet & 40 & W-04-264_P7220009 \\
\hline W-04-264 & Looking at culvert outlet & 245 & W-04-264_P7220010 \\
\hline W-04-265 & Looking upstream from culvert crossing & 0 & W-04-265_P7220011 \\
\hline W-04-265 & Looking downstream from culvert crossing & 150 & W-04-265_P7220012 \\
\hline W-04-265 & Looking at culvert inlet & 100 & W-04-265 P7220013 \\
\hline W-04-265 & Looking at culvert outlet & 90 & W-04-265_P7220014 \\
\hline W-04-266 & Looking upstream from bridge crossing & 110 & W-04-266_P7220015 \\
\hline
\end{tabular}


Appendix 8: List of all photographs taken showing the corresponding site number, descriptive caption, direction faced when taken (azimuth in degrees) and the unique file name for each photograph.

\begin{tabular}{|c|c|c|c|}
\hline $\begin{array}{c}\text { Site } \\
\text { Number }\end{array}$ & Photo Description & Azimuth & File Name \\
\hline W-04-266 & Looking downstream from bridge crossing & 295 & W-04-266_P7220016 \\
\hline W-04-267 & Looking upstream from bridge crossing & 120 & W-04-267_P7220017 \\
\hline W-04-267 & Looking downstream from bridge crossing & 270 & W-04-267_P7220018 \\
\hline W-04-268 & Looking upstream from culvert crossing & 310 & W-04-268_P7220019 \\
\hline W-04-268 & Looking downstream from culvert crossing & 140 & W-04-268_P7220020 \\
\hline W-04-268 & Looking at culvert inlet & 165 & W-04-268_P7220021 \\
\hline W-04-269 & Looking upstream from culvert crossing & 130 & W-04-269_P7220022 \\
\hline W-04-269 & Looking downstream from culvert crossing & 290 & W-04-269_P7220023 \\
\hline W-04-269 & Looking at culvert inlet & 180 & W-04-269_P7220024 \\
\hline W-04-269 & Looking at culvert outlet & 180 & W-04-269_P7220025 \\
\hline W-04-270 & Looking upstream from bridge crossing & 145 & W-04-270_P7220026 \\
\hline W-04-270 & Looking downstream from bridge crossing & 340 & W-04-270_P7220027 \\
\hline W-04-271 & Looking upstream from culvert crossing & 180 & W-04-271_P7230001 \\
\hline W-04-271 & Looking downstream from culvert crossing & 0 & W-04-271_P7230002 \\
\hline W-04-271 & Looking at culvert inlet & 300 & W-04-271_P7230003 \\
\hline W-04-271 & Looking at culvert outlet & 270 & W-04-271_P7230004 \\
\hline W-04-272 & Looking upstream from culvert crossing & 180 & W-04-272_P7230005 \\
\hline W-04-272 & Looking downstream from culvert crossing & 0 & W-04-272_P7230006 \\
\hline W-04-272 & Looking at culvert inlet & 0 & W-04-272_P7230007 \\
\hline W-04-272 & Looking at culvert outlet & 180 & W-04-272_P7230008 \\
\hline W-04-273 & Looking upstream from culvert crossing & 0 & W-04-273_P7230009 \\
\hline W-04-273 & Looking downstream from culvert crossing & 180 & W-04-273_P7230010 \\
\hline W-04-273 & Looking at culvert inlet & 180 & W-04-273_P7230011 \\
\hline W-04-273 & Looking at culvert outlet & 0 & W-04-273_P7230012 \\
\hline W-04-274 & Looking upstream from bridge crossing & 165 & W-04-274_P7230013 \\
\hline W-04-274 & Looking downstream from bridge crossing & 20 & W-04-274_P7230014 \\
\hline W-04-275 & Looking upstream from bridge crossing & 280 & W-04-275_P8030001 \\
\hline W-04-275 & Looking downstream from bridge crossing & 75 & W-04-275_P8030002 \\
\hline W-04-276 & Looking upstream from culvert crossing & 90 & W-04-276_P8030003 \\
\hline W-04-276 & Looking downstream from culvert crossing & 270 & W-04-276_P8030004 \\
\hline W-04-276 & Looking at culvert inlet & 270 & W-04-276_P8030005 \\
\hline W-04-276 & Looking at culvert outlet & 90 & W-04-276_P8030006 \\
\hline W-04-277 & Looking upstream from culvert crossing & 250 & W-04-277_P8030007 \\
\hline
\end{tabular}


Appendix 8: List of all photographs taken showing the corresponding site number, descriptive caption, direction faced when taken (azimuth in degrees) and the unique file name for each photograph.

\begin{tabular}{|c|c|c|c|}
\hline $\begin{array}{c}\text { Site } \\
\text { Number }\end{array}$ & Photo Description & Azimuth & File Name \\
\hline W-04-277 & Looking downstream from culvert crossing & 85 & W-04-277 P8030008 \\
\hline W-04-277 & Looking at culvert inlet & 35 & W-04-277 P8030009 \\
\hline W-04-277 & Looking at culvert outlet & 300 & W-04-277_P8030010 \\
\hline W-04-278 & Looking upstream from left bank & 0 & W-04-278_P8030011 \\
\hline W-04-278 & Looking downstream from left bank & 180 & W-04-278_P8030012 \\
\hline W-04-279 & Looking upstream from culvert crossing & 340 & W-04-279 P8030013 \\
\hline W-04-279 & Looking downstream from culvert crossing & 135 & W-04-279_P8030014 \\
\hline W-04-279 & Looking at culvert inlet & 85 & W-04-279_P8030015 \\
\hline W-04-279 & Looking at culvert outlet & 0 & W-04-279 P8030016 \\
\hline W-04-280 & Looking upstream from culvert crossing & 90 & W-04-280 P8030017 \\
\hline W-04-280 & Looking downstream from culvert crossing & 270 & W-04-280_P8030018 \\
\hline W-04-280 & Looking at culvert inlet & 0 & W-04-280_P8030019 \\
\hline W-04-280 & Looking at culvert outlet & 90 & W-04-280_P8030020 \\
\hline W-04-281 & Looking upstream from culvert crossing & 260 & W-04-281_P8030021 \\
\hline W-04-281 & Looking downstream from culvert crossing & 80 & W-04-281_P8030022 \\
\hline W-04-281 & Looking at culvert inlet & 140 & W-04-281_P8030023 \\
\hline W-04-281 & Looking at culvert outlet & 220 & W-04-281 P8030024 \\
\hline W-04-282 & Looking upstream from culvert crossing & 270 & W-04-282 P8030025 \\
\hline W-04-282 & Looking downstream from culvert crossing & 90 & W-04-282_P8030026 \\
\hline W-04-282 & Looking at culvert inlet & 80 & W-04-282_P8030027 \\
\hline W-04-282 & Looking at culvert outlet & 350 & W-04-282 P8030028 \\
\hline W-04-283 & Looking upstream from bridge crossing & 270 & W-04-283_P8030029 \\
\hline W-04-283 & Looking downstream from bridge crossing & 90 & W-04-283_P8030030 \\
\hline W-04-284 & Looking upstream from culvert crossing & 230 & W-04-284_P8030031 \\
\hline W-04-284 & Looking downstream from culvert crossing & 90 & W-04-284_P8030032 \\
\hline W-04-284 & Looking at culvert inlet & 90 & W-04-284_P8030033 \\
\hline W-04-284 & Looking at culvert outlet & 270 & W-04-284_P8030034 \\
\hline W-04-285 & Looking upstream from culvert crossing & 60 & W-04-285_P8030035 \\
\hline W-04-285 & Looking downstream from culvert crossing & 240 & W-04-285 P8030036 \\
\hline W-04-285 & Looking at culvert inlet & 275 & W-04-285 P8030037 \\
\hline W-04-285 & Looking at culvert outlet & 40 & W-04-285_P8030038 \\
\hline W-04-286 & Looking upstream from ford crossing & 300 & W-04-286 P8030039 \\
\hline W-04-286 & Looking downstream from ford crossing & 140 & W-04-286 P8030040 \\
\hline
\end{tabular}


Appendix 8: List of all photographs taken showing the corresponding site number, descriptive caption, direction faced when taken (azimuth in degrees) and the unique file name for each photograph.

\begin{tabular}{|c|c|c|c|}
\hline $\begin{array}{c}\text { Site } \\
\text { Number }\end{array}$ & Photo Description & Azimuth & File Name \\
\hline W-04-287 & Looking at culvert inlet & 290 & W-04-287_P8030041 \\
\hline W-04-287 & Looking at culvert outlet & 110 & W-04-287-P8030042 \\
\hline W-04-287 & Looking upstream from bridge crossing & 85 & W-04-287_P8030043 \\
\hline W-04-287 & Looking downstream from bridge crossing & 300 & W-04-287_P8030044 \\
\hline W-04-287 & Photo of 1st Sauger & $\mathrm{N} / \mathrm{A}$ & W-04-287_P8030045 \\
\hline W-04-287 & Photo of 1st Sauger & $\mathrm{N} / \mathrm{A}$ & W-04-287 P8030046 \\
\hline W-04-287 & Photo of 2nd Sauger & $\mathrm{N} / \mathrm{A}$ & W-04-287_P8030047 \\
\hline W-04-287 & Photo of 2nd Sauger & N/A & W-04-287_P8030048 \\
\hline W-04-287 & Photo of 2nd Sauger & N/A & W-04-287 P8030049 \\
\hline W-04-287 & Photo of 1st Yellow Perch & $\mathrm{N} / \mathrm{A}$ & W-04-287 P8030050 \\
\hline W-04-287 & Photo of 1st Yellow Perch & $\mathrm{N} / \mathrm{A}$ & W-04-287_P8030051 \\
\hline W-04-287 & Photo of Black Bullhead & $\mathrm{N} / \mathrm{A}$ & W-04-287_P8030052 \\
\hline W-04-288 & Looking upstream from bridge crossing & 270 & W-04-288_P8040001 \\
\hline W-04-288 & Looking downstream from bridge crossing & 90 & W-04-288 P8040002 \\
\hline W-04-289 & Looking upstream from left bank from bottom of riprap dam & 220 & W-04-289_P8040003 \\
\hline W-04-289 & Looking downstream from left bank from bottom of riprap dam & 10 & W-04-289_P8040004 \\
\hline W-04-289 & Photo of schools of bullheads at bottom of riprap dam & N/A & W-04-289 P8040005 \\
\hline W-04-290 & Looking upstream from culvert crossing & 280 & W-04-290 P8040006 \\
\hline W-04-290 & Looking downstream from culvert crossing & 110 & W-04-290_P8040007 \\
\hline W-04-290 & Looking at culvert inlet & 230 & W-04-290_P8040008 \\
\hline W-04-290 & Looking at culvert outlet & 0 & W-04-290 P8040009 \\
\hline W-04-291 & Looking upstream from culvert crossing & 220 & W-04-291_P8040010 \\
\hline W-04-291 & Looking downstream from culvert crossing & 100 & W-04-291_P8040011 \\
\hline W-04-291 & Looking at culvert inlet & 90 & W-04-291_P8040012 \\
\hline W-04-291 & Looking at culvert outlet & 220 & W-04-291_P8040013 \\
\hline W-04-292 & Looking upstream from culvert crossing & 240 & W-04-292 P8040016 \\
\hline W-04-292 & Looking downstream from culvert crossing & 60 & W-04-292_P8040017 \\
\hline W-04-292 & Looking at culvert inlet & 100 & W-04-292_P8040018 \\
\hline W-04-292 & Looking at culvert outlet & 0 & W-04-292 P8040019 \\
\hline W-04-293 & Looking upstream from culvert crossing & 300 & W-04-293 P8040020 \\
\hline W-04-293 & Looking downstream from culvert crossing & 100 & W-04-293_P8040021 \\
\hline W-04-293 & Looking at culvert inlet & 130 & W-04-293 P8040022 \\
\hline W-04-293 & Looking at culvert outlet & 330 & W-04-293 P8040023 \\
\hline
\end{tabular}


Appendix 8: List of all photographs taken showing the corresponding site number, descriptive caption, direction faced when taken (azimuth in degrees) and the unique file name for each photograph.

\begin{tabular}{|c|c|c|c|}
\hline $\begin{array}{c}\text { Site } \\
\text { Number }\end{array}$ & Photo Description & Azimuth & File Name \\
\hline W-04-294 & Looking upstream from bridge crossing & 270 & W-04-294_P8040024 \\
\hline W-04-294 & Photo of cows that have direct access to drain (zoomed in) & 270 & W-04-294 P8040025 \\
\hline W-04-294 & Looking downstream from bridge crossing & 60 & W-04-294_P8040026 \\
\hline W-04-295 & Looking upstream from culvert crossing & 270 & W-04-295_P8040027 \\
\hline W-04-295 & Looking downstream from culvert crossing & 90 & W-04-295_P8040028 \\
\hline W-04-295 & Looking at culvert inlet & 90 & W-04-295_P8040029 \\
\hline W-04-295 & Looking at culvert outlet & 270 & W-04-295_P8040030 \\
\hline W-04-296 & Looking upstream from culvert crossing & 220 & W-04-296_P8040031 \\
\hline W-04-296 & Looking downstream from culvert crossing & 90 & W-04-296_P8040032 \\
\hline W-04-296 & Looking at culvert inlet & 90 & W-04-296_P8040033 \\
\hline W-04-296 & Looking at culvert outlet & 260 & W-04-296_P8040034 \\
\hline W-04-297 & Looking upstream from culvert crossing & 240 & W-04-297_P8050001 \\
\hline W-04-297 & Looking at cattle at river's edge & 240 & W-04-297_P8050002 \\
\hline W-04-297 & Looking downstream from culvert crossing & 85 & W-04-297_P8050003 \\
\hline W-04-297 & Looking at culvert inlet & 110 & W-04-297_P8050004 \\
\hline W-04-297 & Looking at culvert outlet & 220 & W-04-297_P8050005 \\
\hline W-04-297 & Photo of cows in stream & 260 & W-04-297_P8050006 \\
\hline W-04-297 & Photo of cows in stream & 260 & W-04-297_P8050007 \\
\hline W-04-297 & Photo of Jaime \& cows & 300 & W-04-297_P8050008 \\
\hline W-04-297 & Photo of Jaime \& cows & 300 & W-04-297_P8050009 \\
\hline W-04-298 & Looking upstream from bridge crossing & 180 & W-04-298_P8050010 \\
\hline W-04-298 & Looking downstream from bridge crossing & 0 & W-04-298_P8050011 \\
\hline W-04-298 & Looking downstream from bridge crossing, zoomed in on weir & 0 & W-04-298_P8050012 \\
\hline W-04-298 & Looking at weir on downstream side & 180 & W-04-298_P8050013 \\
\hline W-04-299 & Looking upstream from culvert crossing & 210 & W-04-299_P8050014 \\
\hline W-04-299 & Looking downstream from culvert crossing & 35 & W-04-299_P8050015 \\
\hline W-04-299 & Looking at culvert inlet & 90 & W-04-299_P8050016 \\
\hline W-04-299 & Looking at culvert outlet & 150 & W-04-299_P8050017 \\
\hline W-04-299 & Looking at cattle on right bank downstream of culvert structure & 240 & W-04-299_P8050018 \\
\hline W-04-299 & Looking at cattle on left bank on downstream of culvert structure & 50 & W-04-299_P8050019 \\
\hline W-04-300 & Looking upstream from bridge crossing & 320 & W-04-300_P8050020 \\
\hline W-04-300 & Looking downstream from bridge crossing & 120 & W-04-300_P8050021 \\
\hline W-04-301 & Looking at cows on upstream side of bridge crossing & 0 & W-04-301_P8050022 \\
\hline
\end{tabular}


Appendix 8: List of all photographs taken showing the corresponding site number, descriptive caption, direction faced when taken (azimuth in degrees) and the unique file name for each photograph.

\begin{tabular}{|c|c|c|c|}
\hline $\begin{array}{c}\text { Site } \\
\text { Number }\end{array}$ & Photo Description & Azimuth & File Name \\
\hline W-04-301 & Looking downstream from bridge crossing & 150 & W-04-301_P8050023 \\
\hline $\mathrm{X}-04-001$ & Looking upstream from low level crossing & 225 & X-04-001_P5050001 \\
\hline $\mathrm{X}-04-001$ & Looking downstream from low level crossing & 45 & $\mathrm{X}-04-001 \mathrm{P} 5050002$ \\
\hline $\mathrm{X}-04-001$ & Looking upstream at culvert outlets at low level crossing & 220 & X-04-001_P5050003 \\
\hline$X-04-002$ & Looking upstream from dam & 270 & X-04-002_P5050004 \\
\hline $\mathrm{X}-04-002$ & Looking downstream from dam & 90 & X-04-002_P5050005 \\
\hline $\mathrm{X}-04-002$ & Looking upstream at dam & 270 & X-04-002_P5050006 \\
\hline $\mathrm{X}-04-002$ & Looking downstream at dam & 90 & X-04-002_P5050007 \\
\hline $\mathrm{X}-04-003$ & Looking upstream at control structure & 270 & X-04-003_P5050008 \\
\hline $\mathrm{X}-04-003$ & Looking downstream at control structure & 90 & X-04-003_P5050009 \\
\hline $\mathrm{X}-04-003$ & Looking upstream at control structure & 270 & $\mathrm{X}-04-003$ P5050010 \\
\hline $\mathrm{X}-04-004$ & Looking upstream from crossing & 270 & X-04-004_P5050011 \\
\hline $\mathrm{X}-04-004$ & Looking downstream from crossing & 90 & X-04-004_P5050012 \\
\hline$X-04-005$ & Looking upstream from low level crossing & 270 & X-04-005_P5050013 \\
\hline $\mathrm{X}-04-005$ & Looking downstream from low level crossing & 90 & X-04-005_P5050014 \\
\hline $\mathrm{X}-04-005$ & Looking upstream at culverts & 250 & X-04-005_P5050015 \\
\hline $\mathrm{X}-04-006$ & Looking upstream from crossing & 270 & X-04-006_P5060001 \\
\hline $\mathrm{X}-04-006$ & Looking downstream from crossing & 90 & X-04-006_P5060002 \\
\hline $\mathrm{X}-04-006$ & Looking upstream looking under bridge & 290 & X-04-006_P5060003 \\
\hline $\mathrm{X}-04-007$ & Looking upstream from crossing & 270 & X-04-007_P5060004 \\
\hline $\mathrm{X}-04-007$ & Looking downstream from crossing & 90 & X-04-007_P5060005 \\
\hline $\mathrm{X}-04-007$ & Looking upstream at culverts & 250 & X-04-007_P5060006 \\
\hline$X-04-008$ & Looking upstream from crossing & 270 & X-04-008_P5060007 \\
\hline $\mathrm{X}-04-008$ & Looking downstream from crossing & 90 & X-04-008_P5060008 \\
\hline $\mathrm{X}-04-008$ & Looking upstream at culverts & 270 & X-04-008_P5060009 \\
\hline$X-04-009$ & Looking upstream from crossing & 270 & X-04-009_P5060010 \\
\hline $\mathrm{X}-04-009$ & Looking downstream from crossing & 90 & $\mathrm{X}-04-009$ P5060011 \\
\hline $\mathrm{X}-04-009$ & Looking upstream looking at perched culvert & 305 & X-04-009_P5060012 \\
\hline $\mathrm{X}-04-010$ & Looking upstream from crossing & 305 & X-04-010_P5060013 \\
\hline$X-04-010$ & Looking downstream from crossing & 135 & X-04-010_P5060014 \\
\hline $\mathrm{X}-04-010$ & Looking downstream at very small flowing channel & 305 & $\mathrm{X}-04-010+P 5060015$ \\
\hline $\mathrm{X}-04-010$ & Looking upstream at corrugated metal pipe & 305 & X-04-010_P5060016 \\
\hline $\mathrm{X}-04-011$ & Looking downstream from crossing & 90 & X-04-011_P5060017 \\
\hline
\end{tabular}


Appendix 8: List of all photographs taken showing the corresponding site number, descriptive caption, direction faced when taken (azimuth in degrees) and the unique file name for each photograph.

\begin{tabular}{|c|c|c|c|}
\hline $\begin{array}{c}\text { Site } \\
\text { Number }\end{array}$ & Photo Description & Azimuth & File Name \\
\hline $\mathrm{X}-04-011$ & Looking upstream from crossing & 270 & $\begin{array}{lll}X-04-011 & P 5060018\end{array}$ \\
\hline $\mathrm{X}-04-012$ & Looking upstream from crossing & 270 & X-04-012_P5060019 \\
\hline $\mathrm{X}-04-012$ & Looking downstream from crossing & 90 & $\mathrm{X}-04-012$ P5060020 \\
\hline $\mathrm{X}-04-012$ & Looking upstream looking under bridge & 270 & X-04-012_P5060021 \\
\hline$X-04-013$ & Looking upstream from crossing & 270 & $\mathrm{X}-04-013$ P5060022 \\
\hline $\mathrm{X}-04-013$ & Looking downstream from crossing & 125 & $\mathrm{X}-04-013$ P5060023 \\
\hline $\mathrm{X}-04-014$ & Looking upstream from crossing & 240 & X-04-014_P5060024 \\
\hline $\mathrm{X}-04-014$ & Looking downstream from crossing & 120 & $\mathrm{X}-04-014$ P5060025 \\
\hline $\mathrm{X}-04-015$ & Looking upstream from crossing & 270 & $\mathrm{X}-04-015$ P5060026 \\
\hline $\mathrm{X}-04-015$ & Looking downstream from crossing & 90 & X-04-015_P5060027 \\
\hline$X-04-015$ & Looking upstream at culverts & 305 & $\mathrm{X}-04-015$ P5060028 \\
\hline $\mathrm{X}-04-016$ & Looking upstream from below dam & 300 & $\mathrm{X}-04-016$ P5060029 \\
\hline $\mathrm{X}-04-016$ & Looking downstream from above dam & 60 & $\mathrm{X}-04-016$ P5060030 \\
\hline $\mathrm{X}-04-017$ & Looking downstream from dam & 45 & X-04-017_P5060031 \\
\hline $\mathrm{X}-04-017$ & Looking upstream from dam & 225 & $\mathrm{X}-04-017$ P5060032 \\
\hline $\mathrm{X}-04-017$ & Looking at low level crossing & 270 & $\mathrm{X}-04-017$ P5060033 \\
\hline $\mathrm{X}-04-017$ & Looking at corrugated metal pipe at crossing & 45 & $\mathrm{X}-04-017$ P5060034 \\
\hline $\mathrm{X}-04-018$ & Looking upstream from crossing & 185 & X-04-018_P5060035 \\
\hline $\mathrm{X}-04-018$ & Looking downstream from crossing & 40 & X-04-018_P5060036 \\
\hline $\mathrm{X}-04-019$ & Looking upstream from crossing & 225 & $\mathrm{X}-04-019$ P5060037 \\
\hline $\mathrm{X}-04-019$ & Looking downstream from crossing & 5 & $\mathrm{X}-04-019$ P5060038 \\
\hline $\mathrm{X}-04-020$ & Looking downstream from crossing & 45 & X-04-020_P5060039 \\
\hline$X-04-020$ & Looking upstream from crossing & 260 & $\mathrm{X}-04-020 \_\mathrm{P} 5060040$ \\
\hline $\mathrm{X}-04-021$ & Looking upstream from control structure & 270 & $\mathrm{X}-04-021 \quad \mathrm{P} 5060041$ \\
\hline $\mathrm{X}-04-021$ & Looking downstream from control structure & 90 & $\mathrm{X}-04-021 \_\mathrm{P} 5060042$ \\
\hline$X-04-022$ & Looking upstream from crossing & 270 & $\mathrm{X}-04-022 \mathrm{P} 5060043$ \\
\hline $\mathrm{X}-04-022$ & Looking downstream from crossing & 90 & $\mathrm{X}-04-022 \_\mathrm{P} 5060044$ \\
\hline $\mathrm{X}-04-022$ & Looking upstream at culverts & 305 & $\mathrm{X}-04-022$ P5060045 \\
\hline $\mathrm{X}-04-023$ & Looking upstream from crossing & 360 & $\mathrm{X}-04-023-\mathrm{P} 5100001$ \\
\hline $\mathrm{X}-04-023$ & Looking downstream from crossing & 180 & $\mathrm{X}-04-023+\mathrm{P} 5100002$ \\
\hline $\mathrm{X}-04-024$ & Looking upstream from culvert crossing & 300 & $\mathrm{X}-04-024$ P5100003 \\
\hline $\mathrm{X}-04-024$ & Looking downstream from culvert crossing & 105 & $\mathrm{X}-04-024$ P5100004 \\
\hline $\mathrm{X}-04-024$ & Looking upstream at corrugated metal pipe & 280 & X-04-024_P5100005 \\
\hline
\end{tabular}


Appendix 8: List of all photographs taken showing the corresponding site number, descriptive caption, direction faced when taken (azimuth in degrees) and the unique file name for each photograph.

\begin{tabular}{|c|c|c|c|}
\hline $\begin{array}{c}\text { Site } \\
\text { Number }\end{array}$ & Photo Description & Azimuth & File Name \\
\hline $\mathrm{X}-04-025$ & Looking upstream from culvert crossing & 270 & X-04-025_P5100006 \\
\hline$X-04-025$ & Looking downstream at culverts & 90 & $\mathrm{X}-04-025 \mathrm{P} 5100007$ \\
\hline $\mathrm{X}-04-025$ & Looking downstream from culvert crossing & 90 & $\mathrm{X}-04-025 \mathrm{P} 5100008$ \\
\hline $\mathrm{X}-04-026$ & Looking upstream from bridge crossing & 270 & X-04-026_P5100009 \\
\hline $\mathrm{X}-04-026$ & Looking downstream from bridge crossing & 90 & X-04-026_P5100010 \\
\hline $\mathrm{X}-04-027$ & Looking upstream from culvert crossing & 305 & X-04-027_P5100011 \\
\hline $\mathrm{X}-04-027$ & Looking downstream from culvert crossing & 180 & X-04-027_P5100012 \\
\hline $\mathrm{X}-04-028$ & Looking upstream from culvert crossing & 295 & $\mathrm{X}-04-028 \mathrm{P} 5100013$ \\
\hline $\mathrm{X}-04-028$ & Looking downstream from culvert crossing & 125 & X-04-028_P5100014 \\
\hline $\mathrm{X}-04-029$ & Looking upstream from culvert crossing & 270 & X-04-029_P5100015 \\
\hline$X-04-029$ & Looking downstream from culvert crossing & 90 & $\mathrm{X}-04-029$ P5100016 \\
\hline $\mathrm{X}-04-030$ & Looking upstream from low level crossing & 270 & $\mathrm{X}-04-030 \mathrm{P} 5100017$ \\
\hline $\mathrm{X}-04-030$ & Looking downstream from low level crossing & 90 & X-04-030_P5100018 \\
\hline $\mathrm{X}-04-031$ & Looking upstream from culvert crossing & 270 & X-04-031_P5100019 \\
\hline $\mathrm{X}-04-031$ & Looking downstream from culvert crossing & 90 & X-04-031_P5100020 \\
\hline $\mathrm{X}-04-031$ & Looking upstream at culverts & 310 & X-04-031_P5100021 \\
\hline$X-04-032$ & Looking upstream from low level crossing & 270 & $\mathrm{X}-04-032$ P5100022 \\
\hline $\mathrm{X}-04-032$ & Looking downstream from low level crossing & 90 & X-04-032_P5100023 \\
\hline $\mathrm{X}-04-032$ & Looking at flooded low level crossing & 180 & $\mathrm{X}-04-032 \mathrm{P} 5100024$ \\
\hline$X-04-033$ & Looking upstream from bridge crossing & 305 & $\mathrm{X}-04-033 \quad \mathrm{P} 5100025$ \\
\hline $\mathrm{X}-04-033$ & Looking downstream from bridge crossing & 135 & X-04-033_P5100026 \\
\hline $\mathrm{X}-04-033$ & Looking upstream at 11-A North Lateral Drain & 270 & X-04-033_P5100027 \\
\hline$X-04-034$ & Looking upstream from culvert crossing & 180 & $\mathrm{X}-04-034 \mathrm{P} 5100028$ \\
\hline $\mathrm{X}-04-034$ & Looking downstream from culvert crossing & 360 & X-04-034_P5100029 \\
\hline $\mathrm{X}-04-034$ & Looking upstream at perched culverts & 180 & X-04-034_P5100030 \\
\hline$X-04-035$ & Looking upstream from culvert crossing & 180 & $X-04-035$ P5100031 \\
\hline $\mathrm{X}-04-035$ & Looking downstream from culvert crossing & 45 & $\mathrm{X}-04-035 \mathrm{P} 5100032$ \\
\hline $\mathrm{X}-04-035$ & Looking downstream at blocked culverts & 45 & X-04-035_P5100033 \\
\hline $\mathrm{X}-04-036$ & Looking downstream from culvert crossing & 105 & X-04-036_P5140002 \\
\hline $\mathrm{X}-04-036$ & Looking upstream at cement culvert & 270 & X-04-036_P5140003 \\
\hline $\mathrm{X}-04-036$ & Looking upstream from culvert crossing & 305 & $\mathrm{X}-04-036 \_P 5140004$ \\
\hline $\mathrm{X}-04-036$ & Looking downstream at culvert & 45 & X-04-036_P5140005 \\
\hline $\mathrm{X}-04-037$ & Looking upstream from bridge crossing & 305 & X-04-037_P5140006 \\
\hline
\end{tabular}


Appendix 8: List of all photographs taken showing the corresponding site number, descriptive caption, direction faced when taken (azimuth in degrees) and the unique file name for each photograph.

\begin{tabular}{|c|c|c|c|}
\hline $\begin{array}{c}\text { Site } \\
\text { Number }\end{array}$ & Photo Description & Azimuth & File Name \\
\hline $\mathrm{X}-04-037$ & Looking downstream under bridge & 150 & X-04-037 P5140007 \\
\hline $\mathrm{X}-04-037$ & Looking downstream from bridge crossing & 140 & X-04-037_P5140008 \\
\hline $\mathrm{X}-04-037$ & Looking upstream under bridge & 320 & X-04-037_P5140009 \\
\hline $\mathrm{X}-04-038$ & Looking upstream from culvert crossing & 305 & X-04-038_P5140010 \\
\hline $\mathrm{X}-04-038$ & Looking downstream from culvert crossing & 90 & $\mathrm{X}-04-038$ P5140011 \\
\hline $\mathrm{X}-04-038$ & Looking upstream at culverts & 360 & $\mathrm{X}-04-038 \mathrm{P} 5140012$ \\
\hline $\mathrm{X}-04-038$ & Looking downstream at culverts & 180 & X-04-038_P5140013 \\
\hline $\mathrm{X}-04-039$ & Looking upstream from culvert crossing & 305 & $\mathrm{X}-04-039+\mathrm{P} 5140014$ \\
\hline $\mathrm{X}-04-039$ & Looking downstream at perched corrugated metal pipe & 115 & $\mathrm{X}-04-039$ P5140015 \\
\hline $\mathrm{X}-04-039$ & Looking downstream from culvert crossing & 70 & $\mathrm{X}-04-039 \mathrm{P} 5140016$ \\
\hline$X-04-039$ & Looking upstream at culverts & 300 & X-04-039_P5140017 \\
\hline $\mathrm{X}-04-040$ & Looking upstream from culvert crossing & 220 & X-04-040_P5140018 \\
\hline $\mathrm{X}-04-040$ & Looking downstream from culvert crossing & 30 & $\mathrm{X}-04-040$ P5140019 \\
\hline$X-04-040$ & Looking upstream at culverts & 140 & $\mathrm{X}-04-040 \mathrm{P} 5140020$ \\
\hline $\mathrm{X}-04-041$ & Looking upstream from bridge crossing & 315 & X-04-041_P5140021 \\
\hline $\mathrm{X}-04-041$ & Looking downstream under bridge & 90 & $\mathrm{X}-04-041$ P5140022 \\
\hline $\mathrm{X}-04-041$ & Looking downstream from bridge crossing & 135 & $X-04-041$ P5140023 \\
\hline $\mathrm{X}-04-041$ & Looking upstream under bridge & 360 & X-04-041_P5140024 \\
\hline $\mathrm{X}-04-042$ & Looking upstream from culvert crossing & 270 & X-04-042_P5140025 \\
\hline $\mathrm{X}-04-042$ & Looking downstream from culvert crossing & 90 & $\mathrm{X}-04-042 \mathrm{P} 5140026$ \\
\hline $\mathrm{X}-04-042$ & Looking upstream at culverts & 315 & $\mathrm{X}-04-042$ P5140027 \\
\hline $\mathrm{X}-04-042$ & Looking downstream at culverts & 60 & X-04-042_P5140028 \\
\hline$X-04-043$ & Looking upstream from riffle & 320 & X-04-043_P5170001 \\
\hline $\mathrm{X}-04-043$ & Looking downstream from riffle & 130 & $\mathrm{X}-04-043$ P5170002 \\
\hline $\mathrm{X}-04-044$ & Looking upstream from bridge crossing & 360 & $\mathrm{X}-04-044$ P5170003 \\
\hline $\mathrm{X}-04-044$ & Looking downstream under bridge & 210 & X-04-044_P5170004 \\
\hline $\mathrm{X}-04-044$ & Looking downstream from bridge crossing & 180 & X-04-044_P5170005 \\
\hline $\mathrm{X}-04-044$ & Looking upstream under bridge & 305 & X-04-044 P5170006 \\
\hline $\mathrm{X}-04-045$ & Looking at cattle & 305 & $\mathrm{X}-04-045 \quad \mathrm{P} 5170007$ \\
\hline $\mathrm{X}-04-045$ & Looking upstream from culvert crossing & 270 & $\mathrm{X}-04-045 \mathrm{P} 5170008$ \\
\hline $\mathrm{X}-04-045$ & Looking downstream from culvert crossing & 180 & X-04-045_P5170009 \\
\hline $\mathrm{X}-04-045$ & Looking upstream at corrugated metal pipe & 45 & $\mathrm{X}-04-045$ P5170010 \\
\hline$X-04-045$ & Looking downstream at corrugated metal pipe & 120 & $\mathrm{X}-04-045$ P5170011 \\
\hline
\end{tabular}


Appendix 8: List of all photographs taken showing the corresponding site number, descriptive caption, direction faced when taken (azimuth in degrees) and the unique file name for each photograph.

\begin{tabular}{|c|c|c|c|}
\hline $\begin{array}{c}\text { Site } \\
\text { Number }\end{array}$ & Photo Description & Azimuth & File Name \\
\hline $\mathrm{X}-04-046$ & Looking upstream from culvert crossing & 360 & X-04-046_P5170012 \\
\hline$X-04-046$ & Looking downstream at culverts and Kitner Drain from road & 235 & $\mathrm{X}-04-046 \quad \mathrm{P} 5170013$ \\
\hline $\mathrm{X}-04-046$ & Looking downstream from culvert crossing & 180 & $\mathrm{X}-04-046 \quad \mathrm{P} 5170014$ \\
\hline $\mathrm{X}-04-046$ & Looking upstream at culverts & 45 & X-04-046_P5170015 \\
\hline $\mathrm{X}-04-047$ & Looking upstream from bridge crossing & 270 & X-04-047_P5170016 \\
\hline $\mathrm{X}-04-047$ & Looking downstream from bridge crossing & 90 & X-04-047_P5170017 \\
\hline $\mathrm{X}-04-047$ & Looking upstream at bridge & 305 & X-04-047_P5170018 \\
\hline $\mathrm{X}-04-047$ & Looking downstream at cattle impacts & 90 & X-04-047_P5170019 \\
\hline $\mathrm{X}-04-047$ & Looking upstream at dugout pool & 210 & X-04-047_P5170020 \\
\hline $\mathrm{X}-04-048$ & Looking upstream from culvert crossing & 360 & X-04-048_P5180001 \\
\hline$X-04-048$ & Looking downstream from culvert crossing & 180 & $\mathrm{X}-04-048 \quad \mathrm{P} 5180002$ \\
\hline $\mathrm{X}-04-048$ & Looking downstream at culvert inlet & 120 & $\mathrm{X}-04-048 \mathrm{P} 5180003$ \\
\hline $\mathrm{X}-04-048$ & Looking at culvert outlet from left bank & 90 & X-04-048_P5180004 \\
\hline$X-04-049$ & Looking upstream from culvert crossing & 180 & X-04-049_P5180005 \\
\hline $\mathrm{X}-04-049$ & Looking downstream from culvert crossing & 360 & X-04-049_P5180006 \\
\hline $\mathrm{X}-04-049$ & Looking at culvert inlet from right bank & 75 & X-04-049_P5180007 \\
\hline $\mathrm{X}-04-049$ & Looking at culvert outlet from left bank & 250 & X-04-049_P5180008 \\
\hline $\mathrm{X}-04-050$ & Looking upstream from bridge crossing & 260 & X-04-050_P5180009 \\
\hline $\mathrm{X}-04-050$ & Looking downstream from bridge crossing & 120 & $\mathrm{X}-04-050 \mathrm{P} 5180010$ \\
\hline$X-04-051$ & Looking upstream from low bridge crossing & 230 & $\mathrm{X}-04-051$ P5180011 \\
\hline $\mathrm{X}-04-051$ & Looking downstream from low bridge crossing & 60 & X-04-051_P5180012 \\
\hline $\mathrm{X}-04-051$ & Looking upstream at low bridge & 300 & X-04-051_P5180013 \\
\hline $\mathrm{X}-04-051$ & Looking downstream at low bridge & 120 & $\mathrm{X}-04-051+\mathrm{P} 5180014$ \\
\hline $\mathrm{X}-04-051$ & Looking upstream at backed up water caused by beaver dam & 210 & $\mathrm{X}-04-051+P 5180015$ \\
\hline $\mathrm{X}-04-052$ & Looking upstream from bridge crossing & 180 & X-04-052_P5190001 \\
\hline$X-04-052$ & Looking downstream from bridge crossing & 360 & $\mathrm{X}-04-052 \_\mathrm{P} 5190002$ \\
\hline $\mathrm{X}-04-053$ & Looking upstream from culvert crossing & 180 & $\mathrm{X}-04-053+\mathrm{P} 5200003$ \\
\hline $\mathrm{X}-04-053$ & Looking downstream from culvert crossing & 360 & X-04-053_P5200004 \\
\hline $\mathrm{X}-04-053$ & Looking at culvert inlet & 315 & X-04-053_P5200005 \\
\hline $\mathrm{X}-04-053$ & Looking at culvert outlet & 225 & X-04-053_P5200006 \\
\hline $\mathrm{X}-04-053$ & Photo of spawning male White Sucker $455 \mathrm{~mm}$ (released) & $\mathrm{N} / \mathrm{A}$ & X-04-053_P5200007 \\
\hline $\mathrm{X}-04-054$ & Looking upstream from culvert crossing & 180 & X-04-054_P5200008 \\
\hline $\mathrm{X}-04-054$ & Looking downstream from culvert crossing & 360 & X-04-054_P5200009 \\
\hline
\end{tabular}


Appendix 8: List of all photographs taken showing the corresponding site number, descriptive caption, direction faced when taken (azimuth in degrees) and the unique file name for each photograph.

\begin{tabular}{|c|c|c|c|}
\hline $\begin{array}{c}\text { Site } \\
\text { Number }\end{array}$ & Photo Description & Azimuth & File Name \\
\hline $\mathrm{X}-04-054$ & Looking at culvert inlet & 305 & $\mathrm{X}-04-054$ P5200010 \\
\hline $\mathrm{X}-04-054$ & Looking at culvert outlet & 225 & X-04-054_P5200011 \\
\hline $\mathrm{X}-04-055$ & Looking upstream from culvert crossing & 180 & X-04-055_P5200012 \\
\hline $\mathrm{X}-04-055$ & Looking downstream from culvert crossing & 3602 & X-04-055_P5200013 \\
\hline $\mathrm{X}-04-055$ & Looking at culvert inlet & 305 & $X-04-055$ P5200014 \\
\hline $\mathrm{X}-04-055$ & Looking at culvert outlet & 225 & $\mathrm{X}-04-055 \mathrm{P} 5200015$ \\
\hline $\mathrm{X}-04-056$ & Looking upstream from railway bridge crossing & 195 & X-04-056_P5200016 \\
\hline$X-04-056$ & Looking downstream from railway bridge crossing & 45 & $\mathrm{X}-04-056$ P5200017 \\
\hline $\mathrm{X}-04-056$ & Looking upstream on Binden Drain from right bank & 295 & $\mathrm{X}-04-056$ P5200018 \\
\hline $\mathrm{X}-04-057$ & Looking upstream from culvert crossing & 180 & X-04-057_P5200019 \\
\hline$X-04-057$ & Looking downstream from culvert crossing & 360 & X-04-057_P5200020 \\
\hline $\mathrm{X}-04-057$ & Looking at culvert inlet & 315 & X-04-057_P5200021 \\
\hline $\mathrm{X}-04-057$ & Looking at culvert outlet & 135 & $\mathrm{X}-04-057$ P5200022 \\
\hline$X-04-058$ & Looking upstream from culvert crossing & 180 & $\mathrm{X}-04-058$ P5200023 \\
\hline $\mathrm{X}-04-058$ & Looking downstream from culvert crossing & 360 & X-04-058_P5200024 \\
\hline $\mathrm{X}-04-058$ & Looking at culvert inlet & 315 & X-04-058_P5200025 \\
\hline$X-04-058$ & Looking at culvert outlet & 225 & $\mathrm{X}-04-058$ P5200026 \\
\hline $\mathrm{X}-04-059$ & Looking upstream from culvert crossing & 225 & X-04-059_P5200027 \\
\hline $\mathrm{X}-04-059$ & Looking downstream from culvert crossing & 45 & X-04-059_P5200028 \\
\hline $\mathrm{X}-04-059$ & Looking at culvert inlet & 110 & X-04-059_P5200029 \\
\hline $\mathrm{X}-04-059$ & Looking at culvert outlet & 225 & $\mathrm{X}-04-059$ P5200030 \\
\hline $\mathrm{X}-04-060$ & Looking upstream from bridge crossing & 270 & X-04-060_P5200031 \\
\hline$X-04-060$ & Looking downstream from bridge crossing & 90 & $\mathrm{X}-04-060+\mathrm{P} 5200032$ \\
\hline $\mathrm{X}-04-061$ & Looking upstream from bridge crossing & 195 & X-04-061_P5200033 \\
\hline $\mathrm{X}-04-061$ & Looking downstream from bridge crossing & 360 & X-04-061_P5200034 \\
\hline $\mathrm{X}-04-062$ & Looking upstream from bridge crossing & 180 & X-04-062_P5200035 \\
\hline $\mathrm{X}-04-062$ & Looking downstream from bridge crossing & 360 & $\mathrm{X}-04-062 \_\mathrm{P} 5200036$ \\
\hline $\mathrm{X}-04-063$ & Looking upstream from left bank of Westbourne Drain & 180 & $\mathrm{X}-04-063$ P5200037 \\
\hline $\mathrm{X}-04-063$ & Looking downstream from left bank of Westbourne Drain & 350 & $\mathrm{X}-04-063-\mathrm{P} 5200038$ \\
\hline$X-04-063$ & Looking upstream from culvert crossing on 2nd order drain & 90 & $\mathrm{X}-04-063+\mathrm{P} 5200039$ \\
\hline $\mathrm{X}-04-063$ & Looking downstream from culvert crossing on 2 nd order drain & 270 & $\mathrm{X}-04-063+\mathrm{P} 5200040$ \\
\hline $\mathrm{X}-04-063$ & Looking at culvert inlet & 225 & X-04-063 P5200041 \\
\hline$X-04-063$ & Looking at culvert outlet & 135 & X-04-063_P5200042 \\
\hline
\end{tabular}


Appendix 8: List of all photographs taken showing the corresponding site number, descriptive caption, direction faced when taken (azimuth in degrees) and the unique file name for each photograph.

\begin{tabular}{|c|c|c|c|}
\hline $\begin{array}{c}\text { Site } \\
\text { Number }\end{array}$ & Photo Description & Azimuth & File Name \\
\hline $\mathrm{X}-04-064$ & Looking upstream at low level crossing & 180 & X-04-064_P5200043 \\
\hline $\mathrm{X}-04-064$ & Looking downstream at low level crossing & 350 & X-04-064_P5200044 \\
\hline $\mathrm{X}-04-065$ & Looking upstream from bridge crossing & 320 & X-04-065_P5210001 \\
\hline $\mathrm{X}-04-065$ & Looking downstream from bridge crossing & 90 & $\mathrm{X}-04-065 \_\mathrm{P} 5210002$ \\
\hline $\mathrm{X}-04-065$ & Looking at Northern Pike $803 \mathrm{~mm}$ (released) & 225 & X-04-065_P5210003 \\
\hline $\mathrm{X}-04-065$ & Looking at Walleye $297 \mathrm{~mm}$ (released) & $\mathrm{N} / \mathrm{A}$ & X-04-065_P5210004 \\
\hline $\mathrm{X}-04-066$ & Looking upstream from bridge crossing & 230 & X-04-066_P5210005 \\
\hline $\mathrm{X}-04-066$ & Looking downstream from bridge crossing & 30 & X-04-066_P5210006 \\
\hline $\mathrm{X}-04-067$ & Looking upstream from bridge crossing & 360 & X-04-067_P5250007 \\
\hline $\mathrm{X}-04-067$ & Looking downstream from bridge crossing & 180 & X-04-067_P5250008 \\
\hline $\mathrm{X}-04-067$ & Looking upstream at dam & 360 & X-04-067_P5250009 \\
\hline $\mathrm{X}-04-068$ & Looking upstream from right bank & 60 & X-04-068_P5250010 \\
\hline $\mathrm{X}-04-068$ & Looking downstream from right bank & 305 & X-04-068_P5250011 \\
\hline $\mathrm{X}-04-068$ & Looking at spawning male White Sucker- $387 \mathrm{~mm}$ (released) & $\mathrm{N} / \mathrm{A}$ & X-04-068_P5250012 \\
\hline $\mathrm{X}-04-068$ & Looking at spawning male White Sucker 395mm (released) & $\mathrm{N} / \mathrm{A}$ & X-04-068_P5250013 \\
\hline $\mathrm{X}-04-068$ & Looking at spawning male White Sucker $400 \mathrm{~mm}$ (released) & N/A & X-04-068_P5250014 \\
\hline $\mathrm{X}-04-069$ & Looking upstream from riffle & 180 & X-04-069_P5250015 \\
\hline $\mathrm{X}-04-069$ & Looking downstream from riffle & 360 & X-04-069_P5250016 \\
\hline $\mathrm{X}-04-070$ & Looking upstream from bridge crossing & 180 & X-04-070_P5250017 \\
\hline $\mathrm{X}-04-070$ & Looking downstream from bridge crossing & 360 & X-04-070_P5250018 \\
\hline $\mathrm{X}-04-070$ & Looking upstream on 2nd order tributary from bridge crossing & 270 & X-04-070_P5250019 \\
\hline $\mathrm{X}-04-071$ & Looking upstream from right bank & 270 & X-04-071_P5250020 \\
\hline $\mathrm{X}-04-071$ & Looking downstream from right bank & 90 & X-04-071_P5250021 \\
\hline $\mathrm{X}-04-072$ & Looking upstream from right bank & 210 & $\mathrm{X}-04-072 \_P 5250022$ \\
\hline $\mathrm{X}-04-072$ & Looking downstream from right bank & 110 & $\mathrm{X}-04-072 \mathrm{P} 5250023$ \\
\hline$X-04-073$ & Looking upstream from bridge crossing & 290 & $\mathrm{X}-04-073$ P5250024 \\
\hline $\mathrm{X}-04-073$ & Looking downstream from bridge crossing & 125 & X-04-073_P5250025 \\
\hline $\mathrm{X}-04-074$ & Looking upstream from bridge crossing & 270 & X-04-074_P5260001 \\
\hline $\mathrm{X}-04-074$ & Looking downstream from bridge crossing & 90 & X-04-074_P5260002 \\
\hline $\mathrm{X}-04-075$ & Looking upstream from bridge crossing & 45 & $\mathrm{X}-04-075 \mathrm{P} 5260003$ \\
\hline $\mathrm{X}-04-075$ & Looking downstream from bridge crossing & 180 & X-04-075_P5260004 \\
\hline $\mathrm{X}-04-076$ & Looking upstream from bridge crossing & 340 & X-04-076_P5260005 \\
\hline $\mathrm{X}-04-076$ & Looking downstream from bridge crossing & 135 & X-04-076_P5260006 \\
\hline
\end{tabular}


Appendix 8: List of all photographs taken showing the corresponding site number, descriptive caption, direction faced when taken (azimuth in degrees) and the unique file name for each photograph.

\begin{tabular}{|c|c|c|c|}
\hline $\begin{array}{c}\text { Site } \\
\text { Number }\end{array}$ & Photo Description & Azimuth & File Name \\
\hline $\mathrm{X}-04-077$ & Looking upstream from riffle & 180 & X-04-077_P5260007 \\
\hline $\mathrm{X}-04-077$ & Looking downstream from riffle & 30 & X-04-077_P5260008 \\
\hline $\mathrm{X}-04-078$ & Looking upstream from low level crossing & 270 & X-04-078_P5260009 \\
\hline $\mathrm{X}-04-078$ & Looking downstream from low level crossing & 90 & X-04-078_P5260010 \\
\hline $\mathrm{X}-04-078$ & Looking at culvert inlet & 45 & X-04-078_P5260011 \\
\hline $\mathrm{X}-04-078$ & Looking at culvert outlet & 305 & X-04-078_P5260012 \\
\hline $\mathrm{X}-04-079$ & Looking upstream from culvert crossing & 270 & X-04-079_P5260013 \\
\hline $\mathrm{X}-04-079$ & Looking downstream from culvert crossing & 45 & X-04-079_P5260014 \\
\hline $\mathrm{X}-04-080$ & Looking upstream from dam & 270 & X-04-080_P5260015 \\
\hline $\mathrm{X}-04-080$ & Looking downstream from dam & 90 & X-04-080 P5260016 \\
\hline$X-04-080$ & Looking upstream at dam & 305 & X-04-080_P5260017 \\
\hline $\mathrm{X}-04-081$ & Looking upstream from left bank & 305 & X-04-081_P5270001 \\
\hline $\mathrm{X}-04-081$ & Looking downstream from left bank & 120 & X-04-081_P5270002 \\
\hline $\mathrm{X}-04-082$ & Looking upstream from left bank & 180 & X-04-082_P5270003 \\
\hline $\mathrm{X}-04-082$ & Looking downstream from left bank & 45 & X-04-082_P5270004 \\
\hline $\mathrm{X}-04-082$ & Looking upstream on Carr's Creek from right bank & 80 & X-04-082_P5270005 \\
\hline $\mathrm{X}-04-082$ & Looking downstream on Carr's Creek from right bank & 290 & X-04-082_P5270006 \\
\hline $\mathrm{X}-04-083$ & Looking upstream from bridge crossing & 135 & X-04-083_P5270007 \\
\hline $\mathrm{X}-04-083$ & Looking downstream from bridge crossing & 225 & X-04-083_P5270008 \\
\hline $\mathrm{X}-04-084$ & Looking upstream from culvert crossing & 90 & X-04-084_P5270009 \\
\hline $\mathrm{X}-04-084$ & Looking downstream from culvert crossing & 260 & X-04-084_P5270010 \\
\hline $\mathrm{X}-04-084$ & Looking at culvert inlet & 315 & X-04-084_P5270011 \\
\hline $\mathrm{X}-04-084$ & Looking at culvert outlet & 90 & X-04-084_P5270012 \\
\hline $\mathrm{X}-04-085$ & Looking upstream from bridge crossing & 60 & X-04-085_P5270013 \\
\hline $\mathrm{X}-04-085$ & Looking downstream from bridge crossing & 240 & $\mathrm{X}-04-085$ P5270014 \\
\hline$X-04-086$ & Looking upstream on 2nd order tributary from left bank & 295 & X-04-086_P5270015 \\
\hline $\mathrm{X}-04-086$ & Looking upstream on Giroux Drain from right bank & 140 & X-04-086_P5270016 \\
\hline $\mathrm{X}-04-086$ & Looking downstream from culvert crossing & 300 & X-04-086_P5270017 \\
\hline $\mathrm{X}-04-086$ & Looking at culvert outlet & 220 & X-04-086_P5270018 \\
\hline $\mathrm{X}-04-086$ & Looking at culvert inlet & 40 & $\mathrm{X}-04-086 \quad P 5270019$ \\
\hline $\mathrm{X}-04-087$ & Looking upstream from bridge crossing & 305 & X-04-087_P5270020 \\
\hline $\mathrm{X}-04-087$ & Looking downstream from bridge crossing & 135 & X-04-087_P5270021 \\
\hline $\mathrm{X}-04-088$ & Looking upstream from culvert crossing & 180 & X-04-088_P5270022 \\
\hline
\end{tabular}


Appendix 8: List of all photographs taken showing the corresponding site number, descriptive caption, direction faced when taken (azimuth in degrees) and the unique file name for each photograph.

\begin{tabular}{|c|c|c|c|}
\hline $\begin{array}{c}\text { Site } \\
\text { Number }\end{array}$ & Photo Description & Azimuth & File Name \\
\hline $\mathrm{X}-04-088$ & Looking downstream from culvert crossing & 360 & $\begin{array}{l}\mathrm{X}-04-088 \quad \mathrm{P} 5270023 \\
\end{array}$ \\
\hline $\mathrm{X}-04-088$ & Looking at culvert inlet & 325 & X-04-088_P5270024 \\
\hline $\mathrm{X}-04-088$ & Looking at culvert outlet & 225 & X-04-088_P5270025 \\
\hline $\mathrm{X}-04-089$ & Looking upstream from culvert crossing & 90 & X-04-089 P5270026 \\
\hline$X-04-089$ & Looking downstream from culvert crossing & 360 & X-04-089 P5270027 \\
\hline $\mathrm{X}-04-089$ & Looking at perched outlet & 180 & X-04-089_P5270028 \\
\hline $\mathrm{X}-04-089$ & Looking at culvert inlet & 360 & X-04-089_P5270029 \\
\hline $\mathrm{X}-04-090$ & Looking upstream on tributary to Simard Creek & 210 & $\mathrm{X}-04-090$ P5270030 \\
\hline $\mathrm{X}-04-090$ & Looking downstream on tributary to Simard Creek & 360 & $\mathrm{X}-04-090 \quad \mathrm{P} 5270031$ \\
\hline $\mathrm{X}-04-090$ & Upstream on Simard Creek & 180 & X-04-090_P5270032 \\
\hline $\mathrm{X}-04-090$ & Looking downstream on Simard Creek & 90 & X-04-090_P5270033 \\
\hline $\mathrm{X}-04-091$ & Looking upstream from culvert crossing & 110 & X-04-091 P5280001 \\
\hline $\mathrm{X}-04-091$ & Looking downstream from culvert crossing & 340 & X-04-091_P5280002 \\
\hline $\mathrm{X}-04-091$ & Looking at culvert inlet & 280 & X-04-091_P5280003 \\
\hline $\mathrm{X}-04-091$ & Looking at culvert outlet & 100 & X-04-091_P5280004 \\
\hline $\mathrm{X}-04-091$ & Looking at Northern Pike, 382mm (released) & $\mathrm{N} / \mathrm{A}$ & X-04-091 P5280005 \\
\hline $\mathrm{X}-04-092$ & Looking downstream from right bank & 360 & $\mathrm{X}-04-092 \mathrm{P} 5280006$ \\
\hline $\mathrm{X}-04-092$ & Looking upstream from right bank & 180 & X-04-092_P5280007 \\
\hline $\mathrm{X}-04-092$ & Looking at $255 \mathrm{~mm}$ spawning male White Sucker (released) & $\mathrm{N} / \mathrm{A}$ & X-04-092_P5280008 \\
\hline $\mathrm{X}-04-093$ & Looking upstream from culvert crossing & 135 & X-04-093 P5280009 \\
\hline $\mathrm{X}-04-093$ & Looking downstream from culvert crossing & 45 & $\mathrm{X}-04-093 \quad \mathrm{P} 5280010$ \\
\hline $\mathrm{X}-04-093$ & Looking at culvert inlet & 45 & X-04-093_P5280011 \\
\hline $\mathrm{X}-04-093$ & Looking at culvert outlet & 135 & X-04-093_P5280012 \\
\hline $\mathrm{X}-04-094$ & Looking upstream from left bank & 180 & $\mathrm{X}-04-094$ P5280013 \\
\hline $\mathrm{X}-04-094$ & Looking downstream from riffle & 360 & X-04-094_P5280014 \\
\hline$X-04-095$ & Looking upstream from left bank & 305 & $\mathrm{X}-04-095 \mathrm{P} 6010001$ \\
\hline $\mathrm{X}-04-095$ & Looking downstream from left bank & 60 & $\mathrm{X}-04-095+\mathrm{P} 6010002$ \\
\hline $\mathrm{X}-04-096$ & Looking downstream from bridge crossing & 270 & $\mathrm{X}-04-096 \quad \mathrm{P} 6010003$ \\
\hline $\mathrm{X}-04-096$ & Looking upstream from bridge crossing & 60 & $\mathrm{X}-04-096 \quad \mathrm{P} 6010004$ \\
\hline $\mathrm{X}-04-097$ & Looking upstream from culvert crossing & 270 & $\mathrm{X}-04-097 \mathrm{P} 6010005$ \\
\hline $\mathrm{X}-04-097$ & Looking downstream from culvert crossing & 90 & $\mathrm{X}-04-097 \mathrm{P} 6010006$ \\
\hline $\mathrm{X}-04-097$ & Looking at culvert inlet & 120 & $\mathrm{X}-04-097 \quad \mathrm{P} 6010007$ \\
\hline$X-04-097$ & Looking at culvert outlet & 210 & $\mathrm{X}-04-097 \mathrm{P} 6010008$ \\
\hline
\end{tabular}


Appendix 8: List of all photographs taken showing the corresponding site number, descriptive caption, direction faced when taken (azimuth in degrees) and the unique file name for each photograph.

\begin{tabular}{|c|c|c|c|}
\hline $\begin{array}{c}\text { Site } \\
\text { Number }\end{array}$ & Photo Description & Azimuth & File Name \\
\hline $\mathrm{X}-04-097$ & Looking upstream at fished pool & 180 & $\mathrm{X}-04-097 \quad \mathrm{P} 6010009$ \\
\hline $\mathrm{X}-04-097$ & Looking downstream at fished pool & 360 & $\mathrm{X}-04-097 \_\mathrm{P} 6010010$ \\
\hline $\mathrm{X}-04-098$ & Looking downstream from bridge crossing & 270 & X-04-098_P6010011 \\
\hline $\mathrm{X}-04-098$ & Looking upstream from bridge crossing & 110 & $\mathrm{X}-04-098 \_P 6010012$ \\
\hline$X-04-099$ & Looking downstream from bridge crossing & 270 & $\mathrm{X}-04-099 \mathrm{P} 6010013$ \\
\hline X-04-099 & Looking upstream from bridge crossing & 90 & $\mathrm{X}-04-099+\mathrm{P} 6010014$ \\
\hline $\mathrm{X}-04-100$ & Looking upstream from culvert crossing & 270 & X-04-100_P6010015 \\
\hline$X-04-100$ & Looking downstream from culvert crossing & 90 & $\mathrm{X}-04-100-\mathrm{P} 6010016$ \\
\hline $\mathrm{X}-04-100$ & Looking at culvert inlet & 110 & $\mathrm{X}-04-100 \quad \mathrm{P} 6010017$ \\
\hline $\mathrm{X}-04-100$ & Looking at culvert outlet & 225 & $\mathrm{X}-04-100 \mathrm{P} 6010018$ \\
\hline$X-04-100$ & Looking upstream at blockage of fish passage & 225 & $\mathrm{X}-04-100 \mathrm{P} 6010019$ \\
\hline $\mathrm{X}-04-101$ & Looking downstream on Domain Drain at Red River Floodway gates & 240 & X-04-101_P6010020 \\
\hline $\mathrm{X}-04-101$ & Looking upstream from culvert crossing & 270 & $\mathrm{X}-04-101 \mathrm{P} 6010021$ \\
\hline$X-04-101$ & Looking downstream from culvert crossing & 90 & $\mathrm{X}-04-101 \mathrm{P} 6010022$ \\
\hline $\mathrm{X}-04-101$ & Looking at culvert inlet & 120 & X-04-101_P6010023 \\
\hline $\mathrm{X}-04-101$ & Looking at culvert outlet & 45 & $\mathrm{X}-04-101 \quad \mathrm{P} 6010024$ \\
\hline$X-04-102$ & Looking upstream from left bank & 160 & $X-04-102$ P6020001 \\
\hline $\mathrm{X}-04-102$ & Looking downstream from left bank & 305 & $\mathrm{X}-04-102 \mathrm{P} 6020002$ \\
\hline $\mathrm{X}-04-103$ & Looking upstream from left bank & 110 & $\mathrm{X}-04-103 \_\mathrm{P} 6020003$ \\
\hline$X-04-103$ & Looking downstream from left bank & 240 & $X-04-103$ P6020004 \\
\hline $\mathrm{X}-04-103$ & Looking at $643 \mathrm{~mm}$ Common Carp (released) & $\mathrm{N} / \mathrm{A}$ & $\mathrm{X}-04-103 \quad \mathrm{P} 6020007$ \\
\hline $\mathrm{X}-04-104$ & Looking upstream from culvert crossing & 180 & X-04-104_P6020008 \\
\hline$X-04-104$ & Looking downstream from culvert crossing & 325 & X-04-104_P6020009 \\
\hline $\mathrm{X}-04-104$ & Looking at culvert inlet & 305 & $\mathrm{X}-04-104 \_\mathrm{P} 6020010$ \\
\hline $\mathrm{X}-04-104$ & Looking at culvert outlet & 225 & $\mathrm{X}-04-104 \_\mathrm{P} 6020011$ \\
\hline $\mathrm{X}-04-105$ & Looking upstream from culvert crossing & 170 & $\mathrm{X}-04-105 \_\mathrm{P} 6020012$ \\
\hline $\mathrm{X}-04-105$ & Looking downstream from culvert crossing & 290 & X-04-105_P6020013 \\
\hline $\mathrm{X}-04-105$ & Looking at culvert inlet & 315 & $\mathrm{X}-04-105$ P6020014 \\
\hline $\mathrm{X}-04-105$ & Looking at culvert outlet & 90 & $\mathrm{X}-04-105 \quad \mathrm{P} 6020015$ \\
\hline$X-04-106$ & Looking downstream from bridge crossing & 325 & $\mathrm{X}-04-106 \quad \mathrm{P} 6030001$ \\
\hline $\mathrm{X}-04-106$ & Looking upstream from bridge crossing & 180 & $\mathrm{X}-04-106 \_P 6030002$ \\
\hline $\mathrm{X}-04-106$ & Looking at 545mm Common Carp (released) & $\mathrm{N} / \mathrm{A}$ & $\mathrm{X}-04-106 \quad \mathrm{P} 6030003$ \\
\hline$X-04-107$ & Looking upstream from flooded road & 270 & $\mathrm{X}-04-107 \_\mathrm{P} 6030004$ \\
\hline
\end{tabular}


Appendix 8: List of all photographs taken showing the corresponding site number, descriptive caption, direction faced when taken (azimuth in degrees) and the unique file name for each photograph.

\begin{tabular}{|c|c|c|c|}
\hline $\begin{array}{c}\text { Site } \\
\text { Number }\end{array}$ & Photo Description & Azimuth & File Name \\
\hline $\mathrm{X}-04-107$ & Looking downstream from flooded road & 90 & X-04-107_P6030005 \\
\hline $\mathrm{X}-04-107$ & Looking at flooded low level crossing & 180 & $\mathrm{X}-04-107$ P6030006 \\
\hline $\mathrm{X}-04-108$ & Looking upstream from elevated bridge crossing & 180 & X-04-108_P6030007 \\
\hline $\mathrm{X}-04-108$ & Looking downstream from elevated bridge crossing & 360 & X-04-108_P6030008 \\
\hline $\mathrm{X}-04-109$ & Looking at culvert inlet & 150 & X-04-109_P6030009 \\
\hline $\mathrm{X}-04-109$ & Looking upstream from elevated culvert crossing & 305 & X-04-109_P6030010 \\
\hline $\mathrm{X}-04-109$ & Looking downstream from elevated culvert crossing & 110 & X-04-109_P6030011 \\
\hline$X-04-110$ & Looking at culvert outlet & 240 & X-04-110_P6040001 \\
\hline $\mathrm{X}-04-110$ & Looking at cattle tracks on left bank & $\mathrm{N} / \mathrm{A}$ & X-04-110_P6040002 \\
\hline $\mathrm{X}-04-110$ & Looking upstream from culvert crossing & 180 & X-04-110_P6040003 \\
\hline$X-04-110$ & Looking downstream from culvert crossing & 360 & X-04-110_P6040004 \\
\hline $\mathrm{X}-04-110$ & Looking at culvert inlet & 290 & X-04-110_P6040005 \\
\hline $\mathrm{X}-04-110$ & Looking at spawning male Fathead Minnow & $\mathrm{N} / \mathrm{A}$ & X-04-110_P6040006 \\
\hline $\mathrm{X}-04-111$ & Looking upstream from culvert crossing & 290 & X-04-111_P6040009 \\
\hline $\mathrm{X}-04-111$ & Looking downstream from culvert crossing & 110 & X-04-111_P6040010 \\
\hline $\mathrm{X}-04-111$ & Looking at culvert outlet & 340 & X-04-111_P6040011 \\
\hline $\mathrm{X}-04-111$ & Looking at culvert inlet & 160 & X-04-111_P6040012 \\
\hline $\mathrm{X}-04-112$ & Looking upstream from culvert crossing & 180 & $\mathrm{X}-04-112 \mathrm{P} 6040013$ \\
\hline $\mathrm{X}-04-112$ & Looking downstream from culvert crossing & 305 & $\mathrm{X}-04-112 \_\mathrm{P} 6040014$ \\
\hline $\mathrm{X}-04-112$ & Looking at culvert inlet & 250 & $\mathrm{X}-04-112 \_\mathrm{P} 6040015$ \\
\hline $\mathrm{X}-04-112$ & Looking at culvert outlet & 250 & $\mathrm{X}-04-112$ P6040016 \\
\hline $\mathrm{X}-04-113$ & Looking downstream from bridge crossing & 90 & X-04-113_P6070001 \\
\hline$X-04-113$ & Looking upstream from bridge crossing & 270 & $\mathrm{X}-04-113 \_\mathrm{P} 6070002$ \\
\hline $\mathrm{X}-04-114$ & Looking at lowa Darter & $\mathrm{N} / \mathrm{A}$ & X-04-114_P6070005 \\
\hline $\mathrm{X}-04-114$ & Looking at lowa Darter & $\mathrm{N} / \mathrm{A}$ & X-04-114_P6070006 \\
\hline$X-04-114$ & Looking at lowa Darter & $\mathrm{N} / \mathrm{A}$ & X-04-114_P6070007 \\
\hline $\mathrm{X}-04-114$ & Looking upstream from left bank of flooded low level crossing & 210 & $\mathrm{X}-04-114 \_\mathrm{P} 6070010$ \\
\hline $\mathrm{X}-04-114$ & Looking downstream from left bank of flooded low level crossing & 340 & X-04-114_P6070011 \\
\hline $\mathrm{X}-04-114$ & Looking down at exact habitat where lowa Darters were caught & 225 & $\mathrm{X}-04-114 \_\mathrm{P} 6070012$ \\
\hline $\mathrm{X}-04-114$ & Looking at flooded low level crossing & 270 & $\mathrm{X}-04-114 \_\mathrm{P} 6070013$ \\
\hline $\mathrm{X}-04-115$ & Looking upstream from right bank & 260 & X-04-115_P6070014 \\
\hline $\mathrm{X}-04-115$ & Looking downstream from riffle & 85 & $\mathrm{X}-04-115 \_\mathrm{P} 6070015$ \\
\hline $\mathrm{X}-04-115$ & Looking at culvert inlet & 270 & X-04-115_P6070016 \\
\hline
\end{tabular}


Appendix 8: List of all photographs taken showing the corresponding site number, descriptive caption, direction faced when taken (azimuth in degrees) and the unique file name for each photograph.

\begin{tabular}{|c|c|c|c|}
\hline $\begin{array}{c}\text { Site } \\
\text { Number }\end{array}$ & Photo Description & Azimuth & File Name \\
\hline $\mathrm{X}-04-115$ & Looking at Creek Chub & $\mathrm{N} / \mathrm{A}$ & $\mathrm{X}-04-115$ P6070017 \\
\hline $\mathrm{X}-04-115$ & Looking at Common Shiner & $\mathrm{N} / \mathrm{A}$ & X-04-115_P6070018 \\
\hline $\mathrm{X}-04-115$ & Looking at Western Blacknose Dace & $\mathrm{N} / \mathrm{A}$ & X-04-115_P6070019 \\
\hline $\mathrm{X}-04-115$ & Looking at Western Blacknose Dace & $\mathrm{N} / \mathrm{A}$ & $\mathrm{X}-04-115$ P6070020 \\
\hline $\mathrm{X}-04-116$ & Looking upstream from culvert crossing & 180 & X-04-116_P6080001 \\
\hline $\mathrm{X}-04-116$ & Looking downstream from culvert crossing & 360 & X-04-116_P6080002 \\
\hline $\mathrm{X}-04-116$ & Looking at culvert inlet & 45 & X-04-116_P6080003 \\
\hline $\mathrm{X}-04-116$ & Looking at culvert outlet & 120 & X-04-116_P6080004 \\
\hline $\mathrm{X}-04-117$ & Looking upstream from culvert crossing & 180 & $\mathrm{X}-04-117 \mathrm{P} 6080005$ \\
\hline $\mathrm{X}-04-117$ & Looking downstream from culvert crossing & 360 & $\mathrm{X}-04-117$ P6080006 \\
\hline $\mathrm{X}-04-117$ & Looking at culvert inlet & 305 & $\mathrm{X}-04-117=\mathrm{P} 6080007$ \\
\hline $\mathrm{X}-04-117$ & Looking at culvert outlet & 210 & X-04-117_P6080008 \\
\hline $\mathrm{X}-04-118$ & Looking upstream from right bank & 150 & X-04-118_P6080009 \\
\hline $\mathrm{X}-04-118$ & Looking downstream from right bank & 360 & X-04-118_P6080010 \\
\hline $\mathrm{X}-04-119$ & Looking upstream from culvert crossing & 180 & X-04-119 P6080011 \\
\hline $\mathrm{X}-04-119$ & Looking downstream from culvert crossing & 360 & X-04-119_P6080012 \\
\hline $\mathrm{X}-04-119$ & Looking at culvert inlet & 250 & X-04-119_P6080013 \\
\hline $\mathrm{X}-04-119$ & Looking at culvert outlet & 210 & $\mathrm{X}-04-119 \quad \mathrm{P} 6080014$ \\
\hline $\mathrm{X}-04-120$ & Looking upstream from right bank & 270 & X-04-120_P6080019 \\
\hline $\mathrm{X}-04-120$ & Looking downstream from riffle & 120 & X-04-120_P6080020 \\
\hline $\mathrm{X}-04-121$ & Looking downstream from bridge crossing & 225 & $\mathrm{X}-04-121 \_\mathrm{P} 6080021$ \\
\hline $\mathrm{X}-04-121$ & Looking upstream from bridge crossing & 90 & X-04-121_P6080022 \\
\hline $\mathrm{X}-04-121$ & Looking at cattle & 270 & $\mathrm{X}-04-121 \_\mathrm{P} 6080023$ \\
\hline $\mathrm{X}-04-121$ & Looking at 670mm Common Carp (released) & $\mathrm{N} / \mathrm{A}$ & $\mathrm{X}-04-121 \_\mathrm{P} 6080024$ \\
\hline $\mathrm{X}-04-122$ & Looking upstream from culvert crossing & 270 & X-04-122_P6090001 \\
\hline$X-04-122$ & Looking downstream from culvert crossing & 290 & $\mathrm{X}-04-122 \_\mathrm{P} 6090002$ \\
\hline $\mathrm{X}-04-122$ & Looking at culvert inlet & 45 & $\mathrm{X}-04-122 \_\mathrm{P} 6090003$ \\
\hline $\mathrm{X}-04-122$ & Looking at culvert outlet & 315 & $\mathrm{X}-04-122 \_\mathrm{P} 6090004$ \\
\hline $\mathrm{X}-04-123$ & Looking downstream from bridge crossing & 180 & X-04-123_P6090009 \\
\hline$X-04-123$ & Looking upstream from bridge crossing & 360 & X-04-123_P6090010 \\
\hline $\mathrm{X}-04-124$ & Looking upstream from left bank & 195 & X-04-124 P6090011 \\
\hline $\mathrm{X}-04-124$ & Looking downstream from left bank & 340 & X-04-124_P6090012 \\
\hline $\mathrm{X}-04-124$ & Looking at flooded low level crossing & 270 & X-04-124_P6090013 \\
\hline
\end{tabular}


Appendix 8: List of all photographs taken showing the corresponding site number, descriptive caption, direction faced when taken (azimuth in degrees) and the unique file name for each photograph.

\begin{tabular}{|c|c|c|c|}
\hline $\begin{array}{c}\text { Site } \\
\text { Number }\end{array}$ & Photo Description & Azimuth & File Name \\
\hline $\mathrm{X}-04-125$ & Looking upstream from flood control structure & 200 & X-04-125_P6100001 \\
\hline $\mathrm{X}-04-125$ & Looking downstream from flood control structure & 340 & $\mathrm{X}-04-125 \mathrm{P} 6100002$ \\
\hline $\mathrm{X}-04-125$ & Looking upstream at flood control structure & 135 & X-04-125_P6100003 \\
\hline $\mathrm{X}-04-126$ & Looking downstream from bridge crossing & 360 & X-04-126_P6100004 \\
\hline $\mathrm{X}-04-126$ & Looking upstream from bridge crossing & 180 & X-04-126_P6100005 \\
\hline $\mathrm{X}-04-127$ & Looking at dead Common Carp on rocks & $\mathrm{N} / \mathrm{A}$ & X-04-127_P6100006 \\
\hline $\mathrm{X}-04-127$ & Looking at perched outlet & 240 & X-04-127_P6100007 \\
\hline $\mathrm{X}-04-127$ & Looking upstream from culvert crossing & 75 & X-04-127_P6100008 \\
\hline $\mathrm{X}-04-127$ & Looking downstream from culvert crossing & 280 & X-04-127_P6100009 \\
\hline $\mathrm{X}-04-127$ & Looking at culvert inlet & 45 & X-04-127_P6100010 \\
\hline $\mathrm{X}-04-127$ & Looking at 234mm Rock Bass (released) & $\mathrm{N} / \mathrm{A}$ & X-04-127_P6100011 \\
\hline $\mathrm{X}-04-127$ & Looking at $221 \mathrm{~mm}$ Rock Bass (released) & $\mathrm{N} / \mathrm{A}$ & X-04-127_P6100012 \\
\hline $\mathrm{X}-04-127$ & Looking at 262mm Rock Bass (released) & $\mathrm{N} / \mathrm{A}$ & X-04-127_P6100013 \\
\hline $\mathrm{X}-04-127$ & Looking at 347mm Northern Pike (released) & $\mathrm{N} / \mathrm{A}$ & X-04-127_P6100015 \\
\hline $\mathrm{X}-04-127$ & Looking at 539mm Common Carp (released) & $\mathrm{N} / \mathrm{A}$ & X-04-127_P6100016 \\
\hline $\mathrm{X}-04-128$ & Looking upstream from bridge crossing & 270 & X-04-128_P6140001 \\
\hline $\mathrm{X}-04-128$ & Looking downstream from bridge crossing & 90 & X-04-128_P6140002 \\
\hline $\mathrm{X}-04-128$ & Looking at 272mm White Sucker (released) & $\mathrm{N} / \mathrm{A}$ & $\mathrm{X}-04-128+\mathrm{P} 6140003$ \\
\hline $\mathrm{X}-04-128$ & Looking at central mud minnow & $\mathrm{N} / \mathrm{A}$ & X-04-128_P6140004 \\
\hline $\mathrm{X}-04-128$ & Looking at central mud minnow & $\mathrm{N} / \mathrm{A}$ & X-04-128_P6140005 \\
\hline $\mathrm{X}-04-129$ & Looking at culvert outlet & 220 & X-04-129_P6140006 \\
\hline $\mathrm{X}-04-129$ & Looking downstream from left bank & 45 & X-04-129_P6140007 \\
\hline $\mathrm{X}-04-129$ & Looking upstream from culvert crossing & 225 & X-04-129_P6140008 \\
\hline $\mathrm{X}-04-129$ & Looking at culvert inlet & 60 & X-04-129_P6140009 \\
\hline $\mathrm{X}-04-129$ & Looking at $212 \mathrm{~mm}, 249 \mathrm{~mm}$ White Suckers (released) & $\mathrm{N} / \mathrm{A}$ & X-04-129_P6140010 \\
\hline $\mathrm{X}-04-130$ & Looking downstream from right bank & 285 & X-04-130_P6140011 \\
\hline $\mathrm{X}-04-130$ & Looking at left corrugated metal pipe from right bank & 110 & $\mathrm{X}-04-130 \_\mathrm{P} 6140012$ \\
\hline $\mathrm{X}-04-130$ & Looking at right corrugated metal pipe from right bank & 85 & X-04-130_P6140013 \\
\hline $\mathrm{X}-04-130$ & Looking upstream from left bank & 100 & X-04-130_P6140014 \\
\hline $\mathrm{X}-04-130$ & Looking at left corrugated metal pipe from left bank & 210 & X-04-130_P6140015 \\
\hline $\mathrm{X}-04-130$ & Looking at right corrugated metal pipe from left bank & 270 & X-04-130_P6140016 \\
\hline $\mathrm{X}-04-131$ & Looking upstream from culvert crossing & 270 & X-04-131_P6140017 \\
\hline $\mathrm{X}-04-131$ & Looking downstream from culvert crossing & 90 & X-04-131_P6140018 \\
\hline
\end{tabular}


Appendix 8: List of all photographs taken showing the corresponding site number, descriptive caption, direction faced when taken (azimuth in degrees) and the unique file name for each photograph.

\begin{tabular}{|c|c|c|c|}
\hline $\begin{array}{c}\text { Site } \\
\text { Number }\end{array}$ & Photo Description & Azimuth & File Name \\
\hline $\mathrm{X}-04-131$ & Looking at culvert inlet & 305 & X-04-131_P6140019 \\
\hline $\mathrm{X}-04-131$ & Looking at culvert outlet & 225 & X-04-131_P6140020 \\
\hline $\mathrm{X}-04-132$ & Looking upstream from culvert crossing & 270 & X-04-132_P6140021 \\
\hline $\mathrm{X}-04-132$ & Looking downstream from culvert crossing & 90 & X-04-132_P6140022 \\
\hline $\mathrm{X}-04-132$ & Looking at culvert inlet & 45 & X-04-132_P6140023 \\
\hline $\mathrm{X}-04-132$ & Looking at culvert outlet & 305 & X-04-132_P6140024 \\
\hline $\mathrm{X}-04-133$ & Looking upstream from culvert crossing & 120 & X-04-133_P6150025 \\
\hline $\mathrm{X}-04-133$ & Looking downstream from culvert crossing & 310 & X-04-133_P6150026 \\
\hline $\mathrm{X}-04-133$ & Looking at culvert inlet & 20 & X-04-133_P6150027 \\
\hline $\mathrm{X}-04-133$ & Looking at culvert outlet & 120 & X-04-133_P6150028 \\
\hline $\mathrm{X}-04-134$ & Looking upstream from culvert crossing & 90 & X-04-134_P6150029 \\
\hline $\mathrm{X}-04-134$ & Looking downstream from culvert crossing & 270 & X-04-134_P6150030 \\
\hline $\mathrm{X}-04-134$ & Looking at culvert inlet & 225 & X-04-134_P6150031 \\
\hline $\mathrm{X}-04-134$ & Looking at culvert outlet & 135 & X-04-134_P6150032 \\
\hline $\mathrm{X}-04-135$ & Looking upstream from bridge crossing & 135 & $\mathrm{X}-04-135 \mathrm{P} 6150033$ \\
\hline $\mathrm{X}-04-135$ & Looking downstream towards Whitemud River from bridge crossing & 330 & X-04-135_P6150034 \\
\hline$X-04-136$ & Looking upstream from culvert crossing & 225 & X-04-136_P6150035 \\
\hline $\mathrm{X}-04-136$ & Looking downstream from culvert crossing & 45 & X-04-136_P6150036 \\
\hline $\mathrm{X}-04-136$ & Looking at culvert inlet & 120 & X-04-136_P6150037 \\
\hline$X-04-136$ & Looking at culvert outlet & 120 & X-04-136_P6150038 \\
\hline $\mathrm{X}-04-137$ & Looking upstream at reservoir & 135 & X-04-137_P6160001 \\
\hline $\mathrm{X}-04-137$ & Looking downstream from top of dam & 350 & X-04-137_P6160002 \\
\hline $\mathrm{X}-04-137$ & Looking at dam inlet & 160 & X-04-137_P6160003 \\
\hline $\mathrm{X}-04-137$ & Looking at dam outlet & 160 & X-04-137_P6160004 \\
\hline $\mathrm{X}-04-137$ & Looking at Yellow Perch & $\mathrm{N} / \mathrm{A}$ & X-04-137_P6160005 \\
\hline $\mathrm{X}-04-137$ & Looking at Yellow Perch & $\mathrm{N} / \mathrm{A}$ & X-04-137_P6160006 \\
\hline $\mathrm{X}-04-137$ & looking at Walleye sign & 90 & X-04-137_P6160007 \\
\hline $\mathrm{X}-04-138$ & Looking upstream from culvert crossing & 90 & X-04-138_P6160008 \\
\hline $\mathrm{X}-04-138$ & Looking downstream from culvert crossing & 270 & X-04-138_P6160009 \\
\hline $\mathrm{X}-04-138$ & Looking at perched culvert outlet $(0.5 \mathrm{~m})$ & 135 & X-04-138_P6160010 \\
\hline $\mathrm{X}-04-138$ & Looking at Black Bullhead & $\mathrm{N} / \mathrm{A}$ & X-04-138_P6160011 \\
\hline $\mathrm{X}-04-138$ & Looking at brassy minnow & $\mathrm{N} / \mathrm{A}$ & X-04-138_P6160013 \\
\hline$X-04-139$ & Looking upstream from culvert crossing & 340 & X-04-139_P6160014 \\
\hline
\end{tabular}


Appendix 8: List of all photographs taken showing the corresponding site number, descriptive caption, direction faced when taken (azimuth in degrees) and the unique file name for each photograph.

\begin{tabular}{|c|c|c|c|}
\hline $\begin{array}{c}\text { Site } \\
\text { Number }\end{array}$ & Photo Description & Azimuth & File Name \\
\hline $\mathrm{X}-04-139$ & Looking downstream from culvert crossing & 110 & $\begin{array}{l}\mathrm{X}-04-139 \\
\mathrm{P} 6160015\end{array}$ \\
\hline$X-04-139$ & Looking at culvert inlet & 150 & $\mathrm{X}-04-139 \_\mathrm{P} 6160016$ \\
\hline $\mathrm{X}-04-139$ & Looking at culvert outlet & 80 & X-04-139_P6160017 \\
\hline$X-04-140$ & Looking upstream from culvert crossing & 135 & X-04-140_P6160018 \\
\hline$X-04-140$ & Looking downstream from culvert crossing & 325 & $\mathrm{X}-04-140 \quad \mathrm{P} 6160019$ \\
\hline $\mathrm{X}-04-140$ & Looking at culvert inlet & 45 & $\mathrm{X}-04-140 \mathrm{P} 6160020$ \\
\hline $\mathrm{X}-04-140$ & Looking at perched culvert outlet & 225 & X-04-140_P6160021 \\
\hline$X-04-141$ & Looking upstream from culvert crossing & 210 & $\mathrm{X}-04-141 \mathrm{P} 6170001$ \\
\hline $\mathrm{X}-04-141$ & Looking downstream from culvert crossing & 45 & $\mathrm{X}-04-141 \mathrm{P} 6170002$ \\
\hline $\mathrm{X}-04-141$ & Looking at culvert inlet & 90 & $\mathrm{X}-04-141 \mathrm{P} 6170003$ \\
\hline$X-04-141$ & Looking at culvert outlet & 305 & $\mathrm{X}-04-141 \_\mathrm{P} 6170004$ \\
\hline $\mathrm{X}-04-141$ & Looking at group of White Suckers & $\mathrm{N} / \mathrm{A}$ & $\mathrm{X}-04-141 \quad \mathrm{P} 6170005$ \\
\hline $\mathrm{X}-04-142$ & Looking upstream from left bank & 120 & $\mathrm{X}-04-142 \mathrm{P} 6170006$ \\
\hline $\mathrm{X}-04-142$ & Looking downstream from left bank & 210 & $\mathrm{X}-04-142 \mathrm{P} 6170007$ \\
\hline $\mathrm{X}-04-143$ & Looking upstream on west branch from culvert crossing & 350 & $\mathrm{X}-04-143+\mathrm{P} 6170008$ \\
\hline $\mathrm{X}-04-143$ & Looking upstream on east branch from culvert crossing & 10 & $\mathrm{X}-04-143$ P6170009 \\
\hline$X-04-143$ & Looking downstream from culvert crossing & 180 & $X-04-143 \quad P 6170010$ \\
\hline$X-04-144$ & Looking upstream from culvert crossing & 270 & X-04-144_P6170011 \\
\hline $\mathrm{X}-04-144$ & Looking downstream from culvert crossing & 90 & X-04-144_P6170012 \\
\hline$X-04-144$ & Looking at flooded outlet & 90 & $\mathrm{X}-04-144 \mathrm{P} 6170013$ \\
\hline$X-04-145$ & Looking upstream from culvert crossing & 220 & $\mathrm{X}-04-145 \quad \mathrm{P} 6170014$ \\
\hline $\mathrm{X}-04-145$ & Looking at perched culvert inlet (1.5m) & 320 & X-04-145_P6170015 \\
\hline$X-04-145$ & Looking at perched culvert outlet $(2 \mathrm{~m})$ & 285 & X-04-145_P6170016 \\
\hline $\mathrm{X}-04-145$ & Looking at culvert outlet & 210 & $\mathrm{X}-04-145 \quad \mathrm{P} 6170017$ \\
\hline$X-04-145$ & Looking downstream from culvert crossing & 90 & $\mathrm{X}-04-145 \mathrm{P} 6170018$ \\
\hline$X-04-146$ & Looking upstream from culvert crossing & 20 & X-04-146_P6170020 \\
\hline $\mathrm{X}-04-146$ & Looking downstream from culvert crossing & 200 & X-04-146_P6170021 \\
\hline $\mathrm{X}-04-146$ & Looking at culvert inlets & 160 & $\mathrm{X}-04-146 \quad \mathrm{P} 6170022$ \\
\hline$X-04-146$ & Looking at perched outlets $(0.2 \mathrm{~m})$ & 270 & $\mathrm{X}-04-146 \quad \mathrm{P} 6170023$ \\
\hline$X-04-147$ & Looking at perched culvert outlets $(0.5 \mathrm{~m})$ & 305 & $\mathrm{X}-04-147$ P6180001 \\
\hline $\mathrm{X}-04-147$ & Looking downstream from right bank & 205 & $\mathrm{X}-04-147 \_\mathrm{P} 6180002$ \\
\hline $\mathrm{X}-04-147$ & Looking upstream from culvert crossing & 345 & $\mathrm{X}-04-147 \quad \mathrm{P} 6180003$ \\
\hline$X-04-148$ & Looking upstream from bridge crossing & 270 & $\mathrm{X}-04-148 \quad \mathrm{P} 6180004$ \\
\hline
\end{tabular}


Appendix 8: List of all photographs taken showing the corresponding site number, descriptive caption, direction faced when taken (azimuth in degrees) and the unique file name for each photograph.

\begin{tabular}{|c|c|c|c|}
\hline $\begin{array}{c}\text { Site } \\
\text { Number }\end{array}$ & Photo Description & Azimuth & File Name \\
\hline $\mathrm{X}-04-148$ & Looking downstream from bridge crossing & 90 & X-04-148_P6180005 \\
\hline $\mathrm{X}-04-149$ & Looking upstream from bridge crossing & 270 & X-04-149_P6210001 \\
\hline $\mathrm{X}-04-149$ & Looking downstream from bridge crossing & 90 & X-04-149_P6210002 \\
\hline $\mathrm{X}-04-150$ & Looking upstream from bridge crossing & 270 & X-04-150_P6210003 \\
\hline$X-04-150$ & Looking downstream from bridge crossing & 90 & X-04-150_P6210004 \\
\hline $\mathrm{X}-04-150$ & Looking at Finescale Dace & $\mathrm{N} / \mathrm{A}$ & X-04-150_P6210005 \\
\hline $\mathrm{X}-04-150$ & Looking at Finescale Dace & $\mathrm{N} / \mathrm{A}$ & X-04-150_P6210006 \\
\hline$X-04-151$ & Looking upstream from right bank & 160 & $\mathrm{X}-04-151 \_\mathrm{P} 6210007$ \\
\hline $\mathrm{X}-04-151$ & Looking downstream from right bank & 30 & $\mathrm{X}-04-151 \_\mathrm{P} 6210008$ \\
\hline $\mathrm{X}-04-151$ & Looking at culvert outlet & 160 & $\mathrm{X}-04-151 \_\mathrm{P} 6210009$ \\
\hline$X-04-152$ & Looking upstream from culvert crossing & 180 & $\mathrm{X}-04-152 \_\mathrm{P} 6220001$ \\
\hline $\mathrm{X}-04-152$ & Looking downstream from culvert crossing & 360 & $\mathrm{X}-04-152 \_\mathrm{P} 6220002$ \\
\hline $\mathrm{X}-04-152$ & Looking at culvert inlet & 65 & X-04-152_P6220003 \\
\hline$X-04-152$ & Looking at culvert outlet & 120 & X-04-152_P6220004 \\
\hline $\mathrm{X}-04-153$ & Looking upstream from culvert crossing & 360 & X-04-153_P6220005 \\
\hline $\mathrm{X}-04-153$ & Looking downstream from culvert crossing & 150 & X-04-153_P6220006 \\
\hline$X-04-153$ & Looking at culvert inlet & 290 & X-04-153_P6220007 \\
\hline $\mathrm{X}-04-153$ & Looking at culvert outlet & 325 & $\mathrm{X}-04-153 \_\mathrm{P} 6220008$ \\
\hline $\mathrm{X}-04-154$ & Looking upstream from bridge crossing & 285 & X-04-154_P6220009 \\
\hline$X-04-154$ & Looking downstream from bridge crossing & 90 & X-04-154_P6220010 \\
\hline $\mathrm{X}-04-155$ & Looking upstream from culvert crossing & 210 & $\mathrm{X}-04-155 \_\mathrm{P} 6230001$ \\
\hline $\mathrm{X}-04-155$ & Looking downstream from culvert crossing & 45 & $\mathrm{X}-04-155 \_\mathrm{P} 6230002$ \\
\hline $\mathrm{X}-04-155$ & Looking at culvert inlet & 45 & $\mathrm{X}-04-155 \_\mathrm{P} 6230003$ \\
\hline $\mathrm{X}-04-155$ & Looking at culvert outlet & 305 & $\mathrm{X}-04-155 \_\mathrm{P} 6230004$ \\
\hline $\mathrm{X}-04-155$ & Looking at 10 Yellow Perch, fork length from $85-66 \mathrm{~mm}$ (released) & $\mathrm{N} / \mathrm{A}$ & $\mathrm{X}-04-155 \_\mathrm{P} 6230005$ \\
\hline $\mathrm{X}-04-155$ & Looking at Spottail Shiner & $\mathrm{N} / \mathrm{A}$ & $\mathrm{X}-04-155+\mathrm{P} 6230006$ \\
\hline $\mathrm{X}-04-155$ & Looking at Northern Pike & $\mathrm{N} / \mathrm{A}$ & $\mathrm{X}-04-155 \_\mathrm{P} 6230007$ \\
\hline $\mathrm{X}-04-156$ & Looking upstream from culvert crossing & 270 & X-04-156_P6230008 \\
\hline $\mathrm{X}-04-156$ & Looking downstream from culvert crossing & 90 & X-04-156_P6230009 \\
\hline$X-04-156$ & Looking at culvert inlet & 270 & X-04-156_P6230010 \\
\hline $\mathrm{X}-04-156$ & Looking at culvert outlet & 90 & X-04-156_P6230011 \\
\hline $\mathrm{X}-04-157$ & Looking upstream from bridge crossing & 210 & $\mathrm{X}-04-157 \_\mathrm{P} 6230012$ \\
\hline$X-04-157$ & Looking downstream from bridge crossing & 60 & $\mathrm{X}-04-157=\mathrm{P} 6230013$ \\
\hline
\end{tabular}


Appendix 8: List of all photographs taken showing the corresponding site number, descriptive caption, direction faced when taken (azimuth in degrees) and the unique file name for each photograph.

\begin{tabular}{|c|c|c|c|}
\hline $\begin{array}{c}\text { Site } \\
\text { Number }\end{array}$ & Photo Description & Azimuth & File Name \\
\hline $\mathrm{X}-04-158$ & Looking upstream from left bank & 350 & X-04-158_P6230014 \\
\hline $\mathrm{X}-04-158$ & Looking downstream from left bank & 90 & X-04-158_P6230015 \\
\hline $\mathrm{X}-04-159$ & Looking upstream from culvert crossing & 270 & X-04-159_P6230016 \\
\hline $\mathrm{X}-04-159$ & Looking downstream from culvert crossing & 90 & X-04-159_P6230017 \\
\hline$X-04-159$ & Looking at culvert inlet & 45 & X-04-159_P6230018 \\
\hline $\mathrm{X}-04-159$ & Looking at culvert outlet & 320 & X-04-159_P6230019 \\
\hline $\mathrm{X}-04-160$ & Looking upstream from culvert crossing & 270 & X-04-160_P6240001 \\
\hline$X-04-160$ & Looking downstream from culvert crossing & 90 & X-04-160_P6240002 \\
\hline $\mathrm{X}-04-160$ & Looking at culvert inlet & 60 & X-04-160_P6240003 \\
\hline $\mathrm{X}-04-160$ & Looking at culvert outlet & 305 & X-04-160_P6240004 \\
\hline$X-04-161$ & Looking upstream from culvert crossing & 270 & X-04-161_P6240005 \\
\hline $\mathrm{X}-04-161$ & Looking downstream from culvert crossing & 90 & $\mathrm{X}-04-161 \_\mathrm{P} 6240006$ \\
\hline $\mathrm{X}-04-161$ & Looking at culvert inlet & 120 & X-04-161_P6240007 \\
\hline$X-04-161$ & Looking at culvert outlet & 210 & $\mathrm{X}-04-161 \_\mathrm{P} 6240008$ \\
\hline $\mathrm{X}-04-162$ & Looking upstream from left bank & 305 & $\mathrm{X}-04-162 \_\mathrm{P} 6240009$ \\
\hline $\mathrm{X}-04-162$ & Looking downstream from left bank & 150 & X-04-162_P6240010 \\
\hline$X-04-162$ & Looking at perched outlets $(0.4 \mathrm{~m})$ & 285 & $\mathrm{X}-04-162 \_\mathrm{P} 6240011$ \\
\hline $\mathrm{X}-04-162$ & Looking at Longnose Dace & $\mathrm{N} / \mathrm{A}$ & $\mathrm{X}-04-162 \_\mathrm{P} 6240012$ \\
\hline $\mathrm{X}-04-163$ & Looking upstream from left bank & 360 & $\mathrm{X}-04-163 \_P 6250001$ \\
\hline $\mathrm{X}-04-163$ & Looking downstream from left bank & 90 & X-04-163_P6250002 \\
\hline $\mathrm{X}-04-164$ & Looking upstream from culvert crossing & 305 & $\mathrm{X}-04-164 \_\mathrm{P} 6250003$ \\
\hline $\mathrm{X}-04-164$ & Looking downstream from culvert crossing & 120 & X-04-164_P6250004 \\
\hline$X-04-164$ & Looking at culvert inlet & 90 & X-04-164_P6250005 \\
\hline $\mathrm{X}-04-164$ & Looking at culvert outlet & 320 & $\mathrm{X}-04-164 \_\mathrm{P} 6250006$ \\
\hline $\mathrm{X}-04-165$ & Looking upstream from culvert crossing & 305 & $\mathrm{X}-04-165 \_\mathrm{P} 6280001$ \\
\hline$X-04-165$ & Looking downstream from culvert crossing & 110 & $\mathrm{X}-04-165=\mathrm{P} 6280002$ \\
\hline $\mathrm{X}-04-165$ & Looking at culvert inlet & 10 & $\mathrm{X}-04-165 \_\mathrm{P} 6280003$ \\
\hline $\mathrm{X}-04-165$ & Looking at culvert outlet & 340 & X-04-165_P6280004 \\
\hline $\mathrm{X}-04-166$ & Looking upstream from culvert crossing & 180 & X-04-166_P6280005 \\
\hline$X-04-166$ & Looking downstream from culvert crossing & 10 & X-04-166_P6280006 \\
\hline $\mathrm{X}-04-166$ & Looking at culvert inlet & 330 & X-04-166_P6280007 \\
\hline $\mathrm{X}-04-166$ & Looking at culvert outlet & 210 & X-04-166_P6280008 \\
\hline$X-04-167$ & Looking upstream from culvert crossing & 270 & X-04-167_P6280009 \\
\hline
\end{tabular}


Appendix 8: List of all photographs taken showing the corresponding site number, descriptive caption, direction faced when taken (azimuth in degrees) and the unique file name for each photograph.

\begin{tabular}{|c|c|c|c|}
\hline $\begin{array}{c}\text { Site } \\
\text { Number }\end{array}$ & Photo Description & Azimuth & File Name \\
\hline $\mathrm{X}-04-167$ & Looking downstream from culvert crossing & 90 & X-04-167_P6280010 \\
\hline $\mathrm{X}-04-167$ & Looking at culvert inlet & 45 & X-04-167_P6280011 \\
\hline $\mathrm{X}-04-167$ & Looking at culvert outlet & 305 & $\mathrm{X}-04-167 \_\mathrm{P} 6280012$ \\
\hline $\mathrm{X}-04-168$ & Looking upstream from culvert crossing & 270 & X-04-168_P6280013 \\
\hline $\mathrm{X}-04-168$ & Looking downstream from culvert crossing & 90 & X-04-168_P6280014 \\
\hline $\mathrm{X}-04-168$ & Looking at culvert inlet & 135 & X-04-168_P6280015 \\
\hline $\mathrm{X}-04-168$ & Looking at culvert outlet & 315 & X-04-168_P6280016 \\
\hline$X-04-169$ & Looking upstream from culvert crossing & 270 & X-04-169_P6280017 \\
\hline $\mathrm{X}-04-169$ & Looking downstream from culvert crossing & 90 & X-04-169_P6280018 \\
\hline $\mathrm{X}-04-169$ & Looking at culvert inlet & 120 & X-04-169_P6280019 \\
\hline$X-04-169$ & Looking at perched outlets $0.3 \mathrm{~m}$ & 225 & X-04-169_P6280020 \\
\hline $\mathrm{X}-04-170$ & Looking upstream from left bank & 360 & X-04-170_P6290001 \\
\hline $\mathrm{X}-04-170$ & Looking downstream from left bank & 60 & X-04-170_P6290002 \\
\hline $\mathrm{X}-04-171$ & Looking upstream from culvert crossing (Fish Lake) & 270 & X-04-171_P6290003 \\
\hline $\mathrm{X}-04-171$ & Looking downstream from culvert crossing & 90 & X-04-171_P6290004 \\
\hline $\mathrm{X}-04-171$ & Looking down at submerged inlet & $\mathrm{N} / \mathrm{A}$ & X-04-171_P6290005 \\
\hline $\mathrm{X}-04-171$ & Looking at culvert outlet & 305 & X-04-171_P6290006 \\
\hline $\mathrm{X}-04-172$ & Looking upstream from culvert crossing & 180 & $\mathrm{X}-04-172$ P6290008 \\
\hline $\mathrm{X}-04-172$ & Looking downstream from culvert crossing & 360 & X-04-172_P6290009 \\
\hline $\mathrm{X}-04-172$ & Looking at culvert inlet & 45 & X-04-172_P6290010 \\
\hline $\mathrm{X}-04-172$ & Looking at culvert outlet & 225 & X-04-172_P6290011 \\
\hline $\mathrm{X}-04-172$ & Looking at $247 \mathrm{~mm}$ Northern Pike (released) & $\mathrm{N} / \mathrm{A}$ & X-04-172_P6290012 \\
\hline $\mathrm{X}-04-173$ & Looking upstream from culvert crossing & 90 & X-04-173_P6290013 \\
\hline $\mathrm{X}-04-173$ & Looking downstream from culvert crossing & 270 & X-04-173_P6290014 \\
\hline $\mathrm{X}-04-173$ & Looking at culvert inlet & 315 & X-04-173_P6290015 \\
\hline$X-04-173$ & Looking at culvert outlet & 45 & X-04-173_P6290016 \\
\hline $\mathrm{X}-04-174$ & Looking upstream from culvert crossing & 360 & X-04-174_P6290017 \\
\hline $\mathrm{X}-04-174$ & Looking downstream from culvert crossing & 180 & X-04-174_P6290018 \\
\hline $\mathrm{X}-04-174$ & Looking at culvert inlet & 180 & X-04-174_P6290019 \\
\hline$X-04-174$ & Looking at culvert outlet & 360 & X-04-174_P6290020 \\
\hline $\mathrm{X}-04-175$ & Looking upstream from culvert crossing & 180 & X-04-175_P6290021 \\
\hline $\mathrm{X}-04-175$ & Looking downstream from culvert crossing & 360 & X-04-175_P6290022 \\
\hline$X-04-175$ & Looking at culvert inlet & 45 & X-04-175_P6290023 \\
\hline
\end{tabular}


Appendix 8: List of all photographs taken showing the corresponding site number, descriptive caption, direction faced when taken (azimuth in degrees) and the unique file name for each photograph.

\begin{tabular}{|c|c|c|c|}
\hline $\begin{array}{c}\text { Site } \\
\text { Number }\end{array}$ & Photo Description & Azimuth & File Name \\
\hline $\mathrm{X}-04-175$ & Looking at culvert outlet & 120 & X-04-175_P6290024 \\
\hline $\mathrm{X}-04-176$ & Looking upstream from culvert crossing & 270 & X-04-176_P6300001 \\
\hline $\mathrm{X}-04-176$ & Looking downstream from culvert crossing & 90 & X-04-176_P6300002 \\
\hline $\mathrm{X}-04-176$ & Looking at culvert inlet & 45 & X-04-176_P6300003 \\
\hline $\mathrm{X}-04-177$ & Looking upstream from culvert crossing & 195 & X-04-177_P6300004 \\
\hline $\mathrm{X}-04-177$ & Looking downstream from culvert crossing & 30 & X-04-177_P6300005 \\
\hline $\mathrm{X}-04-177$ & Looking at culvert inlet & 45 & $\mathrm{X}-04-177=\mathrm{P} 6300006$ \\
\hline $\mathrm{X}-04-177$ & Looking at culvert outlet & 195 & X-04-177_P6300007 \\
\hline $\mathrm{X}-04-178$ & Looking upstream from culvert crossing & 190 & X-04-178_P7010001 \\
\hline $\mathrm{X}-04-178$ & Looking downstream from culvert crossing & 15 & X-04-178_P7010002 \\
\hline $\mathrm{X}-04-178$ & Looking at culvert inlet & 340 & X-04-178_P7010003 \\
\hline $\mathrm{X}-04-178$ & Looking at culvert outlet & 225 & X-04-178_P7010004 \\
\hline $\mathrm{X}-04-178$ & Looking at Common Carp @ 634, 620 and $2 @$ @ 600-650mm (released) & $\mathrm{N} / \mathrm{A}$ & X-04-178_P7010005 \\
\hline $\mathrm{X}-04-179$ & Looking upstream from culvert crossing & 200 & X-04-179_P7010006 \\
\hline $\mathrm{X}-04-179$ & Looking downstream from culvert crossing & 360 & X-04-179_P7010007 \\
\hline $\mathrm{X}-04-179$ & Looking at culvert inlet & 305 & X-04-179_P7010008 \\
\hline $\mathrm{X}-04-179$ & Looking at culvert outlet & 235 & X-04-179_P7010009 \\
\hline $\mathrm{X}-04-180$ & Looking upstream from culvert crossing & 180 & X-04-180_P7020001 \\
\hline $\mathrm{X}-04-180$ & Looking downstream from culvert crossing & 360 & X-04-180_P7020002 \\
\hline $\mathrm{X}-04-180$ & Looking down at culvert inlet & $\mathrm{N} / \mathrm{A}$ & X-04-180_P7020003 \\
\hline $\mathrm{X}-04-180$ & Looking at culvert outlet & 270 & X-04-180_P7020004 \\
\hline $\mathrm{X}-04-181$ & Looking upstream from left bank & 270 & X-04-181_P7020005 \\
\hline $\mathrm{X}-04-181$ & Looking downstream from left bank & 90 & X-04-181_P7020006 \\
\hline $\mathrm{X}-04-182$ & Looking upstream from culvert crossing & 330 & X-04-182_P7050007 \\
\hline $\mathrm{X}-04-182$ & Looking downstream from culvert crossing & 120 & X-04-182_P7050008 \\
\hline $\mathrm{X}-04-182$ & Looking at culvert inlet & 90 & X-04-182_P7050009 \\
\hline $\mathrm{X}-04-182$ & Looking at culvert outlet & 360 & $\mathrm{X}-04-182 \_\mathrm{P} 7050010$ \\
\hline $\mathrm{X}-04-183$ & Looking upstream from low level crossing & 340 & X-04-183_P7050011 \\
\hline $\mathrm{X}-04-183$ & Looking downstream from low level crossing & 160 & X-04-183_P7050012 \\
\hline $\mathrm{X}-04-183$ & Looking at cattle-impacted crossing & 110 & X-04-183_P7050013 \\
\hline $\mathrm{X}-04-184$ & Looking upstream from culvert crossing & 110 & X-04-184_P7050014 \\
\hline $\mathrm{X}-04-184$ & Looking downstream from culvert crossing & 260 & X-04-184_P7050015 \\
\hline $\mathrm{X}-04-184$ & Looking at culvert inlet & 290 & X-04-184_P7050016 \\
\hline
\end{tabular}


Appendix 8: List of all photographs taken showing the corresponding site number, descriptive caption, direction faced when taken (azimuth in degrees) and the unique file name for each photograph.

\begin{tabular}{|c|c|c|c|}
\hline $\begin{array}{c}\text { Site } \\
\text { Number }\end{array}$ & Photo Description & Azimuth & File Name \\
\hline $\mathrm{X}-04-184$ & Looking at culvert outlet & 110 & $\mathrm{X}-04-184$ P7050017 \\
\hline $\mathrm{X}-04-185$ & Looking upstream from bridge crossing & 180 & $\mathrm{X}-04-185$ P7050018 \\
\hline $\mathrm{X}-04-185$ & Looking downstream from bridge crossing & 190 & $\mathrm{X}-04-185 \_\mathrm{P} 7050019$ \\
\hline $\mathrm{X}-04-185$ & Looking at cattle impact & 215 & X-04-185_P7050020 \\
\hline $\mathrm{X}-04-185$ & Looking at 6 Northern Pike @ 199, 99, 99, 81, 84, and 93mm fork length & $\mathrm{N} / \mathrm{A}$ & $\mathrm{X}-04-185 \_\mathrm{P} 7050021$ \\
\hline $\mathrm{X}-04-185$ & Looking at 7 Yellow Perch @ 93, 71, 73, 68, 69, 66 and 68mm & $\mathrm{N} / \mathrm{A}$ & $\mathrm{X}-04-185$ P7050022 \\
\hline $\mathrm{X}-04-186$ & Looking upstream from bridge crossing & 360 & X-04-186_P7050023 \\
\hline $\mathrm{X}-04-186$ & Looking downstream from bridge crossing & 180 & $\mathrm{X}-04-186 \_\mathrm{P} 7050024$ \\
\hline $\mathrm{X}-04-187$ & Looking upstream from dam & 90 & $\mathrm{X}-04-187 \mathrm{P} 7060001$ \\
\hline $\mathrm{X}-04-187$ & Looking downstream from dam & 270 & X-04-187_P7060002 \\
\hline $\mathrm{X}-04-187$ & Looking at water level staff gauge & 120 & $\mathrm{X}-04-187+\mathrm{P} 7060003$ \\
\hline $\mathrm{X}-04-187$ & Looking at sixteen young of the year Northern Pike (131-90mm) (released) & $\mathrm{N} / \mathrm{A}$ & X-04-187_P7060004 \\
\hline $\mathrm{X}-04-187$ & Looking upstream side of dam & 305 & X-04-187_P7060005 \\
\hline $\mathrm{X}-04-187$ & Looking downstream side of dam & 120 & X-04-187_P7060006 \\
\hline $\mathrm{X}-04-188$ & Looking upstream from culvert crossing & 90 & $\mathrm{X}-04-188$ P7060007 \\
\hline $\mathrm{X}-04-188$ & Looking downstream from culvert crossing & 210 & X-04-188_P7060008 \\
\hline $\mathrm{X}-04-188$ & Looking at culvert inlet & 305 & X-04-188_P7060009 \\
\hline $\mathrm{X}-04-188$ & Looking at culvert outlet & 30 & X-04-188 P7060010 \\
\hline $\mathrm{X}-04-189$ & Looking upstream from culvert crossing & 315 & X-04-189_P7060011 \\
\hline $\mathrm{X}-04-189$ & Looking downstream from culvert crossing & 135 & X-04-189_P7060012 \\
\hline $\mathrm{X}-04-189$ & Looking at culvert inlet & 180 & X-04-189_P7060013 \\
\hline $\mathrm{X}-04-189$ & Looking at culvert outlet & 360 & X-04-189_P7060014 \\
\hline $\mathrm{X}-04-190$ & Looking upstream from culvert crossing at cattle instream & 110 & X-04-190_P7060015 \\
\hline $\mathrm{X}-04-190$ & Looking downstream from culvert crossing & 305 & X-04-190_P7060016 \\
\hline $\mathrm{X}-04-190$ & Looking at culvert inlet & 340 & X-04-190_P7060017 \\
\hline$X-04-190$ & Looking at culvert outlet & 45 & X-04-190_P7060018 \\
\hline $\mathrm{X}-04-191$ & Looking upstream from culvert crossing & 90 & $\mathrm{X}-04-191 \_\mathrm{P} 7060019$ \\
\hline $\mathrm{X}-04-191$ & Looking downstream from culvert crossing & 270 & X-04-191_P7060020 \\
\hline $\mathrm{X}-04-191$ & Looking at culvert inlet & 315 & X-04-191_P7060021 \\
\hline $\mathrm{X}-04-191$ & Looking at culvert outlet & 45 & X-04-191_P7060022 \\
\hline $\mathrm{X}-04-192$ & Looking upstream from culvert crossing & 90 & $\mathrm{X}-04-192$ P7060023 \\
\hline $\mathrm{X}-04-192$ & Looking downstream from culvert crossing & 270 & X-04-192_P7060024 \\
\hline $\mathrm{X}-04-192$ & Looking at culvert inlet & 305 & $\mathrm{X}-04-192 \_\mathrm{P} 7060025$ \\
\hline
\end{tabular}


Appendix 8: List of all photographs taken showing the corresponding site number, descriptive caption, direction faced when taken (azimuth in degrees) and the unique file name for each photograph.

\begin{tabular}{|c|c|c|c|}
\hline $\begin{array}{c}\text { Site } \\
\text { Number }\end{array}$ & Photo Description & Azimuth & File Name \\
\hline $\mathrm{X}-04-192$ & Looking at culvert outlet & 45 & X-04-192_P7060026 \\
\hline $\mathrm{X}-04-192$ & Looking at 682mm Freshwater Drum (released) & $\mathrm{N} / \mathrm{A}$ & $\mathrm{X}-04-192 \_\mathrm{P} 7060027$ \\
\hline $\mathrm{X}-04-192$ & Looking at 682mm Freshwater Drum (released) & $\mathrm{N} / \mathrm{A}$ & X-04-192_P7060028 \\
\hline $\mathrm{X}-04-192$ & Looking at Johnny Darter & $\mathrm{N} / \mathrm{A}$ & X-04-192_P7060029 \\
\hline $\mathrm{X}-04-192$ & Looking at Johnny Darter and Logperch & $\mathrm{N} / \mathrm{A}$ & X-04-192_P7060030 \\
\hline $\mathrm{X}-04-192$ & Looking at Johnny Darter and Logperch & $\mathrm{N} / \mathrm{A}$ & X-04-192_P7060031 \\
\hline $\mathrm{X}-04-193$ & Looking upstream from culvert crossing & 305 & X-04-193_P7070001 \\
\hline $\mathrm{X}-04-193$ & Looking downstream from culvert crossing & 125 & X-04-193_P7070002 \\
\hline $\mathrm{X}-04-193$ & Looking at culvert inlet & 90 & X-04-193_P7070003 \\
\hline $\mathrm{X}-04-193$ & Looking at culvert outlet & 270 & X-04-193_P7070004 \\
\hline $\mathrm{X}-04-194$ & Looking upstream from culvert crossing & 165 & X-04-194_P7070005 \\
\hline $\mathrm{X}-04-194$ & Looking downstream from culvert crossing & 360 & X-04-194_P7070006 \\
\hline $\mathrm{X}-04-194$ & Looking at culvert inlet & 360 & X-04-194_P7070007 \\
\hline$X-04-194$ & Looking at culvert outlet & 170 & X-04-194_P7070008 \\
\hline $\mathrm{X}-04-195$ & Looking upstream from culvert crossing & 180 & X-04-195_P7070009 \\
\hline $\mathrm{X}-04-195$ & Looking downstream from culvert crossing & 360 & X-04-195_P7070010 \\
\hline $\mathrm{X}-04-195$ & Looking at culvert inlet & 45 & X-04-195_P7070011 \\
\hline $\mathrm{X}-04-195$ & Looking at culvert outlet & 120 & $\mathrm{X}-04-195 \quad \mathrm{P} 7070012$ \\
\hline $\mathrm{X}-04-195$ & Looking at rotting adult bullhead found upstream & $\mathrm{N} / \mathrm{A}$ & X-04-195_P7070013 \\
\hline $\mathrm{X}-04-196$ & Looking upstream from culvert crossing & 270 & X-04-196_P7070014 \\
\hline $\mathrm{X}-04-196$ & Looking downstream from culvert crossing & 90 & X-04-196_P7070015 \\
\hline $\mathrm{X}-04-196$ & Looking at culvert inlet & 320 & X-04-196_P7070016 \\
\hline $\mathrm{X}-04-196$ & Looking at culvert outlet & 210 & X-04-196_P7070017 \\
\hline $\mathrm{X}-04-197$ & Looking upstream from culvert crossing & 20 & X-04-197_P7070018 \\
\hline $\mathrm{X}-04-197$ & Looking downstream from culvert crossing & 180 & X-04-197_P7070019 \\
\hline $\mathrm{X}-04-197$ & Looking at culvert inlet & 225 & X-04-197_P7070020 \\
\hline $\mathrm{X}-04-197$ & Looking at culvert outlet & 315 & X-04-197_P7070021 \\
\hline $\mathrm{X}-04-198$ & Looking upstream from culvert crossing & 270 & X-04-198_P7070022 \\
\hline $\mathrm{X}-04-198$ & Looking downstream from culvert crossing & 90 & X-04-198_P7070023 \\
\hline $\mathrm{X}-04-198$ & Looking at culvert inlet & 305 & X-04-198_P7070024 \\
\hline $\mathrm{X}-04-198$ & Looking at culvert outlet & 225 & X-04-198_P7070025 \\
\hline $\mathrm{X}-04-199$ & Looking upstream from left bank & 195 & X-04-199_P7080001 \\
\hline $\mathrm{X}-04-199$ & Looking downstream from left bank & 60 & X-04-199_P7080002 \\
\hline
\end{tabular}


Appendix 8: List of all photographs taken showing the corresponding site number, descriptive caption, direction faced when taken (azimuth in degrees) and the unique file name for each photograph.

\begin{tabular}{|c|c|c|c|}
\hline $\begin{array}{c}\text { Site } \\
\text { Number }\end{array}$ & Photo Description & Azimuth & File Name \\
\hline $\mathrm{X}-04-199$ & Looking at five Yellow Perch @ 84, 88, 122, 88, and 81mm FL & $\mathrm{N} / \mathrm{A}$ & X-04-199_P7080003 \\
\hline$X-04-200$ & Looking upstream from culvert crossing & 110 & $\mathrm{X}-04-200 \_\mathrm{P} 7080004$ \\
\hline$X-04-200$ & Looking downstream from culvert crossing & 340 & $\mathrm{X}-04-200 \_\mathrm{P} 7080005$ \\
\hline$X-04-200$ & Looking at culvert inlet & 360 & X-04-200_P7080006 \\
\hline$X-04-200$ & Looking at culvert outlet & 180 & X-04-200_P7080007 \\
\hline$X-04-200$ & Looking at two Common Carp @ 669 and 497mm (released) & $\mathrm{N} / \mathrm{A}$ & $\mathrm{X}-04-200 \_\mathrm{P} 7080008$ \\
\hline $\mathrm{X}-04-201$ & Looking upstream from culvert crossing & 270 & X-04-201_P7080009 \\
\hline$X-04-201$ & Looking downstream from culvert crossing & 90 & X-04-201_P7080010 \\
\hline $\mathrm{X}-04-201$ & Looking at culvert inlet & 45 & $\mathrm{X}-04-201 \_\mathrm{P} 7080011$ \\
\hline $\mathrm{X}-04-201$ & Looking at culvert outlet & 315 & X-04-201_P7080012 \\
\hline$X-04-202$ & Looking upstream from culvert crossing & 90 & $\mathrm{X}-04-202 \mathrm{P} 7080013$ \\
\hline $\mathrm{X}-04-202$ & Looking downstream from culvert crossing & 270 & X-04-202_P7080014 \\
\hline $\mathrm{X}-04-202$ & Looking at culvert inlet & 120 & X-04-202_P7080015 \\
\hline$X-04-202$ & Looking at culvert outlet & 45 & $\mathrm{X}-04-202 \_\mathrm{P} 7080016$ \\
\hline X-04-203 & Looking upstream from culvert crossing & 90 & X-04-203_P7130001 \\
\hline$X-04-203$ & Looking downstream from culvert crossing & 270 & X-04-203_P7130002 \\
\hline$X-04-203$ & Looking at culvert inlet & 210 & X-04-203_P7130003 \\
\hline$X-04-203$ & Looking at culvert outlet & 135 & $\mathrm{X}-04-203 \_\mathrm{P} 7130004$ \\
\hline$X-04-204$ & Looking upstream from culvert crossing & 330 & X-04-204_P7130005 \\
\hline$X-04-204$ & Looking downstream from culvert crossing & 140 & X-04-204_P7130006 \\
\hline X-04-204 & Looking at culvert inlet & 140 & $\mathrm{X}-04-204 \_\mathrm{P} 7130007$ \\
\hline X-04-204 & Looking at culvert outlet & 360 & X-04-204_P7130008 \\
\hline$X-04-205$ & Looking upstream from left bank & 90 & X-04-205_P7130009 \\
\hline$X-04-205$ & Looking downstream from left bank & 180 & $\mathrm{X}-04-205 \_\mathrm{P} 7130010$ \\
\hline$X-04-206$ & Looking upstream from culvert crossing & 180 & X-04-206_P7130012 \\
\hline$X-04-206$ & Looking at culvert inlet & 90 & X-04-206_P7130013 \\
\hline$X-04-206$ & Looking downstream from culvert crossing & 90 & X-04-206_P7130014 \\
\hline$X-04-206$ & Looking at culvert outlet & 270 & X-04-206_P7130015 \\
\hline X-04-207 & Looking upstream from culvert crossing & 360 & X-04-207_P7130016 \\
\hline X-04-207 & Looking downstream from culvert crossing & 180 & X-04-207_P7130017 \\
\hline $\mathrm{X}-04-207$ & Looking at culvert inlet & 135 & X-04-207_P7130018 \\
\hline$X-04-207$ & Looking at culvert outlet & 305 & X-04-207_P7130019 \\
\hline$X-04-208$ & Looking upstream from bridge crossing & 45 & X-04-208_P7140001 \\
\hline
\end{tabular}


Appendix 8: List of all photographs taken showing the corresponding site number, descriptive caption, direction faced when taken (azimuth in degrees) and the unique file name for each photograph.

\begin{tabular}{|c|c|c|c|}
\hline $\begin{array}{c}\text { Site } \\
\text { Number }\end{array}$ & Photo Description & Azimuth & File Name \\
\hline $\mathrm{X}-04-208$ & Looking downstream from bridge crossing & 200 & X-04-208_P7140002 \\
\hline$X-04-208$ & Looking downstream under bridge & 200 & X-04-208_P7140003 \\
\hline $\mathrm{X}-04-209$ & Looking upstream from bridge crossing & 45 & X-04-209_P7140004 \\
\hline $\mathrm{X}-04-209$ & Looking downstream from bridge crossing & 215 & X-04-209_P7140005 \\
\hline$X-04-209$ & Looking at 304mm TL Burbot (released) & $\mathrm{N} / \mathrm{A}$ & X-04-209_P7140006 \\
\hline $\mathrm{X}-04-210$ & Looking downstream from bridge crossing & $\mathrm{N} / \mathrm{A}$ & X-04-210_P7140007 \\
\hline $\mathrm{X}-04-210$ & Looking upstream from bridge crossing & 360 & X-04-210_P7140008 \\
\hline$X-04-211$ & Looking upstream from culvert crossing & 180 & X-04-211_P7140009 \\
\hline $\mathrm{X}-04-211$ & Looking downstream from culvert crossing & 190 & $\mathrm{X}-04-211 \_\mathrm{P} 7140010$ \\
\hline $\mathrm{X}-04-211$ & Looking at culvert inlet & 55 & $\mathrm{X}-04-211 \_\mathrm{P} 7140011$ \\
\hline$X-04-211$ & Looking at culvert outlet & $\mathrm{N} / \mathrm{A}$ & $\mathrm{X}-04-211 \_\mathrm{P} 7140012$ \\
\hline $\mathrm{X}-04-212$ & Looking upstream from culvert crossing & 305 & $\mathrm{X}-04-212 \_\mathrm{P} 7150013$ \\
\hline $\mathrm{X}-04-212$ & Looking downstream from culvert crossing & 90 & $\mathrm{X}-04-212 \_\mathrm{P} 7150014$ \\
\hline$X-04-212$ & Looking at culvert inlet & 60 & $\mathrm{X}-04-212 \_\mathrm{P} 7150015$ \\
\hline $\mathrm{X}-04-212$ & Looking at culvert outlet & 45 & $\mathrm{X}-04-212 \_\mathrm{P} 7150016$ \\
\hline $\mathrm{X}-04-213$ & Looking upstream from culvert crossing & 270 & X-04-213_P7150017 \\
\hline$X-04-213$ & Looking downstream from culvert crossing & 90 & X-04-213_P7150018 \\
\hline $\mathrm{X}-04-213$ & Looking at culvert inlet & 45 & $\mathrm{X}-04-213 \_\mathrm{P} 7150019$ \\
\hline $\mathrm{X}-04-213$ & Looking at culvert outlet & 305 & $\mathrm{X}-04-213 \_\mathrm{P} 7150020$ \\
\hline$X-04-214$ & Looking upstream from culvert crossing & 27 & $\mathrm{X}-04-214 \_\mathrm{P} 7150021$ \\
\hline $\mathrm{X}-04-214$ & Looking downstream from culvert crossing & 90 & $\mathrm{X}-04-214 \_\mathrm{P} 7150022$ \\
\hline $\mathrm{X}-04-214$ & Looking at culvert inlet & 125 & X-04-214_P7150023 \\
\hline$X-04-214$ & Looking at culvert outlet & 325 & $\mathrm{X}-04-214 \_\mathrm{P} 7150024$ \\
\hline$X-04-214$ & Looking upstream of Mandryk Drain & 180 & X-04-214_P7150025 \\
\hline $\mathrm{X}-04-215$ & Looking upstream from culvert crossing & 270 & $\mathrm{X}-04-215 \_\mathrm{P} 7150026$ \\
\hline$X-04-215$ & Looking downstream from culvert crossing & 90 & $\mathrm{X}-04-215 \_\mathrm{P} 7150027$ \\
\hline $\mathrm{X}-04-215$ & Looking at culvert inlet & 45 & $\mathrm{X}-04-215 \_\mathrm{P} 7150028$ \\
\hline $\mathrm{X}-04-215$ & Looking at culvert outlet & 305 & X-04-215_P7150029 \\
\hline $\mathrm{X}-04-216$ & Looking upstream from left bank & 270 & X-04-216_P7160001 \\
\hline$X-04-216$ & Looking downstream from left bank & 360 & X-04-216_P7160002 \\
\hline $\mathrm{X}-04-217$ & Looking upstream from left bank & 270 & X-04-217_P7160003 \\
\hline $\mathrm{X}-04-217$ & Looking downstream from left bank & 90 & X-04-217_P7160004 \\
\hline$X-04-218$ & Looking upstream from culvert crossing & 225 & X-04-218_P7160006 \\
\hline
\end{tabular}


Appendix 8: List of all photographs taken showing the corresponding site number, descriptive caption, direction faced when taken (azimuth in degrees) and the unique file name for each photograph.

\begin{tabular}{|c|c|c|c|}
\hline $\begin{array}{l}\text { Site } \\
\text { Number }\end{array}$ & Photo Description & Azimuth & File Name \\
\hline $\mathrm{X}-04-218$ & Looking downstream from culvert crossing & 90 & X-04-218_P7160007 \\
\hline$X-04-218$ & Looking at culvert inlet & 135 & $\mathrm{X}-04-218+\mathrm{P} 7160008$ \\
\hline $\mathrm{X}-04-218$ & Looking at culvert outlet & 225 & X-04-218_P7160009 \\
\hline $\mathrm{X}-04-219$ & Looking upstream from culvert crossing & 270 & X-04-219_P7160010 \\
\hline$X-04-219$ & Looking downstream from culvert crossing & 90 & X-04-219_P7160011 \\
\hline $\mathrm{X}-04-219$ & Looking at culvert inlet & 45 & $\mathrm{X}-04-219 \_\mathrm{P} 7160012$ \\
\hline $\mathrm{X}-04-219$ & Looking at culvert outlet & 215 & X-04-219_P7160013 \\
\hline$X-04-220$ & Looking upstream from left bank of Washow Bay Creek & 270 & X-04-220_P7160014 \\
\hline $\mathrm{X}-04-221$ & Looking upstream from left bank & 30 & $\mathrm{X}-04-221 \_\mathrm{P} 7160015$ \\
\hline $\mathrm{X}-04-221$ & Looking downstream from left bank & 150 & $\mathrm{X}-04-221 \_\mathrm{P} 7160016$ \\
\hline $\mathrm{X}-04-221$ & $\begin{array}{l}\text { Looking at } 141 \mathrm{~mm} \text { White Sucker, 147mm Rock Bass and 163mm Common Carp } \\
\text { (released) }\end{array}$ & $\mathrm{N} / \mathrm{A}$ & X-04-221_P7160017 \\
\hline $\mathrm{X}-04-221$ & Looking at four Rock Bass at $68,70,62$ and $61 \mathrm{~mm}$ (released) & $\mathrm{N} / \mathrm{A}$ & $\mathrm{X}-04-221 \_\mathrm{P} 7160018$ \\
\hline $\mathrm{X}-04-222$ & Looking upstream from culvert crossing & 345 & $\mathrm{X}-04-222 \_\mathrm{P} 7190001$ \\
\hline$X-04-222$ & Looking downstream from culvert crossing & 165 & $\mathrm{X}-04-222 \_\mathrm{P} 7190002$ \\
\hline $\mathrm{X}-04-222$ & Looking at culvert inlet & 90 & $\mathrm{X}-04-222 \_\mathrm{P} 7190003$ \\
\hline $\mathrm{X}-04-222$ & Looking at culvert outlet & 360 & $\mathrm{X}-04-222 \_\mathrm{P} 7190004$ \\
\hline $\mathrm{X}-04-222$ & Looking at 2 White Sucker @ 142 and 143mm (released) & $\mathrm{N} / \mathrm{A}$ & $\mathrm{X}-04-222 \_\mathrm{P} 7190005$ \\
\hline $\mathrm{X}-04-223$ & Looking upstream from culvert crossing & 45 & X-04-223_P7200001 \\
\hline $\mathrm{X}-04-223$ & Looking downstream from culvert crossing & 205 & $\mathrm{X}-04-223 \_\mathrm{P} 7200002$ \\
\hline$X-04-223$ & Looking at culvert inlet & 245 & $\mathrm{X}-04-223 \_\mathrm{P} 7200003$ \\
\hline $\mathrm{X}-04-223$ & Looking at culvert outlet & 315 & X-04-223_P7200004 \\
\hline $\mathrm{X}-04-223$ & Looking at $139 \mathrm{~mm}$ White Sucker (released) & $\mathrm{N} / \mathrm{A}$ & X-04-223_P7200005 \\
\hline$X-04-224$ & Looking upstream from culvert crossing & 315 & X-04-224_P7200006 \\
\hline $\mathrm{X}-04-224$ & Looking downstream from culvert crossing & 135 & $\mathrm{X}-04-224 \_\mathrm{P} 7200007$ \\
\hline $\mathrm{X}-04-224$ & Looking at culvert inlet & 25 & X-04-224_P7200008 \\
\hline$X-04-224$ & Looking at culvert outlet & 270 & X-04-224_P7200009 \\
\hline$X-04-225$ & Looking upstream from middle of riffle & 75 & $\mathrm{X}-04-225 \_\mathrm{P} 7200010$ \\
\hline $\mathrm{X}-04-225$ & Looking downstream from middle of riffle & 195 & $\mathrm{X}-04-225 \_\mathrm{P} 7200011$ \\
\hline $\mathrm{X}-04-226$ & Looking upstream from culvert crossing & 255 & X-04-226_P7200012 \\
\hline$X-04-226$ & Looking downstream from culvert crossing & 60 & X-04-226_P7200013 \\
\hline $\mathrm{X}-04-226$ & Looking at culvert inlet & 120 & X-04-226_P7200014 \\
\hline $\mathrm{X}-04-226$ & Looking at culvert outlet & 315 & X-04-226_P7200015 \\
\hline
\end{tabular}


Appendix 8: List of all photographs taken showing the corresponding site number, descriptive caption, direction faced when taken (azimuth in degrees) and the unique file name for each photograph.

\begin{tabular}{|c|c|c|c|}
\hline $\begin{array}{c}\text { Site } \\
\text { Number }\end{array}$ & Photo Description & Azimuth & File Name \\
\hline $\mathrm{X}-04-227$ & Looking upstream from culvert crossing & 30 & $\mathrm{X}-04-227$ P7200016 \\
\hline$X-04-227$ & Looking downstream from culvert crossing & 180 & X-04-227_P7200017 \\
\hline $\mathrm{X}-04-227$ & Looking at culvert inlet & 150 & $\mathrm{X}-04-227 \_\mathrm{P} 7200018$ \\
\hline $\mathrm{X}-04-227$ & Looking at culvert outlet & 45 & X-04-227_P7200019 \\
\hline$X-04-228$ & Looking downstream from culvert crossing & 360 & $\mathrm{X}-04-228 \_\mathrm{P} 7210001$ \\
\hline $\mathrm{X}-04-228$ & Looking upstream from culvert crossing & 100 & X-04-228_P7210002 \\
\hline $\mathrm{X}-04-228$ & Looking at culvert outlet & 120 & X-04-228_P7210003 \\
\hline$X-04-228$ & Looking at culvert inlet & 45 & $\mathrm{X}-04-228 \_\mathrm{P} 7210004$ \\
\hline $\mathrm{X}-04-228$ & Looking at beaver dam obstructing middle corrugated metal pipe & 90 & $\mathrm{X}-04-228 \quad \mathrm{P} 7210005$ \\
\hline $\mathrm{X}-04-229$ & Looking upstream from bridge crossing & 180 & $\mathrm{X}-04-229 \_\mathrm{P} 7210006$ \\
\hline$X-04-229$ & Looking downstream from bridge crossing & 325 & $\mathrm{X}-04-229 \_\mathrm{P} 7210007$ \\
\hline $\mathrm{X}-04-230$ & Looking upstream from culvert crossing & 345 & X-04-230_P7210008 \\
\hline $\mathrm{X}-04-230$ & Looking downstream from culvert crossing & 180 & X-04-230_P7210009 \\
\hline $\mathrm{X}-04-230$ & Looking at culvert inlet & 125 & $\mathrm{X}-04-230 \_\mathrm{P} 7210010$ \\
\hline $\mathrm{X}-04-230$ & Looking at culvert outlet & 295 & $\mathrm{X}-04-230 \_\mathrm{P} 7210011$ \\
\hline $\mathrm{X}-04-231$ & Looking upstream from culvert crossing & 90 & X-04-231_P7210012 \\
\hline $\mathrm{X}-04-231$ & Looking downstream from culvert crossing & 270 & X-04-231_P7210013 \\
\hline $\mathrm{X}-04-231$ & Looking down at culvert inlet (2/3 full) & $\mathrm{N} / \mathrm{A}$ & $\mathrm{X}-04-231 \quad \mathrm{P} 7210014$ \\
\hline $\mathrm{X}-04-231$ & Looking down at culvert outlet & $\mathrm{N} / \mathrm{A}$ & $\mathrm{X}-04-231 \_\mathrm{P} 7210015$ \\
\hline $\mathrm{X}-04-232$ & Looking upstream from culvert crossing & 360 & $\mathrm{X}-04-232 \_\mathrm{P} 7220001$ \\
\hline $\mathrm{X}-04-232$ & Looking downstream from culvert crossing & 180 & X-04-232 P7220002 \\
\hline $\mathrm{X}-04-232$ & Looking at culvert inlet & 135 & X-04-232_P7220003 \\
\hline$X-04-232$ & Looking at culvert outlet & 45 & $\mathrm{X}-04-232 \_\mathrm{P} 7220004$ \\
\hline $\mathrm{X}-04-232$ & Looking at culvert with beaver deceiver & 360 & $\mathrm{X}-04-232 \_\mathrm{P} 7220005$ \\
\hline $\mathrm{X}-04-233$ & Looking upstream from bridge crossing & 40 & X-04-233_P7220006 \\
\hline$X-04-233$ & Looking downstream from bridge crossing & 270 & X-04-233_P7220007 \\
\hline $\mathrm{X}-04-234$ & Looking upstream from right corrugated metal pipe & 90 & $\mathrm{X}-04-234 \_\mathrm{P} 7220008$ \\
\hline $\mathrm{X}-04-234$ & Looking upstream from left corrugated metal pipe & 110 & X-04-234_P7220009 \\
\hline $\mathrm{X}-04-234$ & Looking downstream from right corrugated metal pipe & 280 & X-04-234_P7220010 \\
\hline$X-04-234$ & Looking downstream from left corrugated metal pipe & 260 & X-04-234_P7220011 \\
\hline $\mathrm{X}-04-234$ & Looking at culvert inlet & $\mathrm{N} / \mathrm{A}$ & $\mathrm{X}-04-234 \_\mathrm{P} 7220012$ \\
\hline $\mathrm{X}-04-234$ & Looking at culvert inlet & $\mathrm{N} / \mathrm{A}$ & X-04-234_P7220013 \\
\hline $\mathrm{X}-04-234$ & Looking at culvert outlet & $\mathrm{N} / \mathrm{A}$ & X-04-234_P7220014 \\
\hline
\end{tabular}


Appendix 8: List of all photographs taken showing the corresponding site number, descriptive caption, direction faced when taken (azimuth in degrees) and the unique file name for each photograph.

\begin{tabular}{|c|c|c|c|}
\hline $\begin{array}{c}\text { Site } \\
\text { Number }\end{array}$ & Photo Description & Azimuth & File Name \\
\hline $\mathrm{X}-04-234$ & Looking at culvert outlet & $\mathrm{N} / \mathrm{A}$ & X-04-234_P7220015 \\
\hline $\mathrm{X}-04-235$ & Looking upstream from culvert crossing & 135 & X-04-235 P7220016 \\
\hline $\mathrm{X}-04-235$ & Looking downstream from culvert crossing & 270 & $\mathrm{X}-04-235 \_\mathrm{P} 7220017$ \\
\hline $\mathrm{X}-04-235$ & Looking at culvert inlet & 45 & X-04-235_P7220018 \\
\hline $\mathrm{X}-04-235$ & Looking at culvert outlet & 315 & X-04-235_P7220019 \\
\hline $\mathrm{X}-04-236$ & Looking upstream from culvert crossing & 180 & X-04-236_P7220020 \\
\hline $\mathrm{X}-04-236$ & Looking downstream from culvert crossing & 360 & X-04-236_P7220021 \\
\hline$X-04-236$ & Looking at culvert inlet & 45 & X-04-236_P7220022 \\
\hline $\mathrm{X}-04-236$ & Looking at culvert outlet & 90 & X-04-236_P7220023 \\
\hline $\mathrm{X}-04-237$ & Looking upstream from bridge crossing & 270 & $\mathrm{X}-04-237 \_\mathrm{P} 7220024$ \\
\hline$X-04-237$ & Looking downstream from bridge crossing & 90 & $\mathrm{X}-04-237$ P7220025 \\
\hline $\mathrm{X}-04-238$ & Looking upstream from left bank & 345 & X-04-238_P7220026 \\
\hline $\mathrm{X}-04-238$ & Looking downstream from left bank & 150 & X-04-238_P7220027 \\
\hline$X-04-238$ & Looking upstream from culvert crossing & 345 & X-04-238_P7220028 \\
\hline $\mathrm{X}-04-238$ & Looking downstream from culvert crossing & 150 & X-04-238_P7220029 \\
\hline $\mathrm{X}-04-238$ & Looking at culvert inlet & 150 & X-04-238_P7220030 \\
\hline$X-04-238$ & Looking at culvert outlet & 345 & X-04-238_P7220031 \\
\hline $\mathrm{X}-04-239$ & Looking upstream from right bank & 30 & $\mathrm{X}-04-239 \_\mathrm{P} 7220032$ \\
\hline $\mathrm{X}-04-239$ & Looking downstream from right bank & 180 & X-04-239_P7220033 \\
\hline$X-04-239$ & Looking at three Burbot 273,283 , and $304 \mathrm{~mm}$ TL (released) & $\mathrm{N} / \mathrm{A}$ & X-04-239_P7220034 \\
\hline $\mathrm{X}-04-240$ & Looking upstream from culvert crossing & 300 & X-04-240_P7260001 \\
\hline $\mathrm{X}-04-240$ & Looking downstream from culvert crossing & 120 & $\mathrm{X}-04-240 \_\mathrm{P} 7260002$ \\
\hline$X-04-240$ & Looking at culvert inlet & 135 & $\mathrm{X}-04-240 \_\mathrm{P} 7260003$ \\
\hline $\mathrm{X}-04-240$ & Looking at culvert outlet & 325 & X-04-240_P7260004 \\
\hline $\mathrm{X}-04-241$ & Looking upstream from bridge crossing & 350 & X-04-241_P7260005 \\
\hline$X-04-241$ & Looking downstream from bridge crossing & 135 & $\mathrm{X}-04-241 \_\mathrm{P} 7260006$ \\
\hline $\mathrm{X}-04-241$ & Looking at bridge and dirt road & $\mathrm{N} / \mathrm{A}$ & $\mathrm{X}-04-241 \_\mathrm{P} 7260007$ \\
\hline $\mathrm{X}-04-242$ & Looking upstream from culvert crossing & 315 & X-04-242_P7260008 \\
\hline $\mathrm{X}-04-242$ & Looking downstream from culvert crossing & 90 & X-04-242_P7260009 \\
\hline$X-04-242$ & Looking at culvert inlet & 45 & $\mathrm{X}-04-242 \_\mathrm{P} 7260010$ \\
\hline $\mathrm{X}-04-242$ & Looking at culvert outlet & 360 & X-04-242_P7260011 \\
\hline $\mathrm{X}-04-243$ & Looking upstream from culvert crossing & 315 & X-04-243_P7260012 \\
\hline$X-04-243$ & Looking downstream from culvert crossing & 135 & X-04-243_P7260013 \\
\hline
\end{tabular}


Appendix 8: List of all photographs taken showing the corresponding site number, descriptive caption, direction faced when taken (azimuth in degrees) and the unique file name for each photograph.

\begin{tabular}{|c|c|c|c|}
\hline $\begin{array}{c}\text { Site } \\
\text { Number }\end{array}$ & Photo Description & Azimuth & File Name \\
\hline $\mathrm{X}-04-243$ & Looking at culvert inlet & 135 & $\mathrm{X}-04-243 \quad \mathrm{P} 7260014$ \\
\hline$X-04-243$ & Looking at culvert outlet & 315 & $\mathrm{X}-04-243 \mathrm{P} 7260015$ \\
\hline $\mathrm{X}-04-244$ & Looking upstream from bridge crossing & 360 & X-04-244_P7260016 \\
\hline $\mathrm{X}-04-244$ & Looking downstream from bridge crossing Looking at dam & 190 & X-04-244_P7260017 \\
\hline $\mathrm{X}-04-244$ & Looking down at meter station & 90 & X-04-244_P7260018 \\
\hline $\mathrm{X}-04-244$ & Looking at log jam under bridge & 255 & $\mathrm{X}-04-244$ P7260019 \\
\hline $\mathrm{X}-04-245$ & Looking upstream from bridge crossing looking at dam & 90 & X-04-245_P7260020 \\
\hline $\mathrm{X}-04-245$ & Looking downstream from bridge crossing & 230 & $\mathrm{X}-04-245 \_P 7260021$ \\
\hline $\mathrm{X}-04-245$ & Looking upstream at dam & 90 & $\mathrm{X}-04-245 \mathrm{P} 7260022$ \\
\hline $\mathrm{X}-04-245$ & Looking down at meter station & 180 & $\mathrm{X}-04-245 \mathrm{P} 7260023$ \\
\hline$X-04-246$ & Looking downstream from culvert crossing & 180 & $\mathrm{X}-04-246 \quad \mathrm{P} 7260024$ \\
\hline $\mathrm{X}-04-246$ & Looking at culvert outlet & 360 & X-04-246_P7260025 \\
\hline $\mathrm{X}-04-246$ & Looking at grated culvert inlet $150 \mathrm{~m}$ downstream of culvert crossing & 225 & X-04-246_P7260026 \\
\hline$X-04-246$ & Looking upstream from culvert crossing & 15 & $\mathrm{X}-04-246$ P7260027 \\
\hline $\mathrm{X}-04-246$ & Looking at culvert inlet & 175 & $\mathrm{X}-04-246 \_\mathrm{P} 7260028$ \\
\hline $\mathrm{X}-04-247$ & Looking upstream from culvert crossing & 270 & X-04-247_P7280029 \\
\hline$X-04-247$ & Looking downstream from culvert crossing & 90 & $\mathrm{X}-04-247 \mathrm{P} 7280030$ \\
\hline $\mathrm{X}-04-247$ & Looking at culvert inlet & 45 & $\mathrm{X}-04-247$ P7280031 \\
\hline $\mathrm{X}-04-247$ & Looking at culvert outlet & 360 & X-04-247_P7280032 \\
\hline $\mathrm{X}-04-248$ & Looking upstream from culvert crossing & 290 & $\mathrm{X}-04-248 \_P 7280033$ \\
\hline$X-04-248$ & Looking downstream from culvert crossing & 110 & $\mathrm{X}-04-248 \quad \mathrm{P} 7280034$ \\
\hline $\mathrm{X}-04-248$ & Looking at culvert inlet & 20 & X-04-248_P7280035 \\
\hline$X-04-248$ & Looking at culvert outlet & 340 & $\mathrm{X}-04-248+\mathrm{P} 7280036$ \\
\hline $\mathrm{X}-04-249$ & Looking upstream from bridge crossing & 235 & X-04-249_P7280037 \\
\hline $\mathrm{X}-04-249$ & Looking downstream from bridge crossing & 45 & $\mathrm{X}-04-249 \mathrm{P} 7280038$ \\
\hline$X-04-250$ & Looking upstream from culvert crossing & 270 & $\mathrm{X}-04-250 \mathrm{P} 7280039$ \\
\hline$X-04-250$ & Looking downstream from culvert crossing & 60 & $\mathrm{X}-04-250 \_\mathrm{P} 7280040$ \\
\hline $\mathrm{X}-04-250$ & Looking at culvert inlet & 360 & X-04-250_P7280041 \\
\hline$X-04-250$ & Looking at culvert outlet & 310 & $\mathrm{X}-04-250 \quad \mathrm{P} 7280042$ \\
\hline$X-04-251$ & Looking at dead White Sucker found at outflow & N/A & $\mathrm{X}-04-251 \_\mathrm{P} 7280043$ \\
\hline $\mathrm{X}-04-251$ & Looking downstream from pump outlet & 170 & X-04-251_P7280044 \\
\hline $\mathrm{X}-04-251$ & Looking at pump outlet/inlet & 350 & X-04-251_P7280045 \\
\hline$X-04-251$ & Looking at pump equipment & 170 & $\mathrm{X}-04-251 \mathrm{P} 7280046$ \\
\hline
\end{tabular}


Appendix 8: List of all photographs taken showing the corresponding site number, descriptive caption, direction faced when taken (azimuth in degrees) and the unique file name for each photograph.

\begin{tabular}{|c|c|c|c|}
\hline $\begin{array}{c}\text { Site } \\
\text { Number }\end{array}$ & Photo Description & Azimuth & File Name \\
\hline $\mathrm{X}-04-252$ & Looking downstream from pump outlet & 160 & $\mathrm{X}-04-252$ P7280047 \\
\hline$X-04-252$ & Looking at pump outlet/inlet & 270 & $\mathrm{X}-04-252 \_\mathrm{P} 7280048$ \\
\hline $\mathrm{X}-04-252$ & Looking at pump equipment & 180 & $\mathrm{X}-04-252 \_\mathrm{P} 7280049$ \\
\hline$X-04-253$ & Looking upstream at gradient control riffle from right bank & 45 & X-04-253_P7300001 \\
\hline$X-04-253$ & Looking upstream from riffle on right bank & 90 & $\mathrm{X}-04-253$ P7300002 \\
\hline $\mathrm{X}-04-253$ & Looking downstream from riffle on right bank & 270 & $\mathrm{X}-04-253+\mathrm{P} 7300003$ \\
\hline $\mathrm{X}-04-253$ & Looking at Sauger @ 295, 242 and 203mm fork length (released) & $\mathrm{N} / \mathrm{A}$ & $\mathrm{X}-04-253+P 7300004$ \\
\hline$X-04-253$ & Photo of Shorthead Redhorse $360 \mathrm{~mm}$ before release & $\mathrm{N} / \mathrm{A}$ & X-04-253_P7300005 \\
\hline $\mathrm{X}-04-253$ & Photo of $360 \mathrm{~mm}$ Shorthead Redhorse before release & $\mathrm{N} / \mathrm{A}$ & $\mathrm{X}-04-253$ P7300006 \\
\hline$X-04-253$ & Photo of $288 \mathrm{~mm}$ White Sucker before release & $\mathrm{N} / \mathrm{A}$ & $\mathrm{X}-04-253 \_\mathrm{P} 7300007$ \\
\hline$X-04-254$ & Looking upstream from left bank & 110 & $\mathrm{X}-04-254 \_\mathrm{P} 7300008$ \\
\hline $\mathrm{X}-04-254$ & Looking downstream from left bank & 250 & X-04-254_P7300009 \\
\hline $\mathrm{X}-04-254$ & Looking at gauging control station & 180 & $\mathrm{X}-04-254 \mathrm{P} 7300010$ \\
\hline$X-04-255$ & Looking upstream from riffle on left bank & 100 & $\mathrm{X}-04-255 \mathrm{P} 7300011$ \\
\hline $\mathrm{X}-04-255$ & Looking downstream from riffle on left bank & 280 & $\mathrm{X}-04-255 \_\mathrm{P} 7300012$ \\
\hline$X-04-256$ & Looking upstream at siphon overflow & 135 & X-04-256_P8030001 \\
\hline$X-04-256$ & Looking downstream at siphon outlet & 315 & $\mathrm{X}-04-256 \mathrm{P} 8030002$ \\
\hline $\mathrm{X}-04-256$ & Looking at siphon outlet & 225 & $\mathrm{X}-04-256 \mathrm{P} 8030003$ \\
\hline $\mathrm{X}-04-256$ & Looking upstream from siphon inlet & 135 & $\mathrm{X}-04-256 \_P 8030004$ \\
\hline$X-04-256$ & Looking at siphon inlet & 135 & $\mathrm{X}-04-256 \_P 8030005$ \\
\hline $\mathrm{X}-04-256$ & Looking at left siphon inlet & 135 & $\mathrm{X}-04-256 \mathrm{P} 8030006$ \\
\hline $\mathrm{X}-04-256$ & Looking at right siphon inlet & 135 & $\mathrm{X}-04-256 \_P 8030007$ \\
\hline$X-04-256$ & Looking downstream from siphon overflow & 315 & $\mathrm{X}-04-256 \_\mathrm{P} 8030008$ \\
\hline $\mathrm{X}-04-256$ & Looking at siphon overflow outlets & 180 & $\mathrm{X}-04-256+P 8030009$ \\
\hline $\mathrm{X}-04-256$ & Looking at dead Walleye or Sauger at siphon overflow outlet & $\mathrm{N} / \mathrm{A}$ & $\mathrm{X}-04-256 \mathrm{P} 8030010$ \\
\hline$X-04-256$ & Photo of $333 \mathrm{~mm}$ Channel Catfish before release & $\mathrm{N} / \mathrm{A}$ & $\mathrm{X}-04-256 \_P 8030011$ \\
\hline $\mathrm{X}-04-256$ & Photo of Walleye & $\mathrm{N} / \mathrm{A}$ & $\mathrm{X}-04-256 \_P 8030012$ \\
\hline $\mathrm{X}-04-256$ & Photo of Troutperch & N/A & $\mathrm{X}-04-256$ P8030014 \\
\hline$X-04-256$ & Photo of two Goldeye & $\mathrm{N} / \mathrm{A}$ & $\mathrm{X}-04-256 \mathrm{P} 8030015$ \\
\hline$X-04-256$ & Photo of two Goldeye & N/A & $\mathrm{X}-04-256 \mathrm{P} 8030016$ \\
\hline $\mathrm{X}-04-257$ & Looking upstream from bridge crossing & 80 & $\mathrm{X}-04-257 \_\mathrm{P} 8030017$ \\
\hline $\mathrm{X}-04-257$ & Looking downstream from bridge crossing & 260 & $\mathrm{X}-04-257 \_\mathrm{P} 8030018$ \\
\hline$X-04-258$ & Looking upstream from bridge crossing & 270 & $\mathrm{X}-04-258 \mathrm{P} 8040001$ \\
\hline
\end{tabular}


Appendix 8: List of all photographs taken showing the corresponding site number, descriptive caption, direction faced when taken (azimuth in degrees) and the unique file name for each photograph.

\begin{tabular}{|c|c|c|c|}
\hline $\begin{array}{c}\text { Site } \\
\text { Number }\end{array}$ & Photo Description & Azimuth & File Name \\
\hline $\mathrm{X}-04-258$ & Looking downstream from bridge crossing & 90 & X-04-258_P8040002 \\
\hline $\mathrm{X}-04-259$ & Looking upstream from left bank at flooded low-level crossing & 180 & X-04-259_P8050001 \\
\hline $\mathrm{X}-04-259$ & Looking downstream from left bank at flooded low-level crossing & 360 & X-04-259_P8050002 \\
\hline $\mathrm{X}-04-259$ & Looking at flooded low level crossing from left bank & 270 & X-04-259_P8050003 \\
\hline$X-04-259$ & Looking at Denil fishway & 360 & X-04-259_P8050004 \\
\hline $\mathrm{X}-04-259$ & Looking at Denil fishway from left bank & 270 & X-04-259_P8050005 \\
\hline $\mathrm{X}-04-260$ & Looking upstream from low-level crossing & 235 & X-04-260_P8050006 \\
\hline$X-04-260$ & Looking downstream from low-level crossing & 90 & X-04-260_P8050007 \\
\hline $\mathrm{X}-04-260$ & Looking at culvert inlets & 180 & X-04-260_P8050008 \\
\hline $\mathrm{X}-04-260$ & Looking at culvert outlets & 180 & X-04-260_P8050009 \\
\hline $\mathrm{X}-04-261$ & Looking upstream from low-level crossing & 45 & X-04-261_P8060001 \\
\hline $\mathrm{X}-04-261$ & Looking downstream from low-level crossing & 235 & X-04-261_P8060002 \\
\hline $\mathrm{X}-04-261$ & Looking at culvert inlets & 350 & X-04-261_P8060003 \\
\hline$X-04-261$ & Looking at culvert outlets & 350 & X-04-261_P8060004 \\
\hline $\mathrm{X}-04-262$ & Looking upstream from riffle & 270 & X-04-262_P8060005 \\
\hline $\mathrm{X}-04-262$ & Looking downstream from riffle & 65 & X-04-262_P8060006 \\
\hline $\mathrm{X}-04-262$ & Looking at riffle & 360 & X-04-262_P8060007 \\
\hline $\mathrm{X}-04-262$ & Photo of Logperch $104 \mathrm{~mm}, 103 \mathrm{~mm}, 101 \mathrm{~mm}, 108 \mathrm{~mm}$ before release & $\mathrm{N} / \mathrm{A}$ & $\mathrm{X}-04-262 \_\mathrm{P} 8060008$ \\
\hline $\mathrm{X}-04-263$ & Looking upstream from dam & 170 & X-04-263_P8060011 \\
\hline $\mathrm{X}-04-263$ & Looking downstream from dam & 350 & X-04-263_P8060012 \\
\hline $\mathrm{X}-04-263$ & Looking at dam from left bank & 225 & X-04-263_P8060013 \\
\hline D-05-001 & Looking upstream from bridge crossing & 270 & D-05-001_P4050001 \\
\hline D-05-001 & Looking downstream from bridge crossing & 70 & D-05-001_P4050002 \\
\hline D-05-001 & Looking upstream from bridge crossing & 190 & D-05-001_P4050003 \\
\hline D-05-001 & Looking south under PTH 20 bridge & 180 & D-05-001_P4050004 \\
\hline D-05-001 & Looking downstream from bridge crossing & 30 & D-05-001_P4050005 \\
\hline D-05-001 & Looking downstream from bridge crossing & 30 & D-05-001_P4050006 \\
\hline D-05-002 & Looking upstream from above bridge crossing & 180 & D-05-002_P4050007 \\
\hline D-05-002 & Looking east under bridge & 100 & D-05-002_P4050008 \\
\hline D-05-002 & Looking downstream from bridge crossing & 90 & D-05-002_P4050009 \\
\hline D-05-002 & Looking northwest under bridge & 290 & D-05-002_P4050010 \\
\hline D-05-003 & Looking upstream from culvert crossing & 270 & D-05-003_P4050011 \\
\hline D-05-003 & Looking downstream from culvert crossing at Lake Dauphin & 90 & D-05-003_P4050012 \\
\hline
\end{tabular}


Appendix 8: List of all photographs taken showing the corresponding site number, descriptive caption, direction faced when taken (azimuth in degrees) and the unique file name for each photograph.

\begin{tabular}{|c|c|c|c|}
\hline $\begin{array}{c}\text { Site } \\
\text { Number }\end{array}$ & Photo Description & Azimuth & File Name \\
\hline D-05-004 & White Sucker observed in drain & $\mathrm{N} / \mathrm{A}$ & D-05-004_P4140006 \\
\hline D-05-004 & Looking upstream from culvert crossing & 270 & D-05-004_P4140007 \\
\hline D-05-004 & Looking downstream from culvert crossing & 90 & D-05-004_P4140008 \\
\hline D-05-004 & Looking at culvert outlets & 180 & D-05-004_P4140009 \\
\hline D-05-004 & Looking at culvert inlets & 180 & D-05-004_P4140010 \\
\hline D-05-005 & Looking upstream from culvert crossing & 270 & $\mathrm{D}-05-005 \mathrm{P} 4140011$ \\
\hline D-05-005 & Looking downstream from culvert crossing & 90 & D-05-005_P4140012 \\
\hline D-05-005 & Looking at culvert outlets & 0 & D-05-005_P4140013 \\
\hline D-05-005 & Looking at culvert inlets & 0 & $\mathrm{D}-05-005 \quad \mathrm{P} 4140014$ \\
\hline D-05-006 & Looking upstream from concrete culvert crossing & 270 & $\mathrm{D}-05-006 \mathrm{P} 4140015$ \\
\hline D-05-006 & Looking downstream from concrete culvert crossing & 90 & D-05-006_P4140016 \\
\hline $\mathrm{D}-05-006$ & Looking at culvert inlets & 0 & D-05-006_P4140017 \\
\hline D-05-006 & Looking at culvert outlets & 0 & D-05-006_P4140018 \\
\hline D-05-007 & Looking upstream from bridge crossing & 270 & D-05-007_P4140019 \\
\hline D-05-007 & Looking downstream from bridge crossing & 90 & D-05-007_P4140020 \\
\hline D-05-007 & Cobble and boulders under and downstream from bridge & 180 & D-05-007_P4140021 \\
\hline D-05-007 & Suckers congregating under bridge in drain & N/A & D-05-007 P4140022 \\
\hline $\mathrm{D}-05-008$ & Looking upstream from culvert crossing & 270 & $\mathrm{D}-05-008 \mathrm{P} 4140023$ \\
\hline D-05-008 & Looking downstream from culvert crossing & 90 & D-05-008_P4140024 \\
\hline D-05-008 & Looking at culvert outlets & 0 & D-05-008_P4140025 \\
\hline D-05-008 & Looking at culvert inlets & 180 & D-05-008 P4140026 \\
\hline D-05-009 & Looking upstream from bridge crossing & 270 & D-05-009_P4140027 \\
\hline D-05-009 & Looking downstream from bridge crossing & 90 & D-05-009_P4140028 \\
\hline D-05-011 & Preparing Electrofishing Boat & 270 & D-05-011_P6010001 \\
\hline D-05-011 & Commencing electrofishing & 270 & D-05-011_P6010002 \\
\hline D-05-011 & White Sucker, $330 \mathrm{~mm}$, released & $\mathrm{N} / \mathrm{A}$ & D-05-011_P6010003 \\
\hline D-05-011 & Looking upstream from mid-reach & 190 & D-05-011_P6010004 \\
\hline D-05-011 & Looking southwest at old river channel & 270 & D-05-011_P6010005 \\
\hline $\mathrm{D}-05-011$ & Looking downstream from mid-reach & 330 & D-05-011_P6010006 \\
\hline D-05-011 & Walleye, $246 \mathrm{~mm}$, collected & N/A & D-05-011_P6010008 \\
\hline D-05-011 & Walleye, $586 \mathrm{~mm}$, released & N/A & D-05-011_P6010010 \\
\hline D-05-011 & Troutperch, collected & N/A & D-05-011 P6010011 \\
\hline D-05-011 & White Sucker, $412 \mathrm{~mm}$, released & $\mathrm{N} / \mathrm{A}$ & D-05-011 P6010012 \\
\hline
\end{tabular}


Appendix 8: List of all photographs taken showing the corresponding site number, descriptive caption, direction faced when taken (azimuth in degrees) and the unique file name for each photograph.

\begin{tabular}{|c|c|c|c|}
\hline $\begin{array}{l}\text { Site } \\
\text { Number }\end{array}$ & Photo Description & Azimuth & File Name \\
\hline D-05-011 & White Sucker, $412 \mathrm{~mm}$, released & $\mathrm{N} / \mathrm{A}$ & D-05-011_P6010013 \\
\hline D-05-011 & White Sucker, $421 \mathrm{~mm}$, released & N/A & D-05-011 P6010014 \\
\hline D-05-011 & White Sucker, $386 \mathrm{~mm}$, released & $\mathrm{N} / \mathrm{A}$ & D-05-011 P6010015 \\
\hline D-05-012 & Looking upstream from mid-reach & 180 & D-05-012_P6090001 \\
\hline D-05-012 & Looking downstream from mid-reach & 0 & D-05-012_P6090002 \\
\hline $\mathrm{D}-05-013$ & Looking upstream from immediately below sheet pile weir & 180 & D-05-013_P7200001 \\
\hline D-05-013 & Looking downstream from immediately above sheet pile weir & 90 & D-05-013 P7200002 \\
\hline D-05-013 & Looking south across river at sediment deposition & 180 & D-05-013 P7200003 \\
\hline $\mathrm{D}-05-013$ & Looking south from diversion channel at sediment deposition & 180 & D-05-013_P7200004 \\
\hline D-05-013 & $\begin{array}{l}\text { Part of a panorama looking SW to W across main channel of the Pembina River } \\
\text { noting shale and sediment deposition }\end{array}$ & 220 & D-05-013_P7200005 \\
\hline D-05-013 & $\begin{array}{l}\text { Part of a panorama looking SW to W across main channel of the Pembina River } \\
\text { noting shale and sediment deposition }\end{array}$ & 230 & D-05-013_P7200006 \\
\hline D-05-013 & $\begin{array}{l}\text { Part of a panorama looking SW to W across main channel of the Pembina River } \\
\text { noting shale and sediment deposition }\end{array}$ & 250 & D-05-013_P7200007 \\
\hline D-05-013 & $\begin{array}{l}\text { Part of a panorama looking SW to W across main channel of the Pembina River } \\
\text { noting shale and sediment deposition }\end{array}$ & 270 & D-05-013_P7200008 \\
\hline D-05-014 & Looking north at control structure & 0 & D-05-014_P7200009 \\
\hline D-05-014 & Looking south from control structure & 180 & D-05-014_P7200010 \\
\hline D-05-015 & Looking upstream from crossing at Pelican Lake Diversion Channel & 0 & D-05-015_P7200011 \\
\hline D-05-015 & Looking upstream (south) from crossing at Enhancement Channel & 180 & D-05-015_P7200012 \\
\hline D-05-015 & Intake control structure leading to Pelican lake Diversion Channel & 50 & D-05-015_P7200013 \\
\hline D-05-015 & Culvert outlets from intake control to Pelican Lake Diversion Channel & 270 & D-05-015 P7200014 \\
\hline D-05-015 & $\begin{array}{l}\text { Upstream (south) end of culverts on the Pelican Lake Enhancement Channel leading } \\
\text { to Pelican Lake }\end{array}$ & 180 & D-05-015_P7200015 \\
\hline D-05-015 & White Sucker, $441 \mathrm{~mm}$, released & $\mathrm{N} / \mathrm{A}$ & D-05-015_P7210001 \\
\hline D-05-015 & White Sucker, $404 \mathrm{~mm}$, released & N/A & D-05-015_P7210002 \\
\hline D-05-015 & White Sucker, $404 \mathrm{~mm}$, released & $\mathrm{N} / \mathrm{A}$ & D-05-015_P7210003 \\
\hline D-05-016 & $\begin{array}{l}\text { Looking upstream from Pembina River bridge crossing at sediment deposition on left } \\
\text { bank }\end{array}$ & 270 & D-05-016_P7200016 \\
\hline D-05-016 & Looking downstream from bridge crossing on the Pembina River & 90 & D-05-016_P7200017 \\
\hline D-05-016 & View of fine pulverized shale/sand deposited on bank. & 270 & D-05-016_P7200018 \\
\hline $\mathrm{D}-05-017$ & Part of 4 photo panorama looking upstream from culvert crossing & 270 & D-05-017_P7210004 \\
\hline
\end{tabular}


Appendix 8: List of all photographs taken showing the corresponding site number, descriptive caption, direction faced when taken (azimuth in degrees) and the unique file name for each photograph.

\begin{tabular}{|c|c|c|c|}
\hline $\begin{array}{c}\text { Site } \\
\text { Number }\end{array}$ & Photo Description & Azimuth & File Name \\
\hline D-05-017 & Part of 4 photo panorama looking upstream from culvert crossing & 300 & D-05-017_P7210005 \\
\hline D-05-017 & Part of 4 photo panorama looking upstream from culvert crossing & 330 & D-05-017_P7210006 \\
\hline D-05-017 & $\begin{array}{l}\text { Part of } 4 \text { photo panorama looking upstream from culvert crossing. Note whirlpool at } \\
\text { submerged culvert inlets }\end{array}$ & 45 & D-05-017_P7210007 \\
\hline D-05-017 & Looking at culvert inlets & 30 & D-05-017_P7210008 \\
\hline D-05-017 & Looking downstream from culvert crossing & 150 & D-05-017_P7210009 \\
\hline $\mathrm{D}-05-017$ & Looking at culvert outlets & 270 & D-05-017_P7210010 \\
\hline $\mathrm{D}-05-018$ & Looking upstream from culvert crossing & 0 & $\mathrm{D}-05-018 \mathrm{P} 8030001$ \\
\hline D-05-018 & Looking downstream from culvert crossing & 270 & $\mathrm{D}-05-018 \mathrm{P} 8030002$ \\
\hline D-05-018 & Looking at culvert inlets & 0 & D-05-018_P8030003 \\
\hline $\mathrm{D}-05-018$ & Looking at culvert outlets & 0 & D-05-018_P8030004 \\
\hline D-05-018 & One of 3 Northern Pike released $(153 \mathrm{~mm}-160 \mathrm{~mm})$ & $\mathrm{N} / \mathrm{A}$ & D-05-018_P8030005 \\
\hline D-05-019 & Looking southeast at control structure & 150 & $\mathrm{D}-05-019 \mathrm{P} 8030006$ \\
\hline D-05-019 & Looking southeast at control structure & 150 & D-05-019_P8030007 \\
\hline D-05-019 & $\begin{array}{l}\text { Looking downstream from culvert crossing immediately downstream of control } \\
\text { structure. }\end{array}$ & 290 & D-05-019_P8030008 \\
\hline D-05-019 & Northern Pike $(201 \mathrm{~mm})$ released. & $\mathrm{N} / \mathrm{A}$ & D-05-019_P8030009 \\
\hline $\mathrm{D}-05-020$ & Looking upstream from culvert crossing & 270 & D-05-020_P8040001 \\
\hline D-05-020 & Looking downstream from culvert crossing & 90 & $\mathrm{D}-05-020 \mathrm{P} 8040002$ \\
\hline $\mathrm{D}-05-020$ & Looking at culvert inlets & 180 & $\mathrm{D}-05-020 \mathrm{P} 8040003$ \\
\hline D-05-020 & Looking at culvert outlets & 0 & D-05-020_P8040004 \\
\hline $\mathrm{D}-05-021$ & Looking upstream from culvert crossing & 300 & D-05-021_P8040005 \\
\hline $\mathrm{D}-05-021$ & Looking downstream from culvert crossing & 90 & D-05-021 P8040006 \\
\hline D-05-022 & Looking upstream from culvert crossing & 300 & D-05-022 P8040007 \\
\hline $\mathrm{D}-05-022$ & Looking downstream from culvert crossing & 120 & D-05-022_P8040008 \\
\hline $\mathrm{D}-05-023$ & Looking upstream from culvert crossing at control structure & 270 & D-05-023_P8160001 \\
\hline $\mathrm{D}-05-023$ & Looking downstream from culvert crossing & 90 & $\mathrm{D}-05-023 \mathrm{P} 8160002$ \\
\hline D-05-023 & Control structure inlet & 90 & $\mathrm{D}-05-023 \mathrm{P} 8160003$ \\
\hline $\mathrm{D}-05-023$ & Looking at culvert outlets & 0 & D-05-023_P8160004 \\
\hline D-05-023 & White Sucker released & N/A & D-05-023 P8160005 \\
\hline $\mathrm{D}-05-023$ & White Sucker released & $\mathrm{N} / \mathrm{A}$ & $\mathrm{D}-05-023 \mathrm{P} 8160006$ \\
\hline D-05-023 & White Sucker released & $\mathrm{N} / \mathrm{A}$ & D-05-023_P8160007 \\
\hline D-05-023 & Photo of Northern Pike collected & $\mathrm{N} / \mathrm{A}$ & $\mathrm{D}-05-023 \mathrm{P} 8160008$ \\
\hline
\end{tabular}


Appendix 8: List of all photographs taken showing the corresponding site number, descriptive caption, direction faced when taken (azimuth in degrees) and the unique file name for each photograph.

\begin{tabular}{|c|c|c|c|}
\hline $\begin{array}{c}\text { Site } \\
\text { Number }\end{array}$ & Photo Description & Azimuth & File Name \\
\hline D-05-024 & Looking upstream at weir & 220 & D-05-024_P8160009 \\
\hline D-05-024 & $\begin{array}{l}\text { Looking downstream at shale deposit in the inlet to the Pelican Lake Enhancement } \\
\text { Channel }\end{array}$ & 0 & D-05-024_P8160010 \\
\hline D-05-024 & Looking downstream from $20 \mathrm{~m}$ upstream of weir & 20 & D-05-024_P8160011 \\
\hline $\mathrm{D}-05-025$ & Looking upstream from culvert crossing on Burr Oak Bay Road & 150 & $\mathrm{D}-05-025 \mathrm{P} 8170001$ \\
\hline $\mathrm{D}-05-025$ & Looking downstream from culvert crossing on Burr Oak Bay Road & 300 & D-05-025_P8170002 \\
\hline $\mathrm{D}-05-025$ & Looking SSE at culvert outlet perched $0.5 \mathrm{~m}$ & 120 & D-05-025_P8170003 \\
\hline $\mathrm{D}-05-025$ & Looking NE at culvert inlet & 30 & $\mathrm{D}-05-025$ P8170004 \\
\hline $\mathrm{D}-05-025$ & Looking NW at Henderson Highway crossing culvert inlet & 280 & $\mathrm{D}-05-025 \mathrm{P} 8170005$ \\
\hline $\mathrm{D}-05-025$ & Looking east at Henderson Highway crossing culvert outlet & 90 & D-05-025_P8170006 \\
\hline $\mathrm{D}-05-025$ & Looking downstream from below Henderson Highway crossing & 300 & D-05-025_P8170007 \\
\hline D-05-026 & Looking downstream from culvert crossing & 90 & D-05-026_P8300001 \\
\hline $\mathrm{D}-05-026$ & Looking upstream from culvert crossing & 270 & $\mathrm{D}-05-026 \mathrm{P} 8300002$ \\
\hline D-05-026 & Looking south at culvert inlets & 180 & D-05-026_P8300003 \\
\hline $\mathrm{D}-05-026$ & Looking north at culvert inlets & 0 & D-05-026_P8300004 \\
\hline $\mathrm{D}-05-027$ & Looking downstream at old dam & 90 & $\mathrm{D}-05-027 \mathrm{P} 8300005$ \\
\hline $\mathrm{D}-05-027$ & Looking $\mathrm{N}$ across old dam & 0 & $\mathrm{D}-05-027 \_\mathrm{P} 8300006$ \\
\hline $\mathrm{D}-05-027$ & Looking NE at channel downstream from dam & 45 & D-05-027_P8300007 \\
\hline W-05-001 & Looking upstream from culvert crossing & 270 & W-05-001_P5020001 \\
\hline W-05-001 & Looking downstream from cobble crossing & 90 & W-05-001_P5020002 \\
\hline W-05-001 & Looking down at dead suckers in rocks & $\mathrm{N} / \mathrm{A}$ & W-05-001_P5020003 \\
\hline W-05-001 & The Ashley's doing water quality testing & 35 & W-05-001_P5020004 \\
\hline W-05-001 & The Ashley's electrofishing & 130 & W-05-001 P5020005 \\
\hline W-05-001 & The Ashley's electrofishing from cobble crossing & 220 & W-05-001 P5020006 \\
\hline W-05-001 & Photo of the Ashley's electrofishing upstream from cobble crossing & 270 & W-05-001_P5020007 \\
\hline W-05-002 & Looking upstream from culvert crossing & 270 & W-05-002_P5020008 \\
\hline W-05-002 & Looking downstream from culvert crossing & 90 & W-05-002_P5020009 \\
\hline W-05-002 & Looking at culvert inlet from the road & 60 & W-05-002 P5020010 \\
\hline W-05-002 & Looking at culvert outlet & 320 & W-05-002_P5020011 \\
\hline W-05-003 & Looking upstream from culvert crossing & 270 & W-05-003_P5020012 \\
\hline W-05-003 & Looking downstream from culvert crossing & 90 & W-05-003 P5020013 \\
\hline W-05-003 & Looking at culvert inlet & 90 & W-05-003 P5020014 \\
\hline W-05-003 & Looking at culvert outlet & 270 & W-05-003_P5020015 \\
\hline
\end{tabular}


Appendix 8: List of all photographs taken showing the corresponding site number, descriptive caption, direction faced when taken (azimuth in degrees) and the unique file name for each photograph.

\begin{tabular}{|c|c|c|c|}
\hline $\begin{array}{c}\text { Site } \\
\text { Number }\end{array}$ & Photo Description & Azimuth & File Name \\
\hline W-05-004 & Looking upstream from culvert crossing & 270 & W-05-004_P5020016 \\
\hline W-05-004 & Looking downstream from culvert crossing & 90 & W-05-004_P5020017 \\
\hline W-05-004 & Looking at culvert inlet & 90 & W-05-004 P5020018 \\
\hline W-05-004 & Looking at culvert outlet & 270 & W-05-004_P5020019 \\
\hline W-05-005 & Looking upstream from bridge crossing & 270 & W-05-005_P5020020 \\
\hline W-05-005 & Looking downstream from bridge crossing & 90 & W-05-005_P5020021 \\
\hline W-05-006 & Looking upstream from culvert crossing & 270 & W-05-006_P5020022 \\
\hline W-05-006 & Looking downstream from culvert crossing & 90 & W-05-006 P5020023 \\
\hline W-05-006 & Looking at culvert inlet & 90 & W-05-006_P5020024 \\
\hline W-05-006 & Looking at culvert outlet & 270 & W-05-006_P5020025 \\
\hline W-05-007 & Looking upstream from bridge crossing & 270 & W-05-007_P5020026 \\
\hline W-05-007 & Looking downstream from bridge crossing & 90 & W-05-007_P5020027 \\
\hline W-05-008 & Looking upstream from bridge crossing & 270 & W-05-008_P5020028 \\
\hline W-05-008 & Looking downstream from bridge crossing & 90 & W-05-008_P5020029 \\
\hline W-05-009 & Looking upstream from culvert crossing & 270 & W-05-009_P5020030 \\
\hline W-05-009 & Looking downstream from culvert crossing & 90 & W-05-009_P5020031 \\
\hline W-05-009 & Looking at culvert inlet & 90 & W-05-009_P5020032 \\
\hline W-05-009 & Looking at culvert outlet & 270 & W-05-009_P5020033 \\
\hline W-05-010 & Looking upstream from bridge crossing & 270 & W-05-010_P5020034 \\
\hline W-05-010 & Looking downstream from bridge crossing & 90 & W-05-010 P5020035 \\
\hline W-05-011 & Looking upstream from bridge crossing & 270 & W-05-011_P5020036 \\
\hline W-05-011 & Looking downstream from bridge crossing & 90 & W-05-011_P5020037 \\
\hline W-05-011 & Looking upstream of fish barrier & $\mathrm{N} / \mathrm{A}$ & W-05-011_P5020038 \\
\hline W-05-011 & Photo of bank erosion upstream (300 m upstream) & $\mathrm{N} / \mathrm{A}$ & W-05-011_P5020039 \\
\hline W-05-012 & Looking upstream from culvert crossing & 310 & W-05-012_P5030001 \\
\hline W-05-012 & Looking upstream from culvert at pool & 220 & W-05-012_P5030002 \\
\hline W-05-012 & Looking downstream from culvert crossing & 120 & W-05-012 P5030003 \\
\hline W-05-012 & Photos of suckers by measuring stick (released) & $\mathrm{N} / \mathrm{A}$ & W-05-012_P5030004 \\
\hline W-05-013 & Looking upstream from culvert crossing & 270 & W-05-013_P5030005 \\
\hline W-05-013 & Looking downstream from culvert crossing & 90 & W-05-013_P5030006 \\
\hline W-05-013 & Looking at culvert outlet & 270 & W-05-013 P5030007 \\
\hline W-05-013 & Looking at culvert inlet & 90 & W-05-013_P5030008 \\
\hline W-05-014 & Photo of suckers to be released & $\mathrm{N} / \mathrm{A}$ & W-05-014_P5030009 \\
\hline
\end{tabular}


Appendix 8: List of all photographs taken showing the corresponding site number, descriptive caption, direction faced when taken (azimuth in degrees) and the unique file name for each photograph.

\begin{tabular}{|c|c|c|c|}
\hline $\begin{array}{c}\text { Site } \\
\text { Number }\end{array}$ & Photo Description & Azimuth & File Name \\
\hline W-05-014 & Photo of suckers to be released & $\mathrm{N} / \mathrm{A}$ & W-05-014_P5030010 \\
\hline W-05-014 & Photo of suckers to be released & $\mathrm{N} / \mathrm{A}$ & W-05-014_P5030011 \\
\hline W-05-014 & Looking upstream from bridge crossing & 270 & W-05-014_P5030012 \\
\hline W-05-014 & Looking downstream from bridge crossing & 90 & W-05-014_P5030013 \\
\hline W-05-015 & Looking upstream from bridge crossing & 270 & W-05-015_P5030014 \\
\hline W-05-014 & Looking downstream from bridge crossing & 90 & W-05-014_P5030015 \\
\hline W-05-016 & Looking upstream from culvert crossing & 220 & W-05-016_P5030016 \\
\hline W-05-016 & Looking downstream from culvert crossing & 40 & W-05-016_P5030017 \\
\hline W-05-016 & Looking at culvert inlet & 60 & W-05-016_P5030018 \\
\hline W-05-016 & Looking at culvert outlet & 255 & W-05-016 P5030019 \\
\hline W-05-016 & Photo of sucker on measuring stick (to be released) & N/A & W-05-016_P5030020 \\
\hline W-05-017 & Looking upstream from bridge crossing & 0 & W-05-017_P5040001 \\
\hline W-05-017 & Looking downstream from bridge crossing & 180 & W-05-017_P5040002 \\
\hline W-05-017 & Looking upstream riffle from under bridge & 0 & W-05-017_P5040003 \\
\hline W-05-017 & Photo of a sucker to be released & $\mathrm{N} / \mathrm{A}$ & W-05-017_P5040004 \\
\hline W-05-017 & Photo of a sucker to be released & $\mathrm{N} / \mathrm{A}$ & W-05-017_P5040005 \\
\hline W-05-017 & Photo of the largest sucker captured (to be released) & $\mathrm{N} / \mathrm{A}$ & W-05-017_P5040006 \\
\hline W-05-018 & Looking upstream from culvert crossing & 270 & W-05-018 P5040007 \\
\hline W-05-018 & Looking downstream from culvert crossing & 90 & W-05-018_P5040008 \\
\hline W-05-018 & Looking at culvert inlet & 90 & W-05-018_P5040009 \\
\hline W-05-018 & Looking at culvert outlet & 270 & W-05-018_P5040010 \\
\hline W-05-019 & Looking upstream from culvert crossing & 270 & W-05-019_P5040011 \\
\hline W-05-019 & Looking downstream from culvert crossing & 180 & W-05-019_P5040012 \\
\hline W-05-019 & Looking at culvert inlet & 200 & W-05-019_P5040013 \\
\hline W-05-019 & Looking at culvert outlet & 300 & W-05-019 P5040014 \\
\hline W-05-020 & Looking upstream from culvert crossing & 270 & W-05-020 P5040015 \\
\hline W-05-020 & Looking downstream from the culvert & 90 & W-05-020_P5040016 \\
\hline W-05-021 & Looking upstream from culvert crossing & 270 & W-05-021_P5040017 \\
\hline W-05-021 & Looking downstream from culvert crossing & 90 & W-05-021_P5040018 \\
\hline W-05-021 & Looking at culvert inlet & 90 & W-05-021_P5040019 \\
\hline W-05-021 & Looking at culvert outlet & 200 & W-05-021_P5040020 \\
\hline W-05-022 & Looking upstream from culvert crossing & 320 & W-05-022_P5040021 \\
\hline W-05-022 & Looking downstream from culvert crossing & 130 & W-05-022_P5040022 \\
\hline
\end{tabular}


Appendix 8: List of all photographs taken showing the corresponding site number, descriptive caption, direction faced when taken (azimuth in degrees) and the unique file name for each photograph.

\begin{tabular}{|c|c|c|c|}
\hline $\begin{array}{c}\text { Site } \\
\text { Number }\end{array}$ & Photo Description & Azimuth & File Name \\
\hline W-05-022 & Looking at culvert inlet & 80 & W-05-022 P5040023 \\
\hline W-05-022 & Looking at culvert outlet & 270 & W-05-022 P5040024 \\
\hline W-05-023 & Looking upstream from ford crossing & 270 & W-05-023_P5050001 \\
\hline W-05-023 & Looking downstream from ford crossing & 90 & W-05-023_P5050002 \\
\hline W-05-023 & Looking at culvert outlet & 90 & W-05-023_P5050003 \\
\hline W-05-023 & Looking at culvert inlet & 270 & W-05-023 P5050004 \\
\hline W-05-023 & Looking at perched gated culvert on left bank downstream of ford & 300 & W-05-023_P5050005 \\
\hline W-05-024 & Looking upstream from ford crossing & 270 & W-05-024_P5050006 \\
\hline W-05-024 & Looking downstream from ford crossing & 90 & W-05-024 P5050007 \\
\hline W-05-024 & Looking at culvert outlet & 44 & W-05-024 P5050008 \\
\hline W-05-024 & Looking at culvert inlet & 220 & W-05-024_P5050009 \\
\hline W-05-024 & Photo of Black Bullhead & $\mathrm{N} / \mathrm{A}$ & W-05-024_P5050010 \\
\hline W-05-024 & Photo of Black Bullhead & $\mathrm{N} / \mathrm{A}$ & W-05-024 P5050011 \\
\hline W-05-025 & Looking upstream from culvert crossing & 270 & W-05-025 P5050012 \\
\hline W-05-025 & Looking downstream from culvert crossing & 90 & W-05-025_P5050013 \\
\hline W-05-025 & Looking at culvert inlet & 90 & W-05-025_P5050014 \\
\hline W-05-025 & Looking at culvert outlet & 270 & W-05-025 P5050015 \\
\hline W-05-026 & Looking upstream from culvert crossing & 90 & W-05-026 P5050016 \\
\hline W-05-026 & Looking downstream from culvert crossing & 270 & W-05-026_P5050017 \\
\hline W-05-026 & Looking at culvert inlet & 120 & W-05-026_P5050018 \\
\hline W-05-026 & Looking at culvert outlet & 220 & W-05-026 P5050019 \\
\hline W-05-027 & Looking upstream from left bank & 15 & W-05-027_P5050020 \\
\hline W-05-027 & Looking downstream from left bank & 165 & W-05-027_P5050021 \\
\hline W-05-027 & Looking at culvert inlet & 90 & W-05-027 P5050022 \\
\hline W-05-027 & Looking at culvert outlet & 270 & W-05-027 P5050023 \\
\hline W-05-028 & Looking upstream from bridge crossing & 210 & W-05-028_P5050024 \\
\hline W-05-028 & Looking downstream from bridge crossing & 40 & W-05-028_P5050025 \\
\hline W-05-029 & Looking upstream from culvert crossing & 270 & W-05-029_P5050026 \\
\hline W-05-029 & Looking downstream from culvert crossing & 90 & W-05-029 P5050027 \\
\hline W-05-029 & Looking at culvert inlet & 160 & W-05-029 P5050028 \\
\hline W-05-029 & Looking at culvert outlet & 300 & W-05-029_P5050029 \\
\hline W-05-030 & Looking upstream from culvert crossing & 0 & W-05-030 P5060001 \\
\hline W-05-030 & Looking downstream from culvert crossing & 180 & W-05-030 P5060002 \\
\hline
\end{tabular}


Appendix 8: List of all photographs taken showing the corresponding site number, descriptive caption, direction faced when taken (azimuth in degrees) and the unique file name for each photograph.

\begin{tabular}{|c|c|c|c|}
\hline $\begin{array}{c}\text { Site } \\
\text { Number }\end{array}$ & Photo Description & Azimuth & File Name \\
\hline W-05-030 & Looking at culvert inlet & 45 & W-05-030 P5060003 \\
\hline W-05-031 & Looking upstream from culvert crossing & 180 & W-05-031_P5060004 \\
\hline W-05-031 & Looking downstream from culvert crossing & 0 & W-05-031_P5060005 \\
\hline W-05-031 & Looking at culvert inlet & 20 & W-05-031_P5060006 \\
\hline W-05-031 & Looking at culvert outlet & 150 & W-05-031 P5060007 \\
\hline W-05-032 & Looking upstream from culvert crossing & 225 & W-05-032 P5060008 \\
\hline W-05-032 & Looking downstream from culvert crossing & 45 & W-05-032_P5060009 \\
\hline W-05-032 & Looking at culvert inlet & 45 & W-05-032 P5060010 \\
\hline W-05-032 & Looking at culvert outlet & 270 & W-05-032 P5060011 \\
\hline W-05-032 & Looking at bank scour on right bank upstream of culvert crossing & 105 & W-05-032_P5060012 \\
\hline W-05-033 & Looking upstream bridge crossing & 0 & W-05-033_P5060013 \\
\hline W-05-033 & Looking downstream from bridge crossing & 180 & W-05-033_P5060014 \\
\hline W-05-034 & Looking upstream from culvert crossing & 270 & W-05-034 P5060015 \\
\hline W-05-034 & Looking downstream from culvert crossing & 90 & W-05-034 P5060016 \\
\hline W-05-034 & Looking at culvert inlet & 120 & W-05-034_P5060017 \\
\hline W-05-034 & Looking at culvert outlet & 220 & W-05-034 P5060018 \\
\hline W-05-035 & Looking upstream from culvert crossing & 270 & W-05-035 P5060019 \\
\hline W-05-035 & Looking downstream from culvert crossing & 90 & W-05-035_P5060020 \\
\hline W-05-035 & Looking at culvert inlet & 90 & W-05-035_P5060021 \\
\hline W-05-035 & Looking at culvert outlet & 270 & W-05-035 P5060022 \\
\hline W-05-036 & Looking upstream from culvert crossing & 270 & W-05-036 P5090001 \\
\hline W-05-036 & Looking downstream from culvert crossing & 90 & W-05-036_P5090002 \\
\hline W-05-036 & Looking at culvert inlet & 90 & W-05-036_P5090003 \\
\hline W-05-036 & Looking at culvert outlet & 270 & W-05-036 P5090004 \\
\hline W-05-037 & Looking upstream from culvert crossing & 270 & W-05-037_P5090005 \\
\hline W-05-037 & Looking downstream from culvert crossing & 90 & W-05-037_P5090006 \\
\hline W-05-037 & Looking at culvert inlet & 110 & W-05-037_P5090007 \\
\hline W-05-037 & Looking at culvert outlet & 300 & W-05-037 P5090008 \\
\hline W-05-038 & Looking upstream from culvert crossing & 90 & W-05-038 P5090009 \\
\hline W-05-038 & Looking downstream from culvert crossing & 270 & W-05-038_P5090010 \\
\hline W-05-038 & Looking at culvert inlet & 45 & W-05-038 P5090011 \\
\hline W-05-038 & Looking at culvert outlet & 300 & W-05-038 P5090012 \\
\hline W-05-039 & Looking upstream from culvert crossing & 0 & W-05-039 P5090013 \\
\hline
\end{tabular}


Appendix 8: List of all photographs taken showing the corresponding site number, descriptive caption, direction faced when taken (azimuth in degrees) and the unique file name for each photograph.

\begin{tabular}{|c|c|c|c|}
\hline $\begin{array}{c}\text { Site } \\
\text { Number }\end{array}$ & Photo Description & Azimuth & File Name \\
\hline W-05-039 & Looking downstream from culvert crossing & 180 & W-05-039_P5090014 \\
\hline W-05-039 & Looking at culvert inlet & 120 & W-05-039 P5090015 \\
\hline W-05-039 & Looking at culvert outlet & 60 & W-05-039_P5090016 \\
\hline W-05-040 & Looking upstream from culvert crossing & 270 & W-05-040_P5090017 \\
\hline W-05-040 & Looking downstream from culvert crossing & 90 & W-05-040_P5090018 \\
\hline W-05-040 & Looking at culvert inlet & 350 & W-05-040_P5090019 \\
\hline W-05-040 & Looking at culvert outlet & 320 & W-05-040_P5090020 \\
\hline W-05-041 & Looking upstream from culvert crossing & 270 & W-05-041_P5100001 \\
\hline W-05-041 & Looking downstream from culvert crossing & 90 & W-05-041_P5100002 \\
\hline W-05-041 & Looking at culvert inlet & 90 & W-05-041_P5100003 \\
\hline W-05-041 & Looking at culvert outlet & 270 & W-05-041_P5100004 \\
\hline W-05-042 & Looking upstream from culvert crossing & 315 & W-05-042_P5100005 \\
\hline W-05-042 & Looking downstream from culvert crossing & 135 & W-05-042_P5100006 \\
\hline W-05-042 & Looking at culvert inlet & 105 & W-05-042_P5100007 \\
\hline W-05-042 & Looking at culvert outlet & 315 & W-05-042_P5100008 \\
\hline W-05-043 & Looking upstream from culvert crossing & 250 & W-05-043_P5100009 \\
\hline W-05-043 & Looking downstream from culvert crossing & 60 & W-05-043_P5100010 \\
\hline W-05-043 & Looking at culvert inlet & 270 & W-05-043 P5100011 \\
\hline W-05-043 & Looking at culvert outlet & 0 & W-05-043_P5100012 \\
\hline W-05-044 & Looking downstream from culvert crossing & 330 & W-05-044_P5100013 \\
\hline W-05-044 & Looking upstream from culvert crossing & 120 & W-05-044_P5100014 \\
\hline W-05-044 & Looking at culvert outlet & 210 & W-05-044_P5100015 \\
\hline W-05-044 & Looking at culvert inlet & 30 & W-05-044_P5100016 \\
\hline W-05-044 & Looking at bank slump around tributary culvert on right bank & 90 & W-05-044_P5100017 \\
\hline W-05-045 & Looking upstream from culvert crossing & 0 & W-05-045_P5100018 \\
\hline W-05-045 & Looking downstream from culvert crossing & 180 & W-05-045 P5100019 \\
\hline W-05-045 & Looking at culvert inlet & 30 & W-05-045_P5100020 \\
\hline W-05-045 & Looking at culvert outlet & 150 & W-05-045_P5100021 \\
\hline W-05-046 & Looking upstream from bridge crossing on PR 334 & 270 & W-05-046_P5110001 \\
\hline W-05-046 & Looking downstream from bridge crossing on PR 334 & 90 & W-05-046 P5110002 \\
\hline W-05-047 & Looking upstream from culvert crossing & 320 & W-05-047_P5110003 \\
\hline W-05-047 & Looking downstream from culvert crossing & 90 & W-05-047_P5110004 \\
\hline W-05-047 & Looking at culvert inlet & 60 & W-05-047_P5110005 \\
\hline
\end{tabular}


Appendix 8: List of all photographs taken showing the corresponding site number, descriptive caption, direction faced when taken (azimuth in degrees) and the unique file name for each photograph.

\begin{tabular}{|c|c|c|c|}
\hline $\begin{array}{c}\text { Site } \\
\text { Number }\end{array}$ & Photo Description & Azimuth & File Name \\
\hline W-05-047 & Looking at culvert outlet & 320 & W-05-047 P5110006 \\
\hline W-05-048 & Looking upstream from culvert crossing & 0 & W-05-048_P5110007 \\
\hline W-05-048 & Looking downstream from culvert crossing & 180 & W-05-048_P5110008 \\
\hline W-05-048 & Looking at culvert outlet & 0 & W-05-048_P5110009 \\
\hline W-05-048 & Looking at culvert inlet & 180 & W-05-048 P5110010 \\
\hline W-05-049 & Looking upstream bridge crossing & 75 & W-05-049 P5110011 \\
\hline W-05-049 & Looking downstream bridge crossing & 320 & W-05-049_P5110012 \\
\hline W-05-050 & Looking upstream from culvert crossing & 0 & W-05-050 P5110013 \\
\hline W-05-050 & Looking downstream from culvert crossing & 180 & W-05-050 P5110014 \\
\hline W-05-050 & Looking at culvert inlet & 180 & W-05-050_P5110015 \\
\hline W-05-050 & Looking at culvert outlet & 0 & W-05-050_P5110016 \\
\hline W-05-052 & Looking upstream from culvert crossing & 0 & W-05-052_P5120001 \\
\hline W-05-052 & Looking downstream from culvert crossing & 180 & W-05-052 P5120002 \\
\hline W-05-052 & Looking at culvert inlet & 225 & W-05-052 P5120003 \\
\hline W-05-052 & Looking at culvert outlet & 300 & W-05-052_P5120004 \\
\hline W-05-053 & Looking upstream bridge crossing & 0 & W-05-053 P5120005 \\
\hline W-05-053 & Looking downstream bridge crossing & 180 & W-05-053 P5120006 \\
\hline W-05-054 & Looking upstream from culvert crossing & 330 & W-05-054_P5120007 \\
\hline W-05-054 & Looking downstream from culvert crossing & 150 & W-05-054_P5120008 \\
\hline W-05-054 & Looking at culvert inlet & 180 & W-05-054 P5120009 \\
\hline W-05-054 & Looking at culvert outlet & 310 & W-05-054 P5120010 \\
\hline W-05-054 & Looking at perched culvert and erosion of right bank upstream of culvert crossing & 0 & W-05-054_P5120011 \\
\hline W-05-054 & Looking down at sediment deposition on left bank upstream of culvert crossing & $\mathrm{N} / \mathrm{A}$ & W-05-054_P5120012 \\
\hline W-05-054 & Looking at right bank slump upstream of culvert crossing & 0 & W-05-054 P5120013 \\
\hline W-05-054 & Looking at unstable left bank at downstream end of culvert & 180 & W-05-054_P5120014 \\
\hline W-05-055 & Looking upstream from culvert crossing & 70 & W-05-055_P5160001 \\
\hline W-05-055 & Looking downstream from culvert crossing & 250 & W-05-055_P5160002 \\
\hline W-05-055 & Looking at culvert inlet & 340 & W-05-055 P5160003 \\
\hline W-05-055 & Looking at culvert outlet & 110 & W-05-055 P5160004 \\
\hline W-05-055 & Looking at an example of slumping & 350 & W-05-055 P5160005 \\
\hline W-05-055 & Looking at vegetation upstream & 90 & W-05-055_P5160006 \\
\hline W-05-056 & $\begin{array}{l}\text { Photo of very perched culvert over gorge } 1 \text { mi east of Pelican lake; } 2 \text { mi west- } \\
\text { southwest of Willoughby's Lake }\end{array}$ & $\mathrm{N} / \mathrm{A}$ & W-05-056_P5160007 \\
\hline
\end{tabular}


Appendix 8: List of all photographs taken showing the corresponding site number, descriptive caption, direction faced when taken (azimuth in degrees) and the unique file name for each photograph.

\begin{tabular}{|c|c|c|c|}
\hline $\begin{array}{c}\text { Site } \\
\text { Number }\end{array}$ & Photo Description & Azimuth & File Name \\
\hline W-05-056 & Looking upstream from culvert crossing & 180 & $\begin{array}{ll}W-05-056 & P 5160008\end{array}$ \\
\hline W-05-056 & Looking downstream from culvert crossing & 205 & W-05-056 P5160009 \\
\hline W-05-056 & Looking at culvert inlet (zoomed) & 205 & W-05-056_P5160010 \\
\hline W-05-056 & Looking at culvert outlet & 0 & W-05-056_P5160011 \\
\hline W-05-056 & Example of undercutting & N/A & W-05-056 P5160012 \\
\hline W-05-057 & Looking upstream from bridge crossing & 180 & W-05-057_P5170001 \\
\hline W-05-057 & Looking downstream from bridge crossing & 70 & W-05-057_P5170002 \\
\hline W-05-058 & Looking upstream of road & 170 & W-05-058 P5170003 \\
\hline W-05-058 & Looking downstream stream of road & 350 & W-05-058 P5170004 \\
\hline W-05-058 & Looking at erosion on ford crossing & 90 & W-05-058_P5170005 \\
\hline W-05-058 & Photo of female sucker fork length $154 \mathrm{~mm}$ (released) & N/A & W-05-058_P5170006 \\
\hline W-05-058 & Photo of male sucker fork length $165 \mathrm{~mm}$ (released) & N/A & W-05-058 P5170007 \\
\hline W-05-058 & Photo of male sucker fork length $165 \mathrm{~mm}$ (released) & $\mathrm{N} / \mathrm{A}$ & W-05-058 P5170008 \\
\hline W-05-058 & Photo of male sucker fork length $145 \mathrm{~mm}$ (released) & N/A & W-05-058 P5170009 \\
\hline W-05-059 & Looking upstream from left bank & 80 & W-05-059_P5170010 \\
\hline W-05-059 & Looking downstream from left bank & 280 & W-05-059 P5170011 \\
\hline W-05-060 & Looking upstream from culvert crossing & 180 & W-05-060 P5170012 \\
\hline W-05-060 & Looking downstream from culvert crossing & 90 & W-05-060 P5170013 \\
\hline W-05-060 & Looking at culvert inlet & 90 & W-05-060_P5170014 \\
\hline W-05-060 & Looking at culvert outlet & 180 & W-05-060 P5170015 \\
\hline W-05-061 & Looking upstream from culvert crossing & 180 & W-05-061 P5180001 \\
\hline W-05-061 & Looking downstream from culvert crossing & 0 & W-05-061_P5180002 \\
\hline W-05-061 & Looking at small culvert inlet & 0 & W-05-061 P5180003 \\
\hline W-05-061 & Looking at both culvert inlets & 0 & W-05-061 P5180004 \\
\hline W-05-061 & Looking at culvert outlets & 130 & W-05-061_P5180005 \\
\hline W-05-062 & Looking upstream cement box culvert crossing & 90 & W-05-062_P5180006 \\
\hline W-05-062 & Looking downstream cement box culvert crossing & 270 & W-05-062_P5180007 \\
\hline W-05-062 & Looking at culvert inlet & 270 & W-05-062 P5180008 \\
\hline W-05-062 & Looking at culvert outlet & 45 & W-05-062 P5180009 \\
\hline W-05-063 & Looking upstream from culvert crossing & 90 & W-05-063_P5180010 \\
\hline W-05-063 & Looking downstream from culvert crossing & 270 & W-05-063 P5180011 \\
\hline W-05-063 & Looking at culvert inlet & 45 & W-05-063 P5180012 \\
\hline W-05-063 & Looking at culvert outlet & 90 & W-05-063_P5180013 \\
\hline
\end{tabular}


Appendix 8: List of all photographs taken showing the corresponding site number, descriptive caption, direction faced when taken (azimuth in degrees) and the unique file name for each photograph.

\begin{tabular}{|c|c|c|c|}
\hline $\begin{array}{c}\text { Site } \\
\text { Number }\end{array}$ & Photo Description & Azimuth & File Name \\
\hline W-05-064 & Looking upstream from culvert crossing & 0 & W-05-064_P5180014 \\
\hline W-05-064 & Looking downstream from culvert crossing & 180 & W-05-064 P5180015 \\
\hline W-05-065 & Looking upstream from culvert crossing & 0 & W-05-065_P5180016 \\
\hline W-05-065 & Looking downstream from culvert crossing & 200 & W-05-065_P5180017 \\
\hline W-05-065 & Looking at culvert inlet & 150 & W-05-065_P5180018 \\
\hline W-05-065 & Looking at culvert outlet & 30 & W-05-065_P5180019 \\
\hline W-05-066 & Looking upstream from culvert crossing & 140 & W-05-066_P5180020 \\
\hline W-05-066 & Looking downstream from culvert crossing & 270 & W-05-066_P5180021 \\
\hline W-05-066 & Looking at culvert inlet & 270 & W-05-066_P5180022 \\
\hline W-05-066 & Looking at culvert outlet & 90 & W-05-066 P5180023 \\
\hline W-05-067 & Looking upstream at north tributary & 0 & W-05-067_P5190001 \\
\hline W-05-067 & Looking at east tributary & 90 & W-05-067_P5190002 \\
\hline W-05-067 & Looking downstream from culvert crossing & 180 & W-05-067_P5190003 \\
\hline W-05-067 & Looking at culvert inlet & 150 & W-05-067_P5190004 \\
\hline W-05-067 & Looking at culvert outlet & 45 & W-05-067_P5190005 \\
\hline W-05-068 & Looking upstream from culvert crossing & 90 & W-05-068_P5190006 \\
\hline W-05-068 & Looking downstream from culvert crossing & 160 & W-05-068_P5190007 \\
\hline W-05-068 & Looking at culvert inlet & 160 & W-05-068 P5190008 \\
\hline W-05-068 & Looking at culvert outlet & 110 & W-05-068_P5190009 \\
\hline W-05-069 & Looking upstream from culvert crossing & 45 & W-05-069_P5190010 \\
\hline W-05-069 & Looking downstream from culvert crossing & 225 & W-05-069_P5190011 \\
\hline W-05-069 & Looking at culvert inlet & 180 & W-05-069_P5190012 \\
\hline W-05-069 & Looking at culvert outlet & 0 & W-05-069_P5190013 \\
\hline W-05-070 & Looking upstream from culvert crossing & 90 & W-05-070_P5190014 \\
\hline W-05-070 & Looking downstream from culvert crossing & 270 & W-05-070_P5190015 \\
\hline W-05-070 & Looking at culvert inlet & 320 & W-05-070 P5190016 \\
\hline W-05-070 & Looking at culvert outlet & 150 & W-05-070_P5190017 \\
\hline W-05-071 & Looking upstream from culvert crossing & 0 & W-05-071_P5200001 \\
\hline W-05-071 & Looking downstream from culvert crossing & 180 & W-05-071_P5200002 \\
\hline W-05-071 & Looking at culvert inlet & 30 & W-05-071_P5200003 \\
\hline W-05-071 & Looking at culvert outlet & 210 & W-05-071_P5200004 \\
\hline W-05-072 & Looking upstream from bridge crossing & 115 & W-05-072_P5200005 \\
\hline W-05-072 & Looking downstream from bridge crossing & 300 & W-05-072_P5200006 \\
\hline
\end{tabular}


Appendix 8: List of all photographs taken showing the corresponding site number, descriptive caption, direction faced when taken (azimuth in degrees) and the unique file name for each photograph.

\begin{tabular}{|c|c|c|c|}
\hline $\begin{array}{c}\text { Site } \\
\text { Number }\end{array}$ & Photo Description & Azimuth & File Name \\
\hline W-05-072 & Looking at bank scour on right bank downstream of bridge crossing & 300 & W-05-072_P5200007 \\
\hline W-05-073 & Looking upstream from bridge crossing & 270 & W-05-073 P5240001 \\
\hline W-05-073 & Looking downstream from bridge crossing & 90 & W-05-073_P5240002 \\
\hline W-05-074 & Looking upstream from culvert crossing & 120 & W-05-074_P5240003 \\
\hline W-05-074 & Looking downstream from culvert crossing & 270 & W-05-074_P5240004 \\
\hline W-05-075 & Looking upstream from culvert crossing & 90 & W-05-075_P5240005 \\
\hline W-05-075 & Looking downstream from culvert crossing & 300 & W-05-075_P5240006 \\
\hline W-05-075 & Looking at culvert inlet & 295 & W-05-075_P5240007 \\
\hline W-05-075 & Looking at culvert outlet & 70 & W-05-075_P5240008 \\
\hline W-05-076 & Looking upstream from left bank north of PR 205 & 120 & W-05-076 P5240009 \\
\hline W-05-076 & Looking downstream from left bank north of PR 205 & 300 & W-05-076_P5240010 \\
\hline W-05-076 & Looking at culvert inlet & 300 & W-05-076_P5240011 \\
\hline W-05-076 & Looking at culvert outlet & 120 & W-05-076_P5240012 \\
\hline W-05-077 & Looking upstream from culvert crossing & 90 & W-05-077_P5240013 \\
\hline W-05-077 & Looking downstream from culvert crossing & 270 & W-05-077_P5240014 \\
\hline W-05-077 & Looking at culvert inlet & 320 & W-05-077_P5240015 \\
\hline W-05-077 & Looking at culvert outlet & 140 & W-05-077_P5240016 \\
\hline W-05-078 & Looking upstream from culvert crossing & 180 & W-05-078 P5250001 \\
\hline W-05-078 & Looking downstream from culvert crossing & 0 & W-05-078_P5250002 \\
\hline W-05-078 & Looking at culvert inlet & 30 & W-05-078_P5250003 \\
\hline W-05-078 & Looking at culvert outlet & 160 & W-05-078_P5250004 \\
\hline W-05-079 & Looking upstream from culvert crossing & 270 & W-05-079_P5250005 \\
\hline W-05-079 & Looking downstream from culvert crossing & 90 & W-05-079_P5250006 \\
\hline W-05-079 & Looking at culvert inlet & 150 & W-05-079_P5250007 \\
\hline W-05-079 & Looking at culvert outlet & 200 & W-05-079_P5250008 \\
\hline W-05-080 & Looking at ford crossing & 0 & W-05-080 P5250009 \\
\hline W-05-080 & Looking downstream from ford crossing & 300 & W-05-080_P5250010 \\
\hline W-05-080 & Looking upstream from ford crossing & 80 & W-05-080_P5250011 \\
\hline W-05-081 & Looking upstream from bridge crossing & 40 & W-05-081_P5250012 \\
\hline W-05-081 & Looking downstream from bridge crossing & 220 & W-05-081_P5250013 \\
\hline W-05-082 & Looking upstream from bridge crossing & 180 & W-05-082_P5250014 \\
\hline W-05-082 & Looking downstream from bridge crossing & 0 & W-05-082_P5250015 \\
\hline W-05-083 & Looking at ford crossing & 180 & W-05-083_P5260001 \\
\hline
\end{tabular}


Appendix 8: List of all photographs taken showing the corresponding site number, descriptive caption, direction faced when taken (azimuth in degrees) and the unique file name for each photograph.

\begin{tabular}{|c|c|c|c|}
\hline $\begin{array}{c}\text { Site } \\
\text { Number }\end{array}$ & Photo Description & Azimuth & File Name \\
\hline W-05-083 & Looking upstream from ford crossing & 270 & W-05-083 P5260002 \\
\hline W-05-083 & Looking downstream from ford crossing & 90 & W-05-083_P5260003 \\
\hline W-05-084 & Looking at culvert inlet & 270 & W-05-084_P5260004 \\
\hline W-05-084 & Looking at culvert outlet & 90 & W-05-084_P5260005 \\
\hline W-05-084 & Looking upstream from culvert crossing & 90 & W-05-084 P5260006 \\
\hline W-05-084 & Looking downstream from culvert crossing & 270 & W-05-084_P5260007 \\
\hline W-05-084 & Looking at bank erosion & 220 & W-05-084_P5260008 \\
\hline W-05-085 & Looking upstream from culvert crossing & 180 & W-05-085 P5260009 \\
\hline W-05-085 & Looking downstream from culvert crossing & 0 & W-05-085 P5260010 \\
\hline W-05-085 & Looking at culvert inlet & 0 & W-05-085_P5260011 \\
\hline W-05-085 & Looking at culvert outlet & 180 & W-05-085_P5260012 \\
\hline W-05-086 & Looking upstream from bridge crossing & 260 & W-05-086 P5260013 \\
\hline W-05-086 & Looking downstream from bridge crossing & 100 & W-05-086 P5260014 \\
\hline W-05-086 & Looking downstream from bridge crossing & 100 & W-05-086 P5260015 \\
\hline W-05-087 & Looking upstream from culvert crossing & 0 & W-05-087_P5270001 \\
\hline W-05-087 & Looking downstream from culvert crossing & 180 & W-05-087 P5270002 \\
\hline W-05-087 & Looking at culvert inlet & 225 & W-05-087 P5270003 \\
\hline W-05-087 & Looking at culvert outlet & 145 & W-05-087_P5270004 \\
\hline W-05-088 & Looking upstream from culvert crossing & 340 & W-05-088_P5270005 \\
\hline W-05-088 & Looking downstream from culvert crossing & 180 & W-05-088 P5270006 \\
\hline W-05-088 & Looking at culvert inlet & 220 & W-05-088 P5270007 \\
\hline W-05-088 & Looking at culvert outlet & 45 & W-05-088_P5270008 \\
\hline W-05-089 & Looking upstream bridge crossing & 270 & W-05-089_P5270009 \\
\hline W-05-089 & Looking downstream bridge crossing & 90 & W-05-089 P5270010 \\
\hline W-05-090 & Looking upstream from culvert crossing & 270 & W-05-090 P5270011 \\
\hline W-05-090 & Looking downstream from culvert crossing & 90 & W-05-090 P5270012 \\
\hline W-05-090 & Looking at culvert inlet & 140 & W-05-090_P5270013 \\
\hline W-05-090 & Looking at culvert outlet & 200 & W-05-090 P5270014 \\
\hline W-05-091 & Looking upstream from culvert crossing & 0 & W-05-091 P5270015 \\
\hline W-05-091 & Looking downstream from culvert crossing & 210 & W-05-091_P5270016 \\
\hline W-05-091 & Looking at culvert inlet ( 3 culverts) & 180 & W-05-091 P5270017 \\
\hline W-05-091 & Looking at culvert outlet ( 3 culverts) & 0 & W-05-091 P5270018 \\
\hline W-05-091 & Looking at culvert inlet & 180 & W-05-091_P5270019 \\
\hline
\end{tabular}


Appendix 8: List of all photographs taken showing the corresponding site number, descriptive caption, direction faced when taken (azimuth in degrees) and the unique file name for each photograph.

\begin{tabular}{|c|c|c|c|}
\hline $\begin{array}{c}\text { Site } \\
\text { Number }\end{array}$ & Photo Description & Azimuth & File Name \\
\hline W-05-091 & Looking at culvert outlet & 0 & W-05-091_P5270020 \\
\hline W-05-092 & Looking upstream from bridge crossing & 0 & W-05-092_P5270021 \\
\hline W-05-092 & Looking upstream from bridge crossing & 340 & W-05-092_P5270022 \\
\hline W-05-092 & Looking upstream from bridge crossing & 300 & W-05-092_P5270023 \\
\hline W-05-092 & Looking downstream from bridge crossing & 160 & W-05-092_P5270024 \\
\hline W-05-092 & Looking downstream from bridge crossing & 150 & W-05-092_P5270025 \\
\hline W-05-093 & Looking downstream from bridge crossing & 270 & W-05-093_P5290001 \\
\hline W-05-093 & Looking upstream from bridge crossing & 90 & W-05-093_P5290002 \\
\hline W-05-094 & Looking upstream from culvert crossing & 90 & W-05-094_P5310008 \\
\hline W-05-094 & Looking downstream from culvert crossing & 270 & W-05-094_P5310009 \\
\hline W-05-094 & Looking at culvert inlet & 300 & W-05-094_P5310010 \\
\hline W-05-094 & Looking at culvert outlet & 45 & W-05-094_P5310011 \\
\hline W-05-095 & Looking at culvert inlet & 90 & W-05-095_P5310012 \\
\hline W-05-095 & Looking upstream from grass crossing & 270 & W-05-095_P5310013 \\
\hline W-05-095 & Looking downstream from grass crossing & 90 & W-05-095_P5310014 \\
\hline W-05-095 & Looking at culvert outlet & 300 & W-05-095_P5310015 \\
\hline W-05-096 & Looking upstream from culvert crossing & 270 & W-05-096_P5310016 \\
\hline W-05-096 & Looking downstream from culvert crossing & 90 & W-05-096_P5310017 \\
\hline W-05-096 & Looking at culvert inlet & 300 & W-05-096_P5310018 \\
\hline W-05-096 & Looking at culvert outlet & 150 & W-05-096_P5310019 \\
\hline W-05-097 & Looking upstream from culvert crossing & 115 & W-05-097_P5310020 \\
\hline W-05-097 & Looking upstream from culvert crossing & 150 & W-05-097_P5310021 \\
\hline W-05-097 & Looking upstream from culvert crossing & 180 & W-05-097_P5310022 \\
\hline W-05-097 & Looking upstream from culvert crossing & 230 & W-05-097_P5310023 \\
\hline W-05-097 & Looking downstream from culvert crossing & 0 & W-05-097_P5310024 \\
\hline W-05-098 & Looking upstream from culvert crossing & 180 & W-05-098_P5310025 \\
\hline W-05-098 & Looking downstream from culvert crossing & 0 & W-05-098_P5310026 \\
\hline W-05-098 & Looking at culvert inlet & 30 & W-05-098_P5310027 \\
\hline W-05-098 & Looking at culvert outlet & 120 & W-05-098_P5310028 \\
\hline W-05-099 & Looking upstream from culvert crossing & 180 & W-05-099_P5310029 \\
\hline W-05-099 & Looking downstream from culvert crossing & 0 & W-05-099_P5310030 \\
\hline W-05-099 & Looking at culvert inlet & 30 & W-05-099_P5310031 \\
\hline W-05-099 & Looking at culvert outlet & 140 & W-05-099_P5310032 \\
\hline
\end{tabular}


Appendix 8: List of all photographs taken showing the corresponding site number, descriptive caption, direction faced when taken (azimuth in degrees) and the unique file name for each photograph.

\begin{tabular}{|c|c|c|c|}
\hline $\begin{array}{c}\text { Site } \\
\text { Number }\end{array}$ & Photo Description & Azimuth & File Name \\
\hline W-05-100 & Looking upstream from culvert crossing & 180 & W-05-100 P5310033 \\
\hline W-05-100 & Photo of cattle impacts & 215 & W-05-100 P5310034 \\
\hline W-05-100 & Looking downstream from culvert crossing & 0 & W-05-100_P5310035 \\
\hline W-05-100 & Looking at culvert inlet & 30 & W-05-100_P5310036 \\
\hline W-05-100 & Looking at culvert outlet & 200 & W-05-100 P5310037 \\
\hline W-05-101 & Looking upstream from culvert crossing & 180 & W-05-101_P6010001 \\
\hline W-05-101 & Looking downstream from culvert crossing & 15 & W-05-101_P6010002 \\
\hline W-05-101 & Looking at culvert inlet & 15 & W-05-101_P6010003 \\
\hline W-05-101 & Looking at culvert outlet & 160 & W-05-101 P6010004 \\
\hline W-05-102 & Looking upstream from culvert crossing & 180 & W-05-102_P6010005 \\
\hline W-05-102 & Looking downstream from culvert crossing & 0 & W-05-102_P6010006 \\
\hline W-05-102 & Looking at culvert inlet & 340 & W-05-102_P6010007 \\
\hline W-05-102 & Looking at culvert outlet & 220 & W-05-102 P6010008 \\
\hline W-05-103 & Looking upstream from culvert crossing & 180 & W-05-103 P6010009 \\
\hline W-05-103 & Looking downstream from culvert crossing & 0 & W-05-103_P6010010 \\
\hline W-05-103 & Looking at culvert inlet & 0 & W-05-103 P6010011 \\
\hline W-05-103 & Looking at culvert outlet & 180 & W-05-103 P6010012 \\
\hline W-05-104 & Looking upstream from culvert crossing & 180 & W-05-104_P6010013 \\
\hline W-05-104 & Looking downstream from culvert crossing & 0 & W-05-104_P6010014 \\
\hline W-05-104 & Looking at culvert inlet & 45 & W-05-104 P6010015 \\
\hline W-05-104 & Looking at culvert outlet & 135 & W-05-104 P6010016 \\
\hline W-05-104 & Looking at left bank downstream of culvert crossing & 90 & W-05-104_P6010017 \\
\hline W-05-105 & Looking upstream from culvert crossing at 1 st order tributary & 180 & W-05-105_P6010018 \\
\hline W-05-105 & Looking upstream from culvert crossing at 3rd order tributary & 150 & W-05-105 P6010019 \\
\hline W-05-105 & Looking downstream from culvert crossing & 0 & W-05-105 P6010020 \\
\hline W-05-105 & Looking at culvert inlet & 60 & W-05-105_P6010021 \\
\hline W-05-105 & Looking at culvert outlet & 170 & W-05-105_P6010022 \\
\hline W-05-106 & Looking upstream from culvert crossing & 180 & W-05-106 P6020001 \\
\hline W-05-106 & Looking downstream from culvert crossing & 0 & W-05-106 P6020002 \\
\hline W-05-106 & Looking at culvert inlet & 0 & W-05-106 P6020003 \\
\hline W-05-106 & Looking at culvert outlet & 180 & W-05-106_P6020004 \\
\hline W-05-107 & Looking upstream from culvert crossing & 180 & W-05-107 P6030001 \\
\hline W-05-107 & Looking downstream from culvert crossing & 45 & W-05-107 P6030002 \\
\hline
\end{tabular}


Appendix 8: List of all photographs taken showing the corresponding site number, descriptive caption, direction faced when taken (azimuth in degrees) and the unique file name for each photograph.

\begin{tabular}{|c|c|c|c|}
\hline $\begin{array}{c}\text { Site } \\
\text { Number }\end{array}$ & Photo Description & Azimuth & File Name \\
\hline W-05-107 & Looking at culvert inlet & 45 & $\begin{array}{ll}\mathrm{W}-05-107 & \mathrm{P} 6030003\end{array}$ \\
\hline W-05-107 & Looking at culvert outlet & 230 & W-05-107_P6030004 \\
\hline W-05-108 & Looking upstream from culvert crossing & 120 & W-05-108_P6030005 \\
\hline W-05-108 & Looking downstream from culvert crossing & 310 & W-05-108_P6030006 \\
\hline W-05-108 & Looking at culvert inlet & 260 & W-05-108 P6030007 \\
\hline W-05-108 & Looking at beaver dam from downstream & 145 & W-05-108 P6030008 \\
\hline W-05-108 & Photo of falls (zoomed in) & 180 & W-05-108_P6030009 \\
\hline W-05-108 & Looking at beaver dam from road & 90 & W-05-108 P6030010 \\
\hline W-05-109 & Looking upstream from culvert crossing & 90 & W-05-109 P6030011 \\
\hline W-05-109 & Looking downstream from culvert crossing & 270 & W-05-109_P6030012 \\
\hline W-05-109 & Looking at culvert inlet & 240 & W-05-109_P6030013 \\
\hline W-05-109 & Looking at culvert outlet & 45 & W-05-109 P6030014 \\
\hline W-05-110 & Looking upstream from culvert crossing & 180 & W-05-110 P6040001 \\
\hline W-05-110 & Looking downstream from culvert crossing & 0 & W-05-110 P6040002 \\
\hline W-05-110 & Looking at culvert inlet & 0 & W-05-110_P6040003 \\
\hline W-05-110 & Looking at culvert outlet & 180 & W-05-110 P6040004 \\
\hline W-05-111 & Looking upstream from culvert crossing & 230 & W-05-111 P6040005 \\
\hline W-05-111 & Looking downstream from culvert crossing & 30 & W-05-111_P6040006 \\
\hline W-05-111 & Looking at culvert inlet & 30 & W-05-111_P6040007 \\
\hline W-05-111 & Looking at culvert outlet & 180 & W-05-111 P6040008 \\
\hline W-05-112 & Looking upstream from bridge crossing & 120 & W-05-112 P6040009 \\
\hline W-05-112 & Looking downstream from bridge crossing & 300 & W-05-112_P6040010 \\
\hline W-05-112 & Photo of male sucker, fork length $-178 \mathrm{~mm}$ & $\mathrm{~N} / \mathrm{A}$ & W-05-112_P6040011 \\
\hline W-05-112 & Photo of Black Bullhead, fork length- $191 \mathrm{~mm}$ & N/A & W-05-112 P6040012 \\
\hline W-05-112 & Photo of female sucker, fork length- $190 \mathrm{~mm}$ & $\mathrm{~N} / \mathrm{A}$ & W-05-112_P6040013 \\
\hline W-05-112 & Photo of Northern Pike, fork length- $406 \mathrm{~mm}$ & $\mathrm{~N} / \mathrm{A}$ & W-05-112_P6040014 \\
\hline W-05-113 & Looking upstream from culvert crossing & 270 & W-05-113_P6060001 \\
\hline W-05-113 & Looking downstream from culvert crossing & 90 & W-05-113 P6060002 \\
\hline W-05-113 & Looking at culvert inlet & 140 & W-05-113 P6060004 \\
\hline W-05-113 & Looking at culvert outlet & 220 & W-05-113_P6060005 \\
\hline W-05-114 & Looking upstream from culvert crossing & 80 & W-05-114 P6060006 \\
\hline W-05-114 & Looking downstream from culvert crossing & 270 & W-05-114 P6060007 \\
\hline W-05-114 & Looking at culvert inlet & 270 & W-05-114_P6060008 \\
\hline
\end{tabular}


Appendix 8: List of all photographs taken showing the corresponding site number, descriptive caption, direction faced when taken (azimuth in degrees) and the unique file name for each photograph.

\begin{tabular}{|c|c|c|c|}
\hline $\begin{array}{c}\text { Site } \\
\text { Number }\end{array}$ & Photo Description & Azimuth & File Name \\
\hline W-05-114 & Looking at culvert outlet & 80 & $\begin{array}{l}\mathrm{W}-05-114 \\
\mathrm{P} 6060009\end{array}$ \\
\hline W-05-115 & Looking upstream from culvert crossing & 320 & W-05-115_P6070010 \\
\hline W-05-115 & Looking downstream from culvert crossing & 150 & W-05-115_P6070011 \\
\hline W-05-115 & Looking at culvert inlet & 150 & W-05-115_P6070012 \\
\hline W-05-115 & Looking at culvert outlet & 310 & W-05-115 P6070013 \\
\hline W-05-116 & Looking upstream from culvert crossing & 90 & W-05-116 P6070014 \\
\hline W-05-116 & Looking downstream from culvert crossing & 270 & W-05-116_P6070015 \\
\hline W-05-116 & Looking at culvert inlet & 270 & W-05-116 P6070016 \\
\hline W-05-116 & Looking at culvert outlet & 90 & W-05-116 P6070017 \\
\hline W-05-117 & Looking upstream from culvert crossing & 270 & W-05-117_P6070018 \\
\hline W-05-117 & Looking downstream from culvert crossing & 90 & W-05-117_P6070019 \\
\hline W-05-117 & Looking at culvert inlet & 90 & W-05-117 P6070020 \\
\hline W-05-117 & Looking at culvert outlet & 270 & W-05-117 P6070021 \\
\hline W-05-117 & Photo of bank scour & 300 & W-05-117_P6070022 \\
\hline W-05-117 & Photo of bank scour & 30 & W-05-117_P6070023 \\
\hline W-05-118 & Looking upstream from culvert crossing & 10 & W-05-118 P6080001 \\
\hline W-05-118 & Looking downstream from culvert crossing & 200 & W-05-118 P6080002 \\
\hline W-05-118 & Looking at culvert inlet & 160 & W-05-118_P6080003 \\
\hline W-05-118 & Looking at culvert outlet & 350 & W-05-118_P6080004 \\
\hline W-05-119 & Looking upstream from culvert crossing & 180 & W-05-119 P6080005 \\
\hline W-05-119 & Looking downstream from culvert crossing & 0 & W-05-119 P6080006 \\
\hline W-05-119 & Looking at culvert inlet & 0 & W-05-119_P6080007 \\
\hline W-05-119 & Looking at culvert outlet & 180 & W-05-119_P6080008 \\
\hline W-05-120 & Looking upstream from culvert crossing & 320 & W-05-120 P6090001 \\
\hline W-05-120 & Looking upstream of additional drain & 180 & W-05-120 P6090002 \\
\hline W-05-120 & Looking at culvert inlet & 90 & W-05-120 P6090003 \\
\hline W-05-120 & Looking downstream from culvert crossing & 90 & W-05-120_P6090004 \\
\hline W-05-120 & Looking at culvert outlet & 270 & W-05-120 P6090005 \\
\hline W-05-121 & Looking upstream from culvert crossing & 320 & W-05-121 P6090006 \\
\hline W-05-121 & Looking downstream from culvert crossing & 150 & W-05-121_P6090007 \\
\hline W-05-121 & Looking at culvert inlet & 150 & W-05-121 P6090008 \\
\hline W-05-121 & Looking at culvert outlet & 320 & W-05-121 P6090009 \\
\hline W-05-122 & Looking upstream from bridge crossing & 0 & W-05-122_P6090010 \\
\hline
\end{tabular}


Appendix 8: List of all photographs taken showing the corresponding site number, descriptive caption, direction faced when taken (azimuth in degrees) and the unique file name for each photograph.

\begin{tabular}{|c|c|c|c|}
\hline $\begin{array}{c}\text { Site } \\
\text { Number }\end{array}$ & Photo Description & Azimuth & File Name \\
\hline W-05-122 & Looking downstream from bridge crossing & 180 & W-05-122 P6090011 \\
\hline W-05-123 & Looking upstream of road crossing & $\mathrm{N} / \mathrm{A}$ & W-05-123_P6140001 \\
\hline W-05-123 & Looking downstream stream of road crossing & $\mathrm{N} / \mathrm{A}$ & W-05-123_P6140002 \\
\hline W-05-123 & Photo of dead beaver (zoomed in) & $\mathrm{N} / \mathrm{A}$ & W-05-123_P6140003 \\
\hline W-05-124 & Photo of water flowing over road & 0 & W-05-124 P6140004 \\
\hline W-05-124 & Looking upstream of road crossing & 270 & W-05-124_P6140005 \\
\hline W-05-124 & Looking upstream of road crossing & 90 & W-05-124_P6140006 \\
\hline W-05-125 & Photo of water going over the drain & 90 & W-05-125 P6140007 \\
\hline W-05-126 & Looking upstream from culvert crossing & 0 & W-05-126 P6140008 \\
\hline W-05-126 & Looking downstream from culvert crossing & 180 & W-05-126_P6140009 \\
\hline W-05-126 & Looking at culvert inlet & 180 & W-05-126_P6140010 \\
\hline W-05-126 & Looking at culvert outlet & 0 & W-05-126 P6140011 \\
\hline W-05-127 & Looking upstream from culvert crossing & 90 & W-05-127 P6140012 \\
\hline W-05-127 & Looking upstream from culvert crossing & 340 & W-05-127_P6140013 \\
\hline W-05-127 & Looking downstream from culvert crossing & 300 & W-05-127_P6140014 \\
\hline W-05-127 & Looking at culvert inlet & 280 & W-05-127 P6140015 \\
\hline W-05-127 & Looking at culvert outlet & 70 & W-05-127 P6140016 \\
\hline W-05-128 & Looking upstream from culvert crossing & 160 & W-05-128_P6140017 \\
\hline W-05-128 & Looking downstream from culvert crossing & 300 & W-05-128_P6140018 \\
\hline W-05-128 & Looking at culvert inlet & 320 & W-05-128 P6140019 \\
\hline W-05-128 & Looking at culvert outlet & 130 & W-05-128 P6140020 \\
\hline W-05-129 & Looking upstream from culvert crossing & 270 & W-05-129_P6150001 \\
\hline W-05-129 & Looking downstream from culvert crossing & 90 & W-05-129 P6150002 \\
\hline W-05-129 & Looking at culvert inlet & 260 & W-05-129 P6150003 \\
\hline W-05-129 & Looking at culvert outlet & 60 & W-05-129_P6150004 \\
\hline W-05-129 & Photo of $700 \mathrm{~mm}$ Common Carp & $\mathrm{N} / \mathrm{A}$ & W-05-129_P6150007 \\
\hline W-05-129 & Photo of $700 \mathrm{~mm}$ Common Carp & $\mathrm{N} / \mathrm{A}$ & W-05-129_P6150008 \\
\hline W-05-130 & Looking upstream from culvert crossing & 0 & W-05-130 P6150009 \\
\hline W-05-130 & Looking downstream from culvert crossing & 180 & W-05-130 P6150010 \\
\hline W-05-130 & Looking at culvert inlet & 160 & W-05-130 P6150011 \\
\hline W-05-130 & Looking at culvert outlet & 340 & W-05-130 P6150012 \\
\hline W-05-131 & Looking upstream from culvert crossing & 0 & W-05-131 P6150013 \\
\hline W-05-131 & Looking at tributary to Sylvan Drain & 270 & W-05-131_P6150014 \\
\hline
\end{tabular}


Appendix 8: List of all photographs taken showing the corresponding site number, descriptive caption, direction faced when taken (azimuth in degrees) and the unique file name for each photograph.

\begin{tabular}{|c|c|c|c|}
\hline $\begin{array}{c}\text { Site } \\
\text { Number }\end{array}$ & Photo Description & Azimuth & File Name \\
\hline W-05-131 & Looking at tributary to Sylvan Drain & 270 & $\begin{array}{ll}\mathrm{W}-05-131 & \mathrm{P} 6150015\end{array}$ \\
\hline W-05-131 & Looking downstream from Sylvan Drain culvert crossing & 180 & W-05-131_P6150016 \\
\hline W-05-131 & Looking at culvert inlet & 180 & W-05-131_P6150017 \\
\hline W-05-131 & Looking at culvert outlet & 0 & W-05-131_P6150018 \\
\hline W-05-132 & Looking upstream from culvert crossing & 270 & W-05-132 P6150019 \\
\hline W-05-132 & Looking downstream from culvert crossing & 90 & W-05-132 P6150020 \\
\hline W-05-132 & Looking at culvert inlet & 70 & W-05-132_P6150021 \\
\hline W-05-132 & Looking at culvert outlet & 290 & W-05-132 P6150022 \\
\hline W-05-133 & Looking upstream from right bank dike (20 m from road) & 180 & W-05-133 P6150023 \\
\hline W-05-133 & Looking downstream from right bank dike ( $20 \mathrm{~m}$ from road) & 90 & W-05-133_P6150024 \\
\hline W-05-133 & Looking at flooded field from right bank dike & 230 & W-05-133_P6150025 \\
\hline W-05-133 & Looking upstream 1st order tributary (Shurkas Drain) & 0 & W-05-133_P6150026 \\
\hline W-05-133 & Photo of squirrel on road & $\mathrm{N} / \mathrm{A}$ & W-05-133 P6150027 \\
\hline W-05-133 & Looking at flooded field from right bank dike & 180 & W-05-133 P6150028 \\
\hline W-05-134 & Looking upstream from right bank & 180 & W-05-134_P6150031 \\
\hline W-05-134 & Looking downstream from right bank & 0 & W-05-134 P6150032 \\
\hline W-05-135 & Looking upstream from culvert crossing & 220 & W-05-135 P6160001 \\
\hline W-05-135 & Looking upstream from culvert crossing & 160 & W-05-135_P6160002 \\
\hline W-05-135 & Looking upstream from culvert crossing & 100 & W-05-135_P6160003 \\
\hline W-05-135 & Looking downstream from culvert crossing & 320 & W-05-135 P6160004 \\
\hline W-05-135 & Looking downstream from culvert crossing & 350 & W-05-135 P6160005 \\
\hline W-05-135 & Looking downstream from culvert crossing & 20 & W-05-135_P6160006 \\
\hline W-05-136 & Looking upstream from culvert crossing & 200 & W-05-136_P6160007 \\
\hline W-05-136 & Looking downstream from culvert crossing & 0 & W-05-136 P6160008 \\
\hline W-05-136 & Looking at culvert inlet & 20 & W-05-136_P6160009 \\
\hline W-05-136 & Looking at culvert outlet & 180 & W-05-136_P6160010 \\
\hline W-05-137 & Looking upstream from culvert crossing & 180 & W-05-137_P6160011 \\
\hline W-05-137 & Looking downstream from culvert crossing & 0 & W-05-137 P6160012 \\
\hline W-05-137 & Looking at culvert inlet & 0 & W-05-137 P6160013 \\
\hline W-05-137 & Looking at culvert outlet & 180 & W-05-137_P6160014 \\
\hline W-05-138 & Looking upstream from culvert crossing & 180 & W-05-138 P6160015 \\
\hline W-05-138 & Looking downstream from culvert crossing & 0 & W-05-138 P6160016 \\
\hline W-05-138 & Looking at culvert inlet & 0 & W-05-138_P6160017 \\
\hline
\end{tabular}


Appendix 8: List of all photographs taken showing the corresponding site number, descriptive caption, direction faced when taken (azimuth in degrees) and the unique file name for each photograph.

\begin{tabular}{|c|c|c|c|}
\hline $\begin{array}{c}\text { Site } \\
\text { Number }\end{array}$ & Photo Description & Azimuth & File Name \\
\hline W-05-138 & Looking at culvert outlet & 180 & W-05-138_P6160018 \\
\hline W-05-139 & Looking upstream from culvert crossing & 180 & W-05-139 P6170001 \\
\hline W-05-139 & Looking downstream from culvert crossing & 0 & W-05-139_P6170002 \\
\hline W-05-139 & Looking at culvert inlet & 20 & W-05-139_P6170003 \\
\hline W-05-139 & Looking at culvert outlet & 200 & W-05-139_P6170004 \\
\hline W-05-140 & Looking upstream from culvert crossing & 180 & W-05-140 P6170005 \\
\hline W-05-140 & Looking downstream from culvert crossing & 0 & W-05-140_P6170006 \\
\hline W-05-140 & Looking at culvert inlet & 40 & W-05-140_P6170007 \\
\hline W-05-140 & Looking at culvert outlet & 230 & W-05-140 P6170008 \\
\hline W-05-142 & Looking upstream from culvert crossing & 180 & W-05-142 P6200009 \\
\hline W-05-142 & Looking downstream from culvert crossing & 0 & W-05-142_P6200010 \\
\hline W-05-142 & Looking at culvert inlet & 0 & W-05-142_P6200011 \\
\hline W-05-142 & Looking at culvert outlet & 180 & W-05-142 P6200012 \\
\hline W-05-143 & Looking upstream from culvert crossing & 180 & W-05-143 P6200013 \\
\hline W-05-143 & Looking downstream from culvert crossing & 0 & W-05-143_P6200014 \\
\hline W-05-144 & Looking upstream from culvert crossing & 0 & W-05-144_P6200015 \\
\hline W-05-144 & Looking downstream from culvert crossing & 180 & W-05-144 P6200016 \\
\hline W-05-144 & Looking at culvert inlet & 200 & W-05-144_P6200017 \\
\hline W-05-144 & Looking at culvert outlet & 340 & W-05-144_P6200018 \\
\hline W-05-145 & Looking upstream from culvert crossing & 270 & W-05-145_P6200019 \\
\hline W-05-145 & Looking downstream from culvert crossing & 90 & W-05-145 P6200020 \\
\hline W-05-145 & Looking at culvert inlet & 115 & W-05-145_P6200021 \\
\hline W-05-145 & Looking at culvert outlet & 300 & W-05-145_P6200022 \\
\hline W-05-145 & Looking at vertical culvert & 350 & W-05-145 P6200023 \\
\hline W-05-146 & Looking upstream from culvert crossing & 270 & W-05-146 P6210001 \\
\hline W-05-146 & Looking downstream from culvert crossing & 90 & W-05-146_P6210002 \\
\hline W-05-146 & Looking at culvert inlet & 70 & W-05-146_P6210003 \\
\hline W-05-146 & Looking at pool & 35 & W-05-146_P6210004 \\
\hline W-05-146 & Looking at culvert outlet & 270 & W-05-146 P6210005 \\
\hline W-05-147 & Photo of bison from road & 270 & W-05-147_P6210006 \\
\hline W-05-147 & Photo of bison from road & 270 & W-05-147_P6210007 \\
\hline W-05-147 & Looking upstream from culvert crossing & 300 & W-05-147 P6210008 \\
\hline W-05-147 & Looking downstream from culvert crossing & 120 & W-05-147 P6210009 \\
\hline
\end{tabular}


Appendix 8: List of all photographs taken showing the corresponding site number, descriptive caption, direction faced when taken (azimuth in degrees) and the unique file name for each photograph.

\begin{tabular}{|c|c|c|c|}
\hline $\begin{array}{c}\text { Site } \\
\text { Number }\end{array}$ & Photo Description & Azimuth & File Name \\
\hline W-05-147 & Looking at culvert outlet & 270 & W-05-147_P6210010 \\
\hline W-05-147 & Looking at culvert outlet & 30 & W-05-147_P6210011 \\
\hline W-05-147 & Photo of bison from road & 270 & W-05-147 P6210012 \\
\hline W-05-148 & Looking upstream from bridge crossing & 310 & W-05-148_P6210013 \\
\hline W-05-148 & Looking downstream from bridge crossing & 120 & W-05-148_P6210014 \\
\hline W-05-149 & Looking upstream from culvert crossing & 155 & W-05-149_P6220001 \\
\hline W-05-149 & Looking downstream from culvert crossing & 10 & W-05-149 P6220002 \\
\hline W-05-149 & Looking downstream from culvert crossing & 80 & W-05-149P6220003 \\
\hline W-05-149 & Looking at culvert inlet & 50 & W-05-149_P6220004 \\
\hline W-05-149 & Looking at culvert outlet & 320 & W-05-149_P6220005 \\
\hline W-05-149 & Looking at bank scour/ slump right bank downstream from culverts & 0 & W-05-149 P6220006 \\
\hline W-05-149 & Looking at bank scour/ slump right bank downstream from culverts & 80 & W-05-149_P6220007 \\
\hline W-05-149 & Looking at bank scour/ slump left bank downstream $20 \mathrm{~m}$ from culverts & 30 & W-05-149_P6220008 \\
\hline W-05-150 & Looking upstream from culvert crossing & 270 & W-05-150_P6220009 \\
\hline W-05-150 & Looking downstream from culvert crossing & 90 & W-05-150 P6220010 \\
\hline W-05-150 & Looking at culvert inlet & 90 & W-05-150_P6220011 \\
\hline W-05-150 & Looking at culvert outlet & 270 & W-05-150_P6220012 \\
\hline W-05-150 & Photo of Burbot & $\mathrm{N} / \mathrm{A}$ & W-05-150_P6220013 \\
\hline W-05-150 & Photo of Burbot & $\mathrm{N} / \mathrm{A}$ & W-05-150 P6220014 \\
\hline W-05-151 & Looking upstream from culvert crossing & 270 & W-05-151_P6220015 \\
\hline W-05-151 & Looking downstream from culvert crossing & 90 & W-05-151_P6220016 \\
\hline W-05-151 & Looking at culvert inlet & 90 & W-05-151_P6220017 \\
\hline W-05-152 & Looking upstream from ford crossing & 270 & W-05-152 P6220018 \\
\hline W-05-152 & Looking downstream from ford crossing & 90 & W-05-152_P6220019 \\
\hline W-05-152 & Looking at ford crossing & 0 & W-05-152_P6220020 \\
\hline W-05-152 & Photo of cattle impacts & $\mathrm{N} / \mathrm{A}$ & W-05-152_P6220021 \\
\hline W-05-153 & Looking upstream from culvert crossing & 220 & W-05-153 P6220022 \\
\hline W-05-153 & Looking downstream from culvert crossing & 20 & W-05-153_P6220023 \\
\hline W-05-154 & Looking upstream from bridge crossing & 270 & W-05-154_P6220024 \\
\hline W-05-154 & Looking downstream from bridge crossing & 90 & W-05-154_P6220025 \\
\hline W-05-168 & Looking upstream from PR 470 crossing & 40 & W-05-168 P7110001 \\
\hline W-05-168 & Looking downstream from PR 470 crossing & 240 & W-05-168_P7110002 \\
\hline W-05-169 & Looking upstream from culvert crossing & 180 & W-05-169P7120001 \\
\hline
\end{tabular}


Appendix 8: List of all photographs taken showing the corresponding site number, descriptive caption, direction faced when taken (azimuth in degrees) and the unique file name for each photograph.

\begin{tabular}{|c|c|c|c|}
\hline $\begin{array}{c}\text { Site } \\
\text { Number }\end{array}$ & Photo Description & Azimuth & File Name \\
\hline W-05-169 & Looking downstream from culvert crossing & 0 & W-05-169 P7120002 \\
\hline W-05-169 & Looking at culvert inlet & 200 & W-05-169 P7120003 \\
\hline W-05-169 & Looking at culvert inlet & $\mathrm{N} / \mathrm{A}$ & W-05-169_P7120004 \\
\hline W-05-169 & Looking at culvert outlet & 180 & W-05-169 P7120005 \\
\hline W-05-170 & Looking upstream from culvert crossing & 340 & W-05-170_P7120007 \\
\hline W-05-170 & Looking downstream from culvert crossing & 170 & W-05-170 P7120008 \\
\hline W-05-170 & Looking at culvert inlet & 180 & W-05-170_P7120009 \\
\hline W-05-170 & Looking at culvert outlet & 300 & W-05-170_P7120010 \\
\hline W-05-171 & Looking upstream from culvert crossing & 0 & W-05-171_P7120011 \\
\hline W-05-171 & Looking downstream from culvert crossing & 180 & W-05-171_P7120012 \\
\hline W-05-171 & Looking at culvert inlet & 120 & W-05-171 P7120013 \\
\hline W-05-171 & Looking at culvert outlet & 240 & W-05-171_P7120014 \\
\hline W-05-172 & Looking upstream bridge crossing & 360 & W-05-172_P7120015 \\
\hline W-05-172 & Looking downstream bridge crossing & 180 & W-05-172 P7120016 \\
\hline W-05-174 & Looking upstream at weir & 300 & W-05-174_P7180001 \\
\hline W-05-174 & Looking downstream at weir & 100 & W-05-174_P7180002 \\
\hline W-05-174 & Looking at riffle further upstream & 300 & W-05-174_P7180003 \\
\hline W-05-174 & Looking at 2nd weir further upstream & 300 & W-05-174 P7180004 \\
\hline W-05-175 & Photo of guys canoeing down Boundary Drain & $\mathrm{N} / \mathrm{A}$ & W-05-175_P7180005 \\
\hline W-05-175 & Photo of guys canoeing down Boundary Drain & $\mathrm{N} / \mathrm{A}$ & W-05-175_P7180006 \\
\hline W-05-175 & Looking upstream Boundary Drain & 310 & W-05-175 P7180007 \\
\hline W-05-175 & Looking upstream at 2nd order tributary just north of Dola crossing & 40 & W-05-175_P7180008 \\
\hline W-05-175 & Looking downstream Boundary Drain & 170 & W-05-175_P7180009 \\
\hline W-05-175 & Looking at flooded field southwest of road junction & 170 & W-05-175_P7180010 \\
\hline W-05-176 & Looking upstream from edge of left bank & 270 & W-05-176_P7180011 \\
\hline W-05-176 & Looking downstream from edge of left bank & 90 & W-05-176_P7180012 \\
\hline W-05-177 & Looking upstream from culvert crossing & 270 & W-05-177_P7190001 \\
\hline W-05-177 & Looking downstream from culvert crossing & 90 & W-05-177_P7190002 \\
\hline W-05-177 & Looking at culvert inlet & 90 & W-05-177_P7190003 \\
\hline W-05-177 & Looking at culvert outlet & 270 & W-05-177_P7190004 \\
\hline W-05-178 & Looking upstream from culvert crossing & 270 & W-05-178 P7190005 \\
\hline W-05-178 & Looking downstream from culvert crossing & 90 & W-05-178_P7190006 \\
\hline W-05-178 & Looking at culvert inlet & 75 & W-05-178_P7190007 \\
\hline
\end{tabular}


Appendix 8: List of all photographs taken showing the corresponding site number, descriptive caption, direction faced when taken (azimuth in degrees) and the unique file name for each photograph.

\begin{tabular}{|c|c|c|c|}
\hline $\begin{array}{c}\text { Site } \\
\text { Number }\end{array}$ & Photo Description & Azimuth & File Name \\
\hline W-05-178 & Looking at culvert inlet & 75 & W-05-178_P7190008 \\
\hline W-05-178 & Looking at culvert outlet & 300 & W-05-178_P7190009 \\
\hline W-05-178 & Looking at culvert outlet of south tributary to Meleb Drain & 270 & W-05-178_P7190010 \\
\hline W-05-179 & Looking upstream from culvert crossing & 270 & W-05-179_P7190011 \\
\hline W-05-179 & Looking downstream from culvert crossing & 90 & W-05-179_P7190012 \\
\hline W-05-180 & Looking downstream from culvert crossing & 150 & W-05-180_P7190013 \\
\hline W-05-180 & Looking at culvert inlet & 190 & W-05-180_P7190014 \\
\hline W-05-180 & Looking at culvert outlet & 20 & W-05-180_P7190015 \\
\hline W-05-181 & Looking upstream from culvert crossing & 270 & W-05-181_P7190016 \\
\hline W-05-181 & Looking downstream from culvert crossing & 90 & W-05-181_P7190017 \\
\hline W-05-181 & Looking at culvert inlet & 80 & W-05-181_P7190018 \\
\hline W-05-181 & Looking at culvert outlet & 280 & W-05-181_P7190019 \\
\hline W-05-182 & Looking upstream from left bank & 270 & W-05-182_P7190020 \\
\hline W-05-182 & Looking downstream from left bank & 90 & W-05-182_P7190021 \\
\hline W-05-183 & Looking upstream from culvert crossing & 270 & W-05-183_P7190022 \\
\hline W-05-183 & Looking downstream from culvert crossing & 90 & W-05-183_P7190023 \\
\hline W-05-183 & Looking at culvert inlet & 90 & W-05-183_P7190024 \\
\hline W-05-183 & Looking at culvert outlet & 270 & W-05-183_P7190025 \\
\hline W-05-184 & Looking upstream from culvert crossing & 340 & W-05-184_P7200001 \\
\hline W-05-184 & Looking downstream from culvert crossing & 160 & W-05-184_P7200002 \\
\hline W-05-185 & Looking upstream from culvert crossing & 270 & W-05-185_P7200003 \\
\hline W-05-185 & Looking downstream from culvert crossing & 90 & W-05-185_P7200004 \\
\hline W-05-185 & Looking at culvert inlet & 90 & W-05-185_P7200005 \\
\hline W-05-185 & Looking at culvert outlet & 270 & W-05-185_P7200006 \\
\hline W-05-186 & Looking upstream from left bank & 360 & W-05-186_P7250001 \\
\hline W-05-186 & Looking downstream from left bank & 180 & W-05-186_P7250002 \\
\hline W-05-187 & Looking upstream of road crossing & 20 & W-05-187_P7250003 \\
\hline W-05-187 & Looking downstream stream of road crossing & 200 & W-05-187_P7250004 \\
\hline W-05-187 & Looking at flooded road & 90 & W-05-187_P7250005 \\
\hline W-05-188 & Looking upstream from right bank at washed out culvert crossing & 90 & W-05-188_P7250006 \\
\hline W-05-188 & Looking downstream from right bank at washed out culvert crossing & 270 & W-05-188_P7250007 \\
\hline W-05-188 & Looking upstream of culvert crossing looking at tributary through field & 50 & W-05-188_P7250008 \\
\hline W-05-188 & Looking downstream of culvert crossing looking at drain & 360 & W-05-188_P7250009 \\
\hline
\end{tabular}


Appendix 8: List of all photographs taken showing the corresponding site number, descriptive caption, direction faced when taken (azimuth in degrees) and the unique file name for each photograph.

\begin{tabular}{|c|c|c|c|}
\hline $\begin{array}{c}\text { Site } \\
\text { Number }\end{array}$ & Photo Description & Azimuth & File Name \\
\hline W-05-188 & Looking at washed out culvert from upstream & 280 & $\begin{array}{lll}W-05-188 & \text { P7250010 }\end{array}$ \\
\hline W-05-189 & Looking upstream from culvert crossing & 90 & W-05-189 P7260001 \\
\hline W-05-189 & Looking downstream from culvert crossing & 270 & W-05-189_P7260002 \\
\hline W-05-189 & Looking at culvert inlet & 225 & W-05-189_P7260003 \\
\hline W-05-189 & Looking at culvert outlet & 150 & W-05-189_P7260004 \\
\hline W-05-189 & Photo of Northern Pike (fork length $=145 \mathrm{~mm}$ ) & $\mathrm{N} / \mathrm{A}$ & W-05-189 P7260005 \\
\hline W-05-189 & Photo of young of the year suckers & $\mathrm{N} / \mathrm{A}$ & W-05-189_P7260006 \\
\hline W-05-190 & Looking upstream bridge crossing (3rd order) & 90 & W-05-190_P7260007 \\
\hline W-05-190 & Looking downstream bridge crossing (3rd order) & 270 & W-05-190_P7260008 \\
\hline W-05-190 & Looking upstream on 2nd order (north) tributary & 360 & W-05-190 P7260009 \\
\hline W-05-190 & Looking at culvert inlet of 2 nd order tributary & 240 & W-05-190_P7260010 \\
\hline W-05-190 & Looking at culvert outlet of 2 nd order tributary & 360 & W-05-190_P7260011 \\
\hline W-05-190 & Looking upstream on other 3rd order tributary (south) & 180 & W-05-190_P7260012 \\
\hline W-05-191 & Photo of Ashley P with garter snake & $\mathrm{N} / \mathrm{A}$ & W-05-191_P7260013 \\
\hline W-05-191 & Looking upstream bridge crossing at T-Drain & 90 & W-05-191_P7260014 \\
\hline W-05-191 & Looking upstream at 3rd order tributary & 0 & W-05-191_P7260015 \\
\hline W-05-191 & Looking downstream bridge crossing at T-Drain & 270 & W-05-191_P7260016 \\
\hline W-05-192 & Looking upstream from culvert crossing & 90 & W-05-192 P7260017 \\
\hline W-05-192 & Looking downstream from culvert crossing & 270 & W-05-192_P7260018 \\
\hline W-05-192 & Looking at culvert inlet & 200 & W-05-192_P7260019 \\
\hline W-05-192 & Looking at culvert outlet & 180 & W-05-192_P7260020 \\
\hline W-05-193 & Looking upstream from culvert crossing & 90 & W-05-193_P7260021 \\
\hline W-05-193 & Looking downstream from culvert crossing & 270 & W-05-193_P7260022 \\
\hline W-05-193 & Looking at culvert inlet & 220 & W-05-193_P7260023 \\
\hline W-05-193 & Looking at culvert outlet & 145 & W-05-193 P7260024 \\
\hline W-05-194 & Looking upstream from culvert crossing & 90 & W-05-194_P7270001 \\
\hline W-05-194 & Looking downstream from culvert crossing & 270 & W-05-194_P7270002 \\
\hline W-05-194 & Looking at culvert inlet & 345 & W-05-194_P7270003 \\
\hline W-05-194 & Looking at culvert outlet & 105 & W-05-194 P7270004 \\
\hline W-05-194 & Photo of Burbot & $\mathrm{N} / \mathrm{A}$ & W-05-194_P7270005 \\
\hline W-05-194 & Photo of Burbot & $\mathrm{N} / \mathrm{A}$ & W-05-194_P7270006 \\
\hline W-05-194 & Photo of Burbot & $\mathrm{N} / \mathrm{A}$ & W-05-194_P7270007 \\
\hline W-05-195 & Looking upstream from culvert crossing & 180 & W-05-195_P7270008 \\
\hline
\end{tabular}


Appendix 8: List of all photographs taken showing the corresponding site number, descriptive caption, direction faced when taken (azimuth in degrees) and the unique file name for each photograph.

\begin{tabular}{|c|c|c|c|}
\hline $\begin{array}{c}\text { Site } \\
\text { Number }\end{array}$ & Photo Description & Azimuth & File Name \\
\hline W-05-195 & Looking downstream from culvert crossing & 270 & W-05-195_P7270009 \\
\hline W-05-195 & Looking at culvert inlet & 200 & W-05-195_P7270010 \\
\hline W-05-195 & Looking at culvert outlet & 150 & W-05-195 P7270011 \\
\hline W-05-196 & Looking upstream from culvert crossing & 90 & W-05-196_P7270012 \\
\hline W-05-196 & Looking downstream from culvert crossing & 270 & W-05-196_P7270013 \\
\hline W-05-196 & Looking at culvert inlet & 205 & W-05-196_P7270014 \\
\hline W-05-196 & Looking at culvert outlet & 110 & W-05-196_P7270015 \\
\hline W-05-196 & Looking upstream at Bachman East Drain & 180 & W-05-196 P7270016 \\
\hline W-05-196 & Looking downstream at Bachman East Drain & 360 & W-05-196_P7270017 \\
\hline W-05-196 & Looking at culvert inlets of Bachman East Drain & 45 & W-05-196_P7270018 \\
\hline W-05-196 & Looking at culvert outlets of Bachman East Drain & 180 & W-05-196_P7270019 \\
\hline W-05-197 & Looking upstream at waterfall from left bank & 220 & W-05-197_P7270023 \\
\hline W-05-197 & Looking downstream from left bank & 340 & W-05-197_P7270024 \\
\hline W-05-198 & Looking upstream from culvert crossing & 270 & W-05-198_P7280001 \\
\hline W-05-198 & Looking downstream from culvert crossing & 90 & W-05-198_P7280002 \\
\hline W-05-198 & Looking at culvert inlet & 115 & W-05-198_P7280003 \\
\hline W-05-198 & Looking at culvert outlet & 240 & W-05-198_P7280004 \\
\hline W-05-198 & Looking upstream at cleaned out tributary & 180 & W-05-198_P7280005 \\
\hline W-05-199 & Looking upstream from culvert crossing & 315 & W-05-199_P8020001 \\
\hline W-05-199 & Looking downstream from culvert crossing & 150 & W-05-199_P8020002 \\
\hline W-05-199 & Looking at culvert inlet & 180 & W-05-199_P8020003 \\
\hline W-05-199 & Looking at culvert outlet & 280 & W-05-199_P8020004 \\
\hline W-05-200 & Looking upstream from right bank & 300 & W-05-200_P8020005 \\
\hline W-05-200 & Looking downstream from right bank & 120 & W-05-200 P8020006 \\
\hline W-05-201 & Looking upstream from 1st culvert crossing at northwest tributary & 315 & W-05-201_P8020007 \\
\hline W-05-201 & Looking downstream from 1st culvert crossing at west tributary & 270 & W-05-201_P8020008 \\
\hline W-05-201 & Looking upstream from 2nd culvert crossing & 350 & W-05-201_P8020009 \\
\hline W-05-201 & Looking at culvert inlet west tributary & 90 & W-05-201_P8020010 \\
\hline W-05-201 & Looking at culvert inlet to northwest tributary & 115 & W-05-201_P8020011 \\
\hline W-05-201 & Looking at culvert inlet 2 nd culvert crossing & 140 & W-05-201_P8020012 \\
\hline W-05-201 & Looking downstream 2nd culvert crossing & 270 & W-05-201_P8020013 \\
\hline W-05-202 & Looking at culvert outlet 2 nd culvert crossing & 0 & W-05-202_P8020014 \\
\hline W-05-202 & Looking upstream from culvert crossing & 350 & W-05-202_P8020015 \\
\hline
\end{tabular}


Appendix 8: List of all photographs taken showing the corresponding site number, descriptive caption, direction faced when taken (azimuth in degrees) and the unique file name for each photograph.

\begin{tabular}{|c|c|c|c|}
\hline $\begin{array}{c}\text { Site } \\
\text { Number }\end{array}$ & Photo Description & Azimuth & File Name \\
\hline W-05-202 & Looking downstream from culvert crossing & 165 & W-05-202_P8020016 \\
\hline W-05-202 & Looking at culvert inlet & 220 & W-05-202 P8020017 \\
\hline W-05-202 & Looking at culvert outlet & 350 & W-05-202_P8020018 \\
\hline W-05-203 & Looking upstream bridge crossing & 210 & W-05-203_P8030001 \\
\hline W-05-203 & Looking upstream bridge crossing & 160 & W-05-203_P8030002 \\
\hline W-05-203 & Looking downstream bridge crossing & 80 & W-05-203 P8030003 \\
\hline W-05-204 & Looking upstream from culvert crossing & 0 & W-05-204_P8030004 \\
\hline W-05-204 & Looking downstream from culvert crossing & 180 & W-05-204_P8030005 \\
\hline W-05-204 & Looking at culvert inlet & 220 & W-05-204 P8030006 \\
\hline W-05-204 & Looking at culvert outlet & 100 & W-05-204 P8030007 \\
\hline W-05-204 & Photo of cattle impacts upstream of culvert crossing & 20 & W-05-204_P8030008 \\
\hline W-05-204 & Photo of cattle impacts upstream of culvert crossing & 10 & W-05-204_P8030009 \\
\hline W-05-204 & Photo of cattle impacts downstream of culvert crossing & 200 & W-05-204_P8030010 \\
\hline W-05-204 & Photo of cattle impacts downstream of culvert crossing & 250 & W-05-204 P8030011 \\
\hline W-05-205 & Looking upstream from culvert crossing & 250 & W-05-205_P8030012 \\
\hline W-05-205 & Looking downstream from culvert crossing & 75 & W-05-205_P8030013 \\
\hline W-05-205 & Looking at culvert inlet 1 & 170 & W-05-205 P8030014 \\
\hline W-05-205 & Looking at culvert inlet 2 & 140 & W-05-205 P8030015 \\
\hline W-05-205 & Looking at culvert outlet 1 & 220 & W-05-205_P8030016 \\
\hline W-05-206 & Looking upstream from culvert crossing & 90 & W-05-206_P8030017 \\
\hline W-05-206 & Looking downstream from culvert crossing & 270 & W-05-206 P8030018 \\
\hline W-05-206 & Looking at culvert inlet & 250 & W-05-206_P8030019 \\
\hline W-05-206 & Looking at culvert outlet & 110 & W-05-206_P8030020 \\
\hline W-05-207 & Looking upstream bridge crossing & 45 & W-05-207_P8080001 \\
\hline W-05-207 & Looking downstream bridge crossing & 225 & W-05-207_P8080002 \\
\hline W-05-207 & Photo of YOY bullheads immediately upstream of bridge crossing. & $\mathrm{N} / \mathrm{A}$ & W-05-207_P8080003 \\
\hline W-05-207 & Looking upstream at bank erosion & 85 & W-05-207_P8080004 \\
\hline W-05-207 & Looking upstream at bank erosion & 45 & W-05-207_P8080005 \\
\hline W-05-208 & Looking upstream from quad / walking bridge & 270 & W-05-208 P8100001 \\
\hline W-05-208 & Looking downstream from quad / walking bridge & 45 & W-05-208_P8100002 \\
\hline W-05-208 & Photo of Matt \& Jaime & $\mathrm{N} / \mathrm{A}$ & W-05-208_P8100003 \\
\hline W-05-209 & Looking upstream from culvert crossing & 250 & W-05-209 P8100004 \\
\hline W-05-209 & Looking downstream from culvert crossing & 10 & W-05-209 P8100005 \\
\hline
\end{tabular}


Appendix 8: List of all photographs taken showing the corresponding site number, descriptive caption, direction faced when taken (azimuth in degrees) and the unique file name for each photograph.

\begin{tabular}{|c|c|c|c|}
\hline $\begin{array}{c}\text { Site } \\
\text { Number }\end{array}$ & Photo Description & Azimuth & File Name \\
\hline W-05-209 & Looking at culvert inlet & 160 & W-05-209 P8100006 \\
\hline W-05-209 & Looking at culvert outlet & 100 & W-05-209_P8100007 \\
\hline W-05-210 & Looking upstream from culvert crossing & 270 & W-05-210_P5190018 \\
\hline W-05-210 & Looking downstream from culvert crossing & 90 & W-05-210_P5190019 \\
\hline W-05-210 & Looking at culvert inlet & 0 & W-05-210_P5190020 \\
\hline W-05-210 & Looking at culvert outlet & 180 & W-05-210_P5190021 \\
\hline W-05-211 & Photo of moose & $\mathrm{N} / \mathrm{A}$ & W-05-211_P5310001 \\
\hline W-05-211 & Photo of moose & $\mathrm{N} / \mathrm{A}$ & W-05-211_P5310002 \\
\hline W-05-211 & Photo of moose & $\mathrm{N} / \mathrm{A}$ & W-05-211_P5310003 \\
\hline W-05-211 & Looking upstream from culvert crossing & 210 & W-05-211_P5310004 \\
\hline W-05-211 & Looking downstream from culvert crossing & 0 & W-05-211 P5310005 \\
\hline W-05-211 & Looking at culvert inlet & 180 & W-05-211_P5310006 \\
\hline W-05-211 & Looking at culvert outlet & 210 & W-05-211_P5310007 \\
\hline $\mathrm{X}-05-001$ & Looking upstream from bridge crossing & 150 & $\mathrm{X}-05-001 \mathrm{P} 4270001$ \\
\hline $\mathrm{X}-05-001$ & Looking downstream from bridge crossing & 65 & $\mathrm{X}-05-001 \quad \mathrm{P} 4270002$ \\
\hline $\mathrm{X}-05-002$ & Looking upstream from culvert crossing & 90 & $\mathrm{X}-05-002 \_\mathrm{P} 4270003$ \\
\hline $\mathrm{X}-05-002$ & Looking upstream from culvert crossing & 30 & $\mathrm{X}-05-002 \_\mathrm{P} 4270004$ \\
\hline $\mathrm{X}-05-002$ & Looking downstream from south culvert crossing & 190 & $\mathrm{X}-05-002 \mathrm{P} 4270005$ \\
\hline $\mathrm{X}-05-002$ & Looking downstream from north culvert crossing & 170 & $\mathrm{X}-05-002 \_\mathrm{P} 4270006$ \\
\hline $\mathrm{X}-05-002$ & Looking at outlet of north culvert & 110 & $\mathrm{X}-05-002 \_\mathrm{P} 4270007$ \\
\hline $\mathrm{X}-05-002$ & Looking at outlet of south culvert & 75 & $\mathrm{X}-05-002 \mathrm{P} 4270008$ \\
\hline $\mathrm{X}-05-002$ & Looking at inlet of south culvert & 300 & $\mathrm{X}-05-002 \mathrm{P} 4270009$ \\
\hline $\mathrm{X}-05-002$ & Looking at inlet of north culvert & 245 & $\mathrm{X}-05-002 \mathrm{P} 4270010$ \\
\hline $\mathrm{X}-05-003$ & Looking upstream from culvert crossing & 260 & X-05-003_P4290001 \\
\hline $\mathrm{X}-05-003$ & Looking downstream from culvert crossing & 10 & X-05-003_P4290002 \\
\hline $\mathrm{X}-05-003$ & Looking at culvert inlets & 270 & X-05-003_P4290003 \\
\hline $\mathrm{X}-05-003$ & Looking at culvert outlets & 20 & X-05-003_P4290004 \\
\hline $\mathrm{X}-05-004$ & Looking upstream from culvert crossing & 180 & X-05-004_P4290005 \\
\hline $\mathrm{X}-05-004$ & Looking downstream from culvert crossing & 360 & X-05-004_P4290006 \\
\hline $\mathrm{X}-05-004$ & Looking at culvert inlets & 320 & X-05-004_P4290007 \\
\hline $\mathrm{X}-05-004$ & Looking at culvert outlets & 145 & $\mathrm{X}-05-004 \mathrm{P} 4290008$ \\
\hline $\mathrm{X}-05-005$ & Looking upstream at Barnland Drain & 360 & X-05-005_P4290009 \\
\hline $\mathrm{X}-05-005$ & Looking downstream at Barnland Drain & 90 & $\mathrm{X}-05-005 \mathrm{P} 4290010$ \\
\hline
\end{tabular}


Appendix 8: List of all photographs taken showing the corresponding site number, descriptive caption, direction faced when taken (azimuth in degrees) and the unique file name for each photograph.

\begin{tabular}{|c|c|c|c|}
\hline $\begin{array}{c}\text { Site } \\
\text { Number }\end{array}$ & Photo Description & Azimuth & File Name \\
\hline $\mathrm{X}-05-005$ & Looking at outlet of culvert of 2 nd order tributary & 250 & X-05-005_P4290011 \\
\hline $\mathrm{X}-05-005$ & Looking at culvert inlet of culvert of 2 nd order tributary & 145 & $\mathrm{X}-05-005 \mathrm{P} 4290012$ \\
\hline $\mathrm{X}-05-005$ & upstream at inflow from 2nd order tributary to Barnland Drain & 270 & X-05-005_P4290013 \\
\hline $\mathrm{X}-05-006$ & Looking upstream from bridge crossing & 270 & X-05-006_P4290014 \\
\hline$X-05-006$ & Looking downstream from bridge crossing & 90 & X-05-006_P4290015 \\
\hline $\mathrm{X}-05-007$ & Looking upstream from culvert crossing & 270 & X-05-007_P4290016 \\
\hline $\mathrm{X}-05-007$ & Looking downstream from culvert crossing & 90 & X-05-007_P4290017 \\
\hline $\mathrm{X}-05-007$ & Looking at culvert outlets & 225 & X-05-007_P4290018 \\
\hline $\mathrm{X}-05-007$ & Looking at culvert inlets & 100 & X-05-007_P4290019 \\
\hline $\mathrm{X}-05-007$ & Looking at banked sloughing & $\mathrm{N} / \mathrm{A}$ & X-05-007_P4290020 \\
\hline $\mathrm{X}-05-007$ & Looking at male White Sucker fork length $=480 \mathrm{~mm}$ & $\mathrm{~N} / \mathrm{A}$ & $\mathrm{X}-05-007 \_\mathrm{P} 4290021$ \\
\hline $\mathrm{X}-05-007$ & Looking at female White Sucker fork length $=440 \mathrm{~mm}$ & $\mathrm{~N} / \mathrm{A}$ & X-05-007_P4290022 \\
\hline $\mathrm{X}-05-007$ & Looking at Ashley P. and Ashley W. with White Sucker & $\mathrm{N} / \mathrm{A}$ & X-05-007_P4290023 \\
\hline $\mathrm{X}-05-007$ & Looking at female White Sucker fork length $=430 \mathrm{~mm}$ & 310 & X-05-007_P4290024 \\
\hline $\mathrm{X}-05-008$ & Looking downstream from culvert crossing & 310 & X-05-008_P4290025 \\
\hline $\mathrm{X}-05-008$ & Looking upstream from culvert crossing & 145 & X-05-008_P4290026 \\
\hline$X-05-008$ & Looking upstream at 2nd order tributary & 170 & X-05-008_P4290027 \\
\hline $\mathrm{X}-05-008$ & Looking at culvert outlets & 130 & $\mathrm{X}-05-008+\mathrm{P} 4290028$ \\
\hline $\mathrm{X}-05-008$ & Looking at culvert inlets of 2 nd order tributary & 330 & X-05-008_P4290029 \\
\hline $\mathrm{X}-05-008$ & Looking at culvert inlets & 270 & X-05-008_P4290030 \\
\hline $\mathrm{X}-05-009$ & Looking upstream from culvert crossing at Parker Drain & 325 & X-05-009_P4290031 \\
\hline $\mathrm{X}-05-009$ & Looking upstream at 2nd order tributary & 270 & X-05-009_P4290032 \\
\hline$X-05-009$ & Looking downstream at Parker Drain & 90 & X-05-009_P4290033 \\
\hline $\mathrm{X}-05-010$ & Looking upstream from culvert crossing & 320 & X-05-010_P5030001 \\
\hline $\mathrm{X}-05-010$ & Looking downstream from culvert crossing & 100 & $\mathrm{X}-05-010 \_\mathrm{P} 5030002$ \\
\hline $\mathrm{X}-05-010$ & Looking at culvert outlets & 270 & $\mathrm{X}-05-010 \mathrm{P} 5030003$ \\
\hline $\mathrm{X}-05-010$ & Looking at culvert inlets & 90 & X-05-010_P5030004 \\
\hline $\mathrm{X}-05-011$ & Looking upstream from culvert crossing & 270 & X-05-011_P5030005 \\
\hline $\mathrm{X}-05-011$ & Looking downstream from culvert crossing & 90 & X-05-011_P5030006 \\
\hline $\mathrm{X}-05-011$ & Looking at culvert inlets & 70 & $\mathrm{X}-05-011 \mathrm{P} 5030007$ \\
\hline $\mathrm{X}-05-011$ & Looking at culvert outlets & 290 & $\mathrm{X}-05-011 \_\mathrm{P} 5030008$ \\
\hline $\mathrm{X}-05-011$ & Looking at ford crossing, 1 mile downstream of culvert crossing & 150 & X-05-011_P5030009 \\
\hline $\mathrm{X}-05-011$ & Looking at culvert inlet of ford crossing & 120 & X-05-011_P5030010 \\
\hline
\end{tabular}


Appendix 8: List of all photographs taken showing the corresponding site number, descriptive caption, direction faced when taken (azimuth in degrees) and the unique file name for each photograph.

\begin{tabular}{|c|c|c|c|}
\hline $\begin{array}{c}\text { Site } \\
\text { Number }\end{array}$ & Photo Description & Azimuth & File Name \\
\hline $\mathrm{X}-05-011$ & Looking at outlet of ford crossing & 310 & X-05-011_P5030011 \\
\hline $\mathrm{X}-05-011$ & Looking downstream from ford crossing & 90 & X-05-011_P5030012 \\
\hline $\mathrm{X}-05-012$ & Looking upstream from culvert crossing & 305 & $\mathrm{X}-05-012 \_\mathrm{P} 5030013$ \\
\hline $\mathrm{X}-05-012$ & Looking downstream from culvert crossing & 180 & X-05-012_P5030014 \\
\hline $\mathrm{X}-05-012$ & Looking at culvert inlets & 120 & X-05-012_P5030015 \\
\hline $\mathrm{X}-05-012$ & Looking at culvert outlets & 45 & X-05-012_P5030016 \\
\hline $\mathrm{X}-05-013$ & Looking upstream from culvert crossing & 270 & X-05-013_P5030017 \\
\hline $\mathrm{X}-05-013$ & Looking downstream from culvert crossing & 90 & $\mathrm{X}-05-013 \_\mathrm{P} 5030018$ \\
\hline $\mathrm{X}-05-013$ & Looking at culvert inlets & 45 & X-05-013_P5030019 \\
\hline $\mathrm{X}-05-013$ & Looking at culvert outlets & 165 & X-05-013_P5030020 \\
\hline $\mathrm{X}-05-014$ & Looking upstream from culvert crossing & 205 & X-05-014_P5030021 \\
\hline $\mathrm{X}-05-014$ & Looking downstream from culvert crossing & 110 & X-05-014_P5030022 \\
\hline $\mathrm{X}-05-014$ & Looking at culvert inlets & 45 & X-05-014_P5030023 \\
\hline $\mathrm{X}-05-014$ & Looking at silt deposition & 65 & X-05-014_P5030024 \\
\hline $\mathrm{X}-05-014$ & Looking at culvert outlets & 315 & X-05-014_P5030025 \\
\hline $\mathrm{X}-05-015$ & Looking upstream from culvert crossing & 270 & X-05-015_P5030026 \\
\hline $\mathrm{X}-05-015$ & Looking downstream from culvert crossing & 90 & X-05-015_P5030027 \\
\hline $\mathrm{X}-05-015$ & Looking at culvert inlets & 80 & X-05-015_P5030028 \\
\hline $\mathrm{X}-05-015$ & Looking at culvert outlets & 260 & X-05-015_P5030029 \\
\hline $\mathrm{X}-05-016$ & Looking upstream from culvert crossing & 270 & X-05-016_P5030030 \\
\hline $\mathrm{X}-05-016$ & Looking downstream from culvert crossing & 90 & X-05-016_P5030031 \\
\hline $\mathrm{X}-05-016$ & Looking at culvert inlets & 100 & X-05-016_P5030032 \\
\hline $\mathrm{X}-05-016$ & Looking at culvert outlets & 250 & X-05-016_P5030033 \\
\hline $\mathrm{X}-05-017$ & Looking upstream from culvert crossing & 250 & $\mathrm{X}-05-017$ P5030034 \\
\hline $\mathrm{X}-05-017$ & Looking downstream from culvert crossing & 80 & X-05-017_P5030035 \\
\hline $\mathrm{X}-05-017$ & Looking at culvert outlets & 45 & X-05-017_P5030036 \\
\hline $\mathrm{X}-05-017$ & Looking at culvert inlets & 60 & $\mathrm{X}-05-017=\mathrm{P} 5030037$ \\
\hline $\mathrm{X}-05-017$ & Looking upstream from newly constructed drain & 280 & X-05-017_P5030038 \\
\hline $\mathrm{X}-05-018$ & Looking upstream from culvert crossing & 305 & X-05-018_P5040001 \\
\hline $\mathrm{X}-05-018$ & Looking downstream from culvert crossing & 150 & X-05-018_P5040002 \\
\hline $\mathrm{X}-05-018$ & Looking at culvert inlets & 210 & X-05-018_P5040003 \\
\hline $\mathrm{X}-05-018$ & Looking at culvert outlets & 190 & X-05-018_P5040004 \\
\hline $\mathrm{X}-05-018$ & Photo of a White Sucker (fork length $=410 \mathrm{~mm}$ ) & $\mathrm{N} / \mathrm{A}$ & X-05-018_P5040005 \\
\hline
\end{tabular}


Appendix 8: List of all photographs taken showing the corresponding site number, descriptive caption, direction faced when taken (azimuth in degrees) and the unique file name for each photograph.

\begin{tabular}{|c|c|c|c|}
\hline $\begin{array}{c}\text { Site } \\
\text { Number }\end{array}$ & Photo Description & Azimuth & File Name \\
\hline $\mathrm{X}-05-018$ & Photo of a White Sucker (fork length $=370 \mathrm{~mm}$ ) & $\mathrm{N} / \mathrm{A}$ & X-05-018_P5040006 \\
\hline $\mathrm{X}-05-018$ & Photo of a White Sucker (fork length $=340 \mathrm{~mm}$ ) & $\mathrm{N} / \mathrm{A}$ & $\mathrm{X}-05-018 \mathrm{P} 5040007$ \\
\hline $\mathrm{X}-05-018$ & Photo of a White Sucker (fork length $=320 \mathrm{~mm}$ ) & $\mathrm{N} / \mathrm{A}$ & X-05-018_P5040008 \\
\hline $\mathrm{X}-05-018$ & Photo of a White Sucker (fork length $=280 \mathrm{~mm}$ ) & $\mathrm{N} / \mathrm{A}$ & X-05-018_P5040009 \\
\hline $\mathrm{X}-05-018$ & Photo of a White Sucker (fork length $=250 \mathrm{~mm}$ ) & $\mathrm{N} / \mathrm{A}$ & X-05-018_P5040010 \\
\hline $\mathrm{X}-05-018$ & Photo of a White Sucker (fork length $=230 \mathrm{~mm}$ ) & $\mathrm{N} / \mathrm{A}$ & X-05-018_P5040011 \\
\hline $\mathrm{X}-05-018$ & Photo of a White Sucker (fork length $=250 \mathrm{~mm}$ ) & $\mathrm{N} / \mathrm{A}$ & $\mathrm{X}-05-018+\mathrm{P} 5040012$ \\
\hline $\mathrm{X}-05-018$ & Photo of a White Sucker (fork length $=240 \mathrm{~mm}$ ) & $\mathrm{N} / \mathrm{A}$ & $\mathrm{X}-05-018 \_\mathrm{P} 5040013$ \\
\hline $\mathrm{X}-05-018$ & Photo of a White Sucker (fork length $=180 \mathrm{~mm}$ ) & $\mathrm{N} / \mathrm{A}$ & X-05-018_P5040014 \\
\hline $\mathrm{X}-05-018$ & Photo of a White Sucker (fork length $=120 \mathrm{~mm}$ ) & $\mathrm{N} / \mathrm{A}$ & X-05-018_P5040015 \\
\hline $\mathrm{X}-05-018$ & Photo of a White Sucker & $\mathrm{N} / \mathrm{A}$ & $\mathrm{X}-05-018 \mathrm{P} 5040016$ \\
\hline $\mathrm{X}-05-018$ & Photo of a Central Mudminnow (total length $=100 \mathrm{~mm}$ ) & $\mathrm{N} / \mathrm{A}$ & X-05-018_P5040017 \\
\hline $\mathrm{X}-05-018$ & Looking upstream at riparian vegetation & $\mathrm{N} / \mathrm{A}$ & X-05-018_P5040018 \\
\hline $\mathrm{X}-05-018$ & Looking downstream at riparian vegetation & $\mathrm{N} / \mathrm{A}$ & X-05-018_P5040019 \\
\hline $\mathrm{X}-05-018$ & Looking downstream at riparian vegetation & $\mathrm{N} / \mathrm{A}$ & X-05-018_P5040020 \\
\hline $\mathrm{X}-05-019$ & Looking upstream from bridge crossing & 305 & X-05-019_P5040021 \\
\hline$X-05-019$ & Looking downstream from bridge crossing & 100 & X-05-019_P5040022 \\
\hline $\mathrm{X}-05-019$ & Looking upstream from right bank at sediment deposition & $\mathrm{N} / \mathrm{A}$ & $\mathrm{X}-05-019 \mathrm{P} 5040023$ \\
\hline $\mathrm{X}-05-019$ & Looking at sediment deposition under bridge from right bank & $\mathrm{N} / \mathrm{A}$ & X-05-019_P5040024 \\
\hline $\mathrm{X}-05-019$ & Looking further upstream from bridge & 270 & X-05-019_P5040025 \\
\hline $\mathrm{X}-05-019$ & Looking at sediment deposition downstream of bridge & $\mathrm{N} / \mathrm{A}$ & X-05-019_P5040026 \\
\hline $\mathrm{X}-05-019$ & Looking at sediment deposition downstream of bridge & $\mathrm{N} / \mathrm{A}$ & $\mathrm{X}-05-019+\mathrm{P} 5040027$ \\
\hline$X-05-020$ & Looking upstream from culvert crossing & 270 & $\mathrm{X}-05-020 \_\mathrm{P} 5040028$ \\
\hline $\mathrm{X}-05-020$ & Looking downstream from culvert crossing & 90 & X-05-020_P5040029 \\
\hline $\mathrm{X}-05-020$ & Looking at culvert inlets & 45 & $\mathrm{X}-05-020 \_\mathrm{P} 5040030$ \\
\hline $\mathrm{X}-05-020$ & Looking at culvert outlets & 165 & $\mathrm{X}-05-020 \mathrm{P} 5040031$ \\
\hline $\mathrm{X}-05-020$ & Looking upstream at very small riparian area & 270 & $\mathrm{X}-05-020 \_\mathrm{P} 5040032$ \\
\hline $\mathrm{X}-05-021$ & Looking upstream from bridge crossing & 65 & $\mathrm{X}-05-021 \_P 5040033$ \\
\hline $\mathrm{X}-05-021$ & Looking downstream from bridge crossing & 90 & X-05-021_P5040034 \\
\hline $\mathrm{X}-05-021$ & Photo of Ashley Presenger with White Sucker & N/A & $\mathrm{X}-05-021 \_\mathrm{P} 5040035$ \\
\hline $\mathrm{X}-05-021$ & Looking at White Sucker fork length $=380 \mathrm{~mm}$ & $\mathrm{~N} / \mathrm{A}$ & $\mathrm{X}-05-021 \_\mathrm{P} 5040036$ \\
\hline $\mathrm{X}-05-021$ & Looking downstream at potential barrier to fish passage & 125 & X-05-021_P5040037 \\
\hline $\mathrm{X}-05-021$ & Looking upstream at potential barrier to fish passage & 270 & X-05-021_P5040038 \\
\hline
\end{tabular}


Appendix 8: List of all photographs taken showing the corresponding site number, descriptive caption, direction faced when taken (azimuth in degrees) and the unique file name for each photograph.

\begin{tabular}{|c|c|c|c|}
\hline $\begin{array}{c}\text { Site } \\
\text { Number }\end{array}$ & Photo Description & Azimuth & File Name \\
\hline $\mathrm{X}-05-022$ & Looking upstream from culvert crossing & 305 & X-05-022_P5040039 \\
\hline $\mathrm{X}-05-022$ & Looking downstream from culvert crossing & 110 & $\mathrm{X}-05-022 \_\mathrm{P} 5040040$ \\
\hline $\mathrm{X}-05-022$ & Looking at culvert inlets & 90 & X-05-022_P5040041 \\
\hline $\mathrm{X}-05-022$ & Looking at culvert outlets & 330 & X-05-022_P5040042 \\
\hline $\mathrm{X}-05-023$ & Looking upstream at riffle from right bank & 220 & X-05-023_P5040043 \\
\hline $\mathrm{X}-05-023$ & Looking downstream at riffle from left bank & 30 & X-05-023_P5040044 \\
\hline $\mathrm{X}-05-023$ & Looking at gradient control structure & 180 & X-05-023_P5040045 \\
\hline $\mathrm{X}-05-024$ & Looking upstream at constructed riffle from mid-reach & 270 & X-05-024_P5050001 \\
\hline $\mathrm{X}-05-024$ & Looking upstream at constructed riffle from top of riffle & 270 & X-05-024_P5050002 \\
\hline $\mathrm{X}-05-024$ & Looking downstream at constructed riffle from top of riffle & 100 & X-05-024_P5050003 \\
\hline $\mathrm{X}-05-024$ & Looking at layering of boulders in pool just downstream of riffle & $\mathrm{N} / \mathrm{A}$ & X-05-024_P5050004 \\
\hline $\mathrm{X}-05-024$ & Looking downstream at erosion of banks & 100 & X-05-024_P5050005 \\
\hline $\mathrm{X}-05-024$ & Looking at sediment deposition and eroded banks downstream of riffle & $\mathrm{N} / \mathrm{A}$ & X-05-024_P5050006 \\
\hline $\mathrm{X}-05-024$ & Looking at top of constructed riffle & 240 & X-05-024_P5050007 \\
\hline $\mathrm{X}-05-024$ & Looking at White Sucker released (fork length $=240 \mathrm{~mm}$ ) & $\mathrm{N} / \mathrm{A}$ & X-05-024_P5050008 \\
\hline $\mathrm{X}-05-024$ & Looking at White Sucker released (fork length $=300 \mathrm{~mm}$ ) & $\mathrm{N} / \mathrm{A}$ & X-05-024_P5050009 \\
\hline $\mathrm{X}-05-024$ & Looking downstream from top of reach & 90 & X-05-024_P5050010 \\
\hline $\mathrm{X}-05-025$ & Looking upstream from culvert crossing & 180 & $\mathrm{X}-05-025 \_\mathrm{P} 5050011$ \\
\hline $\mathrm{X}-05-025$ & Looking downstream from culvert crossing & 360 & X-05-025_P5050012 \\
\hline $\mathrm{X}-05-025$ & Looking upstream at Bryson Drain & 90 & X-05-025_P5050013 \\
\hline $\mathrm{X}-05-025$ & Looking at gradient control structure & 270 & X-05-025_P5050014 \\
\hline $\mathrm{X}-05-025$ & Looking upstream at culvert outlets from gradient control structure & 180 & X-05-025_P5050015 \\
\hline $\mathrm{X}-05-025$ & Looking at culvert inlets & 350 & X-05-025_P5050016 \\
\hline $\mathrm{X}-05-025$ & Looking upstream at first order tributary & 270 & X-05-025_P5050017 \\
\hline $\mathrm{X}-05-026$ & Looking upstream from culvert crossing & 295 & X-05-026_P5050018 \\
\hline $\mathrm{X}-05-026$ & Looking downstream from culvert crossing & 110 & X-05-026_P5050019 \\
\hline $\mathrm{X}-05-026$ & Looking at culvert inlets & 120 & X-05-026_P5050020 \\
\hline $\mathrm{X}-05-026$ & Looking at culvert outlets & 235 & X-05-026_P5050021 \\
\hline $\mathrm{X}-05-026$ & Looking at path created by cattle & 35 & X-05-026_P5050022 \\
\hline $\mathrm{X}-05-027$ & Looking upstream from ford crossing & 150 & X-05-027_P5060001 \\
\hline $\mathrm{X}-05-027$ & Looking downstream from ford crossing & 345 & X-05-027_P5060002 \\
\hline $\mathrm{X}-05-027$ & Looking at ford crossing & 90 & X-05-027_P5060003 \\
\hline $\mathrm{X}-05-028$ & Looking upstream from culvert crossing & 165 & X-05-028_P5060004 \\
\hline
\end{tabular}


Appendix 8: List of all photographs taken showing the corresponding site number, descriptive caption, direction faced when taken (azimuth in degrees) and the unique file name for each photograph.

\begin{tabular}{|c|c|c|c|}
\hline $\begin{array}{c}\text { Site } \\
\text { Number }\end{array}$ & Photo Description & Azimuth & File Name \\
\hline $\mathrm{X}-05-028$ & Looking downstream from culvert crossing & 350 & $\begin{array}{lll}X-05-028 & P 5060005\end{array}$ \\
\hline $\mathrm{X}-05-028$ & Looking at culvert inlets & 360 & $\mathrm{X}-05-028 \mathrm{P} 5060006$ \\
\hline $\mathrm{X}-05-028$ & Looking at culvert outlets & 120 & X-05-028_P5060007 \\
\hline $\mathrm{X}-05-029$ & Looking upstream from bridge crossing & 145 & X-05-029_P5060008 \\
\hline $\mathrm{X}-05-029$ & Looking downstream at cattle in drain & 180 & X-05-029_P5060009 \\
\hline $\mathrm{X}-05-029$ & Looking downstream at cattle impacted banks & 260 & $\mathrm{X}-05-029+\mathrm{P} 5060010$ \\
\hline $\mathrm{X}-05-030$ & Looking upstream from culvert crossing & 90 & X-05-030_P5100001 \\
\hline $\mathrm{X}-05-030$ & Looking downstream from culvert crossing & 270 & X-05-030_P5100002 \\
\hline $\mathrm{X}-05-030$ & Looking at culvert inlets & 35 & $\mathrm{X}-05-030$ P5100003 \\
\hline $\mathrm{X}-05-030$ & Looking at culvert outlets & 345 & $\mathrm{X}-05-030 \mathrm{P} 5100004$ \\
\hline $\mathrm{X}-05-031$ & Looking upstream from culvert crossing & 150 & X-05-031_P5100005 \\
\hline $\mathrm{X}-05-031$ & Looking downstream from culvert crossing & 325 & X-05-031_P5100006 \\
\hline $\mathrm{X}-05-031$ & Looking at culvert inlets & 175 & X-05-031_P5100007 \\
\hline$X-05-031$ & Looking at culvert outlets & 180 & $\mathrm{X}-05-031 \_\mathrm{P} 5100008$ \\
\hline $\mathrm{X}-05-032$ & Looking upstream from culvert crossing & 180 & X-05-032_P5110001 \\
\hline $\mathrm{X}-05-032$ & Looking downstream from culvert crossing & 360 & $\mathrm{X}-05-032 \_P 5110002$ \\
\hline $\mathrm{X}-05-032$ & Looking at culvert inlets & 325 & $\mathrm{X}-05-032 \mathrm{P} 5110003$ \\
\hline $\mathrm{X}-05-032$ & Looking at culvert outlets & 150 & $\mathrm{X}-05-032 \mathrm{P} 5110004$ \\
\hline $\mathrm{X}-05-033$ & Looking upstream from right bank & 165 & X-05-033_P5110005 \\
\hline $\mathrm{X}-05-033$ & Looking downstream from left bank & 360 & X-05-033_P5110006 \\
\hline $\mathrm{X}-05-034$ & Looking upstream from culvert crossing & 165 & $\mathrm{X}-05-034 \quad \mathrm{P} 5110007$ \\
\hline $\mathrm{X}-05-034$ & Looking downstream from culvert crossing & 345 & X-05-034_P5110008 \\
\hline $\mathrm{X}-05-034$ & Looking at culvert outlets & 200 & X-05-034_P5110009 \\
\hline $\mathrm{X}-05-034$ & Looking at culvert inlets & 335 & X-05-034_P5110010 \\
\hline $\mathrm{X}-05-035$ & Looking upstream from bridge crossing & 100 & $\mathrm{X}-05-035 \mathrm{P} 5110011$ \\
\hline $\mathrm{X}-05-035$ & Looking downstream from bridge crossing & 270 & $\mathrm{X}-05-035 \mathrm{P} 5110012$ \\
\hline $\mathrm{X}-05-036$ & Looking upstream from culvert crossing & 90 & X-05-036_P5110013 \\
\hline $\mathrm{X}-05-036$ & Looking downstream from culvert crossing & 270 & X-05-036_P5110014 \\
\hline $\mathrm{X}-05-036$ & Looking at culvert inlets & 235 & $\mathrm{X}-05-036 \quad \mathrm{P} 5110015$ \\
\hline $\mathrm{X}-05-036$ & Looking at culvert outlets & 45 & X-05-036_P5110016 \\
\hline $\mathrm{X}-05-036$ & Looking at dead suckers & $\mathrm{N} / \mathrm{A}$ & X-05-036_P5110017 \\
\hline $\mathrm{X}-05-036$ & Looking at dead suckers & $\mathrm{N} / \mathrm{A}$ & $\mathrm{X}-05-036$ P5110018 \\
\hline $\mathrm{X}-05-036$ & Looking at dead suckers & $\mathrm{N} / \mathrm{A}$ & $\mathrm{X}-05-036 \quad \mathrm{P} 5110019$ \\
\hline
\end{tabular}


Appendix 8: List of all photographs taken showing the corresponding site number, descriptive caption, direction faced when taken (azimuth in degrees) and the unique file name for each photograph.

\begin{tabular}{|c|c|c|c|}
\hline $\begin{array}{c}\text { Site } \\
\text { Number }\end{array}$ & Photo Description & Azimuth & File Name \\
\hline $\mathrm{X}-05-037$ & Looking upstream from culvert crossing & 100 & X-05-037_P5120001 \\
\hline $\mathrm{X}-05-037$ & Looking downstream from culvert crossing & 330 & X-05-037_P5120002 \\
\hline $\mathrm{X}-05-037$ & Looking at culvert inlets & 315 & $\mathrm{X}-05-037$ P5120003 \\
\hline $\mathrm{X}-05-037$ & Looking at culvert outlets & 90 & $\mathrm{X}-05-037 \_\mathrm{P} 5120004$ \\
\hline $\mathrm{X}-05-038$ & Looking upstream from the left bank (mid-reach) & 180 & $\mathrm{X}-05-038+\mathrm{P} 5120005$ \\
\hline $\mathrm{X}-05-038$ & Looking downstream from the left bank (mid-reach) & 215 & X-05-038_P5120006 \\
\hline $\mathrm{X}-05-038$ & Photo of a White Sucker (fork length $=220 \mathrm{~mm}$ ) & $\mathrm{N} / \mathrm{A}$ & $\mathrm{X}-05-038+\mathrm{P} 5120007$ \\
\hline $\mathrm{X}-05-039$ & Looking upstream from culvert crossing & 90 & $\mathrm{X}-05-039 \mathrm{P} 5120008$ \\
\hline$X-05-039$ & Looking downstream from culvert crossing & 270 & X-05-039_P5120009 \\
\hline $\mathrm{X}-05-039$ & Looking at culvert inlets & 245 & X-05-039_P5120010 \\
\hline$X-05-039$ & Looking at culvert outlets & 150 & X-05-039_P5120011 \\
\hline $\mathrm{X}-05-040$ & Looking upstream from mid-reach & 165 & $\mathrm{X}-05-040 \mathrm{P} 5120012$ \\
\hline $\mathrm{X}-05-040$ & Looking downstream from mid-reach & 30 & X-05-040_P5120013 \\
\hline $\mathrm{X}-05-041$ & Looking upstream from culvert crossing & 180 & X-05-041_P5120014 \\
\hline $\mathrm{X}-05-041$ & Looking downstream from culvert crossing & 360 & $\mathrm{X}-05-041 \mathrm{P} 5120015$ \\
\hline $\mathrm{X}-05-041$ & Looking at culvert inlets & 305 & X-05-041_P5120016 \\
\hline $\mathrm{X}-05-041$ & Looking at culvert outlets & 215 & X-05-041_P5120017 \\
\hline $\mathrm{X}-05-042$ & Looking upstream from culvert crossing & 110 & $\mathrm{X}-05-042 \_P 5120018$ \\
\hline $\mathrm{X}-05-042$ & Looking downstream from culvert crossing & 345 & $\mathrm{X}-05-042 \mathrm{P} 5120019$ \\
\hline $\mathrm{X}-05-042$ & Looking at culvert inlets & 320 & $\mathrm{X}-05-042 \mathrm{P} 5120020$ \\
\hline $\mathrm{X}-05-042$ & Looking at culvert outlets & 250 & $\mathrm{X}-05-042$ P5120021 \\
\hline $\mathrm{X}-05-042$ & Photo of a White Sucker (fork length $=220 \mathrm{~mm}$ ) & $\mathrm{N} / \mathrm{A}$ & X-05-042_P5120022 \\
\hline$X-05-042$ & Photo of a Central Mudminnow (total length $=190 \mathrm{~mm}$ ) & $\mathrm{N} / \mathrm{A}$ & $X-05-042$ P5120023 \\
\hline $\mathrm{X}-05-043$ & Looking upstream from culvert crossing & 90 & $\mathrm{X}-05-043+\mathrm{P} 5120024$ \\
\hline $\mathrm{X}-05-043$ & Looking downstream from culvert crossing & 280 & X-05-043_P5120025 \\
\hline$X-05-044$ & Looking upstream from culvert crossing & 30 & X-05-044_P5130001 \\
\hline $\mathrm{X}-05-044$ & Looking downstream from culvert crossing & 295 & $\mathrm{X}-05-044 \quad \mathrm{P} 5130002$ \\
\hline $\mathrm{X}-05-044$ & Looking at culvert inlets & 345 & X-05-044_P5130003 \\
\hline $\mathrm{X}-05-044$ & Looking at culvert outlets & 210 & X-05-044_P5130004 \\
\hline $\mathrm{X}-05-045$ & Looking upstream from bridge crossing & 65 & X-05-045_P5130005 \\
\hline $\mathrm{X}-05-045$ & Looking downstream from bridge crossing & 200 & $\mathrm{X}-05-045 \mathrm{P} 5130006$ \\
\hline $\mathrm{X}-05-046$ & Looking upstream from culvert crossing & 45 & X-05-046_P5130008 \\
\hline $\mathrm{X}-05-046$ & Looking downstream from culvert crossing & 150 & X-05-046_P5130009 \\
\hline
\end{tabular}


Appendix 8: List of all photographs taken showing the corresponding site number, descriptive caption, direction faced when taken (azimuth in degrees) and the unique file name for each photograph.

\begin{tabular}{|c|c|c|c|}
\hline $\begin{array}{c}\text { Site } \\
\text { Number }\end{array}$ & Photo Description & Azimuth & File Name \\
\hline $\mathrm{X}-05-046$ & Looking at culvert outlets & 100 & X-05-046_P5130010 \\
\hline $\mathrm{X}-05-046$ & Looking at culvert inlets & 180 & X-05-046_P5130011 \\
\hline $\mathrm{X}-05-047$ & Looking upstream from bridge crossing & 90 & X-05-047_P5130012 \\
\hline $\mathrm{X}-05-047$ & Looking downstream from bridge crossing & 180 & X-05-047_P5130013 \\
\hline $\mathrm{X}-05-048$ & Looking upstream from cement box culvert & 90 & X-05-048_P5140001 \\
\hline $\mathrm{X}-05-048$ & Looking downstream from cement box culvert & 180 & X-05-048_P5140002 \\
\hline $\mathrm{X}-05-048$ & Photo of spawning Fathead Minnow & $\mathrm{N} / \mathrm{A}$ & X-05-048_P5140003 \\
\hline $\mathrm{X}-05-048$ & Photo of spawning Fathead Minnow & $\mathrm{N} / \mathrm{A}$ & X-05-048_P5140004 \\
\hline $\mathrm{X}-05-049$ & Looking upstream from culvert crossing & 90 & X-05-049_P5140005 \\
\hline $\mathrm{X}-05-049$ & Looking downstream from culvert crossing & 270 & X-05-049_P5140006 \\
\hline $\mathrm{X}-05-049$ & Looking at culvert outlets & 100 & X-05-049_P5140007 \\
\hline $\mathrm{X}-05-049$ & Looking at culvert inlets & 240 & X-05-049_P5140008 \\
\hline $\mathrm{X}-05-050$ & Looking upstream from culvert crossing & 90 & X-05-050_P5140009 \\
\hline $\mathrm{X}-05-050$ & Looking downstream from culvert crossing & 270 & X-05-050_P5140010 \\
\hline $\mathrm{X}-05-050$ & Looking at culvert outlets & 100 & X-05-050_P5140011 \\
\hline $\mathrm{X}-05-050$ & Looking at culvert inlets & 240 & X-05-050_P5140012 \\
\hline $\mathrm{X}-05-051$ & Looking upstream from ford crossing & 270 & X-05-051_P5140013 \\
\hline $\mathrm{X}-05-051$ & Looking downstream from ford crossing & 110 & X-05-051_P5140014 \\
\hline $\mathrm{X}-05-051$ & Looking at culvert inlets & 65 & X-05-051_P5140015 \\
\hline $\mathrm{X}-05-051$ & Looking at culvert outlets & 25 & $\mathrm{X}-05-051 \mathrm{P} 5140016$ \\
\hline $\mathrm{X}-05-052$ & Looking upstream from bridge crossing & 305 & X-05-052_P5140017 \\
\hline $\mathrm{X}-05-052$ & Looking downstream from bridge crossing & 100 & X-05-052_P5140020 \\
\hline $\mathrm{X}-05-053$ & Looking upstream at inflow of Westbourne Drain & 160 & X-05-053_P5150001 \\
\hline $\mathrm{X}-05-053$ & Looking downstream at Westbourne Drain & 360 & X-05-053_P5150002 \\
\hline $\mathrm{X}-05-053$ & Looking upstream at Bagot Drain & 280 & X-05-053_P5150003 \\
\hline $\mathrm{X}-05-053$ & Looking at culvert outlets & 330 & X-05-053_P5150004 \\
\hline $\mathrm{X}-05-053$ & Looking at culvert outlets of Westbourne Drain & 180 & X-05-053_P5150005 \\
\hline $\mathrm{X}-05-054$ & Looking upstream from ford crossing & 180 & X-05-054_P5150006 \\
\hline $\mathrm{X}-05-054$ & Looking downstream from ford crossing & 360 & X-05-054_P5150007 \\
\hline $\mathrm{X}-05-055$ & Looking upstream from culvert crossing & 235 & X-05-055_P5150008 \\
\hline $\mathrm{X}-05-055$ & Looking downstream from culvert crossing & 40 & X-05-055_P5150009 \\
\hline $\mathrm{X}-05-055$ & Looking at culvert inlets & 190 & X-05-055_P5150010 \\
\hline $\mathrm{X}-05-055$ & Looking at culvert outlets & 80 & X-05-055_P5150011 \\
\hline
\end{tabular}


Appendix 8: List of all photographs taken showing the corresponding site number, descriptive caption, direction faced when taken (azimuth in degrees) and the unique file name for each photograph.

\begin{tabular}{|c|c|c|c|}
\hline $\begin{array}{c}\text { Site } \\
\text { Number }\end{array}$ & Photo Description & Azimuth & File Name \\
\hline $\mathrm{X}-05-056$ & Looking upstream from culvert crossing & 180 & X-05-056_P5150012 \\
\hline $\mathrm{X}-05-056$ & Looking downstream from culvert crossing & 15 & X-05-056_P5150013 \\
\hline $\mathrm{X}-05-056$ & Looking at culvert inlets & 90 & X-05-056_P5150014 \\
\hline $\mathrm{X}-05-056$ & Looking at culvert outlets & 90 & X-05-056_P5150015 \\
\hline $\mathrm{X}-05-057$ & Looking upstream from culvert crossing & 260 & X-05-057_P5150016 \\
\hline $\mathrm{X}-05-057$ & Looking downstream from culvert crossing & 80 & X-05-057_P5150017 \\
\hline $\mathrm{X}-05-057$ & Looking at culvert outlets & 270 & X-05-057_P5150018 \\
\hline $\mathrm{X}-05-057$ & Looking at culvert inlets & 90 & X-05-057_P5150019 \\
\hline $\mathrm{X}-05-058$ & Looking upstream from bridge crossing & 150 & X-05-058_P5160001 \\
\hline $\mathrm{X}-05-058$ & Looking upstream from bridge crossing & 90 & X-05-058_P5160002 \\
\hline $\mathrm{X}-05-058$ & Looking upstream from bridge crossing & 45 & X-05-058_P5160003 \\
\hline $\mathrm{X}-05-058$ & Looking downstream from bridge crossing & 270 & X-05-058_P5160004 \\
\hline $\mathrm{X}-05-058$ & Looking downstream from bridge crossing & 305 & X-05-058_P5160005 \\
\hline $\mathrm{X}-05-059$ & Looking upstream from culvert crossing & 45 & X-05-059_P5160006 \\
\hline $\mathrm{X}-05-059$ & Looking downstream from culvert crossing & 225 & X-05-059_P5160007 \\
\hline $\mathrm{X}-05-059$ & Looking at culvert inlet & 180 & X-05-059_P5160008 \\
\hline $\mathrm{X}-05-059$ & Looking at culvert outlet & 180 & X-05-059_P5160009 \\
\hline $\mathrm{X}-05-060$ & Looking upstream from culvert crossing & 245 & X-05-060_P5160010 \\
\hline $\mathrm{X}-05-060$ & Looking upstream from culvert crossing & 170 & X-05-060_P5160011 \\
\hline $\mathrm{X}-05-060$ & Looking upstream from culvert crossing & 75 & $\mathrm{X}-05-060 \_\mathrm{P} 5160012$ \\
\hline $\mathrm{X}-05-060$ & Looking downstream from culvert crossing & 260 & X-05-060_P5160013 \\
\hline $\mathrm{X}-05-060$ & Looking downstream from culvert crossing & 35 & X-05-060_P5160014 \\
\hline $\mathrm{X}-05-060$ & Looking at culvert inlet & 80 & X-05-060_P5160015 \\
\hline $\mathrm{X}-05-060$ & Looking at culvert outlet & 80 & X-05-060_P5160016 \\
\hline $\mathrm{X}-05-060$ & Looking at washed out road & 80 & X-05-060_P5160017 \\
\hline $\mathrm{X}-05-060$ & Looking at washed out road & 80 & X-05-060_P5160018 \\
\hline $\mathrm{X}-05-061$ & Looking upstream from culvert crossing & 180 & X-05-061_P5160019 \\
\hline $\mathrm{X}-05-061$ & Looking downstream from culvert crossing & 10 & X-05-061_P5160020 \\
\hline $\mathrm{X}-05-061$ & Looking at culvert inlet & 65 & X-05-061_P5160021 \\
\hline $\mathrm{X}-05-061$ & Looking at culvert outlet & 115 & X-05-061_P5160022 \\
\hline $\mathrm{X}-05-062$ & Looking upstream from culvert crossing & 180 & X-05-062_P5160023 \\
\hline $\mathrm{X}-05-062$ & Looking downstream from culvert crossing & 345 & X-05-062_P5160024 \\
\hline $\mathrm{X}-05-062$ & Looking at culvert outlet & 210 & X-05-062_P5160025 \\
\hline
\end{tabular}


Appendix 8: List of all photographs taken showing the corresponding site number, descriptive caption, direction faced when taken (azimuth in degrees) and the unique file name for each photograph.

\begin{tabular}{|c|c|c|c|}
\hline $\begin{array}{c}\text { Site } \\
\text { Number }\end{array}$ & Photo Description & Azimuth & File Name \\
\hline $\mathrm{X}-05-062$ & Looking at culvert inlet & 100 & X-05-062_P5160026 \\
\hline $\mathrm{X}-05-062$ & Looking at culvert inlet & $\mathrm{N} / \mathrm{A}$ & $\mathrm{X}-05-062 \_\mathrm{P} 5160027$ \\
\hline $\mathrm{X}-05-063$ & Looking upstream from culvert crossing & 235 & $\mathrm{X}-05-063 \quad \mathrm{P} 5160028$ \\
\hline $\mathrm{X}-05-063$ & Looking upstream from culvert crossing & 150 & $\mathrm{X}-05-063+\mathrm{P} 5160029$ \\
\hline$X-05-063$ & Looking downstream from culvert crossing & 360 & $\mathrm{X}-05-063$ P5160030 \\
\hline $\mathrm{X}-05-063$ & Looking at culvert inlet & 90 & X-05-063_P5160031 \\
\hline $\mathrm{X}-05-063$ & Looking at culvert outlet & 100 & $\mathrm{X}-05-063+\mathrm{P} 5160032$ \\
\hline$X-05-064$ & Looking upstream from culvert crossing & 270 & $\mathrm{X}-05-064$ P5160033 \\
\hline $\mathrm{X}-05-064$ & Looking downstream from culvert crossing & 90 & X-05-064_P5160034 \\
\hline $\mathrm{X}-05-064$ & Looking at culvert inlets & 150 & X-05-064_P5160035 \\
\hline$X-05-064$ & Looking at culvert outlets & 165 & X-05-064_P5160036 \\
\hline $\mathrm{X}-05-065$ & Looking upstream from culvert crossing & 180 & $\mathrm{X}-05-065 \mathrm{P} 5160037$ \\
\hline $\mathrm{X}-05-065$ & Looking upstream from culvert crossing & 215 & X-05-065_P5160038 \\
\hline $\mathrm{X}-05-065$ & Looking downstream from culvert crossing & 45 & X-05-065_P5160039 \\
\hline $\mathrm{X}-05-065$ & Looking at culvert outlet & 280 & X-05-065_P5160040 \\
\hline $\mathrm{X}-05-066$ & Looking upstream from culvert crossing & 210 & X-05-066_P5170001 \\
\hline$X-05-066$ & Looking downstream from culvert crossing & 360 & X-05-066_P5170002 \\
\hline $\mathrm{X}-05-066$ & Looking at culvert inlets & 45 & X-05-066_P5170003 \\
\hline $\mathrm{X}-05-066$ & Looking at culvert outlets & 150 & $\mathrm{X}-05-066 \mathrm{P} 5170004$ \\
\hline $\mathrm{X}-05-067$ & Looking upstream from culvert crossing & 235 & $\mathrm{X}-05-067$ P5170005 \\
\hline $\mathrm{X}-05-067$ & Looking downstream from culvert crossing & 45 & X-05-067_P5170006 \\
\hline $\mathrm{X}-05-067$ & Looking at culvert inlets & 45 & X-05-067_P5170007 \\
\hline$X-05-067$ & Looking at culvert outlets & 215 & $\mathrm{X}-05-067 \quad \mathrm{P} 5170008$ \\
\hline $\mathrm{X}-05-068$ & Looking upstream from culvert crossing & 260 & $\mathrm{X}-05-068 \mathrm{P} 5170009$ \\
\hline $\mathrm{X}-05-068$ & Looking downstream from culvert crossing & 90 & X-05-068_P5170010 \\
\hline $\mathrm{X}-05-068$ & Looking at culvert outlets & 225 & X-05-068_P5170011 \\
\hline $\mathrm{X}-05-068$ & Looking at culvert inlets & 100 & $\mathrm{X}-05-068 \quad \mathrm{P} 5170012$ \\
\hline $\mathrm{X}-05-069$ & Looking upstream from bridge crossing & 235 & X-05-069_P5170013 \\
\hline $\mathrm{X}-05-069$ & Looking downstream from bridge crossing & 115 & X-05-069_P5170014 \\
\hline $\mathrm{X}-05-069$ & Looking at slumped banks & 55 & X-05-069_P5170015 \\
\hline $\mathrm{X}-05-070$ & Looking upstream from culvert crossing & 165 & $\mathrm{X}-05-070 \mathrm{P} 5180001$ \\
\hline $\mathrm{X}-05-070$ & Looking downstream from culvert crossing & 360 & $\mathrm{X}-05-070 \_\mathrm{P} 5180002$ \\
\hline $\mathrm{X}-05-070$ & Looking at culvert inlets & 35 & X-05-070_P5180003 \\
\hline
\end{tabular}


Appendix 8: List of all photographs taken showing the corresponding site number, descriptive caption, direction faced when taken (azimuth in degrees) and the unique file name for each photograph.

\begin{tabular}{|c|c|c|c|}
\hline $\begin{array}{c}\text { Site } \\
\text { Number }\end{array}$ & Photo Description & Azimuth & File Name \\
\hline $\mathrm{X}-05-070$ & Looking at culvert outlets & 125 & X-05-070_P5180004 \\
\hline $\mathrm{X}-05-071$ & Looking upstream from culvert crossing & 225 & X-05-071_P5180005 \\
\hline $\mathrm{X}-05-071$ & Looking downstream from culvert crossing & 90 & X-05-071_P5180006 \\
\hline $\mathrm{X}-05-071$ & Looking at culvert inlets & 225 & X-05-071_P5180007 \\
\hline $\mathrm{X}-05-072$ & Looking upstream from culvert crossing & 180 & X-05-072_P5180008 \\
\hline $\mathrm{X}-05-072$ & Looking upstream at the 2nd order tributary & 270 & X-05-072_P5180009 \\
\hline $\mathrm{X}-05-072$ & Looking downstream from culvert crossing & 360 & X-05-072_P5180010 \\
\hline $\mathrm{X}-05-072$ & Looking at culvert inlets & 235 & $\mathrm{X}-05-072 \_\mathrm{P} 5180011$ \\
\hline $\mathrm{X}-05-072$ & Looking at culvert outlets & 150 & X-05-072_P5180012 \\
\hline $\mathrm{X}-05-073$ & Looking upstream from culvert crossing & 180 & X-05-073_P5180013 \\
\hline $\mathrm{X}-05-073$ & Looking downstream from culvert crossing & 335 & X-05-073_P5180014 \\
\hline $\mathrm{X}-05-073$ & Looking at culvert inlet & 320 & X-05-073_P5180015 \\
\hline $\mathrm{X}-05-073$ & Looking at culvert outlet & 245 & X-05-073_P5180016 \\
\hline $\mathrm{X}-05-073$ & Looking down at inlet & $\mathrm{N} / \mathrm{A}$ & X-05-073_P5180017 \\
\hline $\mathrm{X}-05-074$ & Looking upstream from cement box culvert & 150 & X-05-074_P5180018 \\
\hline $\mathrm{X}-05-074$ & Looking downstream from cement box culvert & 305 & X-05-074_P5180019 \\
\hline $\mathrm{X}-05-075$ & Looking upstream from culvert crossing & 270 & X-05-075_P5180020 \\
\hline $\mathrm{X}-05-075$ & Looking downstream from culvert crossing & 90 & X-05-075_P5180021 \\
\hline $\mathrm{X}-05-075$ & Looking upstream at 2nd order tributary & 180 & X-05-075_P5180022 \\
\hline $\mathrm{X}-05-075$ & Looking at culvert inlets & 235 & $\mathrm{X}-05-075+\mathrm{P} 5180023$ \\
\hline $\mathrm{X}-05-075$ & Looking at culvert outlets & 160 & X-05-075_P5180024 \\
\hline $\mathrm{X}-05-076$ & Looking upstream at Rignold Drain from culvert crossing & 180 & X-05-076_P5180025 \\
\hline $\mathrm{X}-05-076$ & Looking downstream from culvert crossing & 360 & X-05-076_P5180026 \\
\hline $\mathrm{X}-05-076$ & Looking at culvert inlet of culvert from Rignold Drain & 10 & X-05-076_P5180027 \\
\hline $\mathrm{X}-05-076$ & Looking at culvert outlets & 180 & X-05-076_P5180028 \\
\hline $\mathrm{X}-05-076$ & Looking at inlets of culverts from the ditch & 345 & X-05-076_P5180029 \\
\hline $\mathrm{X}-05-076$ & Looking upstream at ditch & 270 & X-05-076_P5180030 \\
\hline $\mathrm{X}-05-076$ & Looking upstream at ditch & 180 & X-05-076_P5180031 \\
\hline $\mathrm{X}-05-077$ & Looking upstream from culvert crossing & 210 & X-05-077_P5180032 \\
\hline $\mathrm{X}-05-077$ & Looking downstream from culvert crossing & 30 & X-05-077_P5180033 \\
\hline $\mathrm{X}-05-077$ & Looking at culvert inlets & 75 & X-05-077_P5180034 \\
\hline $\mathrm{X}-05-077$ & Looking at culvert outlets & 235 & X-05-077_P5180035 \\
\hline $\mathrm{X}-05-077$ & Looking down at the dropped culvert & $\mathrm{N} / \mathrm{A}$ & X-05-077_P5180036 \\
\hline
\end{tabular}


Appendix 8: List of all photographs taken showing the corresponding site number, descriptive caption, direction faced when taken (azimuth in degrees) and the unique file name for each photograph.

\begin{tabular}{|c|c|c|c|}
\hline $\begin{array}{c}\text { Site } \\
\text { Number }\end{array}$ & Photo Description & Azimuth & File Name \\
\hline $\mathrm{X}-05-078$ & Looking upstream from culvert crossing & 20 & X-05-078_P5190001 \\
\hline $\mathrm{X}-05-078$ & Looking downstream from culvert crossing & 200 & $\mathrm{X}-05-078 \mathrm{P} 5190002$ \\
\hline $\mathrm{X}-05-078$ & Looking at culvert outlets & 350 & X-05-078_P5190003 \\
\hline $\mathrm{X}-05-078$ & Looking at culvert inlets & 180 & X-05-078_P5190004 \\
\hline $\mathrm{X}-05-079$ & Looking upstream from culvert crossing & 285 & X-05-079_P5190005 \\
\hline $\mathrm{X}-05-079$ & Looking downstream from culvert crossing & 75 & X-05-079_P5190006 \\
\hline $\mathrm{X}-05-079$ & Looking at culvert inlets & 90 & X-05-079_P5190007 \\
\hline $\mathrm{X}-05-079$ & Looking at culvert outlets & 180 & X-05-079_P5190008 \\
\hline $\mathrm{X}-05-080$ & Looking upstream from cement box culvert & 90 & X-05-080_P5190009 \\
\hline $\mathrm{X}-05-080$ & Looking downstream from cement box culvert & 180 & X-05-080_P5190010 \\
\hline$X-05-081$ & Looking upstream from culvert crossing & 180 & $\mathrm{X}-05-081 \_\mathrm{P} 5190011$ \\
\hline $\mathrm{X}-05-081$ & Looking downstream from culvert crossing & 360 & X-05-081_P5190012 \\
\hline $\mathrm{X}-05-081$ & Looking at culvert inlets & 35 & X-05-081_P5190013 \\
\hline $\mathrm{X}-05-081$ & Looking at culvert outlets & 135 & X-05-081_P5190014 \\
\hline $\mathrm{X}-05-082$ & Looking upstream from culvert crossing & 270 & X-05-082_P5190015 \\
\hline $\mathrm{X}-05-082$ & Looking downstream from culvert crossing & 150 & $\mathrm{X}-05-082 \_\mathrm{P} 5190016$ \\
\hline $\mathrm{X}-05-082$ & Looking at culvert inlets & 360 & X-05-082_P5190017 \\
\hline $\mathrm{X}-05-082$ & Looking at culvert outlets & 180 & $\mathrm{X}-05-082 \mathrm{P} 5190018$ \\
\hline $\mathrm{X}-05-083$ & Looking upstream from culvert crossing & 245 & X-05-083_P5190019 \\
\hline $\mathrm{X}-05-083$ & Looking downstream from culvert crossing & 30 & X-05-083_P5190020 \\
\hline $\mathrm{X}-05-083$ & Looking at culvert inlets & 100 & X-05-083_P5190021 \\
\hline $\mathrm{X}-05-083$ & Looking at culvert outlets & 270 & $\mathrm{X}-05-083+\mathrm{P} 5190022$ \\
\hline $\mathrm{X}-05-084$ & Looking upstream from culvert crossing & 270 & X-05-084_P5200001 \\
\hline $\mathrm{X}-05-084$ & Looking downstream from culvert crossing & 90 & X-05-084_P5200002 \\
\hline $\mathrm{X}-05-084$ & Looking at culvert inlet & 95 & X-05-084_P5200003 \\
\hline$X-05-084$ & Looking at culvert outlet & 280 & $\mathrm{X}-05-084 \_\mathrm{P} 5200004$ \\
\hline $\mathrm{X}-05-085$ & Looking upstream from culvert crossing & 270 & X-05-085_P5200005 \\
\hline $\mathrm{X}-05-085$ & Looking downstream from culvert crossing & 90 & X-05-085_P5200006 \\
\hline $\mathrm{X}-05-085$ & Looking at culvert inlets & 60 & X-05-085_P5200007 \\
\hline$X-05-085$ & Looking at culvert outlets & 315 & $\mathrm{X}-05-085 \mathrm{P} 5200008$ \\
\hline $\mathrm{X}-05-086$ & Looking upstream from culvert crossing & 345 & X-05-086_P5200009 \\
\hline $\mathrm{X}-05-086$ & Looking downstream from culvert crossing & 170 & X-05-086_P5200010 \\
\hline $\mathrm{X}-05-086$ & Looking at culvert inlets & 120 & X-05-086_P5200011 \\
\hline
\end{tabular}


Appendix 8: List of all photographs taken showing the corresponding site number, descriptive caption, direction faced when taken (azimuth in degrees) and the unique file name for each photograph.

\begin{tabular}{|c|c|c|c|}
\hline $\begin{array}{c}\text { Site } \\
\text { Number }\end{array}$ & Photo Description & Azimuth & File Name \\
\hline $\mathrm{X}-05-086$ & Looking at culvert outlets & 305 & X-05-086_P5200012 \\
\hline $\mathrm{X}-05-087$ & Looking upstream from culvert crossing & 360 & X-05-087_P5200013 \\
\hline $\mathrm{X}-05-087$ & Looking downstream from culvert crossing & 180 & X-05-087_P5200014 \\
\hline $\mathrm{X}-05-087$ & Looking at culvert inlet & 150 & X-05-087_P5200015 \\
\hline $\mathrm{X}-05-087$ & Looking at culvert outlet & 30 & X-05-087_P5200016 \\
\hline $\mathrm{X}-05-088$ & Looking upstream from bridge crossing & 180 & X-05-088_P5240001 \\
\hline $\mathrm{X}-05-088$ & Looking downstream from bridge crossing & 360 & X-05-088_P5240002 \\
\hline $\mathrm{X}-05-088$ & Looking under bridge from right bank & 160 & $\mathrm{X}-05-088 \_\mathrm{P} 5240003$ \\
\hline $\mathrm{X}-05-089$ & Looking upstream from culvert crossing & 150 & X-05-089_P5250001 \\
\hline $\mathrm{X}-05-089$ & Looking downstream from culvert crossing & 345 & X-05-089_P5250002 \\
\hline $\mathrm{X}-05-089$ & Looking at culvert inlets & 350 & X-05-089_P5250003 \\
\hline $\mathrm{X}-05-089$ & Looking at culvert outlets & 90 & X-05-089_P5250004 \\
\hline $\mathrm{X}-05-090$ & Looking upstream from culvert crossing & 90 & X-05-090_P5250005 \\
\hline $\mathrm{X}-05-090$ & Looking downstream from culvert crossing & 270 & X-05-090_P5250006 \\
\hline $\mathrm{X}-05-090$ & Looking at culvert inlets & 305 & X-05-090_P5250007 \\
\hline $\mathrm{X}-05-090$ & Looking at culvert outlets & 360 & X-05-090_P5250008 \\
\hline $\mathrm{X}-05-091$ & Looking upstream from left culvert crossing & 360 & X-05-091_P5250009 \\
\hline $\mathrm{X}-05-091$ & Looking upstream from right culvert crossing & 360 & X-05-091_P5250010 \\
\hline $\mathrm{X}-05-091$ & Looking downstream from left culvert crossing & 180 & X-05-091_P5250011 \\
\hline $\mathrm{X}-05-091$ & Looking downstream from right culvert crossing & 180 & $\mathrm{X}-05-091 \_\mathrm{P} 5250012$ \\
\hline $\mathrm{X}-05-091$ & Looking at left culvert inlet & 115 & X-05-091_P5250013 \\
\hline $\mathrm{X}-05-091$ & Looking down at right culvert inlet & $\mathrm{N} / \mathrm{A}$ & X-05-091_P5250014 \\
\hline $\mathrm{X}-05-091$ & Looking at left culvert outlet & 315 & X-05-091_P5250015 \\
\hline $\mathrm{X}-05-091$ & Looking at right culvert outlet & 90 & X-05-091_P5250016 \\
\hline $\mathrm{X}-05-092$ & Looking upstream from culvert crossing & 80 & X-05-092_P5250017 \\
\hline $\mathrm{X}-05-092$ & Looking downstream from culvert crossing & 130 & X-05-092_P5250018 \\
\hline $\mathrm{X}-05-092$ & Looking at culvert outlets from $15 \mathrm{~m}$ north of the culverts & 180 & $\mathrm{X}-05-092 \_\mathrm{P} 5250019$ \\
\hline $\mathrm{X}-05-092$ & Looking at culvert inlets & 180 & X-05-092_P5250020 \\
\hline $\mathrm{X}-05-092$ & Looking at culvert outlets & 180 & $\mathrm{X}-05-092 \_\mathrm{P} 5250021$ \\
\hline $\mathrm{X}-05-093$ & Looking upstream from culvert crossing & 270 & X-05-093_P5270001 \\
\hline $\mathrm{X}-05-093$ & Looking downstream from culvert crossing & 90 & X-05-093_P5270002 \\
\hline $\mathrm{X}-05-093$ & Looking at culvert inlets & 325 & X-05-093_P5270003 \\
\hline $\mathrm{X}-05-093$ & Looking at culvert outlets & 235 & X-05-093_P5270004 \\
\hline
\end{tabular}


Appendix 8: List of all photographs taken showing the corresponding site number, descriptive caption, direction faced when taken (azimuth in degrees) and the unique file name for each photograph.

\begin{tabular}{|c|c|c|c|}
\hline $\begin{array}{c}\text { Site } \\
\text { Number }\end{array}$ & Photo Description & Azimuth & File Name \\
\hline $\mathrm{X}-05-094$ & Looking upstream from bridge crossing & 270 & X-05-094_P5270005 \\
\hline $\mathrm{X}-05-094$ & Looking downstream from bridge crossing & 180 & X-05-094_P5270006 \\
\hline $\mathrm{X}-05-095$ & Looking upstream from culvert crossing & 325 & X-05-095_P5300001 \\
\hline $\mathrm{X}-05-095$ & Looking downstream from culvert crossing & 140 & X-05-095_P5300002 \\
\hline $\mathrm{X}-05-095$ & Looking at culvert inlets & 130 & X-05-095_P5300003 \\
\hline $\mathrm{X}-05-095$ & Looking at culvert outlets & 280 & X-05-095_P5300004 \\
\hline $\mathrm{X}-05-096$ & Looking upstream from bridge crossing & 270 & X-05-096_P5300005 \\
\hline $\mathrm{X}-05-096$ & Looking downstream from bridge crossing & 90 & X-05-096_P5300006 \\
\hline $\mathrm{X}-05-097$ & Looking upstream from bridge crossing & 270 & X-05-097_P5300007 \\
\hline $\mathrm{X}-05-097$ & Looking downstream from bridge crossing & 90 & X-05-097_P5300008 \\
\hline $\mathrm{X}-05-098$ & Looking upstream from bridge crossing & 270 & X-05-098_P5300009 \\
\hline $\mathrm{X}-05-098$ & Looking downstream from bridge crossing & 145 & X-05-098_P5300010 \\
\hline $\mathrm{X}-05-099$ & Looking upstream from culvert crossing & 250 & X-05-099_P5300011 \\
\hline $\mathrm{X}-05-099$ & Looking downstream from culvert crossing & 70 & X-05-099_P5300012 \\
\hline $\mathrm{X}-05-099$ & Looking at culvert inlets & 100 & X-05-099_P5300013 \\
\hline $\mathrm{X}-05-099$ & Looking at culvert outlets & 300 & X-05-099_P5300014 \\
\hline $\mathrm{X}-05-100$ & Looking upstream from bridge crossing & 190 & X-05-100_P5310001 \\
\hline $\mathrm{X}-05-100$ & Looking downstream from bridge crossing & 10 & $\mathrm{X}-05-100 \_\mathrm{P} 5310002$ \\
\hline $\mathrm{X}-05-101$ & Looking upstream from culvert crossing & 315 & X-05-101_P5310003 \\
\hline $\mathrm{X}-05-101$ & Looking downstream from culvert crossing & 90 & X-05-101_P5310004 \\
\hline $\mathrm{X}-05-101$ & Looking at culvert inlets & 120 & X-05-101_P5310005 \\
\hline $\mathrm{X}-05-102$ & Looking upstream from bridge crossing & 305 & X-05-102_P5310006 \\
\hline $\mathrm{X}-05-102$ & Looking downstream from bridge crossing & 125 & $\mathrm{X}-05-102 \_\mathrm{P} 5310007$ \\
\hline $\mathrm{X}-05-103$ & Looking upstream from bridge crossing & 240 & X-05-103_P5310008 \\
\hline $\mathrm{X}-05-103$ & Looking downstream from bridge crossing & 40 & X-05-103_P5310009 \\
\hline $\mathrm{X}-05-104$ & Looking upstream from culvert crossing & 250 & X-05-104_P5310010 \\
\hline $\mathrm{X}-05-104$ & Looking downstream from culvert crossing & 60 & X-05-104_P5310011 \\
\hline $\mathrm{X}-05-104$ & Looking at culvert inlets & 120 & X-05-104_P5310012 \\
\hline $\mathrm{X}-05-104$ & Looking at culvert outlets & 265 & X-05-104_P5310013 \\
\hline $\mathrm{X}-05-104$ & Photo of Creek Chub (fork length $=200 \mathrm{~mm}$ ) & $\mathrm{N} / \mathrm{A}$ & X-05-104_P5310014 \\
\hline $\mathrm{X}-05-105$ & Looking upstream from bridge crossing & 225 & X-05-105_P6010001 \\
\hline $\mathrm{X}-05-105$ & Looking downstream from bridge crossing & 80 & X-05-105_P6010002 \\
\hline $\mathrm{X}-05-106$ & Looking upstream from bridge crossing & 270 & X-05-106_P6010003 \\
\hline
\end{tabular}


Appendix 8: List of all photographs taken showing the corresponding site number, descriptive caption, direction faced when taken (azimuth in degrees) and the unique file name for each photograph.

\begin{tabular}{|c|c|c|c|}
\hline $\begin{array}{c}\text { Site } \\
\text { Number }\end{array}$ & Photo Description & Azimuth & File Name \\
\hline $\mathrm{X}-05-106$ & Looking downstream from bridge crossing & 90 & X-05-106_P6010004 \\
\hline $\mathrm{X}-05-107$ & Looking upstream from mid-riffle & 190 & X-05-107_P6010005 \\
\hline $\mathrm{X}-05-107$ & Looking downstream from mid-riffle & 360 & X-05-107_P6010006 \\
\hline $\mathrm{X}-05-107$ & Looking upstream at riffle & 220 & X-05-107_P6010007 \\
\hline$X-05-108$ & Looking upstream from cement box culvert & 265 & X-05-108_P6010008 \\
\hline $\mathrm{X}-05-108$ & Looking downstream from cement box culvert & 80 & X-05-108_P6010009 \\
\hline $\mathrm{X}-05-108$ & Looking at culvert inlets & 105 & X-05-108_P6010010 \\
\hline $\mathrm{X}-05-108$ & Looking at culvert outlets & 270 & X-05-108_P6010011 \\
\hline $\mathrm{X}-05-109$ & Looking upstream from bridge crossing & 245 & X-05-109_P6010012 \\
\hline $\mathrm{X}-05-109$ & Looking downstream from bridge crossing & 40 & X-05-109_P6010013 \\
\hline $\mathrm{X}-05-110$ & Looking upstream from culvert crossing & 270 & X-05-110_P6020001 \\
\hline $\mathrm{X}-05-110$ & Looking downstream from culvert crossing & 90 & X-05-110_P6020002 \\
\hline $\mathrm{X}-05-110$ & Looking at culvert outlet & 60 & X-05-110_P6020003 \\
\hline $\mathrm{X}-05-110$ & Looking at culvert inlets & 330 & X-05-110_P6020004 \\
\hline $\mathrm{X}-05-111$ & Looking upstream from culvert crossing & 220 & X-05-111_P6020005 \\
\hline $\mathrm{X}-05-111$ & Looking downstream from culvert crossing & 40 & X-05-111_P6020006 \\
\hline $\mathrm{X}-05-111$ & Looking at culvert inlets & 350 & X-05-111_P6020007 \\
\hline $\mathrm{X}-05-111$ & Looking at culvert outlets & 170 & $\mathrm{X}-05-111$ P6020008 \\
\hline $\mathrm{X}-05-112$ & Looking upstream from culvert crossing & 320 & $\mathrm{X}-05-112 \_\mathrm{P} 6020009$ \\
\hline $\mathrm{X}-05-112$ & Looking downstream from culvert crossing & 180 & X-05-112_P6020010 \\
\hline $\mathrm{X}-05-112$ & Looking at culvert inlets & 115 & X-05-112_P6020011 \\
\hline $\mathrm{X}-05-112$ & Looking at culvert outlets & 60 & $\mathrm{X}-05-112 \_\mathrm{P} 6020012$ \\
\hline $\mathrm{X}-05-112$ & Looking at ford crossing & 270 & $\mathrm{X}-05-112 \_\mathrm{P} 6020013$ \\
\hline $\mathrm{X}-05-113$ & Looking upstream from culvert crossing & 270 & X-05-113_P6020014 \\
\hline $\mathrm{X}-05-113$ & Looking downstream from culvert crossing & 90 & X-05-113_P6020015 \\
\hline $\mathrm{X}-05-113$ & Looking at culvert inlets & 45 & X-05-113_P6020016 \\
\hline $\mathrm{X}-05-113$ & Looking at culvert outlets & 320 & X-05-113_P6020017 \\
\hline $\mathrm{X}-05-114$ & Looking upstream from bridge crossing & 200 & X-05-114_P6020018 \\
\hline $\mathrm{X}-05-114$ & Looking downstream from bridge crossing & 90 & X-05-114_P6020019 \\
\hline $\mathrm{X}-05-115$ & Looking upstream from culvert crossing & 290 & X-05-115_P6020020 \\
\hline $\mathrm{X}-05-115$ & Looking downstream from culvert crossing & 115 & X-05-115_P6020021 \\
\hline $\mathrm{X}-05-115$ & Looking at culvert inlets & 120 & X-05-115_P6020022 \\
\hline $\mathrm{X}-05-115$ & Looking at culvert outlets & 275 & X-05-115_P6020023 \\
\hline
\end{tabular}


Appendix 8: List of all photographs taken showing the corresponding site number, descriptive caption, direction faced when taken (azimuth in degrees) and the unique file name for each photograph.

\begin{tabular}{|c|c|c|c|}
\hline $\begin{array}{c}\text { Site } \\
\text { Number }\end{array}$ & Photo Description & Azimuth & File Name \\
\hline $\mathrm{X}-05-116$ & Looking upstream from bridge crossing & 315 & X-05-116_P6020024 \\
\hline $\mathrm{X}-05-116$ & Looking downstream from bridge crossing & 115 & $\mathrm{X}-05-116 \_P 6020025$ \\
\hline $\mathrm{X}-05-117$ & Looking upstream from bridge crossing & 220 & $\mathrm{X}-05-117$ P6020026 \\
\hline $\mathrm{X}-05-117$ & Looking downstream from bridge crossing & 30 & $\mathrm{X}-05-117 \_\mathrm{P} 6020027$ \\
\hline $\mathrm{X}-05-118$ & Looking upstream from bridge crossing & 270 & $\mathrm{X}-05-118 \_\mathrm{P} 6060001$ \\
\hline $\mathrm{X}-05-118$ & Looking downstream from bridge crossing & 90 & X-05-118_P6060002 \\
\hline $\mathrm{X}-05-119$ & Looking upstream from culvert crossing & 350 & X-05-119_P6060003 \\
\hline $\mathrm{X}-05-119$ & Looking downstream from culvert crossing & 155 & $\mathrm{X}-05-119 \mathrm{P} 6060004$ \\
\hline $\mathrm{X}-05-119$ & Looking at culvert outlets & 315 & X-05-119_P6060005 \\
\hline $\mathrm{X}-05-119$ & Looking at culvert inlets & 200 & X-05-119_P6060006 \\
\hline$X-05-120$ & Looking upstream from culvert crossing & 270 & $\mathrm{X}-05-120 \quad \mathrm{P} 6060007$ \\
\hline $\mathrm{X}-05-120$ & Looking downstream from culvert crossing & 140 & $\mathrm{X}-05-120 \mathrm{P} 6060008$ \\
\hline $\mathrm{X}-05-120$ & Looking at culvert inlets & 80 & X-05-120_P6060009 \\
\hline $\mathrm{X}-05-120$ & Looking at culvert outlets & 330 & $\mathrm{X}-05-120 \_\mathrm{P} 6060010$ \\
\hline $\mathrm{X}-05-121$ & Looking upstream from culvert crossing & 265 & $\mathrm{X}-05-121 \_\mathrm{P} 6060011$ \\
\hline $\mathrm{X}-05-121$ & Looking downstream from culvert crossing & 10 & $\mathrm{X}-05-121 \_\mathrm{P} 6060012$ \\
\hline$X-05-121$ & Looking at culvert inlets & 30 & $\mathrm{X}-05-121 \_\mathrm{P} 6060013$ \\
\hline $\mathrm{X}-05-121$ & Looking at culvert outlets & 24 & X-05-121_P6060014 \\
\hline $\mathrm{X}-05-122$ & Looking upstream from ford crossing & 220 & $\mathrm{X}-05-122 \mathrm{P} 6060015$ \\
\hline$X-05-122$ & Looking downstream from ford crossing & 130 & $\mathrm{X}-05-122 \mathrm{P} 6060016$ \\
\hline $\mathrm{X}-05-122$ & Looking at Looking at culvert inlets & 30 & $\mathrm{X}-05-122 \_\mathrm{P} 6060017$ \\
\hline $\mathrm{X}-05-122$ & Looking at Looking at culvert outlets & 310 & $\mathrm{X}-05-122 \_\mathrm{P} 6060018$ \\
\hline$X-05-123$ & Looking upstream from culvert crossing & 20 & $\mathrm{X}-05-123+\mathrm{P} 6070001$ \\
\hline $\mathrm{X}-05-123$ & Looking downstream from culvert crossing & 180 & $\mathrm{X}-05-123 \quad \mathrm{P} 6070002$ \\
\hline $\mathrm{X}-05-123$ & Looking at culvert inlets & 160 & X-05-123_P6070003 \\
\hline$X-05-123$ & Looking at culvert outlets & 310 & $X-05-123 \quad P 6070004$ \\
\hline $\mathrm{X}-05-124$ & Looking upstream from bridge crossing & 290 & $\mathrm{X}-05-124 \_\mathrm{P} 6070005$ \\
\hline $\mathrm{X}-05-124$ & Looking downstream from bridge crossing & 130 & X-05-124_P6070006 \\
\hline $\mathrm{X}-05-125$ & Looking upstream from culvert crossing & 160 & X-05-125_P6070007 \\
\hline $\mathrm{X}-05-125$ & Looking downstream from culvert crossing & 360 & $\mathrm{X}-05-125 \_\mathrm{P} 6070008$ \\
\hline $\mathrm{X}-05-125$ & Looking at culvert inlet & 45 & $\mathrm{X}-05-125+\mathrm{P} 6070009$ \\
\hline $\mathrm{X}-05-125$ & Looking at culvert outlet & 220 & X-05-125_P6070010 \\
\hline $\mathrm{X}-05-125$ & Photo of a White Sucker & N/A & $\mathrm{X}-05-125 \_\mathrm{P} 6070011$ \\
\hline
\end{tabular}


Appendix 8: List of all photographs taken showing the corresponding site number, descriptive caption, direction faced when taken (azimuth in degrees) and the unique file name for each photograph.

\begin{tabular}{|c|c|c|c|}
\hline $\begin{array}{c}\text { Site } \\
\text { Number }\end{array}$ & Photo Description & Azimuth & File Name \\
\hline $\mathrm{X}-05-126$ & Looking upstream from culvert crossing & 100 & $\begin{array}{lll}\mathrm{X}-05-126 & \mathrm{P} 6070012\end{array}$ \\
\hline $\mathrm{X}-05-126$ & Looking downstream from culvert crossing & 320 & $\mathrm{X}-05-126 \_\mathrm{P} 6070013$ \\
\hline $\mathrm{X}-05-126$ & Looking at culvert inlets & 170 & $\mathrm{X}-05-126 \_\mathrm{P} 6070014$ \\
\hline $\mathrm{X}-05-126$ & Looking at culvert outlets & 290 & $\mathrm{X}-05-126 \_P 6070015$ \\
\hline$X-05-127$ & Looking upstream from culvert crossing & 140 & $\mathrm{X}-05-127 \quad \mathrm{P} 6090001$ \\
\hline $\mathrm{X}-05-127$ & Looking downstream from culvert crossing & 310 & $\mathrm{X}-05-127 \mathrm{P} 6090002$ \\
\hline $\mathrm{X}-05-127$ & Looking down at culvert inlets & 290 & X-05-127_P6090003 \\
\hline $\mathrm{X}-05-127$ & Looking at culvert outlets & 200 & $\mathrm{X}-05-127 \quad \mathrm{P} 6090004$ \\
\hline $\mathrm{X}-05-128$ & Looking upstream from culvert crossing & 65 & $\mathrm{X}-05-128 \mathrm{P} 6090005$ \\
\hline $\mathrm{X}-05-128$ & Looking further upstream from right bank & 150 & $\mathrm{X}-05-128 \mathrm{P} 6090006$ \\
\hline$X-05-128$ & Looking downstream from culvert crossing & 280 & $\mathrm{X}-05-128+\mathrm{P} 6090007$ \\
\hline $\mathrm{X}-05-128$ & Looking at culvert inlet & 10 & $\mathrm{X}-05-128 \quad \mathrm{P} 6090008$ \\
\hline $\mathrm{X}-05-128$ & Looking at culvert outlet & 100 & $\mathrm{X}-05-128 \quad \mathrm{P} 6090009$ \\
\hline$X-05-129$ & Looking upstream from bridge crossing & 260 & $\mathrm{X}-05-129 \mathrm{P} 6100001$ \\
\hline $\mathrm{X}-05-129$ & Looking downstream from bridge crossing & 30 & $\mathrm{X}-05-129 \mathrm{P} 6100002$ \\
\hline $\mathrm{X}-05-130$ & Looking upstream from culvert crossing & 270 & $\mathrm{X}-05-130 \quad \mathrm{P} 6100003$ \\
\hline$X-05-130$ & Looking downstream from culvert crossing & 90 & $X-05-130 \quad P 6100004$ \\
\hline $\mathrm{X}-05-130$ & Looking at culvert inlets & 45 & X-05-130_P6100005 \\
\hline $\mathrm{X}-05-130$ & Looking at culvert outlets & 315 & $\mathrm{X}-05-130 \_\mathrm{P} 6100006$ \\
\hline $\mathrm{X}-05-131$ & Looking upstream from culvert crossing & 360 & $\mathrm{X}-05-131 \quad \mathrm{P} 6130001$ \\
\hline $\mathrm{X}-05-131$ & Looking downstream from culvert crossing & 270 & $\mathrm{X}-05-131 \mathrm{P} 6130002$ \\
\hline $\mathrm{X}-05-132$ & Looking upstream from culvert crossing & 360 & $\mathrm{X}-05-132 \_\mathrm{P} 6130003$ \\
\hline$X-05-132$ & Looking downstream from culvert crossing & 180 & $\mathrm{X}-05-132 \_\mathrm{P} 6130004$ \\
\hline $\mathrm{X}-05-132$ & Looking at culvert inlets & 245 & $\mathrm{X}-05-132 \mathrm{P} 6130005$ \\
\hline $\mathrm{X}-05-132$ & Looking at culvert outlets & 30 & $\mathrm{X}-05-132 \mathrm{P} 6130006$ \\
\hline$X-05-133$ & Looking upstream from culvert crossing & 30 & $\mathrm{X}-05-133 \mathrm{P} 6130007$ \\
\hline $\mathrm{X}-05-133$ & Looking downstream from culvert crossing & 320 & $\mathrm{X}-05-133 \_\mathrm{P} 6130008$ \\
\hline $\mathrm{X}-05-133$ & Looking at culvert inlet & 300 & $\mathrm{X}-05-133 \quad \mathrm{P} 6130009$ \\
\hline $\mathrm{X}-05-133$ & Looking at culvert outlet & 95 & $\mathrm{X}-05-133-\mathrm{P} 6130010$ \\
\hline$X-05-134$ & Looking upstream from culvert crossing & 330 & $\mathrm{X}-05-134 \mathrm{P} 6130011$ \\
\hline $\mathrm{X}-05-134$ & Looking downstream from culvert crossing & 180 & $\mathrm{X}-05-134 \quad \mathrm{P} 6130012$ \\
\hline $\mathrm{X}-05-134$ & Looking at culvert inlets & 110 & $\mathrm{X}-05-134 \quad \mathrm{P} 6130013$ \\
\hline $\mathrm{X}-05-135$ & Looking upstream from culvert crossing & 360 & $\mathrm{X}-05-135+\mathrm{P} 6130014$ \\
\hline
\end{tabular}


Appendix 8: List of all photographs taken showing the corresponding site number, descriptive caption, direction faced when taken (azimuth in degrees) and the unique file name for each photograph.

\begin{tabular}{|c|c|c|c|}
\hline $\begin{array}{c}\text { Site } \\
\text { Number }\end{array}$ & Photo Description & Azimuth & File Name \\
\hline $\mathrm{X}-05-135$ & Looking downstream from culvert crossing & 185 & $\mathrm{X}-05-135$ P6130015 \\
\hline $\mathrm{X}-05-135$ & Looking at culvert inlet & 120 & X-05-135_P6130016 \\
\hline $\mathrm{X}-05-135$ & Looking at culvert outlet & 35 & X-05-135_P6130017 \\
\hline $\mathrm{X}-05-136$ & Looking upstream from bridge crossing & 270 & X-05-136_P6130018 \\
\hline $\mathrm{X}-05-136$ & Looking downstream from bridge crossing & 110 & X-05-136_P6130019 \\
\hline $\mathrm{X}-05-137$ & Looking upstream from culvert crossing & 290 & X-05-137_P6140001 \\
\hline $\mathrm{X}-05-137$ & Looking downstream from culvert crossing & 80 & $\mathrm{X}-05-137 \_\mathrm{P} 6140002$ \\
\hline $\mathrm{X}-05-137$ & Looking at culvert inlets & 145 & $\mathrm{X}-05-137$ P6140003 \\
\hline $\mathrm{X}-05-137$ & Looking at culvert outlets & 220 & $\mathrm{X}-05-137 \quad \mathrm{P} 6140004$ \\
\hline $\mathrm{X}-05-138$ & Looking upstream from culvert crossing & 300 & X-05-138_P6140005 \\
\hline $\mathrm{X}-05-138$ & Looking downstream from culvert crossing & 45 & X-05-138_P6140006 \\
\hline $\mathrm{X}-05-138$ & Looking at culvert inlet & 90 & X-05-138_P6140007 \\
\hline $\mathrm{X}-05-138$ & Looking at culvert outlet & 310 & X-05-138_P6140008 \\
\hline $\mathrm{X}-05-139$ & Looking upstream from culvert crossing & 240 & X-05-139_P6140009 \\
\hline $\mathrm{X}-05-139$ & Looking downstream from culvert crossing & 40 & X-05-139 P6140010 \\
\hline $\mathrm{X}-05-139$ & Looking at culvert inlets & 210 & X-05-139_P6140011 \\
\hline $\mathrm{X}-05-139$ & Looking at culvert outlets & 155 & X-05-139_P6140012 \\
\hline $\mathrm{X}-05-140$ & Looking upstream from culvert crossing & 190 & X-05-140 P6140013 \\
\hline $\mathrm{X}-05-140$ & Looking downstream from culvert crossing & 360 & X-05-140_P6140014 \\
\hline $\mathrm{X}-05-140$ & Looking at culvert inlet & 45 & X-05-140_P6140015 \\
\hline $\mathrm{X}-05-140$ & Looking at potential barrier to fish passage & 135 & X-05-140_P6140016 \\
\hline $\mathrm{X}-05-140$ & Looking at culvert outlet & 230 & X-05-140_P6140017 \\
\hline $\mathrm{X}-05-141$ & Looking upstream from bridge crossing & 210 & $\mathrm{X}-05-141 \_\mathrm{P} 6140018$ \\
\hline $\mathrm{X}-05-141$ & Looking downstream from bridge crossing & 60 & X-05-141_P6140019 \\
\hline $\mathrm{X}-05-142$ & Looking upstream from culvert crossing & 280 & X-05-142_P6150001 \\
\hline$X-05-142$ & Looking downstream from culvert crossing & 45 & $\mathrm{X}-05-142 \_\mathrm{P} 6150002$ \\
\hline $\mathrm{X}-05-142$ & Looking at culvert inlet & 150 & $\mathrm{X}-05-142 \_\mathrm{P} 6150003$ \\
\hline $\mathrm{X}-05-142$ & Looking at culvert outlet & 220 & $\mathrm{X}-05-142 \_\mathrm{P} 6150004$ \\
\hline $\mathrm{X}-05-143$ & Looking upstream from culvert crossing & 275 & X-05-143_P6150005 \\
\hline $\mathrm{X}-05-143$ & Looking downstream from culvert crossing & 45 & X-05-143_P6150006 \\
\hline $\mathrm{X}-05-143$ & Looking at culvert inlet & 80 & $\mathrm{X}-05-143$ P6150007 \\
\hline $\mathrm{X}-05-143$ & Looking at culvert outlet & 330 & X-05-143_P6150008 \\
\hline $\mathrm{X}-05-144$ & Looking upstream from culvert crossing & 80 & X-05-144_P6150009 \\
\hline
\end{tabular}


Appendix 8: List of all photographs taken showing the corresponding site number, descriptive caption, direction faced when taken (azimuth in degrees) and the unique file name for each photograph.

\begin{tabular}{|c|c|c|c|}
\hline $\begin{array}{c}\text { Site } \\
\text { Number }\end{array}$ & Photo Description & Azimuth & File Name \\
\hline $\mathrm{X}-05-144$ & Looking downstream from culvert crossing & 250 & X-05-144_P6150010 \\
\hline $\mathrm{X}-05-144$ & Looking at culvert outlets & 120 & X-05-144_P6150011 \\
\hline $\mathrm{X}-05-144$ & Photo of Pearl Dace & $\mathrm{N} / \mathrm{A}$ & X-05-144_P6150012 \\
\hline $\mathrm{X}-05-144$ & Photo of Pearl Dace & $\mathrm{N} / \mathrm{A}$ & X-05-144_P6150013 \\
\hline$X-05-144$ & Photo of Pearl Dace & $\mathrm{N} / \mathrm{A}$ & X-05-144_P6150014 \\
\hline $\mathrm{X}-05-144$ & Photo of Pearl Dace & $\mathrm{N} / \mathrm{A}$ & X-05-144_P6150015 \\
\hline $\mathrm{X}-05-145$ & Looking upstream from culvert crossing & 250 & X-05-145_P6150016 \\
\hline $\mathrm{X}-05-145$ & Looking downstream from culvert crossing & 30 & X-05-145_P6150017 \\
\hline $\mathrm{X}-05-145$ & Looking at culvert inlets & 70 & X-05-145_P6150018 \\
\hline $\mathrm{X}-05-145$ & Looking at culvert outlets & 220 & X-05-145_P6150019 \\
\hline $\mathrm{X}-05-145$ & Looking at cement box culvert near old bridge & 110 & X-05-145_P6150020 \\
\hline $\mathrm{X}-05-146$ & Looking upstream at 2nd order tributary to the east & 60 & X-05-146_P6160001 \\
\hline $\mathrm{X}-05-146$ & Looking upstream at 2nd order tributary to the west & 315 & X-05-146_P6160002 \\
\hline $\mathrm{X}-05-146$ & Looking downstream from culvert crossing & 180 & X-05-146_P6160003 \\
\hline $\mathrm{X}-05-146$ & Looking downstream from culvert crossing & 150 & X-05-146_P6160004 \\
\hline $\mathrm{X}-05-146$ & Looking at east culvert outlet & 30 & X-05-146_P6160005 \\
\hline $\mathrm{X}-05-146$ & Looking at west culvert outlet & 300 & X-05-146_P6160006 \\
\hline $\mathrm{X}-05-147$ & Looking upstream from culvert crossing & 260 & $\mathrm{X}-05-147$ P6170001 \\
\hline $\mathrm{X}-05-147$ & Looking upstream at cattle impacts on left bank & 310 & X-05-147_P6170002 \\
\hline $\mathrm{X}-05-147$ & Looking upstream at right bank from culvert crossing & 230 & X-05-147_P6170003 \\
\hline $\mathrm{X}-05-147$ & Looking downstream from culvert crossing & 80 & X-05-147_P6170004 \\
\hline $\mathrm{X}-05-147$ & Looking downstream from culvert crossing & 20 & X-05-147_P6170005 \\
\hline $\mathrm{X}-05-148$ & Looking upstream from bridge crossing & 310 & X-05-148_P6170006 \\
\hline $\mathrm{X}-05-148$ & Looking downstream from bridge crossing & 140 & X-05-148_P6170007 \\
\hline $\mathrm{X}-05-149$ & Looking upstream from culvert crossing & 230 & X-05-149_P6210001 \\
\hline $\mathrm{X}-05-149$ & Looking downstream from culvert crossing & 340 & X-05-149_P6210002 \\
\hline $\mathrm{X}-05-150$ & Looking upstream from culvert crossing & 230 & $\mathrm{X}-05-150 \_\mathrm{P} 6210003$ \\
\hline $\mathrm{X}-05-150$ & Looking downstream from culvert crossing & 60 & X-05-150_P6210004 \\
\hline $\mathrm{X}-05-150$ & Looking at culvert inlet & 100 & X-05-150_P6210005 \\
\hline $\mathrm{X}-05-150$ & Looking at perched outlet & 310 & $\mathrm{X}-05-150 \_\mathrm{P} 6210006$ \\
\hline $\mathrm{X}-05-151$ & Looking upstream from culvert crossing & 300 & X-05-151_P6210007 \\
\hline $\mathrm{X}-05-151$ & Looking downstream from culvert crossing & 90 & X-05-151_P6210008 \\
\hline $\mathrm{X}-05-151$ & Looking at culvert inlet & 45 & $\mathrm{X}-05-151 \_\mathrm{P} 6210009$ \\
\hline
\end{tabular}


Appendix 8: List of all photographs taken showing the corresponding site number, descriptive caption, direction faced when taken (azimuth in degrees) and the unique file name for each photograph.

\begin{tabular}{|c|c|c|c|}
\hline $\begin{array}{c}\text { Site } \\
\text { Number }\end{array}$ & Photo Description & Azimuth & File Name \\
\hline $\mathrm{X}-05-151$ & Looking at culvert outlet & $\mathrm{N} / \mathrm{A}$ & X-05-151_P6210010 \\
\hline$X-05-152$ & Looking upstream from culvert crossing & 240 & $\mathrm{X}-05-152 \_\mathrm{P} 6210011$ \\
\hline $\mathrm{X}-05-152$ & Looking downstream from culvert crossing & 20 & X-05-152_P6210012 \\
\hline $\mathrm{X}-05-152$ & Looking at culvert inlets & 180 & X-05-152_P6210013 \\
\hline$X-05-152$ & Looking at culvert outlets & 150 & X-05-152_P6210014 \\
\hline$X-05-153$ & Looking upstream from culvert crossing & 160 & X-05-153_P6210015 \\
\hline $\mathrm{X}-05-153$ & Looking downstream from culvert crossing & 40 & X-05-153_P6210016 \\
\hline$X-05-153$ & Looking further downstream from left bank & 65 & X-05-153_P6210017 \\
\hline $\mathrm{X}-05-153$ & Looking at culvert outlet & 160 & $\mathrm{X}-05-153 \quad \mathrm{P} 6210018$ \\
\hline $\mathrm{X}-05-153$ & Looking at culvert inlet & 320 & X-05-153_P6210019 \\
\hline$X-05-154$ & Looking upstream from culvert crossing & 260 & X-05-154_P6210020 \\
\hline$X-05-154$ & Looking downstream from culvert crossing & 80 & X-05-154_P6210021 \\
\hline$X-05-154$ & Looking at culvert inlet & 40 & X-05-154_P6210022 \\
\hline$X-05-154$ & Looking at culvert outlet & 300 & X-05-154_P6210023 \\
\hline $\mathrm{X}-05-155$ & Looking upstream from bridge crossing & 295 & X-05-155_P6220005 \\
\hline $\mathrm{X}-05-155$ & Looking downstream from bridge crossing & 65 & X-05-155_P6220006 \\
\hline$X-05-156$ & Looking upstream from culvert crossing & 240 & X-05-156_P6220007 \\
\hline $\mathrm{X}-05-156$ & Looking downstream from culvert crossing & 15 & X-05-156_P6220008 \\
\hline $\mathrm{X}-05-156$ & Looking at culvert inlets & 70 & X-05-156_P6220009 \\
\hline$X-05-156$ & Looking at culvert outlets & 100 & X-05-156_P6220010 \\
\hline $\mathrm{X}-05-157$ & Looking upstream from bridge crossing & 250 & X-05-157_P6220011 \\
\hline$X-05-157$ & Looking downstream from bridge crossing & 50 & X-05-157_P6220012 \\
\hline$X-05-158$ & Looking upstream from culvert crossing & 125 & X-05-158_P6220013 \\
\hline $\mathrm{X}-05-158$ & Looking downstream from culvert crossing & 280 & X-05-158_P6220014 \\
\hline$X-05-158$ & Looking at culvert inlet & 340 & X-05-158_P6220015 \\
\hline$X-05-158$ & Looking at culvert outlet & 150 & X-05-158_P6220016 \\
\hline $\mathrm{X}-05-159$ & Looking upstream from culvert crossing & 130 & X-05-159_P6230001 \\
\hline $\mathrm{X}-05-159$ & Looking downstream from culvert crossing & 290 & X-05-159_P6230002 \\
\hline $\mathrm{X}-05-159$ & Looking at culvert inlet & 350 & X-05-159_P6230003 \\
\hline$X-05-159$ & Looking at culvert outlet & 150 & X-05-159_P6230004 \\
\hline $\mathrm{X}-05-160$ & Looking upstream from culvert crossing & 250 & $\mathrm{X}-05-160 \quad \mathrm{P} 6230005$ \\
\hline$X-05-160$ & Looking downstream from culvert crossing & 40 & X-05-160_P6230006 \\
\hline$X-05-161$ & Looking upstream from culvert crossing & 220 & X-05-161_P6230007 \\
\hline
\end{tabular}


Appendix 8: List of all photographs taken showing the corresponding site number, descriptive caption, direction faced when taken (azimuth in degrees) and the unique file name for each photograph.

\begin{tabular}{|c|c|c|c|}
\hline $\begin{array}{c}\text { Site } \\
\text { Number }\end{array}$ & Photo Description & Azimuth & File Name \\
\hline $\mathrm{X}-05-161$ & Looking downstream from culvert crossing & 10 & X-05-161_P6230008 \\
\hline $\mathrm{X}-05-162$ & Looking upstream from bridge crossing & 180 & $\mathrm{X}-05-162 \_\mathrm{P} 6230009$ \\
\hline $\mathrm{X}-05-162$ & Looking downstream from bridge crossing & 360 & $\mathrm{X}-05-162 \_\mathrm{P} 6230010$ \\
\hline $\mathrm{X}-05-163$ & Looking upstream from culvert crossing & 140 & X-05-163_P6230011 \\
\hline $\mathrm{X}-05-163$ & Looking downstream from culvert crossing & 40 & X-05-163_P6230012 \\
\hline $\mathrm{X}-05-163$ & Looking at culvert inlets & 300 & $\mathrm{X}-05-163 \quad \mathrm{P} 6230013$ \\
\hline $\mathrm{X}-05-163$ & Looking at culvert outlets & $\mathrm{N} / \mathrm{A}$ & $\mathrm{X}-05-163 \_\mathrm{P} 6230014$ \\
\hline $\mathrm{X}-05-164$ & Looking upstream from culvert crossing & 300 & $\mathrm{X}-05-164 \_\mathrm{P} 6230015$ \\
\hline $\mathrm{X}-05-164$ & Looking downstream from culvert crossing & 100 & $\mathrm{X}-05-164 \_\mathrm{P} 6230016$ \\
\hline $\mathrm{X}-05-164$ & Looking at culvert inlets & 45 & $\mathrm{X}-05-164 \_\mathrm{P} 6230017$ \\
\hline $\mathrm{X}-05-164$ & Looking at culvert outlets & 255 & X-05-164_P6230034 \\
\hline $\mathrm{X}-05-165$ & Looking upstream from culvert crossing & 210 & X-05-165_P6270001 \\
\hline $\mathrm{X}-05-165$ & Looking downstream from culvert crossing & 340 & X-05-165_P6270002 \\
\hline $\mathrm{X}-05-165$ & Looking at culvert inlet & 50 & $\mathrm{X}-05-165+\mathrm{P} 6270003$ \\
\hline $\mathrm{X}-05-165$ & Looking at culvert outlet & 135 & $\mathrm{X}-05-165 \_\mathrm{P} 6270004$ \\
\hline $\mathrm{X}-05-166$ & Looking upstream from culvert crossing & 130 & X-05-166_P6270005 \\
\hline $\mathrm{X}-05-166$ & Looking downstream from culvert crossing & 320 & X-05-166_P6270006 \\
\hline $\mathrm{X}-05-166$ & Looking downstream from culvert crossing & 360 & $\mathrm{X}-05-166 \_\mathrm{P} 6270007$ \\
\hline $\mathrm{X}-05-166$ & Looking down at inlet & 150 & X-05-166_P6270008 \\
\hline $\mathrm{X}-05-166$ & Looking at culvert outlet & 270 & X-05-166_P6270009 \\
\hline $\mathrm{X}-05-166$ & Looking at perched outlet & 260 & X-05-166_P6270010 \\
\hline $\mathrm{X}-05-166$ & Looking at perched outlet & 65 & X-05-166_P6270011 \\
\hline $\mathrm{X}-05-167$ & Looking upstream from culvert crossing & 235 & X-05-167_P6280001 \\
\hline $\mathrm{X}-05-167$ & Looking downstream from culvert crossing & 40 & $\mathrm{X}-05-167 \_\mathrm{P} 6280002$ \\
\hline $\mathrm{X}-05-167$ & Looking at left culvert inlet & 45 & $\mathrm{X}-05-167 \_\mathrm{P} 6280003$ \\
\hline$X-05-167$ & Looking at right culvert inlet & 55 & $\mathrm{X}-05-167=\mathrm{P} 6280004$ \\
\hline $\mathrm{X}-05-167$ & Looking at left culvert outlet & 280 & $\mathrm{X}-05-167 \_\mathrm{P} 6280005$ \\
\hline $\mathrm{X}-05-167$ & Looking at right culvert outlet & 250 & $\mathrm{X}-05-167 \_\mathrm{P} 6280006$ \\
\hline $\mathrm{X}-05-168$ & Looking upstream from culvert crossing & 200 & X-05-168_P6280007 \\
\hline $\mathrm{X}-05-168$ & Looking downstream from culvert crossing & 340 & X-05-168_P6280008 \\
\hline $\mathrm{X}-05-168$ & Looking at culvert inlet & 320 & X-05-168_P6280009 \\
\hline $\mathrm{X}-05-168$ & Looking at culvert outlet & 200 & X-05-168_P6280010 \\
\hline$X-05-169$ & Looking upstream from culvert crossing & 70 & X-05-169_P6280011 \\
\hline
\end{tabular}


Appendix 8: List of all photographs taken showing the corresponding site number, descriptive caption, direction faced when taken (azimuth in degrees) and the unique file name for each photograph.

\begin{tabular}{|c|c|c|c|}
\hline $\begin{array}{c}\text { Site } \\
\text { Number }\end{array}$ & Photo Description & Azimuth & File Name \\
\hline $\mathrm{X}-05-169$ & Looking downstream from culvert crossing & 340 & X-05-169_P6280012 \\
\hline$X-05-169$ & Looking at culvert inlets & 360 & $\mathrm{X}-05-169 \_\mathrm{P} 6280013$ \\
\hline $\mathrm{X}-05-169$ & Looking at culvert outlets & 200 & $\mathrm{X}-05-169 \quad \mathrm{P} 6280014$ \\
\hline $\mathrm{X}-05-170$ & Looking upstream from culvert crossing & 180 & $\mathrm{X}-05-170 \_\mathrm{P} 6280015$ \\
\hline$X-05-170$ & Looking downstream from culvert crossing & 280 & $\mathrm{X}-05-170 \_\mathrm{P} 6280016$ \\
\hline$X-05-170$ & Looking at culvert outlet & 150 & $\mathrm{X}-05-170 \_\mathrm{P} 6280017$ \\
\hline $\mathrm{X}-05-171$ & Looking upstream from culvert crossing & 205 & X-05-171_P6280018 \\
\hline$X-05-171$ & Looking downstream from culvert crossing & 350 & $\mathrm{X}-05-171 \_\mathrm{P} 6280019$ \\
\hline $\mathrm{X}-05-171$ & Looking at culvert inlet & 320 & $\mathrm{X}-05-171 \_\mathrm{P} 6280020$ \\
\hline$X-05-171$ & Looking at culvert outlet & 115 & $\mathrm{X}-05-171 \_\mathrm{P} 6280021$ \\
\hline$X-05-172$ & Looking upstream from bridge crossing & 260 & $\mathrm{X}-05-172 \mathrm{P} 6290001$ \\
\hline $\mathrm{X}-05-172$ & Looking downstream from bridge crossing & 80 & $\mathrm{X}-05-172 \mathrm{P} 6290002$ \\
\hline $\mathrm{X}-05-172$ & Looking downstream from $15 \mathrm{~m}$ North of the bridge crossing & 120 & $\mathrm{X}-05-172 \_\mathrm{P} 6290003$ \\
\hline$X-05-172$ & Looking at Northern Pike & $\mathrm{N} / \mathrm{A}$ & X-05-172_P6290004 \\
\hline $\mathrm{X}-05-172$ & Looking at Northern Pike & $\mathrm{N} / \mathrm{A}$ & X-05-172_P6290005 \\
\hline$X-05-173$ & Looking upstream from bridge crossing & 270 & X-05-173_P6290006 \\
\hline $\mathrm{X}-05-173$ & Looking downstream from bridge crossing & 80 & $\mathrm{X}-05-173$ P6290007 \\
\hline $\mathrm{X}-05-174$ & Looking upstream from bridge crossing & 300 & X-05-174_P6300001 \\
\hline $\mathrm{X}-05-174$ & Looking downstream from bridge crossing & 80 & $\mathrm{X}-05-174 \_\mathrm{P} 6300002$ \\
\hline $\mathrm{X}-05-175$ & Looking upstream from bridge crossing & 240 & $\mathrm{X}-05-175 \mathrm{P} 6300003$ \\
\hline $\mathrm{X}-05-175$ & Looking downstream from bridge crossing & 90 & $\mathrm{X}-05-175 \_\mathrm{P} 6300004$ \\
\hline $\mathrm{X}-05-176$ & Looking upstream from mid-reach & 180 & X-05-176_P7040005 \\
\hline$X-05-176$ & Looking downstream from mid-reach & 90 & X-05-176_P7040006 \\
\hline$X-05-177$ & Looking upstream from culvert crossing & 330 & X-05-177_P7040007 \\
\hline $\mathrm{X}-05-177$ & Looking downstream from culvert crossing & 100 & X-05-177_P7040008 \\
\hline$X-05-177$ & Looking at culvert inlet & 360 & $\mathrm{X}-05-177 \_\mathrm{P} 7040009$ \\
\hline $\mathrm{X}-05-177$ & Looking at culvert outlet & 210 & $\mathrm{X}-05-177$ P7040010 \\
\hline $\mathrm{X}-05-178$ & Looking upstream from culvert crossing & 150 & X-05-178_P7050001 \\
\hline $\mathrm{X}-05-178$ & Looking downstream from culvert crossing & 330 & X-05-178_P7050002 \\
\hline$X-05-178$ & Looking at culvert inlets & 70 & X-05-178_P7050003 \\
\hline $\mathrm{X}-05-178$ & Looking at culvert outlets & 95 & $\mathrm{X}-05-178+\mathrm{P} 7050004$ \\
\hline $\mathrm{X}-05-179$ & Looking upstream from culvert crossing & 350 & X-05-179_P7050005 \\
\hline $\mathrm{X}-05-179$ & Looking downstream from culvert crossing & 130 & X-05-179_P7050006 \\
\hline
\end{tabular}


Appendix 8: List of all photographs taken showing the corresponding site number, descriptive caption, direction faced when taken (azimuth in degrees) and the unique file name for each photograph.

\begin{tabular}{|c|c|c|c|}
\hline $\begin{array}{c}\text { Site } \\
\text { Number }\end{array}$ & Photo Description & Azimuth & File Name \\
\hline $\mathrm{X}-05-179$ & Looking downstream (from $\sim 10 \mathrm{~m}$ south of crossing) at cattle impacts & 100 & X-05-179_P7050007 \\
\hline $\mathrm{X}-05-179$ & Looking at culvert inlet & 130 & X-05-179_P7050008 \\
\hline $\mathrm{X}-05-179$ & Looking at culvert outlet & 300 & X-05-179_P7050009 \\
\hline $\mathrm{X}-05-180$ & Looking upstream from culvert crossing & 100 & X-05-180_P7050010 \\
\hline $\mathrm{X}-05-180$ & Looking downstream from culvert crossing & 260 & X-05-180_P7050011 \\
\hline $\mathrm{X}-05-180$ & Looking down at right outlet & $\mathrm{N} / \mathrm{A}$ & $\mathrm{X}-05-180 \_\mathrm{P} 7050012$ \\
\hline $\mathrm{X}-05-181$ & Looking upstream from culvert crossing & 270 & X-05-181_P7060001 \\
\hline $\mathrm{X}-05-181$ & Looking downstream from culvert crossing & 90 & X-05-181_P7060002 \\
\hline $\mathrm{X}-05-181$ & Looking at culvert inlets & 120 & X-05-181_P7060003 \\
\hline $\mathrm{X}-05-181$ & Looking at culvert outlets & 200 & X-05-181_P7060004 \\
\hline $\mathrm{X}-05-182$ & Looking upstream from culvert crossing & 270 & $\mathrm{X}-05-182 \_\mathrm{P} 7060005$ \\
\hline $\mathrm{X}-05-182$ & Looking downstream from culvert crossing & 90 & X-05-182_P7060006 \\
\hline $\mathrm{X}-05-182$ & Looking at culvert inlets & 270 & X-05-182_P7060007 \\
\hline $\mathrm{X}-05-182$ & Looking at culvert outlets & 90 & $\mathrm{X}-05-182 \_\mathrm{P} 7060008$ \\
\hline $\mathrm{X}-05-183$ & Looking upstream from culvert crossing & 290 & X-05-183_P7060009 \\
\hline $\mathrm{X}-05-183$ & Looking downstream from culvert crossing & 100 & X-05-183_P7060010 \\
\hline $\mathrm{X}-05-183$ & Looking at culvert inlets & 140 & X-05-183_P7060011 \\
\hline $\mathrm{X}-05-183$ & Looking at culvert outlets & 350 & $\mathrm{X}-05-183 \_\mathrm{P} 7060012$ \\
\hline $\mathrm{X}-05-184$ & Looking upstream from culvert crossing & 350 & X-05-184_P7060013 \\
\hline $\mathrm{X}-05-184$ & Looking downstream from culvert crossing & 170 & X-05-184_P7060014 \\
\hline $\mathrm{X}-05-184$ & Looking at culvert inlets & 100 & X-05-184_P7060015 \\
\hline $\mathrm{X}-05-184$ & Looking at culvert outlets & 20 & X-05-184_P7060016 \\
\hline $\mathrm{X}-05-184$ & Looking upstream at 2nd order tributary & 90 & X-05-184_P7060017 \\
\hline $\mathrm{X}-05-184$ & Looking upstream at 2nd order tributary & 90 & X-05-184_P7060018 \\
\hline $\mathrm{X}-05-185$ & Looking upstream from culvert crossing & 280 & X-05-185_P7070001 \\
\hline $\mathrm{X}-05-185$ & Looking downstream from culvert crossing & 130 & $\mathrm{X}-05-185+\mathrm{P} 7070002$ \\
\hline $\mathrm{X}-05-185$ & Looking downstream from culvert crossing & 90 & $\mathrm{X}-05-185+\mathrm{P} 7070003$ \\
\hline $\mathrm{X}-05-185$ & Looking at culvert inlets & 150 & X-05-185_P7070004 \\
\hline $\mathrm{X}-05-185$ & Looking at culvert outlets & 330 & X-05-185_P7070005 \\
\hline $\mathrm{X}-05-185$ & Photo of Common Carp released (fork length $=800 \mathrm{~mm}$ ) & $\mathrm{N} / \mathrm{A}$ & $\mathrm{X}-05-185+\mathrm{P} 7070006$ \\
\hline $\mathrm{X}-05-185$ & Photo of Common Carp released (total length $=800 \mathrm{~mm}$ ) & $\mathrm{N} / \mathrm{A}$ & X-05-185_P7070007 \\
\hline $\mathrm{X}-05-186$ & Looking upstream from culvert crossing & 270 & X-05-186_P7070008 \\
\hline $\mathrm{X}-05-186$ & Looking downstream from culvert crossing & 90 & X-05-186_P7070009 \\
\hline
\end{tabular}


Appendix 8: List of all photographs taken showing the corresponding site number, descriptive caption, direction faced when taken (azimuth in degrees) and the unique file name for each photograph.

\begin{tabular}{|c|c|c|c|}
\hline $\begin{array}{c}\text { Site } \\
\text { Number }\end{array}$ & Photo Description & Azimuth & File Name \\
\hline $\mathrm{X}-05-186$ & Looking at culvert inlets & 70 & $\mathrm{X}-05-186 \quad \mathrm{P} 7070010$ \\
\hline$X-05-186$ & Looking at culvert outlets & 200 & $\mathrm{X}-05-186 \quad \mathrm{P} 7070011$ \\
\hline $\mathrm{X}-05-187$ & Looking upstream from culvert crossing & 180 & $\mathrm{X}-05-187 \_\mathrm{P} 7070012$ \\
\hline $\mathrm{X}-05-187$ & Looking downstream from culvert crossing & 360 & $\mathrm{X}-05-187 \mathrm{P} 7070013$ \\
\hline$X-05-187$ & Looking at culvert inlets & 340 & X-05-187_P7070014 \\
\hline $\mathrm{X}-05-187$ & Looking at culvert outlets & 210 & $\mathrm{X}-05-187 \mathrm{P} 7070015$ \\
\hline $\mathrm{X}-05-187$ & Looking at 2nd order tributary & 270 & X-05-187_P7070016 \\
\hline$X-05-188$ & Looking upstream from culvert crossing & 350 & $\mathrm{X}-05-188 \_\mathrm{P} 7070017$ \\
\hline $\mathrm{X}-05-188$ & Looking downstream from culvert crossing & 170 & $\mathrm{X}-05-188 \quad \mathrm{P} 7070038$ \\
\hline$X-05-189$ & Looking upstream from culvert crossing & 90 & X-05-189_P7080001 \\
\hline$X-05-189$ & Looking downstream from culvert crossing & 270 & $\mathrm{X}-05-189 \mathrm{P} 7080002$ \\
\hline $\mathrm{X}-05-189$ & Looking at culvert inlets & 230 & X-05-189_P7080003 \\
\hline $\mathrm{X}-05-189$ & Looking at culvert outlets & 120 & X-05-189_P7080004 \\
\hline$X-05-190$ & Looking upstream from bridge crossing & 80 & $\mathrm{X}-05-190 \mathrm{P} 7110001$ \\
\hline$X-05-190$ & Looking downstream from bridge crossing & 270 & $\mathrm{X}-05-190 \mathrm{P} 7110002$ \\
\hline $\mathrm{X}-05-191$ & Looking upstream from culvert crossing & 280 & X-05-191_P7120002 \\
\hline$X-05-191$ & Looking downstream from culvert crossing & 100 & X-05-191_P7120003 \\
\hline $\mathrm{X}-05-191$ & Looking at culvert inlet & 40 & $\mathrm{X}-05-191 \mathrm{P} 7120004$ \\
\hline $\mathrm{X}-05-191$ & Looking at outlet & 320 & X-05-191_P7120005 \\
\hline$X-05-192$ & Looking upstream from culvert crossing & 330 & $\mathrm{X}-05-192 \mathrm{P} 7130001$ \\
\hline $\mathrm{X}-05-192$ & Looking upstream from culvert crossing & 350 & $\mathrm{X}-05-192 \mathrm{P} 7130002$ \\
\hline $\mathrm{X}-05-192$ & Looking downstream from left outlet & 120 & $\mathrm{X}-05-192 \mathrm{P} 7130003$ \\
\hline$X-05-192$ & Looking downstream from right outlet & 170 & $\mathrm{X}-05-192 \_\mathrm{P} 7130004$ \\
\hline $\mathrm{X}-05-192$ & Looking downstream from culvert crossing & 150 & $\mathrm{X}-05-192 \mathrm{P} 7130005$ \\
\hline $\mathrm{X}-05-192$ & Looking at left culvert inlet & 70 & $\mathrm{X}-05-192 \_\mathrm{P} 7130006$ \\
\hline $\mathrm{X}-05-192$ & Looking at right culvert inlet & 240 & $\mathrm{X}-05-192 \_\mathrm{P} 7130007$ \\
\hline $\mathrm{X}-05-192$ & Looking at left culvert outlet & 10 & $\mathrm{X}-05-192 \_\mathrm{P} 7130008$ \\
\hline $\mathrm{X}-05-192$ & Looking at right culvert outlet & 20 & $\mathrm{X}-05-192 \_\mathrm{P} 7130009$ \\
\hline $\mathrm{X}-05-193$ & Looking upstream from culvert crossing & 300 & X-05-193_P7130010 \\
\hline$X-05-193$ & Looking downstream from culvert crossing & 130 & $\mathrm{X}-05-193 \_\mathrm{P} 7130011$ \\
\hline $\mathrm{X}-05-193$ & Looking at culvert inlets & 90 & $\mathrm{X}-05-193 \mathrm{P} 7130012$ \\
\hline $\mathrm{X}-05-193$ & Looking at culvert outlets & 320 & X-05-193_P7130013 \\
\hline$X-05-194$ & Looking upstream from bridge crossing & 250 & X-05-194_P7130014 \\
\hline
\end{tabular}


Appendix 8: List of all photographs taken showing the corresponding site number, descriptive caption, direction faced when taken (azimuth in degrees) and the unique file name for each photograph.

\begin{tabular}{|c|c|c|c|}
\hline $\begin{array}{c}\text { Site } \\
\text { Number }\end{array}$ & Photo Description & Azimuth & File Name \\
\hline $\mathrm{X}-05-194$ & Looking downstream from bridge crossing & 70 & X-05-194_P7130015 \\
\hline $\mathrm{X}-05-195$ & Looking upstream from culvert crossing & 270 & X-05-195_P7130016 \\
\hline $\mathrm{X}-05-195$ & Looking downstream from culvert crossing & 90 & X-05-195_P7130017 \\
\hline $\mathrm{X}-05-195$ & Looking at culvert inlets & 100 & X-05-195_P7130018 \\
\hline $\mathrm{X}-05-195$ & Looking at culvert outlets & 290 & X-05-195_P7130019 \\
\hline $\mathrm{X}-05-196$ & Looking upstream from culvert crossing & 170 & X-05-196_P7140001 \\
\hline $\mathrm{X}-05-196$ & Looking downstream from culvert crossing & 50 & X-05-196_P7140002 \\
\hline $\mathrm{X}-05-196$ & Looking at culvert inlets & 335 & X-05-196_P7140003 \\
\hline $\mathrm{X}-05-196$ & Looking at culvert outlets & 170 & X-05-196_P7140004 \\
\hline $\mathrm{X}-05-196$ & Looking upstream at 2nd order tributary & 270 & X-05-196_P7140005 \\
\hline $\mathrm{X}-05-196$ & Looking at culvert inlets of 2nd order tributary & 360 & X-05-196_P7140006 \\
\hline $\mathrm{X}-05-196$ & Looking at culvert outlets of 2nd order tributary & 230 & X-05-196_P7140007 \\
\hline $\mathrm{X}-05-197$ & Looking upstream from culvert crossing & 310 & X-05-197_P7140008 \\
\hline $\mathrm{X}-05-197$ & Looking downstream from culvert crossing & 145 & X-05-197_P7140009 \\
\hline $\mathrm{X}-05-197$ & Looking at culvert inlets & 150 & X-05-197_P7140010 \\
\hline $\mathrm{X}-05-197$ & Looking at culvert outlets & 250 & X-05-197_P7140011 \\
\hline $\mathrm{X}-05-198$ & Looking upstream from left bank of ford crossing & 300 & X-05-198_P7140012 \\
\hline $\mathrm{X}-05-198$ & Looking downstream from left bank of ford crossing & 70 & X-05-198_P7140013 \\
\hline $\mathrm{X}-05-198$ & Looking at gravel ford crossing & 340 & X-05-198_P7140014 \\
\hline $\mathrm{X}-05-199$ & Looking upstream from culvert crossing & 230 & X-05-199_P7140015 \\
\hline $\mathrm{X}-05-199$ & Looking downstream from culvert crossing & 20 & X-05-199_P7140016 \\
\hline $\mathrm{X}-05-199$ & Looking at culvert inlet & 40 & X-05-199_P7140017 \\
\hline $\mathrm{X}-05-199$ & Looking at culvert outlet & 140 & X-05-199_P7140018 \\
\hline $\mathrm{X}-05-200$ & Looking upstream from bridge crossing & 170 & X-05-200_P7150001 \\
\hline $\mathrm{X}-05-200$ & Looking downstream from bridge crossing & 40 & X-05-200_P7150002 \\
\hline $\mathrm{X}-05-201$ & Looking upstream from culvert crossing & 220 & X-05-201_P8030001 \\
\hline $\mathrm{X}-05-201$ & Looking downstream from culvert crossing & 20 & X-05-201_P8030002 \\
\hline $\mathrm{X}-05-201$ & Looking at culvert inlets & 50 & X-05-201_P8030003 \\
\hline $\mathrm{X}-05-201$ & Looking at culvert outlets & 170 & X-05-201_P8030004 \\
\hline $\mathrm{X}-05-202$ & Looking upstream from culvert crossing & 150 & X-05-202_P8030005 \\
\hline $\mathrm{X}-05-202$ & Looking upstream from east of crossing & 185 & X-05-202_P8030006 \\
\hline $\mathrm{X}-05-202$ & Looking downstream from culvert crossing & 320 & X-05-202_P8030007 \\
\hline$X-05-202$ & Looking at culvert inlet & 360 & X-05-202_P8030008 \\
\hline
\end{tabular}


Appendix 8: List of all photographs taken showing the corresponding site number, descriptive caption, direction faced when taken (azimuth in degrees) and the unique file name for each photograph.

\begin{tabular}{|c|c|c|c|}
\hline $\begin{array}{c}\text { Site } \\
\text { Number }\end{array}$ & Photo Description & Azimuth & File Name \\
\hline $\mathrm{X}-05-202$ & Looking at culvert outlet & 160 & $\mathrm{X}-05-202 \mathrm{P} 8030009$ \\
\hline $\mathrm{X}-05-203$ & Looking upstream from bridge crossing at Pembina River & 250 & $\mathrm{X}-05-203 \_P 8030010$ \\
\hline $\mathrm{X}-05-203$ & Looking upstream from bridge crossing at North Pembina River & 360 & $\mathrm{X}-05-203+\mathrm{P} 8030011$ \\
\hline $\mathrm{X}-05-203$ & Looking downstream from bridge crossing & 115 & $\mathrm{X}-05-203 \_P 8030012$ \\
\hline$X-05-203$ & Looking at cattle impacts & 125 & $\mathrm{X}-05-203 \mathrm{P} 8030013$ \\
\hline $\mathrm{X}-05-203$ & Looking at cattle impacts & 115 & $\mathrm{X}-05-203+\mathrm{P} 8030014$ \\
\hline $\mathrm{X}-05-203$ & Looking at cattle impacts & 110 & $\mathrm{X}-05-203+\mathrm{P} 8030015$ \\
\hline $\mathrm{X}-05-204$ & Looking upstream from culvert crossing & 270 & $\mathrm{X}-05-204 \mathrm{P} 8040001$ \\
\hline $\mathrm{X}-05-204$ & Looking downstream from culvert crossing & 90 & $\mathrm{X}-05-204 \mathrm{P} 8040002$ \\
\hline $\mathrm{X}-05-204$ & Looking at culvert inlet & 45 & X-05-204_P8040003 \\
\hline$X-05-204$ & Looking at culvert outlet & 360 & $\mathrm{X}-05-204 \_\mathrm{P} 8040004$ \\
\hline $\mathrm{X}-05-204$ & Photo of Yellow Perch & $\mathrm{N} / \mathrm{A}$ & $\mathrm{X}-05-204 \mathrm{P} 8040005$ \\
\hline $\mathrm{X}-05-204$ & Photo of Ashley and Ashley with Yellow Perch & $\mathrm{N} / \mathrm{A}$ & $\mathrm{X}-05-204 \mathrm{P} 8040008$ \\
\hline$X-05-204$ & Photo of Ashley and Ashley with Yellow Perch & N/A & $\mathrm{X}-05-204 \mathrm{P} 8040009$ \\
\hline $\mathrm{X}-05-205$ & Looking downstream from culvert crossing & 300 & $\mathrm{X}-05-205 \_\mathrm{P} 8040010$ \\
\hline $\mathrm{X}-05-205$ & Looking upstream from culvert crossing & 90 & $\mathrm{X}-05-205$ P8040011 \\
\hline$X-05-205$ & Looking at culvert outlet & 330 & $\mathrm{X}-05-205 \mathrm{P} 8040012$ \\
\hline $\mathrm{X}-05-205$ & Looking at culvert inlet & 210 & X-05-205_P8040013 \\
\hline $\mathrm{X}-05-205$ & Looking upstream at 1 st order tributary & 360 & X-05-205_P8040014 \\
\hline$X-05-206$ & Looking upstream from culvert crossing & 350 & $\mathrm{X}-05-206 \mathrm{P} 8040015$ \\
\hline $\mathrm{X}-05-206$ & Looking downstream from culvert crossing & 150 & $\mathrm{X}-05-206 \quad \mathrm{P} 8040016$ \\
\hline $\mathrm{X}-05-206$ & Looking at culvert inlet & 100 & X-05-206_P8040017 \\
\hline$X-05-206$ & Looking at culvert outlet & 180 & $\mathrm{X}-05-206 \_\mathrm{P} 8040018$ \\
\hline $\mathrm{X}-05-207$ & Looking upstream from right bank & 150 & $\mathrm{X}-05-207 \quad \mathrm{P} 8040021$ \\
\hline $\mathrm{X}-05-207$ & Looking downstream from right bank & 310 & $\mathrm{X}-05-207 \_\mathrm{P} 8040022$ \\
\hline$X-05-208$ & Looking upstream from culvert crossing & 340 & $\mathrm{X}-05-208 \mathrm{P} 8040023$ \\
\hline $\mathrm{X}-05-208$ & Looking downstream from 1st culvert crossing & 90 & $\mathrm{X}-05-208 \_\mathrm{P} 8040024$ \\
\hline $\mathrm{X}-05-208$ & Looking downstream from 2 nd culvert crossing & 140 & $\mathrm{X}-05-208 \quad \mathrm{P} 8040025$ \\
\hline$X-05-208$ & Looking at first set of culvert inlets & 20 & $\mathrm{X}-05-208 \quad \mathrm{P} 8040026$ \\
\hline$X-05-208$ & Looking at first set of culvert outlets & 300 & $\mathrm{X}-05-208 \mathrm{P} 8040027$ \\
\hline $\mathrm{X}-05-208$ & Looking at second set of culvert inlets & 100 & $\mathrm{X}-05-208 \mathrm{P} 8040028$ \\
\hline $\mathrm{X}-05-208$ & Looking at second set of culvert outlets & 360 & $\mathrm{X}-05-208 \quad \mathrm{P} 8040029$ \\
\hline $\mathrm{X}-05-208$ & Photo of Ashley P. and Vaughn fishing & $\mathrm{N} / \mathrm{A}$ & $\mathrm{X}-05-208+\mathrm{P} 8040030$ \\
\hline
\end{tabular}


Appendix 8: List of all photographs taken showing the corresponding site number, descriptive caption, direction faced when taken (azimuth in degrees) and the unique file name for each photograph.

\begin{tabular}{|c|c|c|c|}
\hline $\begin{array}{c}\text { Site } \\
\text { Number }\end{array}$ & Photo Description & Azimuth & File Name \\
\hline $\mathrm{X}-05-208$ & Photo of Ashley W. and Vaughn fishing & $\mathrm{N} / \mathrm{A}$ & X-05-208_P8040031 \\
\hline$X-05-208$ & Photo of Ashley W. and Vaughn fishing & $\mathrm{N} / \mathrm{A}$ & $\mathrm{X}-05-208 \mathrm{P} 8040032$ \\
\hline $\mathrm{X}-05-210$ & Looking upstream from culvert crossing & 265 & X-05-210_P6220001 \\
\hline $\mathrm{X}-05-210$ & Looking downstream from culvert crossing & 150 & X-05-210_P6220002 \\
\hline$X-05-210$ & Looking at culvert inlets & 100 & X-05-210_P6220003 \\
\hline $\mathrm{X}-05-210$ & Looking at culvert outlets & 290 & $\mathrm{X}-05-210 \mathrm{P} 6220004$ \\
\hline D-06-001 & Looking upstream from mid-reach & 180 & D-06-001_P7060001 \\
\hline $\mathrm{D}-06-001$ & Looking downstream from mid-reach & 0 & D-06-001_P7060002 \\
\hline D-06-001 & Looking downstream under bridge & 0 & D-06-001 P7060003 \\
\hline W-06-001 & Looking upstream from culvert crossing & 90 & W-06-001_P5090001 \\
\hline W-06-001 & Looking downstream from culvert crossing & 270 & W-06-001_P5090002 \\
\hline W-06-001 & Looking at culvert inlets & 10 & W-06-001_P5090003 \\
\hline W-06-001 & Looking at culvert outlets & 295 & W-06-001_P5090004 \\
\hline W-06-001 & Photo of eroded / damaged crossing & 0 & W-06-001_P5090005 \\
\hline W-06-002 & Looking upstream from culvert crossing & 270 & W-06-002_P5090006 \\
\hline W-06-002 & Looking downstream from culvert crossing & 45 & W-06-002_P5090007 \\
\hline W-06-002 & Looking at culvert inlets & 15 & W-06-002 P5090008 \\
\hline W-06-002 & Looking at culvert outlets & 345 & W-06-002 P5090009 \\
\hline W-06-003 & Looking upstream from bridge crossing & 20 & W-06-003_P5090010 \\
\hline W-06-003 & Looking downstream from bridge crossing & 210 & W-06-003_P5090011 \\
\hline W-06-004 & Looking upstream from bridge crossing & 40 & W-06-004 P5090012 \\
\hline W-06-004 & Looking downstream from bridge crossing & 130 & W-06-004_P5090013 \\
\hline W-06-005 & Looking upstream from culvert crossing & 20 & W-06-005_P5090014 \\
\hline W-06-005 & Looking upstream from culvert crossing & 330 & W-06-005_P5090015 \\
\hline W-06-005 & Looking upstream from culvert crossing & 300 & W-06-005 P5090016 \\
\hline W-06-005 & Looking downstream from culvert crossing & 250 & W-06-005_P5090017 \\
\hline W-06-005 & Looking downstream from culvert crossing & 200 & W-06-005_P5090018 \\
\hline W-06-005 & Looking downstream from culvert crossing & 160 & W-06-005_P5090019 \\
\hline W-06-005 & Looking at west culvert inlet from bank & 330 & W-06-005 P5090020 \\
\hline W-06-006 & Looking upstream from culvert crossing & 0 & W-06-006 P5100001 \\
\hline W-06-006 & Looking downstream from culvert crossing & 180 & W-06-006_P5100002 \\
\hline W-06-006 & Looking at culvert inlets & 350 & W-06-006 P5100003 \\
\hline W-06-006 & Looking at culvert outlets & 350 & W-06-006 P5100004 \\
\hline
\end{tabular}


Appendix 8: List of all photographs taken showing the corresponding site number, descriptive caption, direction faced when taken (azimuth in degrees) and the unique file name for each photograph.

\begin{tabular}{|c|c|c|c|}
\hline $\begin{array}{c}\text { Site } \\
\text { Number }\end{array}$ & Photo Description & Azimuth & File Name \\
\hline W-06-007 & Looking upstream from culvert crossing & 0 & W-06-007 P5100005 \\
\hline W-06-007 & Looking downstream from culvert crossing & 180 & W-06-007_P5100006 \\
\hline W-06-007 & Looking at culvert inlets & 250 & W-06-007_P5100007 \\
\hline W-06-007 & Looking at culvert outlets & 280 & W-06-007_P5100008 \\
\hline W-06-008 & Looking upstream from culvert crossing & 170 & W-06-008_P5100009 \\
\hline W-06-008 & Looking downstream from culvert crossing & 0 & W-06-008 P5100010 \\
\hline W-06-008 & Looking at culvert inlet & 85 & W-06-008_P5100011 \\
\hline W-06-008 & Looking at culvert outlet & 110 & W-06-008_P5100012 \\
\hline W-06-009 & Looking upstream from culvert crossing & 45 & W-06-009 P5100013 \\
\hline W-06-009 & Looking downstream from culvert crossing & 315 & W-06-009 P5100014 \\
\hline W-06-009 & Looking at culvert inlet & 160 & W-06-009_P5100015 \\
\hline W-06-009 & Looking at culvert outlet & 60 & W-06-009_P5100016 \\
\hline W-06-010 & Looking upstream from culvert crossing & 270 & W-06-010_P5100017 \\
\hline W-06-010 & Looking downstream from culvert crossing & 90 & W-06-010_P5100018 \\
\hline W-06-010 & Looking at culvert inlet & 90 & W-06-010_P5100019 \\
\hline $\mathrm{W}-06-010$ & Looking at culvert outlet & 270 & W-06-010_P5100020 \\
\hline W-06-010 & Looking at culvert outlet & 270 & W-06-010 P5100021 \\
\hline W-06-011 & Looking upstream from culvert crossing & 340 & W-06-011_P5110001 \\
\hline W-06-011 & Looking downstream from culvert crossing & 140 & W-06-011_P5110002 \\
\hline W-06-011 & Looking at culvert inlets & 90 & W-06-011_P5110003 \\
\hline W-06-011 & Looking at culvert outlets & 300 & W-06-011_P5110004 \\
\hline W-06-012 & Looking upstream from culvert crossing & 45 & W-06-012_P5110005 \\
\hline W-06-012 & Looking downstream from culvert crossing & 180 & W-06-012_P5110006 \\
\hline W-06-012 & Looking at culvert inlet & 220 & W-06-012_P5110007 \\
\hline W-06-012 & Looking at culvert outlet & 300 & W-06-012 P5110008 \\
\hline W-06-013 & Looking upstream from bridge crossing & 0 & W-06-013 P5110009 \\
\hline W-06-013 & Looking downstream from bridge crossing & 180 & W-06-013_P5110010 \\
\hline W-06-013 & Wide angle photo of crossing from road & 270 & W-06-013_P5110011 \\
\hline W-06-014 & Looking upstream from culvert crossing & 315 & W-06-014 P5110012 \\
\hline W-06-014 & Looking downstream from culvert crossing & 110 & W-06-014_P5110013 \\
\hline W-06-014 & Looking at culvert inlet (North) & 90 & W-06-014_P5110014 \\
\hline W-06-014 & Looking at culvert inlet (middle) & 90 & W-06-014_P5110015 \\
\hline W-06-014 & Looking at culvert outlet (North) & 270 & W-06-014 P5110016 \\
\hline
\end{tabular}


Appendix 8: List of all photographs taken showing the corresponding site number, descriptive caption, direction faced when taken (azimuth in degrees) and the unique file name for each photograph.

\begin{tabular}{|c|c|c|c|}
\hline $\begin{array}{c}\text { Site } \\
\text { Number }\end{array}$ & Photo Description & Azimuth & File Name \\
\hline W-06-014 & Looking at culvert outlet (middle) & 270 & W-06-014_P5110017 \\
\hline W-06-014 & Looking at culvert outlet (South) & 270 & W-06-014 P5110018 \\
\hline W-06-014 & Looking at culvert inlet (South) & 90 & W-06-014_P5110019 \\
\hline W-06-015 & Looking upstream from bridge crossing & 0 & W-06-015_P5110020 \\
\hline W-06-015 & Looking downstream from bridge crossing & 180 & W-06-015_P5110021 \\
\hline W-06-016 & Looking upstream from bridge crossing & 70 & W-06-016_P5110022 \\
\hline W-06-016 & Looking downstream from bridge crossing & 270 & W-06-016_P5110023 \\
\hline W-06-017 & Looking downstream from bridge crossing & 180 & W-06-017_P5120001 \\
\hline W-06-018 & Looking upstream from culvert crossing & 90 & W-06-018_P5120002 \\
\hline W-06-018 & Looking downstream from culvert crossing & 270 & W-06-018 P5120003 \\
\hline W-06-018 & Looking at culvert inlet & 220 & W-06-018_P5120004 \\
\hline W-06-018 & Looking at culvert outlet & 110 & W-06-018_P5120005 \\
\hline W-06-018 & Looking at the Northern Pike that was released & $\mathrm{N} / \mathrm{A}$ & W-06-018_P5120006 \\
\hline W-06-019 & Looking upstream in the third order tributary & 165 & W-06-019_P5150001 \\
\hline W-06-019 & Looking upstream in the second order tributary & 85 & W-06-019_P5150002 \\
\hline W-06-019 & Looking downstream from the bridge crossing & 260 & W-06-019_P5150003 \\
\hline W-06-019 & Looking at gated bridge from the road & 140 & W-06-019_P5150004 \\
\hline W-06-019 & Looking at other bridge from the road & 220 & W-06-019 P5150005 \\
\hline W-06-019 & Looking at school of shiners and Common Carp & $\mathrm{N} / \mathrm{A}$ & W-06-019_P5150006 \\
\hline W-06-019 & Looking at school of shiners and Common Carp & N/A & W-06-019_P5150007 \\
\hline W-06-019 & Looking at school of shiners and Common Carp & $\mathrm{N} / \mathrm{A}$ & W-06-019_P5150008 \\
\hline W-06-019 & Looking at school of shiners and Common Carp & $\mathrm{N} / \mathrm{A}$ & W-06-019_P5150009 \\
\hline W-06-019 & Looking at school of shiners and Common Carp & N/A & W-06-019_P5150010 \\
\hline W-06-019 & Looking at school of shiners and Common Carp & $\mathrm{N} / \mathrm{A}$ & W-06-019_P5150011 \\
\hline W-06-019 & Looking at school of shiners and Common Carp & $\mathrm{N} / \mathrm{A}$ & W-06-019_P5150012 \\
\hline W-06-019 & Looking at school of shiners and Common Carp & N/A & W-06-019 P5150013 \\
\hline W-06-020 & Looking at cow in the stream & 90 & W-06-020_P5150014 \\
\hline W-06-020 & Looking upstream from the culvert crossing & 270 & W-06-020_P5150015 \\
\hline W-06-020 & Looking downstream from the culvert crossing & 60 & W-06-020_P5150016 \\
\hline W-06-020 & Looking at culvert inlet & 0 & W-06-020 P5150017 \\
\hline W-06-020 & Looking at culvert outlet & 320 & W-06-020_P5150018 \\
\hline W-06-021 & Looking upstream from the culvert crossing & 270 & W-06-021_P5160001 \\
\hline W-06-021 & Looking downstream form the culvert crossing & 90 & W-06-021_P5160002 \\
\hline
\end{tabular}


Appendix 8: List of all photographs taken showing the corresponding site number, descriptive caption, direction faced when taken (azimuth in degrees) and the unique file name for each photograph.

\begin{tabular}{|c|c|c|c|}
\hline $\begin{array}{c}\text { Site } \\
\text { Number }\end{array}$ & Photo Description & Azimuth & File Name \\
\hline W-06-021 & Looking at culvert inlet & 45 & W-06-021 P5160003 \\
\hline W-06-021 & Looking at culvert outlet & 330 & W-06-021_P5160004 \\
\hline W-06-022 & Looking upstream from ford crossing & 270 & W-06-022_P5160005 \\
\hline W-06-022 & Looking downstream form ford crossing & 90 & W-06-022_P5160006 \\
\hline W-06-023 & Released sucker & N/A & W-06-023 P5160007 \\
\hline W-06-023 & Looking upstream from the culvert crossing & 330 & W-06-023 P5160008 \\
\hline W-06-023 & Looking downstream from the culvert crossing & 170 & W-06-023_P5160009 \\
\hline W-06-023 & Looking at culvert inlet & 150 & W-06-023 P5160010 \\
\hline W-06-023 & Looking at culvert outlet & 210 & W-06-023 P5160011 \\
\hline W-06-024 & Looking at cattle impacts upstream of culvert crossing & 270 & W-06-024_P5160012 \\
\hline W-06-024 & Looking at cattle impacts upstream of culvert crossing & 270 & W-06-024_P5160013 \\
\hline W-06-024 & Looking downstream of cattle crossing & 90 & W-06-024_P5160014 \\
\hline W-06-024 & Looking at culvert inlet & 45 & W-06-024 P5160015 \\
\hline W-06-024 & Looking at culvert outlet & 345 & W-06-024_P5160016 \\
\hline W-06-025 & Looking upstream from culvert crossing & 90 & W-06-025_P5170001 \\
\hline W-06-025 & Looking downstream from culvert crossing & 270 & W-06-025 P5170002 \\
\hline W-06-025 & Looking at culvert inlet & 250 & W-06-025 P5170003 \\
\hline W-06-025 & Looking at culvert outlet & 130 & W-06-025_P5170004 \\
\hline W-06-025 & Looking at released suckers & $\mathrm{N} / \mathrm{A}$ & W-06-025_P5170005 \\
\hline W-06-025 & Looking at released suckers & N/A & W-06-025 P5170006 \\
\hline W-06-026 & Looking upstream from culvert crossing & 90 & W-06-026 P5170007 \\
\hline W-06-026 & Looking downstream from culvert crossing & 270 & W-06-026_P5170008 \\
\hline W-06-026 & Looking at culvert inlet & 315 & W-06-026_P5170009 \\
\hline W-06-026 & Looking at culvert outlet & 45 & W-06-026 P5170010 \\
\hline W-06-027 & Looking downstream from culvert crossing & 270 & W-06-027 P5170011 \\
\hline W-06-027 & Looking upstream from culvert crossing & 90 & W-06-027_P5170012 \\
\hline W-06-027 & Looking at culvert inlet & 315 & W-06-027_P5170013 \\
\hline W-06-027 & Looking at culvert outlet & 45 & W-06-027 P5170014 \\
\hline W-06-027 & Looking at cattle impacts on the culvert crossing & 250 & W-06-027 P5170015 \\
\hline W-06-027 & Looking at the released sucker & N/A & W-06-027_P5170016 \\
\hline W-06-028 & Looking upstream from the culvert crossing & 270 & W-06-028 P5170017 \\
\hline W-06-028 & Looking downstream from the culvert crossing & 180 & W-06-028 P5170018 \\
\hline W-06-028 & Looking at culvert inlet & 210 & W-06-028_P5170019 \\
\hline
\end{tabular}


Appendix 8: List of all photographs taken showing the corresponding site number, descriptive caption, direction faced when taken (azimuth in degrees) and the unique file name for each photograph.

\begin{tabular}{|c|c|c|c|}
\hline $\begin{array}{c}\text { Site } \\
\text { Number }\end{array}$ & Photo Description & Azimuth & File Name \\
\hline W-06-028 & Looking at culvert outlet & 40 & W-06-028_P5170020 \\
\hline W-06-028 & Photo of released Northern Pike & $\mathrm{N} / \mathrm{A}$ & W-06-028 P5170021 \\
\hline W-06-029 & Looking upstream from bridge crossing & 250 & W-06-029_P5230001 \\
\hline W-06-029 & Looking downstream from bridge crossing & 70 & W-06-029_P5230002 \\
\hline W-06-029 & Photo of released suckers & $\mathrm{N} / \mathrm{A}$ & W-06-029_P5230003 \\
\hline W-06-030 & Looking upstream from bridge crossing & 275 & W-06-030_P5230004 \\
\hline W-06-030 & Looking downstream from bridge crossing & 90 & W-06-030_P5230005 \\
\hline W-06-031 & Looking upstream from bridge crossing & 270 & W-06-031_P5230006 \\
\hline W-06-031 & Looking downstream from bridge crossing & 90 & W-06-031_P5230007 \\
\hline W-06-031 & Looking at dredging/control structure & 310 & W-06-031_P5230008 \\
\hline W-06-031 & Looking at dredging/control structure & 310 & W-06-031_P5230009 \\
\hline W-06-032 & Looking upstream from culvert crossing & 0 & W-06-032_P5240001 \\
\hline W-06-032 & Looking downstream from culvert crossing & 180 & W-06-032_P5240002 \\
\hline W-06-032 & Looking at culvert inlet & 240 & W-06-032_P5240003 \\
\hline W-06-032 & Looking at culvert outlet & 0 & W-06-032_P5240004 \\
\hline W-06-033 & Looking upstream from culvert crossing & 240 & W-06-033_P5240005 \\
\hline W-06-033 & Looking downstream from culvert crossing & 40 & W-06-033_P5240006 \\
\hline W-06-033 & Looking at culvert inlets & 30 & W-06-033 P5240007 \\
\hline W-06-033 & Looking at culvert outlets & 230 & W-06-033_P5240008 \\
\hline W-06-034 & Looking upstream from culvert crossing & 90 & W-06-034_P5240009 \\
\hline W-06-034 & Looking downstream from culvert crossing & 270 & W-06-034_P5240010 \\
\hline W-06-034 & Looking at culvert inlets & 315 & W-06-034_P5240011 \\
\hline W-06-034 & Looking at culvert outlets & 170 & W-06-034_P5240012 \\
\hline W-06-035 & Looking upstream from culvert crossing & 90 & W-06-035_P5240013 \\
\hline W-06-035 & Looking downstream from culvert crossing & 180 & W-06-035 P5240014 \\
\hline W-06-036 & Looking upstream from culvert crossing & 150 & W-06-036_P5250001 \\
\hline W-06-036 & Looking downstream from culvert crossing & 300 & W-06-036_P5250002 \\
\hline W-06-036 & Looking at culvert inlet & 240 & W-06-036_P5250003 \\
\hline W-06-036 & Looking at culvert outlet & 160 & W-06-036_P5250004 \\
\hline W-06-037 & Looking upstream from culvert crossing & 180 & W-06-037_P5250005 \\
\hline W-06-037 & Looking downstream from culvert crossing & 0 & W-06-037_P5250006 \\
\hline W-06-037 & Looking at culvert inlets & 340 & W-06-037_P5250007 \\
\hline W-06-037 & Looking at culvert outlets & 210 & W-06-037_P5250008 \\
\hline
\end{tabular}


Appendix 8: List of all photographs taken showing the corresponding site number, descriptive caption, direction faced when taken (azimuth in degrees) and the unique file name for each photograph.

\begin{tabular}{|c|c|c|c|}
\hline $\begin{array}{c}\text { Site } \\
\text { Number }\end{array}$ & Photo Description & Azimuth & File Name \\
\hline W-06-038 & Looking upstream bridge crossing & 180 & W-06-038_P5250009 \\
\hline W-06-038 & Looking downstream bridge crossing & 0 & W-06-038 P5250010 \\
\hline W-06-039 & Looking upstream from culvert crossing & 90 & W-06-039_P5260001 \\
\hline W-06-039 & Looking downstream from culvert crossing & 270 & W-06-039_P5260002 \\
\hline W-06-039 & Looking at culvert inlets & 170 & W-06-039_P5260003 \\
\hline W-06-039 & Looking at culvert outlets & 190 & W-06-039_P5260004 \\
\hline W-06-040 & Looking upstream from culvert crossing & 160 & W-06-040_P5260005 \\
\hline W-06-040 & Looking downstream from culvert crossing & 350 & W-06-040_P5260006 \\
\hline W-06-040 & Looking at culvert inlets & 220 & W-06-040_P5260007 \\
\hline W-06-040 & Looking at culvert outlets & 45 & W-06-040 P5260008 \\
\hline W-06-041 & Looking upstream from culvert crossing & 180 & W-06-041_P5260009 \\
\hline W-06-041 & Looking downstream from culvert crossing & 0 & W-06-041_P5260010 \\
\hline W-06-041 & Looking at culvert inlet & 260 & W-06-041_P5260011 \\
\hline W-06-041 & Looking at culvert outlet & 170 & W-06-041_P5260012 \\
\hline W-06-042 & Looking upstream from culvert crossing & 180 & W-06-042_P5260013 \\
\hline W-06-042 & Looking downstream from culvert crossing & 0 & W-06-042_P5260014 \\
\hline W-06-042 & Looking at culvert inlet & $\mathrm{N} / \mathrm{A}$ & W-06-042_P5260015 \\
\hline W-06-042 & Looking at culvert outlet & $\mathrm{N} / \mathrm{A}$ & W-06-042 P5260016 \\
\hline W-06-043 & Looking upstream from culvert crossing & 75 & W-06-043_P5260017 \\
\hline W-06-043 & Looking downstream from culvert crossing & 0 & W-06-043_P5260018 \\
\hline W-06-043 & Looking at culvert inlets & 270 & W-06-043_P5260019 \\
\hline W-06-043 & Looking at culvert outlets & 265 & W-06-043_P5260020 \\
\hline W-06-044 & Looking upstream from culvert crossing & 200 & W-06-044_P5260021 \\
\hline W-06-044 & Looking downstream from culvert crossing & 70 & W-06-044_P5260022 \\
\hline W-06-044 & Looking at culvert inlet & 340 & W-06-044_P5260023 \\
\hline W-06-044 & Looking at culvert outlet & 290 & W-06-044_P5260024 \\
\hline W-06-045 & Looking upstream from culvert crossing & 180 & W-06-045_P5260025 \\
\hline W-06-045 & Looking downstream from culvert crossing & 0 & W-06-045_P5260026 \\
\hline W-06-045 & Looking at culvert inlets & 60 & W-06-045_P5260027 \\
\hline W-06-045 & Looking at culvert outlets & 130 & W-06-045 P5260028 \\
\hline W-06-046 & Looking upstream from culvert crossing & 150 & W-06-046_P5270001 \\
\hline W-06-046 & Looking downstream from culvert crossing & 340 & W-06-046_P5270002 \\
\hline W-06-046 & Looking at culvert inlet & 340 & W-06-046_P5270003 \\
\hline
\end{tabular}


Appendix 8: List of all photographs taken showing the corresponding site number, descriptive caption, direction faced when taken (azimuth in degrees) and the unique file name for each photograph.

\begin{tabular}{|c|c|c|c|}
\hline $\begin{array}{c}\text { Site } \\
\text { Number }\end{array}$ & Photo Description & Azimuth & File Name \\
\hline W-06-046 & Looking at culvert outlet & 180 & W-06-046_P5270004 \\
\hline W-06-047 & Looking upstream from crossing & 150 & W-06-047 P5270005 \\
\hline W-06-047 & Looking downstream from crossing & 330 & W-06-047_P5270006 \\
\hline W-06-047 & Looking at inlet & 30 & W-06-047_P5270007 \\
\hline W-06-047 & Looking at outlet & 250 & W-06-047_P5270008 \\
\hline W-06-047 & Looking at water control structure & 130 & W-06-047 P5270009 \\
\hline W-06-048 & Looking upstream from culvert crossing & 90 & W-06-048_P5270010 \\
\hline W-06-048 & Looking downstream from culvert crossing & 285 & W-06-048_P5270011 \\
\hline W-06-048 & Looking at culvert inlet & 345 & W-06-048 P5270012 \\
\hline W-06-048 & Looking at culvert outlet & 60 & W-06-048 P5270013 \\
\hline W-06-049 & Looking upstream from culvert crossing & 270 & W-06-049_P5270014 \\
\hline W-06-049 & Looking downstream from culvert crossing & 85 & W-06-049_P5270015 \\
\hline W-06-049 & Looking at culvert inlets & 330 & W-06-049_P5270016 \\
\hline W-06-049 & Looking at culvert outlets & 290 & W-06-049 P5270017 \\
\hline W-06-049 & Looking at bank erosion downstream of culverts & 100 & W-06-049_P5270018 \\
\hline W-06-049 & Looking upstream of washout & 200 & W-06-049_P5270019 \\
\hline W-06-049 & Looking downstream of washout & 160 & W-06-049 P5270020 \\
\hline W-06-049 & Looking downstream of washout (wide angle) & 90 & W-06-049 P5270021 \\
\hline W-06-050 & Looking upstream from culvert crossing & 150 & W-06-050_P5300001 \\
\hline W-06-050 & Looking downstream from culvert crossing & 330 & W-06-050_P5300002 \\
\hline W-06-050 & Looking at culvert inlets & 290 & W-06-050 P5300003 \\
\hline W-06-050 & Looking at culvert outlets & 140 & W-06-050_P5300004 \\
\hline W-06-051 & Looking upstream from box culvert crossing & 220 & W-06-051_P5300005 \\
\hline W-06-051 & Looking downstream from box culvert crossing & 30 & W-06-051_P5300006 \\
\hline W-06-051 & Looking at box culvert inlet & 30 & W-06-051_P5300007 \\
\hline W-06-051 & Looking at box culvert outlet & 220 & W-06-051_P5300008 \\
\hline W-06-052 & Looking upstream from culvert crossing & 200 & W-06-052_P5300009 \\
\hline W-06-052 & Looking downstream from culvert crossing & 40 & W-06-052_P5300010 \\
\hline W-06-052 & Looking at culvert inlet & 40 & W-06-052 P5300011 \\
\hline W-06-052 & Looking at culvert outlet & 200 & W-06-052 P5300012 \\
\hline W-06-053 & Looking upstream from culvert crossing & 260 & W-06-053_P5300013 \\
\hline W-06-053 & Looking downstream from culvert crossing & 80 & W-06-053 P5300014 \\
\hline W-06-053 & Looking at culvert inlet & 80 & W-06-053 P5300015 \\
\hline
\end{tabular}


Appendix 8: List of all photographs taken showing the corresponding site number, descriptive caption, direction faced when taken (azimuth in degrees) and the unique file name for each photograph.

\begin{tabular}{|c|c|c|c|}
\hline $\begin{array}{c}\text { Site } \\
\text { Number }\end{array}$ & Photo Description & Azimuth & File Name \\
\hline W-06-053 & Looking at culvert outlet & 260 & $\begin{array}{l}\mathrm{W}-06-053 \quad \mathrm{P} 5300016 \\
\end{array}$ \\
\hline W-06-054 & Looking upstream from culvert crossing & 0 & W-06-054_P5300017 \\
\hline W-06-054 & Looking downstream from culvert crossing & 180 & W-06-054_P5300018 \\
\hline W-06-054 & Looking at culvert inlets & 200 & W-06-054_P5300019 \\
\hline W-06-054 & Looking at culvert outlets & 330 & W-06-054 P5300020 \\
\hline W-06-055 & Looking upstream from culvert crossing & 340 & W-06-055 P5300021 \\
\hline W-06-055 & Looking downstream from culvert crossing & 200 & W-06-055_P5300022 \\
\hline W-06-056 & Looking upstream from culvert crossing & 90 & W-06-056 P5310001 \\
\hline W-06-056 & Looking downstream from culvert crossing & 270 & W-06-056 P5310002 \\
\hline W-06-056 & Looking at culvert outlet & 160 & W-06-056_P5310003 \\
\hline W-06-056 & Looking downstream from culvert crossing & 160 & W-06-056_P5310004 \\
\hline W-06-056 & Looking downstream from culvert crossing & 160 & W-06-056 P5310005 \\
\hline W-06-056 & Looking downstream from culvert crossing & 160 & W-06-056 P5310006 \\
\hline W-06-056 & Looking downstream from culvert crossing & 160 & W-06-056 P5310007 \\
\hline W-06-056 & Looking downstream from culvert crossing & 160 & W-06-056_P5310008 \\
\hline W-06-056 & Looking downstream from culvert crossing & 160 & W-06-056 P5310009 \\
\hline W-06-056 & Looking at culvert inlet & 180 & W-06-056 P5310010 \\
\hline W-06-056 & Looking upstream from culvert crossing & 170 & W-06-056 P5310011 \\
\hline W-06-057 & Looking upstream from culvert crossing & 260 & W-06-057_P5310012 \\
\hline W-06-057 & Looking downstream from culvert crossing & 50 & W-06-057 P5310013 \\
\hline W-06-057 & Looking at culvert inlet & 60 & W-06-057 P5310014 \\
\hline W-06-057 & Looking at culvert outlet & 200 & W-06-057_P5310015 \\
\hline W-06-058 & Looking upstream from culvert crossing & 270 & W-06-058 P6010001 \\
\hline W-06-058 & Looking at South culvert inlet from overhead & $\mathrm{N} / \mathrm{A}$ & W-06-058 P6010002 \\
\hline W-06-058 & Looking at North culvert inlet from overhead & $\mathrm{N} / \mathrm{A}$ & W-06-058_P6010003 \\
\hline W-06-058 & Looking downstream from culvert crossing & 90 & W-06-058_P6010004 \\
\hline W-06-058 & Looking at culvert outlet & 270 & W-06-058_P6010005 \\
\hline W-06-059 & Looking upstream from culvert crossing & 360 & W-06-059 P6010006 \\
\hline W-06-059 & Looking downstream from culvert crossing & 180 & W-06-059 P6010007 \\
\hline W-06-059 & Looking at culvert inlet & 165 & W-06-059P6010008 \\
\hline W-06-059 & Looking at culvert outlet & 85 & W-06-059 P6010009 \\
\hline W-06-060 & Looking upstream from culvert crossing & 345 & W-06-060 P6010010 \\
\hline W-06-060 & Looking downstream from culvert crossing & 160 & W-06-060_P6010011 \\
\hline
\end{tabular}


Appendix 8: List of all photographs taken showing the corresponding site number, descriptive caption, direction faced when taken (azimuth in degrees) and the unique file name for each photograph.

\begin{tabular}{|c|c|c|c|}
\hline $\begin{array}{c}\text { Site } \\
\text { Number }\end{array}$ & Photo Description & Azimuth & File Name \\
\hline W-06-060 & Looking at culvert inlets & 160 & W-06-060_P6010012 \\
\hline W-06-060 & Looking at culvert outlets & 345 & W-06-060_P6010013 \\
\hline W-06-061 & Looking upstream of project from PTH 110 & 300 & W-06-061_P6060001 \\
\hline W-06-061 & Looking upstream wide angle & 240 & W-06-061_P6060002 \\
\hline W-06-061 & Looking downstream from gravel crossing & 180 & W-06-061_P6060003 \\
\hline W-06-061 & Looking at geotextile on the Left bank & 190 & W-06-061_P6060004 \\
\hline W-06-061 & Looking at geotextile on the Left bank & 170 & W-06-061_P6060005 \\
\hline W-06-061 & Looking upstream from gravel crossing & 340 & W-06-061_P6060006 \\
\hline W-06-061 & Looking at Sediment barrier on the Right bank & 200 & W-06-061_P6060007 \\
\hline W-06-061 & Looking at revegetation & 180 & W-06-061_P6060008 \\
\hline W-06-062 & Looking upstream from culvert crossing & 325 & W-06-062_P6060009 \\
\hline W-06-062 & Looking downstream from culvert crossing & 100 & W-06-062_P6060010 \\
\hline W-06-062 & Looking at culvert inlet & 60 & W-06-062_P6060011 \\
\hline W-06-062 & Looking at culvert outlet & 245 & W-06-062_P6060012 \\
\hline W-06-063 & Looking upstream from bridge crossing & 180 & W-06-063_P6070001 \\
\hline W-06-063 & Looking downstream from bridge crossing & 0 & W-06-063_P6070002 \\
\hline W-06-064 & Looking upstream from bridge crossing & 200 & W-06-064_P6070003 \\
\hline W-06-064 & Looking downstream from bridge crossing & 5 & W-06-064_P6070004 \\
\hline W-06-065 & Looking upstream from bridge crossing & 180 & W-06-065_P6070005 \\
\hline W-06-065 & Looking downstream from bridge crossing & 0 & W-06-065_P6070006 \\
\hline W-06-066 & Looking upstream from culvert crossing & 260 & W-06-066_P6070007 \\
\hline W-06-066 & Looking downstream from culvert crossing & 120 & W-06-066_P6070008 \\
\hline W-06-066 & Looking at culvert inlets & 130 & W-06-066_P6070009 \\
\hline W-06-066 & Looking at culvert outlets & 295 & W-06-066_P6070010 \\
\hline W-06-067 & Looking upstream from culvert crossing & 10 & W-06-067_P6080001 \\
\hline W-06-067 & Looking upstream from culvert crossing & 110 & W-06-067_P6080002 \\
\hline W-06-067 & Looking downstream from culvert crossing & 270 & W-06-067_P6080003 \\
\hline W-06-067 & Looking at culvert inlets & 250 & W-06-067_P6080004 \\
\hline W-06-068 & Looking upstream from culvert crossing & 295 & W-06-068_P6080005 \\
\hline W-06-068 & Looking downstream from culvert crossing & 105 & W-06-068_P6080006 \\
\hline W-06-068 & Looking at culvert inlets & 75 & W-06-068_P6080007 \\
\hline W-06-068 & Looking at culvert outlets & 260 & W-06-068_P6080008 \\
\hline W-06-069 & Looking upstream from bridge crossing & 180 & W-06-069_P6120001 \\
\hline
\end{tabular}


Appendix 8: List of all photographs taken showing the corresponding site number, descriptive caption, direction faced when taken (azimuth in degrees) and the unique file name for each photograph.

\begin{tabular}{|c|c|c|c|}
\hline $\begin{array}{c}\text { Site } \\
\text { Number }\end{array}$ & Photo Description & Azimuth & File Name \\
\hline W-06-069 & Looking downstream from bridge crossing & 0 & W-06-069_P6120002 \\
\hline W-06-070 & Looking upstream from bridge crossing & 90 & W-06-070 P6120003 \\
\hline W-06-070 & Looking downstream from bridge crossing & 270 & W-06-070_P6120004 \\
\hline W-06-071 & Looking upstream from culvert crossing & 10 & W-06-071_P6130001 \\
\hline W-06-071 & Looking downstream from culvert crossing & 200 & W-06-071_P6130002 \\
\hline W-06-071 & Looking at culvert inlets & 205 & W-06-071_P6130003 \\
\hline W-06-071 & Looking at culvert outlets & 310 & W-06-071_P6130004 \\
\hline W-06-072 & Looking upstream from culvert crossing & 310 & W-06-072_P6130005 \\
\hline W-06-072 & Looking downstream from culvert crossing & 125 & W-06-072_P6130006 \\
\hline W-06-072 & Looking at culvert inlets & 90 & W-06-072 P6130007 \\
\hline W-06-072 & Looking at culvert outlets & 300 & W-06-072_P6130008 \\
\hline W-06-073 & Looking upstream from culvert crossing & 340 & W-06-073_P6130009 \\
\hline W-06-073 & Looking downstream from culvert crossing & 150 & W-06-073_P6130010 \\
\hline W-06-073 & Looking at culvert inlet (covered in mud) & 90 & W-06-073_P6130011 \\
\hline W-06-073 & Looking at culvert outlet & 270 & W-06-073_P6130012 \\
\hline W-06-074 & Looking upstream from culvert crossing & 270 & W-06-074_P6130013 \\
\hline W-06-074 & Looking downstream from culvert crossing & 90 & W-06-074_P6130014 \\
\hline W-06-074 & Looking at culvert inlets & 120 & W-06-074_P6130015 \\
\hline W-06-074 & Looking at culvert outlets & 200 & W-06-074_P6130016 \\
\hline W-06-075 & Looking upstream from culvert crossing & 260 & W-06-075_P6130017 \\
\hline W-06-075 & Looking downstream from culvert crossing & 80 & W-06-075_P6130018 \\
\hline W-06-075 & Looking at culvert inlet & 130 & W-06-075_P6130019 \\
\hline W-06-075 & Looking at culvert outlet & 230 & W-06-075_P6130020 \\
\hline W-06-076 & Looking upstream from culvert crossing & 325 & W-06-076_P6130021 \\
\hline W-06-076 & Looking upstream from culvert crossing & 210 & W-06-076 P6130022 \\
\hline W-06-076 & Looking downstream from culvert crossing & 75 & W-06-076 P6130023 \\
\hline W-06-076 & Looking at culvert inlets & 160 & W-06-076_P6130024 \\
\hline W-06-076 & Looking at culvert outlets & 210 & W-06-076_P6130025 \\
\hline W-06-077 & Looking upstream from culvert crossing & 300 & W-06-077_P6130026 \\
\hline W-06-077 & Looking downstream from culvert crossing & 90 & W-06-077_P6130027 \\
\hline W-06-077 & Looking at culvert inlets & 70 & W-06-077_P6130028 \\
\hline W-06-077 & Looking at culvert outlets & 290 & W-06-077_P6130029 \\
\hline W-06-078 & Looking upstream bridge crossing & 90 & W-06-078_P6140001 \\
\hline
\end{tabular}


Appendix 8: List of all photographs taken showing the corresponding site number, descriptive caption, direction faced when taken (azimuth in degrees) and the unique file name for each photograph.

\begin{tabular}{|c|c|c|c|}
\hline $\begin{array}{c}\text { Site } \\
\text { Number }\end{array}$ & Photo Description & Azimuth & File Name \\
\hline W-06-078 & Looking downstream bridge crossing & 270 & $\begin{array}{ll}\mathrm{W}-06-078 & \mathrm{P} 6140002\end{array}$ \\
\hline W-06-078 & Photo of released Northern Pike & $\mathrm{N} / \mathrm{A}$ & W-06-078 P6140003 \\
\hline W-06-079 & Looking upstream from culvert crossing & 100 & W-06-079_P6140004 \\
\hline W-06-079 & Looking downstream from culvert crossing & 240 & W-06-079_P6140005 \\
\hline W-06-079 & Looking at culvert inlets & 195 & W-06-079_P6140006 \\
\hline W-06-079 & Looking at culvert outlets & 60 & W-06-079 P6140007 \\
\hline W-06-080 & Looking upstream bridge crossing & 90 & W-06-080_P6140008 \\
\hline W-06-080 & Looking downstream bridge crossing & 270 & W-06-080_P6140009 \\
\hline W-06-081 & Looking upstream unnamed tributary & 270 & W-06-081_P6150001 \\
\hline W-06-081 & Looking downstream Bachman Drain & 90 & W-06-081_P6150002 \\
\hline W-06-081 & Looking upstream Bachman Drain & 180 & W-06-081_P6150003 \\
\hline W-06-082 & Looking upstream from culvert crossing & 270 & W-06-082_P6150004 \\
\hline W-06-082 & Looking downstream from culvert crossing & 90 & W-06-082_P6150005 \\
\hline W-06-082 & Looking at culvert inlets & 85 & W-06-082_P6150006 \\
\hline W-06-082 & Looking at culvert outlets & 285 & W-06-082_P6150007 \\
\hline W-06-083 & Dave with students & 200 & W-06-083_P6200001 \\
\hline W-06-083 & Dave with students & 350 & W-06-083 P6200002 \\
\hline W-06-083 & Looking upstream from ford crossing & 180 & W-06-083 P6200003 \\
\hline W-06-083 & Looking downstream of ford crossing & 0 & W-06-083_P6200004 \\
\hline W-06-084 & Looking upstream from culvert crossing & 180 & W-06-084_P6210001 \\
\hline W-06-084 & Looking downstream from culvert crossing & 0 & W-06-084_P6210002 \\
\hline W-06-084 & Looking at culvert inlet & 180 & W-06-084_P6210003 \\
\hline W-06-084 & Looking at culvert outlet & 0 & W-06-084_P6210004 \\
\hline W-06-085 & Looking upstream from north culvert crossing & 260 & W-06-085_P6210005 \\
\hline W-06-085 & Looking upstream from south culvert crossing & 260 & W-06-085 P6210006 \\
\hline W-06-085 & Looking downstream from north culvert crossing & 80 & W-06-085 P6210007 \\
\hline W-06-085 & Looking downstream from south culvert crossing & 60 & W-06-085_P6210008 \\
\hline W-06-085 & Looking at culvert inlet & 45 & W-06-085_P6210009 \\
\hline W-06-085 & Looking at culvert outlet & 290 & W-06-085 P6210010 \\
\hline W-06-086 & Looking upstream from north culvert crossing & 100 & W-06-086 P6210011 \\
\hline W-06-086 & Looking upstream from south culvert crossing & 110 & W-06-086_P6210012 \\
\hline W-06-086 & Looking downstream from road & 280 & W-06-086_P6210013 \\
\hline W-06-086 & Looking downstream from north culvert crossing & 270 & W-06-086 P6210014 \\
\hline
\end{tabular}


Appendix 8: List of all photographs taken showing the corresponding site number, descriptive caption, direction faced when taken (azimuth in degrees) and the unique file name for each photograph.

\begin{tabular}{|c|c|c|c|}
\hline $\begin{array}{c}\text { Site } \\
\text { Number }\end{array}$ & Photo Description & Azimuth & File Name \\
\hline W-06-086 & Looking at north culvert inlet from overhead & 245 & W-06-086_P6210015 \\
\hline W-06-086 & Looking at machinery tracks on left bank upstream of culvert crossing & 180 & W-06-086_P6210016 \\
\hline W-06-086 & Looking at south culvert inlet from overhead & 270 & W-06-086_P6210017 \\
\hline W-06-086 & Looking at both culvert inlets from $20 \mathrm{~m}$ upstream of crossing & 315 & W-06-086_P6210018 \\
\hline W-06-086 & Looking further upstream from 20 m upstream of crossing & 95 & W-06-086_P6210019 \\
\hline W-06-086 & Looking downstream from top of north culvert & 270 & W-06-086_P6210020 \\
\hline W-06-086 & Looking downstream from top of north culvert & 270 & W-06-086_P6210021 \\
\hline W-06-086 & Looking at north culvert outlet & 85 & W-06-086_P6210022 \\
\hline W-06-086 & Looking at both culvert outlets & 90 & W-06-086_P6210023 \\
\hline W-06-086 & Looking at south culvert outlet (zoomed) & 90 & W-06-086_P6210024 \\
\hline W-06-087 & Looking upstream bridge crossing & 140 & W-06-087_P6220001 \\
\hline W-06-087 & Looking downstream bridge crossing & 320 & W-06-087_P6220002 \\
\hline W-06-087 & Looking upstream under bridge & 140 & W-06-087_P6220003 \\
\hline W-06-087 & Looking south at bank armouring and sediment control & 180 & W-06-087_P6220004 \\
\hline W-06-088 & Looking upstream from culvert crossing & 15 & W-06-088_P6220005 \\
\hline W-06-088 & Looking downstream from culvert crossing & 175 & W-06-088_P6220006 \\
\hline W-06-088 & Looking at culvert inlet & 195 & W-06-088_P6220007 \\
\hline W-06-088 & Looking at culvert outlet & 15 & W-06-088_P6220008 \\
\hline W-06-088 & Photo of released Northern Pike & $\mathrm{N} / \mathrm{A}$ & W-06-088_P6220009 \\
\hline W-06-088 & Photo of released Northern Pike & $\mathrm{N} / \mathrm{A}$ & W-06-088_P6220010 \\
\hline W-06-088 & Photo of released Northern Pike & $\mathrm{N} / \mathrm{A}$ & W-06-088_P6220011 \\
\hline W-06-088 & Photo of released Northern Pike & $\mathrm{N} / \mathrm{A}$ & W-06-088_P6220012 \\
\hline W-06-089 & Looking upstream bridge crossing & 80 & W-06-089_P6220013 \\
\hline W-06-089 & Looking downstream bridge crossing & 260 & W-06-089_P6220014 \\
\hline W-06-090 & Looking upstream bridge crossing & 180 & W-06-090_P6220015 \\
\hline W-06-090 & Looking downstream bridge crossing & 0 & W-06-090_P6220016 \\
\hline W-06-091 & Looking upstream from culvert crossing & 180 & W-06-091_P6230001 \\
\hline W-06-091 & Looking downstream from culvert crossing & 0 & W-06-091_P6230002 \\
\hline W-06-091 & Looking at culvert inlet & 45 & W-06-091_P6230003 \\
\hline W-06-091 & Looking at culvert outlet & 210 & W-06-091_P6230004 \\
\hline W-06-092 & Looking upstream from bridge crossing & 280 & W-06-092_P6230005 \\
\hline W-06-092 & Looking downstream from bridge crossing & 100 & W-06-092_P6230006 \\
\hline W-06-092 & Looking from bridge down at beaver dam west side of bridge & 270 & W-06-092_P6230007 \\
\hline
\end{tabular}


Appendix 8: List of all photographs taken showing the corresponding site number, descriptive caption, direction faced when taken (azimuth in degrees) and the unique file name for each photograph.

\begin{tabular}{|c|c|c|c|}
\hline $\begin{array}{c}\text { Site } \\
\text { Number }\end{array}$ & Photo Description & Azimuth & File Name \\
\hline W-06-092 & Looking at beaver dam from right bank east side of bridge & 75 & W-06-092 P6230008 \\
\hline W-06-093 & Looking upstream from culvert crossing & 190 & W-06-093 P6230009 \\
\hline W-06-093 & Looking downstream from culvert crossing & 10 & W-06-093_P6230010 \\
\hline W-06-093 & Looking at culvert inlets & 345 & W-06-093_P6230011 \\
\hline W-06-093 & Looking at culvert outlets & 240 & W-06-093_P6230012 \\
\hline W-06-094 & Looking upstream from $5 \mathrm{~m}$ north of culvert inlet at Kris Johnson Drain & 180 & W-06-094_P6270001 \\
\hline W-06-094 & Looking upstream from $5 \mathrm{~m}$ north of culvert inlet at unnamed tributary & 0 & W-06-094_P6270002 \\
\hline W-06-094 & Looking downstream from culvert crossing & 90 & W-06-094_P6270003 \\
\hline W-06-094 & Looking at culvert inlets & 135 & W-06-094_P6270004 \\
\hline W-06-094 & Looking at culvert outlets & 250 & W-06-094_P6270005 \\
\hline W-06-095 & Looking upstream Blind Bay & 180 & W-06-095_P6270006 \\
\hline W-06-095 & Looking upstream unnamed tributary southwest from crossing & 270 & W-06-095_P6270007 \\
\hline W-06-095 & Looking upstream unclassified drain southeast from crossing & 90 & W-06-095_P6270008 \\
\hline W-06-095 & Looking upstream unclassified drain northwest from crossing & 270 & W-06-095 P6270009 \\
\hline W-06-095 & Looking upstream unnamed tributary northeast of crossing & 90 & W-06-095_P6270010 \\
\hline W-06-095 & Looking downstream Blind Bay & 0 & W-06-095_P6270011 \\
\hline W-06-095 & Looking at culvert inlets & 315 & W-06-095 P6270012 \\
\hline W-06-095 & Looking at algae bloom above culvert outlets & 240 & W-06-095 P6270013 \\
\hline W-06-096 & Looking upstream from culvert crossing & 180 & W-06-096_P6270014 \\
\hline W-06-096 & Looking downstream from culvert crossing & 0 & W-06-096_P6270015 \\
\hline W-06-096 & Looking at culvert inlets & 35 & W-06-096_P6270016 \\
\hline W-06-096 & Looking at culvert outlets & 150 & W-06-096_P6270017 \\
\hline W-06-097 & Looking upstream from culvert crossing & 270 & W-06-097_P6270018 \\
\hline W-06-097 & Looking downstream from culvert crossing & 90 & W-06-097_P6270019 \\
\hline W-06-097 & Looking at culvert inlets & 45 & W-06-097_P6270020 \\
\hline W-06-097 & Looking at culvert outlets & 290 & W-06-097_P6270021 \\
\hline W-06-097 & Photo of Northern Pike & $\mathrm{N} / \mathrm{A}$ & W-06-097_P6270022 \\
\hline W-06-097 & Photo of Northern Pike & $\mathrm{N} / \mathrm{A}$ & W-06-097_P6270023 \\
\hline W-06-098 & Looking upstream from culvert crossing & 350 & W-06-098 P6280001 \\
\hline W-06-098 & Looking downstream from culvert crossing & 160 & W-06-098 P6280002 \\
\hline W-06-098 & Looking at culvert inlets & 190 & W-06-098_P6280003 \\
\hline W-06-098 & Looking at culvert outlets & 320 & W-06-098_P6280004 \\
\hline W-06-099 & Looking upstream from culvert crossing & 340 & W-06-099 P6280005 \\
\hline
\end{tabular}


Appendix 8: List of all photographs taken showing the corresponding site number, descriptive caption, direction faced when taken (azimuth in degrees) and the unique file name for each photograph.

\begin{tabular}{|c|c|c|c|}
\hline $\begin{array}{c}\text { Site } \\
\text { Number }\end{array}$ & Photo Description & Azimuth & File Name \\
\hline W-06-099 & Looking downstream from culvert crossing & 160 & W-06-099_P6280006 \\
\hline W-06-099 & Looking at culvert inlets & 200 & W-06-099 P6280007 \\
\hline W-06-099 & Looking at culvert outlets & 0 & W-06-099_P6280008 \\
\hline W-06-099 & Photo of dead \& decaying Common Carp & 200 & W-06-100_P6280009 \\
\hline W-06-099 & Photo of dead \& decaying Common Carp & 0 & W-06-100_P6280010 \\
\hline W-06-100 & Looking upstream from bridge crossing & 80 & W-06-101_P6280011 \\
\hline W-06-100 & Looking downstream from bridge crossing & 255 & W-06-101_P6280012 \\
\hline W-06-101 & Looking upstream from box culvert crossing & 90 & W-06-101_P6280013 \\
\hline W-06-101 & Looking upstream from box culvert crossing & 45 & W-06-101 P6280014 \\
\hline W-06-101 & Looking downstream from box culvert crossing & 270 & W-06-101_P6280015 \\
\hline W-06-101 & Looking at culvert inlet & 300 & W-06-101_P6280016 \\
\hline W-06-101 & Looking at culvert outlet & 40 & W-06-101_P6280017 \\
\hline W-06-102 & Looking upstream from culvert & 130 & W-06-102 P6280018 \\
\hline W-06-102 & Looking upstream the 2 nd order tributary & 0 & W-06-102 P6280019 \\
\hline W-06-102 & Looking downstream from culvert & 285 & W-06-102_P6280020 \\
\hline W-06-102 & Looking at culvert inlet & 350 & W-06-102_P6280021 \\
\hline W-06-102 & Looking at culvert outlet & 25 & W-06-102 P6280022 \\
\hline W-06-103 & Looking upstream from culvert & 110 & W-06-103 P6280023 \\
\hline W-06-103 & Looking downstream from culvert & 285 & W-06-103_P6280024 \\
\hline W-06-103 & Looking at culvert inlet & 15 & W-06-103_P6280025 \\
\hline W-06-103 & Looking at culvert outlet & 160 & W-06-103 P6280026 \\
\hline W-06-104 & Looking upstream from the right bank & 290 & W-06-104_P6290001 \\
\hline W-06-104 & Looking downstream from the left bank & 70 & W-06-104_P6290002 \\
\hline W-06-105 & Looking upstream from the culvert crossing & 270 & W-06-105 P6290003 \\
\hline W-06-105 & Looking downstream from the culvert crossing & 90 & W-06-105 P6290004 \\
\hline W-06-105 & Looking at the culvert inlet & 115 & W-06-105_P6290005 \\
\hline W-06-105 & Looking at the culvert outlet & 235 & W-06-105_P6290006 \\
\hline W-06-106 & Looking upstream from the culvert crossing & 45 & W-06-106_P6290007 \\
\hline W-06-106 & Looking downstream from the culvert crossing & 135 & W-06-106 P6290008 \\
\hline W-06-106 & Looking at the culvert inlet & 40 & W-06-106 P6290009 \\
\hline W-06-106 & Looking at the culvert outlet & 240 & W-06-106_P6290010 \\
\hline W-06-107 & Looking upstream from bridge crossing & 0 & W-06-107 P7040001 \\
\hline W-06-107 & Looking downstream from bridge crossing & 180 & W-06-107 P7040002 \\
\hline
\end{tabular}


Appendix 8: List of all photographs taken showing the corresponding site number, descriptive caption, direction faced when taken (azimuth in degrees) and the unique file name for each photograph.

\begin{tabular}{|c|c|c|c|}
\hline $\begin{array}{c}\text { Site } \\
\text { Number }\end{array}$ & Photo Description & Azimuth & File Name \\
\hline W-06-108 & Looking upstream from the culvert crossing & 340 & W-06-108_P7040003 \\
\hline W-06-108 & Looking downstream from the culvert crossing & 180 & W-06-108 P7040004 \\
\hline W-06-108 & Looking at the culvert inlet & 225 & W-06-108_P7040005 \\
\hline W-06-108 & Looking at the culvert outlet & 340 & W-06-108_P7040006 \\
\hline W-06-109 & Looking upstream from culvert crossing & 90 & W-06-109_P7040007 \\
\hline W-06-109 & Looking downstream from culvert crossing & 270 & W-06-109_P7040008 \\
\hline W-06-109 & Looking at culvert inlet & 330 & W-06-109_P7040009 \\
\hline W-06-109 & Looking at culvert outlet & 45 & W-06-109_P7040010 \\
\hline W-06-110 & Looking upstream from bridge crossing & 280 & W-06-110_P7050001 \\
\hline W-06-110 & Looking downstream from bridge crossing & 80 & W-06-110 P7050002 \\
\hline W-06-111 & Looking upstream from bridge crossing & 300 & W-06-111_P7050003 \\
\hline W-06-111 & Looking upstream from bridge crossing & 250 & W-06-111_P7050004 \\
\hline W-06-111 & Looking downstream from bridge crossing & 95 & W-06-111_P7050005 \\
\hline W-06-112 & Looking upstream from culvert crossing & 250 & W-06-112_P7050006 \\
\hline W-06-112 & Looking downstream from culvert crossing & 80 & W-06-112_P7050007 \\
\hline W-06-112 & Looking at culvert inlet & 70 & W-06-112_P7050008 \\
\hline W-06-112 & Looking at culvert outlet & 330 & W-06-112_P7050009 \\
\hline W-06-113 & Looking upstream from culvert crossing & 270 & W-06-113 P7060001 \\
\hline W-06-113 & Looking downstream from culvert crossing & 90 & W-06-113_P7060002 \\
\hline W-06-113 & Looking at culvert inlet & 15 & W-06-113_P7060003 \\
\hline W-06-113 & Looking at culvert outlet & 330 & W-06-113_P7060004 \\
\hline W-06-113 & Looking at the culvert crossing from the road & 0 & W-06-113_P7060005 \\
\hline W-06-113 & Looking at the culvert crossing from the road & 180 & W-06-113_P7060006 \\
\hline W-06-113 & Looking at the culvert crossing from the road (wide angle) & 320 & W-06-113_P7060007 \\
\hline W-06-114 & Looking upstream from the culvert crossing & 270 & W-06-114_P7100001 \\
\hline W-06-114 & Looking downstream from the culvert crossing & 90 & W-06-114_P7100002 \\
\hline W-06-114 & Looking at culvert outlet & 270 & W-06-114_P7100003 \\
\hline W-06-114 & Looking at culvert inlet & 90 & W-06-114_P7100004 \\
\hline W-06-114 & Wide angle shot of the washed out crossing & 0 & W-06-114_P7100005 \\
\hline W-06-114 & Photo of replacement culvert behind bushes south of washout & 10 & W-06-114_P7100006 \\
\hline W-06-115 & Looking upstream from the bridge crossing & 270 & W-06-115_P7100007 \\
\hline W-06-115 & Looking downstream from the bridge crossing & 90 & W-06-115_P7100008 \\
\hline W-06-116 & Looking upstream from culvert crossing & 180 & W-06-116_P7110001 \\
\hline
\end{tabular}


Appendix 8: List of all photographs taken showing the corresponding site number, descriptive caption, direction faced when taken (azimuth in degrees) and the unique file name for each photograph.

\begin{tabular}{|c|c|c|c|}
\hline $\begin{array}{c}\text { Site } \\
\text { Number }\end{array}$ & Photo Description & Azimuth & File Name \\
\hline W-06-116 & Looking downstream from culvert crossing & 0 & $\begin{array}{l}\mathrm{W}-06-116 \quad \text { P7110002 } \\
\end{array}$ \\
\hline W-06-118 & Looking upstream from culvert crossing & 180 & W-06-118_P7110003 \\
\hline W-06-118 & Looking downstream from culvert crossing & 0 & W-06-118_P7110004 \\
\hline W-06-118 & Looking at culvert inlets & 70 & W-06-118_P7110005 \\
\hline W-06-118 & Looking at culvert outlets & 180 & W-06-118 P7110006 \\
\hline W-06-117 & Looking upstream bridge from crossing & 270 & W-06-117 P7100009 \\
\hline W-06-117 & Looking downstream from bridge crossing & 120 & W-06-117_P7100010 \\
\hline W-06-119 & Looking upstream ford crossing & 210 & W-06-119 P7110007 \\
\hline W-06-119 & Looking downstream ford crossing & 15 & W-06-119 P7110008 \\
\hline W-06-119 & Looking at ford inlet & 70 & W-06-119_P7110009 \\
\hline W-06-119 & Looking at ford outlet & 180 & W-06-119_P7110010 \\
\hline W-06-119 & Photo of released Burbot & $\mathrm{N} / \mathrm{A}$ & W-06-119_P7110011 \\
\hline W-06-120 & Looking upstream bridge from crossing & 0 & W-06-120 P7120001 \\
\hline W-06-120 & Looking downstream from bridge crossing & 180 & W-06-120 P7120002 \\
\hline W-06-121 & Looking downstream bridge from crossing & 270 & W-06-121_P7120003 \\
\hline W-06-121 & Looking upstream from bridge crossing & 90 & W-06-121 P7120004 \\
\hline W-06-121 & Looking further upstream from bridge crossing & 90 & W-06-121 P7120005 \\
\hline W-06-122 & Looking upstream from culvert crossing & 270 & W-06-122_P7120006 \\
\hline W-06-122 & Looking downstream from culvert crossing & 90 & W-06-122_P7120007 \\
\hline W-06-122 & Looking at culvert inlets & 130 & W-06-122 P7120008 \\
\hline W-06-122 & Looking at culvert outlets & 190 & W-06-122 P7120009 \\
\hline W-06-123 & Looking upstream bridge from crossing & 180 & W-06-123_P7120010 \\
\hline W-06-123 & Looking downstream from bridge crossing & 0 & W-06-123_P7120011 \\
\hline W-06-124 & Looking upstream bridge crossing & 270 & W-06-124 P7180001 \\
\hline W-06-124 & Looking downstream bridge crossing & 90 & W-06-124_P7180002 \\
\hline W-06-125 & Looking upstream from culvert crossing & 180 & W-06-125 P7180003 \\
\hline W-06-125 & Looking downstream from culvert crossing & 0 & W-06-125_P7180004 \\
\hline W-06-125 & Looking at culvert inlet & 0 & W-06-125 P7180005 \\
\hline W-06-125 & Looking at culvert outlet & 180 & W-06-125 P7180006 \\
\hline W-06-126 & Looking upstream bridge crossing & 0 & W-06-126_P7180007 \\
\hline W-06-126 & Looking downstream bridge crossing & 180 & W-06-126 P7180008 \\
\hline W-06-126 & Photo of released Smallmouth Bass & $\mathrm{N} / \mathrm{A}$ & W-06-126 P7180009 \\
\hline W-06-126 & Photo of released sculpin & $\mathrm{N} / \mathrm{A}$ & W-06-126_P7180010 \\
\hline
\end{tabular}


Appendix 8: List of all photographs taken showing the corresponding site number, descriptive caption, direction faced when taken (azimuth in degrees) and the unique file name for each photograph.

\begin{tabular}{|c|c|c|c|}
\hline $\begin{array}{c}\text { Site } \\
\text { Number }\end{array}$ & Photo Description & Azimuth & File Name \\
\hline W-06-127 & Looking upstream from culvert crossing & 90 & W-06-127_P7180011 \\
\hline W-06-127 & Looking downstream from culvert crossing & 270 & W-06-127 P7180012 \\
\hline W-06-127 & Looking at culvert inlet & 0 & W-06-127_P7180013 \\
\hline W-06-127 & Looking at culvert outlet & 110 & W-06-127_P7180014 \\
\hline W-06-128 & Looking at beaver dam & $\mathrm{N} / \mathrm{A}$ & W-06-128_P7180015 \\
\hline W-06-128 & Looking at beaver dam & $\mathrm{N} / \mathrm{A}$ & W-06-128_P7180016 \\
\hline W-06-128 & Looking at beaver dam & $\mathrm{N} / \mathrm{A}$ & W-06-128_P7180017 \\
\hline W-06-128 & Looking upstream from culvert crossing & 130 & W-06-128_P7180018 \\
\hline W-06-128 & Looking downstream from culvert crossing & 330 & W-06-128_P7180019 \\
\hline W-06-128 & Looking at culvert inlets & 200 & W-06-128_P7180020 \\
\hline W-06-128 & Looking at culvert outlets & 300 & W-06-128_P7180021 \\
\hline W-06-129 & Looking upstream from culvert crossing & 140 & W-06-129_P7180022 \\
\hline W-06-129 & Looking downstream from culvert crossing & 320 & W-06-129_P7180023 \\
\hline W-06-129 & Looking at culvert inlets & 340 & W-06-129_P7180024 \\
\hline W-06-129 & Looking at culvert outlets & 240 & W-06-129_P7180025 \\
\hline W-06-130 & Looking upstream bridge crossing & 20 & W-06-130_P7190001 \\
\hline W-06-130 & Looking downstream bridge crossing & 160 & W-06-130_P7190002 \\
\hline W-06-131 & Looking upstream bridge crossing & 80 & W-06-131_P7190003 \\
\hline W-06-131 & Looking upstream bridge crossing & 110 & W-06-131_P7190004 \\
\hline W-06-131 & Looking upstream bridge crossing & 140 & W-06-131_P7190005 \\
\hline W-06-131 & Looking downstream bridge crossing & 260 & W-06-131_P7190006 \\
\hline W-06-131 & Looking downstream bridge crossing & 280 & W-06-131_P7190007 \\
\hline W-06-131 & Looking downstream bridge crossing & 300 & W-06-131_P7190008 \\
\hline W-06-132 & Looking upstream from culvert crossing & 160 & W-06-132_P7190009 \\
\hline W-06-132 & Looking downstream from culvert crossing & 0 & W-06-132_P7190010 \\
\hline W-06-132 & Looking downstream from culvert crossing & 260 & W-06-132_P7190011 \\
\hline W-06-133 & Looking at released suckers & $\mathrm{N} / \mathrm{A}$ & W-06-133_P7190012 \\
\hline W-06-134 & Looking upstream bridge crossing & 310 & W-06-134_P7200001 \\
\hline W-06-134 & Looking downstream bridge crossing & 90 & W-06-134_P7200002 \\
\hline W-06-134 & Looking directly down from west side of bridge (downstream) & $\mathrm{N} / \mathrm{A}$ & W-06-134_P7200003 \\
\hline W-06-134 & Looking upstream from 10 m downstream of bridge (under bridge) & 60 & W-06-134_P7200004 \\
\hline W-06-134 & Looking upstream from 10 m downstream of bridge (under bridge) & 90 & W-06-134_P7200005 \\
\hline W-06-135 & Looking upstream bridge crossing & 140 & W-06-135_P7200006 \\
\hline
\end{tabular}


Appendix 8: List of all photographs taken showing the corresponding site number, descriptive caption, direction faced when taken (azimuth in degrees) and the unique file name for each photograph.

\begin{tabular}{|c|c|c|c|}
\hline $\begin{array}{c}\text { Site } \\
\text { Number }\end{array}$ & Photo Description & Azimuth & File Name \\
\hline W-06-135 & Looking upstream bridge crossing & 210 & W-06-135 P7200007 \\
\hline W-06-135 & Looking upstream bridge crossing & 250 & W-06-135 P7200008 \\
\hline W-06-135 & Looking downstream bridge crossing & 40 & W-06-135_P7200009 \\
\hline W-06-135 & Looking downstream bridge crossing & 0 & W-06-135_P7200010 \\
\hline W-06-135 & Looking downstream bridge crossing & 340 & W-06-135_P7200011 \\
\hline W-06-136 & Looking downstream from left bank & 90 & W-06-136 P7200012 \\
\hline W-06-136 & Looking upstream from left bank & 270 & W-06-136_P7200013 \\
\hline W-06-137 & Looking upstream from culvert crossing & 150 & W-06-137_P7200014 \\
\hline W-06-137 & Looking downstream from culvert crossing & 270 & W-06-137_P7200015 \\
\hline W-06-137 & Looking at culvert inlets & 330 & W-06-137_P7200016 \\
\hline W-06-137 & Looking at culvert outlets & 20 & W-06-137 P7200017 \\
\hline W-06-138 & Looking upstream from right bank at riffle/dam & 0 & W-06-138_P8010001 \\
\hline W-06-138 & Looking downstream from right bank at riffle/dam & 180 & W-06-138_P8010002 \\
\hline W-06-138 & Looking at beginning of oxbow from right bank at riffle/dam & 90 & W-06-138 P8010003 \\
\hline W-06-139 & Looking downstream from right bank at dam/weir & 0 & W-06-139_P8010004 \\
\hline W-06-139 & Looking at dam from right bank & 90 & W-06-139_P8010005 \\
\hline W-06-139 & Photo of Northern Pike on ruler (released) & $\mathrm{N} / \mathrm{A}$ & W-06-139_P8010006 \\
\hline W-06-140 & Looking upstream from right bank at OG dam & 270 & W-06-140 P8010007 \\
\hline W-06-140 & Looking downstream from right bank at OG dam & 90 & W-06-140_P8010008 \\
\hline W-06-140 & Looking at OG dam from right bank & 180 & W-06-140_P8010009 \\
\hline W-06-141 & Looking upstream from dam & 330 & W-06-141 P8010010 \\
\hline W-06-141 & Looking downstream from dam & 90 & W-06-141_P8010011 \\
\hline W-06-141 & Looking north at dam & 0 & W-06-141_P8010012 \\
\hline W-06-141 & Looking north at dam & 0 & W-06-141_P8010013 \\
\hline W-06-141 & Photo of released Northern Pike & $\mathrm{N} / \mathrm{A}$ & W-06-141_P8010015 \\
\hline W-06-141 & Photo of released Northern Pike & $\mathrm{N} / \mathrm{A}$ & W-06-141_P8010016 \\
\hline W-06-141 & Photo of released Shorthead Redhorse & $\mathrm{N} / \mathrm{A}$ & W-06-141_P8010017 \\
\hline W-06-141 & Photo of released Shorthead Redhorse & $\mathrm{N} / \mathrm{A}$ & W-06-141_P8010018 \\
\hline W-06-141 & Photo of released Shorthead Redhorse & $\mathrm{N} / \mathrm{A}$ & W-06-141_P8010019 \\
\hline W-06-141 & Photo of released Shorthead Redhorse & $\mathrm{N} / \mathrm{A}$ & W-06-141_P8010020 \\
\hline W-06-141 & Looking upstream from bridge crossing & 300 & W-06-141_P8020001 \\
\hline W-06-142 & Looking downstream from bridge crossing & 90 & W-06-142_P8020002 \\
\hline W-06-142 & Looking under bridge & 0 & W-06-142_P8020003 \\
\hline
\end{tabular}


Appendix 8: List of all photographs taken showing the corresponding site number, descriptive caption, direction faced when taken (azimuth in degrees) and the unique file name for each photograph.

\begin{tabular}{|c|l|c|c|}
\hline $\begin{array}{c}\text { Site } \\
\text { Number }\end{array}$ & \multicolumn{1}{|c|}{ Photo Description } & Azimuth & File Name \\
\hline W-06-142 & Looking upstream from bridge crossing & 270 & W-06-142_P8080001 \\
\hline W-06-143 & Looking downstream from bridge crossing & 90 & W-06-143_P8080002 \\
\hline W-06-143 & Photo of Freshwater Drum (released) & N/A & W-06-143_P8080003 \\
\hline W-06-143 & Photo of Vaughn releasing Freshwater Drum & 205 & W-06-143_P8080004 \\
\hline W-06-143 & Looking upstream from bridge crossing & 290 & W-06-143_P8080005 \\
\hline W-06-144 & Looking downstream from bridge crossing & 90 & W-06-144_P8080006 \\
\hline W-06-144 & Looking upstream from culvert crossing & 270 & W-06-144_P8080007 \\
\hline W-06-145 & Looking downstream from culvert crossing & 90 & W-06-145_P8080008 \\
\hline W-06-145 & Looking at culvert inlet & 15 & W-06-145_P8080009 \\
\hline W-06-145 & Looking at culvert outlet & 190 & W-06-145_P8080010 \\
\hline W-06-145 & Looking upstream from bridge crossing & 110 & W-06-145_P8090001 \\
\hline W-06-146 & Looking downstream from bridge crossing & 30 & W-06-146_P8090002 \\
\hline
\end{tabular}

